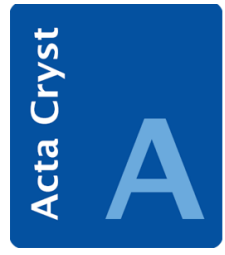

FOUNDATIONS

ADVANCES

Volume 71 (2015)

Supporting information for article:

Axial point groups: rank 1, 2, 3 and 4 property tensor tables

Daniel B. Litvin 


\section{LINE GROUPS}

\section{RANK 1,2,3 AND 4 PROPERTY TENSOR TABLES}

Daniel B. Litvin

Department of Physics, Eberly College of Science, The Pennsylvania State University, Penn State Berks, P.O. Box 7009, Reading, PA 19610-6009, U.S.A., u3c@psu.edu

Contents

Rank 1, 2, 3, and 4 Property Tensors

Printed Matrix Format of Rank 1, 2, 3, and 4 Property Tensors

Line Group Series and Line Groups for $n=1,2,3$, 4 and $\infty$

Property Tensor Tables

Choose Tensor Rank and then Point Group Type

Rank 1 Tensors

Rank 2 Tensors

Rank 3 Tensors

Rank 4 Tensors 


\section{Property Tensor Tables}

Rank 1 Property Tensor Tables

Table Format:

\begin{tabular}{cc|c|c|c|}
\multicolumn{1}{c}{1} & $\mathrm{e}$ & $\mathrm{a}$ & $\mathrm{ae}$ \\
$\mathrm{V} \mathrm{V}$ & $\mathrm{eV}$ & $\mathrm{aV}$ & $\mathrm{aeV}$ \\
\hline
\end{tabular}

\begin{tabular}{|c|c|c|}
\hline Series & $\mathrm{n}=1$ & $\mathrm{n}>1$ \\
\hline $\begin{array}{l}\mathbf{C}_{\mathrm{n}} \\
\mathbf{C}_{\mathrm{nv}}\end{array}$ & $\begin{array}{l}\mathbf{C}_{1}=1 \\
\mathbf{C}_{1 \mathrm{v}}=\mathbf{m}_{\mathrm{y}}\end{array}$ & $\begin{array}{l}\mathbf{C}_{\infty}=\infty \\
\mathbf{C}_{\infty v}=\infty \mathbf{m m}\end{array}$ \\
\hline $\begin{array}{l}\mathbf{S}_{2 \mathrm{n}} \\
\mathbf{C}_{\mathrm{nh}} \\
\mathbf{D}_{\mathrm{n}}\end{array}$ & $\begin{array}{l}\mathbf{S}_{2}=\overline{\mathbf{1}} \\
\mathbf{C}_{1 \mathrm{~h}}=\mathbf{m}_{\mathrm{z}} \\
\mathbf{D}_{1}=\mathbf{2}_{\mathrm{y}}\end{array}$ & $\begin{array}{l}\mathbf{C}_{\infty h}=\infty / \mathbf{m} \\
\mathbf{C}_{\infty h}=\infty / \mathbf{m} \\
\mathbf{D}_{\infty}=\infty \mathbf{2}\end{array}$ \\
\hline $\mathbf{D}_{\text {nd }}$ & $\mathbf{D}_{1 \mathrm{~d}}=\mathbf{2}_{\mathrm{y}} / \mathbf{m}_{\mathrm{y}}$ & $\mathbf{D}_{\infty \mathrm{h}}=\infty / \mathbf{m m m}$ \\
\hline $\mathbf{D}_{\mathrm{nh}}$ & $\mathbf{D}_{1 \mathrm{~h}}=\mathbf{2}_{\mathrm{x}} \mathbf{m}_{\mathrm{y}} \mathbf{m}_{\mathrm{z}}$ & $\mathbf{D}_{\infty \mathrm{h}}=\infty / \mathrm{mmm}$ \\
\hline $\mathbf{C}_{2 n}\left(\mathbf{C}_{n}\right)$ & $\mathbf{C}_{2}\left(\mathbf{C}_{1}\right)=\mathbf{2}_{\mathrm{z}}(\mathbf{1})=\mathbf{2}_{\mathrm{z}}^{\prime}$ & $\mathrm{C}_{\infty} 1^{\prime}=\infty 1^{\prime}$ \\
\hline $\begin{array}{l}\mathbf{S}_{2 n}\left(\mathbf{C}_{n}\right) \\
\mathbf{C}_{n h}\left(\mathbf{C}_{n}\right)\end{array}$ & $\begin{array}{l}\mathbf{S}_{2}\left(\mathbf{C}_{1}\right)=\overline{\mathbf{1}}(\mathbf{1})=\overline{\mathbf{1}}^{\prime} \\
\mathbf{C}_{\mathrm{th}}\left(\mathbf{C}_{1}\right)=\mathbf{m}_{\mathbf{z}(\mathbf{1})=\mathbf{m}_{\mathbf{z}}}\end{array}$ & $\begin{array}{l}\mathbf{C}_{\infty h}\left(\mathbf{C}_{\infty}\right)=\infty / \mathbf{m}^{\prime} \\
\mathbf{C}_{\infty \mathrm{h}}\left(\mathbf{C}_{\infty}\right)=\infty / \mathbf{m}^{\prime}\end{array}$ \\
\hline $\begin{array}{l}\mathbf{C}_{2 \mathrm{nh}}\left(\mathbf{S}_{2 \mathrm{n}}\right) \\
\mathbf{C}_{2 \mathrm{nh}}\left(\mathbf{C}_{\mathrm{nh}}\right) \\
\mathbf{D}_{\mathrm{n}}\left(\mathbf{C}_{\mathrm{n}}\right) \\
\mathbf{D}_{2 \mathrm{n}}\left(\mathbf{D}_{\mathrm{n}}\right) \\
\mathbf{C}_{\mathrm{nv}}\left(\mathbf{C}_{\mathrm{n}}\right) \\
\mathbf{C}_{2 \mathrm{nv}}\left(\mathbf{C}_{\mathrm{nv}}\right)\end{array}$ & $\begin{array}{l}\mathbf{C}_{2 \mathrm{~h}}\left(\mathbf{S}_{2}\right)=\mathbf{2}_{\mathrm{z}} / \mathbf{m}_{\mathrm{z}}(\overline{\mathbf{1}})=\mathbf{2}_{\mathrm{z}}{ }^{\prime} / \mathbf{m}_{\mathrm{z}}^{\prime} \\
\mathbf{C}_{2 \mathrm{~h}}\left(\mathbf{C}_{1 \mathrm{~h}}\right)=\mathbf{2}_{\mathrm{z}} / \mathbf{m}_{\mathrm{z}}\left(\mathbf{m}_{\mathrm{z}}\right)=\mathbf{2}_{\mathrm{z}} \mathbf{z}^{\prime} / \mathbf{m}_{\mathrm{z}} \\
\mathbf{D}_{1}\left(\mathbf{C}_{1}\right)=\mathbf{2}_{\mathrm{y}}(\mathbf{1})=\mathbf{2}_{\mathrm{y}}{ }^{\prime} \\
\mathbf{D}_{2}\left(\mathbf{D}_{1}\right)=\mathbf{2}_{\mathrm{x}} \mathbf{2}_{\mathrm{y}} \mathbf{2}\left(\mathbf{2}_{\mathrm{y}}\right)=\mathbf{2}_{\mathrm{x}} \mathbf{2}_{\mathbf{y}} \mathbf{2}_{\mathrm{z}}^{\prime} \\
\mathbf{C}_{1 \mathrm{v}}\left(\mathbf{C}_{1}\right)=\mathbf{m}_{\mathrm{y}}(\mathbf{1})=\mathbf{m}_{\mathrm{y}} \\
\mathbf{C}_{2 \mathrm{v}}\left(\mathbf{C}_{1 \mathrm{v}}\right)=\mathbf{2}_{\mathrm{z}} \mathbf{m}_{\mathrm{x}} \mathbf{m}_{\mathrm{y}}\left(\mathbf{m}_{\mathrm{y}}\right)=\mathbf{2}_{\mathrm{z}} \mathbf{m}_{\mathrm{x}} \mathbf{m}_{\mathrm{y}}\end{array}$ & $\begin{array}{l}\mathbf{C}_{\infty h} \mathbf{1}^{\prime}=\infty / \mathbf{m} \mathbf{1}^{\prime} \\
\mathbf{C}_{\infty \mathrm{h}} \mathbf{1}^{\prime}=\infty / \mathbf{m} \mathbf{1}^{\prime} \\
\mathbf{D}_{\infty}\left(\mathbf{C}_{\infty}\right)=\infty \mathbf{2}^{\prime} \\
\mathbf{D}_{\infty} \mathbf{1}^{\prime}=\infty \mathbf{2} \mathbf{1}^{\prime} \\
\mathbf{C}_{\infty v}\left(\mathbf{C}_{\infty}\right)=\infty \mathbf{m}^{\prime} \mathbf{m}^{\prime} \\
\mathbf{C}_{\infty v} \mathbf{1}^{\prime}=\infty \mathbf{m m} \mathbf{1}^{\prime}\end{array}$ \\
\hline $\begin{array}{l}\mathbf{D}_{\mathrm{nd}}\left(\mathbf{S}_{2 \mathrm{n}}\right) \\
\mathbf{D}_{\mathrm{nd}}\left(\mathbf{D}_{\mathrm{n}}\right) \\
\mathbf{D}_{\mathrm{nd}}\left(\mathbf{C}_{\mathrm{nv}}\right)\end{array}$ & $\begin{array}{l}\mathbf{D}_{1 \mathrm{~d}}\left(\mathbf{S}_{2}\right)=\mathbf{2}_{\mathrm{y}} / \mathbf{m}_{\mathrm{y}}(\overline{\mathbf{1}})=\mathbf{2}_{\mathrm{y}} \mathrm{y}^{\prime} / \mathbf{m}_{\mathrm{y}}{ }^{\prime} \\
\mathbf{D}_{1 \mathrm{~d}}\left(\mathbf{D}_{1}\right)=\mathbf{2}_{\mathrm{y}} / \mathbf{m}_{\mathrm{y}}\left(\mathbf{2}_{\mathrm{y}}\right)=\mathbf{2} \mathbf{y}_{\mathrm{y}} / \mathbf{m}_{\mathrm{y}} \\
\mathbf{D}_{1 \mathrm{~d}}\left(\mathbf{C}_{1 \mathrm{v}}\right)=\mathbf{2}_{\mathrm{y}} / \mathbf{m}_{\mathrm{y}}\left(\mathbf{m}_{\mathrm{y}}\right)=\mathbf{2}_{\mathrm{y}} / \mathbf{m}_{\mathrm{y}}\end{array}$ & $\begin{array}{l}\mathbf{D}_{\infty \mathrm{h}}\left(\mathbf{C}_{\infty \mathrm{h}}\right)=\infty / \mathbf{m m}^{\prime} \mathbf{m}^{\prime} \\
\mathbf{D}_{\infty \mathrm{h}}\left(\mathbf{D}_{\infty}\right)=\infty / \mathbf{m}^{\prime} \mathbf{m}^{\prime} \mathbf{m}^{\prime} \\
\mathbf{D}_{\infty h}\left(\mathbf{C}_{\infty \mathrm{V}}\right)=\infty / \mathbf{m}^{\prime} \mathbf{m m}\end{array}$ \\
\hline $\begin{array}{l}\mathbf{D}_{\mathrm{nh}}\left(\mathbf{C}_{\mathrm{nh}}\right) \\
\mathbf{D}_{\mathrm{nh}}\left(\mathbf{D}_{\mathrm{n}}\right) \\
\mathbf{D}_{\mathrm{nh}}\left(\mathbf{C}_{\mathrm{nv}}\right) \\
\mathbf{D}_{2 \mathrm{nh}}\left(\mathbf{D}_{\mathrm{nd}}\right) \\
\mathbf{D}_{2 \mathrm{nh}}\left(\mathbf{D}_{\mathrm{nh}}\right)\end{array}$ & 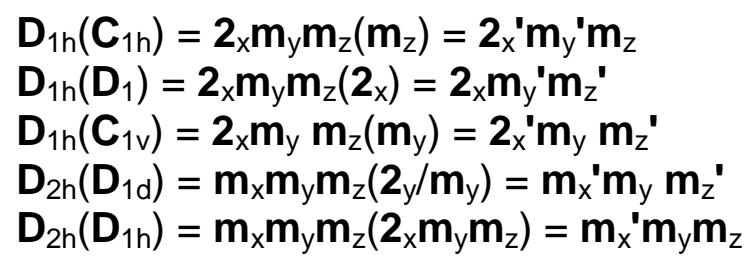 & $\begin{array}{l}\mathbf{D}_{\infty h}\left(\mathbf{C}_{\infty h}\right)=\infty / \mathbf{m m}^{\prime} \mathbf{m}^{\prime} \\
\mathbf{D}_{\infty h}\left(\mathbf{D}_{\infty}\right)=\infty / \mathbf{m}^{\prime} \mathbf{m}^{\prime} \mathbf{m}^{\prime} \\
\mathbf{D}_{\infty h}\left(\mathbf{C}_{\infty \mathrm{v}}\right)=\infty / \mathbf{m}^{\prime} \mathbf{m} \mathbf{m} \\
\mathbf{D}_{\infty h} \mathbf{1}^{\prime}=\infty / \mathbf{m m m} \mathbf{1}^{\prime} \\
\mathbf{D}_{\infty h} \mathbf{1}^{\prime}=\infty / \mathbf{m m m} \mathbf{1}^{\prime}\end{array}$ \\
\hline
\end{tabular}


Rank 2 Property Tensor Tables

Table Format:

\begin{tabular}{c|c|c|c|c|}
\multicolumn{1}{c}{} & \multicolumn{1}{c}{1} & \multicolumn{2}{c}{$\mathrm{e}$} & $\mathrm{a}$ \\
$\mathrm{V}^{2}$ & $\mathrm{~V}^{2}$ & $\mathrm{eV}^{2}$ & $\mathrm{a} \mathrm{V}^{2}$ & $\mathrm{aeV}$ \\
\cline { 3 - 5 }$\left[\mathrm{V}^{2}\right]$ & {$\left[\mathrm{V}^{2}\right]$} & $\mathrm{e}\left[\mathrm{V}^{2}\right]$ & $\mathrm{a}\left[\mathrm{V}^{2}\right]$ & $\mathrm{ae}\left[\mathrm{V}^{2}\right]$ \\
\cline { 2 - 5 }$\left\{\mathrm{V}^{2}\right\}$ & $\left\{\mathrm{V}^{2}\right\}$ & $\mathrm{e}\left\{\mathrm{V}^{2}\right\}$ & $\mathrm{a}\left\{\mathrm{V}^{2}\right\}$ & $\mathrm{ae}\left\{\mathrm{V}^{2}\right\}$ \\
\cline { 2 - 5 } & &
\end{tabular}

Series $\quad n=1$

$$
\mathrm{n}=2 \quad \mathrm{n}>2
$$

$\mathrm{C}_{\mathrm{n}} \quad \mathrm{C}_{1}=\mathbf{1}$

$$
\mathbf{C}_{2}=\mathbf{2}_{\mathbf{z}}
$$
$\mathbf{C}_{\mathrm{nv}} \quad \mathbf{C}_{1 \mathrm{v}}=\mathrm{m}_{\mathrm{y}}$

$$
\mathbf{C}_{2 v}=\mathbf{2}_{z} \mathbf{m}_{x} \mathbf{m}_{\mathrm{y}} \mathbf{C}_{\infty \mathrm{v}}=\infty \mathbf{m m}
$$

$\mathbf{S}_{2 \mathrm{n}} \quad \mathbf{S}_{2}=\overline{1}$

$$
\mathrm{S}_{4}=\overline{\mathbf{4}}_{\mathrm{z}}
$$

$\mathbf{C}_{\mathrm{nh}} \quad \mathbf{C}_{1 \mathrm{~h}}=\mathbf{m}_{\mathrm{z}}$

$$
\mathbf{C}_{\infty h}=\infty / \mathbf{m}
$$

$$
\mathrm{C}_{2 \mathrm{~h}}=\mathbf{2} / \mathrm{m}_{\mathrm{z}}
$$

$\mathbf{D}_{\mathrm{n}} \quad \mathbf{D}_{1}=\mathbf{2} \mathrm{y}$

$$
\mathbf{C}_{\infty \mathrm{h}}=\infty / \mathbf{m}
$$

$$
D_{2}=2 \mathbf{x}_{\mathbf{y}} \mathbf{2}_{\mathrm{z}}
$$

$$
\mathbf{D}_{\infty}=\infty \mathbf{2}
$$

$\mathbf{D}_{\text {nd }} \quad \mathbf{D}_{1 \mathrm{~d}}=\mathbf{2}_{\mathrm{y}} / \mathrm{m}_{\mathrm{y}}$

$$
\mathbf{D}_{2 \mathrm{~d}}=\overline{\mathbf{4}}_{\mathrm{z}} \mathbf{m}_{\mathrm{x}} \mathbf{2}_{\mathrm{xy}} \mathbf{D}_{\propto \mathrm{h}}=\infty / \mathbf{m m m}
$$

$\mathbf{D}_{\mathrm{nh}} \quad \mathbf{D}_{1 \mathrm{~h}}=\mathbf{2}_{\mathrm{x}} \mathbf{m}_{\mathrm{y}} \mathbf{m}_{\mathrm{z}}$

$$
\mathbf{D}_{2 \mathrm{~h}}=\mathbf{m}_{\mathbf{x}} \mathbf{m}_{\mathrm{y}} \mathbf{m}_{\mathrm{z}} \mathbf{D}_{\infty \mathrm{h}}=\infty / \mathbf{m m m}
$$

$\mathrm{C}_{2 \mathrm{n}}\left(\mathrm{C}_{\mathrm{n}}\right) \quad \mathrm{C}_{2}\left(\mathrm{C}_{1}\right)=\mathbf{2}_{\mathrm{z}}(\mathbf{1})=\mathbf{2}_{\mathrm{z}}{ }^{\prime}$

$$
\begin{array}{r}
\mathbf{C}_{4}\left(\mathbf{C}_{2}\right)=\mathbf{4}_{\mathbf{z}}\left(\mathbf{2}_{\mathrm{z}}\right)=\mathbf{4}_{z^{\prime}} \\
\mathbf{C}_{\infty} \mathbf{1}^{\prime}=\infty \mathbf{1}^{\prime}
\end{array}
$$

$\mathbf{S}_{2 n}\left(\mathbf{C}_{\mathrm{n}}\right) \quad \mathbf{S}_{2}\left(\mathbf{C}_{1}\right)=\overline{\mathbf{1}}(\mathbf{1})=\overline{\mathbf{1}}^{\prime}$

$$
\begin{aligned}
& \mathbf{S}_{4}\left(\mathbf{C}_{2}\right)=\overline{\mathbf{4}}_{\mathbf{z}}\left(\mathbf{2}_{\mathbf{z}}\right)=\overline{\mathbf{4}}_{\mathbf{z}}{ }^{\prime} \\
& \mathbf{C}_{\infty h}\left(\mathbf{C}_{\infty}\right)=\infty / \mathbf{m}^{\prime} \\
& \left.\mathbf{C}_{\mathrm{nh}}\left(\mathbf{C}_{\mathrm{n}}\right) \quad \mathbf{C}_{\mathrm{ih}}\left(\mathbf{C}_{1}\right)=\mathbf{m}_{\mathrm{z}} \mathbf{1}\right)=\mathbf{m}_{\mathrm{z}}^{\prime} \\
& C_{2 h}\left(C_{2}\right)=2_{z} / m_{z}\left(2_{z}\right)=2 z_{z} / m_{z}{ }^{\prime} \\
& \mathbf{C}_{\infty h}\left(\mathbf{C}_{\infty}\right)=\infty / \mathbf{m}^{\prime} \\
& \mathbf{C}_{2 \mathrm{nh}}\left(\mathbf{S}_{2 \mathrm{n}}\right) \quad \mathbf{C}_{2 \mathrm{~h}}\left(\mathbf{S}_{2}\right)=\mathbf{2}_{\mathrm{z}} / \mathbf{m}_{\mathrm{z}}(\overline{\mathbf{1}})=\mathbf{2}_{\mathrm{z}}{ }^{\prime} / \mathbf{m}_{\mathbf{z}}{ }^{\prime} \\
& C_{4 h}\left(S_{4}\right)=4_{z} / m_{z}\left(\overline{4}_{z}\right)=4_{z}^{\prime} / m_{z}^{\prime} \\
& \mathrm{C}_{\infty \mathrm{h}} \mathbf{1}^{\prime}=\infty / \mathrm{m}^{\prime}
\end{aligned}
$$


Series

$$
\mathrm{n}=1
$$

Rank 2

$$
\mathrm{n}=2
$$

$$
\mathrm{n}>2
$$

$\mathbf{C}_{2 n h}\left(\mathbf{C}_{\mathrm{nh}}\right)$

$C_{2 h}\left(C_{1 h}\right)=2 z / m_{z}\left(m_{z}\right)=2{ }_{z} / m_{z}$

$$
C_{4 h}\left(C_{2 h}\right)=4_{z} / m_{z}\left(2_{z} / m_{z}\right)=4_{z}{ }^{\prime} / m_{z}
$$

$\mathbf{D}_{\mathrm{n}}\left(\mathbf{C}_{\mathrm{n}}\right)$

$$
D_{1}\left(C_{1}\right)=2_{y}(1)=2 y^{\prime}
$$

$$
\mathrm{C}_{\infty \mathrm{h}} \mathbf{1}^{\prime}=\infty / \mathrm{m}^{\prime}
$$

$$
D_{2}\left(C_{2}\right)=2 x 2_{y} 2_{z}\left(2_{z}\right)=2_{x}{ }^{\prime} 2_{y}{ }^{\prime} 2_{z}
$$

$$
\mathbf{D}_{\infty}\left(\mathbf{C}_{\infty}\right)=\infty \mathbf{2}^{\prime}
$$

$D_{2 n}\left(D_{n}\right) \quad D_{2}\left(D_{1}\right)=2{ }_{x}{ }_{y} 2\left(2_{y}\right)=2_{x}{ }^{\prime} 2_{y} 2_{z}{ }^{\prime}$

$$
D_{4}\left(D_{2}\right)=42_{x} 2_{x y}\left(2 x 2_{y} 2_{z}\right)=4 z^{\prime} 22_{x y}^{\prime}
$$

$\mathbf{C}_{\mathrm{nv}}\left(\mathbf{C}_{\mathrm{n}}\right) \quad \mathbf{C}_{1 \mathrm{v}}\left(\mathbf{C}_{1}\right)=\mathbf{m}_{\mathrm{y}}(\mathbf{1})=\mathbf{m}_{\mathrm{y}}{ }^{\prime}$

$$
D_{\infty} 1^{\prime}=\infty 21^{\prime}
$$

$$
C_{2 v}\left(C_{2}\right)={ }_{2} m_{x} m_{y}\left(2_{z}\right)=2{ }_{z} m_{x}{ }^{\prime} m_{y}{ }^{\prime}
$$

$$
\mathbf{C}_{\infty \mathrm{v}}\left(\mathbf{C}_{\infty}\right)=\infty \mathbf{m}^{\prime} \mathbf{m}^{\prime}
$$

$\mathbf{C}_{2 \mathrm{nv}}\left(\mathbf{C}_{\mathrm{nv}}\right) \quad \mathbf{C}_{2 \mathrm{v}}\left(\mathbf{C}_{1 \mathrm{v}}\right)=\mathbf{2}_{\mathrm{z}} \mathrm{m}_{\mathrm{x}} \mathbf{m}_{\mathrm{y}}\left(\mathbf{m}_{\mathrm{y}}\right)=\mathbf{2}_{\mathrm{z}}{ }^{\prime} \mathbf{m}_{\mathrm{x}}{ }^{\prime} \mathbf{m}_{\mathrm{y}}$

$$
\mathbf{C}_{4 \mathrm{v}}\left(\mathbf{C}_{2 \mathrm{v}}\right)=\mathbf{4}_{\mathrm{z}} \mathrm{m}_{\mathrm{x}} \mathrm{m}_{\mathrm{xy}}\left(\mathbf{2}_{\mathrm{z}} \mathrm{m}_{\mathrm{x}} \mathrm{m}_{\mathrm{y}}\right)=\mathbf{4}_{\mathrm{z}}{ }^{\prime} \mathrm{m}_{\mathrm{x}} \mathrm{m}_{\mathrm{xy}}{ }^{\prime}
$$

$$
\mathrm{C}_{\infty \mathrm{V}} \mathbf{1}^{\prime}=\infty \mathrm{mm}^{\prime}{ }^{\prime}
$$

$\mathbf{D}_{\mathrm{nd}}\left(\mathbf{S}_{2 \mathrm{n}}\right)$

$D_{1 \mathrm{~d}}\left(S_{2}\right)=2_{\mathrm{y}} / \mathrm{m}_{\mathrm{y}}(\overline{\mathbf{1}})=\mathbf{2}_{\mathrm{y}}{ }^{\prime} / \mathrm{m}_{\mathrm{y}}{ }^{\prime}$

$$
D_{2 \mathrm{~d}}\left(\mathbf{S}_{4}\right)=\overline{\mathbf{4}}_{z} \mathrm{~m}_{\mathrm{x}} \mathbf{2}_{\mathrm{xy}}\left(\overline{\mathbf{4}}_{\mathrm{z}}\right)=\overline{\mathbf{4}}_{\mathrm{z}} \mathrm{m}_{\mathrm{x}}{ }^{\prime} \mathbf{2}_{\mathrm{xy}}{ }^{\prime}
$$

$\mathbf{D}_{\mathrm{nd}}\left(\mathbf{D}_{\mathrm{n}}\right)$

$$
\mathbf{D}_{\infty \mathrm{h}}\left(\mathbf{C}_{\infty \mathrm{h}}\right)=\infty / \mathrm{mm}^{\prime} \mathbf{m}^{\prime}
$$

$$
\begin{aligned}
& D_{2 d}\left(D_{2}\right)=\overline{4}_{z} m_{x} \mathbf{2}_{x y}\left(2_{x y} \mathbf{2}_{\bar{x} y} \mathbf{2}_{z}\right)=\overline{4}_{z}^{\prime} m_{x}^{\prime} \mathbf{2}_{x y} \\
& \mathbf{D}_{\infty \mathrm{h}}\left(\mathbf{D}_{\infty}\right)=\infty / \mathbf{m}^{\prime} \mathbf{m}^{\prime} \mathbf{m}^{\prime} \\
& \mathbf{D}_{\mathrm{nd}}\left(\mathbf{C}_{\mathrm{nv}}\right) \quad \mathbf{D}_{1 \mathrm{~d}}\left(\mathbf{C}_{1 \mathrm{v}}\right)=\mathbf{2}_{\mathrm{y}} / \mathrm{m}_{\mathrm{y}}\left(\mathrm{m}_{\mathrm{y}}\right)=\mathbf{2}_{\mathrm{y}}{ }^{\prime} / \mathbf{m}_{\mathrm{y}} \\
& D_{2 \mathrm{~d}}\left(C_{2 v}\right)=\overline{4}_{z} m_{x} 2_{x y}\left(2_{z} m_{x} m_{y}\right)=\overline{4}_{z}{ }^{\prime} m_{x} 2_{x y}{ }^{\prime} \\
& \mathbf{D}_{\infty \mathrm{h}}\left(\mathbf{C}_{\infty \mathrm{v}}\right)=\infty / \mathbf{m}^{\prime} \mathbf{m m} \\
& \mathbf{D}_{\mathrm{nh}}\left(\mathbf{C}_{\mathrm{nh}}\right) \quad \mathbf{D}_{1 \mathrm{~h}}\left(\mathbf{C}_{1 \mathrm{~h}}\right)=\mathbf{2}_{\mathrm{x}} \mathbf{m}_{\mathrm{y}} \mathbf{m}_{\mathrm{z}}\left(\mathbf{m}_{\mathrm{z}}\right)=\mathbf{2}_{\mathrm{x}}{ }^{\prime} \mathbf{m}_{\mathrm{y}}{ }^{\prime} \mathbf{m}_{\mathrm{z}} \\
& D_{2 h}\left(C_{2 h}\right)=m_{x} m_{y} m_{z}\left(2_{z} / m_{z}\right)=m_{x}^{\prime} m_{y}{ }^{\prime} m_{z} \\
& \mathbf{D}_{\infty \mathrm{h}}\left(\mathbf{C}_{\infty \mathrm{h}}\right)=\infty / \mathbf{m m}^{\prime} \mathbf{m}^{\prime} \\
& D_{\mathrm{nh}}\left(D_{\mathrm{n}}\right) \quad D_{1 \mathrm{~h}}\left(D_{1}\right)=\mathbf{2}_{\mathrm{x}} \mathrm{m}_{\mathrm{y}} \mathrm{m}_{\mathrm{z}}\left(\mathbf{2}_{\mathrm{x}}\right)=\mathbf{2}_{\mathrm{x}} \mathrm{m}_{\mathrm{y}}{ }^{\prime} \mathrm{m}_{\mathrm{z}}{ }^{\prime} \\
& D_{2 h}\left(D_{2}\right)=m_{x} m_{y} m_{z}\left(2_{x} 2_{y} 2_{z}\right)=m_{x}{ }^{\prime} m_{y}{ }^{\prime} m_{z}{ }^{\prime} \\
& \mathbf{D}_{\infty \mathrm{h}}\left(\mathbf{D}_{\infty}\right)=\infty / \mathrm{m}^{\prime} \mathrm{m}^{\prime} \mathbf{m}^{\prime} \\
& \mathbf{D}_{\mathrm{nh}}\left(\mathbf{C}_{\mathrm{nv}}\right) \quad \mathbf{D}_{1 \mathrm{~h}}\left(\mathbf{C}_{1 \mathrm{v}}\right)=\mathbf{2}_{\mathrm{x}} \mathbf{m}_{\mathrm{y}} \mathbf{m}_{\mathrm{z}}\left(\mathbf{m}_{\mathrm{y}}\right)=\mathbf{2}_{\mathrm{x}}{ }^{\prime} \mathbf{m}_{\mathrm{y}} \mathbf{m}_{\mathrm{z}}^{\prime} \\
& D_{2 h}\left(C_{2 v}\right)=m_{x} m_{y} m_{z}\left(2_{z} m_{x} m_{y}\right)=m_{x} m_{y} m_{z}^{\prime} \\
& D_{\infty \mathrm{h}}\left(\mathbf{C}_{\infty \mathrm{V}}\right)=\infty / \mathbf{m}^{\prime} \mathbf{m m} \\
& \mathbf{D}_{2 \mathrm{nh}}\left(\mathbf{D}_{\mathrm{nd}}\right) \quad \mathbf{D}_{2 \mathrm{~h}}\left(\mathbf{D}_{1 \mathrm{~d}}\right)=\mathbf{m}_{\mathrm{x}} \mathbf{m}_{\mathrm{y}} \mathbf{m}_{\mathrm{z}}\left(\mathbf{2}_{\mathrm{y}} / \mathbf{m}_{\mathrm{y}}\right)=\mathbf{m}_{\mathrm{x}}{ }^{\prime} \mathbf{m}_{\mathrm{y}} \mathbf{m}_{\mathrm{z}}{ }^{\prime} \\
& D_{4 h}\left(D_{2 d}\right)=4_{z} / m_{z} m_{x} m_{x y}\left(\overline{4}_{z} m_{x} 2_{x y}\right)=4_{z}^{\prime} / m_{z}{ }^{\prime} m_{x} m_{x y}{ }^{\prime} \\
& \mathrm{D}_{\infty \mathrm{h}} \mathbf{1}^{\prime}=\infty / \mathrm{mmm}^{\prime} \\
& \mathbf{D}_{2 \mathrm{nh}}\left(\mathbf{D}_{\mathrm{nh}}\right) \quad \mathbf{D}_{2 \mathrm{~h}}\left(\mathbf{D}_{1 \mathrm{~h}}\right)=\mathbf{m}_{\mathrm{x}} \mathbf{m}_{\mathbf{y}} \mathbf{m}_{\mathrm{z}}\left(\mathbf{2}_{\mathrm{x}} \mathbf{m}_{\mathbf{y}} \mathbf{m}_{\mathrm{z}}\right)=\mathbf{m}_{\mathrm{x}} \mathbf{\prime m}_{\mathbf{y}} \mathbf{m}_{\mathrm{z}} \\
& D_{4 h}\left(D_{2 h}\right)=4_{z} / m_{z} m_{x} m_{x y}\left(m_{x} m_{y} m_{z}\right)=4_{z}^{\prime} / m_{z} m_{x} m_{x y}{ }^{\prime} \\
& D_{\infty h} 1^{\prime}=\infty / \mathrm{mmm1}^{\prime}
\end{aligned}
$$


Rank 3 Property Tensor Tables

Table Format:

\begin{tabular}{c|c|c|c|c|}
\multicolumn{1}{c}{1} & \multicolumn{1}{c}{$\mathrm{e}$} & $\mathrm{a}$ & $\mathrm{ae}$ \\
\cline { 2 - 5 } $\mathrm{V}^{3}$ & $\mathrm{~V}^{3}$ & $\mathrm{eV}$ & $\mathrm{a} \mathrm{V}^{3}$ & $\mathrm{aeV}$ \\
{$\left[\mathrm{V}^{3}\right]$} & {$\left[\mathrm{V}^{3}\right]$} & $\mathrm{e}\left[\mathrm{V}^{3}\right]$ & $\mathrm{a}\left[\mathrm{V}^{3}\right]$ & $\mathrm{ae}\left[\mathrm{V}^{3}\right]$ \\
\cline { 2 - 5 } $\mathrm{V}\left[\mathrm{V}^{2}\right]$ & $\mathrm{V}\left[\mathrm{V}^{2}\right]$ & $\mathrm{eV}\left[\mathrm{V}^{2}\right]$ & $\mathrm{aV}\left[\mathrm{V}^{2}\right]$ & $\mathrm{aeV}\left[\mathrm{V}^{2}\right]$ \\
\cline { 2 - 5 }$\left\{\mathrm{V}^{2}\right\} \mathrm{V}$ & $\left\{\mathrm{V}^{2}\right\} \mathrm{V}$ & $\mathrm{e}\left\{\mathrm{V}^{2}\right\} \mathrm{V}$ & $\mathrm{a}\left\{\mathrm{V}^{2}\right\} \mathrm{V}$ & $\mathrm{ae}\left\{\mathrm{V}^{2}\right\} \mathrm{V}$ \\
\cline { 2 - 5 } & & &
\end{tabular}

Series $\quad n=1$

$$
n=2 \quad n=3 \quad n
$$

$\mathrm{C}_{\mathrm{n}} \quad \mathrm{C}_{1}=\mathbf{1}$

$$
\mathbf{C}_{2}=\mathbf{2}_{\mathbf{z}}
$$

$$
\mathbf{C}_{3}=\mathbf{3}_{\mathrm{z}}
$$

$\mathbf{C}_{\mathrm{nv}} \quad \mathbf{C}_{1 \mathrm{v}}=\mathbf{m}_{\mathrm{y}}$

$$
\mathbf{C}_{2 v}=\mathbf{2}_{z} \mathbf{m}_{\times} \mathbf{m}_{\mathrm{y}} \mathbf{C}_{3 \mathrm{v}}=\mathbf{3}_{\mathrm{z}} \mathbf{m}_{\mathrm{x}} \quad \mathbf{C}_{\infty \mathrm{v}}=\infty \mathbf{m m}
$$

$\mathbf{S}_{2 \mathrm{n}} \quad \mathbf{S}_{2}=\overline{\mathbf{1}}$

$$
\mathrm{S}_{4}=\overline{\mathbf{4}}_{\mathrm{z}}
$$

$$
\mathbf{S}_{6}=\overline{\mathbf{3}}_{\mathbf{z}}
$$

$$
\mathbf{C}_{\infty}=\infty
$$

$\mathbf{C}_{\mathrm{nh}} \quad \mathbf{C}_{\mathrm{ih}}=\mathbf{m}_{\mathrm{z}}$

$$
C_{2 h}=2 / m_{z}
$$$$
\mathbf{C}_{\infty h}=\infty / \mathbf{m}
$$

$\mathbf{D}_{\mathrm{n}} \quad \mathbf{D}_{1}=\mathbf{2}_{\mathrm{y}}$

$$
\mathbf{C}_{3 \mathrm{~h}}=\overline{\mathbf{6}}_{\mathrm{z}}
$$$$
\mathbf{C}_{\infty \mathrm{h}}=\infty / \mathbf{m}
$$

$$
D_{2}=2 \mathbf{x}_{\mathbf{y}} \mathbf{2}_{\mathrm{z}}
$$$$
D_{3}=3_{z} 2_{x}
$$$$
\mathbf{D}_{\infty}=\infty \mathbf{2}
$$

$\mathbf{D}_{\text {nd }} \quad \mathbf{D}_{1 \mathrm{~d}}=\mathbf{2}_{\mathrm{y}} / \mathrm{m}_{\mathrm{y}}$

$$
\begin{array}{r}
\mathbf{D}_{2 \mathrm{~d}}=\overline{\mathbf{4}}_{\mathbf{z}} \mathbf{m}_{\mathrm{x}} \mathbf{2}_{\mathrm{xy}} \\
\mathbf{D}_{3 \mathrm{~d}}=\overline{\mathbf{3}}_{z} \mathbf{m}_{\mathrm{x}}
\end{array}
$$

$\mathbf{D}_{\mathrm{nh}} \quad \mathbf{D}_{1 \mathrm{~h}}=\mathbf{2}_{\mathrm{x}} \mathbf{m}_{\mathrm{y}} \mathbf{m}_{\mathrm{z}}$

$$
\mathbf{D}_{\infty \mathrm{h}}=\infty / \mathrm{mmm}
$$

$$
\begin{aligned}
& \mathbf{D}_{2 \mathrm{~h}}=\mathbf{m}_{\mathbf{x}} \mathbf{m}_{\mathbf{y}} \mathbf{m}_{\mathrm{z}} \\
& \mathbf{D}_{3 \mathrm{~h}}=\overline{\mathbf{6}}_{\mathbf{z}} \mathbf{m}_{\mathbf{2}} \mathbf{2}_{1} \mathbf{D}_{\propto \mathrm{h}}=\infty / \mathbf{m m m}
\end{aligned}
$$


Series

$$
\mathrm{n}=1
$$

$$
\mathrm{n}=2
$$

$$
\mathrm{n}=3
$$

$\mathbf{C}_{2 n}\left(\mathbf{C}_{\mathrm{n}}\right) \quad \mathbf{C}_{2}\left(\mathbf{C}_{1}\right)=\mathbf{2}_{\mathrm{z}}(\mathbf{1})=\mathbf{2}_{\mathrm{z}}^{\prime}$

$$
\mathrm{n}>3
$$

$$
\mathbf{C}_{4}\left(\mathbf{C}_{2}\right)=\mathbf{4}_{\mathrm{z}}\left(\mathbf{2}_{\mathrm{z}}\right)=\mathbf{4}_{z^{\prime}}
$$

$$
C_{6}\left(C_{3}\right)=6_{z}\left(3_{z}\right)=6_{z}^{\prime}
$$

$$
\mathbf{C}_{\infty} 1^{\prime}=\infty 1^{\prime}
$$

$S_{2 n}\left(C_{n}\right) \quad S_{2}\left(C_{1}\right)=\overline{1}(\mathbf{1})=\overline{1}^{\prime}$

$$
\begin{aligned}
& \mathbf{S}_{4}\left(\mathbf{C}_{2}\right)=\overline{\mathbf{4}}_{\mathbf{z}}\left(\mathbf{2}_{\mathrm{z}}\right)=\overline{\mathbf{4}}_{\mathbf{z}}{ }^{\prime} \\
& \mathbf{S}_{6}\left(\mathbf{C}_{3}\right)=\overline{\mathbf{3}}_{\mathbf{z}}\left(\mathbf{3}_{z}\right)=\overline{\mathbf{3}}_{\mathbf{z}}{ }^{\prime} \mathbf{C}_{\infty \mathrm{h}}\left(\mathbf{C}_{\infty}\right)=\infty / \mathbf{m}^{\prime}
\end{aligned}
$$

$\mathbf{C}_{n h}\left(\mathbf{C}_{n}\right) \quad \mathbf{C}_{1 h}\left(\mathbf{C}_{1}\right)=\mathbf{m}_{z}(\mathbf{1})=\mathbf{m}_{z^{\prime}}$

$$
\begin{aligned}
& \mathbf{C}_{2 h}\left(\mathbf{C}_{2}\right)=\mathbf{2}_{\mathrm{z}} / \mathbf{m}_{\mathrm{z}}\left(\mathbf{2}_{\mathrm{z}}\right)=\mathbf{2}_{\mathrm{z}} / \mathbf{m}_{\mathbf{z}^{\prime}} \\
& \mathbf{C}_{3 \mathrm{~h}}\left(\mathbf{C}_{3}\right)=\overline{\mathbf{6}}_{\mathbf{z}}\left(\mathbf{3}_{\mathrm{z}}\right)=\overline{\mathbf{6}}_{\mathrm{z}}{ }^{\prime} \\
& \mathbf{C}_{\infty \mathrm{h}}\left(\mathbf{C}_{\infty}\right)=\infty / \mathbf{m}^{\prime}
\end{aligned}
$$

$\mathbf{C}_{2 n h}\left(\mathbf{S}_{2 \mathrm{n}}\right) \quad \mathbf{C}_{2 \mathrm{~h}}\left(\mathbf{S}_{2}\right)=\mathbf{2}_{\mathrm{z}} / \mathbf{m}_{\mathrm{z}}(\overline{\mathbf{1}})=\mathbf{2}_{\mathbf{z}}{ }^{\prime} / \mathbf{m}_{\mathbf{z}^{\prime}}$

$$
\begin{aligned}
& \mathbf{C}_{4 h}\left(S_{4}\right)=4_{z} / m_{z}\left(\overline{4}_{z}\right)=4_{z}{ }^{\prime} / m_{z}{ }^{\prime} \\
& C_{6 h}\left(S_{6}\right)=6_{z} / m_{z}\left(\overline{3}_{z}\right)=6_{z} z^{\prime} / m_{z}{ }^{\prime} \\
& C_{\infty h} 1^{\prime}=\infty / m 1^{\prime}
\end{aligned}
$$

$\mathbf{C}_{2 n h}\left(\mathbf{C}_{n h}\right) \quad \mathbf{C}_{2 h}\left(\mathbf{C}_{1 h}\right)=\mathbf{2}_{\mathrm{z}} / \mathbf{m}_{\mathrm{z}}\left(\mathbf{m}_{\mathrm{z}}\right)=\mathbf{2}_{\mathrm{z}}{ }^{\prime} / \mathbf{m}_{\mathrm{z}}$

$$
\begin{aligned}
& C_{4 h}\left(C_{2 h}\right)=4_{z} / m_{z}\left(2_{z} / m_{z}\right)= 4_{z} z^{\prime} / m_{z} \\
& C_{6 h}\left(C_{3 h}\right)=6_{z} / m_{z}\left(3_{z} / m_{z}\right)=6_{z}{ }^{\prime} / m_{z} \\
& C_{\infty h} 1^{\prime}=\infty / \mathbf{m}^{\prime}
\end{aligned}
$$

$\mathrm{D}_{\mathrm{n}}\left(\mathrm{C}_{\mathrm{n}}\right) \quad \mathrm{D}_{1}\left(\mathrm{C}_{1}\right)=\mathbf{2}_{\mathrm{y}}(\mathbf{1})=\mathbf{2}_{\mathrm{y}} \mathrm{y}^{\prime}$

$$
\begin{aligned}
D_{2}\left(C_{2}\right) & =2 x 2_{y} 2_{z}\left(2_{z}\right)=2 x^{\prime} 2^{\prime} 2_{z} \\
D_{3}\left(C_{3}\right)=3 & 3_{z} 2_{x}\left(3_{z}\right)=3 z_{z}{ }^{\prime}
\end{aligned}
$$

$$
\begin{aligned}
& D_{2 n}\left(D_{n}\right) \quad D_{2}\left(D_{1}\right)=2 x_{x} \mathbf{y}^{2} 2\left(2_{y}\right)=2 x^{\prime} \mathbf{2}_{y} 2_{z}^{\prime} \\
& D_{4}\left(D_{2}\right)=4_{z} 2_{x} 2_{x y}\left(2 x 2_{y} 2_{z}\right)=4_{z}{ }^{\prime} 22_{x y}^{\prime} \\
& D_{6}\left(D_{3}\right)=6_{z} 2_{x} 2_{1}\left(3_{z} 2_{x}\right)=6_{z}{ }^{\prime} 2_{x} 2_{1}{ }^{\prime} \\
& \mathbf{C}_{\mathrm{nv}}\left(\mathbf{C}_{\mathrm{n}}\right) \quad \mathbf{C}_{1 \mathrm{v}}\left(\mathbf{C}_{1}\right)=\mathbf{m}_{\mathrm{y}}(\mathbf{1})=\mathbf{m}_{\mathrm{y}}{ }^{\prime} \\
& D_{\infty} 1^{\prime}=\infty 21^{\prime} \\
& C_{2 v}\left(C_{2}\right)=2_{z} m_{x} m_{y}\left(2_{z}\right)=\mathbf{2}_{z} m_{x}{ }^{\prime} m_{y}^{\prime} \\
& C_{3 v}\left(C_{3}\right)=3_{z} m_{x}\left(3_{z}\right)=3_{z} m_{x}^{\prime} \\
& C_{2 n v}\left(C_{n v}\right) \quad C_{2 v}\left(C_{1 v}\right)=2_{z} m_{x} m_{y}\left(m_{y}\right)=2_{z}{ }^{\prime} m_{x}^{\prime} m_{y} \\
& \mathbf{C}_{\infty V}\left(\mathbf{C}_{\infty}\right)=\infty \mathbf{m}^{\prime} \mathbf{m}^{\prime} \\
& C_{4 v}\left(C_{2 v}\right)=4_{z} m_{x} m_{x y}\left(2_{z} m_{x} m_{y}\right)=4_{z}^{\prime} m_{x} m_{x y}{ }^{\prime} \\
& C_{6 v}\left(C_{3 v}\right)=6_{z} m_{x} m_{1}\left(3_{z} m_{x}\right)=6_{z}^{\prime} m_{x} m_{1}^{\prime} \\
& \mathrm{C}_{\infty \mathrm{V}} \mathbf{1}^{\prime}=\infty \mathrm{mm} \mathbf{1}^{\prime} \\
& \mathbf{D}_{\mathrm{nd}}\left(\mathbf{S}_{2 \mathrm{n}}\right) \quad \mathbf{D}_{1 \mathrm{~d}}\left(\mathbf{S}_{2}\right)=\mathbf{2}_{\mathrm{y}} / \mathbf{m}_{\mathrm{y}}(\overline{\mathbf{1}})=\mathbf{2}_{\mathrm{y}}{ }^{\prime} / \mathbf{m}_{\mathbf{y}^{\prime}} \\
& D_{2 d}\left(S_{4}\right)=\overline{4}_{z} m_{x} 2_{x y}\left(\overline{4}_{z}\right)=\overline{4}_{z} m_{x}{ }^{\prime} \mathbf{2}_{x y}{ }^{\prime} \\
& \mathbf{D}_{3 \mathrm{~d}}\left(\mathbf{S}_{6}\right)=\overline{\mathbf{3}}_{\mathbf{z}} \mathbf{m}_{\mathrm{x}}\left(\overline{\mathbf{3}}_{\mathbf{z}}\right)=\overline{\mathbf{3}}_{\mathbf{z}} \mathbf{m}_{\mathrm{x}}{ }^{\prime} \\
& \mathbf{D}_{\infty h}\left(\mathbf{C}_{\infty h}\right)=\infty / \mathbf{m m}^{\prime} \mathbf{m}^{\prime}
\end{aligned}
$$


Series

$$
\mathrm{n}=1
$$

$$
\mathrm{n}=2
$$

$$
\mathrm{n}=3
$$

$$
\mathrm{n}>3
$$

$\mathbf{D}_{\text {nd }}\left(\mathbf{D}_{\mathrm{n}}\right)$

$D_{1 \mathrm{~d}}\left(D_{1}\right)=2_{\mathrm{y}} / \mathrm{m}_{\mathrm{y}}\left(\mathbf{2}_{\mathrm{y}}\right)=\mathbf{2}_{\mathrm{y}} / \mathrm{m}_{\mathrm{y}}{ }^{\prime}$

$$
D_{2 d}\left(D_{2}\right)=\overline{4}_{z} m_{x} 2_{x y}\left(2_{x y} \mathbf{2}_{\bar{x} \mathbf{y}} \mathbf{2}_{z}\right)=\overline{4}_{z}{ }^{\prime} m_{x}{ }^{\prime} 2_{x y}
$$

$$
\mathbf{D}_{3 \mathrm{~d}}\left(\mathbf{D}_{3}\right)=\overline{\mathbf{3}}_{\mathbf{z}} \mathbf{m}_{\mathrm{x}}\left(\mathbf{3}_{\mathrm{z}} \mathbf{2}_{\mathrm{x}}\right)=\overline{\mathbf{3}}_{\mathbf{z}}{ }^{\prime} \mathbf{m}_{\mathrm{x}}{ }^{\prime}
$$

$\mathbf{D}_{\text {nd }}\left(\mathbf{C}_{\mathrm{nv}}\right) \quad \mathbf{D}_{1 \mathrm{~d}}\left(\mathbf{C}_{1 \mathrm{v}}\right)=\mathbf{2}_{\mathrm{y}} / \mathbf{m}_{\mathrm{y}}\left(\mathbf{m}_{\mathrm{y}}\right)=\mathbf{2}_{\mathrm{y}} / \mathbf{m}_{\mathrm{y}}$

$$
\mathbf{D}_{\infty h}\left(\mathbf{D}_{\infty}\right)=\infty / \mathbf{m}^{\prime} \mathbf{m}^{\prime} \mathbf{m}^{\prime}
$$$$
D_{2 \mathrm{~d}}\left(C_{2 \mathrm{v}}\right)=\overline{4}_{\mathrm{z}} \mathbf{m}_{\mathrm{x}} \mathbf{2}_{\mathrm{xy}}\left(\mathbf{2}_{\mathrm{z}} \mathbf{m}_{\mathrm{x}} \mathbf{m}_{\mathrm{y}}\right)=\overline{\mathbf{4}}_{\mathrm{z}}{ }^{\prime} \mathbf{m}_{\mathrm{x}} \mathbf{2}_{\mathrm{xy}}{ }^{\prime}
$$

$$
\begin{aligned}
\mathbf{D}_{3 \mathrm{~d}}\left(\mathbf{C}_{3 \mathrm{v}}\right)=\overline{\mathbf{3}}_{\mathrm{z}} \mathbf{m}_{\mathrm{x}}\left(\mathbf{3}_{\mathrm{z}} \mathbf{m}_{\mathrm{x}}\right)=\overline{\mathbf{3}}_{\mathrm{z}}{ }^{\prime} \mathbf{m}_{\mathrm{x}} \\
\mathbf{D}_{\infty \mathrm{h}}\left(\mathbf{C}_{\infty \mathrm{v}}\right)=\infty / \mathbf{m}^{\prime} \mathbf{m m}
\end{aligned}
$$

$\mathbf{D}_{\mathrm{nh}}\left(\mathbf{C}_{\mathrm{nh}}\right)$

$$
\mathbf{D}_{1 \mathrm{~h}}\left(\mathbf{C}_{1 \mathrm{~h}}\right)=\mathbf{2}_{\mathrm{x}} \mathbf{m}_{\mathrm{y}} \mathbf{m}_{\mathrm{z}}\left(\mathbf{m}_{\mathrm{z}}\right)=\mathbf{2 x}_{\mathrm{x}} \mathbf{m}_{\mathrm{y}} \mathbf{m}_{\mathrm{z}}
$$

$$
\mathbf{D}_{2 \mathrm{~h}}\left(\mathbf{C}_{2 \mathrm{~h}}\right)=\mathbf{m}_{\mathrm{x}} \mathbf{m}_{\mathbf{y}} \mathbf{m}_{\mathrm{z}}\left(\mathbf{2}_{\mathrm{z}} / \mathbf{m}_{\mathrm{z}}\right)=\mathbf{m}_{\mathrm{x}} \mathbf{m}_{\mathrm{y}} \mathbf{m}_{\mathrm{z}}
$$

$$
D_{3 h}\left(C_{3 h}\right)=\overline{\boldsymbol{\sigma}}_{z} m_{x} \mathbf{2}_{1}\left(\overline{\boldsymbol{\sigma}}_{z}\right)=\overline{\boldsymbol{\sigma}}_{\mathbf{z}} \mathrm{m}_{\mathrm{x}}{ }^{\prime} \mathbf{2}_{1}{ }^{\prime}
$$

$\mathbf{D}_{\text {nh }}\left(\mathbf{D}_{\mathrm{n}}\right)$

$$
\begin{aligned}
& \mathbf{D}_{1 \mathrm{~h}}\left(\mathbf{D}_{1}\right)=\mathbf{2}_{\mathrm{x}} \mathbf{m}_{\mathrm{y}} \mathbf{m}_{\mathrm{z}}\left(\mathbf{2}_{\mathrm{x}}\right)=\mathbf{2}_{\mathrm{x}} \mathbf{m}_{\mathrm{y}}{ }^{\prime} \mathbf{m}_{\mathrm{z}}{ }^{\prime} \\
& \mathbf{D}_{2 \mathrm{~h}}\left(\mathbf{D}_{2}\right)=\mathbf{m}_{\mathrm{x}} \mathbf{m}_{\mathrm{y}} \mathbf{m}_{\mathrm{z}}\left(\mathbf{2}_{\mathrm{x}} \mathbf{2}_{\mathrm{y}} \mathbf{2}_{\mathrm{z}}\right)=\mathbf{m}_{\mathrm{x}}{ }^{\prime} \mathbf{m}_{\mathrm{y}}{ }^{\prime} \mathbf{m}_{\mathrm{z}}{ }^{\prime} \\
& \mathbf{D}_{3 \mathrm{~h}}\left(\mathbf{D}_{3}\right)=\overline{\mathbf{6}}_{\mathrm{z}} \mathbf{m}_{\mathrm{x}} \mathbf{2}_{1}\left(\mathbf{3}_{\mathrm{z}} \mathbf{2}_{1}\right)=\overline{\mathbf{6}}_{\mathrm{z}}{ }^{\prime} \mathbf{m}_{\mathrm{x}} \mathbf{2}_{1}
\end{aligned}
$$$$
\mathbf{D}_{\infty h}\left(\mathbf{C}_{\infty h}\right)=\infty / \mathbf{m m}^{\prime} \mathbf{m}^{\prime}
$$

$\mathbf{D}_{\mathrm{nh}}\left(\mathbf{C}_{\mathrm{nv}}\right) \quad \mathbf{D}_{1 \mathrm{~h}}\left(\mathbf{C}_{1 \mathrm{v}}\right)=\mathbf{2}_{\mathrm{x}} \mathbf{m}_{\mathrm{y}} \mathbf{m}_{\mathrm{z}}\left(\mathbf{m}_{\mathrm{y}}\right)=\mathbf{2}_{\mathrm{x}}{ }^{\prime} \mathbf{m}_{\mathrm{y}} \mathbf{m}_{\mathrm{z}}{ }^{\prime}$

$$
\mathbf{D}_{\infty h}\left(\mathbf{D}_{\infty}\right)=\infty / \mathbf{m}^{\prime} \mathbf{m}^{\prime} \mathbf{m}^{\prime}
$$$$
\mathbf{D}_{2 \mathrm{~h}}\left(\mathbf{C}_{2 \mathrm{v}}\right)=\mathbf{m}_{\mathrm{x}} \mathbf{m}_{\mathrm{y}} \mathbf{m}_{\mathrm{z}}\left(\mathbf{2}_{\mathrm{z}} \mathbf{m}_{\mathrm{x}} \mathbf{m}_{\mathrm{y}}\right)=\mathbf{m}_{\mathrm{x}} \mathbf{m}_{\mathrm{y}} \mathbf{m}_{\mathrm{z}}
$$

$$
\begin{aligned}
\mathbf{D}_{3 \mathrm{~h}}\left(\mathbf{C}_{3 \mathrm{v}}\right)=\overline{\mathbf{6}}_{\mathrm{z}} \mathbf{m}_{\mathrm{x}} \mathbf{2}_{1}\left(\mathbf{3}_{\mathrm{z}} \mathbf{m}_{\mathrm{x}}\right)=\overline{\mathbf{6}}_{\mathrm{z}}{ }^{\prime} \mathbf{m}_{\mathrm{x}} \mathbf{2}_{1}{ }^{\prime} \\
\mathbf{D}_{\infty \mathrm{h}}\left(\mathbf{C}_{\infty \mathrm{v}}\right)=\infty / \mathbf{m}^{\prime} \mathbf{m m}
\end{aligned}
$$

$\mathbf{D}_{2 n h}\left(\mathbf{D}_{\text {nd }}\right) \quad \mathbf{D}_{2 \mathrm{~h}}\left(\mathbf{D}_{1 \mathrm{~d}}\right)=\mathbf{m}_{\mathrm{x}} \mathbf{m}_{\mathrm{y}} \mathbf{m}_{\mathrm{z}}\left(\mathbf{2}_{\mathbf{y}} / \mathbf{m}_{\mathrm{y}}\right)=\mathbf{m}_{\mathrm{x}} \mathbf{m}_{\mathrm{y}} \mathbf{m}_{\mathrm{z}}{ }^{\prime}$

$$
\begin{gathered}
\mathbf{D}_{4 \mathrm{~h}}\left(\mathbf{D}_{2 \mathrm{~d}}\right)=\mathbf{4}_{\mathrm{z}} / \mathbf{m}_{\mathrm{z}} \mathbf{m}_{\mathrm{x}} \mathbf{m}_{\mathrm{xy}}\left(\overline{\mathbf{4}}_{\mathrm{z}} \mathbf{m}_{\mathrm{x}} \mathbf{2}_{\mathrm{xy}}\right)=\mathbf{4}_{z^{\prime}} / \mathbf{m}_{\mathrm{z}}{ }^{\prime} \mathbf{m}_{\mathrm{x}} \mathbf{m}_{\mathrm{xy}}{ }^{\prime} \\
\mathbf{D}_{6 \mathrm{~h}}\left(\mathbf{D}_{3 \mathrm{~d}}\right)=\mathbf{6}_{\mathrm{z}} / \mathbf{m}_{\mathrm{z}} \mathbf{m}_{\mathrm{x}} \mathbf{m}_{1}\left(\overline{\mathbf{3}}_{z} \mathbf{m}_{\mathrm{x}}\right)=\mathbf{6}_{z^{\prime}} / \mathbf{m}_{\mathrm{z}}{ }^{\prime} \mathbf{m}_{\mathrm{x}} \mathbf{m}_{1^{\prime}} \mathbf{D}_{\infty \mathrm{h}} \mathbf{1}^{\prime}=\infty / \mathbf{m m m} \mathbf{1}^{\prime}
\end{gathered}
$$

$\mathbf{D}_{2 n h}\left(\mathbf{D}_{n h}\right) \quad \mathbf{D}_{2 h}\left(\mathbf{D}_{1 \mathrm{~h}}\right)=\mathbf{m}_{\mathrm{x}} \mathbf{m}_{\mathbf{y}} \mathbf{m}_{\mathbf{z}}\left(\mathbf{2}_{\mathbf{x}} \mathbf{m}_{\mathbf{y}} \mathbf{m}_{\mathrm{z}}\right)=\mathbf{m}_{\mathrm{x}} \mathbf{m}_{\mathbf{y}} \mathbf{m}_{\mathrm{z}}$

$$
D_{4 h}\left(D_{2 h}\right)=4_{z} / m_{z} m_{x} m_{x y}\left(m_{x} m_{y} m_{z}\right)=4_{z}{ }^{\prime} / m_{z} m_{x} m_{x y}{ }^{\prime}
$$

$$
\begin{gathered}
\mathbf{D}_{6 \mathrm{~h}}\left(\mathbf{D}_{3 \mathrm{~h}}\right)=\mathbf{6}_{\mathrm{z}} / \mathbf{m}_{\mathrm{z}} \mathbf{m}_{\mathrm{x}} \mathbf{m}_{1}\left(\overline{\mathbf{6}}_{\mathrm{z}} \mathbf{m}_{\mathrm{x}} \mathbf{2}_{1}\right)=\mathbf{6}_{\mathrm{z}}{ }^{\prime} / \mathbf{m}_{\mathrm{z}} \mathbf{m}_{\mathrm{x}} \mathbf{m}_{1}{ }^{\prime} \\
\mathbf{D}_{\infty \mathrm{h}} \mathbf{1}^{\prime}=\infty / \mathbf{m m m} \mathbf{1}^{\prime}
\end{gathered}
$$


Rank 4 Property Tensor Tables

\begin{tabular}{|c|c|c|c|c|c|}
\hline & & 1 & e & $a$ & ae \\
\hline \multirow{2}{*}{ Table } & $\mathrm{V}^{4}$ & $\mathrm{~V}^{4}$ & $\mathrm{eV}^{4}$ & $\mathrm{aV}^{4}$ & $\mathrm{aeV}^{4}$ \\
\hline & {$\left[\mathrm{V}^{4}\right]$} & {$\left[\mathrm{V}^{4}\right]$} & $e\left[V^{4}\right]$ & $\mathrm{a}\left[\mathrm{V}^{4}\right]$ & $\mathrm{ae}\left[\mathrm{V}^{4}\right]$ \\
\hline \multirow{5}{*}{ Format: } & $\mathrm{V}\left[\mathrm{V}^{3}\right]$ & $\mathrm{V}\left[\mathrm{V}^{3}\right]$ & $\mathrm{eV}\left[\mathrm{V}^{3}\right]$ & $\mathrm{aV}\left[\mathrm{V}^{3}\right]$ & $\mathrm{aeV}\left[\mathrm{V}^{3}\right]$ \\
\hline & {$\left[\left[\mathrm{V}^{2}\right]^{2}\right]$} & {$\left[\left[\mathrm{V}^{2}\right]^{2}\right]$} & $\mathrm{e}\left[\left[\mathrm{V}^{2}\right]^{2}\right]$ & $a\left[\left[\mathrm{~V}^{2}\right]^{2}\right]$ & $\mathrm{ae}\left[\left[\mathrm{V}^{2}\right]^{2}\right]$ \\
\hline & {$\left[\mathrm{V}^{2}\right]^{2}$} & {$\left[\mathrm{~V}^{2}\right]^{2}$} & $e\left[V^{2}\right]^{2}$ & $\mathrm{a}\left[\mathrm{V}^{2}\right]^{2}$ & $\mathrm{ae}\left[\mathrm{V}^{2}\right]^{2}$ \\
\hline & {$\left[\left(\mathrm{V}^{2}\right)\right]^{2}$} & {$\left[\left(\mathrm{~V}^{2}\right)\right]^{2}$} & $\mathrm{e}\left[\left(\mathrm{V}^{2}\right)\right]^{2}$ & $\mathrm{a}\left[\left(\mathrm{V}^{2}\right)\right]^{2}$ & $\mathrm{ae}\left[\left(\mathrm{V}^{2}\right)\right]^{2}$ \\
\hline & {$\left[\mathrm{V}^{2}\right] \mathrm{V}^{2}$} & {$\left[\mathrm{~V}^{2}\right] \mathrm{V}^{2}$} & $e\left[V^{2}\right] V^{2}$ & $a\left[V^{2}\right] V^{2}$ & $a e\left[V^{2}\right] V^{2}$ \\
\hline
\end{tabular}

Series $\quad n=1$

$$
n=2
$$

$$
\mathrm{n}=3
$$

$$
\mathrm{n}=4
$$

$\mathrm{C}_{\mathrm{n}} \quad \mathrm{C}_{1}=\mathbf{1}$

$$
\begin{array}{lll}
\mathbf{C}_{2}=\mathbf{2}_{\mathrm{z}} & \mathrm{C}_{3}=\mathbf{3}_{\mathrm{z}} \quad \mathrm{C}_{4}=\mathbf{4}_{\mathrm{z}}
\end{array}
$$

$$
\mathrm{n}>4
$$

$$
\begin{aligned}
& \mathbf{C}_{\mathrm{nv}} \quad \mathbf{C}_{1 \mathrm{v}}=\mathbf{m}_{\mathrm{y}} \\
& \mathbf{C}_{2 \mathrm{v}}=\mathbf{2}_{\mathrm{z}} \mathbf{m}_{\mathrm{x}} \mathbf{m}_{\mathrm{y}} \\
& \mathbf{C}_{3 v}=\mathbf{3}_{z} \mathbf{m}_{x} \\
& \mathbf{C}_{4 v}=\mathbf{4}_{z} \mathbf{m}_{x} \mathbf{m}_{x y} \mathbf{C}_{\infty \mathrm{v}}=\infty \mathbf{m m} \\
& \mathbf{S}_{2 \mathrm{n}} \quad \mathbf{S}_{2}=\overline{1} \\
& S_{4}=\overline{4}_{z} \\
& \mathbf{S}_{6}=\overline{\mathbf{3}}_{\mathbf{z}} \\
& \mathbf{S}_{8}=\overline{\mathbf{8}}_{z} \\
& \mathbf{C}_{\infty h}=\infty / \mathbf{m}
\end{aligned}
$$


Series $\quad n=1$

Rank 4

$$
\mathrm{n}=2
$$

$$
\mathrm{n}=3
$$

$$
\mathrm{n}=4
$$

$\mathbf{C}_{\mathrm{nh}} \quad \mathbf{C}_{1 \mathrm{~h}}=\mathbf{m}_{\mathrm{z}}$

$$
\begin{aligned}
& \mathrm{C}_{2 \mathrm{~h}}=\mathbf{2} / \mathrm{m}_{\mathrm{z}} \\
& \mathbf{D}_{\mathrm{n}} \quad \mathbf{D}_{1}=\mathbf{2} \mathrm{y} \\
& \mathrm{C}_{3 \mathrm{~h}}=\overline{\boldsymbol{6}}_{\mathrm{z}} \\
& C_{4 h}=4_{z} / m_{z} \\
& \mathbf{C}_{\infty \mathrm{h}}=\infty / \mathbf{m} \\
& D_{2}=2 x{ }^{2} 2_{z} \\
& D_{3}=32_{x} \\
& D_{4}=4_{z} 2_{x} 2_{x y} \\
& \mathbf{D}_{\infty}=\infty \mathbf{2} \\
& \mathrm{D}_{\text {nd }} \quad \mathrm{D}_{1 \mathrm{~d}}=\mathbf{2}_{\mathrm{y}} / \mathrm{m}_{\mathrm{y}} \\
& D_{2 d}=\overline{4}_{z} m_{x} 2_{x y} \\
& \mathbf{D}_{3 d}=\overline{\mathbf{3}}_{z} \mathbf{m}_{x} \\
& \mathbf{D}_{4 \mathrm{~d}}=\overline{\mathbf{8}}_{\mathrm{z}} \mathbf{m}_{\mathrm{x} 1} \mathbf{2}_{1} \\
& \mathbf{D}_{\alpha \mathrm{h}}=\infty / \mathrm{mmm} \\
& D_{3 h}=\overline{6}_{z} m_{x} 2_{1} \\
& D_{4 h}=4_{z} / m_{z} m_{x} m_{x y} \\
& D_{\infty \mathrm{h}}=\infty / \mathrm{mmm} \\
& \mathbf{C}_{2 \mathrm{n}}\left(\mathbf{C}_{\mathrm{n}}\right) \quad \mathrm{C}_{2}\left(\mathbf{C}_{1}\right)=\mathbf{2}_{\mathrm{z}}(\mathbf{1})=\mathbf{2}_{\mathbf{z}^{\prime}} \\
& \mathrm{C}_{4}\left(\mathrm{C}_{2}\right)=\mathbf{4}_{\mathrm{z}}\left(2_{\mathrm{z}}\right)=\mathbf{4}_{\mathrm{z}^{\prime}} \\
& \mathrm{C}_{6}\left(\mathrm{C}_{3}\right)=\mathbf{6}_{\mathrm{z}}\left(\mathbf{3}_{\mathrm{z}}\right)=\mathbf{6}_{\mathrm{z}}{ }^{\prime} \\
& \mathrm{C}_{8}\left(\mathrm{C}_{4}\right)=\mathbf{8}_{\mathrm{z}}\left(\mathbf{4}_{\mathrm{z}}\right)=\mathbf{8}_{z^{\prime}} \\
& \mathrm{C}_{\infty} 1^{\prime}=\infty 1^{\prime} \\
& \mathbf{S}_{8}\left(\mathbf{C}_{4}\right)=\overline{\mathbf{8}}_{z}\left(\mathbf{4}_{z}\right)=\overline{\mathbf{8}}_{z}^{\prime}
\end{aligned}
$$

$\mathrm{n}>4$ 
Series $\quad n=1$

$$
\mathrm{n}=2
$$

$$
n=3 \quad n=4
$$

$\mathrm{n}>4$

$\mathbf{C}_{2 n h}\left(\mathbf{S}_{2 \mathrm{n}}\right) \quad \mathbf{C}_{2 \mathrm{~h}}\left(\mathbf{S}_{2}\right)=\mathbf{2}_{\mathrm{z}} / \mathbf{m}_{\mathrm{z}}(\overline{\mathbf{1}})=\mathbf{2}_{\mathrm{z}}{ }^{\prime} / \mathbf{m}_{\mathbf{z}}{ }^{\prime}$

$$
\mathrm{C}_{4 h}\left(S_{4}\right)=4_{z} / m_{z}\left(\overline{4}_{z}\right)=4_{z}^{\prime} / m_{z}^{\prime}
$$

$$
\mathrm{C}_{6 \mathrm{~h}}\left(S_{6}\right)=\mathbf{6}_{\mathrm{z}} / \mathrm{m}_{\mathrm{z}}\left(\overline{3}_{\mathrm{z}}\right)=\mathbf{6}_{\mathrm{z}}{ }^{\prime} / \mathrm{m}_{\mathrm{z}}{ }^{\prime}
$$

$$
\mathrm{C}_{8 \mathrm{~h}}\left(\mathrm{~S}_{8}\right)=\mathbf{8}_{\mathrm{z}} / \mathrm{m}_{\mathrm{z}}\left(\overline{\mathbf{8}}_{\mathbf{z}}\right)=\mathbf{8}_{\mathrm{z}^{\prime}} / \mathrm{m}_{\mathrm{z}}{ }^{\prime}
$$

$\mathbf{C}_{2 n h}\left(C_{n h}\right) \quad C_{2 h}\left(C_{1 h}\right)=2 z_{z} / m_{z}\left(m_{z}\right)=\mathbf{2 z}_{z} / \mathbf{m}_{z}$

$$
\mathrm{C}_{\infty \mathrm{h}} \mathbf{1}^{\prime}=\infty / \mathrm{m} \mathbf{1}^{\prime}
$$

$$
\begin{aligned}
& C_{4 h}\left(C_{2 h}\right)=4_{z} / m_{z}\left(2_{z} / m_{z}\right)=4_{z}^{\prime} / m_{z} \\
& \mathrm{C}_{6 \mathrm{~h}}\left(\mathrm{C}_{3 \mathrm{~h}}\right)=6_{\mathrm{z}} / \mathrm{m}_{\mathrm{z}}\left(3_{\mathrm{z}} / \mathrm{m}_{\mathrm{z}}\right)=6_{\mathrm{z}}{ }^{\prime} / \mathrm{m}_{\mathrm{z}} \\
& C_{8 h}\left(C_{4 h}\right)=8_{z} / m_{z}\left(4_{z} / m_{z}\right)=8_{z}^{\prime} / m_{z} \\
& \mathrm{C}_{\infty \mathrm{h}} \mathbf{1}^{\prime}=\infty / \mathrm{m} \mathbf{1}^{\prime}
\end{aligned}
$$

$\mathbf{D}_{\mathrm{n}}\left(\mathbf{C}_{\mathrm{n}}\right) \quad \mathrm{D}_{1}\left(\mathbf{C}_{1}\right)=\mathbf{2}_{\mathrm{y}}(\mathbf{1})=\mathbf{2}_{\mathrm{y}}{ }^{\prime}$

$$
\begin{aligned}
& D_{2}\left(C_{2}\right)=2 x 2_{y} 2_{z}\left(2_{z}\right)=2 x^{\prime} 2_{y}^{\prime} 2_{z} \\
& D_{3}\left(C_{3}\right)=32^{2}\left(3_{z}\right)=3 z^{\prime}{ }^{\prime} \\
& D_{4}\left(C_{4}\right)=4_{z} 2_{x} 2_{x y}\left(4_{z}\right)=4_{z} 2_{x}^{\prime} 2_{x y}{ }^{\prime}
\end{aligned}
$$

$D_{2 n}\left(D_{n}\right) \quad D_{2}\left(D_{1}\right)=2{ }_{x}{ }_{y} 2\left(2_{y}\right)=2 x^{\prime}{ }^{\prime}{ }^{\prime} 2^{\prime}$

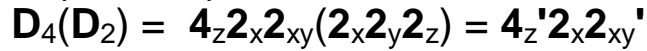

$$
\begin{aligned}
& D_{6}\left(D_{3}\right)=6_{z} 2_{x} 2_{1}\left(3{ }_{z} 2_{x}\right)=6_{z}{ }^{\prime} 22_{x}{ }^{\prime}
\end{aligned}
$$$$
D_{8}\left(D_{4}\right)=8_{z} 2_{x 1} 2_{1}\left(4_{z} 2_{\times 1} 2_{x 2}\right)=8_{z}{ }^{\prime} 2_{x 1} 2_{1}{ }^{\prime}
$$

$\mathbf{C}_{\mathrm{nv}}\left(\mathbf{C}_{\mathrm{n}}\right) \quad \mathbf{C}_{1 \mathrm{v}}\left(\mathbf{C}_{1}\right)=\mathbf{m}_{\mathrm{y}}(\mathbf{1})=\mathbf{m}_{\mathrm{y}}{ }^{\prime}$

$$
D_{\infty} 1^{\prime}=\infty 21^{\prime}
$$$$
C_{2 v}\left(C_{2}\right)=2_{z} m_{x} m_{y}\left(2_{z}\right)=2_{z} m_{x}{ }^{\prime} m_{y}^{\prime}
$$$$
\mathbf{C}_{3 \mathrm{v}}\left(\mathbf{C}_{3}\right)=\mathbf{3}_{\mathrm{z}} \mathbf{m}_{\mathrm{x}}\left(\mathbf{3}_{\mathrm{z}}\right)=\mathbf{3}_{\mathrm{z}} \mathbf{m}_{\mathrm{x}}{ }^{\prime}
$$

$\mathbf{C}_{2 n v}\left(C_{n v}\right) \quad C_{2 v}\left(C_{1 v}\right)=2_{z} m_{x} m_{y}\left(m_{y}\right)=2_{z}^{\prime} m_{x}{ }^{\prime} m_{y}$

$$
\begin{aligned}
& C_{4 v}\left(C_{4}\right)=4_{z} m_{x} m_{x y}\left(4_{z}\right)=\mathbf{4}_{z} m_{x}{ }^{\prime} m_{x y}{ }^{\prime} \\
& \mathbf{C}_{\infty \mathrm{V}}\left(\mathbf{C}_{\infty}\right)=\infty \mathbf{m}^{\prime} \mathbf{m}^{\prime}
\end{aligned}
$$$$
C_{4 v}\left(C_{2 v}\right)=4_{z} m_{x} m_{x y}\left(2_{z} m_{x} m_{y}\right)=4_{z}^{\prime} m_{x} m_{x y}{ }^{\prime}
$$$$
C_{6 v}\left(C_{3 v}\right)=6_{z} m_{x} m_{1}\left(3_{z} m_{x}\right)=6_{z}{ }^{\prime} m_{x} m_{1}{ }^{\prime}
$$$$
C_{8 v}\left(C_{4 v}\right)=8_{z} m_{x 1} m_{1}\left(4_{z} m_{x} m_{x y}\right)=8_{z}^{\prime} m_{x 1} m_{1}^{\prime}
$$$$
\mathrm{C}_{\infty \vee} 1^{\prime}=\infty \mathrm{mm} 1^{\prime}
$$

$\mathbf{D}_{\mathrm{nd}}\left(\mathbf{S}_{2 \mathrm{n}}\right) \quad \mathbf{D}_{1 \mathrm{~d}}\left(\mathbf{S}_{2}\right)=\mathbf{2}_{\mathrm{y}} / \mathbf{m}_{\mathrm{y}}(\overline{\mathbf{1}})=\mathbf{2}_{\mathrm{y}}{ }^{\prime} / \mathbf{m}_{\mathrm{y}}{ }^{\prime}$

$$
\begin{aligned}
& D_{2 d}\left(S_{4}\right)=\overline{\mathbf{4}}_{z} \mathbf{m}_{x} \mathbf{2}_{\mathrm{xy}}\left(\overline{\mathbf{4}}_{\mathbf{z}}\right)=\overline{\mathbf{4}}_{\mathbf{z}} \mathbf{m}_{\mathrm{x}} \mathbf{2}_{\mathrm{xy}}{ }^{\prime} \\
& \mathbf{D}_{3 \mathrm{~d}}\left(\mathbf{S}_{6}\right)=\overline{\mathbf{3}}_{z} \mathbf{m}_{\mathrm{x}}\left(\overline{\mathbf{3}}_{\mathbf{z}}\right)=\overline{\mathbf{3}}_{\mathrm{z}} \mathbf{m}_{\mathrm{x}}{ }^{\prime} \\
& \mathbf{D}_{4 \mathrm{~d}}\left(\mathbf{S}_{8}\right)=\overline{\mathbf{8}}_{\mathbf{z}} \mathbf{m}_{\mathrm{x} 1} \mathbf{2}_{1}\left(\overline{\mathbf{8}}_{\mathbf{z}}\right)=\overline{\mathbf{8}}_{\mathbf{z}} \mathbf{m}_{\mathrm{x} 1}{ }^{\prime} \mathbf{2}_{1}{ }^{\prime} \\
& \mathbf{D}_{\infty h}\left(\mathbf{C}_{\infty h}\right)=\infty / \mathbf{m m}^{\prime} \mathbf{m}^{\prime}
\end{aligned}
$$


Series $\quad n=1$

$$
\mathrm{n}=2
$$

$$
\mathrm{n}=3
$$

$$
\mathrm{n}=4
$$

$\mathrm{n}>4$

$\mathbf{D}_{\text {nd }}\left(\mathbf{D}_{\mathrm{n}}\right)$

$$
\begin{aligned}
& \mathbf{D}_{1 \mathrm{~d}}\left(\mathbf{D}_{1}\right)=\mathbf{2}_{\mathrm{y}} / \mathbf{m}_{\mathrm{y}}\left(\mathbf{2}_{\mathrm{y}}\right)=\mathbf{2}_{\mathrm{y}} / \mathbf{m}_{\mathrm{y}}{ }^{\prime} \\
& \mathbf{D}_{2 \mathrm{~d}}\left(\mathbf{D}_{2}\right)=\overline{4}_{z} \mathbf{m}_{\mathrm{x}} \mathbf{2}_{\mathrm{xy}}\left(\mathbf{2}_{\mathrm{xy}} \mathbf{2}_{\overline{\mathbf{x}} \mathbf{y}} \mathbf{2}_{\mathbf{z}}\right)=\overline{\mathbf{4}}_{\mathrm{z}}{ }^{\prime} \mathbf{m}_{\mathrm{x}} \mathbf{2}_{\mathrm{xy}} \\
& \mathbf{D}_{3 \mathrm{~d}}\left(\mathbf{D}_{3}\right)=\overline{\mathbf{3}}_{\mathbf{z}} \mathbf{m}_{\mathrm{x}}\left(\mathbf{3}_{\mathrm{z}} \mathbf{2}_{\mathrm{x}}\right)=\overline{\mathbf{3}}_{\mathbf{z}}{ }^{\prime} \mathbf{m}_{\mathrm{x}}{ }^{\prime} \\
& \mathbf{D}_{4 \mathrm{~d}}\left(\mathbf{D}_{4}\right)=\overline{\mathbf{8}}_{\mathbf{z}} \mathbf{m}_{\mathrm{x} 1} \mathbf{2}_{1}\left(\mathbf{4}_{\mathrm{z}} \mathbf{2}_{\mathrm{x}} \mathbf{2}_{\mathrm{xy}}\right)=\overline{\mathbf{8}}_{\mathbf{z}}{ }^{\prime} \mathbf{m}_{\mathrm{x} 1} \mathbf{2}_{1} \\
& \mathbf{D}_{\infty h}\left(\mathbf{D}_{\infty}\right)=\infty / \mathbf{m}^{\prime} \mathbf{m}^{\prime} \mathbf{m}^{\prime}
\end{aligned}
$$

$D_{\text {nd }}\left(C_{n v}\right) \quad D_{1 \mathrm{~d}}\left(C_{1 v}\right)=\mathbf{2}_{\mathrm{y}} / \mathbf{m}_{\mathrm{y}}\left(\mathbf{m}_{\mathrm{y}}\right)=\mathbf{2}_{\mathrm{y}}{ }^{\prime} / \mathbf{m}_{\mathrm{y}}$

$$
\begin{aligned}
& D_{2 d}\left(C_{2 v}\right)=\overline{4}_{z} m_{x} 2_{x y}\left(2_{z} m_{x} m_{y}\right)=\overline{4}_{z}{ }^{\prime} m_{x} 2_{x y}{ }^{\prime} \\
& \mathbf{D}_{3 \mathrm{~d}}\left(\mathbf{C}_{3 \mathrm{v}}\right)=\overline{\mathbf{3}}_{\mathrm{z}} \mathbf{m}_{\mathrm{x}}\left(\mathbf{3}_{\mathrm{z}} \mathbf{m}_{\mathrm{x}}\right)=\overline{\mathbf{3}}_{\mathrm{z}}{ }^{\prime} \mathbf{m}_{\mathrm{x}} \\
& D_{4 \mathrm{~d}}\left(C_{4 \mathrm{v}}\right)=\overline{\mathbf{8}}_{\mathrm{z}} \mathbf{m}_{\mathrm{x} 1} \mathbf{2}_{1}\left(\mathbf{4}_{\mathrm{z}} \mathbf{m}_{\mathrm{x} 1} \mathbf{m}_{\mathrm{x} 3}\right)=\overline{\mathbf{8}}_{\mathrm{z}}{ }^{\prime} \mathbf{m}_{\mathrm{x} 1} \mathbf{2} \\
& \mathbf{D}_{\infty \mathrm{h}}\left(\mathbf{C}_{\infty \mathrm{v}}\right)=\infty / \mathbf{m}^{\prime} \mathbf{m m}
\end{aligned}
$$

$\mathbf{D}_{\mathrm{nh}}\left(\mathbf{C}_{\mathrm{nh}}\right) \quad \mathbf{D}_{1 \mathrm{~h}}\left(\mathbf{C}_{1 \mathrm{~h}}\right)=\mathbf{2}_{\mathrm{x}} \mathbf{m}_{\mathbf{y}} \mathbf{m}_{\mathrm{z}}\left(\mathbf{m}_{\mathrm{z}}\right)=\mathbf{2}_{\mathrm{x}}^{\prime} \mathbf{m}_{\mathrm{y}}^{\prime} \mathbf{m}_{\mathrm{z}}$

$$
\begin{aligned}
& \mathbf{D}_{2 \mathrm{~h}}\left(\mathbf{C}_{2 \mathrm{~h}}\right)=\mathbf{m}_{\mathrm{x}} \mathbf{m}_{\mathbf{y}} \mathbf{m}_{\mathrm{z}}\left(\mathbf{2}_{\mathrm{z}} / \mathbf{m}_{\mathrm{z}}\right)=\mathbf{m}_{\mathrm{x}}{ }^{\prime} \mathbf{m}_{\mathrm{y}}{ }^{\prime} \mathbf{m}_{\mathrm{z}} \\
& D_{3 h}\left(C_{3 h}\right)=\overline{\boldsymbol{\sigma}}_{z} m_{x} \mathbf{2}_{1}\left(\overline{\boldsymbol{\sigma}}_{\mathrm{z}}\right)=\overline{\boldsymbol{\sigma}}_{\mathrm{z}} \mathrm{m}_{\mathrm{x}} \mathbf{2}^{\prime}{ }^{\prime} \\
& \mathbf{D}_{4 \mathrm{~h}}\left(\mathbf{C}_{4 \mathrm{~h}}\right)=\mathbf{4}_{\mathrm{z}} / \mathbf{m}_{\mathrm{z}} \mathbf{m}_{\mathrm{x}} \mathbf{m}_{\mathrm{xy}}\left(\mathbf{4}_{\mathrm{z}} / \mathbf{m}_{\mathrm{z}}\right)=\mathbf{4}_{\mathrm{z}} / \mathbf{m}_{\mathrm{z}} \mathbf{m}_{\mathrm{x}} \mathbf{m}_{\mathrm{xy}}{ }^{\prime} \\
& \mathbf{D}_{\mathrm{nh}}\left(\mathbf{D}_{\mathrm{n}}\right) \quad \mathbf{D}_{1 \mathrm{~h}}\left(\mathbf{D}_{1}\right)=\mathbf{2}_{\mathrm{x}} \mathbf{m}_{\mathrm{y}} \mathbf{m}_{\mathrm{z}}\left(\mathbf{2}_{\mathrm{x}}\right)=\mathbf{2}_{\mathrm{x}} \mathbf{m}_{\mathrm{y}}{ }^{\prime} \mathbf{m}_{\mathrm{z}}{ }^{\prime} \\
& \mathbf{D}_{\infty \mathrm{h}}\left(\mathbf{C}_{\infty \mathrm{h}}\right)=\infty / \mathbf{m m}^{\prime} \mathbf{m}^{\prime}
\end{aligned}
$$$$
D_{2 h}\left(D_{2}\right)=m_{x} m_{y} m_{z}\left(2_{x} 2_{y} 2_{z}\right)=m_{x}^{\prime} m_{y}^{\prime} m_{z}^{\prime}
$$$$
D_{3 h}\left(D_{3}\right)=\overline{6}_{z} m_{x} 2_{1}\left(3_{z} 2_{1}\right)=\overline{6}_{z}{ }^{\prime} m_{x}{ }^{\prime} 2_{1}
$$$$
D_{4 h}\left(D_{4}\right)=4_{z} / m_{z} m_{x} m_{x y}\left(4_{z} 2_{x} 2_{x y}\right)=4_{z} / m_{z}^{\prime} m_{x}^{\prime} m_{x y}{ }^{\prime}
$$$$
\mathbf{D}_{\infty \mathrm{h}}\left(\mathbf{D}_{\infty}\right)=\infty / \mathbf{m}^{\prime} \mathbf{m}^{\prime} \mathbf{m}^{\prime}
$$

$$
\begin{aligned}
& \mathbf{D}_{\mathrm{nh}}\left(\mathbf{C}_{\mathrm{nv}}\right) \quad \mathbf{D}_{\mathrm{1h}}\left(\mathbf{C}_{1 \mathrm{v}}\right)=\mathbf{2}_{\mathrm{x}} \mathbf{m}_{\mathrm{y}} \mathbf{m}_{\mathrm{z}}\left(\mathbf{m}_{\mathrm{y}}\right)=\mathbf{2}_{\mathrm{x}}{ }^{\prime} \mathbf{m}_{\mathrm{y}} \mathbf{m}_{\mathrm{z}}{ }^{\prime} \\
& D_{2 h}\left(C_{2 v}\right)=m_{x} m_{y} m_{z}\left(2_{z} m_{x} m_{y}\right)=m_{x} m_{y} m_{z}^{\prime} \\
& D_{3 h}\left(C_{3 v}\right)=\overline{6}_{z} m_{x} 2_{1}\left(3_{z} m_{x}\right)=\overline{6}_{z}{ }^{\prime} m_{x} 2_{1}{ }^{\prime} \\
& D_{4 h}\left(C_{4 v}\right)=4_{z} / m_{z} m_{x} m_{x y}\left(4_{z} m_{x} m_{x y}\right)=4_{z} / m_{z} m_{x} m_{x y} \\
& \mathbf{D}_{2 n h}\left(\mathbf{D}_{\text {nd }}\right) \quad \mathbf{D}_{2 \mathrm{~h}}\left(\mathbf{D}_{1 \mathrm{~d}}\right)=\mathbf{m}_{\mathrm{x}} \mathbf{m}_{\mathrm{y}} \mathbf{m}_{\mathrm{z}}\left(\mathbf{2}_{\mathbf{y}} / \mathbf{m}_{\mathrm{y}}\right)=\mathbf{m}_{\mathrm{x}}{ }^{\prime} \mathbf{m}_{\mathrm{y}} \mathbf{m}_{\mathrm{z}}{ }^{\prime} \\
& \mathbf{D}_{\infty h}\left(\mathbf{C}_{\infty \mathrm{V}}\right)=\infty / \mathbf{m}^{\prime} \mathbf{m m} \\
& D_{4 h}\left(D_{2 \mathrm{~d}}\right)=\mathbf{4}_{z} / \mathbf{m}_{\mathrm{z}} \mathbf{m}_{\mathrm{x}} \mathbf{m}_{\mathrm{xy}}\left(\overline{4}_{\mathrm{z}} \mathbf{m}_{\mathrm{x}} \mathbf{2}_{\mathrm{xy}}\right)=\mathbf{4}_{\mathrm{z}}{ }^{\prime} / \mathbf{m}_{\mathrm{z}}{ }^{\prime} \mathbf{m}_{\mathrm{x}} \mathbf{m}_{\mathrm{xy}}{ }^{\prime} \\
& D_{6 h}\left(D_{3 \mathrm{~d}}\right)=\mathbf{6}_{\mathrm{z}} / \mathbf{m}_{\mathrm{z}} \mathbf{m}_{\mathrm{x}} \mathbf{m}_{1}\left(\overline{3}_{\mathrm{z}} \mathbf{m}_{\mathrm{x}}\right)=\mathbf{6}_{\mathrm{z}}{ }^{\prime} / \mathbf{m}_{\mathrm{z}}{ }^{\prime} \mathbf{m}_{\mathrm{x}} \mathbf{m}_{1}{ }^{\prime} \\
& C_{8 h}\left(D_{4 d}\right)=\mathbf{8}_{z} / m_{z} m_{x 1} m_{1}\left(\overline{8}_{z} m_{x 1} 2_{1}\right)=\mathbf{8}_{z}^{\prime} / \mathbf{m}_{z}^{\prime} m_{x 1} m_{1}^{\prime} \\
& D_{\infty h} \mathbf{1}^{\prime}=\infty / \mathbf{m m m}^{\prime} \\
& D_{4 h}\left(D_{2 h}\right)=4_{z} / m_{z} m_{x} m_{x y}\left(m_{x} m_{y} m_{z}\right)=4_{z}^{\prime} / m_{z} m_{x} m_{x y}{ }^{\prime} \\
& \mathbf{D}_{6 \mathrm{~h}}\left(\mathbf{D}_{3 \mathrm{~h}}\right)=\mathbf{6}_{\mathrm{z}} / \mathbf{m}_{\mathrm{z}} \mathbf{m}_{\mathrm{x}} \mathbf{m}_{1}\left(\overline{\mathbf{6}}_{\mathrm{z}} \mathbf{m}_{\mathrm{x}} \mathbf{2}_{1}\right)=\mathbf{6}_{\mathrm{z}}{ }^{\prime} / \mathbf{m}_{\mathrm{z}} \mathbf{m}_{\mathrm{x}} \mathbf{m}_{1}{ }^{\prime} \\
& \mathbf{D}_{8 \mathrm{~h}}\left(\mathbf{D}_{4 \mathrm{~h}}\right)=\mathbf{8}_{\mathrm{z}} / \mathbf{m}_{\mathrm{z}} \mathbf{m}_{\mathrm{x} 1} \mathbf{m}_{1}\left(\mathbf{4}_{\mathrm{z}} / \mathbf{m}_{\mathrm{z}} \mathbf{m}_{\times 1} \mathbf{m}_{\mathrm{x} 2}\right)=\mathbf{8}_{\mathrm{z}}{ }^{\prime} / \mathbf{m}_{\mathrm{z}} \mathbf{m}_{\mathrm{x} 1} \mathbf{m}^{\prime}{ }^{\prime} \\
& D_{\infty h} \mathbf{1}^{\prime}=\infty / \mathbf{m m m}^{\prime}
\end{aligned}
$$




\section{Rank 1, 2, 3, and 4 Property Tensors}

Rank 1:

V

$\mathrm{eV}$

$\mathrm{aV}$

$\mathrm{aeV}$

Rank 2:

$\mathrm{V}^{2}$

$\mathrm{eV}^{2}$

$\mathrm{aV}^{2}$

$\mathrm{aeV}^{2}$

$\left[\mathrm{V}^{2}\right]$

$$
\mathrm{e}\left[\mathrm{V}^{2}\right]
$$

$\mathrm{a}\left[\mathrm{V}^{2}\right]$

$\mathrm{ae}\left[\mathrm{V}^{2}\right]$

$\mathrm{ij}=\mathrm{ji}$

$\left\{\mathrm{V}^{2}\right\}$

$e\left\{V^{2}\right\}$

$\mathrm{a}\left\{\mathrm{V}^{2}\right\}$

$\mathrm{ae}\left\{\mathrm{V}^{2}\right\}$

$\mathrm{ij}=-\mathrm{ji}$

Rank 3:

$\mathrm{V}^{3}$

$\left[\mathrm{V}^{3}\right]$

$\mathrm{V}\left[\mathrm{V}^{2}\right]$

$\mathrm{eV}^{3}$

$\mathrm{aV}^{3}$

$\mathrm{aeV}^{3}$

$\left\{\mathrm{V}^{2}\right\} \mathrm{V}$

$$
\mathrm{e}\left[\mathrm{V}^{3}\right]
$$

$\mathrm{a}\left[\mathrm{V}^{3}\right]$

$\mathrm{ae}\left[\mathrm{V}^{3}\right]$

symmetric wrt all indicies

Rank 4:

$\mathrm{V}^{4}$

$$
\mathrm{eV}^{4}
$$

$\mathrm{aV}^{4}$

$\mathrm{aeV}^{4}$

$\left[\mathrm{V}^{4}\right]$

$\mathrm{e}\left[\mathrm{V}^{4}\right]$

$\mathrm{a}\left[\mathrm{V}^{4}\right]$

$\mathrm{ae}\left[\mathrm{V}^{4}\right]$

symmetric wrt all indicies

$\mathrm{V}\left[\mathrm{V}^{3}\right]$

$\mathrm{eV}\left[\mathrm{V}^{3}\right]$

$\mathrm{aV}\left[\mathrm{V}^{3}\right.$ ]

$\mathrm{aeV}\left[\mathrm{V}^{3}\right]$

ilk=ikl

$\left[\left[\mathrm{V}^{2}\right]^{2}\right]$

$\mathrm{e}\left[\left[\mathrm{V}^{2}\right]^{2}\right]$

$\mathrm{a}\left[\left[\mathrm{V}^{2}\right]^{2}\right]$

$\mathrm{ae}\left[\left[\mathrm{V}^{2}\right]^{2}\right]$

symmetric wrt to last three indicies

$\left[\mathrm{V}^{2}\right]^{2}$

$e\left[V^{2}\right]^{2}$

$\mathrm{a}\left[\mathrm{V}^{2}\right]^{2}$

$\mathrm{ae}\left[\mathrm{V}^{2}\right]^{2}$

$i j k l=j i k l=i j \mid k=k l i j$

$\left[\left(\mathrm{V}^{2}\right)\right]^{2}$

$\mathrm{e}\left[\left(\mathrm{V}^{2}\right)\right]^{2}$

$\mathrm{a}\left[\left(\mathrm{V}^{2}\right)\right]^{2}$

$\mathrm{ae}\left[\left(\mathrm{V}^{2}\right)\right]^{2}$

$i j k l=j i k l=i j \mid k$

$\left[\mathrm{V}^{2}\right] \mathrm{V}^{2}$

$\mathrm{e}\left[\mathrm{V}^{2}\right] \mathrm{V}^{2}$

$\mathrm{a}\left[\mathrm{V}^{2}\right] \mathrm{v}^{2}$

$\mathrm{ae}\left[\mathrm{V}^{2}\right] \mathrm{V}^{2}$

$k l i j=i j k l$

$\mathrm{jikl}=\mathrm{ijk} \mid$ 
Rank 1 tensors:

$\left(\begin{array}{c}1 \\ e \\ a \\ a e\end{array}\right) v\left(\begin{array}{l}x \\ y \\ z\end{array}\right)$

Rank 2 tensors:

$\left(\begin{array}{c}1 \\ e \\ a \\ a e\end{array}\right) V^{2} \quad\left(\begin{array}{ccc}x x & x y & x z \\ y x & y y & y z \\ z x & z y & z z\end{array}\right)$

$\left(\begin{array}{c}1 \\ e \\ a \\ a e\end{array}\right)\left[V^{2}\right] \quad\left(\begin{array}{ccc}x x & x y & x z \\ x y & y y & y z \\ x z & y z & z z\end{array}\right) \quad i j=j i$

$\left(\begin{array}{c}1 \\ e \\ a \\ a e\end{array}\right)\left\{V^{2}\right\} \quad\left(\begin{array}{ccc}0 & x y & x z \\ -x y & 0 & y z \\ -x z & -y z & 0\end{array}\right) \quad i j=-j i$

Rank 3 tensors:

$\left(\begin{array}{c}1 \\ e \\ a \\ a e\end{array}\right) V^{3} \quad\left(\begin{array}{lllllllll}x x x & x y y & x z z & x y z & x z x & x x y & x z y & x x z & x y x \\ y x x & y y y & y z z & y y z & y z x & y x y & y z y & y x z & y y x \\ z x x & z y y & z z z & z y z & z z x & z x y & z z y & z x z & z y x\end{array}\right)$

$\left(\begin{array}{c}1 \\ e \\ a \\ a e\end{array}\right)\left[V^{3}\right] \quad\left(\begin{array}{llll}x x x & x y y & x z z & x y z \\ y x x & y y y & y z z & \\ z x x & z y y & z z z\end{array}\right) \quad$ symmetric with respect to all indicies 


$$
\begin{aligned}
& \left(\begin{array}{c}
1 \\
e \\
a \\
a e
\end{array}\right) V\left[V^{2}\right] \quad\left(\begin{array}{llllll}
x x x & x y y & x z z & x y z & x x z & x x y \\
y x x & y y y & y z z & y y z & y x z & y x y \\
z x x & z y y & z z z & z y z & z x z & z x y
\end{array}\right) \quad \text { ilk =ikl } \\
& \left(\begin{array}{c}
1 \\
e \\
a \\
a e
\end{array}\right)\left\{V^{2}\right\} V \quad\left(\begin{array}{lll}
y z x & y z y & y z z \\
z x x & z x y & z x z \\
x y x & x y y & x y z
\end{array}\right) \quad \text { jik }=-i j k
\end{aligned}
$$


Rank 4 tensors:

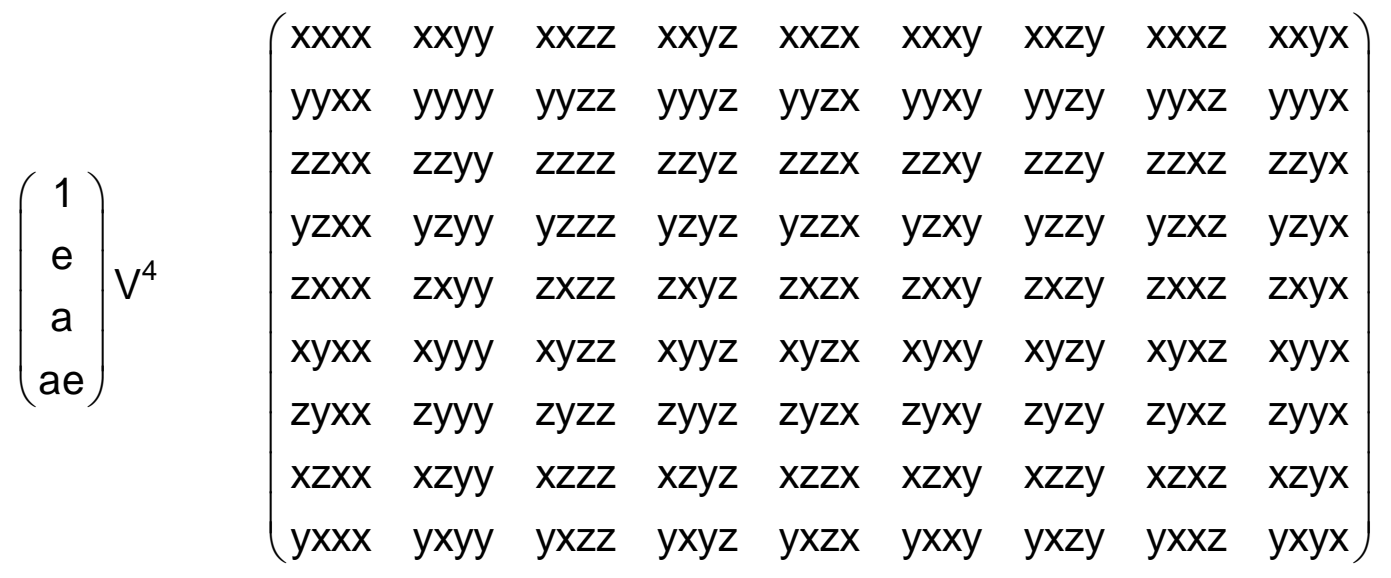

$\left(\begin{array}{c}1 \\ e \\ a \\ a e\end{array}\right)\left[V^{4}\right] \quad\left(\begin{array}{lllll}x x x x & x y y y & x z z z & y y z z & x x y z \\ y x x x & y y y y & y z z z & z z x x & y y x z \\ z x x x & z y y y & z z z z & x x y y & z z x y\end{array}\right) \quad$ symmetric with respect to all indicies

$\left(\begin{array}{c}1 \\ e \\ a \\ a e\end{array}\right) V\left[V^{3}\right] \quad\left(\begin{array}{llllllllll}x x x x & x y y y & x z z z & x x y y & x y z z & x z x x & x x z z & x y x x & x z y y & x x y z \\ y x x x & y y y y & y z z z & y x y y & y y z z & y z x x & y x z z & y y x x & y z y y & y x y z \\ z x x x & z y y y & z z z z & z x y y & z y z z & z z x x & z x z z & z y x x & z z y y & z x y z\end{array}\right)$

symmetric with respect to last three indicies

$\left(\begin{array}{c}1 \\ e \\ a \\ a e\end{array}\right)\left[\left[\mathrm{V}^{2}\right]^{2}\right] \quad\left(\begin{array}{cccccc}x x x x & x x y y & x x z z & x x y z & x x x z & x x x y \\ & y y y y & y y z z & y y y z & y y x z & y y x y \\ & & z z z z & z z y z & z z x z & z z x y \\ & & & y z y z & y z x z & y z x y \\ & & & & x z x z & x z x y \\ & & & & & x y x y\end{array}\right) \quad \quad i j k l=j i k l=i j l k=k l i j$

$\left(\begin{array}{c}1 \\ e \\ a \\ a e\end{array}\right)\left[V^{2}\right]^{2}\left(\begin{array}{llllll}x x x x & x x y y & x x z z & x x y z & x x x z & x x x y \\ y y x x & y y y y & y y z z & y y y z & y y x z & y y x y \\ z z x x & z z y y & z z z z & z z y z & z z x z & z z x y \\ y z x x & y z y y & y z z z & y z y z & y z x z & y z x y \\ x z x x & x z y y & x z z z & x z y z & x z x z & x z x y \\ x y x x & x y y y & x y z z & x y y z & x y x z & x y x y\end{array}\right) \quad \quad i j k l=j i k l=i j l k$ 

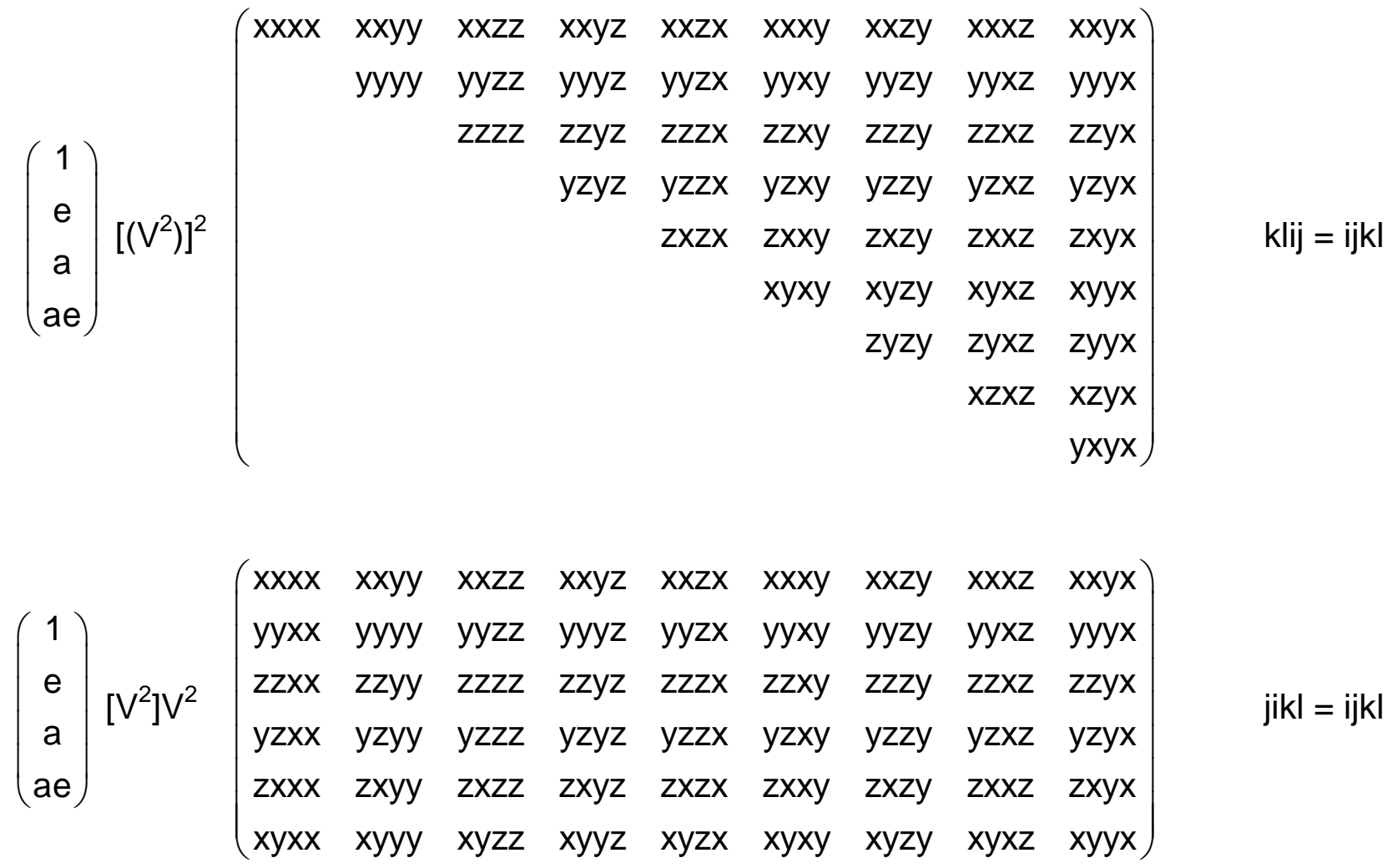
Line Group Series and Line Groups for $n=1,2,3,4$ and $\infty$

\begin{tabular}{|c|c|}
\hline $\mathbf{C}_{n}$ & $\begin{array}{l}\mathbf{C}_{1}=\mathbf{1} \\
\mathbf{C}_{2}=\mathbf{2}_{\mathrm{z}} \\
\mathbf{C}_{3}=\mathbf{3}_{\mathbf{z}} \\
\mathbf{C}_{4}=\mathbf{4}_{\mathbf{z}} \\
\mathbf{C}_{\infty}=\infty\end{array}$ \\
\hline $\mathbf{C}_{\mathrm{nv}}$ & $\begin{array}{l}\mathbf{C}_{1 v}=\mathbf{m}_{\mathrm{y}} \\
\mathbf{C}_{2 \mathrm{v}}=\mathbf{2}_{\mathrm{z}} \mathbf{m}_{\mathrm{x}} \mathbf{m}_{\mathrm{y}} \\
\mathbf{C}_{3 \mathrm{v}}=\mathbf{3}_{\mathrm{z}} \mathbf{m}_{\mathrm{x}} \\
\mathbf{C}_{4 \mathrm{v}}=\mathbf{4}_{\mathrm{z}} \mathbf{m}_{\mathrm{x}} \mathbf{m}_{\mathrm{x}} \\
\mathbf{C}_{{ }_{\mathrm{w}}}=\infty \mathbf{m}\end{array}$ \\
\hline $\mathbf{C}_{\mathrm{nh}}$ & $\begin{array}{l}\mathbf{C}_{1 \mathrm{~h}}=\mathbf{m}_{\mathrm{z}} \\
\mathbf{C}_{2 \mathrm{~h}}=\mathbf{2}_{\mathrm{z}} / \mathbf{m}_{\mathrm{z}} \\
\mathbf{C}_{3 \mathrm{~h}}=\overline{\mathbf{6}}_{\mathrm{z}} \\
\mathbf{C}_{4 \mathrm{~h}}=\mathbf{4}_{\mathrm{z}} / \mathbf{m}_{\mathrm{z}} \\
\mathbf{C}_{\infty \mathrm{h}}=\infty / \mathbf{m}\end{array}$ \\
\hline
\end{tabular}

$\mathbf{S}_{2 n}$

$\mathbf{S}_{2}=\overline{1}$

$\mathbf{S}_{4}=\overline{\mathbf{4}}_{z}$

$\mathbf{S}_{6}=\overline{\mathbf{3}}_{z}$

$\mathrm{S}_{8}=\overline{\mathbf{8}}_{\mathrm{z}}$

$\mathbf{C}_{\infty \mathrm{h}}=\infty / \mathrm{m}$

$\mathbf{D}_{\mathrm{n}}$

$$
\begin{aligned}
& \mathbf{D}_{1}=\mathbf{2}_{\mathrm{y}} \\
& \mathbf{D}_{2}=\mathbf{2}_{\mathrm{x}} \mathbf{2} \mathbf{2} \mathbf{z}_{\mathrm{z}} \\
& \mathbf{D}_{3}=\mathbf{3}_{\mathrm{z}} \mathbf{2}_{\mathrm{x}} \\
& \mathbf{D}_{4}=\mathbf{4}_{\mathrm{z}} \mathbf{2}_{\mathrm{x}} \mathbf{2}_{\mathrm{x}} \mathrm{y} \\
& \mathbf{D}_{\infty}=\infty \mathbf{2}
\end{aligned}
$$

$\mathbf{D}_{\text {nd }}$

$D_{1 d}=2 y / m_{y}$

$D_{2 d}=\overline{4}_{z} m_{x} \mathbf{2}_{x y}$

$\mathbf{D}_{3 \mathrm{~d}}=\overline{\mathbf{3}}_{\mathrm{z}} \mathbf{m}_{\mathrm{x}}$

$\mathbf{D}_{4 \mathrm{~d}}=\overline{\mathbf{8}}_{\mathrm{z}} \mathbf{m}_{\mathrm{x} 1} \mathbf{2}_{1}$

$\mathbf{D}_{\alpha \mathrm{h}}=\infty / \mathrm{mmm}$

$\mathbf{D}_{\text {nh }}$

$\mathbf{D}_{1 \mathrm{~h}}=\mathbf{2}_{\mathrm{x}} \mathbf{m}_{\mathrm{y}} \mathbf{m}_{\mathrm{z}}$

$\mathbf{D}_{2 \mathrm{~h}}=\mathbf{m}_{\mathrm{x}} \mathbf{m}_{\mathbf{y}} \mathbf{m}_{\mathrm{z}}$

$D_{3 \mathrm{~h}}=\overline{\mathbf{6}}_{\mathrm{z}} \mathrm{m}_{\mathrm{x}} \mathbf{2}_{1}$

$\mathbf{D}_{4 \mathrm{~h}}=\mathbf{4}_{\mathrm{z}} / \mathbf{m}_{\mathrm{z}} \mathbf{m}_{\mathrm{x}} \mathbf{m}_{\mathrm{xy}}$

$\mathbf{D}_{\alpha \mathrm{h}}=\infty / \mathrm{mmm}$ 

$\mathbf{C}_{2 n}\left(\mathbf{C}_{n}\right)$
$\mathrm{C}_{2}\left(\mathrm{C}_{1}\right)=\mathbf{2}_{\mathrm{z}}(\mathbf{1})=\mathbf{2}_{\mathrm{z}}^{\prime}$
$\mathbf{C}_{4}\left(C_{2}\right)=\mathbf{4}_{\mathrm{z}}\left(\mathbf{2}_{\mathrm{z}}\right)=\mathbf{4}_{z^{\prime}}$
$\mathrm{C}_{6}\left(\mathrm{C}_{3}\right)=\mathbf{6}_{\mathrm{z}}\left(\mathbf{3}_{\mathrm{z}}\right)=\mathbf{6}_{\mathrm{z}^{\prime}}{ }^{\prime}$
$\mathrm{C}_{8}\left(\mathrm{C}_{4}\right)=\mathbf{8}_{\mathrm{z}}\left(\mathbf{4}_{\mathrm{z}}\right)=\mathbf{8}_{\mathrm{z}}{ }^{\prime}$
$\mathrm{C}_{\infty} \mathbf{1}^{\prime}=\infty \mathbf{1}^{\prime}$
$\mathbf{S}_{2 n}\left(\mathbf{C}_{n}\right)$
$S_{2}\left(C_{1}\right)=\overline{1}(1)=\overline{1}^{\prime}$
$\mathbf{S}_{4}\left(\mathbf{C}_{2}\right)=\overline{\mathbf{4}}_{\mathbf{z}}\left(\mathbf{2}_{\mathrm{z}}\right)=\overline{\mathbf{4}}_{\mathbf{z}}{ }^{\prime}$
$\mathbf{S}_{6}\left(\mathbf{C}_{3}\right)=\overline{\mathbf{3}}_{\mathbf{z}}\left(\mathbf{3}_{\mathbf{z}}\right)=\overline{\mathbf{3}}_{\mathbf{z}}{ }^{\prime}$
$\mathrm{S}_{8}\left(\mathbf{C}_{4}\right)=\overline{\mathbf{8}}_{z}\left(\mathbf{4}_{z}\right)=\overline{\mathbf{8}}_{z}^{\prime}$
$\mathbf{C}_{\infty \mathrm{h}}\left(\mathbf{C}_{\infty}\right)=\infty / \mathbf{m}^{\prime}$
$\mathbf{C}_{\mathrm{nh}}\left(\mathbf{C}_{\mathrm{n}}\right)$
$\mathbf{C}_{1 \mathrm{~h}}\left(\mathbf{C}_{1}\right)=\mathbf{m}_{\mathrm{z}}(\mathbf{1})=\mathbf{m}_{\mathbf{z}}{ }^{\prime}$
$C_{2 h}\left(C_{2}\right)=2_{z} / m_{z}\left(2_{z}\right)=2{ }_{z} / m_{z}{ }^{\prime}$
$\mathbf{C}_{3 h}\left(\mathbf{C}_{3}\right)=\overline{\boldsymbol{\sigma}}_{\mathbf{z}}\left(\mathbf{3}_{\mathrm{z}}\right)=\overline{\mathbf{6}}_{\mathbf{z}}{ }^{\prime}$
$C_{4 h}\left(C_{4}\right)=4_{z} / m_{z}\left(4_{z}\right)=4_{z} / m_{z}^{\prime}$
$\mathbf{C}_{\infty h}\left(\mathbf{C}_{\infty}\right)=\infty / \mathbf{m}^{\prime}$
$\mathbf{C}_{2 n h}\left(\mathbf{S}_{2 n}\right)$
$\mathrm{C}_{2 \mathrm{~h}}\left(\mathrm{~S}_{2}\right)=\mathbf{2}_{\mathrm{z}} / \mathbf{m}_{\mathrm{z}}(\overline{\mathbf{1}})=\mathbf{2}_{\mathrm{z}} \mathrm{z}^{\prime} / \mathrm{m}_{\mathrm{z}}{ }^{\prime}$
$C_{4 h}\left(S_{4}\right)=4_{z} / m_{z}\left(\overline{4}_{z}\right)=4_{z}{ }^{\prime} / m_{z}{ }^{\prime}$
$\mathrm{C}_{6 \mathrm{~h}}\left(\mathrm{~S}_{6}\right)=\boldsymbol{6}_{\mathrm{z}} / \mathrm{m}_{\mathrm{z}}\left(\overline{\mathbf{3}}_{\mathrm{z}}\right)=\mathbf{6}_{\mathrm{z}}{ }^{\prime} / \mathrm{m}_{\mathrm{z}}{ }^{\prime}$
$\mathrm{C}_{8 \mathrm{~h}}\left(\mathbf{S}_{8}\right)=\mathbf{8}_{\mathrm{z}} / \mathrm{m}_{\mathrm{z}}\left(\overline{\mathbf{8}}_{\mathrm{z}}\right)=\mathbf{8}_{\mathrm{z}^{\prime}} / \mathrm{m}_{\mathrm{z}}{ }^{\prime}$
$\mathrm{C}_{\infty \mathrm{h}} \mathbf{1}^{\prime}=\infty / \mathrm{m1}^{\prime}$
$\mathbf{C}_{2 n h}\left(\mathbf{C}_{n h}\right)$
$\mathbf{C}_{2 \mathrm{~h}}\left(\mathbf{C}_{1 \mathrm{~h}}\right)=\mathbf{2}_{\mathrm{z}} / \mathbf{m}_{\mathrm{z}}\left(\mathbf{m}_{\mathrm{z}}\right)=\mathbf{2}_{\mathrm{z}}{ }^{\prime} / \mathbf{m}_{\mathrm{z}}$
$C_{4 h}\left(C_{2 h}\right)=4_{z} / m_{z}\left(2_{z} / m_{z}\right)=4_{z}^{\prime} / m_{z}$
$\mathrm{C}_{6 \mathrm{~h}}\left(\mathrm{C}_{3 \mathrm{~h}}\right)=\mathbf{6}_{\mathrm{z}} / \mathrm{m}_{\mathrm{z}}\left(\mathbf{3}_{\mathrm{z}} / \mathrm{m}_{\mathrm{z}}\right)=\mathbf{6}_{\mathrm{z}} / \mathrm{m}_{\mathrm{z}}$
$\mathbf{C}_{8 \mathrm{~h}}\left(\mathbf{C}_{4 \mathrm{~h}}\right)=\mathbf{8}_{\mathrm{z}} / \mathbf{m}_{\mathrm{z}}\left(\mathbf{4}_{\mathrm{z}} / \mathbf{m}_{\mathrm{z}}\right)=\mathbf{8}_{\mathrm{z}}{ }^{\prime} / \mathbf{m}_{\mathrm{z}}$
$\mathrm{C}_{\infty h} \mathbf{1}^{\prime}=\infty / \mathrm{m}^{\prime}$
$\mathbf{D}_{\mathrm{n}}\left(\mathbf{C}_{\mathrm{n}}\right)$
$\mathrm{D}_{1}\left(\mathrm{C}_{1}\right)=\mathbf{2}_{\mathrm{y}}(\mathbf{1})=\mathbf{2}_{\mathrm{y}} \mathrm{y}^{\prime}$
$D_{2}\left(C_{2}\right)=22^{2} 2_{z}\left(2_{z}\right)=2 x^{\prime} 2_{y}^{\prime} 2_{z}$
$\mathbf{D}_{3}\left(C_{3}\right)=\mathbf{3}_{\mathrm{z}} \mathbf{2}_{\mathrm{x}}\left(\mathbf{3}_{\mathrm{z}}\right)=\mathbf{3}_{\mathrm{z}} \mathbf{2}_{\mathrm{x}}{ }^{\prime}$
$D_{4}\left(C_{4}\right)=4_{z} \mathbf{2}_{x} \mathbf{2}_{\mathrm{xy}}\left(\mathbf{4}_{\mathrm{z}}\right)=\mathbf{4}_{\mathrm{z}} \mathbf{2}^{\prime} \mathbf{2}_{\mathrm{xy}}{ }^{\prime}$
$\mathbf{D}_{\infty}\left(\mathbf{C}_{\infty}\right)=\infty \mathbf{2}^{\prime}$
$\mathbf{D}_{2 n}\left(\mathbf{D}_{n}\right)$

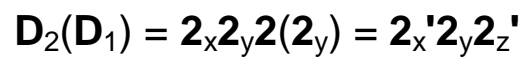
$D_{4}\left(D_{2}\right)=4_{z} 2_{x} 2_{x y}\left(2 x 2_{y} 2_{z}\right)=4_{z}{ }^{\prime} 2_{x} 2_{x y}{ }^{\prime}$
$D_{6}\left(D_{3}\right)=6_{z} \mathbf{2}_{x} \mathbf{2}_{1}\left(3_{z} \mathbf{2}_{\mathrm{x}}\right)=\mathbf{6}_{\mathrm{z}}{ }^{\prime} \mathbf{2}_{\mathrm{x}} \mathbf{2}^{\prime}{ }^{\prime}$
$D_{8}\left(D_{4}\right)=8_{z} 2_{x 1} 2_{1}\left(4_{z} 2_{x 1} 2_{x 2}\right)=8_{z}{ }^{\prime} 2_{\times 1} 2_{1}{ }^{\prime}$
$D_{\infty} 1^{\prime}=\infty 21^{\prime}$ 


$$
\begin{aligned}
& \mathbf{C}_{\mathrm{nv}}\left(\mathbf{C}_{\mathrm{n}}\right) \quad \mathbf{C}_{1 \mathrm{v}}\left(\mathbf{C}_{1}\right)=\mathbf{m}_{\mathrm{y}}(\mathbf{1})=\mathbf{m}_{\mathrm{y}}{ }^{\prime} \\
& C_{2 v}\left(C_{2}\right)=\mathbf{2}_{z} m_{x} m_{y}\left(2_{z}\right)=\mathbf{2}_{z} m_{x}^{\prime} m_{y}^{\prime} \\
& C_{3 v}\left(C_{3}\right)=3_{z} m_{x}\left(3_{z}\right)=3_{z} m_{x}^{\prime} \\
& C_{4 v}\left(C_{4}\right)=4_{z} m_{x} m_{x y}\left(4_{z}\right)=4_{z} m_{x}{ }^{\prime} m_{x y}{ }^{\prime} \\
& \mathbf{C}_{\infty v}\left(\mathbf{C}_{\infty}\right)=\infty \mathbf{m}^{\prime} \mathbf{m}^{\prime} \\
& \mathbf{C}_{2 n v}\left(C_{n v}\right) \quad C_{2 v}\left(C_{1 v}\right)=2_{z} m_{x} m_{y}\left(m_{y}\right)=2_{z}{ }^{\prime} m_{x}^{\prime} m_{y} \\
& \mathbf{C}_{4 \mathrm{v}}\left(\mathbf{C}_{2 \mathrm{v}}\right)=\mathbf{4}_{\mathrm{z}} \mathbf{m}_{\mathrm{x}} \mathbf{m}_{\mathrm{xy}}\left(\mathbf{2}_{\mathrm{z}} \mathbf{m}_{\mathrm{x}} \mathbf{m}_{\mathrm{y}}\right)=\mathbf{4}_{\mathrm{z}}{ }^{\prime} \mathbf{m}_{\mathrm{x}} \mathbf{m}_{\mathrm{xy}}{ }^{\prime} \\
& C_{6 v}\left(C_{3 v}\right)=6_{z} m_{x} m_{1}\left(3_{z} m_{x}\right)=6_{z}^{\prime} m_{x} m_{1}^{\prime} \\
& \mathbf{C}_{8 \mathrm{v}}\left(\mathbf{C}_{4 \mathrm{v}}\right)=\mathbf{8}_{\mathrm{z}} \mathbf{m}_{\mathrm{x} 1} \mathbf{m}_{1}\left(\mathbf{4}_{\mathrm{z}} \mathbf{m}_{\mathrm{x}} \mathbf{m}_{\mathrm{xy}}\right)=\mathbf{8}_{\mathrm{z}} \mathbf{m}_{\mathrm{x} 1} \mathbf{m}_{1}{ }^{\prime} \\
& \mathrm{C}_{\infty v} 1^{\prime}=\infty \mathrm{mm} 1^{\prime} \\
& \mathbf{D}_{\mathrm{nd}}\left(\mathbf{S}_{2 \mathrm{n}}\right) \\
& D_{1 \mathrm{~d}}\left(S_{2}\right)=\mathbf{2}_{\mathrm{y}} / \mathbf{m}_{\mathrm{y}}(\overline{\mathbf{1}})=\mathbf{2 y}_{\mathrm{y}}{ }^{\prime} / \mathbf{m}_{\mathrm{y}}{ }^{\prime} \\
& D_{2 d}\left(S_{4}\right)=\overline{4}_{z} m_{x} 2_{x y}\left(\overline{4}_{z}\right)=\overline{4}_{z} m_{x}{ }^{\prime} \mathbf{2}_{x y}{ }^{\prime} \\
& \mathbf{D}_{3 \mathrm{~d}}\left(\mathbf{S}_{6}\right)=\overline{\mathbf{3}}_{z} \mathbf{m}_{\mathrm{x}}\left(\overline{\mathbf{3}}_{z}\right)=\overline{\mathbf{3}}_{z} \mathbf{m}_{x}{ }^{\prime} \\
& \mathbf{D}_{4 \mathrm{~d}}\left(\mathbf{S}_{8}\right)=\overline{\mathbf{8}}_{\mathrm{z}} \mathbf{m}_{\mathrm{x} \mathbf{1}} \mathbf{2}_{1}\left(\overline{\mathbf{8}}_{\mathrm{z}}\right)=\overline{\mathbf{8}}_{\mathrm{z}} \mathbf{m}_{\mathrm{x} 1} \mathbf{2}_{1}^{\prime} \\
& \mathbf{D}_{\infty \mathrm{h}}\left(\mathbf{C}_{\infty \mathrm{h}}\right)=\infty / \mathbf{m m}^{\prime} \mathbf{m}^{\prime} \\
& \mathbf{D}_{\text {nd }}\left(\mathbf{D}_{\mathrm{n}}\right) \\
& D_{1 \mathrm{~d}}\left(D_{1}\right)=2_{\mathrm{y}} / \mathrm{m}_{\mathrm{y}}\left(2_{\mathrm{y}}\right)=2_{\mathrm{y}} / \mathrm{m}_{\mathrm{y}}{ }^{\prime} \\
& D_{2 d}\left(D_{2}\right)=\overline{4}_{z} m_{x} 2_{x y}\left(2_{x y} 2_{\bar{x} y} 2_{z}\right)=\overline{4}_{z}{ }^{\prime} m_{x}{ }^{\prime} 2_{x y} \\
& \mathbf{D}_{3 \mathrm{~d}}\left(\mathbf{D}_{3}\right)=\overline{\mathbf{3}}_{\mathbf{z}} \mathbf{m}_{\mathrm{x}}\left(\mathbf{3}_{\mathrm{z}} \mathbf{2}_{\mathrm{x}}\right)=\overline{\mathbf{3}}_{\mathrm{z}}{ }^{\prime} \mathbf{m}_{\mathrm{x}}{ }^{\prime} \\
& D_{4 d}\left(D_{4}\right)=\overline{8}_{z} m_{x 1} 2_{1}\left(4_{z} 2_{x} 2_{x y}\right)=\overline{8}_{z}{ }^{\prime} m_{x 1} \mathbf{2}_{1} \\
& \mathbf{D}_{\infty h}\left(\mathbf{D}_{\infty}\right)=\infty / \mathbf{m}^{\prime} \mathbf{m}^{\prime} \mathbf{m}^{\prime} \\
& \mathbf{D}_{\mathrm{nd}}\left(\mathbf{C}_{\mathrm{nv}}\right) \\
& D_{1 \mathrm{~d}}\left(C_{1 \mathrm{v}}\right)=\mathbf{2}_{\mathrm{y}} / \mathbf{m}_{\mathrm{y}}\left(\mathbf{m}_{\mathrm{y}}\right)=\mathbf{2 y}_{\mathrm{y}} / \mathbf{m}_{\mathrm{y}} \\
& D_{2 \mathrm{~d}}\left(\mathbf{C}_{2 \mathrm{v}}\right)=\overline{\mathbf{4}}_{\mathrm{z}} \mathbf{m}_{\mathrm{x}} \mathbf{2}_{\mathrm{xy}}\left(\mathbf{2}_{\mathrm{z}} \mathbf{m}_{\mathrm{x}} \mathbf{m}_{\mathrm{y}}\right)=\overline{\mathbf{4}}_{\mathrm{z}}{ }^{\prime} \mathbf{m}_{\mathrm{x}} \mathbf{2}_{\mathrm{xy}}{ }^{\prime} \\
& \mathbf{D}_{3 \mathrm{~d}}\left(\mathbf{C}_{3 \mathrm{v}}\right)=\overline{\mathbf{3}}_{\mathrm{z}} \mathbf{m}_{\mathrm{x}}\left(\mathbf{3}_{\mathrm{z}} \mathbf{m}_{\mathrm{x}}\right)=\overline{\mathbf{3}}_{\mathrm{z}}{ }^{\prime} \mathbf{m}_{\mathrm{x}} \\
& \mathbf{D}_{4 \mathrm{~d}}\left(\mathbf{C}_{4 \mathrm{v}}\right)=\overline{\mathbf{8}}_{\mathbf{z}} \mathbf{m}_{\mathrm{x} 1} \mathbf{2}_{1}\left(\mathbf{4}_{\mathrm{z}} \mathbf{m}_{\mathrm{x} 1} \mathbf{m}_{\times 3}\right)=\overline{\mathbf{8}}_{\mathbf{z}} \mathbf{m}_{\mathrm{x} \mathbf{1}} \mathbf{2} \\
& \mathbf{D}_{\infty h}\left(\mathbf{C}_{\infty \mathrm{V}}\right)=\infty / \mathbf{m}^{\prime} \mathbf{m m} \\
& \mathbf{D}_{\mathrm{nh}}\left(\mathbf{C}_{\mathrm{nh}}\right) \\
& \mathbf{D}_{1 \mathrm{~h}}\left(\mathbf{C}_{1 \mathrm{~h}}\right)=\mathbf{2}_{\mathrm{x}} \mathbf{m}_{\mathrm{y}} \mathbf{m}_{\mathrm{z}}\left(\mathbf{m}_{\mathrm{z}}\right)=\mathbf{2} \mathbf{2}_{\mathrm{x}} \mathbf{m}_{\mathrm{y}} \mathbf{m}_{\mathrm{z}} \\
& \mathbf{D}_{2 \mathrm{~h}}\left(\mathbf{C}_{2 \mathrm{~h}}\right)=\mathbf{m}_{\mathrm{x}} \mathbf{m}_{\mathrm{y}} \mathbf{m}_{\mathrm{z}}\left(\mathbf{2}_{\mathrm{z}} / \mathbf{m}_{\mathrm{z}}\right)=\mathbf{m}_{\mathrm{x}} \mathbf{m}_{\mathrm{y}}{ }^{\prime} \mathbf{m}_{\mathrm{z}} \\
& D_{3 h}\left(C_{3 h}\right)=\overline{\mathbf{6}}_{z} m_{x} \mathbf{2}_{1}\left(\overline{\boldsymbol{6}}_{z}\right)=\overline{\mathbf{6}}_{z} \mathbf{m}_{x}{ }^{\prime} \mathbf{2}_{1}{ }^{\prime} \\
& D_{4 h}\left(C_{4 h}\right)=\mathbf{4}_{z} / m_{z} m_{x} m_{x y}\left(\mathbf{4}_{z} / m_{z}\right)=\mathbf{4}_{z} / m_{z} m_{x}^{\prime} m_{x y}{ }^{\prime} \\
& \mathbf{D}_{\infty \mathrm{h}}\left(\mathbf{C}_{\infty \mathrm{h}}\right)=\infty / \mathbf{m m}^{\prime} \mathbf{m}^{\prime} \\
& D_{1 \mathrm{~h}}\left(D_{1}\right)=\mathbf{2}_{\mathrm{x}} \mathbf{m}_{\mathrm{y}} \mathbf{m}_{\mathrm{z}}\left(\mathbf{2}_{\mathrm{x}}\right)=\mathbf{2}_{\mathrm{x}} \mathbf{m}_{\mathrm{y}}{ }^{\prime} \mathbf{m}_{\mathrm{z}}{ }^{\prime} \\
& D_{2 h}\left(D_{2}\right)=m_{x} m_{y} m_{z}\left(2 \mathbf{x}^{2} \mathbf{y}_{\mathbf{y}} \mathbf{2}_{\mathrm{z}}\right)=\mathbf{m}_{\mathrm{x}}{ }^{\prime} \mathbf{m}_{\mathrm{y}}{ }^{\prime} \mathbf{m}_{\mathrm{z}}{ }^{\prime} \\
& D_{3 h}\left(D_{3}\right)=\overline{6}_{z} m_{x} 2_{1}\left(3_{z} 2_{1}\right)=\overline{6}_{z}{ }^{\prime} m_{x}{ }^{\prime} \mathbf{2}_{1} \\
& D_{4 h}\left(D_{4}\right)=4_{z} / m_{z} m_{x} m_{x y}\left(4_{z} 2_{x} 2_{x y}\right)=4_{z} / m_{z}^{\prime} m_{x}^{\prime} m_{x y} \\
& D_{\infty h}\left(D_{\infty}\right)=\infty / \mathbf{m}^{\prime} \mathbf{m}^{\prime} \mathbf{m}^{\prime}
\end{aligned}
$$




$$
\begin{aligned}
& \mathbf{D}_{\mathrm{nh}}\left(\mathbf{C}_{\mathrm{nv}}\right) \quad \mathbf{D}_{1 \mathrm{~h}}\left(\mathbf{C}_{1 \mathrm{v}}\right)=\mathbf{2}_{\mathrm{x}} \mathbf{m}_{\mathrm{y}} \mathbf{m}_{\mathrm{z}}\left(\mathbf{m}_{\mathrm{y}}\right)=\mathbf{2}_{\mathrm{x}}{ }^{\prime} \mathbf{m}_{\mathrm{y}} \mathbf{m}_{\mathrm{z}}{ }^{\prime} \\
& D_{2 h}\left(C_{2 v}\right)=m_{x} m_{y} m_{z}\left(2_{z} m_{x} m_{y}\right)=m_{x} m_{y} m_{z}^{\prime} \\
& D_{3 h}\left(C_{3 v}\right)=\overline{6}_{z} m_{x} 2_{1}\left(3_{z} m_{x}\right)=\overline{6}_{z}{ }^{\prime} m_{x} 2_{1}{ }^{\prime} \\
& D_{4 h}\left(C_{4 v}\right)=4_{z} / m_{z} m_{x} m_{x y}\left(4_{z} m_{x} m_{x y}\right)=4_{z} / m_{z} m_{x} m_{x y} \\
& \mathbf{D}_{\infty h}\left(\mathbf{C}_{\infty \mathrm{v}}\right)=\infty / \mathbf{m}^{\prime} \mathbf{m m} \\
& \mathbf{D}_{2 \mathrm{nh}}\left(\mathbf{D}_{\mathrm{nd}}\right) \quad \mathbf{D}_{2 \mathrm{~h}}\left(\mathbf{D}_{1 \mathrm{~d}}\right)=\mathbf{m}_{\mathrm{x}} \mathbf{m}_{\mathrm{y}} \mathbf{m}_{\mathrm{z}}\left(\mathbf{2}_{\mathbf{y}} / \mathbf{m}_{\mathrm{y}}\right)=\mathbf{m}_{\mathrm{x}}{ }^{\prime} \mathbf{m}_{\mathrm{y}} \mathbf{m}_{\mathbf{z}}{ }^{\prime} \\
& D_{4 h}\left(D_{2 \mathrm{~d}}\right)=4_{z} / m_{z} m_{x} m_{x y}\left(\overline{4}_{z} m_{x} 2_{x y}\right)=4_{z}{ }^{\prime} / m_{z}{ }^{\prime} m_{x} m_{x y}{ }^{\prime} \\
& D_{6 h}\left(D_{3 \mathrm{~d}}\right)=\mathbf{6}_{\mathrm{z}} / \mathrm{m}_{\mathrm{z}} \mathrm{m}_{\mathrm{x}} \mathrm{m}_{1}\left(\overline{3}_{\mathrm{z}} \mathrm{m}_{\mathrm{x}}\right)=\mathbf{6}_{\mathrm{z}}{ }^{\prime} / \mathrm{m}_{\mathrm{z}}{ }^{\prime} \mathrm{m}_{\mathrm{x}} \mathrm{m}_{1}{ }^{\prime} \\
& C_{8 h}\left(D_{4 d}\right)=8_{z} / m_{z} m_{x 1} m_{1}\left(\overline{8}_{z} m_{x 1} 2_{1}\right)=8_{z}^{\prime} / m_{z}^{\prime} m_{x 1} m_{1}^{\prime} \\
& D_{\infty h} \mathbf{1}^{\prime}=\infty / \mathrm{mmm1}^{\prime} \\
& \mathbf{D}_{2 \mathrm{nh}}\left(\mathbf{D}_{\mathrm{nh}}\right) \quad \mathbf{D}_{2 \mathrm{~h}}\left(\mathbf{D}_{1 \mathrm{~h}}\right)=\mathbf{m}_{\mathrm{x}} \mathbf{m}_{\mathbf{y}} \mathbf{m}_{\mathrm{z}}\left(\mathbf{2}_{\mathrm{x}} \mathbf{m}_{\mathbf{y}} \mathbf{m}_{\mathrm{z}}\right)=\mathbf{m}_{\mathrm{x}} \mathbf{m}_{\mathrm{y}} \mathbf{m}_{\mathrm{z}} \\
& D_{4 h}\left(D_{2 h}\right)=4_{z} / m_{z} m_{x} m_{x y}\left(m_{x} m_{y} m_{z}\right)=4_{z}^{\prime} / m_{z} m_{x} m_{x y}{ }^{\prime} \\
& D_{6 h}\left(D_{3 h}\right)=6_{z} / m_{z} m_{x} m_{1}\left(\overline{6}_{z} m_{x} 2_{1}\right)=6_{z}{ }^{\prime} / m_{z} m_{x} m_{1}{ }^{\prime} \\
& D_{8 h}\left(D_{4 h}\right)=8_{z} / m_{z} m_{x 1} m_{1}\left(4_{z} / m_{z} m_{x 1} m_{x 2}\right)=8_{z}{ }^{\prime} / m_{z} m_{x 1} m_{1}{ }^{\prime} \\
& D_{\infty \mathrm{h}} \mathbf{1}^{\prime}=\infty / \mathrm{mmm}^{\prime}
\end{aligned}
$$



$\begin{array}{ll}\mathrm{C}_{\mathrm{n}} & \mathrm{C}_{1}=1\end{array}$

$$
\mathrm{n}=1
$$

$$
\begin{gathered}
1 \text { e a ae } \\
\text { V( } \left.\begin{array}{c}
x \\
y \\
z
\end{array}\right)\left(\begin{array}{l}
x \\
y \\
z
\end{array}\right)\left(\begin{array}{l}
x \\
y \\
z
\end{array}\right)\left(\begin{array}{l}
x \\
y \\
z
\end{array}\right)
\end{gathered}
$$
$\mathbf{C}_{\infty}=\infty^{\mathrm{n}>1 ;}$

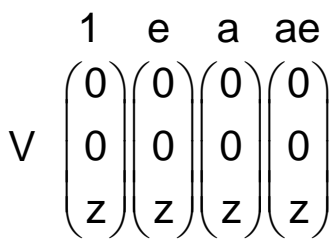

$$
\mathrm{n}=1
$$

$\mathbf{C}_{\mathrm{nv}} \quad \mathbf{C}_{1 \mathrm{v}}=\mathbf{m}_{\mathrm{y}}$

$$
\begin{gathered}
1 \text { e a ae } \\
\text { V( } \left.\begin{array}{c}
x \\
0 \\
z
\end{array}\right)\left(\begin{array}{l}
0 \\
y \\
0
\end{array}\right)\left(\begin{array}{l}
x \\
0 \\
z
\end{array}\right)\left(\begin{array}{l}
0 \\
y \\
0
\end{array}\right)
\end{gathered}
$$

$n>1$
$\mathbf{C}_{\infty v}=\infty \mathbf{m m}$

$$
\mathrm{n}=1
$$

$\mathbf{S}_{2 n}$

$$
\mathbf{S}_{2}=\overline{1}
$$

$$
\begin{gathered}
1 \text { e a ae } \\
V\left(\begin{array}{l}
0 \\
0 \\
0
\end{array}\right)\left(\begin{array}{l}
x \\
y \\
z
\end{array}\right)\left(\begin{array}{l}
0 \\
0 \\
0
\end{array}\right)\left(\begin{array}{l}
x \\
y \\
z
\end{array}\right)
\end{gathered}
$$

$$
\mathrm{n}>1
$$

$$
\mathbf{C}_{\infty \mathrm{h}}=\infty / \mathbf{m}
$$

1
$V\left(\begin{array}{l}0 \\ 0 \\ 0\end{array}\right)\left(\begin{array}{l}0 \\ 0 \\ z\end{array}\right)\left(\begin{array}{l}0 \\ 0 \\ 0\end{array}\right)\left(\begin{array}{l}0 \\ 0 \\ z\end{array}\right)$

$$
\begin{array}{r}
\mathbf{C}_{\mathrm{nh}} \quad \mathbf{C}_{1 \mathrm{~h}}=\mathbf{m}_{\mathrm{z}} \\
\mathrm{n}=1 \\
1\left(\begin{array}{l}
\mathrm{x} \\
\mathrm{y} \\
0
\end{array}\right)\left(\begin{array}{l}
0 \\
0 \\
\mathrm{z}
\end{array}\right)\left(\begin{array}{l}
\mathrm{x} \\
\mathrm{y} \\
0
\end{array}\right)\left(\begin{array}{l}
0 \\
0 \\
z
\end{array}\right)
\end{array}
$$

$$
\begin{aligned}
& n>1 \\
& \mathbf{C}_{\infty h}=\infty / \mathbf{m} \\
& \begin{array}{c}
1 \quad \mathrm{e} \\
\mathrm{V} \\
\left(\begin{array}{l}
0 \\
0 \\
0 \\
0
\end{array}\right)\left(\begin{array}{l}
0 \\
0 \\
z
\end{array}\right)\left(\begin{array}{l}
0 \\
0 \\
0
\end{array}\right)\left(\begin{array}{l}
0 \\
0 \\
z
\end{array}\right)
\end{array}
\end{aligned}
$$



$D_{\mathrm{n}}$
$\mathbf{D}_{1}=\mathbf{2}_{\mathrm{y}}$
$\mathrm{n}=1$
$\mathbf{D}_{\infty}=\infty \mathbf{2}$
1 e
$V\left(\begin{array}{l}0 \\ y \\ 0\end{array}\right)\left(\begin{array}{l}0 \\ y \\ 0\end{array}\right)\left(\begin{array}{l}0 \\ y \\ 0\end{array}\right)\left(\begin{array}{l}0 \\ y \\ 0\end{array}\right)$
$1 \quad \mathrm{e}$
$\mathrm{V}\left(\begin{array}{l}0 \\ 0 \\ 0 \\ 0\end{array}\right)\left(\begin{array}{l}0 \\ 0 \\ 0\end{array}\right)\left(\begin{array}{l}0 \\ 0 \\ 0\end{array}\right)\left(\begin{array}{l}0 \\ 0 \\ 0\end{array}\right)$
$n=1$
$D_{\text {nd }} \quad D_{1 d}=2_{y} / m_{y}$
$\mathrm{n}>1$
$\mathbf{D}_{\alpha \mathrm{h}}=\infty / \mathrm{mmm}$

$$
\begin{gathered}
1 \quad \text { e } \\
\text { V a a } \\
\qquad\left(\begin{array}{l}
0 \\
0 \\
0
\end{array}\right)\left(\begin{array}{l}
0 \\
y \\
0
\end{array}\right)\left(\begin{array}{l}
0 \\
0 \\
0
\end{array}\right)\left(\begin{array}{l}
0 \\
y \\
0
\end{array}\right)
\end{gathered}
$$
$1 \quad \mathrm{e}$
$\mathrm{v}\left(\begin{array}{l}0 \\ 0 \\ 0\end{array}\right)\left(\begin{array}{l}0 \\ 0 \\ 0\end{array}\right)\left(\begin{array}{l}0 \\ 0 \\ 0\end{array}\right)\left(\begin{array}{l}0 \\ 0 \\ 0\end{array}\right)$
$D_{\text {nh }}$

$$
\mathrm{n}=1
$$
$D_{1 \mathrm{~h}}=\mathbf{2}_{\mathrm{x}} \mathbf{m}_{\mathrm{y}} \mathbf{m}_{\mathrm{z}}$
$n>1$
$\mathbf{D}_{\alpha \mathrm{h}}=\infty / \mathrm{mmm}$

$$
\begin{gathered}
1 \quad \mathrm{e} \\
\mathrm{V} \\
\left(\begin{array}{l}
\mathrm{x} \\
0 \\
0
\end{array}\right)\left(\begin{array}{l}
0 \\
0 \\
0 \\
0
\end{array}\right)\left(\begin{array}{l}
\mathrm{x} \\
0 \\
0
\end{array}\right)\left(\begin{array}{l}
0 \\
0 \\
0
\end{array}\right)
\end{gathered}
$$
$1 \quad \mathrm{e}$
$\mathrm{V}\left(\begin{array}{l}0 \\ 0 \\ 0 \\ 0\end{array}\right)\left(\begin{array}{l}0 \\ 0 \\ 0\end{array}\right)\left(\begin{array}{l}0 \\ 0 \\ 0\end{array}\right)\left(\begin{array}{l}0 \\ 0 \\ 0\end{array}\right)$
$\mathbf{C}_{2 n}\left(\mathbf{C}_{n}\right)$

$$
\begin{aligned}
& \begin{array}{r}
\mathrm{n}=1 \\
\mathbf{C}_{2}\left(\mathbf{C}_{1}\right)=\mathbf{2}_{\mathrm{z}}(\mathbf{1})=\mathbf{2}_{\mathbf{z}^{\prime}}
\end{array}
\end{aligned}
$$

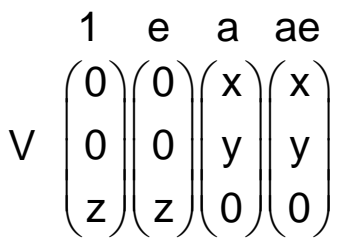$$
\mathbf{C}_{\infty} \mathbf{1}^{\prime}=\infty \mathbf{1}^{\prime}
$$

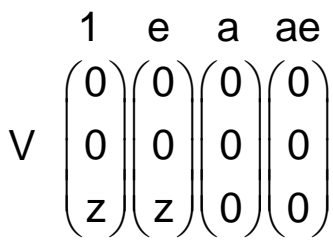




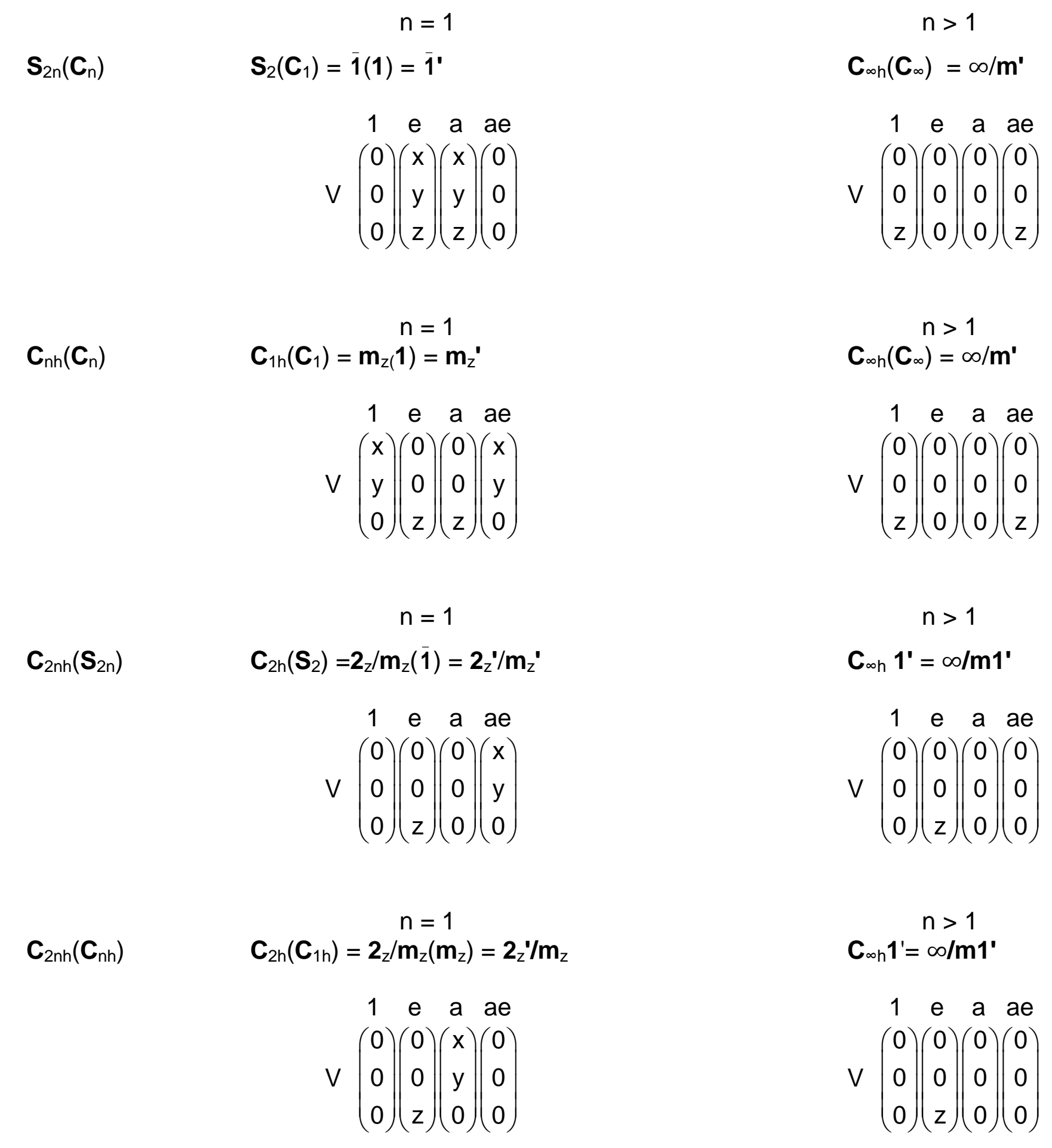



$\mathbf{D}_{n}\left(\mathbf{C}_{n}\right)$
$\mathrm{n}=1$
$\mathbf{D}_{1}\left(\mathbf{C}_{1}\right)=\mathbf{2}_{\mathbf{y}}(\mathbf{1})=\mathbf{2 y}^{\prime}$
$\mathrm{n}>1$
$\mathbf{D}_{\infty}\left(\mathbf{C}_{\infty}\right)=\infty \mathbf{2}^{\prime}$

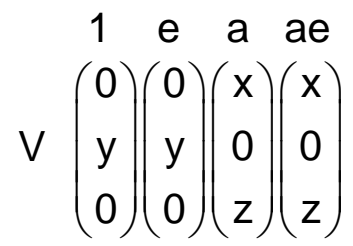
1 e a ae
$V\left(\begin{array}{l}0 \\ 0 \\ 0\end{array}\right)\left(\begin{array}{l}0 \\ 0 \\ 0\end{array}\right)\left(\begin{array}{l}0 \\ 0 \\ z\end{array}\right)\left(\begin{array}{l}0 \\ 0 \\ z\end{array}\right)$
$\mathbf{D}_{2 \mathrm{n}}\left(\mathbf{D}_{\mathrm{n}}\right)$
$\mathrm{n}=1$
$\mathbf{D}_{2}\left(\mathbf{D}_{1}\right)=\mathbf{2}_{\mathrm{x}} \mathbf{2} \mathbf{2}\left(\mathbf{2}_{\mathrm{y}}\right)=\mathbf{2}_{\mathrm{x}} \mathbf{2}_{\mathrm{y}} \mathbf{2}_{\mathrm{z}}{ }^{\prime}$
$\mathrm{n}>1$
$\mathbf{D}_{\infty} \mathbf{1}^{\prime}=\infty \mathbf{2 1}$
$1 \quad \mathrm{e} \quad \mathrm{a}$ ae
$\mathrm{V}\left(\begin{array}{l}0 \\ 0 \\ 0\end{array}\right)\left(\begin{array}{l}0 \\ 0 \\ 0\end{array}\right)\left(\begin{array}{l}0 \\ \mathrm{y} \\ 0\end{array}\right)\left(\begin{array}{l}0 \\ \mathrm{y} \\ 0\end{array}\right)$
1
$V$
$V\left(\begin{array}{l}0 \\ 0 \\ 0\end{array}\right)\left(\begin{array}{l}0 \\ 0 \\ 0\end{array}\right)\left(\begin{array}{l}0 \\ 0 \\ 0 \\ 0\end{array}\right)\left(\begin{array}{l}0 \\ 0 \\ 0\end{array}\right)$
$\mathbf{C}_{\mathrm{nv}}\left(\mathbf{C}_{\mathrm{n}}\right)$
$\mathrm{n}=1$
$\mathbf{C}_{1 \mathrm{v}}\left(\mathbf{C}_{1}\right)=\mathbf{m}_{\mathrm{y}}(\mathbf{1})=\mathrm{m}_{\mathrm{y}}{ }^{\prime}$

$$
\begin{gathered}
1 \quad \text { e a ae } \\
v\left(\begin{array}{l}
x \\
0 \\
z
\end{array}\right)\left(\begin{array}{l}
0 \\
y \\
0
\end{array}\right)\left(\begin{array}{l}
0 \\
y \\
0
\end{array}\right)\left(\begin{array}{l}
x \\
0 \\
z
\end{array}\right)
\end{gathered}
$$
$\mathbf{C}_{2 \text { nv }}\left(\mathbf{C}_{\text {nv }}\right)$
$n=1$
$\mathbf{C}_{2 \mathrm{v}}\left(\mathbf{C}_{1 \mathrm{v}}\right)=\mathbf{2}_{\mathrm{z}} \mathbf{m}_{\mathrm{x}} \mathbf{m}_{\mathrm{y}}\left(\mathbf{m}_{\mathrm{y}}\right)=\mathbf{2}_{\mathrm{z}}{ }^{\prime} \mathbf{m}_{\mathrm{x}}{ }^{\prime} \mathbf{m}_{\mathrm{y}}$

$$
\begin{aligned}
& 1 \text { e a ae } \\
& \text { V }\left(\begin{array}{l}
0 \\
0 \\
z
\end{array}\right)\left(\begin{array}{l}
0 \\
0 \\
0
\end{array}\right)\left(\begin{array}{l}
x \\
0 \\
0
\end{array}\right)\left(\begin{array}{l}
0 \\
y \\
0
\end{array}\right)
\end{aligned}
$$

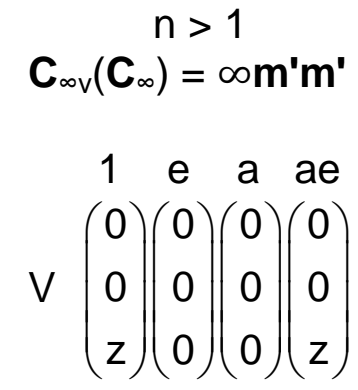
$\mathrm{n}>1$
$\mathbf{C}_{\infty v} \mathbf{1}^{\prime}=\infty \mathbf{m m} \mathbf{1}^{\prime}$
$1 \quad$ e
$V$
$V\left(\begin{array}{l}0 \\ 0 \\ z\end{array}\right)\left(\begin{array}{l}0 \\ 0 \\ 0\end{array}\right)\left(\begin{array}{l}0 \\ 0 \\ 0\end{array}\right)\left(\begin{array}{l}0 \\ 0 \\ 0\end{array}\right)$ 


$$
\mathrm{n}=1
$$

$\mathbf{D}_{\text {nd }}\left(\mathbf{S}_{2 n}\right)$

$$
D_{1 d}\left(S_{2}\right)=2_{y} / m_{y}(\overline{1})=2 y^{\prime} / m_{y}{ }^{\prime}
$$$$
1 \text { e a ae }
$$$$
v\left(\begin{array}{l}
0 \\
0 \\
0
\end{array}\right)\left(\begin{array}{l}
0 \\
y \\
0
\end{array}\right)\left(\begin{array}{l}
0 \\
0 \\
0
\end{array}\right)\left(\begin{array}{l}
x \\
0 \\
z
\end{array}\right)
$$

$$
n=1
$$

$\mathbf{D}_{\text {nd }}\left(\mathbf{D}_{\mathrm{n}}\right)$

$$
\mathbf{D}_{1 \mathrm{~d}}\left(\mathbf{D}_{1}\right)=\mathbf{2}_{\mathrm{y}} / \mathbf{m}_{\mathrm{y}}\left(\mathbf{2}_{\mathrm{y}}\right)=\mathbf{2}_{\mathrm{y}} / \mathbf{m}_{\mathrm{y}}{ }^{\prime}
$$

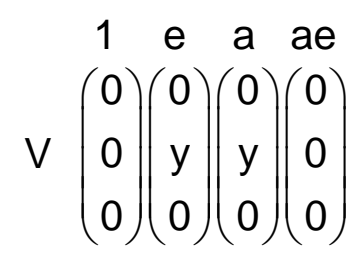

$\mathbf{D}_{\text {nd }}\left(\mathbf{C}_{\text {nv }}\right)$

$\mathbf{D}_{\mathrm{nh}}\left(\mathbf{C}_{\mathrm{nh}}\right)$

$$
n=1
$$

$$
\mathbf{D}_{1 \mathrm{~h}}\left(\mathbf{C}_{1 \mathrm{~h}}\right)=\mathbf{2}_{\mathrm{x}} \mathbf{m}_{\mathrm{y}} \mathbf{m}_{\mathrm{z}}\left(\mathbf{m}_{\mathrm{z}}\right)=\mathbf{2 x}_{\mathrm{x}} \mathbf{m}_{\mathrm{y}} \mathbf{m}_{\mathrm{z}}
$$

$$
\begin{gathered}
1 \quad \begin{array}{c}
\mathrm{a} \\
\mathrm{V}
\end{array} \\
\left(\begin{array}{l}
\mathrm{x} \\
0 \\
0
\end{array}\right)\left(\begin{array}{l}
0 \\
0 \\
0
\end{array}\right)\left(\begin{array}{l}
0 \\
\mathrm{y} \\
0
\end{array}\right)\left(\begin{array}{l}
0 \\
0 \\
z
\end{array}\right)
\end{gathered}
$$

$n>1$
$\mathbf{D}_{\infty h}\left(\mathbf{C}_{\infty h}\right)=\infty / \mathbf{m m}^{\prime} \mathbf{m}^{\prime}$

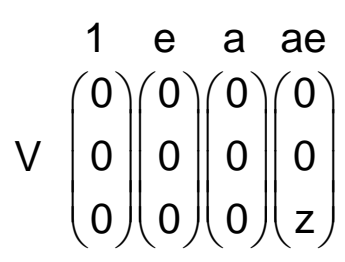

$\begin{aligned} \mathrm{n} & >1 \\ \mathbf{D}_{\infty h}\left(\mathbf{D}_{\infty}\right) & =\infty / \mathbf{m}^{\prime} \mathbf{m}^{\prime} \mathbf{m}^{\prime}\end{aligned}$

$1 \quad$ e a
V $\left(\begin{array}{l}0 \\ 0 \\ 0\end{array}\right)\left(\begin{array}{l}0 \\ 0 \\ 0\end{array}\right)\left(\begin{array}{l}0 \\ 0 \\ 0\end{array}\right)\left(\begin{array}{l}0 \\ 0 \\ 0\end{array}\right)$

$\begin{aligned} \mathrm{n} & >1 \\ \mathbf{D}_{\infty \mathrm{h}}\left(\mathbf{C}_{\infty \mathrm{v}}\right) & =\infty / \mathbf{m}^{\prime} \mathbf{m m}\end{aligned}$

1
$\mathrm{v}$
$\left(\begin{array}{l}0 \\ 0 \\ 0\end{array}\right)\left(\begin{array}{l}0 \\ 0 \\ 0\end{array}\right)\left(\begin{array}{l}0 \\ 0 \\ z\end{array}\right)\left(\begin{array}{l}0 \\ 0 \\ 0\end{array}\right)$

$\begin{aligned} \mathrm{n} & >1 \\ \mathbf{D}_{\infty \mathrm{h}}\left(\mathbf{C}_{\infty \mathrm{h}}\right) & =\infty / \mathbf{m m}^{\prime} \mathbf{m}^{\prime}\end{aligned}$

1 e a ae
V $\left(\begin{array}{l}0 \\ 0 \\ 0\end{array}\right)\left(\begin{array}{l}0 \\ 0 \\ 0\end{array}\right)\left(\begin{array}{l}0 \\ 0 \\ 0\end{array}\right)\left(\begin{array}{l}0 \\ 0 \\ z\end{array}\right)$ 

$\mathbf{D}_{\mathrm{nh}}\left(\mathbf{D}_{\mathrm{n}}\right)$
$\mathbf{D}_{1 \mathrm{~h}}\left(\mathbf{D}_{1}\right)=\mathbf{2}_{\mathrm{x}} \mathbf{m}_{\mathrm{y}} \mathbf{m}_{\mathrm{z}}\left(\mathbf{2}_{\mathrm{x}}\right)=\mathbf{2} \mathrm{x} \mathbf{m}_{\mathrm{y}} \mathbf{m}_{\mathrm{z}}$
$\mathbf{D}_{\infty h}\left(\mathbf{D}_{\infty}{ }^{\mathrm{n}}=\infty / \mathbf{m}^{\prime} \mathbf{m}^{\prime} \mathbf{m}^{\prime}\right.$
$1 \quad$ e a ae
$V\left(\begin{array}{l}x \\ 0 \\ 0\end{array}\right)\left(\begin{array}{l}0 \\ 0 \\ 0\end{array}\right)\left(\begin{array}{l}0 \\ 0 \\ 0\end{array}\right)\left(\begin{array}{l}x \\ 0 \\ 0\end{array}\right)$
$1 \quad e \quad a$ ae
$V\left(\begin{array}{l}0 \\ 0 \\ 0\end{array}\right)\left(\begin{array}{l}0 \\ 0 \\ 0\end{array}\right)\left(\begin{array}{l}0 \\ 0 \\ 0\end{array}\right)\left(\begin{array}{l}0 \\ 0 \\ 0\end{array}\right)$
$n=1$
$\mathbf{D}_{\text {nh }}\left(\mathbf{C}_{\text {nv }}\right)$

$$
\begin{aligned}
& \begin{aligned}
\mathbf{D}_{1 \mathrm{~h}}\left(\mathbf{C}_{1 \mathrm{v}}\right)= & \begin{array}{c}
\mathrm{n}=1 \\
\mathbf{m}_{\mathrm{y}}
\end{array} \\
\mathbf{m}_{\mathrm{z}}\left(\mathbf{m}_{\mathrm{y}}\right) & \\
1 \text { e } & \text { a } \text { ae }
\end{aligned} \\
& v\left(\begin{array}{l}
x \\
0 \\
0
\end{array}\right)\left(\begin{array}{l}
0 \\
0 \\
0
\end{array}\right)\left(\begin{array}{l}
0 \\
0 \\
z
\end{array}\right)\left(\begin{array}{l}
0 \\
y \\
0
\end{array}\right)
\end{aligned}
$$
$\begin{aligned} \mathrm{n} & >1 \\ \mathbf{D}_{\infty h}\left(\mathbf{C}_{\infty \mathrm{v}}\right) & =\infty / \mathbf{m}^{\prime} \mathbf{m m}\end{aligned}$

$$
n=1
$$
$\mathbf{D}_{2 \mathrm{nh}}\left(\mathbf{D}_{\mathrm{nd}}\right)$

$$
\mathbf{D}_{2 \mathrm{~h}}\left(\mathbf{D}_{1 \mathrm{~d}}\right)=\mathbf{m}_{\mathrm{x}} \mathbf{m}_{\mathbf{y}} \mathbf{m}_{\mathrm{z}}\left(\mathbf{2}_{\mathbf{y}} / \mathbf{m}_{\mathrm{y}}\right)=\mathbf{m}_{\mathrm{x}} \mathbf{m}_{\mathrm{y}} \mathbf{m}_{\mathrm{z}}{ }^{\prime}
$$
1 e a ae
$\mathrm{V}\left(\begin{array}{l}0 \\ 0 \\ 0\end{array}\right)\left(\begin{array}{l}0 \\ 0 \\ 0\end{array}\right)\left(\begin{array}{l}0 \\ 0 \\ z\end{array}\right)\left(\begin{array}{l}0 \\ 0 \\ 0\end{array}\right)$$$
\begin{gathered}
1 \quad e \quad a \text { ae } \\
V\left(\begin{array}{l}
0 \\
0 \\
0
\end{array}\right)\left(\begin{array}{l}
0 \\
0 \\
0
\end{array}\right)\left(\begin{array}{l}
0 \\
0 \\
0
\end{array}\right)\left(\begin{array}{l}
0 \\
y \\
0
\end{array}\right)
\end{gathered}
$$$$
\begin{gathered}
1 \quad e \quad a \text { ae } \\
V\left(\begin{array}{l}
0 \\
0 \\
0
\end{array}\right)\left(\begin{array}{l}
0 \\
0 \\
0
\end{array}\right)\left(\begin{array}{l}
0 \\
0 \\
0
\end{array}\right)\left(\begin{array}{l}
0 \\
y \\
0
\end{array}\right)
\end{gathered}
$$
$D_{\infty \mathrm{h}} \mathbf{1}^{\prime}=\infty / \mathbf{m m m}^{\prime}$
$\mathbf{D}_{2 \mathrm{nh}}\left(\mathbf{D}_{\mathrm{nh}}\right)$

$$
\mathrm{n}=1
$$
$\mathbf{D}_{2 \mathrm{~h}}\left(\mathbf{D}_{1 \mathrm{~h}}\right)=\mathbf{m}_{\mathbf{x}} \mathbf{m}_{\mathbf{y}} \mathbf{m}_{\mathbf{z}}\left(\mathbf{2}_{\mathbf{x}} \mathbf{m}_{\mathrm{y}} \mathbf{m}_{\mathrm{z}}\right)=\mathbf{m}_{\mathbf{x}} \mathbf{m}_{\mathbf{y}} \mathbf{m}_{\mathbf{z}}$
$\mathrm{n}>1$
$\mathbf{D}_{\infty \mathrm{h}} \mathbf{1}^{\prime}=\infty / \mathbf{m m m} \mathbf{1}^{\prime}$

$$
\begin{gathered}
1 \quad \text { e a ae } \\
V\left(\begin{array}{l}
0 \\
0 \\
0
\end{array}\right)\left(\begin{array}{l}
0 \\
0 \\
0
\end{array}\right)\left(\begin{array}{l}
x \\
0 \\
0
\end{array}\right)\left(\begin{array}{l}
0 \\
0 \\
0
\end{array}\right)
\end{gathered}
$$

$$
\begin{gathered}
1 \text { e a ae } \\
V\left(\begin{array}{l}
0 \\
0 \\
0
\end{array}\right)\left(\begin{array}{l}
0 \\
0 \\
0
\end{array}\right)\left(\begin{array}{l}
0 \\
0 \\
0
\end{array}\right)\left(\begin{array}{l}
0 \\
0 \\
0
\end{array}\right)
\end{gathered}
$$


$\begin{array}{rlr} & \mathrm{n}=1 & \mathrm{n}=2 \\ \mathbf{c}_{\mathrm{n}} & \mathbf{c}_{1}=\mathbf{1} & \mathbf{C}_{2}=\mathbf{2}_{2}\end{array}$

$V^{2} \quad\left(\begin{array}{ccc}x x & x y & x z \\ y x & y y & y z\end{array}\right)\left(\begin{array}{ccc}x x & x y & x z \\ y x & y y & y z \\ z x & z y & z z\end{array}\right)\left(\begin{array}{ccc}x x & x y & x z \\ y x & y y & y z \\ z x & z y & z z\end{array}\right)\left(\begin{array}{ccc}x x & x y & x z \\ y x & y y & y z \\ z x & z y & z z\end{array}\right)$ $\left(\begin{array}{lll}z x & z y & z z\end{array}\right)\left(\begin{array}{lll}z x & z y & z z\end{array}\right)\left(\begin{array}{lll}z x & z y & z z\end{array}\right)\left(\begin{array}{lll}z x & z y & z z\end{array}\right)$

$\left[V^{2}\right]\left(\begin{array}{lll}x x & x y & x z \\ x y & y y & y z \\ x z & y z & z z\end{array}\right)\left(\begin{array}{lll}x x & x y & x z \\ x y & y y & y z \\ x z & y z & z z\end{array}\right)\left(\begin{array}{lll}x x & x y & x z \\ x y & y y & y z \\ x z & y z & z z\end{array}\right)\left(\begin{array}{ccc}x x & x y & x z \\ x y & y y & y z \\ x z & y z & z z\end{array}\right)$

$\left\{V^{2}\right\} \quad\left(\begin{array}{ccc}0 & x y & x z \\ -x y & 0 & y z \\ -x z & -y z & 0\end{array}\right)\left(\begin{array}{ccc}0 & x y & x z \\ -x y & 0 & y z \\ -x z & -y z & 0\end{array}\right)\left(\begin{array}{ccc}0 & x y & x z \\ -x y & 0 & y z \\ -x z & -y z & 0\end{array}\right)\left(\begin{array}{ccc}0 & x y & x z \\ -x y & 0 & y z \\ -x z & -y z & 0\end{array}\right)$

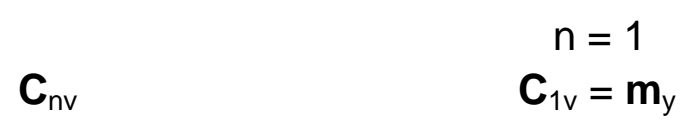

$V^{2} \quad\left(\begin{array}{ccc}x x & 0 & x z \\ 0 & y y & 0 \\ z x & 0 & z z\end{array}\right)\left(\begin{array}{ccc}0 & x y & 0 \\ y x & 0 & y z \\ 0 & z y & 0\end{array}\right)\left(\begin{array}{ccc}x x & 0 & x z \\ 0 & y y & 0 \\ z x & 0 & z z\end{array}\right)\left(\begin{array}{ccc}0 & x y & 0 \\ y x & 0 & y z \\ 0 & z y & 0\end{array}\right)$

$\left[V^{2}\right] \quad\left(\begin{array}{ccc}x x & 0 & x z \\ 0 & y y & 0 \\ x z & 0 & z z\end{array}\right)\left(\begin{array}{ccc}0 & x y & 0 \\ x y & 0 & y z \\ 0 & y z & 0\end{array}\right)\left(\begin{array}{ccc}x x & 0 & x z \\ 0 & y y & 0 \\ x z & 0 & z z\end{array}\right)\left(\begin{array}{ccc}0 & x y & 0 \\ x y & 0 & y z \\ 0 & y z & 0\end{array}\right)$

$\left\{V^{2}\right\} \quad\left(\begin{array}{ccc}0 & 0 & x z \\ 0 & 0 & 0 \\ -x z & 0 & 0\end{array}\right)\left(\begin{array}{ccc}0 & x y & 0 \\ -x y & 0 & y z \\ 0 & -y z & 0\end{array}\right)\left(\begin{array}{ccc}0 & 0 & x z \\ 0 & 0 & 0 \\ -x z & 0 & 0\end{array}\right)\left(\begin{array}{ccc}0 & x y & 0 \\ -x y & 0 & y z \\ 0 & -y z & 0\end{array}\right)$

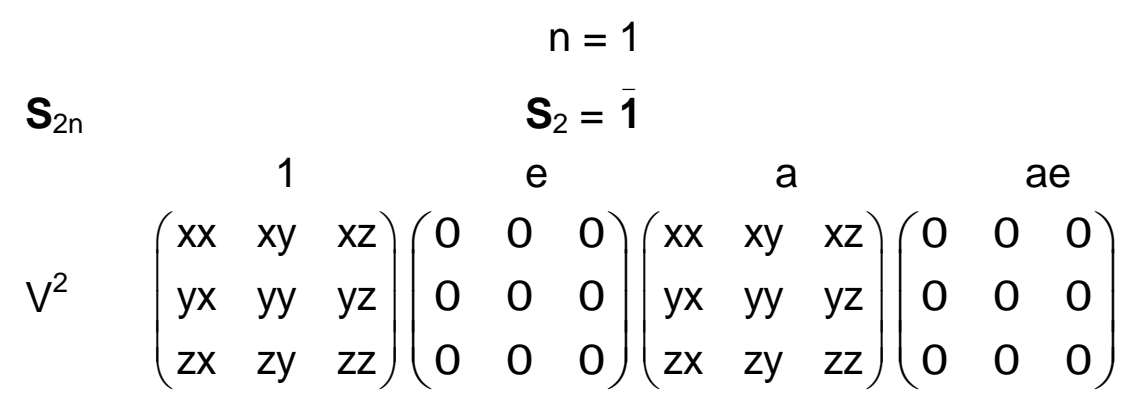

$\left[V^{2}\right]\left(\begin{array}{ccc}x x & x y & x z \\ x y & y y & y z \\ x z & y z & z z\end{array}\right)\left(\begin{array}{ccc}0 & 0 & 0 \\ 0 & 0 & 0 \\ 0 & 0 & 0\end{array}\right)\left(\begin{array}{lll}x x & x y & x z \\ x y & y y & y z \\ x z & y z & z z\end{array}\right)\left(\begin{array}{ccc}0 & 0 & 0 \\ 0 & 0 & 0 \\ 0 & 0 & 0\end{array}\right)$

$\left\{V^{2}\right\} \quad\left(\begin{array}{ccc}0 & x y & x z \\ -x y & 0 & y z \\ -x z & -y z & 0\end{array}\right)\left(\begin{array}{ccc}0 & 0 & 0 \\ 0 & 0 & 0 \\ 0 & 0 & 0\end{array}\right)\left(\begin{array}{ccc}0 & x y & x z \\ -x y & 0 & y z \\ -x z & -y z & 0\end{array}\right)\left(\begin{array}{lll}0 & 0 & 0 \\ 0 & 0 & 0 \\ 0 & 0 & 0\end{array}\right)$

$$
\mathrm{n}=2
$$

$\left(\begin{array}{ccc}x x & x y & 0 \\ y x & y y & 0\end{array}\right)\left(\begin{array}{ccc}x x & x y & 0 \\ y x & y y & 0 \\ 0 & 0 & z z\end{array}\right)\left(\begin{array}{ccc}x x & x y & 0 \\ y x & y y & 0 \\ 0 & 0 & z z\end{array}\right)\left(\begin{array}{ccc}x x & x y & 0 \\ y x & y y & 0 \\ 0 & 0 & z z\end{array}\right)$

$\left(\begin{array}{ccc}0 & 0 & z z\end{array}\right)\left(\begin{array}{ccc}0 & 0 & z z\end{array}\right)\left(\begin{array}{ccc}0 & 0 & z z\end{array}\right)\left(\begin{array}{ccc}0 & 0 & z z\end{array}\right)$

$\left(\begin{array}{ccc}x x & x y & 0 \\ x y & y y & 0 \\ 0 & 0 & z z\end{array}\right)\left(\begin{array}{ccc}x x & x y & 0 \\ x y & y y & 0 \\ 0 & 0 & z z\end{array}\right)\left(\begin{array}{ccc}x x & x y & 0 \\ x y & y y & 0 \\ 0 & 0 & z z\end{array}\right)\left(\begin{array}{ccc}x x & x y & 0 \\ x y & y y & 0 \\ 0 & 0 & z z\end{array}\right)$

$\left(\begin{array}{ccc}0 & x y & 0 \\ -x y & 0 & 0 \\ 0 & 0 & 0\end{array}\right)\left(\begin{array}{ccc}0 & x y & 0 \\ -x y & 0 & 0 \\ 0 & 0 & 0\end{array}\right)\left(\begin{array}{ccc}0 & x y & 0 \\ -x y & 0 & 0 \\ 0 & 0 & 0\end{array}\right)\left(\begin{array}{ccc}0 & x y & 0 \\ -x y & 0 & 0 \\ 0 & 0 & 0\end{array}\right)$

$$
\mathbf{C}_{2 \mathrm{v}}=\mathbf{2}_{\mathrm{z}} \mathbf{m}_{\mathrm{x}} \mathbf{m}_{\mathrm{y}}
$$

$\left(\begin{array}{ccc}x x & 0 & 0 \\ 0 & y y & 0 \\ 0 & 0 & z z\end{array}\right)\left(\begin{array}{ccc}0 & x y & 0 \\ y x & 0 & 0 \\ 0 & 0 & 0\end{array}\right)\left(\begin{array}{ccc}x x & 0 & 0 \\ 0 & y y & 0 \\ 0 & 0 & z z\end{array}\right)\left(\begin{array}{ccc}0 & x y & 0 \\ y x & 0 & 0 \\ 0 & 0 & 0\end{array}\right)$

$\left(\begin{array}{ccc}x x & 0 & 0 \\ 0 & y y & 0 \\ 0 & 0 & z z\end{array}\right)\left(\begin{array}{ccc}0 & x y & 0 \\ x y & 0 & 0 \\ 0 & 0 & 0\end{array}\right)\left(\begin{array}{ccc}x x & 0 & 0 \\ 0 & y y & 0 \\ 0 & 0 & z z\end{array}\right)\left(\begin{array}{ccc}0 & x y & 0 \\ x y & 0 & 0 \\ 0 & 0 & 0\end{array}\right)$

$\left(\begin{array}{lll}0 & 0 & 0 \\ 0 & 0 & 0 \\ 0 & 0 & 0\end{array}\right)\left(\begin{array}{ccc}0 & x y & 0 \\ -x y & 0 & 0 \\ 0 & 0 & 0\end{array}\right)\left(\begin{array}{lll}0 & 0 & 0 \\ 0 & 0 & 0 \\ 0 & 0 & 0\end{array}\right)\left(\begin{array}{ccc}0 & x y & 0 \\ -x y & 0 & 0 \\ 0 & 0 & 0\end{array}\right)$

$\left(\begin{array}{lll}0 & 0 & 0 \\ 0 & 0 & 0 \\ 0 & 0 & 0\end{array}\right)\left(\begin{array}{ccc}0 & x y & 0 \\ -x y & 0 & 0 \\ 0 & 0 & 0\end{array}\right)\left(\begin{array}{ccc}0 & 0 & 0 \\ 0 & 0 & 0 \\ 0 & 0 & 0\end{array}\right)\left(\begin{array}{ccc}0 & x y & 0 \\ -x y & 0 & 0 \\ 0 & 0 & 0\end{array}\right)$

$\left(\begin{array}{ccc}0 & x y & 0 \\ -x y & 0 & 0 \\ 0 & 0 & 0\end{array}\right)\left(\begin{array}{ccc}0 & x y & 0 \\ -x y & 0 & 0 \\ 0 & 0 & 0\end{array}\right)\left(\begin{array}{ccc}0 & x y & 0 \\ -x y & 0 & 0 \\ 0 & 0 & 0\end{array}\right)\left(\begin{array}{ccc}0 & x y & 0 \\ -x y & 0 & 0 \\ 0 & 0 & 0\end{array}\right)$

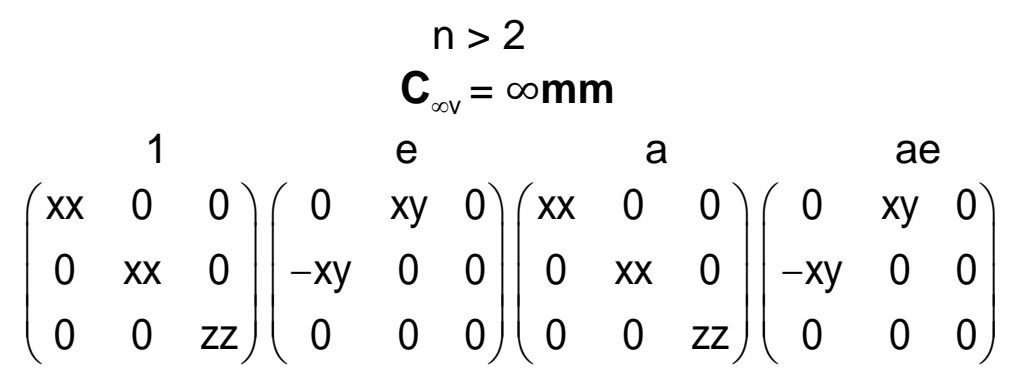

$\left(\begin{array}{ccc}x x & 0 & 0 \\ 0 & x x & 0 \\ 0 & 0 & z z\end{array}\right)\left(\begin{array}{ccc}0 & 0 & 0 \\ 0 & 0 & 0 \\ 0 & 0 & 0\end{array}\right)\left(\begin{array}{ccc}x x & 0 & 0 \\ 0 & x x & 0 \\ 0 & 0 & z z\end{array}\right)\left(\begin{array}{ccc}0 & 0 & 0 \\ 0 & 0 & 0 \\ 0 & 0 & 0\end{array}\right)$

$$
\mathrm{n}>2
$$

$$
\mathrm{C}_{\infty \mathrm{h}}=\infty / \mathrm{m}
$$

$\left(\begin{array}{ccc}x x & x y & 0 \\ -x y & x x & 0 \\ 0 & 0 & z z\end{array}\right)\left(\begin{array}{ccc}x x & x y & 0 \\ x y & -x x & 0 \\ 0 & 0 & 0\end{array}\right)\left(\begin{array}{ccc}x x & x y & 0 \\ -x y & x x & 0 \\ 0 & 0 & z z\end{array}\right)\left(\begin{array}{ccc}x x & x y & 0 \\ x y & -x x & 0 \\ 0 & 0 & 0\end{array}\right)$

$\left(\begin{array}{ccc}x x & x y & 0 \\ -x y & x x & 0 \\ 0 & 0 & z z\end{array}\right)\left(\begin{array}{ccc}0 & 0 & 0 \\ 0 & 0 & 0 \\ 0 & 0 & 0\end{array}\right)\left(\begin{array}{ccc}x x & x y & 0 \\ -x y & x x & 0 \\ 0 & 0 & z z\end{array}\right)\left(\begin{array}{ccc}0 & 0 & 0 \\ 0 & 0 & 0 \\ 0 & 0 & 0\end{array}\right)$

$\left(\begin{array}{ccc}x x & 0 & 0 \\ 0 & x x & 0 \\ 0 & 0 & z z\end{array}\right)\left(\begin{array}{ccc}x x & x y & 0 \\ x y & -x x & 0 \\ 0 & 0 & 0\end{array}\right)\left(\begin{array}{ccc}x x & 0 & 0 \\ 0 & x x & 0 \\ 0 & 0 & z z\end{array}\right)\left(\begin{array}{ccc}x x & x y & 0 \\ x y & -x x & 0 \\ 0 & 0 & 0\end{array}\right)$

$\left(\begin{array}{ccc}x x & 0 & 0 \\ 0 & x x & 0 \\ 0 & 0 & z z\end{array}\right)\left(\begin{array}{ccc}0 & 0 & 0 \\ 0 & 0 & 0 \\ 0 & 0 & 0\end{array}\right)\left(\begin{array}{ccc}x x & 0 & 0 \\ 0 & x x & 0 \\ 0 & 0 & z z\end{array}\right)\left(\begin{array}{ccc}0 & 0 & 0 \\ 0 & 0 & 0 \\ 0 & 0 & 0\end{array}\right)$

$\left(\begin{array}{ccc}0 & x y & 0 \\ -x y & 0 & 0 \\ 0 & 0 & 0\end{array}\right)\left(\begin{array}{ccc}0 & 0 & 0 \\ 0 & 0 & 0 \\ 0 & 0 & 0\end{array}\right)\left(\begin{array}{ccc}0 & x y & 0 \\ -x y & 0 & 0 \\ 0 & 0 & 0\end{array}\right)\left(\begin{array}{ccc}0 & 0 & 0 \\ 0 & 0 & 0 \\ 0 & 0 & 0\end{array}\right)$ $\left(\begin{array}{ccc}0 & x y & 0 \\ -x y & 0 & 0 \\ 0 & 0 & 0\end{array}\right)\left(\begin{array}{lll}0 & 0 & 0 \\ 0 & 0 & 0 \\ 0 & 0 & 0\end{array}\right)\left(\begin{array}{ccc}0 & x y & 0 \\ -x y & 0 & 0 \\ 0 & 0 & 0\end{array}\right)\left(\begin{array}{lll}0 & 0 & 0 \\ 0 & 0 & 0 \\ 0 & 0 & 0\end{array}\right)$ 


$$
\begin{array}{ccc} 
& \mathrm{n}=1 & \mathrm{n}=2 \\
\mathbf{c}_{\mathrm{nh}} & \mathbf{c}_{1 \mathrm{~h}}=\mathbf{m}_{\mathrm{z}} & \mathbf{c}_{2 \mathrm{~h}}=\mathbf{2} / \mathbf{m}_{\mathrm{z}}
\end{array}
$$

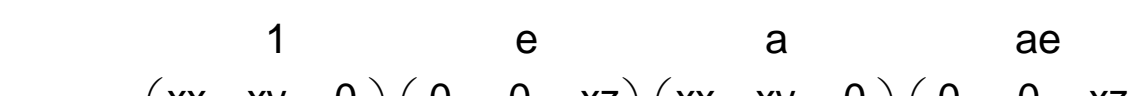
$V^{2} \quad\left(\begin{array}{ccc}x x & x y & 0 \\ y x & y y & 0 \\ 0 & 0 & z z\end{array}\right)\left(\begin{array}{ccc}0 & 0 & x z \\ 0 & 0 & y z \\ z x & z y & 0\end{array}\right)\left(\begin{array}{ccc}x x & x y & 0 \\ y x & y y & 0 \\ 0 & 0 & z z\end{array}\right)\left(\begin{array}{ccc}0 & 0 & x z \\ 0 & 0 & y z \\ z x & z y & 0\end{array}\right)$ $\left[V^{2}\right] \quad\left(\begin{array}{ccc}x x & x y & 0 \\ x y & y y & 0 \\ 0 & 0 & z z\end{array}\right)\left(\begin{array}{ccc}0 & 0 & x z \\ 0 & 0 & y z \\ x z & y z & 0\end{array}\right)\left(\begin{array}{ccc}x x & x y & 0 \\ x y & y y & 0 \\ 0 & 0 & z z\end{array}\right)\left(\begin{array}{ccc}0 & 0 & x z \\ 0 & 0 & y z \\ x z & y z & 0\end{array}\right)$ $\left\{\mathrm{V}^{2}\right\} \quad\left(\begin{array}{ccc}0 & \mathrm{xy} & 0 \\ -\mathrm{xy} & 0 & 0 \\ 0 & 0 & 0\end{array}\right)\left(\begin{array}{ccc}0 & 0 & \mathrm{xz} \\ 0 & 0 & \mathrm{yz} \\ -\mathrm{xz} & -\mathrm{yz} & 0\end{array}\right)\left(\begin{array}{ccc}0 & \mathrm{xy} & 0 \\ -\mathrm{xy} & 0 & 0 \\ 0 & 0 & 0\end{array}\right)\left(\begin{array}{ccc}0 & 0 & \mathrm{xz} \\ 0 & 0 & y z \\ -\mathrm{xz} & -\mathrm{yz} & 0\end{array}\right)$

$D_{n}$

$\mathrm{V}^{2} \quad\left(\begin{array}{ccc}x x & 0 & x z \\ 0 & y y & 0 \\ z x & 0 & z z\end{array}\right)\left(\begin{array}{ccc}x x & 0 & x z \\ 0 & y y & 0 \\ z x & 0 & z z\end{array}\right)\left(\begin{array}{ccc}x x & 0 & x z \\ 0 & y y & 0 \\ z x & 0 & z z\end{array}\right)\left(\begin{array}{ccc}x x & 0 & x z \\ 0 & y y & 0 \\ z x & 0 & z z\end{array}\right)$

$\left[V^{2}\right] \quad\left(\begin{array}{ccc}x x & 0 & x z \\ 0 & y y & 0 \\ x z & 0 & z z\end{array}\right)\left(\begin{array}{ccc}x x & 0 & x z \\ 0 & y y & 0 \\ x z & 0 & z z\end{array}\right)\left(\begin{array}{ccc}x x & 0 & x z \\ 0 & y y & 0 \\ x z & 0 & z z\end{array}\right)\left(\begin{array}{ccc}x x & 0 & x z \\ 0 & y y & 0 \\ x z & 0 & z z\end{array}\right)$

$\left\{V^{2}\right\} \quad\left(\begin{array}{ccc}0 & 0 & x z \\ 0 & 0 & 0 \\ -x z & 0 & 0\end{array}\right)\left(\begin{array}{ccc}0 & 0 & x z \\ 0 & 0 & 0 \\ -x z & 0 & 0\end{array}\right)\left(\begin{array}{ccc}0 & 0 & x z \\ 0 & 0 & 0 \\ -x z & 0 & 0\end{array}\right)\left(\begin{array}{ccc}0 & 0 & x z \\ 0 & 0 & 0 \\ -x z & 0 & 0\end{array}\right)$ $\left(\begin{array}{ccc}x x & x y & 0 \\ y x & y y & 0 \\ 0 & 0 & z z\end{array}\right)\left(\begin{array}{ccc}0 & 0 & 0 \\ 0 & 0 & 0 \\ 0 & 0 & 0\end{array}\right)\left(\begin{array}{ccc}x x & x y & 0 \\ y x & y y & 0 \\ 0 & 0 & z z\end{array}\right)\left(\begin{array}{ccc}0 & 0 & 0 \\ 0 & 0 & 0 \\ 0 & 0 & 0\end{array}\right)$

$\left(\begin{array}{ccc}x x & x y & 0 \\ x y & y y & 0 \\ 0 & 0 & z z\end{array}\right)\left(\begin{array}{ccc}0 & 0 & 0 \\ 0 & 0 & 0 \\ 0 & 0 & 0\end{array}\right)\left(\begin{array}{ccc}x x & x y & 0 \\ x y & y y & 0 \\ 0 & 0 & z z\end{array}\right)\left(\begin{array}{ccc}0 & 0 & 0 \\ 0 & 0 & 0 \\ 0 & 0 & 0\end{array}\right)$ $\left(\begin{array}{ccc}0 & x y & 0 \\ -x y & 0 & 0 \\ 0 & 0 & 0\end{array}\right)\left(\begin{array}{ccc}0 & 0 & 0 \\ 0 & 0 & 0 \\ 0 & 0 & 0\end{array}\right)\left(\begin{array}{ccc}0 & x y & 0 \\ -x y & 0 & 0 \\ 0 & 0 & 0\end{array}\right)\left(\begin{array}{lll}0 & 0 & 0 \\ 0 & 0 & 0 \\ 0 & 0 & 0\end{array}\right)$

$$
\mathrm{n}=2
$$$$
D_{2}=2 x 2_{y} 2_{z}
$$

$\left(\begin{array}{ccc}x x & 0 & 0 \\ 0 & y y & 0 \\ 0 & 0 & z z\end{array}\right)\left(\begin{array}{ccc}x x & 0 & 0 \\ 0 & y y & 0 \\ 0 & 0 & z z\end{array}\right)\left(\begin{array}{ccc}x x & 0 & 0 \\ 0 & y y & 0 \\ 0 & 0 & z z\end{array}\right)\left(\begin{array}{ccc}x x & 0 & 0 \\ 0 & y y & 0 \\ 0 & 0 & z z\end{array}\right)$

$\left(\begin{array}{ccc}x x & 0 & 0 \\ 0 & y y & 0 \\ 0 & 0 & z z\end{array}\right)\left(\begin{array}{ccc}x x & 0 & 0 \\ 0 & y y & 0 \\ 0 & 0 & z z\end{array}\right)\left(\begin{array}{ccc}x x & 0 & 0 \\ 0 & y y & 0 \\ 0 & 0 & z z\end{array}\right)\left(\begin{array}{ccc}x x & 0 & 0 \\ 0 & y y & 0 \\ 0 & 0 & z z\end{array}\right)$

$\left(\begin{array}{lll}0 & 0 & 0 \\ 0 & 0 & 0 \\ 0 & 0 & 0\end{array}\right)\left(\begin{array}{lll}0 & 0 & 0 \\ 0 & 0 & 0 \\ 0 & 0 & 0\end{array}\right)\left(\begin{array}{lll}0 & 0 & 0 \\ 0 & 0 & 0 \\ 0 & 0 & 0\end{array}\right)\left(\begin{array}{lll}0 & 0 & 0 \\ 0 & 0 & 0 \\ 0 & 0 & 0\end{array}\right)$

$$
n>2
$$$$
\mathbf{C}_{\infty \mathrm{h}}=\infty / \mathrm{m}
$$

$\left(\begin{array}{ccc}x x & x y & 0 \\ -x y & x x & 0 \\ 0 & 0 & z z\end{array}\right)\left(\begin{array}{ccc}0 & 0 & 0 \\ 0 & 0 & 0 \\ 0 & 0 & 0\end{array}\right)\left(\begin{array}{ccc}x x & x y & 0 \\ -x y & x x & 0 \\ 0 & 0 & z z\end{array}\right)\left(\begin{array}{ccc}0 & 0 & 0 \\ 0 & 0 & 0 \\ 0 & 0 & 0\end{array}\right)$

$\left(\begin{array}{ccc}x x & 0 & 0 \\ 0 & x x & 0 \\ 0 & 0 & z z\end{array}\right)\left(\begin{array}{ccc}0 & 0 & 0 \\ 0 & 0 & 0 \\ 0 & 0 & 0\end{array}\right)\left(\begin{array}{ccc}x x & 0 & 0 \\ 0 & x x & 0 \\ 0 & 0 & z z\end{array}\right)\left(\begin{array}{lll}0 & 0 & 0 \\ 0 & 0 & 0 \\ 0 & 0 & 0\end{array}\right)$

$\left(\begin{array}{ccc}0 & x y & 0 \\ -x y & 0 & 0 \\ 0 & 0 & 0\end{array}\right)\left(\begin{array}{ccc}0 & 0 & 0 \\ 0 & 0 & 0 \\ 0 & 0 & 0\end{array}\right)\left(\begin{array}{ccc}0 & x y & 0 \\ -x y & 0 & 0 \\ 0 & 0 & 0\end{array}\right)\left(\begin{array}{lll}0 & 0 & 0 \\ 0 & 0 & 0 \\ 0 & 0 & 0\end{array}\right)$

$$
\begin{aligned}
\mathrm{n} & >2 \\
\mathbf{D}_{\infty} & =\infty 2
\end{aligned}
$$

$\left(\begin{array}{ccc}x x & 0 & 0 \\ 0 & x x & 0 \\ 0 & 0 & z z\end{array}\right)\left(\begin{array}{ccc}x x & 0 & 0 \\ 0 & x x & 0 \\ 0 & 0 & z z\end{array}\right)\left(\begin{array}{ccc}x x & 0 & 0 \\ 0 & x x & 0 \\ 0 & 0 & z z\end{array}\right)\left(\begin{array}{ccc}x x & 0 & 0 \\ 0 & x x & 0 \\ 0 & 0 & z z\end{array}\right)$

$\left(\begin{array}{ccc}x x & 0 & 0 \\ 0 & x x & 0 \\ 0 & 0 & z z\end{array}\right)\left(\begin{array}{ccc}x x & 0 & 0 \\ 0 & x x & 0 \\ 0 & 0 & z z\end{array}\right)\left(\begin{array}{ccc}x x & 0 & 0 \\ 0 & x x & 0 \\ 0 & 0 & z z\end{array}\right)\left(\begin{array}{ccc}x x & 0 & 0 \\ 0 & x x & 0 \\ 0 & 0 & z z\end{array}\right)$

$\left(\begin{array}{lll}0 & 0 & 0 \\ 0 & 0 & 0 \\ 0 & 0 & 0\end{array}\right)\left(\begin{array}{lll}0 & 0 & 0 \\ 0 & 0 & 0 \\ 0 & 0 & 0\end{array}\right)\left(\begin{array}{lll}0 & 0 & 0 \\ 0 & 0 & 0 \\ 0 & 0 & 0\end{array}\right)\left(\begin{array}{lll}0 & 0 & 0 \\ 0 & 0 & 0 \\ 0 & 0 & 0\end{array}\right)$

\section{$\mathrm{n}>2$ \\ $\mathbf{D}_{\infty \mathrm{h}}=\infty / \mathbf{m m m}$}

\section{$\mathbf{D}_{2 \mathrm{~d}}=\overline{\mathbf{4}}_{\mathrm{z}} \mathbf{m}_{\mathrm{x}} \mathbf{2}_{\mathrm{xy}}$}

$\left(\begin{array}{ccc}x x & 0 & 0 \\ 0 & x x & 0 \\ 0 & 0 & z z\end{array}\right)\left(\begin{array}{ccc}0 & x y & 0 \\ x y & 0 & 0 \\ 0 & 0 & 0\end{array}\right)\left(\begin{array}{ccc}x x & 0 & 0 \\ 0 & x x & 0 \\ 0 & 0 & z z\end{array}\right)\left(\begin{array}{ccc}0 & x y & 0 \\ x y & 0 & 0 \\ 0 & 0 & 0\end{array}\right)$

$\left(\begin{array}{ccc}x x & 0 & 0 \\ 0 & x x & 0 \\ 0 & 0 & z z\end{array}\right)\left(\begin{array}{ccc}0 & 0 & 0 \\ 0 & 0 & 0 \\ 0 & 0 & 0\end{array}\right)\left(\begin{array}{ccc}x x & 0 & 0 \\ 0 & x x & 0 \\ 0 & 0 & z z\end{array}\right)\left(\begin{array}{ccc}0 & 0 & 0 \\ 0 & 0 & 0 \\ 0 & 0 & 0\end{array}\right)$

$\left(\begin{array}{ccc}x x & 0 & 0 \\ 0 & x x & 0 \\ 0 & 0 & z z\end{array}\right)\left(\begin{array}{ccc}0 & x y & 0 \\ x y & 0 & 0 \\ 0 & 0 & 0\end{array}\right)\left(\begin{array}{ccc}x x & 0 & 0 \\ 0 & x x & 0 \\ 0 & 0 & z z\end{array}\right)\left(\begin{array}{ccc}0 & x y & 0 \\ x y & 0 & 0 \\ 0 & 0 & 0\end{array}\right)$

$\left(\begin{array}{ccc}x x & 0 & 0 \\ 0 & x x & 0 \\ 0 & 0 & z z\end{array}\right)\left(\begin{array}{ccc}0 & 0 & 0 \\ 0 & 0 & 0 \\ 0 & 0 & 0\end{array}\right)\left(\begin{array}{ccc}x x & 0 & 0 \\ 0 & x x & 0 \\ 0 & 0 & z z\end{array}\right)\left(\begin{array}{ccc}0 & 0 & 0 \\ 0 & 0 & 0 \\ 0 & 0 & 0\end{array}\right)$

$\left(\begin{array}{lll}0 & 0 & 0 \\ 0 & 0 & 0 \\ 0 & 0 & 0\end{array}\right)\left(\begin{array}{lll}0 & 0 & 0 \\ 0 & 0 & 0 \\ 0 & 0 & 0\end{array}\right)\left(\begin{array}{lll}0 & 0 & 0 \\ 0 & 0 & 0 \\ 0 & 0 & 0\end{array}\right)\left(\begin{array}{lll}0 & 0 & 0 \\ 0 & 0 & 0 \\ 0 & 0 & 0\end{array}\right)$ $\left(\begin{array}{lll}0 & 0 & 0 \\ 0 & 0 & 0 \\ 0 & 0 & 0\end{array}\right)\left(\begin{array}{lll}0 & 0 & 0 \\ 0 & 0 & 0 \\ 0 & 0 & 0\end{array}\right)\left(\begin{array}{lll}0 & 0 & 0 \\ 0 & 0 & 0 \\ 0 & 0 & 0\end{array}\right)\left(\begin{array}{lll}0 & 0 & 0 \\ 0 & 0 & 0 \\ 0 & 0 & 0\end{array}\right)$ 
$\mathbf{D}_{\text {nh }}$

$$
\mathrm{n}=1
$$

$D_{1 \mathrm{~h}}=\mathbf{2}_{\mathrm{x}} \mathbf{m}_{\mathrm{y}} \mathbf{m}_{\mathrm{z}}$

$V^{2} \quad\left(\begin{array}{ccc}x x & 0 & 0 \\ 0 & y y & 0 \\ 0 & 0 & z z\end{array}\right)\left(\begin{array}{ccc}0 & 0 & 0 \\ 0 & 0 & y z \\ 0 & z y & 0\end{array}\right)\left(\begin{array}{ccc}x x & 0 & 0 \\ 0 & y y & 0 \\ 0 & 0 & z z\end{array}\right)\left(\begin{array}{ccc}0 & 0 & 0 \\ 0 & 0 & y z \\ 0 & z y & 0\end{array}\right)$

$\left[V^{2}\right] \quad\left(\begin{array}{ccc}x x & 0 & 0 \\ 0 & y y & 0 \\ 0 & 0 & z z\end{array}\right)\left(\begin{array}{ccc}0 & 0 & 0 \\ 0 & 0 & y z \\ 0 & y z & 0\end{array}\right)\left(\begin{array}{ccc}x x & 0 & 0 \\ 0 & y y & 0 \\ 0 & 0 & z z\end{array}\right)\left(\begin{array}{ccc}0 & 0 & 0 \\ 0 & 0 & y z \\ 0 & y z & 0\end{array}\right)$

$\left\{V^{2}\right\} \quad\left(\begin{array}{ccc}0 & 0 & 0 \\ 0 & 0 & 0 \\ 0 & 0 & 0\end{array}\right)\left(\begin{array}{ccc}0 & 0 & 0 \\ 0 & 0 & y z \\ 0 & -y z & 0\end{array}\right)\left(\begin{array}{ccc}0 & 0 & 0 \\ 0 & 0 & 0 \\ 0 & 0 & 0\end{array}\right)\left(\begin{array}{ccc}0 & 0 & 0 \\ 0 & 0 & y z \\ 0 & -y z & 0\end{array}\right)$

$\mathrm{n}=1$

$\mathbf{C}_{2 n}\left(\mathbf{C}_{n}\right)$

$C_{2}\left(C_{1}\right)=2_{z}(1)=2_{z}{ }^{\prime}$

$V^{2} \quad\left(\begin{array}{ccc}x x & x y & 0 \\ y x & y y & 0 \\ 0 & 0 & z z\end{array}\right)\left(\begin{array}{ccc}x x & x y & 0 \\ y x & y y & 0 \\ 0 & 0 & z z\end{array}\right)\left(\begin{array}{ccc}0 & 0 & x z \\ 0 & 0 & y z \\ z x & z y & 0\end{array}\right)\left(\begin{array}{ccc}0 & 0 & x z \\ 0 & 0 & y z \\ z x & z y & 0\end{array}\right)$

$\left[V^{2}\right] \quad\left(\begin{array}{ccc}x x & x y & 0 \\ x y & y y & 0 \\ 0 & 0 & z z\end{array}\right)\left(\begin{array}{ccc}x x & x y & 0 \\ x y & y y & 0 \\ 0 & 0 & z z\end{array}\right)\left(\begin{array}{ccc}0 & 0 & x z \\ 0 & 0 & y z \\ x z & y z & 0\end{array}\right)\left(\begin{array}{ccc}0 & 0 & x z \\ 0 & 0 & y z \\ x z & y z & 0\end{array}\right)$

$\left\{V^{2}\right\} \quad\left(\begin{array}{ccc}0 & x y & 0 \\ -x y & 0 & 0 \\ 0 & 0 & 0\end{array}\right)\left(\begin{array}{ccc}0 & x y & 0 \\ -x y & 0 & 0 \\ 0 & 0 & 0\end{array}\right)\left(\begin{array}{ccc}0 & 0 & x z \\ 0 & 0 & y z \\ -x z & -y z & 0\end{array}\right)\left(\begin{array}{ccc}0 & 0 & x z \\ 0 & 0 & y z \\ -x z & -y z & 0\end{array}\right)$

$$
\mathrm{n}=1
$$

$\mathbf{S}_{2 n}\left(\mathbf{C}_{n}\right)$

$$
\mathbf{S}_{2}\left(\mathbf{C}_{1}\right)=\overline{\mathbf{1}}(\mathbf{1})=\overline{\mathbf{1}}^{\prime}
$$

$V^{2} \quad\left(\begin{array}{lll}x x & x y & x z \\ y x & y y & y z \\ z x & z y & z z\end{array}\right)\left(\begin{array}{lll}0 & 0 & 0 \\ 0 & 0 & 0 \\ 0 & 0 & 0\end{array}\right)\left(\begin{array}{lll}0 & 0 & 0 \\ 0 & 0 & 0 \\ 0 & 0 & 0\end{array}\right)\left(\begin{array}{lll}x x & x y & x z \\ y x & y y & y z \\ z x & z y & z z\end{array}\right)$

$\left[V^{2}\right]\left(\begin{array}{lll}x x & x y & x z \\ x y & y y & y z \\ x z & y z & z z\end{array}\right)\left(\begin{array}{lll}0 & 0 & 0 \\ 0 & 0 & 0 \\ 0 & 0 & 0\end{array}\right)\left(\begin{array}{lll}0 & 0 & 0 \\ 0 & 0 & 0 \\ 0 & 0 & 0\end{array}\right)\left(\begin{array}{lll}x x & x y & x z \\ x y & y y & y z \\ x z & y z & z z\end{array}\right)$

$\left\{V^{2}\right\} \quad\left(\begin{array}{ccc}0 & x y & x z \\ -x y & 0 & y z \\ -x z & -y z & 0\end{array}\right)\left(\begin{array}{lll}0 & 0 & 0 \\ 0 & 0 & 0 \\ 0 & 0 & 0\end{array}\right)\left(\begin{array}{lll}0 & 0 & 0 \\ 0 & 0 & 0 \\ 0 & 0 & 0\end{array}\right)\left(\begin{array}{ccc}0 & x y & x z \\ -x y & 0 & y z \\ -x z & -y z & 0\end{array}\right)$ $\mathrm{n}=2$

$\mathbf{D}_{2 \mathrm{~h}}=\mathbf{m}_{\mathrm{x}} \mathbf{m}_{\mathrm{y}} \mathbf{m}_{\mathrm{z}}$

$\left(\begin{array}{ccc}x x & 0 & 0 \\ 0 & y y & 0 \\ 0 & 0 & z z\end{array}\right)\left(\begin{array}{ccc}0 & 0 & 0 \\ 0 & 0 & 0 \\ 0 & 0 & 0\end{array}\right)\left(\begin{array}{ccc}x x & 0 & 0 \\ 0 & y y & 0 \\ 0 & 0 & z z\end{array}\right)\left(\begin{array}{ccc}0 & 0 & 0 \\ 0 & 0 & 0 \\ 0 & 0 & 0\end{array}\right)$

$\left(\begin{array}{ccc}\mathrm{xx} & 0 & 0 \\ 0 & \mathrm{yy} & 0 \\ 0 & 0 & \mathrm{zz}\end{array}\right)\left(\begin{array}{ccc}0 & 0 & 0 \\ 0 & 0 & 0 \\ 0 & 0 & 0\end{array}\right)\left(\begin{array}{ccc}\mathrm{xx} & 0 & 0 \\ 0 & \mathrm{yy} & 0 \\ 0 & 0 & \mathrm{zz}\end{array}\right)\left(\begin{array}{ccc}0 & 0 & 0 \\ 0 & 0 & 0 \\ 0 & 0 & 0\end{array}\right)$

$\left(\begin{array}{lll}0 & 0 & 0 \\ 0 & 0 & 0 \\ 0 & 0 & 0\end{array}\right)\left(\begin{array}{lll}0 & 0 & 0 \\ 0 & 0 & 0 \\ 0 & 0 & 0\end{array}\right)\left(\begin{array}{lll}0 & 0 & 0 \\ 0 & 0 & 0 \\ 0 & 0 & 0\end{array}\right)\left(\begin{array}{lll}0 & 0 & 0 \\ 0 & 0 & 0 \\ 0 & 0 & 0\end{array}\right)$

$\mathrm{n}=2$

$C_{4}\left(C_{2}\right)=4_{z}\left(2_{z}\right)=4_{z}^{\prime}$

$\left(\begin{array}{ccc}x x & x y & 0 \\ -x y & x x & 0 \\ 0 & 0 & z z\end{array}\right)\left(\begin{array}{ccc}x x & x y & 0 \\ -x y & x x & 0 \\ 0 & 0 & z z\end{array}\right)\left(\begin{array}{ccc}x x & x y & 0 \\ x y & -x x & 0 \\ 0 & 0 & 0\end{array}\right)\left(\begin{array}{ccc}x x & x y & 0 \\ x y & -x x & 0 \\ 0 & 0 & 0\end{array}\right)$

$\left(\begin{array}{ccc}x x & 0 & 0 \\ 0 & x x & 0 \\ 0 & 0 & z z\end{array}\right)\left(\begin{array}{ccc}x x & 0 & 0 \\ 0 & x x & 0 \\ 0 & 0 & z z\end{array}\right)\left(\begin{array}{ccc}x x & x y & 0 \\ x y & -x x & 0 \\ 0 & 0 & 0\end{array}\right)\left(\begin{array}{ccc}x x & x y & 0 \\ x y & -x x & 0 \\ 0 & 0 & 0\end{array}\right)$

$\left(\begin{array}{ccc}0 & x y & 0 \\ -x y & 0 & 0 \\ 0 & 0 & 0\end{array}\right)\left(\begin{array}{ccc}0 & x y & 0 \\ -x y & 0 & 0 \\ 0 & 0 & 0\end{array}\right)\left(\begin{array}{lll}0 & 0 & 0 \\ 0 & 0 & 0 \\ 0 & 0 & 0\end{array}\right)\left(\begin{array}{lll}0 & 0 & 0 \\ 0 & 0 & 0 \\ 0 & 0 & 0\end{array}\right)$

$$
\mathrm{n}=2
$$

$\mathrm{S}_{4}\left(\mathrm{C}_{2}\right)=\overline{\mathbf{4}}_{\mathrm{z}}\left(\mathbf{2}_{\mathrm{z}}\right)=\overline{\mathbf{4}}_{\mathrm{z}}{ }^{\prime}$

$\left(\begin{array}{ccc}x x & x y & 0 \\ -x y & x x & 0 \\ 0 & 0 & z z\end{array}\right)\left(\begin{array}{ccc}x x & x y & 0 \\ x y & -x x & 0 \\ 0 & 0 & 0\end{array}\right)\left(\begin{array}{ccc}x x & x y & 0 \\ x y & -x x & 0 \\ 0 & 0 & 0\end{array}\right)\left(\begin{array}{ccc}x x & x y & 0 \\ -x y & x x & 0 \\ 0 & 0 & z z\end{array}\right)$

$\left(\begin{array}{ccc}x x & 0 & 0 \\ 0 & x x & 0 \\ 0 & 0 & z z\end{array}\right)\left(\begin{array}{ccc}x x & x y & 0 \\ x y & -x x & 0 \\ 0 & 0 & 0\end{array}\right)\left(\begin{array}{ccc}x x & x y & 0 \\ x y & -x x & 0 \\ 0 & 0 & 0\end{array}\right)\left(\begin{array}{ccc}x x & 0 & 0 \\ 0 & x x & 0 \\ 0 & 0 & z z\end{array}\right)$

$\left(\begin{array}{ccc}0 & x y & 0 \\ -x y & 0 & 0 \\ 0 & 0 & 0\end{array}\right)\left(\begin{array}{lll}0 & 0 & 0 \\ 0 & 0 & 0 \\ 0 & 0 & 0\end{array}\right)\left(\begin{array}{lll}0 & 0 & 0 \\ 0 & 0 & 0 \\ 0 & 0 & 0\end{array}\right)\left(\begin{array}{ccc}0 & x y & 0 \\ -x y & 0 & 0 \\ 0 & 0 & 0\end{array}\right)$

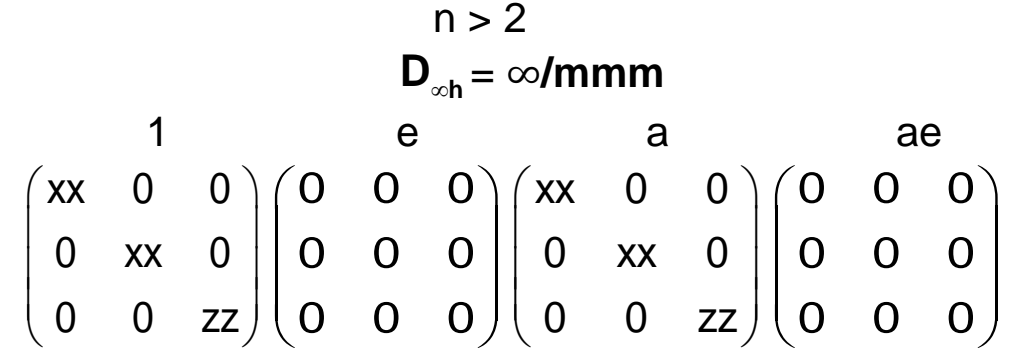

$\left(\begin{array}{ccc}x x & 0 & 0 \\ 0 & x x & 0 \\ 0 & 0 & z z\end{array}\right)\left(\begin{array}{ccc}0 & 0 & 0 \\ 0 & 0 & 0 \\ 0 & 0 & 0\end{array}\right)\left(\begin{array}{ccc}x x & 0 & 0 \\ 0 & x x & 0 \\ 0 & 0 & z z\end{array}\right)\left(\begin{array}{ccc}0 & 0 & 0 \\ 0 & 0 & 0 \\ 0 & 0 & 0\end{array}\right)$

$\left(\begin{array}{lll}0 & 0 & 0 \\ 0 & 0 & 0 \\ 0 & 0 & 0\end{array}\right)\left(\begin{array}{lll}0 & 0 & 0 \\ 0 & 0 & 0 \\ 0 & 0 & 0\end{array}\right)\left(\begin{array}{lll}0 & 0 & 0 \\ 0 & 0 & 0 \\ 0 & 0 & 0\end{array}\right)\left(\begin{array}{lll}0 & 0 & 0 \\ 0 & 0 & 0 \\ 0 & 0 & 0\end{array}\right)$ $\left(\begin{array}{ccc}x x & x y & 0 \\ -x y & x x & 0 \\ 0 & 0 & z z\end{array}\right)\left(\begin{array}{ccc}x x & x y & 0 \\ -x y & x x & 0 \\ 0 & 0 & z z\end{array}\right)\left(\begin{array}{ccc}0 & 0 & 0 \\ 0 & 0 & 0 \\ 0 & 0 & 0\end{array}\right)\left(\begin{array}{ccc}0 & 0 & 0 \\ 0 & 0 & 0 \\ 0 & 0 & 0\end{array}\right)$

$\left(\begin{array}{ccc}x x & 0 & 0 \\ 0 & x x & 0 \\ 0 & 0 & z z\end{array}\right)\left(\begin{array}{ccc}x x & 0 & 0 \\ 0 & x x & 0 \\ 0 & 0 & z z\end{array}\right)\left(\begin{array}{ccc}0 & 0 & 0 \\ 0 & 0 & 0 \\ 0 & 0 & 0\end{array}\right)\left(\begin{array}{lll}0 & 0 & 0 \\ 0 & 0 & 0 \\ 0 & 0 & 0\end{array}\right)$

$\left(\begin{array}{ccc}0 & x y & 0 \\ -x y & 0 & 0 \\ 0 & 0 & 0\end{array}\right)\left(\begin{array}{ccc}0 & x y & 0 \\ -x y & 0 & 0 \\ 0 & 0 & 0\end{array}\right)\left(\begin{array}{lll}0 & 0 & 0 \\ 0 & 0 & 0 \\ 0 & 0 & 0\end{array}\right)\left(\begin{array}{lll}0 & 0 & 0 \\ 0 & 0 & 0 \\ 0 & 0 & 0\end{array}\right)$

$$
\begin{gathered}
\mathrm{n}>2 \\
\mathbf{C}_{\infty \mathrm{h}}\left(\mathbf{C}_{\infty}\right)=\infty / \mathbf{m}^{\prime}
\end{gathered}
$$

$\left(\begin{array}{ccc}x x & x y & 0 \\ -x y & x x & 0 \\ 0 & 0 & z z\end{array}\right)\left(\begin{array}{ccc}0 & 0 & 0 \\ 0 & 0 & 0 \\ 0 & 0 & 0\end{array}\right)\left(\begin{array}{ccc}0 & 0 & 0 \\ 0 & 0 & 0 \\ 0 & 0 & 0\end{array}\right)\left(\begin{array}{ccc}x x & x y & 0 \\ -x y & x x & 0 \\ 0 & 0 & z z\end{array}\right)$

$\left(\begin{array}{ccc}x x & 0 & 0 \\ 0 & x x & 0 \\ 0 & 0 & z z\end{array}\right)\left(\begin{array}{lll}0 & 0 & 0 \\ 0 & 0 & 0 \\ 0 & 0 & 0\end{array}\right)\left(\begin{array}{ccc}0 & 0 & 0 \\ 0 & 0 & 0 \\ 0 & 0 & 0\end{array}\right)\left(\begin{array}{ccc}x x & 0 & 0 \\ 0 & x x & 0 \\ 0 & 0 & z z\end{array}\right)$

$\left(\begin{array}{ccc}0 & x y & 0 \\ -x y & 0 & 0 \\ 0 & 0 & 0\end{array}\right)\left(\begin{array}{lll}0 & 0 & 0 \\ 0 & 0 & 0 \\ 0 & 0 & 0\end{array}\right)\left(\begin{array}{lll}0 & 0 & 0 \\ 0 & 0 & 0 \\ 0 & 0 & 0\end{array}\right)\left(\begin{array}{ccc}0 & x y & 0 \\ -x y & 0 & 0 \\ 0 & 0 & 0\end{array}\right)$ 
$\mathrm{n}=1$

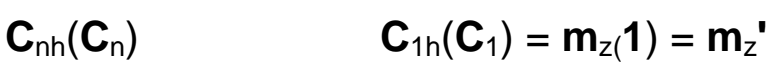

$V^{2} \quad\left(\begin{array}{ccc}x x & x y & 0 \\ y x & y y & 0 \\ 0 & 0 & z z\end{array}\right)\left(\begin{array}{ccc}0 & 0 & x z \\ 0 & 0 & y z \\ z x & z y & 0\end{array}\right)\left(\begin{array}{ccc}0 & 0 & x z \\ 0 & 0 & y z \\ z x & z y & 0\end{array}\right)\left(\begin{array}{ccc}x x & x y & 0 \\ y x & y y & 0 \\ 0 & 0 & z z\end{array}\right)$

$\left[V^{2}\right] \quad\left(\begin{array}{ccc}x x & x y & 0 \\ x y & y y & 0 \\ 0 & 0 & z z\end{array}\right)\left(\begin{array}{ccc}0 & 0 & x z \\ 0 & 0 & y z \\ x z & y z & 0\end{array}\right)\left(\begin{array}{ccc}0 & 0 & x z \\ 0 & 0 & y z \\ x z & y z & 0\end{array}\right)\left(\begin{array}{ccc}x x & x y & 0 \\ x y & y y & 0 \\ 0 & 0 & z z\end{array}\right)$

$\left\{V^{2}\right\} \quad\left(\begin{array}{ccc}0 & x y & 0 \\ -x y & 0 & 0 \\ 0 & 0 & 0\end{array}\right)\left(\begin{array}{ccc}0 & 0 & x z \\ 0 & 0 & y z \\ -x z & -y z & 0\end{array}\right)\left(\begin{array}{ccc}0 & 0 & x z \\ 0 & 0 & y z \\ -x z & -y z & 0\end{array}\right)\left(\begin{array}{ccc}0 & x y & 0 \\ -x y & 0 & 0 \\ 0 & 0 & 0\end{array}\right)$

$\mathrm{n}=1$

$\mathbf{C}_{2 n h}\left(\mathbf{S}_{2 n}\right) \quad \mathbf{C}_{2 h}\left(\mathbf{S}_{2}\right)=\mathbf{2}_{z} / \mathbf{m}_{z}(\overline{\mathbf{1}})=\mathbf{2}_{z}{ }^{\prime} / \mathbf{m}_{z}^{\prime}$

$\mathrm{V}^{2} \quad\left(\begin{array}{ccc}\mathrm{xx} & \mathrm{xy} & 0 \\ \mathrm{yx} & \mathrm{yy} & 0 \\ 0 & 0 & \mathrm{zz}\end{array}\right)\left(\begin{array}{ccc}0 & 0 & 0 \\ 0 & 0 & 0 \\ 0 & 0 & 0\end{array}\right)\left(\begin{array}{ccc}0 & 0 & \mathrm{xz} \\ 0 & 0 & y z \\ z x & z y & 0\end{array}\right)\left(\begin{array}{ccc}0 & 0 & 0 \\ 0 & 0 & 0 \\ 0 & 0 & 0\end{array}\right)$

$\left[V^{2}\right] \quad\left(\begin{array}{ccc}x x & x y & 0 \\ x y & y y & 0 \\ 0 & 0 & z z\end{array}\right)\left(\begin{array}{ccc}0 & 0 & 0 \\ 0 & 0 & 0 \\ 0 & 0 & 0\end{array}\right)\left(\begin{array}{ccc}0 & 0 & x z \\ 0 & 0 & y z \\ x z & y z & 0\end{array}\right)\left(\begin{array}{ccc}0 & 0 & 0 \\ 0 & 0 & 0 \\ 0 & 0 & 0\end{array}\right)$

$\left\{V^{2}\right\} \quad\left(\begin{array}{ccc}0 & x y & 0 \\ -x y & 0 & 0 \\ 0 & 0 & 0\end{array}\right)\left(\begin{array}{ccc}0 & 0 & 0 \\ 0 & 0 & 0 \\ 0 & 0 & 0\end{array}\right)\left(\begin{array}{ccc}0 & 0 & x z \\ 0 & 0 & y z \\ -x z & -y z & 0\end{array}\right)\left(\begin{array}{ccc}0 & 0 & 0 \\ 0 & 0 & 0 \\ 0 & 0 & 0\end{array}\right)$

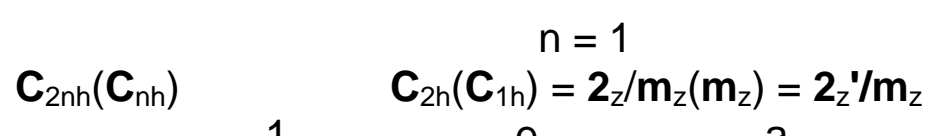

$\mathrm{V}^{2} \quad\left(\begin{array}{ccc}\mathrm{xx} & \mathrm{xy} & 0 \\ \mathrm{yx} & \mathrm{yy} & 0 \\ 0 & 0 & \mathrm{zz}\end{array}\right)\left(\begin{array}{ccc}0 & 0 & 0 \\ 0 & 0 & 0 \\ 0 & 0 & 0\end{array}\right)\left(\begin{array}{ccc}0 & 0 & 0 \\ 0 & 0 & 0 \\ 0 & 0 & 0\end{array}\right)\left(\begin{array}{ccc}0 & 0 & \mathrm{xz} \\ 0 & 0 & y z \\ \mathrm{zx} & \mathrm{zy} & 0\end{array}\right)$

$\left[V^{2}\right] \quad\left(\begin{array}{ccc}x x & x y & 0 \\ x y & y y & 0 \\ 0 & 0 & z z\end{array}\right)\left(\begin{array}{ccc}0 & 0 & 0 \\ 0 & 0 & 0 \\ 0 & 0 & 0\end{array}\right)\left(\begin{array}{ccc}0 & 0 & 0 \\ 0 & 0 & 0 \\ 0 & 0 & 0\end{array}\right)\left(\begin{array}{ccc}0 & 0 & x z \\ 0 & 0 & y z \\ x z & y z & 0\end{array}\right)$

$\left\{V^{2}\right\} \quad\left(\begin{array}{ccc}0 & x y & 0 \\ -x y & 0 & 0 \\ 0 & 0 & 0\end{array}\right)\left(\begin{array}{lll}0 & 0 & 0 \\ 0 & 0 & 0 \\ 0 & 0 & 0\end{array}\right)\left(\begin{array}{lll}0 & 0 & 0 \\ 0 & 0 & 0 \\ 0 & 0 & 0\end{array}\right)\left(\begin{array}{ccc}0 & 0 & x z \\ 0 & 0 & y z \\ -x z & -y z & 0\end{array}\right)$ $\mathrm{n}=2$
$\mathbf{C}_{2 h}\left(\mathbf{C}_{2}\right)=\mathbf{2}_{\mathbf{z}} / \mathbf{m}_{\mathbf{z}}\left(\mathbf{2}_{\mathbf{z}}\right)=\mathbf{2}_{\mathbf{z}} / \mathbf{m}_{\mathbf{z}}$

$\left(\begin{array}{ccc}x x & x y & 0 \\ y x & y y & 0 \\ 0 & 0 & z z\end{array}\right)\left(\begin{array}{ccc}0 & 0 & 0 \\ 0 & 0 & 0 \\ 0 & 0 & 0\end{array}\right)\left(\begin{array}{ccc}0 & 0 & 0 \\ 0 & 0 & 0 \\ 0 & 0 & 0\end{array}\right)\left(\begin{array}{ccc}x x & x y & 0 \\ y x & y y & 0 \\ 0 & 0 & z z\end{array}\right)$

$\left(\begin{array}{ccc}x x & x y & 0 \\ x y & y y & 0 \\ 0 & 0 & z z\end{array}\right)\left(\begin{array}{ccc}0 & 0 & 0 \\ 0 & 0 & 0 \\ 0 & 0 & 0\end{array}\right)\left(\begin{array}{ccc}0 & 0 & 0 \\ 0 & 0 & 0 \\ 0 & 0 & 0\end{array}\right)\left(\begin{array}{ccc}x x & x y & 0 \\ x y & y y & 0 \\ 0 & 0 & z z\end{array}\right)$

$\left(\begin{array}{ccc}0 & x y & 0 \\ -x y & 0 & 0 \\ 0 & 0 & 0\end{array}\right)\left(\begin{array}{lll}0 & 0 & 0 \\ 0 & 0 & 0 \\ 0 & 0 & 0\end{array}\right)\left(\begin{array}{lll}0 & 0 & 0 \\ 0 & 0 & 0 \\ 0 & 0 & 0\end{array}\right)\left(\begin{array}{ccc}0 & x y & 0 \\ -x y & 0 & 0 \\ 0 & 0 & 0\end{array}\right)$

$\mathrm{n}=2$

\section{$\mathrm{C}_{4 h}\left(S_{4}\right)=\mathbf{4}_{z} / \mathrm{m}_{\mathrm{z}}\left(\overline{4}_{\mathrm{z}}\right)=\mathbf{4}_{z^{\prime}} / \mathrm{m}_{\mathrm{z}}{ }^{\prime}$}

$\begin{array}{cccc}1 & e & a & a e\end{array}$ $\left(\begin{array}{lll}x x & x y & 0 \\ -x y & x x & 0\end{array}\right)\left(\begin{array}{ccc}0 & 0 & 0 \\ 0 & 0 & 0\end{array}\right)\left(\begin{array}{ccc}0 & 0 & 0 \\ 0 & 0 & 0\end{array}\right)\left(\begin{array}{lll}x x & x y & 0 \\ x y & -x x & 0\end{array}\right)$ $\left.\begin{array}{ccc}-x y & x x & 0 \\ 0 & 0 & z z\end{array}\right)\left(\begin{array}{ccc}0 & 0 & 0 \\ 0 & 0 & 0\end{array}\right)\left(\begin{array}{lll}0 & 0 & 0 \\ 0 & 0 & 0\end{array}\right)\left(\begin{array}{ccc}x y & -x x & 0 \\ 0 & 0 & 0\end{array}\right)$

$\left(\begin{array}{ccc}x x & 0 & 0 \\ 0 & x x & 0 \\ 0 & 0 & z z\end{array}\right)\left(\begin{array}{ccc}0 & 0 & 0 \\ 0 & 0 & 0 \\ 0 & 0 & 0\end{array}\right)\left(\begin{array}{ccc}0 & 0 & 0 \\ 0 & 0 & 0 \\ 0 & 0 & 0\end{array}\right)\left(\begin{array}{ccc}x x & x y & 0 \\ x y & -x x & 0 \\ 0 & 0 & 0\end{array}\right)$

$\left(\begin{array}{ccc}0 & x y & 0 \\ -x y & 0 & 0 \\ 0 & 0 & 0\end{array}\right)\left(\begin{array}{lll}0 & 0 & 0 \\ 0 & 0 & 0 \\ 0 & 0 & 0\end{array}\right)\left(\begin{array}{lll}0 & 0 & 0 \\ 0 & 0 & 0 \\ 0 & 0 & 0\end{array}\right)\left(\begin{array}{lll}0 & 0 & 0 \\ 0 & 0 & 0 \\ 0 & 0 & 0\end{array}\right)$

\section{$\mathrm{n}=2$}

$C_{4 h}\left(C_{2 h}\right)=4_{z} / m_{z}\left(2_{z} / m_{z}\right)=4_{z}^{\prime} / m_{z}$

$\left(\begin{array}{ccc}x x & x y & 0 \\ -x y & x x & 0 \\ 0 & 0 & z z\end{array}\right)\left(\begin{array}{ccc}0 & 0 & 0 \\ 0 & 0 & 0 \\ 0 & 0 & 0\end{array}\right)\left(\begin{array}{ccc}x x & x y & 0 \\ x y & -x x & 0 \\ 0 & 0 & 0\end{array}\right)\left(\begin{array}{ccc}0 & 0 & 0 \\ 0 & 0 & 0 \\ 0 & 0 & 0\end{array}\right)$

$\left(\begin{array}{ccc}x x & 0 & 0 \\ 0 & x x & 0 \\ 0 & 0 & z z\end{array}\right)\left(\begin{array}{ccc}0 & 0 & 0 \\ 0 & 0 & 0 \\ 0 & 0 & 0\end{array}\right)\left(\begin{array}{ccc}x x & x y & 0 \\ x y & -x x & 0 \\ 0 & 0 & 0\end{array}\right)\left(\begin{array}{ccc}0 & 0 & 0 \\ 0 & 0 & 0 \\ 0 & 0 & 0\end{array}\right)$

$\left(\begin{array}{ccc}0 & x y & 0 \\ -x y & 0 & 0 \\ 0 & 0 & 0\end{array}\right)\left(\begin{array}{lll}0 & 0 & 0 \\ 0 & 0 & 0 \\ 0 & 0 & 0\end{array}\right)\left(\begin{array}{lll}0 & 0 & 0 \\ 0 & 0 & 0 \\ 0 & 0 & 0\end{array}\right)\left(\begin{array}{lll}0 & 0 & 0 \\ 0 & 0 & 0 \\ 0 & 0 & 0\end{array}\right)$ $\left(\begin{array}{ccc}0 & x y & 0 \\ -x y & 0 & 0 \\ 0 & 0 & 0\end{array}\right)\left(\begin{array}{lll}0 & 0 & 0 \\ 0 & 0 & 0 \\ 0 & 0 & 0\end{array}\right)\left(\begin{array}{lll}0 & 0 & 0 \\ 0 & 0 & 0 \\ 0 & 0 & 0\end{array}\right)\left(\begin{array}{lll}0 & 0 & 0 \\ 0 & 0 & 0 \\ 0 & 0 & 0\end{array}\right)$

$\mathrm{n}>2$

$\mathbf{C}_{\infty \mathbf{h}}\left(\mathbf{C}_{\infty}\right)=\infty / \mathbf{m}^{\prime}$

$\left(\begin{array}{ccc}x x & x y & 0 \\ -x y & x x & 0 \\ 0 & 0 & z z\end{array}\right)\left(\begin{array}{lll}0 & 0 & 0 \\ 0 & 0 & 0 \\ 0 & 0 & 0\end{array}\right)\left(\begin{array}{ccc}0 & 0 & 0 \\ 0 & 0 & 0 \\ 0 & 0 & 0\end{array}\right)\left(\begin{array}{ccc}x x & x y & 0 \\ -x y & x x & 0 \\ 0 & 0 & z z\end{array}\right)$

$\left(\begin{array}{ccc}x x & 0 & 0 \\ 0 & x x & 0 \\ 0 & 0 & z z\end{array}\right)\left(\begin{array}{lll}0 & 0 & 0 \\ 0 & 0 & 0 \\ 0 & 0 & 0\end{array}\right)\left(\begin{array}{ccc}0 & 0 & 0 \\ 0 & 0 & 0 \\ 0 & 0 & 0\end{array}\right)\left(\begin{array}{ccc}x x & 0 & 0 \\ 0 & x x & 0 \\ 0 & 0 & z z\end{array}\right)$

$\left(\begin{array}{ccc}0 & x y & 0 \\ -x y & 0 & 0 \\ 0 & 0 & 0\end{array}\right)\left(\begin{array}{lll}0 & 0 & 0 \\ 0 & 0 & 0 \\ 0 & 0 & 0\end{array}\right)\left(\begin{array}{lll}0 & 0 & 0 \\ 0 & 0 & 0 \\ 0 & 0 & 0\end{array}\right)\left(\begin{array}{ccc}0 & x y & 0 \\ -x y & 0 & 0 \\ 0 & 0 & 0\end{array}\right)$

$n>2$

\section{$\mathbf{C}_{\infty h} \mathbf{1}^{\prime}=\infty / \mathbf{m} \mathbf{1}^{\prime}$}

$\left(\begin{array}{ccc}x x & x y & 0 \\ -x y & x x & 0 \\ 0 & 0 & z z\end{array}\right)\left(\begin{array}{ccc}0 & 0 & 0 \\ 0 & 0 & 0 \\ 0 & 0 & 0\end{array}\right)\left(\begin{array}{lll}0 & 0 & 0 \\ 0 & 0 & 0 \\ 0 & 0 & 0\end{array}\right)\left(\begin{array}{lll}0 & 0 & 0 \\ 0 & 0 & 0 \\ 0 & 0 & 0\end{array}\right)$

$\left(\begin{array}{ccc}x x & 0 & 0 \\ 0 & x x & 0 \\ 0 & 0 & z z\end{array}\right)\left(\begin{array}{lll}0 & 0 & 0 \\ 0 & 0 & 0 \\ 0 & 0 & 0\end{array}\right)\left(\begin{array}{lll}0 & 0 & 0 \\ 0 & 0 & 0 \\ 0 & 0 & 0\end{array}\right)\left(\begin{array}{lll}0 & 0 & 0 \\ 0 & 0 & 0 \\ 0 & 0 & 0\end{array}\right)$

$n>2$

$\mathbf{C}_{\infty \mathrm{h}} \mathbf{1}^{\prime}=\infty / \mathrm{m} \mathbf{1}^{\prime}$

$\left(\begin{array}{ccc}x x & x y & 0 \\ -x y & x x & 0 \\ 0 & 0 & z z\end{array}\right)\left(\begin{array}{ccc}0 & 0 & 0 \\ 0 & 0 & 0 \\ 0 & 0 & 0\end{array}\right)\left(\begin{array}{ccc}0 & 0 & 0 \\ 0 & 0 & 0 \\ 0 & 0 & 0\end{array}\right)\left(\begin{array}{lll}0 & 0 & 0 \\ 0 & 0 & 0 \\ 0 & 0 & 0\end{array}\right)$

$\left(\begin{array}{ccc}\mathrm{xx} & 0 & 0 \\ 0 & \mathrm{xx} & 0 \\ 0 & 0 & \mathrm{zz}\end{array}\right)\left(\begin{array}{lll}0 & 0 & 0 \\ 0 & 0 & 0 \\ 0 & 0 & 0\end{array}\right)\left(\begin{array}{lll}0 & 0 & 0 \\ 0 & 0 & 0 \\ 0 & 0 & 0\end{array}\right)\left(\begin{array}{lll}0 & 0 & 0 \\ 0 & 0 & 0 \\ 0 & 0 & 0\end{array}\right)$

$\left(\begin{array}{ccc}0 & x y & 0 \\ -x y & 0 & 0 \\ 0 & 0 & 0\end{array}\right)\left(\begin{array}{lll}0 & 0 & 0 \\ 0 & 0 & 0 \\ 0 & 0 & 0\end{array}\right)\left(\begin{array}{lll}0 & 0 & 0 \\ 0 & 0 & 0 \\ 0 & 0 & 0\end{array}\right)\left(\begin{array}{lll}0 & 0 & 0 \\ 0 & 0 & 0 \\ 0 & 0 & 0\end{array}\right)$ 
$\mathbf{D}_{\mathrm{n}}\left(\mathbf{C}_{\mathrm{n}}\right)$ $n=1$

$V^{2} \quad\left(\begin{array}{ccc}x x & 0 & x z \\ 0 & y y & 0 \\ z x & 0 & z z\end{array}\right)\left(\begin{array}{ccc}x x & 0 & x z \\ 0 & y y & 0 \\ z x & 0 & z z\end{array}\right)\left(\begin{array}{ccc}0 & x y & 0 \\ y x & 0 & y z \\ 0 & z y & 0\end{array}\right)\left(\begin{array}{ccc}0 & x y & 0 \\ y x & 0 & y z \\ 0 & z y & 0\end{array}\right)$ $\left[V^{2}\right] \quad\left(\begin{array}{ccc}x x & 0 & x z \\ 0 & y y & 0 \\ x z & 0 & z z\end{array}\right)\left(\begin{array}{ccc}x x & 0 & x z \\ 0 & y y & 0 \\ x z & 0 & z z\end{array}\right)\left(\begin{array}{ccc}0 & x y & 0 \\ x y & 0 & y z \\ 0 & y z & 0\end{array}\right)\left(\begin{array}{ccc}0 & x y & 0 \\ x y & 0 & y z \\ 0 & y z & 0\end{array}\right)$

$\left\{V^{2}\right\} \quad\left(\begin{array}{ccc}0 & 0 & x z \\ 0 & 0 & 0 \\ -x z & 0 & 0\end{array}\right)\left(\begin{array}{ccc}0 & 0 & x z \\ 0 & 0 & 0 \\ -x z & 0 & 0\end{array}\right)\left(\begin{array}{ccc}0 & x y & 0 \\ -x y & 0 & y z \\ 0 & -y z & 0\end{array}\right)\left(\begin{array}{ccc}0 & x y & 0 \\ -x y & 0 & y z \\ 0 & -y z & 0\end{array}\right)$ $\left(\begin{array}{lll}-x z & 0 & 0\end{array}\right)\left(\begin{array}{ccc}-x z & 0 & 0\end{array}\right)\left(\begin{array}{ccc}0 & -y z & 0\end{array}\right)\left(\begin{array}{ccc}0 & -y z & 0\end{array}\right)$

$\mathbf{D}_{2 \mathrm{n}}\left(\mathbf{D}_{\mathrm{n}}\right)$

$$
\mathrm{n}=1
$$

$D_{2 n}\left(D_{n}\right) \quad D_{2}\left(D_{1}\right)=2 \times{ }^{2} \mathbf{y} 2\left(\mathbf{2}_{y}\right)=2 x^{\prime} \mathbf{2}_{y} \mathbf{2}^{\prime}$

$\mathrm{V}^{2} \quad\left(\begin{array}{ccc}\mathrm{xx} & 0 & 0 \\ 0 & \mathrm{yy} & 0 \\ 0 & 0 & \mathrm{zz}\end{array}\right)\left(\begin{array}{ccc}\mathrm{xx} & 0 & 0 \\ 0 & \mathrm{yy} & 0 \\ 0 & 0 & \mathrm{zz}\end{array}\right)\left(\begin{array}{ccc}0 & 0 & \mathrm{xz} \\ 0 & 0 & 0 \\ \mathrm{zx} & 0 & 0\end{array}\right)\left(\begin{array}{ccc}0 & 0 & \mathrm{xz} \\ 0 & 0 & 0 \\ \mathrm{zx} & 0 & 0\end{array}\right)$

$\left[V^{2}\right] \quad\left(\begin{array}{ccc}x x & 0 & 0 \\ 0 & y y & 0 \\ 0 & 0 & z z\end{array}\right)\left(\begin{array}{ccc}x x & 0 & 0 \\ 0 & y y & 0 \\ 0 & 0 & z z\end{array}\right)\left(\begin{array}{ccc}0 & 0 & x z \\ 0 & 0 & 0 \\ x z & 0 & 0\end{array}\right)\left(\begin{array}{ccc}0 & 0 & x z \\ 0 & 0 & 0 \\ x z & 0 & 0\end{array}\right)$

$\left\{V^{2}\right\} \quad\left(\begin{array}{lll}0 & 0 & 0 \\ 0 & 0 & 0 \\ 0 & 0 & 0\end{array}\right)\left(\begin{array}{lll}0 & 0 & 0 \\ 0 & 0 & 0 \\ 0 & 0 & 0\end{array}\right)\left(\begin{array}{ccc}0 & 0 & x z \\ 0 & 0 & 0 \\ -x z & 0 & 0\end{array}\right)\left(\begin{array}{ccc}0 & 0 & x z \\ 0 & 0 & 0 \\ -x z & 0 & 0\end{array}\right)$

$\mathrm{n}=1$

$\mathrm{C}_{\mathrm{nv}}\left(\mathrm{C}_{\mathrm{n}}\right)$

$\mathbf{C}_{1 \mathrm{v}}\left(\mathbf{C}_{1}\right)=\mathbf{m}_{\mathrm{y}}(\mathbf{1})=\mathbf{m}_{\mathrm{y}^{\prime}}$

$V^{2} \quad\left(\begin{array}{ccc}x x & 0 & x z \\ 0 & y y & 0 \\ z x & 0 & z z\end{array}\right)\left(\begin{array}{ccc}0 & x y & 0 \\ y x & 0 & y z \\ 0 & z y & 0\end{array}\right)\left(\begin{array}{ccc}0 & x y & 0 \\ y x & 0 & y z \\ 0 & z y & 0\end{array}\right)\left(\begin{array}{ccc}x x & 0 & x z \\ 0 & y y & 0 \\ z x & 0 & z z\end{array}\right)$

$\left[V^{2}\right] \quad\left(\begin{array}{ccc}x x & 0 & x z \\ 0 & y y & 0 \\ x z & 0 & z z\end{array}\right)\left(\begin{array}{ccc}0 & x y & 0 \\ x y & 0 & y z \\ 0 & y z & 0\end{array}\right)\left(\begin{array}{ccc}0 & x y & 0 \\ x y & 0 & y z \\ 0 & y z & 0\end{array}\right)\left(\begin{array}{ccc}x x & 0 & x z \\ 0 & y y & 0 \\ x z & 0 & z z\end{array}\right)$

$\left\{V^{2}\right\} \quad\left(\begin{array}{ccc}0 & 0 & x z \\ 0 & 0 & 0 \\ -x z & 0 & 0\end{array}\right)\left(\begin{array}{ccc}0 & x y & 0 \\ -x y & 0 & y z \\ 0 & -y z & 0\end{array}\right)\left(\begin{array}{ccc}0 & x y & 0 \\ -x y & 0 & y z \\ 0 & -y z & 0\end{array}\right)\left(\begin{array}{ccc}0 & 0 & x z \\ 0 & 0 & 0 \\ -x z & 0 & 0\end{array}\right)\left(\begin{array}{ccc}0 & 0 & 0 \\ 0 & 0 & 0 \\ 0 & 0 & 0\end{array}\right)\left(\begin{array}{ccc}0 & x y & 0 \\ -x y & 0 & 0 \\ 0 & 0 & 0\end{array}\right)\left(\begin{array}{ccc}0 & x y & 0 \\ -x y & 0 & 0 \\ 0 & 0 & 0\end{array}\right)\left(\begin{array}{lll}0 & 0 & 0 \\ 0 & 0 & 0 \\ 0 & 0 & 0\end{array}\right)$
$D_{2}\left(C_{2}\right)=2{ }_{x} 2_{y} 2_{z}\left(2_{z}\right)=2_{x}^{\prime} 2_{y}^{\prime} 2_{z}$

$\left(\begin{array}{ccc}x x & 0 & 0 \\ 0 & y y & 0 \\ 0 & 0 & z z\end{array}\right)\left(\begin{array}{ccc}x x & 0 & 0 \\ 0 & y y & 0 \\ 0 & 0 & z z\end{array}\right)\left(\begin{array}{ccc}0 & x y & 0 \\ y x & 0 & 0 \\ 0 & 0 & 0\end{array}\right)\left(\begin{array}{ccc}0 & x y & 0 \\ y x & 0 & 0 \\ 0 & 0 & 0\end{array}\right)$

$\left(\begin{array}{ccc}x x & 0 & 0 \\ 0 & y y & 0 \\ 0 & 0 & z z\end{array}\right)\left(\begin{array}{ccc}x x & 0 & 0 \\ 0 & y y & 0 \\ 0 & 0 & z z\end{array}\right)\left(\begin{array}{ccc}0 & x y & 0 \\ x y & 0 & 0 \\ 0 & 0 & 0\end{array}\right)\left(\begin{array}{ccc}0 & x y & 0 \\ x y & 0 & 0 \\ 0 & 0 & 0\end{array}\right)$

$\left(\begin{array}{lll}0 & 0 & 0 \\ 0 & 0 & 0 \\ 0 & 0 & 0\end{array}\right)\left(\begin{array}{lll}0 & 0 & 0 \\ 0 & 0 & 0 \\ 0 & 0 & 0\end{array}\right)\left(\begin{array}{ccc}0 & x y & 0 \\ -x y & 0 & 0 \\ 0 & 0 & 0\end{array}\right)\left(\begin{array}{ccc}0 & x y & 0 \\ -x y & 0 & 0 \\ 0 & 0 & 0\end{array}\right)$

$$
\mathrm{n}=2
$$

$D_{4}\left(D_{2}\right)=4_{z} 2_{x} 2_{x y}\left(2 x{ }^{y} 2_{z}\right)=4_{z}{ }^{\prime} 2_{x} 2_{x y}{ }^{\prime}$

$\left(\begin{array}{ccc}x x & 0 & 0 \\ 0 & x x & 0 \\ 0 & 0 & z z\end{array}\right)\left(\begin{array}{ccc}x x & 0 & 0 \\ 0 & x x & 0 \\ 0 & 0 & z z\end{array}\right)\left(\begin{array}{ccc}x x & 0 & 0 \\ 0 & -x x & 0 \\ 0 & 0 & 0\end{array}\right)\left(\begin{array}{ccc}x x & 0 & 0 \\ 0 & -x x & 0 \\ 0 & 0 & 0\end{array}\right)$

$\left(\begin{array}{ccc}x x & 0 & 0 \\ 0 & x x & 0 \\ 0 & 0 & z z\end{array}\right)\left(\begin{array}{ccc}x x & 0 & 0 \\ 0 & x x & 0 \\ 0 & 0 & z z\end{array}\right)\left(\begin{array}{ccc}x x & 0 & 0 \\ 0 & -x x & 0 \\ 0 & 0 & 0\end{array}\right)\left(\begin{array}{ccc}x x & 0 & 0 \\ 0 & -x x & 0 \\ 0 & 0 & 0\end{array}\right)$

$\left(\begin{array}{lll}0 & 0 & 0 \\ 0 & 0 & 0 \\ 0 & 0 & 0\end{array}\right)\left(\begin{array}{lll}0 & 0 & 0 \\ 0 & 0 & 0 \\ 0 & 0 & 0\end{array}\right)\left(\begin{array}{lll}0 & 0 & 0 \\ 0 & 0 & 0 \\ 0 & 0 & 0\end{array}\right)\left(\begin{array}{lll}0 & 0 & 0 \\ 0 & 0 & 0 \\ 0 & 0 & 0\end{array}\right)$

$$
\mathrm{n}=2
$$

$\mathbf{C}_{2 \mathrm{v}}\left(\mathbf{C}_{2}\right)=\mathbf{2}_{\mathrm{z}} \mathbf{m}_{\mathrm{x}} \mathbf{m}_{\mathrm{y}}\left(\mathbf{2}_{\mathrm{z}}\right)=\mathbf{2}_{\mathrm{z}} \mathbf{m}_{\mathrm{x}}{ }^{\prime} \mathbf{m}_{\mathrm{y}}{ }^{\prime}$

$\left(\begin{array}{ccc}x x & 0 & 0 \\ 0 & y y & 0 \\ 0 & 0 & z z\end{array}\right)\left(\begin{array}{ccc}0 & x y & 0 \\ y x & 0 & 0 \\ 0 & 0 & 0\end{array}\right)\left(\begin{array}{ccc}0 & x y & 0 \\ y x & 0 & 0 \\ 0 & 0 & 0\end{array}\right)\left(\begin{array}{ccc}x x & 0 & 0 \\ 0 & y y & 0 \\ 0 & 0 & z z\end{array}\right)$

$\left(\begin{array}{ccc}x x & 0 & 0 \\ 0 & y y & 0\end{array}\right)\left(\begin{array}{ccc}0 & x y & 0\end{array}\right)\left(\begin{array}{ccc}0 & x y & 0\end{array}\right)\left(\begin{array}{ccc}x x & 0 & 0\end{array}\right)$

$\left(\begin{array}{ccc}x x & 0 & 0 \\ 0 & y y & 0 \\ 0 & 0 & z z\end{array}\right)\left(\begin{array}{ccc}0 & x y & 0 \\ x y & 0 & 0 \\ 0 & 0 & 0\end{array}\right)\left(\begin{array}{ccc}0 & x y & 0 \\ x y & 0 & 0 \\ 0 & 0 & 0\end{array}\right)\left(\begin{array}{ccc}x x & 0 & 0 \\ 0 & y y & 0 \\ 0 & 0 & z z\end{array}\right)$ $\left(\begin{array}{ccc}x x & 0 & 0 \\ 0 & x x & 0 \\ 0 & 0 & z z\end{array}\right)\left(\begin{array}{ccc}0 & 0 & 0 \\ 0 & 0 & 0 \\ 0 & 0 & 0\end{array}\right)\left(\begin{array}{ccc}0 & 0 & 0 \\ 0 & 0 & 0 \\ 0 & 0 & 0\end{array}\right)\left(\begin{array}{ccc}x x & 0 & 0 \\ 0 & x x & 0 \\ 0 & 0 & z z\end{array}\right)$

$\left(\begin{array}{lll}0 & 0 & 0 \\ 0 & 0 & 0 \\ 0 & 0 & 0\end{array}\right)\left(\begin{array}{ccc}0 & x y & 0 \\ -x y & 0 & 0 \\ 0 & 0 & 0\end{array}\right)\left(\begin{array}{ccc}0 & x y & 0 \\ -x y & 0 & 0 \\ 0 & 0 & 0\end{array}\right)\left(\begin{array}{lll}0 & 0 & 0 \\ 0 & 0 & 0 \\ 0 & 0 & 0\end{array}\right)$

$D_{(\mathrm{C})}$

$\mathrm{D}_{\infty}\left(\mathbf{C}_{\infty}\right)=\infty \mathbf{2}^{\prime}$
$\left(\begin{array}{ccc}\mathrm{xx} & 0 & 0 \\ 0 & \mathrm{xx} & 0 \\ 0 & 0 & \mathrm{zz}\end{array}\right)\left(\begin{array}{ccc}\mathrm{xx} & 0 & 0 \\ 0 & \mathrm{xx} & 0 \\ 0 & 0 & \mathrm{zz}\end{array}\right)\left(\begin{array}{ccc}0 & \mathrm{xy} & 0 \\ -\mathrm{xy} & 0 & 0 \\ 0 & 0 & 0\end{array}\right)\left(\begin{array}{ccc}0 & \mathrm{xy} & 0 \\ -\mathrm{xy} & 0 & 0 \\ 0 & 0 & 0\end{array}\right)$

$\left(\begin{array}{ccc}x x & 0 & 0 \\ 0 & x x & 0 \\ 0 & 0 & z z\end{array}\right)\left(\begin{array}{ccc}x x & 0 & 0 \\ 0 & x x & 0 \\ 0 & 0 & z z\end{array}\right)\left(\begin{array}{ccc}0 & 0 & 0 \\ 0 & 0 & 0 \\ 0 & 0 & 0\end{array}\right)\left(\begin{array}{lll}0 & 0 & 0 \\ 0 & 0 & 0 \\ 0 & 0 & 0\end{array}\right)$

$\left(\begin{array}{lll}0 & 0 & 0 \\ 0 & 0 & 0 \\ 0 & 0 & 0\end{array}\right)\left(\begin{array}{lll}0 & 0 & 0 \\ 0 & 0 & 0 \\ 0 & 0 & 0\end{array}\right)\left(\begin{array}{ccc}0 & x y & 0 \\ -x y & 0 & 0 \\ 0 & 0 & 0\end{array}\right)\left(\begin{array}{ccc}0 & x y & 0 \\ -x y & 0 & 0 \\ 0 & 0 & 0\end{array}\right)$

$$
\begin{aligned}
n & >2 \\
D_{\infty} \mathbf{1}^{\prime} & =\infty \mathbf{2 1}
\end{aligned}
$$

$\left(\begin{array}{ccc}x x & 0 & 0 \\ 0 & x x & 0 \\ 0 & 0 & z z\end{array}\right)\left(\begin{array}{ccc}x x & 0 & 0 \\ 0 & x x & 0 \\ 0 & 0 & z z\end{array}\right)\left(\begin{array}{ccc}0 & 0 & 0 \\ 0 & 0 & 0 \\ 0 & 0 & 0\end{array}\right)\left(\begin{array}{ccc}0 & 0 & 0 \\ 0 & 0 & 0 \\ 0 & 0 & 0\end{array}\right)$

$\left(\begin{array}{ccc}x x & 0 & 0 \\ 0 & x x & 0 \\ 0 & 0 & z z\end{array}\right)\left(\begin{array}{ccc}x x & 0 & 0 \\ 0 & x x & 0 \\ 0 & 0 & z z\end{array}\right)\left(\begin{array}{ccc}0 & 0 & 0 \\ 0 & 0 & 0 \\ 0 & 0 & 0\end{array}\right)\left(\begin{array}{lll}0 & 0 & 0 \\ 0 & 0 & 0 \\ 0 & 0 & 0\end{array}\right)$

$\left(\begin{array}{lll}0 & 0 & 0 \\ 0 & 0 & 0 \\ 0 & 0 & 0\end{array}\right)\left(\begin{array}{lll}0 & 0 & 0 \\ 0 & 0 & 0 \\ 0 & 0 & 0\end{array}\right)\left(\begin{array}{lll}0 & 0 & 0 \\ 0 & 0 & 0 \\ 0 & 0 & 0\end{array}\right)\left(\begin{array}{lll}0 & 0 & 0 \\ 0 & 0 & 0 \\ 0 & 0 & 0\end{array}\right)$

$n>2$

$\mathbf{C}_{\infty \mathrm{vv}}\left(\mathbf{C}_{\infty}\right)=\infty \mathbf{m}^{\prime} \mathbf{m}^{\prime}$

$\left(\begin{array}{ccc}x x & 0 & 0 \\ 0 & x x & 0 \\ 0 & 0 & z z\end{array}\right)\left(\begin{array}{ccc}0 & x y & 0 \\ -x y & 0 & 0 \\ 0 & 0 & 0\end{array}\right)\left(\begin{array}{ccc}0 & x y & 0 \\ -x y & 0 & 0 \\ 0 & 0 & 0\end{array}\right)\left(\begin{array}{ccc}x x & 0 & 0 \\ 0 & x x & 0 \\ 0 & 0 & z z\end{array}\right)$ 
$n=1$
$\mathbf{C}_{2 n v}\left(C_{n v}\right) \quad \mathbf{C}_{2 v}\left(\mathbf{C}_{1 \mathrm{v}}\right)=\mathbf{2}_{\mathrm{z}} \mathbf{m}_{\mathrm{x}} \mathbf{m}_{\mathrm{y}}\left(\mathbf{m}_{\mathrm{y}}\right)=\mathbf{2}_{\mathrm{z}} \mathbf{m}_{\mathrm{x}} \mathbf{m}_{\mathrm{y}}$

$\mathrm{V}^{2} \quad\left(\begin{array}{ccc}\mathrm{xx} & 0 & 0 \\ 0 & y y & 0 \\ 0 & 0 & \mathrm{zz}\end{array}\right)\left(\begin{array}{ccc}0 & \mathrm{xy} & 0 \\ \mathrm{yx} & 0 & 0 \\ 0 & 0 & 0\end{array}\right)\left(\begin{array}{ccc}0 & 0 & \mathrm{xz} \\ 0 & 0 & 0 \\ \mathrm{zx} & 0 & 0\end{array}\right)\left(\begin{array}{ccc}0 & 0 & 0 \\ 0 & 0 & y z \\ 0 & z y & 0\end{array}\right)$

$\left[V^{2}\right] \quad\left(\begin{array}{ccc}x x & 0 & 0 \\ 0 & y y & 0 \\ 0 & 0 & z z\end{array}\right)\left(\begin{array}{ccc}0 & x y & 0 \\ x y & 0 & 0 \\ 0 & 0 & 0\end{array}\right)\left(\begin{array}{ccc}0 & 0 & x z \\ 0 & 0 & 0 \\ x z & 0 & 0\end{array}\right)\left(\begin{array}{ccc}0 & 0 & 0 \\ 0 & 0 & y z \\ 0 & y z & 0\end{array}\right)$

$\left\{\mathrm{V}^{2}\right\} \quad\left(\begin{array}{lll}0 & 0 & 0 \\ 0 & 0 & 0 \\ 0 & 0 & 0\end{array}\right)\left(\begin{array}{ccc}0 & x y & 0 \\ -x y & 0 & 0 \\ 0 & 0 & 0\end{array}\right)\left(\begin{array}{ccc}0 & 0 & x z \\ 0 & 0 & 0 \\ -x z & 0 & 0\end{array}\right)\left(\begin{array}{ccc}0 & 0 & 0 \\ 0 & 0 & y z \\ 0 & -y z & 0\end{array}\right)$

$$
\mathrm{n}=1
$$

$\mathbf{D}_{\mathrm{nd}}\left(\mathbf{S}_{2 \mathrm{n}}\right) \quad \mathbf{D}_{1 \mathrm{~d}}\left(\mathbf{S}_{2}\right)=\mathbf{2}_{\mathrm{y}} / \mathbf{m}_{\mathrm{y}}(\overline{\mathbf{1}})=\mathbf{2}_{\mathrm{y}}{ }^{\prime} / \mathbf{m}_{\mathrm{y}}{ }^{\prime}$

$\mathrm{V}^{2} \quad\left(\begin{array}{ccc}x x & 0 & x z \\ 0 & y y & 0 \\ z x & 0 & z z\end{array}\right)\left(\begin{array}{ccc}0 & 0 & 0 \\ 0 & 0 & 0 \\ 0 & 0 & 0\end{array}\right)\left(\begin{array}{ccc}0 & x y & 0 \\ y x & 0 & y z \\ 0 & z y & 0\end{array}\right)\left(\begin{array}{ccc}0 & 0 & 0 \\ 0 & 0 & 0 \\ 0 & 0 & 0\end{array}\right)$

$\left[V^{2}\right] \quad\left(\begin{array}{ccc}x x & 0 & x z \\ 0 & y y & 0 \\ x z & 0 & z z\end{array}\right)\left(\begin{array}{ccc}0 & 0 & 0 \\ 0 & 0 & 0 \\ 0 & 0 & 0\end{array}\right)\left(\begin{array}{ccc}0 & x y & 0 \\ x y & 0 & y z \\ 0 & y z & 0\end{array}\right)\left(\begin{array}{lll}0 & 0 & 0 \\ 0 & 0 & 0 \\ 0 & 0 & 0\end{array}\right)$

$\left\{V^{2}\right\} \quad\left(\begin{array}{ccc}0 & 0 & x z \\ 0 & 0 & 0 \\ -x z & 0 & 0\end{array}\right)\left(\begin{array}{lll}0 & 0 & 0 \\ 0 & 0 & 0 \\ 0 & 0 & 0\end{array}\right)\left(\begin{array}{ccc}0 & x y & 0 \\ -x y & 0 & y z \\ 0 & -y z & 0\end{array}\right)\left(\begin{array}{lll}0 & 0 & 0 \\ 0 & 0 & 0 \\ 0 & 0 & 0\end{array}\right)$

$$
\mathrm{n}=1
$$

$\mathbf{D}_{\mathrm{nd}}\left(\mathbf{D}_{\mathrm{n}}\right)$

$$
D_{1 d}\left(D_{1}\right)=2_{y} / m_{y}\left(2_{y}\right)=2_{y} / m_{y}{ }^{\prime}
$$

ae

$v^{2} \quad\left(\begin{array}{ccc}x x & 0 & x z \\ 0 & y y & 0 \\ z x & 0 & z z\end{array}\right)\left(\begin{array}{ccc}0 & 0 & 0 \\ 0 & 0 & 0 \\ 0 & 0 & 0\end{array}\right)\left(\begin{array}{ccc}0 & 0 & 0 \\ 0 & 0 & 0 \\ 0 & 0 & 0\end{array}\right)\left(\begin{array}{ccc}x x & 0 & x z \\ 0 & y y & 0 \\ z x & 0 & z z\end{array}\right)$

$\left[V^{2}\right] \quad\left(\begin{array}{ccc}x x & 0 & x z \\ 0 & y y & 0 \\ x z & 0 & z z\end{array}\right)\left(\begin{array}{ccc}0 & 0 & 0 \\ 0 & 0 & 0 \\ 0 & 0 & 0\end{array}\right)\left(\begin{array}{ccc}0 & 0 & 0 \\ 0 & 0 & 0 \\ 0 & 0 & 0\end{array}\right)\left(\begin{array}{ccc}x x & 0 & x z \\ 0 & y y & 0 \\ x z & 0 & z z\end{array}\right)$

$\left\{V^{2}\right\} \quad\left(\begin{array}{ccc}0 & 0 & x z \\ 0 & 0 & 0 \\ -x z & 0 & 0\end{array}\right)\left(\begin{array}{lll}0 & 0 & 0 \\ 0 & 0 & 0 \\ 0 & 0 & 0\end{array}\right)\left(\begin{array}{lll}0 & 0 & 0 \\ 0 & 0 & 0 \\ 0 & 0 & 0\end{array}\right)\left(\begin{array}{ccc}0 & 0 & x z \\ 0 & 0 & 0 \\ -x z & 0 & 0\end{array}\right)$ $\mathrm{n}=2$

$C_{4 v}\left(C_{2 v}\right)=4_{z} m_{x} m_{x y}\left(2_{z} m_{x} m_{y}\right)=4_{z}{ }^{\prime} m_{x} m_{x y}$

$\left(\begin{array}{ccc}x x & 0 & 0 \\ 0 & x x & 0 \\ 0 & 0 & z z\end{array}\right)\left(\begin{array}{ccc}0 & x y & 0 \\ -x y & 0 & 0 \\ 0 & 0 & 0\end{array}\right)\left(\begin{array}{ccc}x x & 0 & 0 \\ 0 & -x x & 0 \\ 0 & 0 & 0\end{array}\right)\left(\begin{array}{ccc}0 & x y & 0 \\ x y & 0 & 0 \\ 0 & 0 & 0\end{array}\right)$

$\left(\begin{array}{ccc}x x & 0 & 0 \\ 0 & x x & 0 \\ 0 & 0 & z z\end{array}\right)\left(\begin{array}{ccc}0 & 0 & 0 \\ 0 & 0 & 0 \\ 0 & 0 & 0\end{array}\right)\left(\begin{array}{ccc}x x & 0 & 0 \\ 0 & -x x & 0 \\ 0 & 0 & 0\end{array}\right)\left(\begin{array}{ccc}0 & x y & 0 \\ x y & 0 & 0 \\ 0 & 0 & 0\end{array}\right)$

$\left(\begin{array}{lll}0 & 0 & 0 \\ 0 & 0 & 0 \\ 0 & 0 & 0\end{array}\right)\left(\begin{array}{ccc}0 & x y & 0 \\ -x y & 0 & 0 \\ 0 & 0 & 0\end{array}\right)\left(\begin{array}{lll}0 & 0 & 0 \\ 0 & 0 & 0 \\ 0 & 0 & 0\end{array}\right)\left(\begin{array}{lll}0 & 0 & 0 \\ 0 & 0 & 0 \\ 0 & 0 & 0\end{array}\right)$

$$
\mathrm{n}=2
$$

$$
\mathbf{D}_{2 \mathrm{~d}}\left(\mathbf{S}_{4}\right)=\overline{\mathbf{4}}_{\mathrm{z}} \mathbf{m}_{\mathrm{x}} \mathbf{2}_{\mathrm{xy}}\left(\overline{\mathbf{4}}_{\mathrm{z}}\right)=\overline{\mathbf{4}}_{\mathrm{z}} \mathbf{m}_{\mathrm{x}} \mathbf{2}_{\mathrm{xy}}{ }^{\prime}
$$

$\left(\begin{array}{ccc}x x & 0 & 0 \\ 0 & x x & 0 \\ 0 & 0 & z z\end{array}\right)\left(\begin{array}{ccc}0 & x y & 0 \\ x y & 0 & 0 \\ 0 & 0 & 0\end{array}\right)\left(\begin{array}{ccc}0 & x y & 0 \\ -x y & 0 & 0 \\ 0 & 0 & 0\end{array}\right)\left(\begin{array}{ccc}x x & 0 & 0 \\ 0 & -x x & 0 \\ 0 & 0 & 0\end{array}\right)$

$\left(\begin{array}{ccc}x x & 0 & 0 \\ 0 & x x & 0 \\ 0 & 0 & z z\end{array}\right)\left(\begin{array}{ccc}0 & x y & 0 \\ x y & 0 & 0 \\ 0 & 0 & 0\end{array}\right)\left(\begin{array}{ccc}0 & 0 & 0 \\ 0 & 0 & 0 \\ 0 & 0 & 0\end{array}\right)\left(\begin{array}{ccc}x x & 0 & 0 \\ 0 & -x x & 0 \\ 0 & 0 & 0\end{array}\right)$

$\left(\begin{array}{lll}0 & 0 & 0 \\ 0 & 0 & 0 \\ 0 & 0 & 0\end{array}\right)\left(\begin{array}{lll}0 & 0 & 0 \\ 0 & 0 & 0 \\ 0 & 0 & 0\end{array}\right)\left(\begin{array}{ccc}0 & x y & 0 \\ -x y & 0 & 0 \\ 0 & 0 & 0\end{array}\right)\left(\begin{array}{lll}0 & 0 & 0 \\ 0 & 0 & 0 \\ 0 & 0 & 0\end{array}\right)$

$$
\mathrm{n}=2
$$

$D_{2 d}\left(D_{2}\right)=\overline{4}_{z} m_{x} 2_{x y}\left(2_{x y} \mathbf{2}_{\bar{x} y} \mathbf{2}_{z}\right)=\overline{4}_{z}{ }^{\prime} m_{x}{ }^{\prime} \mathbf{2}_{x y}$

$\left(\begin{array}{ccc}x x & 0 & 0 \\ 0 & x x & 0 \\ 0 & 0 & z z\end{array}\right)\left(\begin{array}{ccc}0 & x y & 0 \\ x y & 0 & 0 \\ 0 & 0 & 0\end{array}\right)\left(\begin{array}{ccc}0 & x y & 0 \\ x y & 0 & 0 \\ 0 & 0 & 0\end{array}\right)\left(\begin{array}{ccc}x x & 0 & 0 \\ 0 & x x & 0 \\ 0 & 0 & z z\end{array}\right)$

$\left(\begin{array}{ccc}x x & 0 & 0 \\ 0 & x x & 0 \\ 0 & 0 & z z\end{array}\right)\left(\begin{array}{ccc}0 & x y & 0 \\ x y & 0 & 0 \\ 0 & 0 & 0\end{array}\right)\left(\begin{array}{ccc}0 & x y & 0 \\ x y & 0 & 0 \\ 0 & 0 & 0\end{array}\right)\left(\begin{array}{ccc}x x & 0 & 0 \\ 0 & x x & 0 \\ 0 & 0 & z z\end{array}\right)$

$\left(\begin{array}{lll}0 & 0 & 0 \\ 0 & 0 & 0 \\ 0 & 0 & 0\end{array}\right)\left(\begin{array}{lll}0 & 0 & 0 \\ 0 & 0 & 0 \\ 0 & 0 & 0\end{array}\right)\left(\begin{array}{lll}0 & 0 & 0 \\ 0 & 0 & 0 \\ 0 & 0 & 0\end{array}\right)\left(\begin{array}{lll}0 & 0 & 0 \\ 0 & 0 & 0 \\ 0 & 0 & 0\end{array}\right)$ $n>2$

$\mathrm{C}_{\infty \mathrm{V}} \mathbf{1}^{\prime}=\infty \mathrm{mm} \mathbf{1}^{\prime}$

$\left(\begin{array}{ccc}x x & 0 & 0 \\ 0 & x x & 0 \\ 0 & 0 & z z\end{array}\right)\left(\begin{array}{ccc}0 & x y & 0 \\ -x y & 0 & 0 \\ 0 & 0 & 0\end{array}\right)\left(\begin{array}{ccc}0 & 0 & 0 \\ 0 & 0 & 0 \\ 0 & 0 & 0\end{array}\right)\left(\begin{array}{ccc}0 & 0 & 0 \\ 0 & 0 & 0 \\ 0 & 0 & 0\end{array}\right)$

$\left(\begin{array}{ccc}x x & 0 & 0 \\ 0 & x x & 0 \\ 0 & 0 & z z\end{array}\right)\left(\begin{array}{lll}0 & 0 & 0 \\ 0 & 0 & 0 \\ 0 & 0 & 0\end{array}\right)\left(\begin{array}{lll}0 & 0 & 0 \\ 0 & 0 & 0 \\ 0 & 0 & 0\end{array}\right)\left(\begin{array}{lll}0 & 0 & 0 \\ 0 & 0 & 0 \\ 0 & 0 & 0\end{array}\right)$

$\left(\begin{array}{lll}0 & 0 & 0 \\ 0 & 0 & 0 \\ 0 & 0 & 0\end{array}\right)\left(\begin{array}{ccc}0 & x y & 0 \\ -x y & 0 & 0 \\ 0 & 0 & 0\end{array}\right)\left(\begin{array}{lll}0 & 0 & 0 \\ 0 & 0 & 0 \\ 0 & 0 & 0\end{array}\right)\left(\begin{array}{lll}0 & 0 & 0 \\ 0 & 0 & 0 \\ 0 & 0 & 0\end{array}\right)$

\section{$n>2$}

\section{$\mathbf{D}_{\infty h}\left(\mathbf{C}_{\infty h}\right)=\infty / \mathbf{m m}^{\prime} \mathbf{m}^{\prime}$}

$\left(\begin{array}{ccc}x x & 0 & 0 \\ 0 & x x & 0 \\ 0 & 0 & z z\end{array}\right)\left(\begin{array}{ccc}0 & 0 & 0 \\ 0 & 0 & 0 \\ 0 & 0 & 0\end{array}\right)\left(\begin{array}{ccc}0 & x y & 0 \\ -x y & 0 & 0 \\ 0 & 0 & 0\end{array}\right)\left(\begin{array}{ccc}0 & 0 & 0 \\ 0 & 0 & 0 \\ 0 & 0 & 0\end{array}\right)$

$\left(\begin{array}{ccc}x x & 0 & 0 \\ 0 & x x & 0 \\ 0 & 0 & z z\end{array}\right)\left(\begin{array}{lll}0 & 0 & 0 \\ 0 & 0 & 0 \\ 0 & 0 & 0\end{array}\right)\left(\begin{array}{lll}0 & 0 & 0 \\ 0 & 0 & 0 \\ 0 & 0 & 0\end{array}\right)\left(\begin{array}{lll}0 & 0 & 0 \\ 0 & 0 & 0 \\ 0 & 0 & 0\end{array}\right)$

$\left(\begin{array}{lll}0 & 0 & 0 \\ 0 & 0 & 0\end{array}\right)\left(\begin{array}{lll}0 & 0 & 0 \\ 0 & 0 & 0\end{array}\right)\left(\begin{array}{ccc}0 & x y & 0 \\ -x y & 0 & 0\end{array}\right)\left(\begin{array}{lll}0 & 0 & 0 \\ 0 & 0 & 0\end{array}\right)$ $\left(\begin{array}{lll}0 & 0 & 0 \\ 0 & 0 & 0\end{array}\right)\left(\begin{array}{lll}0 & 0 & 0 \\ 0 & 0 & 0\end{array}\right)\left(\begin{array}{ccc}-x y & 0 & 0 \\ 0 & 0 & 0\end{array}\right)\left(\begin{array}{lll}0 & 0 & 0 \\ 0 & 0 & 0 \\ 0 & 0 & 0\end{array}\right)$

\section{$\mathbf{D}_{\infty h}\left(\mathbf{D}_{\infty}\right)=\infty / \mathbf{m}^{\prime} \mathbf{m}^{\prime} \mathbf{m}^{\prime}$}

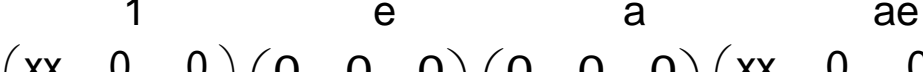
$\left(\begin{array}{ccc}x x & 0 & 0 \\ 0 & x x & 0\end{array}\right)\left(\begin{array}{ccc}0 & 0 & 0 \\ 0 & 0 & 0 \\ 0 & 0 & z z\end{array}\right)\left(\begin{array}{ccc}0 & 0 & 0 \\ 0 & 0 & 0 \\ 0 & 0 & 0\end{array}\right)\left(\begin{array}{ccc}x x & 0 & 0 \\ 0 & x x & 0\end{array}\right)$ $\left(\begin{array}{lll}0 & 0 & z z\end{array}\right)\left(\begin{array}{lll}0 & 0 & 0\end{array}\right)\left(\begin{array}{lll}0 & 0 & 0\end{array}\right)\left(\begin{array}{lll}0 & 0 & z z\end{array}\right)$

$\left(\begin{array}{ccc}x x & 0 & 0 \\ 0 & x x & 0 \\ 0 & 0 & z z\end{array}\right)\left(\begin{array}{lll}0 & 0 & 0 \\ 0 & 0 & 0 \\ 0 & 0 & 0\end{array}\right)\left(\begin{array}{ccc}0 & 0 & 0 \\ 0 & 0 & 0 \\ 0 & 0 & 0\end{array}\right)\left(\begin{array}{ccc}x x & 0 & 0 \\ 0 & x x & 0 \\ 0 & 0 & z z\end{array}\right)$

$\left(\begin{array}{lll}0 & 0 & 0 \\ 0 & 0 & 0 \\ 0 & 0 & 0\end{array}\right)\left(\begin{array}{lll}0 & 0 & 0 \\ 0 & 0 & 0 \\ 0 & 0 & 0\end{array}\right)\left(\begin{array}{lll}0 & 0 & 0 \\ 0 & 0 & 0 \\ 0 & 0 & 0\end{array}\right)\left(\begin{array}{lll}0 & 0 & 0 \\ 0 & 0 & 0 \\ 0 & 0 & 0\end{array}\right)$ 


$$
n=1
$$

$\mathbf{D}_{\text {nd }}\left(\mathbf{C}_{\mathrm{nv}}\right) \quad \mathbf{D}_{1 \mathrm{~d}}\left(\mathbf{C}_{1 \mathrm{v}}\right)=\mathbf{2}_{\mathrm{y}} / \mathrm{m}_{\mathrm{y}}\left(\mathrm{m}_{\mathrm{y}}\right)=\mathbf{2}_{\mathrm{y}}{ }^{1} / \mathbf{m}_{\mathrm{y}}$

V $^{2} \quad\left(\begin{array}{ccc}x x & 0 & x z \\ 0 & y y & 0 \\ z x & 0 & z z\end{array}\right)\left(\begin{array}{ccc}0 & 0 & 0 \\ 0 & 0 & 0 \\ 0 & 0 & 0\end{array}\right)\left(\begin{array}{ccc}0 & 0 & 0 \\ 0 & 0 & 0 \\ 0 & 0 & 0\end{array}\right)\left(\begin{array}{ccc}0 & x y & 0 \\ y x & 0 & y z \\ 0 & z y & 0\end{array}\right)$

$\left[V^{2}\right] \quad\left(\begin{array}{ccc}x x & 0 & x z \\ 0 & y y & 0 \\ x z & 0 & z z\end{array}\right)\left(\begin{array}{ccc}0 & 0 & 0 \\ 0 & 0 & 0 \\ 0 & 0 & 0\end{array}\right)\left(\begin{array}{ccc}0 & 0 & 0 \\ 0 & 0 & 0 \\ 0 & 0 & 0\end{array}\right)\left(\begin{array}{ccc}0 & x y & 0 \\ x y & 0 & y z \\ 0 & y z & 0\end{array}\right)$

$\left\{V^{2}\right\} \quad\left(\begin{array}{ccc}0 & 0 & x z \\ 0 & 0 & 0 \\ -x z & 0 & 0\end{array}\right)\left(\begin{array}{lll}0 & 0 & 0 \\ 0 & 0 & 0 \\ 0 & 0 & 0\end{array}\right)\left(\begin{array}{lll}0 & 0 & 0 \\ 0 & 0 & 0 \\ 0 & 0 & 0\end{array}\right)\left(\begin{array}{ccc}0 & x y & 0 \\ -x y & 0 & y z \\ 0 & -y z & 0\end{array}\right)$

$\mathbf{D}_{1 \mathrm{~h}}\left(\mathbf{C}_{1 \mathrm{~h}}\right)=\mathbf{2}_{\mathrm{x}} \mathbf{m}_{\mathrm{y}} \mathbf{m}_{\mathrm{z}}\left(\mathbf{m}_{\mathrm{z}}\right)=\mathbf{2}_{\mathrm{x}}{ }^{\prime} \mathbf{m}_{\mathbf{y}} \mathbf{C}_{\mathrm{z}}$

$V^{2} \quad\left(\begin{array}{ccc}x x & 0 & 0 \\ 0 & y y & 0 \\ 0 & 0 & z z\end{array}\right)\left(\begin{array}{ccc}0 & 0 & 0 \\ 0 & 0 & y z \\ 0 & z y & 0\end{array}\right)\left(\begin{array}{ccc}0 & x y & 0 \\ y x & 0 & 0 \\ 0 & 0 & 0\end{array}\right)\left(\begin{array}{ccc}0 & 0 & x z \\ 0 & 0 & 0 \\ z x & 0 & 0\end{array}\right)$

$\left(\begin{array}{lll}x x & 0 & 0\end{array}\right)\left(\begin{array}{ccc}0 & 0 & 0\end{array}\right)\left(\begin{array}{lll}0 & x y & 0\end{array}\right)\left(\begin{array}{ccc}0 & 0 & x z\end{array}\right)$

$\left[\mathrm{V}^{2}\right] \quad\left(\begin{array}{ccc}0 & y y & 0 \\ 0 & 0 & z z\end{array}\right)\left(\begin{array}{ccc}0 & 0 & y z \\ 0 & y z & 0\end{array}\right)\left(\begin{array}{ccc}x y & 0 & 0 \\ 0 & 0 & 0\end{array}\right)\left(\begin{array}{ccc}0 & 0 & x z \\ 0 & 0 & 0 \\ x z & 0 & 0\end{array}\right)$

$\left\{V^{2}\right\} \quad\left(\begin{array}{lll}0 & 0 & 0 \\ 0 & 0 & 0 \\ 0 & 0 & 0\end{array}\right)\left(\begin{array}{ccc}0 & 0 & 0 \\ 0 & 0 & y z \\ 0 & -y z & 0\end{array}\right)\left(\begin{array}{ccc}0 & x y & 0 \\ -x y & 0 & 0 \\ 0 & 0 & 0\end{array}\right)\left(\begin{array}{ccc}0 & 0 & x z \\ 0 & 0 & 0 \\ -x z & 0 & 0\end{array}\right)$

$D_{\mathrm{nh}}\left(D_{\mathrm{n}}\right) \quad \mathbf{D}_{1 \mathrm{~h}}\left(\mathbf{D}_{1}\right)=\mathbf{2}_{\mathrm{x}} \mathbf{m}_{\mathrm{y}} \mathbf{m}_{\mathrm{z}}\left(\mathbf{2}_{\mathrm{x}}\right)=\mathbf{2}_{\mathrm{x}} \mathbf{m}_{\mathrm{y}} \mathbf{m}_{\mathrm{z}}$

$\mathrm{V}^{2} \quad\left(\begin{array}{ccc}\mathrm{xx} & 0 & 0 \\ 0 & \mathrm{yy} & 0 \\ 0 & 0 & \mathrm{zz}\end{array}\right)\left(\begin{array}{ccc}0 & 0 & 0 \\ 0 & 0 & \mathrm{yz} \\ 0 & \mathrm{zy} & 0\end{array}\right)\left(\begin{array}{ccc}0 & 0 & 0 \\ 0 & 0 & y z \\ 0 & z y & 0\end{array}\right)\left(\begin{array}{ccc}\mathrm{xx} & 0 & 0 \\ 0 & y y & 0 \\ 0 & 0 & z z\end{array}\right)$

$\left[V^{2}\right] \quad\left(\begin{array}{ccc}x x & 0 & 0 \\ 0 & y y & 0 \\ 0 & 0 & z z\end{array}\right)\left(\begin{array}{ccc}0 & 0 & 0 \\ 0 & 0 & y z \\ 0 & y z & 0\end{array}\right)\left(\begin{array}{ccc}0 & 0 & 0 \\ 0 & 0 & y z \\ 0 & y z & 0\end{array}\right)\left(\begin{array}{ccc}x x & 0 & 0 \\ 0 & y y & 0 \\ 0 & 0 & z z\end{array}\right)$

$\left\{V^{2}\right\} \quad\left(\begin{array}{ccc}0 & 0 & 0 \\ 0 & 0 & 0 \\ 0 & 0 & 0\end{array}\right)\left(\begin{array}{ccc}0 & 0 & 0 \\ 0 & 0 & y z \\ 0 & -y z & 0\end{array}\right)\left(\begin{array}{ccc}0 & 0 & 0 \\ 0 & 0 & y z \\ 0 & -y z & 0\end{array}\right)\left(\begin{array}{ccc}0 & 0 & 0 \\ 0 & 0 & 0 \\ 0 & 0 & 0\end{array}\right)$ $\mathrm{n}=2$

$D_{2 d}\left(C_{2 v}\right)=\overline{4}_{z} m_{x} 2_{x y}\left(2_{z} m_{x} m_{y}\right)=\overline{4}_{z} ' m_{x} 2_{x y} '$

$\left(\begin{array}{ccc}x x & 0 & 0 \\ 0 & x x & 0 \\ 0 & 0 & z z\end{array}\right)\left(\begin{array}{ccc}0 & x y & 0 \\ x y & 0 & 0 \\ 0 & 0 & 0\end{array}\right)\left(\begin{array}{ccc}x x & 0 & 0 \\ 0 & -x x & 0 \\ 0 & 0 & 0\end{array}\right)\left(\begin{array}{ccc}0 & x y & 0 \\ -x y & 0 & 0 \\ 0 & 0 & 0\end{array}\right)$

$\mathbf{D}_{\infty \mathrm{h}}\left(\mathbf{C}_{\infty \mathrm{cv}}\right)=\infty / \mathbf{m}^{\prime} \mathbf{m m}$

$\left(\begin{array}{ccc}x x & 0 & 0 \\ 0 & x x & 0 \\ 0 & 0 & z z\end{array}\right)\left(\begin{array}{ccc}0 & 0 & 0 \\ 0 & 0 & 0 \\ 0 & 0 & 0\end{array}\right)\left(\begin{array}{ccc}0 & 0 & 0 \\ 0 & 0 & 0 \\ 0 & 0 & 0\end{array}\right)\left(\begin{array}{ccc}0 & x y & 0 \\ -x y & 0 & 0 \\ 0 & 0 & 0\end{array}\right)$

$\left(\begin{array}{ccc}x x & 0 & 0 \\ 0 & x x & 0 \\ 0 & 0 & z z\end{array}\right)\left(\begin{array}{ccc}0 & x y & 0 \\ x y & 0 & 0 \\ 0 & 0 & 0\end{array}\right)\left(\begin{array}{ccc}x x & 0 & 0 \\ 0 & -x x & 0 \\ 0 & 0 & 0\end{array}\right)\left(\begin{array}{ccc}0 & 0 & 0 \\ 0 & 0 & 0 \\ 0 & 0 & 0\end{array}\right)$

$\left(\begin{array}{ccc}x x & 0 & 0 \\ 0 & x x & 0 \\ 0 & 0 & z z\end{array}\right)\left(\begin{array}{lll}0 & 0 & 0 \\ 0 & 0 & 0 \\ 0 & 0 & 0\end{array}\right)\left(\begin{array}{lll}0 & 0 & 0 \\ 0 & 0 & 0 \\ 0 & 0 & 0\end{array}\right)\left(\begin{array}{lll}0 & 0 & 0 \\ 0 & 0 & 0 \\ 0 & 0 & 0\end{array}\right)$

$\left(\begin{array}{lll}0 & 0 & 0 \\ 0 & 0 & 0 \\ 0 & 0 & 0\end{array}\right)\left(\begin{array}{lll}0 & 0 & 0 \\ 0 & 0 & 0 \\ 0 & 0 & 0\end{array}\right)\left(\begin{array}{lll}0 & 0 & 0 \\ 0 & 0 & 0 \\ 0 & 0 & 0\end{array}\right)\left(\begin{array}{ccc}0 & x y & 0 \\ -x y & 0 & 0 \\ 0 & 0 & 0\end{array}\right)$

$\left(\begin{array}{lll}0 & 0 & 0 \\ 0 & 0 & 0 \\ 0 & 0 & 0\end{array}\right)\left(\begin{array}{lll}0 & 0 & 0 \\ 0 & 0 & 0 \\ 0 & 0 & 0\end{array}\right)\left(\begin{array}{lll}0 & 0 & 0 \\ 0 & 0 & 0 \\ 0 & 0 & 0\end{array}\right)\left(\begin{array}{ccc}0 & x y & 0 \\ -x y & 0 & 0 \\ 0 & 0 & 0\end{array}\right)$

\section{$n=2$
$D_{2 h}\left(C_{2 h}\right)=m_{x} m_{y} m_{z}\left(\mathbf{z}_{z} / m_{z}\right)=m_{x}{ }^{\prime} m_{y}{ }^{\prime} m_{z}$}

$\begin{array}{cccc}1 & e & a & a e\end{array}$ $\left(\begin{array}{ccc}x x & 0 & 0 \\ 0 & y & 0\end{array}\right)\left(\begin{array}{lll}0 & 0 & 0 \\ 0 & 0 & 0\end{array}\right)\left(\begin{array}{lll}0 & x y & 0 \\ y x & 0 & 0\end{array}\right)\left(\begin{array}{lll}0 & 0 & 0 \\ 0 & 0 & 0\end{array}\right)$ $\left(\begin{array}{ccc}0 & y y & 0 \\ 0 & 0 & z z\end{array}\right)\left(\begin{array}{ccc}0 & 0 & 0 \\ 0 & 0 & 0\end{array}\right)\left(\begin{array}{ccc}y x & 0 & 0 \\ 0 & 0 & 0\end{array}\right)\left(\begin{array}{ccc}0 & 0 & 0 \\ 0 & 0 & 0\end{array}\right)$ $\left(\begin{array}{lll}x x & 0 & 0 \\ 0 & y y & 0\end{array}\right)\left(\begin{array}{lll}0 & 0 & 0 \\ 0 & 0 & 0\end{array}\right)\left(\begin{array}{ccc}0 & x y & 0 \\ x y & 0 & 0\end{array}\right)\left(\begin{array}{lll}0 & 0 & 0 \\ 0 & 0 & 0\end{array}\right)$ $\left.\begin{array}{ccc}0 & y y & 0 \\ 0 & 0 & z z\end{array}\right)\left(\begin{array}{lll}0 & 0 & 0 \\ 0 & 0 & 0\end{array}\right)\left(\begin{array}{ccc}x y & 0 & 0 \\ 0 & 0 & 0\end{array}\right)\left(\begin{array}{ccc}0 & 0 & 0 \\ 0 & 0 & 0\end{array}\right)$ $\left(\begin{array}{lll}0 & 0 & 0\end{array}\right)\left(\begin{array}{llll}0 & 0 & 0\end{array}\right)\left(\begin{array}{llll}0 & x y & 0\end{array}\right)\left(\begin{array}{lll}0 & 0 & 0\end{array}\right)$ $\left(\begin{array}{lll}0 & 0 & 0 \\ 0 & 0 & 0\end{array}\right)\left(\begin{array}{lll}0 & 0 & 0 \\ 0 & 0 & 0\end{array}\right)\left(\begin{array}{ccc}0 & x y & 0 \\ -x y & 0 & 0\end{array}\right)\left(\begin{array}{lll}0 & 0 & 0 \\ 0 & 0 & 0\end{array}\right)$ $\left(\begin{array}{lll}0 & 0 & 0 \\ 0 & 0 & 0\end{array}\right)\left(\begin{array}{lll}0 & 0 & 0 \\ 0 & 0 & 0\end{array}\right)\left(\begin{array}{ccc}-x y & 0 & 0 \\ 0 & 0 & 0\end{array}\right)\left(\begin{array}{lll}0 & 0 & 0 \\ 0 & 0 & 0\end{array}\right)$

$$
\mathrm{n}=2
$$

$\mathbf{D}_{2 \mathrm{~h}}\left(\mathbf{D}_{2}\right)=\mathbf{m}_{\mathrm{x}} \mathbf{m}_{\mathrm{y}} \mathbf{m}_{\mathrm{z}}\left(\mathbf{2}_{\mathrm{x}} \mathbf{2}_{\mathrm{y}} \mathbf{2}_{\mathrm{z}}\right)=\mathbf{m}_{\mathrm{x}}{ }^{\prime} \mathbf{m}_{\mathrm{y}}{ }^{\prime} \mathbf{m}_{\mathrm{z}}{ }^{\prime}$

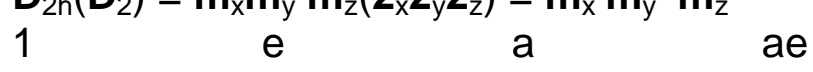
$\left(\begin{array}{ccc}x x & 0 & 0 \\ 0 & y y & 0\end{array}\right)\left(\begin{array}{ccc}0 & 0 & 0 \\ 0 & 0 & 0\end{array}\right)\left(\begin{array}{lll}0 & 0 & 0 \\ 0 & 0 & 0\end{array}\right)\left(\begin{array}{ccc}x x & 0 & 0 \\ 0 & y y & 0\end{array}\right)$ $\left(\begin{array}{ccc}0 & y y & 0 \\ 0 & 0 & z z\end{array}\right)\left(\begin{array}{ccc}0 & 0 & 0 \\ 0 & 0 & 0\end{array}\right)\left(\begin{array}{ccc}0 & 0 & 0 \\ 0 & 0 & 0\end{array}\right)\left(\begin{array}{ccc}x x & 0 & 0 \\ 0 & y y & 0 \\ 0 & 0 & z z\end{array}\right)$ $\left(\begin{array}{ccc}x x & 0 & 0 \\ 0 & y y & 0 \\ 0 & 0 & z z\end{array}\right)\left(\begin{array}{ccc}0 & 0 & 0 \\ 0 & 0 & 0 \\ 0 & 0 & 0\end{array}\right)\left(\begin{array}{ccc}0 & 0 & 0 \\ 0 & 0 & 0 \\ 0 & 0 & 0\end{array}\right)\left(\begin{array}{ccc}x x & 0 & 0 \\ 0 & y y & 0 \\ 0 & 0 & z z\end{array}\right)$ $\left(\begin{array}{lll}0 & 0 & 0 \\ 0 & 0 & 0 \\ 0 & 0 & 0\end{array}\right)\left(\begin{array}{lll}0 & 0 & 0 \\ 0 & 0 & 0 \\ 0 & 0 & 0\end{array}\right)\left(\begin{array}{lll}0 & 0 & 0 \\ 0 & 0 & 0 \\ 0 & 0 & 0\end{array}\right)\left(\begin{array}{lll}0 & 0 & 0 \\ 0 & 0 & 0 \\ 0 & 0 & 0\end{array}\right)$

\section{$\begin{aligned} & n>2 \\ & \mathbf{D}_{\infty \mathrm{h}}\left(\mathbf{C}_{\infty \mathrm{h}}\right)=\infty / \mathbf{m m}^{\prime} \mathbf{m}^{\prime}\end{aligned}$}

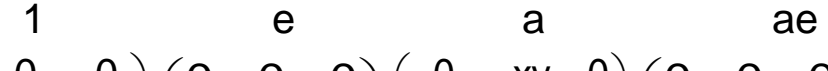
$\left(\begin{array}{ccc}x x & 0 & 0 \\ 0 & x x & 0\end{array}\right)\left(\begin{array}{ccc}0 & 0 & 0 \\ 0 & 0 & 0\end{array}\right)\left(\begin{array}{ccc}0 & x y & 0 \\ -x y & 0 & 0\end{array}\right)\left(\begin{array}{ccc}0 & 0 & 0 \\ 0 & 0 & 0\end{array}\right)$ $\left.\begin{array}{ccc}0 & x x & 0 \\ 0 & 0 & z z\end{array}\right)\left(\begin{array}{ccc}0 & 0 & 0 \\ 0 & 0 & 0\end{array}\right)\left(\begin{array}{ccc}-x y & 0 & 0 \\ 0 & 0 & 0\end{array}\right)\left(\begin{array}{ccc}0 & 0 & 0 \\ 0 & 0 & 0\end{array}\right)$ $\left(\begin{array}{ccc}x x & 0 & 0 \\ 0 & x x & 0\end{array}\right)\left(\begin{array}{lll}0 & 0 & 0 \\ 0 & 0 & 0\end{array}\right)\left(\begin{array}{lll}0 & 0 & 0 \\ 0 & 0 & 0\end{array}\right)\left(\begin{array}{lll}0 & 0 & 0 \\ 0 & 0 & 0\end{array}\right)$ $\left(\begin{array}{ccc}0 & x x & 0 \\ 0 & 0 & z z\end{array}\right)\left(\begin{array}{lll}0 & 0 & 0 \\ 0 & 0 & 0\end{array}\right)\left(\begin{array}{lll}0 & 0 & 0 \\ 0 & 0 & 0\end{array}\right)\left(\begin{array}{lll}0 & 0 & 0 \\ 0 & 0 & 0\end{array}\right)$ $\left(\begin{array}{lll}0 & 0 & 0\end{array}\right)\left(\begin{array}{lll}0 & 0 & 0\end{array}\right)\left(\begin{array}{ccc}0 & x y & 0 \\ 0 & 0 & 0\end{array}\right)\left(\begin{array}{lll}0 & 0 & 0\end{array}\right)$

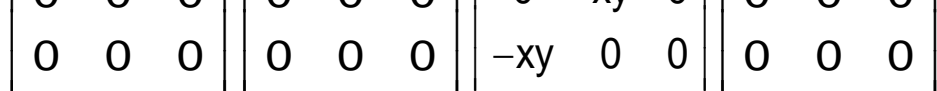
$\left(\begin{array}{lll}0 & 0 & 0\end{array}\right)\left(\begin{array}{lll}0 & 0 & 0\end{array}\right)\left(\begin{array}{lll}0 & 0 & 0\end{array}\right)\left(\begin{array}{lll}0 & 0 & 0\end{array}\right)$

$$
\begin{aligned}
& \left(\begin{array}{ccc}
x x & 0 & 0 \\
0 & x x & 0 \\
0 & 0 & z z
\end{array}\right)\left(\begin{array}{lll}
0 & 0 & 0 \\
0 & 0 & 0 \\
0 & 0 & 0
\end{array}\right)\left(\begin{array}{lll}
0 & 0 & 0 \\
0 & 0 & 0 \\
0 & 0 & 0
\end{array}\right)\left(\begin{array}{ccc}
x x & 0 & 0 \\
0 & x x & 0 \\
0 & 0 & z z
\end{array}\right) \\
& \left(\begin{array}{ccc}
x x & 0 & 0 \\
0 & x x & 0 \\
0 & 0 & z z
\end{array}\right)\left(\begin{array}{lll}
0 & 0 & 0 \\
0 & 0 & 0 \\
0 & 0 & 0
\end{array}\right)\left(\begin{array}{lll}
0 & 0 & 0 \\
0 & 0 & 0 \\
0 & 0 & 0
\end{array}\right)\left(\begin{array}{ccc}
x x & 0 & 0 \\
0 & x x & 0 \\
0 & 0 & z z
\end{array}\right) \\
& \left(\begin{array}{ccc}
0 & 0 & 0 \\
0 & 0 & 0 \\
0 & 0 & 0
\end{array}\right)\left(\begin{array}{lll}
0 & 0 & 0 \\
0 & 0 & 0 \\
0 & 0 & 0
\end{array}\right)\left(\begin{array}{lll}
0 & 0 & 0 \\
0 & 0 & 0 \\
0 & 0 & 0
\end{array}\right)\left(\begin{array}{ccc}
0 & 0 & 0 \\
0 & 0 & 0 \\
0 & 0 & 0
\end{array}\right)
\end{aligned}
$$


$n=1$

$\mathbf{D}_{\mathrm{nh}}\left(\mathbf{C}_{\mathrm{nv}}\right) \quad \mathbf{D}_{1 \mathrm{~h}}\left(\mathbf{C}_{1 \mathrm{v}}\right)=\mathbf{2}_{\mathrm{x}} \mathbf{m}_{\mathrm{y}} \mathbf{m}_{\mathrm{z}}\left(\mathbf{m}_{\mathrm{y}}\right)=\mathbf{2}_{\mathrm{x}}{ }^{\prime} \mathbf{m}_{\mathrm{y}} \mathbf{m}_{\mathrm{z}}{ }^{\prime}$

$\mathrm{V}^{2} \quad\left(\begin{array}{ccc}x x & 0 & 0 \\ 0 & y y & 0 \\ 0 & 0 & z z\end{array}\right)\left(\begin{array}{ccc}0 & 0 & 0 \\ 0 & 0 & y z \\ 0 & z y & 0\end{array}\right)\left(\begin{array}{ccc}0 & 0 & x z \\ 0 & 0 & 0 \\ z x & 0 & 0\end{array}\right)\left(\begin{array}{ccc}0 & x y & 0 \\ y x & 0 & 0 \\ 0 & 0 & 0\end{array}\right)$

$\left[V^{2}\right] \quad\left(\begin{array}{ccc}x x & 0 & 0 \\ 0 & y y & 0 \\ 0 & 0 & z z\end{array}\right)\left(\begin{array}{ccc}0 & 0 & 0 \\ 0 & 0 & y z \\ 0 & y z & 0\end{array}\right)\left(\begin{array}{ccc}0 & 0 & x z \\ 0 & 0 & 0 \\ x z & 0 & 0\end{array}\right)\left(\begin{array}{ccc}0 & x y & 0 \\ x y & 0 & 0 \\ 0 & 0 & 0\end{array}\right)$

$\left\{\mathrm{V}^{2}\right\} \quad\left(\begin{array}{ccc}0 & 0 & 0 \\ 0 & 0 & 0 \\ 0 & 0 & 0\end{array}\right)\left(\begin{array}{ccc}0 & 0 & 0 \\ 0 & 0 & y z \\ 0 & -y z & 0\end{array}\right)\left(\begin{array}{ccc}0 & 0 & x z \\ 0 & 0 & 0 \\ -x z & 0 & 0\end{array}\right)\left(\begin{array}{ccc}0 & x y & 0 \\ -x y & 0 & 0 \\ 0 & 0 & 0\end{array}\right)$

$$
\mathrm{n}=1
$$

$\mathbf{D}_{2 \mathrm{nh}}\left(\mathbf{D}_{\mathrm{nd}}\right) \quad \mathbf{D}_{2 \mathrm{~h}}\left(\mathbf{D}_{1 \mathrm{~d}}\right)=\mathbf{m}_{\mathrm{x}} \mathbf{m}_{\mathbf{y}} \mathbf{m}_{\mathrm{z}}\left(\mathbf{2}_{\mathbf{y}} / \mathbf{m}_{\mathbf{y}}\right)=\mathbf{m}_{\mathrm{x}} \mathbf{m}_{\mathbf{y}} \mathbf{m}_{\mathbf{z}}{ }^{\prime}$

$$
\begin{array}{ccc}
1 & \text { e } & \text { a }
\end{array}
$$

$\mathrm{V}^{2} \quad\left(\begin{array}{ccc}\mathrm{xx} & 0 & 0 \\ 0 & \mathrm{yy} & 0 \\ 0 & 0 & \mathrm{zz}\end{array}\right)\left(\begin{array}{ccc}0 & 0 & 0 \\ 0 & 0 & 0 \\ 0 & 0 & 0\end{array}\right)\left(\begin{array}{ccc}0 & 0 & \mathrm{xz} \\ 0 & 0 & 0 \\ \mathrm{zx} & 0 & 0\end{array}\right)\left(\begin{array}{ccc}0 & 0 & 0 \\ 0 & 0 & 0 \\ 0 & 0 & 0\end{array}\right)$

$\left[V^{2}\right] \quad\left(\begin{array}{ccc}x x & 0 & 0 \\ 0 & y y & 0 \\ 0 & 0 & z z\end{array}\right)\left(\begin{array}{ccc}0 & 0 & 0 \\ 0 & 0 & 0 \\ 0 & 0 & 0\end{array}\right)\left(\begin{array}{ccc}0 & 0 & x z \\ 0 & 0 & 0 \\ x z & 0 & 0\end{array}\right)\left(\begin{array}{ccc}0 & 0 & 0 \\ 0 & 0 & 0 \\ 0 & 0 & 0\end{array}\right)$

$\left\{V^{2}\right\} \quad\left(\begin{array}{lll}0 & 0 & 0 \\ 0 & 0 & 0 \\ 0 & 0 & 0\end{array}\right)\left(\begin{array}{lll}0 & 0 & 0 \\ 0 & 0 & 0 \\ 0 & 0 & 0\end{array}\right)\left(\begin{array}{ccc}0 & 0 & x z \\ 0 & 0 & 0 \\ -x z & 0 & 0\end{array}\right)\left(\begin{array}{lll}0 & 0 & 0 \\ 0 & 0 & 0 \\ 0 & 0 & 0\end{array}\right)$

$$
\mathrm{n}=1
$$

$\mathbf{D}_{2 \mathrm{nh}}\left(\mathbf{D}_{\mathrm{nh}}\right) \quad \mathbf{D}_{2 \mathrm{~h}}\left(\mathbf{D}_{1 \mathrm{~h}}\right)=\mathbf{m}_{\mathrm{x}} \mathbf{m}_{\mathbf{y}} \mathbf{m}_{\mathrm{z}}\left(\mathbf{2}_{\mathbf{x}} \mathbf{m}_{\mathbf{y}} \mathbf{m}_{\mathrm{z}}\right)=\mathbf{m}_{\mathrm{x}}{ }^{\prime} \mathbf{m}_{\mathbf{y}} \mathbf{m}_{\mathrm{z}}$ $\begin{array}{llll}1 & \text { e } & \text { a } & \text { ae }\end{array}$ $\mathrm{V}^{2} \quad\left(\begin{array}{ccc}\mathrm{xx} & 0 & 0 \\ 0 & \mathrm{yy} & 0 \\ 0 & 0 & \mathrm{zz}\end{array}\right)\left(\begin{array}{ccc}0 & 0 & 0 \\ 0 & 0 & 0 \\ 0 & 0 & 0\end{array}\right)\left(\begin{array}{ccc}0 & 0 & 0 \\ 0 & 0 & 0 \\ 0 & 0 & 0\end{array}\right)\left(\begin{array}{ccc}0 & 0 & 0 \\ 0 & 0 & y z \\ 0 & z y & 0\end{array}\right)$

$\left[V^{2}\right] \quad\left(\begin{array}{ccc}x x & 0 & 0 \\ 0 & y y & 0 \\ 0 & 0 & z z\end{array}\right)\left(\begin{array}{ccc}0 & 0 & 0 \\ 0 & 0 & 0 \\ 0 & 0 & 0\end{array}\right)\left(\begin{array}{ccc}0 & 0 & 0 \\ 0 & 0 & 0 \\ 0 & 0 & 0\end{array}\right)\left(\begin{array}{ccc}0 & 0 & 0 \\ 0 & 0 & y z \\ 0 & y z & 0\end{array}\right)$

$\left\{V^{2}\right\} \quad\left(\begin{array}{lll}0 & 0 & 0 \\ 0 & 0 & 0 \\ 0 & 0 & 0\end{array}\right)\left(\begin{array}{lll}0 & 0 & 0 \\ 0 & 0 & 0 \\ 0 & 0 & 0\end{array}\right)\left(\begin{array}{ccc}0 & 0 & 0 \\ 0 & 0 & 0 \\ 0 & 0 & 0\end{array}\right)\left(\begin{array}{ccc}0 & 0 & 0 \\ 0 & 0 & y z \\ 0 & -y z & 0\end{array}\right)$ $n=2$
$\mathbf{D}_{2 h}\left(\mathbf{C}_{2 \mathrm{v}}\right)=\mathbf{m}_{\mathrm{x}} \mathbf{m}_{\mathrm{y}} \mathbf{m}_{\mathrm{z}}\left(\mathbf{2}_{\mathrm{z}} \mathbf{m}_{\mathrm{x}} \mathbf{m}_{\mathrm{y}}\right)=\mathbf{m}_{\mathrm{x}} \mathbf{m}_{\mathrm{y}} \mathbf{m}_{\mathrm{z}}$

$\left(\begin{array}{ccc}x x & 0 & 0 \\ 0 & y y & 0 \\ 0 & 0 & z z\end{array}\right)\left(\begin{array}{lll}0 & 0 & 0 \\ 0 & 0 & 0 \\ 0 & 0 & 0\end{array}\right)\left(\begin{array}{ccc}0 & 0 & 0 \\ 0 & 0 & 0 \\ 0 & 0 & 0\end{array}\right)\left(\begin{array}{ccc}0 & x y & 0 \\ y x & 0 & 0 \\ 0 & 0 & 0\end{array}\right)$

$\left(\begin{array}{ccc}x x & 0 & 0 \\ 0 & y y & 0 \\ 0 & 0 & z z\end{array}\right)\left(\begin{array}{ccc}0 & 0 & 0 \\ 0 & 0 & 0 \\ 0 & 0 & 0\end{array}\right)\left(\begin{array}{ccc}0 & 0 & 0 \\ 0 & 0 & 0 \\ 0 & 0 & 0\end{array}\right)\left(\begin{array}{ccc}0 & x y & 0 \\ x y & 0 & 0 \\ 0 & 0 & 0\end{array}\right)$

$\left(\begin{array}{lll}0 & 0 & 0 \\ 0 & 0 & 0 \\ 0 & 0 & 0\end{array}\right)\left(\begin{array}{lll}0 & 0 & 0 \\ 0 & 0 & 0 \\ 0 & 0 & 0\end{array}\right)\left(\begin{array}{lll}0 & 0 & 0 \\ 0 & 0 & 0 \\ 0 & 0 & 0\end{array}\right)\left(\begin{array}{ccc}0 & x y & 0 \\ -x y & 0 & 0 \\ 0 & 0 & 0\end{array}\right)$

$$
\mathrm{n}=2
$$

$D_{4 h}\left(D_{2 d}\right)=4_{z} / m_{z} m_{x} m_{x y}\left(\overline{4}_{z} m_{x} 2_{x y}\right)=4_{z}^{\prime} / m_{z}^{\prime} m_{x} m_{x y}{ }^{\prime}$

$\left(\begin{array}{ccc}x x & 0 & 0 \\ 0 & x x & 0 \\ 0 & 0 & z z\end{array}\right)\left(\begin{array}{ccc}0 & 0 & 0 \\ 0 & 0 & 0 \\ 0 & 0 & 0\end{array}\right)\left(\begin{array}{ccc}0 & 0 & 0 \\ 0 & 0 & 0 \\ 0 & 0 & 0\end{array}\right)\left(\begin{array}{ccc}0 & x y & 0 \\ x y & 0 & 0 \\ 0 & 0 & 0\end{array}\right)$

$\left(\begin{array}{ccc}x x & 0 & 0 \\ 0 & x x & 0 \\ 0 & 0 & z z\end{array}\right)\left(\begin{array}{ccc}0 & 0 & 0 \\ 0 & 0 & 0 \\ 0 & 0 & 0\end{array}\right)\left(\begin{array}{lll}0 & 0 & 0 \\ 0 & 0 & 0 \\ 0 & 0 & 0\end{array}\right)\left(\begin{array}{ccc}0 & x y & 0 \\ x y & 0 & 0 \\ 0 & 0 & 0\end{array}\right)$

$\left(\begin{array}{lll}0 & 0 & 0 \\ 0 & 0 & 0 \\ 0 & 0 & 0\end{array}\right)\left(\begin{array}{lll}0 & 0 & 0 \\ 0 & 0 & 0 \\ 0 & 0 & 0\end{array}\right)\left(\begin{array}{lll}0 & 0 & 0 \\ 0 & 0 & 0 \\ 0 & 0 & 0\end{array}\right)\left(\begin{array}{lll}0 & 0 & 0 \\ 0 & 0 & 0 \\ 0 & 0 & 0\end{array}\right)$

$$
\mathrm{n}=2
$$

$D_{4 h}\left(D_{2 h}\right)=4_{z} / m_{z} m_{x} m_{x y}\left(m_{x} m_{y} m_{z}\right)=4_{z}^{\prime} / m_{z} m_{x} m_{x y}{ }^{\prime}$

$$
\begin{aligned}
& \left(\begin{array}{ccc}
x x & 0 & 0 \\
0 & x x & 0 \\
0 & 0 & z z
\end{array}\right)\left(\begin{array}{lll}
0 & 0 & 0 \\
0 & 0 & 0 \\
0 & 0 & 0
\end{array}\right)\left(\begin{array}{ccc}
x x & 0 & 0 \\
0 & -x x & 0 \\
0 & 0 & 0
\end{array}\right)\left(\begin{array}{lll}
0 & 0 & 0 \\
0 & 0 & 0 \\
0 & 0 & 0
\end{array}\right) \\
& \left(\begin{array}{ccc}
x x & 0 & 0 \\
0 & x x & 0 \\
0 & 0 & z z
\end{array}\right)\left(\begin{array}{lll}
0 & 0 & 0 \\
0 & 0 & 0 \\
0 & 0 & 0
\end{array}\right)\left(\begin{array}{ccc}
x x & 0 & 0 \\
0 & -x x & 0 \\
0 & 0 & 0
\end{array}\right)\left(\begin{array}{lll}
0 & 0 & 0 \\
0 & 0 & 0 \\
0 & 0 & 0
\end{array}\right) \\
& \left(\begin{array}{lll}
0 & 0 & 0 \\
0 & 0 & 0 \\
0 & 0 & 0
\end{array}\right)\left(\begin{array}{lll}
0 & 0 & 0 \\
0 & 0 & 0 \\
0 & 0 & 0
\end{array}\right)\left(\begin{array}{ccc}
0 & 0 & 0 \\
0 & 0 & 0 \\
0 & 0 & 0
\end{array}\right)\left(\begin{array}{lll}
0 & 0 & 0 \\
0 & 0 & 0 \\
0 & 0 & 0
\end{array}\right)
\end{aligned}
$$

$\mathrm{n}>2$

n>
$\left(\begin{array}{ccc}x x & 0 & 0 \\ 0 & x x & 0 \\ 0 & 0 & z z\end{array}\right)\left(\begin{array}{ccc}0 & 0 & 0 \\ 0 & 0 & 0 \\ 0 & 0 & 0\end{array}\right)\left(\begin{array}{ccc}0 & 0 & 0 \\ 0 & 0 & 0 \\ 0 & 0 & 0\end{array}\right)\left(\begin{array}{ccc}0 & x y & 0 \\ -x y & 0 & 0 \\ 0 & 0 & 0\end{array}\right)$

$\left(\begin{array}{ccc}x x & 0 & 0 \\ 0 & x x & 0 \\ 0 & 0 & z z\end{array}\right)\left(\begin{array}{lll}0 & 0 & 0 \\ 0 & 0 & 0 \\ 0 & 0 & 0\end{array}\right)\left(\begin{array}{lll}0 & 0 & 0 \\ 0 & 0 & 0 \\ 0 & 0 & 0\end{array}\right)\left(\begin{array}{lll}0 & 0 & 0 \\ 0 & 0 & 0 \\ 0 & 0 & 0\end{array}\right)$

$\left(\begin{array}{lll}0 & 0 & 0 \\ 0 & 0 & 0 \\ 0 & 0 & 0\end{array}\right)\left(\begin{array}{lll}0 & 0 & 0 \\ 0 & 0 & 0 \\ 0 & 0 & 0\end{array}\right)\left(\begin{array}{lll}0 & 0 & 0 \\ 0 & 0 & 0 \\ 0 & 0 & 0\end{array}\right)\left(\begin{array}{ccc}0 & x y & 0 \\ -x y & 0 & 0 \\ 0 & 0 & 0\end{array}\right)$

$\left(\begin{array}{ccc}x x & 0 & 0 \\ 0 & x x & 0 \\ 0 & 0 & z z\end{array}\right)\left(\begin{array}{ccc}0 & 0 & 0 \\ 0 & 0 & 0 \\ 0 & 0 & 0\end{array}\right)\left(\begin{array}{lll}0 & 0 & 0 \\ 0 & 0 & 0 \\ 0 & 0 & 0\end{array}\right)\left(\begin{array}{lll}0 & 0 & 0 \\ 0 & 0 & 0 \\ 0 & 0 & 0\end{array}\right)$
$\left(\begin{array}{ccc}x x & 0 & 0 \\ 0 & x x & 0 \\ 0 & 0 & z z\end{array}\right)\left(\begin{array}{lll}0 & 0 & 0 \\ 0 & 0 & 0 \\ 0 & 0 & 0\end{array}\right)\left(\begin{array}{lll}0 & 0 & 0 \\ 0 & 0 & 0 \\ 0 & 0 & 0\end{array}\right)\left(\begin{array}{lll}0 & 0 & 0 \\ 0 & 0 & 0 \\ 0 & 0 & 0\end{array}\right)$
$\left(\begin{array}{ccc}0 & 0 & 0 \\ 0 & 0 & 0 \\ 0 & 0 & 0\end{array}\right)\left(\begin{array}{lll}0 & 0 & 0 \\ 0 & 0 & 0 \\ 0 & 0 & 0\end{array}\right)\left(\begin{array}{lll}0 & 0 & 0 \\ 0 & 0 & 0 \\ 0 & 0 & 0\end{array}\right)\left(\begin{array}{lll}0 & 0 & 0 \\ 0 & 0 & 0 \\ 0 & 0 & 0\end{array}\right)$

$\mathrm{n}>2$

$\mathbf{D}_{\infty h} \mathbf{1}^{\prime}=\infty / \mathbf{m m m 1}$
$\left(\begin{array}{ccc}x x & 0 & 0 \\ 0 & x x & 0 \\ 0 & 0 & z z\end{array}\right)\left(\begin{array}{ccc}0 & 0 & 0 \\ 0 & 0 & 0 \\ 0 & 0 & 0\end{array}\right)\left(\begin{array}{ccc}0 & 0 & 0 \\ 0 & 0 & 0 \\ 0 & 0 & 0\end{array}\right)\left(\begin{array}{lll}0 & 0 & 0 \\ 0 & 0 & 0 \\ 0 & 0 & 0\end{array}\right)$

$\left(\begin{array}{ccc}x x & 0 & 0 \\ 0 & x x & 0 \\ 0 & 0 & z z\end{array}\right)\left(\begin{array}{lll}0 & 0 & 0 \\ 0 & 0 & 0 \\ 0 & 0 & 0\end{array}\right)\left(\begin{array}{lll}0 & 0 & 0 \\ 0 & 0 & 0 \\ 0 & 0 & 0\end{array}\right)\left(\begin{array}{lll}0 & 0 & 0 \\ 0 & 0 & 0 \\ 0 & 0 & 0\end{array}\right)$

$\left(\begin{array}{lll}0 & 0 & 0 \\ 0 & 0 & 0 \\ 0 & 0 & 0\end{array}\right)\left(\begin{array}{lll}0 & 0 & 0 \\ 0 & 0 & 0 \\ 0 & 0 & 0\end{array}\right)\left(\begin{array}{lll}0 & 0 & 0 \\ 0 & 0 & 0 \\ 0 & 0 & 0\end{array}\right)\left(\begin{array}{lll}0 & 0 & 0 \\ 0 & 0 & 0 \\ 0 & 0 & 0\end{array}\right)$ 


\section{$\mathrm{n}=1 \quad \mathrm{C}_{1}=1$}

$\left.\begin{array}{llllllllll}x & x x x & x y y & x z z & x y z & x z x & x x y & x z y & x x z & x y x \\ y x x & y y y & y z z & y y z & y z x & y x y & y z y & y x z & y y x\end{array}\right)$ $\begin{array}{lllllllll}y x x & y y y & y z z & y y z & y z x & y x y & y z y & y x z & y y x\end{array}$

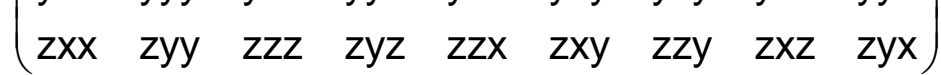

$\left[V^{3}\right]\left(\begin{array}{llll}x x x & x y y & x z z & x y z \\ y x x & y y y & y z z & \\ z x x & z y y & z z z\end{array}\right)$

$\begin{array}{llllll}x x x & x y y & x z z & x y z & x x z & x x y\end{array}$

$V\left[V^{2}\right] \quad \begin{array}{llllll}x x x & y y y & y z z & y y z & y x z & y x y\end{array}$

$\begin{array}{llllll}z x x & z y y & z z z & z y z & z x z & z x y\end{array}$

$\left\{V^{2}\right\} V\left(\begin{array}{ccc}y z x & y z y & y z z \\ z x x & z x y & z x z \\ x y x & x y y & x y z\end{array}\right)$

$\mathrm{n}=2 \quad \mathrm{C}_{2}=\mathbf{2}_{\mathrm{z}}$

$\mathrm{V}^{3} \quad\left(\begin{array}{rrrrrrrrr}0 & 0 & 0 & x y z & x z x & 0 & x z y & x x z & 0 \\ 0 & 0 & 0 & y y z & y z x & 0 & y z y & y x z & 0 \\ z x x & z y y & z z z & 0 & 0 & z x y & 0 & 0 & z y x\end{array}\right)$

$\left[V^{3}\right]\left(\begin{array}{rrrr}0 & 0 & 0 & x y z \\ 0 & 0 & 0 & \\ z x x & z y y & z z z & \end{array}\right)$

$V\left[V^{2}\right]\left(\begin{array}{rrrrrr}0 & 0 & 0 & x y z & x x z & 0 \\ 0 & 0 & 0 & y y z & y x z & 0 \\ z x x & z y y & z z z & 0 & 0 & z x y\end{array}\right)$

$\left\{V^{2}\right\} V\left(\begin{array}{ccc}y z x & y z y & 0 \\ z x x & z x y & 0 \\ 0 & 0 & x y z\end{array}\right)$ $\left(\begin{array}{lllllllll}x x x & x y y & x z z & x y z & x z x & x x y & x z y & x x z & x y x\end{array}\right.$ $\begin{array}{lllllllll}y x x & y y y & y z z & y y z & y z x & y x y & y z y & y x z & y y x\end{array}$ $\begin{array}{lllllllll}z x x & z y y & z z z & z y z & z z x & \text { zxy } & \text { zzy } & \text { zxz } & \text { zyx }\end{array}$

$\left(\begin{array}{llll}x x x & x y y & x z z & x y z \\ y x x & y y y & y z z & \\ z x x & z y y & z z z & \end{array}\right)$

$\left(\begin{array}{llllll}x x x & x y y & x z z & x y z & x x z & x x y\end{array}\right)$

$\left.\begin{array}{llllll}y x x & y y y & y z z & y y z & y x z & y x y\end{array}\right)$

$\left(\begin{array}{llllll}z x x & z y y & z z z & z y z & z x z & z x y\end{array}\right)$

$\left(\begin{array}{ccc}y z x & y z y & y z z \\ z x x & z x y & z x z \\ x y x & x y y & x y z\end{array}\right)$ $a$

$\left(\begin{array}{lllllllll}x x x & x y y & x z z & x y z & x z x & x x y & x z y & x x z & x y x\end{array}\right.$ $\begin{array}{lllllllll}y x x & y y y & y z z & y y z & y z x & y x y & y z y & y x z & y y x\end{array}$

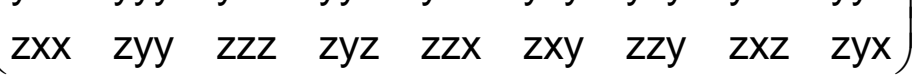

$\left(\begin{array}{llll}x x x & x y y & x z z & x y z \\ y x x & y y y & y z z & \\ z x x & z y y & z z z\end{array}\right)$

$\left(\begin{array}{llllll}x x x & x y y & x z z & x y z & x x z & x x y\end{array}\right)$

$\begin{array}{llllll}y x x & y y y & y z z & y y z & y x z & y x y\end{array}$

$\left(\begin{array}{llllll}z x x & z y y & z z z & z y z & z x z & z x y\end{array}\right)$

$\left(\begin{array}{ccc}y z x & y z y & y z z \\ z x x & z x y & z x z \\ x y x & x y y & x y z\end{array}\right)$ se

$\begin{array}{lllllllll}x x x & x y y & x z z & x y z & x z x & x x y & x z y & x x z & x y x \\ y x x & y y y & y z z & y y z & y z x & y x y & y z y & y x z & y y x\end{array}$ $\begin{array}{lllllllll}y x x & y y y & y z z & y y z & y z x & y x y & y z y & y x z & y y x\end{array}$

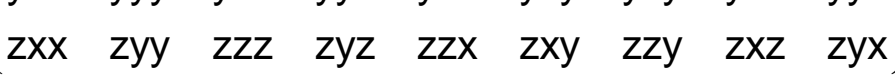

$\left(\begin{array}{llll}x x x & x y y & x z z & x y z \\ y x x & y y y & y z z & \\ z x x & z y y & z z z & \end{array}\right)$

$\left(\begin{array}{llllll}x x x & x y y & x z z & x y z & x x z & x x y\end{array}\right)$ $\begin{array}{llllll}y x x & y y y & y z z & y y z & y x z & y x y\end{array}$ zxx $\quad$ zyy $\quad$ zzz $\quad$ zyz $\quad z x z \quad$ zxy

$\left(\begin{array}{lll}y z x & y z y & y z z \\ z x x & z x y & z x z\end{array}\right)$ $\begin{array}{lll}2 x y x & x y y & x y z\end{array}$ $\left(\begin{array}{lllllllll}0 & 0 & 0 & x y z & x z x & 0 & x z y & x x z & 0 \\ 0 & 0 & 0 & y y z & y z x & 0 & y z y & y x z & 0\end{array}\right)$ $\begin{array}{lllllllll}0 & 0 & 0 & y y z & y z x & 0 & y z y & y x z & 0\end{array}$

$\left(\begin{array}{rrrr}0 & 0 & 0 & x y z \\ 0 & 0 & 0 & \\ z x x & z y y & z z z & \end{array}\right)$

$\left(\begin{array}{rrrrrr}0 & 0 & 0 & x y z & x x z & 0 \\ 0 & 0 & 0 & y y z & y x z & 0 \\ z x x & z y y & z z z & 0 & 0 & z x y\end{array}\right)$

$\left(\begin{array}{ccc}y z x & y z y & 0 \\ z x x & z x y & 0 \\ 0 & 0 & x y z\end{array}\right)$ $\left(\begin{array}{rrrr}0 & 0 & 0 & x y z \\ 0 & 0 & 0 & \\ z x x & z y y & z z z & \end{array}\right)$

$\left(\begin{array}{ccc}y z x & y z y & 0 \\ z x x & z x y & 0 \\ 0 & 0 & x y z\end{array}\right)$ ae

$\left(\begin{array}{lllllllll}0 & 0 & 0 & x y z & x z x & 0 & x z y & x x z & 0 \\ 0 & 0 & 0 & y y z & y z x & 0 & y z y & y x z & 0\end{array}\right) \quad\left(\begin{array}{lllllllll}0 & 0 & 0 & x y z & x z x & 0 & x z y & x x z & 0 \\ 0 & 0 & 0 & y y z & y z x & 0 & y z y & y x z & 0\end{array}\right)$ $\begin{array}{lllllllll}0 & 0 & 0 & y y z & y z x & 0 & y z y & y x z & 0\end{array}$

$\left(\begin{array}{rrrr}0 & 0 & 0 & x y z \\ 0 & 0 & 0 & \\ z x x & z y y & z z z & \end{array}\right)$

$\left(\begin{array}{rrrrrr}0 & 0 & 0 & x y z & x x z & 0 \\ 0 & 0 & 0 & y y z & y x z & 0 \\ z x x & z y y & z z z & 0 & 0 & z x y\end{array}\right)$

$\left(\begin{array}{rrrrrr}0 & 0 & 0 & x y z & x x z & 0 \\ 0 & 0 & 0 & y y z & y x z & 0 \\ z x x & z y y & z z z & 0 & 0 & z x y\end{array}\right)$

$\left(\begin{array}{ccc}y z x & y z y & 0 \\ z x x & z x y & 0 \\ 0 & 0 & x y z\end{array}\right)$ 
$\mathrm{n}=3 \quad \mathrm{C}_{3}=\mathbf{3}_{2}$

$V^{3} \quad\left(\begin{array}{rrrrrrrrr}x x x & -x x x & 0 & x y z & x z x & -y y y & -y z x & x x z & -y y y \\ -y y y & y y y & 0 & y y z & y z x & -x x x & x z x & -x y z & -x x x \\ z x x & z x x & z z z & 0 & 0 & z x y & 0 & 0 & -z x y\end{array}\right)$

$\left[V^{3}\right]\left(\begin{array}{rrrr}x x x & -x x x & 0 & 0 \\ -y y y & y y y & 0 & \\ z x x & z x x & z z z & \end{array}\right)$

$V\left[V^{2}\right]\left(\begin{array}{rrrrrr}x x x & -x x x & 0 & x y z & x x z & -y y y \\ -y y y & y y y & 0 & x x z & -x y z & -x x x \\ z x x & z x x & z z z & 0 & 0 & 0\end{array}\right)$

$\left\{V^{2}\right\} \bigvee\left(\begin{array}{ccc}y z x & y z y & 0 \\ -y z y & y z x & 0 \\ 0 & 0 & x y z\end{array}\right)$

$n>3 \quad \mathbf{C}_{\infty}=\infty$

$V^{3} \quad\left(\begin{array}{rrrrrrrrr}0 & 0 & 0 & x y z & x z x & 0 & -y z x & x x z & 0 \\ 0 & 0 & 0 & y y z & y z x & 0 & x z x & -x y z & 0 \\ z x x & z x x & z z z & 0 & 0 & z x y & 0 & 0 & -z x y\end{array}\right)$

$\left[V^{3}\right]\left(\begin{array}{rrrr}0 & 0 & 0 & 0 \\ 0 & 0 & 0 & \\ z x x & z x x & z z z & \end{array}\right)$

$V\left[V^{2}\right]\left(\begin{array}{rrrrrr}0 & 0 & 0 & x y z & x x z & 0 \\ 0 & 0 & 0 & x x z & -x y z & 0 \\ z x x & z x x & z z z & 0 & 0 & 0\end{array}\right)$

$\left\{V^{2}\right\} \bigvee\left(\begin{array}{ccc}y z x & y z y & 0 \\ -y z y & y z x & 0 \\ 0 & 0 & x y z\end{array}\right)$

\section{e}

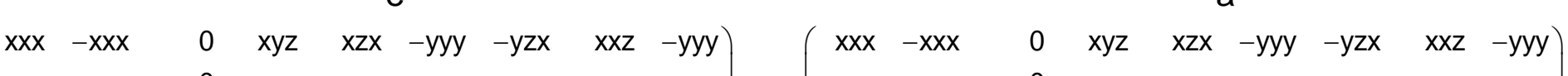
$\left.\begin{array}{rrrrrrrrr}-y y y & y y y & 0 & y y z & y z x & -x x x & x z x & -x y z & -x x x \\ z x x & z x x & z z z & 0 & 0 & z x y & 0 & 0 & -z x y\end{array}\right) \quad\left(\begin{array}{rrrrrrrrr}-y y y & y y y & 0 & y y z & y z x & -x x x & x z x & -x y z & -x x x \\ z x x & z x x & z z z & 0 & 0 & z x y & 0 & 0 & -z x y\end{array}\right)$

$\left(\begin{array}{rrrr}x x x & -x x x & 0 & 0 \\ -y y y & y y y & 0 & \\ z x x & z x x & z z z & \end{array}\right)$

$\left(\begin{array}{rrrr}x x x & -x x x & 0 & 0 \\ -y y y & y y y & 0 & \\ z x x & z x x & z z z & \end{array}\right)$

$\left(\begin{array}{rrrrrr}x x x & -x x x & 0 & x y z & x x z & -y y y \\ -y y y & y y y & 0 & x x z & -x y z & -x x x \\ z x x & z x x & z z z & 0 & 0 & 0\end{array}\right)$

$\left(\begin{array}{rrrrrr}x x x & -x x x & 0 & x y z & x x z & -y y y \\ -y y y & y y y & 0 & x x z & -x y z & -x x x \\ z x x & z x x & z z z & 0 & 0 & 0\end{array}\right)$

$\left(\begin{array}{ccc}y z x & y z y & 0 \\ -y z y & y z x & 0 \\ 0 & 0 & x y z\end{array}\right)$ $\left.\begin{array}{ccccccccc}x x x & -x x x & 0 & x y z & x z x & -y y y & -y z x & x x z & -y y y \\ -y y y & y y y & 0 & y y z & y z x & -x y x & x z x & -x y z & -x x x\end{array}\right)$ $\left.\begin{array}{rrrrrrrrr}-y y y & y y y & 0 & y y z & y z x & -x x x & x z x & -x y z & -x x x \\ z x x & z x x & z z z & 0 & 0 & z x y & 0 & 0 & -z x y\end{array}\right)$

$\left.\begin{array}{rrrrr}x x x & -x x x & 0 & 0 \\ -y y y & y y y & 0 & \\ z x x & z x x & z z z & \end{array}\right)$

$\left(\begin{array}{rrrrrr}x x x & -x x x & 0 & x y z & x x z & -y y y \\ -y y y & y y y & 0 & x x z & -x y z & -x x x \\ z x x & z x x & z z z & 0 & 0 & 0\end{array}\right)$

$\left(\begin{array}{ccc}y z x & y z y & 0 \\ -y z y & y z x & 0 \\ 0 & 0 & x y z\end{array}\right)$ e

$\left(\begin{array}{ccccccccc}0 & 0 & 0 & x y z & x z x & 0 & -y z x & x x z & 0 \\ 0 & 0 & 0 & y y z & y z x & 0 & x z x & -x y z & 0\end{array}\right)$ $\left.\begin{array}{rrrrrrrrr}0 & 0 & 0 & y y z & y z x & 0 & x z x & -x y z & 0 \\ z x x & z x x & z z z & 0 & 0 & z x y & 0 & 0 & -z x y\end{array}\right)$

$\left(\begin{array}{rrrr}0 & 0 & 0 & 0 \\ 0 & 0 & 0 & \\ z x x & z x x & z z z & \end{array}\right)$

$\left(\begin{array}{rrrrrr}0 & 0 & 0 & x y z & x x z & 0 \\ 0 & 0 & 0 & x x z & -x y z & 0 \\ z x x & z x x & z z z & 0 & 0 & 0\end{array}\right)$

$\left(\begin{array}{ccc}y z x & y z y & 0 \\ -y z y & y z x & 0 \\ 0 & 0 & x y z\end{array}\right)$ $\left(\begin{array}{rrrrrrrr}0 & 0 & 0 & x y z & x z x & 0 & -y z x & x x z \\ 0 & 0 & 0 & y y z & y z x & 0 & x z x & -x y z\end{array}\right.$ $\left.\begin{array}{rrrrrrrrr}0 & 0 & 0 & y y z & y z x & 0 & x z x & -x y z & 0 \\ z x x & z x x & z z z & 0 & 0 & z x y & 0 & 0 & -z x y\end{array}\right)$

$\left(\begin{array}{rrrr}0 & 0 & 0 & 0 \\ 0 & 0 & 0 & \\ z x x & z x x & z z z & \end{array}\right)$

$\left(\begin{array}{rrrr}0 & 0 & 0 & 0 \\ 0 & 0 & 0 & \\ z x x & z x x & z z z & \end{array}\right)$

$\left(\begin{array}{rrrrrr}0 & 0 & 0 & x y z & x x z & 0 \\ 0 & 0 & 0 & x x z & -x y z & 0 \\ z x x & z x x & z z z & 0 & 0 & 0\end{array}\right)$

$\left(\begin{array}{rrrrrr}0 & 0 & 0 & x y z & x x z & 0 \\ 0 & 0 & 0 & x x z & -x y z & 0 \\ z x x & z x x & z z z & 0 & 0 & 0\end{array}\right)$

$\left(\begin{array}{ccc}y z x & y z y & 0 \\ -y z y & y z x & 0 \\ 0 & 0 & x y z\end{array}\right)$ $\left(\begin{array}{ccc}y z x & y z y & 0 \\ -y z y & y z x & 0 \\ 0 & 0 & x y z\end{array}\right)$ 
$\mathrm{n}=1 \quad \mathbf{C}_{1 \mathrm{v}}=\mathbf{m}_{\mathrm{y}}$

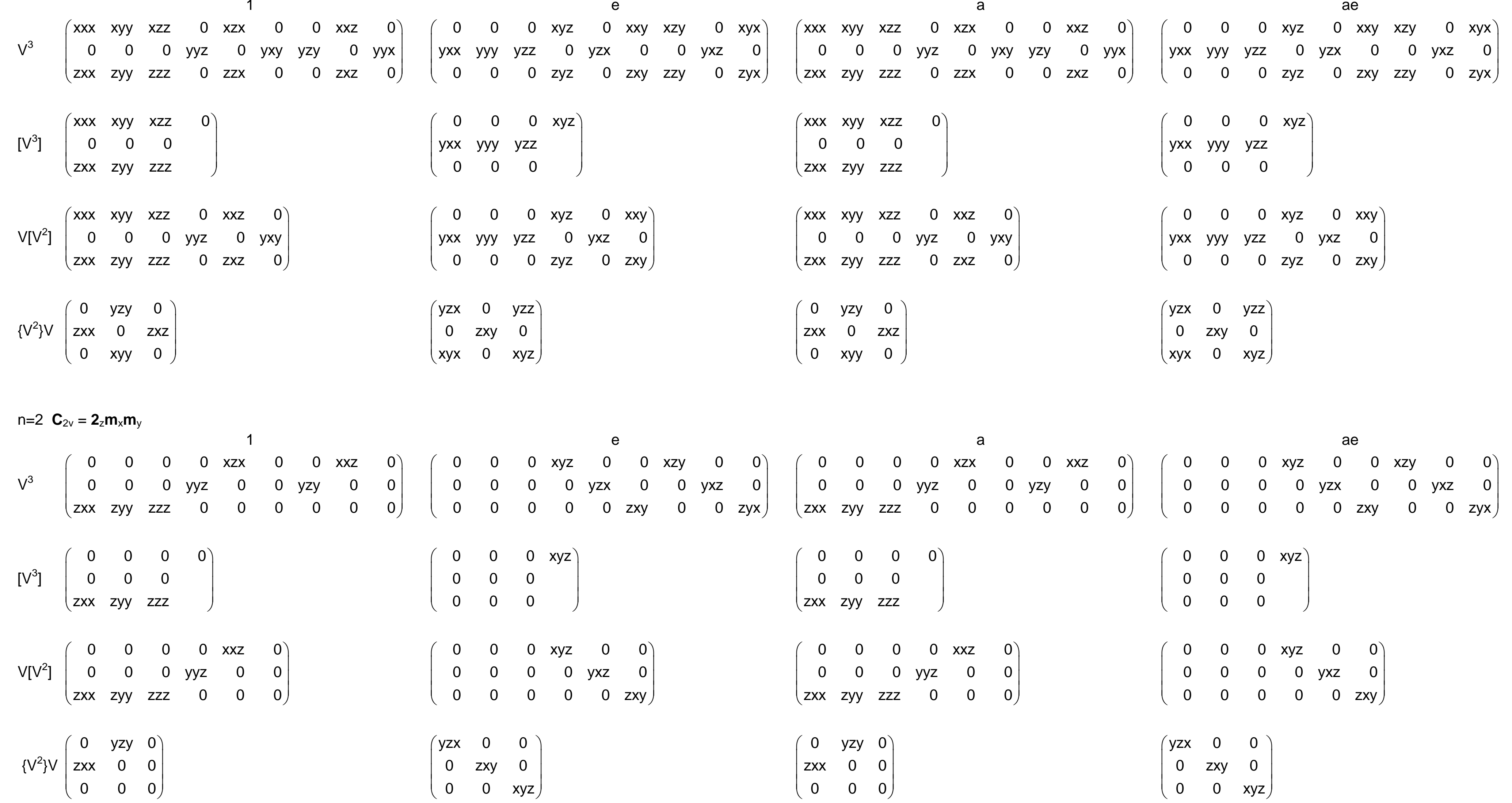


$n=3 \quad C_{3 v}=3_{z} m_{x}$

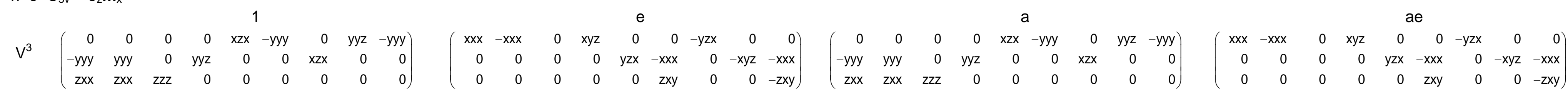
$\left[\mathrm{V}^{3}\right]\left(\begin{array}{rrrr}0 & 0 & 0 & 0 \\ -y y y & y y y & 0 & \\ z x x & z x x & z z z & \end{array}\right)$

$\left(\begin{array}{rrrr}x x x & -x x x & 0 & 0 \\ 0 & 0 & 0 & \\ 0 & 0 & 0 & \end{array}\right)$

$\left(\begin{array}{rrrr}0 & 0 & 0 & 0 \\ -y y y & y y y & 0 & \\ z x x & z x x & z z z & \end{array}\right)$

$V\left[V^{2}\right]\left(\begin{array}{rrrrrr}0 & 0 & 0 & 0 & x x z & -y y y \\ -y y y & y y y & 0 & x x z & 0 & 0 \\ z x x & z y y & z z z & 0 & 0 & 0\end{array}\right)$

$\left(\begin{array}{rrrrrr}x x x & -x x x & 0 & x y z & 0 & 0 \\ 0 & 0 & 0 & 0 & -x y z & -x x x \\ 0 & 0 & 0 & 0 & 0 & 0\end{array}\right)$

$\left(\begin{array}{rrrrrr}0 & 0 & 0 & 0 & x x z & -y y y \\ -y y y & y y y & 0 & x x z & 0 & 0 \\ z x x & z y y & z z z & 0 & 0 & 0\end{array}\right)$

$\left(\begin{array}{rrrr}x x x & -x x x & 0 & 0 \\ 0 & 0 & 0 & \\ 0 & 0 & 0 & \end{array}\right)$

$\left\{\mathrm{V}^{2}\right\} \bigvee\left(\begin{array}{ccc}0 & y z y & 0 \\ -y z y & 0 & 0 \\ 0 & 0 & 0\end{array}\right)$

$\left(\begin{array}{ccc}y z x & 0 & 0 \\ 0 & y z x & 0 \\ 0 & 0 & x y z\end{array}\right)$

$\left(\begin{array}{ccc}0 & y z y & 0 \\ -y z y & 0 & 0 \\ 0 & 0 & 0\end{array}\right)$

$\left(\begin{array}{rrrrrr}x x x & -x x x & 0 & x y z & 0 & 0 \\ 0 & 0 & 0 & 0 & -x y z & -x x x \\ 0 & 0 & 0 & 0 & 0 & 0\end{array}\right)$

$\left(\begin{array}{ccc}y z x & 0 & 0 \\ 0 & y z x & 0 \\ 0 & 0 & x y z\end{array}\right)$

$\mathrm{n}>3 \quad \mathbf{C}_{\infty \mathrm{v}}=\infty \mathbf{m m}$

$\mathrm{V}^{3} \quad\left(\begin{array}{rrrrrrrrr}0 & 0 & 0 & 0 & \mathrm{xzx} & 0 & 0 & \mathrm{yyz} & 0 \\ 0 & 0 & 0 & y y z & 0 & 0 & \mathrm{xzx} & 0 & 0 \\ \mathrm{zxx} & \mathrm{zxx} & \mathrm{zzz} & 0 & 0 & 0 & 0 & 0 & 0\end{array}\right) \quad\left(\begin{array}{rrrrrrrrr}0 & 0 & 0 & \mathrm{xyz} & 0 & 0 & -\mathrm{yzx} & 0 & 0 \\ 0 & 0 & 0 & 0 & \mathrm{yzx} & 0 & 0 & -\mathrm{xyz} & 0 \\ 0 & 0 & 0 & 0 & 0 & \mathrm{zxy} & 0 & 0 & -\mathrm{zxy}\end{array}\right)$

$\left[\mathrm{V}^{3}\right]\left(\begin{array}{rrrr}0 & 0 & 0 & 0 \\ 0 & 0 & 0 & \\ \mathrm{zxx} & \mathrm{zxx} & \mathrm{zzz} & \end{array}\right)$

$\left(\begin{array}{rrrr}0 & 0 & 0 & 0 \\ 0 & 0 & 0 & \\ z x x & z x x & z z z & \end{array}\right)$

$\left(\begin{array}{rrrrrrrrr}0 & 0 & 0 & 0 & x z x & 0 & 0 & y y z & 0 \\ 0 & 0 & 0 & y y z & 0 & 0 & x z x & 0 & 0 \\ z x x & z x x & z z z & 0 & 0 & 0 & 0 & 0 & 0\end{array}\right)$
$\left(\begin{array}{rrrr}0 & 0 & 0 & 0 \\ 0 & 0 & 0 & \\ z x x & z x x & z z z & \end{array}\right)$

ae

$V\left[V^{2}\right]\left(\begin{array}{rrrrrr}0 & 0 & 0 & 0 & x x z & 0 \\ 0 & 0 & 0 & x x z & 0 & 0 \\ z x x & z x x & z z z & 0 & 0 & 0\end{array}\right)$

$\left(\begin{array}{rrrrrr}0 & 0 & 0 & x y z & 0 & 0 \\ 0 & 0 & 0 & 0 & -x y z & 0 \\ 0 & 0 & 0 & 0 & 0 & 0\end{array}\right)$

$\left(\begin{array}{rrrrrr}0 & 0 & 0 & 0 & x x z & 0 \\ 0 & 0 & 0 & x x z & 0 & 0 \\ z x x & z x x & z z z & 0 & 0 & 0\end{array}\right)$

$\left(\begin{array}{ccc}y z x & 0 & 0 \\ 0 & y z x & 0 \\ 0 & 0 & x y z\end{array}\right)$

$\left(\begin{array}{ccc}0 & y z y & 0 \\ -y z y & 0 & 0 \\ 0 & 0 & 0\end{array}\right)$

$\left(\begin{array}{ccc}y z x & 0 & 0 \\ 0 & y z x & 0 \\ 0 & 0 & x y z\end{array}\right)$

$\left(\begin{array}{rrrrrr}0 & 0 & 0 & x y z & 0 & 0 \\ 0 & 0 & 0 & 0 & -x y z & 0 \\ 0 & 0 & 0 & 0 & 0 & 0\end{array}\right)$

$\left\{V^{2}\right\} \vee\left(\begin{array}{ccc}0 & y z y & 0 \\ -y z y & 0 & 0 \\ 0 & 0 & 0\end{array}\right)$

$\begin{array}{rrrr}0 & -y z x & 0 & 0 \\ 0 & 0 & -x y z & 0\end{array}$ 
$\mathrm{n}=1 \quad \mathbf{S}_{2}=\overline{1}$

$\mathrm{V}^{3} \quad\left(\begin{array}{lllllllll}0 & 0 & 0 & 0 & 0 & 0 & 0 & 0 & 0 \\ 0 & 0 & 0 & 0 & 0 & 0 & 0 & 0 & 0 \\ 0 & 0 & 0 & 0 & 0 & 0 & 0 & 0 & 0\end{array}\right)$

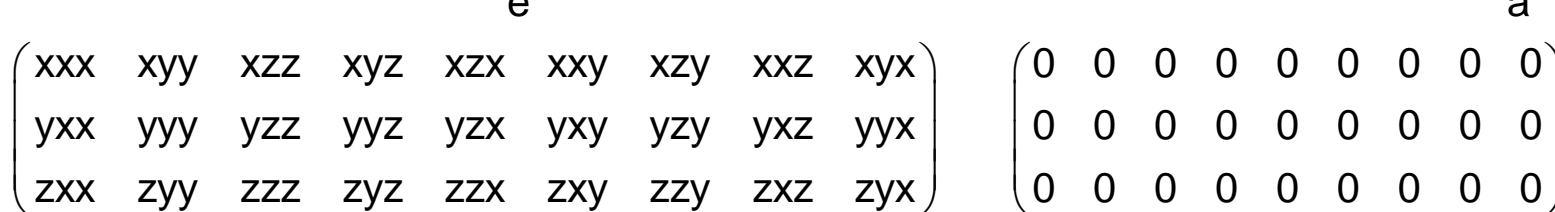

a

$\left(\begin{array}{lllllllll}x x x & x y y & x z z & x y z & x z x & x x y & x z y & x x z & x y x\end{array}\right.$

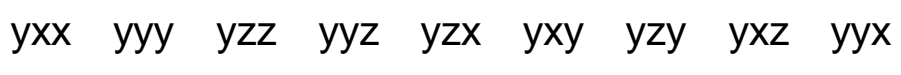
$\begin{array}{llllllllll} & z x x & z y y & z z z & z y z & z z x & \text { zxy } & \text { zzy } & \text { zxz } & \text { zyx }\end{array}$

$\left[\mathrm{V}^{3}\right] \quad\left(\begin{array}{llll}0 & 0 & 0 & 0 \\ 0 & 0 & 0 & \\ 0 & 0 & 0 & \end{array}\right)$

$\left(\begin{array}{llll}x x x & x y y & x z z & x y z \\ y x x & y y y & y z z & \\ z x x & z y y & z z z & \end{array}\right)$

$\left(\begin{array}{llll}0 & 0 & 0 & 0 \\ 0 & 0 & 0 & \\ 0 & 0 & 0\end{array}\right)$

$V\left[V^{2}\right]\left(\begin{array}{llllll}0 & 0 & 0 & 0 & 0 & 0 \\ 0 & 0 & 0 & 0 & 0 & 0 \\ 0 & 0 & 0 & 0 & 0 & 0\end{array}\right)$

$\left(\begin{array}{llllll}x x x & x y y & x z z & x y z & x x z & x x y\end{array}\right)$

$\left.\begin{array}{llllll}y x x & y y y & y z z & y y z & y x z & y x y \\ z x x & z y y & z z z & z y z & z x z & z x y\end{array}\right)$

$\left(\begin{array}{llllll}0 & 0 & 0 & 0 & 0 & 0 \\ 0 & 0 & 0 & 0 & 0 & 0 \\ 0 & 0 & 0 & 0 & 0 & 0\end{array}\right)$

$\begin{array}{llllll}0 & 0 & 0 & 0 & 0 & 0\end{array}$

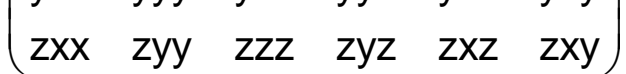

$\begin{array}{llllll}0 & 0 & 0 & 0 & 0 & 0\end{array}$

$$
\left\{V^{2}\right\} V\left(\begin{array}{lll}
0 & 0 & 0 \\
0 & 0 & 0 \\
0 & 0 & 0
\end{array}\right)
$$

$\left(\begin{array}{ccc}y z x & y z y & y z z \\ z x x & z x y & z x z \\ x y x & x y y & x y z\end{array}\right)$

$\left(\begin{array}{lll}0 & 0 & 0 \\ 0 & 0 & 0 \\ 0 & 0 & 0\end{array}\right)$

$\left(\begin{array}{llll}x x x & x y y & x z z & x y z \\ y x x & y y y & y z z & \\ z x x & z y y & z z z\end{array}\right)$

$\left(\begin{array}{llllll}x x x & x y y & x z z & x y z & x x z & x x y\end{array}\right)$ $\begin{array}{llllll}y x x & y y y & y z z & y y z & y x z & y x y\end{array}$ zxx $\quad$ zyy $\quad z z z \quad z y z \quad z x z \quad z x y)$

$\left(\begin{array}{ccc}y z x & y z y & y z z \\ z x x & z x y & z x z \\ x y x & x y y & x y z\end{array}\right)$

\section{$\mathrm{n}=2 \mathbf{S}_{4}=\overline{\mathbf{4}}_{z}$}

$V^{3} \quad\left(\begin{array}{rrrrrrrrr}0 & 0 & 0 & x y z & x z x & 0 & y z x & -y y z & 0 \\ 0 & 0 & 0 & y y z & y z x & 0 & -x z & x y z & 0 \\ z x x & -z x x & 0 & 0 & 0 & z x y & 0 & 0 & z x y\end{array}\right)$

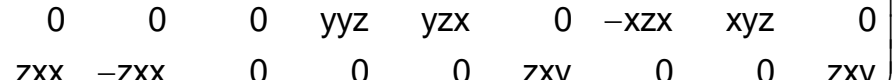

$\left[V^{3}\right]\left(\begin{array}{rrrr}0 & 0 & 0 & x y z \\ 0 & 0 & 0 & \\ z x x & -z x x & 0 & \end{array}\right)$

$\left(\begin{array}{rrrr}0 & 0 & 0 & 0 \\ 0 & 0 & 0 & \\ z x x & z x x & z z z & \end{array}\right)$

$V\left[V^{2}\right]\left(\begin{array}{rrrrrr}0 & 0 & 0 & x y z & x x z & 0 \\ 0 & 0 & 0 & -x x z & x y z & 0 \\ z x x & -z x x & 0 & 0 & 0 & z x y\end{array}\right)$

$\left(\begin{array}{rrrrrr}0 & 0 & 0 & x y z & x x z & 0 \\ 0 & 0 & 0 & x x z & -x y z & 0 \\ z x x & z x x & z z z & 0 & 0 & 0\end{array}\right)$

$\left(\begin{array}{rrrrrr}0 & 0 & 0 & x y z & x x z & 0 \\ 0 & 0 & 0 & -x x z & x y z & 0 \\ z x x & -z x x & 0 & 0 & 0 & z x y\end{array}\right)$

$\left\{V^{2}\right\} V\left(\begin{array}{ccc}y z x & y z y & 0 \\ y z y & -y z x & 0 \\ 0 & 0 & 0\end{array}\right)$

$\left(\begin{array}{ccc}y z x & y z y & 0 \\ -y z y & y z x & 0 \\ 0 & 0 & x y z\end{array}\right)$

$\left(\begin{array}{ccc}y z x & y z y & 0 \\ y z y & -y z x & 0 \\ 0 & 0 & 0\end{array}\right)$

$\left(\begin{array}{ccc}y z x & y z y & 0 \\ -y z y & y z x & 0 \\ 0 & 0 & x y z\end{array}\right)$ 
$\mathrm{n}=3 \quad \mathbf{S}_{6}=\overline{\mathbf{3}}_{z}$

$\mathrm{V}^{3} \quad\left(\begin{array}{lllllllll}0 & 0 & 0 & 0 & 0 & 0 & 0 & 0 & 0 \\ 0 & 0 & 0 & 0 & 0 & 0 & 0 & 0 & 0 \\ 0 & 0 & 0 & 0 & 0 & 0 & 0 & 0 & 0\end{array}\right)$

$\left[\mathrm{V}^{3}\right]\left(\begin{array}{llll}0 & 0 & 0 & 0 \\ 0 & 0 & 0 & \\ 0 & 0 & 0 & \end{array}\right)$

$V\left[V^{2}\right]\left(\begin{array}{llllll}0 & 0 & 0 & 0 & 0 & 0 \\ 0 & 0 & 0 & 0 & 0 & 0 \\ 0 & 0 & 0 & 0 & 0 & 0\end{array}\right)$

$\left(\begin{array}{llllll}0 & 0 & 0 & 0 & 0 & 0\end{array}\right)$

$\left\{V^{2}\right\} V\left(\begin{array}{lll}0 & 0 & 0 \\ 0 & 0 & 0 \\ 0 & 0 & 0\end{array}\right)$

$\mathrm{n}>3 \quad \mathrm{C}_{o \mathrm{~h}}=\infty / \mathrm{m}$

$V^{3} \quad\left(\begin{array}{lllllllll}0 & 0 & 0 & 0 & 0 & 0 & 0 & 0 & 0 \\ 0 & 0 & 0 & 0 & 0 & 0 & 0 & 0 & 0 \\ 0 & 0 & 0 & 0 & 0 & 0 & 0 & 0 & 0\end{array}\right)$

$\left[\mathrm{V}^{3}\right]\left(\begin{array}{llll}0 & 0 & 0 & 0 \\ 0 & 0 & 0 & \\ 0 & 0 & 0 & \end{array}\right)$

$V\left[V^{2}\right]\left(\begin{array}{llllll}0 & 0 & 0 & 0 & 0 & 0 \\ 0 & 0 & 0 & 0 & 0 & 0 \\ 0 & 0 & 0 & 0 & 0 & 0\end{array}\right)$

$\left\{\mathrm{V}^{2}\right\} \mathrm{V}\left(\begin{array}{ccc}0 & 0 & 0 \\ 0 & 0 & 0 \\ 0 & 0 & 0\end{array}\right)$

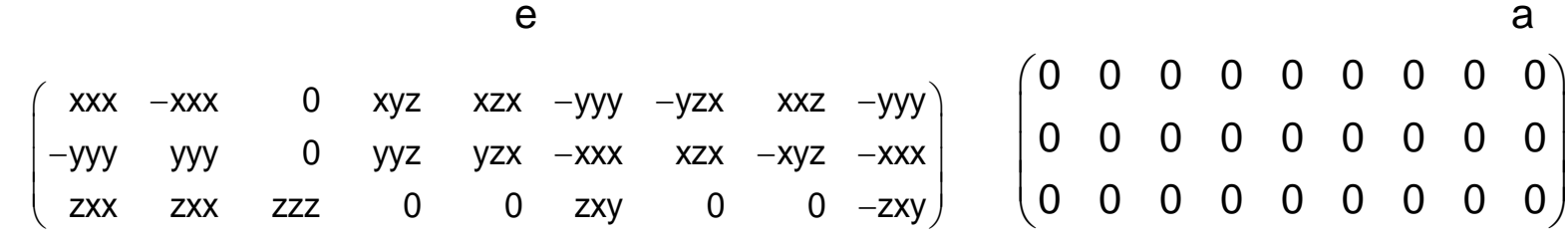

$\left(\begin{array}{rrrr}x x x & -x x x & 0 & 0 \\ -y y y & y y y & 0 & \\ z x x & z x x & z z z & \end{array}\right)$

$\left(\begin{array}{llll}0 & 0 & 0 & 0 \\ 0 & 0 & 0 & \\ 0 & 0 & 0 & \end{array}\right)$

$\left(\begin{array}{rrrrrr}x x x & -x x x & 0 & x y z & x x z & -y y y \\ -y y y & y y y & 0 & x x z & -x y z & -x x x \\ z x x & z x x & z z z & 0 & 0 & 0\end{array}\right)$

$\left(\begin{array}{llllll}0 & 0 & 0 & 0 & 0 & 0 \\ 0 & 0 & 0 & 0 & 0 & 0 \\ 0 & 0 & 0 & 0 & 0 & 0\end{array}\right)$

$\left(\begin{array}{ccc}y z x & y z y & 0 \\ -y z y & y z x & 0 \\ 0 & 0 & x y z\end{array}\right)$

$\left(\begin{array}{lll}0 & 0 & 0 \\ 0 & 0 & 0 \\ 0 & 0 & 0\end{array}\right)$

$\left(\begin{array}{rrrrrrrrr}0 & 0 & 0 & x y z & x z x & 0 & -y z x & y y z & 0 \\ 0 & 0 & 0 & y y z & y z x & 0 & x z x & -x y z & 0 \\ z x x & z x x & z z z & 0 & 0 & z x y & 0 & 0 & -z x y\end{array}\right) \quad\left(\begin{array}{rrrrrrrrr}0 & 0 & 0 & 0 & 0 & 0 & 0 & 0 & 0 \\ 0 & 0 & 0 & 0 & 0 & 0 & 0 & 0 & 0 \\ 0 & 0 & 0 & 0 & 0 & 0 & 0 & 0 & 0\end{array}\right)$

$\left(\begin{array}{rrrr}0 & 0 & 0 & 0 \\ 0 & 0 & 0 & \\ z x x & z x x & z z z & \end{array}\right)$

$\left(\begin{array}{llll}0 & 0 & 0 & 0 \\ 0 & 0 & 0 & \\ 0 & 0 & 0\end{array}\right)$

$\left(\begin{array}{rrrrrr}0 & 0 & 0 & x y z & x x z & 0 \\ 0 & 0 & 0 & x x z & -x y z & 0 \\ z x x & z x x & z z z & 0 & 0 & 0\end{array}\right)$

$\left(\begin{array}{llllll}0 & 0 & 0 & 0 & 0 & 0 \\ 0 & 0 & 0 & 0 & 0 & 0 \\ 0 & 0 & 0 & 0 & 0 & 0\end{array}\right)$

$\left(\begin{array}{ccc}y z x & y z y & 0 \\ -y z y & y z x & 0 \\ 0 & 0 & x y z\end{array}\right)$ ae

$\left(\begin{array}{lllllllll}x x x & -x x x & 0 & x y z & x z x & -y y y & -y z x & x x z & -y y y \\ -y y y & y y & 0 & y y z & y z & -x y x & x z x & -x y z & -x y x\end{array}\right)$ $\left.\begin{array}{rrrrrrrrr}-y y y & y y y & 0 & y y z & y z x & -x x x & x z x & -x y z & -x x x \\ z x x & z x x & z z z & 0 & 0 & z x y & 0 & 0 & -z x y\end{array}\right)$

$\left(\begin{array}{rrrr}x x x & -x x x & 0 & 0 \\ -y y y & y y y & 0 & \\ z x x & z x x & z z z & \end{array}\right)$

$\left(\begin{array}{rrrrrr}x x x & -x x x & 0 & x y z & x x z & -y y y \\ -y y y & y y y & 0 & x x z & -x y z & -x x x \\ z x x & z x x & z z z & 0 & 0 & 0\end{array}\right)$

$\left(\begin{array}{ccc}y z x & y z y & 0 \\ -y z y & y z x & 0 \\ 0 & 0 & x y z\end{array}\right)$

$\left(\begin{array}{rrrrrrrrr}0 & 0 & 0 & x y z & x z x & 0 & -y z x & y y z & 0 \\ 0 & 0 & 0 & y y z & y z x & 0 & x z x & -x y z & 0 \\ z x x & z x x & z z z & 0 & 0 & z x y & 0 & 0 & -z x y\end{array}\right)$

$\left(\begin{array}{rrrr}0 & 0 & 0 & 0 \\ 0 & 0 & 0 & \\ z x x & z x x & z z z & \end{array}\right)$

$\left(\begin{array}{rrrrrr}0 & 0 & 0 & x y z & x x z & 0 \\ 0 & 0 & 0 & x x z & -x y z & 0 \\ z x x & z x x & z z z & 0 & 0 & 0\end{array}\right)$

$\left(\begin{array}{ccc}y z x & y z y & 0 \\ -y z y & y z x & 0 \\ 0 & 0 & x y z\end{array}\right)$ 
$\mathrm{n}=1 \quad \mathbf{C}_{\mathrm{in}}=\mathbf{m}_{\mathrm{z}}$

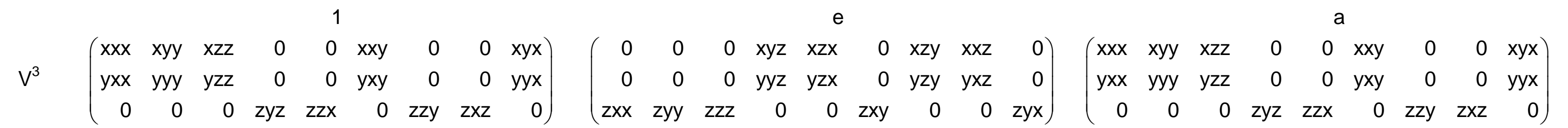
$\left[V^{3}\right]\left(\begin{array}{rrrr}x x x & x y y & x z z & 0 \\ y x x & y y y & y z z & \\ 0 & 0 & 0\end{array}\right)$ $\left(\begin{array}{rrrr}0 & 0 & 0 & x y z \\ 0 & 0 & 0 & \\ z x x & z y y & z z z\end{array}\right)$ $\left(\begin{array}{rrrr}x x x & x y y & x z z & 0 \\ y x x & y y y & y z z & \\ 0 & 0 & 0 & \end{array}\right)$ $\left.V^{2} V^{2}\right]\left(\begin{array}{rrrrrr}x x x & x y y & x z z & 0 & 0 & x x y \\ y x x & y y y & y z z & 0 & 0 & y x y \\ 0 & 0 & 0 & z y z & z x z & 0\end{array}\right)$ $\left(\begin{array}{rrrrrr}0 & 0 & 0 & x y z & x x z & 0 \\ 0 & 0 & 0 & y y z & y x z & 0 \\ z x x & z y y & z z z & 0 & 0 & z x y\end{array}\right)$ $\left(\begin{array}{rrrrrr}x x x & x y y & x z z & 0 & 0 & x x y \\ y x x & y y y & y z z & 0 & 0 & y x y \\ 0 & 0 & 0 & z y z & z x z & 0\end{array}\right)$ $\left\{V^{2}\right\} V\left(\begin{array}{ccc}0 & 0 & y z z \\ 0 & 0 & z x z \\ x y x & x y y & 0\end{array}\right)$ $\left(\begin{array}{ccc}y z x & y z y & 0 \\ z x x & z x y & 0 \\ 0 & 0 & x y z\end{array}\right)$ $\left(\begin{array}{ccc}0 & 0 & y z z \\ 0 & 0 & z x z \\ x y x & x y y & 0\end{array}\right)$ $\left(\begin{array}{lllllllll}0 & 0 & 0 & x y z & x z x & 0 & x z y & x x z & 0 \\ 0 & 0 & 0 & y y z & y z x & 0 & y z y & y x z & 0\end{array}\right)$ $\left(\begin{array}{rrrrrrrrr}0 & 0 & 0 & y y z & y z x & 0 & y z y & y x z & 0 \\ z x x & z y y & z z z & 0 & 0 & z x y & 0 & 0 & z y x\end{array}\right)$ $\left(\begin{array}{rrrr}0 & 0 & 0 & x y z \\ 0 & 0 & 0 & \\ z x x & z y y & z z z & \end{array}\right)$ $\mathrm{n}=2 \quad \mathrm{C}_{2 \mathrm{~h}}=2 / \mathrm{m}_{\mathrm{z}}$ $V^{3} \quad\left(\begin{array}{lllllllll}0 & 0 & 0 & 0 & 0 & 0 & 0 & 0 & 0 \\ 0 & 0 & 0 & 0 & 0 & 0 & 0 & 0 & 0 \\ 0 & 0 & 0 & 0 & 0 & 0 & 0 & 0 & 0\end{array}\right)$ $\left(\begin{array}{rrrrrrrrr}0 & 0 & 0 & x y z & x z x & 0 & x z y & x x z & 0 \\ 0 & 0 & 0 & y y z & y z x & 0 & y z y & y x z & 0 \\ z x x & z y y & z z z & 0 & 0 & z x y & 0 & 0 & z y x\end{array}\right)\left(\begin{array}{lllllllll}0 & 0 & 0 & 0 & 0 & 0 & 0 & 0 & 0 \\ 0 & 0 & 0 & 0 & 0 & 0 & 0 & 0 & 0 \\ 0 & 0 & 0 & 0 & 0 & 0 & 0 & 0 & 0\end{array}\right)$ $\left(\begin{array}{rrrrrr}0 & 0 & 0 & x y z & x x z & 0 \\ 0 & 0 & 0 & y y z & y x z & 0 \\ z x x & z y y & z z z & 0 & 0 & z x y\end{array}\right)$ $\left[\mathrm{V}^{3}\right]\left(\begin{array}{llll}0 & 0 & 0 & 0 \\ 0 & 0 & 0 & \\ 0 & 0 & 0 & \end{array}\right)$ $\left(\begin{array}{rrrr}0 & 0 & 0 & x y z \\ 0 & 0 & 0 & \\ z x x & z y y & z z z & \end{array}\right)$

$\left(\begin{array}{llll}0 & 0 & 0 & 0 \\ 0 & 0 & 0 & \\ 0 & 0 & 0 & \end{array}\right)$

$$
\left(\begin{array}{llllll}
0 & 0 & 0 & 0 & 0 & 0 \\
0 & 0 & 0 & 0 & 0 & 0
\end{array}\right)
$$

$V\left[V^{2}\right]\left(\begin{array}{llllll}0 & 0 & 0 & 0 & 0 & 0 \\ 0 & 0 & 0 & 0 & 0 & 0 \\ 0 & 0 & 0 & 0 & 0 & 0\end{array}\right)$ $\left\{V^{2}\right\} V\left(\begin{array}{ccc}0 & 0 & 0 \\ 0 & 0 & 0 \\ 0 & 0 & 0\end{array}\right)$ $\left(\begin{array}{lll}0 & 0 & 0 \\ 0 & 0 & 0\end{array}\right)$ $\left(\begin{array}{rrrrrr}0 & 0 & 0 & x y z & x x z & 0 \\ 0 & 0 & 0 & y y z & y x z & 0 \\ z x x & z y y & z z z & 0 & 0 & z x y\end{array}\right)$

$\left(\begin{array}{llllll}0 & 0 & 0 & 0 & 0 & 0 \\ 0 & 0 & 0 & 0 & 0 & 0 \\ 0 & 0 & 0 & 0 & 0 & 0\end{array}\right)$

$\left(\begin{array}{ccc}y z x & y z y & 0 \\ z x x & z x y & 0 \\ 0 & 0 & x y z\end{array}\right)$ $\left(\begin{array}{lll}0 & 0 & 0 \\ 0 & 0 & 0 \\ 0 & 0 & 0\end{array}\right)$ $\left(\begin{array}{ccc}y z x & y z y & 0 \\ z x x & z x y & 0 \\ 0 & 0 & x y z\end{array}\right)$

ae

$\left(\begin{array}{rrrrrrrrr}0 & 0 & 0 & x y z & x z x & 0 & x z y & x x z & 0 \\ 0 & 0 & 0 & y y z & y z x & 0 & y z y & y x z & 0 \\ z x x & z y y & z z z & 0 & 0 & z x y & 0 & 0 & z y x\end{array}\right)$

$\left(\begin{array}{rrrr}0 & 0 & 0 & x y z \\ 0 & 0 & 0 & \\ z x x & z y y & z z z & \end{array}\right)$

$\left(\begin{array}{rrrrrr}0 & 0 & 0 & x y z & x x z & 0 \\ 0 & 0 & 0 & y y z & y x z & 0 \\ z x x & z y y & z z z & 0 & 0 & z x y\end{array}\right)$

$\left(\begin{array}{ccc}y z x & y z y & 0 \\ z x x & z x y & 0 \\ 0 & 0 & x y z\end{array}\right)$ 
$\mathrm{n}=3 \quad \mathrm{C}_{3 \mathrm{~h}}=\overline{\mathbf{6}}_{2}$ $\left(\begin{array}{rrrrrrrrr}0 & 0 & 0 & x y z & x z x & 0 & -y z x & y y z & 0 \\ 0 & 0 & 0 & y y z & y z x & 0 & x z x & -x y z & 0 \\ z x x & z x x & z z z & 0 & 0 & z x y & 0 & 0 & -z x y\end{array}\right)\left(\begin{array}{rrrrrrrrr}0 & 0 & 0 & 0 & 0 & 0 & 0 & 0 & 0 \\ 0 & 0 & 0 & 0 & 0 & 0 & 0 & 0 & 0 \\ 0 & 0 & 0 & 0 & 0 & 0 & 0 & 0 & 0\end{array}\right)$
ae

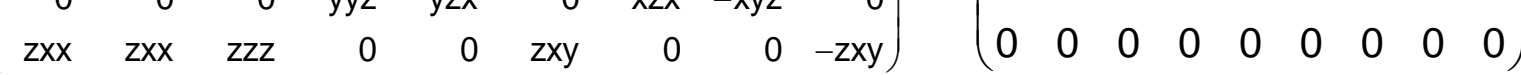 $\left[V^{3}\right]\left(\begin{array}{llll}0 & 0 & 0 & 0 \\ 0 & 0 & 0 \\ 0 & 0 & 0\end{array}\right)$ $V\left[V^{2}\right]\left(\begin{array}{llllll}0 & 0 & 0 & 0 & 0 & 0 \\ 0 & 0 & 0 & 0 & 0 & 0 \\ 0 & 0 & 0 & 0 & 0 & 0\end{array}\right)$ $\left(\begin{array}{rrrr}0 & 0 & 0 & 0 \\ 0 & 0 & 0 & \\ z x x & z x x & z z z & \end{array}\right)$ $\left(\begin{array}{llll}0 & 0 & 0 & 0 \\ 0 & 0 & 0 & \\ 0 & 0 & 0\end{array}\right)$ $\left(\begin{array}{rrrrrr}0 & 0 & 0 & x y z & x x z & 0 \\ 0 & 0 & 0 & x x z & -x y z & 0 \\ z x x & z x x & z z z & 0 & 0 & 0\end{array}\right)$ $\left(\begin{array}{llllll}0 & 0 & 0 & 0 & 0 & 0 \\ 0 & 0 & 0 & 0 & 0 & 0 \\ 0 & 0 & 0 & 0 & 0 & 0\end{array}\right)$ $\left\{V^{2}\right\} V\left(\begin{array}{lll}0 & 0 & 0 \\ 0 & 0 & 0 \\ 0 & 0 & 0\end{array}\right)$
$\left(\begin{array}{lll}0 & 0 & 0 \\ 0 & 0 & 0 \\ 0 & 0 & 0\end{array}\right)$
$\left(\begin{array}{rrrrrrrrr}0 & 0 & 0 & x y z & x z x & 0 & -y z x & y y z & 0 \\ 0 & 0 & 0 & y y z & y z x & 0 & x z x & -x y z & 0 \\ z x x & z x x & z z z & 0 & 0 & z x y & 0 & 0 & -z x y\end{array}\right)$
$\left(\begin{array}{rrrr}0 & 0 & 0 & 0 \\ 0 & 0 & 0 & \\ z x x & z x x & z z z & \end{array}\right)$
$\left(\begin{array}{rrrrrr}0 & 0 & 0 & x y z & x x z & 0 \\ 0 & 0 & 0 & x x z & -x y z & 0 \\ z x x & z x x & z z z & 0 & 0 & 0\end{array}\right)$ $\left(\begin{array}{ccc}y z x & y z y & 0 \\ -y z y & y z x & 0 \\ 0 & 0 & x y z\end{array}\right)$ 
$\mathrm{n}=1 \quad \mathrm{D}_{1}=\mathbf{2}_{\mathrm{y}}$

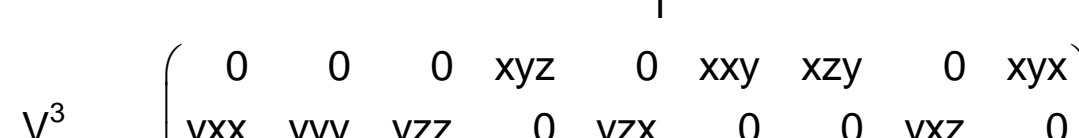
$V^{3} \quad\left(\begin{array}{rrrrrrrrr}y x x & y y y & y z z & 0 & y z x & 0 & 0 & y x z & 0 \\ 0 & 0 & 0 & z y z & 0 & z x y & z z y & 0 & z y x\end{array}\right)$ $\left[V^{3}\right] \quad\left(\begin{array}{rrrr}0 & 0 & 0 & x y z \\ y x x & y y y & y z z & \\ 0 & 0 & 0\end{array}\right)$

$V\left[V^{2}\right]\left(\begin{array}{rrrrrr}0 & 0 & 0 & x y z & 0 & x x y \\ y x x & y y y & y z z & 0 & y x z & 0 \\ 0 & 0 & 0 & z y z & 0 & z x y\end{array}\right)$

$\left\{V^{2}\right\} \bigvee\left(\begin{array}{ccc}y z x & 0 & y z z \\ 0 & z x y & 0 \\ x y x & 0 & x y z\end{array}\right)$

$\mathrm{n}=2 \quad \mathbf{D}_{2}=\mathbf{2} \mathbf{x}_{\mathbf{2}} \mathbf{y}_{\mathrm{z}}$

$V^{3} \quad\left(\begin{array}{rrrrrrrrr}0 & 0 & 0 & x y z & 0 & 0 & x z y & 0 & 0 \\ 0 & 0 & 0 & 0 & y z x & 0 & 0 & y x z & 0 \\ 0 & 0 & 0 & 0 & 0 & z x y & 0 & 0 & z y x\end{array}\right) \quad\left(\begin{array}{l}0 \\ 0 \\ 0\end{array}\right.$

$\left[V^{3}\right]\left(\begin{array}{llll}0 & 0 & 0 & x y z \\ 0 & 0 & 0 & \\ 0 & 0 & 0 & \end{array}\right)$

$V\left[V^{2}\right]\left(\begin{array}{rrrrrr}0 & 0 & 0 & \mathrm{xyz} & 0 & 0 \\ 0 & 0 & 0 & 0 & \mathrm{yxz} & 0 \\ 0 & 0 & 0 & 0 & 0 & \mathrm{zxy}\end{array}\right)$

$\left\{\mathrm{V}^{2}\right\} \vee\left(\begin{array}{ccc}\mathrm{yzx} & 0 & 0 \\ 0 & \mathrm{zxy} & 0 \\ 0 & 0 & \mathrm{xyz}\end{array}\right)$

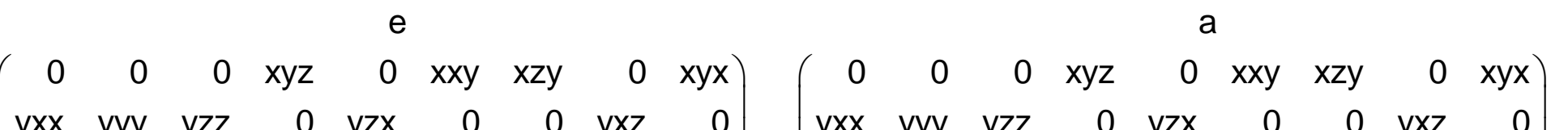
$\left(\begin{array}{rrrrrrrrr}\mathrm{yxx} & \mathrm{yyy} & \mathrm{yzz} & 0 & \mathrm{yzx} & 0 & 0 & \mathrm{yxz} & 0 \\ 0 & 0 & 0 & \mathrm{zyz} & 0 & \mathrm{zxy} & \mathrm{zzy} & 0 & \mathrm{zyx}\end{array}\right)\left(\begin{array}{rrrrrrrrr}\mathrm{yxx} & \mathrm{yyy} & \mathrm{yzz} & 0 & \mathrm{yzx} & 0 & 0 & \mathrm{yxz} & 0 \\ 0 & 0 & 0 & \mathrm{zyz} & 0 & \mathrm{zxy} & \mathrm{zzy} & 0 & \mathrm{zyx}\end{array}\right)$

$\left(\begin{array}{rrrr}0 & 0 & 0 & x y z \\ y x x & y y y & y z z & \\ 0 & 0 & 0\end{array}\right)$

$\left(\begin{array}{rrrrrr}0 & 0 & 0 & x y z & 0 & x x y \\ y x x & y y y & y z z & 0 & y x z & 0 \\ 0 & 0 & 0 & z y z & 0 & z x y\end{array}\right)$

$\left(\begin{array}{ccc}y z x & 0 & y z z \\ 0 & z x y & 0 \\ x y x & 0 & x y z\end{array}\right)$ $\left(\begin{array}{rrrr}0 & 0 & 0 & x y z \\ y x x & y y y & y z z & \\ 0 & 0 & 0\end{array}\right)$

$\left(\begin{array}{rrrrrr}0 & 0 & 0 & x y z & 0 & x x y \\ y x x & y y y & y z z & 0 & y x z & 0 \\ 0 & 0 & 0 & z y z & 0 & z x y\end{array}\right)$

$\left(\begin{array}{ccc}y z x & 0 & y z z \\ 0 & z x y & 0 \\ x y x & 0 & x y z\end{array}\right)$ ae

$\left(\begin{array}{ccccccccc}0 & 0 & 0 & x y z & 0 & x x y & x z y & 0 & x y x \\ y x & y y & y z & 0 & y z & 0 & 0 & y x z & 0\end{array}\right)$

$\begin{array}{rrrrrrrrrr}y \mathrm{yx} & \mathrm{yyy} & \mathrm{yzz} & 0 & \mathrm{yzx} & 0 & 0 & \mathrm{yxz} & 0 \\ 0 & 0 & 0 & \mathrm{zyz} & 0 & \mathrm{zxy} & \mathrm{zzy} & 0 & \mathrm{zyx}\end{array}$

$\left(\begin{array}{rrrr}0 & 0 & 0 & x y z \\ y x x & y y y & y z z & \\ 0 & 0 & 0\end{array}\right)$

$\left(\begin{array}{rrrrrr}0 & 0 & 0 & x y z & 0 & x x y \\ y x x & y y y & y z z & 0 & y x z & 0 \\ 0 & 0 & 0 & z y z & 0 & z x y\end{array}\right)$

$\left(\begin{array}{ccc}y z x & 0 & y z z \\ 0 & z x y & 0 \\ x y x & 0 & x y z\end{array}\right)$

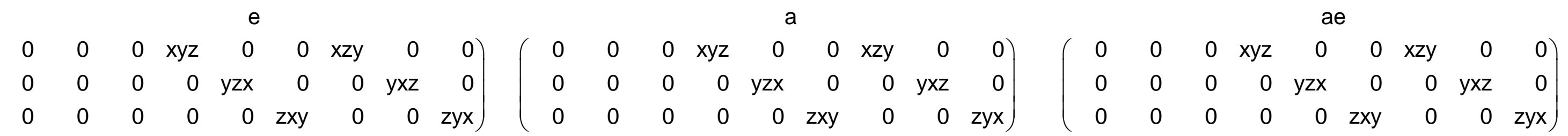

$\left(\begin{array}{llll}0 & 0 & 0 & \mathrm{xyz} \\ 0 & 0 & 0 & \\ 0 & 0 & 0 & \end{array}\right)$

$\left(\begin{array}{llll}0 & 0 & 0 & \mathrm{xyz} \\ 0 & 0 & 0 & \\ 0 & 0 & 0 & \end{array}\right)$

$\left(\begin{array}{llll}0 & 0 & 0 & \mathrm{xyz} \\ 0 & 0 & 0 & \\ 0 & 0 & 0 & \end{array}\right)$

$\left(\begin{array}{rrrrrr}0 & 0 & 0 & x y z & 0 & 0 \\ 0 & 0 & 0 & 0 & y x z & 0 \\ 0 & 0 & 0 & 0 & 0 & z x y\end{array}\right)$

$\left(\begin{array}{rrrrrr}0 & 0 & 0 & x y z & 0 & 0 \\ 0 & 0 & 0 & 0 & y x z & 0 \\ 0 & 0 & 0 & 0 & 0 & z x y\end{array}\right)$

$\left(\begin{array}{rrrrrr}0 & 0 & 0 & x y z & 0 & 0 \\ 0 & 0 & 0 & 0 & y x z & 0 \\ 0 & 0 & 0 & 0 & 0 & z x y\end{array}\right)$

$\left(\begin{array}{ccc}y z x & 0 & 0 \\ 0 & z x y & 0 \\ 0 & 0 & x y z\end{array}\right)$

$\left(\begin{array}{ccc}\mathrm{yzx} & 0 & 0 \\ 0 & \mathrm{zxy} & 0 \\ 0 & 0 & \mathrm{xyz}\end{array}\right)$

$\left(\begin{array}{ccc}y z x & 0 & 0 \\ 0 & z x y & 0 \\ 0 & 0 & x y z\end{array}\right)$ 
$\mathrm{n}=3 \quad \mathrm{D}_{3}=\mathbf{3}_{\mathrm{z}} \mathbf{2}_{\mathrm{x}}$

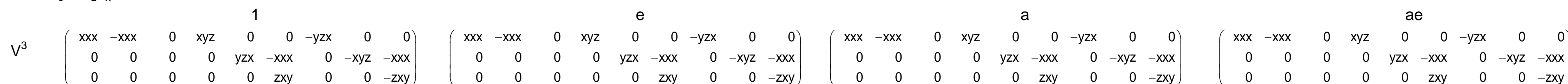

$\left[\mathrm{V}^{3}\right]\left(\begin{array}{rrrr}\mathrm{xxx} & -\mathrm{xxx} & 0 & 0 \\ 0 & 0 & 0 & \\ 0 & 0 & 0 & \end{array}\right)$

$\left(\begin{array}{rrrr}x x x & -x x x & 0 & 0 \\ 0 & 0 & 0 & \\ 0 & 0 & 0 & \end{array}\right)$

$\left(\begin{array}{rrrr}x x x & -x x x & 0 & 0 \\ 0 & 0 & 0 & \\ 0 & 0 & 0 & \end{array}\right)$

$\left(\begin{array}{rrrr}x x x & -x x x & 0 & 0 \\ 0 & 0 & 0 & \\ 0 & 0 & 0 & \end{array}\right)$

$V\left[V^{2}\right]\left(\begin{array}{rrrrrr}0 & 0 & 0 & x y z & 0 & -y y y \\ -y y y & y y y & 0 & 0 & -x y z & 0 \\ 0 & 0 & 0 & 0 & 0 & 0\end{array}\right)$

$\left(\begin{array}{rrrrrr}0 & 0 & 0 & x y z & 0 & -y y y \\ -y y y & y y y & 0 & 0 & -x y z & 0 \\ 0 & 0 & 0 & 0 & 0 & 0\end{array}\right)$

$\left(\begin{array}{rrrrrr}0 & 0 & 0 & x y z & 0 & -y y y \\ -y y y & y y y & 0 & 0 & -x y z & 0 \\ 0 & 0 & 0 & 0 & 0 & 0\end{array}\right)$

$\left(\begin{array}{rrrrrr}0 & 0 & 0 & x y z & 0 & -y y y \\ -y y y & y y y & 0 & 0 & -x y z & 0 \\ 0 & 0 & 0 & 0 & 0 & 0\end{array}\right)$

$\left\{\mathrm{V}^{2}\right\} \mathrm{V}\left(\begin{array}{ccc}\mathrm{yzx} & 0 & 0 \\ 0 & \mathrm{yzx} & 0 \\ 0 & 0 & \mathrm{xyz}\end{array}\right)$

$\left(\begin{array}{ccc}y z x & 0 & 0 \\ 0 & y z x & 0 \\ 0 & 0 & x y z\end{array}\right)$

$\left(\begin{array}{ccc}y z x & 0 & 0 \\ 0 & y z x & 0 \\ 0 & 0 & x y z\end{array}\right)$

$\left(\begin{array}{ccc}y z x & 0 & 0 \\ 0 & y z x & 0 \\ 0 & 0 & x y z\end{array}\right)$

$n>3 \quad D_{\infty}=\infty 2$

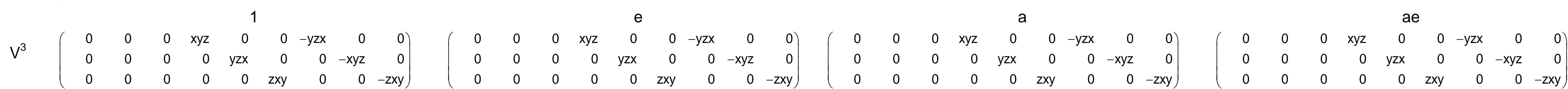

$\left[V^{3}\right]\left(\begin{array}{llll}0 & 0 & 0 & 0 \\ 0 & 0 & 0 & \\ 0 & 0 & 0 & \end{array}\right)$

$\left(\begin{array}{llll}0 & 0 & 0 & 0 \\ 0 & 0 & 0 & \\ 0 & 0 & 0 & \end{array}\right)$

$\left(\begin{array}{llll}0 & 0 & 0 & 0 \\ 0 & 0 & 0 & \\ 0 & 0 & 0 & \end{array}\right)$

$\left(\begin{array}{llll}0 & 0 & 0 & 0 \\ 0 & 0 & 0 & \\ 0 & 0 & 0 & \end{array}\right)$

$V\left[V^{2}\right]\left(\begin{array}{rrrrrr}0 & 0 & 0 & x y z & 0 & 0 \\ 0 & 0 & 0 & 0 & -x y z & 0 \\ 0 & 0 & 0 & 0 & 0 & 0\end{array}\right)$

$\left(\begin{array}{rrrrrr}0 & 0 & 0 & x y z & 0 & 0 \\ 0 & 0 & 0 & 0 & -x y z & 0 \\ 0 & 0 & 0 & 0 & 0 & 0\end{array}\right)$

$\left(\begin{array}{rrrrrr}0 & 0 & 0 & x y z & 0 & 0 \\ 0 & 0 & 0 & 0 & -x y z & 0 \\ 0 & 0 & 0 & 0 & 0 & 0\end{array}\right)$

$\left(\begin{array}{rrrrrr}0 & 0 & 0 & x y z & 0 & 0 \\ 0 & 0 & 0 & 0 & -x y z & 0 \\ 0 & 0 & 0 & 0 & 0 & 0\end{array}\right)$

$\left\{V^{2}\right\} V\left(\begin{array}{ccc}y z x & 0 & 0 \\ 0 & y z x & 0 \\ 0 & 0 & x y z\end{array}\right)$

$\left(\begin{array}{ccc}y z x & 0 & 0 \\ 0 & y z x & 0 \\ 0 & 0 & x y z\end{array}\right)$

$\left(\begin{array}{ccc}y z x & 0 & 0 \\ 0 & y z x & 0 \\ 0 & 0 & x y z\end{array}\right)$

$\left(\begin{array}{ccc}y z x & 0 & 0 \\ 0 & y z x & 0 \\ 0 & 0 & x y z\end{array}\right)$ 


\section{$\mathrm{n}=1 \quad \mathbf{D}_{1 \mathrm{~d}}=\mathbf{2}_{\mathrm{y}} / \mathbf{m}_{\mathrm{y}}$}

$\left(\begin{array}{lllllllll}0 & 0 & 0 & 0 & 0 & 0 & 0 & 0 & 0 \\ 0 & 0 & 0 & 0 & 0 & 0 & 0 & 0 & 0\end{array}\right)$

$\mathrm{V}^{3} \quad\left(\begin{array}{lllllllll}0 & 0 & 0 & 0 & 0 & 0 & 0 & 0 & 0 \\ 0 & 0 & 0 & 0 & 0 & 0 & 0 & 0 & 0\end{array}\right)$

$\left(\begin{array}{lllllllll}0 & 0 & 0 & 0 & 0 & 0 & 0 & 0 & 0\end{array}\right)$

$\left[\mathrm{V}^{3}\right] \quad\left(\begin{array}{llll}0 & 0 & 0 & 0 \\ 0 & 0 & 0 & \\ 0 & 0 & 0 & \end{array}\right)$

$\left.\mathrm{V}^{2}\right]\left(\begin{array}{llllll}0 & 0 & 0 & 0 & 0 & 0 \\ 0 & 0 & 0 & 0 & 0 & 0 \\ 0 & 0 & 0 & 0 & 0 & 0\end{array}\right)$

$\left(\begin{array}{llllll}0 & 0 & 0 & 0 & 0 & 0 \\ 0 & 0 & 0 & 0 & 0 & 0\end{array}\right)$

$\left\{V^{2}\right\} \vee\left(\begin{array}{ccc}0 & 0 & 0 \\ 0 & 0 & 0 \\ 0 & 0 & 0\end{array}\right)$

$\mathrm{n}=2 \quad \mathbf{D}_{2 \mathrm{~d}}=\overline{\mathbf{4}}_{\mathbf{z}} \mathbf{m}_{\mathrm{x}} \mathbf{2}_{\mathrm{xy}}$

$V^{3} \quad\left(\begin{array}{rrrrrrrrr}0 & 0 & 0 & 0 & x z x & 0 & 0 & -y y z & 0 \\ 0 & 0 & 0 & y y z & 0 & 0 & -x z x & 0 & 0 \\ z x x & -z x x & 0 & 0 & 0 & 0 & 0 & 0 & 0\end{array}\right)$

$\left[\mathrm{V}^{3}\right] \quad\left(\begin{array}{rrrr}0 & 0 & 0 & 0 \\ 0 & 0 & 0 & \\ \mathrm{zxx} & -\mathrm{zxx} & 0 & \end{array}\right)$

$\left(\begin{array}{llll}0 & 0 & 0 & 0 \\ 0 & 0 & 0 & \\ 0 & 0 & 0\end{array}\right)$

$V\left[V^{2}\right]\left(\begin{array}{rrrrrr}0 & 0 & 0 & 0 & x x z & 0 \\ 0 & 0 & 0 & -x x z & 0 & 0 \\ z x x & -z x x & 0 & 0 & 0 & 0\end{array}\right)$

$\left\{V^{2}\right\} \vee\left(\begin{array}{ccc}0 & y z y & 0 \\ y z y & 0 & 0 \\ 0 & 0 & 0\end{array}\right)$ $\left(\begin{array}{rrrrrrrrr}0 & 0 & 0 & x y z & 0 & x x y & x z y & 0 & x y x \\ y x x & y y y & y z z & 0 & y z x & 0 & 0 & y x z & 0 \\ 0 & 0 & 0 & z y z & 0 & z x y & z z y & 0 & z y x\end{array}\right)\left(\begin{array}{lllllllll}0 & 0 & 0 & 0 & 0 & 0 & 0 & 0 & 0 \\ 0 & 0 & 0 & 0 & 0 & 0 & 0 & 0 & 0 \\ 0 & 0 & 0 & 0 & 0 & 0 & 0 & 0 & 0\end{array}\right)$

$\left(\begin{array}{llll}0 & 0 & 0 & 0 \\ 0 & 0 & 0 & \\ 0 & 0 & 0 & \end{array}\right)$

$\left(\begin{array}{rrrrrr}0 & 0 & 0 & x y z & 0 & x x y \\ y x x & y y y & y z z & 0 & y x z & 0 \\ 0 & 0 & 0 & z y z & 0 & z x y\end{array}\right)$

$\left(\begin{array}{llllll}0 & 0 & 0 & 0 & 0 & 0 \\ 0 & 0 & 0 & 0 & 0 & 0 \\ 0 & 0 & 0 & 0 & 0 & 0\end{array}\right)$

$\left(\begin{array}{ccc}y z x & 0 & y z z \\ 0 & z x y & 0 \\ x y x & 0 & x y z\end{array}\right)$

$\left(\begin{array}{lll}0 & 0 & 0 \\ 0 & 0 & 0 \\ 0 & 0 & 0\end{array}\right)$ $\left(\begin{array}{rrrrrrrrr}0 & 0 & 0 & x y z & 0 & x x y & x z y & 0 & x y x \\ y x x & y y y & y z z & 0 & y z x & 0 & 0 & y x z & 0 \\ 0 & 0 & 0 & z y z & 0 & z x y & z z y & 0 & z y x\end{array}\right)$

$\left(\begin{array}{rrrr}0 & 0 & 0 & x y z \\ y x x & y y y & y z z & \\ 0 & 0 & 0 & \end{array}\right)$

$\left(\begin{array}{rrrrrr}0 & 0 & 0 & x y z & 0 & x x y \\ y x x & y y y & y z z & 0 & y x z & 0 \\ 0 & 0 & 0 & z y z & 0 & z x y\end{array}\right)$

$\left(\begin{array}{ccc}y z x & 0 & y z z \\ 0 & z x y & 0 \\ x y x & 0 & x y z\end{array}\right)$

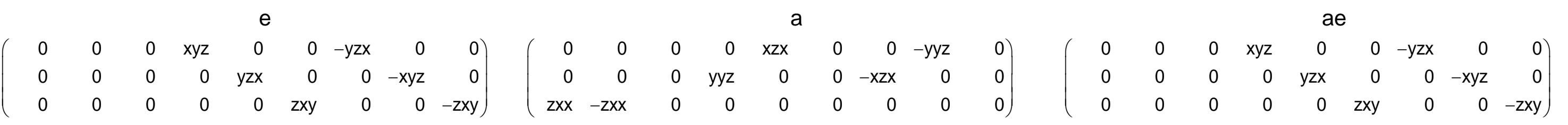

$\left(\begin{array}{rrrr}0 & 0 & 0 & 0 \\ 0 & 0 & 0 & \\ z x x & -z x x & 0 & \end{array}\right)$

$\left(\begin{array}{llll}0 & 0 & 0 & 0 \\ 0 & 0 & 0 & \\ 0 & 0 & 0 & \end{array}\right)$

$\left(\begin{array}{rrrrrr}0 & 0 & 0 & 0 & x x z & 0 \\ 0 & 0 & 0 & -x x z & 0 & 0 \\ z x x & -z x x & 0 & 0 & 0 & 0\end{array}\right)$

$\left(\begin{array}{ccc}0 & y z y & 0 \\ y z y & 0 & 0 \\ 0 & 0 & 0\end{array}\right)$ $\left(\begin{array}{rrrrrr}0 & 0 & 0 & x y z & 0 & 0 \\ 0 & 0 & 0 & 0 & -x y z & 0 \\ 0 & 0 & 0 & 0 & 0 & 0\end{array}\right)$

$\left(\begin{array}{ccc}y z x & 0 & 0 \\ 0 & y z x & 0 \\ 0 & 0 & x y z\end{array}\right)$ 
$V^{3} \quad\left(\begin{array}{lllllllll}0 & 0 & 0 & 0 & 0 & 0 & 0 & 0 & 0 \\ 0 & 0 & 0 & 0 & 0 & 0 & 0 & 0 & 0 \\ 0 & 0 & 0 & 0 & 0 & 0 & 0 & 0 & 0\end{array}\right)$

$$
\left(\begin{array}{rrrrrrrrr}
x x x & -x x x & 0 & x y z & 0 & 0 & -y z x & 0 & 0 \\
0 & 0 & 0 & 0 & y z x & -x x x & 0 & -x y z & -x x x \\
0 & 0 & 0 & 0 & 0 & z x y & 0 & 0 & -z x y
\end{array}\right)\left(\begin{array}{rrrrrrrrr}
0 & 0 & 0 & 0 & 0 & 0 & 0 & 0 & 0 \\
0 & 0 & 0 & 0 & 0 & 0 & 0 & 0 & 0 \\
0 & 0 & 0 & 0 & 0 & 0 & 0 & 0 & 0
\end{array}\right)
$$$$
\left[\mathrm{V}^{3}\right]\left(\begin{array}{llll}
0 & 0 & 0 & 0 \\
0 & 0 & 0 & \\
0 & 0 & 0 &
\end{array}\right)
$$

$V\left[V^{2}\right]\left(\begin{array}{llllll}0 & 0 & 0 & 0 & 0 & 0 \\ 0 & 0 & 0 & 0 & 0 & 0 \\ 0 & 0 & 0 & 0 & 0 & 0\end{array}\right)$

$\left(\begin{array}{llllll}0 & 0 & 0 & 0 & 0 & 0 \\ 0 & 0 & 0 & 0 & 0 & 0\end{array}\right.$

$\left\{V^{2}\right\} V\left(\begin{array}{lll}0 & 0 & 0 \\ 0 & 0 & 0 \\ 0 & 0 & 0\end{array}\right)$

$\mathrm{n}>3 \quad \mathbf{D}_{o \mathrm{~h}}=\infty / \mathbf{m m m}$

$\vee^{3} \quad\left(\begin{array}{lllllllll}0 & 0 & 0 & 0 & 0 & 0 & 0 & 0 & 0 \\ 0 & 0 & 0 & 0 & 0 & 0 & 0 & 0 & 0 \\ 0 & 0 & 0 & 0 & 0 & 0 & 0 & 0 & 0\end{array}\right)$

$\begin{array}{lllllllll}0 & 0 & 0 & 0 & 0 & 0 & 0 & 0 & 0\end{array}$

$\left[V^{3}\right]\left(\begin{array}{llll}0 & 0 & 0 & 0 \\ 0 & 0 & 0 & \\ 0 & 0 & 0 & \end{array}\right)$

$V\left[V^{2}\right]\left(\begin{array}{llllll}0 & 0 & 0 & 0 & 0 & 0 \\ 0 & 0 & 0 & 0 & 0 & 0 \\ 0 & 0 & 0 & 0 & 0 & 0\end{array}\right)$

$\left(\begin{array}{llllll}0 & 0 & 0 & 0 & 0 & 0\end{array}\right.$

$\left\{\mathrm{V}^{2}\right\} \mathrm{V}\left(\begin{array}{ccc}0 & 0 & 0 \\ 0 & 0 & 0 \\ 0 & 0 & 0\end{array}\right)$

$\left(\begin{array}{rrrr}x x x & -x x x & 0 & 0 \\ 0 & 0 & 0 & \\ 0 & 0 & 0 & \end{array}\right) \quad\left(\begin{array}{llll}0 & 0 & 0 & 0 \\ 0 & 0 & 0 & \\ 0 & 0 & 0\end{array}\right)$

$\left(\begin{array}{rrrrrr}x x x & -x x x & 0 & x y z & 0 & 0 \\ 0 & 0 & 0 & 0 & -x y z & -x x x \\ 0 & 0 & 0 & 0 & 0 & 0\end{array}\right)$

$\left(\begin{array}{llllll}0 & 0 & 0 & 0 & 0 & 0 \\ 0 & 0 & 0 & 0 & 0 & 0 \\ 0 & 0 & 0 & 0 & 0 & 0\end{array}\right)$

$\left(\begin{array}{ccc}y z x & 0 & 0 \\ 0 & y z x & 0 \\ 0 & 0 & x y z\end{array}\right)$

$\left(\begin{array}{lll}0 & 0 & 0 \\ 0 & 0 & 0 \\ 0 & 0 & 0\end{array}\right)$

$\left(\begin{array}{rrrrrrrrr}0 & 0 & 0 & x y z & 0 & 0 & -y z x & 0 & 0 \\ 0 & 0 & 0 & 0 & y z x & 0 & 0 & -x y z & 0 \\ 0 & 0 & 0 & 0 & 0 & z x y & 0 & 0 & -z x y\end{array}\right) \quad\left(\begin{array}{rrrrrrrrr}0 & 0 & 0 & 0 & 0 & 0 & 0 & 0 & 0 \\ 0 & 0 & 0 & 0 & 0 & 0 & 0 & 0 & 0 \\ 0 & 0 & 0 & 0 & 0 & 0 & 0 & 0 & 0\end{array}\right)$

$\left(\begin{array}{llll}0 & 0 & 0 & 0 \\ 0 & 0 & 0 & \\ 0 & 0 & 0 & \end{array}\right)$

$\left(\begin{array}{llll}0 & 0 & 0 & 0 \\ 0 & 0 & 0 & \\ 0 & 0 & 0\end{array}\right)$

$\left(\begin{array}{rrrrrr}0 & 0 & 0 & x y z & 0 & 0 \\ 0 & 0 & 0 & 0 & -x y z & 0 \\ 0 & 0 & 0 & 0 & 0 & 0\end{array}\right)$

$\left(\begin{array}{llllll}0 & 0 & 0 & 0 & 0 & 0 \\ 0 & 0 & 0 & 0 & 0 & 0 \\ 0 & 0 & 0 & 0 & 0 & 0\end{array}\right)$

$\left(\begin{array}{ccc}y z x & 0 & 0 \\ 0 & z x y & 0 \\ 0 & 0 & x y z\end{array}\right)$

$\left(\begin{array}{lll}0 & 0 & 0 \\ 0 & 0 & 0 \\ 0 & 0 & 0\end{array}\right)$ ae

$\left(\begin{array}{rrrrrrrrr}x x x & -x x x & 0 & x y z & 0 & 0 & -y z x & 0 & 0 \\ 0 & 0 & 0 & 0 & y z x & -x x x & 0 & -x y z & -x x x \\ 0 & 0 & 0 & 0 & 0 & z x y & 0 & 0 & -z x y\end{array}\right)$

$\left(\begin{array}{rrrr}x x x & -x x x & 0 & 0 \\ 0 & 0 & 0 & \\ 0 & 0 & 0 & \end{array}\right)$

$\left(\begin{array}{rrrrrr}x x x & -x x x & 0 & x y z & 0 & 0 \\ 0 & 0 & 0 & 0 & -x y z & -x x x \\ 0 & 0 & 0 & 0 & 0 & 0\end{array}\right)$

$\left(\begin{array}{ccc}y z x & 0 & 0 \\ 0 & y z x & 0 \\ 0 & 0 & x y z\end{array}\right)$

$\left(\begin{array}{rrrrrrrrr}0 & 0 & 0 & x y z & 0 & 0 & -y z x & 0 & 0 \\ 0 & 0 & 0 & 0 & y z x & 0 & 0 & -x y z & 0 \\ 0 & 0 & 0 & 0 & 0 & z x y & 0 & 0 & -z x y\end{array}\right)$

$\left(\begin{array}{llll}0 & 0 & 0 & 0 \\ 0 & 0 & 0 & \\ 0 & 0 & 0 & \end{array}\right)$

$\left(\begin{array}{rrrrrr}0 & 0 & 0 & x y z & 0 & 0 \\ 0 & 0 & 0 & 0 & -x y z & 0 \\ 0 & 0 & 0 & 0 & 0 & 0\end{array}\right)$

$\left(\begin{array}{ccc}y z x & 0 & 0 \\ 0 & z x y & 0 \\ 0 & 0 & x y z\end{array}\right)$ 


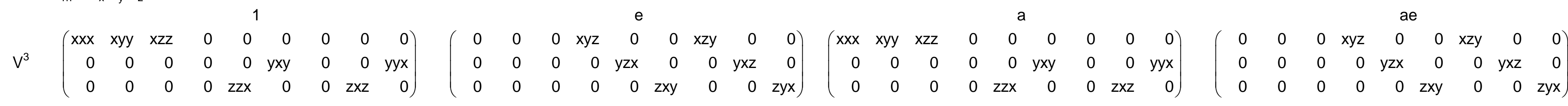
$\left[V^{3}\right]\left(\begin{array}{rrrr}x x x & x y y & x z z & 0 \\ 0 & 0 & 0 & \\ 0 & 0 & 0 & \end{array}\right)$ $\left(\begin{array}{llll}0 & 0 & 0 & x y z \\ 0 & 0 & 0 & \\ 0 & 0 & 0 & \end{array}\right)$ $\left(\begin{array}{rrrr}x x x & x y y & x z z & 0 \\ 0 & 0 & 0 & \\ 0 & 0 & 0 & \end{array}\right)$ $\left(\begin{array}{llll}0 & 0 & 0 & x y z \\ 0 & 0 & 0 & \\ 0 & 0 & 0 & \end{array}\right)$ $V\left[V^{2}\right]\left(\begin{array}{rrrrrr}x x x & x y y & x z z & 0 & 0 & 0 \\ 0 & 0 & 0 & 0 & 0 & y x y \\ 0 & 0 & 0 & 0 & z x z & 0\end{array}\right)$ $\left(\begin{array}{rrrrrr}0 & 0 & 0 & x y z & 0 & 0 \\ 0 & 0 & 0 & 0 & y x z & 0 \\ 0 & 0 & 0 & 0 & 0 & z x y\end{array}\right)$ $\left(\begin{array}{rrrrrr}x x x & x y y & x z z & 0 & 0 & 0 \\ 0 & 0 & 0 & 0 & 0 & y x y \\ 0 & 0 & 0 & 0 & z x z & 0\end{array}\right)$ $\left(\begin{array}{rrrrrr}0 & 0 & 0 & \mathrm{xyz} & 0 & 0 \\ 0 & 0 & 0 & 0 & \mathrm{yxz} & 0 \\ 0 & 0 & 0 & 0 & 0 & \mathrm{zxy}\end{array}\right)$ $\left\{V^{2}\right\} V\left(\begin{array}{ccc}0 & 0 & 0 \\ 0 & 0 & z x z \\ 0 & x y y & 0\end{array}\right)$ $\left(\begin{array}{ccc}y z x & 0 & 0 \\ 0 & z x y & 0 \\ 0 & 0 & x y z\end{array}\right)$ $\left(\begin{array}{ccc}0 & 0 & 0 \\ 0 & 0 & z x z \\ 0 & x y y & 0\end{array}\right)$ $\left(\begin{array}{ccc}y z x & 0 & 0 \\ 0 & z x y & 0 \\ 0 & 0 & x y z\end{array}\right)$ $\mathrm{n}=2 \quad \mathbf{D}_{2 \mathrm{~h}}=\mathbf{m}_{\mathrm{x}} \mathbf{m}_{\mathrm{y}} \mathbf{m}_{\mathrm{z}}$ $V^{3} \quad\left(\begin{array}{lllllllll}0 & 0 & 0 & 0 & 0 & 0 & 0 & 0 & 0 \\ 0 & 0 & 0 & 0 & 0 & 0 & 0 & 0 & 0 \\ 0 & 0 & 0 & 0 & 0 & 0 & 0 & 0 & 0\end{array}\right)$ $\left(\begin{array}{rrrrrrrrr}0 & 0 & 0 & x y z & 0 & 0 & x z y & 0 & 0 \\ 0 & 0 & 0 & 0 & y z x & 0 & 0 & y x z & 0 \\ 0 & 0 & 0 & 0 & 0 & z x y & 0 & 0 & z y x\end{array}\right)\left(\begin{array}{lllllllll}0 & 0 & 0 & 0 & 0 & 0 & 0 & 0 & 0 \\ 0 & 0 & 0 & 0 & 0 & 0 & 0 & 0 & 0 \\ 0 & 0 & 0 & 0 & 0 & 0 & 0 & 0 & 0\end{array}\right)$ $\left(\begin{array}{rrrrrrrrr}0 & 0 & 0 & x y z & 0 & 0 & x z y & 0 & 0 \\ 0 & 0 & 0 & 0 & y z x & 0 & 0 & y x z & 0 \\ 0 & 0 & 0 & 0 & 0 & z x y & 0 & 0 & z y x\end{array}\right)$ $\left[V^{3}\right]\left(\begin{array}{llll}0 & 0 & 0 & 0 \\ 0 & 0 & 0 & \\ 0 & 0 & 0 & \end{array}\right)$ $\left(\begin{array}{llll}0 & 0 & 0 & x y z \\ 0 & 0 & 0 & \\ 0 & 0 & 0 & \end{array}\right)$ $\left(\begin{array}{llll}0 & 0 & 0 & x y z \\ 0 & 0 & 0 & \\ 0 & 0 & 0 & \end{array}\right)$ $V\left[V^{2}\right]\left(\begin{array}{llllll}0 & 0 & 0 & 0 & 0 & 0 \\ 0 & 0 & 0 & 0 & 0 & 0 \\ 0 & 0 & 0 & 0 & 0 & 0\end{array}\right)$ $\left(\begin{array}{rrrrrr}0 & 0 & 0 & x y z & 0 & 0 \\ 0 & 0 & 0 & 0 & y x z & 0 \\ 0 & 0 & 0 & 0 & 0 & z x y\end{array}\right)$ $\left(\begin{array}{llll}0 & 0 & 0 & 0 \\ 0 & 0 & 0 & \\ 0 & 0 & 0 & \end{array}\right)$ $\left\{V^{2}\right\} V\left(\begin{array}{ccc}0 & 0 & 0 \\ 0 & 0 & 0 \\ 0 & 0 & 0\end{array}\right)$ $\left(\begin{array}{ccc}y z x & 0 & 0 \\ 0 & z x y & 0 \\ 0 & 0 & x y z\end{array}\right)$ $\left(\begin{array}{llllll}0 & 0 & 0 & 0 & 0 & 0 \\ 0 & 0 & 0 & 0 & 0 & 0 \\ 0 & 0 & 0 & 0 & 0 & 0\end{array}\right)$ $\left(\begin{array}{rrrrrr}0 & 0 & 0 & x y z & 0 & 0 \\ 0 & 0 & 0 & 0 & y x z & 0 \\ 0 & 0 & 0 & 0 & 0 & z x y\end{array}\right)$ $\left(\begin{array}{lll}0 & 0 & 0 \\ 0 & 0 & 0 \\ 0 & 0 & 0\end{array}\right)$ $\left(\begin{array}{ccc}y z x & 0 & 0 \\ 0 & z x y & 0 \\ 0 & 0 & x y z\end{array}\right)$ 


\section{$\mathrm{n}=3 \mathrm{D}_{3 \mathrm{~h}}=\overline{\mathbf{6}}_{2} \mathrm{~m}_{\mathrm{x}} \mathbf{2}_{1}$}

$V^{3} \quad\left(\begin{array}{rrrrrrrrr}0 & 0 & 0 & 0 & 0 & -y y y & 0 & 0 & -y y y \\ -y y y & y y y & 0 & 0 & 0 & 0 & 0 & 0 & 0 \\ 0 & 0 & 0 & 0 & 0 & 0 & 0 & 0 & 0\end{array}\right)$

$\left[\mathrm{V}^{3}\right] \quad\left(\begin{array}{rrrr}0 & 0 & 0 & 0 \\ -y y y & \text { yyy } & 0 & \\ 0 & 0 & 0 & \end{array}\right)$

$V\left[V^{2}\right]\left(\begin{array}{rrrrrr}0 & 0 & 0 & 0 & 0 & -y y y \\ -y y y & y y y & 0 & 0 & 0 & 0 \\ 0 & 0 & 0 & 0 & 0 & 0\end{array}\right)$

$\left\{V^{2}\right\} V\left(\begin{array}{ccc}0 & 0 & 0 \\ 0 & 0 & 0 \\ 0 & 0 & 0\end{array}\right)$

$\mathrm{n}>3 \quad \mathbf{D}_{\alpha \mathrm{h}}=\infty / \mathbf{m m m}$

$\mathrm{V}^{3} \quad\left(\begin{array}{lllllllll}0 & 0 & 0 & 0 & 0 & 0 & 0 & 0 & 0 \\ 0 & 0 & 0 & 0 & 0 & 0 & 0 & 0 & 0 \\ 0 & 0 & 0 & 0 & 0 & 0 & 0 & 0 & 0\end{array}\right)$

$\left[V^{3}\right] \quad\left(\begin{array}{llll}0 & 0 & 0 & 0 \\ 0 & 0 & 0 & \\ 0 & 0 & 0 & \end{array}\right)$

$V\left[V^{2}\right]\left(\begin{array}{llllll}0 & 0 & 0 & 0 & 0 & 0 \\ 0 & 0 & 0 & 0 & 0 & 0 \\ 0 & 0 & 0 & 0 & 0 & 0\end{array}\right)$

$\left\{V^{2}\right\} V\left(\begin{array}{lll}0 & 0 & 0 \\ 0 & 0 & 0 \\ 0 & 0 & 0\end{array}\right)$

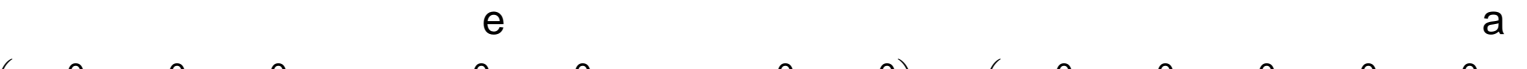

0

ae

$\begin{array}{llll}0 & -y z x & 0 & 0\end{array}$

$\left.\begin{array}{rrrrrrrrr}0 & 0 & 0 & x y z & 0 & 0 & -y z x & 0 & 0 \\ 0 & 0 & 0 & 0 & y z x & 0 & 0 & -x y z & 0 \\ 0 & 0 & 0 & 0 & 0 & z x y & 0 & 0 & -z x y\end{array}\right)$

$\left(\begin{array}{llll}0 & 0 & 0 & 0 \\ 0 & 0 & 0 & \\ 0 & 0 & 0\end{array}\right)$

$\left(\begin{array}{rrrr}0 & 0 & 0 & 0 \\ -y y y & \text { yyy } & 0 & \\ 0 & 0 & 0 & \end{array}\right)$

$\left(\begin{array}{llll}0 & 0 & 0 & 0 \\ 0 & 0 & 0 & \\ 0 & 0 & 0 & \end{array}\right)$

$\left(\begin{array}{rrrrrr}0 & 0 & 0 & x y z & 0 & 0 \\ 0 & 0 & 0 & 0 & -x y z & 0 \\ 0 & 0 & 0 & 0 & 0 & 0\end{array}\right)$

$\left(\begin{array}{rrrrrr}0 & 0 & 0 & 0 & 0 & -y y y \\ -y y y & \text { yyy } & 0 & 0 & 0 & 0 \\ 0 & 0 & 0 & 0 & 0 & 0\end{array}\right)$

$\left(\begin{array}{rrrrrr}0 & 0 & 0 & x y z & 0 & 0 \\ 0 & 0 & 0 & 0 & -x y z & 0 \\ 0 & 0 & 0 & 0 & 0 & 0\end{array}\right)$

$\left(\begin{array}{ccc}y z x & 0 & 0 \\ 0 & y z x & 0 \\ 0 & 0 & x y z\end{array}\right)$

$\left(\begin{array}{lll}0 & 0 & 0 \\ 0 & 0 & 0 \\ 0 & 0 & 0\end{array}\right)$

$\left(\begin{array}{ccc}y z x & 0 & 0 \\ 0 & y z x & 0 \\ 0 & 0 & x y z\end{array}\right)$

ae

$\left(\begin{array}{rrrrrrrrr}0 & 0 & 0 & x y z & 0 & 0 & -y z x & 0 & 0 \\ 0 & 0 & 0 & 0 & y z x & 0 & 0 & -x y z & 0 \\ 0 & 0 & 0 & 0 & 0 & z x y & 0 & 0 & -z x y\end{array}\right)$

$\left(\begin{array}{rrrrrrrrr}0 & 0 & 0 & x y z & 0 & 0 & -y z x & 0 & 0 \\ 0 & 0 & 0 & 0 & y z x & 0 & 0 & -x y z & 0 \\ 0 & 0 & 0 & 0 & 0 & z x y & 0 & 0 & -z x y\end{array}\right) \quad\left(\begin{array}{rrrrrrrrr}0 & 0 & 0 & 0 & 0 & 0 & 0 & 0 & 0 \\ 0 & 0 & 0 & 0 & 0 & 0 & 0 & 0 & 0 \\ 0 & 0 & 0 & 0 & 0 & 0 & 0 & 0 & 0\end{array}\right)$

$\left(\begin{array}{llll}0 & 0 & 0 & 0 \\ 0 & 0 & 0 & \\ 0 & 0 & 0\end{array}\right)$

$\left(\begin{array}{llll}0 & 0 & 0 & 0 \\ 0 & 0 & 0 & \\ 0 & 0 & 0\end{array}\right)$

$\left(\begin{array}{llll}0 & 0 & 0 & 0 \\ 0 & 0 & 0 & \\ 0 & 0 & 0 & \end{array}\right)$

$\left(\begin{array}{rrrrrr}0 & 0 & 0 & x y z & 0 & 0 \\ 0 & 0 & 0 & 0 & -x y z & 0 \\ 0 & 0 & 0 & 0 & 0 & 0\end{array}\right)$

$\left(\begin{array}{llllll}0 & 0 & 0 & 0 & 0 & 0 \\ 0 & 0 & 0 & 0 & 0 & 0 \\ 0 & 0 & 0 & 0 & 0 & 0\end{array}\right)$

$\left(\begin{array}{rrrrrr}0 & 0 & 0 & x y z & 0 & 0 \\ 0 & 0 & 0 & 0 & -x y z & 0 \\ 0 & 0 & 0 & 0 & 0 & 0\end{array}\right)$

$\left(\begin{array}{ccc}y z x & 0 & 0 \\ 0 & z x y & 0 \\ 0 & 0 & x y z\end{array}\right)$

$\left(\begin{array}{lll}0 & 0 & 0 \\ 0 & 0 & 0 \\ 0 & 0 & 0\end{array}\right)$

$\left(\begin{array}{ccc}y z x & 0 & 0 \\ 0 & z x y & 0 \\ 0 & 0 & x y z\end{array}\right)$ 
$\mathrm{n}=1 \quad \mathbf{C}_{2}\left(\mathbf{C}_{1}\right)=\mathbf{2}_{\mathrm{z}}(\mathbf{1})=\mathbf{2}_{\mathrm{z}}{ }^{\prime}$

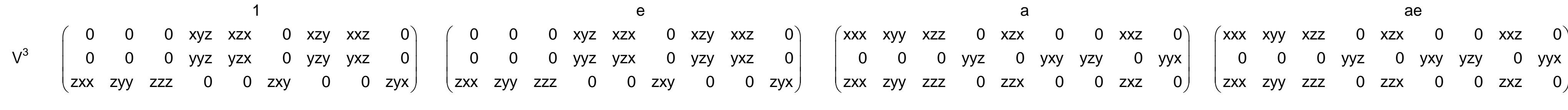
$\left[\mathrm{V}^{3}\right]\left(\begin{array}{rrrr}0 & 0 & 0 & x y z \\ 0 & 0 & 0 & \\ z x x & z y y & z z z & \end{array}\right)$ $\left(\begin{array}{rrrr}0 & 0 & 0 & x y z \\ 0 & 0 & 0 & \\ z x x & z y y & z z z & \end{array}\right)$ $\left(\begin{array}{rrrr}x x x & x y y & x z z & 0 \\ 0 & 0 & 0 & \\ z x x & z y y & z z z & \end{array}\right)$ $V\left[V^{2}\right]\left(\begin{array}{rrrrrr}0 & 0 & 0 & x y z & x x z & 0 \\ 0 & 0 & 0 & y y z & y x z & 0 \\ z x x & z y y & z z z & 0 & 0 & z x y\end{array}\right)$ $\left(\begin{array}{rrrrrr}0 & 0 & 0 & x y z & x x z & 0 \\ 0 & 0 & 0 & y y z & y x z & 0 \\ z x x & z y y & z z z & 0 & 0 & z x y\end{array}\right)$ $\left(\begin{array}{rrrrrr}x x x & x y y & x z z & 0 & x x z & 0 \\ 0 & 0 & 0 & y y z & 0 & y x y \\ z x x & z y y & z z z & 0 & z x z & 0\end{array}\right)$ $\left(\begin{array}{rrrr}x x x & x y y & x z z & 0 \\ 0 & 0 & 0 & \\ z x x & z y y & z z z & \end{array}\right)$ $\left\{V^{2}\right\} V\left(\begin{array}{ccc}y z x & y z y & 0 \\ z x x & z x y & 0 \\ 0 & 0 & x y z\end{array}\right)$ $\left(\begin{array}{ccc}y z x & y z y & 0 \\ z x x & z x y & 0 \\ 0 & 0 & x y z\end{array}\right)$ $\left(\begin{array}{ccc}0 & y z y & 0 \\ z x x & 0 & z x z \\ 0 & x y y & 0\end{array}\right)$ $\left(\begin{array}{rrrrrr}x x x & x y y & x z z & 0 & x x z & 0 \\ 0 & 0 & 0 & y y z & 0 & y x y \\ z x x & z y y & z z z & 0 & z x z & 0\end{array}\right)$

$\mathrm{n}=2 \quad \mathbf{C}_{4}\left(\mathrm{C}_{2}\right)=\mathbf{4}_{\mathrm{z}}\left(\mathbf{2}_{\mathrm{z}}\right)=\mathbf{4}_{\mathrm{z}}{ }^{\prime}$

$\mathrm{V}^{3} \quad\left(\begin{array}{rrrrrrrrr}0 & 0 & 0 & x y z & x z x & 0 & -y z x & y y z & 0 \\ 0 & 0 & 0 & y y z & y z x & 0 & x z x & -x y z & 0 \\ z x x & z y y & z z z & 0 & 0 & z x y & 0 & 0 & -z x y\end{array}\right)$ $\left[\mathrm{V}^{3}\right] \quad\left(\begin{array}{rrrr}0 & 0 & 0 & 0 \\ 0 & 0 & 0 & \\ z x x & z x x & z z z & \end{array}\right)$

$\left(\begin{array}{rrrr}0 & 0 & 0 & 0 \\ 0 & 0 & 0 & \\ z x x & z x x & z z z & \end{array}\right)$

$V\left[V^{2}\right]\left(\begin{array}{rrrrrr}0 & 0 & 0 & x y z & x x z & 0 \\ 0 & 0 & 0 & x x z & -x y z & 0 \\ z x x & z y y & z z z & 0 & 0 & 0\end{array}\right)$

$\left(\begin{array}{rrrrrr}0 & 0 & 0 & x y z & x x z & 0 \\ 0 & 0 & 0 & x x z & -x y z & 0 \\ z x x & z y y & z z z & 0 & 0 & 0\end{array}\right)$

$\left(\begin{array}{rrrrrr}0 & 0 & 0 & x y z & x x z & 0 \\ 0 & 0 & 0 & -x x z & x y z & 0 \\ z x x & -z x x & 0 & 0 & 0 & z x y\end{array}\right)$

$\left(\begin{array}{ccc}0 & y z y & 0 \\ z x x & 0 & z x z \\ 0 & x y y & 0\end{array}\right)$

$\left\{V^{2}\right\} V\left(\begin{array}{ccc}y z x & y z y & 0 \\ -y z y & y z x & 0 \\ 0 & 0 & x y z\end{array}\right)$ $\left(\begin{array}{ccc}y z x & y z y & 0 \\ -y z y & y z x & 0 \\ 0 & 0 & x y z\end{array}\right)$ $\left(\begin{array}{ccc}y z x & y z y & 0 \\ y z y & -y z x & 0 \\ 0 & 0 & 0\end{array}\right)$ $\left(\begin{array}{ccc}y z x & y z y & 0 \\ y z y & -y z x & 0 \\ 0 & 0 & 0\end{array}\right)$ 
$n=3 \quad C_{6}\left(C_{3}\right)=6_{z}\left(3_{z}\right)=6_{z}^{\prime}$

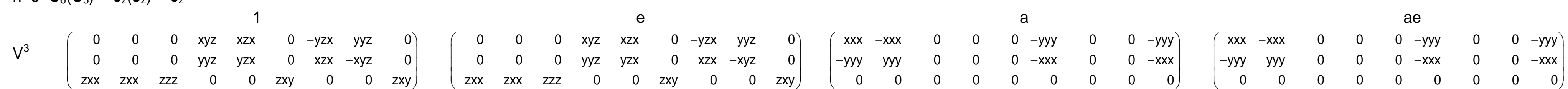

$\left[\mathrm{V}^{3}\right]\left(\begin{array}{rrrr}0 & 0 & 0 & 0 \\ 0 & 0 & 0 & \\ \mathrm{zxx} & \mathrm{zxx} & \mathrm{zzz} & \end{array}\right)$

$\left(\begin{array}{rrrr}0 & 0 & 0 & 0 \\ 0 & 0 & 0 & \\ z x x & z x x & z z z & \end{array}\right)$

$\left(\begin{array}{rrrr}x x x & -x x x & 0 & 0 \\ -y y y & y y y & 0 & \\ 0 & 0 & 0 & \end{array}\right)$

$\left(\begin{array}{rrrr}x x x & -x x x & 0 & 0 \\ -y y y & y y y & 0 & \\ 0 & 0 & 0 & \end{array}\right)$

$V\left[V^{2}\right]\left(\begin{array}{rrrrrr}0 & 0 & 0 & x y z & x x z & 0 \\ 0 & 0 & 0 & x x z & -x y z & 0 \\ z x x & z x x & z z z & 0 & 0 & 0\end{array}\right)$

$\left(\begin{array}{rrrrrr}0 & 0 & 0 & x y z & x x z & 0 \\ 0 & 0 & 0 & x x z & -x y z & 0 \\ z x x & z x x & z z z & 0 & 0 & 0\end{array}\right)$

$\left(\begin{array}{rrrrrr}x x x & -x x x & 0 & 0 & 0 & -y y y \\ -y y y & y y y & 0 & 0 & 0 & -x x x \\ 0 & 0 & 0 & 0 & 0 & 0\end{array}\right)$

$\left(\begin{array}{rrrrrr}x x x & -x x x & 0 & 0 & 0 & -y y y \\ -y y y & y y y & 0 & 0 & 0 & -x x x \\ 0 & 0 & 0 & 0 & 0 & 0\end{array}\right)$

$\left\{V^{2}\right\} \bigvee\left(\begin{array}{ccc}y z x & y z y & 0 \\ -y z y & y z x & 0 \\ 0 & 0 & x y z\end{array}\right)$

$\left(\begin{array}{ccc}y z x & y z y & 0 \\ -y z y & y z x & 0 \\ 0 & 0 & x y z\end{array}\right)$

$\left(\begin{array}{lll}0 & 0 & 0 \\ 0 & 0 & 0 \\ 0 & 0 & 0\end{array}\right)$

$\left(\begin{array}{lll}0 & 0 & 0 \\ 0 & 0 & 0 \\ 0 & 0 & 0\end{array}\right)$

$n>3 \quad \mathbf{C}_{\infty} \mathbf{1}^{\prime}=\infty \mathbf{1}^{\prime}$

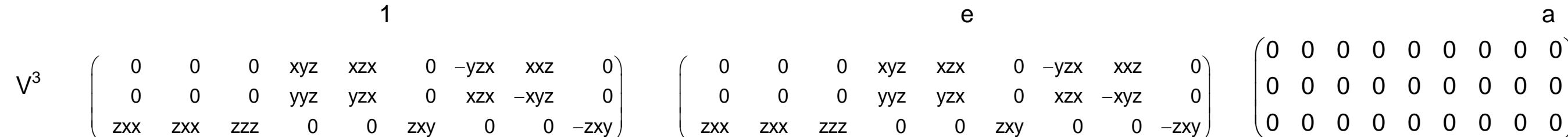

$\left(\begin{array}{lllllllll}0 & 0 & 0 & 0 & 0 & 0 & 0 & 0 & 0 \\ 0 & 0 & 0 & 0 & 0 & 0 & 0 & 0 & 0 \\ 0 & 0 & 0 & 0 & 0 & 0 & 0 & 0 & 0\end{array}\right)$

$\left[V^{3}\right]\left(\begin{array}{rrrr}0 & 0 & 0 & 0 \\ 0 & 0 & 0 & \\ z x x & z x x & z z z\end{array}\right)$

$\left(\begin{array}{rrrr}0 & 0 & 0 & 0 \\ 0 & 0 & 0 & \\ z x x & z x x & z z z & \end{array}\right)$

$\left(\begin{array}{llll}0 & 0 & 0 & 0 \\ 0 & 0 & 0 & \\ 0 & 0 & 0 & \end{array}\right)$

$\left(\begin{array}{llll}0 & 0 & 0 & 0 \\ 0 & 0 & 0 & \\ 0 & 0 & 0\end{array}\right)$

$V\left[V^{2}\right]\left(\begin{array}{rrrrrr}0 & 0 & 0 & x y z & x x z & 0 \\ 0 & 0 & 0 & x x z & -x y z & 0 \\ z x x & z x x & z z z & 0 & 0 & 0\end{array}\right)$

$\left(\begin{array}{rrrrrr}0 & 0 & 0 & x y z & x x z & 0 \\ 0 & 0 & 0 & x x z & -x y z & 0 \\ z x x & z x x & z z z & 0 & 0 & 0\end{array}\right)$

$\left(\begin{array}{llllll}0 & 0 & 0 & 0 & 0 & 0 \\ 0 & 0 & 0 & 0 & 0 & 0 \\ 0 & 0 & 0 & 0 & 0 & 0\end{array}\right)$

$\left\{V^{2}\right\} \bigvee\left(\begin{array}{ccc}y z x & y z y & 0 \\ -y z y & y z x & 0 \\ 0 & 0 & x y z\end{array}\right)$

$\left(\begin{array}{ccc}y z x & y z y & 0 \\ -y z y & y z x & 0 \\ 0 & 0 & x y z\end{array}\right)$

$\left(\begin{array}{lll}0 & 0 & 0 \\ 0 & 0 & 0 \\ 0 & 0 & 0\end{array}\right)$

$\left(\begin{array}{llllll}0 & 0 & 0 & 0 & 0 & 0 \\ 0 & 0 & 0 & 0 & 0 & 0 \\ 0 & 0 & 0 & 0 & 0 & 0\end{array}\right)$

$\begin{array}{llllll}0 & 0 & 0 & 0 & 0 & 0\end{array}$

$\left(\begin{array}{lll}0 & 0 & 0 \\ 0 & 0 & 0 \\ 0 & 0 & 0\end{array}\right)$ 
$\mathrm{n}=1 \quad \mathbf{S}_{2}\left(\mathbf{C}_{1}\right)=\overline{\mathbf{1}}(\mathbf{1})=\overline{\mathbf{1}}{ }^{\prime}$

$\mathrm{V}^{3} \quad\left(\begin{array}{lllllllll}0 & 0 & 0 & 0 & 0 & 0 & 0 & 0 & 0 \\ 0 & 0 & 0 & 0 & 0 & 0 & 0 & 0 & 0 \\ 0 & 0 & 0 & 0 & 0 & 0 & 0 & 0 & 0\end{array}\right)$

$\left[\mathrm{V}^{3}\right] \quad\left(\begin{array}{llll}0 & 0 & 0 & 0 \\ 0 & 0 & 0 & \\ 0 & 0 & 0\end{array}\right)$

$V\left[V^{2}\right]\left(\begin{array}{llllll}0 & 0 & 0 & 0 & 0 & 0 \\ 0 & 0 & 0 & 0 & 0 & 0 \\ 0 & 0 & 0 & 0 & 0 & 0\end{array}\right)$

$\left(\begin{array}{llllll}0 & 0 & 0 & 0 & 0 & 0\end{array}\right)$

$\left\{V^{2}\right\} V\left(\begin{array}{ccc}0 & 0 & 0 \\ 0 & 0 & 0 \\ 0 & 0 & 0\end{array}\right)$

$\mathrm{n}=2 \quad \mathbf{S}_{4}\left(\mathbf{C}_{2}\right)=\overline{\mathbf{4}}_{\mathrm{z}}\left(\mathbf{2}_{\mathrm{z}}\right)=\overline{\mathbf{4}}_{\mathrm{z}}{ }^{\prime}$

$V^{3} \quad\left(\begin{array}{rrrrrrrrr}0 & 0 & 0 & x y z & x z x & 0 & y z x & -y y z & 0 \\ 0 & 0 & 0 & y y z & y z x & 0 & -x z x & x y z & 0 \\ z x x & -z x x & 0 & 0 & 0 & z x y & 0 & 0 & z x y\end{array}\right)$

$\left[\mathrm{V}^{3}\right]\left(\begin{array}{rrrr}0 & 0 & 0 & \mathrm{xyz} \\ 0 & 0 & 0 & \\ \mathrm{zxx} & -\mathrm{zxx} & 0 & \end{array}\right)$

$V\left[V^{2}\right]\left(\begin{array}{rrrrrr}0 & 0 & 0 & x y z & x x z & 0 \\ 0 & 0 & 0 & -x x z & x y z & 0 \\ z x x & -z x x & 0 & 0 & 0 & z x y\end{array}\right)$

$\left\{V^{2}\right\} \bigvee\left(\begin{array}{ccc}y z x & y z y & 0 \\ y z y & -y z x & 0 \\ 0 & 0 & 0\end{array}\right)$ $\left(\begin{array}{lllllllll}x x x & x y y & x z z & x y z & x z x & x x y & x z y & x x z & x y x \\ y x x & y y y & y z z & y y z & y z x & y x y & y z y & y x z & y y x \\ z x x & z y y & z z z & z y z & z z x & z x y & z z y & z x z & z y x\end{array}\right)$ $\begin{array}{lllllllll}z x x & z y y & z z z & z y z & z z x & z x y & z z y & z x z & z y x\end{array}$

$\left(\begin{array}{llll}x x x & x y y & x z z & x y z \\ y x x & y y y & y z z & \\ z x x & z y y & z z z & \end{array}\right)$

$\left(\begin{array}{llllll}x x x & x y y & x z z & x y z & x x z & x x y \\ y x x & y y y & y z z & y y z & y x z & y x y\end{array}\right)$

$\begin{array}{llllll}y x x & y y y & y z z & y y z & y x z & y x y\end{array}$

$\left(\begin{array}{llllll}z x x & z y y & z z z & z y z & z x z & z x y\end{array}\right)$

$\left(\begin{array}{ccc}y z x & y z y & y z z \\ z x x & z x y & z x z \\ x y x & x y y & x y z\end{array}\right)$ $a$

$\left(\begin{array}{lllllllll}x x x & x y y & x z z & x y z & x z x & x x y & x z y & x x z & x y x\end{array}\right.$ $\begin{array}{lllllllll}y x x & y y y & y z z & y y z & y z x & y x y & y z y & y x z & y y x\end{array}$ $\begin{array}{llllllllll}z x x & z y y & z z z & z y z & z z x & z x y & z z y & z x z & z y x\end{array}$

$\left(\begin{array}{llll}x x x & x y y & x z z & x y z \\ y x x & y y y & y z z & \\ z x x & z y y & z z z\end{array}\right)$

$\left(\begin{array}{llllll}x x x & x y y & x z z & x y z & x x z & x x y \\ y x x & y y y & y z z & y y z & y x z & y x y \\ z x x & z y y & z z z & z y z & z x z & z x y\end{array}\right)$

$\left(\begin{array}{lll}y z x & y z y & y z z \\ z x x & z x y & z x z \\ x y x & x y y & x y z\end{array}\right)$ ae

$\left(\begin{array}{lllllllll}0 & 0 & 0 & 0 & 0 & 0 & 0 & 0 & 0 \\ 0 & 0 & 0 & 0 & 0 & 0 & 0 & 0 & 0 \\ 0 & 0 & 0 & 0 & 0 & 0 & 0 & 0 & 0\end{array}\right)$

$\left(\begin{array}{llll}0 & 0 & 0 & 0 \\ 0 & 0 & 0 & \\ 0 & 0 & 0 & \end{array}\right)$

$\left(\begin{array}{llllll}0 & 0 & 0 & 0 & 0 & 0 \\ 0 & 0 & 0 & 0 & 0 & 0 \\ 0 & 0 & 0 & 0 & 0 & 0\end{array}\right)$

$\left(\begin{array}{lll}0 & 0 & 0 \\ 0 & 0 & 0 \\ 0 & 0 & 0\end{array}\right)$ $e$

$\left(\begin{array}{lllllllll}0 & 0 & 0 & x y z & x z x & 0 & -y z x & y y z & 0 \\ 0 & 0 & 0 & y y z & y z x & 0 & x z & -x y z & 0 \\ z x x & z y & z z z\end{array} \quad\left(\begin{array}{ccccccccc}0 & 0 & 0 & x y z & x z x & 0 & -y z x & y y z & 0 \\ 0 & 0 & 0 & y z & y z & 0 & x z x & -x y z & 0\end{array}\right.\right.$ $\left.\begin{array}{rrrrrrrrr}0 & 0 & 0 & y y z & y z x & 0 & x z x & -x y z & 0 \\ z x x & z y y & z z z & 0 & 0 & z x y & 0 & 0 & -z x y\end{array}\right)\left(\begin{array}{rrrrrrrrr}0 & 0 & 0 & y y z & y z x & 0 & x z x & -x y z & 0 \\ z x x & z y & z z z & 0 & 0 & z x y & 0 & 0 & -z x y\end{array}\right)$

$\left(\begin{array}{rrrr}0 & 0 & 0 & 0 \\ 0 & 0 & 0 & \\ z x x & z x x & z z z & \end{array}\right)$

$\left(\begin{array}{rrrrrr}0 & 0 & 0 & x y z & x x z & 0 \\ 0 & 0 & 0 & x x z & -x y z & 0 \\ z x x & z y y & z z z & 0 & 0 & 0\end{array}\right)$

$\left(\begin{array}{ccc}y z x & y z y & 0 \\ -y z y & y z x & 0 \\ 0 & 0 & x y z\end{array}\right)$ $\left(\begin{array}{rrrr}0 & 0 & 0 & 0 \\ 0 & 0 & 0 & \\ z x x & z x x & z z z & \end{array}\right)$

$\left(\begin{array}{rrrrrr}0 & 0 & 0 & x y z & x x z & 0 \\ 0 & 0 & 0 & x x z & -x y z & 0 \\ z x x & z y y & z z z & 0 & 0 & 0\end{array}\right)$

$\left(\begin{array}{ccc}y z x & y z y & 0 \\ -y z y & y z x & 0 \\ 0 & 0 & x y z\end{array}\right)$ ae

$\left(\begin{array}{ccccccccc}0 & 0 & 0 & x y z & x z x & 0 & y z x & -y y z & 0 \\ 0 & 0 & 0 & y z & y z x & 0 & -x z x & x y z & 0\end{array}\right)$ $\left.\begin{array}{rrrrrrrrr}0 & 0 & 0 & y y z & y z x & 0 & -x z x & x y z & 0 \\ z x x & -z x x & 0 & 0 & 0 & z x y & 0 & 0 & z x y\end{array}\right)$

$\left(\begin{array}{rrrr}0 & 0 & 0 & x y z \\ 0 & 0 & 0 & \\ z x x & -z x x & 0 & \end{array}\right)$

$\left(\begin{array}{rrrrrr}0 & 0 & 0 & x y z & x x z & 0 \\ 0 & 0 & 0 & -x x z & x y z & 0 \\ z x x & -z x x & 0 & 0 & 0 & z x y\end{array}\right)$

$\left(\begin{array}{ccc}y z x & y z y & 0 \\ y z y & -y z x & 0 \\ 0 & 0 & 0\end{array}\right)$ 
$\mathrm{n}=3 \quad \mathbf{S}_{6}\left(\mathbf{C}_{3}\right)=\overline{\mathbf{3}}_{\mathbf{z}}\left(\mathbf{3}_{\mathrm{z}}\right)=\overline{\mathbf{3}}_{\mathbf{z}}{ }^{\prime}$

$V^{3} \quad\left(\begin{array}{lllllllll}0 & 0 & 0 & 0 & 0 & 0 & 0 & 0 & 0 \\ 0 & 0 & 0 & 0 & 0 & 0 & 0 & 0 & 0 \\ 0 & 0 & 0 & 0 & 0 & 0 & 0 & 0 & 0\end{array}\right)$

$\left[\mathrm{V}^{3}\right]\left(\begin{array}{llll}0 & 0 & 0 & 0 \\ 0 & 0 & 0 & \\ 0 & 0 & 0 & \end{array}\right)$

$\mathrm{V}\left[\mathrm{V}^{2}\right]\left(\begin{array}{llllll}0 & 0 & 0 & 0 & 0 & 0 \\ 0 & 0 & 0 & 0 & 0 & 0 \\ 0 & 0 & 0 & 0 & 0 & 0\end{array}\right)$

$\left(\begin{array}{llllll}0 & 0 & 0 & 0 & 0 & 0\end{array}\right.$

$\left\{V^{2}\right\} V\left(\begin{array}{ccc}0 & 0 & 0 \\ 0 & 0 & 0 \\ 0 & 0 & 0\end{array}\right)$

$\mathrm{n}>3 \quad \mathbf{C}_{\infty \mathrm{h}}\left(\mathbf{C}_{\infty}\right)=\infty / \mathbf{m}^{\prime}$

$\mathrm{V}^{3} \quad\left(\begin{array}{lllllllll}0 & 0 & 0 & 0 & 0 & 0 & 0 & 0 & 0 \\ 0 & 0 & 0 & 0 & 0 & 0 & 0 & 0 & 0 \\ 0 & 0 & 0 & 0 & 0 & 0 & 0 & 0 & 0\end{array}\right)$

$\left[\mathrm{V}^{3}\right]\left(\begin{array}{llll}0 & 0 & 0 & 0 \\ 0 & 0 & 0 & \\ 0 & 0 & 0 & \end{array}\right)$

$V\left[V^{2}\right]\left(\begin{array}{llllll}0 & 0 & 0 & 0 & 0 & 0 \\ 0 & 0 & 0 & 0 & 0 & 0 \\ 0 & 0 & 0 & 0 & 0 & 0\end{array}\right)$

$\left\{V^{2}\right\} V\left(\begin{array}{ccc}0 & 0 & 0 \\ 0 & 0 & 0 \\ 0 & 0 & 0\end{array}\right)$ $\left(\begin{array}{rrrrrrrrr}x x x & -x x x & 0 & x y z & x z x & -y y y & -y z x & x x z & -y y y \\ -y y y & y y y & 0 & y y z & y z x & -x x x & x z x & -x y z & -x x x \\ z x x & z x x & z z z & 0 & 0 & z x y & 0 & 0 & -z x y\end{array}\right) \quad\left(\begin{array}{rrrrrrrrr}x x x & -x x x & 0 & x y z & x z x & -y y y & -y z x & x x z & -y y y \\ -y y y & y y y & 0 & y y z & y z x & -x x x & x z x & -x y z & -x x x \\ z x x & z x x & z z z & 0 & 0 & z x y & 0 & 0 & -z x y\end{array}\right) \quad\left(\begin{array}{rrrrrrrrr}0 & 0 & 0 & 0 & 0 & 0 & 0 & 0 & 0 \\ 0 & 0 & 0 & 0 & 0 & 0 & 0 & 0 & 0 \\ 0 & 0 & 0 & 0 & 0 & 0 & 0 & 0 & 0\end{array}\right)$

$\left(\begin{array}{rrrr}x x x & -x x x & 0 & 0 \\ -y y y & y y y & 0 & \\ z x x & z x x & z z z & \end{array}\right)$

$\left(\begin{array}{rrrrrr}x x x & -x x x & 0 & x y z & x x z & -y y y \\ -y y y & y y y & 0 & x x z & -x y z & -x x x \\ z x x & z x x & z z z & 0 & 0 & 0\end{array}\right)$

$\left(\begin{array}{rrrr}x x x & -x x x & 0 & 0 \\ -y y y & y y y & 0 & \\ z x x & z x x & z z z\end{array}\right)$

$\left(\begin{array}{llll}0 & 0 & 0 & 0 \\ 0 & 0 & 0 & \\ 0 & 0 & 0\end{array}\right)$

$\left(\begin{array}{rrrrrr}x x x & -x x x & 0 & x y z & x x z & -y y y \\ -y y y & y y y & 0 & x x z & -x y z & -x x x \\ z x x & z x x & z z z & 0 & 0 & 0\end{array}\right)$

$\left(\begin{array}{llllll}0 & 0 & 0 & 0 & 0 & 0 \\ 0 & 0 & 0 & 0 & 0 & 0\end{array}\right)$

$\left.\begin{array}{llllll}0 & 0 & 0 & 0 & 0 & 0 \\ 0 & 0 & 0 & 0 & 0 & 0\end{array}\right)$

$\left(\begin{array}{ccc}y z x & y z y & 0 \\ -y z y & y z x & 0 \\ 0 & 0 & x y z\end{array}\right)$

$\left(\begin{array}{ccc}y z x & y z y & 0 \\ -y z y & y z x & 0 \\ 0 & 0 & x y z\end{array}\right)$

$\left(\begin{array}{lll}0 & 0 & 0 \\ 0 & 0 & 0 \\ 0 & 0 & 0\end{array}\right)$

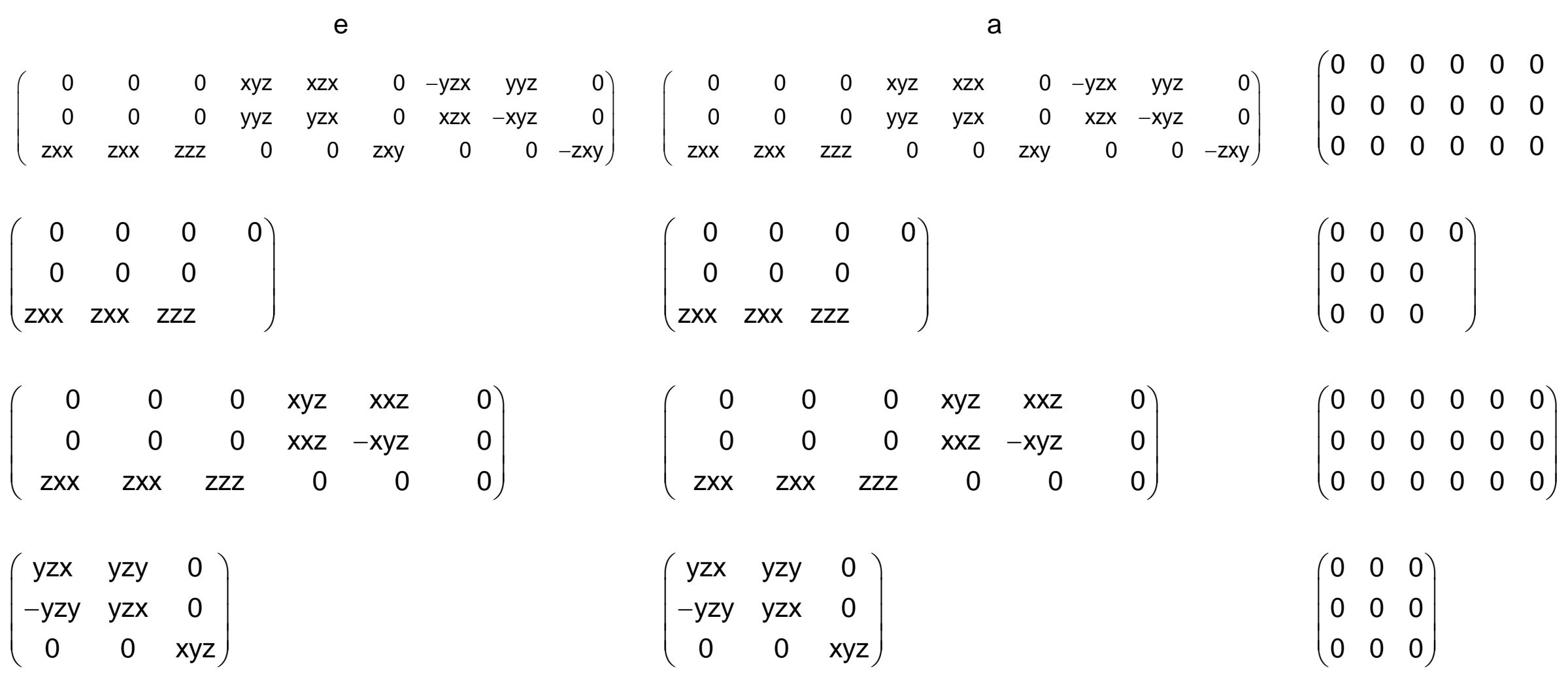


$\mathrm{n}=1 \quad \mathbf{C}_{1 \mathrm{~h}}\left(\mathbf{C}_{1}\right)=\mathbf{m}_{\mathrm{z}}(\mathbf{1})=\mathbf{m}_{\mathrm{z}}{ }^{\prime}$

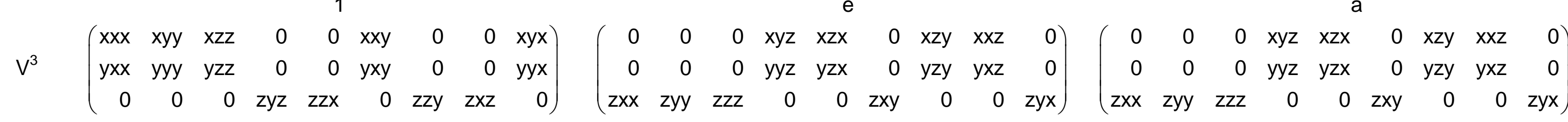

$\left[V^{3}\right]\left(\begin{array}{rrrr}x x x & x y y & x z z & 0 \\ y x x & y y y & y z z & \\ 0 & 0 & 0\end{array}\right)$

$V\left[V^{2}\right]\left(\begin{array}{rrrrrr}x x x & x y y & x z z & 0 & 0 & x x y \\ y x x & y y y & y z z & 0 & 0 & y x y \\ 0 & 0 & 0 & z y z & z x z & 0\end{array}\right)$

$\left\{V^{2}\right\} V\left(\begin{array}{ccc}0 & 0 & y z z \\ 0 & 0 & z x z \\ x y x & x y y & 0\end{array}\right)$

$\mathrm{n}=2 \quad \mathbf{C}_{2 \mathrm{~h}}\left(\mathbf{C}_{2}\right)=\mathbf{z}_{\mathrm{z}} / \mathbf{m}_{\mathrm{z}}\left(\mathbf{2}_{\mathrm{z}}\right)=\mathbf{2}_{\mathrm{z}} / \mathbf{m}_{\mathrm{z}}{ }^{\prime}$

$\mathrm{V}^{3} \quad\left(\begin{array}{lllllllll}0 & 0 & 0 & 0 & 0 & 0 & 0 & 0 & 0 \\ 0 & 0 & 0 & 0 & 0 & 0 & 0 & 0 & 0 \\ 0 & 0 & 0 & 0 & 0 & 0 & 0 & 0 & 0\end{array}\right)$

$\left[\mathrm{V}^{3}\right]\left(\begin{array}{llll}0 & 0 & 0 & 0 \\ 0 & 0 & 0 & \\ 0 & 0 & 0 & \end{array}\right)$

$V\left[V^{2}\right]\left(\begin{array}{llllll}0 & 0 & 0 & 0 & 0 & 0 \\ 0 & 0 & 0 & 0 & 0 & 0 \\ 0 & 0 & 0 & 0 & 0 & 0\end{array}\right)$

$\left\{V^{2}\right\} V\left(\begin{array}{lll}0 & 0 & 0 \\ 0 & 0 & 0 \\ 0 & 0 & 0\end{array}\right)$ $\left(\begin{array}{rrrr}0 & 0 & 0 & x y z \\ 0 & 0 & 0 & \\ z x x & z y y & z z z & \end{array}\right)$

$\left(\begin{array}{rrrrrr}0 & 0 & 0 & x y z & x x z & 0 \\ 0 & 0 & 0 & y y z & y x z & 0 \\ z x x & z y y & z z z & 0 & 0 & z x y\end{array}\right)$

$\left(\begin{array}{ccc}y z x & y z y & 0 \\ z x x & z x y & 0 \\ 0 & 0 & x y z\end{array}\right)$

$\left(\begin{array}{rrrr}0 & 0 & 0 & x y z \\ 0 & 0 & 0 & \\ z x x & z y y & z z z & \end{array}\right)$

$\left(\begin{array}{rrrrrr}0 & 0 & 0 & x y z & x x z & 0 \\ 0 & 0 & 0 & y y z & y x z & 0 \\ z x x & z y y & z z z & 0 & 0 & z x y\end{array}\right)$ $\left(\begin{array}{ccc}y z x & y z y & 0 \\ z x x & z x y & 0 \\ 0 & 0 & x y z\end{array}\right)$

$\left(\begin{array}{rrrrrrrrr}0 & 0 & 0 & x y z & x z x & 0 & x z y & x x z & 0 \\ 0 & 0 & 0 & y y z & y z x & 0 & y z y & y x z & 0 \\ z x x & z y y & z z z & 0 & 0 & z x y & 0 & 0 & z y x\end{array}\right)$

$\left(\begin{array}{rrrrrrrrr}0 & 0 & 0 & x y z & x z x & 0 & x z y & x x z & 0 \\ 0 & 0 & 0 & y y z & y z x & 0 & y z y & y x z & 0 \\ z x x & z y y & z z z & 0 & 0 & z x y & 0 & 0 & z y x\end{array}\right)$

$\left(\begin{array}{rrrr}0 & 0 & 0 & x y z \\ 0 & 0 & 0 & \\ z x x & z y y & z z z & \end{array}\right)$

$\left(\begin{array}{rrrr}0 & 0 & 0 & x y z \\ 0 & 0 & 0 & \\ z x x & z y y & z z z & \end{array}\right)$

$\left(\begin{array}{rrrrrr}0 & 0 & 0 & x y z & x x z & 0 \\ 0 & 0 & 0 & y y z & y x z & 0 \\ z x x & z y y & z z z & 0 & 0 & z x y\end{array}\right)$

$\left(\begin{array}{rrrrrr}0 & 0 & 0 & x y z & x x z & 0 \\ 0 & 0 & 0 & y y z & y x z & 0 \\ z x x & z y y & z z z & 0 & 0 & z x y\end{array}\right)$

$\left(\begin{array}{ccc}y z x & y z y & 0 \\ z x x & z x y & 0 \\ 0 & 0 & x y z\end{array}\right)$

$\left(\begin{array}{ccc}y z x & y z y & 0 \\ z x x & z x y & 0 \\ 0 & 0 & x y z\end{array}\right)$ $\left(\begin{array}{rrrrrrrrr}x x x & x y y & x z z & 0 & 0 & x x y & 0 & 0 & x y x \\ y x x & y y y & y z z & 0 & 0 & y x y & 0 & 0 & y y x \\ 0 & 0 & 0 & z y z & z z x & 0 & z z y & z x z & 0\end{array}\right)$

$\left(\begin{array}{rrrr}x x x & x y y & x z z & 0 \\ y x x & y y y & y z z & \\ 0 & 0 & 0 & \end{array}\right)$

$\left(\begin{array}{rrrrrr}x x x & x y y & x z z & 0 & 0 & x x y \\ y x x & y y y & y z z & 0 & 0 & y x y \\ 0 & 0 & 0 & z y z & z x z & 0\end{array}\right)$

$\left(\begin{array}{ccc}0 & 0 & y z z \\ 0 & 0 & z x z \\ x y x & x y y & 0\end{array}\right)$

$\left(\begin{array}{lllllllll}0 & 0 & 0 & 0 & 0 & 0 & 0 & 0 & 0 \\ 0 & 0 & 0 & 0 & 0 & 0 & 0 & 0 & 0 \\ 0 & 0 & 0 & 0 & 0 & 0 & 0 & 0 & 0\end{array}\right)$

$\left(\begin{array}{llll}0 & 0 & 0 & 0 \\ 0 & 0 & 0 & \\ 0 & 0 & 0 & \end{array}\right)$

$\left(\begin{array}{llllll}0 & 0 & 0 & 0 & 0 & 0 \\ 0 & 0 & 0 & 0 & 0 & 0 \\ 0 & 0 & 0 & 0 & 0 & 0\end{array}\right)$

$\left(\begin{array}{lll}0 & 0 & 0 \\ 0 & 0 & 0 \\ 0 & 0 & 0\end{array}\right)$ 
$\mathrm{n}=3 \quad \mathbf{C}_{3 n}\left(\mathbf{C}_{3}\right)=\overline{\mathbf{6}}_{z}\left(\mathbf{3}_{z}\right)=\overline{\mathbf{6}}_{z}{ }^{\prime}$
$V^{3} \quad\left(\begin{array}{rrllllllr}x x x & -x x x & 0 & 0 & 0 & -y y y & 0 & 0 & -y y y \\ -y y y & y y y & 0 & 0 & 0 & -x x x & 0 & 0 & -x x x \\ 0 & 0 & 0 & 0 & 0 & 0 & 0 & 0 & 0\end{array}\right)$
ae
$\left[\mathrm{V}^{3}\right] \quad\left(\begin{array}{rrrr}\mathrm{xxx} & -\mathrm{xxx} & 0 & 0 \\ -y y y & y y y & 0 & \\ 0 & 0 & 0 & \end{array}\right)$
$V\left[V^{2}\right]\left(\begin{array}{rrrrrr}x x x & -x x x & 0 & 0 & 0 & -y y y \\ -y y y & y y y & 0 & 0 & 0 & -x x x \\ 0 & 0 & 0 & 0 & 0 & 0\end{array}\right)$
$\left\{V^{2}\right\} V\left(\begin{array}{ccc}0 & 0 & 0 \\ 0 & 0 & 0 \\ 0 & 0 & 0\end{array}\right)$
$\left(\begin{array}{ccc}y z x & y z y & 0 \\ -y z y & y z x & 0 \\ 0 & 0 & x y z\end{array}\right)$
$\left(\begin{array}{rrrr}0 & 0 & 0 & 0 \\ 0 & 0 & 0 & \\ z x x & z x x & z z z & \end{array}\right)$
$\left(\begin{array}{rrrrrr}0 & 0 & 0 & x y z & x x z & 0 \\ 0 & 0 & 0 & x x z & -x y z & 0 \\ z x x & z x x & z z z & 0 & 0 & 0\end{array}\right)$
$\left(\begin{array}{rrrr}0 & 0 & 0 & 0 \\ 0 & 0 & 0 & \\ z x x & z x x & z z z & \end{array}\right)$
$\left(\begin{array}{rrrrrr}0 & 0 & 0 & x y z & x x z & 0 \\ 0 & 0 & 0 & x x z & -x y z & 0 \\ z x x & z x x & z z z & 0 & 0 & 0\end{array}\right)$
$\left(\begin{array}{rrrrrr}x x x & -x x x & 0 & 0 & 0 & -y y y \\ -y y y & y y y & 0 & 0 & 0 & -x x x \\ 0 & 0 & 0 & 0 & 0 & 0\end{array}\right)$
$\left(\begin{array}{ccc}y z x & y z y & 0 \\ -y z y & y z x & 0 \\ 0 & 0 & x y z\end{array}\right)$
$\left(\begin{array}{lll}0 & 0 & 0 \\ 0 & 0 & 0 \\ 0 & 0 & 0\end{array}\right)$

$\mathrm{n}>3 \quad \mathbf{C}_{\infty \mathrm{h}}\left(\mathbf{C}_{\infty}\right)=\infty / \mathbf{m}^{\prime}$

$\mathrm{V}^{3} \quad\left(\begin{array}{lllllllll}0 & 0 & 0 & 0 & 0 & 0 & 0 & 0 & 0 \\ 0 & 0 & 0 & 0 & 0 & 0 & 0 & 0 & 0 \\ 0 & 0 & 0 & 0 & 0 & 0 & 0 & 0 & 0\end{array}\right)$

$\left[V^{3}\right]\left(\begin{array}{llll}0 & 0 & 0 & 0 \\ 0 & 0 & 0 & \\ 0 & 0 & 0 & \end{array}\right)$

$V\left[V^{2}\right]\left(\begin{array}{llllll}0 & 0 & 0 & 0 & 0 & 0 \\ 0 & 0 & 0 & 0 & 0 & 0 \\ 0 & 0 & 0 & 0 & 0 & 0\end{array}\right)$

$\left\{V^{2}\right\} V\left(\begin{array}{lll}0 & 0 & 0 \\ 0 & 0 & 0 \\ 0 & 0 & 0\end{array}\right)$ $\left(\begin{array}{rrrrrrrrr}0 & 0 & 0 & x y z & x z x & 0 & -y z x & y y z & 0 \\ 0 & 0 & 0 & y y z & y z x & 0 & x z x & -x y z & 0 \\ z x x & z x x & z z z & 0 & 0 & z x y & 0 & 0 & -z x y\end{array}\right) \quad\left(\begin{array}{rrrrrrrrrr}0 & 0 & 0 & x y z & x z x & 0 & -y z x & y y z & 0 \\ 0 & 0 & 0 & y y z & y z x & 0 & x z x & -x y z & 0 \\ z x x & z x x & z z z & 0 & 0 & z x y & 0 & 0 & -z x y\end{array}\right) \quad\left(\begin{array}{rrrrrrrrr}0 & 0 & 0 & 0 & 0 & 0 & 0 & 0 & 0 \\ 0 & 0 & 0 & 0 & 0 & 0 & 0 & 0 & 0 \\ 0 & 0 & 0 & 0 & 0 & 0 & 0 & 0 & 0\end{array}\right)$

$\left(\begin{array}{rrrr}0 & 0 & 0 & 0 \\ 0 & 0 & 0 & \\ z x x & z x x & z z z & \end{array}\right)$

$\left(\begin{array}{rrrr}0 & 0 & 0 & 0 \\ 0 & 0 & 0 & \\ z x x & z x x & z z z & \end{array}\right)$

$\left(\begin{array}{llll}0 & 0 & 0 & 0 \\ 0 & 0 & 0 & \\ 0 & 0 & 0 & \end{array}\right)$

$\left(\begin{array}{rrrrrr}0 & 0 & 0 & x y z & x x z & 0 \\ 0 & 0 & 0 & x x z & -x y z & 0 \\ z x x & z x x & z z z & 0 & 0 & 0\end{array}\right)$

$\left(\begin{array}{rrrrrr}0 & 0 & 0 & x y z & x x z & 0 \\ 0 & 0 & 0 & x x z & -x y z & 0 \\ z x x & z x x & z z z & 0 & 0 & 0\end{array}\right)$

$\left(\begin{array}{llllll}0 & 0 & 0 & 0 & 0 & 0 \\ 0 & 0 & 0 & 0 & 0 & 0 \\ 0 & 0 & 0 & 0 & 0 & 0\end{array}\right)$

$\left(\begin{array}{ccc}y z x & y z y & 0 \\ -y z y & y z x & 0 \\ 0 & 0 & x y z\end{array}\right)$ $\left(\begin{array}{lll}0 & 0 & 0 \\ 0 & 0 & 0 \\ 0 & 0 & 0\end{array}\right)$ 
$\mathrm{n}=1 \quad \mathbf{C}_{2 \mathrm{~h}}\left(\mathbf{S}_{2}\right)=\mathbf{2}_{\mathrm{z}} / \mathbf{m}_{\mathrm{z}}(\overline{\mathbf{1}})=\mathbf{2}_{\mathbf{z}}{ }^{\prime} / \mathbf{m}_{\mathbf{z}}{ }^{\prime}$

$\mathrm{V}^{3} \quad\left(\begin{array}{lllllllll}0 & 0 & 0 & 0 & 0 & 0 & 0 & 0 & 0 \\ 0 & 0 & 0 & 0 & 0 & 0 & 0 & 0 & 0 \\ 0 & 0 & 0 & 0 & 0 & 0 & 0 & 0 & 0\end{array}\right)$ $\left(\begin{array}{rrrrrrrrr}0 & 0 & 0 & x y z & x z x & 0 & x z y & x x z & 0 \\ 0 & 0 & 0 & y y z & y z x & 0 & y z y & y x z & 0 \\ z x x & z y y & z z z & 0 & 0 & z x y & 0 & 0 & z y x\end{array}\right)\left(\begin{array}{lllllllll}0 & 0 & 0 & 0 & 0 & 0 & 0 & 0 & 0 \\ 0 & 0 & 0 & 0 & 0 & 0 & 0 & 0 & 0 \\ 0 & 0 & 0 & 0 & 0 & 0 & 0 & 0 & 0\end{array}\right)$

$\left[\mathrm{V}^{3}\right]\left(\begin{array}{llll}0 & 0 & 0 & 0 \\ 0 & 0 & 0 & \\ 0 & 0 & 0 & \end{array}\right)$

$V\left[V^{2}\right]\left(\begin{array}{llllll}0 & 0 & 0 & 0 & 0 & 0 \\ 0 & 0 & 0 & 0 & 0 & 0 \\ 0 & 0 & 0 & 0 & 0 & 0\end{array}\right)$

$\left(\begin{array}{llllll}0 & 0 & 0 & 0 & 0 & 0\end{array}\right)$

$\left\{V^{2}\right\} V\left(\begin{array}{ccc}0 & 0 & 0 \\ 0 & 0 & 0 \\ 0 & 0 & 0\end{array}\right)$

$\mathrm{n}=2 \quad \mathrm{C}_{4 \mathrm{~h}}\left(\mathrm{~S}_{4}\right)=\mathbf{4}_{\mathrm{z}} / \mathrm{m}_{\mathrm{z}}\left(\overline{\mathbf{4}}_{\mathrm{z}}\right)=\mathbf{4}_{\mathrm{z}}{ }^{\prime} / \mathrm{m}_{\mathrm{z}}{ }^{\prime}$

$\left(\begin{array}{ccccccccc}0 & 0 & 0 & 0 & 0 & 0 & 0 & 0 & 0 \\ 0 & 0 & 0 & 0 & 0 & 0 & 0 & 0 & 0\end{array}\right)$

$V^{3} \quad\left(\begin{array}{lllllllll}0 & 0 & 0 & 0 & 0 & 0 & 0 & 0 & 0 \\ 0 & 0 & 0 & 0 & 0 & 0 & 0 & 0 & 0 \\ 0 & 0 & 0 & 0 & 0 & 0 & 0 & 0 & 0\end{array}\right)$

$\left[\mathrm{V}^{3}\right]\left(\begin{array}{llll}0 & 0 & 0 & 0 \\ 0 & 0 & 0 & \\ 0 & 0 & 0 & \end{array}\right)$

$\left(\begin{array}{llllll}0 & 0 & 0 & 0 & 0 & 0 \\ 0 & 0 & 0 & 0 & 0 & 0\end{array}\right)$

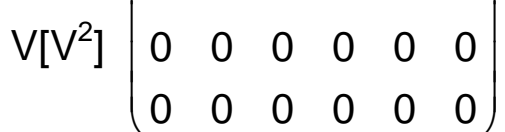

$\left\{V^{2}\right\} V\left(\begin{array}{ccc}0 & 0 & 0 \\ 0 & 0 & 0 \\ 0 & 0 & 0\end{array}\right)$

$\left(\begin{array}{lll}0 & 0 & 0 \\ 0 & 0 & 0\end{array}\right)$ $\left(\begin{array}{rrrr}0 & 0 & 0 & x y z \\ 0 & 0 & 0 & \\ z x x & z y y & z z z\end{array}\right)$

$\left(\begin{array}{llll}0 & 0 & 0 & 0 \\ 0 & 0 & 0 & \\ 0 & 0 & 0 & \end{array}\right)$

$\left(\begin{array}{rrrrrr}0 & 0 & 0 & x y z & x x z & 0 \\ 0 & 0 & 0 & y y z & y x z & 0 \\ z x x & z y y & z z z & 0 & 0 & z x y\end{array}\right)$

$\left(\begin{array}{llllll}0 & 0 & 0 & 0 & 0 & 0 \\ 0 & 0 & 0 & 0 & 0 & 0 \\ 0 & 0 & 0 & 0 & 0 & 0\end{array}\right)$

$\left(\begin{array}{ccc}y z x & y z y & 0 \\ z x x & z x y & 0 \\ 0 & 0 & x y z\end{array}\right)$

$\left(\begin{array}{lll}0 & 0 & 0 \\ 0 & 0 & 0 \\ 0 & 0 & 0\end{array}\right)$ $\left(\begin{array}{rrrrrrrrr}x x x & x y y & x z z & 0 & 0 & x x y & 0 & 0 & x y x \\ y x x & y y y & y z z & 0 & 0 & y x y & 0 & 0 & y y x \\ 0 & 0 & 0 & z y z & z z x & 0 & z z y & z x z & 0\end{array}\right)$

$\left(\begin{array}{rrrr}x x x & x y y & x z z & 0 \\ y x x & y y y & y z z & \\ 0 & 0 & 0\end{array}\right)$

$\left(\begin{array}{rrrrrr}x x x & x y y & x z z & 0 & 0 & x x y \\ y x x & y y y & y z z & 0 & 0 & y x y \\ 0 & 0 & 0 & z y z & z x z & 0\end{array}\right)$

$\left(\begin{array}{ccc}0 & 0 & y z z \\ 0 & 0 & z x z \\ x y x & x y y & 0\end{array}\right)$ $\left(\begin{array}{rrrrrrrrr}0 & 0 & 0 & x y z & x z x & 0 & -y z x & y y z & 0 \\ 0 & 0 & 0 & y y z & y z x & 0 & x z x & -x y z & 0 \\ z x x & z y y & z z z & 0 & 0 & z x y & 0 & 0 & -z x y\end{array}\right) \quad\left(\begin{array}{rrrrrrrrrr}0 & 0 & 0 & x y z & x z x & 0 & y z x & -y y z & 0 \\ 0 & 0 & 0 & y y z & y z x & 0 & -x z x & x y z & 0 \\ z x x & -z x x & 0 & 0 & 0 & z x y & 0 & 0 & z x y\end{array}\right) \quad\left(\begin{array}{rrrrrrrrr}0 & 0 & 0 & 0 & 0 & 0 & 0 & 0 & 0 \\ 0 & 0 & 0 & 0 & 0 & 0 & 0 & 0 & 0 \\ 0 & 0 & 0 & 0 & 0 & 0 & 0 & 0 & 0\end{array}\right)$

$\left(\begin{array}{rrrr}0 & 0 & 0 & 0 \\ 0 & 0 & 0 & \\ z x x & z x x & z z z & \end{array}\right)$

$\left(\begin{array}{rrrrrr}0 & 0 & 0 & x y z & x x z & 0 \\ 0 & 0 & 0 & x x z & -x y z & 0 \\ z x x & z y y & z z z & 0 & 0 & 0\end{array}\right)$

$\left(\begin{array}{rrrr}0 & 0 & 0 & x y z \\ 0 & 0 & 0 & \\ z x x & -z x x & 0 & \end{array}\right)$

$\left(\begin{array}{llll}0 & 0 & 0 & 0 \\ 0 & 0 & 0 & \\ 0 & 0 & 0 & \end{array}\right)$

$\left(\begin{array}{rrrrrr}0 & 0 & 0 & x y z & x x z & 0 \\ 0 & 0 & 0 & -x x z & x y z & 0 \\ z x x & -z x x & 0 & 0 & 0 & z x y\end{array}\right)$

$\left(\begin{array}{llllll}0 & 0 & 0 & 0 & 0 & 0 \\ 0 & 0 & 0 & 0 & 0 & 0 \\ 0 & 0 & 0 & 0 & 0 & 0\end{array}\right)$

$\left(\begin{array}{ccc}y z x & y z y & 0 \\ -y z y & y z x & 0 \\ 0 & 0 & x y z\end{array}\right)$ $\left(\begin{array}{ccc}y z x & y z y & 0 \\ y z y & -y z x & 0 \\ 0 & 0 & 0\end{array}\right)$

$\left(\begin{array}{lll}0 & 0 & 0 \\ 0 & 0 & 0 \\ 0 & 0 & 0\end{array}\right)$ 


$$
\left(\begin{array}{rrrrrrrrr}
0 & 0 & 0 & x y z & x z x & 0 & -y z x & y y z & 0 \\
0 & 0 & 0 & y y z & y z x & 0 & x z x & -x y z & 0 \\
z x x & z x x & z z z & 0 & 0 & z x y & 0 & 0 & -z x y
\end{array}\right)
$$

$\left(\begin{array}{lllllllll}0 & 0 & 0 & 0 & 0 & 0 & 0 & 0 & 0 \\ 0 & 0 & 0 & 0 & 0 & 0 & 0 & 0 & 0\end{array}\right)$

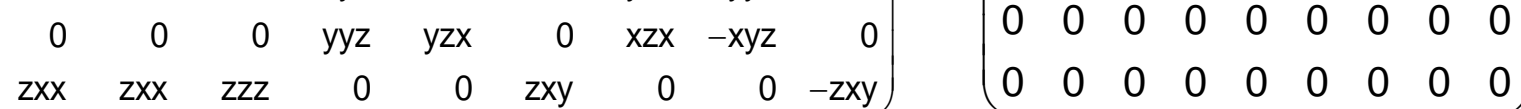

$\left[\mathrm{V}^{3}\right]\left(\begin{array}{llll}0 & 0 & 0 & 0 \\ 0 & 0 & 0 & \\ 0 & 0 & 0 & \end{array}\right)$

$V\left[V^{2}\right]\left(\begin{array}{llllll}0 & 0 & 0 & 0 & 0 & 0 \\ 0 & 0 & 0 & 0 & 0 & 0 \\ 0 & 0 & 0 & 0 & 0 & 0\end{array}\right)$

$\left\{V^{2}\right\} V\left(\begin{array}{lll}0 & 0 & 0 \\ 0 & 0 & 0 \\ 0 & 0 & 0\end{array}\right)$

$\mathrm{n}>3 \quad \mathbf{C}_{\infty \mathrm{h}} \mathbf{1}^{\prime}=\infty / \mathbf{m}^{\prime}$

$\left(\begin{array}{lllllllll}0 & 0 & 0 & 0 & 0 & 0 & 0 & 0 & 0 \\ 0 & 0 & 0 & 0 & 0 & 0 & 0 & 0 & 0\end{array}\right)$ $\begin{array}{llllllllll}0 & 0 & 0 & 0 & 0 & 0 & 0 & 0\end{array}$

$\left(\begin{array}{lllllllll}0 & 0 & 0 & 0 & 0 & 0 & 0 & 0 & 0\end{array}\right)$

$\left[\mathrm{V}^{3}\right]\left(\begin{array}{llll}0 & 0 & 0 & 0 \\ 0 & 0 & 0 & \\ 0 & 0 & 0 & \end{array}\right)$

$V\left[V^{2}\right]\left(\begin{array}{llllll}0 & 0 & 0 & 0 & 0 & 0 \\ 0 & 0 & 0 & 0 & 0 & 0 \\ 0 & 0 & 0 & 0 & 0 & 0\end{array}\right)$

$\left\{V^{2}\right\} V\left(\begin{array}{ccc}0 & 0 & 0 \\ 0 & 0 & 0 \\ 0 & 0 & 0\end{array}\right)$

$\left(\begin{array}{lll}0 & 0 & 0 \\ 0 & 0 & 0\end{array}\right)$ $\left(\begin{array}{rrrr}0 & 0 & 0 & 0 \\ 0 & 0 & 0 & \\ z x x & z x x & z z z & \end{array}\right)$

$\left(\begin{array}{llll}0 & 0 & 0 & 0 \\ 0 & 0 & 0 & \\ 0 & 0 & 0\end{array}\right)$

$\left(\begin{array}{rrrrrr}0 & 0 & 0 & x y z & x x z & 0 \\ 0 & 0 & 0 & x x z & -x y z & 0 \\ z x x & z x x & z z z & 0 & 0 & 0\end{array}\right)$

$\left(\begin{array}{llllll}0 & 0 & 0 & 0 & 0 & 0 \\ 0 & 0 & 0 & 0 & 0 & 0 \\ 0 & 0 & 0 & 0 & 0 & 0\end{array}\right)$

$\left(\begin{array}{ccc}y z x & y z y & 0 \\ -y z y & y z x & 0 \\ 0 & 0 & x y z\end{array}\right)$

$\left(\begin{array}{lll}0 & 0 & 0 \\ 0 & 0 & 0 \\ 0 & 0 & 0\end{array}\right)$

$\left(\begin{array}{rrrrrrrrr}0 & 0 & 0 & x y z & x z x & 0 & -y z x & y y z & 0 \\ 0 & 0 & 0 & y y z & y z x & 0 & x z x & -x y z & 0 \\ z x x & z x x & z z z & 0 & 0 & z x y & 0 & 0 & -z x y\end{array}\right) \quad\left(\begin{array}{rrrrrrrrr}0 & 0 & 0 & 0 & 0 & 0 & 0 & 0 & 0 \\ 0 & 0 & 0 & 0 & 0 & 0 & 0 & 0 & 0 \\ 0 & 0 & 0 & 0 & 0 & 0 & 0 & 0 & 0\end{array}\right)$

$\left(\begin{array}{llll}0 & 0 & 0 & 0 \\ 0 & 0 & 0 & \\ 0 & 0 & 0 & \end{array}\right)$

$\left(\begin{array}{rrrr}0 & 0 & 0 & 0 \\ 0 & 0 & 0 & \\ z x x & z x x & z z z & \end{array}\right)$

$\left(\begin{array}{llllll}0 & 0 & 0 & 0 & 0 & 0\end{array}\right.$

$\left(\begin{array}{rrrrrr}0 & 0 & 0 & x y z & x x z & 0 \\ 0 & 0 & 0 & x x z & -x y z & 0 \\ z x x & z x x & z z z & 0 & 0 & 0\end{array}\right)$

$\left(\begin{array}{llllll}0 & 0 & 0 & 0 & 0 & 0 \\ 0 & 0 & 0 & 0 & 0 & 0 \\ 0 & 0 & 0 & 0 & 0 & 0\end{array}\right)$

$\left(\begin{array}{ccc}y z x & y z y & 0 \\ -y z y & y z x & 0 \\ 0 & 0 & x y z\end{array}\right)$ $\left(\begin{array}{rrrrrrrrr}x x x & -x x x & 0 & 0 & 0 & -y y y & 0 & 0 & -y y y \\ -y y y & y y y & 0 & 0 & 0 & -x x x & 0 & 0 & -x x x \\ 0 & 0 & 0 & 0 & 0 & 0 & 0 & 0 & 0\end{array}\right)$

$\left(\begin{array}{rrrr}x x x & -x x x & 0 & 0 \\ -y y y & y y y & 0 & \\ 0 & 0 & 0\end{array}\right)$

$\left(\begin{array}{rrrrrr}x x x & -x x x & 0 & 0 & 0 & -y y y \\ -y y y & y y y & 0 & 0 & 0 & -x x x \\ 0 & 0 & 0 & 0 & 0 & 0\end{array}\right)$

$\left(\begin{array}{lll}0 & 0 & 0 \\ 0 & 0 & 0 \\ 0 & 0 & 0\end{array}\right)$

$\left(\begin{array}{lllllllll}0 & 0 & 0 & 0 & 0 & 0 & 0 & 0 & 0 \\ 0 & 0 & 0 & 0 & 0 & 0 & 0 & 0 & 0\end{array}\right.$

$\begin{array}{lllllllll}0 & 0 & 0 & 0 & 0 & 0 & 0 & 0 & 0\end{array}$

$\left(\begin{array}{llll}0 & 0 & 0 & 0 \\ 0 & 0 & 0 & \\ 0 & 0 & 0 & \end{array}\right)$

$\left(\begin{array}{llllll}0 & 0 & 0 & 0 & 0 & 0 \\ 0 & 0 & 0 & 0 & 0 & 0 \\ 0 & 0 & 0 & 0 & 0 & 0\end{array}\right)$

$\left(\begin{array}{lll}0 & 0 & 0 \\ 0 & 0 & 0 \\ 0 & 0 & 0\end{array}\right)$ 
$\mathrm{n}=1 \quad \mathbf{C}_{2 \mathrm{~h}}\left(\mathbf{C}_{1 \mathrm{~h}}\right)=\mathbf{2}_{\mathrm{z}} / \mathbf{m}_{\mathrm{z}}\left(\mathbf{m}_{\mathrm{z}}\right)=\mathbf{2}_{\mathrm{z}}^{\prime} / \mathbf{m}_{\mathrm{z}}$

$V^{3} \quad\left(\begin{array}{lllllllll}0 & 0 & 0 & 0 & 0 & 0 & 0 & 0 & 0 \\ 0 & 0 & 0 & 0 & 0 & 0 & 0 & 0 & 0 \\ 0 & 0 & 0 & 0 & 0 & 0 & 0 & 0 & 0\end{array}\right)$ $\left(\begin{array}{lllllllll}0 & 0 & 0 & 0 & 0 & 0 & 0 & 0 & 0\end{array}\right)$

$\left[\mathrm{V}^{3}\right]\left(\begin{array}{llll}0 & 0 & 0 & 0 \\ 0 & 0 & 0 & \\ 0 & 0 & 0 & \end{array}\right)$

$\left(\begin{array}{llllll}0 & 0 & 0 & 0 & 0 & 0 \\ 0 & 0 & 0 & 0 & 0 & 0\end{array}\right)$

$V\left[V^{2}\right] \quad\left(\begin{array}{llllll}0 & 0 & 0 & 0 & 0 & 0 \\ 0 & 0 & 0 & 0 & 0 & 0\end{array}\right)$

$\left(\begin{array}{llllll}0 & 0 & 0 & 0 & 0 & 0\end{array}\right)$

$\left\{V^{2}\right\} V\left(\begin{array}{lll}0 & 0 & 0 \\ 0 & 0 & 0 \\ 0 & 0 & 0\end{array}\right)$

$n=2 \quad C_{4 h}\left(C_{2 h}\right)=4_{z} / m_{z}\left(2_{z} / m_{z}\right)=4_{z}^{\prime} / m_{z}$

$\mathrm{V}^{3} \quad\left(\begin{array}{lllllllll}0 & 0 & 0 & 0 & 0 & 0 & 0 & 0 & 0 \\ 0 & 0 & 0 & 0 & 0 & 0 & 0 & 0 & 0 \\ 0 & 0 & 0 & 0 & 0 & 0 & 0 & 0 & 0\end{array}\right)$

$\left[V^{3}\right] \quad\left(\begin{array}{llll}0 & 0 & 0 & 0 \\ 0 & 0 & 0 & \\ 0 & 0 & 0 & \end{array}\right)$

$V\left[V^{2}\right]\left(\begin{array}{llllll}0 & 0 & 0 & 0 & 0 & 0 \\ 0 & 0 & 0 & 0 & 0 & 0 \\ 0 & 0 & 0 & 0 & 0 & 0\end{array}\right)$

$\left\{V^{2}\right\} V\left(\begin{array}{lll}0 & 0 & 0 \\ 0 & 0 & 0 \\ 0 & 0 & 0\end{array}\right)$ $\left(\begin{array}{rrrrrrrrr}0 & 0 & 0 & x y z & x z x & 0 & x z y & x x z & 0 \\ 0 & 0 & 0 & y y z & y z x & 0 & y z y & y x z & 0 \\ z x x & z y y & z z z & 0 & 0 & z x y & 0 & 0 & z y x\end{array}\right)\left(\begin{array}{rrrrrrrrr}x x x & x y y & x z z & 0 & 0 & x x y & 0 & 0 & x y x \\ y x x & y y y & y z z & 0 & 0 & y x y & 0 & 0 & y y x \\ 0 & 0 & 0 & z y z & z z x & 0 & z z y & z x z & 0\end{array}\right) \quad\left(\begin{array}{rrrrrrrrr}0 & 0 & 0 & 0 & 0 & 0 & 0 & 0 & 0 \\ 0 & 0 & 0 & 0 & 0 & 0 & 0 & 0 & 0 \\ 0 & 0 & 0 & 0 & 0 & 0 & 0 & 0 & 0\end{array}\right)$

$\left(\begin{array}{rrrr}0 & 0 & 0 & x y z \\ 0 & 0 & 0 & \\ z x x & z y y & z z z\end{array}\right)$

$\left(\begin{array}{rrrr}x x x & x y y & x z z & 0 \\ y x x & y y y & y z z & \\ 0 & 0 & 0\end{array}\right)$

$\left(\begin{array}{llll}0 & 0 & 0 & 0 \\ 0 & 0 & 0 & \\ 0 & 0 & 0\end{array}\right)$

$\left(\begin{array}{rrrrrr}0 & 0 & 0 & x y z & x x z & 0 \\ 0 & 0 & 0 & y y z & y x z & 0 \\ z x x & z y y & z z z & 0 & 0 & z x y\end{array}\right)$

$\left(\begin{array}{rrrrrr}x x x & x y y & x z z & 0 & 0 & x x y \\ y x x & y y y & y z z & 0 & 0 & y x y \\ 0 & 0 & 0 & z y z & z x z & 0\end{array}\right)$

$\left(\begin{array}{llllll}0 & 0 & 0 & 0 & 0 & 0 \\ 0 & 0 & 0 & 0 & 0 & 0 \\ 0 & 0 & 0 & 0 & 0 & 0\end{array}\right)$

$\left(\begin{array}{ccc}y z x & y z y & 0 \\ z x x & z x y & 0 \\ 0 & 0 & x y z\end{array}\right)$

$\left(\begin{array}{ccc}0 & 0 & y z z \\ 0 & 0 & z x z \\ x y x & x y y & 0\end{array}\right)$

$\left(\begin{array}{lll}0 & 0 & 0 \\ 0 & 0 & 0 \\ 0 & 0 & 0\end{array}\right)$ $\left(\begin{array}{rrrrrrrrr}0 & 0 & 0 & x y z & x z x & 0 & -y z x & y y z & 0 \\ 0 & 0 & 0 & y y z & y z x & 0 & x z x & -x y z & 0 \\ z x x & z y y & z z z & 0 & 0 & z x y & 0 & 0 & -z x y\end{array}\right) \quad\left(\begin{array}{rrrrrrrrr}0 & 0 & 0 & 0 & 0 & 0 & 0 & 0 & 0 \\ 0 & 0 & 0 & 0 & 0 & 0 & 0 & 0 & 0 \\ 0 & 0 & 0 & 0 & 0 & 0 & 0 & 0 & 0\end{array}\right)$

$\left(\begin{array}{rrrr}0 & 0 & 0 & 0 \\ 0 & 0 & 0 & \\ z x x & z x x & z z z & \end{array}\right)$

$\left(\begin{array}{llll}0 & 0 & 0 & 0 \\ 0 & 0 & 0 & \\ 0 & 0 & 0 & \end{array}\right)$

$\left(\begin{array}{rrrrrr}0 & 0 & 0 & x y z & x x z & 0 \\ 0 & 0 & 0 & x x z & -x y z & 0 \\ z x x & z y y & z z z & 0 & 0 & 0\end{array}\right)$

$\left(\begin{array}{llllll}0 & 0 & 0 & 0 & 0 & 0 \\ 0 & 0 & 0 & 0 & 0 & 0 \\ 0 & 0 & 0 & 0 & 0 & 0\end{array}\right)$

$\left(\begin{array}{ccc}y z x & y z y & 0 \\ -y z y & y z x & 0 \\ 0 & 0 & x y z\end{array}\right)$ $\left(\begin{array}{lll}0 & 0 & 0 \\ 0 & 0 & 0 \\ 0 & 0 & 0\end{array}\right)$ $\left(\begin{array}{rrrrrrrrr}0 & 0 & 0 & x y z & x z x & 0 & y z x & -y y z & 0 \\ 0 & 0 & 0 & y y z & y z x & 0 & -x z x & x y z & 0 \\ z x x & -z x x & 0 & 0 & 0 & z x y & 0 & 0 & z x y\end{array}\right)$

$\left(\begin{array}{rrrr}0 & 0 & 0 & x y z \\ 0 & 0 & 0 & \\ z x x & -z x x & 0 & \end{array}\right)$

$\left(\begin{array}{rrrrrr}0 & 0 & 0 & x y z & x x z & 0 \\ 0 & 0 & 0 & -x x z & x y z & 0 \\ z x x & -z x x & 0 & 0 & 0 & z x y\end{array}\right)$

$\left(\begin{array}{ccc}y z x & y z y & 0 \\ y z y & -y z x & 0 \\ 0 & 0 & 0\end{array}\right)$ 
$\mathrm{n}>3 \quad \mathbf{C}_{\infty \mathrm{h}} \mathbf{1}^{\prime}=\infty / \mathbf{m} \mathbf{1}^{\prime}$

$V^{3} \quad\left(\begin{array}{lllllllll}0 & 0 & 0 & 0 & 0 & 0 & 0 & 0 & 0 \\ 0 & 0 & 0 & 0 & 0 & 0 & 0 & 0 & 0 \\ 0 & 0 & 0 & 0 & 0 & 0 & 0 & 0 & 0\end{array}\right)$ 
$\mathrm{n}=1 \quad \mathrm{D}_{1}\left(\mathrm{C}_{1}\right) \quad=\mathbf{2}_{\mathrm{y}}(\mathbf{1})=\mathbf{2}_{\mathrm{y}^{\prime}}$
$V^{3} \quad\left(\begin{array}{rrrrrrrrr}0 & 0 & 0 & x y z & 0 & x x y & x z y & 0 & x y x \\ y x x & y y y & y z z & 0 & y z x & 0 & 0 & y x z & 0 \\ 0 & 0 & 0 & z y z & 0 & z x y & z z y & 0 & z y x\end{array}\right)$
$\left(\begin{array}{rrrrrrrrr}0 & 0 & 0 & x y z & 0 & x x y & x z y & 0 & x y x \\ y x x & y y y & y z z & 0 & y z x & 0 & 0 & y x z & 0 \\ 0 & 0 & 0 & z y z & 0 & z x y & z z y & 0 & z y x\end{array}\right)$
$\left(\begin{array}{cccccc}x x x & x y y & x z z & 0 & x z x^{a} \\ 0 & 0 & 0 & y y z & 0 & y x y\end{array}\right.$
a
$\left[\vee^{3}\right]\left(\begin{array}{rrrr}0 & 0 & 0 & x y z \\ y x x & y y y & y z z & \\ 0 & 0 & 0 & \end{array}\right)$
$\left(\begin{array}{rrrr}0 & 0 & 0 & x y z \\ y x x & y y y & y z z & \\ 0 & 0 & 0 & \end{array}\right)$
$\left(\begin{array}{rrrrrrrrr}x & 0 & 0 & y y z & 0 & y x y & y z y & 0 & y y x \\ z x x & z y y & z z z & 0 & z z x & 0 & 0 & z x z & 0\end{array}\right)$
$\left(\begin{array}{ccccccccc}x x x & x y y & x z z & 0 & x z x & 0 & 0 & x x z & 0 \\ 0 & 0 & 0 & y y z & 0 & y x y & y z y & 0 & y y x\end{array}\right)$
$\left.\begin{array}{rrrrrrrrr}0 & 0 & 0 & y y z & 0 & y x y & y z y & 0 & y y x \\ z x x & z y y & z z z & 0 & z z x & 0 & 0 & z x z & 0\end{array}\right)$
$V\left[V^{2}\right]\left(\begin{array}{rrrrrr}0 & 0 & 0 & x y z & 0 & x x y \\ y x x & y y y & y z z & 0 & y x z & 0 \\ 0 & 0 & 0 & z y z & 0 & z x y\end{array}\right)$
$\left(\begin{array}{rrrrrr}0 & 0 & 0 & x y z & 0 & x x y \\ y x x & y y y & y z z & 0 & y x z & 0 \\ 0 & 0 & 0 & z y z & 0 & z x y\end{array}\right)$
$\left(\begin{array}{rrrr}x x x & x y y & x z z & 0 \\ 0 & 0 & 0 & \\ z x x & z y y & z z z & \end{array}\right)$
$\left(\begin{array}{rrrr}x x x & x y y & x z z & 0 \\ 0 & 0 & 0 & \\ z x x & z y y & z z z & \end{array}\right)$
$\left\{V^{2}\right\} \vee\left(\begin{array}{ccc}y z x & 0 & y z z \\ 0 & z x y & 0 \\ x y x & 0 & x y z\end{array}\right)$
$\left(\begin{array}{ccc}y z x & 0 & y z z \\ 0 & z x y & 0 \\ x y x & 0 & x y z\end{array}\right)$
$\left(\begin{array}{rrrrrr}x x x & x y y & x z z & 0 & x x z & 0 \\ 0 & 0 & 0 & y y z & 0 & y x y \\ z x x & z y y & z z z & 0 & z x z & 0\end{array}\right)$
$\left(\begin{array}{rrrrrr}x x x & x y y & x z z & 0 & x x z & 0 \\ 0 & 0 & 0 & y y z & 0 & y x y \\ z x x & z y y & z z z & 0 & z x z & 0\end{array}\right)$
$\left(\begin{array}{ccc}0 & y z y & 0 \\ z x x & 0 & z x z \\ 0 & x y y & 0\end{array}\right)$
$\left(\begin{array}{ccc}0 & y z y & 0 \\ z x x & 0 & z x z \\ 0 & x y y & 0\end{array}\right)$
$\mathrm{n}=2 \quad \mathrm{D}_{2}\left(\mathrm{C}_{2}\right)=\mathbf{2} \mathbf{x}_{\mathrm{y}} \mathbf{2}_{\mathrm{z}}\left(\mathbf{2}_{\mathrm{z}}\right)=\mathbf{2} \mathbf{x}^{\prime} \mathbf{2}^{\prime} \mathbf{2}_{\mathrm{z}}$

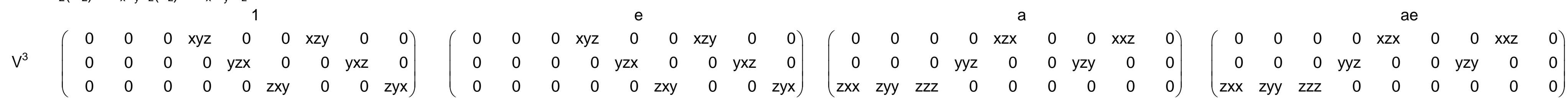
$\left[\mathrm{V}^{3}\right]\left(\begin{array}{cccc}0 & 0 & 0 & \mathrm{xyz} \\ 0 & 0 & 0 & \\ 0 & 0 & 0 & \end{array}\right) \quad\left(\begin{array}{cccc}0 & 0 & 0 & \mathrm{xyz} \\ 0 & 0 & 0 & \\ 0 & 0 & 0 & \end{array}\right)$
$\left(\begin{array}{rrrr}0 & 0 & 0 & 0 \\ 0 & 0 & 0 & \\ \text { zxx } & \text { zyy } & \text { zzz } & \end{array}\right)$
$\left(\begin{array}{rrrr}0 & 0 & 0 & 0 \\ 0 & 0 & 0 & \\ \text { zxx } & \text { zyy } & \text { zzz } & \end{array}\right)$
$V\left[V^{2}\right]\left(\begin{array}{rrrrrr}0 & 0 & 0 & x y z & 0 & 0 \\ 0 & 0 & 0 & 0 & y x z & 0 \\ 0 & 0 & 0 & 0 & 0 & z x y\end{array}\right)$
$\left(\begin{array}{rrrrrr}0 & 0 & 0 & x y z & 0 & 0 \\ 0 & 0 & 0 & 0 & y x z & 0 \\ 0 & 0 & 0 & 0 & 0 & z x y\end{array}\right)$
$\left(\begin{array}{rrrrrr}0 & 0 & 0 & 0 & x x z & 0 \\ 0 & 0 & 0 & y y z & 0 & 0 \\ z x x & z y y & z z z & 0 & 0 & 0\end{array}\right)$
$\left(\begin{array}{rrrrrr}0 & 0 & 0 & 0 & x x z & 0 \\ 0 & 0 & 0 & y y z & 0 & 0 \\ z x x & z y y & z z z & 0 & 0 & 0\end{array}\right)$
$\left\{V^{2}\right\} V\left(\begin{array}{ccc}y z x & 0 & 0 \\ 0 & z x y & 0 \\ 0 & 0 & x y z\end{array}\right)$
$\left(\begin{array}{ccc}y z x & 0 & 0 \\ 0 & z x y & 0 \\ 0 & 0 & x y z\end{array}\right)$
$\left(\begin{array}{ccc}0 & y z y & 0 \\ z x x & 0 & 0 \\ 0 & 0 & 0\end{array}\right)$
$\left(\begin{array}{ccc}0 & y z y & 0 \\ z x x & 0 & 0 \\ 0 & 0 & 0\end{array}\right)$

ae 
$\mathrm{n}=3 \quad \mathbf{D}_{3}\left(\mathbf{C}_{3}\right)=\mathbf{3}_{\mathrm{z}} \mathbf{2}_{\mathrm{x}}\left(\mathbf{3}_{\mathrm{z}}\right)=\mathbf{3}_{\mathrm{z}} \mathbf{2}_{\mathrm{x}}{ }^{\prime}$

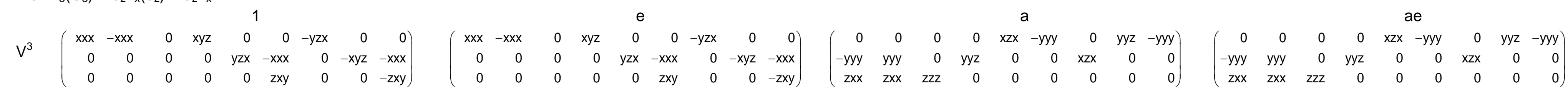
$\left[\mathrm{V}^{3}\right]\left(\begin{array}{rrrr}\mathrm{xxx} & -\mathrm{xxx} & 0 & 0 \\ 0 & 0 & 0 & \\ 0 & 0 & 0 & \end{array}\right)$

$\left(\begin{array}{rrrr}x x x & -x x x & 0 & 0 \\ 0 & 0 & 0 & \\ 0 & 0 & 0 & \end{array}\right)$

$\left(\begin{array}{rrrr}0 & 0 & 0 & 0 \\ -y y y & y y y & 0 & \\ z x x & z x x & z z z & \end{array}\right)$

$\left(\begin{array}{rrrr}0 & 0 & 0 & 0 \\ -y y y & y y y & 0 & \\ z x x & z x x & z z z & \end{array}\right)$

$\left.\mathrm{V} \mathrm{V}^{2}\right]\left(\begin{array}{rrrrrr}\mathrm{xxx} & -\mathrm{xxx} & 0 & \mathrm{xyz} & 0 & 0 \\ 0 & 0 & 0 & 0 & -\mathrm{xyz} & -\mathrm{xxx} \\ 0 & 0 & 0 & 0 & 0 & 0\end{array}\right)$

$\left(\begin{array}{rrrrrr}x x x & -x x x & 0 & x y z & 0 & 0 \\ 0 & 0 & 0 & 0 & -x y z & -x x x \\ 0 & 0 & 0 & 0 & 0 & 0\end{array}\right)$

$\left(\begin{array}{rrrrrr}0 & 0 & 0 & 0 & x x z & -y y y \\ -y y y & y y y & 0 & x x z & 0 & 0 \\ z x x & z y y & z z z & 0 & 0 & 0\end{array}\right)$

$\left(\begin{array}{rrrrrr}0 & 0 & 0 & 0 & x x z & -y y y \\ -y y y & y y y & 0 & x x z & 0 & 0 \\ z x x & z y y & z z z & 0 & 0 & 0\end{array}\right)$

$\left\{V^{2}\right\} V\left(\begin{array}{ccc}y z x & 0 & 0 \\ 0 & y z x & 0 \\ 0 & 0 & x y z\end{array}\right)$

$\left(\begin{array}{ccc}y z x & 0 & 0 \\ 0 & y z x & 0 \\ 0 & 0 & x y z\end{array}\right)$

$\left(\begin{array}{ccc}0 & y z y & 0 \\ -y z y & 0 & 0 \\ 0 & 0 & 0\end{array}\right)$

$\left(\begin{array}{ccc}0 & y z y & 0 \\ -y z y & 0 & 0 \\ 0 & 0 & 0\end{array}\right)$

$\mathrm{n}>3 \quad \mathbf{D}_{\infty}\left(\mathbf{C}_{\infty}\right)=\infty \mathbf{2}^{\prime}$

$\mathrm{V}^{3} \quad\left(\begin{array}{rrrrrrrrr}0 & 0 & 0 & \mathrm{xyz} & 0 & 0 & -\mathrm{yzx} & 0 & 0 \\ 0 & 0 & 0 & 0 & \mathrm{yzx} & 0 & 0 & -\mathrm{xyz} & 0 \\ 0 & 0 & 0 & 0 & 0 & \mathrm{zxy} & 0 & 0 & -\mathrm{zxy}\end{array}\right) \quad\left(\begin{array}{rrrrrrrrr}0 & 0 & 0 & \mathrm{xyz} & 0 & 0 & -\mathrm{yzx} & 0 & 0 \\ 0 & 0 & 0 & 0 & \mathrm{yzx} & 0 & 0 & -\mathrm{xyz} & 0 \\ 0 & 0 & 0 & 0 & 0 & \mathrm{zxy} & 0 & 0 & -\mathrm{zxy}\end{array}\right)$

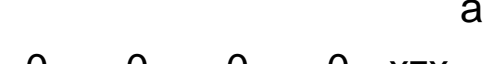

$\begin{array}{lllllllll}0 & 0 & 0 & 0 & x z x & 0 & 0 & y y z & 0 \\ 0 & 0 & 0 & y y z & 0 & 0 & x z x & 0 & 0\end{array} \quad\left(\begin{array}{cccccccccc}0 & 0 & 0 & 0 & x z x & 0 & 0 & y y z & 0 \\ 0 & 0 & 0 & y y z & 0 & 0 & x z x & 0 & 0\end{array}\right.$

$\left[\mathrm{V}^{3}\right]\left(\begin{array}{rrrr}0 & 0 & 0 & 0 \\ 0 & 0 & 0 & \\ \mathrm{zxx} & \mathrm{zxx} & \mathrm{zzz} & \end{array}\right)$

$\left(\begin{array}{rrrr}0 & 0 & 0 & 0 \\ 0 & 0 & 0 & \\ z x x & z x x & z z z & \end{array}\right)$

$\left(\begin{array}{rrrr}0 & 0 & 0 & 0 \\ 0 & 0 & 0 & \\ z x x & z x x & z z z & \end{array}\right)$

$\left(\begin{array}{rrrr}0 & 0 & 0 & 0 \\ 0 & 0 & 0 & \\ z x x & z x x & z z z & \end{array}\right)$

$\mathrm{V}\left[\mathrm{V}^{2}\right]\left(\begin{array}{rrrrrr}0 & 0 & 0 & \mathrm{xyz} & 0 & 0 \\ 0 & 0 & 0 & 0 & -x y z & 0 \\ 0 & 0 & 0 & 0 & 0 & 0\end{array}\right)$

$\left(\begin{array}{rrrrrr}0 & 0 & 0 & x y z & 0 & 0 \\ 0 & 0 & 0 & 0 & -x y z & 0 \\ 0 & 0 & 0 & 0 & 0 & 0\end{array}\right)$

$\left(\begin{array}{rrrrrr}0 & 0 & 0 & 0 & x x z & 0 \\ 0 & 0 & 0 & x x z & 0 & 0 \\ z x x & z x x & z z z & 0 & 0 & 0\end{array}\right)$

$\left(\begin{array}{rrrrrr}0 & 0 & 0 & 0 & x x z & 0 \\ 0 & 0 & 0 & x x z & 0 & 0 \\ z x x & z x x & z z z & 0 & 0 & 0\end{array}\right)$

$\left\{V^{2}\right\} V\left(\begin{array}{ccc}y z x & 0 & 0 \\ 0 & y z x & 0 \\ 0 & 0 & x y z\end{array}\right)$

$\left(\begin{array}{ccc}y z x & 0 & 0 \\ 0 & y z x & 0 \\ 0 & 0 & x y z\end{array}\right)$

$\left(\begin{array}{ccc}0 & y z y & 0 \\ -y z y & 0 & 0 \\ 0 & 0 & 0\end{array}\right)$

$\left(\begin{array}{ccc}0 & y z y & 0 \\ -y z y & 0 & 0 \\ 0 & 0 & 0\end{array}\right)$ 
$\mathrm{n}=1 \quad \mathbf{D}_{2}\left(\mathbf{D}_{1}\right)=\mathbf{2}_{\mathrm{x}} \mathbf{2}_{\mathrm{y}} \mathbf{2}\left(\mathbf{2}_{\mathrm{y}}\right)=\mathbf{2}_{\mathrm{x}}{ }^{\prime} \mathbf{y}_{\mathrm{y}} \mathbf{2}^{\prime}{ }^{\prime}$

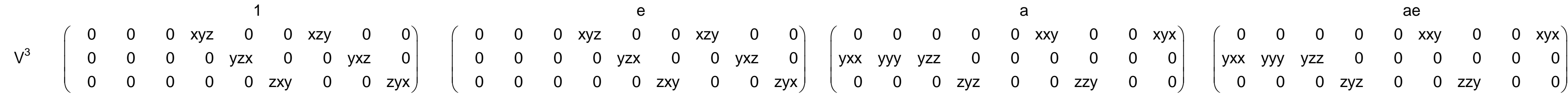

$\left[\mathrm{V}^{3}\right] \quad\left(\begin{array}{llll}0 & 0 & 0 & \mathrm{xyz} \\ 0 & 0 & 0 & \\ 0 & 0 & 0 & \end{array}\right)$

$\left(\begin{array}{llll}0 & 0 & 0 & x y z \\ 0 & 0 & 0 & \\ 0 & 0 & 0 & \end{array}\right)$

$\left(\begin{array}{rrrr}0 & 0 & 0 & 0 \\ y x x & y y y & y z z & \\ 0 & 0 & 0 & \end{array}\right)$

$\left(\begin{array}{rrrr}0 & 0 & 0 & 0 \\ y x x & y y y & y z z & \\ 0 & 0 & 0 & \end{array}\right)$

$V\left[V^{2}\right]\left(\begin{array}{rrrrrr}0 & 0 & 0 & x y z & 0 & 0 \\ 0 & 0 & 0 & 0 & y x z & 0 \\ 0 & 0 & 0 & 0 & 0 & z x y\end{array}\right)$

$\left(\begin{array}{rrrrrr}0 & 0 & 0 & x y z & 0 & 0 \\ 0 & 0 & 0 & 0 & y x z & 0 \\ 0 & 0 & 0 & 0 & 0 & z x y\end{array}\right)$

$\left(\begin{array}{rrrrrr}0 & 0 & 0 & 0 & 0 & x x y \\ y x x & y y y & y z z & 0 & 0 & 0 \\ 0 & 0 & 0 & z y z & 0 & 0\end{array}\right)$

$\left(\begin{array}{rrrrrr}0 & 0 & 0 & 0 & 0 & x x y \\ y x x & y y y & y z z & 0 & 0 & 0 \\ 0 & 0 & 0 & z y z & 0 & 0\end{array}\right)$

$\left\{V^{2}\right\} V\left(\begin{array}{ccc}y z x & 0 & 0 \\ 0 & z x y & 0 \\ 0 & 0 & x y z\end{array}\right)$

$\left(\begin{array}{ccc}y z x & 0 & 0 \\ 0 & z x y & 0 \\ 0 & 0 & x y z\end{array}\right)$

$\left(\begin{array}{ccc}0 & 0 & y z z \\ 0 & 0 & 0 \\ x y x & 0 & 0\end{array}\right)$

$\left(\begin{array}{ccc}0 & 0 & y z z \\ 0 & 0 & 0 \\ x y x & 0 & 0\end{array}\right)$

$\mathrm{n}=2 \quad \mathrm{D}_{4}\left(\mathrm{D}_{2}\right)=\mathbf{4}_{\mathrm{z}} \mathbf{2}_{\mathrm{x}} \mathbf{2}_{\mathrm{xy}}\left(\mathbf{2}_{\mathrm{x}} \mathbf{2}_{\mathrm{y}} \mathbf{2}_{\mathrm{z}}\right)=\mathbf{4}_{\mathrm{z}} \mathbf{2}_{\mathrm{x}} \mathbf{2}_{\mathrm{xy}}{ }^{\prime}$

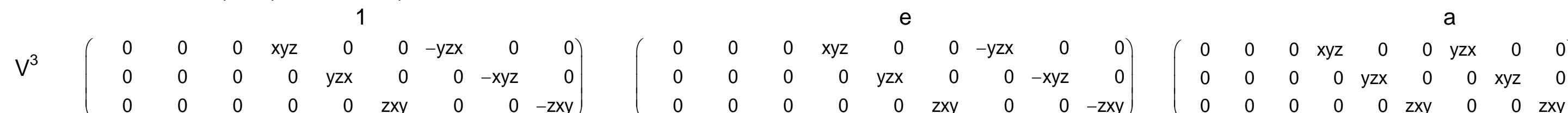

$\left[\mathrm{V}^{3}\right] \quad\left(\begin{array}{llll}0 & 0 & 0 & 0 \\ 0 & 0 & 0 \\ 0 & 0 & 0\end{array}\right)$

$\left(\begin{array}{llll}0 & 0 & 0 & 0 \\ 0 & 0 & 0 \\ 0 & 0 & 0\end{array}\right)$

$\left(\begin{array}{llll}0 & 0 & 0 & x y z \\ 0 & 0 & 0 & \\ 0 & 0 & 0 & \end{array}\right)$

$\left(\begin{array}{llll}0 & 0 & 0 & x y z \\ 0 & 0 & 0 & \\ 0 & 0 & 0\end{array}\right)$

$V\left[V^{2}\right]\left(\begin{array}{rrrrrr}0 & 0 & 0 & x y z & 0 & 0 \\ 0 & 0 & 0 & 0 & -x y z & 0 \\ 0 & 0 & 0 & 0 & 0 & 0\end{array}\right)$

$\left(\begin{array}{rrrrrr}0 & 0 & 0 & x y z & 0 & 0 \\ 0 & 0 & 0 & 0 & -x y z & 0 \\ 0 & 0 & 0 & 0 & 0 & 0\end{array}\right)$

$\left(\begin{array}{rrrrrr}0 & 0 & 0 & x y z & 0 & 0 \\ 0 & 0 & 0 & 0 & x y z & 0 \\ 0 & 0 & 0 & 0 & 0 & z x y\end{array}\right)$

$\left(\begin{array}{rrrrrr}0 & 0 & 0 & x y z & 0 & 0 \\ 0 & 0 & 0 & 0 & x y z & 0 \\ 0 & 0 & 0 & 0 & 0 & z x y\end{array}\right)$

$\left\{V^{2}\right\} V\left(\begin{array}{ccc}y z x & 0 & 0 \\ 0 & y z x & 0 \\ 0 & 0 & x y z\end{array}\right)$

$\left(\begin{array}{ccc}y z x & 0 & 0 \\ 0 & y z x & 0 \\ 0 & 0 & x y z\end{array}\right)$

$\left(\begin{array}{ccc}y z x & 0 & 0 \\ 0 & -y z x & 0 \\ 0 & 0 & 0\end{array}\right)$

$\left(\begin{array}{ccc}y z x & 0 & 0 \\ 0 & -y z x & 0 \\ 0 & 0 & 0\end{array}\right)$ 


\section{$\mathrm{n}=3 \quad \mathrm{D}_{6}\left(\mathrm{D}_{3}\right)=\mathbf{6}_{\mathrm{z}} \mathbf{2}_{\mathrm{x}} \mathbf{2}_{1}\left(\mathbf{3}_{\mathrm{z}} \mathbf{2}_{\mathrm{x}}\right)=\mathbf{6}_{\mathrm{z}} \mathbf{2}_{\mathrm{x}} \mathbf{2}^{\prime}{ }^{\prime}$}
$\mathrm{V}^{3} \quad\left(\begin{array}{rrrrrrrrr}0 & 0 & 0 & x y z & 0 & 0 & -y z x & 0 & 0 \\ 0 & 0 & 0 & 0 & y z x & 0 & 0 & -x y z & 0 \\ 0 & 0 & 0 & 0 & 0 & z x y & 0 & 0 & -z x y\end{array}\right)$
$\left(\begin{array}{rrrrrrrrr}0 & 0 & 0 & x y z & 0 & 0 & -y z x & 0 & 0 \\ 0 & 0 & 0 & 0 & y z x & 0 & 0 & -x y z & 0 \\ 0 & 0 & 0 & 0 & 0 & z x y & 0 & 0 & -z x y\end{array}\right)$
$\left(\begin{array}{rr}x x x & -x x x \\ 0 & 0 \\ 0 & 0\end{array}\right.$
a
$\left[V^{3}\right]\left(\begin{array}{llll}0 & 0 & 0 & 0 \\ 0 & 0 & 0 & \\ 0 & 0 & 0 & \end{array}\right)$
$\left(\begin{array}{llll}0 & 0 & 0 & 0 \\ 0 & 0 & 0 & \\ 0 & 0 & 0 & \end{array}\right)$
$\left(\begin{array}{rrrr}x x x & -x x x & 0 & 0 \\ 0 & 0 & 0 & \\ 0 & 0 & 0 & \end{array}\right)$
$V\left[V^{2}\right]\left(\begin{array}{rrrrrr}0 & 0 & 0 & x y z & 0 & 0 \\ 0 & 0 & 0 & 0 & -x y z & 0 \\ 0 & 0 & 0 & 0 & 0 & 0\end{array}\right)$
$\left(\begin{array}{rrrrrr}0 & 0 & 0 & x y z & 0 & 0 \\ 0 & 0 & 0 & 0 & -x y z & 0 \\ 0 & 0 & 0 & 0 & 0 & 0\end{array}\right)$
$\left(\begin{array}{rrrrrr}x x x & -x x x & 0 & 0 & 0 & 0 \\ 0 & 0 & 0 & 0 & 0 & -x x x \\ 0 & 0 & 0 & 0 & 0 & 0\end{array}\right)$
$\left(\begin{array}{rrrrrrrrr}\mathrm{xxx} & -\mathrm{xxx} & 0 & 0 & 0 & 0 & 0 & 0 & 0 \\ 0 & 0 & 0 & 0 & 0 & -\mathrm{xxx} & 0 & 0 & -\mathrm{xxx} \\ 0 & 0 & 0 & 0 & 0 & 0 & 0 & 0 & 0\end{array}\right)$
$\left\{V^{2}\right\} \vee\left(\begin{array}{ccc}y z x & 0 & 0 \\ 0 & y z x & 0 \\ 0 & 0 & x y z\end{array}\right)$
$\left(\begin{array}{ccc}y z x & 0 & 0 \\ 0 & y z x & 0 \\ 0 & 0 & x y z\end{array}\right)$
$\left(\begin{array}{lll}0 & 0 & 0 \\ 0 & 0 & 0 \\ 0 & 0 & 0\end{array}\right)$
$\left(\begin{array}{rrrr}x x x & -x x x & 0 & 0 \\ 0 & 0 & 0 & \\ 0 & 0 & 0 & \end{array}\right)$
$\left(\begin{array}{rrrrrr}x x x & -x x x & 0 & 0 & 0 & 0 \\ 0 & 0 & 0 & 0 & 0 & -x x x \\ 0 & 0 & 0 & 0 & 0 & 0\end{array}\right)$
$\left(\begin{array}{lll}0 & 0 & 0 \\ 0 & 0 & 0 \\ 0 & 0 & 0\end{array}\right)$

$n>3 \quad D_{\infty} 1^{\prime}=\infty 21^{\prime}$

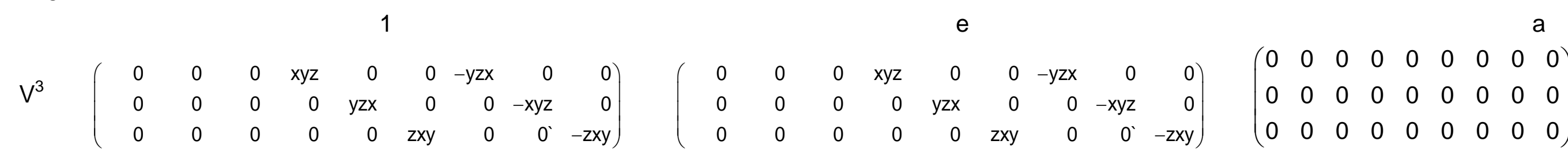

$\begin{array}{lllllllll}0 & 0 & 0 & 0 & 0\end{array}$

$\left(\begin{array}{lllllllll}0 & 0 & 0 & 0 & 0 & 0 & 0 & 0 & 0\end{array}\right.$

$\begin{array}{llllllllll}0 & 0 & 0 & 0 & 0 & 0 & 0 & 0 & 0\end{array}$

$\left[V^{3}\right]\left(\begin{array}{rrrr}0 & 0 & 0 & 0 \\ 0 & 0 & 0 & \\ z x x & z x x & z z z & \end{array}\right)$

$\left(\begin{array}{rrrr}0 & 0 & 0 & 0 \\ 0 & 0 & 0 & \\ z x x & z x x & z z z & \end{array}\right)$

$\left(\begin{array}{llll}0 & 0 & 0 & 0 \\ 0 & 0 & 0 & \\ 0 & 0 & 0 & \end{array}\right)$

$\left(\begin{array}{llll}0 & 0 & 0 & 0 \\ 0 & 0 & 0 & \\ 0 & 0 & 0 & \end{array}\right)$

$V\left[V^{2}\right]\left(\begin{array}{rrrrrr}0 & 0 & 0 & x y z & 0 & 0 \\ 0 & 0 & 0 & 0 & -x y z & 0 \\ 0 & 0 & 0 & 0 & 0 & 0\end{array}\right)$

$\left(\begin{array}{rrrrrr}0 & 0 & 0 & x y z & 0 & 0 \\ 0 & 0 & 0 & 0 & -x y z & 0 \\ 0 & 0 & 0 & 0 & 0 & 0\end{array}\right)$

$\left(\begin{array}{llllll}0 & 0 & 0 & 0 & 0 & 0 \\ 0 & 0 & 0 & 0 & 0 & 0 \\ 0 & 0 & 0 & 0 & 0 & 0\end{array}\right)$

$\left(\begin{array}{llllll}0 & 0 & 0 & 0 & 0 & 0 \\ 0 & 0 & 0 & 0 & 0 & 0 \\ 0 & 0 & 0 & 0 & 0 & 0\end{array}\right)$

$\left\{\mathrm{V}^{2}\right\} \mathrm{V}\left(\begin{array}{ccc}\mathrm{yzx} & 0 & 0 \\ 0 & \mathrm{yzx} & 0 \\ 0 & 0 & \mathrm{xyz}\end{array}\right)$

$\left(\begin{array}{ccc}y z x & 0 & 0 \\ 0 & y z x & 0 \\ 0 & 0 & x y z\end{array}\right)$

$\left(\begin{array}{lll}0 & 0 & 0 \\ 0 & 0 & 0 \\ 0 & 0 & 0\end{array}\right)$

$\left(\begin{array}{lll}0 & 0 & 0 \\ 0 & 0 & 0 \\ 0 & 0 & 0\end{array}\right)$ 
$\mathrm{n}=1 \quad \mathbf{C}_{1 \mathrm{v}}\left(\mathbf{C}_{1}\right)=\mathbf{m}_{\mathrm{y}}(\mathbf{1})=\mathbf{m}_{\mathrm{y}}{ }^{\prime}$

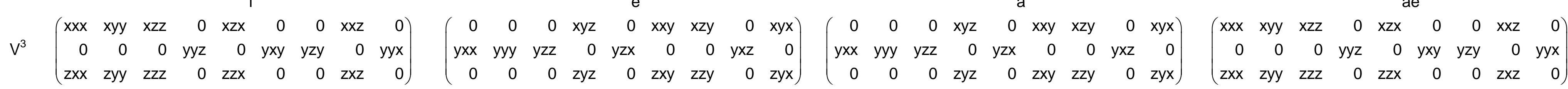

$$
\begin{aligned}
& {\left[V^{3}\right]\left(\begin{array}{rrrr}
x x x & x y y & x z z & 0 \\
0 & 0 & 0 & \\
z x x & z y y & z z z &
\end{array}\right) \quad\left(\begin{array}{rrrr}
0 & 0 & 0 & x y z \\
y x x & y y y & y z z & \\
0 & 0 & 0
\end{array}\right) \quad\left(\begin{array}{rrrr}
0 & 0 & 0 & x y z \\
y x x & y y y & y z z & 0 \\
0 & 0 & 0
\end{array}\right)} \\
& V\left[V^{2}\right]\left(\begin{array}{rrrrrr}
x x x & x y y & x z z & 0 & x x z & 0 \\
0 & 0 & 0 & y y z & 0 & y x y \\
z x x & z y y & z z z & 0 & z x z & 0
\end{array}\right) \\
& \left(\begin{array}{rrrrrr}
0 & 0 & 0 & x y z & 0 & x x y \\
y x x & y y y & y z z & 0 & y x z & 0 \\
0 & 0 & 0 & z y z & 0 & z x y
\end{array}\right) \\
& \left(\begin{array}{rrrrrr}
0 & 0 & 0 & x y z & 0 & x x y \\
y x x & y y y & y z z & 0 & y x z & 0 \\
0 & 0 & 0 & z y z & 0 & z x y
\end{array}\right) \\
& \left(\begin{array}{rrrr}
x x x & x y y & x z z & 0 \\
0 & 0 & 0 & \\
z x x & z y y & z z z &
\end{array}\right) \\
& \left\{V^{2}\right\} V\left(\begin{array}{ccc}
0 & y z y & 0 \\
z x x & 0 & z x z \\
0 & x y y & 0
\end{array}\right) \\
& \left(\begin{array}{ccc}
y z x & 0 & y z z \\
0 & z x y & 0 \\
x y x & 0 & x y z
\end{array}\right) \\
& \left(\begin{array}{ccc}
y z x & 0 & y z z \\
0 & z x y & 0 \\
x y x & 0 & x y z
\end{array}\right) \\
& \left(\begin{array}{rrrrrr}
x x x & x y y & x z z & 0 & x x z & 0 \\
0 & 0 & 0 & y y z & 0 & y x y \\
z x x & z y y & z z z & 0 & z x z & 0
\end{array}\right) \\
& \left(\begin{array}{ccc}
0 & y z y & 0 \\
z x x & 0 & z x z \\
0 & x y y & 0
\end{array}\right)
\end{aligned}
$$

\section{$\mathrm{n}=2 \quad \mathbf{C}_{2 \mathrm{v}}\left(\mathbf{C}_{2}\right)=\mathbf{2}_{\mathrm{z}} \mathbf{m}_{\mathrm{x}} \mathbf{m}_{\mathrm{y}}\left(\mathbf{2}_{\mathrm{z}}\right)=\mathbf{2}_{\mathrm{z}} \mathbf{m}_{\mathrm{x}}{ }^{\prime} \mathbf{m}_{\mathrm{y}}{ }^{\prime}$}

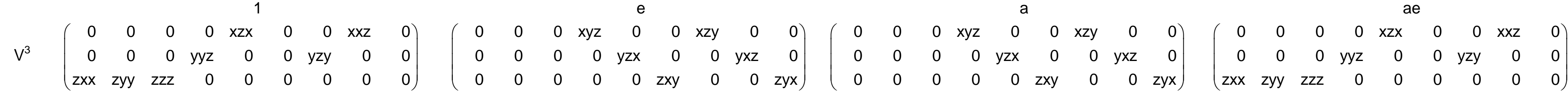

$$
\left[V^{3}\right]\left(\begin{array}{rrrr}
0 & 0 & 0 & 0 \\
0 & 0 & 0 & \\
\text { zxx } & \text { zyy } & z z z &
\end{array}\right)
$$$$
\left(\begin{array}{llll}
0 & 0 & 0 & x y z \\
0 & 0 & 0 & \\
0 & 0 & 0 &
\end{array}\right)
$$$$
\left(\begin{array}{llll}
0 & 0 & 0 & x y z \\
0 & 0 & 0 & \\
0 & 0 & 0 &
\end{array}\right)
$$$$
V\left[V^{2}\right]\left(\begin{array}{rrrrrr}
0 & 0 & 0 & 0 & x x z & 0 \\
0 & 0 & 0 & y y z & 0 & 0 \\
z x x & z y y & z z z & 0 & 0 & 0
\end{array}\right)
$$$$
\left(\begin{array}{rrrrrr}
0 & 0 & 0 & x y z & 0 & 0 \\
0 & 0 & 0 & 0 & y x z & 0 \\
0 & 0 & 0 & 0 & 0 & z x y
\end{array}\right)
$$$$
\left(\begin{array}{rrrrrr}
0 & 0 & 0 & x y z & 0 & 0 \\
0 & 0 & 0 & 0 & y x z & 0 \\
0 & 0 & 0 & 0 & 0 & z x y
\end{array}\right)
$$$$
\left(\begin{array}{rrrr}
0 & 0 & 0 & 0 \\
0 & 0 & 0 & \\
\text { zxx } & \text { zyy } & z z z &
\end{array}\right)
$$

$$
\left\{V^{2}\right\} \vee\left(\begin{array}{ccc}
0 & y z y & 0 \\
z x x & 0 & 0 \\
0 & 0 & 0
\end{array}\right)
$$$$
\left(\begin{array}{ccc}
y z x & 0 & 0 \\
0 & z x y & 0 \\
0 & 0 & x y z
\end{array}\right)
$$$$
\left(\begin{array}{ccc}
y z x & 0 & 0 \\
0 & z x y & 0 \\
0 & 0 & x y z
\end{array}\right)
$$$$
\left(\begin{array}{rrrrrr}
0 & 0 & 0 & 0 & x x z & 0 \\
0 & 0 & 0 & y y z & 0 & 0 \\
z x x & z y y & z z z & 0 & 0 & 0
\end{array}\right)
$$$$
\left(\begin{array}{ccc}
0 & y z y & 0 \\
z x x & 0 & 0 \\
0 & 0 & 0
\end{array}\right)
$$ 
$\mathrm{n}=3 \quad \mathbf{C}_{3 \mathrm{v}}\left(\mathbf{C}_{3}\right)=\mathbf{3}_{\mathrm{z}} \mathbf{m}_{\mathrm{x}}\left(\mathbf{3}_{\mathrm{z}}\right)=\mathbf{3}_{\mathrm{z}} \mathbf{m}_{\mathrm{x}}{ }^{\prime}$

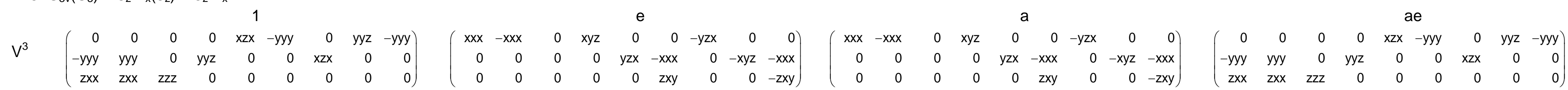

$\left[V^{3}\right]\left(\begin{array}{rrrr}0 & 0 & 0 & 0 \\ -y y y & y y y & 0 & \\ z x x & z x x & z z z & \end{array}\right)$

$\left(\begin{array}{rrrr}x x x & -x x x & 0 & 0 \\ 0 & 0 & 0 & \\ 0 & 0 & 0 & \end{array}\right)$

$\left(\begin{array}{rrrr}x x x & -x x x & 0 & 0 \\ 0 & 0 & 0 & \\ 0 & 0 & 0 & \end{array}\right)$

$\left(\begin{array}{rrrr}0 & 0 & 0 & 0 \\ -y y y & y y y & 0 & \\ z x x & z x x & z z z & \end{array}\right)$

$V\left[V^{2}\right]\left(\begin{array}{rrrrrr}0 & 0 & 0 & 0 & x x z & -y y y \\ -y y y & y y y & 0 & x x z & 0 & 0 \\ z x x & z y y & z z z & 0 & 0 & 0\end{array}\right)$

$\left(\begin{array}{rrrrrr}x x x & -x x x & 0 & x y z & 0 & 0 \\ 0 & 0 & 0 & 0 & -x y z & -x x x \\ 0 & 0 & 0 & 0 & 0 & 0\end{array}\right)$

$\left(\begin{array}{rrrrrr}\mathrm{xxx} & -\mathrm{xxx} & 0 & \mathrm{xyz} & 0 & 0 \\ 0 & 0 & 0 & 0 & -\mathrm{xyz} & -\mathrm{xxx} \\ 0 & 0 & 0 & 0 & 0 & 0\end{array}\right)$

$\left(\begin{array}{rrrrrr}0 & 0 & 0 & 0 & x x z & -y y y \\ -y y y & y y y & 0 & x x z & 0 & 0 \\ z x x & z y y & z z z & 0 & 0 & 0\end{array}\right)$

$\left\{\mathrm{V}^{2}\right\} \vee\left(\begin{array}{ccc}0 & y z y & 0 \\ -y z y & 0 & 0 \\ 0 & 0 & 0\end{array}\right)$

$\left(\begin{array}{ccc}y z x & 0 & 0 \\ 0 & y z x & 0 \\ 0 & 0 & x y z\end{array}\right)$

$\left(\begin{array}{ccc}y z x & 0 & 0 \\ 0 & y z x & 0 \\ 0 & 0 & x y z\end{array}\right)$

$\left(\begin{array}{ccc}0 & y z y & 0 \\ -y z y & 0 & 0 \\ 0 & 0 & 0\end{array}\right)$

$n>3 \quad \mathbf{C}_{\infty \mathrm{v}}\left(\mathbf{C}_{\infty}\right)=\infty \mathbf{m}^{\prime} \mathbf{m}^{\prime}$

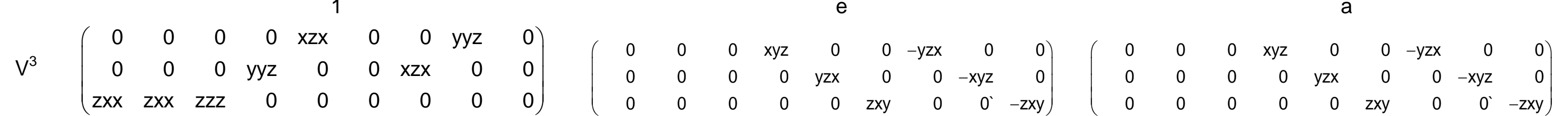

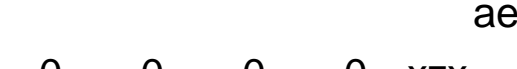

$\left[V^{3}\right]\left(\begin{array}{rrrr}0 & 0 & 0 & 0 \\ 0 & 0 & 0 & \\ z x x & z x x & z z z & \end{array}\right)$

$\left(\begin{array}{rrrr}0 & 0 & 0 & 0 \\ 0 & 0 & 0 & \\ z x x & z x x & z z z & \end{array}\right)$

$\left(\begin{array}{rrrr}0 & 0 & 0 & 0 \\ 0 & 0 & 0 & \\ z x x & z x x & z z z & \end{array}\right)$

$\left(\begin{array}{rrrr}0 & 0 & 0 & 0 \\ 0 & 0 & 0 & \\ z x x & z x x & z z z & \end{array}\right)$

$V\left[V^{2}\right]\left(\begin{array}{rrrrrr}0 & 0 & 0 & 0 & x x z & 0 \\ 0 & 0 & 0 & x x z & 0 & 0 \\ z x x & z x x & z z z & 0 & 0 & 0\end{array}\right)$

$\left(\begin{array}{rrrrrr}0 & 0 & 0 & x y z & 0 & 0 \\ 0 & 0 & 0 & 0 & -x y z & 0 \\ 0 & 0 & 0 & 0 & 0 & 0\end{array}\right)$

$\left(\begin{array}{rrrrrr}0 & 0 & 0 & x y z & 0 & 0 \\ 0 & 0 & 0 & 0 & -x y z & 0 \\ 0 & 0 & 0 & 0 & 0 & 0\end{array}\right)$

$\left(\begin{array}{rrrrrr}0 & 0 & 0 & 0 & x x z & 0 \\ 0 & 0 & 0 & x x z & 0 & 0 \\ z x x & z x x & z z z & 0 & 0 & 0\end{array}\right)$

$\left\{V^{2}\right\} \vee\left(\begin{array}{ccc}0 & y z y & 0 \\ -y z y & 0 & 0 \\ 0 & 0 & 0\end{array}\right)$

$\left(\begin{array}{ccc}y z x & 0 & 0 \\ 0 & y z x & 0 \\ 0 & 0 & x y z\end{array}\right)$

$\left(\begin{array}{ccc}y z x & 0 & 0 \\ 0 & y z x & 0 \\ 0 & 0 & x y z\end{array}\right)$

$\left(\begin{array}{ccc}0 & y z y & 0 \\ -y z y & 0 & 0 \\ 0 & 0 & 0\end{array}\right)$ 
$\mathrm{n}=1 \quad \mathbf{C}_{2 \mathrm{v}}\left(\mathbf{C}_{\mathrm{iv}}\right)=\mathbf{2}_{\mathrm{z}} \mathbf{m}_{\mathrm{x}} \mathbf{m}_{\mathrm{y}}\left(\mathbf{m}_{\mathrm{y}}\right)=\mathbf{2}_{\mathrm{z}}{ }^{\prime} \mathbf{m}_{\mathrm{x}}{ }^{\prime} \mathbf{m}_{\mathrm{y}}$

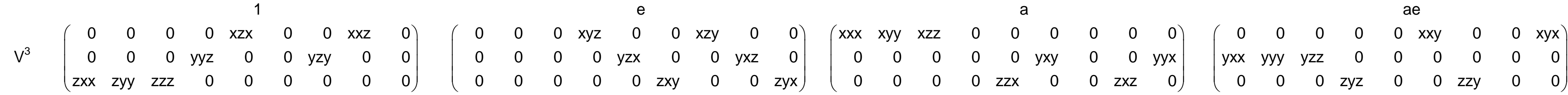

$\left[V^{3}\right] \quad\left(\begin{array}{rrrr}0 & 0 & 0 & 0 \\ 0 & 0 & 0 & \\ z x x & z y y & z z z & \end{array}\right)$

$\left(\begin{array}{llll}0 & 0 & 0 & x y z \\ 0 & 0 & 0 & \\ 0 & 0 & 0 & \end{array}\right)$

$\left(\begin{array}{rrrr}x x x & x y y & x z z & 0 \\ 0 & 0 & 0 & \\ 0 & 0 & 0 & \end{array}\right)$

$\left(\begin{array}{rrrr}0 & 0 & 0 & 0 \\ y x x & y y y & y z z & \\ 0 & 0 & 0 & \end{array}\right)$

$\left.V^{2} V^{2}\right]\left(\begin{array}{rrrrrr}0 & 0 & 0 & 0 & x x z & 0 \\ 0 & 0 & 0 & y y z & 0 & 0 \\ z x x & z y y & z z z & 0 & 0 & 0\end{array}\right)$

$\left(\begin{array}{rrrrrr}0 & 0 & 0 & x y z & 0 & 0 \\ 0 & 0 & 0 & 0 & y x z & 0 \\ 0 & 0 & 0 & 0 & 0 & z x y\end{array}\right)$

$\left(\begin{array}{rrrrrr}x x x & x y y & x z z & 0 & 0 & 0 \\ 0 & 0 & 0 & 0 & 0 & y x y \\ 0 & 0 & 0 & 0 & z x z & 0\end{array}\right)$

$\left(\begin{array}{rrrrrr}0 & 0 & 0 & 0 & 0 & x x y \\ y x x & y y y & y z z & 0 & 0 & 0 \\ 0 & 0 & 0 & z y z & 0 & 0\end{array}\right)$

$\left\{V^{2}\right\} V\left(\begin{array}{ccc}0 & y z y & 0 \\ z x x & 0 & 0 \\ 0 & 0 & 0\end{array}\right)$

$\left(\begin{array}{ccc}y z x & 0 & 0 \\ 0 & z x y & 0 \\ 0 & 0 & x y z\end{array}\right)$

$\left(\begin{array}{ccc}0 & 0 & 0 \\ 0 & 0 & z x z \\ 0 & x y y & 0\end{array}\right)$

$\left(\begin{array}{ccc}0 & 0 & y z z \\ 0 & 0 & 0 \\ x y x & 0 & 0\end{array}\right)$

$\mathrm{n}=2 \quad \mathbf{C}_{4 \mathrm{v}}\left(\mathbf{C}_{2 \mathrm{v}}\right)=\mathbf{4}_{\mathrm{z}} \mathbf{m}_{\mathrm{x}} \mathbf{m}_{\mathrm{xy}}\left(\mathbf{2}_{\mathrm{z}} \mathbf{m}_{\mathrm{x}} \mathbf{m}_{\mathrm{y}}\right)=\mathbf{4}_{\mathrm{z}} \mathbf{m}_{\mathrm{x}} \mathbf{m}_{\mathrm{xy}}{ }^{\prime}$

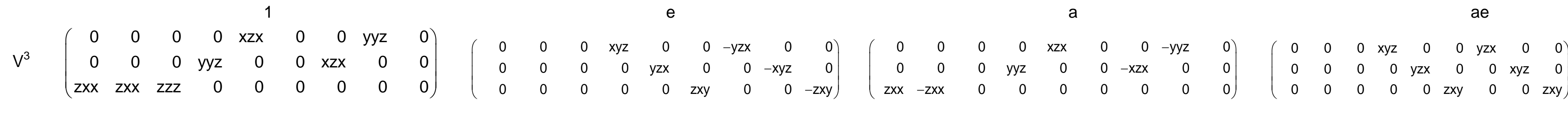
$\left[V^{3}\right]\left(\begin{array}{rrrr}0 & 0 & 0 & 0 \\ 0 & 0 & 0 & \\ \text { zxx } & \text { zyy } & \text { zzz } & \end{array}\right)$
$\left(\begin{array}{llll}0 & 0 & 0 & 0 \\ 0 & 0 & 0 & \\ 0 & 0 & 0 & \end{array}\right)$

$V\left[V^{2}\right]\left(\begin{array}{rrrrrr}0 & 0 & 0 & 0 & x x z & 0 \\ 0 & 0 & 0 & x x z & 0 & 0 \\ z x x & z x x & z z z & 0 & 0 & 0\end{array}\right)$

$\left(\begin{array}{rrrrrr}0 & 0 & 0 & x y z & 0 & 0 \\ 0 & 0 & 0 & 0 & -x y z & 0 \\ 0 & 0 & 0 & 0 & 0 & 0\end{array}\right)$

$\left(\begin{array}{rrrr}0 & 0 & 0 & 0 \\ 0 & 0 & 0 & \\ z x x & -z x x & 0 & \end{array}\right)$

$\left(\begin{array}{llll}0 & 0 & 0 & x y z \\ 0 & 0 & 0 & \\ 0 & 0 & 0 & \end{array}\right)$

$\left\{V^{2}\right\} \vee\left(\begin{array}{ccc}0 & y z y & 0 \\ -y z y & 0 & 0 \\ 0 & 0 & 0\end{array}\right)$

$\left(\begin{array}{ccc}y z x & 0 & 0 \\ 0 & y z x & 0 \\ 0 & 0 & x y z\end{array}\right)$

$\left(\begin{array}{rrrrrr}0 & 0 & 0 & 0 & x x z & 0 \\ 0 & 0 & 0 & -x x z & 0 & 0 \\ z x x & -z x x & 0 & 0 & 0 & 0\end{array}\right)$

$\left(\begin{array}{rrrrrr}0 & 0 & 0 & x y z & 0 & 0 \\ 0 & 0 & 0 & 0 & x y z & 0 \\ 0 & 0 & 0 & 0 & 0 & z x y\end{array}\right)$

$\left(\begin{array}{ccc}0 & y z y & 0 \\ y z y & 0 & 0 \\ 0 & 0 & 0\end{array}\right)$

$\left(\begin{array}{ccc}y z x & 0 & 0 \\ 0 & -y z x & 0 \\ 0 & 0 & 0\end{array}\right)$ 
$\mathrm{n}=1 \quad \mathrm{D}_{1 \mathrm{~d}}\left(\mathbf{S}_{2}\right)=\mathbf{2}_{\mathrm{y}} / \mathbf{m}_{\mathrm{y}}(\overline{\mathbf{1}})=\mathbf{2 y}^{\prime} / \mathbf{m}_{\mathrm{y}}{ }^{\prime}$

$\mathrm{V}^{3} \quad\left(\begin{array}{ccccccccc}0 & 0 & 0 & 0 & 0 & 0 & 0 & 0 & 0 \\ 0 & 0 & 0 & 0 & 0 & 0 & 0 & 0 & 0 \\ 0 & 0 & 0 & 0 & 0 & 0 & 0 & 0 & 0\end{array}\right)$ $\left(\begin{array}{rrrrrrrrr}0 & 0 & 0 & x y z & 0 & x x y & x z y & 0 & x y x \\ y x x & y y y & y z z & 0 & y z x & 0 & 0 & y x z & 0 \\ 0 & 0 & 0 & z y z & 0 & z x y & z z y & 0 & z y x\end{array}\right)\left(\begin{array}{lllllllll}0 & 0 & 0 & 0 & 0 & 0 & 0 & 0 & 0 \\ 0 & 0 & 0 & 0 & 0 & 0 & 0 & 0 & 0 \\ 0 & 0 & 0 & 0 & 0 & 0 & 0 & 0 & 0\end{array}\right)$ $\left(\begin{array}{rrrrrrrrr}x x x & x y y & x z z & 0 & x z x & 0 & 0 & x x z & 0 \\ 0 & 0 & 0 & y y z & 0 & y x y & y z y & 0 & y y x \\ z x x & z y y & z z z & 0 & z z x & 0 & 0 & z x z & 0\end{array}\right)$ $\left[V^{3}\right]\left(\begin{array}{llll}0 & 0 & 0 & 0 \\ 0 & 0 & 0 & \\ 0 & 0 & 0 & \end{array}\right)$ $\left(\begin{array}{rrrr}0 & 0 & 0 & x y z \\ y x x & y y y & y z z & \\ 0 & 0 & 0 & \end{array}\right)$ $\left(\begin{array}{llll}0 & 0 & 0 & 0 \\ 0 & 0 & 0 & \\ 0 & 0 & 0 & \end{array}\right)$ $V\left[V^{2}\right]\left(\begin{array}{llllll}0 & 0 & 0 & 0 & 0 & 0 \\ 0 & 0 & 0 & 0 & 0 & 0 \\ 0 & 0 & 0 & 0 & 0 & 0\end{array}\right)$ $\left(\begin{array}{rrrrrr}0 & 0 & 0 & x y z & 0 & x x y \\ y x x & y y y & y z z & 0 & y x z & 0 \\ 0 & 0 & 0 & z y z & 0 & z x y\end{array}\right)$

$\left(\begin{array}{llllll}0 & 0 & 0 & 0 & 0 & 0 \\ 0 & 0 & 0 & 0 & 0 & 0 \\ 0 & 0 & 0 & 0 & 0 & 0\end{array}\right)$ $\left(\begin{array}{llllll}0 & 0 & 0 & 0 & 0 & 0\end{array}\right)$

$\begin{array}{llllll}0 & 0 & 0 & 0 & 0 & 0 \\ 0 & 0 & 0 & 0 & 0 & 0\end{array}$

$\left\{V^{2}\right\} V\left(\begin{array}{ccc}0 & 0 & 0 \\ 0 & 0 & 0 \\ 0 & 0 & 0\end{array}\right)$

$\left(\begin{array}{ccc}y z x & 0 & y z z \\ 0 & z x y & 0 \\ x y x & 0 & x y z\end{array}\right)$

$\left(\begin{array}{lll}0 & 0 & 0 \\ 0 & 0 & 0 \\ 0 & 0 & 0\end{array}\right)$

$\left(\begin{array}{rrrr}x x x & x y y & x z z & 0 \\ 0 & 0 & 0 & \\ z x x & z y y & z z z & \end{array}\right)$

$\left(\begin{array}{rrrrrr}x x x & x y y & x z z & 0 & x x z & 0 \\ 0 & 0 & 0 & y y z & 0 & y x y \\ z x x & z y y & z z z & 0 & z x z & 0\end{array}\right)$

$\mathrm{n}=2 \quad \mathbf{D}_{2 \mathrm{~d}}\left(\mathbf{S}_{4}\right)=\overline{\mathbf{4}}_{\mathbf{z}} \mathbf{m}_{\mathrm{x}} \mathbf{2}_{\mathrm{xy}}\left(\overline{\mathbf{4}}_{\mathbf{z}}\right)=\overline{\mathbf{4}}_{\mathbf{z}} \mathbf{m}_{x^{\prime}} \mathbf{2}_{\mathrm{xy}}{ }^{\prime}$

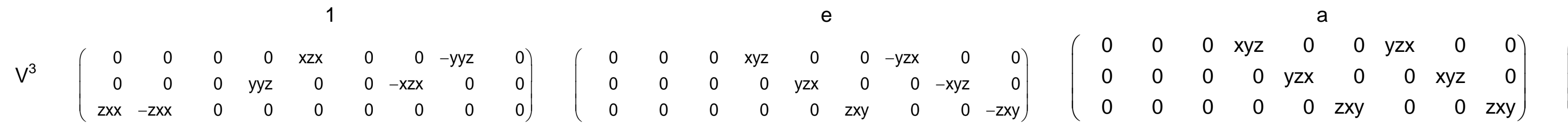
$\left(\begin{array}{ccc}0 & y z y & 0 \\ z x x & 0 & z x z \\ 0 & x y y & 0\end{array}\right)$

$\left[\mathrm{V}^{3}\right] \quad\left(\begin{array}{rrrr}0 & 0 & 0 & 0 \\ 0 & 0 & 0 & \\ \mathrm{zxx} & -\mathrm{zxx} & 0\end{array}\right)$

$\left(\begin{array}{llll}0 & 0 & 0 & 0 \\ 0 & 0 & 0 & \\ 0 & 0 & 0 & \end{array}\right)$

$\left(\begin{array}{llll}0 & 0 & 0 & x y z \\ 0 & 0 & 0 & \\ 0 & 0 & 0 & \end{array}\right)$

$V\left[V^{2}\right]\left(\begin{array}{rrrrrr}0 & 0 & 0 & 0 & x x z & 0 \\ 0 & 0 & 0 & -x x z & 0 & 0 \\ z x x & -z x x & 0 & 0 & 0 & 0\end{array}\right)$

$\left(\begin{array}{rrrrrr}0 & 0 & 0 & x y z & 0 & 0 \\ 0 & 0 & 0 & 0 & -x y z & 0 \\ 0 & 0 & 0 & 0 & 0 & 0\end{array}\right)$

$\left(\begin{array}{rrrrrr}0 & 0 & 0 & x y z & 0 & 0 \\ 0 & 0 & 0 & 0 & x y z & 0 \\ 0 & 0 & 0 & 0 & 0 & z x y\end{array}\right)$

$\left(\begin{array}{rrrrrrrrr}0 & 0 & 0 & 0 & x z x & 0 & 0 & y y z & 0 \\ 0 & 0 & 0 & y y z & 0 & 0 & x z x & 0 & 0 \\ z x x & \text { zxx } & \text { zzz } & 0 & 0 & 0 & 0 & 0 & 0\end{array}\right)$

$\left\{V^{2}\right\} V\left(\begin{array}{ccc}0 & y z y & 0 \\ y z y & 0 & 0 \\ 0 & 0 & 0\end{array}\right)$

$\left(\begin{array}{ccc}y z x & 0 & 0 \\ 0 & y z x & 0 \\ 0 & 0 & x y z\end{array}\right)$

$\left(\begin{array}{ccc}y z x & 0 & 0 \\ 0 & -y z x & 0 \\ 0 & 0 & 0\end{array}\right)$

$\left(\begin{array}{rrrr}0 & 0 & 0 & 0 \\ 0 & 0 & 0 & \\ \text { zxx } & \text { zyy } & \text { zzz } & \end{array}\right)$

$\left(\begin{array}{rrrrrr}0 & 0 & 0 & 0 & x x z & 0 \\ 0 & 0 & 0 & x x z & 0 & 0 \\ z x x & z x x & z z z & 0 & 0 & 0\end{array}\right)$

$\left(\begin{array}{ccc}0 & y z y & 0 \\ -y z y & 0 & 0 \\ 0 & 0 & 0\end{array}\right)$ 
$\mathrm{n}=3 \quad \mathbf{D}_{3 d}\left(\mathbf{S}_{6}\right)=\overline{\mathbf{3}}_{z} \mathbf{m}_{x}\left(\overline{\mathbf{3}}_{z}\right)=\overline{\mathbf{3}}_{z} \mathbf{m}_{x}{ }^{\prime}$

$\mathrm{V}^{3}\left(\begin{array}{lllllllll}0 & 0 & 0 & 0 & 0 & 0 & 0 & 0 & 0 \\ 0 & 0 & 0 & 0 & 0 & 0 & 0 & 0 & 0 \\ 0 & 0 & 0 & 0 & 0 & 0 & 0 & 0 & 0\end{array}\right)$

$\left[\mathrm{V}^{3}\right]\left(\begin{array}{llll}0 & 0 & 0 & 0 \\ 0 & 0 & 0 & \\ 0 & 0 & 0 & \end{array}\right)$

$V\left[V^{2}\right]\left(\begin{array}{llllll}0 & 0 & 0 & 0 & 0 & 0 \\ 0 & 0 & 0 & 0 & 0 & 0 \\ 0 & 0 & 0 & 0 & 0 & 0\end{array}\right)$

$\left(\begin{array}{llllll}0 & 0 & 0 & 0 & 0 & 0\end{array}\right)$

$\left\{V^{2}\right\} V\left(\begin{array}{ccc}0 & 0 & 0 \\ 0 & 0 & 0 \\ 0 & 0 & 0\end{array}\right)$

$\mathrm{n}>3 \quad \mathbf{D}_{\propto h}\left(\mathbf{C}_{\propto h}\right)=\infty / \mathbf{m m}^{\prime} \mathbf{m}^{\prime}$

$\left(\begin{array}{ccccccccc}0 & 0 & 0 & 0 & 0 & 0 & 0 & 0 & 0 \\ 0 & 0 & 0 & 0 & 0 & 0 & 0 & 0 & 0\end{array}\right)$

$V^{3} \quad\left(\begin{array}{lllllllll}0 & 0 & 0 & 0 & 0 & 0 & 0 & 0 & 0 \\ 0 & 0 & 0 & 0 & 0 & 0 & 0 & 0 & 0\end{array}\right)$

$\left(\begin{array}{lllllllll}0 & 0 & 0 & 0 & 0 & 0 & 0 & 0 & 0\end{array}\right)$

$\left[V^{3}\right]\left(\begin{array}{llll}0 & 0 & 0 & 0 \\ 0 & 0 & 0 & \\ 0 & 0 & 0 & \end{array}\right)$

$V\left[V^{2}\right]\left(\begin{array}{llllll}0 & 0 & 0 & 0 & 0 & 0 \\ 0 & 0 & 0 & 0 & 0 & 0 \\ 0 & 0 & 0 & 0 & 0 & 0\end{array}\right)$

$\left\{V^{2}\right\} V\left(\begin{array}{ccc}0 & 0 & 0 \\ 0 & 0 & 0 \\ 0 & 0 & 0\end{array}\right)$

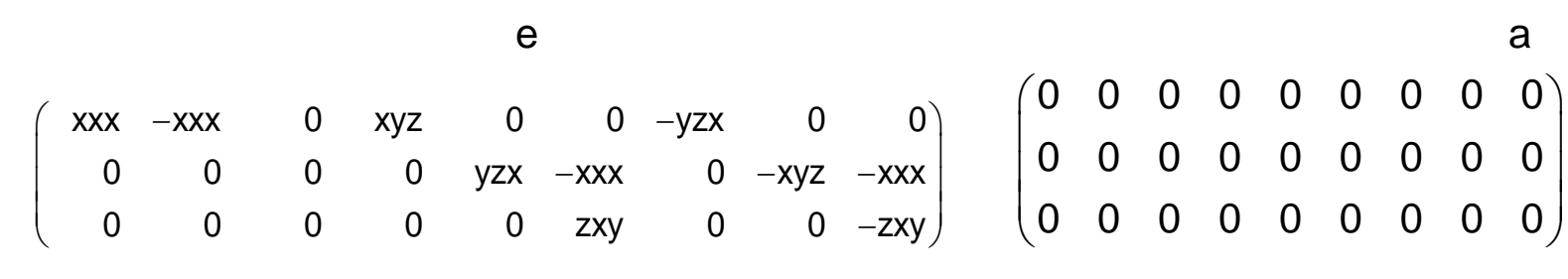

$\left(\begin{array}{rrrr}x x x & -x x x & 0 & 0 \\ 0 & 0 & 0 & \\ 0 & 0 & 0 & \end{array}\right)$

$\left(\begin{array}{llll}0 & 0 & 0 & 0 \\ 0 & 0 & 0 & \\ 0 & 0 & 0\end{array}\right)$

$\left(\begin{array}{rrrrrr}0 & 0 & 0 & x y z & 0 & -y y y \\ -y y y & y y y & 0 & 0 & -x y z & 0 \\ 0 & 0 & 0 & 0 & 0 & 0\end{array}\right)$

$\left(\begin{array}{llllll}0 & 0 & 0 & 0 & 0 & 0 \\ 0 & 0 & 0 & 0 & 0 & 0 \\ 0 & 0 & 0 & 0 & 0 & 0\end{array}\right)$

$\left(\begin{array}{ccc}y z x & 0 & 0 \\ 0 & y z x & 0 \\ 0 & 0 & x y z\end{array}\right)$

$\left(\begin{array}{lll}0 & 0 & 0 \\ 0 & 0 & 0 \\ 0 & 0 & 0\end{array}\right)$

$\left(\begin{array}{rrrrrrrrr}0 & 0 & 0 & x y z & 0 & 0 & -y z x & 0 & 0 \\ 0 & 0 & 0 & 0 & y z x & 0 & 0 & -x y z & 0 \\ 0 & 0 & 0 & 0 & 0 & z x y & 0 & 0 & -z x y\end{array}\right) \quad\left(\begin{array}{rrrrrrrrr}0 & 0 & 0 & 0 & 0 & 0 & 0 & 0 & 0 \\ 0 & 0 & 0 & 0 & 0 & 0 & 0 & 0 & 0 \\ 0 & 0 & 0 & 0 & 0 & 0 & 0 & 0 & 0\end{array}\right)$

$\left(\begin{array}{llll}0 & 0 & 0 & 0 \\ 0 & 0 & 0 & \\ 0 & 0 & 0 & \end{array}\right)$

$\left(\begin{array}{rrrr}0 & 0 & 0 & 0 \\ 0 & 0 & 0 & \\ z x x & z x x & z z z & \end{array}\right)$

$\left(\begin{array}{llllll}0 & 0 & 0 & 0 & 0 & 0 \\ 0 & 0 & 0 & 0 & 0 & 0 \\ 0 & 0 & 0 & 0 & 0 & 0\end{array}\right)$

$\left(\begin{array}{ccc}y z x & 0 & 0 \\ 0 & y z x & 0 \\ 0 & 0 & x y z\end{array}\right)$

\section{ae}

$\left(\begin{array}{rrrrrrrrr}0 & 0 & 0 & 0 & x z x & -y y y & 0 & y y z & -y y y \\ -y y y & y y & 0 & y y z & 0 & 0 & x z x & 0 & 0 \\ z & \end{array}\right.$ $\begin{array}{rrrrrrrrr}-y y y & y y y & 0 & y y z & 0 & 0 & x z x & 0 & 0 \\ z x x & z x x & z z z & 0 & 0 & 0 & 0 & 0 & 0\end{array}$

$\left(\begin{array}{rrrr}0 & 0 & 0 & 0 \\ -y y y & y y y & 0 & \\ z x x & z x x & z z z & \end{array}\right)$

$\left(\begin{array}{rrrrrr}0 & 0 & 0 & 0 & x x z & -y y y \\ -y y y & y y y & 0 & x x z & 0 & 0 \\ z x x & z y y & z z z & 0 & 0 & 0\end{array}\right)$

$\left(\begin{array}{ccc}0 & y z y & 0 \\ -y z y & 0 & 0 \\ 0 & 0 & 0\end{array}\right)$ $\left(\begin{array}{rrrrrrrrr}0 & 0 & 0 & 0 & x z x & 0 & 0 & y y z & 0 \\ 0 & 0 & 0 & y y z & 0 & 0 & x z x & 0 & 0 \\ z x x & z x x & z z z & 0 & 0 & 0 & 0 & 0 & 0\end{array}\right)$

$\left(\begin{array}{rrrr}0 & 0 & 0 & 0 \\ 0 & 0 & 0 & \\ z x x & z x x & z z z & \end{array}\right)$

$\left(\begin{array}{rrrrrr}0 & 0 & 0 & 0 & x x z & 0 \\ 0 & 0 & 0 & x x z & 0 & 0 \\ z x x & z x x & z z z & 0 & 0 & 0\end{array}\right)$

$\left(\begin{array}{ccc}0 & y z y & 0 \\ -y z y & 0 & 0 \\ 0 & 0 & 0\end{array}\right)$ 
$\mathrm{n}=1 \quad \mathbf{D}_{1 \mathrm{~d}}\left(\mathbf{D}_{1}\right)=\mathbf{2}_{\mathrm{y}} / \mathbf{m}_{\mathrm{y}}\left(\mathbf{2}_{\mathrm{y}}\right)=\mathbf{2}_{\mathrm{y}} / \mathbf{m}_{\mathrm{y}}{ }^{\prime}$

$\mathrm{V}^{3} \quad\left(\begin{array}{lllllllll}0 & 0 & 0 & 0 & 0 & 0 & 0 & 0 & 0 \\ 0 & 0 & 0 & 0 & 0 & 0 & 0 & 0 & 0 \\ 0 & 0 & 0 & 0 & 0 & 0 & 0 & 0 & 0\end{array}\right)$

$\left(\begin{array}{rrrrrrrrr}0 & 0 & 0 & x y z & 0 & x x y & x z y & 0 & x y x \\ y x x & y y y & y z z & 0 & y z x & 0 & 0 & y x z & 0 \\ 0 & 0 & 0 & z y z & 0 & z x y & z z y & 0 & z y x\end{array}\right) \quad\left(\begin{array}{rrrrrrrrr}0 & 0 & 0 & x y z & 0 & x x y & x z y & 0 & x y x \\ y x x & y y y & y z z & 0 & y z x & 0 & 0 & y x z & 0 \\ 0 & 0 & 0 & z y z & 0 & z x y & z z y & 0 & z y x\end{array}\right) \quad\left(\begin{array}{lllllllll}0 & 0 & 0 & 0 & 0 & 0 & 0 & 0 & 0 \\ 0 & 0 & 0 & 0 & 0 & 0 & 0 & 0 & 0 \\ 0 & 0 & 0 & 0 & 0 & 0 & 0 & 0 & 0\end{array}\right)$

$\left[\mathrm{V}^{3}\right]\left(\begin{array}{llll}0 & 0 & 0 & 0 \\ 0 & 0 & 0 & \\ 0 & 0 & 0 & \end{array}\right)$

$\left(\begin{array}{rrrr}0 & 0 & 0 & x y z \\ y x x & y y y & y z z & \\ 0 & 0 & 0 & \end{array}\right)$

$\left(\begin{array}{rrrr}0 & 0 & 0 & x y z \\ y x x & y y y & y z z & \\ 0 & 0 & 0 & \end{array}\right)$

$\left(\begin{array}{llll}0 & 0 & 0 & 0 \\ 0 & 0 & 0 & \\ 0 & 0 & 0 & \end{array}\right)$

$\left(\begin{array}{llllll}0 & 0 & 0 & 0 & 0 & 0 \\ 0 & 0 & 0 & 0 & 0 & 0\end{array}\right)$

$V\left[V^{2}\right]\left(\begin{array}{llllll}0 & 0 & 0 & 0 & 0 & 0 \\ 0 & 0 & 0 & 0 & 0 & 0 \\ 0 & 0 & 0 & 0 & 0 & 0\end{array}\right)$

$\left(\begin{array}{rrrrrr}0 & 0 & 0 & x y z & 0 & x x y \\ y x x & y y y & y z z & 0 & y x z & 0 \\ 0 & 0 & 0 & z y z & 0 & z x y\end{array}\right)$

$\left(\begin{array}{rrrrrr}0 & 0 & 0 & x y z & 0 & x x y \\ y x x & y y y & y z z & 0 & y x z & 0 \\ 0 & 0 & 0 & z y z & 0 & z x y\end{array}\right)$

$\left(\begin{array}{llllll}0 & 0 & 0 & 0 & 0 & 0 \\ 0 & 0 & 0 & 0 & 0 & 0 \\ 0 & 0 & 0 & 0 & 0 & 0\end{array}\right)$

$\left\{V^{2}\right\} V\left(\begin{array}{lll}0 & 0 & 0 \\ 0 & 0 & 0 \\ 0 & 0 & 0\end{array}\right)$

$\left(\begin{array}{ccc}y z x & 0 & y z z \\ 0 & z x y & 0 \\ x y x & 0 & x y z\end{array}\right)$

$\left(\begin{array}{ccc}y z x & 0 & y z z \\ 0 & z x y & 0 \\ x y x & 0 & x y z\end{array}\right)$

$\left(\begin{array}{lll}0 & 0 & 0 \\ 0 & 0 & 0 \\ 0 & 0 & 0\end{array}\right)$

$\mathrm{n}=2 \quad \mathbf{D}_{2 \mathrm{~d}}\left(\mathbf{D}_{2}\right)=\overline{\mathbf{4}}_{\mathrm{z}} \mathbf{m}_{\mathrm{x}} \mathbf{2}_{\mathrm{xy}}\left(\mathbf{2}_{\mathrm{xy}} \mathbf{2}_{\overline{\mathrm{x} y}} \mathbf{2}_{\mathrm{z}}\right)=\overline{\mathbf{4}}_{\mathrm{z}}{ }^{\prime} \mathbf{m}_{\mathrm{x}} \mathbf{2}_{\mathrm{xy}}$

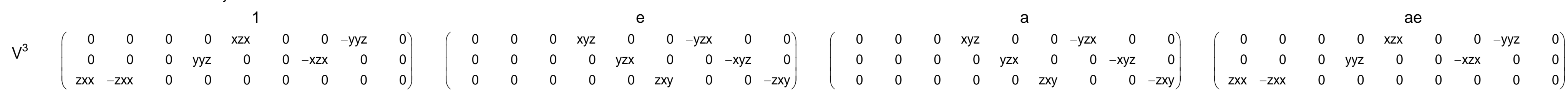

$\left[\mathrm{V}^{3}\right] \quad\left(\begin{array}{rrrr}0 & 0 & 0 & 0 \\ 0 & 0 & 0 & \\ \mathrm{zxx} & -\mathrm{zxx} & 0 & \end{array}\right) \quad\left(\begin{array}{llll}0 & 0 & 0 & 0 \\ 0 & 0 & 0 \\ 0 & 0 & 0\end{array}\right) \quad\left(\begin{array}{llll}0 & 0 & 0 & 0 \\ 0 & 0 & 0 \\ 0 & 0 & 0\end{array}\right)$

$V\left[V^{2}\right]\left(\begin{array}{rrrrrr}0 & 0 & 0 & 0 & x x z & 0 \\ 0 & 0 & 0 & -x x z & 0 & 0 \\ z x x & -z x x & 0 & 0 & 0 & 0\end{array}\right)$

$\left(\begin{array}{rrrrrr}0 & 0 & 0 & x y z & 0 & 0 \\ 0 & 0 & 0 & 0 & -x y z & 0 \\ 0 & 0 & 0 & 0 & 0 & 0\end{array}\right)$

$\left(\begin{array}{rrrrrr}0 & 0 & 0 & x y z & 0 & 0 \\ 0 & 0 & 0 & 0 & -x y z & 0 \\ 0 & 0 & 0 & 0 & 0 & 0\end{array}\right)$

$\left(\begin{array}{rrrr}0 & 0 & 0 & 0 \\ 0 & 0 & 0 & \\ z x x & -z x x & 0 & \end{array}\right)$

$\left\{V^{2}\right\} \bigvee\left(\begin{array}{ccc}0 & y z y & 0 \\ y z y & 0 & 0 \\ 0 & 0 & 0\end{array}\right)$

$\left(\begin{array}{ccc}y z x & 0 & 0 \\ 0 & y z x & 0 \\ 0 & 0 & x y z\end{array}\right)$

$\left(\begin{array}{ccc}y z x & 0 & 0 \\ 0 & y z x & 0 \\ 0 & 0 & x y z\end{array}\right)$

$\left(\begin{array}{rrrrrr}0 & 0 & 0 & 0 & x x z & 0 \\ 0 & 0 & 0 & -x x z & 0 & 0 \\ z x x & -z x x & 0 & 0 & 0 & 0\end{array}\right)$

$\left(\begin{array}{ccc}0 & y z y & 0 \\ y z y & 0 & 0 \\ 0 & 0 & 0\end{array}\right)$ 
$\left(\begin{array}{ccccccccc}0 & 0 & 0 & 0 & 0 & 0 & 0 & 0 & 0 \\ 0 & 0 & 0 & 0 & 0 & 0 & 0 & 0 & 0\end{array}\right)$

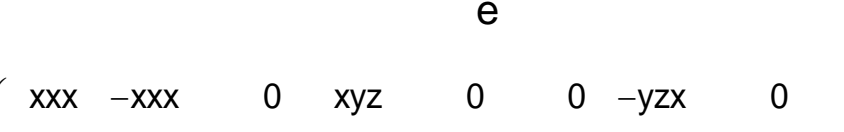

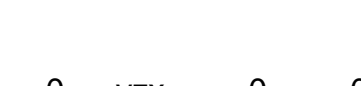

$\left(\begin{array}{lllllllll}0 & 0 & 0 & 0 & 0 & 0 & 0 & 0\end{array}\right.$ $\left.\begin{array}{lllllllll}0 & 0 & 0 & 0 & 0 & 0 & 0 & 0 & 0 \\ 0 & 0 & 0 & 0 & 0 & 0 & 0 & 0 & 0\end{array}\right)$ $\left(\begin{array}{rrrrrrrrr}\mathrm{xxx} & -\mathrm{xxx} & 0 & \mathrm{xyz} & 0 & 0 & -\mathrm{yzx} & 0 & 0 \\ 0 & 0 & 0 & 0 & \mathrm{yzx} & -\mathrm{xxx} & 0 & -\mathrm{xyz} & -\mathrm{xxx} \\ 0 & 0 & 0 & 0 & 0 & \mathrm{zxy} & 0 & 0 & -\mathrm{zxy}\end{array}\right) \quad\left(\begin{array}{rrrrrrrrr}\mathrm{xxx} & -\mathrm{xxx} & 0 & \mathrm{xyz} & 0 & 0 & -\mathrm{yzx} & 0 & 0 \\ 0 & 0 & 0 & 0 & \mathrm{yzx} & -\mathrm{xxx} & 0 & -\mathrm{xyz} & -\mathrm{xxx} \\ 0 & 0 & 0 & 0 & 0 & \mathrm{zxy} & 0 & 0 & -\mathrm{zxy}\end{array}\right)$ $\left.\begin{array}{lllllllll}0 & 0 & 0 & 0 & 0 & 0 & 0 & 0 & 0 \\ 0 & 0 & 0 & 0 & 0 & 0 & 0 & 0 & 0\end{array}\right)$ $\left[\mathrm{V}^{3}\right] \quad\left(\begin{array}{llll}0 & 0 & 0 & 0 \\ 0 & 0 & 0 & \\ 0 & 0 & 0 & \end{array}\right)$ $V\left[V^{2}\right]\left(\begin{array}{llllll}0 & 0 & 0 & 0 & 0 & 0 \\ 0 & 0 & 0 & 0 & 0 & 0 \\ 0 & 0 & 0 & 0 & 0 & 0\end{array}\right)$ $\left(\begin{array}{rrrr}x x x & -x x x & 0 & 0 \\ 0 & 0 & 0 & \\ 0 & 0 & 0 & \end{array}\right)$ $\left(\begin{array}{rrrr}x x x & -x x x & 0 & 0 \\ 0 & 0 & 0 & \\ 0 & 0 & 0 & \end{array}\right)$ $\left(\begin{array}{llll}0 & 0 & 0 & 0 \\ 0 & 0 & 0 & \\ 0 & 0 & 0 & \end{array}\right)$ $\left(\begin{array}{rrrrrr}0 & 0 & 0 & x y z & 0 & -y y y \\ -y y y & y y y & 0 & 0 & -x y z & 0 \\ 0 & 0 & 0 & 0 & 0 & 0\end{array}\right)$ $\left(\begin{array}{rrrrrr}0 & 0 & 0 & x y z & 0 & -y y y \\ -y y y & \text { yyy } & 0 & 0 & -x y z & 0 \\ 0 & 0 & 0 & 0 & 0 & 0\end{array}\right)$ $\left(\begin{array}{llllll}0 & 0 & 0 & 0 & 0 & 0 \\ 0 & 0 & 0 & 0 & 0 & 0 \\ 0 & 0 & 0 & 0 & 0 & 0\end{array}\right)$ $\left\{V^{2}\right\} V\left(\begin{array}{ccc}0 & 0 & 0 \\ 0 & 0 & 0 \\ 0 & 0 & 0\end{array}\right)$ $\left(\begin{array}{ccc}y z x & 0 & 0 \\ 0 & y z x & 0 \\ 0 & 0 & x y z\end{array}\right)$ $\left(\begin{array}{ccc}y z x & 0 & 0 \\ 0 & y z x & 0 \\ 0 & 0 & x y z\end{array}\right)$ 
$\mathrm{V}^{3} \quad\left(\begin{array}{ccccccccc}0 & 0 & 0 & 0 & 0 & 0 & 0 & 0 & 0 \\ 0 & 0 & 0 & 0 & 0 & 0 & 0 & 0 & 0 \\ 0 & 0 & 0 & 0 & 0 & 0 & 0 & 0 & 0\end{array}\right)$ $\left(\begin{array}{rrrrrrrrr}0 & 0 & 0 & x y z & 0 & x x y & x z y & 0 & x y x \\ y x x & y y y & y z z & 0 & y z x & 0 & 0 & y x z & 0 \\ 0 & 0 & 0 & z y z & 0 & z x y & z z y & 0 & z y x\end{array}\right)\left(\begin{array}{rrrrrrrrr}x x x & x y y & x z z & 0 & x z x & 0 & 0 & x x z & 0 \\ 0 & 0 & 0 & y y z & 0 & y x y & y z y & 0 & y y x \\ z x x & z y y & z z z & 0 & z z x & 0 & 0 & z x z & 0\end{array}\right) \quad\left(\begin{array}{lllllllll}0 & 0 & 0 & 0 & 0 & 0 & 0 & 0 & 0 \\ 0 & 0 & 0 & 0 & 0 & 0 & 0 & 0 & 0 \\ 0 & 0 & 0 & 0 & 0 & 0 & 0 & 0 & 0\end{array}\right)$ $\left[\mathrm{V}^{3}\right]\left(\begin{array}{llll}0 & 0 & 0 & 0 \\ 0 & 0 & 0 & \\ 0 & 0 & 0\end{array}\right)$ $\left(\begin{array}{rrrr}0 & 0 & 0 & x y z \\ y x x & y y y & y z z & \\ 0 & 0 & 0 & \end{array}\right)$

$\left(\begin{array}{llllll}0 & 0 & 0 & 0 & 0 & 0\end{array}\right)$

$V\left[V^{2}\right]\left(\begin{array}{llllll}0 & 0 & 0 & 0 & 0 & 0 \\ 0 & 0 & 0 & 0 & 0 & 0\end{array}\right)$ $\left(\begin{array}{rrrrrr}0 & 0 & 0 & x y z & 0 & x x y \\ y x x & y y y & y z z & 0 & y x z & 0 \\ 0 & 0 & 0 & z y z & 0 & z x y\end{array}\right)$ $\left(\begin{array}{rrrr}x x x & x y y & x z z & 0 \\ 0 & 0 & 0 & \\ z x x & z y y & z z z & \end{array}\right)$

$\left(\begin{array}{llll}0 & 0 & 0 & 0 \\ 0 & 0 & 0 & \\ 0 & 0 & 0 & \end{array}\right)$

$\left(\begin{array}{llllll}0 & 0 & 0 & 0 & 0 & 0 \\ 0 & 0 & 0 & 0 & 0 & 0\end{array}\right)$ $\left(\begin{array}{rrrrrr}x x x & x y y & x z z & 0 & x x z & 0 \\ 0 & 0 & 0 & y y z & 0 & y x y \\ z x x & z y y & z z z & 0 & z x z & 0\end{array}\right)$ $\left(\begin{array}{llllll}0 & 0 & 0 & 0 & 0 & 0 \\ 0 & 0 & 0 & 0 & 0 & 0 \\ 0 & 0 & 0 & 0 & 0 & 0\end{array}\right)$ $\left\{V^{2}\right\} V\left(\begin{array}{lll}0 & 0 & 0 \\ 0 & 0 & 0 \\ 0 & 0 & 0\end{array}\right)$ $\left(\begin{array}{ccc}y z x & 0 & y z z \\ 0 & z x y & 0 \\ x y x & 0 & x y z\end{array}\right)$ $\left(\begin{array}{ccc}0 & y z y & 0 \\ z x x & 0 & z x z \\ 0 & x y y & 0\end{array}\right)$ $\left(\begin{array}{lll}0 & 0 & 0 \\ 0 & 0 & 0 \\ 0 & 0 & 0\end{array}\right)$

$\mathrm{n}=2 \quad \mathbf{D}_{2 \mathrm{~d}}\left(\mathbf{C}_{2 \mathrm{v}}\right)=\overline{\mathbf{4}}_{\mathrm{z}} \mathbf{m}_{\mathrm{x}} \mathbf{2}_{\mathrm{xy}}\left(\mathbf{2}_{\mathrm{z}} \mathbf{m}_{\mathrm{x}} \mathbf{m}_{\mathrm{y}}\right)=\overline{\mathbf{4}}_{\mathrm{z}} \cdot \mathbf{m}_{\mathrm{x}} \mathbf{2}_{\mathrm{xy}} \mathrm{y}^{\prime}$ $\mathrm{V}^{3} \quad\left(\begin{array}{rrrrrrrrr}0 & 0 & 0 & 0 & \mathrm{xzx} & 0 & 0 & -\mathrm{yyz} & 0 \\ 0 & 0 & 0 & y y z & 0 & 0 & -x z x & 0 & 0 \\ z x x & -z x x & 0 & 0 & 0 & 0 & 0 & 0 & 0\end{array}\right) \quad\left(\begin{array}{rrrrrrrrr}0 & 0 & 0 & x y z & 0 & 0 & -y z x & 0 & 0 \\ 0 & 0 & 0 & 0 & y z x & 0 & 0 & -x y z & 0 \\ 0 & 0 & 0 & 0 & 0 & z x y & 0 & 0 & -z x y\end{array}\right)$ $\left[\mathrm{V}^{3}\right]\left(\begin{array}{rrrr}0 & 0 & 0 & 0 \\ 0 & 0 & 0 & \\ \mathrm{zxx} & -\mathrm{zxx} & 0 & \end{array}\right)$ $V\left[V^{2}\right]\left(\begin{array}{rrrrrr}0 & 0 & 0 & 0 & x x z & 0 \\ 0 & 0 & 0 & -x x z & 0 & 0 \\ z x x & -z x x & 0 & 0 & 0 & 0\end{array}\right)$ $\left\{V^{2}\right\} \bigvee\left(\begin{array}{ccc}0 & y z y & 0 \\ y z y & 0 & 0 \\ 0 & 0 & 0\end{array}\right)$ $\left(\begin{array}{llll}0 & 0 & 0 & 0 \\ 0 & 0 & 0 & \\ 0 & 0 & 0 & \end{array}\right)$

$\left(\begin{array}{rrrrrr}0 & 0 & 0 & x y z & 0 & 0 \\ 0 & 0 & 0 & 0 & -x y z & 0 \\ 0 & 0 & 0 & 0 & 0 & 0\end{array}\right)$ $\left(\begin{array}{ccc}y z x & 0 & 0 \\ 0 & y z x & 0 \\ 0 & 0 & x y z\end{array}\right)$ $a$

$\left(\begin{array}{rrrrrrrrr}0 & 0 & 0 & 0 & x z x & 0 & 0 & y y z & 0 \\ 0 & 0 & 0 & y y z & 0 & 0 & x z x & 0 & 0 \\ z x x & z x x & z z z & 0 & 0 & 0 & 0 & 0 & 0\end{array}\right) \quad\left(\begin{array}{rrrrrrrrr}0 & 0 & 0 & x y z & 0 & 0 & y z x & 0 & 0 \\ 0 & 0 & 0 & 0 & y z x & 0 & 0 & x y z & 0 \\ 0 & 0 & 0 & 0 & 0 & z x y & 0 & 0 & z x y\end{array}\right)$

$\left(\begin{array}{rrrr}0 & 0 & 0 & 0 \\ 0 & 0 & 0 & \\ z x x & z y y & z z z & \end{array}\right)$

$\left(\begin{array}{llll}0 & 0 & 0 & x y z \\ 0 & 0 & 0 & \\ 0 & 0 & 0\end{array}\right)$

$\left(\begin{array}{rrrrrr}0 & 0 & 0 & 0 & x x z & 0 \\ 0 & 0 & 0 & x x z & 0 & 0 \\ z x x & z x x & z z z & 0 & 0 & 0\end{array}\right)$

$\left(\begin{array}{rrrrrr}0 & 0 & 0 & x y z & 0 & 0 \\ 0 & 0 & 0 & 0 & x y z & 0 \\ 0 & 0 & 0 & 0 & 0 & z x y\end{array}\right)$

$\left(\begin{array}{ccc}0 & y z y & 0 \\ -y z y & 0 & 0 \\ 0 & 0 & 0\end{array}\right)$ $\left(\begin{array}{ccc}y z x & 0 & 0 \\ 0 & -y z x & 0 \\ 0 & 0 & 0\end{array}\right)$ 
$\mathrm{n}=3 \quad \mathbf{D}_{3 \mathrm{~d}}\left(\mathbf{C}_{3 \mathrm{v}}\right)=\overline{\mathbf{3}}_{\mathrm{z}} \mathbf{m}_{\mathrm{x}}\left(\mathbf{3}_{\mathrm{z}} \mathbf{m}_{\mathrm{x}}\right)=\overline{\mathbf{3}}_{\mathrm{z}}{ }^{\prime} \mathbf{m}_{\mathrm{x}}$

$\vee^{3} \quad\left(\begin{array}{lllllllll}0 & 0 & 0 & 0 & 0 & 0 & 0 & 0 & 0 \\ 0 & 0 & 0 & 0 & 0 & 0 & 0 & 0 & 0 \\ 0 & 0 & 0 & 0 & 0 & 0 & 0 & 0 & 0\end{array}\right)$

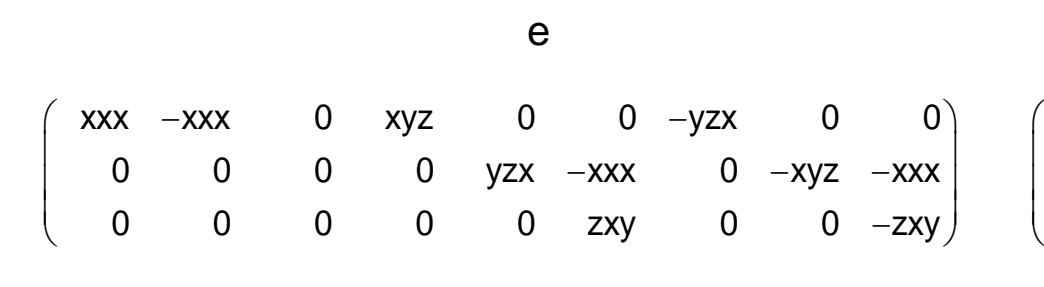

$\left(\begin{array}{rrrr}x x x & -x x x & 0 & 0 \\ 0 & 0 & 0 & \\ 0 & 0 & 0 & \end{array}\right)$

$\left(\begin{array}{rrrrrr}0 & 0 & 0 & x y z & 0 & -y y y \\ -y y y & y y y & 0 & 0 & -x y z & 0 \\ 0 & 0 & 0 & 0 & 0 & 0\end{array}\right)$

$\left(\begin{array}{ccc}y z x & 0 & 0 \\ 0 & y z x & 0 \\ 0 & 0 & x y z\end{array}\right)$ $\left(\begin{array}{rrrrrrrrr}0 & 0 & 0 & 0 & x z x & -y y y & 0 & y y z & -y y y \\ -y y y & y y y & 0 & y y z & 0 & 0 & x z x & 0 & 0 \\ z x x & z x x & z z z & 0 & 0 & 0 & 0 & 0 & 0\end{array}\right)$

$\left(\begin{array}{rrrr}0 & 0 & 0 & 0 \\ -y y y & y y y & 0 & \\ z x x & z x x & z z z & \end{array}\right)$

$\left(\begin{array}{rrrrrr}0 & 0 & 0 & 0 & x x z & -y y y \\ -y y y & y y y & 0 & x x z & 0 & 0 \\ z x x & z y y & z z z & 0 & 0 & 0\end{array}\right)$

$\left(\begin{array}{ccc}0 & y z y & 0 \\ -y z y & 0 & 0 \\ 0 & 0 & 0\end{array}\right)$ $\left(\begin{array}{lllllllll}0 & 0 & 0 & 0 & 0 & 0 & 0 & 0 & 0 \\ 0 & 0 & 0 & 0 & 0 & 0 & 0 & 0 & 0 \\ 0 & 0 & 0 & 0 & 0 & 0 & 0 & 0 & 0\end{array}\right)$

$\left(\begin{array}{llll}0 & 0 & 0 & 0 \\ 0 & 0 & 0 & \\ 0 & 0 & 0 & \end{array}\right)$

$\left(\begin{array}{llllll}0 & 0 & 0 & 0 & 0 & 0 \\ 0 & 0 & 0 & 0 & 0 & 0 \\ 0 & 0 & 0 & 0 & 0 & 0\end{array}\right)$

$\left(\begin{array}{lll}0 & 0 & 0 \\ 0 & 0 & 0 \\ 0 & 0 & 0\end{array}\right)$

$n>3 \quad \mathbf{D}_{\propto h}\left(\mathbf{C}_{\infty \mathrm{v}}\right)=\infty / \mathbf{m}^{\prime} \mathbf{m m}$

$\mathrm{V}^{3} \quad\left(\begin{array}{lllllllll}0 & 0 & 0 & 0 & 0 & 0 & 0 & 0 & 0 \\ 0 & 0 & 0 & 0 & 0 & 0 & 0 & 0 & 0 \\ 0 & 0 & 0 & 0 & 0 & 0 & 0 & 0 & 0\end{array}\right)$

$\left(\begin{array}{rrrrrrrrr}0 & 0 & 0 & x y z & 0 & 0 & -y z x & 0 & 0 \\ 0 & 0 & 0 & 0 & y z x & 0 & 0 & -x y z & 0 \\ 0 & 0 & 0 & 0 & 0 & z x y & 0 & 0 & -z x y\end{array}\right) \quad\left(\begin{array}{rrrrrrrrr}0 & 0 & 0 & 0 & x z x & 0 & 0 & y y z & 0 \\ 0 & 0 & 0 & y y z & 0 & 0 & x z x & 0 & 0 \\ z x x & z x x & z z z & 0 & 0 & 0 & 0 & 0 & 0\end{array}\right) \quad\left(\begin{array}{lllllllll}0 & 0 & 0 & 0 & 0 & 0 & 0 & 0 & 0 \\ 0 & 0 & 0 & 0 & 0 & 0 & 0 & 0 & 0 \\ 0 & 0 & 0 & 0 & 0 & 0 & 0 & 0 & 0\end{array}\right)$

$\left[\mathrm{V}^{3}\right]\left(\begin{array}{llll}0 & 0 & 0 & 0 \\ 0 & 0 & 0 & \\ 0 & 0 & 0 & \end{array}\right)$

$\left(\begin{array}{rrrr}0 & 0 & 0 & 0 \\ 0 & 0 & 0 & \\ z x x & z x x & z z z & \end{array}\right)$

$\left(\begin{array}{rrrr}0 & 0 & 0 & 0 \\ 0 & 0 & 0 & \\ z x x & z x x & z z z & \end{array}\right)$

$\left(\begin{array}{llll}0 & 0 & 0 & 0 \\ 0 & 0 & 0 & \\ 0 & 0 & 0 & \end{array}\right)$

$V\left[V^{2}\right]\left(\begin{array}{llllll}0 & 0 & 0 & 0 & 0 & 0 \\ 0 & 0 & 0 & 0 & 0 & 0 \\ 0 & 0 & 0 & 0 & 0 & 0\end{array}\right)$

$\left\{V^{2}\right\} V\left(\begin{array}{ccc}0 & 0 & 0 \\ 0 & 0 & 0 \\ 0 & 0 & 0\end{array}\right)$

$\left(\begin{array}{rrrrrr}0 & 0 & 0 & x y z & 0 & 0 \\ 0 & 0 & 0 & 0 & -x y z & 0 \\ 0 & 0 & 0 & 0 & 0 & 0\end{array}\right)$

$\left(\begin{array}{rrrrrr}0 & 0 & 0 & 0 & x x z & 0 \\ 0 & 0 & 0 & x x z & 0 & 0 \\ z x x & z x x & z z z & 0 & 0 & 0\end{array}\right)$

$\left(\begin{array}{llllll}0 & 0 & 0 & 0 & 0 & 0 \\ 0 & 0 & 0 & 0 & 0 & 0 \\ 0 & 0 & 0 & 0 & 0 & 0\end{array}\right)$

$\left(\begin{array}{ccc}y z x & 0 & 0 \\ 0 & y z x & 0 \\ 0 & 0 & x y z\end{array}\right)$

$\left(\begin{array}{ccc}0 & y z y & 0 \\ -y z y & 0 & 0 \\ 0 & 0 & 0\end{array}\right)$

$\left(\begin{array}{lll}0 & 0 & 0 \\ 0 & 0 & 0 \\ 0 & 0 & 0\end{array}\right)$ 
$\mathrm{n}=1 \quad \mathbf{D}_{1 \mathrm{~h}}\left(\mathbf{C}_{1 \mathrm{~h}}\right)=\mathbf{2}_{\mathrm{x}} \mathbf{m}_{\mathrm{y}} \mathbf{m}_{\mathrm{z}}\left(\mathbf{m}_{\mathrm{z}}\right)=\mathbf{2}_{\mathrm{x}}{ }^{\prime} \mathbf{m}_{\mathrm{y}}{ }^{\prime} \mathbf{m}_{\mathrm{z}}$

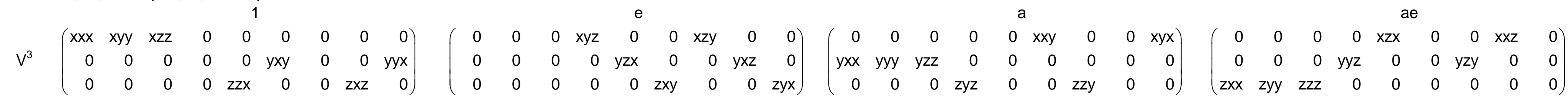

$\left[\mathrm{V}^{3}\right]\left(\begin{array}{rrrr}x x x & x y y & x z z & 0 \\ 0 & 0 & 0 & \\ 0 & 0 & 0 & \end{array}\right)$

$\left(\begin{array}{llll}0 & 0 & 0 & x y z \\ 0 & 0 & 0 & \\ 0 & 0 & 0 & \end{array}\right)$

$\left(\begin{array}{rrrr}0 & 0 & 0 & 0 \\ y x x & y y y & y z z & \\ 0 & 0 & 0 & \end{array}\right)$

$\left(\begin{array}{rrrr}0 & 0 & 0 & 0 \\ 0 & 0 & 0 & \\ z x x & z y y & z z z & \end{array}\right)$

$V\left[V^{2}\right]\left(\begin{array}{rrrrrr}x x x & x y y & x z z & 0 & 0 & 0 \\ 0 & 0 & 0 & 0 & 0 & y x y \\ 0 & 0 & 0 & 0 & z x z & 0\end{array}\right)$

$\left(\begin{array}{rrrrrr}0 & 0 & 0 & x y z & 0 & 0 \\ 0 & 0 & 0 & 0 & y x z & 0 \\ 0 & 0 & 0 & 0 & 0 & z x y\end{array}\right)$

$\left(\begin{array}{rrrrrr}0 & 0 & 0 & 0 & 0 & x x y \\ y x x & y y y & y z z & 0 & 0 & 0 \\ 0 & 0 & 0 & z y z & 0 & 0\end{array}\right)$

$\left(\begin{array}{rrrrrr}0 & 0 & 0 & 0 & x x z & 0 \\ 0 & 0 & 0 & y y z & 0 & 0 \\ z x x & z y y & z z z & 0 & 0 & 0\end{array}\right)$

$\left\{V^{2}\right\} V\left(\begin{array}{ccc}0 & 0 & 0 \\ 0 & 0 & z x z \\ 0 & x y y & 0\end{array}\right)$

$\left(\begin{array}{ccc}y z x & 0 & 0 \\ 0 & z x y & 0 \\ 0 & 0 & x y z\end{array}\right)$

$\left(\begin{array}{ccc}0 & 0 & y z z \\ 0 & 0 & 0 \\ x y x & 0 & 0\end{array}\right)$

$\left(\begin{array}{ccc}0 & y z y & 0 \\ z x x & 0 & 0 \\ 0 & 0 & 0\end{array}\right)$

$\mathrm{n}=2 \quad \mathbf{D}_{2 \mathrm{~h}}\left(\mathbf{C}_{2 \mathrm{~h}}\right)=\mathbf{m}_{\mathrm{x}} \mathbf{m}_{\mathrm{y}} \mathbf{m}_{\mathrm{z}}\left(\mathbf{2}_{\mathrm{z}} / \mathbf{m}_{\mathrm{z}}\right)=\mathbf{m}_{\mathrm{x}}{ }^{\prime} \mathbf{m}_{\mathrm{y}}{ }^{\prime} \mathbf{m}_{\mathrm{z}}$

$\mathrm{V}^{3} \quad\left(\begin{array}{lllllllll}0 & 0 & 0 & 0 & 0 & 0 & 0 & 0 & 0 \\ 0 & 0 & 0 & 0 & 0 & 0 & 0 & 0 & 0 \\ 0 & 0 & 0 & 0 & 0 & 0 & 0 & 0 & 0\end{array}\right)$

$\left(\begin{array}{rrrrrrrrr}0 & 0 & 0 & x y z & 0 & 0 & x z y & 0 & 0 \\ 0 & 0 & 0 & 0 & y z x & 0 & 0 & y x z & 0 \\ 0 & 0 & 0 & 0 & 0 & z x y & 0 & 0 & z y x\end{array}\right)\left(\begin{array}{lllllllll}0 & 0 & 0 & 0 & 0 & 0 & 0 & 0 & 0 \\ 0 & 0 & 0 & 0 & 0 & 0 & 0 & 0 & 0 \\ 0 & 0 & 0 & 0 & 0 & 0 & 0 & 0 & 0\end{array}\right)$

$\left(\begin{array}{rrrrrrrrr}0 & 0 & 0 & 0 & x z x & 0 & 0 & x x z & 0 \\ 0 & 0 & 0 & y y z & 0 & 0 & y z y & 0 & 0 \\ z x x & \text { zyy } & \text { zzz } & 0 & 0 & 0 & 0 & 0 & 0\end{array}\right)$

$\left[\mathrm{V}^{3}\right]\left(\begin{array}{llll}0 & 0 & 0 & 0 \\ 0 & 0 & 0 & \\ 0 & 0 & 0 & \end{array}\right)$

$\left(\begin{array}{llll}0 & 0 & 0 & x y z \\ 0 & 0 & 0 & \\ 0 & 0 & 0 & \end{array}\right)$

$\left(\begin{array}{llll}0 & 0 & 0 & 0 \\ 0 & 0 & 0 & \\ 0 & 0 & 0 & \end{array}\right)$

$V\left[V^{2}\right]\left(\begin{array}{llllll}0 & 0 & 0 & 0 & 0 & 0 \\ 0 & 0 & 0 & 0 & 0 & 0 \\ 0 & 0 & 0 & 0 & 0 & 0\end{array}\right)$

$\left(\begin{array}{rrrrrr}0 & 0 & 0 & x y z & 0 & 0 \\ 0 & 0 & 0 & 0 & y x z & 0 \\ 0 & 0 & 0 & 0 & 0 & z x y\end{array}\right)$

$\left(\begin{array}{llllll}0 & 0 & 0 & 0 & 0 & 0 \\ 0 & 0 & 0 & 0 & 0 & 0 \\ 0 & 0 & 0 & 0 & 0 & 0\end{array}\right)$

$\left(\begin{array}{rrrr}0 & 0 & 0 & 0 \\ 0 & 0 & 0 & \\ z x x & z y y & z z z & \end{array}\right)$

$\left\{V^{2}\right\} V\left(\begin{array}{ccc}0 & 0 & 0 \\ 0 & 0 & 0 \\ 0 & 0 & 0\end{array}\right)$

$\left(\begin{array}{ccc}y z x & 0 & 0 \\ 0 & z x y & 0 \\ 0 & 0 & x y z\end{array}\right)$

$\left(\begin{array}{lll}0 & 0 & 0 \\ 0 & 0 & 0 \\ 0 & 0 & 0\end{array}\right)$

$\left(\begin{array}{rrrrrr}0 & 0 & 0 & 0 & x x z & 0 \\ 0 & 0 & 0 & y y z & 0 & 0 \\ z x x & z y y & z z z & 0 & 0 & 0\end{array}\right)$

$\left(\begin{array}{ccc}0 & y z y & 0 \\ z x x & 0 & 0 \\ 0 & 0 & 0\end{array}\right)$ 
$\mathrm{n}=3 \quad \mathbf{D}_{3 h}\left(\mathbf{C}_{3 h}\right)=\overline{\mathbf{6}}_{z} \mathbf{m}_{x} \mathbf{2}_{1}\left(\overline{\mathbf{6}}_{z}\right)=\overline{\mathbf{6}}_{z} \mathbf{m}_{x}{ }^{\prime} \mathbf{2}_{1}{ }^{\prime}$

$\mathrm{V}^{3} \quad\left(\begin{array}{cccccc}0 & 0 & 0 & 0 & 0 & -y y y\end{array}\right.$ $V^{3} \quad\left(\begin{array}{rrrrrrrrr}0 & 0 & 0 & 0 & 0 & -y y y & 0 & 0 & -y y y \\ -y y y & y y y & 0 & 0 & 0 & 0 & 0 & 0 & 0 \\ 0 & 0 & 0 & 0 & 0 & 0 & 0 & 0 & 0\end{array}\right)$

$\left[V^{3}\right] \quad\left(\begin{array}{rrrr}0 & 0 & 0 & 0 \\ -y y y & y y y & 0 & \\ 0 & 0 & 0 & \end{array}\right)$

$V\left[V^{2}\right]\left(\begin{array}{rrrrrr}0 & 0 & 0 & 0 & 0 & -y y y \\ -y y y & y y y & 0 & 0 & 0 & 0 \\ 0 & 0 & 0 & 0 & 0 & 0\end{array}\right)$

$\left\{V^{2}\right\} V\left(\begin{array}{lll}0 & 0 & 0 \\ 0 & 0 & 0 \\ 0 & 0 & 0\end{array}\right)$

$\mathrm{n}>3 \quad \mathbf{D}_{\infty h}\left(\mathbf{C}_{\infty h}\right)=\infty / \mathbf{m m} \mathbf{m}^{\prime}$

$\mathrm{V}^{3} \quad\left(\begin{array}{ccccccccc}0 & 0 & 0 & 0 & 0 & 0 & 0 & 0 & 0 \\ 0 & 0 & 0 & 0 & 0 & 0 & 0 & 0 & 0 \\ 0 & 0 & 0 & 0 & 0 & 0 & 0 & 0 & 0\end{array}\right)$

$\left[\mathrm{V}^{3}\right]\left(\begin{array}{llll}0 & 0 & 0 & 0 \\ 0 & 0 & 0 & \\ 0 & 0 & 0 & \end{array}\right)$

$V\left[V^{2}\right]\left(\begin{array}{llllll}0 & 0 & 0 & 0 & 0 & 0 \\ 0 & 0 & 0 & 0 & 0 & 0 \\ 0 & 0 & 0 & 0 & 0 & 0\end{array}\right)$

$\left(\begin{array}{llllll}0 & 0 & 0 & 0 & 0 & 0 \\ 0 & 0 & 0 & 0 & 0 & 0\end{array}\right)$

$\left\{V^{2}\right\} V\left(\begin{array}{lll}0 & 0 & 0 \\ 0 & 0 & 0 \\ 0 & 0 & 0\end{array}\right)$

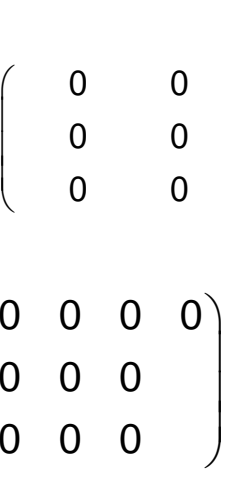

$\left(\begin{array}{rrrrrr}0 & 0 & 0 & x y z & 0 & 0 \\ 0 & 0 & 0 & 0 & -x y z & 0 \\ 0 & 0 & 0 & 0 & 0 & 0\end{array}\right)$

$\left(\begin{array}{rrrrrr}\mathrm{xxx} & -\mathrm{xxx} & 0 & 0 & 0 & 0 \\ 0 & 0 & 0 & 0 & 0 & -\mathrm{xxx} \\ 0 & 0 & 0 & 0 & 0 & 0\end{array}\right)$

$\left(\begin{array}{ccc}y z x & 0 & 0 \\ 0 & y z x & 0 \\ 0 & 0 & x y z\end{array}\right)$

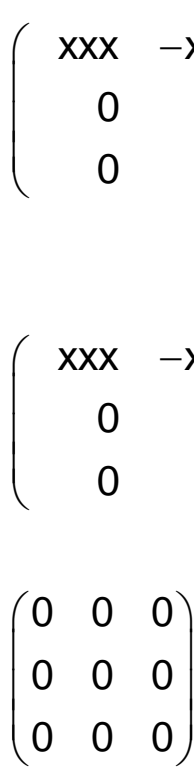

a

ae

$\left.\begin{array}{rrrrrrrrr}0 & 0 & 0 & 0 & x z x & 0 & 0 & y y z & 0 \\ 0 & 0 & 0 & y y z & 0 & 0 & x z x & 0 & 0\end{array}\right)$ $\begin{array}{lllllllll}z x x & z x x & z z z & 0 & 0 & 0 & 0 & 0 & 0\end{array}$

$\left(\begin{array}{rrrr}0 & 0 & 0 & 0 \\ 0 & 0 & 0 & \\ z x x & z y y & z z z & \end{array}\right)$

$\left(\begin{array}{rrrrrr}0 & 0 & 0 & 0 & x x z & 0 \\ 0 & 0 & 0 & x x z & 0 & 0 \\ z x x & z x x & z z z & 0 & 0 & 0\end{array}\right)$

$\left(\begin{array}{ccc}0 & y z y & 0 \\ -y z y & 0 & 0 \\ 0 & 0 & 0\end{array}\right)$ $\left(\begin{array}{rrrrrrrrr}0 & 0 & 0 & x y z & 0 & 0 & -y z x & 0 & 0 \\ 0 & 0 & 0 & 0 & y z x & 0 & 0 & -x y z & 0 \\ 0 & 0 & 0 & 0 & 0 & z x y & 0 & 0 & -z x y\end{array}\right) \quad\left(\begin{array}{rrrrrrrrr}0 & 0 & 0 & 0 & 0 & 0 & 0 & 0 & 0 \\ 0 & 0 & 0 & 0 & 0 & 0 & 0 & 0 & 0 \\ 0 & 0 & 0 & 0 & 0 & 0 & 0 & 0 & 0\end{array}\right)$

$\left(\begin{array}{rrrr}0 & 0 & 0 & 0 \\ 0 & 0 & 0 & \\ z x x & z x x & z z z & \end{array}\right)$

$\left(\begin{array}{rrrrrr}0 & 0 & 0 & x y z & 0 & 0 \\ 0 & 0 & 0 & 0 & -x y z & 0 \\ 0 & 0 & 0 & 0 & 0 & 0\end{array}\right)$

$\left(\begin{array}{ccc}y z x & 0 & 0 \\ 0 & y z x & 0 \\ 0 & 0 & x y z\end{array}\right)$ $\left(\begin{array}{llll}0 & 0 & 0 & 0 \\ 0 & 0 & 0 & \\ 0 & 0 & 0\end{array}\right)$

$\left(\begin{array}{llllll}0 & 0 & 0 & 0 & 0 & 0 \\ 0 & 0 & 0 & 0 & 0 & 0 \\ 0 & 0 & 0 & 0 & 0 & 0\end{array}\right)$

$\left(\begin{array}{lll}0 & 0 & 0 \\ 0 & 0 & 0 \\ 0 & 0 & 0\end{array}\right)$ $\left(\begin{array}{rrrrrrrrr}0 & 0 & 0 & 0 & x z x & 0 & 0 & y y z & 0 \\ 0 & 0 & 0 & y y z & 0 & 0 & x z x & 0 & 0 \\ z x x & z x x & z z z & 0 & 0 & 0 & 0 & 0 & 0\end{array}\right)$

$\left(\begin{array}{rrrr}0 & 0 & 0 & 0 \\ 0 & 0 & 0 & \\ z x x & z x x & z z z & \end{array}\right)$

$\left(\begin{array}{rrrrrr}0 & 0 & 0 & 0 & x x z & 0 \\ 0 & 0 & 0 & x x z & 0 & 0 \\ z x x & z x x & z z z & 0 & 0 & 0\end{array}\right)$

$\left(\begin{array}{ccc}0 & y z y & 0 \\ -y z y & 0 & 0 \\ 0 & 0 & 0\end{array}\right)$ 
$\mathrm{n}=1 \quad \mathbf{D}_{1 \mathrm{~h}}\left(\mathbf{D}_{1}\right)=\mathbf{2}_{\mathrm{x}} \mathbf{m}_{\mathrm{y}} \mathbf{m}_{\mathrm{z}}\left(\mathbf{2}_{\mathrm{x}}\right)=\underset{\mathbf{x}}{\mathbf{2}_{\mathrm{x}} \mathbf{m}_{\mathrm{y}}{ }^{\prime} \mathbf{m}_{\mathrm{z}}{ }^{\prime}}$

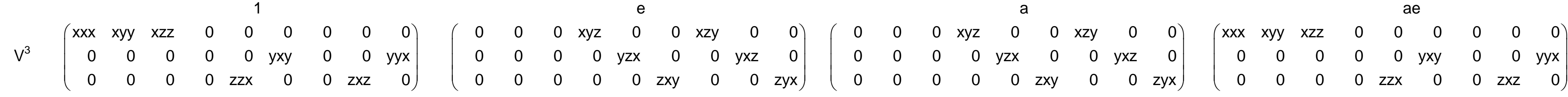

$\left[V^{3}\right] \quad\left(\begin{array}{rrrr}x x x & x y y & x z z & 0 \\ 0 & 0 & 0 & \\ 0 & 0 & 0 & \end{array}\right)$

$\left(\begin{array}{llll}0 & 0 & 0 & x y z \\ 0 & 0 & 0 & \\ 0 & 0 & 0 & \end{array}\right)$

$\left(\begin{array}{llll}0 & 0 & 0 & x y z \\ 0 & 0 & 0 & \\ 0 & 0 & 0 & \end{array}\right)$

$\left(\begin{array}{rrrr}x x x & x y y & x z z & 0 \\ 0 & 0 & 0 & \\ 0 & 0 & 0 & \end{array}\right)$

$V\left[V^{2}\right]\left(\begin{array}{rrrrrr}x x x & x y y & x z z & 0 & 0 & 0 \\ 0 & 0 & 0 & 0 & 0 & y x y \\ 0 & 0 & 0 & 0 & z x z & 0\end{array}\right)$

$\left(\begin{array}{rrrrrr}0 & 0 & 0 & x y z & 0 & 0 \\ 0 & 0 & 0 & 0 & y x z & 0 \\ 0 & 0 & 0 & 0 & 0 & z x y\end{array}\right)$

$\left(\begin{array}{rrrrrr}0 & 0 & 0 & x y z & 0 & 0 \\ 0 & 0 & 0 & 0 & y x z & 0 \\ 0 & 0 & 0 & 0 & 0 & z x y\end{array}\right)$

$\left(\begin{array}{rrrrrr}x x x & x y y & x z z & 0 & 0 & 0 \\ 0 & 0 & 0 & 0 & 0 & y x y \\ 0 & 0 & 0 & 0 & z x z & 0\end{array}\right)$

$\left\{V^{2}\right\} V\left(\begin{array}{ccc}0 & 0 & 0 \\ 0 & 0 & z x z \\ 0 & x y y & 0\end{array}\right)$

$\left(\begin{array}{ccc}y z x & 0 & 0 \\ 0 & z x y & 0 \\ 0 & 0 & x y z\end{array}\right)$

$\left(\begin{array}{ccc}y z x & 0 & 0 \\ 0 & z x y & 0 \\ 0 & 0 & x y z\end{array}\right)$

$\left(\begin{array}{ccc}0 & 0 & 0 \\ 0 & 0 & z x z \\ 0 & x y y & 0\end{array}\right)$

$\mathrm{n}=2 \quad \mathbf{D}_{2 \mathrm{~h}}\left(\mathbf{D}_{2}\right)=\mathbf{m}_{\mathrm{x}} \mathbf{m}_{\mathrm{y}} \mathbf{m}_{\mathrm{z}}\left(\mathbf{2}_{\mathrm{x}} \mathbf{2}_{\mathrm{y}} \mathbf{2}_{\mathrm{z}}\right)=\mathbf{m}_{\mathrm{x}}{ }^{\prime} \mathbf{m}_{\mathrm{y}}{ }^{\prime} \mathbf{m}_{\mathrm{z}}{ }^{\prime}$

$\mathrm{V}^{3} \quad\left(\begin{array}{lllllllll}0 & 0 & 0 & 0 & 0 & 0 & 0 & 0 & 0 \\ 0 & 0 & 0 & 0 & 0 & 0 & 0 & 0 & 0 \\ 0 & 0 & 0 & 0 & 0 & 0 & 0 & 0 & 0\end{array}\right)$

$\left[\mathrm{V}^{3}\right]\left(\begin{array}{llll}0 & 0 & 0 & 0 \\ 0 & 0 & 0 & \\ 0 & 0 & 0 & \end{array}\right)$

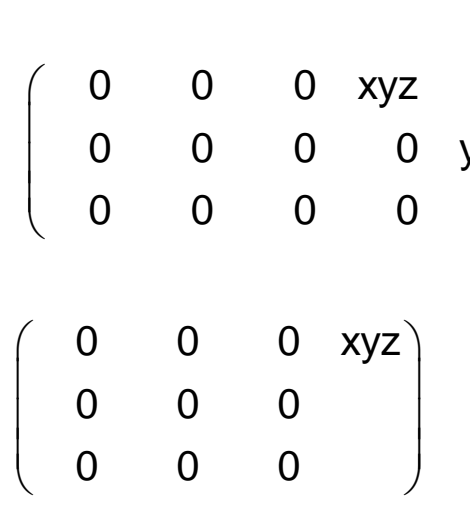

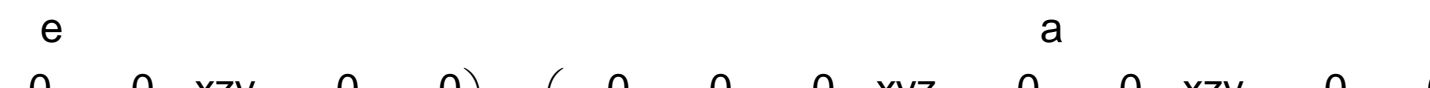
$\left.\begin{array}{rrrrr}y z x & 0 & 0 & y x z & 0 \\ 0 & z x y & 0 & 0 & z y x\end{array}\right)\left(\begin{array}{rrrrrrrrr}0 & 0 & 0 & x y z & 0 & 0 & x z y & 0 & 0 \\ 0 & 0 & 0 & 0 & y z x & 0 & 0 & y x z & 0 \\ 0 & 0 & 0 & 0 & 0 & z x y & 0 & 0 & z y x\end{array}\right)$

$\left(\begin{array}{lllllllll}0 & 0 & 0 & 0 & 0 & 0 & 0 & 0 & 0 \\ 0 & 0 & 0 & 0 & 0 & 0 & 0 & 0 & 0\end{array}\right)$

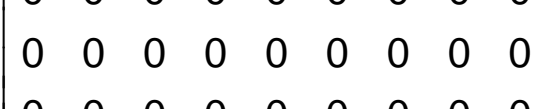

$V\left[V^{2}\right]\left(\begin{array}{llllll}0 & 0 & 0 & 0 & 0 & 0 \\ 0 & 0 & 0 & 0 & 0 & 0 \\ 0 & 0 & 0 & 0 & 0 & 0\end{array}\right)$

$\left(\begin{array}{rrrrrr}0 & 0 & 0 & x y z & 0 & 0 \\ 0 & 0 & 0 & 0 & y x z & 0 \\ 0 & 0 & 0 & 0 & 0 & z x y\end{array}\right)$

$\left(\begin{array}{llll}0 & 0 & 0 & x y z \\ 0 & 0 & 0 & \\ 0 & 0 & 0 & \end{array}\right)$

$\left(\begin{array}{llll}0 & 0 & 0 & 0 \\ 0 & 0 & 0 & \\ 0 & 0 & 0 & \end{array}\right)$

$\left(\begin{array}{llllll}0 & 0 & 0 & 0 & 0 & 0\end{array}\right)$

$\left(\begin{array}{ccc}y z x & 0 & 0 \\ 0 & z x y & 0 \\ 0 & 0 & x y z\end{array}\right)$

$\left(\begin{array}{rrrrrr}0 & 0 & 0 & x y z & 0 & 0 \\ 0 & 0 & 0 & 0 & y x z & 0 \\ 0 & 0 & 0 & 0 & 0 & z x y\end{array}\right)$

$\left(\begin{array}{llllll}0 & 0 & 0 & 0 & 0 & 0 \\ 0 & 0 & 0 & 0 & 0 & 0 \\ 0 & 0 & 0 & 0 & 0 & 0\end{array}\right)$

$\left\{V^{2}\right\} V\left(\begin{array}{ccc}0 & 0 & 0 \\ 0 & 0 & 0 \\ 0 & 0 & 0\end{array}\right)$

$\left(\begin{array}{ccc}y z x & 0 & 0 \\ 0 & z x y & 0 \\ 0 & 0 & x y z\end{array}\right)$

$\left(\begin{array}{lll}0 & 0 & 0 \\ 0 & 0 & 0 \\ 0 & 0 & 0\end{array}\right)$ 


\section{$\mathrm{n}=3 \quad \mathbf{D}_{3 h}\left(\mathbf{D}_{3}\right)=\overline{\mathbf{6}}_{\mathbf{z}} \mathbf{m}_{\mathrm{x}} \mathbf{2}_{1}\left(\mathbf{3}_{\mathrm{z}} \mathbf{2}_{1}\right)=\overline{\mathbf{6}}_{\mathbf{z}}{ }^{\prime} \mathbf{m}_{\mathrm{x}} \mathbf{2}_{1}$}

$V^{3} \quad\left(\begin{array}{ccccc}0 & 0 & 0 & 0 & 0\end{array}\right.$

$V^{3} \quad\left(\begin{array}{rrrrrrrrr}0 & 0 & 0 & 0 & 0 & -y y y & 0 & 0 & -y y y \\ -y y y & y y y & 0 & 0 & 0 & 0 & 0 & 0 & 0 \\ 0 & 0 & 0 & 0 & 0 & 0 & 0 & 0 & 0\end{array}\right)$

$\left[\mathrm{V}^{3}\right] \quad\left(\begin{array}{rrrr}0 & 0 & 0 & 0 \\ -y y y & \text { yyy } & 0 & \\ 0 & 0 & 0 & \end{array}\right)$

$V\left[V^{2}\right]\left(\begin{array}{rrrrrr}0 & 0 & 0 & 0 & 0 & -y y y \\ -y y y & y y y & 0 & 0 & 0 & 0 \\ 0 & 0 & 0 & 0 & 0 & 0\end{array}\right)$

$\left\{V^{2}\right\} V\left(\begin{array}{lll}0 & 0 & 0 \\ 0 & 0 & 0 \\ 0 & 0 & 0\end{array}\right)$

$n>3 \quad \mathbf{D}_{\infty h}\left(\mathbf{D}_{\infty}\right)=\infty / \mathbf{m}^{\prime} \mathbf{m}^{\prime} \mathbf{m}^{\prime}$

$V^{3} \quad\left(\begin{array}{lllllllll}0 & 0 & 0 & 0 & 0 & 0 & 0 & 0 & 0 \\ 0 & 0 & 0 & 0 & 0 & 0 & 0 & 0 & 0 \\ 0 & 0 & 0 & 0 & 0 & 0 & 0 & 0 & 0\end{array}\right)$

$\left[\mathrm{V}^{3}\right]\left(\begin{array}{llll}0 & 0 & 0 & 0 \\ 0 & 0 & 0 & \\ 0 & 0 & 0 & \end{array}\right)$

$V\left[V^{2}\right]\left(\begin{array}{llllll}0 & 0 & 0 & 0 & 0 & 0 \\ 0 & 0 & 0 & 0 & 0 & 0 \\ 0 & 0 & 0 & 0 & 0 & 0\end{array}\right)$

$\left\{V^{2}\right\} V\left(\begin{array}{lll}0 & 0 & 0 \\ 0 & 0 & 0 \\ 0 & 0 & 0\end{array}\right)$ $\left(\begin{array}{rrrrrrrrr}0 & 0 & 0 & x y z & 0 & 0 & -y z x & 0 & 0 \\ 0 & 0 & 0 & 0 & y z x & 0 & 0 & -x y z & 0 \\ 0 & 0 & 0 & 0 & 0 & z x y & 0 & 0 & -z x y\end{array}\right)\left(\begin{array}{lll}0 & 0 \\ 0 & 0 \\ 0 & 0\end{array}\right.$
$\left(\begin{array}{rrrr}0 & 0 & 0 & 0 \\ 0 & 0 & 0 & \\ 0 & 0 & 0\end{array}\right)$

$\left.\begin{array}{cccccc}x y z & 0 & 0 & -y z x & 0 & 0 \\ 0 & y z x & 0 & 0 & -x y z & 0\end{array}\right)$

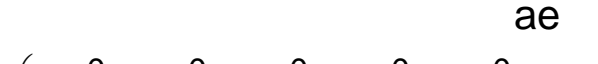

$\left(\begin{array}{rrrrrrrrr}0 & 0 & 0 & 0 & 0 & -y y y & 0 & 0 & -y y y \\ -y y y & \text { yyy } & 0 & 0 & 0 & 0 & 0 & 0 & 0 \\ 0 & 0 & 0 & 0 & 0 & 0 & 0 & 0 & 0\end{array}\right)$

$\left(\begin{array}{rrrrrr}0 & 0 & 0 & x y z & 0 & 0 \\ 0 & 0 & 0 & 0 & -x y z & 0 \\ 0 & 0 & 0 & 0 & 0 & 0\end{array}\right)$

$\left(\begin{array}{rrrrrr}0 & 0 & 0 & x y z & 0 & 0 \\ 0 & 0 & 0 & 0 & -x y z & 0 \\ 0 & 0 & 0 & 0 & 0 & 0\end{array}\right)$

$\left(\begin{array}{rrrr}0 & 0 & 0 & 0 \\ -y y y & \text { yyy } & 0 & \\ 0 & 0 & 0 & \end{array}\right)$

$\left(\begin{array}{rrrrrr}0 & 0 & 0 & 0 & 0 & -y y y \\ -y y y & y y y & 0 & 0 & 0 & 0 \\ 0 & 0 & 0 & 0 & 0 & 0\end{array}\right)$

$\left(\begin{array}{ccc}y z x & 0 & 0 \\ 0 & y z x & 0 \\ 0 & 0 & x y z\end{array}\right)$

$\left(\begin{array}{ccc}y z x & 0 & 0 \\ 0 & y z x & 0 \\ 0 & 0 & x y z\end{array}\right)$

$\left(\begin{array}{lll}0 & 0 & 0 \\ 0 & 0 & 0 \\ 0 & 0 & 0\end{array}\right)$

$\left.\begin{array}{rrrrrrrrr}0 & 0 & 0 & x y z & 0 & 0 & -y z x & 0 & 0 \\ 0 & 0 & 0 & 0 & y z x & 0 & 0 & -x y z & 0 \\ 0 & 0 & 0 & 0 & 0 & z x y & 0 & 0 & -z x y\end{array}\right) \quad\left(\begin{array}{rrrrrrrrrr}0 & 0 & 0 & x y z & 0 & 0 & -y z x & 0 & 0 \\ 0 & 0 & 0 & 0 & y z x & 0 & 0 & -x y z & 0 \\ 0 & 0 & 0 & 0 & 0 & z x y & 0 & 0 & -z x y\end{array}\right) \quad\left(\begin{array}{rrrrrrrrr}0 & 0 & 0 & 0 & 0 & 0 & 0 & 0 & 0 \\ 0 & 0 & 0 & 0 & 0 & 0 & 0 & 0 & 0 \\ 0 & 0 & 0 & 0 & 0 & 0 & 0 & 0 & 0\end{array}\right)$

$\left(\begin{array}{rrrr}0 & 0 & 0 & 0 \\ 0 & 0 & 0 & \\ z x x & z x x & z z z & \end{array}\right)$

$\left(\begin{array}{rrrr}0 & 0 & 0 & 0 \\ 0 & 0 & 0 & \\ z x x & z x x & z z z & \end{array}\right)$

$\left(\begin{array}{llll}0 & 0 & 0 & 0 \\ 0 & 0 & 0 & \\ 0 & 0 & 0 & \end{array}\right)$

$\left(\begin{array}{rrrrrr}0 & 0 & 0 & x y z & 0 & 0 \\ 0 & 0 & 0 & 0 & -x y z & 0 \\ 0 & 0 & 0 & 0 & 0 & 0\end{array}\right)$

$\left(\begin{array}{rrrrrr}0 & 0 & 0 & x y z & 0 & 0 \\ 0 & 0 & 0 & 0 & -x y z & 0 \\ 0 & 0 & 0 & 0 & 0 & 0\end{array}\right)$

$\left(\begin{array}{llllll}0 & 0 & 0 & 0 & 0 & 0 \\ 0 & 0 & 0 & 0 & 0 & 0 \\ 0 & 0 & 0 & 0 & 0 & 0\end{array}\right)$

$\left(\begin{array}{ccc}y z x & 0 & 0 \\ 0 & y z x & 0 \\ 0 & 0 & x y z\end{array}\right)$

$\left(\begin{array}{ccc}y z x & 0 & 0 \\ 0 & y z x & 0 \\ 0 & 0 & x y z\end{array}\right)$

$\left(\begin{array}{lll}0 & 0 & 0 \\ 0 & 0 & 0 \\ 0 & 0 & 0\end{array}\right)$ 
$\mathrm{n}=1 \quad \mathbf{D}_{1 \mathrm{~h}}\left(\mathbf{C}_{1 \mathrm{v}}\right)=\mathbf{2}_{\mathrm{x}} \mathbf{m}_{\mathrm{y}} \mathbf{m}_{\mathrm{z}}\left(\mathbf{m}_{\mathrm{y}}\right)=\mathbf{2}_{\mathrm{x}} \mathbf{m}_{\mathrm{y}} \mathbf{m}_{\mathrm{z}}{ }^{\prime}$

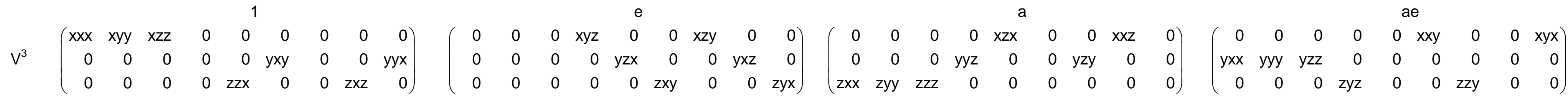

$\left[\mathrm{V}^{3}\right] \quad\left(\begin{array}{rrrr}x x x & x y y & x z z & 0 \\ 0 & 0 & 0 & \\ 0 & 0 & 0 & \end{array}\right)$

$\left(\begin{array}{llll}0 & 0 & 0 & x y z \\ 0 & 0 & 0 & \\ 0 & 0 & 0 & \end{array}\right)$

$\left(\begin{array}{rrrr}0 & 0 & 0 & 0 \\ 0 & 0 & 0 & \\ z x x & z y y & z z z & \end{array}\right)$

$\left(\begin{array}{rrrr}0 & 0 & 0 & 0 \\ y x x & y y y & y z z & \\ 0 & 0 & 0 & \end{array}\right)$

$V\left[V^{2}\right]\left(\begin{array}{rrrrrr}x x x & x y y & x z z & 0 & 0 & 0 \\ 0 & 0 & 0 & 0 & 0 & y x y \\ 0 & 0 & 0 & 0 & z x z & 0\end{array}\right)$

$\left(\begin{array}{rrrrrr}0 & 0 & 0 & x y z & 0 & 0 \\ 0 & 0 & 0 & 0 & y x z & 0 \\ 0 & 0 & 0 & 0 & 0 & z x y\end{array}\right)$

$\left(\begin{array}{rrrrrr}0 & 0 & 0 & 0 & x x z & 0 \\ 0 & 0 & 0 & y y z & 0 & 0 \\ z x x & z y y & z z z & 0 & 0 & 0\end{array}\right)$

$\left(\begin{array}{rrrrrr}0 & 0 & 0 & 0 & 0 & x x y \\ y x x & y y y & y z z & 0 & 0 & 0 \\ 0 & 0 & 0 & z y z & 0 & 0\end{array}\right)$

$\left\{V^{2}\right\} V\left(\begin{array}{ccc}0 & 0 & 0 \\ 0 & 0 & z x z \\ 0 & x y y & 0\end{array}\right)$

$\left(\begin{array}{ccc}y z x & 0 & 0 \\ 0 & z x y & 0 \\ 0 & 0 & x y z\end{array}\right)$

$\left(\begin{array}{ccc}0 & y z y & 0 \\ z x x & 0 & 0 \\ 0 & 0 & 0\end{array}\right)$

$\left(\begin{array}{ccc}0 & 0 & y z z \\ 0 & 0 & 0 \\ x y x & 0 & 0\end{array}\right)$

$\mathrm{n}=2 \quad \mathbf{D}_{\mathrm{hh}}\left(\mathbf{C}_{2 \mathrm{v}}\right)=\mathbf{m}_{\mathrm{x}} \mathbf{m}_{\mathrm{y}} \mathbf{m}_{\mathrm{z}}\left(\mathbf{2}_{\mathrm{z}} \mathbf{m}_{\mathrm{x}} \mathbf{m}_{\mathrm{y}}\right)=\mathbf{m}_{\mathrm{x}} \mathbf{m}_{\mathrm{y}} \mathbf{m}_{\mathrm{z}}$

$\mathrm{V}^{3} \quad\left(\begin{array}{lllllllll}0 & 0 & 0 & 0 & 0 & 0 & 0 & 0 & 0 \\ 0 & 0 & 0 & 0 & 0 & 0 & 0 & 0 & 0 \\ 0 & 0 & 0 & 0 & 0 & 0 & 0 & 0 & 0\end{array}\right)$

$\left(\begin{array}{rrrrrrrrr}0 & 0 & 0 & x y z & 0 & 0 & x z y & 0 & 0 \\ 0 & 0 & 0 & 0 & y z x & 0 & 0 & y x z & 0 \\ 0 & 0 & 0 & 0 & 0 & z x y & 0 & 0 & z y x\end{array}\right) \quad\left(\begin{array}{rrrrrrrrr}0 & 0 & 0 & 0 & x z x & 0 & 0 & x x z & 0 \\ 0 & 0 & 0 & y y z & 0 & 0 & y z y & 0 & 0 \\ z x x & z y y & z z z & 0 & 0 & 0 & 0 & 0 & 0\end{array}\right) \quad\left(\begin{array}{lllllllll}0 & 0 & 0 & 0 & 0 & 0 & 0 & 0 & 0 \\ 0 & 0 & 0 & 0 & 0 & 0 & 0 & 0 & 0 \\ 0 & 0 & 0 & 0 & 0 & 0 & 0 & 0 & 0\end{array}\right)$

$\left[\mathrm{V}^{3}\right]\left(\begin{array}{llll}0 & 0 & 0 & 0 \\ 0 & 0 & 0 \\ 0 & 0 & 0\end{array}\right)$

$\left(\begin{array}{llll}0 & 0 & 0 & x y z \\ 0 & 0 & 0 & \\ 0 & 0 & 0\end{array}\right)$

$\left(\begin{array}{rrrr}0 & 0 & 0 & 0 \\ 0 & 0 & 0 & \\ z x x & z y y & z z z\end{array}\right)$

$\left(\begin{array}{llll}0 & 0 & 0 & 0 \\ 0 & 0 & 0 & \\ 0 & 0 & 0 & \end{array}\right)$

$V\left[V^{2}\right]\left(\begin{array}{llllll}0 & 0 & 0 & 0 & 0 & 0 \\ 0 & 0 & 0 & 0 & 0 & 0 \\ 0 & 0 & 0 & 0 & 0 & 0\end{array}\right)$

$\left(\begin{array}{rrrrrr}0 & 0 & 0 & x y z & 0 & 0 \\ 0 & 0 & 0 & 0 & y x z & 0 \\ 0 & 0 & 0 & 0 & 0 & z x y\end{array}\right)$

$\left(\begin{array}{rrrrrr}0 & 0 & 0 & 0 & x x z & 0 \\ 0 & 0 & 0 & y y z & 0 & 0 \\ z x x & z y y & z z z & 0 & 0 & 0\end{array}\right)$

$\left(\begin{array}{llllll}0 & 0 & 0 & 0 & 0 & 0 \\ 0 & 0 & 0 & 0 & 0 & 0 \\ 0 & 0 & 0 & 0 & 0 & 0\end{array}\right)$

$\left\{V^{2}\right\} V\left(\begin{array}{lll}0 & 0 & 0 \\ 0 & 0 & 0 \\ 0 & 0 & 0\end{array}\right)$

$\left(\begin{array}{ccc}y z x & 0 & 0 \\ 0 & z x y & 0 \\ 0 & 0 & x y z\end{array}\right)$

$\left(\begin{array}{ccc}0 & y z y & 0 \\ z x x & 0 & 0 \\ 0 & 0 & 0\end{array}\right)$

$\left(\begin{array}{lll}0 & 0 & 0 \\ 0 & 0 & 0 \\ 0 & 0 & 0\end{array}\right)$ 
$\mathrm{n}=1 \quad \mathbf{D}_{2 \mathrm{~h}}\left(\mathbf{D}_{1 \mathrm{~d}}\right)=\mathbf{m}_{\mathrm{x}} \mathbf{m}_{\mathrm{y}} \mathbf{m}_{\mathrm{z}}\left(\mathbf{2}_{\mathrm{y}} / \mathbf{m}_{\mathrm{y}}\right)=\mathbf{m}_{\mathrm{x}}{ }^{\prime} \mathbf{m}_{\mathrm{y}} \mathbf{m}_{\mathrm{z}}{ }^{\prime}$

$V^{3} \quad\left(\begin{array}{lllllllll}0 & 0 & 0 & 0 & 0 & 0 & 0 & 0 & 0 \\ 0 & 0 & 0 & 0 & 0 & 0 & 0 & 0 & 0 \\ 0 & 0 & 0 & 0 & 0 & 0 & 0 & 0 & 0\end{array}\right)$

$\left[\mathrm{V}^{3}\right]\left(\begin{array}{llll}0 & 0 & 0 & 0 \\ 0 & 0 & 0 & \\ 0 & 0 & 0 & \end{array}\right)$

$\left(\begin{array}{llllll}0 & 0 & 0 & 0 & 0 & 0 \\ 0 & 0 & 0 & 0 & 0 & 0\end{array}\right)$

$V\left[V^{2}\right]\left(\begin{array}{llllll}0 & 0 & 0 & 0 & 0 & 0 \\ 0 & 0 & 0 & 0 & 0 & 0 \\ 0 & 0 & 0 & 0 & 0 & 0\end{array}\right)$

$\left\{V^{2}\right\} V\left(\begin{array}{ccc}0 & 0 & 0 \\ 0 & 0 & 0 \\ 0 & 0 & 0\end{array}\right)$

$\mathrm{n}=2 \mathbf{D}_{4 \mathrm{~h}}\left(\mathbf{D}_{2 \mathrm{~d}}\right)=\mathbf{4}_{\mathrm{z}} / \mathbf{m}_{\mathrm{z}} \mathbf{m}_{\mathrm{x}} \mathbf{m}_{\mathrm{xy}}\left(\overline{\mathbf{4}}_{\mathrm{z}} \mathbf{m}_{\mathrm{x}} \mathbf{2}_{\mathrm{xy}}\right)=\mathbf{4}_{\mathrm{z}}{ }^{\prime} / \mathbf{m}_{\mathrm{z}}{ }^{\prime} \mathbf{m}_{\mathrm{x}} \mathbf{m}_{\mathrm{xy}}{ }^{\prime}$

$\mathrm{V}^{3} \quad\left(\begin{array}{lllllllll}0 & 0 & 0 & 0 & 0 & 0 & 0 & 0 & 0 \\ 0 & 0 & 0 & 0 & 0 & 0 & 0 & 0 & 0 \\ 0 & 0 & 0 & 0 & 0 & 0 & 0 & 0 & 0\end{array}\right)$

$\left[\mathrm{V}^{3}\right]\left(\begin{array}{llll}0 & 0 & 0 & 0 \\ 0 & 0 & 0 & \\ 0 & 0 & 0 & \end{array}\right)$

$V\left[V^{2}\right]\left(\begin{array}{llllll}0 & 0 & 0 & 0 & 0 & 0 \\ 0 & 0 & 0 & 0 & 0 & 0 \\ 0 & 0 & 0 & 0 & 0 & 0\end{array}\right)$

$\left\{V^{2}\right\} V\left(\begin{array}{lll}0 & 0 & 0 \\ 0 & 0 & 0 \\ 0 & 0 & 0\end{array}\right)$ $\left(\begin{array}{rrrrrrrrr}0 & 0 & 0 & x y z & 0 & 0 & x z y & 0 & 0 \\ 0 & 0 & 0 & 0 & y z x & 0 & 0 & y x z & 0 \\ 0 & 0 & 0 & 0 & 0 & z x y & 0 & 0 & z y x\end{array}\right)\left(\begin{array}{lllllllll}0 & 0 & 0 & 0 & 0 & 0 & 0 & 0 & 0 \\ 0 & 0 & 0 & 0 & 0 & 0 & 0 & 0 & 0 \\ 0 & 0 & 0 & 0 & 0 & 0 & 0 & 0 & 0\end{array}\right)$

$\left(\begin{array}{llll}0 & 0 & 0 & x y z \\ 0 & 0 & 0 & \\ 0 & 0 & 0 & \end{array}\right)$

$\left(\begin{array}{llll}0 & 0 & 0 & 0 \\ 0 & 0 & 0 & \\ 0 & 0 & 0 & \end{array}\right)$

$\left(\begin{array}{rrrrrr}0 & 0 & 0 & x y z & 0 & 0 \\ 0 & 0 & 0 & 0 & y x z & 0 \\ 0 & 0 & 0 & 0 & 0 & z x y\end{array}\right)$

$\left(\begin{array}{llllll}0 & 0 & 0 & 0 & 0 & 0 \\ 0 & 0 & 0 & 0 & 0 & 0 \\ 0 & 0 & 0 & 0 & 0 & 0\end{array}\right)$

$\left(\begin{array}{ccc}y z x & 0 & 0 \\ 0 & z x y & 0 \\ 0 & 0 & x y z\end{array}\right)$

$\left(\begin{array}{lll}0 & 0 & 0 \\ 0 & 0 & 0 \\ 0 & 0 & 0\end{array}\right)$ $\left(\begin{array}{rrrrrrrrr}0 & 0 & 0 & 0 & 0 & x x y & 0 & 0 & x y x \\ y x x & y y y & y z z & 0 & 0 & 0 & 0 & 0 & 0 \\ 0 & 0 & 0 & z y z & 0 & 0 & z z y & 0 & 0\end{array}\right)$

$\left(\begin{array}{rrrr}0 & 0 & 0 & 0 \\ y x x & y y y & y z z & \\ 0 & 0 & 0 & \end{array}\right)$

$\left(\begin{array}{rrrrrr}0 & 0 & 0 & 0 & 0 & x x y \\ y x x & y y y & y z z & 0 & 0 & 0 \\ 0 & 0 & 0 & z y z & 0 & 0\end{array}\right)$

$\left(\begin{array}{ccc}0 & 0 & y z z \\ 0 & 0 & 0 \\ x y x & 0 & 0\end{array}\right)$ $\left(\begin{array}{rrrrrrrrr}0 & 0 & 0 & x y z & 0 & 0 & -y z x & 0 & 0 \\ 0 & 0 & 0 & 0 & y z x & 0 & 0 & -x y z & 0 \\ 0 & 0 & 0 & 0 & 0 & z x y & 0 & 0 & -z x y\end{array}\right) \quad\left(\begin{array}{rrrrrrrrr}0 & 0 & 0 & 0 & x z x & 0 & 0 & -y y z & 0 \\ 0 & 0 & 0 & y y z & 0 & 0 & -x z x & 0 & 0 \\ z x x & -z x x & 0 & 0 & 0 & 0 & 0 & 0 & 0\end{array}\right) \quad\left(\begin{array}{rrrrrrrrr}0 & 0 & 0 & 0 & 0 & 0 & 0 & 0 & 0 \\ 0 & 0 & 0 & 0 & 0 & 0 & 0 & 0 & 0 \\ 0 & 0 & 0 & 0 & 0 & 0 & 0 & 0 & 0\end{array}\right)$

$\left(\begin{array}{llll}0 & 0 & 0 & 0 \\ 0 & 0 & 0 & \\ 0 & 0 & 0\end{array}\right)$

$\left(\begin{array}{rrrrrr}0 & 0 & 0 & x y z & 0 & 0 \\ 0 & 0 & 0 & 0 & -x y z & 0 \\ 0 & 0 & 0 & 0 & 0 & 0\end{array}\right)$

$\left(\begin{array}{ccc}\mathrm{yzx} & 0 & 0 \\ 0 & \mathrm{yzx} & 0 \\ 0 & 0 & \mathrm{xyz}\end{array}\right)$ $\left(\begin{array}{rrrr}0 & 0 & 0 & 0 \\ 0 & 0 & 0 & \\ z x x & -z x x & 0 & \end{array}\right)$

$\left(\begin{array}{rrrrrr}0 & 0 & 0 & 0 & x x z & 0 \\ 0 & 0 & 0 & -x x z & 0 & 0 \\ z x x & -z x x & 0 & 0 & 0 & 0\end{array}\right)$

$\left(\begin{array}{ccc}0 & y z y & 0 \\ y z y & 0 & 0 \\ 0 & 0 & 0\end{array}\right)$ $\left(\begin{array}{llll}0 & 0 & 0 & 0 \\ 0 & 0 & 0 & \\ 0 & 0 & 0 & \end{array}\right)$

$\left(\begin{array}{llllll}0 & 0 & 0 & 0 & 0 & 0 \\ 0 & 0 & 0 & 0 & 0 & 0 \\ 0 & 0 & 0 & 0 & 0 & 0\end{array}\right)$

$\left(\begin{array}{lll}0 & 0 & 0 \\ 0 & 0 & 0 \\ 0 & 0 & 0\end{array}\right)$ 
$\mathrm{n}=3 \quad \mathbf{D}_{6 \mathrm{~h}}\left(\mathbf{D}_{3 \mathrm{~d}}\right)=\mathbf{6}_{\mathrm{z}} / \mathbf{m}_{z} \mathbf{m}_{x} \mathbf{m}_{1}\left(\overline{\mathbf{3}}_{z} \mathbf{m}_{\mathrm{x}}\right)=\mathbf{6}_{\mathrm{z}}{ }^{\prime} / \mathbf{m}_{\mathrm{z}}{ }^{\prime} \mathbf{m}_{x} \mathbf{m}_{1}{ }^{\prime}$

$\mathrm{V}^{3} \quad\left(\begin{array}{lllllllll}0 & 0 & 0 & 0 & 0 & 0 & 0 & 0 & 0 \\ 0 & 0 & 0 & 0 & 0 & 0 & 0 & 0 & 0 \\ 0 & 0 & 0 & 0 & 0 & 0 & 0 & 0 & 0\end{array}\right)$

$\left[\mathrm{V}^{3}\right]\left(\begin{array}{llll}0 & 0 & 0 & 0 \\ 0 & 0 & 0 & \\ 0 & 0 & 0 & \end{array}\right)$

$V\left[V^{2}\right]\left(\begin{array}{llllll}0 & 0 & 0 & 0 & 0 & 0 \\ 0 & 0 & 0 & 0 & 0 & 0 \\ 0 & 0 & 0 & 0 & 0 & 0\end{array}\right)$

$\left(\begin{array}{llllll}0 & 0 & 0 & 0 & 0 & 0\end{array}\right)$

$\left\{V^{2}\right\} V\left(\begin{array}{lll}0 & 0 & 0 \\ 0 & 0 & 0 \\ 0 & 0 & 0\end{array}\right)$

$n>3 \quad D_{\infty h} \mathbf{1}^{\prime}=\infty / \mathbf{m m m}^{\prime}$

$\vee^{3} \quad\left(\begin{array}{lllllllll}0 & 0 & 0 & 0 & 0 & 0 & 0 & 0 & 0 \\ 0 & 0 & 0 & 0 & 0 & 0 & 0 & 0 & 0 \\ 0 & 0 & 0 & 0 & 0 & 0 & 0 & 0 & 0\end{array}\right)$

$\left[V^{3}\right]\left(\begin{array}{llll}0 & 0 & 0 & 0 \\ 0 & 0 & 0 & \\ 0 & 0 & 0 & \end{array}\right)$

$V\left[V^{2}\right]\left(\begin{array}{llllll}0 & 0 & 0 & 0 & 0 & 0 \\ 0 & 0 & 0 & 0 & 0 & 0 \\ 0 & 0 & 0 & 0 & 0 & 0\end{array}\right)$

$\left\{V^{2}\right\} V\left(\begin{array}{lll}0 & 0 & 0 \\ 0 & 0 & 0 \\ 0 & 0 & 0\end{array}\right)$ $\left(\begin{array}{rrrrrrrrr}0 & 0 & 0 & x y z & 0 & 0 & -y z x & 0 & 0 \\ 0 & 0 & 0 & 0 & y z x & 0 & 0 & -x y z & 0 \\ 0 & 0 & 0 & 0 & 0 & z x y & 0 & 0 & -z x y\end{array}\right)\left(\begin{array}{rrrrrrrrr}0 & 0 & 0 & 0 & 0 & 0 & 0 & 0 & 0 \\ 0 & 0 & 0 & 0 & 0 & 0 & 0 & 0 & 0 \\ 0 & 0 & 0 & 0 & 0 & 0 & 0 & 0 & 0\end{array}\right)$

$\left(\begin{array}{llll}0 & 0 & 0 & 0 \\ 0 & 0 & 0 & \\ 0 & 0 & 0 & \end{array}\right)$

$\left(\begin{array}{rrrrrr}0 & 0 & 0 & x y z & 0 & 0 \\ 0 & 0 & 0 & 0 & -x y z & 0 \\ 0 & 0 & 0 & 0 & 0 & 0\end{array}\right)$

$\left(\begin{array}{llll}0 & 0 & 0 & 0 \\ 0 & 0 & 0 & \\ 0 & 0 & 0 & \end{array}\right)$

$\left(\begin{array}{llllll}0 & 0 & 0 & 0 & 0 & 0 \\ 0 & 0 & 0 & 0 & 0 & 0 \\ 0 & 0 & 0 & 0 & 0 & 0\end{array}\right)$

$\left(\begin{array}{ccc}y z x & 0 & 0 \\ 0 & y z x & 0 \\ 0 & 0 & x y z\end{array}\right)$ ae

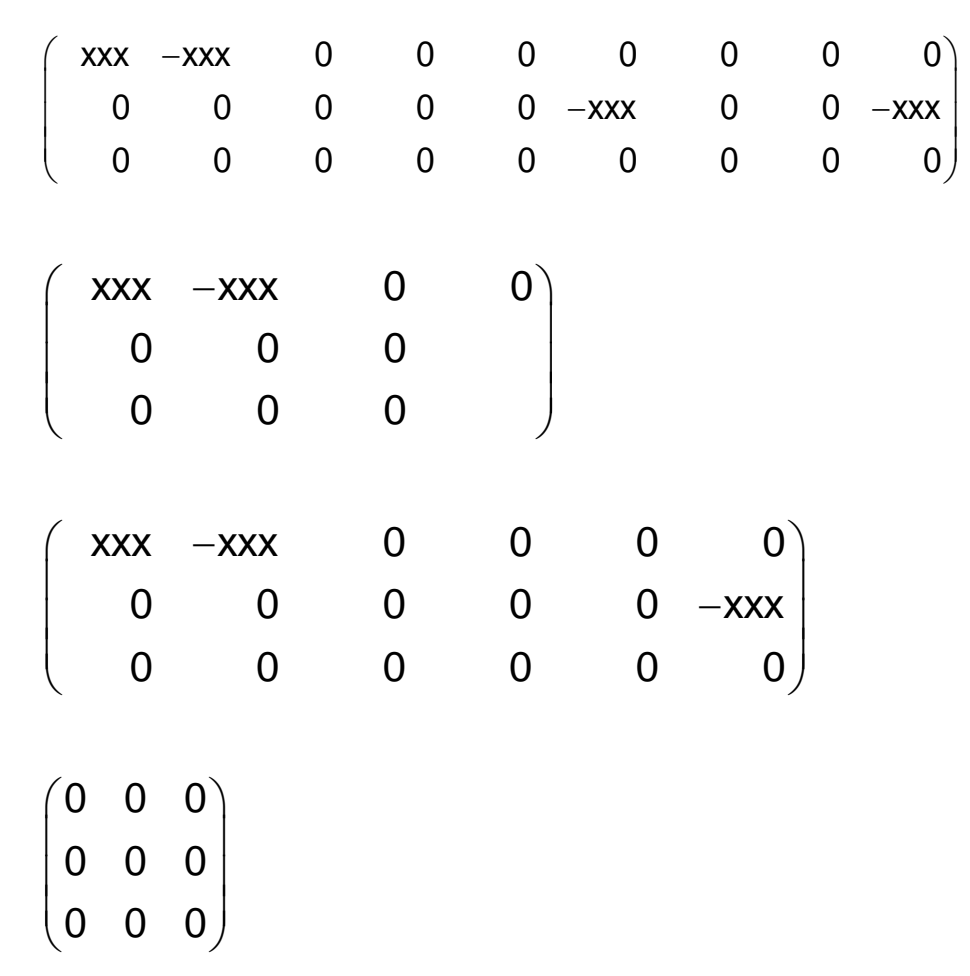

$\left(\begin{array}{rrrr}x x x & -x x x & 0 & 0 \\ 0 & 0 & 0 & \\ 0 & 0 & 0 & \end{array}\right)$

$\left(\begin{array}{rrrrrr}\mathrm{xxx} & -\mathrm{xxx} & 0 & 0 & 0 & 0 \\ 0 & 0 & 0 & 0 & 0 & -\mathrm{xxx} \\ 0 & 0 & 0 & 0 & 0 & 0\end{array}\right)$

$\left(\begin{array}{lll}0 & 0 & 0 \\ 0 & 0 & 0 \\ 0 & 0 & 0\end{array}\right)$ $\left(\begin{array}{lllllllll}0 & 0 & 0 & 0 & 0 & 0 & 0 & 0 & 0 \\ 0 & 0 & 0 & 0 & 0 & 0 & 0 & 0 & 0 \\ 0 & 0 & 0 & 0 & 0 & 0 & 0 & 0 & 0\end{array}\right)$

$\left(\begin{array}{llll}0 & 0 & 0 & 0 \\ 0 & 0 & 0 & \\ 0 & 0 & 0 & \end{array}\right)$

$\left(\begin{array}{llllll}0 & 0 & 0 & 0 & 0 & 0 \\ 0 & 0 & 0 & 0 & 0 & 0 \\ 0 & 0 & 0 & 0 & 0 & 0\end{array}\right)$

$\left(\begin{array}{lll}0 & 0 & 0 \\ 0 & 0 & 0 \\ 0 & 0 & 0\end{array}\right)$

$\left(\begin{array}{rrrrrrrrr}0 & 0 & 0 & x y z & 0 & 0 & -y z x & 0 & 0 \\ 0 & 0 & 0 & 0 & y z x & 0 & 0 & -x y z & 0 \\ 0 & 0 & 0 & 0 & 0 & z x y & 0 & 0 & -z x y\end{array}\right) \quad\left(\begin{array}{lllllllll}0 & 0 & 0 & 0 & 0 & 0 & 0 & 0 & 0 \\ 0 & 0 & 0 & 0 & 0 & 0 & 0 & 0 & 0 \\ 0 & 0 & 0 & 0 & 0 & 0 & 0 & 0 & 0\end{array}\right)$

$\left(\begin{array}{llll}0 & 0 & 0 & 0 \\ 0 & 0 & 0 & \\ 0 & 0 & 0 & \end{array}\right)$

$\left(\begin{array}{llllll}0 & 0 & 0 & 0 & 0 & 0 \\ 0 & 0 & 0 & 0 & 0 & 0 \\ 0 & 0 & 0 & 0 & 0 & 0\end{array}\right)$

$\left(\begin{array}{lll}0 & 0 & 0 \\ 0 & 0 & 0 \\ 0 & 0 & 0\end{array}\right)$

$\left(\begin{array}{ccc}y z x & 0 & 0 \\ 0 & y z x & 0 \\ 0 & 0 & x y z\end{array}\right)$ 
$\mathrm{n}=1 \quad \mathbf{D}_{2 h}\left(\mathbf{D}_{1 h}\right)=\mathbf{m}_{x} \mathbf{m}_{\mathbf{y}} \mathbf{m}_{\mathbf{z}}\left(\mathbf{2}_{\mathbf{x}} \mathbf{m}_{\mathbf{y}} \mathbf{m}_{\mathrm{z}}\right)=\mathbf{m}_{x}{ }^{\prime} \mathbf{m}_{\mathbf{y}} \mathbf{m}_{\mathrm{z}}$

$\mathrm{V}^{3} \quad\left(\begin{array}{lllllllll}0 & 0 & 0 & 0 & 0 & 0 & 0 & 0 & 0 \\ 0 & 0 & 0 & 0 & 0 & 0 & 0 & 0 & 0 \\ 0 & 0 & 0 & 0 & 0 & 0 & 0 & 0 & 0\end{array}\right)$

$\left(\begin{array}{rrrrrrrrr}0 & 0 & 0 & x y z & 0 & 0 & x z y & 0 & 0 \\ 0 & 0 & 0 & 0 & y z x & 0 & 0 & y x z & 0 \\ 0 & 0 & 0 & 0 & 0 & z x y & 0 & 0 & z y x\end{array}\right) \quad\left(\begin{array}{rrrrrrrrr}x x x & x y y & x z z & 0 & 0 & 0 & 0 & 0 & 0 \\ 0 & 0 & 0 & 0 & 0 & y x y & 0 & 0 & y y x \\ 0 & 0 & 0 & 0 & z z x & 0 & 0 & z x z & 0\end{array}\right) \quad\left(\begin{array}{rrrrrrrrr}0 & 0 & 0 & 0 & 0 & 0 & 0 & 0 & 0 \\ 0 & 0 & 0 & 0 & 0 & 0 & 0 & 0 & 0 \\ 0 & 0 & 0 & 0 & 0 & 0 & 0 & 0 & 0\end{array}\right)$

$\left[\mathrm{V}^{3}\right]\left(\begin{array}{llll}0 & 0 & 0 & 0 \\ 0 & 0 & 0 & \\ 0 & 0 & 0 & \end{array}\right)$

$\left(\begin{array}{llll}0 & 0 & 0 & x y z \\ 0 & 0 & 0 & \\ 0 & 0 & 0 & \end{array}\right)$

$\left(\begin{array}{rrrr}x x x & x y y & x z z & 0 \\ 0 & 0 & 0 & \\ 0 & 0 & 0 & \end{array}\right)$

$\left(\begin{array}{llll}0 & 0 & 0 & 0 \\ 0 & 0 & 0 & \\ 0 & 0 & 0 & \end{array}\right)$

$\left(\begin{array}{llllll}0 & 0 & 0 & 0 & 0 & 0 \\ 0 & 0 & 0 & 0 & 0 & 0\end{array}\right)$

$V\left[V^{2}\right]\left(\begin{array}{llllll}0 & 0 & 0 & 0 & 0 & 0 \\ 0 & 0 & 0 & 0 & 0 & 0 \\ 0 & 0 & 0 & 0 & 0 & 0\end{array}\right)$

$\left(\begin{array}{rrrrrr}0 & 0 & 0 & x y z & 0 & 0 \\ 0 & 0 & 0 & 0 & y x z & 0 \\ 0 & 0 & 0 & 0 & 0 & z x y\end{array}\right)$

$\left(\begin{array}{rrrrrr}x x x & x y y & x z z & 0 & 0 & 0 \\ 0 & 0 & 0 & 0 & 0 & y x y \\ 0 & 0 & 0 & 0 & z x z & 0\end{array}\right)$

$\left(\begin{array}{llllll}0 & 0 & 0 & 0 & 0 & 0 \\ 0 & 0 & 0 & 0 & 0 & 0 \\ 0 & 0 & 0 & 0 & 0 & 0\end{array}\right)$

$\left\{V^{2}\right\} V\left(\begin{array}{lll}0 & 0 & 0 \\ 0 & 0 & 0 \\ 0 & 0 & 0\end{array}\right)$

$\left(\begin{array}{ccc}y z x & 0 & 0 \\ 0 & z x y & 0 \\ 0 & 0 & x y z\end{array}\right)$

$\left(\begin{array}{ccc}0 & 0 & 0 \\ 0 & 0 & z x z \\ 0 & x y y & 0\end{array}\right)$

$\left(\begin{array}{lll}0 & 0 & 0 \\ 0 & 0 & 0 \\ 0 & 0 & 0\end{array}\right)$

$\mathrm{n}=2 \quad \mathbf{D}_{4 h}\left(\mathbf{D}_{2 \mathrm{~h}}\right)=\mathbf{4}_{\mathrm{z}} / \mathbf{m}_{\mathrm{z}} \mathbf{m}_{\mathrm{x}} \mathbf{m}_{\mathrm{xy}}\left(\mathbf{m}_{\mathrm{x}} \mathbf{m}_{\mathrm{y}} \mathbf{m}_{\mathrm{z}}\right)=\mathbf{4}_{z^{\prime}} / \mathbf{m}_{\mathrm{z}} \mathbf{m}_{\mathrm{x}} \mathbf{m}_{\mathrm{xy}}{ }^{\prime}$

$\mathrm{V}^{3} \quad\left(\begin{array}{lllllllll}0 & 0 & 0 & 0 & 0 & 0 & 0 & 0 & 0 \\ 0 & 0 & 0 & 0 & 0 & 0 & 0 & 0 & 0 \\ 0 & 0 & 0 & 0 & 0 & 0 & 0 & 0 & 0\end{array}\right)$

$\left[\mathrm{V}^{3}\right]\left(\begin{array}{llll}0 & 0 & 0 & 0 \\ 0 & 0 & 0 & \\ 0 & 0 & 0 & \end{array}\right)$

$\left(\begin{array}{rrrrrrrrr}0 & 0 & 0 & x y z & 0 & 0 & -y z x & 0 & 0 \\ 0 & 0 & 0 & 0 & y z x & 0 & 0 & -x y z & 0 \\ 0 & 0 & 0 & 0 & 0 & z x y & 0 & 0 & -z x y\end{array}\right) \quad\left(\begin{array}{rrrrrrrrr}0 & 0 & 0 & 0 & 0 & 0 & 0 & 0 & 0 \\ 0 & 0 & 0 & 0 & 0 & 0 & 0 & 0 & 0 \\ 0 & 0 & 0 & 0 & 0 & 0 & 0 & 0 & 0\end{array}\right)$

ae

$V\left[V^{2}\right]\left(\begin{array}{llllll}0 & 0 & 0 & 0 & 0 & 0 \\ 0 & 0 & 0 & 0 & 0 & 0 \\ 0 & 0 & 0 & 0 & 0 & 0\end{array}\right)$

$\left\{V^{2}\right\} V\left(\begin{array}{ccc}0 & 0 & 0 \\ 0 & 0 & 0 \\ 0 & 0 & 0\end{array}\right)$

$\left(\begin{array}{llll}0 & 0 & 0 & 0 \\ 0 & 0 & 0 & \\ 0 & 0 & 0 & \end{array}\right)$

$\left(\begin{array}{llll}0 & 0 & 0 & 0 \\ 0 & 0 & 0 & \\ 0 & 0 & 0 & \end{array}\right)$

$\left(\begin{array}{rrrrrr}0 & 0 & 0 & x y z & 0 & 0 \\ 0 & 0 & 0 & 0 & -x y z & 0 \\ 0 & 0 & 0 & 0 & 0 & 0\end{array}\right)$

$\left(\begin{array}{llllll}0 & 0 & 0 & 0 & 0 & 0 \\ 0 & 0 & 0 & 0 & 0 & 0 \\ 0 & 0 & 0 & 0 & 0 & 0\end{array}\right)$

$\left(\begin{array}{ccc}y z x & 0 & 0 \\ 0 & y z x & 0 \\ 0 & 0 & x y z\end{array}\right)$

$\left(\begin{array}{lll}0 & 0 & 0 \\ 0 & 0 & 0 \\ 0 & 0 & 0\end{array}\right)$

$\left(\begin{array}{rrrrrrrrr}0 & 0 & 0 & 0 & x z x & 0 & 0 & -y y z & 0 \\ 0 & 0 & 0 & y y z & 0 & 0 & -x z x & 0 & 0 \\ z x x & -z x x & 0 & 0 & 0 & 0 & 0 & 0 & 0\end{array}\right)$

$\left(\begin{array}{rrrr}0 & 0 & 0 & 0 \\ 0 & 0 & 0 & \\ z x x & -z x x & 0 & \end{array}\right)$

$\left(\begin{array}{rrrrrr}0 & 0 & 0 & 0 & x x z & 0 \\ 0 & 0 & 0 & -x x z & 0 & 0 \\ z x x & -z x x & 0 & 0 & 0 & 0\end{array}\right)$

$\left(\begin{array}{ccc}0 & y z y & 0 \\ y z y & 0 & 0 \\ 0 & 0 & 0\end{array}\right)$ 
$\mathrm{n}=3 \quad \mathbf{D}_{6 \mathrm{~h}}\left(\mathbf{D}_{3 \mathrm{~h}}\right)=\mathbf{6}_{\mathrm{z}} / \mathbf{m}_{\mathrm{z}} \mathbf{m}_{\mathrm{x}} \mathbf{m}_{1}\left(\overline{\mathbf{6}}_{\mathrm{z}} \mathbf{m}_{\mathrm{x}} \mathbf{2}_{1}\right)=\mathbf{6}_{\mathrm{z}}{ }^{\prime} / \mathbf{m}_{\mathrm{z}} \mathbf{m}_{\mathrm{x}} \mathbf{m}_{1}{ }^{\prime}$
$\mathrm{V}^{3}\left(\begin{array}{ccccccccc}0 & 0 & 0 & 0 & 0 & 0 & 0 & 0 & 0 \\ 0 & 0 & 0 & 0 & 0 & 0 & 0 & 0 & 0 \\ 0 & 0 & 0 & 0 & 0 & 0 & 0 & 0 & 0\end{array}\right)$
e

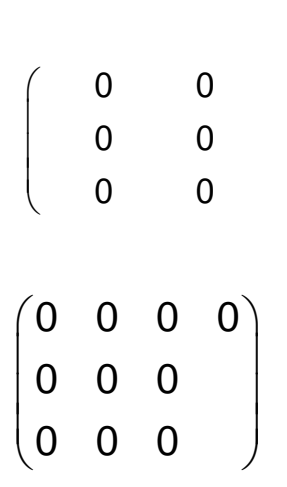
a
$\left[\mathrm{V}^{3}\right]\left(\begin{array}{llll}0 & 0 & 0 & 0 \\ 0 & 0 & 0 & \\ 0 & 0 & 0 & \end{array}\right)$
$V\left[V^{2}\right]\left(\begin{array}{llllll}0 & 0 & 0 & 0 & 0 & 0 \\ 0 & 0 & 0 & 0 & 0 & 0 \\ 0 & 0 & 0 & 0 & 0 & 0\end{array}\right)$
$\left\{\mathrm{V}^{2}\right\} \mathrm{V}\left(\begin{array}{lll}0 & 0 & 0 \\ 0 & 0 & 0 \\ 0 & 0 & 0\end{array}\right)$
$\left(\begin{array}{rrrrrr}0 & 0 & 0 & x y z & 0 & 0 \\ 0 & 0 & 0 & 0 & -x y z & 0 \\ 0 & 0 & 0 & 0 & 0 & 0\end{array}\right)$
$\left(\begin{array}{rrrrrr}\mathrm{xxx} & -\mathrm{xxx} & 0 & 0 & 0 & 0 \\ 0 & 0 & 0 & 0 & 0 & -\mathrm{xxx} \\ 0 & 0 & 0 & 0 & 0 & 0\end{array}\right)$
$\left(\begin{array}{llllll}0 & 0 & 0 & 0 & 0 & 0 \\ 0 & 0 & 0 & 0 & 0 & 0 \\ 0 & 0 & 0 & 0 & 0 & 0\end{array}\right)$
$\left(\begin{array}{ccc}y z x & 0 & 0 \\ 0 & y z x & 0 \\ 0 & 0 & x y z\end{array}\right)$
$\left(\begin{array}{lll}0 & 0 & 0 \\ 0 & 0 & 0 \\ 0 & 0 & 0\end{array}\right)$
$\left(\begin{array}{lll}0 & 0 & 0 \\ 0 & 0 & 0 \\ 0 & 0 & 0\end{array}\right)$
$\left(\begin{array}{llll}0 & 0 & 0 & 0 \\ 0 & 0 & 0 & \\ 0 & 0 & 0 & \end{array}\right)$

ae

$n>3 \quad D_{\propto h} \mathbf{1}^{\prime}=\infty / \mathbf{m m m}^{\prime}$

$\mathrm{V}^{3} \quad\left(\begin{array}{lllllllll}0 & 0 & 0 & 0 & 0 & 0 & 0 & 0 & 0 \\ 0 & 0 & 0 & 0 & 0 & 0 & 0 & 0 & 0 \\ 0 & 0 & 0 & 0 & 0 & 0 & 0 & 0 & 0\end{array}\right)$

$\left(\begin{array}{rrrrrrrrr}0 & 0 & 0 & x y z & 0 & 0 & -y z x & 0 & 0 \\ 0 & 0 & 0 & 0 & y z x & 0 & 0 & -x y z & 0 \\ 0 & 0 & 0 & 0 & 0 & z x y & 0 & 0 & -z x y\end{array}\right) \quad\left(\begin{array}{rrrrrrrrr}0 & 0 & 0 & 0 & 0 & 0 & 0 & 0 & 0 \\ 0 & 0 & 0 & 0 & 0 & 0 & 0 & 0 & 0 \\ 0 & 0 & 0 & 0 & 0 & 0 & 0 & 0 & 0\end{array}\right)$

$\left(\begin{array}{llll}0 & 0 & 0 & 0 \\ 0 & 0 & 0 & \\ 0 & 0 & 0 & \end{array}\right)$

$\left(\begin{array}{rrrr}0 & 0 & 0 & 0 \\ 0 & 0 & 0 & \\ z x x & z x x & z z z & \end{array}\right)$

$\left(\begin{array}{llllll}0 & 0 & 0 & 0 & 0 & 0 \\ 0 & 0 & 0 & 0 & 0 & 0 \\ 0 & 0 & 0 & 0 & 0 & 0\end{array}\right)$

$\left(\begin{array}{rrrrrr}0 & 0 & 0 & x y z & 0 & 0 \\ 0 & 0 & 0 & 0 & -x y z & 0 \\ 0 & 0 & 0 & 0 & 0 & 0\end{array}\right)$

$\left(\begin{array}{ccc}y z x & 0 & 0 \\ 0 & y z x & 0 \\ 0 & 0 & x y z\end{array}\right)$ $\left(\begin{array}{lll}0 & 0 & 0 \\ 0 & 0 & 0 \\ 0 & 0 & 0\end{array}\right)$ $\left(\begin{array}{lllllllll}0 & 0 & 0 & 0 & 0 & 0 & 0 & 0 & 0 \\ 0 & 0 & 0 & 0 & 0 & 0 & 0 & 0 & 0 \\ 0 & 0 & 0 & 0 & 0 & 0 & 0 & 0 & 0\end{array}\right)$

$\left(\begin{array}{llll}0 & 0 & 0 & 0 \\ 0 & 0 & 0 & \\ 0 & 0 & 0 & \end{array}\right)$

$\left(\begin{array}{llllll}0 & 0 & 0 & 0 & 0 & 0 \\ 0 & 0 & 0 & 0 & 0 & 0\end{array}\right)$

$\left.0 \begin{array}{llllll}0 & 0 & 0 & 0 & 0 & 0 \\ 0 & 0 & 0 & 0 & 0 & 0\end{array}\right)$

$\left(\begin{array}{lll}0 & 0 & 0 \\ 0 & 0 & 0 \\ 0 & 0 & 0\end{array}\right)$ 


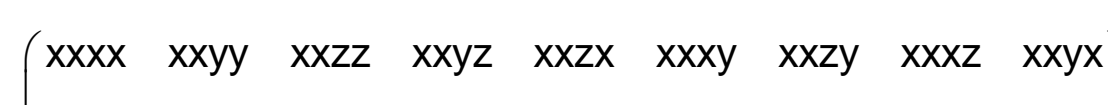
yyxx yyyy yyzz yyyz yyzx yyxy

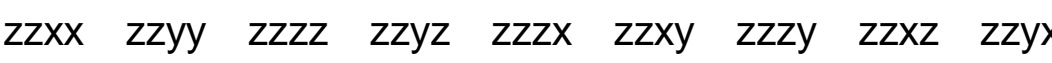
$\begin{array}{lllllllll}y z x x & y z y y & y z z z & y z y z & y z z x & y z x y & y z z y & y z x z & y z y x\end{array}$ $\begin{array}{llllllllll}z x x x & z x y y & z x z z & z x y z & z x z x & z x x y & z x z y & z x x z & z x y x\end{array}$

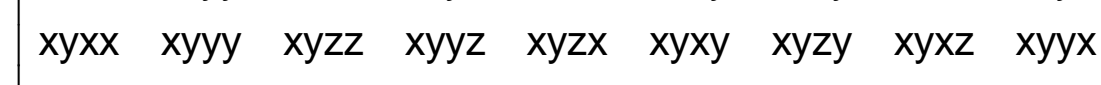
zyxx zyyy zyzz zyyz zyzx zyxy zyzy zyxz zyyx $\begin{array}{lllllllll}x z x x & x z y y & x z z z & x z y z & x z z x & x z x y & x z z y & x z x z & x z y x\end{array}$ $\begin{array}{lllllllll}y x x x & y x y y & y x z z & y x y z & y x z x & y x x y & y x z y & y x x z & y x y x\end{array}$

$\left.V^{4}\right]\left(\begin{array}{lllll}x x x x & x y y y & x z z z & y y z z & x x y z \\ y x x & y y y & y z z & z z x & y y x z\end{array}\right)$ $\left(\begin{array}{lllll}y x x x & y y y y & y z z z & z z x x & y y x z \\ z x x x & z y y y & z z z z & x x y y & z z x y\end{array}\right)$ $\begin{array}{lllllllll}x x x x & x x y y & x x z z & x x y z & x x z x & x x x y & x x z y & x x x z & x x y x \\ y x x & y y y & y z z & y y z & y y z x & y x y & y y z & y x x z & y y x\end{array}$ yyxx yyyy yyzz

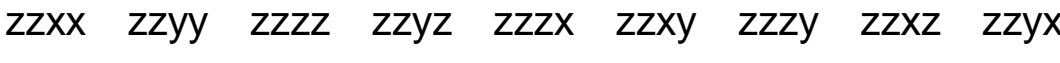
yzxx yzyy $\begin{array}{lllllllll}z x x x & z x y y & z x z z & z x y z & z x z x & z x x y & z x z y & z x x z & z x y x \\ x y x x & x y y & x y z z & x y z & x y z x & x y y & x y z y & x y z & x y x\end{array}$ $\begin{array}{lllllllllll} & x y x x & x y y y & x y z z & x y y z & x y z x & \text { xyxy } & \text { xyzy } & \text { xyxz } & \text { xyyx }\end{array}$ zyxx zyyy zyzz zyyz zyzx zyxy zyzy $z y x z$ zyyx $\begin{array}{lllllllll}x z x x & x z y y & x z z z & x z y z & x z z x & x z x y & x z z y & x z x z & x z y x\end{array}$ $\begin{array}{lllllllll}y x x x & y x y y & y x z z & y x y z & y x z x & y x x y & y x z y & y x x z & y x y x\end{array}$

$\left(\begin{array}{lllll}x x x x & x y y y & x z z z & y y z z & x x y z \\ y x x x & y y y y & y z z z & z z x x & y y x z \\ z x x x & z y y y & z z z & x x y y & z z x y\end{array}\right)$ $\left(\begin{array}{lllll}\text { yxxx } & \text { yyyy } & y z z z & z z x x & y y x z \\ z x x x & z y y y & z z z z & x x y y & z z x y\end{array}\right)$ $\left(\begin{array}{lllllllll}x x x x & x x y y & x x z z & x x y z & x x z x & x x x y & x x z y & x x x z & x x y x \\ y y x & y y y & y y z & y y y z & y z x & y y x & y y z y & y x z & y y y x\end{array}\right)$ yyxx yyyy yyzz

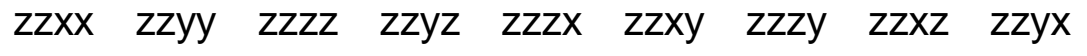

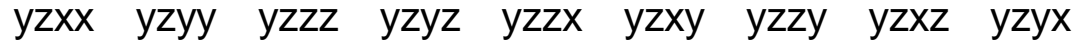
$\begin{array}{lllllllll}z x x x & z x y y & z x z z & z x y z & z x z x & z x x y & z x z y & z x x z & z x y x\end{array}$ $\begin{array}{llllllllll} & x y x x & x y y y & x y z z & x y y z & x y z x & x y x y & x y z y & x y x z & x y y x\end{array}$ zyxx zyyy zyzz zyyz zyzx zyxy $\begin{array}{lllllllll}x z x x & x z y y & x z z z & x z y z & x z z x & x z x y & x z z y & x z x z & x z y x\end{array}$

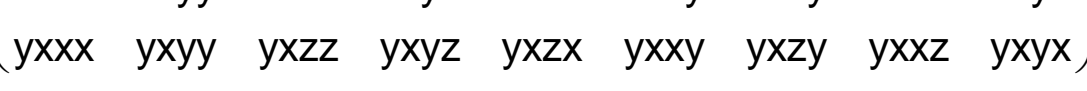

$\left(\begin{array}{lllll}x x x x & x y y y & x z z z & y y z z & x x y z \\ y x x x & y y y y & y z z z & z z x x & y y x z \\ z x x x & z y y y & z z z & x x y y & z z x y\end{array}\right)$ $\left(\begin{array}{lllll}y x x x & y y y y & y z z z & z z x x & y y x z \\ z x x x & z y y y & z z z z & x x y y & z z x y\end{array}\right)$ $\left(\begin{array}{lllllllll}x x x x & x x y y & x x z z & x x y z & x x z x & x x x y & x x z y & x x x z & x x y x\end{array}\right.$ yyxx yyyy

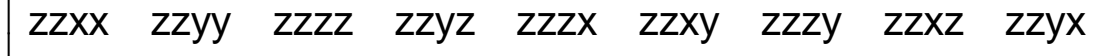
$\begin{array}{llllllllllllll} & y z x x & y z y y & y z z z & y z y z & y z z x & y z x y & y z z y & y z x z & y z y x\end{array}$

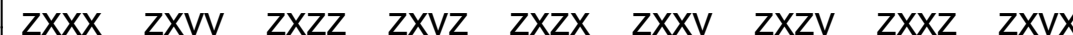

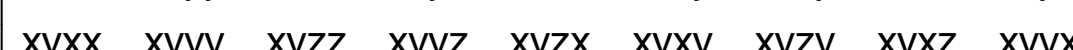

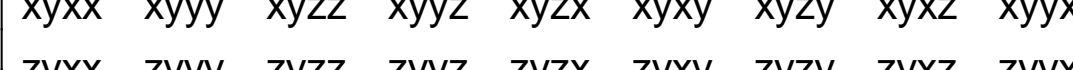

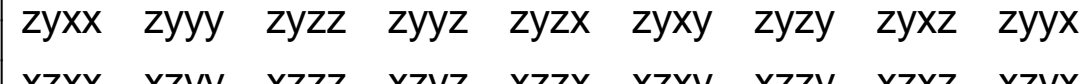
$\left(\begin{array}{lllllllll}x z x x & x z y y & x z z z & x z y z & x z z x & x z x y & x z z y & x z x z & x z y x \\ y x x x & y x y y & y x z z & y x y z & y x z x & y x x y & y x z y & y x x z & y x y x\end{array}\right)$

$\begin{array}{lllll}x x x x & x y y y & x z z z & y y z z & x x y z\end{array}$

$\begin{array}{lllll}\text { yxxx } & \text { yyyy } & y z z z & z z x x & y y x z \\ z x x x & z y y y & z z z z & x x y y & z z x y\end{array}$

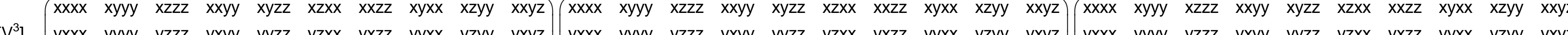

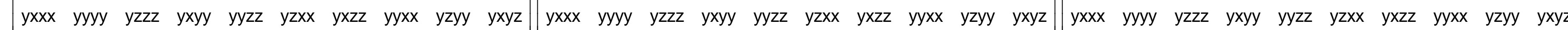

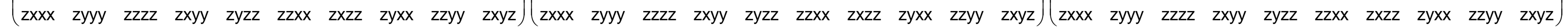

$\left(\begin{array}{llllllllll}x x x x & x y y y & x z z z & x x y y & x y z z & x z x x & x x z z & x y x x & x z y y & x x y z\end{array}\right)$ $\begin{array}{llllllllll}x x x x & y y y y & y z z z & y x y y & y y z z & y z x x & y x z z & y y x x & y z y y & y x y z\end{array}$ $\left(\begin{array}{llllll}x x x x & x x y y & x x z z & x x y z & x x x z & x x x y\end{array}\right)$ yyyy $\begin{array}{llll} & y y z & z z x z & z z x y\end{array}$ $\begin{array}{lll}y z y z & y z x z & y z x y \\ & x z x z & x z x y\end{array}$ $\begin{array}{ll}x z x z & x z x y \\ & x y x y\end{array}$

$$
\left(\begin{array}{cccccc}
x x x x & x x y y & x x z z & x x y z & x x x z & x x x y \\
& y y y y & y y z z & y y y z & y y x z & y y x y \\
& & z z z z & z z y z & z z x z & z z x y \\
& & & y z y z & y z x z & y z x y \\
& & & & x z x z & x z x y \\
& & & & & x y x y
\end{array}\right)
$$

$\begin{array}{llllll}x x x x & x x y y & x x z z & x x y z & x x x z & x x x y\end{array}$ yyxx yyyy

$\begin{array}{llllll}y y x x & y y y & y z z & y y z & y y z & y y x y \\ y z x & \text { zzyy } & z z z z & z z y z & z z x z & z z x y\end{array}$

yzxx yzyy yzzz $\quad y z y z \quad y z x z \quad y z x y$

$\begin{array}{llllll}x z x x & x z y y & x z z z & x z y z & x z x z & x z x y\end{array}$

$\begin{array}{llllll}x y x x & x y y y & x y z z & x y y z & x y x z & x y x y\end{array}$

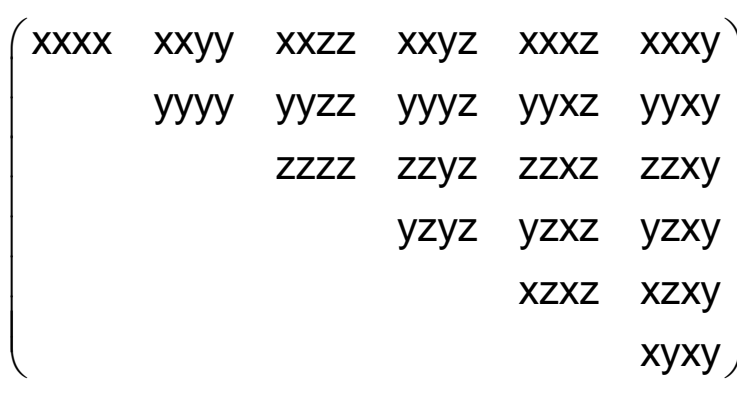

$\begin{array}{llllll}x x x x & x x y y & x x z z & x x y z & x x x z & x x x y\end{array}$ $\begin{array}{llllll}x y y x x & y y y y & y y z z & y y y z & y y x z & y y x y\end{array}$ zzxx zzyy zzzz $\quad$ zzyz $\quad$ zzxz $\quad$ zzxy $\begin{array}{llllll}z z x x & y z y y & y z z z & y z y z & y z x z & y z x y\end{array}$ $\begin{array}{llllll}x z x x & x z y y & x z z z & x z y z & x z x z & x z x y\end{array}$ $\begin{array}{llllll}x y x x & x y y y & x y z z & x y y z & x y x z & x y x y\end{array}$

$\begin{array}{llllll}x x x x & x x y y & x x z z & x x y z & x x x z & x x x y \\ y x x & y y y & y y z z & y y z & y y x z & y y x\end{array}$ $\begin{array}{llllll}\text { yyxx } & \text { yyyy } & \text { yyzz } & \text { yyyz } & \text { yyxz } & \text { yyxy }\end{array}$

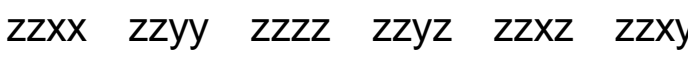
yzxx $\quad$ yzyy $\quad y z z z$ yzyz $\quad y z x z \quad y z x y$ $\begin{array}{llllll}x z x x & x z y y & x z z z & x z y z & x z x z & x z x y\end{array}$ $\begin{array}{llllll}x z x x & x z y y & x z z z & x z y z & x z x z & x z x y \\ x y x x & x y y y & x y z z & x y y z & x y x z & x y x y\end{array}$
$\left(\begin{array}{llllll}x x x x & x x y y & x x z z & x x y z & x x x z & x x x y\end{array}\right.$ yyyy $\quad$ yyzz $\quad$ yyyz $\quad y y x z \quad$ yyxy $\begin{array}{llll}z z z z & z z y z & z z x z & z z x y\end{array}$ $y z y z \quad y z x z \quad y z x y$ $\begin{array}{rr}x z x z & x z x y \\ x y x y\end{array}$

$\left(\begin{array}{llllll}x x x x & x x y y & x x z z & x x y z & x x x z & x x x y\end{array}\right.$ $\begin{array}{llllll}x y y x x & y y y y & y y z z & y y y z & y y x z & y y x y\end{array}$

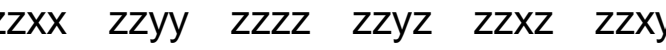
$\begin{array}{llllll}y z x x & y z y y & y z z z & y z y z & y z x z & y z x y\end{array}$ $\begin{array}{llllll}x z x x & x z y y & x z z z & x z y z & x z x z & x z x y\end{array}$ $\begin{array}{llllll}x y x x & x y y y & x y z z & x y y z & x y x z & x y x y\end{array}$ 
$\mathrm{n}=1 \quad \mathbf{C}_{1}=\mathbf{1}$

$\left(\begin{array}{lllllllll}x x x x & x x y y & x x z z & x x y z & x x z x & x x x y & x x z y & x x x z & x x y x\end{array}\right)$ yyyy $\quad$ yyzz zzzz zzyz zzzx zzxy zzzy $\quad$ zzxz $z z y x$ $\begin{array}{llllll}y z y z & y z z x & y z x y & y z z y & y z x z & y z y x \\ & z x z x & z x x y & z x z y & z x x z & z x y x\end{array}$ $\begin{array}{llllll}z x z x & z x x y & z x z y & z x x z & z x y x\end{array}$ $\begin{array}{llll}x y x y & x y z y & x y x z & x y y x\end{array}$ $\begin{array}{lll}\text { zyzy } & z y x z & z y y x \\ & x z x z & x z y x\end{array}$ $y x y x$

$\left(\begin{array}{lllllllll}x x x x & x x y y & x x z z & x x y z & x x z x & x x x y & x x z y & x x x z & x x y x \\ y x x & y y y & y y z & y y y z & y y z x & y x y & y z y & y y z & y y y\end{array}\right.$ yyxx yyyy yyzz yyyz yyzx $\left[\begin{array}{l|lllllllll}{\left[^{2}\right] V^{2}} & z z x x & z z y y & z z z z & z z y z & z z z x & z z x y & z z z y & z z x z & z z y x \\ & y z x x & y z y y & y z z z & y z y z & y z z x & y z x y & y z z y & y z x z & y z y x \\ & z x x & z x y y & z x z & z x y & z x z x & z x y & z z y & z x z & z x y x\end{array}\right.$ $\begin{array}{llllllllll}y z x x & y z y y & y z z z & y z y z & y z z x & y z x y & y z z y & y z x z & y z y x \\ z x x x & z x y y & z x z z & z x y z & z x z x & z x x y & z x z y & z x x z & z x y x \\ x y x & x y y y & x y z & x y y & x y z x & x y x & x y z y & x y z & x y y\end{array}$ $\begin{array}{llllllllll} & x y x x & x y y y & x y z z & x y y z & x y z x & x y x y & x y z y & x y x z & x y y x\end{array}$

$\mathbf{c}_{n}$

a

$\left(\begin{array}{lllllllll}x x x x & x x y y & x x z z & x x y z & x x z x & x x x y & x x z y & x x x z & x x y x\end{array}\right)$ yyyy yyzz yyyz yyzx zzzz zzyz $\quad$ zzzx $\quad$ zzxy zzzy $z z x z \quad$ zzyx $\begin{array}{llllll}y z y z & y z z x & y z x y & y z z y & y z x z & y z y x \\ & z x z x & z x x y & z x z y & z x y z & z x y x\end{array}$ $\begin{array}{llllll}z x z x & z x x y & z x z y & z x x z & z x y x\end{array}$ $\begin{array}{llll}2 x y x y & x y z y & x y x z & x y y x\end{array}$ $\begin{array}{lll}\text { zyzy } & z y x z & z y y x \\ & x z x z & x z y x\end{array}$ $\begin{array}{ll}x z X z & x z y x \\ y x y x\end{array}$

$\left(\begin{array}{lllllllll}x x x x & x x y y & x x z z & x x y z & x x z x & x x x y & x x z y & x x x z & x x y x\end{array}\right)$

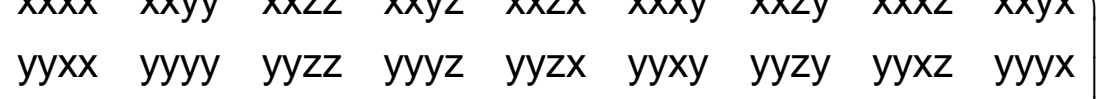
$\begin{array}{lllllllll}\text { zzxx } & \text { zzyy } & \text { zzzz } & \text { zzyz } & \text { zzzx } & \text { zzxy } & \text { zzzy } & \text { zzxz } & \text { zzyx }\end{array}$ $\begin{array}{lllllllll}z z x x & z z y y & \text { zzzz } & \text { zzyz } & \text { zzzx } & \text { zzxy } & \text { zzzy } & \text { zZxz } & \text { zzyx } \\ \text { yzxx } & \text { yzyy } & \text { yzzz } & \text { yzyz } & \text { yzzx } & \text { yzxy } & \text { yzzy } & \text { yzxz } & \text { yzyx } \\ z x x x & z x y y & z x z & z x y z & z x z x & z x x y & z x z y & z x z & z x y x\end{array}$ $\begin{array}{lllllllll}y z x x & y z y y & y z z z & y z y z & y z z x & y z x y & y z z y & y z x z & y z y x \\ z x x x & z x y y & z x z z & z x y z & z x z x & z x x y & z x z y & z x x z & z x y x \\ x y x & x y y & x y z z & x y z & x y z & x y x y & x y z y & x y z & x y x\end{array}$ $\begin{array}{lllllllll}z x x x & z x y y & z x z z & z x y z & z x z x & z x x y & z x z y & z x x z & z x y x \\ x y x x & x y y y & x y z z & x y y z & x y z x & \text { xyxy } & x y z y & x y x z & x y y x\end{array}$ $\left(\begin{array}{lllllllll}x x x x & x x y y & x x z z & x x y z & x x z x & x x x y & x x z y & x x x z & x x y x\end{array}\right)$ yyyy $\begin{array}{lllllll}\text { zzzz } & \text { zzyz } & \text { zzzx } & \text { zzxy } & \text { zzzy } & \text { zzxz } & \text { zzyx }\end{array}$ $\begin{array}{llllll}y z y z & y z z x & y z x y & y z z y & y z x z & y z y x\end{array}$ $\begin{array}{lllll}z x z x & z x x y & z x z y & z x x z & z x y x\end{array}$ $\begin{array}{llll}x y x y & x y z y & x y x z & x y y x\end{array}$ $\begin{array}{llll}\text { zyzy } & z y x z & z y y x\end{array}$ $\begin{array}{ll}z y x z & z y y x \\ x z x z & x z y x\end{array}$ $y x y x$

$\left(\begin{array}{lllllllll}x x x x & x x y y & x x z z & x x y z & x x z x & x x x y & x x z y & x x x z & x x y x\end{array}\right)$

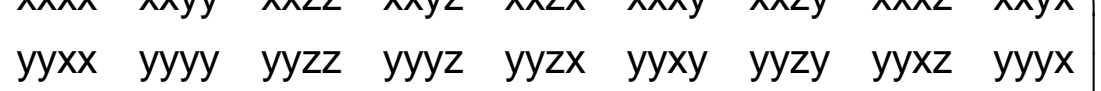
zzxx zzyy zzzz zzyz $\quad$ zzzx $\quad$ zzxy zzzy $\quad$ zzxz $\quad$ zzyx yzxx yzyy yzzz yzyz $\begin{array}{lllllllll}z x x x & z x y y & z x z z & z x y z & z x z x & z x x y & z x z y & z x x z & z x y x\end{array}$

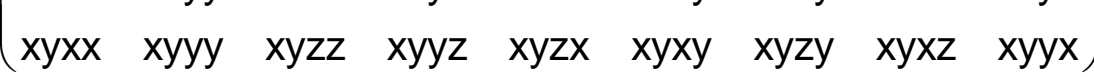

ae

$\left(\begin{array}{lllllllll}x x x x & x x y y & x x z z & x x y z & x x z x & x x x y & x x z y & x x x z & x x y x\end{array}\right.$ yyyy $\begin{array}{llllllll}z z z z & z z y z & z z z x & z z x y & z z z y & z z x z & z z y x\end{array}$

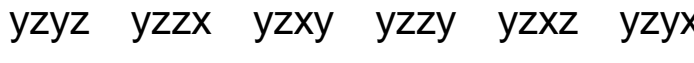
$\begin{array}{lllll}z x z x & z x x y & z x z y & z x x z & z x y x\end{array}$ $\begin{array}{llll}x y x y & x y z y & x y x z & x y y x\end{array}$ $\begin{array}{ccc}\text { zyzy } & z y x z & z y y x \\ & x z x z & x z y x \\ & & y x y x\end{array}$

$\begin{array}{lllllllll}x x x x & x x y y & x x z z & x x y z & x x z x & x x x y & x x z y & x x x z & x x y x\end{array}$ yyxx yyyy yyzz yyyz yyzx yyxy yyzy $y y x z$ yyyx zzxx zzyy zzzz zzyz $z z z x$ zzxy zzzy $\quad$ zzxz $\quad$ zzyx yzxx yzyy $\begin{array}{lllllllll}z x x x & z x y y & z x z z & z x y z & z x z x & z x x y & z x z y & z x x z & z x y x\end{array}$ $\begin{array}{lllllllll}\text { xyxx } & x y y y & x y z z & x y y z & x y z x & x y x y & x y z y & x y x z & x y y x\end{array}$ 
$\left(\begin{array}{lllllllll}x x x x & x x y y & x x z z & 0 & 0 & x x x y & 0 & 0 & x x y x \\ y y x & y y y & y z z & 0 & 0 & y y x y & 0 & 0 & x y x\end{array}\right)$ $\begin{array}{llllllllll}\text { yyxx } & \text { yyyy } & \text { yyzz } & 0 & 0 & \text { yyxy } & 0 & 0 & \text { yyyx } \\ \text { zzxx } & z z y & z z z z & 0 & 0 & z z x y & 0 & 0 & z z y x\end{array}$ $\begin{array}{lllllllll}\text { zzxx } & \text { zzyy } & z z z z & 0 & 0 & \text { zzxy } & 0 & 0 & \text { zzyx } \\ 0 & 0 & 0 & y z y z & y z z x & 0 & y z z y & y z x z & 0\end{array}$ $\begin{array}{lllllllll}0 & 0 & 0 & y z y z & y z z x & 0 & y z z y & y z x z & 0 \\ 0 & 0 & 0 & z x y z & z x z x & 0 & z x z y & z x y z & 0\end{array}$ $\begin{array}{ccccccccc}0 & 0 & 0 & z x y z & z x z x & 0 & z x z y & z x x z & 0 \\ x y x x & x y y y & x y z z & 0 & 0 & x y x y & 0 & 0 & x y y x \\ 0 & 0 & 0 & z y y z & z y z x & 0 & z y z y & z y x z & 0\end{array}$ $\begin{array}{ccccccccc}0 & 0 & 0 & \text { zyyz } & \text { zyzx } & 0 & \text { zyzy } & \text { zyxz } & 0\end{array}$ $\begin{array}{lllllllll}0 & 0 & 0 & z y y z & z y z x & 0 & z y z y & z y x z & 0 \\ 0 & 0 & 0 & x z y z & x z z x & 0 & x z z y & x z x z & 0\end{array}$ $\begin{array}{ccccccccc}0 & 0 & 0 & x z y z & x z z x & 0 & x z z y & x z x z & 0 \\ y x x x & y x y y & y x z z & 0 & 0 & y x x y & 0 & 0 & y x y x\end{array}$

$\left.V^{4}\right] \quad\left(\begin{array}{ccccc}x x x x & x y y y & 0 & y y z z & 0 \\ y x x x & y y y y & 0 & z z x x & 0 \\ 0 & 0 & z z z z & x x y y & z z x y\end{array}\right)$

$\left(\begin{array}{ccccc}\mathrm{xxxx} & \mathrm{xyyy} & 0 & \mathrm{yyzz} & 0 \\ \mathrm{yxxx} & \mathrm{yyyy} & 0 & \mathrm{zzxx} & 0 \\ 0 & 0 & \mathrm{zzzz} & \mathrm{xxyy} & \mathrm{zzxy}\end{array}\right)$

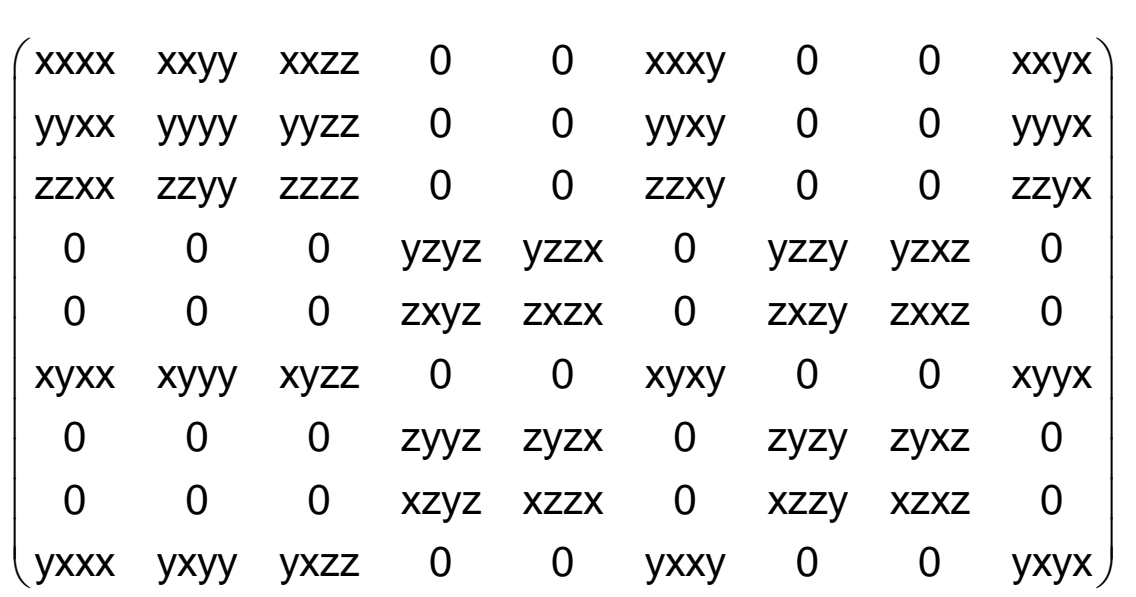

$\left(\begin{array}{ccccc}x x x x & x y y y & 0 & y y z z & 0 \\ y x x x & y y y y & 0 & z z x x & 0 \\ 0 & 0 & z z z z & x x y y & z z x y\end{array}\right)$ $\left(\begin{array}{lllllllll}x x x x & x x y y & x x z z & 0 & 0 & x x x y & 0 & 0 & x x y x \\ y y x x & y y y & y z z & 0 & 0 & y x y & 0 & 0 & x y x\end{array}\right.$ $\begin{array}{lllllllll}\text { yyxx } & \text { yyyy } & \text { yyzz } & 0 & 0 & \text { yyxy } & 0 & 0 & \text { yyyx } \\ z z x & z y y & z z z & 0 & 0 & z x y & 0 & 0 & z y x\end{array}$ $\begin{array}{llllllllll}\text { zzxx } & \text { zzyy } & \text { zzzz } & 0 & 0 & \text { zzxy } & 0 & 0 & z z y x\end{array}$ $\begin{array}{lllllllll}0 & 0 & 0 & y z y z & y z z x & 0 & y z z y & y z x z & 0 \\ 0 & 0 & 0 & z x y z & z x z & 0 & z x z y & z x y z & 0\end{array}$ $\begin{array}{lllllllll}0 & 0 & 0 & z x y z & z x z x & 0 & z x z y & z x x z & 0\end{array}$ $\begin{array}{lllllllll}\text { xyxx } & \text { xyyy } & \text { xyzz } & 0 & 0 & \text { xyxy } & 0 & 0 & \text { xyyx }\end{array}$ $\begin{array}{ccccccccc}0 & 0 & 0 & \text { zyyz } & \text { zyzx } & 0 & \text { zyzy } & \text { zyxz } & 0\end{array}$ $\begin{array}{lllllllll}0 & 0 & 0 & z y y z & z y z x & 0 & z y z y & z y x z & 0 \\ 0 & 0 & 0 & x z y z & x z z x & 0 & x z z y & x z x z & 0\end{array}$ $\begin{array}{ccccccccc}0 & 0 & 0 & x z y z & x z z x & 0 & x z z y & x z x z & 0 \\ y x x x & y x y y & y x z z & 0 & 0 & y x x y & 0 & 0 & y x y x\end{array}$ $\left(\begin{array}{ccccc}x x x x & x y y y & 0 & y y z z & 0 \\ y x x x & y y y y & 0 & z z x x & 0 \\ 0 & 0 & z z z z & x x y & z z x y\end{array}\right)$ $\left(\begin{array}{ccccc}x x x x & y y y & 0 & z z x x & 0 \\ 0 & 0 & z z z z & x x y y & z z x y\end{array}\right)$

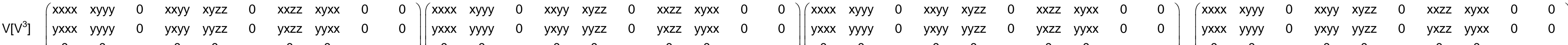

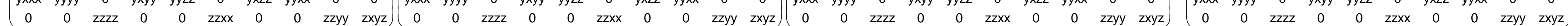

$\left[\left[^{2}\right]^{2}\right]\left(\begin{array}{cccccc}x x x x & x x y y & x x z z & 0 & 0 & x x x y \\ & y y y y & y y z z & 0 & 0 & y y x y \\ & & z z z z & 0 & 0 & z z x y \\ & & & y z y z & y z x z & 0 \\ & & & & x z x z & 0 \\ & & & & & \text { xyxy }\end{array}\right)$ $\left.\begin{array}{llllll}x y y x x & y y y y & y y z z & 0 & 0 & y y x y\end{array}\right)$

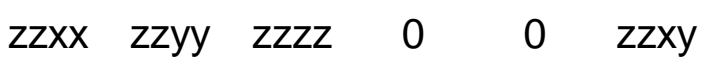
$\begin{array}{cccccc}0 & 0 & 0 & y z y z & y z x z & 0 \\ 0 & 0 & 0 & x z y z & x z x z & 0\end{array}$

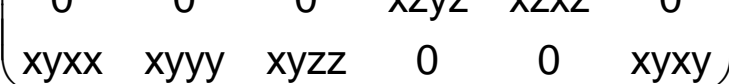

$$
\left(\begin{array}{cccccc}
x x x x & x x y y & x x z z & 0 & 0 & x x x y \\
& y y y y & y y z z & 0 & 0 & y y x y \\
& & z z z z & 0 & 0 & z z x y \\
& & & y z y z & y z x z & 0 \\
& & & & x z x z & 0 \\
& & & & & x y x y
\end{array}\right)
$$

$\left(\begin{array}{llllll}x x x x & x x y y & x x z z & 0 & 0 & x x x y \\ y y x x & y y y y & y y z z & 0 & 0 & y y x y\end{array}\right)$ $\begin{array}{llllll}x y y x x & \text { yyyy } & y y z z & 0 & 0 & \text { yyxy } \\ z z x & z z y y & z z z z & 0 & 0 & z z x y\end{array}$

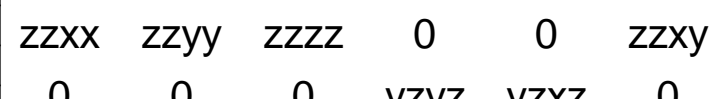
$\begin{array}{llllll}0 & 0 & 0 & y z y z & y z x z & 0\end{array}$ $\begin{array}{cccccc}0 & 0 & 0 & x z y z & x z x z & 0\end{array}$

$$
\left(\begin{array}{cccccc}
x x x x & x x y y & x x z z & 0 & 0 & x x x y \\
& y y y y & y y z z & 0 & 0 & y y x y \\
& & z z z z & 0 & 0 & z z x y \\
& & & y z y z & y z x z & 0 \\
& & & & x z x z & 0 \\
& & & & & x y x y
\end{array}\right)
$$

$\left(\begin{array}{llllll}x x x x & x x y y & x x z z & 0 & 0 & x x x y \\ y y x x & y y y y & y y z z & 0 & 0 & y y x y \\ z z x x & z z y & z z z z & 0 & 0 & z z x y\end{array}\right)$ $\left.\begin{array}{cccccc}\text { yyxx } & \text { yyyy } & \text { yyzz } & 0 & 0 & \text { yyxy } \\ z z x x & z z y y & z z z z & 0 & 0 & z z x y \\ 0 & 0 & 0 & y z y z & y z x z & 0\end{array}\right)$ $\begin{array}{cccccc}\text { zzxx } & \text { zzyy } & \text { zzzz } & 0 & 0 & \text { zzxy } \\ 0 & 0 & 0 & y z y z & y z x z & 0 \\ 0 & 0 & 0 & x z y z & x z x z & 0\end{array}$ $\begin{array}{llllll}0 & 0 & 0 & y z y z & y z x z & 0 \\ 0 & 0 & 0 & x z y z & x z x z & 0\end{array}$ $\begin{array}{cccccc}0 & 0 & 0 & x z y z & x z x z & 0 \\ x y x x & x y y y & x y z z & 0 & 0 & x y x y\end{array}$

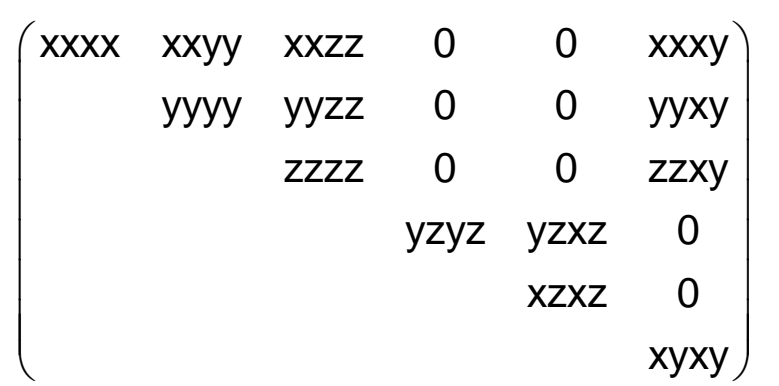

$\left(\begin{array}{llllll}x x x x & x x y y & x x z z & 0 & 0 & x x x y \\ y y x & y y y & y z z & 0 & 0 & y y x y\end{array}\right)$ $\begin{array}{llllll}\text { yyxx } & \text { yyyy } & y y z z & 0 & 0 & \text { yyxy } \\ z z x x & z z y & z z z z & 0 & 0 & z z x y\end{array}$ $\begin{array}{llllll}\text { zzxx } & \text { zzyy } & \text { zzzz } & 0 & 0 & \text { zzxy } \\ 0 & 0 & 0 & y z y & y z x z & 0\end{array}$ $\begin{array}{llllll}0 & 0 & 0 & y z y z & y z x z & 0\end{array}$ $\begin{array}{llllll}0 & 0 & 0 & x z y z & x z x z & 0\end{array}$ 


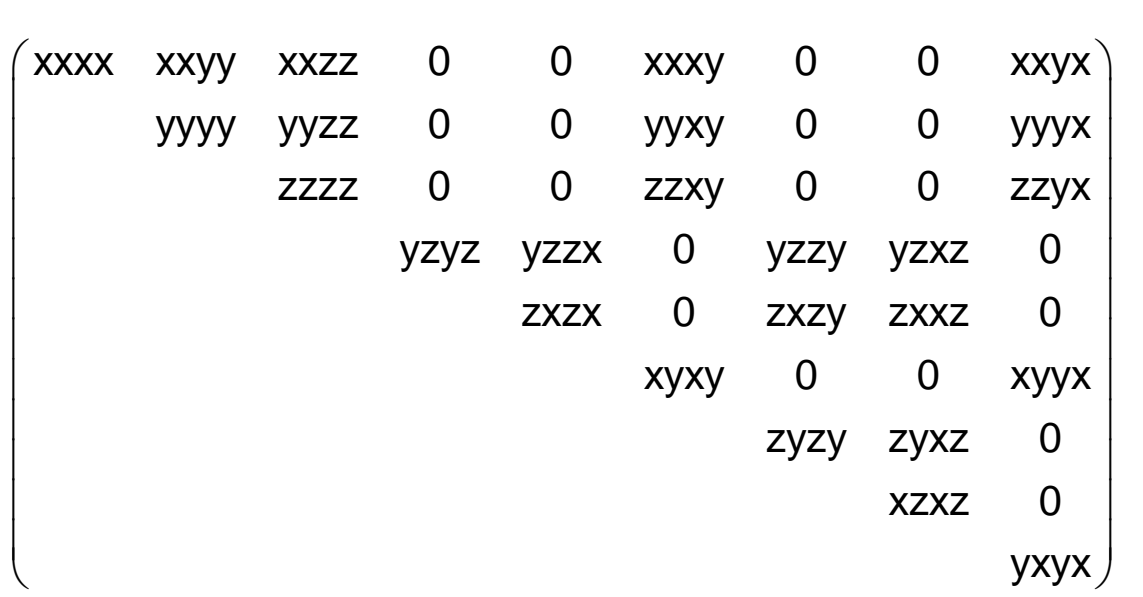

$\left(\begin{array}{lllllllll}x x x x & x x y y & x x z z & 0 & 0 & x x x y & 0 & 0 & x x y x\end{array}\right)$ $\begin{array}{lllllllll}\text { yyxx } & \text { yyyy } & \text { yyzz } & 0 & 0 & \text { yyxy } & 0 & 0 & \text { yyyx } \\ \text { zzxx } & \text { zzyy } & \text { zzzz } & 0 & 0 & \text { zzxy } & 0 & 0 & \text { zzyx }\end{array}$ $\begin{array}{lllllllll}0 & 0 & 0 & y z y z & y z z x & 0 & y z z y & y z x z & 0\end{array}$ $\begin{array}{lllllllll}0 & 0 & 0 & z x y z & z x z x & 0 & z x z y & z x x z & 0\end{array}$

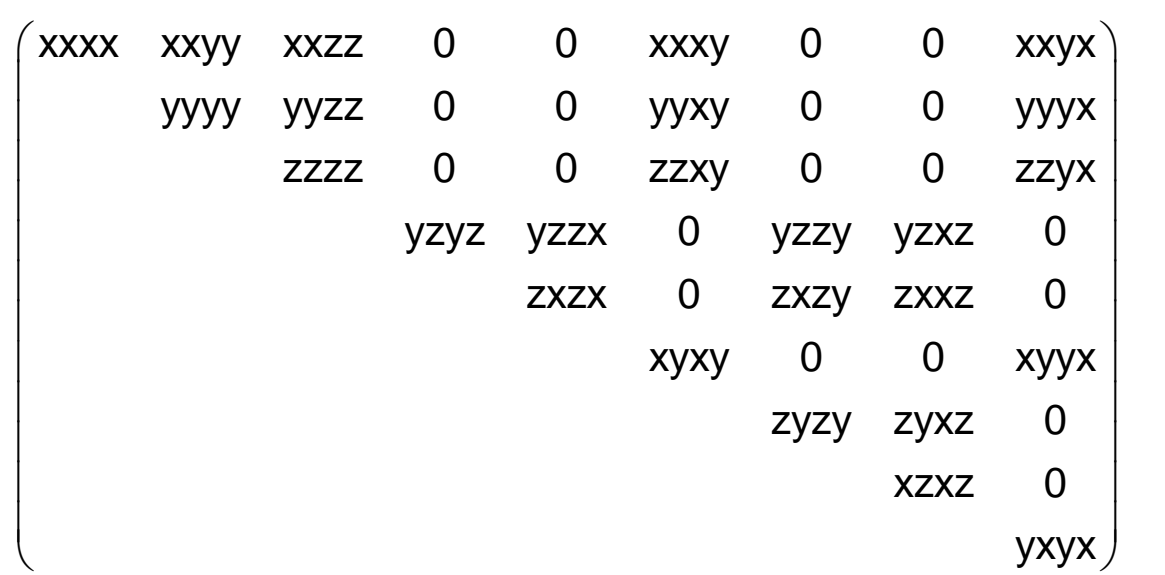

$\left(\begin{array}{lllllllll}x x x x & x x y y & x x z z & 0 & 0 & x x x y & 0 & 0 & x x y x\end{array}\right)$ $\left.\begin{array}{lllllllll}\operatorname{yyxx} & \text { yyyy } & \text { yyzz } & 0 & 0 & \text { yyxy } & 0 & 0 & \text { yyyx } \\ \text { zzxx } & \text { zzyy } & \text { zzzz } & 0 & 0 & \text { zzxy } & 0 & 0 & \text { zzyx } \\ 0 & 0 & 0 & y z y z & y z z & 0 & y z z y & y z z z & 0\end{array}\right)$ $\begin{array}{lllllllll}0 & 0 & 0 & y z y z & y z z x & 0 & y z z y & y z x z & 0\end{array}$ $\begin{array}{lllllllll}0 & 0 & 0 & z x y z & z x z x & 0 & z x z y & z x x z & 0\end{array}$ $\left(\begin{array}{lllllllll}x x x x & x x y y & x x z z & 0 & 0 & x x x y & 0 & 0 & x x y x\end{array}\right.$ $\begin{array}{llllllll}\text { yyyy } & y y z z & 0 & 0 & \text { yyxy } & 0 & 0 & \text { yyyx } \\ & z z z z & 0 & 0 & z z x y & 0 & 0 & z z x\end{array}$ $\begin{array}{lcccccc}z z z z & 0 & 0 & z z x y & 0 & 0 & z z y x \\ & y z y z & y z z x & 0 & y z z y & y z x z & 0\end{array}$ $\begin{array}{cccccc}y z y z & y z z x & 0 & y z z y & y z x z & 0 \\ & z x z x & 0 & z x z y & z x y z & 0\end{array}$ $\begin{array}{llll}x y y x y & 0 & 0 & x y y x\end{array}$ $\begin{array}{lll}\text { zyzy } & \text { zyxz } & 0 \\ & x z x z & 0\end{array}$

$\left(\begin{array}{lllllllll}x x x x & x x y y & x x z z & 0 & 0 & x x x y & 0 & 0 & x x y x\end{array}\right.$ $\begin{array}{lllllllll}\text { yyxx } & \text { yyyy } & \text { yyzz } & 0 & 0 & \text { yyxy } & 0 & 0 & \text { yyyx } \\ z z x x & z z y y & z z z z & 0 & 0 & z z y & 0 & 0 & z z x\end{array}$ $\begin{array}{lllllllll}z z x x & z z y y & z z z z & 0 & 0 & z z x y & 0 & 0 & z z y x\end{array}$ $\begin{array}{lllllllll}0 & 0 & 0 & y z y z & y z z x & 0 & y z z y & y z x z & 0\end{array}$ $\begin{array}{lllllllll}0 & 0 & 0 & z x y z & z x z x & 0 & z x z y & z x x z & 0\end{array}$

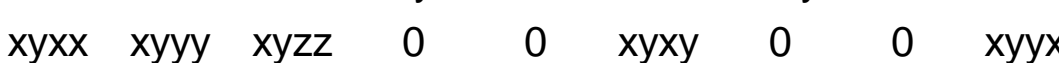




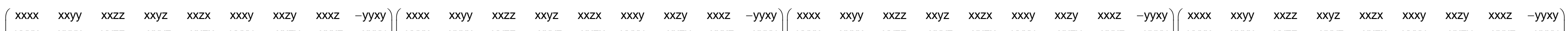

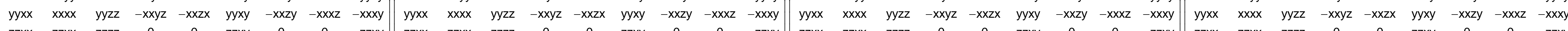

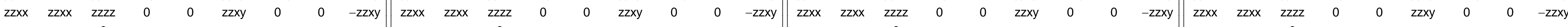

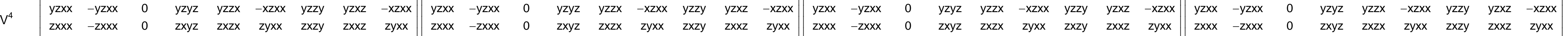

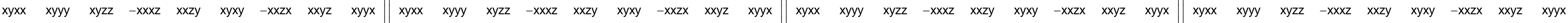

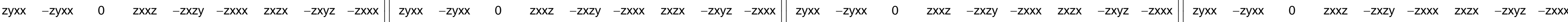

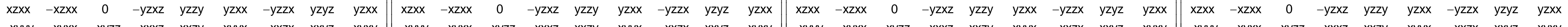

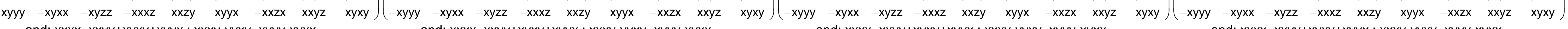
and: $x x x x=x x y y+x y x y+x y y x ; x x x y-y y x y=x y y y-x y x x \quad$ and: $x x x x=x x y y+x y x y+x y y x ; x x x y-y y x y=x y y y-x y x x$ and: $x x x x=x x y y+x y x y+x y y x ; x x x y-y y x y=x y y y-x y x x$ and: $x x x x=x x y y+x y x y+x y y x ; x x x y-y y x y=x y y y-x y x x$

$\left[V^{4}\right]\left(\begin{array}{ccccc}3 x x y y & 0 & 0 & y y z z & x x y z \\ 0 & 3 x x y y & 0 & y y z z & -z x x x \\ z x x x & -x x y z & z z z z & x x y y & 0\end{array}\right)$

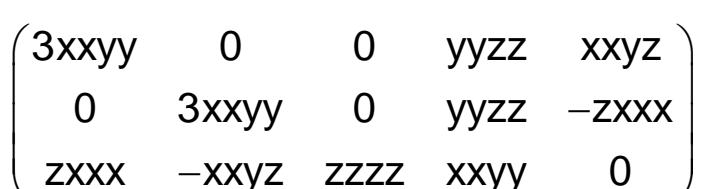

$\left(\begin{array}{ccccc}3 x x y y & 0 & 0 & y y z z & x x y z \\ 0 & 3 x x y y & 0 & y y z z & -z x x x \\ z x x x & -x x y z & z z z z & x x y y & 0\end{array}\right)$

$\left(\begin{array}{ccccc}3 x x y y & 0 & 0 & y y z z & x x y z \\ 0 & 3 x x y y & 0 & y y z z & -z x x x \\ z x x x & -x x y z & z z z z & x x y y & 0\end{array}\right)$

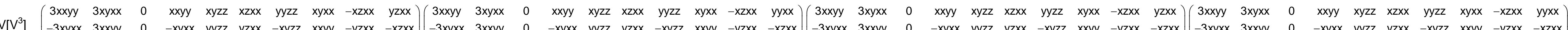

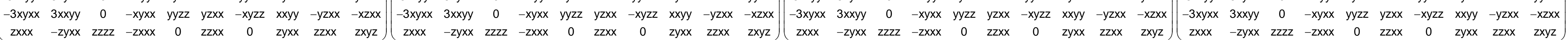

$\left[\left[\mathrm{V}^{2}\right]^{2}\right]\left(\begin{array}{cccccc}\mathrm{xxxx} & \mathrm{xxyy} & \mathrm{xxzz} & \mathrm{xxyz} & -\mathrm{yyxz} & 0 \\ & \mathrm{xxxx} & \mathrm{xxzz} & -\mathrm{xxyz} & \mathrm{yyxz} & 0 \\ & & \mathrm{zzzz} & 0 & 0 & 0 \\ & & & \mathrm{yzyz} & 0 & \mathrm{yyxz} \\ & & & & \mathrm{yzyz} & \mathrm{xxyz} \\ & & & & & \mathrm{xyxy}\end{array}\right)$

and $x y x y=1 / 2(x x x x-x x y y)$

$\left(\begin{array}{llllll}x x x x & x x y y & x x z z & x x y z & -y y x z & x x x y\end{array}\right.$ $\begin{array}{llllll}x x y y & x x x x & x x z z & -x x y z & y y x z & -x x x y\end{array}$ $\begin{array}{llllll}z z x x & z z x x & z z z z & 0 & 0 & 0\end{array}$

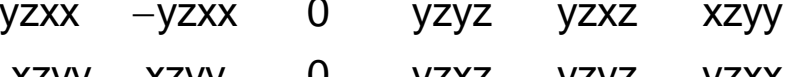
$\begin{array}{llllll}-x z y y & x z y y & 0 & y z x z & y z y z & y z x x \\ -x x x y & x x x y & 0 & y y x z & x x y z & x y x y\end{array}$

and $x y x y=1 / 2(x x x x-x x y y)$

$$
\left(\begin{array}{cccccc}
x x x x & x x y y & x x z z & x x y z & -y y x z & 0 \\
& x x x x & x x z z & -x x y z & y y x z & 0 \\
& & z z z z & 0 & 0 & 0 \\
& & & y z y z & 0 & y y x z \\
& & & & y z y z & x x y z \\
& & & & & x y x y
\end{array}\right)
$$

and $x y x y=1 / 2(x x x x-x x y y)$

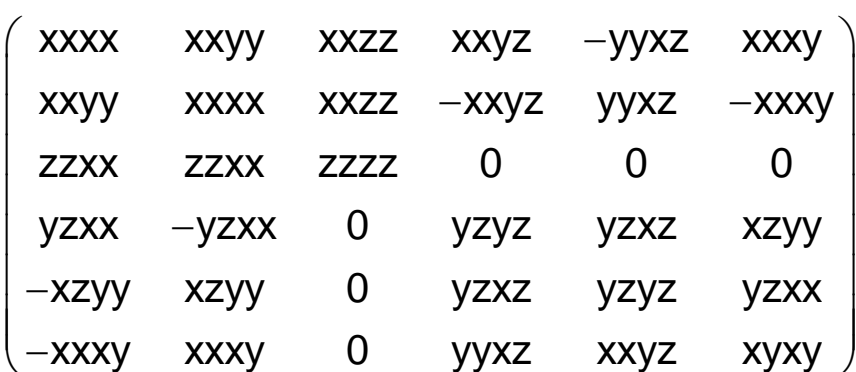

and $x y x y=1 / 2(x x x x-x x y y)$

$$
\left(\begin{array}{cccccc}
x x x x & x x y y & x x z z & x x y z & -y y x z & 0 \\
& x x x x & x x z z & -x x y z & y y x z & 0 \\
& & z z z z & 0 & 0 & 0 \\
& & & y z y z & 0 & y y x z \\
& & & & y z y z & x x y z \\
& & & & & x y x y
\end{array}\right)
$$

and $x y x y=1 / 2(x x x x-x x y y)$

$\left(\begin{array}{llllll}x x x x & x x y y & x x z z & x x y z & -y y x z & x x x y \\ x x y & x x y & x x z & -x x y z & y y x z & -x x y\end{array}\right)$ $\begin{array}{llllll}x x y y & x x x x & x x z z & -x x y z & y y x z & -x x x y\end{array}$ $\begin{array}{lllllll}z z x x & z z x x & z z z z & 0 & 0 & 0\end{array}$ $\begin{array}{llllll}y z x x & -y z x x & 0 & y z y z & y z x z & x z y y\end{array}$ $\begin{array}{llllll}-x z y y & x z y y & 0 & y z x z & y z y z & y z x x\end{array}$ $\begin{array}{llllll}-x x x y & x x x y & 0 & y y x z & x x y z & x y x y\end{array}$

and $x y x y=1 / 2(x x x x-x x y y)$

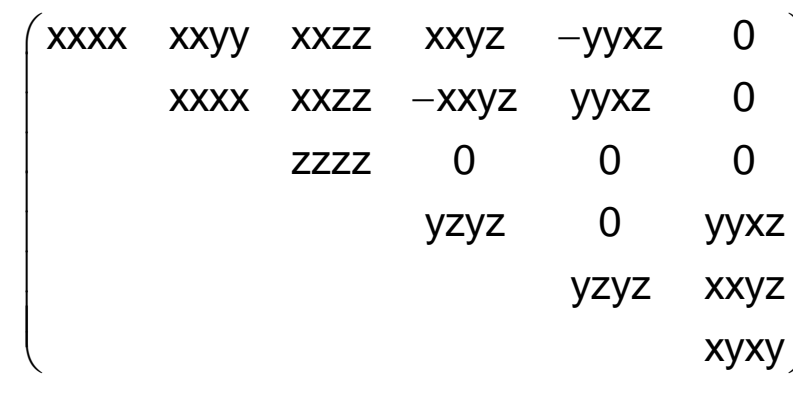

and $x y x y=1 / 2(x x x x-x x y y)$

$\left(\begin{array}{cccccc}x x x x & x x y y & x x z z & x x y z & -y y x z & x x x y\end{array}\right)$ $\begin{array}{llllll}x x y y & x x x x & x x z z & -x x y z & y y x z & -x x x y\end{array}$ $\begin{array}{cccccc}z z x x & z z x x & z z z z & 0 & 0 & 0\end{array}$ $\begin{array}{cccccc}y z x x & -y z x x & 0 & y z y z & y z x z & x z y y \\ -x z y y & x z y & 0 & y z x z & y z y z & y z x\end{array}$ $\begin{array}{llllll}-x z y y & x z y y & 0 & y z x z & y z y z & y z x x\end{array}$ $\begin{array}{llllll}-x x x y & x x x y & 0 & y y x z & x x y z & x y x y\end{array}$

and $x y x y=1 / 2(x x x x-x x y y)$ 


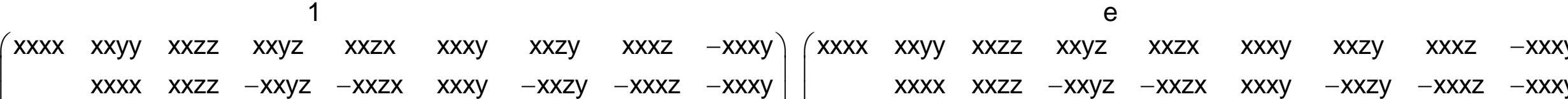
$\begin{array}{lllllll}z z z z & 0 & 0 & z z x y & 0 & 0 & -z z x y\end{array}$ $\begin{array}{llllll}y z y z & y z z x & -x x x z & y z z y & 0 & -x x x z\end{array}$ $\begin{array}{llllll}z x z x & x x z y & 0 & y z z y & x x z y\end{array}$ $\begin{array}{llll}x y x y & -x x z x & x x y z & x y y x\end{array}$ $z x z x \quad-y z z x-x x z x$

$\left[\left(\mathrm{V}^{2}\right)\right]^{2}$ $\begin{array}{cc}\mathrm{yzyz} & \mathrm{xxyz} \\ & \mathrm{xyxy}\end{array}$

and $x x x x=x x y y+y z y z+z y z y$ $\left.\begin{array}{llcccccc}x x x x & x x z z & -x x y z & -x x z x & x x x y & -x x z y & -x x x z & -x x x y \\ & z z z & 0 & 0 & z z y & 0 & 0 & -z z x y\end{array}\right)$ $\begin{array}{lllllll}z z z z & 0 & 0 & z z x y & 0 & 0 & -z z x y\end{array}$ $\begin{array}{cccccc}y z y z & y z z x & -x x x z & y z z y & 0 & -x x x z\end{array}$ $\begin{array}{lllll}z x z x & x x z y & 0 & y z z y & x x z y\end{array}$ $\begin{array}{llll}x y x y & -x x z x & x x y z & x y y x\end{array}$ $\begin{array}{lll}z x z x & -y z z x & -x x z x\end{array}$

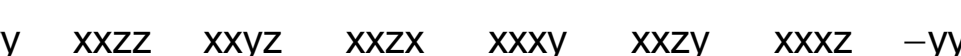

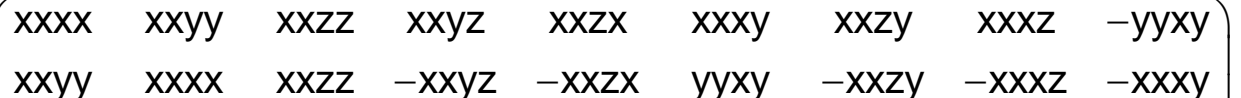
$\left[V^{2}\right] V^{2} \quad$\begin{tabular}{ccccccccc}
$z z x x$ & $z z x x$ & $z z z z$ & 0 & 0 & $z z x y$ & 0 & 0 & $-z z x y$ \\
\hline
\end{tabular} $\begin{array}{ccccccccc}y z z x x & -y z x x & 0 & y z y z & y z z x & -z x x x & z x z x & -z x y z & -z x y x \\ z x x x & -z x x x & 0 & z x y z & z x z x & y z x x & -y z z x & y z y z & y z x x \\ x y x x & -x y x x & 0 & -x x y z & x x y & x y y & -x x z & x y z & x y x y\end{array}$

and $x x x x=x x y y+y z y z+z y z y$

)

and $x x x x=x x y y+y z y z+z y z y$ 
e

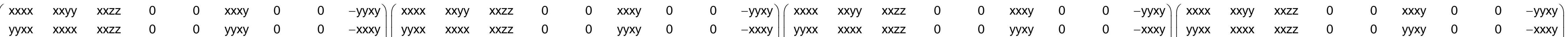

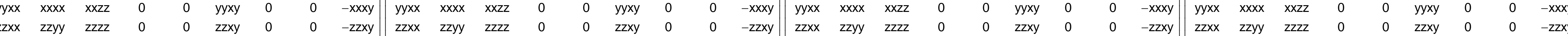

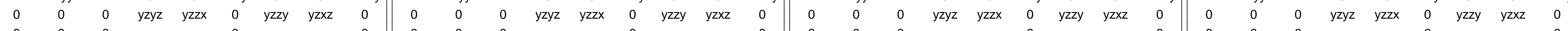

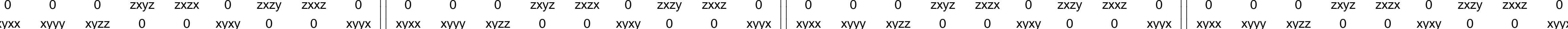

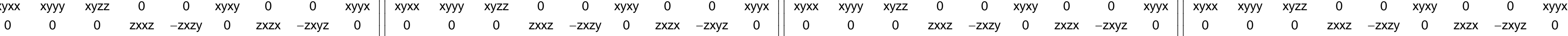

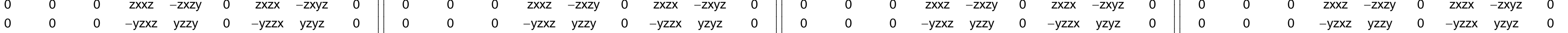

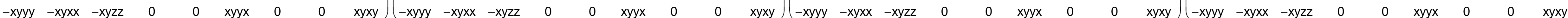
$\left[V^{4}\right] \quad\left(\begin{array}{ccccc}x x x x & -y x x x & 0 & y y z z & 0 \\ y x x x & x x x x & 0 & y y z z & 0 \\ 0 & 0 & z z z z & x x y y & 0\end{array}\right)$
$\left(\begin{array}{ccccc}x x x x & -y x x x & 0 & y y z z & 0 \\ y x x x & x x x x & 0 & y y z z & 0 \\ 0 & 0 & z z z z & x x y y & 0\end{array}\right)$
$\left(\begin{array}{ccccc}\mathrm{xxxx} & -\mathrm{yxxx} & 0 & \mathrm{yyzz} & 0 \\ \mathrm{yxxx} & \mathrm{xxxx} & 0 & \mathrm{yyzz} & 0 \\ 0 & 0 & \mathrm{zzzz} & \mathrm{xxyy} & 0\end{array}\right)$
$\left(\begin{array}{ccccc}\mathrm{xxxx} & -\mathrm{yxxx} & 0 & \mathrm{yyzz} & 0 \\ \mathrm{yxxx} & \mathrm{xxxx} & 0 & \mathrm{yyzz} & 0 \\ 0 & 0 & \mathrm{zzzz} & \mathrm{xxyy} & 0\end{array}\right)$

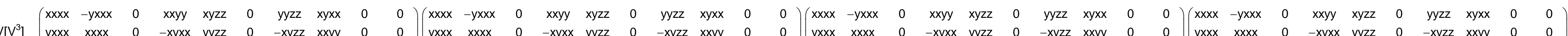

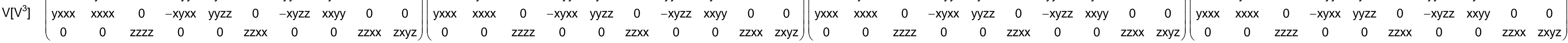

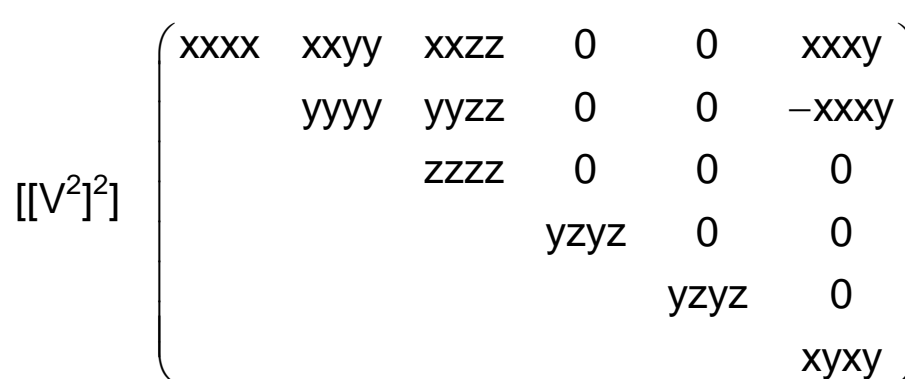

$\left[\mathrm{V}^{2}\right]^{2}\left(\begin{array}{cccccc}\mathrm{xxxx} & \mathrm{xxyy} & \mathrm{xxzz} & 0 & 0 & \mathrm{xxxy} \\ \mathrm{yyyx} & \mathrm{xxxx} & \mathrm{xxzz} & 0 & 0 & -\mathrm{xxxy} \\ \mathrm{zzxx} & \mathrm{zzxx} & \mathrm{zzzz} & 0 & 0 & 0 \\ 0 & 0 & 0 & \mathrm{yzyz} & \mathrm{yzxz} & 0 \\ 0 & 0 & 0 & -\mathrm{yzxz} & \mathrm{yzyz} & 0 \\ \mathrm{xyxx} & -\mathrm{xyxx} & 0 & 0 & 0 & \mathrm{xyxy}\end{array}\right)$

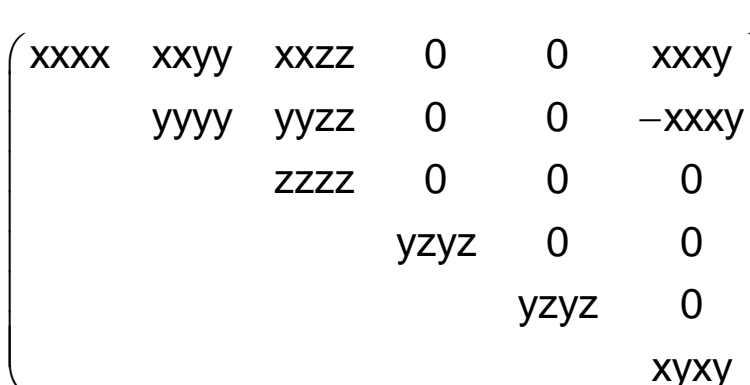

$\left(\begin{array}{cccccc}x x x x & x x y y & x x z z & 0 & 0 & x x x y \\ y y x x & x x x x & x x z z & 0 & 0 & -x x x y \\ z z x x & z z x x & z z z z & 0 & 0 & 0 \\ 0 & 0 & 0 & y z y z & y z x z & 0 \\ 0 & 0 & 0 & -y z x z & y z y z & 0 \\ x y x x & -x y x x & 0 & 0 & 0 & x y x y\end{array}\right)$

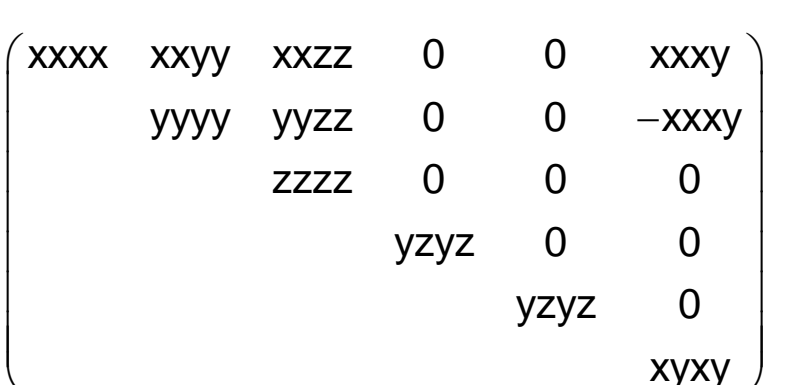

$\left(\begin{array}{cccccc}x x x x & x x y y & x x z z & 0 & 0 & x x x y \\ y y x x & x x x x & x x z z & 0 & 0 & -x x x y \\ z z x x & z z x x & z z z z & 0 & 0 & 0 \\ 0 & 0 & 0 & y z y z & y z x z & 0 \\ 0 & 0 & 0 & -y z x z & y z y z & 0 \\ x y x x & -x y x x & 0 & 0 & 0 & x y x y\end{array}\right)$

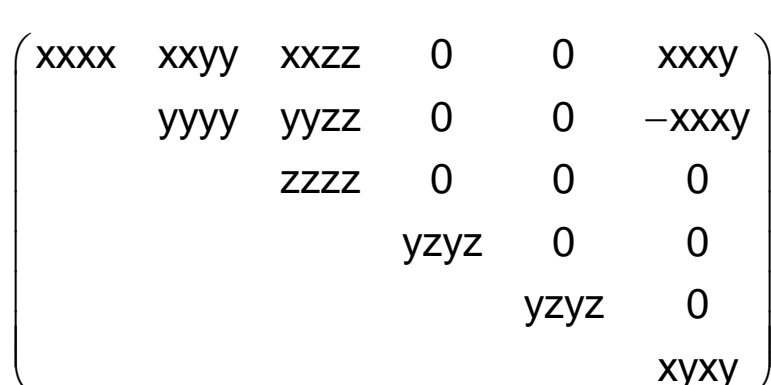

$\left(\begin{array}{cccccc}x x x x & x x y y & x x z z & 0 & 0 & x x x y \\ y y x x & x x x x & x x z z & 0 & 0 & -x x x y \\ z z x x & z z x x & z z z z & 0 & 0 & 0 \\ 0 & 0 & 0 & y z y z & y z x z & 0 \\ 0 & 0 & 0 & -y z x z & y z y z & 0 \\ x y x x & -x y x x & 0 & 0 & 0 & x y x y\end{array}\right)$ 


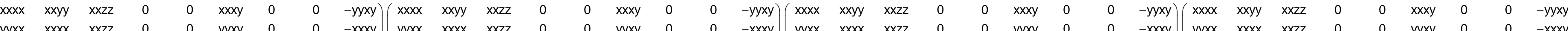

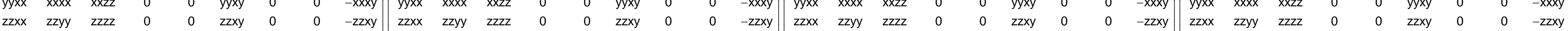

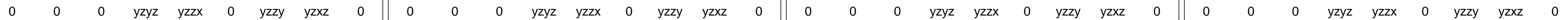

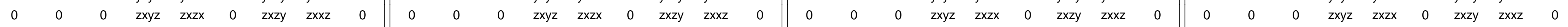

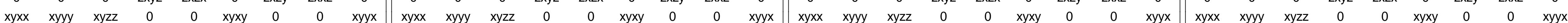

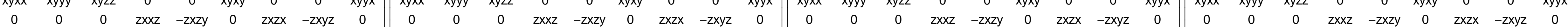

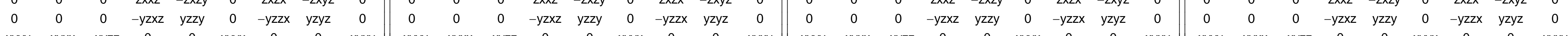

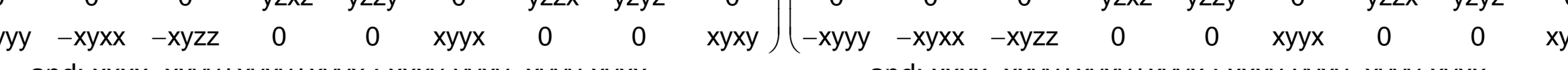
and: $x x x x=x x y y+x y x y+x y y x \cdot x x x y-y y x y=x y y y-x y x x$

$\left(\begin{array}{ccccccccc}0 & 0 & 0 & -y z x z & y z z y & 0 & -y z z x & y z y z & 0 \\ -x y y y & -x y x x & -x y z z & 0 & 0 & x y y x & 0 & 0 & x y x y\end{array}\right)$ and: $x x x x=x x y y+x y x y+x y y x ; x x x y-y y x y=x y y y-x y x x$

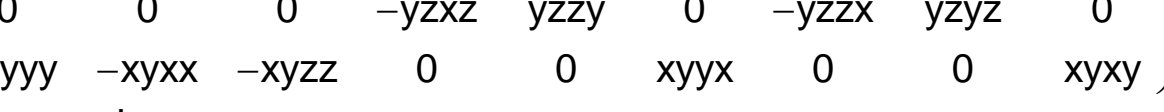
$\left[V^{4}\right] \quad\left(\begin{array}{ccccc}3 x x y y & 0 & 0 & y y z z & 0 \\ 0 & 3 x x y y & 0 & y y z z & 0 \\ 0 & 0 & z z z z & x x y y & 0\end{array}\right)$

$$
\left(\begin{array}{ccccc}
3 x x y y & 0 & 0 & y y z z & 0 \\
0 & 3 x x y y & 0 & y y z z & 0 \\
0 & 0 & z z z z & x x y y & 0
\end{array}\right)
$$

$$
\left(\begin{array}{ccccc}
3 x x y y & 0 & 0 & y y z z & 0 \\
0 & 3 x x y y & 0 & y y z z & 0 \\
0 & 0 & z z z z & x x y y & 0
\end{array}\right)
$$$$
\left(\begin{array}{ccccc}
3 x x y y & 0 & 0 & y y z z & 0 \\
0 & 3 x x y y & 0 & y y z z & 0 \\
0 & 0 & z z z z & x x y y & 0
\end{array}\right)
$$

$V\left[V^{3}\right] \quad\left(\begin{array}{cccccccccc}3 x x y y & 3 x y x x & 0 & x x y y & x y z z & 0 & y y z z & x y x x & 0 & 0 \\ -3 x y x x & 3 x x y y & 0 & -x y x x & y y z z & 0 & -x y z z & x x y y & 0 & 0\end{array}\right.$ $\left(\begin{array}{llllllllll}3 x x y y & 3 x y x x & 0 & x x y y & x y z z & 0 & y y z z & x y x x & 0 & 0\end{array}\right)$ $\left(\begin{array}{llllllllll}3 x x y y & 3 x y x x & 0 & x x y y & x y z z & 0 & y y z z & x y x x & 0 & 0 \\ 3 x y x & 3 x y y & 0 & -x y x & y z z & 0 & -x y z & x x y & 0 & 0\end{array}\right)$ $\left(\begin{array}{cccccccccc}3 x x y y & 3 x y x x & 0 & x x y y & x y z z & 0 & y y z z & x y x x & 0 & 0 \\ -3 x y x & 3 x x y & 0 & -x y x & y y z & 0 & -x y & x y & 0 & 0\end{array}\right)$ $\left(\begin{array}{cccccccccc}-3 x y x x & 3 x x y y & 0 & -x y x x & y y z z & 0 & -x y z z & x x y y & 0 & 0 \\ 0 & 0 & z z z z & 0 & 0 & z z x x & 0 & 0 & z z x x & z x y z\end{array}\right)$

$\left.\left[V^{2}\right]^{2}\right]\left(\begin{array}{cccccc}x x x x & x x y y & x x z z & 0 & 0 & 0 \\ & x x x x & x x z z & 0 & 0 & 0 \\ & & z z z z & 0 & 0 & 0 \\ & & & y z y z & 0 & 0 \\ & & & & y z y z & 0 \\ & & & & & x y x y\end{array}\right)$

and $x y x y=1 / 2(x x x x-x x y y)$

$\left[V^{2}\right]^{2}\left(\begin{array}{cccccc}x x x x & x x y y & x x z z & 0 & 0 & x x x y \\ x x y y & x x x x & x x z z & 0 & 0 & -x x x y \\ z z x x & z z x x & z z z z & 0 & 0 & 0 \\ 0 & 0 & 0 & y z y z & y z x z & 0 \\ 0 & 0 & 0 & -y z x z & y z y z & 0 \\ -x x x y & x x x y & 0 & 0 & 0 & x y x y\end{array}\right)$

and $x y x y=1 / 2(x x x x-x x y y)$

$$
\left(\begin{array}{cccccc}
x x x x & x x y y & x x z z & 0 & 0 & 0 \\
& x x x x & x x z z & 0 & 0 & 0 \\
& & z z z z & 0 & 0 & 0 \\
& & & y z y z & 0 & 0 \\
& & & & y z y z & 0 \\
& & & & & x y x y
\end{array}\right)
$$

and $x y x y=1 / 2(x x x x-x x y y)$

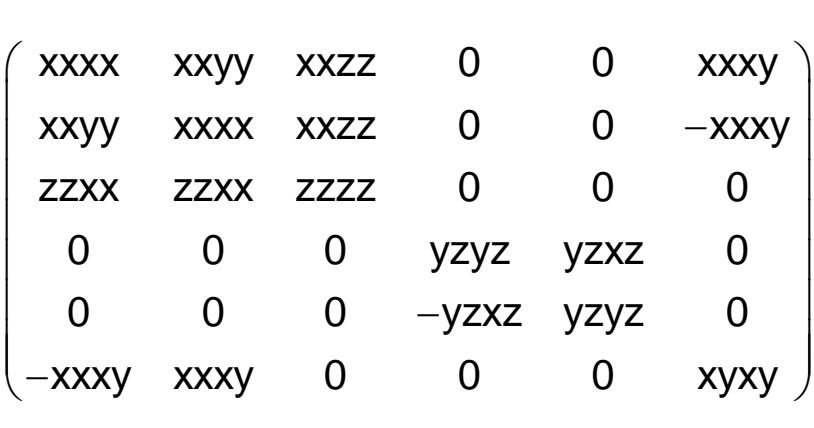

and $x y x y=1 / 2(x x x x-x x y y)$

$$
\left(\begin{array}{cccccc}
x x x x & x x y y & x x z z & 0 & 0 & 0 \\
& x x x x & x x z z & 0 & 0 & 0 \\
& & z z z z & 0 & 0 & 0 \\
& & & y z y z & 0 & 0 \\
& & & & y z y z & 0 \\
& & & & & x y x y
\end{array}\right)
$$

and $x y x y=1 / 2(x x x x-x x y y)$

$\left(\begin{array}{cccccc}x x x x & x x y y & x x z z & 0 & 0 & x x x y \\ x x y y & x x x x & x x z z & 0 & 0 & -x x x y \\ z z x x & z z x x & z z z z & 0 & 0 & 0 \\ 0 & 0 & 0 & y z y z & y z x z & 0 \\ 0 & 0 & 0 & -y z x z & y z y z & 0 \\ -x x x y & x x x y & 0 & 0 & 0 & x y x y\end{array}\right)$

and $x y x y=1 / 2(x x x x-x x y y)$

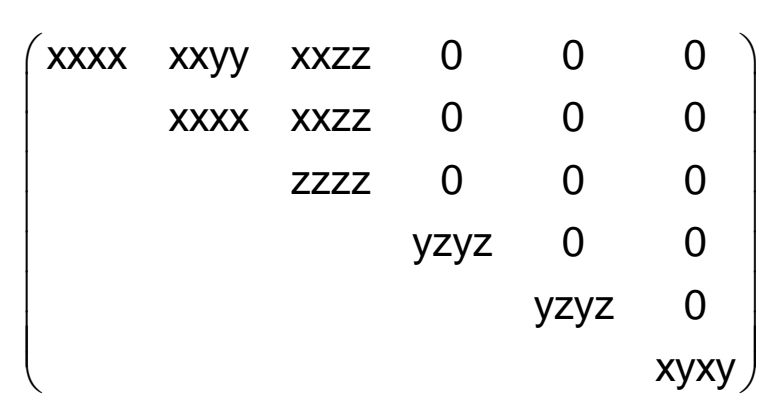

and $x y x y=1 / 2(x x x x-x x y y)$

$\left(\begin{array}{cccccc}x x x x & x x y y & x x z z & 0 & 0 & x x x y \\ x x y y & x x x x & x x z z & 0 & 0 & -x x x y \\ z z x x & z z x x & z z z z & 0 & 0 & 0 \\ 0 & 0 & 0 & y z y z & y z x z & 0 \\ 0 & 0 & 0 & -y z x z & y z y z & 0 \\ -x x x y & x x x y & 0 & 0 & 0 & x y x y\end{array}\right)$

and $x y x y=1 / 2(x x x x-x x y y)$ 
$n>4 \quad \mathbf{C}_{\infty}=\infty$

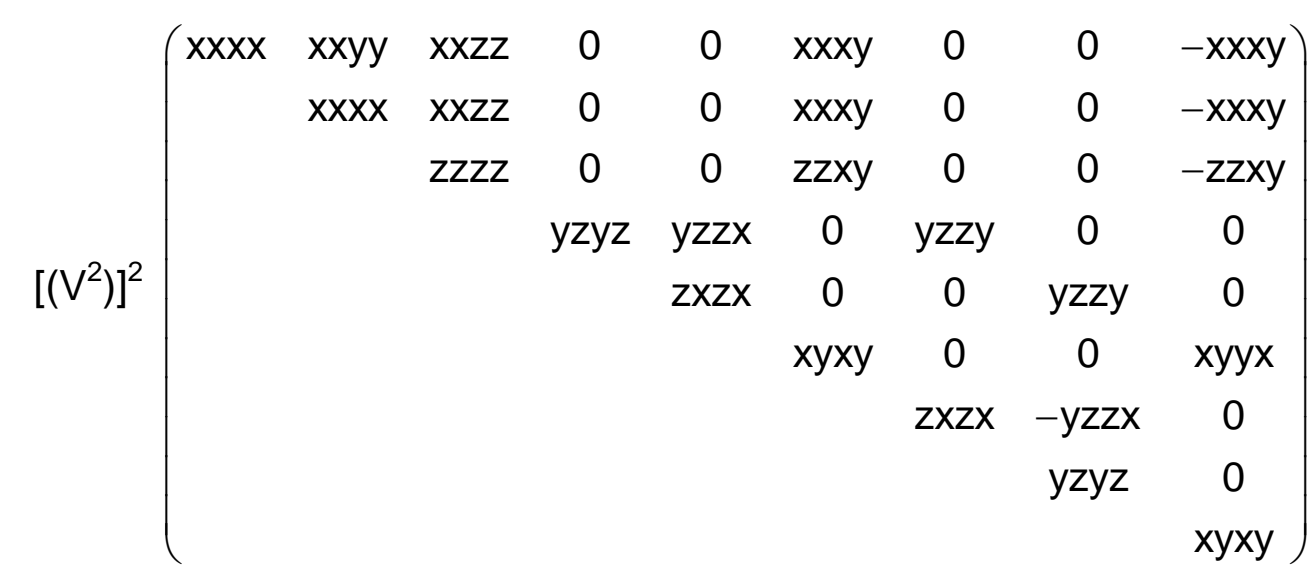

and $x x x x=x x y y+y z y z+z y z y$

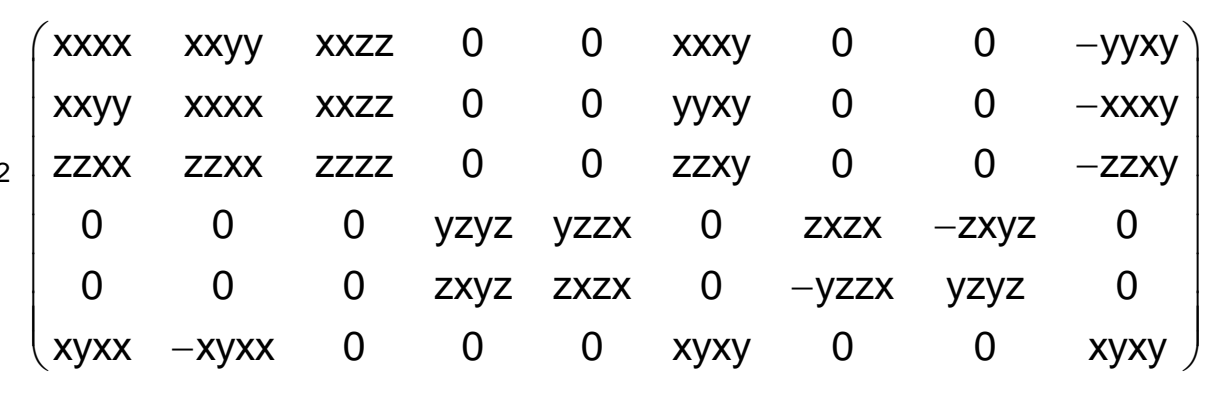

and $x x x x=x x y y+2 x y x y ; y y x y-x x x y=2 x y x x$ e

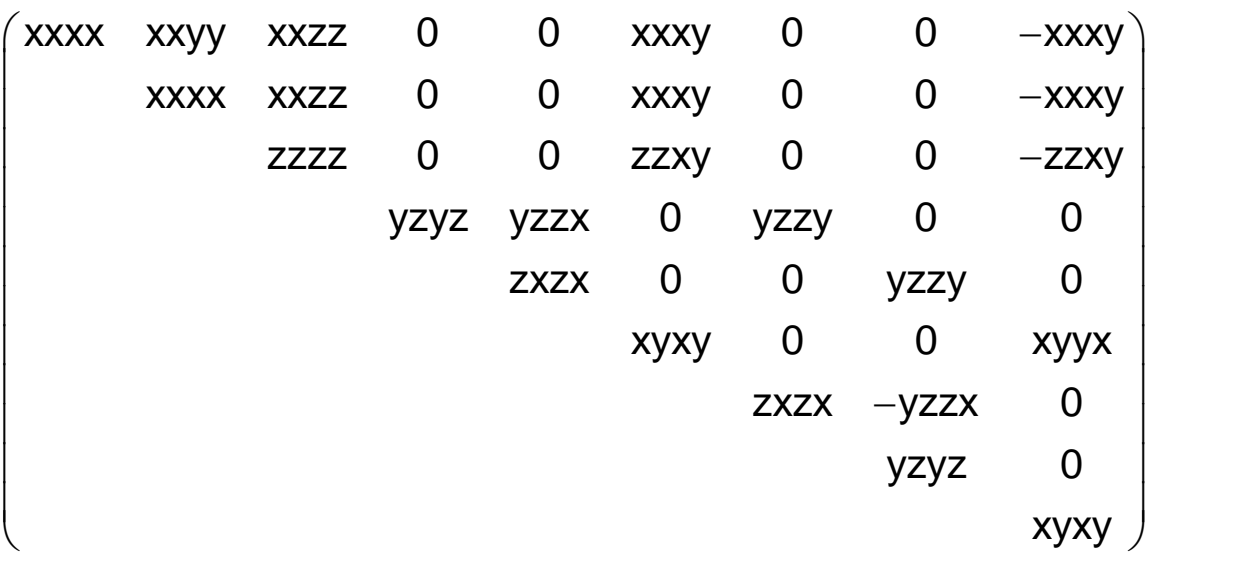

and $x x x x=x x y y+y z y z+z y z y$

$\left.\begin{array}{lllllllll}x x y y & x x x x & x x z z & 0 & 0 & y y x y & 0 & 0 & -x y x y \\ z z x & z z x x & z z z & 0 & 0 & z z x y & 0 & 0 & -z z y\end{array}\right)\left(\begin{array}{ccccccccc}x x x x & x x y y & x x z z & 0 & 0 & x x x y & 0 & 0 & -y y x y \\ x x y y & x x x x & x x z z & 0 & 0 & y y x y & 0 & 0 & -x x y \\ z z x & z z x x & z z z & 0 & 0 & z z x y & 0 & 0 & -z z x y\end{array}\right)$ $\begin{array}{lllllllll}z z x x & z z x x & z z z z & 0 & 0 & z z x y & 0 & 0 & -z z x y\end{array} \mid \begin{array}{llllllllll}x x y y & x x x x & x x z z & 0 & 0 & y y x y & 0 & 0 & -x x x y \\ z z x x & z z x x & z z z z & 0 & 0 & z z x y & 0 & 0 & -z z x y\end{array}$ $\begin{array}{ccccccccc}0 & 0 & 0 & y z y z & y z z x & 0 & z x z x & -z x y z & 0 \\ 0 & 0 & 0 & z x y z & z x z x & 0 & -y z z & y z y z & 0\end{array}$ $\left(\begin{array}{ccccccccc}0 & 0 & 0 & z x y z & z x z x & 0 & -y z z x & y z y z & 0 \\ x y x x & -x y x x & 0 & 0 & 0 & x y x y & 0 & 0 & x y x y\end{array}\right)$

and $x x x x=x x y y+2 x y x y ; y y x y-x x x y=2 x y x x$

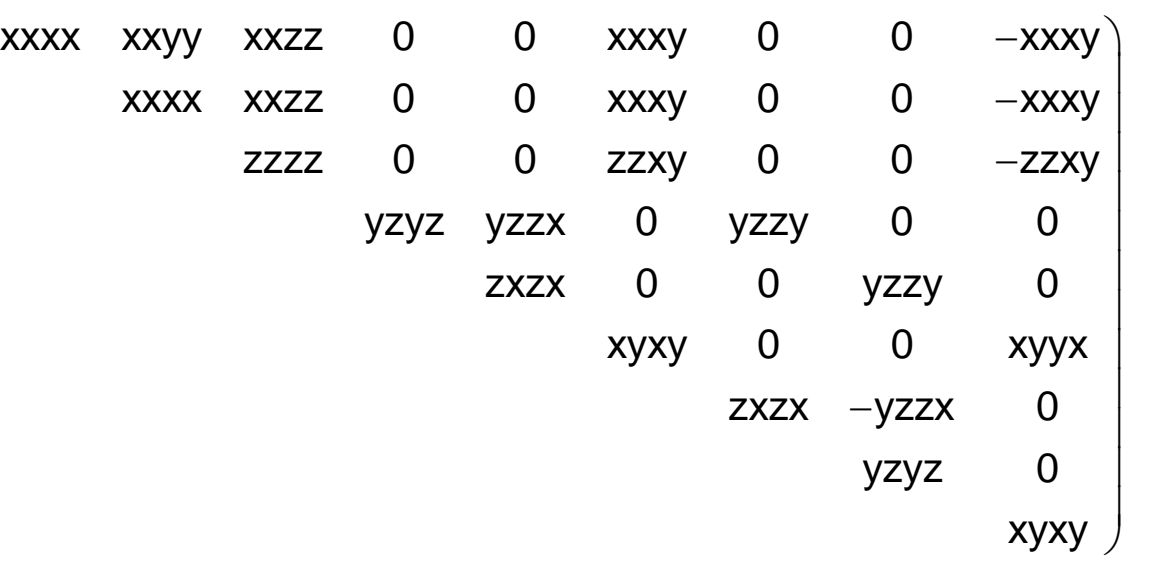

and $x x x x=x x y y+y z y z+z y z y$ $\begin{array}{ccccccccc}0 & 0 & 0 & z x y z & z x z x & 0 & -y z z x & y z y z & 0 \\ x y x x & -x y x x & 0 & 0 & 0 & x y x y & 0 & 0 & x y x y\end{array}$

and $x x x x=x x y y+2 x y x y ; y y x y-x x x y=2 x y x x$

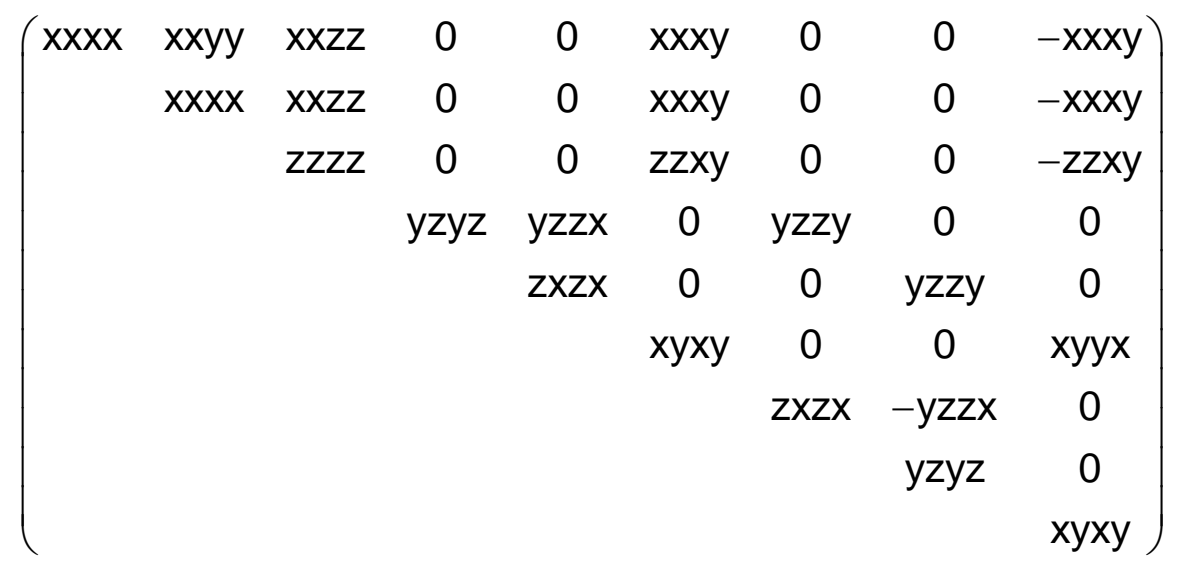

and $x x x x=x x y y+y z y z+z y z y$

$\left(\begin{array}{lllllllll}x x x x & x x y y & x x z z & 0 & 0 & x x x y & 0 & 0 & -y y x y \\ x x y & x x x & x x z z & 0 & 0 & y x y & 0 & 0 & -x x y\end{array}\right)$ $\left.\begin{array}{lllllllll}x x y y & x x x x & x x z z & 0 & 0 & y y x y & 0 & 0 & -x x x y \\ z z x x & z z x x & z z z z & 0 & 0 & z z x y & 0 & 0 & -z z x y \\ 0 & 0 & 0 & y z y z & y z z x & 0 & z x z x & -z x y z & 0\end{array}\right)$ $\begin{array}{ccccccccc}z z x x & z z x x & z z z z & 0 & 0 & z z x y & 0 & 0 & -z z x y \\ 0 & 0 & 0 & z z y z & y z z x & 0 & z x z x & -z x z & 0\end{array}$ $\left.\begin{array}{ccccccccc}0 & 0 & 0 & y z y z & y z z x & 0 & z x z x & -z x y z & 0 \\ 0 & 0 & 0 & z x y z & z x z x & 0 & -y z z x & y z y z & 0 \\ x y x x & -x y x x & 0 & 0 & 0 & x y x y & 0 & 0 & x y x y\end{array}\right)\left(\begin{array}{cccccccccc}0 & 0 & 0 & y z y z & y z z x & 0 & z x z x & -z x y z & 0 \\ 0 & 0 & 0 & z x y z & z x z x & 0 & -y z z x & y z y z & 0 \\ x y x x & -x y x x & 0 & 0 & 0 & x y x y & 0 & 0 & x y x y\end{array}\right.$

and $x x x x=x x y y+2 x y x y ; y y x y-x x x y=2 x y x x$ 


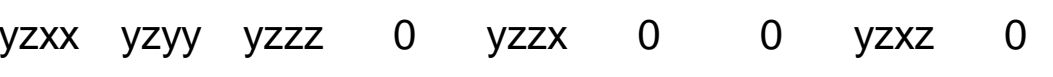
$\begin{array}{lllllllll}0 & 0 & 0 & z x y z & 0 & z x x y & z x z y & 0 & z x y x\end{array}$ $\begin{array}{lllllllll}\text { xyxx } & \text { xyyy } & \text { xyzz } & 0 & \text { xyzx } & 0 & 0 & x y x z & 0\end{array}$ $\begin{array}{lllllllll}\text { zyxx } & \text { zyyy } & \text { zyzz } & 0 & \text { zyzx } & 0 & 0 & \text { zyxz } & 0\end{array}$ $\begin{array}{cccccccccc}\text { zyxx } & \text { zyyy } & \text { zyzz } & 0 & \text { zyzx } & 0 & 0 & z y x z & 0 \\ 0 & 0 & 0 & x z y z & 0 & \text { xzxy } & x z z y & 0 & \text { xzyx }\end{array}$ $\begin{array}{lllllllll}0 & 0 & 0 & y x y z & 0 & y x x y & y x z y & 0 & y x y x\end{array}$

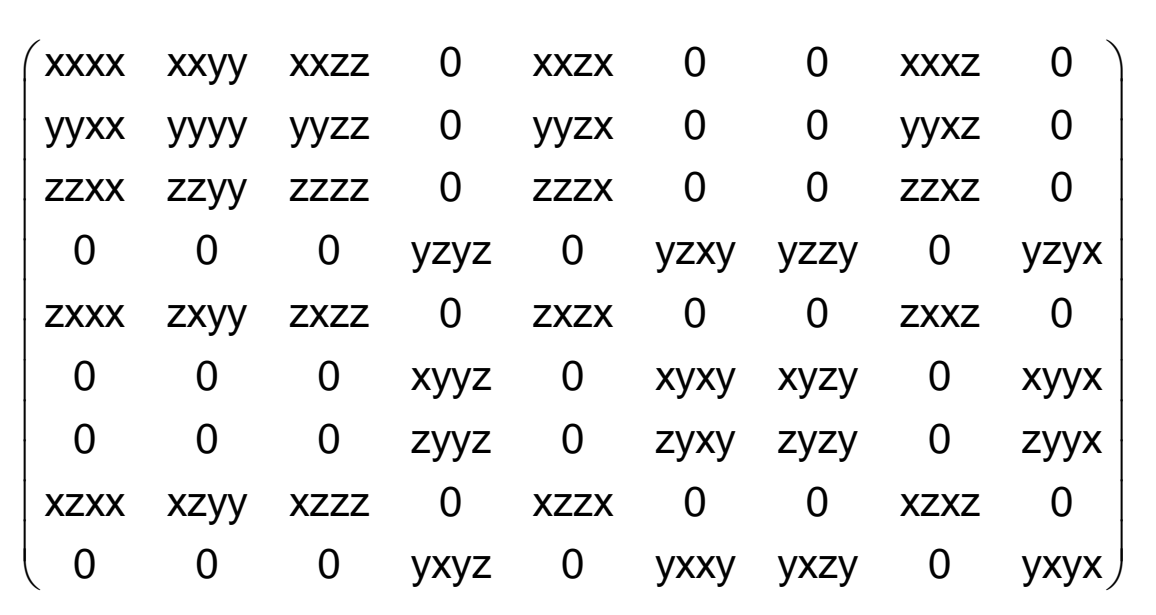
$\left(\begin{array}{lllllllll}0 & 0 & 0 & x x y z & 0 & x x x y & x x z y & 0 & x x y x \\ 0 & 0 & 0 & y y z & 0 & y x y & y y z & 0 & y y x\end{array}\right.$ $\begin{array}{lllllllll}0 & 0 & 0 & y y y z & 0 & \text { yyxy } & \text { yyzy } & 0 & \text { yyyx } \\ 0 & 0 & 0 & z z y z & 0 & z x y & z z z & 0 & z y x\end{array}$ $\begin{array}{ccccccccc}0 & 0 & 0 & z z y z & 0 & z z x y & z z z y & 0 & z z y x \\ y z x x & y z y y & y z z z & 0 & y z z x & 0 & 0 & y z x z & 0 \\ 0 & 0 & 0 & z x y z & 0 & z x y y & z x z y & 0 & z x y x\end{array}$ $\begin{array}{llllllllll}0 & 0 & 0 & z x y z & 0 & z x x y & z x z y & 0 & z x y x\end{array}$

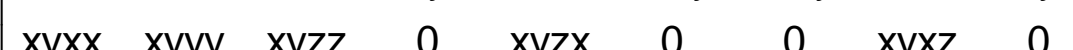

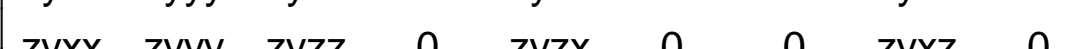

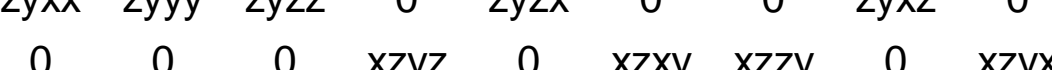
$\begin{array}{ccccccccc}0 & 0 & 0 & x z y z & 0 & x z x y & x z z y & 0 & x z y x \\ y x x x & y x y y & y x z z & 0 & y x z x & 0 & 0 & y x x z & 0\end{array}$

$\left[V^{4}\right]\left(\begin{array}{ccccc}x x x x & 0 & x z z z & y y z z & 0 \\ 0 & y y y y & 0 & z z x x & y y x z \\ z x x x & 0 & z z z z & x x y y & 0\end{array}\right)$ $\left(\begin{array}{ccccc}0 & x y y y & 0 & 0 & x x y z \\ y x x x & 0 & y z z z & 0 & 0 \\ 0 & z y y y & 0 & 0 & z z x y\end{array}\right)$

$\left(\begin{array}{ccccc}x x x x & 0 & x z z z & y y z z & 0 \\ 0 & y y y y & 0 & z z x x & y y x z \\ z x x x & 0 & z z z z & x x y y & 0\end{array}\right)$ $\left(\begin{array}{ccccc}0 & x y y y & 0 & 0 & x x y z \\ y x x x & 0 & y z z z & 0 & 0 \\ 0 & \text { zyyy } & 0 & 0 & z z x y\end{array}\right)$

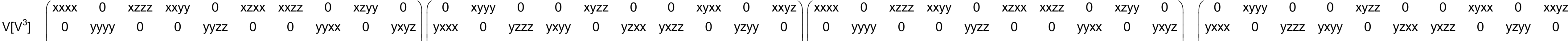

$\left.\left[V^{2}\right]^{2}\right]\left(\begin{array}{cccccc}x x x x & x x y y & x x z z & 0 & x x x z & 0 \\ & y y y y & y y z z & 0 & y y x z & 0 \\ & & z z z z & 0 & z z x z & 0 \\ & & & y z y z & 0 & y z x y \\ & & & & x z x z & 0 \\ & & & & & x y x y\end{array}\right)$

$\left(\begin{array}{llllll}x x x x & x x y y & x x z z & 0 & x x x z & 0 \\ y y x x & y y y & y y z z & 0 & y y z & 0\end{array}\right)$ $\left.\begin{array}{cccccc}x y y x x & y y y y & y y z z & 0 & y y x z & 0\end{array}\right)$ $\begin{array}{lccccc}z z x x & z z y y & z z z z & 0 & z z x z & 0 \\ 0 & 0 & 0 & y z y z & 0 & y z x y\end{array}$ $\begin{array}{llllll}x z x x & x z y y & x z z z & 0 & x z x z & 0\end{array}$ $\begin{array}{llllll}x y x x & x y y y & x y z z & x y y z & 0 & x y x y\end{array}$

$$
\left(\begin{array}{cccccc}
0 & 0 & 0 & \text { xxyz } & 0 & \mathrm{xxxy} \\
& 0 & 0 & \mathrm{yyyz} & 0 & \mathrm{yyxy} \\
& & 0 & \mathrm{zzyz} & 0 & \mathrm{zzxy} \\
& & & 0 & \mathrm{yzxz} & 0 \\
& & & & 0 & \mathrm{xzxy} \\
& & & & & 0
\end{array}\right)
$$

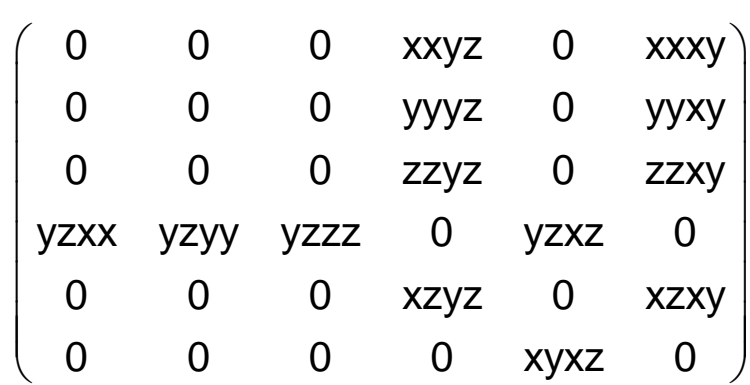

$$
\left(\begin{array}{cccccc}
x x x x & x x y y & x x z z & 0 & x x x z & 0 \\
& y y y y & y y z z & 0 & y y z z & 0 \\
& & z z z z & 0 & z z x z & 0 \\
& & & y z y z & 0 & y z x y \\
& & & & x z x z & 0 \\
& & & & & x y x y
\end{array}\right)
$$

$\left(\begin{array}{llllll}x x x x & x x y y & x x z z & 0 & x x x z & 0\end{array}\right)$ $\left(\begin{array}{llllll}x y x x x & y y y y & y y z z & 0 & y y x z & 0 \\ z z x x & z z y y & z z z z & 0 & z z x z & 0\end{array}\right)$ $\begin{array}{llllll}z z x x & z z y y & z z z z & 0 & z z x z & 0\end{array}$ $\begin{array}{cccccc}0 & 0 & 0 & y z y z & 0 & y z x y\end{array}$ $\begin{array}{llllll}x z x x & x z y y & x z z z & 0 & x z x z & 0\end{array}$

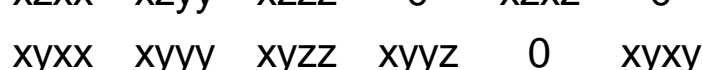

$$
\left(\begin{array}{cccccc}
0 & 0 & 0 & \text { xxyz } & 0 & \text { xxxy } \\
& 0 & 0 & \text { yyyz } & 0 & y y x y \\
& & 0 & z z y z & 0 & z z x y \\
& & & 0 & y z x z & 0 \\
& & & & 0 & x z x y \\
& & & & & 0
\end{array}\right)
$$

$\left(\begin{array}{cccccc}0 & 0 & 0 & x x y z & 0 & x x x y \\ 0 & 0 & 0 & y y y z & 0 & y y x y \\ 0 & 0 & 0 & z z y z & 0 & z z x y \\ y z x x & y z y y & y z z z & 0 & y z x z & 0 \\ 0 & 0 & 0 & x z y z & 0 & x z x y \\ 0 & 0 & 0 & 0 & x y x z & 0\end{array}\right)$


1

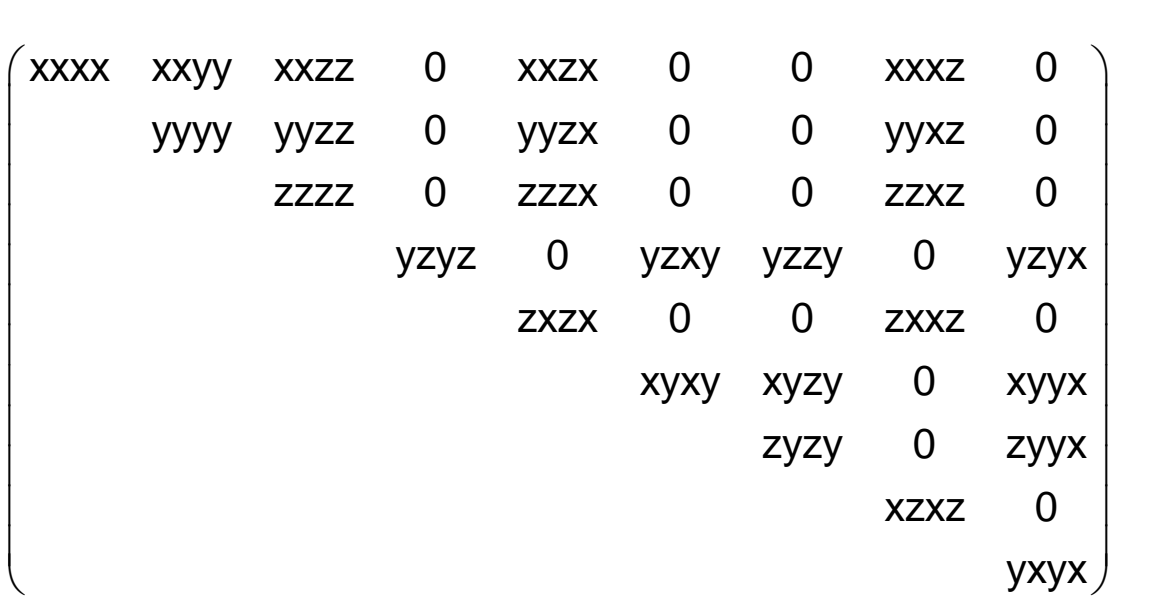

$$
\left(\begin{array}{l}
0 \\
3
\end{array}\right.
$$$$
\left(\begin{array}{ccccccccc}
0 & 0 & 0 & x x y z & 0 & \text { xxxy } & \text { xxzy } & 0 & \text { xxyx } \\
& 0 & 0 & y y y z & 0 & \text { yyxy } & \text { yyzy } & 0 & y y y x \\
& & 0 & z z y z & 0 & \text { zzxy } & \text { zzzy } & 0 & \text { zzyx } \\
& & & 0 & y z z x & 0 & 0 & y z x z & 0 \\
& & & & 0 & \text { zxxy } & \text { zxzy } & 0 & \text { zxyx } \\
& & & & & 0 & 0 & \text { xyxz } & 0 \\
& & & & & & 0 & z y x z & 0 \\
& & & & & & & 0 & \text { xzyx } \\
& & & & & & & 0
\end{array}\right)
$$

$\left(\begin{array}{lllllllll}x x x x & x x y y & x x z z & 0 & x x z x & 0 & 0 & x x x z & 0\end{array}\right)$ $\left(\begin{array}{lllllllll}\text { yyxx } & \text { yyyy } & y y z z & 0 & \text { yyzx } & 0 & 0 & \text { yyxz } & 0 \\ z z x x & \text { zzyy } & z z z z & 0 & \text { zzzx } & 0 & 0 & z z x z & 0\end{array}\right)$

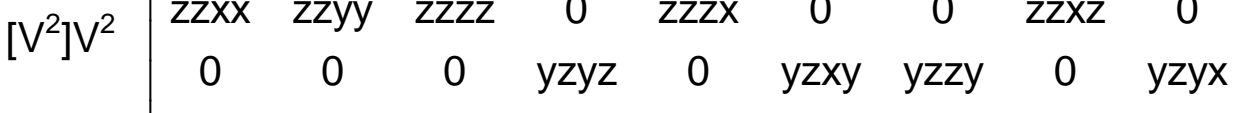
$\begin{array}{ccccccccc}\text { zxxx } & z x y y & z x z z & 0 & z x z x & 0 & 0 & z x x z & 0 \\ 0 & 0 & 0 & x y y z & 0 & x y x & x y z y & 0 & x y y x\end{array}$ $\left(\begin{array}{lllllllll}0 & 0 & 0 & x x y z & 0 & x x x y & x x z y & 0 & x x y x \\ 0 & 0 & 0 & x y y z & 0 & x y x y & y z y & 0 & 0\end{array}\right.$

$\left(\begin{array}{lllllllll}0 & 0 & 0 & \text { yyyz } & 0 & \text { yyxy } & \text { yyzy } & 0 & \text { yyyx } \\ 0 & 0 & 0 & z z y z & 0 & \text { zzy } & z z z & 0 & z z x\end{array}\right.$ $\begin{array}{lllllllll}0 & 0 & 0 & z z y z & 0 & z z x y & z z z y & 0 & z z y x\end{array}$

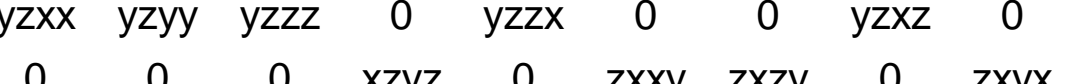

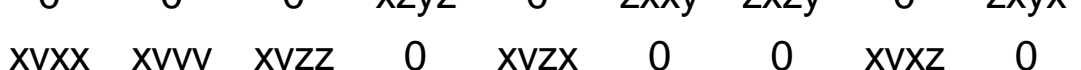

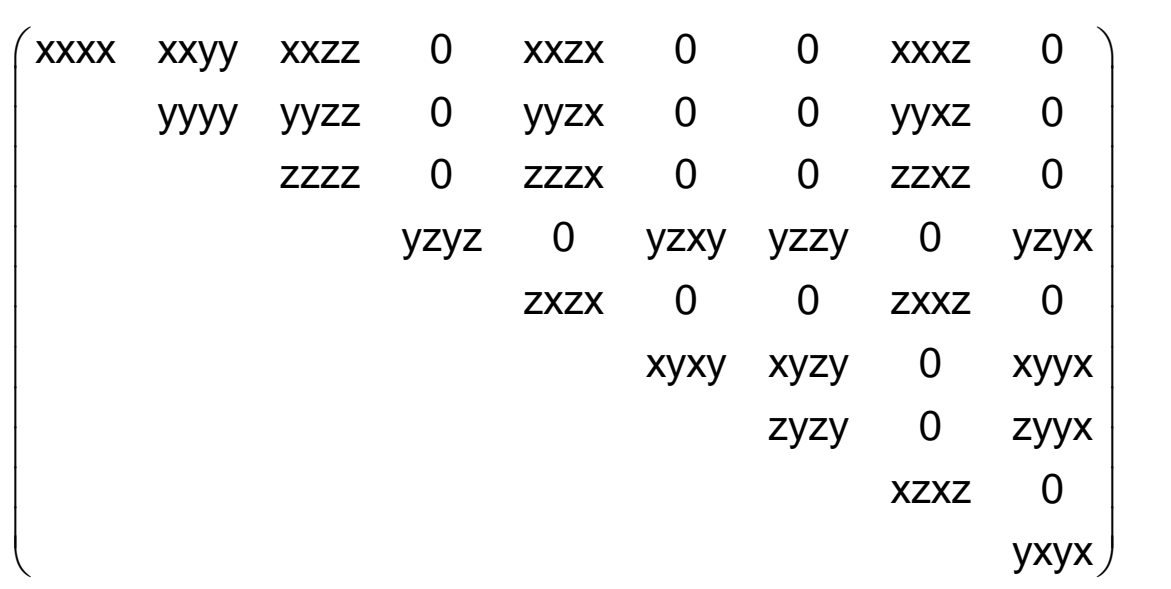

$\left(\begin{array}{lllllllll}0 & 0 & 0 & x x y z & 0 & x x x y & x x z y & 0 & x x y x \\ & 0 & 0 & y y y z & 0 & y y x y & y y z & 0 & y y x\end{array}\right)$

$\begin{array}{llllllll}0 & 0 & \text { yyyz } & 0 & \text { yyxy } & \text { yyzy } & 0 & \text { yyyx }\end{array}$ $\begin{array}{lllllll}0 & z z y z & 0 & z z x y & z z z y & 0 & z z y x\end{array}$ $\begin{array}{cccccc}0 & y z z x & 0 & 0 & y z x z & 0 \\ 0 & z x x y & z x z y & 0 & z x y x\end{array}$ $\begin{array}{llll}0 & 0 & x y x z & 0\end{array}$ $\begin{array}{ccc}0 & x y x z & 0 \\ 0 & z y x z & 0 \\ 0 & x z y x\end{array}$

$\left(\begin{array}{lllllllll}x x x x & x x y y & x x z z & 0 & x x z x & 0 & 0 & x x x z & 0 \\ y x x & y y y & y y z z & 0 & y y z x & 0 & 0 & y y z z & 0\end{array}\right)$ $\left.\begin{array}{lllllllll}\text { yyxx } & \text { yyyy } & y y z z & 0 & \text { yyzx } & 0 & 0 & y y x z & 0 \\ z z x x & z z y y & z z z z & 0 & z z z x & 0 & 0 & z z x z & 0\end{array}\right)$ $\begin{array}{ccccccccc}\text { zzxx } & \text { zzyy } & \text { zzzz } & 0 & z z z x & 0 & 0 & \text { zzxz } & 0 \\ 0 & 0 & 0 & y z y z & 0 & y z x y & y z z y & 0 & y z y x\end{array}$ $\begin{array}{ccccccccc}z x x x & z x y y & z x z z & 0 & z x z x & 0 & 0 & z x x z & 0 \\ 0 & 0 & 0 & x y z & 0 & x y x y & x y z y & 0 & x y y x\end{array}$ 
e

$\left(\begin{array}{ccccccccc}\text { xxxx } & \text { xxyy } & \text { xxzz } & 0 & 0 & 0 & 0 & 0 & 0 \\ \text { yyxx } & \text { yyyy } & \text { yyzz } & 0 & 0 & 0 & 0 & 0 & 0 \\ \text { zzxx } & \text { zzyy } & \text { zzzz } & 0 & 0 & 0 & 0 & 0 & 0 \\ 0 & 0 & 0 & \text { yzyz } & 0 & 0 & \text { yzzy } & 0 & 0 \\ 0 & 0 & 0 & 0 & \text { zxzx } & 0 & 0 & \text { zxxz } & 0 \\ 0 & 0 & 0 & 0 & 0 & x y x y & 0 & 0 & \text { xyyx } \\ 0 & 0 & 0 & z y y z & 0 & 0 & z y z y & 0 & 0 \\ 0 & 0 & 0 & 0 & x z z x & 0 & 0 & \text { xzxz } & 0 \\ 0 & 0 & 0 & 0 & 0 & \text { yxxy } & 0 & 0 & y x y x\end{array}\right)$

$\left[V^{4}\right] \quad\left(\begin{array}{ccccc}x x x x & 0 & 0 & y y z z & 0 \\ 0 & y y y y & 0 & z z x x & 0 \\ 0 & 0 & z z z z & x x y y & 0\end{array}\right)$

$\left(\begin{array}{ccccccccc}0 & 0 & 0 & 0 & 0 & \text { xxxy } & 0 & 0 & \text { xxyx } \\ 0 & 0 & 0 & 0 & 0 & \text { yyxy } & 0 & 0 & \text { yyyx } \\ 0 & 0 & 0 & 0 & 0 & \text { zzxy } & 0 & 0 & \text { zzyx } \\ 0 & 0 & 0 & 0 & \text { yzzx } & 0 & 0 & y z x z & 0 \\ 0 & 0 & 0 & \text { zxyz } & 0 & 0 & \text { zxzy } & 0 & 0 \\ x y x x & x y y y & x y z z & 0 & 0 & 0 & 0 & 0 & 0 \\ 0 & 0 & 0 & 0 & z y z x & 0 & 0 & \text { zyxz } & 0 \\ 0 & 0 & 0 & x z y z & 0 & 0 & x z z y & 0 & 0 \\ y x x x & y x y y & y x z z & 0 & 0 & 0 & 0 & 0 & 0\end{array}\right)$

$\left(\begin{array}{ccccc}0 & x y y y & 0 & 0 & 0 \\ y x x x & 0 & 0 & 0 & 0 \\ 0 & 0 & 0 & 0 & z z x y\end{array}\right)$

$\left(\begin{array}{ccccc}x x x x & 0 & 0 & y y z z & 0 \\ 0 & y y y y & 0 & z z x x & 0 \\ 0 & 0 & z z z z & x x y y & 0\end{array}\right)$

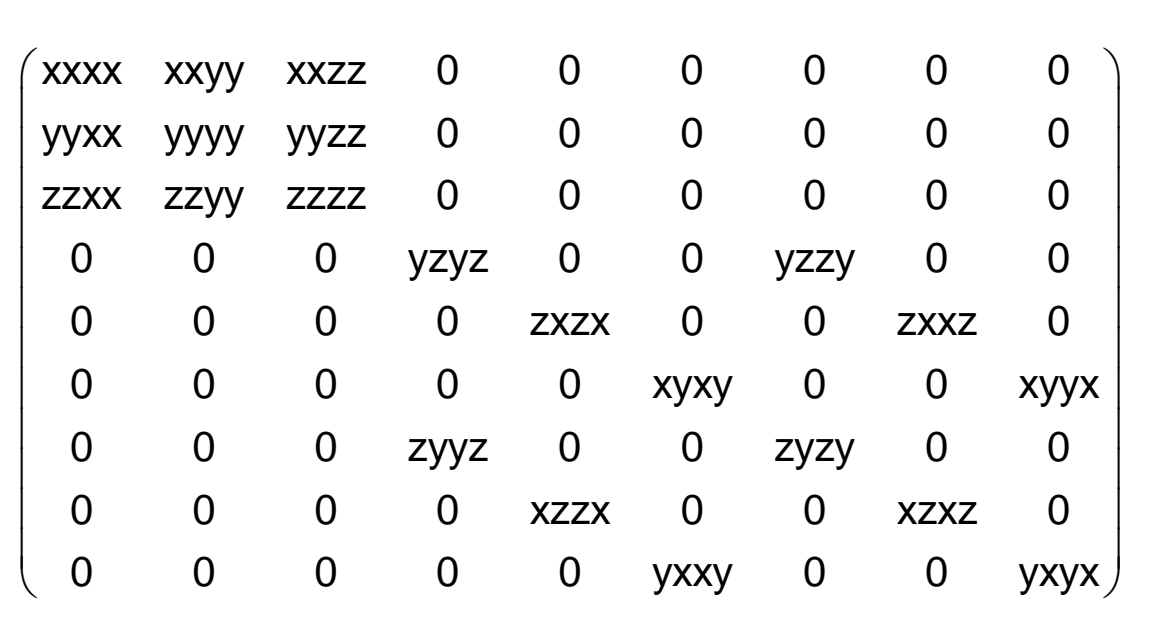

$\left(\begin{array}{ccccccccc}0 & 0 & 0 & 0 & 0 & \text { xxxy } & 0 & 0 & \text { xxyx } \\ 0 & 0 & 0 & 0 & 0 & \text { yyxy } & 0 & 0 & \text { yyyx } \\ 0 & 0 & 0 & 0 & 0 & z z x y & 0 & 0 & \text { zzyx } \\ 0 & 0 & 0 & 0 & \text { yzzx } & 0 & 0 & \text { yzxz } & 0 \\ 0 & 0 & 0 & \text { zxyz } & 0 & 0 & \text { zxzy } & 0 & 0 \\ \text { xyxx } & \text { xyyy } & x y z z & 0 & 0 & 0 & 0 & 0 & 0 \\ 0 & 0 & 0 & 0 & z y z x & 0 & 0 & z y x z & 0 \\ 0 & 0 & 0 & \text { xzyz } & 0 & 0 & \text { xzzy } & 0 & 0 \\ \text { yxxx } & \text { yxyy } & \text { yxzz } & 0 & 0 & 0 & 0 & 0 & 0\end{array}\right)$

$\left(\begin{array}{ccccc}0 & x y y y & 0 & 0 & 0 \\ y x x x & 0 & 0 & 0 & 0 \\ 0 & 0 & 0 & 0 & z z x y\end{array}\right)$

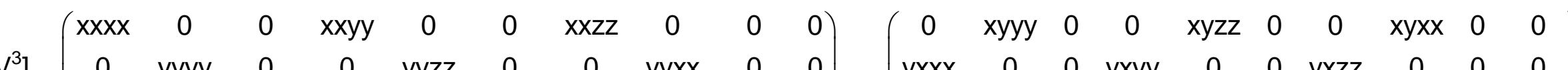

$\left.\mathrm{V}^{3} \mathrm{~V}^{3}\right]\left(\begin{array}{cccccccccc}0 & \mathrm{yyyy} & 0 & 0 & \mathrm{yyzz} & 0 & 0 & \mathrm{yyxx} & 0 & 0 \\ 0 & 0 & \mathrm{zzzz} & 0 & 0 & \mathrm{zzxx} & 0 & 0 & \mathrm{zzyy} & 0\end{array}\right)\left(\begin{array}{cccccccccc}0 \mathrm{yxxx} & 0 & 0 & \mathrm{yxyy} & 0 & 0 & \mathrm{yxzz} & 0 & 0 & 0 \\ 0 & 0 & 0 & 0 & 0 & 0 & 0 & 0 & 0 & \mathrm{zxyz}\end{array}\right)$

$\left[\left[V^{2}\right]^{2}\right]\left(\begin{array}{cccccc}x x x x & x x y y & x x z z & 0 & 0 & 0 \\ & y y y y & y y z z & 0 & 0 & 0 \\ & & z z z z & 0 & 0 & 0 \\ & & & \text { yzyz } & 0 & 0 \\ & & & & x z x z & 0 \\ & & & & & \text { xyxy }\end{array}\right)$

$\left(\begin{array}{cccccc}0 & 0 & 0 & 0 & 0 & \mathrm{xxxy} \\ & 0 & 0 & 0 & 0 & \mathrm{yyxy} \\ & & 0 & 0 & 0 & \mathrm{zzxy} \\ & & & 0 & \mathrm{yzxz} & 0 \\ & & & & 0 & 0 \\ & & & & & 0\end{array}\right)$

$\left[V^{2}\right]^{2}\left(\begin{array}{cccccc}x x x x & x x y y & x x z z & 0 & 0 & 0 \\ y y x x & y y y y & y y z z & 0 & 0 & 0 \\ z z x x & z z y y & z z z z & 0 & 0 & 0 \\ 0 & 0 & 0 & y z y z & 0 & 0 \\ 0 & 0 & 0 & 0 & x z x z & 0 \\ 0 & 0 & 0 & 0 & 0 & x y x y\end{array}\right)$ $\left(\begin{array}{cccccccccc}x x x x & 0 & 0 & x x y y & 0 & 0 & x x z z & 0 & 0 & 0 \\ 0 & y y y y & 0 & 0 & y y z z & 0 & 0 & y y x x & 0 & 0 \\ 0 & 0 & z z z & 0 & 0 & z z x x & 0 & 0 & z z y & 0\end{array}\right) \quad\left(\begin{array}{cccccccccc}0 & x y y y & 0 & 0 & x y z z & 0 & 0 & x y x x & 0 & 0 \\ y x x x & 0 & 0 & y x y y & 0 & 0 & y x z z & 0 & 0 & 0 \\ 0 & 0 & 0 & 0 & 0 & 0 & 0 & 0 & 0 & z x y z\end{array}\right)$

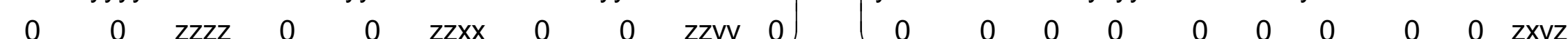

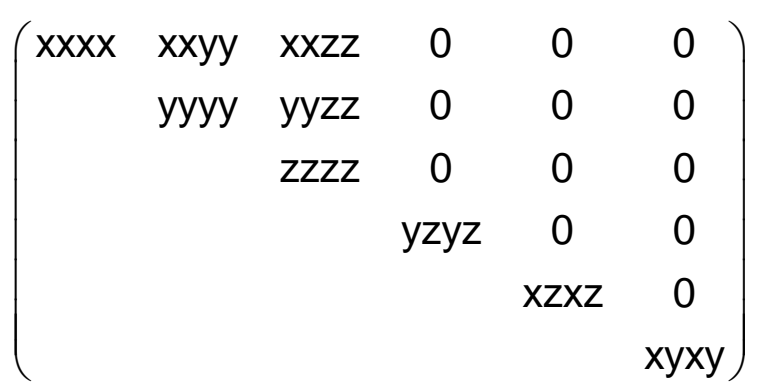

$\left(\begin{array}{cccccc}0 & 0 & 0 & 0 & 0 & \mathrm{xxxy} \\ & 0 & 0 & 0 & 0 & \mathrm{yyxy} \\ & & 0 & 0 & 0 & \mathrm{zzxy} \\ & & & 0 & \mathrm{yzxz} & 0 \\ & & & & 0 & 0 \\ & & & & & 0\end{array}\right)$

$\left(\begin{array}{cccccc}\text { xxxx } & \text { xxyy } & \text { xxzz } & 0 & 0 & 0 \\ \text { yyxx } & \text { yyyy } & \text { yyzz } & 0 & 0 & 0 \\ \text { zzxx } & \text { zzyy } & \text { zzzz } & 0 & 0 & 0 \\ 0 & 0 & 0 & \text { yzyz } & 0 & 0 \\ 0 & 0 & 0 & 0 & \text { xzxz } & 0 \\ 0 & 0 & 0 & 0 & 0 & \text { xyxy }\end{array}\right)$ $\left(\begin{array}{cccccc}0 & 0 & 0 & 0 & 0 & \mathrm{xxxy} \\ 0 & 0 & 0 & 0 & 0 & \mathrm{yyxy} \\ 0 & 0 & 0 & 0 & 0 & \mathrm{zzxy} \\ 0 & 0 & 0 & 0 & \mathrm{yzxz} & 0 \\ 0 & 0 & 0 & \mathrm{xzyz} & 0 & 0 \\ 0 & 0 & 0 & 0 & 0 & 0\end{array}\right)$ 
$\mathrm{n}=2 \quad \mathbf{C}_{2 \mathrm{v}}=\mathbf{2}_{z} \mathbf{m}_{x} \mathbf{m}_{\mathrm{y}}$

$\mathrm{c}_{\mathrm{nv}}$

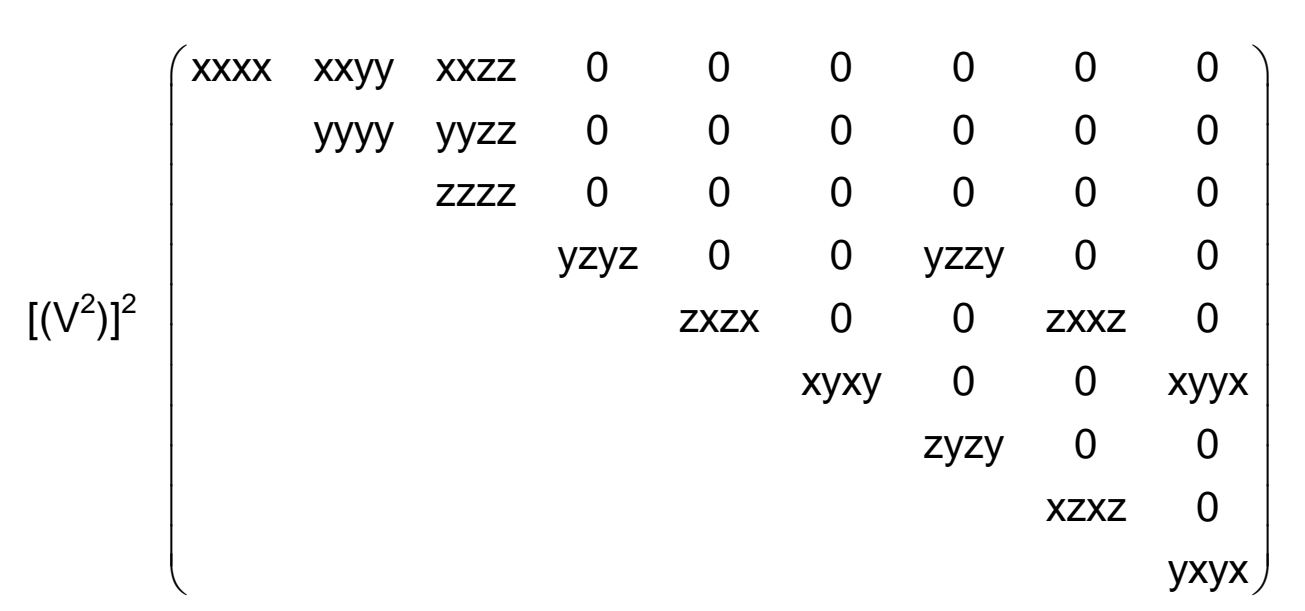

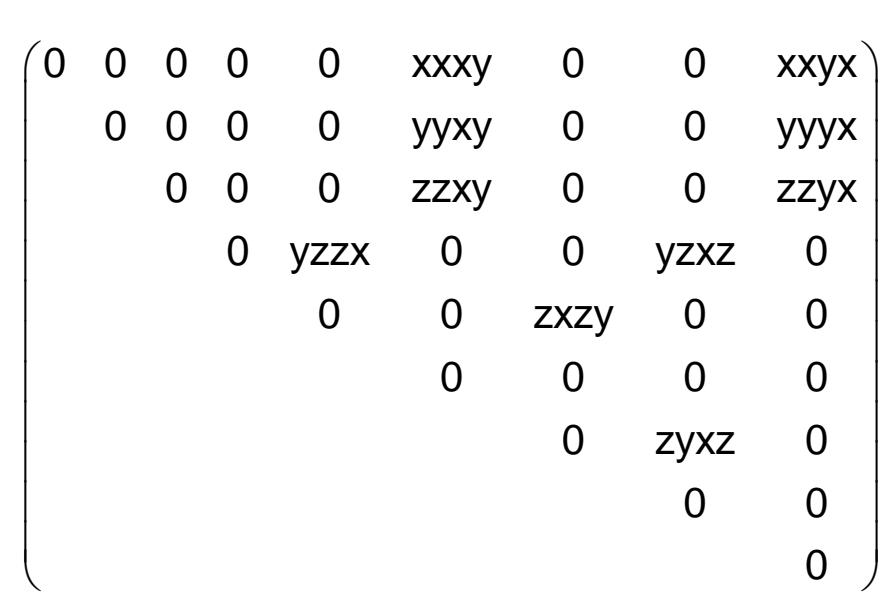

$\left[\mathrm{V}^{2}\right] \mathrm{V}^{2}\left(\begin{array}{ccccccccc}\mathrm{x} x \mathrm{x} & \mathrm{x} x \mathrm{y} y & \mathrm{xxzz} & 0 & 0 & 0 & 0 & 0 & 0 \\ \mathrm{yyxx} & \mathrm{yyyy} & \mathrm{yyzz} & 0 & 0 & 0 & 0 & 0 & 0 \\ \mathrm{zzxx} & \mathrm{zzyy} & \mathrm{zzzz} & 0 & 0 & 0 & 0 & 0 & 0 \\ 0 & 0 & 0 & \mathrm{yzyz} & 0 & 0 & \mathrm{yzzy} & 0 & 0 \\ 0 & 0 & 0 & 0 & \mathrm{zxzx} & 0 & 0 & \mathrm{zxxz} & 0 \\ 0 & 0 & 0 & 0 & 0 & \mathrm{xyxy} & 0 & 0 & \mathrm{xyyx}\end{array}\right)$

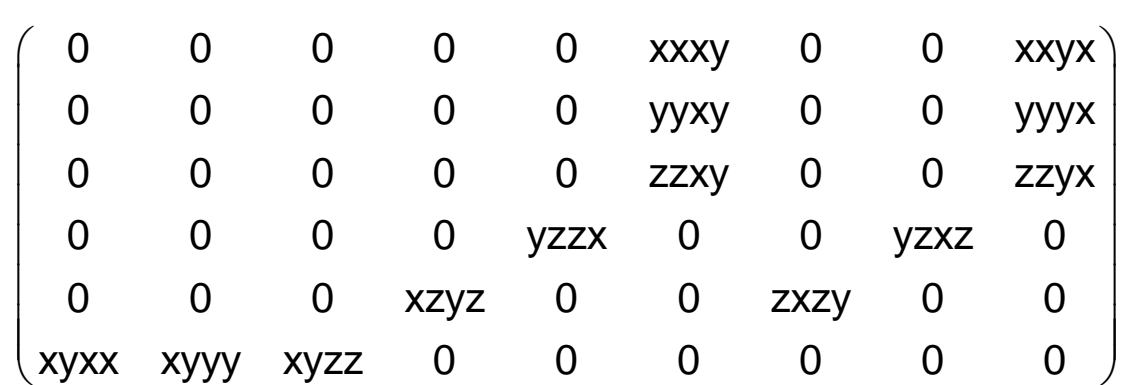

$\left(\begin{array}{ccccccccc}\text { xxxx } & \text { xxyy } & \text { xxzz } & 0 & 0 & 0 & 0 & 0 & 0 \\ & \text { yyyy } & \text { yyzz } & 0 & 0 & 0 & 0 & 0 & 0 \\ & & \text { zzzz } & 0 & 0 & 0 & 0 & 0 & 0 \\ & & & \text { yzyz } & 0 & 0 & \text { yzzy } & 0 & 0 \\ & & & & \text { zxzx } & 0 & 0 & \text { zxxz } & 0 \\ & & & & & \text { xyxy } & 0 & 0 & \text { xyyx } \\ & & & & & & \text { zyzy } & 0 & 0 \\ & & & & & & & \text { xzxz } & 0 \\ & & & & & & & & \text { yxyx }\end{array}\right)$

$\left(\begin{array}{lllllllll}x x x x & x x y y & x x z z & 0 & 0 & 0 & 0 & 0 & 0\end{array}\right)$

$\left.\begin{array}{lllllllll}\text { yyxx } & \text { yyyy } & \text { yyzz } & 0 & 0 & 0 & 0 & 0 & 0 \\ z z x x & \text { zzyy } & \text { zzzz } & 0 & 0 & 0 & 0 & 0 & 0 \\ 0 & 0 & 0 & y z y z & 0 & 0 & y z z y & 0 & 0\end{array}\right)$ $\begin{array}{ccccccccc}\text { zzxx } & \text { zzyy } & \text { zzzz } & 0 & 0 & 0 & 0 & 0 & 0 \\ 0 & 0 & 0 & \text { yzyz } & 0 & 0 & \text { yzzy } & 0 & 0\end{array}$

$\begin{array}{ccccccccc}0 & 0 & 0 & 0 & z x z x & 0 & 0 & z x x z & 0 \\ 0 & 0 & 0 & 0 & 0 & x y x y & 0 & 0 & x y y x\end{array}$

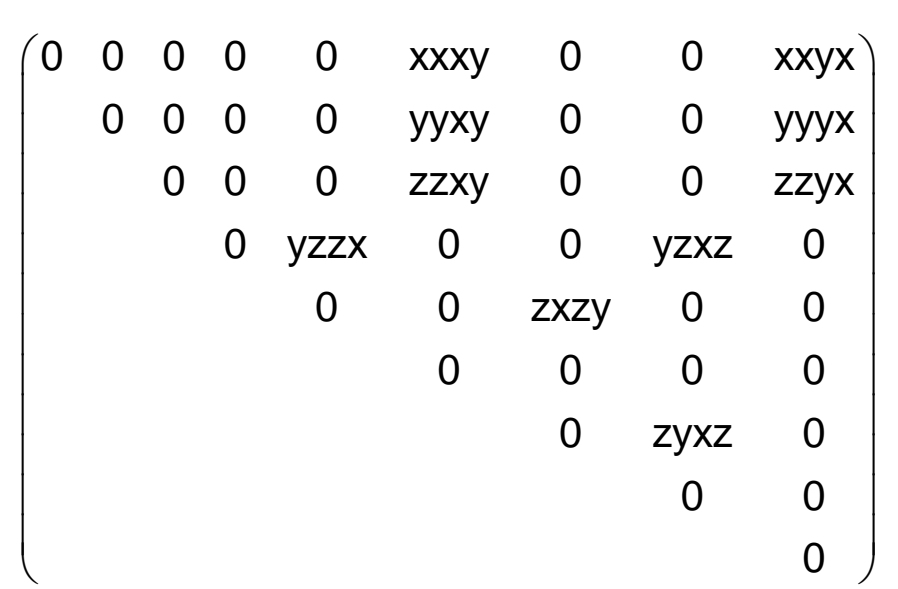

$\left(\begin{array}{ccccccccc}0 & 0 & 0 & 0 & 0 & \text { xxxy } & 0 & 0 & \text { xxyx } \\ 0 & 0 & 0 & 0 & 0 & \text { yyxy } & 0 & 0 & \text { yyyx } \\ 0 & 0 & 0 & 0 & 0 & \text { zzxy } & 0 & 0 & \text { zzyx } \\ 0 & 0 & 0 & 0 & \text { yzzx } & 0 & 0 & \text { yzxz } & 0 \\ 0 & 0 & 0 & \text { xzyz } & 0 & 0 & \text { zxzy } & 0 & 0 \\ \text { xyxx } & \text { xyyy } & \text { xyzz } & 0 & 0 & 0 & 0 & 0 & 0\end{array}\right)$ 
$\mathrm{V}^{4}\left(\begin{array}{ccccccccc}\mathrm{xxxx} & \mathrm{xxyy} & \mathrm{xxzz} & \mathrm{xxyz} & 0 & 0 & \mathrm{xxzy} & 0 & 0 \\ \mathrm{yyxx} & \mathrm{xxxx} & \mathrm{xxzz} & -\mathrm{xxyz} & 0 & 0 & -\mathrm{xxzy} & 0 & 0 \\ \mathrm{zzxx} & \mathrm{zzxx} & \mathrm{zzzz} & 0 & 0 & 0 & 0 & 0 & 0 \\ \mathrm{yzxx} & -\mathrm{yzxx} & 0 & \mathrm{yzyz} & 0 & 0 & \mathrm{yzzy} & 0 & 0 \\ 0 & 0 & 0 & 0 & \mathrm{zxzx} & \mathrm{zyxx} & 0 & \mathrm{zxxz} & \mathrm{zyxx} \\ 0 & 0 & 0 & 0 & \mathrm{xxzy} & \mathrm{xyxy} & 0 & \mathrm{xxyz} & \mathrm{xyyx} \\ \mathrm{zyxx} & -\mathrm{zyxx} & 0 & \mathrm{zxxz} & 0 & 0 & \mathrm{zxzx} & 0 & 0 \\ 0 & 0 & 0 & 0 & \mathrm{yzzy} & \mathrm{yzxx} & 0 & \mathrm{yzyz} & \mathrm{yzxx} \\ 0 & 0 & 0 & 0 & \mathrm{xxzy} & \mathrm{xyyx} & 0 & \mathrm{xxyz} & \mathrm{xyxy}\end{array}\right)$
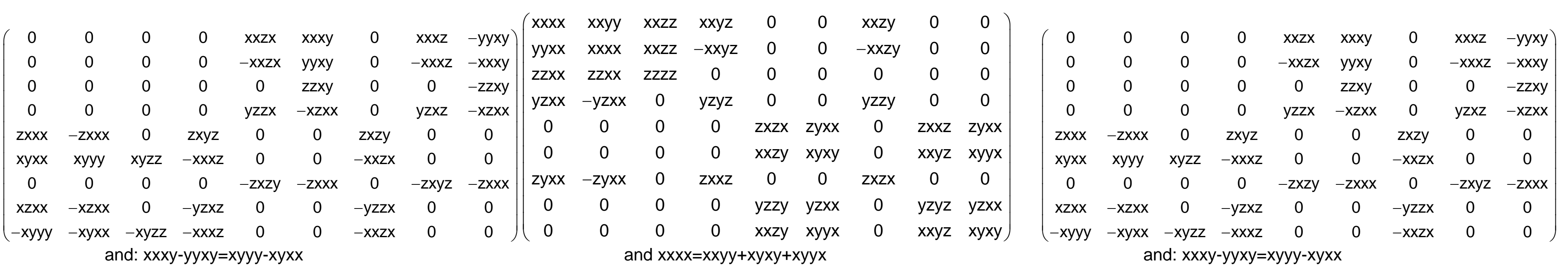

$\left[V^{4}\right] \quad\left(\begin{array}{ccccc}3 x x y y & 0 & 0 & y y z z & x x y z \\ 0 & 3 x x y y & 0 & y y z z & 0 \\ 0 & -x x y z & z z z z & x x y y & 0\end{array}\right)$
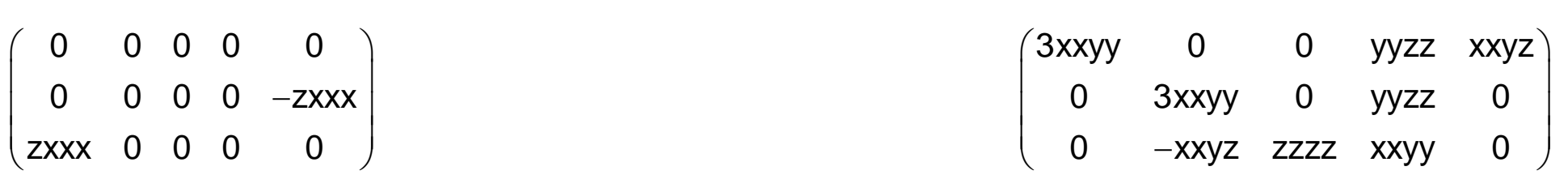

$\left(\begin{array}{ccccc}0 & 0 & 0 & 0 & 0 \\ 0 & 0 & 0 & 0 & -z x x x \\ z x x x & 0 & 0 & 0 & 0\end{array}\right)$

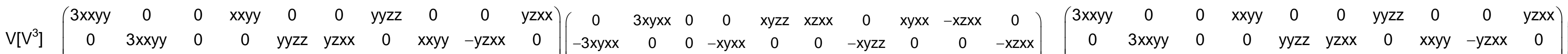
$\left.\begin{array}{cccccccccc}0 & 3 x y x x & 0 & 0 & x y z z & x z x x & 0 & x y x x & -x z x x & 0 \\ -3 x y x x & 0 & 0 & -x y x x & 0 & 0 & -x y z z & 0 & 0 & -x z x x \\ z x x x & 0 & 0 & -z x x x & 0 & 0 & 0 & 0 & 0 & z x y z\end{array}\right)$

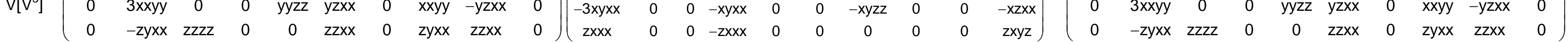

$\left.\left[\mathrm{V}^{2}\right]^{2}\right]\left(\begin{array}{cccccc}x x x x & x x y y & x x z z & x x y z & 0 & 0 \\ & x x x x & x x z z & -x x y z & 0 & 0 \\ & & z z z z & 0 & 0 & 0 \\ & & & y z y z & 0 & 0 \\ & & & & y z y z & x x y z \\ & & & & & x y x y\end{array}\right)$

and $x y x y=1 / 2(x x x x-x x y y)$

$\left[V^{2}\right]^{2}\left(\begin{array}{cccccc}x x x x & x x y y & x x z z & x x y z & 0 & 0 \\ x x y y & x x x x & x x z z & -x x y z & 0 & 0 \\ z z x x & z z x x & z z z z & 0 & 0 & 0 \\ y z x x & -y z x x & 0 & y z y z & 0 & 0 \\ 0 & 0 & 0 & 0 & y z y z & y z x x \\ 0 & 0 & 0 & 0 & x x y z & x y x y\end{array}\right)$

and $x y x y=1 / 2(x x x x-x x y y)$
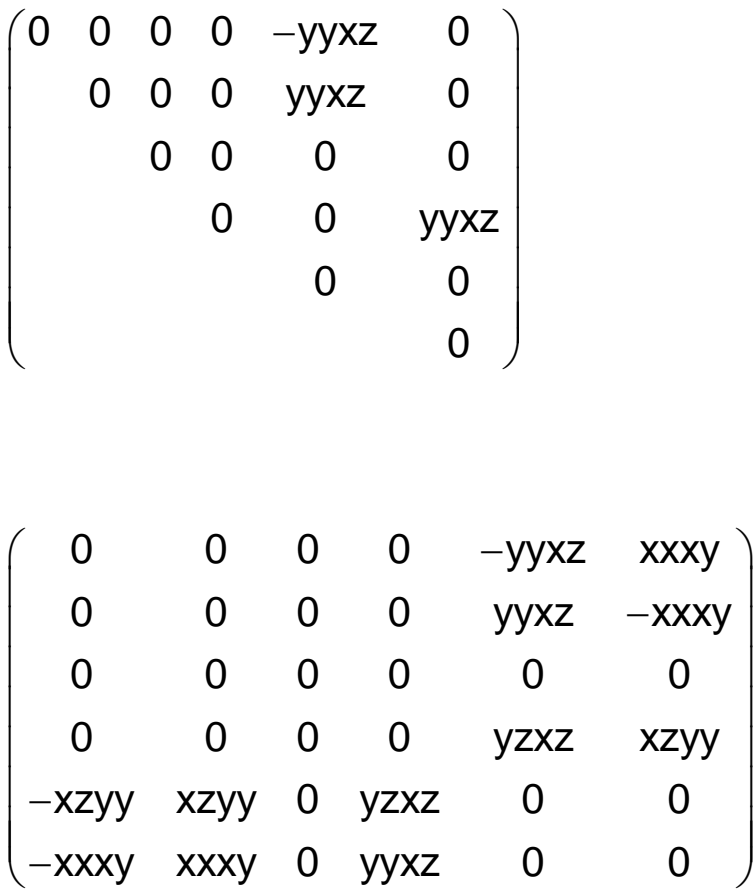

$\begin{array}{llllll}0 & 0 & 0 & 0 & y z x z & x z y \\ -x z y y & x z y y & 0 & y z x z & 0 & 0 \\ -x x y & x x x y & 0 & y y x z & 0 & 0\end{array}$

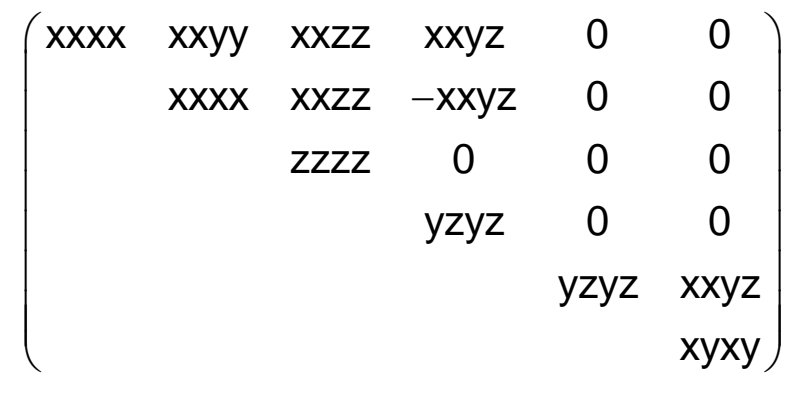

and $x y x y=1 / 2(x x x x-x x y y)$

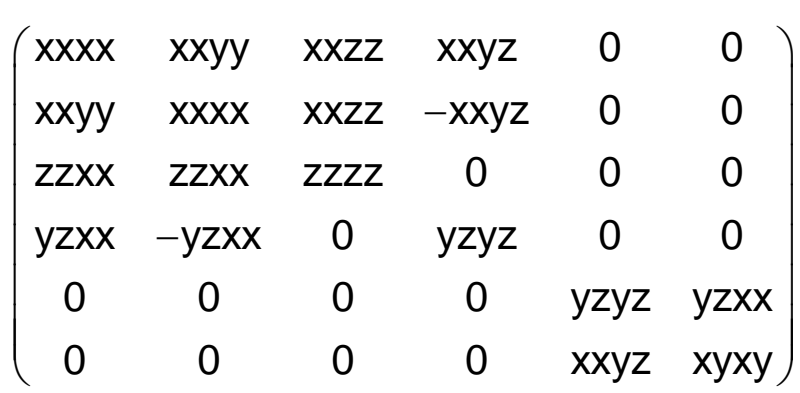

and $x y x y=1 / 2(x x x x-x x y y)$

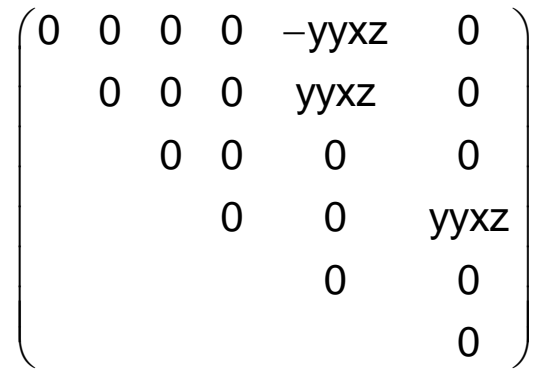

$\left(\begin{array}{cccccc}0 & 0 & 0 & 0 & -y y x z & x x x y \\ 0 & 0 & 0 & 0 & y y x z & -x x x y \\ 0 & 0 & 0 & 0 & 0 & 0 \\ 0 & 0 & 0 & 0 & y z x z & x z y y \\ -x z y y & x z y y & 0 & y z x z & 0 & 0 \\ -x x x y & x x x y & 0 & y y x z & 0 & 0\end{array}\right)$ 


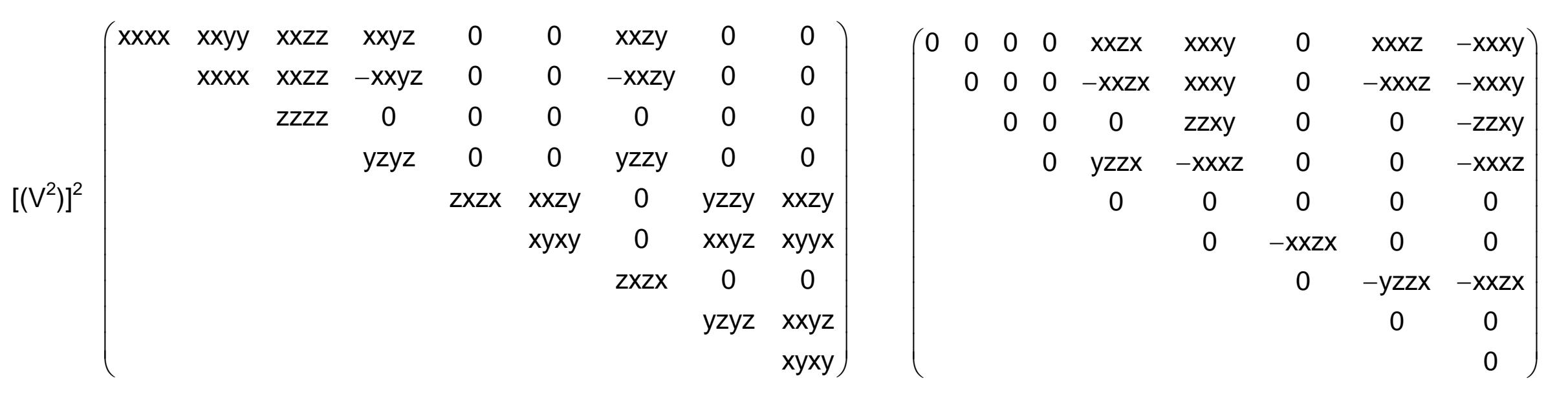

add: $x x x x=x x y y+x y x y+x y y x$

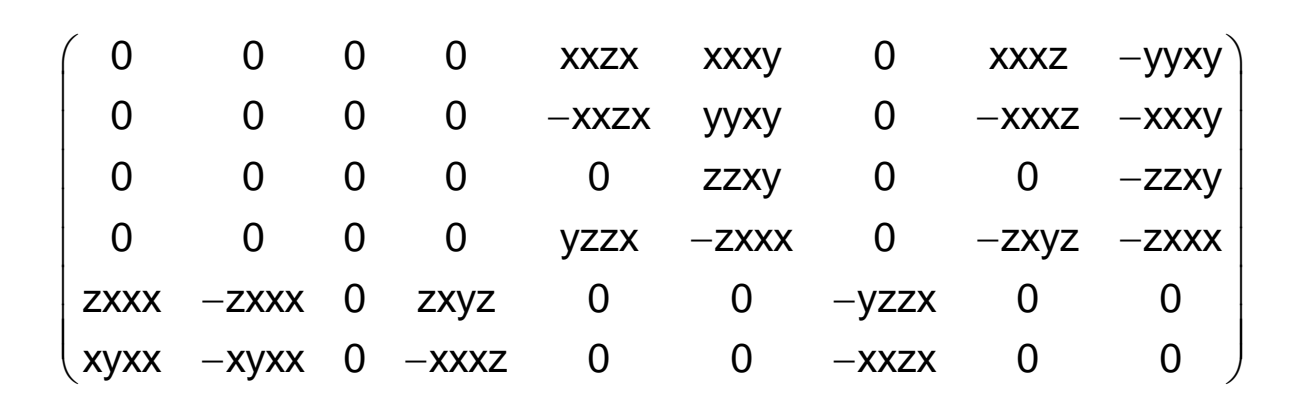

and: $y y x y-x x x y=2 x y x x$ a

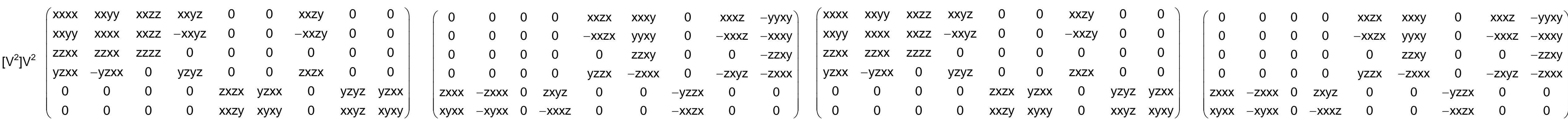
add: $x x x x=x x y y+2 x y x y$ add: $x x x x=x x y y+2 x y x y$

and: $y y x y-x x x y=2 x y x x$ 
e

$\left(\begin{array}{ccccccccc}0 & 0 & 0 & 0 & 0 & x x x y & 0 & 0 & -y y x y \\ 0 & 0 & 0 & 0 & 0 & y y x y & 0 & 0 & -x x x y \\ 0 & 0 & 0 & 0 & 0 & z z x y & 0 & 0 & -z z x y \\ 0 & 0 & 0 & 0 & y z z x & 0 & 0 & y z x z & 0 \\ 0 & 0 & 0 & z x y z & 0 & 0 & z x z y & 0 & 0 \\ x y x x & x y y y & x y z z & 0 & 0 & 0 & 0 & 0 & 0 \\ 0 & 0 & 0 & 0 & -z x z y & 0 & 0 & -z x y z & 0 \\ 0 & 0 & 0 & -y z x z & 0 & 0 & -y z z x & 0 & 0 \\ -x y y y & -x y x x & -x y z z & 0 & 0 & 0 & 0 & 0 & 0\end{array}\right)\left(\begin{array}{ccccccccc}x x x x & x x y y & x x z z & 0 & 0 & 0 & 0 & 0 & 0 \\ x x y y & x x x x & x x z z & 0 & 0 & 0 & 0 & 0 & 0 \\ z z x x & z z x x & z z z z & 0 & 0 & 0 & 0 & 0 & 0 \\ 0 & 0 & 0 & y z y z & 0 & 0 & y z z y & 0 & 0 \\ 0 & 0 & 0 & 0 & z x z x & 0 & 0 & z x x z & 0 \\ 0 & 0 & 0 & 0 & 0 & x y x y & 0 & 0 & x y y x \\ 0 & 0 & 0 & z x x z & 0 & 0 & z x z x & 0 & 0 \\ 0 & 0 & 0 & 0 & \text { yzzy } & 0 & 0 & y z y z & 0 \\ 0 & 0 & 0 & 0 & 0 & x y y x & 0 & 0 & x y x y\end{array}\right)$

$\left[V^{4}\right] \quad\left(\begin{array}{ccccc}x x x x & 0 & 0 & y y z z & 0 \\ 0 & x x x x & 0 & y y z z & 0 \\ 0 & 0 & z z z z & x x y y & 0\end{array}\right)$

$\left(\begin{array}{ccccc}0 & -y x x x & 0 & 0 & 0 \\ y x x x & 0 & 0 & 0 & 0 \\ 0 & 0 & 0 & 0 & 0\end{array}\right)$

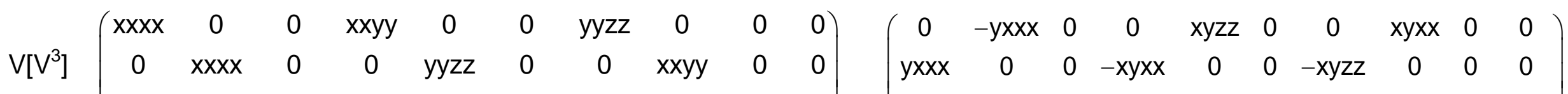

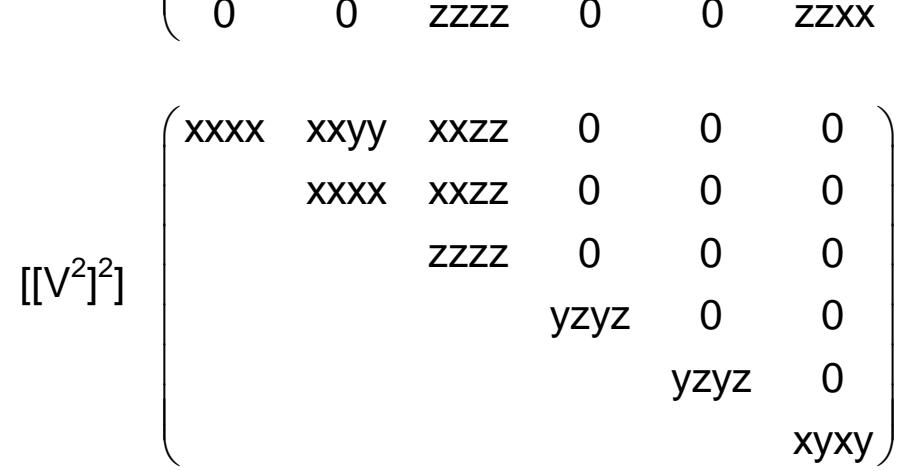

$\begin{array}{llllll}x x x x & x x y y & x x z z & 0 & 0 & 0 \\ x x y & x x x x & x z z & 0 & 0 & 0\end{array}$

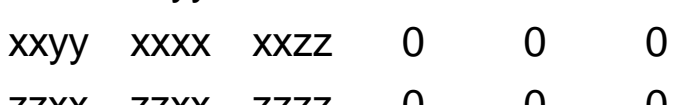

$\begin{array}{cccccc}z z x x & z z x x & z z z z & 0 & 0 & 0 \\ 0 & 0 & 0 & y z y z & 0 & 0\end{array}$

$\begin{array}{cccccc}0 & 0 & 0 & 0 & y z y z & 0 \\ 0 & 0 & 0 & 0 & 0 & x y x y\end{array}$

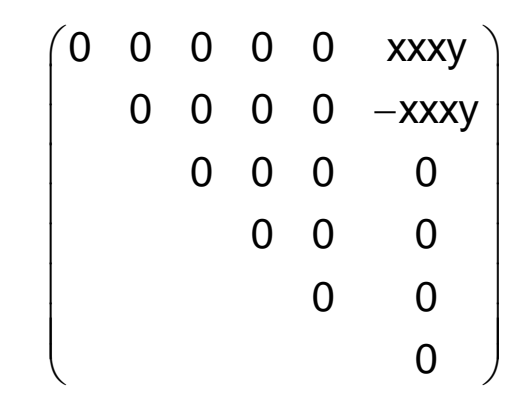

$\left(\begin{array}{cccccc}0 & 0 & 0 & 0 & 0 & \text { xxxy } \\ 0 & 0 & 0 & 0 & 0 & -x x x y \\ 0 & 0 & 0 & 0 & 0 & 0 \\ 0 & 0 & 0 & 0 & y z x z & 0 \\ 0 & 0 & 0 & -y z x z & 0 & 0 \\ x y x x & -x y x x & 0 & 0 & 0 & 0\end{array}\right)$ $\left(\begin{array}{ccccc}\mathrm{xxxx} & 0 & 0 & \mathrm{yyzz} & 0 \\ 0 & \mathrm{xxxx} & 0 & \mathrm{yyzz} & 0 \\ 0 & 0 & \mathrm{zzzz} & \mathrm{xxyy} & 0\end{array}\right)$

$\left(\begin{array}{cccccccccc}x x x x & 0 & 0 & x x y y & 0 & 0 & y y z z & 0 & 0 & 0 \\ 0 & x x x & 0 & 0 & y y z z & 0 & 0 & x x y y & 0 & 0\end{array}\right)$

$\begin{array}{lllccccccc}0 & x x x x & 0 & 0 & y y z z & 0 & 0 & x x y y & 0 & 0\end{array}$

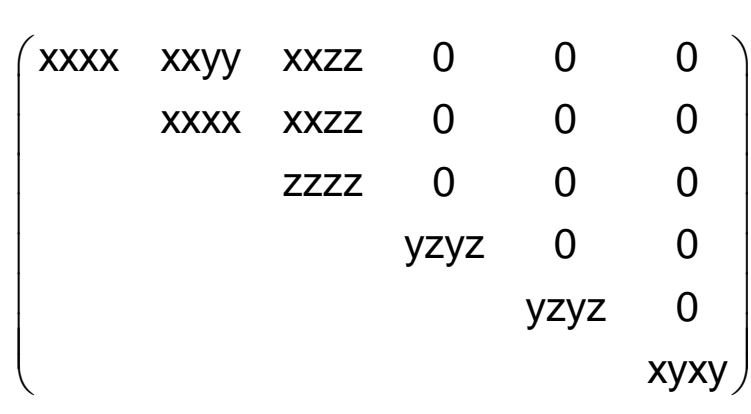

$\left(\begin{array}{cccccc}x x x x & x x y y & x x z z & 0 & 0 & 0 \\ x x y y & x x x x & x x z z & 0 & 0 & 0 \\ z z x x & z z x x & z z z z & 0 & 0 & 0 \\ 0 & 0 & 0 & y z y z & 0 & 0 \\ 0 & 0 & 0 & 0 & y z y z & 0 \\ 0 & 0 & 0 & 0 & 0 & x y x y\end{array}\right)$

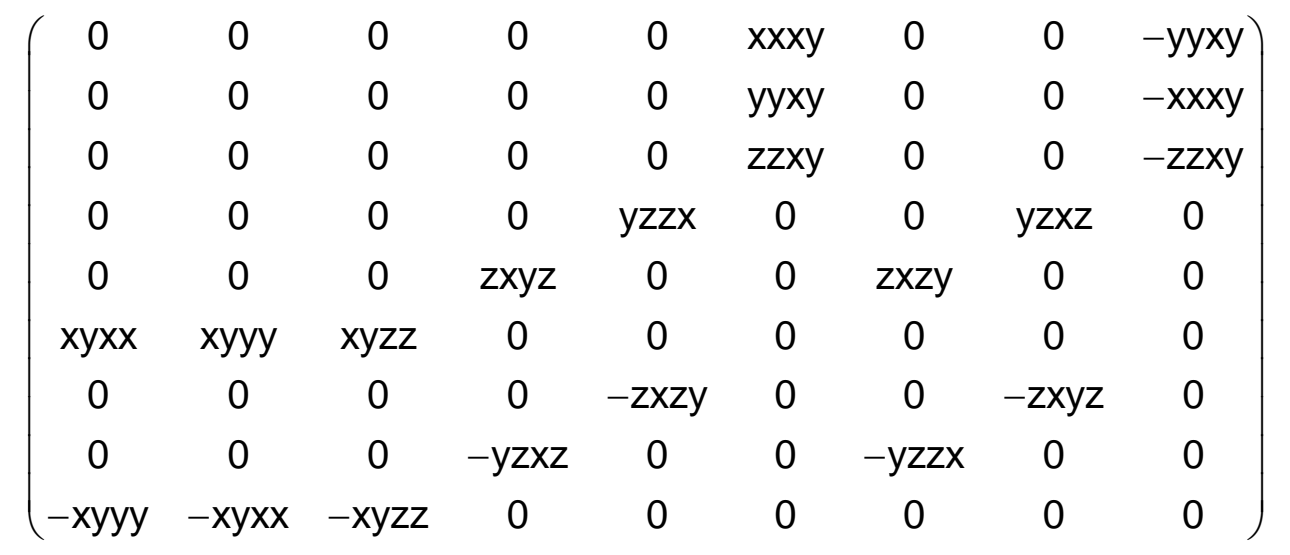

$\left(\begin{array}{ccccc}0 & -y x x x & 0 & 0 & 0 \\ y x x x & 0 & 0 & 0 & 0 \\ 0 & 0 & 0 & 0 & 0\end{array}\right)$

$\left(\begin{array}{cccccccccc}0 & -y \mathrm{xxx} & 0 & 0 & \mathrm{xyzz} & 0 & 0 & \mathrm{xyxx} & 0 & 0 \\ \mathrm{yxxx} & 0 & 0 & -\mathrm{xyxx} & 0 & 0 & -\mathrm{xyzz} & 0 & 0 & 0 \\ 0 & 0 & 0 & 0 & 0 & 0 & 0 & 0 & 0 & z x y z\end{array}\right)$

$\left(\begin{array}{cccccc}0 & 0 & 0 & 0 & 0 & \mathrm{xxxy} \\ & 0 & 0 & 0 & 0 & -\mathrm{xxxy} \\ & & 0 & 0 & 0 & 0 \\ & & & 0 & 0 & 0 \\ & & & & 0 & 0 \\ & & & & & 0\end{array}\right)$

$\left(\begin{array}{cccccc}0 & 0 & 0 & 0 & 0 & \text { xxxy } \\ 0 & 0 & 0 & 0 & 0 & -x x x y \\ 0 & 0 & 0 & 0 & 0 & 0 \\ 0 & 0 & 0 & 0 & y z x z & 0 \\ 0 & 0 & 0 & -y z x z & 0 & 0 \\ x y x x & -x y x x & 0 & 0 & 0 & 0\end{array}\right)$ 
$\mathrm{n}=4 \quad \mathrm{C}_{4 \mathrm{v}}=\mathbf{4}_{\mathrm{z}} \mathrm{m}_{\times} \mathbf{m}_{\mathrm{xy}}$

$c_{n v}$

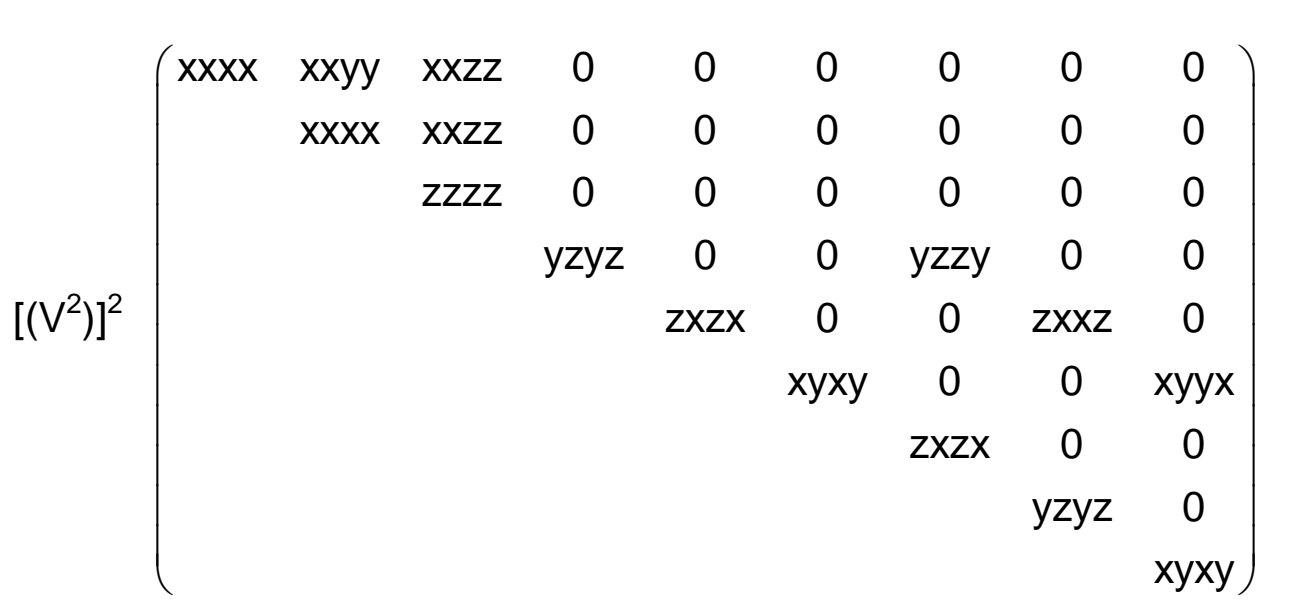

$\left(\begin{array}{ccccccccc}0 & 0 & 0 & 0 & 0 & x x x y & 0 & 0 & -y y x y \\ & 0 & 0 & 0 & 0 & y x y & 0 & 0 & -x y y\end{array}\right)$

$\left.\begin{array}{llllllll}0 & 0 & 0 & 0 & \text { yyxy } & 0 & 0 & -x x x y \\ & 0 & 0 & 0 & z z x y & 0 & 0 & -z z x y\end{array}\right)$

$\begin{array}{llclll}0 & y z z x & 0 & 0 & 0 & 0\end{array}$

$\begin{array}{lllll}0 & 0 & 0 & 0 & 0\end{array}$

$\begin{array}{cccc}0 & 0 & 0 & 0 \\ 0 & -y z z x & 0 \\ & 0 & 0 \\ & & & 0\end{array}$

$\left[\mathrm{V}^{2}\right] \mathrm{V}^{2}\left(\begin{array}{ccccccccc}\mathrm{xxxx} & \mathrm{xxyy} & \mathrm{xxzz} & 0 & 0 & 0 & 0 & 0 & 0 \\ \mathrm{xxyy} & \mathrm{xxxx} & \mathrm{xxzz} & 0 & 0 & 0 & 0 & 0 & 0 \\ \mathrm{zzxx} & \mathrm{zzxx} & \mathrm{zzzz} & 0 & 0 & 0 & 0 & 0 & 0 \\ 0 & 0 & 0 & \mathrm{yzyz} & 0 & 0 & \mathrm{zxzx} & 0 & 0 \\ 0 & 0 & 0 & 0 & \mathrm{zxzx} & 0 & 0 & \mathrm{yzyz} & 0 \\ 0 & 0 & 0 & 0 & 0 & \mathrm{xyxy} & 0 & 0 & \mathrm{xyxy}\end{array}\right)$

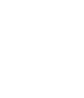

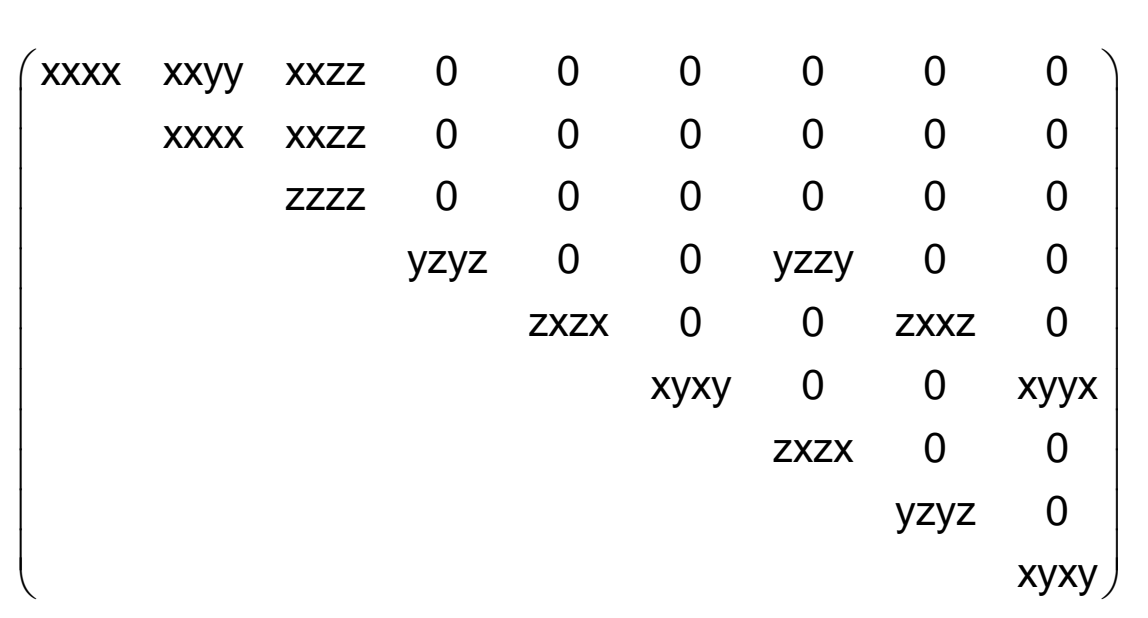

$\left(\begin{array}{lllllllll}x x x x & x x y y & x x z z & 0 & 0 & 0 & 0 & 0 & 0 \\ x x y y & x x x x & x x z z & 0 & 0 & 0 & 0 & 0 & 0 \\ z z x x & z z x & z z z z & 0 & 0 & 0 & 0 & 0 & 0\end{array}\right)$

$\begin{array}{lllllllll}x x y y & x x x x & x x z z & 0 & 0 & 0 & 0 & 0 & 0 \\ z z x x & z z x x & z z z z & 0 & 0 & 0 & 0 & 0 & 0\end{array}$

$\begin{array}{lllllllll}0 & 0 & 0 & y z y z & 0 & 0 & z x z x & 0 & 0\end{array}$

$\begin{array}{ccccccccc}0 & 0 & 0 & y z z z & 0 & 0 & 2 x z x & 0 & 0 \\ 0 & 0 & 0 & 0 & z x z x & 0 & 0 & y z y z & 0 \\ 0 & 0 & 0 & 0 & 0 & x y x y & 0 & 0 & x y x y\end{array}$

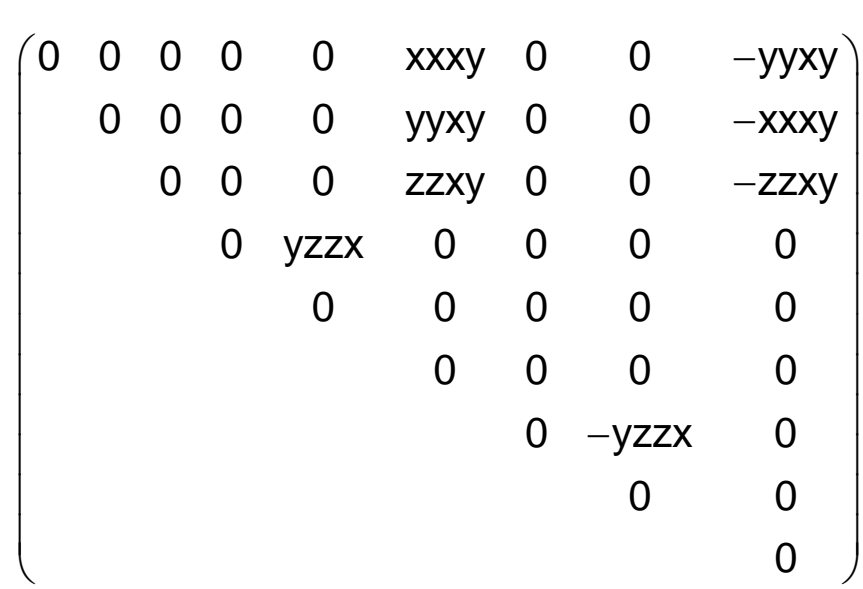

$\left(\begin{array}{ccccccccc}0 & 0 & 0 & 0 & 0 & \text { xxxy } & 0 & 0 & - \text { yyxy } \\ 0 & 0 & 0 & 0 & 0 & \text { yyxy } & 0 & 0 & - \text { xxxy } \\ 0 & 0 & 0 & 0 & 0 & \text { zzxy } & 0 & 0 & - \text { zzxy } \\ 0 & 0 & 0 & 0 & \text { yzzx } & 0 & 0 & -z x y z & 0 \\ 0 & 0 & 0 & \text { zxyz } & 0 & 0 & - \text { yzzx } & 0 & 0 \\ \text { xyxx } & - \text { xyxx } & 0 & 0 & 0 & 0 & 0 & 0 & 0\end{array}\right)$ 
$\mathrm{V}^{4}\left(\begin{array}{ccccccccc}\mathrm{x} x \mathrm{x} x & \mathrm{xxyy} & \mathrm{xxzz} & 0 & 0 & 0 & 0 & 0 & 0 \\ \mathrm{xxyy} & \mathrm{xxxx} & \mathrm{xxzz} & 0 & 0 & 0 & 0 & 0 & 0 \\ \mathrm{zzxx} & \mathrm{zzxx} & \mathrm{zzzz} & 0 & 0 & 0 & 0 & 0 & 0 \\ 0 & 0 & 0 & \mathrm{yzyz} & 0 & 0 & \mathrm{yzzy} & 0 & 0 \\ 0 & 0 & 0 & 0 & \mathrm{zxzx} & 0 & 0 & \mathrm{zxxz} & 0 \\ 0 & 0 & 0 & 0 & 0 & \mathrm{xyxy} & 0 & 0 & \mathrm{xyyx} \\ 0 & 0 & 0 & \mathrm{zxxz} & 0 & 0 & \mathrm{zxzx} & 0 & 0 \\ 0 & 0 & 0 & 0 & \mathrm{yzzy} & 0 & 0 & \mathrm{yzyz} & 0 \\ 0 & 0 & 0 & 0 & 0 & \mathrm{xyyx} & 0 & 0 & \mathrm{xyxy}\end{array}\right)$

e

a

$\left[V^{4}\right] \quad\left(\begin{array}{ccccc}3 x x y y & 0 & 0 & y y z z & 0 \\ 0 & 3 x x y y & 0 & y y z z & 0 \\ 0 & 0 & z z z z & x x y y & 0\end{array}\right)$
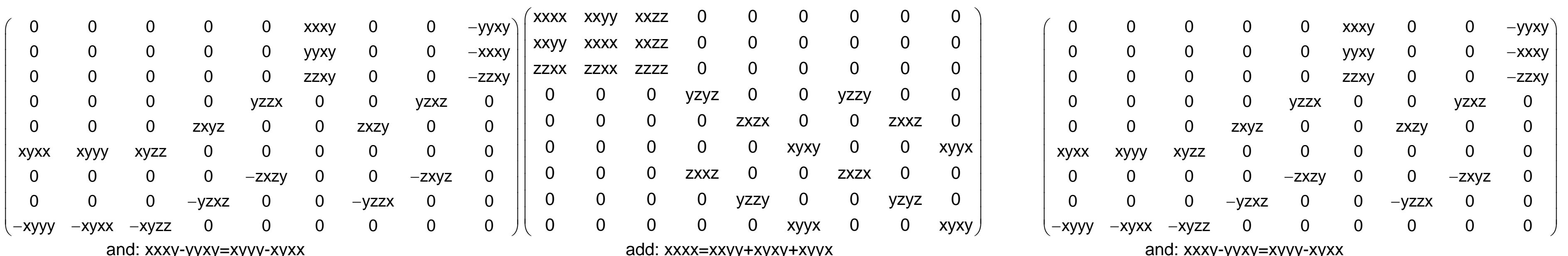

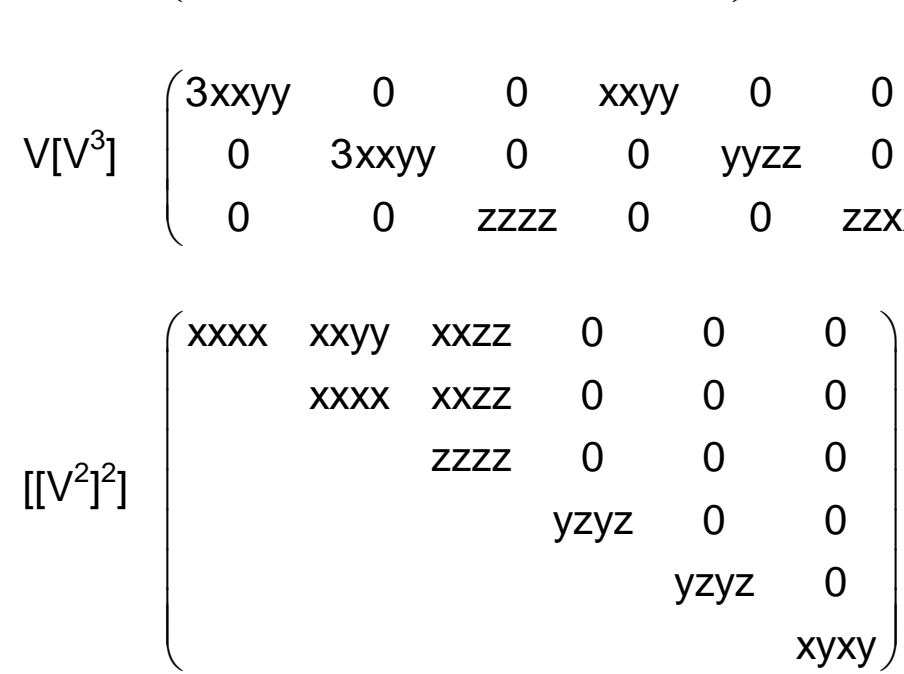

$\left(\begin{array}{lllll}0 & 0 & 0 & 0 & 0 \\ 0 & 0 & 0 & 0 & 0 \\ 0 & 0 & 0 & 0 & 0\end{array}\right)$

$\left(\begin{array}{ccccc}3 x x y y & 0 & 0 & y y z z & 0 \\ 0 & 3 x x y y & 0 & y y z z & 0 \\ 0 & 0 & z z z z & x x y y & 0\end{array}\right)$

$\left(\begin{array}{lllll}0 & 0 & 0 & 0 & 0 \\ 0 & 0 & 0 & 0 & 0 \\ 0 & 0 & 0 & 0 & 0\end{array}\right)$

$\left(\begin{array}{cccccccccc}3 x x y y & 0 & 0 & x x y y & 0 & 0 & y y z z & 0 & 0 & 0 \\ 0 & 3 x x y y & 0 & 0 & y y z z & 0 & 0 & x x y y & 0 & 0 \\ 0 & 0 & z z z z & 0 & 0 & z z x x & 0 & 0 & z z x & 0\end{array}\right)\left(\begin{array}{cccccccccc}0 & 3 x y x x & 0 & 0 & x y z z & 0 & 0 & x y x x & 0 & 0 \\ -3 x y x x & 0 & 0 & -x y x x & 0 & 0 & -x y z z & 0 & 0 & 0 \\ 0 & 0 & 0 & 0 & 0 & 0 & 0 & 0 & 0 & z x y\end{array}\right)$

$\left.\begin{array}{cccccccccc}0 & 0 & \text { zzzz } & 0 & 0 & \text { zzxx } & 0 & 0 & \text { zzxx } & 0\end{array}\right)\left(\begin{array}{lll}0 & 0\end{array}\right.$

$$
\left(\begin{array}{cccccc}
x x x x & x x y y & x x z z & 0 & 0 & 0 \\
& x x x x & x x z z & 0 & 0 & 0 \\
& & z z z z & 0 & 0 & 0 \\
& & & y z y z & 0 & 0 \\
& & & & y z y z & 0 \\
& & & & & \text { xyxy }
\end{array}\right)
$$

and $x y x y=1 / 2(x x x x-x x y y)$

$\left(\begin{array}{cccccc}x x x x & x x y y & x x z z & 0 & 0 & 0 \\ x x y y & x x x x & x x z z & 0 & 0 & 0 \\ \text { zzxx } & z z x x & z z z z & 0 & 0 & 0 \\ 0 & 0 & 0 & y z y z & 0 & 0 \\ 0 & 0 & 0 & 0 & y z y z & 0 \\ 0 & 0 & 0 & 0 & 0 & x y x y\end{array}\right)$

and $x y x y=1 / 2(x x x x-x x y y)$

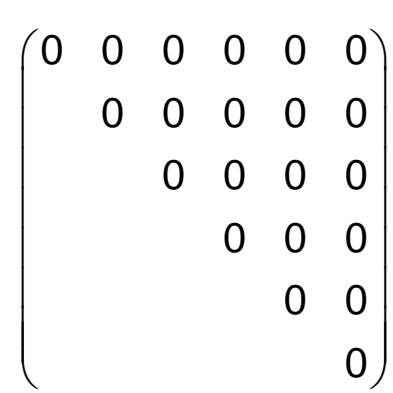

$\left(\begin{array}{cccccc}0 & 0 & 0 & 0 & 0 & x x x y \\ 0 & 0 & 0 & 0 & 0 & -x x x y \\ 0 & 0 & 0 & 0 & 0 & 0 \\ 0 & 0 & 0 & 0 & y z x z & 0 \\ 0 & 0 & 0 & -y z x z & 0 & 0 \\ -x x x y & x x x y & 0 & 0 & 0 & 0\end{array}\right)$

and $x y x y=1 / 2(x x x x-x x y y)$

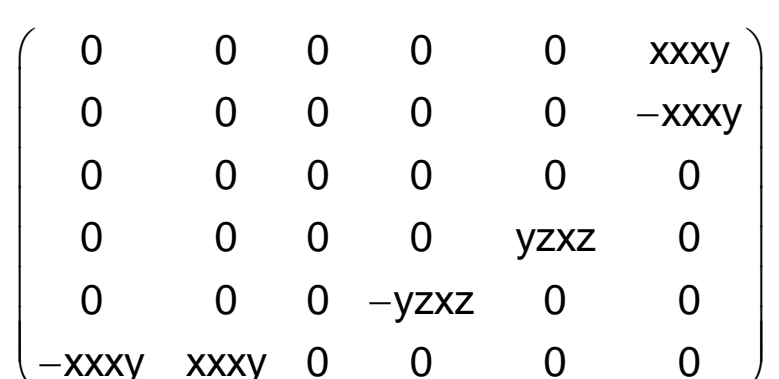

$\begin{array}{cccccc}-x x x y & x x x y & 0 & 0 & 0 & 0\end{array}$ 
$\mathrm{n}>4 \quad \mathrm{C}=\infty \mathrm{mm}$

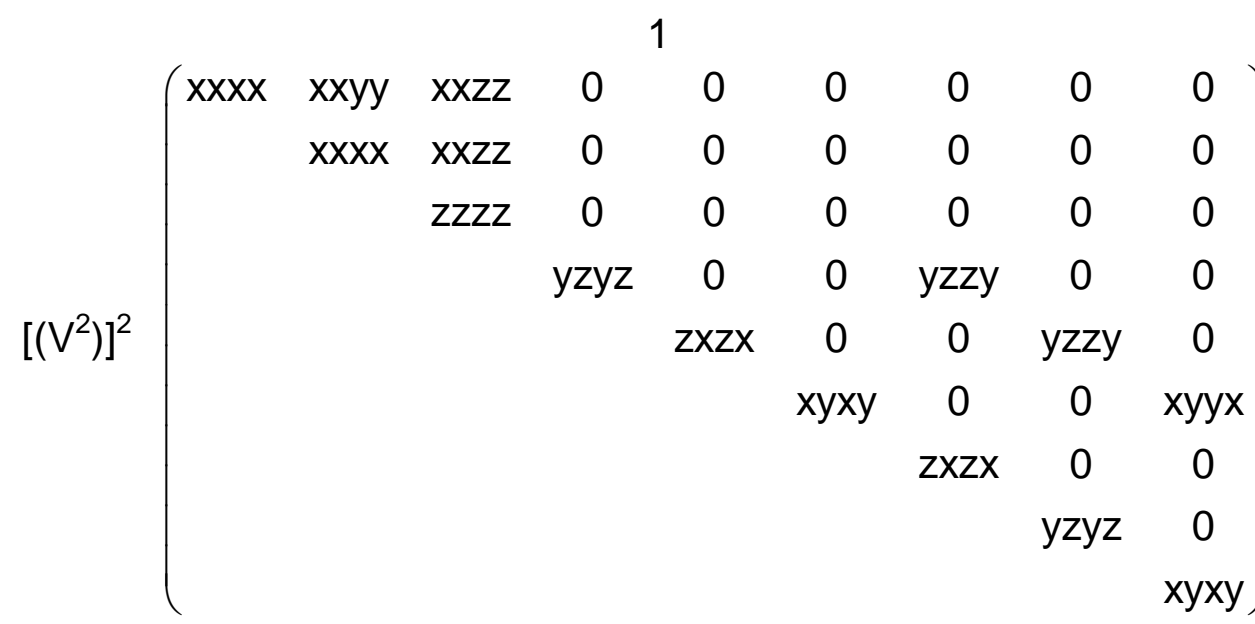

and $x x x x=x x y y+y z y z+z y z y$

$\left[\mathrm{V}^{2}\right] \mathrm{V}^{2}\left(\begin{array}{ccccccccc}\mathrm{xxxx} & \mathrm{xxyy} & \mathrm{xxzz} & 0 & 0 & 0 & 0 & 0 & 0 \\ \mathrm{xxyy} & \mathrm{xxxx} & \mathrm{xxzz} & 0 & 0 & 0 & 0 & 0 & 0 \\ \mathrm{zzxx} & \mathrm{zzxx} & \mathrm{zzzz} & 0 & 0 & 0 & 0 & 0 & 0 \\ 0 & 0 & 0 & \mathrm{yzyz} & 0 & 0 & \mathrm{zxzx} & 0 & 0 \\ 0 & 0 & 0 & 0 & \mathrm{zxzx} & 0 & 0 & \mathrm{yzyz} & 0 \\ 0 & 0 & 0 & 0 & 0 & \mathrm{xyxy} & 0 & 0 & \mathrm{xyxy}\end{array}\right)$

and: $x x x x=x x y y+2 x y x y$

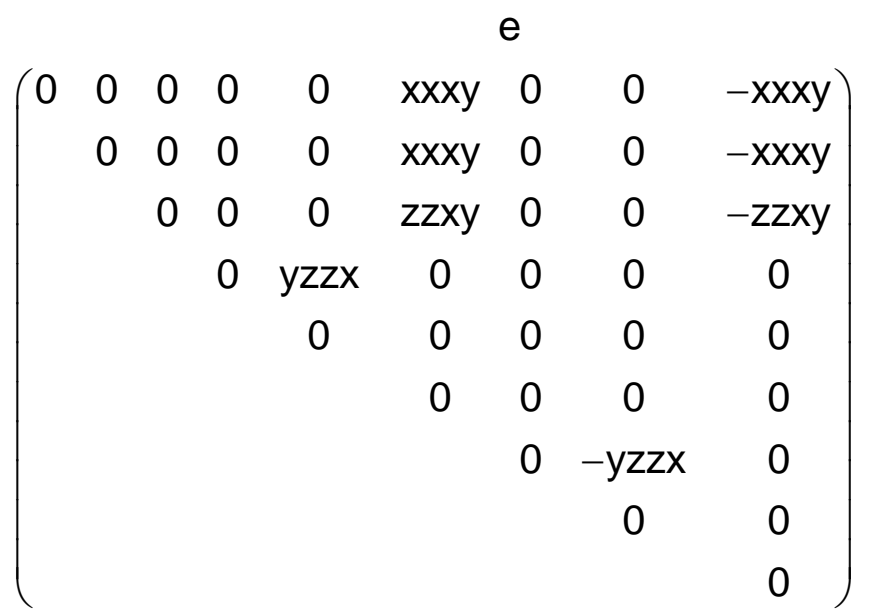

$\left(\begin{array}{ccccccccc}0 & 0 & 0 & 0 & 0 & \text { xxxy } & 0 & 0 & -y y x y \\ 0 & 0 & 0 & 0 & 0 & \text { yyxy } & 0 & 0 & - \text { xxxy } \\ 0 & 0 & 0 & 0 & 0 & z z x y & 0 & 0 & -z z x y \\ 0 & 0 & 0 & 0 & y z z x & 0 & 0 & -z x y z & 0 \\ 0 & 0 & 0 & z x y z & 0 & 0 & -y z z x & 0 & 0 \\ \text { xyxx } & -x y x x & 0 & 0 & 0 & 0 & 0 & 0 & 0\end{array}\right)$

and: $y y x y-x x x y=2 x y x x$
$C_{n v}$
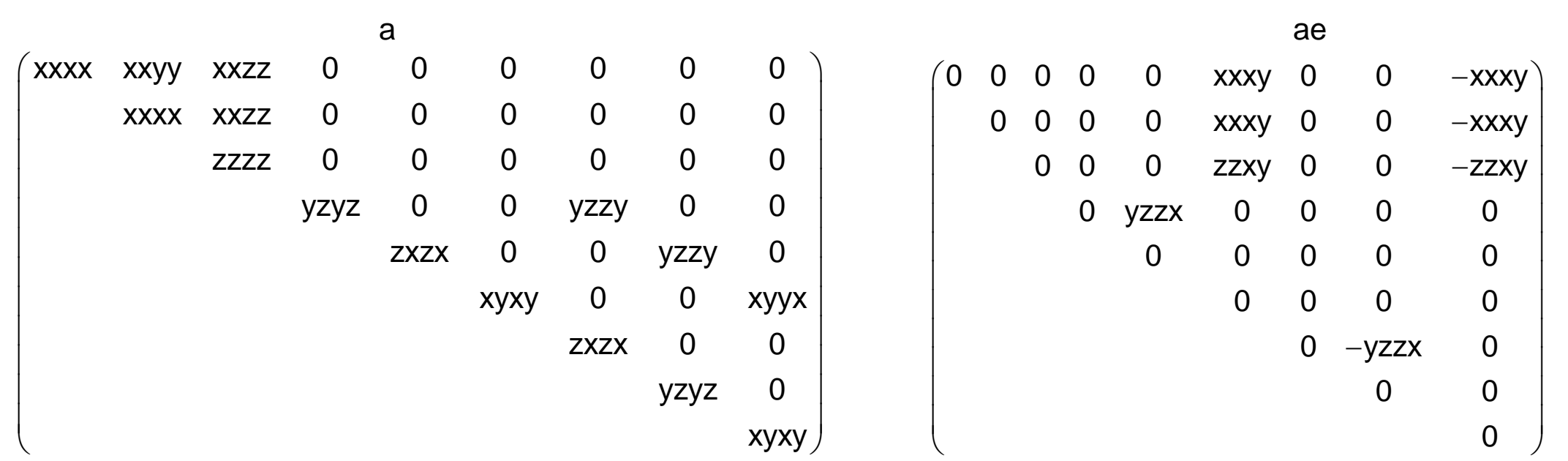

and $x x x x=x x y y+y z y z+z y z y$

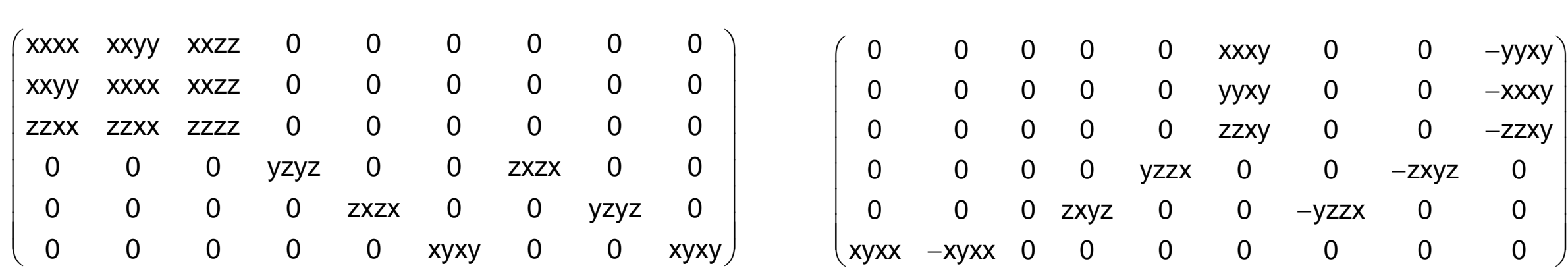

and: $x x x x=x x y y+2 x y x y$ and: $y y x y-x x x y=2 x y x x$ 
$\begin{array}{lllllllll}x x x x & x x y y & x x z z & x x y z & x x z x & x x x y & x x z y & x x x z & x x y x\end{array}$

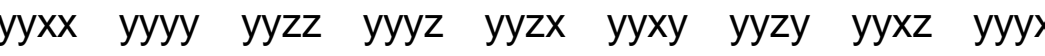
$\begin{array}{lllllllll}z z x x & \text { zzyy } & z z z z & z z y z & \text { zzzx } & \text { zzxy } & \text { zzzy } & \text { zzxz } & \text { zzyx } \\ \text { yzxx } & \text { yzyy } & \text { yzzz } & \text { yzyz } & \text { yzzx } & \text { yzxy } & \text { yzzy } & \text { yzxz } & \text { yzyx }\end{array}$ $\begin{array}{llllllllll}z x x x & z x y y & z x z z & z x y z & z x z x & z x x y & z x z y & z x x z & z x y x\end{array}$

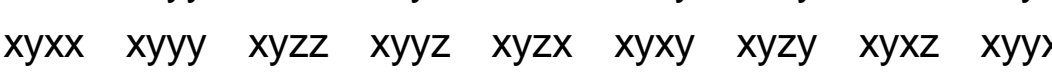
zyxx zyyy zyzz zyyz zyzx zyxy zyzy zyxz zyyx $\begin{array}{lllllllll}x z x x & x z y y & x z z z & x z y z & x z z x & x z x y & x z z y & x z x z & x z y x\end{array}$ $\begin{array}{lllllllll}y x x x & y x y y & y x z z & y x y z & y x z x & y x x y & y x z y & y x x z & y x y x\end{array}$

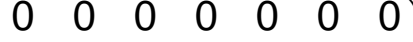
$\left(\begin{array}{llllllllll}0 & 0 & 0 & 0 & 0 & 0 & 0 & 0 & 0 \\ 0 & 0 & 0 & 0 & 0 & 0 & 0 & 0 & 0 \\ 0 & 0 & 0 & 0 & 0 & 0 & 0 & 0 & 0\end{array}\right.$ $\begin{array}{lllllllll}0 & 0 & 0 & 0 & 0 & 0 & 0 & 0 & 0 \\ 0 & 0 & 0 & 0 & 0 & 0 & 0 & 0 & 0\end{array}$ $\begin{array}{llllllllll}0 & 0 & 0 & 0 & 0 & 0 & 0 & 0 & 0\end{array}$ $\begin{array}{lllllllllllll}0 & 0 & 0 & 0 & 0 & 0 & 0 & 0 & 0 \\ 0 & 0 & 0 & 0 & 0 & 0 & 0 & 0 & 0\end{array}$ $\begin{array}{lllllllllllll}0 & 0 & 0 & 0 & 0 & 0 & 0 & 0 & 0 \\ 0 & 0 & 0 & 0 & 0 & 0 & 0 & 0 & 0\end{array}$ $\begin{array}{llllllllll}0 & 0 & 0 & 0 & 0 & 0 & 0 & 0 & 0 \\ 0 & 0 & 0 & 0 & 0 & 0 & 0 & 0 & 0\end{array}$ $\begin{array}{lllllllll}0 & 0 & 0 & 0 & 0 & 0 & 0 & 0 & 0 \\ 0 & 0 & 0 & 0 & 0 & 0 & 0 & 0 & 0\end{array}$ $\left(\begin{array}{lllllllll}0 & 0 & 0 & 0 & 0 & 0 & 0 & 0 & 0\end{array}\right)$

$\left.V^{4}\right] \quad\left(\begin{array}{lllll}x x x x & x y y y & x z z z & y y z z & x x y z \\ y x x x & y y y y & y z z z & z z x x & y y x z \\ z x y x & z y y & z z z z & x x y & z z x y\end{array}\right)$

$\left(\begin{array}{lllll}0 & 0 & 0 & 0 & 0 \\ 0 & 0 & 0 & 0 & 0 \\ 0 & 0 & 0 & 0 & 0\end{array}\right)$

$\left(\begin{array}{lllll}0 & 0 & 0 & 0 & 0\end{array}\right.$

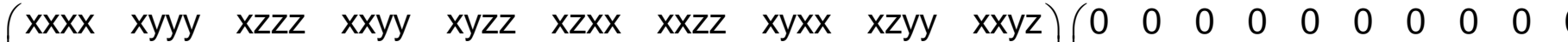

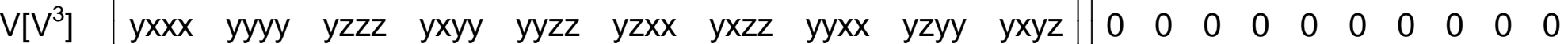

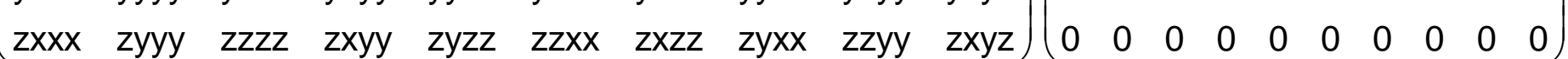

$\left.\left[\mathrm{V}^{2}\right]^{2}\right]$

$\begin{array}{llllll}x x x x & x x y y & x x z z & x x y z & x x x z & x x x y\end{array}$

yyyy

$\begin{array}{llll}2 z y z & z z x z & z z x y\end{array}$

$\begin{array}{lll}y z y z & y z x z & y z x y \\ & x z x z & x z x y\end{array}$

$x y x y$
$x y x y$

$\begin{array}{llllll}x x x x & x x y y & x x z z & x x y z & x x x z & x x x y\end{array}$

yyxx yyyy yyzz $\quad$ yyyz $\quad y y x z \quad$ yyxy

$\begin{array}{llllll}z z x x & z z y y & z z z z & z z y z & z z x z & z z x y\end{array}$

yzxx yzyy $y z z z$ yzyz $y z x z \quad y z x y$

$\begin{array}{llllll}x z x x & x z y y & x z z z & x z y z & x z x z & x z x y \\ (x y x & x y y y & x y z & x y y z & x y z & x y x y\end{array}$

$\begin{array}{llllll}x y x x & x y y y & x y z z & x y y z & x y x z & x y x y\end{array}$

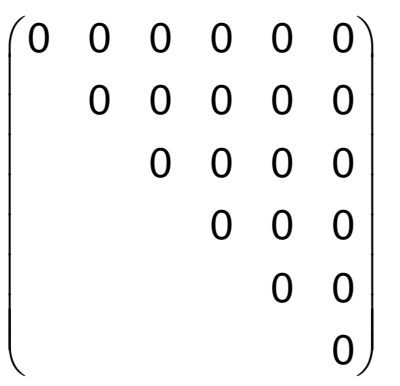

$\left(\begin{array}{llllll}0 & 0 & 0 & 0 & 0 & 0 \\ 0 & 0 & 0 & 0 & 0 & 0\end{array}\right.$

$\begin{array}{llllll}0 & 0 & 0 & 0 & 0 & 0 \\ 0 & 0 & 0 & 0 & 0 & 0\end{array}$

$\begin{array}{llllll}0 & 0 & 0 & 0 & 0 & 0 \\ 0 & 0 & 0 & 0 & 0 & 0\end{array}$

$\begin{array}{llllll}0 & 0 & 0 & 0 & 0 & 0 \\ 0 & 0 & 0 & 0 & 0 & 0\end{array}$

$0 \begin{array}{llllll}0 & 0 & 0 & 0 & 0 & 0 \\ 0 & 0 & 0 & 0 & 0 & 0\end{array}$ $\begin{array}{lllllllll}x x x x & x x y y & x x z z & x x y z & x x z & x x x y & x x z y & x x x z & x x y x\end{array}$

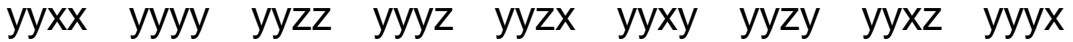
$\begin{array}{lllllllll}\text { zzxx } & \text { zzyy } & \text { zzzz } & \text { zzyz } & \text { zzzx } & \text { zzxy } & \text { zzzy } & \text { zzxz } & \text { zzyx } \\ \text { yzxx } & \text { yzyy } & \text { yzzz } & \text { yzyz } & \text { yzzx } & \text { yzxy } & \text { yzzy } & \text { yzxz } & \text { yzyx }\end{array}$ yzxx yzyy yzzz yzyz yzzx $\begin{array}{lllllllll}z x x x & z x y y & z x z z & z x y z & z x z x & z x x y & z x z y & z x x z & z x y x\end{array}$

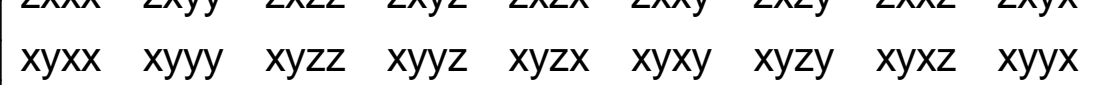
$\begin{array}{lllllllll}x y y x & x y y & x y z z & x y y z & x y z x & x y x y & x y z y & x y x z & x y y x \\ z y x x & z y y y & z y z z & z y y z & z y z x & z y x y & z y z y & z y x z & z y y x\end{array}$ $\begin{array}{lllllllll}x z x x & x z y y & x z z z & x z y z & x z z x & x z x y & x z z y & x z x z & x z y x\end{array}$

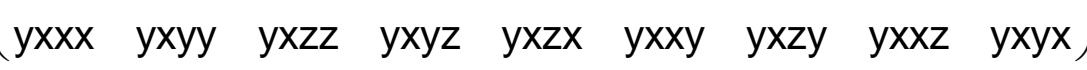

$\left(\begin{array}{lllll}x x x x & x y y y & x z z z & y y z z & x x y z \\ y x x x & y y y y & y z z z & z z x x & x y x z \\ z x x x & z y y & z z z z & x x y & z z x y\end{array}\right)$ $\begin{array}{lllll}\text { yxxx } & y y y y & y z z z & z z x x & y y x z\end{array}$

$\left.\begin{array}{llll}0 & 0 & 0 & 0 \\ 0 & 0 & 0 & 0\end{array}\right)$

$\left(\begin{array}{llllllllllll}x x x x & x y y y & x z z z & x x y y & x y z z & x z x x & x x z z & x y x x & x z y y & x x y z\end{array}\right)\left(\begin{array}{llllllllll}0 & 0 & 0 & 0 & 0 & 0 & 0 & 0 & 0 & 0\end{array}\right)$ $\left(\begin{array}{lllllllllll}x x x x & y y y y & y z z z & y x y y & y y z z & y z x x & y x z z & y y x x & y z y y & y x y z\end{array}\left(\begin{array}{lllllllllll}0 & 0 & 0 & 0 & 0 & 0 & 0 & 0 & 0 & 0 & 0 \\ 0 & 0 & 0 & 0 & 0 & 0 & 0 & 0 & 0 & 0\end{array}\right)\right.$ $\left.\begin{array}{lllllllllll}\text { zxxx } & \text { zyyy } & \text { zzzz } & \text { zxyy } & \text { zyzz } & z z x x & z x z z & \text { zyxx } & \text { zzyy } & \text { zxyz }\end{array}\right)\left(\begin{array}{llllllllll}0 & 0 & 0 & 0 & 0 & 0 & 0 & 0 & 0 & 0\end{array}\right.$

$\left(\begin{array}{llllll}x x x x & x x y y & x x z z & x x y z & x x x z & x x x y\end{array}\right)$

yyy $\begin{array}{llll}z z z z & z z y z & z z x z & z z x y\end{array}$ $\begin{array}{lll}y z y z & y z x z & y z x y \\ & x z z z & x z x y\end{array}$ $x z x y$
$x y x y$

$\left(\begin{array}{llllll}x x x x & x x y y & x x z z & x x y z & x x x z & x x x y\end{array}\right)$ yyxx $\quad$ yyyy $\quad$ yyzz $\quad$ yyyz $\quad$ yyxz $\quad$ yyxy $\begin{array}{llllll}\text { zzxx } & \text { zzyy } & \text { zzzz } & \text { zzyz } & \text { zzxz } & \text { zzxy }\end{array}$ $y z x x$ yzyy $y z z z \quad y z y z \quad y z x z \quad y z x y$

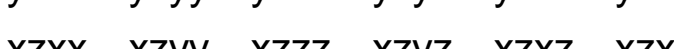
$\begin{array}{llllll}x z x x & x z y y & x z z z & x z y z & x z x z & x z x y \\ x y x x & x y y y & x y z z & x y y z & x y x z & x y x y\end{array} \mid$ $\begin{array}{lllllllll}0 & 0 & 0 & 0 & 0 & 0 & 0 & 0 & 0\end{array}$

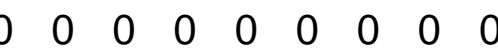
$\begin{array}{llllllll}0 & 0 & 0 & 0 & 0 & 0 & 0 & 0 \\ 0 & 0 & 0 & 0 & 0 & 0 & 0 & 0\end{array}$ $\begin{array}{lllllllll}0 & 0 & 0 & 0 & 0 & 0 & 0 & 0 & 0 \\ 0 & 0 & 0 & 0 & 0 & 0 & 0 & 0 & 0\end{array}$ $\begin{array}{llllllllll}0 & 0 & 0 & 0 & 0 & 0 & 0 & 0 & 0 \\ 0 & 0 & 0 & 0 & 0 & 0 & 0 & 0 & 0\end{array}$ $\begin{array}{llllllllll}0 & 0 & 0 & 0 & 0 & 0 & 0 & 0 & 0 \\ 0 & 0 & 0 & 0 & 0 & 0 & 0 & 0 & 0\end{array}$ $\begin{array}{lllllllll}0 & 0 & 0 & 0 & 0 & 0 & 0 & 0 & 0 \\ 0 & 0 & 0 & 0 & 0 & 0 & 0 & 0 & 0\end{array}$ $\begin{array}{lllllllll}0 & 0 & 0 & 0 & 0 & 0 & 0 & 0 & 0\end{array}$

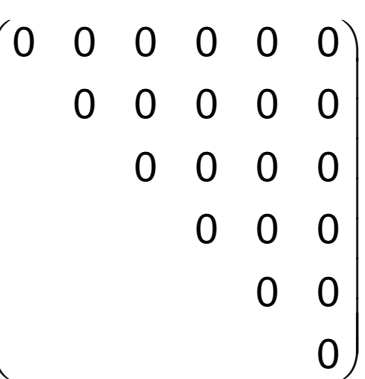

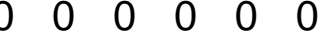
$\begin{array}{llllll}0 & 0 & 0 & 0 & 0 & 0 \\ 0 & 0 & 0 & 0 & 0 & 0\end{array}$ $\begin{array}{llllll}0 & 0 & 0 & 0 & 0 & 0 \\ 0 & 0 & 0 & 0 & 0 & 0\end{array}$ $\begin{array}{llllll}0 & 0 & 0 & 0 & 0 & 0\end{array}$ $\begin{array}{llllll}0 & 0 & 0 & 0 & 0 & 0 \\ 0 & 0 & 0 & 0 & 0 & 0\end{array}$ $\begin{array}{llllll}0 & 0 & 0 & 0 & 0 & 0\end{array}$ 
$\begin{array}{llllllllll}x x x x & x x y y & x x z z & x x y z & x x z x & x x x y & x x z y & x x x z & x x y x\end{array}$

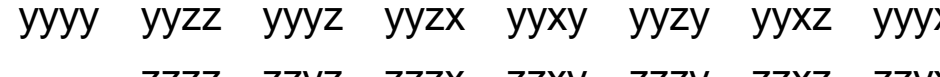
$\begin{array}{lllllll}z z z z & \text { zzyz } & \text { zzzx } & \text { zzxy } & \text { zzzy } & \text { zzxz } & \text { zzyx }\end{array}$ $\begin{array}{llllll}y z y z & y z z x & y z x y & y z z y & y z x z & y z y x\end{array}$ $\begin{array}{llllll}z x z x & z x x y & z x z y & z x x z & z x y x\end{array}$ $\begin{array}{llll}x y x y & x y z y & x y x z & x y y x \\ z y z y & z y x z & z y y x \\ & & x z z & x z y x\end{array}$ $\begin{array}{lll}z y z y & z y x z & z y y x \\ x z x z & x z y x \\ & & y x y x\end{array}$ $y x y x$

$\begin{array}{llllll}0 & 0 & 0 & 0 & 0 & 0 \\ 0 & 0 & 0 & 0 & 0 & 0\end{array}$

$\begin{array}{lllll}0 & 0 & 0 & 0 & 0\end{array}$

$\begin{array}{lllll}0 & 0 & 0 & 0 & 0\end{array}$

$\begin{array}{llll}0 & 0 & 0 & 0 \\ & 0 & 0 & 0\end{array}$

$\begin{array}{lll}0 & 0 & 0 \\ & 0 & 0 \\ & & 0\end{array}$

0

$\begin{array}{lllllllll}x x x x & x x y y & x x z z & x x y z & x x z x & x x x y & x x z y & x x x z & x x y x\end{array}$ yyxx yyyy yyzz yyyz yyzx yyxy $\left[V^{2}\right] V^{2} \quad \begin{array}{llllllllll}z z x x & z z y y & z z z z & z z y z & z z z x & z z x y & z z z y & z z x z & z z y x \\ y z x x & y z y & y z z z & y z y z & y z z x & y z x y & y z z y & y z x z & y z y x \\ & z x x x & z x y & z x z z & z x y z & z x z & z x x y & z x z y & z x z & z x y\end{array}$

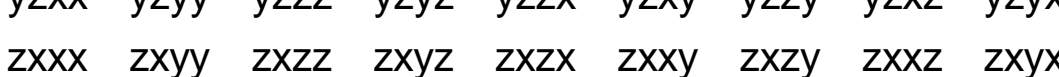

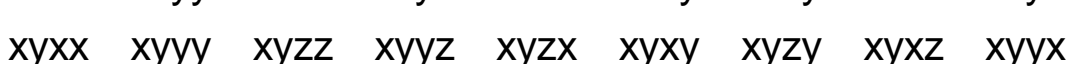

$\left(\begin{array}{lllllllll}0 & 0 & 0 & 0 & 0 & 0 & 0 & 0 & 0 \\ 0 & 0 & 0 & 0 & 0 & 0 & 0 & 0 & 0\end{array}\right)$

$\begin{array}{lllllllll}0 & 0 & 0 & 0 & 0 & 0 & 0 & 0 & 0 \\ 0 & 0 & 0 & 0 & 0 & 0 & 0 & 0 & 0\end{array}$

$\begin{array}{llllllllll}0 & 0 & 0 & 0 & 0 & 0 & 0 & 0 & 0 \\ 0 & 0 & 0 & 0 & 0 & 0 & 0 & 0 & 0\end{array}$

$0 \begin{array}{llllllllll}0 & 0 & 0 & 0 & 0 & 0 & 0 & 0 & 0 \\ 0 & 0 & 0 & 0 & 0 & 0 & 0 & 0\end{array}$

$\begin{array}{llllllllll}0 & 0 & 0 & 0 & 0 & 0 & 0 & 0 & 0\end{array}$

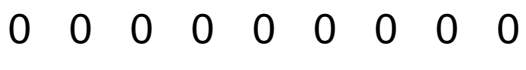

$\begin{array}{lllllllll}x x x x & x x y y & x x z z & x x y z & x x z x & x x x y & x x z y & x x x z & x x y x\end{array}$ yyyy $\begin{array}{lllllll}z z z z & \text { zzyz } & \text { zzzx } & \text { zzxy } & \text { zzzy } & \text { zzxz } & \text { zzyx }\end{array}$ $y z y z \quad y z z x \quad y z x y \quad y z z y \quad y z x z \quad y z y x$ $\begin{array}{lllll}z x z x & z x x y & z x z y & z x x z & z x y x\end{array}$ $\begin{array}{llll}x y x y & x y z y & x y x z & x y y x\end{array}$ $\begin{array}{llll}\text { zyzy } & z y x z & z y y x\end{array}$ $\begin{array}{rr}x z x z & x z y x \\ y x y x\end{array}$ yyxx yyyy yyzz

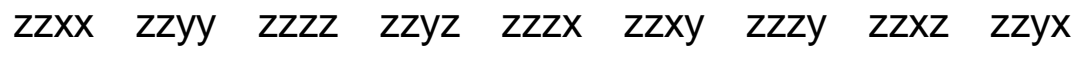

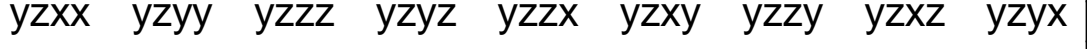
$\begin{array}{llllllllll}z x x x & z x y y & z x z z & z x y z & z x z x & z x x y & z x z y & z x x z & z x y x\end{array}$

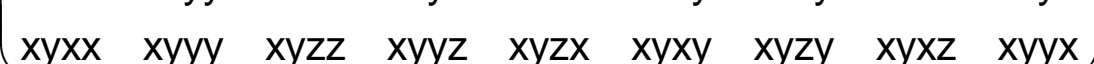
$0 \begin{array}{llllllll}0 & 0 & 0 & 0 & 0 & 0 & 0 \\ 0 & 0 & 0 & 0 & 0 & 0 & 0\end{array}$ $\begin{array}{llllll}0 & 0 & 0 & 0 & 0 & 0\end{array}$ $\begin{array}{lllll}0 & 0 & 0 & 0 & 0\end{array}$ $\begin{array}{lllll}0 & 0 & 0 & 0\end{array}$ $\begin{array}{llll}0 & 0 & 0 \\ 0 & 0 & 0\end{array}$ 0 0

$\left(\begin{array}{lllllllll}0 & 0 & 0 & 0 & 0 & 0 & 0 & 0 & 0 \\ 0 & 0 & 0 & 0 & 0 & 0 & 0 & 0 & 0\end{array}\right.$ $\begin{array}{lllllllll}0 & 0 & 0 & 0 & 0 & 0 & 0 & 0 & 0 \\ 0 & 0 & 0 & 0 & 0 & 0 & 0 & 0 & 0\end{array}$ $\begin{array}{llllllllll}0 & 0 & 0 & 0 & 0 & 0 & 0 & 0 & 0 \\ 0 & 0 & 0 & 0 & 0 & 0 & 0 & 0 & 0\end{array}$ $\begin{array}{llllllllll}0 & 0 & 0 & 0 & 0 & 0 & 0 & 0 & 0 \\ 0 & 0 & 0 & 0 & 0 & 0 & 0 & 0 & 0\end{array}$ $\begin{array}{lllllllll}0 & 0 & 0 & 0 & 0 & 0 & 0 & 0 & 0 \\ 0 & 0 & 0 & 0 & 0 & 0 & 0 & 0 & 0\end{array}$ $\begin{array}{llllllllll}0 & 0 & 0 & 0 & 0 & 0 & 0 & 0 & 0\end{array}$ 


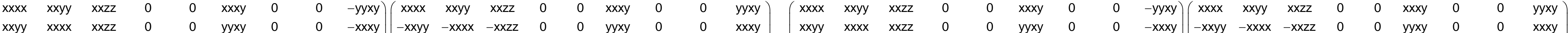

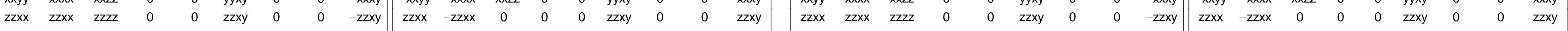

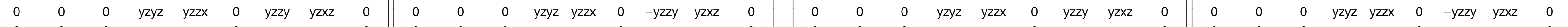

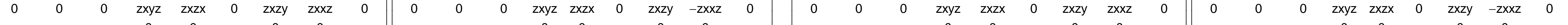

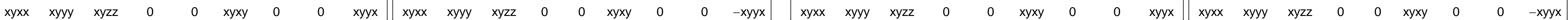

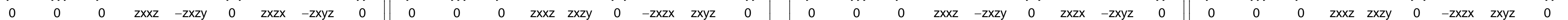

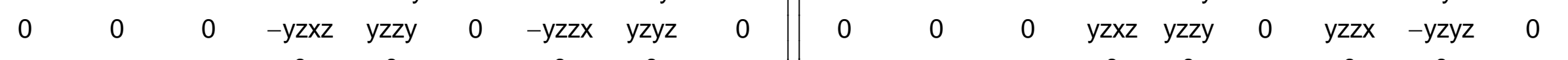

$\left[V^{4}\right] \quad\left(\begin{array}{ccccc}x x x x & -y x x x & 0 & y y z z & 0 \\ y x x x & x x x x & 0 & y y z z & 0 \\ 0 & 0 & z z z z & x x y y & 0\end{array}\right)$

$$
\left(\begin{array}{ccccc}
x x x x & y x x x & 0 & y y z z & 0 \\
y x x x & -x x x x & 0 & -y y z z & 0 \\
0 & 0 & 0 & 0 & z z x y
\end{array}\right)
$$

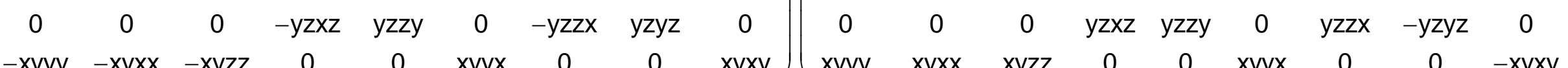

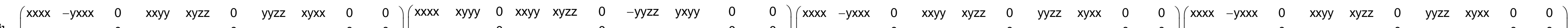

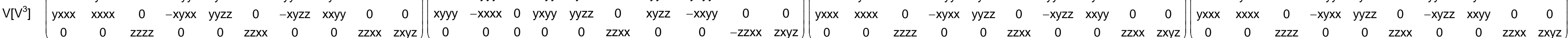

$$
\left.\left[\mathrm{V}^{2}\right]^{2}\right]\left(\begin{array}{cccccc}
\mathrm{xxxx} & \mathrm{xxyy} & \mathrm{xxzz} & 0 & 0 & \mathrm{xxxy} \\
& \mathrm{xxxx} & \mathrm{xxzz} & 0 & 0 & -\mathrm{xxxy} \\
& & \mathrm{zzzz} & 0 & 0 & 0 \\
& & & \mathrm{yzyz} & 0 & 0 \\
& & & & \mathrm{yzyz} & 0 \\
& & & & & \mathrm{xyxy}
\end{array}\right)
$$

$\left[\mathrm{V}^{2}\right]^{2}\left(\begin{array}{cccccc}x \mathrm{x} x \mathrm{x} & \mathrm{xxyy} & \mathrm{xxzz} & 0 & 0 & \mathrm{xxxy} \\ \mathrm{xxyy} & \mathrm{xxxx} & \mathrm{xxzz} & 0 & 0 & -\mathrm{xxxy} \\ \mathrm{zzxx} & \mathrm{zzxx} & \mathrm{zzzz} & 0 & 0 & 0 \\ 0 & 0 & 0 & \mathrm{yzyz} & \mathrm{yzxz} & 0 \\ 0 & 0 & 0 & -\mathrm{yzxz} & \mathrm{yzyz} & 0 \\ -\mathrm{xxxy} & \mathrm{xxxy} & 0 & 0 & 0 & \mathrm{xyxy}\end{array}\right)$

$$
\left(\begin{array}{cccccc}
x x x x & 0 & x x z z & 0 & 0 & x x x y \\
& -x x x x & -x x z z & 0 & 0 & x x x y \\
& & 0 & 0 & 0 & z z x y \\
& & & y z y z & y z x z & 0 \\
& & & & -y z y z & 0 \\
& & & & & 0
\end{array}\right)
$$

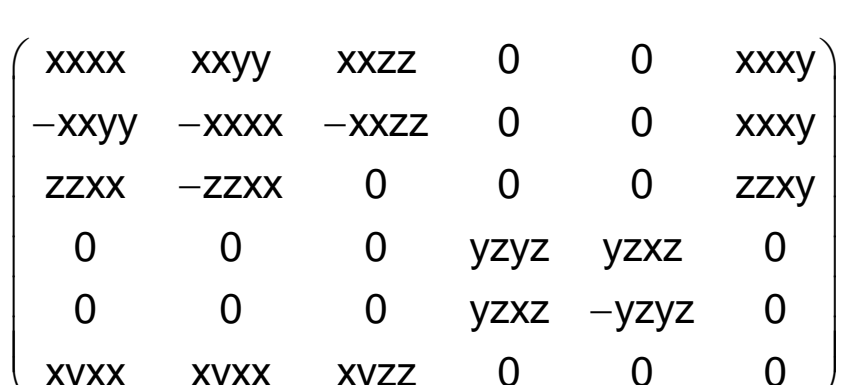

$$
\left(\begin{array}{cccccc}
x x x x & x x y y & x x z z & 0 & 0 & x x x y \\
& x x x x & x x z z & 0 & 0 & -x x x y \\
& & z z z z & 0 & 0 & 0 \\
& & & y z y z & 0 & 0 \\
& & & & y z y z & 0 \\
& & & & & \text { xyxy }
\end{array}\right)
$$

$$
\left(\begin{array}{cccccc}
\text { xxxx } & x x y y & x x z z & 0 & 0 & x x x y \\
x x y y & x x x x & x x z z & 0 & 0 & -x x x y \\
\text { zzxx } & \text { zzxx } & z z z z & 0 & 0 & 0 \\
0 & 0 & 0 & y z y z & y z x z & 0 \\
0 & 0 & 0 & -y z x z & y z y z & 0 \\
-x x x y & x x x y & 0 & 0 & 0 & x y x y
\end{array}\right)
$$

$\left(\begin{array}{ccccc}\mathrm{xxxx} & \mathrm{yxxx} & 0 & \mathrm{yyzz} & 0 \\ \mathrm{yxxx} & -\mathrm{xxxx} & 0 & -\mathrm{yyzz} & 0 \\ 0 & 0 & 0 & 0 & z z x y\end{array}\right)$

$$
\left(\begin{array}{cccccc}
x x x x & x x y y & x x z z & 0 & 0 & x x x y \\
-x x y y & -x x x x & -x x z z & 0 & 0 & x x x y \\
z z x x & -z z x x & 0 & 0 & 0 & z z x y \\
0 & 0 & 0 & y z y z & y z x z & 0 \\
0 & 0 & 0 & y z x z & -y z y z & 0 \\
x y x x & x y x x & x y z z & 0 & 0 & 0
\end{array}\right)
$$


$\left.\begin{array}{ccccccccc}0 & 0 & 0 & 0 & 0 & 0 & 0 & 0 & 0 \\ 0 & 0 & 0 & 0 & 0 & 0 & 0 & 0 & 0\end{array}\right)$

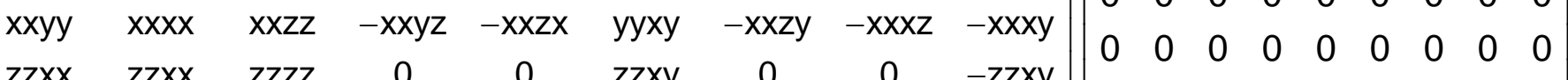

$V^{4} \quad \begin{array}{lllllllllll}z z x x & -y z x x & 0 & y z y z & y z z x & -x z x x & y z z y & y z x z & -x z x x \\ z x y x & -z x x & 0 & 2 x y z & z x x & z y x x & 2 x y & z x z & z y x y\end{array} \mid \begin{array}{lllllllll}0 & 0 & 0 & 0 & 0 & 0 & 0 & 0 & 0 \\ 0 & 0 & 0 & 0 & 0 & 0 & 0 & 0 & 0\end{array}$ $\begin{array}{llllllllll}z x x x & -z x x x & 0 & z x y z & z x z x & z y x x & z x z y & z x x z & z y x x\end{array} \mid \begin{array}{llllllllll}0 & 0 & 0 & 0 & 0 & 0 & 0 & 0 & 0\end{array}$

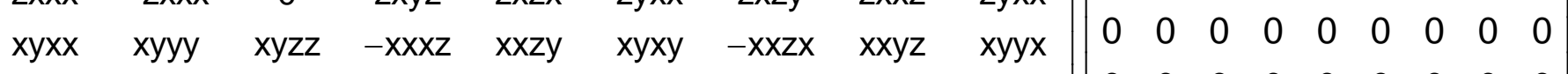
\begin{tabular}{llllllllll|lllllllll}
$z y x x$ & $-z y x x$ & 0 & $z x x z$ & $-z x z y$ & $-z x x x$ & $z x z x$ & $-z x y z$ & $-z x x x$ & 0 & 0 & 0 & 0 & 0 & 0 & 0 & 0 & 0 \\
0 & 0 & 0 & 0 & 0 & 0 & 0 & 0 & 0
\end{tabular} \begin{tabular}{llllllllll|lllllllll}
$x z x x$ & $-x z x x$ & 0 & $-y z x z$ & $y z z y$ & $y z x x$ & $-y z z x$ & $y z y z$ & $y z x x$ & 0 & 0 & 0 & 0 & 0 & 0 & 0 & 0 & 0 \\
$-x y y$ & $-x y x$ & $-x y z z$ & $-x x z$ & $x x y$ & $x y x$ & $-x x z$ & $x y z$ & $x y x y$ & 0 & 0 & 0 & 0 & 0 & 0 & 0 & 0
\end{tabular} $\begin{array}{lllllllllllllllllllllll}-x y y y & -x y x x & -x y z z & -x x x z & x x z y & x y y x & -x x z x & x x y z & x y x y & 0 & 0 & 0 & 0 & 0 & 0 & 0 & 0 & 0\end{array}$ and: $x x x x=x x y y+x y x y+x y y x ; x x x y-y y x y=x y y y-x y x x$

$\left[V^{4}\right] \quad\left(\begin{array}{ccccc}3 x x y y & 0 & 0 & y y z z & x x y z \\ 0 & 3 x x y y & 0 & y y z z & -z x x x \\ z x x x & -x x y z & z z z z & x x y y & 0\end{array}\right)$

$\left(\begin{array}{lllll}0 & 0 & 0 & 0 & 0 \\ 0 & 0 & 0 & 0 & 0 \\ 0 & 0 & 0 & 0 & 0\end{array}\right)$

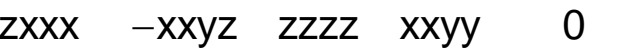

$\left.\begin{array}{lllllllllll}3 x x y y & 3 x y x x & 0 & x x y y & x y z z & x z x x & y y z z & x y x x & -x z x x & y z x x\end{array}\right)\left(\begin{array}{cccccccccc}0 & 0 & 0 & 0 & 0 & 0 & 0 & 0 & 0 & 0 \\ 0 & 0 & 0 & 0 & 0 & 0 & 0 & 0 & 0 & 0\end{array}\right)$ $\left(\begin{array}{ccccccccccc}-3 x y x x & 3 x x y y & 0 & -x y x x & y y z z & y z x x & -x y z z & x x y y & -y z x x & -x z x x \\ 2 x x & -2 y x & z z z & -2 x x & 0 & z z x & 0 & z y x & z z x & z x y z\end{array}\right)\left(\begin{array}{llllllllll}0 & 0 & 0 & 0 & 0 & 0 & 0 & 0 & 0 & 0 \\ 0 & 0 & 0 & 0 & 0 & 0 & 0 & 0 & 0 & 0\end{array}\right)$ $\left.\begin{array}{llllllllll}z x x x & -z y x x & z z z z & -z x x x & 0 & z z x x & 0 & z y x x & z z x x & z x y z\end{array}\right)\left(\begin{array}{llllllllll}0 & 0 & 0 & 0 & 0 & 0 & 0 & 0 & 0 & 0\end{array}\right.$

$\left.\left[\mathrm{V}^{2}\right]^{2}\right]$

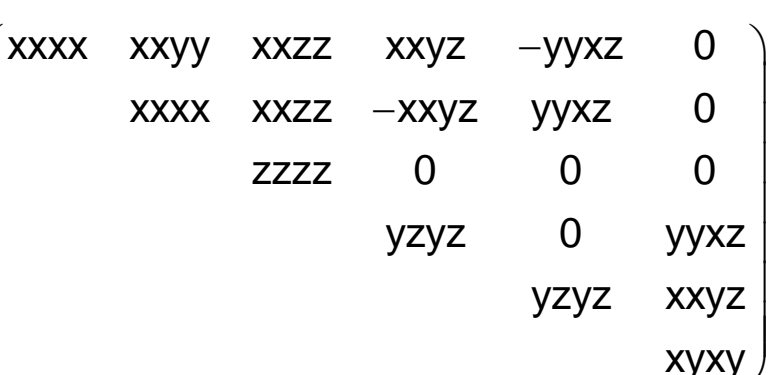

and $x y x y=1 / 2(x x x x-x x y y)$

$\left(\begin{array}{cccccc}x x x x & x x y y & x x z z & x x y z & -y y x z & x x x y \\ x y y & x x y & x x z & -x x y z & y y z & -x x y\end{array}\right)$ $\begin{array}{llllll}x x y y & x x x x & x x z z & -x x y z & y y x z & -x x x y\end{array}$ $\begin{array}{cccccc}z z x x & z z x x & z z z z & 0 & 0 & 0 \\ y z x x & -y z x x & 0 & y z y z & y z x z & x z y y \\ -x z y y & x z y y & 0 & y z x z & y z y z & y z x x\end{array}$ $\begin{array}{cccccc}y z x x & -y z x x & 0 & y z y z & y z x z & x z y y \\ -x z y y & x z y y & 0 & y z x z & y z y z & y z x y\end{array}$ $\begin{array}{llllll}-x z y y & x z y y & 0 & y z x z & y z y z & y z x x \\ -x x x y & x x x y & 0 & y y x z & x x y z & x y x y\end{array}$

and $x y x y=1 / 2(x x x x-x x y y)$

$$
\left(\begin{array}{llllll}
0 & 0 & 0 & 0 & 0 & 0 \\
& 0 & 0 & 0 & 0 & 0 \\
& & 0 & 0 & 0 & 0 \\
& & & 0 & 0 & 0 \\
& & & & 0 & 0 \\
& & & & & 0
\end{array}\right)
$$

$\left(\begin{array}{llllll}0 & 0 & 0 & 0 & 0 & 0 \\ 0 & 0 & 0 & 0 & 0 & 0\end{array}\right)$ $\begin{array}{llllll}0 & 0 & 0 & 0 & 0 & 0 \\ 0 & 0 & 0 & 0 & 0 & 0\end{array}$ $\begin{array}{llllll}0 & 0 & 0 & 0 & 0 & 0 \\ 0 & 0 & 0 & 0 & 0 & 0\end{array}$ $\begin{array}{llllll}0 & 0 & 0 & 0 & 0 & 0 \\ 0 & 0 & 0 & 0 & 0 & 0\end{array}$ $\begin{array}{llllll}0 & 0 & 0 & 0 & 0 & 0\end{array}$

$\begin{array}{llll}x x x y & x x z y & x x x z & -y y x y\end{array}\left(\begin{array}{lllllllll}0 & 0 & 0 & 0 & 0 & 0 & 0 & 0 & 0 \\ 0 & 0 & 0 & 0 & 0 & 0 & 0 & 0 & 0\end{array}\right)$ $\begin{array}{llll}x y y x y & -x x z y & -x x x z & -y y x y \\ 2 x y y & 0 & 0 & -z z y y\end{array} \mid\left(\begin{array}{lllllllll}0 & 0 & 0 & 0 & 0 & 0 & 0 & 0 & 0 \\ 0 & 0 & 0 & 0 & 0 & 0 & 0 & 0 & 0\end{array}\right)$ $\begin{array}{lllllllll}z z x x & z z x x & z z z z & 0 & 0 & z z x y & 0 & 0 & -z z x y\end{array} \mid \begin{array}{lllllllll}0 & 0 & 0 & 0 & 0 & 0 & 0 & 0 & 0\end{array}$

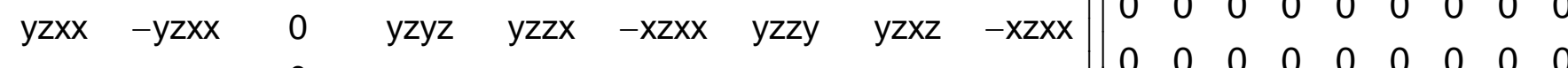
$\begin{array}{llllllllll}z x x x & -z x x x & 0 & z x y z & z x z x & z y x x & z x z y & z x x z & z y x x\end{array} \mid \begin{array}{llllllllll}0 & 0 & 0 & 0 & 0 & 0 & 0 & 0 & 0 \\ 0 & 0 & 0 & 0 & 0 & 0 & 0 & 0 & 0\end{array}$

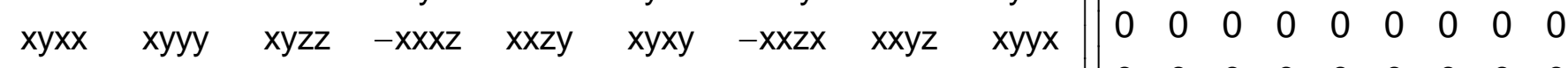
$\begin{array}{llllllllll}z y x x & -z y x x & 0 & z x x z & -z x z y & -z x x x & z x z x & -z x y z & -z x x x & \mid\end{array} \mid \begin{array}{llllllllll}0 & 0 & 0 & 0 & 0 & 0 & 0 & 0 & 0\end{array}$

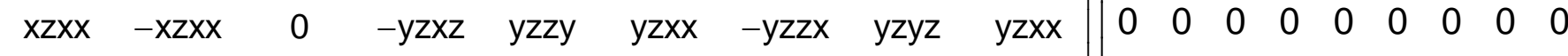
$\left.\begin{array}{llllllllll}-x y y y & -x y x x & -x y z z & -x x x z & x x z y & x y y x & -x x z x & x x y z & x y x y\end{array}\right)\left(\begin{array}{llllllllllllll}0 & 0 & 0 & 0 & 0 & 0 & 0 & 0 & 0\end{array}\right)$ and: $x x x x=x x y y+x y x y+x y y x ; x x x y-y y x y=x y y y-x y x x$

$\left(\begin{array}{ccccc}3 x x y y & 0 & 0 & y y z z & x x y z \\ 0 & 3 x x y y & 0 & y y z z & -z x x x \\ z x x x & -x x y z & z z z z & x x y y & 0\end{array}\right) \quad\left(\begin{array}{ccccc}0 & 0 & 0 & 0 & 0 \\ 0 & 0 & 0 & 0 & 0 \\ 0 & 0 & 0 & 0 & 0\end{array}\right)$

$\left(\begin{array}{llllllllll}3 x x y y & 3 x y x x & 0 & x x y y & x y z z & x z x x & y y z z & x y x x & -x z x x & y z x x \\ 3 x y x & 3 x y & 0 & -x y x & y z z & y z x & -x y z & x y y & -z z x & -x z x\end{array}\right)\left(\begin{array}{cccccccccc}0 & 0 & 0 & 0 & 0 & 0 & 0 & 0 & 0 & 0 \\ 0 & 0 & 0 & 0 & 0 & 0 & 0 & 0 & 0 & 0 \\ 0 & 0 & 0 & 0 & 0 & 0 & 0 & 0 & 0\end{array}\right)$ $\left.\begin{array}{llllllllllll}-3 x y x x & 3 x x y y & 0 & -x y x & y y z z & y z x x & -x y z z & x x y y & -y z x x & -x z x x\end{array}\right)\left(\begin{array}{llllllllll}0 & 0 & 0 & 0 & 0 & 0 & 0 & 0 & 0 & 0 \\ 0 & 0 & 0 & 0 & 0 & 0 & 0 & 0 & 0 & 0\end{array}\right)$ $\left.\begin{array}{lllllllllll}z x x x & -z y x x & z z z z & -z x x x & 0 & z z x x & 0 & z y x x & z z x x & z x y z\end{array}\right)\left(\begin{array}{llllllllll}0 & 0 & 0 & 0 & 0 & 0 & 0 & 0 & 0 & 0\end{array}\right)$

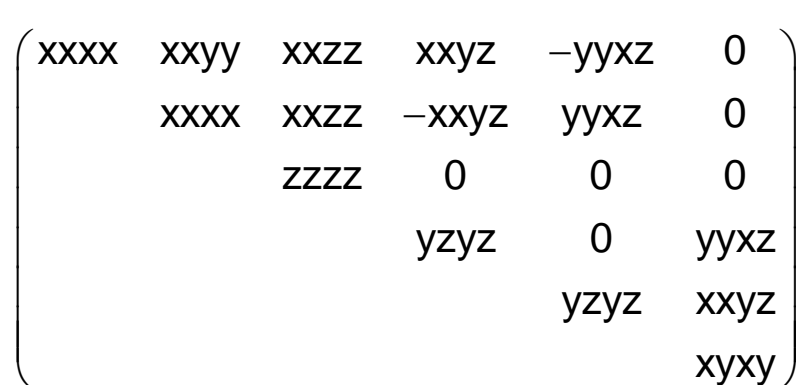

and $x y x y=1 / 2(x x x x-x x y y)$

$\left(\begin{array}{cccccc}x x x x & x x y y & x x z z & x x y z & -y y x z & x x x y \\ x x y y & x x y & x x z & -x x y z & y y x z & -x x y\end{array}\right)$ $\left.\begin{array}{cccccc}x x y y & x x x x & x x z z & -x x y z & y y x z & -x x x y \\ z z x x & z z x x & z z z z & 0 & 0 & 0\end{array}\right)$

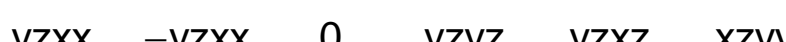

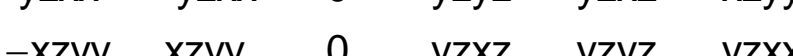
$\begin{array}{llllll}-x z y y & x z y y & 0 & y z x z & y z y z & y z x x \\ -x x x y & x x x y & 0 & y y x z & x x y z & x y x y\end{array}$

and $x y x y=1 / 2(x x x x-x x y y)$

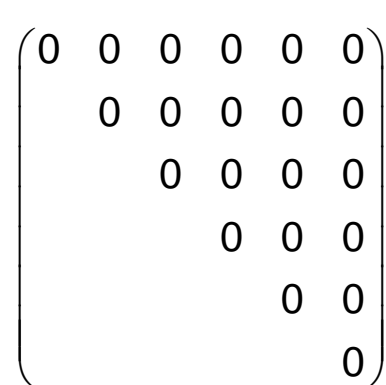

$\begin{array}{llllll}0 & 0 & 0 & 0 & 0 & 0 \\ 0 & 0 & 0 & 0 & 0 & 0\end{array}$ $\left(\begin{array}{llllll}0 & 0 & 0 & 0 & 0 & 0 \\ 0 & 0 & 0 & 0 & 0 & 0 \\ 0 & 0 & 0 & 0 & -1\end{array}\right.$ $\begin{array}{llllll}0 & 0 & 0 & 0 & 0 & 0\end{array}$ $\left(\begin{array}{llllll}0 & 0 & 0 & 0 & 0 & 0\end{array}\right.$ $\begin{array}{llllll}0 & 0 & 0 & 0 & 0 & 0\end{array}$ 
$\mathrm{n}=3 \quad \mathbf{S}_{6}=\overline{\mathbf{3}}_{2}$

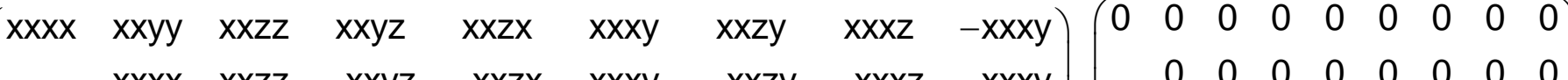

$$
\begin{aligned}
& \begin{array}{lllllllll}
x x x x & x x z z & -x x y z & -x x z x & x x x y & -x x z y & -x x x z & -x x y y
\end{array} \mid \\
& \begin{array}{llllll}
0 & 0 & 0 & 0 & 0 & 0
\end{array} \\
& \begin{array}{lllllll}
2 x z x & x x z y & 0 & y z z y & x x z y
\end{array} \mid \begin{array}{lllllll}
y & 0 & 0 & 0 & 0 & 0
\end{array} \\
& \begin{array}{cccc}
x y x y & -x x z x & x x y z & x y y x \\
& z x z x & -y z z x & -x x z x
\end{array}\left|\begin{array}{rrrrr}
0 & 0 & 0 & 0 \\
& & 0 & 0 & 0
\end{array}\right| \\
& \left.\begin{array}{rr}
y z y z & x x y z \\
x y x y
\end{array}\right)\left(\begin{array}{rrr}
0 & 0 \\
& & 0
\end{array}\right.
\end{aligned}
$$

and $x x x x=x x y y+y z y z+z y z y$

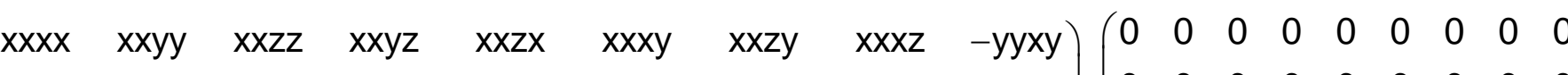
$\left.\begin{array}{llllllllll}x x y y & x x x x & x x z z & -x x y z & -x x z x & y y x y & -x x z y & -x x x z & -x x x y \\ z x x & z z x x & z z z & 0 & 0 & z x y & 0 & 0 & -z z y\end{array} \mid \begin{array}{lllllllll}0 & 0 & 0 & 0 & 0 & 0 & 0 & 0 & 0 \\ 0 & 0 & 0 & 0 & 0 & 0 & 0 & 0 & 0\end{array}\right)$

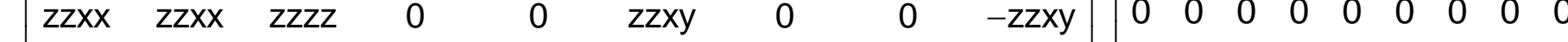
$\begin{array}{llllllllll}y z x x & -y z x x & 0 & y z y z & y z z x & -z x x x & z x z x & -z x y z & -z x x x \\ z x x & -z x x & 0 & z x y z & z z x & y z x & -y z z & y z y z & y z x x\end{array} \mid \begin{array}{llllllllll}0 & 0 & 0 & 0 & 0 & 0 & 0 & 0 & 0 \\ 0 & 0 & 0 & 0 & 0 & 0 & 0 & 0 & 0\end{array}$ $\begin{array}{lllllllll}z x x x & -z x x x & 0 & z x y z & z x z x & y z x x & -y z z x & y z y z & y z x x \\ x y x & -x y x & 0 & -x y z & x x z y & x x y & -x x z & x y z & x y x y\end{array} \mid\left(\begin{array}{lllllllll}0 & 0 & 0 & 0 & 0 & 0 & 0 & 0 & 0 \\ 0 & 0 & 0 & 0 & 0 & 0 & 0 & 0 & 0\end{array}\right.$ $\left.\begin{array}{lllllllll}x y x x & -x y x x & 0 & -x x x z & x x z y & x y x y & -x x z x & x x y z & x y x y\end{array}\right)\left(\begin{array}{lllllllll}0 & 0 & 0 & 0 & 0 & 0 & 0 & 0 & 0\end{array}\right.$ and $x x x x=x x y y+2 x y x y ; y y x y-x x x y=2 x y x x$

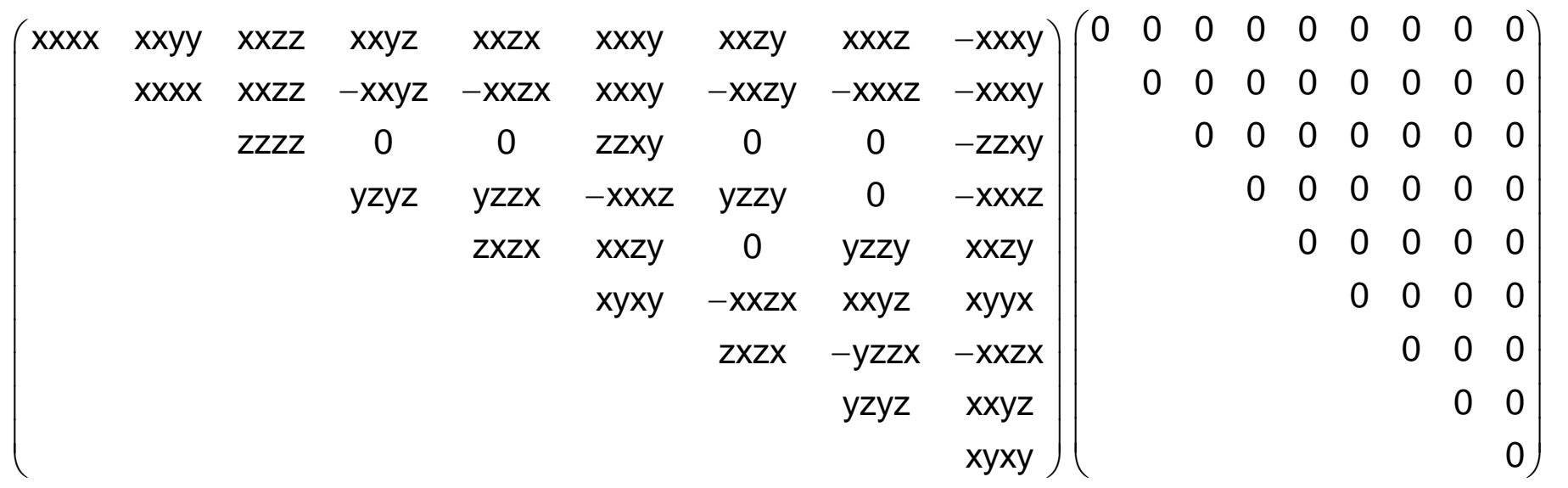

and $x x x x=x x y y+y z y z+z y z y$

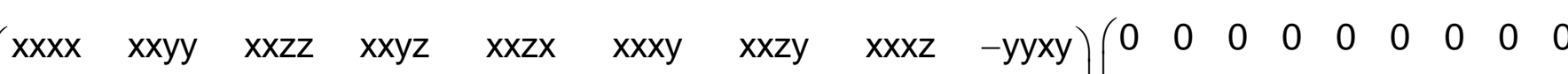

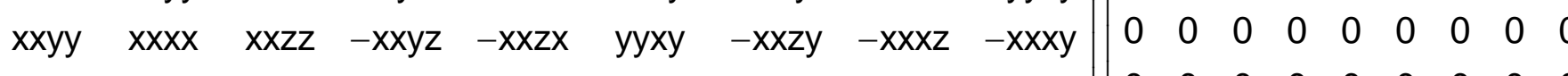
$\begin{array}{llllllccc}z z x x & z z x x & z z z z & 0 & 0 & z z x y & 0 & 0 & -z z x y\end{array} \mid \begin{array}{cccccccccc}0 & 0 & 0 & 0 & 0 & 0 & 0 & 0 & 0 \\ 0 & 0 & 0 & 0 & 0 & 0 & 0 & 0 & 0\end{array}$

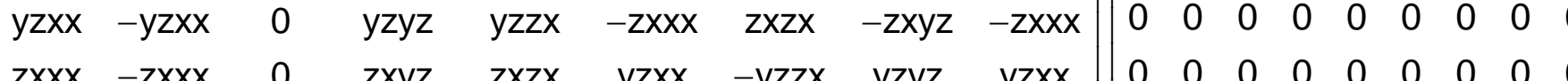
$\left.\begin{array}{cccccccccc}z x x x & -z x x x & 0 & z x y z & z x z x & y z x x & -y z z x & y z y z & y z x x \\ x y x x & -x y x x & 0 & -x x x z & x x z y & x y x y & -x x z x & x x y z & x y x y\end{array}\right)\left(\begin{array}{cccccccccc}0 & 0 & 0 & 0 & 0 & 0 & 0 & 0 & 0 \\ 0 & 0 & 0 & 0 & 0 & 0 & 0 & 0 & 0\end{array}\right)$ and $x x x x=x x y y+2 x y x y ; y y x y-x x x y=2 x y x x$ 


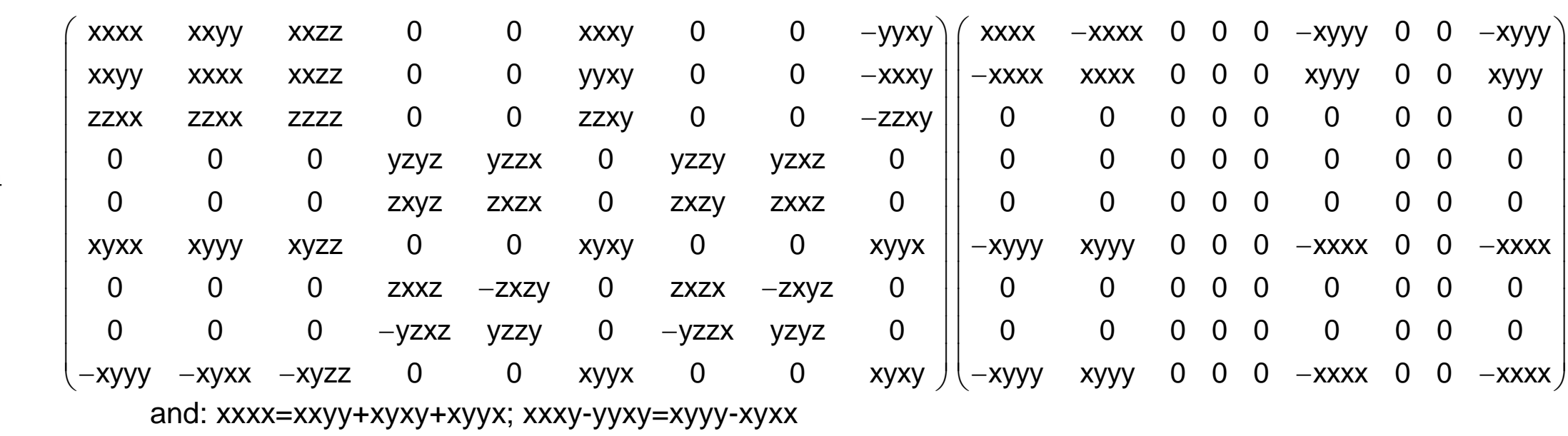
$\left[V^{4}\right]\left(\begin{array}{ccccc}3 x x y y & 0 & 0 & y y z z & 0 \\ 0 & 3 x x y y & 0 & y y z z & 0 \\ 0 & 0 & z z z z & x x y y & 0\end{array}\right)$

$$
\left(\begin{array}{ccccc}
x x x x & x y y y & 0 & 0 & 0 \\
-x y y y & x x x x & 0 & 0 & 0 \\
0 & 0 & 0 & -x x x x & 0
\end{array}\right)
$$

$\left(\begin{array}{llllllllll}3 x x y y & 3 x y x x & 0 & x x y y & x y z z & 0 & y y z z & x y x x & 0 & 0 \\ -3 x y x & 3 x y & 0 & -x y x & y z z & 0 & -x y z & x x y & 0 & 0\end{array}\right)\left(\begin{array}{cccccccccc}x y y y & 0 & -x x x x & 0 & 0 & 0 & -x y y y & 0 & 0\end{array}\right)$

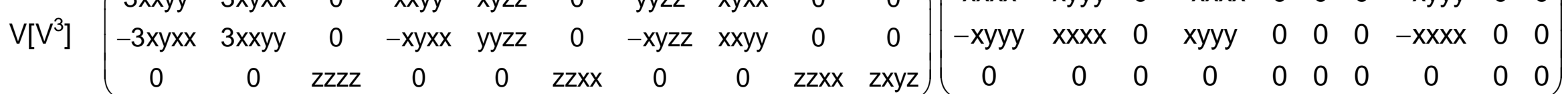
$\left.\left[\mathrm{V}^{2}\right]^{2}\right]\left(\begin{array}{cccccc}\mathrm{xxxx} & \mathrm{xxyy} & \mathrm{xxzz} & 0 & 0 & 0 \\ & \mathrm{xxxx} & \mathrm{xxzz} & 0 & 0 & 0 \\ & & \mathrm{zzzz} & 0 & 0 & 0 \\ & & & \mathrm{yzyz} & 0 & 0 \\ & & & & \mathrm{yzyz} & 0 \\ & & & & & \mathrm{xyxy}\end{array}\right)$

$$
\left(\begin{array}{cccccc}
x x x x & -x x x x & 0 & 0 & 0 & -x y y y \\
& x x x x & 0 & 0 & 0 & x y y y \\
& & 0 & 0 & 0 & 0 \\
& & & 0 & 0 & 0 \\
& & & & 0 & 0 \\
& & & & & -x x x x
\end{array}\right)
$$

add: $x y x y=1 / 2(x x x x-x x y y)$

$\left[V^{2}\right]^{2}\left(\begin{array}{cccccc}x x x x & x x y y & x x z z & 0 & 0 & x x x y \\ x x y y & x x x x & x x z z & 0 & 0 & -x x x y \\ z z x x & z z x x & z z z z & 0 & 0 & 0 \\ 0 & 0 & 0 & y z y z & y z x z & 0 \\ 0 & 0 & 0 & -y z x z & y z y z & 0 \\ -x x x y & x x x y & 0 & 0 & 0 & x y x y\end{array}\right)$

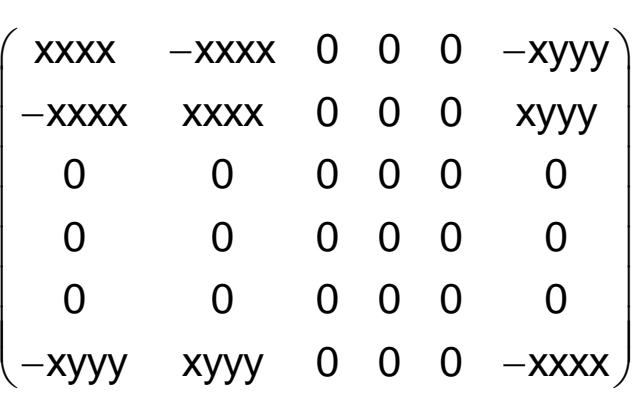

add: $x y x y=1 / 2(x x x x-x x y y)$ $\left(\begin{array}{ccccccccc}x x x x & x x y y & x x z z & 0 & 0 & x x x y & 0 & 0 & -y y x y \\ x x y y & x x x x & x x z z & 0 & 0 & y y x y & 0 & 0 & -x x x y \\ z z x x & z z x x & z z z z & 0 & 0 & z z x y & 0 & 0 & -z z x y \\ 0 & 0 & 0 & y z y z & y z z x & 0 & y z z y & y z x z & 0 \\ 0 & 0 & 0 & z x y z & z x z x & 0 & z x z y & z x x z & 0 \\ x y x x & x y y y & x y z z & 0 & 0 & x y x y & 0 & 0 & x y y x \\ 0 & 0 & 0 & z x x z & -z x z y & 0 & z x z x & -z x y z & 0 \\ 0 & 0 & 0 & -y z x z & y z z y & 0 & -y z z x & y z y z & 0 \\ -x y y y & -x y x x & -x y z z & 0 & 0 & x y y x & 0 & 0 & x y x y\end{array}\right)\left(\begin{array}{ccccccccc}x x x x & -x x x x & 0 & 0 & 0 & -x y y y & 0 & 0 & -x y y y \\ -x x x x & x x x x & 0 & 0 & 0 & x y y y & 0 & 0 & x y y y \\ 0 & 0 & 0 & 0 & 0 & 0 & 0 & 0 & 0 \\ 0 & 0 & 0 & 0 & 0 & 0 & 0 & 0 & 0 \\ 0 & 0 & 0 & 0 & 0 & 0 & 0 & 0 & 0 \\ -x y y y y & x y y y & 0 & 0 & 0 & -x x x x & 0 & 0 & -x x x x \\ 0 & 0 & 0 & 0 & 0 & 0 & 0 & 0 & 0 \\ 0 & 0 & 0 & 0 & 0 & 0 & 0 & 0 & 0 \\ -x y y y & x y y y & 0 & 0 & 0 & -x x x x & 0 & 0 & -x x x x\end{array}\right)$
and $x x x x=x x y y+x y x y+x y y x ; x x x y-y y x y=x y y y-x y x x$

$\left(\begin{array}{ccccc}3 x x y y & 0 & 0 & y y z z & 0 \\ 0 & 3 x x y y & 0 & y y z z & 0 \\ 0 & 0 & z z z z & x x y y & 0\end{array}\right) \quad\left(\begin{array}{ccccc}x x x x & x y y y & 0 & 0 & 0 \\ -x y y y & x x x x & 0 & 0 & 0 \\ 0 & 0 & 0 & -x x x x & 0\end{array}\right)$

$\left(\begin{array}{llllllllll}3 x x y y & 3 x y x x & 0 & x x y y & x y z z & 0 & y y z z & x y x x & 0 & 0\end{array}\right)\left(\begin{array}{llllllllll}x x x x & x y y y & 0 & -x x x x & 0 & 0 & 0 & -x y y y & 0 & 0\end{array}\right)$

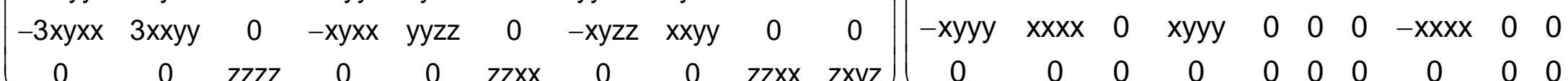

$$
\left(\begin{array}{cccccc}
x x x x & x x y y & x x z z & 0 & 0 & 0 \\
& x x x x & x x z z & 0 & 0 & 0 \\
& & z z z z & 0 & 0 & 0 \\
& & & y z y z & 0 & 0 \\
& & & & y z y z & 0 \\
& & & & & \text { xyxy }
\end{array}\right)
$$

add: $x y x y=1 / 2(x x x x-x x y y)$

$\left(\begin{array}{cccccc}x x x x & x x y y & x x z z & 0 & 0 & x x x y \\ x x y y & x x x x & x x z z & 0 & 0 & -x x x y \\ z z x x & z z x x & z z z z & 0 & 0 & 0 \\ 0 & 0 & 0 & y z y z & y z x z & 0 \\ 0 & 0 & 0 & -y z x z & y z y z & 0 \\ -x x x y & x x x y & 0 & 0 & 0 & x y x y\end{array}\right)$

add: $x y x y=1 / 2(x x x x-x x y y)$
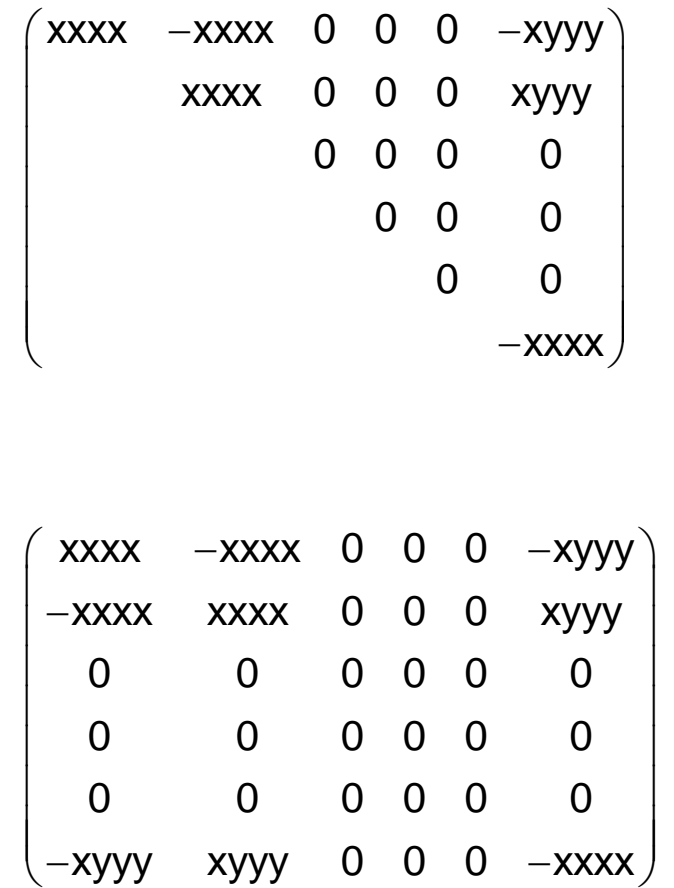
$\mathrm{n}=4 \quad \mathrm{~S}_{8}=\overline{\mathbf{8}}_{z}$

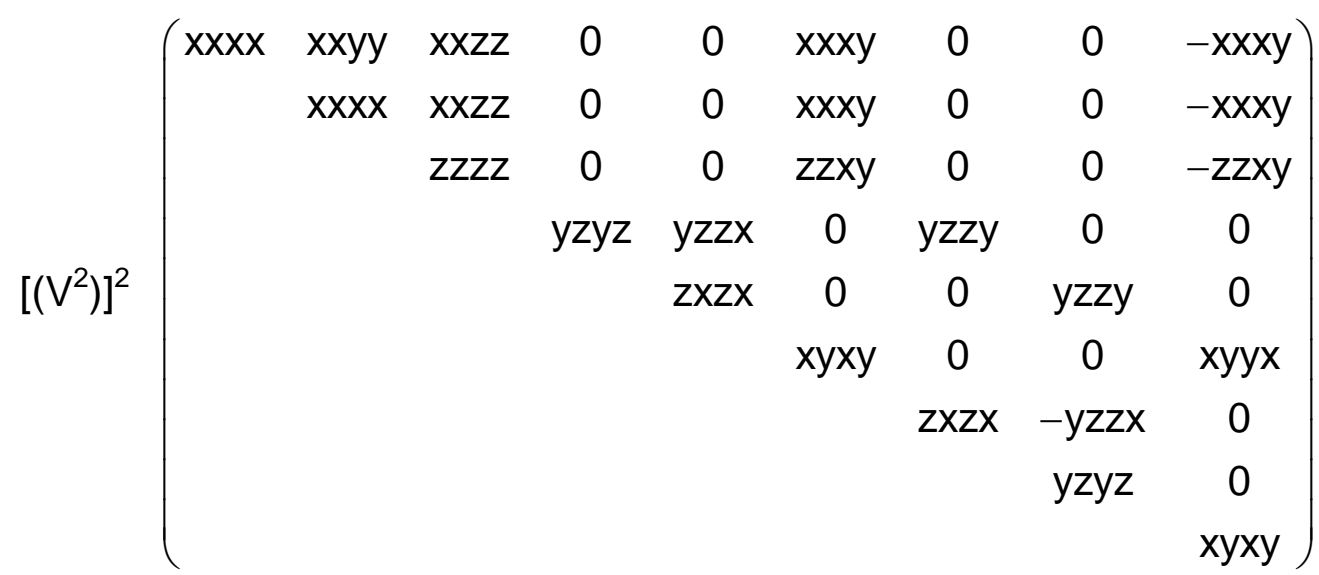

$\begin{array}{lllllllll}x x x x & -x x x x & 0 & 0 & 0 & -x y y y & 0 & 0 & -x y y y\end{array}$ $\begin{array}{llllllll}x x x x & 0 & 0 & 0 & -x y y y & 0 & 0 & -x y y y\end{array}$ $\begin{array}{lllllll}0 & 0 & 0 & 0 & 0 & 0 & 0\end{array}$ $\begin{array}{llllll}0 & 0 & 0 & 0 & 0 & 0\end{array}$ $\begin{array}{lllll}0 & 0 & 0 & 0 & 0\end{array}$ $\begin{array}{rrrrr}-x x x x & 0 & 0 & -x x x x\end{array}$ $\begin{array}{ccc}0 & 0 & 0 \\ & 0 & 0\end{array}$

add: $x x x x=x x y y+x y x y+x y y x$

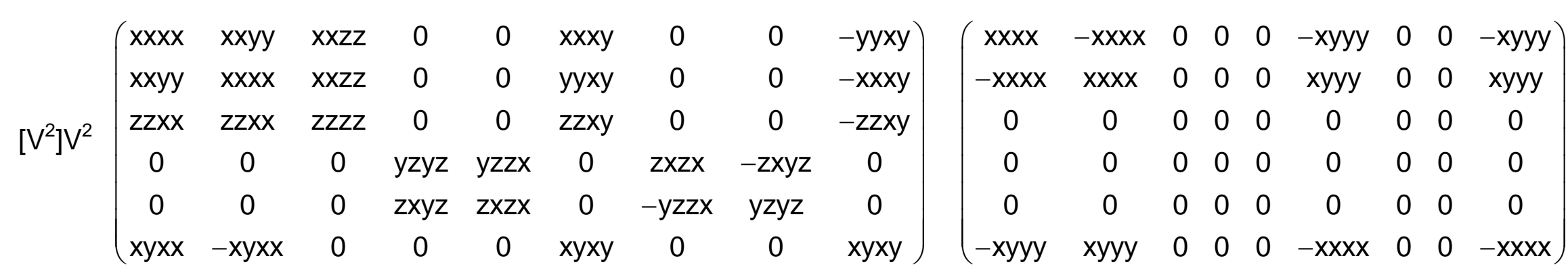

add: $x x x x=x x y y+2 x y x y ; y y x y-x x x y=2 x y x x$
$\mathbf{S}_{2 n}$

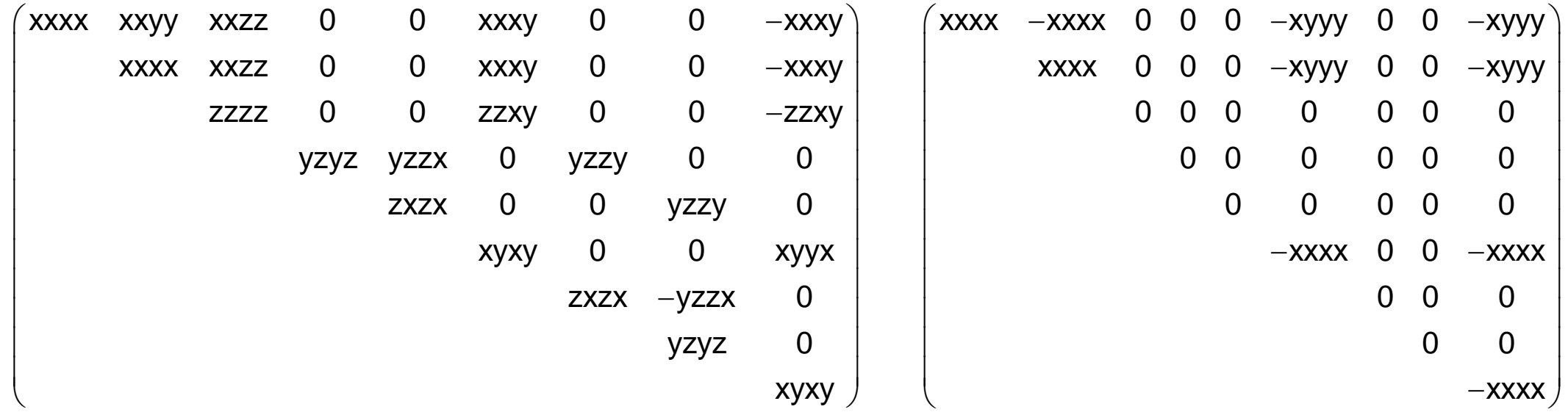

add: $x x x x=x x y y+x y x y+x y y x$

$\left(\begin{array}{ccccccccc}x x x x & x x y y & x x z z & 0 & 0 & x x x y & 0 & 0 & -y y x y \\ x x y y & x x x x & x x z z & 0 & 0 & y y x y & 0 & 0 & -x x x y \\ z z x x & z z x x & z z z z & 0 & 0 & z z x y & 0 & 0 & -z z x y \\ 0 & 0 & 0 & y z y z & y z z x & 0 & z x z x & -z x y z & 0 \\ 0 & 0 & 0 & z x y z & z x z x & 0 & -y z z x & y z y z & 0 \\ x y x x & -x y x x & 0 & 0 & 0 & x y x y & 0 & 0 & x y x y\end{array}\right)\left(\begin{array}{ccccccccc}x x x x & -x x x x & 0 & 0 & 0 & -x y y y & 0 & 0 & -x y y y \\ -x x x x & x x x x & 0 & 0 & 0 & x y y y & 0 & 0 & x y y y \\ 0 & 0 & 0 & 0 & 0 & 0 & 0 & 0 & 0 \\ 0 & 0 & 0 & 0 & 0 & 0 & 0 & 0 & 0 \\ 0 & 0 & 0 & 0 & 0 & 0 & 0 & 0 & 0 \\ -x y y y & x y y y & 0 & 0 & 0 & -x x x x & 0 & 0 & -x x x x\end{array}\right)$

add: $x x x x=x x y y+2 x y x y ; y y x y-x x x y=2 x y x x$ 


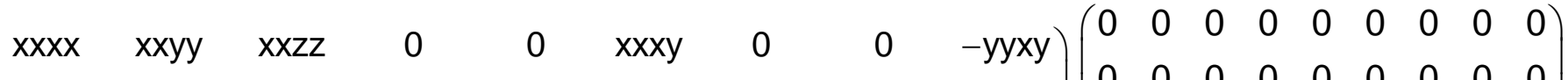

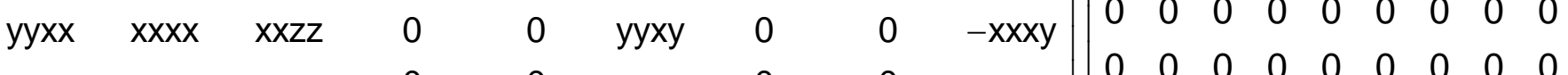
$\begin{array}{llllllllllllllllllll} & z z x x & z z y y & z z z z & 0 & 0 & z z x y & 0 & 0 & 0 & -z z x y & 0 & 0 & 0 & 0 & 0 & 0 & 0 & 0 & 0 \\ 0 & 0 & 0 & 0 & 0 & 0 & 0 & 0 & 0\end{array}$

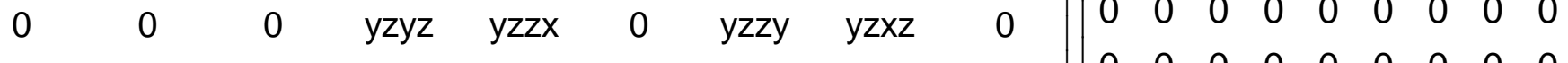

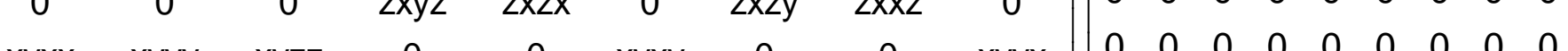

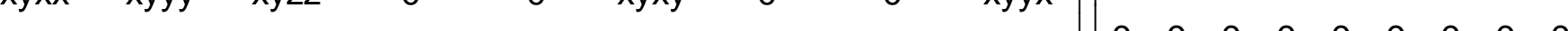
\begin{tabular}{lllllllll|llllllllll}
0 & 0 & 0 & $z x x z$ & $-z x z y$ & 0 & $z x z x$ & $-z x y z$ & 0 & 0 & 0 & 0 & 0 & 0 & 0 & 0 & 0 & 0 \\
0 & 0 & 0 & $-y z x z$ & $y z z$ & 0 & $-y z z x$ & $y z y z$ & 0 & 0 & 0 & 0 & 0 & 0 & 0 & 0 & 0 & 0
\end{tabular}

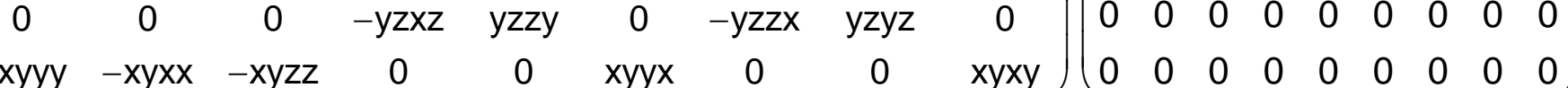
and: $x x x x=x x y y+x y x y+x y y x ; x x x y-y y x y=x y y y-x y x x$

$\left[V^{4}\right] \quad\left(\begin{array}{ccccc}3 x x y y & 0 & 0 & y y z z & 0 \\ 0 & 3 x x y y & 0 & y y z z & 0 \\ 0 & 0 & z z z z & x x y y & 0\end{array}\right)$

$\left(\begin{array}{lllll}0 & 0 & 0 & 0 & 0 \\ 0 & 0 & 0 & 0 & 0 \\ 0 & 0 & 0 & 0 & 0\end{array}\right)$

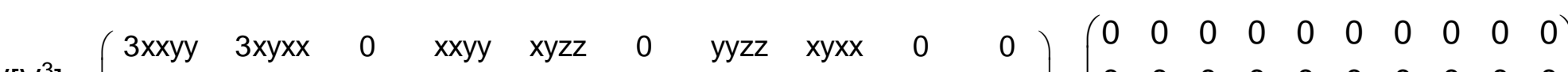

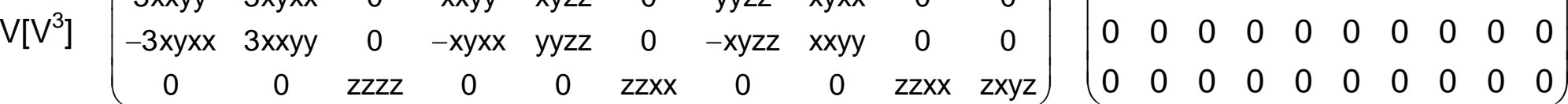

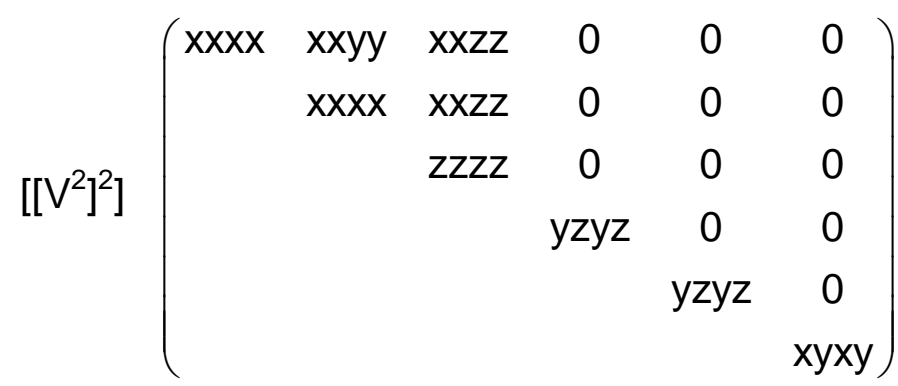

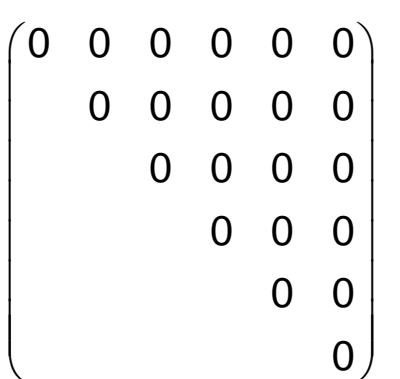

and $x y x y=1 / 2(x x x x-x x y y)$

$\left.\begin{array}{cccccc}x x x x & x x y y & x x z z & 0 & 0 & x x x y \\ x x y y & x x x y & x x z z & 0 & 0 & -x x y\end{array}\right)$

$\begin{array}{cccccc}x x y y & x x x x & x x z z & 0 & 0 & -x x x y \\ z z x x & z z x x & z z z z & 0 & 0 & 0\end{array}$

$\begin{array}{lccccc}0 & 0 & 0 & y z y z & y z x z & 0\end{array}$

$\begin{array}{llllll}0 & 0 & 0 & -y z x z & y z y z & 0\end{array}$

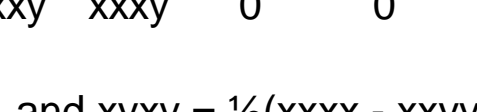

$\left(\begin{array}{llllll}0 & 0 & 0 & 0 & 0 & 0 \\ 0 & 0 & 0 & 0 & 0 & 0\end{array}\right)$ $\begin{array}{llllll}0 & 0 & 0 & 0 & 0 & 0 \\ 0 & 0 & 0 & 0 & 0 & 0 \\ 0 & 0 & 0 & 0 & 0 & 0\end{array}$ $0 \begin{array}{llllll}0 & 0 & 0 & 0 & 0 & 0 \\ 0 & 0 & 0 & 0 & 0 & 0\end{array}$ $\begin{array}{llllll}0 & 0 & 0 & 0 & 0 & 0 \\ 0 & 0 & 0 & 0 & 0 & 0\end{array}$ $\left(\begin{array}{llllll}0 & 0 & 0 & 0 & 0 & 0\end{array}\right)$

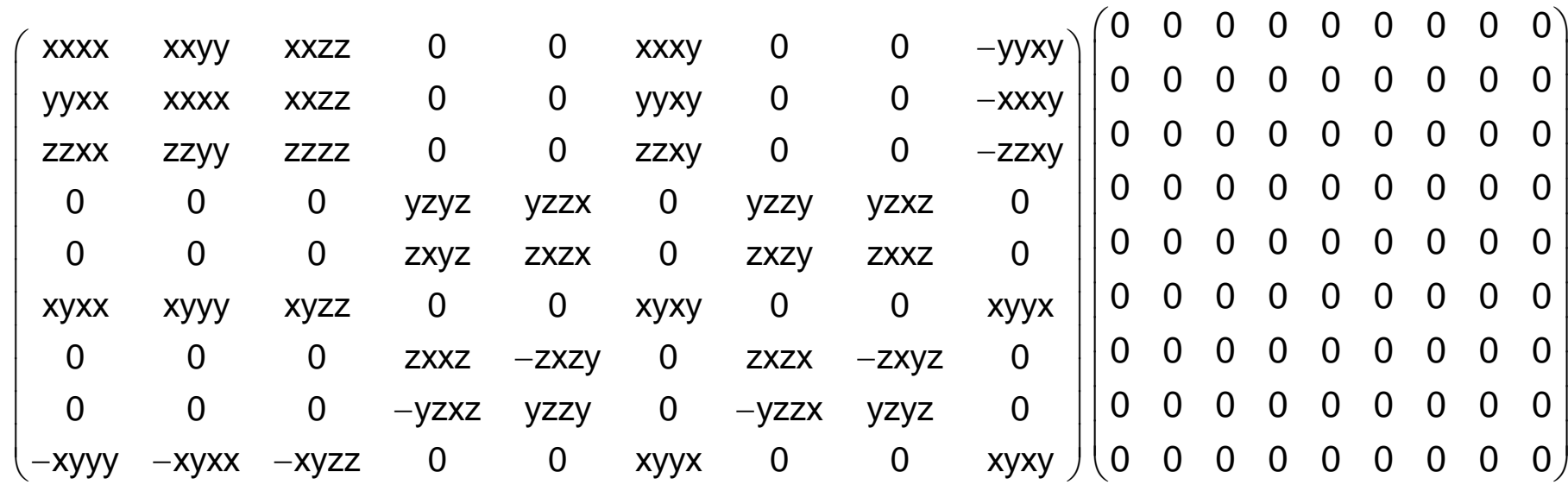

and: $x x x x=x x y y+x y x y+x y y x ; x x x y-y y x y=x y y y-x y x x$

$\left(\begin{array}{ccccc}3 x x y y & 0 & 0 & y y z z & 0 \\ 0 & 3 x x y y & 0 & y y z z & 0 \\ 0 & 0 & z z z z & x x y y & 0\end{array}\right) \quad\left(\begin{array}{ccccc}0 & 0 & 0 & 0 & 0 \\ 0 & 0 & 0 & 0 & 0 \\ 0 & 0 & 0 & 0 & 0\end{array}\right)$

$\left(\begin{array}{llllllllll}3 x x y y & 3 x y x x & 0 & x x y y & x y z z & 0 & y y z z & x y x x & 0 & 0\end{array}\right)\left(\begin{array}{llllllllll}0 & 0 & 0 & 0 & 0 & 0 & 0 & 0 & 0 & 0 \\ 0 & 0 & 0 & 0 & 0 & 0 & 0 & 0 & 0 & 0\end{array}\right)$

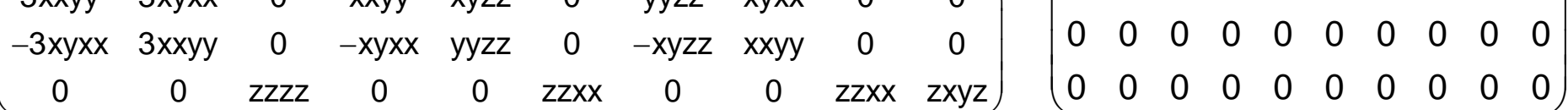

$$
\left(\begin{array}{cccccc}
x x x x & x x y y & x x z z & 0 & 0 & 0 \\
& x x x x & x x z z & 0 & 0 & 0 \\
& & z z z z & 0 & 0 & 0 \\
& & & y z y z & 0 & 0 \\
& & & & y z y z & 0 \\
& & & & & \text { xyxy }
\end{array}\right)
$$

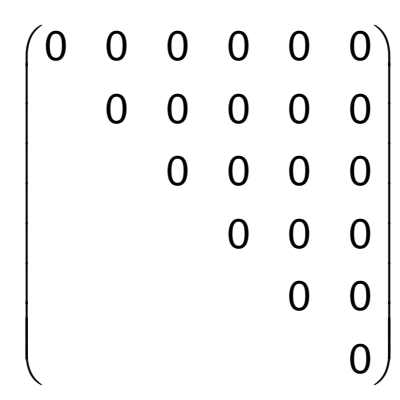

and $x y x y=1 / 2(x x x x-x x y y)$

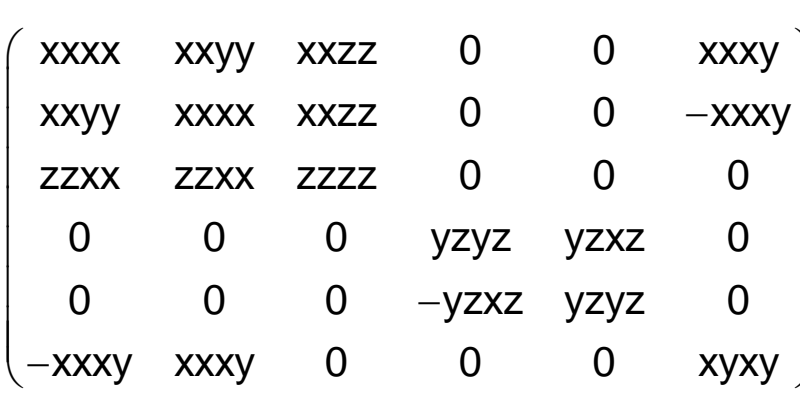

$\left(\begin{array}{llllll}0 & 0 & 0 & 0 & 0 & 0 \\ 0 & 0 & 0 & 0 & 0 & 0\end{array}\right.$

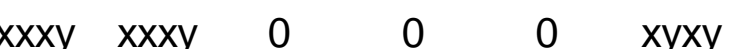

and $x y x y=1 / 2(x x x x-x x y y)$ 


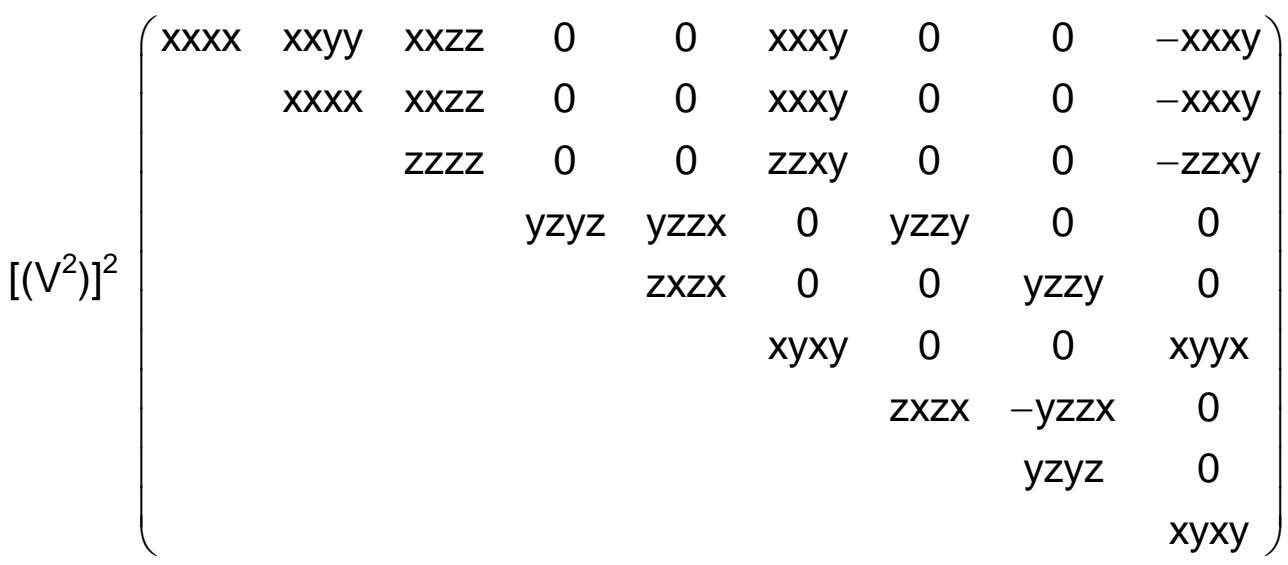

and $x x x x=x x y y+y z y z+z y z y$

$\left(\begin{array}{lllllllll}x x x x & x x y y & x x z z & 0 & 0 & x x x y & 0 & 0 & -y y x y \\ x x y & x x x & x x z z & 0 & 0 & y x y & 0 & 0 & -x x y\end{array}\left(\begin{array}{ccccccccc}0 & 0 & 0 & 0 & 0 & 0 & 0 & 0 & 0 \\ 0 & 0 & 0 & 0 & 0 & 0 & 0 & 0 & 0\end{array}\right)\right.$ $\begin{array}{llllllllll}x x y y & x x x x & x x z z & 0 & 0 & y y x y & 0 & 0 & -x x x y\end{array} \mid\left(\begin{array}{lllllllll}0 & 0 & 0 & 0 & 0 & 0 & 0 & 0 & 0 \\ 0 & 0 & 0 & 0 & 0 & 0 & 0 & 0 & 0\end{array}\right.$

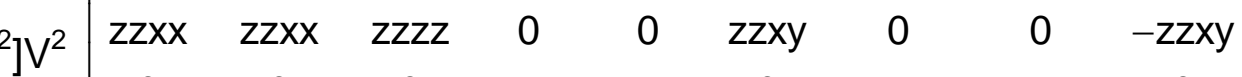
$\begin{array}{lllllllll}0 & 0 & 0 & y z y z & y z z x & 0 & z x z x & -z x y z & 0 \\ 0 & 0 & 0 & z x y & z x z & 0 & -y z z x & y y z & 0\end{array}$ $\begin{array}{lllllllll}0 & 0 & 0 & z x y z & z x z x & 0 & -y z z x & y z y z & 0\end{array}$

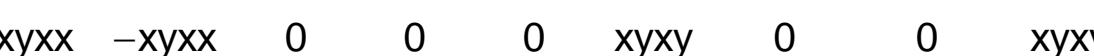

and $x x x x=x x y y+2 x y x y ; y y x y-x x x y=2 x y x x$ $\begin{array}{lllllllll}0 & 0 & 0 & 0 & 0 & 0 & 0 & 0 & 0 \\ & 0 & 0 & 0 & 0 & 0 & 0 & 0 & 0\end{array}$

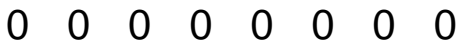

$\begin{array}{llllll}0 & 0 & 0 & 0 & 0 & 0 \\ 0 & 0 & 0 & 0 & 0 & 0\end{array}$

$\begin{array}{llllll}0 & 0 & 0 & 0 & 0 & 0\end{array}$

$\begin{array}{llll}0 & 0 & 0 & 0 \\ 0 & 0 & 0 & 0\end{array}$

$\begin{array}{lll}0 & 0 & 0 \\ & 0 & 0\end{array}$

0

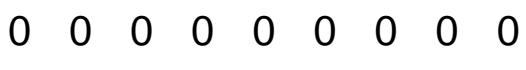
$\begin{array}{llllllllllll}0 & 0 & 0 & 0 & 0 & 0 & 0 & 0 & 0 \\ 0 & 0 & 0 & 0 & 0 & 0 & 0 & 0 & 0\end{array}$ $\begin{array}{llllllllll}0 & 0 & 0 & 0 & 0 & 0 & 0 & 0 & 0 \\ 0 & 0 & 0 & 0 & 0 & 0 & 0 & 0 & 0\end{array}$

$\begin{array}{llllllllll}0 & 0 & 0 & 0 & 0 & 0 & 0 & 0 & 0 \\ 0 & 0 & 0 & 0 & 0 & 0 & 0 & 0\end{array}$

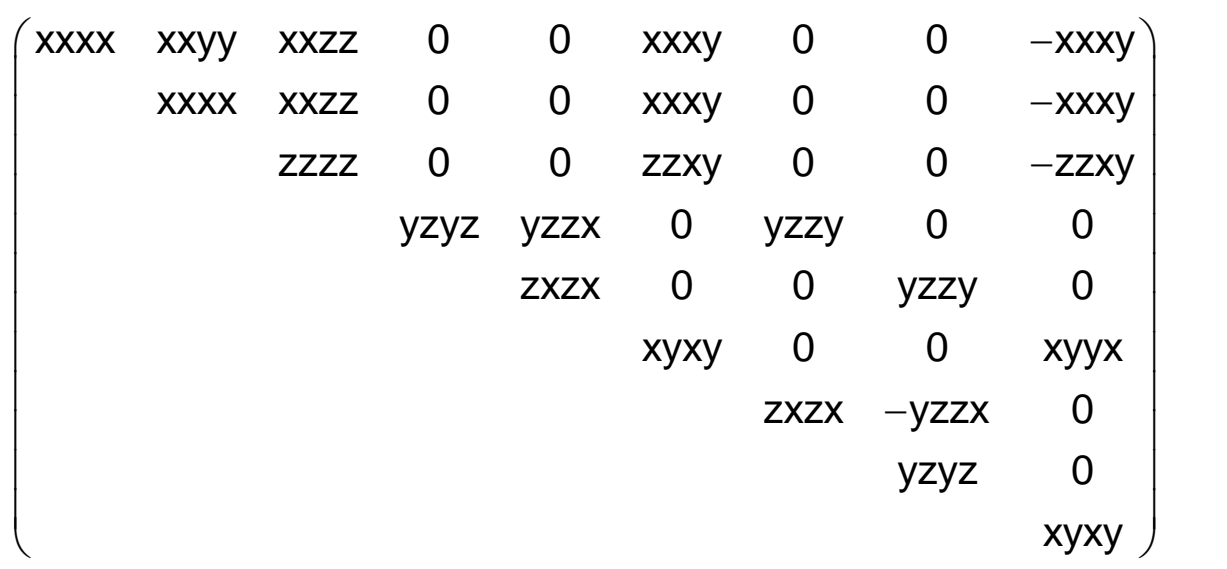

$\left(\begin{array}{lllllllll}0 & 0 & 0 & 0 & 0 & 0 & 0 & 0 & 0 \\ & 0 & 0 & 0 & 0 & 0 & 0 & 0 & 0\end{array}\right)$

$\begin{array}{llllllll}0 & 0 & 0 & 0 & 0 & 0 & 0 & 0\end{array}$

$0 \begin{array}{llllll}0 & 0 & 0 & 0 & 0 & 0 \\ 0 & 0 & 0 & 0 & 0 & 0\end{array}$ $\begin{array}{lllllll}0 & 0 & 0 & 0 & 0 \\ 0 & 0 & 0 & 0 & 0\end{array}$ $\begin{array}{llll}0 & 0 & 0 & 0\end{array}$ $\begin{array}{lll}0 & 0 & 0 \\ 0 & 0\end{array}$

and $x x x x=x x y y+y z y z+z y z y$

$\left(\begin{array}{lllllllll}x x x x & x x y y & x x z z & 0 & 0 & x x x y & 0 & 0 & -y y x y \\ x y y & x x x x & x z z & 0 & 0 & x y y & 0 & 0 & -x x y\end{array}\left(\begin{array}{ccccccccc}0 & 0 & 0 & 0 & 0 & 0 & 0 & 0 & 0 \\ 0 & 0 & 0 & 0 & 0 & 0 & 0 & 0 & 0\end{array}\right)\right.$ $\left.\begin{array}{lllllllll}x x y y & x x x x & x x z z & 0 & 0 & y y x y & 0 & 0 & -x x x y \\ 0 x x & z z x x & z z z & 0 & 0 & z x y & 0 & 0 & -z z x y\end{array}\right)\left(\begin{array}{llllllllll}0 & 0 & 0 & 0 & 0 & 0 & 0 & 0 & 0 \\ 0 & 0 & 0 & 0 & 0 & 0 & 0 & 0 & 0 \\ 0 & 0 & 0 & 0 & 0 & 0 & 0 & 0 & 0\end{array}\right)$

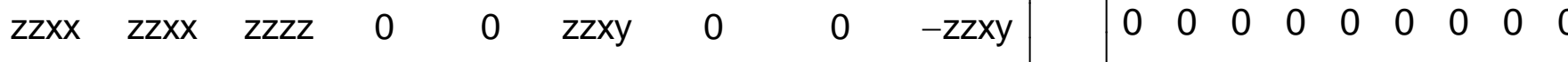
$\begin{array}{lllllllll}0 & 0 & 0 & y z y z & y z z x & 0 & z x z x & -z x y z & 0 \\ 0 & 0 & 0 & z x y z & z x z x & 0 & -y z z & y z y z & 0\end{array} \mid \begin{array}{llllllllll}0 & 0 & 0 & 0 & 0 & 0 & 0 & 0 & 0 \\ 0 & 0 & 0 & 0 & 0 & 0 & 0 & 0 & 0\end{array}$ $\left.\begin{array}{ccccccccc}0 & 0 & 0 & z x y z & z x z x & 0 & -y z z x & y z y z & 0\end{array}\right)\left(\begin{array}{lllllllll}0 & 0 & 0 & 0 & 0 & 0 & 0 & 0 & 0 \\ 0 & 0 & 0 & 0 & 0 & 0 & 0 & 0 & 0\end{array}\right)$ $\left.\begin{array}{lllllllll}x y x x & -x y x x & 0 & 0 & 0 & x y x y & 0 & 0 & x y x y\end{array}\right)\left(\begin{array}{lllllllll}0 & 0 & 0 & 0 & 0 & 0 & 0 & 0 & 0\end{array}\right.$

and $x x x x=x x y y+2 x y x y ; y y x y-x x x y=2 x y x x$ 


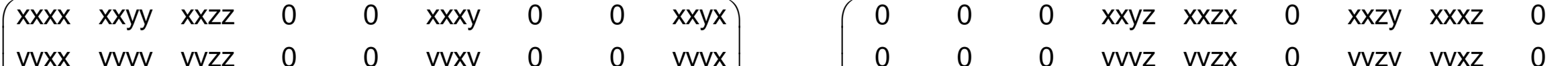
$\begin{array}{lllllllll}y y x x & y y y y & y y z z & 0 & 0 & y y x y & 0 & 0 & y y y x \\ 2 z x x & z z y & z z z z & 0 & 0 & z z x & 0 & 0 & z z y x\end{array}$ $\begin{array}{lllllllll}\text { zzxx } & \text { zzyy } & z z z z & 0 & 0 & z z x y & 0 & 0 & z z y x\end{array}$ $\begin{array}{lllllllll}0 & 0 & 0 & y z y z & y z z x & 0 & y z z y & y z x z & 0\end{array}$ $\begin{array}{lllllllll}0 & 0 & 0 & z x y z & z x z x & 0 & z x z y & z x x z & 0\end{array}$ $\begin{array}{lllllllll}\text { xyxx } & \text { xyyy } & \text { xyzz } & 0 & 0 & \text { xyxy } & 0 & 0 & \text { xyyx }\end{array}$ $\begin{array}{lllllllll}0 & 0 & 0 & \text { zyyz } & \text { zyzx } & 0 & \text { zyzy } & \text { zyxz } & 0\end{array}$ $\begin{array}{lllllllll}0 & 0 & 0 & x z y z & x z z x & 0 & x z z y & x z x z & 0\end{array}$ $\begin{array}{lllllllll}y \mathrm{yxx} & \mathrm{yxyy} & \mathrm{yxzz} & 0 & 0 & \mathrm{yxxy} & 0 & 0 & \mathrm{yxyx}\end{array}$

$\left[V^{4}\right] \quad\left(\begin{array}{ccccc}x x x x & x y y y & 0 & y y z z & 0 \\ y x x x & y y y y & 0 & z z x x & 0 \\ 0 & 0 & z z z z & x x y y & z z x y\end{array}\right)$ $\begin{array}{lllllllll}0 & 0 & 0 & z z y z & z z z x & 0 & \text { zzzy } & z z x z & 0\end{array}$ $\begin{array}{lllllllll}\text { yzxx } & y z y y & y z z z & 0 & 0 & y z x y & 0 & 0 & y z y x\end{array}$ $\begin{array}{lllllllll}z x x x & z x y y & z x z z & 0 & 0 & z x x y & 0 & 0 & z x y x\end{array}$ $\begin{array}{lllllllll}0 & 0 & 0 & \text { xyyz } & \text { xyzx } & 0 & \text { xyzy } & \text { xyxz } & 0\end{array}$

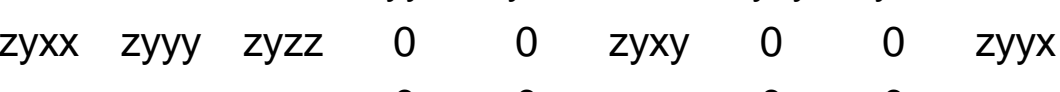
$\begin{array}{ccccccccc}x z x x & x z y y & x z z z & 0 & 0 & x z x y & 0 & 0 & x z y x\end{array}$

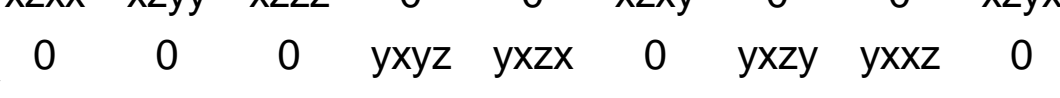

\section{$\begin{array}{ccccc}0 & 0 & x z z z & 0 & x x y z \\ 0 & 0 & y z z z & 0 & y y x z\end{array}$}

$\left(\begin{array}{ccccc}0 & 0 & x z z & 0 & x y z \\ 0 & 0 & y z z z & 0 & y y x z\end{array}\right.$ $\begin{array}{lllllllll}x x x x & x x y y & x x z z & 0 & 0 & x x x y & 0 & 0 & x x y x \\ y x x & y y y & y y z z & 0 & 0 & y y x y & 0 & 0 & x y y\end{array}$

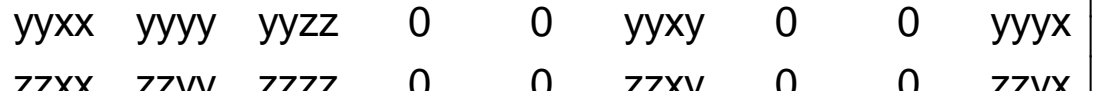
$\begin{array}{llll}0 & 0 & & \\ y z y z & y z z x & 0 & 0\end{array}$

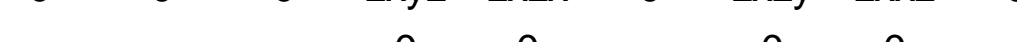
$\begin{array}{llllllllll}0 & 0 & 0 & 0 & 0 & 0 & 0 & 0 & 0 & 0\end{array}$ $\begin{array}{llllllllll}0 & 0 & 0 & \text { zyyz } & \text { zyzx } & 0 & \text { zyzy } & \text { zyxz } & 0 \\ 0 & 0 & 0 & x z y z & x z z x & 0 & x z y & x z z & 0\end{array}$ $\begin{array}{ccccccccc}0 & 0 & 0 & x z y z & x z z x & 0 & x z z y & x z x z & 0 \\ y x x x & y x y y & y x z z & 0 & 0 & y x x y & 0 & 0 & y x y x\end{array}$ $\left(\begin{array}{ccccc}x x x x & x y y y & 0 & y y z z & 0 \\ y x x x & y y y y & 0 & z z x x & 0 \\ 0 & 0 & z z z z & x x y & z z x y\end{array}\right)$ $\left.\begin{array}{ccccc}x & 0 & z z z z & x x y y & z z x y\end{array}\right)$ $\left(\begin{array}{lllllllll}0 & 0 & 0 & x x y z & x x z x & 0 & x x z y & x x x z & 0 \\ 0 & 0 & 0 & y y z & y y z x & 0 & y y y & y y x z & 0\end{array}\right)$ $\left.\begin{array}{ccccccccc}0 & 0 & 0 & x x y z & x x z x & 0 & x x y & x x x z & 0 \\ 0 & 0 & 0 & y y y z & y y z x & 0 & y y z y & y y x z & 0 \\ y & 0 & z z y z & z z z x & 0 & z z z y & z z x z & 0\end{array}\right)$ $\begin{array}{lllllllll}y z x x & y z y y & y z z z & 0 & 0 & y z x y & 0 & 0 & y z y x\end{array}$ $\begin{array}{lllllllll}z x x x & z x y y & z x z z & 0 & 0 & z x x y & 0 & 0 & z x y x\end{array}$ $\begin{array}{lcccccccc}0 & 0 & 0 & x y y z & x y z x & 0 & x y z y & x y x z & 0\end{array}$

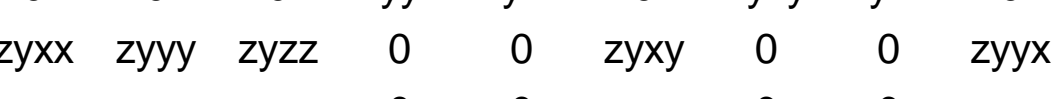
$\begin{array}{ccccccccc}x z x x & x z y y & x z z z & 0 & 0 & x z x y & 0 & 0 & x z y x\end{array}$ $\begin{array}{lllllllll}0 & 0 & 0 & y x y z & y x z x & 0 & y x z y & y x x z & 0\end{array}$

$\left(\begin{array}{ccccc}0 & 0 & x z z z & 0 & x x y z \\ 0 & 0 & y z z z & 0 & y y x z \\ z x x x & z y y y & 0 & 0 & 0\end{array}\right)$

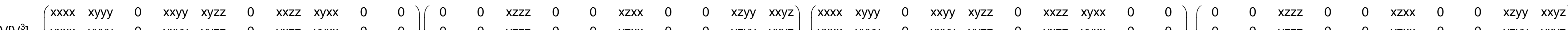

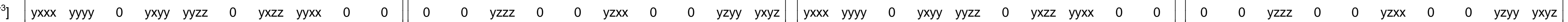

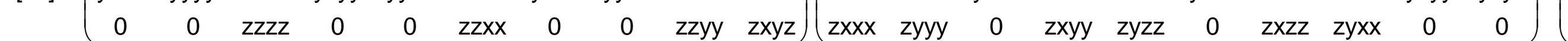

$\left[\left[\mathrm{V}^{2}\right]^{2}\right]$ $\left(\begin{array}{cccccc}x x x x & x x y y & x x z z & 0 & 0 & x x x y \\ & \text { yyyy } & \text { yyzz } & 0 & 0 & y y x y \\ & & \text { zzzz } & 0 & 0 & \text { zzxy } \\ & & & y z y z & y z x z & 0 \\ & & & & x z x z & 0 \\ & & & & & \text { xyxy }\end{array}\right)$

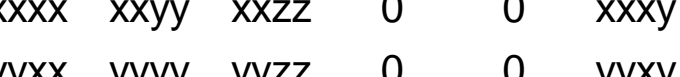

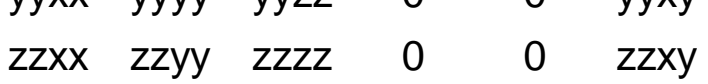
$\begin{array}{llllll}0 & 0 & 0 & y z y z & y z x z & 0 \\ 0 & 0 & 0 & x z y z & x z x z & 0\end{array}$ $\begin{array}{llllll}0 & 0 & 0 & x z y z & x z x z & 0\end{array}$

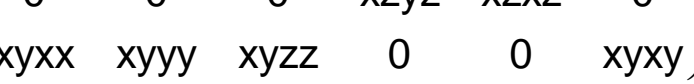

$$
\left(\begin{array}{cccccc}
\text { xxxx } & \text { xxyy } & \text { xxzz } & 0 & 0 & \text { xxxy } \\
& \text { yyyy } & \text { yyzz } & 0 & 0 & \text { yyxy } \\
& & \text { zzzz } & 0 & 0 & \text { zzxy } \\
& & & y z y z & y z x z & 0 \\
& & & & x z x z & 0 \\
& & & & & \text { xyxy }
\end{array}\right)
$$

$\begin{array}{llllll}x x x x & x x y y & x x z z & 0 & 0 & x x x y \\ y y x & y y y & y y z z & 0 & 0 & y x y\end{array}$ $\begin{array}{llllll}x y y x x & y y y y & y y z z & 0 & 0 & y y x y \\ z z x x & z z y & z z z z & 0 & 0 & z z x y\end{array}$ $\begin{array}{cccccc}\text { zzxx } & \text { zzyy } & \text { zzzz } & 0 & 0 & \text { zzxy }\end{array}$ $\begin{array}{cccccc}0 & 0 & 0 & y z y z & y z x z & 0 \\ 0 & 0 & 0 & x y z & x z x z & 0\end{array}$ $\begin{array}{cccccc}0 & 0 & 0 & x z y z & x z x z & 0 \\ x y x x & x y y y & x y z z & 0 & 0 & x y x y\end{array}$

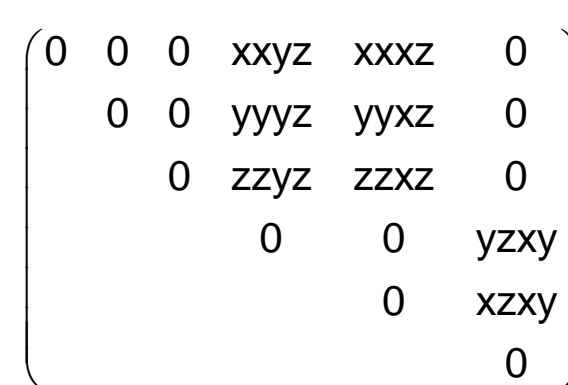

$\left(\begin{array}{cccccc}0 & 0 & 0 & \text { xxyz } & \text { xxxz } & 0 \\ 0 & 0 & 0 & \text { yyyz } & \text { yyxz } & 0 \\ 0 & 0 & 0 & z z y z & z z x z & 0 \\ y z x x & y z y y & y z z z & 0 & 0 & y z x y \\ \text { xzxx } & x z y y & x z z z & 0 & 0 & x z x y \\ 0 & 0 & 0 & \text { xyyz } & \text { xyxz } & 0\end{array}\right)$

$\left(\begin{array}{cccccc}0 & 0 & 0 & x x y z & x x x z & 0 \\ 0 & 0 & 0 & y y y z & y y x z & 0 \\ 0 & 0 & 0 & z z y z & z z x z & 0 \\ y z x x & y z y y & y z z z & 0 & 0 & y z x y \\ x z x x & x z y y & x z z z & 0 & 0 & x z x y \\ 0 & 0 & 0 & x y y z & x y x z & 0\end{array}\right)$


$n=1 \quad C_{1 h}=m_{2}$

1

$$
\begin{aligned}
& \begin{array}{lllllllll}
x x x x & x x y y & x x z z & 0 & 0 & x x x y & 0 & 0 & x x y x \\
& y y y y & y y z z & 0 & 0 & y y x y & 0 & 0 & y y y x
\end{array} \\
& \begin{array}{llllllll}
\text { yyyy } & y y z z & 0 & 0 & y y x y & 0 & 0 & y y y x \\
& \text { zzzz } & 0 & 0 & z z x y & 0 & 0 & z z y x
\end{array} \\
& \begin{array}{llllll}
y z y z & y z z x & 0 & y z z y & y z x z & 0
\end{array} \\
& \begin{array}{lllll}
z x z x & 0 & z x z y & z x x z & 0
\end{array} \\
& \begin{array}{cccc}
x y x y & 0 & 0 & x y y x \\
z y z y & z y x z & 0 \\
& x z x z & 0
\end{array} \\
& \begin{array}{cc}
x z x z & 0 \\
y x y x
\end{array}
\end{aligned}
$$

(

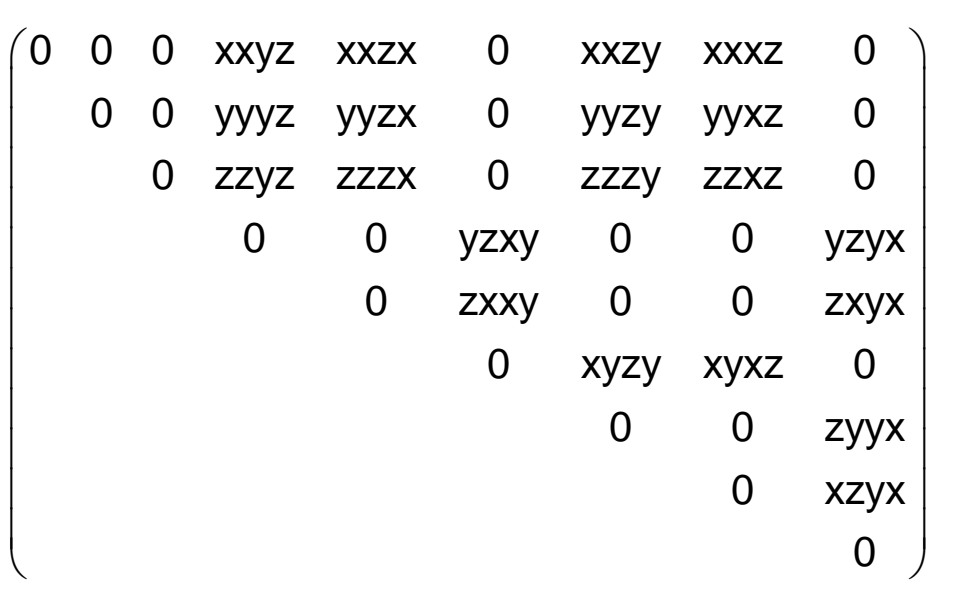
$\left.\begin{array}{lllllllll}x x x x & x x y y & x x z z & 0 & 0 & x x x y & 0 & 0 & x x y x \\ & y y y & y y z z & 0 & 0 & y y x y & 0 & 0 & y y x\end{array}\right)$ $\begin{array}{llllllll}\text { yyyy } & y y z z & 0 & 0 & \text { yyxy } & 0 & 0 & \text { yyyx }\end{array}$ $\begin{array}{lllllll}z z z z & 0 & 0 & z z x y & 0 & 0 & z z y x\end{array}$ $\begin{array}{llllll}y z y z & y z z x & 0 & y z z y & y z x z & 0\end{array}$ $\begin{array}{ccccc}z x z x & 0 & z x z y & z x x z & 0\end{array}$ $\begin{array}{cccc}x y x y & 0 & 0 & x y y x \\ & z y z y & z y x z & 0 \\ & x z x z & 0\end{array}$

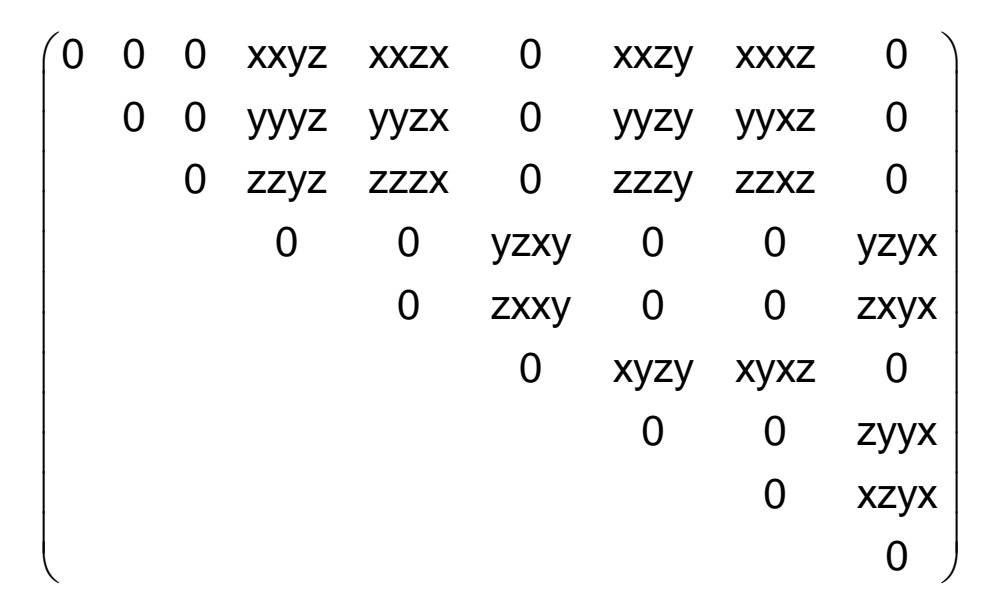

$\left(\begin{array}{ccccccccc}x x x x & x x y y & x x z z & 0 & 0 & x x x y & 0 & 0 & x x y x \\ y y x x & y y y y & y y z z & 0 & 0 & y y x y & 0 & 0 & y y y x \\ z z x x & z z y y & z z z z & 0 & 0 & z z x y & 0 & 0 & z z y x \\ 0 & 0 & 0 & y z y z & y z z x & 0 & y z z y & y z x z & 0 \\ 0 & 0 & 0 & z x y z & z x z x & 0 & z x z y & z x x z & 0 \\ \text { xyxx } & x y y y & x y z z & 0 & 0 & x y x y & 0 & 0 & x y y x\end{array}\right)$

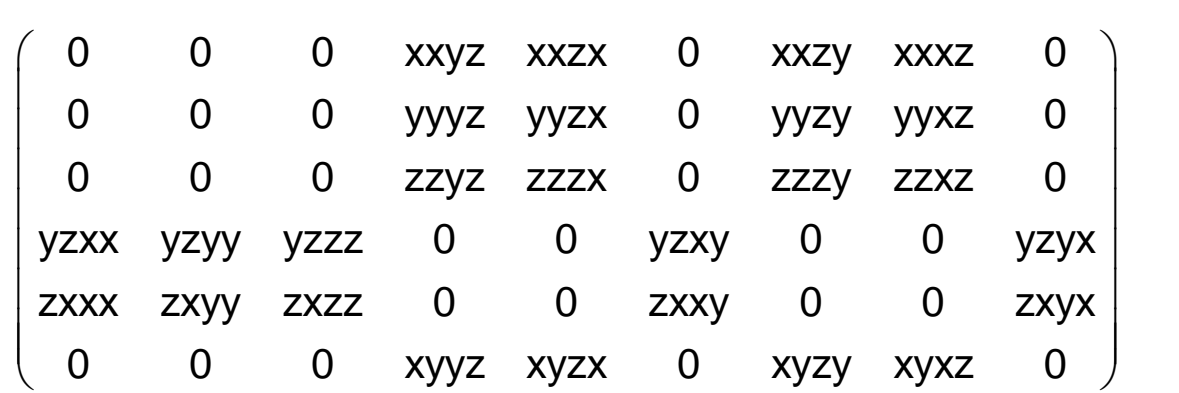

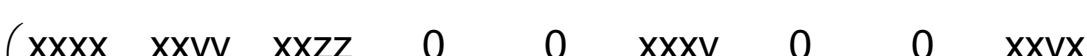

$\left(\begin{array}{lllllllll}x x x x & x x y y & x x z z & 0 & 0 & x x x y & 0 & 0 & x x y x \\ y y x x & y y y y & y y z z & 0 & 0 & y y x y & 0 & 0 & y y y x \\ z z x & z z y & z z z z & 0 & 0 & z z x y & 0 & 0 & z z y x\end{array}\right)$ $\begin{array}{llllllllll}\text { zzxx } & \text { zzyy } & \text { zzzz } & 0 & 0 & \text { zzxy } & 0 & 0 & z z y x\end{array}$ $\begin{array}{lllllllll}0 & 0 & 0 & y z y z & y z z x & 0 & y z z y & y z x z & 0\end{array}$ $\begin{array}{lllllllll}0 & 0 & 0 & z x y z & z x z x & 0 & z x z y & z x x z & 0\end{array}$ $\left(\begin{array}{ccccccccc}0 & 0 & 0 & x x y z & x x z x & 0 & \text { xxzy } & \text { xxxz } & 0 \\ 0 & 0 & 0 & \text { yyyz } & \text { yyzx } & 0 & \text { yyzy } & \text { yyxz } & 0 \\ 0 & 0 & 0 & \text { zzyz } & \text { zzzx } & 0 & \text { zzzy } & \text { zzxz } & 0 \\ \text { yzxx } & \text { yzyy } & \text { yzzz } & 0 & 0 & \text { yzxy } & 0 & 0 & \text { yzyx } \\ \text { zxxx } & \text { zxyy } & \text { zxzz } & 0 & 0 & \text { zxxy } & 0 & 0 & \text { zxyx } \\ 0 & 0 & 0 & \text { xyyz } & \text { xyzx } & 0 & \text { xyzy } & \text { xyxz } & 0\end{array}\right)$ 
1

$\left(\begin{array}{lllllllll}x x x x & x x y y & x x z z & 0 & 0 & x x x y & 0 & 0 & x x y x \\ y y x x & y y y & y y z & 0 & 0 & y x y & 0 & 0 & x y x\end{array}\right.$ $\left.\begin{array}{lllllllll}\text { yyxx } & \text { yyyy } & \text { yyzz } & 0 & 0 & \text { yyxy } & 0 & 0 & \text { yyyx } \\ \text { zzxx } & \text { zzyy } & \text { zzzz } & 0 & 0 & \text { zzxy } & 0 & 0 & \text { zzyx } \\ 0 & 0 & 0 & y z y z & y z z x & 0 & y z z y & y z x z & 0\end{array}\right)$

$\mathrm{v}^{4} \quad \begin{array}{ccccccccc}0 & 0 & 0 & \mathrm{yzyz} & \mathrm{yzzx} & 0 & \mathrm{yzzy} & \mathrm{yzxz} & 0 \\ 0 & 0 & 0 & \mathrm{zxyz} & \mathrm{zxzx} & 0 & \mathrm{zxzy} & \mathrm{zxxz} & 0\end{array}$

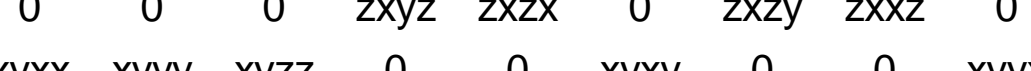

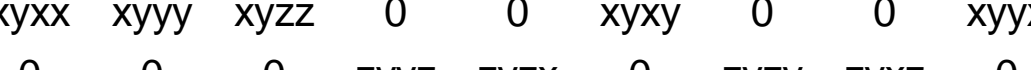

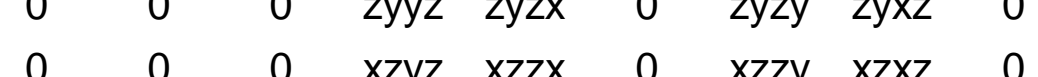
$\begin{array}{ccccccccc}0 & 0 & 0 & x z y z & x z z x & 0 & x z z y & x z x z & 0 \\ y x x x & y x y y & y x z z & 0 & 0 & y x x y & 0 & 0 & y x y x\end{array}$

$\left(\begin{array}{lllllllll}0 & 0 & 0 & 0 & 0 & 0 & 0 & 0 & 0 \\ 0 & 0 & 0 & 0 & 0 & 0 & 0 & 0 & 0\end{array}\right)$ $\begin{array}{lllllllll}0 & 0 & 0 & 0 & 0 & 0 & 0 & 0 & 0 \\ 0 & 0 & 0 & 0 & 0 & 0 & 0 & 0 & 0\end{array}$ $0 \begin{array}{lllllllll}0 & 0 & 0 & 0 & 0 & 0 & 0 & 0 & 0 \\ 0 & 0 & 0 & 0 & 0 & 0 & 0 & 0 & 0\end{array}$ $0 \begin{array}{lllllllll}0 & 0 & 0 & 0 & 0 & 0 & 0 & 0 & 0 \\ 0 & 0 & 0 & 0 & 0 & 0 & 0 & 0 & 0\end{array}$ $0 \begin{array}{llllllllll}0 & 0 & 0 & 0 & 0 & 0 & 0 & 0 & 0 \\ 0 & 0 & 0 & 0 & 0 & 0 & 0 & 0 & 0\end{array}$ $\begin{array}{llllllllll}0 & 0 & 0 & 0 & 0 & 0 & 0 & 0 & 0 \\ 0 & 0 & 0 & 0 & 0 & 0 & 0 & 0 & 0 & 0\end{array}$ $\begin{array}{llllllllll}0 & 0 & 0 & 0 & 0 & 0 & 0 & 0 & 0 \\ 0 & 0 & 0 & 0 & 0 & 0 & 0 & 0 & 0\end{array}$ $\begin{array}{lllllllllllll}0 & 0 & 0 & 0 & 0 & 0 & 0 & 0 & 0\end{array}$ $\left(\begin{array}{lllllllll}0 & 0 & 0 & 0 & 0 & 0 & 0 & 0 & 0 \\ 0 & 0 & 0 & 0 & 0 & 0 & 0 & 0 & 0\end{array}\right)$

$\left[V^{4}\right]\left(\begin{array}{ccccc}x x x x & x y y y & 0 & y y z z & 0 \\ y x x x & y y y y & 0 & z z x x & 0 \\ 0 & 0 & z z z z & x x y y & z z x y\end{array}\right)$

$$
\left(\begin{array}{lllll}
0 & 0 & 0 & 0 & 0 \\
0 & 0 & 0 & 0 & 0 \\
0 & 0 & 0 & 0 & 0
\end{array}\right)
$$

$\left(\begin{array}{llllllllll}x x x x & x y y y & 0 & x x y y & x y z z & 0 & x x z z & x y x x & 0 & 0 \\ y x x x & y y y y & 0 & y x y y & y y z z & 0 & y x z z & y y x x & 0 & 0\end{array}\right)\left(\begin{array}{llllllllll}0 & 0 & 0 & 0 & 0 & 0 & 0 & 0 & 0 & 0 \\ 0 & 0 & 0 & 0 & 0 & 0 & 0 & 0 & 0 & 0 \\ 0 & 0 & 0 & 0 & 0 & 0 & 0 & 0 & 0\end{array}\right)$ VIV] $\left(\begin{array}{ccccccccccc}x & y x x & y y y y & 0 & y x y y & y y z z & 0 & y x z z & y y x x & 0 & 0 \\ 0 & 0 & z z z z & 0 & 0 & z z x & 0 & 0 & z z y & z x y z\end{array}\right)\left(\begin{array}{lllllllllll}0 & 0 & 0 & 0 & 0 & 0 & 0 & 0 & 0 & 0 \\ 0 & 0 & 0 & 0 & 0 & 0 & 0 & 0 & 0 & 0\end{array}\right)$
$\left[\left[V^{2}\right]^{2}\right]\left(\begin{array}{cccccc}x x x x & x x y y & x x z z & 0 & 0 & x x x y \\ & y y y y & y y z z & 0 & 0 & y y x y \\ & & z z z z & 0 & 0 & z z x y \\ & & & y z y z & y z x z & 0 \\ & & & & x z x z & 0 \\ & & & & & x y x y\end{array}\right)$

$\left(\begin{array}{llllll}x x x x & x x y y & x x z z & 0 & 0 & x x x y \\ y y x x & y y y y & y y z z & 0 & 0 & y y x y \\ z z x x & z z y y & z z z & 0 & 0 & z z x y\end{array}\right)$

$\begin{array}{llllll}z z x x & z z y y & z z z z & 0 & 0 & z z x y\end{array}$ $\begin{array}{llllll}0 & 0 & 0 & y z y z & y z x z & 0 \\ 0 & 0 & 0 & x z y & x z z & 0\end{array}$ $\begin{array}{cccccc}0 & 0 & 0 & x z y z & x z x z & 0\end{array}$

$$
\left(\begin{array}{llllll}
0 & 0 & 0 & 0 & 0 & 0 \\
& 0 & 0 & 0 & 0 & 0 \\
& & 0 & 0 & 0 & 0 \\
& & & 0 & 0 & 0 \\
& & & & 0 & 0 \\
& & & & & 0
\end{array}\right)
$$

$\left(\begin{array}{llllll}0 & 0 & 0 & 0 & 0 & 0 \\ 0 & 0 & 0 & 0 & 0 & 0\end{array}\right.$ $\begin{array}{llllll}0 & 0 & 0 & 0 & 0 & 0 \\ 0 & 0 & 0 & 0 & 0 & 0 \\ 0 & 0 & 0 & 0 & 0 & 0\end{array}$ $\begin{array}{lllllll}0 & 0 & 0 & 0 & 0 & 0\end{array}$ $\begin{array}{llllll}0 & 0 & 0 & 0 & 0 & 0\end{array}$ $\begin{array}{llllll}0 & 0 & 0 & 0 & 0 & 0 \\ 0 & 0 & 0 & 0 & 0 & 0\end{array}$

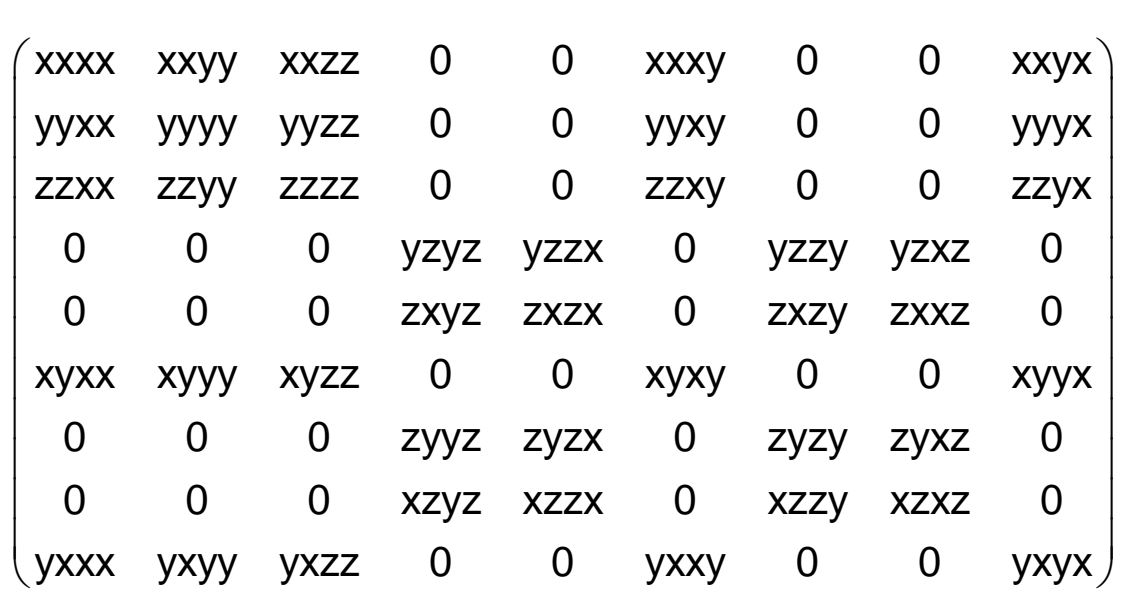

$\left(\begin{array}{lllll}x x x x & x y y y & 0 & y y z z & 0 \\ y x x x & y y y y & 0 & z z x x & 0\end{array}\right)$

$\left(\begin{array}{ccccc}\operatorname{yxxx} & y y y y & 0 & z z x x & 0 \\ 0 & 0 & z z z z & x x y y & z z x y\end{array}\right)$

$\left(\begin{array}{llllllll}0 & 0 & 0 & 0 & 0 & 0 & 0 & 0 \\ 0 & 0 & 0 & 0 & 0 & 0 & 0 & 0\end{array}\right.$ $\begin{array}{lllllllll}0 & 0 & 0 & 0 & 0 & 0 & 0 & 0 & 0 \\ 0 & 0 & 0 & 0 & 0 & 0 & 0 & 0 & 0\end{array}$ $\begin{array}{lllllllll}0 & 0 & 0 & 0 & 0 & 0 & 0 & 0 & 0 \\ 0 & 0 & 0 & 0 & 0 & 0 & 0 & 0 & 0 \\ 0 & 0 & 0 & 0 & 0 & 0 & 0 & 0 & 0\end{array}$ $\begin{array}{llllllllll}0 & 0 & 0 & 0 & 0 & 0 & 0 & 0 & 0 \\ 0 & 0 & 0 & 0 & 0 & 0 & 0\end{array}$ $\begin{array}{lllllllll}0 & 0 & 0 & 0 & 0 & 0 & 0 & 0 & 0 \\ 0 & 0 & 0 & 0 & 0 & 0\end{array}$

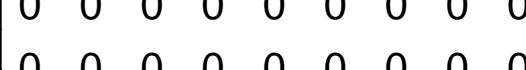
$\left(\begin{array}{lllllllllll}0 & 0 & 0 & 0 & 0 & 0 & 0 & 0 & 0 \\ 0 & 0 & 0 & 0 & 0 & 0 & 0 & 0 & 0 \\ 0 & 0 & 0 & 0 & 0 & 0 & 0 & 0 & 0\end{array}\right.$ $\left(\begin{array}{llllllllll}0 & 0 & 0 & 0 & 0 & 0 & 0 & 0 & 0 \\ 0 & 0 & 0 & 0 & 0 & 0 & 0 & 0 & 0\end{array}\right.$

$\left(\begin{array}{llllllllll}x x x x & x y y y & 0 & x x y y & x y z z & 0 & x x z z & x y x x & 0 & 0 \\ y x x x & y y y y & 0 & y x y y & y y z z & 0 & y x z & y y x x & 0 & 0\end{array}\right)\left(\begin{array}{llllllllll}0 & 0 & 0 & 0 & 0 & 0 & 0 & 0 & 0 & 0 \\ 0 & 0 & 0 & 0 & 0 & 0 & 0 & 0 & 0 & 0 \\ 0 & 0 & 0 & 0 & 0 & 0 & 0 & 0 & 0\end{array}\right)$

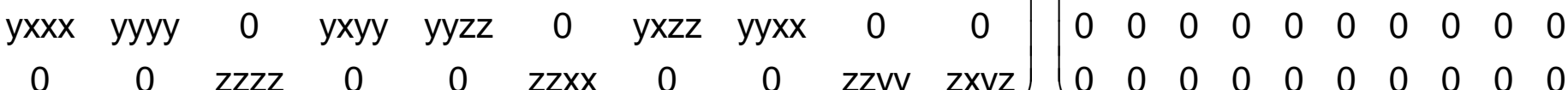

$$
\left(\begin{array}{cccccc}
x x x x & x x y y & x x z z & 0 & 0 & \text { xxxy } \\
& \text { yyyy } & \text { yyzz } & 0 & 0 & y y x y \\
& & z z z z & 0 & 0 & z z x y \\
& & & y z y z & y z x z & 0 \\
& & & & x z x z & 0 \\
& & & & & \text { xyxy }
\end{array}\right)
$$

$\left(\begin{array}{llllll}x x x x & x x y y & x x z z & 0 & 0 & x x x y \\ y x x & y y y & y y z z & 0 & 0 & y y x y\end{array}\right.$ $\begin{array}{llllll}\text { yyxx } & \text { yyyy } & \text { yyzz } & 0 & 0 & \text { yyxy } \\ z z x x & z z y y & z z z z & 0 & 0 & z z x y\end{array}$ $\begin{array}{cccccc}\text { zzxx } & \text { zzyy } & \text { zzzz } & 0 & 0 & \text { zzxy } \\ 0 & 0 & 0 & y z y z & y z x z & 0\end{array}$ $\begin{array}{llllll}0 & 0 & 0 & y z y z & y z x z & 0 \\ 0 & 0 & 0 & x z z & x z x z & 0\end{array}$ $\left(\begin{array}{cccccc}0 & 0 & 0 & x z y z & x z x z & 0 \\ x y x x & x y y y & x y z z & 0 & 0 & x y x y\end{array}\right.$

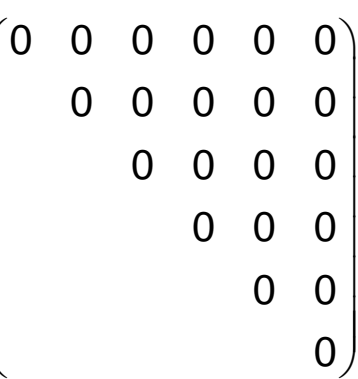

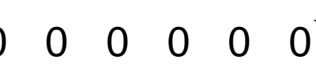

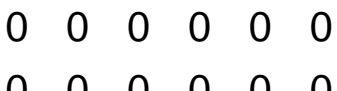
$\begin{array}{lllllll}0 & 0 & 0 & 0 & 0 & 0 \\ 0 & 0 & 0 & 0 & 0 & 0 \\ 0 & 0 & 0 & 0 & 0 & 0\end{array}$ $\begin{array}{lllllll}0 & 0 & 0 & 0 & 0 & 0 \\ 0 & 0 & 0 & 0 & 0 & 0 \\ 0 & 0 & 0 & 0 & 0\end{array}$ $\left(\begin{array}{llllll}0 & 0 & 0 & 0 & 0 & 0 \\ 0 & 0 & 0 & 0 & 0 & 0\end{array}\right.$ 


$$
\begin{aligned}
& \left(\begin{array}{lllllllll}
x x x x & x x y y & x x z z & 0 & 0 & x x x y & 0 & 0 & x x y x \\
& y y y y & y y z z & 0 & 0 & y y x y & 0 & 0 & y y x
\end{array}\right) \\
& \begin{array}{llllllll}
\text { yyyy } & \text { yyzz } & 0 & 0 & \text { yyxy } & 0 & 0 & \text { yyy } \\
& \text { zzzz } & 0 & 0 & \text { zzxy } & 0 & 0 & \text { zzyx }
\end{array} \\
& \begin{array}{llllll}
y z y z & y z z x & 0 & y z z y & y z x z & 0
\end{array} \\
& \begin{array}{lllll}
z x z x & 0 & z x z y & z x x z & 0
\end{array} \\
& \begin{array}{llll}
\text { xyxy } & 0 & 0 & x y y x
\end{array} \\
& \begin{array}{lll}
z y z y & z y x z & 0 \\
x z x z & 0
\end{array}
\end{aligned}
$$

$\left(\begin{array}{lllllllll}0 & 0 & 0 & 0 & 0 & 0 & 0 & 0 & 0 \\ 0 & 0 & 0 & 0 & 0 & 0 & 0 & 0\end{array}\right.$

$0 \begin{array}{llllllll}0 & 0 & 0 & 0 & 0 & 0 & 0 & 0\end{array}$ $\begin{array}{lllllll}0 & 0 & 0 & 0 & 0 & 0 & 0\end{array}$ $\begin{array}{llllll}0 & 0 & 0 & 0 & 0 & 0 \\ 0 & 0 & 0 & 0\end{array}$ $\begin{array}{lllll}0 & 0 & 0 & 0 & 0\end{array}$ $\begin{array}{llll}0 & 0 & 0 & 0\end{array}$ $\begin{array}{lll}0 & 0 \\ 0 & 0 \\ & 0\end{array}$

$\left(\begin{array}{lllllllll}x x x x & x x y y & x x z z & 0 & 0 & x x x y & 0 & 0 & x x y x \\ y x x & y y y & y y z z & 0 & 0 & y y y & 0 & 0 & y y x\end{array}\right.$ $\begin{array}{lllllllll}\text { yyxx } & y y y y & y y z z & 0 & 0 & \text { yyxy } & 0 & 0 & \text { yyyx } \\ z z x x & z y y & z z z & 0 & 0 & z z y & 0 & 0 & z y x\end{array}$

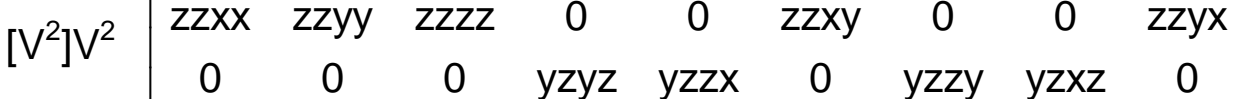
$\begin{array}{ccccccccc}0 & 0 & 0 & z x y z & z x z x & 0 & z x z y & z x x z & 0\end{array}$ $\begin{array}{llllllll} & 0 & & 0 & 0 & & & \end{array}$

$\left(\begin{array}{lllllllll}0 & 0 & 0 & 0 & 0 & 0 & 0 & 0 & 0 \\ 0 & 0 & 0 & 0 & 0 & 0 & 0 & 0 & 0\end{array}\right.$ $\left.\begin{array}{lllllllll}0 & 0 & 0 & 0 & 0 & 0 & 0 & 0 & 0 \\ 0 & 0 & 0 & 0 & 0 & 0 & 0 & 0 & 0 \\ 0 & 0 & 0 & 0 & 0 & 0 & 0 & 0 & 0\end{array}\right)$ $\begin{array}{lllllllll}0 & 0 & 0 & 0 & 0 & 0 & 0 & 0 & 0 \\ 0 & 0 & 0 & 0 & 0 & 0 & 0 & 0 & 0\end{array}$ $\begin{array}{lllllllll}0 & 0 & 0 & 0 & 0 & 0 & 0 & 0 & 0 \\ 0 & 0 & 0 & 0 & 0 & 0 & 0 & 0 & 0\end{array}$

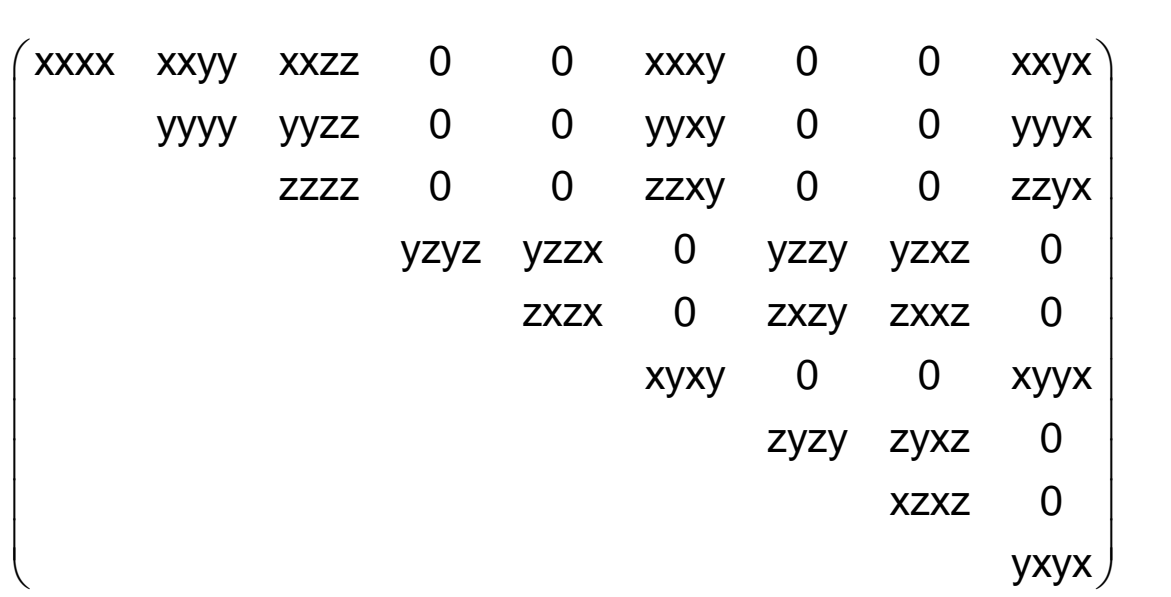

$\left(\begin{array}{lllllllll}x x x x & x x y y & x x z z & 0 & 0 & x x x y & 0 & 0 & x x y x\end{array}\right.$ $\begin{array}{lllllllll}\text { yyxx } & \text { yyyy } & \text { yyzz } & 0 & 0 & \text { yyxy } & 0 & 0 & \text { yyyx } \\ \text { zxx } & \text { zzy } & \text { zzzz } & 0 & 0 & z x y & 0 & 0 & z y x\end{array}$ $\begin{array}{lllllllll}z z x x & z z y y & z z z z & 0 & 0 & z z x y & 0 & 0 & z z y x\end{array}$ $\begin{array}{lllllllll}0 & 0 & 0 & y z y z & y z z x & 0 & y z z y & y z x z & 0\end{array}$ $\begin{array}{ccccccccc}0 & 0 & 0 & z x y z & z x z x & 0 & z x z y & z x x z & 0\end{array}$ $\left(\begin{array}{llllllll}0 & 0 & 0 & 0 & 0 & 0 & 0 & 0 \\ 0 & 0 & 0 & 0 & 0 & 0 & 0 & 0\end{array}\right.$ $\left.\begin{array}{llllllll}0 & 0 & 0 & 0 & 0 & 0 & 0 & 0\end{array}\right)$ $0 \begin{array}{lllllll}0 & 0 & 0 & 0 & 0 & 0 & 0\end{array}$ $\begin{array}{llllll}0 & 0 & 0 & 0 & 0 & 0 \\ 0 & 0 & 0 & 0 & 0 & 0\end{array}$ $\begin{array}{lllll}0 & 0 & 0 & 0 \\ 0 & 0 & 0 & 0\end{array}$

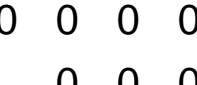
$\begin{array}{lll}0 & 0 & 0 \\ 0 & 0 \\ & & 0\end{array}$ $\left(\begin{array}{lllllllll}0 & 0 & 0 & 0 & 0 & 0 & 0 & 0 & 0 \\ 0 & 0 & 0 & 0 & 0 & 0 & 0 & 0 & 0\end{array}\right)$ $\begin{array}{lllllllll}0 & 0 & 0 & 0 & 0 & 0 & 0 & 0 & 0 \\ 0 & 0 & 0 & 0 & 0 & 0 & 0 & 0 & 0\end{array}$ $\begin{array}{lllllllll}0 & 0 & 0 & 0 & 0 & 0 & 0 & 0 & 0 \\ 0 & 0 & 0 & 0 & 0 & 0 & 0 & 0 & 0\end{array}$ $\begin{array}{llllllllll}0 & 0 & 0 & 0 & 0 & 0 & 0 & 0 & 0 \\ 0 & 0 & 0 & 0 & 0 & 0 & 0 & 0 & 0\end{array}$ $\begin{array}{lllllllll}0 & 0 & 0 & 0 & 0 & 0 & 0 & 0 & 0\end{array}$ 


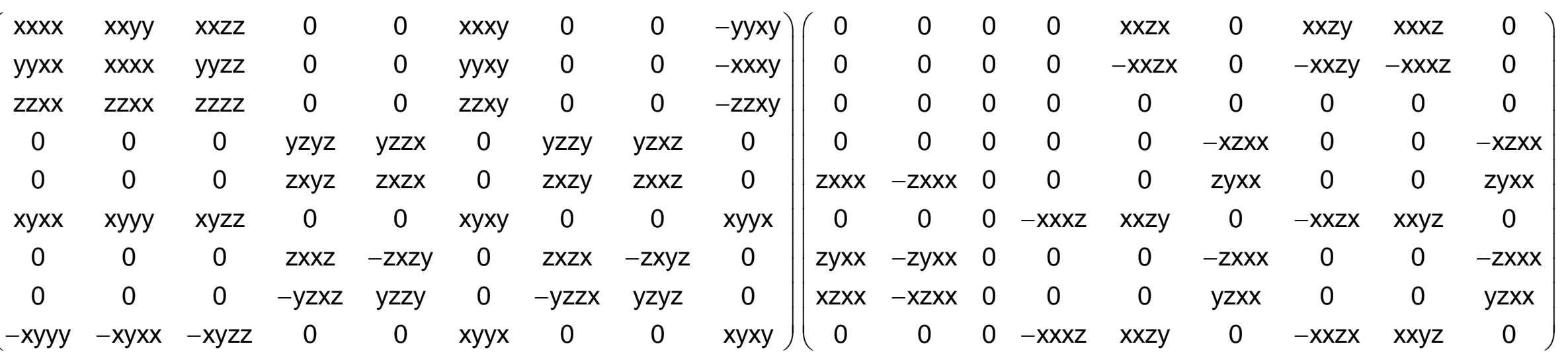
and: $x x x x=x x y y+x y x y+x y y x ; x x x y-y y x y=x y y-x y$

$\left[V^{4}\right] \quad\left(\begin{array}{ccccc}3 x x y y & 0 & 0 & y y z z & 0 \\ 0 & 3 x x y y & 0 & y y z z & 0 \\ 0 & 0 & z z z z & x x y y & 0\end{array}\right)$ $\left(\begin{array}{ccccc}0 & 0 & 0 & 0 & x x y z \\ 0 & 0 & 0 & 0 & -z x x x \\ z x x x & -x x y z & 0 & 0 & 0\end{array}\right)$

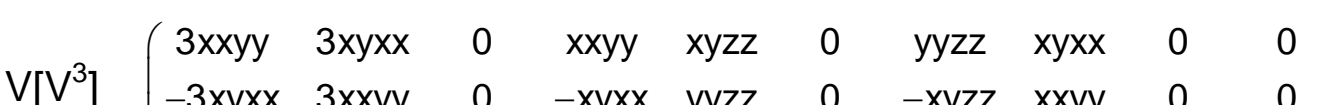

$V\left[V^{3}\right]\left(\begin{array}{cccccccccc}-3 x y x & 3 x x y y & 0 & -x y x & y y z z & 0 & -x y z z & x x y y & 0 & 0 \\ 0 & 0 & z z z z & 0 & 0 & z z x x & 0 & 0 & z z x x & z x y z\end{array}\right)$

$\left[\left[V^{2}\right]^{2}\right]\left(\begin{array}{cccccc}x x x x & x x y y & x x z z & 0 & 0 & 0 \\ & x x x x & x x z z & 0 & 0 & 0 \\ & & z z z z & 0 & 0 & 0 \\ & & & y z y z & 0 & 0 \\ & & & & y z y z & 0 \\ & & & & & \text { xyxy }\end{array}\right)$

and $x y x y=1 / 2(x x x x-x x y y)$

$\left[V^{2}\right]^{2}\left(\begin{array}{cccccc}x x x x & x x y y & x x z z & 0 & 0 & x x x y \\ x x y y & x x x x & x x z z & 0 & 0 & -x x x y \\ z z x x & z z x x & z z z z & 0 & 0 & 0 \\ 0 & 0 & 0 & y z y z & y z x z & 0 \\ 0 & 0 & 0 & y z x z & y z y z & 0 \\ -x x x y & x x x y & 0 & 0 & 0 & x y x y\end{array}\right)$

$\begin{array}{cccccccccc}0 & 0 & 0 & 0 & 0 & x z x x & 0 & 0 & -x z x x & y z x x \\ 0 & 0 & 0 & 0 & 0 & y z x & 0 & 0 & -y z x & -x z x\end{array}$

$\begin{array}{llllllllll}0 & 0 & 0 & 0 & 0 & 0 & 0 & 0 & & \end{array}$

$\left(\begin{array}{cccccc}0 & 0 & 0 & x x y z & -y y x z & 0 \\ & 0 & 0 & -x x y z & y y x z & 0 \\ & & 0 & 0 & 0 & 0 \\ & & & 0 & 0 & y y x z \\ & & & & 0 & x x y z \\ & & & & & 0\end{array}\right)$

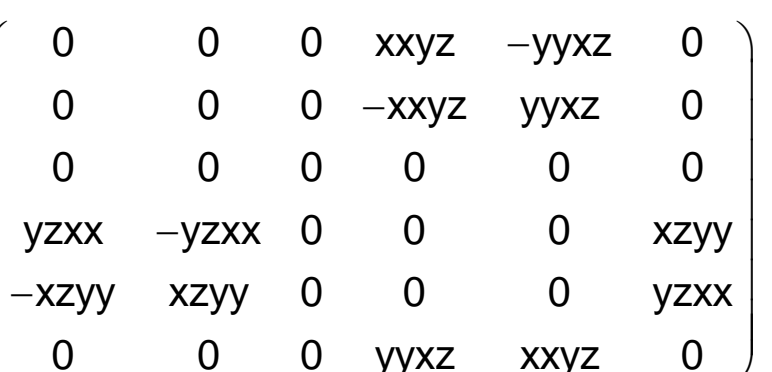

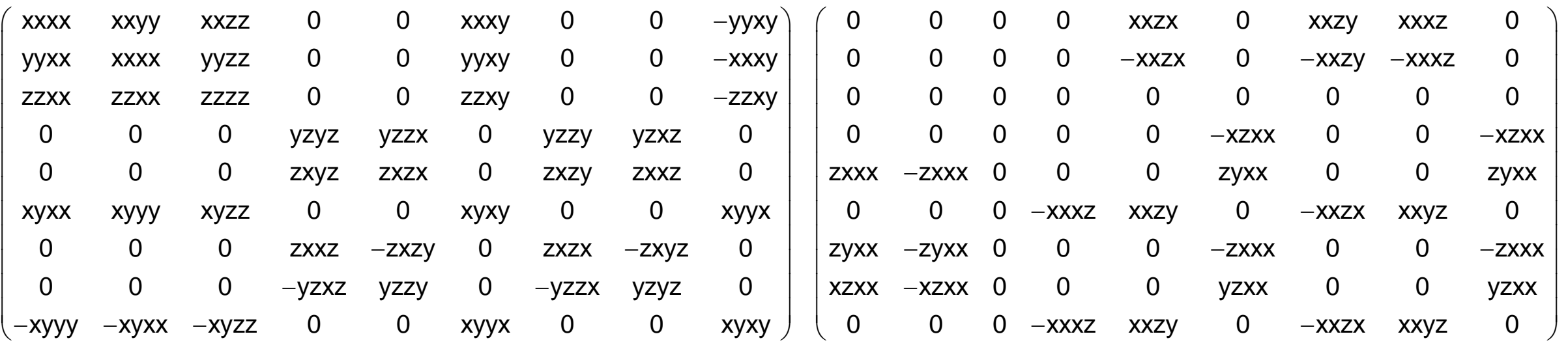

and: $x x x x=x x y y+x y x y+x y y x ; x x x y-y y x y=x y y y-x y x x$

$\left(\begin{array}{ccccc}3 x x y y & 0 & 0 & y y z z & 0 \\ 0 & 3 x x y y & 0 & y y z z & 0 \\ 0 & 0 & z z z z & x x y y & 0\end{array}\right) \quad\left(\begin{array}{ccccc}0 & 0 & 0 & 0 & x x y z \\ 0 & 0 & 0 & 0 & -z x x x \\ z x x x & -x x y z & 0 & 0 & 0\end{array}\right)$

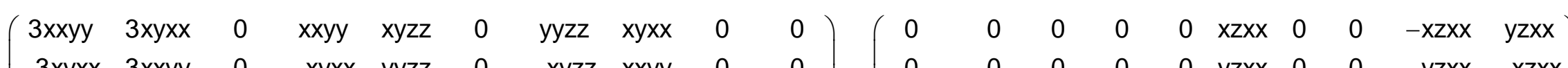

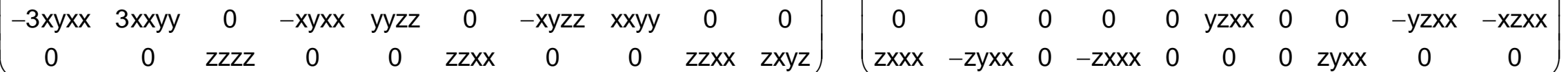

$$
\left(\begin{array}{cccccc}
x x x x & x x y y & x x z z & 0 & 0 & 0 \\
& x x x x & x x z z & 0 & 0 & 0 \\
& & z z z z & 0 & 0 & 0 \\
& & & y z y z & 0 & 0 \\
& & & & y z y z & 0 \\
& & & & & \text { xyxy }
\end{array}\right)
$$

and $x y x y=1 / 2(x x x x-x x y y)$

$\left(\begin{array}{cccccc}x x x x & x x y y & x x z z & 0 & 0 & x x x y \\ x x y y & x x x x & x x z z & 0 & 0 & -x x x y \\ z z x x & z z x x & z z z z & 0 & 0 & 0 \\ 0 & 0 & 0 & y z y z & y z x z & 0 \\ 0 & 0 & 0 & y z x z & y z y z & 0 \\ -x x x y & x x x y & 0 & 0 & 0 & x y x y\end{array}\right)$

and $x y x y=1 / 2(x x x x-x x y y)$
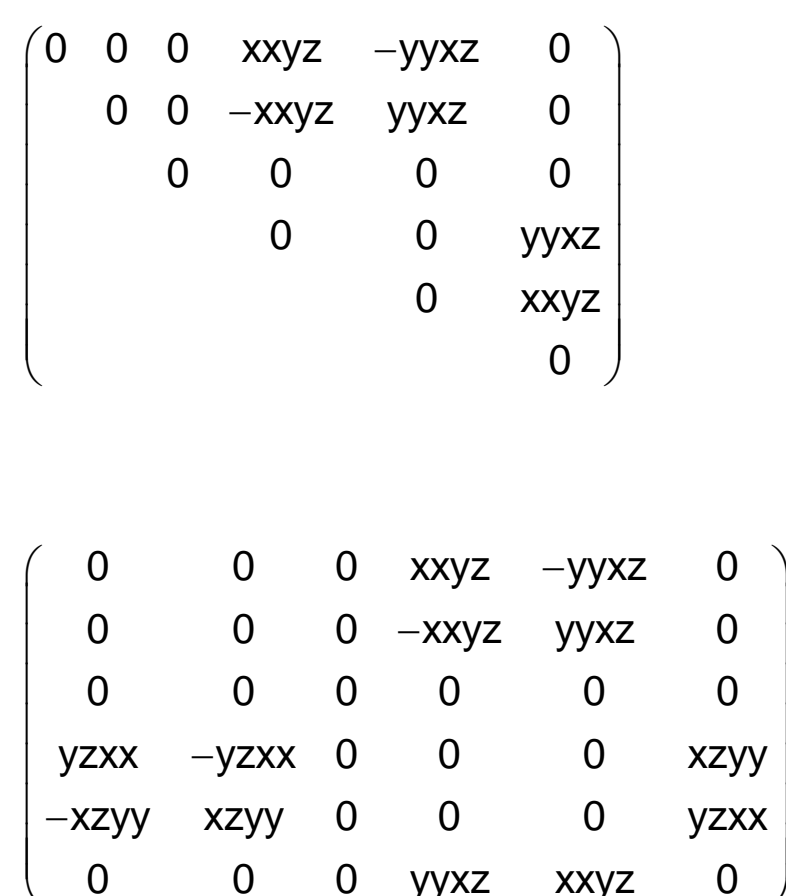

and $x y x y=1 / 2(x x x x-x x y y)$ 
$\mathrm{n}=3 \quad \mathbf{C}_{3 \mathrm{~h}}=\overline{\mathbf{6}}_{z}$

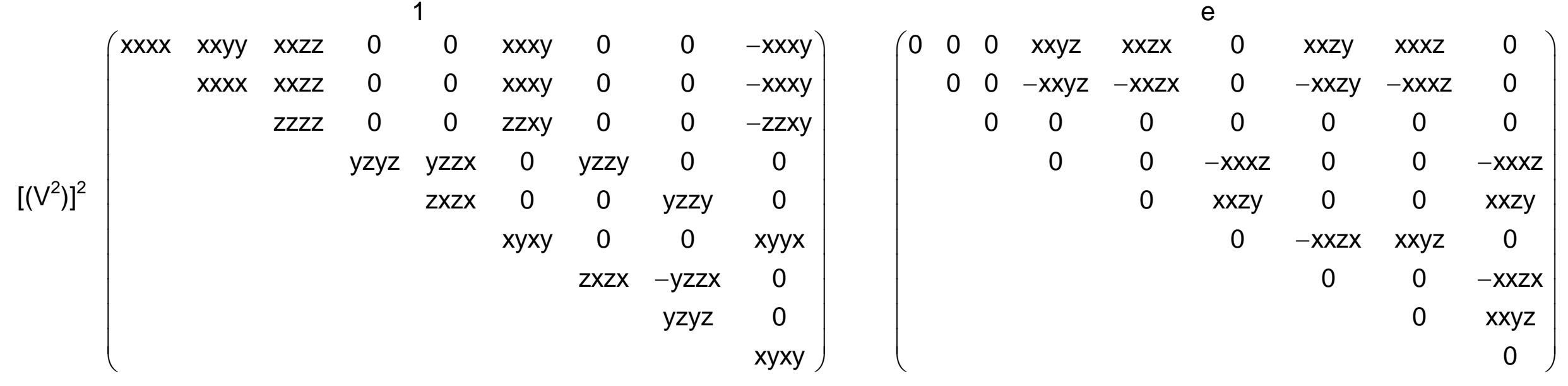

and $x x x x=x x y y+y z y z+z y z y$

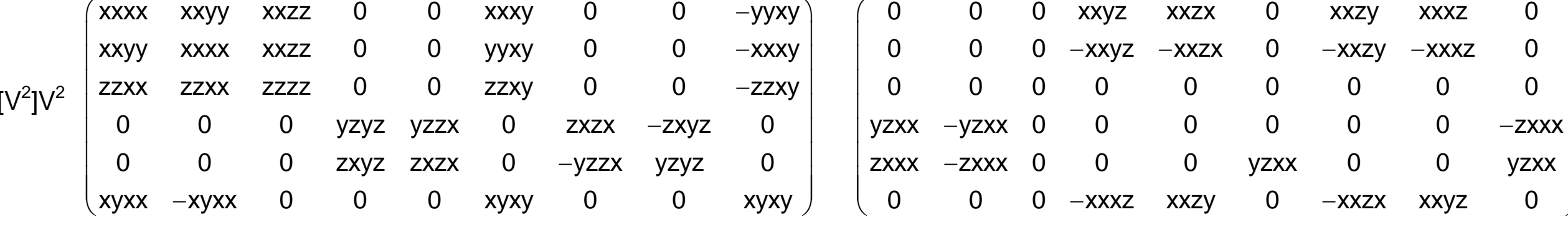

and $x x x x=x x y y+2 x y x y ; y y x y-x x x y=2 x y x x$

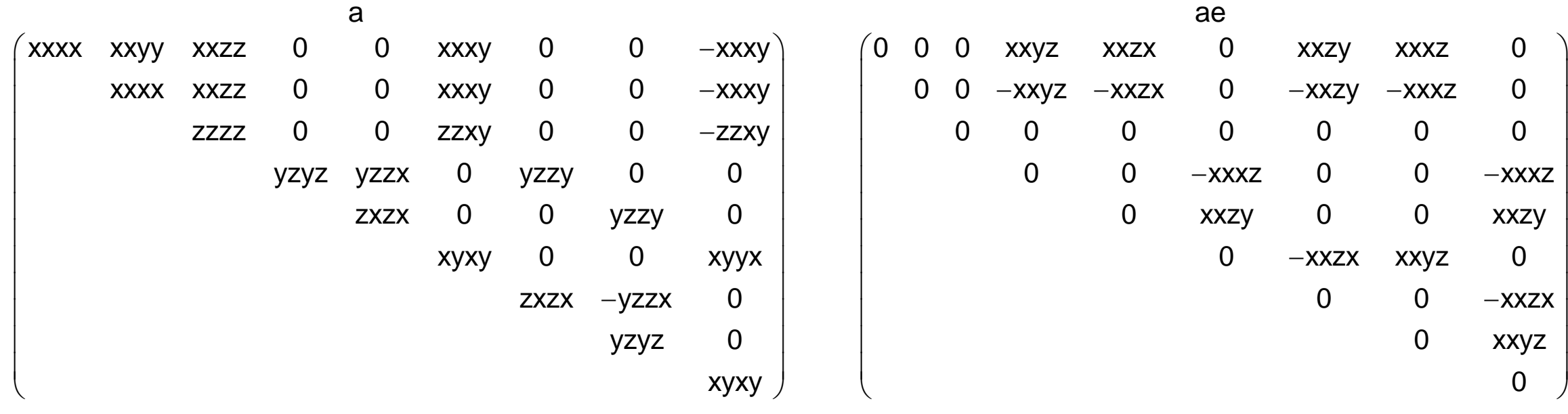

and $x x x x=x x y y+y z y z+z y z y$

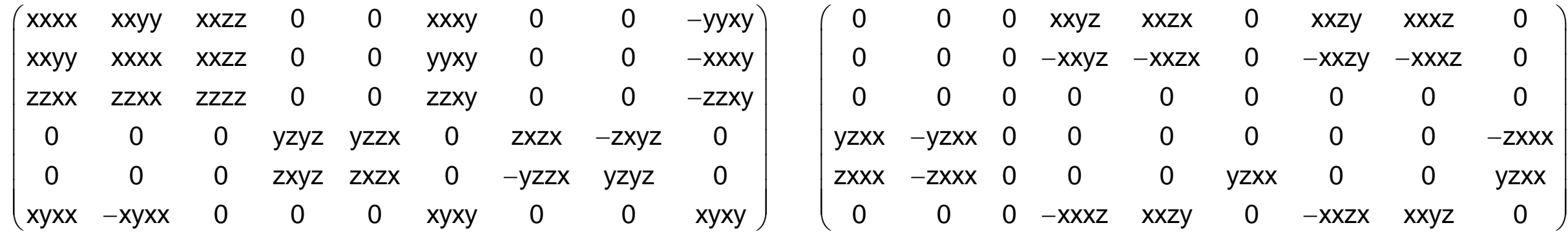
and $x x x x=x x y y+2 x y x y ; y y x y-x x x y=2 x y x x$ 


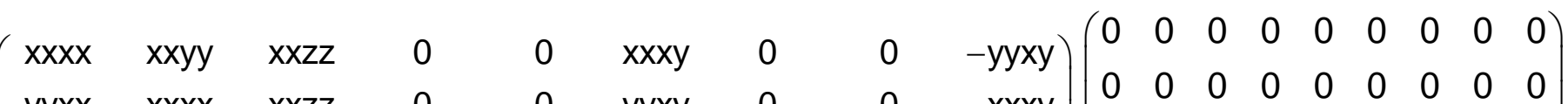

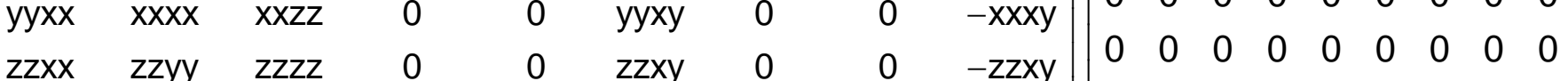
\begin{tabular}{lllllllll|lllllllll}
0 & 0 & 0 & $y z y z$ & $y z z x$ & 0 & $y z z y$ & $y z x z$ & 0 & 0 & 0 & 0 & 0 & 0 & 0 & 0 & 0 & 0
\end{tabular} $\begin{array}{llllllllllllllllll}0 & 0 & 0 & z x y z & z x z x & 0 & z x z y & z x x z & 0 & 0 & 0 & 0 & 0 & 0 & 0 & 0 & 0 & 0\end{array}$

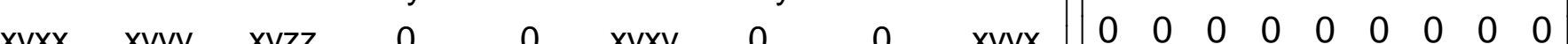

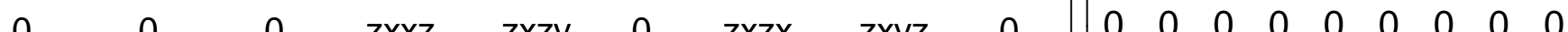

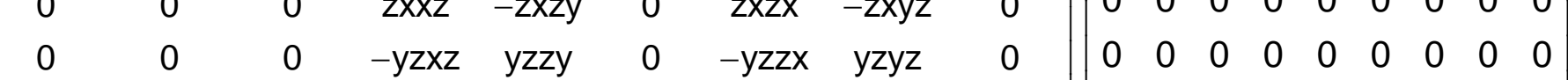

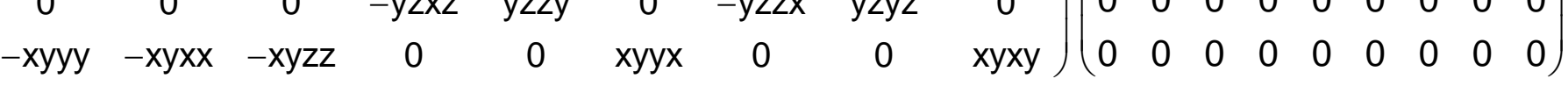

$\left.{ }^{4}{ }^{4}\right]\left(\begin{array}{ccccc}x x x x & -y x x x & 0 & y y z z & 0 \\ y x x x & x x x x & 0 & y y z z & 0 \\ 0 & 0 & z z z z & x x y y & 0\end{array}\right)$

$$
\left(\begin{array}{lllll}
0 & 0 & 0 & 0 & 0 \\
0 & 0 & 0 & 0 & 0 \\
0 & 0 & 0 & 0 & 0
\end{array}\right)
$$

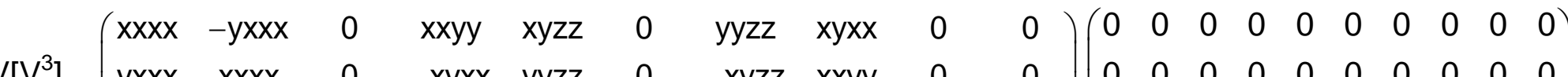

$$
\left[\begin{array}{cccccc}
\text { xxxx } & \text { xxyy } & \text { xxzz } & 0 & 0 & \text { xxxy } \\
& \text { yyyy } & \text { yyzz } & 0 & 0 & -x x x y \\
& & z z z z & 0 & 0 & 0 \\
& & & y z y z & 0 & 0 \\
& & & & y z y z & 0 \\
& & & & & \text { xyxy }
\end{array}\right)
$$

$$
\left(\begin{array}{cccccc}
x x x x & x x y y & x x z z & 0 & 0 & x x x y \\
y x x & x x y x & x z z & 0 & 0 & -x x y
\end{array}\right.
$$

$$
\left(\begin{array}{llllll}
0 & 0 & 0 & 0 & 0 & 0 \\
& 0 & 0 & 0 & 0 & 0 \\
& & 0 & 0 & 0 & 0 \\
& & & 0 & 0 & 0 \\
& & & & 0 & 0 \\
& & & & & 0
\end{array}\right)
$$

$\left(\begin{array}{llllll}0 & 0 & 0 & 0 & 0 & 0 \\ 0 & 0 & 0 & 0 & 0 & 0\end{array}\right.$ $\left.\begin{array}{llllll}0 & 0 & 0 & 0 & 0 & 0 \\ 0 & 0 & 0 & 0 & 0 & 0 \\ 0 & 0 & 0 & 0 & 0 & 0\end{array}\right)$ $0 \begin{array}{llllll}0 & 0 & 0 & 0 & 0 & 0 \\ 0 & 0 & 0 & 0 & 0 & 0\end{array}$ $\begin{array}{llllll}0 & 0 & 0 & 0 & 0 & 0 \\ 0 & 0 & 0 & 0 & 0 & 0\end{array}$ $\begin{array}{llllll}0 & 0 & 0 & 0 & 0 & 0\end{array}$

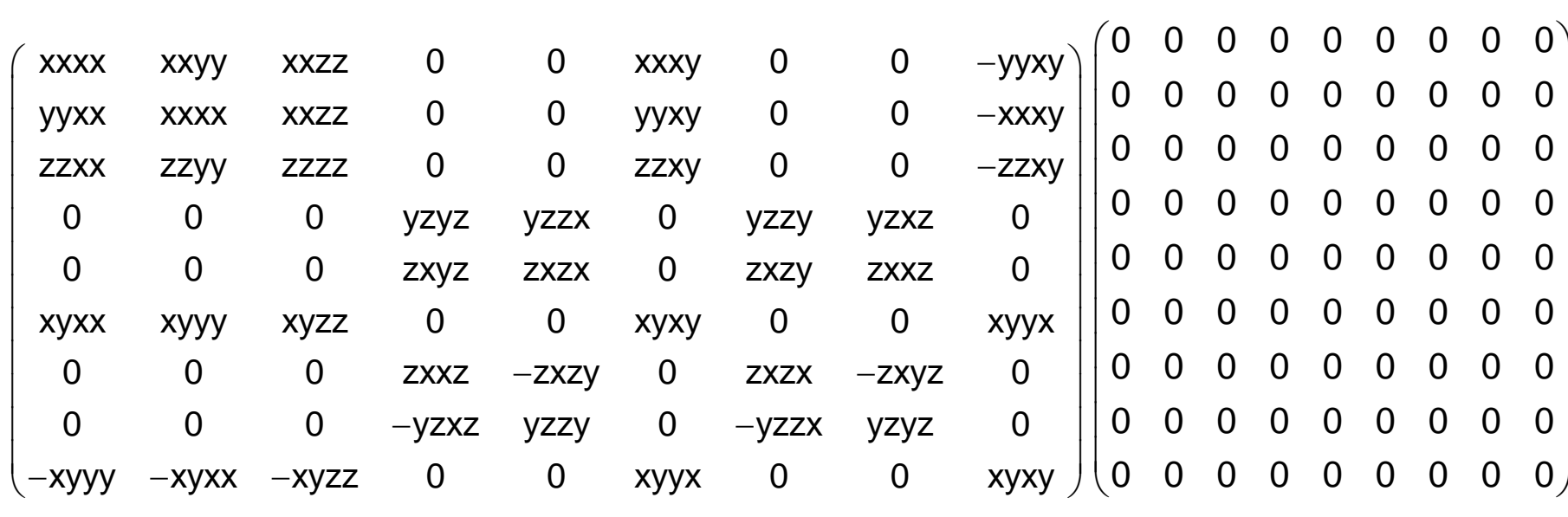

$\left(\begin{array}{ccccc}x x x x & -y x x x & 0 & y y z z & 0 \\ y x x x & x x x x & 0 & y y z z & 0 \\ 0 & 0 & z z z z & x x y y & 0\end{array}\right)$ $\left(\begin{array}{lllll}0 & 0 & 0 & 0 & 0 \\ 0 & 0 & 0 & 0 & 0 \\ 0 & 0 & 0 & 0 & 0\end{array}\right)$

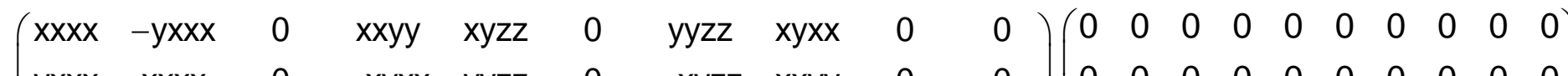
$\begin{array}{llllllllll}y x x x & x x x x & 0 & -x y x x & y y z z & 0 & -x y z z & x x y y & 0 & 0\end{array} 0\left(\begin{array}{llllllllll}0 & 0 & 0 & 0 & 0 & 0 & 0 & 0 & 0 & 0 \\ 0 & 0 & 0 & 0 & 0 & 0 & 0 & 0 & 0 & 0\end{array}\right)$

$$
\left(\begin{array}{cccccc}
x x x x & x x y y & x x z z & 0 & 0 & x x x y \\
& \text { yyyy } & \text { yyzz } & 0 & 0 & -x x x y \\
& & \text { zzzz } & 0 & 0 & 0 \\
& & & y z y z & 0 & 0 \\
& & & & y z y z & 0 \\
& & & & & \text { xyxy }
\end{array}\right)
$$

$\left(\begin{array}{cccccc}x x x x & x x y y & x x z z & 0 & 0 & x x x y \\ y y x x & x x x x & x x z z & 0 & 0 & -x x x y \\ z z x x & z z x x & z z z z & 0 & 0 & 0 \\ 0 & 0 & 0 & y z y z & y z x z & 0 \\ 0 & 0 & 0 & -y z x z & y z y z & 0 \\ x y x x & -x y x x & 0 & 0 & 0 & x y x y\end{array}\right)$

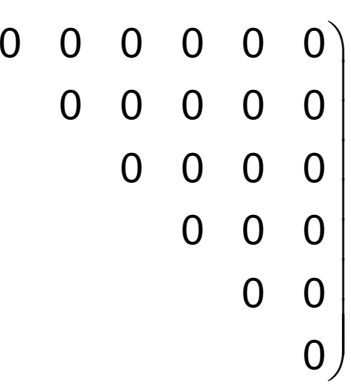

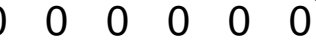
$\begin{array}{llllll}0 & 0 & 0 & 0 & 0 & 0 \\ 0 & 0 & 0 & 0 & 0 & 0\end{array}$

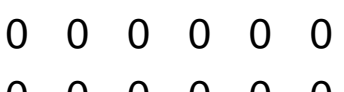
$\begin{array}{llllll}0 & 0 & 0 & 0 & 0 & 0\end{array}$ $\begin{array}{llllll}0 & 0 & 0 & 0 & 0 & 0 \\ 0 & 0 & 0 & 0 & 0 & 0\end{array}$ 
$\left[\left(\mathrm{V}^{2}\right)\right]^{2}$

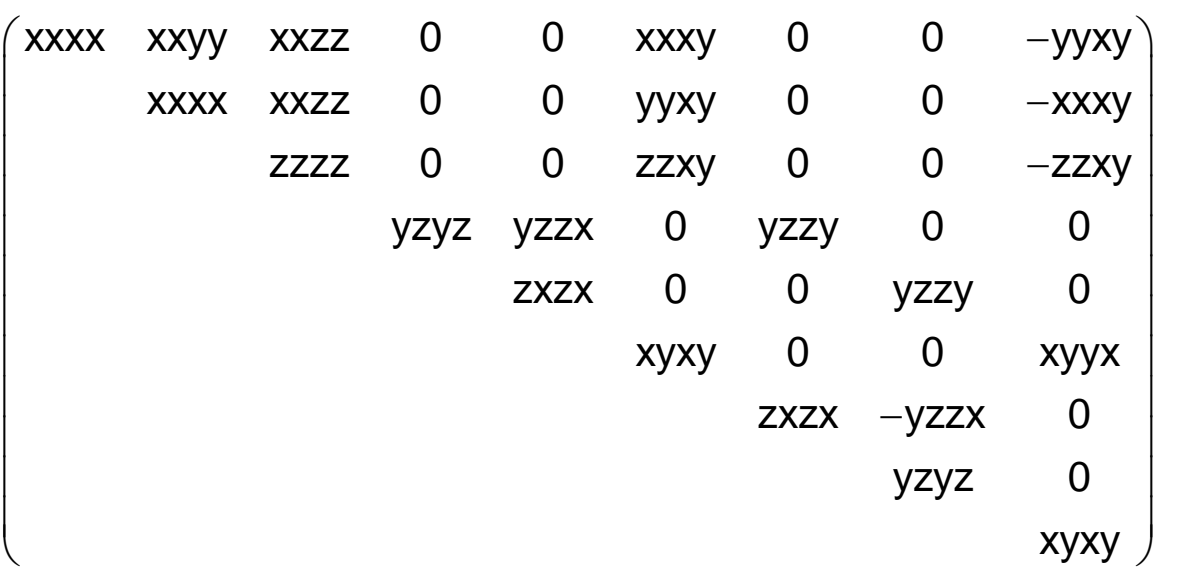

$\left(\begin{array}{lllllllll}0 & 0 & 0 & 0 & 0 & 0 & 0 & 0 & 0\end{array}\right.$ $\begin{array}{llllllll}0 & 0 & 0 & 0 & 0 & 0 & 0 & 0\end{array}$

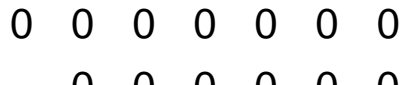
$0 \begin{array}{llllll}0 & 0 & 0 & 0 & 0 & 0\end{array}$ $\begin{array}{lllll}0 & 0 & 0 & 0 & 0\end{array}$ $\begin{array}{llll}0 & 0 & 0 & 0 \\ 0 & 0 & 0\end{array}$

$\left(\begin{array}{lllllllll}x x x x & x x y y & x x z z & 0 & 0 & x x x y & 0 & 0 & -y y x y \\ y x x & x x y x & x x z & 0 & 0 & y x y & 0 & 0 & -x x y\end{array}\right)\left(\begin{array}{lllllllll}0 & 0 & 0 & 0 & 0 & 0 & 0 & 0 & 0 \\ 0 & 0 & 0 & 0 & 0 & 0 & 0 & 0 & 0\end{array}\right)$ $\left.\begin{array}{lllllllll}\mathrm{yyxx} & \mathrm{xxxx} & \mathrm{xxzz} & 0 & 0 & \mathrm{yyxy} & 0 & 0 & -\mathrm{xxxy}\end{array} \mid \begin{array}{lllllllll}0 & 0 & 0 & 0 & 0 & 0 & 0 & 0 & 0 \\ 0 & 0 & 0 & 0 & 0 & 0 & 0 & 0 & 0\end{array}\right)$

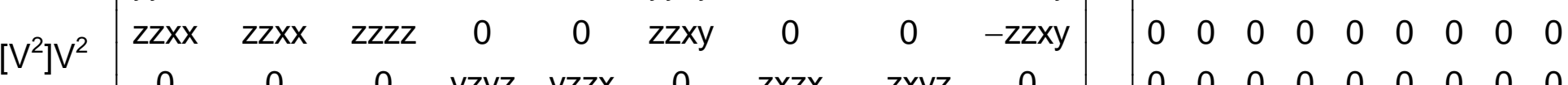
$\left.\begin{array}{ccccccccc}0 & 0 & 0 & y z y z & y z z x & 0 & z x z x & -z x y z & 0 \\ 0 & 0 & 0 & z x y z & z x z x & 0 & -y z z x & y z y z & 0 \\ x y y x & -x y x & 0 & 0 & 0 & x y x & 0 & 0 & x y y\end{array}\right)\left(\begin{array}{lllllllll}0 & 0 & 0 & 0 & 0 & 0 & 0 & 0 & 0 \\ 0 & 0 & 0 & 0 & 0 & 0 & 0 & 0 & 0 \\ 0 & 0 & 0 & 0 & 0 & 0 & 0 & 0 & 0\end{array}\right)$

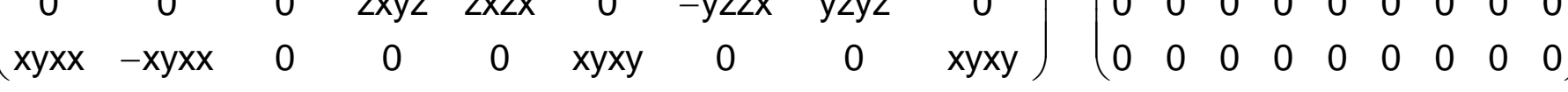

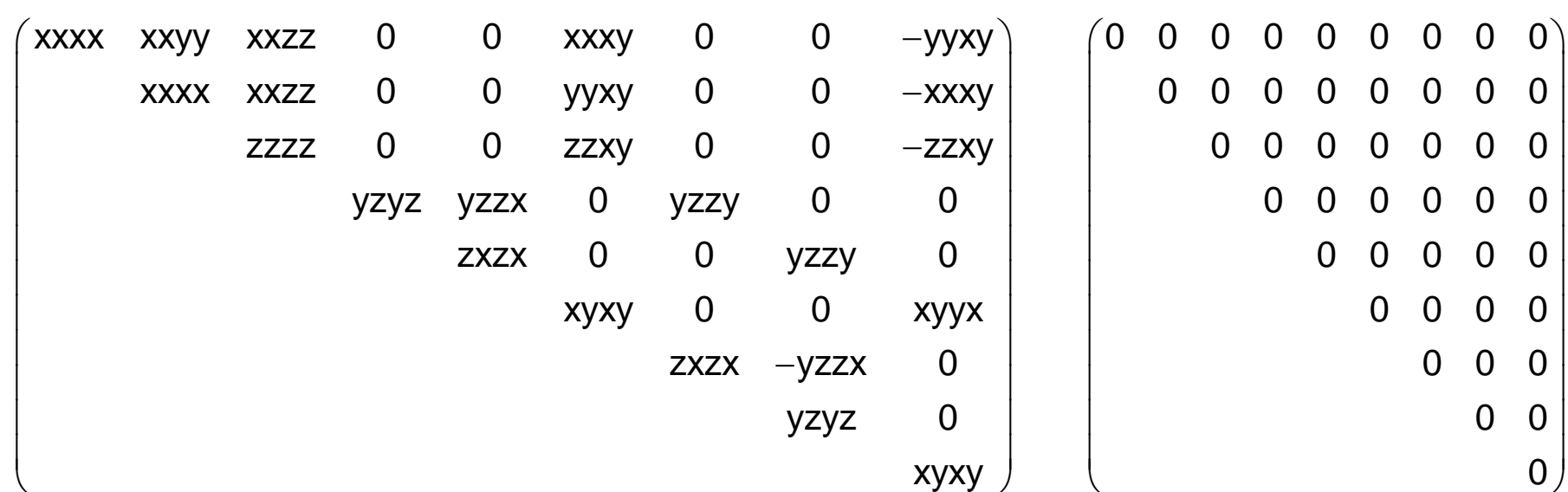

$\left(\begin{array}{ccccccccc}\mathrm{xxxx} & \mathrm{x} x \mathrm{y} y & \mathrm{xxzz} & 0 & 0 & \mathrm{xxxy} & 0 & 0 & -\mathrm{yyxy} \\ \mathrm{yyxx} & \mathrm{xxxx} & \mathrm{xxzz} & 0 & 0 & \mathrm{yyxy} & 0 & 0 & -\mathrm{xxxy} \\ \mathrm{zzxx} & \mathrm{zzxx} & \mathrm{zzzz} & 0 & 0 & \mathrm{zzxy} & 0 & 0 & -\mathrm{zzxy} \\ 0 & 0 & 0 & \mathrm{yzyz} & \mathrm{yzzx} & 0 & \mathrm{zxzx} & -\mathrm{zxyz} & 0 \\ 0 & 0 & 0 & \mathrm{zxyz} & \mathrm{zxzx} & 0 & -\mathrm{yzzx} & \mathrm{yzzyz} & 0 \\ \mathrm{xyxx} & -\mathrm{xyxx} & 0 & 0 & 0 & \mathrm{xyxy} & 0 & 0 & \mathrm{xyxy}\end{array}\right)\left(\begin{array}{ccccccccc}0 & 0 & 0 & 0 & 0 & 0 & 0 & 0 & 0 \\ 0 & 0 & 0 & 0 & 0 & 0 & 0 & 0 & 0 \\ 0 & 0 & 0 & 0 & 0 & 0 & 0 & 0 & 0 \\ 0 & 0 & 0 & 0 & 0 & 0 & 0 & 0 & 0 \\ 0 & 0 & 0 & 0 & 0 & 0 & 0 & 0 & 0 \\ 0 & 0 & 0 & 0 & 0 & 0 & 0 & 0 & 0\end{array}\right)$




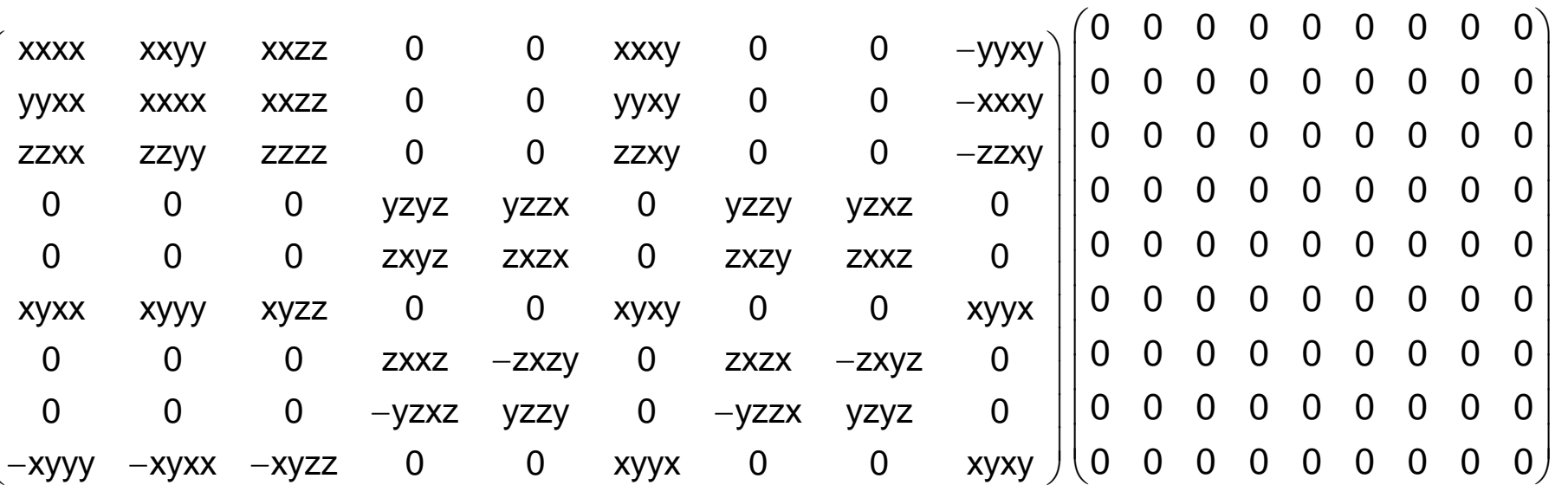

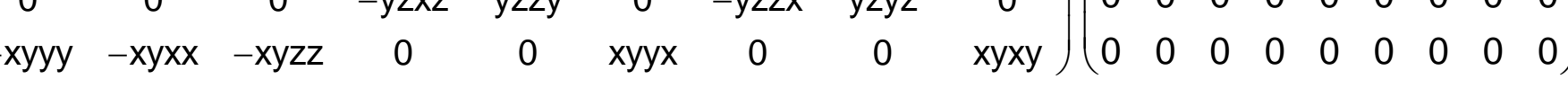
and: $x x x x=x x y y+x y x y+x y y x ; x x x y-y y x y=x y y y-x y x x$
$\left[\mathrm{V}^{4}\right]$
$\left(\begin{array}{ccccc}3 x x y y & 0 & 0 & y y z z & 0 \\ 0 & 3 x x y y & 0 & y y z z & 0 \\ 0 & 0 & z z z & x x y & 0\end{array}\right)$
$\left(\begin{array}{lllll}0 & 0 & 0 & 0 & 0 \\ 0 & 0 & 0 & 0 & 0 \\ 0 & 0 & 0 & 0 & 0\end{array}\right)$
$\begin{array}{llll}0 & \text { zzzz } & \text { xxyy } & 0\end{array}$
$0 \begin{array}{lllll}0 & 0 & 0 & 0 & 0\end{array}$

$\left.V^{2} V^{3}\right]\left(\begin{array}{cccccccccc}3 x x y y & 3 x y x x & 0 & x x y y & x y z z & 0 & y y z z & x y x x & 0 & 0 \\ 3 x y x & 3 x y y & 0 & x y x x & y y z & 0 & -x y z z & x y y & 0 & 0 \\ 0 & 0 & 2 z z z & 0 & 0 & z z x x & 0 & 0 & z x x & z x y z\end{array}\right)\left(\begin{array}{cccccccccc}0 & 0 & 0 & 0 & 0 & 0 & 0 & 0 & 0 & 0 \\ 0 & 0 & 0 & 0 & 0 & 0 & 0 & 0 & 0 & 0 \\ 0 & 0 & 0 & 0 & 0 & 0 & 0 & 0 & 0 & 0\end{array}\right)$ $\left(\begin{array}{cccccccccc}-3 x y x x & 3 x x y y & 0 & -x y x x & y y z z & 0 & -x y z z & x x y y & 0 & 0 \\ 0 & 0 & z z z z & 0 & 0 & z z x x & 0 & 0 & z z x x & z x y z\end{array}\right)\left(\begin{array}{ccccccccccc}0 & 0 & 0 & 0 & 0 & 0 & 0 & 0 & 0 & 0\end{array}\right)$

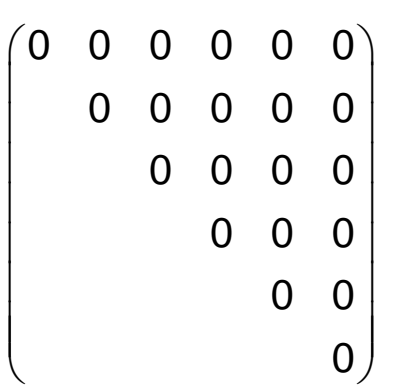

$\left(\begin{array}{llllll}0 & 0 & 0 & 0 & 0 & 0 \\ 0 & 0 & 0 & 0 & 0 & 0\end{array}\right.$ $\begin{array}{llllll}0 & 0 & 0 & 0 & 0 & 0 \\ 0 & 0 & 0 & 0 & 0 & 0\end{array}$ $\begin{array}{llllll}0 & 0 & 0 & 0 & 0 & 0 \\ 0 & 0 & 0 & 0 & 0 & 0\end{array}$ $0 \begin{array}{llllll}0 & 0 & 0 & 0 & 0 & 0 \\ 0 & 0 & 0 & 0 & 0 & 0\end{array}$

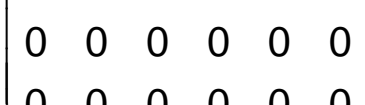

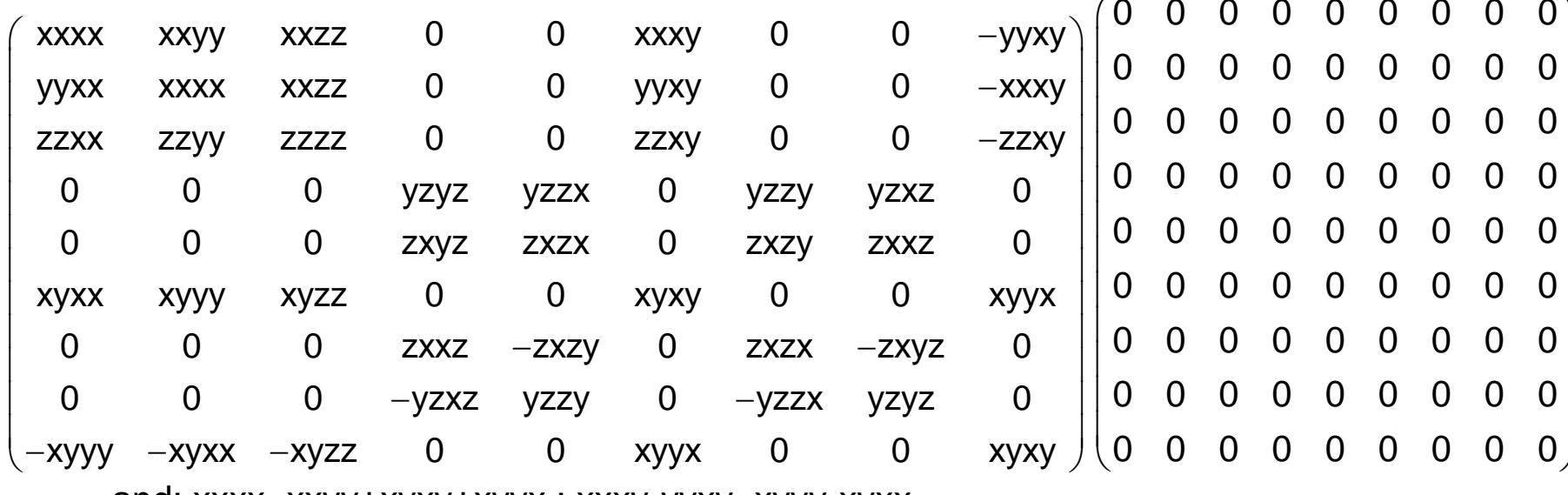
and: $x x x x=x x y y+x y x y+x y y x ; x x x y-y y x y=x y y y-x y x x$

$\left(\begin{array}{ccccc}3 x x y y & 0 & 0 & y y z z & 0 \\ 0 & 3 x x y y & 0 & y y z z & 0 \\ 0 & 0 & z z z z & x x y y & 0\end{array}\right)$

$\left(\begin{array}{lllll}0 & 0 & 0 & 0 & 0 \\ 0 & 0 & 0 & 0 & 0 \\ 0 & 0 & 0 & 0 & 0\end{array}\right)$ $\left(\begin{array}{lllll}0 & 0 & 0 & 0 & 0 \\ 0 & 0 & 0 & 0 & 0 \\ 0 & 0 & 0 & 0 & 0\end{array}\right)$

$\left(\begin{array}{llllllllll}3 x x y y & 3 x y x x & 0 & x x y y & x y z z & 0 & y y z z & x y x x & 0 & 0 \\ -3 x y x & 3 x y y & 0 & -x y x & y y z & 0 & -x y z z & x y y & 0 & 0\end{array}\right)$

$\left(\begin{array}{llllllllll}0 & 0 & 0 & 0 & 0 & 0 & 0 & 0 & 0 & 0 \\ 0 & 0 & 0 & 0 & 0 & 0 & 0 & 0 & 0 & 0\end{array}\right)$ $\left.\begin{array}{llllllllll}-3 x y x x & 3 x x y y & 0 & -x y x x & y y z z & 0 & -x y z z & x x y y & 0 & 0 \\ 0 & 0 & z z z & 0 & 0 & z x x & 0 & 0 & z z x & z x y z\end{array}\right)\left(\begin{array}{llllllllll}0 & 0 & 0 & 0 & 0 & 0 & 0 & 0 & 0 & 0 \\ 0 & 0 & 0 & 0 & 0 & 0 & 0 & 0 & 0 & 0\end{array}\right)$ $\left.\begin{array}{lllllllllll}0 & 0 & z z z z & 0 & 0 & z z x x & 0 & 0 & z z x x & z x y z\end{array}\right)\left(\begin{array}{lllllllllll}0 & 0 & 0 & 0 & 0 & 0 & 0 & 0 & 0 & 0\end{array}\right)$

$$
\left(\begin{array}{cccccc}
x x x x & x x y y & x x z z & 0 & 0 & 0 \\
& x x x x & x x z z & 0 & 0 & 0 \\
& & z z z z & 0 & 0 & 0 \\
& & & y z y z & 0 & 0 \\
& & & & y z y z & 0
\end{array}\right)
$$

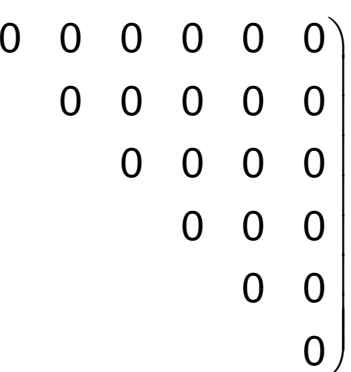

and $x y x y=1 / 2(x x x x-x x y y)$

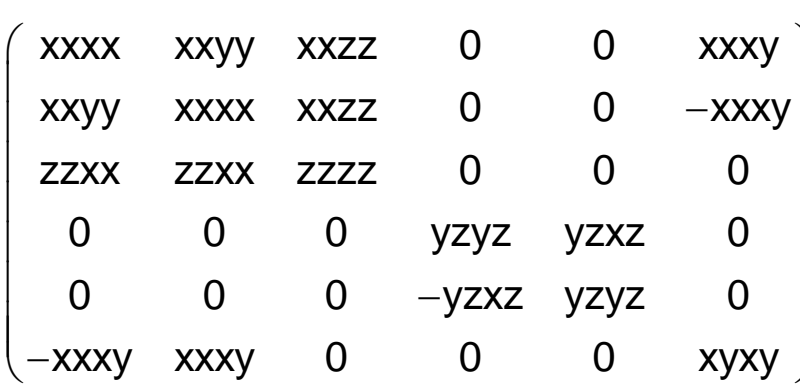

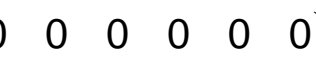

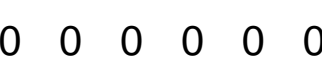

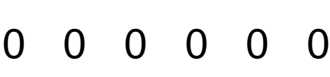

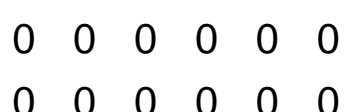

$\left(\begin{array}{llllll}0 & 0 & 0 & 0 & 0 & 0 \\ 0 & 0 & 0 & 0 & 0 & 0 \\ 0 & 0 & 0\end{array}\right.$

and $x y x y=1 / 2(x x x x-x x y y)$

and $x y x y=1 / 2(x x x x-x x y y)$ 


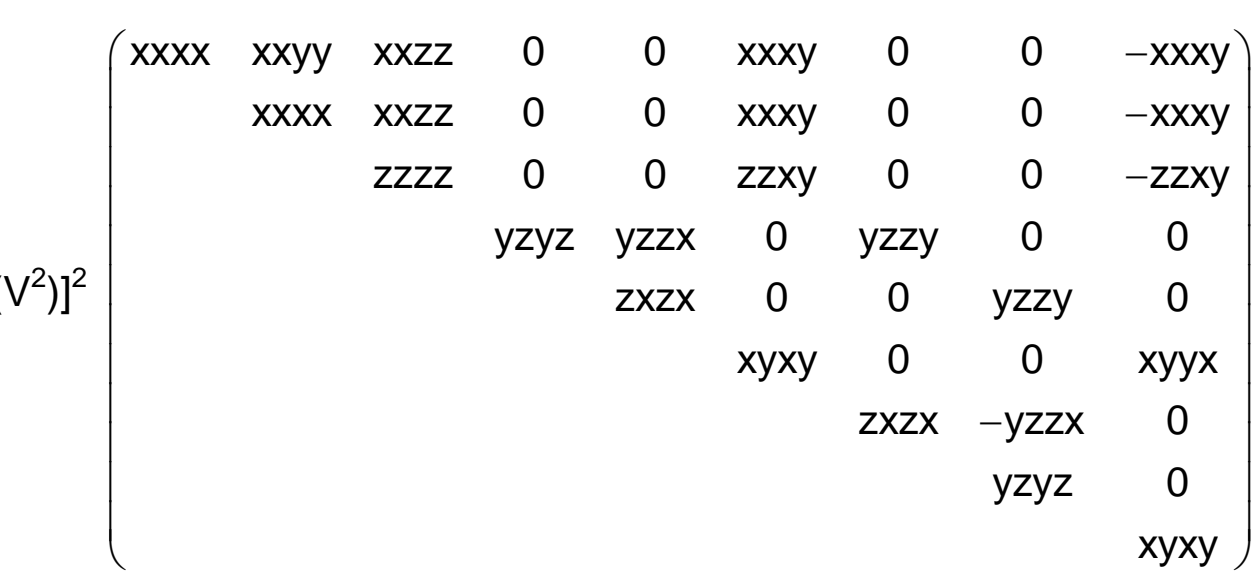

$\left(\begin{array}{lllllllll}0 & 0 & 0 & 0 & 0 & 0 & 0 & 0 & 0 \\ & 0 & 0 & 0 & 0 & 0 & 0 & 0 & 0 \\ & & 0 & 0 & 0 & 0 & 0 & 0 & 0\end{array}\right)$ $\begin{array}{lllllll}0 & 0 & 0 & 0 & 0 & 0 & 0\end{array}$ $0 \begin{array}{lllll}0 & 0 & 0 & 0 & 0\end{array}$ $0 \begin{array}{lllll}0 & 0 & 0 & 0 & 0 \\ 0 & 0 & 0\end{array}$ $\begin{array}{llll}0 & 0 & 0 & 0\end{array}$ $\begin{array}{lll}0 & 0 & 0 \\ & 0 & 0\end{array}$

and $x x x x=x x y y+y z y z+z y z y$

$\left(\begin{array}{lllllllll}x x x x & x x y y & x x z z & 0 & 0 & x x x y & 0 & 0 & -y y x y \\ x x y y & x y x & x x z z & 0 & 0 & y x y & 0 & 0 & -x y y\end{array}\right)\left(\begin{array}{ccccccccc}0 & 0 & 0 & 0 & 0 & 0 & 0 & 0 & 0 \\ 0 & 0 & 0 & 0 & 0 & 0 & 0 & 0 & 0 \\ 0 & 0 & 0 & 0 & 0 & 0 & 0 & 0 & 0\end{array}\right)$

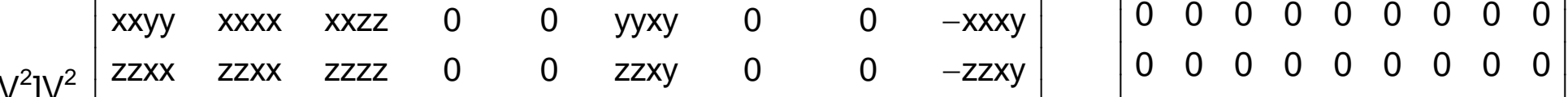

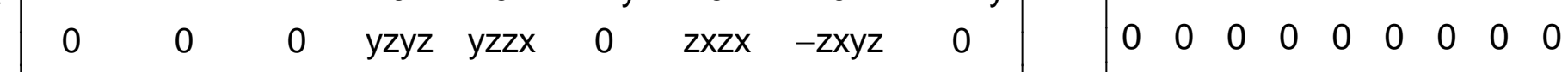
$\left(\begin{array}{ccccccccc}0 & 0 & 0 & z x y z & z x z x & 0 & -y z z x & y z y z & 0 \\ x y x x & -x y x x & 0 & 0 & 0 & x y x y & 0 & 0 & x y x y\end{array}\right) \quad\left(\begin{array}{ccccccccc}0 & 0 & 0 & 0 & 0 & 0 & 0 & 0 & 0 \\ 0 & 0 & 0 & 0 & 0 & 0 & 0 & 0 & 0\end{array}\right.$

and $x x x x=x x y y+2 x y x y ; y y x y-x x x y=2 x y x x$

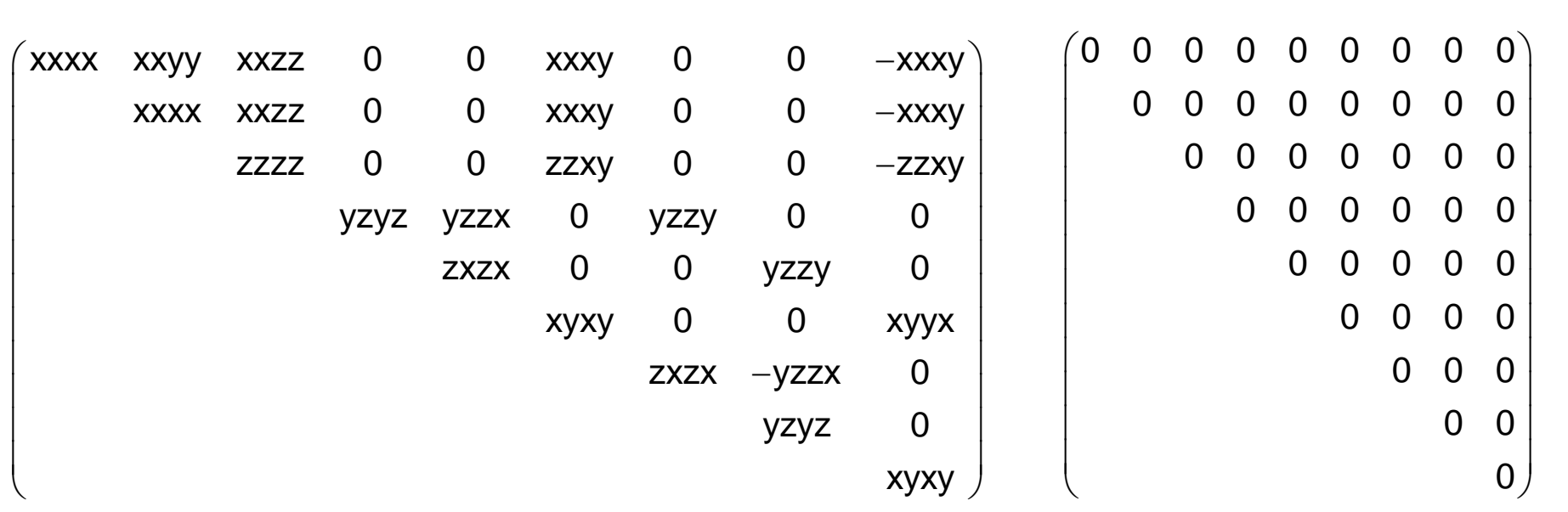

and $x x x x=x x y y+y z y z+z y z y$

$\left(\begin{array}{ccccccccc}x x x x & x x y y & x x z z & 0 & 0 & x x x y & 0 & 0 & -y y x y \\ x x y y & x x x x & x x z z & 0 & 0 & y y x y & 0 & 0 & -x x x y \\ z z x x & z z x x & z z z z & 0 & 0 & z z x y & 0 & 0 & -z z x y \\ 0 & 0 & 0 & y z y z & y z z x & 0 & z x z x & -z x y z & 0 \\ 0 & 0 & 0 & z x y z & z x z x & 0 & -y z z x & y z y z & 0 \\ x y x x & -x y x x & 0 & 0 & 0 & x y x y & 0 & 0 & x y x y\end{array}\right)\left(\begin{array}{ccccccccc}0 & 0 & 0 & 0 & 0 & 0 & 0 & 0 & 0 \\ 0 & 0 & 0 & 0 & 0 & 0 & 0 & 0 & 0 \\ 0 & 0 & 0 & 0 & 0 & 0 & 0 & 0 & 0 \\ 0 & 0 & 0 & 0 & 0 & 0 & 0 & 0 & 0 \\ 0 & 0 & 0 & 0 & 0 & 0 & 0 & 0 & 0 \\ 0 & 0 & 0 & 0 & 0 & 0 & 0 & 0 & 0\end{array}\right)$

and $x x x x=x x y y+2 x y x y ; y y x y-x x x y=2 x y x x$ 
$\left[V^{4}\right]\left(\begin{array}{ccccc}\mathrm{xxxx} & 0 & \mathrm{xzzz} & \mathrm{yyzz} & 0 \\ 0 & \mathrm{yyyy} & 0 & \mathrm{zzxx} & \mathrm{yyxz} \\ \mathrm{zxxx} & 0 & \mathrm{zzzz} & \mathrm{xxyy} & 0\end{array}\right)$

$\begin{array}{lllllllll}x x x x & x x y y & x x z z & 0 & x x z x & 0 & 0 & x x x z & 0\end{array}$

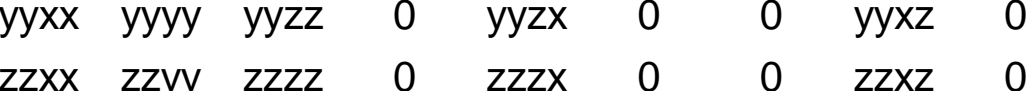

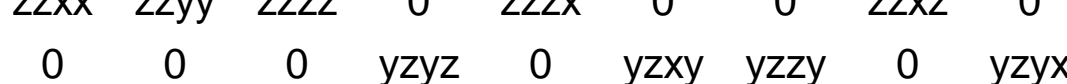
$\begin{array}{lllllllll}z x x x & z x y y & z x z z & 0 & z x z x & 0 & 0 & z x x z & 0\end{array}$ $\begin{array}{lllllllll}0 & 0 & 0 & \text { xyyz } & 0 & \text { xyxy } & \text { xyzy } & 0 & \text { xyyx }\end{array}$ $\begin{array}{lllllllll}0 & 0 & 0 & \text { zyyz } & 0 & \text { zyxy } & \text { zyzy } & 0 & \text { zyyx }\end{array}$ $\begin{array}{lllllllll}x z x x & x z y y & x z z z & 0 & x z z x & 0 & 0 & x z x z & 0\end{array}$ $\begin{array}{lllllllll}0 & 0 & 0 & y x y z & 0 & y x x y & y x z y & 0 & y x y x\end{array}$ $\left(\begin{array}{ccccc}x x x x & 0 & x z z z & y y z z & 0 \\ 0 & y y y y & 0 & z z x x & y y x z \\ z x x x & 0 & z z z z & x x y y & 0\end{array}\right)$ $\left(\begin{array}{lllllllll}x x x x & x x y y & x x z z & 0 & x x z x & 0 & 0 & x x x z & 0 \\ y y x x & y y y & y y z z & 0 & y y z & 0 & 0 & y x z & 0\end{array}\right)$ $\begin{array}{lllllllll}\text { yyxx } & y y y y & y y z z & 0 & y y z x & 0 & 0 & y y x z & 0\end{array}$ $\begin{array}{lllllllll}z z x x & z z y y & z z z z & 0 & z z z x & 0 & 0 & z z x z & 0\end{array}$ $\begin{array}{lllllllll}0 & 0 & 0 & y z y z & 0 & y z x y & y z z y & 0 & y z y x\end{array}$ $\begin{array}{lllllllll}z x x x & z x y y & z x z z & 0 & z x z x & 0 & 0 & z x x z & 0\end{array}$ $\begin{array}{lllllllll}0 & 0 & 0 & \text { xyyz } & 0 & \text { xyxy } & \text { xyzy } & 0 & \text { xyyx }\end{array}$ $\begin{array}{lllllllll}0 & 0 & 0 & \text { zyyz } & 0 & \text { zyxy } & \text { zyzy } & 0 & \text { zyyx }\end{array}$ $\begin{array}{ccccccccc}x z x x & x z y y & x z z z & 0 & x z z x & 0 & 0 & x z x z & 0\end{array}$ $\begin{array}{llllll}y x y z & 0 & y x x y & y x z y & 0 & y x y x\end{array}$

$\left(\begin{array}{ccccc}x x x x & 0 & x z z z & y y z z & 0 \\ 0 & y y y y & 0 & z z x x & y y x z \\ z x x x & 0 & z z z z & x x y y & 0\end{array}\right)$ $\left(\begin{array}{lllllllll}x x x x & x x y y & x x z z & 0 & x x z x & 0 & 0 & x x x z & 0\end{array}\right)$ $\begin{array}{llllllllll}y y x x & y y y y & y y z z & 0 & y y z x & 0 & 0 & y y x z & 0\end{array}$ $\begin{array}{llllllllll}z z x x & z z y y & z z z z & 0 & z z z x & 0 & 0 & z z x z & 0\end{array}$ $\begin{array}{lllllllll}0 & 0 & 0 & y z y z & 0 & y z x y & y z z y & 0 & y z y x\end{array}$ $\begin{array}{lllllllll}z x x x & z x y y & z x z z & 0 & z x z x & 0 & 0 & z x x z & 0\end{array}$ $\begin{array}{lllllllll}0 & 0 & 0 & x y y z & 0 & x y x y & x y z y & 0 & x y y x\end{array}$ $\begin{array}{ccccccccc}0 & 0 & 0 & z y y z & 0 & z y x y & z y z y & 0 & z y y x\end{array}$ $\begin{array}{ccccccccc}x z x x & x z y y & x z z z & 0 & x z z x & 0 & 0 & x z x z & 0\end{array}$ $\begin{array}{lllllllll}0 & 0 & 0 & y x y z & 0 & y x x y & y x z y & 0 & y x y x\end{array}$ $\left.\begin{array}{ccccc}x x x x & 0 & x z z z & y y z z & 0 \\ 0 & y y y y & 0 & z z x x & y y x z\end{array}\right)$ $\begin{array}{llllll}z x x x & 0 & z z z z & x x y y & 0\end{array}$

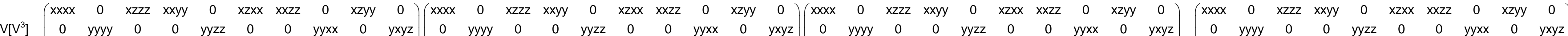

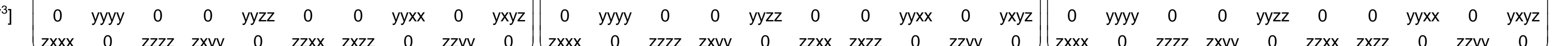
$\left(\begin{array}{cccccc}x x x x & x x y y & x x z z & 0 & x x x z & 0 \\ & y y y y & y y z z & 0 & y y x z & 0 \\ & & z z z z & 0 & z z x z & 0 \\ & & & y z y z & 0 & y z x y \\ & & & & x z x z & 0 \\ & & & & & x y x y\end{array}\right)$

$\left.\begin{array}{llllll}x x x x & x x y y & x x z z & 0 & x x x z & 0 \\ y x x & y y y & y y z z & 0 & y y x z & 0\end{array}\right)$ $\left(\begin{array}{llllll}x y x x & y y y y & y y z z & 0 & y y x z & 0\end{array}\right)$ $\begin{array}{lllllllllll} & y y x x & y y y & y z y y & z z z z & 0 & z z z z & 0\end{array}$ $\begin{array}{cccccc}0 & 0 & 0 & y z y z & 0 & y z x y\end{array}$

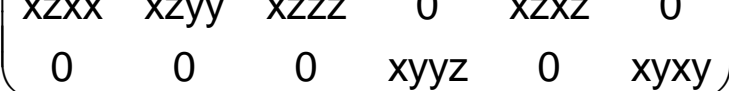

$$
\left(\begin{array}{cccccc}
\text { xxxx } & \text { xxyy } & x x z z & 0 & x x x z & 0 \\
& \text { yyyy } & y y z z & 0 & y y x z & 0 \\
& & z z z z & 0 & z z x z & 0 \\
& & & y z y z & 0 & y z x y \\
& & & & x z x z & 0 \\
& & & & & x y x y
\end{array}\right)
$$

$$
\left(\begin{array}{cccccc}
\text { xxxx } & \text { xxyy } & \text { xxzz } & 0 & \text { xxxz } & 0 \\
& \text { yyyy } & \text { yyzz } & 0 & \text { yyxz } & 0 \\
& & \text { zzzz } & 0 & \text { zzxz } & 0 \\
& & & y z y z & 0 & \text { yzxy } \\
& & & & \text { xzxz } & 0 \\
& & & & & \text { xyxy }
\end{array}\right)
$$

$\left(\begin{array}{cccccc}x \mathrm{xxx} & \mathrm{xxyy} & \mathrm{xxzz} & 0 & \mathrm{xxxz} & 0 \\ \mathrm{yyxx} & \mathrm{yyyy} & \mathrm{yyzz} & 0 & \mathrm{yyxz} & 0 \\ \mathrm{zzxx} & \mathrm{zzyy} & \mathrm{zzzz} & 0 & \mathrm{zzxz} & 0 \\ 0 & 0 & 0 & \mathrm{yzyz} & 0 & \mathrm{yzxy} \\ \mathrm{xzxx} & \mathrm{xzyy} & \mathrm{xzzz} & 0 & \mathrm{xzxz} & 0 \\ 0 & 0 & 0 & \mathrm{xyyz} & 0 & \mathrm{xyxy}\end{array}\right)$ $\begin{array}{llllll}x x x x & x x y y & x x z z & 0 & x x x z & 0 \\ y x x & y y y y & y y z & 0 & y y x z & 0\end{array}$

$\left.\begin{array}{llllll}y y x x & y y y y & y y z z & 0 & y y x z & 0\end{array}\right)$ $\begin{array}{llllll}\text { zzxx } & \text { zzyy } & \text { zzzz } & 0 & \text { zzxz } & 0\end{array}$ $\begin{array}{cccccc}0 & 0 & 0 & y z y z & 0 & y z x y\end{array}$ $\begin{array}{cccccc}x z x x & x z y y & x z z z & 0 & x z x z & 0\end{array}$ $\begin{array}{lllllllllll}z x x x & 0 & z z z z & z x y y & 0 & z z x x & z x z z & 0 & \text { zzyy } & 0\end{array}$ $\begin{array}{lllll}\text { yyyy } & y y z z & 0 & \text { yyxz } & 0\end{array}$ $\begin{array}{llll}z z z z & 0 & z z x z & 0\end{array}$ $y z y z \quad \begin{array}{cc}0 & y z x y \\ x z x z & 0\end{array}$ $x y x y$

$\left(\begin{array}{llllll}x x x x & x x y y & x x z z & 0 & x x x z & 0 \\ y y x & y y y & y y z z & 0 & x y x z & 0\end{array}\right)$ $\left(\begin{array}{cccccc}x y x x & x y y y & x y z z & 0 & x x z z & 0 \\ z z x x & z z y & z z z & 0 & y y x z & 0\end{array}\right.$ $\begin{array}{llllll}z z x x & z z y y & z z z z & 0 & z z x z & 0\end{array}$ $\begin{array}{cccccc}0 & 0 & 0 & y z y z & 0 & y z x y\end{array}$ $\begin{array}{cccccc}x z x x & x z y y & x z z z & 0 & x z x z & 0\end{array}$ 
$\mathrm{n}=1 \quad \mathbf{D}_{1}=\mathbf{2}_{\mathrm{y}}$ $\left(\begin{array}{ccccccccc}\text { xxxx } & \text { xxyy } & \text { xxzz } & 0 & \text { xxzx } & 0 & 0 & \text { xxxz } & 0 \\ & \text { yyyy } & \text { yyzz } & 0 & \text { yyzx } & 0 & 0 & \text { yyxz } & 0 \\ & & \text { zzzz } & 0 & \text { zzzx } & 0 & 0 & \text { zzxz } & 0 \\ & & & \text { yzyz } & 0 & \text { yzxy } & \text { yzzy } & 0 & \text { yzyx } \\ & & & & \text { zxzx } & 0 & 0 & \text { zxxz } & 0 \\ & & & & & \text { xyxy } & \text { xyzy } & 0 & \text { xyyx } \\ & & & & & & \text { zyzy } & 0 & \text { zyyx } \\ & & & & & & \text { xzxz } & 0 \\ & & & & & & & & \text { yxyx }\end{array}\right)$

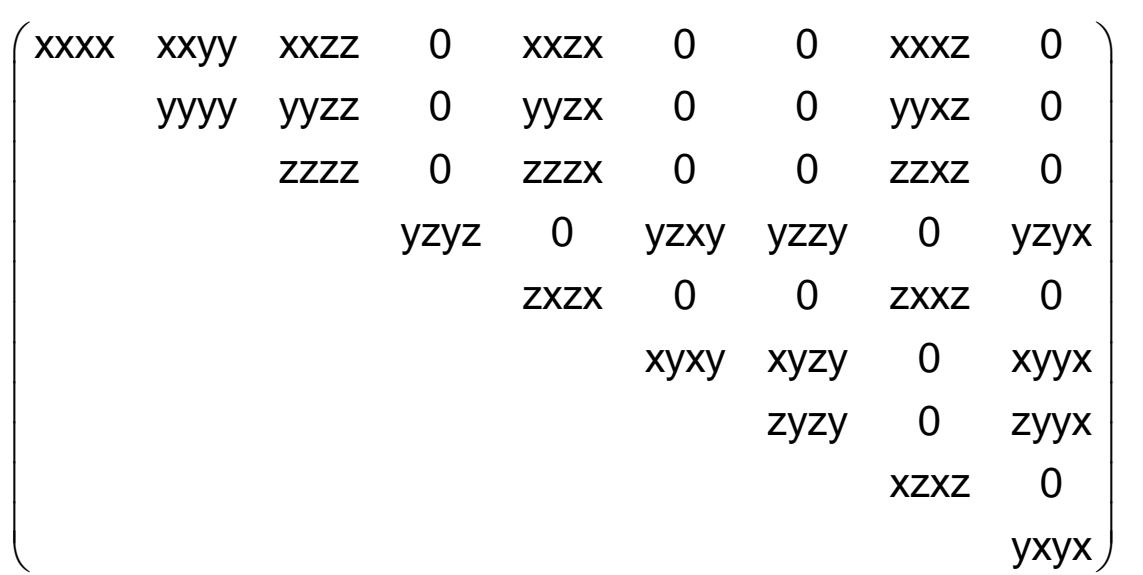

$\mathrm{D}_{\mathrm{n}}$

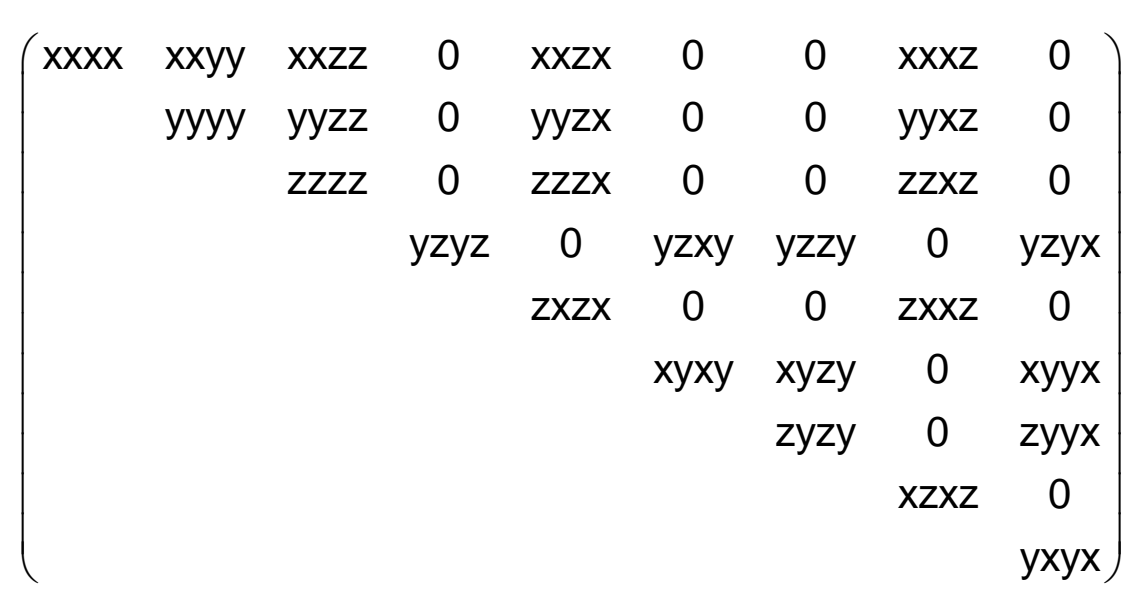

$\begin{array}{lllllllll}x x x x & x x y y & x x z z & 0 & x x z x & 0 & 0 & x x x z & 0\end{array}$

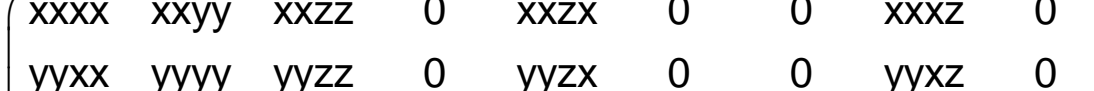
$\left(\begin{array}{llllllllll}\mathrm{yyxx} & y y y y & y y z z & 0 & y y z x & 0 & 0 & y y x z & 0 \\ z z x x & z z y y & z z z z & 0 & z z z x & 0 & 0 & z z x z & 0\end{array}\right)$ $\begin{array}{ccccccccc}0 & 0 & 0 & y z y z & 0 & y z x y & y z z y & 0 & y z y x\end{array}$

$\begin{array}{ccccccccc}z x x x & z x y y & z x z z & 0 & z x z x & 0 & 0 & z x x z & 0 \\ 0 & 0 & 0 & x y y z & 0 & x y x y & x y z y & 0 & \text { xyyx }\end{array}$

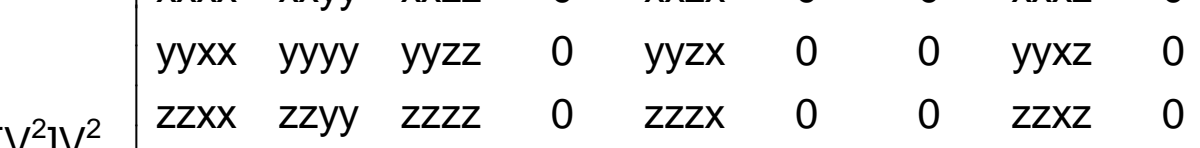
$\begin{array}{lllllllll}0 & 0 & 0 & y z y z & 0 & y z x y & y z z y & 0 & y z y x\end{array}$ $\begin{array}{ccccccccc}z x x x & z x y y & z x z z & 0 & z x z x & 0 & 0 & z x x z & 0 \\ 0 & 0 & 0 & x y y z & 0 & x y x y & x y z y & 0 & x y y x\end{array}$

$\left(\begin{array}{lllllllll}x x x x & x x y y & x x z z & 0 & x x z x & 0 & 0 & x x x z & 0 \\ y y x x & y y y & y y z z & 0 & y y z x & 0 & 0 & y y x z & 0\end{array}\right)$ $\left.\begin{array}{ccccccccc}\text { yyxx } & y y y y & y y z z & 0 & y y z x & 0 & 0 & y y x z & 0 \\ z z x x & z z y y & z z z z & 0 & z z z x & 0 & 0 & z z x z & 0\end{array}\right)$ $\begin{array}{lllllllll}0 & 0 & 0 & y z y z & 0 & y z x y & y z z y & 0 & y z y x\end{array}$ $\begin{array}{ccccccccc}\text { zxxx } & \text { zxyy } & \text { zxzz } & 0 & \text { zxzx } & 0 & 0 & \text { zxxz } & 0 \\ 0 & 0 & 0 & x y y z & 0 & x y x y & x y z y & 0 & x y y x\end{array}$

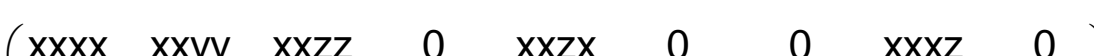
$\begin{array}{llllllllll}\text { yyxx } & \text { yyyy } & \text { yyzz } & 0 & \text { yyzx } & 0 & 0 & \text { yyxz } & 0 \\ \text { zzxx } & z z y y & z z z z & 0 & \text { zzzx } & 0 & 0 & \text { zzxz } & 0\end{array}$ $\begin{array}{ccccccccc}0 & 0 & 0 & y z y z & 0 & y z x y & y z z y & 0 & y z y x\end{array}$ $\begin{array}{ccccccccc}\text { zxxx } & \text { zxyy } & \text { zxzz } & 0 & \text { zxzx } & 0 & 0 & z x x z & 0 \\ 0 & 0 & 0 & x y z & 0 & x y x y & x y z y & 0 & x y y x\end{array}$ 
$\left(\begin{array}{ccccccccc}\text { xxxx } & \text { xxyy } & \text { xxzz } & 0 & 0 & 0 & 0 & 0 & 0 \\ \text { yyxx } & \text { yyyy } & \text { yyzz } & 0 & 0 & 0 & 0 & 0 & 0 \\ \text { zzxx } & \text { zzyy } & \text { zzzz } & 0 & 0 & 0 & 0 & 0 & 0 \\ 0 & 0 & 0 & \text { yzyz } & 0 & 0 & \text { yzzy } & 0 & 0 \\ 0 & 0 & 0 & 0 & \text { zxzx } & 0 & 0 & \text { zxxz } & 0 \\ 0 & 0 & 0 & 0 & 0 & \text { xyxy } & 0 & 0 & \text { xyyx } \\ 0 & 0 & 0 & z y y z & 0 & 0 & \text { zyzy } & 0 & 0 \\ 0 & 0 & 0 & 0 & x z z x & 0 & 0 & \text { xzxz } & 0 \\ 0 & 0 & 0 & 0 & 0 & \text { yxxy } & 0 & 0 & y x y x\end{array}\right)$

$\left[\mathrm{V}^{4}\right]\left(\begin{array}{ccccc}\mathrm{xxxx} & 0 & 0 & \mathrm{yyzz} & 0 \\ 0 & \mathrm{yyyy} & 0 & \mathrm{zzxx} & 0 \\ 0 & 0 & \mathrm{zzzz} & \mathrm{xxyy} & 0\end{array}\right)$

$\left.\mathrm{V} \mathrm{V}^{3}\right] \quad\left(\begin{array}{cccccccccc}\mathrm{xxxx} & 0 & 0 & \mathrm{xxyy} & 0 & 0 & \mathrm{xxzz} & 0 & 0 & 0 \\ 0 & \mathrm{yyyy} & 0 & 0 & \mathrm{yyzz} & 0 & 0 & \mathrm{yyxx} & 0 & 0 \\ 0 & 0 & z z z z & 0 & 0 & z z x x & 0 & 0 & z z y & 0\end{array}\right)$

$\left[\left[V^{2}\right]^{2}\right]\left(\begin{array}{cccccc}x x x x & x x y y & x x z z & 0 & 0 & 0 \\ & y y y y & y y z z & 0 & 0 & 0 \\ & & z z z z & 0 & 0 & 0 \\ & & & \text { yzyz } & 0 & 0 \\ & & & & x z x z & 0 \\ & & & & & \text { xyxy }\end{array}\right)$

$\left[V^{2}\right]^{2}\left(\begin{array}{cccccc}x x x x & x x y y & x x z z & 0 & 0 & 0 \\ y y x x & y y y y & y y z z & 0 & 0 & 0 \\ z z x x & z z y y & z z z z & 0 & 0 & 0 \\ 0 & 0 & 0 & y z y z & 0 & 0 \\ 0 & 0 & 0 & 0 & x z x z & 0 \\ 0 & 0 & 0 & 0 & 0 & x y x y\end{array}\right)$ e

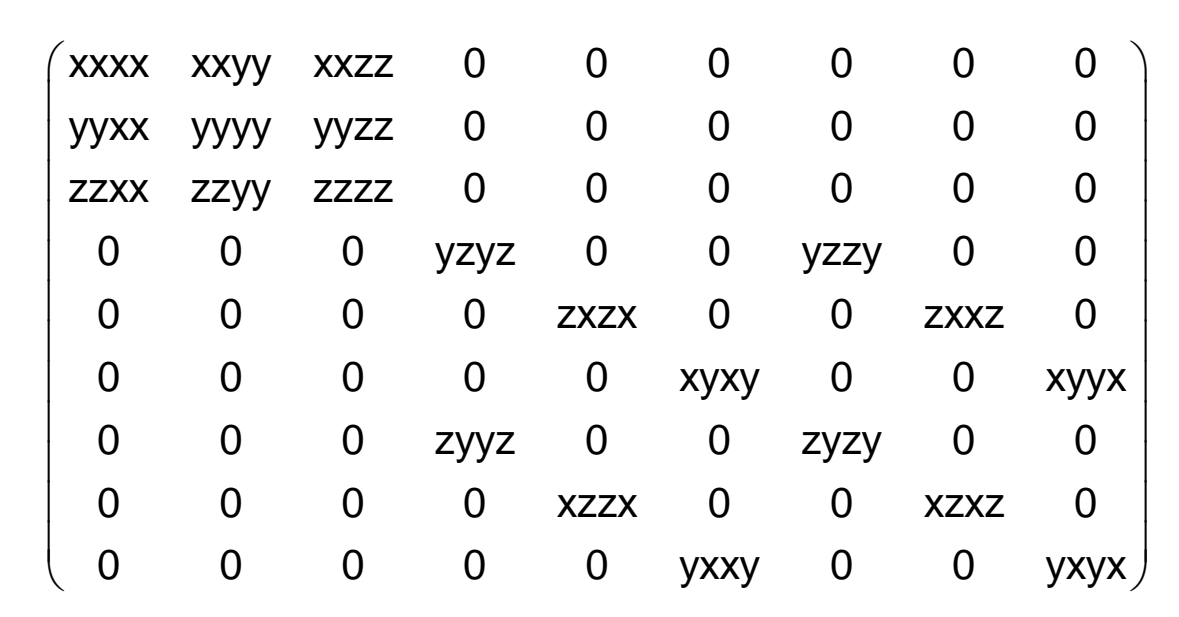

$\left(\begin{array}{ccccc}x x x x & 0 & 0 & y y z z & 0 \\ 0 & y y y y & 0 & z z x x & 0 \\ 0 & 0 & z z z z & x x y y & 0\end{array}\right)$

$\left(\begin{array}{cccccccccc}x x x x & 0 & 0 & x x y y & 0 & 0 & x x z z & 0 & 0 & 0 \\ 0 & y y y & 0 & 0 & y y z & 0 & 0 & y y x & 0 & 0\end{array}\right)$ $\left(\begin{array}{cccccccccc}0 & \text { yyyy } & 0 & 0 & \text { yyzz } & 0 & 0 & y y x x & 0 & 0 \\ 0 & 0 & z z z z & 0 & 0 & z z x & 0 & 0 & z z y & 0\end{array}\right)$

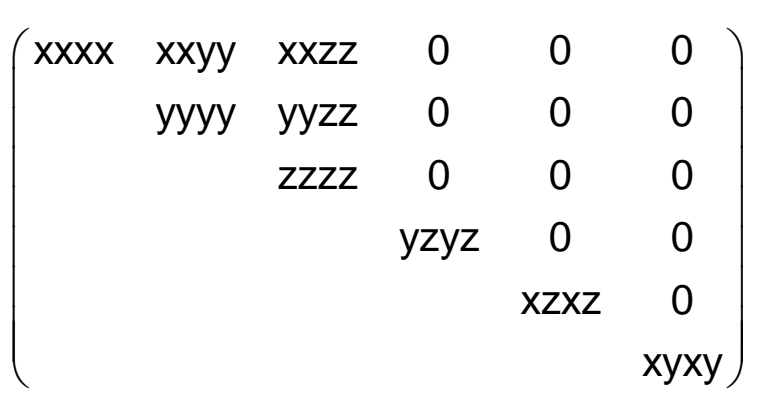

$\left(\begin{array}{cccccc}\text { xxxx } & \text { xxyy } & \text { xxzz } & 0 & 0 & 0 \\ \text { yyxx } & \text { yyyy } & \text { yyzz } & 0 & 0 & 0 \\ \text { zzxx } & \text { zzyy } & \text { zzzz } & 0 & 0 & 0 \\ 0 & 0 & 0 & \text { yzyz } & 0 & 0 \\ 0 & 0 & 0 & 0 & \text { xzxz } & 0 \\ 0 & 0 & 0 & 0 & 0 & \text { xyxy }\end{array}\right)$

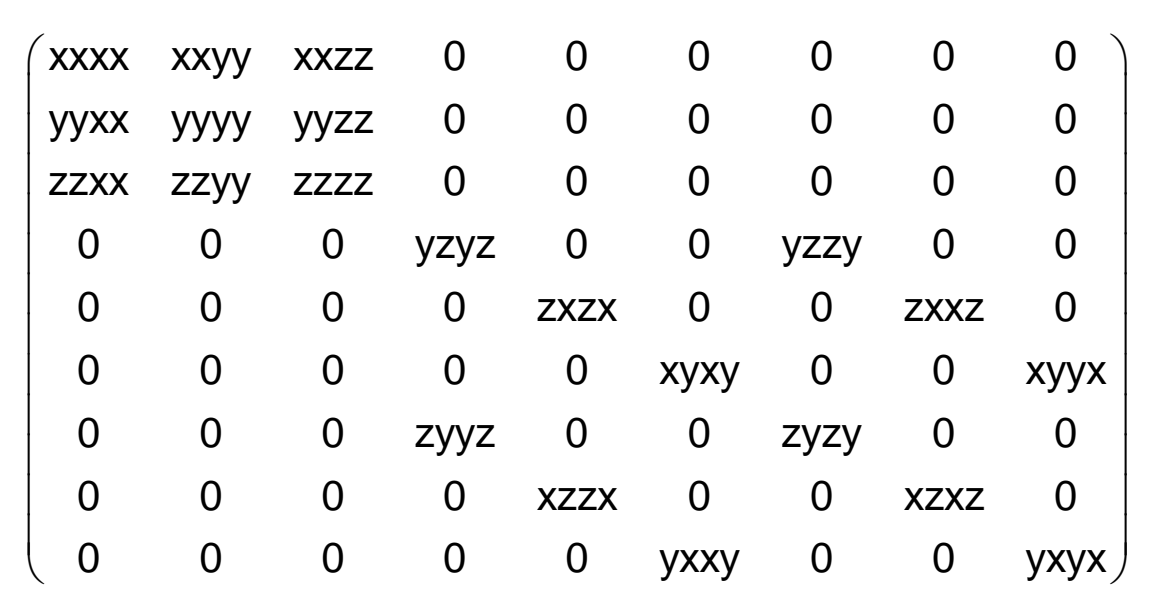

$\left(\begin{array}{lllllllll}x x x x & x x y y & x x z z & 0 & 0 & 0 & 0 & 0 & 0 \\ y y x x & y y y & y z z & 0 & 0 & 0 & 0 & 0 & 0\end{array}\right)$ $\left(\begin{array}{lllllllll}\text { yyxx } & \text { yyyy } & \text { yyzz } & 0 & 0 & 0 & 0 & 0 & 0 \\ z z x x & \text { zzyy } & \text { zzzz } & 0 & 0 & 0 & 0 & 0 & 0 \\ 0 & 0 & 0 & y z y z & 0 & 0 & y z z & 0 & 0\end{array}\right)$ $\begin{array}{lllllllll}0 & 0 & 0 & y z y z & 0 & 0 & \text { yzzy } & 0 & 0\end{array}$ $\begin{array}{lllllllll}0 & 0 & 0 & 0 & z x z x & 0 & 0 & z x x z & 0\end{array}$ $\begin{array}{lllllllll}0 & 0 & 0 & 0 & 0 & x y x y & 0 & 0 & x y y x\end{array}$ $\begin{array}{lllllllll}0 & 0 & 0 & \text { zyyz } & 0 & 0 & \text { zyzy } & 0 & 0 \\ 0 & 0 & 0 & 0 & x z z x & 0 & 0 & x z z z & 0\end{array}$ $\left.\begin{array}{ccccccccc}0 & 0 & 0 & 0 & \mathrm{xzzx} & 0 & 0 & \mathrm{xzxz} & 0 \\ 0 & 0 & 0 & 0 & 0 & \mathrm{yxxy} & 0 & 0 & \mathrm{yxyx}\end{array}\right)$ $\left(\begin{array}{ccccc}x x x x & 0 & 0 & y y z z & 0 \\ 0 & y y y y & 0 & z z x x & 0 \\ 0 & 0 & z z z z & x x y y & 0\end{array}\right)$

$\left(\begin{array}{ccccc}\mathrm{xxxx} & 0 & 0 & \mathrm{yyzz} & 0 \\ 0 & \mathrm{yyyy} & 0 & \mathrm{zzxx} & 0 \\ 0 & 0 & \mathrm{zzzz} & \mathrm{xxyy} & 0\end{array}\right)$

$\left(\begin{array}{cccccccccc}x x x x & 0 & 0 & x x y y & 0 & 0 & x x z z & 0 & 0 & 0 \\ 0 & 0 & 0 & 0 & y y z z & 0 & 0 & y y y x & 0 & 0\end{array}\right) \quad\left(\begin{array}{cccccccccc}x x x x & 0 & 0 & x x y y & 0 & 0 & x x z z & 0 & 0 & 0 \\ 0 & y y y y & 0 & 0 & y y z z & 0 & 0 & y y y x x & 0 & 0\end{array}\right)$

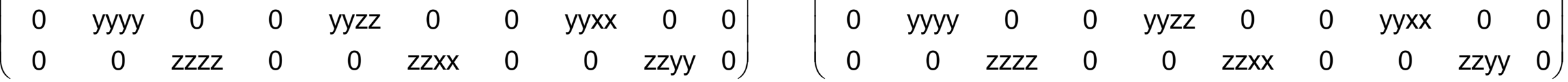

$\left(\begin{array}{cccccc}x x x x & x x y y & x x z z & 0 & 0 & 0 \\ & \text { yyyy } & \text { yyzz } & 0 & 0 & 0 \\ & & \text { zzzz } & 0 & 0 & 0 \\ & & & y z y z & 0 & 0 \\ & & & & x z x z & 0 \\ & & & & & \text { xyxy }\end{array}\right)$

$\left(\begin{array}{llllll}x x x x & x x y y & x x z z & 0 & 0 & 0 \\ y y x x & y y y y & y y z z & 0 & 0 & 0 \\ z z x x & z z y & z z z z & 0 & 0 & 0\end{array}\right)$ $\left.\begin{array}{cccccc}\text { yyxx } & \text { yyyy } & y y z z & 0 & 0 & 0 \\ z z x x & z z y y & z z z z & 0 & 0 & 0 \\ 0 & 0 & 0 & y z y z & 0 & 0\end{array}\right)$ $\begin{array}{lccccc}0 & 0 & 0 & y z y z & 0 & 0\end{array}$ $\begin{array}{cccccc}0 & 0 & 0 & y z y z & 0 & 0 \\ 0 & 0 & 0 & 0 & x z x z & 0\end{array}$ $\left(\begin{array}{cccccc}\text { xxxx } & \text { xxyy } & \text { xxzz } & 0 & 0 & 0 \\ & \text { yyyy } & \text { yyzz } & 0 & 0 & 0 \\ & & \text { zzzz } & 0 & 0 & 0 \\ & & & y z y z & 0 & 0 \\ & & & & \text { xzxz } & 0 \\ & & & & & \text { xyxy }\end{array}\right)$

$\left(\begin{array}{llllll}x x x x & x x y y & x x z z & 0 & 0 & 0 \\ y y x x & y y y y & y y z z & 0 & 0 & 0 \\ z z x x & z z y & z z z z & 0 & 0 & 0\end{array}\right)$ $\left.\begin{array}{llllll}\text { yyxx } & \text { yyyy } & \text { yyzz } & 0 & 0 & 0 \\ z z x x & z z y y & z z z z & 0 & 0 & 0 \\ 0 & 0 & 0 & y z y z & 0 & 0\end{array}\right)$ $\begin{array}{llllll}0 & 0 & 0 & y z y z & 0 & 0\end{array}$ $\begin{array}{cccccc}0 & 0 & 0 & y z y z & 0 & 0 \\ 0 & 0 & 0 & 0 & x z x z & 0 \\ 0 & 0 & 0 & 0 & 0 & x y y\end{array}$ 


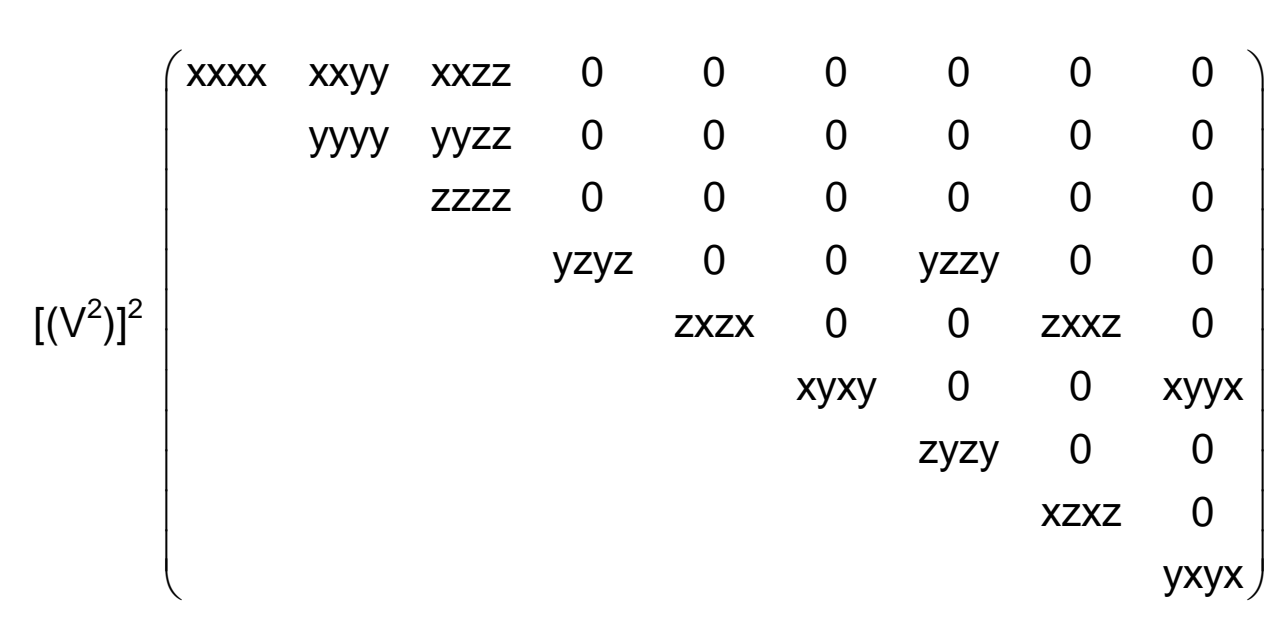

$\left[\mathrm{V}^{2}\right] \mathrm{V}^{2}\left(\begin{array}{ccccccccc}\mathrm{xxxx} & \mathrm{x} x \mathrm{y} y & \mathrm{xxzz} & 0 & 0 & 0 & 0 & 0 & 0 \\ \mathrm{yyxx} & \mathrm{yyyy} & \mathrm{yyzz} & 0 & 0 & 0 & 0 & 0 & 0 \\ \mathrm{zzxx} & \mathrm{zzyy} & \mathrm{zzzz} & 0 & 0 & 0 & 0 & 0 & 0 \\ 0 & 0 & 0 & \mathrm{yzyz} & 0 & 0 & \mathrm{yzzy} & 0 & 0 \\ 0 & 0 & 0 & 0 & \mathrm{zxzx} & 0 & 0 & \mathrm{zxxz} & 0 \\ 0 & 0 & 0 & 0 & 0 & \mathrm{xyxy} & 0 & 0 & \mathrm{xyyx}\end{array}\right)$

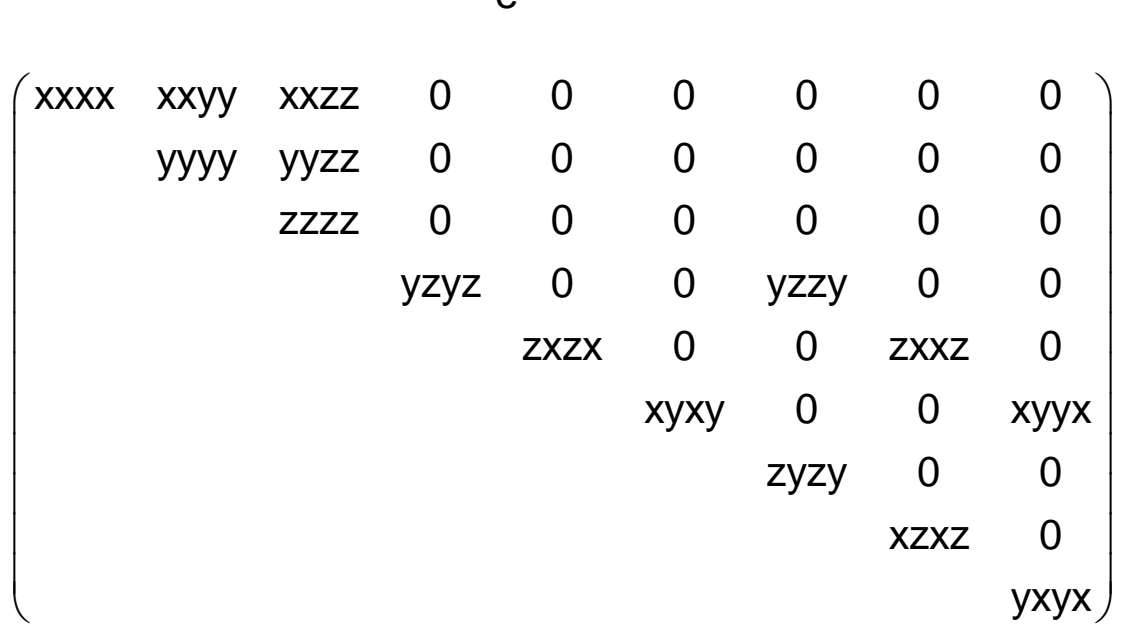

$\left(\begin{array}{ccccccccc}\text { xxxx } & \text { xxyy } & \text { xxzz } & 0 & 0 & 0 & 0 & 0 & 0 \\ \text { yyxx } & \text { yyyy } & \text { yyzz } & 0 & 0 & 0 & 0 & 0 & 0 \\ \text { zzxx } & \text { zzyy } & \text { zzzz } & 0 & 0 & 0 & 0 & 0 & 0 \\ 0 & 0 & 0 & \text { yzyz } & 0 & 0 & \text { yzzy } & 0 & 0 \\ 0 & 0 & 0 & 0 & \text { zxzx } & 0 & 0 & \text { zxxz } & 0 \\ 0 & 0 & 0 & 0 & 0 & \text { xyxy } & 0 & 0 & \text { xyyx }\end{array}\right)$

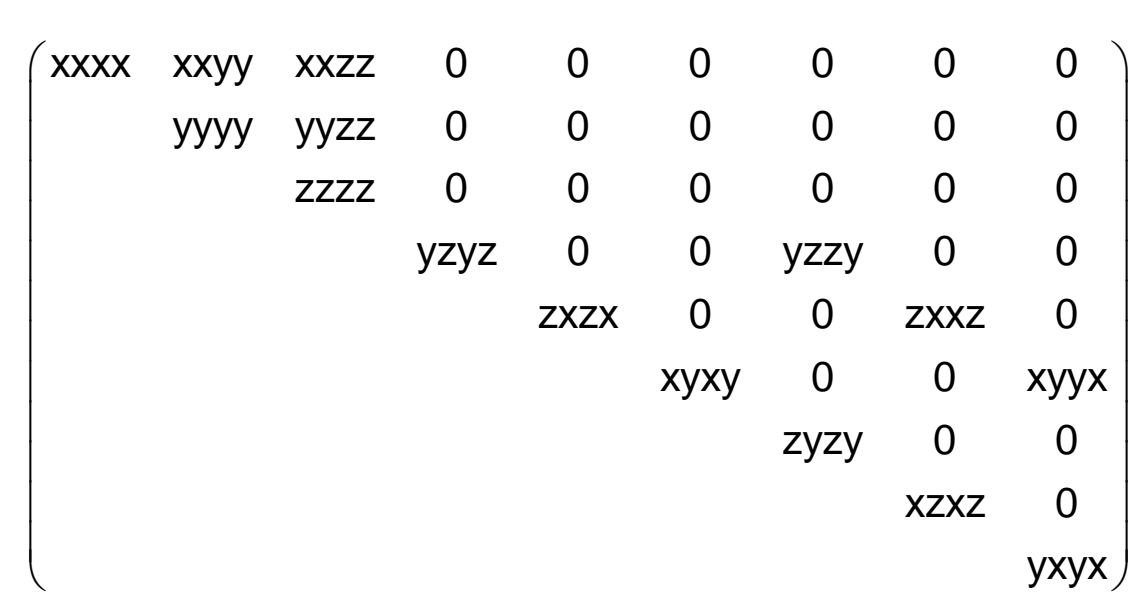

$\left(\begin{array}{ccccccccc}\text { xxxx } & \text { xxyy } & \text { xxzz } & 0 & 0 & 0 & 0 & 0 & 0 \\ \text { yyxx } & \text { yyyy } & \text { yyzz } & 0 & 0 & 0 & 0 & 0 & 0 \\ \text { zzxx } & \text { zzyy } & \text { zzzz } & 0 & 0 & 0 & 0 & 0 & 0 \\ 0 & 0 & 0 & y z y z & 0 & 0 & \text { yzzy } & 0 & 0 \\ 0 & 0 & 0 & 0 & \text { zxzx } & 0 & 0 & \text { zxxz } & 0 \\ 0 & 0 & 0 & 0 & 0 & \text { xyxy } & 0 & 0 & \text { xyyx }\end{array}\right)$

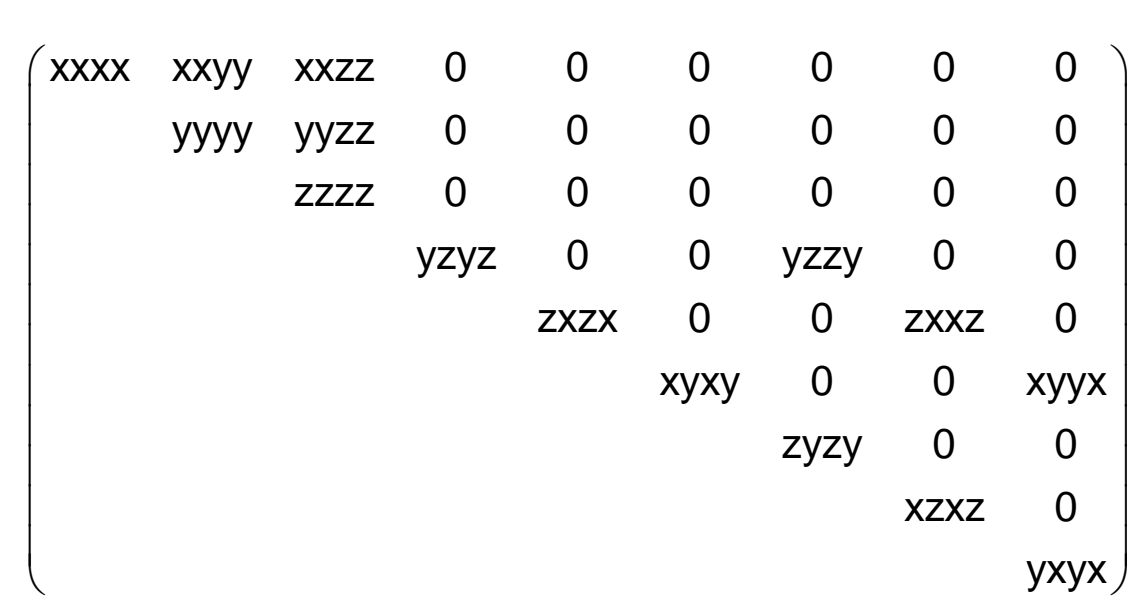

$\left(\begin{array}{ccccccccc}\text { xxxx } & \text { xxyy } & \text { xxzz } & 0 & 0 & 0 & 0 & 0 & 0 \\ \text { yyxx } & \text { yyyy } & \text { yyzz } & 0 & 0 & 0 & 0 & 0 & 0 \\ \text { zzxx } & \text { zzyy } & \text { zzzz } & 0 & 0 & 0 & 0 & 0 & 0 \\ 0 & 0 & 0 & \text { yzyz } & 0 & 0 & \text { yzzy } & 0 & 0 \\ 0 & 0 & 0 & 0 & \text { zxzx } & 0 & 0 & \text { zxxz } & 0 \\ 0 & 0 & 0 & 0 & 0 & \text { xyxy } & 0 & 0 & \text { xyyx }\end{array}\right)$ 
1

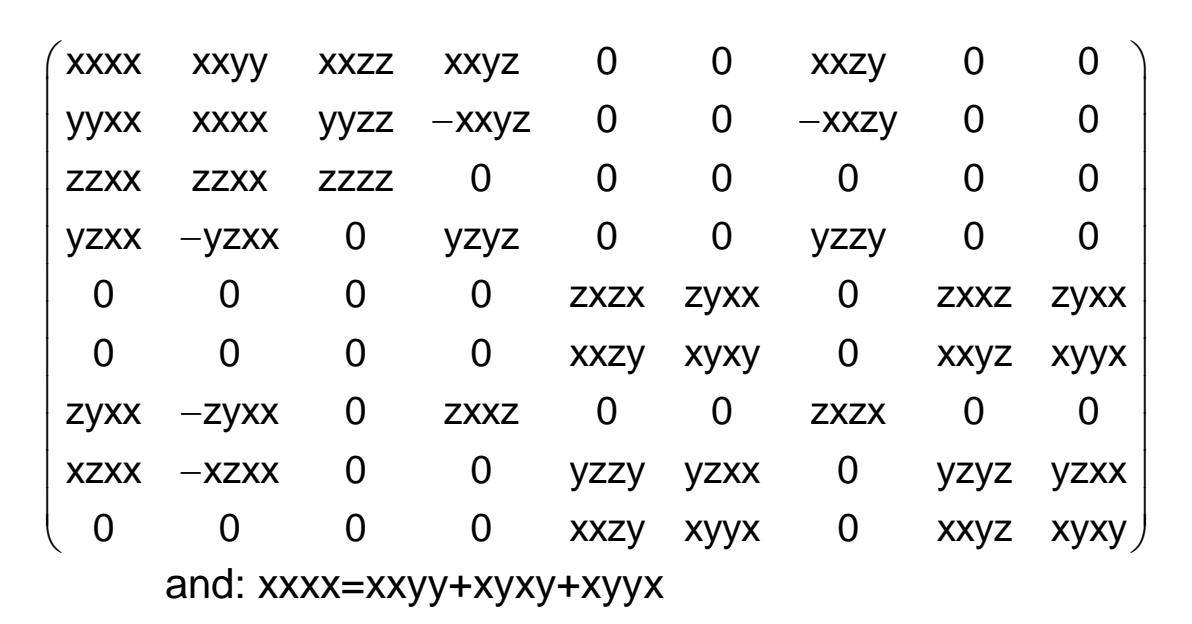

$\left[V^{4}\right] \quad\left(\begin{array}{ccccc}3 x x y y & 0 & 0 & y y z z & x x y z \\ 0 & 3 x x y y & 0 & y y z z & 0 \\ 0 & -x x y z & z z z z & x x y y & 0\end{array}\right)$

$V\left[V^{3}\right]\left(\begin{array}{cccccccccc}3 x x y y & 0 & 0 & x x y y & 0 & 0 & y y z z & 0 & 0 & y z x x \\ 0 & 3 x x y y & 0 & 0 & y y z z & y z x x & 0 & x x y y & -y z x & 0 \\ 0 & -z y x x & z z z z & 0 & 0 & z z x x & 0 & z y x x & z z x x & 0\end{array}\right)$

$\left[\left[^{2}\right]^{2}\right]\left(\begin{array}{cccccc}x x x x & x x y y & x x z z & x x y z & 0 & 0 \\ & x x x x & x x z z & -x y y z & 0 & 0 \\ & & z z z z & 0 & 0 & 0 \\ & & & y z y z & 0 & 0 \\ & & & & y z y z & x x y z \\ & & & & & x y x y\end{array}\right)$

and $x y x y=1 / 2(x x x x-x x y y)$

$\left[V^{2}\right]^{2}\left(\begin{array}{cccccc}x x x x & x x y y & x x z z & x x y z & 0 & 0 \\ x x y y & x x x x & x x z z & -x x y z & 0 & 0 \\ z z x x & z z x x & z z z z & 0 & 0 & 0 \\ y z x x & -y z x x & 0 & y z y z & 0 & 0 \\ 0 & 0 & 0 & 0 & y z y z & y z x x \\ 0 & 0 & 0 & 0 & x x y z & x y x y\end{array}\right)$

and $x y x y=1 / 2(x x x x-x x y y)$ $\left(\begin{array}{lllllllll}x x x x & x x y y & x x z z & x x y z & 0 & 0 & x x z y & 0 & 0\end{array}\right.$ $\left.\begin{array}{ccccccccc}\mathrm{yyxx} & \mathrm{xxxx} & \mathrm{yyzz} & -\mathrm{xxyz} & 0 & 0 & -\mathrm{xxzy} & 0 & 0 \\ \mathrm{zzxx} & \mathrm{zzxx} & \mathrm{zzzz} & 0 & 0 & 0 & 0 & 0 & 0 \\ y z x x & -y z x x & 0 & y z y z & 0 & 0 & y z z y & 0 & 0\end{array}\right)$ $\begin{array}{ccccccccc}\mathrm{zzxx} & \mathrm{zzxx} & \mathrm{zzzz} & 0 & 0 & 0 & 0 & 0 & 0 \\ \mathrm{yzxx} & -\mathrm{yzxx} & 0 & \mathrm{yzyz} & 0 & 0 & y z z y & 0 & 0 \\ 0 & 0 & 0 & 0 & z x z x & z y x & 0 & z x z & z y x x\end{array}$ $\begin{array}{ccccccccc}y z x x & -y z x x & 0 & y z y z & 0 & 0 & y z z y & 0 & 0 \\ 0 & 0 & 0 & 0 & z x z x & z y x x & 0 & z x x z & z y x x \\ 0 & 0 & 0 & 0 & x z z y & x y x & 0 & x x y z & x y y\end{array}$ $\begin{array}{ccccccccc}0 & 0 & 0 & 0 & z x z x & z y x x & 0 & z x x z & z y x x \\ 0 & 0 & 0 & 0 & x x z y & x y x y & 0 & x x y z & x y y x \\ z y x x & -z y x x & 0 & z x z & 0 & 0 & z x z & 0 & 0\end{array}$

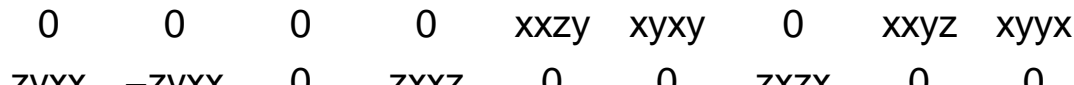
$\begin{array}{ccccccccc}z y x x & -z y x x & 0 & z x x z & 0 & 0 & z x z x & 0 & 0 \\ 0 & 0 & 0 & 0 & x z y & y z x x & 0 & y z y z & y z x x\end{array}$ $\begin{array}{ccccccccc}\mathrm{xzxx} & -\mathrm{xzxx} & 0 & 0 & \mathrm{yzzy} & \mathrm{yzxx} & 0 & \mathrm{yzyz} & \mathrm{yzxx} \\ 0 & 0 & 0 & 0 & \mathrm{x} x \mathrm{y} y & \mathrm{xyyx} & 0 & \mathrm{xxyz} & \mathrm{xyxy}\end{array} \mid$ and: $x x x x=x x y y+x y x y+x y y x$

$\left(\begin{array}{ccccc}3 x x y y & 0 & 0 & y y z z & x x y z \\ 0 & 3 x x y y & 0 & y y z z & 0 \\ 0 & -x x y z & z z z z & x x y y & 0\end{array}\right)$

$\left(\begin{array}{cccccccccc}3 x x y y & 0 & 0 & x x y y & 0 & 0 & y y z z & 0 & 0 & y z x x \\ 0 & 3 x x y y & 0 & 0 & y y z z & y z x x & 0 & x x y y & -y z z x & 0 \\ 0 & -z y x x & z z z z & 0 & 0 & z z x x & 0 & \text { zyxy } & z z x x & 0\end{array}\right) \quad\left(\begin{array}{ccccccccccc}3 x x y y & 0 & 0 & x x y y & 0 & 0 & y y z z & 0 & 0 & y z x x \\ 0 & 3 x x y y & 0 & 0 & y y z z & y z x x & 0 & x x y y & -y z x x & 0 \\ 0 & -z y x x & z z z z & 0 & 0 & z z x x & 0 & z y x x & z z x x & 0\end{array}\right)$ $\left.\begin{array}{llllllllll}0 & 3 x x y y & 0 & 0 & y y z z & y z x x & 0 & x x y y & -y z x x & 0 \\ 0 & -z y x x & z z z z & 0 & 0 & z z x x & 0 & z y y x & z z x x & 0\end{array}\right)$

$\left(\begin{array}{lllllllll}x x x x & x x y y & x x z z & x x y z & 0 & 0 & x x z y & 0 & 0 \\ 7 y x x & x y x & y y z z & -x x y z & 0 & 0 & -x x y & 0 & 0\end{array}\right)$ $\left(\begin{array}{ccccccccc}\mathrm{yyxx} & \mathrm{xxxx} & \mathrm{yyzz} & -\mathrm{xxyz} & 0 & 0 & -\mathrm{xxzy} & 0 & 0 \\ \mathrm{zzxx} & \mathrm{zzxx} & \mathrm{zzzz} & 0 & 0 & 0 & 0 & 0 & 0 \\ \mathrm{yzx} & -\mathrm{yzx} & 0 & y z y z & 0 & 0 & \mathrm{yzzy} & 0 & 0\end{array}\right)$ $\begin{array}{lllllllll}y z x x & -y z x x & 0 & y z y z & 0 & 0 & y z z y & 0 & 0\end{array}$ $\begin{array}{lllllllll}0 & 0 & 0 & 0 & \mathrm{zxzx} & \mathrm{zy} x \mathrm{x} & 0 & \mathrm{zxxz} & \mathrm{zy} \mathrm{yx}\end{array}$ $\begin{array}{ccccccccc}0 & 0 & 0 & 0 & x x z y & x y x y & 0 & x x y z & x y y x \\ z y x x & -z y x x & 0 & z x y z & 0 & 0 & z x z x & 0 & 0\end{array}$ $\begin{array}{ccccccccc}z y x x & -z y x x & 0 & z x x z & 0 & 0 & z x z x & 0 & 0 \\ x z x & -x z x & 0 & 0 & y z z y & y z x x & 0 & y z y z & y z x x\end{array}$ $\begin{array}{ccccccccc}x z x x & -x z x x & 0 & 0 & y z z y & y z x x & 0 & y z y z & y z x x \\ 0 & 0 & 0 & 0 & x y z y & x y y x & 0 & x x y z & x y x y\end{array}$ and: $x x x x=x x y y+x y x y+x y y x$

$\left(\begin{array}{ccccc}3 x x y y & 0 & 0 & y y z z & x x y z \\ 0 & 3 x x y y & 0 & y y z z & 0 \\ 0 & -x x y z & z z z z & x x y & 0\end{array}\right)$

$\left.\begin{array}{ccccc}0 & 3 x x y y & 0 & y y z z & 0 \\ 0 & -x x y z & z z z z & x x y y & 0\end{array}\right)$

$$
\left(\begin{array}{cccccc}
x x x x & x x y y & x x z z & x x y z & 0 & 0 \\
& x x x x & x x z z & -x x y z & 0 & 0 \\
& & z z z z & 0 & 0 & 0 \\
& & & y z y z & 0 & 0 \\
& & & & y z y z & x x y z \\
& & & & & x y x y
\end{array}\right)
$$

and $x y x y=1 / 2(x x x x-x x y y)$

and $x y x y=1 / 2(x x x x-x x y y)$

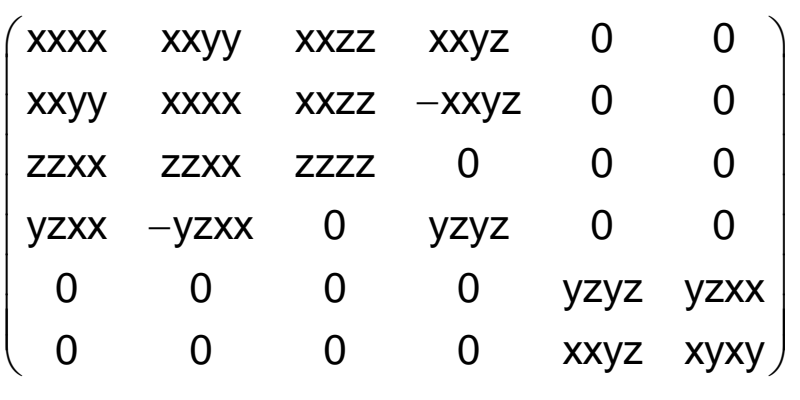

and $x y x y=1 / 2(x x x x-x x y y)$ $\left(\begin{array}{cccccc}x x x x & x x y y & x x z z & x x y z & 0 & 0 \\ x x y y & x x x x & x x z z & -x x y z & 0 & 0 \\ z z x x & z z x x & z z z z & 0 & 0 & 0 \\ y z x x & -y z x x & 0 & y z y z & 0 & 0 \\ 0 & 0 & 0 & 0 & y z y z & y z x x \\ 0 & 0 & 0 & 0 & x x y z & x y x y\end{array}\right)$

and $x y x y=1 / 2(x x x x-x x y y)$ $\left(\begin{array}{lllllllll}x x x x & x x y y & x x z z & x x y z & 0 & 0 & x x z y & 0 & 0 \\ \text { yyx } & x x y x & y y z & -x y z & 0 & 0 & -x z y & 0 & 0\end{array}\right)$ $\left.\begin{array}{ccccccccc}y y x x & x x x x & y y z z & -x x y z & 0 & 0 & -x x z y & 0 & 0 \\ z z x x & z z x x & z z z z & 0 & 0 & 0 & 0 & 0 & 0 \\ y z x x & -y z x & 0 & y z y z & 0 & 0 & y z z y & 0 & 0\end{array}\right)$ $\begin{array}{llllllllll}y z z x & z z x x & z z z z & 0 & 0 & 0 & 0 & 0 & 0 \\ y z x x & -y z x x & 0 & y z y z & 0 & 0 & y z z y & 0 & 0\end{array}$ $\begin{array}{ccccccccc}y z x x & -y z x x & 0 & y z y z & 0 & 0 & y z z y & 0 & 0 \\ 0 & 0 & 0 & 0 & z x z x & z y x x & 0 & z x x z & z y x x \\ 0 & 0 & 0 & 0 & x x y y & x y x y & 0 & x x y z & x y x\end{array} \mid$

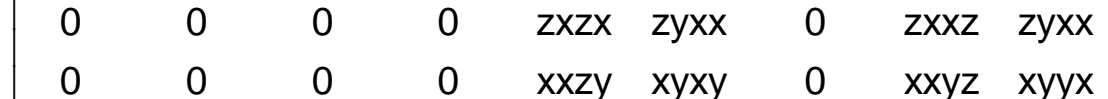
$\begin{array}{ccccccccc}z y x x & -z y x x & 0 & z x x z & 0 & 0 & z x z x & 0 & 0\end{array}$ $\left(\begin{array}{ccccccccc}x z x x & -x z x x & 0 & 0 & y z z y & y z x x & 0 & y z y z & y z x x \\ 0 & 0 & 0 & 0 & x x z y & x y y x & 0 & x x y z & x y x y\end{array}\right)$ and: $x x x x=x x y y+x y x y+x y y x$

$\left(\begin{array}{ccccc}3 x x y y & 0 & 0 & y y z z & x x y z \\ 0 & 3 x x y y & 0 & y y z z & 0 \\ 0 & -x x y z & z z z z & x y y & 0\end{array}\right)$

$\left(\begin{array}{llllll}0 & 3 x x y y & 0 & y y z z & 0 \\ 0 & -x x y z & z z z z & x x y y & 0\end{array}\right)$

$\left(\begin{array}{cccccccccc}3 x x y y & 0 & 0 & x x y y & 0 & 0 & y y z z & 0 & 0 & y z x x \\ 0 & 3 x x y y & 0 & 0 & y y z & y z x x & 0 & x x y y & -y z x x & 0\end{array}\right)$

$\left(\begin{array}{cccccccccc}0 & 3 x x y y & 0 & 0 & y y z z & y z x x & 0 & x x y y & -y z x x & 0 \\ 0 & -z y y x & z z z z & 0 & 0 & z z x x & 0 & z y y x & z z x x & 0\end{array}\right)$

$$
\left(\begin{array}{cccccc}
x x x x & x x y y & x x z z & x x y z & 0 & 0 \\
& x x x x & x x z z & -x x y z & 0 & 0 \\
& & z z z z & 0 & 0 & 0 \\
& & & y z y z & 0 & 0 \\
& & & & y z y z & x x y z \\
& & & & & x y x y
\end{array}\right)
$$

and $x y x y=1 / 2(x x x x-x x y y)$

$\left(\begin{array}{cccccc}x x x x & x x y y & x x z z & x x y z & 0 & 0 \\ x x y y & x x x x & x x z z & -x x y z & 0 & 0 \\ z z x x & z z x x & z z z z & 0 & 0 & 0 \\ y z x x & -y z x x & 0 & y z y z & 0 & 0 \\ 0 & 0 & 0 & 0 & y z y z & y z x x \\ 0 & 0 & 0 & 0 & x x y z & x y x y\end{array}\right)$

and $x y x y=1 / 2(x x x x-x x y y)$ 
$n=3 \quad D_{3}=\mathbf{3}_{2} \mathbf{2}_{\mathrm{x}}$

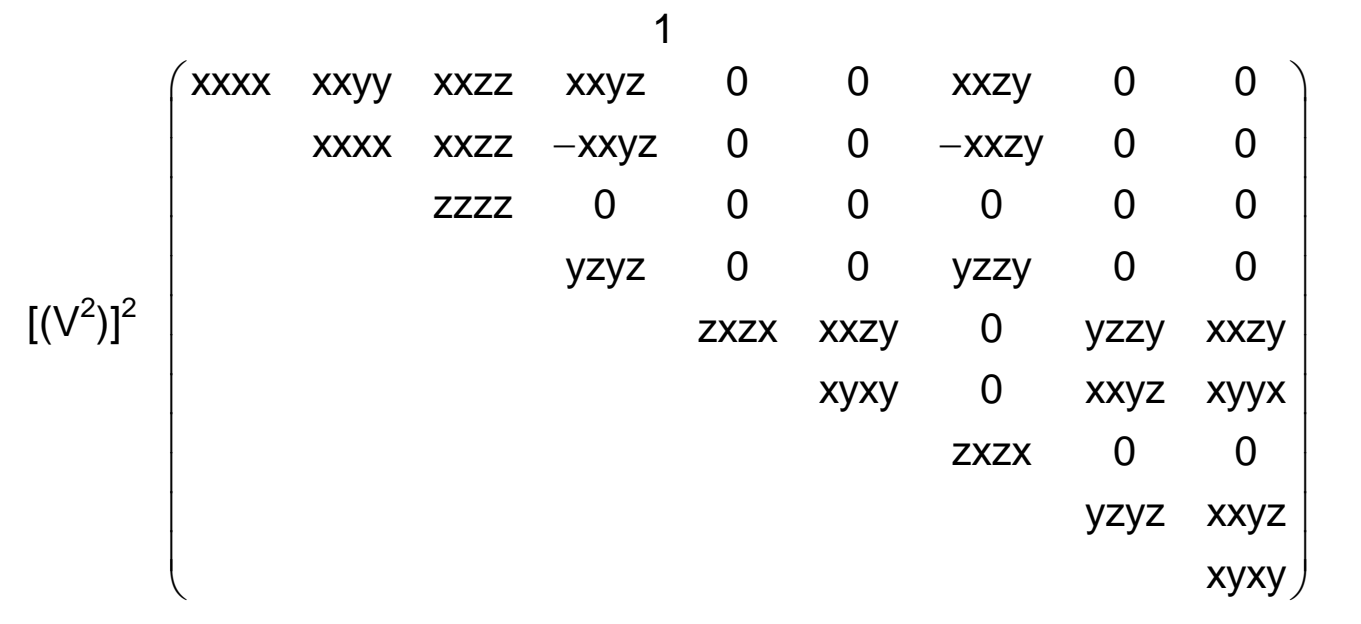

and $x x x x=x x y y+y z y z+z y z y$

\begin{abstract}
$\begin{array}{lllllllll}x x x x & x x y y & x x z z & x x y z & 0 & 0 & x x z y & 0 & 0 \\ x x y y & x x y x & x x z z & -x x y z & 0 & 0 & -x x z y & 0 & 0\end{array}$ $\left(\begin{array}{cccccccccc}x x y y & x x x x & x x z z & -x x y z & 0 & 0 & -x x z y & 0 & 0 \\ z z x x & z z x x & z z z z & 0 & 0 & 0 & 0 & 0 & 0 \\ y z x x & -y z x x & 0 & y z y z & 0 & 0 & z x z x & 0 & 0\end{array}\right)$

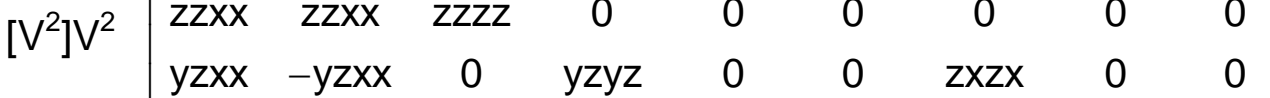
$\left(\begin{array}{ccccccccc}\operatorname{yz} x x & 0 & 0 & 0 & z x z x & y z x x & 0 & y z y z & y z x x \\ 0 & 0 & 0 & 0 & x x z y & x y x & 0 & x y z & x y x\end{array}\right)$

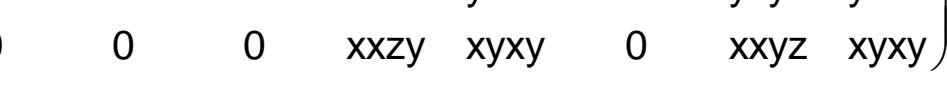
and $x x x x=x x y y+2 x y x y$

$\mathrm{D}_{\mathrm{n}}$

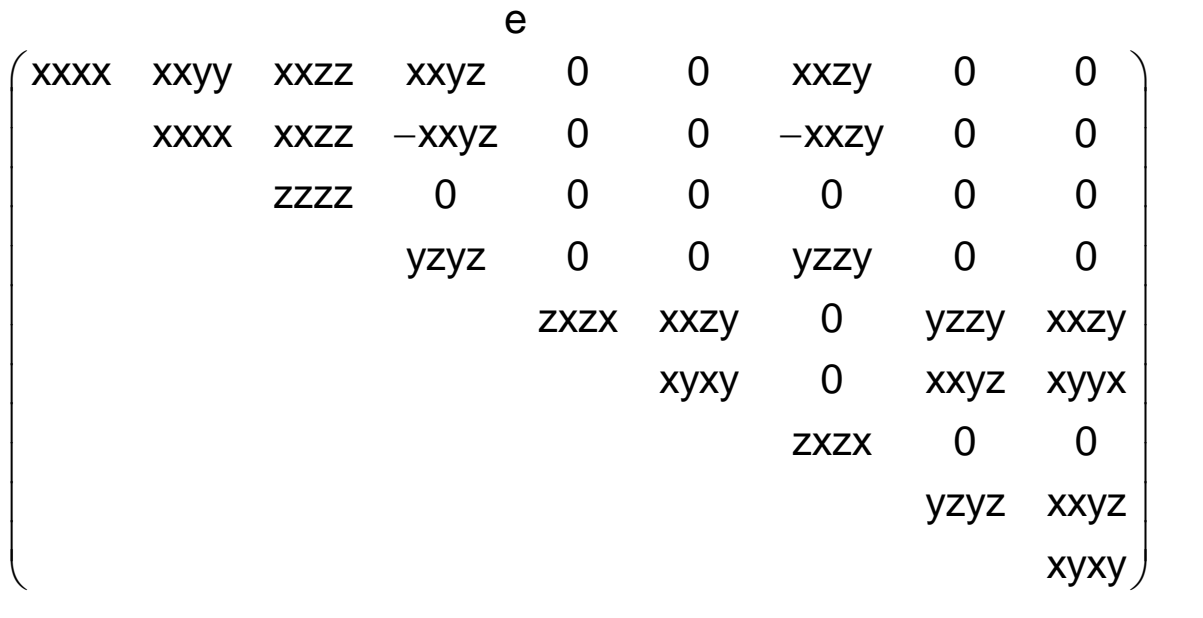

and $x x x x=x x y y+y z y z+z y z y$

$\left(\begin{array}{lllllllll}x x x x & x x y y & x x z z & x x y z & 0 & 0 & x x z y & 0 & 0 \\ x y y & x x x x & x x z z & -x x y z & 0 & 0 & -x x z y & 0 & 0\end{array}\right)$ $\left.\begin{array}{lllllllll}x x y y & x x x x & x x z z & -x x y z & 0 & 0 & -x x z y & 0 & 0\end{array}\right)$ $\begin{array}{lllllllll}z z x x & z z x x & z z z z & 0 & 0 & 0 & 0 & 0 & 0\end{array}$ $\begin{array}{lllllllll}y z x x & -y z x x & 0 & y z y z & 0 & 0 & z x z x & 0 & 0\end{array}$ $\begin{array}{ccccccccc}0 & 0 & 0 & 0 & z x z x & y z x x & 0 & y z y z & y z x x \\ 0 & 0 & 0 & 0 & x x z y & x y x y & 0 & x x y z & x y x\end{array}$ $\begin{array}{lllllll}0 & 0 & x x z y & x y x y & 0 & x x y z & x y x y\end{array}$ and $x x x x=x x y y+2 x y x y$

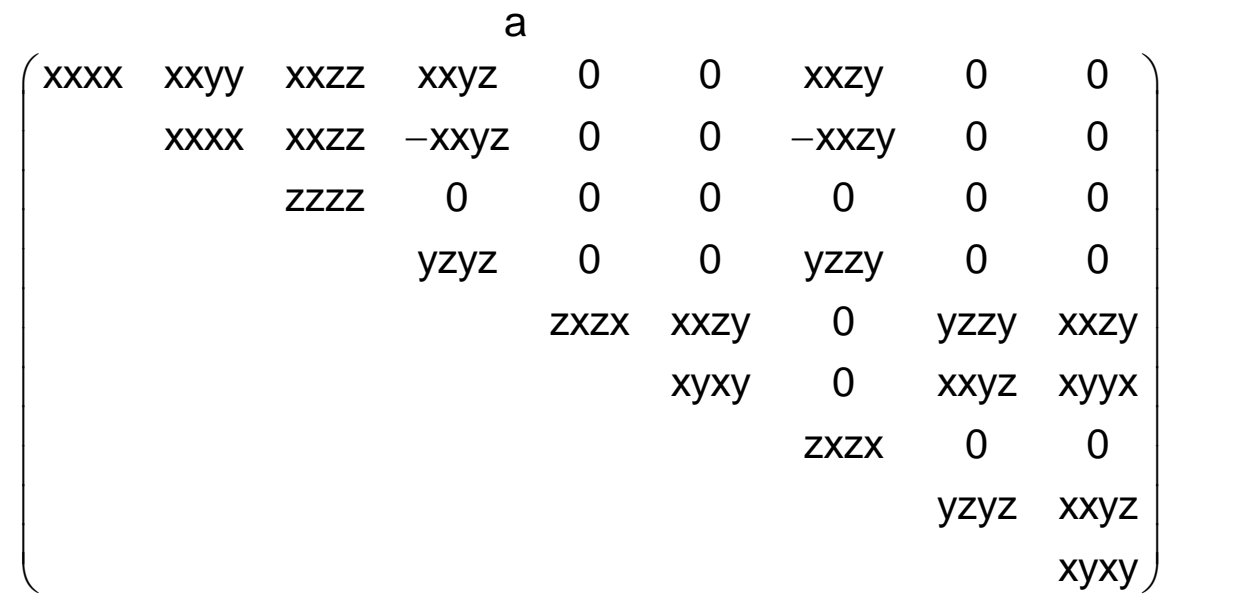

and $x x x x=x x y y+y z y z+z y z y$

$\left(\begin{array}{lllllllll}x x x x & x x y y & x x z z & x x y z & 0 & 0 & x x z y & 0 & 0 \\ x x y & x x y x & x y z & -x x y z & 0 & 0 & -x x y & 0 & 0\end{array}\right)$ $\left(\begin{array}{ccccccccc}x x x y y & x x x x & x x z z & -x x y z & 0 & 0 & -x x z y & 0 & 0 \\ z z x x & z z x x & z z z z & 0 & 0 & 0 & 0 & 0 & 0 \\ y z x x & -y z x & 0 & y z y z & 0 & 0 & z x z x & 0 & 0\end{array}\right)$ $\begin{array}{ccccccccc}z z x x & z z x x & z z z z & 0 & 0 & 0 & 0 & 0 & 0 \\ y z x x & -y z x x & 0 & y z y z & 0 & 0 & z x z x & 0 & 0\end{array}$ $\left(\begin{array}{ccccccccc}\mathrm{yzxx} & -\mathrm{yzx} x & 0 & \mathrm{yzyz} & 0 & 0 & \mathrm{zxzx} & 0 & 0 \\ 0 & 0 & 0 & 0 & \mathrm{zxzx} & \mathrm{yzxx} & 0 & \mathrm{yzyz} & \mathrm{yzxx} \\ 0 & 0 & 0 & 0 & x \mathrm{z} x & \mathrm{x} x \mathrm{c} & 0 & \mathrm{xyyz} & \mathrm{x} x \mathrm{y}\end{array}\right.$ $\begin{array}{ccccccccc}0 & 0 & 0 & 0 & z x z x & y z x x & 0 & y z y z & y z x x \\ 0 & 0 & 0 & 0 & x x z y & x y x y & 0 & x x y z & x y x y\end{array}$ and $x x x x=x x y y+2 x y x y$

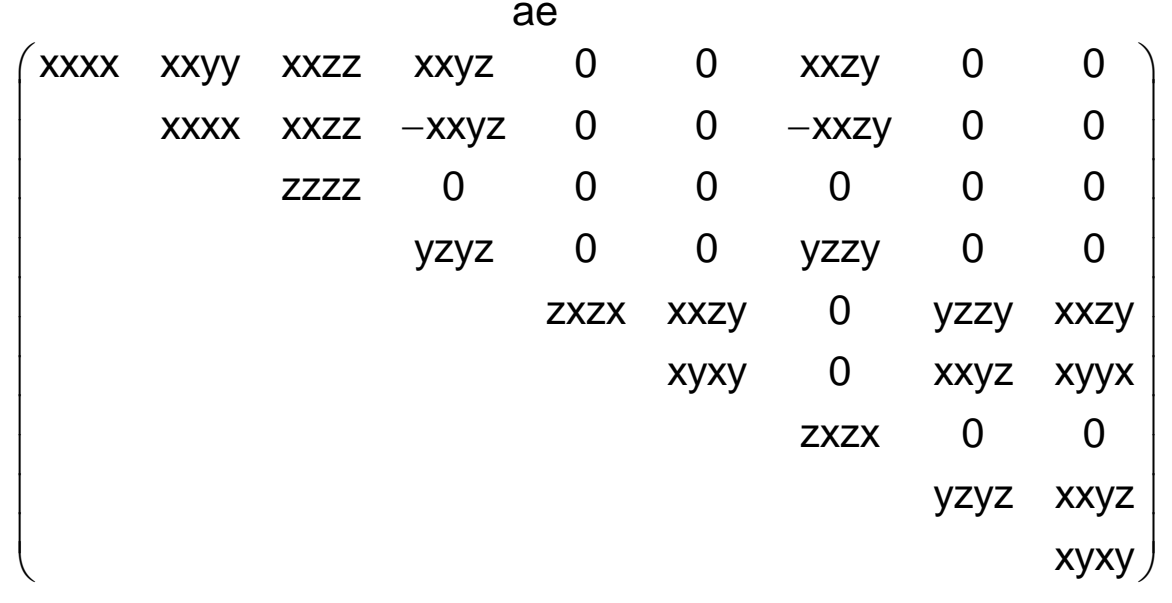

and $x x x x=x x y y+y z y z+z y z y$

$\left(\begin{array}{lllllllll}x x x x & x x y y & x x z z & x x y z & 0 & 0 & x x z y & 0 & 0 \\ x x y & x x x & x x z & -x x y & 0 & 0 & -x x z y & 0 & 0\end{array}\right)$ $\begin{array}{lllllllll}x x y y & x x x x & x x z z & -x x y z & 0 & 0 & -x x z y & 0 & 0\end{array}$ $\begin{array}{lllllllll}z z x x & z z x x & z z z z & 0 & 0 & 0 & 0 & 0 & 0\end{array}$ $\begin{array}{ccccccccc}y z x x & -y z x x & 0 & y z y z & 0 & 0 & z x z x & 0 & 0 \\ 0 & 0 & 0 & 0 & z x z x & y z x x & 0 & y z y z & y z x x \\ 0 & 0 & 0 & 0 & x x z y & x y x & 0 & x y z & x y x\end{array}$ $\left.\begin{array}{cccccccccc}0 & 0 & 0 & 0 & z x z x & y z x x & 0 & y z y z & y z x x \\ 0 & 0 & 0 & 0 & x x z y & x y x y & 0 & x x y z & x y x y\end{array}\right)$ and $x x x x=x x y y+2 x y x y$ 
$\mathrm{V}^{4}\left(\begin{array}{ccccccccc}\mathrm{xxxx} & \mathrm{xxyy} & \mathrm{xxzz} & 0 & 0 & 0 & 0 & 0 & 0 \\ \mathrm{yyxx} & \mathrm{xxxx} & \mathrm{xxzz} & 0 & 0 & 0 & 0 & 0 & 0 \\ \mathrm{zzxx} & \mathrm{zzyy} & \mathrm{zzzz} & 0 & 0 & 0 & 0 & 0 & 0 \\ 0 & 0 & 0 & \mathrm{yzyz} & 0 & 0 & \mathrm{yzzy} & 0 & 0 \\ 0 & 0 & 0 & 0 & \mathrm{zxzx} & 0 & 0 & \mathrm{zxxz} & 0 \\ 0 & 0 & 0 & 0 & 0 & \mathrm{xyxy} & 0 & 0 & \mathrm{xyyx} \\ 0 & 0 & 0 & \mathrm{zxxz} & 0 & 0 & \mathrm{zxzx} & 0 & 0 \\ 0 & 0 & 0 & 0 & \mathrm{yzzy} & 0 & 0 & \mathrm{yzyz} & 0 \\ 0 & 0 & 0 & 0 & 0 & \mathrm{xyyx} & 0 & 0 & \mathrm{xyxy}\end{array}\right)$

$\left[V^{4}\right] \quad\left(\begin{array}{ccccc}x x x x & 0 & 0 & y y z z & 0 \\ 0 & x x x x & 0 & y y z z & 0 \\ 0 & 0 & z z z z & x x y y & 0\end{array}\right)$

$V\left[V^{3}\right]\left(\begin{array}{cccccccccc}x x x x & 0 & 0 & x x y y & 0 & 0 & y y z z & 0 & 0 & 0 \\ 0 & x x x x & 0 & 0 & y z z & 0 & 0 & x x y y & 0 & 0 \\ 0 & 0 & z z z z & 0 & 0 & z z x & 0 & 0 & z z x & 0\end{array}\right)$ ] $\left(\begin{array}{cccccccccc}0 & \mathrm{xxxx} & 0 & 0 & \mathrm{yyzz} & 0 & 0 & \mathrm{xxyy} & 0 & 0 \\ 0 & 0 & \mathrm{zzzz} & 0 & 0 & \mathrm{zzxx} & 0 & 0 & \mathrm{zzxx} & 0\end{array}\right)$ $\left(\begin{array}{cccccccccc}x x x x & 0 & 0 & x x y y & 0 & 0 & y y z z & 0 & 0 & 0 \\ 0 & x x y x & 0 & 0 & y y z z & 0 & 0 & x x y y & 0 & 0 \\ 0 & 0 & z z z & 0 & 0 & z z x & 0 & 0 & z z x & 0\end{array}\right)$ $\left.\begin{array}{cccccccccc}0 & \mathrm{xxxx} & 0 & 0 & \mathrm{yyzz} & 0 & 0 & \mathrm{xxyy} & 0 & 0 \\ 0 & 0 & \mathrm{zzzz} & 0 & 0 & \mathrm{zzxx} & 0 & 0 & \mathrm{zzxx} & 0\end{array}\right)$

$\left[\left[^{2}\right]^{2}\right]\left(\begin{array}{cccccc}x x x x & x x y y & x x z z & 0 & 0 & 0 \\ & \text { yyyy } & y y z z & 0 & 0 & 0 \\ & & z z z z & 0 & 0 & 0 \\ & & & y z y z & 0 & 0 \\ & & & & \text { yzyz } & 0 \\ & & & & & \text { xyxy }\end{array}\right)$

$\left[V^{2}\right]^{2}\left(\begin{array}{cccccc}x x x x & x x y y & x x z z & 0 & 0 & 0 \\ y y x x & x x x x & x x z z & 0 & 0 & 0 \\ z z x x & z z x x & z z z z & 0 & 0 & 0 \\ 0 & 0 & 0 & y z y z & 0 & 0 \\ 0 & 0 & 0 & 0 & y z y z & 0 \\ 0 & 0 & 0 & 0 & 0 & x y x y\end{array}\right)$

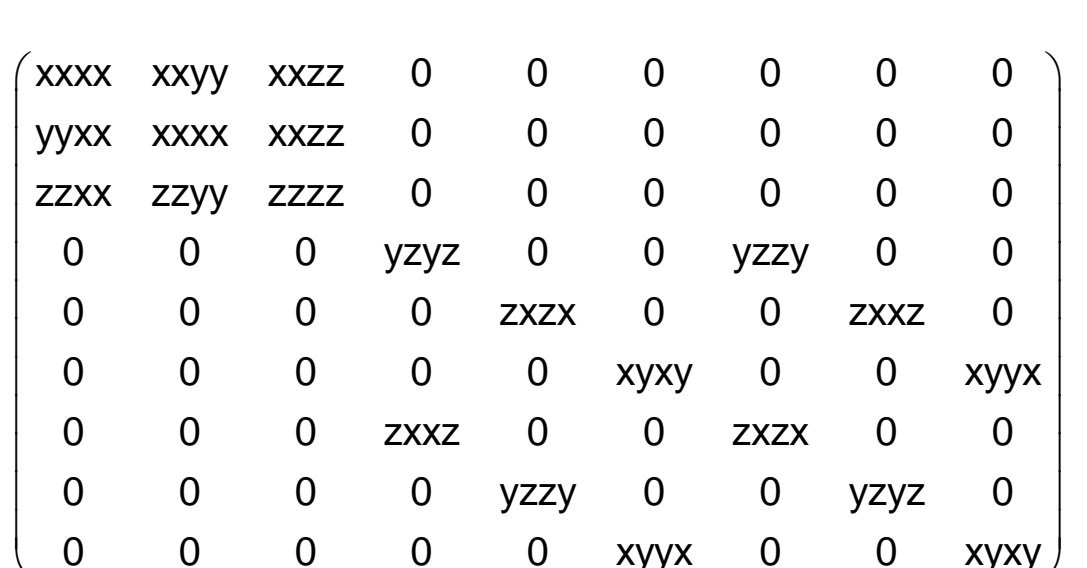

$\left(\begin{array}{ccccc}x x x x & 0 & 0 & y y z z & 0 \\ 0 & x x x x & 0 & y y z z & 0 \\ 0 & 0 & z z z z & x x y y & 0\end{array}\right)$

$\left(\begin{array}{cccccccccc}x x x x & 0 & 0 & x x y y & 0 & 0 & y y z z & 0 & 0 & 0\end{array}\right)$ $\left(\begin{array}{cccccccccc}0 & \mathrm{xxxx} & 0 & 0 & \mathrm{yyzz} & 0 & 0 & \mathrm{xxyy} & 0 & 0 \\ 0 & 0 & \mathrm{zzzz} & 0 & 0 & \mathrm{zzxx} & 0 & 0 & \mathrm{zzxx} & 0\end{array}\right)$

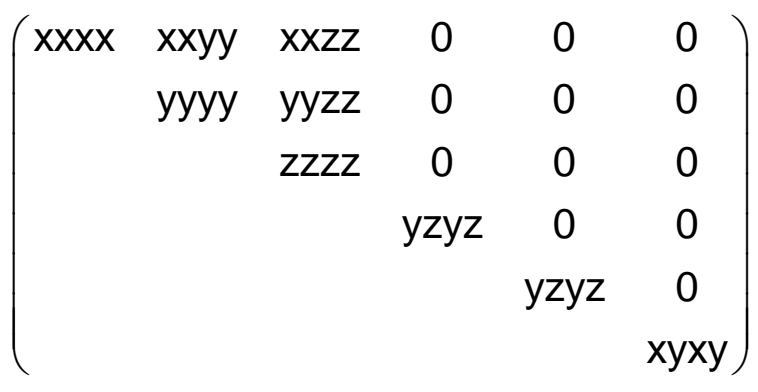

$\left(\begin{array}{llllll}x x x x & x x y y & x x z z & 0 & 0 & 0 \\ y y x x & x x x x & x x z z & 0 & 0 & 0\end{array}\right)$ $\begin{array}{llllll}x y x x & x x x x & x x z z & 0 & 0 & 0\end{array}$ $\begin{array}{cccccc}z z x x & z z x x & z z z z & 0 & 0 & 0 \\ 0 & 0 & 0 & y z z z & 0 & 0\end{array}$ $\begin{array}{cccccc}0 & 0 & 0 & \text { yzyz } & 0 & 0 \\ 0 & 0 & 0 & 0 & \text { yzyz } & 0 \\ 0 & 0 & 0 & 0 & 0 & x y y\end{array}$

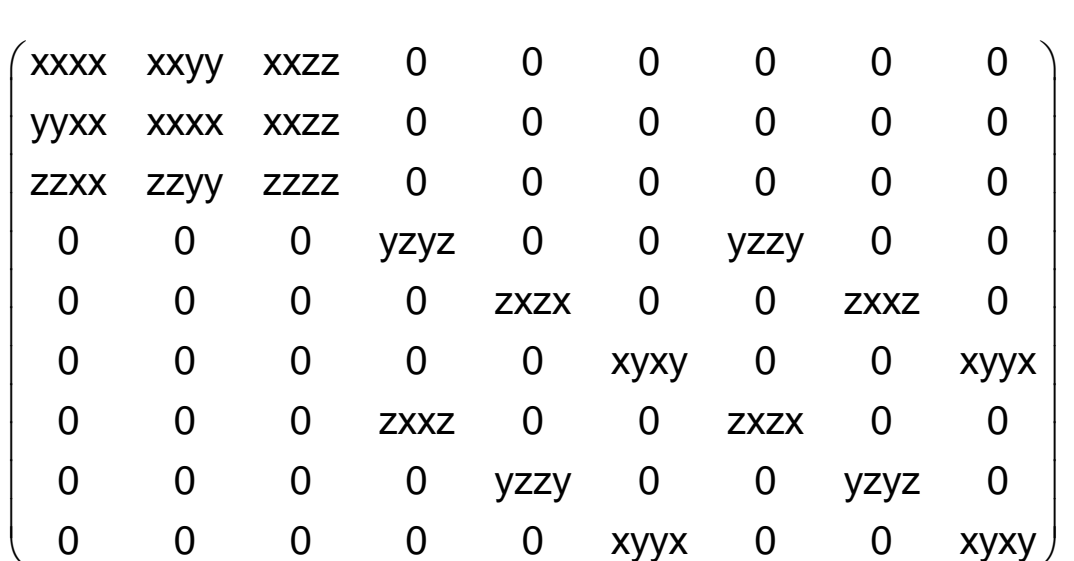

$\left(\begin{array}{lllllllll}x x x x & x x y y & x x z z & 0 & 0 & 0 & 0 & 0 & 0 \\ y x x & x x y x & x z z z & 0 & 0 & 0 & 0 & 0 & 0\end{array}\right)$ $\left.\begin{array}{lllllllll}x y x x & x x x x & x x z z & 0 & 0 & 0 & 0 & 0 & 0 \\ z z x x & z z y y & z z z z & 0 & 0 & 0 & 0 & 0 & 0\end{array}\right)$ $\begin{array}{ccccccccc}\text { zzxx } & \text { zzyy } & \text { zzzz } & 0 & 0 & 0 & 0 & 0 & 0 \\ 0 & 0 & 0 & y z y z & 0 & 0 & y z z y & 0 & 0 \\ 0 & 0 & 0 & 0 & z x z x & 0 & 0 & z x x z & 0\end{array}$ $\begin{array}{ccccccccc}0 & 0 & 0 & y z y z & 0 & 0 & y z z y & 0 & 0 \\ 0 & 0 & 0 & 0 & z x z x & 0 & 0 & z x x z & 0 \\ 0 & 0 & 0 & 0 & 0 & x y x & 0 & 0 & x y x\end{array}$ $\begin{array}{ccccccccc}0 & 0 & 0 & 0 & z x z x & 0 & 0 & z x x z & 0 \\ 0 & 0 & 0 & 0 & 0 & x y x y & 0 & 0 & x y y x \\ 0 & 0 & 0 & z x z & 0 & 0 & z x z & 0 & 0\end{array} \mid$ $\begin{array}{ccccccccc}0 & 0 & 0 & z x x z & 0 & 0 & z x z x & 0 & 0\end{array}$ $\begin{array}{ccccccccc}0 & 0 & 0 & 0 & y z z y & 0 & 0 & y z y z & 0 \\ 0 & 0 & 0 & 0 & 0 & x y x & 0 & 0 & x y y\end{array}$ $\left(\begin{array}{ccccc}x x x x & 0 & 0 & y y z z & 0 \\ 0 & x x x x & 0 & y y z z & 0 \\ 0 & 0 & z z z z & x x y & 0\end{array}\right)$ $\left(\begin{array}{ccccc}\mathrm{xxxx} & 0 & 0 & \mathrm{yyzz} & 0 \\ 0 & \mathrm{xxxx} & 0 & \mathrm{yyzz} & 0 \\ 0 & 0 & \mathrm{zzzz} & \mathrm{xxyy} & 0\end{array}\right)$

$\left(\begin{array}{llllllllll}x x x x & 0 & 0 & x x y y & 0 & 0 & y y z z & 0 & 0 & 0\end{array}\right)$ $\begin{array}{cccccccccc}0 & \mathrm{xxxx} & 0 & 0 & \mathrm{yyzz} & 0 & 0 & \mathrm{xxyy} & 0 & 0 \\ 0 & 0 & \mathrm{zzzz} & 0 & 0 & \mathrm{zzxx} & 0 & 0 & \mathrm{zzxx} & 0\end{array}$

$$
\left(\begin{array}{cccccc}
\text { xxxx } & \text { xxyy } & \text { xxzz } & 0 & 0 & 0 \\
& \text { yyyy } & \text { yyzz } & 0 & 0 & 0 \\
& & \text { zzzz } & 0 & 0 & 0 \\
& & & y z y z & 0 & 0 \\
& & & & \text { yzyz } & 0 \\
& & & & & \text { xyxy }
\end{array}\right)
$$

$\left(\begin{array}{llllll}x x x x & x x y y & x x z z & 0 & 0 & 0\end{array}\right.$ $\left(\begin{array}{cccccc}x x x x & x x y y & x x z z & 0 & 0 & 0 \\ y y x x & x x x x & x x z z & 0 & 0 & 0 \\ z z x x & z z x x & z z z z & 0 & 0 & 0 \\ 0 & 0 & 0 & y z y z & 0 & 0 \\ 0 & 0 & 0 & 0 & y z y z & 0 \\ 0 & 0 & 0 & 0 & 0 & x y x y\end{array}\right)$

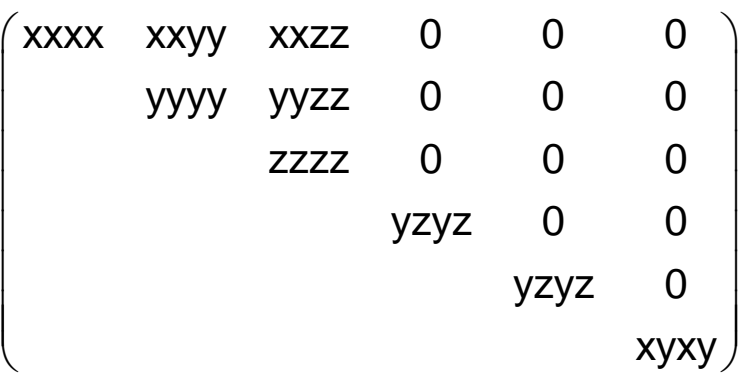

$\left(\begin{array}{llllll}x x x x & x x y y & x x z z & 0 & 0 & 0\end{array}\right)$ $\begin{array}{llllll}y y x x & x x x x & x x z z & 0 & 0 & 0\end{array}$ $\begin{array}{llllll}z z x x & z z x x & z z z z & 0 & 0 & 0 \\ 0 & 0 & 0 & y z y z & 0 & 0\end{array}$ $\begin{array}{cccccc}0 & 0 & 0 & y z y z & 0 & 0 \\ 0 & 0 & 0 & 0 & y z y z & 0\end{array}$ $\begin{array}{cccccc}0 & 0 & 0 & 0 & y z y z & 0 \\ 0 & 0 & 0 & 0 & 0 & x y x y\end{array}$ 
$\mathrm{n}=4 \quad \mathrm{D}_{4}=\mathbf{4}_{\mathbf{z}} \mathbf{2}_{\mathbf{x}} \mathbf{2}_{\mathrm{xy}}$

$\left[\left(\mathrm{V}^{2}\right)\right]^{2}$

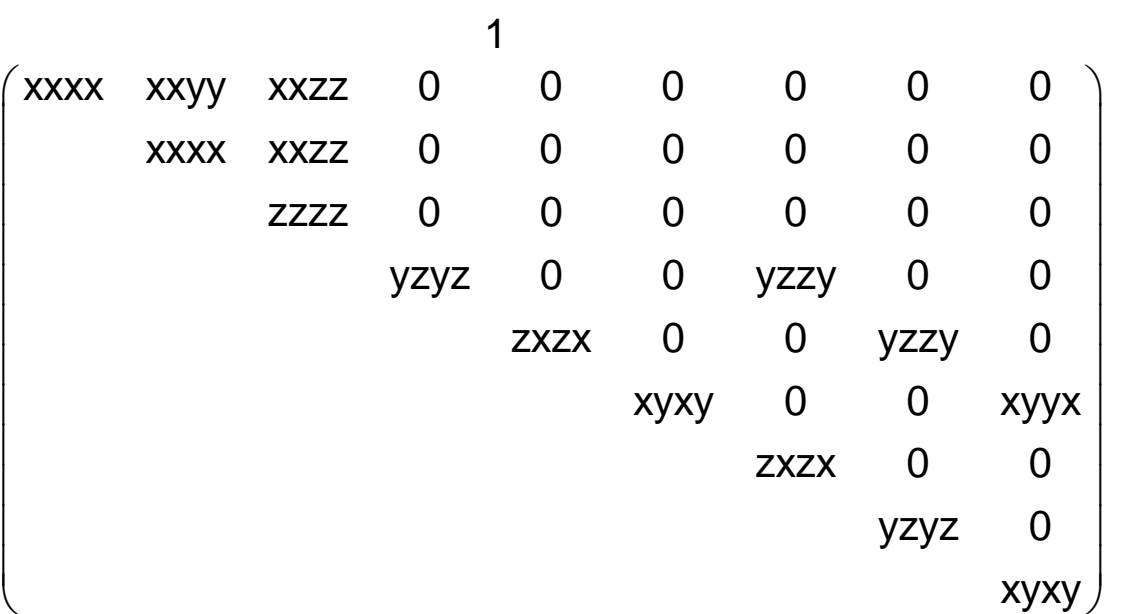

$\left(\begin{array}{lllllllll}x x x x & x x y y & x x z z & 0 & 0 & 0 & 0 & 0 & 0 \\ \text { Wxx } & x y x & x x z z & 0 & 0 & 0 & 0 & 0 & 0\end{array}\right)$ $\begin{array}{lllllllll}y y x x & x x x x & x x z z & 0 & 0 & 0 & 0 & 0 & 0\end{array}$ $\left[\mathrm{V}^{2}\right] \mathrm{V}^{2} \quad \begin{array}{cccccccccc} & \mathrm{zzxx} & \mathrm{zzxx} & \mathrm{zzzz} & 0 & 0 & 0 & 0 & 0 & 0 \\ 0 & 0 & 0 & y z y z & 0 & 0 & z x z x & 0 & 0\end{array}$ $\left(\begin{array}{ccccccccc}0 & 0 & 0 & y z y z & 0 & 0 & z x z x & 0 & 0 \\ 0 & 0 & 0 & 0 & z x z x & 0 & 0 & y z y z & 0 \\ 0 & 0 & 0 & 0 & 0 & x y x & 0 & 0 & x y y\end{array}\right)$

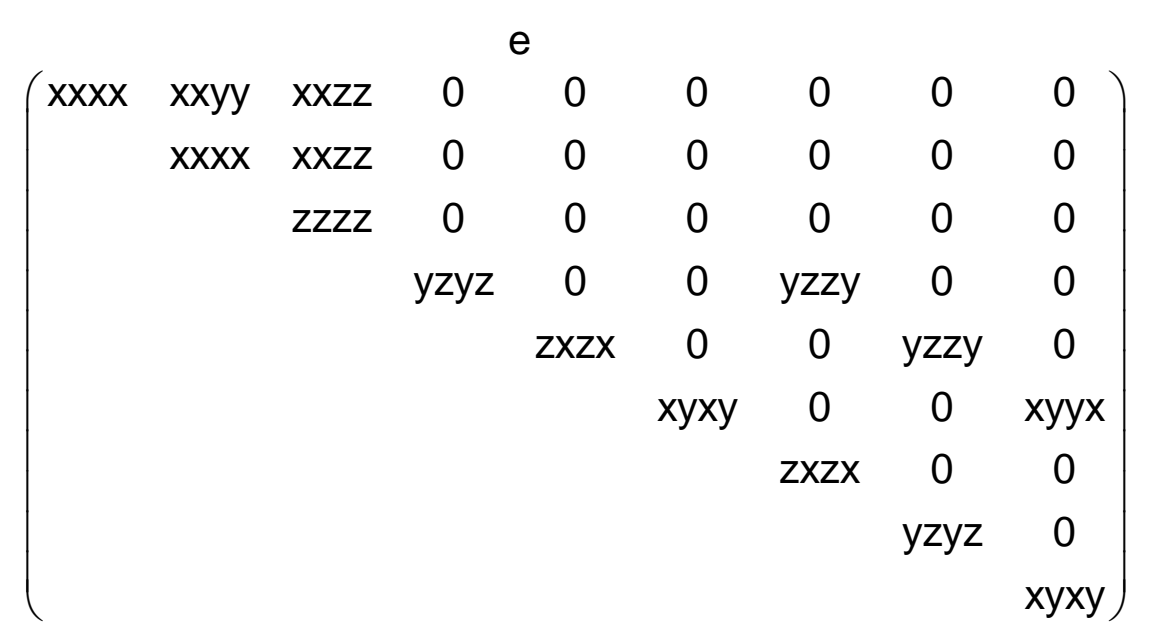

$\left(\begin{array}{ccccccccc}\text { xxxx } & \text { xxyy } & \text { xxzz } & 0 & 0 & 0 & 0 & 0 & 0 \\ \text { yyxx } & \text { xxxx } & \text { xxzz } & 0 & 0 & 0 & 0 & 0 & 0 \\ \text { zzxx } & \text { zzxx } & \text { zzzz } & 0 & 0 & 0 & 0 & 0 & 0 \\ 0 & 0 & 0 & \text { yzyz } & 0 & 0 & \text { zxzx } & 0 & 0 \\ 0 & 0 & 0 & 0 & \text { zxzx } & 0 & 0 & \text { yzyz } & 0 \\ 0 & 0 & 0 & 0 & 0 & \text { xyxy } & 0 & 0 & \text { xyxy }\end{array}\right)$
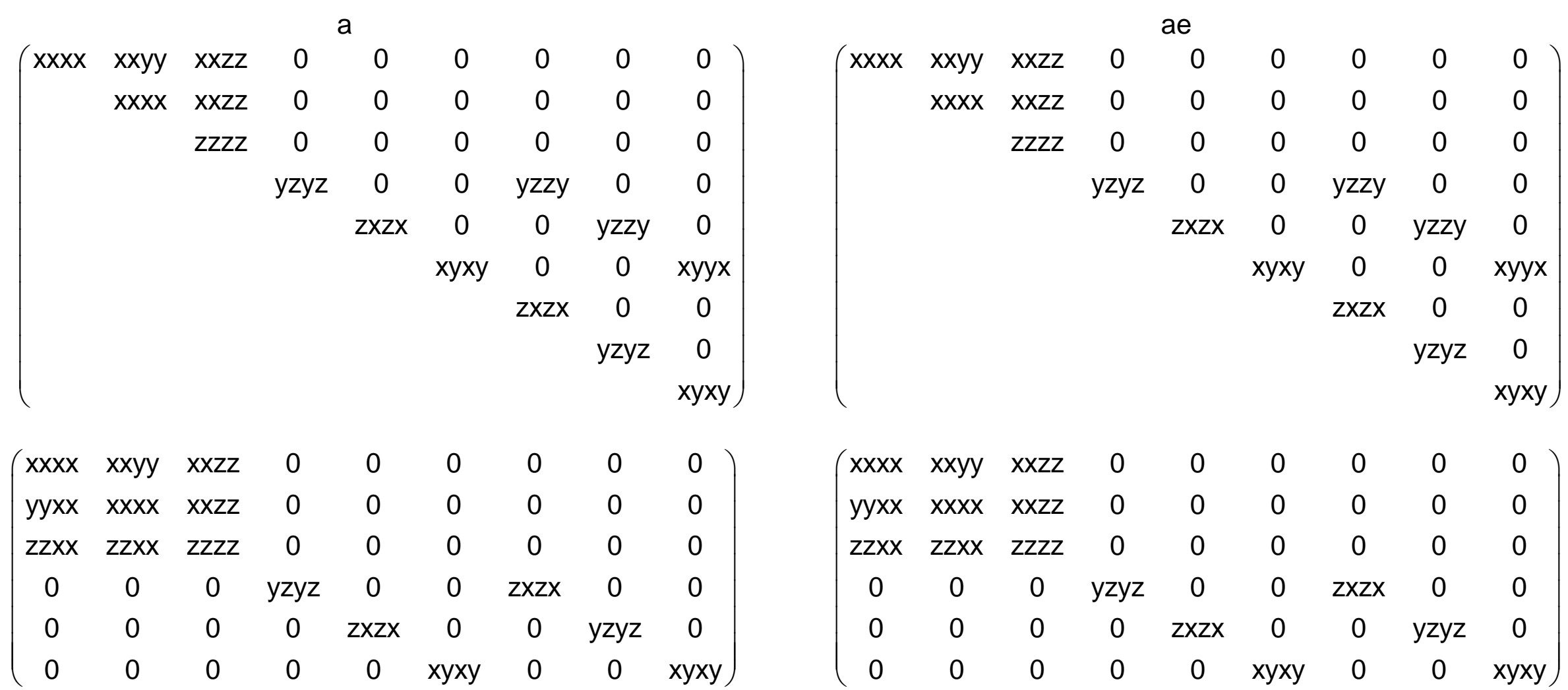
$\mathrm{V}^{4}\left(\begin{array}{ccccccccc}\mathrm{xxxx} & \mathrm{xxyy} & \mathrm{xxzz} & 0 & 0 & 0 & 0 & 0 & 0 \\ \mathrm{yyxx} & \mathrm{xxxx} & \mathrm{xxzz} & 0 & 0 & 0 & 0 & 0 & 0 \\ \mathrm{zzxx} & \mathrm{zzyy} & \mathrm{zzzz} & 0 & 0 & 0 & 0 & 0 & 0 \\ 0 & 0 & 0 & \mathrm{yzyz} & 0 & 0 & \mathrm{yzzy} & 0 & 0 \\ 0 & 0 & 0 & 0 & \mathrm{zxzx} & 0 & 0 & \mathrm{zxxz} & 0 \\ 0 & 0 & 0 & 0 & 0 & \mathrm{xyxy} & 0 & 0 & \mathrm{xyyx} \\ 0 & 0 & 0 & \mathrm{zxxz} & 0 & 0 & \mathrm{zxzx} & 0 & 0 \\ 0 & 0 & 0 & 0 & \mathrm{yzzy} & 0 & 0 & \mathrm{yzyz} & 0 \\ 0 & 0 & 0 & 0 & 0 & \mathrm{xyyy} & 0 & 0 & \mathrm{xyxy}\end{array}\right)$

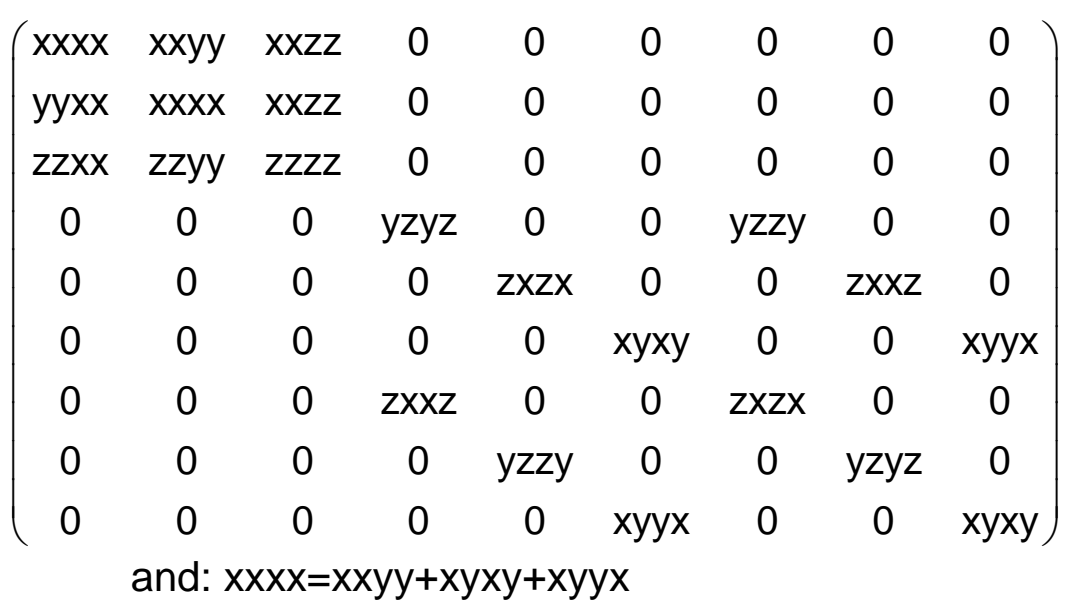

[V $\left.V^{4}\right]\left(\begin{array}{ccccc}3 x x y y & 0 & 0 & y y z z & 0 \\ 0 & 3 x x y y & 0 & y y z z & 0 \\ 0 & 0 & z z z z & x x y y & 0\end{array}\right)$

$\left.V_{[} V^{3}\right]\left(\begin{array}{cccccccccc}3 x x y y & 0 & 0 & x x y y & 0 & 0 & y y z z & 0 & 0 & 0 \\ 0 & 3 x x y y & 0 & 0 & y y z z & 0 & 0 & x x y y & 0 & 0 \\ 0 & 0 & 2 z z & 0 & 0 & z z x & 0 & 0 & z z x & 0\end{array}\right)$

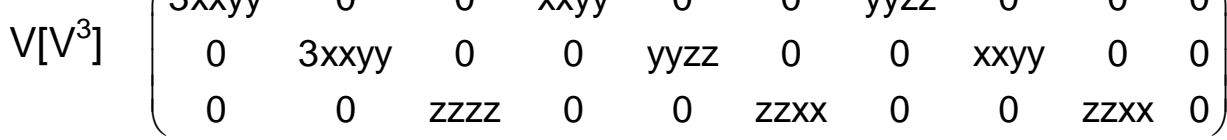

$\left.\left[\mathrm{V}^{2}\right]^{2}\right]\left(\begin{array}{cccccc}\mathrm{x} x \mathrm{x} & \mathrm{xxyy} & \mathrm{xxzz} & 0 & 0 & 0 \\ & \mathrm{xxxx} & \mathrm{xxzz} & 0 & 0 & 0 \\ & & \mathrm{zzzz} & 0 & 0 & 0 \\ & & & \mathrm{yzyz} & 0 & 0 \\ & & & & \mathrm{yzyz} & 0 \\ & & & & & \mathrm{xyxy}\end{array}\right)$

and $x y x y=1 / 2(x x x x-x x y y)$

$\left[V^{2}\right]^{2}\left(\begin{array}{cccccc}x x x x & x x y y & x x z z & 0 & 0 & 0 \\ x x y y & x x x x & x x z z & 0 & 0 & 0 \\ z z x x & z z x x & z z z z & 0 & 0 & 0 \\ 0 & 0 & 0 & y z y z & 0 & 0 \\ 0 & 0 & 0 & 0 & y z y z & 0 \\ 0 & 0 & 0 & 0 & 0 & x y x y\end{array}\right)$

and $x y x y=1 / 2(x x x x-x x y y)$

$\left(\begin{array}{ccccc}3 x x y y & 0 & 0 & y y z z & 0 \\ 0 & 3 x x y y & 0 & y y z z & 0 \\ 0 & 0 & z z z z & x x y y & 0\end{array}\right)$

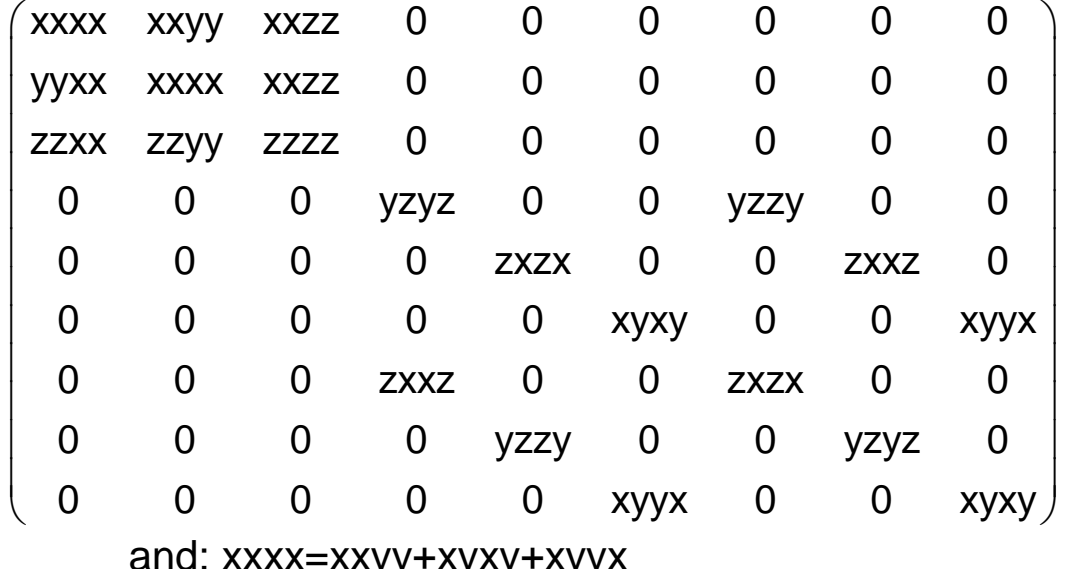

$\left(\begin{array}{lllllllll}x x x x & x x y y & x x z z & 0 & 0 & 0 & 0 & 0 & 0 \\ \text { wyx } & x y x & x z z & 0 & 0 & 0 & 0 & 0 & 0\end{array}\right)$ $\left.\begin{array}{lllllllll}\mathrm{yyxx} & \mathrm{xxxx} & \mathrm{xxzz} & 0 & 0 & 0 & 0 & 0 & 0 \\ z z x \mathrm{x} & z z y y & z z z z & 0 & 0 & 0 & 0 & 0 & 0\end{array}\right)$ $\begin{array}{lllllllll}0 & 0 & 0 & y z y z & 0 & 0 & y z z y & 0 & 0\end{array}$ $\begin{array}{ccccccccc}0 & 0 & 0 & 0 & z x z x & 0 & 0 & z x x z & 0\end{array}$ $\begin{array}{lllllllll}0 & 0 & 0 & 0 & z x z x & 0 & 0 & z x y & 0 \\ 0 & 0 & 0 & 0 & 0 & x y x y & 0 & 0 & x\end{array}$

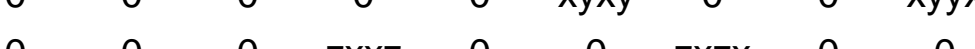
$\begin{array}{ccccccccc}0 & 0 & 0 & z x x z & 0 & 0 & z x z x & 0 & 0 \\ 0 & 0 & 0 & 0 & y z z y & 0 & 0 & y z y z & 0\end{array}$ $\begin{array}{ccccccccc}0 & 0 & 0 & 0 & y z z y & 0 & 0 & y z y z & 0 \\ 0 & 0 & 0 & 0 & 0 & x y y x & 0 & 0 & x y x y\end{array}$

$\left(\begin{array}{cccccccccc}3 x x y y & 0 & 0 & x x y y & 0 & 0 & y y z z & 0 & 0 & 0 \\ 0 & 3 x x y y & 0 & 0 & y y z z & 0 & 0 & x x y y & 0 & 0 \\ 0 & 0 & z z z & 0 & 0 & z z x & 0 & 0 & z z x & 0\end{array}\right)$ $\left(\begin{array}{cccccccccc}0 & 3 x x y y & 0 & 0 & y y z z & 0 & 0 & x x y y & 0 & 0 \\ 0 & 0 & z z z z & 0 & 0 & z z x x & 0 & 0 & z z x x & 0\end{array}\right)$

$\left(\begin{array}{ccccc}3 x x y y & 0 & 0 & y y z z & 0 \\ 0 & 3 x x y y & 0 & y y z z & 0 \\ 0 & 0 & z z z z & x x y y & 0\end{array}\right)$

$\left(\begin{array}{cccccccccc}3 x x y y & 0 & 0 & x x y y & 0 & 0 & y y z z & 0 & 0 & 0 \\ 0 & 3 x x y y & 0 & 0 & y y z z & 0 & 0 & x x y y & 0 & 0 \\ 0 & 0 & z z z & 0 & 0 & z z x & 0 & 0 & z z x & 0\end{array}\right)$ $\left.\begin{array}{cccccccccc}0 & 3 x x y y & 0 & 0 & y y z z & 0 & 0 & \text { xxyy } & 0 & 0 \\ 0 & 0 & z z z z & 0 & 0 & z z x x & 0 & 0 & z z x x & 0\end{array}\right)$

$$
\left(\begin{array}{cccccc}
x x x x & x x y y & x x z z & 0 & 0 & 0 \\
& x x x x & x x z z & 0 & 0 & 0 \\
& & z z z z & 0 & 0 & 0 \\
& & & y z y z & 0 & 0 \\
& & & & y z y z & 0 \\
& & & & & x y x y
\end{array}\right)
$$

and $x y x y=1 / 2(x x x x-x x y y)$

$\left(\begin{array}{cccccc}\text { xxxx } & x x y y & x x z z & 0 & 0 & 0 \\ \text { xxyy } & x x x x & x x z z & 0 & 0 & 0 \\ \text { zzxx } & \text { zzxx } & \text { zzzz } & 0 & 0 & 0 \\ 0 & 0 & 0 & y z y z & 0 & 0 \\ 0 & 0 & 0 & 0 & y z y z & 0 \\ 0 & 0 & 0 & 0 & 0 & x y x y\end{array}\right)$

and $x y x y=1 / 2(x x x x-x x y y)$ $\left(\begin{array}{ccccc}3 x x y y & 0 & 0 & y y z z & 0 \\ 0 & 3 x x y y & 0 & y y z z & 0 \\ 0 & 0 & z z z z & x x y y & 0\end{array}\right)$

$\left(\begin{array}{cccccccccc}3 x x y y & 0 & 0 & x x y y & 0 & 0 & y y z z & 0 & 0 & 0 \\ 0 & 3 x x y y & 0 & 0 & y y z z & 0 & 0 & x x y y & 0 & 0 \\ 0 & 0 & 2 z z z & 0 & 0 & z z x & 0 & 0 & z z x & 0\end{array}\right)$ $\left.\begin{array}{cccccccccc}0 & 3 x x y y & 0 & 0 & y y z z & 0 & 0 & x x y y & 0 & 0 \\ 0 & 0 & z z z z & 0 & 0 & z z x x & 0 & 0 & z z x x & 0\end{array}\right)$

$\left(\begin{array}{cccccc}x x x x & x x y y & x x z z & 0 & 0 & 0 \\ & x x x x & x x z z & 0 & 0 & 0 \\ & & z z z z & 0 & 0 & 0 \\ & & & y z y z & 0 & 0 \\ & & & & y z y z & 0 \\ & & & & & x y x y\end{array}\right)$

and $x y x y=1 / 2(x x x x-x x y y)$

$\left(\begin{array}{cccccc}x x x x & x x y y & x x z z & 0 & 0 & 0 \\ x x y y & x x x x & x x z z & 0 & 0 & 0 \\ z z x x & z z x x & z z z z & 0 & 0 & 0 \\ 0 & 0 & 0 & y z y z & 0 & 0 \\ 0 & 0 & 0 & 0 & y z y z & 0 \\ 0 & 0 & 0 & 0 & 0 & x y x y\end{array}\right)$

and $x y x y=1 / 2(x x x x-x x y y)$ $\left(\begin{array}{lllll}x x x x & x x y y & x x z z & 0 & 0\end{array}\right.$ $\left.\begin{array}{llllll}x x y y & x x x x & x x z z & 0 & 0 & 0 \\ z x x & z z x & z z z & 0 & 0 & 0\end{array}\right)$ $\begin{array}{lllllll}z z x x & z z x x & z z z z & 0 & 0 & 0 \\ 0 & 0 & 0 & y z y z & 0 & 0\end{array}$ $\begin{array}{cccccc}0 & 0 & 0 & y z y z & 0 & 0 \\ 0 & 0 & 0 & 0 & y z y z & 0 \\ 0 & 0 & 0 & 0 & 0 & x y y\end{array}$

and $x y x y=1 / 2(x x x x-x x y y)$ 
$n>4 \quad \mathbf{D}_{x}=\infty 2$

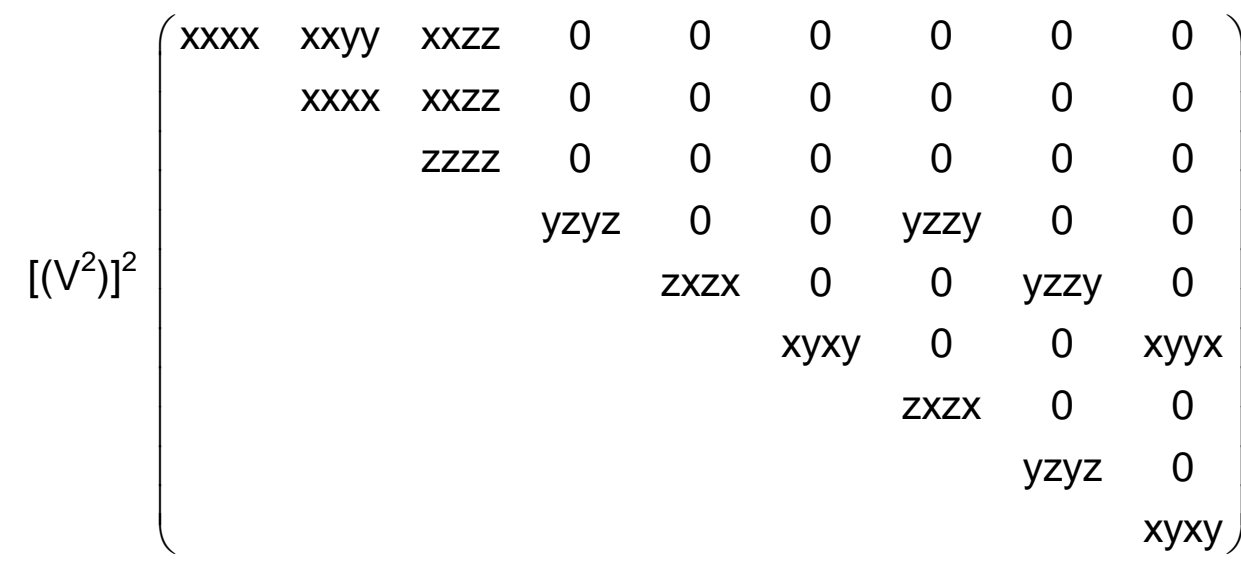

and $x x x x=x x y y+y z y z+z y z y$

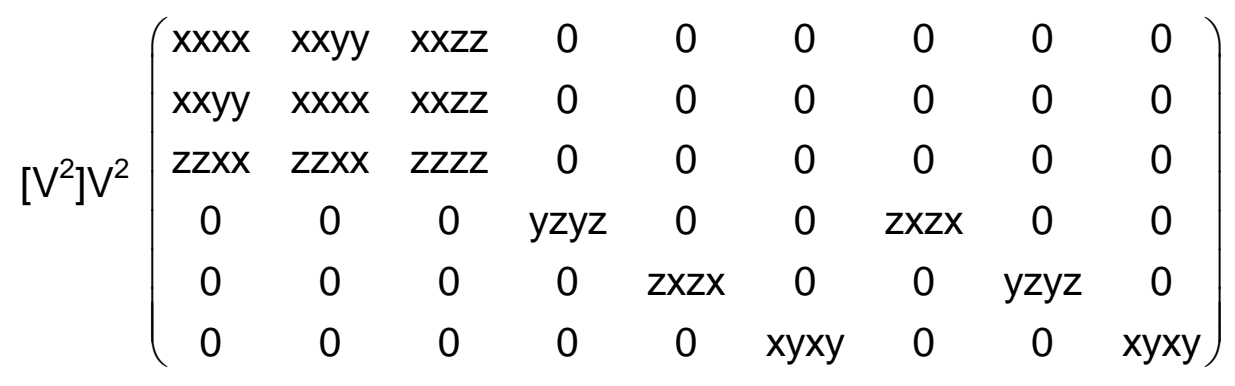

and $x x x x=x x y y+2 x y x y$ $\begin{array}{lllllllll}x x x x & x x y y & x x z z & 0 & 0 & 0 & 0 & 0 & 0\end{array}$ $\left.\begin{array}{llllllll}x x x x & x x z z & 0 & 0 & 0 & 0 & 0 & 0\end{array}\right)$ $\begin{array}{lllllll}z z z z & 0 & 0 & 0 & 0 & 0 & 0\end{array}$ $\begin{array}{llllll}\text { yzyz } & 0 & 0 & \text { yzzy } & 0 & 0\end{array}$ $\begin{array}{lllll}z x z x & 0 & 0 & y z z y & 0\end{array}$ $\begin{array}{cccc}x y x y & 0 & 0 & x y y x\end{array}$ $\begin{array}{ccc}0 & 0 \\ y z y z & 0\end{array}$ and $x x x x=x x y y+y z y z+z y z y$

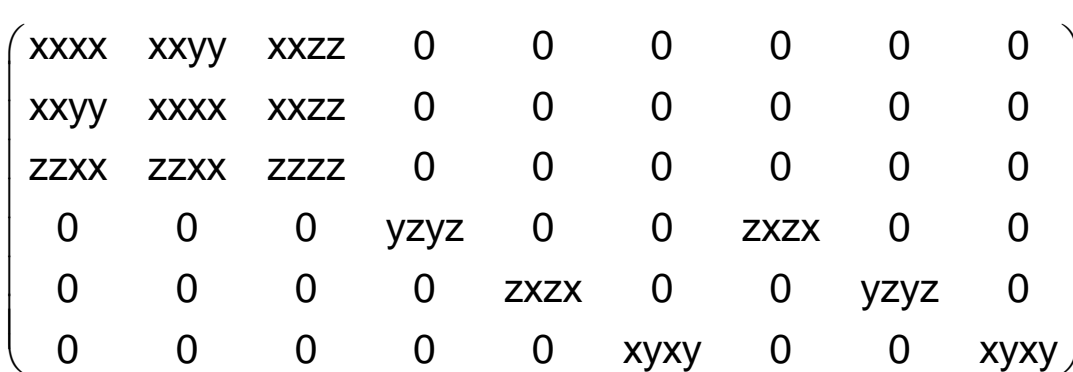

and $x x x x=x x y y+2 x y x y$
$D_{n}$

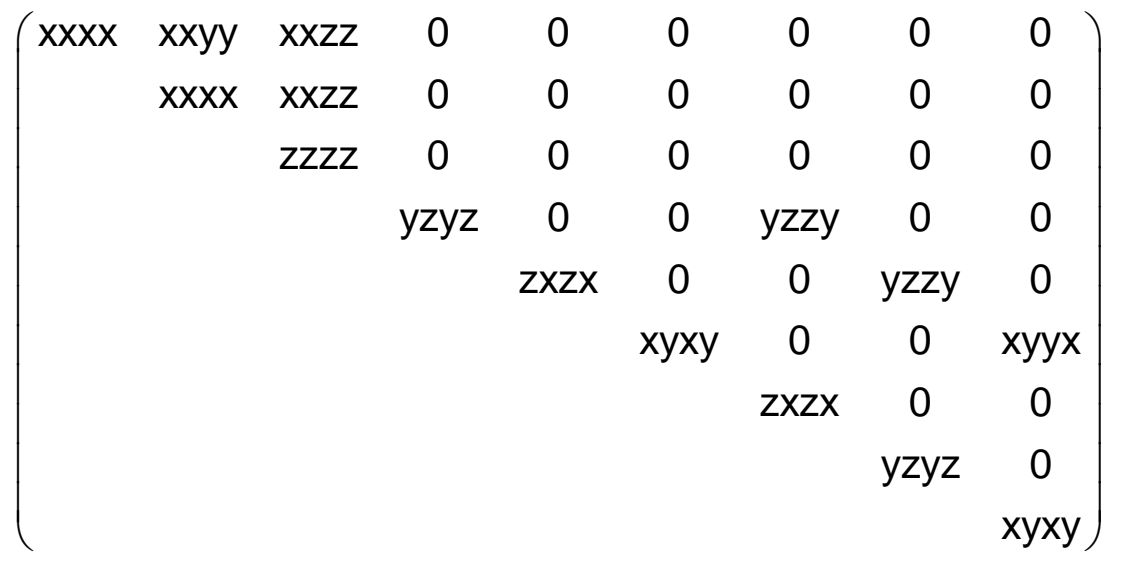

and $x x x x=x x y y+y z y z+z y z y$ $\begin{array}{lllllllll}x x y y & x x x x & x x z z & 0 & 0 & 0 & 0 & 0 & 0\end{array}$ $\begin{array}{llllllllll}z z x x & z z x x & z z z z & 0 & 0 & 0 & 0 & 0 & 0\end{array}$ $\left.\begin{array}{ccccccccc}0 & 0 & 0 & y z y z & 0 & 0 & z x z x & 0 & 0 \\ 0 & 0 & 0 & 0 & z x z x & 0 & 0 & y z y z & 0 \\ 0 & 0 & 0 & 0 & 0 & x y y & 0 & 0 & x y y\end{array}\right)$ $\begin{array}{ccccccccc}0 & 0 & 0 & 0 & \operatorname{zxzx} & 0 & 0 & \mathrm{yzyz} & 0 \\ 0 & 0 & 0 & 0 & 0 & \mathrm{xyxy} & 0 & 0 & \mathrm{xyxy}\end{array}$ and $x x x x=x x y y+2 x y x y$

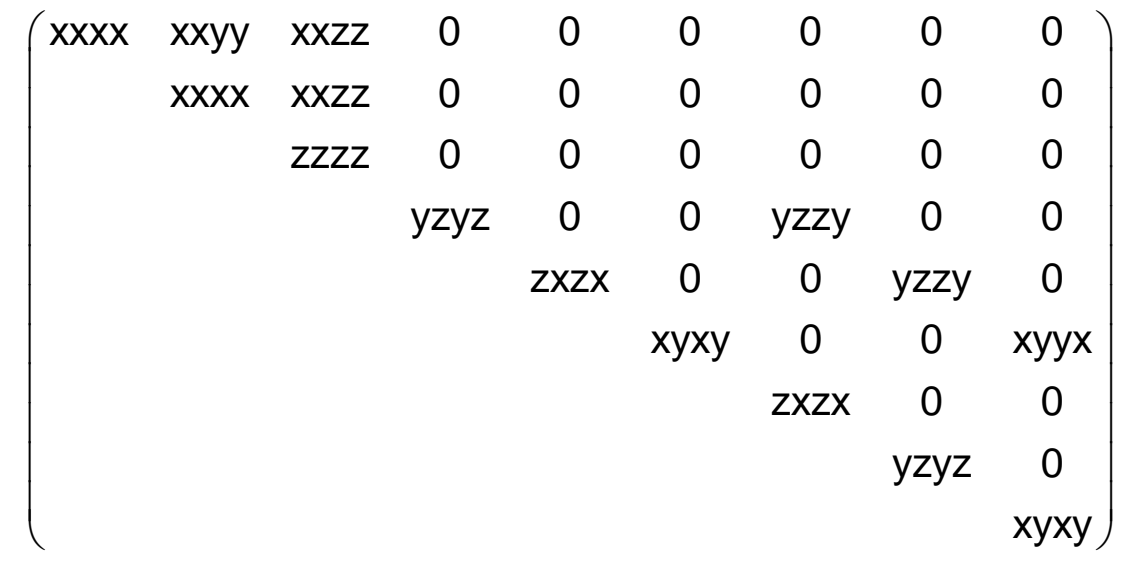

and $x x x x=x x y y+y z y z+z y z y$ $\left(\begin{array}{ccccccccc}\text { xxxx } & \text { xxyy } & \text { xxzz } & 0 & 0 & 0 & 0 & 0 & 0 \\ \text { xxyy } & \text { xxxx } & \text { xxzz } & 0 & 0 & 0 & 0 & 0 & 0 \\ \text { zzxx } & \text { zzxx } & \text { zzzz } & 0 & 0 & 0 & 0 & 0 & 0 \\ 0 & 0 & 0 & \text { yzyz } & 0 & 0 & z x z x & 0 & 0 \\ 0 & 0 & 0 & 0 & z x z x & 0 & 0 & \text { yzyz } & 0 \\ 0 & 0 & 0 & 0 & 0 & \text { xyxy } & 0 & 0 & \text { xyxy }\end{array}\right)$ and $x x x x=x x y y+2 x y x y$ 
1

$\left(\begin{array}{lllllllll}x x x x & x x y y & x x z z & 0 & x x z x & 0 & 0 & x x x z & 0 \\ x y x x & y y y & y y z z & 0 & y z x & 0 & 0 & y x z & 0\end{array}\right)$ $\left.\begin{array}{lllllllll}y y x x & y y y y & y y z z & 0 & y y z x & 0 & 0 & y y x z & 0\end{array}\right)$ $\begin{array}{ccccccccc}\text { zzxx } & \text { zzyy } & \text { zzzz } & 0 & \text { zzzx } & 0 & 0 & z z x z & 0 \\ 0 & 0 & 0 & y z y z & 0 & y z y & y z z y & 0 & y z y x\end{array}$ $\begin{array}{lllllllll}0 & 0 & 0 & y z y z & 0 & y z x y & y z z y & 0 & y z y x\end{array}$ $\begin{array}{lllllllll}0 & 0 & 0 & 0 & z x z x & 0 & 0 & z x x z & 0\end{array}$ $\begin{array}{cccccccccc}0 & 0 & 0 & z y y z & 0 & z y x y & z y z y & 0 & z y y x \\ x z z x & x z y & x z z & 0 & x z z x & 0 & 0 & x z x z & 0\end{array}$

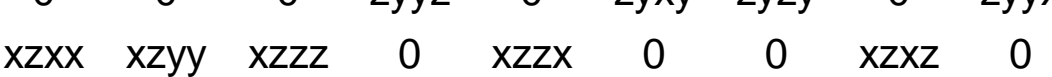
$\begin{array}{ccccccccc}x z x x & x z y y & x z z z & 0 & x z z x & 0 & 0 & x z x z & 0 \\ 0 & 0 & 0 & y x y z & 0 & y x x y & y x z y & 0 & y x y x\end{array}$

$\left[V^{4}\right]\left(\begin{array}{ccccc}\mathrm{xxxx} & 0 & \mathrm{xzzz} & \mathrm{yyzz} & 0 \\ 0 & \mathrm{yyyy} & 0 & \mathrm{zzxx} & \mathrm{yyxz} \\ \mathrm{zxxx} & 0 & \mathrm{zzzz} & \mathrm{xxyy} & 0\end{array}\right)$

$\left(\begin{array}{lllllllll}0 & 0 & 0 & 0 & 0 & 0 & 0 & 0 & 0\end{array}\right.$ $\begin{array}{lllllllll}0 & 0 & 0 & 0 & 0 & 0 & 0 & 0 & 0 \\ 0 & 0 & 0 & 0 & 0 & 0 & 0 & 0 & 0\end{array}$ $0 \begin{array}{lllllllll}0 & 0 & 0 & 0 & 0 & 0 & 0 & 0 & 0 \\ 0 & 0 & 0 & 0 & 0 & 0 & 0 & 0 & 0\end{array}$ $0 \begin{array}{llllllllll}0 & 0 & 0 & 0 & 0 & 0 & 0 & 0 & 0 \\ 0 & 0 & 0 & 0 & 0 & 0 & 0 & 0 & 0\end{array}$ $0 \begin{array}{lllllllll}0 & 0 & 0 & 0 & 0 & 0 & 0 & 0 & 0 \\ 0 & 0 & 0 & 0 & 0 & 0 & 0 & 0 & 0\end{array}$ $0 \begin{array}{lllllllll}0 & 0 & 0 & 0 & 0 & 0 & 0 & 0 & 0 \\ 0 & 0 & 0 & 0 & 0 & 0 & 0\end{array}$

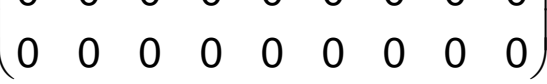

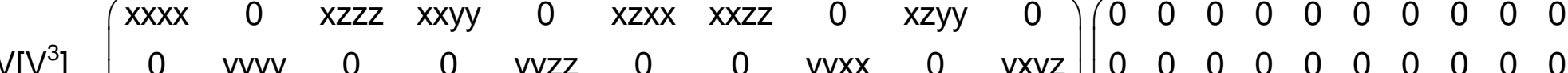

$\left(\begin{array}{ccccccccccc}z x x x & 0 & \text { zzzz } & \text { zxyy } & 0 & \text { zzxx } & \text { zxzz } & 0 & \text { zzyy } & 0\end{array}\right)\left(\begin{array}{lllllllllll}0 & 0 & 0 & 0 & 0 & 0 & 0 & 0 & 0 & 0\end{array}\right)$
$\left.\left[V^{2}\right]^{2}\right]$ $\left(\begin{array}{cccccc}x x x x & x x y y & x x z z & 0 & x x x z & 0 \\ & \text { yyyy } & y y z z & 0 & y y x z & 0 \\ & & z z z z & 0 & z z x z & 0 \\ & & & y z y z & 0 & y z x y \\ & & & & x z x z & 0 \\ & & & & & x y x y\end{array}\right)$

$\left.\begin{array}{llllll}x x x x & x x y y & x x z z & 0 & x x x z & 0 \\ y y x x & y y y & y z z & 0 & y x z & 0\end{array}\right)$ $\left.\begin{array}{cccccc}\mathrm{yyxx} & y y y y & y y z z & 0 & y y x z & 0 \\ z z x x & z z y y & z z z z & 0 & z z x z & 0\end{array}\right)$ $\begin{array}{lllllll}x z x x & x z y y & x z z z & 0 & x z z z & 0\end{array}$ $\begin{array}{llllll}\text { xyxx } & \text { xyyy } & x y z z & x y y z & 0 & x y x y\end{array}$

$$
\left(\begin{array}{lllll}
0 & 0 & 0 & 0 & 0 \\
0 & 0 & 0 & 0 & 0 \\
0 & 0 & 0 & 0 & 0
\end{array}\right)
$$

$\begin{array}{llllll}0 & 0 & 0 & 0 & 0 & 0 \\ 0 & 0 & 0 & 0 & 0 & 0\end{array}$ $\left.\begin{array}{lllllllll}x x x x & x x y y & x x z z & 0 & x x z x & 0 & 0 & x x x z & 0 \\ y x x & y y y & y z z & 0 & y y z x & 0 & 0 & y y z z & 0\end{array}\right)\left(\begin{array}{lllllllll}0 & 0 & 0 & 0 & 0 & 0 & 0 & 0 & 0 \\ 0 & 0 & 0 & 0 & 0 & 0 & 0 & 0 & 0\end{array}\right)$

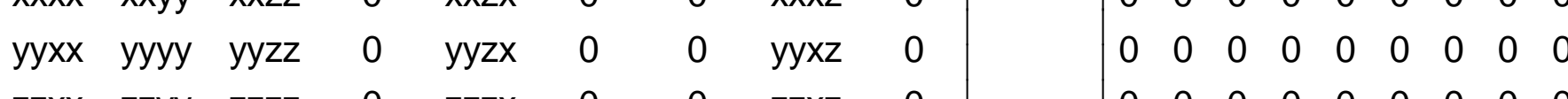
$\begin{array}{lllllllll}\text { zzxx } & \text { zzyy } & z z z z & 0 & \text { zzzx } & 0 & 0 & z z x z & 0\end{array}$ $\begin{array}{lllllllll}0 & 0 & 0 & y z y z & 0 & y z x y & y z z y & 0 & y z y x\end{array}$ $\begin{array}{llllllllll}z x x x & z x y y & z x z z & 0 & z x z x & 0 & 0 & z x x z & 0\end{array}$ $\begin{array}{lllllllll}0 & 0 & 0 & x y y z & 0 & x y x y & x y z y & 0 & x y y x\end{array}$ $\begin{array}{lllllllll}0 & 0 & 0 & \text { zyyz } & 0 & \text { zyxy } & \text { zyzy } & 0 & \text { zyyx }\end{array}$ $\begin{array}{lllllllll}x z x x & x z y y & x z z z & 0 & x z z x & 0 & 0 & x z x z & 0\end{array}$ $\begin{array}{lllllllll}0 & 0 & 0 & y x y z & 0 & y x x y & y x z y & 0 & y x y x\end{array}$ $0 \begin{array}{llllllllll}0 & 0 & 0 & 0 & 0 & 0 & 0 & 0 & 0 \\ 0 & 0 & 0 & 0 & 0 & 0 & 0 & 0 & 0\end{array}$ $\begin{array}{lllllllll}0 & 0 & 0 & 0 & 0 & 0 & 0 & 0 & 0 \\ 0 & 0 & 0 & 0 & 0 & 0 & 0 & 0 & 0\end{array}$ $0 \begin{array}{llllllllll}0 & 0 & 0 & 0 & 0 & 0 & 0 & 0 & 0 \\ 0 & 0 & 0 & 0 & 0 & 0 & 0 & 0 & 0\end{array}$ $\begin{array}{lllllllll}0 & 0 & 0 & 0 & 0 & 0 & 0 & 0 & 0 \\ 0 & 0 & 0 & 0 & 0 & 0 & 0 & 0 & 0\end{array}$ $\left(\begin{array}{lllllllllll}0 & 0 & 0 & 0 & 0 & 0 & 0 & 0 & 0 \\ 0 & 0 & 0 & 0 & 0 & 0 & 0 & 0 & 0 \\ 0 & 0 & 0 & 0 & 0 & 0 & 0 & 0 & 0\end{array}\right.$ $\left(\begin{array}{ccccc}x x x x & 0 & x z z z & y y z z & 0 \\ 0 & y y y y & 0 & z z x x & y y x z \\ z x x x & 0 & z z z z & x x y y & 0\end{array}\right) \quad\left(\begin{array}{ccccc}0 & 0 & 0 & 0 & 0 \\ 0 & 0 & 0 & 0 & 0 \\ 0 & 0 & 0 & 0 & 0\end{array}\right)$

$\left(\begin{array}{cccccccccc}x x x x & 0 & x z z z & x x y y & 0 & x z x x & x x z z & 0 & x z y y & 0 \\ 0 & y y y & 0 & 0 & y y z z & 0 & 0 & y y x x & 0 & y x y z\end{array}\right)\left(\begin{array}{cccccccccc}0 & 0 & 0 & 0 & 0 & 0 & 0 & 0 & 0 & 0 \\ 0 & 0 & 0 & 0 & 0 & 0 & 0 & 0 & 0 & 0\end{array}\right)$ $\left.\begin{array}{lllllllllll}z x x x & 0 & z z z z & z x y y & 0 & z z x x & z x z z & 0 & z z y y & 0\end{array}\right)\left(\begin{array}{llllllllll}0 & 0 & 0 & 0 & 0 & 0 & 0 & 0 & 0 & 0\end{array}\right.$

$$
\begin{aligned}
& \left(\begin{array}{llllll}
x x x x & x x y y & x x z z & 0 & x x x z & 0 \\
& y y y y & y y z z & 0 & y y x z & 0
\end{array}\right) \\
& \begin{array}{lllll}
x y y y & y y z z & 0 & y y x z & 0
\end{array} \\
& \begin{array}{ccc}
y z y z & 0 & y z x y
\end{array} \\
& \left.\begin{array}{cc}
x z x z & 0 \\
x y x y
\end{array}\right)
\end{aligned}
$$

$\left(\begin{array}{llllll}x x x x & x x y y & x x z z & 0 & x x x z & 0 \\ y x x & y y y & y y z z & 0 & y y z & 0\end{array}\right)$ $\left(\begin{array}{cccccc}\operatorname{yyxx} & \text { yyyy } & y y z z & 0 & y y x z & 0 \\ z z x x & z z y y & z z z z & 0 & z z x z & 0 \\ 0 & 0 & 0 & y z y z & 0 & y z x y\end{array}\right)$ $\begin{array}{llllll}z z x x & z z y y & z z z z & 0 & z z x z & 0\end{array}$ $\begin{array}{cccccc}0 & 0 & 0 & y z y z & 0 & y z x y\end{array}$ $\begin{array}{llllll}x z x x & x z y y & x z z z & 0 & x z x z & 0\end{array}$ $\begin{array}{cccccc}x z x x & x z y y & x z z z & 0 & x z x z & 0 \\ x y x x & x y y y & x y z z & x y y z & 0 & x y x y\end{array}$

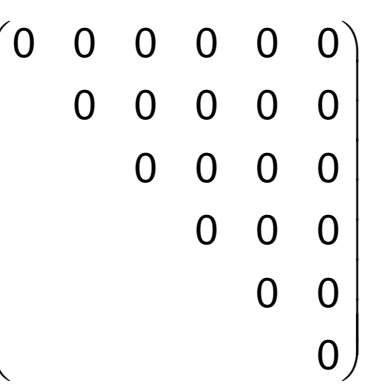

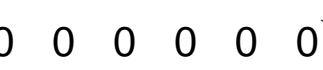
$0 \begin{array}{llllll}0 & 0 & 0 & 0 & 0 & 0 \\ 0 & 0 & 0 & 0 & 0 & 0 \\ 0 & 0 & 0 & 0 & 0 & 0\end{array}$ $\begin{array}{lllllll}0 & 0 & 0 & 0 & 0 & 0 \\ 0 & 0 & 0 & 0 & 0 & 0 \\ 0 & 0 & 0 & 0 & 0 & 0\end{array}$ $\begin{array}{lllllll}0 & 0 & 0 & 0 & 0 & 0 \\ 0 & 0 & 0 & 0 & 0 & 0 \\ 0 & 0 & 0 & 0 & 0\end{array}$

$\left(\begin{array}{llllll}0 & 0 & 0 & 0 & 0 & 0 \\ 0 & 0 & 0 & 0 & 0 & 0\end{array}\right)$ 
1

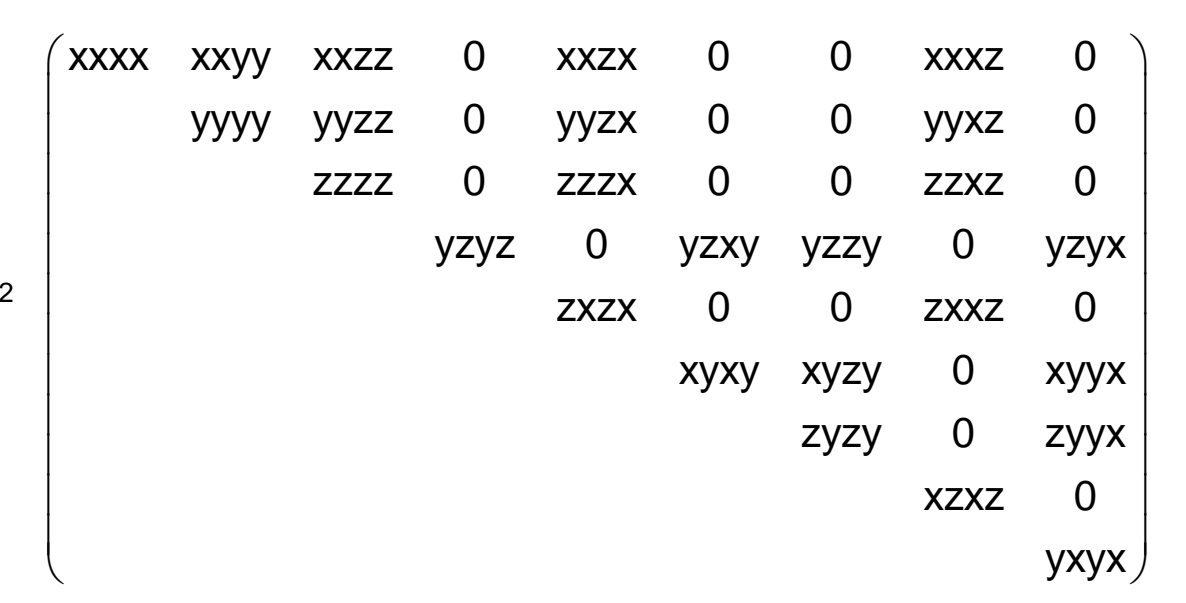

$\begin{array}{llllllll}0 & 0 & 0 & 0 & 0 & 0 & 0 & 0\end{array}$ $0 \begin{array}{lllllll}0 & 0 & 0 & 0 & 0 & 0 & 0\end{array}$ $\begin{array}{llllll}0 & 0 & 0 & 0 & 0 & 0\end{array}$ $0 \begin{array}{lllll}0 & 0 & 0 & 0 & 0\end{array}$ $0 \begin{array}{llll}0 & 0 & 0 & 0\end{array}$ $\begin{array}{lll}0 & 0 & 0 \\ & 0 & 0 \\ & & 0\end{array}$ $\left.V^{2}\right] V^{2}\left(\begin{array}{ccccccccc}x x x x & x x y y & x x z z & 0 & x x z x & 0 & 0 & x x x z & 0 \\ y y x x & y y y y & y y z z & 0 & y y z x & 0 & 0 & y y x z & 0 \\ z z x x & z z y y & z z z z & 0 & z z z x & 0 & 0 & z z x z & 0 \\ 0 & 0 & 0 & y z y z & 0 & y z x y & y z z y & 0 & y z y x \\ z x x x & z x y y & z x z z & 0 & z x z x & 0 & 0 & z x x z & 0 \\ 0 & 0 & 0 & x y y z & 0 & x y x y & x y z y & 0 & \text { xyyx }\end{array}\right)$

$\left(\begin{array}{lllllllll}0 & 0 & 0 & 0 & 0 & 0 & 0 & 0 & 0 \\ 0 & 0 & 0 & 0 & 0 & 0 & 0 & 0 & 0\end{array}\right)$ $\begin{array}{lllllllll}0 & 0 & 0 & 0 & 0 & 0 & 0 & 0 & 0 \\ 0 & 0 & 0 & 0 & 0 & 0 & 0 & 0 & 0\end{array}$

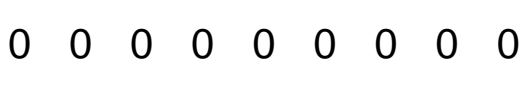
$0 \begin{array}{lllllllll}0 & 0 & 0 & 0 & 0 & 0 & 0 & 0 & 0 \\ 0 & 0 & 0 & 0 & 0 & 0 & 0 & 0 & 0\end{array}$ $\begin{array}{lllllllll}0 & 0 & 0 & 0 & 0 & 0 & 0 & 0 & 0 \\ 0 & 0 & 0 & 0 & 0 & 0 & 0 & 0 & 0\end{array}$ $\left(\begin{array}{lllllllll}0 & 0 & 0 & 0 & 0 & 0 & 0 & 0 & 0\end{array}\right)$
$\left(\begin{array}{lllllllll}x x x x & x x y y & x x z z & 0 & x x z x & 0 & 0 & x x x z & 0\end{array}\right.$

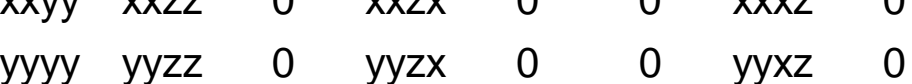 $\begin{array}{lllllll}z z z z & 0 & z z z x & 0 & 0 & z z x z & 0\end{array}$ $\begin{array}{llllll}\text { yzyz } & 0 & y z x y & y z z y & 0 & y z y x\end{array}$ $\begin{array}{lllll}z x z x & 0 & 0 & z x x z & 0\end{array}$ $\begin{array}{llll}\text { xyxy } & \text { xyzy } & 0 & \text { xyyx }\end{array}$ $\begin{array}{lll}\text { zyzy } & 0 & z y y x \\ & x z x z & 0\end{array}$ $\begin{array}{ll}0 x x z & 0 \\ y x y x\end{array}$ yxyx

$\left(\begin{array}{llllllll}0 & 0 & 0 & 0 & 0 & 0 & 0 & 0 \\ 0 & 0 & 0 & 0 & 0 & 0 & 0\end{array}\right.$ $\begin{array}{lllllllll}0 & 0 & 0 & 0 & 0 & 0 & 0 & 0\end{array}$ $0 \begin{array}{lllllll}0 & 0 & 0 & 0 & 0 & 0 & 0\end{array}$ $0 \begin{array}{llllll}0 & 0 & 0 & 0 & 0 & 0 \\ 0 & 0 & 0\end{array}$ $\begin{array}{lllll}0 & 0 & 0 & 0 & 0 \\ & 0 & 0 & 0 & 0\end{array}$

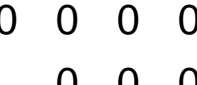
$\begin{array}{lll}0 & 0 & 0 \\ & 0 & 0 \\ & & 0\end{array}$

$\left(\begin{array}{lllllllll}x x x x & x x y y & x x z z & 0 & x x z x & 0 & 0 & x x x z & 0\end{array}\right)$ $\begin{array}{lllllllll}\text { yyxx } & y y y y & y y z z & 0 & y y z x & 0 & 0 & y y x z & 0 \\ z z x x & z z y y & z z z z & 0 & z z z x & 0 & 0 & z z x z & 0\end{array}$ $\begin{array}{lllllllll}0 & 0 & 0 & \text { yzyz } & 0 & \text { yzxy } & \text { yzzy } & 0 & \text { yzyx }\end{array}$ $\begin{array}{ccccccccc}\text { zxxx } & \text { zxyy } & \text { zxzz } & 0 & z x z x & 0 & 0 & z x x z & 0 \\ 0 & 0 & 0 & x y y z & 0 & x y x y & x y z y & 0 & x y y x\end{array}$ $\left(\begin{array}{lllllllll}0 & 0 & 0 & 0 & 0 & 0 & 0 & 0 & 0 \\ 0 & 0 & 0 & 0 & 0 & 0 & 0 & 0 & 0\end{array}\right)$ $\begin{array}{llllllllll}0 & 0 & 0 & 0 & 0 & 0 & 0 & 0 & 0 \\ 0 & 0 & 0 & 0 & 0 & 0 & 0 & 0 & 0\end{array}$ $\begin{array}{lllllllll}0 & 0 & 0 & 0 & 0 & 0 & 0 & 0 & 0 \\ 0 & 0 & 0 & 0 & 0 & 0 & 0 & 0 & 0\end{array}$ $\begin{array}{lllllllll}0 & 0 & 0 & 0 & 0 & 0 & 0 & 0 & 0\end{array}$ $\begin{array}{lllllllll}0 & 0 & 0 & 0 & 0 & 0 & 0 & 0 & 0 \\ 0 & 0 & 0 & 0 & 0 & 0 & 0 & 0 & 0\end{array}$ 


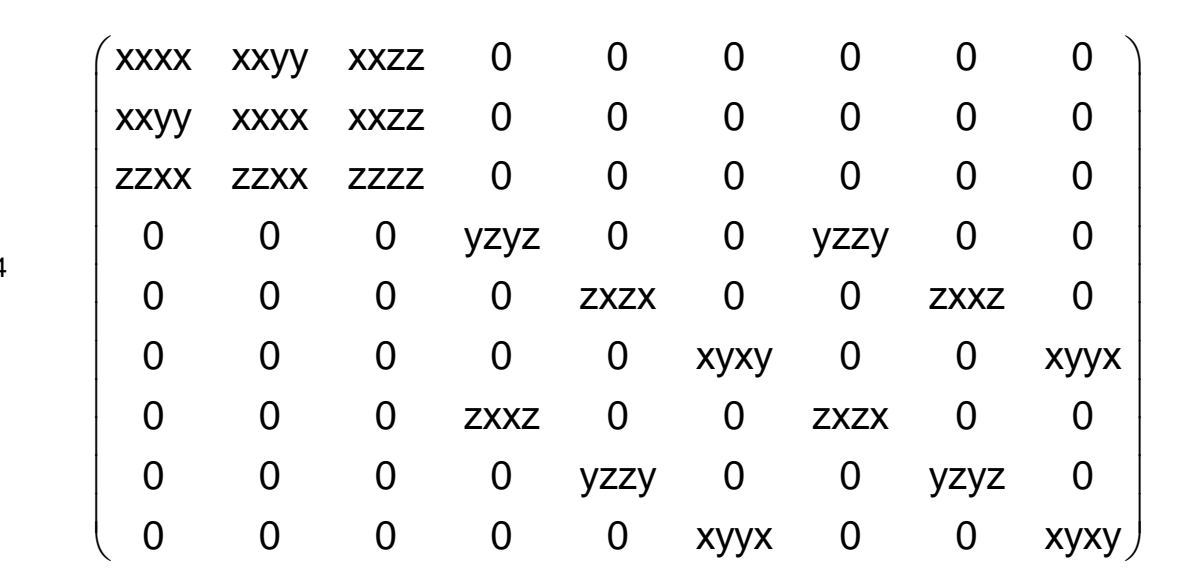

$\left(\begin{array}{lllllllll}0 & 0 & 0 & 0 & 0 & x x x y & 0 & 0 & y y x y \\ 0 & 0 & 0 & 0 & 0 & y y x y & 0 & 0 & x x x y \\ 0 & 0 & 0 & 0 & 0 & z z x y & 0 & 0 & z x y\end{array}\right)$ $\begin{array}{cccccccccc}0 & 0 & 0 & 0 & 0 & y y x y & 0 & 0 & x x x y \\ 0 & 0 & 0 & 0 & 0 & z z x y & 0 & 0 & z z x y \\ 0 & 0 & 0 & 0 & y z z & 0 & 0 & y z x z & 0\end{array}$ $\begin{array}{ccccccccc}0 & 0 & 0 & 0 & y z z x & 0 & 0 & y z x z & 0 \\ 0 & 0 & 0 & z x y & 0 & 0 & z x y & 0 & 0\end{array}$ $\begin{array}{lllllllll}0 & 0 & 0 & z x y z & 0 & 0 & z x z y & 0 & 0\end{array}$ $\begin{array}{lllllllll}\text { xyxx } & \text { xyyy } & \text { xyzz } & 0 & 0 & 0 & 0 & 0 & 0\end{array}$

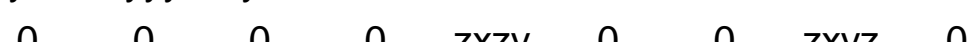
$\begin{array}{cccccccccc}0 & 0 & 0 & 0 & z x z y & 0 & 0 & z x y z & 0 \\ x & 0 & 0 & y z x z & 0 & 0 & y z z x & 0 & 0\end{array}$ $\begin{array}{ccccccccc}0 & 0 & 0 & y z x z & 0 & 0 & y z z x & 0 & 0 \\ x y y y & x y x x & x y z z & 0 & 0 & 0 & 0 & 0 & 0\end{array}$

$\left[V^{4}\right] \quad\left(\begin{array}{ccccc}\mathrm{xxxx} & 0 & 0 & \mathrm{yyzz} & 0 \\ 0 & \mathrm{xxxx} & 0 & \mathrm{yyzz} & 0 \\ 0 & 0 & \mathrm{zzzz} & \mathrm{xxyy} & 0\end{array}\right)$ $\left(\begin{array}{ccccc}0 & y x x x & 0 & 0 & 0 \\ y x x x & 0 & 0 & 0 & 0 \\ 0 & 0 & 0 & 0 & z z x y\end{array}\right)$ $\left.\mathrm{V}^{3}\right]\left(\begin{array}{cccccccccc}\mathrm{xxxx} & 0 & 0 & \mathrm{xxyy} & 0 & 0 & \mathrm{yyzz} & 0 & 0 & 0 \\ 0 & \mathrm{xxxx} & 0 & 0 & \mathrm{yyzz} & 0 & 0 & \mathrm{xxyy} & 0 & 0 \\ 0 & 0 & \mathrm{zzzz} & 0 & 0 & \mathrm{zzxx} & 0 & 0 & \mathrm{zzxx} & 0\end{array}\right)$

$\begin{array}{llllllllll}0 & \text { xyyy } & 0 & 0 & \text { xyzz } & 0 & 0 & \text { yxyy } & 0 & 0\end{array}$

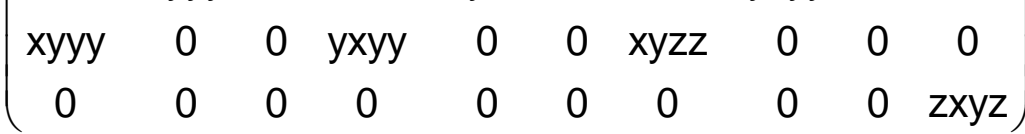

$\left[\left[V^{2}\right]^{2}\right]\left(\begin{array}{cccccc}x x x x & x x y y & x x z z & 0 & 0 & 0 \\ & x x x x & x x z z & 0 & 0 & 0 \\ & & z z z z & 0 & 0 & 0 \\ & & & y z y z & 0 & 0 \\ & & & & y z y z & 0 \\ & & & & & x y x y\end{array}\right)$

$\left(\begin{array}{llllll}x x x x & x x y y & x x z z & 0 & 0 & 0 \\ x x y y & x x x & x z z & 0 & 0 & 0\end{array}\right)$

$\left[\mathrm{V}^{2}\right]^{2}$

$\left.\begin{array}{llllll}x x y y & x x x x & x x z z & 0 & 0 & 0\end{array}\right)$

$\begin{array}{cccccc}z z x x & z z x x & z z z z & 0 & 0 & 0 \\ 0 & 0 & 0 & y z y z & 0 & 0\end{array}$

$\begin{array}{cccccc}0 & 0 & 0 & y z y z & 0 & 0 \\ 0 & 0 & 0 & 0 & y z y z & 0 \\ 0 & 0 & 0 & 0 & 0 & x y x y\end{array}$

$$
\left(\begin{array}{cccccc}
0 & 0 & 0 & 0 & 0 & x x x y \\
& 0 & 0 & 0 & 0 & x x x y \\
& & 0 & 0 & 0 & z z x y \\
& & & 0 & y z x z & 0 \\
& & & & 0 & 0 \\
& & & & & 0
\end{array}\right)
$$

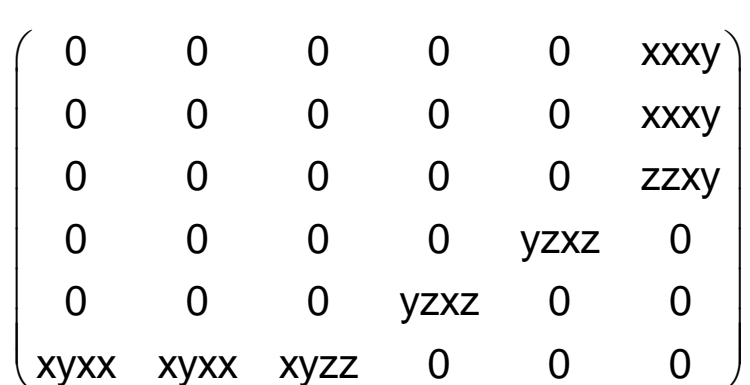

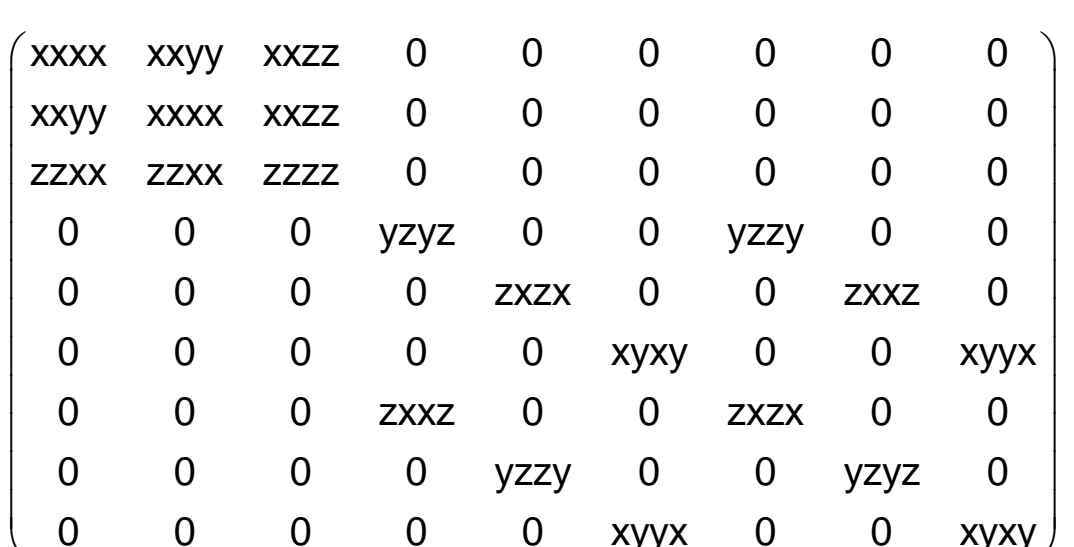

$\left(\begin{array}{ccccc}x x x x & 0 & 0 & y y z z & 0 \\ 0 & x x x x & 0 & y y z z & 0 \\ 0 & 0 & z z z z & x x y & 0\end{array}\right)$

$\begin{array}{lllll}0 & 0 & \text { zzzz } & \text { xxyy } & 0\end{array}$

$\left(\begin{array}{cccccccccc}x x x x & 0 & 0 & x x y y & 0 & 0 & y y z z & 0 & 0 & 0 \\ 0 & x y x x & 0 & 0 & y y z z & 0 & 0 & x x y y & 0 & 0 \\ 0 & 0 & z z z z & 0 & 0 & z z x x & 0 & 0 & z z x & 0\end{array}\right)$ $\left(\begin{array}{cccccccccc}0 & \mathrm{xxxx} & 0 & 0 & \mathrm{yyzz} & 0 & 0 & \mathrm{xxyy} & 0 & 0 \\ 0 & 0 & \mathrm{zzzz} & 0 & 0 & \mathrm{zzxx} & 0 & 0 & \mathrm{zzxx} & 0\end{array}\right)$

$$
\left(\begin{array}{cccccc}
x x x x & x x y y & x x z z & 0 & 0 & 0 \\
& x x x x & x x z z & 0 & 0 & 0 \\
& & z z z z & 0 & 0 & 0 \\
& & & y z y z & 0 & 0 \\
& & & & y z y z & 0 \\
& & & & & \text { xyxy }
\end{array}\right)
$$

$\left(\begin{array}{llllll}x x x x & x x y y & x x z z & 0 & 0 & 0\end{array}\right)$

$\begin{array}{llllll}x x y y & x x x x & x x z z & 0 & 0 & 0 \\ z z x x & z z x x & z z z z & 0 & 0 & 0\end{array}$

$\begin{array}{ccccccc}z z x x & z z x x & z z z z & 0 & 0 & 0 \\ 0 & 0 & 0 & y z y z & 0 & 0\end{array}$

$\left(\begin{array}{cccccc}0 & 0 & 0 & 0 & y z y z & 0 \\ 0 & 0 & 0 & 0 & 0 & x y x y\end{array}\right.$

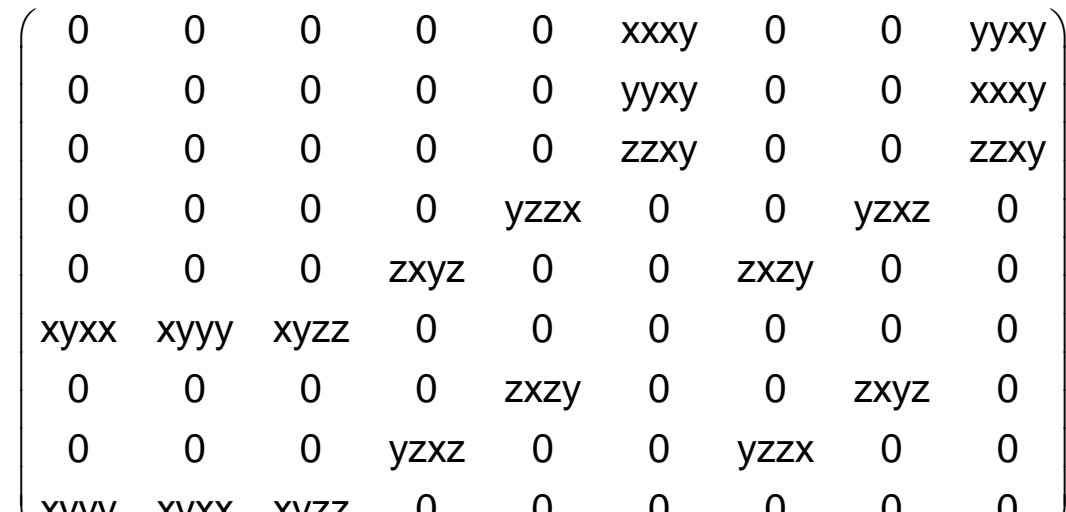

$\left(\begin{array}{ccccc}0 & \mathrm{yxxx} & 0 & 0 & 0 \\ \mathrm{yxxx} & 0 & 0 & 0 & 0 \\ 0 & 0 & 0 & 0 & z z x y\end{array}\right)$

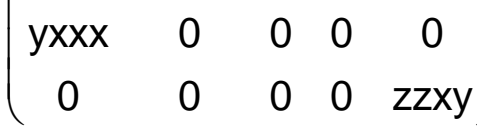

$\begin{array}{llllllllll}0 & \text { xyyy } & 0 & 0 & \text { xyzz } & 0 & 0 & \text { yxyy } & 0 & 0\end{array}$

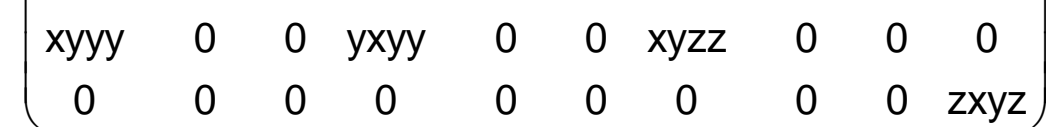

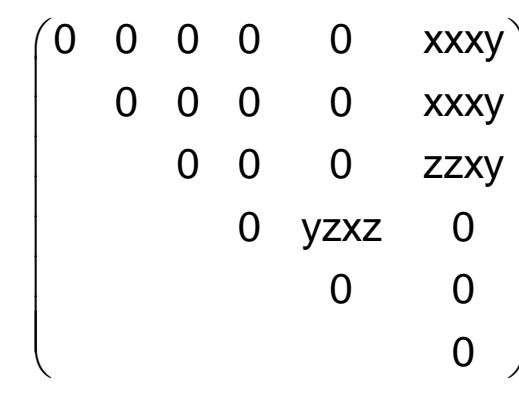

$\left(\begin{array}{cccccc}0 & 0 & 0 & 0 & 0 & x x x y \\ 0 & 0 & 0 & 0 & 0 & x x x y \\ 0 & 0 & 0 & 0 & 0 & \text { zzxy } \\ 0 & 0 & 0 & 0 & y z x z & 0 \\ 0 & 0 & 0 & y z x z & 0 & 0 \\ x y x x & x y x x & x y z z & 0 & 0 & 0\end{array}\right)$ 
$\mathrm{n}=2 \quad \mathbf{D}_{2 \mathrm{~d}}=\overline{\mathbf{4}}_{\mathrm{z}} \mathbf{m}_{\mathrm{x}} \mathbf{2}_{\mathrm{xy}}$

e

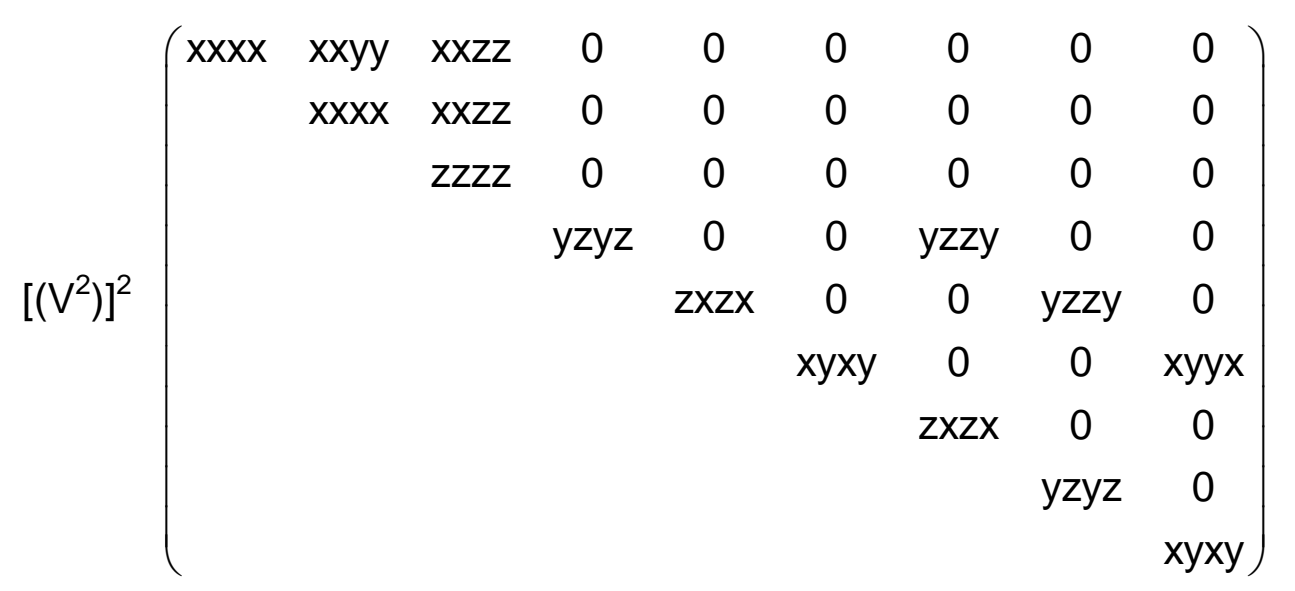

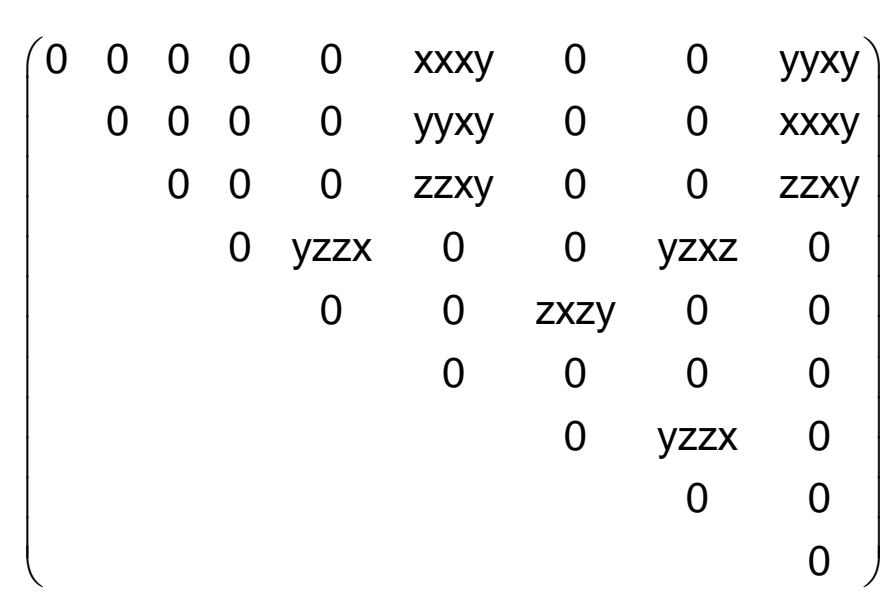

$\left[\mathrm{V}^{2}\right] \mathrm{V}^{2}\left(\begin{array}{ccccccccc}\mathrm{xxxx} & \mathrm{xxyy} & \mathrm{xxzz} & 0 & 0 & 0 & 0 & 0 & 0 \\ \mathrm{xxyy} & \mathrm{xxxx} & \mathrm{xxzz} & 0 & 0 & 0 & 0 & 0 & 0 \\ \mathrm{zzxx} & \mathrm{zzxx} & \mathrm{zzzz} & 0 & 0 & 0 & 0 & 0 & 0 \\ 0 & 0 & 0 & \mathrm{yzyz} & 0 & 0 & \mathrm{zxzx} & 0 & 0 \\ 0 & 0 & 0 & 0 & \mathrm{zxzx} & 0 & 0 & \mathrm{yzyz} & 0 \\ 0 & 0 & 0 & 0 & 0 & \mathrm{xyxy} & 0 & 0 & \mathrm{xyxy}\end{array}\right)$
$D_{\text {nd }}$

a

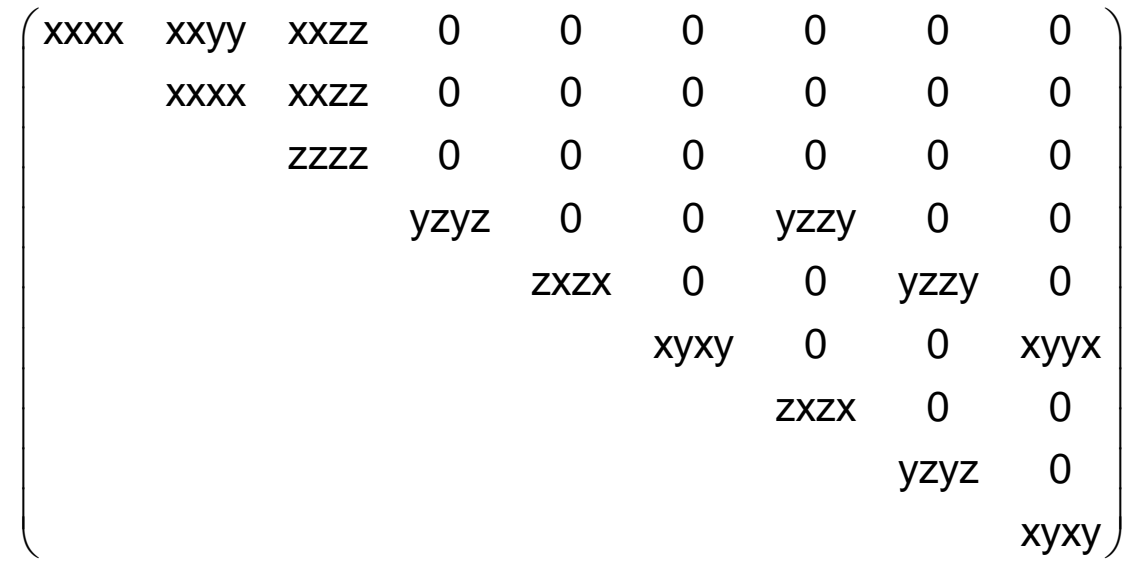

$\left(\begin{array}{ccccccccc}\text { xxxx } & \text { xxyy } & \text { xxzz } & 0 & 0 & 0 & 0 & 0 & 0 \\ \text { xxyy } & \text { xxxx } & \text { xxzz } & 0 & 0 & 0 & 0 & 0 & 0 \\ \text { zzxx } & \text { zzxx } & \text { zzzz } & 0 & 0 & 0 & 0 & 0 & 0 \\ 0 & 0 & 0 & y z y z & 0 & 0 & \text { zxzx } & 0 & 0 \\ 0 & 0 & 0 & 0 & \text { zxzx } & 0 & 0 & \text { yzyz } & 0 \\ 0 & 0 & 0 & 0 & 0 & \text { xyxy } & 0 & 0 & \text { xyxy }\end{array}\right)$

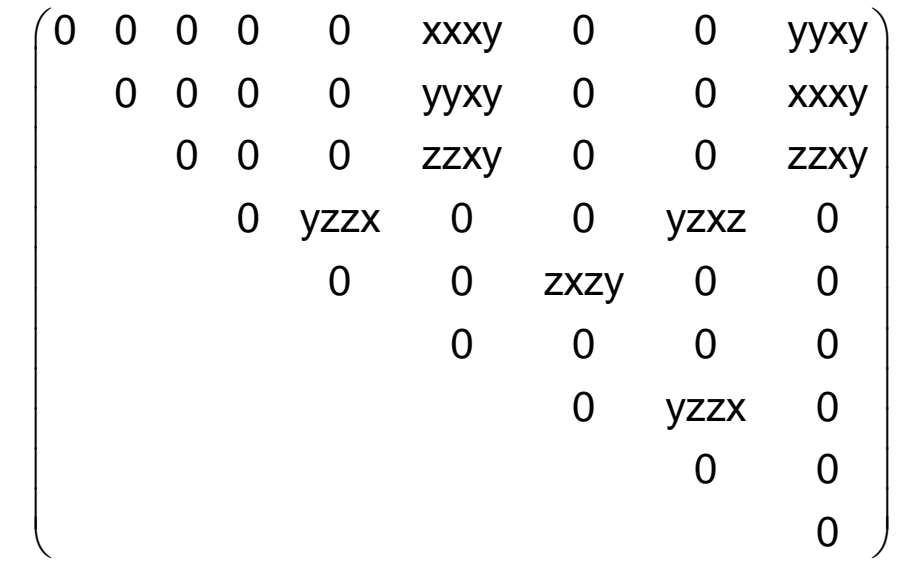

$\left(\begin{array}{ccccccccc}0 & 0 & 0 & 0 & 0 & \text { xxxy } & 0 & 0 & \text { yyxy } \\ 0 & 0 & 0 & 0 & 0 & \text { yyxy } & 0 & 0 & \text { xxxy } \\ 0 & 0 & 0 & 0 & 0 & \text { zzxy } & 0 & 0 & \text { zzxy } \\ 0 & 0 & 0 & 0 & \text { yzzx } & 0 & 0 & \text { zxyz } & 0 \\ 0 & 0 & 0 & \text { zxyz } & 0 & 0 & \text { yzzx } & 0 & 0 \\ \text { xyxx } & \text { xyxx } & \text { xyzz } & 0 & 0 & 0 & 0 & 0 & 0\end{array}\right)$ 
$\mathrm{V}^{4}\left(\begin{array}{ccccccccc}\text { xxxx } & \text { xxyy } & x x z z & x x y z & 0 & 0 & x x z y & 0 & 0 \\ x x y y & x x x x & x x z z & -x x y z & 0 & 0 & -x x z y & 0 & 0 \\ z z x x & z z x x & z z z z & 0 & 0 & 0 & 0 & 0 & 0 \\ y z x x & -y z x x & 0 & y z y z & 0 & 0 & y z z y & 0 & 0 \\ 0 & 0 & 0 & 0 & z x z x & z y x x & 0 & z x x z & z y x x \\ 0 & 0 & 0 & 0 & x x z y & x y x y & 0 & x x y z & x y y x \\ z y x x & -z y x x & 0 & z x x z & 0 & 0 & z x z x & 0 & 0 \\ 0 & 0 & 0 & 0 & y z z y & y z x x & 0 & y z y z & y z x x \\ 0 & 0 & 0 & 0 & x x z y & x y y x & 0 & x x y z & x y x y\end{array}\right)$

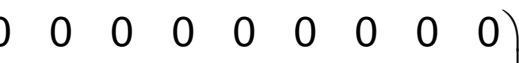
$0 \begin{array}{llllllllll}0 & 0 & 0 & 0 & 0 & 0 & 0 & 0 & 0\end{array}$ $0 \begin{array}{llllllllll}0 & 0 & 0 & 0 & 0 & 0 & 0 & 0 & 0 \\ 0 & 0 & 0 & 0 & 0 & 0 & 0 & 0 & 0\end{array}$ $\begin{array}{lllllllll}0 & 0 & 0 & 0 & 0 & 0 & 0 & 0 & 0 \\ 0 & 0 & 0 & 0 & 0 & 0 & 0 & 0\end{array}$

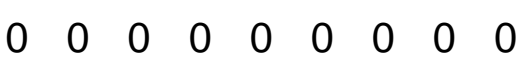
$\begin{array}{lllllllll}0 & 0 & 0 & 0 & 0 & 0 & 0 & 0 & 0 \\ 0 & 0 & 0 & 0 & 0 & 0 & 0 & 0 & 0\end{array}$

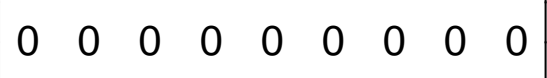

$\left(\begin{array}{lllllllll}0 & 0 & 0 & 0 & 0 & 0 & 0 & 0 & 0 \\ 0 & 0 & 0 & 0 & 0 & 0 & 0 & 0 & 0\end{array}\right.$ and: $x x x x=x x y y+x y x y+x y y x$

$\left(\begin{array}{lllll}0 & 0 & 0 & 0 & 0 \\ 0 & 0 & 0 & 0 & 0 \\ 0 & 0 & 0 & 0 & 0\end{array}\right)$

$\left(\begin{array}{lllll}0 & 0 & 0 & 0 & 0 \\ 0 & 0 & 0 & 0 & 0\end{array}\right.$

$\left[V^{4}\right] \quad\left(\begin{array}{ccccc}3 x x y y & 0 & 0 & y y z z & x x y z \\ 0 & 3 x x y y & 0 & y y z z & 0 \\ 0 & -x x y z & z z z z & x x y y & 0\end{array}\right)$

$\mathrm{V}\left[\mathrm{V}^{3}\right]\left(\begin{array}{cccccccccc}3 x x y y & 0 & 0 & x x y y & 0 & 0 & y y z z & 0 & 0 & y z x x \\ 0 & 3 x x y y & 0 & 0 & y y z z & y z x x & 0 & x x y y & -y z x x & 0 \\ 0 & -z y x x & z z z & 0 & 0 & z z x x & 0 & z y x x & z z x & 0\end{array}\right)$

$\left(\begin{array}{llllllllll}0 & 0 & 0 & 0 & 0 & 0 & 0 & 0 & 0 & 0 \\ 0 & 0 & 0 & 0 & 0 & 0 & 0 & 0 & 0 & 0\end{array}\right)$ $\left(\begin{array}{llllllllll}0 & 0 & 0 & 0 & 0 & 0 & 0 & 0 & 0 & 0 \\ 0 & 0 & 0 & 0 & 0 & 0 & 0 & 0 & 0 & 0\end{array}\right)$ $\begin{array}{ccccccccccc}0 & -z y x x & z z z z & 0 & 0 & z z x x & 0 & z y x x & z z x x & 0\end{array}$

$\left[\left[^{2}\right]^{2}\right]\left(\begin{array}{cccccc}x x x x & x x y y & x x z z & x x y z & 0 & 0 \\ & x x x x & x x z z & -x x y z & 0 & 0 \\ & & z z z z & 0 & 0 & 0 \\ & & & y z y z & 0 & 0 \\ & & & & y z y z & x x y z \\ & & & & & x y x y\end{array}\right)$

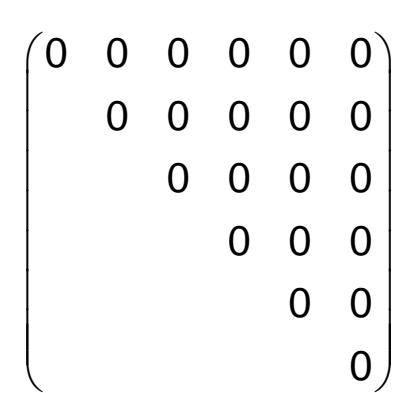

and $x y x y=1 / 2(x x x x-x x y y)$

$\left(\begin{array}{cccccc}x x x x & x x y y & x x z z & x x y z & 0 & 0 \\ x x y y & x x x x & x x z z & -x x y z & 0 & 0 \\ z z x x & z z x x & z z z z & 0 & 0 & 0 \\ y z x x & -y z x x & 0 & y z y z & 0 & 0 \\ 0 & 0 & 0 & 0 & y z y z & y z x x \\ 0 & 0 & 0 & 0 & x y z & x y x\end{array}\right)$

and $x y x y=1 / 2(x x x x-x x y y)$

$\left(\begin{array}{llllll}0 & 0 & 0 & 0 & 0 & 0 \\ 0 & 0 & 0 & 0 & 0 & 0 \\ 0 & 0 & 0 & 0 & 0\end{array}\right.$

$\left.\begin{array}{llllll}0 & 0 & 0 & 0 & 0 & 0 \\ 0 & 0 & 0 & 0 & 0 & 0 \\ 0 & 0 & 0 & 0 & 0 & 0\end{array}\right)$

$0 \begin{array}{llllll}0 & 0 & 0 & 0 & 0 & 0\end{array}$

$\begin{array}{llllll}0 & 0 & 0 & 0 & 0 & 0 \\ 0 & 0 & 0 & 0 & 0 & 0\end{array}$

$\begin{array}{llllll}0 & 0 & 0 & 0 & 0 & 0 \\ 0 & 0 & 0 & 0 & 0 & 0\end{array}$

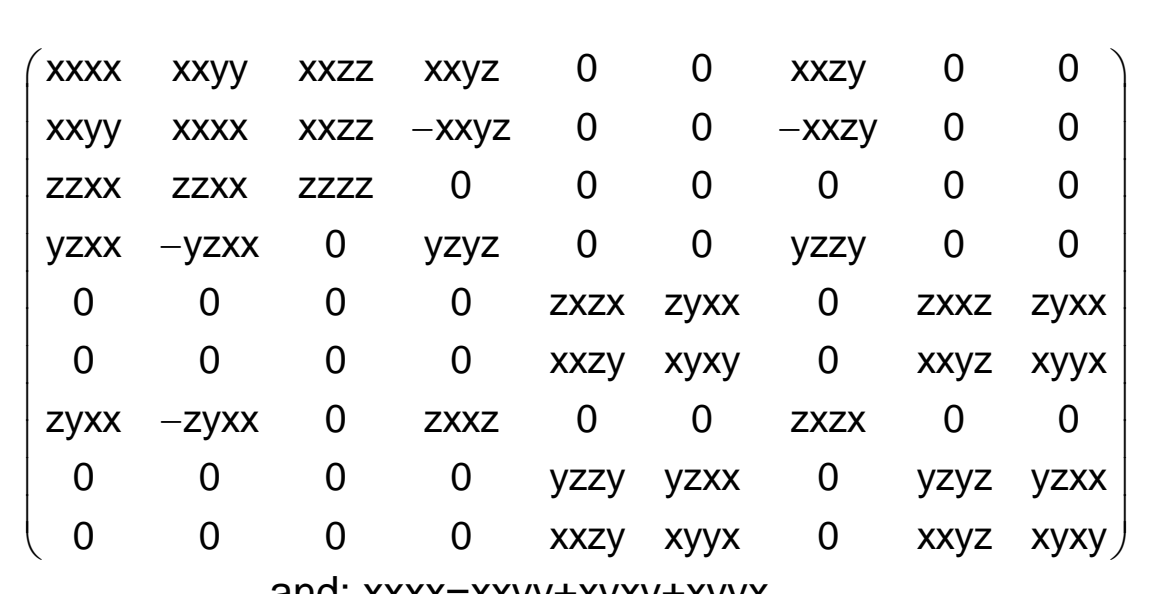

$\left(\begin{array}{lllllllll}0 & 0 & 0 & 0 & 0 & 0 & 0 & 0 & 0 \\ 0 & 0 & 0 & 0 & 0 & 0 & 0 & 0 & 0\end{array}\right.$ $\left(\begin{array}{llllllllll}0 & 0 & 0 & 0 & 0 & 0 & 0 & 0 & 0 \\ 0 & 0 & 0 & 0 & 0 & 0 & 0 & 0 & 0 \\ 0 & 0 & 0 & 0 & 0 & 0 & 0 & 0 & 0 & 0\end{array}\right)$ $\begin{array}{llllllllll}0 & 0 & 0 & 0 & 0 & 0 & 0 & 0 & 0 \\ 0 & 0 & 0 & 0 & 0 & 0 & 0 & 0 & 0\end{array}$ $\begin{array}{lllllllll}0 & 0 & 0 & 0 & 0 & 0 & 0 & 0 & 0 \\ 0 & 0 & 0 & 0 & 0 & 0 & 0 & 0 & 0\end{array}$ $0 \begin{array}{lllllllll}0 & 0 & 0 & 0 & 0 & 0 & 0 & 0 & 0 \\ 0 & 0 & 0 & 0 & 0 & 0 & 0 & 0 & 0\end{array}$ $\begin{array}{llllllllll}0 & 0 & 0 & 0 & 0 & 0 & 0 & 0 & 0 \\ 0 & 0 & 0 & 0 & 0 & 0 & 0 & 0 & 0\end{array}$ $\begin{array}{lllllllll}0 & 0 & 0 & 0 & 0 & 0 & 0 & 0 & 0 \\ 0 & 0 & 0 & 0 & 0 & 0 & 0 & 0 & 0\end{array}$

$\begin{array}{llllllllll}0 & 0 & 0 & 0 & 0 & 0 & 0 & 0 & 0 \\ 0 & 0 & 0 & 0 & 0 & 0 & 0 & 0 & 0\end{array}$ $\begin{array}{llll}0 & 0 & y z z & y z x \\ a & 0 & x x y & x y y x\end{array}$

$\left(\begin{array}{ccccc}3 x x y y & 0 & 0 & y y z z & x x y z \\ 0 & 3 x x y y & 0 & y y z z & 0 \\ 0 & -x x y z & z z z z & x x y y & 0\end{array}\right) \quad\left(\begin{array}{ccccc}0 & 0 & 0 & 0 & 0 \\ 0 & 0 & 0 & 0 & 0 \\ 0 & 0 & 0 & 0 & 0\end{array}\right)$

$\left(\begin{array}{cccccccccc}3 x x y y & 0 & 0 & x x y y & 0 & 0 & y y z z & 0 & 0 & y z x x \\ 0 & 3 x x y y & 0 & 0 & y y z z & y z x x & 0 & x x y y & -y z x x & 0 \\ 0 & -2 y x x & z z z z & 0 & 0 & z z x x & 0 & z y x & z z x & 0\end{array}\right)$

$\left(\begin{array}{llllllllll}0 & 0 & 0 & 0 & 0 & 0 & 0 & 0 & 0 & 0 \\ 0 & 0 & 0 & 0 & 0 & 0 & 0 & 0 & 0 & 0\end{array}\right)$ $\left(\begin{array}{cccccccccc}0 & 3 x x y y & 0 & 0 & y y z z & y z x x & 0 & x x y y & -y z x x & 0 \\ 0 & -z y x x & z z z z & 0 & 0 & z z x x & 0 & z y x x & z z x x & 0\end{array}\right)$

$\left(\begin{array}{lllllllllll}0 & 0 & 0 & 0 & 0 & 0 & 0 & 0 & 0 & 0 \\ 0 & 0 & 0 & 0 & 0 & 0 & 0 & 0 & 0 & 0 \\ 0 & 0 & 0 & 0 & 0 & 0 & 0 & 0 & 0 & 0\end{array}\right)$
$\left(\begin{array}{cccccc}x x x x & x x y y & x x z z & x x y z & 0 & 0 \\ & x x x x & x x z z & -x x y z & 0 & 0\end{array}\right)$

$\left.\begin{array}{c}0 \\ 0 \\ 0 \\ 0 \\ x x y z \\ x y x y\end{array}\right)$

$\left(\begin{array}{llllll}0 & 0 & 0 & 0 & 0 & 0 \\ & 0 & 0 & 0 & 0 & 0 \\ & & 0 & 0 & 0 & 0 \\ & & & 0 & 0 & 0 \\ & & & & 0 & 0 \\ & & & & & 0\end{array}\right)$

and $x y x y=1 / 2(x x x x-x x y y)$

$\left(\begin{array}{cccccc}x x x x & x x y y & x x z z & x x y z & 0 & 0 \\ x x y y & x x x x & x x z z & -x x y z & 0 & 0 \\ z z x x & z z x x & z z z z & 0 & 0 & 0 \\ y z x x & -y z x x & 0 & y z y z & 0 & 0 \\ 0 & 0 & 0 & 0 & y z y z & y z x x \\ 0 & 0 & 0 & 0 & x x y z & x y x y\end{array}\right)$

$\left.\begin{array}{llllll}0 & 0 & 0 & 0 & 0 & 0 \\ 0 & 0 & 0 & 0 & 0 & 0 \\ 0 & 0 & 0 & 0 & 0 & 0\end{array}\right)$

$\left.0 \begin{array}{llllll}0 & 0 & 0 & 0 & 0 & 0 \\ 0 & 0 & 0 & 0 & 0 & 0 \\ 0 & 0 & 0 & 0 & 0 & 0\end{array}\right)$

$0 \begin{array}{llllll}0 & 0 & 0 & 0 & 0 & 0 \\ 0 & 0 & 0 & 0 & 0 & 0 \\ 0 & 0 & 0 & 0 & 0\end{array}$

$\begin{array}{lllllll}0 & 0 & 0 & 0 & 0 & 0 \\ 0 & 0 & 0 & 0 & 0 & 0\end{array}$

and $x y x y=1 / 2(x x x x-x x y y)$ 


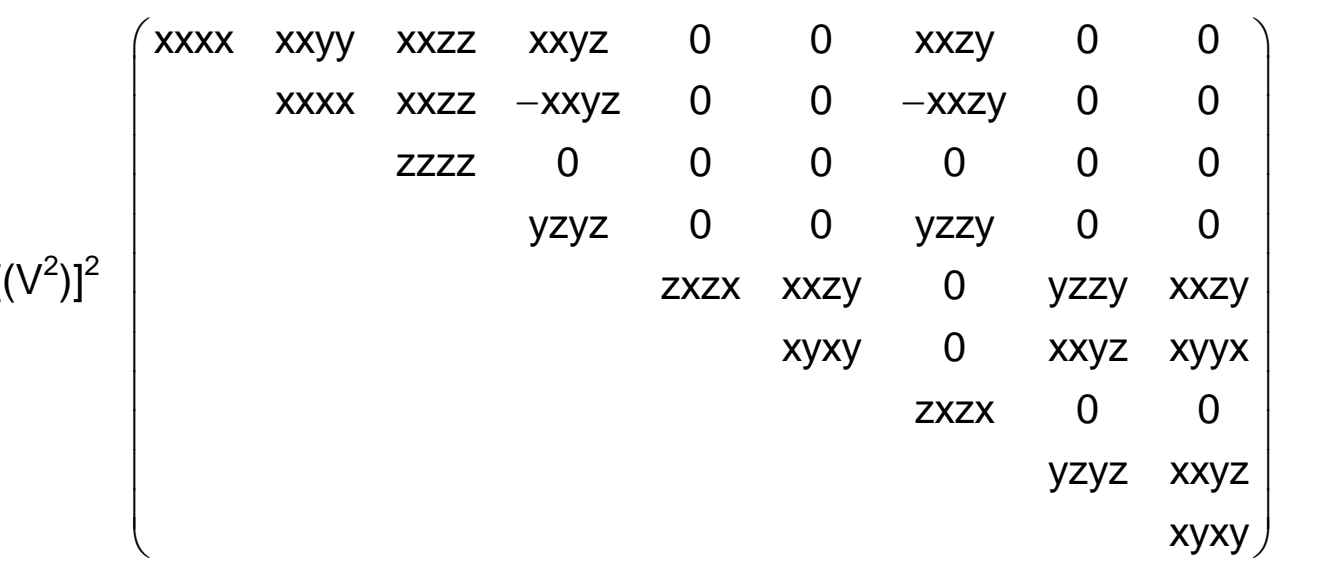

and $x x x x=x x y y+y z y z+z y z y$ $\left[\mathrm{V}^{2}\right] \mathrm{V}^{2}\left(\begin{array}{ccccccccc}\mathrm{x} x \mathrm{x} x & \mathrm{xxyy} & \mathrm{xxzz} & \mathrm{xxyz} & 0 & 0 & \mathrm{xxzy} & 0 & 0 \\ \mathrm{xxyy} & \mathrm{xxxx} & \mathrm{xxzz} & -\mathrm{xxyz} & 0 & 0 & -\mathrm{xxzy} & 0 & 0 \\ \mathrm{zzxx} & \mathrm{zzxx} & \mathrm{zzzz} & 0 & 0 & 0 & 0 & 0 & 0 \\ \mathrm{yzxx} & -\mathrm{yzxx} & 0 & \mathrm{yzyz} & 0 & 0 & \mathrm{zxzx} & 0 & 0 \\ 0 & 0 & 0 & 0 & \mathrm{zxzx} & \mathrm{yzxx} & 0 & \mathrm{yzyz} & \mathrm{yzxx} \\ 0 & 0 & 0 & 0 & \mathrm{xxzy} & \mathrm{xyxy} & 0 & \mathrm{xxyz} & \mathrm{xyxy}\end{array}\right) \quad\left(\begin{array}{ccccccccc}0 & 0 & 0 & 0 & 0 & 0 & 0 & 0 & 0 \\ 0 & 0 & 0 & 0 & 0 & 0 & 0 & 0 & 0 \\ 0 & 0 & 0 & 0 & 0 & 0 & 0 & 0 & 0 \\ 0 & 0 & 0 & 0 & 0 & 0 & 0 & 0 & 0 \\ 0 & 0 & 0 & 0 & 0 & 0 & 0 & 0 & 0 \\ 0 & 0 & 0 & 0 & 0 & 0 & 0 & 0 & 0\end{array}\right)$ and $x x x x=x x y y+2 x y x y$ $\left(\begin{array}{lllllllll}0 & 0 & 0 & 0 & 0 & 0 & 0 & 0 & 0 \\ 0 & 0 & 0 & 0 & 0 & 0 & 0 & 0 & 0\end{array}\right)$

$\begin{array}{lllllll}0 & 0 & 0 & 0 & 0 & 0 & 0\end{array}$ $\begin{array}{llllll}0 & 0 & 0 & 0 & 0 & 0 \\ 0 & 0 & 0 & 0 & 0 & 0\end{array}$ $\begin{array}{lllll}0 & 0 & 0 & 0 & 0 \\ 0 & 0 & 0 & 0 & 0\end{array} \mid$ $\begin{array}{lllll}0 & 0 & 0 & 0 & 0 \\ 0 & 0 & 0 & 0\end{array}$ $\begin{array}{rrr}0 & 0 & 0 \\ & 0 & 0\end{array}$ $\begin{array}{ll}0 & 0 \\ & 0\end{array}$

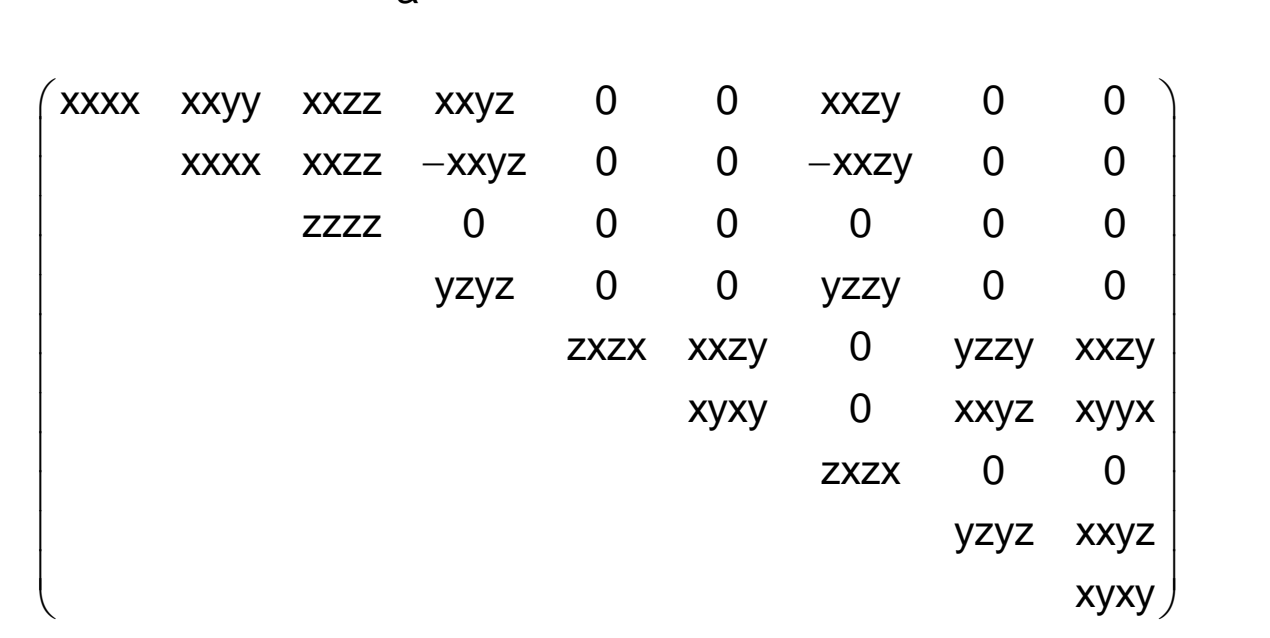

and $x x x x=x x y y+y z y z+z y z y$

$\left(\begin{array}{ccccccccc}x x x x & x x y y & x x z z & x x y z & 0 & 0 & x x z y & 0 & 0 \\ x x y y & x x x x & x x z z & -x x y z & 0 & 0 & -x x z y & 0 & 0 \\ z z x x & z z x x & z z z z & 0 & 0 & 0 & 0 & 0 & 0 \\ y z x x & -y z x x & 0 & y z y z & 0 & 0 & z x z x & 0 & 0 \\ 0 & 0 & 0 & 0 & z x z x & y z x x & 0 & y z y z & y z x x \\ 0 & 0 & 0 & 0 & x x z y & x y x y & 0 & x x y z & x y x y\end{array}\right) \quad\left(\begin{array}{ccccccccc}0 & 0 & 0 & 0 & 0 & 0 & 0 & 0 & 0 \\ 0 & 0 & 0 & 0 & 0 & 0 & 0 & 0 & 0 \\ 0 & 0 & 0 & 0 & 0 & 0 & 0 & 0 & 0 \\ 0 & 0 & 0 & 0 & 0 & 0 & 0 & 0 & 0 \\ 0 & 0 & 0 & 0 & 0 & 0 & 0 & 0 & 0 \\ 0 & 0 & 0 & 0 & 0 & 0 & 0 & 0 & 0\end{array}\right)$

and $x x x x=x x y y+2 x y x y$

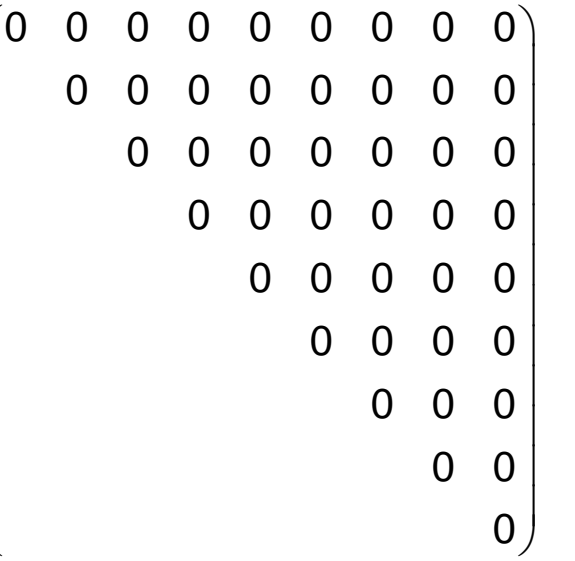

$\left(\begin{array}{ccccccccc}x x x x & x x y y & x x z z & x x y z & 0 & 0 & x x z y & 0 & 0 \\ x x y y & x x x x & x x z z & -x x y z & 0 & 0 & -x x z y & 0 & 0 \\ z z x x & z z x x & z z z z & 0 & 0 & 0 & 0 & 0 & 0 \\ y z x x & -y z x x & 0 & y z y z & 0 & 0 & z x z x & 0 & 0 \\ 0 & 0 & 0 & 0 & z x z x & y z x x & 0 & y z y z & y z x x \\ 0 & 0 & 0 & 0 & x x z y & x y x y & 0 & x x y z & x y x y\end{array}\right) \quad\left(\begin{array}{ccccccccc}0 & 0 & 0 & 0 & 0 & 0 & 0 & 0 & 0 \\ 0 & 0 & 0 & 0 & 0 & 0 & 0 & 0 & 0 \\ 0 & 0 & 0 & 0 & 0 & 0 & 0 & 0 & 0 \\ 0 & 0 & 0 & 0 & 0 & 0 & 0 & 0 & 0 \\ 0 & 0 & 0 & 0 & 0 & 0 & 0 & 0 & 0 \\ 0 & 0 & 0 & 0 & 0 & 0 & 0 & 0 & 0\end{array}\right)$ 
$\left.\begin{array}{ccccccccc}x x x x & x x y y & x x z z & 0 & 0 & 0 & 0 & 0 & 0 \\ x x y y & x x x x & x x z z & 0 & 0 & 0 & 0 & 0 & 0 \\ z z x x & z z x x & z z z z & 0 & 0 & 0 & 0 & 0 & 0 \\ 0 & 0 & 0 & y z y z & 0 & 0 & y z z y & 0 & 0 \\ 0 & 0 & 0 & 0 & z x z x & 0 & 0 & z x x z & 0 \\ 0 & 0 & 0 & 0 & 0 & x y x y & 0 & 0 & x y y x \\ 0 & 0 & 0 & z x x z & 0 & 0 & z x z x & 0 & 0 \\ 0 & 0 & 0 & 0 & y z z y & 0 & 0 & y z y z & 0 \\ 0 & 0 & 0 & 0 & 0 & x y y x & 0 & 0 & \text { xyxy }\end{array}\right)$

$\left(\begin{array}{lllllllll}0 & 0 & 0 & 0 & 0 & -x y y y & 0 & 0 & -x y y y \\ 0 & 0 & 0 & 0 & 0 & -x y y & 0 & 0 & x y y\end{array}\right)$ $\left(\begin{array}{ccccccccc}0 & 0 & 0 & 0 & 0 & -x y y & 0 & 0 & -x y y \\ 0 & 0 & 0 & 0 & 0 & x y y y & 0 & 0 & x y y \\ 0 & 0 & 0 & 0 & 0 & 0 & 0 & 0 & 0\end{array}\right.$ $\begin{array}{llllllllll}0 & 0 & 0 & 0 & 0 & 0 & 0 & 0 & 0 & 0 \\ 0 & 0 & 0 & 0 & 0 & 0 & 0\end{array}$ $\begin{array}{llllllllll}0 & 0 & 0 & 0 & 0 & 0 & 0 & 0 & 0 & 0\end{array}$ $\begin{array}{llllllllll}0 & 0 & 0 & 0 & 0 & 0 & 0 & 0 & 0 & 0\end{array}$ $\begin{array}{lllllllll}0 & 0 & 0 & 0 & 0 & 0 & 0 & 0 & 0\end{array}$ $\begin{array}{lllllllll}0 & 0 & 0 & 0 & 0 & 0 & 0 & 0 & 0 \\ -x y y y & x y y & 0 & 0 & 0 & 0 & 0 & 0 & 0\end{array}$

$\left.V^{4}\right] \quad\left(\begin{array}{ccccc}3 x x y y & 0 & 0 & y y z z & 0 \\ 0 & 3 x x y y & 0 & y y z z & 0 \\ 0 & 0 & z z z z & x x y y & 0\end{array}\right)$ $\left(\begin{array}{ccccc}0 & x y y y & 0 & 0 & 0 \\ -x y y y & 0 & 0 & 0 & 0 \\ 0 & 0 & 0 & 0 & 0\end{array}\right)$

$\mathrm{V}\left[\mathrm{V}^{3}\right]\left(\begin{array}{cccccccccc}3 x x y y & 0 & 0 & x x y y & 0 & 0 & y y z z & 0 & 0 & 0 \\ 0 & 3 x x y y & 0 & 0 & y y z z & 0 & 0 & x x y y & 0 & 0 \\ 0 & 0 & z z z z & 0 & 0 & z z x x & 0 & 0 & z z x x & 0\end{array}\right)$

$\begin{array}{llllllllll}0 & \text { xyyy } & 0 & 0 & 0 & 0 & 0 & -x y y y & 0 & 0\end{array}$

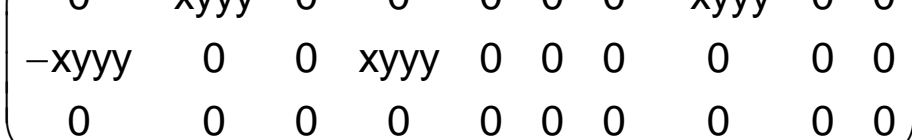

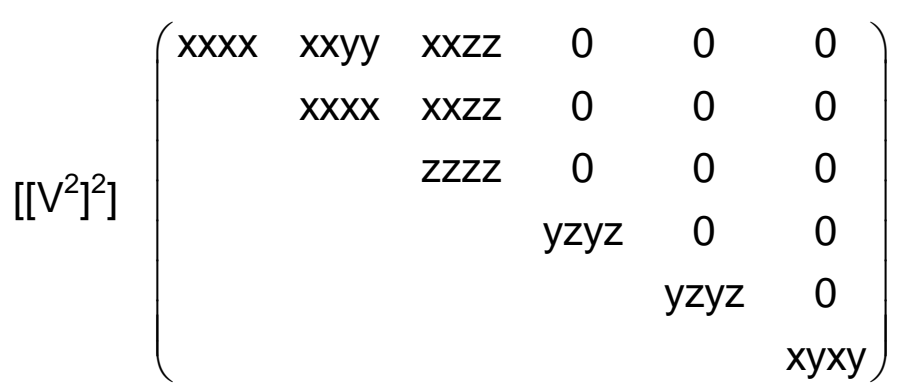

$$
\left(\begin{array}{cccccc}
0 & 0 & 0 & 0 & 0 & -x y y y \\
& 0 & 0 & 0 & 0 & x y y y \\
& & 0 & 0 & 0 & 0 \\
& & & 0 & 0 & 0 \\
& & & & 0 & 0 \\
& & & & & 0
\end{array}\right)
$$

add: $x y x y=1 / 2(x x x x-x x y y)$
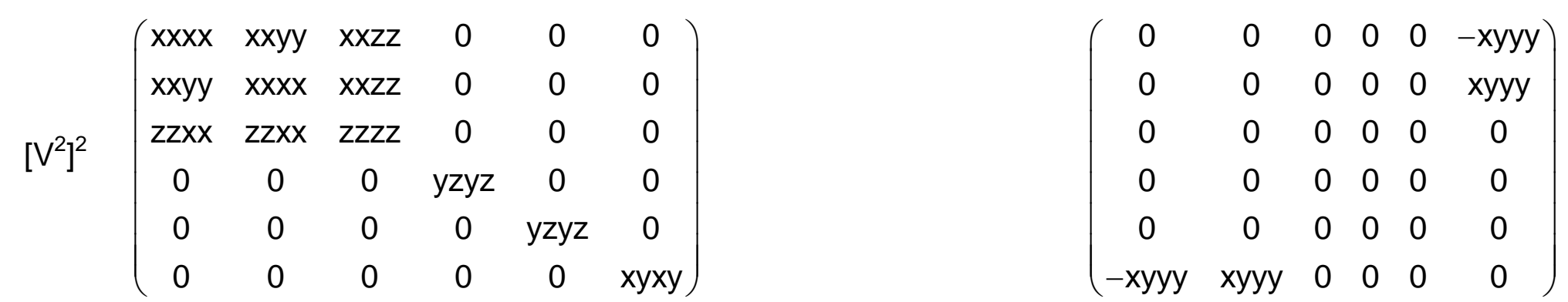

add: $x y x y=1 / 2(x x x x-x x y y)$
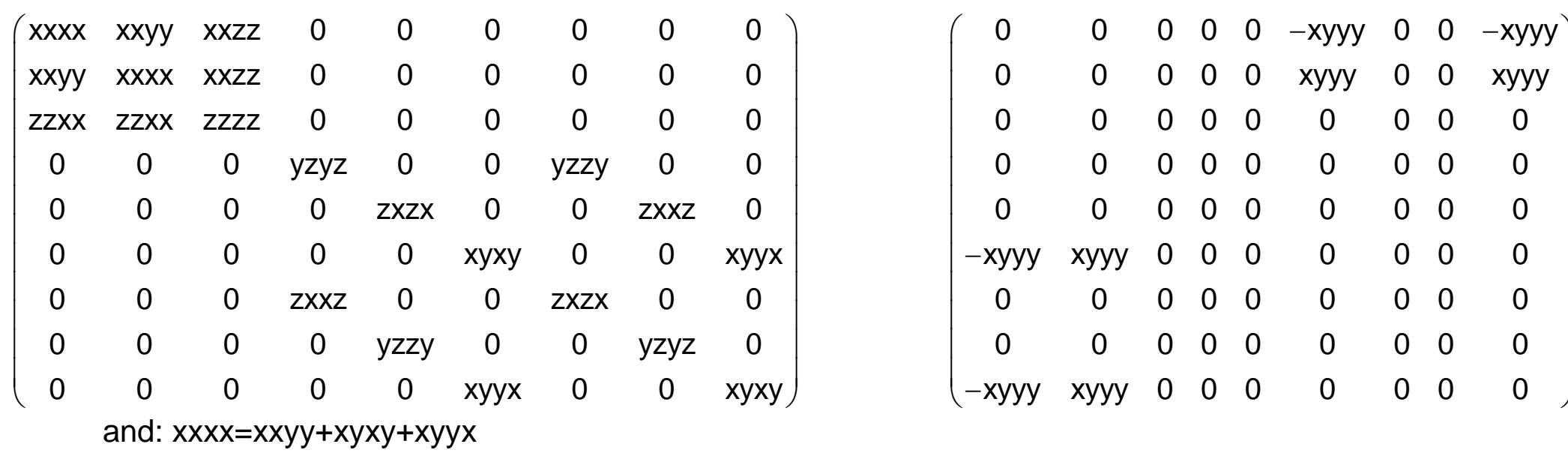

$\left(\begin{array}{ccccc}3 x x y y & 0 & 0 & y y z z & 0 \\ 0 & 3 x x y y & 0 & y y z z & 0 \\ 0 & 0 & z z z z & x x y y & 0\end{array}\right)$

$\left(\begin{array}{ccccc}0 & \text { xyyy } & 0 & 0 & 0 \\ -x y y y & 0 & 0 & 0 & 0 \\ 0 & 0 & 0 & 0 & 0\end{array}\right)$

$\left(\begin{array}{cccccccccc}3 x x y y & 0 & 0 & x x y y & 0 & 0 & y y z z & 0 & 0 & 0 \\ 0 & 3 x y y & 0 & 0 & y y z & 0 & 0 & x x y y & 0 & 0\end{array}\right)$

$\left(\begin{array}{cccccccccc}0 & \text { xyyy } & 0 & 0 & 0 & 0 & 0 & -x y y y & 0 & 0 \\ -x y y y & 0 & 0 & x y y & 0 & 0 & 0 & 0 & 0 & 0\end{array}\right)$ $\left(\begin{array}{cccccccccc}3 x x y y & 0 x y y y & 0 & 0 & y y z z & 0 & 0 & x x y y & 0 & 0 \\ 0 & 0 & \text { zzzz } & 0 & 0 & z z x x & 0 & 0 & z z x x & 0\end{array}\right)$

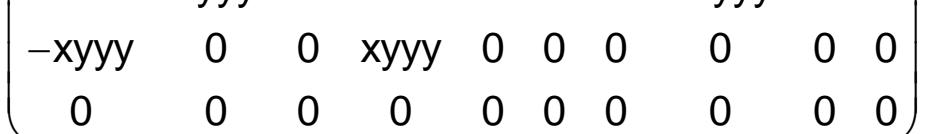

$$
\left(\begin{array}{cccccc}
x x x x & x x y y & x x z z & 0 & 0 & 0 \\
& \text { xxxx } & \text { xxzz } & 0 & 0 & 0 \\
& & \text { zzzz } & 0 & 0 & 0 \\
& & & y z y z & 0 & 0 \\
& & & & y z y z & 0 \\
& & & & & \text { xyxy }
\end{array}\right)
$$

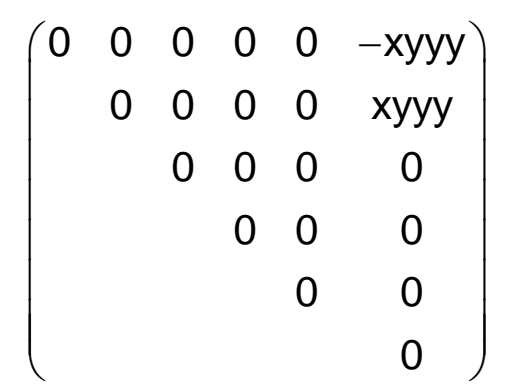

add: $x y x y=1 / 2(x x x x-x x y y)$
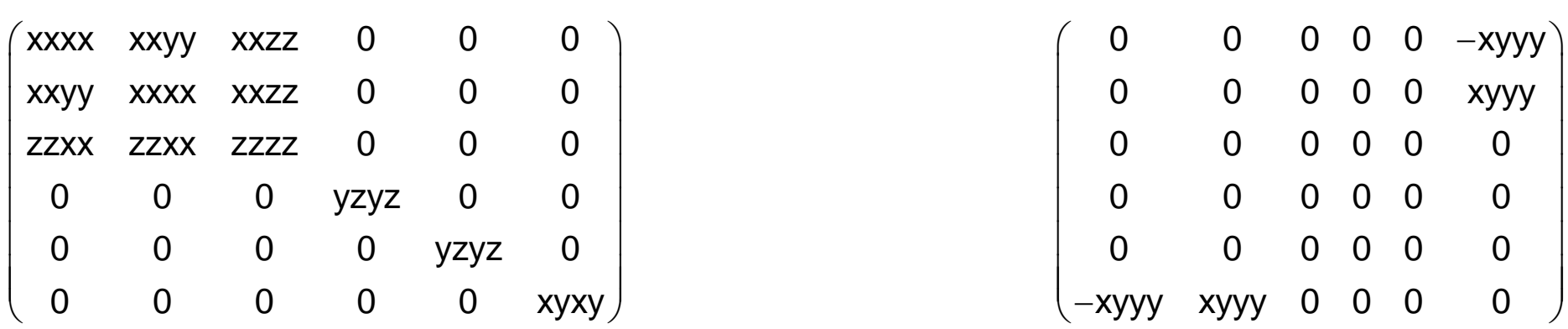

add: $x y x y=1 / 2(x x x x-x x y y)$ 
$\mathrm{n}=4 \quad \mathbf{D}_{4 \mathrm{~d}}=\overline{\mathbf{8}}_{\mathrm{z}} \mathbf{m}_{\mathrm{x}} \mathbf{2}_{1}$

$\left[\left(\mathrm{V}^{2}\right)\right]^{2}$

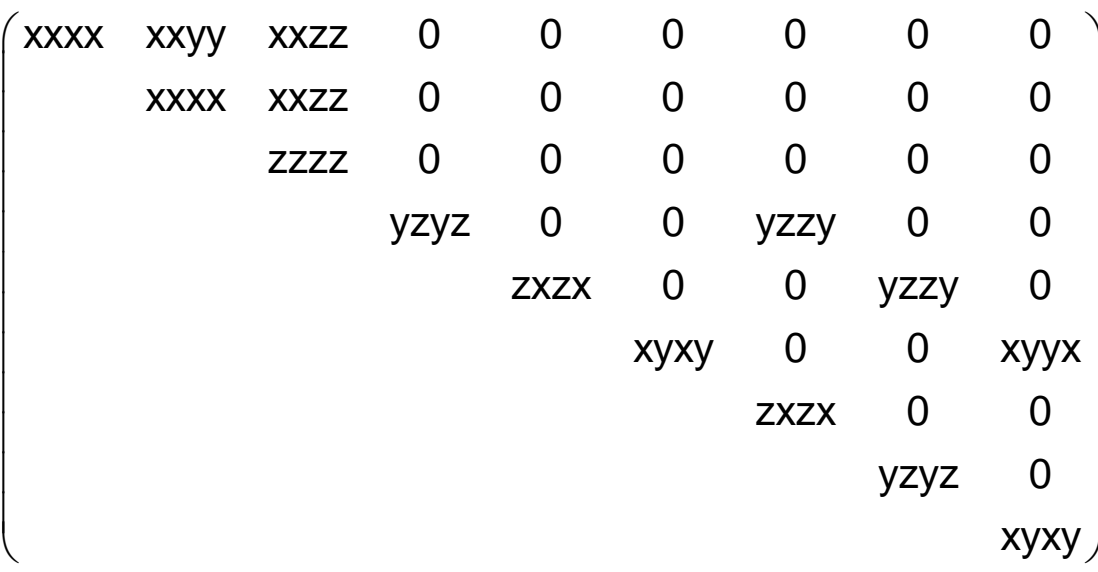

add: $x x x x=x x y y+x y x y+x y y x$

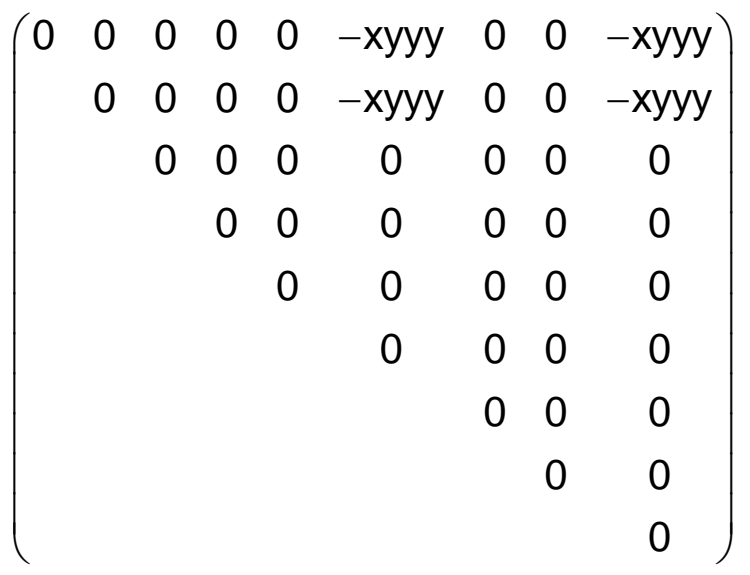

$\left[\mathrm{V}^{2}\right] \mathrm{V}^{2}\left(\begin{array}{ccccccccc}\mathrm{x} x \mathrm{x} & \mathrm{xxyy} & \mathrm{xxzz} & 0 & 0 & 0 & 0 & 0 & 0 \\ \mathrm{xxyy} & \mathrm{xxxx} & \mathrm{xxzz} & 0 & 0 & 0 & 0 & 0 & 0 \\ \mathrm{zzxx} & \mathrm{zzxx} & \mathrm{zzzz} & 0 & 0 & 0 & 0 & 0 & 0 \\ 0 & 0 & 0 & \mathrm{yzyz} & 0 & 0 & \mathrm{zxzx} & 0 & 0 \\ 0 & 0 & 0 & 0 & \mathrm{zxzx} & 0 & 0 & \mathrm{yzyz} & 0 \\ 0 & 0 & 0 & 0 & 0 & \mathrm{xyxy} & 0 & 0 & \text { xyxy }\end{array}\right)$

$\left(\begin{array}{ccccccccc}0 & 0 & 0 & 0 & 0 & -x y y y & 0 & 0 & -x y y y \\ 0 & 0 & 0 & 0 & 0 & x y y y & 0 & 0 & x y y y \\ 0 & 0 & 0 & 0 & 0 & 0 & 0 & 0 & 0 \\ 0 & 0 & 0 & 0 & 0 & 0 & 0 & 0 & 0 \\ 0 & 0 & 0 & 0 & 0 & 0 & 0 & 0 & 0 \\ -x y y y & x y y y & 0 & 0 & 0 & 0 & 0 & 0 & 0\end{array}\right)$

add: $x x x x=x x y y+2 x y x y$
$D_{n d}$

ae
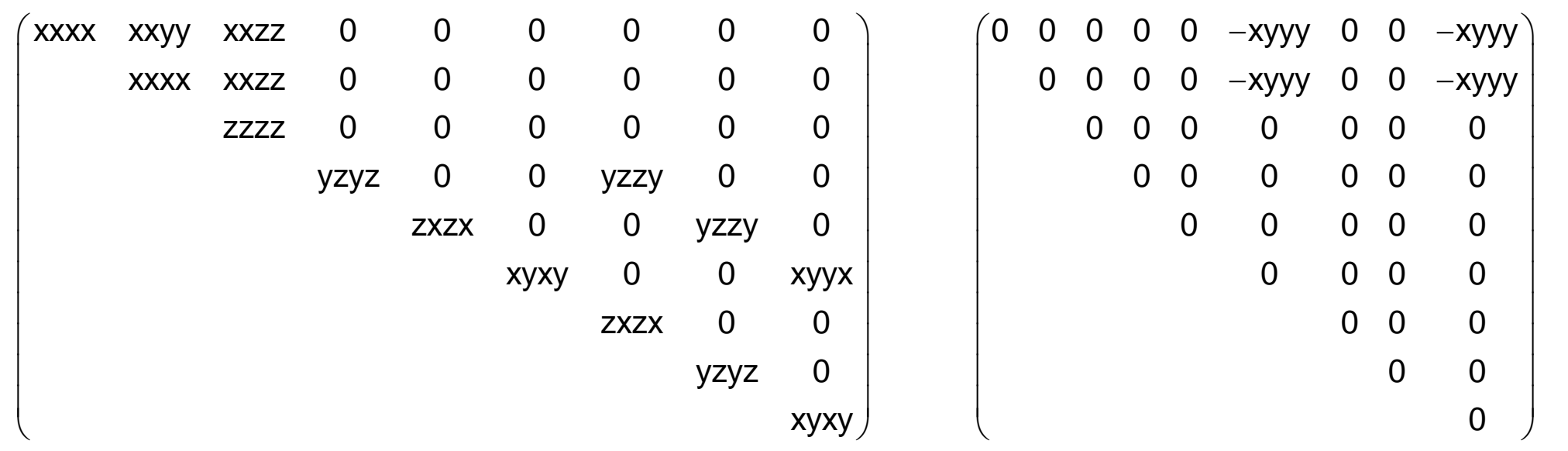

add: $x x x x=x x y y+x y x y+x y y x$

$\left(\begin{array}{ccccccccc}\text { xxxx } & \text { xxyy } & \text { xxzz } & 0 & 0 & 0 & 0 & 0 & 0 \\ \text { xxyy } & \text { xxxx } & \text { xxzz } & 0 & 0 & 0 & 0 & 0 & 0 \\ \text { zzxx } & \text { zzxx } & \text { zzzz } & 0 & 0 & 0 & 0 & 0 & 0 \\ 0 & 0 & 0 & y z y z & 0 & 0 & \text { zxzx } & 0 & 0 \\ 0 & 0 & 0 & 0 & \text { zxzx } & 0 & 0 & y z y z & 0 \\ 0 & 0 & 0 & 0 & 0 & \text { xyxy } & 0 & 0 & \text { xyxy }\end{array}\right)$

$\begin{array}{llllllll}0 & 0 & 0 & 0 & -x y y y & 0 & 0 & -x y y y\end{array}$ $\begin{array}{lllllllll}0 & 0 & 0 & 0 & 0 & \text { xyyy } & 0 & 0 & \text { xyyy } \\ 0 & 0 & 0 & 0 & 0 & 0 & 0 & 0 & 0\end{array}$ $\begin{array}{lllllllll}0 & 0 & 0 & 0 & 0 & 0 & 0 & 0 & 0 \\ 0 & 0 & 0 & 0 & 0 & 0 & 0 & 0 & 0\end{array}$ $\begin{array}{lllllllll}0 & 0 & 0 & 0 & 0 & 0 & 0 & 0 & 0\end{array}$

add: $x x x x=x x y y+2 x y x y$ 
$\mathrm{V}^{4}\left(\begin{array}{ccccccccc}\mathrm{xxxx} & \mathrm{xxyy} & \mathrm{xxzz} & 0 & 0 & 0 & 0 & 0 & 0 \\ \mathrm{yyxx} & \mathrm{xxxx} & \mathrm{xxzz} & 0 & 0 & 0 & 0 & 0 & 0 \\ \mathrm{zzxx} & \mathrm{zzyy} & \mathrm{zzzz} & 0 & 0 & 0 & 0 & 0 & 0 \\ 0 & 0 & 0 & \mathrm{yzyz} & 0 & 0 & \mathrm{yzzy} & 0 & 0 \\ 0 & 0 & 0 & 0 & \mathrm{zxzx} & 0 & 0 & \mathrm{zxxz} & 0 \\ 0 & 0 & 0 & 0 & 0 & \mathrm{xyxy} & 0 & 0 & \mathrm{xyyx} \\ 0 & 0 & 0 & \mathrm{zxxz} & 0 & 0 & \mathrm{zxzx} & 0 & 0 \\ 0 & 0 & 0 & 0 & \mathrm{yzzy} & 0 & 0 & \mathrm{yzyz} & 0 \\ 0 & 0 & 0 & 0 & 0 & \mathrm{xyyx} & 0 & 0 & \mathrm{xyxy}\end{array}\right)$

$\begin{array}{lllllllll}0 & 0 & 0 & 0 & 0 & 0 & 0 & 0 & 0 \\ 0 & 0 & 0 & 0 & 0 & 0 & 0 & 0 & 0\end{array}$ $0 \begin{array}{lllllllll}0 & 0 & 0 & 0 & 0 & 0 & 0 & 0 & 0 \\ 0 & 0 & 0 & 0 & 0 & 0 & 0 & 0 & 0\end{array}$

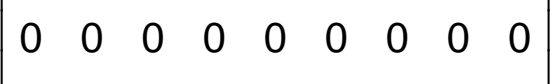

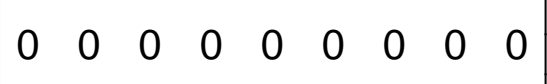
$\begin{array}{llllllllll}0 & 0 & 0 & 0 & 0 & 0 & 0 & 0 & 0\end{array}$ $0 \begin{array}{lllllllll}0 & 0 & 0 & 0 & 0 & 0 & 0 & 0 & 0 \\ 0 & 0 & 0 & 0 & 0 & 0 & 0 & 0 & 0\end{array}$ $0 \begin{array}{llllllllll}0 & 0 & 0 & 0 & 0 & 0 & 0 & 0 & 0 \\ 0 & 0 & 0 & 0 & 0 & 0 & 0 & 0 & 0\end{array}$ $0 \begin{array}{llllllllll}0 & 0 & 0 & 0 & 0 & 0 & 0 & 0 & 0 \\ 0 & 0 & 0 & 0 & 0 & 0 & 0 & 0 & 0\end{array}$ $\begin{array}{lllllllll}0 & 0 & 0 & 0 & 0 & 0 & 0 & 0 & 0\end{array}$

$\left[\mathrm{V}^{4}\right] \quad\left(\begin{array}{ccccc}3 x x y y & 0 & 0 & y y z z & 0 \\ 0 & 3 x x y y & 0 & y y z z & 0 \\ 0 & 0 & z z z z & x x y y & 0\end{array}\right)$

$\left(\begin{array}{lllll}0 & 0 & 0 & 0 & 0 \\ 0 & 0 & 0 & 0 & 0 \\ 0 & 0 & 0 & 0 & 0\end{array}\right)$

$\begin{array}{lllll}0 & 0 & 0 & 0 & 0\end{array}$

$\left(\begin{array}{llllllllll}0 & 0 & 0 & 0 & 0 & 0 & 0 & 0 & 0 & 0 \\ 0 & 0 & 0 & 0 & 0 & 0 & 0 & 0 & 0 & 0\end{array}\right)$ $\left.\begin{array}{llllllllll}0 & 0 & 0 & 0 & 0 & 0 & 0 & 0 & 0 & 0 \\ 0 & 0 & 0 & 0 & 0 & 0 & 0 & 0 & 0 & 0\end{array}\right)$

V $\left.\left[V^{3}\right] \quad \begin{array}{cccccccccc}3 x x y y & 0 & 0 & x x y y & 0 & 0 & y y z z & 0 & 0 & 0 \\ 0 & 3 x x y y & 0 & 0 & y y z z & 0 & 0 & x x y y & 0 & 0 \\ 0 & 0 & z z z & 0 & 0 & z z x x & 0 & 0 & z z x & 0\end{array}\right)$

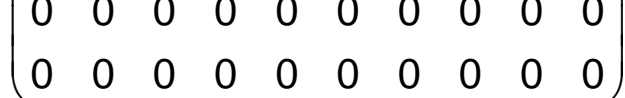

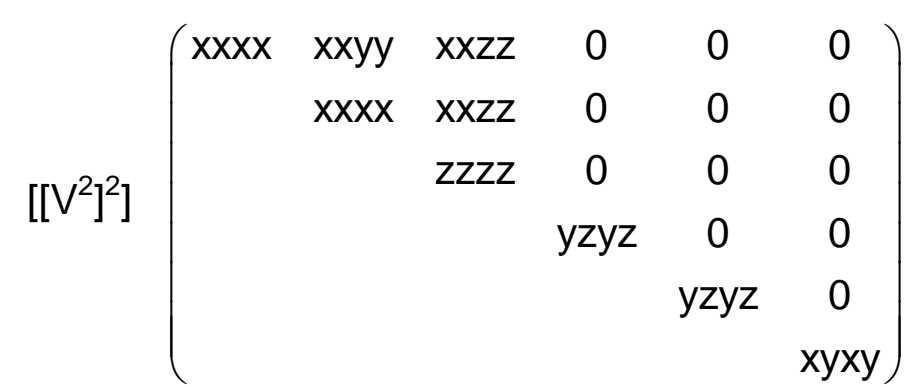

\begin{tabular}{|c|c|c|c|}
\hline & & & \\
\hline & & & 0 \\
\hline & 0 & & \\
\hline & & & 0 \\
\hline & & & \\
\hline
\end{tabular}

and $x y x y=1 / 2(x x x x-x x y y)$

$\left[\mathrm{V}^{2}\right]^{2}$ $\left(\begin{array}{cccccc}\text { xxxx } & x x y y & x x z z & 0 & 0 & 0 \\ x x y y & x x x x & x x z z & 0 & 0 & 0 \\ z z x x & z z x x & z z z z & 0 & 0 & 0 \\ 0 & 0 & 0 & y z y z & 0 & 0 \\ 0 & 0 & 0 & 0 & y z y z & 0 \\ 0 & 0 & 0 & 0 & 0 & x y x y\end{array}\right)$

and $x y x y=1 / 2(x x x x-x x y y)$

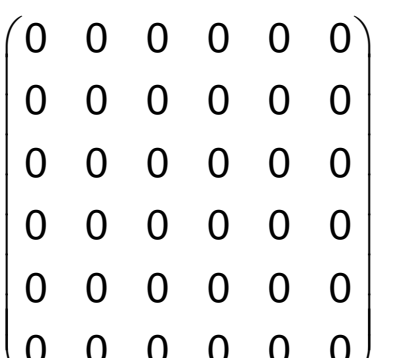

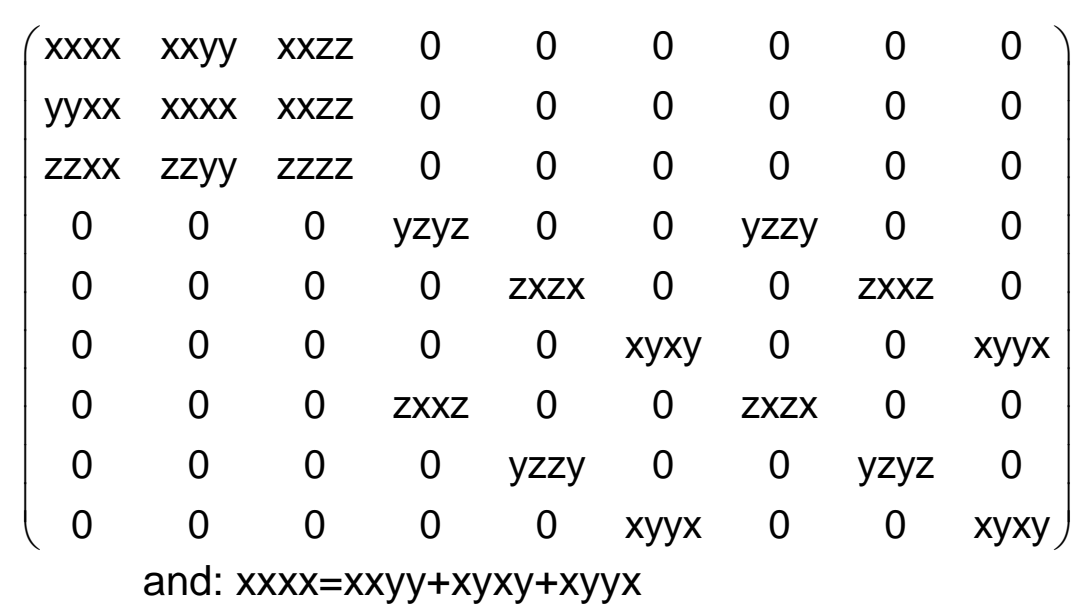

$\left(\begin{array}{lllllllll}0 & 0 & 0 & 0 & 0 & 0 & 0 & 0 & 0\end{array}\right)$ $\left.\begin{array}{llllllllll}0 & 0 & 0 & 0 & 0 & 0 & 0 & 0 & 0 \\ 0 & 0 & 0 & 0 & 0 & 0 & 0 & 0 & 0 \\ 0 & 0 & 0 & 0 & 0 & 0 & 0 & 0 & 0\end{array}\right)$ $\begin{array}{lllllllllll}0 & 0 & 0 & 0 & 0 & 0 & 0 & 0 & 0 \\ 0 & 0 & 0 & 0 & 0 & 0 & 0 & 0 & 0 \\ 0 & 0 & 0 & 0 & 0 & 0 & 0\end{array}$ $\begin{array}{llllllllllll}0 & 0 & 0 & 0 & 0 & 0 & 0 & 0 & 0 \\ 0 & 0 & 0 & 0 & 0 & 0 & 0 & 0 & 0 \\ 0 & 0 & 0 & 0 & 0 & 0\end{array}$ $\begin{array}{llllllllllll}0 & 0 & 0 & 0 & 0 & 0 & 0 & 0 & 0 \\ 0 & 0 & 0 & 0 & 0 & 0 & 0 & 0 & 0\end{array}$ $\begin{array}{llllllllll}0 & 0 & 0 & 0 & 0 & 0 & 0 & 0 & 0 \\ 0 & 0 & 0 & 0 & 0 & 0 & 0 & 0 & 0\end{array}$ $\begin{array}{lllllllll}0 & 0 & 0 & 0 & 0 & 0 & 0 & 0 & 0 \\ 0 & 0 & 0 & 0 & 0 & 0 & 0 & 0 & 0\end{array}$ $\begin{array}{llllllllll}0 & 0 & 0 & 0 & 0 & 0 & 0 & 0 & 0\end{array}$

$\left(\begin{array}{ccccc}3 x x y y & 0 & 0 & y y z z & 0 \\ 0 & 3 x x y y & 0 & y y z z & 0 \\ 0 & 0 & z z z z & x x y y & 0\end{array}\right)$

$\left(\begin{array}{lllll}0 & 0 & 0 & 0 & 0 \\ 0 & 0 & 0 & 0 & 0 \\ 0 & 0 & 0 & 0 & 0\end{array}\right)$ $\left(\begin{array}{lllll}0 & 0 & 0 & 0 & 0 \\ 0 & 0 & 0 & 0 & 0\end{array}\right)$

$\left(\begin{array}{cccccccccc}3 x x y y & 0 & 0 & x x y y & 0 & 0 & y y z z & 0 & 0 & 0 \\ 0 & 3 x x y y & 0 & 0 & y y z z & 0 & 0 & x x y y & 0 & 0 \\ 0 & 0 & z z z z & 0 & 0 & z z x & 0 & 0 & z z x & 0\end{array}\right)$

$\left(\begin{array}{llllllllll}0 & 0 & 0 & 0 & 0 & 0 & 0 & 0 & 0 & 0 \\ 0 & 0 & 0 & 0 & 0 & 0 & 0 & 0 & 0 & 0\end{array}\right)$

$\begin{array}{llllllllll}0 & 0 & 0 & 0 & 0 & 0 & 0 & 0 & 0 & 0 \\ 0 & 0 & 0 & 0 & 0 & 0 & 0 & 0 & 0 & 0\end{array}$

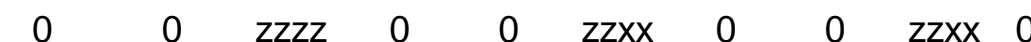

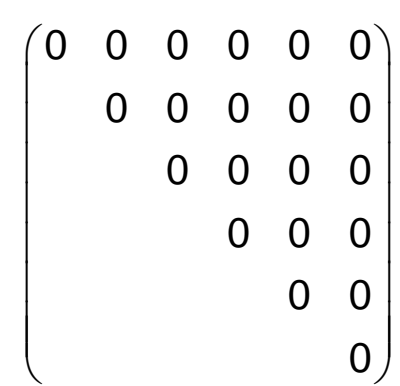

and $x y x y=1 / 2(x x x x-x x y y)$

$$
\left(\begin{array}{cccccc}
x x x x & x x y y & x x z z & 0 & 0 & 0 \\
& x x x x & x x z z & 0 & 0 & 0 \\
& & z z z z & 0 & 0 & 0 \\
& & & y z y z & 0 & 0 \\
& & & & y z y z & 0 \\
& & & & & \\
& & & & & \\
& & & & &
\end{array}\right.
$$

$\left(\begin{array}{cccccc}x x x x & x x y y & x x z z & 0 & 0 & 0 \\ x x y y & x x x x & x x z z & 0 & 0 & 0 \\ z z x x & z z x x & z z z z & 0 & 0 & 0 \\ 0 & 0 & 0 & y z y z & 0 & 0 \\ 0 & 0 & 0 & 0 & y z y z & 0 \\ 0 & 0 & 0 & 0 & 0 & x y x y\end{array}\right)$

$\left(\begin{array}{llllll}0 & 0 & 0 & 0 & 0 & 0 \\ 0 & 0 & 0 & 0 & 0 & 0\end{array}\right)$

$\begin{array}{llllll}0 & 0 & 0 & 0 & 0 & 0 \\ 0 & 0 & 0 & 0 & 0 & 0 \\ 0 & 0 & 0 & 0 & 0 & 0\end{array}$

$\begin{array}{lllllll}0 & 0 & 0 & 0 & 0 & 0 \\ 0 & 0 & 0 & 0 & 0\end{array}$

$\begin{array}{llllll}0 & 0 & 0 & 0 & 0 & 0 \\ 0 & 0 & 0 & 0 & 0 & 0\end{array}$

and $x y x y=1 / 2(x x x x-x x y y)$ 
a

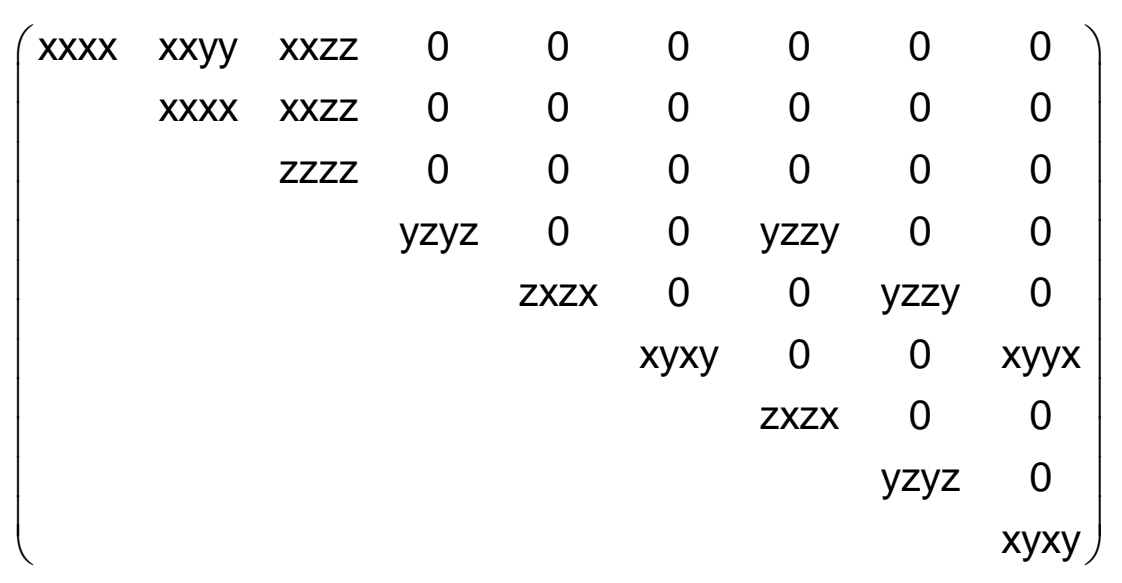

and $x x x x=x x y y+y z y z+z y z y$

$\left(\begin{array}{lllllllll}x x x x & x x y y & x x z z & 0 & 0 & 0 & 0 & 0 & 0 \\ x x y y & x x x x & x x z z & 0 & 0 & 0 & 0 & 0 & 0\end{array}\right) \quad\left(\begin{array}{lllllllll}0 & 0 & 0 & 0 & 0 & 0 & 0 & 0 & 0 \\ 0 & 0 & 0 & 0 & 0 & 0 & 0 & 0 & 0 \\ 0 & 0 & 0 & 0 & 0 & 0 & 0 & 0 & 0\end{array}\right)$

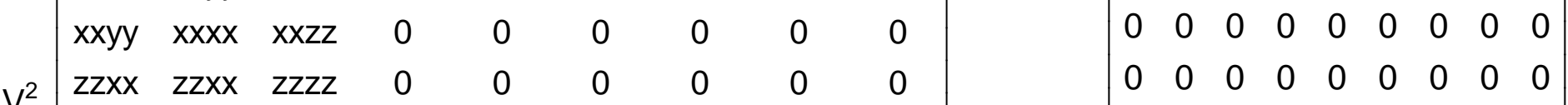

$\left[\begin{array}{lllllllll}0 & 0 & 0 & y z y z & 0 & 0 & z x z x & 0 & 0 \\ 0 & 0 & 0 & 0 & 2 x z x & 0 & 0 & y z y z & 0\end{array} \mid \begin{array}{llllllllllll}0 & 0 & 0 & 0 & 0 & 0 & 0 & 0 & 0 & 0 \\ 0 & 0 & 0 & 0 & 0 & 0 & 0 & 0 & 0 \\ 0 & 0 & 0 & 0 & 0 & 0 & 0 & 0 & 0\end{array}\right.$

$\begin{array}{lllllllll}0 & 0 & 0 & 0 & 0 & 0 & 0 & 0 & 0 \\ 0 & 0 & 0 & 0 & 0 & 0 & 0 & 0 & 0 \\ 0 & 0 & 0 & 0 & 0 & 0 & 0 & 0 & 0\end{array}$

$\begin{array}{llllllllll}0 & 0 & 0 & 0 & 0 & 0 & 0 & 0 & 0\end{array}$

and $x x x x=x x y y+2 x y x y$

and $x x x x=x x y y+y z y z+z y z y$

$\left(\begin{array}{lllllllll}0 & 0 & 0 & 0 & 0 & 0 & 0 & 0 & 0\end{array}\right.$ $\begin{array}{llllllll}0 & 0 & 0 & 0 & 0 & 0 & 0 & 0\end{array}$

$\begin{array}{lllllll}0 & 0 & 0 & 0 & 0 & 0 & 0\end{array}$ $\begin{array}{lllll}0 & 0 & 0 & 0 & 0 \\ 0 & 0 & 0 & 0 & 0\end{array}$ $\begin{array}{lllll}0 & 0 & 0 & 0 & 0 \\ 0 & 0 & 0 & 0 & 0\end{array}$ $\begin{array}{llll}0 & 0 & 0 \\ 0 & 0 & 0\end{array}$ $\begin{array}{lll}0 & 0 & 0 \\ & 0 & 0 \\ & & 0\end{array}$

$\left(\begin{array}{ccccccccc}x x x x & x x y y & x x z z & 0 & 0 & 0 & 0 & 0 & 0 \\ x x y y & x x x x & x x z z & 0 & 0 & 0 & 0 & 0 & 0 \\ z z x x & z z x x & z z z z & 0 & 0 & 0 & 0 & 0 & 0 \\ 0 & 0 & 0 & y z y z & 0 & 0 & z x z x & 0 & 0 \\ 0 & 0 & 0 & 0 & z x z x & 0 & 0 & y z y z & 0 \\ 0 & 0 & 0 & 0 & 0 & x y x y & 0 & 0 & \text { xyxy }\end{array}\right) \quad\left(\begin{array}{ccccccccc}0 & 0 & 0 & 0 & 0 & 0 & 0 & 0 & 0 \\ 0 & 0 & 0 & 0 & 0 & 0 & 0 & 0 & 0 \\ 0 & 0 & 0 & 0 & 0 & 0 & 0 & 0 & 0 \\ 0 & 0 & 0 & 0 & 0 & 0 & 0 & 0 & 0 \\ 0 & 0 & 0 & 0 & 0 & 0 & 0 & 0 & 0 \\ 0 & 0 & 0 & 0 & 0 & 0 & 0 & 0 & 0\end{array}\right)$


$\left(\begin{array}{cccccccccc}x x x x & 0 & 0 & x x y y & 0 & 0 & x x z z & 0 & 0 & 0\end{array}\right)$ $\left(\begin{array}{lllll}0 & \text { zyyy } & 0 & 0 & 0\end{array}\right)$

$$
\left(\begin{array}{cccccc}
x x x x & x x y y & x x z z & 0 & 0 & 0 \\
& \text { yyyy } & y y z z & 0 & 0 & 0 \\
& & z z z z & 0 & 0 & 0 \\
& & & y z y z & 0 & 0 \\
& & & & x z x z & 0 \\
& & & & & \text { xyxy }
\end{array}\right)
$$

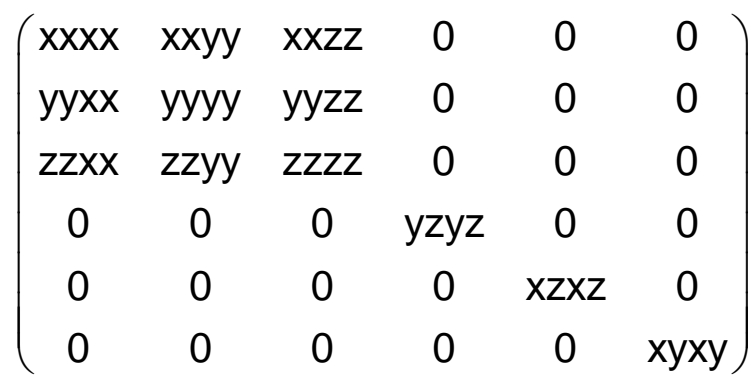

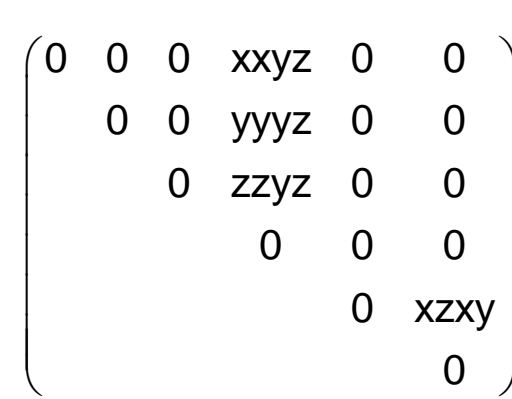

$\left(\begin{array}{cccccc}0 & 0 & 0 & x x y z & 0 & 0 \\ 0 & 0 & 0 & y y y z & 0 & 0 \\ 0 & 0 & 0 & z z y z & 0 & 0 \\ y z x x & y z y y & y z z z & 0 & 0 & 0 \\ 0 & 0 & 0 & 0 & 0 & x z x y \\ 0 & 0 & 0 & 0 & x y x z & 0\end{array}\right)$ 
$\mathrm{n}=1 \quad \mathbf{D}_{1 \mathrm{~h}}=\mathbf{2}_{\mathrm{x}} \mathbf{m}_{\mathrm{y}} \mathbf{m}_{\mathrm{z}}$

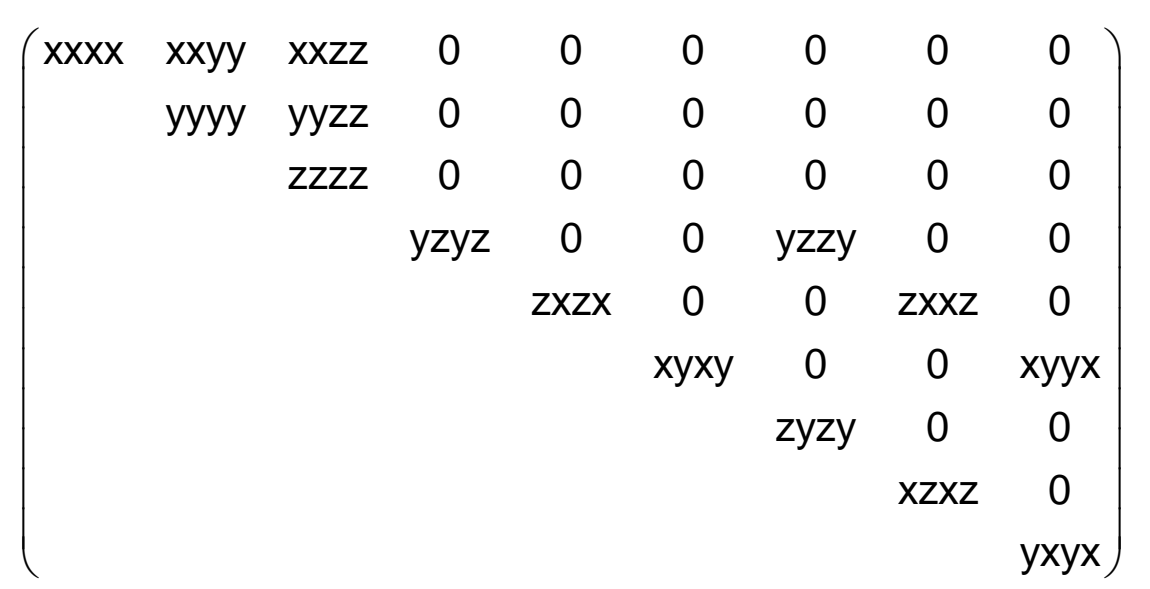

$$
\text { ( }
$$

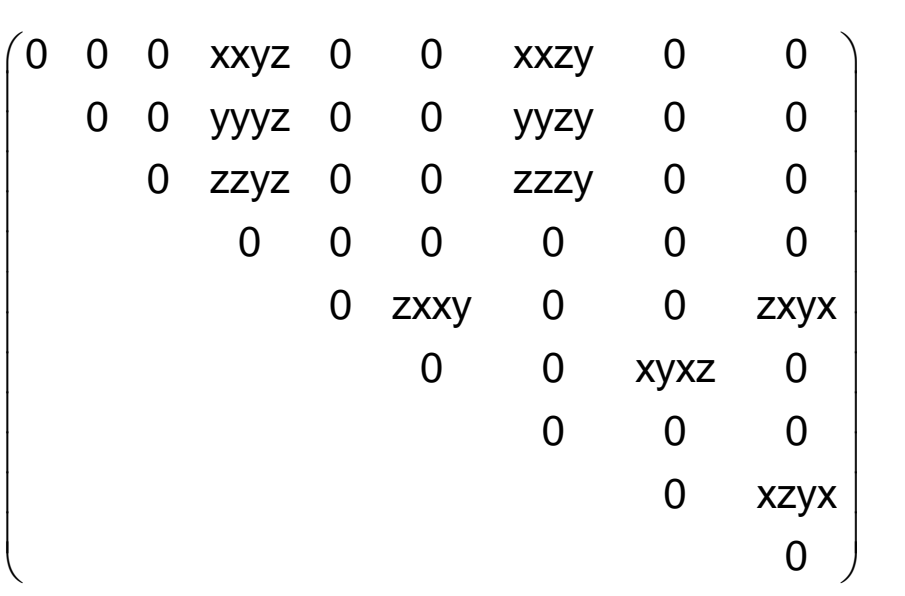

$D_{\text {nh }}$ ae

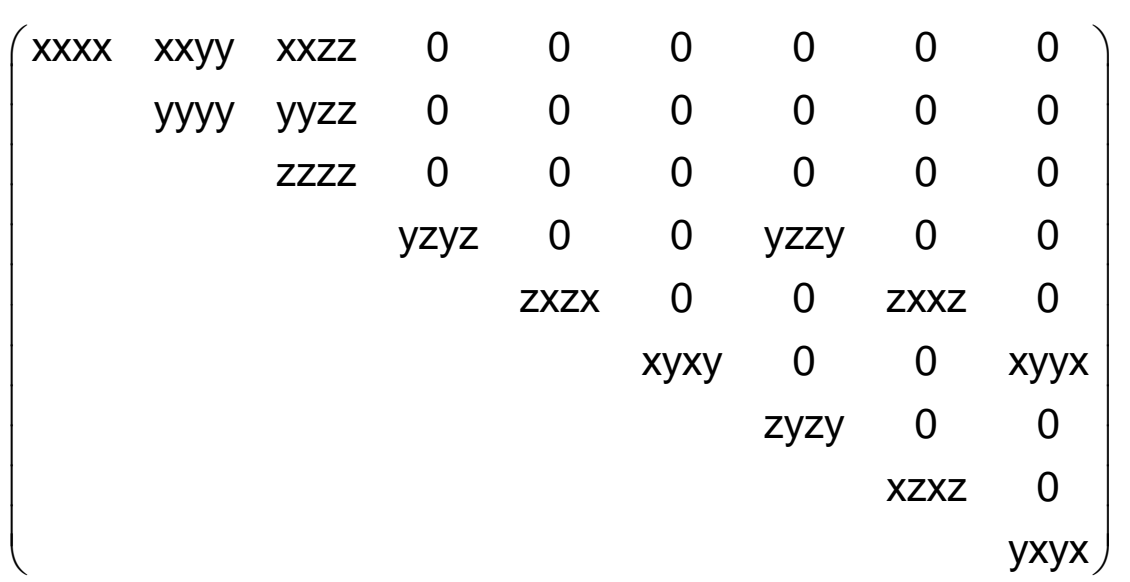

$\left(\begin{array}{lllllllll}0 & 0 & 0 & x x y z & 0 & 0 & x x z y & 0 & 0 \\ & 0 & 0 & y y y z & 0 & 0 & y y z y & 0 & 0\end{array}\right)$

$\begin{array}{llllllll}0 & 0 & \text { yyyz } & 0 & 0 & \text { yyzy } & 0 & 0\end{array}$ $\begin{array}{cccccc}\text { zzyz } & 0 & 0 & \text { zzzy } & 0 & 0 \\ 0 & 0 & 0 & 0 & 0 & 0\end{array}$ $\begin{array}{ccccc}0 & 0 & 0 & 0 & 0 \\ 0 & z x y y & 0 & 0 & z x y x\end{array}$ $\begin{array}{cccc}0 & 0 & x y x z & 0\end{array}$ $\begin{array}{ccc}0 & x y x z & 0 \\ 0 & 0 & 0 \\ 0 & x z y x\end{array}$

$\left(\begin{array}{ccccccccc}\text { xxxx } & \text { xxyy } & \text { xxzz } & 0 & 0 & 0 & 0 & 0 & 0 \\ \text { yyxx } & \text { yyyy } & \text { yyzz } & 0 & 0 & 0 & 0 & 0 & 0 \\ \text { zzxx } & \text { zzyy } & \text { zzzz } & 0 & 0 & 0 & 0 & 0 & 0 \\ 0 & 0 & 0 & \text { yzyz } & 0 & 0 & \text { yzzy } & 0 & 0 \\ 0 & 0 & 0 & 0 & \text { zxzx } & 0 & 0 & \text { zxxz } & 0 \\ 0 & 0 & 0 & 0 & 0 & \text { xyxy } & 0 & 0 & \text { xyyx }\end{array}\right) \quad\left(\begin{array}{cccccccccc}0 & 0 & 0 & \text { xxyz } & 0 & 0 & \text { xxzy } & 0 & 0 \\ 0 & 0 & 0 & \text { yyyz } & 0 & 0 & \text { yyzy } & 0 & 0 \\ 0 & 0 & 0 & \text { zzyz } & 0 & 0 & \text { zzzy } & 0 & 0 \\ \text { yzxx } & \text { yzyy } & \text { yzzz } & 0 & 0 & 0 & 0 & 0 & 0 \\ 0 & 0 & 0 & 0 & 0 & \text { zxxy } & 0 & 0 & \text { zxyx } \\ 0 & 0 & 0 & 0 & \text { xyzx } & 0 & 0 & \text { xyxz } & 0\end{array}\right)$ $\left(\begin{array}{lllllllll}x x x x & x x y y & x x z z & 0 & 0 & 0 & 0 & 0 & 0 \\ y y x x & y y y y & y y z z & 0 & 0 & 0 & 0 & 0 & 0\end{array}\right)$

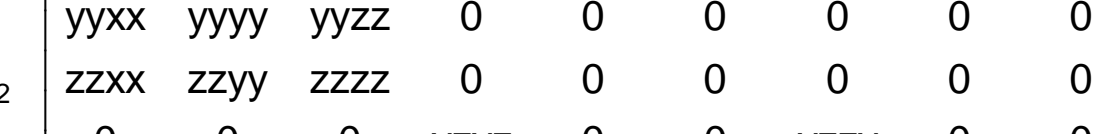

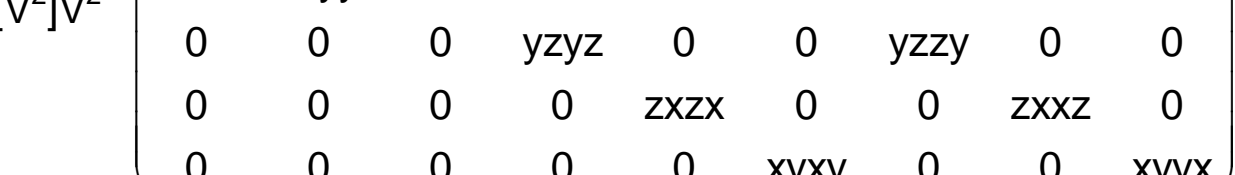

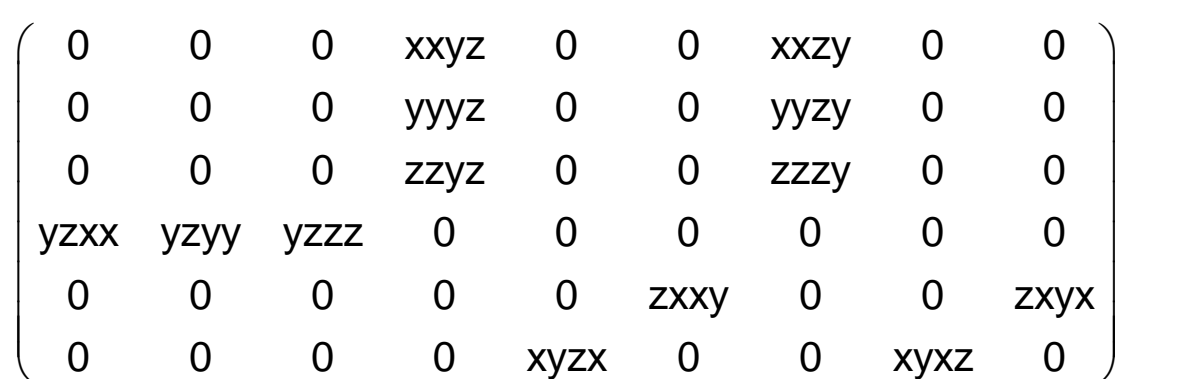




$\left(\begin{array}{ccccccccc}\text { xxxx } & \text { xxyy } & \text { xxzz } & 0 & 0 & 0 & 0 & 0 & 0 \\ \text { yyxx } & \text { yyyy } & \text { yyzz } & 0 & 0 & 0 & 0 & 0 & 0 \\ \text { zzxx } & \text { zzyy } & \text { zzzz } & 0 & 0 & 0 & 0 & 0 & 0 \\ 0 & 0 & 0 & \text { yzyz } & 0 & 0 & \text { yzzy } & 0 & 0 \\ 0 & 0 & 0 & 0 & \text { zxzx } & 0 & 0 & \text { zxxz } & 0 \\ 0 & 0 & 0 & 0 & 0 & \text { xyxy } & 0 & 0 & \text { xyyx } \\ 0 & 0 & 0 & \text { zyyz } & 0 & 0 & \text { zyzy } & 0 & 0 \\ 0 & 0 & 0 & 0 & \text { xzzx } & 0 & 0 & \text { xzxz } & 0 \\ 0 & 0 & 0 & 0 & 0 & \text { yxxy } & 0 & 0 & \text { yxyx }\end{array}\right)$

$\left[V^{4}\right] \quad\left(\begin{array}{ccccc}x x x x & 0 & 0 & y y z z & 0 \\ 0 & y y y y & 0 & z z x x & 0 \\ 0 & 0 & z z z z & x x y y & 0\end{array}\right)$

$\left(\begin{array}{lllllllll}0 & 0 & 0 & 0 & 0 & 0 & 0 & 0 & 0 \\ 0 & 0 & 0 & 0 & 0 & 0 & 0 & 0 & 0\end{array}\right)$ $\begin{array}{lllllllll}0 & 0 & 0 & 0 & 0 & 0 & 0 & 0 & 0 \\ 0 & 0 & 0 & 0 & 0 & 0 & 0 & 0 & 0\end{array}$ $0 \begin{array}{lllllllll}0 & 0 & 0 & 0 & 0 & 0 & 0 & 0 & 0 \\ 0 & 0 & 0 & 0 & 0 & 0 & 0 & 0 & 0\end{array}$ $\begin{array}{lllllllll}0 & 0 & 0 & 0 & 0 & 0 & 0 & 0 & 0 \\ 0 & 0 & 0 & 0 & 0 & 0 & 0 & 0 & 0\end{array}$ $0 \begin{array}{lllllllll}0 & 0 & 0 & 0 & 0 & 0 & 0 & 0 & 0 \\ 0 & 0 & 0 & 0 & 0 & 0 & 0 & 0 & 0\end{array}$ $0 \begin{array}{llllllllll}0 & 0 & 0 & 0 & 0 & 0 & 0 & 0 & 0 \\ 0 & 0 & 0 & 0 & 0 & 0 & 0 & 0 & 0\end{array}$ $\begin{array}{llllllllll}0 & 0 & 0 & 0 & 0 & 0 & 0 & 0 & 0 & 0\end{array}$ $\begin{array}{lllllllll}0 & 0 & 0 & 0 & 0 & 0 & 0 & 0 & 0 \\ 0 & 0 & 0 & 0 & 0 & 0 & 0 & 0 & 0\end{array}$ $\left(\begin{array}{lllllllll}0 & 0 & 0 & 0 & 0 & 0 & 0 & 0 & 0 \\ 0 & 0 & 0 & 0 & 0 & 0 & 0 & 0 & 0\end{array}\right)$

$\left.V^{3}\right]\left(\begin{array}{cccccccccc}x x x x & 0 & 0 & x x y y & 0 & 0 & x x z z & 0 & 0 & 0 \\ 0 & y y y y & 0 & 0 & y y z z & 0 & 0 & y y x x & 0 & 0 \\ 0 & 0 & z z z z & 0 & 0 & z z x & 0 & 0 & z z y y & 0\end{array}\right)$ $\left(\begin{array}{lllll}0 & 0 & 0 & 0 & 0 \\ 0 & 0 & 0 & 0 & 0 \\ 0 & 0 & 0 & 0 & 0\end{array}\right)$ $\begin{array}{lllll}0 & 0 & 0 & 0 & 0\end{array}$

$\begin{array}{llllllllll}0 & 0 & 0 & 0 & 0 & 0 & 0 & 0 & 0 & 0 \\ 0 & 0 & 0 & 0 & 0 & 0 & 0 & 0 & 0 & 0\end{array}$ $\left.\begin{array}{llllllllll}0 & 0 & 0 & 0 & 0 & 0 & 0 & 0 & 0 & 0 \\ 0 & 0 & 0 & 0 & 0 & 0 & 0 & 0 & 0 & 0\end{array}\right)$

$$
\left[\left[^{2}\right]^{2}\right]\left(\begin{array}{cccccc}
\text { xxxx } & \text { xxyy } & \text { xxzz } & 0 & 0 & 0 \\
& \text { yyyy } & \text { yyzz } & 0 & 0 & 0 \\
& & \text { zzzz } & 0 & 0 & 0 \\
& & & y z y z & 0 & 0 \\
& & & & \text { xzxz } & 0 \\
& & & & & \text { xyxy }
\end{array}\right)
$$

$\left[V^{2}\right]^{2}\left(\begin{array}{cccccc}x x x x & x x y y & x x z z & 0 & 0 & 0 \\ y y x x & y y y y & y y z z & 0 & 0 & 0 \\ z z x x & z z y y & z z z z & 0 & 0 & 0 \\ 0 & 0 & 0 & y z y z & 0 & 0 \\ 0 & 0 & 0 & 0 & x z x z & 0 \\ 0 & 0 & 0 & 0 & 0 & x y x y\end{array}\right)$

$$
\left(\begin{array}{llllll}
0 & 0 & 0 & 0 & 0 & 0 \\
& 0 & 0 & 0 & 0 & 0 \\
& & 0 & 0 & 0 & 0 \\
& & & 0 & 0 & 0 \\
& & & & 0 & 0 \\
& & & & & \\
& & & & & 0
\end{array}\right)
$$

$\left(\begin{array}{llllll}0 & 0 & 0 & 0 & 0 & 0 \\ 0 & 0 & 0 & 0 & 0 & 0\end{array}\right.$ $\begin{array}{lllllll}0 & 0 & 0 & 0 & 0 & 0 \\ 0 & 0 & 0 & 0 & 0 & 0 \\ 0 & 0 & 0 & 0 & 0 & 0\end{array}$ $\begin{array}{llllll}0 & 0 & 0 & 0 & 0 & 0\end{array}$ $\begin{array}{llllll}0 & 0 & 0 & 0 & 0 & 0 \\ 0 & 0 & 0 & 0 & 0\end{array}$ $\left(\begin{array}{lllllll}0 & 0 & 0 & 0 & 0 & 0 \\ 0 & 0 & 0 & 0 & 0 & 0 & 0\end{array}\right.$ $\left(\begin{array}{ccccccccc}\text { xxxx } & \text { xxyy } & \text { xxzz } & 0 & 0 & 0 & 0 & 0 & 0 \\ \text { yyxx } & \text { yyyy } & \text { yyzz } & 0 & 0 & 0 & 0 & 0 & 0 \\ \text { zzxx } & \text { zzyy } & \text { zzzz } & 0 & 0 & 0 & 0 & 0 & 0 \\ 0 & 0 & 0 & \text { yzyz } & 0 & 0 & \text { yzzy } & 0 & 0 \\ 0 & 0 & 0 & 0 & \text { zxzx } & 0 & 0 & \text { zxxz } & 0 \\ 0 & 0 & 0 & 0 & 0 & x y x y & 0 & 0 & \text { xyyx } \\ 0 & 0 & 0 & z y y z & 0 & 0 & \text { zyzy } & 0 & 0 \\ 0 & 0 & 0 & 0 & \text { xzzx } & 0 & 0 & \text { xzxz } & 0 \\ 0 & 0 & 0 & 0 & 0 & \text { yxxy } & 0 & 0 & \text { yxyx }\end{array}\right)$

$\left(\begin{array}{ccccc}x x x x & 0 & 0 & y y z z & 0 \\ 0 & y y y y & 0 & z z x x & 0 \\ 0 & 0 & z z z z & x x y y & 0\end{array}\right)$

$\left(\begin{array}{cccccccccc}x x x x & 0 & 0 & x x y y & 0 & 0 & x x z z & 0 & 0 & 0 \\ 0 & 0 & 0 & 0 & y z z & 0 & 0 & y y x & 0 & 0\end{array}\right) \quad\left(\begin{array}{cccccccccc}0 & 0 & 0 & 0 & 0 & 0 & 0 & 0 & 0 & 0 \\ 0 & 0 & 0 & 0 & 0 & 0 & 0 & 0 & 0 & 0 \\ 0 & 0 & 0 & 0 & 0 & 0 & 0 & 0 & 0 & 0\end{array}\right)$

$\left.\begin{array}{cccccccccc}0 & \text { yyyy } & 0 & 0 & y y z z & 0 & 0 & y y x x & 0 & 0 \\ 0 & 0 & z z z z & 0 & 0 & z z x x & 0 & 0 & z z y y & 0\end{array}\right) \quad\left(\begin{array}{cccccccccc}0 & 0 & 0 & 0 & 0 & 0 & 0 & 0 & 0 & 0 \\ 0 & 0 & 0 & 0 & 0 & 0 & 0 & 0 & 0 & 0\end{array}\right)$

$$
\left(\begin{array}{cccccc}
\text { xxxx } & \text { xxyy } & \text { xxzz } & 0 & 0 & 0 \\
& \text { yyyy } & \text { yyzz } & 0 & 0 & 0 \\
& & \text { zzzz } & 0 & 0 & 0 \\
& & & y z y z & 0 & 0 \\
& & & & x z x z & 0 \\
& & & & & \text { xyxy }
\end{array}\right)
$$

$\left(\begin{array}{cccccc}\text { xxxx } & \text { xxyy } & \text { xxzz } & 0 & 0 & 0 \\ \text { yyxx } & \text { yyyy } & \text { yyzz } & 0 & 0 & 0 \\ \text { zzxx } & \text { zzyy } & \text { zzzz } & 0 & 0 & 0 \\ 0 & 0 & 0 & \text { yzyz } & 0 & 0 \\ 0 & 0 & 0 & 0 & \text { xzxz } & 0 \\ 0 & 0 & 0 & 0 & 0 & \text { xyxy }\end{array}\right)$

$\left(\begin{array}{lllllllll}0 & 0 & 0 & 0 & 0 & 0 & 0 & 0 & 0 \\ 0 & 0 & 0 & 0 & 0 & 0 & 0 & 0 & 0\end{array}\right)$ $\begin{array}{lllllllll}0 & 0 & 0 & 0 & 0 & 0 & 0 & 0 & 0 \\ 0 & 0 & 0 & 0 & 0 & 0 & 0 & 0 & 0\end{array}$ $\begin{array}{lllllllll}0 & 0 & 0 & 0 & 0 & 0 & 0 & 0 & 0 \\ 0 & 0 & 0 & 0 & 0 & 0 & 0 & 0 & 0 \\ 0 & 0 & 0 & 0 & 0 & 0 & 0 & 0 & 0\end{array}$ $\begin{array}{llllllllll}0 & 0 & 0 & 0 & 0 & 0 & 0 & 0 & 0 \\ 0 & 0 & 0 & 0 & 0 & 0 & 0 & 0 & 0\end{array}$ $0 \begin{array}{llllllllll}0 & 0 & 0 & 0 & 0 & 0 & 0 & 0 & 0 \\ 0 & 0 & 0 & 0 & 0 & 0 & 0 & 0\end{array}$ $\begin{array}{lllllllll}0 & 0 & 0 & 0 & 0 & 0 & 0 & 0 & 0 \\ 0 & 0 & 0 & 0 & 0 & 0 & 0 & 0 & 0\end{array}$ $\begin{array}{lllllllll}0 & 0 & 0 & 0 & 0 & 0 & 0 & 0 & 0\end{array}$ $\left(\begin{array}{lllll}0 & 0 & 0 & 0 & 0 \\ 0 & 0 & 0 & 0 & 0 \\ 0 & 0 & 0 & 0 & 0\end{array}\right)$

$\begin{array}{lllll}0 & 0 & 0 & 0 & 0\end{array}$

$$
\begin{aligned}
& \left.\begin{array}{lllll}
0 & 0 & 0 & 0 & 0 \\
0 & 0 & 0 & 0 & 0
\end{array}\right) \\
& 0
\end{aligned}
$$

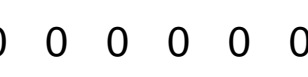

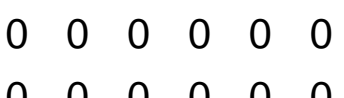
$\begin{array}{llllll}0 & 0 & 0 & 0 & 0 & 0 \\ 0 & 0 & 0 & 0\end{array}$ $\begin{array}{llllll}0 & 0 & 0 & 0 & 0 & 0 \\ 0 & 0 & 0 & 0 & 0 & 0\end{array}$ $\left(\begin{array}{llllll}0 & 0 & 0 & 0 & 0 & 0 \\ 0 & 0 & 0 & 0 & 0 & 0\end{array}\right.$ 


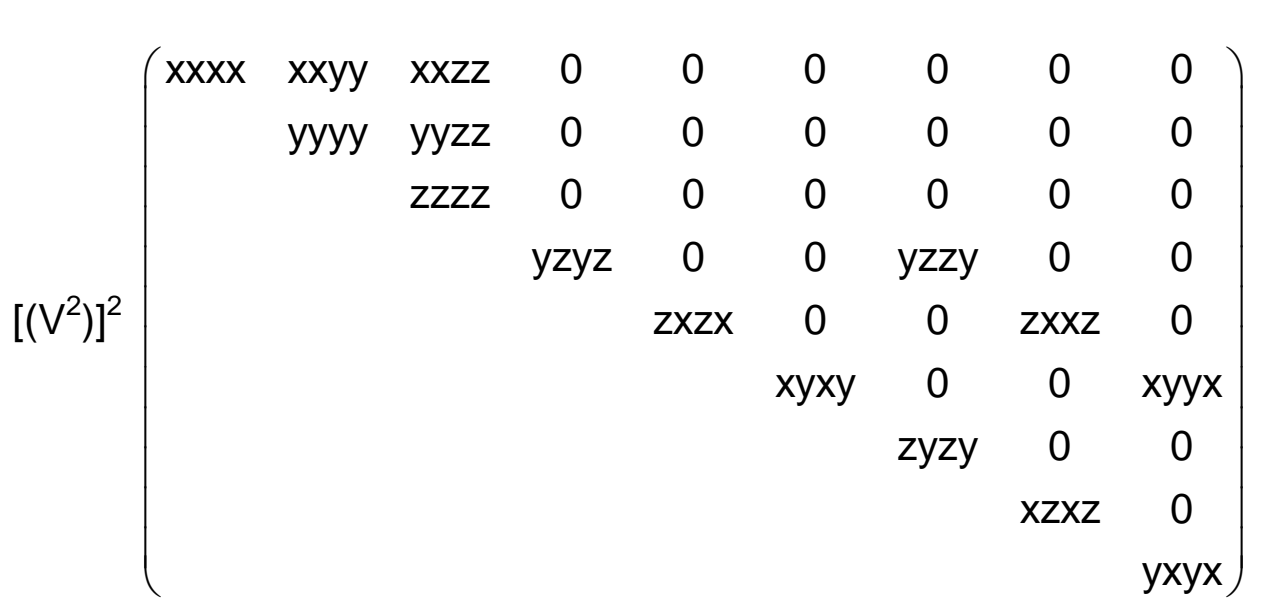

$\left[\mathrm{V}^{2}\right] \mathrm{V}^{2}\left(\begin{array}{ccccccccc}\mathrm{xxxx} & \mathrm{xxyy} & \mathrm{xxzz} & 0 & 0 & 0 & 0 & 0 & 0 \\ \mathrm{yyxx} & \mathrm{yyyy} & \mathrm{yyzz} & 0 & 0 & 0 & 0 & 0 & 0 \\ \mathrm{zzxx} & \mathrm{zzyy} & \mathrm{zzzz} & 0 & 0 & 0 & 0 & 0 & 0 \\ 0 & 0 & 0 & \mathrm{yzyz} & 0 & 0 & \mathrm{yzzy} & 0 & 0 \\ 0 & 0 & 0 & 0 & \mathrm{zxzx} & 0 & 0 & \mathrm{zxxz} & 0 \\ 0 & 0 & 0 & 0 & 0 & \mathrm{xyxy} & 0 & 0 & \mathrm{xyyx}\end{array}\right)$ $\left(\begin{array}{lllllllll}0 & 0 & 0 & 0 & 0 & 0 & 0 & 0 & 0\end{array}\right.$

$\begin{array}{llllllll}0 & 0 & 0 & 0 & 0 & 0 & 0 & 0\end{array}$ $0 \begin{array}{lllllll}0 & 0 & 0 & 0 & 0 & 0 & 0 \\ & 0 & 0 & 0 & 0 & 0 & 0\end{array}$ $\begin{array}{lllll}0 & 0 & 0 & 0 & 0\end{array}$ $\begin{array}{llll}0 & 0 & 0 & 0\end{array}$ $\begin{array}{llll}0 & 0 & 0 \\ & 0 & 0\end{array}$

0
0
0
0
0
0
0
0
0

$\left(\begin{array}{lllllllll}0 & 0 & 0 & 0 & 0 & 0 & 0 & 0 & 0 \\ 0 & 0 & 0 & 0 & 0 & 0 & 0 & 0 & 0\end{array}\right)$

$0 \begin{array}{lllllllll}0 & 0 & 0 & 0 & 0 & 0 & 0 & 0 & 0 \\ 0 & 0 & 0 & 0 & 0 & 0 & 0 & 0 & 0\end{array}$

$\begin{array}{lllllllll}0 & 0 & 0 & 0 & 0 & 0 & 0 & 0 & 0\end{array}$

$0 \begin{array}{lllllllll}0 & 0 & 0 & 0 & 0 & 0 & 0 & 0 & 0 \\ 0 & 0 & 0 & 0 & 0 & 0 & 0 & 0 & 0\end{array}$

$\begin{array}{lllllllll}0 & 0 & 0 & 0 & 0 & 0 & 0 & 0 & 0 \\ 0 & 0 & 0 & 0 & 0 & 0 & 0 & 0 & 0\end{array}$ a

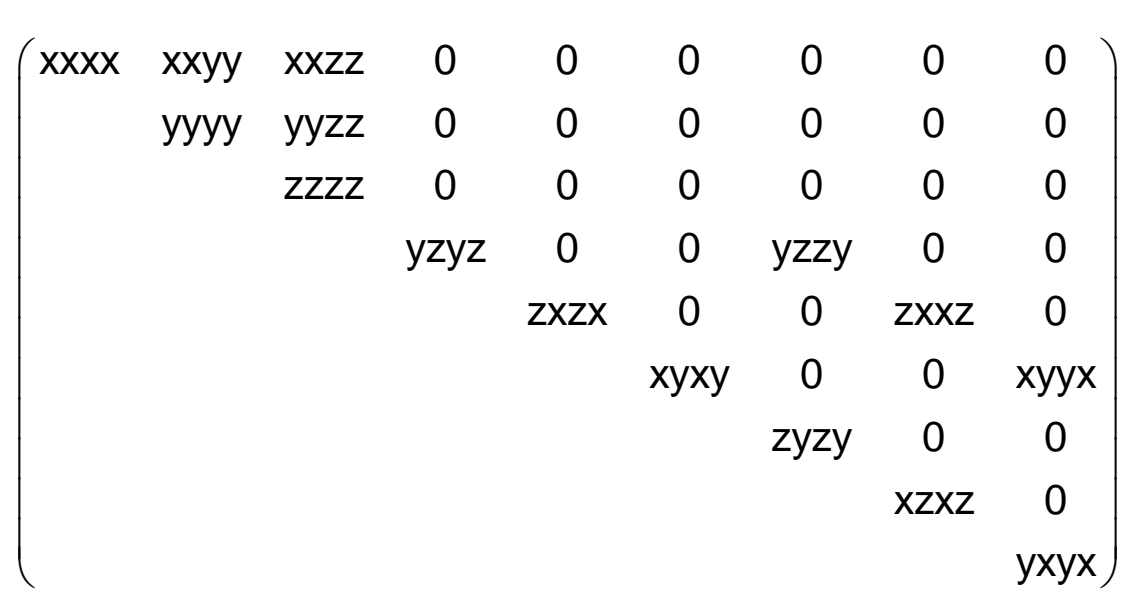

$\left(\begin{array}{lllllllll}0 & 0 & 0 & 0 & 0 & 0 & 0 & 0 & 0 \\ & 0 & 0 & 0 & 0 & 0 & 0 & 0 & 0\end{array}\right)$

$\begin{array}{llllllll}0 & 0 & 0 & 0 & 0 & 0 & 0 & 0\end{array}$

$\begin{array}{llllllll}0 & 0 & 0 & 0 & 0 & 0 & 0\end{array}$

$\begin{array}{llllll}0 & 0 & 0 & 0 & 0 & 0 \\ 0 & 0 & 0 & 0 & 0 & 0\end{array}$

$\begin{array}{lllll}0 & 0 & 0 & 0 & 0\end{array}$

$\begin{array}{llll}0 & 0 & 0 & 0 \\ & 0 & 0 & 0\end{array}$

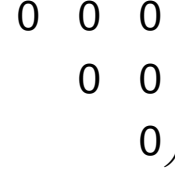

$\left(\begin{array}{ccccccccc}\text { xxxx } & \text { xxyy } & \text { xxzz } & 0 & 0 & 0 & 0 & 0 & 0 \\ \text { yyxx } & \text { yyyy } & \text { yyzz } & 0 & 0 & 0 & 0 & 0 & 0 \\ z z x x & z z y y & z z z z & 0 & 0 & 0 & 0 & 0 & 0 \\ 0 & 0 & 0 & y z y z & 0 & 0 & y z z y & 0 & 0 \\ 0 & 0 & 0 & 0 & z x z x & 0 & 0 & \text { zxxz } & 0 \\ 0 & 0 & 0 & 0 & 0 & x y x y & 0 & 0 & \text { xyyx }\end{array}\right) \quad\left(\begin{array}{ccccccccc}0 & 0 & 0 & 0 & 0 & 0 & 0 & 0 & 0 \\ 0 & 0 & 0 & 0 & 0 & 0 & 0 & 0 & 0 \\ 0 & 0 & 0 & 0 & 0 & 0 & 0 & 0 & 0 \\ 0 & 0 & 0 & 0 & 0 & 0 & 0 & 0 & 0 \\ 0 & 0 & 0 & 0 & 0 & 0 & 0 & 0 & 0 \\ 0 & 0 & 0 & 0 & 0 & 0 & 0 & 0 & 0\end{array}\right)$ 


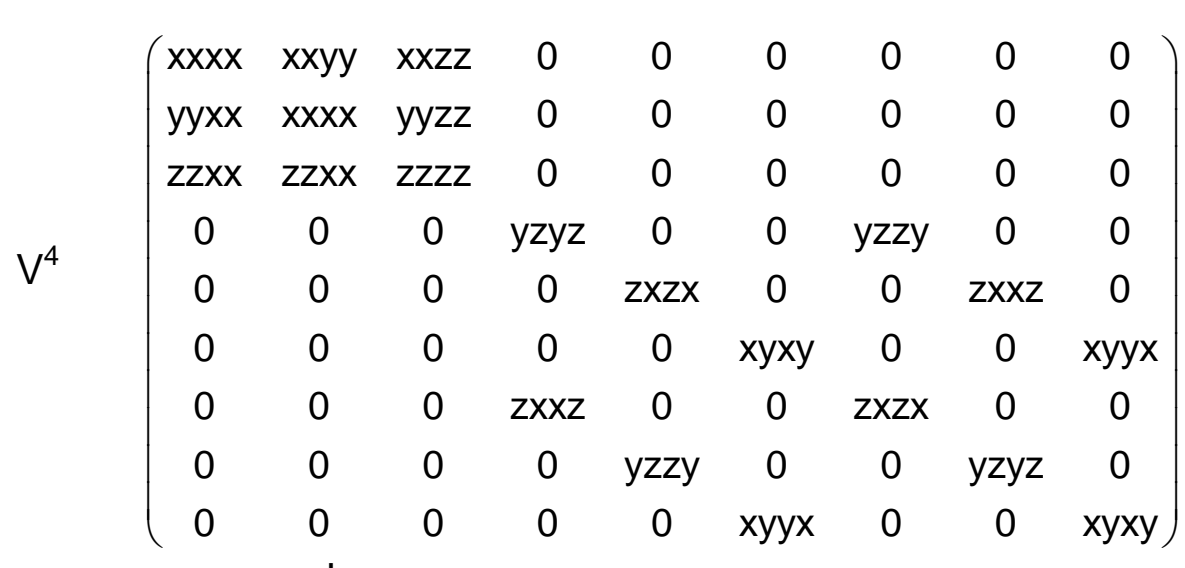
and: $x x x x=x x y y+x y x y+x y y x$

$$
\left[V^{4}\right] \quad\left(\begin{array}{ccccc}
3 x x y y & 0 & 0 & y y z z & 0 \\
0 & 3 x x y y & 0 & y y z z & 0 \\
0 & 0 & z z z z & x x y y & 0
\end{array}\right)
$$

$V\left[V^{3}\right]\left(\begin{array}{cccccccccc}3 x x y y & 0 & 0 & x x y y & 0 & 0 & y y z z & 0 & 0 & 0 \\ 0 & 3 x x y y & 0 & 0 & y y z z & 0 & 0 & x x y y & 0 & 0 \\ 0 & 0 & z z z & 0 & 0 & z z x & 0 & 0 & z z x & 0\end{array}\right)$

$$
\left.\left[V^{2}\right]^{2}\right]\left(\begin{array}{cccccc}
x x x x & x x y y & x x z z & 0 & 0 & 0 \\
& x x x x & x x z z & 0 & 0 & 0 \\
& & z z z z & 0 & 0 & 0 \\
& & & y z y z & 0 & 0 \\
& & & & y z y z & 0 \\
& & & & & x y x y
\end{array}\right)
$$

and $x y x y=1 / 2(x x x x-x x y y)$

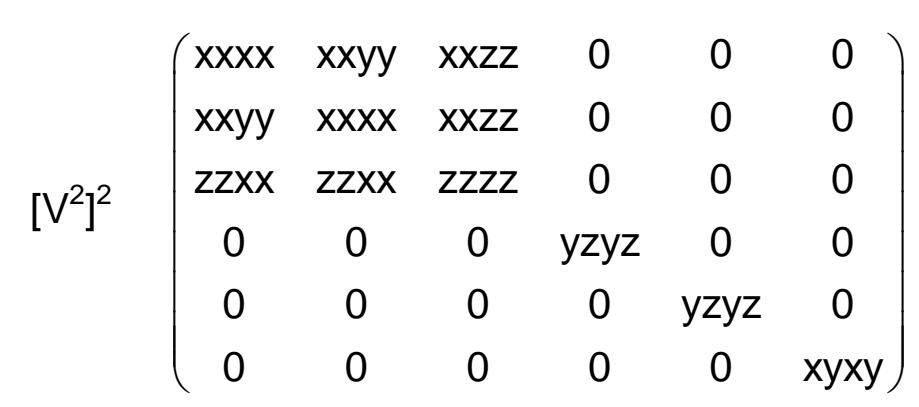

and $x y x y=1 / 2(x x x x-x x y y)$ $\left(\begin{array}{ccccccccc}0 & 0 & 0 & 0 & x x z x & 0 & 0 & x x x z & 0 \\ 0 & 0 & 0 & 0 & -x x z x & 0 & 0 & -x x x z & 0 \\ 0 & 0 & 0 & 0 & 0 & 0 & 0 & 0 & 0 \\ 0 & 0 & 0 & 0 & 0 & -x z x x & 0 & 0 & -x z x x \\ z x x x & -z x x x & 0 & 0 & 0 & 0 & 0 & 0 & 0 \\ 0 & 0 & 0 & -x x x z & 0 & 0 & -x x z x & 0 & 0 \\ 0 & 0 & 0 & 0 & 0 & -z x x x & 0 & 0 & -z x x x \\ x z x x & -x z x x & 0 & 0 & 0 & 0 & 0 & 0 & 0 \\ 0 & 0 & 0 & -x x x z & 0 & 0 & -x x z x & 0 & 0\end{array}\right)$

$a$

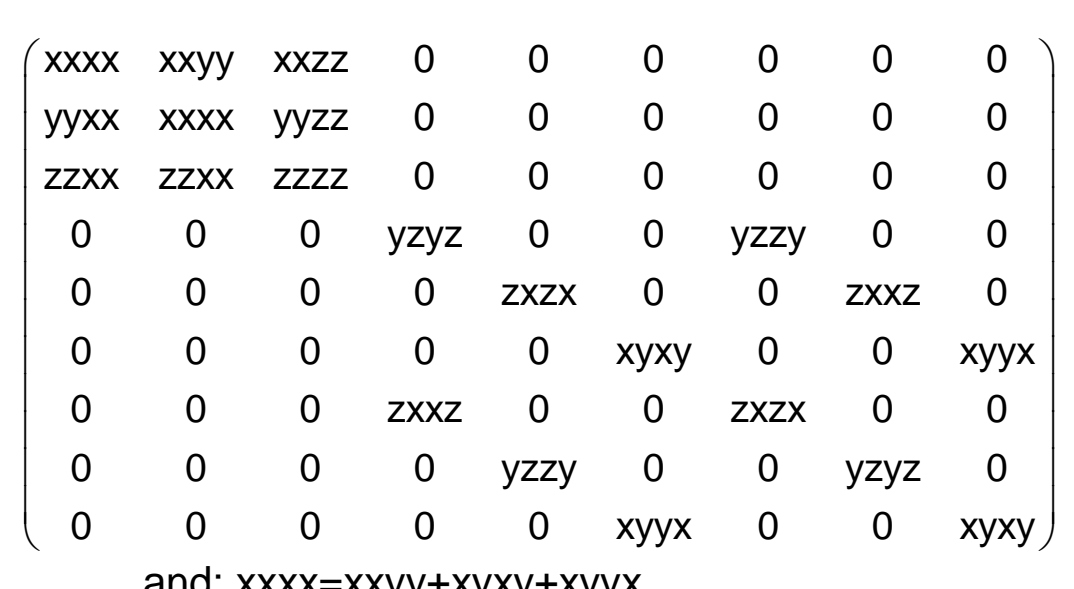

$$
\left(\begin{array}{ccccc}
0 & 0 & 0 & 0 & 0 \\
0 & 0 & 0 & 0 & -z x x x \\
z x x x & 0 & 0 & 0 & 0
\end{array}\right)
$$
$\left(\begin{array}{cccccccccc}0 & 0 & 0 & 0 & 0 & x z x x & 0 & 0 & -x z x x & 0 \\ 0 & 0 & 0 & 0 & 0 & 0 & 0 & 0 & 0 & -x z x x \\ z x x x & 0 & 0 & -z x x x & 0 & 0 & 0 & 0 & 0 & 0\end{array}\right)$

$$
\left(\begin{array}{cccccc}
0 & 0 & 0 & 0 & -y y x z & 0 \\
& 0 & 0 & 0 & y y x z & 0 \\
& & 0 & 0 & 0 & 0 \\
& & & 0 & 0 & y y x z \\
& & & & 0 & 0 \\
& & & & & 0
\end{array}\right)
$$

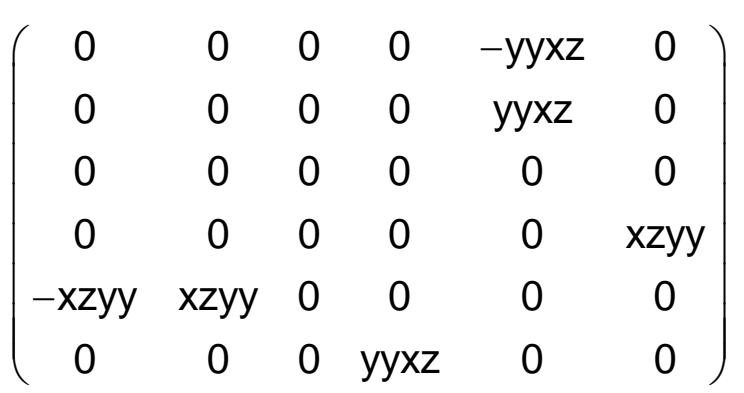

$$
\left(\begin{array}{ccccc}
3 x x y y & 0 & 0 & y y z z & 0 \\
0 & 3 x x y y & 0 & y y z z & 0 \\
0 & 0 & z z z z & x x y y & 0
\end{array}\right)
$$

$\left(\begin{array}{cccccccccc}3 x x y y & 0 & 0 & x x y y & 0 & 0 & y y z z & 0 & 0 & 0 \\ 0 & 3 x x y y & 0 & 0 & y y z z & 0 & 0 & x x y y & 0 & 0 \\ 0 & 0 & z z z z & 0 & 0 & z z x x & 0 & 0 & z z x & 0\end{array}\right)$

$\left(\begin{array}{cccccccccc}0 & 3 x x y y & 0 & 0 & y y z z & 0 & 0 & x x y y & 0 & 0 \\ 0 & 0 & z z z z & 0 & 0 & z z x x & 0 & 0 & \text { zzxx } & 0\end{array}\right)$

$$
\left(\begin{array}{cccccc}
x x x x & x x y y & x x z z & 0 & 0 & 0 \\
& x x x x & x x z z & 0 & 0 & 0 \\
& & z z z z & 0 & 0 & 0 \\
& & & y z y z & 0 & 0 \\
& & & & y z y z & 0 \\
& & & & & \\
& & & & & \\
& & & & &
\end{array}\right.
$$

and $x y x y=1 / 2(x x x x-x x y y)$

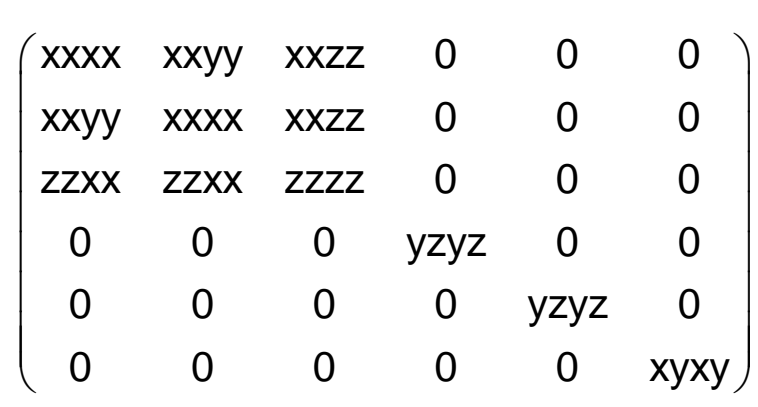

and $x y x y=1 / 2(x x x x-x x y y)$

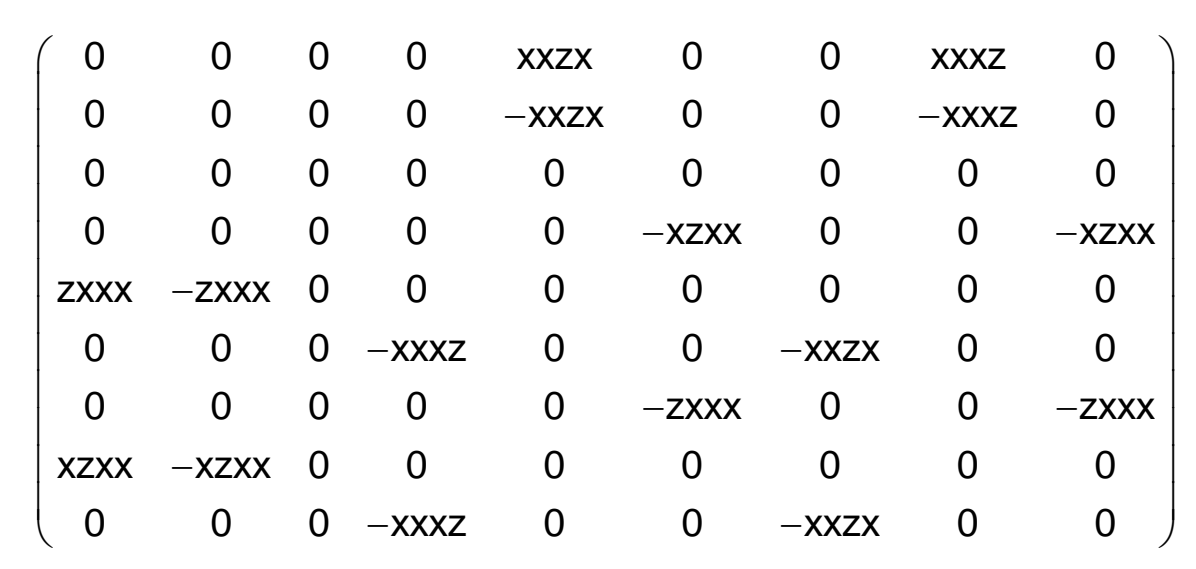

$$
\left(\begin{array}{ccccc}
0 & 0 & 0 & 0 & 0 \\
0 & 0 & 0 & 0 & -z x x x \\
z x x x & 0 & 0 & 0 & 0
\end{array}\right)
$$

$\left(\begin{array}{cccccccccc}0 & 0 & 0 & 0 & 0 & x z x x & 0 & 0 & -x z x x & 0 \\ 0 & 0 & 0 & 0 & 0 & 0 & 0 & 0 & 0 & -x z x x \\ z x x y x & 0 & 0 & -7 x x x & 0 & 0 & 0 & 0 & 0 & 0\end{array}\right)$

$\begin{array}{ccccccc}0 & 0 & 0 & 0 & -y & 0\end{array}$

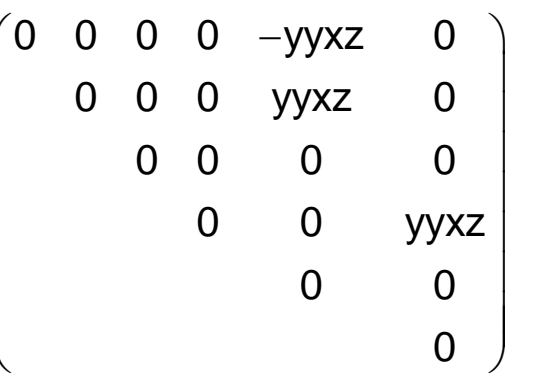

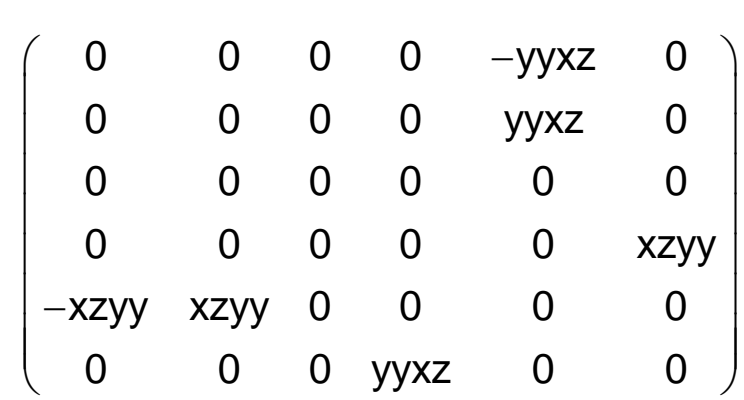




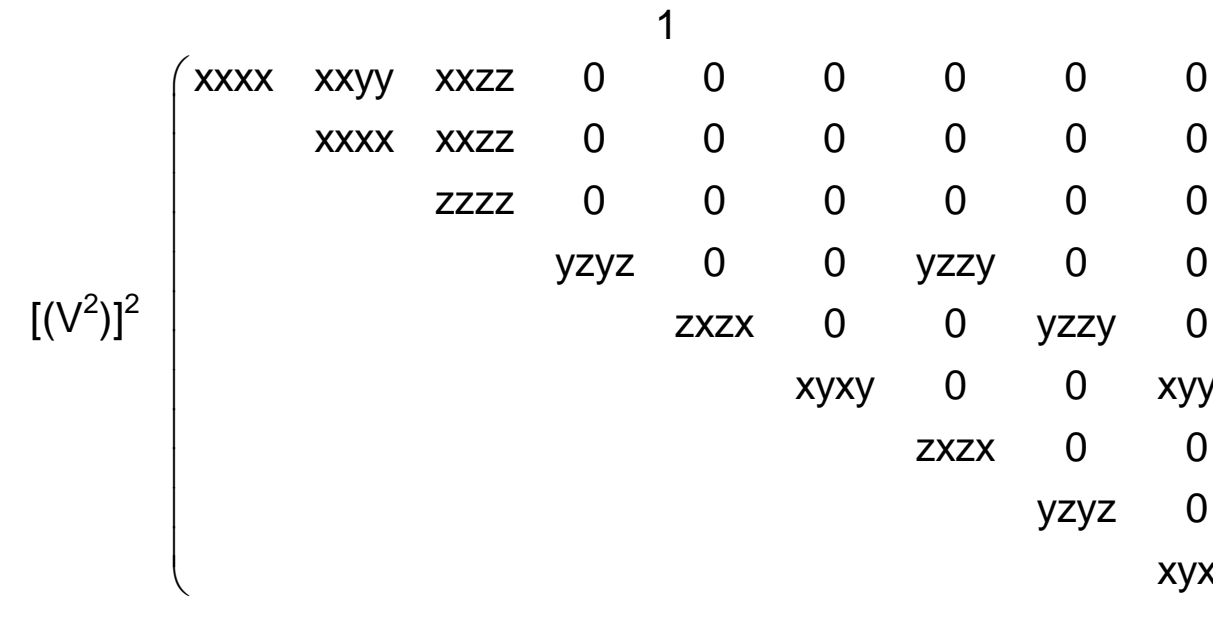

and $x x x x=x x y y+y z y z+z y z y$

$\left[\mathrm{V}^{2}\right] \mathrm{V}^{2}\left(\begin{array}{ccccccccc}\mathrm{x} x \mathrm{x} x & \mathrm{xxyy} & \mathrm{xxzz} & 0 & 0 & 0 & 0 & 0 & 0 \\ \mathrm{xxyy} & \mathrm{xxxx} & \mathrm{xxzz} & 0 & 0 & 0 & 0 & 0 & 0 \\ \mathrm{zzxx} & \mathrm{zzxx} & \mathrm{zzzz} & 0 & 0 & 0 & 0 & 0 & 0 \\ 0 & 0 & 0 & \mathrm{yzyz} & 0 & 0 & \mathrm{zxzx} & 0 & 0 \\ 0 & 0 & 0 & 0 & \mathrm{zxzx} & 0 & 0 & \mathrm{yzyz} & 0 \\ 0 & 0 & 0 & 0 & 0 & \mathrm{xyxy} & 0 & 0 & \mathrm{xyxy}\end{array}\right)$

and $x x x x=x x y y+2 x y x y$

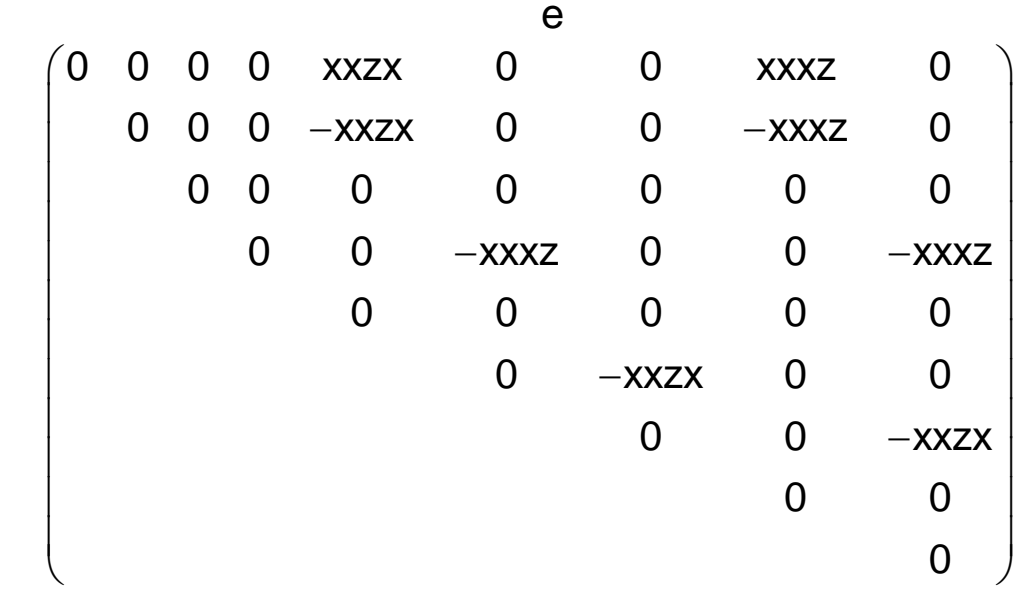

$\left(\begin{array}{ccccccccc}0 & 0 & 0 & 0 & \text { xxzx } & 0 & 0 & \text { xxxz } & 0 \\ 0 & 0 & 0 & 0 & -x x z x & 0 & 0 & -x x x z & 0 \\ 0 & 0 & 0 & 0 & 0 & 0 & 0 & 0 & 0 \\ 0 & 0 & 0 & 0 & 0 & 0 & 0 & 0 & -z x x x \\ z x x x & -z x x x & 0 & 0 & 0 & 0 & 0 & 0 & 0 \\ 0 & 0 & 0 & -x x x z & 0 & 0 & -x x z x & 0 & 0\end{array}\right)$

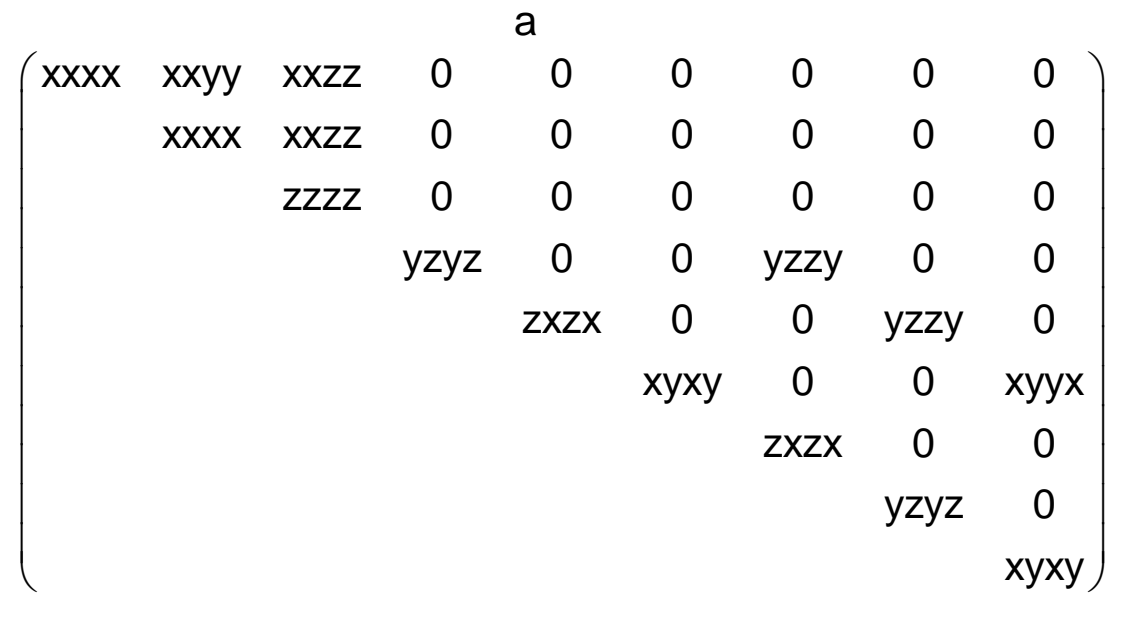

and $x x x x=x x y y+y z y z+z y z y$

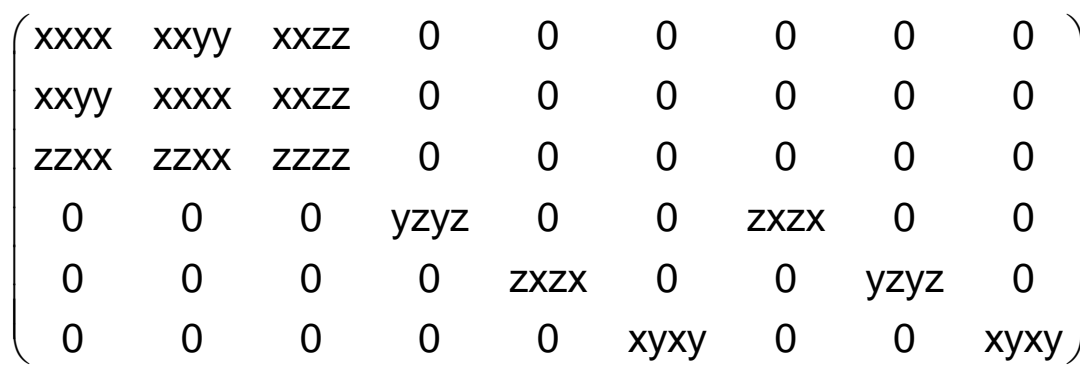

and $x x x x=x x y y+2 x y x y$

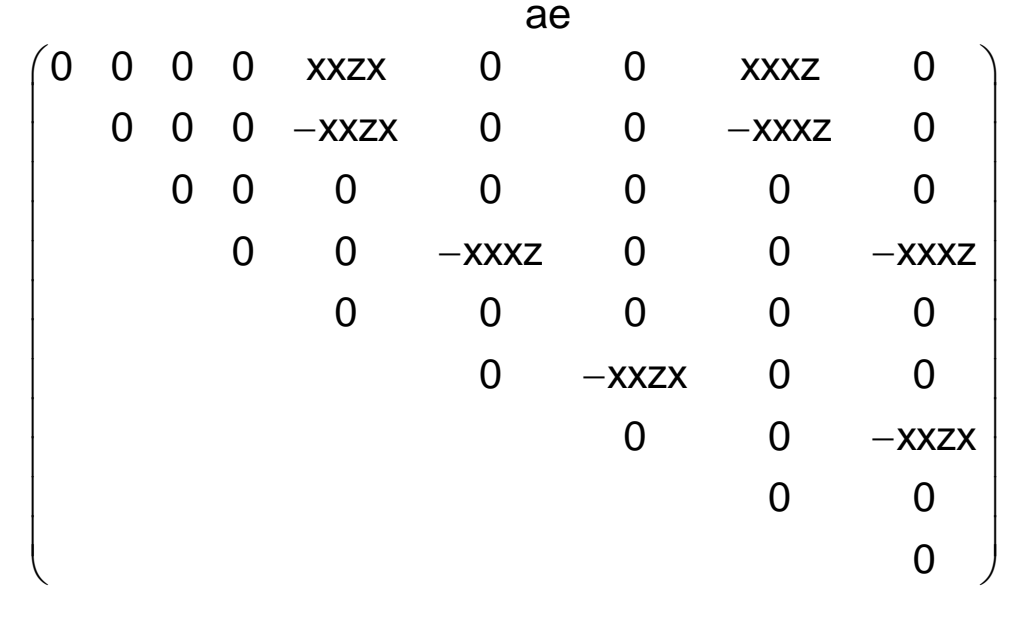

$\left(\begin{array}{ccccccccc}0 & 0 & 0 & 0 & \text { xxzx } & 0 & 0 & \text { xxxz } & 0 \\ 0 & 0 & 0 & 0 & -x x z x & 0 & 0 & -x x x z & 0 \\ 0 & 0 & 0 & 0 & 0 & 0 & 0 & 0 & 0 \\ 0 & 0 & 0 & 0 & 0 & 0 & 0 & 0 & -z x x x \\ z x x x & -z x x x & 0 & 0 & 0 & 0 & 0 & 0 & 0 \\ 0 & 0 & 0 & -x x x z & 0 & 0 & -x x z x & 0 & 0\end{array}\right)$ 


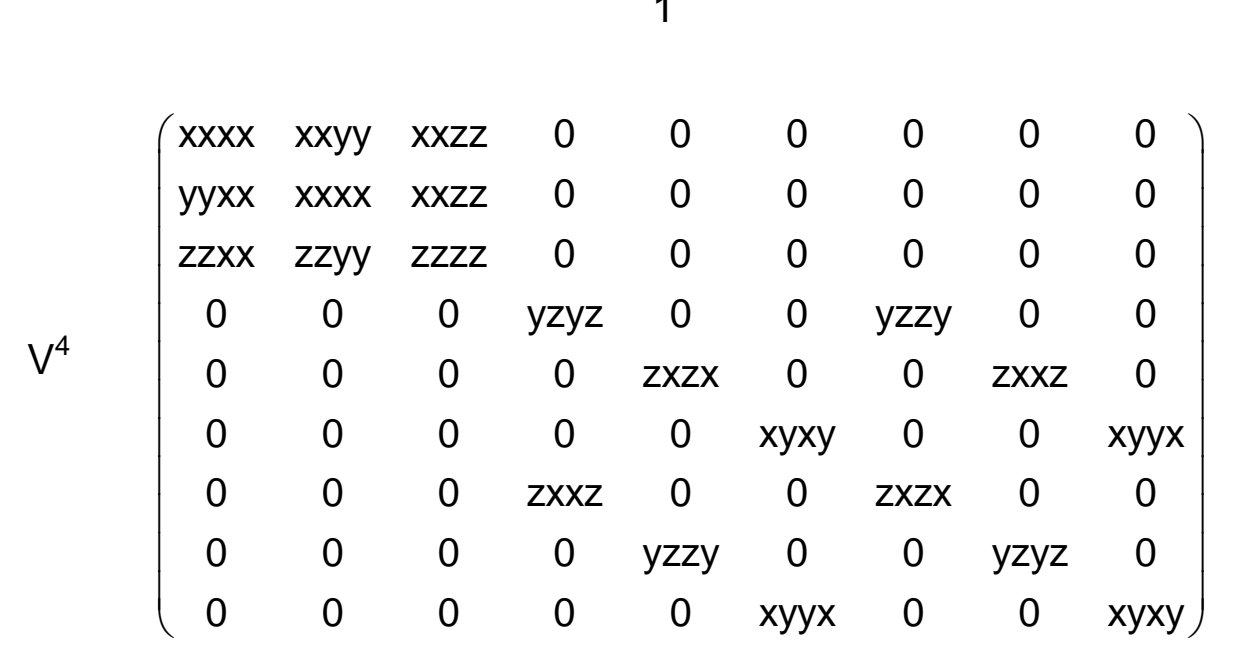

$\left(\begin{array}{lllllllll}0 & 0 & 0 & 0 & 0 & 0 & 0 & 0 & 0 \\ 0 & 0 & 0 & 0 & 0 & 0 & 0 & 0 & 0\end{array}\right)$ $\begin{array}{lllllllll}0 & 0 & 0 & 0 & 0 & 0 & 0 & 0 & 0 \\ 0 & 0 & 0 & 0 & 0 & 0 & 0 & 0 & 0\end{array}$ $\begin{array}{lllllllll}0 & 0 & 0 & 0 & 0 & 0 & 0 & 0 & 0 \\ 0 & 0 & 0 & 0 & 0 & 0 & 0 & 0 & 0\end{array}$ $\begin{array}{lllllllll}0 & 0 & 0 & 0 & 0 & 0 & 0 & 0 & 0 \\ 0 & 0 & 0 & 0 & 0 & 0 & 0 & 0 & 0\end{array}$ $0 \begin{array}{lllllllll}0 & 0 & 0 & 0 & 0 & 0 & 0 & 0 & 0 \\ 0 & 0 & 0 & 0 & 0 & 0 & 0 & 0 & 0\end{array}$ $\begin{array}{lllllllll}0 & 0 & 0 & 0 & 0 & 0 & 0 & 0 & 0\end{array}$ $\begin{array}{llllllllll}0 & 0 & 0 & 0 & 0 & 0 & 0 & 0 & 0\end{array}$ $\begin{array}{lllllllll}0 & 0 & 0 & 0 & 0 & 0 & 0 & 0 & 0 \\ 0 & 0 & 0 & 0 & 0 & 0 & 0 & 0 & 0\end{array}$ $\left(\begin{array}{lllllllll}0 & 0 & 0 & 0 & 0 & 0 & 0 & 0 & 0 \\ 0 & 0 & 0 & 0 & 0 & 0 & 0 & 0 & 0\end{array}\right)$

$\left[V^{4}\right] \quad\left(\begin{array}{ccccc}\mathrm{xxxx} & 0 & 0 & \mathrm{yyzz} & 0 \\ 0 & \mathrm{xxxx} & 0 & \mathrm{yyzz} & 0 \\ 0 & 0 & \mathrm{zzzz} & \mathrm{xxyy} & 0\end{array}\right)$

$\left(\begin{array}{lllll}0 & 0 & 0 & 0 & 0 \\ 0 & 0 & 0 & 0 & 0 \\ 0 & 0 & 0 & 0 & 0\end{array}\right)$

$\begin{array}{lllll}0 & 0 & 0 & 0 & 0\end{array}$

$\mathrm{V}\left[\mathrm{V}^{3}\right] \quad\left(\begin{array}{cccccccccc}\mathrm{xxxx} & 0 & 0 & \mathrm{xxyy} & 0 & 0 & \mathrm{yyzz} & 0 & 0 & 0 \\ 0 & \mathrm{xxxx} & 0 & 0 & \mathrm{yyzz} & 0 & 0 & \mathrm{xxyy} & 0 & 0 \\ 0 & 0 & z z z z & 0 & 0 & z z x x & 0 & 0 & z z x x & 0\end{array}\right)$

$\begin{array}{llllllllll}0 & 0 & 0 & 0 & 0 & 0 & 0 & 0 & 0 & 0 \\ 0 & 0 & 0 & 0 & 0 & 0 & 0 & 0 & 0 & 0\end{array}$ $\left(\begin{array}{llllllllll}0 & 0 & 0 & 0 & 0 & 0 & 0 & 0 & 0 & 0 \\ 0 & 0 & 0 & 0 & 0 & 0 & 0 & 0 & 0 & 0\end{array}\right)$

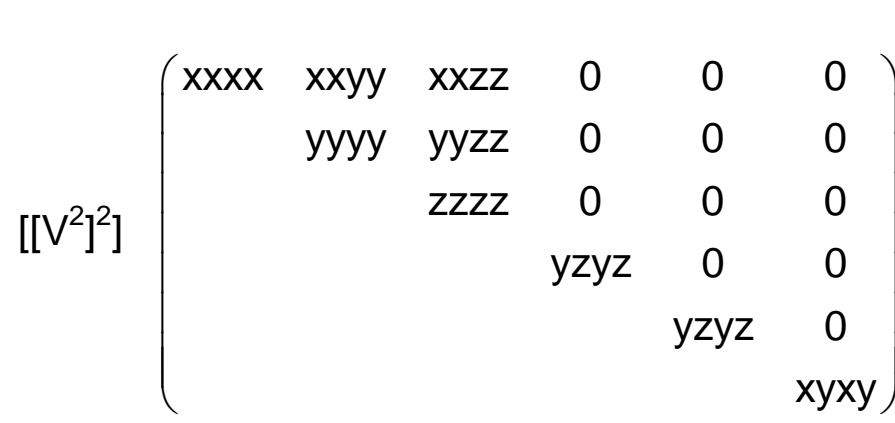
$\begin{array}{llllllllll}0 & 0 & 0 & 0 & 0 & 0 & 0 & 0 & 0 & 0\end{array}$

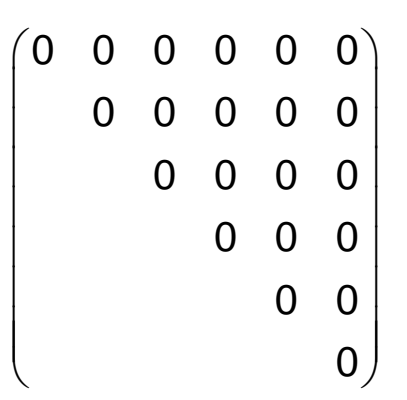

$\left(\begin{array}{llllll}0 & 0 & 0 & 0 & 0 & 0 \\ 0 & 0 & 0 & 0 & 0 & 0\end{array}\right)$ $\begin{array}{llllll}0 & 0 & 0 & 0 & 0 & 0 \\ 0 & 0 & 0 & 0 & 0 & 0 \\ 0 & 0 & 0 & 0 & 0 & 0\end{array}$ $0 \begin{array}{lllllll}0 & 0 & 0 & 0 & 0 & 0\end{array}$ $\begin{array}{llllll}0 & 0 & 0 & 0 & 0 & 0 \\ 0 & 0 & 0 & 0 & 0\end{array}$ $\begin{array}{llllll}0 & 0 & 0 & 0 & 0 & 0 \\ 0 & 0 & 0 & 0 & 0 & 0\end{array}$

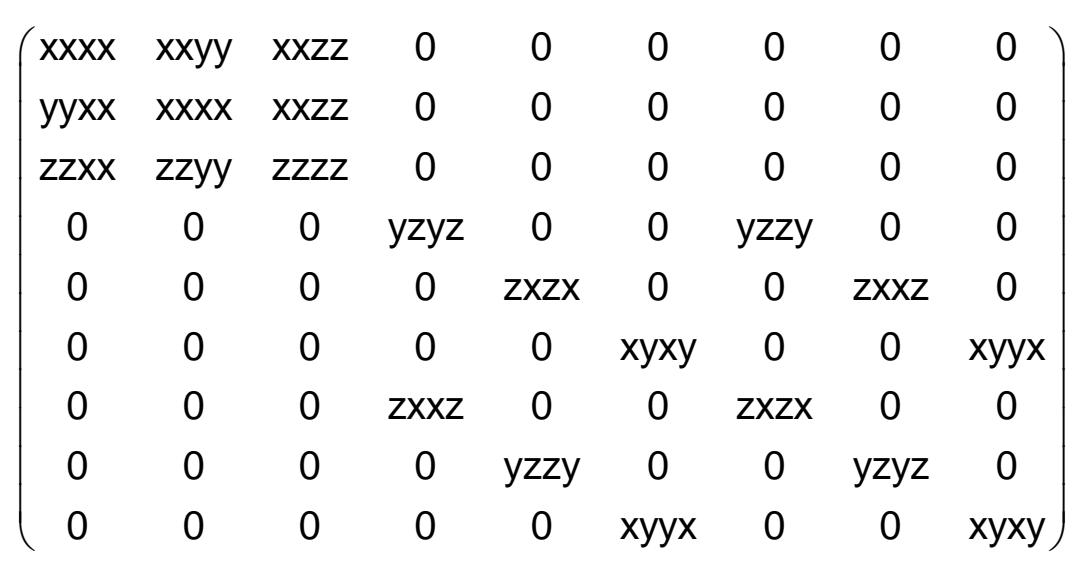

$\left(\begin{array}{ccccc}x x x x & 0 & 0 & y y z z & 0 \\ 0 & x x x x & 0 & y y z z & 0 \\ 0 & 0 & z z z z & x x y y & 0\end{array}\right)$

$\left(\begin{array}{cccccccccc}x x x x & 0 & 0 & x x y y & 0 & 0 & y y z z & 0 & 0 & 0 \\ 0 & x x x x & 0 & 0 & y y z z & 0 & 0 & x x y y & 0 & 0 \\ 0 & 0 & z z z & 0 & 0 & z z x & 0 & 0 & z z x & 0\end{array}\right)$

$\begin{array}{llllllllll}0 & 0 & z z z z & 0 & 0 & z z x x & 0 & 0 & z z x x & 0\end{array}$

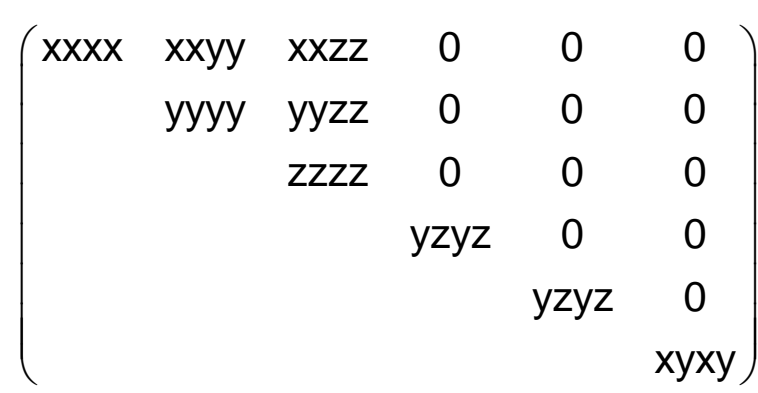

$\left(\begin{array}{cccccc}x x x x & x x y y & x x z z & 0 & 0 & 0 \\ y y x x & x x x x & x x z z & 0 & 0 & 0 \\ z z x x & z z x x & z z z z & 0 & 0 & 0 \\ 0 & 0 & 0 & y z y z & 0 & 0 \\ 0 & 0 & 0 & 0 & y z y z & 0 \\ 0 & 0 & 0 & 0 & 0 & x y x y\end{array}\right)$ $\left(\begin{array}{lllllllll}0 & 0 & 0 & 0 & 0 & 0 & 0 & 0 & 0 \\ 0 & 0 & 0 & 0 & 0 & 0 & 0 & 0 & 0\end{array}\right)$ $\begin{array}{lllllllll}0 & 0 & 0 & 0 & 0 & 0 & 0 & 0 & 0 \\ 0 & 0 & 0 & 0 & 0 & 0 & 0 & 0 & 0\end{array}$ $\begin{array}{llllllllll}0 & 0 & 0 & 0 & 0 & 0 & 0 & 0 & 0 \\ 0 & 0 & 0 & 0 & 0 & 0 & 0 & 0 & 0 \\ 0 & 0 & 0 & 0 & 0 & 0 & 0 & 0 & 0\end{array}$ $\begin{array}{llllllllll}0 & 0 & 0 & 0 & 0 & 0 & 0 & 0 & 0\end{array}$ $\begin{array}{llllllllll}0 & 0 & 0 & 0 & 0 & 0 & 0 & 0 & 0 \\ 0 & 0 & 0 & 0 & 0 & 0 & 0 & 0 & 0\end{array}$

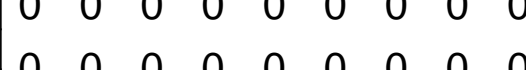
$\left(\begin{array}{lllllllll}0 & 0 & 0 & 0 & 0 & 0 & 0 & 0 & 0 \\ 0 & 0 & 0 & 0 & 0 & 0 & 0 & 0 & 0 \\ 0 & 0 & 0 & 0 & 0 & 0 & 0 & 0 & 0\end{array}\right.$ $\begin{array}{llllllllll}0 & 0 & 0 & 0 & 0 & 0 & 0 & 0 & 0\end{array}$

$\left(\begin{array}{lllll}0 & 0 & 0 & 0 & 0 \\ 0 & 0 & 0 & 0 & 0 \\ 0 & 0 & 0 & 0 & 0\end{array}\right)$

$\begin{array}{lllll}0 & 0 & 0 & 0 & 0\end{array}$

$\left(\begin{array}{llllllllll}0 & 0 & 0 & 0 & 0 & 0 & 0 & 0 & 0 & 0 \\ 0 & 0 & 0 & 0 & 0 & 0 & 0 & 0 & 0 & 0 \\ 0 & 0 & 0 & 0 & 0 & 0 & 0 & 0 & 0 & 0\end{array}\right)$ $\left(\begin{array}{llllllllll}0 & 0 & 0 & 0 & 0 & 0 & 0 & 0 & 0 & 0 \\ 0 & 0 & 0 & 0 & 0 & 0 & 0 & 0 & 0 & 0\end{array}\right)$ $\left(\begin{array}{lllllllllll}0 & 0 & 0 & 0 & 0 & 0 & 0 & 0 & 0 & 0\end{array}\right.$

$$
\left(\begin{array}{llllll}
0 & 0 & 0 & 0 & 0 & 0 \\
& 0 & 0 & 0 & 0 & 0 \\
& & 0 & 0 & 0 & 0 \\
& & & 0 & 0 & 0 \\
& & & & 0 & 0 \\
& & & & & 0
\end{array}\right)
$$

$\left(\begin{array}{llllll}0 & 0 & 0 & 0 & 0 & 0 \\ 0 & 0 & 0 & 0 & 0 & 0\end{array}\right.$ $0 \begin{array}{llllll}0 & 0 & 0 & 0 & 0 & 0 \\ 0 & 0 & 0 & 0 & 0 & 0\end{array}$ $\begin{array}{llllll}0 & 0 & 0 & 0 & 0 & 0 \\ 0 & 0 & 0 & 0 & 0 & 0\end{array}$ $0 \begin{array}{llllll}0 & 0 & 0 & 0 & 0 & 0\end{array}$ $\begin{array}{llllll}0 & 0 & 0 & 0 & 0 & 0 \\ 0 & 0 & 0 & 0 & 0 & 0\end{array}$ $\begin{array}{llllll}0 & 0 & 0 & 0 & 0 & 0\end{array}$ 


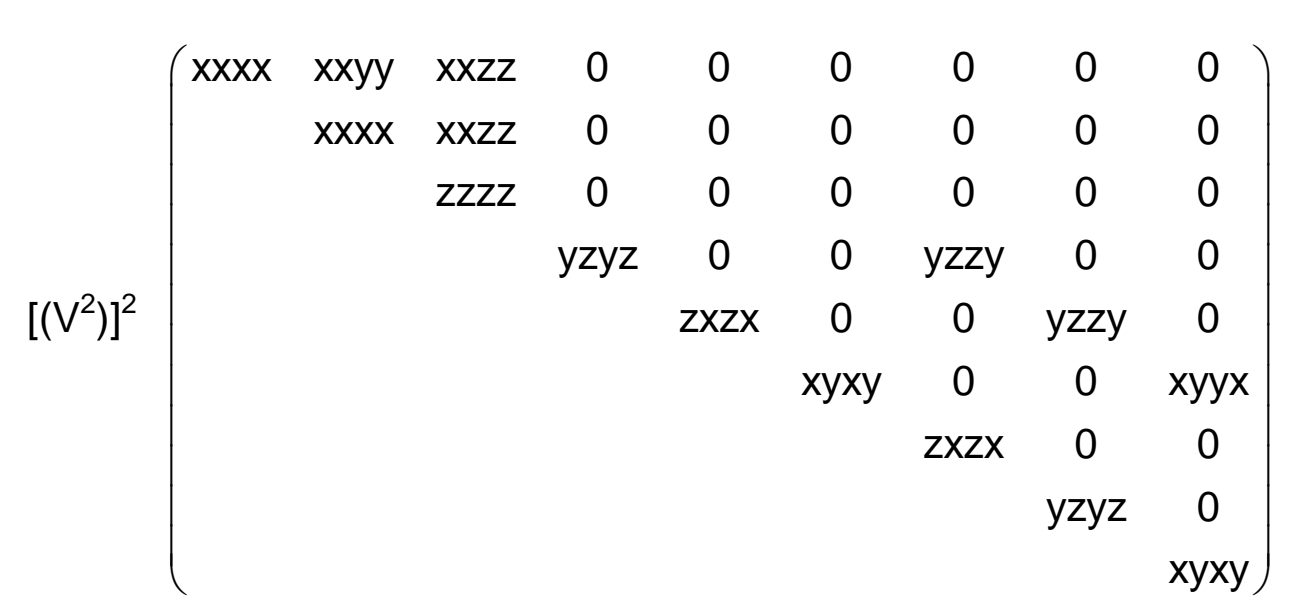

$\begin{array}{llllllll}0 & 0 & 0 & 0 & 0 & 0 & 0 & 0\end{array}$

$\left[\mathrm{V}^{2}\right] \mathrm{V}^{2}\left(\begin{array}{ccccccccc}\mathrm{xxxx} & \mathrm{xxyy} & \mathrm{xxzz} & 0 & 0 & 0 & 0 & 0 & 0 \\ \mathrm{yyxx} & \mathrm{xxxx} & \mathrm{xxzz} & 0 & 0 & 0 & 0 & 0 & 0 \\ \mathrm{zzxx} & \mathrm{zzxx} & \mathrm{zzzz} & 0 & 0 & 0 & 0 & 0 & 0 \\ 0 & 0 & 0 & \mathrm{yzyz} & 0 & 0 & \mathrm{zxzx} & 0 & 0 \\ 0 & 0 & 0 & 0 & \mathrm{zxzx} & 0 & 0 & \mathrm{yzyz} & 0 \\ 0 & 0 & 0 & 0 & 0 & \mathrm{xyxy} & 0 & 0 & \mathrm{xyxy}\end{array}\right)$ $\left(\begin{array}{lllllllll}0 & 0 & 0 & 0 & 0 & 0 & 0 & 0 & 0\end{array}\right.$ $\begin{array}{lllllll}0 & 0 & 0 & 0 & 0 & 0 & 0\end{array}$ $\begin{array}{llllll}0 & 0 & 0 & 0 & 0 & 0\end{array}$ $\begin{array}{lllll}0 & 0 & 0 & 0 & 0\end{array}$ $\begin{array}{llll}0 & 0 & 0 & 0\end{array}$ $\begin{array}{lll}0 & 0 & 0 \\ & 0 & 0\end{array}$

0
0
0
0
0
0
0
0
0

$\left(\begin{array}{lllllllll}0 & 0 & 0 & 0 & 0 & 0 & 0 & 0 & 0 \\ 0 & 0 & 0 & 0 & 0 & 0 & 0 & 0 & 0\end{array}\right)$

$0 \begin{array}{lllllllll}0 & 0 & 0 & 0 & 0 & 0 & 0 & 0 & 0 \\ 0 & 0 & 0 & 0 & 0 & 0 & 0 & 0 & 0\end{array}$

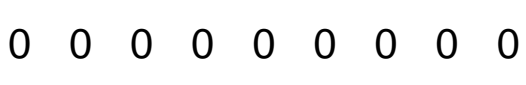

$0 \begin{array}{lllllllll}0 & 0 & 0 & 0 & 0 & 0 & 0 & 0 & 0 \\ 0 & 0 & 0 & 0 & 0 & 0 & 0 & 0 & 0\end{array}$

$\begin{array}{lllllllll}0 & 0 & 0 & 0 & 0 & 0 & 0 & 0 & 0 \\ 0 & 0 & 0 & 0 & 0 & 0 & 0 & 0 & 0\end{array}$ a

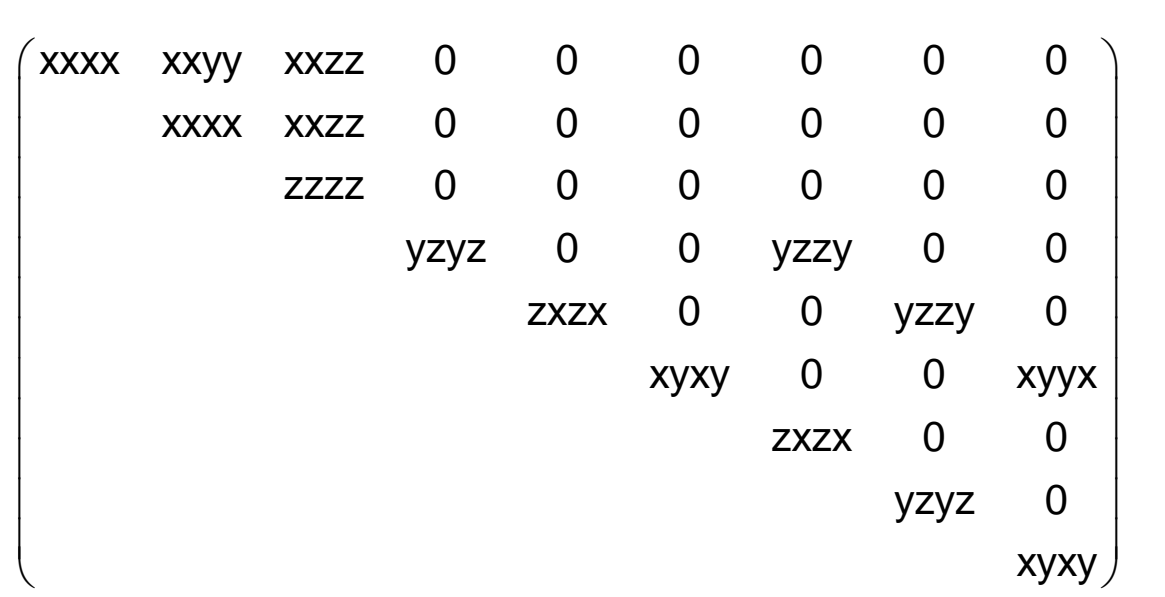

$\left(\begin{array}{lllllllll}0 & 0 & 0 & 0 & 0 & 0 & 0 & 0 & 0 \\ & 0 & 0 & 0 & 0 & 0 & 0 & 0 & 0\end{array}\right)$

$\begin{array}{llllllll}0 & 0 & 0 & 0 & 0 & 0 & 0 & 0\end{array}$

$\begin{array}{llllllll}0 & 0 & 0 & 0 & 0 & 0 & 0\end{array}$

$\begin{array}{llllll}0 & 0 & 0 & 0 & 0 & 0\end{array}$

$0 \begin{array}{lllll}0 & 0 & 0 & 0 & 0\end{array}$

$\begin{array}{llll}0 & 0 & 0 & 0 \\ & 0 & 0 & 0\end{array}$

$\begin{array}{lll}0 & 0 & 0 \\ & 0 & 0 \\ & & 0\end{array}$

$\left(\begin{array}{lllllllll}x x x x & x x y y & x x z z & 0 & 0 & 0 & 0 & 0 & 0 \\ y & x & x\end{array}\right)$

$\begin{array}{lllllllll}y y x x & x x x x & x x z z & 0 & 0 & 0 & 0 & 0 & 0 \\ z z x x & z z x x & z z z z & 0 & 0 & 0 & 0 & 0 & 0\end{array}$

$\begin{array}{ccccccccc}0 & 0 & 0 & y z y z & 0 & 0 & z x z x & 0 & 0\end{array}$

$\begin{array}{ccccccccc}0 & 0 & 0 & 0 & z x z x & 0 & 0 & y z y z & 0 \\ 0 & 0 & 0 & 0 & 0 & x y x y & 0 & 0 & x y x y\end{array}$ $\begin{array}{lllllllll}0 & 0 & 0 & 0 & 0 & 0 & 0 & 0 & 0 \\ 0 & 0 & 0 & 0 & 0 & 0 & 0 & 0 & 0\end{array}$

$\left.\begin{array}{lllllllll}0 & 0 & 0 & 0 & 0 & 0 & 0 & 0 & 0 \\ 0 & 0 & 0 & 0 & 0 & 0 & 0 & 0 & 0 \\ 0 & 0 & 0 & 0 & 0 & 0 & 0 & 0 & 0\end{array}\right)$ $\begin{array}{llllllllll}0 & 0 & 0 & 0 & 0 & 0 & 0 & 0 & 0 \\ 0 & 0 & 0 & 0 & 0 & 0 & 0 & 0 & 0 \\ 0 & 0 & 0 & 0 & 0 & 0 & 0 & 0 & 0\end{array}$ $\begin{array}{lllllllll}0 & 0 & 0 & 0 & 0 & 0 & 0 & 0 & 0 \\ 0 & 0 & 0 & 0 & 0 & 0 & 0 & 0 & 0\end{array}$ $\left(\begin{array}{llllllllll}0 & 0 & 0 & 0 & 0 & 0 & 0 & 0 & 0 \\ 0 & 0 & 0 & 0 & 0 & 0 & 0 & 0 & 0\end{array}\right.$ 
$\mathrm{V}^{4}\left(\begin{array}{ccccccccc}\mathrm{xxxx} & \mathrm{xxyy} & \mathrm{xxzz} & 0 & 0 & 0 & 0 & 0 & 0 \\ \mathrm{yyxx} & \mathrm{xxxx} & \mathrm{xxzz} & 0 & 0 & 0 & 0 & 0 & 0 \\ \mathrm{zzxx} & \mathrm{zzyy} & \mathrm{zzzz} & 0 & 0 & 0 & 0 & 0 & 0 \\ 0 & 0 & 0 & \mathrm{yzyz} & 0 & 0 & \mathrm{yzzy} & 0 & 0 \\ 0 & 0 & 0 & 0 & \mathrm{zxzx} & 0 & 0 & \mathrm{zxxz} & 0 \\ 0 & 0 & 0 & 0 & 0 & \mathrm{xyxy} & 0 & 0 & \mathrm{xyyx} \\ 0 & 0 & 0 & \mathrm{zxxz} & 0 & 0 & \mathrm{zxzx} & 0 & 0 \\ 0 & 0 & 0 & 0 & \mathrm{yzzy} & 0 & 0 & \mathrm{yzyz} & 0 \\ 0 & 0 & 0 & 0 & 0 & \mathrm{xyyx} & 0 & 0 & \mathrm{xyxy}\end{array}\right)$

$\begin{array}{lllllllll}0 & 0 & 0 & 0 & 0 & 0 & 0 & 0 & 0 \\ 0 & 0 & 0 & 0 & 0 & 0 & 0 & 0 & 0\end{array}$ $0 \begin{array}{lllllllll}0 & 0 & 0 & 0 & 0 & 0 & 0 & 0 & 0 \\ 0 & 0 & 0 & 0 & 0 & 0 & 0 & 0 & 0\end{array}$

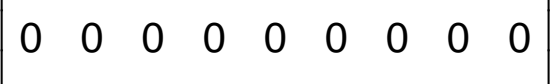

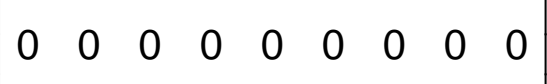
$\begin{array}{llllllllll}0 & 0 & 0 & 0 & 0 & 0 & 0 & 0 & 0\end{array}$ $0 \begin{array}{lllllllll}0 & 0 & 0 & 0 & 0 & 0 & 0 & 0 & 0 \\ 0 & 0 & 0 & 0 & 0 & 0 & 0 & 0 & 0\end{array}$ $0 \begin{array}{llllllllll}0 & 0 & 0 & 0 & 0 & 0 & 0 & 0 & 0 \\ 0 & 0 & 0 & 0 & 0 & 0 & 0 & 0 & 0\end{array}$ $0 \begin{array}{llllllllll}0 & 0 & 0 & 0 & 0 & 0 & 0 & 0 & 0 \\ 0 & 0 & 0 & 0 & 0 & 0 & 0 & 0 & 0\end{array}$ $\begin{array}{lllllllll}0 & 0 & 0 & 0 & 0 & 0 & 0 & 0 & 0\end{array}$

$\left[\mathrm{V}^{4}\right] \quad\left(\begin{array}{ccccc}3 x x y y & 0 & 0 & y y z z & 0 \\ 0 & 3 x x y y & 0 & y y z z & 0 \\ 0 & 0 & z z z z & x x y y & 0\end{array}\right)$

$\left(\begin{array}{lllll}0 & 0 & 0 & 0 & 0 \\ 0 & 0 & 0 & 0 & 0 \\ 0 & 0 & 0 & 0 & 0\end{array}\right)$

$\begin{array}{lllll}0 & 0 & 0 & 0 & 0\end{array}$

$\left(\begin{array}{llllllllll}0 & 0 & 0 & 0 & 0 & 0 & 0 & 0 & 0 & 0 \\ 0 & 0 & 0 & 0 & 0 & 0 & 0 & 0 & 0 & 0\end{array}\right)$ $\left.\begin{array}{llllllllll}0 & 0 & 0 & 0 & 0 & 0 & 0 & 0 & 0 & 0 \\ 0 & 0 & 0 & 0 & 0 & 0 & 0 & 0 & 0 & 0\end{array}\right)$

V $\left.\left[V^{3}\right] \quad \begin{array}{cccccccccc}3 x x y y & 0 & 0 & x x y y & 0 & 0 & y y z z & 0 & 0 & 0 \\ 0 & 3 x x y y & 0 & 0 & y y z z & 0 & 0 & x x y y & 0 & 0 \\ 0 & 0 & z z z & 0 & 0 & z z x x & 0 & 0 & z z x & 0\end{array}\right)$

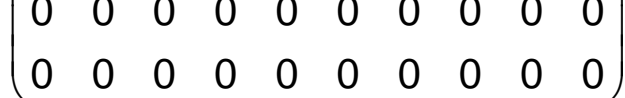

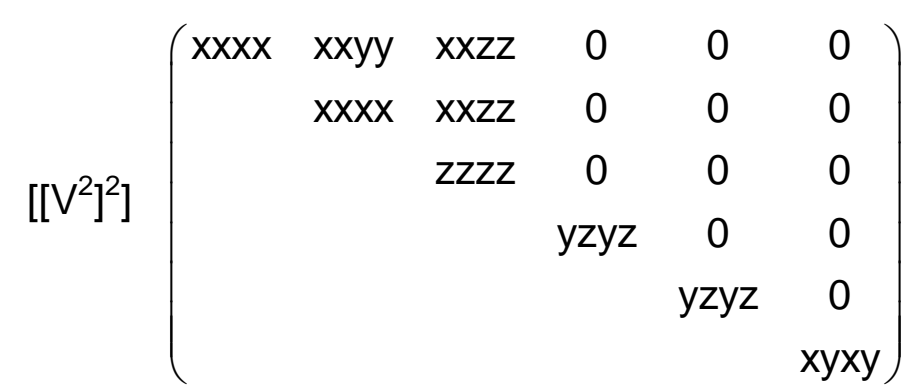

\begin{tabular}{|c|c|c|c|}
\hline & & & \\
\hline & & & 0 \\
\hline & 0 & & \\
\hline & & & 0 \\
\hline & & & \\
\hline
\end{tabular}

and $x y x y=1 / 2(x x x x-x x y y)$

$\left[\mathrm{V}^{2}\right]^{2}$ $\left(\begin{array}{cccccc}\text { xxxx } & x x y y & x x z z & 0 & 0 & 0 \\ x x y y & x x x x & x x z z & 0 & 0 & 0 \\ z z x x & z z x x & z z z z & 0 & 0 & 0 \\ 0 & 0 & 0 & y z y z & 0 & 0 \\ 0 & 0 & 0 & 0 & y z y z & 0 \\ 0 & 0 & 0 & 0 & 0 & x y x y\end{array}\right)$

and $x y x y=1 / 2(x x x x-x x y y)$

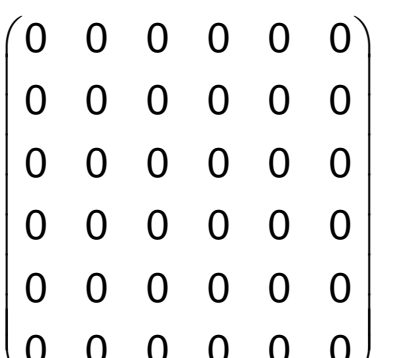

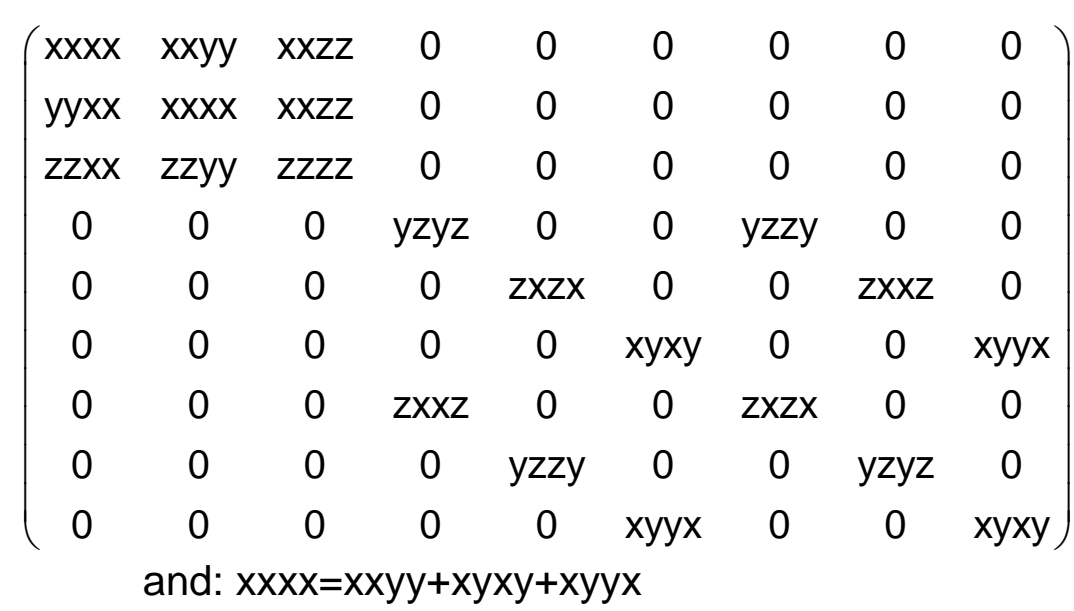

$\left(\begin{array}{lllllllll}0 & 0 & 0 & 0 & 0 & 0 & 0 & 0 & 0\end{array}\right)$ $\left.\begin{array}{llllllllll}0 & 0 & 0 & 0 & 0 & 0 & 0 & 0 & 0 \\ 0 & 0 & 0 & 0 & 0 & 0 & 0 & 0 & 0 \\ 0 & 0 & 0 & 0 & 0 & 0 & 0 & 0 & 0\end{array}\right)$ $\begin{array}{lllllllllll}0 & 0 & 0 & 0 & 0 & 0 & 0 & 0 & 0 \\ 0 & 0 & 0 & 0 & 0 & 0 & 0 & 0 & 0 \\ 0 & 0 & 0 & 0 & 0 & 0 & 0\end{array}$ $\begin{array}{llllllllllll}0 & 0 & 0 & 0 & 0 & 0 & 0 & 0 & 0 \\ 0 & 0 & 0 & 0 & 0 & 0 & 0 & 0 & 0 \\ 0 & 0 & 0 & 0 & 0 & 0\end{array}$ $\begin{array}{llllllllllll}0 & 0 & 0 & 0 & 0 & 0 & 0 & 0 & 0 \\ 0 & 0 & 0 & 0 & 0 & 0 & 0 & 0 & 0\end{array}$ $\begin{array}{llllllllll}0 & 0 & 0 & 0 & 0 & 0 & 0 & 0 & 0 \\ 0 & 0 & 0 & 0 & 0 & 0 & 0 & 0 & 0\end{array}$ $\begin{array}{lllllllll}0 & 0 & 0 & 0 & 0 & 0 & 0 & 0 & 0 \\ 0 & 0 & 0 & 0 & 0 & 0 & 0 & 0 & 0\end{array}$ $\begin{array}{llllllllll}0 & 0 & 0 & 0 & 0 & 0 & 0 & 0 & 0\end{array}$

$\left(\begin{array}{ccccc}3 x x y y & 0 & 0 & y y z z & 0 \\ 0 & 3 x x y y & 0 & y y z z & 0 \\ 0 & 0 & z z z z & x x y y & 0\end{array}\right)$

$\left(\begin{array}{lllll}0 & 0 & 0 & 0 & 0 \\ 0 & 0 & 0 & 0 & 0 \\ 0 & 0 & 0 & 0 & 0\end{array}\right)$ $\left(\begin{array}{lllll}0 & 0 & 0 & 0 & 0 \\ 0 & 0 & 0 & 0 & 0\end{array}\right)$

$\left(\begin{array}{cccccccccc}3 x x y y & 0 & 0 & x x y y & 0 & 0 & y y z z & 0 & 0 & 0 \\ 0 & 3 x x y y & 0 & 0 & y y z z & 0 & 0 & x x y y & 0 & 0 \\ 0 & 0 & z z z z & 0 & 0 & z z x & 0 & 0 & z z x & 0\end{array}\right)$

$\left(\begin{array}{llllllllll}0 & 0 & 0 & 0 & 0 & 0 & 0 & 0 & 0 & 0 \\ 0 & 0 & 0 & 0 & 0 & 0 & 0 & 0 & 0 & 0\end{array}\right)$

$\begin{array}{llllllllll}0 & 0 & 0 & 0 & 0 & 0 & 0 & 0 & 0 & 0 \\ 0 & 0 & 0 & 0 & 0 & 0 & 0 & 0 & 0 & 0\end{array}$

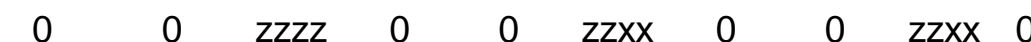

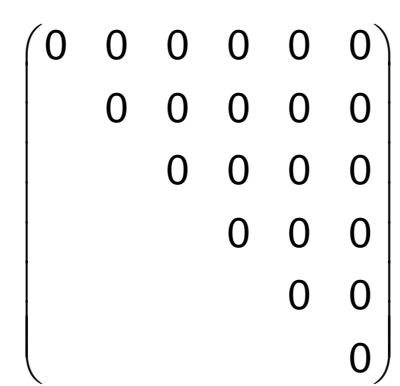

and $x y x y=1 / 2(x x x x-x x y y)$

$$
\left(\begin{array}{cccccc}
x x x x & x x y y & x x z z & 0 & 0 & 0 \\
& x x x x & x x z z & 0 & 0 & 0 \\
& & z z z z & 0 & 0 & 0 \\
& & & y z y z & 0 & 0 \\
& & & & y z y z & 0 \\
& & & & & \\
& & & & & \\
& & & & &
\end{array}\right.
$$

$\left(\begin{array}{cccccc}x x x x & x x y y & x x z z & 0 & 0 & 0 \\ x x y y & x x x x & x x z z & 0 & 0 & 0 \\ z z x x & z z x x & z z z z & 0 & 0 & 0 \\ 0 & 0 & 0 & y z y z & 0 & 0 \\ 0 & 0 & 0 & 0 & y z y z & 0 \\ 0 & 0 & 0 & 0 & 0 & x y x y\end{array}\right)$

$\left(\begin{array}{llllll}0 & 0 & 0 & 0 & 0 & 0 \\ 0 & 0 & 0 & 0 & 0 & 0\end{array}\right)$

$\begin{array}{llllll}0 & 0 & 0 & 0 & 0 & 0 \\ 0 & 0 & 0 & 0 & 0 & 0 \\ 0 & 0 & 0 & 0 & 0 & 0\end{array}$

$\begin{array}{lllllll}0 & 0 & 0 & 0 & 0 & 0 \\ 0 & 0 & 0 & 0 & 0\end{array}$

$\begin{array}{llllll}0 & 0 & 0 & 0 & 0 & 0 \\ 0 & 0 & 0 & 0 & 0 & 0\end{array}$

and $x y x y=1 / 2(x x x x-x x y y)$ 
a

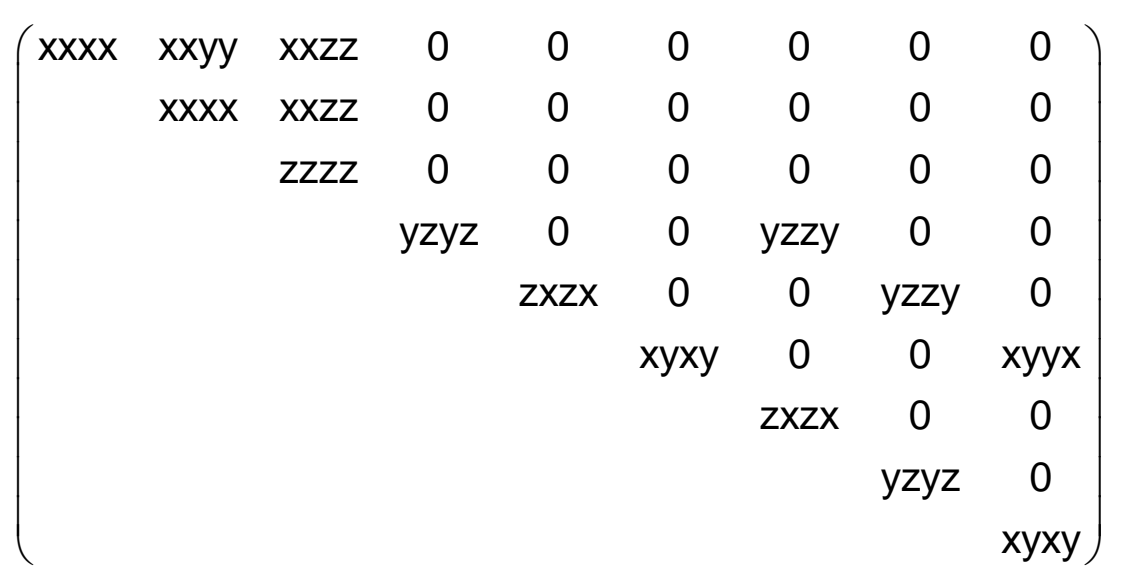

and $x x x x=x x y y+y z y z+z y z y$

$\left(\begin{array}{lllllllll}x x x x & x x y y & x x z z & 0 & 0 & 0 & 0 & 0 & 0 \\ x x y y & x x x x & x x z z & 0 & 0 & 0 & 0 & 0 & 0\end{array}\right) \quad\left(\begin{array}{lllllllll}0 & 0 & 0 & 0 & 0 & 0 & 0 & 0 & 0 \\ 0 & 0 & 0 & 0 & 0 & 0 & 0 & 0 & 0 \\ 0 & 0 & 0 & 0 & 0 & 0 & 0 & 0 & 0\end{array}\right)$

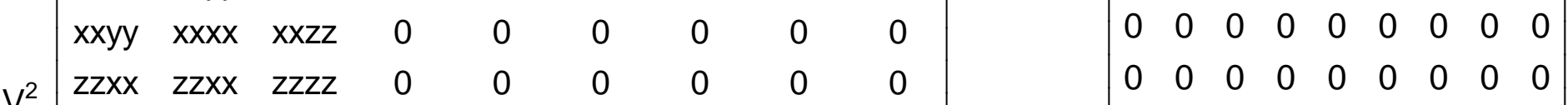

$\left[\begin{array}{lllllllll}0 & 0 & 0 & y z y z & 0 & 0 & z x z x & 0 & 0 \\ 0 & 0 & 0 & 0 & 2 x z x & 0 & 0 & y z y z & 0\end{array} \mid \begin{array}{llllllllllll}0 & 0 & 0 & 0 & 0 & 0 & 0 & 0 & 0 & 0 \\ 0 & 0 & 0 & 0 & 0 & 0 & 0 & 0 & 0 \\ 0 & 0 & 0 & 0 & 0 & 0 & 0 & 0 & 0\end{array}\right.$

$\begin{array}{lllllllll}0 & 0 & 0 & 0 & 0 & 0 & 0 & 0 & 0 \\ 0 & 0 & 0 & 0 & 0 & 0 & 0 & 0 & 0 \\ 0 & 0 & 0 & 0 & 0 & 0 & 0 & 0 & 0\end{array}$

$\begin{array}{llllllllll}0 & 0 & 0 & 0 & 0 & 0 & 0 & 0 & 0\end{array}$

and $x x x x=x x y y+2 x y x y$

and $x x x x=x x y y+y z y z+z y z y$

$\left(\begin{array}{lllllllll}0 & 0 & 0 & 0 & 0 & 0 & 0 & 0 & 0\end{array}\right.$ $\begin{array}{llllllll}0 & 0 & 0 & 0 & 0 & 0 & 0 & 0\end{array}$

$\begin{array}{lllllll}0 & 0 & 0 & 0 & 0 & 0 & 0\end{array}$ $\begin{array}{lllll}0 & 0 & 0 & 0 & 0 \\ 0 & 0 & 0 & 0 & 0\end{array}$ $\begin{array}{lllll}0 & 0 & 0 & 0 & 0 \\ 0 & 0 & 0 & 0 & 0\end{array}$ $\begin{array}{llll}0 & 0 & 0 \\ 0 & 0 & 0\end{array}$ $\begin{array}{lll}0 & 0 & 0 \\ & 0 & 0 \\ & & 0\end{array}$

$\left(\begin{array}{ccccccccc}x x x x & x x y y & x x z z & 0 & 0 & 0 & 0 & 0 & 0 \\ x x y y & x x x x & x x z z & 0 & 0 & 0 & 0 & 0 & 0 \\ z z x x & z z x x & z z z z & 0 & 0 & 0 & 0 & 0 & 0 \\ 0 & 0 & 0 & y z y z & 0 & 0 & z x z x & 0 & 0 \\ 0 & 0 & 0 & 0 & z x z x & 0 & 0 & y z y z & 0 \\ 0 & 0 & 0 & 0 & 0 & x y x y & 0 & 0 & \text { xyxy }\end{array}\right) \quad\left(\begin{array}{ccccccccc}0 & 0 & 0 & 0 & 0 & 0 & 0 & 0 & 0 \\ 0 & 0 & 0 & 0 & 0 & 0 & 0 & 0 & 0 \\ 0 & 0 & 0 & 0 & 0 & 0 & 0 & 0 & 0 \\ 0 & 0 & 0 & 0 & 0 & 0 & 0 & 0 & 0 \\ 0 & 0 & 0 & 0 & 0 & 0 & 0 & 0 & 0 \\ 0 & 0 & 0 & 0 & 0 & 0 & 0 & 0 & 0\end{array}\right)$


$\begin{array}{lllllllll}x x x x & x x y y & x x z z & 0 & 0 & x x x y & 0 & 0 & x x y x \\ y y x & y y y & y y z & 0 & 0 & y x y & 0 & 0 & y y x\end{array}$ $\begin{array}{lllllllll}\text { yyxx } & \text { yyyy } & y y z z & 0 & 0 & \text { yyxy } & 0 & 0 & \text { yyyx } \\ z z x x & z z y & z z z z & 0 & 0 & z x y & 0 & 0 & z z x\end{array}$ $\begin{array}{lllllllll}\text { zzxx } & \text { zzyy } & \text { zzzz } & 0 & 0 & \text { zzxy } & 0 & 0 & \text { zzyx }\end{array}$

$\begin{array}{lllllllll}0 & 0 & 0 & y z y z & y z z x & 0 & y z z y & y z x z & 0\end{array}$ $\begin{array}{lllllllll}0 & 0 & 0 & z x y z & z x z x & 0 & z x z y & z x x z & 0\end{array}$ $\begin{array}{lllllllll}\text { xyxx } & \text { xyyy } & \text { xyzz } & 0 & 0 & \text { xyxy } & 0 & 0 & \text { xyyx }\end{array}$ $\begin{array}{lllllllll}0 & 0 & 0 & \text { zyyz } & \text { zyzx } & 0 & \text { zyzy } & \text { zyxz } & 0\end{array}$ $\begin{array}{lllllllll}0 & 0 & 0 & x z y z & x z z x & 0 & x z z y & x z x z & 0\end{array}$ $\begin{array}{lllllllll}y \mathrm{yxx} & \mathrm{yxyy} & \mathrm{yxzz} & 0 & 0 & \mathrm{yxxy} & 0 & 0 & \mathrm{yxyx}\end{array}$

$\left[V^{4}\right] \quad\left(\begin{array}{ccccc}x x x x & x y y y & 0 & y y z z & 0 \\ y x x x & y y y y & 0 & z z x x & 0 \\ 0 & 0 & z z z z & x x y y & z z x y\end{array}\right)$

$\left(\begin{array}{lllllllll}x x x x & x x y y & x x z z & 0 & 0 & x x x y & 0 & 0 & x x y x \\ y x x & y y y & y y z & 0 & 0 & y x y & 0 & 0 & x y x\end{array}\right)$ $\begin{array}{lllllllll}\text { yyxx } & \text { yyyy } & y y z z & 0 & 0 & \text { yyxy } & 0 & 0 & \text { yyyx } \\ \text { zzxx } & z z y & z z z & 0 & 0 & z z x y & 0 & 0 & z z y x\end{array}$ $\begin{array}{ccccccccc}\text { zzxx } & \text { zzyy } & \text { zzzz } & 0 & 0 & \text { zzxy } & 0 & 0 & \text { zzyx }\end{array}$ $\begin{array}{lllllllll}0 & 0 & 0 & y z y z & y z z x & 0 & y z z y & y z x z & 0\end{array}$

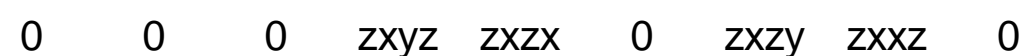
$\begin{array}{lllllllll} & 0 & 0 & 0 & 0 & 0 & & \end{array}$ $\begin{array}{lllllllll}0 & 0 & 0 & \text { zyyz } & \text { zyzx } & 0 & \text { zyzy } & \text { zyxz } & 0\end{array}$ $\begin{array}{lllllllll}0 & 0 & 0 & x z y z & x z z x & 0 & x z z y & x z x z & 0\end{array}$ $\begin{array}{lllllllll}y x x x & y x y y & y x z z & 0 & 0 & y x x y & 0 & 0 & y x y x\end{array}$

$\left(\begin{array}{ccccc}x x x x & x y y y & 0 & y y z z & 0 \\ y x x x & y y y y & 0 & z z x x & 0 \\ 0 & 0 & z z z z & x x y y & z z x y\end{array}\right)$ $\begin{array}{lllllllll}0 & 0 & 0 & x x y z & x x z x & 0 & x x z y & x x x z & 0 \\ 0 & 0 & 0 & y y z & x y z x & 0 & y y z & y y z & 0\end{array}$

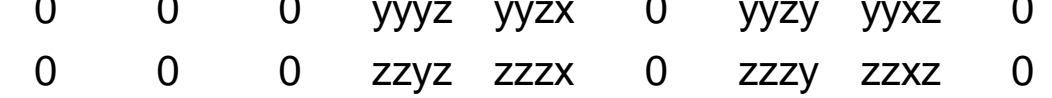

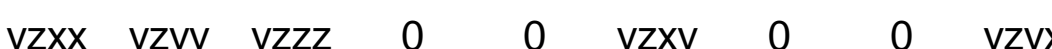
$\begin{array}{lllllllll}z x x x & z x y y & z x z z & 0 & 0 & z x x y & 0 & 0 & z x y x\end{array}$ $\begin{array}{lllllllll}0 & 0 & 0 & x y y z & x y z x & 0 & x y z y & x y x z & 0\end{array}$ $\begin{array}{lllllllll}\text { zyxx } & \text { zyyy } & \text { zyzz } & 0 & 0 & \text { zyxy } & 0 & 0 & \text { zyyx }\end{array}$ $\left(\begin{array}{ccccccccc}\mathrm{xzxx} & \mathrm{xzyy} & \mathrm{xzzz} & 0 & 0 & \mathrm{xzxy} & 0 & 0 & \mathrm{xzyx} \\ 0 & 0 & 0 & \mathrm{yxyz} & \mathrm{yxzx} & 0 & \mathrm{yxzy} & \mathrm{yxxz} & 0\end{array}\right)$

$\left(\begin{array}{ccccc}0 & 0 & x z z z & 0 & x x y z \\ 0 & 0 & y z z z & 0 & y y x z \\ z x x x & z y y y & 0 & 0 & 0\end{array}\right)$ $\left(\begin{array}{lllllllll}0 & 0 & 0 & x x y z & x x z x & 0 & x x z y & x x x z & 0 \\ 0 & 0 & 0 & y y z & y y z x & 0 & y y y & y y x z & 0\end{array}\right)$ $\begin{array}{lllllllll}0 & 0 & 0 & \text { yyyz } & \text { yyzx } & 0 & \text { yyzy } & \text { yyxz } & 0 \\ 0 & 0 & 0 & z z y & z z z x & 0 & z z z & z z x z & 0\end{array}$ $\begin{array}{lllllllll}0 & 0 & 0 & z z y z & z z z x & 0 & z z z y & z z x z & 0\end{array}$ $\begin{array}{lllllllll}\text { yzxx } & y z y y & y z z z & 0 & 0 & y z x y & 0 & 0 & y z y x\end{array}$ $\begin{array}{lllllllll}z x x x & z x y y & z x z z & 0 & 0 & z x x y & 0 & 0 & z x y x\end{array}$ $\begin{array}{lllllllll}0 & 0 & 0 & x y y z & x y z x & 0 & x y z y & x y x z & 0\end{array}$ $\begin{array}{lllllllll}\text { zyxx } & \text { zyyy } & \text { zyzz } & 0 & 0 & \text { zyxy } & 0 & 0 & \text { zyyx }\end{array}$ $\begin{array}{lllllllll}x z x x & x z y y & x z z z & 0 & 0 & x z x y & 0 & 0 & x z y x\end{array}$ $\begin{array}{lllllllll}0 & 0 & 0 & y x y z & y x z x & 0 & y x z y & y x x z & 0\end{array}$

$\left(\begin{array}{ccccc}0 & 0 & x z z z & 0 & x x y z \\ 0 & 0 & y z z z & 0 & y y x z \\ z x x x & z y y y & 0 & 0 & 0\end{array}\right)$

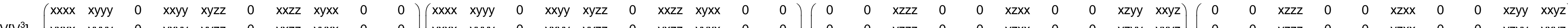

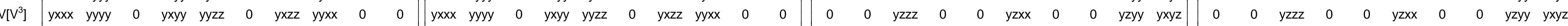

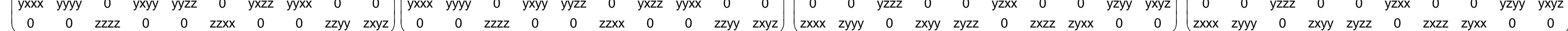

$\left.\left[V^{2}\right]^{2}\right]\left(\begin{array}{cccccc}x x x x & x x y y & x x z z & 0 & 0 & x x x y \\ & \text { yyyy } & y y z z & 0 & 0 & y y x y \\ & & z z z z & 0 & 0 & z z x y \\ & & & y z y z & y z x z & 0 \\ & & & & x z x z & 0 \\ & & & & & x y x y\end{array}\right)$

$\begin{array}{llllll}x x x x & x x y y & x x z z & 0 & 0 & x x x y\end{array}$

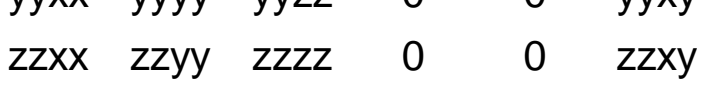
$\begin{array}{lllllll}0 & 0 & 0 & y z y z & y z x z & 0 \\ 0 & 0 & 0 & y z y z & x y z z & 0\end{array}$ $\begin{array}{llllll}0 & 0 & 0 & x z y z & x z x z & 0\end{array}$ $\begin{array}{llllll}\text { xyxx } & \text { xyyy } & \text { xyzz } & 0 & 0 & \text { xyxy }\end{array}$

$$
\left(\begin{array}{cccccc}
x x x x & \text { xxyy } & \text { xxzz } & 0 & 0 & \text { xxxy } \\
& \text { yyyy } & \text { yyzz } & 0 & 0 & \text { yyxy } \\
& & \text { zzzz } & 0 & 0 & \text { zzxy } \\
& & & y z y z & y z x z & 0 \\
& & & & x z x z & 0 \\
& & & & & \text { xyxy }
\end{array}\right)
$$

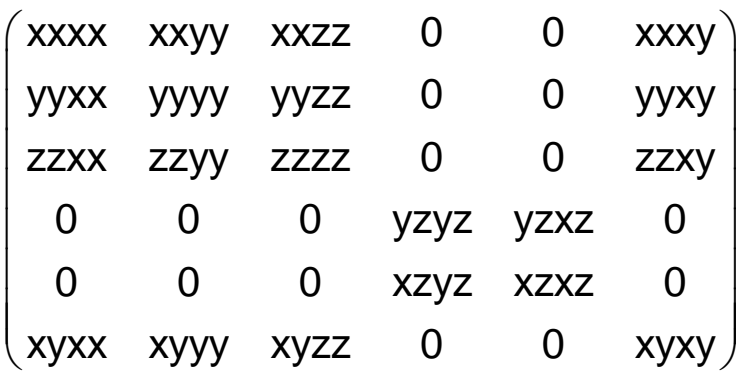

$$
\left(\begin{array}{cccccc}
0 & 0 & 0 & x x y z & x x x z & 0 \\
& 0 & 0 & y y y z & y y x z & 0 \\
& & 0 & z z y z & z z x z & 0 \\
& & & 0 & 0 & y z x y \\
& & & & 0 & x z x y \\
& & & & & 0
\end{array}\right)
$$

$\left(\begin{array}{cccccc}0 & 0 & 0 & \text { xxyz } & \text { xxxz } & 0 \\ 0 & 0 & 0 & \text { yyyz } & \text { yyxz } & 0 \\ 0 & 0 & 0 & z z y z & z z x z & 0 \\ y z x x & y z y y & y z z z & 0 & 0 & y z x y \\ x z x x & x z y y & x z z z & 0 & 0 & x z x y \\ 0 & 0 & 0 & \text { xyyz } & \text { xyxz } & 0\end{array}\right)$

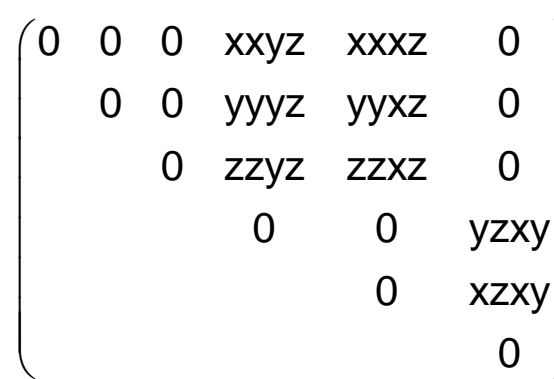

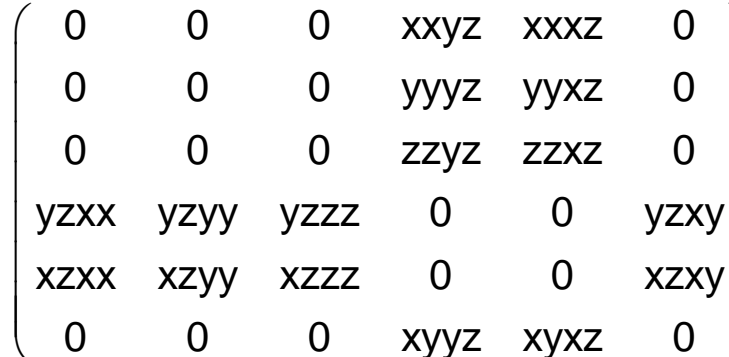




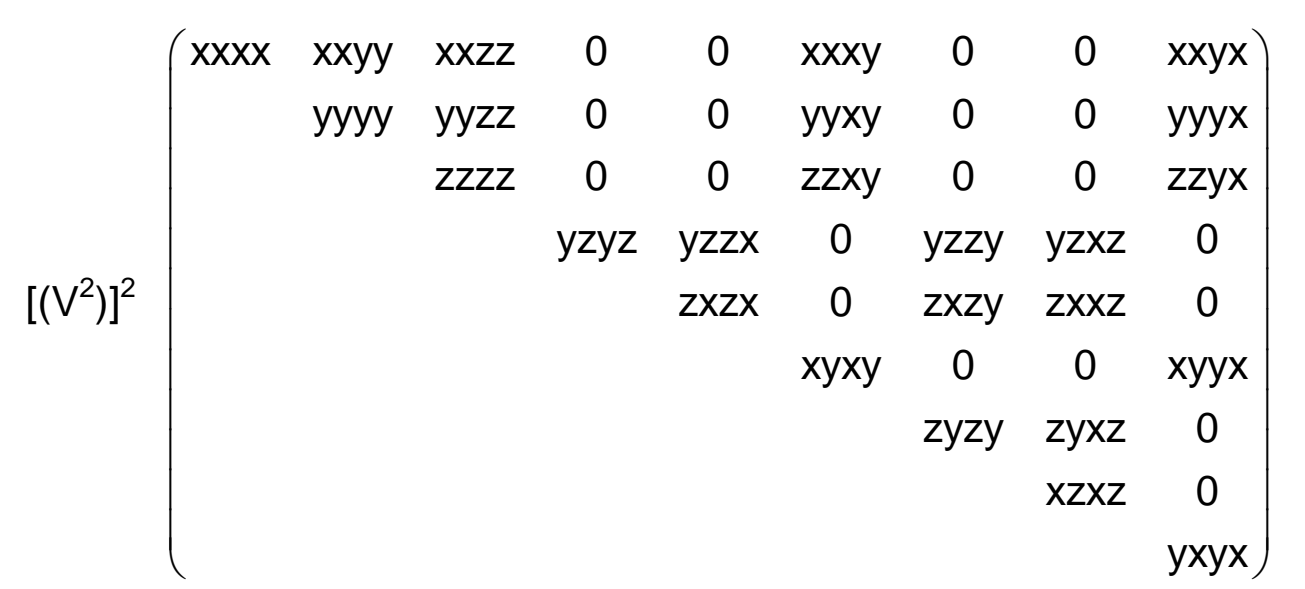
$\left.\begin{array}{lllllllll}x x x x & x x y y & x x z z & 0 & 0 & x x x y & 0 & 0 & x x y x \\ & y y y & y y z z & 0 & 0 & y y x y & 0 & 0 & y y x\end{array}\right)$
$\begin{array}{lllllllll}\text { yyyy } & y y z z & 0 & 0 & y y x y & 0 & 0 & y y y x\end{array}$
$\begin{array}{lllllll}z z z z & 0 & 0 & z z x y & 0 & 0 & z z y x\end{array}$ $\begin{array}{lllllll}0 y z y z & y z z x & 0 & y z z y & y z x z & 0\end{array}$ $\begin{array}{lllll}z x z x & 0 & z x z y & z x x z & 0\end{array}$ $\begin{array}{lccc}x y x y & 0 & 0 & x y y x\end{array}$ $\begin{array}{lll}\text { zyzy } & \text { zyxz } & 0 \\ & x z x z & 0\end{array}$

$\left[\mathrm{V}^{2}\right] \mathrm{V}^{2}\left(\begin{array}{ccccccccc}\mathrm{x} x \mathrm{x} & \mathrm{xxyy} & \mathrm{xxzz} & 0 & 0 & \mathrm{xxxy} & 0 & 0 & \mathrm{xxyx} \\ \mathrm{yyxx} & \mathrm{yyyy} & \mathrm{yyzz} & 0 & 0 & \mathrm{yyxy} & 0 & 0 & \mathrm{yyyx} \\ \mathrm{zzxx} & \mathrm{zzyy} & \mathrm{zzzz} & 0 & 0 & \mathrm{zzxy} & 0 & 0 & \mathrm{zzyx} \\ 0 & 0 & 0 & \mathrm{yzyz} & \mathrm{yzzx} & 0 & \mathrm{yzzy} & \mathrm{yzzz} & 0 \\ 0 & 0 & 0 & \mathrm{zxyz} & \mathrm{zxzx} & 0 & \mathrm{zxzy} & \mathrm{zxxz} & 0 \\ \mathrm{xyxx} & \mathrm{xyyy} & \mathrm{xyzz} & 0 & 0 & \mathrm{xyxy} & 0 & 0 & \mathrm{xyyx}\end{array}\right)$
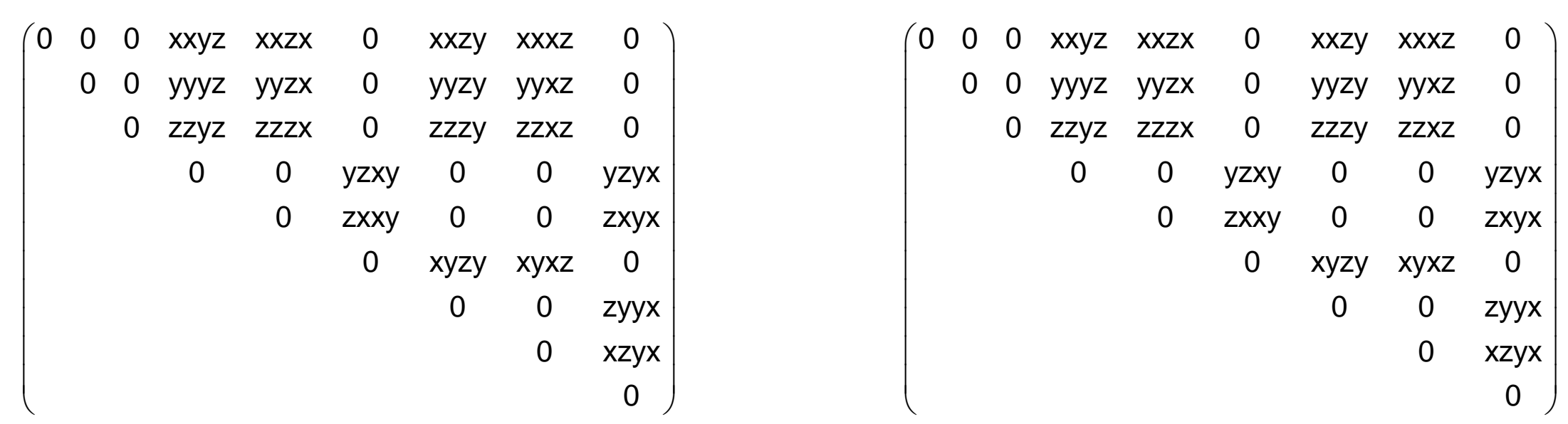

$\left(\begin{array}{lllllllll}0 & 0 & 0 & x x y z & x x z x & 0 & x x z y & x x x z & 0\end{array}\right)$ $\begin{array}{ccccccccc}0 & 0 & 0 & y y y z & y y z x & 0 & \text { yyzy } & \text { yyxz } & 0 \\ 0 & 0 & 0 & \text { zzyz } & \text { zzzx } & 0 & \text { zzzy } & \text { zzxz } & 0\end{array}$ $\begin{array}{lllllllll}\text { yzxx } & \text { yzyy } & \text { yzzz } & 0 & 0 & \text { yzxy } & 0 & 0 & \text { yzyx }\end{array}$ $\begin{array}{ccccccccc}z x x x & z x y y & z x z z & 0 & 0 & z x x y & 0 & 0 & z x y x\end{array}$

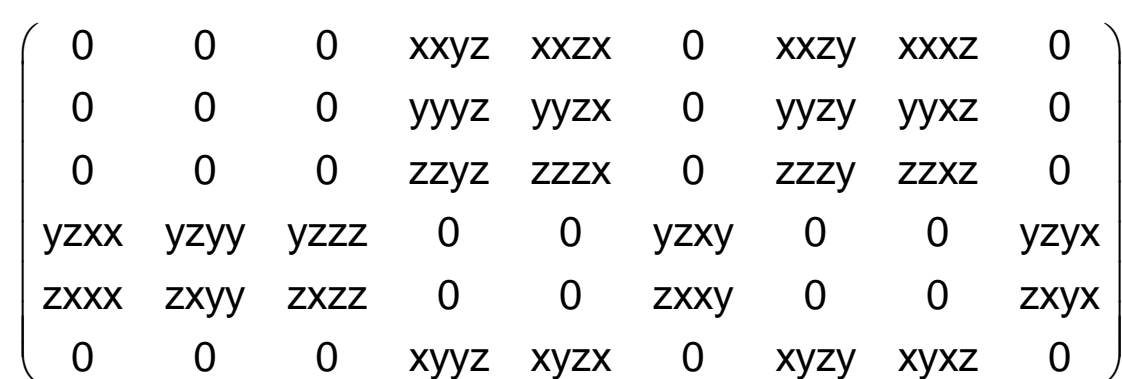




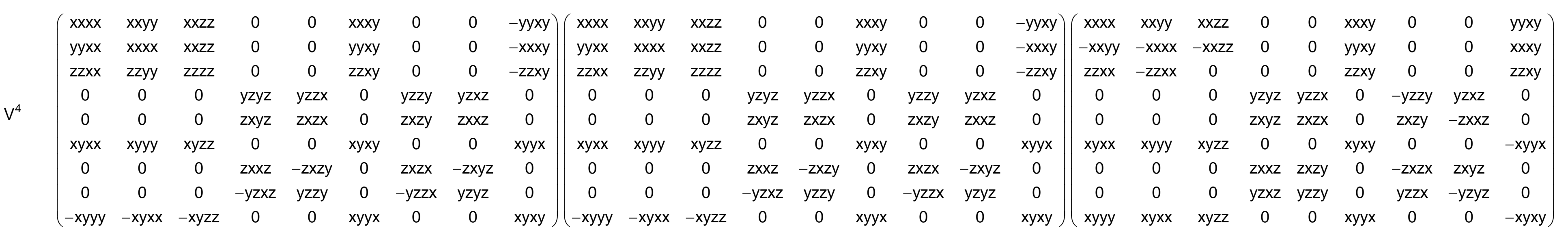
$\left[V^{4}\right] \quad\left(\begin{array}{ccccc}\mathrm{xxxx} & -\mathrm{yxxx} & 0 & \mathrm{yyzz} & 0 \\ \mathrm{yxxx} & \mathrm{xxxx} & 0 & \mathrm{yyzz} & 0 \\ 0 & 0 & \mathrm{zzzz} & \mathrm{xxyy} & 0\end{array}\right)$
$\left(\begin{array}{ccccc}\mathrm{xxxx} & -\mathrm{yxxx} & 0 & \mathrm{yyzz} & 0 \\ \mathrm{yxxx} & \mathrm{xxxx} & 0 & \mathrm{yyzz} & 0 \\ 0 & 0 & \mathrm{zzzz} & \mathrm{xxyy} & 0\end{array}\right)$
$\left(\begin{array}{ccccc}\mathrm{xxxx} & \mathrm{yxxx} & 0 & \mathrm{yyzz} & 0 \\ \mathrm{yxxx} & -\mathrm{xxxx} & 0 & -\mathrm{yyzz} & 0 \\ 0 & 0 & 0 & 0 & \mathrm{zzxy}\end{array}\right)$

$\left(\begin{array}{ccccccccc}x x x x & x x y y & x x z z & 0 & 0 & x x x y & 0 & 0 & y y x y \\ -x x y y & -x x x x & -x x z z & 0 & 0 & y y x y & 0 & 0 & x x x y \\ z z x x & -z z x x & 0 & 0 & 0 & z z x y & 0 & 0 & z z x y \\ 0 & 0 & 0 & y z y z & y z z x & 0 & -y z z y & y z x z & 0 \\ 0 & 0 & 0 & z x y z & z x z x & 0 & z x z y & -z x x z & 0 \\ x y x x & x y y y & x y z z & 0 & 0 & x y x y & 0 & 0 & -x y y x \\ 0 & 0 & 0 & z x x z & z x z y & 0 & -z x z x & z x y z & 0 \\ 0 & 0 & 0 & y z x z & y z z y & 0 & y z z x & -y z y z & 0 \\ x y y y & x y x x & x y z z & 0 & 0 & x y y x & 0 & 0 & -x y x y\end{array}\right)$

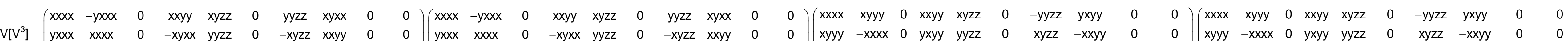

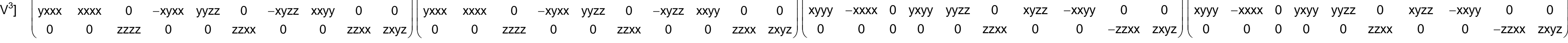

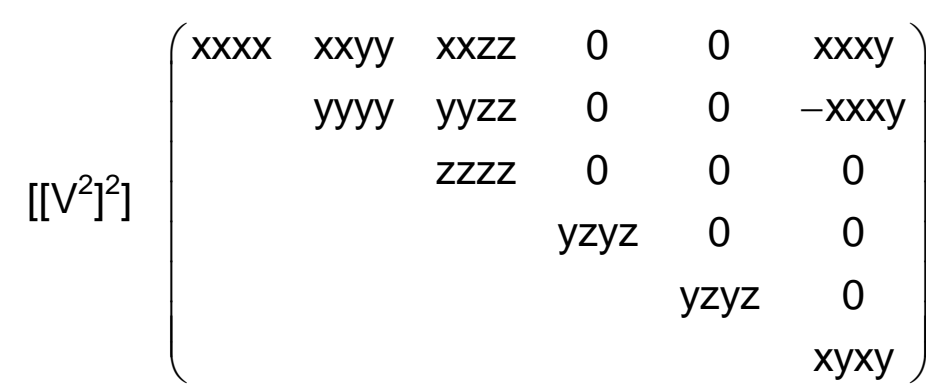

$\left[V^{2}\right]^{2}\left(\begin{array}{cccccc}x x x x & x x y y & x x z z & 0 & 0 & x x x y \\ y y x x & x x x x & x x z z & 0 & 0 & -x x x y \\ z z x x & z z x x & z z z z & 0 & 0 & 0 \\ 0 & 0 & 0 & y z y z & y z x z & 0 \\ 0 & 0 & 0 & -y z x z & y z y z & 0 \\ x y x x & -x y x x & 0 & 0 & 0 & x y x y\end{array}\right)$

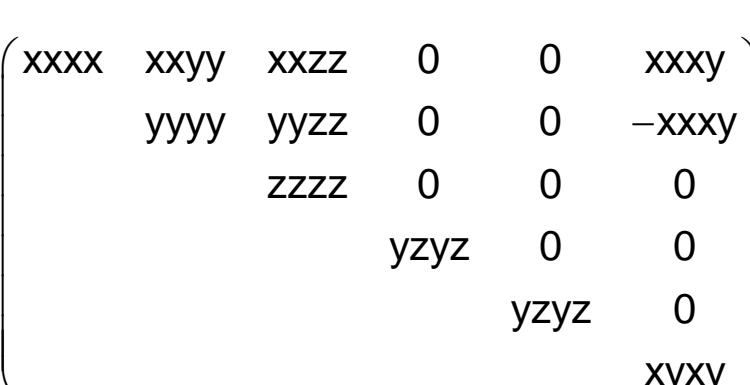

$\left(\begin{array}{cccccc}x x x x & x x y y & x x z z & 0 & 0 & x x x y \\ y y x x & x x x x & x x z z & 0 & 0 & -x x x y \\ z z x x & z z x x & z z z z & 0 & 0 & 0 \\ 0 & 0 & 0 & y z y z & y z x z & 0 \\ 0 & 0 & 0 & -y z x z & y z y z & 0 \\ \text { xyxx } & -x y x x & 0 & 0 & 0 & x y x y\end{array}\right)$

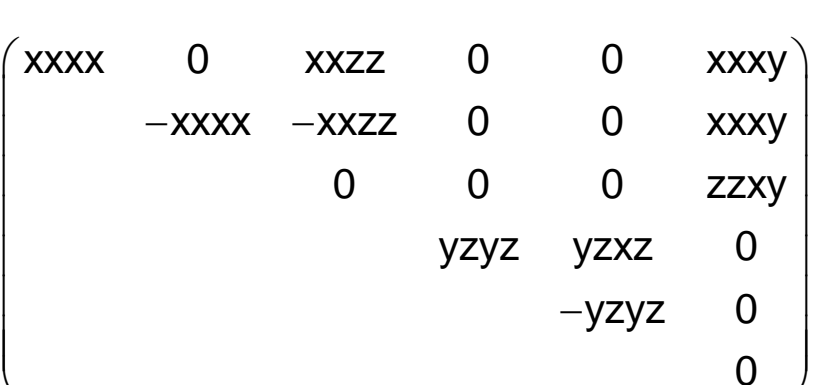

$\left(\begin{array}{cccccc}x x x x & x x y y & x x z z & 0 & 0 & x x x y \\ -x x y y & -x x x x & -x x z z & 0 & 0 & x x x y \\ z z x x & -z z x x & 0 & 0 & 0 & z z x y \\ 0 & 0 & 0 & y z y z & y z x z & 0 \\ 0 & 0 & 0 & y z x z & -y z y z & 0 \\ x y x x & x y x x & x y z z & 0 & 0 & 0\end{array}\right)$

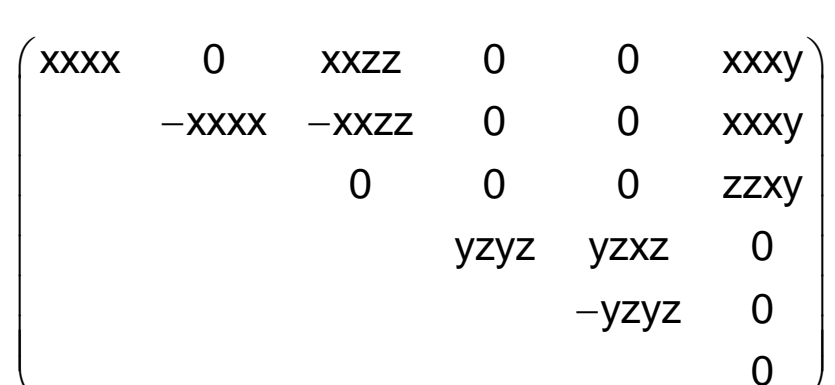

$\left(\begin{array}{cccccc}x x x x & x x y y & x x z z & 0 & 0 & x x x y \\ -x x y y & -x x x x & -x x z z & 0 & 0 & x x x y \\ z z x x & -z z x x & 0 & 0 & 0 & z z x y \\ 0 & 0 & 0 & y z y z & y z x z & 0 \\ 0 & 0 & 0 & y z x z & -y z y z & 0 \\ x y x x & x y x x & x y z z & 0 & 0 & 0\end{array}\right)$ 


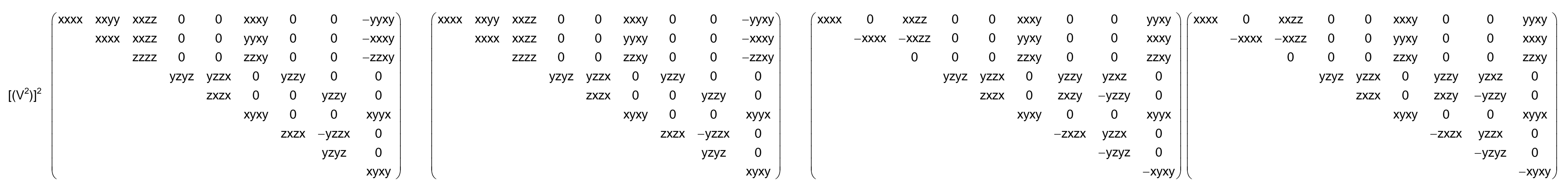

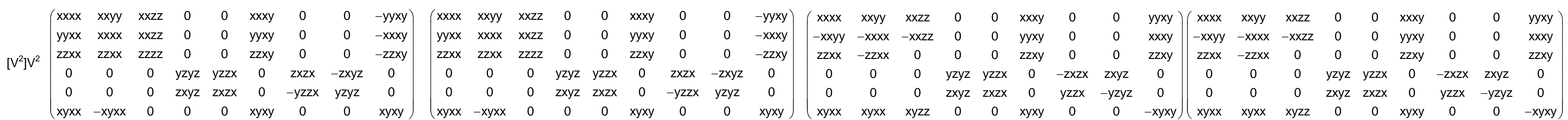




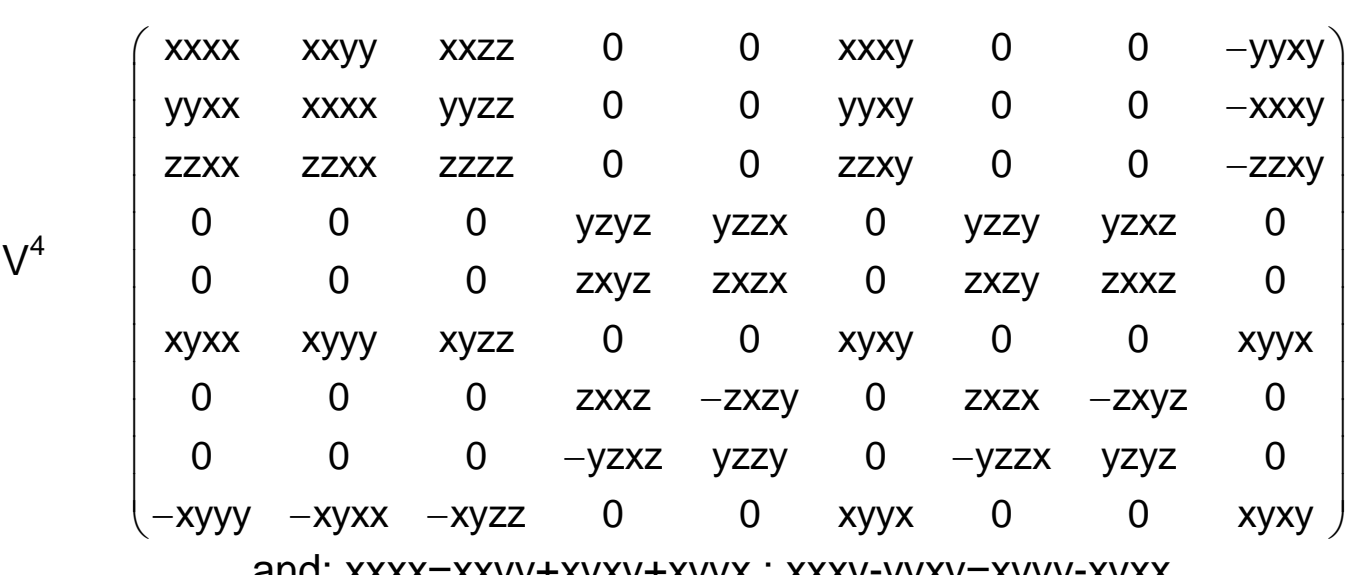
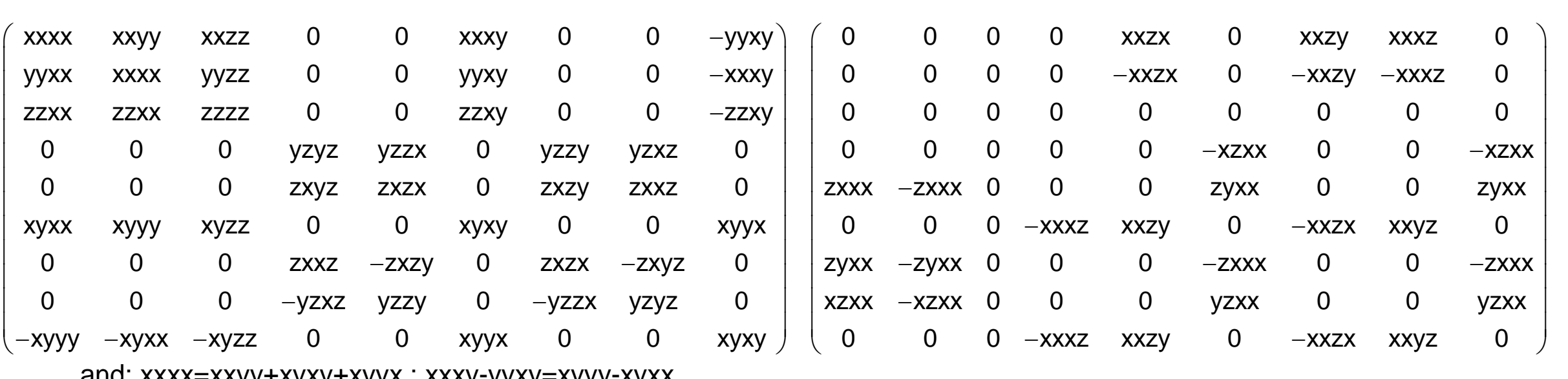
$\left(\begin{array}{ccccccccc}0 & 0 & 0 & 0 & x x z x & 0 & x x z y & x x x z & 0 \\ 0 & 0 & 0 & 0 & -x x z x & 0 & -x x z y & -x x x z & 0 \\ 0 & 0 & 0 & 0 & 0 & 0 & 0 & 0 & 0 \\ 0 & 0 & 0 & 0 & 0 & -x z x x & 0 & 0 & -x z x x \\ z x x x x & -z x x x & 0 & 0 & 0 & z y x x & 0 & 0 & z y x x \\ 0 & 0 & 0 & -x x x z & x x z y & 0 & -x x z x & x x y z & 0 \\ z y x x & -z y x x & 0 & 0 & 0 & -z x x x & 0 & 0 & -z x x x \\ \text { xzxx } & -x z x x & 0 & 0 & 0 & y z x x & 0 & 0 & y z x x \\ 0 & 0 & 0 & -x x x z & x x z y & 0 & -x x z x & x x y z & 0\end{array}\right)$ and: $x x x x=x x y y+x y x y+x y y x ; x x x y-y y x y=x y y y-x y x x$ and: $x x x x=x x y y+x y x y+x y y x ; x x x y-y y x y=x y y y-x y x x$

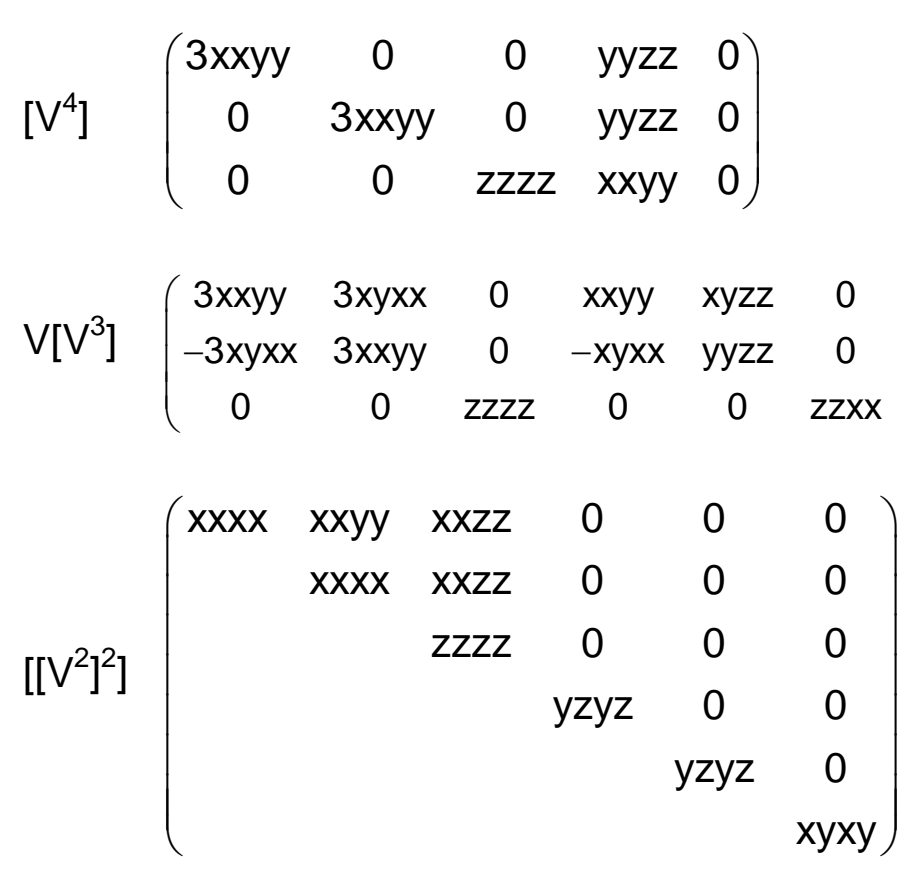

and $x y x y=1 / 2(x x x x-x x y y)$

$\left[V^{2}\right]^{2}\left(\begin{array}{cccccc}x x x x & x x y y & x x z z & 0 & 0 & x x x y \\ x x y y & x x x x & x x z z & 0 & 0 & -x x x y \\ z z x x & z z x x & z z z z & 0 & 0 & 0 \\ 0 & 0 & 0 & y z y z & y z x z & 0 \\ 0 & 0 & 0 & y z x z & y z y z & 0 \\ -x x x y & x x x y & 0 & 0 & 0 & x y x y\end{array}\right)$

and $x y x y=1 / 2(x x x x-x x y y)$

$$
\left(\begin{array}{ccccc}
3 x x y y & 0 & 0 & y y z z & 0 \\
0 & 3 x x y y & 0 & y y z z & 0 \\
0 & 0 & z z z z & x x y y & 0
\end{array}\right)
$$$$
\left(\begin{array}{ccccc}
0 & 0 & 0 & 0 & x x y z \\
0 & 0 & 0 & 0 & -z x x x \\
z x x x & -x x y z & 0 & 0 & 0
\end{array}\right)
$$

$\left(\begin{array}{ccccc}0 & 0 & 0 & 0 & x x y z \\ 0 & 0 & 0 & 0 & -z x x x \\ z x x x & -x x y z & 0 & 0 & 0\end{array}\right)$

$\left(\begin{array}{cccccccccc}0 & 0 & 0 & 0 & 0 & x z x x & 0 & 0 & -x z z x & y z x x \\ 0 & 0 & 0 & 0 & 0 & y z x x & 0 & 0 & -y z x x & -x z x x \\ 2 x x & -2 y x x & 0 & -z x x & 0 & 0 & 0 & & & \end{array}\right.$ $\begin{array}{llllllllll}2 x x x & -z y x x & 0 & -z x x x & 0 & 0 & 0 & z y x x & 0 & 0\end{array}$

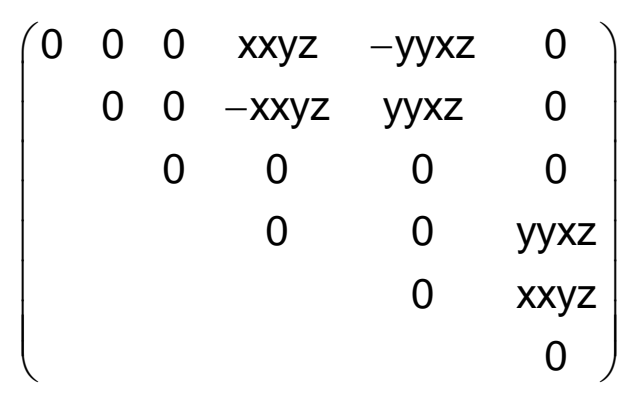

and $x y x y=1 / 2(x x x x-x x y y)$
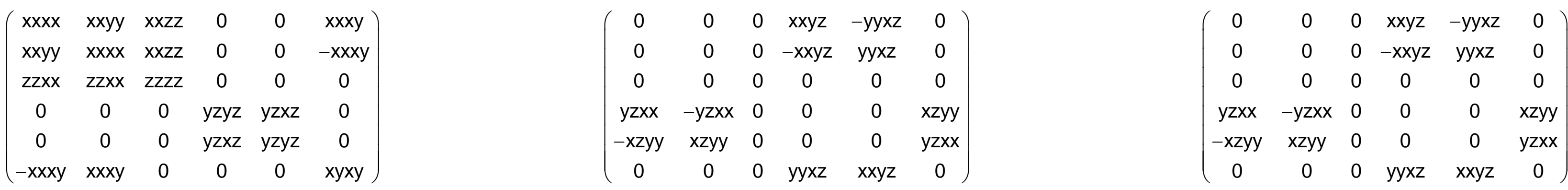

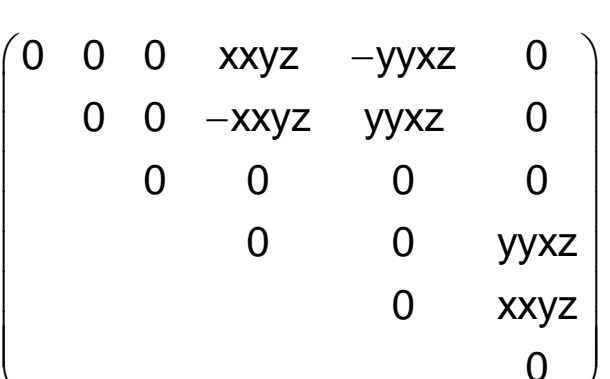

$x y=1 / 2(x x x x-x x y y)$ 


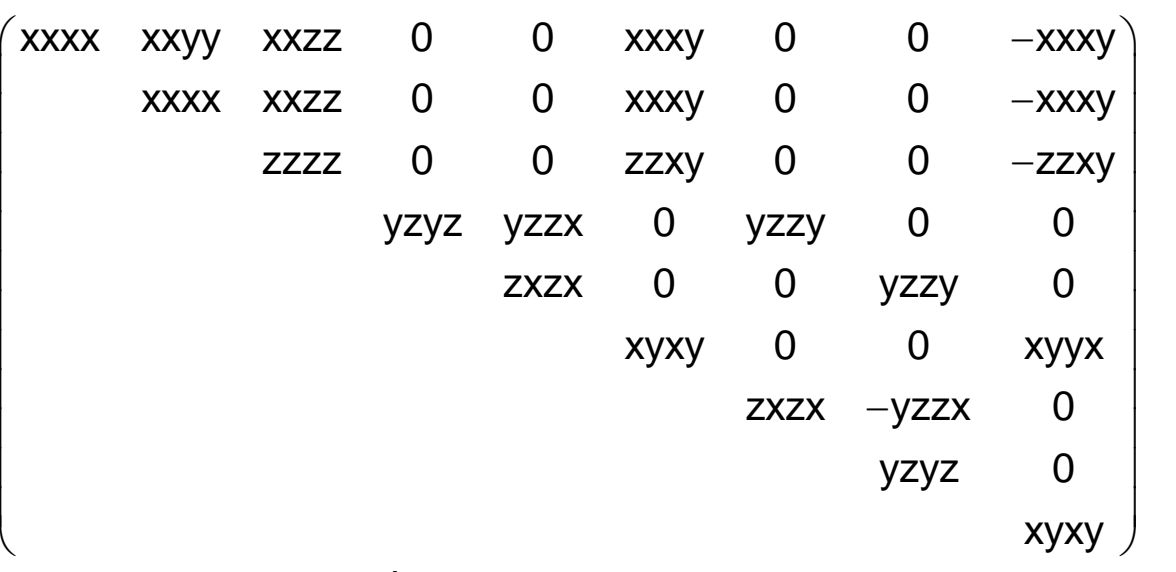

and $x x x x=x x y y+y z y z+z y z y$
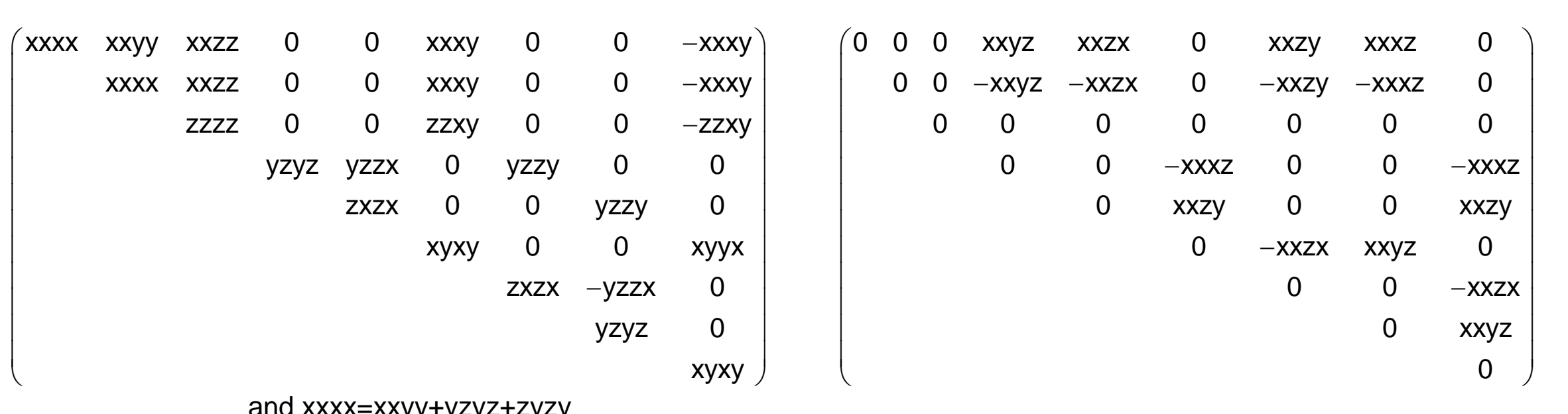

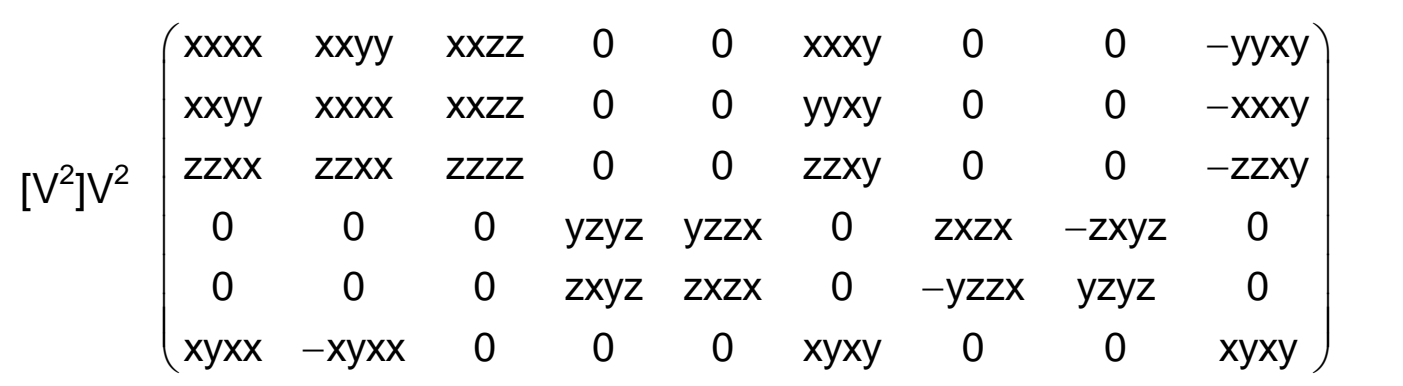

and $x x x x=x x y y+2 x y x y$; $y y x y-x x x y=2 x y x x$

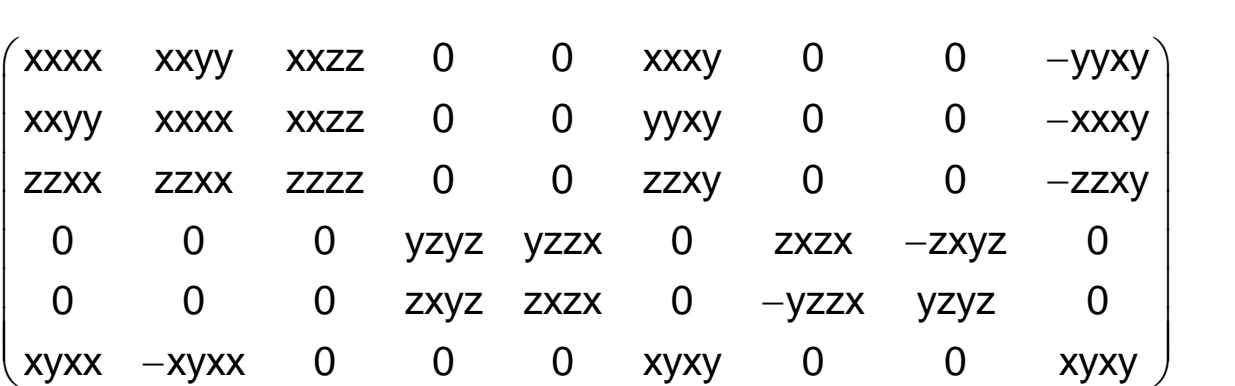
$\left(\begin{array}{ccccccccc}0 & 0 & 0 & x x y z & x x z x & 0 & x x z y & x x x z & 0 \\ 0 & 0 & 0 & -x x y z & -x x z x & 0 & -x x z y & -x x x z & 0 \\ 0 & 0 & 0 & 0 & 0 & 0 & 0 & 0 & 0 \\ y z x x & -y z x x & 0 & 0 & 0 & 0 & 0 & 0 & -z x x x \\ z x x x & -z x x x & 0 & 0 & 0 & y z x x & 0 & 0 & y z x x \\ 0 & 0 & 0 & -x x x z & x x z y & 0 & -x x z x & x x y z & 0\end{array}\right)$

and $x x x x=x x y y+2 x y x y ; y y x y-x x x y=2 x y x x$

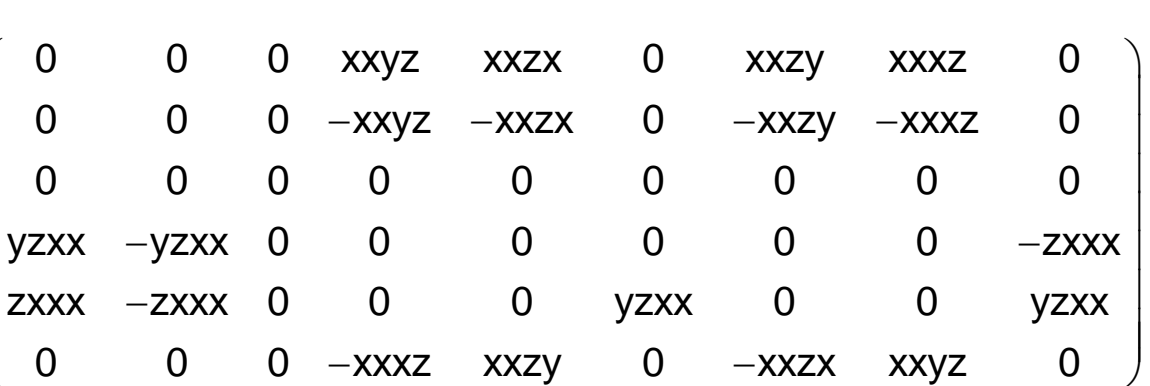

$\left.\begin{array}{ccccccccc}0 & 0 & 0 & x x y z & x x z x & 0 & x x z y & x x x z & 0 \\ 0 & 0 & 0 & -x x y z & -x x z x & 0 & -x x z y & -x x x z & 0 \\ 0 & 0 & 0 & 0 & 0 & 0 & 0 & 0 & 0 \\ y z x x & -y z x x & 0 & 0 & 0 & 0 & 0 & 0 & -z x x x \\ z x x x & -z x x x & 0 & 0 & 0 & y z x x & 0 & 0 & y z x x \\ 0 & 0 & 0 & -x x x z & x x z y & 0 & -x x z x & x x y z & 0\end{array}\right)$ 


\begin{tabular}{|c|c|c|c|c|c|c|c|c|c|c|c|c|c|c|c|c|c|c|c|c|c|c|c|c|}
\hline xx & xxyy & $x x z z$ & 0 & 0 & xxxy & 0 & 0 & $-y y x y)$ & xxxx & xxyy & xxzz & 0 & 0 & xxxy & 0 & 0 & $-y y x y)$ & $x x x x$ & $-x x x x$ & $\begin{array}{lll}0 & 0\end{array}$ & 0 & -хууy & & $-x y y y$ \\
\hline & $x x x x$ & yyzz & 0 & 0 & yyxy & 0 & 0 & $-x x x y$ & $y y x x$ & $x x x x$ & yyzz & 0 & 0 & yухy & 0 & 0 & $-x x x y$ & $-x x x x$ & $x x x x$ & $\begin{array}{lll}0 & 0\end{array}$ & 0 & xyyy & & xyyy \\
\hline & zzxx & zzzz & 0 & 0 & zzxy & 0 & 0 & $-z z x y$ & zzxx & zzxx & zZzZ & 0 & & zzxy & & & -zzxy & 0 & 0 & $\begin{array}{llc}0 & 0\end{array}$ & 0 & 0 & & D \\
\hline 0 & 0 & 0 & yzyz & $y z z x$ & 0 & yzzy & $y z x z$ & 0 & 0 & 0 & 0 & yzyz & yzzx & 0 & yzzy & $y z x z$ & 0 & $c$ & 0 & & 0 & 0 & & \\
\hline 0 & 0 & 0 & zxyz & $z x z x$ & 0 & zxzy & $z x x z$ & 0 & 0 & 0 & & & $z x z x$ & 0 & & $z x x z$ & & 0 & 0 & & 0 & 0 & & \\
\hline IXx & xyyy & xyzz & 0 & 0 & xyxy & 0 & 0 & xyyx & xyxx & xyyy & xyzz & 0 & 0 & хуху & 0 & 0 & xyyx & -xyyy & xyyy & 0 & 0 & $-x x x x$ & & 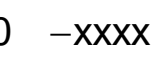 \\
\hline & 0 & 0 & $z x x z$ & $-z x z y$ & 0 & $z x z x$ & $-z x y z$ & 0 & 0 & 0 & 0 & $z x x z$ & $-z x z y$ & 0 & zxzx & $-z x y z$ & & 0 & 0 & & 0 & 0 & & \\
\hline & 0 & 0 & $-y z x z$ & yzzy & 0 & -yzzx & yzyz & 0 & 0 & 0 & 0 & $-y z x z$ & yzzy & 0 & $-y z z x$ & yzyz & 0 & & 0 & & & 0 & & \\
\hline
\end{tabular}

$\left(\begin{array}{ccccccccc}x x x x & -x x x x & 0 & 0 & 0 & -x y y y & 0 & 0 & -x y y y \\ -x x x & x x x & 0 & 0 & 0 & x y y & 0 & 0 & x y y\end{array}\right)$ $\begin{array}{ccccccccc}-x x x x & x x x x & 0 & 0 & 0 & x y y y & 0 & 0 & x y y y\end{array}$ $\begin{array}{lllllclll}0 & 0 & 0 & 0 & 0 & 0 & 0 & 0 & 0 \\ 0 & 0 & 0 & 0 & 0 & 0 & 0 & 0 & 0\end{array}$ $\begin{array}{lllllllll}0 & 0 & 0 & 0 & 0 & 0 & 0 & 0 & 0 \\ 0 & 0 & 0 & 0 & 0 & 0 & 0 & 0 & 0\end{array}$ $\begin{array}{lllllllll}0 & 0 & 0 & 0 & 0 & 0 & 0 & 0 & 0 \\ -x y y & x y y & 0 & 0 & 0 & -x & 0\end{array}$ $\begin{array}{llllllllll}0 & -x y y y & \text { xyyy } & 0 & 0 & 0 & -x x x x & 0 & 0 & -x x x x\end{array}$ $\begin{array}{lllllllll}0 & 0 & 0 & 0 & 0 & 0 & 0 & 0 & 0 \\ 0 & 0 & 0 & 0 & 0 & 0 & 0 & 0 & 0\end{array}$ -xyyy xyyy

$\begin{array}{ccccccccc}0 & 0 & 0 & -y z x z & y z y & 0 & -y z z x & y z y z & 0 \\ \text { and: } x x x y=x x y y+x y x y+x y y x ; & x y x y y-y y x y=x y y y-x y x x & & \end{array}$

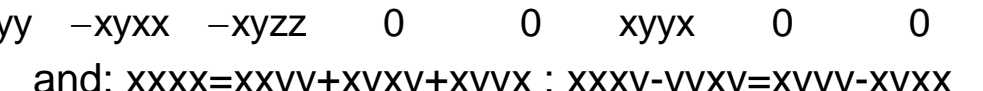

$\left(\begin{array}{ccccc}x x x x & x y y y & 0 & 0 & 0 \\ -x y y y & x x x x & 0 & 0 & 0 \\ 0 & 0 & 0 & -x x x x & 0\end{array}\right)$

$\left(\begin{array}{ccccc}x x x x & x y y y & 0 & 0 & 0 \\ -x y y y & x x x x & 0 & 0 & 0 \\ 0 & 0 & 0 & -x x x x & 0\end{array}\right)$

$\left(\begin{array}{cccccc}0 & 0 & 0 & -x x x x & 0\end{array}\right)$

$\left(\begin{array}{llllllllll}x x x x & x y y y & 0 & -x x x x & 0 & 0 & 0 & -x y y y & 0 & 0\end{array}\right.$ $\begin{array}{llllllllll}-x y y y & x x x x & 0 & x y y y & 0 & 0 & 0 & -x x x x & 0 & 0\end{array}$

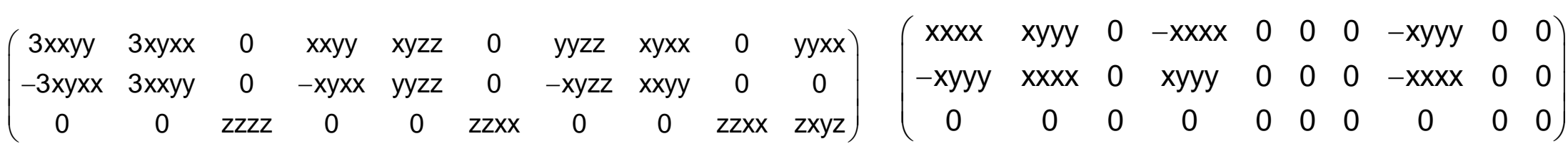

$\left(\begin{array}{cccccccccc}-x & 0 & 0 & 0 & 0 & 0 & 0 & 0 & 0 & 0\end{array}\right)$

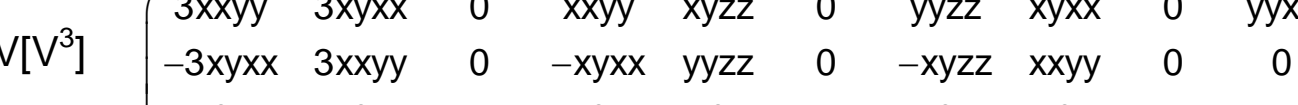

$$
\left(\begin{array}{cccccc}
x x x x & x x y y & x x z z & 0 & 0 & 0 \\
& x x x x & x x z z & 0 & 0 & 0 \\
& & z z z z & 0 & 0 & 0 \\
& & & y z y z & 0 & 0 \\
& & & & y z y z & 0 \\
& & & & & \text { xyxy }
\end{array}\right)
$$
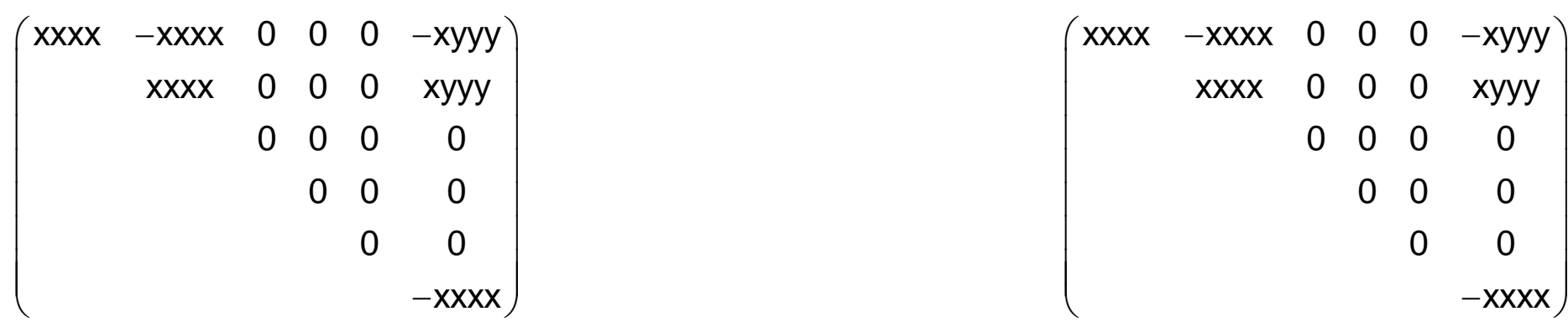

and $x y x y=1 / 2(x x x x-x x y y)$

$\left(\begin{array}{cccccc}\text { xxxx } & x x y y & x x z z & 0 & 0 & x x x y \\ x x y y & x x x x & x x z z & 0 & 0 & -x x x y \\ z z x x & z z x x & z z z z & 0 & 0 & 0 \\ 0 & 0 & 0 & y z y z & y z x z & 0 \\ 0 & 0 & 0 & y z x z & y z y z & 0 \\ -x x x y & x x x y & 0 & 0 & 0 & x y x y\end{array}\right)$

$\left(\begin{array}{cccccc}x x x x & -x x x x & 0 & 0 & 0 & -x y y y \\ -x x x x & x x x x & 0 & 0 & 0 & x y y y \\ 0 & 0 & 0 & 0 & 0 & 0 \\ 0 & 0 & 0 & 0 & 0 & 0 \\ 0 & 0 & 0 & 0 & 0 & 0 \\ -x y y y & x y y y & 0 & 0 & 0 & -x x x x\end{array}\right)$

$\left(\begin{array}{cccccc}x x x x & -x x x x & 0 & 0 & 0 & -x y y y \\ -x x x x & x x x x & 0 & 0 & 0 & x y y y \\ 0 & 0 & 0 & 0 & 0 & 0 \\ 0 & 0 & 0 & 0 & 0 & 0 \\ 0 & 0 & 0 & 0 & 0 & 0 \\ -x y y y & x y y y & 0 & 0 & 0 & -x x x x\end{array}\right)$

and $x y x y=1 / 2(x x x x-x x y y)$

and $x y x y=1 / 2(x x x x-x x y y)$ 


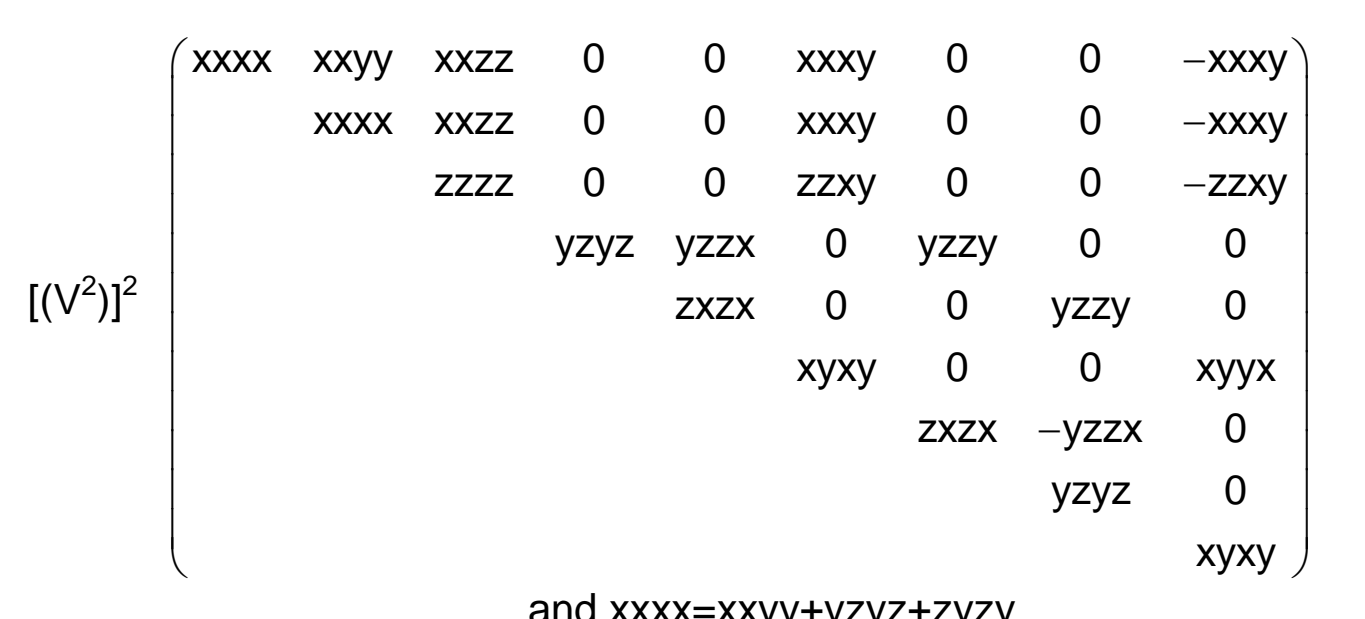

and $x x x x=x x y y+y z y z+z y z y$ $\begin{array}{lllllllll}x x y y & x x x x & x x z z & 0 & 0 & y y x y & 0 & 0 & -x x x y \\ z x x & z x x x & z z z z & 0 & 0 & z z x y & 0 & 0 & -z x y\end{array}$

$\left[\mathrm{V}^{2}\right] \mathrm{V}^{2} \quad \begin{array}{cccccccccc}\mathrm{zzxx} & \mathrm{zzxx} & \mathrm{zzzz} & 0 & 0 & \mathrm{zzxy} & 0 & 0 & -\mathrm{zzxy} \\ 0 & 0 & 0 & \mathrm{yzyz} & \mathrm{yzzx} & 0 & \mathrm{zxzx} & -\mathrm{zxyz} & 0 \\ 0 & 0 & 0 & \mathrm{zxyz} & \mathrm{zxzx} & 0 & -\mathrm{yzzx} & \mathrm{yzyz} & 0\end{array}$ $\left.\begin{array}{ccccccccc}0 & 0 & 0 & y z y z & y z z x & 0 & z x z x & -z x y z & 0 \\ 0 & 0 & 0 & z x y z & z x z x & 0 & -y z z x & y z y z & 0 \\ x y x x & -x y x x & 0 & 0 & 0 & x y x & 0 & 0 & x y x y\end{array}\right)$

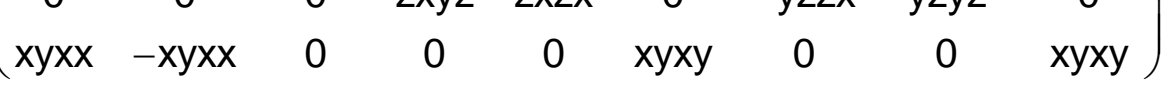
and $x x x x=x x y y+2 x y x y ; y y x y-x x x y=2 x y x x$

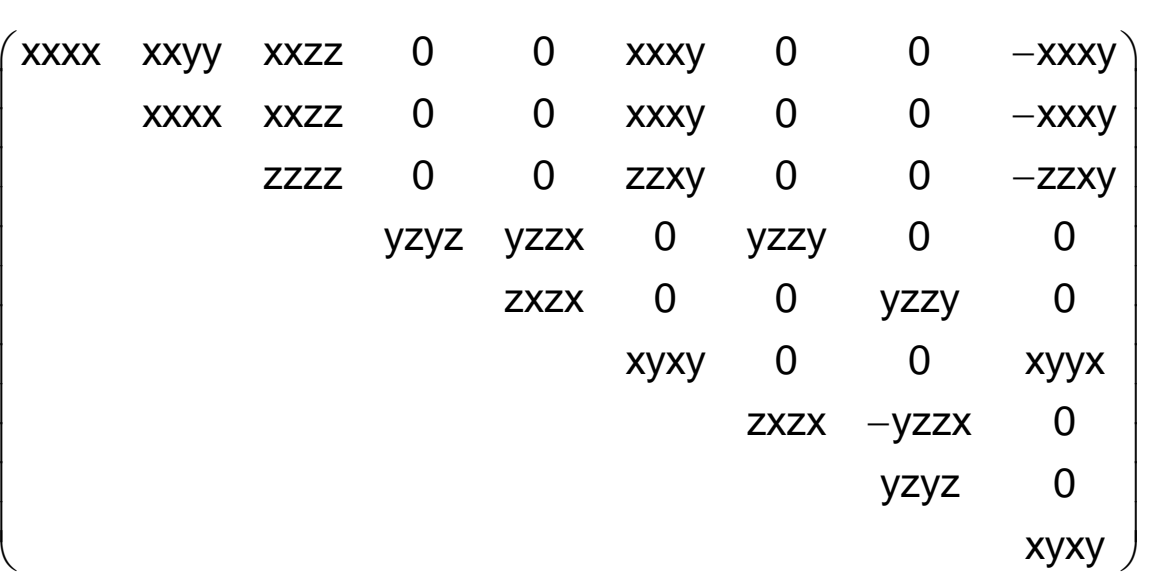

and $x x x x=x x y y+y z y z+z y z y$

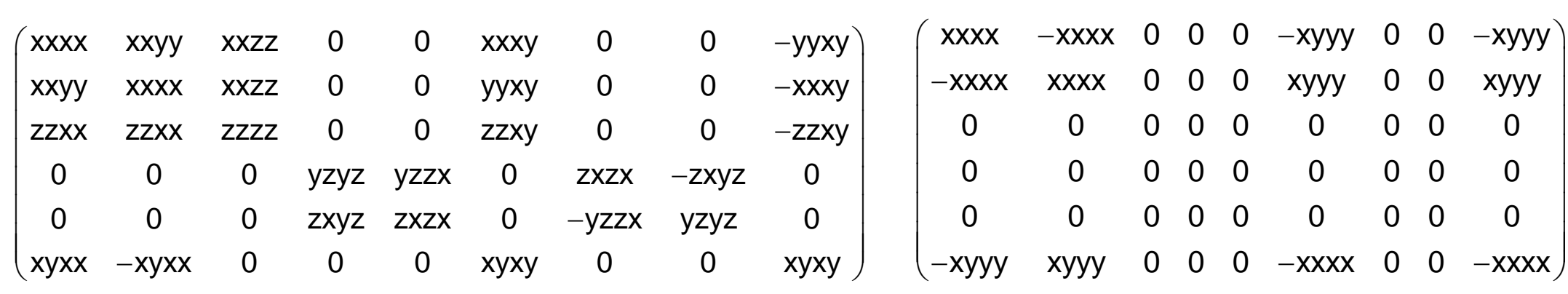

and $x x x x=x x y y+2 x y x y ; y y x y-x x x y=2 x y x x$ $\left(\begin{array}{lllllllll}x x x x & -x x x x & 0 & 0 & 0 & -x y y y & 0 & 0 & -x y y y \\ x & x x x & 0 & 0 & 0 & -x y y & 0 & 0 & -x y y\end{array}\right)$

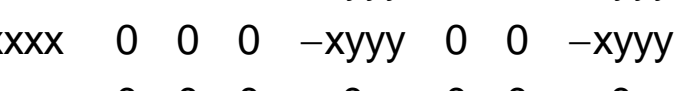
$\begin{array}{lllllll}0 & 0 & 0 & 0 & 0 & 0 & 0\end{array}$ $\begin{array}{llllll}0 & 0 & 0 & 0 & 0 & 0\end{array}$ $\begin{array}{ccccc}0 & 0 & 0 & 0 & 0 \\ -x \times x x & 0 & 0 & -x \times x x\end{array}$ $\begin{array}{rrrr}0 & 0 & 0\end{array}$ 0
0
$-x x x x$

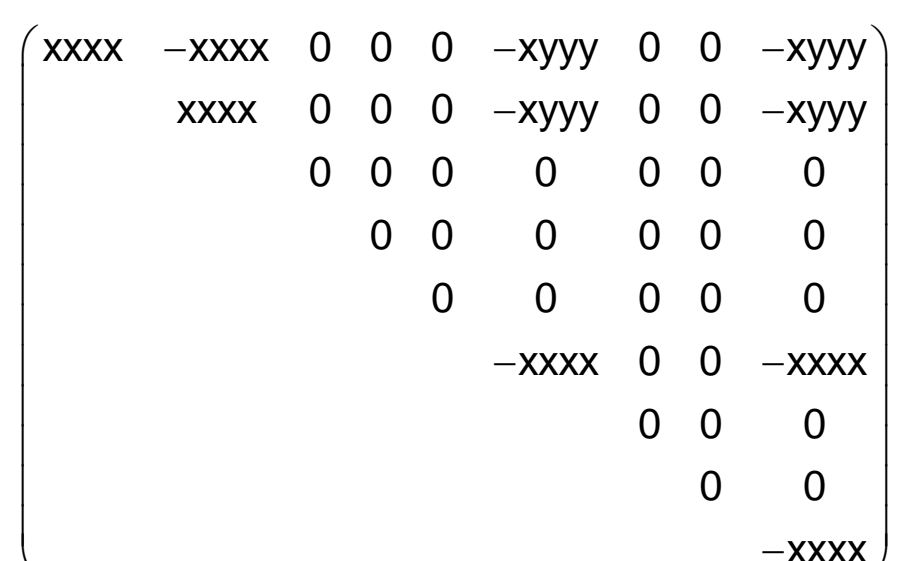

$\left(\begin{array}{lllllllll}x x x x & -x x x x & 0 & 0 & 0 & -x y y y & 0 & 0 & -x y y y \\ -x x x & x x x & 0 & 0 & 0 & x y y & 0 & 0 & x y y y\end{array}\right.$ $\left(\begin{array}{llllllllll}x x x x & -x x x x & x x x x & 0 & 0 & 0 & x y y y & 0 & 0 & x y y y\end{array}\right)$ $\begin{array}{lllllllll}0 & 0 & 0 & 0 & 0 & 0 & 0 & 0 & 0\end{array}$ $\begin{array}{lllllllll}0 & 0 & 0 & 0 & 0 & 0 & 0 & 0 & 0\end{array}$ $\begin{array}{ccccccccc}0 & 0 & 0 & 0 & 0 & 0 & 0 & 0 & 0\end{array}$ $\begin{array}{llllllllll}- \text {-xyyy } & \text { xyyy } & 0 & 0 & 0 & -x x x x & 0 & 0 & -x x x x\end{array}$ 


$\left(\begin{array}{ccccccccc}x x x x & x x y y & x x z z & 0 & 0 & x x x y & 0 & 0 & -y y x y \\ y y x x & x x x x & y y z z & 0 & 0 & y y x y & 0 & 0 & -x x x y \\ z z x x & z z x x & z z z z & 0 & 0 & z z x y & 0 & 0 & -z z x y \\ 0 & 0 & 0 & y z y z & y z z x & 0 & y z z y & y z x z & 0 \\ 0 & 0 & 0 & z x y z & z x z x & 0 & z x z y & z x x z & 0 \\ x y x x & x y y y & x y z z & 0 & 0 & x y x y & 0 & 0 & x y y x \\ 0 & 0 & 0 & z x x z & -z x z y & 0 & z x z x & -z x y z & 0 \\ 0 & 0 & 0 & -y z x z & y z z y & 0 & - \text { yzzx } & \text { yzyz } & 0 \\ -x y y y & -x y x x & -x y z z & 0 & 0 & x y y x & 0 & 0 & x y x y\end{array}\right)$

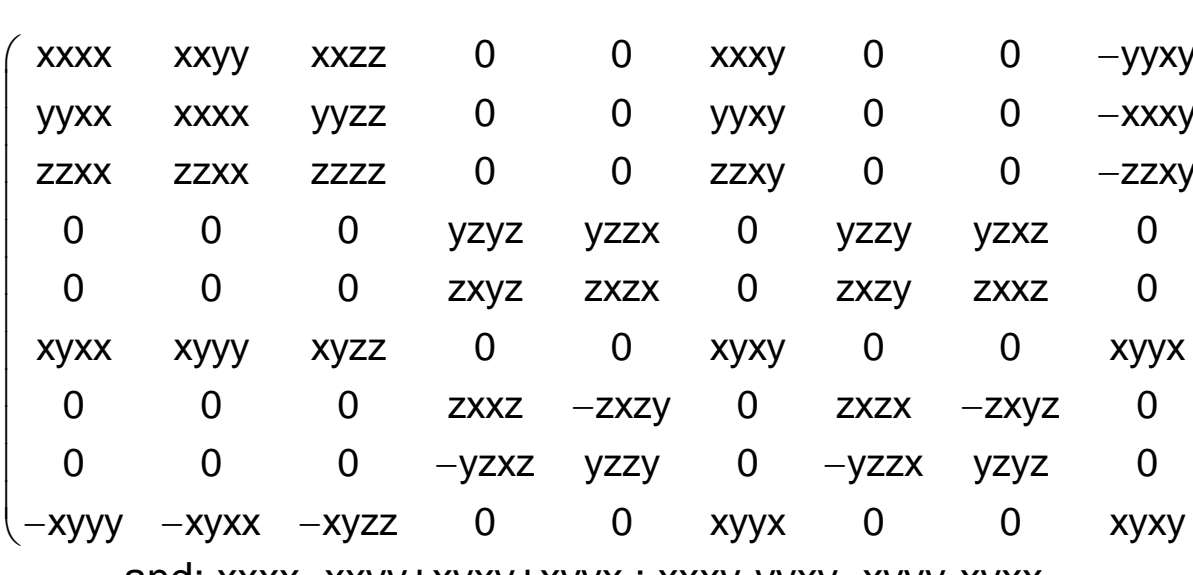
and: $x x x x=x x y y+x y x y+x y y x ; x x x y-y y x y=x y y y-x y x x$ and: $x x x x=x x y y+x y x y+x y y x ; x x x y-y y x y=x y y y-x y x x$

$\left[V^{4}\right] \quad\left(\begin{array}{ccccc}3 x x y y & 0 & 0 & y y z z & 0 \\ 0 & 3 x x y y & 0 & y y z z & 0 \\ 0 & 0 & z z z z & x x y y & 0\end{array}\right)$

$$
\left(\begin{array}{ccccc}
3 x x y y & 0 & 0 & y y z z & 0 \\
0 & 3 x x y y & 0 & y y z z & 0 \\
0 & 0 & z z z z & x x y y & 0
\end{array}\right)
$$$$
\left(\begin{array}{lllll}
0 & 0 & 0 & 0 & 0 \\
0 & 0 & 0 & 0 & 0 \\
0 & 0 & 0 & 0 & 0
\end{array}\right)
$$

$V\left[V^{3}\right] \quad\left(\begin{array}{cccccccccc}3 x x y y & 3 x y x x & 0 & x x y y & x y z z & 0 & y y z z & x y x x & 0 & y y x x \\ -3 x y x x & 3 x x y y & 0 & -x y x x & y y z z & 0 & -x y z z & x x y y & 0 & 0 \\ 0 & 0 & 2 z z & 0 & 0 & z z x & 0 & 0 & z z x & 2 x y z\end{array}\right)$

$\left(\begin{array}{cccccccccc}3 x x y y & 3 x y x x & 0 & x x y y & x y z z & 0 & y y z z & x y x x & 0 & y y x x \\ 3 x y x x & 3 x x y y & 0 & -x y x x & y y z z & 0 & -x y z z & x x y y & 0 & 0 \\ 0 & 0 & z z z z & 0 & 0 & z z x & 0 & 0 & z z x & z x y z\end{array}\right)$

$\left(\begin{array}{llllllllll}0 & 0 & 0 & 0 & 0 & 0 & 0 & 0 & 0 & 0 \\ 0 & 0 & 0 & 0 & 0 & 0 & 0 & 0 & 0 & 0 \\ 0 & 0 & 0 & 0 & 0 & 0 & 0 & 0 & 0 & 0\end{array}\right)$

$\left(\begin{array}{lllllllll}0 & 0 & 0 & 0 & 0 & 0 & 0 & 0 & 0 \\ 0 & 0 & 0 & 0 & 0 & 0 & 0 & 0 & 0 \\ 0 & 0 & 0 & 0 & 0 & 0 & 0 & 0 & 0\end{array}\right.$

$\begin{array}{lllllllll}0 & 0 & 0 & 0 & 0 & 0 & 0 & 0 & 0\end{array}$

$\begin{array}{llllllllll}0 & 0 & 0 & 0 & 0 & 0 & 0 & 0 & 0 \\ 0 & 0 & 0 & 0 & 0 & 0 & 0 & 0\end{array}$

$\begin{array}{llllllllll}0 & 0 & 0 & 0 & 0 & 0 & 0 & 0 & 0 \\ 0 & 0 & 0 & 0 & 0 & 0 & 0 & 0 & 0\end{array}$

$\begin{array}{llllllllll}0 & 0 & 0 & 0 & 0 & 0 & 0 & 0 & 0 \\ 0 & 0 & 0 & 0 & 0 & 0 & 0 & 0 & 0\end{array}$

$0 \begin{array}{lllllllll}0 & 0 & 0 & 0 & 0 & 0 & 0 & 0 & 0 \\ 0 & 0 & 0 & 0 & 0 & 0 & 0 & 0 & 0\end{array}$

$\begin{array}{llllllllll}0 & 0 & 0 & 0 & 0 & 0 & 0 & 0 & 0 \\ 0 & 0 & 0 & 0 & 0 & 0 & 0 & 0 & 0\end{array}$

$\left(\begin{array}{cccccccccc}-3 x y x x & 3 x x y y & 0 & -x y x x & y y z z & 0 & -x y z z & x x y y & 0 & 0 \\ 0 & 0 & z z z z & 0 & 0 & z z x x & 0 & 0 & z z x x & z x y z\end{array}\right)$

$\left(\begin{array}{lllllllllll}0 & 0 & 0 & 0 & 0 & 0 & 0 & 0 & 0 & 0 \\ 0 & 0 & 0 & 0 & 0 & 0 & 0 & 0 & 0 & 0 \\ 0 & 0 & 0 & 0 & 0 & 0 & 0 & 0 & 0 & 0\end{array}\right)$

$$
\left(\begin{array}{cccccc}
x x x x & \text { xxyy } & \text { xxzz } & 0 & 0 & 0 \\
& \text { xxxx } & \text { xxzz } & 0 & 0 & 0 \\
& & \text { zzzz } & 0 & 0 & 0 \\
& & & y z y z & 0 & 0 \\
& & & & \text { yzyz } & 0 \\
& & & & & \text { xyxy }
\end{array}\right)
$$

$$
\left(\begin{array}{llllll}
0 & 0 & 0 & 0 & 0 & 0 \\
& 0 & 0 & 0 & 0 & 0 \\
& & 0 & 0 & 0 & 0 \\
& & & 0 & 0 & 0 \\
& & & & 0 & 0 \\
& & & & & 0
\end{array}\right)
$$

and $x y x y=1 / 2(x x x x-x x y y)$

and $x y x y=1 / 2(x x x x-x x y y)$

$\left(\begin{array}{cccccc}x x x x & x x y y & x x z z & 0 & 0 & x x x y \\ x x y y & x x x x & x x z z & 0 & 0 & -x x x y \\ \text { zzxx } & \text { zzxx } & \text { zzzz } & 0 & 0 & 0 \\ 0 & 0 & 0 & y z y z & y z x z & 0 \\ 0 & 0 & 0 & y z x z & y z y z & 0 \\ -x x x y & x x x y & 0 & 0 & 0 & x y x y\end{array}\right)$

and $x y x y=1 / 2(x x x x-x x y y)$ $\left(\begin{array}{llllll}0 & 0 & 0 & 0 & 0 & 0 \\ 0 & 0 & 0 & 0 & 0 & 0\end{array}\right)$

$\begin{array}{llllll}0 & 0 & 0 & 0 & 0 & 0 \\ 0 & 0 & 0 & 0 & 0 & 0\end{array}$

$\begin{array}{llllll}0 & 0 & 0 & 0 & 0 & 0 \\ 0 & 0 & 0 & 0 & 0 & 0\end{array}$ $0 \begin{array}{llllll}0 & 0 & 0 & 0 & 0 & 0 \\ 0 & 0 & 0 & 0 & 0 & 0\end{array}$ $\begin{array}{llllll}0 & 0 & 0 & 0 & 0 & 0 \\ 0 & 0 & 0 & 0 & 0 & 0\end{array}$

$\begin{array}{llllll}0 & 0 & 0 & 0 & 0 & 0\end{array}$ $\left(\begin{array}{llllllllll}0 & 0 & 0 & 0 & 0 & 0 & 0 & 0 & 0 \\ 0 & 0 & 0 & 0 & 0 & 0 & 0 & 0 & 0\end{array}\right)$ $\begin{array}{llllllllllll}0 & 0 & 0 & 0 & 0 & 0 & 0 & 0 & 0 \\ 0 & 0 & 0 & 0 & 0 & 0 & 0 & 0 & 0\end{array}$ $\begin{array}{llllllllll}0 & 0 & 0 & 0 & 0 & 0 & 0 & 0 & 0\end{array}$ $\begin{array}{llllllllll}0 & 0 & 0 & 0 & 0 & 0 & 0 & 0 & 0\end{array}$ $\begin{array}{lllllllll}0 & 0 & 0 & 0 & 0 & 0 & 0 & 0 & 0 \\ 0 & 0 & 0 & 0 & 0 & 0 & 0 & 0 & 0\end{array}$ $\begin{array}{lllllllll}0 & 0 & 0 & 0 & 0 & 0 & 0 & 0 & 0 \\ 0 & 0 & 0 & 0 & 0 & 0 & 0 & 0 & 0\end{array}$ $\begin{array}{lllllllll}0 & 0 & 0 & 0 & 0 & 0 & 0 & 0 & 0 \\ 0 & 0 & 0 & 0 & 0 & 0 & 0 & 0 & 0\end{array}$

$\left(\begin{array}{lllll}0 & 0 & 0 & 0 & 0 \\ 0 & 0 & 0 & 0 & 0 \\ 0 & 0 & 0 & 0 & 0\end{array}\right)$ $\begin{array}{lllll}0 & 0 & 0 & 0 & 0\end{array}$

$\left(\begin{array}{llllllllll}0 & 0 & 0 & 0 & 0 & 0 & 0 & 0 & 0 & 0 \\ 0 & 0 & 0 & 0 & 0 & 0 & 0 & 0 & 0 & 0\end{array}\right)$ $\left.\begin{array}{llllllllll}0 & 0 & 0 & 0 & 0 & 0 & 0 & 0 & 0 & 0 \\ 0 & 0 & 0 & 0 & 0 & 0 & 0 & 0 & 0 & 0\end{array}\right)$ $\begin{array}{lllllllllll}0 & 0 & 0 & 0 & 0 & 0 & 0 & 0 & 0 & 0\end{array}$

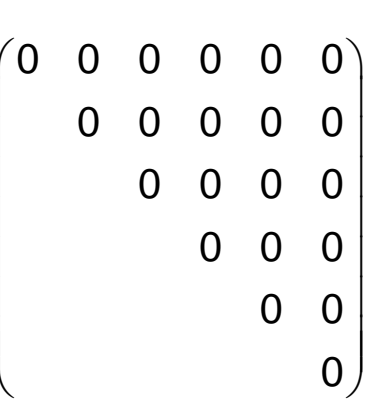

$\left(\begin{array}{llllll}0 & 0 & 0 & 0 & 0 & 0 \\ 0 & 0 & 0 & 0 & 0 & 0\end{array}\right)$ $\begin{array}{llllll}0 & 0 & 0 & 0 & 0 & 0 \\ 0 & 0 & 0 & 0 & 0 & 0\end{array}$ $0 \begin{array}{llllll}0 & 0 & 0 & 0 & 0 & 0 \\ 0 & 0 & 0 & 0 & 0 & 0\end{array}$ $\begin{array}{llllll}0 & 0 & 0 & 0 & 0 & 0 \\ 0 & 0 & 0 & 0 & 0 & 0 \\ 0 & 0 & 0 & 0 & 0 & 0\end{array}$ $\begin{array}{llllll}0 & 0 & 0 & 0 & 0 & 0 \\ 0 & 0 & 0 & 0 & 0 & 0\end{array}$ $\begin{array}{llllll}0 & 0 & 0 & 0 & 0 & 0\end{array}$ 


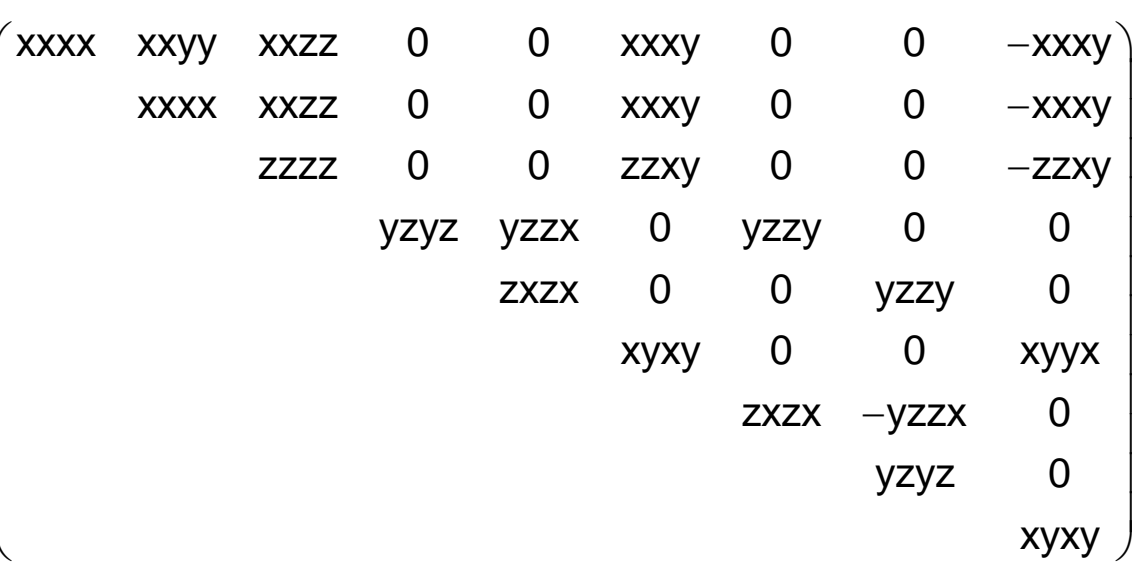

and $x x x x=x x y y+y z y z+z y z y$

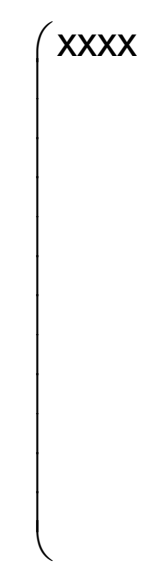

$\begin{array}{llllllll}x x x x & x x y y & x x z z & 0 & 0 & 0 & x x y y & 0\end{array}$ $\left(\begin{array}{lllllllll}x x y y & x x x x & x x z z & 0 & 0 & y y x y & 0 & 0 & -x x x y \\ z z x y & z z x x & z z z z & 0 & 0 & z z x y & 0 & 0 & -z x y\end{array}\right)$

$\left[\mathrm{V}^{2}\right] \mathrm{V}^{2} \begin{array}{cccccccccc}2 z x x & 0 & 0 & y z y z & y z z x & 0 & z x z x & -z x y z & 0 \\ 0 & 0 & 0 & z x y z & z x z x & 0 & -y z z & y z y z & 0\end{array}$ $\begin{array}{ccccccccc}0 & 0 & 0 & y z y z & y z z x & 0 & z x z x & -z x y z & 0 \\ 0 & 0 & 0 & z x y z & z x z x & 0 & -y z z x & y z y z & 0\end{array}$

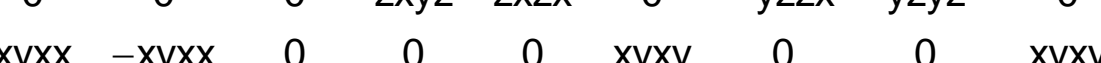
and $x x x x=x x y y+2 x y x y ; y y x y-x x x y=2 x y x x$

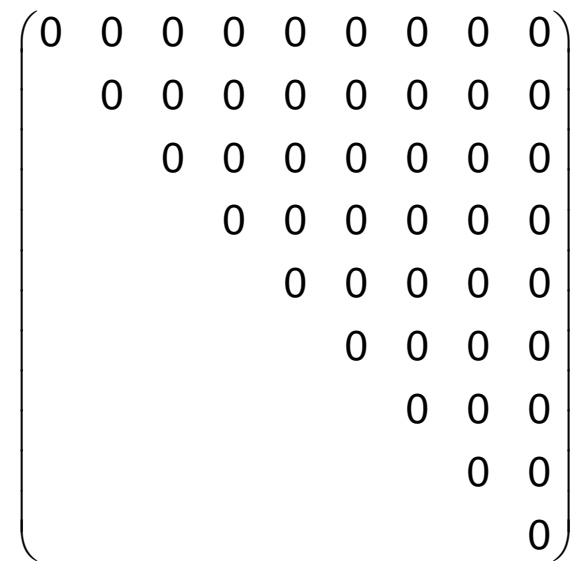

\section{and $x x x x=x x y y+y z y z+z y z y$}

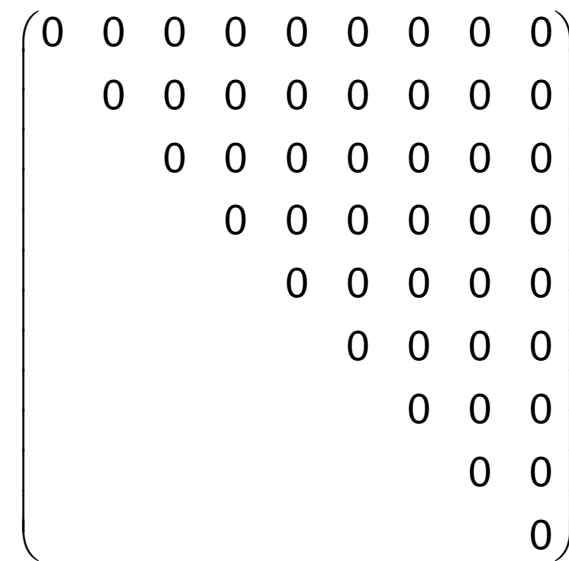

$\left(\begin{array}{lllllllll}0 & 0 & 0 & 0 & 0 & 0 & 0 & 0 & 0 \\ 0 & 0 & 0 & 0 & 0 & 0 & 0 & 0 & 0 \\ 0 & 0 & 0 & 0 & 0\end{array}\right)$ $\left.\begin{array}{llllllllllllll}0 & 0 & 0 & 0 & 0 & 0 & 0 & 0 & 0 \\ 0 & 0 & 0 & 0 & 0 & 0 & 0 & 0 & 0\end{array}\right)$ $\begin{array}{lllllllll}0 & 0 & 0 & 0 & 0 & 0 & 0 & 0 & 0 \\ 0 & 0 & 0 & 0 & 0 & 0 & 0 & 0 & 0\end{array}$ $\begin{array}{llllllllll}0 & 0 & 0 & 0 & 0 & 0 & 0 & 0 & 0 \\ 0 & 0 & 0 & 0 & 0 & 0 & 0 & 0 & 0\end{array}$ $\begin{array}{lllllllll}0 & 0 & 0 & 0 & 0 & 0 & 0 & 0 & 0 \\ 0 & 0 & 0 & 0 & 0 & 0 & 0 & 0 & 0\end{array}$ $\left(\begin{array}{lllllllll}0 & 0 & 0 & 0 & 0 & 0 & 0 & 0 & 0 \\ 0 & 0 & 0 & 0 & 0 & 0 & 0 & 0 & 0 \\ 0 & 0 & 0 & 0 & 0 & 0 & 0 & 0 & 0\end{array}\right)$

$\begin{array}{ccccccccc}0 & 0 & 0 & y z y z & y z z x & 0 & z x z x & -z x y z & 0 \\ 0 & 0 & 0 & z x y z & z x z x & 0 & -y z z x & y z y z & 0\end{array} \mid\left(\begin{array}{ccccccccc}0 & 0 & 0 & 0 & 0 & 0 & 0 & 0 & 0 \\ 0 & 0 & 0 & 0 & 0 & 0 & 0 & 0 & 0 \\ 0 & 0 & 0 & 0 & 0 & 0 & 0 & 0 & 0\end{array}\right)$

and $x x x x=x x y y+2 x y x y ; y y x y-x x x y=2 x y x x$ 
$\begin{array}{lllllllll}x x x x & x x y y & x x z z & x x y z & x x z & x x x y & x x z y & x x x z & x x y x\end{array}$

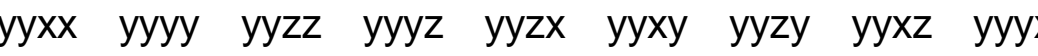

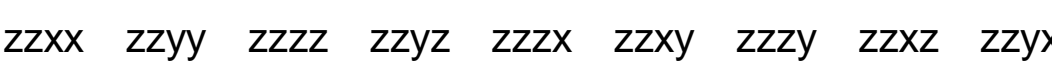
yzxx yzyy yzzz $y z y z$ yzzx

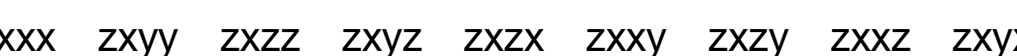

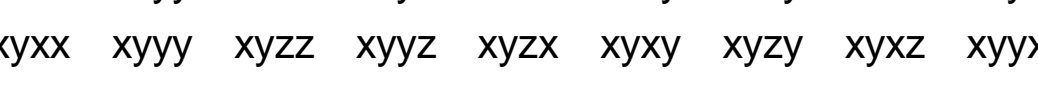
zyxx zyyy zyzz zyyz zyzx $\begin{array}{llllllllll}x y x x & x z y y & x z z z & x z y z & x z z x & x z x y & x z z y & x z x z & x z y x\end{array}$ $\begin{array}{lllllllll}y x x x x & y x y y & y x z z & y x y z & y x z x & y x x y & y x z y & y x x z & y x y x\end{array}$

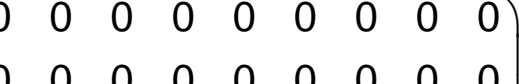
$\begin{array}{llllllllllll}0 & 0 & 0 & 0 & 0 & 0 & 0 & 0 & 0\end{array}$ $\begin{array}{lllllllllllllll}0 & 0 & 0 & 0 & 0 & 0 & 0 & 0 & 0\end{array}$ $\begin{array}{llllllllll}0 & 0 & 0 & 0 & 0 & 0 & 0 & 0 & 0\end{array}$ $\begin{array}{llllllllll}0 & 0 & 0 & 0 & 0 & 0 & 0 & 0 & 0 \\ 0 & 0 & 0 & 0 & 0 & 0 & 0 & 0 & 0 \\ 0 & 0 & 0 & 0 & 0 & 0 & 0 & 0 & 0\end{array}$ $\begin{array}{lllllllll}0 & 0 & 0 & 0 & 0 & 0 & 0 & 0 & 0 \\ 0 & 0 & 0 & 0 & 0 & 0 & 0 & 0 & 0\end{array}$ $\begin{array}{lllllllll}0 & 0 & 0 & 0 & 0 & 0 & 0 & 0 & 0 \\ 0 & 0 & 0 & 0 & 0 & 0 & 0 & 0 & 0\end{array}$ $\begin{array}{lllllllll}0 & 0 & 0 & 0 & 0 & 0 & 0 & 0 & 0 \\ 0 & 0 & 0 & 0 & 0 & 0 & 0 & 0 & 0\end{array}$ $\begin{array}{lllllllll}0 & 0 & 0 & 0 & 0 & 0 & 0 & 0 & 0\end{array}$

$\left(\begin{array}{lllll}x x x x & x y y y & x z z z & y y z z & x x y z \\ y x x x & y y y & y z z z & z z x x & y y x z \\ z x x x & z y y & z z z & x x y & z z y y\end{array}\right)$

$\left(\begin{array}{lllll}0 & 0 & 0 & 0 & 0 \\ 0 & 0 & 0 & 0 & 0 \\ 0 & 0 & 0 & 0 & 0\end{array}\right)$

$0 \begin{array}{lllll}0 & 0 & 0 & 0 & 0\end{array}$

$\begin{array}{lllll}z x x x & z y y y & z z z z & x x y y & z z x y\end{array}$

$\left(\begin{array}{lllllllllllllllllllllll}x x x x & x y y y & x z z z & x x y y & x y z z & x z x x & x x z z & x y x x & x z y y & x x y z \\ y x x x & y y y & y z z & y x y & y y z z & y z x x & y x z z & 0 & 0 & 0 & 0 & 0 & 0 & 0 & 0 & 0 \\ 0 & 0 & 0 & 0 & 0 & 0 & 0 & 0 & 0 & 0\end{array}\right)$

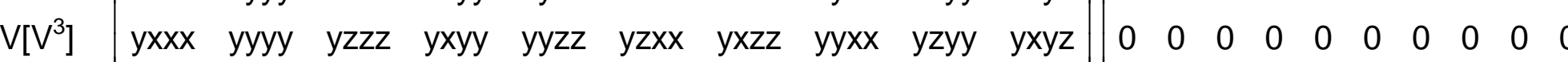

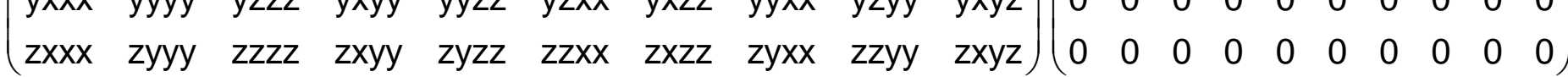

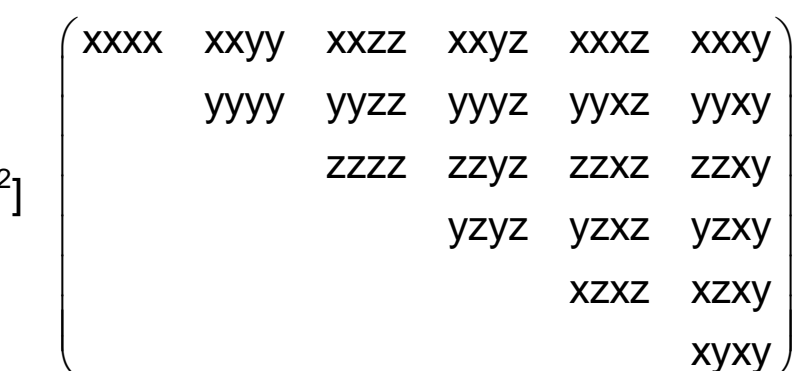$$
x y x y
$$

$$
\begin{aligned}
& \begin{array}{llllll}
0 & 0 & 0 & 0 & 0 & 0 \\
0 & 0 & 0 & 0 & 0
\end{array} \\
& \begin{array}{llll}
0 & 0 & 0 & 0 \\
0 & 0 & 0 & 0
\end{array} \\
& \begin{array}{llll}
0 & 0 & 0 & 0
\end{array} \\
& \begin{array}{lll}
0 & 0 & 0 \\
& 0 & 0
\end{array}
\end{aligned}
$$

$\begin{array}{llllll}x x x x & x x y y & x x z z & x x y z & x x x z & x x x y \\ y x x & y y y & y y z & y y y z & y x z & y y x y\end{array}$ yyxx yyyy

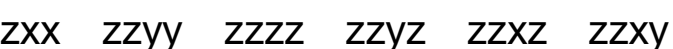
yzxx $\quad y z y y \quad y z z z \quad y z y z \quad y z x z \quad y z x y$ $\begin{array}{llllll}x z x x & x z y y & x z z z & x z y z & x z x z & x z x y \\ x y x x & x y y y & x y z z & x y y z & x y x z & x y x y\end{array}$

$\left(\begin{array}{llllll}0 & 0 & 0 & 0 & 0 & 0 \\ 0 & 0 & 0 & 0 & 0 & 0 \\ 0 & 0 & 0 & 0 & 0 & 0\end{array}\right)$

$\left(\begin{array}{llllll}0 & 0 & 0 & 0 & 0 & 0 \\ 0 & 0 & 0 & 0 & 0 & 0 \\ 0 & 0 & 0 & 0 & 0 & 0\end{array}\right.$

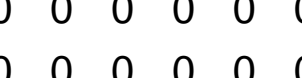

$\begin{array}{llllll}0 & 0 & 0 & 0 & 0 & 0 \\ 0 & 0 & 0 & 0 & 0 & 0\end{array}$

$\begin{array}{llllll}0 & 0 & 0 & 0 & 0 & 0 \\ 0 & 0 & 0 & 0 & 0 & 0\end{array}$ $\left(\begin{array}{lllllllll}0 & 0 & 0 & 0 & 0 & 0 & 0 & 0 & 0 \\ 0 & 0 & 0 & 0 & 0 & 0 & 0 & 0 & 0\end{array}\right)$

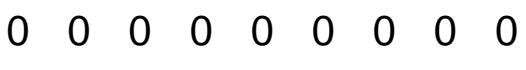

$0 \begin{array}{lllllllll}0 & 0 & 0 & 0 & 0 & 0 & 0 & 0 & 0 \\ 0 & 0 & 0 & 0 & 0 & 0 & 0 & 0 & 0\end{array}$

$\begin{array}{llllllllll}0 & 0 & 0 & 0 & 0 & 0 & 0 & 0 & 0 \\ 0 & 0 & 0 & 0 & 0 & 0 & 0 & 0 & 0\end{array}$

$\begin{array}{llllllllll}0 & 0 & 0 & 0 & 0 & 0 & 0 & 0 & 0 \\ 0 & 0 & 0 & 0 & 0 & 0 & 0 & 0 & 0\end{array}$

$\begin{array}{llllllllll}0 & 0 & 0 & 0 & 0 & 0 & 0 & 0 & 0\end{array}$

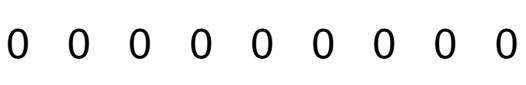

$\begin{array}{lllllllll}0 & 0 & 0 & 0 & 0 & 0 & 0 & 0 & 0 \\ 0 & 0 & 0 & 0 & 0 & 0 & 0 & 0 & 0\end{array}$

$\left(\begin{array}{lllll}0 & 0 & 0 & 0 & 0 \\ 0 & 0 & 0 & 0 & 0 \\ 0 & 0 & 0 & 0 & 0\end{array}\right)$

$\begin{array}{llllll}0 & 0 & 0 & 0 & 0\end{array}$

$\left(\begin{array}{llllllllll}0 & 0 & 0 & 0 & 0 & 0 & 0 & 0 & 0 & 0 \\ 0 & 0 & 0 & 0 & 0 & 0 & 0 & 0 & 0 & 0\end{array}\right.$ $\left.\begin{array}{lllllllllll}0 & 0 & 0 & 0 & 0 & 0 & 0 & 0 & 0 & 0 \\ 0 & 0 & 0 & 0 & 0 & 0 & 0 & 0 & 0 & 0\end{array}\right)$ $\begin{array}{lllllllllll}0 & 0 & 0 & 0 & 0 & 0 & 0 & 0 & 0 & 0\end{array}$

$$
\left(\begin{array}{llllll}
0 & 0 & 0 & 0 & 0 & 0 \\
& 0 & 0 & 0 & 0 & 0 \\
& & 0 & 0 & 0 & 0 \\
& & & 0 & 0 & 0 \\
& & & & 0 & 0
\end{array}\right)
$$

$\left(\begin{array}{llllll}0 & 0 & 0 & 0 & 0 & 0 \\ 0 & 0 & 0 & 0 & 0 & 0\end{array}\right)$

$\begin{array}{llllll}0 & 0 & 0 & 0 & 0 & 0 \\ 0 & 0 & 0 & 0 & 0 & 0\end{array}$

$0 \begin{array}{llllll}0 & 0 & 0 & 0 & 0 & 0 \\ 0 & 0 & 0 & 0 & 0 & 0\end{array}$

$\begin{array}{llllll}0 & 0 & 0 & 0 & 0 & 0 \\ 0 & 0 & 0 & 0 & 0 & 0\end{array}$

$\begin{array}{llllll}0 & 0 & 0 & 0 & 0 & 0 \\ 0 & 0 & 0 & 0 & 0 & 0\end{array}$

$\begin{array}{llllll}0 & 0 & 0 & 0 & 0 & 0\end{array}$ $\begin{array}{lllllllll}x x x x & x x y y & x x z z & x x y z & x x z x & x x x y & x x z y & x x x z & x x y x\end{array}$ yyxx yyyy

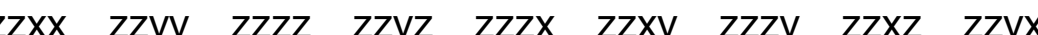
yzxx yzyy yzzz yzyz yzzx yzxy yzzy yzxz yzyx

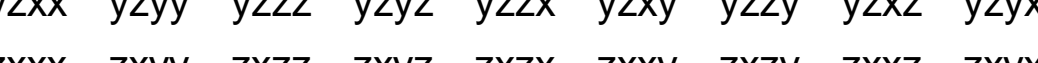

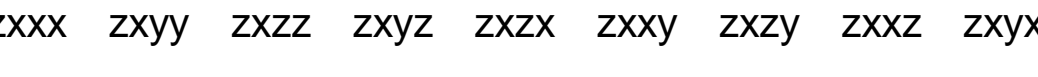

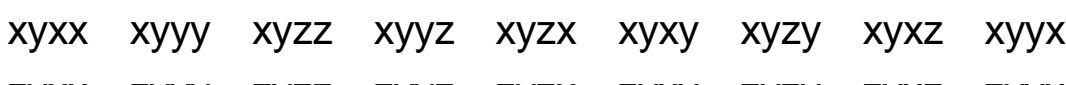
zyxx zyyy zyzz zyyz zyzx zyxy zyzy zyxz $\quad$ zyyx

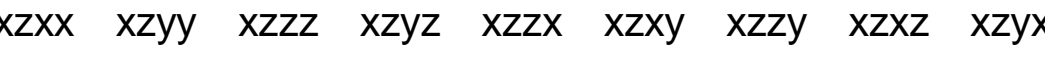

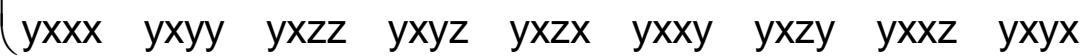

$\left(\begin{array}{lllll}x x x x & x y y y & x z z z & y y z z & x x y z \\ y x x x & y y y y & y z z z & z z x x & y y x z \\ z x x x & z y y y & z z z z & x x y y & z z x y\end{array}\right)$

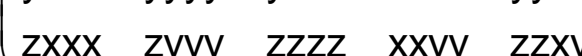

$\left(\begin{array}{llllllllll}x x x x & x y y y & x z z z & x x y y & x y z z & x z x x & x x z z & x y x x & x z y y & x x y z\end{array}\right.$

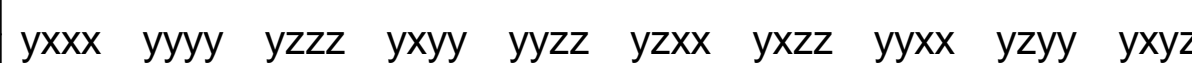
$\begin{array}{lllllllllll}\text { zxxx } & \text { zyyy } & \text { zzzz } & \text { zxyy } & \text { zyzz } & \text { zzxx } & \text { zxzz } & \text { zyxx } & \text { zzyy } & \text { zxyz }\end{array}$

$$
\left(\begin{array}{cccccc}
x x x x & x x y y & x x z z & x x y z & x x x z & x x x y \\
& y y y y & y y z z & y y y z & y y x z & y y x y \\
& & z z z z & z z y z & z z x z & z z x y \\
& & & y z y z & y z x z & y z x y \\
& & & & x z x z & x z x y \\
& & & & & x y x y
\end{array}\right)
$$

$\begin{array}{llllll}x x x x & x x y y & x x z z & x x y z & x x x z & x x x y \\ x y x x & y y y y & y y z & y y y z & y y z z & y y x y\end{array}$ $\begin{array}{llllll}x y x x & y y y y & y y z z & y y y z & y y x z & y y x y\end{array}$ zzxx $\quad$ zzyy $\quad$ zzzz $\quad$ zzyz $\quad z z x z \quad z z x y$ yzxx yzyy yzzz yzyz $y z x z \quad y z x y$ $\begin{array}{llllll}x z x x & x z y y & x z z z & x z y z & x z x z & x z x y\end{array}$ $\begin{array}{llllll}x y x x & x y y y & x y z z & x y y z & x y x z & x y x y\end{array}$ 


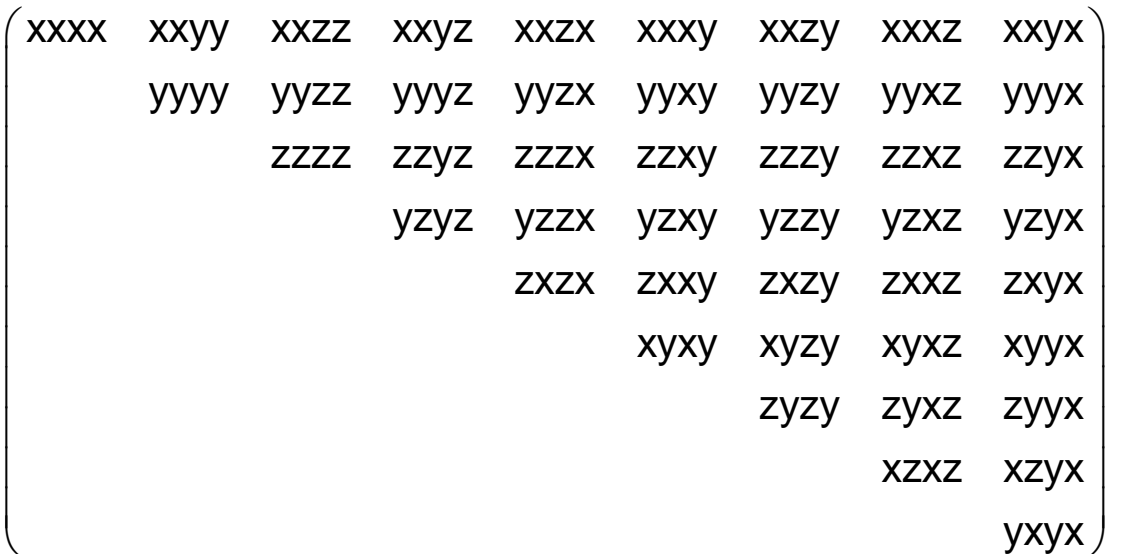

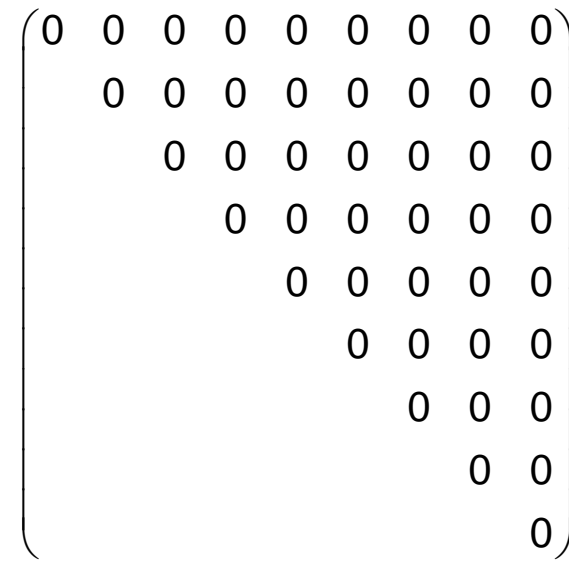

$\begin{array}{lllllllll}0 & 0 & 0 & 0 & 0 & 0 & 0 & 0 & 0 \\ 0 & 0 & 0 & 0 & 0 & 0 & 0 & 0 & 0\end{array}$ $\begin{array}{lllllllll}0 & 0 & 0 & 0 & 0 & 0 & 0 & 0 & 0 \\ 0 & 0 & 0 & 0 & 0 & 0 & 0 & 0 & 0\end{array}$

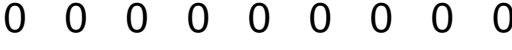
$\begin{array}{lllllllll}0 & 0 & 0 & 0 & 0 & 0 & 0 & 0 & 0 \\ 0 & 0 & 0 & 0 & 0 & 0 & 0 & 0 & 0\end{array}$ $\begin{array}{llllllllll}0 & 0 & 0 & 0 & 0 & 0 & 0 & 0 & 0 \\ 0 & 0 & 0 & 0 & 0 & 0 & 0 & 0 & 0 & 0\end{array}$ $\begin{array}{lllllllll}0 & 0 & 0 & 0 & 0 & 0 & 0 & 0 & 0 \\ 0 & 0 & 0 & 0 & 0 & 0 & 0 & 0\end{array}$ $\left(\begin{array}{lllllllll}x x x x & x x y y & x x z z & x x y z & x x z x & x x x y & x x z y & x x x z & x x y x\end{array}\right)$ yyyy $\begin{array}{lllllll}z z z z & z z y z & z z z x & z z x y & z z z y & z z x z & z z y x\end{array}$

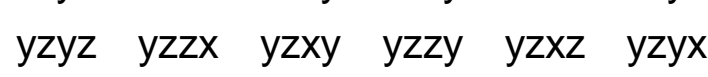
$\begin{array}{lllll}z x z x & z x x y & z x z y & z x x z & z x y x\end{array}$ $\begin{array}{llll}x y x y & x y z y & x y x z & x y y x\end{array}$ zyzy $\quad$ zyxz $\quad z y y x$ $\begin{array}{rr}x z x z & x z y x \\ y x y x\end{array}$ $\left(\begin{array}{lllllllll}x x x x & x x y y & x x z z & x x y z & x x z x & x x x y & x x z y & x x x z & x x y x \\ y y x x & \text { yyyy } & y y z z & \text { yyyz } & \text { yyzx } & \text { yyxy } & \text { yyzy } & \text { yyxz } & \text { yyyx } \\ 2 z x x & z z y y & z z z z & z y z & z z z & z x x y & z z y & z z x z & z z y x\end{array}\right.$ zzxx zzyy zzzz zzyz zzzx zzxy zzzy zzxz zzyx yzxx yzyy yzzz yzyz yzzx yzxy yzzy $\begin{array}{llllllllll}z x x x & z x y y & z x z z & z x y z & z x z x & z x x y & z x z y & z x x z & z x y x\end{array}$ $\left(\begin{array}{lllllllll}0 & 0 & 0 & 0 & 0 & 0 & 0 & 0 & 0 \\ 0 & 0 & 0 & 0 & 0 & 0 & 0 & 0 & 0\end{array}\right)$ $\left.\begin{array}{lllllllll}0 & 0 & 0 & 0 & 0 & 0 & 0 & 0 & 0 \\ 0 & 0 & 0 & 0 & 0 & 0 & 0 & 0 & 0 \\ 0 & 0 & 0 & 0 & 0 & 0 & 0 & 0 & 0\end{array}\right)$ $0 \begin{array}{llllllllll}0 & 0 & 0 & 0 & 0 & 0 & 0 & 0 & 0 \\ 0 & 0 & 0 & 0 & 0 & 0 & 0 & 0 & 0\end{array}$

$\begin{array}{llllllllll}0 & 0 & 0 & 0 & 0 & 0 & 0 & 0 & 0 \\ 0 & 0 & 0 & 0 & 0 & 0 & 0 & 0 & 0\end{array}$

$\begin{array}{llllllllll}0 & 0 & 0 & 0 & 0 & 0 & 0 & 0 & 0\end{array}$ $\begin{array}{lllllllll}x x x x & x x y y & x x z z & x x y z & x x z x & x x x y & x x z y & x x x z & x x y x\end{array}$ yyxx yyyy yyzz zzxx zzyy zzzz zzyz zzzx zzxy zzzy $z z x z$ zzyx

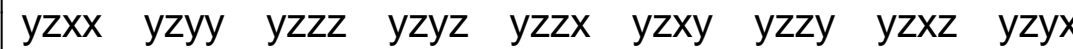

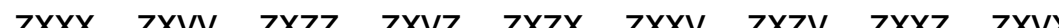
$\left(\begin{array}{lllllllll}\text { zxxx } & z x y y & \text { zxzz } & \text { zxyz } & \text { zxzx } & \text { zxxy } & \text { zxzy } & \text { zxxz } & \text { zxyx } \\ \text { xyxx } & \text { xyyy } & \text { xyzz } & \text { xyyz } & \text { xyzx } & \text { xyxy } & \text { xyzy } & \text { xyxz } & \text { xyyx }\end{array}\right)$ 


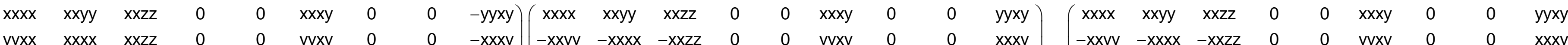

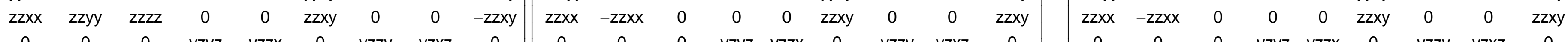
\begin{tabular}{llllllllll|lllllllll|l|llllllll}
0 & 0 & 0 & $y z y z$ & $y z z x$ & 0 & $y z z y$ & $y z x z$ & 0 & 0 & 0 & 0 & $y z y z$ & $y z z x$ & 0 & $-y z z y$ & $y z x z$ & 0 & 0 & 0 & 0 & $y z y z$ & $y z z x$ & 0 & $-y z z y$ & $y z x z$ & 0 \\
0 & 0 & 0 & $z x y z$ & $z x z x$ & 0 & $z x z y$ & $z x y z$ & 0 & 0 & 0 & 0 & $z x y z$ & $z x z x$ & 0 & $z x z y$ & $-z x y z$ & 0 & 0 & 0 & 0 & $z x y z$ & $z x z x$ & 0 & $z x z y$ & $-z x y z$ & 0
\end{tabular} \begin{tabular}{lllllllll|lllllllll|lllllllll} 
xyxx & $x y y y$ & $x y z z$ & 0 & 0 & $x y x y$ & 0 & 0 & $x y y x$ & $x y x x$ & $x y y y$ & $x y z z$ & 0 & 0 & $x y x y$ & 0 & 0 & $-x y y x$ & $x y x x$ & $x y y y$ & $x y z z$ & 0 & 0 & $x y x y$ & 0 & 0 & $-x y y x$ \\
\hline
\end{tabular}

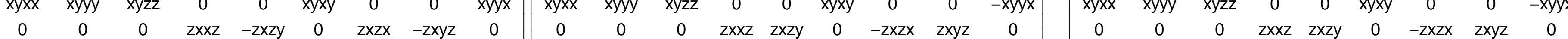
$\left(\begin{array}{lllllllll|lllllllllllllllllll}0 & 0 & 0 & -y z x z & y z z y & 0 & -y z z x & y z y z & 0 & 0 & 0 & 0 & y z x z & y z z y & 0 & y z z x & -y z y z & 0 & 0 & 0 & 0 & y z x z & y z z y & 0 & y z z x & -y z y z & 0 \\ -x y y & -x y x & -x y z z & 0 & 0 & x y x & 0 & 0 & x y y\end{array}\right.$

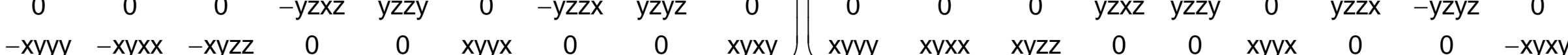

$\left[V^{4}\right] \quad\left(\begin{array}{ccccc}x x x x & -y x x x & 0 & y y z z & 0 \\ y x x x & x x x x & 0 & y y z z & 0 \\ 0 & 0 & z z z z & x x y y & 0\end{array}\right)$

$$
\left(\begin{array}{ccccc}
x x x x & y x x x & 0 & y y z z & 0 \\
y x x x & -x x x x & 0 & -y y z z & 0 \\
0 & 0 & 0 & 0 & z z x y
\end{array}\right)
$$$$
\left(\begin{array}{ccccc}
\operatorname{xxxx} & y x x x & 0 & y y z z & 0 \\
y x x x & -x x x x & 0 & -y y z z & 0 \\
0 & 0 & 0 & 0 & z z x y
\end{array}\right)
$$

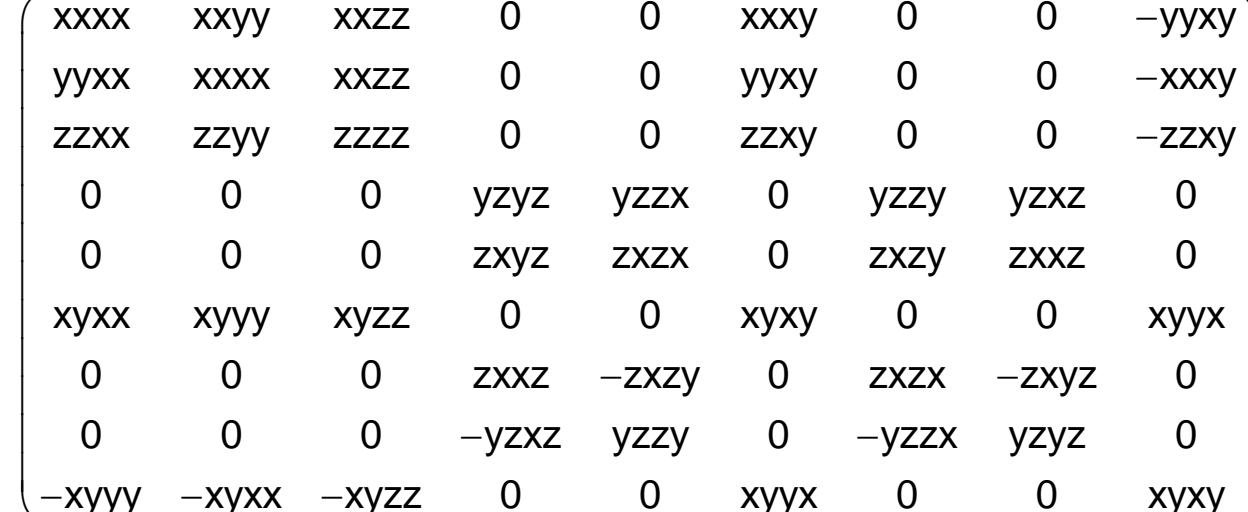

$\left(\begin{array}{ccccc}x x x x & -y x x x & 0 & y y z z & 0 \\ y x x x & x x x x & 0 & y y z z & 0 \\ 0 & 0 & z z z z & x x y y & 0\end{array}\right)$ $\left(\begin{array}{ccccc}y x x x & x x x x & 0 & y y z z & 0 \\ 0 & 0 & z z z z & x x y y & 0\end{array}\right)$

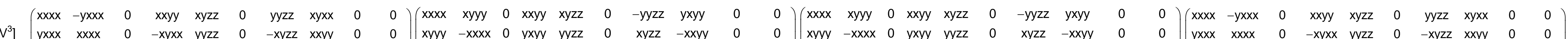

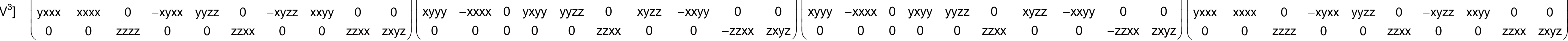

$$
\left[\left[V^{2}\right]^{2}\right]\left(\begin{array}{cccccc}
x x x x & x x y y & x x z z & 0 & 0 & x x x y \\
& y y y y & y y z z & 0 & 0 & -x x x y \\
& & z z z z & 0 & 0 & 0 \\
& & & y z y z & 0 & 0 \\
& & & & y z y z & 0 \\
& & & & & \\
& & & & &
\end{array}\right.
$$

$\left[\mathrm{V}^{2}\right]^{2}\left(\begin{array}{cccccc}\mathrm{xxxx} & \mathrm{xxyy} & \mathrm{xxzz} & 0 & 0 & \mathrm{xxxy} \\ \mathrm{yyxx} & \mathrm{xxxx} & \mathrm{xxzz} & 0 & 0 & -\mathrm{xxxy} \\ \mathrm{zzxx} & \mathrm{zzxx} & \mathrm{zzzz} & 0 & 0 & 0 \\ 0 & 0 & 0 & \mathrm{yzyz} & \mathrm{yzxz} & 0 \\ 0 & 0 & 0 & -\mathrm{yzxz} & \mathrm{yzyz} & 0 \\ \mathrm{xyxx} & -\mathrm{xyxx} & 0 & 0 & 0 & \mathrm{xyxy}\end{array}\right)$

$$
\left(\begin{array}{cccccc}
x x x x & 0 & x x z z & 0 & 0 & x x x y \\
& -x x x x & -x x z z & 0 & 0 & x x x y \\
& 0 & 0 & 0 & z z x y \\
& & y z y z & y z x z & 0 \\
& & & -y z y z & 0 \\
& & & & & 0
\end{array}\right)
$$

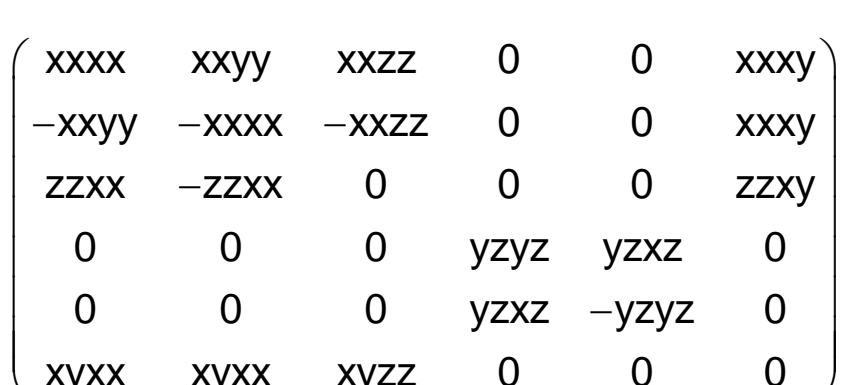

$$
\left(\begin{array}{cccccc}
\mathrm{xxxx} & 0 & \mathrm{xxzz} & 0 & 0 & \mathrm{xxxy} \\
& -\mathrm{xxxx} & -\mathrm{xxzz} & 0 & 0 & \mathrm{xxxy} \\
& 0 & 0 & 0 & \mathrm{zzxy} \\
& & \mathrm{yzyz} & \mathrm{yzxz} & 0 \\
& & & & -\mathrm{yzyz} & 0 \\
& & & & & 0
\end{array}\right)
$$

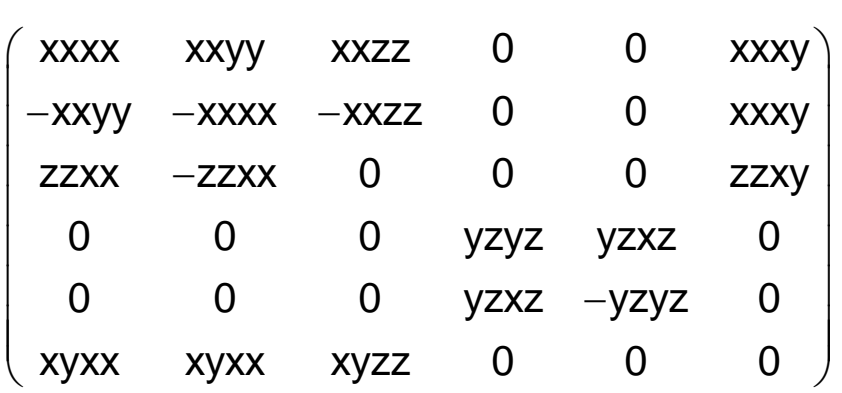

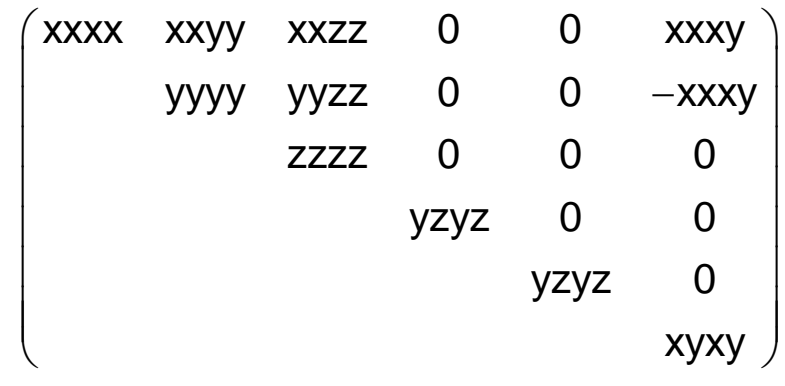

$\left(\begin{array}{cccccc}x x x x & x x y y & x x z z & 0 & 0 & x x x y \\ y y x x & x x x x & x x z z & 0 & 0 & -x x x y \\ z z x x & z z x x & z z z z & 0 & 0 & 0 \\ 0 & 0 & 0 & y z y z & y z x z & 0 \\ 0 & 0 & 0 & -y z x z & y z y z & 0 \\ x y x x & -x y x x & 0 & 0 & 0 & x y x y\end{array}\right)$ 


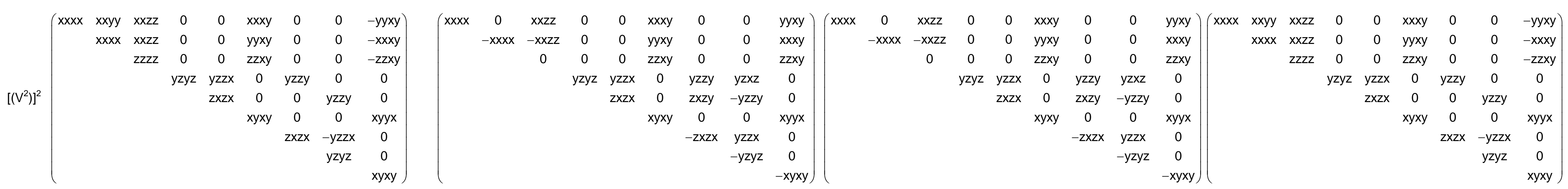

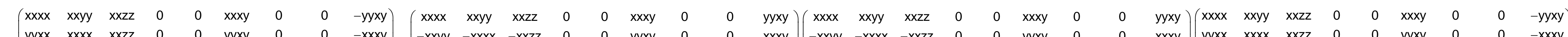

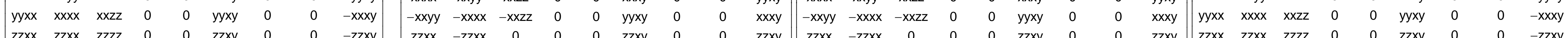

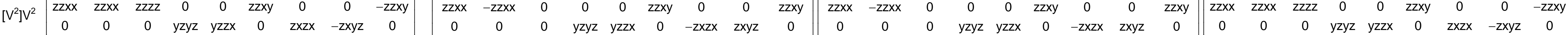

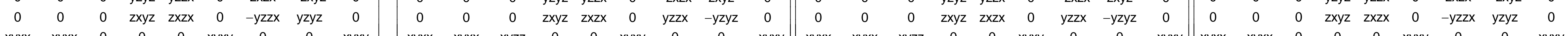


$\left(\begin{array}{lllllllll}x x x x & x x y y & x x z z & x x y z & x x z x & x x x y & x x z y & x x x z & -y y x y \\ y x x & x x y & y y z & -x x y & -x z x & y y x & -x y & -x x z & -x x y\end{array}\left(\begin{array}{ccccccccc}0 & 0 & 0 & 0 & 0 & 0 & 0 & 0 & 0 \\ 0 & 0 & 0 & 0 & 0 & 0 & 0 & 0 & 0 \\ 0 & 0 & 0 & 0 & 0 & 0 & 0 & 0 & 0\end{array}\right)\right.$

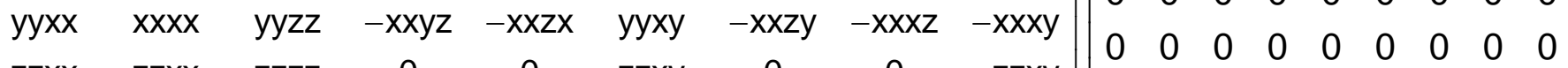
$\begin{array}{llllllllll}z z x x & z z x x & z z z z & 0 & 0 & z z x y & 0 & 0 & -z z x y & 0 \\ y & 0 & 0 & 0 & 0 & 0 & 0 & 0 & 0 & 0\end{array}$

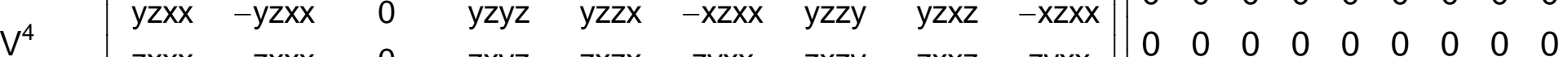

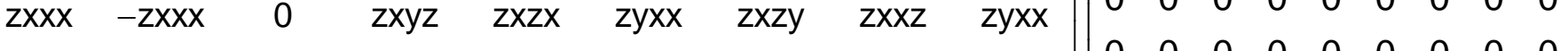
$\begin{array}{llllllllllllllllllllll}x y x x & x y y y & x y z z & -x x x z & x x z y & x y x y & -x x z x & x x y z & x y y x & 0 & 0 & 0 & 0 & 0 & 0 & 0 & 0 & 0\end{array}$ $\begin{array}{lllllllllllllllllll}z y x x & -z y x x & 0 & z x x z & -z x z y & -z x x x & z x z x & -z x y z & -z x x x & 0 & 0 & 0 & 0 & 0 & 0 & 0 & 0 & 0\end{array}$ $\left.\begin{array}{ccccccccc}x z x x & -x z x x & 0 & -y z x z & y z z y & y z x x & -y z z x & y z y z & y z x x \\ -x y y y & -x y x x & -x y z z & -x y x z & x x z y & x y y x & -x x z x & x x y z & x y x y\end{array} \mid \begin{array}{llllllllll}0 & 0 & 0 & 0 & 0 & 0 & 0 & 0 & 0 \\ 0 & 0 & 0 & 0 & 0 & 0 & 0 & 0 & 0\end{array}\right)$ and: $x x x x=x x y y+x y x y+x y y x ; x x x y-y y x y=x y y y-x y x x$

$\left[V^{4}\right] \quad\left(\begin{array}{ccccc}3 x x y y & 0 & 0 & y y z z & x x y z \\ 0 & 3 x x y y & 0 & y y z z & -z x x x \\ z x x x & -x x y z & z z z z & x x y y & 0\end{array}\right)$

$$
\left(\begin{array}{lllll}
0 & 0 & 0 & 0 & 0 \\
0 & 0 & 0 & 0 & 0 \\
0 & 0 & 0 & 0 & 0
\end{array}\right)
$$$$
\begin{array}{lllll}
0 x x x & -x x y z & z z z z & x x y y & 0
\end{array}
$$

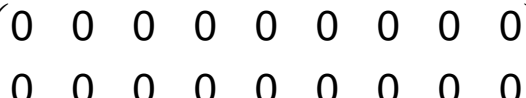

$0 \begin{array}{lllllllll}0 & 0 & 0 & 0 & 0 & 0 & 0 & 0 & 0 \\ 0 & 0 & 0 & 0 & 0 & 0 & 0 & 0 & 0 \\ 0 & 0 & 0 & 0 & 0\end{array}$

$\begin{array}{lllllllllll}0 & 0 & 0 & 0 & 0 & 0 & 0 & 0 & 0 \\ 0 & 0 & 0 & 0 & 0 & 0 & 0 & 0 & 0\end{array}$

$\begin{array}{lllllllll}0 & 0 & 0 & 0 & 0 & 0 & 0 & 0 & 0 \\ 0 & 0 & 0 & 0 & 0 & 0 & 0 & 0 & 0\end{array}$

$\begin{array}{llllllllll}0 & 0 & 0 & 0 & 0 & 0 & 0 & 0 & 0\end{array}$

$0 \begin{array}{llllllllll}0 & 0 & 0 & 0 & 0 & 0 & 0 & 0 & 0\end{array}$

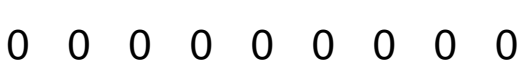

$\begin{array}{llllllllll}0 & 0 & 0 & 0 & 0 & 0 & 0 & 0 & 0\end{array}$

$\left(\begin{array}{lllll}0 & 0 & 0 & 0 & 0 \\ 0 & 0 & 0 & 0 & 0 \\ 0 & 0 & 0 & 0 & 0\end{array}\right)$

$\begin{array}{lllll}0 & 0 & 0 & 0 & 0\end{array}$

$\left(\begin{array}{llllllllll}0 & 0 & 0 & 0 & 0 & 0 & 0 & 0 & 0 & 0 \\ 0 & 0 & 0 & 0 & 0 & 0 & 0 & 0 & 0 & 0\end{array}\right.$

$0 \begin{array}{lllllllllll}0 & 0 & 0 & 0 & 0 & 0 & 0 & 0 & 0 & 0 \\ 0 & 0 & 0 & 0 & 0 & 0 & 0 & 0 & 0\end{array}$

$\left(\begin{array}{cccccccccc}3 x x y y & 3 x y x x & 0 & x x y y & x y z z & x z x x & y y z z & x y x x & -x z x x & y y x x \\ -3 x y x x & 3 x x y y & 0 & -x y y x & y y z z & y z x x & -x y z z & x x y y & -y z x x & -x z x y \\ 2 x y & -2 y x & 2 z z & -2 x x & 0 & z z x & 0 & z y x & z z x & z x y z\end{array}\right)\left(\begin{array}{cccccccccc}0 & 0 & 0 & 0 & 0 & 0 & 0 & 0 & 0 & 0 \\ 0 & 0 & 0 & 0 & 0 & 0 & 0 & 0 & 0 & 0 \\ 0 & 0 & 0 & 0 & 0 & 0 & 0 & 0 & 0 & 0\end{array}\right)$

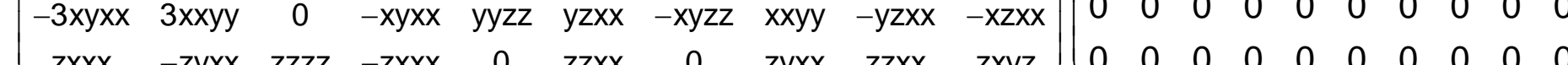

$\begin{array}{llllllllll}0 & 0 & 0 & 0 & 0 & 0 & 0 & 0 & 0 & 0\end{array}$

$$
\left(\begin{array}{cccccc}
x x x x & x x y y & x x z z & x x y z & -y y x z & 0 \\
& x x x x & x x z z & -x x y z & y y x z & 0 \\
& & z z z z & 0 & 0 & 0 \\
& & & y z y z & 0 & y y x z \\
& & & & y z y z & x x y z \\
& & & & & x y x y
\end{array}\right)
$$

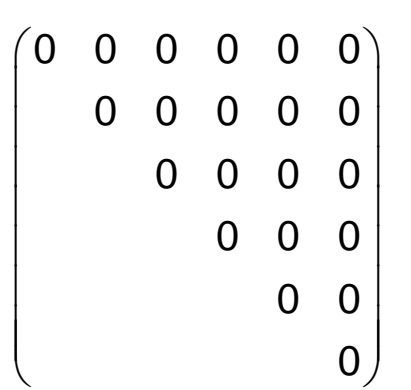

and $x y x y=1 / 2(x x x x-x x y y)$

$\begin{array}{llllll}x x x x & x x y y & x x z z & x x y z & -y y x z & x x x y\end{array}$

$\begin{array}{llllll}x x y y & x x x x & x x z z & -x x y z & y y x z & -x x x y\end{array}$

$\begin{array}{cccccc}z z x x & z z x x & z z z z & 0 & 0 & 0 \\ y z x x & -y z x x & 0 & y z y z & y z x z & x z y y \\ -x x y y & x z y y & 0 & y z x z & y z y z & y z x x\end{array}$

$\begin{array}{llllll}-x z y y & x z y y & 0 & y z x z & y z y z & y z x x \\ -x x y y & x x y & 0 & y y z z & x x y z & x y x\end{array}$

$\begin{array}{llllll}-x x x y & x x x y & 0 & y y x z & x x y z & x y x y\end{array}$

and $x y x y=1 / 2(x x x x-x x y y)$

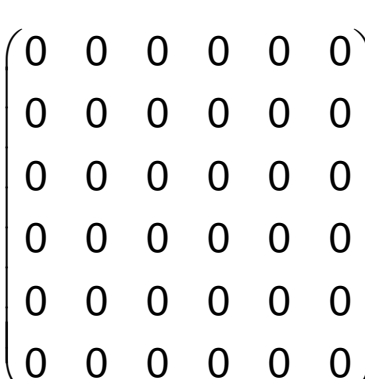

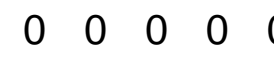

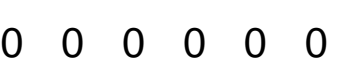

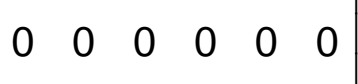

$\begin{array}{llllll}0 & 0 & 0 & 0 & 0 & 0 \\ 0 & 0 & 0 & 0 & 0 & 0 \\ 0 & 0 & 0 & 0 & 0 & 0\end{array}$

$\left(\begin{array}{lllllll}0 & 0 & 0 & 0 & 0 & 0 \\ 0 & 0 & 0 & 0 & 0 \\ 0 & 0 & 0 & 0 & 0\end{array}\right.$

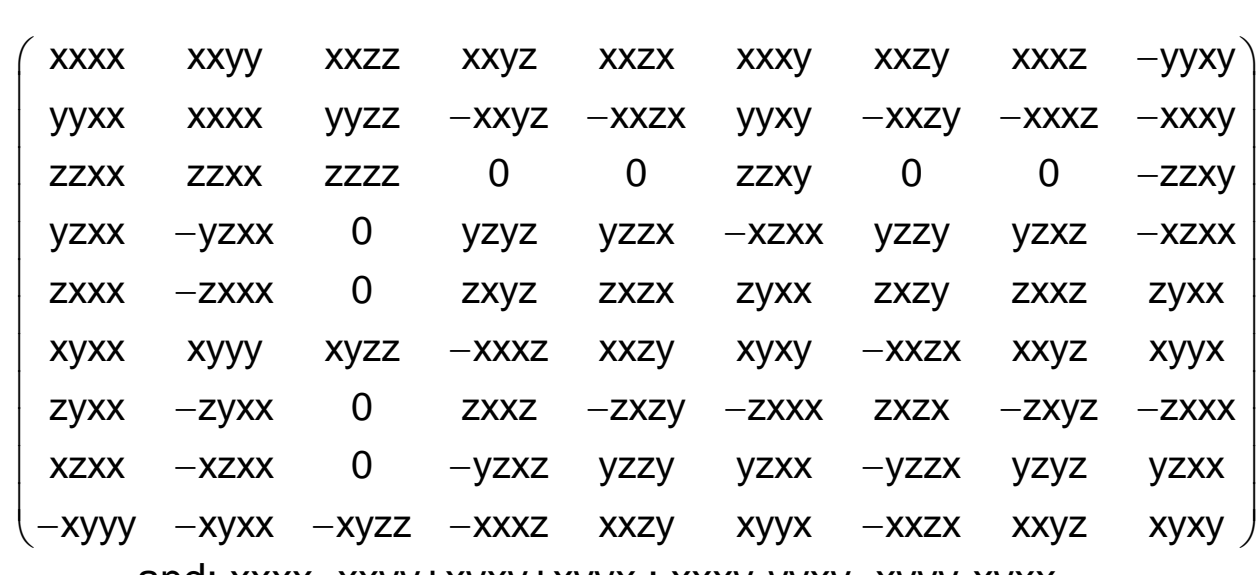

and: $x x x x=x x y y+x y x y+x y y x ; x x x y-y y x y=x y y y-x y x x$

$\begin{array}{ccccc}3 x x y y & 0 & 0 & y y z z & x x y z \\ 0 & 3 x x y y & 0 & y y z z & -z x x x\end{array}$

$\left.\begin{array}{ccccc}0 & 3 x x y y & 0 & y y z z & -z x x x \\ z x x x & -x x y z & z z z z & x x y y & 0\end{array}\right)$

$\begin{array}{llllllllll}3 x x y y & 3 x y x x & 0 & x x y y & x y z z & x z x x & y y z z & x y x x & -x z x x & y y x x\end{array}$ $\begin{array}{llllllllll}-3 x y x x & 3 x x y y & 0 & -x y x x & y y z z & y z x x & -x y z z & x x y y & -y z x x & -x z x x\end{array}$ $\begin{array}{llllllllll}z x x x & -z y x x & z z z z & -z x x x & 0 & z z x x & 0 & z y x x & z z x x & z x y z\end{array}$

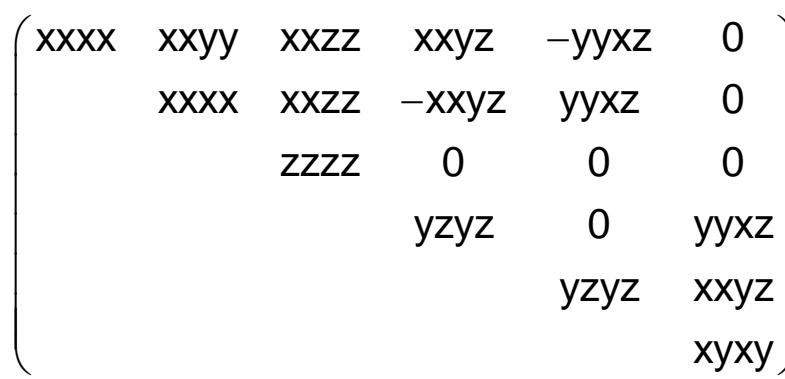

and $x y x y=1 / 2(x x x x-x x y y)$

$\begin{array}{llllll}x x x x & x x y y & x x z z & x x y z & -y y x z & x x x y\end{array}$

$\left.\begin{array}{cccccc}x x y y & x x x x & x x z z & -x x y z & y y x z & -x x x y \\ z z x & z z x & z z z z & 0 & 0 & 0\end{array}\right)$ $\begin{array}{lllllll}2 z x x & z z x x & z z z z & 0 & 0 & 0 \\ y z x & -y z x x & 0 & y z y z & y z x z & x z y y\end{array}$ $\begin{array}{cccccc}y z x x & -y z x x & 0 & y z y z & y z x z & x z y y \\ -x z y y & x z y y & 0 & y z x z & y z y z & y z x x\end{array}$

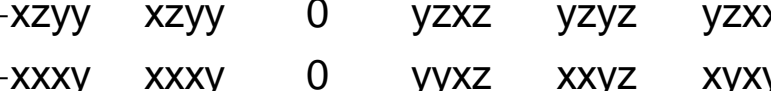

and $x y x y=1 / 2(x x x x-x x y y)$ 


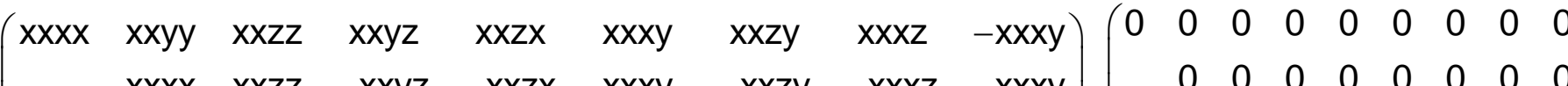

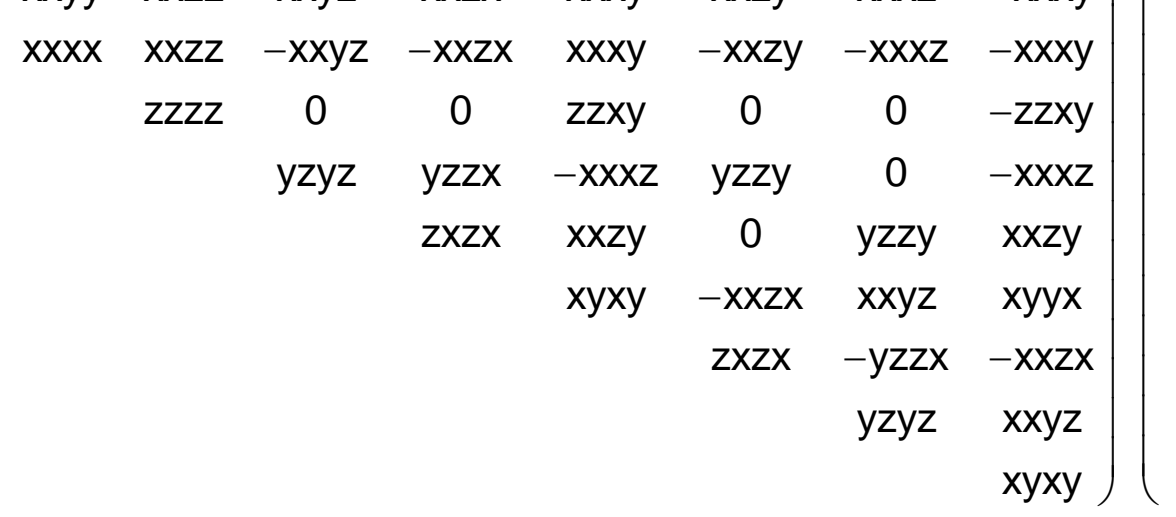

$\begin{array}{llllllll}0 & 0 & 0 & 0 & 0 & 0 & 0 \\ 0 & 0 & 0 & 0 & 0 & 0 & 0\end{array}$

$\begin{array}{llllll}0 & 0 & 0 & 0 & 0 & 0\end{array}$

$\begin{array}{lllll}0 & 0 & 0 & 0 & 0\end{array}$

$\begin{array}{llll}0 & 0 & 0 & 0\end{array}$

$0 \begin{array}{lll}0 & 0 & 0\end{array}$

and $x x x x=x x y y+y z y z+z y z y$

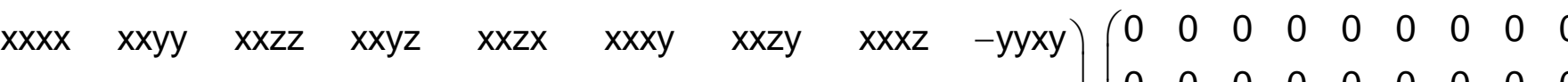

$\left.\begin{array}{lllllllll}x x y y & x x x x & x x z z & -x x y z & -x x z x & y y x y & -x x z y & -x x x z & -x x x y\end{array} \mid \begin{array}{lllllllll}0 & 0 & 0 & 0 & 0 & 0 & 0 & 0 & 0 \\ 0 & 0 & 0 & 0 & 0 & 0 & 0 & 0 & 0\end{array}\right)$

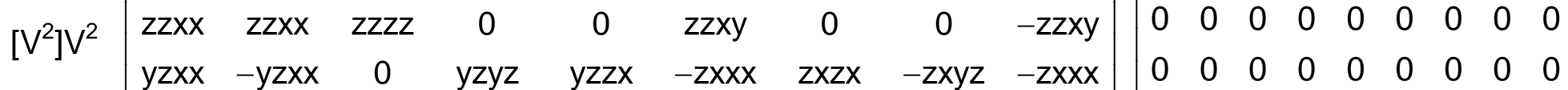

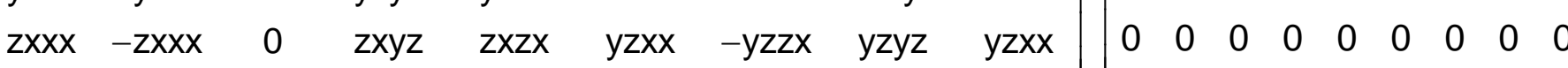
$\left.\begin{array}{lllllllll}x y x x & -x y x x & 0 & -x x x z & x x z y & x y x y & -x x z x & x x y z & x y x y\end{array}\right)\left(\begin{array}{llllllllll}0 & 0 & 0 & 0 & 0 & 0 & 0 & 0 & 0\end{array}\right.$

and $x x x x=x x y y+2 x y x y ; y y x y-x x x y=2 x y x x$

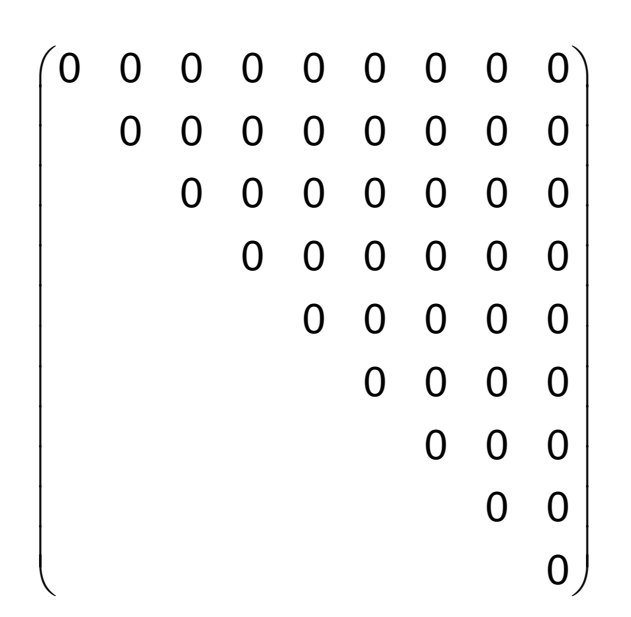

$\left(\begin{array}{lllllllll}0 & 0 & 0 & 0 & 0 & 0 & 0 & 0 & 0 \\ 0 & 0 & 0 & 0 & 0 & 0 & 0 & 0 & 0\end{array}\right.$ $0 \begin{array}{lllllllll}0 & 0 & 0 & 0 & 0 & 0 & 0 & 0 & 0 \\ 0 & 0 & 0 & 0 & 0 & 0 & 0 & 0 & 0\end{array}$

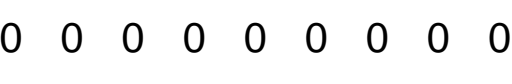

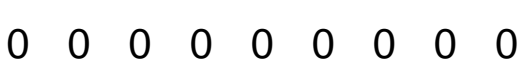
$0 \begin{array}{lllllllll}0 & 0 & 0 & 0 & 0 & 0 & 0 & 0 & 0 \\ 0 & 0 & 0 & 0 & 0 & 0 & 0 & 0\end{array}$ $\begin{array}{llllllllll}0 & 0 & 0 & 0 & 0 & 0 & 0 & 0 & 0\end{array}$

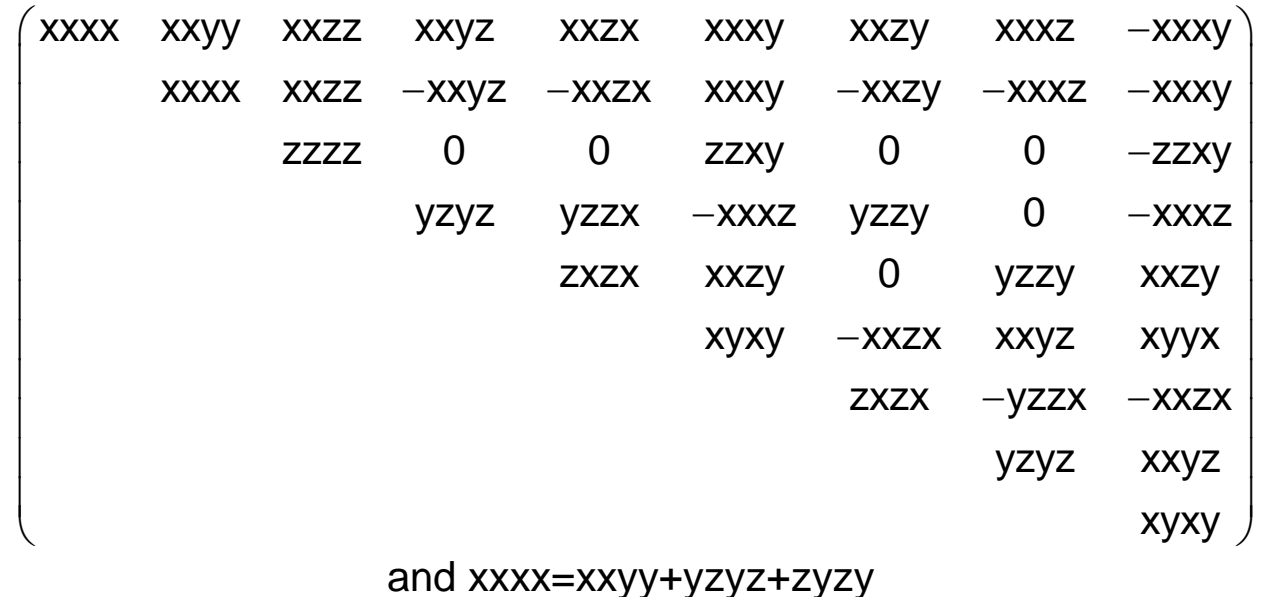

and $x x x x=x x y y+y z y z+z y z y$

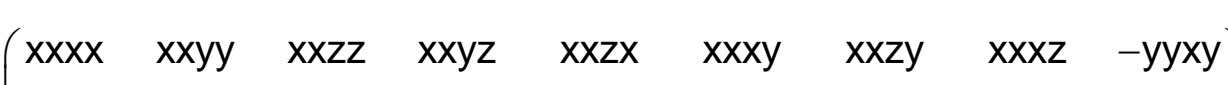
$\begin{array}{lllllllll}x x y y & x x x x & x x z z & -x x y z & -x x z x & y y x y & -x x z y & -x x x z & -x x x y\end{array}$ $\begin{array}{llllccccc}z z x x & z z x x & z z z z & 0 & 0 & z z x y & 0 & 0 & -z z x y \\ y z x x & -y z x x & 0 & y z y z & y z z x & -z x x x & z x z x & -z x y z & -z x x x\end{array}$ $\begin{array}{lllllllll}y z x x & -y z x x & 0 & y z y z & y z z x & -z x x x & z x z x & -z x y z & -z x x x \\ 2 x x & -z x x & 0 & z x y z & z x z x & y z x & -y z z & y z y z & y z x x\end{array}$ $\begin{array}{ccccccccc}z x x x & -z x x x & 0 & z x y z & z x z x & y z x x & -y z z x & y z y z & y z x x \\ x y x x & -x y x x & 0 & -x x y z & x x z y & x y x y & -x x z x & x x y z & x y x y\end{array} \mid$ and $x x x x=x x y y+2 x y x y ; y y x y-x x x y=2 x y x x$ 


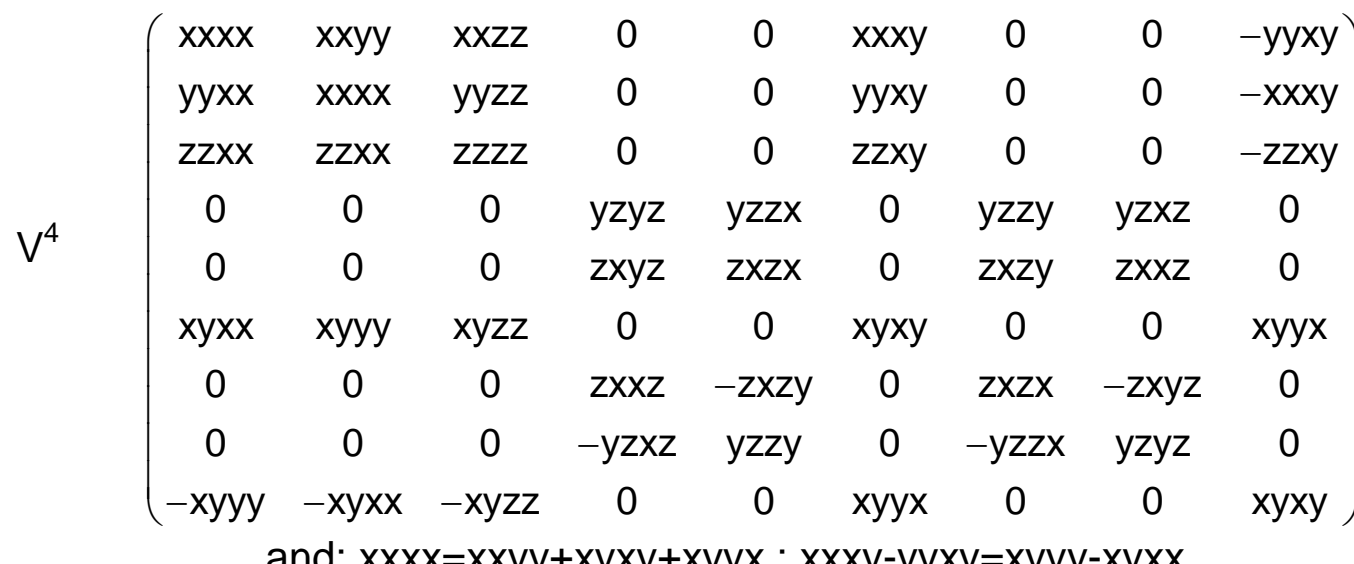

$\left[V^{4}\right]\left(\begin{array}{ccccc}3 x x y y & 0 & 0 & y y z z & 0 \\ 0 & 3 x x y y & 0 & y y z z & 0 \\ 0 & 0 & z z z z & x x y y & 0\end{array}\right)$

$\left.\begin{array}{llllllllll}3 x x y y & 3 x y x x & 0 & x x y y & x y z z & 0 & y y z z & x y x x & 0 & y y x x\end{array}\right)\left(\begin{array}{ccccccccccc}x x x x & x y y y & 0 & -x x x x & 0 & 0 & 0 & -x y y y & 0 & 0 \\ -x y y y & x y x & 0 & x y y & 0 & 0 & 0 & -x y x & 0 & 0\end{array}\right)$

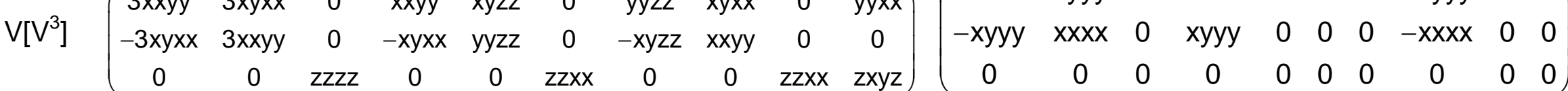

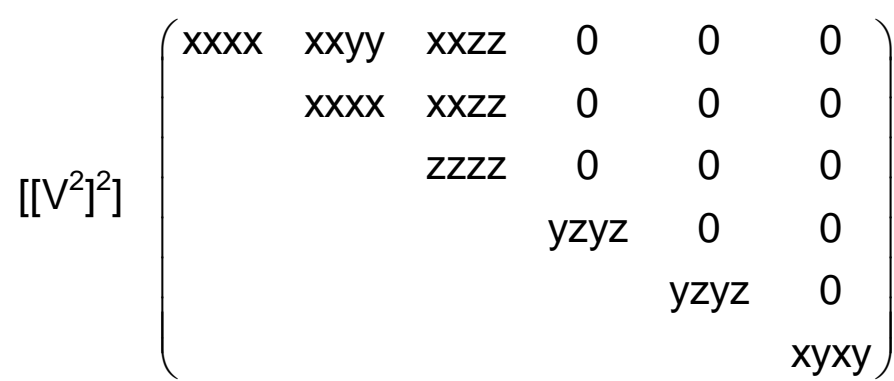

and $x y x y=1 / 2(x x x x-x x y y)$

$\left[\mathrm{V}^{2}\right]^{2}\left(\begin{array}{cccccc}\mathrm{x} x \mathrm{xx} & \mathrm{xxyy} & \mathrm{xxzz} & 0 & 0 & \mathrm{xxxy} \\ \mathrm{xxyy} & \mathrm{xxxx} & \mathrm{xxzz} & 0 & 0 & -\mathrm{xxxy} \\ \mathrm{zzxx} & \mathrm{zzxx} & \mathrm{zzzz} & 0 & 0 & 0 \\ 0 & 0 & 0 & \mathrm{yzyz} & \mathrm{yzxz} & 0 \\ 0 & 0 & 0 & \mathrm{yzxz} & \mathrm{yzyz} & 0 \\ -\mathrm{xxxy} & \mathrm{xxxy} & 0 & 0 & 0 & \mathrm{xyxy}\end{array}\right)$

and $x y x y=1 / 2(x x x x-x x y y)$

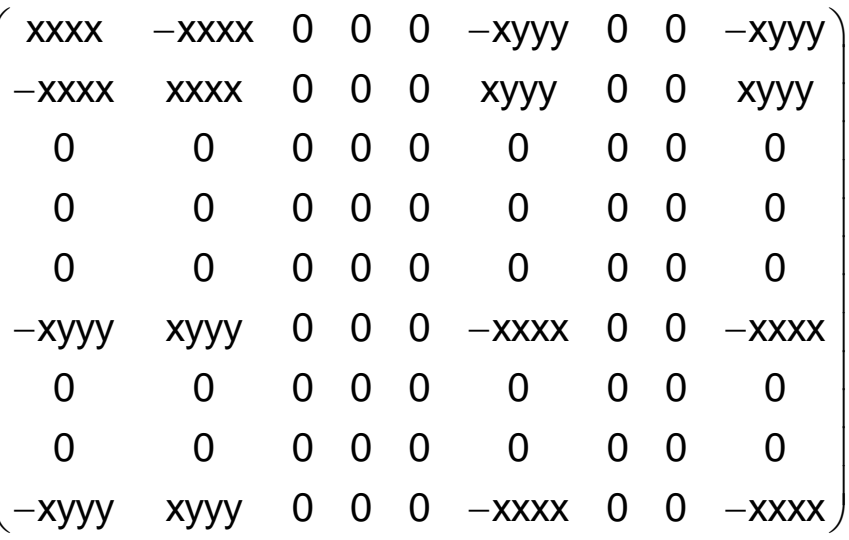

$$
\left(\begin{array}{ccccc}
x x x x & x y y y & 0 & 0 & 0 \\
-x y y y & x x x x & 0 & 0 & 0 \\
0 & 0 & 0 & -x x x x & 0
\end{array}\right)
$$

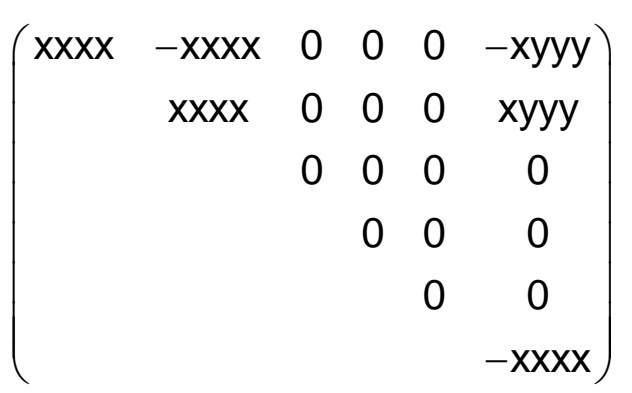

$\begin{array}{lllllllll}x x x x & -x x x x & 0 & 0 & 0 & -x y y y & 0 & 0 & -x y y y \\ -x x x x & x x x x & 0 & 0 & 0 & x y y & 0 & 0 & x y y\end{array}$ $\begin{array}{cccccccccc}-x x x x & x x x x & 0 & 0 & 0 & x y y y & 0 & 0 & x y y y\end{array}$

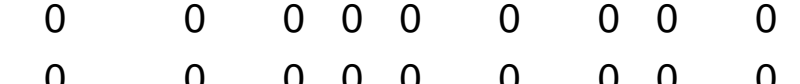
$\begin{array}{lllllllll}0 & 0 & 0 & 0 & 0 & 0 & 0 & 0 & 0\end{array}$ $\begin{array}{llllllllll}- \text {-xyyy } & \text { xyyy } & 0 & 0 & 0 & -x x x x & 0 & 0 & -x x x x\end{array}$ $\begin{array}{ccccccccc}0 & 0 & 0 & 0 & 0 & 0 & 0 & 0 & 0 \\ 0 & 0 & 0 & 0 & 0 & 0 & 0 & 0 & 0\end{array}$ $\begin{array}{ccccccccc}0 & 0 & 0 & 0 & 0 & 0 & 0 & 0 & 0 \\ -x y y & x y y & 0 & 0 & 0 & -x & 0\end{array}$ $\begin{array}{ccccccccc}-x y y y & x y y y & 0 & 0 & 0 & -x x x x & 0 & 0 & -x x x x\end{array}$

$\left(\begin{array}{ccccc}x x x x & x y y y & 0 & 0 & 0 \\ -x y y y & x x x x & 0 & 0 & 0 \\ 0 & 0 & 0 & -x x x x & 0\end{array}\right)$

$\left.\begin{array}{llll}0 & 0 & -x \mathrm{xxx} & 0\end{array}\right)$

$\left(\begin{array}{llllllllll}x x x x & x y y y & 0 & -x x x x & 0 & 0 & 0 & -x y y y & 0 & 0 \\ -x y y & x x x x & 0 & x y y y & 0 & 0 & 0 & -x x x x & 0 & 0\end{array}\right)$ $\left(\begin{array}{cccccccccc}-\mathrm{xyyy} & \mathrm{xxxx} & 0 & \mathrm{xyyy} & 0 & 0 & 0 & -\mathrm{xxxx} & 0 & 0 \\ 0 & 0 & 0 & 0 & 0 & 0 & 0 & 0 & 0 & 0\end{array}\right)$

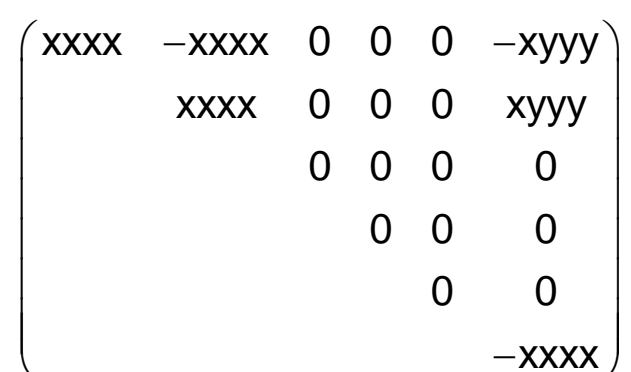

$$
\left(\begin{array}{cccccc}
\operatorname{xxxx} & -x x x x & 0 & 0 & 0 & -x y y y \\
-x x x x & x x x x & 0 & 0 & 0 & x y y y \\
0 & 0 & 0 & 0 & 0 & 0 \\
0 & 0 & 0 & 0 & 0 & 0 \\
0 & 0 & 0 & 0 & 0 & 0 \\
-x y y y & x y y y & 0 & 0 & 0 & -x x x x
\end{array}\right)
$$$$
\left(\begin{array}{cccccc}
x x x x & -x x x x & 0 & 0 & 0 & -x y y y \\
-x x x x & x x x x & 0 & 0 & 0 & x y y y \\
0 & 0 & 0 & 0 & 0 & 0 \\
0 & 0 & 0 & 0 & 0 & 0 \\
0 & 0 & 0 & 0 & 0 & 0 \\
-x y y y & x y y y & 0 & 0 & 0 & -x x x x
\end{array}\right)
$$

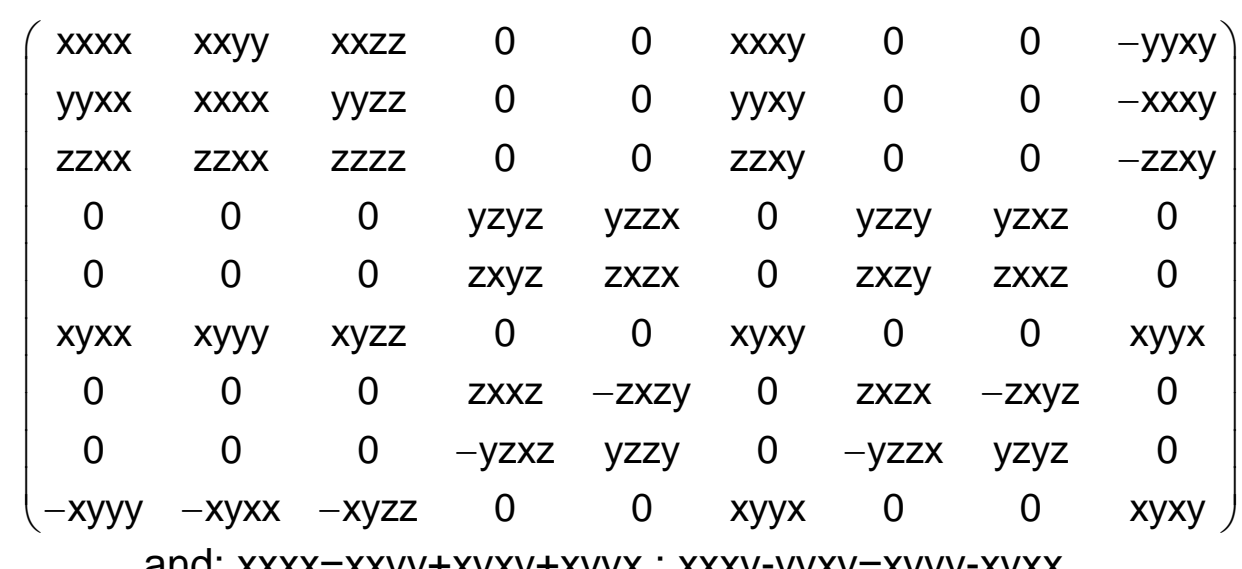

and: $x x x x=x x y y+x y x y+x y y x ; x x x y-y y x y=x y y-x y y x$

$\left(\begin{array}{ccccc}3 x x y y & 0 & 0 & y y z z & 0 \\ 0 & 3 x x y & 0 & y y z & 0\end{array}\right)$

$\left(\begin{array}{ccccc}0 & 3 x x y y & 0 & y y z z & 0 \\ 0 & 0 & z z z & x x y & 0\end{array}\right)$

$\left.\begin{array}{cccccccccc}3 x x y y & 3 x y x x & 0 & x x y y & x y z z & 0 & y y z z & x y x x & 0 & y y x x \\ -3 y x x & 3 x x y & 0 & -x y y x & y y z z & 0 & -x y z z & x x y y & 0 & 0\end{array}\right)$ $\left.\begin{array}{cccccccccc}-3 x y x x & 3 x x y y & 0 & -x y x & y y z z & 0 & -x y z z & x x y y & 0 & 0 \\ 0 & 0 & z z z z & 0 & 0 & z z x x & 0 & 0 & z z x x & z x y z\end{array}\right)$

$$
\left(\begin{array}{cccccc}
\text { xxxx } & \text { xxyy } & \text { xxzz } & 0 & 0 & 0 \\
& \text { xxxx } & \text { xxzz } & 0 & 0 & 0 \\
& & \text { zzzz } & 0 & 0 & 0 \\
& & & y z y z & 0 & 0 \\
& & & & y z y z & 0 \\
& & & & & \text { xyxy }
\end{array}\right)
$$

and $x y x y=1 / 2(x x x x-x x y y)$

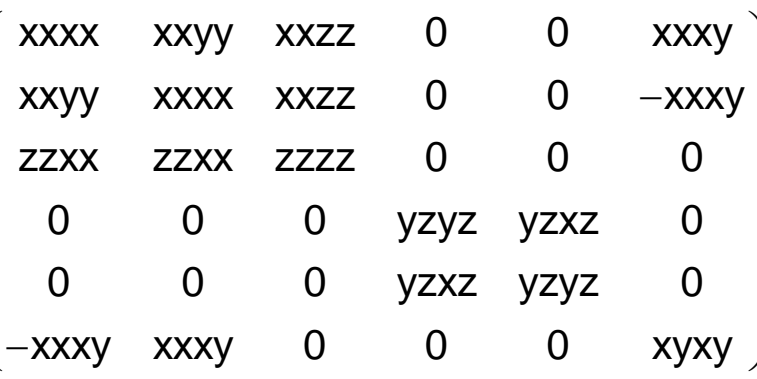

and $x y x y=1 / 2(x x x x-x x y y)$ 


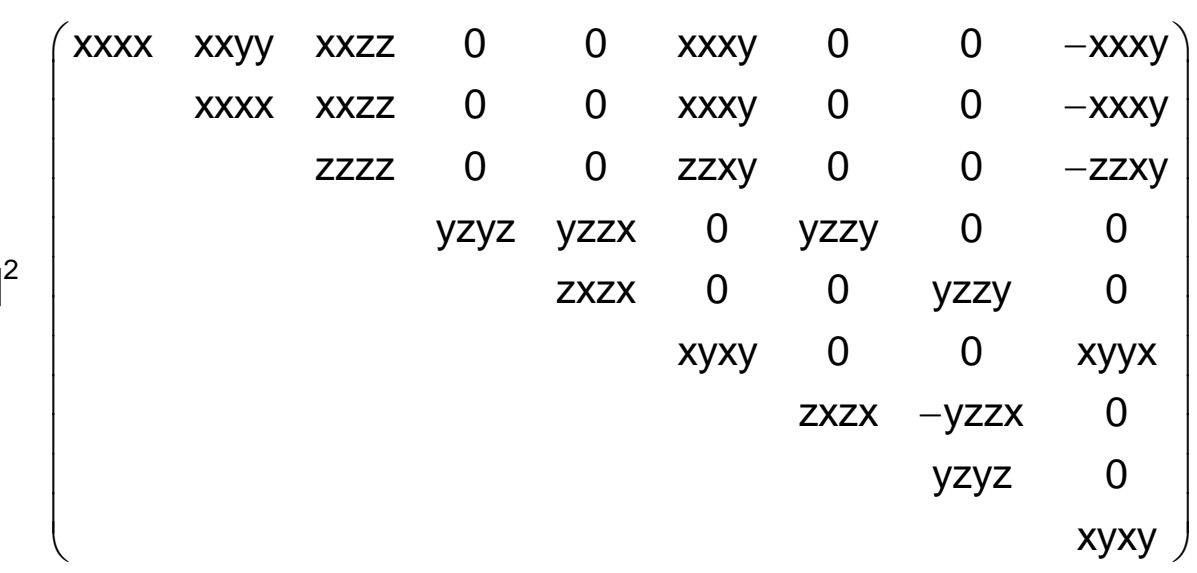

and $x x x x=x x y y+y z y z+z y z y$

$\begin{array}{lllllllll}x x x x & x x y y & x x z z & 0 & 0 & x x x y & 0 & 0 & -y y x y \\ x x y y & x y x & x x z z & 0 & 0 & y x y & 0 & 0 & -x x y\end{array}$ $\left.\begin{array}{lllllllll}x x y y y & x x x x & x x z z & 0 & 0 & y y x y & 0 & 0 & -x x x y \\ z z x x & z z x x & z z z z & 0 & 0 & z z x y & 0 & 0 & -z z x y\end{array}\right)$ $\left[\mathrm{V}^{2}\right] \mathrm{V}^{2} \quad \mathrm{zz} x \mathrm{x}$

$\begin{array}{cccccccccc}0 & 0 & 0 & y z y z & y z z x & 0 & z x z x & -z x y z & 0\end{array}$ $\begin{array}{ccccccccc}0 & 0 & 0 & z x y z & z x z x & 0 & -y z z x & y z y z & 0\end{array}$

and $x x x x=x x y y+2 x y x y ; y y x y-x x x y=2 x y x x$ $\begin{array}{lllllllll}x x x x & -x x x x & 0 & 0 & 0 & -x y y y & 0 & 0 & -x y y y\end{array}$

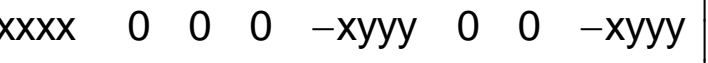
$\begin{array}{lllllll}0 & 0 & 0 & 0 & 0 & 0 & 0\end{array}$ $\begin{array}{llllll}0 & 0 & 0 & 0 & 0 & 0\end{array}$ $\begin{array}{lllll}0 & 0 & 0 & 0 & 0\end{array}$ $-x x x x \quad 0 \quad 0 \quad-x x x x$ $\begin{array}{lll}0 & 0 & 0 \\ & 0 & 0\end{array}$ $-x \mathrm{xxx}$

$\left(\begin{array}{lllllllll}x x x x & -x x x x & 0 & 0 & 0 & -x y y y & 0 & 0 & -x y y y \\ -x x x x & x x x x & 0 & 0 & 0 & x y y & 0 & 0 & x y y\end{array}\right.$ $\begin{array}{ccccccccc}-x x x x & x x x x & 0 & 0 & 0 & x y y y & 0 & 0 & x y y y\end{array}$ $\begin{array}{lllllllll}0 & 0 & 0 & 0 & 0 & 0 & 0 & 0 & 0 \\ 0 & 0 & 0 & 0 & 0 & 0 & 0 & 0 & 0\end{array}$ $\begin{array}{ccccccccc}0 & 0 & 0 & 0 & 0 & 0 & 0 & 0 & 0 \\ 0 & 0 & 0 & 0 & 0 & 0 & 0 & 0 & 0 \\ 0 & 0 & 0 & 0 & 0 & 0 & 0 & 0 & 0\end{array}$ $\begin{array}{llllllllll}-x y y y & x y y y & 0 & 0 & 0 & -x x y x & 0 & 0 & -x x y x\end{array}$ $\left(\begin{array}{lllllllll}x x x x & -x x x x & 0 & 0 & 0 & -x y y y & 0 & 0 & -x y y y \\ x & -x x x & 0 & 0 & 0 & -x y y & 0 & 0 & -x y y\end{array}\right)$

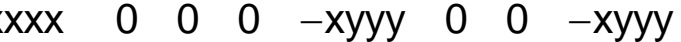
$\begin{array}{lllllll}0 & 0 & 0 & 0 & 0 & 0 & 0\end{array}$ $\begin{array}{llllll}0 & 0 & 0 & 0 & 0 & 0\end{array}$ $\begin{array}{lllll}0 & 0 & 0 & 0 & 0\end{array}$ $\begin{array}{lllll}-x & 0 & 0 & 0 & 0\end{array}$ $\begin{array}{ccc}0 & 0 & 0\end{array}$ 0 $\begin{array}{lllllllll}-x x x x & x x x x & 0 & 0 & 0 & x y y y & 0 & 0 & x y y y\end{array}$ $\begin{array}{lllllllll}0 & 0 & 0 & 0 & 0 & 0 & 0 & 0 & 0\end{array}$ $\begin{array}{lllllllll}0 & 0 & 0 & 0 & 0 & 0 & 0 & 0 & 0 \\ 0 & 0 & 0 & 0 & 0 & 0 & 0 & 0 & 0\end{array}$ $\begin{array}{lllllllll}0 & 0 & 0 & 0 & 0 & 0 & 0 & 0 & 0\end{array}$ $\begin{array}{llllllllll}- \text {-xyyy } & \text { xyyy } & 0 & 0 & 0 & -x x x x & 0 & 0 & -x x x x\end{array}$

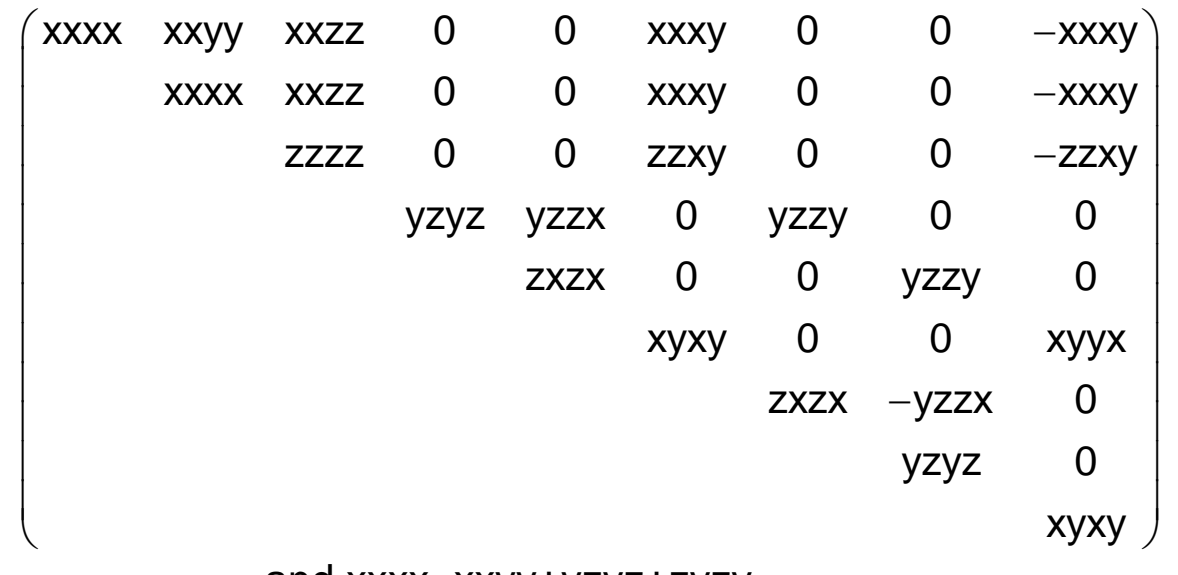

and $x x x x=x x y y+y z y z+z y z y$

$\begin{array}{llll}x x x x & x x y y & x x z z & 0\end{array}$ $\left.\begin{array}{lllllllll}x x y y & x x x x & x x z z & 0 & 0 & y y x y & 0 & 0 & -x x x y \\ z z x x & z z x & z z z & 0 & 0 & z z y & 0 & 0 & -z z x y\end{array}\right)$ $\begin{array}{llllllllll}z z x x & z z x x & z z z z & 0 & 0 & z z x y & 0 & 0 & -z z x y \\ 0 & 0 & 0 & y z y & y z z x & 0 & z x z x & -z x y z & 0\end{array}$ $\begin{array}{lllllllll}0 & 0 & 0 & y z y z & y z z x & 0 & z x z x & -z x y z & 0 \\ 0 & 0 & 0 & z x y & z x z x & 0 & -y z z & y z y & 0\end{array}$ $\begin{array}{ccccccccc}0 & 0 & 0 & \text { zxyz } & z x z x & 0 & -y z z x & y z y z & 0 \\ 0 y x x & -x y y x & 0 & 0 & 0 & x y x y & 0 & 0 & x y x y\end{array}$ and $x x x x=x x y y+2 x y x y ; y y x y-x x x y=2 x y x x$ 


\begin{tabular}{|c|c|c|c|c|c|c|c|c|c|c|c|c|c|}
\hline$(x x$ & xxyy & $x x z z$ & 0 & 0 & $x x x y$ & 0 & 0 & $-y y x y)$ & 0 & $\begin{array}{ll}0 & 0\end{array}$ & 0 & 0 & \\
\hline & $x x x x$ & xxzz & 0 & 0 & yyxy & $c$ & 0 & $-x x x y$ & 0 & 0 & $\begin{array}{ll}0 & 0\end{array}$ & 0 & 0 \\
\hline & zzyy & zzzz & 0 & 0 & zzxy & 0 & 0 & $-z z x y$ & 0 & & 0 & & \\
\hline & 0 & 0 & yzyz & yzzx & 0 & yzzy & $y z x z$ & 0 & 0 & $\begin{array}{ll}0 & 0\end{array}$ & $\begin{array}{lll}0 & 0\end{array}$ & 0 & $\begin{array}{ll}0 & 0\end{array}$ \\
\hline & 0 & 0 & $z x y z$ & $z x z x$ & 0 & zxzy & $z x x z$ & 0 & 0 & $\begin{array}{ll}0 & 0\end{array}$ & $\begin{array}{ll}0 & 0\end{array}$ & 0 & $\begin{array}{ll}0 & 0\end{array}$ \\
\hline & xyyy & xyzz & 0 & 0 & xyxy & 0 & 0 & xyyx & 0 & $\begin{array}{ll}0 & 0\end{array}$ & $\begin{array}{ll}0 & 0\end{array}$ & 0 & 0 \\
\hline & 0 & 0 & $z x x z$ & -zxzy & 0 & $z x z x$ & $-z x y z$ & & 0 & $\begin{array}{ll}0 & 0\end{array}$ & $\begin{array}{ll}0 & 0\end{array}$ & 0 & 0 \\
\hline & 0 & 0 & $-y z x z$ & yzzy & 0 & $-y z z x$ & yzyz & 0 & 0 & $\begin{array}{ll}0 & 0\end{array}$ & 00 & 0 & \\
\hline
\end{tabular}
and: $x x x x=x x y y+x y x y+x y y x ; x x x y-y y x y=x y y y-x y x x$

$\left[V^{4}\right] \quad\left(\begin{array}{ccccc}3 x x y y & 0 & 0 & y y z z & 0 \\ 0 & 3 x x y y & 0 & y y z z & 0 \\ 0 & 0 & z z z z & x x y y & 0\end{array}\right)$

$\left(\begin{array}{lllll}0 & 0 & 0 & 0 & 0 \\ 0 & 0 & 0 & 0 & 0 \\ 0 & 0 & 0 & 0 & 0\end{array}\right)$

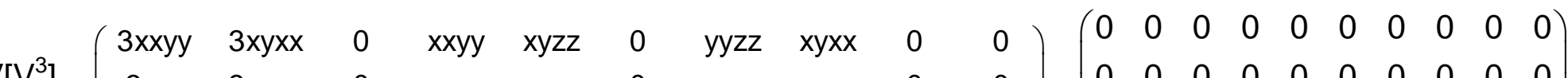

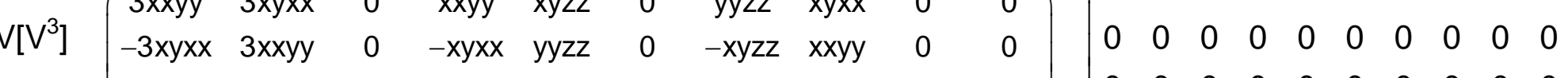

$\left[\left[V^{2}\right]^{2}\right]\left(\begin{array}{cccccc}x x x x & x x y y & x x z z & 0 & 0 & 0 \\ & x x x x & x x z z & 0 & 0 & 0 \\ & & z z z z & 0 & 0 & 0 \\ & & & y z y z & 0 & 0 \\ & & & & y z y z & 0 \\ & & & & & x y x y\end{array}\right)$

$\left(\begin{array}{llllllllll}0 & 0 & 0 & 0 & 0 & 0 & 0 & 0 & 0 & 0 \\ 0 & 0 & 0 & 0 & 0 & 0 & 0 & 0 & 0 & 0\end{array}\right)$

and $x y x y=1 / 2(x x x x-x x y y)$

$\left[\mathrm{V}^{2}\right]^{2}\left(\begin{array}{cccccc}x x x x & x x y y & x x z z & 0 & 0 & x x x y \\ x x y y & x x x x & x x z z & 0 & 0 & -x x x y \\ z z x x & z z x x & z z z z & 0 & 0 & 0 \\ 0 & 0 & 0 & y z y z & y z x z & 0 \\ 0 & 0 & 0 & -y z x z & y z y z & 0 \\ -x x x y & x x x y & 0 & 0 & 0 & x y x y\end{array}\right)$

and $x y x y=1 / 2(x x x x-x x y y)$
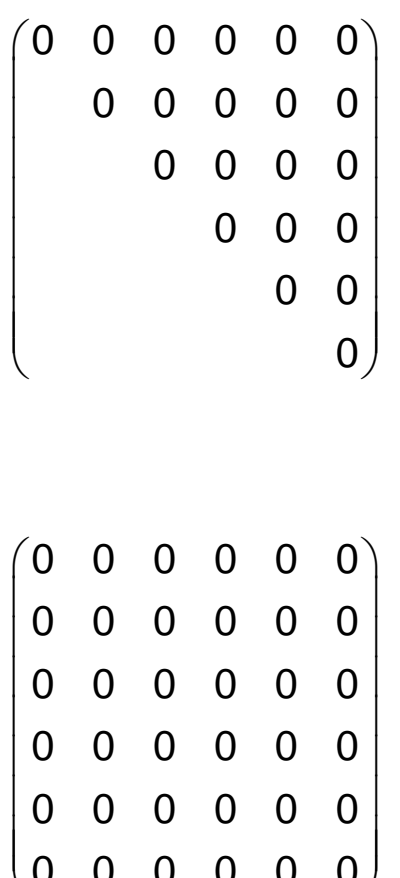

$\left(\begin{array}{llllllll}0 & 0 & 0 & 0 & 0 & 0 & 0 & 0 \\ 0 & 0 & 0 & 0 & 0 & 0 & 0 & 0\end{array}\right.$

$0 \begin{array}{lllllllll}0 & 0 & 0 & 0 & 0 & 0 & 0 & 0 & 0 \\ 0 & 0 & 0 & 0 & 0 & 0 & 0 & 0 & 0\end{array}$

$0 \begin{array}{llllllllll}0 & 0 & 0 & 0 & 0 & 0 & 0 & 0 & 0 \\ 0 & 0 & 0 & 0 & 0 & 0 & 0 & 0\end{array}$

$\begin{array}{llllllllll}0 & 0 & 0 & 0 & 0 & 0 & 0 & 0 & 0 \\ 0 & 0 & 0 & 0 & 0 & 0 & 0 & 0\end{array}$

$\begin{array}{llllllllll}0 & 0 & 0 & 0 & 0 & 0 & 0 & 0 & 0 \\ 0 & 0 & 0 & 0 & 0 & 0 & 0\end{array}$

$\begin{array}{llllllllll}0 & 0 & 0 & 0 & 0 & 0 & 0 & 0 & 0\end{array}$

$0 \begin{array}{lllllllll}0 & 0 & 0 & 0 & 0 & 0 & 0 & 0 & 0 \\ 0 & 0 & 0 & 0 & 0 & 0 & 0 & 0 & 0\end{array}$

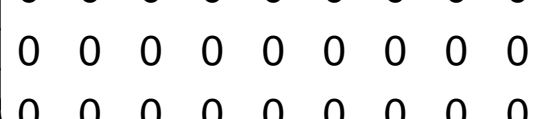

$\begin{array}{llllllllll}0 & 0 & 0 & 0 & 0 & 0 & 0 & 0 & 0\end{array}$

$\left(\begin{array}{lllll}0 & 0 & 0 & 0 & 0 \\ 0 & 0 & 0 & 0 & 0 \\ 0 & 0 & 0 & 0 & 0\end{array}\right)$

$\begin{array}{llllllllll}0 & 0 & 0 & 0 & 0 & 0 & 0 & 0 & 0 & 0 \\ 0 & 0 & 0 & 0 & 0 & 0 & 0 & 0 & 0 & 0\end{array}$

$\left(\begin{array}{llllllllll}0 & 0 & 0 & 0 & 0 & 0 & 0 & 0 & 0 & 0 \\ 0 & 0 & 0 & 0 & 0 & 0 & 0 & 0 & 0 & 0\end{array}\right)$

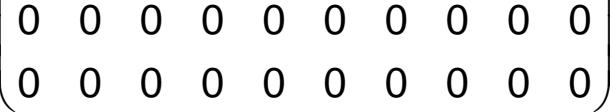

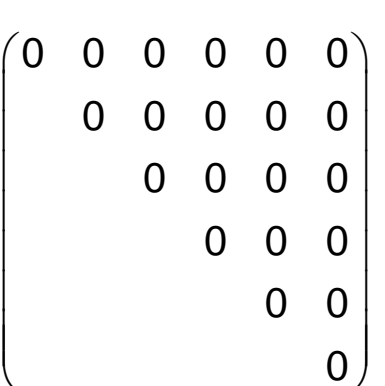

$\left(\begin{array}{llllll}0 & 0 & 0 & 0 & 0 & 0 \\ 0 & 0 & 0 & 0 & 0 & 0\end{array}\right.$

$0 \begin{array}{llllll}0 & 0 & 0 & 0 & 0 & 0 \\ 0 & 0 & 0 & 0 & 0 & 0\end{array}$

$0 \begin{array}{llllll}0 & 0 & 0 & 0 & 0 & 0 \\ 0 & 0 & 0 & 0 & 0 & 0\end{array}$

$\begin{array}{llllll}0 & 0 & 0 & 0 & 0 & 0 \\ 0 & 0 & 0 & 0 & 0 & 0\end{array}$

$\begin{array}{llllll}0 & 0 & 0 & 0 & 0 & 0 \\ 0 & 0 & 0 & 0 & 0 & 0\end{array}$

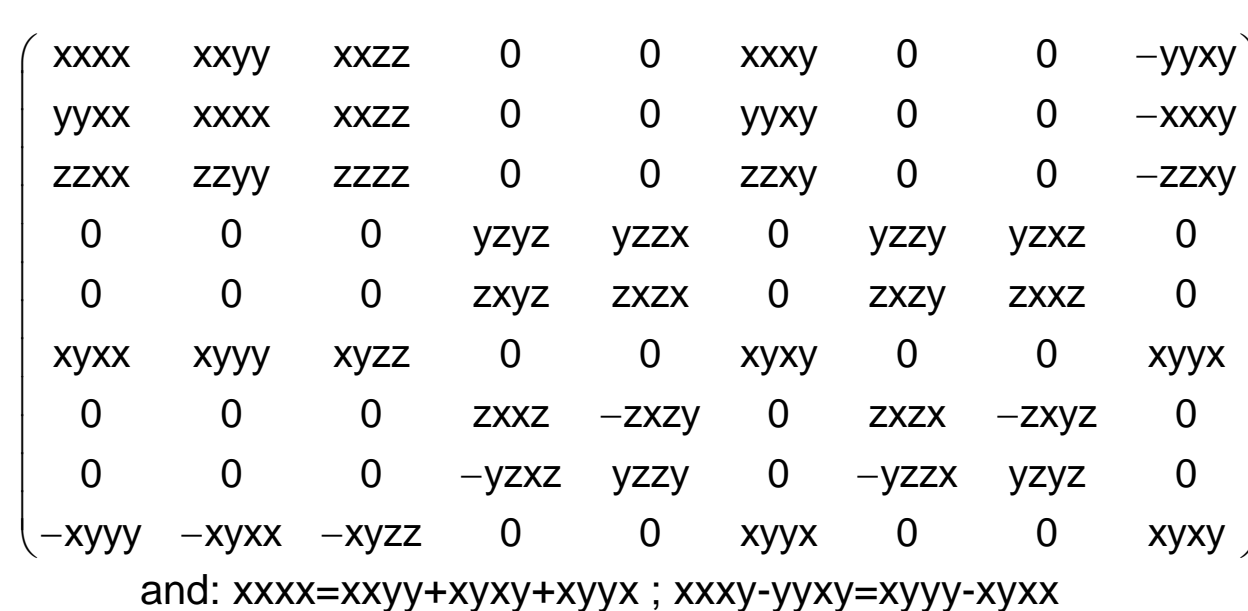

$\left(\begin{array}{ccccc}3 x x y y & 0 & 0 & y y z z & 0 \\ 0 & 3 x x y y & 0 & y y z z & 0 \\ 0 & 0 & z z z z & x x y y & 0\end{array}\right)$

$\left(\begin{array}{ccccc}0 & 3 x x y y & 0 & y y z z & 0 \\ 0 & 0 & z z z z & x x y y & 0\end{array}\right)$

$\left(\begin{array}{cccccccccc}3 x x y y & 3 x y x x & 0 & x x y y & x y z z & 0 & y y z z & x y x x & 0 & 0 \\ 3 x y x x & 3 x x y & 0 & -x y x & y y z & 0 & -x y z & x x y & 0 & 0\end{array}\right)$

$\left.\begin{array}{cccccccccc}-3 x y x x & 3 x x y y & 0 & -x y x x & y y z z & 0 & -x y z z & x x y y & 0 & 0 \\ 0 & 0 & z z z z & 0 & 0 & z z x x & 0 & 0 & z z x x & z x y z\end{array}\right)$

$\left(\begin{array}{cccccc}x x x x & x x y y & x x z z & 0 & 0 & 0 \\ & x x x x & x x z z & 0 & 0 & 0 \\ & & z z z z & 0 & 0 & 0 \\ & & & y z y z & 0 & 0 \\ & & & & y z y z & 0 \\ & & & & & \\ & & & & & \end{array}\right.$

and $x y x y=1 / 2(x x x x-x x y y)$

$\left(\begin{array}{cccccc}x x x x & x x y y & x x z z & 0 & 0 & x x x y \\ x x y y & x x x x & x x z z & 0 & 0 & -x x x y \\ z z x x & z z x x & z z z z & 0 & 0 & 0 \\ 0 & 0 & 0 & y z y z & y z x z & 0 \\ 0 & 0 & 0 & -y z x z & y z y z & 0 \\ -x x x y & x x x y & 0 & 0 & 0 & x y x y\end{array}\right)$

and $x y x y=1 / 2(x x x x-x x y y)$ 


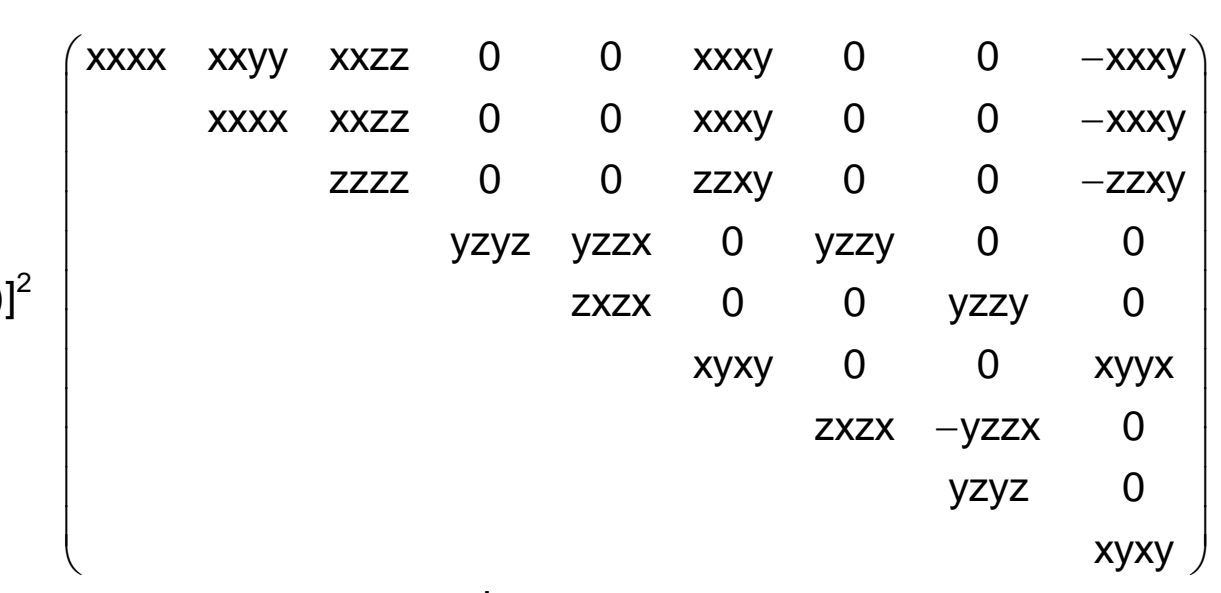

$\left(\begin{array}{lllllllll}0 & 0 & 0 & 0 & 0 & 0 & 0 & 0 & 0 \\ & 0 & 0 & 0 & 0 & 0 & 0 & 0 & 0\end{array}\right)$

$\begin{array}{lllllll}0 & 0 & 0 & 0 & 0 & 0 & 0\end{array}$

$\begin{array}{llllll}0 & 0 & 0 & 0 & 0 & 0\end{array}$

$\begin{array}{llllll}0 & 0 & 0 & 0 & 0\end{array}$

$\begin{array}{lllll}0 & 0 & 0 & 0 \\ 0 & 0 & 0 & 0\end{array}$

$\begin{array}{lll}0 & 0 & 0\end{array}$

and $x x x x=x x y y+y z y z+z y z y$

$\left[\mathrm{V}^{2}\right] \mathrm{V}^{2}\left(\begin{array}{ccccccccc}\mathrm{x} x \mathrm{x} x & \mathrm{xxyy} & \mathrm{xxzz} & 0 & 0 & \mathrm{xxxy} & 0 & 0 & -\mathrm{yyxy} \\ \mathrm{xxyy} & \mathrm{xxxx} & \mathrm{xxzz} & 0 & 0 & \mathrm{yyxy} & 0 & 0 & -\mathrm{xxxy} \\ \mathrm{zzxx} & \mathrm{zzxx} & \mathrm{zzzz} & 0 & 0 & \mathrm{zzxy} & 0 & 0 & -\mathrm{zzxy} \\ 0 & 0 & 0 & \mathrm{yzyz} & \mathrm{yzzx} & 0 & \mathrm{zxzx} & -\mathrm{zxyz} & 0 \\ 0 & 0 & 0 & \mathrm{zxyz} & \mathrm{zxzx} & 0 & -\mathrm{yzzx} & \mathrm{yzyz} & 0 \\ \mathrm{xyxx} & -\mathrm{xyxx} & 0 & 0 & 0 & \mathrm{xyxy} & 0 & 0 & \mathrm{xyxy}\end{array}\right) \quad\left(\begin{array}{ccccccccc}0 & 0 & 0 & 0 & 0 & 0 & 0 & 0 & 0 \\ 0 & 0 & 0 & 0 & 0 & 0 & 0 & 0 & 0 \\ 0 & 0 & 0 & 0 & 0 & 0 & 0 & 0 & 0 \\ 0 & 0 & 0 & 0 & 0 & 0 & 0 & 0 & 0 \\ 0 & 0 & 0 & 0 & 0 & 0 & 0 & 0 & 0 \\ 0 & 0 & 0 & 0 & 0 & 0 & 0 & 0 & 0\end{array}\right)$

and $x x x x=x x y y+2 x y x y ; y y x y-x x x y=2 x y x x$

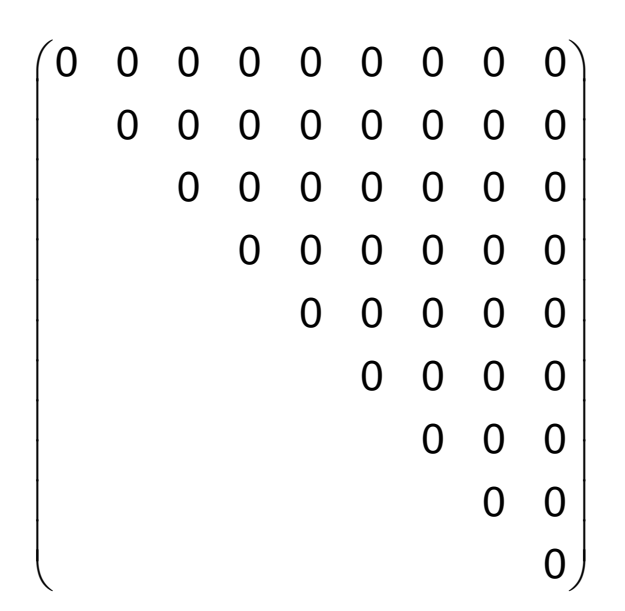

$\left(\begin{array}{lllllllll}0 & 0 & 0 & 0 & 0 & 0 & 0 & 0 & 0 \\ 0 & 0 & 0 & 0 & 0 & 0 & 0 & 0 & 0\end{array}\right.$

$0 \begin{array}{lllllllll}0 & 0 & 0 & 0 & 0 & 0 & 0 & 0 & 0 \\ 0 & 0 & 0 & 0 & 0 & 0 & 0 & 0 & 0\end{array}$

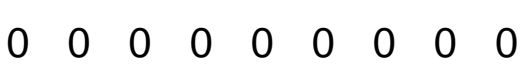

$\begin{array}{lllllllll}0 & 0 & 0 & 0 & 0 & 0 & 0 & 0 & 0\end{array}$

$\left(\begin{array}{lllllllll}0 & 0 & 0 & 0 & 0 & 0 & 0 & 0 & 0 \\ 0 & 0 & 0 & 0 & 0 & 0 & 0 & 0 & 0\end{array}\right)$

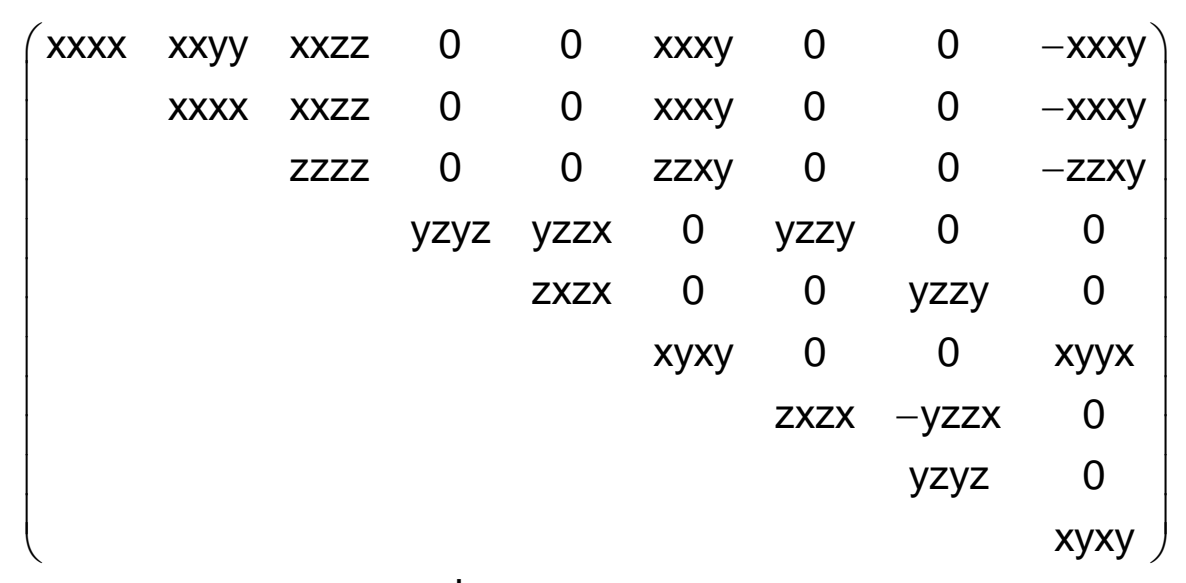

and $x x x x=x x y y+y z y z+z y z y$

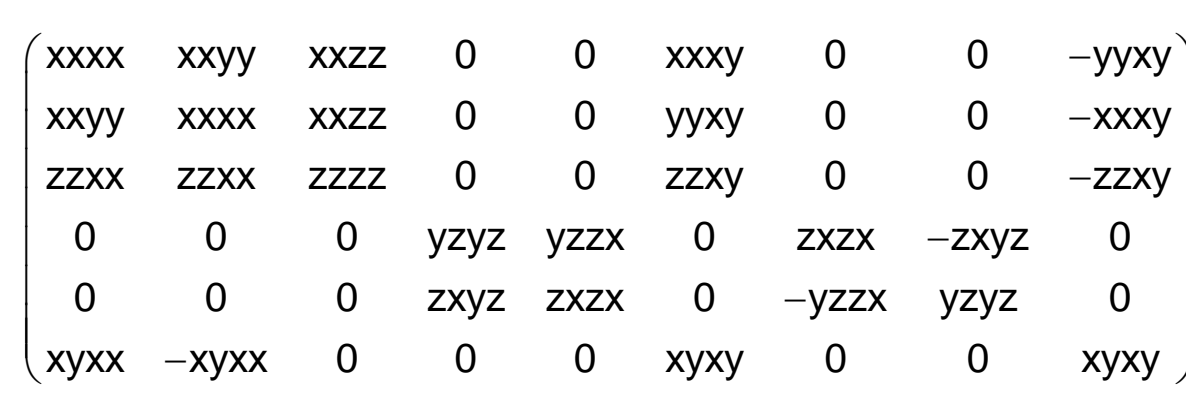

and $x x x x=x x y y+2 x y x y ; y y x y-x x x y=2 x y x x$ 
$\left(\begin{array}{lllllllll}x x x x & x x y y & x x z z & 0 & 0 & x x x y & 0 & 0 & x x y x \\ y y x & y y y & y y z z & 0 & 0 & y y y & 0 & 0 & x y y x\end{array}\right)$ $\left.\begin{array}{lllllllll}\text { yyxx } & \text { yyyy } & \text { yyzz } & 0 & 0 & \text { yyxy } & 0 & 0 & \text { yyyx } \\ \text { zzxx } & \text { zzyy } & \text { zzzz } & 0 & 0 & \text { zzxy } & 0 & 0 & \text { zzyx } \\ 0 & 0 & 0 & y z y z & y z z x & 0 & y z z y & y z z z & 0\end{array}\right)$ $\begin{array}{lllllllll}0 & 0 & 0 & y z y z & y z z x & 0 & y z z y & y z x z & 0\end{array}$

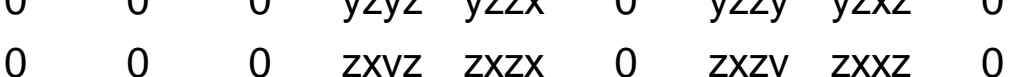

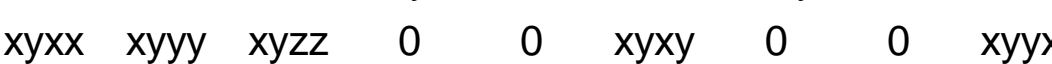

$\begin{array}{lllllllll}0 & 0 & 0 & \text { zyyz } & \text { zyzx } & 0 & \text { zyzy } & \text { zyxz } & 0\end{array}$

$\begin{array}{lllllllll}0 & 0 & 0 & x z y z & x z z x & 0 & x z z y & x z x z & 0\end{array}$

$\begin{array}{lllllllll}y x x x & y x y y & y x z z & 0 & 0 & y x x y & 0 & 0 & y x y x\end{array}$

$\left[V^{4}\right] \quad\left(\begin{array}{ccccc}x x x x & x y y y & 0 & y y z z & 0 \\ y x x x & y y y y & 0 & z z x x & 0 \\ 0 & 0 & z z z z & x x y y & z z x y\end{array}\right)$ $\left(\begin{array}{lllllllll}0 & 0 & 0 & x x y z & x x z x & 0 & x x z y & x x x z & 0\end{array}\right)$

$\begin{array}{lllllllll}0 & 0 & 0 & \text { yyyz } & \text { yyzx } & 0 & \text { yyzy } & \text { yyxz } & 0 \\ 0 & 0 & 0 & \text { zzyz } & \text { zzzx } & 0 & \text { zzzy } & \text { zzxz } & 0\end{array}$

$\begin{array}{lllllllll}\text { yzxx } & y z y y & y z z z & 0 & 0 & y z x y & 0 & 0 & \text { yzyx }\end{array}$

$\begin{array}{lllllllll}2 x z x & y z y y & y z z z & 0 & 0 & y z x y & 0 & 0 & y z y x \\ 0 & z x y y & z x z z & 0 & 0 & z x x y & 0 & 0 & z x y x\end{array}$

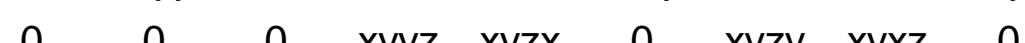

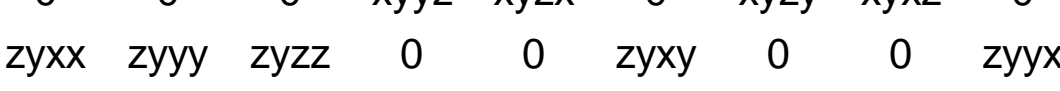

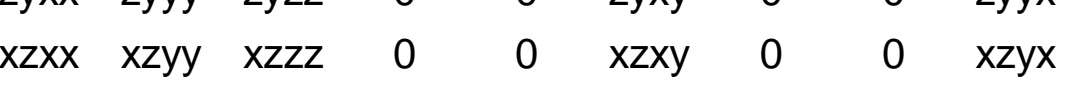

$\begin{array}{ccccccccc}0 & 0 & 0 & y x y z & y x z x & 0 & y x z y & y x x z & 0\end{array}$

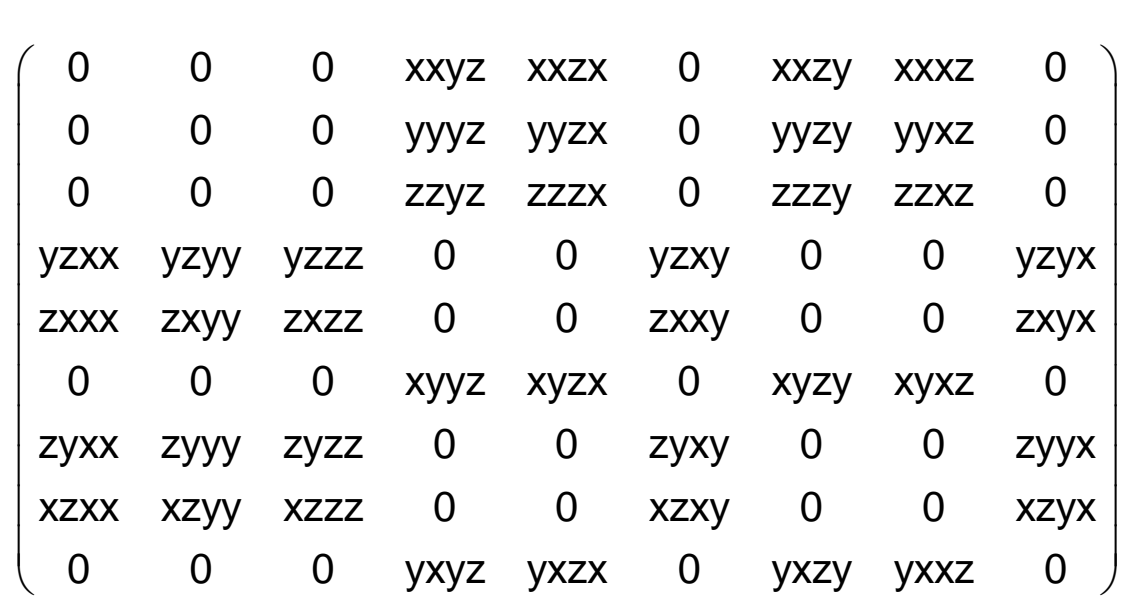

$\left(\begin{array}{ccccc}0 & 0 & x z z z & 0 & x x y z \\ 0 & 0 & y z z z & 0 & y y x z \\ z x x x & z y y y & 0 & 0 & 0\end{array}\right)$ $\left(\begin{array}{lllllllll}x x x x & x x y y & x x z z & 0 & 0 & x x x y & 0 & 0 & x x y x \\ y y x x & y y y & y y z & 0 & 0 & y y x y & 0 & 0 & y y x\end{array}\right.$ $\begin{array}{lllllllll}\text { yyxx } & \text { yyyy } & \text { yyzz } & 0 & 0 & \text { yyxy } & 0 & 0 & \text { yyyx } \\ \text { zzxx } & \text { zzyy } & \text { zzz } & 0 & 0 & z z x & 0 & 0 & z z y\end{array}$ $\begin{array}{llllllllll}z z x x & z z y y & z z z z & 0 & 0 & z z x y & 0 & 0 & z z y x\end{array}$ $\begin{array}{lllllllll}0 & 0 & 0 & y z y z & y z z x & 0 & y z z y & y z x z & 0\end{array}$ $\begin{array}{lllllllll}0 & 0 & 0 & z x y z & z x z x & 0 & z x z y & z x x z & 0\end{array}$

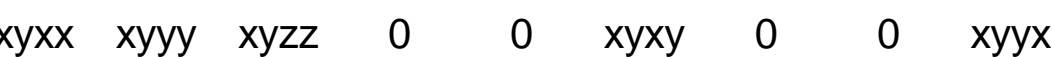
$\begin{array}{ccccccccc}0 & 0 & 0 & x z y z & x z z x & 0 & x z z y & x z x z & 0 \\ y x x x & y x y y & y x z z & 0 & 0 & y x x y & 0 & 0 & y x y x\end{array}$

$\left(\begin{array}{ccccc}x x x x & x y y y & 0 & y y z z & 0 \\ y x x x & y y y y & 0 & z z x x & 0 \\ 0 & 0 & z z z z & x x y y & z z x y\end{array}\right)$ $\begin{array}{lllllllll}0 & 0 & 0 & \text { zyyz } & z y z x & 0 & \text { zyzy } & \text { zyxz } & 0\end{array}$

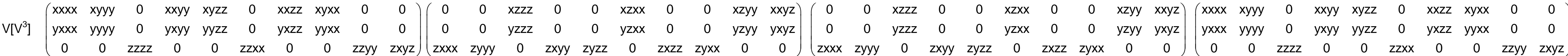

$\left[\left[^{2}\right]^{2}\right]\left(\begin{array}{cccccc}x x x x & x x y y & x x z z & 0 & 0 & x x x y \\ & y y y y & y y z z & 0 & 0 & y y x y \\ & & z z z z & 0 & 0 & z z x y \\ & & & y z y z & y z x z & 0 \\ & & & & x z x z & 0 \\ & & & & & x y x y\end{array}\right)$ $\left(\begin{array}{cccccc}x y x x x & x x y y & x y z z & 0 & 0 & x x y y \\ z z x x & z z y & y y z & 0 & 0 & y y x y\end{array}\right)$ zzxx zzyy zzzz $00 \quad 0 \quad$ zzxy $\begin{array}{cccccc}0 & 0 & 0 & y z y z & y z x z & 0 \\ 0 & 0 & 0 & x z y z & x z x z & 0\end{array}$ $\begin{array}{llllll}\text { xyxx } & \text { xyyy } & \text { xyzz } & 0 & 0 & x y x y\end{array}$

$$
\left(\begin{array}{cccccc}
0 & 0 & 0 & \text { xxyz } & \text { xxxz } & 0 \\
& 0 & 0 & \text { yyyz } & \text { yyxz } & 0 \\
& & 0 & z z y z & z z x z & 0 \\
& & & 0 & 0 & y z x y \\
& & & & 0 & x z x y \\
& & & & & 0
\end{array}\right)
$$

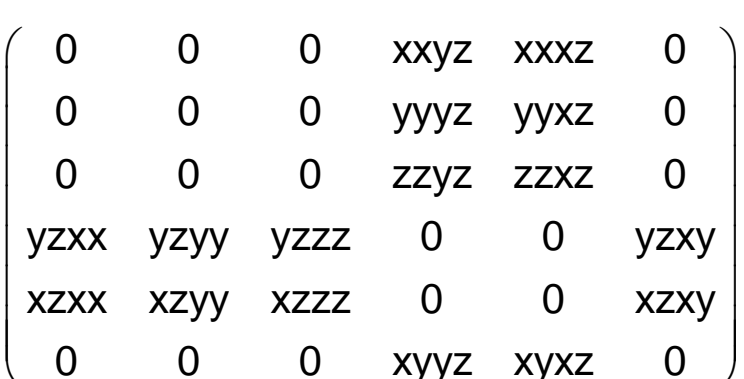

$$
\left(\begin{array}{cccccc}
0 & 0 & 0 & \text { xxyz } & \text { xxxz } & 0 \\
& 0 & 0 & \text { yyyz } & \text { yyxz } & 0 \\
& & 0 & \text { zzyz } & \text { zzxz } & 0 \\
& & & 0 & 0 & y z x y \\
& & & & 0 & x z x y \\
& & & & & 0
\end{array}\right)
$$

$\left(\begin{array}{cccccc}0 & 0 & 0 & x x y z & x x x z & 0 \\ 0 & 0 & 0 & \text { yyyz } & y y x z & 0 \\ 0 & 0 & 0 & \text { zzyz } & \text { zzxz } & 0 \\ \text { yzxx } & y z y y & y z z z & 0 & 0 & y z x y \\ \text { xzxx } & x z y y & x z z z & 0 & 0 & x z x y \\ 0 & 0 & 0 & x y y z & x y x z & 0\end{array}\right)$

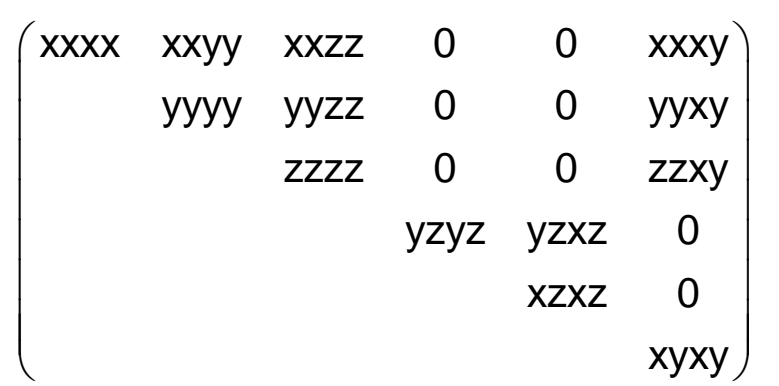
$\begin{array}{llllll}x y x x & y y y y & y y z z & 0 & 0 & y y x y \\ z z x x & z y y & z z z z & 0 & 0 & z z x y\end{array}$ $\begin{array}{llllll}z z x x & z z y y & z z z z & 0 & 0 & z z x y\end{array}$ $\begin{array}{llllll}0 & 0 & 0 & y z y z & y z x z & 0\end{array}$ $\begin{array}{llllll}0 & 0 & 0 & x z y z & x z x z & 0\end{array}$ $\left(\begin{array}{llllll}x x x x & x x y y & x x z z & 0 & 0 & x x x y\end{array}\right)$ 


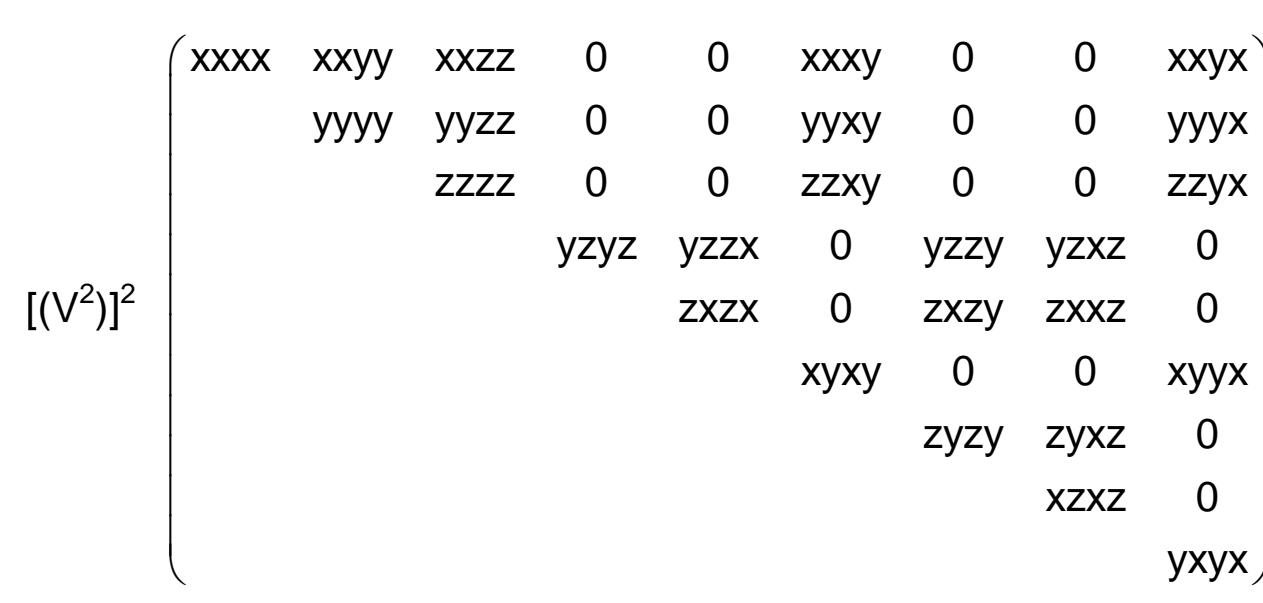

$\left(\begin{array}{lllllllll}x x x x & x x y y & x x z z & 0 & 0 & x x x y & 0 & 0 & x x y x \\ y y x & y y y & y z z & 0 & 0 & x y y & 0 & 0 & x y x\end{array}\right.$

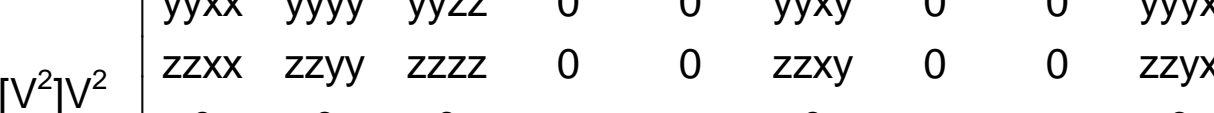
$\begin{array}{lllllllll}0 & 0 & 0 & y z y z & y z z x & 0 & y z z y & y z x z & 0\end{array}$ $\begin{array}{ccccccccc}0 & 0 & 0 & z x y z & z x z x & 0 & z x z y & z x x z & 0 \\ x y x x & x y y y & x y z z & 0 & 0 & x y x y & 0 & 0 & x y y x\end{array}$ $\begin{array}{lllllllll}0 & 0 & 0 & x x y z & x x z x & 0 & x x z y & x x x z & 0\end{array}$

$\begin{array}{llllllll}0 & 0 & y y y z & y y z x & 0 & \text { yyzy } & \text { yyxz } & 0\end{array}$

$\begin{array}{lllllll}0 & z z y z & z z z x & 0 & z z z y & z z x z & 0\end{array}$

$\begin{array}{llllll}0 & 0 & y z x y & 0 & 0 & y z y x\end{array}$

$\begin{array}{lllll}0 & z x x y & 0 & 0 & z x y x\end{array}$

$\begin{array}{llll}0 & \text { xyzy } & \mathrm{xyxz} & 0\end{array}$

$\begin{array}{lll}0 & \text { zyyx } \\ 0 & \mathrm{xzyx}\end{array}$

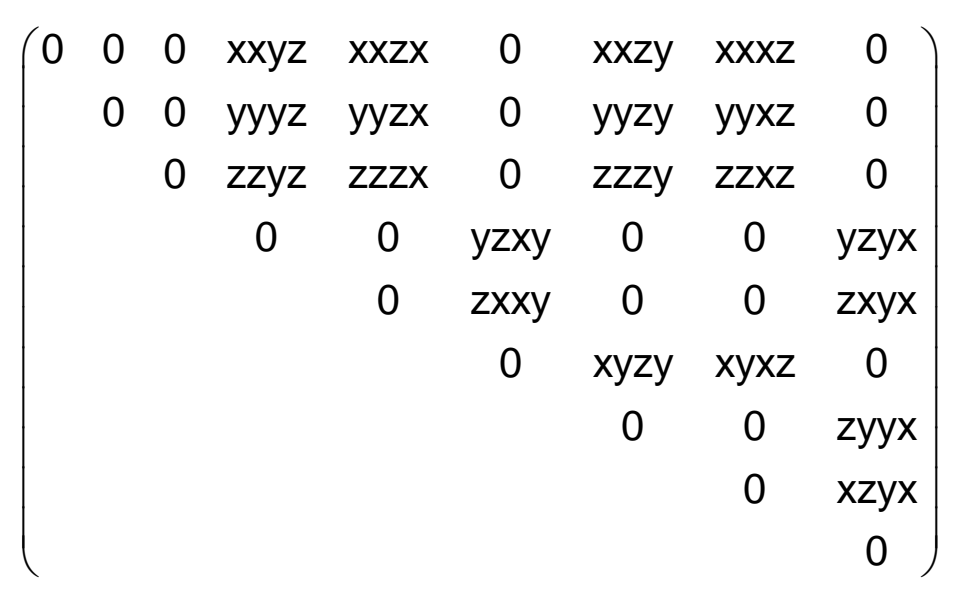

$\left(\begin{array}{ccccccccc}0 & 0 & 0 & x x y z & x x z x & 0 & x x z y & x x x z & 0 \\ 0 & 0 & 0 & y y y z & y y z x & 0 & y y z y & y y x z & 0 \\ 0 & 0 & 0 & z z y z & z z z x & 0 & \text { zzzy } & \text { zzxz } & 0 \\ y z x x & y z y y & y z z z & 0 & 0 & \text { yzxy } & 0 & 0 & \text { yzyx } \\ \text { zxxx } & \text { zxyy } & \text { zxzz } & 0 & 0 & \text { zxxy } & 0 & 0 & \text { zxyx } \\ 0 & 0 & 0 & \text { xyyz } & \text { xyzx } & 0 & \text { xyzy } & \text { xyxz } & 0\end{array}\right)$

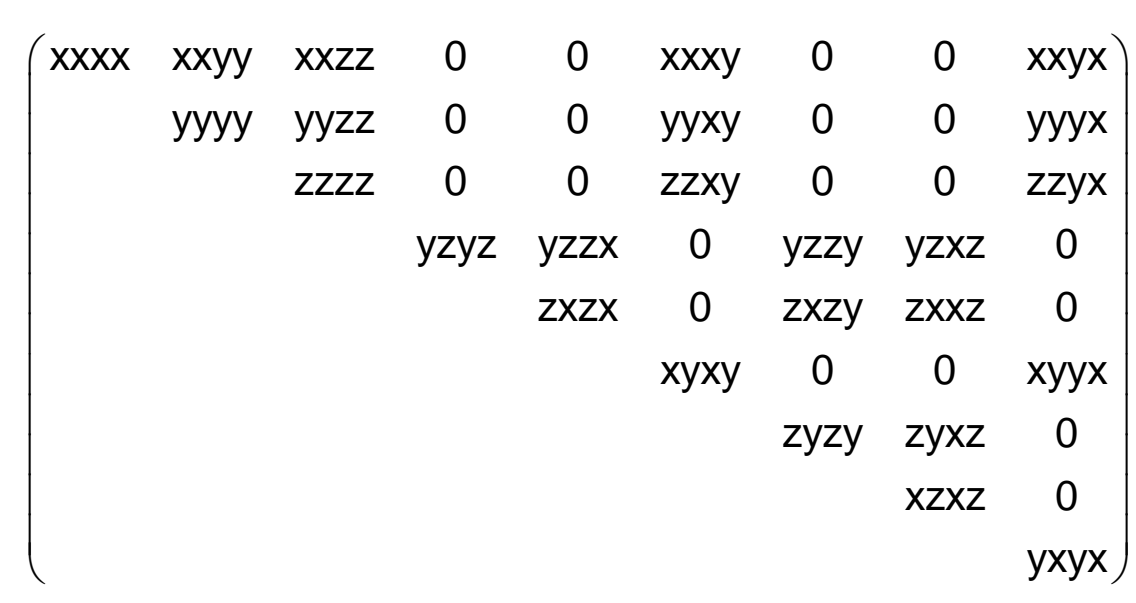

$\left(\begin{array}{lllllllll}x x x x & x x y y & x x z z & 0 & 0 & x x x y & 0 & 0 & x x y x\end{array}\right)$ $\begin{array}{lllllllll}\text { yyxx } & y y y y & y y z z & 0 & 0 & \text { yyxy } & 0 & 0 & \text { yyyx } \\ z z x & z y y & z z z & 0 & 0 & z z y & 0 & 0 & z z x\end{array}$ $\begin{array}{lllllllll}\text { zzxx } & \text { zzyy } & \text { zzzz } & 0 & 0 & \text { zzxy } & 0 & 0 & \text { zzyx } \\ 0 & 0 & 0 & y z y z & y z z x & 0 & y z y & y z x z & 0\end{array}$ $\begin{array}{lllllllll}0 & 0 & 0 & y z y z & y z z x & 0 & y z z y & y z x z & 0\end{array}$ $\begin{array}{ccccccccc}0 & 0 & 0 & z x y z & z x z x & 0 & z x z y & z x x z & 0 \\ x y x x & x y y y & x y z z & 0 & 0 & x y x y & 0 & 0 & x y y x\end{array}$ 
$\left(\begin{array}{lllllllll}x x x x & x x y y & x x z z & 0 & 0 & x x x y & 0 & 0 & x x y x \\ y x x & y y y & y y z z & 0 & 0 & y y y & 0 & 0 & y y x\end{array}\right)$ $\left.\begin{array}{lllllllll}\text { yyxx } & \text { yyyy } & \text { yyzz } & 0 & 0 & \text { yyxy } & 0 & 0 & \text { yyyx } \\ z z x x & z z y y & z z z z & 0 & 0 & z z x y & 0 & 0 & z z y x \\ 0 & 0 & 0 & y z y z & y z z x & 0 & y z z y & y z x z & 0\end{array}\right)$

$\mathrm{v}^{4} \quad \begin{array}{ccccccccc}0 & 0 & 0 & \mathrm{yzyz} & \mathrm{yzzx} & 0 & \mathrm{yzzy} & \mathrm{yzxz} & 0 \\ 0 & 0 & 0 & \mathrm{zxyz} & \mathrm{zxzx} & 0 & \mathrm{zxzy} & \mathrm{zxxz} & 0\end{array}$

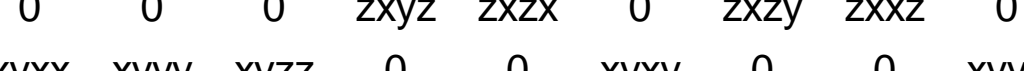

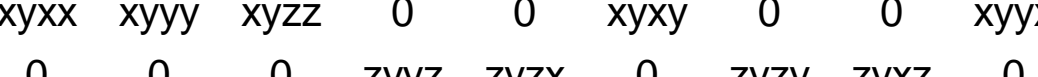

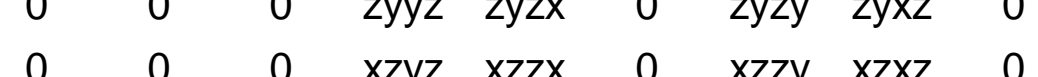
$\begin{array}{ccccccccc}0 & 0 & 0 & x z y z & x z z x & 0 & x z z y & x z x z & 0 \\ y x x x & y x y y & y x z z & 0 & 0 & y x x y & 0 & 0 & y x y x\end{array}$

$\left[V^{4}\right] \quad\left(\begin{array}{ccccc}x x x x & x y y y & 0 & y y z z & 0 \\ y x x x & y y y y & 0 & z z x x & 0 \\ 0 & 0 & z z z z & x x y y & z z x y\end{array}\right)$

$\begin{array}{lllllllll}0 & 0 & 0 & 0 & 0 & 0 & 0 & 0 & 0 \\ 0 & 0 & 0 & 0 & 0 & 0 & 0 & 0 & 0\end{array}$ $\begin{array}{lllllllll}0 & 0 & 0 & 0 & 0 & 0 & 0 & 0 & 0 \\ 0 & 0 & 0 & 0 & 0 & 0 & 0 & 0 & 0\end{array}$

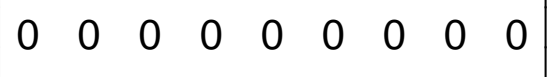

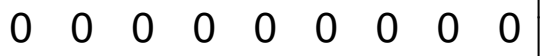
$0 \begin{array}{lllllllll}0 & 0 & 0 & 0 & 0 & 0 & 0 & 0 & 0 \\ 0 & 0 & 0 & 0 & 0 & 0 & 0 & 0 & 0\end{array}$ $\begin{array}{lllllllll}0 & 0 & 0 & 0 & 0 & 0 & 0 & 0 & 0 \\ 0 & 0 & 0 & 0 & 0 & 0 & 0 & 0 & 0\end{array}$ $\begin{array}{lllllllllll}0 & 0 & 0 & 0 & 0 & 0 & 0 & 0 & 0\end{array}$ $\begin{array}{lllllllllll}0 & 0 & 0 & 0 & 0 & 0 & 0 & 0 & 0 \\ 0 & 0 & 0 & 0 & 0 & 0 & 0 & 0 & 0\end{array}$ $\left(\begin{array}{lllllllll}0 & 0 & 0 & 0 & 0 & 0 & 0 & 0 & 0 \\ 0 & 0 & 0 & 0 & 0 & 0 & 0 & 0 & 0\end{array}\right)$

$$
\left(\begin{array}{lllll}
0 & 0 & 0 & 0 & 0 \\
0 & 0 & 0 & 0 & 0 \\
0 & 0 & 0 & 0 & 0
\end{array}\right)
$$

$\left(\begin{array}{llllllllll}x x x x & x y y y & 0 & x x y y & x y z z & 0 & x x z z & x y x x & 0 & 0 \\ y x x & y & 0 & y x y & y y z & 0 & y x z z & y x x & 0 & 0\end{array}\right)\left(\begin{array}{cccccccccc}0 & 0 & 0 & 0 & 0 & 0 & 0 & 0 & 0 & 0 \\ 0 & 0 & 0 & 0 & 0 & 0 & 0 & 0 & 0 & 0\end{array}\right)$

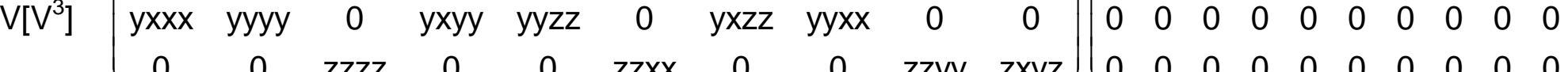

$\left.\left[\mathrm{V}^{2}\right]^{2}\right]\left(\begin{array}{cccccc}\mathrm{xxxx} & \mathrm{xxyy} & \mathrm{xxzz} & 0 & 0 & \mathrm{xxxy} \\ & \mathrm{yyyy} & \mathrm{yyzz} & 0 & 0 & \mathrm{yyxy} \\ & & \mathrm{zzzz} & 0 & 0 & \mathrm{zzxy} \\ & & & \mathrm{yzyz} & \mathrm{yzxz} & 0 \\ & & & & \mathrm{xzxz} & 0 \\ & & & & & \mathrm{xyxy}\end{array}\right)$

$\left(\begin{array}{llllll}x x x x & x x y y & x x z z & 0 & 0 & x x x y \\ y y x x & y y y y & y y z z & 0 & 0 & y y x y \\ z z x x & z z y y & z z z & 0 & 0 & z z x y\end{array}\right)$

$\begin{array}{llllll}\text { zzxx } & \text { zzyy } & \text { zzzz } & 0 & 0 & \text { zzxy } \\ 0 & 0 & 0 & y z y & y z z z & 0\end{array}$ $\begin{array}{llllll}0 & 0 & 0 & y z y z & y z x z & 0 \\ 0 & 0 & 0 & x z y z & x z z & 0\end{array}$

$\begin{array}{lllccc}\text { xyxx } & x y y y & x y z z & 0 & 0 & x y x y\end{array}$

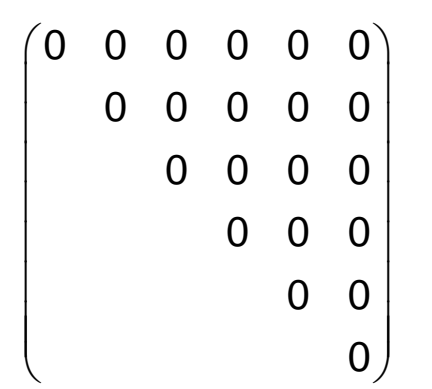

$\left(\begin{array}{llllll}0 & 0 & 0 & 0 & 0 & 0 \\ 0 & 0 & 0 & 0 & 0 & 0\end{array}\right.$

$\begin{array}{llllll}0 & 0 & 0 & 0 & 0 & 0 \\ 0 & 0 & 0 & 0 & 0 & 0\end{array}$

$\begin{array}{llllll}0 & 0 & 0 & 0 & 0 & 0 \\ 0 & 0 & 0 & 0 & 0 & 0\end{array}$

$\begin{array}{llllll}0 & 0 & 0 & 0 & 0 & 0 \\ 0 & 0 & 0 & 0 & 0 & 0\end{array}$

$\begin{array}{llllll}0 & 0 & 0 & 0 & 0 & 0 \\ 0 & 0 & 0 & 0 & 0 & 0\end{array}$ $\begin{array}{lllllllll}0 & 0 & 0 & 0 & 0 & 0 & 0 & 0 & 0 \\ 0 & 0 & 0 & 0 & 0 & 0 & 0 & 0 & 0\end{array}$ $\begin{array}{lllllllll}0 & 0 & 0 & 0 & 0 & 0 & 0 & 0 & 0 \\ 0 & 0 & 0 & 0 & 0 & 0 & 0 & 0 & 0\end{array}$ $\begin{array}{llllllllll}0 & 0 & 0 & 0 & 0 & 0 & 0 & 0 & 0 \\ 0 & 0 & 0 & 0 & 0 & 0 & 0 & 0 & 0 \\ 0 & 0 & 0 & 0 & 0 & 0 & 0 & 0 & 0\end{array}$ $\begin{array}{lllllllll}0 & 0 & 0 & 0 & 0 & 0 & 0 & 0 & 0 \\ 0 & 0 & 0 & 0 & 0 & 0 & 0 & 0 & 0\end{array}$ $\begin{array}{llllllllll}0 & 0 & 0 & 0 & 0 & 0 & 0 & 0 & 0 \\ 0 & 0 & 0 & 0 & 0 & 0 & 0 & 0 & 0\end{array}$ $\begin{array}{llllllllll}0 & 0 & 0 & 0 & 0 & 0 & 0 & 0 & 0\end{array}$ $0 \begin{array}{llllllllll}0 & 0 & 0 & 0 & 0 & 0 & 0 & 0 & 0 \\ 0 & 0 & 0 & 0 & 0 & 0 & 0 & 0\end{array}$ $\begin{array}{llllllllll}0 & 0 & 0 & 0 & 0 & 0 & 0 & 0 & 0\end{array}$

$\left(\begin{array}{lllll}0 & 0 & 0 & 0 & 0 \\ 0 & 0 & 0 & 0 & 0 \\ 0 & 0 & 0 & 0 & 0\end{array}\right)$

$\left.\begin{array}{llllllllll}0 & 0 & 0 & 0 & 0 & 0 & 0 & 0 & 0 & 0 \\ 0 & 0 & 0 & 0 & 0 & 0 & 0 & 0 & 0 & 0\end{array}\right)$ $\left(\begin{array}{llllllllll}0 & 0 & 0 & 0 & 0 & 0 & 0 & 0 & 0 & 0 \\ 0 & 0 & 0 & 0 & 0 & 0 & 0 & 0 & 0 & 0\end{array}\right)$ $\begin{array}{lllllllllll}0 & 0 & 0 & 0 & 0 & 0 & 0 & 0 & 0 & 0\end{array}$

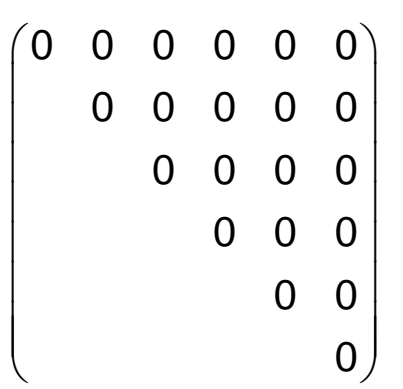

$\left(\begin{array}{llllll}0 & 0 & 0 & 0 & 0 & 0 \\ 0 & 0 & 0 & 0 & 0 & 0 \\ 0 & 0 & 0 & 0 & 0 & 0\end{array}\right)$ $\left(\begin{array}{llllll}0 & 0 & 0 & 0 & 0 & 0 \\ 0 & 0 & 0 & 0 & 0 & 0 \\ 0 & 0 & 0 & 0 & 0 & 0 \\ 0 & 0 & 0 & 0 & 0 & 0\end{array}\right)$ $0 \begin{array}{llllll}0 & 0 & 0 & 0 & 0 \\ 0 & 0 & 0 & 0 & 0 & 0\end{array}$ $\begin{array}{llllll}0 & 0 & 0 & 0 & 0 & 0\end{array}$ $\begin{array}{llllll}0 & 0 & 0 & 0 & 0 & 0 \\ 0 & 0 & 0 & 0 & 0 & 0\end{array}$ $\begin{array}{lllllllll}x x x x & x x y y & x x z z & 0 & 0 & x x x y & 0 & 0 & x x y x \\ y x x & y y y & y y z & 0 & 0 & y x y & 0 & 0 & y y x\end{array}$

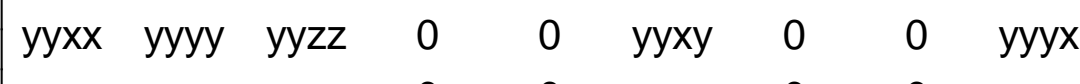
$\begin{array}{ccccccccc}z z x x & z z y y & z z z z & 0 & 0 & z z x y & 0 & 0 & z z y x\end{array}$ $\begin{array}{lllllllll}0 & 0 & 0 & y z y z & y z z x & 0 & y z z y & y z x z & 0\end{array}$ $\begin{array}{llllllllll}0 & 0 & 0 & z x y z & z x z x & 0 & z x z y & z x x z & 0\end{array}$

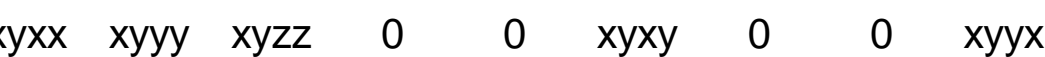
$\begin{array}{lllllllll}0 & 0 & 0 & \text { zyyz } & z y z x & 0 & \text { zyzy } & \text { zyxz } & 0\end{array}$ $\begin{array}{ccccccccc}0 & 0 & 0 & x z y z & x z z x & 0 & x z z y & x z x z & 0 \\ y x x x & y x y y & y x z z & 0 & 0 & y x x y & 0 & 0 & y x y x\end{array}$

$\left(\begin{array}{ccccc}x x x x & x y y y & 0 & y y z z & 0 \\ y x x x & y y y y & 0 & z z x x & 0 \\ 0 & 0 & z z z & x x y y & z z x y\end{array}\right)$

$\left(\begin{array}{ccccc}\operatorname{yxxx} & y y y y & 0 & z z x x & 0 \\ 0 & 0 & z z z z & x x y y & z z x y\end{array}\right)$

$\left(\begin{array}{llllllllll}x x x x & x y y y & 0 & x x y y & x y z z & 0 & x x z z & x y x x & 0 & 0 \\ x x x x & y y y y & 0 & y x y y & y y z z & 0 & y x z z & y y x x & 0 & 0 \\ 0 & 0 & z z z z & 0 & 0 & z z x & 0 & 0 & z z y y & z x y z\end{array}\right)$

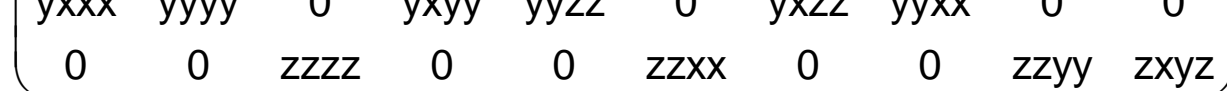

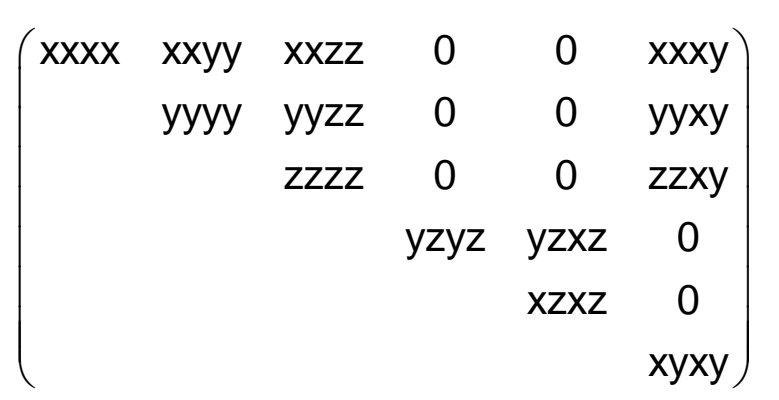

$\left(\begin{array}{llllll}x x x x & x x y y & x x z z & 0 & 0 & x x x y \\ y x x & y y y & y y z z & 0 & 0 & y y x y\end{array}\right)$ $\begin{array}{llllll}\text { yyxx } & \text { yyyy } & y y z z & 0 & 0 & \text { yyxy } \\ z z x x & z z y & z z z z & 0 & 0 & z z x y\end{array}$ $\begin{array}{llllll}z z x x & z z y y & z z z z & 0 & 0 & z z x y\end{array}$ $\begin{array}{llllll}0 & 0 & 0 & y z y z & y z x z & 0\end{array}$ $\begin{array}{llllll}0 & 0 & 0 & x z y z & x z x z & 0\end{array}$ $\begin{array}{llllll}\text { xyxx } & x y y y & x y z z & 0 & 0 & x y x y\end{array}$ 


$$
1
$$

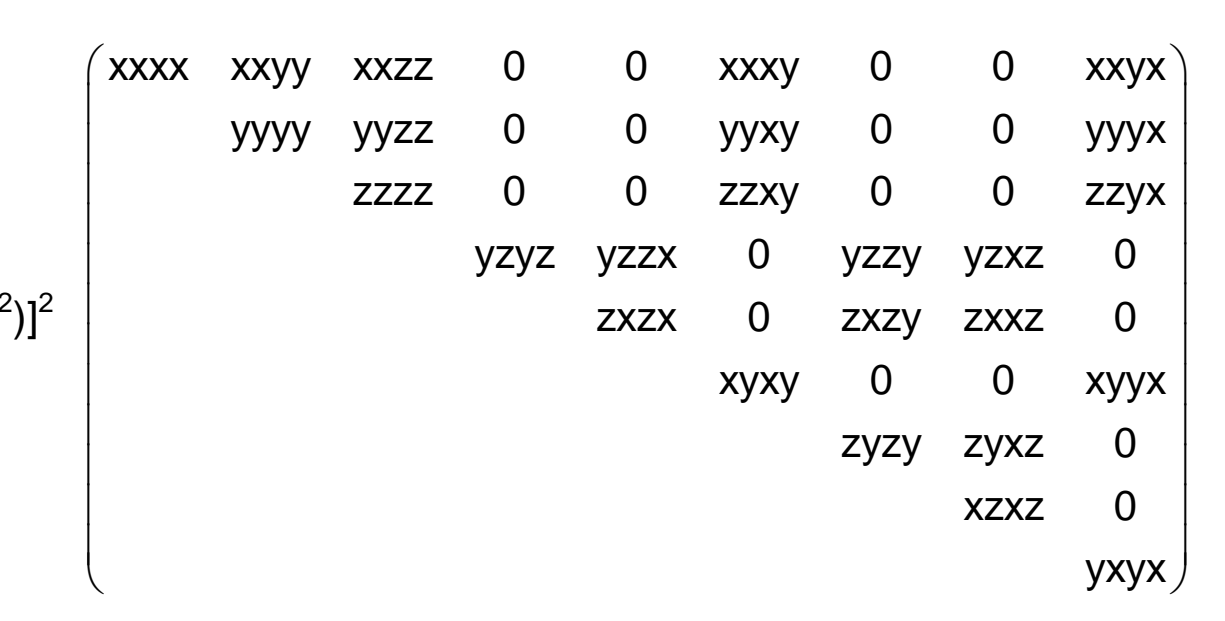

$\left(\begin{array}{lllllllll}0 & 0 & 0 & 0 & 0 & 0 & 0 & 0 & 0\end{array}\right)$

$\begin{array}{llllllll}0 & 0 & 0 & 0 & 0 & 0 & 0 & 0\end{array}$ $\begin{array}{lllllll}0 & 0 & 0 & 0 & 0 & 0 & 0\end{array}$ $\begin{array}{llllll}0 & 0 & 0 & 0 & 0 & 0\end{array}$ $\begin{array}{lllll}0 & 0 & 0 & 0 & 0\end{array}$ $\begin{array}{llll}0 & 0 & 0 & 0\end{array}$ $0 \begin{array}{lll}0 & 0 & 0 \\ 0 & 0 & 0\end{array}$

$\left(\begin{array}{lllllllll}x x x x & x x y y & x x z z & 0 & 0 & x x x y & 0 & 0 & x x y x \\ y x x & y w y & y y z z & 0 & 0 & y x y & 0 & 0 & x y x\end{array}\right)$

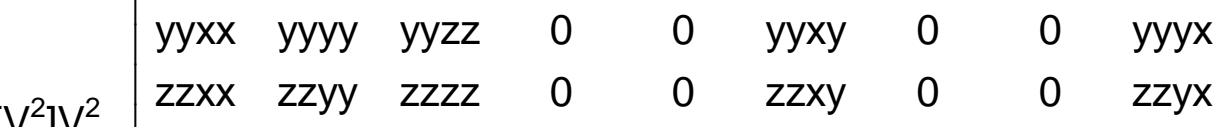
$\begin{array}{ccccccccc}\text { zzxx } & z z y y & z z z z & 0 & 0 & z z x y & 0 & 0 & z z y x\end{array}$ $\begin{array}{lllllllll}0 & 0 & 0 & y z y z & y z z x & 0 & y z z y & y z x z & 0\end{array}$ $\begin{array}{ccccccccc}0 & 0 & 0 & z x y z & z x z x & 0 & z x z y & z x x z & 0 \\ x y x x & x y y y & x y z z & 0 & 0 & x y x y & 0 & 0 & x y y x\end{array}$

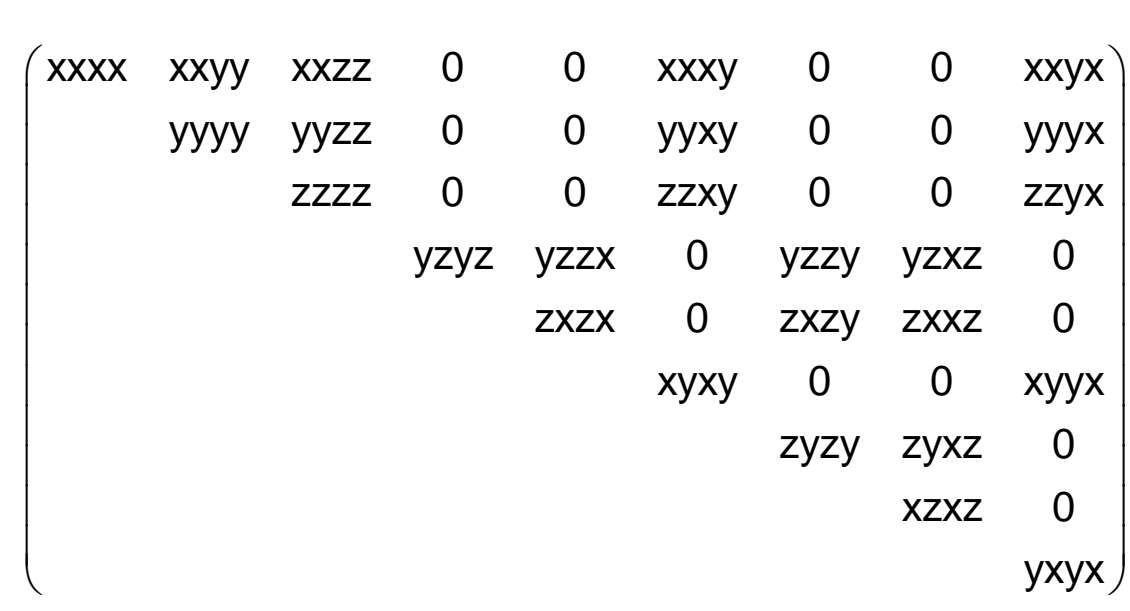

$\left(\begin{array}{lllllllll}0 & 0 & 0 & 0 & 0 & 0 & 0 & 0 & 0 \\ 0 & 0 & 0 & 0 & 0 & 0 & 0 & 0 & 0\end{array}\right.$

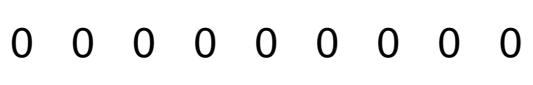

$0 \begin{array}{lllllllll}0 & 0 & 0 & 0 & 0 & 0 & 0 & 0 & 0 \\ 0 & 0 & 0 & 0 & 0 & 0 & 0 & 0 & 0\end{array}$

$\begin{array}{llllllllll}0 & 0 & 0 & 0 & 0 & 0 & 0 & 0 & 0 \\ 0 & 0 & 0 & 0 & 0 & 0 & 0 & 0 & 0\end{array}$

$\begin{array}{lllllllll}0 & 0 & 0 & 0 & 0 & 0 & 0 & 0 & 0 \\ 0 & 0 & 0 & 0 & 0 & 0 & 0 & 0 & 0\end{array}$

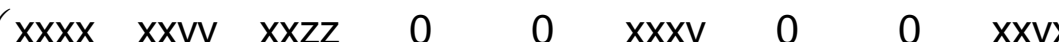
$\left.\begin{array}{lllllllll}\text { yyxx } & \text { yyyy } & \text { yyzz } & 0 & 0 & \text { yyxy } & 0 & 0 & \text { yyyx } \\ z z x x & z y y & z z z & 0 & 0 & z z y & 0 & 0 & z z x\end{array}\right)$ $\begin{array}{lllllllll}\text { zzxx } & \text { zzyy } & \text { zzzz } & 0 & 0 & \text { zzxy } & 0 & 0 & \text { zzyx }\end{array}$ $\begin{array}{lllllllll}0 & 0 & 0 & y z y z & y z z x & 0 & y z z y & y z x z & 0\end{array}$ $\begin{array}{ccccccccc}0 & 0 & 0 & z x y z & z x z x & 0 & z x z y & z x x z & 0 \\ x y y x & x y y y & x y z z & 0 & 0 & x y x y & 0 & 0 & x y y x\end{array}$ 
$\left.\mathrm{V}^{3} \mathrm{~V}^{3}\right]\left(\begin{array}{cccccccccc}3 x x y y & 3 x y x x & 0 & x x y y & x y z z & 0 & y y z z & x y x x & 0 & y y x x \\ -3 x y x x & 3 x x y y & 0 & -x y x & y y z & 0 & -x y z z & x x y y & 0 & 0 \\ 0 & 0 & z z z z & 0 & 0 & z z x x & 0 & 0 & z z x & z x y z\end{array}\right)$ $\left(\begin{array}{cccccccccc}-3 x y y x & 3 x x y y & 0 & -x y x x & y y z z & 0 & -x y z z & x x y y & 0 & 0 \\ 0 & 0 & z z z z & 0 & 0 & z z x x & 0 & 0 & z z x x & z x y z\end{array}\right)$
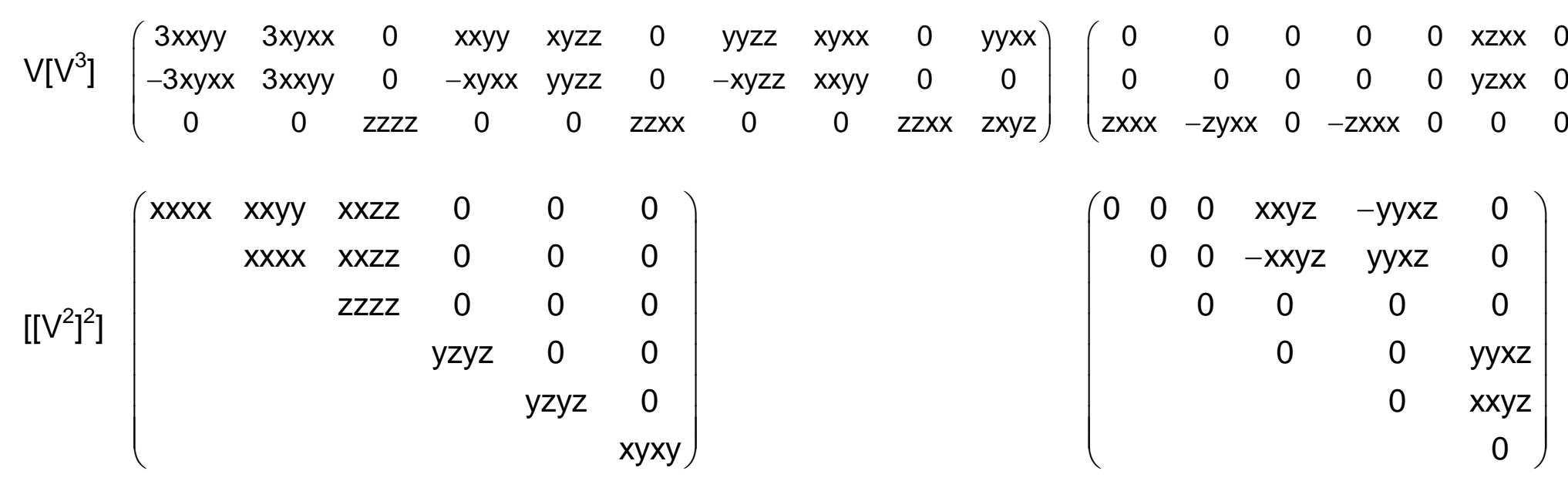

and $x y x y=1 / 2(x x x x-x x y y)$

$\left[V^{2}\right]^{2}\left(\begin{array}{cccccc}x x x x & x x y y & x x z z & 0 & 0 & x x x y \\ x x y y & x x x x & x x z z & 0 & 0 & -x x x y \\ z z x x & z z x x & z z z z & 0 & 0 & 0 \\ 0 & 0 & 0 & y z y z & y z x z & 0 \\ 0 & 0 & 0 & y z x z & y z y z & 0 \\ -x x x y & x x x y & 0 & 0 & 0 & x y x y\end{array}\right)$

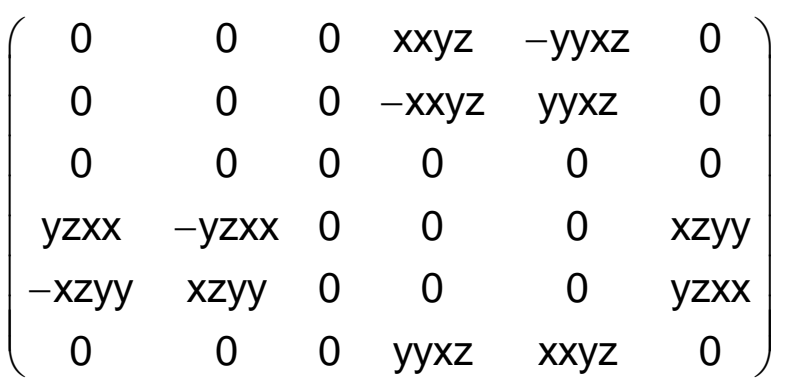

and $x y x y=1 / 2(x x x x-x x y y)$

$\left(\begin{array}{ccccccccc}0 & 0 & 0 & 0 & \text { xxzx } & 0 & \text { xxzy } & \text { xxxz } & 0 \\ 0 & 0 & 0 & 0 & -x x z x & 0 & -x x z y & -x x x z & 0 \\ 0 & 0 & 0 & 0 & 0 & 0 & 0 & 0 & 0 \\ 0 & 0 & 0 & 0 & 0 & -x z x x & 0 & 0 & -x z x x \\ z x x x & -z x x x & 0 & 0 & 0 & z y x x & 0 & 0 & z y x x \\ 0 & 0 & 0 & -x x x z & x x z y & 0 & -x x z x & x x y z & 0 \\ z y x x & -z y x x & 0 & 0 & 0 & -z x x x & 0 & 0 & -z x x x \\ \text { xzxx } & -x z x x & 0 & 0 & 0 & y z x x & 0 & 0 & y z x x \\ 0 & 0 & 0 & -x x x z & x x z y & 0 & -x x z x & x x y z & 0\end{array}\right)$

$\left(\begin{array}{lllllllll}x x x x & x x y y & x x z z & 0 & 0 & x x x y & 0 & 0 & -y y x y \\ \text { yxx } & x x x y & y y z z & 0 & 0 & y y x & 0 & 0 & -x x y\end{array}\right)$ $\begin{array}{ccccccccc}\mathrm{yyxx} & \mathrm{xxxx} & \mathrm{yyzz} & 0 & 0 & \mathrm{yyxy} & 0 & 0 & -\mathrm{xxxy} \\ \mathrm{zzxx} & \mathrm{zzxx} & \mathrm{zzzz} & 0 & 0 & \mathrm{zzxy} & 0 & 0 & -\mathrm{zzxy} \\ 0 & 0 & 0 & y z y z & y z z x & 0 & \mathrm{yzzy} & \mathrm{yzxz} & 0\end{array}$ $\begin{array}{lllllllll}0 & 0 & 0 & y z y z & y z z x & 0 & y z z y & y z x z & 0\end{array}$ $\begin{array}{lllllllll}0 & 0 & 0 & z x y z & z x z x & 0 & z x z y & z x x z & 0\end{array}$ $\begin{array}{ccccccccc}x y x x & x y y y & x y z z & 0 & 0 & x y x y & 0 & 0 & x y y x \\ 0 & 0 & 0 & z x y z & -z x z y & 0 & z x z x & -z x y z & 0\end{array}$ $\begin{array}{ccccccccc}0 & 0 & 0 & -y z x z & y z z y & 0 & -y z z x & y z y z & 0 \\ -x y y y & -x y x x & -x y z z & 0 & 0 & x y y & 0 & 0 & x y x\end{array}$ and: $x x x x=x x y y+x y x y+x y y x ; x x x y-y y x y=x y y y-x y x x$

$\left(\begin{array}{ccccc}0 & 0 & 0 & 0 & x x y z \\ 0 & 0 & 0 & 0 & -z x x x \\ z x x x & -x x y z & 0 & 0 & 0\end{array}\right)$

$\left(\begin{array}{cccccccccc}0 & 0 & 0 & 0 & 0 & x z x x & 0 & 0 & -x z x x & y z x x \\ 0 & 0 & 0 & 0 & 0 & y z x & 0 & 0 & -y z x & -x z x\end{array}\right)$ $\left(\begin{array}{cccccccccc}0 & 0 & 0 & 0 & 0 & y z x x & 0 & 0 & -y z x x & -x z x x \\ 2 z x y x & -2 y x x & 0 & -z x y x & 0 & 0 & 0 & z y x & 0 & 0\end{array}\right)$

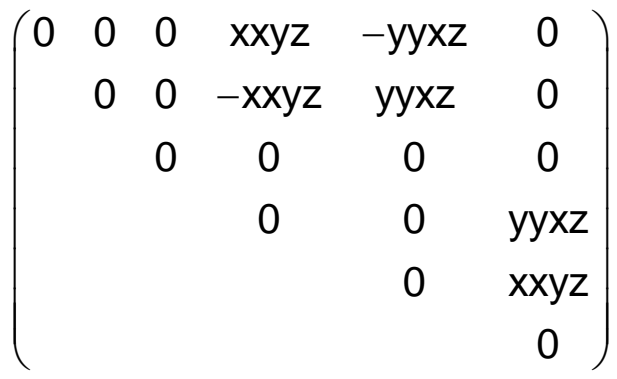

$\left(\begin{array}{cccccc}0 & 0 & 0 & x x y z & -y y x z & 0 \\ 0 & 0 & 0 & -x x y z & y y x z & 0 \\ 0 & 0 & 0 & 0 & 0 & 0 \\ y z x x & -y z x x & 0 & 0 & 0 & x z y y \\ -x z y y & x z y y & 0 & 0 & 0 & y z x x \\ 0 & 0 & 0 & y y x z & x x y z & 0\end{array}\right)$

$\left(\begin{array}{ccccc}3 x x y y & 0 & 0 & y y z z & 0 \\ 0 & 3 x x y & 0 & y y z & 0\end{array}\right)$

$\left(\begin{array}{ccccc}0 & 3 x x y y & 0 & y y z z & 0 \\ 0 & 0 & z z z z & x x y y & 0\end{array}\right)$

$\left(\begin{array}{cccccccccc}3 x x y y & 3 x y x x & 0 & x x y y & x y z z & 0 & y y z z & x y x x & 0 & y y x x \\ 3 x y y x & 3 x x y y & 0 & -x y y x & y y z z & 0 & -x y z z & x x y y & 0 & 0 \\ 0 & 0 & 2 z z & 0 & 0 & z z x & 0 & 0 & z z x & z x y z\end{array}\right)$ $\begin{array}{llllllllll}0 & 0 & z z z z & 0 & 0 & z z x x & 0 & 0 & z z x x & z x y z\end{array}$

$$
\left(\begin{array}{cccccc}
x x x x & x x y y & x x z z & 0 & 0 & 0 \\
& \text { xxxx } & \text { xxzz } & 0 & 0 & 0 \\
& & \text { zzzz } & 0 & 0 & 0 \\
& & & y z y z & 0 & 0 \\
& & & & y z y z & 0 \\
& & & & & \text { xyxy }
\end{array}\right)
$$

and $x y x y=1 / 2(x x x x-x x y y)$

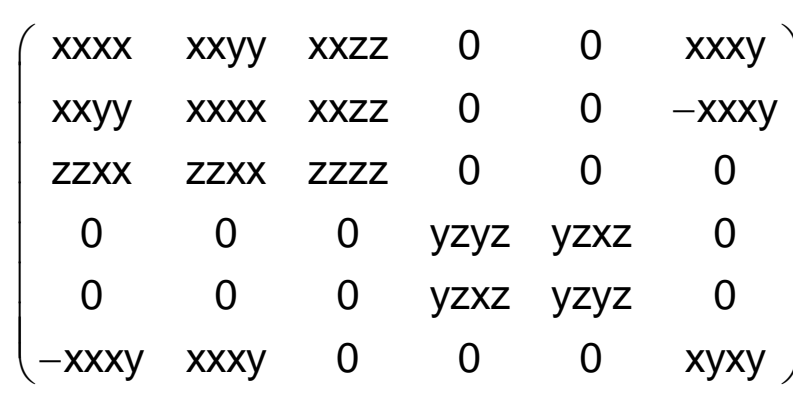

and $x y x y=1 / 2(x x x x-x x y y)$ 


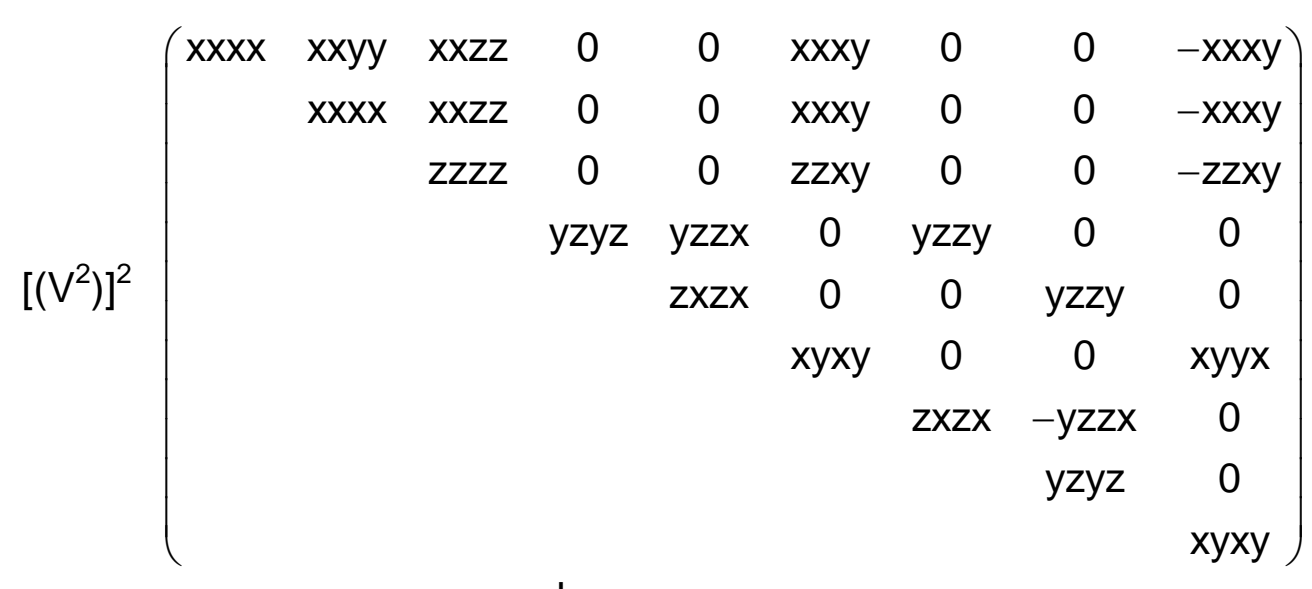

and $x x x x=x x y y+y z y z+z y z y$ e

$\left(\begin{array}{ccccccccc}0 & 0 & 0 & x x y z & x x z x & 0 & x x z y & x x x z & 0 \\ & 0 & 0 & -x x y z & -x x z x & 0 & -x x z y & -x x y z & 0\end{array}\right)$

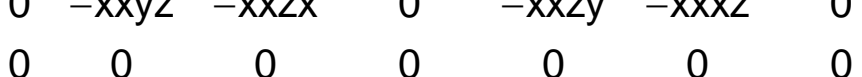

$\begin{array}{ccccc}0 & -x x x z & 0 & 0 & -x x x z \\ 0 & x x z y & 0 & 0 & x x z y \\ & 0 & -x x z x & x x y z & 0\end{array}$

$\begin{array}{cccc}0 & -x x z x & x x y z & 0 \\ 0 & 0 & -x x z x\end{array}$

$\begin{array}{ccc}0 & 0 & -x x z x \\ & 0 & x x y z\end{array}$

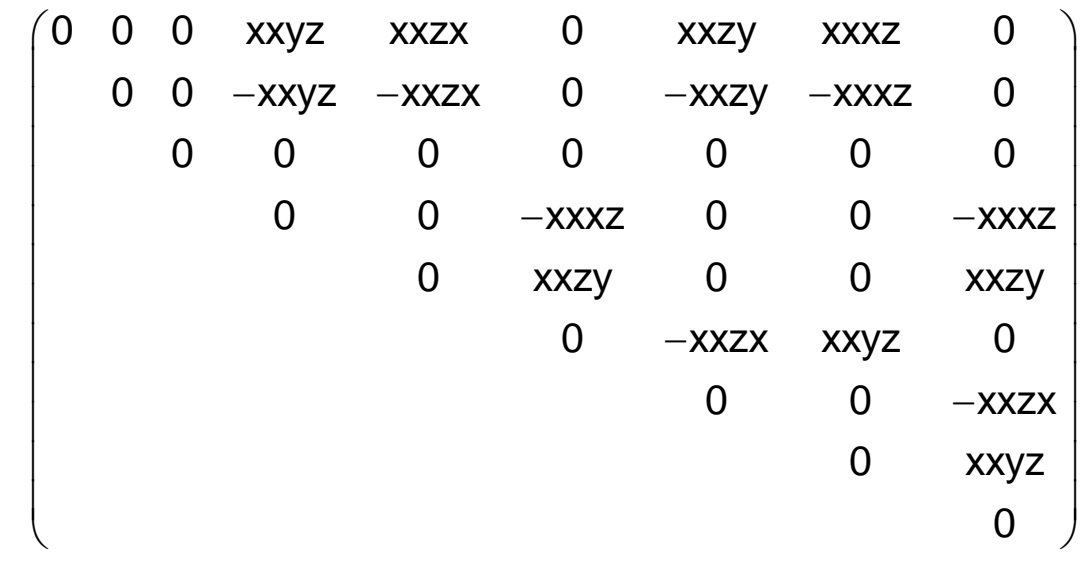

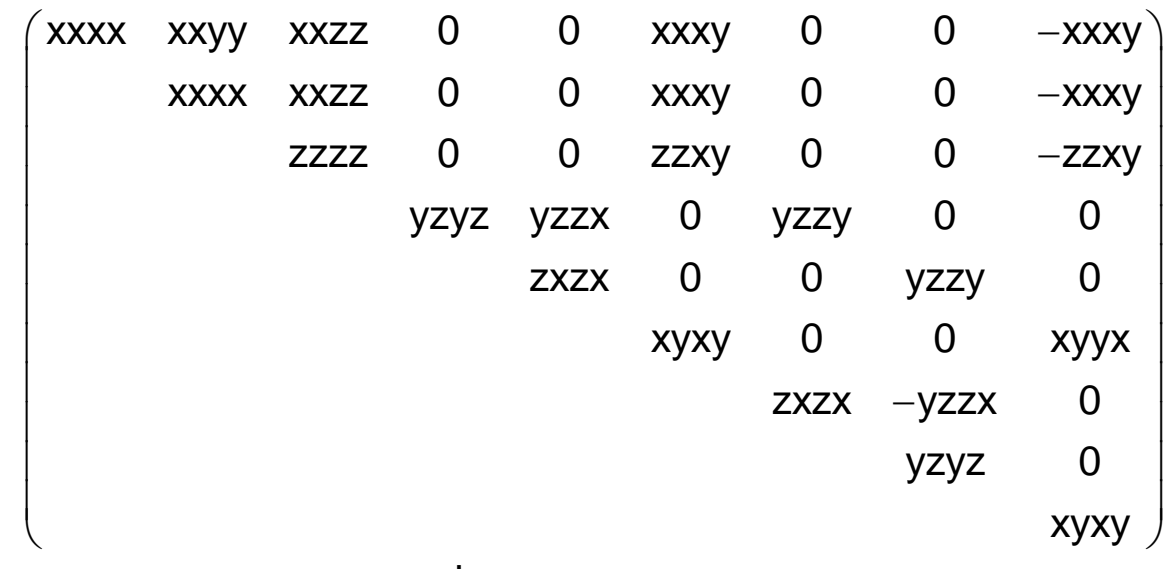

and $x x x x=x x y y+y z y z+z y z y$

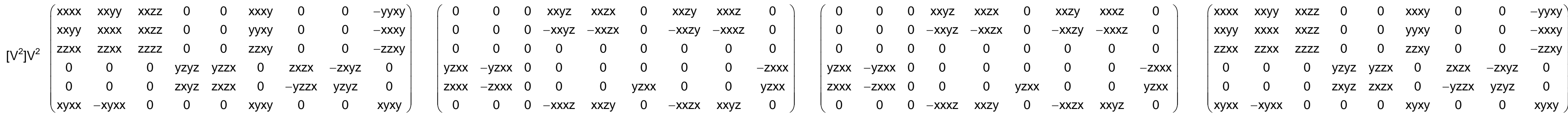
and $x x x x=x x y y+2 x y x y ; y y x y-x x x y=2 x y x x$ and $x x x x=x x y y+2 x y x y ; y y x y-x x x y=2 x y x x$ 
$\left(\begin{array}{ccccccccc}x x x x & x x y y & x x z z & 0 & 0 & x x x y & 0 & 0 & -y y x y \\ y y x x & x x x x & x x z z & 0 & 0 & y y x y & 0 & 0 & -x x x y \\ z z x x & z z y y & z z z z & 0 & 0 & z z x y & 0 & 0 & -z z x y \\ 0 & 0 & 0 & y z y z & y z z x & 0 & y z z y & y z x z & 0 \\ 0 & 0 & 0 & z x y z & z x z x & 0 & z x z y & z x x z & 0 \\ x y x x & x y y y & x y z z & 0 & 0 & x y x y & 0 & 0 & x y y x \\ 0 & 0 & 0 & z x x z & -z x z y & 0 & z x z x & -z x y z & 0 \\ 0 & 0 & 0 & -y z x z & y z z y & 0 & -y z z x & y z y z & 0 \\ -x y y y & -x y x x & -x y z z & 0 & 0 & x y y x & 0 & 0 & x y x y\end{array}\right)$

$\begin{array}{lllllllll}0 & 0 & 0 & 0 & 0 & 0 & 0 & 0 & 0 \\ 0 & 0 & 0 & 0 & 0 & 0 & 0 & 0 & 0\end{array}$

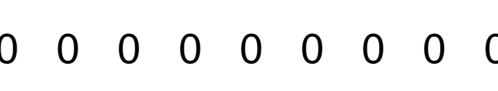

$\left[V^{4}\right]\left(\begin{array}{ccccc}x x x x & -y x x x & 0 & y y z z & 0 \\ y x x x & x x x x & 0 & y y z z & 0 \\ 0 & 0 & z z z z & x x y y & 0\end{array}\right)$

$V^{\prime}\left[V^{3}\right]\left(\begin{array}{cccccccccc}x x x x & -y x x x & 0 & x x y y & x y z z & 0 & y y z z & x y x x & 0 & 0 \\ y x x x & x x x x & 0 & -x y x x & y y z z & 0 & -x y z z & x x y y & 0 & 0 \\ 0 & 0 & z z z z & 0 & 0 & z z x & 0 & 0 & z z x x & z x y z\end{array}\right)$ $\begin{array}{llllllllll}0 & 0 & 0 & 0 & 0 & 0 & 0 & 0 & 0 \\ 0 & 0 & 0 & 0 & 0 & 0 & 0 & 0 & 0\end{array}$ $\begin{array}{lllllllllll}0 & 0 & 0 & 0 & 0 & 0 & 0 & 0 & 0 \\ 0 & 0 & 0 & 0 & 0\end{array}$

$\begin{array}{lllllllll}0 & 0 & 0 & 0 & 0 & 0 & 0 & 0 & 0 \\ 0 & 0 & 0 & 0 & 0 & 0 & 0 & 0\end{array}$ $\begin{array}{llllllllll}0 & 0 & 0 & 0 & 0 & 0 & 0 & 0 & 0 \\ 0 & 0 & 0 & 0 & 0 & 0 & 0 & 0 & 0\end{array}$ $\begin{array}{lllllllll}0 & 0 & 0 & 0 & 0 & 0 & 0 & 0 & 0 \\ 0 & 0 & 0 & 0 & 0 & 0 & 0 & 0 & 0\end{array}$

$\left(\begin{array}{lllll}0 & 0 & 0 & 0 & 0 \\ 0 & 0 & 0 & 0 & 0 \\ 0 & 0 & 0 & 0 & 0\end{array}\right)$ $\begin{array}{lllll}0 & 0 & 0 & 0 & 0 \\ 0 & 0 & 0 & 0\end{array}$

$\left(\begin{array}{llllllllll}0 & 0 & 0 & 0 & 0 & 0 & 0 & 0 & 0 & 0 \\ 0 & 0 & 0 & 0 & 0 & 0 & 0 & 0 & 0 & 0\end{array}\right)$ $\left.\begin{array}{llllllllll}0 & 0 & 0 & 0 & 0 & 0 & 0 & 0 & 0 & 0 \\ 0 & 0 & 0 & 0 & 0 & 0 & 0 & 0 & 0 & 0\end{array}\right)$ $\begin{array}{llllllllll}0 & 0 & 0 & 0 & 0 & 0 & 0 & 0 & 0 & 0\end{array}$

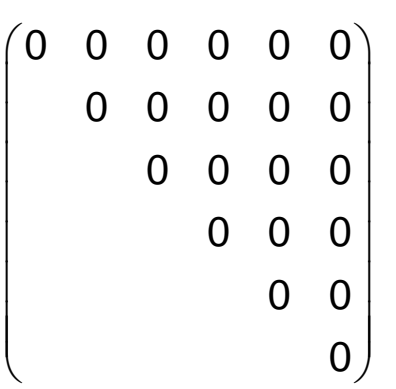

$\left(\begin{array}{llllll}0 & 0 & 0 & 0 & 0 & 0 \\ 0 & 0 & 0 & 0 & 0 & 0 \\ 0 & 0 & 0 & 0 & 0 & 0\end{array}\right)$ $\left.\begin{array}{llllll}0 & 0 & 0 & 0 & 0 & 0 \\ 0 & 0 & 0 & 0 & 0 & 0 \\ 0 & 0 & 0 & 0 & 0 & 0\end{array}\right)$ $0 \begin{array}{llllll}0 & 0 & 0 & 0 & 0 & 0\end{array}$ $0 \begin{array}{llllll}0 & 0 & 0 & 0 & 0 & 0 \\ 0 & 0 & 0 & 0 & 0 & 0\end{array}$ $\begin{array}{lllllllll}0 & 0 & 0 & 0 & 0 & 0 & 0 & 0 & 0 \\ 0 & 0 & 0 & 0 & 0 & 0 & 0 & 0 & 0\end{array}$ $\begin{array}{llllllllll}0 & 0 & 0 & 0 & 0 & 0 & 0 & 0 & 0 \\ 0 & 0 & 0 & 0 & 0 & 0 & 0 & 0 & 0 \\ 0 & 0 & 0 & 0 & 0 & 0 & 0 & 0 & 0\end{array}$ $\begin{array}{llllllllll}0 & 0 & 0 & 0 & 0 & 0 & 0 & 0 & 0 \\ 0 & 0 & 0 & 0 & 0 & 0 & 0 & 0\end{array}$ $\begin{array}{llllllllll}0 & 0 & 0 & 0 & 0 & 0 & 0 & 0 & 0 \\ 0 & 0 & 0 & 0 & 0 & 0 & 0 & 0 & 0\end{array}$ $\begin{array}{llllllllll}0 & 0 & 0 & 0 & 0 & 0 & 0 & 0 & 0 \\ 0 & 0 & 0 & 0 & 0 & 0\end{array}$ $\begin{array}{llllllllll}0 & 0 & 0 & 0 & 0 & 0 & 0 & 0 & 0 \\ 0 & 0 & 0 & 0 & 0 & 0 & 0 & 0 & 0\end{array}$ $\begin{array}{llllllllll}0 & 0 & 0 & 0 & 0 & 0 & 0 & 0 & 0 \\ 0 & 0 & 0 & 0 & 0 & 0 & 0 & 0 & 0\end{array}$ $\left(\begin{array}{lllll}0 & 0 & 0 & 0 & 0 \\ 0 & 0 & 0 & 0 & 0 \\ 0 & 0 & 0 & 0 & 0\end{array}\right)$

$\left(\begin{array}{llllllllll}0 & 0 & 0 & 0 & 0 & 0 & 0 & 0 & 0 & 0 \\ 0 & 0 & 0 & 0 & 0 & 0 & 0 & 0 & 0 & 0\end{array}\right)$ $\left(\begin{array}{lllllllllll}0 & 0 & 0 & 0 & 0 & 0 & 0 & 0 & 0 & 0 \\ 0 & 0 & 0 & 0 & 0 & 0 & 0 & 0 & 0 & 0\end{array}\right)$

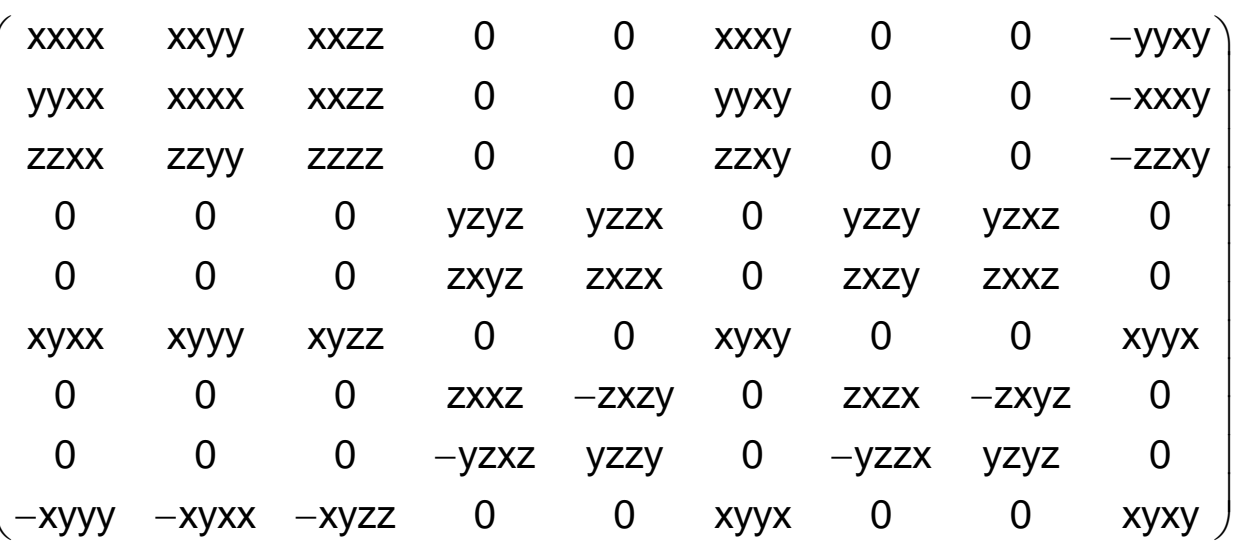

$\left(\begin{array}{ccccc}x x x x & -y x x x & 0 & y y z z & 0 \\ y x x x & x x x x & 0 & y y z z & 0 \\ 0 & 0 & z z z z & x x y y & 0\end{array}\right)$

$\left(\begin{array}{ccccc}\mathrm{yxxx} & \mathrm{xxxx} & 0 & \mathrm{yyzz} & 0 \\ 0 & 0 & \mathrm{zzzz} & \mathrm{xxyy} & 0\end{array}\right)$

$\left(\begin{array}{cccccccccc}x x x x & -y x x x & 0 & x x y y & x y z z & 0 & y y z z & x y x x & 0 & 0 \\ x x & x x x & 0 & -x y x & y y z & 0 & -x y z & x x y y & 0 & 0\end{array}\right)$ $\left(\begin{array}{cccccccccc}y x x x x & x x x x & 0 & -x y x x & y y z z & 0 & -x y z z & x x y y & 0 & 0 \\ 0 & 0 & z z z z & 0 & 0 & z z x x & 0 & 0 & z z x x & z x y z\end{array}\right)$

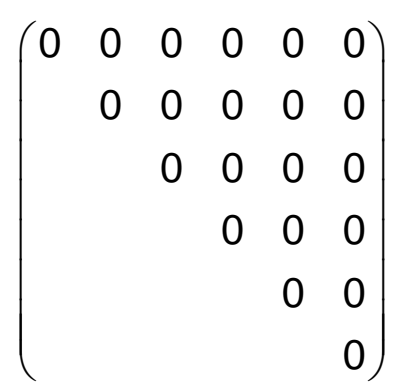

$$
\left(\begin{array}{cccccc}
\text { xxxx } & \text { xxyy } & \text { xxzz } & 0 & 0 & \text { xxxy } \\
& \text { yyyy } & \text { yyzz } & 0 & 0 & -x x x y \\
& & \text { zzzz } & 0 & 0 & 0 \\
& & & \text { yzyz } & 0 & 0 \\
& & & & y z y z & 0 \\
& & & & & \text { xyxy }
\end{array}\right)
$$

$\left(\begin{array}{llllll}0 & 0 & 0 & 0 & 0 & 0 \\ 0 & 0 & 0 & 0 & 0 & 0 \\ 0 & 0 & 0 & 0 & 0 & 0\end{array}\right)$ $\begin{array}{llllll}0 & 0 & 0 & 0 & 0 & 0\end{array}$ $0 \begin{array}{llllll}0 & 0 & 0 & 0 & 0 & 0\end{array}$ $\begin{array}{llllll}0 & 0 & 0 & 0 & 0 & 0 \\ 0 & 0 & 0 & 0 & 0\end{array}$ $\begin{array}{llllll}0 & 0 & 0 & 0 & 0 & 0 \\ 0 & 0 & 0 & 0 & 0 & 0\end{array}$ $\left(\begin{array}{cccccc}x x x x & x x y y & x x z z & 0 & 0 & x x x y \\ y y x x & x x x x & x x z z & 0 & 0 & -x x x y \\ z z x x & z z x x & z z z z & 0 & 0 & 0 \\ 0 & 0 & 0 & y z y z & y z x z & 0 \\ 0 & 0 & 0 & -y z x z & y z y z & 0 \\ x y x x & -x y x x & 0 & 0 & 0 & x y x y\end{array}\right)$ 


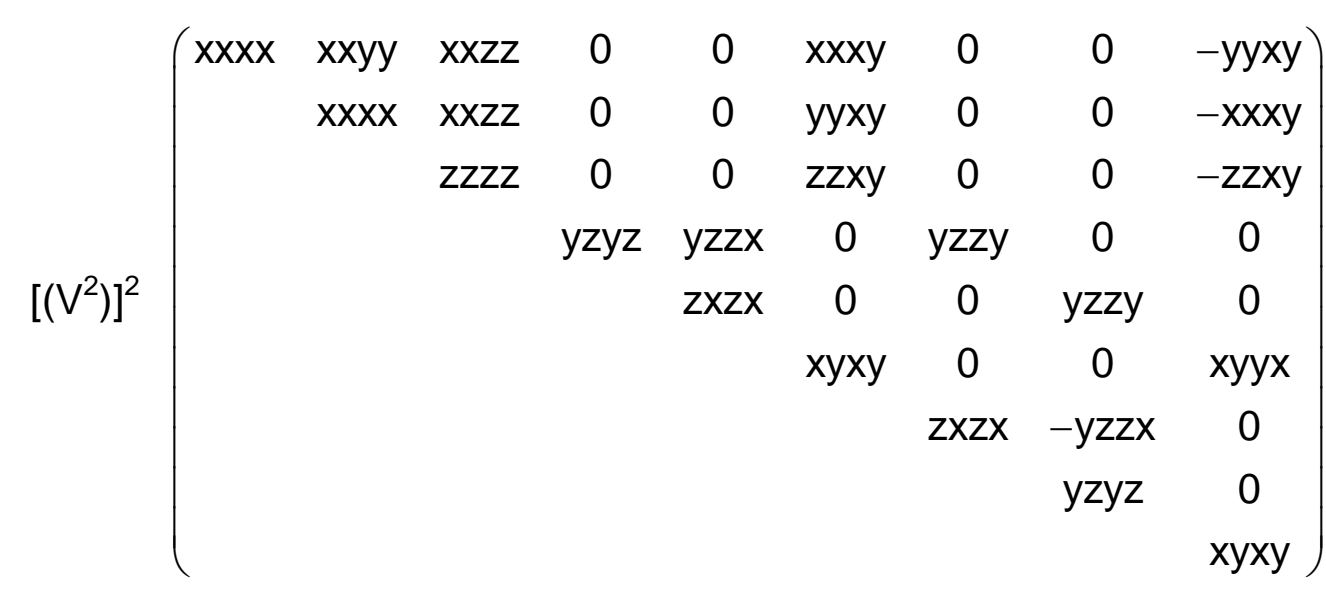

$\left(\begin{array}{lllllllll}0 & 0 & 0 & 0 & 0 & 0 & 0 & 0 & 0\end{array}\right.$ $0 \begin{array}{lllllll}0 & 0 & 0 & 0 & 0 & 0 & 0\end{array}$

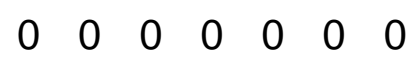
$\begin{array}{llllll}0 & 0 & 0 & 0 & 0 & 0\end{array}$ $\begin{array}{lllll}0 & 0 & 0 & 0 & 0 \\ & 0 & 0 & 0 & 0\end{array}$ $\begin{array}{llll}0 & 0 & 0 & 0\end{array}$ $\begin{array}{lll}0 & 0 & 0 \\ 0 & 0 & 0 \\ & 0 & 0\end{array}$

$\left(\begin{array}{lllllllll}x x x x & x x y y & x x z z & 0 & 0 & x x x y & 0 & 0 & -y y x y \\ y y x & x x y & x x z & 0 & 0 & y x y & 0 & 0 & -x x y\end{array}\right)\left(\begin{array}{ccccccccc}0 & 0 & 0 & 0 & 0 & 0 & 0 & 0 & 0 \\ 0 & 0 & 0 & 0 & 0 & 0 & 0 & 0 & 0\end{array}\right)$

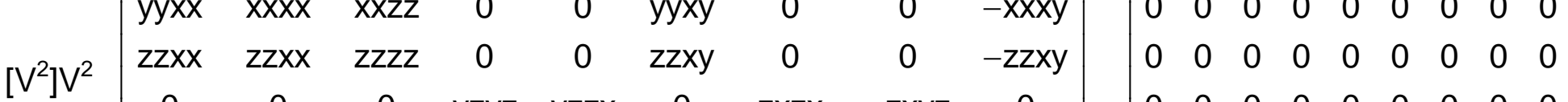

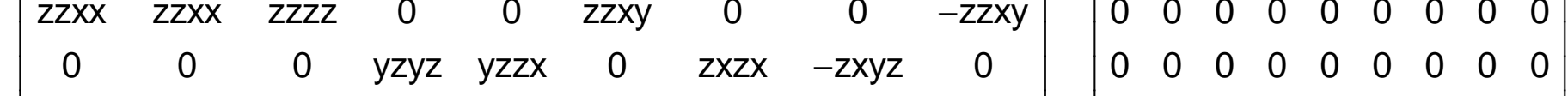
$\begin{array}{lllllllll}0 & 0 & 0 & z x y z & z x z x & 0 & -y z z x & y z y z & 0\end{array} \mid\left(\begin{array}{llllllllll}0 & 0 & 0 & 0 & 0 & 0 & 0 & 0 & 0 \\ 0 & 0 & 0 & 0 & 0 & 0 & 0 & 0 & 0 \\ 0 & 0 & 0 & 0 & 0 & 0 & 0 & 0 & 0\end{array}\right)$ $\left.\begin{array}{lllllllll}\text { xyxx } & -x y x x & 0 & 0 & 0 & x y x y & 0 & 0 & x y x y\end{array}\right)\left(\begin{array}{llllllllll}0 & 0 & 0 & 0 & 0 & 0 & 0 & 0 & 0\end{array}\right.$

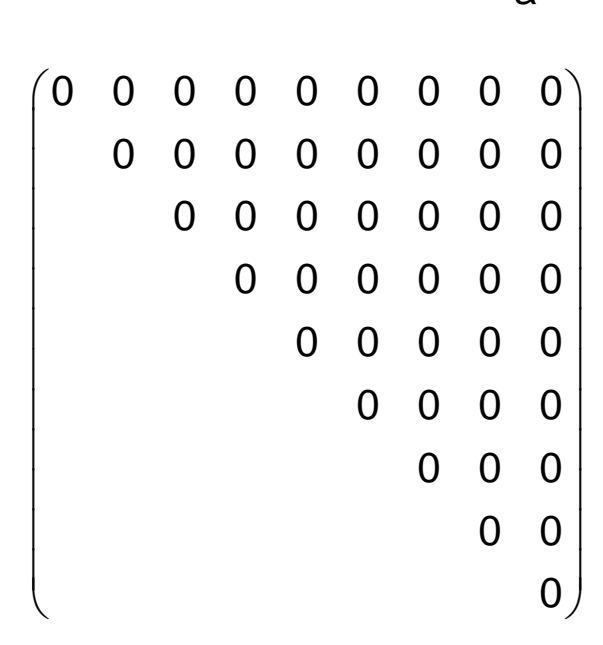

$\left(\begin{array}{lllllllll}0 & 0 & 0 & 0 & 0 & 0 & 0 & 0 & 0 \\ 0 & 0 & 0 & 0 & 0 & 0 & 0 & 0 & 0\end{array}\right.$

$\begin{array}{lllllllll}0 & 0 & 0 & 0 & 0 & 0 & 0 & 0 & 0 \\ 0 & 0 & 0 & 0 & 0 & 0 & 0 & 0 & 0\end{array}$

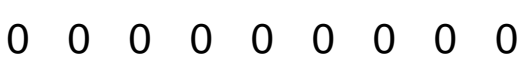

$0 \begin{array}{lllllllll}0 & 0 & 0 & 0 & 0 & 0 & 0 & 0 & 0 \\ 0 & 0 & 0 & 0 & 0 & 0 & 0 & 0 & 0\end{array}$

$\begin{array}{lllllllll}0 & 0 & 0 & 0 & 0 & 0 & 0 & 0 & 0\end{array}$

$0 \begin{array}{lllllllll}0 & 0 & 0 & 0 & 0 & 0 & 0 & 0 & 0\end{array}$

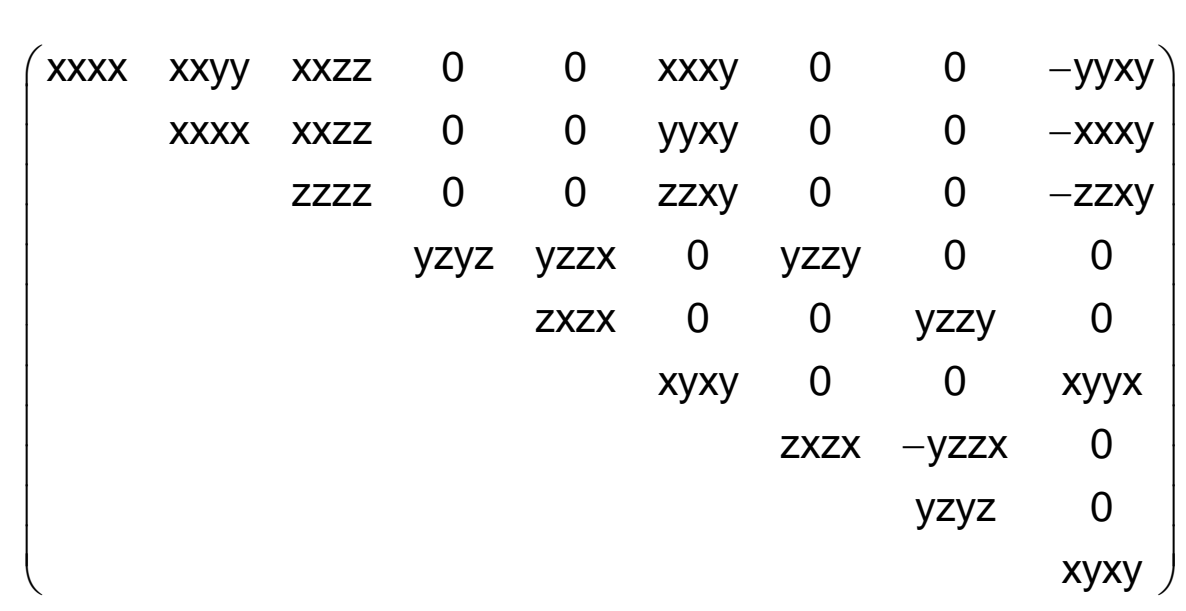

$\left(\begin{array}{ccccccccc}\text { xxxx } & \text { xxyy } & \text { xxzz } & 0 & 0 & \text { xxxy } & 0 & 0 & - \text { yyxy } \\ \text { yyxx } & \text { xxxx } & \text { xxzz } & 0 & 0 & \text { yyxy } & 0 & 0 & -x x x y \\ z z x x & z z x x & z z z z & 0 & 0 & z z x y & 0 & 0 & -z z x y \\ 0 & 0 & 0 & y z y z & y z z x & 0 & \text { zxzx } & -z x y z & 0 \\ 0 & 0 & 0 & \text { zxyz } & \text { zxzx } & 0 & -y z z x & \text { yzyz } & 0 \\ \text { xyxx } & -x y x x & 0 & 0 & 0 & \text { xyxy } & 0 & 0 & \text { xyxy }\end{array}\right)$ 


\begin{tabular}{|c|c|c|c|c|c|c|c|c|c|c|c|c|c|}
\hline$(x x$ & xxyy & $x x z z$ & 0 & 0 & $x x x y$ & 0 & 0 & $-y y x y$ & 0 & $\begin{array}{ll}0 & 0\end{array}$ & 0 & 0 & \\
\hline & $x x x x$ & $x x z z$ & 0 & 0 & yyxy & $c$ & 0 & $-x x x y$ & 0 & 0 & $\begin{array}{ll}0 & 0\end{array}$ & 0 & 0 \\
\hline & zzyy & zzzz & 0 & 0 & zzxy & 0 & 0 & $-z z x y$ & 0 & & 0 & & \\
\hline & 0 & 0 & yzyz & yzzx & 0 & yzzy & $y z x z$ & 0 & 0 & $\begin{array}{ll}0 & 0\end{array}$ & $\begin{array}{lll}0 & 0\end{array}$ & 0 & $\begin{array}{lll}0 & 0\end{array}$ \\
\hline & 0 & 0 & zxyz & $z x z x$ & 0 & zxzy & $z x x z$ & 0 & 0 & $\begin{array}{ll}0 & 0\end{array}$ & $\begin{array}{ll}0 & 0\end{array}$ & 0 & $\begin{array}{ll}0 & 0\end{array}$ \\
\hline & xyyy & xyzz & 0 & 0 & xyxy & 0 & 0 & xyyx & 0 & $\begin{array}{ll}0 & 0\end{array}$ & $\begin{array}{ll}0 & 0\end{array}$ & 0 & 0 \\
\hline & 0 & 0 & $z x x z$ & -zxzy & 0 & $z x z x$ & $-z x y z$ & & 0 & $\begin{array}{ll}0 & 0\end{array}$ & $\begin{array}{ll}0 & 0\end{array}$ & 0 & 0 \\
\hline & 0 & 0 & $-y z x z$ & yzzy & 0 & $-y z z x$ & yzyz & 0 & 0 & $\begin{array}{ll}0 & 0\end{array}$ & 00 & 0 & \\
\hline
\end{tabular}
and: $x x x x=x x y y+x y x y+x y y x ; x x x y-y y x y=x y y y-x y x x$

$\left[V^{4}\right] \quad\left(\begin{array}{ccccc}3 x x y y & 0 & 0 & y y z z & 0 \\ 0 & 3 x x y y & 0 & y y z z & 0 \\ 0 & 0 & z z z z & x x y y & 0\end{array}\right)$

$\left(\begin{array}{lllll}0 & 0 & 0 & 0 & 0 \\ 0 & 0 & 0 & 0 & 0 \\ 0 & 0 & 0 & 0 & 0\end{array}\right)$

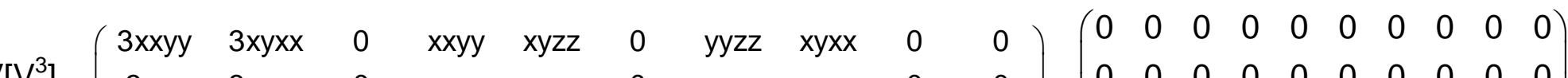

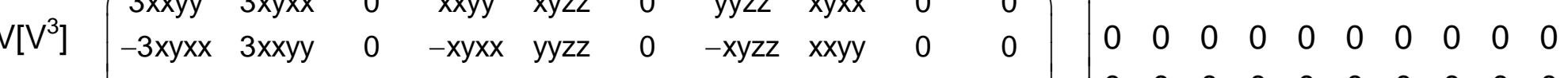

$\left[\left[V^{2}\right]^{2}\right]\left(\begin{array}{cccccc}x x x x & x x y y & x x z z & 0 & 0 & 0 \\ & x x x x & x x z z & 0 & 0 & 0 \\ & & z z z z & 0 & 0 & 0 \\ & & & y z y z & 0 & 0 \\ & & & & y z y z & 0 \\ & & & & & x y x y\end{array}\right)$

$\left(\begin{array}{llllllllll}0 & 0 & 0 & 0 & 0 & 0 & 0 & 0 & 0 & 0 \\ 0 & 0 & 0 & 0 & 0 & 0 & 0 & 0 & 0 & 0\end{array}\right)$

and $x y x y=1 / 2(x x x x-x x y y)$

$\left[\mathrm{V}^{2}\right]^{2}\left(\begin{array}{cccccc}x x x x & x x y y & x x z z & 0 & 0 & x x x y \\ x x y y & x x x x & x x z z & 0 & 0 & -x x x y \\ z z x x & z z x x & z z z z & 0 & 0 & 0 \\ 0 & 0 & 0 & y z y z & y z x z & 0 \\ 0 & 0 & 0 & -y z x z & y z y z & 0 \\ -x x x y & x x x y & 0 & 0 & 0 & x y x y\end{array}\right)$

and $x y x y=1 / 2(x x x x-x x y y)$

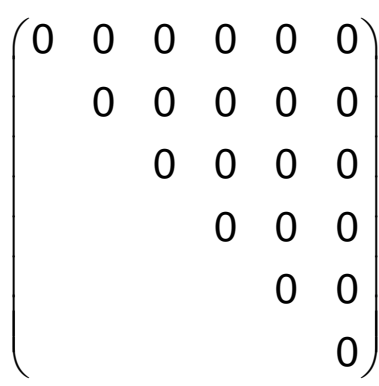

$\left(\begin{array}{llllll}0 & 0 & 0 & 0 & 0 & 0 \\ 0 & 0 & 0 & 0 & 0 & 0 \\ 0 & 0 & 0 & 0 & 0 & 0 \\ 0 & 0 & 0 & 0 & 0 & 0 \\ 0 & 0 & 0 & 0 & 0 & 0 \\ 0 & 0 & 0 & 0 & 0 & 0\end{array}\right)$

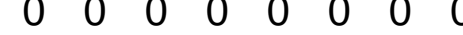

$0 \begin{array}{lllllllll}0 & 0 & 0 & 0 & 0 & 0 & 0 & 0 & 0 \\ 0 & 0 & 0 & 0 & 0 & 0 & 0 & 0 & 0\end{array}$

$\begin{array}{lllllllll}0 & 0 & 0 & 0 & 0 & 0 & 0 & 0 & 0 \\ 0 & 0 & 0 & 0 & 0 & 0 & 0 & 0 & 0\end{array}$

$\begin{array}{llllllllll}0 & 0 & 0 & 0 & 0 & 0 & 0 & 0 & 0 \\ 0 & 0 & 0 & 0 & 0 & 0 & 0\end{array}$

$\begin{array}{llllllllll}0 & 0 & 0 & 0 & 0 & 0 & 0 & 0 & 0\end{array}$

$\begin{array}{lllllllll}0 & 0 & 0 & 0 & 0 & 0 & 0 & 0 & 0\end{array}$

$\begin{array}{lllllllll}0 & 0 & 0 & 0 & 0 & 0 & 0 & 0 & 0 \\ 0 & 0 & 0 & 0 & 0 & 0 & 0 & 0 & 0\end{array}$

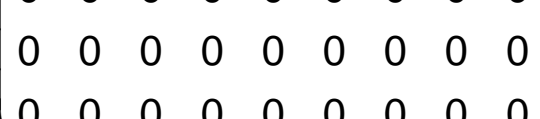

$\begin{array}{llllllllll}0 & 0 & 0 & 0 & 0 & 0 & 0 & 0 & 0\end{array}$

$\left(\begin{array}{lllll}0 & 0 & 0 & 0 & 0 \\ 0 & 0 & 0 & 0 & 0 \\ 0 & 0 & 0 & 0 & 0\end{array}\right)$

$\begin{array}{llllllllll}0 & 0 & 0 & 0 & 0 & 0 & 0 & 0 & 0 & 0 \\ 0 & 0 & 0 & 0 & 0 & 0 & 0 & 0 & 0 & 0\end{array}$

$\left(\begin{array}{llllllllll}0 & 0 & 0 & 0 & 0 & 0 & 0 & 0 & 0 & 0 \\ 0 & 0 & 0 & 0 & 0 & 0 & 0 & 0 & 0 & 0\end{array}\right)$

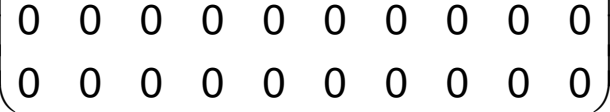

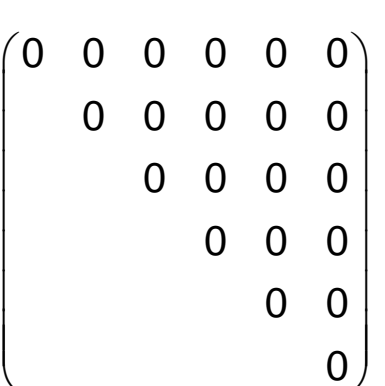

$\left(\begin{array}{llllll}0 & 0 & 0 & 0 & 0 & 0 \\ 0 & 0 & 0 & 0 & 0 & 0\end{array}\right)$

$0 \begin{array}{llllll}0 & 0 & 0 & 0 & 0 & 0 \\ 0 & 0 & 0 & 0 & 0 & 0\end{array}$

$\begin{array}{llllll}0 & 0 & 0 & 0 & 0 & 0 \\ 0 & 0 & 0 & 0 & 0 & 0\end{array}$

$\begin{array}{llllll}0 & 0 & 0 & 0 & 0 & 0 \\ 0 & 0 & 0 & 0 & 0 & 0\end{array}$

$\begin{array}{llllll}0 & 0 & 0 & 0 & 0 & 0 \\ 0 & 0 & 0 & 0 & 0 & 0\end{array}$

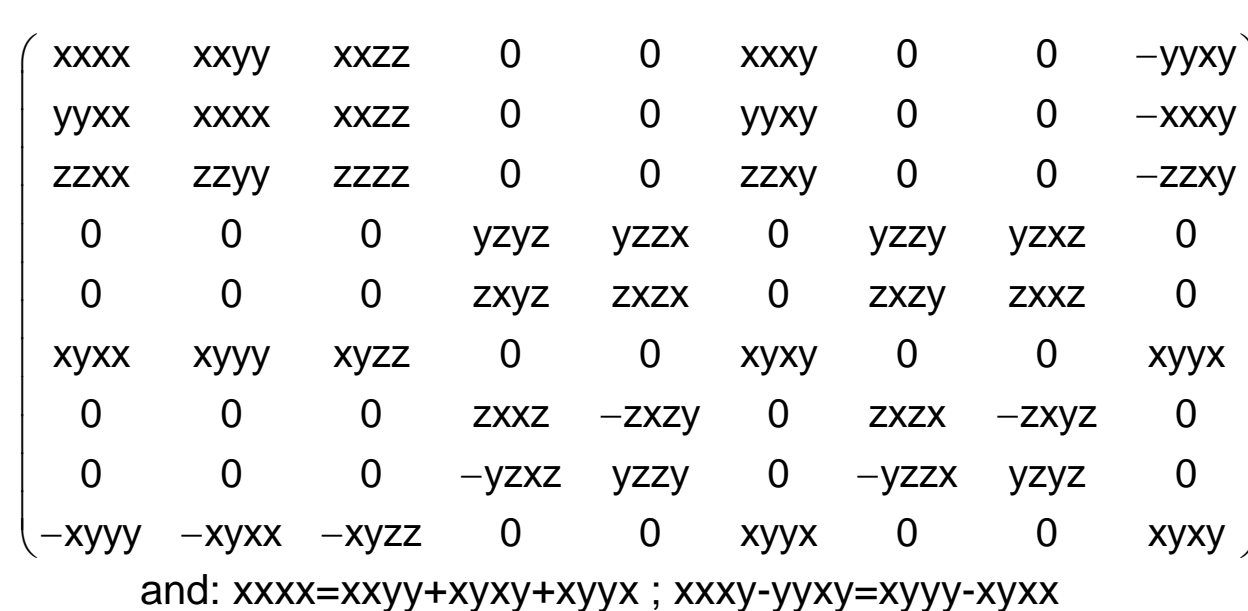

$\left(\begin{array}{ccccc}3 x x y y & 0 & 0 & y y z z & 0 \\ 0 & 3 x x y y & 0 & y y z z & 0 \\ 0 & 0 & z z z z & x x y y & 0\end{array}\right)$

$\left(\begin{array}{ccccc}0 & 3 x x y y & 0 & y y z z & 0 \\ 0 & 0 & z z z z & x x y y & 0\end{array}\right)$

$\left(\begin{array}{cccccccccc}3 x x y y & 3 x y x x & 0 & x x y y & x y z z & 0 & y y z z & x y x x & 0 & 0 \\ 3 x y x x & 3 x x y & 0 & -x y x & y y z & 0 & -x y z & x x y & 0 & 0\end{array}\right)$

$\left.\begin{array}{cccccccccc}-3 x y x x & 3 x x y y & 0 & -x y x x & y y z z & 0 & -x y z z & x x y y & 0 & 0 \\ 0 & 0 & z z z z & 0 & 0 & z z x x & 0 & 0 & z z x x & z x y z\end{array}\right)$

$\left(\begin{array}{cccccc}x x x x & x x y y & x x z z & 0 & 0 & 0 \\ & x x x x & x x z z & 0 & 0 & 0 \\ & & z z z z & 0 & 0 & 0 \\ & & & y z y z & 0 & 0 \\ & & & & y z y z & 0 \\ & & & & & \\ & & & & & \end{array}\right.$

and $x y x y=1 / 2(x x x x-x x y y)$

$\left(\begin{array}{cccccc}x x x x & x x y y & x x z z & 0 & 0 & x x x y \\ x x y y & x x x x & x x z z & 0 & 0 & -x x x y \\ z z x x & z z x x & z z z z & 0 & 0 & 0 \\ 0 & 0 & 0 & y z y z & y z x z & 0 \\ 0 & 0 & 0 & -y z x z & y z y z & 0 \\ -x x x y & x x x y & 0 & 0 & 0 & x y x y\end{array}\right)$

and $x y x y=1 / 2(x x x x-x x y y)$ 
$\left[\left(\mathrm{V}^{2}\right)\right]^{2}$

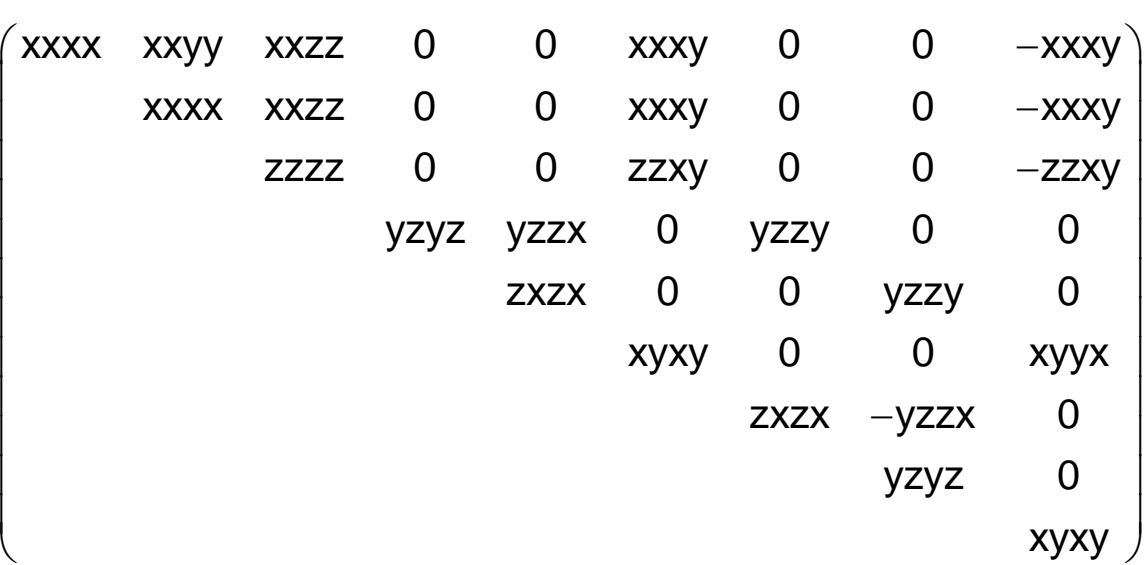

and $x x x x=x x y y+y z y z+z y z y$ $\left(\begin{array}{lllllllll}0 & 0 & 0 & 0 & 0 & 0 & 0 & 0 & 0 \\ & 0 & 0 & 0 & 0 & 0 & 0 & 0 & 0\end{array}\right)$

$\begin{array}{llllllll}0 & 0 & 0 & 0 & 0 & 0 & 0 & 0\end{array}$ $\begin{array}{lllllll}0 & 0 & 0 & 0 & 0 & 0 & 0\end{array}$ $\begin{array}{lllll}0 & 0 & 0 & 0 & 0 \\ 0 & 0 & 0 & 0 & 0\end{array}$ $\begin{array}{lllll}0 & 0 & 0 & 0 & 0\end{array}$ $\begin{array}{llll}0 & 0 & 0 & 0\end{array}$ $\begin{array}{lll}0 & 0 & 0 \\ & 0 & 0\end{array}$ 0

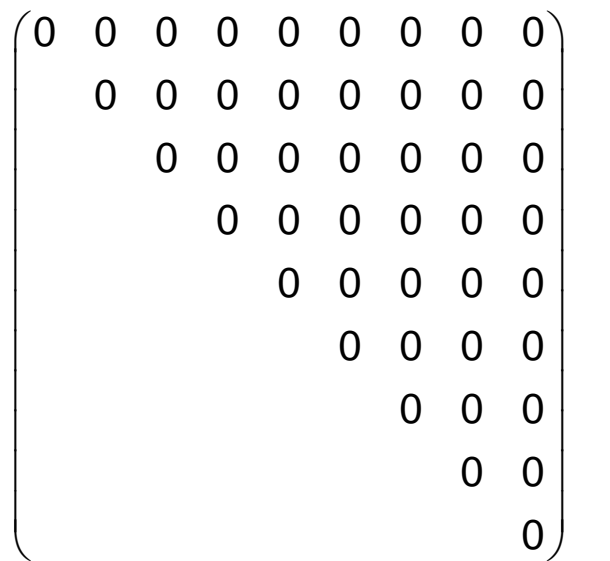

$\begin{array}{lllllllll}0 & 0 & 0 & 0 & 0 & 0 & 0 & 0 & 0 \\ 0 & 0 & 0 & 0 & 0 & 0 & 0 & 0 & 0\end{array}$ $\left.\begin{array}{llllllllll}0 & 0 & 0 & 0 & 0 & 0 & 0 & 0 & 0 \\ 0 & 0 & 0 & 0 & 0 & 0 & 0 & 0 & 0 \\ 0 & 0 & 0 & 0 & 0 & 0 & 0 & 0 & 0\end{array}\right)$ $0 \begin{array}{lllllllll}0 & 0 & 0 & 0 & 0 & 0 & 0 & 0 & 0 \\ 0 & 0 & 0 & 0 & 0 & 0 & 0 & 0 & 0\end{array}$ $\begin{array}{lllllllll}0 & 0 & 0 & 0 & 0 & 0 & 0 & 0 & 0\end{array}$ $\begin{array}{llllllllll}0 & 0 & 0 & 0 & 0 & 0 & 0 & 0 & 0 \\ 0 & 0 & 0 & 0 & 0 & 0 & 0 & 0 & 0\end{array}$

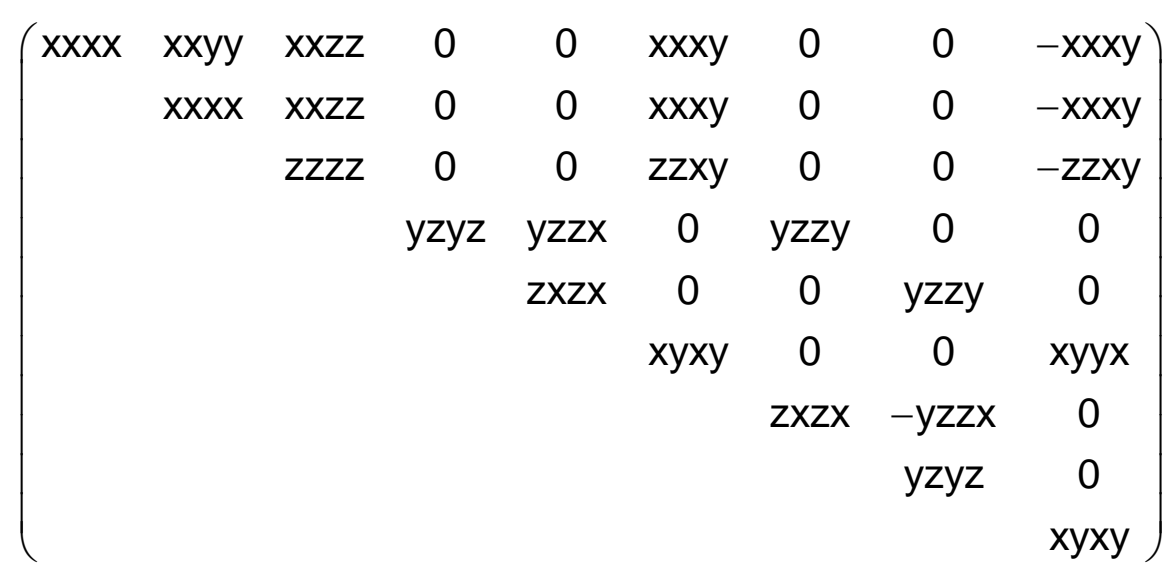

and $x x x x=x x y y+y z y z+z y z y$

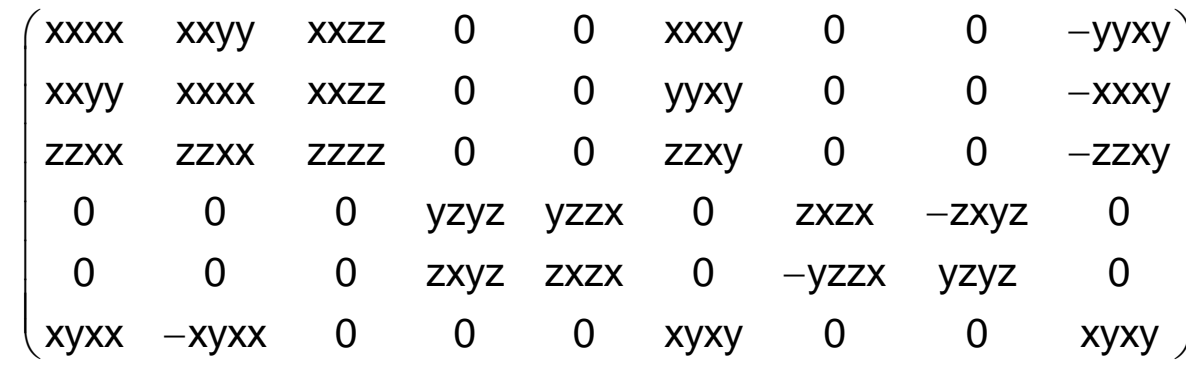

and $x x x x=x x y y+2 x y x y ; y y x y-x x x y=2 x y x x$ 
1

$\left(\begin{array}{lllllllll}x x x x & x x y y & x x z z & 0 & 0 & x x x y & 0 & 0 & x x y x \\ y y x & y y y & y y z z & 0 & 0 & y y x y & 0 & 0 & x y y x\end{array}\right.$ $\begin{array}{lllllllll}x y x x & y y y y & y y z z & 0 & 0 & y y x y & 0 & 0 & y y y x \\ z z x & z y y & z z z & 0 & 0 & z z x & 0 & 0 & z z x\end{array}$ $\begin{array}{lllllllll}\text { zzxx } & z z y y & z z z z & 0 & 0 & \text { zzxy } & 0 & 0 & \text { zzyx }\end{array}$

$\begin{array}{lllllllll}0 & 0 & 0 & y z y z & y z z x & 0 & y z z y & y z x z & 0\end{array}$ $\begin{array}{lllllllll}0 & 0 & 0 & z x y z & z x z x & 0 & z x z y & z x x z & 0\end{array}$

$\begin{array}{lllllllll}\text { xyxx } & \text { xyyy } & \text { xyzz } & 0 & 0 & \text { xyxy } & 0 & 0 & \text { xyyx }\end{array}$

$\begin{array}{lllllllll}0 & 0 & 0 & \text { zyyz } & z y z x & 0 & \text { zyzy } & \text { zyxz } & 0 \\ 0 & 0 & 0 & x z y z & x z z x & 0 & x z z & x z x z & 0\end{array}$

$\begin{array}{lllllllll}0 & 0 & 0 & x z y z & x z z x & 0 & x z z y & x z x z & 0\end{array}$

$\begin{array}{lllllllll}y x x x & y x y y & y x z z & 0 & 0 & y x x y & 0 & 0 & y x y x\end{array}$ $\left(\begin{array}{lllllllll}0 & 0 & 0 & 0 & 0 & 0 & 0 & 0 & 0 \\ 0 & 0 & 0 & 0 & 0 & 0 & 0 & 0 & 0 \\ 0 & 0 & 0 & 0 & 0 & 0 & 0 & 0 & 0\end{array}\right.$ $0 \begin{array}{llllllllll}0 & 0 & 0 & 0 & 0 & 0 & 0 & 0 & 0 \\ 0 & 0 & 0 & 0 & 0 & 0 & 0 & 0 & 0\end{array}$ $\begin{array}{llllllllll}0 & 0 & 0 & 0 & 0 & 0 & 0 & 0 & 0 \\ 0 & 0 & 0 & 0 & 0 & 0 & 0 & 0\end{array}$ $0 \begin{array}{llllllllll}0 & 0 & 0 & 0 & 0 & 0 & 0 & 0 & 0 \\ 0 & 0 & 0 & 0 & 0 & 0 & 0 & 0\end{array}$ $\begin{array}{lllllllll}0 & 0 & 0 & 0 & 0 & 0 & 0 & 0 & 0\end{array}$ $\begin{array}{llllllllll}0 & 0 & 0 & 0 & 0 & 0 & 0 & 0 & 0\end{array}$ $\begin{array}{llllllllll}0 & 0 & 0 & 0 & 0 & 0 & 0 & 0 & 0\end{array}$

$\left.{ }^{4}\right] \quad\left(\begin{array}{ccccc}x x x x & x y y y & 0 & y y z z & 0 \\ y x x x & y y y y & 0 & z z x x & 0 \\ 0 & 0 & z z z z & x x y y & z z x y\end{array}\right)$

$\left(\begin{array}{lllll}0 & 0 & 0 & 0 & 0 \\ 0 & 0 & 0 & 0 & 0 \\ 0 & 0 & 0 & 0 & 0\end{array}\right)$

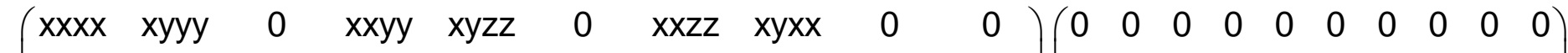

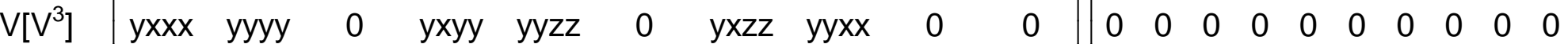
$\left(\begin{array}{ccccccccccc}0 & 0 & z z z z & 0 & 0 & \text { zzxx } & 0 & 0 & \text { zzyy } & \text { zxyz }\end{array}\right)\left(\begin{array}{lllllllllll}0 & 0 & 0 & 0 & 0 & 0 & 0 & 0 & 0 & 0\end{array}\right)$
$\left[\left[\mathrm{V}^{2}\right]^{2}\right]$

$\left(\begin{array}{cccccc}x x x x & x x y y & x x z z & 0 & 0 & x x x y \\ & y y y y & y y z z & 0 & 0 & y y x y \\ & & z z z z & 0 & 0 & z z x y \\ & & & y z y z & y z x z & 0 \\ & & & & x z x z & 0 \\ & & & & & x y x y\end{array}\right)$

$\left(\begin{array}{llllll}x x x x & x x y y & x x z z & 0 & 0 & x x x y \\ y y x & y y y & y y z z & 0 & 0 & y y x y\end{array}\right)$

zzxx zzyy zzzz 0 co 0 zzxy

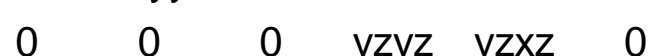

$\begin{array}{llllll}0 & 0 & 0 & x z y z & x z x z & 0\end{array}$

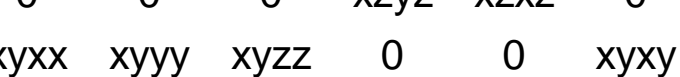

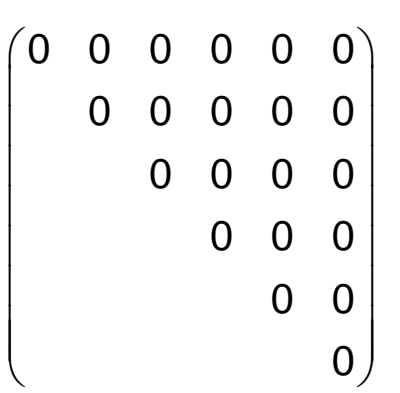

$\left(\begin{array}{llllll}0 & 0 & 0 & 0 & 0 & 0 \\ 0 & 0 & 0 & 0 & 0 & 0\end{array}\right.$

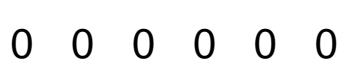

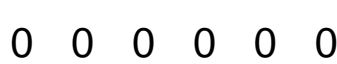

$0 \begin{array}{llllll}0 & 0 & 0 & 0 & 0 & 0 \\ 0 & 0 & 0 & 0 & 0 & 0\end{array}$

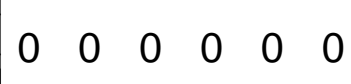

$\begin{array}{llllll}0 & 0 & 0 & 0 & 0 & 0 \\ 0 & 0 & 0 & 0 & 0\end{array}$ $\left(\begin{array}{ccccccccc}0 & 0 & 0 & x x y z & x x z x & 0 & x x z y & x x x z & 0 \\ 0 & 0 & 0 & \text { yyyz } & \text { yyzx } & 0 & \text { yyzy } & \text { yyxz } & 0 \\ 0 & 0 & 0 & z z y z & \text { zzzx } & 0 & \text { zzzy } & z z x z & 0 \\ y z x x & y z y y & y z z z & 0 & 0 & y z x y & 0 & 0 & y z y x \\ z x x x & z x y y & z x z z & 0 & 0 & z x x y & 0 & 0 & z x y x \\ 0 & 0 & 0 & x y y z & x y z x & 0 & x y z y & x y x z & 0 \\ z y x x & z y y y & z y z z & 0 & 0 & z y x y & 0 & 0 & z y y x \\ x z x x & x z y y & x z z z & 0 & 0 & x z x y & 0 & 0 & x z y x \\ 0 & 0 & 0 & y x y z & y x z x & 0 & y x z y & y x x z & 0\end{array}\right)$

$\left(\begin{array}{lllllllll}0 & 0 & 0 & 0 & 0 & 0 & 0 & 0 & 0 \\ 0 & 0 & 0 & 0 & 0 & 0 & 0 & 0 & 0\end{array}\right)$ $\left(\begin{array}{lllllllll}0 & 0 & 0 & 0 & 0 & 0 & 0 & 0 & 0 \\ 0 & 0 & 0 & 0 & 0 & 0 & 0 & 0 & 0 \\ 0 & 0 & 0 & 0 & 0 & 0 & 0 & 0 & 0 \\ 0 & 0 & 0 & 0 & 0 & 0 & 0 & 0 & 0\end{array}\right.$ $0 \begin{array}{lllllllll}0 & 0 & 0 & 0 & 0 & 0 & 0 & 0 & 0 \\ 0 & 0 & 0 & 0 & 0 & 0 & 0 & 0 & 0\end{array}$ $\begin{array}{llllllllll}0 & 0 & 0 & 0 & 0 & 0 & 0 & 0 & 0 \\ 0 & 0 & 0 & 0 & 0 & 0 & 0 & 0 & 0\end{array}$ $\begin{array}{llllllllll}0 & 0 & 0 & 0 & 0 & 0 & 0 & 0 & 0\end{array}$ $\begin{array}{llllllllll}0 & 0 & 0 & 0 & 0 & 0 & 0 & 0 & 0 \\ 0 & 0 & 0 & 0\end{array}$

$\begin{array}{llllllllll}0 & 0 & 0 & 0 & 0 & 0 & 0 & 0 & 0\end{array}$ $\begin{array}{llllllllll}0 & 0 & 0 & 0 & 0 & 0 & 0 & 0 & 0 \\ 0 & 0 & 0 & 0 & 0 & 0 & 0 & 0 & 0\end{array}$

$\left(\begin{array}{ccccc}0 & 0 & x z z z & 0 & x x y z \\ 0 & 0 & y z z z & 0 & y y x z \\ z x x x & z y y y & 0 & 0 & 0\end{array}\right)$

$\left(\begin{array}{lllll}0 & 0 & 0 & 0 & 0 \\ 0 & 0 & 0 & 0 & 0 \\ 0 & 0 & 0 & 0 & 0\end{array}\right)$

$\left(\begin{array}{llllllllll}0 & 0 & x z z z & 0 & 0 & x z x x & 0 & 0 & x z y y & x x y z\end{array}\right)\left(\begin{array}{llllllllll}0 & 0 & 0 & 0 & 0 & 0 & 0 & 0 & 0 & 0 \\ 0 & 0 & y z z z & 0 & 0 & y z x & 0 & 0 & y z y & y x y z\end{array}\right)$

$\left.\begin{array}{llllllllll}0 & 0 & y z z z & 0 & 0 & y z x x & 0 & 0 & y z y y & y x y z\end{array}\right)\left(\begin{array}{llllllllll}0 & 0 & 0 & 0 & 0 & 0 & 0 & 0 & 0 & 0 \\ 0 & 0 & 0 & 0 & 0 & 0 & 0 & 0 & 0 & 0 \\ 0 & 0 & 0 & 0 & 0 & 0 & 0 & 0 & 0 & 0\end{array}\right)$

$\left.\begin{array}{lllllllllll}2 x x x & z y y y & 0 & z x y y & z y z z & 0 & z x z z & z y x x & 0 & 0\end{array}\right)\left(\begin{array}{llllllllll}0 & 0 & 0 & 0 & 0 & 0 & 0 & 0 & 0 & 0\end{array}\right.$

$$
\left(\begin{array}{cccccc}
0 & 0 & 0 & x x y z & x x x z & 0 \\
& 0 & 0 & y y y z & y y x z & 0 \\
& & 0 & z z y z & z z x z & 0 \\
& & & 0 & 0 & y z x y \\
& & & & 0 & x z x y \\
& & & & & 0
\end{array}\right)
$$

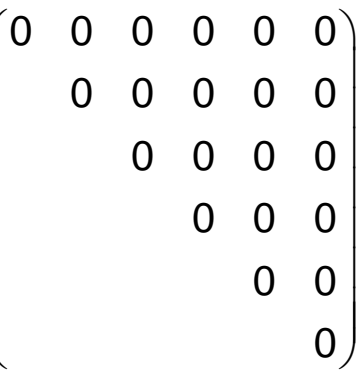

$\left(\begin{array}{cccccc}0 & 0 & 0 & \text { xxyz } & \text { xxxz } & 0 \\ 0 & 0 & 0 & \text { yyyz } & \text { yyxz } & 0 \\ 0 & 0 & 0 & z z y z & z z x z & 0 \\ \text { yzxx } & \text { yzyy } & \text { yzzz } & 0 & 0 & \text { yzxy } \\ \text { xzxx } & \text { xzyy } & \text { xzzz } & 0 & 0 & \text { xzxy } \\ 0 & 0 & 0 & \text { xyyz } & \text { xyxz } & 0\end{array}\right)$

$\left(\begin{array}{llllll}0 & 0 & 0 & 0 & 0 & 0 \\ 0 & 0 & 0 & 0\end{array}\right)$

$\begin{array}{llllll}0 & 0 & 0 & 0 & 0 & 0 \\ 0 & 0 & 0 & 0 & 0 & 0\end{array}$

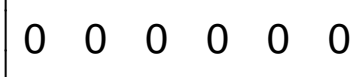

$\begin{array}{llllll}0 & 0 & 0 & 0 & 0 & 0 \\ 0 & 0 & 0 & 0 & 0 & 0\end{array}$

$\begin{array}{llllll}0 & 0 & 0 & 0 & 0 & 0 \\ 0 & 0 & 0 & 0 & 0 & 0\end{array}$ 


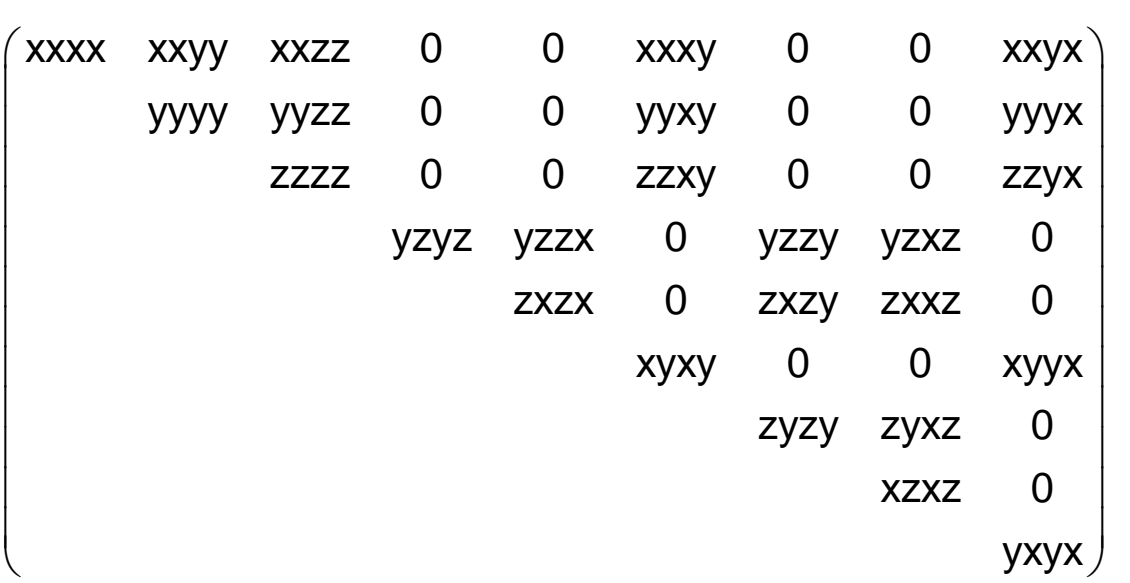

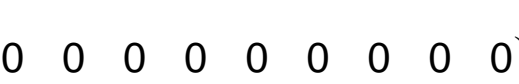
$\begin{array}{llllllll}0 & 0 & 0 & 0 & 0 & 0 & 0 & 0\end{array}$ $0 \begin{array}{lllllll}0 & 0 & 0 & 0 & 0 & 0 & 0\end{array}$ $\begin{array}{llllll}0 & 0 & 0 & 0 & 0 & 0\end{array}$ $\begin{array}{lllll}0 & 0 & 0 & 0 & 0 \\ 0 & 0\end{array}$ $\begin{array}{lllll}0 & 0 & 0 & 0 \\ 0 & 0 & 0 & 0\end{array}$ $\begin{array}{llll}0 & 0 & 0\end{array}$ $\begin{array}{lll}0 & 0 & 0 \\ & 0 & 0 \\ & & 0\end{array}$ $\begin{array}{lllllllll}\text { yyxx } & y y y y & y y z z & 0 & 0 & \text { yyxy } & 0 & 0 & \text { yyyx } \\ z z x x & z z y & z z z & 0 & 0 & z z y & 0 & 0 & z z y\end{array}$

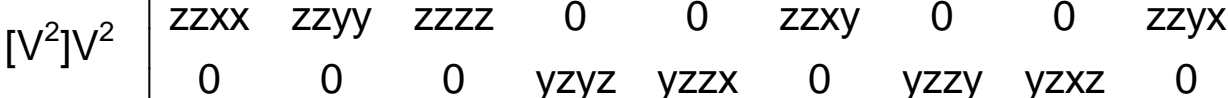
$\begin{array}{ccccccccc}0 & 0 & 0 & z x y z & z x z x & 0 & z x z y & z x x z & 0\end{array}$

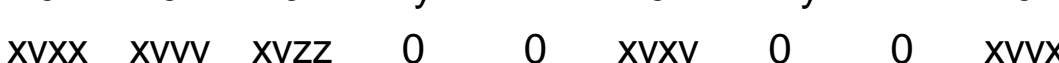

$$
\left.\begin{array}{ccccccccc}
0 & 0 & 0 & x x y z & x x z x & 0 & x x z y & x x x z & 0 \\
& 0 & 0 & y y y z & y y z x & 0 & y y z y & y y x z & 0 \\
& & 0 & z z y z & z z z x & 0 & z z z y & z z x z & 0 \\
& & & 0 & 0 & y z x y & 0 & 0 & y z y x \\
& & & & 0 & z x x y & 0 & 0 & z x y x \\
& & & & & 0 & x y z y & x y x z & 0 \\
& & & & & & 0 & 0 & \text { zyyx } \\
& & & & & & 0 & \text { xzyx } \\
& & & & & & & & 0
\end{array}\right)
$$
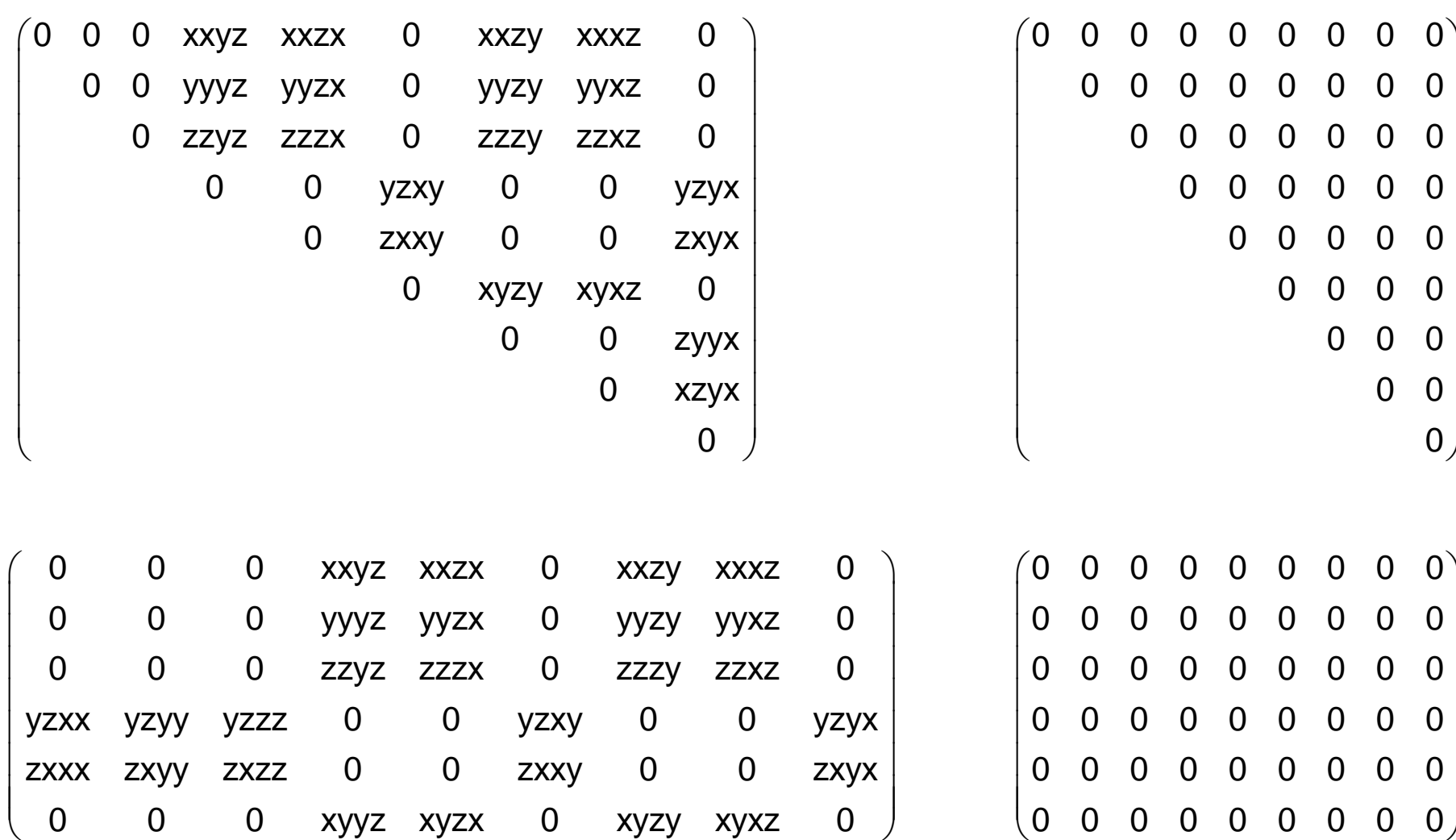

$\left(\begin{array}{lllllllll}0 & 0 & 0 & 0 & 0 & 0 & 0 & 0 & 0 \\ 0 & 0 & 0 & 0 & 0 & 0 & 0 & 0 & 0\end{array}\right)$ $\left.\begin{array}{llllllllll}0 & 0 & 0 & 0 & 0 & 0 & 0 & 0 & 0 \\ 0 & 0 & 0 & 0 & 0 & 0 & 0 & 0 & 0 \\ 0 & 0 & 0 & 0 & 0 & 0 & 0 & 0 & 0\end{array}\right)$ $\begin{array}{llllllllll}0 & 0 & 0 & 0 & 0 & 0 & 0 & 0 & 0 \\ 0 & 0 & 0 & 0 & 0 & 0 & 0 & 0 & 0 \\ 0 & 0 & 0 & 0 & 0 & 0 & 0 & 0 & 0\end{array}$ $\begin{array}{llllllllll}0 & 0 & 0 & 0 & 0 & 0 & 0 & 0 & 0 \\ 0 & 0 & 0 & 0 & 0 & 0 & 0 & 0 & 0\end{array}$ $\begin{array}{llllllllll}0 & 0 & 0 & 0 & 0 & 0 & 0 & 0 & 0\end{array}$ 
ae

$\mathrm{V}^{4}$

\begin{tabular}{|c|c|c|c|c|c|c|c|c|}
\hline$x x x x$ & xxyy & $x x z z$ & 0 & 0 & $x x x y$ & 0 & 0 & $-y y x y$ \\
\hline /yxx & $x x x x$ & $x x z z$ & 0 & 0 & yyxy & 0 & 0 & $-x x x y$ \\
\hline$z x x$ & zzyy & zzzz & 0 & 0 & $z z x y$ & 0 & 0 & -zzxy \\
\hline 0 & 0 & 0 & yzyz & yzzx & 0 & yzzy & $y z x z$ & \\
\hline 0 & 0 & 0 & zxyz & zxzx & 0 & zxzy & $z x x z$ & \\
\hline$x y x x$ & xyyy & xyzz & 0 & 0 & $x y x y$ & 0 & 0 & $x y y x$ \\
\hline 0 & 0 & 0 & $z x x z$ & $-z x z y$ & 0 & $z x z x$ & $-z x y z$ & \\
\hline 0 & 0 & 0 & $-y z x z$ & yzzy & 0 & $-y z z x$ & yzyz & 0 \\
\hline & $-x y x x$ & $-x y z z$ & 0 & 0 & xyyx & 0 & 0 & $\mathrm{VXy}$ \\
\hline
\end{tabular}

$\left[V^{4}\right] \quad\left(\begin{array}{ccccc}\mathrm{x} x \mathrm{xx} & -\mathrm{yxxx} & 0 & \mathrm{yyzz} & 0 \\ \mathrm{yxxx} & \mathrm{xxxx} & 0 & \mathrm{yyzz} & 0 \\ 0 & 0 & z z z & \mathrm{xxy} & 0\end{array}\right)$

$\begin{array}{lllll}0 & 0 & \text { zzzz } & \text { xxyy } & 0\end{array}$

$\left.V^{3}\right]\left(\begin{array}{cccccccccc}x x x x & -y x x x & 0 & x x y y & x y z z & 0 & y y z z & x y x x & 0 & 0 \\ y y x & x x x & 0 & -x y x x & y z z & 0 & -x y z z & x y y & 0 & 0 \\ 0 & 0 & z z z z & 0 & 0 & z z x x & 0 & 0 & z z x x & z x y z\end{array}\right)$

$\mathrm{V}\left[\mathrm{V}^{3}\right] \quad\left(\begin{array}{cccccccccc}\mathrm{yxxx} & \mathrm{xxxx} & 0 & -x y x x & \mathrm{yyzz} & 0 & -\mathrm{xyzz} & \mathrm{xxyy} & 0 & 0 \\ 0 & 0 & \mathrm{zzzz} & 0 & 0 & \mathrm{zzxx} & 0 & 0 & \mathrm{zzxx} & \mathrm{zxyz}\end{array}\right)$

$\left[\left[V^{2}\right]^{2}\right]\left(\begin{array}{cccccc}x x x x & x x y y & x x z z & 0 & 0 & x x x y \\ & \text { yyyy } & y y z z & 0 & 0 & -x x x y \\ & & z z z z & 0 & 0 & 0 \\ & & & y z y z & 0 & 0 \\ & & & & y z y z & 0 \\ & & & & & \text { xyxy }\end{array}\right)$

$\left[\mathrm{V}^{2}\right]^{2}$ $\begin{array}{lllllllll}0 & 0 & 0 & 0 & 0 & 0 & 0 & 0 & 0 \\ 0 & 0 & 0 & 0 & 0 & 0 & 0 & 0 & 0\end{array}$

$\begin{array}{lllllllll}0 & 0 & 0 & 0 & 0 & 0 & 0 & 0 & 0 \\ 0 & 0 & 0 & 0 & 0 & 0 & 0 & 0 & 0\end{array}$

$\begin{array}{lllllllll}0 & 0 & 0 & 0 & 0 & 0 & 0 & 0 & 0 \\ 0 & 0 & 0 & 0 & 0 & 0 & 0 & 0 & 0\end{array}$

$\begin{array}{llllllllll}0 & 0 & 0 & 0 & 0 & 0 & 0 & 0 & 0 \\ 0 & 0 & 0 & 0 & 0 & 0 & 0 & 0 & 0\end{array}$

$\begin{array}{lllllllllll}0 & 0 & 0 & 0 & 0 & 0 & 0 & 0 & 0 \\ 0 & 0 & 0 & 0 & 0 & 0 & 0 & 0 & 0\end{array}$

$\begin{array}{llllllllll}0 & 0 & 0 & 0 & 0 & 0 & 0 & 0 & 0 \\ 0 & 0 & 0 & 0 & 0 & 0 & 0 & 0 & 0 \\ 0 & 0 & 0 & 0 & 0 & 0 & 0 & 0 & 0\end{array}$

$\begin{array}{llllllllll}0 & 0 & 0 & 0 & 0 & 0 & 0 & 0 & 0 \\ 0 & 0 & 0 & 0 & 0 & 0 & 0 & 0 & 0\end{array}$

$\begin{array}{llllllllll}0 & 0 & 0 & 0 & 0 & 0 & 0 & 0 & 0\end{array}$

$\left(\begin{array}{lllll}0 & 0 & 0 & 0 & 0 \\ 0 & 0 & 0 & 0 & 0 \\ 0 & 0 & 0 & 0 & 0\end{array}\right)$

$\begin{array}{llllllllll}0 & 0 & 0 & 0 & 0 & 0 & 0 & 0 & 0 & 0\end{array}$

$\left.\begin{array}{llllllllll}0 & 0 & 0 & 0 & 0 & 0 & 0 & 0 & 0 & 0 \\ 0 & 0 & 0 & 0 & 0 & 0 & 0 & 0 & 0 & 0\end{array}\right)$

$\left(\begin{array}{llllll}0 & 0 & 0 & 0 & 0 & 0 \\ & 0 & 0 & 0 & 0 & 0 \\ & & 0 & 0 & 0 & 0 \\ & & & 0 & 0 & 0 \\ & & & & 0 & 0 \\ & & & & & 0\end{array}\right)$

$\left(\begin{array}{llllll}0 & 0 & 0 & 0 & 0 & 0 \\ 0 & 0 & 0 & 0 & 0 & 0 \\ 0 & 0 & 0 & 0 & 0 & 0\end{array}\right)$

$\left(\begin{array}{llllll}0 & 0 & 0 & 0 & 0 & 0 \\ 0 & 0 & 0 & 0 & 0 & 0 \\ 0 & 0 & 0 & 0 & 0 & 0\end{array}\right.$

$\begin{array}{llllll}0 & 0 & 0 & 0 & 0 & 0 \\ 0 & 0 & 0 & 0 & 0 & 0\end{array}$

$\begin{array}{llllll}0 & 0 & 0 & 0 & 0 & 0 \\ 0 & 0 & 0 & 0 & 0 & 0 \\ 0 & 0 & 0 & 0 & 0 & 0\end{array}$

$\left(\begin{array}{llllll}0 & 0 & 0 & 0 & 0 & 0 \\ 0 & 0 & 0 & 0 & 0 & 0\end{array}\right)$ $\left(\begin{array}{lllllllll}0 & 0 & 0 & 0 & 0 & 0 & 0 & 0 & 0 \\ 0 & 0 & 0 & 0 & 0 & 0 & 0 & 0 & 0\end{array}\right)$

$\begin{array}{lllllllll}0 & 0 & 0 & 0 & 0 & 0 & 0 & 0 & 0 \\ 0 & 0 & 0 & 0 & 0 & 0 & 0 & 0 & 0\end{array}$

$\begin{array}{llllllllll}0 & 0 & 0 & 0 & 0 & 0 & 0 & 0 & 0 \\ 0 & 0 & 0 & 0 & 0 & 0 & 0 & 0 & 0\end{array}$

$\begin{array}{llllllllll}0 & 0 & 0 & 0 & 0 & 0 & 0 & 0 & 0\end{array}$

$\begin{array}{llllllllll}0 & 0 & 0 & 0 & 0 & 0 & 0 & 0 & 0 \\ 0 & 0 & 0 & 0 & 0 & 0 & 0 & 0 & 0\end{array}$

$\begin{array}{lllllllll}0 & 0 & 0 & 0 & 0 & 0 & 0 & 0 & 0 \\ 0 & 0 & 0 & 0 & 0 & 0 & 0 & 0 & 0\end{array}$

$\begin{array}{llllllllll}0 & 0 & 0 & 0 & 0 & 0 & 0 & 0 & 0 \\ 0 & 0 & 0 & 0 & 0 & 0 & 0 & 0 & 0\end{array}$

$\left(\begin{array}{lllll}0 & 0 & 0 & 0 & 0 \\ 0 & 0 & 0 & 0 & 0 \\ 0 & 0 & 0 & 0 & 0\end{array}\right)$

$\begin{array}{llllll}0 & 0 & 0 & 0 & 0 \\ 0 & 0 & 0 & 0 & 0\end{array}$

$\left(\begin{array}{llllllllll}0 & 0 & 0 & 0 & 0 & 0 & 0 & 0 & 0 & 0 \\ 0 & 0 & 0 & 0 & 0 & 0 & 0 & 0 & 0 & 0\end{array}\right.$ $\left(\begin{array}{llllllllll}0 & 0 & 0 & 0 & 0 & 0 & 0 & 0 & 0 & 0 \\ 0 & 0 & 0 & 0 & 0 & 0 & 0 & 0 & 0 & 0\end{array}\right)$

$\begin{array}{llllllllll}0 & 0 & 0 & 0 & 0 & 0 & 0 & 0 & 0 & 0\end{array}$

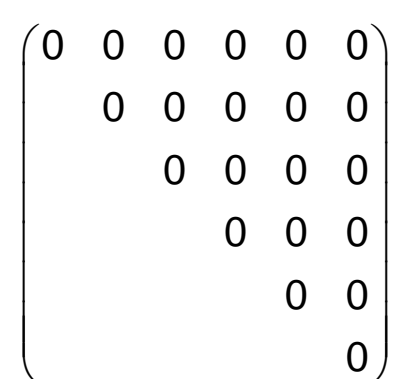

$\left(\begin{array}{llllll}0 & 0 & 0 & 0 & 0 & 0 \\ 0 & 0 & 0 & 0 & 0 & 0\end{array}\right)$

$\begin{array}{llllll}0 & 0 & 0 & 0 & 0 & 0 \\ 0 & 0 & 0 & 0 & 0 & 0\end{array}$

$\begin{array}{llllll}0 & 0 & 0 & 0 & 0 & 0 \\ 0 & 0 & 0 & 0 & 0\end{array}$

$\begin{array}{llllll}0 & 0 & 0 & 0 & 0 & 0 \\ 0 & 0 & 0 & 0 & 0 & 0\end{array}$

$\begin{array}{llllll}0 & 0 & 0 & 0 & 0 & 0 \\ 0 & 0 & 0 & 0 & 0 & 0 \\ 0 & 0 & 0 & 0 & 0 & 0\end{array}$

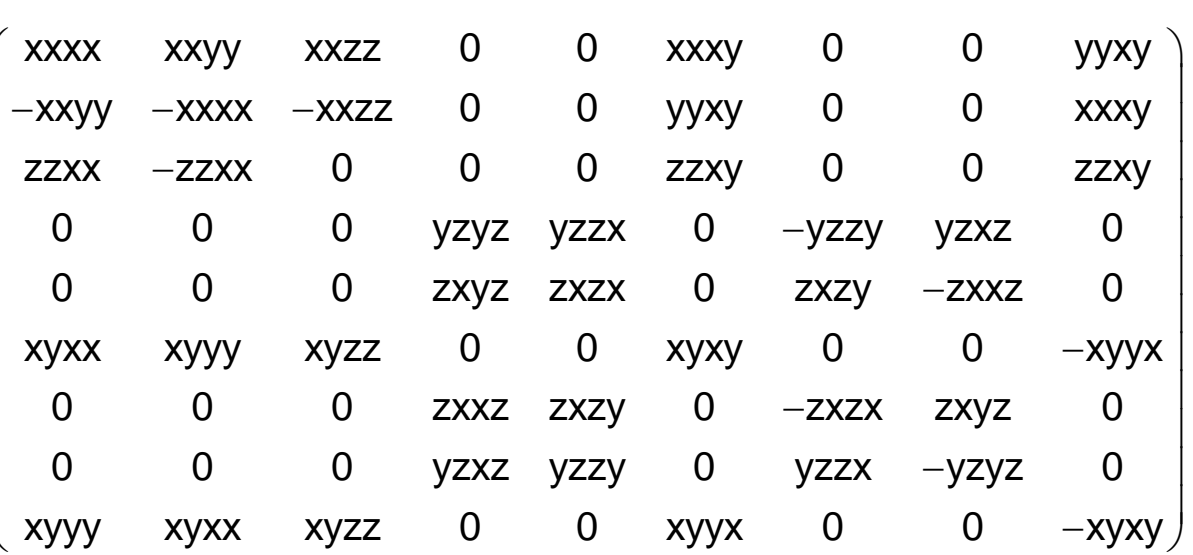

$\left(\begin{array}{ccccc}x x x x & y x x x & 0 & y y z z & 0 \\ y x x x & -x x x x & 0 & -y y z z & 0 \\ 0 & 0 & 0 & 0 & z z x y\end{array}\right)$

$\left.\begin{array}{ccccc}\mathrm{yxxx} & -\mathrm{xxxx} & 0 & -\mathrm{yyzz} & 0 \\ 0 & 0 & 0 & 0 & \mathrm{zzxy}\end{array}\right)$

$\left(\begin{array}{llllllllll}x x x x & x y y y & 0 & x x y y & x y z z & 0 & -y y z z & y x y y & 0 & 0 \\ x y y & -x y x x & 0 & y y y & y y z & 0 & \text { xyzz } & -x y y & 0 & 0\end{array}\right)$

$\left(\begin{array}{cccccccccc}x \mathrm{xyyy} & -\mathrm{xxxx} & 0 & \mathrm{yxyy} & \mathrm{yyzz} & 0 & \mathrm{xyzz} & -\mathrm{xxyy} & 0 & 0 \\ 0 & 0 & 0 & 0 & 0 & \mathrm{zzxx} & 0 & 0 & -\mathrm{zzxx} & \mathrm{zxyz}\end{array}\right)$

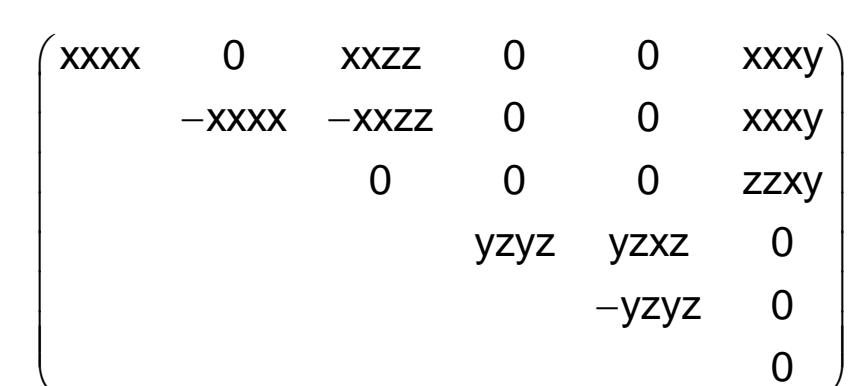

$\left(\begin{array}{cccccc}x x x x & x x y y & x x z z & 0 & 0 & x x x y \\ -x x y y & -x x x x & -x x z z & 0 & 0 & x x x y \\ z z x x & -z z x x & 0 & 0 & 0 & z z x y \\ 0 & 0 & 0 & y z y z & y z x z & 0 \\ 0 & 0 & 0 & y z x z & -y z y z & 0 \\ \text { xyxx } & x y x x & x y z z & 0 & 0 & 0\end{array}\right)$ 


$$
\begin{aligned}
& \begin{array}{lllllllll}
x x x x & x x y y & x x z z & 0 & 0 & x x x y & 0 & 0 & -y y x y \\
& x x y x & x x z z & 0 & 0 & y y x y & 0 & 0 & -x x y
\end{array} \\
& \begin{array}{lllllllll}
x x x x & x x z z & 0 & 0 & y y x y & 0 & 0 & -x x x y
\end{array} \\
& \begin{array}{lllllll}
z z z z & 0 & 0 & z z x y & 0 & 0 & -z z x y
\end{array}
\end{aligned}
$$

$\left(\begin{array}{lllllllll}0 & 0 & 0 & 0 & 0 & 0 & 0 & 0 & 0 \\ & 0 & 0 & 0 & 0 & 0 & 0 & 0 & 0\end{array}\right)$

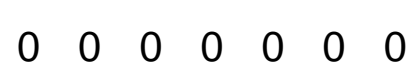

$0 \begin{array}{llllll}0 & 0 & 0 & 0 & 0 & 0\end{array}$

$\begin{array}{llll}0 & 0 & 0 & 0\end{array}$

$\begin{array}{lll}0 & 0 & 0 \\ & 0 & 0\end{array}$

$0)$

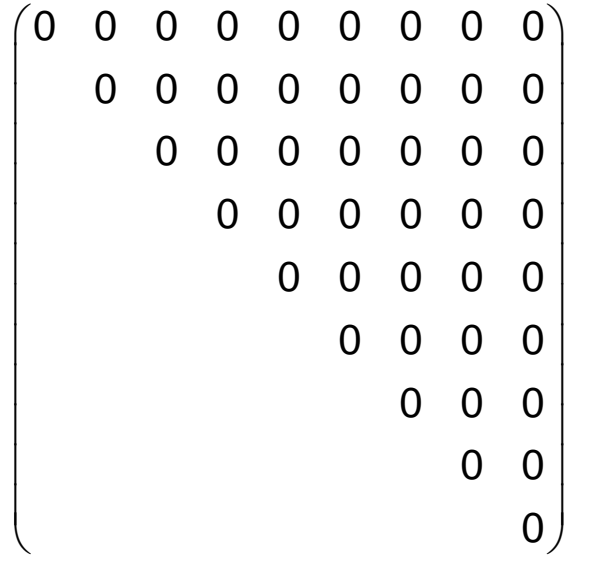

$\left(\begin{array}{lllllllll}0 & 0 & 0 & 0 & 0 & 0 & 0 & 0 & 0\end{array}\right)$

$0 \begin{array}{lllllllll}0 & 0 & 0 & 0 & 0 & 0 & 0 & 0 & 0 \\ 0 & 0 & 0 & 0 & 0 & 0 & 0 & 0 & 0\end{array}$

$0 \begin{array}{lllllllll}0 & 0 & 0 & 0 & 0 & 0 & 0 & 0 & 0 \\ 0 & 0 & 0 & 0 & 0 & 0 & 0 & 0 & 0\end{array}$

$\begin{array}{lllllllll}0 & 0 & 0 & 0 & 0 & 0 & 0 & 0 & 0\end{array}$

$0 \begin{array}{llllllllll}0 & 0 & 0 & 0 & 0 & 0 & 0 & 0 & 0 \\ 0 & 0 & 0 & 0 & 0 & 0 & 0 & 0\end{array}$

$\begin{array}{llllllllll}0 & 0 & 0 & 0 & 0 & 0 & 0 & 0 & 0\end{array}$

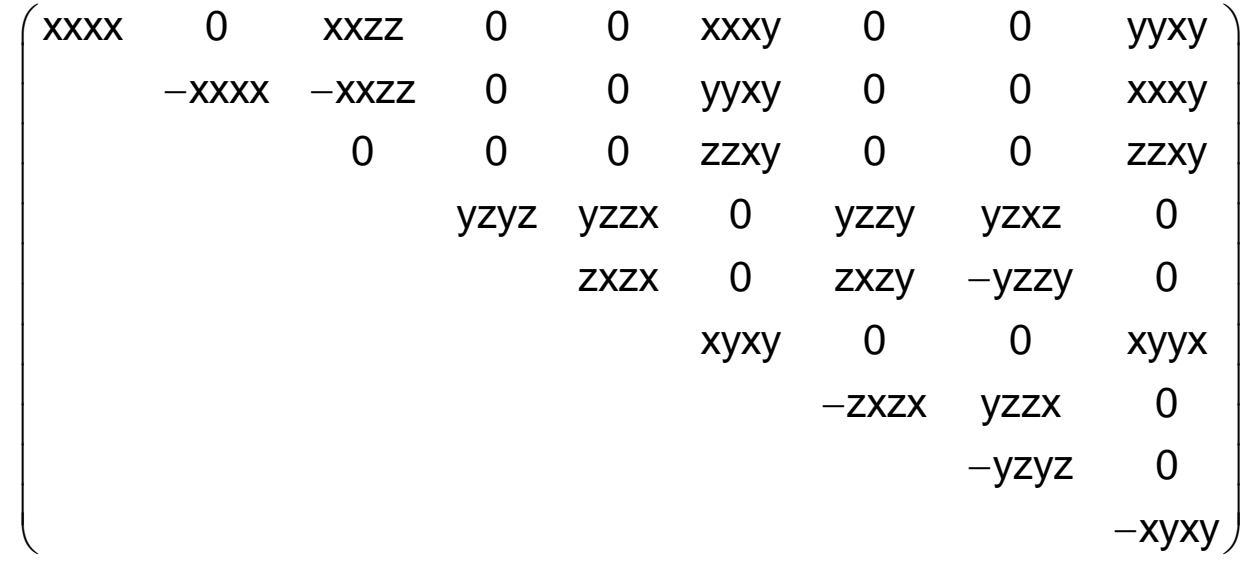

$\left(\begin{array}{ccccccccc}\text { xxxx } & x x y y & x x z z & 0 & 0 & x x x y & 0 & 0 & y y x y \\ -x x y y & -x x x x & -x x z z & 0 & 0 & y y x y & 0 & 0 & x x x y \\ z z x x & -z z x x & 0 & 0 & 0 & z z x y & 0 & 0 & z z x y \\ 0 & 0 & 0 & y z y z & y z z x & 0 & -z x z x & z x y z & 0 \\ 0 & 0 & 0 & z x y z & z x z x & 0 & y z z x & -y z y z & 0 \\ \text { xyxx } & x y x x & x y z z & 0 & 0 & x y x y & 0 & 0 & -x y x y\end{array}\right)$ 


$\left(\begin{array}{ccccccccc}x x x x & x x y y & x x z z & 0 & 0 & x x x y & 0 & 0 & -y y x y \\ y y x x & x x x x & y y z z & 0 & 0 & y y x y & 0 & 0 & -x x x y \\ z z x x & z z x x & z z z z & 0 & 0 & z z x y & 0 & 0 & -z z x y \\ 0 & 0 & 0 & y z y z & y z z x & 0 & y z z y & y z x z & 0 \\ 0 & 0 & 0 & z x y z & z x z x & 0 & z x z y & z x x z & 0 \\ x y x x & x y y y & x y z z & 0 & 0 & x y x y & 0 & 0 & x y y x \\ 0 & 0 & 0 & z x x z & -z x z y & 0 & z x z x & -z x y z & 0 \\ 0 & 0 & 0 & -y z x z & y z z y & 0 & -y z z x & y z y z & 0 \\ -x y y y & -x y x x & -x y z z & 0 & 0 & x y y x & 0 & 0 & x y x y\end{array}\right)\left(\begin{array}{ccccccccc}0 & 0 & 0 & 0 & 0 & 0 & 0 & 0 & 0 \\ 0 & 0 & 0 & 0 & 0 & 0 & 0 & 0 & 0 \\ 0 & 0 & 0 & 0 & 0 & 0 & 0 & 0 & 0 \\ 0 & 0 & 0 & 0 & 0 & 0 & 0 & 0 & 0 \\ 0 & 0 & 0 & 0 & 0 & 0 & 0 & 0 & 0 \\ 0 & 0 & 0 & 0 & 0 & 0 & 0 & 0 & 0 \\ 0 & 0 & 0 & 0 & 0 & 0 & 0 & 0 & 0 \\ 0 & 0 & 0 & 0 & 0 & 0 & 0 & 0 & 0 \\ 0 & 0 & 0 & 0 & 0 & 0 & 0 & 0 & 0\end{array}\right)$

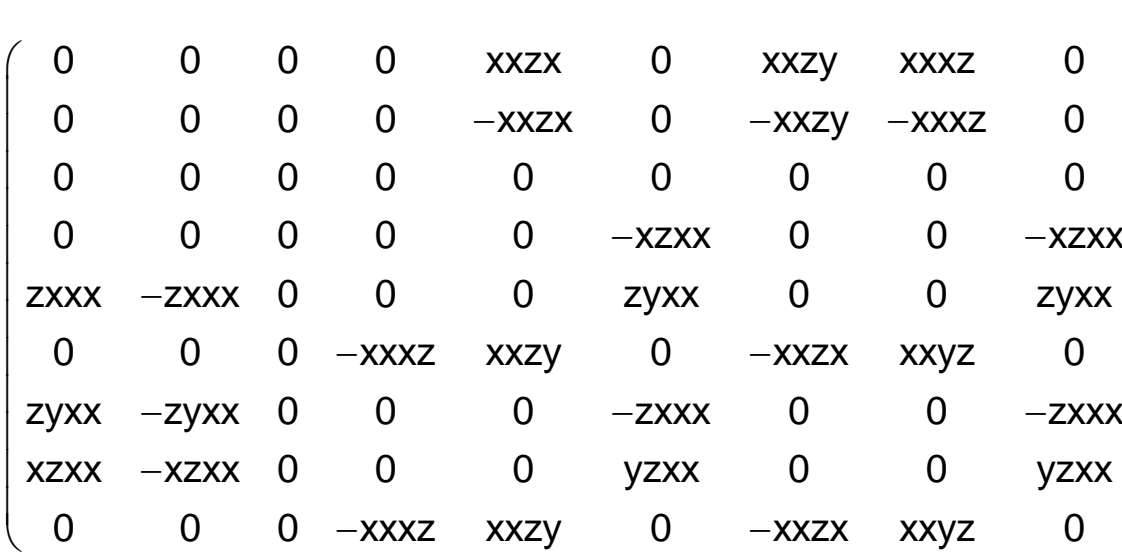
and. $x x x x=x x y+x y x y+x y$

$\left[V^{4}\right] \quad\left(\begin{array}{ccccc}3 x x y y & 0 & 0 & y y z z & 0 \\ 0 & 3 x x y y & 0 & y y z z & 0 \\ 0 & 0 & z z z z & x x y y & 0\end{array}\right)$

$\left(\begin{array}{llllllllll}3 x x y y & 3 x y x x & 0 & x x y y & x y z z & 0 & y y z z & x y x x & 0 & y y x x\end{array}\right)\left(\begin{array}{cccccccccc}0 & 0 & 0 & 0 & 0 & 0 & 0 & 0 & 0 & 0 \\ 0 & 0 & 0 & 0 & 0 & 0 & 0 & 0 & 0 & 0\end{array}\right)$

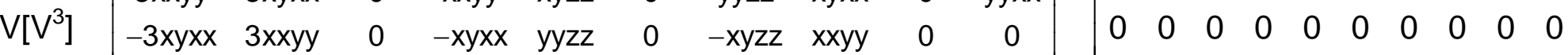
$\left(\begin{array}{ccccccccccc}-3 x y x x & 3 x x y y & 0 & -x y x x & y y z z & 0 & -x y z z & x x y y & 0 & 0 \\ 0 & 0 & z z z z & 0 & 0 & z z x x & 0 & 0 & z z x x & z x y z\end{array}\right)\left(\begin{array}{cccccccccc}0 & 0 & 0 & 0 & 0 & 0 & 0 & 0 & 0 & 0\end{array}\right)$

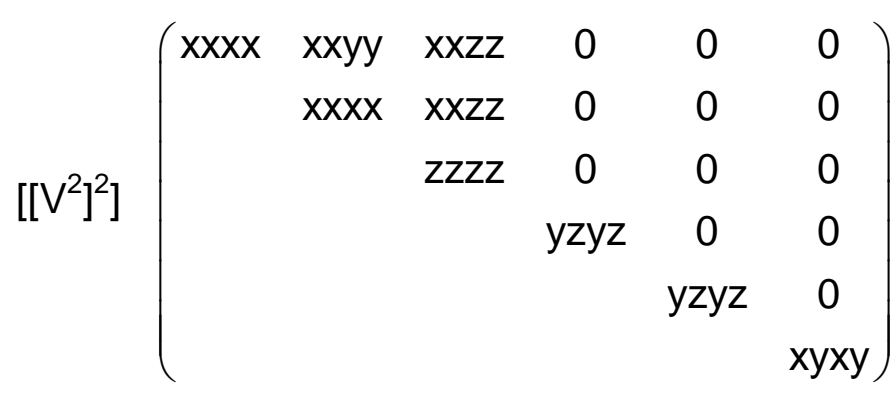

and $x y x y=1 / 2(x x x x-x x y y)$

$\left[\mathrm{V}^{2}\right]^{2}\left(\begin{array}{cccccc}x \mathrm{x} x \mathrm{x} & \mathrm{xxyy} & \mathrm{xxzz} & 0 & 0 & \mathrm{xxxy} \\ \mathrm{xxyy} & \mathrm{xxxx} & \mathrm{xxzz} & 0 & 0 & -\mathrm{xxxy} \\ \mathrm{zzxx} & \mathrm{zzxx} & \mathrm{zzzz} & 0 & 0 & 0 \\ 0 & 0 & 0 & \mathrm{yzyz} & \mathrm{yzxz} & 0 \\ 0 & 0 & 0 & \mathrm{yzxz} & \mathrm{yzyz} & 0 \\ -\mathrm{xxxy} & \mathrm{xxxy} & 0 & 0 & 0 & \mathrm{xyxy}\end{array}\right)$

and $x y x y=1 / 2(x x x x-x x y y)$

$$
\left(\begin{array}{lllll}
0 & 0 & 0 & 0 & 0 \\
0 & 0 & 0 & 0 & 0 \\
0 & 0 & 0 & 0 & 0
\end{array}\right)
$$

$\left(\begin{array}{ccccc}0 & 0 & 0 & 0 & x x y z \\ 0 & 0 & 0 & 0 & -z x x x \\ z x x x & -x x y z & 0 & 0 & 0\end{array}\right)$

$\left(\begin{array}{llllllllll}0 & 0 & 0 & 0 & 0 & x z x x & 0 & 0 & -x z x x & y z x x \\ 0 & 0 & 0 & 0 & 0 & y z x & 0 & 0 & -y z x & -x z x\end{array}\right)$ $\left(\begin{array}{cccccccccc}0 & 0 & 0 & 0 & 0 & y z x x & 0 & 0 & -y z x x & -x z x x \\ 2 z x x & -2 y y x & 0 & -2 x x & 0 & 0 & 0 & z y x & 0 & 0\end{array}\right)$ $\begin{array}{llllllllll} & 0 & 0 & 0 & 0 & 0 & 0 & 0 & 0 & 0\end{array}$

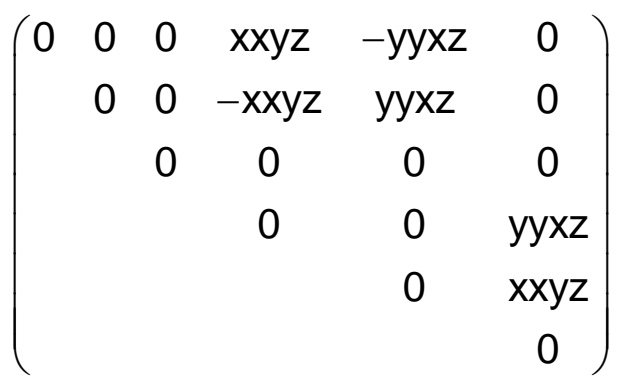

$$
\begin{array}{llll}
0 & 0 & 0 & 0 \\
& 0 & 0 & 0 \\
& & & 0 \\
& & & 0 \\
& & & 0
\end{array}
$$

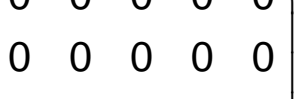

$\left.\begin{array}{ll}0 & 0 \\ & 0\end{array}\right)$

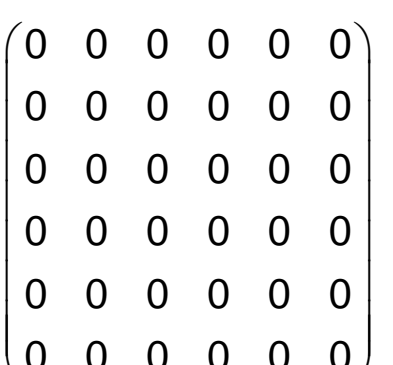

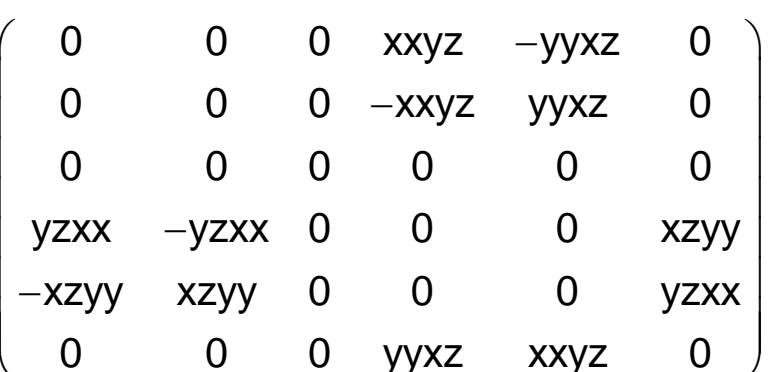

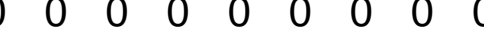
$\begin{array}{lllllllll}0 & 0 & 0 & 0 & 0 & 0 & 0 & 0 & 0 \\ 0 & 0 & 0 & 0 & 0 & 0 & 0 & 0 & 0\end{array}$ $\begin{array}{llllllllll}0 & 0 & 0 & 0 & 0 & 0 & 0 & 0 & 0 \\ 0 & 0 & 0 & 0 & 0 & 0 & 0 & 0 & 0\end{array}$

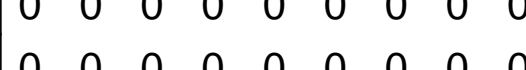
$\begin{array}{lllllllll}0 & 0 & 0 & 0 & 0 & 0 & 0 & 0 & 0\end{array}$ $\begin{array}{llllllllll}0 & 0 & 0 & 0 & 0 & 0 & 0 & 0 & 0\end{array}$ $\begin{array}{lllllllll}0 & 0 & 0 & 0 & 0 & 0 & 0 & 0 & 0 \\ 0 & 0 & 0 & 0 & 0 & 0 & 0 & 0 & 0\end{array}$ $\begin{array}{llllllllll}0 & 0 & 0 & 0 & 0 & 0 & 0 & 0 & 0\end{array}$

$\left(\begin{array}{lllll}0 & 0 & 0 & 0 & 0 \\ 0 & 0 & 0 & 0 & 0 \\ 0 & 0 & 0 & 0 & 0\end{array}\right)$ $\left(\begin{array}{lllll}0 & 0 & 0 & 0 & 0 \\ 0 & 0 & 0 & 0 & 0 \\ 0 & 0 & 0 & 0 & 0\end{array}\right)$

$\left(\begin{array}{llllllllll}0 & 0 & 0 & 0 & 0 & 0 & 0 & 0 & 0 & 0 \\ 0 & 0 & 0 & 0 & 0 & 0 & 0 & 0 & 0 & 0\end{array}\right)$ $\left.\begin{array}{llllllllll}0 & 0 & 0 & 0 & 0 & 0 & 0 & 0 & 0 & 0 \\ 0 & 0 & 0 & 0 & 0 & 0 & 0 & 0 & 0 & 0\end{array}\right)$ $\begin{array}{llllllllll}0 & 0 & 0 & 0 & 0 & 0 & 0 & 0 & 0 & 0\end{array}$

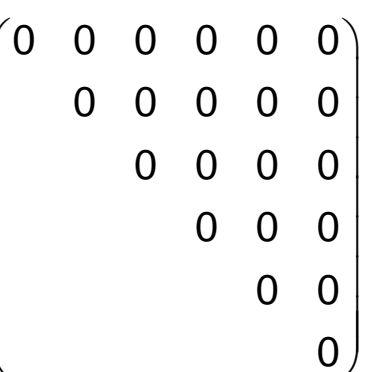

$\left(\begin{array}{llllll}0 & 0 & 0 & 0 & 0 & 0 \\ 0 & 0 & 0 & 0 & 0 & 0\end{array}\right)$ $\left(\begin{array}{llllll}0 & 0 & 0 & 0 & 0 & 0 \\ 0 & 0 & 0 & 0 & 0 & 0\end{array}\right.$ $\begin{array}{llllll}0 & 0 & 0 & 0 & 0 & 0 \\ 0 & 0 & 0 & 0 & 0 & 0\end{array}$ $\begin{array}{llllll}0 & 0 & 0 & 0 & 0 & 0 \\ 0 & 0 & 0 & 0 & 0 & 0\end{array}$ $\begin{array}{llllll}0 & 0 & 0 & 0 & 0 & 0 \\ 0 & 0 & 0 & 0 & 0 & 0\end{array}$ $\begin{array}{llllll}0 & 0 & 0 & 0 & 0 & 0\end{array}$ 


$\left(\begin{array}{ccccccccc}x x x x & x x y y & x x z z & 0 & 0 & x x x y & 0 & 0 & -y y x y \\ y y x x & x x x x & y y z z & 0 & 0 & y y x y & 0 & 0 & -x x x y \\ z z x x & z z x x & z z z z & 0 & 0 & z z x y & 0 & 0 & -z z x y \\ 0 & 0 & 0 & y z y z & y z z x & 0 & y z z y & y z x z & 0 \\ 0 & 0 & 0 & z x y z & z x z x & 0 & z x z y & z x x z & 0 \\ x y x x & x y y y & x y z z & 0 & 0 & x y x y & 0 & 0 & x y y x \\ 0 & 0 & 0 & z x x z & -z x z y & 0 & z x z x & -z x y z & 0 \\ 0 & 0 & 0 & -y z x z & y z z y & 0 & -y z z x & y z y z & 0 \\ -x y y y & -x y x x & -x y z z & 0 & 0 & x y y x & 0 & 0 & x y x y\end{array}\right)\left(\begin{array}{ccccccccc}0 & 0 & 0 & 0 & 0 & 0 & 0 & 0 & 0 \\ 0 & 0 & 0 & 0 & 0 & 0 & 0 & 0 & 0 \\ 0 & 0 & 0 & 0 & 0 & 0 & 0 & 0 & 0 \\ 0 & 0 & 0 & 0 & 0 & 0 & 0 & 0 & 0 \\ 0 & 0 & 0 & 0 & 0 & 0 & 0 & 0 & 0 \\ 0 & 0 & 0 & 0 & 0 & 0 & 0 & 0 & 0 \\ 0 & 0 & 0 & 0 & 0 & 0 & 0 & 0 & 0 \\ 0 & 0 & 0 & 0 & 0 & 0 & 0 & 0 & 0 \\ 0 & 0 & 0 & 0 & 0 & 0 & 0 & 0 & 0\end{array}\right)$

$\left(\begin{array}{lllllllll}0 & 0 & 0 & 0 & 0 & 0 & 0 & 0 & 0 \\ 0 & 0 & 0 & 0 & 0 & 0 & 0 & 0 & 0\end{array}\right.$ $0 \begin{array}{lllllllll}0 & 0 & 0 & 0 & 0 & 0 & 0 & 0 & 0 \\ 0 & 0 & 0 & 0 & 0 & 0 & 0 & 0 & 0\end{array}$

$\begin{array}{lllllllll}0 & 0 & 0 & 0 & 0 & 0 & 0 & 0 & 0 \\ 0 & 0 & 0 & 0 & 0 & 0 & 0 & 0 & 0\end{array}$

$0 \begin{array}{llllllllll}0 & 0 & 0 & 0 & 0 & 0 & 0 & 0 & 0 \\ 0 & 0 & 0 & 0 & 0 & 0 & 0 & 0\end{array}$ $0 \begin{array}{llllllllll}0 & 0 & 0 & 0 & 0 & 0 & 0 & 0 & 0 \\ 0 & 0 & 0 & 0 & 0 & 0 & 0 & 0\end{array}$ $\begin{array}{llllllllll}0 & 0 & 0 & 0 & 0 & 0 & 0 & 0 & 0 \\ 0 & 0 & 0 & 0 & 0 & 0 & 0 & 0 & 0\end{array}$ $\begin{array}{llllllllll}0 & 0 & 0 & 0 & 0 & 0 & 0 & 0 & 0\end{array}$

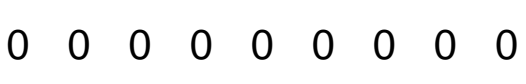
$\begin{array}{llllllllll}0 & 0 & 0 & 0 & 0 & 0 & 0 & 0 & 0\end{array}$ and: $x x x x=x x y y+x y x y+x y y x ; x x x y-y y x y=x y y y-x y x x$

$\left(\begin{array}{lllll}0 & 0 & 0 & 0 & 0 \\ 0 & 0 & 0 & 0 & 0 \\ 0 & 0 & 0 & 0 & 0\end{array}\right)$

$\left(\begin{array}{llllllllll}0 & 0 & 0 & 0 & 0 & 0 & 0 & 0 & 0 & 0 \\ 0 & 0 & 0 & 0 & 0 & 0 & 0 & 0 & 0 & 0\end{array}\right)$ $\begin{array}{lllllllllll}0 & 0 & 0 & 0 & 0 & 0 & 0 & 0 & 0 & 0 \\ 0 & 0 & 0 & 0 & 0 & 0 & 0 & 0 & 0\end{array}$ $\begin{array}{lllllllllll}0 & 0 & 0 & 0 & 0 & 0 & 0 & 0 & 0 & 0\end{array}$

V[V $\left.V^{3}\right] \quad\left(\begin{array}{cccccccccc}3 x x y y & 3 x y x x & 0 & x x y y & x y z z & 0 & \text { yyzz } & \text { xyxx } & 0 & \text { yyxx } \\ -3 x y x x & 3 x x y y & 0 & -x y x x & y y z z & 0 & -x y z z & x x y y & 0 & 0 \\ 0 & 0 & 2 z z & 0 & 0 & 2 x x & 0 & 0 & z x x & z x y z\end{array}\right)\left(\begin{array}{cccccccccc}0 & 0 & 0 & 0 & 0 & 0 & 0 & 0 & 0 & 0 \\ 0 & 0 & 0 & 0 & 0 & 0 & 0 & 0 & 0 & 0 \\ 0 & 0 & 0 & 0 & 0 & 0 & 0 & 0 & 0 & 0\end{array}\right)$ $\left(\begin{array}{ccccccccccc}0 & 0 & z z z z & 0 & 0 & z z x x & 0 & 0 & z z x x & z x y z\end{array}\right)\left(\begin{array}{lllllllllll}0 & 0 & 0 & 0 & 0 & 0 & 0 & 0 & 0 & 0\end{array}\right)$$$
\left(\begin{array}{cccccc}
x x x x & x x y y & x x z z & 0 & 0 & 0 \\
& x x x x & x x z z & 0 & 0 & 0 \\
& & z z z z & 0 & 0 & 0 \\
& & & y z y z & 0 & 0 \\
& & & & y z y z & 0 \\
& & & & & \\
& & & & &
\end{array}\right.
$$

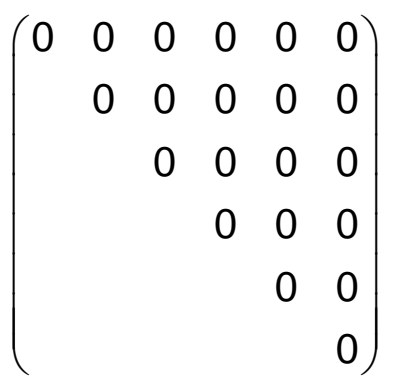

and $x y x y=1 / 2(x x x x-x x y y)$

$\begin{array}{llllll}x x x x & x x y y & x x z z & 0 & 0 & x x x y \\ x x y & x y x & x y z & 0 & 0 & -x x y\end{array}$

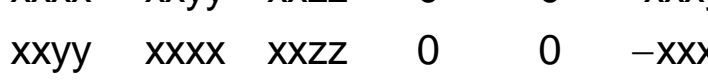

$\begin{array}{cccccc}\operatorname{zzxx} & \mathrm{zZXx} & \mathrm{zzZz} & 0 & 0 & 0 \\ 0 & 0 & 0 & \mathrm{yzyz} & \mathrm{yzxz} & 0 \\ 0 & 0 & 0 & \mathrm{yzz} & \mathrm{yzyz} & 0\end{array}$

$\left.\begin{array}{cccccc}0 & 0 & 0 & y z x z & y z y z & 0 \\ -x x x y & x x y & 0 & 0 & 0 & x y y\end{array}\right)$

and $x y x y=1 / 2(x x x x-x x y y)$

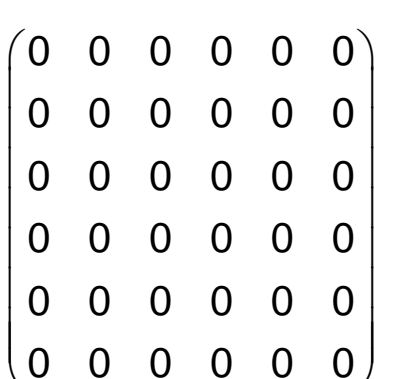

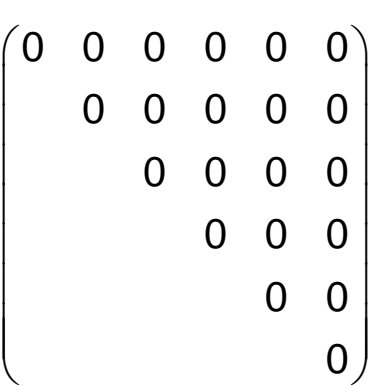

$\left(\begin{array}{llllll}0 & 0 & 0 & 0 & 0 & 0 \\ 0 & 0 & 0 & 0 & 0 & 0\end{array}\right)$

$\begin{array}{llllll}0 & 0 & 0 & 0 & 0 & 0 \\ 0 & 0 & 0 & 0 & 0 & 0\end{array}$

$\begin{array}{llllll}0 & 0 & 0 & 0 & 0 & 0\end{array}$

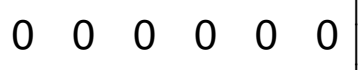

$\begin{array}{llllll}0 & 0 & 0 & 0 & 0 & 0 \\ 0 & 0 & 0 & 0 & 0 & 0\end{array}$

$\begin{array}{llllll}0 & 0 & 0 & 0 & 0 & 0\end{array}$ $\left(\begin{array}{ccccccccc}x x x x & -x x x x & 0 & 0 & 0 & -x y y y & 0 & 0 & -x y y y \\ -x x x x & x x x x & 0 & 0 & 0 & x y y y & 0 & 0 & x y y y \\ 0 & 0 & 0 & 0 & 0 & 0 & 0 & 0 & 0 \\ 0 & 0 & 0 & 0 & 0 & 0 & 0 & 0 & 0 \\ 0 & 0 & 0 & 0 & 0 & 0 & 0 & 0 & 0 \\ -x y y y & x y y y & 0 & 0 & 0 & -x x x x & 0 & 0 & -x x x x \\ 0 & 0 & 0 & 0 & 0 & 0 & 0 & 0 & 0 \\ 0 & 0 & 0 & 0 & 0 & 0 & 0 & 0 & 0 \\ -x y y y & x y y y & 0 & 0 & 0 & -x x x x & 0 & 0 & -x x x x\end{array}\right)$

$\left(\begin{array}{ccccc}x x x x & x y y y & 0 & 0 & 0 \\ -x y y y & x x y x & 0 & 0 & 0 \\ 0 & 0 & 0 & -x x x x & 0\end{array}\right)$

$\left(\begin{array}{ccccc}-\mathrm{xyyy} & \mathrm{xxxx} & 0 & 0 & 0 \\ 0 & 0 & 0 & -\mathrm{xxxx} & 0\end{array}\right)$

$\left(\begin{array}{llllllllll}x x x x & x y y y & 0 & -x x x x & 0 & 0 & 0 & -x y y y & 0 & 0 \\ -x y y & \text { xxxx } & 0 & x y y y & 0 & 0 & 0 & -x x x x & 0 & 0\end{array}\right)$ $\left.\begin{array}{cccccccccc}-x y y y & x x x x & 0 & x y y y & 0 & 0 & 0 & -x x x x & 0 & 0 \\ 0 & 0 & 0 & 0 & 0 & 0 & 0 & 0 & 0 & 0\end{array}\right)$
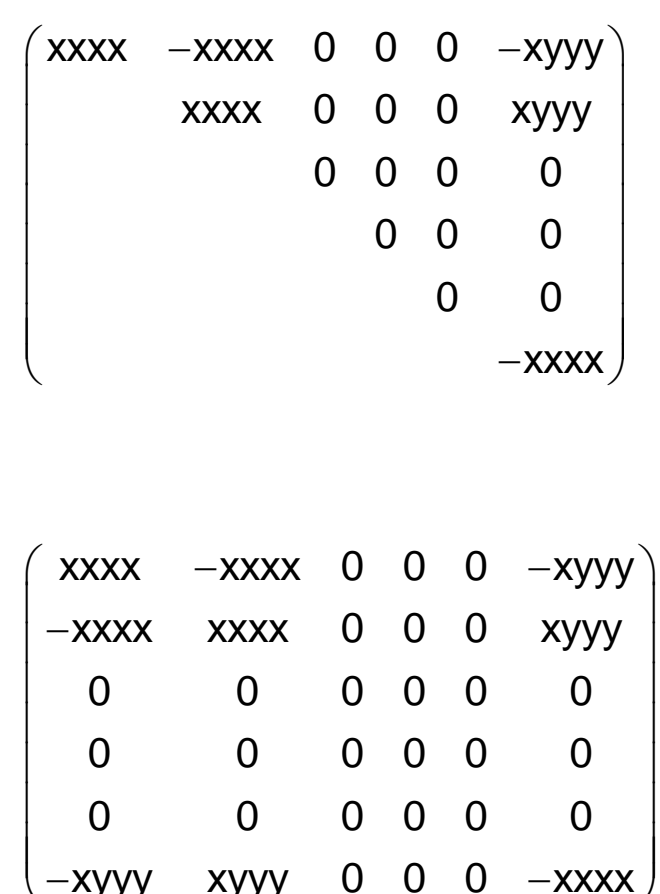


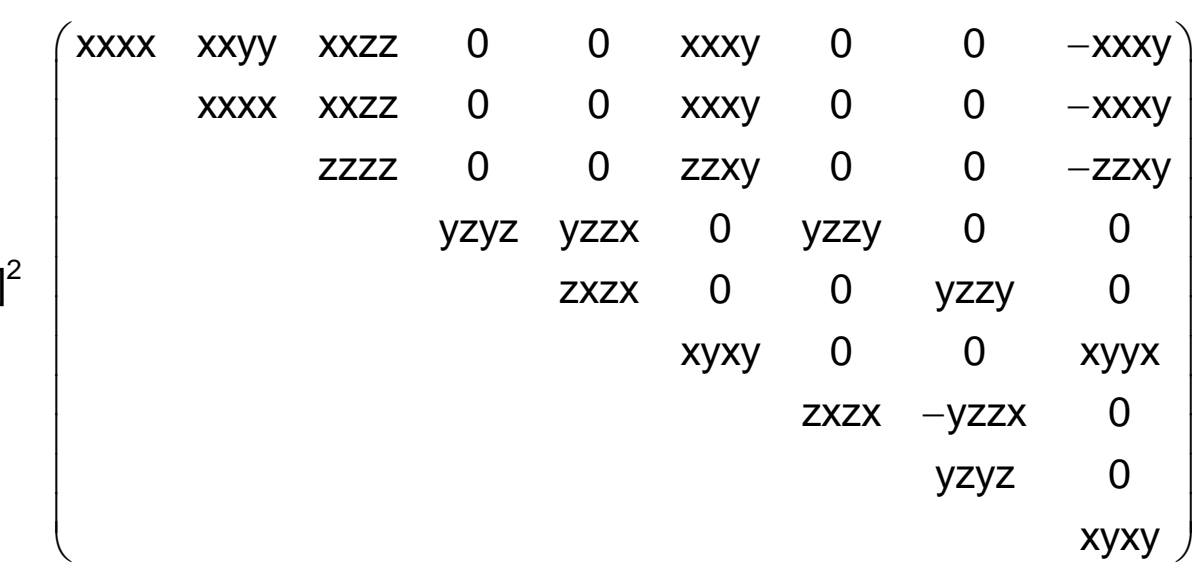

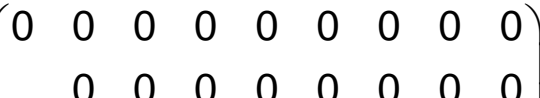

$\begin{array}{lllllll}0 & 0 & 0 & 0 & 0 & 0 & 0\end{array}$

$0 \begin{array}{lllll}0 & 0 & 0 & 0 & 0\end{array}$

$0 \begin{array}{lllll}0 & 0 & 0 & 0 & 0 \\ 0 & 0 & -1\end{array}$ $0 \begin{array}{llll}0 & 0 & 0 \\ 0 & 0 & 0\end{array}$

$\begin{array}{lll}0 & 0 & 0 \\ & 0 & 0\end{array}$

and $x x x x=x x y y+y z y z+z y z y$

$\left(\begin{array}{lllllllll}x x x x & x x y y & x x z z & 0 & 0 & x x x y & 0 & 0 & -y y x y \\ x x y y & x x x & x x z z & 0 & 0 & y x y & 0 & 0 & -x x y\end{array}\right)\left(\begin{array}{ccccccccc}0 & 0 & 0 & 0 & 0 & 0 & 0 & 0 & 0 \\ 0 & 0 & 0 & 0 & 0 & 0 & 0 & 0 & 0\end{array}\right)$

$\left.\begin{array}{llllllllll}x x y y & x x x x & x x z z & 0 & 0 & y y x y & 0 & 0 & -x x x y\end{array} \mid \begin{array}{lllllllll}0 & 0 & 0 & 0 & 0 & 0 & 0 & 0 & 0 \\ 0 & 0 & 0 & 0 & 0 & 0 & 0 & 0 & 0\end{array}\right)$

$\begin{array}{ccccccccc}z z x x & z z x x & z z z z & 0 & 0 & z z x y & 0 & 0 & -z z x y\end{array} \mid \begin{array}{lllllllll}0 & 0 & 0 & 0 & 0 & 0 & 0 & 0 & 0\end{array}$

$\begin{array}{lllllllll}0 & 0 & 0 & y z y z & y z z x & 0 & z x z x & -z x y z & 0 \\ 0 & 0 & 0 & z x z & z x z & 0 & -y z z & y z y z & 0\end{array} \mid \begin{array}{lllllllll}0 & 0 & 0 & 0 & 0 & 0 & 0 & 0 & 0 \\ 0 & 0 & 0 & 0 & 0 & 0 & 0 & 0 & 0\end{array}$

$\left.\begin{array}{ccccccccc}0 & 0 & 0 & z x y z & z x z x & 0 & -y z z x & y z y z & 0 \\ x y x x & -x y x x & 0 & 0 & 0 & x y x y & 0 & 0 & x y x y\end{array}\right) \quad\left(\begin{array}{ccccccccc}0 & 0 & 0 & 0 & 0 & 0 & 0 & 0 & 0 \\ 0 & 0 & 0 & 0 & 0 & 0 & 0 & 0 & 0\end{array}\right.$

and $x x x x=x x y y+2 x y x y ; y y x y-x x x y=2 x y x x$

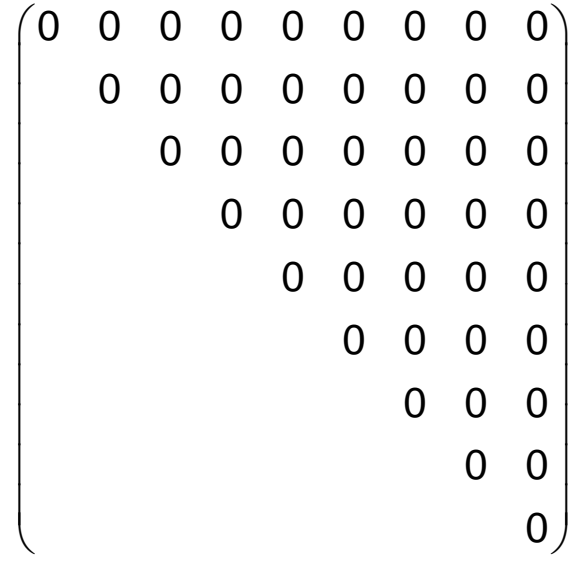

$\begin{array}{lllllllll}0 & 0 & 0 & 0 & 0 & 0 & 0 & 0 & 0\end{array}$

$0 \begin{array}{lllllllll}0 & 0 & 0 & 0 & 0 & 0 & 0 & 0 & 0\end{array}$

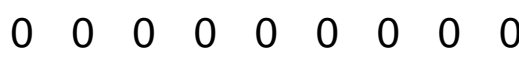

$\begin{array}{lllllllll}0 & 0 & 0 & 0 & 0 & 0 & 0 & 0 & 0\end{array}$

$0 \begin{array}{llllllllll}0 & 0 & 0 & 0 & 0 & 0 & 0 & 0 & 0 \\ 0 & 0 & 0 & 0 & 0 & 0 & 0 & 0 & 0\end{array}$

$\begin{array}{llllllllll}0 & 0 & 0 & 0 & 0 & 0 & 0 & 0 & 0\end{array}$

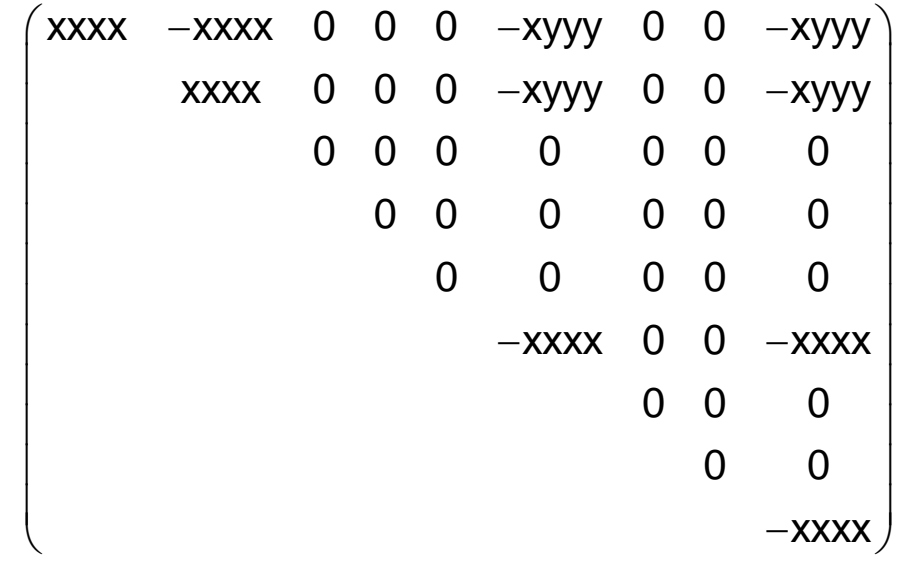

$\left(\begin{array}{ccccccccc}x x x x & -x x x x & 0 & 0 & 0 & -x y y y & 0 & 0 & -x y y y\end{array}\right)$ $\begin{array}{lllllllll}-x x x x & x x x x & 0 & 0 & 0 & x y y y & 0 & 0 & x y y y \\ 0 & 0 & 0 & 0 & 0 & 0 & 0 & 0 & 0\end{array}$ $\begin{array}{llllllll}0 & 0 & 0 & 0 & 0 & 0 & 0 & 0 \\ 0 & 0 & 0 & 0 & 0 & 0 & 0 & 0\end{array}$ $\begin{array}{llllllll}0 & 0 & 0 & 0 & 0 & 0 & 0 & 0\end{array}$ $\begin{array}{llllllll}0 & 0 & 0 & 0 & 0 & 0 & 0 & 0\end{array}$

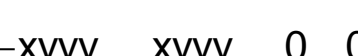




$\left(\begin{array}{ccccccccc}x x x x & x x y y & x x z z & 0 & 0 & x x x y & 0 & 0 & -y y x y \\ y y x x & x x x x & x x z z & 0 & 0 & y y x y & 0 & 0 & -x x x y \\ z z x x & z z y y & z z z z & 0 & 0 & z z x y & 0 & 0 & -z z x y \\ 0 & 0 & 0 & y z y z & y z z x & 0 & y z z y & y z x z & 0 \\ 0 & 0 & 0 & z x y z & z x z x & 0 & z x z y & z x x z & 0 \\ x y x x & x y y y & x y z z & 0 & 0 & x y x y & 0 & 0 & x y y x \\ 0 & 0 & 0 & z x x z & -z x z y & 0 & z x z x & -z x y z & 0 \\ 0 & 0 & 0 & -y z x z & y z z y & 0 & -y z z x & y z y z & 0 \\ -x y y y & -x y x x & -x y z z & 0 & 0 & x y y x & 0 & 0 & x y x y\end{array}\right)\left(\begin{array}{lllllllll}0 & 0 & 0 & 0 & 0 & 0 & 0 & 0 & 0 \\ 0 & 0 & 0 & 0 & 0 & 0 & 0 & 0 & 0 \\ 0 & 0 & 0 & 0 & 0 & 0 & 0 & 0 & 0 \\ 0 & 0 & 0 & 0 & 0 & 0 & 0 & 0 & 0 \\ 0 & 0 & 0 & 0 & 0 & 0 & 0 & 0 & 0 \\ 0 & 0 & 0 & 0 & 0 & 0 & 0 & 0 & 0 \\ 0 & 0 & 0 & 0 & 0 & 0 & 0 & 0 & 0 \\ 0 & 0 & 0 & 0 & 0 & 0 & 0 & 0 & 0 \\ 0 & 0 & 0 & 0 & 0 & 0 & 0 & 0 & 0\end{array}\right)$
and: $x x x x=x x y y+x y x y+x y y x ; x x x y-y y x y=x y y y-x y x x$
$\left[\mathrm{V}^{4}\right]$
$\left(\begin{array}{ccccc}3 x x y y & 0 & 0 & y y z z & 0 \\ 0 & 3 x x y y & 0 & y y z z & 0 \\ 0 & 0 & 2 z z z & x y y & 0\end{array}\right)$
$\begin{array}{llll}0 & \text { zzzz } & \mathrm{xxyy} & 0\end{array}$
$\left(\begin{array}{lllll}0 & 0 & 0 & 0 & 0 \\ 0 & 0 & 0 & 0 & 0 \\ 0 & 0 & 0 & 0 & 0\end{array}\right)$

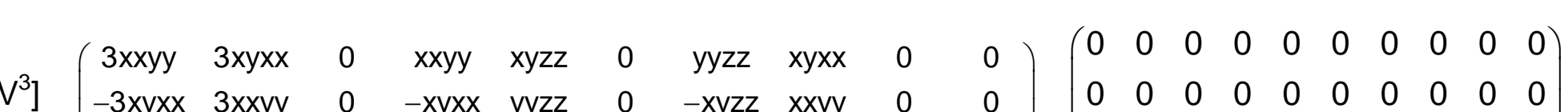

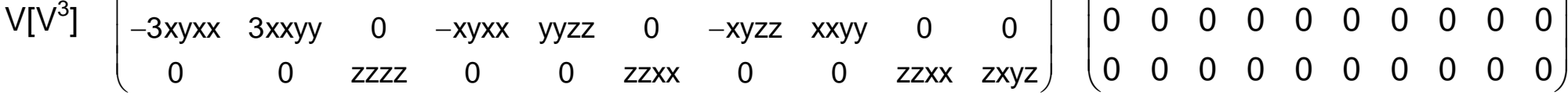

$\left[\left[^{2}\right]^{2}\right]\left(\begin{array}{cccccc}x x x x & x x y y & x x z z & 0 & 0 & 0 \\ & x x x x & x x z z & 0 & 0 & 0 \\ & & z z z z & 0 & 0 & 0 \\ & & & y z y z & 0 & 0 \\ & & & & y z y z & 0 \\ & & & & & x y x y\end{array}\right)$

$\left(\begin{array}{llllll}0 & 0 & 0 & 0 & 0 & 0 \\ & 0 & 0 & 0 & 0 & 0 \\ & & 0 & 0 & 0 & 0 \\ & & & 0 & 0 & 0 \\ & & & & 0 & 0 \\ & & & & & 0\end{array}\right)$

and $x y x y=1 / 2(x x x x-x x y y)$ $\left(\begin{array}{llllll}0 & 0 & 0 & 0 & 0 & 0 \\ 0 & 0 & 0 & 0 & 0 & 0\end{array}\right)$

$\begin{array}{llllll}0 & 0 & 0 & 0 & 0 & 0 \\ 0 & 0 & 0 & 0 & 0 & 0\end{array}$

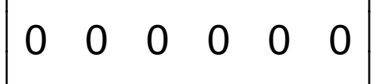

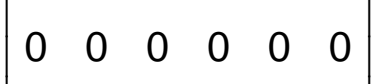

$\begin{array}{llllll}0 & 0 & 0 & 0 & 0 & 0 \\ 0 & 0 & 0 & 0 & 0 & 0\end{array}$ $\left(\begin{array}{lllllllll}0 & 0 & 0 & 0 & 0 & 0 & 0 & 0 & 0 \\ 0 & 0 & 0 & 0 & 0 & 0 & 0 & 0 & 0\end{array}\right)$ $\left.\begin{array}{lllllllll}0 & 0 & 0 & 0 & 0 & 0 & 0 & 0 & 0 \\ 0 & 0 & 0 & 0 & 0 & 0 & 0 & 0 & 0\end{array}\right)$ $\begin{array}{lllllllll}0 & 0 & 0 & 0 & 0 & 0 & 0 & 0 & 0 \\ 0 & 0 & 0 & 0 & 0 & 0 & 0 & 0 & 0\end{array}$

$0 \begin{array}{lllllllll}0 & 0 & 0 & 0 & 0 & 0 & 0 & 0 & 0 \\ 0 & 0 & 0 & 0 & 0 & 0 & 0 & 0 & 0\end{array}$ $0 \begin{array}{llllllllll}0 & 0 & 0 & 0 & 0 & 0 & 0 & 0 & 0 \\ 0 & 0 & 0 & 0 & 0 & 0 & 0\end{array}$ $0 \begin{array}{lllllllll}0 & 0 & 0 & 0 & 0 & 0 & 0 & 0 & 0 \\ 0 & 0 & 0 & 0 & 0 & 0 & 0 & 0 & 0\end{array}$ $0 \begin{array}{llllllllll}0 & 0 & 0 & 0 & 0 & 0 & 0 & 0 & 0\end{array}$

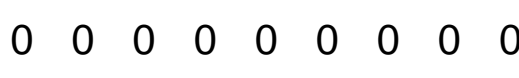
$\begin{array}{llllllllll}0 & 0 & 0 & 0 & 0 & 0 & 0 & 0 & 0\end{array}$

$\left(\begin{array}{lllll}0 & 0 & 0 & 0 & 0 \\ 0 & 0 & 0 & 0 & 0 \\ 0 & 0 & 0 & 0 & 0\end{array}\right)$

$\left(\begin{array}{llllllllll}0 & 0 & 0 & 0 & 0 & 0 & 0 & 0 & 0 & 0 \\ 0 & 0 & 0 & 0 & 0 & 0 & 0 & 0 & 0 & 0\end{array}\right)$ $\left(\begin{array}{lllllllllll}0 & 0 & 0 & 0 & 0 & 0 & 0 & 0 & 0 & 0 \\ 0 & 0 & 0 & 0 & 0 & 0 & 0 & 0 & 0 & 0\end{array}\right)$ $\begin{array}{lllllllllll}0 & 0 & 0 & 0 & 0 & 0 & 0 & 0 & 0 & 0\end{array}$

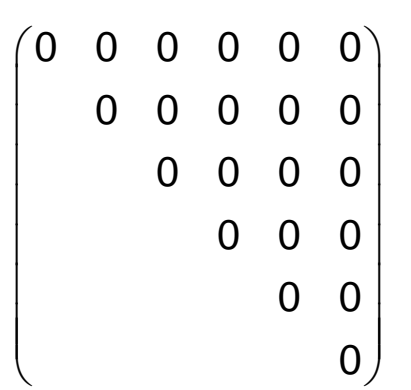

$\left(\begin{array}{llllll}0 & 0 & 0 & 0 & 0 & 0 \\ 0 & 0 & 0 & 0 & 0 & 0\end{array}\right)$

$\begin{array}{llllll}0 & 0 & 0 & 0 & 0 & 0 \\ 0 & 0 & 0 & 0 & 0 & 0\end{array}$

$\begin{array}{llllll}0 & 0 & 0 & 0 & 0 & 0 \\ 0 & 0 & 0 & 0 & 0 & 0\end{array}$

$\begin{array}{llllll}0 & 0 & 0 & 0 & 0 & 0 \\ 0 & 0 & 0 & 0 & 0 & 0\end{array}$ $\begin{array}{llllll}0 & 0 & 0 & 0 & 0 & 0 \\ 0 & 0 & 0 & 0 & 0 & 0\end{array}$ $\left(\begin{array}{lllllllll}0 & 0 & 0 & 0 & 0 & 0 & 0 & 0 & 0 \\ 0 & 0 & 0 & 0 & 0 & 0 & 0 & 0 & 0\end{array}\right)$ $\left(\begin{array}{llllllllll}0 & 0 & 0 & 0 & 0 & 0 & 0 & 0 & 0 \\ 0 & 0 & 0 & 0 & 0 & 0 & 0 & 0 & 0\end{array}\right)$ $\begin{array}{lllllllll}0 & 0 & 0 & 0 & 0 & 0 & 0 & 0 & 0 \\ 0 & 0 & 0 & 0 & 0 & 0 & 0 & 0 & 0 \\ 0 & 0 & 0 & 0 & 0 & 0 & 0\end{array}$ $\begin{array}{lllllllllll}0 & 0 & 0 & 0 & 0 & 0 & 0 & 0 & 0 \\ 0 & 0 & 0 & 0 & 0 & 0 & 0 & 0 & 0\end{array}$ $\begin{array}{llllllllll}0 & 0 & 0 & 0 & 0 & 0 & 0 & 0 & 0 & 0\end{array}$ $\begin{array}{llllllllll}0 & 0 & 0 & 0 & 0 & 0 & 0 & 0 & 0 \\ 0 & 0 & 0 & 0 & 0 & 0 & 0 & 0 & 0\end{array}$ $\begin{array}{lllllllll}0 & 0 & 0 & 0 & 0 & 0 & 0 & 0 & 0 \\ 0 & 0 & 0 & 0 & 0 & 0 & 0 & 0 & 0\end{array}$ $\begin{array}{lllllllll}0 & 0 & 0 & 0 & 0 & 0 & 0 & 0 & 0\end{array}$

$\left(\begin{array}{lllll}0 & 0 & 0 & 0 & 0 \\ 0 & 0 & 0 & 0 & 0 \\ 0 & 0 & 0 & 0 & 0\end{array}\right)$ $\left(\begin{array}{lllll}0 & 0 & 0 & 0 & 0 \\ 0 & 0 & 0 & 0 & 0\end{array}\right)$

$\left(\begin{array}{llllllllll}0 & 0 & 0 & 0 & 0 & 0 & 0 & 0 & 0 & 0 \\ 0 & 0 & 0 & 0 & 0 & 0 & 0 & 0 & 0 & 0\end{array}\right)$ $\left(\begin{array}{llllllllll}0 & 0 & 0 & 0 & 0 & 0 & 0 & 0 & 0 & 0 \\ 0 & 0 & 0 & 0 & 0 & 0 & 0 & 0 & 0 & 0\end{array}\right)$ $\begin{array}{llllllllll}0 & 0 & 0 & 0 & 0 & 0 & 0 & 0 & 0 & 0\end{array}$

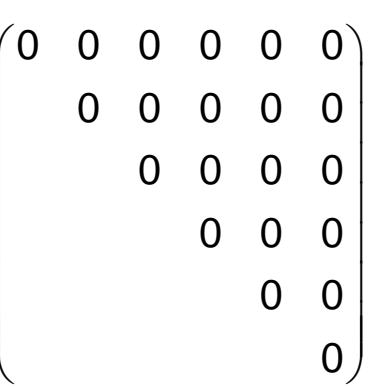

$\begin{array}{llllll}0 & 0 & 0 & 0 & 0 & 0 \\ 0 & 0 & 0 & 0 & 0 & 0\end{array}$ $\begin{array}{llllll}0 & 0 & 0 & 0 & 0 & 0 \\ 0 & 0 & 0 & 0 & 0 & 0\end{array}$ $\begin{array}{llllll}0 & 0 & 0 & 0 & 0 & 0 \\ 0 & 0 & 0 & 0 & 0\end{array}$ $0 \begin{array}{llllll}0 & 0 & 0 & 0 & 0 & 0 \\ 0 & 0 & 0 & 0 & 0 & 0\end{array}$ $\begin{array}{llllll}0 & 0 & 0 & 0 & 0 & 0 \\ 0 & 0 & 0 & 0 & 0 & 0\end{array}$

and $x y x y=1 / 2(x x x x-x x y y)$ 


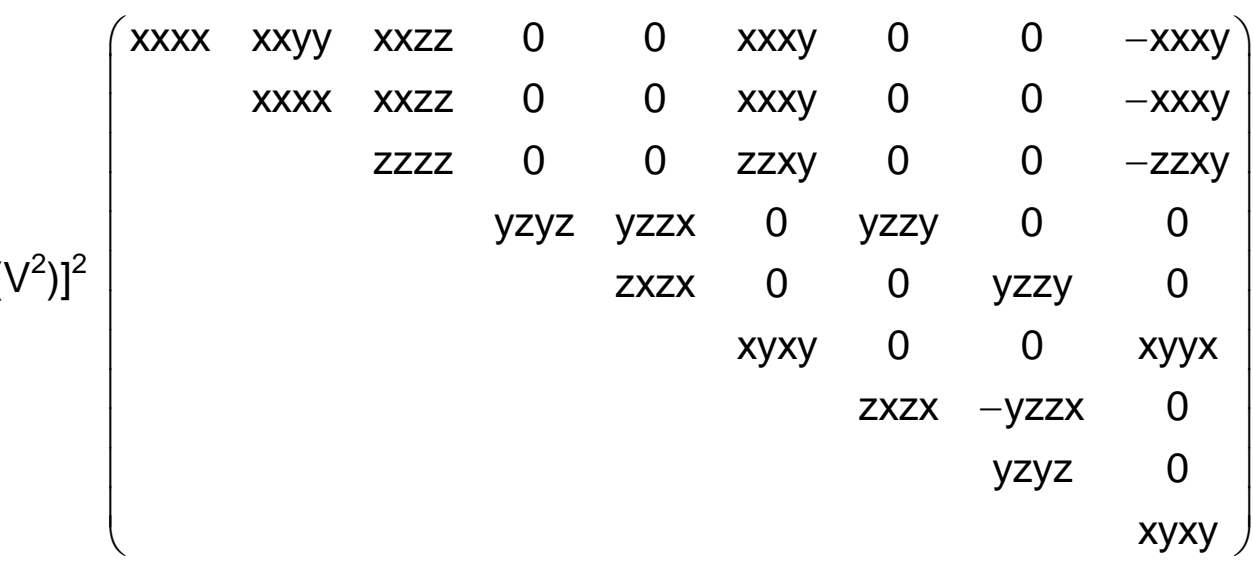

$\left(\begin{array}{lllllllll}0 & 0 & 0 & 0 & 0 & 0 & 0 & 0 & 0 \\ & 0 & 0 & 0 & 0 & 0 & 0 & 0 & 0\end{array}\right)$ $0 \begin{array}{lllllll}0 & 0 & 0 & 0 & 0 & 0 \\ 0 & 0 & 0\end{array}$ $0 \begin{array}{lllll}0 & 0 & 0 & 0 & 0\end{array}$ $0 \begin{array}{lllll}0 & 0 & 0 & 0 & 0 \\ 0 & 0 & 0\end{array}$ $0 \begin{array}{lll}0 & 0 & 0 \\ 0 & 0 & 0\end{array}$ $\begin{array}{lll}0 & 0 & 0\end{array}$ $\begin{array}{ll}0 & 0 \\ & 0\end{array}$

and $x x x x=x x y y+y z y z+z y z y$

$\left(\begin{array}{lllllllll}x x x x & x x y y & x x z z & 0 & 0 & x x x y & 0 & 0 & -y y x y \\ x x y y & x y x & x x z z & 0 & 0 & y x y & 0 & 0 & -x x y\end{array}\right)\left(\begin{array}{ccccccccc}0 & 0 & 0 & 0 & 0 & 0 & 0 & 0 & 0 \\ 0 & 0 & 0 & 0 & 0 & 0 & 0 & 0 & 0 \\ 0 & 0 & 0 & 0 & 0 & 0 & 0 & 0 & 0\end{array}\right)$ $\left(\begin{array}{lllllllll}x x y y & x x x x & x x z z & 0 & 0 & y y x y & 0 & 0 & -x x x y \\ z z x x & z z x x & z z z z & 0 & 0 & z z x y & 0 & 0 & -z z x y\end{array} \mid \begin{array}{llllllllll}0 & 0 & 0 & 0 & 0 & 0 & 0 & 0 & 0 \\ 0 & 0 & 0 & 0 & 0 & 0 & 0 & 0 & 0 \\ 0 & 0 & 0 & 0 & 0 & 0 & 0 & 0 & 0\end{array}\right.$ $\left[V^{2}\right] V^{2} \quad \begin{array}{ccccccccc}z z x x & z z x x & z z z z & 0 & 0 & z z x y & 0 & 0 & -z z x y \\ 0 & 0 & 0 & y z y z & y z z x & 0 & z x z x & -z x y z & 0\end{array} \mid$ $\left.\begin{array}{lllllllll}0 & 0 & 0 & z x y z & z x z x & 0 & -y z z x & y z y z & 0\end{array}\right) \quad\left(\begin{array}{lllllllllll}0 & 0 & 0 & 0 & 0 & 0 & 0 & 0 & 0 \\ 0 & 0 & 0 & 0 & 0 & 0 & 0 & 0 & 0 \\ 0 & 0 & 0 & 0 & 0 & 0 & 0 & 0 & 0\end{array}\right)$ $\left.\begin{array}{lllllllll}x y x x & -x y x x & 0 & 0 & 0 & x y x y & 0 & 0 & x y x y\end{array}\right) \quad\left(\begin{array}{lllllllll}0 & 0 & 0 & 0 & 0 & 0 & 0 & 0 & 0\end{array}\right)$

and $x x x x=x x y y+2 x y x y ; y y x y-x x x y=2 x y x x$

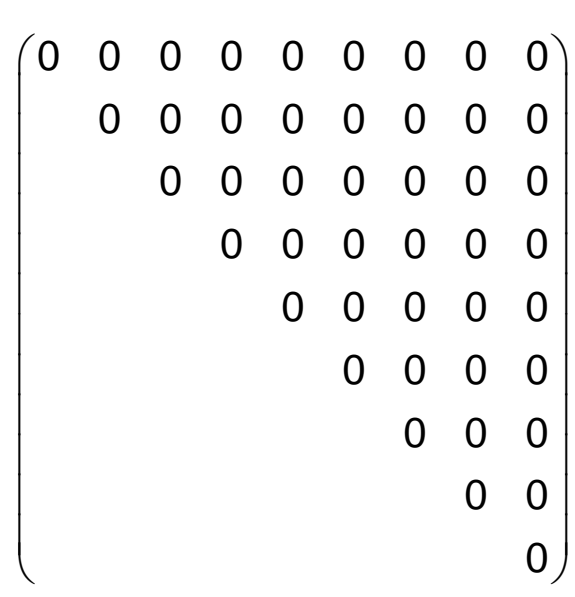

$\left(\begin{array}{lllllllll}0 & 0 & 0 & 0 & 0 & 0 & 0 & 0 & 0 \\ & 0 & 0 & 0 & 0 & 0 & 0 & 0 & 0\end{array}\right)$ $\begin{array}{lllllll}0 & 0 & 0 & 0 & 0 & 0 & 0\end{array}$ $\begin{array}{llllll}0 & 0 & 0 & 0 & 0 & 0\end{array}$ $\begin{array}{lllll}0 & 0 & 0 & 0 & 0\end{array}$ $\begin{array}{llll}0 & 0 & 0 & 0 \\ & 0 & 0 & 0\end{array}$ $\begin{array}{lll}0 & 0 & 0 \\ & 0 & 0 \\ & & 0\end{array}$

$\left(\begin{array}{lllllllll}0 & 0 & 0 & 0 & 0 & 0 & 0 & 0 & 0 \\ 0 & 0 & 0 & 0 & 0 & 0 & 0 & 0 & 0\end{array}\right.$ $\begin{array}{lllllllll}0 & 0 & 0 & 0 & 0 & 0 & 0 & 0 & 0 \\ 0 & 0 & 0 & 0 & 0 & 0 & 0 & 0 & 0\end{array}$ $\begin{array}{lllllllll}0 & 0 & 0 & 0 & 0 & 0 & 0 & 0 & 0\end{array}$ $\begin{array}{lllllllll}0 & 0 & 0 & 0 & 0 & 0 & 0 & 0 & 0 \\ 0 & 0 & 0 & 0 & 0 & 0 & 0 & 0 & 0\end{array}$ $\begin{array}{llllllllll}0 & 0 & 0 & 0 & 0 & 0 & 0 & 0 & 0\end{array}$ $\begin{array}{llllllllll}0 & 0 & 0 & 0 & 0 & 0 & 0 & 0 & 0\end{array}$ $\begin{array}{lllllllll}0 & 0 & 0 & 0 & 0 & 0 & 0 & 0 & 0 \\ 0 & 0 & 0 & 0 & 0 & 0 & 0 & 0 & 0\end{array}$ $\begin{array}{lllllllll}0 & 0 & 0 & 0 & 0 & 0 & 0 & 0 & 0 \\ 0 & 0 & 0 & 0 & 0 & 0 & 0 & 0 & 0\end{array}$ $\begin{array}{lllllllll}0 & 0 & 0 & 0 & 0 & 0 & 0 & 0 & 0 \\ 0 & 0 & 0 & 0 & 0 & 0 & 0 & 0 & 0\end{array}$ $\begin{array}{lllllllll}0 & 0 & 0 & 0 & 0 & 0 & 0 & 0 & 0 \\ 0 & 0 & 0 & 0 & 0 & 0 & 0 & 0 & 0\end{array}$ $\begin{array}{lllllllll}0 & 0 & 0 & 0 & 0 & 0 & 0 & 0 & 0 \\ 0 & 0 & 0 & 0 & 0 & 0 & 0 & 0 & 0\end{array}$ 
$\left(\begin{array}{lllllllll}0 & 0 & 0 & 0 & 0 & 0 & 0 & 0 & 0 \\ 0 & 0 & 0 & 0 & 0 & 0 & 0 & 0 & 0\end{array}\right)$ $\begin{array}{lllllllll}0 & 0 & 0 & 0 & 0 & 0 & 0 & 0 & 0 \\ 0 & 0 & 0 & 0 & 0 & 0 & 0 & 0 & 0\end{array}$ $0 \begin{array}{lllllllll}0 & 0 & 0 & 0 & 0 & 0 & 0 & 0 & 0 \\ 0 & 0 & 0 & 0 & 0 & 0 & 0 & 0 & 0\end{array}$ $\begin{array}{lllllllll}0 & 0 & 0 & 0 & 0 & 0 & 0 & 0 & 0 \\ 0 & 0 & 0 & 0 & 0 & 0 & 0 & 0 & 0\end{array}$ $\begin{array}{llllllllll}0 & 0 & 0 & 0 & 0 & 0 & 0 & 0 & 0 \\ 0 & 0 & 0 & 0 & 0 & 0 & 0 & 0 & 0\end{array}$ $\begin{array}{llllllllll}0 & 0 & 0 & 0 & 0 & 0 & 0 & 0 & 0 \\ 0 & 0 & 0 & 0 & 0 & 0 & 0 & 0 & 0 & 0\end{array}$ $\begin{array}{llllllllllllll}0 & 0 & 0 & 0 & 0 & 0 & 0 & 0 & 0 \\ 0 & 0 & 0 & 0 & 0 & 0 & 0 & 0 & 0\end{array}$ $\left(\begin{array}{lllllllll}0 & 0 & 0 & 0 & 0 & 0 & 0 & 0 & 0 \\ 0 & 0 & 0 & 0 & 0 & 0 & 0 & 0 & 0 \\ 0 & 0 & 0 & 0 & 0 & 0 & 0 & 0 & 0\end{array}\right)$ $\left(\begin{array}{lllllllll}0 & 0 & 0 & 0 & 0 & 0 & 0 & 0 & 0 \\ 0 & 0 & 0 & 0 & 0 & 0 & 0 & 0 & 0\end{array}\right)$ $\begin{array}{ccccccccc}y x x x & y x y y & y x z z & 0 & 0 & y x x y & 0 & 0 & y x y x\end{array}$

$$
\left(\begin{array}{lllll}
0 & 0 & 0 & 0 & 0 \\
0 & 0 & 0 & 0 & 0 \\
0 & 0 & 0 & 0 & 0
\end{array}\right)
$$

$\left[V^{4}\right]\left(\begin{array}{ccccc}x x x x & x y y y & 0 & y y z z & 0 \\ y x x x & y y y y & 0 & z z x x & 0 \\ 0 & 0 & z z z z & x x y y & z z x y\end{array}\right)$

$\left(\begin{array}{llllllllll}x x x x & x y y y & 0 & x x y y & x y z z & 0 & x x z z & x y x x & 0 & 0 \\ y x x & y y y & 0 & y x y y & y y z & 0 & y x z z & y x x & 0 & 0\end{array}\right)\left(\begin{array}{llllllllll}0 & 0 & 0 & 0 & 0 & 0 & 0 & 0 & 0 & 0 \\ 0 & 0 & 0 & 0 & 0 & 0 & 0 & 0 & 0 & 0 \\ 0 & 0 & 0 & 0 & 0 & 0 & 0 & 0 & 0\end{array}\right)$

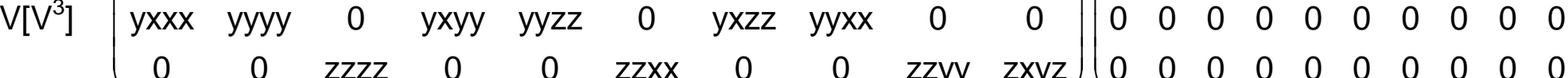

$\left[\left[V^{2}\right]^{2}\right]\left(\begin{array}{cccccc}x x x x & x x y y & x x z z & 0 & 0 & x x x y \\ & y y y y & y y z z & 0 & 0 & y y x y \\ & & z z z z & 0 & 0 & z z x y \\ & & & y z y z & y z x z & 0 \\ & & & & x z x z & 0 \\ & & & & & x y x y\end{array}\right)$

$\left(\begin{array}{llllll}x x x x & x x y y & x x z z & 0 & 0 & x x x y \\ y y x x & y y y y & y y z z & 0 & 0 & y y x y \\ z z x x & z z y y & z z z z & 0 & 0 & z z x y\end{array}\right)$

$\begin{array}{llllll}z z x x & z z y y & z z z z & 0 & 0 & z z x y\end{array}$ $\begin{array}{llllll}0 & 0 & 0 & y z y z & y z x z & 0\end{array}$ $\begin{array}{cccccc}0 & 0 & 0 & x z y z & x z x z & 0\end{array}$

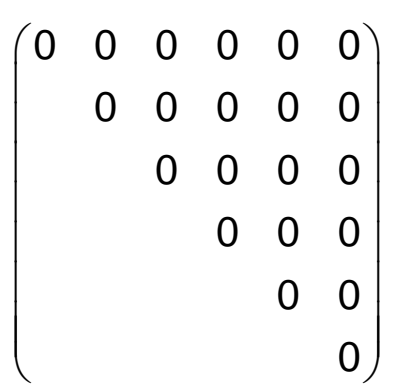

$\left(\begin{array}{llllll}0 & 0 & 0 & 0 & 0 & 0 \\ 0 & 0 & 0 & 0 & 0 & 0\end{array}\right)$ $\begin{array}{llllll}0 & 0 & 0 & 0 & 0 & 0 \\ 0 & 0 & 0 & 0 & 0 & 0 \\ 0 & 0 & 0 & 0 & 0 & 0\end{array}$ $0 \begin{array}{lllllll}0 & 0 & 0 & 0 & 0 & 0\end{array}$ $\begin{array}{llllll}0 & 0 & 0 & 0 & 0 & 0\end{array}$ $\begin{array}{llllll}0 & 0 & 0 & 0 & 0 & 0 \\ 0 & 0 & 0 & 0 & 0 & 0 \\ 0 & 0 & 0 & 0 & 0 & 0\end{array}$ $\begin{array}{lllllllll}0 & 0 & 0 & 0 & 0 & 0 & 0 & 0 & 0 \\ 0 & 0 & 0 & 0 & 0 & 0 & 0 & 0 & 0\end{array}$ $0 \begin{array}{lllllllll}0 & 0 & 0 & 0 & 0 & 0 & 0 & 0 & 0 \\ 0 & 0 & 0 & 0 & 0 & 0 & 0 & 0 & 0\end{array}$ $0 \begin{array}{lllllllll}0 & 0 & 0 & 0 & 0 & 0 & 0 & 0 & 0 \\ 0 & 0 & 0 & 0 & 0 & 0 & 0 & 0 & 0\end{array}$ $\begin{array}{lllllllll}0 & 0 & 0 & 0 & 0 & 0 & 0 & 0 & 0 \\ 0 & 0 & 0 & 0 & 0 & 0 & 0 & 0 & 0\end{array}$ $\begin{array}{lllllllll}0 & 0 & 0 & 0 & 0 & 0 & 0 & 0 & 0 \\ 0 & 0 & 0 & 0 & 0 & 0 & 0 & 0\end{array}$ $\begin{array}{llllllllll}0 & 0 & 0 & 0 & 0 & 0 & 0 & 0 & 0\end{array}$ $\begin{array}{llllllllll}0 & 0 & 0 & 0 & 0 & 0 & 0 & 0 & 0\end{array}$ $0 \begin{array}{llllllllll}0 & 0 & 0 & 0 & 0 & 0 & 0 & 0 & 0 \\ 0 & 0 & 0 & 0 & 0 & 0 & 0\end{array}$ $\begin{array}{llllllllll}0 & 0 & 0 & 0 & 0 & 0 & 0 & 0 & 0\end{array}$

$\left(\begin{array}{lllll}0 & 0 & 0 & 0 & 0 \\ 0 & 0 & 0 & 0 & 0 \\ 0 & 0 & 0 & 0 & 0\end{array}\right)$ $\begin{array}{lllll}0 & 0 & 0 & 0 & 0\end{array}$

$\left(\begin{array}{llllllllll}0 & 0 & 0 & 0 & 0 & 0 & 0 & 0 & 0 & 0 \\ 0 & 0 & 0 & 0 & 0 & 0 & 0 & 0 & 0 & 0\end{array}\right)$ $\left(\begin{array}{llllllllll}0 & 0 & 0 & 0 & 0 & 0 & 0 & 0 & 0 & 0 \\ 0 & 0 & 0 & 0 & 0 & 0 & 0 & 0 & 0 & 0\end{array}\right)$ $\begin{array}{lllllllllll}0 & 0 & 0 & 0 & 0 & 0 & 0 & 0 & 0 & 0\end{array}$

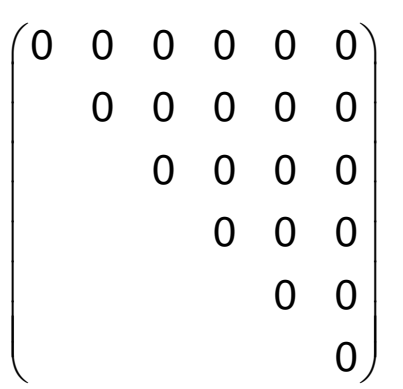

$\left(\begin{array}{llllll}0 & 0 & 0 & 0 & 0 & 0 \\ 0 & 0 & 0 & 0 & 0 & 0 \\ 0 & 0 & 0 & 0 & 0 & 0\end{array}\right)$ $\left.\begin{array}{llllll}0 & 0 & 0 & 0 & 0 & 0 \\ 0 & 0 & 0 & 0 & 0 & 0 \\ 0 & 0 & 0 & 0 & 0 & 0\end{array}\right)$ $0 \begin{array}{llllll}0 & 0 & 0 & 0 & 0 & 0 \\ 0 & 0 & 0 & 0 & 0\end{array}$ $\begin{array}{llllll}0 & 0 & 0 & 0 & 0 & 0\end{array}$ $\begin{array}{llllll}0 & 0 & 0 & 0 & 0 & 0 \\ 0 & 0 & 0 & 0 & 0 & 0\end{array}$ $\left(\begin{array}{lllllllll}0 & 0 & 0 & x x y z & x x z x & 0 & x x z y & x x x z & 0 \\ 0 & 0 & 0 & y y y z & y z x & 0 & y y z y & y x z & 0\end{array}\right)$ $\left.\begin{array}{lllllllll}0 & 0 & 0 & \text { yyyz } & y y z x & 0 & y y z y & y y x z & 0 \\ 0 & 0 & 0 & z z y & z z z x & 0 & z z y & z z x z & 0\end{array}\right)$ $\begin{array}{lllllllll}\text { yzxx } & \text { yzyy } & \text { yzzz } & 0 & 0 & y z x y & 0 & 0 & y z y x \\ z x x x & z x y & z x z z & 0 & 0 & z x y & 0 & 0 & z x y x\end{array}$ $\begin{array}{lllllllll}z x x x & z x y y & z x z z & 0 & 0 & z x x y & 0 & 0 & z x y x\end{array}$ $\begin{array}{lllllllll}0 & 0 & 0 & x y y z & x y z x & 0 & x y z y & x y x z & 0\end{array}$

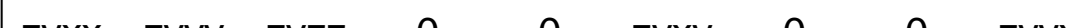

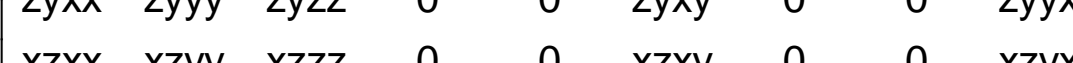
$\begin{array}{ccccccccc}x z x x & x z y y & x z z z & 0 & 0 & x z x y & 0 & 0 & x z y x \\ 0 & 0 & 0 & y x y z & y x z x & 0 & y x z y & y x x z & 0\end{array}$

$\left(\begin{array}{ccccc}0 & 0 & x z z z & 0 & x x y z \\ 0 & 0 & y z z z & 0 & y y x z \\ z x x x & z y y & 0 & 0 & 0\end{array}\right)$ zxxx $\quad$ zyyy $\quad 0 \quad 00 \quad 0$

$\left(\begin{array}{cccccccccc}0 & 0 & x z z z & 0 & 0 & x z x x & 0 & 0 & x z y y & x x y z \\ 0 & 0 & y z z & 0 & 0 & y z x x & 0 & 0 & y z y y & y x y z\end{array}\right.$

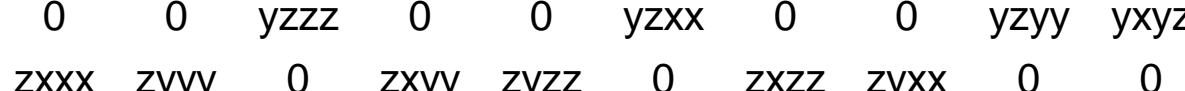

$$
\left(\begin{array}{cccccc}
0 & 0 & 0 & x x y z & x x x z & 0 \\
& 0 & 0 & y y y z & y y x z & 0 \\
& & 0 & z z y z & z z x z & 0 \\
& & & 0 & 0 & y z x y \\
& & & & 0 & x z x y \\
& & & & & 0
\end{array}\right)
$$

$\left(\begin{array}{cccccc}0 & 0 & 0 & x x y z & x x x z & 0 \\ 0 & 0 & 0 & y y y z & y y x z & 0 \\ 0 & 0 & 0 & \text { zzyz } & \text { zzxz } & 0 \\ y z x x & y z y y & y z z z & 0 & 0 & y z x y \\ x z x x & x z y y & x z z z & 0 & 0 & \text { xzxy } \\ 0 & 0 & 0 & x y y z & x y x z & 0\end{array}\right)$ 


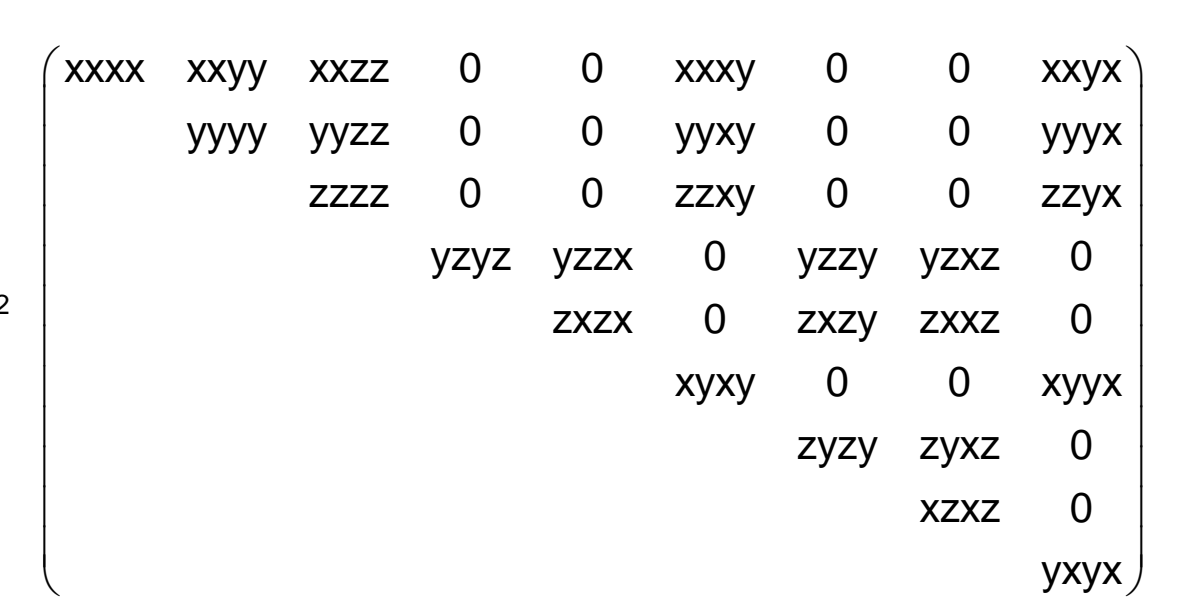

$\left(\begin{array}{lllllllll}0 & 0 & 0 & 0 & 0 & 0 & 0 & 0 & 0\end{array}\right)$

$0 \begin{array}{llllllll}0 & 0 & 0 & 0 & 0 & 0 & 0 & 0\end{array}$

$\begin{array}{lllllll}0 & 0 & 0 & 0 & 0 & 0 & 0 \\ 0 & 0 & 0 & 0 & 0 & 0\end{array}$

$\begin{array}{lllll}0 & 0 & 0 & 0 & 0\end{array}$

$\begin{array}{llll}0 & 0 & 0 & 0\end{array}$

$\begin{array}{lll}0 & 0 & 0 \\ & 0 & 0\end{array}$

yxyx

0

$\left(\begin{array}{lllllllll}x x x x & x x y y & x x z z & 0 & 0 & x x x y & 0 & 0 & x x y x \\ y y x & y y y & y y z z & 0 & 0 & y x y & 0 & 0 & y y x\end{array}\right)$

$\left.\left[V^{2}\right] V^{2} \quad \begin{array}{ccccccccc}x y x x & z z y y & z z z z & 0 & 0 & z z x y & 0 & 0 & z z y x \\ 0 & 0 & 0 & y z y z & y z z x & 0 & y z z & y z z z & 0\end{array}\right)$ $\begin{array}{ccccccccc}0 & 0 & 0 & y z y z & y z z x & 0 & y z z y & y z x z & 0\end{array}$ $\begin{array}{ccccccccc}0 & 0 & 0 & z x y z & z x z x & 0 & z x z y & z x x z & 0\end{array}$ $\begin{array}{lllllllll}\text { xyxx } & \text { xyyy } & \text { xyzz } & 0 & 0 & \text { xyxy } & 0 & 0 & \text { xyyx }\end{array}$ $\left(\begin{array}{lllllllll}0 & 0 & 0 & 0 & 0 & 0 & 0 & 0 & 0 \\ 0 & 0 & 0 & 0 & 0 & 0 & 0 & 0 & 0 \\ 0 & 0 & 0 & 0 & 0 & 0 & 0 & 0 & 0\end{array}\right)$

$\left(\begin{array}{lllllllll}0 & 0 & 0 & 0 & 0 & 0 & 0 & 0 & 0 \\ 0 & 0 & 0 & 0 & 0 & 0 & 0 & 0 & 0 \\ 0 & 0 & 0 & 0 & 0 & 0 & 0 & 0 & 0\end{array}\right)$

$\begin{array}{lllllllllllll}0 & 0 & 0 & 0 & 0 & 0 & 0 & 0 & 0 \\ 0 & 0 & 0 & 0 & 0 & 0 & 0 & 0 & 0\end{array}$

$\begin{array}{llllllllll}0 & 0 & 0 & 0 & 0 & 0 & 0 & 0 & 0\end{array}$

$\begin{array}{lllllllll}0 & 0 & 0 & 0 & 0 & 0 & 0 & 0 & 0 \\ 0 & 0 & 0 & 0 & 0 & 0 & 0 & 0 & 0\end{array}$ $\left(\begin{array}{lllllllll}0 & 0 & 0 & 0 & 0 & 0 & 0 & 0 & 0\end{array}\right)$

$\begin{array}{llllllll}0 & 0 & 0 & 0 & 0 & 0 & 0 & 0\end{array}$

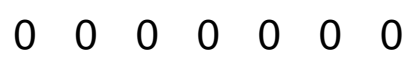

$\begin{array}{llllll}0 & 0 & 0 & 0 & 0 & 0\end{array}$

$\begin{array}{lllll}0 & 0 & 0 & 0 \\ 0 & 0 & 0 & 0\end{array}$

$\begin{array}{rrr}0 & 0 & 0 \\ & 0 & 0\end{array}$

$\left.\begin{array}{ll}0 & 0 \\ & 0\end{array}\right)$

$\begin{array}{lllllllll}0 & 0 & 0 & 0 & 0 & 0 & 0 & 0 & 0 \\ 0 & 0 & 0 & 0 & 0 & 0 & 0 & 0 & 0\end{array}$

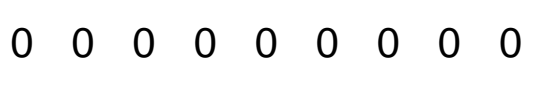

$0 \begin{array}{lllllllll}0 & 0 & 0 & 0 & 0 & 0 & 0 & 0 & 0 \\ 0 & 0 & 0 & 0 & 0 & 0 & 0 & 0 & 0\end{array}$

$\begin{array}{llllllllll}0 & 0 & 0 & 0 & 0 & 0 & 0 & 0 & 0\end{array}$

$\begin{array}{lllllllll}0 & 0 & 0 & 0 & 0 & 0 & 0 & 0 & 0 \\ 0 & 0 & 0 & 0 & 0 & 0 & 0 & 0 & 0\end{array}$

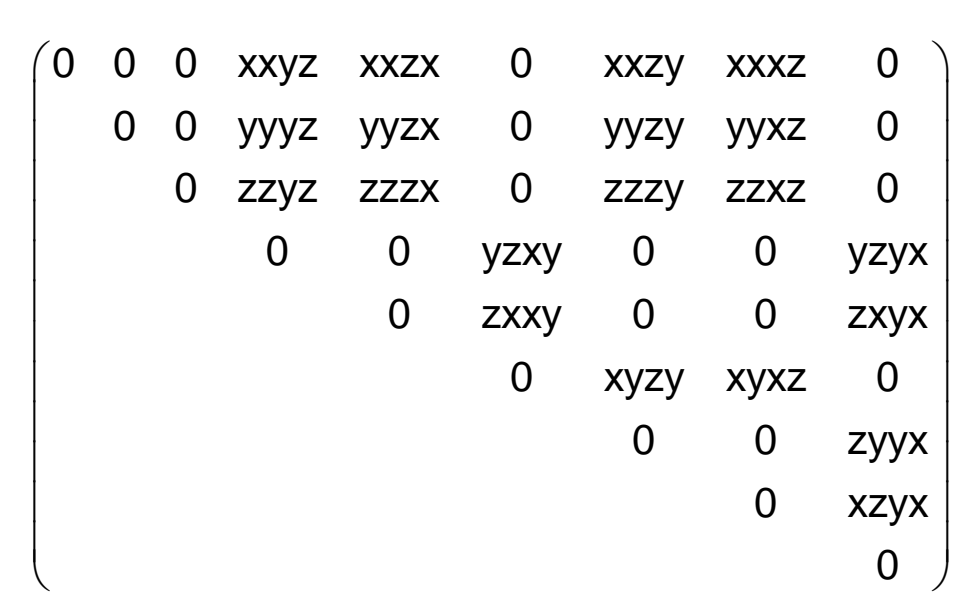

$\left(\begin{array}{ccccccccc}0 & 0 & 0 & \text { xxyz } & \text { xxzx } & 0 & \text { xxzy } & \text { xxxz } & 0 \\ 0 & 0 & 0 & \text { yyyz } & \text { yyzx } & 0 & \text { yyzy } & \text { yyxz } & 0 \\ 0 & 0 & 0 & \text { zzyz } & \text { zzzx } & 0 & \text { zzzy } & \text { zzxz } & 0 \\ \text { yzxx } & \text { yzyy } & \text { yzzz } & 0 & 0 & \text { yzxy } & 0 & 0 & \text { yzyx } \\ \text { zxxx } & \text { zxyy } & \text { zxzz } & 0 & 0 & \text { zxxy } & 0 & 0 & \text { zxyx } \\ 0 & 0 & 0 & \text { xyyz } & \text { xyzx } & 0 & \text { xyzy } & \text { xyxz } & 0\end{array}\right)$ 
$\begin{array}{lllllllll}0 & 0 & 0 & 0 & 0 & 0 & 0 & 0 & 0 \\ 0 & 0 & 0 & 0 & 0 & 0 & 0 & 0 & 0\end{array}$ $\begin{array}{lllllllll}0 & 0 & 0 & 0 & 0 & 0 & 0 & 0 & 0 \\ 0 & 0 & 0 & 0 & 0 & 0 & 0 & 0 & 0\end{array}$

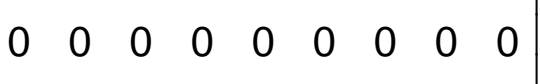
$\begin{array}{lllllllll}0 & 0 & 0 & 0 & 0 & 0 & 0 & 0 & 0 \\ 0 & 0 & 0 & 0 & 0 & 0 & 0 & 0 & 0\end{array}$

$\begin{array}{llllllllll}0 & 0 & 0 & 0 & 0 & 0 & 0 & 0 & 0 \\ 0 & 0 & 0 & 0 & 0 & 0 & 0 & 0 & 0\end{array}$

$\begin{array}{llllllllll}0 & 0 & 0 & 0 & 0 & 0 & 0 & 0 & 0 \\ 0 & 0 & 0 & 0 & 0 & 0 & 0 & 0 & 0\end{array}$ $\begin{array}{lllllllll}0 & 0 & 0 & 0 & 0 & 0 & 0 & 0 & 0 \\ 0 & 0 & 0 & 0 & 0 & 0 & 0 & 0 & 0 \\ 0 & 0 & 0 & 0 & 0 & 0 & 0 & 0 & 0\end{array} \mid$

$\begin{array}{lllllllll}0 & 0 & 0 & 0 & 0 & 0 & 0 & 0 & 0\end{array}$

$\left[V^{4}\right]\left(\begin{array}{ccccc}x x x x & -y x x x & 0 & y y z z & 0 \\ y x x x & x x x x & 0 & y y z z & 0 \\ 0 & 0 & z z z z & x x y y & 0\end{array}\right)$

$\left(\begin{array}{lllll}0 & 0 & 0 & 0 & 0 \\ 0 & 0 & 0 & 0 & 0 \\ 0 & 0 & 0 & 0 & 0\end{array}\right)$

$\left.V^{3}\right]\left(\begin{array}{cccccccccc}x x x x & -y x x x & 0 & x x y y & x y z z & 0 & y y z z & x y x x & 0 & 0 \\ y x x x & x x x x & 0 & -x y x x & y y z z & 0 & -x y z z & x x y y & 0 & 0 \\ 0 & 0 & z z z z & 0 & 0 & z z x & 0 & 0 & z z x x & z x y z\end{array}\right)$

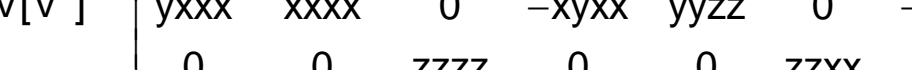

$\left(\begin{array}{llllllllll}0 & 0 & 0 & 0 & 0 & 0 & 0 & 0 & 0 & 0 \\ 0 & 0 & 0 & 0 & 0 & 0 & 0 & 0 & 0 & 0\end{array}\right.$ $\left(\begin{array}{llllllllll}0 & 0 & 0 & 0 & 0 & 0 & 0 & 0 & 0 & 0 \\ 0 & 0 & 0 & 0 & 0 & 0 & 0 & 0 & 0 & 0\end{array}\right)$ $\begin{array}{llllllllll}0 & 0 & 0 & 0 & 0 & 0 & 0 & 0 & 0 & 0\end{array}$

$\left(\begin{array}{cccccc}x x x x & \text { xxyy } & \text { xxzz } & 0 & 0 & \text { xxxy } \\ & \text { yyyy } & \text { yyzz } & 0 & 0 & -x x x y \\ & & \text { zzzz } & 0 & 0 & 0 \\ & & & y z y z & 0 & 0 \\ & & & & y z y z & 0 \\ & & & & & \text { xyxy }\end{array}\right)$

$\left(\begin{array}{llllll}x x x & x x y y & x x z z & 0 & 0 & x\end{array}\right.$

$\begin{array}{llllll}x y y x x & x x x x & x x z z & 0 & 0 & -x x x y\end{array}$

$\begin{array}{cccccc}z z x x & \text { zzxx } & \text { zzzz } & 0 & 0 & 0 \\ 0 & 0 & 0 & y z y z & y z x z & 0 \\ 0 & 0 & 0 & -y z z z & y z y z & 0\end{array}$

$\begin{array}{cccccc}0 & 0 & 0 & y z y z & y z x z & 0 \\ 0 & 0 & 0 & -y z x z & y z y z & 0\end{array}$

$$
\left(\begin{array}{llllll}
0 & 0 & 0 & 0 & 0 & 0 \\
& 0 & 0 & 0 & 0 & 0 \\
& & 0 & 0 & 0 & 0 \\
& & & 0 & 0 & 0 \\
& & & & 0 & 0 \\
& & & & & \\
& & & & & 0
\end{array}\right)
$$

$\left(\begin{array}{llllll}0 & 0 & 0 & 0 & 0 & 0 \\ 0 & 0 & 0 & 0 & 0 & 0\end{array}\right)$ $\begin{array}{llllll}0 & 0 & 0 & 0 & 0 & 0 \\ 0 & 0 & 0 & 0 & 0 & 0 \\ 0 & 0 & 0 & 0 & 0 & 0\end{array}$

$0 \begin{array}{llllll}0 & 0 & 0 & 0 & 0 & 0\end{array}$

$\begin{array}{llllll}0 & 0 & 0 & 0 & 0 & 0 \\ 0 & 0 & 0 & 0 & 0 & 0\end{array}$ $\left(\begin{array}{ccccccccc}\text { xxxx } & x x y y & x x z z & 0 & 0 & x x x y & 0 & 0 & y y x y \\ -x x y y & -x x x x & -x x z & 0 & 0 & y y x y & 0 & 0 & x x y y \\ z z x x & -z z x x & 0 & 0 & 0 & z z x y & 0 & 0 & z z x y \\ 0 & 0 & 0 & y z y z & y z z x & 0 & -y z z y & y z x z & 0 \\ 0 & 0 & 0 & z x y z & z x z x & 0 & z x z y & -z x x z & 0 \\ x y x x & x y y y & x y z z & 0 & 0 & x y x y & 0 & 0 & -x y y x \\ 0 & 0 & 0 & z x x z & z x z y & 0 & -z x z x & z x y z & 0 \\ 0 & 0 & 0 & y z x z & y z z y & 0 & y z z x & -y z y z & 0 \\ x y y y & x y x x & x y z z & 0 & 0 & x y y x & 0 & 0 & -x y x y\end{array}\right)\left(\begin{array}{ccccccccc}0 & 0 & 0 & 0 & 0 & 0 & 0 & 0 & 0 \\ 0 & 0 & 0 & 0 & 0 & 0 & 0 & 0 & 0 \\ 0 & 0 & 0 & 0 & 0 & 0 & 0 & 0 & 0 \\ 0 & 0 & 0 & 0 & 0 & 0 & 0 & 0 & 0 \\ 0 & 0 & 0 & 0 & 0 & 0 & 0 & 0 & 0 \\ 0 & 0 & 0 & 0 & 0 & 0 & 0 & 0 & 0 \\ 0 & 0 & 0 & 0 & 0 & 0 & 0 & 0 & 0 \\ 0 & 0 & 0 & 0 & 0 & 0 & 0 & 0 & 0 \\ 0 & 0 & 0 & 0 & 0 & 0 & 0 & 0 & 0\end{array}\right)$

$\left(\begin{array}{ccccc}x x x x & y x x x & 0 & y y z z & 0 \\ y x x x & -x x x x & 0 & -y y z z & 0 \\ 0 & 0 & 0 & 0 & z z x y\end{array}\right)$

$\left(\begin{array}{lllll}0 & 0 & 0 & 0 & 0 \\ 0 & 0 & 0 & 0 & 0 \\ 0 & 0 & 0 & 0 & 0\end{array}\right)$

$\begin{array}{lllll}0 & 0 & 0 & 0 & 0\end{array}$

$\left(\begin{array}{llllllllll}x x x x & x y y y & 0 & x x y y & x y z z & 0 & -y y z z & y x y y & 0 & 0 \\ 0 & -x y & 0 & y x y & y z z & 0 & x y z z & -x y y & 0 & 0\end{array}\right)\left(\begin{array}{cccccccccc}0 & 0 & 0 & 0 & 0 & 0 & 0 & 0 & 0 & 0 \\ 0 & 0 & 0 & 0 & 0 & 0 & 0 & 0 & 0 & 0 \\ 0 & 0 & 0 & 0 & 0 & 0 & 0 & 0 & 0\end{array}\right)$

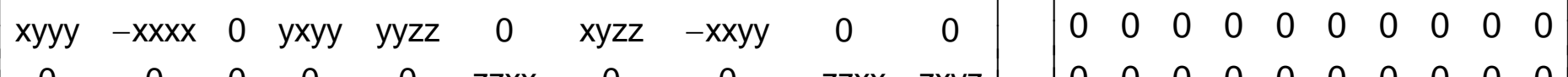

$$
\left(\begin{array}{cccccc}
\mathrm{xxxx} & 0 & \mathrm{xxzz} & 0 & 0 & \mathrm{xxxy} \\
& -\mathrm{xxxx} & -\mathrm{xxzz} & 0 & 0 & \mathrm{xxxy} \\
& & 0 & 0 & 0 & \mathrm{zzxy} \\
& & \mathrm{yzyz} & \mathrm{yzxz} & 0 \\
& & & & -\mathrm{yzzz} & 0 \\
& & & & & 0
\end{array}\right)
$$

$\left(\begin{array}{cccccc}x x x x & x x y y & x x z z & 0 & 0 & x x x y \\ -x x y y & -x x x x & -x x z z & 0 & 0 & x x x y \\ z z x x & -z z x x & 0 & 0 & 0 & z z x y \\ 0 & 0 & 0 & y z y z & y z x z & 0 \\ 0 & 0 & 0 & y z x z & -y z y z & 0 \\ x y x x & x y x x & x y z z & 0 & 0 & 0\end{array}\right)$

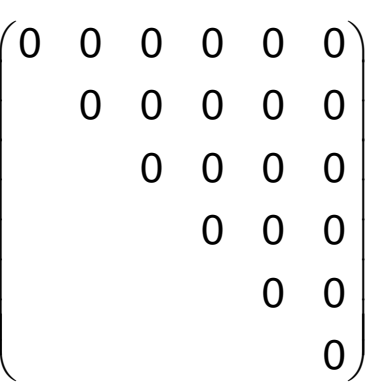

$\begin{array}{llllll}0 & 0 & 0 & 0 & 0 & 0 \\ 0 & 0 & 0 & 0 & 0 & 0\end{array}$ $\begin{array}{llllll}0 & 0 & 0 & 0 & 0 & 0 \\ 0 & 0 & 0 & 0 & 0 & 0\end{array}$ $\begin{array}{llllll}0 & 0 & 0 & 0 & 0 & 0 \\ 0 & 0 & 0 & 0 & 0 & 0\end{array}$ $\begin{array}{llllll}0 & 0 & 0 & 0 & 0 & 0 \\ 0 & 0 & 0 & 0 & 0 & 0\end{array}$ $\begin{array}{llllll}0 & 0 & 0 & 0 & 0 & 0 \\ 0 & 0 & 0 & 0 & 0 & 0\end{array}$ 
$\left[\left(\mathrm{V}^{2}\right)\right]^{2}$

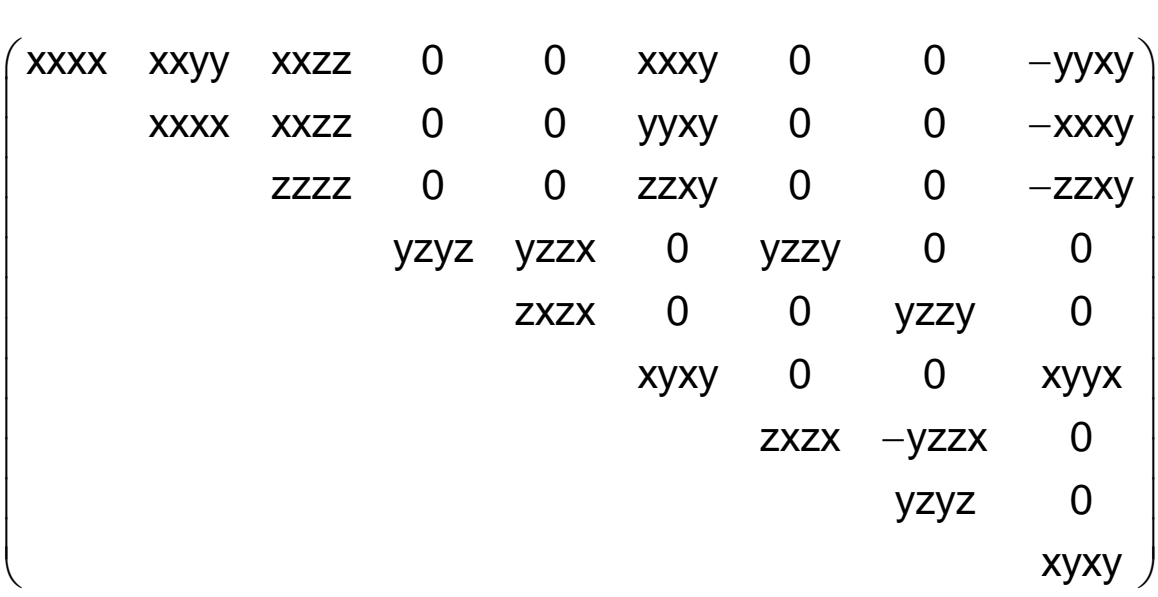

$\begin{array}{lllllllll}0 & 0 & 0 & 0 & 0 & 0 & 0 & 0 & 0 \\ & 0 & 0 & 0 & 0 & 0 & 0 & 0 & 0\end{array}$ $\begin{array}{llllllll}0 & 0 & 0 & 0 & 0 & 0 & 0 & 0\end{array}$

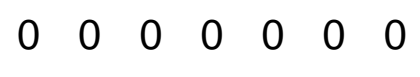

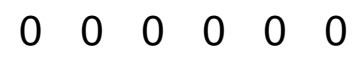
$0 \begin{array}{llll}0 & 0 & 0 & 0 \\ 0 & 0 & 0\end{array}$ $\begin{array}{llll}0 & 0 & 0 & 0 \\ -1\end{array}$ $\begin{array}{lll}0 & 0 & 0 \\ & 0 & 0 \\ 0 & & 0\end{array}$

$\left(\begin{array}{lllllllll}x x x x & x x y y & x x z z & 0 & 0 & x x x y & 0 & 0 & -y y x y \\ y y x & x y x & x x z & 0 & 0 & y x y & 0 & 0 & -x x y\end{array}\right)\left(\begin{array}{lllllllll}0 & 0 & 0 & 0 & 0 & 0 & 0 & 0 & 0 \\ 0 & 0 & 0 & 0 & 0 & 0 & 0 & 0 & 0\end{array}\right)$ $\begin{array}{lllllllll}\mathrm{yyxx} & \mathrm{xxxx} & \mathrm{xxzz} & 0 & 0 & \mathrm{yyxy} & 0 & 0 & -\mathrm{xxxy} \\ \mathrm{zzxx} & z z x x & z z z z & 0 & 0 & z z x y & 0 & 0 & -z z x y\end{array} \mid\left(\begin{array}{lllllllll}0 & 0 & 0 & 0 & 0 & 0 & 0 & 0 & 0 \\ 0 & 0 & 0 & 0 & 0 & 0 & 0 & 0 & 0 \\ 0 & 0 & 0 & 0 & 0 & 0 & 0 & 0 & 0 \\ 0 & 0 & 0 & 0 & 0 & 0 & 0 & 0 & 0\end{array}\right)$

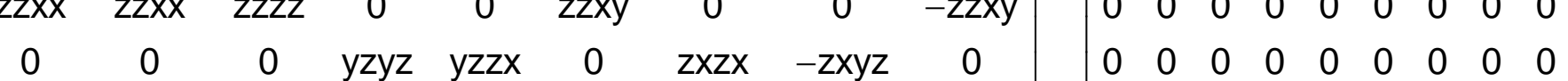
$\begin{array}{ccccccccc}0 & 0 & 0 & y z y z & y z z x & 0 & z x z x & -z x y z & 0 \\ 0 & 0 & 0 & z x y z & z x z x & 0 & -y z z x & y z y z & 0\end{array} \mid\left(\begin{array}{lllllllll}0 & 0 & 0 & 0 & 0 & 0 & 0 & 0 & 0 \\ 0 & 0 & 0 & 0 & 0 & 0 & 0 & 0 & 0 \\ 0 & 0 & 0 & 0 & 0 & 0 & 0 & 0 & 0\end{array}\right)$ $\left.\begin{array}{lllllllll}\text { xyxx } & -x y x x & 0 & 0 & 0 & x y x y & 0 & 0 & x y x y\end{array}\right)\left(\begin{array}{lllllllll}0 & 0 & 0 & 0 & 0 & 0 & 0 & 0 & 0\end{array}\right.$

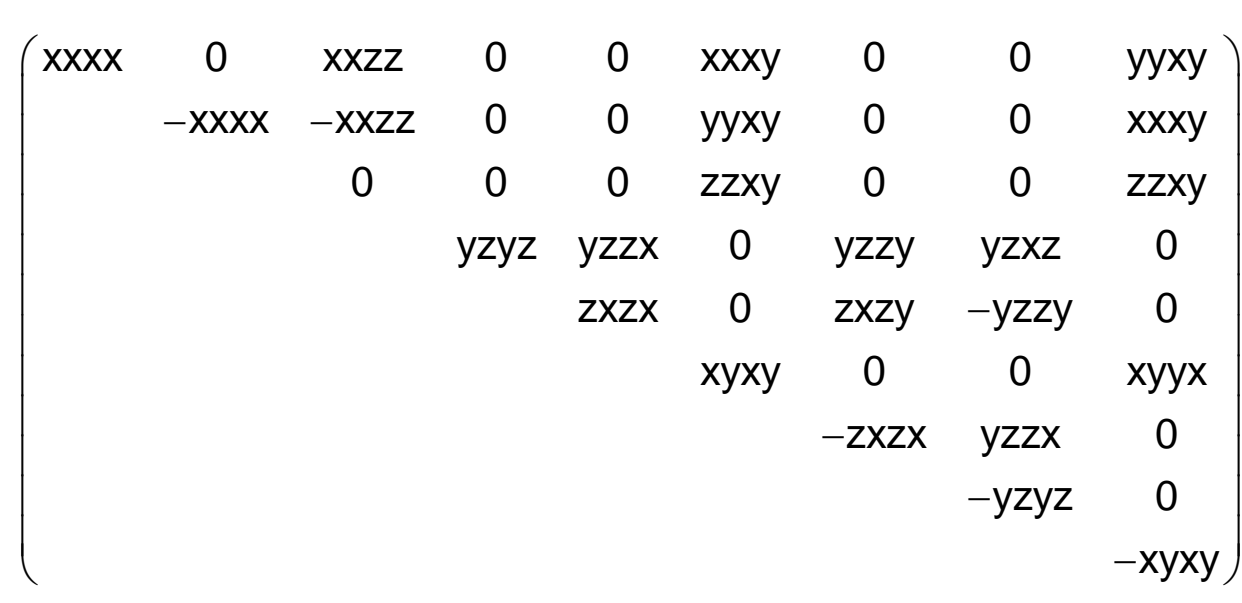

$\left(\begin{array}{llllllll}0 & 0 & 0 & 0 & 0 & 0 & 0 & 0 \\ 0 & 0 & 0 & 0 & 0 & 0 & 0 & 0\end{array}\right.$ $\begin{array}{lllllllll}0 & 0 & 0 & 0 & 0 & 0 & 0 & 0\end{array}$ $0 \begin{array}{lllllll}0 & 0 & 0 & 0 & 0 & 0 \\ 0 & 0 & 0 & 0 & 0 & 0 \\ 0 & 0 & 0\end{array}$ $0 \begin{array}{llll}0 & 0 & 0 & 0 \\ 0 & 0 & -1\end{array}$ $0 \begin{array}{lll}0 & 0 & 0 \\ 0 & 0 & -1\end{array}$ $\begin{array}{lll}0 & 0 & 0 \\ & 0 & 0\end{array}$

$\left(\begin{array}{ccccccccc}\text { xxxx } & \text { xxyy } & \text { xxzz } & 0 & 0 & \text { xxxy } & 0 & 0 & \text { yyxy } \\ -x x y y & -x x x x & -x x z z & 0 & 0 & y y x y & 0 & 0 & x x x y \\ z z x x & -z z x x & 0 & 0 & 0 & z z x y & 0 & 0 & z z x y \\ 0 & 0 & 0 & y z y z & y z z x & 0 & -z x z x & z x y z & 0 \\ 0 & 0 & 0 & z x y z & z x z x & 0 & y z z x & -y z y z & 0 \\ x y x x & x y x x & x y z z & 0 & 0 & x y x y & 0 & 0 & -x y x y\end{array}\right)$

$\left(\begin{array}{lllllllll}0 & 0 & 0 & 0 & 0 & 0 & 0 & 0 & 0\end{array}\right)$ $0 \begin{array}{lllllllll}0 & 0 & 0 & 0 & 0 & 0 & 0 & 0 & 0 \\ 0 & 0 & 0 & 0 & 0 & 0 & 0 & 0 & 0\end{array}$ $\begin{array}{lllllllll}0 & 0 & 0 & 0 & 0 & 0 & 0 & 0 & 0 \\ 0 & 0 & 0 & 0 & 0 & 0 & 0 & 0 & 0\end{array}$ $0 \begin{array}{llllllllll}0 & 0 & 0 & 0 & 0 & 0 & 0 & 0 & 0 \\ 0 & 0 & 0 & 0 & 0 & 0 & 0 & 0 & 0\end{array}$ $\begin{array}{lllllllll}0 & 0 & 0 & 0 & 0 & 0 & 0 & 0 & 0 \\ 0 & 0 & 0 & 0 & 0 & 0 & 0 & 0 & 0\end{array}$ 

$\mathbb{V}^{4}\left(\begin{array}{ccccccccc}x x x x x & x x y y & x x z z & 0 & 0 & x x x y & 0 & 0 & -y y x y \\ y y x x & x x x x & y y z z & 0 & 0 & y y x y & 0 & 0 & -x x x y \\ z z x x & z z x x & z z z z & 0 & 0 & z z x y & 0 & 0 & -z z x y \\ 0 & 0 & 0 & y z y z & y z z x & 0 & y z z y & y z x z & 0 \\ 0 & 0 & 0 & z x y z & z x z x & 0 & z x z y & z x x z & 0 \\ x y y x & x y y y & x y z z & 0 & 0 & x y x y & 0 & 0 & x y y x \\ 0 & 0 & 0 & z x x z & -z x z y & 0 & z x z x & -z x y z & 0 \\ 0 & 0 & 0 & -y z x z & y z z y & 0 & -y z z x & y z y z & 0 \\ -x y y y & -x y x x & -x y z z & 0 & 0 & x y y x & 0 & 0 & x y x y\end{array}\right)$

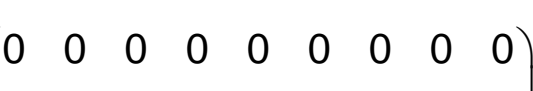

$\begin{array}{lllllllll}0 & 0 & 0 & 0 & 0 & 0 & 0 & 0 & 0 \\ 0 & 0 & 0 & 0 & 0 & 0 & 0 & 0 & 0\end{array}$

$\begin{array}{llllllllll}0 & 0 & 0 & 0 & 0 & 0 & 0 & 0 & 0 \\ 0 & 0 & 0 & 0 & 0 & 0 & 0 & 0 & 0 \\ 0 & 0 & 0 & 0 & 0 & 0 & 0 & 0 & 0\end{array}$

$\begin{array}{llllllllll}0 & 0 & 0 & 0 & 0 & 0 & 0 & 0 & 0\end{array}$

$\begin{array}{llllllllll}0 & 0 & 0 & 0 & 0 & 0 & 0 & 0 & 0\end{array}$

$\begin{array}{lllllllll}0 & 0 & 0 & 0 & 0 & 0 & 0 & 0 & 0 \\ 0 & 0 & 0 & 0 & 0 & 0 & 0 & 0 & 0\end{array}$

$\left(\begin{array}{lllllllll}0 & 0 & 0 & 0 & 0 & 0 & 0 & 0 & 0 \\ 0 & 0 & 0 & 0 & 0 & 0 & 0 & 0 & 0\end{array}\right.$

$\left[V^{4}\right]\left(\begin{array}{ccccc}3 x x y y & 0 & 0 & y y z z & 0 \\ 0 & 3 x x y y & 0 & y y z z & 0 \\ 0 & 0 & z z z z & x x y y & 0\end{array}\right)$

$$
\left(\begin{array}{lllll}
0 & 0 & 0 & 0 & 0 \\
0 & 0 & 0 & 0 & 0 \\
0 & 0 & 0 & 0 & 0
\end{array}\right)
$$

$\left.V_{V} V^{3}\right]\left(\begin{array}{cccccccccc}3 x x y y & 3 x y x x & 0 & x x y y & x y z z & 0 & y y z z & x y x x & 0 & y y x x \\ -3 x y x x & 3 x x y y & 0 & -x y x & y y z & 0 & -x y z z & x x y y & 0 & 0 \\ 0 & 0 & z z z z & 0 & 0 & z z x x & 0 & 0 & z z x & z x y z\end{array}\right)\left(\begin{array}{cccccccccc}0 & 0 & 0 & 0 & 0 & 0 & 0 & 0 & 0 & 0 \\ 0 & 0 & 0 & 0 & 0 & 0 & 0 & 0 & 0 & 0 \\ 0 & 0 & 0 & 0 & 0 & 0 & 0 & 0 & 0 & 0\end{array}\right)$

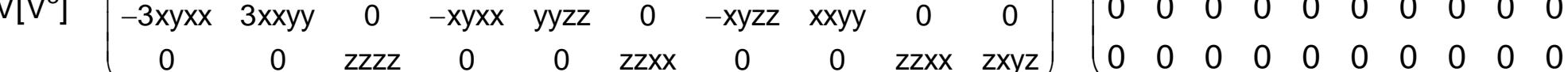

$\left[\left[V^{2}\right]^{2}\right]\left(\begin{array}{cccccc}x x x x & x x y y & x x z z & 0 & 0 & 0 \\ & x x x x & x x z z & 0 & 0 & 0 \\ & & z z z z & 0 & 0 & 0 \\ & & & y z y z & 0 & 0 \\ & & & & y z y z & 0 \\ & & & & & x y x y\end{array}\right)$

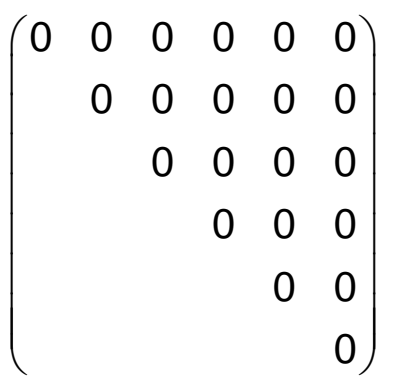

and $x y x y=1 / 2(x x x x-x x y y)$

$\left[V^{2}\right]^{2}\left(\begin{array}{cccccc}x x x x & x x y y & x x z z & 0 & 0 & x x x y \\ x x y y & x x x x & x x z z & 0 & 0 & -x x x y \\ z z x x & z z x x & z z z z & 0 & 0 & 0 \\ 0 & 0 & 0 & y z y z & y z x z & 0 \\ 0 & 0 & 0 & y z x z & y z y z & 0 \\ -x x x y & x x x y & 0 & 0 & 0 & x y x y\end{array}\right)$

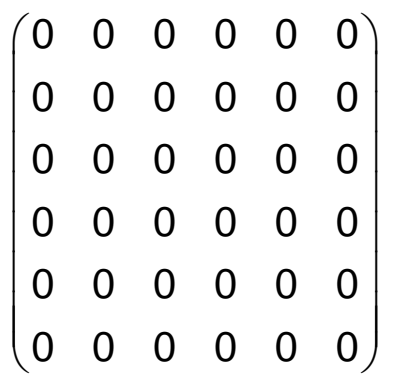

and $x y x y=1 / 2(x x x x-x x y y)$ $\left(\begin{array}{lllllllll}0 & 0 & 0 & 0 & 0 & 0 & 0 & 0 & 0 \\ 0 & 0 & 0 & 0 & 0 & 0 & 0 & 0 & 0\end{array}\right)$ $\begin{array}{lllllllll}0 & 0 & 0 & 0 & 0 & 0 & 0 & 0 & 0 \\ 0 & 0 & 0 & 0 & 0 & 0 & 0 & 0 & 0\end{array}$ $0 \begin{array}{lllllllll}0 & 0 & 0 & 0 & 0 & 0 & 0 & 0 & 0 \\ 0 & 0 & 0 & 0 & 0 & 0 & 0 & 0 & 0\end{array}$ $0 \begin{array}{lllllllll}0 & 0 & 0 & 0 & 0 & 0 & 0 & 0 & 0 \\ 0 & 0 & 0 & 0 & 0 & 0 & 0 & 0 & 0\end{array}$ $\begin{array}{lllllllll}0 & 0 & 0 & 0 & 0 & 0 & 0 & 0 & 0 \\ 0 & 0 & 0 & 0 & 0 & 0 & 0 & 0\end{array}$ $\begin{array}{llllllllll}0 & 0 & 0 & 0 & 0 & 0 & 0 & 0 & 0 \\ 0 & 0 & 0 & 0 & 0 & 0 & 0 & 0 & 0 & 0\end{array}$ $\begin{array}{llllllllll}0 & 0 & 0 & 0 & 0 & 0 & 0 & 0 & 0\end{array}$ $0 \begin{array}{llllllllll}0 & 0 & 0 & 0 & 0 & 0 & 0 & 0 & 0 \\ 0 & 0 & 0 & 0 & 0 & 0 & 0 & 0\end{array}$ $\begin{array}{llllllllll}0 & 0 & 0 & 0 & 0 & 0 & 0 & 0 & 0\end{array}$

$$
\left(\begin{array}{lllll}
0 & 0 & 0 & 0 & 0 \\
0 & 0 & 0 & 0 & 0 \\
0 & 0 & 0 & 0 & 0
\end{array}\right)
$$

$\left(\begin{array}{llllllllll}0 & 0 & 0 & 0 & 0 & 0 & 0 & 0 & 0 & 0 \\ 0 & 0 & 0 & 0 & 0 & 0 & 0 & 0 & 0 & 0\end{array}\right)$ $\left(\begin{array}{lllllllllll}0 & 0 & 0 & 0 & 0 & 0 & 0 & 0 & 0 & 0 \\ 0 & 0 & 0 & 0 & 0 & 0 & 0 & 0 & 0 & 0\end{array}\right)$

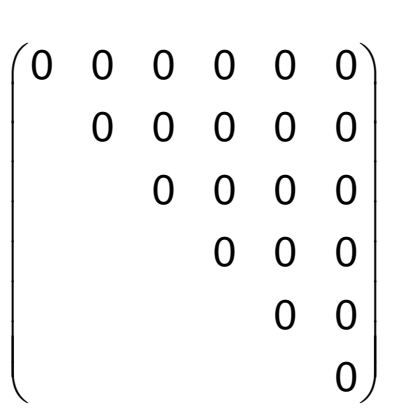

$$
\left(\begin{array}{llllll}
0 & 0 & 0 & 0 & 0 & 0 \\
0 & 0 & 0 & 0 & 0 & 0 \\
0 & 0 & 0 & 0 & 0 & 0 \\
0 & 0 & 0 & 0 & 0 & 0 \\
0 & 0 & 0 & 0 & 0 & 0 \\
0 & 0 & 0 & 0 & 0 & 0
\end{array}\right)
$$

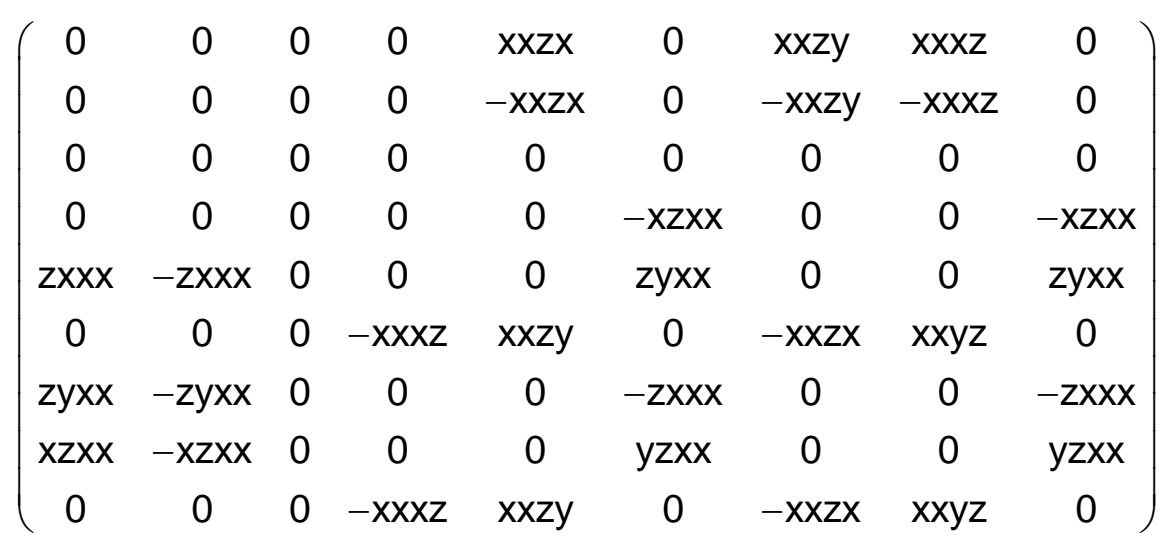

$\left(\begin{array}{ccccc}0 & 0 & 0 & 0 & x x y z \\ 0 & 0 & 0 & 0 & -z x x x \\ z x x x & -x x y z & 0 & 0 & 0\end{array}\right)$

$\left(\begin{array}{llllllllll}0 & 0 & 0 & 0 & 0 & x z x x & 0 & 0 & -x z x x & y z x x \\ 0 & 0 & 0 & 0 & 0 & y z x & 0 & 0 & -y z x & -x z x\end{array}\right)$ $\left(\begin{array}{cccccccccc}0 & 0 & 0 & 0 & 0 & y z x x & 0 & 0 & -y z x x & -x z x x \\ 2 x x x x & -z y x x & 0 & -z x x x & 0 & 0 & 0 & z y x x & 0 & 0\end{array}\right)$
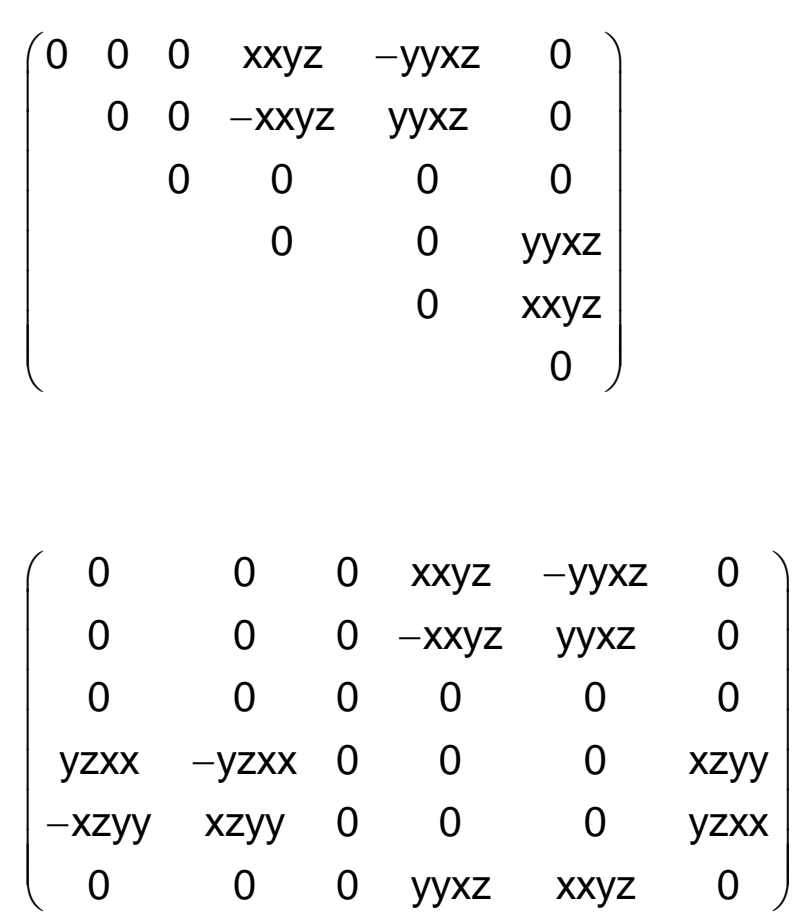


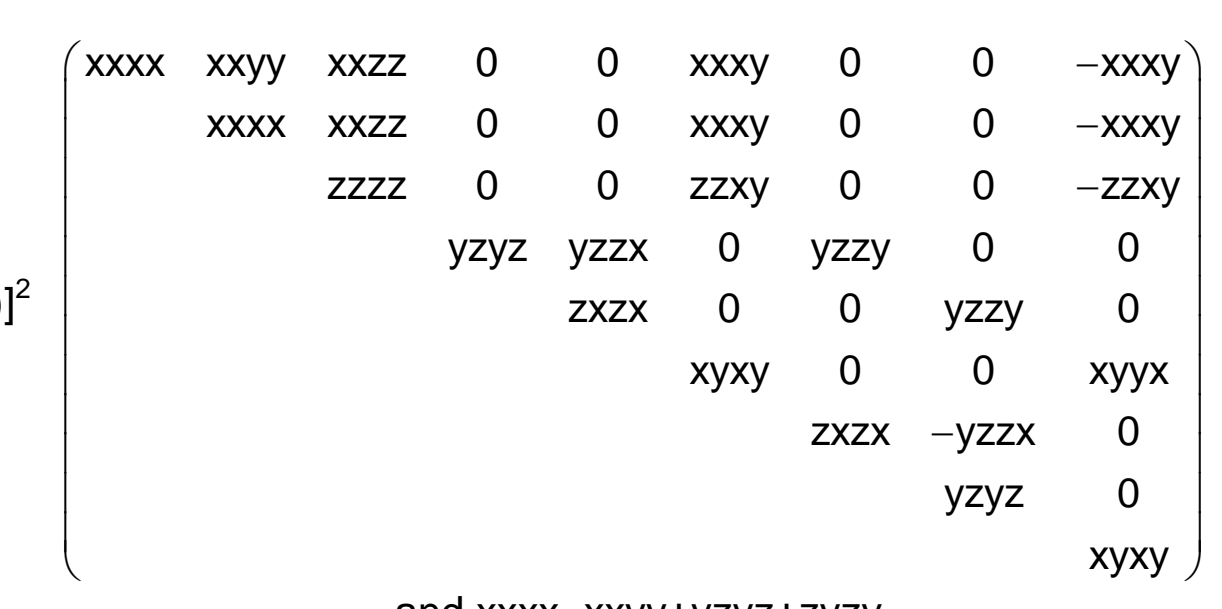

$\left(\begin{array}{lllllllll}0 & 0 & 0 & 0 & 0 & 0 & 0 & 0 & 0\end{array}\right.$ $\begin{array}{llllllll}0 & 0 & 0 & 0 & 0 & 0 & 0 & 0\end{array}$

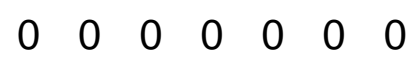

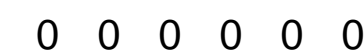
$\begin{array}{lllll}0 & 0 & 0 & 0 & 0\end{array}$ $0 \begin{array}{llll}0 & 0 & 0 & 0\end{array}$ $\begin{array}{lll}0 & 0 & 0 \\ & 0 & 0 \\ 0\end{array}$ and $x x x x=x x y y+y z y z+z y z y$

$\left(\begin{array}{lllllllll}x x x x & x x y y & x x z z & 0 & 0 & x x x y & 0 & 0 & -y y x y \\ x y y & x x x & x z z & 0 & 0 & y y x & 0 & 0 & -x x y\end{array}\right)\left(\begin{array}{ccccccccc}0 & 0 & 0 & 0 & 0 & 0 & 0 & 0 & 0 \\ 0 & 0 & 0 & 0 & 0 & 0 & 0 & 0 & 0\end{array}\right)$ $\begin{array}{lllllllll}x x y y & x x x x & x x z z & 0 & 0 & y y x y & 0 & 0 & -x x x y\end{array} \mid \begin{array}{llllllllll}x & 0 & 0 & 0 & 0 & 0 & 0 & 0 & 0\end{array}$

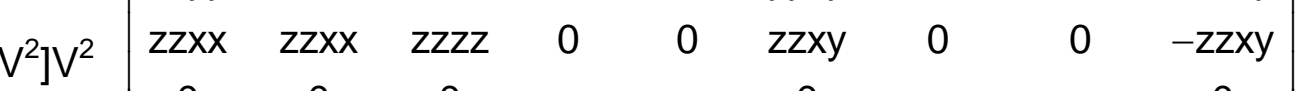
$\begin{array}{lllllllll}0 & 0 & 0 & y z y z & y z z x & 0 & z x z x & -z x y z & 0\end{array}$ $\begin{array}{ccccccccc}0 & 0 & 0 & z x y z & z x z x & 0 & -y z z x & y z y z & 0 \\ x y x x & -x y x x & 0 & 0 & 0 & x y x y & 0 & 0 & x y x y\end{array}$ $\begin{array}{llllllllll}0 & 0 & 0 & 0 & 0 & 0 & 0 & 0 & 0 \\ 0 & 0 & 0 & 0 & 0 & 0 & 0 & 0\end{array}$ $\begin{array}{lllllllll}0 & 0 & 0 & 0 & 0 & 0 & 0 & 0 & 0 \\ 0 & 0 & 0 & 0 & 0 & 0 & 0 & 0\end{array}$ $0 \begin{array}{lllllllll}0 & 0 & 0 & 0 & 0 & 0 & 0 & 0 & 0 \\ 0 & 0 & 0 & 0 & 0 & 0 & 0 & 0 & 0\end{array}$ $\begin{array}{lllllllll}0 & 0 & 0 & 0 & 0 & 0 & 0 & 0 & 0\end{array}$ and $x x x x=x x y y+2 x y x y ; y y x y-x x x y=2 x y x x$
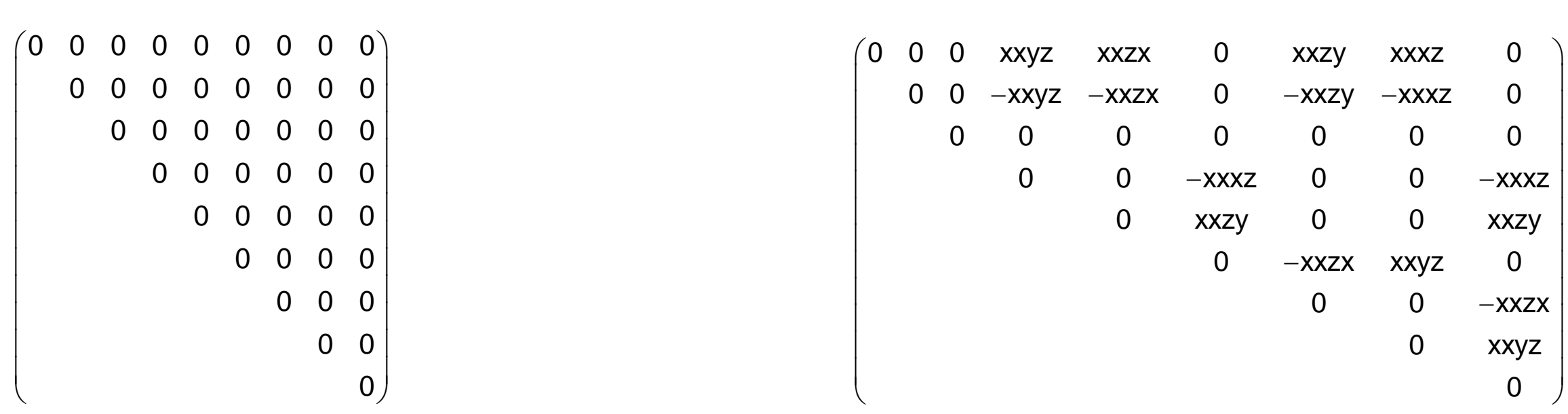

$\left(\begin{array}{lllllllll}0 & 0 & 0 & 0 & 0 & 0 & 0 & 0 & 0 \\ 0 & 0 & 0 & 0 & 0 & 0 & 0 & 0 & 0\end{array}\right.$

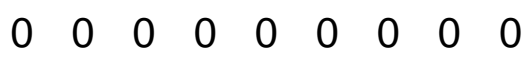

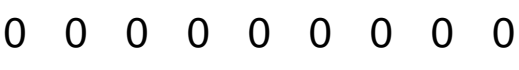
$0 \begin{array}{lllllllll}0 & 0 & 0 & 0 & 0 & 0 & 0 & 0 & 0 \\ 0 & 0 & 0 & 0 & 0 & 0 & 0 & 0\end{array}$ $\begin{array}{llllllllll}0 & 0 & 0 & 0 & 0 & 0 & 0 & 0 & 0\end{array}$ $\left(\begin{array}{lllllllll}0 & 0 & 0 & 0 & 0 & 0 & 0 & 0 & 0\end{array}\right.$

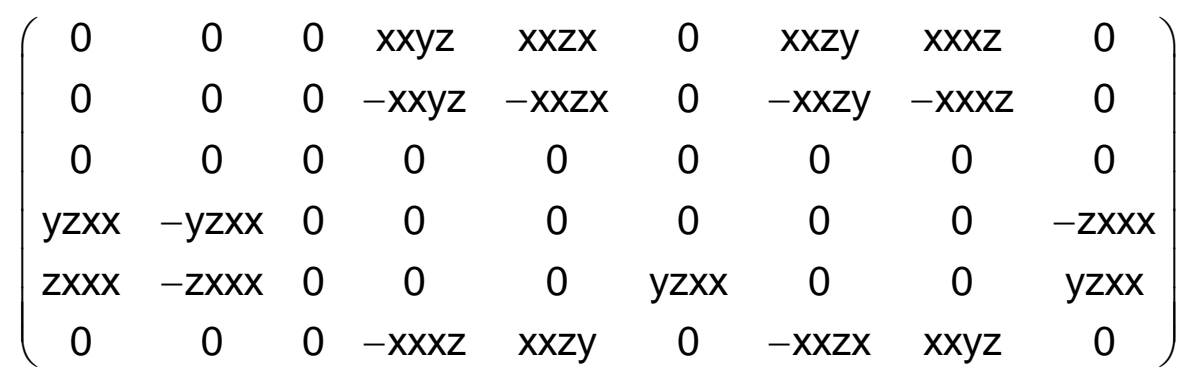




$\left(\begin{array}{ccccccccc}x x x x & x x y y & x x z z & 0 & 0 & x x x y & 0 & 0 & -y y x y \\ y y x x & x x x x & y y z z & 0 & 0 & y y x y & 0 & 0 & -x x y \\ z z x x & z z x x & z z z z & 0 & 0 & z z x y & 0 & 0 & -z z x y \\ 0 & 0 & 0 & y z y z & y z z x & 0 & y z z y & y z x z & 0 \\ 0 & 0 & 0 & z x y z & z x z x & 0 & z x z y & z x x z & 0 \\ x y x x & x y y y & x y z z & 0 & 0 & x y x y & 0 & 0 & x y y x \\ 0 & 0 & 0 & z x x z & -z x z y & 0 & z x z x & -z x y z & 0 \\ 0 & 0 & 0 & -y z x z & y z z y & 0 & -y z z x & y z y z & 0 \\ -x y y y & -x y x x & -x y z z & 0 & 0 & x y y x & 0 & 0 & x y x y\end{array}\right)\left(\begin{array}{ccccccccc}0 & 0 & 0 & 0 & 0 & 0 & 0 & 0 & 0 \\ 0 & 0 & 0 & 0 & 0 & 0 & 0 & 0 & 0 \\ 0 & 0 & 0 & 0 & 0 & 0 & 0 & 0 & 0 \\ 0 & 0 & 0 & 0 & 0 & 0 & 0 & 0 & 0 \\ 0 & 0 & 0 & 0 & 0 & 0 & 0 & 0 & 0 \\ 0 & 0 & 0 & 0 & 0 & 0 & 0 & 0 & 0 \\ 0 & 0 & 0 & 0 & 0 & 0 & 0 & 0 & 0 \\ 0 & 0 & 0 & 0 & 0 & 0 & 0 & 0 & 0 \\ 0 & 0 & 0 & 0 & 0 & 0 & 0 & 0 & 0\end{array}\right)$
and: $x x x x=x x y y+x y x y+x y y x ; x x x y-y y x y=x y y y-x y x x$

$\left[V^{4}\right] \quad\left(\begin{array}{ccccc}3 x x y y & 0 & 0 & y y z z & 0 \\ 0 & 3 x x y y & 0 & y y z z & 0 \\ 0 & 0 & z z z z & x x y & 0\end{array}\right)$

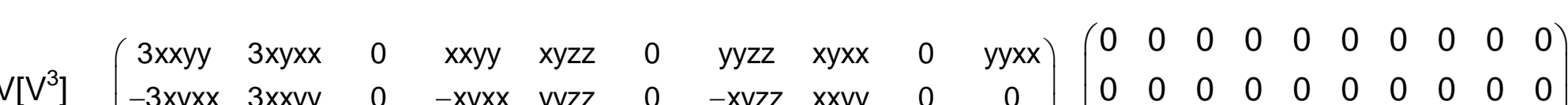

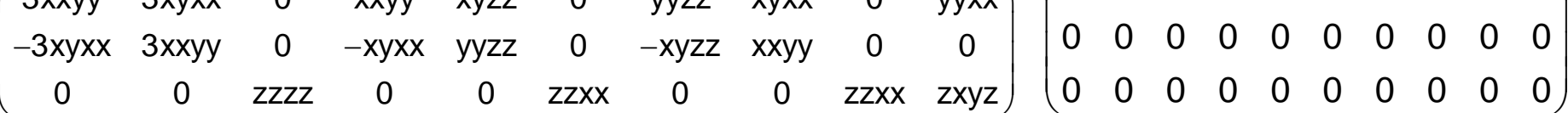

$\left[\left[V^{2}\right]^{2}\right]\left(\begin{array}{cccccc}x x x x & x x y y & x x z z & 0 & 0 & 0 \\ & x x x x & x x z z & 0 & 0 & 0 \\ & & z z z z & 0 & 0 & 0 \\ & & & y z y z & 0 & 0 \\ & & & & y z y z & 0 \\ & & & & & x y x y\end{array}\right)$

and $x y x y=1 / 2(x x x x-x x y y)$

$\left[V^{2}\right]^{2}\left(\begin{array}{cccccc}x x x x & x x y y & x x z z & 0 & 0 & x x x y \\ x x y y & x x x x & x x z z & 0 & 0 & -x x x y \\ z z x x & z z x x & z z z z & 0 & 0 & 0 \\ 0 & 0 & 0 & y z y z & y z x z & 0 \\ 0 & 0 & 0 & y z x z & y z y z & 0 \\ -x x x y & x x x y & 0 & 0 & 0 & x y x y\end{array}\right)$

and $x y x y=1 / 2(x x x x-x x y y)$ $\left(\begin{array}{lllll}0 & 0 & 0 & 0 & 0 \\ 0 & 0 & 0 & 0 & 0 \\ 0 & 0 & 0 & 0 & 0\end{array}\right)$

$\begin{array}{lllll}0 & 0 & 0 & 0 & 0\end{array}$

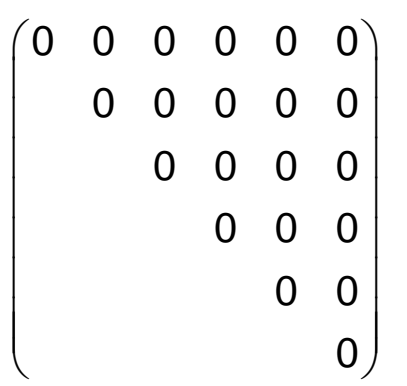

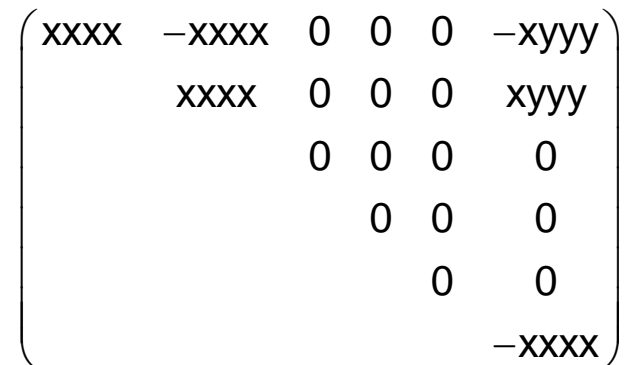

$\left(\begin{array}{llllll}0 & 0 & 0 & 0 & 0 & 0 \\ 0 & 0 & 0 & 0 & 0 & 0 \\ 0 & 0 & 0 & 0 & 0 & 0 \\ 0 & 0 & 0 & 0 & 0 & 0 \\ 0 & 0 & 0 & 0 & 0 & 0 \\ 0 & 0 & 0 & 0 & 0 & 0\end{array}\right)$

$\left(\begin{array}{ccccccccc}\text { xxxx } & -x x x x & 0 & 0 & 0 & -x y y y & 0 & 0 & -x y y y \\ -x x x x & x x x x & 0 & 0 & 0 & x y y y & 0 & 0 & x y y y \\ 0 & 0 & 0 & 0 & 0 & 0 & 0 & 0 & 0 \\ 0 & 0 & 0 & 0 & 0 & 0 & 0 & 0 & 0 \\ 0 & 0 & 0 & 0 & 0 & 0 & 0 & 0 & 0 \\ -x y y y & x y y y & 0 & 0 & 0 & -x x x x & 0 & 0 & -x x x x \\ 0 & 0 & 0 & 0 & 0 & 0 & 0 & 0 & 0 \\ 0 & 0 & 0 & 0 & 0 & 0 & 0 & 0 & 0 \\ -x y y y & x y y y & 0 & 0 & 0 & -x x x x & 0 & 0 & -x x x x\end{array}\right)$

$\left(\begin{array}{ccccc}x x x x & x y y y & 0 & 0 & 0 \\ -x y y y & x x x x & 0 & 0 & 0 \\ 0 & 0 & 0 & -x x x x & 0\end{array}\right)$

$\left(\begin{array}{llllllllll}x x x x & x y y y & 0 & -x x x x & 0 & 0 & 0 & -x y y y & 0 & 0 \\ -x y y & x x x x & 0 & x y y & 0 & 0 & 0 & -x y x & 0 & 0\end{array}\right)$ $\left(\begin{array}{cccccccccc}-x y y y & x x x x & 0 & x y y y & 0 & 0 & 0 & -x x x x & 0 & 0 \\ 0 & 0 & 0 & 0 & 0 & 0 & 0 & 0 & 0 & 0\end{array}\right)$

$\left(\begin{array}{lllllllll}0 & 0 & 0 & 0 & 0 & 0 & 0 & 0 & 0 \\ 0 & 0 & 0 & 0 & 0 & 0 & 0 & 0 & 0\end{array}\right)$

$\begin{array}{lllllllll}0 & 0 & 0 & 0 & 0 & 0 & 0 & 0 & 0 \\ 0 & 0 & 0 & 0 & 0 & 0 & 0 & 0 & 0\end{array}$

$\begin{array}{lllllllll}0 & 0 & 0 & 0 & 0 & 0 & 0 & 0 & 0 \\ 0 & 0 & 0 & 0 & 0 & 0 & 0 & 0 & 0\end{array}$

$0 \begin{array}{lllllllll}0 & 0 & 0 & 0 & 0 & 0 & 0 & 0 & 0 \\ 0 & 0 & 0 & 0 & 0 & 0 & 0 & 0 & 0\end{array}$

$\begin{array}{lllllllll}0 & 0 & 0 & 0 & 0 & 0 & 0 & 0 & 0\end{array}$

$\begin{array}{llllllllll}0 & 0 & 0 & 0 & 0 & 0 & 0 & 0 & 0 \\ 0 & 0 & 0 & 0 & 0 & 0 & 0 & 0 & 0\end{array}$

$\begin{array}{lllllllll}0 & 0 & 0 & 0 & 0 & 0 & 0 & 0 & 0 \\ 0 & 0 & 0 & 0 & 0 & 0 & 0 & 0 & 0\end{array}$

$\begin{array}{lllllllll}0 & 0 & 0 & 0 & 0 & 0 & 0 & 0 & 0 \\ 0 & 0 & 0 & 0 & 0 & 0 & 0 & 0 & 0\end{array}$

$\left(\begin{array}{lllll}0 & 0 & 0 & 0 & 0 \\ 0 & 0 & 0 & 0 & 0\end{array}\right)$

$\left(\begin{array}{lllll}0 & 0 & 0 & 0 & 0 \\ 0 & 0 & 0 & 0 & 0\end{array}\right)$

$\left.\begin{array}{lllll}0 & 0 & 0 & 0 & 0\end{array}\right)$

$\left(\begin{array}{llllllllll}0 & 0 & 0 & 0 & 0 & 0 & 0 & 0 & 0 & 0 \\ 0 & 0 & 0 & 0 & 0 & 0 & 0 & 0 & 0 & 0\end{array}\right)$ $\left.\begin{array}{llllllllll}0 & 0 & 0 & 0 & 0 & 0 & 0 & 0 & 0 & 0 \\ 0 & 0 & 0 & 0 & 0 & 0 & 0 & 0 & 0 & 0\end{array}\right)$

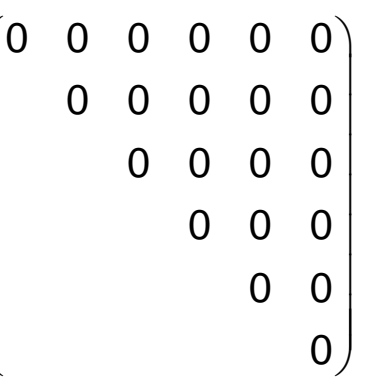

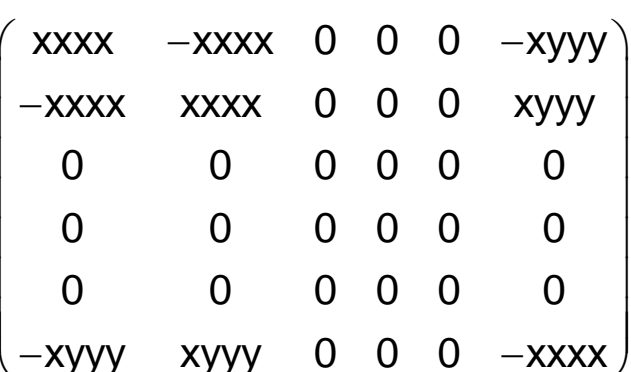

$\left(\begin{array}{llllll}0 & 0 & 0 & 0 & 0 & 0 \\ 0 & 0 & 0 & 0 & 0 & 0\end{array}\right)$ $\begin{array}{llllll}0 & 0 & 0 & 0 & 0 & 0\end{array}$ $\begin{array}{lllllll}0 & 0 & 0 & 0 & 0 & 0 \\ 0 & 0 & 0 & 0 & 0 & 0\end{array}$ $\begin{array}{lllllll}0 & 0 & 0 & 0 & 0 & 0 \\ 0 & 0 & 0 & 0 & 0 & 0\end{array}$ $\begin{array}{llllll}0 & 0 & 0 & 0 & 0 & 0 \\ 0 & 0 & 0 & 0 & 0 & 0\end{array}$ 


$$
\text { a }
$$$$
\left(\begin{array}{ccccccccc}
x x x x & -x x x x & 0 & 0 & 0 & -x y y y & 0 & 0 & -x y y y \\
& x x x x & 0 & 0 & 0 & -x y y y & 0 & 0 & -x y y y \\
& & 0 & 0 & 0 & 0 & 0 & 0 & 0 \\
& & & 0 & 0 & 0 & 0 & 0 & 0 \\
& & & 0 & 0 & 0 & 0 & 0 \\
& & & & -x x x x & 0 & 0 & -x x x x \\
& & & & & 0 & 0 & 0 \\
& & & & & & & 0 & 0 \\
& & & & & & & & -x x x
\end{array}\right)
$$

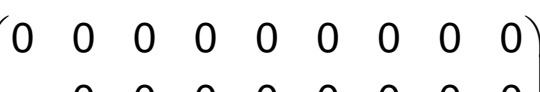
$\begin{array}{llllllll}0 & 0 & 0 & 0 & 0 & 0 & 0 & 0\end{array}$

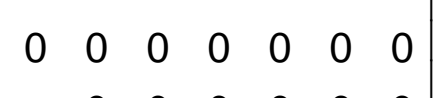
$0 \begin{array}{lllll}0 & 0 & 0 & 0 & 0\end{array}$ $0 \begin{array}{lllll}0 & 0 & 0 & 0 \\ 0 & 0 & 0\end{array}$ $0 \begin{array}{lll}0 & 0 & 0 \\ 0 & 0 & 0\end{array}$ $0 \begin{array}{lll}0 & 0 & 0 \\ & 0 & 0\end{array}$ and $x x x x=x x y y+y z y z+z y z y$

$\left(\begin{array}{lllllllll}x x x x & x x y y & x x z z & 0 & 0 & x x x y & 0 & 0 & -y y x y \\ x y y & x x x & x z z & 0 & 0 & y y x y & 0 & 0 & -x x y\end{array}\right)\left(\begin{array}{lllllllll}0 & 0 & 0 & 0 & 0 & 0 & 0 & 0 & 0 \\ 0 & 0 & 0 & 0 & 0 & 0 & 0 & 0 & 0\end{array}\right)$ $\left.\begin{array}{lllllllll}x x y y & x x x x & x x z z & 0 & 0 & y y x y & 0 & 0 & -x x x y \\ z x x & x z x x & z z z & 0 & 0 & z x y & 0 & 0 & -z x y\end{array}\right)\left(\begin{array}{cccccccccc}0 & 0 & 0 & 0 & 0 & 0 & 0 & 0 & 0 \\ 0 & 0 & 0 & 0 & 0 & 0 & 0 & 0 & 0 \\ 0 & 0 & 0 & 0 & 0 & 0 & 0 & 0 & 0\end{array}\right.$ $\begin{array}{ccccccccc}0 & 2 z x x & z z z z & 0 & 0 & z z x y & 0 & 0 & -z z x y \\ 0 & 0 & 0 & y z y z & y z z x & 0 & z x z x & -z x y z & 0\end{array}$ $\begin{array}{lllllll}0 & y z y z & y z z x & 0 & 2 x z x & -z x y z & 0 \\ 0 & 0 & 0 & 0 & -y z z x & y z y z & 0\end{array} \mid \begin{array}{llllllllll}0 & 0 & 0 & 0 & 0 & 0 & 0 & 0 & 0 \\ 0 & 0 & 0 & 0 & 0 & 0 & 0 & 0 & 0 \\ 0 & 0 & 0 & 0 & 0 & 0 & 0 & 0 & 0\end{array}$

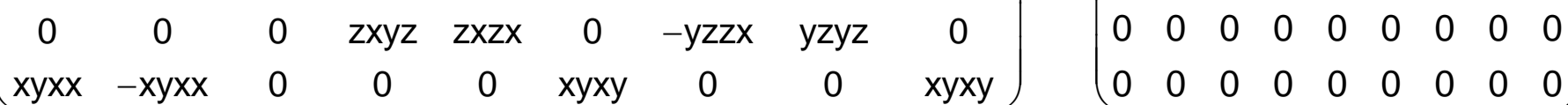

\section{and $x x x x=x x y y+2 x y x y ; y y x y-x x x y=2 x y x x$}

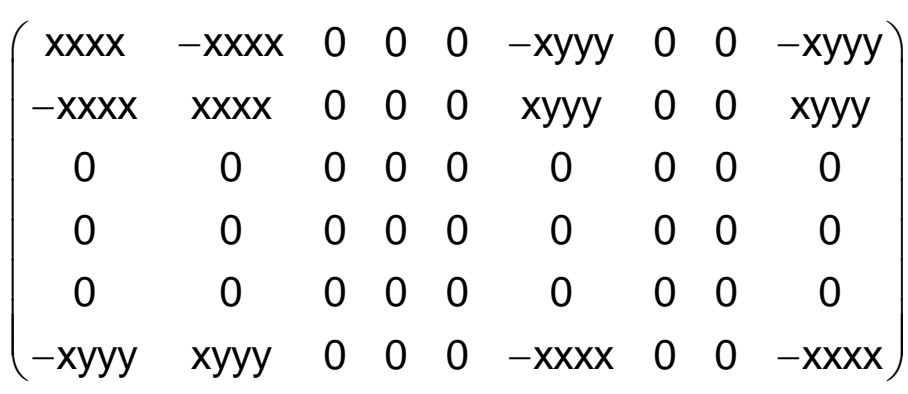

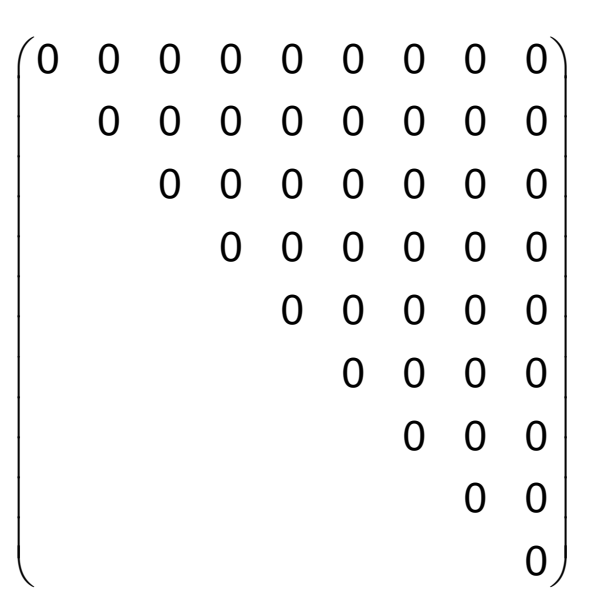
$\begin{array}{llllllllll}0 & 0 & 0 & 0 & 0 & 0 & 0 & 0 & 0 \\ 0 & 0 & 0 & 0 & 0 & 0 & 0 & 0 & 0\end{array}$ $\begin{array}{lllllllll}0 & 0 & 0 & 0 & 0 & 0 & 0 & 0 & 0 \\ 0 & 0 & 0 & 0 & 0 & 0 & 0 & 0 & 0\end{array}$

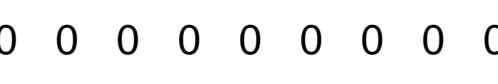
$\begin{array}{lllllllll}0 & 0 & 0 & 0 & 0 & 0 & 0 & 0 & 0 \\ 0 & 0 & 0 & 0 & 0 & 0 & 0 & 0 & 0\end{array}$ 


\begin{tabular}{|c|c|c|c|c|c|c|c|c|c|c|c|c|c|}
\hline$x x x$ & xxyy & $x x z z$ & 0 & 0 & $x x x y$ & 0 & 0 & $-y y x y$ & 0 & 00 & 0 & U & 0 \\
\hline$y x x$ & $x x x x$ & xxzz & 0 & 0 & yуxy & 0 & 0 & $-x x x y$ & 0 & 0 & & & 0 \\
\hline zXX & zzyy & zzzz & 0 & 0 & zzxy & 0 & 0 & $-z z x y$ & J & 0 & & U & 0 \\
\hline & 0 & 0 & yzyz & yzzx & & yzzy & yzxz & & 0 & $\begin{array}{ll}0 & 0\end{array}$ & 0 & 0 & 0 \\
\hline & 0 & 0 & zxyz & $z x z x$ & 0 & zxzy & $z x x z$ & 0 & 0 & 0 & 0 & 0 & \\
\hline $\mathrm{yxx}$ & xyyy & xyzz & 0 & 0 & xyxy & 0 & 0 & xyyx & & & 0 & & 0 \\
\hline 0 & 0 & 0 & $z x x z$ & $-z x z y$ & 0 & zxzx & $-z x y z$ & 0 & 0 & 0 & 0 & 0 & 0 \\
\hline & 0 & 0 & $-y z x z$ & yzzy & 0 & -yzzx & yzyz & & 0 & & & 0 & \\
\hline & $x y x$ & xyzz & & & xyyx & & & & & & & & \\
\hline
\end{tabular}
and: $x x x x=x x y y+x y x y+x y y x ; x x x y-y y x y=x y y y-x y x x$

$\left[V^{4}\right] \quad\left(\begin{array}{ccccc}3 x x y y & 0 & 0 & y y z z & 0 \\ 0 & 3 x x y y & 0 & y y z z & 0 \\ 0 & 0 & z z z z & x x y y & 0\end{array}\right)$

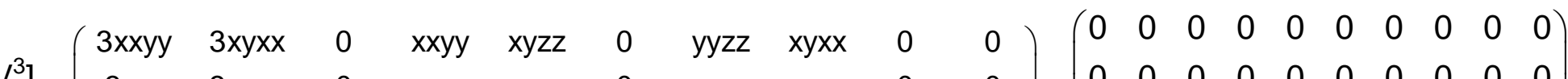

$\left.\left.\mathrm{V}^{2} \mathrm{~V}^{3}\right] \quad \begin{array}{cccccccccccccccccccccc}-3 x y x x & 3 x x y y & 0 & -x y x & y y z z & 0 & -x y z z & x x y y & 0 & 0 & 0 & 0 & 0 & 0 & 0 & 0 & 0 & 0 & 0 & 0\end{array}\right)$

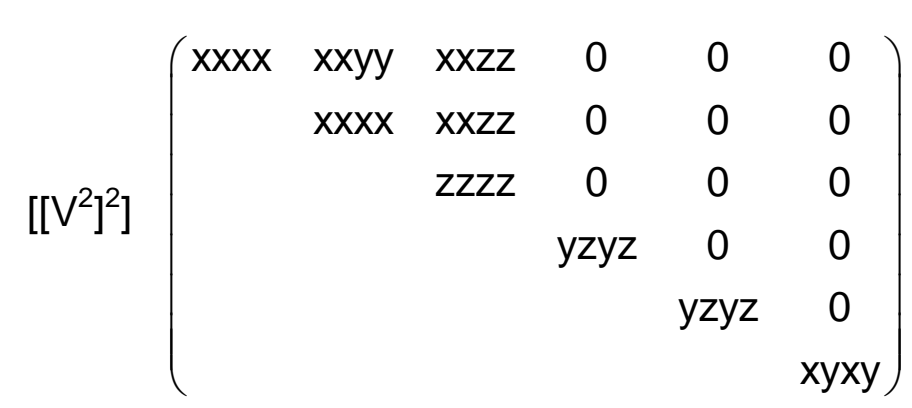

and $x y x y=1 / 2(x x x x-x x y y)$

$\left[\mathrm{V}^{2}\right]^{2}$

$\left(\begin{array}{cccccc}x x x x & x x y y & x x z z & 0 & 0 & x x x y \\ x x y y & x x x x & x x z z & 0 & 0 & -x x x y \\ z z x x & z z x x & z z z z & 0 & 0 & 0 \\ 0 & 0 & 0 & y z y z & y z x z & 0 \\ 0 & 0 & 0 & -y z x z & y z y z & 0 \\ -x x x y & x x x y & 0 & 0 & 0 & x y x y\end{array}\right)$

and $x y x y=1 / 2(x x x x-x x y y)$

$$
\left(\begin{array}{lllll}
0 & 0 & 0 & 0 & 0 \\
0 & 0 & 0 & 0 & 0 \\
0 & 0 & 0 & 0 & 0
\end{array}\right)
$$

$\left(\begin{array}{llllllllll}0 & 0 & 0 & 0 & 0 & 0 & 0 & 0 & 0 & 0 \\ 0 & 0 & 0 & 0 & 0 & 0 & 0 & 0 & 0 & 0\end{array}\right)$

$\begin{array}{lllllllll}0 & 0 & 0 & 0 & 0 & 0 & 0 & 0 & 0 \\ 0 & 0 & 0 & 0 & 0 & 0 & 0 & 0 & 0\end{array}$

$0 \begin{array}{lllllllll}0 & 0 & 0 & 0 & 0 & 0 & 0 & 0 & 0 \\ 0 & 0 & 0 & 0 & 0 & 0 & 0 & 0 & 0\end{array}$

$0 \begin{array}{lllllllll}0 & 0 & 0 & 0 & 0 & 0 & 0 & 0 & 0 \\ 0 & 0 & 0 & 0 & 0 & 0 & 0 & 0 & 0\end{array}$

$\begin{array}{llllllllll}0 & 0 & 0 & 0 & 0 & 0 & 0 & 0 & 0 \\ 0 & 0 & 0 & 0 & 0 & 0 & 0\end{array}$

$0 \begin{array}{llllllllll}0 & 0 & 0 & 0 & 0 & 0 & 0 & 0 & 0 \\ 0 & 0 & 0 & 0 & 0 & 0 & 0 & 0\end{array}$

$\begin{array}{llllllllll}0 & 0 & 0 & 0 & 0 & 0 & 0 & 0 & 0\end{array}$

$0 \begin{array}{lllllllll}0 & 0 & 0 & 0 & 0 & 0 & 0 & 0 & 0 \\ 0 & 0 & 0 & 0 & 0 & 0 & 0 & 0 & 0\end{array}$

$0 \begin{array}{lllllllll}0 & 0 & 0 & 0 & 0 & 0 & 0 & 0 & 0 \\ 0 & 0 & 0 & 0 & 0 & 0 & 0 & 0 & 0\end{array}$

$\begin{array}{llllllllll}0 & 0 & 0 & 0 & 0 & 0 & 0 & 0 & 0\end{array}$

$\left(\begin{array}{lllll}0 & 0 & 0 & 0 & 0 \\ 0 & 0 & 0 & 0 & 0 \\ 0 & 0 & 0 & 0 & 0\end{array}\right)$

$\left(\begin{array}{llllllllll}0 & 0 & 0 & 0 & 0 & 0 & 0 & 0 & 0 & 0 \\ 0 & 0 & 0 & 0 & 0 & 0 & 0 & 0 & 0 & 0\end{array}\right)$

$\left(\begin{array}{llllllllll}0 & 0 & 0 & 0 & 0 & 0 & 0 & 0 & 0 & 0 \\ 0 & 0 & 0 & 0 & 0 & 0 & 0 & 0 & 0 & 0 \\ 0 & 0 & 0 & 0 & 0 & 0 & 0 & 0\end{array}\right)$

$\left(\begin{array}{llllllllll}0 & 0 & 0 & 0 & 0 & 0 & 0 & 0 & 0 & 0 \\ 0 & 0 & 0 & 0 & 0 & 0 & 0 & 0 & 0 & 0 \\ 0 & 0 & 0 & 0 & 0 & 0 & 0 & 0 & 0 & 0\end{array}\right)$

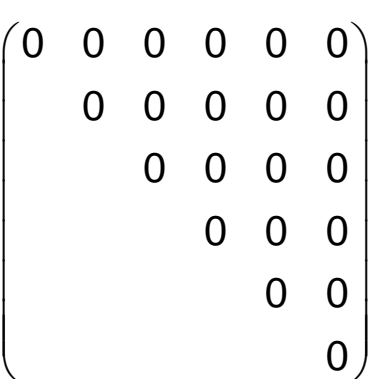

$\begin{array}{llll}0 & 0 & 0 & 0\end{array}$

$\begin{array}{llll}0 & 0 & 0 & 0 \\ & 0 & 0 & 0\end{array}$

$\begin{array}{lll}0 & 0 & 0 \\ & 0 & 0\end{array}$

0

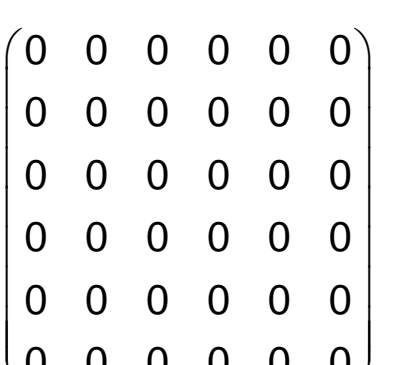

$\left(\begin{array}{llllll}0 & 0 & 0 & 0 & 0 & 0 \\ 0 & 0 & 0 & 0 & 0 & 0\end{array}\right)$

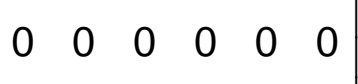

$0 \begin{array}{llllll}0 & 0 & 0 & 0 & 0 & 0 \\ 0 & 0 & 0 & 0 & 0 & 0\end{array}$

$0 \begin{array}{llllll}0 & 0 & 0 & 0 & 0 & 0 \\ 0 & 0 & 0 & 0 & 0 & 0\end{array}$

$\begin{array}{llllll}0 & 0 & 0 & 0 & 0 & 0 \\ 0 & 0 & 0 & 0 & 0 & 0\end{array}$

$\begin{array}{llllll}0 & 0 & 0 & 0 & 0 & 0\end{array}$ $\left(\begin{array}{lllllllll}0 & 0 & 0 & 0 & 0 & 0 & 0 & 0 & 0 \\ 0 & 0 & 0 & 0 & 0 & 0 & 0 & 0 & 0\end{array}\right)$

$\begin{array}{lllllllll}0 & 0 & 0 & 0 & 0 & 0 & 0 & 0 & 0 \\ 0 & 0 & 0 & 0 & 0 & 0 & 0 & 0 & 0\end{array}$

$\begin{array}{lllllllll}0 & 0 & 0 & 0 & 0 & 0 & 0 & 0 & 0\end{array}$ $\begin{array}{llllllllll}0 & 0 & 0 & 0 & 0 & 0 & 0 & 0 & 0\end{array}$ $\begin{array}{lllllllll}0 & 0 & 0 & 0 & 0 & 0 & 0 & 0 & 0\end{array}$ $\begin{array}{llllllllll}0 & 0 & 0 & 0 & 0 & 0 & 0 & 0 & 0\end{array}$ $\begin{array}{lllllllll}0 & 0 & 0 & 0 & 0 & 0 & 0 & 0 & 0 \\ 0 & 0 & 0 & 0 & 0 & 0 & 0 & 0 & 0\end{array}$ $\begin{array}{lllllllll}0 & 0 & 0 & 0 & 0 & 0 & 0 & 0 & 0 \\ 0 & 0 & 0 & 0 & 0 & 0 & 0 & 0 & 0\end{array}$

$\begin{array}{llllllllll}0 & 0 & 0 & 0 & 0 & 0 & 0\end{array}$

$\left(\begin{array}{lllll}0 & 0 & 0 & 0 & 0 \\ 0 & 0 & 0 & 0 & 0 \\ 0 & 0 & 0 & 0 & 0\end{array}\right)$

$\begin{array}{llllll}0 & 0 & 0 & 0 & 0 & 0 \\ 0 & 0 & 0 & 0\end{array}$

$\begin{array}{llllllllll}0 & 0 & 0 & 0 & 0 & 0 & 0 & 0 & 0 & 0 \\ 0 & 0 & 0 & 0 & 0 & 0 & 0 & 0 & 0 & 0\end{array}$

$\left(\begin{array}{llllllllll}0 & 0 & 0 & 0 & 0 & 0 & 0 & 0 & 0 & 0 \\ 0 & 0 & 0 & 0 & 0 & 0 & 0 & 0 & 0 & 0\end{array}\right)$

$\begin{array}{lllllllllll}0 & 0 & 0 & 0 & 0 & 0 & 0 & 0 & 0 & 0\end{array}$

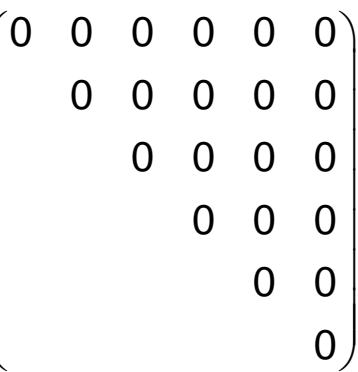

$\left(\begin{array}{llllll}0 & 0 & 0 & 0 & 0 & 0 \\ 0 & 0 & 0 & 0 & 0 & 0\end{array}\right.$

$\begin{array}{llllll}0 & 0 & 0 & 0 & 0 & 0 \\ 0 & 0 & 0 & 0 & 0 & 0\end{array}$

$\begin{array}{llllll}0 & 0 & 0 & 0 & 0 & 0 \\ 0 & 0 & 0 & 0 & 0 & 0\end{array}$

$\begin{array}{llllll}0 & 0 & 0 & 0 & 0 & 0 \\ 0 & 0 & 0 & 0 & 0 & 0\end{array}$

$\begin{array}{llllll}0 & 0 & 0 & 0 & 0 & 0 \\ 0 & 0 & 0 & 0 & 0 & 0\end{array}$

$\begin{array}{llllll}0 & 0 & 0 & 0 & 0 & 0 \\ 0 & 0 & 0 & 0 & 0 & 0\end{array}$ 


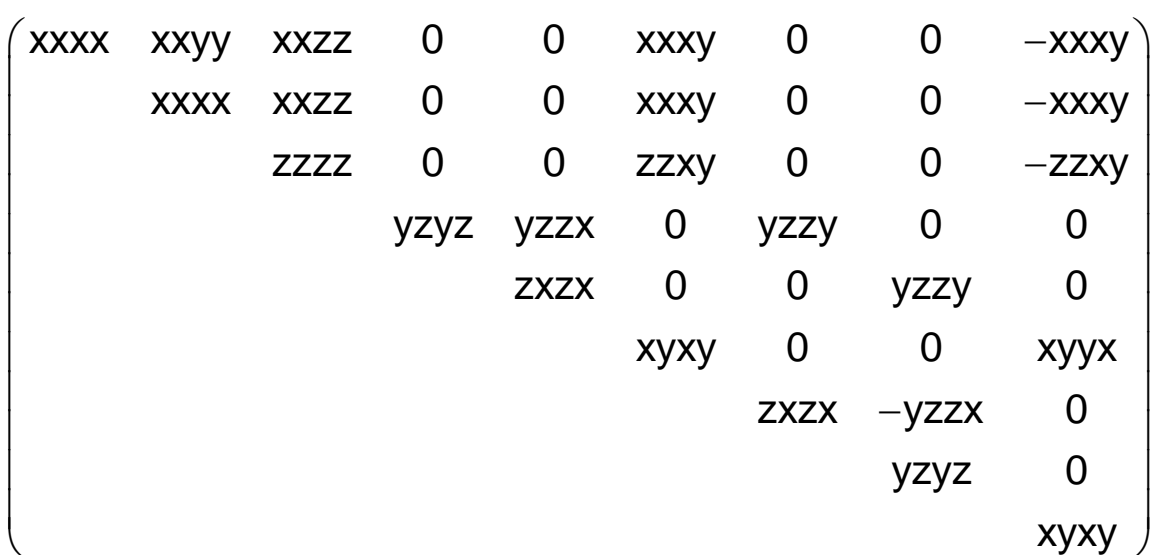

$\left(\begin{array}{lllllllll}0 & 0 & 0 & 0 & 0 & 0 & 0 & 0 & 0 \\ & 0 & 0 & 0 & 0 & 0 & 0 & 0 & 0\end{array}\right)$

$\begin{array}{llllllll}0 & 0 & 0 & 0 & 0 & 0 & 0 & 0\end{array}$

$\begin{array}{llllll}0 & 0 & 0 & 0 & 0 & 0 \\ 0 & 0 & 0 & 0 & 0 & 0\end{array}$

$\begin{array}{llllll}0 & 0 & 0 & 0 & 0 & 0 \\ & 0 & 0 & 0 & 0 & 0\end{array}$

$0 \begin{array}{llll}0 & 0 & 0 \\ 0 & 0 & 0\end{array}$

$\begin{array}{lll}0 & 0 & 0 \\ & 0 & 0\end{array}$

0
0

and $x x x x=x x y y+y z y z+z y z y$

$\left(\begin{array}{llllllllll}x x x x & x x y y & x x z z & 0 & 0 & x x x y & 0 & 0 & -y y x y \\ x y y & x x a x & x x z & 0 & 0 & y x y & 0 & 0 & -x y\end{array}\right)\left(\begin{array}{ccccccccc}0 & 0 & 0 & 0 & 0 & 0 & 0 & 0 & 0 \\ 0 & 0 & 0 & 0 & 0 & 0 & 0 & 0 & 0\end{array}\right)$

$\left.\begin{array}{lllllllll}x x y y & x x x x & x x z z & 0 & 0 & y y x y & 0 & 0 & -x x x y \\ z z x y & z z x & z z z z & 0 & 0 & z x y & 0 & 0 & -z x y\end{array} \mid \begin{array}{lllllllll}0 & 0 & 0 & 0 & 0 & 0 & 0 & 0 & 0 \\ 0 & 0 & 0 & 0 & 0 & 0 & 0 & 0 & 0\end{array}\right)$

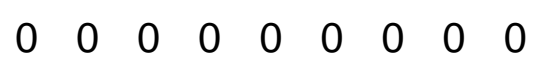

$\begin{array}{llllllllllll}0 & 0 & 0 & 0 & 0 & 0 & 0 & 0 & 0 \\ 0 & 0 & 0 & 0 & 0 & 0 & 0 & 0 & 0\end{array}$

$0 \begin{array}{lllllllll}0 & 0 & 0 & 0 & 0 & 0 & 0 & 0 & 0 \\ 0 & 0 & 0 & 0 & 0 & 0 & 0 & 0 & 0\end{array}$

$\begin{array}{lllllllll}0 & 0 & 0 & y z y z & y z z x & 0 & z x z x & -z x y z & 0\end{array}$

$\begin{array}{ccccccccc}0 & 0 & 0 & z x y z & z x z x & 0 & -y z z x & y z y z & 0\end{array}$

$\begin{array}{lllllllll}\text { xyxx } & -x y x x & 0 & 0 & 0 & \text { xyxy } & 0 & 0 & \text { xyxy }\end{array}$

$\begin{array}{lllllllll}0 & 0 & 0 & 0 & 0 & 0 & 0 & 0 & 0\end{array}$

and $x x x x=x x y y+2 x y x y ; y y x y-x x x y=2 x y x x$

$$
\left(\begin{array}{lllllllll}
0 & 0 & 0 & 0 & 0 & 0 & 0 & 0 & 0 \\
& 0 & 0 & 0 & 0 & 0 & 0 & 0 & 0 \\
& & 0 & 0 & 0 & 0 & 0 & 0 & 0 \\
& & & 0 & 0 & 0 & 0 & 0 & 0 \\
& & & & 0 & 0 & 0 & 0 & 0 \\
& & & & & 0 & 0 & 0 & 0 \\
& & & & & & 0 & 0 & 0 \\
& & & & & & & 0 & 0 \\
& & & & & & & & \\
& & & & & & & 0
\end{array}\right)
$$

$\left(\begin{array}{lllllllll}0 & 0 & 0 & 0 & 0 & 0 & 0 & 0 & 0 \\ 0 & 0 & 0 & 0 & 0 & 0 & 0 & 0 & 0\end{array}\right.$

$0 \begin{array}{lllllllll}0 & 0 & 0 & 0 & 0 & 0 & 0 & 0 & 0 \\ 0 & 0 & 0 & 0 & 0 & 0 & 0 & 0 & 0\end{array}$

$0 \begin{array}{lllllllll}0 & 0 & 0 & 0 & 0 & 0 & 0 & 0 & 0 \\ 0 & 0 & 0 & 0 & 0 & 0 & 0 & 0 & 0\end{array}$

$0 \begin{array}{lllllllll}0 & 0 & 0 & 0 & 0 & 0 & 0 & 0 & 0 \\ 0 & 0 & 0 & 0 & 0 & 0 & 0 & 0 & 0\end{array}$

$0 \begin{array}{lllllllll}0 & 0 & 0 & 0 & 0 & 0 & 0 & 0 & 0 \\ 0 & 0 & 0 & 0 & 0 & 0 & 0 & 0 & 0\end{array}$

$\begin{array}{lllllllll}0 & 0 & 0 & 0 & 0 & 0 & 0 & 0 & 0\end{array}$ $\left(\begin{array}{lllllllll}0 & 0 & 0 & 0 & 0 & 0 & 0 & 0 & 0 \\ & 0 & 0 & 0 & 0 & 0 & 0 & 0 & 0\end{array}\right)$

$\begin{array}{llllllll}0 & 0 & 0 & 0 & 0 & 0 & 0 & 0\end{array}$

$\begin{array}{lllllll}0 & 0 & 0 & 0 & 0 & 0 & 0\end{array}$

$\begin{array}{llllll}0 & 0 & 0 & 0 & 0 & 0\end{array}$

$\begin{array}{lllll}0 & 0 & 0 & 0 & 0 \\ 0 & 0 & 0 & 0\end{array}$

$\begin{array}{llll}0 & 0 & 0\end{array}$

$\begin{array}{lll}0 & 0 & 0 \\ & 0 & 0 \\ & & 0\end{array}$

$\left.\begin{array}{ll}0 & 0 \\ & 0\end{array}\right)$ $\left(\begin{array}{lllllllll}0 & 0 & 0 & 0 & 0 & 0 & 0 & 0 & 0 \\ 0 & 0 & 0 & 0 & 0 & 0 & 0 & 0 & 0\end{array}\right.$

$\begin{array}{lllllllll}0 & 0 & 0 & 0 & 0 & 0 & 0 & 0 & 0 \\ 0 & 0 & 0 & 0 & 0 & 0 & 0 & 0 & 0\end{array}$

$\begin{array}{lllllllll}0 & 0 & 0 & 0 & 0 & 0 & 0 & 0 & 0 \\ 0 & 0 & 0 & 0 & 0 & 0 & 0 & 0 & 0 \\ 0 & 0 & 0 & 0 & 0 & 0 & 0 & 0 & 0\end{array}$

$\begin{array}{llllllllll}0 & 0 & 0 & 0 & 0 & 0 & 0 & 0 & 0\end{array}$

$\begin{array}{llllllllll}0 & 0 & 0 & 0 & 0 & 0 & 0 & 0 & 0\end{array}$ 
$\begin{array}{lllllllll}x x x x & x x y y & x x z z & 0 & x x z x & 0 & 0 & x x x z & 0 \\ y y x & y y y & y y z & 0 & y y z x & 0 & 0 & y y x z & 0\end{array}$ $\begin{array}{llllllllll}y y x x & y y y y & y y z z & 0 & y y z x & 0 & 0 & y y x z & 0\end{array}$ $\begin{array}{llllllllll}z z x x & z z y y & z z z z & 0 & z z z x & 0 & 0 & z z x z & 0\end{array}$

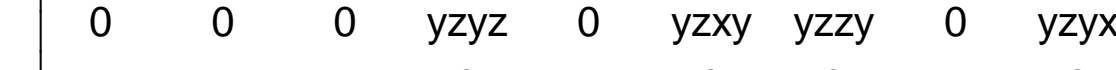
$\begin{array}{lllllllll}z x x x & z x y y & z x z z & 0 & z x z x & 0 & 0 & z x x z & 0\end{array}$ $\begin{array}{ccccccccc}0 & 0 & 0 & x y y z & 0 & x y x y & x y z y & 0 & x y y x\end{array}$ $\begin{array}{ccccccccc}0 & 0 & 0 & x y y z & 0 & x y x y & x y z y & 0 & x y y x \\ 0 & 0 & 0 & z y y z & 0 & z y x y & z y z y & 0 & z y y x \\ x & x y y & x z z z & 0 & x z z x & 0 & 0 & x z x z & 0\end{array}$ $\begin{array}{lllllllll}x z x x & x z y y & x z z z & 0 & x z z x & 0 & 0 & x z x z & 0\end{array}$ $\begin{array}{ccccccccc}0 & 0 & 0 & y x y z & 0 & y x x y & y x z y & 0 & y x y x\end{array}$

$\left[V^{4}\right]\left(\begin{array}{ccccc}\mathrm{xxxx} & 0 & \mathrm{xzzz} & \mathrm{yyzz} & 0 \\ 0 & \mathrm{yyyy} & 0 & \mathrm{zzxx} & \mathrm{yyxz} \\ \mathrm{zxxx} & 0 & \mathrm{zzzz} & \mathrm{xxyy} & 0\end{array}\right)$

$\left(\begin{array}{lllllllll}x x x x & x x y y & x x z z & 0 & x x z x & 0 & 0 & x x x z & 0 \\ y y x & y y y & y y z z & 0 & y y z x & 0 & 0 & x y x z & 0\end{array}\right)$ $\begin{array}{llllllllll}y y x x & y y y y & y y z z & 0 & y y z x & 0 & 0 & y y x z & 0\end{array}$ $\begin{array}{lllllllll}\text { zzxx } & z z y y & z z z z & 0 & z z z x & 0 & 0 & z z x z & 0\end{array}$ $\begin{array}{lllllllll}0 & 0 & 0 & y z y z & 0 & y z x y & y z z y & 0 & y z y x\end{array}$ $\begin{array}{lllllllll}2 x x x & z x y y & z x z z & 0 & z x z x & 0 & 0 & z x x z & 0 \\ 0 & 0 & 0 & x y y z & 0 & x y x y & x y z y & 0 & x y x\end{array}$ $\begin{array}{lllllllll}0 & 0 & 0 & x y y z & 0 & x y x y & x y z y & 0 & x y y x \\ 0 & 0 & 0 & z y z & 0 & \text { zyxy } & z y z & 0 & z y x\end{array}$ $\begin{array}{lllllllll}0 & 0 & 0 & \text { zyyz } & 0 & \text { zyxy } & \text { zyzy } & 0 & \text { zyyx }\end{array}$ $\begin{array}{llllllllll}x z x x & x z y y & x z z z & 0 & x z z x & 0 & 0 & x z x z & 0\end{array}$ $\begin{array}{lllllllll}0 & 0 & 0 & y x y z & 0 & y x x y & y x z y & 0 & y x y x\end{array}$

$\left(\begin{array}{ccccc}\mathrm{xxxx} & 0 & \mathrm{xzzz} & \mathrm{yyzz} & 0 \\ 0 & \mathrm{yyyy} & 0 & \mathrm{zzxx} & \mathrm{yyxz} \\ \mathrm{zxxx} & 0 & \mathrm{zzzz} & \mathrm{xxyy} & 0\end{array}\right)$ $\left(\begin{array}{lllllllll}0 & 0 & 0 & x x y z & 0 & x x x y & x x z y & 0 & x x y x \\ 0 & 0 & 0 & y y z & 0 & y y x & y y z & 0 & y y x\end{array}\right)$ $\begin{array}{lllllllll}0 & 0 & 0 & y y y z & 0 & y y x y & y y z y & 0 & y y y x \\ 0 & 0 & 0 & z z y z & 0 & z z x y & z z z y & 0 & z z y x\end{array}$ $\begin{array}{lllllllll}\text { yzxx } & \text { yzyy } & \text { yzzz } & 0 & \text { yzzx } & 0 & 0 & \text { yzxz } & 0\end{array}$ $\begin{array}{lllllllll}0 & 0 & 0 & z x y z & 0 & z x x y & z x z y & 0 & z x y x\end{array}$ $\begin{array}{lllllllll}\text { xyxx } & x y y y & x y z z & 0 & x y z x & 0 & 0 & x y x z & 0\end{array}$

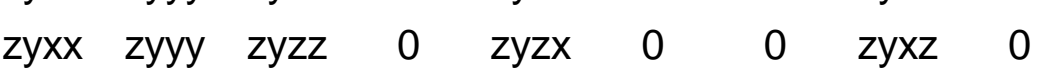
$\begin{array}{lllllllll}0 & 0 & 0 & x z y z & 0 & x z x y & x z z y & 0 & x z y x\end{array}$ $\left.\begin{array}{lllllllll}y x x x & y x y y & y x z z & 0 & y x z x & 0 & 0 & y x x z & 0\end{array}\right)$

$\left(\begin{array}{ccccc}0 & x y y y & 0 & 0 & x x y z \\ y x x x & 0 & y z z z & 0 & 0 \\ 0 & z y y y & 0 & 0 & z z x y\end{array}\right)$ $\left(\begin{array}{lllllllll}0 & 0 & 0 & x x y z & 0 & x x x y & x x z y & 0 & x x y x \\ 0 & 0 & 0 & y y z & 0 & y x y & y y z & 0 & y y x\end{array}\right.$ $\begin{array}{lllllllll}0 & 0 & 0 & \text { yyyz } & 0 & \text { yyxy } & \text { yyzy } & 0 & \text { yyyx } \\ 0 & 0 & 0 & z z y z & 0 & \text { zzxy } & \text { zzzy } & 0 & \text { zzyx }\end{array}$ $\begin{array}{llllllll}0 & 0 & z z y z & 0 & z z x y & z z z y & 0 & z z y x\end{array}$ $\begin{array}{cccccccc}0 y z y y & y z z z & 0 & y z z x & 0 & 0 & y z x z & 0\end{array}$ $\begin{array}{llllllll}0 & 0 & z x y z & 0 & z x x y & z x z y & 0 & z x y x\end{array}$ $\begin{array}{lllllllll}\text { xyxx } & \text { xyyy } & x y z z & 0 & x y z x & 0 & 0 & x y x z & 0\end{array}$ $\begin{array}{lllllllll}z y x x & z y y y & z y z z & 0 & z y z x & 0 & 0 & z y x z & 0\end{array}$ $\begin{array}{lllllllll}0 & 0 & 0 & x z y z & 0 & x z x y & x z z y & 0 & x z y x\end{array}$ $\begin{array}{lllllllll}y x x x & y x y y & y x z z & 0 & y x z x & 0 & 0 & y x x z & 0\end{array}$

$\left(\begin{array}{ccccc}0 & \text { xyyy } & 0 & 0 & \text { xxyz } \\ y x x x & 0 & y z z z & 0 & 0 \\ 0 & \text { zyyy } & 0 & 0 & z z x y\end{array}\right)$

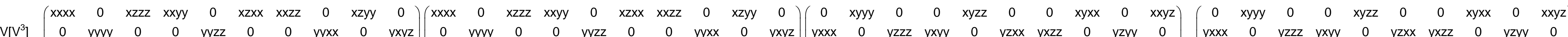

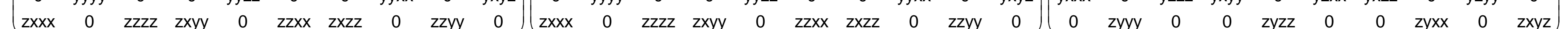

$\left.\left[\mathrm{V}^{2}\right]^{2}\right]$

$$
\begin{aligned}
& \left.\begin{array}{lllll}
x x y y & x x z z & 0 & x x x z & 0 \\
\text { yyyy } & y y z z & 0 & y y x z & 0
\end{array}\right) \\
& \begin{array}{cccc}
x z z z & 0 & y y x z & 0 \\
z z z z & 0 & z z x z & 0
\end{array} \\
& \begin{array}{ccc}
y z y z & 0 & y z x y \\
x z x z & 0
\end{array} \\
& \text { xyxy }
\end{aligned}
$$

$\left[\mathrm{V}^{2}\right]^{2}$

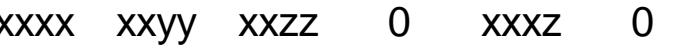

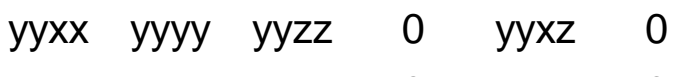

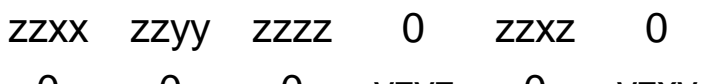
$\begin{array}{llllll}0 & 0 & 0 & y z y z & 0 & y z x y\end{array}$ $\left(\begin{array}{cccccc}x z x & x z y y & x z z z & 0 & x z x z & 0 \\ 0 & 0 & 0 & x y y z & 0 & x y x y\end{array}\right)$

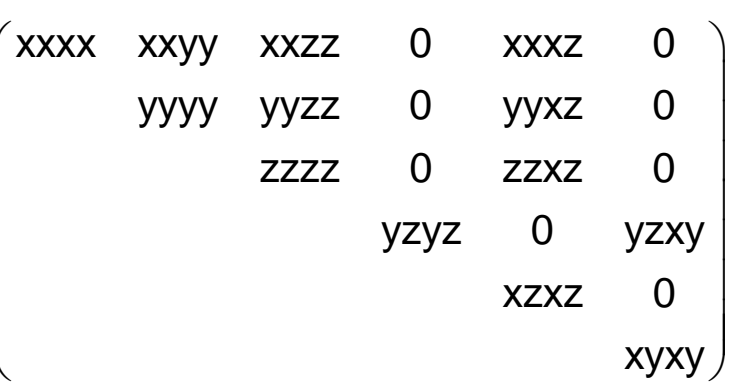

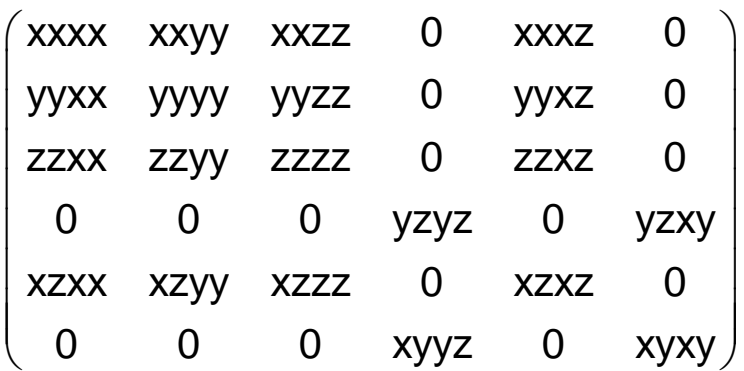

$$
\begin{aligned}
& \left(\begin{array}{llllll}
0 & 0 & 0 & \text { xxyz } & 0 & \text { xxxy } \\
& 0 & 0 & \text { yyyz } & 0 & \text { yyxy }
\end{array}\right. \\
& \begin{array}{llll}
0 & z z y z & 0 & z z x y
\end{array} \\
& \begin{array}{ccc}
0 & y z x z & 0 \\
0 & x z x y
\end{array}
\end{aligned}
$$

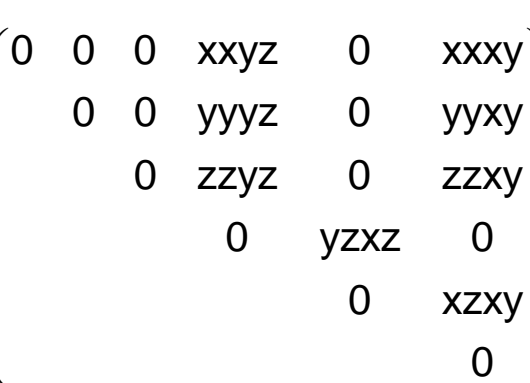$$
\left(\begin{array}{cccccc}
0 & 0 & 0 & \text { xxyz } & 0 & \text { xxxy } \\
0 & 0 & 0 & \text { yyyz } & 0 & \text { yyxy } \\
0 & 0 & 0 & z z y z & 0 & z z x y \\
y z x x & y z y y & y z z z & 0 & y z x z & 0 \\
0 & 0 & 0 & x z y z & 0 & \text { xzxy } \\
0 & 0 & 0 & 0 & x y x z & 0
\end{array}\right)
$$$$
\left(\begin{array}{cccccc}
0 & 0 & 0 & \text { xxyz } & 0 & \text { xxxy } \\
0 & 0 & 0 & \text { yyyz } & 0 & \text { yyxy } \\
0 & 0 & 0 & \text { zzyz } & 0 & \text { zzxy } \\
\text { yzxx } & y z y y & y z z z & 0 & y z x z & 0 \\
0 & 0 & 0 & \text { xzyz } & 0 & \text { xzxy } \\
0 & 0 & 0 & 0 & \text { xyxz } & 0
\end{array}\right)
$$ 


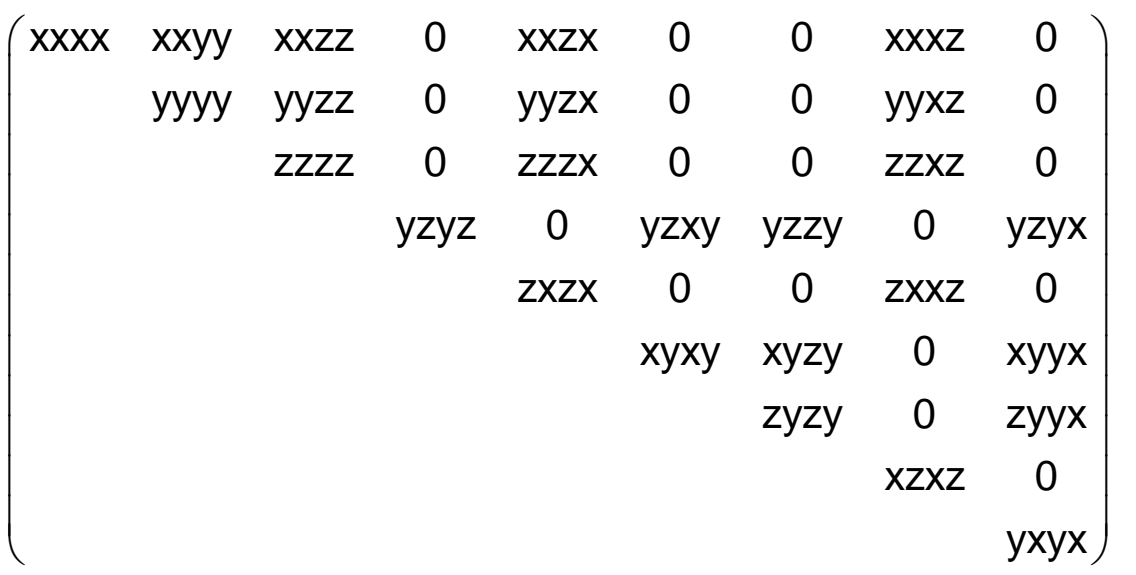

$$
\begin{aligned}
& \begin{array}{lllllllll}
x x x x & x x y y & x x z z & 0 & x x z x & 0 & 0 & x x x z & 0 \\
& y y y & y y z & 0 & y y z x & 0 & 0 & y y x z & 0
\end{array} \\
& \left.\begin{array}{lllllllll}
\text { yyyy } & y y z z & 0 & y y z x & 0 & 0 & y y x z & 0
\end{array}\right) \\
& \begin{array}{lllllll}
z z z z & 0 & z z z x & 0 & 0 & z z x z & 0
\end{array} \\
& \begin{array}{llllll}
y z y z & 0 & y z x y & y z z y & 0 & y z y x
\end{array} \\
& \begin{array}{ccccc}
z x z x & 0 & 0 & z x x z & 0 \\
x y x y & x y z y & 0 & x y y x \\
& & z y z y & 0 & z y x
\end{array} \\
& \begin{array}{ccc}
\text { zyzy } & 0 & z y y x \\
x z x z & 0
\end{array} \\
& \begin{array}{c}
0 \\
y x y x
\end{array}
\end{aligned}
$$

ae

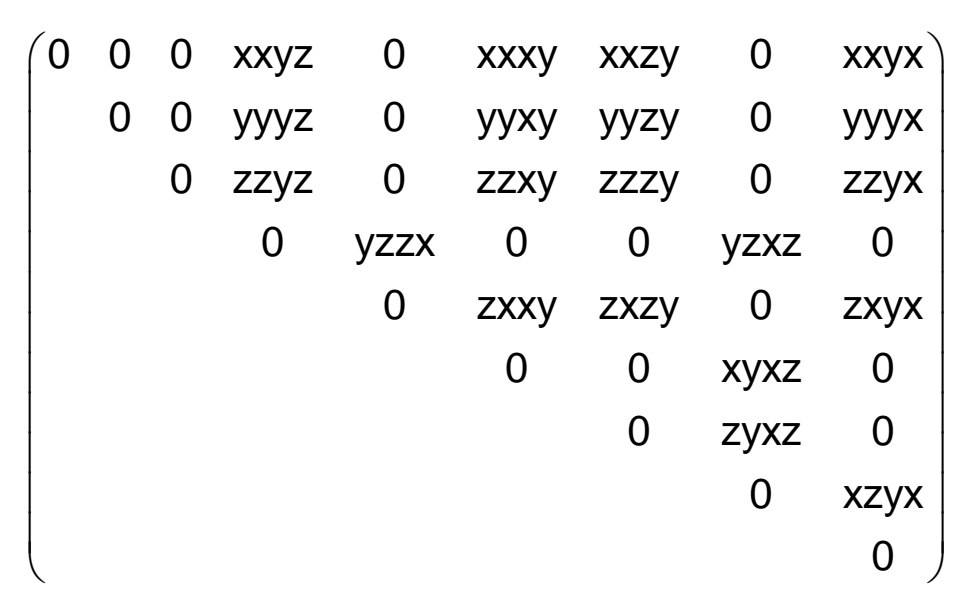

$\left(\begin{array}{lllllllll}x x x x & x x y y & x x z z & 0 & x x z x & 0 & 0 & x x x z & 0\end{array}\right)$ $\left.\begin{array}{lllllllll}\text { yyxx } & y y y y & y y z z & 0 & y y z x & 0 & 0 & y y x z & 0\end{array}\right)$ $\left[\mathrm{V}^{2}\right] \mathrm{V}^{2} \quad \begin{array}{ccccccccc}\mathrm{zzxx} & \mathrm{zzyy} & \mathrm{zzzz} & 0 & \mathrm{zzzx} & 0 & 0 & \mathrm{zzxz} & 0 \\ 0 & 0 & 0 & \mathrm{yzyz} & 0 & \mathrm{yzxy} & \mathrm{yzzy} & 0 & \mathrm{yzyx}\end{array}$ $\begin{array}{lllllllll}z x x x & z x y y & z x z z & 0 & z x z x & 0 & 0 & z x x z & 0\end{array}$

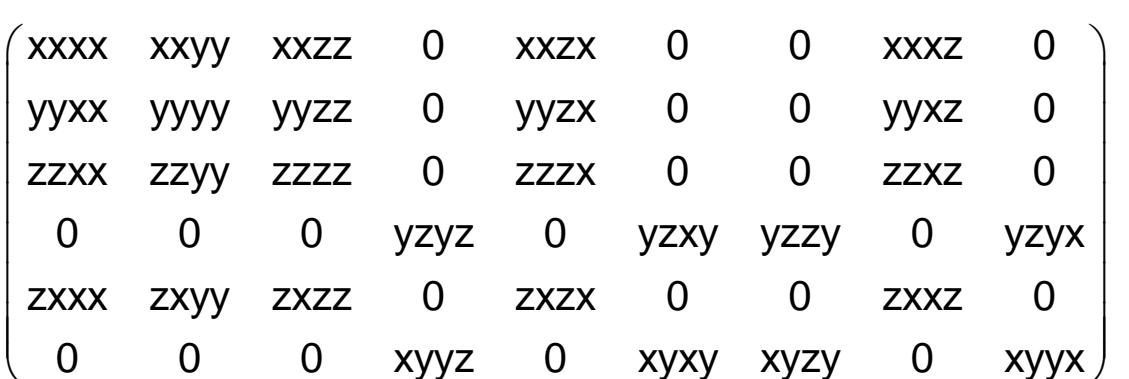

$$
\begin{aligned}
& \begin{array}{lllllllll}
0 & 0 & x x y z & 0 & x x x y & x x z y & 0 & x x y x \\
0 & 0 & y y z & 0 & y y x & y y z y & 0 & y y y x
\end{array} \\
& \begin{array}{lllllll}
0 & y y y z & 0 & \text { yyxy } & y y z y & 0 & y y y x \\
0 & z z y z & 0 & z z x y & z z z y & 0 & z z y x
\end{array} \\
& \begin{array}{llllll}
0 & y z z x & 0 & 0 & y z x z & 0
\end{array} \\
& \begin{array}{lllll}
0 & z x x y & z x z y & 0 & z x y x
\end{array} \\
& \begin{array}{ccc}
0 & \mathrm{xyxz} & 0 \\
0 & \mathrm{zyxz} & 0 \\
& 0 & \mathrm{xzyx}
\end{array} \\
& 0
\end{aligned}
$$

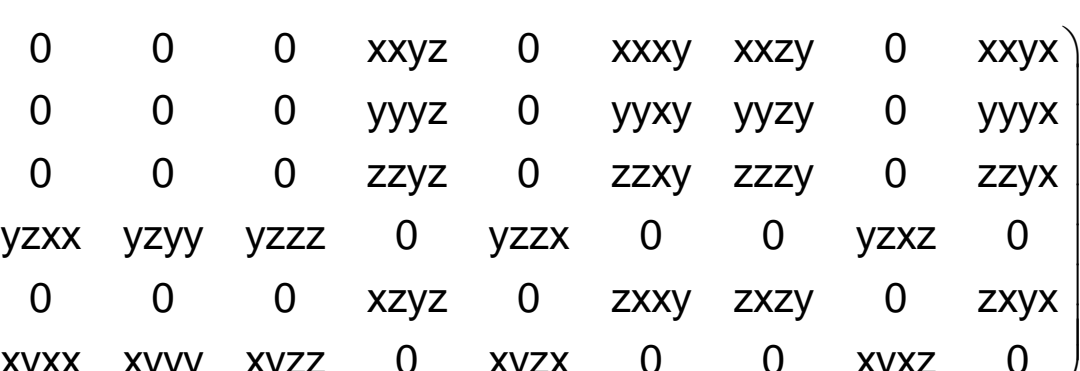



$\left[V^{4}\right] \quad\left(\begin{array}{ccccc}x x x x & 0 & 0 & y y z z & 0 \\ 0 & y y y y & 0 & z z x x & 0 \\ 0 & 0 & z z z z & x x y y & 0\end{array}\right)$

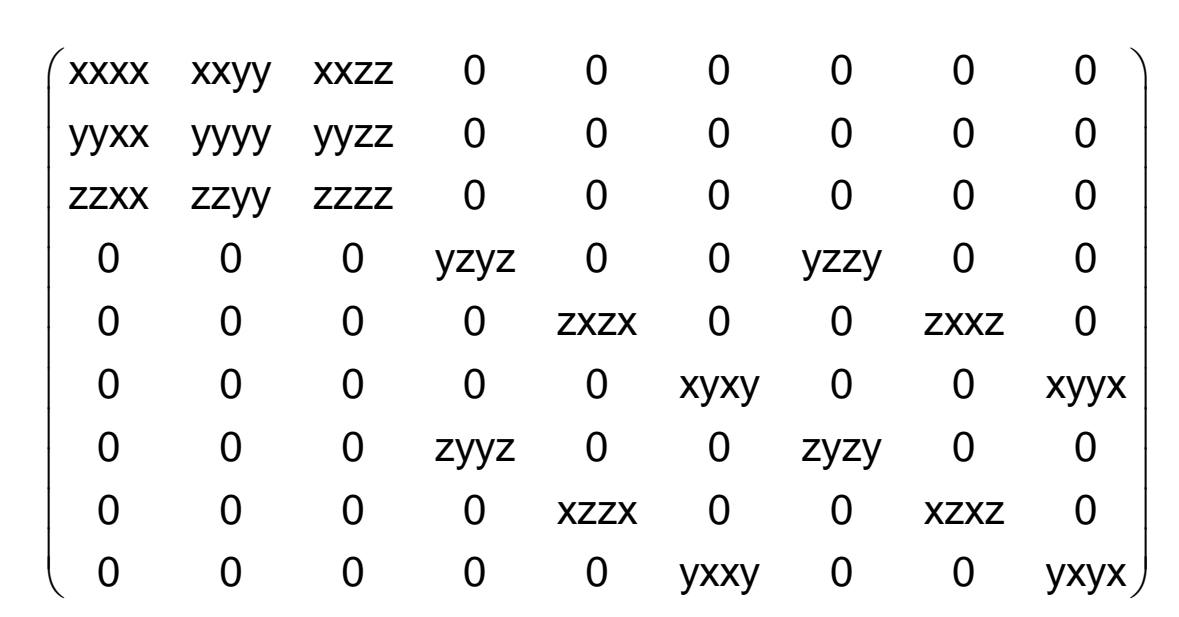
$\left(\begin{array}{ccccccccc}0 & 0 & 0 & 0 & 0 & \text { xxxy } & 0 & 0 & \text { xxyx } \\ 0 & 0 & 0 & 0 & 0 & \text { yyxy } & 0 & 0 & \text { yyyx } \\ 0 & 0 & 0 & 0 & 0 & \text { zzxy } & 0 & 0 & \text { zzyx } \\ 0 & 0 & 0 & 0 & \text { yzzx } & 0 & 0 & \text { yzxz } & 0 \\ 0 & 0 & 0 & \text { zxyz } & 0 & 0 & \text { zxzy } & 0 & 0 \\ \text { xyxx } & \text { xyyy } & \text { xyzz } & 0 & 0 & 0 & 0 & 0 & 0 \\ 0 & 0 & 0 & 0 & \text { zyzx } & 0 & 0 & \text { zyxz } & 0 \\ 0 & 0 & 0 & x z y z & 0 & 0 & \text { xzzy } & 0 & 0 \\ \text { yxxx } & \text { yxyy } & \text { yxzz } & 0 & 0 & 0 & 0 & 0 & 0\end{array}\right)$
$\left(\begin{array}{ccccccccc}0 & 0 & 0 & 0 & 0 & \text { xxxy } & 0 & 0 & \text { xxyx } \\ 0 & 0 & 0 & 0 & 0 & \text { yyxy } & 0 & 0 & \text { yyyx } \\ 0 & 0 & 0 & 0 & 0 & z z x y & 0 & 0 & \text { zzyx } \\ 0 & 0 & 0 & 0 & y z z x & 0 & 0 & y z x z & 0 \\ 0 & 0 & 0 & \text { zxyz } & 0 & 0 & \text { zxzy } & 0 & 0 \\ \text { xyxx } & x y y y & x y z z & 0 & 0 & 0 & 0 & 0 & 0 \\ 0 & 0 & 0 & 0 & \text { zyzx } & 0 & 0 & z y x z & 0 \\ 0 & 0 & 0 & \text { xzyz } & 0 & 0 & \text { xzzy } & 0 & 0 \\ \text { yxxx } & \text { yxyy } & \text { yxzz } & 0 & 0 & 0 & 0 & 0 & 0\end{array}\right)$

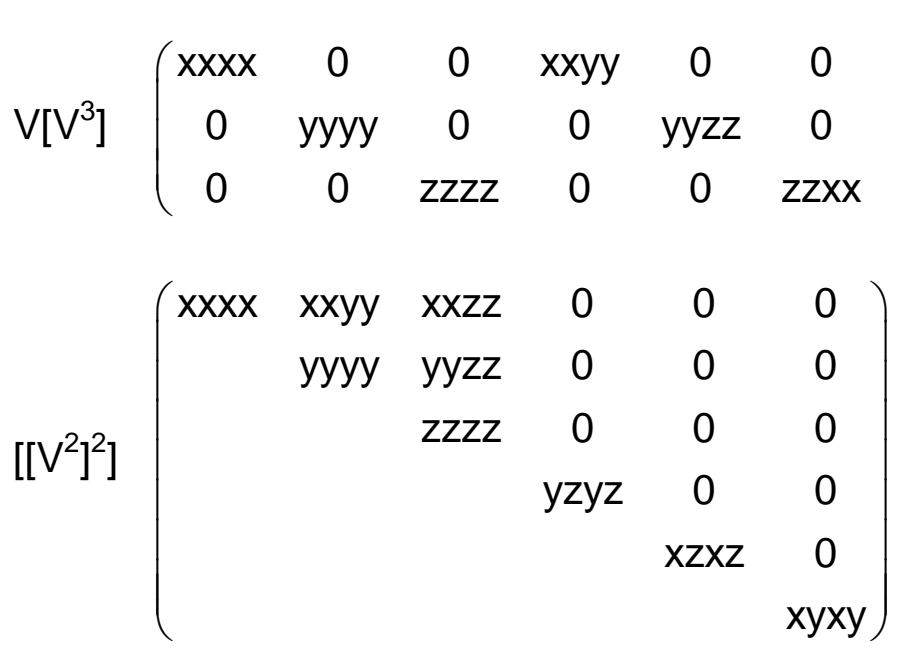
$\left(\begin{array}{ccccc}x x x x & 0 & 0 & y y z z & 0 \\ 0 & y y y y & 0 & z z x x & 0 \\ 0 & 0 & z z z z & x x y y & 0\end{array}\right)$
$\left(\begin{array}{ccccc}0 & x y y y & 0 & 0 & 0 \\ y x x x & 0 & 0 & 0 & 0 \\ 0 & 0 & 0 & 0 & z z x y\end{array}\right)$
$\left(\begin{array}{ccccc}0 & x y y y & 0 & 0 & 0 \\ y x x x & 0 & 0 & 0 & 0 \\ 0 & 0 & 0 & 0 & z z x y\end{array}\right)$
$\left(\begin{array}{cccccccccc}\text { xxxx } & 0 & 0 & \text { xxyy } & 0 & 0 & \text { xxzz } & 0 & 0 & 0 \\ 0 & y y y y & 0 & 0 & y y z z & 0 & 0 & y y x x & 0 & 0 \\ 0 & 0 & z z z z & 0 & 0 & z z x x & 0 & 0 & z z y y & 0\end{array}\right)$
$\left(\begin{array}{cccccccccc}0 & x y y y & 0 & 0 & x y z z & 0 & 0 & x y x x & 0 & 0 \\ y x x x & 0 & 0 & y x y y & 0 & 0 & y x z z & 0 & 0 & 0 \\ 0 & 0 & 0 & 0 & 0 & 0 & 0 & 0 & 0 & z x y z\end{array}\right)$
$\left(\begin{array}{cccccccccc}0 & x y y y & 0 & 0 & x y z z & 0 & 0 & x y x x & 0 & 0 \\ \operatorname{yxxx} & 0 & 0 & y x y y & 0 & 0 & y x z z & 0 & 0 & 0 \\ 0 & 0 & 0 & 0 & 0 & 0 & 0 & 0 & 0 & z x y z\end{array}\right)$

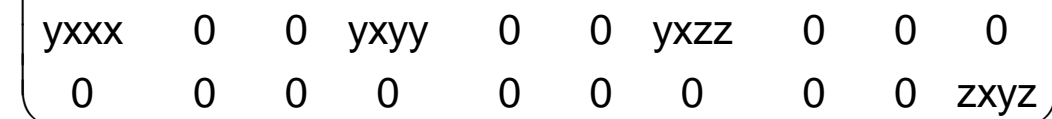
$\left[V^{2}\right]^{2}\left(\begin{array}{cccccc}x x x x & x x y y & x x z z & 0 & 0 & 0 \\ y y x x & y y y y & y y z z & 0 & 0 & 0 \\ z z x x & z z y y & z z z z & 0 & 0 & 0 \\ 0 & 0 & 0 & y z y z & 0 & 0 \\ 0 & 0 & 0 & 0 & x z x z & 0 \\ 0 & 0 & 0 & 0 & 0 & x y x y\end{array}\right)$
$\left(\begin{array}{cccccc}x x x x & x x y y & x x z z & 0 & 0 & 0 \\ & \text { yyyy } & \text { yyzz } & 0 & 0 & 0 \\ & & z z z z & 0 & 0 & 0 \\ & & & y z y z & 0 & 0 \\ & & & & x z x z & 0 \\ & & & & & \text { xyxy }\end{array}\right)$
$\left(\begin{array}{cccccc}\text { xxxx } & \text { xxyy } & \text { xxzz } & 0 & 0 & 0 \\ \text { yyxx } & \text { yyyy } & \text { yyzz } & 0 & 0 & 0 \\ \text { zzxx } & \text { zzyy } & \text { zzzz } & 0 & 0 & 0 \\ 0 & 0 & 0 & \text { yzyz } & 0 & 0 \\ 0 & 0 & 0 & 0 & x z x z & 0 \\ 0 & 0 & 0 & 0 & 0 & \text { xyxy }\end{array}\right)$

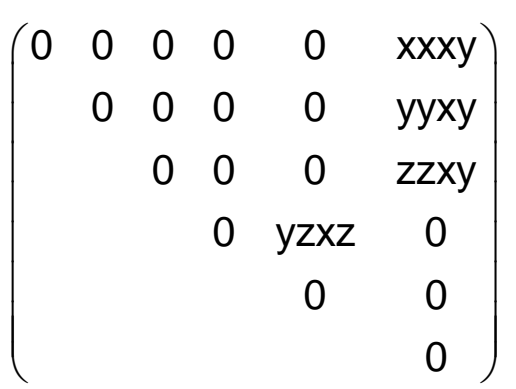
$\left(\begin{array}{llllll}0 & 0 & 0 & 0 & 0 & x x x y \\ 0 & 0 & 0 & 0 & 0 & y y x y \\ 0 & 0 & 0 & 0 & 0 & z x y y\end{array}\right)$ $\begin{array}{llllll}0 & 0 & 0 & 0 & 0 & \text { yyxy } \\ 0 & 0 & 0 & 0 & 0 & z z x y\end{array}$ $\begin{array}{cccccc}0 & 0 & 0 & 0 & 0 & z z x y \\ 0 & 0 & 0 & 0 & y z x & 0\end{array}$ $\begin{array}{llllll}0 & 0 & 0 & 0 & y z x z & 0\end{array}$ $\begin{array}{llllll}0 & 0 & 0 & x z y z & 0 & 0\end{array}$

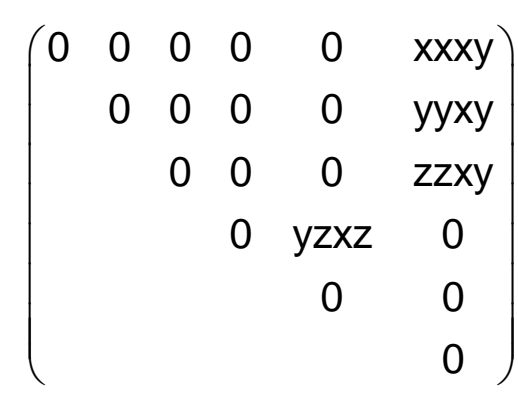
$\left(\begin{array}{cccccc}0 & 0 & 0 & 0 & 0 & \mathrm{xxxy} \\ 0 & 0 & 0 & 0 & 0 & \mathrm{yyxy} \\ 0 & 0 & 0 & 0 & 0 & \mathrm{zzxy} \\ 0 & 0 & 0 & 0 & \mathrm{yzxz} & 0 \\ 0 & 0 & 0 & \mathrm{xzyz} & 0 & 0 \\ 0 & 0 & 0 & 0 & 0 & 0\end{array}\right)$ 


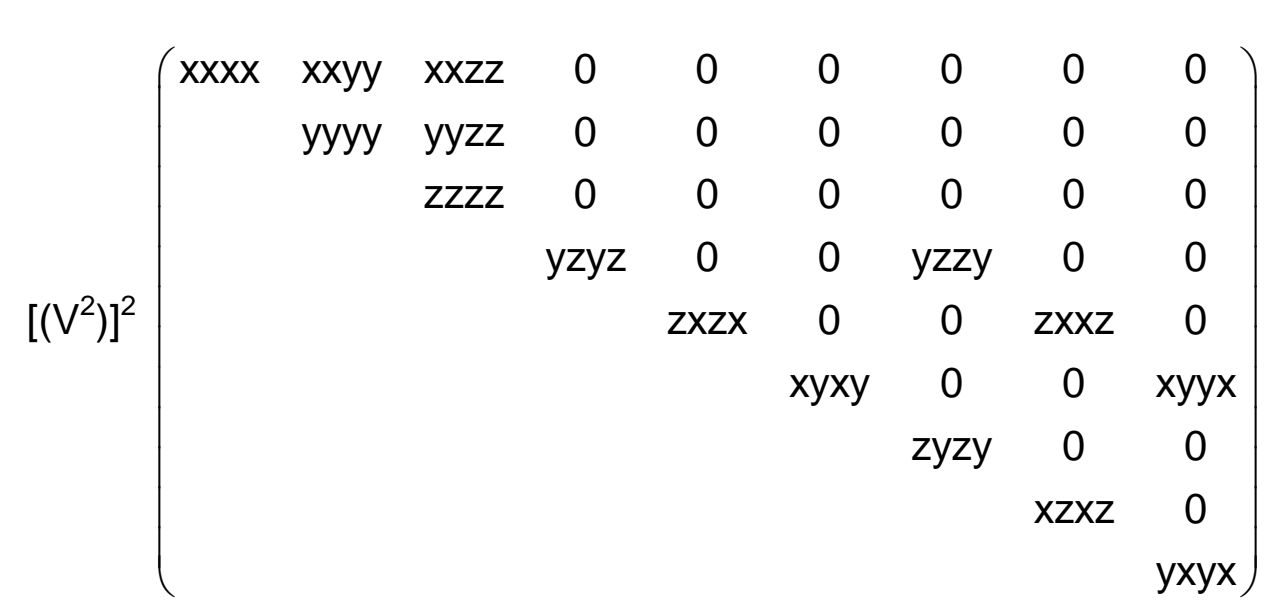

$\left[\mathrm{V}^{2}\right] \mathrm{V}^{2}\left(\begin{array}{ccccccccc}\mathrm{xxxx} & \mathrm{x} x \mathrm{y} y & \mathrm{xxzz} & 0 & 0 & 0 & 0 & 0 & 0 \\ \mathrm{yyxx} & \mathrm{yyyy} & \mathrm{yyzz} & 0 & 0 & 0 & 0 & 0 & 0 \\ \mathrm{zzxx} & \mathrm{zzyy} & \mathrm{zzzz} & 0 & 0 & 0 & 0 & 0 & 0 \\ 0 & 0 & 0 & \mathrm{yzyz} & 0 & 0 & \mathrm{yzzy} & 0 & 0 \\ 0 & 0 & 0 & 0 & \mathrm{zxzx} & 0 & 0 & \mathrm{zxxz} & 0 \\ 0 & 0 & 0 & 0 & 0 & \mathrm{xyxy} & 0 & 0 & \mathrm{xyyx}\end{array}\right)$
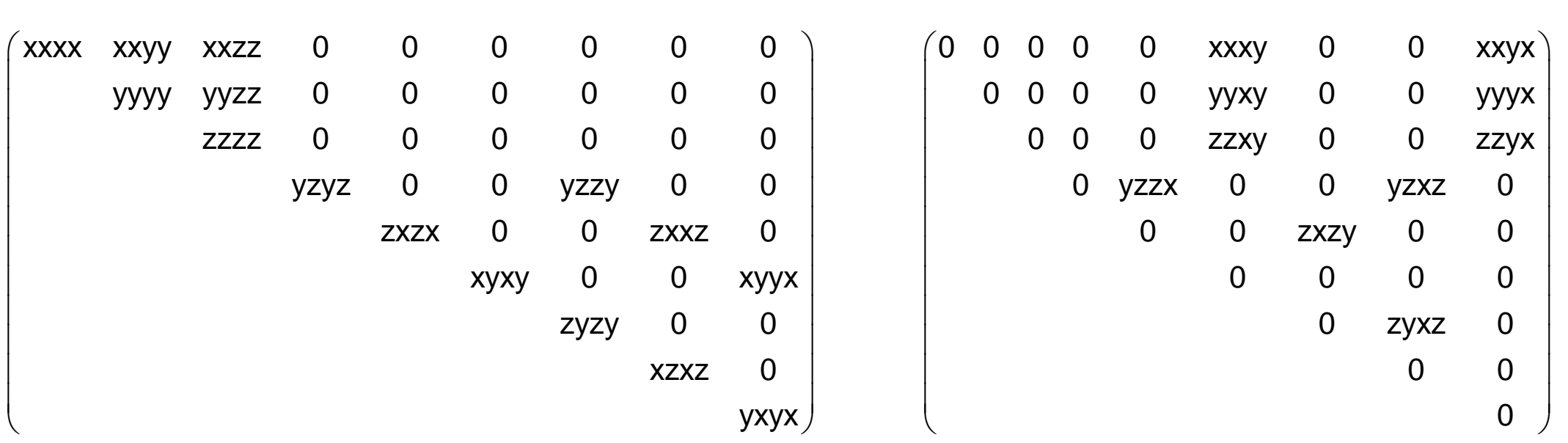

$\left(\begin{array}{ccccccccc}\text { xxxx } & \text { xxyy } & \text { xxzz } & 0 & 0 & 0 & 0 & 0 & 0 \\ \text { yyxx } & \text { yyyy } & \text { yyzz } & 0 & 0 & 0 & 0 & 0 & 0 \\ \text { zzxx } & \text { zzyy } & \text { zzzz } & 0 & 0 & 0 & 0 & 0 & 0 \\ 0 & 0 & 0 & \text { yzyz } & 0 & 0 & \text { yzzy } & 0 & 0 \\ 0 & 0 & 0 & 0 & \text { zxzx } & 0 & 0 & \text { zxxz } & 0 \\ 0 & 0 & 0 & 0 & 0 & \text { xyxy } & 0 & 0 & \text { xyyx }\end{array}\right)$ $\left(\begin{array}{ccccccccc}0 & 0 & 0 & 0 & 0 & \text { xxxy } & 0 & 0 & \text { xxyx } \\ 0 & 0 & 0 & 0 & 0 & \text { yyxy } & 0 & 0 & \text { yyyx } \\ 0 & 0 & 0 & 0 & 0 & \text { zzxy } & 0 & 0 & \text { zzyx } \\ 0 & 0 & 0 & 0 & \text { yzzx } & 0 & 0 & \text { yzxz } & 0 \\ 0 & 0 & 0 & \text { xzyz } & 0 & 0 & \text { zxzy } & 0 & 0 \\ \text { xyxx } & \text { xyyy } & \text { xyzz } & 0 & 0 & 0 & 0 & 0 & 0\end{array}\right)$

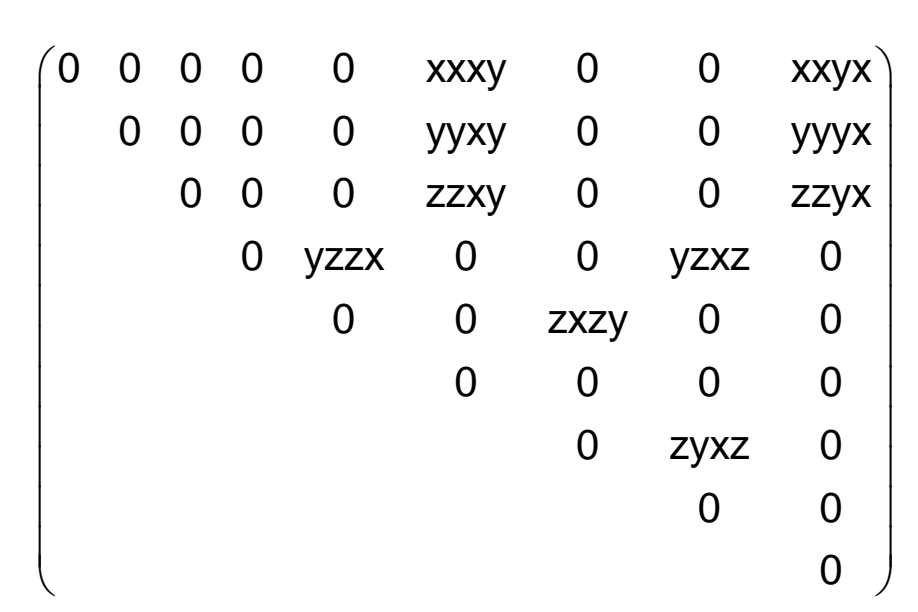

$\left(\begin{array}{ccccccccc}0 & 0 & 0 & 0 & 0 & \text { xxxy } & 0 & 0 & \text { xxyx } \\ 0 & 0 & 0 & 0 & 0 & \text { yyxy } & 0 & 0 & \text { yyyx } \\ 0 & 0 & 0 & 0 & 0 & \text { zzxy } & 0 & 0 & \text { zzyx } \\ 0 & 0 & 0 & 0 & \text { yzzx } & 0 & 0 & \text { yzxz } & 0 \\ 0 & 0 & 0 & \text { xzyz } & 0 & 0 & \text { zxzy } & 0 & 0 \\ \text { xyxx } & \text { xyyy } & \text { xyzz } & 0 & 0 & 0 & 0 & 0 & 0\end{array}\right)$ 
$\begin{array}{lllllllll}x x x x & x x y y & x x z z & x x y z & 0 & 0 & x x z y & 0 & 0 \\ y y x & x x y x & y z z & -x y z & 0 & 0 & -x x y & 0 & 0\end{array}$ $\left(\begin{array}{ccccccccc}\mathrm{yyxxx} & \mathrm{xxxx} & \mathrm{yyzz} & -\mathrm{xxyz} & 0 & 0 & -\mathrm{xxzy} & 0 & 0 \\ \mathrm{zzxx} & \mathrm{zzxx} & \mathrm{zzzz} & 0 & 0 & 0 & 0 & 0 & 0 \\ y z x & -\mathrm{yzx} & 0 & y z y z & 0 & 0 & y z z y & 0 & 0\end{array}\right)$ $\begin{array}{lllllllll}0 & 0 & 0 & 0 & z x z x & z y x x & 0 & z x x z & z y x x\end{array}$ $\begin{array}{ccccccccc}0 & 0 & 0 & 0 & x x z y & x y x y & 0 & x x y z & x y y x\end{array}$ $\begin{array}{ccccccccc}z y x x & -z y x x & 0 & z x x z & 0 & 0 & z x z x & 0 & 0\end{array}$ $\begin{array}{ccccccccc}x z x x & -x z x x & 0 & 0 & y z z y & y z x x & 0 & y z y z & y z x x \\ 0 & 0 & 0 & 0 & x y z y & x y y x & 0 & x x y z & x y x y\end{array}$ and: $x x x x=x x y y+x y x y+x y y x$

$\left[V^{4}\right] \quad\left(\begin{array}{ccccc}3 x x y y & 0 & 0 & y y z z & x x y z \\ 0 & 3 x x y y & 0 & y y z z & 0 \\ 0 & -x x y z & z z z z & x x y y & 0\end{array}\right)$

$\left.V_{[} V^{3}\right]\left(\begin{array}{cccccccccc}3 x x y y & 0 & 0 & x x y y & 0 & 0 & y y z z & 0 & 0 & y z x x \\ 0 & 3 x x y y & 0 & 0 & y y z z & y z x x & 0 & x x y y & -y z x x & 0 \\ 0 & -z y x x & z z z z & 0 & 0 & z z x x & 0 & z y x x & z z x x & 0\end{array}\right)$

$\left.\left[\mathrm{IV}^{2}\right]^{2}\right]\left(\begin{array}{cccccc}\mathrm{xxxx} & \mathrm{xxyy} & \mathrm{xxzz} & \mathrm{xxyz} & 0 & 0 \\ & \mathrm{xxxx} & \mathrm{xxzz} & -\mathrm{xxyz} & 0 & 0 \\ & & \mathrm{zzzz} & 0 & 0 & 0 \\ & & & \mathrm{yzyz} & 0 & 0 \\ & & & & \mathrm{yzyz} & \mathrm{xxyz} \\ & & & & & \mathrm{xyxy}\end{array}\right)$

and $x y x y=1 / 2(x x x x-x x y y)$

$\left[V^{2}\right]^{2}\left(\begin{array}{cccccc}x x x x & x x y y & x x z z & x x y z & 0 & 0 \\ x x y y & x x x x & x x z z & -x x y z & 0 & 0 \\ z z x x & z z x x & z z z z & 0 & 0 & 0 \\ y z x x & -y z x x & 0 & y z y z & 0 & 0 \\ 0 & 0 & 0 & 0 & y z y z & y z x x \\ 0 & 0 & 0 & 0 & x x y z & x y x y\end{array}\right)$

and $x y x y=1 / 2(x x x x-x x y y)$ $\left(\begin{array}{lllllllll}x x x x & x x y y & x x z z & x x y z & 0 & 0 & x x z y & 0 & 0\end{array}\right)$ $\left.\begin{array}{ccccccccc}x y y x x & x x x x & y y z z & -x x y z & 0 & 0 & -x x z y & 0 & 0 \\ z z x x & z z x x & z z z z & 0 & 0 & 0 & 0 & 0 & 0 \\ y z x x & -y z x x & 0 & y z y z & 0 & 0 & y z z y & 0 & 0\end{array}\right)$ $\begin{array}{ccccccccc}z z x x & z z x x & z z z z & 0 & 0 & 0 & 0 & 0 & 0 \\ y z x x & -y z x x & 0 & y z y z & 0 & 0 & y z z y & 0 & 0 \\ 0 & 0 & 0 & 0 & z x z & z y x & 0 & z x y z & z y x x\end{array}$ $\begin{array}{ccccccccc}\mathrm{yzxx} & -\mathrm{yzxx} & 0 & \mathrm{yzyz} & 0 & 0 & \mathrm{yzzy} & 0 & 0 \\ 0 & 0 & 0 & 0 & \mathrm{zxzx} & \mathrm{zyyx} & 0 & \mathrm{zxxz} & \mathrm{zyxx} \\ 0 & 0 & 0 & 0 & \mathrm{x} z \mathrm{y} & \mathrm{xyy} & 0 & \mathrm{x} x \mathrm{z} & \mathrm{xyyx}\end{array}$ $\begin{array}{ccccccccc}0 & 0 & 0 & 0 & z x z x & z y x x & 0 & z x x z & z y x x \\ 0 & 0 & 0 & 0 & x x z y & x y x y & 0 & x x y z & x y y x \\ z y x x & -z y x x & 0 & z x y z & 0 & 0 & z x z & 0 & 0\end{array}$ $\begin{array}{ccccccccc}0 & 0 & 0 & 0 & x x z y & x y x y & 0 & x x y z & x y y x \\ z y x x & -z y x x & 0 & z x z & 0 & 0 & z x z x & 0 & 0 \\ x z x x & -x z x x & 0 & 0 & y z z y & y z x x & 0 & y z y z & y z x x\end{array}$ $\begin{array}{ccccccccc}z y x x & -z y x x & 0 & z x x z & 0 & 0 & z x z x & 0 & 0 \\ x z x x & -x z x & 0 & 0 & y z z y & y z x x & 0 & y z y z & y z x x\end{array}$ $\left(\begin{array}{ccccccccc}\mathrm{xzxx} & -\mathrm{xzxx} & 0 & 0 & \mathrm{yzzy} & \mathrm{yzxx} & 0 & \mathrm{yzyz} & \mathrm{yzxx} \\ 0 & 0 & 0 & 0 & \mathrm{x} x z \mathrm{x} & \mathrm{xyyx} & 0 & \mathrm{xxyz} & \mathrm{xyxy}\end{array}\right)$ and: $x x x x=x x y y+x y x y+x y y x$

$\left(\begin{array}{ccccc}3 x x y y & 0 & 0 & y y z z & x x y z \\ 0 & 3 x x y y & 0 & y y z z & 0 \\ 0 & -x x y z & z z z z & x x y y & 0\end{array}\right)$

$\left(\begin{array}{cccccccccc}3 x x y y & 0 & 0 & x x y y & 0 & 0 & y y z z & 0 & 0 & y z x x \\ 0 & 3 x y y y & 0 & 0 & y y z z & y z z x & 0 & x x y y & -y z z x & 0\end{array}\right)$ $\left(\begin{array}{ccccccccccc}0 & 3 x x y y & 0 & 0 & y y z z & y z x x & 0 & x x y y & -y z x x & 0 \\ 0 & -z y x x & z z z z & 0 & 0 & z z x x & 0 & z y x x & z z x x & 0\end{array}\right)$

$$
\left(\begin{array}{cccccc}
x x x x & x x y y & x x z z & x x y z & 0 & 0 \\
& x x x x & x x z z & -x x y z & 0 & 0 \\
& & z z z z & 0 & 0 & 0 \\
& & & y z y z & 0 & 0 \\
& & & & y z y z & x x y z \\
& & & & & x y x y
\end{array}\right)
$$

and $x y x y=1 / 2(x x x x-x x y y)$

$\left(\begin{array}{cccccc}x x x x & x x y y & x x z z & x x y z & 0 & 0 \\ x x y y & x x x x & x x z z & -x x y z & 0 & 0 \\ z z x x & z z x x & z z z z & 0 & 0 & 0 \\ y z x x & -y z x x & 0 & y z y z & 0 & 0 \\ 0 & 0 & 0 & 0 & y z y z & y z x x \\ 0 & 0 & 0 & 0 & x x y z & x y x y\end{array}\right)$

$$
\left(\begin{array}{cccccc}
0 & 0 & 0 & 0 & -y y x z & x x x y \\
0 & 0 & 0 & 0 & y y x z & -x x x y \\
0 & 0 & 0 & 0 & 0 & 0 \\
0 & 0 & 0 & 0 & y z x z & x z y y \\
-x z y y & x z y y & 0 & y z x z & 0 & 0 \\
-x x x y & x x x y & 0 & y y x z & 0 & 0
\end{array}\right)
$$

and $x y x y=1 / 2(x x x x-x x y y)$ $\left(\begin{array}{cccccc}0 & 0 & 0 & 0 & -\mathrm{yyxz} & 0 \\ & 0 & 0 & 0 & \mathrm{yyxz} & 0 \\ & & 0 & 0 & 0 & 0 \\ & & & 0 & 0 & \mathrm{yyxz} \\ & & & & 0 & 0 \\ & & & & & 0\end{array}\right)$

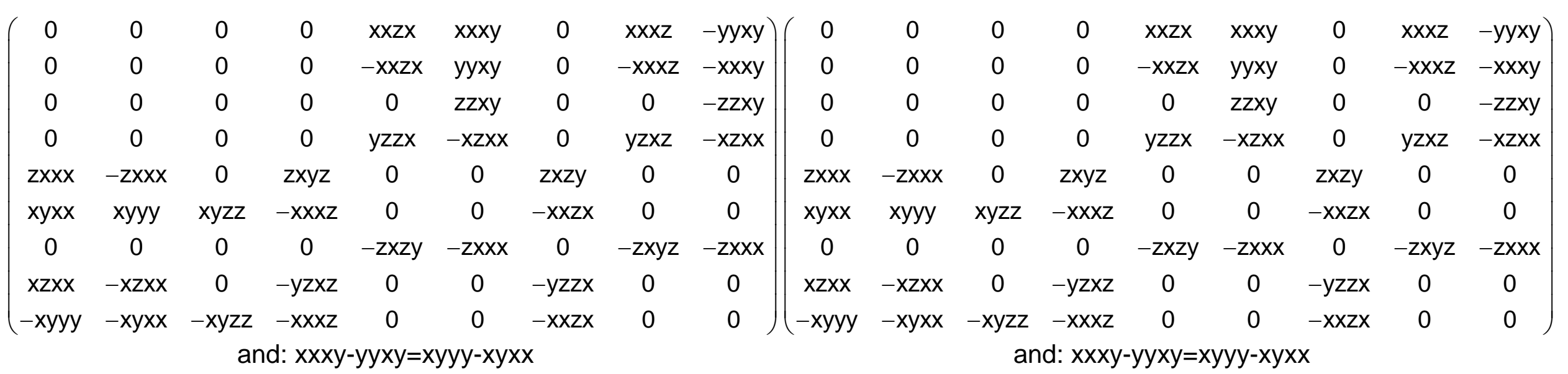
$\left(\begin{array}{ccccc}0 & 0 & 0 & 0 & 0 \\ 0 & 0 & 0 & 0 & -z x x x \\ z x x x & 0 & 0 & 0 & 0\end{array}\right)$
$\left(\begin{array}{ccccc}0 & 0 & 0 & 0 & 0 \\ 0 & 0 & 0 & 0 & -z x x x \\ z x x x & 0 & 0 & 0 & 0\end{array}\right)$

$\left(\begin{array}{cccccccccc}0 & 3 x y x x & 0 & 0 & x y z z & x z x x & 0 & x y x x & -x z x x & 0 \\ -3 x y y x & 0 & 0 & -x y y x & 0 & 0 & -x y z z & 0 & 0 & -x z y x\end{array}\right)$ $\left.\begin{array}{cccccccccc}0 & 3 x y x x & 0 & 0 & x y z z & x z x x & 0 & x y x x & -x z x x & 0 \\ 3 x y y x & 0 & 0 & -x y x x & 0 & 0 & -x y z z & 0 & 0 & -x z x x \\ z x x & 0 & 0 & -z x x x & 0 & 0 & 0 & 0 & 0 & z x y z\end{array}\right)$

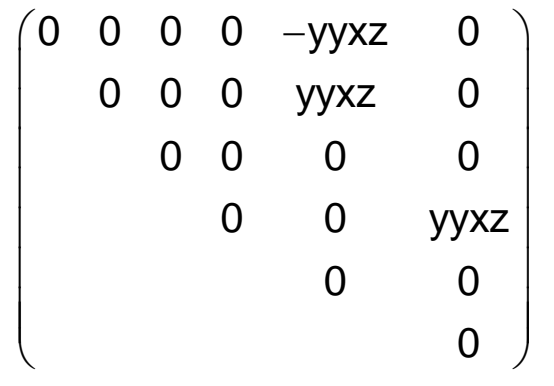

$\left(\begin{array}{cccccc}0 & 0 & 0 & 0 & -y y x z & x x x y \\ 0 & 0 & 0 & 0 & y y x z & -x x x y \\ 0 & 0 & 0 & 0 & 0 & 0 \\ 0 & 0 & 0 & 0 & y z x z & x z y y \\ -x z y y & x z y y & 0 & y z x z & 0 & 0 \\ -x x x y & x x x y & 0 & y y x z & 0 & 0\end{array}\right)$




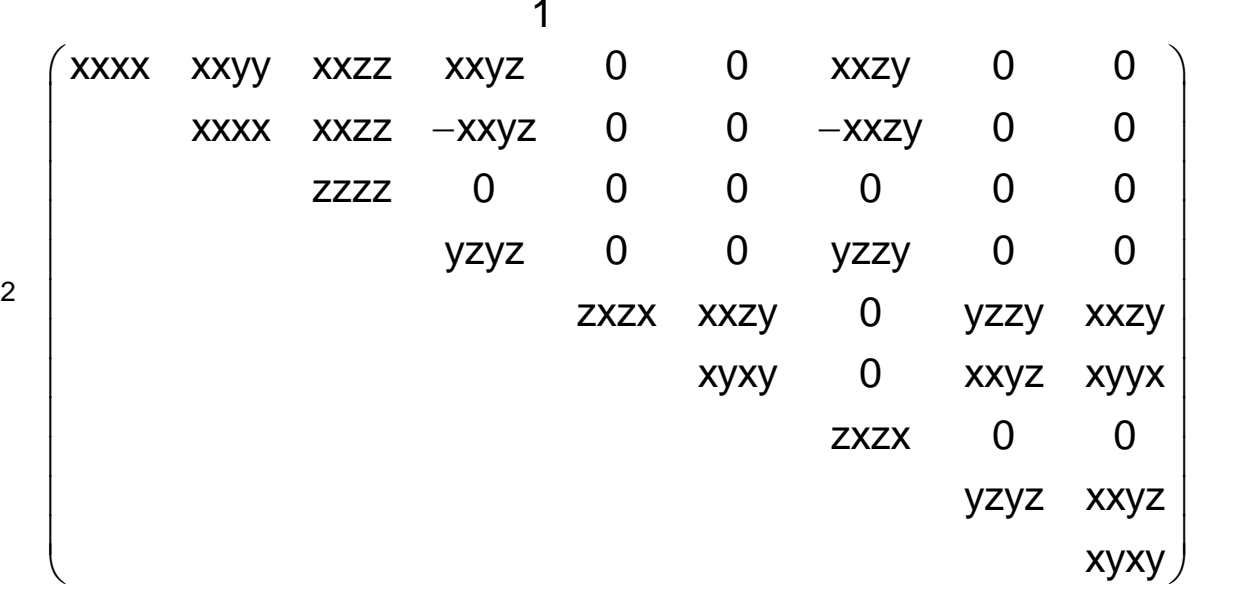

and $x x x x=x x y y+y z y z+z y z y$

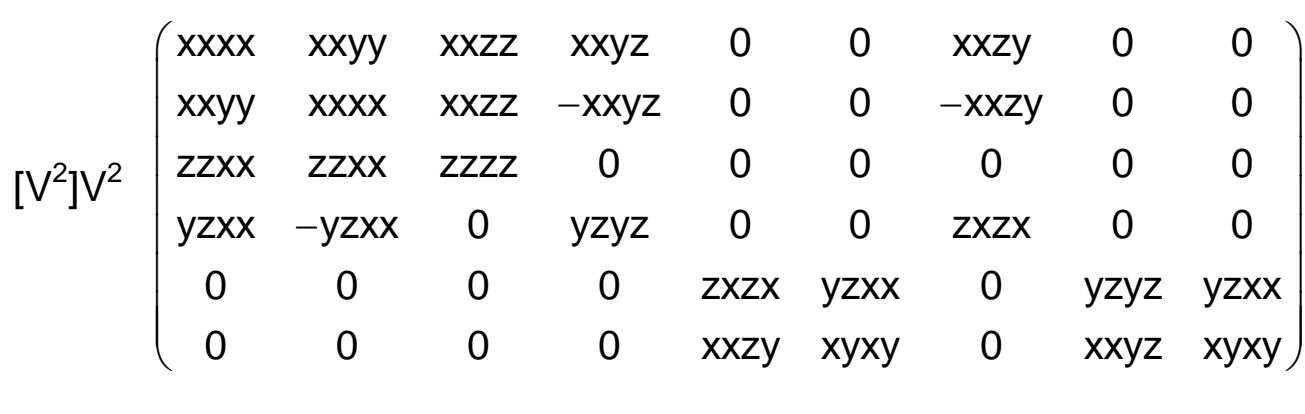

and $x x x x=x x y y+2 x y x y$

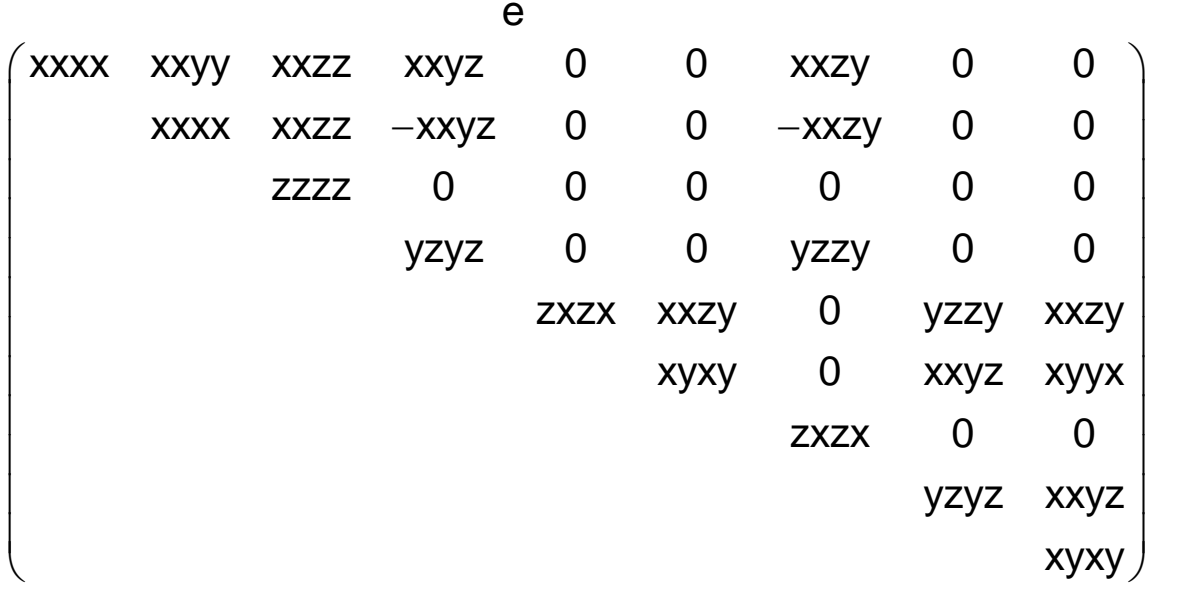

and $x x x x=x x y y+y z y z+z y z y$

$\left(\begin{array}{lllllllll}x x x x & x x y y & x x z z & x x y z & 0 & 0 & x x z y & 0 & 0 \\ x y y & x x x x & x x z z & -x y z & 0 & 0 & -x x z y & 0 & 0\end{array}\right)$ $\begin{array}{lllllllll}x x y y & x x x x & x x z z & -x x y z & 0 & 0 & -x x z y & 0 & 0\end{array}$ $\begin{array}{lllllllll}z z x x & \text { zzxx } & \text { zzzz } & 0 & 0 & 0 & 0 & 0 & 0\end{array}$ $\begin{array}{lllllllll}y z x x & -y z x x & 0 & y z y z & 0 & 0 & z x z x & 0 & 0\end{array}$ $\begin{array}{ccccccccc}0 & 0 & 0 & 0 & z x z x & y z x x & 0 & y z y z & y z x x \\ 0 & 0 & 0 & 0 & x x z y & x y x y & 0 & x y z & x y x\end{array}$ $\begin{array}{lll}0 & 0 & x x z y\end{array}$ and $x x x x=x x y y+2 x y x y$
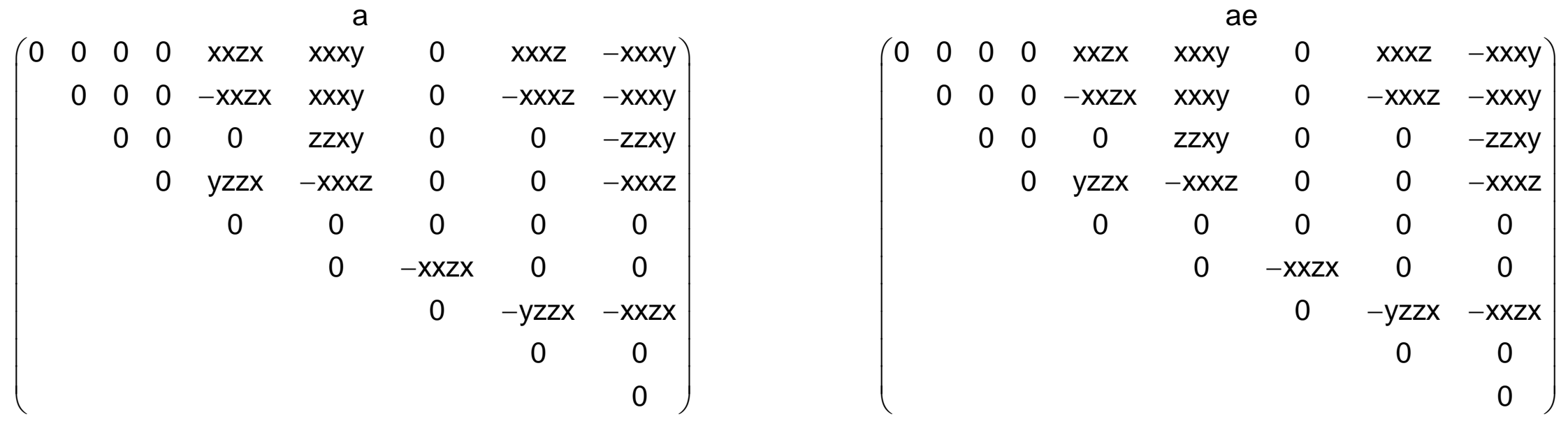
$\left(\begin{array}{lllllllll}0 & 0 & 0 & 0 & x x z x & x x x y & 0 & x x x z & -y y x y \\ 0 & 0 & 0 & 0 & -x x z & y x y & 0 & -x x y z & -x x y y\end{array}\right)$ $\left.\begin{array}{cccccccccc}0 & 0 & 0 & 0 & -x x z x & y y x y & 0 & -x x x z & -x x x y \\ 0 & 0 & 0 & 0 & 0 & z z x y & 0 & 0 & -z x y\end{array}\right)$ $\begin{array}{lllllllll}0 & 0 & 0 & 0 & y z z x & -z x x x & 0 & -z x y z & -z x x x\end{array}$ $\begin{array}{lllllllll}z x x x & -z x x x & 0 & z x y z & 0 & 0 & -y z z x & 0 & 0\end{array}$ $\begin{array}{llllllll}x y x x & -x y x x & 0 & -x x x z & 0 & 0 & -x x z x & 0\end{array}$ and: $y y x y-x x x y=2 x y x x$

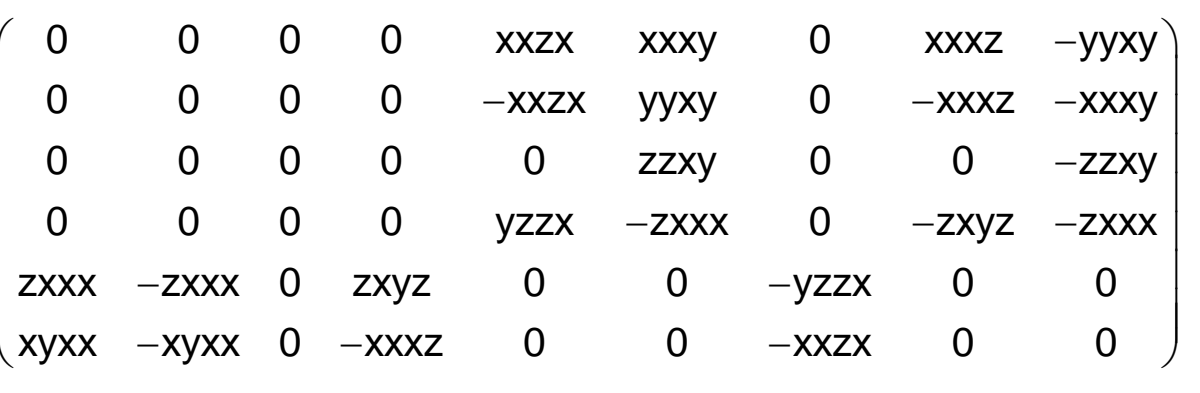
and: $y y x y-x x x y=2 x y x x$


$\mathrm{V}^{4}\left(\begin{array}{ccccccccc}\mathrm{xxxx} & \mathrm{xxyy} & \mathrm{xxzz} & 0 & 0 & 0 & 0 & 0 & 0 \\ \mathrm{yyxx} & \mathrm{xxxx} & \mathrm{xxzz} & 0 & 0 & 0 & 0 & 0 & 0 \\ \mathrm{zzxx} & \mathrm{zzyy} & \mathrm{zzzz} & 0 & 0 & 0 & 0 & 0 & 0 \\ 0 & 0 & 0 & \mathrm{yzyz} & 0 & 0 & \mathrm{yzzy} & 0 & 0 \\ 0 & 0 & 0 & 0 & \mathrm{zxzx} & 0 & 0 & \mathrm{zxxz} & 0 \\ 0 & 0 & 0 & 0 & 0 & \mathrm{xyxy} & 0 & 0 & \mathrm{xyyx} \\ 0 & 0 & 0 & \mathrm{zxxz} & 0 & 0 & \mathrm{zxzx} & 0 & 0 \\ 0 & 0 & 0 & 0 & \mathrm{yzzy} & 0 & 0 & \mathrm{yzyz} & 0 \\ 0 & 0 & 0 & 0 & 0 & \mathrm{xyyx} & 0 & 0 & \mathrm{xyxy}\end{array}\right)$

$\left[V^{4}\right] \quad\left(\begin{array}{ccccc}\mathrm{xxxx} & 0 & 0 & \mathrm{yyzz} & 0 \\ 0 & \mathrm{xxxx} & 0 & \mathrm{yyzz} & 0 \\ 0 & 0 & \mathrm{zzzz} & \mathrm{xxyy} & 0\end{array}\right)$

$\mathrm{V}\left[\mathrm{V}^{3}\right] \quad\left(\begin{array}{cccccccccc}\mathrm{xxxx} & 0 & 0 & \mathrm{xxyy} & 0 & 0 & \mathrm{yyzz} & 0 & 0 & 0 \\ 0 & \mathrm{xxxx} & 0 & 0 & \mathrm{yyzz} & 0 & 0 & \mathrm{xxyy} & 0 & 0 \\ 0 & 0 & z \mathrm{z} z & 0 & 0 & z z x x & 0 & 0 & z z x & 0\end{array}\right)$

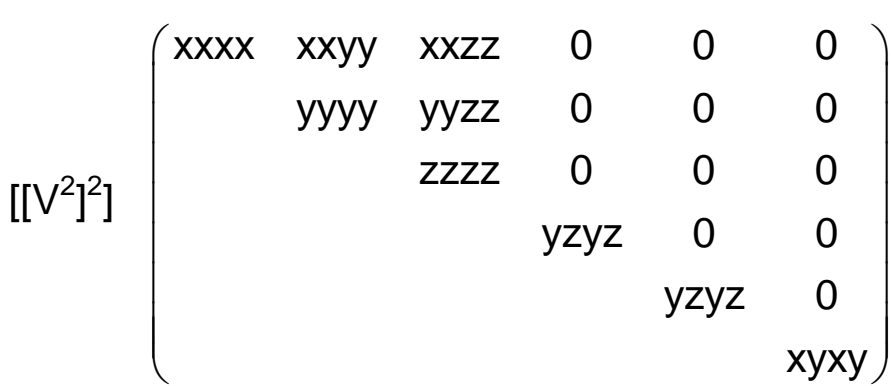

$\left[\mathrm{V}^{2}\right]^{2}\left(\begin{array}{cccccc}\mathrm{xxxx} & \mathrm{xxyy} & \mathrm{xxzz} & 0 & 0 & 0 \\ \mathrm{yyxx} & \mathrm{xxxx} & \mathrm{xxzz} & 0 & 0 & 0 \\ \mathrm{zzxx} & \mathrm{zzxx} & \mathrm{zzzz} & 0 & 0 & 0 \\ 0 & 0 & 0 & \mathrm{yzyz} & 0 & 0 \\ 0 & 0 & 0 & 0 & \mathrm{yzyz} & 0 \\ 0 & 0 & 0 & 0 & 0 & \mathrm{xyxy}\end{array}\right)$

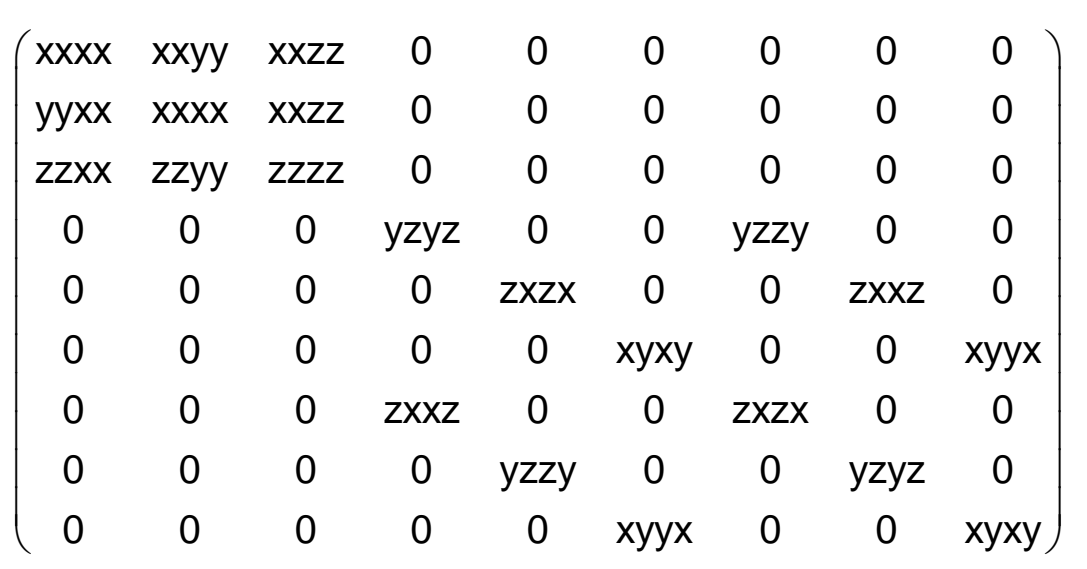

$\left(\begin{array}{ccccc}\mathrm{xxxx} & 0 & 0 & \mathrm{yyzz} & 0 \\ 0 & \mathrm{xxxx} & 0 & \mathrm{yyzz} & 0 \\ 0 & 0 & \mathrm{zzzz} & \mathrm{xxyy} & 0\end{array}\right)$

$\left(\begin{array}{cccccccccc}x x x x & 0 & 0 & x x y y & 0 & 0 & y y z z & 0 & 0 & 0\end{array}\right)$ $\left(\begin{array}{cccccccccc}x x x x & 0 & 0 & x x y y & 0 & 0 & y y z z & 0 & 0 & 0 \\ 0 & x x x x & 0 & 0 & y y z z & 0 & 0 & x x y y & 0 & 0 \\ 0 & 0 & z z z z & 0 & 0 & z z x & 0 & 0 & z z x x & 0\end{array}\right)$ $\left(\begin{array}{cccccc}x x x x & x x y y & x x z z & 0 & 0 & 0 \\ & \text { yyyy } & \text { yyzz } & 0 & 0 & 0 \\ & & \text { zzzz } & 0 & 0 & 0 \\ & & y z y z & 0 & 0 \\ & & & & y z y z & 0 \\ & & & & & \text { xyxy }\end{array}\right)$

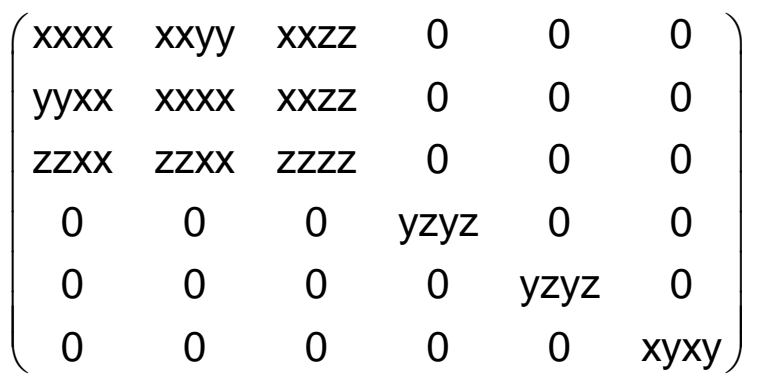

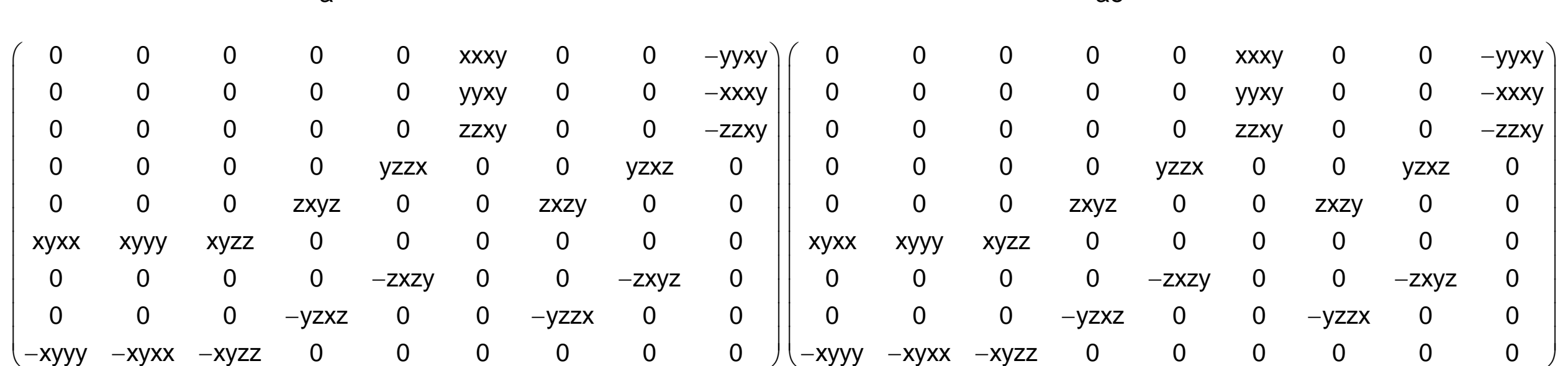

$\left(\begin{array}{ccccc}0 & -y x x x & 0 & 0 & 0 \\ y x x x & 0 & 0 & 0 & 0 \\ 0 & 0 & 0 & 0 & 0\end{array}\right)$

$\left(\begin{array}{ccccc}0 & -\mathrm{yxxx} & 0 & 0 & 0 \\ \mathrm{yxxx} & 0 & 0 & 0 & 0 \\ 0 & 0 & 0 & 0 & 0\end{array}\right)$

$\left(\begin{array}{cccccccccc}0 & -y x x x & 0 & 0 & x y z z & 0 & 0 & x y x x & 0 & 0 \\ y x a x & 0 & 0 & -x y x & 0 & 0 & -x y z z & 0 & 0 & 0\end{array}\right)$ $\left.\begin{array}{cccccccccc}y x x x & 0 & 0 & -x y x x & 0 & 0 & -x y z z & 0 & 0 & 0\end{array}\right)$

$\left(\begin{array}{cccccccccc}0 & -y x x x & 0 & 0 & x y z z & 0 & 0 & x y x x & 0 & 0\end{array}\right)$ $\left.\begin{array}{cccccccccc}\operatorname{yxxx} & 0 & 0 & -x y x x & 0 & 0 & -x y z z & 0 & 0 & 0 \\ 0 & 0 & 0 & 0 & 0 & 0 & 0 & 0 & 0 & z x y z\end{array}\right)$

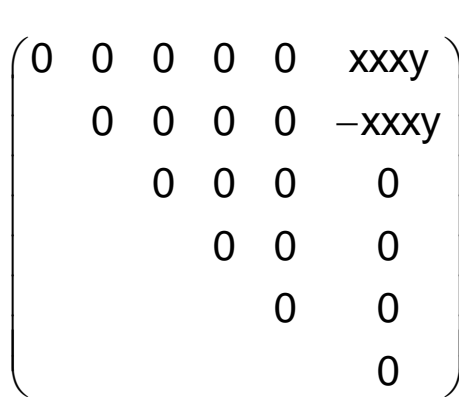

$\left(\begin{array}{cccccc}0 & 0 & 0 & 0 & 0 & x x x y \\ 0 & 0 & 0 & 0 & 0 & -x x x y \\ 0 & 0 & 0 & 0 & 0 & 0 \\ 0 & 0 & 0 & 0 & y z x z & 0 \\ 0 & 0 & 0 & -y z x z & 0 & 0 \\ x y x x & -x y x x & 0 & 0 & 0 & 0\end{array}\right)$

$\left(\begin{array}{lllll}0 & 0 & 0 & 0 & x x x y \\ 0 & 0 & 0 & 0 & -x x y\end{array}\right.$

$\begin{array}{llllll}0 & 0 & 0 & -x x x y \\ 0 & 0 & 0 & x\end{array}$

$\begin{array}{llll}0 & 0 & 0\end{array}$

$\left.\begin{array}{ll}0 & 0 \\ 0 & 0\end{array}\right)$

$\left(\begin{array}{cccccc}0 & 0 & 0 & 0 & 0 & x x x y \\ 0 & 0 & 0 & 0 & 0 & -x x x y \\ 0 & 0 & 0 & 0 & 0 & 0 \\ 0 & 0 & 0 & 0 & y z x z & 0 \\ 0 & 0 & 0 & -y z x z & 0 & 0 \\ x y x x & -x y x x & 0 & 0 & 0 & 0\end{array}\right)$ 
$n=4 \quad D_{4}\left(C_{4}\right)=4_{z} 2_{x}{ }^{2}{ }_{x y}\left(\mathbf{4}_{z}\right)=\mathbf{4}_{z} \mathbf{2}^{\prime} \mathbf{2}_{x y^{\prime}}$

\begin{tabular}{|c|c|c|c|c|c|c|c|c|c|c|c|c|c|c|c|c|c|c|c|c|c|c|c|c|c|c|c|c|c|c|c|c|c|c|c|c|}
\hline & & & & I & & & & & & & & & & & & & & & & & & $\mathrm{a}$ & & & & & & & & & & $\mathrm{ae}$ & & & & \\
\hline$(x x x x$ & $x x \quad$ xxyy & xxzz & 0 & 0 & 0 & 0 & 0 & 0 & $(x x x x$ & xxyy & $x x z z$ & 0 & 0 & 0 & 0 & 0 & 0 & $\left(\begin{array}{ll}0 & 0\end{array}\right.$ & $\begin{array}{lll}0 & 0 & 0\end{array}$ & 0 & $x x x$ & xy 0 & 0 & $-y y x y$ & & & $\left(\begin{array}{ll}0 & 0\end{array}\right.$ & 0 & 0 & 0 & $x x x y$ & $\begin{array}{l}y \\
y\end{array}$ & 0 & $-y y x y$ & & \\
\hline & $x x x$ & $x x z z$ & 0 & 0 & 0 & 0 & 0 & 0 & & $x x x x$ & $x x z z$ & 0 & 0 & 0 & 0 & 0 & 0 & 0 & $\begin{array}{lll}0 & 0 & 0\end{array}$ & 0 & yyx & $\begin{array}{ll}x y & 0\end{array}$ & 0 & $-x x x y$ & & & 0 & 0 & 0 & 0 & yyxy & $\begin{array}{l}y \\
y\end{array}$ & 0 & $-x x x y$ & & \\
\hline & & zzzz & 0 & 0 & 0 & 0 & 0 & 0 & & & zzzz & 0 & 0 & 0 & 0 & 0 & 0 & & $\begin{array}{ll}0 & 0\end{array}$ & 0 & $z z x$ & $\begin{array}{ll}x y & 0\end{array}$ & 0 & $-z z x y$ & & & & 0 & 0 & 0 & $z z x y$ & $\begin{array}{l}y \\
y\end{array}$ & 0 & $-z z x y$ & & \\
\hline & & & yzyz & 0 & 0 & yzzy & 0 & 0 & & & & yzyz & 0 & 0 & yzzy & 0 & 0 & & 0 & $y z z x$ & $x$ & 0 & 0 & 0 & & & & & $0 y$ & yzzx & 0 & 0 & 0 & 0 & & \\
\hline$\left[\left(\mathrm{V}^{2}\right)\right]^{2}$ & & & & $z x z x$ & 0 & 0 & yzzy & 0 & & & & & $z x z x$ & 0 & 0 & yzzy & 0 & & & 0 & 0 & 0 & 0 & 0 & & & & & & 0 & 0 & 0 & 0 & 0 & & \\
\hline & & & & & xyxy & 0 & 0 & xyyx & & & & & & xyxy & 0 & 0 & xyyx & & & & 0 & 0 & 0 & 0 & & & & & & & 0 & 0 & 0 & 0 & & \\
\hline & & & & & & $z x z x$ & 0 & 0 & & & & & & & $z x z x$ & 0 & 0 & & & & & 0 & $-y z z x$ & 0 & & & & & & & & 0 & $-y z z x$ & 0 & & \\
\hline & & & & & & & yzyz & 0 & & & & & & & & yzyz & 0 & & & & & & 0 & 0 & & & & & & & & & 0 & 0 & & \\
\hline & & & & & & & & xyxy & & & & & & & & & xyxy & & & & & & & 0 & & & & & & & & & & 0 & & \\
\hline$(x x x x$ & $x x$ xxyy & xxzz & 0 & 0 & 0 & 0 & 0 & 0 & $(x x x x$ & xxyy & $x x z z$ & 0 & 0 & 0 & 0 & 0 & 0 & 0 & 0 & 0 & 0 & 0 & xxxy & 0 & 0 & $-y y x y)$ & 0 & & 0 & 0 & 0 & 0 & xxxy & 0 & 0 & $-y y x y$ \\
\hline$z z x x$ & $\begin{array}{ll}x x & z z x x\end{array}$ & zzzz & 0 & 0 & 0 & 0 & 0 & 0 & $z z x x$ & $z z x x$ & zzzz & 0 & 0 & 0 & 0 & 0 & 0 & 0 & 0 & 0 & 0 & 0 & $z z x y$ & 0 & 0 & $-z z x y$ & 0 & & 0 & 0 & 0 & 0 & zzxy & 0 & 0 & $-z z x y$ \\
\hline 0 & 0 & 0 & yzyz & 0 & 0 & $z x z x$ & 0 & 0 & 0 & 0 & 0 & yzyz & 0 & 0 & $z x z x$ & 0 & 0 & 0 & 0 & 0 & 0 & $y z z x$ & 0 & 0 & $-z x y z$ & 0 & 0 & & 0 & 0 & 0 & $y z z x$ & 0 & 0 & $-z x y z$ & 0 \\
\hline 0 & 0 & 0 & 0 & $z x z x$ & 0 & 0 & yzyz & 0 & 0 & 0 & 0 & 0 & $z x z x$ & 0 & 0 & yzyz & 0 & 0 & 0 & $0 z$ & $z x y z$ & 0 & 0 & $-y z z x$ & 0 & 0 & 0 & & 0 & $0 z$ & zxyz & 0 & 0 & $-y z z x$ & 0 & 0 \\
\hline 0 & 0 & 0 & 0 & 0 & xyxy & 0 & 0 & xyxy & 0 & 0 & 0 & 0 & 0 & xyxy & 0 & 0 & xyxy & $x y x x$ & - $x y x x$ & 0 & 0 & 0 & 0 & 0 & 0 & 0 & $x y x x$ & $\begin{array}{lll}x & -x y\end{array}$ & $y x x$ & 0 & 0 & 0 & 0 & 0 & 0 & 0 \\
\hline
\end{tabular}




\section{(a)}

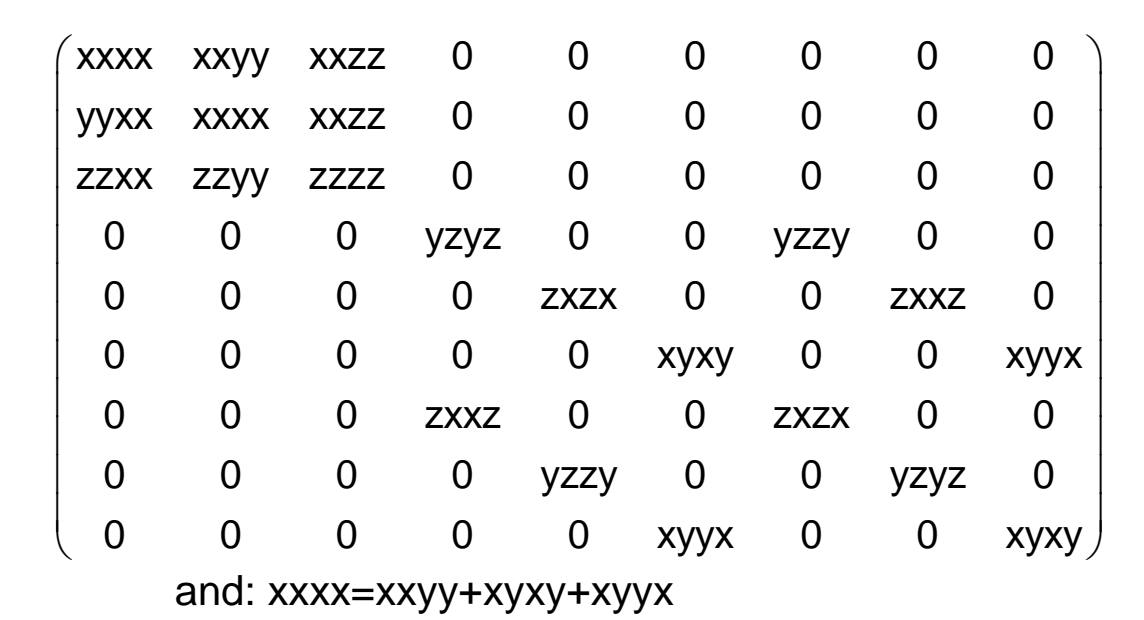

[V $\left.V^{4}\right]\left(\begin{array}{ccccc}3 x x y y & 0 & 0 & y y z z & 0 \\ 0 & 3 x x y y & 0 & y y z z & 0 \\ 0 & 0 & z z z z & x x y y & 0\end{array}\right)$

V $\left[V^{3}\right]\left(\begin{array}{cccccccccc}3 x x y y & 0 & 0 & x x y y & 0 & 0 & y y z z & 0 & 0 & 0 \\ 0 & 0 x y y & 0 & 0 & y y z z & 0 & 0 & x y y & 0 & 0\end{array}\right)$

$\left.\mathrm{V}^{3} \mathrm{~V}^{3}\right]\left(\begin{array}{cccccccccc}3 x x y & 3 x x y & 0 & 0 & y y z z & 0 & 0 & x x y y & 0 & 0 \\ 0 & 0 & z z z z & 0 & 0 & z z x x & 0 & 0 & z z x x & 0\end{array}\right)$

$\left.\left[\mathrm{V}^{2}\right]^{2}\right]\left(\begin{array}{cccccc}\mathrm{x} x \mathrm{x} & \mathrm{xxyy} & \mathrm{xxzz} & 0 & 0 & 0 \\ & \mathrm{xxxx} & \mathrm{xxzz} & 0 & 0 & 0 \\ & & \mathrm{zzzz} & 0 & 0 & 0 \\ & & & \mathrm{yzyz} & 0 & 0 \\ & & & & \mathrm{yzyz} & 0 \\ & & & & & \mathrm{xyxy}\end{array}\right)$

and $x y x y=1 / 2(x x x x-x x y y)$

$\left[\mathrm{V}^{2}\right]^{2}\left(\begin{array}{cccccc}\mathrm{x} x \mathrm{x} & \mathrm{xxyy} & \mathrm{xxzz} & 0 & 0 & 0 \\ \mathrm{xxyy} & \mathrm{xxxx} & \mathrm{xxzz} & 0 & 0 & 0 \\ \mathrm{zzxx} & \mathrm{zzxx} & \mathrm{zzzz} & 0 & 0 & 0 \\ 0 & 0 & 0 & \mathrm{yzyz} & 0 & 0 \\ 0 & 0 & 0 & 0 & \mathrm{yzyz} & 0 \\ 0 & 0 & 0 & 0 & 0 & \mathrm{xyxy}\end{array}\right)$

and $x y x y=1 / 2(x x x x-x x y y)$

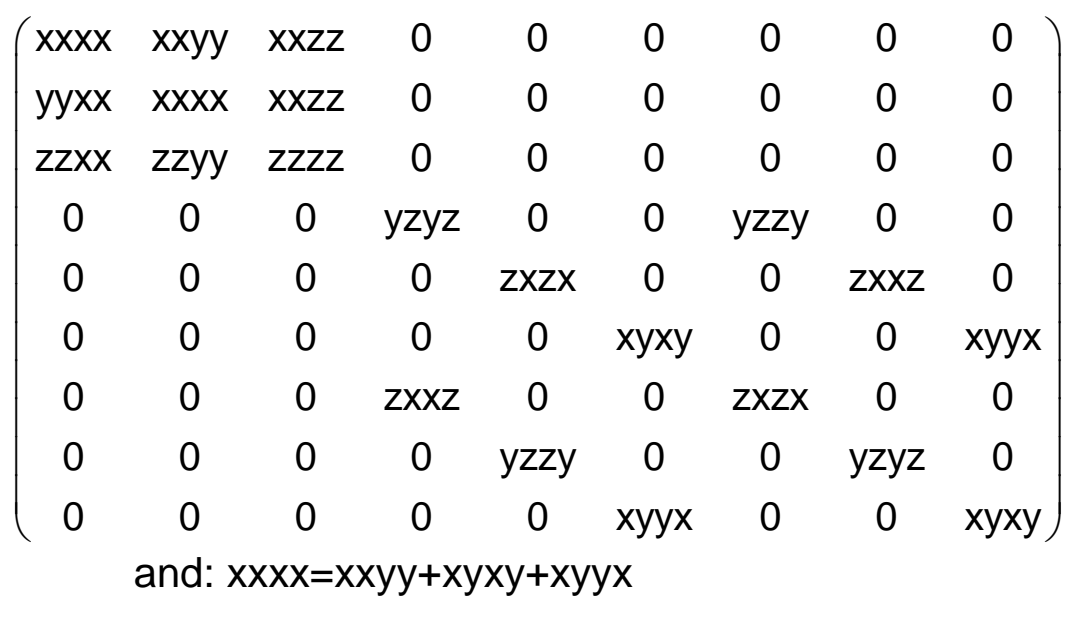

$\left(\begin{array}{ccccc}3 x x y y & 0 & 0 & y y z z & 0 \\ 0 & 3 x x y y & 0 & y y z z & 0 \\ 0 & 0 & z z z z & x x y y & 0\end{array}\right)$

$\left(\begin{array}{cccccccccc}3 x x y y & 0 & 0 & x x y y & 0 & 0 & y y z z & 0 & 0 & 0 \\ 0 & 3 x x y y & 0 & 0 & y y z z & 0 & 0 & x x y y & 0 & 0 \\ 0 & 0 & z z z & 0 & 0 & z z x & 0 & 0 & z z x & 0\end{array}\right)$ $\left(\begin{array}{cccccccccc}0 & 3 x x y y & 0 & 0 & y y z z & 0 & 0 & x x y y & 0 & 0 \\ 0 & 0 & z z z z & 0 & 0 & z z x x & 0 & 0 & z z x x & 0\end{array}\right)$

$$
\left(\begin{array}{cccccc}
x x x x & x x y y & x x z z & 0 & 0 & 0 \\
& x x x x & x x z z & 0 & 0 & 0 \\
& & z z z z & 0 & 0 & 0 \\
& & & y z y z & 0 & 0 \\
& & & & y z y z & 0 \\
& & & & & \\
& & & & &
\end{array}\right.
$$

and $x y x y=1 / 2(x x x x-x x y y)$

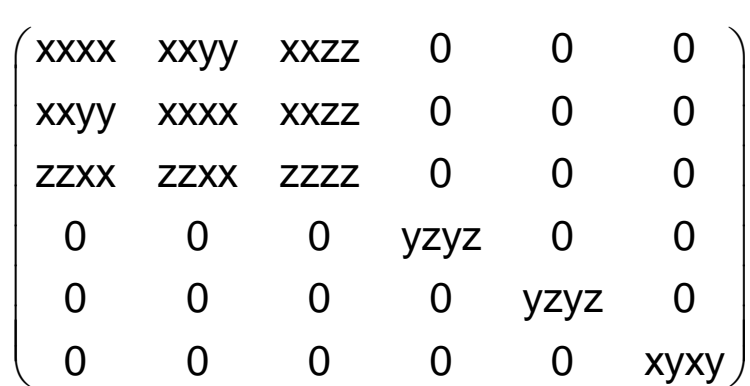

and $x y x y=1 / 2(x x x x-x x y y)$

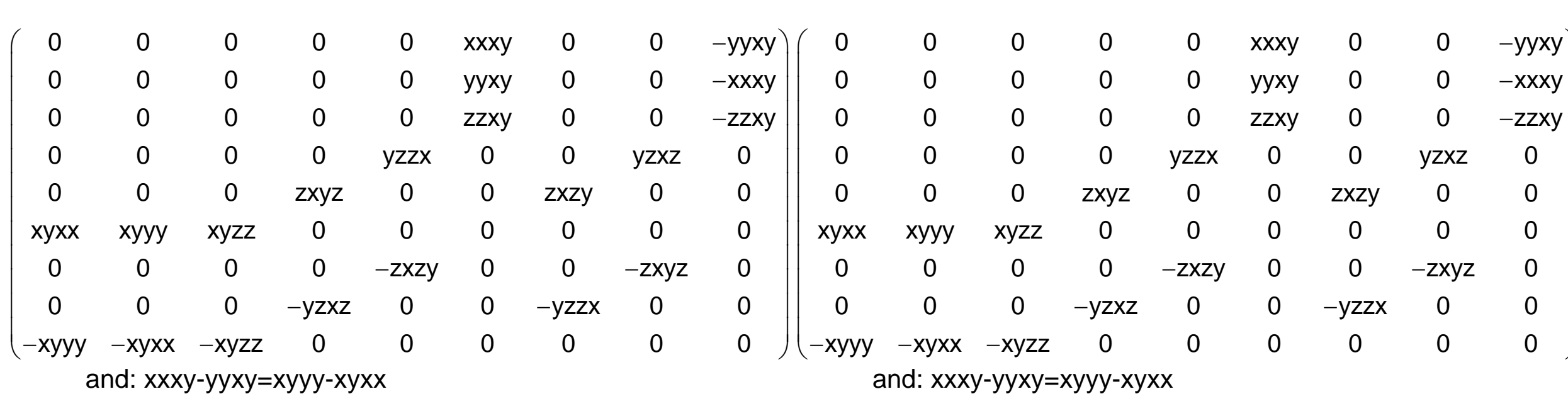

$\left(\begin{array}{lllll}0 & 0 & 0 & 0 & 0 \\ 0 & 0 & 0 & 0 & 0 \\ 0 & 0 & 0 & 0 & 0\end{array}\right)$

$\begin{array}{lllll}0 & 0 & 0 & 0 & 0\end{array}$

$\left(\begin{array}{lllll}0 & 0 & 0 & 0 & 0 \\ 0 & 0 & 0 & 0 & 0 \\ 0 & 0 & 0 & 0 & 0\end{array}\right)$

$\left(\begin{array}{lllll}0 & 0 & 0 & 0 & 0 \\ 0 & 0 & 0 & 0 & 0\end{array}\right)$

$\left(\begin{array}{cccccccccc}0 & 3 x y x x & 0 & 0 & x y z z & 0 & 0 & x y x x & 0 & 0 \\ -3 x y x & 0 & 0 & -x y y x & 0 & 0 & -x y z z & 0 & 0 & 0\end{array}\right)$

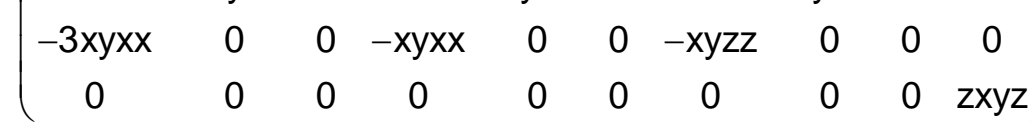
$\left(\begin{array}{cccccccccc}0 & 3 x y x x & 0 & 0 & x y z z & 0 & 0 & x y x x & 0 & 0 \\ -3 x y x x & 0 & 0 & -x y x x & 0 & 0 & -x y z z & 0 & 0 & 0\end{array}\right)$

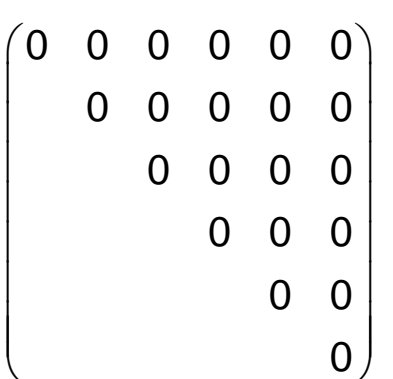
$\left(\begin{array}{llllll}0 & 0 & 0 & 0 & 0 & 0 \\ & 0 & 0 & 0 & 0 & 0 \\ & & 0 & 0 & 0 & 0 \\ & & & 0 & 0 & 0 \\ & & & & 0 & 0 \\ & & & & & 0\end{array}\right)$

$\left(\begin{array}{cccccc}0 & 0 & 0 & 0 & 0 & \text { xxxy } \\ 0 & 0 & 0 & 0 & 0 & -x x x y \\ 0 & 0 & 0 & 0 & 0 & 0 \\ 0 & 0 & 0 & 0 & y z x z & 0 \\ 0 & 0 & 0 & -y z x z & 0 & 0 \\ -x x x y & x x x y & 0 & 0 & 0 & 0\end{array}\right)$ $\left(\begin{array}{cccccc}0 & 0 & 0 & 0 & 0 & x x x y \\ 0 & 0 & 0 & 0 & 0 & -x x x y \\ 0 & 0 & 0 & 0 & 0 & 0 \\ 0 & 0 & 0 & 0 & y z x z & 0 \\ 0 & 0 & 0 & -y z x z & 0 & 0 \\ -x x x y & x x x y & 0 & 0 & 0 & 0\end{array}\right)$ 


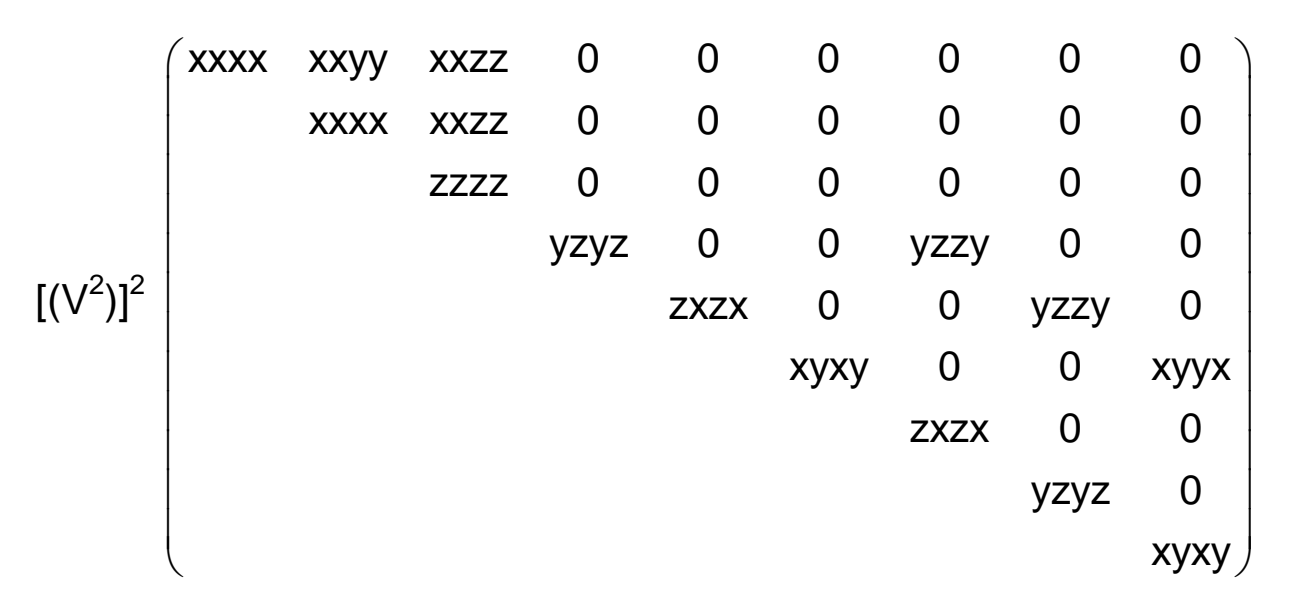

and $x x x x=x x y y+y z y z+z y z y$

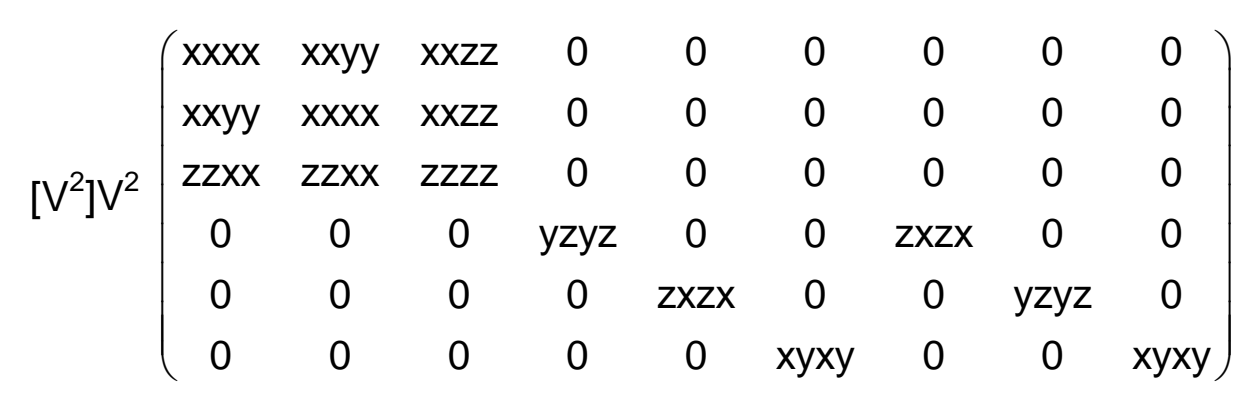

and $x x x x=x x y y+2 x y x y$

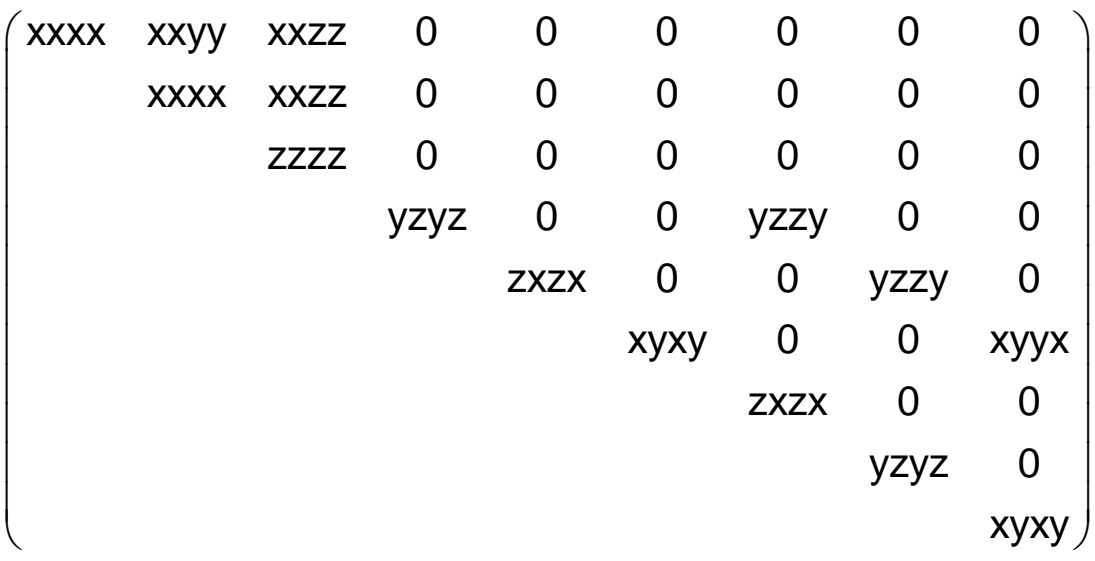

and $x x x x=x x y y+y z y z+z y z y$

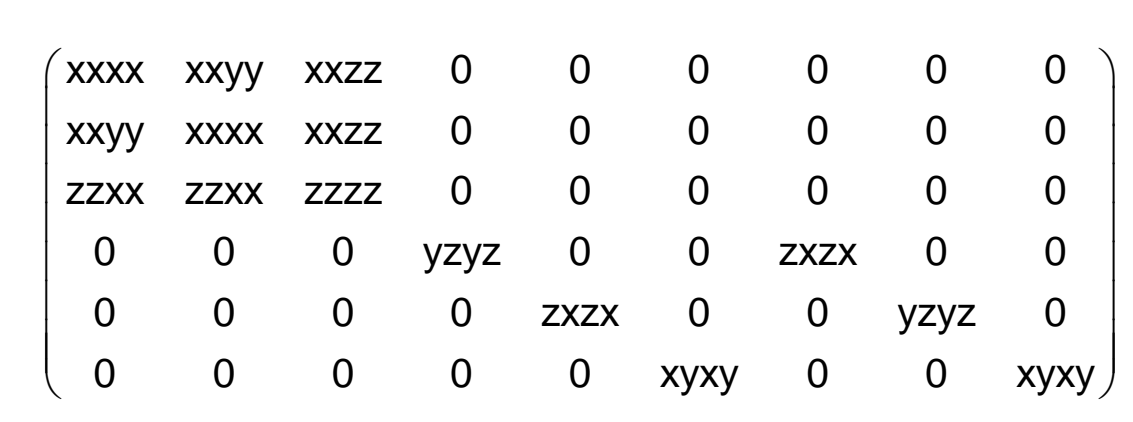

and $x x x x=x x y y+2 x y x y$
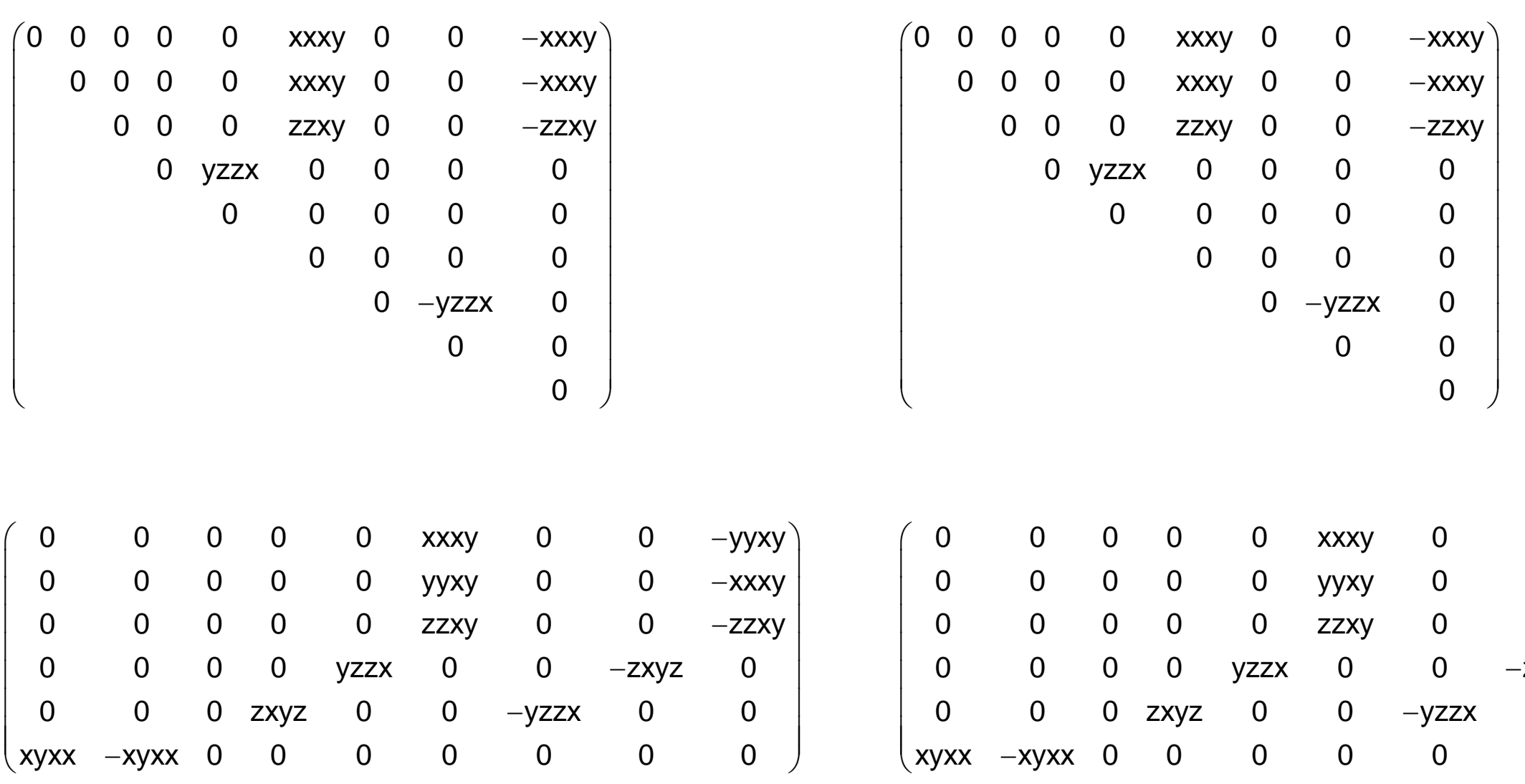

and: $y y x y-x x x y=2 x y x x$

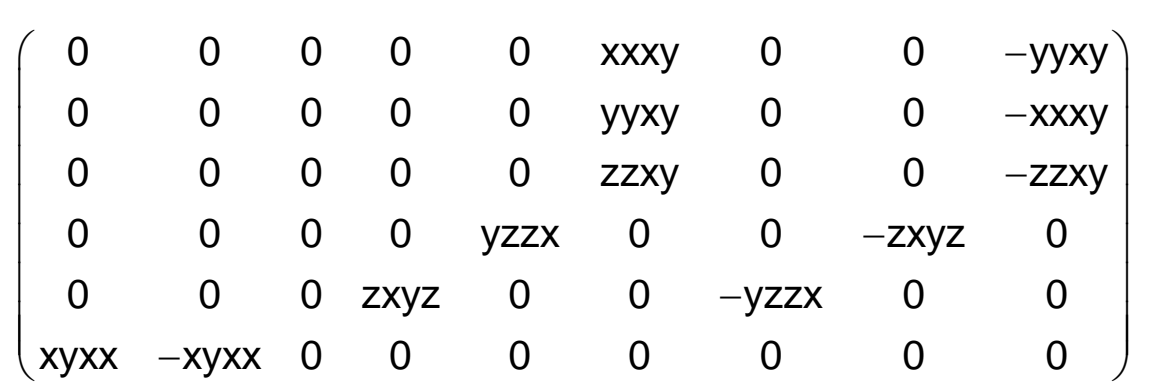

and $x x x x=x x y y+2 x y x y$ 
e

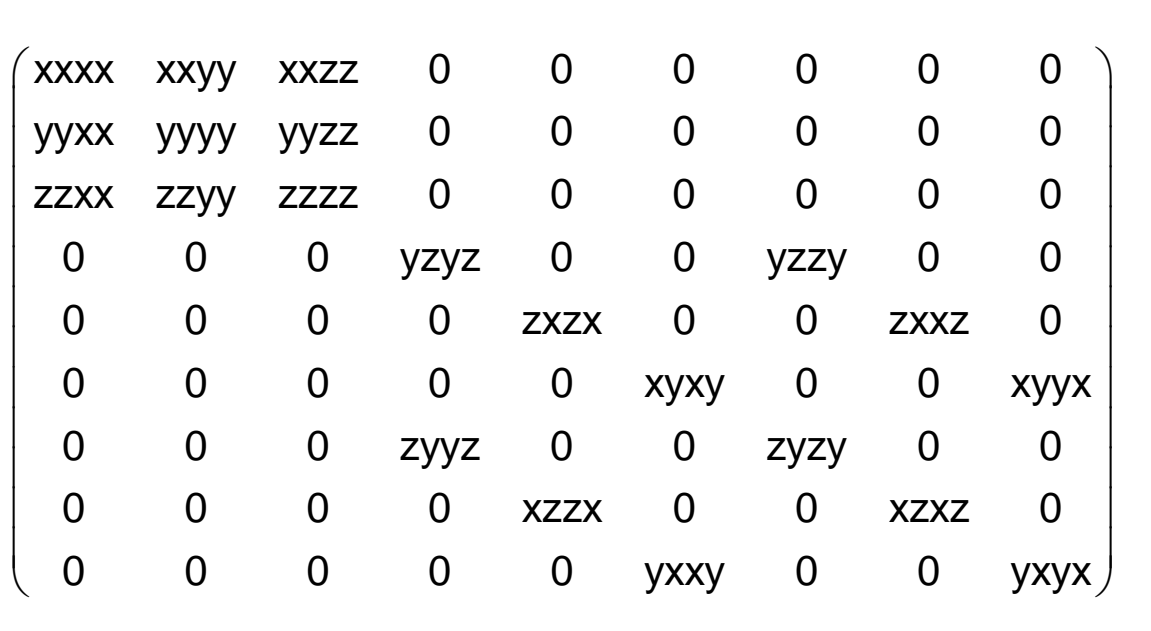

$\left(\begin{array}{ccccc}x x x x & 0 & 0 & y y z z & 0 \\ 0 & y y y y & 0 & z z x x & 0 \\ 0 & 0 & z z z z & x x y y & 0\end{array}\right)$

$\left(\begin{array}{cccccccccc}x x x x & 0 & 0 & x x y y & 0 & 0 & x x z z & 0 & 0 & 0 \\ 0 & y y y & 0 & 0 & y y z z & 0 & 0 & y y x x & 0 & 0\end{array}\right)$ $\left(\begin{array}{cccccccccc}0 & y y y y & 0 & 0 & y y z z & 0 & 0 & \text { yyxx } & 0 & 0 \\ 0 & 0 & z z z z & 0 & 0 & z z x x & 0 & 0 & z z y y & 0\end{array}\right)$

$\left(\begin{array}{cccccc}\text { xxxx } & \text { xxyy } & \text { xxzz } & 0 & 0 & 0 \\ & \text { yyyy } & \text { yyzz } & 0 & 0 & 0 \\ & & \text { zzzz } & 0 & 0 & 0 \\ & & & \text { yzyz } & 0 & 0 \\ & & & & \text { xzxz } & 0 \\ & & & & & \text { xyxy }\end{array}\right)$ $\left(\begin{array}{llllll}x x x x & x x y y & x x z z & 0 & 0 & 0 \\ y y x x & y y y y & y y z z & 0 & 0 & 0 \\ z z x & z z y y & z z z z & 0 & 0 & 0\end{array}\right)$ $\begin{array}{cccccc}\text { zzxx } & \text { zzyy } & \text { zzzz } & 0 & 0 & 0 \\ 0 & 0 & 0 & y z y z & 0 & 0 \\ 0 & 0 & 0 & 0 & x z x z & 0\end{array}$ a

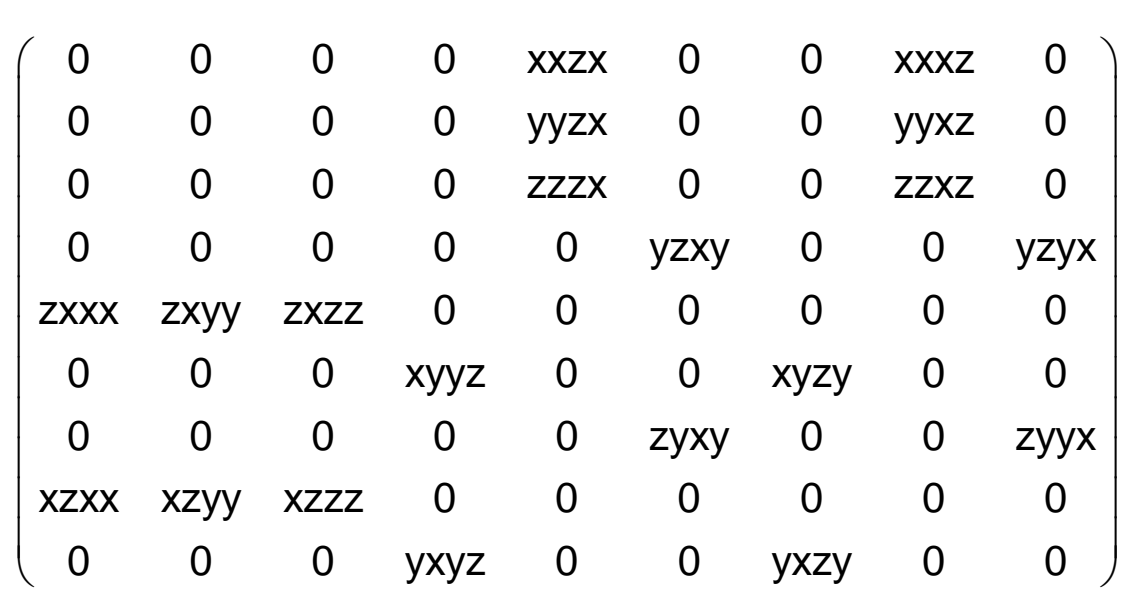

$\left(\begin{array}{ccccc}0 & 0 & x z z z & 0 & 0 \\ 0 & 0 & 0 & 0 & y y x z \\ z x x x & 0 & 0 & 0 & 0\end{array}\right)$

$\left(\begin{array}{ccccccccc}0 & 0 & 0 & 0 & \text { xxzx } & 0 & 0 & \text { xxxz } & 0 \\ 0 & 0 & 0 & 0 & \text { yyzx } & 0 & 0 & \text { yyxz } & 0 \\ 0 & 0 & 0 & 0 & \text { zzzx } & 0 & 0 & \text { zzxz } & 0 \\ 0 & 0 & 0 & 0 & 0 & y z x y & 0 & 0 & \text { yzyx } \\ \text { zxxx } & z x y y & z x z z & 0 & 0 & 0 & 0 & 0 & 0 \\ 0 & 0 & 0 & x y y z & 0 & 0 & x y z y & 0 & 0 \\ 0 & 0 & 0 & 0 & 0 & \text { zyxy } & 0 & 0 & \text { zyyx } \\ \text { xzxx } & \text { xzyy } & \text { xzzz } & 0 & 0 & 0 & 0 & 0 & 0 \\ 0 & 0 & 0 & \text { yxyz } & 0 & 0 & \text { yxzy } & 0 & 0\end{array}\right)$

$\left(\begin{array}{ccccc}0 & 0 & x z z z & 0 & 0 \\ 0 & 0 & 0 & 0 & y y x z \\ z x x x & 0 & 0 & 0 & 0\end{array}\right)$

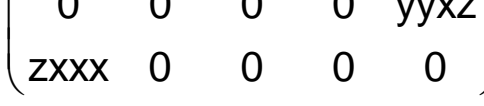

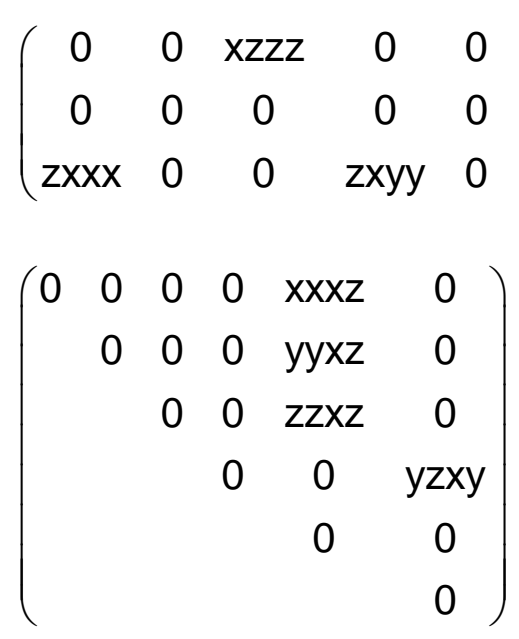

$\left(\begin{array}{cccccc}0 & 0 & 0 & 0 & x x x z & 0 \\ 0 & 0 & 0 & 0 & y y x z & 0 \\ 0 & 0 & 0 & 0 & \text { zzxz } & 0 \\ 0 & 0 & 0 & 0 & 0 & y z x y \\ x z x x & x z y y & x z z z & 0 & 0 & 0 \\ 0 & 0 & 0 & x y y z & 0 & 0\end{array}\right)$ $\left(\begin{array}{cccccccccc}0 & 0 & x z z z & 0 & 0 & x z x x & 0 & 0 & x z y y & 0 \\ 0 & 0 & 0 & 0 & 0 & 0 & 0 & 0 & 0 & y x y z\end{array}\right)$ $\left(\begin{array}{cccccccccc}0 & 0 & 0 & 0 & 0 & 0 & 0 & 0 & 0 & y x y z \\ z x x x & 0 & 0 & z x y y & 0 & 0 & z x z z & 0 & 0 & 0\end{array}\right)$

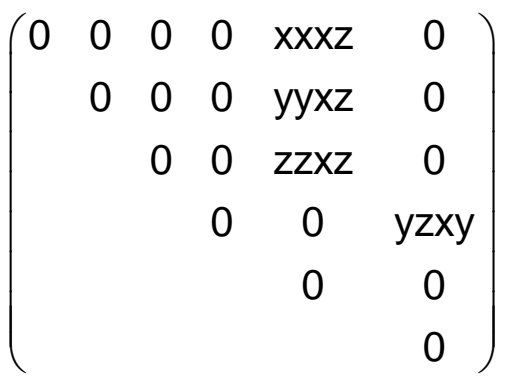
$\left(\begin{array}{cccccc}0 & 0 & 0 & 0 & x x x z & 0 \\ 0 & 0 & 0 & 0 & y y x z & 0 \\ 0 & 0 & 0 & 0 & \text { zzxz } & 0 \\ 0 & 0 & 0 & 0 & 0 & \text { yzxy } \\ \text { xzxx } & x z y y & x z z z & 0 & 0 & 0 \\ 0 & 0 & 0 & x y y z & 0 & 0\end{array}\right)$ 
a
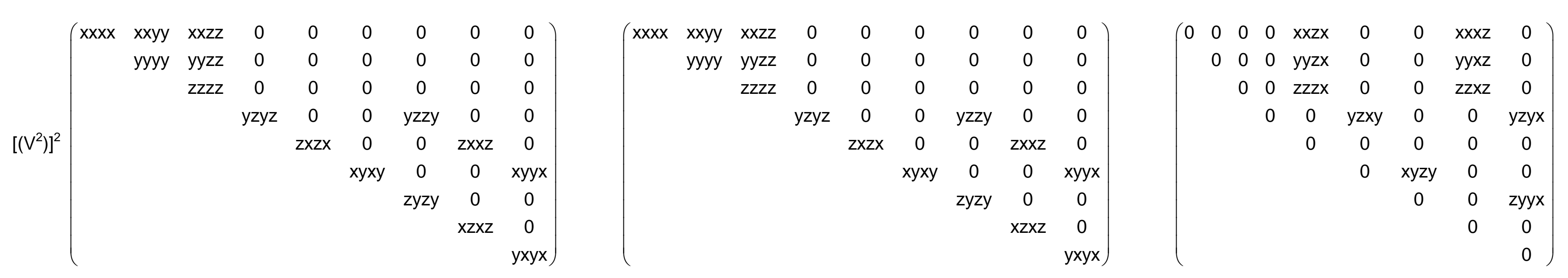

$\left(\begin{array}{ccccccccc}\text { xxxx } & \text { xxyy } & \text { xxzz } & 0 & 0 & 0 & 0 & 0 & 0 \\ \text { yyxx } & \text { yyyy } & \text { yyzz } & 0 & 0 & 0 & 0 & 0 & 0 \\ \text { zzxx } & \text { zzyy } & \text { zzzz } & 0 & 0 & 0 & 0 & 0 & 0 \\ 0 & 0 & 0 & \text { yzyz } & 0 & 0 & \text { yzzy } & 0 & 0 \\ 0 & 0 & 0 & 0 & \text { zxzx } & 0 & 0 & \text { zxxz } & 0 \\ 0 & 0 & 0 & 0 & 0 & \text { xyxy } & 0 & 0 & \text { xyyx }\end{array}\right)$ $\left(\begin{array}{ccccccccc}0 & 0 & 0 & 0 & \text { xxzx } & 0 & 0 & \text { xxxz } & 0 \\ 0 & 0 & 0 & 0 & \text { yyzx } & 0 & 0 & \text { yyxz } & 0 \\ 0 & 0 & 0 & 0 & \text { zzzx } & 0 & 0 & \text { zzxz } & 0 \\ 0 & 0 & 0 & 0 & 0 & \text { yzxy } & 0 & 0 & \text { yzyx } \\ \text { zxxx } & \text { zxyy } & \text { zxzz } & 0 & 0 & 0 & 0 & 0 & 0 \\ 0 & 0 & 0 & \text { xyyz } & 0 & 0 & \text { xyzy } & 0 & 0\end{array}\right)$

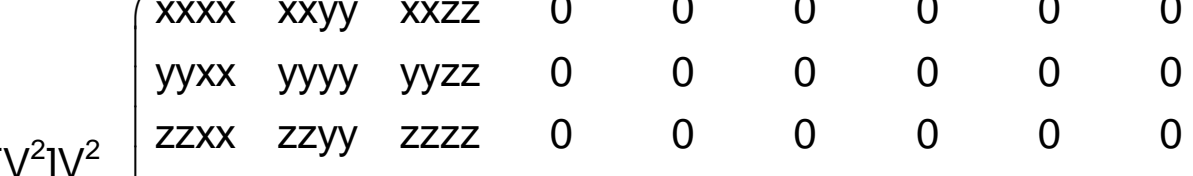
$\begin{array}{ccccccccc}z & 0 & 0 & y z y z & 0 & 0 & y z z y & 0 & 0 \\ 0 & 0 & 0 & 0 & z x z & 0 & 0 & z x z & 0\end{array}$ $\left(\begin{array}{ccccccccc}0 & 0 & 0 & 0 & \text { zxzx } & 0 & 0 & \text { zxxz } & 0 \\ 0 & 0 & 0 & 0 & 0 & \text { xyxy } & 0 & 0 & \text { xyyx }\end{array}\right)$ ae

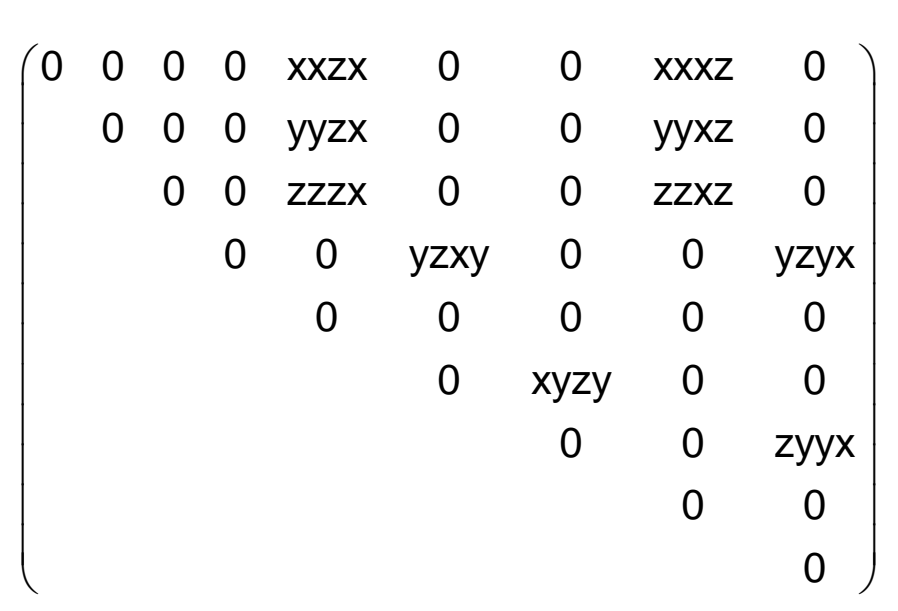

$\left(\begin{array}{ccccccccc}0 & 0 & 0 & 0 & \text { xxzx } & 0 & 0 & \text { xxxz } & 0 \\ 0 & 0 & 0 & 0 & \text { yyzx } & 0 & 0 & \text { yyxz } & 0 \\ 0 & 0 & 0 & 0 & \text { zzzx } & 0 & 0 & \text { zzxz } & 0 \\ 0 & 0 & 0 & 0 & 0 & \text { yzxy } & 0 & 0 & \text { yzyx } \\ \text { zxxx } & \text { zxyy } & \text { zxzz } & 0 & 0 & 0 & 0 & 0 & 0 \\ 0 & 0 & 0 & \text { xyyz } & 0 & 0 & \text { xyzy } & 0 & 0\end{array}\right)$ 


\section{1}

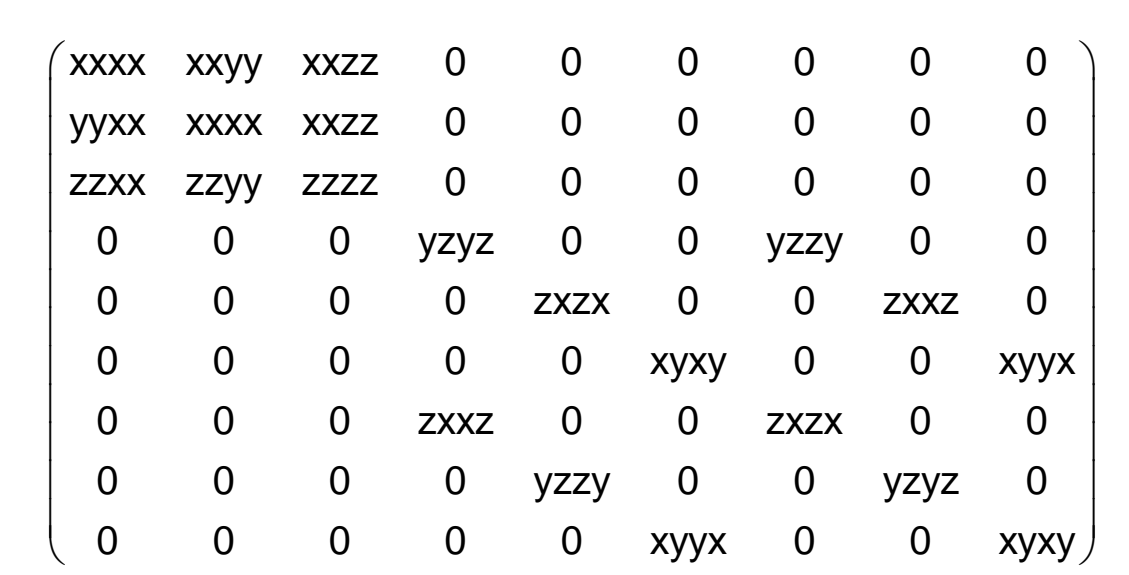

$\left[V^{4}\right] \quad\left(\begin{array}{ccccc}x x x x & 0 & 0 & y y z z & 0 \\ 0 & x x x x & 0 & y y z z & 0 \\ 0 & 0 & z z z z & x x y y & 0\end{array}\right)$

$\left.V^{3}\right]\left(\begin{array}{cccccccccc}x x x x & 0 & 0 & x x y y & 0 & 0 & y y z z & 0 & 0 & 0 \\ 0 & x x x & 0 & 0 & y y z & 0 & 0 & x x y & 0 & 0 \\ 0 & 0 & z z z & 0 & 0 & z z x x & 0 & 0 & z z x x & 0\end{array}\right)$ $V\left[V^{3}\right] \quad\left(\begin{array}{cccccccccc}0 & x x x x & 0 & 0 & y y z z & 0 & 0 & x x y y & 0 & 0 \\ 0 & 0 & z z z z & 0 & 0 & z z x x & 0 & 0 & z z x x & 0\end{array}\right)$

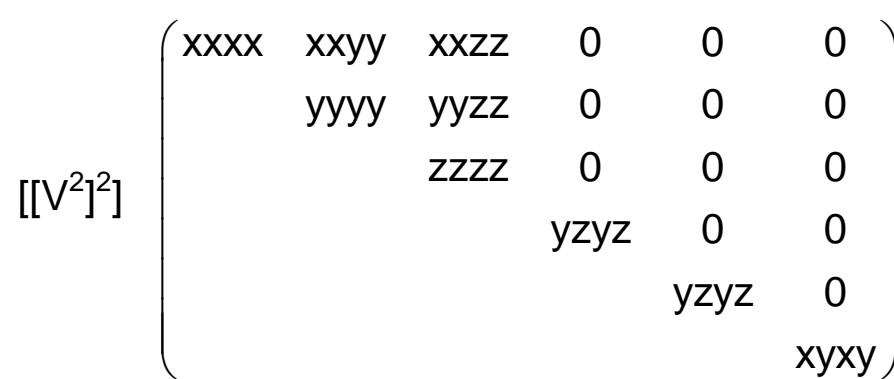

$\left[V^{2}\right]^{2}\left(\begin{array}{cccccc}x x x x & x x y y & x x z z & 0 & 0 & 0 \\ y y x x & x x x x & x x z z & 0 & 0 & 0 \\ z z x x & z z x x & z z z z & 0 & 0 & 0 \\ 0 & 0 & 0 & y z y z & 0 & 0 \\ 0 & 0 & 0 & 0 & y z y z & 0 \\ 0 & 0 & 0 & 0 & 0 & x y x y\end{array}\right)$

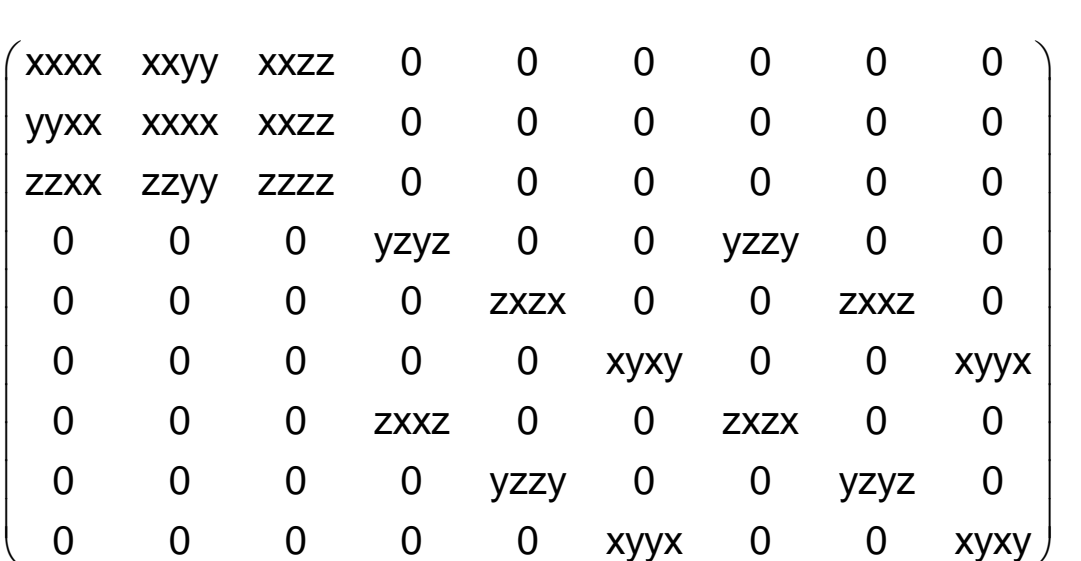

$\left(\begin{array}{ccccc}x x x x & 0 & 0 & y y z z & 0 \\ 0 & x x x x & 0 & y y z z & 0 \\ 0 & 0 & z z z z & x x y y & 0\end{array}\right)$

$\left(\begin{array}{llllllllll}x x x x & 0 & 0 & x x y y & 0 & 0 & y y z z & 0 & 0 & 0\end{array}\right)$ $\left(\begin{array}{cccccccccc}0 & \mathrm{xxxx} & 0 & 0 & \mathrm{yyzz} & 0 & 0 & \mathrm{xxyy} & 0 & 0 \\ 0 & 0 & \mathrm{zzzz} & 0 & 0 & \mathrm{zzxx} & 0 & 0 & \mathrm{zzxx} & 0\end{array}\right)$

$$
\left(\begin{array}{cccccc}
\text { xxxx } & \text { xxyy } & \text { xxzz } & 0 & 0 & 0 \\
& \text { yyyy } & \text { yyzz } & 0 & 0 & 0 \\
& & \text { zzzz } & 0 & 0 & 0 \\
& & & \text { yzyz } & 0 & 0 \\
& & & & \text { yzyz } & 0 \\
& & & & & \text { xyxy }
\end{array}\right)
$$

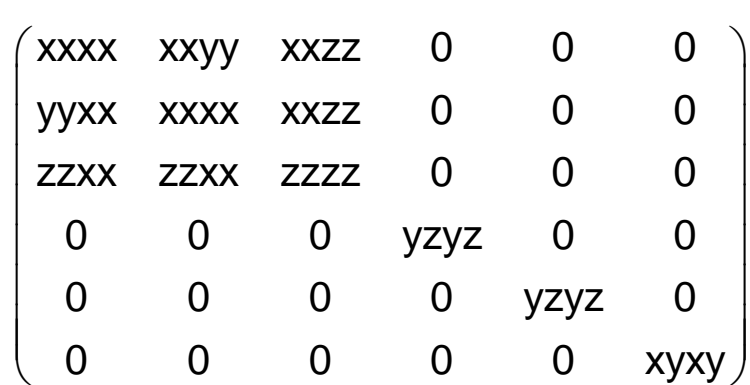

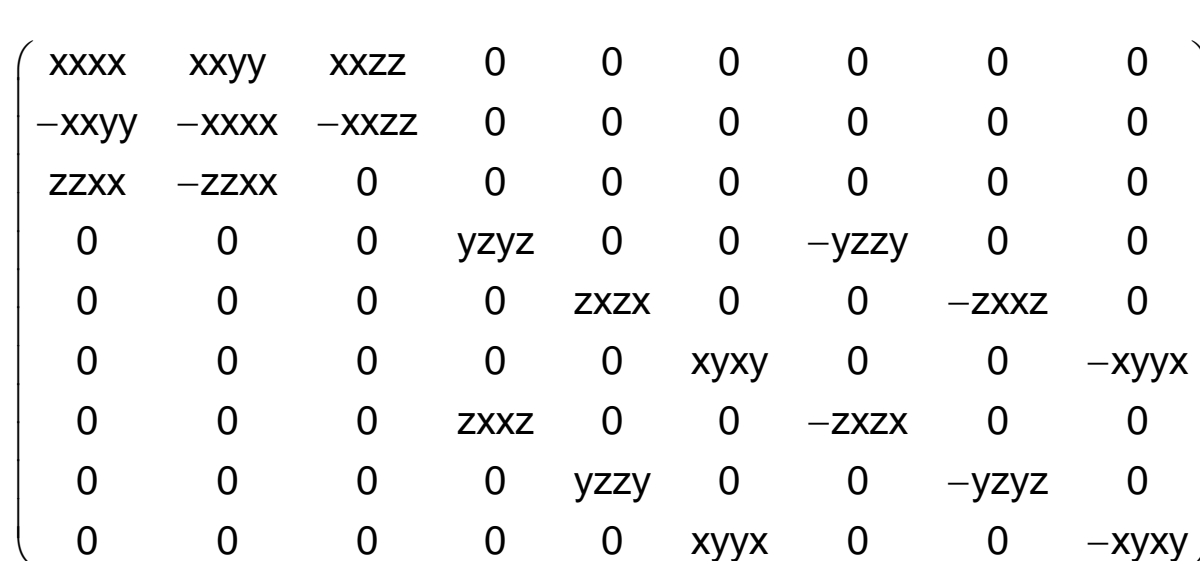

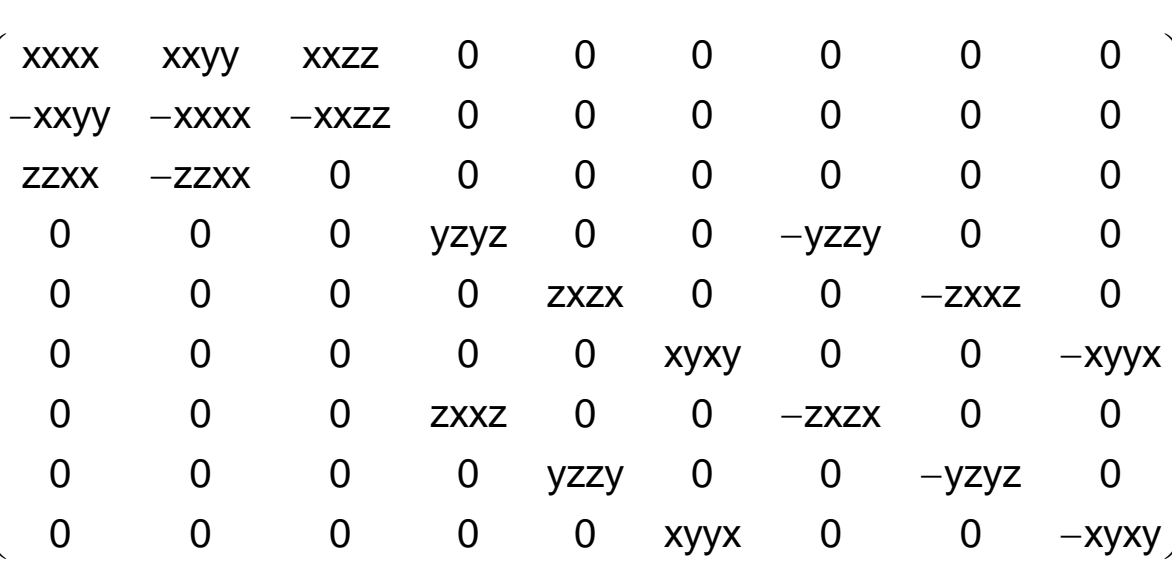

$\left(\begin{array}{ccccc}\mathrm{xxxx} & 0 & 0 & \mathrm{yyzz} & 0 \\ 0 & -\mathrm{xxxx} & 0 & -\mathrm{yyzz} & 0 \\ 0 & 0 & 0 & 0 & 0\end{array}\right)$

$\left(\begin{array}{ccccc}x x x x & 0 & 0 & y y z z & 0 \\ 0 & -x x x x & 0 & -y y z z & 0 \\ 0 & 0 & 0 & 0 & 0\end{array}\right)$

$\left(\begin{array}{cccccccccc}x x x x & 0 & 0 & x x y y & 0 & 0 & -y y z z & 0 & 0 & 0 \\ 0 & -x x x & 0 & 0 & y y z z & 0 & 0 & -x x y & 0 & 0\end{array}\right)\left(\begin{array}{ccccccccccc}x x x x & 0 & 0 & x x y y & 0 & 0 & -y y z z & 0 & 0 & 0\end{array}\right)$

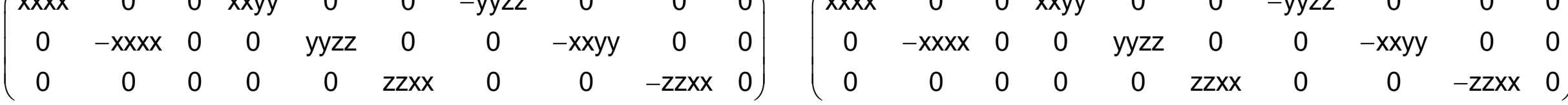

$\left(\begin{array}{cccccc}x x x x & 0 & x x z z & 0 & 0 & 0 \\ & -x x x x & -x x z z & 0 & 0 & 0 \\ & & 0 & 0 & 0 & 0 \\ & & y z y z & 0 & 0 \\ & & & -y z y z & 0 \\ & & & & & 0\end{array}\right)$

$\left(\begin{array}{cccccc}\mathrm{xxxx} & 0 & \mathrm{xxzz} & 0 & 0 & 0 \\ & -\mathrm{xxxx} & -\mathrm{xxzz} & 0 & 0 & 0 \\ & & 0 & 0 & 0 \\ & & \mathrm{yzyz} & 0 & 0 \\ & & & & -\mathrm{yzyz} & 0 \\ & & & & & 0\end{array}\right)$

$\left(\begin{array}{cccccc}\text { xxxx } & x x y y & x x z z & 0 & 0 & 0 \\ -x x y y & -x x x x & -x x z z & 0 & 0 & 0 \\ z z x x & -z z x x & 0 & 0 & 0 & 0 \\ 0 & 0 & 0 & y z y z & 0 & 0 \\ 0 & 0 & 0 & 0 & -y z y z & 0 \\ 0 & 0 & 0 & 0 & 0 & 0\end{array}\right)$ $\left(\begin{array}{cccccc}\text { xxxx } & \text { xxyy } & \text { xxzz } & 0 & 0 & 0 \\ -x x y y & -x x x x & -x x z z & 0 & 0 & 0 \\ \text { zzxx } & -z z x x & 0 & 0 & 0 & 0 \\ 0 & 0 & 0 & y z y z & 0 & 0 \\ 0 & 0 & 0 & 0 & -y z y z & 0 \\ 0 & 0 & 0 & 0 & 0 & 0\end{array}\right)$ 

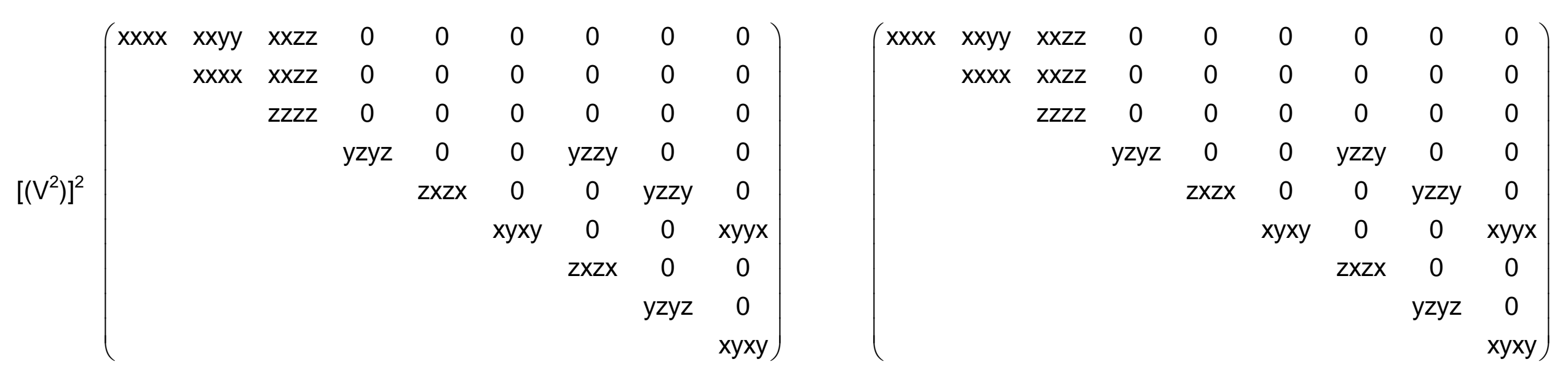

$\left[V^{2}\right] \mathrm{V}^{2}\left(\begin{array}{ccccccccc}\mathrm{x} x \mathrm{x} & \mathrm{xxyy} & \mathrm{xxzz} & 0 & 0 & 0 & 0 & 0 & 0 \\ \mathrm{yyxx} & \mathrm{xxxx} & \mathrm{xxzz} & 0 & 0 & 0 & 0 & 0 & 0 \\ \mathrm{zzxx} & \mathrm{zzxx} & \mathrm{zzzz} & 0 & 0 & 0 & 0 & 0 & 0 \\ 0 & 0 & 0 & \mathrm{yzyz} & 0 & 0 & \mathrm{zxzx} & 0 & 0 \\ 0 & 0 & 0 & 0 & \mathrm{zxzx} & 0 & 0 & \mathrm{yzyz} & 0 \\ 0 & 0 & 0 & 0 & 0 & \mathrm{xyxy} & 0 & 0 & \text { xyxy }\end{array}\right)$

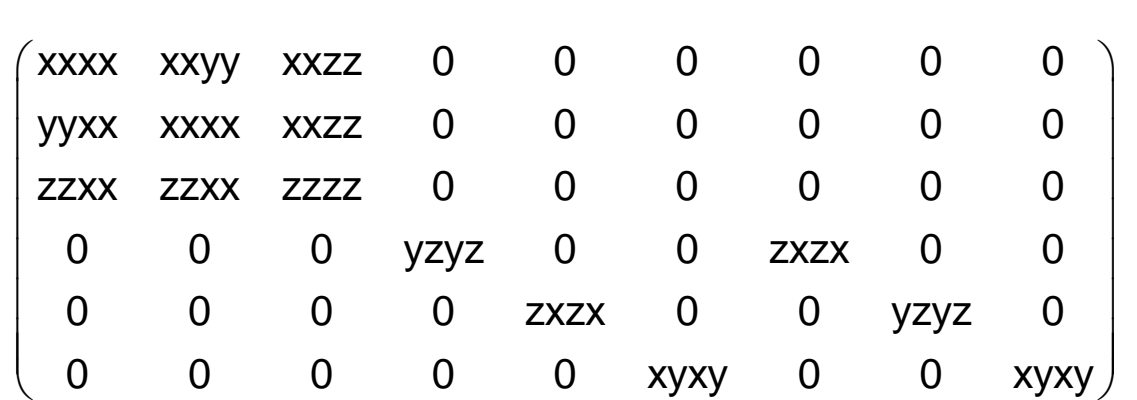

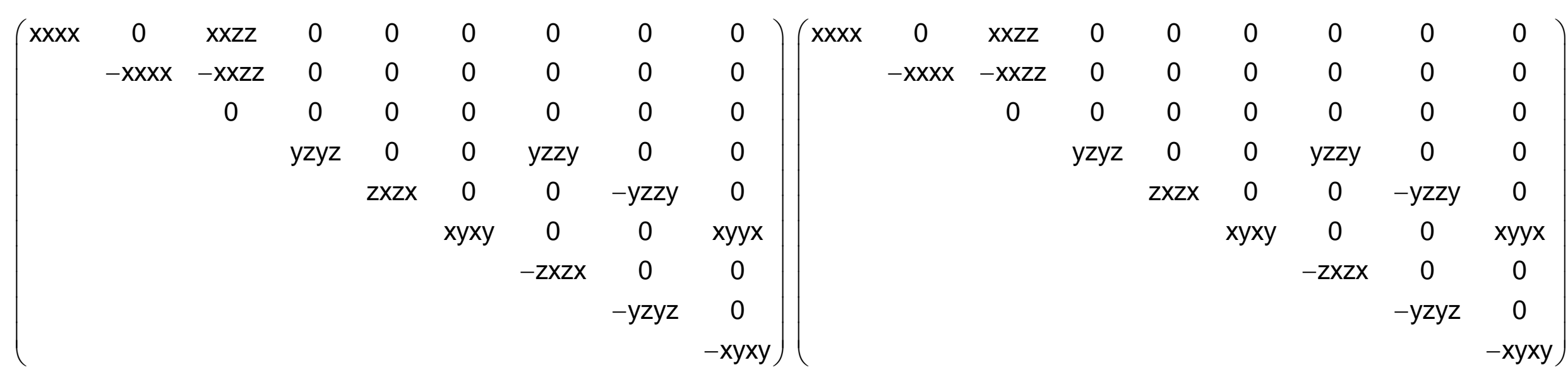

$\left(\begin{array}{ccccccccc}\text { xxxx } & \text { xxyy } & \text { xxzz } & 0 & 0 & 0 & 0 & 0 & 0 \\ - \text {-xxyy } & - \text {-xxxx } & - \text {-xxzz } & 0 & 0 & 0 & 0 & 0 & 0 \\ \text { zzxx } & -z z x x & 0 & 0 & 0 & 0 & 0 & 0 & 0 \\ 0 & 0 & 0 & \text { yzyz } & 0 & 0 & -z x z x & 0 & 0 \\ 0 & 0 & 0 & 0 & \text { zxzx } & 0 & 0 & - \text { yzyz } & 0 \\ 0 & 0 & 0 & 0 & 0 & \text { xyxy } & 0 & 0 & - \text { xyxy }\end{array}\right)$

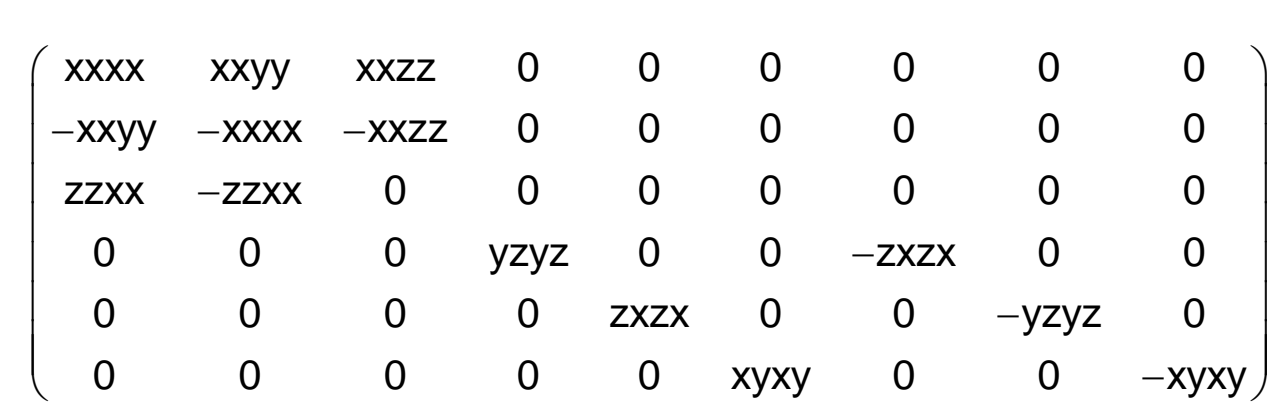


$\mathrm{V}^{4}\left(\begin{array}{ccccccccc}\text { xxxx } & \text { xxyy } & \text { xxzz } & 0 & 0 & 0 & 0 & 0 & 0 \\ \text { yyxx } & \text { xxxx } & \text { yyzz } & 0 & 0 & 0 & 0 & 0 & 0 \\ \text { zzxx } & z z x x & z z z z & 0 & 0 & 0 & 0 & 0 & 0 \\ 0 & 0 & 0 & y z y z & 0 & 0 & y z z y & 0 & 0 \\ 0 & 0 & 0 & 0 & z x z x & 0 & 0 & z x x z & 0 \\ 0 & 0 & 0 & 0 & 0 & x y x y & 0 & 0 & x y y x \\ 0 & 0 & 0 & z x x z & 0 & 0 & z x z x & 0 & 0 \\ 0 & 0 & 0 & 0 & y z z y & 0 & 0 & y z y z & 0 \\ 0 & 0 & 0 & 0 & 0 & x y y x & 0 & 0 & x y x y\end{array}\right)$

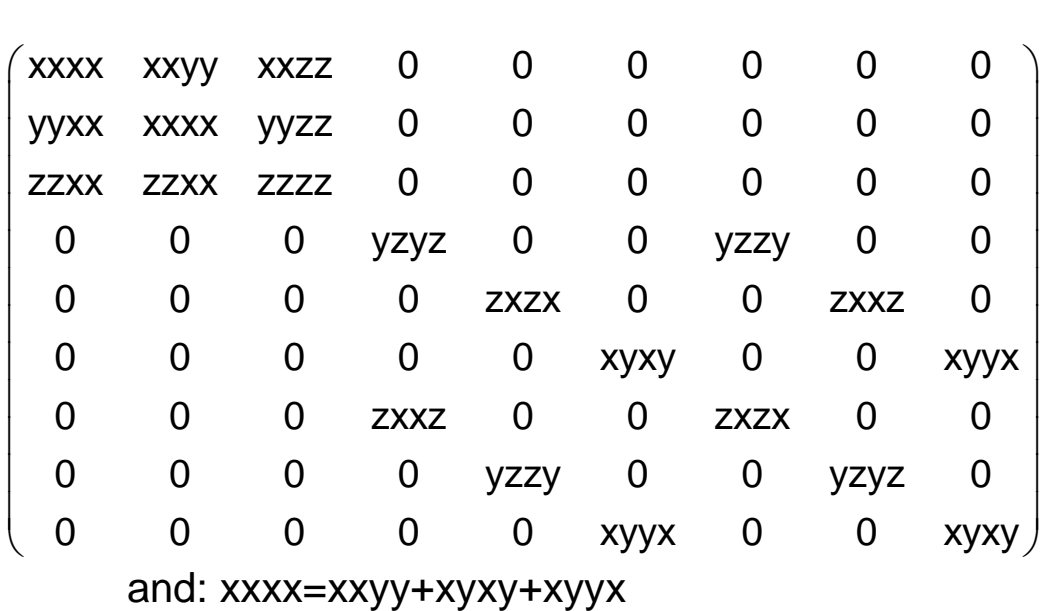

$\left[V^{4}\right] \quad\left(\begin{array}{ccccc}3 x x y y & 0 & 0 & y y z z & 0 \\ 0 & 3 x x y y & 0 & y y z z & 0 \\ 0 & 0 & z z z z & x x y y & 0\end{array}\right)$

$\left.\mathrm{V}_{[} \mathrm{V}^{3}\right]\left(\begin{array}{cccccccccc}3 \mathrm{xxyyy} & 0 & 0 & \mathrm{xxyy} & 0 & 0 & \mathrm{yyzz} & 0 & 0 & \mathrm{yyxx} \\ 0 & 3 \mathrm{xxyy} & 0 & 0 & \mathrm{yyzz} & 0 & 0 & \mathrm{xxyy} & 0 & 0 \\ 0 & 0 & \mathrm{zzzz} & 0 & 0 & \mathrm{zzxx} & 0 & 0 & \mathrm{zzxx} & 0\end{array}\right)$

$$
\left(\begin{array}{ccccc}
3 x x y y & 0 & 0 & y y z z & 0 \\
0 & 3 x x y y & 0 & \text { yyzz } & 0 \\
0 & 0 & z z z z & x x y y & 0
\end{array}\right)
$$

$\left(\begin{array}{cccccccccc}3 x x y y & 0 & 0 & x x y y & 0 & 0 & y y z z & 0 & 0 & y y x x \\ 0 & 3 x x y y & 0 & 0 & y y z z & 0 & 0 & x x y y & 0 & 0 \\ 0 & 0 & z z z z & 0 & 0 & z z x x & 0 & 0 & z z x x & 0\end{array}\right)$$$
\left[\left[V^{2}\right]^{2}\right]\left(\begin{array}{cccccc}
x x x x & x x y y & x x z z & 0 & 0 & 0 \\
& x x x x & x x z z & 0 & 0 & 0 \\
& & z z z z & 0 & 0 & 0 \\
& & & y z y z & 0 & 0 \\
& & & & y z y z & 0 \\
& & & & & x y x y
\end{array}\right)
$$

and $x y x y=1 / 2(x x x x-x x y y)$

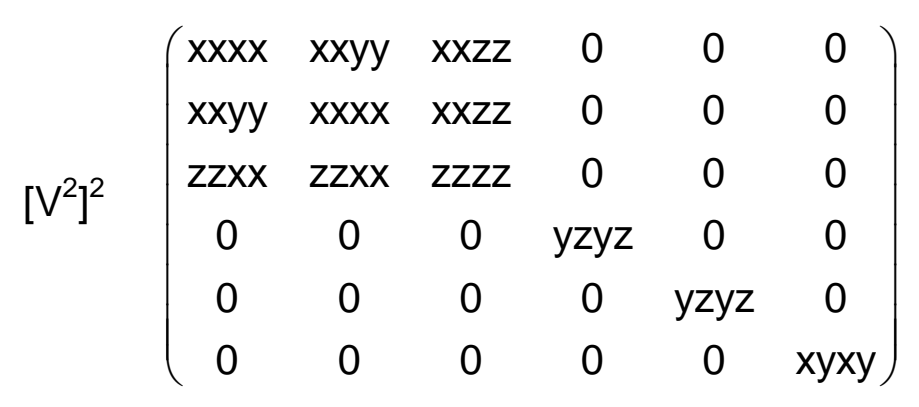

and $x y x y=1 / 2(x x x x-x x y y)$

$$
\left(\begin{array}{cccccc}
x x x x & x x y y & x x z z & 0 & 0 & 0 \\
& x x x x & x x z z & 0 & 0 & 0 \\
& & z z z z & 0 & 0 & 0 \\
& & & y z y z & 0 & 0 \\
& & & & y z y z & 0 \\
& & & & & \text { xyxy }
\end{array}\right)
$$

and $x y x y=1 / 2(x x x x-x x y y)$

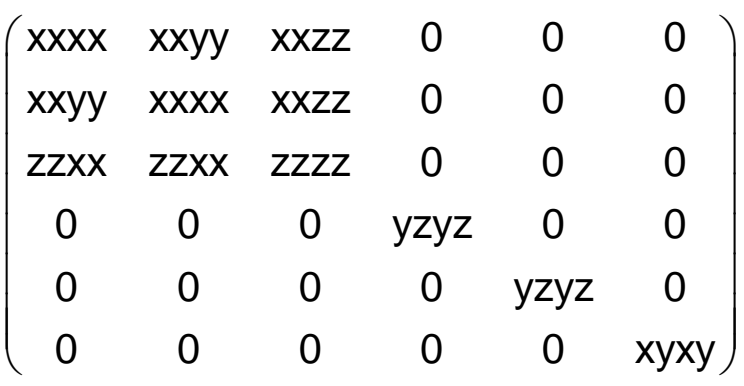

and $x y x y=1 / 2(x x x x-x x y y)$

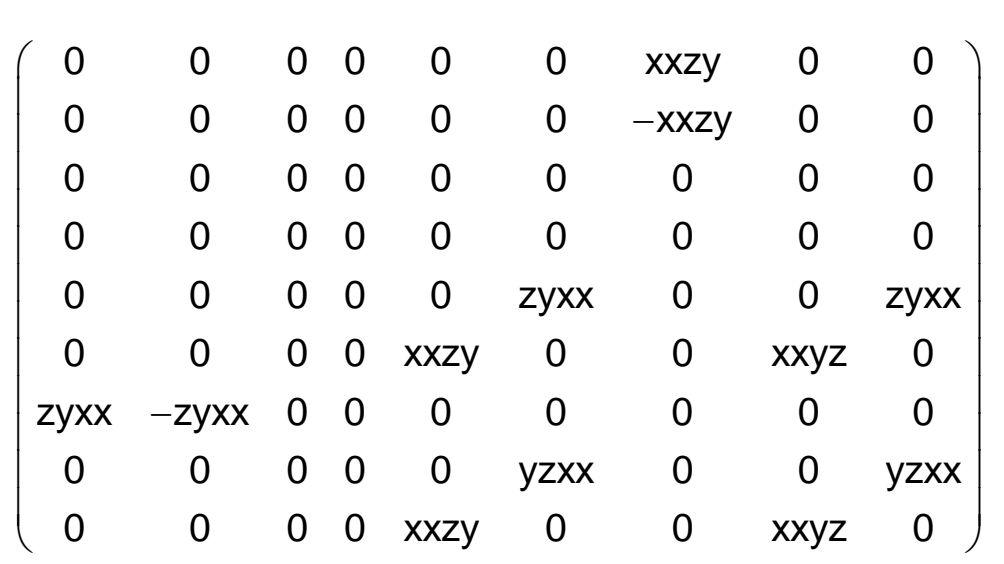$$
\left(\begin{array}{ccccc}
0 & 0 & 0 & 0 & \mathrm{xxyz} \\
0 & 0 & 0 & 0 & 0 \\
0 & -\mathrm{xxyz} & 0 & 0 & 0
\end{array}\right)
$$

$$
\left(\begin{array}{cccccccccc}
0 & 0 & 0 & 0 & 0 & 0 & 0 & 0 & 0 & \mathrm{yzxx} \\
0 & 0 & 0 & 0 & 0 & \mathrm{yzxx} & 0 & 0 & -\mathrm{yzxx} & 0 \\
0 & -\mathrm{zyxx} & 0 & 0 & 0 & 0 & 0 & \mathrm{zyxx} & 0 & 0
\end{array}\right)
$$

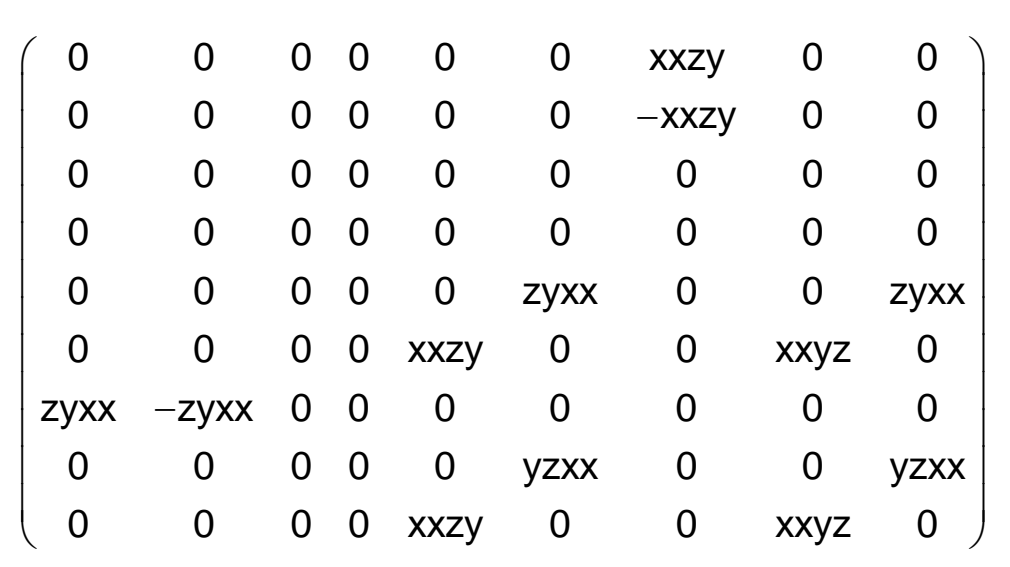

$\left(\begin{array}{ccccc}0 & 0 & 0 & 0 & \mathrm{xxyz} \\ 0 & 0 & 0 & 0 & 0 \\ 0 & -\mathrm{xxyz} & 0 & 0 & 0\end{array}\right)$

$\left(\begin{array}{cccccccccc}0 & 0 & 0 & 0 & 0 & 0 & 0 & 0 & 0 & \mathrm{yzxx} \\ 0 & 0 & 0 & 0 & 0 & \mathrm{yzxx} & 0 & 0 & -\mathrm{yzxx} & 0 \\ 0 & -2 \mathrm{yx} & 0 & 0 & 0 & 0 & 0 & \mathrm{zyx} & 0 & 0\end{array}\right)$

$\left(\begin{array}{ccccccccccc}0 & 0 & 0 & 0 & 0 & 0 & 0 & 0 & 0 & y z x \\ 0 & -z y x & 0 & 0 & 0 & 0 & 0 & z y x x & 0 & 0\end{array}\right.$

$\left(\begin{array}{cccccc}0 & 0 & 0 & \text { xxyz } & 0 & 0 \\ & 0 & 0 & -x x y z & 0 & 0 \\ & & 0 & 0 & 0 & 0 \\ & & & 0 & 0 & 0 \\ & & & & 0 & x x y z \\ & & & & & 0\end{array}\right)$

$\left(\begin{array}{cccccc}0 & 0 & 0 & \text { xxyz } & 0 & 0 \\ & 0 & 0 & -x x y z & 0 & 0 \\ & & 0 & 0 & 0 & 0 \\ & & & 0 & 0 & 0 \\ & & & & 0 & \text { xxyz } \\ & & & & & 0\end{array}\right)$

$\left(\begin{array}{cccccc}0 & 0 & 0 & \text { xxyz } & 0 & 0 \\ 0 & 0 & 0 & -x x y z & 0 & 0 \\ 0 & 0 & 0 & 0 & 0 & 0 \\ y z x x & -y z x x & 0 & 0 & 0 & 0 \\ 0 & 0 & 0 & 0 & 0 & y z x x \\ 0 & 0 & 0 & 0 & \text { xxyz } & 0\end{array}\right)$

$\left(\begin{array}{cccccc}0 & 0 & 0 & x x y z & 0 & 0 \\ 0 & 0 & 0 & -x x y z & 0 & 0 \\ 0 & 0 & 0 & 0 & 0 & 0 \\ y z x x & -y z x x & 0 & 0 & 0 & 0 \\ 0 & 0 & 0 & 0 & 0 & y z x x \\ 0 & 0 & 0 & 0 & \text { xxyz } & 0\end{array}\right)$ 


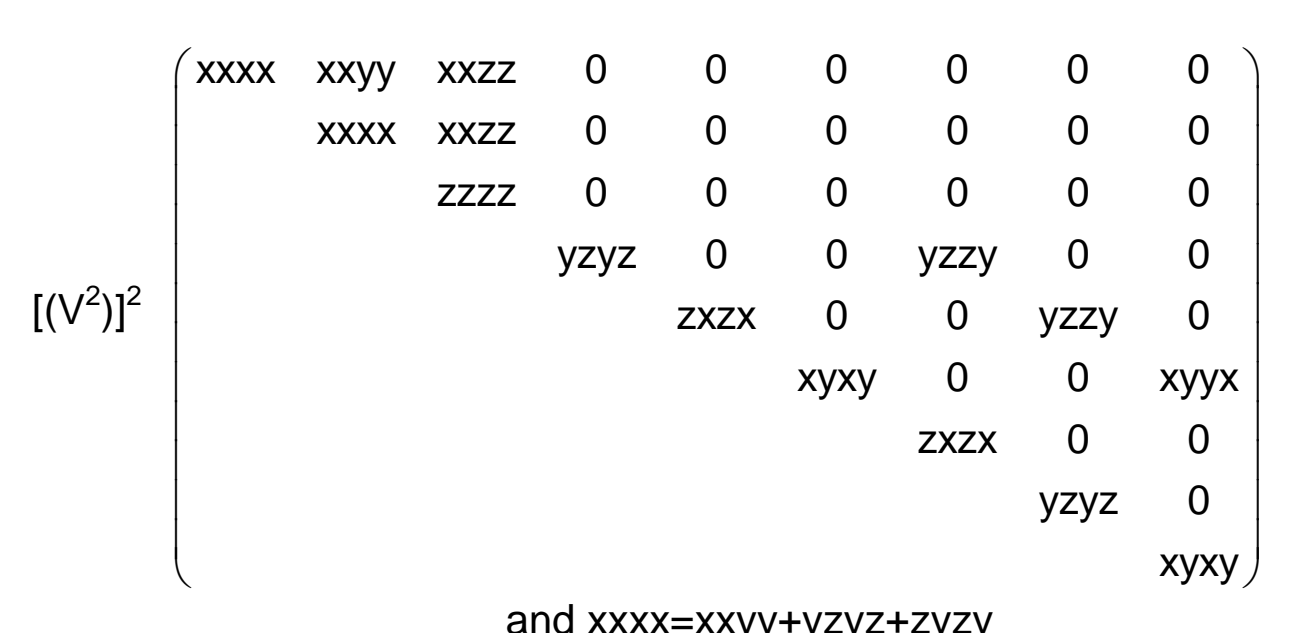

and $x x x x=x x y y+y z y z+z y z y$

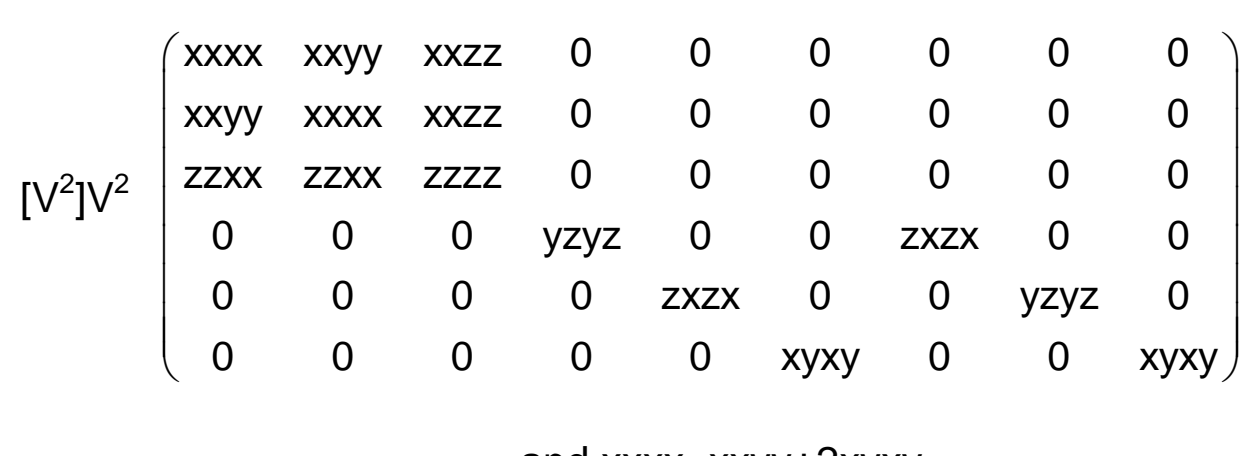

and $x x x x=x x y y+2 x y x y$ e

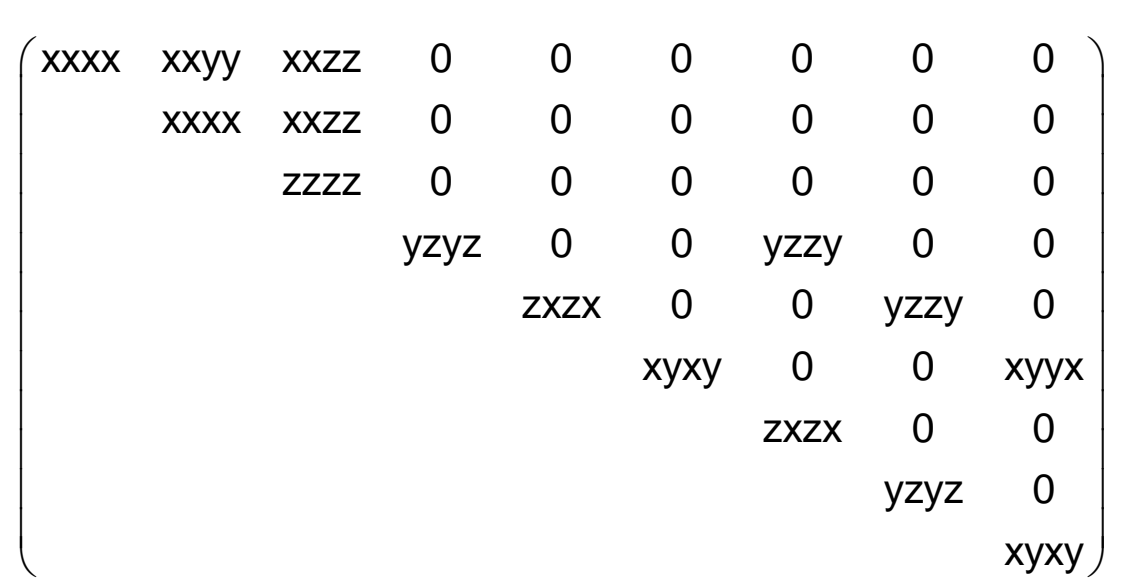

and $x x x x=x x y y+y z y z+z y z y$

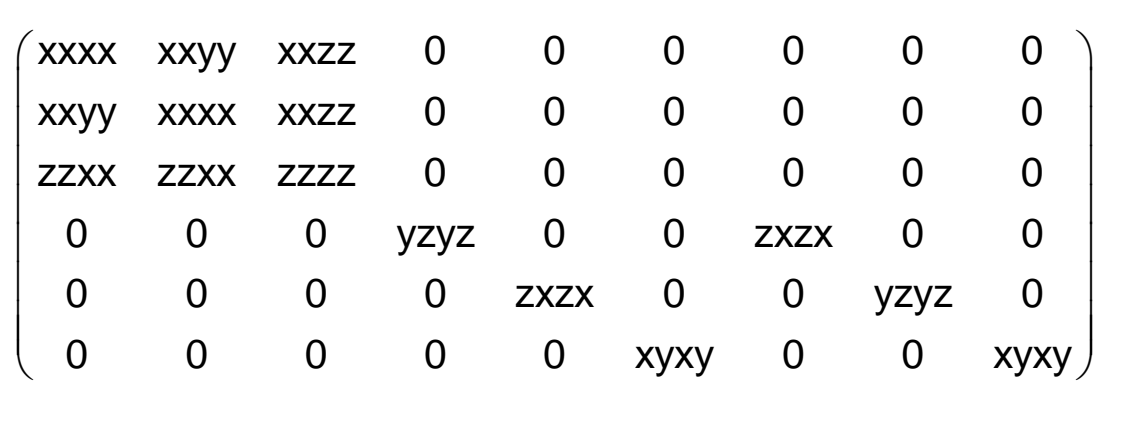

$\left(\begin{array}{ccccccccc}0 & 0 & 0 & x x y z & 0 & 0 & x x z y & 0 & 0 \\ 0 & 0 & 0 & -x x y z & 0 & 0 & -x x z y & 0 & 0 \\ 0 & 0 & 0 & 0 & 0 & 0 & 0 & 0 & 0 \\ y z x x & -y z x x & 0 & 0 & 0 & 0 & 0 & 0 & 0 \\ 0 & 0 & 0 & 0 & 0 & y z x x & 0 & 0 & y z x x \\ 0 & 0 & 0 & 0 & \text { xxzy } & 0 & 0 & \text { xxyz } & 0\end{array}\right)$ ae

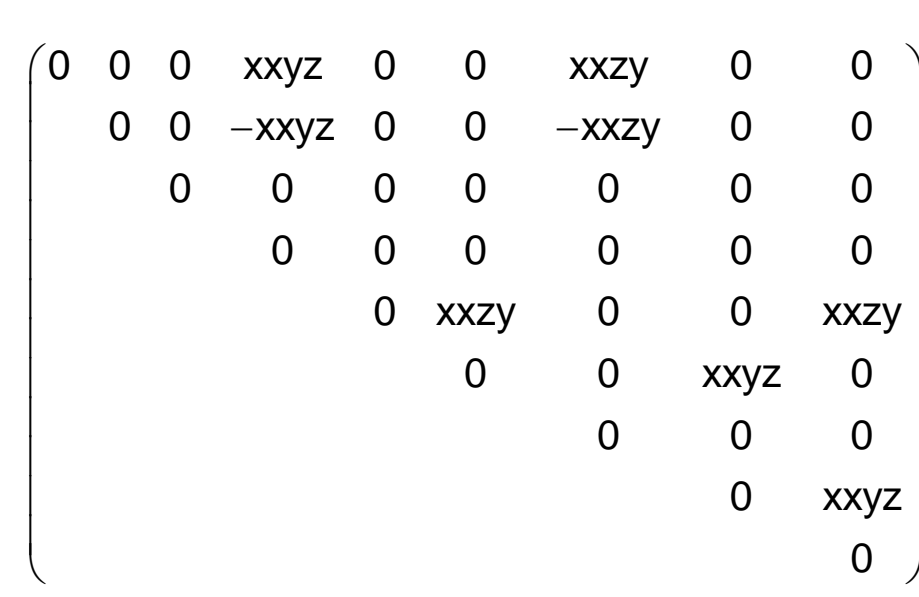

and $x x x x=x x y y+2 x y x y$ 


\section{1}

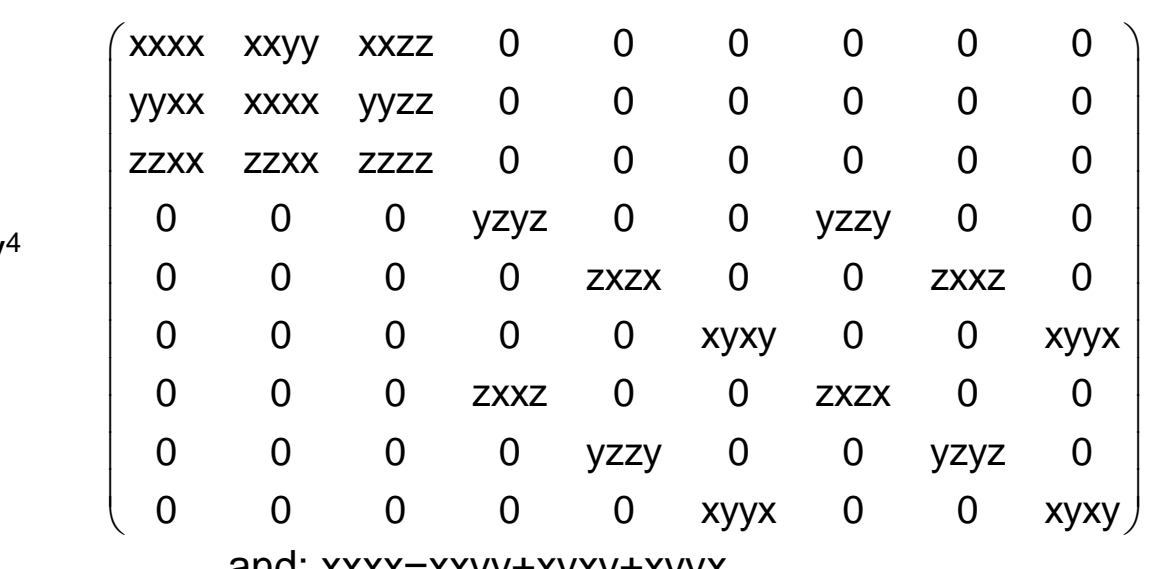

and: $x x x x=x x y y$

$\left[V^{4}\right] \quad\left(\begin{array}{ccccc}3 x x y y & 0 & 0 & y y z z & 0 \\ 0 & 3 x x y y & 0 & y y z z & 0 \\ 0 & 0 & z z z z & x x y y & 0\end{array}\right)$

$\left.\mathrm{V}^{3} \mathrm{~V}^{3}\right] \quad\left(\begin{array}{cccccccccc}3 \mathrm{xxxyy} & 0 & 0 & \mathrm{xxyy} & 0 & 0 & \mathrm{yyzz} & 0 & 0 & \mathrm{yyxx} \\ 0 & 3 \mathrm{xxyy} & 0 & 0 & \mathrm{yyzz} & 0 & 0 & \mathrm{xxyy} & 0 & 0 \\ 0 & 0 & \mathrm{zzzz} & 0 & 0 & \mathrm{zzxx} & 0 & 0 & \mathrm{zzxx} & 0\end{array}\right)$

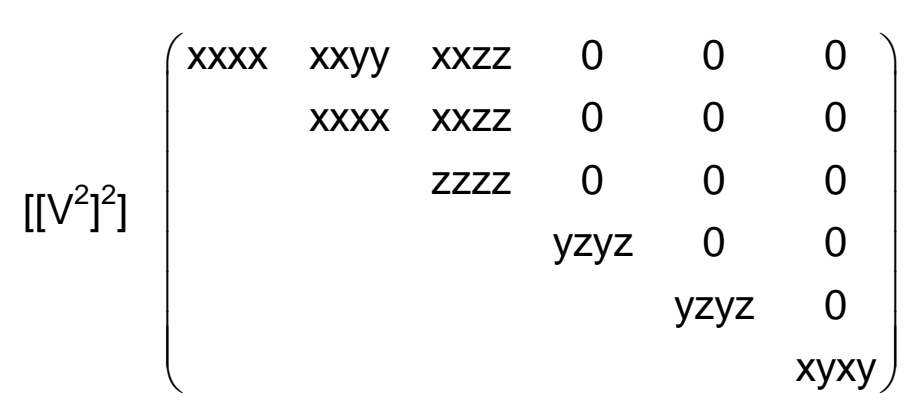

and $x y x y=1 / 2(x x x x-x x y y)$

$\left[\mathrm{V}^{2}\right]^{2}\left(\begin{array}{cccccc}x x x x & x x y y & x x z z & 0 & 0 & 0 \\ x x y y & x y x x & x x z z & 0 & 0 & 0 \\ z z x x & z z x x & z z z z & 0 & 0 & 0 \\ 0 & 0 & 0 & y z y z & 0 & 0 \\ 0 & 0 & 0 & 0 & y z y z & 0 \\ 0 & 0 & 0 & 0 & 0 & x y x y\end{array}\right)$

and $x y x y=1 / 2(x x x x-x x y y)$

$\left(\begin{array}{ccccc}3 x x y y & 0 & 0 & y y z z & 0 \\ 0 & 3 x x y y & 0 & y y z z & 0 \\ 0 & 0 & z z z z & x x y y & 0\end{array}\right)$

and $x y x y=1 / 2(x x x x-x x y y)$
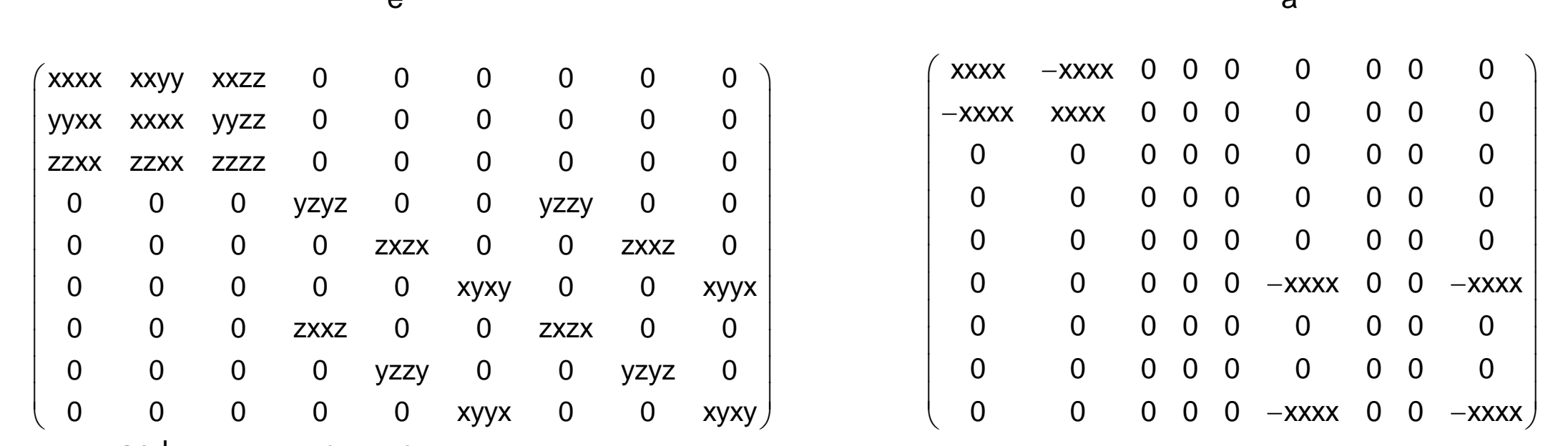

$\left(\begin{array}{ccccc}0 & 3 x x y y & 0 & y y z z & 0 \\ 0 & 0 & z z z z & x x y y & 0\end{array}\right)$

$\left(\begin{array}{ccccc}\mathrm{xxxx} & 0 & 0 & 0 & 0 \\ 0 & \mathrm{xxxx} & 0 & 0 & 0 \\ 0 & 0 & 0 & -\mathrm{xxxx} & 0\end{array}\right)$

$\left(\begin{array}{cccccccccc}3 x x y y & 0 & 0 & x x y y & 0 & 0 & y y z z & 0 & 0 & y y x x \\ 0 & 3 x x y y & 0 & 0 & y y z z & 0 & 0 & x x y y & 0 & 0 \\ 0 & 0 & 2 z z z & 0 & 0 & z z x & 0 & 0 & z z x & 0\end{array}\right)$

$\left(\begin{array}{cccccccccc}x x x x & 0 & 0 & -x x x x & 0 & 0 & 0 & 0 & 0 & 0 \\ 0 & x x y x & 0 & 0 & 0 & 0 & 0 & -x x x x & 0 & 0\end{array}\right)$

$\left(\begin{array}{cccccccccc}0 & x x x x & 0 & 0 & 0 & 0 & 0 & -x x x x & 0 & 0 \\ 0 & 0 & 0 & 0 & 0 & 0 & 0 & 0 & 0 & 0\end{array}\right)$

$\left(\begin{array}{cccccc}x x x x & x x y y & x x z z & 0 & 0 & 0 \\ & x x x x & x x z z & 0 & 0 & 0 \\ & & z z z z & 0 & 0 & 0 \\ & & & y z y z & 0 & 0 \\ & & & & y z y z & 0 \\ & & & & & x y x y\end{array}\right)$

$\left(\begin{array}{cccccc}\mathrm{xxxx} & -\mathrm{xxxx} & 0 & 0 & 0 & 0 \\ & \mathrm{xxxx} & 0 & 0 & 0 & 0 \\ & & 0 & 0 & 0 & 0 \\ & & & 0 & 0 & 0 \\ & & & & 0 & 0 \\ & & & & & -x \mathrm{xxx}\end{array}\right)$

and $x y x y=1 / 2(x x x x-x x y y)$

$\left(\begin{array}{cccccc}x x x x & x x y y & x x z z & 0 & 0 & 0 \\ x x y y & x x x x & x x z z & 0 & 0 & 0 \\ z z x x & z z x x & z z z z & 0 & 0 & 0 \\ 0 & 0 & 0 & y z y z & 0 & 0 \\ 0 & 0 & 0 & 0 & y z y z & 0 \\ 0 & 0 & 0 & 0 & 0 & x y x y\end{array}\right)$

$\left(\begin{array}{cccccc}x x x x & -x x x x & 0 & 0 & 0 & 0 \\ -x x x x & x x x x & 0 & 0 & 0 & 0 \\ 0 & 0 & 0 & 0 & 0 & 0 \\ 0 & 0 & 0 & 0 & 0 & 0 \\ 0 & 0 & 0 & 0 & 0 & 0 \\ 0 & 0 & 0 & 0 & 0 & -x x x x\end{array}\right)$ $\left(\begin{array}{lllllllll}x x x x & -x x x x & 0 & 0 & 0 & 0 & 0 & 0 & 0 \\ -x & x & x\end{array}\right.$

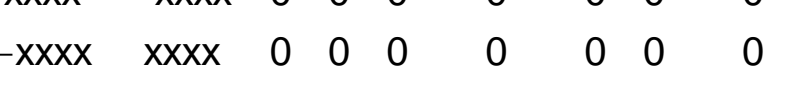
$\begin{array}{llllllll}0 & 0 & 0 & 0 & 0 & 0 & 0 & 0\end{array}$ $\begin{array}{llllllll}0 & 0 & 0 & 0 & 0 & 0 & 0\end{array}$ $\begin{array}{lllllll}0 & 0 & 0 & 0 & 0 & 0 & 0\end{array}$ $\begin{array}{lllllll}0 & 0 & 0 & -x \mathrm{xxx} & 0 & 0 & -\mathrm{xxxx} \\ 0 & 0 & 0 & 0 & 0 & 0 & 0\end{array}$ $\begin{array}{ccccccc}0 & 0 & 0 & 0 & 0 & 0 & 0 \\ 0 & 0 & 0 & 0 & 0 & 0 & 0\end{array}$

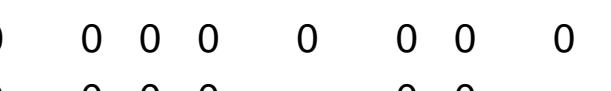

$\begin{array}{lllllllll}0 & 0 & 0 & 0 & -x x x x & 0 & 0 & -x x x x\end{array}$

$\left(\begin{array}{ccccc}x x x x & 0 & 0 & 0 & 0 \\ 0 & x x x x & 0 & 0 & 0 \\ 0 & 0 & 0 & -x x x x & 0\end{array}\right)$

$\left(\begin{array}{cccccccccc}x x x x & 0 & 0 & -x x x x & 0 & 0 & 0 & 0 & 0 & 0\end{array}\right)$ $\left.\begin{array}{lllllllllll}0 & \mathrm{xxxx} & 0 & 0 & 0 & 0 & 0 & -\mathrm{xxxx} & 0 & 0 \\ 0 & 0 & 0 & 0 & 0 & 0 & 0 & 0 & 0 & 0\end{array}\right)$

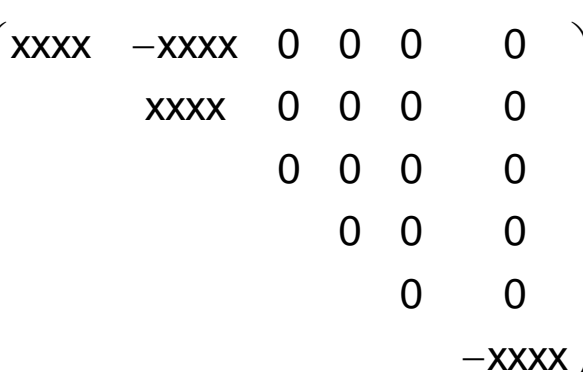

$-x x x x$

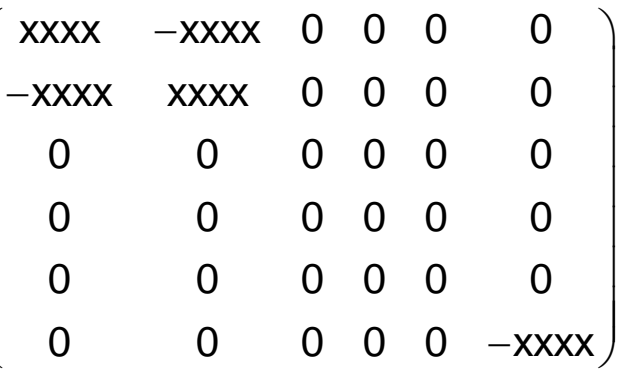




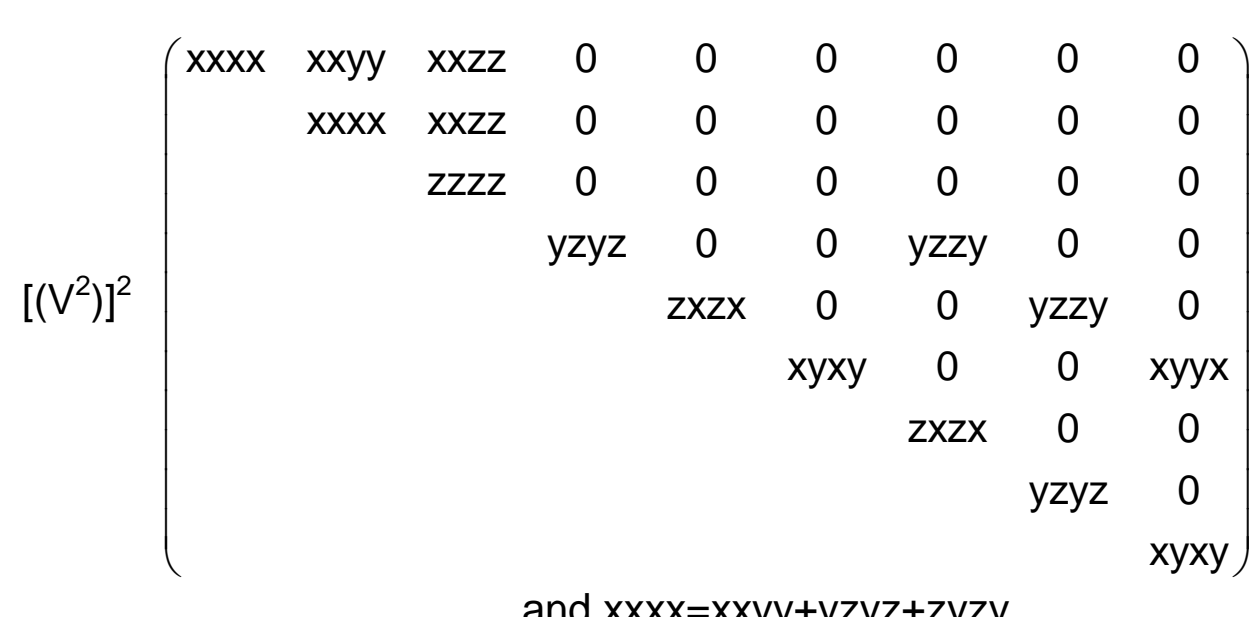

$\left[\mathrm{V}^{2}\right] \mathrm{V}^{2}\left(\begin{array}{ccccccccc}\mathrm{x} x \mathrm{x} & \mathrm{xxyy} & \mathrm{xxzz} & 0 & 0 & 0 & 0 & 0 & 0 \\ \mathrm{xxyy} & \mathrm{xxxx} & \mathrm{xxzz} & 0 & 0 & 0 & 0 & 0 & 0 \\ \mathrm{zzxx} & \mathrm{zzxx} & \mathrm{zzzz} & 0 & 0 & 0 & 0 & 0 & 0 \\ 0 & 0 & 0 & \mathrm{yzyz} & 0 & 0 & \mathrm{zxzx} & 0 & 0 \\ 0 & 0 & 0 & 0 & \mathrm{zxzx} & 0 & 0 & \mathrm{yzyz} & 0 \\ 0 & 0 & 0 & 0 & 0 & \mathrm{xyxy} & 0 & 0 & \mathrm{xyxy}\end{array}\right)$

and $x x x x=x x y y+2 x y x y$

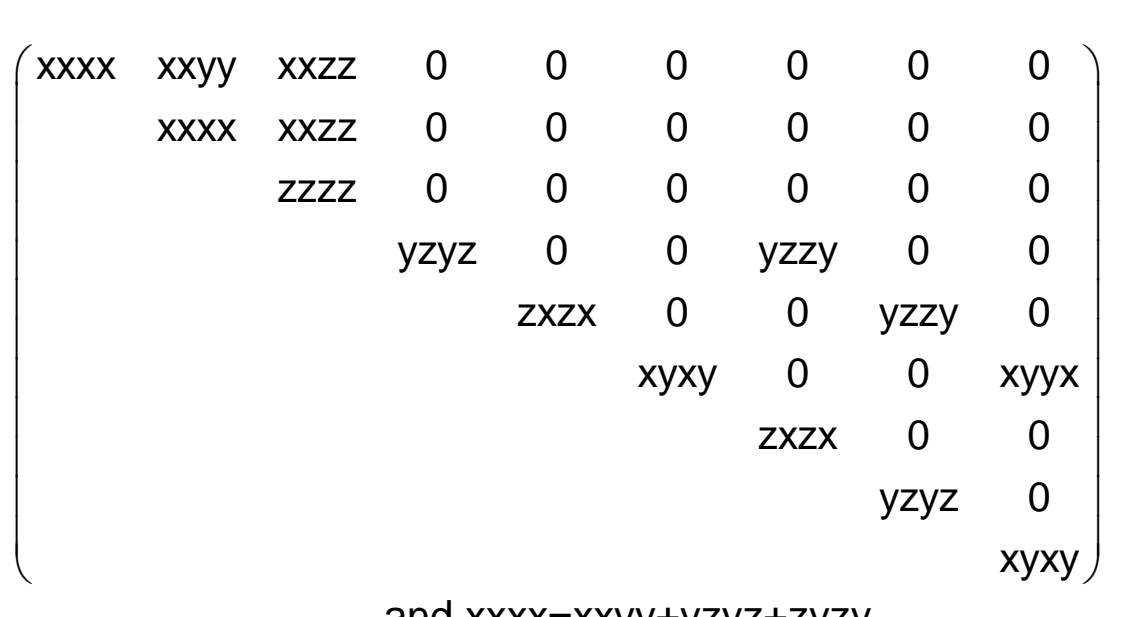

and $x x x x=x x y y+y z y z+z y z y$

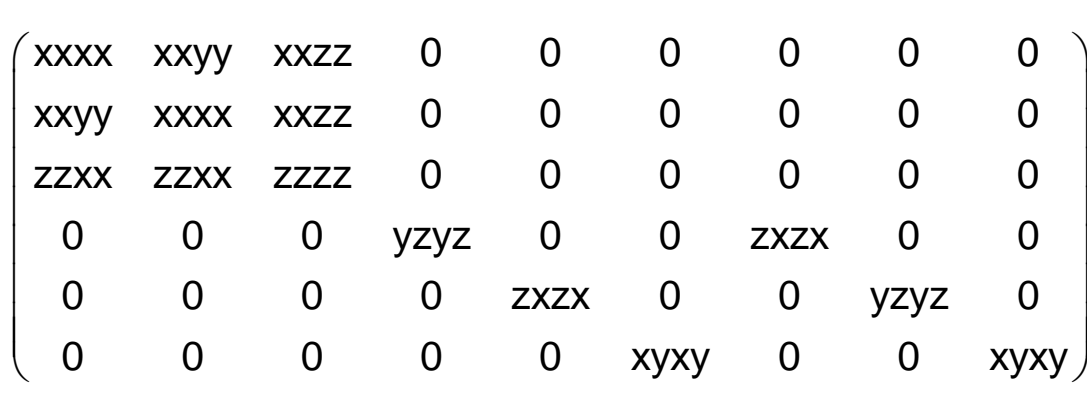

and $x x x x=x x y y+2 x y x y$

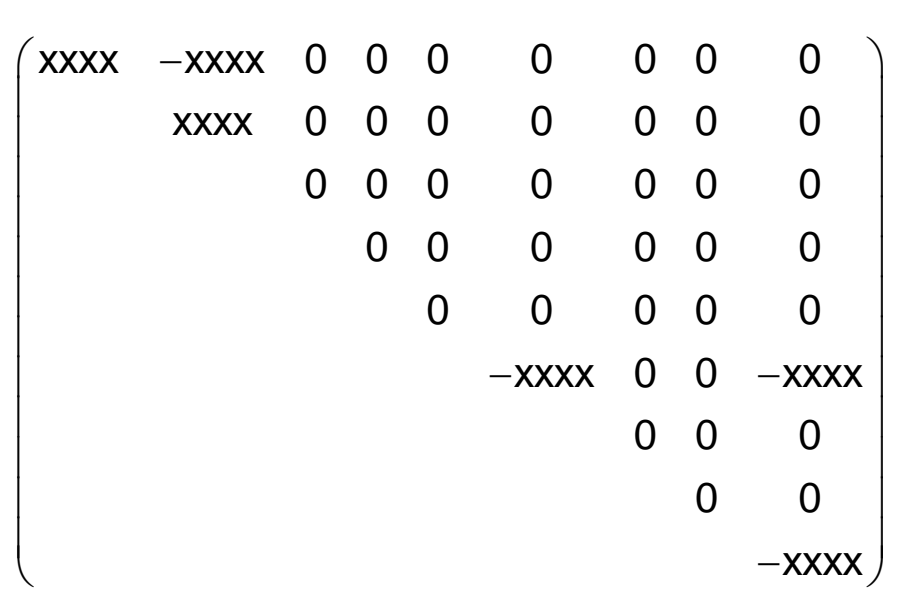

$\left(\begin{array}{ccccccccc}x x x x & -x x x x & 0 & 0 & 0 & 0 & 0 & 0 & 0 \\ -x x x x & x x x x & 0 & 0 & 0 & 0 & 0 & 0 & 0 \\ 0 & 0 & 0 & 0 & 0 & 0 & 0 & 0 & 0 \\ 0 & 0 & 0 & 0 & 0 & 0 & 0 & 0 & 0 \\ 0 & 0 & 0 & 0 & 0 & 0 & 0 & 0 & 0 \\ 0 & 0 & 0 & 0 & 0 & -x x x x & 0 & 0 & -x x x x\end{array}\right)$

$\begin{array}{ccccccccccc}x \times x x & -x x y x & 0 & 0 & 0 & 0 & 0 & 0 & -1 & 0\end{array}$

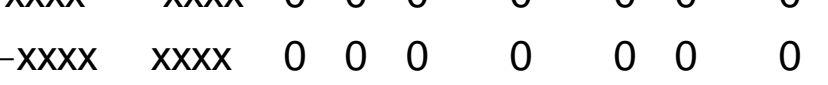

$\begin{array}{lllllllll}0 & 0 & 0 & 0 & 0 & 0 & 0 & 0 & 0 \\ 0 & 0 & 0 & 0 & 0 & 0 & 0 & 0 & 0\end{array}$

$\begin{array}{lllllllll}0 & 0 & 0 & 0 & 0 & 0 & 0 & 0 & 0 \\ 0 & 0 & 0 & 0 & 0 & 0 & 0 & 0 & 0\end{array}$

$\begin{array}{cccccccc}0 & 0 & 0 & 0 & 0 & 0 & 0 & 0 \\ 0 & 0 & 0 & 0 & -x x x x & 0 & 0 & -x x x x\end{array}$ 
1

$\left(\begin{array}{lllllllll}x x x x & x x y y & x x z z & 0 & 0 & 0 & 0 & 0 & 0 \\ x y x & x y x & x y z & 0 & 0 & 0 & 0 & 0 & 0\end{array}\right)$ $\begin{array}{llllllllll}\text { zzxx } & z z y y & z z z z & 0 & 0 & 0 & 0 & 0 & 0\end{array}$

$\mathrm{v}^{4} \quad \begin{array}{ccccccccc}0 & 0 & 0 & \mathrm{yzyz} & 0 & 0 & \mathrm{yzzy} & 0 & 0 \\ 0 & 0 & 0 & 0 & \mathrm{zxzx} & 0 & 0 & \mathrm{zxxz} & 0 \\ 0 & 0 & 0 & 0 & 0 & \mathrm{xyxy} & 0 & 0 & \mathrm{xyyx}\end{array}$ $\begin{array}{ccccccccc}0 & 0 & 0 & 0 & z x z x & 0 & 0 & z x x z & 0\end{array}$ $\begin{array}{lllllllll}0 & 0 & 0 & 0 & 0 & x y x y & 0 & 0 & x y y x \\ 0 & 0 & 0 & z x z & 0 & 0 & z x x & 0 & 0\end{array}$ $\left.\begin{array}{ccccccccc}0 & 0 & 0 & 0 & 0 & x y x y & 0 & 0 & x y y x \\ 0 & 0 & 0 & 0 & y z z y & 0 & z x z x & 0 & 0 \\ 0 & 0 & 0 & 0 & 0 & x y y x & 0 & 0 & x y x y\end{array}\right)$ $\begin{array}{ccccccccc}0 & 0 & 0 & 0 & y z z y & 0 & 0 & y z y z & 0 \\ 0 & 0 & 0 & 0 & 0 & x y y x & 0 & 0 & x y x y\end{array}$ and: $x x x x=x x y y+x y x y+x y y x$

$\left[V^{4}\right]\left(\begin{array}{ccccc}3 x x y y & 0 & 0 & y y z z & 0 \\ 0 & 3 x x y y & 0 & y y z z & 0 \\ 0 & 0 & z z z z & x x y y & 0\end{array}\right)$

$\mathrm{V}\left[V^{3}\right]\left(\begin{array}{cccccccccc}3 x x y y & 0 & 0 & x x y y & 0 & 0 & y y z z & 0 & 0 & 0 \\ 0 & 3 x x y y & 0 & 0 & y y z z & 0 & 0 & x x y y & 0 & 0 \\ 0 & 0 & z z z & 0 & 0 & z z x & 0 & 0 & z z x & 0\end{array}\right)$

$$
\left.\left[V^{2}\right]^{2}\right]\left(\begin{array}{cccccc}
x x x x & x x y y & x x z z & 0 & 0 & 0 \\
& x x x x & x x z z & 0 & 0 & 0 \\
& & z z z z & 0 & 0 & 0 \\
& & & y z y z & 0 & 0 \\
& & & & y z y z & 0 \\
& & & & & \text { xyxy }
\end{array}\right)
$$

and $x y x y=1 / 2(x x x x-x x y y)$

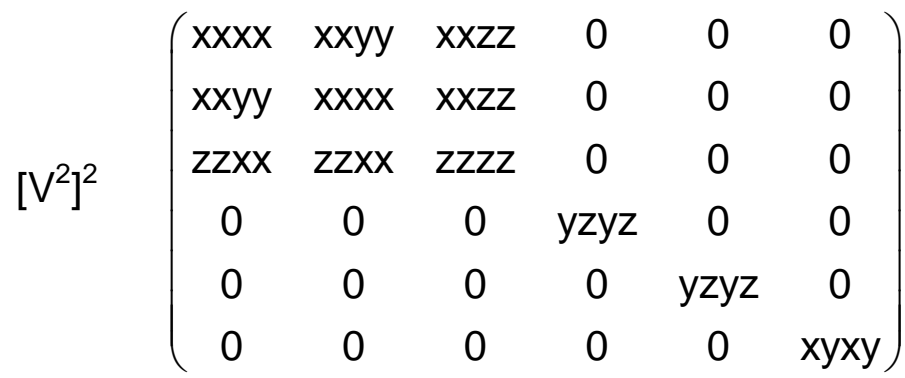

and $x y x y=1 / 2(x x x x-x x y y)$

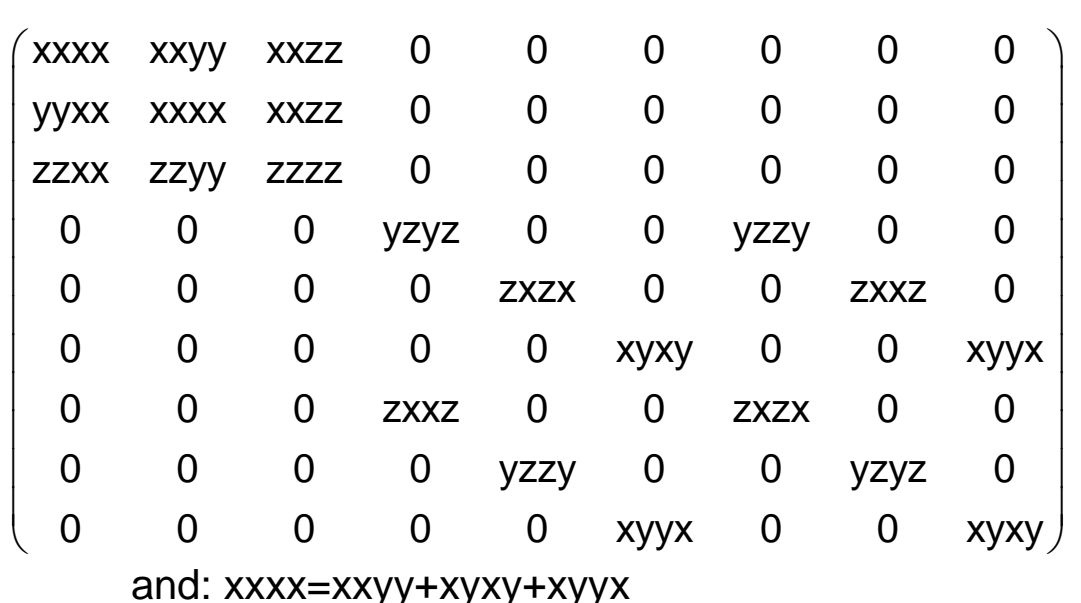

$$
\left(\begin{array}{ccccc}
3 x x y y & 0 & 0 & y y z z & 0 \\
0 & 3 x x y y & 0 & y y z z & 0 \\
0 & 0 & z z z z & x x y y & 0
\end{array}\right)
$$

$\left(\begin{array}{cccccccccc}3 x x y y & 0 & 0 & x x y y & 0 & 0 & y y z z & 0 & 0 & 0 \\ 0 & 3 x x y y & 0 & 0 & y y z z & 0 & 0 & x x y y & 0 & 0 \\ 0 & 0 & 2 z z & 0 & 0 & z z x & 0 & 0 & z x & 0\end{array}\right)$

$\left(\begin{array}{llllllllll}0 & 0 & 0 & 0 & 0 & 0 & 0 & 0 & 0 & 0 \\ 0 & 0 & 0 & 0 & 0 & 0 & 0 & 0 & 0 & 0 \\ 0 & 0 & 0 & 0 & 0 & 0 & 0 & 0 & 0 & 0\end{array}\right)$

$\left(\begin{array}{llllllllllll}0 & 0 & 0 & 0 & 0 & 0 & 0 & 0 & 0 & 0 \\ 0 & 0 & 0 & 0 & 0 & 0 & 0 & 0 & 0 & 0 \\ 0 & 0 & 0 & 0 & 0 & 0 & 0 & 0 & 0 & 0\end{array}\right)$

$\begin{array}{lllllllll}0 & 0 & 0 & 0 & 0 & 0 & 0 & 0 & 0 \\ 0 & 0 & 0 & 0 & 0 & 0 & 0 & 0 & 0 \\ 0 & 0 & 0 & 0 & 0 & 0\end{array}$

$\begin{array}{llllllllll}0 & 0 & 0 & 0 & 0 & 0 & 0 & 0 & 0 \\ 0 & 0 & 0 & 0 & 0 & 0 & 0 & 0\end{array}$

$\begin{array}{llllllllll}0 & 0 & 0 & 0 & 0 & 0 & 0 & 0 & 0 \\ 0 & 0 & 0 & 0 & 0 & 0 & 0\end{array}$

$\begin{array}{llllllllll}0 & 0 & 0 & 0 & 0 & 0 & 0 & 0 & 0 \\ 0 & 0 & 0 & 0 & 0 & 0 & 0 & 0\end{array}$

$\begin{array}{llllllllll}0 & 0 & 0 & 0 & 0 & 0 & 0 & 0 & 0\end{array}$

$\begin{array}{lllllllll}0 & 0 & 0 & 0 & 0 & 0 & 0 & 0 & 0 \\ 0 & 0 & 0 & 0 & 0 & 0 & 0 & 0 & 0\end{array}$

$\begin{array}{llllllllll}0 & 0 & 0 & 0 & 0 & 0 & 0 & 0 & 0 \\ 0 & 0 & 0 & 0 & 0 & 0 & 0 & 0 & 0\end{array}$

$\left(\begin{array}{lllll}0 & 0 & 0 & 0 & 0 \\ 0 & 0 & 0 & 0 & 0 \\ 0 & 0 & 0 & 0 & 0\end{array}\right)$

$\left(\begin{array}{lllll}0 & 0 & 0 & 0 & 0 \\ 0 & 0 & 0 & 0 & 0 \\ 0 & 0 & 0 & 0 & 0\end{array}\right)$

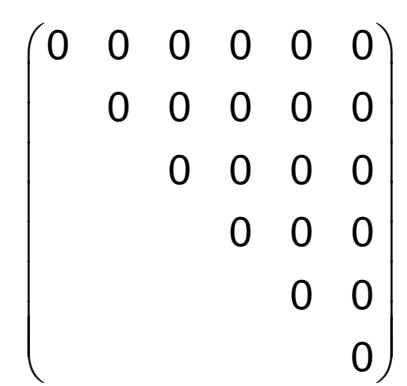

$\left(\begin{array}{cccccc}x x x x & x x y y & x x z z & 0 & 0 & 0 \\ & \text { xxxx } & \text { xxzz } & 0 & 0 & 0 \\ & & \text { zzzz } & 0 & 0 & 0 \\ & & & y z y z & 0 & 0 \\ & & & & y z y z & 0 \\ & & & & & \text { xyxy }\end{array}\right)$

and $x y x y=1 / 2(x x x x-x x y y)$

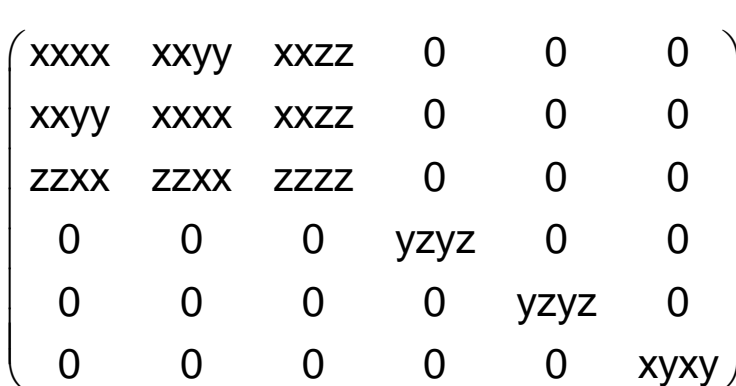

$\left(\begin{array}{llllll}0 & 0 & 0 & 0 & 0 & 0 \\ 0 & 0 & 0 & 0 & 0 & 0\end{array}\right)$

$\left.\begin{array}{llllll}0 & 0 & 0 & 0 & 0 & 0 \\ 0 & 0 & 0 & 0 & 0 & 0\end{array}\right)$

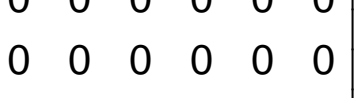

$\begin{array}{llllll}0 & 0 & 0 & 0 & 0 & 0 \\ 0 & 0 & 0 & 0 & 0 & 0\end{array}$

and $x y x y=1 / 2(x x x x-x x y y)$ $\left(\begin{array}{lllllllll}0 & 0 & 0 & 0 & 0 & 0 & 0 & 0 & 0 \\ 0 & 0 & 0 & 0 & 0 & 0 & 0 & 0 & 0\end{array}\right)$

$\left.\begin{array}{llllllllll}0 & 0 & 0 & 0 & 0 & 0 & 0 & 0 & 0 \\ 0 & 0 & 0 & 0 & 0 & 0 & 0 & 0 & 0\end{array}\right)$

$\begin{array}{llllllllll}0 & 0 & 0 & 0 & 0 & 0 & 0 & 0 & 0 \\ 0 & 0 & 0 & 0 & 0 & 0 & 0 & 0 & 0\end{array}$

$\begin{array}{llllllllllll}0 & 0 & 0 & 0 & 0 & 0 & 0 & 0 & 0 \\ 0 & 0 & 0 & 0 & 0 & 0 & 0 & 0 & 0 \\ 0 & 0 & 0 & 0 & 0 & 0 & 0 & 0\end{array}$

$\begin{array}{lllllllll}0 & 0 & 0 & 0 & 0 & 0 & 0 & 0 & 0\end{array}$

$\begin{array}{lllllllll}0 & 0 & 0 & 0 & 0 & 0 & 0 & 0 & 0 \\ 0 & 0 & 0 & 0 & 0 & 0 & 0 & 0 & 0\end{array}$

$\begin{array}{lllllllll}0 & 0 & 0 & 0 & 0 & 0 & 0 & 0 & 0 \\ 0 & 0 & 0 & 0 & 0 & 0 & 0 & 0 & 0\end{array}$

$\left(\begin{array}{lllll}0 & 0 & 0 & 0 & 0 \\ 0 & 0 & 0 & 0 & 0 \\ 0 & 0 & 0 & 0 & 0\end{array}\right)$

$\begin{array}{llllll}0 & 0 & 0 & 0 & 0 \\ 0 & 0 & 0 & 0 & 0\end{array}$

$\begin{array}{llllllllll}0 & 0 & 0 & 0 & 0 & 0 & 0 & 0 & 0 & 0 \\ 0 & 0 & 0 & 0 & 0 & 0 & 0 & 0 & 0 & 0\end{array}$

$\begin{array}{llllllllll}0 & 0 & 0 & 0 & 0 & 0 & 0 & 0 & 0 & 0 \\ 0 & 0 & 0 & 0 & 0 & 0 & 0 & 0 & 0 & 0\end{array}$

$\begin{array}{llllllllll}0 & 0 & 0 & 0 & 0 & 0 & 0 & 0 & 0 & 0\end{array}$

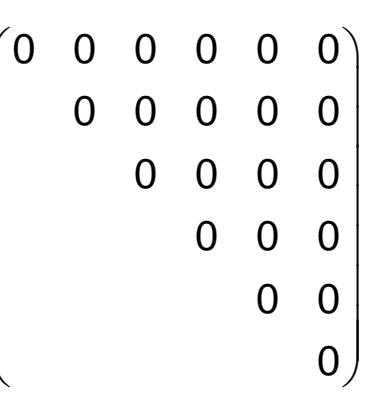

$\left(\begin{array}{llllll}0 & 0 & 0 & 0 & 0 & 0 \\ 0 & 0 & 0 & 0 & 0 & 0\end{array}\right)$

$\begin{array}{llllll}0 & 0 & 0 & 0 & 0 & 0 \\ 0 & 0 & 0 & 0 & 0 & 0\end{array}$

$0 \begin{array}{llllll}0 & 0 & 0 & 0 & 0 & 0 \\ 0 & 0 & 0 & 0 & 0 & 0\end{array}$

$\begin{array}{llllll}0 & 0 & 0 & 0 & 0 & 0 \\ 0 & 0 & 0 & 0 & 0 & 0\end{array}$

$\begin{array}{llllll}0 & 0 & 0 & 0 & 0 & 0 \\ 0 & 0 & 0 & 0 & 0 & 0\end{array}$ 


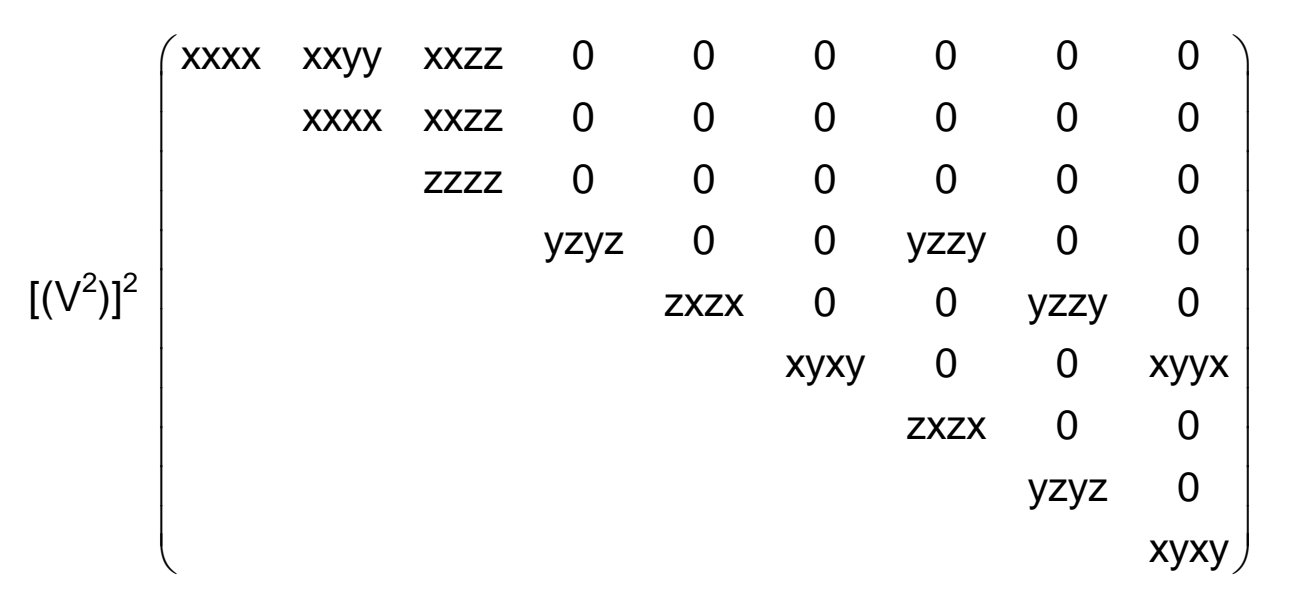

and $x x x x=x x y y+y z y z+z y z y$

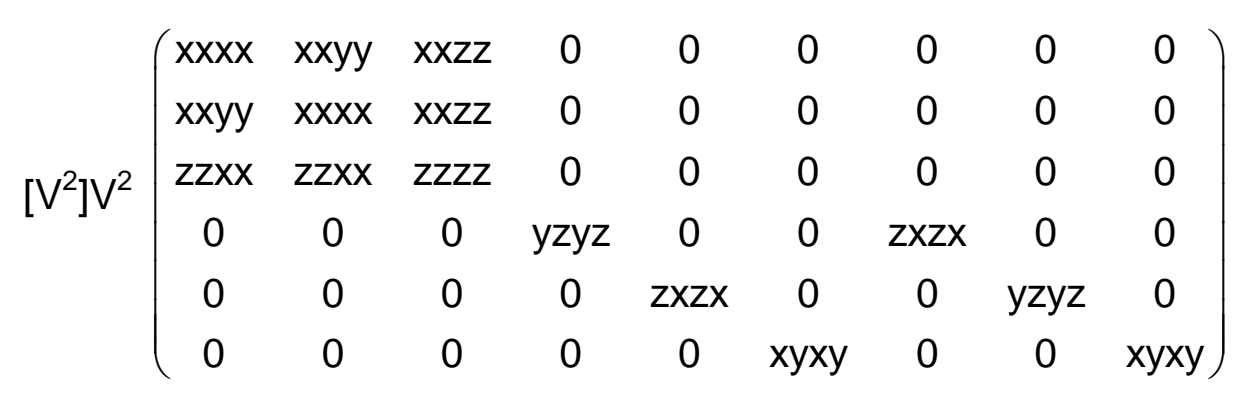

and $x x x x=x x y y+2 x y x y$

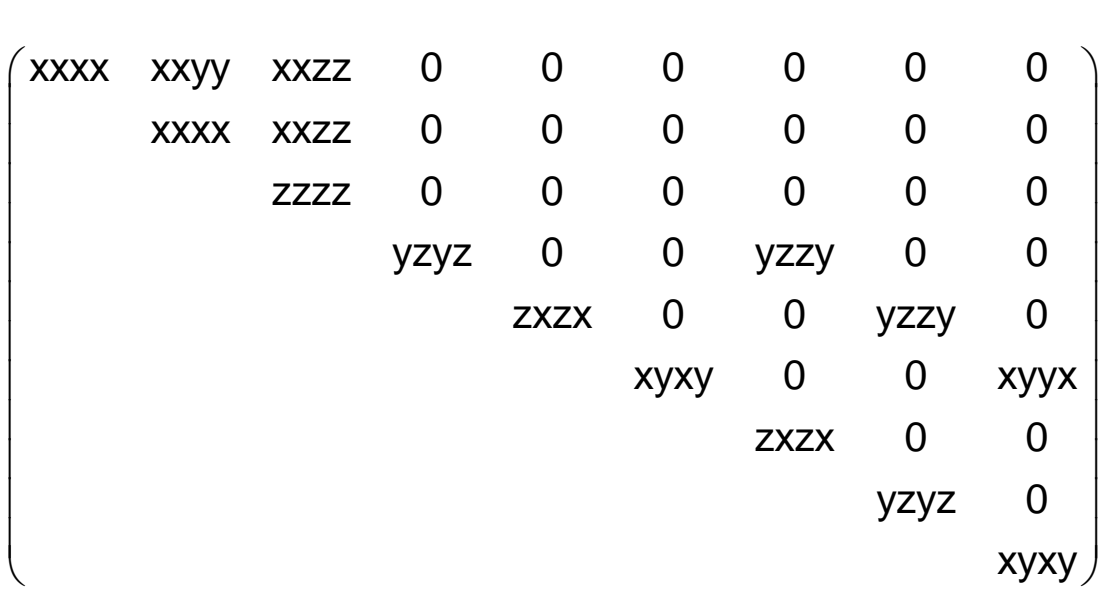

and $x x x x=x x y y+y z y z+z y z y$

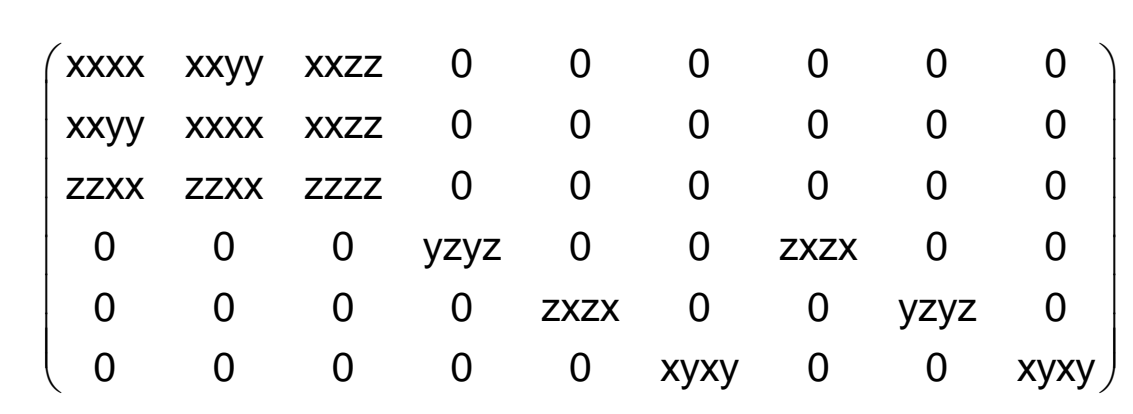

and $x x x x=x x y y+2 x y x y$

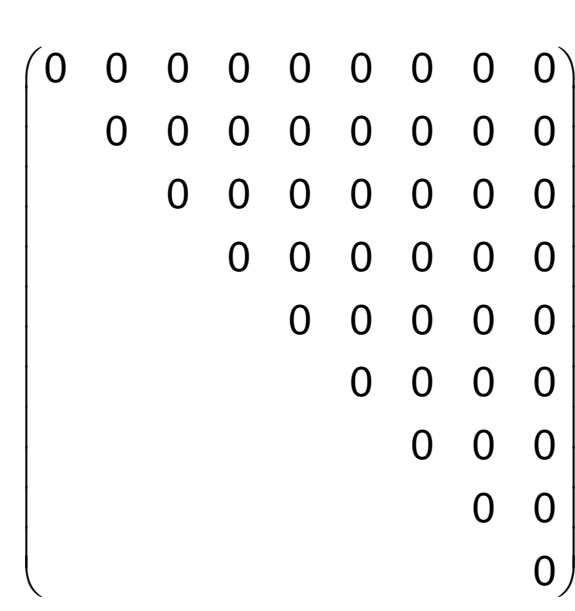

$\left(\begin{array}{lllllllll}0 & 0 & 0 & 0 & 0 & 0 & 0 & 0 & 0 \\ 0 & 0 & 0 & 0 & 0 & 0 & 0 & 0 & 0\end{array}\right.$ $\begin{array}{lllllllll}0 & 0 & 0 & 0 & 0 & 0 & 0 & 0 & 0\end{array}$ $\begin{array}{llllllllll}0 & 0 & 0 & 0 & 0 & 0 & 0 & 0 & 0 \\ 0 & 0 & 0 & 0 & 0 & 0 & 0 & 0 & 0\end{array}$ $0 \begin{array}{lllllllll}0 & 0 & 0 & 0 & 0 & 0 & 0 & 0 & 0 \\ 0 & 0 & 0 & 0 & 0 & 0 & 0 & 0 & 0\end{array}$ $\left(\begin{array}{lllllllll}0 & 0 & 0 & 0 & 0 & 0 & 0 & 0 & 0 \\ 0 & 0 & 0 & 0 & 0 & 0 & 0 & 0 & 0\end{array}\right)$

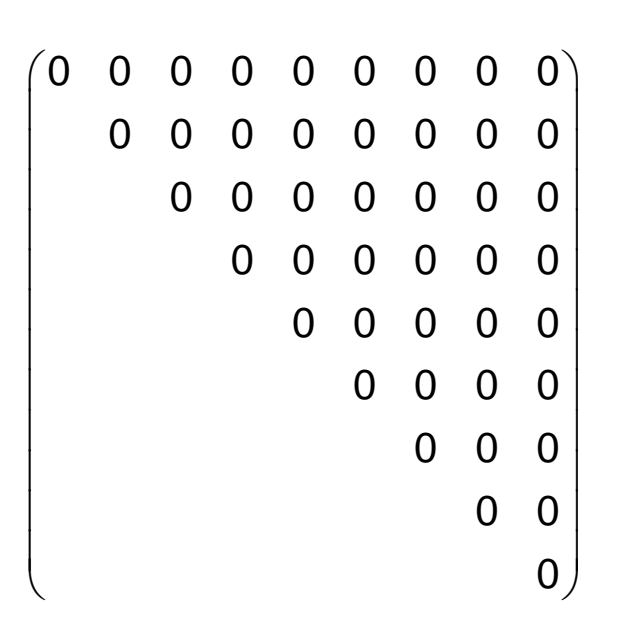

$\left(\begin{array}{lllllllll}0 & 0 & 0 & 0 & 0 & 0 & 0 & 0 & 0 \\ 0 & 0 & 0 & 0 & 0 & 0 & 0 & 0 & 0\end{array}\right.$ $\begin{array}{lllllllll}0 & 0 & 0 & 0 & 0 & 0 & 0 & 0 & 0 \\ 0 & 0 & 0 & 0 & 0 & 0 & 0 & 0 & 0\end{array}$ $\begin{array}{lllllllll}0 & 0 & 0 & 0 & 0 & 0 & 0 & 0 & 0 \\ 0 & 0 & 0 & 0 & 0 & 0 & 0 & 0 & 0\end{array}$ $\begin{array}{lllllllll}0 & 0 & 0 & 0 & 0 & 0 & 0 & 0 & 0 \\ 0 & 0 & 0 & 0 & 0 & 0 & 0 & 0 & 0\end{array}$ $\begin{array}{lllllllll}0 & 0 & 0 & 0 & 0 & 0 & 0 & 0 & 0 \\ 0 & 0 & 0 & 0 & 0 & 0 & 0 & 0 & 0\end{array}$

$$
\left.\begin{array}{l}
0 \\
0 \\
0 \\
0 \\
0 \\
0 \\
0 \\
0 \\
0
\end{array}\right)
$$


$\left(\begin{array}{lllllllll}x x x x & x x y y & x x z z & 0 & x x z x & 0 & 0 & x x x z & 0 \\ y y x x & y y y & y y z z & 0 & y z x & 0 & 0 & y x z & 0\end{array}\right)$ $\left.\begin{array}{lllllllll}\mathrm{yyxx} & \mathrm{yyyy} & \mathrm{yyzz} & 0 & \mathrm{yyzx} & 0 & 0 & \mathrm{yyxz} & 0 \\ \mathrm{zzxx} & \mathrm{zzyy} & \mathrm{zzzz} & 0 & \mathrm{zzzx} & 0 & 0 & \mathrm{zzxz} & 0 \\ 0 & 0 & 0 & \mathrm{yzyz} & 0 & \mathrm{yzx} & \mathrm{yzzy} & 0 & \mathrm{yzyx}\end{array}\right)$ $\begin{array}{lllllllll}0 & 0 & 0 & y z y z & 0 & y z x y & y z z y & 0 & y z y x\end{array}$

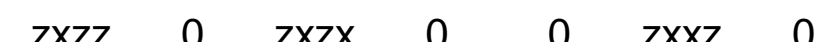
$\begin{array}{lllllllll}0 & 0 & 0 & x y y z & 0 & x y x y & x y z y & 0 & \text { xyyx } \\ 0 & 0 & 0 & z y z & 0 & \text { zyxy } & z y z & 0 & z y x\end{array}$ $\begin{array}{lllllllll}0 & 0 & 0 & z y y z & 0 & \text { zyxy } & \text { zyzy } & 0 & \text { zyyx }\end{array}$ $\begin{array}{ccccccccc}x z x x & x z y y & x z z z & 0 & x z z x & 0 & 0 & 0 & 0\end{array}$ $\begin{array}{lllllllll}0 & 0 & 0 & y x y z & 0 & y x x y & y x z y & 0 & y x y x\end{array}$

$\left[\mathrm{V}^{4}\right]$ $\left(\begin{array}{ccccc}x x x x & 0 & x z z & y y z z & 0 \\ z x x x & y y y y & 0 & z z x x & y y x z \\ & 0 & z z z & x x y y & 0\end{array}\right)$ $\left(\begin{array}{cccccccccc}y x x x & y x y y & y x z z & 0 & y x z x & 0 & 0 & y x x z & 0\end{array}\right)$

$\left(\begin{array}{lllllllll}0 & 0 & 0 & x x y z & 0 & x x x y & x x z y & 0 & x x y x \\ 0 & 0 & 0 & y y z & 0 & y x y & y y z & 0 & y y x\end{array}\right)$ \begin{tabular}{cccccccccc}
0 & 0 & 0 & $y y y z$ & 0 & yyxy & yyzy & 0 & yyyx \\
0 & 0 & 0 & $z z y z$ & 0 & zzxy & zzzy & 0 & zzyx \\
\hline$z z x$ & $y z y y$ & $y z z z$ & 0 & $y z z x$ & 0 & 0 & $y z x z$ & 0
\end{tabular} $\begin{array}{lllllllll}y z x x & y z y y & y z z z & 0 & y z z x & 0 & 0 & y z x z & 0\end{array}$ $\begin{array}{lllllllll}0 & 0 & 0 & z x y z & 0 & z x x y & z x z y & 0 & z x y x\end{array}$

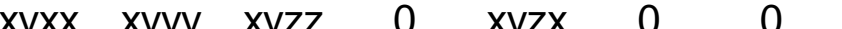

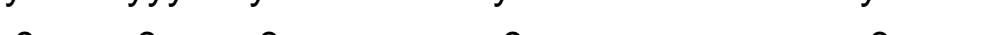
$\left(\begin{array}{ccccc}0 & x y y y & 0 & 0 & x x y z \\ y x x x & 0 & y z z z & 0 & 0 \\ 0 & z y y y & 0 & 0 & z z x y\end{array}\right)$ $\left(\begin{array}{lllllllll}0 & 0 & 0 & x x y z & 0 & x x x y & x x z y & 0 & x x y x \\ 0 & 0 & 0 & y y y z & 0 & y y x & x y y & 0 & y x y x\end{array}\right)$ $\left.\begin{array}{llllllllll}0 & 0 & 0 & y y y z & 0 & \text { yyxy } & \text { yyzy } & 0 & \text { yyyx } \\ 0 & 0 & 0 & z z y z & 0 & \text { zzxy } & \text { zzzy } & 0 & \text { zzyx } \\ y z x x & y z y & y z z z & 0 & y z z x & 0 & 0 & y z z z & 0\end{array}\right)$ $\begin{array}{lllllllll}y z x x & y z y y & y z z z & 0 & y z z x & 0 & 0 & y z x z & 0\end{array}$ $\begin{array}{llllllllll}0 & 0 & 0 & z x y z & 0 & z x x y & z x z y & 0 & z x y x\end{array}$ $\begin{array}{lllllllll}x y x x & x y y y & x y z z & 0 & x y z x & 0 & 0 & x y x z & 0\end{array}$ $\begin{array}{lllllllll}\text { zyxx } & \text { zyyy } & \text { zyzz } & 0 & \text { zyzx } & 0 & 0 & \text { zyxz } & 0\end{array}$ $\begin{array}{lllllllll}0 & 0 & 0 & x z y z & 0 & x z x y & x z z y & 0 & x z y x\end{array}$ $\begin{array}{lllllllll}y x x x & y x y y & y x z z & 0 & y x z x & 0 & 0 & y x x z & 0\end{array}$ $\left(\begin{array}{ccccc}0 & x y y y & 0 & 0 & x x y z \\ y x x x & 0 & y z z z & 0 & 0 \\ 0 & z y y y & 0 & 0 & z z x y\end{array}\right)$ $\left(\begin{array}{lllllllll}x x x x & x x y y & x x z z & 0 & x x z x & 0 & 0 & x x x z & 0 \\ x y x x & y y y & y y z z & 0 & y y z x & 0 & 0 & y y x z & 0\end{array}\right.$ $\begin{array}{llllllllll}\text { yyxx } & y y y y & y y z z & 0 & y y z x & 0 & 0 & y y x z & 0\end{array}$ $\begin{array}{llllllllll}\text { zzxx } & z z y y & z z z z & 0 & z z z x & 0 & 0 & z z x z & 0\end{array}$ $\begin{array}{ccccccccc}0 & 0 & 0 & y z y z & 0 & y z x y & y z z y & 0 & y z y x\end{array}$ $\begin{array}{lllllllll}z x x x & z x y y & z x z z & 0 & z x z x & 0 & 0 & z x x z & 0\end{array}$ $\begin{array}{lllllllll}0 & 0 & 0 & x y y z & 0 & x y x y & x y z y & 0 & x y y x\end{array}$ $\begin{array}{lllllllll}0 & 0 & 0 & z y y z & 0 & \text { zyxy } & \text { zyzy } & 0 & \text { zyyx }\end{array}$

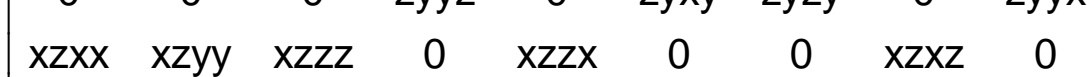
$\begin{array}{ccccccccc}0 & 0 & 0 & y x y z & 0 & y x x y & y x z y & 0 & y x y x\end{array}$ $\left(\begin{array}{ccccc}x x x x & 0 & x z z z & y y z z & 0 \\ 0 & y y y y & 0 & z z x x & y y x z \\ z x x x & 0 & z z z z & x x y y & 0\end{array}\right)$

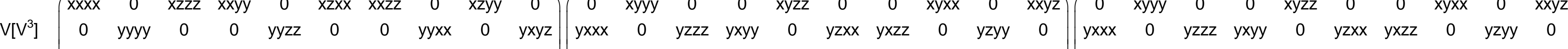
$\left.\begin{array}{lllllllllll}z x x x & 0 & z z z z & z x y y & 0 & z z x x & z x z z & 0 & \text { zzyy } & 0\end{array}\right)\left(\begin{array}{lllllllllllllll}0 & \text { zyyy } & 0 & 0 & \text { zyzz } & 0 & 0 & \text { zyxx } & 0 & \text { zxyz }\end{array}\right)\left(\begin{array}{lllllll}\text { zyyy } & 0 & 0 & \text { zyzz } & 0\end{array}\right.$

$\left(\begin{array}{llllllllll}x x x x & 0 & x z z z & x x y y & 0 & x z x x & x x z z & 0 & x z y y & 0\end{array}\right)$

$\left[\left[V^{2}\right]^{2}\right]\left(\begin{array}{cccccc}x x x x & x x y y & x x z z & 0 & x x x z & 0 \\ & y y y y & y y z z & 0 & y y x z & 0 \\ & & z z z z & 0 & z z x z & 0 \\ & & & y z y z & 0 & y z x y \\ & & & & x z x z & 0 \\ & & & & & x y x y\end{array}\right)$

$\left[\mathrm{V}^{2}\right]^{2}$

$\begin{array}{llll}x x x x & x x y y & x x z z & 0\end{array}$ $\left.\begin{array}{cccccc}y y x x & y y y y & y y z z & 0 & y y x z & 0 \\ z z x x & z z y y & z z z z & 0 & z z x z & 0 \\ 0 & 0 & 0 & y z y z & 0 & y z x y\end{array}\right)$

$\begin{array}{llllll}0 & 0 & 0 & y z y z & 0 & y z x y \\ x z x & x z y y & x z z & 0 & x z x z & 0\end{array}$ $\begin{array}{cccccc}x z x x & x z y y & x z z z & 0 & x z x z & 0\end{array}$

$$
\left(\begin{array}{cccccc}
0 & 0 & 0 & x x y z & 0 & x x x y \\
& 0 & 0 & y y y z & 0 & y y x y \\
& & 0 & z z y z & 0 & z z x y \\
& & & 0 & y z x z & 0 \\
& & & & 0 & x z x y \\
& & & & & 0
\end{array}\right)
$$

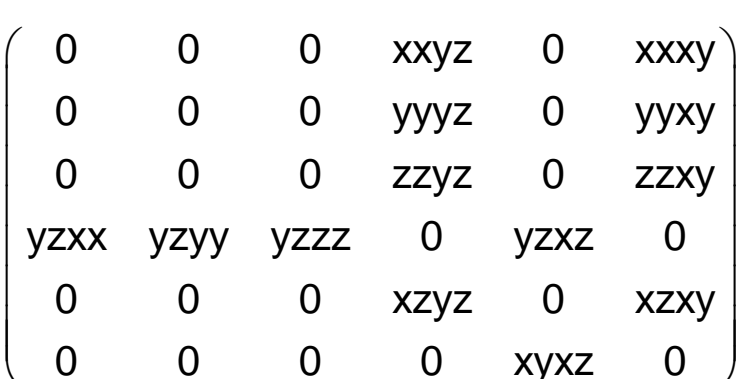

$$
\left(\begin{array}{cccccc}
0 & 0 & 0 & \text { xxyz } & 0 & \text { xxxy } \\
& 0 & 0 & y y z z & 0 & y y x y \\
& & 0 & z z y z & 0 & z z x y \\
& & & 0 & y z x z & 0 \\
& & & & 0 & x z x y \\
& & & & & 0
\end{array}\right)
$$

$\left(\begin{array}{cccccc}0 & 0 & 0 & x x y z & 0 & \text { xxxy } \\ 0 & 0 & 0 & \text { yyyz } & 0 & \text { yyxy } \\ 0 & 0 & 0 & \text { zzyz } & 0 & \text { zzxy } \\ \text { yzxx } & \text { yzyy } & \text { yzzz } & 0 & y z x z & 0 \\ 0 & 0 & 0 & \text { xzyz } & 0 & \text { xzxy } \\ 0 & 0 & 0 & 0 & \text { xyxz } & 0\end{array}\right)$ $\left(\begin{array}{cccccccccc}x & y y y y & 0 & 0 & y y z z & 0 & 0 & y y x x & 0 & y x y z \\ z x x x & 0 & z z z z & z x y y & 0 & z z x x & z x z z & 0 & z z y y & 0\end{array}\right)$

$$
\left(\begin{array}{cccccc}
x x x x & x x y y & x x z z & 0 & x x x z & 0 \\
& y y y y & y y z z & 0 & y y x z & 0 \\
& & z z z z & 0 & z z x z & 0 \\
& & & y z y z & 0 & y z x y \\
& & & & x z x z & 0 \\
& & & & & x y x y
\end{array}\right)
$$

$\left(\begin{array}{llllll}x x x x & x x y y & x x z z & 0 & x x x z & 0\end{array}\right)$ $\left.\begin{array}{llllll}y y x x & y y y y & y y z z & 0 & y y x z & 0 \\ z z x & z z y y & z z z z & 0 & z z x z & 0\end{array}\right)$ $\begin{array}{lllllll}z z x x & z z y y & z z z z & 0 & z z x z & 0\end{array}$ $\begin{array}{cccccc}0 & 0 & 0 & y z y z & 0 & y z x y\end{array}$ $\begin{array}{lllllll}x z x x & x z y y & x z z z & 0 & x z x z & 0\end{array}$ 

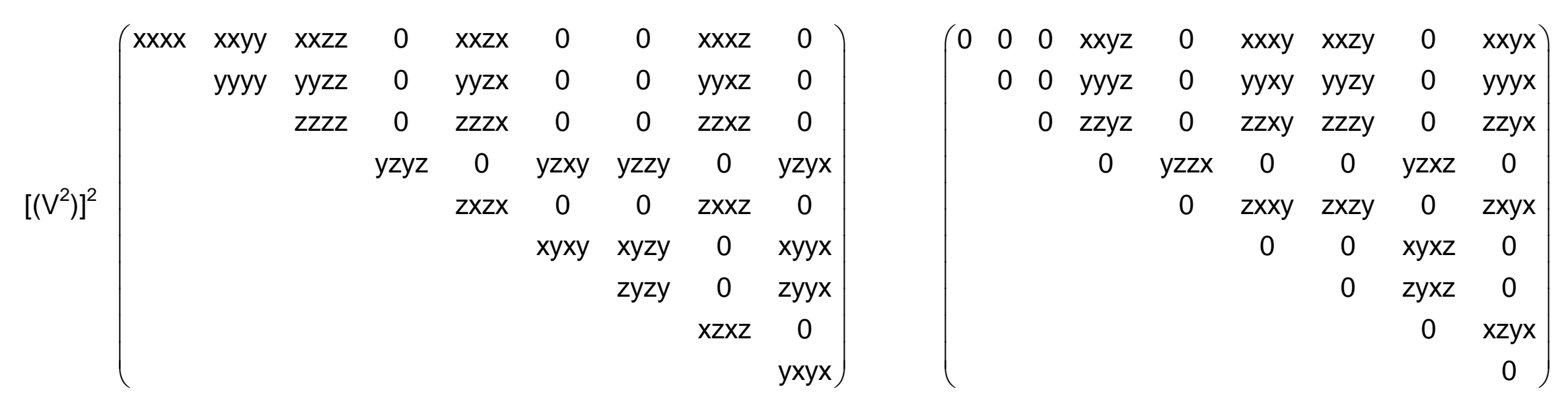

$\left(\begin{array}{lllllllll}x x x x & x x y y & x x z z & 0 & x x z x & 0 & 0 & x x x z & 0 \\ y y x & y y y y & y z z & 0 & y y z x & 0 & 0 & y y x z & 0\end{array}\right)$ $\left.\begin{array}{lllllllll}\text { yyxx } & y y y y & y y z z & 0 & y y z x & 0 & 0 & y y x z & 0\end{array}\right)$ $\left[V^{2}\right] V^{2} \quad \begin{array}{ccccccccc}2 z x x & z z y y & z z z z & 0 & z z z x & 0 & 0 & z z x z & 0\end{array}$ $\begin{array}{ccccccccc}0 & 0 & 0 & y z y z & 0 & y z x y & y z z y & 0 & y z y x \\ z x x x & z x y y & z x z z & 0 & z x z x & 0 & 0 & z x x z & 0\end{array}$ $\begin{array}{ccccccccc}z x x x & z x y y & z x z z & 0 & z x z x & 0 & 0 & z x x z & 0 \\ 0 & 0 & 0 & x y y z & 0 & x y x y & x y z y & 0 & x y y x\end{array}$ a
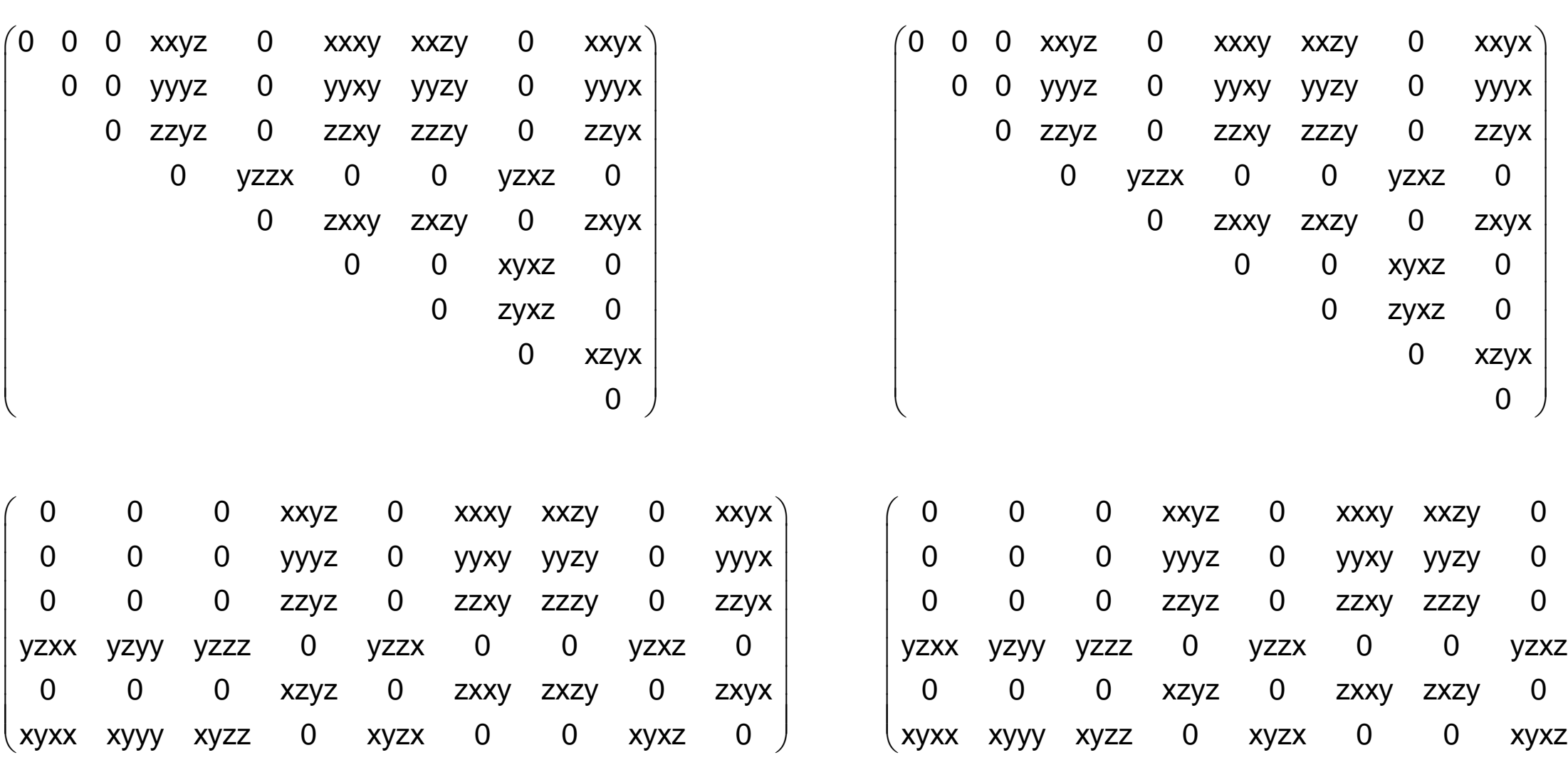

$\left(\begin{array}{lllllllll}0 & 0 & 0 & x x y z & 0 & x x x y & x x z y & 0 & x x y x \\ 0 & 0 & 0 & x y z & 0 & y y x & x y y & 0 & y x y\end{array}\right)$

$\begin{array}{lllllllll}0 & 0 & 0 & \text { yyyz } & 0 & \text { yyxy } & \text { yyzy } & 0 & \text { yyyx } \\ 0 & 0 & 0 & z y z & 0 & \text { zzxy } & \text { zzzy } & 0 & \text { zzy }\end{array}$ $\begin{array}{lllllllll}0 & 0 & 0 & z z y z & 0 & z z x y & z z z y & 0 & z z y x\end{array}$ $\begin{array}{ccccccccc}\text { yzxx } & y z y y & y z z z & 0 & y z z x & 0 & 0 & y z x z & 0 \\ 0 & 0 & 0 & x z y z & 0 & z x y y & z x z y & 0 & \end{array}$ $\left(\begin{array}{ccccccccc}0 & 0 & 0 & x z y z & 0 & z x x y & z x z y & 0 & z x y x \\ x y x x & x y y y & x y z z & 0 & x y z x & 0 & 0 & x y x z & 0\end{array}\right)$

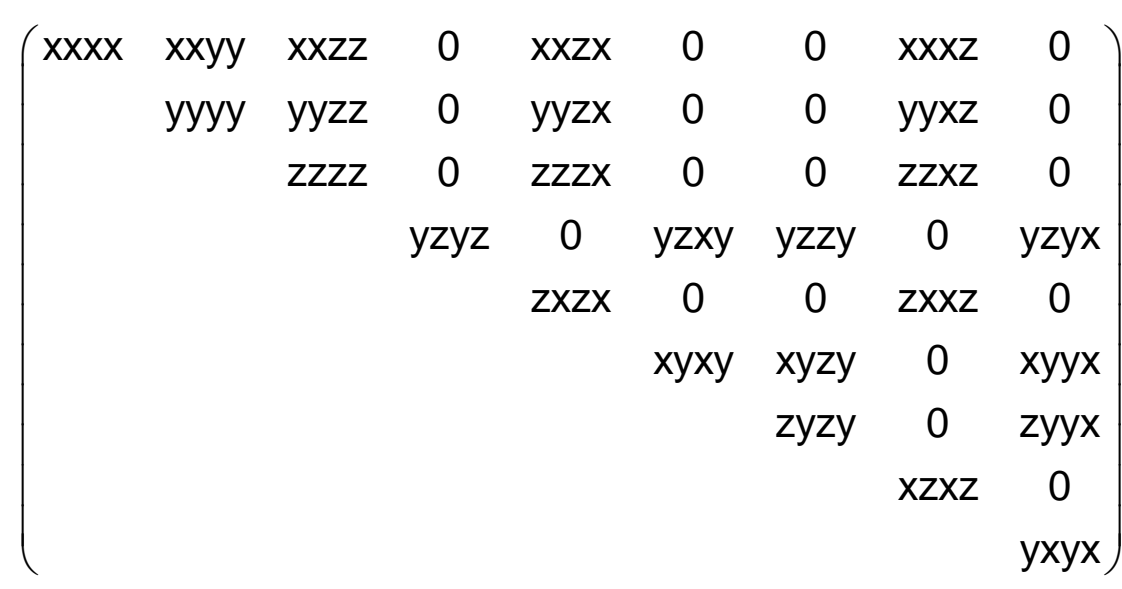

$\left(\begin{array}{lllllllll}x x x x & x x y y & x x z z & 0 & x x z x & 0 & 0 & x x x z & 0 \\ y y x x & y y y & y y z z & 0 & y z x & 0 & 0 & y x z & 0\end{array}\right)$

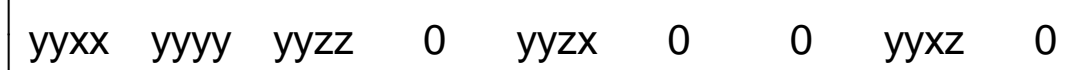
$\begin{array}{lllllllll}z z x x & z z y y & z z z z & 0 & z z z x & 0 & 0 & z z x z & 0\end{array}$ $\begin{array}{ccccccccc}0 & 0 & 0 & y z y z & 0 & y z x y & y z z y & 0 & y z y x\end{array}$ $\left(\begin{array}{ccccccccc}z x x x & z x y y & z x z z & 0 & z x z x & 0 & 0 & z x x z & 0 \\ 0 & 0 & 0 & x y y z & 0 & x y x y & x y z y & 0 & x y y x\end{array}\right.$ 
e

$\left(\begin{array}{ccccccccc}\text { xxxx } & \text { xxyy } & \text { xxzz } & 0 & 0 & 0 & 0 & 0 & 0 \\ \text { yyxx } & \text { yyyy } & \text { yyzz } & 0 & 0 & 0 & 0 & 0 & 0 \\ \text { zzxx } & \text { zzyy } & \text { zzzz } & 0 & 0 & 0 & 0 & 0 & 0 \\ 0 & 0 & 0 & \text { yzyz } & 0 & 0 & \text { yzzy } & 0 & 0 \\ 0 & 0 & 0 & 0 & \text { zxzx } & 0 & 0 & \text { zxxz } & 0 \\ 0 & 0 & 0 & 0 & 0 & x y x y & 0 & 0 & x y y x \\ 0 & 0 & 0 & z y y z & 0 & 0 & z y z y & 0 & 0 \\ 0 & 0 & 0 & 0 & x z z x & 0 & 0 & \text { xzxz } & 0 \\ 0 & 0 & 0 & 0 & 0 & \text { yxxy } & 0 & 0 & \text { yxyx }\end{array}\right)$

$\left[V^{4}\right] \quad\left(\begin{array}{ccccc}x x x x & 0 & 0 & y y z z & 0 \\ 0 & y y y y & 0 & z z x x & 0 \\ 0 & 0 & z z z z & x x y y & 0\end{array}\right)$

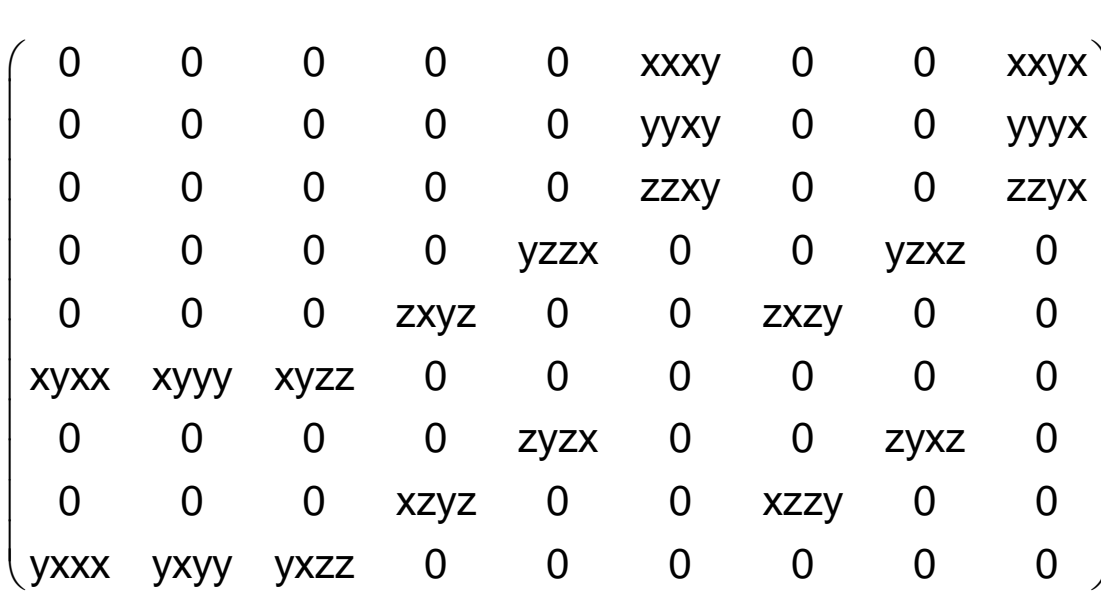

$\left(\begin{array}{ccccc}0 & x y y y & 0 & 0 & 0 \\ y x x x & 0 & 0 & 0 & 0 \\ 0 & 0 & 0 & 0 & z z x y\end{array}\right)$

$\mathrm{V}\left[\mathrm{V}^{3}\right] \quad\left(\begin{array}{cccccccccc}\mathrm{xxxx} & 0 & 0 & \mathrm{xxyy} & 0 & 0 & \mathrm{xxzz} & 0 & 0 & 0 \\ 0 & \mathrm{yyyy} & 0 & 0 & \mathrm{yyzz} & 0 & 0 & \mathrm{yyxx} & 0 & 0 \\ 0 & 0 & z z z & 0 & 0 & z z x & 0 & 0 & z z y & 0\end{array}\right)$

$\left(\begin{array}{llllllllll}0 & x y y y & 0 & 0 & x y z z & 0 & 0 & x y x x & 0 & 0\end{array}\right)$

VIV] $\quad \begin{array}{cccccccccc}0 & y y y y & 0 & 0 & y y z z & 0 & 0 & y y x x & 0 & 0 \\ 0 & 0 & z z z z & 0 & 0 & z z x x & 0 & 0 & z z y y & 0\end{array}$

$\left[\left[^{2}\right]^{2}\right]\left(\begin{array}{cccccc}\text { xxxx } & \text { xxyy } & \text { xxzz } & 0 & 0 & 0 \\ & \text { yyyy } & \text { yyzz } & 0 & 0 & 0 \\ & & \text { zzzz } & 0 & 0 & 0 \\ & & & \text { yzyz } & 0 & 0 \\ & & & & \text { xzxz } & 0 \\ & & & & & \text { xyxy }\end{array}\right)$

$\left.\mathbb{N}^{2}\right]^{2}\left(\begin{array}{cccccc}x x x x & x x y y & x x z z & 0 & 0 & 0 \\ y y x x & y y y y & y y z z & 0 & 0 & 0 \\ z z x x & z z y y & z z z z & 0 & 0 & 0 \\ 0 & 0 & 0 & y z y z & 0 & 0 \\ 0 & 0 & 0 & 0 & x z x z & 0 \\ 0 & 0 & 0 & 0 & 0 & x y x y\end{array}\right)$ $\left(\begin{array}{cccccccccc}0 \mathrm{yxxx} & 0 & 0 & \mathrm{yxyy} & 0 & 0 & \mathrm{yxzz} & 0 & 0 & 0 \\ 0 & 0 & 0 & 0 & 0 & 0 & 0 & 0 & 0 & \mathrm{zxyz}\end{array}\right)$

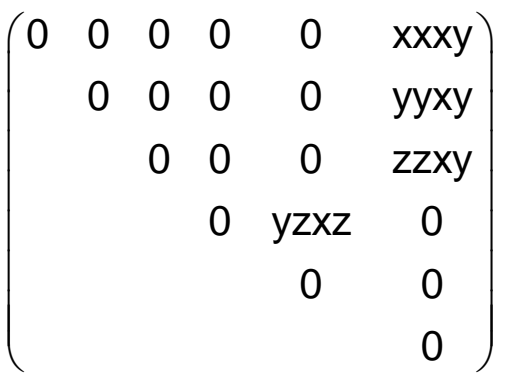

$\left(\begin{array}{llllll}0 & 0 & 0 & 0 & 0 & x x x y \\ 0 & 0 & 0 & 0 & 0 & y x y\end{array}\right)$

$\begin{array}{llllll}0 & 0 & 0 & 0 & 0 & \text { yyxy } \\ 0 & 0 & 0 & 0 & 0 & z z x y\end{array}$

$\begin{array}{cccccc}0 & 0 & 0 & 0 & 0 & z z x y\end{array}$

$\left.\begin{array}{cccccc}0 & 0 & 0 & 0 & 0 & z z x \\ 0 & 0 & 0 & 0 & y z x z & 0 \\ 0 & 0 & 0 & x z y z & 0 & 0 \\ 0 & 0 & 0 & 0 & 0 & 0\end{array}\right)$

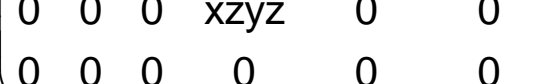

a

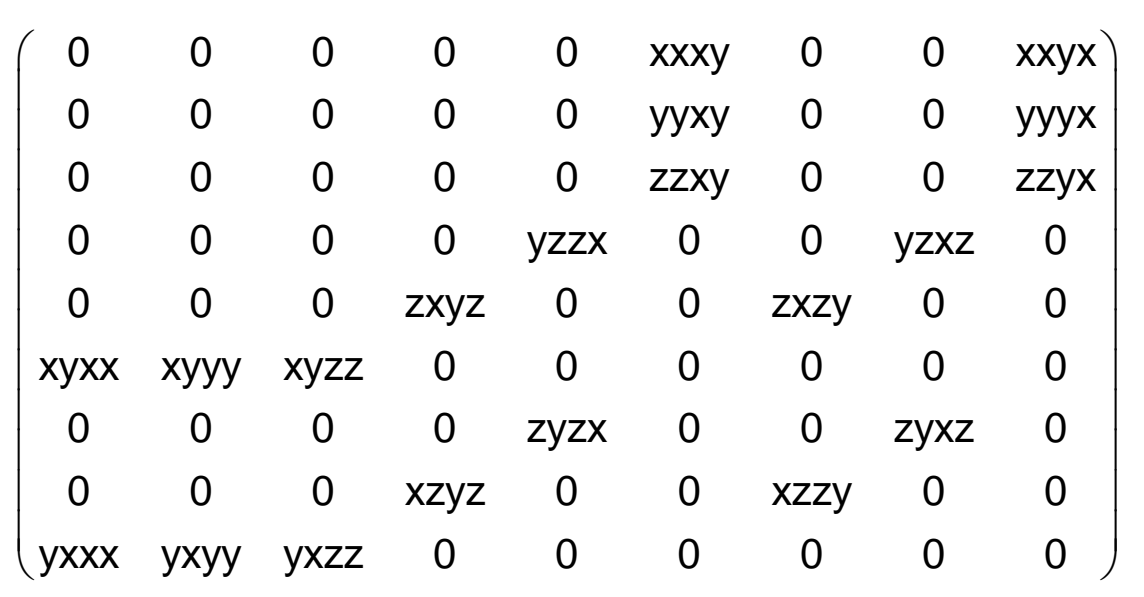

$\left(\begin{array}{ccccc}0 & x y y y & 0 & 0 & 0 \\ y x x x & 0 & 0 & 0 & 0 \\ 0 & 0 & 0 & 0 & z z x y\end{array}\right)$

$\left(\begin{array}{cccccccccc}0 & \text { xyyy } & 0 & 0 & x y z z & 0 & 0 & x y x x & 0 & 0 \\ 0 & 0 & 0 & y x y & 0 & 0 & y x z & 0 & 0 & 0\end{array}\right)$

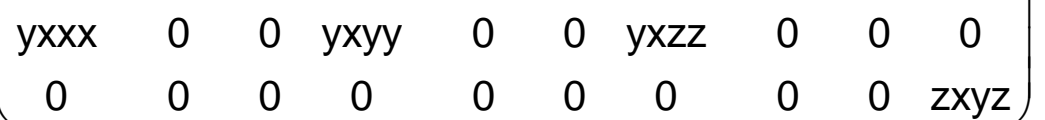

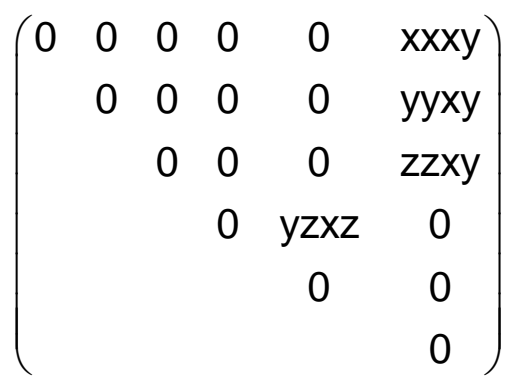

$\left(\begin{array}{llllll}0 & 0 & 0 & 0 & 0 & x x x y \\ 0 & 0 & 0 & 0 & 0 & y x y\end{array}\right.$ $\begin{array}{llllll}0 & 0 & 0 & 0 & 0 & y y x y \\ 0 & 0 & 0 & 0 & 0 & z x y\end{array}$ $\begin{array}{cccccc}0 & 0 & 0 & 0 & 0 & z z x y \\ 0 & 0 & 0 & 0 & y z x z & 0 \\ 0 & 0 & 0 & x z y z & 0 & 0\end{array}$ $\begin{array}{llllcl}0 & 0 & 0 & x z y z & 0 & 0\end{array}$ $\begin{array}{llccccc}0 & 0 & 0 & x z y z & 0 & 0 \\ 0 & 0 & 0 & 0 & 0 & 0\end{array}$ $\left(\begin{array}{lllllllll}x x x x & x x y y & x x z z & 0 & 0 & 0 & 0 & 0 & 0 \\ y x x & y y y & y y z z & 0 & 0 & 0 & 0 & 0 & 0\end{array}\right)$ $\begin{array}{lllllllll}\mathrm{yyxx} & \mathrm{yyyy} & \mathrm{yyzz} & 0 & 0 & 0 & 0 & 0 & 0 \\ \mathrm{zzxx} & \mathrm{zzyy} & \mathrm{zzzz} & 0 & 0 & 0 & 0 & 0 & 0\end{array}$ $\begin{array}{lllllllll}0 & 0 & 0 & y z y z & 0 & 0 & y z z y & 0 & 0\end{array}$ $\begin{array}{ccccccccc}0 & 0 & 0 & 0 & z x z x & 0 & 0 & z x x z & 0 \\ 0 & 0 & 0 & 0 & 0 & x y x y & 0 & 0 & x y y x \\ 0 & 0 & 0 & z w z & 0 & 0 & z y z y & 0 & 0\end{array}$

$\begin{array}{lllllllll}0 & 0 & 0 & \text { zyyz } & 0 & 0 & \text { zyzy } & 0 & 0\end{array}$ $\begin{array}{ccccccccc}0 & 0 & 0 & z y y z & 0 & 0 & z y z y & 0 & 0 \\ 0 & 0 & 0 & 0 & x z z x & 0 & 0 & x z x z & 0\end{array}$ $\begin{array}{lllllllll}0 & 0 & 0 & 0 & 0 & y \mathrm{xxy} & 0 & 0 & \mathrm{yxyx}\end{array}$

$\left(\begin{array}{ccccc}x x x x & 0 & 0 & y y z z & 0 \\ 0 & y y y y & 0 & z z x x & 0 \\ 0 & 0 & z z z z & x x y y & 0\end{array}\right)$

$\left(\begin{array}{cccccccccc}x x x x & 0 & 0 & x x y y & 0 & 0 & x x z z & 0 & 0 & 0 \\ 0 & y y y y & 0 & 0 & y y z z & 0 & 0 & y y x x & 0 & 0\end{array}\right)$ $\left(\begin{array}{cccccccccc}0 & y y y y & 0 & 0 & y y z z & 0 & 0 & y y x x & 0 & 0 \\ 0 & 0 & z z z z & 0 & 0 & z z x x & 0 & 0 & z z y y & 0\end{array}\right)$

$$
\left(\begin{array}{cccccc}
x x x x & x x y y & x x z z & 0 & 0 & 0 \\
& \text { yyyy } & \text { yyzz } & 0 & 0 & 0 \\
& & \text { zzzz } & 0 & 0 & 0 \\
& & & y z y z & 0 & 0 \\
& & & & \text { xzxz } & 0 \\
& & & & & \text { xyxy }
\end{array}\right)
$$

$\left(\begin{array}{llllll}x x x x & x x y y & x x z z & 0 & 0 & 0 \\ y x x & y y y & y y z z & 0 & 0 & 0\end{array}\right)$ $\begin{array}{llllll}\text { yyxx } & y y y y & y y z z & 0 & 0 & 0\end{array}$ $\begin{array}{llllll}\text { zzxx } & \text { zzyy } & \text { zzzz } & 0 & 0 & 0\end{array}$ $\begin{array}{cccccc}0 & 0 & 0 & y z y z & 0 & 0 \\ 0 & 0 & 0 & 0 & x z x z & 0\end{array}$ $\left.\begin{array}{cccccc}0 & 0 & 0 & 0 & x z x z & 0 \\ 0 & 0 & 0 & 0 & 0 & x y x y\end{array}\right)$ 
$\mathrm{n}=2 \quad \mathbf{C}_{2 \mathrm{v}}\left(\mathbf{C}_{2}\right)=\mathbf{2}_{\mathrm{z}} \mathbf{m}_{x} \mathbf{m}_{\mathrm{y}}\left(\mathbf{2}_{\mathrm{z}}\right)=\mathbf{2}_{\mathrm{z}} \mathbf{m}_{\mathrm{x}}{ }^{\prime} \mathbf{m}_{\mathrm{y}}{ }^{\prime}$

$\mathbf{C}_{\mathrm{nv}}\left(\mathbf{C}_{\mathrm{n}}\right)$

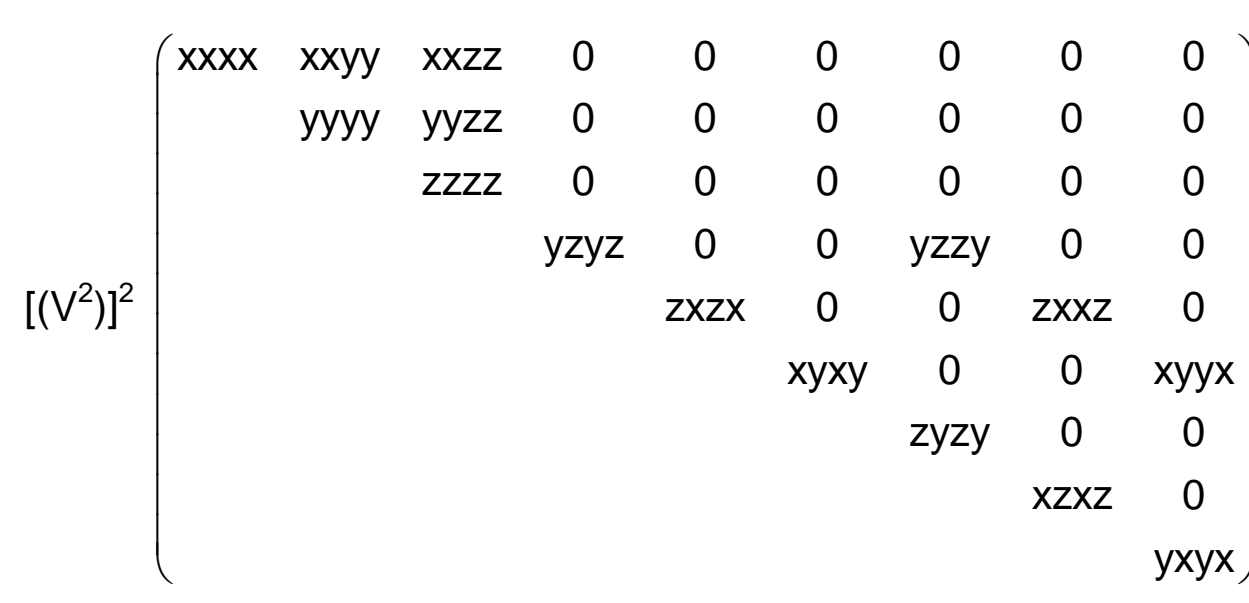

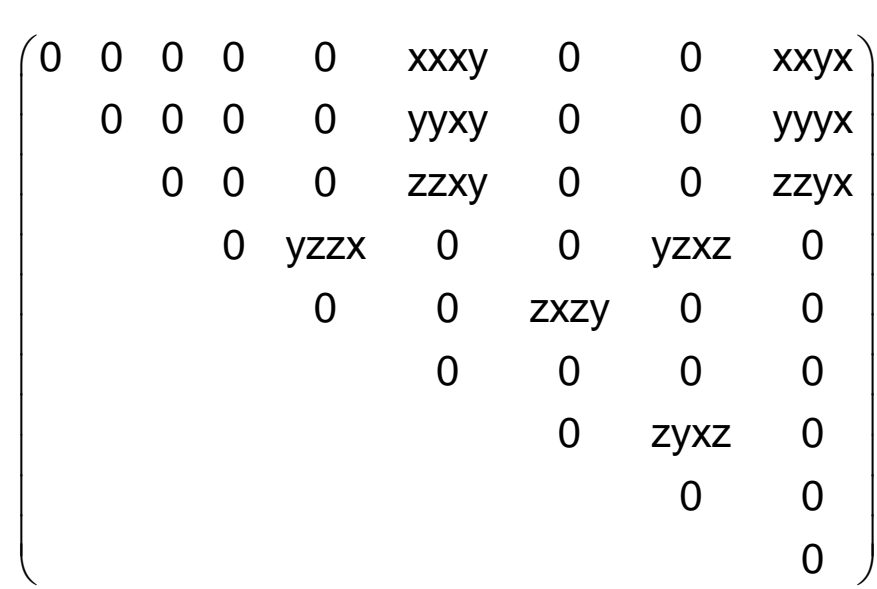

$\left[\mathrm{V}^{2}\right] \mathrm{V}^{2}\left(\begin{array}{ccccccccc}\mathrm{x} x \mathrm{x} & \mathrm{x} x \mathrm{y} y & \mathrm{xxzz} & 0 & 0 & 0 & 0 & 0 & 0 \\ \mathrm{yyxx} & \mathrm{yyyy} & \mathrm{yyzz} & 0 & 0 & 0 & 0 & 0 & 0 \\ \mathrm{zzxx} & \mathrm{zzyy} & \mathrm{zzzz} & 0 & 0 & 0 & 0 & 0 & 0 \\ 0 & 0 & 0 & \mathrm{yzyz} & 0 & 0 & \mathrm{yzzy} & 0 & 0 \\ 0 & 0 & 0 & 0 & \mathrm{zxzx} & 0 & 0 & \mathrm{zxxz} & 0 \\ 0 & 0 & 0 & 0 & 0 & \mathrm{xyxy} & 0 & 0 & \mathrm{xyyx}\end{array}\right)$

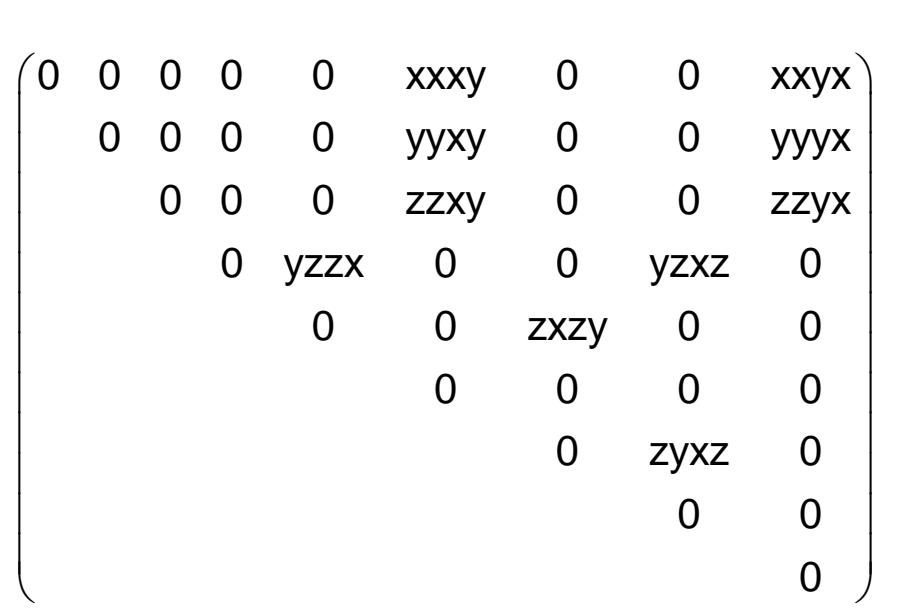

$\left(\begin{array}{ccccccccc}0 & 0 & 0 & 0 & 0 & \text { xxxy } & 0 & 0 & \text { xxyx } \\ 0 & 0 & 0 & 0 & 0 & \text { yyxy } & 0 & 0 & \text { yyyx } \\ 0 & 0 & 0 & 0 & 0 & \text { zzxy } & 0 & 0 & \text { zzyx } \\ 0 & 0 & 0 & 0 & \text { yzzx } & 0 & 0 & \text { yzxz } & 0 \\ 0 & 0 & 0 & \text { xzyz } & 0 & 0 & \text { zxzy } & 0 & 0 \\ \text { xyxx } & \text { xyyy } & \text { xyzz } & 0 & 0 & 0 & 0 & 0 & 0\end{array}\right)$

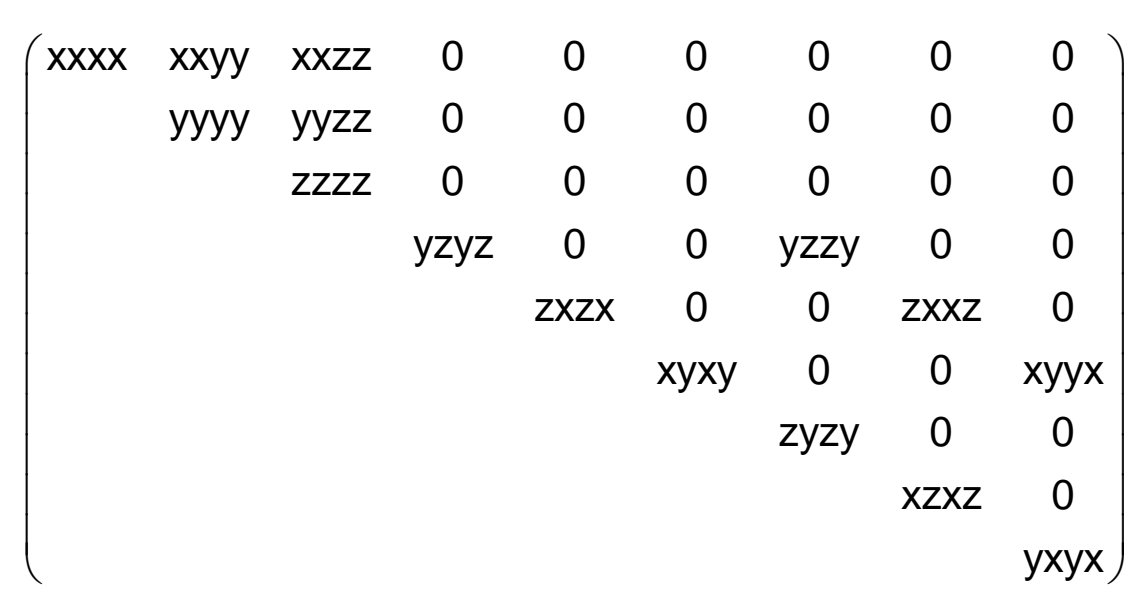

$\left(\begin{array}{ccccccccc}\text { xxxx } & \text { xxyy } & \text { xxzz } & 0 & 0 & 0 & 0 & 0 & 0 \\ \text { yyxx } & \text { yyyy } & \text { yyzz } & 0 & 0 & 0 & 0 & 0 & 0 \\ \text { zzxx } & \text { zzyy } & \text { zzzz } & 0 & 0 & 0 & 0 & 0 & 0 \\ 0 & 0 & 0 & \text { yzyz } & 0 & 0 & \text { yzzy } & 0 & 0 \\ 0 & 0 & 0 & 0 & \text { zxzx } & 0 & 0 & \text { zxxz } & 0 \\ 0 & 0 & 0 & 0 & 0 & \text { xyxy } & 0 & 0 & \text { xyyx }\end{array}\right)$ 

and: $x x x x=x x y y+x y x y+x y y x$

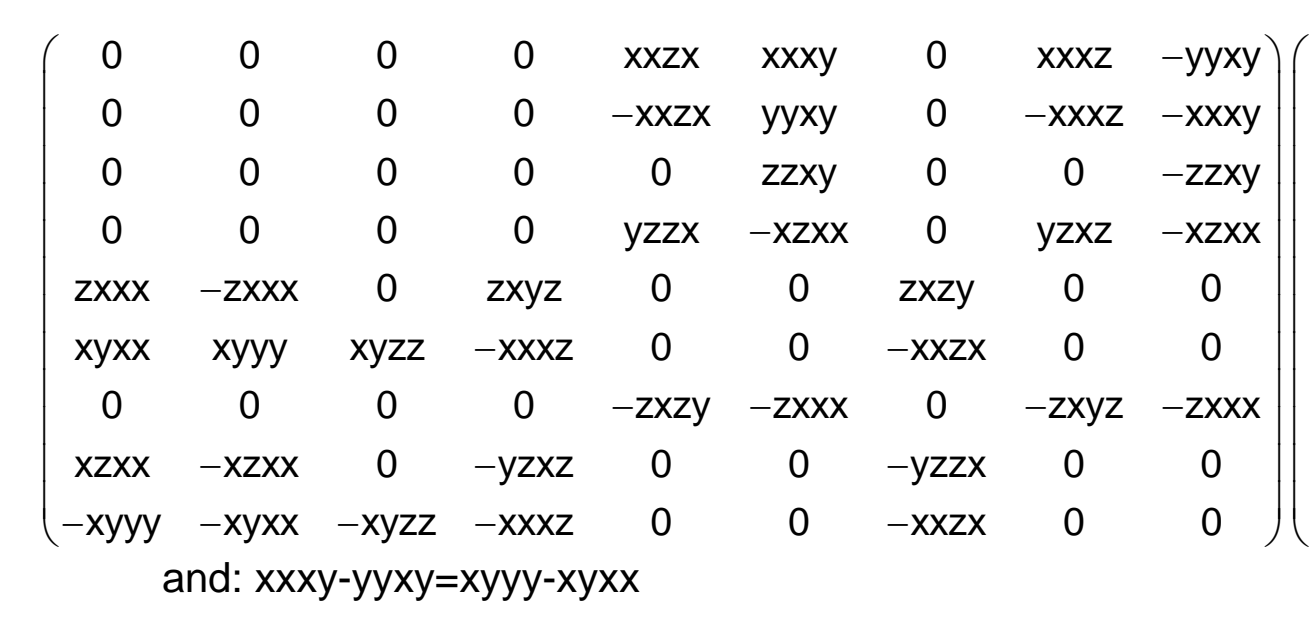

$\begin{array}{lllll}x x z x & x x x y & 0 & x x x z & -y y x y \\ -x x z x & y y x y & 0 & -x x y z & -x x y y\end{array}\left(\begin{array}{ccccccccc}x x x x & x x y y & x x z z & x x y z & 0 & 0 & x x z y & 0 & 0 \\ y y x x & x y x x & y y z z & -x y y z & 0 & 0 & -x x z y & 0 & 0\end{array}\right.$ $\left.\begin{array}{ccccc|ccccccccc}-x x z x & y y x y & 0 & -x x x z & -x x x y & y y x x & x x x x & y y z z & -x x y z & 0 & 0 & -x x z y & 0 & 0 \\ 0 & z z x y & 0 & 0 & -z z x y & z z x x & z z x x & z z z z & 0 & 0 & 0 & 0 & 0 & 0\end{array}\right)$ \begin{tabular}{lllll|lllllllll}
$y z z x$ & $-x z x x$ & 0 & $y z x z$ & $-x z x x$ & $y z x x$ & $-y z x x$ & 0 & $y z y z$ & 0 & 0 & $y z z y$ & 0 & 0
\end{tabular} \begin{tabular}{llllll|lllllllll}
$z x y z$ & 0 & 0 & $z x z y$ & 0 & 0 & 0 & 0 & 0 & 0 & $z x z x$ & $z y x x$ & 0 & $z x x z$ & $z y x x$
\end{tabular}

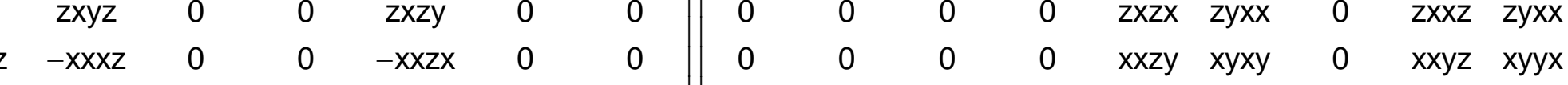

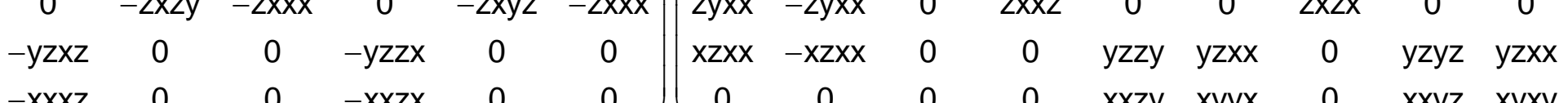
and: $x x x y-y y x y=x y y y-x y x x$

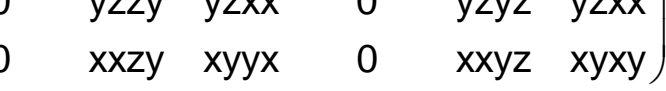
and: $x x x x=x x y y+x y x y+x y y x$

$\left[V^{4}\right] \quad\left(\begin{array}{ccccc}3 x x y y & 0 & 0 & y y z z & x x y z \\ 0 & 3 x x y y & 0 & y y z z & 0 \\ 0 & -x x y z & z z z z & x x y y & 0\end{array}\right)$

$\left(\begin{array}{ccccc}0 & 0 & 0 & 0 & 0 \\ 0 & 0 & 0 & 0 & -z x x x \\ z x x x & 0 & 0 & 0 & 0\end{array}\right)$

$\left(\begin{array}{ccccc}0 & 0 & 0 & 0 & 0 \\ 0 & 0 & 0 & 0 & -z x x x \\ z x x x & 0 & 0 & 0 & 0\end{array}\right)$

$\left(\begin{array}{ccccc}3 x x y y & 0 & 0 & y y z z & x x y z \\ 0 & 3 x x y y & 0 & y y z z & 0 \\ 0 & -x x y z & z z z z & x x y y & 0\end{array}\right)$

$\left.V_{V} V^{3}\right]\left(\begin{array}{cccccccccc}3 x x y y & 0 & 0 & x x y y & 0 & 0 & y y z z & 0 & 0 & y z x x \\ 0 & 3 x x y y & 0 & 0 & y y z z & y z x x & 0 & x x y y & -y z x x & 0 \\ 0 & -z y x x & z z z z & 0 & 0 & z z x & 0 & z y x & z z x & 0\end{array}\right)$ $\left(\begin{array}{cccccccccc}0 & 3 x y x x & 0 & 0 & x y z z & x z x x & 0 & x y x x & -x z x x & 0 \\ -3 x y x x & 0 & 0 & -x y x x & 0 & 0 & -x y z z & 0 & 0 & -x z x x \\ z x x x & 0 & 0 & -z x x x & 0 & 0 & 0 & 0 & 0 & z x y z\end{array}\right)\left(\begin{array}{ccccccccccc}0 & 3 x y x x & 0 & 0 & x y z z & x z x x & 0 & x y x x & -x z x x & 0 \\ -3 x y x x & 0 & 0 & -x y x x & 0 & 0 & -x y z z & 0 & 0 & -x z x x \\ z x x x & 0 & 0 & -z x x x & 0 & 0 & 0 & 0 & 0 & z x y z\end{array}\right)\left(\begin{array}{ccccccccccc}3 x x y y & 0 & 0 & x x y y & 0 & 0 & y y z z & 0 & 0 & y z x x \\ 0 & 3 x x y y & 0 & 0 & y y z z & y z x x & 0 & x x y y & -y z x x & 0 \\ 0 & -z y x x & z z z z & 0 & 0 & z z x x & 0 & z y x x & z z x x & 0\end{array}\right)$ $\left(\begin{array}{cccccccccc}0 & 3 x y x x & 0 & 0 & x y z z & x z x x & 0 & x y x x & -x z x x & 0 \\ -3 x y x x & 0 & 0 & -x y x x & 0 & 0 & -x y z z & 0 & 0 & -x z x x \\ z x x x & 0 & 0 & -z x x x & 0 & 0 & 0 & 0 & 0 & z x y z\end{array}\right)\left(\begin{array}{ccccccccccc}0 & 3 x y x x & 0 & 0 & x y z z & x z x x & 0 & x y x x & -x z x x & 0 \\ -3 x y x x & 0 & 0 & -x y x x & 0 & 0 & -x y z z & 0 & 0 & -x z x x \\ z x x x & 0 & 0 & -z x x x & 0 & 0 & 0 & 0 & 0 & z x y z\end{array}\right)\left(\begin{array}{ccccccccccc}3 x x y y & 0 & 0 & x x y y & 0 & 0 & y y z z & 0 & 0 & y z x x \\ 0 & 3 x x y y & 0 & 0 & y y z z & y z x x & 0 & x x y y & -y z x x & 0 \\ 0 & -z y x x & z z z z & 0 & 0 & z z x x & 0 & z y x x & z z x x & 0\end{array}\right)$ $\left(\begin{array}{cccccccccc}0 & 3 x y x x & 0 & 0 & x y z z & x z x x & 0 & x y x x & -x z x x & 0 \\ -3 x y x x & 0 & 0 & -x y x x & 0 & 0 & -x y z z & 0 & 0 & -x z x x \\ z x x x & 0 & 0 & -z x x x & 0 & 0 & 0 & 0 & 0 & z x y z\end{array}\right)\left(\begin{array}{ccccccccccc}0 & 3 x y x x & 0 & 0 & x y z z & x z x x & 0 & x y x x & -x z x x & 0 \\ -3 x y x x & 0 & 0 & -x y x x & 0 & 0 & -x y z z & 0 & 0 & -x z x x \\ z x x x & 0 & 0 & -z x x x & 0 & 0 & 0 & 0 & 0 & z x y z\end{array}\right)\left(\begin{array}{ccccccccccc}3 x x y y & 0 & 0 & x x y y & 0 & 0 & y y z z & 0 & 0 & y z x x \\ 0 & 3 x x y y & 0 & 0 & y y z z & y z x x & 0 & x x y y & -y z x x & 0 \\ 0 & -z y x x & z z z z & 0 & 0 & z z x x & 0 & z y x x & z z x x & 0\end{array}\right)$

$\left[\left[V^{2}\right]^{2}\right]\left(\begin{array}{cccccc}x x x x & x x y y & x x z z & x x y z & 0 & 0 \\ & x x x x & x x z z & -x x y z & 0 & 0 \\ & & z z z z & 0 & 0 & 0 \\ & & & y z y z & 0 & 0 \\ & & & & y z y z & x x y z \\ & & & & & x y x y\end{array}\right)$
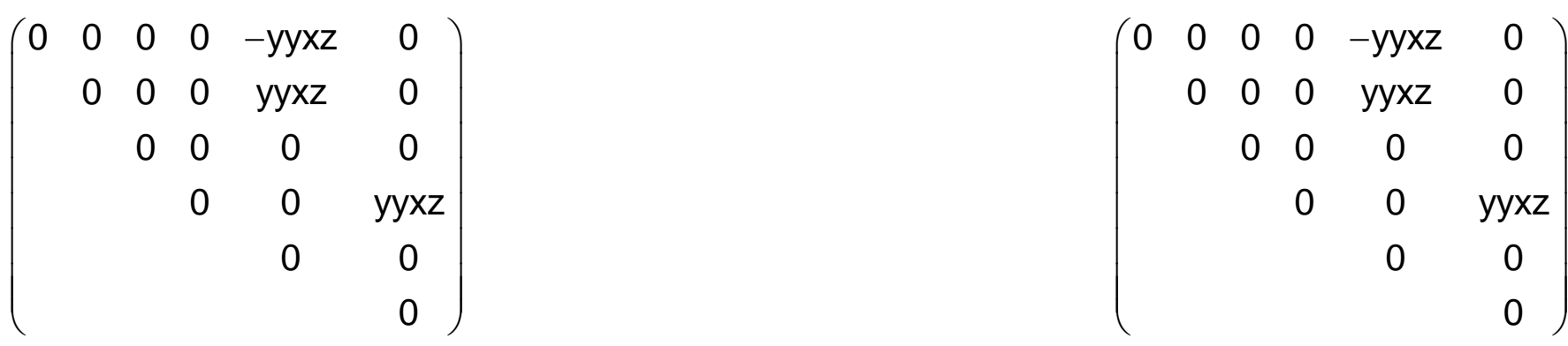

and $x y x y=1 / 2(x x x x-x x y y)$

$\left[V^{2}\right]^{2}\left(\begin{array}{cccccc}x x x x & x x y y & x x z z & x x y z & 0 & 0 \\ x x y y & x x x x & x x z z & -x x y z & 0 & 0 \\ z z x x & z z x x & z z z z & 0 & 0 & 0 \\ y z x x & -y z x x & 0 & y z y z & 0 & 0 \\ 0 & 0 & 0 & 0 & y z y z & y z x x \\ 0 & 0 & 0 & 0 & x x y z & x y x y\end{array}\right)$
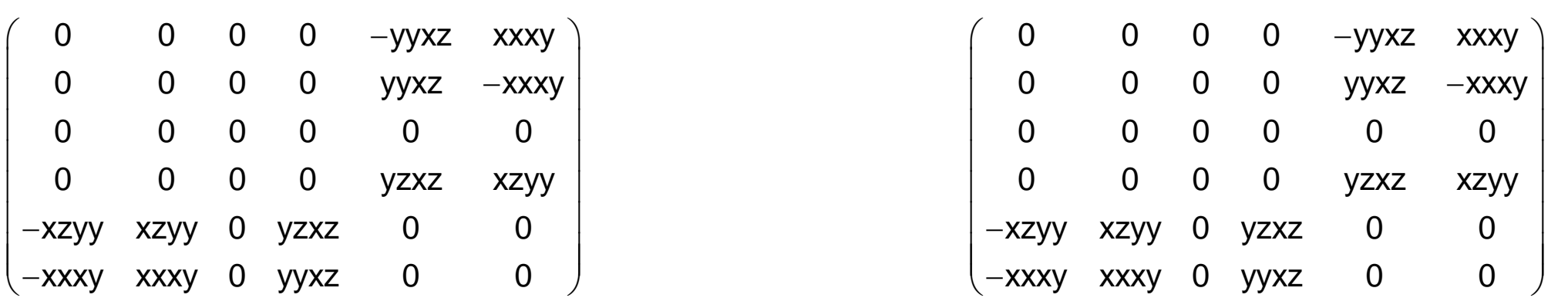

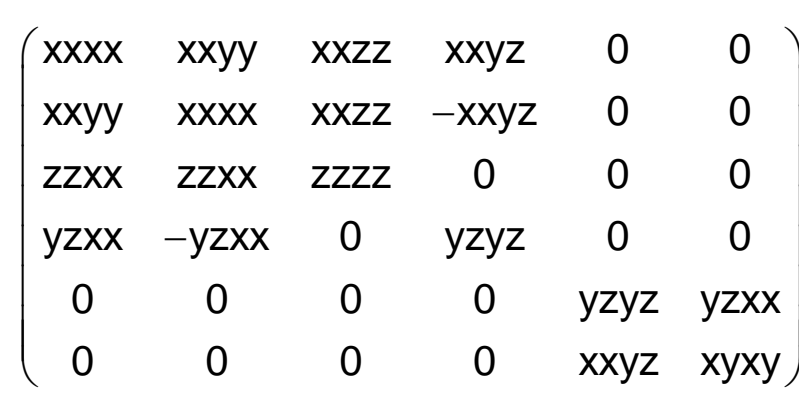

and $x y x y=1 / 2(x x x x-x x y y)$ and $x y x y=1 / 2(x x x x-x x y y)$

and $x y x y=1 / 2(x x x x-x x y y)$ 


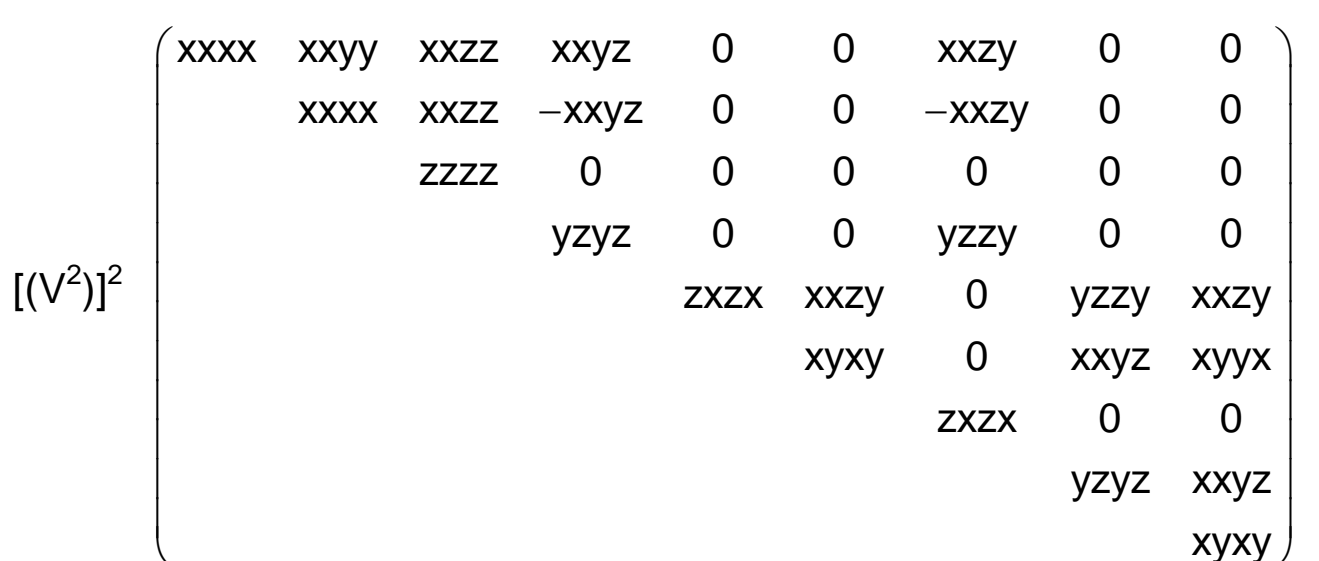

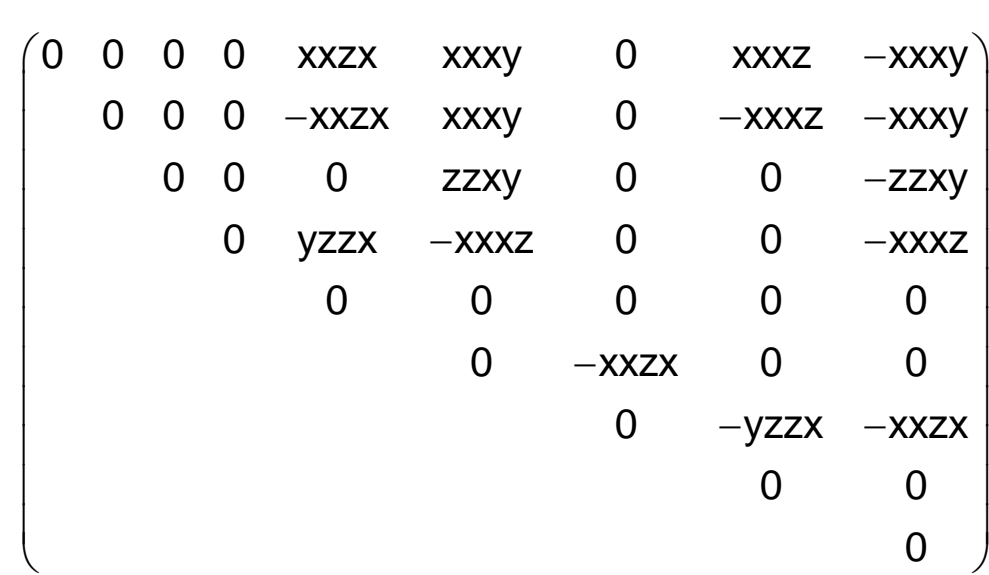

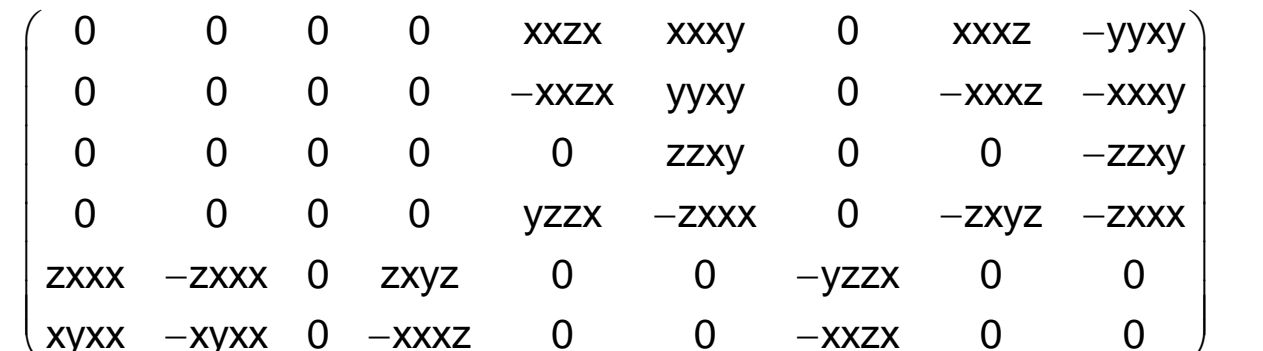

$\begin{array}{ccccccccc}z x x x & -z x x x & 0 & z x y z & 0 & 0 & -y z z x & 0 & 0 \\ x y x x & -x y x x & 0 & -x x y z & 0 & 0 & -x x z x & 0 & 0\end{array}$

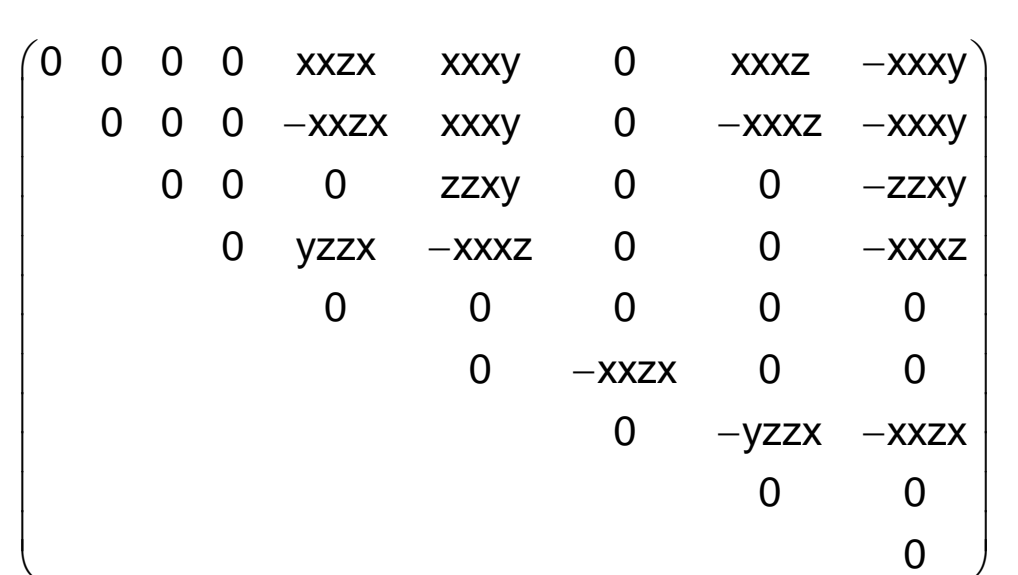

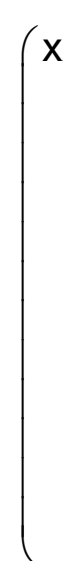

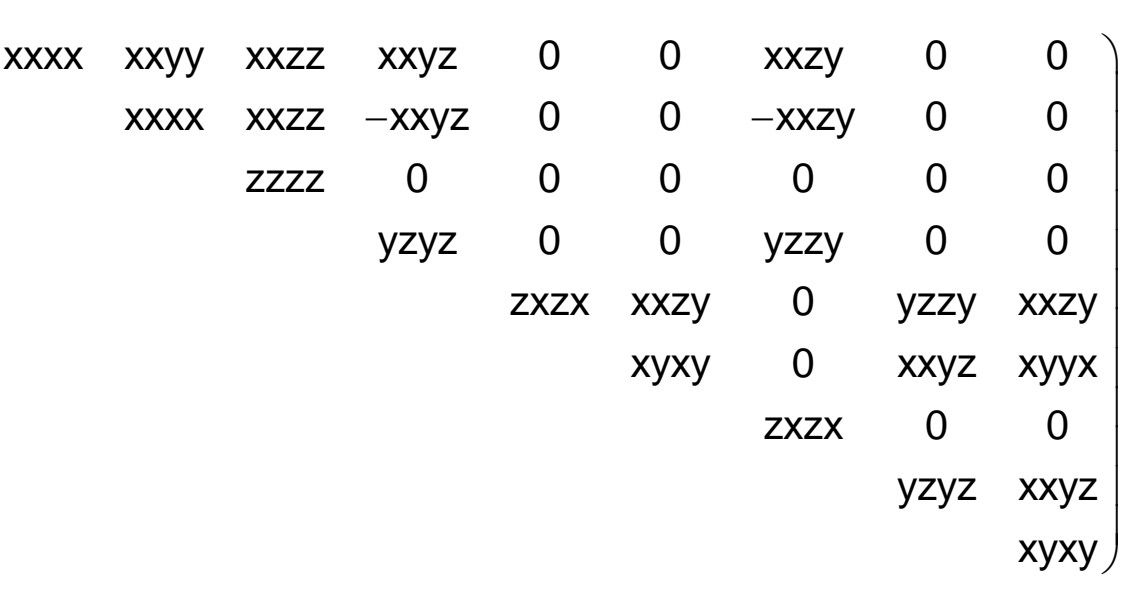

and $x x x x=x x y y+y z y z+z y z y$

$\left[\mathrm{V}^{2}\right] \mathrm{V}^{2}\left(\begin{array}{ccccccccc}\mathrm{x} x \mathrm{y} & \mathrm{xxxx} & \mathrm{xxzz} & -\mathrm{xxyz} & 0 & 0 & -x \mathrm{xzy} & 0 & 0 \\ \mathrm{zzxx} & \mathrm{zzxx} & \mathrm{zzzz} & 0 & 0 & 0 & 0 & 0 & 0 \\ \mathrm{yzxx} & -\mathrm{yzxx} & 0 & \mathrm{yzyz} & 0 & 0 & \mathrm{zxzx} & 0 & 0 \\ 0 & 0 & 0 & 0 & \mathrm{zxzx} & \mathrm{yzxx} & 0 & \mathrm{yzyz} & \mathrm{yzxx} \\ 0 & 0 & 0 & 0 & \mathrm{x} z \mathrm{y} & \mathrm{xyy} & 0 & \mathrm{xxyz} & \mathrm{xyy}\end{array}\right)$

and $x x x x=x x y y+2 x y x y$

and: $y y x y-x x x y=2 x y x x$

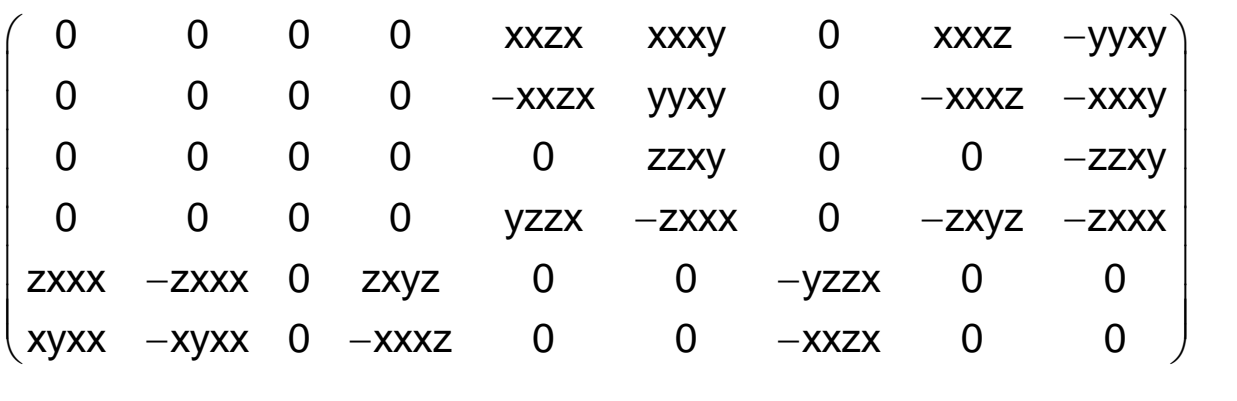

and: $y y x y-x x x y=2 x y x x$ $\left.\begin{array}{lllllllll}x x x x & x x y y & x x z z & x x y z & 0 & 0 & x x z y & 0 & 0 \\ x x y y & x x y x & x x z z & -x x y z & 0 & 0 & -x x z y & 0 & 0\end{array}\right)$ $\left.\begin{array}{ccccccccc}x x x y & x x x x & x x z z & -x x y z & 0 & 0 & -x x z y & 0 & 0 \\ z z x x & z z x x & z z z z & 0 & 0 & 0 & 0 & 0 & 0 \\ y z x x & -y z x & 0 & y z y z & 0 & 0 & z x z x & 0 & 0\end{array}\right)$ $\begin{array}{lllllllll}y z x x & -y z x x & 0 & y z y z & 0 & 0 & z x z x & 0 & 0\end{array}$

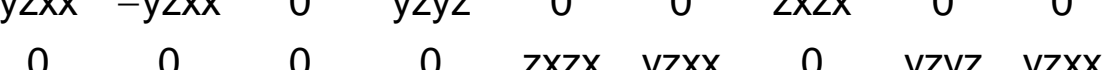
$\begin{array}{cccccccc}0 & 0 & 0 & z x z x & y z x x & 0 & y z y z & y z x x \\ 0 & 0 & 0 & x x z y & x y x y & 0 & x x y z & x y x y\end{array}$ and $x x x x=x x y y+2 x y x y$ 
$\left(\begin{array}{lllllllll}x x x x & x x y y & x x z z & 0 & 0 & 0 & 0 & 0 & 0 \\ y x x & x x x & x x z z & 0 & 0 & 0 & 0 & 0 & 0\end{array}\right)$

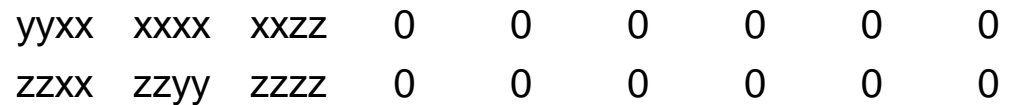

\begin{tabular}{l|ccccccccc}
$\mathrm{V}^{4}$ & 0 & 0 & 0 & $\mathrm{yzyz}$ & 0 & 0 & $\mathrm{yzzy}$ & 0 & 0 \\
0 & 0 & 0 & 0 & $\mathrm{zxzx}$ & 0 & 0 & $\mathrm{zxxz}$ & 0
\end{tabular} $\begin{array}{ccccccccc}0 & 0 & 0 & 0 & 0 & x y x y & 0 & 0 & x y y x \\ 0 & 0 & 0 & z x y z & 0 & 0 & z x z & 0 & 0\end{array}$ $\left.\begin{array}{ccccccccc}0 & 0 & 0 & z x x z & 0 & 0 & z x z x & 0 & 0 \\ 0 & 0 & 0 & 0 & y z z y & 0 & 0 & y z y z & 0 \\ 0 & 0 & 0 & 0 & 0 & x y y x & 0 & 0 & x y x y\end{array}\right)$ $\begin{array}{lllllllll}0 & 0 & 0 & 0 & 0 & \text { xyyx } & 0 & 0 & \text { xyxy }\end{array}$ $\left(\begin{array}{ccccccccc}0 & 0 & 0 & 0 & 0 & \text { xxxy } & 0 & 0 & - \text { yyxy } \\ 0 & 0 & 0 & 0 & 0 & \text { yyxy } & 0 & 0 & - \text {-xxy } \\ 0 & 0 & 0 & 0 & 0 & \text { zzxy } & 0 & 0 & - \text { zzxy } \\ 0 & 0 & 0 & 0 & \text { yzzx } & 0 & 0 & \text { yzxz } & 0 \\ 0 & 0 & 0 & \text { zxyz } & 0 & 0 & \text { zxzy } & 0 & 0 \\ \text { xyxx } & \text { xyyy } & \text { xyzz } & 0 & 0 & 0 & 0 & 0 & 0 \\ 0 & 0 & 0 & 0 & -z x z y & 0 & 0 & -z x y z & 0 \\ 0 & 0 & 0 & -y z x z & 0 & 0 & -y z z x & 0 & 0 \\ -x y y y & -x y x x & -x y z z & 0 & 0 & 0 & 0 & 0 & 0\end{array}\right)$ $\left(\begin{array}{ccccccccc}0 & 0 & 0 & 0 & 0 & \text { xxxy } & 0 & 0 & - \text { yyxy } \\ 0 & 0 & 0 & 0 & 0 & \text { yyxy } & 0 & 0 & - \text { xxxy } \\ 0 & 0 & 0 & 0 & 0 & \text { zzxy } & 0 & 0 & - \text { zzxy } \\ 0 & 0 & 0 & 0 & \text { yzzx } & 0 & 0 & \text { yzxz } & 0 \\ 0 & 0 & 0 & \text { zxyz } & 0 & 0 & \text { zxzy } & 0 & 0 \\ \text { xyxx } & \text { xyyy } & \text { xyzz } & 0 & 0 & 0 & 0 & 0 & 0 \\ 0 & 0 & 0 & 0 & -z x z y & 0 & 0 & -z x y z & 0 \\ 0 & 0 & 0 & -y z x z & 0 & 0 & -y z z x & 0 & 0 \\ -x y y y & -x y x x & -x y z z & 0 & 0 & 0 & 0 & 0 & 0\end{array}\right)$

$\begin{array}{lllllllll}x x x x & x x y y & x x z z & 0 & 0 & 0 & 0 & 0 & 0 \\ y x x & x y x & x x z & 0 & 0 & 0 & 0 & 0 & 0\end{array}$ $\left.\begin{array}{ccccccccc}y y x x & x x x x & x x z z & 0 & 0 & 0 & 0 & 0 & 0 \\ z z x x & z z y y & z z z z & 0 & 0 & 0 & 0 & 0 & 0 \\ 0 & 0 & 0 & y z y z & 0 & 0 & y z z & 0 & 0\end{array}\right)$ $\begin{array}{ccccccccc}0 & 0 & 0 & \text { yzyz } & 0 & 0 & \text { yzzy } & 0 & 0 \\ 0 & 0 & 0 & 0 & z x x & 0 & 0 & z x y z & 0 \\ 0 & 0 & 0 & 0 & 0 & x y x y & 0 & 0 & x y y x\end{array}$ $\begin{array}{ccccccccc}0 & 0 & 0 & 0 & z x z x & 0 & 0 & z x x z & 0 \\ 0 & 0 & 0 & 0 & 0 & x y x y & 0 & 0 & x y y x \\ 0 & 0 & 0 & z x y z & 0 & 0 & z x z x & 0 & 0\end{array}$ $\begin{array}{ccccccccc}0 & 0 & 0 & 0 & 0 & x y x y & 0 & 0 & x y y x \\ 0 & 0 & 0 & z x x z & 0 & 0 & z x z x & 0 & 0 \\ 0 & 0 & 0 & 0 & y z z & 0 & 0 & y z y z & 0\end{array}$ $\begin{array}{ccccccccc}0 & 0 & 0 & z x x z & 0 & 0 & z x z x & 0 & 0 \\ 0 & 0 & 0 & 0 & y z z y & 0 & 0 & y z y z & 0 \\ 0 & 0 & 0 & 0 & 0 & x y x & 0 & 0 & x y x y\end{array}$ $\left[V^{4}\right] \quad\left(\begin{array}{ccccc}x x x x & 0 & 0 & y y z z & 0 \\ 0 & x x x x & 0 & y y z z & 0 \\ 0 & 0 & z z z z & x x y y & 0\end{array}\right)$ $\left(\begin{array}{ccccc}0 & -y x x x & 0 & 0 & 0 \\ y x x x & 0 & 0 & 0 & 0 \\ 0 & 0 & 0 & 0 & 0\end{array}\right)$ $\left(\begin{array}{ccccc}0 & -y x x x & 0 & 0 & 0 \\ y x x x & 0 & 0 & 0 & 0 \\ 0 & 0 & 0 & 0 & 0\end{array}\right)$ $\left(\begin{array}{ccccc}\mathrm{xxxx} & 0 & 0 & \mathrm{yyzz} & 0 \\ 0 & \mathrm{xxxx} & 0 & \mathrm{yyzz} & 0 \\ 0 & 0 & \mathrm{zzzz} & \mathrm{xxyy} & 0\end{array}\right)$

$V\left[V^{3}\right]\left(\begin{array}{cccccccccc}\mathrm{xxxx} & 0 & 0 & \mathrm{xxyy} & 0 & 0 & \mathrm{yyzz} & 0 & 0 & 0 \\ 0 & \mathrm{xxxx} & 0 & 0 & \mathrm{yyzz} & 0 & 0 & \mathrm{xxyy} & 0 & 0 \\ 0 & 0 & z z z z & 0 & 0 & z z x & 0 & 0 & z z x x & 0\end{array}\right)$ $\left(\begin{array}{cccccccccc}0 & -y x x x & 0 & 0 & x y z z & 0 & 0 & x y x x & 0 & 0 \\ y x x & 0 & 0 & -x y y x & 0 & 0 & -x y z z & 0 & 0 & 0\end{array}\right)$

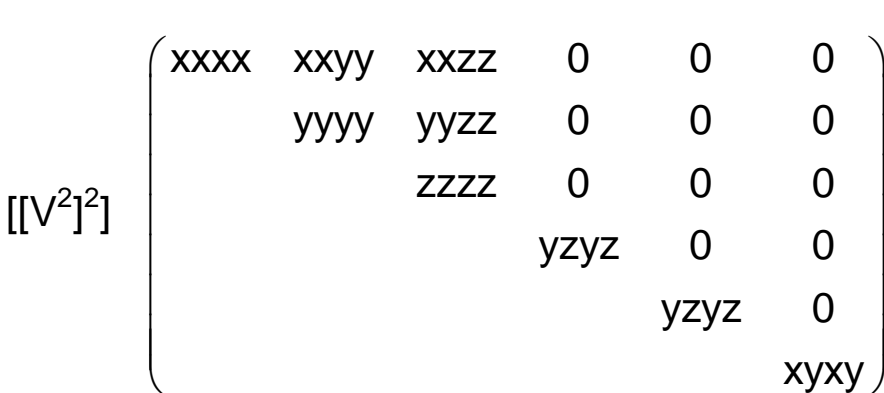
$\left(\begin{array}{cccccccccc}0 & 0 & 0 & 0 & 0 & 0 & 0 & 0 & 0 & z x y z\end{array}\right.$

$\left(\begin{array}{cccccccccc}0 & -y x x x & 0 & 0 & x y z z & 0 & 0 & x y x x & 0 & 0 \\ 0 & 0 & 0 & -x y x & 0 & 0 & -x y z z & 0 & 0 & 0\end{array}\right)$ $\left.\begin{array}{cccccccccc}y x x x & 0 & 0 & -x y x x & 0 & 0 & -x y z z & 0 & 0 & 0 \\ 0 & 0 & 0 & 0 & 0 & 0 & 0 & 0 & 0 & z x y z\end{array}\right)$

$$
\begin{aligned}
& \begin{array}{llllll}
0 & 0 & 0 & 0 & 0 & x x x y \\
0 & 0 & 0 & 0 & -x x y
\end{array} \\
& \begin{array}{lllll}
0 & 0 & 0 & 0 & -x x x y
\end{array} \\
& \begin{array}{llll}
0 & 0 & 0 & 0 \\
& 0 & 0 & 0
\end{array} \\
& \begin{array}{lll}
0 & 0 & 0 \\
& 0 & 0 \\
& & \\
& & 0
\end{array}
\end{aligned}
$$

$\left[\mathrm{V}^{2}\right]^{2}\left(\begin{array}{cccccc}\mathrm{x} x \mathrm{x} x & \mathrm{xxyy} & \mathrm{xxzz} & 0 & 0 & 0 \\ \mathrm{yyxx} & \mathrm{xxxx} & \mathrm{xxzz} & 0 & 0 & 0 \\ \mathrm{zzxx} & \mathrm{zzxx} & \mathrm{zzzz} & 0 & 0 & 0 \\ 0 & 0 & 0 & \mathrm{yzyz} & 0 & 0 \\ 0 & 0 & 0 & 0 & \mathrm{yzyz} & 0 \\ 0 & 0 & 0 & 0 & 0 & \mathrm{xyxy}\end{array}\right)$

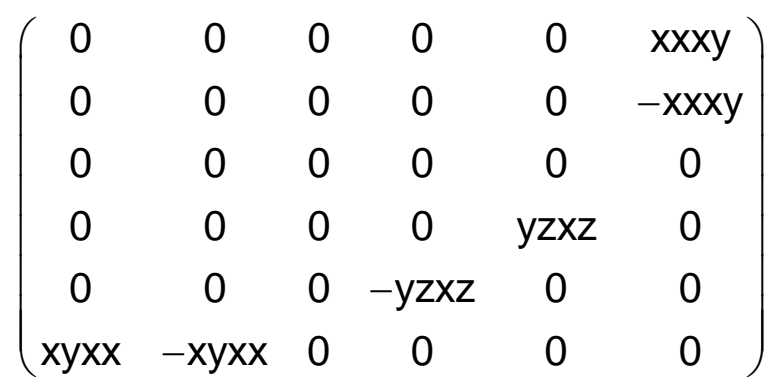

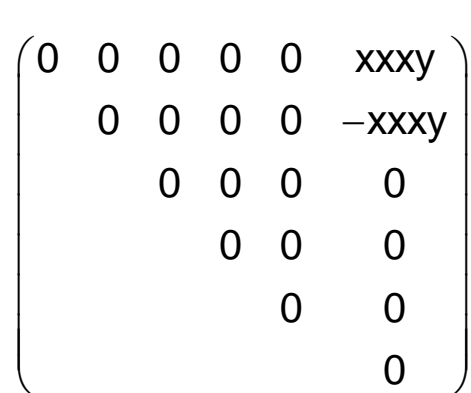

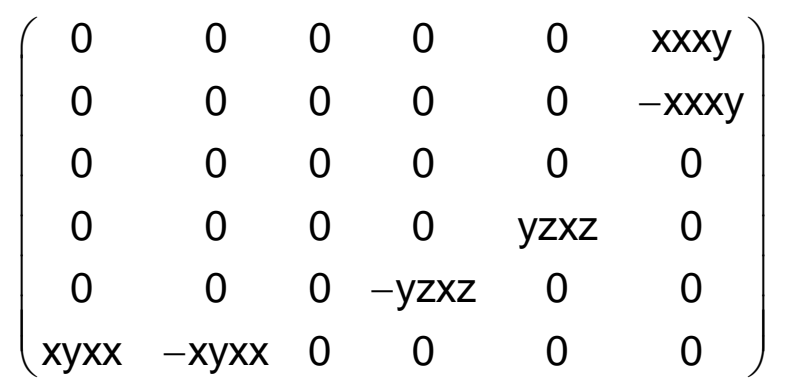

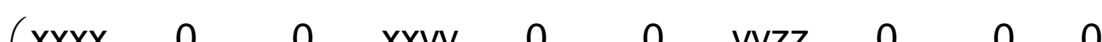
$\left.\begin{array}{cccccccccc}0 & x x x x & 0 & 0 & y y z z & 0 & 0 & x x y y & 0 & 0 \\ 0 & 0 & z z z z & 0 & 0 & z z x x & 0 & 0 & z z x x & 0\end{array}\right)$

$\left(\begin{array}{cccccc}x x x x & x x y y & x x z z & 0 & 0 & 0 \\ & \text { yyyy } & \text { yyzz } & 0 & 0 & 0 \\ & & \text { zzzz } & 0 & 0 & 0 \\ & & & y z y z & 0 & 0 \\ & & & & y z y z & 0 \\ & & & & & \text { xyxy }\end{array}\right)$

$\left(\begin{array}{cccccc}\text { xxxx } & x x y y & x x z z & 0 & 0 & 0 \\ y y x x & x x x x & x x z z & 0 & 0 & 0 \\ z z x x & z z x x & z z z z & 0 & 0 & 0 \\ 0 & 0 & 0 & y z y z & 0 & 0 \\ 0 & 0 & 0 & 0 & y z y z & 0 \\ 0 & 0 & 0 & 0 & 0 & x y x y\end{array}\right)$



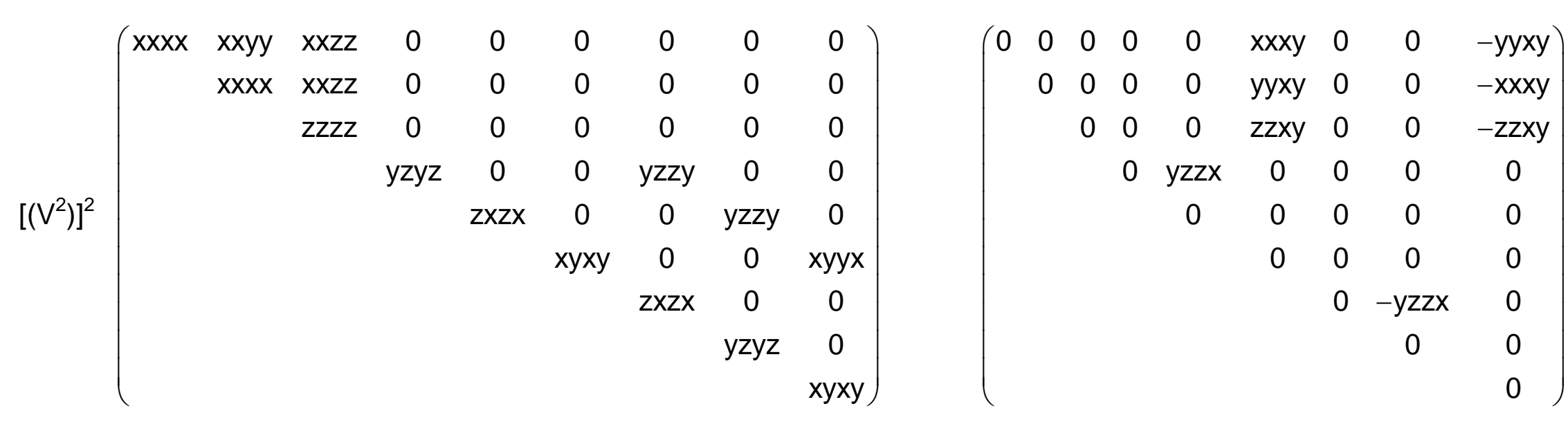

$\left[\mathrm{V}^{2}\right] \mathrm{V}^{2}\left(\begin{array}{ccccccccc}\mathrm{x} x \mathrm{x} & \mathrm{xxyy} & \mathrm{xxzz} & 0 & 0 & 0 & 0 & 0 & 0 \\ \mathrm{yyxx} & \mathrm{xxxx} & \mathrm{xxzz} & 0 & 0 & 0 & 0 & 0 & 0 \\ \mathrm{zzxx} & \mathrm{zzxx} & \mathrm{zzzz} & 0 & 0 & 0 & 0 & 0 & 0 \\ 0 & 0 & 0 & \mathrm{yzyz} & 0 & 0 & \mathrm{zxzx} & 0 & 0 \\ 0 & 0 & 0 & 0 & \mathrm{zxzx} & 0 & 0 & \mathrm{yzyz} & 0 \\ 0 & 0 & 0 & 0 & 0 & \mathrm{xyxy} & 0 & 0 & \mathrm{xyxy}\end{array}\right)$
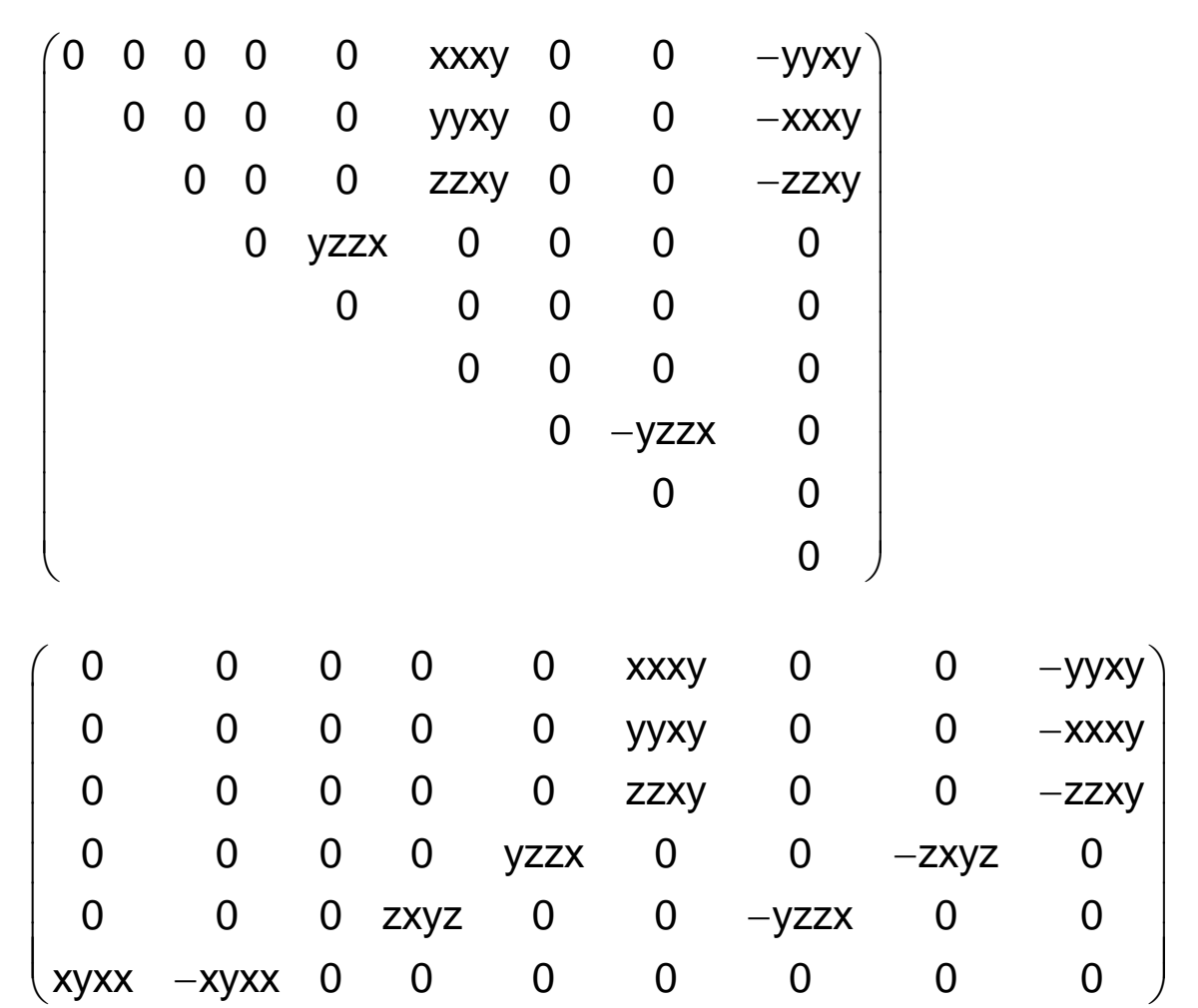

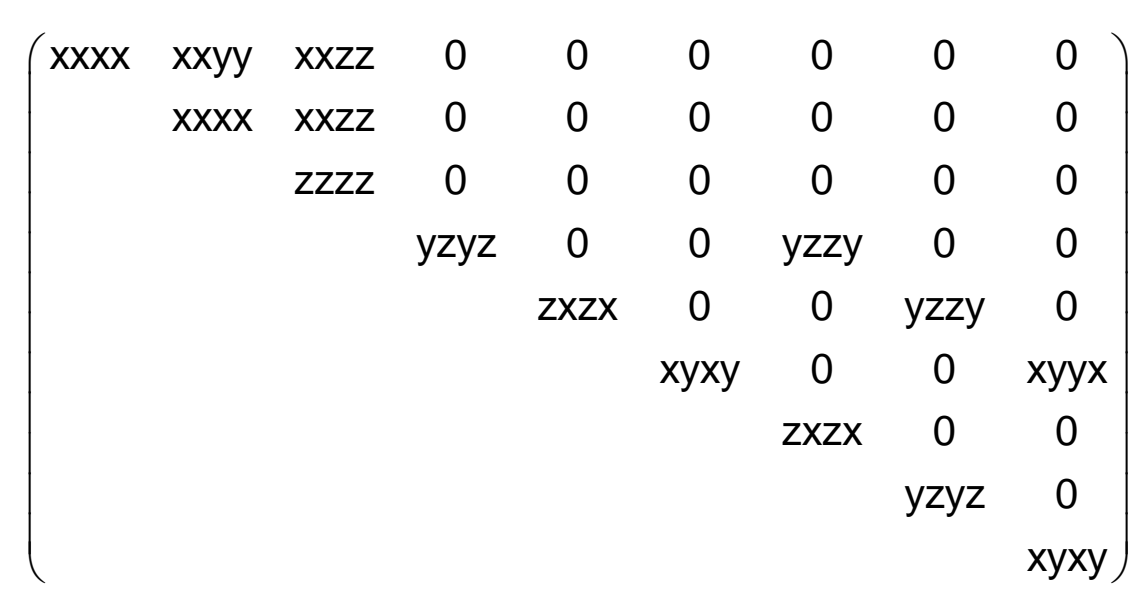

$\left(\begin{array}{lllllllll}x x x x & x x y y & x x z z & 0 & 0 & 0 & 0 & 0 & 0 \\ \text { yyxx } & x y x x & x x z & 0 & 0 & 0 & 0 & 0 & 0\end{array}\right.$ $\begin{array}{ccccccccc}y y x x & x x x x & x x z z & 0 & 0 & 0 & 0 & 0 & 0 \\ z z x x & z z x x & z z z z & 0 & 0 & 0 & 0 & 0 & 0\end{array}$ $\begin{array}{lllllllll}0 & 0 & 0 & y z y z & 0 & 0 & z x z x & 0 & 0\end{array}$ $\begin{array}{ccccccccc}0 & 0 & 0 & 0 & z x z x & 0 & 0 & \text { yzyz } & 0 \\ 0 & 0 & 0 & 0 & 0 & x y x y & 0 & 0 & x y x y\end{array}$ 
e

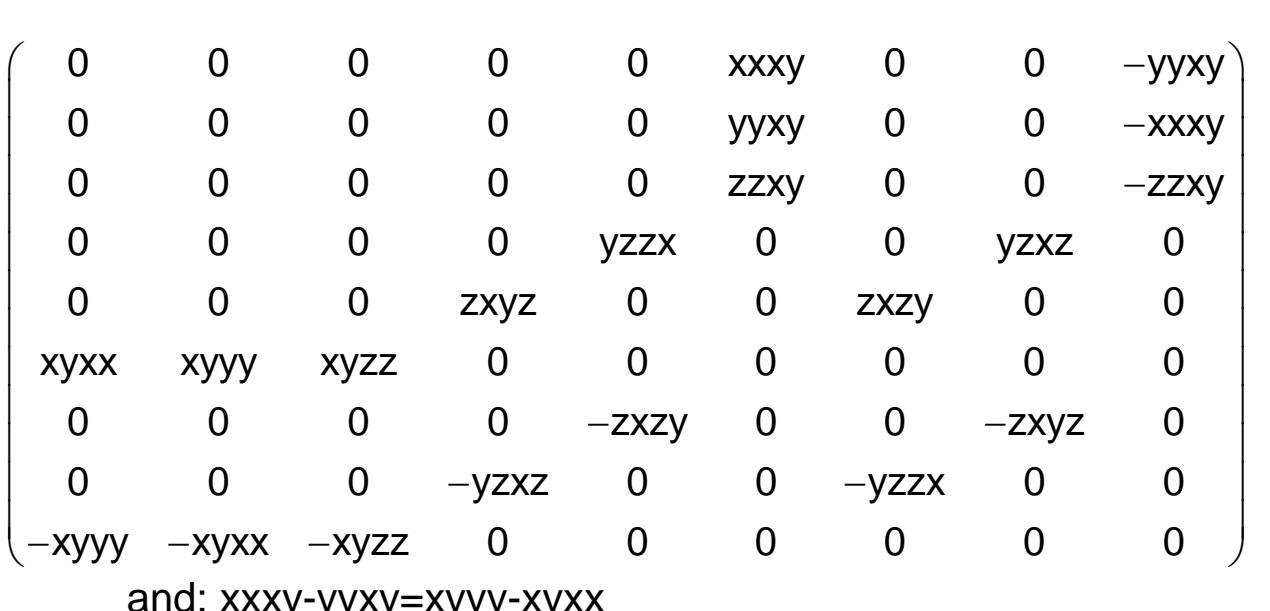

and: $x x x y-y y x y=x y y y-x y x x$

\begin{abstract}
$\left(\begin{array}{lllll}0 & 0 & 0 & 0 & 0 \\ 0 & 0 & 0 & 0 & 0 \\ 0 & 0 & 0 & 0 & 0\end{array}\right)$
\end{abstract}
$\begin{array}{lllll}0 & 0 & 0 & 0 & 0\end{array}$

$\left[V^{4}\right] \quad\left(\begin{array}{ccccc}3 x x y y & 0 & 0 & y y z z & 0 \\ 0 & 3 x x y y & 0 & y y z z & 0 \\ 0 & 0 & z z z z & x x y y & 0\end{array}\right)$

$\left.\mathrm{V}^{3} \mathrm{~V}^{3}\right]\left(\begin{array}{cccccccccc}3 \mathrm{xx} y \mathrm{y} & 0 & 0 & \mathrm{xxyy} & 0 & 0 & \mathrm{yyzz} & 0 & 0 & 0 \\ 0 & 3 x \mathrm{x} y \mathrm{y} & 0 & 0 & \mathrm{yyzz} & 0 & 0 & \mathrm{xxyy} & 0 & 0 \\ 0 & 0 & 2 z z & 0 & 0 & z & 0 & 0 & z z x & 0\end{array}\right)$

$\begin{array}{llllllllll}0 & 3 x y x x & 0 & 0 & x y z z & 0 & 0 & x y x x & 0 & 0 \\ -3 x y x & 0 & 0 & -x y x & 0 & 0 & -x y z z & 0 & 0 & 0\end{array}$ $\left.\begin{array}{cccccccccc}-3 x y x x & 0 & 0 & -x y x x & 0 & 0 & -x y z z & 0 & 0 & 0\end{array}\right)$ $\left(\begin{array}{llllllllll}0 & 0 & z z z z & 0 & 0 & z z x x & 0 & 0 & z z x x & 0\end{array}\right.$

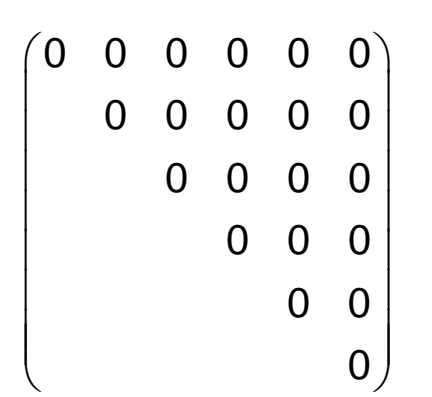

and $x y x y=1 / 2(x x x x-x x y y)$

$\left[\mathrm{V}^{2}\right]^{2}\left(\begin{array}{cccccc}\mathrm{xxxx} & \mathrm{xxyy} & \mathrm{xxzz} & 0 & 0 & 0 \\ \mathrm{xxyy} & \mathrm{xxxx} & \mathrm{xxzz} & 0 & 0 & 0 \\ \mathrm{zzxx} & \mathrm{zzxx} & \mathrm{zzzz} & 0 & 0 & 0 \\ 0 & 0 & 0 & \mathrm{yzyz} & 0 & 0 \\ 0 & 0 & 0 & 0 & \mathrm{yzyz} & 0 \\ 0 & 0 & 0 & 0 & 0 & \mathrm{xyxy}\end{array}\right)$

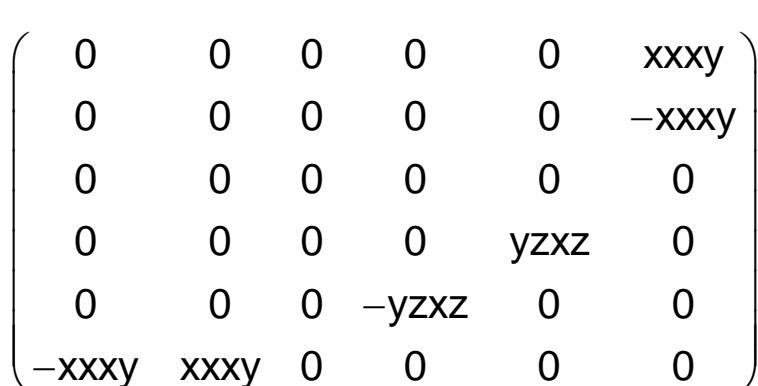

and $x y x y=1 / 2(x x x x-x x y y)$ $\left(\begin{array}{ccccccccc}0 & 0 & 0 & 0 & 0 & \text { xxxy } & 0 & 0 & - \text { yyxy } \\ 0 & 0 & 0 & 0 & 0 & \text { yyxy } & 0 & 0 & - \text {-xxy } \\ 0 & 0 & 0 & 0 & 0 & \text { zzxy } & 0 & 0 & - \text { zzxy } \\ 0 & 0 & 0 & 0 & \text { yzzx } & 0 & 0 & \text { yzxz } & 0 \\ 0 & 0 & 0 & \text { zxyz } & 0 & 0 & \text { zxzy } & 0 & 0 \\ \text { xyxx } & \text { xyyy } & \text { xyzz } & 0 & 0 & 0 & 0 & 0 & 0 \\ 0 & 0 & 0 & 0 & -z x z y & 0 & 0 & -z x y z & 0 \\ 0 & 0 & 0 & -y z x z & 0 & 0 & -y z z x & 0 & 0 \\ -x y y y & -x y x x & -x y z z & 0 & 0 & 0 & 0 & 0 & 0\end{array}\right)$ $\left(\begin{array}{ccccccccc}\text { xxxx } & \text { xxyy } & \text { xxzz } & 0 & 0 & 0 & 0 & 0 & 0 \\ \text { yyxx } & \text { xxxx } & \text { xxzz } & 0 & 0 & 0 & 0 & 0 & 0 \\ \text { zzxx } & \text { zzyy } & \text { zzzz } & 0 & 0 & 0 & 0 & 0 & 0 \\ 0 & 0 & 0 & y z y z & 0 & 0 & \text { yzzy } & 0 & 0 \\ 0 & 0 & 0 & 0 & \text { zxzx } & 0 & 0 & \text { zxxz } & 0 \\ 0 & 0 & 0 & 0 & 0 & \text { xyxy } & 0 & 0 & \text { xyyx } \\ 0 & 0 & 0 & \text { zxxz } & 0 & 0 & \text { zxzx } & 0 & 0 \\ 0 & 0 & 0 & 0 & \text { yzzy } & 0 & 0 & \text { yzyz } & 0 \\ 0 & 0 & 0 & 0 & 0 & \text { xyyx } & 0 & 0 & \text { xyxy }\end{array}\right)$

and: $x x x x=x x y y+x y x y+x y y x$ $\left(\begin{array}{lllll}0 & 0 & 0 & 0 & 0 \\ 0 & 0 & 0 & 0 & 0 \\ 0 & 0 & 0 & 0 & 0\end{array}\right)$

$\left(\begin{array}{lllll}0 & 0 & 0 & 0 & 0 \\ 0 & 0 & 0 & 0 & 0\end{array}\right)$

$\left(\begin{array}{llllllllll}0 & 3 x y x x & 0 & 0 & x y z z & 0 & 0 & x y x x & 0 & 0\end{array}\right)$

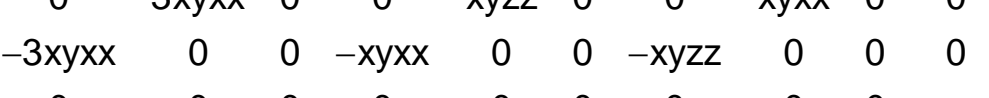

$$
\left(\begin{array}{llllll}
0 & 0 & 0 & 0 & 0 & 0 \\
& 0 & 0 & 0 & 0 & 0 \\
& & 0 & 0 & 0 & 0 \\
& & & 0 & 0 & 0 \\
& & & & 0 & 0 \\
& & & & & \\
& & & & & 0
\end{array}\right)
$$

$$
\left(\begin{array}{ccccc}
3 x x y y & 0 & 0 & y y z z & 0 \\
0 & 3 x x y y & 0 & y y z z & 0 \\
0 & 0 & z z z z & x x y y & 0
\end{array}\right)
$$

$\left(\begin{array}{cccccccccc}3 x x y y & 0 & 0 & x x y y & 0 & 0 & y y z z & 0 & 0 & 0 \\ 0 & 3 x x y y & 0 & 0 & y y z z & 0 & 0 & x x y y & 0 & 0 \\ 0 & 0 & 2 z z & 0 & 0 & z z x & 0 & 0 & z z x & 0\end{array}\right)$

$\left.\begin{array}{cccccccccc}0 & 3 x x y y & 0 & 0 & y y z z & 0 & 0 & x x y y & 0 & 0 \\ 0 & 0 & z z z z & 0 & 0 & z z x x & 0 & 0 & z z x x & 0\end{array}\right)$

$$
\left(\begin{array}{cccccc}
x x x x & x x y y & x x z z & 0 & 0 & 0 \\
& x x x x & x x z z & 0 & 0 & 0 \\
& & z z z z & 0 & 0 & 0 \\
& & & y z y z & 0 & 0 \\
& & & & y z y z & 0 \\
& & & & & x y x y
\end{array}\right)
$$

and $x y x y=1 / 2(x x x x-x x y y)$

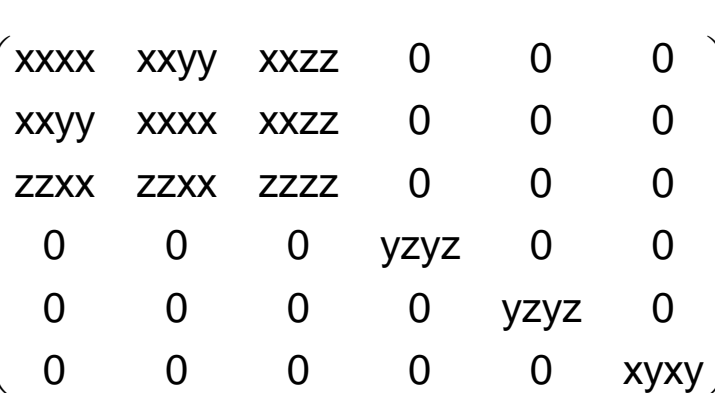

and $x y x y=1 / 2(x x x x-x x y y)$ 


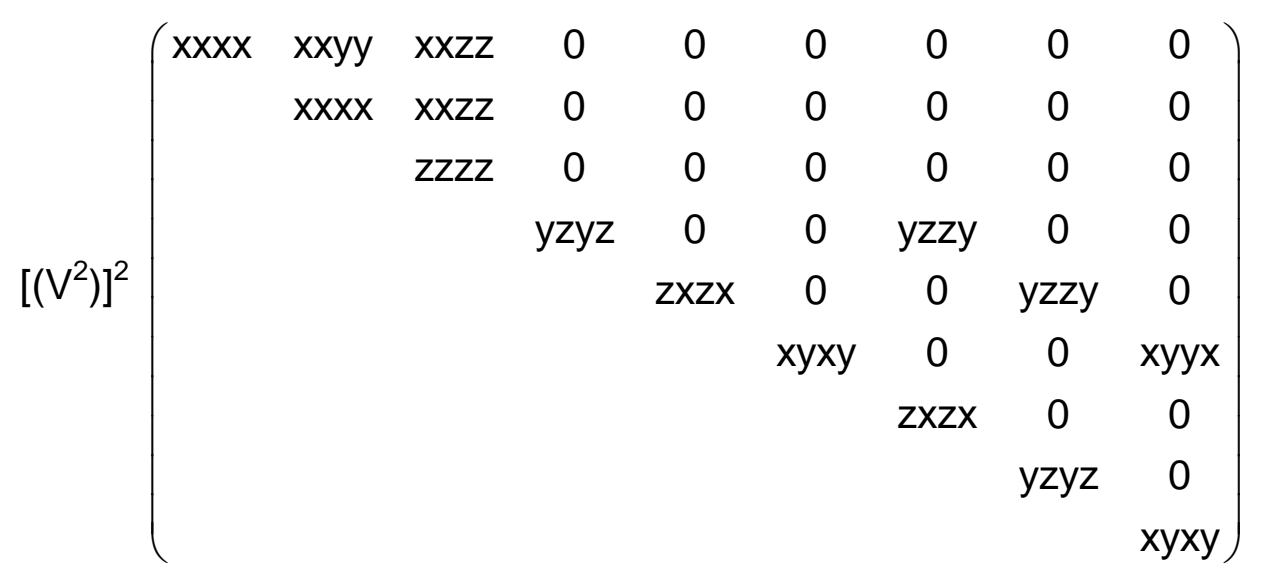

and $x x x x=x x y y+y z y z+z y z y$

$\left[\mathrm{V}^{2}\right] \mathrm{V}^{2}\left(\begin{array}{ccccccccc}\mathrm{x} x \mathrm{x} & \mathrm{xxyy} & \mathrm{xxzz} & 0 & 0 & 0 & 0 & 0 & 0 \\ \mathrm{xxyy} & \mathrm{xxxx} & \mathrm{xxzz} & 0 & 0 & 0 & 0 & 0 & 0 \\ \mathrm{zzxx} & \mathrm{zzxx} & \mathrm{zzzz} & 0 & 0 & 0 & 0 & 0 & 0 \\ 0 & 0 & 0 & \mathrm{yzyz} & 0 & 0 & \mathrm{zxzx} & 0 & 0 \\ 0 & 0 & 0 & 0 & \mathrm{zxzx} & 0 & 0 & \mathrm{yzyz} & 0 \\ 0 & 0 & 0 & 0 & 0 & \mathrm{xyxy} & 0 & 0 & \text { xyxy }\end{array}\right)$

and $x x x x=x x y y+2 x y x y$ $\left(\begin{array}{lllllllll}0 & 0 & 0 & 0 & 0 & x x x y & 0 & 0 & -x x x y \\ & 0 & 0 & 0 & 0 & x x x y & 0 & 0 & -x x y \\ & & 0 & 0 & 0 & \text { zxxy } & 0 & 0 & -z z x y\end{array}\right)$

$\left.\begin{array}{cccccccc}0 & 0 & 0 & 0 & x x x y & 0 & 0 & -x x x y \\ & 0 & 0 & 0 & z z x y & 0 & 0 & -z z x y\end{array}\right)$

$\begin{array}{llllll}0 & y z z x & 0 & 0 & 0 & 0\end{array}$

$\begin{array}{lllll}0 & 0 & 0 & 0 & 0\end{array}$

$\begin{array}{ccc}0 & 0 & 0 \\ 0 & -y z z x & 0 \\ & 0 & 0 \\ & & 0\end{array}$

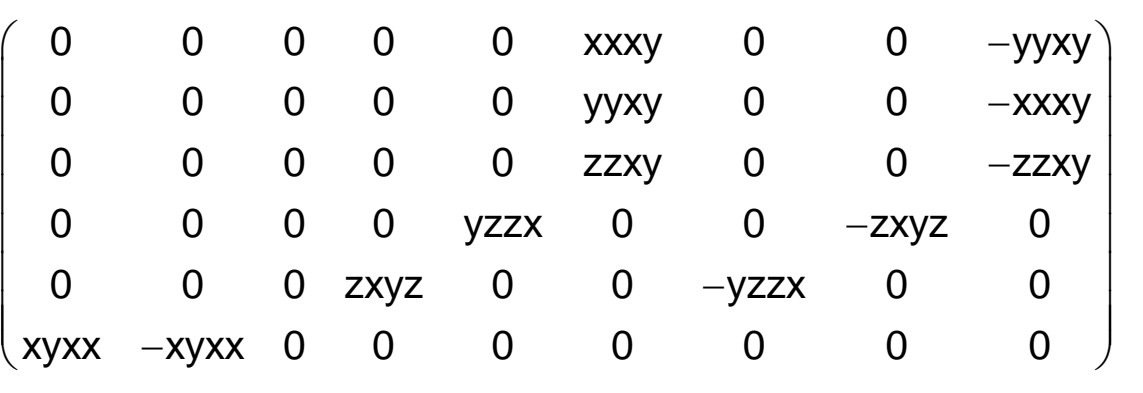

and: $y y x y-x x x y=2 x y x x$

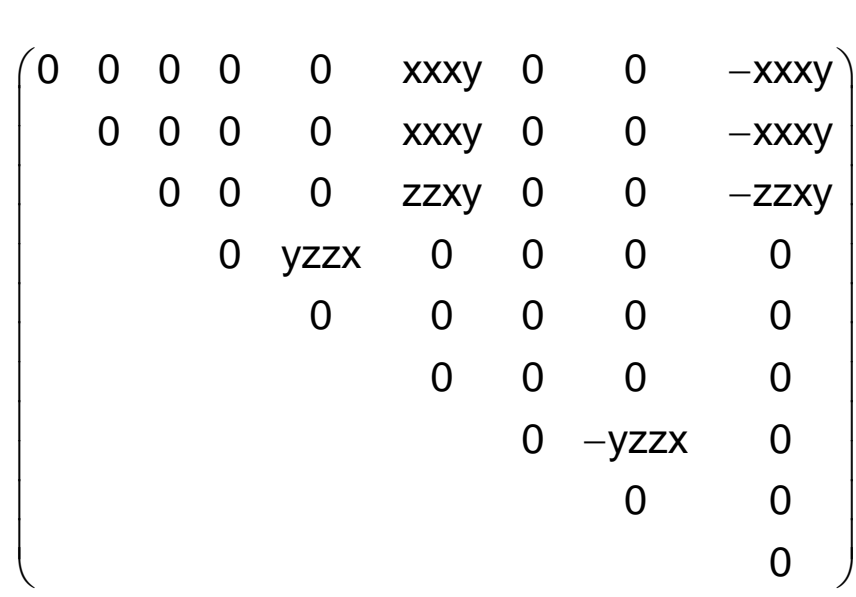

$\left(\begin{array}{ccccccccc}0 & 0 & 0 & 0 & 0 & \text { xxxy } & 0 & 0 & - \text { yyxy } \\ 0 & 0 & 0 & 0 & 0 & \text { yyxy } & 0 & 0 & -x x x y \\ 0 & 0 & 0 & 0 & 0 & z z x y & 0 & 0 & -z z x y \\ 0 & 0 & 0 & 0 & y z z x & 0 & 0 & -z x y z & 0 \\ 0 & 0 & 0 & \text { zxyz } & 0 & 0 & -y z z x & 0 & 0 \\ x y x x & -x y x x & 0 & 0 & 0 & 0 & 0 & 0 & 0\end{array}\right)$

and: $y y x y-x x x y=2 x y x x$

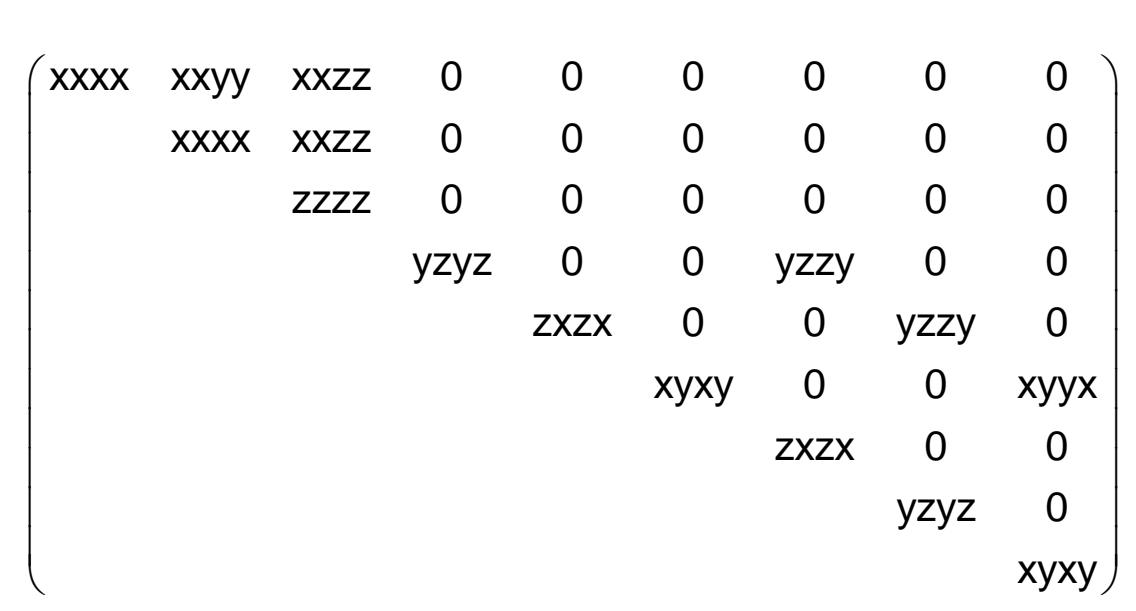

and $x x x x=x x y y+y z y z+z y z y$

$\left(\begin{array}{lllllllll}x x x x & x x y y & x x z z & 0 & 0 & 0 & 0 & 0 & 0 \\ x x y & x x x & x x z z & 0 & 0 & 0 & 0 & 0 & 0\end{array}\right)$ $\begin{array}{lllllllll}x x y y & x x x x & x x z z & 0 & 0 & 0 & 0 & 0 & 0\end{array}$ $\begin{array}{lllllllll}z z x x & z z x x & z z z z & 0 & 0 & 0 & 0 & 0 & 0\end{array}$ $\begin{array}{ccccccccc}0 & 0 & 0 & y z y z & 0 & 0 & z x z x & 0 & 0 \\ 0 & 0 & 0 & 0 & z x z x & 0 & 0 & y z y z & 0\end{array}$ $\left(\begin{array}{ccccccccc}0 & 0 & 0 & 0 & z x z x & 0 & 0 & y z y z & 0 \\ 0 & 0 & 0 & 0 & 0 & x y x y & 0 & 0 & x y x y\end{array}\right)$ and $x x x x=x x y y+2 x y x y$ 
1

$\left(\begin{array}{ccccccccc}\text { xxxx } & \text { xxyy } & \text { xxzz } & 0 & 0 & 0 & 0 & 0 & 0 \\ \text { yyxx } & \text { yyyy } & \text { yyzz } & 0 & 0 & 0 & 0 & 0 & 0 \\ \text { zzxx } & \text { zzyy } & \text { zzzz } & 0 & 0 & 0 & 0 & 0 & 0 \\ 0 & 0 & 0 & \text { yzyz } & 0 & 0 & \text { yzzy } & 0 & 0 \\ 0 & 0 & 0 & 0 & \text { zxzx } & 0 & 0 & \text { zxxz } & 0 \\ 0 & 0 & 0 & 0 & 0 & x y x y & 0 & 0 & x y y x \\ 0 & 0 & 0 & z y y z & 0 & 0 & \text { zyzy } & 0 & 0 \\ 0 & 0 & 0 & 0 & \text { xzzx } & 0 & 0 & \text { xzxz } & 0 \\ 0 & 0 & 0 & 0 & 0 & \text { yxxy } & 0 & 0 & \text { yxyx }\end{array}\right)$

$\left[V^{4}\right] \quad\left(\begin{array}{ccccc}x x x x & 0 & 0 & y y z z & 0 \\ 0 & y y y y & 0 & z z x x & 0 \\ 0 & 0 & z z z z & x x y y & 0\end{array}\right)$

$\left.V_{[V}{ }^{3}\right]\left(\begin{array}{cccccccccc}x x x x & 0 & 0 & x x y y & 0 & 0 & x x z z & 0 & 0 & 0 \\ 0 & y y y y & 0 & 0 & y y z z & 0 & 0 & y y x x & 0 & 0 \\ 0 & 0 & z z z z & 0 & 0 & z z x & 0 & 0 & z z y & 0\end{array}\right)$

$\left(\begin{array}{llllllllll}0 & 0 & z z z z & 0 & 0 & z z x x & 0 & 0 & \text { zzyy } & 0\end{array}\right)$

$\left[\mathrm{V}^{2}\right]^{2}$

$\left(\begin{array}{cccccc}x x x x & \text { xxyy } & \text { xxzz } & 0 & 0 & 0 \\ & \text { yyyy } & \text { yyzz } & 0 & 0 & 0 \\ & & \text { zzzz } & 0 & 0 & 0 \\ & & & \text { yzyz } & 0 & 0 \\ & & & & \text { xzxz } & 0 \\ & & & & & \text { xyxy }\end{array}\right)$

$\left[\mathrm{V}^{2}\right]^{2}$

$\left(\begin{array}{cccccc}\text { xxxx } & \text { xxyy } & \text { xxzz } & 0 & 0 & 0 \\ \text { yyxx } & \text { yyyy } & \text { yyzz } & 0 & 0 & 0 \\ \text { zzxx } & \text { zzyy } & \text { zzzz } & 0 & 0 & 0 \\ 0 & 0 & 0 & y z y z & 0 & 0 \\ 0 & 0 & 0 & 0 & \text { xzxz } & 0 \\ 0 & 0 & 0 & 0 & 0 & \text { xyxy }\end{array}\right)$

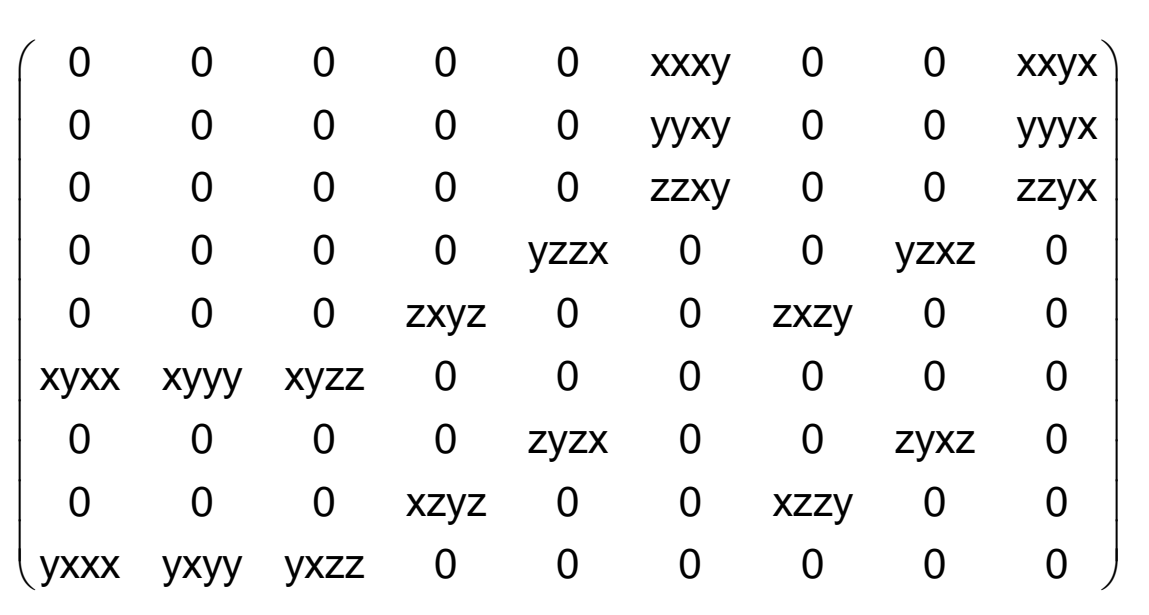

$\left(\begin{array}{ccccc}0 & x y y y & 0 & 0 & 0 \\ y x x x & 0 & 0 & 0 & 0 \\ 0 & 0 & 0 & 0 & z z x y\end{array}\right)$

$\left(\begin{array}{cccccccccc}0 & x y y y & 0 & 0 & x y z z & 0 & 0 & x y x x & 0 & 0 \\ y x x x & 0 & 0 & y x y y & 0 & 0 & y x z z & 0 & 0 & 0\end{array}\right)$

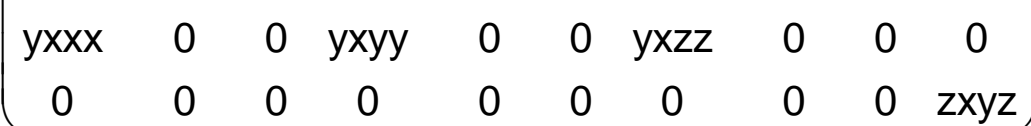

$$
\begin{aligned}
& \left(\begin{array}{cccccc}
0 & 0 & 0 & 0 & 0 & x x x y \\
0 & 0 & 0 & 0 & y x y
\end{array}\right) \\
& \begin{array}{llll}
0 & 0 & 0 & y y x y \\
0 & 0 & 0 & z z x y
\end{array} \\
& \begin{array}{ccc}
0 & 0 & z z x y \\
0 & y z x z & 0 \\
0 & 0
\end{array}
\end{aligned}
$$

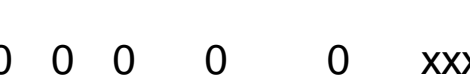

$\begin{array}{llllll}0 & 0 & 0 & 0 & 0 & \text { yyxy } \\ 0 & 0 & 0 & 0 & 0 & z z x y\end{array}$

$\begin{array}{llllll}0 & 0 & 0 & 0 & 0 & z z x y \\ 0 & 0 & 0 & 0 & y z x z\end{array}$

$\begin{array}{llllll}0 & 0 & 0 & 0 & y z x z & 0 \\ 0 & 0 & 0 & x z y z & 0 & 0\end{array}$

$\begin{array}{llllll}0 & 0 & 0 & x z y z & 0 & 0 \\ 0 & 0 & 0 & 0 & 0 & 0\end{array}$

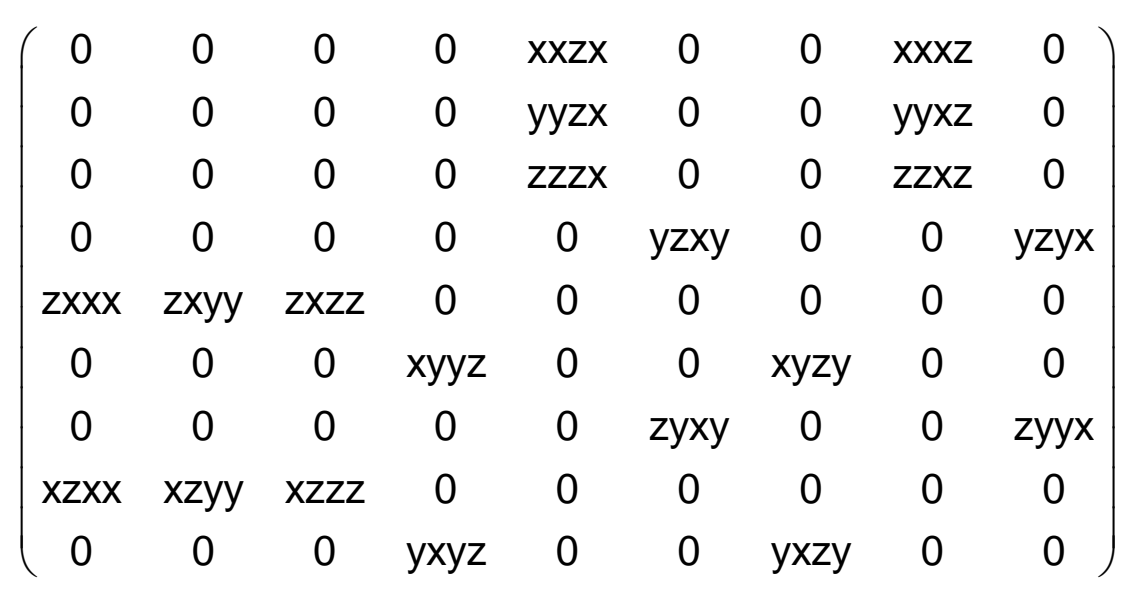

$\left(\begin{array}{ccccccccc}0 & 0 & 0 & \text { xxyz } & 0 & 0 & \text { xxzy } & 0 & 0 \\ 0 & 0 & 0 & \text { yyyz } & 0 & 0 & \text { yyzy } & 0 & 0 \\ 0 & 0 & 0 & \text { zzyz } & 0 & 0 & \text { zzzy } & 0 & 0 \\ \text { yzxx } & \text { yzyy } & \text { yzzz } & 0 & 0 & 0 & 0 & 0 & 0 \\ 0 & 0 & 0 & 0 & 0 & \text { zxxy } & 0 & 0 & \text { zxyx } \\ 0 & 0 & 0 & 0 & \text { xyzx } & 0 & 0 & \text { xyxz } & 0 \\ \text { zyxx } & \text { zyyy } & \text { zyzz } & 0 & 0 & 0 & 0 & 0 & 0 \\ 0 & 0 & 0 & 0 & 0 & \text { xzxy } & 0 & 0 & \text { xzyx } \\ 0 & 0 & 0 & 0 & \text { yxzx } & 0 & 0 & \text { yxxz } & 0\end{array}\right)$

$\left(\begin{array}{ccccc}0 & 0 & \mathrm{xzzz} & 0 & 0 \\ 0 & 0 & 0 & 0 & \mathrm{yyxz} \\ \mathrm{zxxx} & 0 & 0 & 0 & 0\end{array}\right)$

$\left(\begin{array}{cccccccccc}0 & 0 & x z z z & 0 & 0 & x z x x & 0 & 0 & x z y y & 0 \\ 0 & 0 & 0 & 0 & 0 & 0 & 0 & 0 & 0 & y x y z\end{array}\right)$

$\left.\begin{array}{cccccccccc}0 & 0 & 0 & 0 & 0 & 0 & 0 & 0 & 0 & y x y z \\ z x x x & 0 & 0 & z x y & 0 & 0 & z x z z & 0 & 0 & 0\end{array}\right)$

$$
\left(\begin{array}{cccccc}
0 & 0 & 0 & 0 & \mathrm{xxxz} & 0 \\
& 0 & 0 & 0 & \mathrm{yyxz} & 0 \\
& & 0 & 0 & \mathrm{zzxz} & 0 \\
& & & 0 & 0 & \mathrm{yzxy} \\
& & & & 0 & 0 \\
& & & & & 0
\end{array}\right)
$$

$\left(\begin{array}{ccccc}0 & 0 & 0 & 0 & x x y z \\ 0 & 0 & y z z z & 0 & 0 \\ 0 & z y y y & 0 & 0 & 0\end{array}\right)$

$\left(\begin{array}{cccccccccc}0 & 0 & 0 & 0 & 0 & 0 & 0 & 0 & 0 & x x y z \\ 0 & 0 & y z z z & 0 & 0 & y z x x & 0 & 0 & y z y y & 0 \\ 0 & z y y & 0 & 0 & z y z z & 0 & 0 & z y x x & 0 & 0\end{array}\right)$

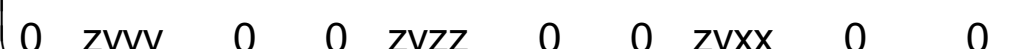

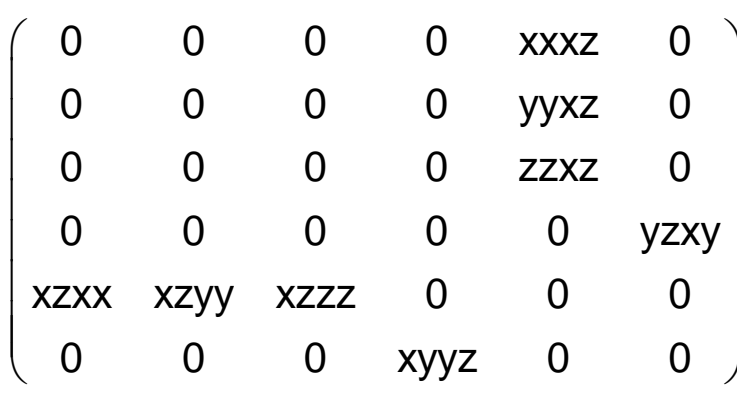

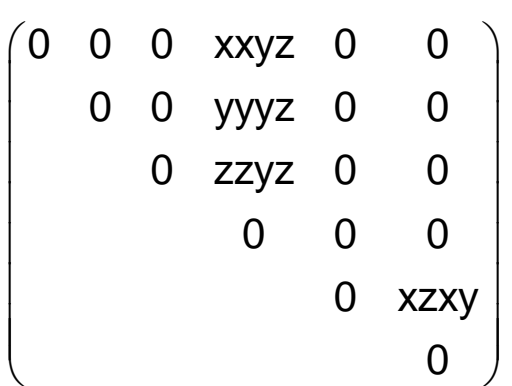

$\left(\begin{array}{cccccc}0 & 0 & 0 & \text { xxyz } & 0 & 0 \\ 0 & 0 & 0 & \text { yyyz } & 0 & 0 \\ 0 & 0 & 0 & \text { zzyz } & 0 & 0 \\ \text { yzxx } & \text { yzyy } & \text { yzzz } & 0 & 0 & 0 \\ 0 & 0 & 0 & 0 & 0 & \text { xzxy } \\ 0 & 0 & 0 & 0 & \text { xyxz } & 0\end{array}\right)$ 
$\mathrm{n}=1 \quad \mathbf{C}_{2 \mathrm{v}}\left(\mathbf{C}_{\mathrm{vv}}\right)=\mathbf{2}_{\mathrm{z}} \mathbf{m}_{\mathrm{x}} \mathbf{m}_{\mathrm{y}}\left(\mathbf{m}_{\mathrm{y}}\right)=\mathbf{2}_{\mathrm{z}} \mathbf{m}_{\mathrm{x}} \mathbf{m}_{\mathrm{y}}$

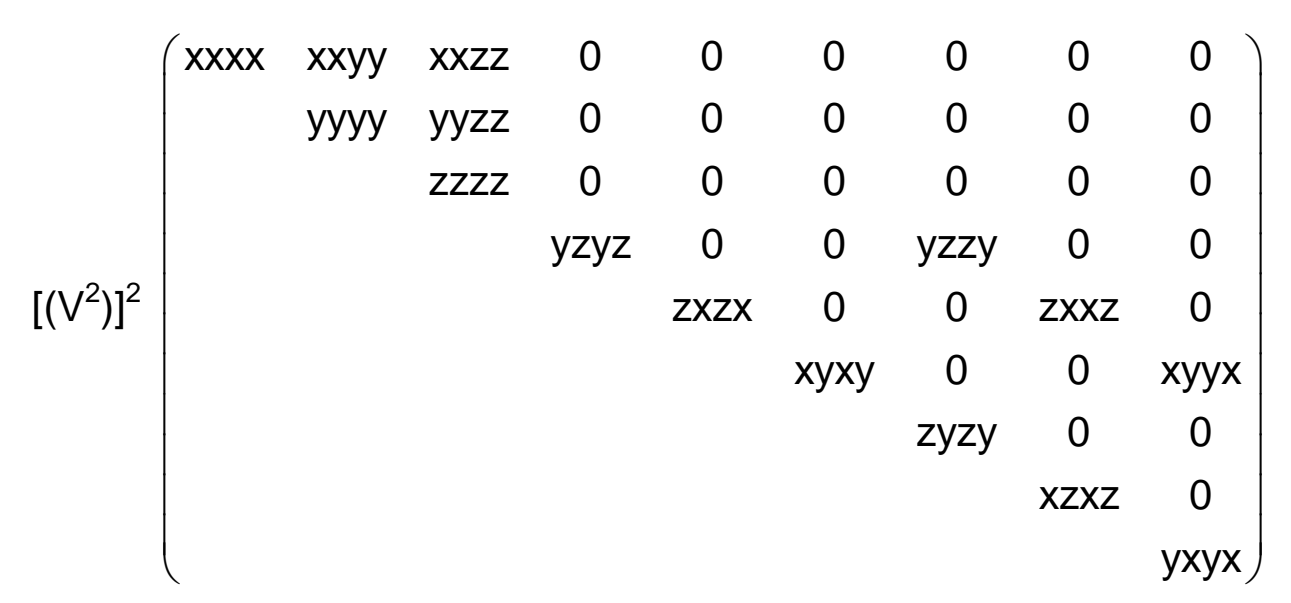

$$
\left(\begin{array}{ccccccccc}
0 & 0 & 0 & 0 & 0 & \text { xxxy } & 0 & 0 & \text { xxyx } \\
& 0 & 0 & 0 & 0 & \text { yyxy } & 0 & 0 & y y y x \\
& & 0 & 0 & 0 & \text { zzxy } & 0 & 0 & \text { zzyx } \\
& & & 0 & y z z x & 0 & 0 & y z x z & 0 \\
& & & & 0 & 0 & z x z y & 0 & 0 \\
& & & & & 0 & 0 & 0 & 0 \\
& & & & & & 0 & z y x z & 0 \\
& & & & & & & 0 & 0 \\
& & & & & & & & 0
\end{array}\right)
$$

$\left[V^{2}\right] V^{2}\left(\begin{array}{ccccccccc}x x x x & x x y y & x x z z & 0 & 0 & 0 & 0 & 0 & 0 \\ \text { yyxx } & \text { yyyy } & \text { yyzz } & 0 & 0 & 0 & 0 & 0 & 0 \\ z z x x & z z y y & \text { zzzz } & 0 & 0 & 0 & 0 & 0 & 0 \\ 0 & 0 & 0 & y z y z & 0 & 0 & y z z y & 0 & 0 \\ 0 & 0 & 0 & 0 & z x z x & 0 & 0 & \text { zxxz } & 0 \\ 0 & 0 & 0 & 0 & 0 & \text { xyxy } & 0 & 0 & \text { xyyx }\end{array}\right)$

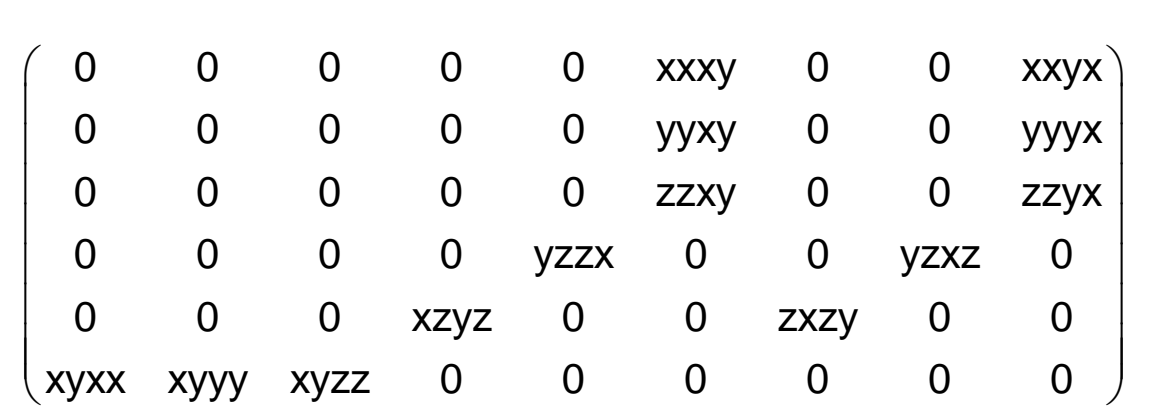

$\mathbf{C}_{2 n v}\left(\mathbf{C}_{\mathrm{nv}}\right)$

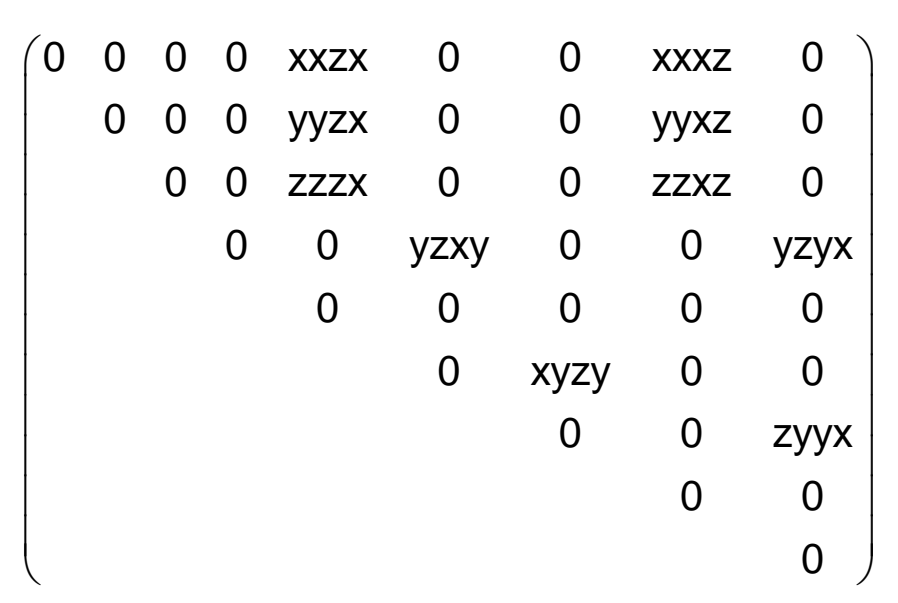

$\left(\begin{array}{ccccccccc}0 & 0 & 0 & 0 & \text { xxzx } & 0 & 0 & \text { xxxz } & 0 \\ 0 & 0 & 0 & 0 & \text { yyzx } & 0 & 0 & \text { yyxz } & 0 \\ 0 & 0 & 0 & 0 & \text { zzzx } & 0 & 0 & \text { zzxz } & 0 \\ 0 & 0 & 0 & 0 & 0 & y z x y & 0 & 0 & \text { yzyx } \\ \text { zxxx } & \text { zxyy } & \text { zxzz } & 0 & 0 & 0 & 0 & 0 & 0 \\ 0 & 0 & 0 & \text { xyyz } & 0 & 0 & \text { xyzy } & 0 & 0\end{array}\right) \quad\left(\begin{array}{ccccccccc}0 & 0 & 0 & \text { xxyz } & 0 & 0 & \text { xxzy } & 0 & 0 \\ 0 & 0 & 0 & \text { yyyz } & 0 & 0 & \text { yyzy } & 0 & 0 \\ 0 & 0 & 0 & \text { zzyz } & 0 & 0 & \text { zzzy } & 0 & 0 \\ \text { yzxx } & \text { yzyy } & \text { yzzz } & 0 & 0 & 0 & 0 & 0 & 0 \\ 0 & 0 & 0 & 0 & 0 & \text { zxxy } & 0 & 0 & \text { zxyx } \\ 0 & 0 & 0 & 0 & \text { xyzx } & 0 & 0 & \text { xyxz } & 0\end{array}\right)$

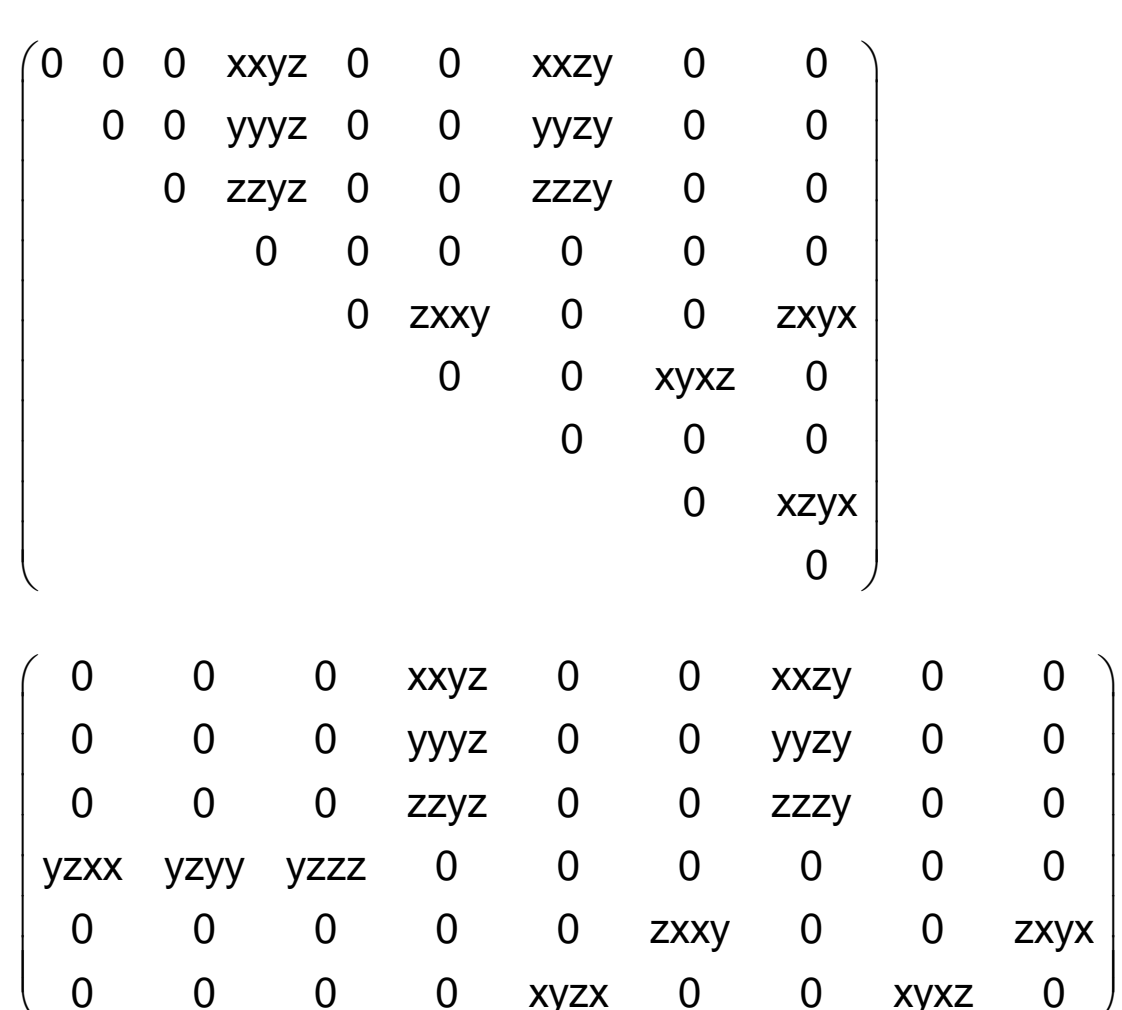


a

$V^{4}\left(\begin{array}{ccccccccc}x x x x & x x y y & x x z z & 0 & 0 & 0 & 0 & 0 & 0 \\ y y x x & x x x x & x x z z & 0 & 0 & 0 & 0 & 0 & 0 \\ \text { zzxx } & \text { zzyy } & \text { zzzz } & 0 & 0 & 0 & 0 & 0 & 0 \\ 0 & 0 & 0 & y z y z & 0 & 0 & y z z y & 0 & 0 \\ 0 & 0 & 0 & 0 & z x z x & 0 & 0 & z x x z & 0 \\ 0 & 0 & 0 & 0 & 0 & x y x y & 0 & 0 & x y y x \\ 0 & 0 & 0 & z x x z & 0 & 0 & z x z x & 0 & 0 \\ 0 & 0 & 0 & 0 & y z z y & 0 & 0 & y z y z & 0 \\ 0 & 0 & 0 & 0 & 0 & x y y x & 0 & 0 & x y x y\end{array}\right)$

$\left(\begin{array}{ccccccccc}0 & 0 & 0 & 0 & 0 & \text { xxxy } & 0 & 0 & -y y x y \\ 0 & 0 & 0 & 0 & 0 & \text { yyxy } & 0 & 0 & -x x x y \\ 0 & 0 & 0 & 0 & 0 & \text { zzxy } & 0 & 0 & -z z x y \\ 0 & 0 & 0 & 0 & y z z x & 0 & 0 & y z x z & 0 \\ 0 & 0 & 0 & \text { zxyz } & 0 & 0 & \text { zxzy } & 0 & 0 \\ \text { xyxx } & x y y y & x y z z & 0 & 0 & 0 & 0 & 0 & 0 \\ 0 & 0 & 0 & 0 & -z x z y & 0 & 0 & -z x y z & 0 \\ 0 & 0 & 0 & -y z x z & 0 & 0 & -y z z x & 0 & 0 \\ -x y y y & -x y x x & -x y z z & 0 & 0 & 0 & 0 & 0 & 0\end{array}\right)$

$\left(\begin{array}{ccccccccc}x x x x & x x y y & x x z z & 0 & 0 & 0 & 0 & 0 & 0 \\ -x x y y & -x x y & -x x z z & 0 & 0 & 0 & 0 & 0 & 0\end{array}\right)\left(\begin{array}{llllllllll}0 & 0 & 0 & 0 & 0 & x x x y & 0 & 0 & y y x y \\ 0 & 0 & 0 & 0 & 0 & y y x & 0 & 0 & x x y\end{array}\right)$

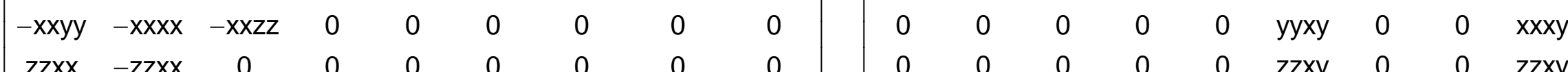
$\begin{array}{ccccccccc}z z x x & -z z x x & 0 & 0 & 0 & 0 & 0 & 0 & 0 \\ 0 & 0 & 0 & y z y z & 0 & 0 & -y z z y & 0 & 0 \\ 0 & 0 & 0 & 0 & z x z x & 0 & 0 & -z x z z & 0\end{array} \mid \begin{array}{lllllllll}0 & 0 & 0 & 0 & 0 & z z x y & 0 & 0 & z z x y \\ 0 & 0 & 0 & 0 & y z z x & 0 & 0 & y z x z & 0 \\ 0 & 0 & 0 & z x y & 0 & 0 & z x y & 0 & 0\end{array}$ $\begin{array}{ccccccccc}0 & 0 & 0 & y z y z & 0 & 0 & -y z z y & 0 & 0 \\ 0 & 0 & 0 & 0 & z x z x & 0 & 0 & -z x y z & 0\end{array} \mid \begin{array}{lllllllll}0 & 0 & 0 & 0 & y z z x & 0 & 0 & y z x z & 0 \\ 0 & 0 & 0 & z x y z & 0 & 0 & z x z y & 0 & 0\end{array}$ $\begin{array}{ccccccccc}0 & 0 & 0 & 0 & 0 & x y x y & 0 & 0 & -x y y x\end{array} \mid \begin{array}{cccccccccc}0 & 0 & 0 & z x y z & 0 & 0 & \text { zxzy } & 0 & 0 \\ \text { xyxx } & x y y y & x y z z & 0 & 0 & 0 & 0 & 0 & 0 \\ 0 & 0 & 0 & 0 & 0 & 2 x z y & 0 & 0 & 2 x y z & 0\end{array}$ $\left.\begin{array}{lllcccccc}0 & 0 & 0 & z x x z & 0 & 0 & -z x z x & 0 & 0 \\ 0 & 0 & 0 & 0 & y z y & 0 & 0 & -y z y z & 0 \\ 0 & 0 & 0 & 0 & 0 & x y x & 0 & 0 & -x y x y\end{array}\right)\left(\begin{array}{ccccccccc}0 & 0 & 0 & 0 & z x z y & 0 & 0 & z x y z & 0 \\ 0 & 0 & 0 & y z z & 0 & 0 & y z z x & 0 & 0\end{array}\right.$ $\left.\begin{array}{ccccccccc}0 & 0 & 0 & 0 & \text { yzzy } & 0 & 0 & -y z y z & 0 \\ 0 & 0 & 0 & 0 & 0 & x y y x & 0 & 0 & -x y x y\end{array}\right)\left(\begin{array}{ccccccccc}0 & 0 & 0 & y z x z & 0 & 0 & y z z x & 0 & 0 \\ x y y y & x y x x & x y z z & 0 & 0 & 0 & 0 & 0 & 0\end{array}\right.$

$\left[V^{4}\right]\left(\begin{array}{ccccc}\mathrm{xxxx} & 0 & 0 & \mathrm{yyzz} & 0 \\ 0 & \mathrm{xxxx} & 0 & \mathrm{yyzz} & 0 \\ 0 & 0 & \mathrm{zzzz} & \mathrm{xxyy} & 0\end{array}\right)$ $\left(\begin{array}{ccccc}0 & -y x x x & 0 & 0 & 0 \\ y x x x & 0 & 0 & 0 & 0 \\ 0 & 0 & 0 & 0 & 0\end{array}\right)$

$\left(\begin{array}{ccccc}\mathrm{xxxx} & 0 & 0 & \mathrm{yyzz} & 0 \\ 0 & -\mathrm{xxxx} & 0 & -\mathrm{yyzz} & 0 \\ 0 & 0 & 0 & 0 & 0\end{array}\right)$

$\left(\begin{array}{ccccc}0 & \mathrm{yxxx} & 0 & 0 & 0 \\ \mathrm{y} x \mathrm{xx} & 0 & 0 & 0 & 0 \\ 0 & 0 & 0 & 0 & z z x y\end{array}\right)$

$V\left[V^{3}\right]\left(\begin{array}{cccccccccc}x x x x & 0 & 0 & x x y y & 0 & 0 & y y z z & 0 & 0 & 0 \\ 0 & x x x x & 0 & 0 & y y z z & 0 & 0 & x x y y & 0 & 0 \\ 0 & 0 & z z z z & 0 & 0 & z z x & 0 & 0 & z z x & 0\end{array}\right)$

$\left(\begin{array}{cccccccccc}0 & -y x x x & 0 & 0 & x y z z & 0 & 0 & x y x x & 0 & 0\end{array}\right)$

$\left(\begin{array}{llllllllll}0 & 0 & z z z z & 0 & 0 & z z x x & 0 & 0 & z z x x & 0\end{array}\right)$ $\begin{array}{cccccccccc}0 & 0 & 0 & 0 & 0 & 0 & 0 & 0 & 0 & \text { zxyz }\end{array}$

$\left(\begin{array}{cccccccc}x x x x & 0 & 0 & x x y y & 0 & 0 & -y y z z & 0\end{array}\right.$

$\left(\begin{array}{llllllllll}0 & \text { xyyy } & 0 & 0 & \text { xyzz } & 0 & 0 & y x y y & 0 & 0 \\ x y y & 0 & 0\end{array}\right)$

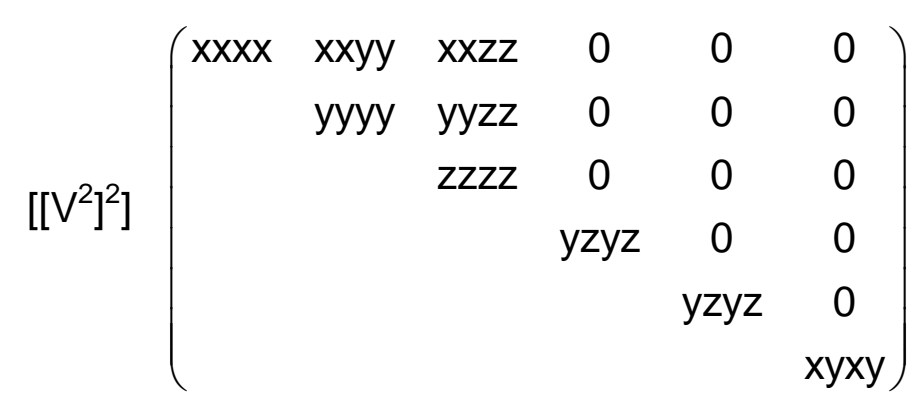

$\left(\begin{array}{cccccc}0 & 0 & 0 & 0 & 0 & \mathrm{xxxy} \\ & 0 & 0 & 0 & 0 & -\mathrm{xxxy} \\ & & 0 & 0 & 0 & 0 \\ & & & 0 & 0 & 0 \\ & & & & 0 & 0 \\ & & & & & 0\end{array}\right)$

$\left[V^{2}\right]^{2}\left(\begin{array}{cccccc}x x x x & x x y y & x x z z & 0 & 0 & 0 \\ y y x x & x x x x & x x z z & 0 & 0 & 0 \\ z z x x & z z x x & z z z z & 0 & 0 & 0 \\ 0 & 0 & 0 & y z y z & 0 & 0 \\ 0 & 0 & 0 & 0 & y z y z & 0 \\ 0 & 0 & 0 & 0 & 0 & x y x y\end{array}\right)$

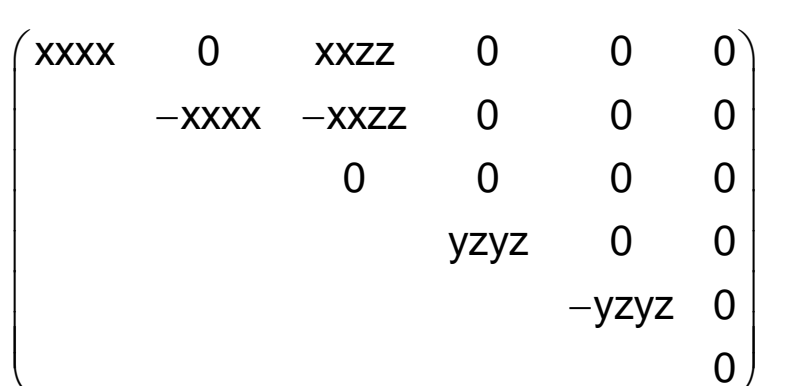

$\left(\begin{array}{cccccc}\text { xxxx } & \text { xxyy } & \text { xxzz } & 0 & 0 & 0 \\ -x x y y & -x x x x & -x x z z & 0 & 0 & 0 \\ \text { zzxx } & -z z x x & 0 & 0 & 0 & 0 \\ 0 & 0 & 0 & y z y z & 0 & 0 \\ 0 & 0 & 0 & 0 & -y z y z & 0 \\ 0 & 0 & 0 & 0 & 0 & 0\end{array}\right)$

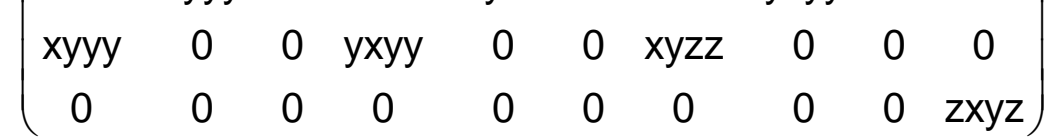

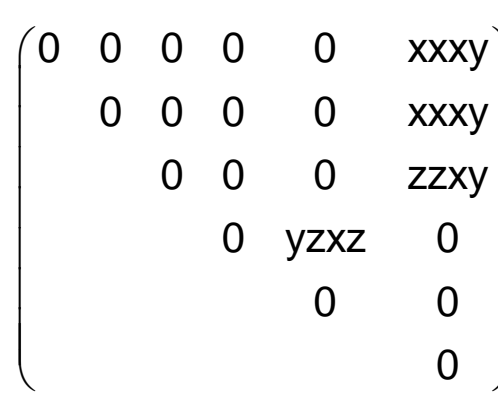

$\left(\begin{array}{cccccc}0 & 0 & 0 & 0 & 0 & x x x y \\ 0 & 0 & 0 & 0 & 0 & x x x y \\ 0 & 0 & 0 & 0 & 0 & z z x y \\ 0 & 0 & 0 & 0 & y z x z & 0 \\ 0 & 0 & 0 & y z x z & 0 & 0 \\ x y x x & x y x x & x y z z & 0 & 0 & 0\end{array}\right)$ 
$\mathrm{n}=2 \quad \mathbf{C}_{4 \mathrm{v}}\left(\mathbf{C}_{2 \mathrm{v}}\right)=\mathbf{4}_{z} \mathbf{m}_{\times} \mathbf{m}_{\mathrm{xy}}\left(\mathbf{2}_{z} \mathbf{m}_{\times} \mathbf{m}_{\mathrm{y}}\right)=\mathbf{4}_{z}^{\prime} \mathbf{m}_{\times} \mathbf{m}_{\mathrm{xy}}$

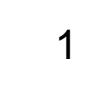

e

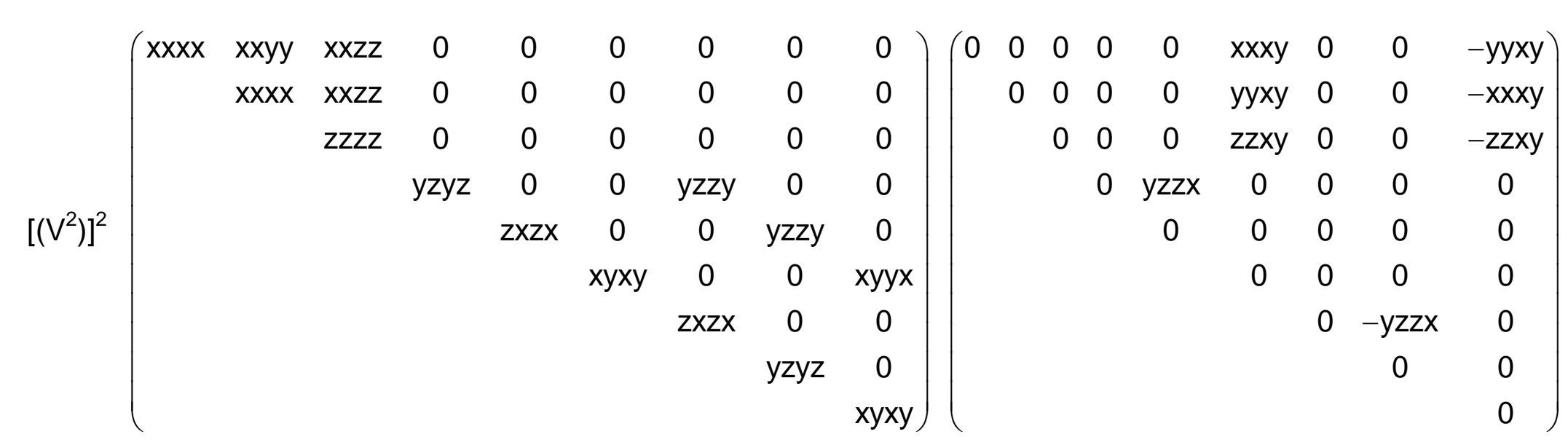

$\left[\mathrm{V}^{2}\right] \mathrm{V}^{2}\left(\begin{array}{ccccccccc}\mathrm{xxxx} & \mathrm{xxyy} & \mathrm{xxzz} & 0 & 0 & 0 & 0 & 0 & 0 \\ \mathrm{yyxx} & \mathrm{xxxx} & \mathrm{zxzz} & 0 & 0 & 0 & 0 & 0 & 0 \\ \mathrm{zzxx} & \mathrm{zzxx} & \mathrm{zzzz} & 0 & 0 & 0 & 0 & 0 & 0 \\ 0 & 0 & 0 & \mathrm{yzyz} & 0 & 0 & \mathrm{zxzx} & 0 & 0 \\ 0 & 0 & 0 & 0 & \mathrm{zxzx} & 0 & 0 & \mathrm{yzyz} & 0 \\ 0 & 0 & 0 & 0 & 0 & \mathrm{xyxy} & 0 & 0 & \mathrm{xyxy}\end{array}\right)\left(\begin{array}{ccccccccc}0 & 0 & 0 & 0 & 0 & \mathrm{xxxy} & 0 & 0 & -\mathrm{yyxy} \\ 0 & 0 & 0 & 0 & 0 & \mathrm{yyxy} & 0 & 0 & -\mathrm{xxxy} \\ 0 & 0 & 0 & 0 & 0 & \mathrm{zzxy} & 0 & 0 & -\mathrm{zzxy} \\ 0 & 0 & 0 & 0 & \mathrm{yzzzx} & 0 & 0 & -\mathrm{zxyz} & 0 \\ 0 & 0 & 0 & \mathrm{zxyz} & 0 & 0 & -\mathrm{yzzx} & 0 & 0 \\ \mathrm{xyxx} & -\mathrm{xyyx} & 0 & 0 & 0 & 0 & 0 & 0 & 0\end{array}\right)$
$\mathbf{C}_{2 n v}\left(\mathbf{C}_{\mathrm{nv}}\right)$

a

$\left(\begin{array}{ccccccccc}\text { xxxx } & \text { xxyy } & \text { xxzz } & 0 & 0 & 0 & 0 & 0 & 0 \\ - \text { xxyy } & - \text {-xxxx } & -\mathrm{xxzz} & 0 & 0 & 0 & 0 & 0 & 0 \\ \mathrm{zzxx} & -\mathrm{zzxx} & 0 & 0 & 0 & 0 & 0 & 0 & 0 \\ 0 & 0 & 0 & \mathrm{yzyz} & 0 & 0 & -\mathrm{zxzx} & 0 & 0 \\ 0 & 0 & 0 & 0 & \mathrm{zxzx} & 0 & 0 & -\mathrm{yzyz} & 0 \\ 0 & 0 & 0 & 0 & 0 & \text { xyxy } & 0 & 0 & -\mathrm{xyxy}\end{array}\right) \quad\left(\begin{array}{ccccccccc}0 & 0 & 0 & 0 & 0 & \mathrm{xxxy} & 0 & 0 & \mathrm{yyxy} \\ 0 & 0 & 0 & 0 & 0 & \mathrm{yyxy} & 0 & 0 & \mathrm{xxxy} \\ 0 & 0 & 0 & 0 & 0 & \mathrm{zzxy} & 0 & 0 & \mathrm{zzxy} \\ 0 & 0 & 0 & 0 & \mathrm{yzzzx} & 0 & 0 & \mathrm{zxyz} & 0 \\ 0 & 0 & 0 & \mathrm{zxyz} & 0 & 0 & \mathrm{yzzx} & 0 & 0 \\ \mathrm{xyxx} & \mathrm{xyyx} & \mathrm{xyzz} & 0 & 0 & 0 & 0 & 0 & 0\end{array}\right)$



$\left(\begin{array}{ccccccccc}x x x x & x x y y & x x z z & 0 & 0 & 0 & 0 & 0 & 0 \\ y y x x & x x x x & y y z z & 0 & 0 & 0 & 0 & 0 & 0 \\ z z x x & z z x x & z z z z & 0 & 0 & 0 & 0 & 0 & 0 \\ 0 & 0 & 0 & y z y z & 0 & 0 & y z z y & 0 & 0 \\ 0 & 0 & 0 & 0 & z x z x & 0 & 0 & z x x z & 0 \\ 0 & 0 & 0 & 0 & 0 & x y x y & 0 & 0 & x y y x \\ 0 & 0 & 0 & z x x z & 0 & 0 & z x z x & 0 & 0 \\ 0 & 0 & 0 & 0 & y z z y & 0 & 0 & y z y z & 0 \\ 0 & 0 & 0 & 0 & 0 & x y y x & 0 & 0 & x y x y\end{array}\right)$
and: $x x x x=x x y y+x y x y+x y y x$

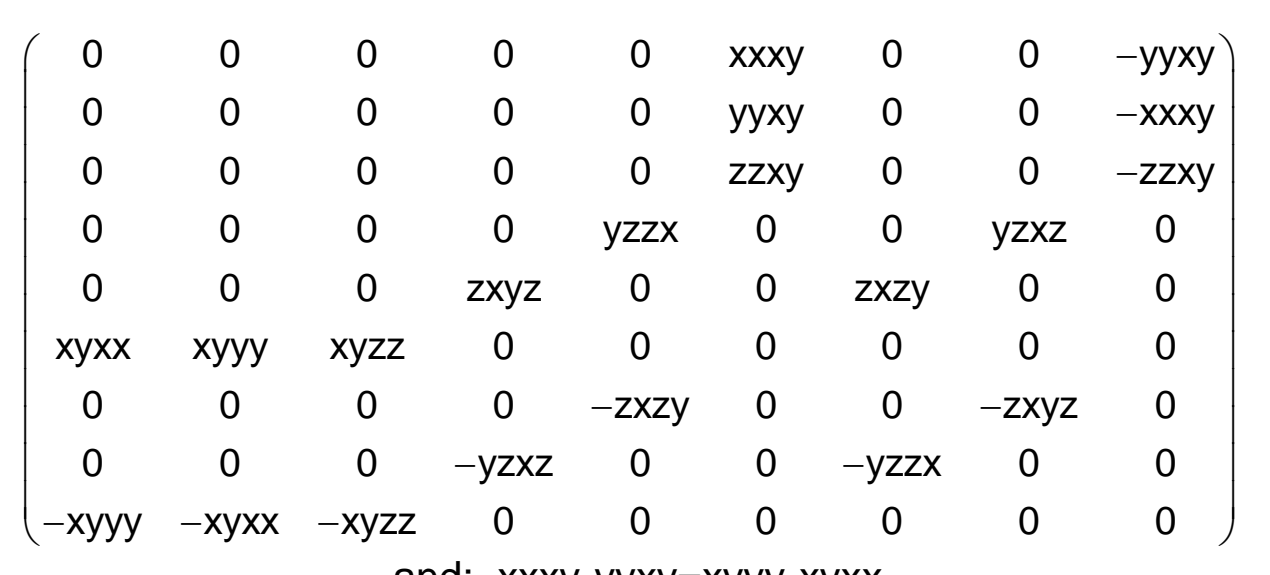
$\left[\mathrm{V}^{4}\right]\left(\begin{array}{ccccc}3 \mathrm{xxyy} & 0 & 0 & \mathrm{yyzz} & 0 \\ 0 & 3 \mathrm{xxyy} & 0 & \mathrm{yyzz} & 0 \\ 0 & 0 & \mathrm{zzzz} & \mathrm{xxyy} & 0\end{array}\right)$
$\left(\begin{array}{lllll}0 & 0 & 0 & 0 & 0 \\ 0 & 0 & 0 & 0 & 0 \\ 0 & 0 & 0 & 0 & 0\end{array}\right)$

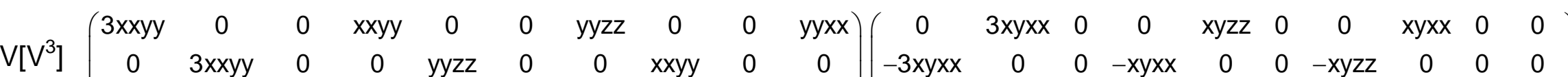

$\left.\left[\mathrm{V}^{2}\right]^{2}\right]\left(\begin{array}{cccccc}\mathrm{xxxx} & \mathrm{xxyy} & \mathrm{xxzz} & 0 & 0 & 0 \\ & \mathrm{xxxx} & \mathrm{xxzz} & 0 & 0 & 0 \\ & & \mathrm{zzzz} & 0 & 0 & 0 \\ & & & \mathrm{yzyz} & 0 & 0 \\ & & & & \mathrm{yzyz} & 0 \\ & & & & & \mathrm{xyxy}\end{array}\right)$

and $x y x y=1 / 2(x x x x-x x y y)$

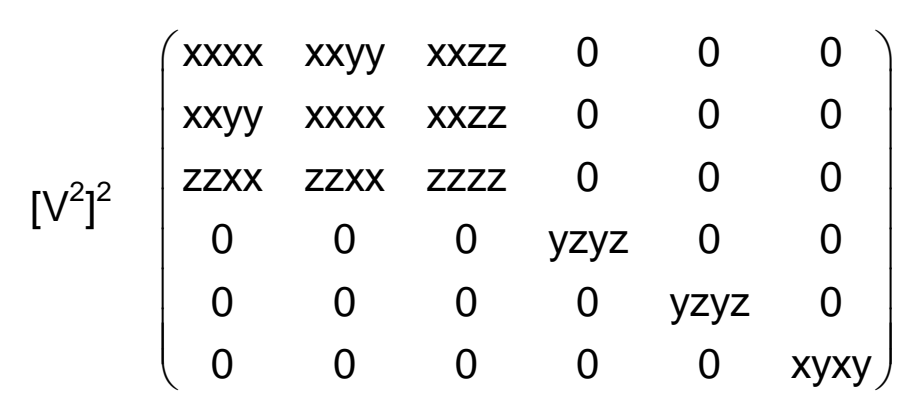

and $x y x y=1 / 2(x x x x-x x y y)$

$$
\left(\begin{array}{llllll}
0 & 0 & 0 & 0 & 0 & 0 \\
& 0 & 0 & 0 & 0 & 0 \\
& & 0 & 0 & 0 & 0 \\
& & & 0 & 0 & 0 \\
& & & & 0 & 0 \\
& & & & & 0
\end{array}\right)
$$

and $x y x y=1 / 2(x x x x-x x y y)$

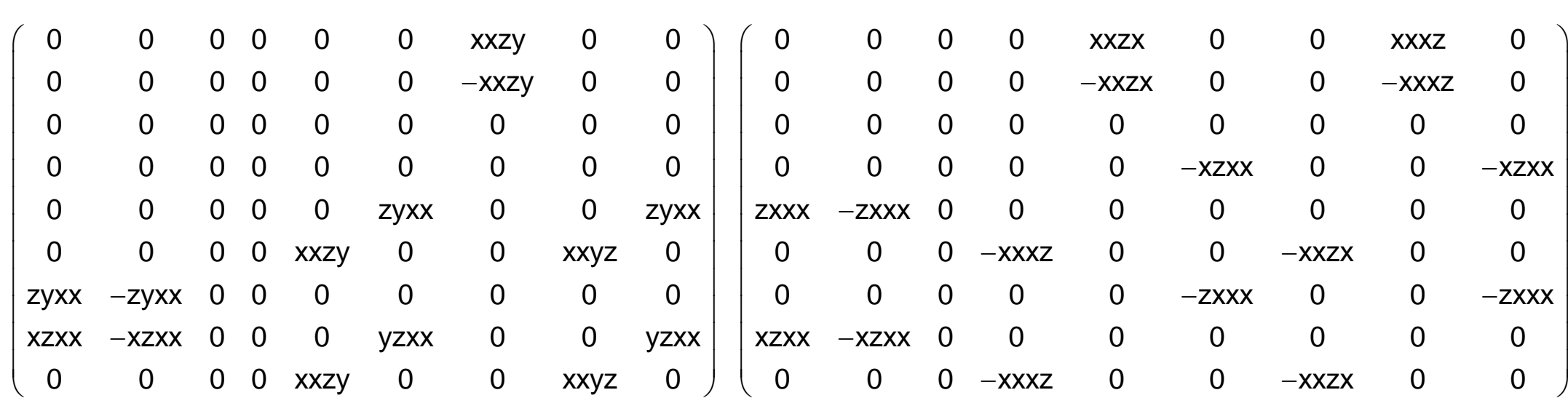

$\left(\begin{array}{ccccc}0 & 0 & 0 & 0 & x x y z \\ 0 & 0 & 0 & 0 & 0 \\ 0 & -x x y z & 0 & 0 & 0\end{array}\right)$

$\left(\begin{array}{cccccccccc}0 & 0 & 0 & 0 & 0 & 0 & 0 & 0 & 0 & y z x x \\ 0 & 0 & 0 & 0 & 0 & y z x x & 0 & 0 & -y z x x & 0 \\ 0 & -z y x x & 0 & 0 & 0 & 0 & 0 & z y x x & 0 & 0\end{array}\right)$ $\left.\begin{array}{cccccccccccc}0 & 0 & 0 & 0 & 0 & y z x x & 0 & 0 & -y z x x & 0 \\ 0 & -z y x x & 0 & 0 & 0 & 0 & 0 & z y x x & 0 & 0\end{array}\right)$

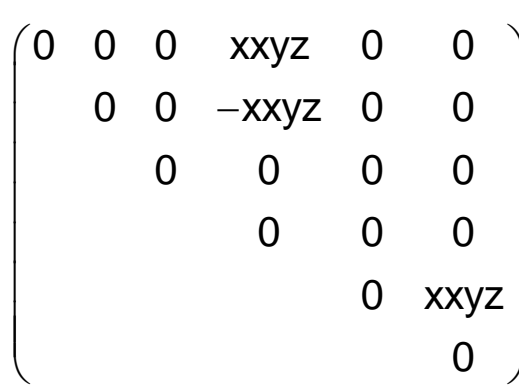

$\left(\begin{array}{ccccc}0 & 0 & 0 & 0 & 0 \\ 0 & 0 & 0 & 0 & -z x x x \\ z x x x & 0 & 0 & 0 & 0\end{array}\right)$

$\left(\begin{array}{cccccccccc}0 & 0 & 0 & 0 & 0 & x z x x & 0 & 0 & -x z x x & 0 \\ 0 & 0 & 0 & 0 & 0 & 0 & 0 & 0 & 0 & -x z x x \\ z x x x & 0 & 0 & -z x x x & 0 & 0 & 0 & 0 & 0 & 0\end{array}\right)$

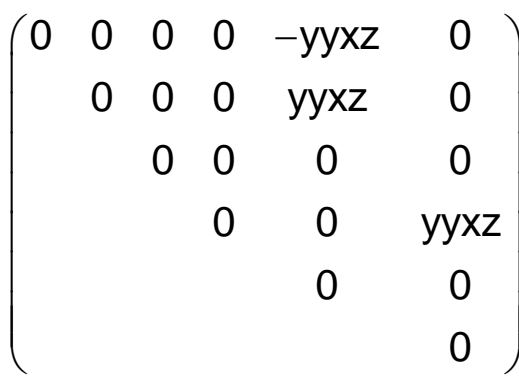

$\left(\begin{array}{cccccc}0 & 0 & 0 & \text { xxyz } & 0 & 0 \\ 0 & 0 & 0 & -x x y z & 0 & 0 \\ 0 & 0 & 0 & 0 & 0 & 0 \\ y z x x & -y z x x & 0 & 0 & 0 & 0 \\ 0 & 0 & 0 & 0 & 0 & y z x x \\ 0 & 0 & 0 & 0 & \text { xxyz } & 0\end{array}\right)$ $\left(\begin{array}{cccccc}0 & 0 & 0 & 0 & -y y x z & 0 \\ 0 & 0 & 0 & 0 & y y x z & 0 \\ 0 & 0 & 0 & 0 & 0 & 0 \\ 0 & 0 & 0 & 0 & 0 & x z y y \\ -x z y y & x z y y & 0 & 0 & 0 & 0 \\ 0 & 0 & 0 & y y x z & 0 & 0\end{array}\right)$ 


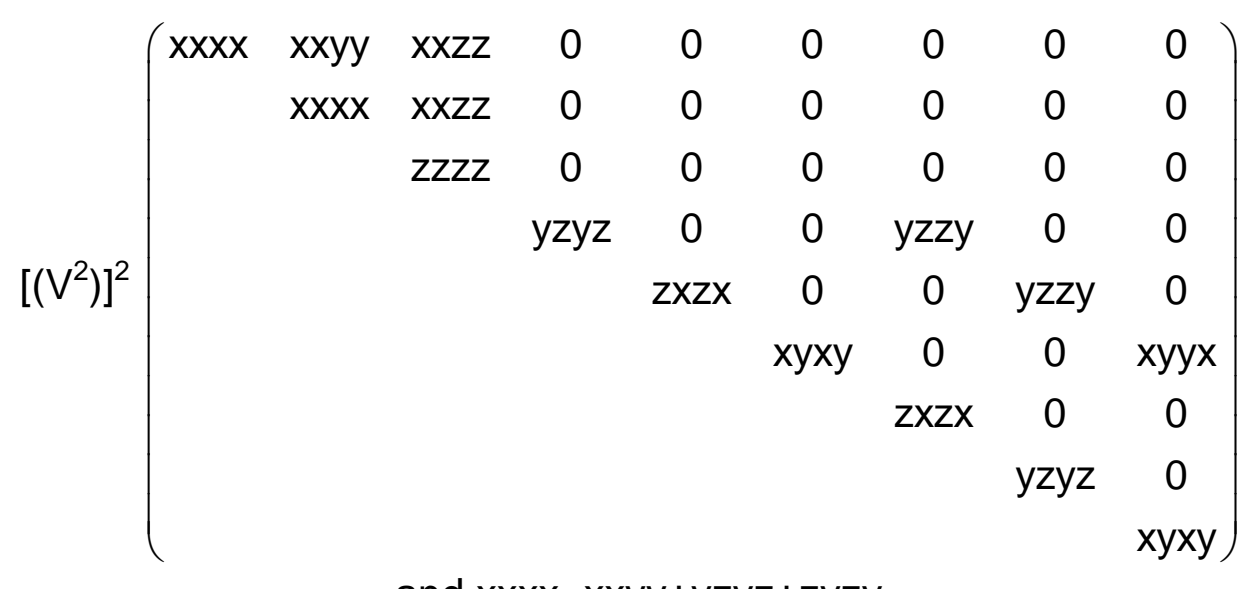

and $x x x x=x x y y+y z y z+z y z y$

$\left(\begin{array}{lllllllll}x x x x & x x y y & x x z z & 0 & 0 & 0 & 0 & 0 & 0 \\ x x y y & x x x & x x z z & 0 & 0 & 0 & 0 & 0\end{array}\right)$ $\left[\mathrm{V}^{2}\right] \mathrm{V}^{2}\left(\begin{array}{ccccccccc}\mathrm{x} x \mathrm{xzxy} & \mathrm{xxxx} & \mathrm{xxzz} & 0 & 0 & 0 & 0 & 0 & 0 \\ & \mathrm{zzxx} & \mathrm{zzzz} & 0 & 0 & 0 & 0 & 0 & 0\end{array}\right)$ $\left(\begin{array}{ccccccccc}0 & 0 & 0 & y z y z & 0 & 0 & z x z x & 0 & 0 \\ 0 & 0 & 0 & 0 & z x z x & 0 & 0 & y z y z & 0 \\ 0 & 0 & 0 & 0 & 0 & x y y & 0 & 0 & x y x y\end{array}\right)$ and $x x x x=x x y y+2 x y x y$
$\mathbf{C}_{2 n v}\left(\mathbf{C}_{\mathrm{nv}}\right)$

$$
\left(\begin{array}{ccccccccc}
0 & 0 & 0 & 0 & 0 & x x x y & 0 & 0 & -x x x y \\
& 0 & 0 & 0 & 0 & x x x y & 0 & 0 & -x x y \\
& & 0 & 0 & 0 & z z x y & 0 & 0 & -z z x y \\
& & & 0 & y z z x & 0 & 0 & 0 & 0 \\
& & & & 0 & 0 & 0 & 0 & 0 \\
& & & & & 0 & 0 & 0 & 0 \\
& & & & & & 0 & -y z z x & 0 \\
& & & & & & & 0 & 0 \\
& & & & & & & & 0
\end{array}\right)
$$

$$
\left(\begin{array}{ccccccccc}
0 & 0 & 0 & \text { xxyz } & 0 & 0 & \text { xxzy } & 0 & 0 \\
& 0 & 0 & -x x y z & 0 & 0 & -x x z y & 0 & 0 \\
& & 0 & 0 & 0 & 0 & 0 & 0 & 0 \\
& & & 0 & 0 & 0 & 0 & 0 & 0 \\
& & & & 0 & x x z y & 0 & 0 & x x z y \\
& & & & & 0 & 0 & x x y z & 0 \\
& & & & & & 0 & 0 & 0 \\
& & & & & & & 0 & x x y z \\
& & & & & & & & 0
\end{array}\right)
$$
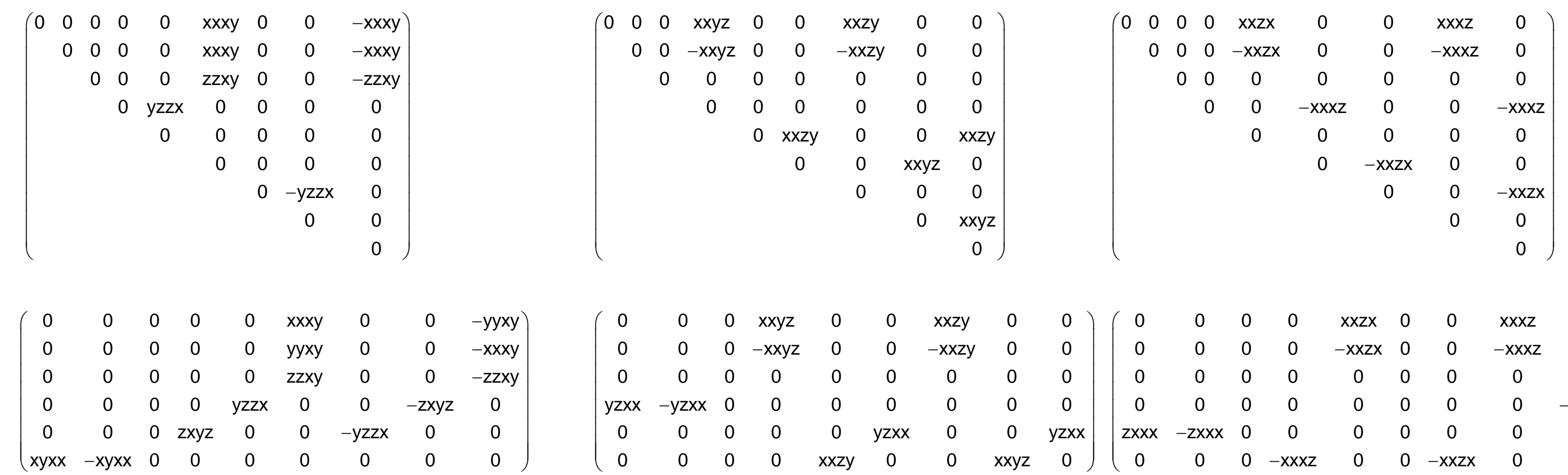

$\left(\begin{array}{ccccccccc}0 & 0 & 0 & x x y z & 0 & 0 & x x z y & 0 & 0 \\ 0 & 0 & 0 & -x x y z & 0 & 0 & -x x z y & 0 & 0 \\ 0 & 0 & 0 & 0 & 0 & 0 & 0 & 0 & 0 \\ y z x x & -y z x x & 0 & 0 & 0 & 0 & 0 & 0 & 0 \\ 0 & 0 & 0 & 0 & 0 & y z x x & 0 & 0 & y z x x \\ 0 & 0 & 0 & 0 & x x z y & 0 & 0 & x x y z & 0\end{array}\right)\left(\begin{array}{ccccccccc}0 & 0 & 0 & 0 & x x z x & 0 & 0 & x x x z & 0 \\ 0 & 0 & 0 & 0 & -x x z x & 0 & 0 & -x x x z & 0 \\ 0 & 0 & 0 & 0 & 0 & 0 & 0 & 0 & 0 \\ 0 & 0 & 0 & 0 & 0 & 0 & 0 & 0 & -z x x x \\ z x x x & -z x x x & 0 & 0 & 0 & 0 & 0 & 0 & 0 \\ 0 & 0 & 0 & -x x x z & 0 & 0 & -x x z x & 0 & 0\end{array}\right)$

and $y y x y-x x x y=2 x y x x$ 
$\mathrm{V}^{4}\left(\begin{array}{ccccccccc}\mathrm{xxxx} & \mathrm{xxyy} & \mathrm{xxzz} & 0 & 0 & 0 & 0 & 0 & 0 \\ \mathrm{yyxx} & \mathrm{xxxx} & \mathrm{yyzz} & 0 & 0 & 0 & 0 & 0 & 0 \\ \mathrm{zzxx} & \mathrm{zzxx} & \mathrm{zzzz} & 0 & 0 & 0 & 0 & 0 & 0 \\ 0 & 0 & 0 & \mathrm{yzyz} & 0 & 0 & \mathrm{yzzy} & 0 & 0 \\ 0 & 0 & 0 & 0 & \mathrm{zxzx} & 0 & 0 & \mathrm{zxxz} & 0 \\ 0 & 0 & 0 & 0 & 0 & \mathrm{xyxy} & 0 & 0 & \mathrm{xyyx} \\ 0 & 0 & 0 & \mathrm{zxxz} & 0 & 0 & \mathrm{zxzx} & 0 & 0 \\ 0 & 0 & 0 & 0 & \mathrm{yzzy} & 0 & 0 & \mathrm{yzyz} & 0 \\ 0 & 0 & 0 & 0 & 0 & \mathrm{xyyx} & 0 & 0 & \mathrm{xyxy}\end{array}\right)$ and $x x x y-y y x y=x y y y-x y x x$

$\left[V^{4}\right]\left(\begin{array}{ccccc}3 x x y y & 0 & 0 & y y z z & 0 \\ 0 & 3 x x y y & 0 & y y z z & 0 \\ 0 & 0 & z z z z & x x y y & 0\end{array}\right)$

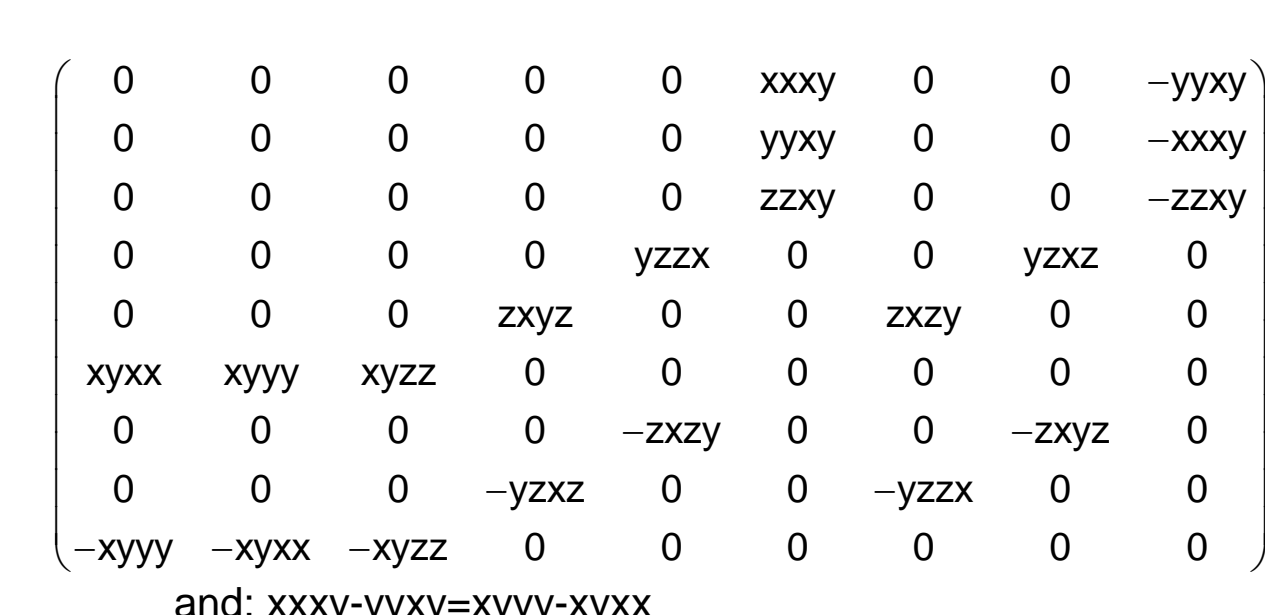

and: $x x x y-y y x y=x y y y-x y x x$

$\left(\begin{array}{lllll}0 & 0 & 0 & 0 & 0 \\ 0 & 0 & 0 & 0 & 0 \\ 0 & 0 & 0 & 0 & 0\end{array}\right)$

$\left(\begin{array}{lllll}0 & 0 & 0 & 0 & 0 \\ 0 & 0 & 0 & 0 & 0 \\ 0 & 0 & 0 & 0 & 0\end{array}\right)$

$\left(\begin{array}{ccccc}\mathrm{xxxx} & 0 & 0 & 0 & 0 \\ 0 & \mathrm{xxxx} & 0 & 0 & 0 \\ 0 & 0 & 0 & -x \mathrm{xxx} & 0\end{array}\right)$

$V\left[V^{3}\right] \quad\left(\begin{array}{cccccccccc}3 x x y y & 0 & 0 & x x y y & 0 & 0 & y y z z & 0 & 0 & y y x x \\ 0 & 3 x x y y & 0 & 0 & y y z z & 0 & 0 & x x y y & 0 & 0 \\ 0 & 0 & z z z & 0 & 0 & z z x & 0 & 0 & z z x & 0\end{array}\right)$

$\left.\left[\mathrm{V}^{2}\right]^{2}\right]\left(\begin{array}{cccccc}\mathrm{xxxx} & \mathrm{xxyy} & \mathrm{xxzz} & 0 & 0 & 0 \\ & \mathrm{xxxx} & \mathrm{xxzz} & 0 & 0 & 0 \\ & & \mathrm{zzzz} & 0 & 0 & 0 \\ & & & \mathrm{yzyz} & 0 & 0 \\ & & & & \mathrm{yzyz} & 0 \\ & & & & & \mathrm{xyxy}\end{array}\right)$

and $x y x y=1 / 2(x x x x-x x y y)$

$\left[\mathrm{V}^{2}\right]^{2}\left(\begin{array}{cccccc}\mathrm{xxxx} & \mathrm{xxyy} & \mathrm{xxzz} & 0 & 0 & 0 \\ \mathrm{xxyy} & \mathrm{xxxx} & \mathrm{xxzz} & 0 & 0 & 0 \\ \mathrm{zzxx} & \mathrm{zzxx} & \mathrm{zzzz} & 0 & 0 & 0 \\ 0 & 0 & 0 & \mathrm{yzyz} & 0 & 0 \\ 0 & 0 & 0 & 0 & \mathrm{yzyz} & 0 \\ 0 & 0 & 0 & 0 & 0 & \mathrm{xyxy}\end{array}\right)$

and $x y x y=1 / 2(x x x x-x x y y)$ $\left(\begin{array}{cccccccccc}0 & 3 x y x x & 0 & 0 & x y z z & 0 & 0 & x y x x & 0 & 0 \\ -3 x y x x & 0 & 0 & -x y x x & 0 & 0 & -x y z z & 0 & 0 & 0\end{array}\right)$ $\begin{array}{cccccccccc}-3 x y x x & 0 & 0 & -x y x x & 0 & 0 & -x y z z & 0 & 0 & 0 \\ 0 & 0 & 0 & 0 & 0 & 0 & 0 & 0 & 0 & z x y z\end{array}$

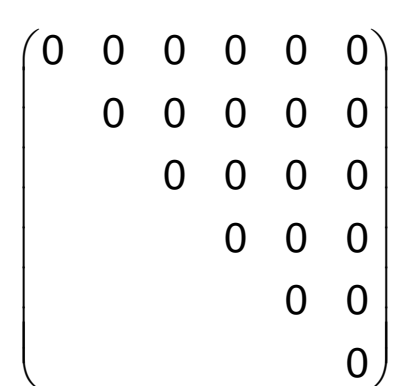

$\left(\begin{array}{cccccccccc}x x x x & 0 & 0 & -x x x x & 0 & 0 & 0 & 0 & 0 & 0 \\ 0 & x x x & 0 & 0 & 0 & 0 & 0 & -x x x x & 0 & 0\end{array}\right)$ $\left.\begin{array}{llllllllll}0 & \mathrm{x} x \mathrm{xx} & 0 & 0 & 0 & 0 & 0 & -\mathrm{xxxx} & 0 & 0 \\ 0 & 0 & 0 & 0 & 0 & 0 & 0 & 0 & 0 & 0\end{array}\right)$

$\left(\begin{array}{cccccc}x x x x & -x x x x & 0 & 0 & 0 & 0 \\ & x x x x & 0 & 0 & 0 & 0 \\ & 0 & 0 & 0 & 0 \\ & & & 0 & 0 & 0 \\ & & & & 0 & 0 \\ & & & & & -x x x x\end{array}\right)$

$\left(\begin{array}{cccccc}x x x x & -x x x x & 0 & 0 & 0 & 0 \\ -x x x x & x x x x & 0 & 0 & 0 & 0 \\ 0 & 0 & 0 & 0 & 0 & 0 \\ 0 & 0 & 0 & 0 & 0 & 0 \\ 0 & 0 & 0 & 0 & 0 & 0 \\ 0 & 0 & 0 & 0 & 0 & -x x x x\end{array}\right)$

$\left(\begin{array}{cccccc}0 & 0 & 0 & 0 & 0 & x x x y \\ 0 & 0 & 0 & 0 & 0 & -x x x y \\ 0 & 0 & 0 & 0 & 0 & 0 \\ 0 & 0 & 0 & 0 & y z x z & 0 \\ 0 & 0 & 0 & y z x z & 0 & 0 \\ -x x x y & x x x y & 0 & 0 & 0 & 0\end{array}\right)$

$\begin{array}{llll}0 & 0 & 0 & -x x x x\end{array}$

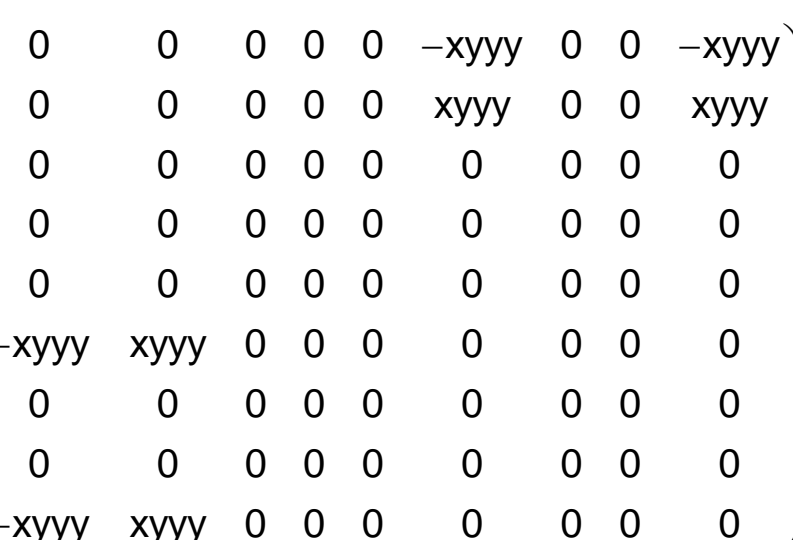

$\left(\begin{array}{ccccc}0 & \text { xyyy } & 0 & 0 & 0 \\ -x y y y & 0 & 0 & 0 & 0 \\ 0 & 0 & 0 & 0 & 0\end{array}\right)$

$\left(\begin{array}{cccccccccc}0 & \text { xyyy } & 0 & 0 & 0 & 0 & 0 & -x y y y & 0 & 0 \\ -x y y & 0 & 0 & x y y & 0 & 0 & 0 & 0 & 0 & 0\end{array}\right)$ $\left.\begin{array}{cccccccccc}-x y y y & 0 & 0 & x y y y & 0 & 0 & 0 & 0 & 0 & 0 \\ 0 & 0 & 0 & 0 & 0 & 0 & 0 & 0 & 0 & 0\end{array}\right)$

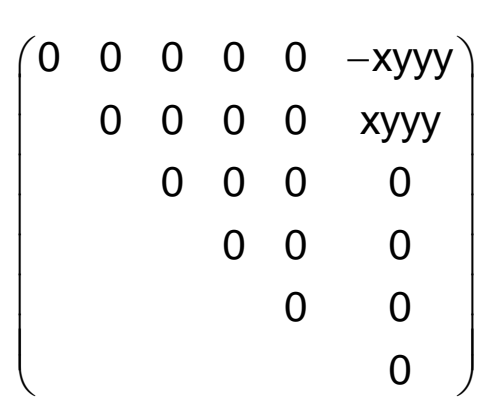

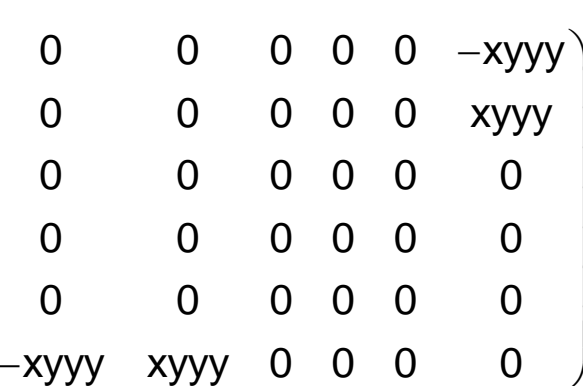




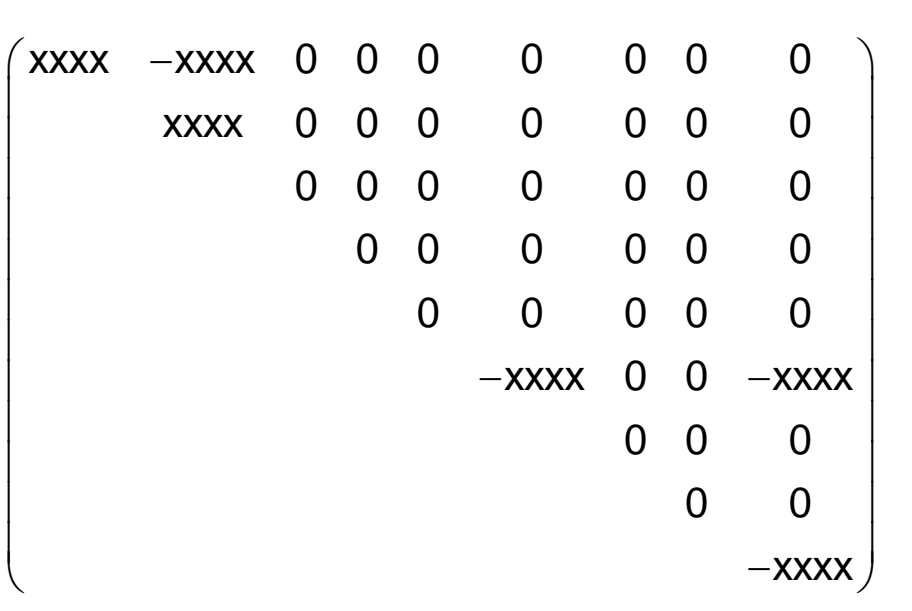

$\left[\mathrm{V}^{2}\right] \mathrm{V}^{2}\left(\begin{array}{ccccccccc}\mathrm{x} x \mathrm{xx} & \mathrm{xxyy} & \mathrm{xxzz} & 0 & 0 & 0 & 0 & 0 & 0 \\ \mathrm{xxyy} & \mathrm{xxxx} & \mathrm{xxzz} & 0 & 0 & 0 & 0 & 0 & 0 \\ \mathrm{zzxx} & \mathrm{zzxx} & \mathrm{zzzz} & 0 & 0 & 0 & 0 & 0 & 0 \\ 0 & 0 & 0 & \mathrm{yzyz} & 0 & 0 & \mathrm{zxzx} & 0 & 0 \\ 0 & 0 & 0 & 0 & \mathrm{zxzx} & 0 & 0 & \mathrm{yzyz} & 0 \\ 0 & 0 & 0 & 0 & 0 & \mathrm{xyxy} & 0 & 0 & \text { xyxy }\end{array}\right)($ and $x x x x=x x y y+2 x y x y$ $\left(\begin{array}{ccccccccc}x x x x & -x x x x & 0 & 0 & 0 & 0 & 0 & 0 & 0 \\ -x x x x & x x x x & 0 & 0 & 0 & 0 & 0 & 0 & 0 \\ 0 & 0 & 0 & 0 & 0 & 0 & 0 & 0 & 0 \\ 0 & 0 & 0 & 0 & 0 & 0 & 0 & 0 & 0 \\ 0 & 0 & 0 & 0 & 0 & 0 & 0 & 0 & 0 \\ 0 & 0 & 0 & 0 & 0 & -x x x x & 0 & 0 & -x x x x\end{array}\right)$ and $y y x y-x x x y=2 x y x x$

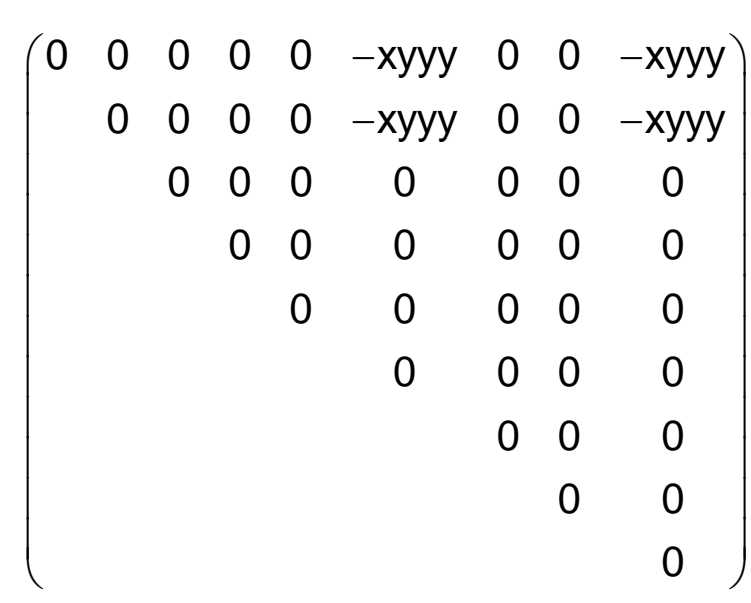

$\left(\begin{array}{ccccccccc}0 & 0 & 0 & 0 & 0 & -x y y y & 0 & 0 & -x y y y \\ 0 & 0 & 0 & 0 & 0 & x y y y & 0 & 0 & \text { xyyy } \\ 0 & 0 & 0 & 0 & 0 & 0 & 0 & 0 & 0 \\ 0 & 0 & 0 & 0 & 0 & 0 & 0 & 0 & 0 \\ 0 & 0 & 0 & 0 & 0 & 0 & 0 & 0 & 0 \\ -x y y y & x y y y & 0 & 0 & 0 & 0 & 0 & 0 & 0\end{array}\right)$ 

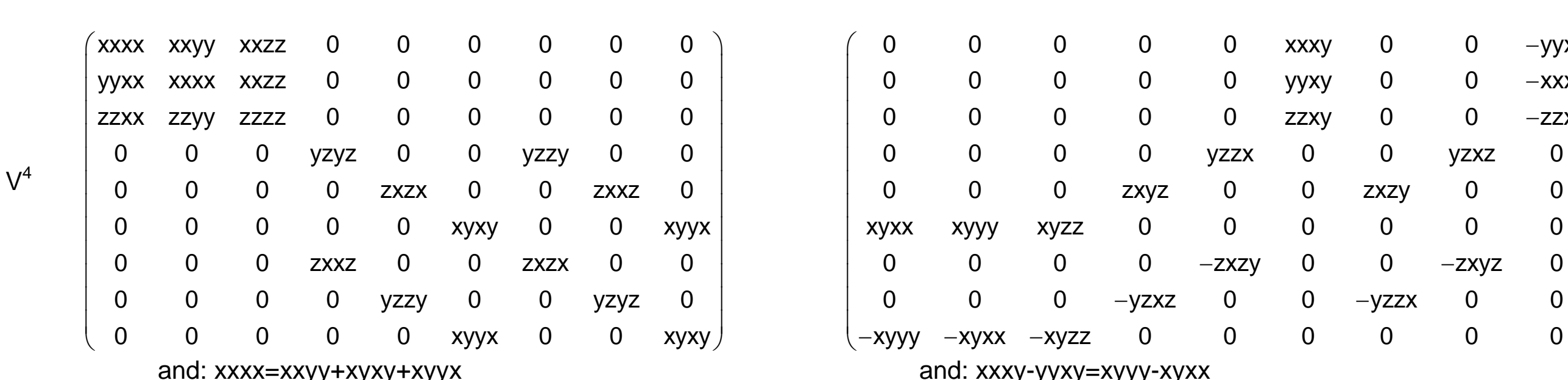
and: $x x x x=x x y y+x y x y+x y y x$

$\left[V^{4}\right] \quad\left(\begin{array}{ccccc}3 x x y y & 0 & 0 & y y z z & 0 \\ 0 & 3 x x y y & 0 & y y z z & 0 \\ 0 & 0 & z z z z & x x y y & 0\end{array}\right)$

$\left(\begin{array}{lllll}0 & 0 & 0 & 0 & 0 \\ 0 & 0 & 0 & 0 & 0 \\ 0 & 0 & 0 & 0 & 0\end{array}\right)$

$\begin{array}{lllll}0 & 0 & 0 & 0 & 0 \\ 0 & 0 & 0 & 0 & 0\end{array}$

$\left.\mathrm{V}^{3} \mathrm{~V}^{3}\right]\left(\begin{array}{cccccccccc}3 x x y y & 0 & 0 & x x y y & 0 & 0 & y y z z & 0 & 0 & 0 \\ 0 & 3 x x y y & 0 & 0 & y y z z & 0 & 0 & x x y y & 0 & 0 \\ 0 & 0 & z z z & 0 & 0 & z z x & 0 & 0 & z z x & 0\end{array}\right)$

$\left(\begin{array}{cccccccccc}0 & 3 x y x x & 0 & 0 & x y z z & 0 & 0 & x y x x & 0 & 0 \\ -3 x y x & 0 & 0 & -x y y x & 0 & 0 & -x y z z & 0 & 0 & 0\end{array}\right)$

$\left.\begin{array}{cccccccccc}-3 x y x x & 0 & 0 & -x y x x & 0 & 0 & -x y z z & 0 & 0 & 0 \\ 0 & 0 & 0 & 0 & 0 & 0 & 0 & 0 & 0 & 2 x y z\end{array}\right)$

$\left(\begin{array}{llllll}0 & 0 & 0 & 0 & 0 & 0 \\ & 0 & 0 & 0 & 0 & 0 \\ & & 0 & 0 & 0 & 0 \\ & & & 0 & 0 & 0 \\ & & & & 0 & 0 \\ & & & & & 0\end{array}\right)$

and $x y x y=1 / 2(x x x x-x x y y)$

$\left[\mathrm{V}^{2}\right]^{2}\left(\begin{array}{cccccc}\mathrm{xxxx} & \mathrm{xxyy} & \mathrm{xxzz} & 0 & 0 & 0 \\ \mathrm{xxyy} & \mathrm{xxxx} & \mathrm{xxzz} & 0 & 0 & 0 \\ \mathrm{zzxx} & \mathrm{zzxx} & \mathrm{zzzz} & 0 & 0 & 0 \\ 0 & 0 & 0 & \mathrm{yzyz} & 0 & 0 \\ 0 & 0 & 0 & 0 & \mathrm{yzyz} & 0 \\ 0 & 0 & 0 & 0 & 0 & \mathrm{xyxy}\end{array}\right)$

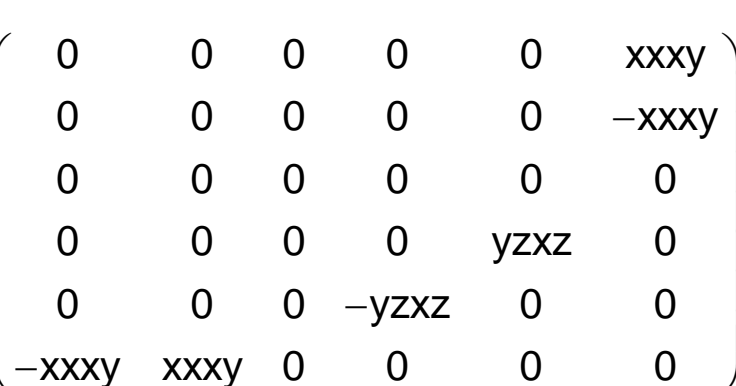

$\left(\begin{array}{lllllllll}0 & 0 & 0 & 0 & 0 & 0 & 0 & 0 & 0 \\ 0 & 0 & 0 & 0 & 0 & 0 & 0 & 0 & 0\end{array}\right)$ $\begin{array}{lllllllll}0 & 0 & 0 & 0 & 0 & 0 & 0 & 0 & 0 \\ 0 & 0 & 0 & 0 & 0 & 0 & 0 & 0 & 0\end{array}$ $\begin{array}{lllllllll}0 & 0 & 0 & 0 & 0 & 0 & 0 & 0 & 0 \\ 0 & 0 & 0 & 0 & 0 & 0 & 0 & 0 & 0\end{array}$ $\begin{array}{lllllllll}0 & 0 & 0 & 0 & 0 & 0 & 0 & 0 & 0 \\ 0 & 0 & 0 & 0 & 0 & 0 & 0 & 0 & 0\end{array}$ $\begin{array}{lllllllll}0 & 0 & 0 & 0 & 0 & 0 & 0 & 0 & 0 \\ 0 & 0 & 0 & 0 & 0 & 0 & 0 & 0 & 0\end{array}$ $\begin{array}{llllllllll}0 & 0 & 0 & 0 & 0 & 0 & 0 & 0 & 0 \\ 0 & 0 & 0 & 0 & 0 & 0 & 0 & 0 & 0\end{array}$ $\begin{array}{lllllllll}0 & 0 & 0 & 0 & 0 & 0 & 0 & 0 & 0\end{array}$ $\begin{array}{lllllllll}0 & 0 & 0 & 0 & 0 & 0 & 0 & 0 & 0 \\ 0 & 0 & 0 & 0 & 0 & 0 & 0 & 0 & 0\end{array}$

$\left(\begin{array}{lllll}0 & 0 & 0 & 0 & 0 \\ 0 & 0 & 0 & 0 & 0 \\ 0 & 0 & 0 & 0 & 0\end{array}\right)$ $\left(\begin{array}{lllll}0 & 0 & 0 & 0 & 0 \\ 0 & 0 & 0 & 0 & 0\end{array}\right)$

$\left(\begin{array}{llllllllll}0 & 0 & 0 & 0 & 0 & 0 & 0 & 0 & 0 & 0 \\ 0 & 0 & 0 & 0 & 0 & 0 & 0 & 0 & 0 & 0\end{array}\right)$ $\begin{array}{lllllllllll}0 & 0 & 0 & 0 & 0 & 0 & 0 & 0 & 0 & 0 \\ 0 & 0 & 0 & 0 & 0 & 0 & 0 & 0 & 0 & 0\end{array}$ $\begin{array}{lllllllllll}0 & 0 & 0 & 0 & 0 & 0 & 0 & 0 & 0 & 0\end{array}$

$\left(\begin{array}{llllllllll}0 & 0 & 0 & 0 & 0 & 0 & 0 & 0 & 0 & 0 \\ 0 & 0 & 0 & 0 & 0 & 0 & 0 & 0 & 0 & 0\end{array}\right)$ $\begin{array}{lllllllllll}0 & 0 & 0 & 0 & 0 & 0 & 0 & 0 & 0 & 0\end{array}$

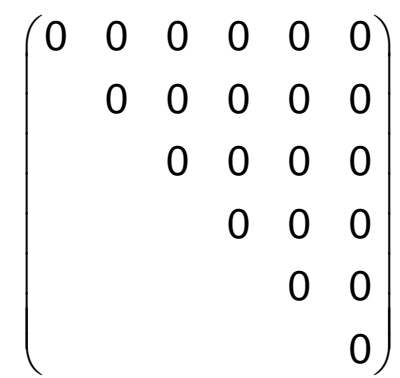

$\begin{array}{llll}0 & 0 & 0 & 0\end{array}$

$\begin{array}{lll}0 & 0 & 0 \\ & 0 & 0 \\ & & 0\end{array}$

$\begin{array}{ll}0 & 0 \\ & 0\end{array}$

$\begin{array}{lllll}0 & 0 & 0 & 0 & 0 \\ 0 & 0 & 0 & 0\end{array}$ $\begin{array}{llllll}0 & 0 & 0 & 0 & 0 & 0\end{array}$ $\begin{array}{llllll}0 & 0 & 0 & 0 & 0 & 0 \\ 0 & 0 & 0 & 0 & 0 & 0\end{array}$ $\begin{array}{llllll}0 & 0 & 0 & 0 & 0 & 0 \\ 0 & 0 & 0 & 0 & 0 & 0\end{array}$ $\begin{array}{llllll}0 & 0 & 0 & 0 & 0 & 0 \\ 0 & 0 & 0 & 0 & 0 & 0\end{array}$ $\begin{array}{llllll}0 & 0 & 0 & 0 & 0 & 0\end{array}$ 
$n>4 \quad \mathbf{C}_{\alpha v} 1^{\prime}=\infty \mathbf{m m} 1$

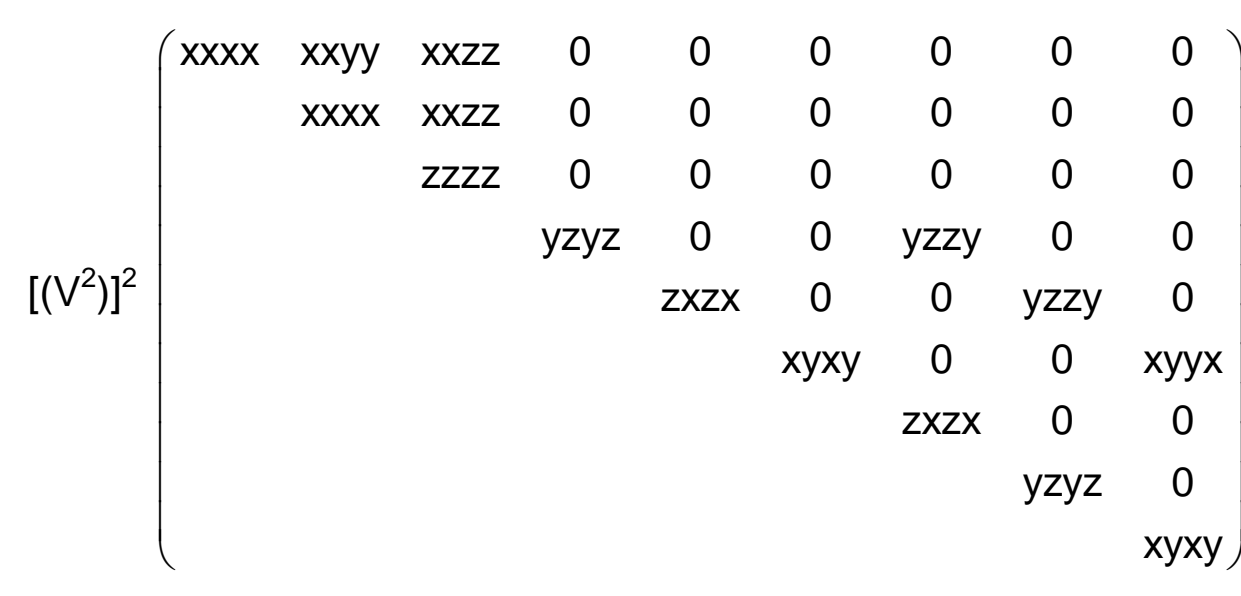

and $x x x x=x x y y+y z y z+z y z y$

$\left[\mathrm{V}^{2}\right] \mathrm{V}^{2}\left(\begin{array}{ccccccccc}\mathrm{x} x \mathrm{x} & \mathrm{xxyy} & \mathrm{xxzz} & 0 & 0 & 0 & 0 & 0 & 0 \\ \mathrm{xxyy} & \mathrm{xxxx} & \mathrm{xxzz} & 0 & 0 & 0 & 0 & 0 & 0 \\ \mathrm{zzxx} & \mathrm{zzxx} & \mathrm{zzzz} & 0 & 0 & 0 & 0 & 0 & 0 \\ 0 & 0 & 0 & \mathrm{yzyz} & 0 & 0 & \mathrm{zxzx} & 0 & 0 \\ 0 & 0 & 0 & 0 & \mathrm{zxzx} & 0 & 0 & \mathrm{yzyz} & 0 \\ 0 & 0 & 0 & 0 & 0 & \mathrm{xyxy} & 0 & 0 & \mathrm{xyxy}\end{array}\right)$
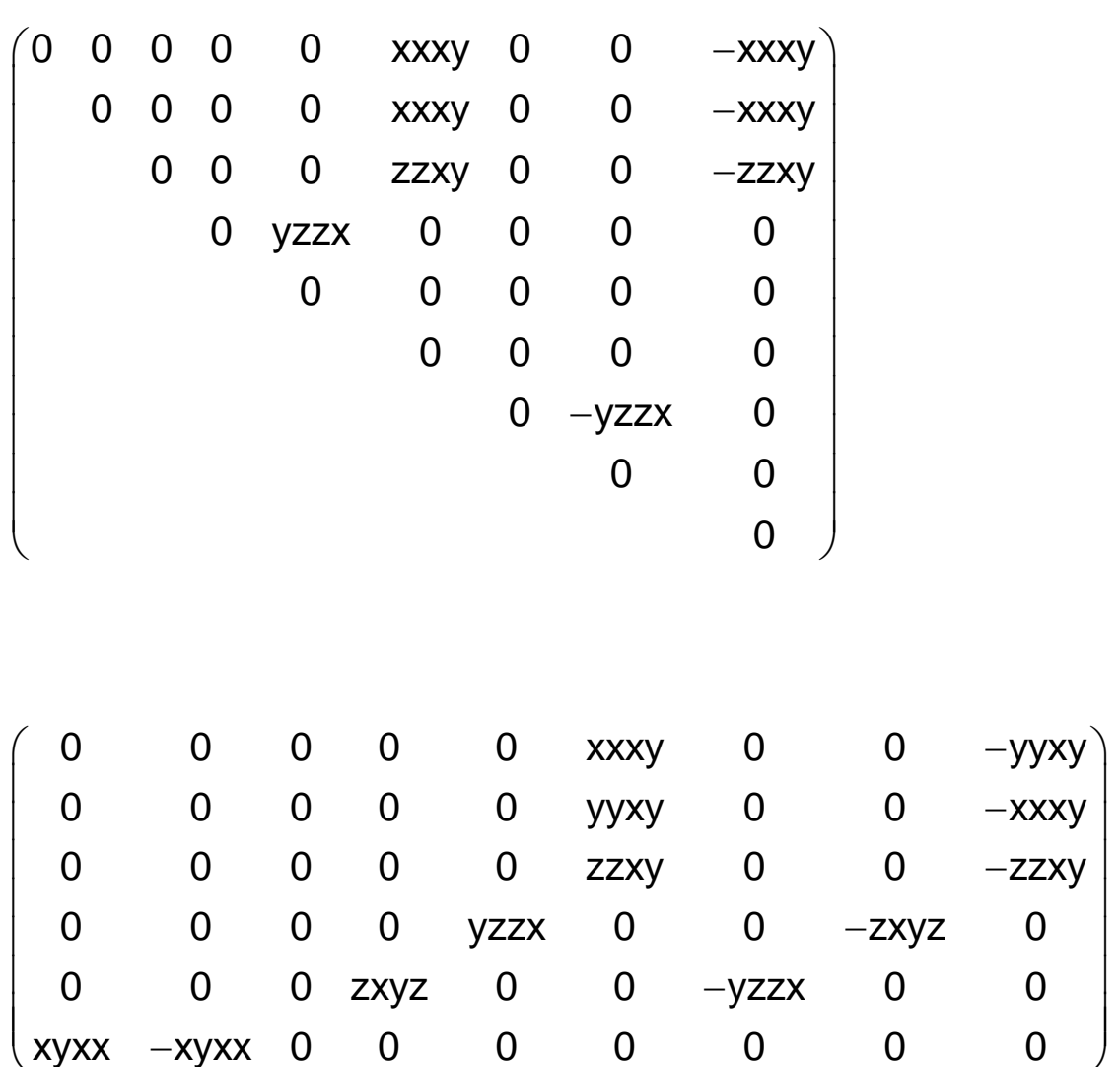

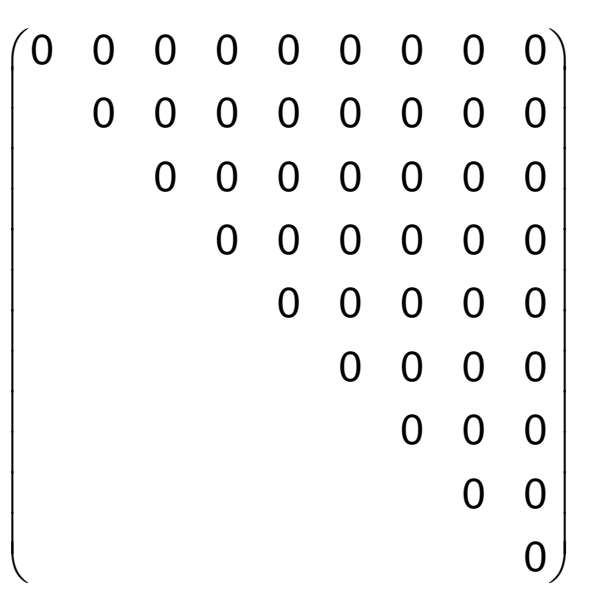

$\left(\begin{array}{lllllllll}0 & 0 & 0 & 0 & 0 & 0 & 0 & 0 & 0 \\ 0 & 0 & 0 & 0 & 0 & 0\end{array}\right.$ $\begin{array}{lllllllll}0 & 0 & 0 & 0 & 0 & 0 & 0 & 0 & 0 \\ 0 & 0 & 0 & 0 & 0 & 0 & 0 & 0 & 0\end{array}$ $0 \begin{array}{lllllllll}0 & 0 & 0 & 0 & 0 & 0 & 0 & 0 & 0 \\ 0 & 0 & 0 & 0 & 0 & 0 & 0 & 0 & 0\end{array}$ $\begin{array}{llllllllll}0 & 0 & 0 & 0 & 0 & 0 & 0 & 0 & 0 \\ 0 & 0 & 0 & 0 & 0 & 0 & 0 & 0 & 0\end{array}$ $\left(\begin{array}{lllllllll}0 & 0 & 0 & 0 & 0 & 0 & 0 & 0 & 0 \\ 0 & 0 & 0 & 0 & 0 & 0 & 0 & 0 & 0\end{array}\right)$ $\left(\begin{array}{lllllllll}0 & 0 & 0 & 0 & 0 & 0 & 0 & 0 & 0\end{array}\right)$

$\begin{array}{llllllll}0 & 0 & 0 & 0 & 0 & 0 & 0 & 0\end{array}$

$0 \begin{array}{lllllll}0 & 0 & 0 & 0 & 0 & 0 & 0\end{array}$

$\begin{array}{llllll}0 & 0 & 0 & 0 & 0 & 0 \\ 0 & 0 & 0 & 0 & 0\end{array}$

$\begin{array}{llll}0 & 0 & 0 & 0\end{array}$

$\begin{array}{lll}0 & 0 & 0 \\ & 0 & 0 \\ & & 0\end{array}$

$\begin{array}{ll}0 & 0 \\ & 0\end{array}$

$\begin{array}{llllllll}0 & 0 & 0 & 0 & 0 & 0 & 0 & 0 \\ 0 & 0 & 0 & 0 & 0 & 0 & 0 & 0\end{array}$ $\begin{array}{lllllllll}0 & 0 & 0 & 0 & 0 & 0 & 0 & 0 & 0 \\ 0 & 0 & 0 & 0 & 0 & 0 & 0 & 0 & 0\end{array}$ $\begin{array}{lllllllll}0 & 0 & 0 & 0 & 0 & 0 & 0 & 0 & 0 \\ 0 & 0 & 0 & 0 & 0 & 0 & 0 & 0 & 0\end{array}$

$\begin{array}{llllllllll}0 & 0 & 0 & 0 & 0 & 0 & 0 & 0 & 0 \\ 0 & 0 & 0 & 0 & 0 & 0 & 0 & 0 & 0\end{array}$

$\begin{array}{lllllllll}0 & 0 & 0 & 0 & 0 & 0 & 0 & 0 & 0 \\ 0 & 0 & 0 & 0 & 0 & 0 & 0 & 0 & 0\end{array}$ 


\section{1}

e a

$\left(\begin{array}{lllllllll}x x x x & x x y y & x x z z & 0 & 0 & 0 & 0 & 0 & 0 \\ \text { yyxx } & \text { yyyy } & \text { yyzz } & 0 & 0 & 0 & 0 & 0 & 0 \\ z z x x & z z y y & z z z z & 0 & 0 & 0 & 0 & 0 & 0\end{array}\right)$ $\begin{array}{ccccccccc}\text { zzxx } & \text { zzyy } & \text { zzzz } & 0 & 0 & 0 & 0 & 0 & 0 \\ 0 & 0 & 0 & y z y z & 0 & 0 & y z z y & 0 & 0\end{array}$

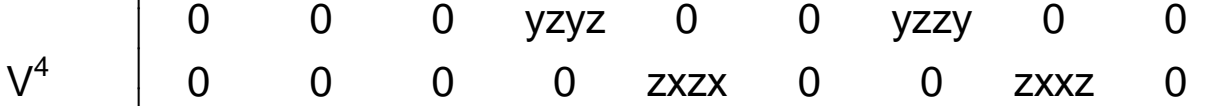
$\begin{array}{ccccccccc}0 & 0 & 0 & y z y z & 0 & 0 & y z z y & 0 & 0 \\ 0 & 0 & 0 & 0 & z x z x & 0 & 0 & z x x z & 0 \\ 0 & 0 & 0 & 0 & 0 & x y x y & 0 & 0 & x y y x\end{array}$ $\begin{array}{lllllllll}0 & 0 & 0 & 0 & z x z x & 0 & 0 & z x y z & 0 \\ 0 & 0 & 0 & 0 & 0 & x y x y & 0 & 0 & x y y x \\ 0 & 0 & 0 & z y y z & 0 & 0 & z y z y & 0 & 0\end{array}$ $\begin{array}{ccccccccc}0 & 0 & 0 & z y y z & 0 & 0 & z y z y & 0 & 0 \\ 0 & 0 & 0 & 0 & x z z x & 0 & 0 & x z x z & 0\end{array}$ $\begin{array}{lllllllll}0 & 0 & 0 & 0 & 0 & y x x y & 0 & 0 & y x y x\end{array}$

$\left[\mathrm{V}^{4}\right] \quad\left(\begin{array}{ccccc}\mathrm{xxxx} & 0 & 0 & \mathrm{yyzz} & 0 \\ 0 & y y y y & 0 & \mathrm{zzxx} & 0 \\ 0 & 0 & z z z z & \mathrm{xxyy} & 0\end{array}\right)$

$\left(\begin{array}{lllllllll}0 & 0 & 0 & 0 & 0 & 0 & 0 & 0 & 0 \\ 0 & 0 & 0 & 0 & 0 & 0 & 0 & 0 & 0\end{array}\right)$ $0 \begin{array}{lllllllll}0 & 0 & 0 & 0 & 0 & 0 & 0 & 0 & 0 \\ 0 & 0 & 0 & 0 & 0 & 0 & 0 & 0 & 0\end{array}$ $0 \begin{array}{llllllllll}0 & 0 & 0 & 0 & 0 & 0 & 0 & 0 & 0 \\ 0 & 0 & 0 & 0 & 0 & 0 & 0 & 0 & 0\end{array}$ $0 \begin{array}{llllllllll}0 & 0 & 0 & 0 & 0 & 0 & 0 & 0 & 0 \\ 0 & 0 & 0 & 0 & 0 & 0 & 0 & 0 & 0\end{array}$ $\begin{array}{lllllllll}0 & 0 & 0 & 0 & 0 & 0 & 0 & 0 & 0 \\ 0 & 0 & 0 & 0 & 0 & 0 & 0 & 0 & 0\end{array}$ $0 \begin{array}{llllllllll}0 & 0 & 0 & 0 & 0 & 0 & 0 & 0 & 0 \\ 0 & 0 & 0 & 0 & 0 & 0 & 0 & 0 & 0\end{array}$ $\begin{array}{lllllllll}0 & 0 & 0 & 0 & 0 & 0 & 0 & 0 & 0 \\ 0 & 0 & 0 & 0 & 0 & 0 & 0 & 0 & 0\end{array}$ $\begin{array}{lllllllll}0 & 0 & 0 & 0 & 0 & 0 & 0 & 0 & 0\end{array}$

$\left.\mathrm{V}^{3} \mathrm{~V}^{3}\right]\left(\begin{array}{cccccccccc}\mathrm{xxxx} & 0 & 0 & \mathrm{xxyy} & 0 & 0 & \mathrm{xxzz} & 0 & 0 & 0 \\ 0 & \mathrm{yyyy} & 0 & 0 & \mathrm{yyzz} & 0 & 0 & \mathrm{yyxx} & 0 & 0 \\ 0 & 0 & z z z z & 0 & 0 & z z x x & 0 & 0 & z z y & 0\end{array}\right)$ $\left(\begin{array}{lllll}0 & 0 & 0 & 0 & 0 \\ 0 & 0 & 0 & 0 & 0 \\ 0 & 0 & 0 & 0 & 0\end{array}\right)$

$\begin{array}{llllll}0 & 0 & 0 & 0 & 0 & 0 \\ 0 & 0 & 0 & 0\end{array}$

$\begin{array}{llllllllll}0 & 0 & 0 & 0 & 0 & 0 & 0 & 0 & 0 & 0 \\ 0 & 0 & 0 & 0 & 0 & 0 & 0 & 0 & 0 & 0\end{array}$ $\left.\begin{array}{llllllllll}0 & 0 & 0 & 0 & 0 & 0 & 0 & 0 & 0 & 0 \\ 0 & 0 & 0 & 0 & 0 & 0 & 0 & 0 & 0 & 0\end{array}\right)$

$$
\left(\begin{array}{llllll}
0 & 0 & 0 & 0 & 0 & 0 \\
& 0 & 0 & 0 & 0 & 0 \\
& & 0 & 0 & 0 & 0 \\
& & & 0 & 0 & 0 \\
& & & & 0 & 0 \\
& & & & & 0
\end{array}\right)
$$

$\left(\begin{array}{llllll}0 & 0 & 0 & 0 & 0 & 0 \\ 0 & 0 & 0 & 0 & 0 & 0 \\ 0 & 0 & 0 & 0 & 0 & 0\end{array}\right)$ $\begin{array}{lllllll}0 & 0 & 0 & 0 & 0 & 0 \\ 0 & 0 & 0 & 0 & 0\end{array}$ $0 \begin{array}{llllll}0 & 0 & 0 & 0 & 0 & 0\end{array}$ $\begin{array}{llllll}0 & 0 & 0 & 0 & 0 & 0 \\ 0 & 0 & 0 & 0 & 0 & 0\end{array}$ $0 \begin{array}{llllll}0 & 0 & 0 & 0 & 0 & 0\end{array}$

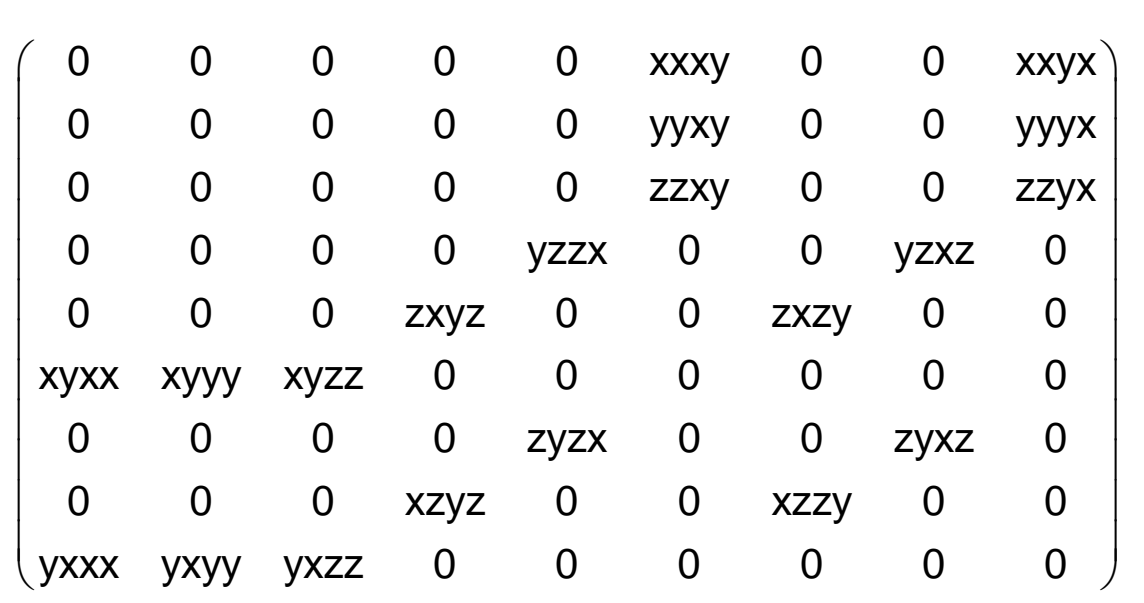

$\left(\begin{array}{ccccc}0 & x y y y & 0 & 0 & 0 \\ y x x x & 0 & 0 & 0 & 0 \\ 0 & 0 & 0 & 0 & z z x y\end{array}\right)$

$\left(\begin{array}{cccccccccc}0 & x y y y & 0 & 0 & x y z z & 0 & 0 & x y x x & 0 & 0 \\ y x x x & 0 & 0 & y y y & 0 & 0 & y x z & 0 & 0 & 0\end{array}\right)$

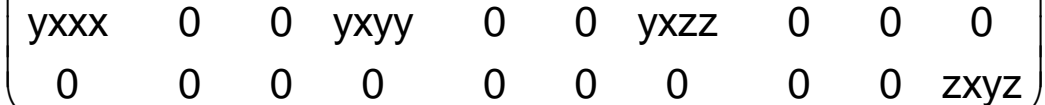

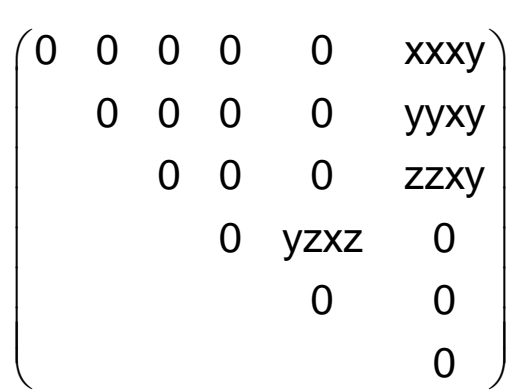

$\left(\begin{array}{llllll}0 & 0 & 0 & 0 & 0 & x x x y \\ 0 & 0 & 0 & 0 & 0 & y y x y \\ 0 & 0 & 0 & 0 & 0 & z x y y\end{array}\right)$ $\begin{array}{llllll}0 & 0 & 0 & 0 & 0 & \text { yyxy } \\ 0 & 0 & 0 & 0 & 0 & z z x y \\ 0 & 0 & 0 & 0 & v y x z & 0\end{array}$ $\begin{array}{llllll}0 & 0 & 0 & 0 & 0 & z z x y\end{array}$ $\begin{array}{llllll}0 & 0 & 0 & 0 & y z x z & 0 \\ 0 & 0 & 0 & x z y & 0\end{array}$ $\begin{array}{lllccc}0 & 0 & 0 & x z y z & 0 & 0\end{array}$ $\left(\begin{array}{lllllllll}0 & 0 & 0 & 0 & 0 & 0 & 0 & 0 & 0 \\ 0 & 0 & 0 & 0 & 0 & 0 & 0 & 0 & 0\end{array}\right.$ $\begin{array}{lllllllll}0 & 0 & 0 & 0 & 0 & 0 & 0 & 0 & 0 \\ 0 & 0 & 0 & 0 & 0 & 0 & 0 & 0 & 0\end{array}$ $\begin{array}{llllllllll}0 & 0 & 0 & 0 & 0 & 0 & 0 & 0 & 0 \\ 0 & 0 & 0 & 0 & 0 & 0 & 0 & 0 & 0 \\ 0 & 0 & 0 & 0 & 0 & 0 & 0 & 0 & 0\end{array}$ $\begin{array}{llllllllll}0 & 0 & 0 & 0 & 0 & 0 & 0 & 0 & 0 \\ 0 & 0 & 0 & 0 & 0 & 0 & 0 & 0 & 0 & 0\end{array}$ $\begin{array}{llllllllll}0 & 0 & 0 & 0 & 0 & 0 & 0 & 0 & 0\end{array}$ $\begin{array}{llllllllll}0 & 0 & 0 & 0 & 0 & 0 & 0 & 0 & 0\end{array}$ $\begin{array}{lllllllll}0 & 0 & 0 & 0 & 0 & 0 & 0 & 0 & 0 \\ 0 & 0 & 0 & 0 & 0 & 0 & 0 & 0 & 0\end{array}$ $\begin{array}{llllllllll}0 & 0 & 0 & 0 & 0 & 0 & 0 & 0 & 0\end{array}$

$\left(\begin{array}{lllll}0 & 0 & 0 & 0 & 0 \\ 0 & 0 & 0 & 0 & 0 \\ 0 & 0 & 0 & 0 & 0\end{array}\right)$ $\left.\begin{array}{lllll}0 & 0 & 0 & 0 & 0\end{array}\right)$

$\left(\begin{array}{llllllllll}0 & 0 & 0 & 0 & 0 & 0 & 0 & 0 & 0 & 0 \\ 0 & 0 & 0 & 0 & 0 & 0 & 0 & 0 & 0 & 0\end{array}\right.$ $\left.\begin{array}{llllllllll}0 & 0 & 0 & 0 & 0 & 0 & 0 & 0 & 0 & 0 \\ 0 & 0 & 0 & 0 & 0 & 0 & 0 & 0 & 0 & 0\end{array}\right)$ $\begin{array}{lllllllllll}0 & 0 & 0 & 0 & 0 & 0 & 0 & 0 & 0 & 0\end{array}$

$\left(\begin{array}{llllll}y y x x & y y y y & y y z z & 0 & 0 & 0 \\ z z x x & z z y y & z z z z & 0 & 0 & 0 \\ 0 & 0 & 0 & y z z z & 0 & 0\end{array}\right)$ $\left(\begin{array}{cccccc}0 & 0 & 0 & y z y z & 0 & 0 \\ 0 & 0 & 0 & 0 & x z x z & 0 \\ 0 & 0 & 0 & 0 & 0 & x y x y\end{array}\right.$

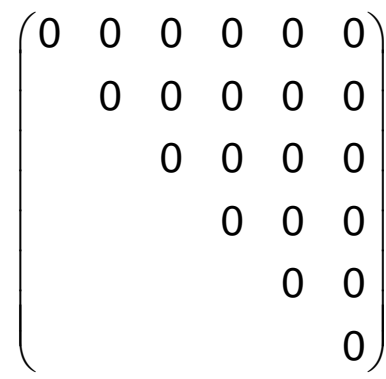

$\left(\begin{array}{llllll}0 & 0 & 0 & 0 & 0 & 0 \\ 0 & 0 & 0 & 0 & 0 & 0 \\ 0 & 0 & 0 & 0 & 0 & 0\end{array}\right)$ $\begin{array}{lllllll}0 & 0 & 0 & 0 & 0 & 0 \\ 0 & 0 & 0 & 0 & 0 & 0\end{array}$ $0 \begin{array}{llllll}0 & 0 & 0 & 0 & 0 & 0 \\ 0 & 0 & 0 & 0\end{array}$ $\begin{array}{llllll}0 & 0 & 0 & 0 & 0 & 0 \\ 0 & 0 & 0\end{array}$ $\begin{array}{llllll}0 & 0 & 0 & 0 & 0 & 0 \\ 0 & 0 & 0 & 0 & 0 & 0\end{array}$ 


$$
\left(\begin{array}{ccccccccc}
\text { xxxx } & \text { xxyy } & \text { xxzz } & 0 & 0 & 0 & 0 & 0 & 0 \\
& \text { yyyy } & \text { yzzz } & 0 & 0 & 0 & 0 & 0 & 0 \\
& & \text { zzzz } & 0 & 0 & 0 & 0 & 0 & 0 \\
& & & \text { yzyz } & 0 & 0 & \text { yzzy } & 0 & 0 \\
& & & & \text { zxzx } & 0 & 0 & \text { zxxz } & 0 \\
& & & & & \text { xyxy } & 0 & 0 & \text { xyyx } \\
& & & & & & \text { zyzy } & 0 & 0 \\
& & & & & & & \text { xzxz } & 0 \\
& & & & & & & & \text { yxyx }
\end{array}\right)
$$

$\left(\begin{array}{lllllllll}0 & 0 & 0 & 0 & 0 & 0 & 0 & 0 & 0 \\ \hline & 0 & 0 & 0 & 0 & 0 & 0 & 0 & 0\end{array}\right.$ $\begin{array}{llllllll}0 & 0 & 0 & 0 & 0 & 0 & 0 & 0\end{array}$

$\begin{array}{lllllll}0 & 0 & 0 & 0 & 0 & 0 & 0\end{array}$

$\begin{array}{llllll}0 & 0 & 0 & 0 & 0 & 0\end{array}$

$\begin{array}{lllll}0 & 0 & 0 & 0 & 0\end{array}$

$\begin{array}{llll}0 & 0 & 0 & 0\end{array}$

$\begin{array}{lll}0 & 0 & 0 \\ & 0 & 0\end{array}$

0

$\left.\mathrm{V}^{2}\right] \mathrm{V}^{2}\left(\begin{array}{ccccccccc}\mathrm{xxxx} & \mathrm{xxyy} & \mathrm{xxzz} & 0 & 0 & 0 & 0 & 0 & 0 \\ \mathrm{yyxx} & \mathrm{yyyy} & \mathrm{yyzz} & 0 & 0 & 0 & 0 & 0 & 0 \\ \mathrm{zzxx} & \mathrm{zzyy} & \mathrm{zzzz} & 0 & 0 & 0 & 0 & 0 & 0 \\ 0 & 0 & 0 & \mathrm{yzyz} & 0 & 0 & \mathrm{yzzy} & 0 & 0 \\ 0 & 0 & 0 & 0 & \mathrm{zxzx} & 0 & 0 & \mathrm{zxxz} & 0 \\ 0 & 0 & 0 & 0 & 0 & \mathrm{xyxy} & 0 & 0 & \mathrm{xyyx}\end{array}\right)$ $\begin{array}{lllllllll}0 & 0 & 0 & 0 & 0 & 0 & 0 & 0 & 0 \\ 0 & 0 & 0 & 0 & 0 & 0 & 0 & 0 & 0\end{array}$ $\begin{array}{lllllllll}0 & 0 & 0 & 0 & 0 & 0 & 0 & 0 & 0 \\ 0 & 0 & 0 & 0 & 0 & 0 & 0 & 0 & 0\end{array}$

$\begin{array}{lllllllll}0 & 0 & 0 & 0 & 0 & 0 & 0 & 0 & 0 \\ 0 & 0 & 0 & 0 & 0 & 0 & 0 & 0 & 0\end{array}$

$\begin{array}{lllllllll}0 & 0 & 0 & 0 & 0 & 0 & 0 & 0 & 0\end{array}$

$\begin{array}{lllllllll}0 & 0 & 0 & 0 & 0 & 0 & 0 & 0 & 0\end{array}$

$$
\begin{aligned}
& \left(\begin{array}{lllllllll}
0 & 0 & 0 & 0 & 0 & x x x y & 0 & 0 & x x y x \\
& 0 & 0 & 0 & 0 & y y x y & 0 & 0 & y y y x
\end{array}\right. \\
& \begin{array}{llllllll}
0 & 0 & 0 & 0 & \text { yyxy } & 0 & 0 & \text { yyyx }
\end{array} \\
& \begin{array}{ccccccc}
0 & 0 & 0 & z z x y & 0 & 0 & z z y x
\end{array} \\
& \begin{array}{llllll}
0 & y z z x & 0 & 0 & y z x z & 0
\end{array} \\
& \begin{array}{ccccc}
0 & 0 & \text { zxzy } & 0 & 0 \\
0 & 0 & 0 & 0
\end{array} \\
& \begin{array}{cccc}
0 & 0 & 0 & 0 \\
0 & z y x z & 0
\end{array} \\
& \begin{array}{ccc}
0 & \text { zyxz } & 0 \\
0 & 0 \\
& & 0
\end{array}
\end{aligned}
$$

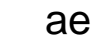

ae

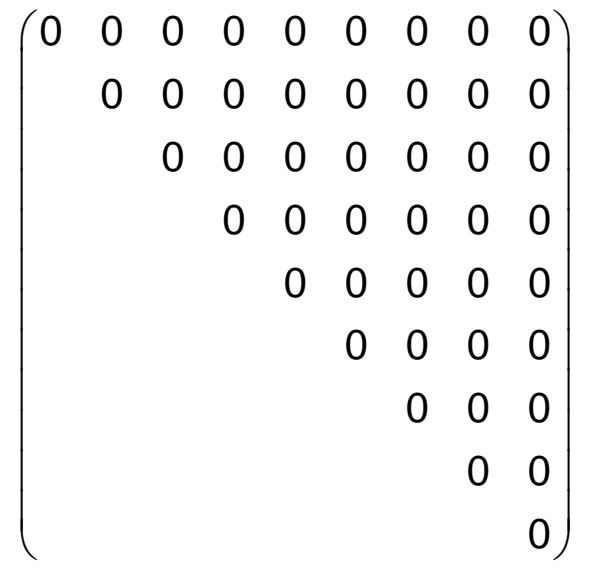

$\left(\begin{array}{ccccccccc}0 & 0 & 0 & 0 & 0 & \text { xxxy } & 0 & 0 & \text { xxyx } \\ 0 & 0 & 0 & 0 & 0 & \text { yyxy } & 0 & 0 & \text { yyyx } \\ 0 & 0 & 0 & 0 & 0 & \text { zzxy } & 0 & 0 & \text { zzyx } \\ 0 & 0 & 0 & 0 & \text { yzzx } & 0 & 0 & \text { yzxz } & 0 \\ 0 & 0 & 0 & \text { xzyz } & 0 & 0 & \text { zxzy } & 0 & 0 \\ \text { xyxx } & \text { xyyy } & \text { xyzz } & 0 & 0 & 0 & 0 & 0 & 0\end{array}\right)$

$\left(\begin{array}{lllllllll}0 & 0 & 0 & 0 & 0 & 0 & 0 & 0 & 0 \\ 0 & 0 & 0 & 0 & 0 & 0 & 0 & 0 & 0\end{array}\right)$ $\left.\begin{array}{lllllllll}0 & 0 & 0 & 0 & 0 & 0 & 0 & 0 & 0 \\ 0 & 0 & 0 & 0 & 0 & 0 & 0 & 0 & 0 \\ 0 & 0 & 0 & 0 & 0 & 0 & 0 & 0 & 0\end{array}\right)$

$\begin{array}{lllllllll}0 & 0 & 0 & 0 & 0 & 0 & 0 & 0 & 0\end{array}$

$\begin{array}{llllllllll}0 & 0 & 0 & 0 & 0 & 0 & 0 & 0 & 0 \\ 0 & 0 & 0 & 0 & 0 & 0 & 0 & 0 & 0\end{array}$

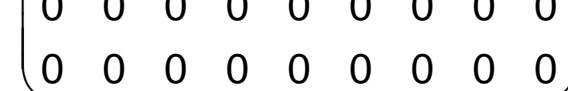


$\mathrm{V}^{4}\left(\begin{array}{ccccccccc}\mathrm{x} x \mathrm{x} x & \mathrm{xxyy} & \mathrm{xxzz} & 0 & 0 & 0 & 0 & 0 & 0 \\ \mathrm{yyxx} & \mathrm{xxxx} & \mathrm{xxzz} & 0 & 0 & 0 & 0 & 0 & 0 \\ \mathrm{zzxx} & \mathrm{zzyy} & \mathrm{zzzz} & 0 & 0 & 0 & 0 & 0 & 0 \\ 0 & 0 & 0 & \mathrm{yzyz} & 0 & 0 & \mathrm{yzzy} & 0 & 0 \\ 0 & 0 & 0 & 0 & \mathrm{zxzx} & 0 & 0 & \mathrm{zxxz} & 0 \\ 0 & 0 & 0 & 0 & 0 & \mathrm{xyxy} & 0 & 0 & \mathrm{xyyx} \\ 0 & 0 & 0 & \mathrm{zxxz} & 0 & 0 & \mathrm{zxzx} & 0 & 0 \\ 0 & 0 & 0 & 0 & \mathrm{yzzy} & 0 & 0 & \mathrm{yzyz} & 0 \\ 0 & 0 & 0 & 0 & 0 & \mathrm{xyyx} & 0 & 0 & \mathrm{xyxy}\end{array}\right)$

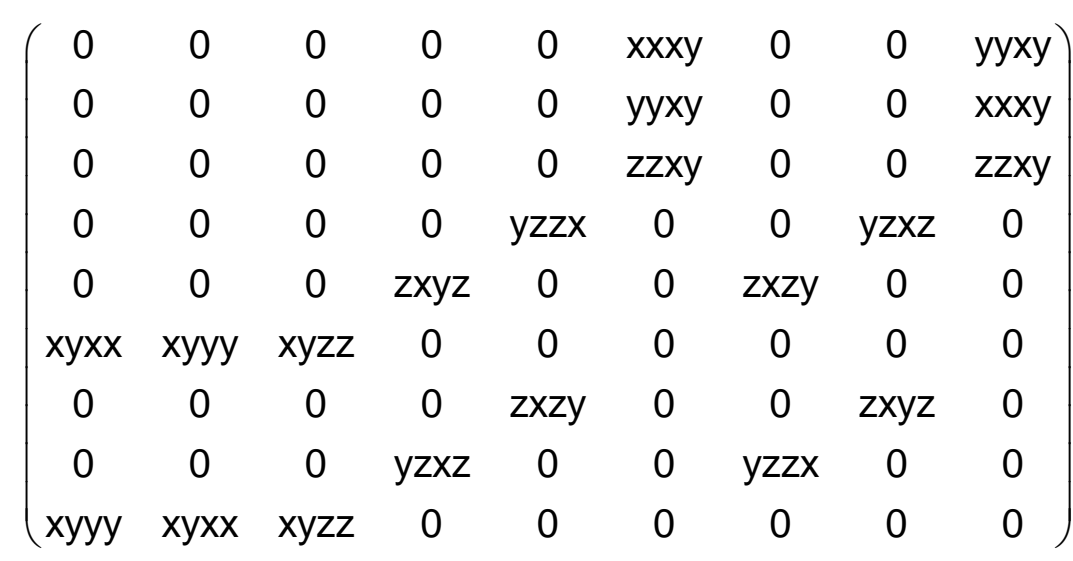

$\left[V^{4}\right] \quad\left(\begin{array}{ccccc}x x x x & 0 & 0 & y y z z & 0 \\ 0 & x x x x & 0 & y y z z & 0 \\ 0 & 0 & z z z z & x x y y & 0\end{array}\right)$

$\mathrm{V}\left[\mathrm{V}^{3}\right]\left(\begin{array}{cccccccccc}\mathrm{xxxx} & 0 & 0 & \mathrm{xxyy} & 0 & 0 & \mathrm{yyzz} & 0 & 0 & 0 \\ 0 & \mathrm{xxxx} & 0 & 0 & \mathrm{yyzz} & 0 & 0 & \mathrm{xxyy} & 0 & 0 \\ 0 & 0 & z z z z & 0 & 0 & z z x x & 0 & 0 & z z x x & 0\end{array}\right)$

$\left.\begin{array}{llllllllll}0 & 0 & z z z z & 0 & 0 & z z x x & 0 & 0 & z z x x & 0\end{array}\right)$

$\left[\left[^{2}\right]^{2}\right]\left(\begin{array}{cccccc}\text { xxxx } & \text { xxyy } & \text { xxzz } & 0 & 0 & 0 \\ & \text { yyyy } & \text { yyzz } & 0 & 0 & 0 \\ & & \text { zzzz } & 0 & 0 & 0 \\ & & & \text { yzyz } & 0 & 0 \\ & & & & \text { yzyz } & 0 \\ & & & & & \text { xyxy }\end{array}\right)$

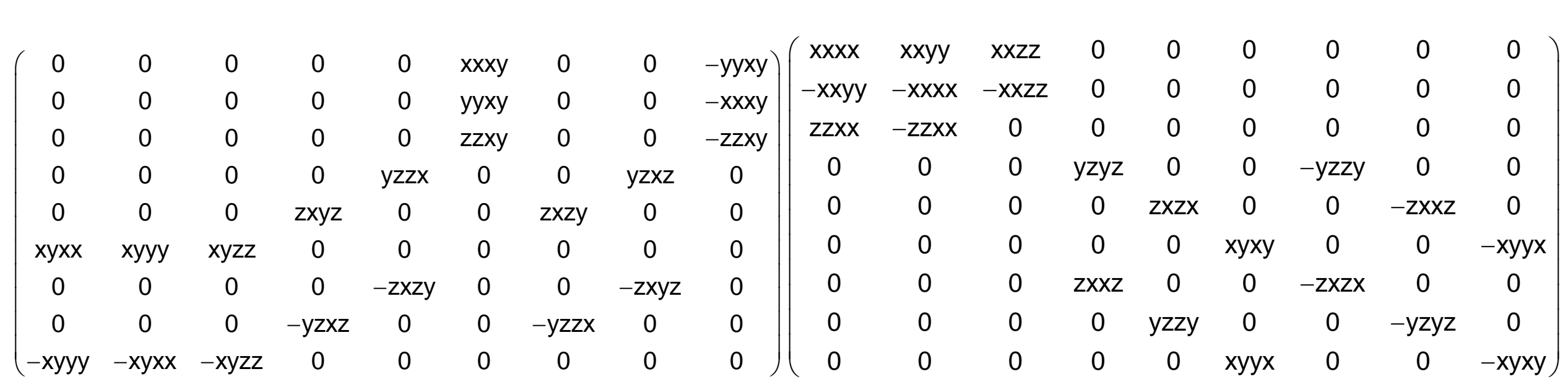

$\left[\mathrm{V}^{2}\right]^{2}\left(\begin{array}{cccccc}\mathrm{x} x \mathrm{x} & \mathrm{xxyy} & \mathrm{xxzz} & 0 & 0 & 0 \\ \mathrm{yyxx} & \mathrm{xxxx} & \mathrm{xxzz} & 0 & 0 & 0 \\ \mathrm{zzxx} & \mathrm{zzxx} & \mathrm{zzzz} & 0 & 0 & 0 \\ 0 & 0 & 0 & \mathrm{yzyz} & 0 & 0 \\ 0 & 0 & 0 & 0 & \mathrm{yzyz} & 0 \\ 0 & 0 & 0 & 0 & 0 & \mathrm{xyxy}\end{array}\right)$

$\left(\begin{array}{ccccc}0 & y x x x & 0 & 0 & 0 \\ y x x x & 0 & 0 & 0 & 0 \\ 0 & 0 & 0 & 0 & z z x y\end{array}\right)$

$\left(\begin{array}{llllllllll}0 & x y y y & 0 & 0 & x y z z & 0 & 0 & y x y y & 0 & 0 \\ x y y & 0 & 0 & y x y & 0 & 0 & x y z z & 0 & 0 & 0\end{array}\right)$

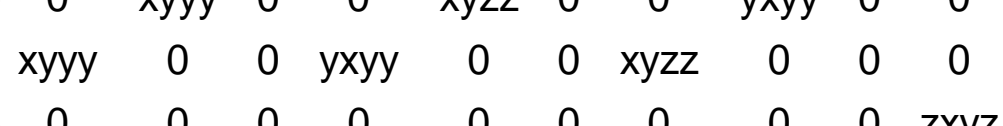

$$
\left(\begin{array}{cccccc}
0 & 0 & 0 & 0 & 0 & x x x y \\
& 0 & 0 & 0 & 0 & x x x y \\
& & 0 & 0 & 0 & z z x y \\
& & & 0 & y z x z & 0 \\
& & & & 0 & 0 \\
& & & & & 0
\end{array}\right)
$$

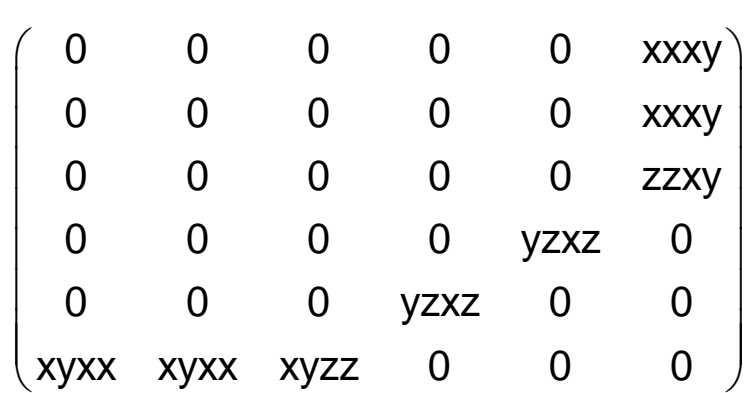

$\left(\begin{array}{cccccc}0 & 0 & 0 & 0 & 0 & \text { xxxy } \\ 0 & 0 & 0 & 0 & 0 & -x x x y \\ 0 & 0 & 0 & 0 & 0 & 0 \\ 0 & 0 & 0 & 0 & y z x z & 0 \\ 0 & 0 & 0 & -y z x z & 0 & 0 \\ x y x x & -x y x x & 0 & 0 & 0 & 0\end{array}\right)$

$\left(\begin{array}{cccccc}x x x x & x x y y & x x z z & 0 & 0 & 0 \\ -x x y y & -x x x x & -x x z z & 0 & 0 & 0 \\ z z x x & -z z x x & 0 & 0 & 0 & 0 \\ 0 & 0 & 0 & y z y z & 0 & 0 \\ 0 & 0 & 0 & 0 & -y z y z & 0 \\ 0 & 0 & 0 & 0 & 0 & 0\end{array}\right)$

$\left(\begin{array}{ccccc}0 & -y x x x & 0 & 0 & 0 \\ y x x x & 0 & 0 & 0 & 0 \\ 0 & 0 & 0 & 0 & 0\end{array}\right)$

$\left(\begin{array}{rrrrrrrrrr}0 & -y x x x & 0 & 0 & x y z z & 0 & 0 & x y x x & 0 & 0 \\ y x x x & 0 & 0 & -x y x x & 0 & 0 & -x y z z & 0 & 0 & 0\end{array}\right)$

$\left.\begin{array}{ccccccccccc}\mathrm{y} x \mathrm{xx} & 0 & 0 & -\mathrm{xyxx} & 0 & 0 & -\mathrm{xyzz} & 0 & 0 & 0\end{array}\right)$

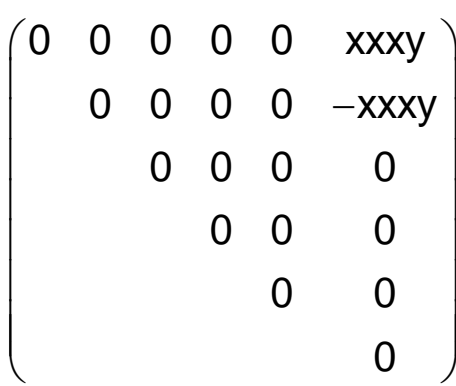

$\left(\begin{array}{ccccc}x x x x & 0 & 0 & y y z z & 0 \\ 0 & -x x x x & 0 & -y y z z & 0 \\ 0 & 0 & 0 & 0 & 0\end{array}\right)$

$\left(\begin{array}{cccccccccc}x x x x & 0 & 0 & x x y y & 0 & 0 & -y y z z & 0 & 0 & 0 \\ 0 & -x x x x & 0 & 0 & y z z & 0 & 0 & -x y y & 0 & 0\end{array}\right)$

$\left.\begin{array}{cccccccccc}0 & -x x x x & 0 & 0 & y y z z & 0 & 0 & -x x y y & 0 & 0 \\ 0 & 0 & 0 & 0 & 0 & z z x x & 0 & 0 & -z z x & 0\end{array}\right)$

$\left(\begin{array}{cccccc}x x x x & 0 & x x z z & 0 & 0 & 0 \\ & -x x x x & -x x z z & 0 & 0 & 0 \\ & & 0 & 0 & 0 & 0 \\ & & y z y z & 0 & 0 \\ & & & & -y z y z & 0 \\ & & & & & 0\end{array}\right)$

$\begin{array}{cccccc}0 & 0 & 0 & y z x z & 0 & 0 \\ x y x x & x y x x & x y z z & 0 & 0 & 0\end{array}$

$\left.\begin{array}{cccccc}0 & 0 & 0 & -y z x z & 0 & 0 \\ x y x x & -x y x x & 0 & 0 & 0 & 0\end{array}\right) \quad\left(\begin{array}{cccccc}0 & 0 & 0 & 0 & -y z y z & 0 \\ 0 & 0 & 0 & 0 & 0 & 0\end{array}\right)$ 


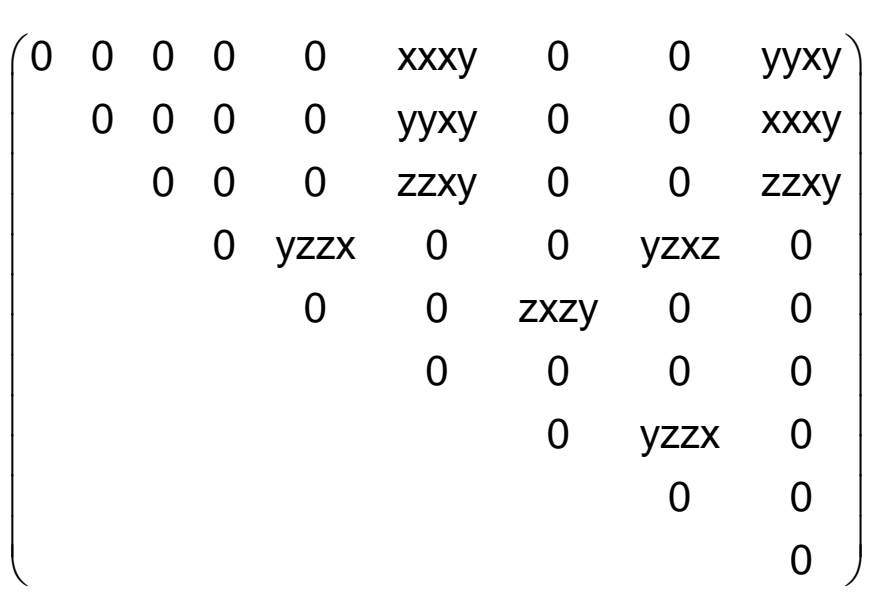

$\left(\begin{array}{lllllllll}x x x x & x x y y & x x z z & 0 & 0 & 0 & 0 & 0 & 0 \\ y y x x & x x x x & x x z z & 0 & 0 & 0 & 0 & 0 & 0\end{array}\right)$ $\left(\begin{array}{lllllllll}y y x x & x x x x & x x z z & 0 & 0 & 0 & 0 & 0 & 0 \\ z z x x & z z x x & z z z z & 0 & 0 & 0 & 0 & 0 & 0\end{array}\right)$ $\left[V^{2}\right] V^{2} \mid \begin{array}{ccccccccc}0 & 0 & 0 & y z y z & 0 & 0 & z x z x & 0 & 0\end{array}$ $\begin{array}{ccccccccc}0 & 0 & 0 & 0 & z x z x & 0 & 0 & y z y z & 0 \\ 0 & 0 & 0 & 0 & 0 & x y y & 0 & 0 & x y x\end{array}$

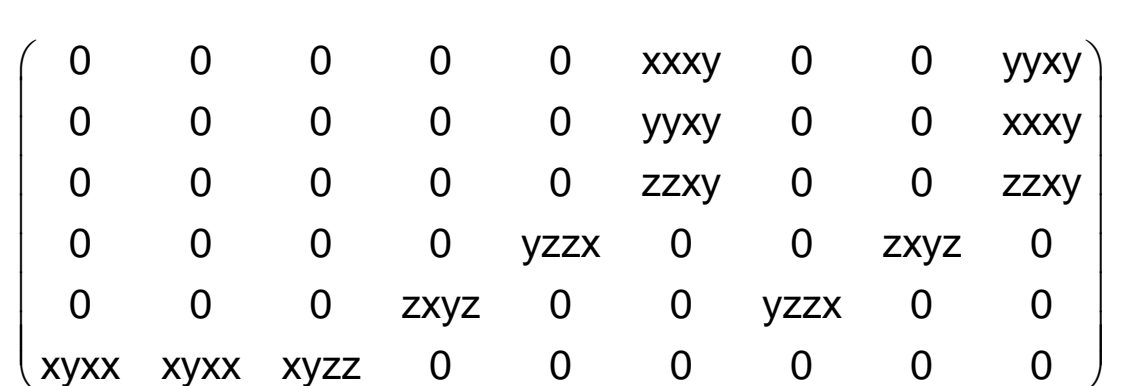

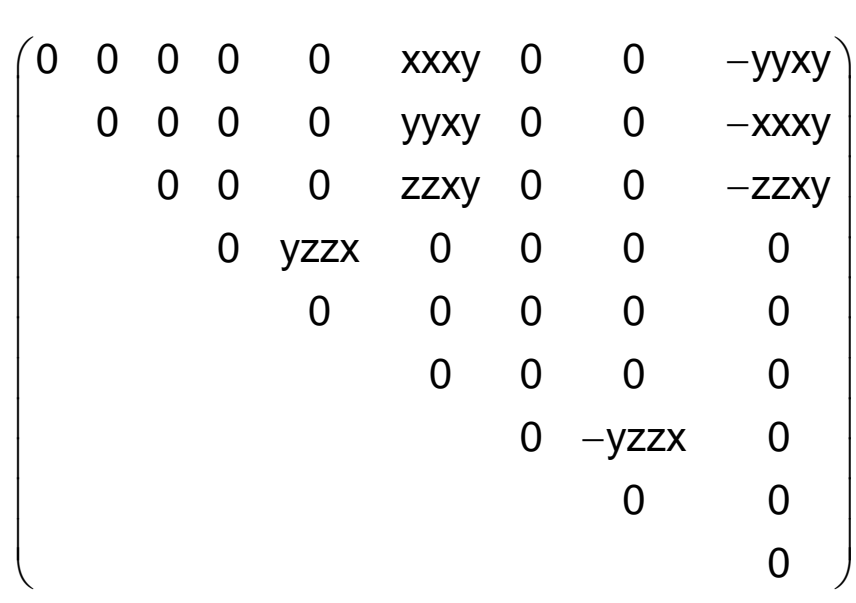

$\left(\begin{array}{ccccccccc}0 & 0 & 0 & 0 & 0 & \text { xxxy } & 0 & 0 & -y y x y \\ 0 & 0 & 0 & 0 & 0 & \text { yyxy } & 0 & 0 & -x x x y \\ 0 & 0 & 0 & 0 & 0 & \text { zzxy } & 0 & 0 & - \text { zzxy } \\ 0 & 0 & 0 & 0 & \text { yzzx } & 0 & 0 & -z x y z & 0 \\ 0 & 0 & 0 & \text { zxyz } & 0 & 0 & -y z z x & 0 & 0 \\ \text { xyxx } & - \text {-xyxx } & 0 & 0 & 0 & 0 & 0 & 0 & 0\end{array}\right)$
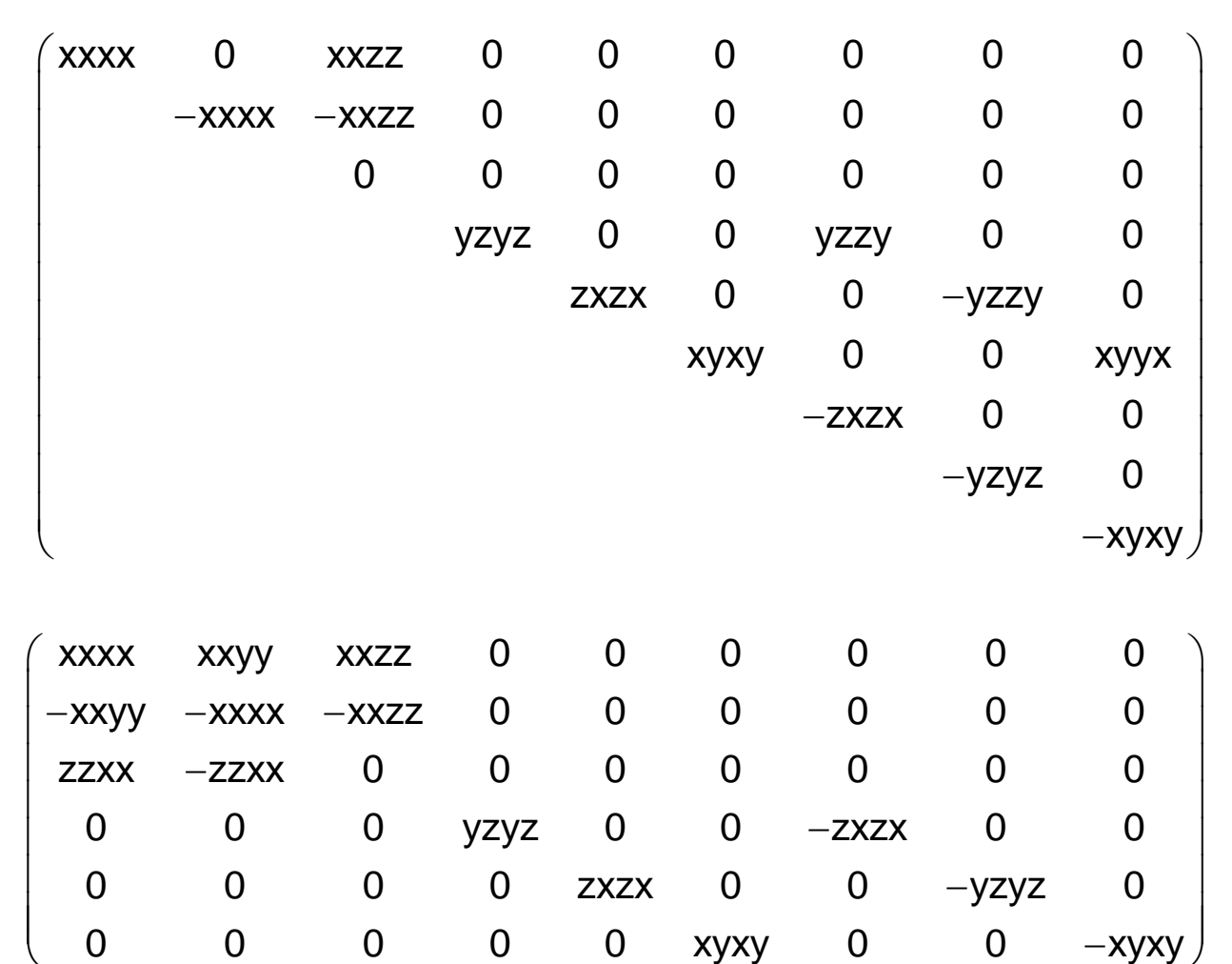
$\left(\begin{array}{ccccccccc}x x x x & x x y y & x x z z & x x y z & 0 & 0 & x x z y & 0 & 0 \\ y y x x & x x y x & y y z z & -x x y z & 0 & 0 & -x x z y & 0 & 0 \\ 2 z x x & z z x y & z z z & 0 & 0 & 0 & 0 & 0 & 0\end{array}\right)$ $\left(\begin{array}{ccccccccc}\mathrm{yyxx} & \mathrm{xxxx} & \mathrm{yyzz} & -x \mathrm{xxyz} & 0 & 0 & -x x z y & 0 & 0 \\ z z x x & z z x x & z z z z & 0 & 0 & 0 & 0 & 0 & 0 \\ y z x x & -y z x & 0 & y z y z & 0 & 0 & y z y & 0 & 0\end{array}\right)$ $\begin{array}{ccccccccc}y z x x & -y z x x & 0 & y z y z & 0 & 0 & y z z y & 0 & 0 \\ 0 & 0 & 0 & 0 & z z z x & z y x x & 0 & z x y z & z y x x\end{array}$ $\begin{array}{ccccccccc}0 & 0 & 0 & 0 & z x z x & z y x x & 0 & z x x z & z y x x \\ 0 & 0 & 0 & 0 & x x z & x y x y & 0 & x y z & x y x\end{array}$ $\begin{array}{llllllllll}0 & 0 & 0 & 0 & z x z x & z y x x & 0 & z x y z & z y x x \\ 0 & 0 & 0 & 0 & x x z y & x y x y & 0 & x x y z & x y y x\end{array}$ \begin{tabular}{ccccccccc}
\hline$z y x x$ & $-z y x x$ & 0 & $z x x z$ & 0 & 0 & $z x z x$ & 0 & 0
\end{tabular} $\left(\begin{array}{ccccccccc}\mathrm{xzxx} & -\mathrm{xzzx} & 0 & 0 & \mathrm{yzzy} & \mathrm{yzxx} & 0 & \mathrm{yzyz} & \mathrm{yzxx} \\ 0 & 0 & 0 & 0 & \mathrm{xxzy} & \mathrm{xyyx} & 0 & \mathrm{xxyz} & \mathrm{xyxy}\end{array}\right)$

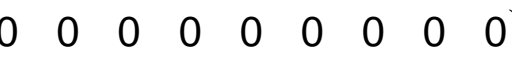
$\begin{array}{llllllllll}0 & 0 & 0 & 0 & 0 & 0 & 0 & 0 & 0 \\ 0 & 0 & 0 & 0 & 0 & 0 & 0 & 0 & 0\end{array}$ $\begin{array}{llllllllll}0 & 0 & 0 & 0 & 0 & 0 & 0 & 0 & 0 \\ 0 & 0 & 0 & 0 & 0 & 0 & 0 & 0 & 0 & 0\end{array}$ $\begin{array}{llllllllll}0 & 0 & 0 & 0 & 0 & 0 & 0 & 0 & 0\end{array}$ $\left(\begin{array}{lllllllll}0 & 0 & 0 & 0 & 0 & 0 & 0 & 0 & 0 \\ 0 & 0 & 0 & 0 & 0 & 0 & 0 & 0 & 0 \\ 0 & 0 & 0 & 0 & 0 & 0 & 0 & 0 & 0\end{array}\right)$ $\left(\begin{array}{lllllllll}0 & 0 & 0 & 0 & 0 & 0 & 0 & 0 & 0 \\ 0 & 0 & 0 & 0 & 0 & 0 & 0 & 0 & 0\end{array}\right)$

\section{and: $x x x x=x x y y+x y x y+x y y x$}

$\left[V^{4}\right] \quad\left(\begin{array}{ccccc}3 x x y y & 0 & 0 & y y z z & x x y z \\ 0 & 3 x x y y & 0 & y y z z & 0 \\ 0 & -x x y z & z z z z & x x y y & 0\end{array}\right)$

$\left(\begin{array}{lllll}0 & 0 & 0 & 0 & 0 \\ 0 & 0 & 0 & 0 & 0 \\ 0 & 0 & 0 & 0 & 0\end{array}\right)$

$\left(\begin{array}{lllll}0 & 0 & 0 & 0 & 0 \\ 0 & 0 & 0 & 0 & 0\end{array}\right)$

$\left.V^{3}\right]\left(\begin{array}{cccccccccc}3 x x y y & 0 & 0 & x x y y & 0 & 0 & y y z z & 0 & 0 & y z x x \\ 0 & 3 x x y y & 0 & 0 & y y z z & y z x x & 0 & x x y y & -y z x x & 0 \\ 0 & -z y x x & z z z z & 0 & 0 & z z x x & 0 & z y y x & z z x x & 0\end{array}\right)$

$\left(\begin{array}{llllllllll}0 & 0 & 0 & 0 & 0 & 0 & 0 & 0 & 0 & 0 \\ 0 & 0 & 0 & 0 & 0 & 0 & 0 & 0 & 0 & 0\end{array}\right.$

$\left[\left[^{2}\right]^{2}\right]\left(\begin{array}{cccccc}x x x x & x x y y & x x z z & x x y z & 0 & 0 \\ & x x x x & x x z z & -x x y z & 0 & 0 \\ & & z z z z & 0 & 0 & 0 \\ & & & y z y z & 0 & 0 \\ & & & & y z y z & x x y z \\ & & & & & x y x y\end{array}\right)$

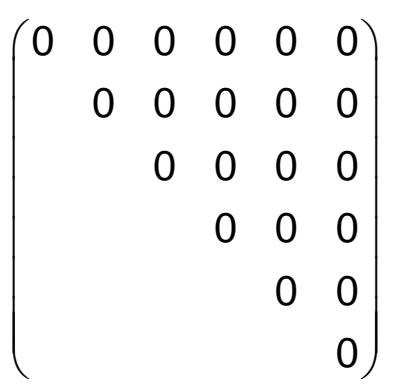

and $x y x y=1 / 2(x x x x-x x y y)$

$\begin{array}{llllll}x x x x & x x y y & x x z z & x x y z & 0 & 0 \\ x x y y & x x x x & x x z z & -x x y z & 0 & 0\end{array}$ $\left(\begin{array}{llllll}x x x y y & x x x x & x x z z & -x x y z & 0 & 0 \\ z z x x & z z x x & z z z z & 0 & 0 & 0 \\ y z x x & -y z x x & 0 & y z y z & 0 & 0\end{array}\right)$

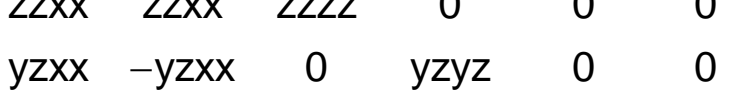

$\left(\begin{array}{cccccc}0 & 0 & 0 & 0 & y z y z & y z x x \\ 0 & 0 & 0 & 0 & x x y z & x y x y\end{array}\right)$

and $x y x y=1 / 2(x x x x-x x y y)$

$\left(\begin{array}{llllll}0 & 0 & 0 & 0 & 0 & 0 \\ 0 & 0 & 0 & 0 & 0 & 0 \\ 0 & 0 & 0 & 0 & 0 & 0 \\ 0 & 0 & 0 & 0 & 0 & 0 \\ 0 & 0 & 0 & 0 & 0 & 0 \\ 0 & 0 & 0 & 0 & 0 & 0\end{array}\right)$ $\begin{array}{lllllllll}0 & 0 & 0 & 0 & 0 & 0 & 0 & 0 & 0 \\ 0 & 0 & 0 & 0 & 0 & 0 & 0 & 0 & 0\end{array}$ $\begin{array}{llllllllll}0 & 0 & 0 & 0 & 0 & 0 & 0 & 0 & 0\end{array}$ $\left(\begin{array}{lllllllllll}0 & 0 & 0 & 0 & 0 & 0 & 0 & 0 & 0 & 0 \\ 0 & 0 & 0 & 0 & 0 & 0 & 0 & 0 & 0 & 0\end{array}\right)$

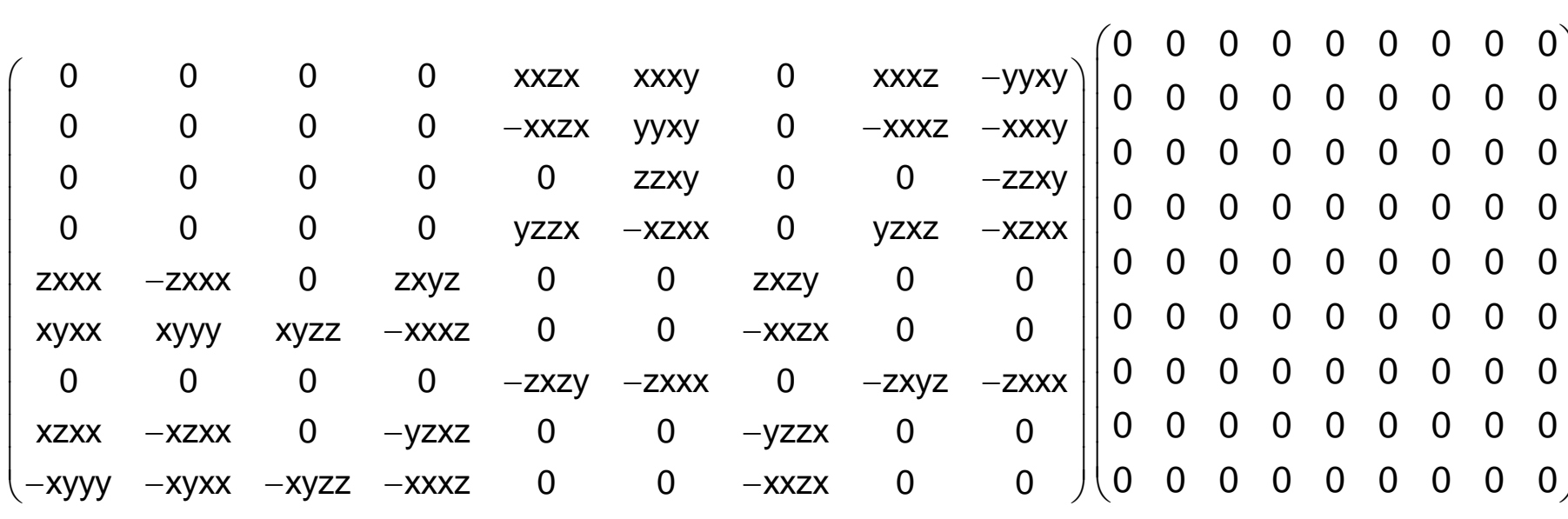

\section{and: $x x x y-y y x y=x y y y-x y x x$}
$\left(\begin{array}{ccccc}0 & 0 & 0 & 0 & 0 \\ 0 & 0 & 0 & 0 & -z x x x \\ z x x x & 0 & 0 & 0 & 0\end{array}\right)$
$\left(\begin{array}{lllll}0 & 0 & 0 & 0 & 0 \\ 0 & 0 & 0 & 0 & 0\end{array}\right)$
$\left(\begin{array}{lllll}0 & 0 & 0 & 0 & 0 \\ 0 & 0 & 0 & 0 & 0\end{array}\right)$

$\left(\begin{array}{cccccccccc}0 & 3 x y x x & 0 & 0 & x y z z & x z x x & 0 & x y x x & -x z x x & 0 \\ -3 x y x x & 0 & 0 & -x y x x & 0 & 0 & -x y z z & 0 & 0 & -x z x x \\ z x x x & 0 & 0 & -z x x x & 0 & 0 & 0 & 0 & 0 & z x y z\end{array}\right)\left(\begin{array}{cccccccccc}0 & 0 & 0 & 0 & 0 & 0 & 0 & 0 & 0 & 0 \\ 0 & 0 & 0 & 0 & 0 & 0 & 0 & 0 & 0 & 0 \\ 0 & 0 & 0 & 0 & 0 & 0 & 0 & 0 & 0 & 0\end{array}\right)$

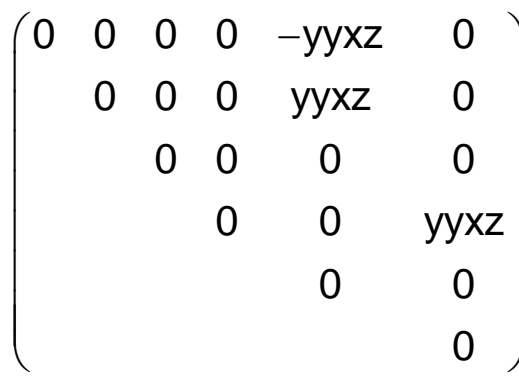

$\left(\begin{array}{llllll}0 & 0 & 0 & 0 & 0 & 0 \\ & 0 & 0 & 0 & 0 & 0 \\ & & 0 & 0 & 0 & 0 \\ & & & 0 & 0 & 0 \\ & & & & 0 & 0 \\ & & & & & 0\end{array}\right)$ $\left(\begin{array}{llllll}0 & 0 & 0 & 0 & 0 & 0 \\ 0 & 0 & 0 & 0 & 0 & 0\end{array}\right)$ $\begin{array}{llllll}0 & 0 & 0 & 0 & 0 & 0 \\ 0 & 0 & 0 & 0 & 0 & 0\end{array}$ $\begin{array}{llllll}0 & 0 & 0 & 0 & 0 & 0 \\ 0 & 0 & 0 & 0 & 0 & 0 \\ 0 & 0 & 0 & 0 & 0 & 0\end{array}$ $0 \begin{array}{llllll}0 & 0 & 0 & 0 & 0 & 0 \\ 0 & 0 & 0 & 0 & 0\end{array}$ $\begin{array}{lllllll}0 & 0 & 0 & 0 & 0 & 0\end{array}$ 


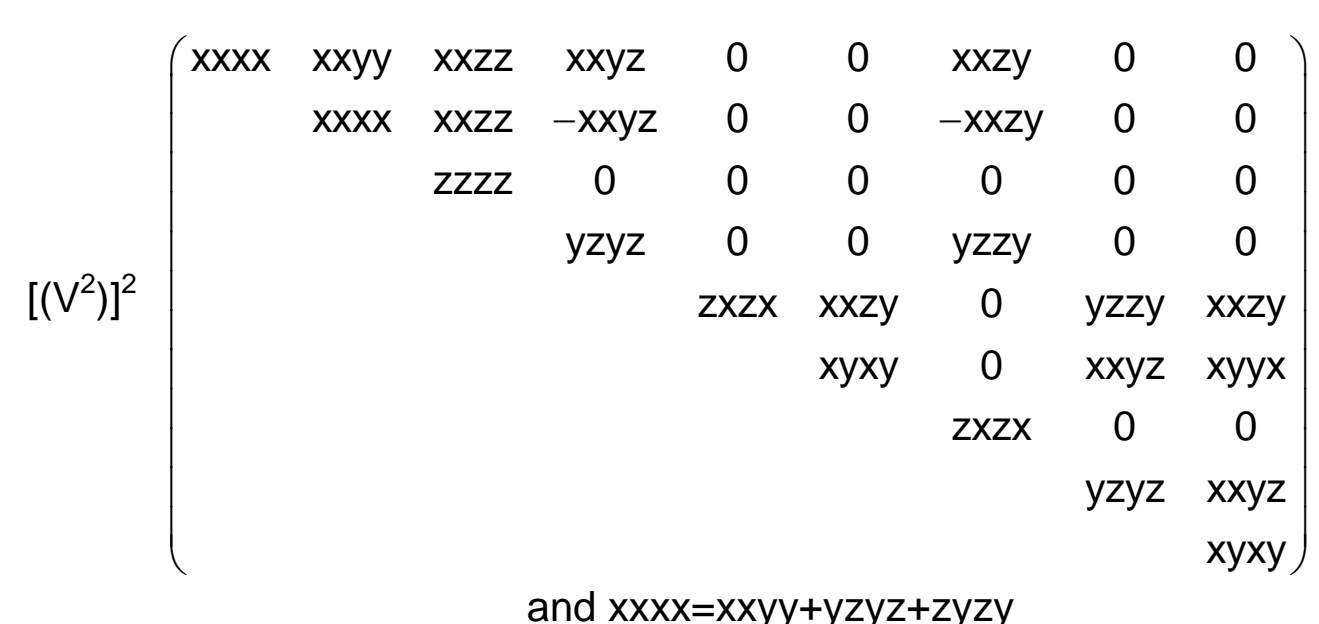

$\left(\begin{array}{lllllllll}0 & 0 & 0 & 0 & 0 & 0 & 0 & 0 & 0\end{array}\right)$

$\begin{array}{llllllll}0 & 0 & 0 & 0 & 0 & 0 & 0 & 0\end{array}$

$\begin{array}{lllllll}0 & 0 & 0 & 0 & 0 & 0 & 0\end{array}$

$\begin{array}{llllll}0 & 0 & 0 & 0 & 0 & 0 \\ & 0 & 0 & 0 & 0 & 0\end{array}$

$\begin{array}{lllll}0 & 0 & 0 & 0 & 0\end{array}$

$\begin{array}{llll}0 & 0 & 0 & 0\end{array}$

$\begin{array}{lll}0 & 0 & 0 \\ & 0 & 0 \\ & & 0\end{array}$

and $x x x x=x x y y+y z y z+z y z y$

$\left[V^{2}\right] V^{2}\left(\begin{array}{ccccccccc}x x x x & x x y y & x x z z & x x y z & 0 & 0 & x x z y & 0 & 0 \\ x x y y & x x x x & x x z z & -x x y z & 0 & 0 & -x x z y & 0 & 0 \\ z z x x & z z x x & z z z z & 0 & 0 & 0 & 0 & 0 & 0 \\ y z x x & -y z x x & 0 & y z y z & 0 & 0 & z x z x & 0 & 0 \\ 0 & 0 & 0 & 0 & z x z x & y z x x & 0 & y z y z & y z x x \\ 0 & 0 & 0 & 0 & x x z y & x y x y & 0 & x x y z & x y x y\end{array}\right)$

$\begin{array}{lllllllll}0 & 0 & 0 & 0 & 0 & 0 & 0 & 0 & 0 \\ 0 & 0 & 0 & 0 & 0 & 0 & 0 & 0 & 0\end{array}$ $\begin{array}{lllllllll}0 & 0 & 0 & 0 & 0 & 0 & 0 & 0 & 0 \\ 0 & 0 & 0 & 0 & 0 & 0 & 0 & 0 & 0\end{array}$ $\begin{array}{lllllllll}0 & 0 & 0 & 0 & 0 & 0 & 0 & 0 & 0 \\ 0 & 0 & 0 & 0 & 0 & 0 & 0 & 0 & 0\end{array}$

$0 \begin{array}{lllllllll}0 & 0 & 0 & 0 & 0 & 0 & 0 & 0 & 0 \\ 0 & 0 & 0 & 0 & 0 & 0 & 0 & 0 & 0\end{array}$ $\begin{array}{lllllllll}0 & 0 & 0 & 0 & 0 & 0 & 0 & 0 & 0\end{array}$
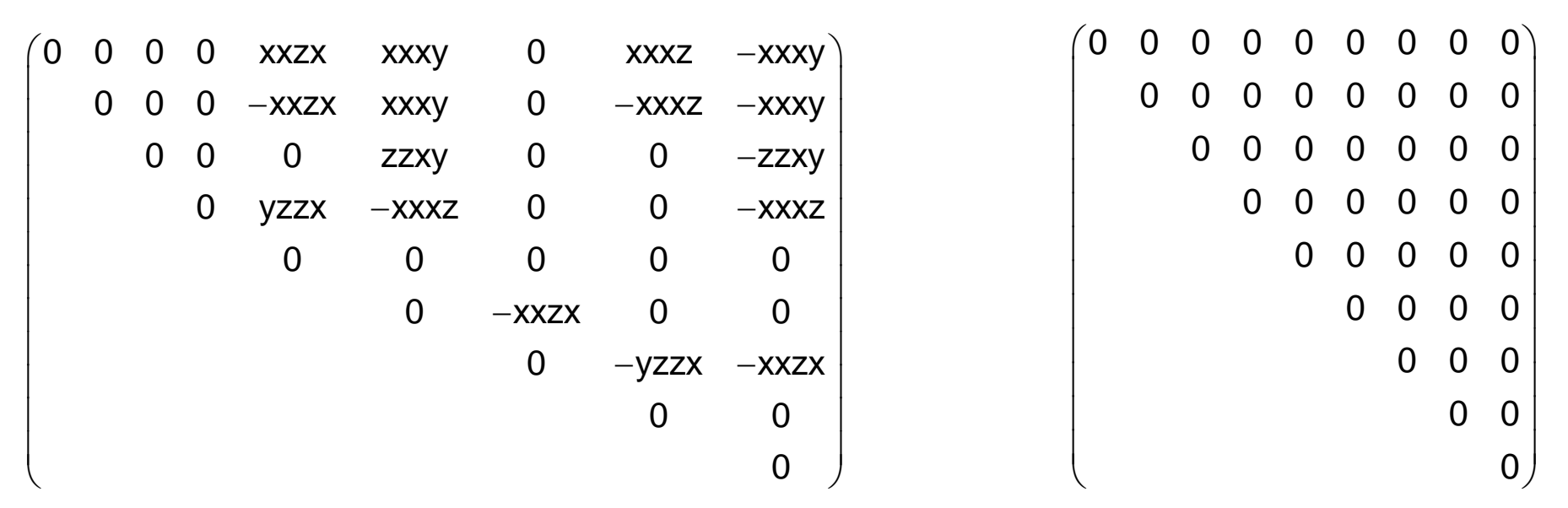

$\left(\begin{array}{ccccccccc}0 & 0 & 0 & 0 & x x z x & x x x y & 0 & x x x z & -y y x y \\ 0 & 0 & 0 & 0 & -x x z x & y y x y & 0 & -x x x z & -x x x y \\ 0 & 0 & 0 & 0 & 0 & z z x y & 0 & 0 & -z z x y \\ 0 & 0 & 0 & 0 & y z z x & -z x x x & 0 & -z x y z & -z x x x \\ z x x x & -z x x x & 0 & z x y z & 0 & 0 & -y z z x & 0 & 0 \\ x y x x & -x y x x & 0 & -x x x z & 0 & 0 & -x x z x & 0 & 0\end{array}\right)\left(\begin{array}{ccccccccc}0 & 0 & 0 & 0 & 0 & 0 & 0 & 0 & 0 \\ 0 & 0 & 0 & 0 & 0 & 0 & 0 & 0 & 0 \\ 0 & 0 & 0 & 0 & 0 & 0 & 0 & 0 & 0 \\ 0 & 0 & 0 & 0 & 0 & 0 & 0 & 0 & 0 \\ 0 & 0 & 0 & 0 & 0 & 0 & 0 & 0 & 0 \\ 0 & 0 & 0 & 0 & 0 & 0 & 0 & 0 & 0\end{array}\right)$

and: $y y x y-x x x y=2 x y x x$ 
$\mathrm{V}^{4}\left(\begin{array}{ccccccccc}\mathrm{xxxx} & \mathrm{xxyy} & \mathrm{xxzz} & 0 & 0 & 0 & 0 & 0 & 0 \\ \mathrm{yyxx} & \mathrm{xxxx} & \mathrm{yyzz} & 0 & 0 & 0 & 0 & 0 & 0 \\ \mathrm{zzxx} & \mathrm{zzxx} & \mathrm{zzzz} & 0 & 0 & 0 & 0 & 0 & 0 \\ 0 & 0 & 0 & \mathrm{yzyz} & 0 & 0 & \mathrm{yzzy} & 0 & 0 \\ 0 & 0 & 0 & 0 & \mathrm{zxzx} & 0 & 0 & \mathrm{zxxz} & 0 \\ 0 & 0 & 0 & 0 & 0 & \mathrm{xyxy} & 0 & 0 & \mathrm{xyyx} \\ 0 & 0 & 0 & \mathrm{zxxz} & 0 & 0 & \mathrm{zxzx} & 0 & 0 \\ 0 & 0 & 0 & 0 & \mathrm{yzzy} & 0 & 0 & \mathrm{yzyz} & 0 \\ 0 & 0 & 0 & 0 & 0 & \mathrm{xyyx} & 0 & 0 & \mathrm{xyxy}\end{array}\right)$

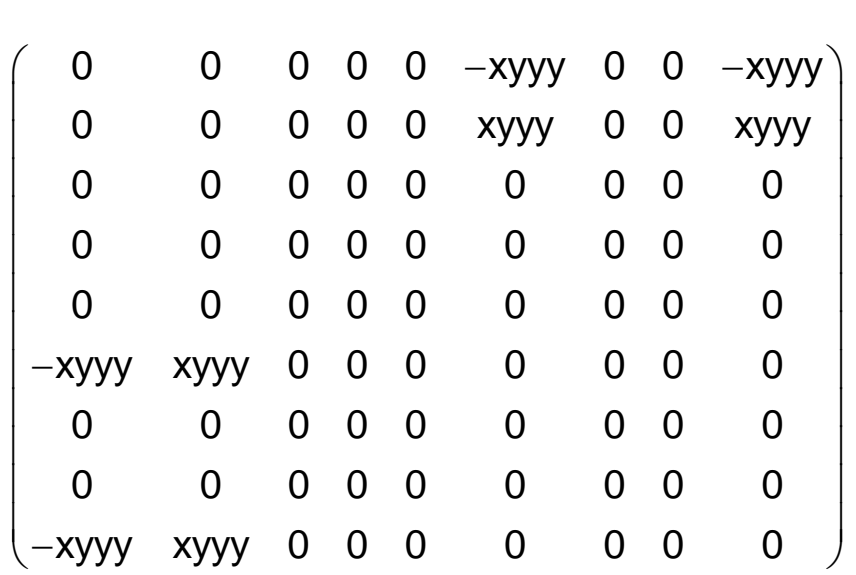

$\left.\mathrm{V}^{4}\right]\left(\begin{array}{ccccc}3 x x y y & 0 & 0 & y y z z & 0 \\ 0 & 3 x x y y & 0 & y y z z & 0 \\ 0 & 0 & z z z z & x x y y & 0\end{array}\right)$

$\left(\begin{array}{ccccc}0 & x y y y & 0 & 0 & 0 \\ -x y y y & 0 & 0 & 0 & 0 \\ 0 & 0 & 0 & 0 & 0\end{array}\right)$

$\mathrm{V}\left[\mathrm{V}^{3}\right] \quad\left(\begin{array}{cccccccccc}3 \mathrm{xxyy} & 0 & 0 & \mathrm{xxyy} & 0 & 0 & \mathrm{yyzz} & 0 & 0 & \mathrm{yyxx} \\ 0 & 3 \mathrm{xxyy} & 0 & 0 & \mathrm{yyzz} & 0 & 0 & \mathrm{xxyy} & 0 & 0 \\ 0 & 0 & \mathrm{zzzz} & 0 & 0 & \mathrm{zzxx} & 0 & 0 & \mathrm{zzxx} & 0\end{array}\right)$

$\begin{array}{llllllllll}0 & \text { xyyy } & 0 & 0 & 0 & 0 & 0 & -x y y y & 0 & 0\end{array}$

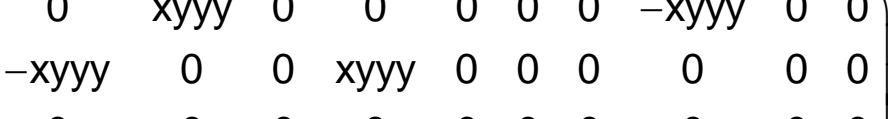

$\left[\left[^{2}\right]^{2}\right]\left(\begin{array}{cccccc}x x x x & x x y y & x x z z & 0 & 0 & 0 \\ & x x x x & x x z z & 0 & 0 & 0 \\ & & z z z z & 0 & 0 & 0 \\ & & & y z y z & 0 & 0 \\ & & & & y z y z & 0 \\ & & & & & \text { xyxy }\end{array}\right)$

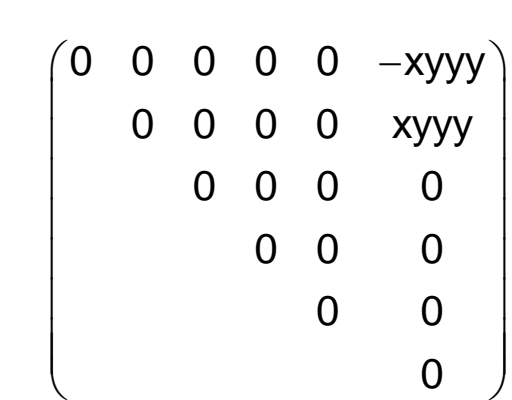

and $x y x y=1 / 2(x x x x-x x y y)$

$\left[\mathrm{V}^{2}\right]^{2}\left(\begin{array}{cccccc}\mathrm{xxxx} & \mathrm{xxyy} & \mathrm{xxzz} & 0 & 0 & 0 \\ \mathrm{xxyy} & \mathrm{xxxx} & \mathrm{xxzz} & 0 & 0 & 0 \\ \mathrm{zzxx} & \mathrm{zzxx} & \mathrm{zzzz} & 0 & 0 & 0 \\ 0 & 0 & 0 & \mathrm{yzyz} & 0 & 0 \\ 0 & 0 & 0 & 0 & \mathrm{yzyz} & 0 \\ 0 & 0 & 0 & 0 & 0 & \mathrm{xyxy}\end{array}\right)$

and $x y x y=1 / 2(x x x x-x x y y)$

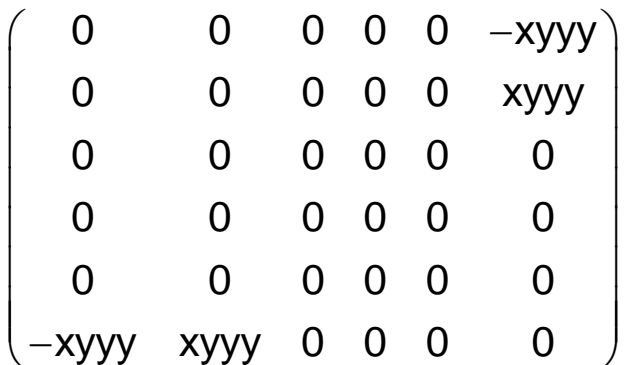

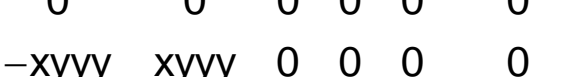

$\left(\begin{array}{ccccccccc}0 & 0 & 0 & 0 & 0 & x x x y & 0 & 0 & -y y x y \\ 0 & 0 & 0 & 0 & 0 & y y x y & 0 & 0 & -x x x y \\ 0 & 0 & 0 & 0 & 0 & z z x y & 0 & 0 & -z z x y \\ 0 & 0 & 0 & 0 & y z z x & 0 & 0 & y z x z & 0 \\ 0 & 0 & 0 & z x y z & 0 & 0 & z x z y & 0 & 0 \\ x y x x & x y y y & x y z z & 0 & 0 & 0 & 0 & 0 & 0 \\ 0 & 0 & 0 & 0 & -z x z y & 0 & 0 & -z x y z & 0 \\ 0 & 0 & 0 & -y z x z & 0 & 0 & -y z z x & 0 & 0 \\ -x y y y & -x y x x & -x y z z & 0 & 0 & 0 & 0 & 0 & 0\end{array}\right)\left(\begin{array}{ccccccccc}x x x x x & -x x x x & 0 & 0 & 0 & 0 & 0 & 0 & 0 \\ -x x x x & x x x x & 0 & 0 & 0 & 0 & 0 & 0 & 0 \\ 0 & 0 & 0 & 0 & 0 & 0 & 0 & 0 & 0 \\ 0 & 0 & 0 & 0 & 0 & 0 & 0 & 0 & 0 \\ 0 & 0 & 0 & 0 & 0 & 0 & 0 & 0 & 0 \\ 0 & 0 & 0 & 0 & 0 & -x x x x & 0 & 0 & -x x x x \\ 0 & 0 & 0 & 0 & 0 & 0 & 0 & 0 & 0 \\ 0 & 0 & 0 & 0 & 0 & 0 & 0 & 0 & 0 \\ 0 & 0 & 0 & 0 & 0 & -x x x x & 0 & 0 & -x x x x\end{array}\right)$
and: $x x x y-y y x y=x y y y-x y x x$

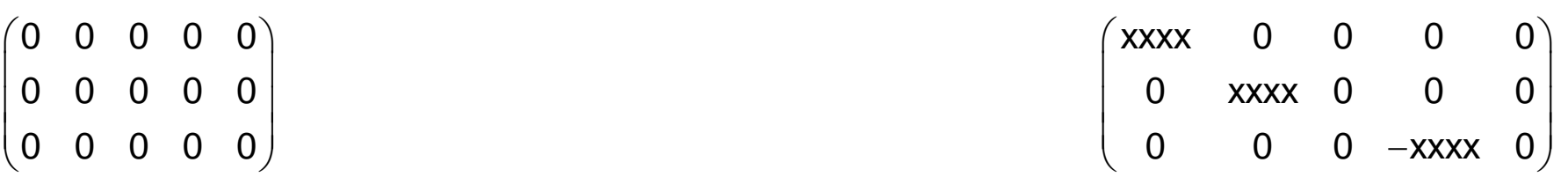




\begin{abstract}
e
\end{abstract}

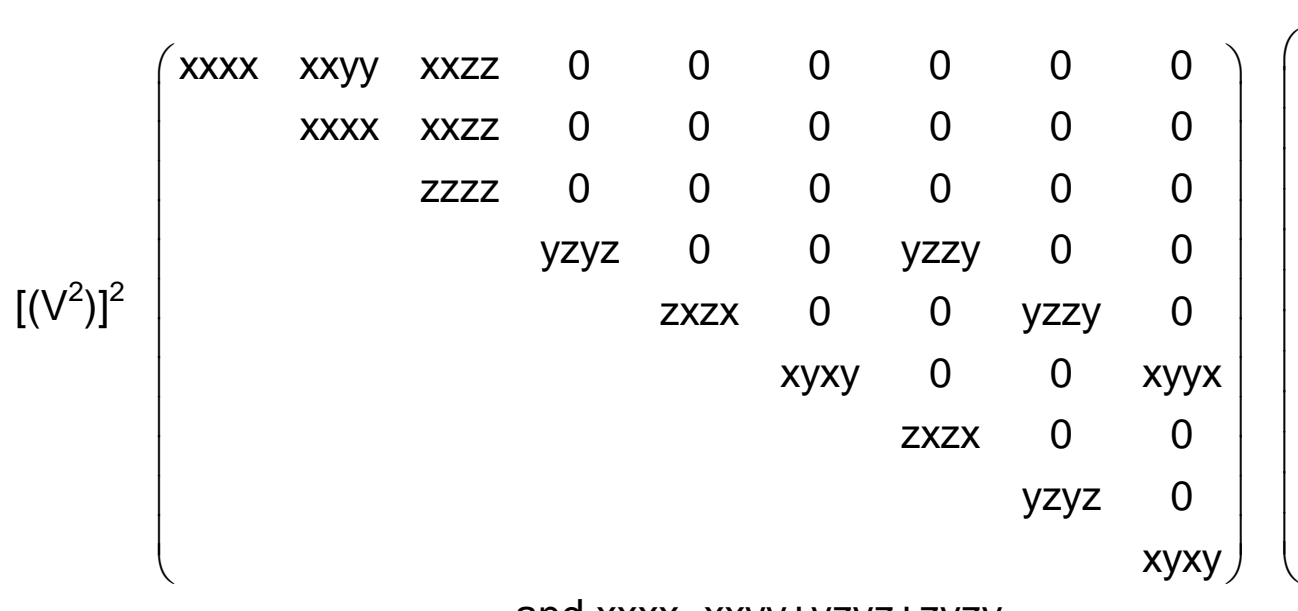

$\left(\begin{array}{lllllllll}0 & 0 & 0 & 0 & 0 & -x y y y & 0 & 0 & -x y y y \\ & 0 & 0 & 0 & 0 & -x y y y & 0 & 0 & -x y y\end{array}\right)$

$\begin{array}{cccccccc}0 & 0 & 0 & 0 & -x y y y & 0 & 0 & -x y y y \\ 0 & 0 & 0 & 0 & 0 & 0 & 0\end{array}$

$\begin{array}{lllllll}0 & 0 & 0 & 0 & 0 & 0 & 0 \\ & 0 & 0 & 0 & 0 & 0 & 0\end{array}$

$\begin{array}{lllll}0 & 0 & 0 & 0 & 0\end{array}$

$\begin{array}{llll}0 & 0 & 0 & 0\end{array}$

$\begin{array}{lll}0 & 0 & 0 \\ & 0 & 0\end{array}$

and $x x x x=x x y y+y z y z+z y z y$

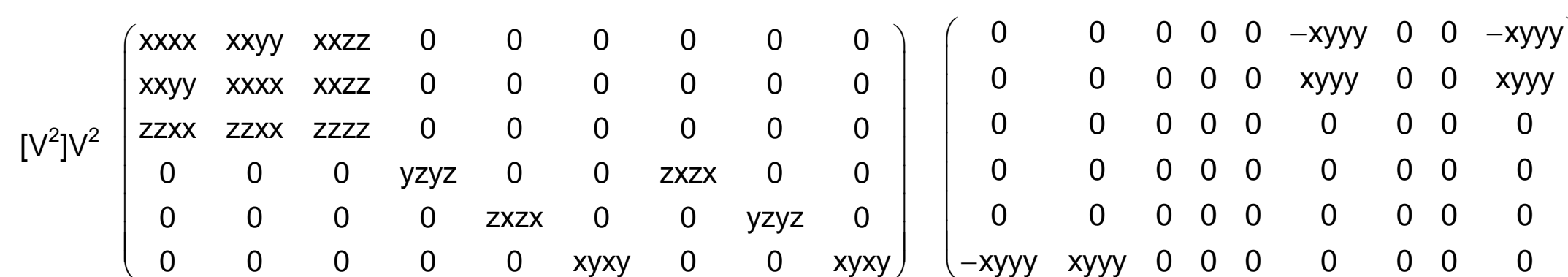

and $x x x x=x x y y+2 x y x y$
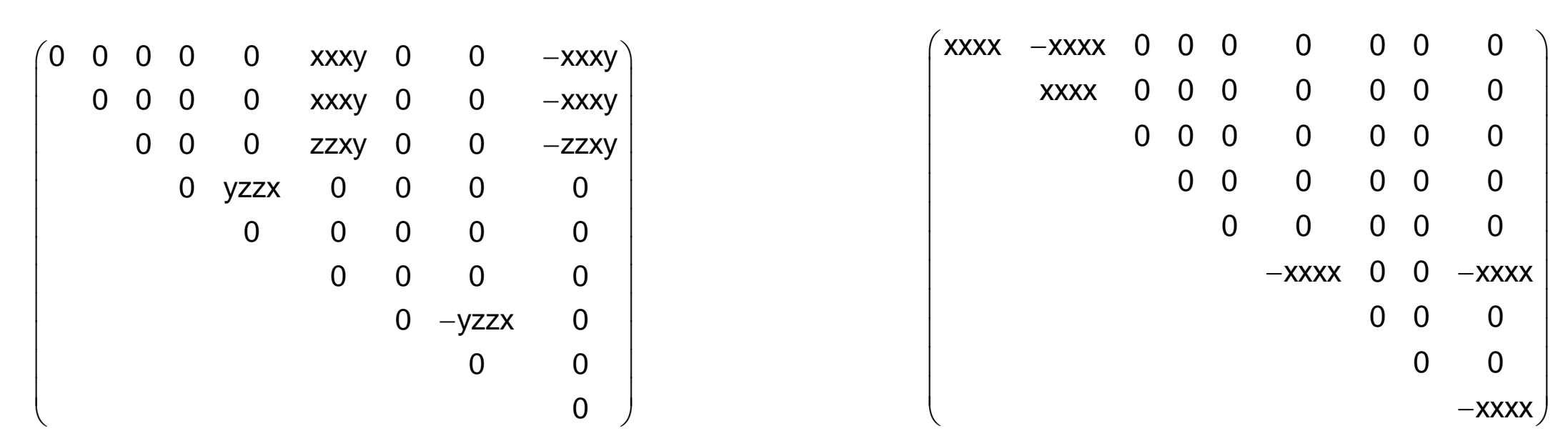

$\left(\begin{array}{ccccccccc}0 & 0 & 0 & 0 & 0 & x x x y & 0 & 0 & -y y x y \\ 0 & 0 & 0 & 0 & 0 & y y x y & 0 & 0 & -x x x y \\ 0 & 0 & 0 & 0 & 0 & z z x y & 0 & 0 & -z z x y \\ 0 & 0 & 0 & 0 & y z z x & 0 & 0 & -z x y z & 0 \\ 0 & 0 & 0 & z x y z & 0 & 0 & -y z z x & 0 & 0 \\ x y x x & -x y x x & 0 & 0 & 0 & 0 & 0 & 0 & 0\end{array}\right)$

$\left(\begin{array}{ccccccccc}x x x x & -x x x x & 0 & 0 & 0 & 0 & 0 & 0 & 0 \\ -x x x x & x x x x & 0 & 0 & 0 & 0 & 0 & 0 & 0 \\ 0 & 0 & 0 & 0 & 0 & 0 & 0 & 0 & 0 \\ 0 & 0 & 0 & 0 & 0 & 0 & 0 & 0 & 0 \\ 0 & 0 & 0 & 0 & 0 & 0 & 0 & 0 & 0 \\ 0 & 0 & 0 & 0 & 0 & -x x x x & 0 & 0 & -x x x x\end{array}\right)$

and $y y x y-x x x y=2 x y x x$ 
$\left.\begin{array}{lllllllll}x x x x & x x y y & x x z z & 0 & 0 & 0 & 0 & 0 & 0 \\ 7 y x x & x x y x & x x z z & 0 & 0 & 0 & 0 & 0 & 0\end{array}\right)$ $\left(\begin{array}{ccccccccc}x y y x x & x x y x x & x x z z & 0 & 0 & 0 & 0 & 0 & 0 \\ z z x x & z z y y & z z z z & 0 & 0 & 0 & 0 & 0 & 0 \\ 0 & 0 & 0 & y z y z & 0 & 0 & y z z y & 0 & 0\end{array}\right)$

$\begin{array}{llllllllll}\vee^{4} & 0 & 0 & 0 & y z y z & 0 & 0 & y z z y & 0 & 0 \\ 0 & 0 & 0 & 0 & z x z x & 0 & 0 & z x z & 0\end{array}$ $\begin{array}{ccccccccc}0 & 0 & 0 & 0 & z x z x & 0 & 0 & z x x z & 0\end{array}$ $\begin{array}{ccccccccc}0 & 0 & 0 & 0 & 0 & x y x y & 0 & 0 & x y y x \\ 0 & 0 & 0 & z x y z & 0 & 0 & z x z & 0 & 0 \\ 0 & 0 & 0 & 0 & y z z y & 0 & 0 & y z y z & 0\end{array}$ $\left.\begin{array}{lllllllll}0 & 0 & 0 & z x x z & 0 & 0 & z x z x & 0 & 0 \\ 0 & 0 & 0 & 0 & y z y & 0 & 0 & y z y z & 0 \\ 0 & 0 & 0 & 0 & 0 & x y y x & 0 & 0 & x y x y\end{array}\right)$

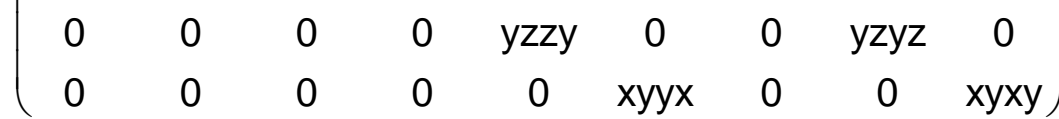
and: $x x x x=x x y y+x y x y+x y y x$

$\left[\mathrm{V}^{4}\right] \quad\left(\begin{array}{ccccc}3 \mathrm{xxyy} & 0 & 0 & \mathrm{yyzz} & 0 \\ 0 & 3 \mathrm{xxyy} & 0 & \mathrm{yyzz} & 0 \\ 0 & 0 & z z z z & \mathrm{xxyy} & 0\end{array}\right)$

$\left(\begin{array}{lllllllll}0 & 0 & 0 & 0 & 0 & 0 & 0 & 0 & 0 \\ 0 & 0 & 0 & 0 & 0 & 0 & 0 & 0 & 0\end{array}\right)$ $\left.\begin{array}{lllllllll}0 & 0 & 0 & 0 & 0 & 0 & 0 & 0 & 0 \\ 0 & 0 & 0 & 0 & 0 & 0 & 0 & 0 & 0\end{array}\right)$ $0 \begin{array}{llllllllll}0 & 0 & 0 & 0 & 0 & 0 & 0 & 0 & 0 \\ 0 & 0 & 0 & 0 & 0 & 0 & 0 & 0 & 0\end{array}$ $\begin{array}{lllllllll}0 & 0 & 0 & 0 & 0 & 0 & 0 & 0 & 0 \\ 0 & 0 & 0 & 0 & 0 & 0 & 0 & 0 & 0\end{array}$

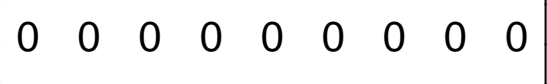
$\begin{array}{lllllllll}0 & 0 & 0 & 0 & 0 & 0 & 0 & 0 & 0 \\ 0 & 0 & 0 & 0 & 0 & 0 & 0 & 0 & 0\end{array}$ $\begin{array}{lllllllll}0 & 0 & 0 & 0 & 0 & 0 & 0 & 0 & 0 \\ 0 & 0 & 0 & 0 & 0 & 0 & 0 & 0 & 0\end{array}$ $0 \begin{array}{llllllllll}0 & 0 & 0 & 0 & 0 & 0 & 0 & 0 & 0 \\ 0 & 0 & 0 & 0 & 0 & 0 & 0 & 0\end{array}$ $\begin{array}{lllllllll}0 & 0 & 0 & 0 & 0 & 0 & 0 & 0 & 0\end{array}$

$\left(\begin{array}{lllll}0 & 0 & 0 & 0 & 0 \\ 0 & 0 & 0 & 0 & 0 \\ 0 & 0 & 0 & 0 & 0\end{array}\right)$

$\left(\begin{array}{lllll}0 & 0 & 0 & 0 & 0 \\ 0 & 0 & 0 & 0 & 0 \\ 0 & 0 & 0 & 0 & 0\end{array}\right.$

$\left(\begin{array}{llllllllll}0 & 0 & 0 & 0 & 0 & 0 & 0 & 0 & 0 & 0 \\ 0 & 0 & 0 & 0 & 0 & 0 & 0 & 0 & 0 & 0\end{array}\right)$ $\left(\begin{array}{llllllllll}0 & 0 & 0 & 0 & 0 & 0 & 0 & 0 & 0 & 0 \\ 0 & 0 & 0 & 0 & 0 & 0 & 0 & 0 & 0 & 0\end{array}\right)$

$\left.V_{[} V^{3}\right]\left(\begin{array}{cccccccccc}3 x x y y & 0 & 0 & x x y y & 0 & 0 & y y z z & 0 & 0 & 0 \\ 0 & 3 x y y & 0 & 0 & y y z z & 0 & 0 & x x y y & 0 & 0\end{array}\right)$ $\left(\begin{array}{cccccc}x x x x & x x y y & x x z z & 0 & 0 & 0 \\ & x x x x & x x z z & 0 & 0 & 0 \\ & & z z z z & 0 & 0 & 0 \\ & & & y z y z & 0 & 0 \\ & & & & y z y z & 0 \\ & & & & & x y x y\end{array}\right)$

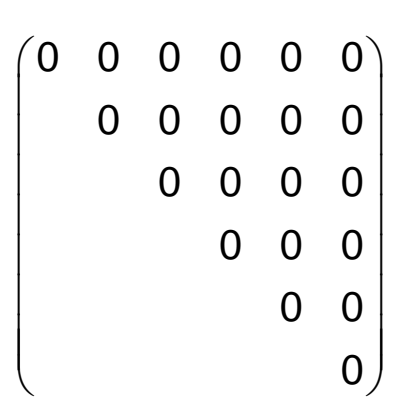

and $x y x y=1 / 2(x x x x-x x y y)$

$\left(\begin{array}{cccccc}x x x x & x x y y & x x z z & 0 & 0 & 0 \\ x y y & x x y & x x z z & 0 & 0 & 0\end{array}\right)$ $\left.\begin{array}{llllll}x x y y & x x x x & x x z z & 0 & 0 & 0 \\ z z x x & z z x x & z z z z & 0 & 0 & 0\end{array}\right)$ $\begin{array}{ccccccc}2 & 0 & 0 & y z y z & 0 & 0 \\ 0 & 0 & 0 & 0 & y z y z & 0\end{array}$ $\begin{array}{cccccc}0 & 0 & 0 & y z y z & 0 & 0 \\ 0 & 0 & 0 & 0 & y z y z & 0\end{array}$

and $x y x y=1 / 2(x x x x-x x y y)$ $\left(\begin{array}{llllll}0 & 0 & 0 & 0 & 0 & 0 \\ 0 & 0 & 0 & 0 & 0 & 0\end{array}\right)$

$\left(\begin{array}{llllll}0 & 0 & 0 & 0 & 0 & 0 \\ 0 & 0 & 0 & 0 & 0 & 0 \\ 0 & 0 & 0 & 0 & 0 & 0\end{array}\right.$

$0 \begin{array}{llllll}0 & 0 & 0 & 0 & 0 & 0 \\ 0 & 0 & 0 & 0 & 0 & 0 \\ 0 & 0 & 0 & 0 & -1\end{array}$

$\begin{array}{llllll}0 & 0 & 0 & 0 & 0 & 0 \\ 0 & 0 & 0 & 0 & 0 & 0 \\ 0 & 0 & 0 & 0 & 0 & 0\end{array}$

$\begin{array}{llllll}0 & 0 & 0 & 0 & 0 & 0 \\ 0 & 0 & 0 & 0 & 0 & 0\end{array}$

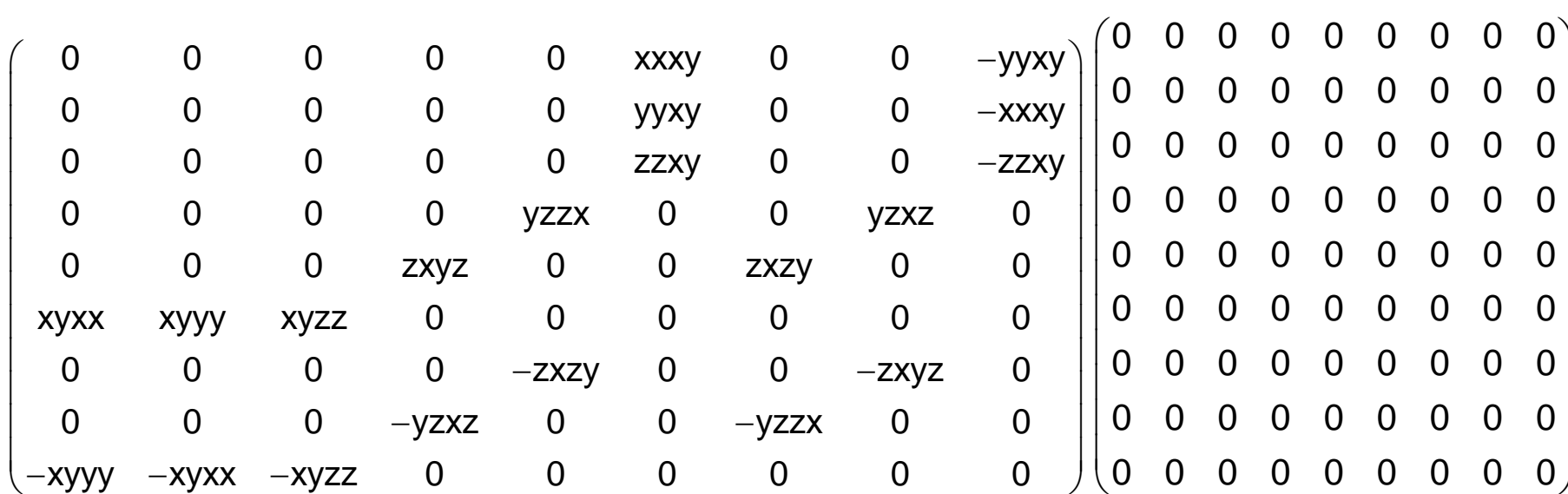

and: $x x x y-y y x y=x y y y-x y x x$

$\left(\begin{array}{lllll}0 & 0 & 0 & 0 & 0 \\ 0 & 0 & 0 & 0 & 0 \\ 0 & 0 & 0 & 0 & 0\end{array}\right) \quad\left(\begin{array}{lllll}0 & 0 & 0 & 0 & 0 \\ 0 & 0 & 0 & 0 & 0 \\ 0 & 0 & 0 & 0 & 0\end{array}\right)$

$\left(\begin{array}{cccccccccc}0 & 3 x y x x & 0 & 0 & x y z z & 0 & 0 & x y x x & 0 & 0 \\ -3 x y x x & 0 & 0 & -x y x x & 0 & 0 & -x y z z & 0 & 0 & 0\end{array}\right)$

$\left(\begin{array}{llllllllll}0 & 0 & 0 & 0 & 0 & 0 & 0 & 0 & 0 & 0 \\ 0 & 0 & 0 & 0 & 0 & 0 & 0 & 0 & 0 & 0\end{array}\right)$

$\left(\begin{array}{llllllllll}0 & 0 & 0 & 0 & 0 & 0 & 0 & 0 & 0 & 0 \\ 0 & 0 & 0 & 0 & 0 & 0 & 0 & 0 & 0 & 0\end{array}\right)$

$\begin{array}{llllllllll}0 & 0 & 0 & 0 & 0 & 0 & 0 & 0 & 0 & z x y z\end{array}$

$\left(\begin{array}{llllll}0 & 0 & 0 & 0 & 0 & 0 \\ & 0 & 0 & 0 & 0 & 0 \\ & & 0 & 0 & 0 & 0 \\ & & & 0 & 0 & 0 \\ & & & & 0 & 0 \\ & & & & & 0\end{array}\right)$

$$
\begin{aligned}
& \left.\begin{array}{lllll}
0 & 0 & 0 & 0 & 0 \\
0 & 0 & 0 & 0 & 0
\end{array}\right) \\
& \begin{array}{llll}
0 & 0 & 0 & 0
\end{array} \\
& \begin{array}{lll}
0 & 0 & 0 \\
& 0 & 0
\end{array} \\
& 0
\end{aligned}
$$

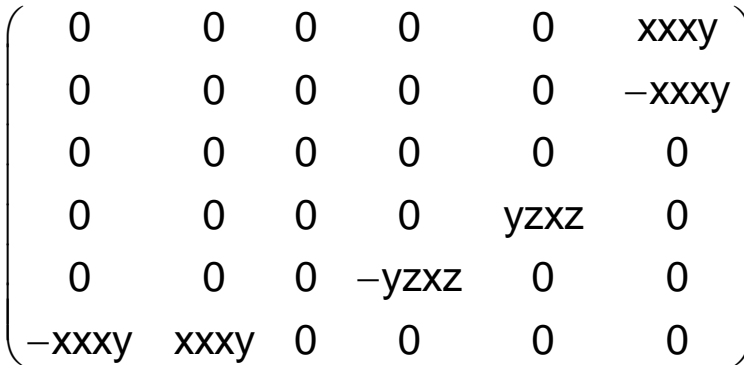

$\left(\begin{array}{llllll}0 & 0 & 0 & 0 & 0 & 0 \\ 0 & 0 & 0 & 0 & 0 & 0\end{array}\right)$ $\begin{array}{llllll}0 & 0 & 0 & 0 & 0 & 0 \\ 0 & 0 & 0 & 0 & 0 & 0\end{array}$ $\begin{array}{llllll}0 & 0 & 0 & 0 & 0 & 0 \\ 0 & 0 & 0 & 0 & 0 & 0\end{array}$ $\begin{array}{llllll}0 & 0 & 0 & 0 & 0 & 0 \\ 0 & 0 & 0 & 0 & 0 & 0 \\ 0 & 0 & 0 & 0 & 0\end{array}$ $\left(\begin{array}{llllll}0 & 0 & 0 & 0 & 0 & 0 \\ 0 & 0 & 0 & 0 & 0 & 0\end{array}\right.$ 


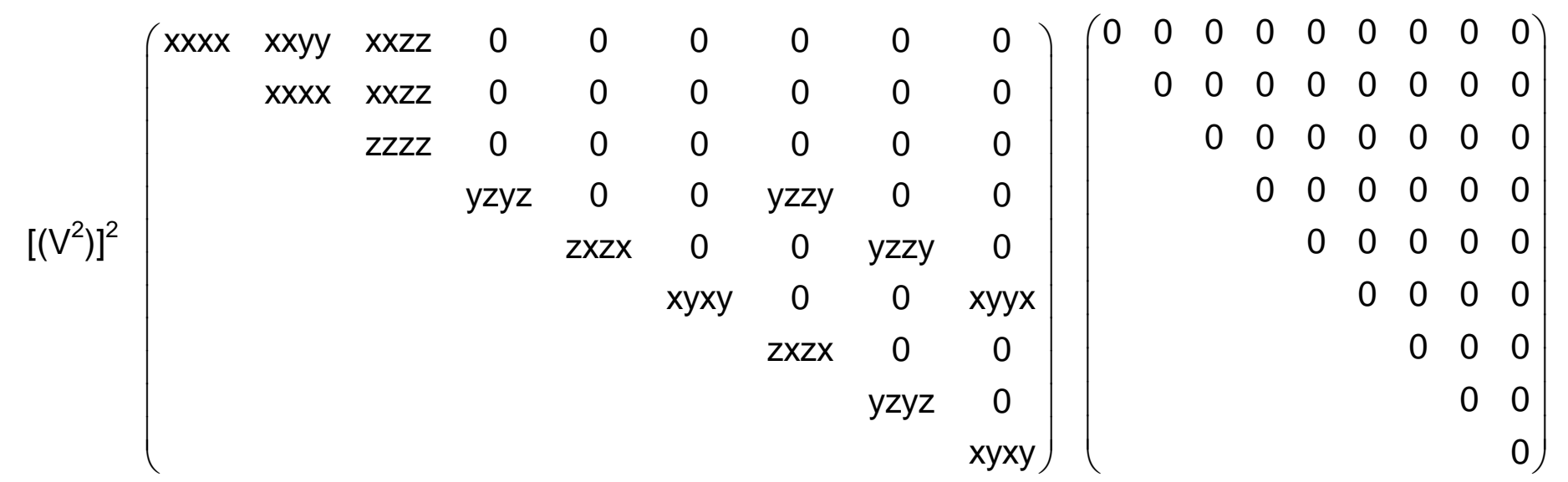

and $x x x x=x x y y+y z y z+z y z y$

$\left[V^{2}\right] V^{2}\left(\begin{array}{ccccccccc}\operatorname{xxxx} & x x y y & x x z z & 0 & 0 & 0 & 0 & 0 & 0 \\ x x y y & x x x x & x x z z & 0 & 0 & 0 & 0 & 0 & 0 \\ z z x x & z z x x & z z z z & 0 & 0 & 0 & 0 & 0 & 0 \\ 0 & 0 & 0 & y z y z & 0 & 0 & z x z x & 0 & 0 \\ 0 & 0 & 0 & 0 & z x z x & 0 & 0 & y z y z & 0 \\ 0 & 0 & 0 & 0 & 0 & x y x y & 0 & 0 & x y x y\end{array}\right)\left(\begin{array}{ccccccccc}0 & 0 & 0 & 0 & 0 & 0 & 0 & 0 & 0 \\ 0 & 0 & 0 & 0 & 0 & 0 & 0 & 0 & 0 \\ 0 & 0 & 0 & 0 & 0 & 0 & 0 & 0 & 0 \\ 0 & 0 & 0 & 0 & 0 & 0 & 0 & 0 & 0 \\ 0 & 0 & 0 & 0 & 0 & 0 & 0 & 0 & 0 \\ 0 & 0 & 0 & 0 & 0 & 0 & 0 & 0 & 0\end{array}\right)$

and $x x x x=x x y y+2 x y x y$

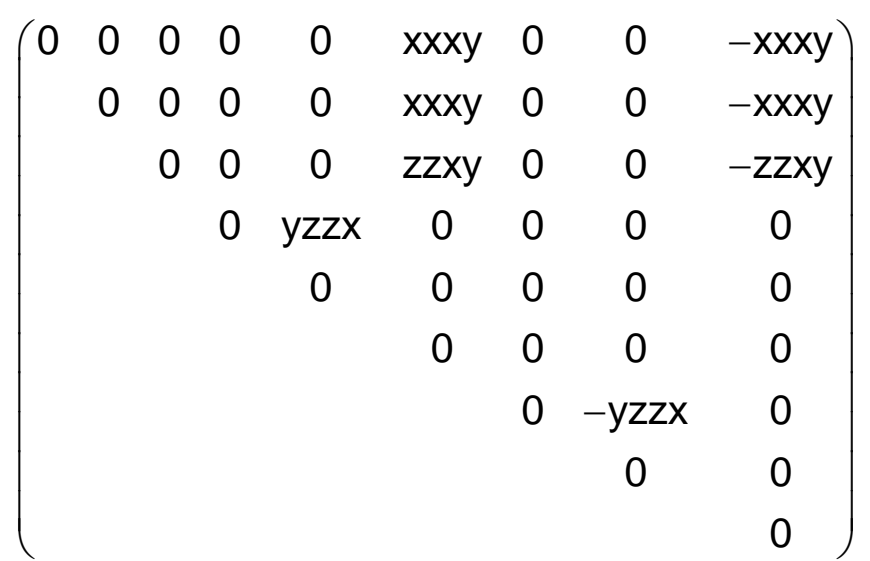

$\left(\begin{array}{ccccccccc}0 & 0 & 0 & 0 & 0 & \text { xxxy } & 0 & 0 & - \text { yyxy } \\ 0 & 0 & 0 & 0 & 0 & \text { yyxy } & 0 & 0 & - \text {-xxxy } \\ 0 & 0 & 0 & 0 & 0 & \text { zzxy } & 0 & 0 & - \text { zzxy } \\ 0 & 0 & 0 & 0 & \text { yzzx } & 0 & 0 & -z x y z & 0 \\ 0 & 0 & 0 & \text { zxyz } & 0 & 0 & -y z z x & 0 & 0 \\ \text { xyxx } & -x y x x & 0 & 0 & 0 & 0 & 0 & 0 & 0\end{array}\right)$

and: $y y x y-x x x y=2 x y x x$

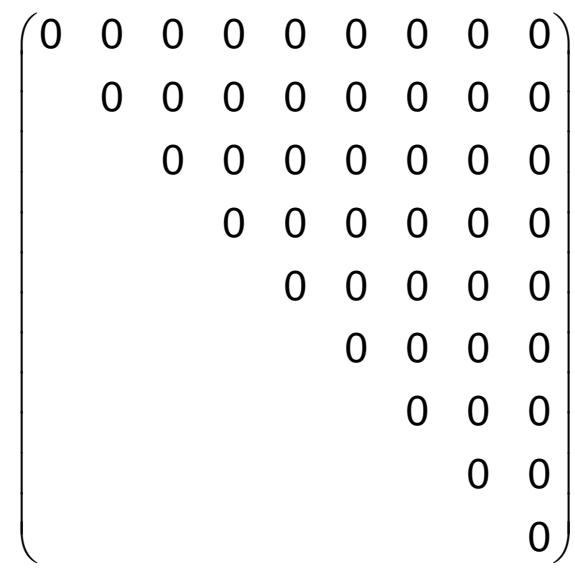

$\left(\begin{array}{lllllllll}0 & 0 & 0 & 0 & 0 & 0 & 0 & 0 & 0 \\ 0 & 0 & 0 & 0 & 0 & 0 & 0 & 0 & 0\end{array}\right)$

$\begin{array}{lllllllll}0 & 0 & 0 & 0 & 0 & 0 & 0 & 0 & 0\end{array}$

$\begin{array}{lllllllll}0 & 0 & 0 & 0 & 0 & 0 & 0 & 0 & 0 \\ 0 & 0 & 0 & 0 & 0 & 0 & 0 & 0 & 0\end{array}$

$\begin{array}{lllllllll}0 & 0 & 0 & 0 & 0 & 0 & 0 & 0 & 0 \\ 0 & 0 & 0 & 0 & 0 & 0 & 0 & 0 & 0\end{array}$

$\begin{array}{lllllllll}0 & 0 & 0 & 0 & 0 & 0 & 0 & 0 & 0 \\ 0 & 0 & 0 & 0 & 0 & 0 & 0 & 0 & 0\end{array}$

$\begin{array}{llllllllll}0 & 0 & 0 & 0 & 0 & 0 & 0 & 0 & 0\end{array}$ 


\section{1}

$\left(\begin{array}{lllllllll}0 & 0 & 0 & 0 & 0 & 0 & 0 & 0 & 0 \\ 0 & 0 & 0 & 0 & 0 & 0 & 0 & 0 & 0\end{array}\right)$

$\left(\begin{array}{lllllllll}x x x x & x x y y & x x z z & 0 & 0 & 0 & 0 & 0 & 0 \\ y y x x & y y y y & y z z & 0 & 0 & 0 & 0 & 0 & 0\end{array}\right)$

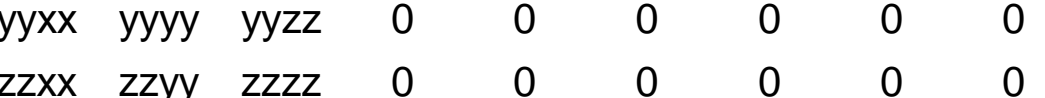

$v^{4} \quad \begin{array}{ccccccccc}0 & 0 & 0 & y z y z & 0 & 0 & y z z y & 0 & 0 \\ 0 & 0 & 0 & 0 & z x z & 0 & 0 & z x z & 0 \\ 0 & 0 & 0 & 0 & 0 & x y x y & 0 & 0 & x y x\end{array} \mid$

$\begin{array}{ccccccccc}0 & 0 & 0 & 0 & z x z x & 0 & 0 & z x x z & 0 \\ 0 & 0 & 0 & 0 & 0 & x y x y & 0 & 0 & x y y x\end{array}$

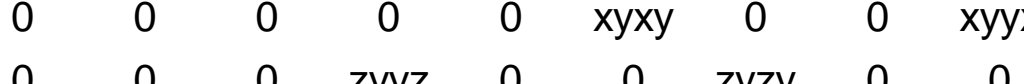

$\begin{array}{ccccccccc}0 & 0 & 0 & 0 & x z z x & 0 & 0 & x z x z & 0 \\ 0 & 0 & 0 & 0 & 0 & y x x y & 0 & 0 & \end{array}$

$\left.\mathrm{V}^{4}\right] \quad\left(\begin{array}{ccccc}\mathrm{xxxx} & 0 & 0 & \mathrm{yyzz} & 0 \\ 0 & \mathrm{yyyy} & 0 & \mathrm{zzxx} & 0 \\ 0 & 0 & \mathrm{zzzz} & \mathrm{xxyy} & 0\end{array}\right)$

$\left(\begin{array}{lllllllll}0 & 0 & 0 & 0 & 0 & 0 & 0 & 0 & 0 \\ 0 & 0 & 0 & 0 & 0 & 0 & 0 & 0 & 0 \\ 0 & 0 & 0 & 0 & 0 & 0 & 0 & 0 & 0 \\ 0 & 0 & 0 & 0 & 0 & 0 & 0 & 0 & 0\end{array}\right)$

$0 \begin{array}{llllllllll}0 & 0 & 0 & 0 & 0 & 0 & 0 & 0 & 0 \\ 0 & 0 & 0 & 0 & 0 & 0 & 0 & 0 & 0\end{array}$

$0 \begin{array}{llllllllll}0 & 0 & 0 & 0 & 0 & 0 & 0 & 0 & 0 \\ 0 & 0 & 0 & 0 & 0 & 0 & 0 & 0\end{array}$

$\begin{array}{llllllllll}0 & 0 & 0 & 0 & 0 & 0 & 0 & 0 & 0 \\ 0 & 0 & 0 & 0 & 0 & 0 & 0 & 0 & 0\end{array}$

$0 \begin{array}{lllllllll}0 & 0 & 0 & 0 & 0 & 0 & 0 & 0 & 0 \\ 0 & 0 & 0 & 0 & 0 & 0 & 0 & 0 & 0\end{array}$

$\begin{array}{lllllllll}0 & 0 & 0 & 0 & 0 & 0 & 0 & 0 & 0 \\ 0 & 0 & 0 & 0 & 0 & 0 & 0 & 0 & 0\end{array}$

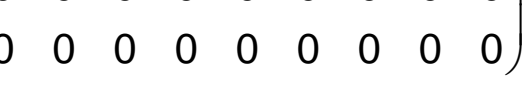

$\mathrm{V}\left[\mathrm{V}^{3}\right] \quad\left(\begin{array}{cccccccccc}\mathrm{x} x \mathrm{xx} & 0 & 0 & \mathrm{xxyy} & 0 & 0 & \mathrm{xxzz} & 0 & 0 & 0 \\ 0 & \mathrm{yyyy} & 0 & 0 & \mathrm{yyzz} & 0 & 0 & \mathrm{yyxx} & 0 & 0 \\ 0 & 0 & \mathrm{zzz} & 0 & 0 & \mathrm{zzxx} & 0 & 0 & \mathrm{z} y \mathrm{y} & 0\end{array}\right)$

$\left(\begin{array}{lllll}0 & 0 & 0 & 0 & 0 \\ 0 & 0 & 0 & 0 & 0 \\ 0 & 0 & 0 & 0 & 0\end{array}\right)$

$V^{\prime}\left[V^{0}\right]\left(\begin{array}{ccccccccccc}0 & y y y y & 0 & 0 & y y z z & 0 & 0 & y y x x & 0 & 0 \\ 0 & 0 & z z z z & 0 & 0 & z z x x & 0 & 0 & z z y y & 0\end{array}\right)$

$\left(\begin{array}{llllllllll}0 & 0 & 0 & 0 & 0 & 0 & 0 & 0 & 0 & 0 \\ 0 & 0 & 0 & 0 & 0 & 0 & 0 & 0 & 0 & 0\end{array}\right)$

$\left(\begin{array}{lllllllllll}0 & 0 & 0 & 0 & 0 & 0 & 0 & 0 & 0 & 0\end{array}\right.$

$\begin{array}{llllllllll}0 & 0 & 0 & 0 & 0 & 0 & 0 & 0 & 0 & 0\end{array}$

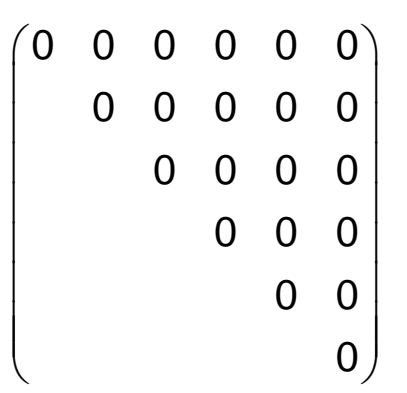

$\left(\begin{array}{llllll}0 & 0 & 0 & 0 & 0 & 0 \\ 0 & 0 & 0 & 0 & 0 & 0\end{array}\right)$

$\begin{array}{llllll}0 & 0 & 0 & 0 & 0 & 0 \\ 0 & 0 & 0 & 0 & 0 & 0 \\ 0 & 0 & 0 & 0 & 0 & 0\end{array}$

$\begin{array}{llllll}0 & 0 & 0 & 0 & 0 & 0 \\ 0 & 0 & 0 & 0 & 0 & 0 \\ 0 & 0 & 0 & 0\end{array}$

$\left(\begin{array}{llllll}0 & 0 & 0 & 0 & 0 & 0 \\ 0 & 0 & 0 & 0 & 0 & 0 \\ 0 & 0 & 0 & 0\end{array}\right.$

$\begin{array}{llllll}0 & 0 & 0 & 0 & 0 & 0 \\ 0 & 0 & 0 & 0 & 0 & 0\end{array}$

$\left(\begin{array}{llllll}x x x x & x x y y & x x z z & 0 & 0 & 0 \\ y y x x & y y y y & y y z z & 0 & 0 & 0 \\ z z x x & z z y y & z z z z & 0 & 0 & 0\end{array}\right)$

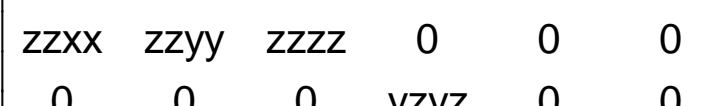
$\left(\begin{array}{cccccc}0 & 0 & 0 & 0 & x z x z & 0 \\ 0 & 0 & 0 & 0 & 0 & x y x y\end{array}\right)$ $\begin{array}{lllllllll}0 & 0 & 0 & 0 & 0 & 0 & 0 & 0 & 0 \\ 0 & 0 & 0 & 0 & 0 & 0 & 0 & 0 & 0\end{array}$

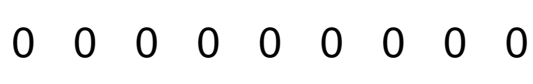
$0 \begin{array}{llllllllll}0 & 0 & 0 & 0 & 0 & 0 & 0 & 0 & 0 \\ 0 & 0 & 0 & 0 & 0 & 0 & 0 & 0 & 0\end{array}$ $0 \begin{array}{llllllllllll}0 & 0 & 0 & 0 & 0 & 0 & 0 & 0 & 0 \\ 0 & 0 & 0 & 0 & 0 & 0 & 0 & 0 & 0\end{array}$ $\begin{array}{llllllllll}0 & 0 & 0 & 0 & 0 & 0 & 0 & 0 & 0 \\ 0 & 0 & 0 & 0 & 0 & 0 & 0 & 0 & 0\end{array}$

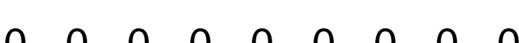
$0 \begin{array}{lllllllll}0 & 0 & 0 & 0 & 0 & 0 & 0 & 0 & 0 \\ 0 & 0 & 0 & 0 & 0 & 0 & 0 & 0 & 0\end{array}$ $0 \begin{array}{lllllllll}0 & 0 & 0 & 0 & 0 & 0 & 0 & 0 & 0\end{array}$ $\begin{array}{llllllllll}0 & 0 & 0 & 0 & 0 & 0 & 0 & 0 & 0\end{array}$

\section{$\left(\begin{array}{lllll}0 & 0 & 0 & 0 & 0 \\ 0 & 0 & 0 & 0 & 0 \\ 0 & 0 & 0 & 0 & 0\end{array}\right)$}

$\left(\begin{array}{llllllllll}0 & 0 & 0 & 0 & 0 & 0 & 0 & 0 & 0 & 0 \\ 0 & 0 & 0 & 0 & 0 & 0 & 0 & 0 & 0 & 0\end{array}\right)$ $\left.\begin{array}{lllllllllll}0 & 0 & 0 & 0 & 0 & 0 & 0 & 0 & 0 & 0 \\ 0 & 0 & 0 & 0 & 0 & 0 & 0 & 0 & 0 & 0\end{array}\right)$ $\begin{array}{lllllllllll}0 & 0 & 0 & 0 & 0 & 0 & 0 & 0 & 0 & 0\end{array}$

$$
\left(\begin{array}{llllll}
0 & 0 & 0 & 0 & 0 & 0 \\
& 0 & 0 & 0 & 0 & 0 \\
& & 0 & 0 & 0 & 0 \\
& & & 0 & 0 & 0 \\
& & & & 0 & 0 \\
& & & & & 0
\end{array}\right)
$$

$\left(\begin{array}{llllll}0 & 0 & 0 & 0 & 0 & 0 \\ 0 & 0 & 0 & 0 & 0 & 0\end{array}\right)$ $\left(\begin{array}{llllll}0 & 0 & 0 & 0 & 0 & 0 \\ 0 & 0 & 0 & 0 & 0 & 0 \\ 0 & 0 & 0 & 0 & 0 & 0\end{array}\right)$ $\begin{array}{llllll}0 & 0 & 0 & 0 & 0 & 0 \\ 0 & 0 & 0\end{array}$ $\begin{array}{llllll}0 & 0 & 0 & 0 & 0 & 0 \\ 0 & 0 & 0 & 0 & 0 & 0\end{array}$ $\left(\begin{array}{llllll}0 & 0 & 0 & 0 & 0 & 0 \\ 0 & 0 & 0 & 0 & 0 & 0\end{array}\right)$

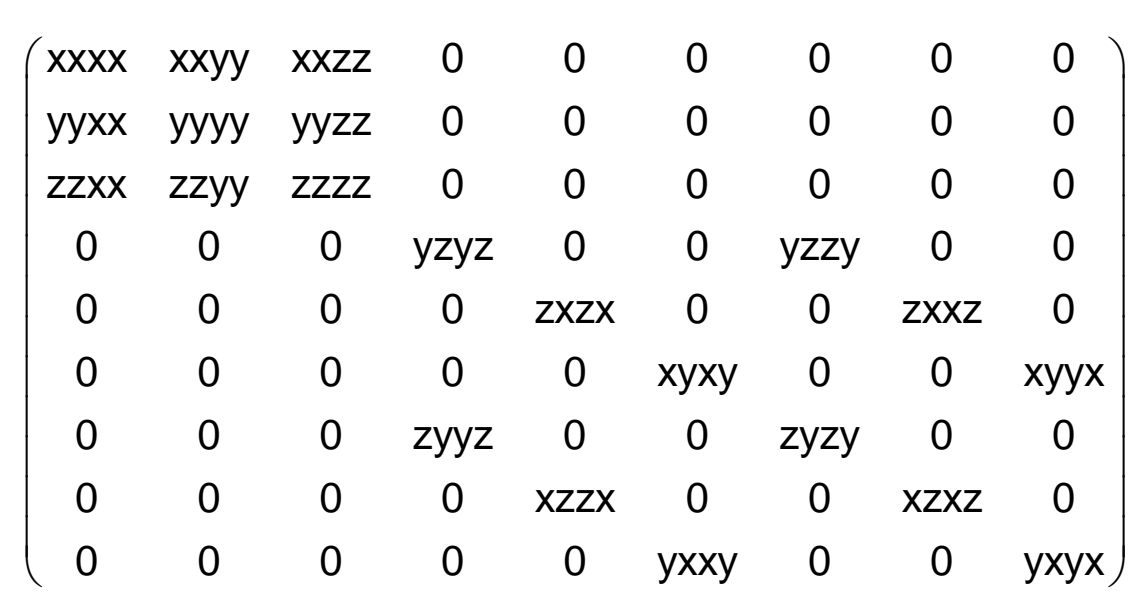

$\left(\begin{array}{ccccc}x x x x & 0 & 0 & y y z z & 0 \\ 0 & y y y y & 0 & z z x x & 0 \\ 0 & 0 & z z z z & x x y y & 0\end{array}\right)$

$\left(\begin{array}{ccccc}0 & 0 & z z z z & x x y y & 0\end{array}\right)$

$\left(\begin{array}{cccccccccc}\mathrm{xxxx} & 0 & 0 & \mathrm{xxyy} & 0 & 0 & \mathrm{xxzz} & 0 & 0 & 0 \\ 0 & \mathrm{yyyy} & 0 & 0 & \mathrm{yyzz} & 0 & 0 & \mathrm{yyxx} & 0 & 0 \\ 0 & 0 & \mathrm{zzzz} & 0 & 0 & \mathrm{zzxx} & 0 & 0 & \mathrm{zzy} & 0\end{array}\right)$

$\begin{array}{llllllllll}0 & 0 & z z z z & 0 & 0 & z z x x & 0 & 0 & \text { zzyy } & 0\end{array}$

$\left(\begin{array}{cccccc}x x x x & x x y y & x x z z & 0 & 0 & 0 \\ & \text { yyyy } & \text { yyzz } & 0 & 0 & 0 \\ & & \text { zzzz } & 0 & 0 & 0 \\ & & & y z y z & 0 & 0 \\ & & & & x z x z & 0 \\ & & & & & \text { xyxy }\end{array}\right)$

$\left(\begin{array}{llllll}x x x x & x x y y & x x z z & 0 & 0 & 0 \\ y y x x & y y y & y y z z & 0 & 0 & 0 \\ z z x x & z z y y & z z z z & 0 & 0 & 0\end{array}\right)$

$\begin{array}{llllll}\text { zzxx } & \text { zzyy } & \text { zzzz } & 0 & 0 & 0\end{array}$

$\begin{array}{cccccc}0 & 0 & 0 & y z y z & 0 & 0\end{array}$

$\begin{array}{cccccc}0 & 0 & 0 & y z y z & 0 & 0 \\ 0 & 0 & 0 & 0 & x z x z & 0 \\ 0 & 0 & 0 & 0 & 0 & x y x y\end{array}$ 


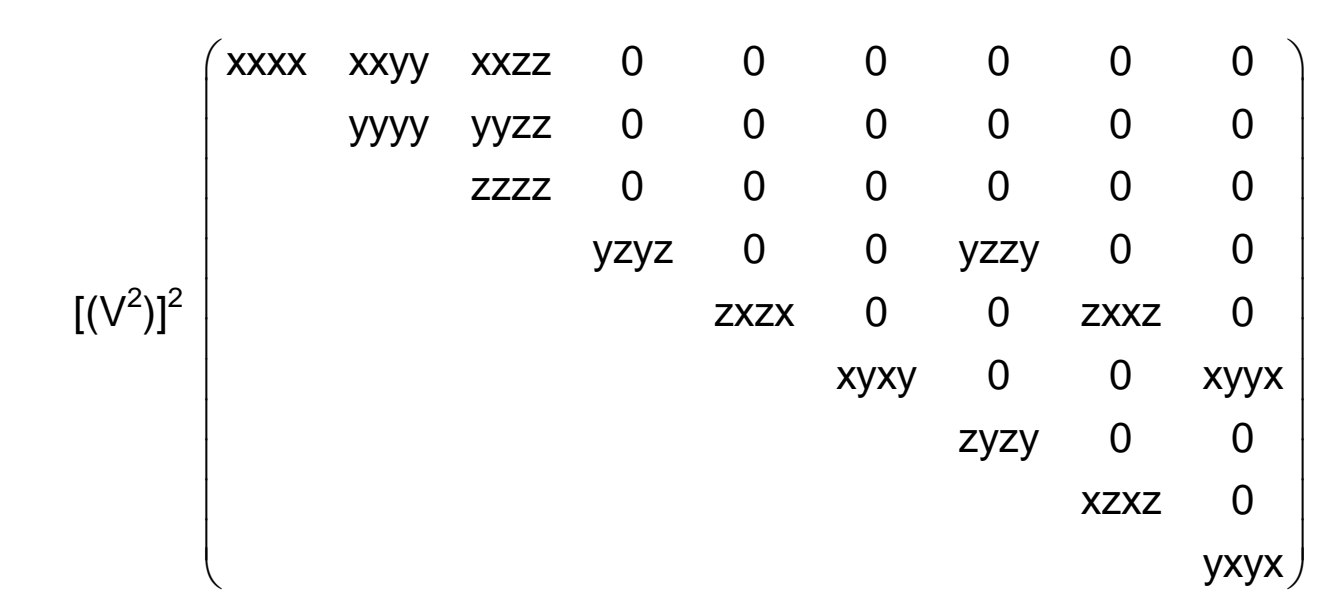

$\left(\begin{array}{lllllllll}0 & 0 & 0 & 0 & 0 & 0 & 0 & 0 & 0\end{array}\right)$

$\begin{array}{llllllll}0 & 0 & 0 & 0 & 0 & 0 & 0 & 0\end{array}$

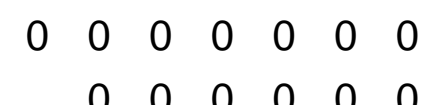

$\begin{array}{lllll}0 & 0 & 0 & 0 & 0\end{array}$

$\begin{array}{lllll}0 & 0 & 0 & 0\end{array}$

$\begin{array}{lll}0 & 0 & 0 \\ & 0 & 0\end{array}$

$\left(\begin{array}{lllllllll}x x x x & x x y y & x x z z & 0 & 0 & 0 & 0 & 0 & 0 \\ y x x & y y y & y y z z & 0 & 0 & 0 & 0 & 0 & 0\end{array}\right)\left(\begin{array}{ccccccccc}0 & 0 & 0 & 0 & 0 & 0 & 0 & 0 & 0 \\ 0 & 0 & 0 & 0 & 0 & 0 & 0 & 0\end{array}\right)$

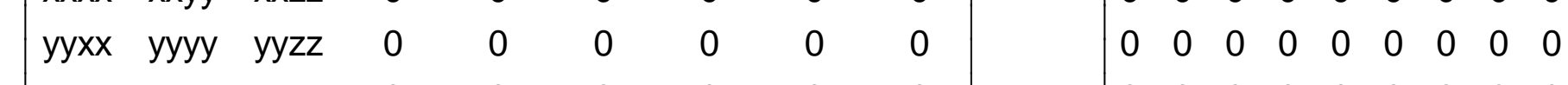

$\mathrm{V}^{2} \quad \begin{array}{ccccccccc}\text { zzxx } & z z y y & z z z z & 0 & 0 & 0 & 0 & 0 & 0 \\ 0 & 0 & 0 & y z y z & 0 & 0 & y z z y & 0 & 0 \\ 0 & 0 & 0 & 0 & z x z x & 0 & 0 & z x x z & 0\end{array} \mid \quad \quad \begin{array}{ccccccccc}0 & 0 & 0 & 0 & 0 & 0 & 0 & 0 & 0 \\ 0 & 0 & 0 & 0 & 0 & 0 & 0 & 0 & 0 \\ 0 & 0 & 0 & 0 & 0 & 0 & 0 & 0 & 0\end{array}$

$\left(\begin{array}{ccccccccc}0 & 0 & 0 & y z y z & 0 & 0 & y z z y & 0 & 0 \\ 0 & 0 & 0 & 0 & z x z x & 0 & 0 & z x x z & 0 \\ 0 & 0 & 0 & 0 & 0 & x y x y & 0 & 0 & x y y x\end{array}\right) \quad\left(\begin{array}{lllllllll}0 & 0 & 0 & 0 & 0 & 0 & 0 & 0 & 0 \\ 0 & 0 & 0 & 0 & 0 & 0 & 0 & 0 & 0 \\ 0 & 0 & 0 & 0 & 0 & 0 & 0 & 0 & 0 \\ 0 & 0 & 0 & 0 & 0 & 0 & 0 & 0 & 0\end{array}\right)$

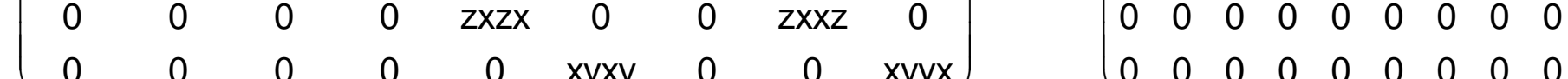

$\left(\begin{array}{lllllllll}0 & 0 & 0 & 0 & 0 & 0 & 0 & 0 & 0\end{array}\right.$

$\begin{array}{llllllll}0 & 0 & 0 & 0 & 0 & 0 & 0 & 0 \\ 0 & 0 & 0 & 0 & 0 & 0 & 0\end{array}$

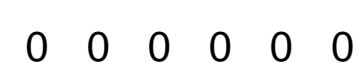

$\begin{array}{lllll}0 & 0 & 0 & 0 & 0\end{array}$

$\begin{array}{llll}0 & 0 & 0 & 0\end{array}$

$\begin{array}{lll}0 & 0 \\ 0 & 0\end{array}$

$\left(\begin{array}{lllllllll}0 & 0 & 0 & 0 & 0 & 0 & 0 & 0 & 0 \\ 0 & 0 & 0 & 0 & 0 & 0 & 0 & 0 & 0\end{array}\right.$

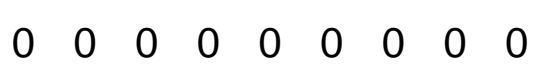

$0 \begin{array}{lllllllll}0 & 0 & 0 & 0 & 0 & 0 & 0 & 0 & 0 \\ 0 & 0 & 0 & 0 & 0 & 0 & 0 & 0 & 0\end{array}$

$0 \begin{array}{lllllllll}0 & 0 & 0 & 0 & 0 & 0 & 0 & 0 & 0 \\ 0 & 0 & 0 & 0 & 0 & 0 & 0 & 0 & 0\end{array}$

$\begin{array}{llllllllll}0 & 0 & 0 & 0 & 0 & 0 & 0 & 0 & 0 \\ 0 & 0 & 0 & 0 & 0 & 0 & 0 & 0 & 0\end{array}$

$\begin{array}{lllllllll}0 & 0 & 0 & 0 & 0 & 0 & 0 & 0 & 0\end{array}$

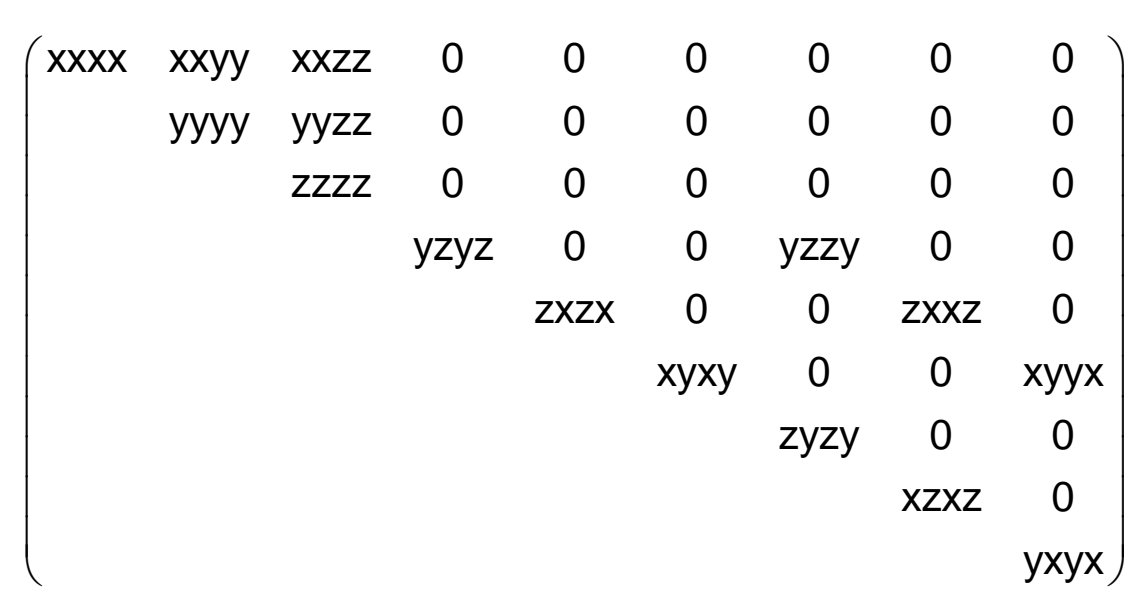

$\left(\begin{array}{lllllllll}x x x x & x x y y & x x z z & 0 & 0 & 0 & 0 & 0 & 0 \\ y y x x & y y y & y y z z & 0 & 0 & 0 & 0 & 0 & 0\end{array}\right.$ $\begin{array}{lllllllll}\text { yyxx } & \text { yyyy } & y y z z & 0 & 0 & 0 & 0 & 0 & 0 \\ \text { zzx } & \text { zzyy } & \text { zzzz } & 0 & 0 & 0 & 0 & 0 & 0\end{array}$ $\begin{array}{lllllllll}z z x x & z z y y & z z z z & 0 & 0 & 0 & 0 & 0 & 0\end{array}$ $\begin{array}{ccccccccc}0 & 0 & 0 & y z y z & 0 & 0 & y z z y & 0 & 0\end{array}$ $\begin{array}{ccccccccc}0 & 0 & 0 & 0 & z x z x & 0 & 0 & z x x z & 0 \\ 0 & 0 & 0 & 0 & 0 & x y x y & 0 & 0 & x y y x\end{array}$ 

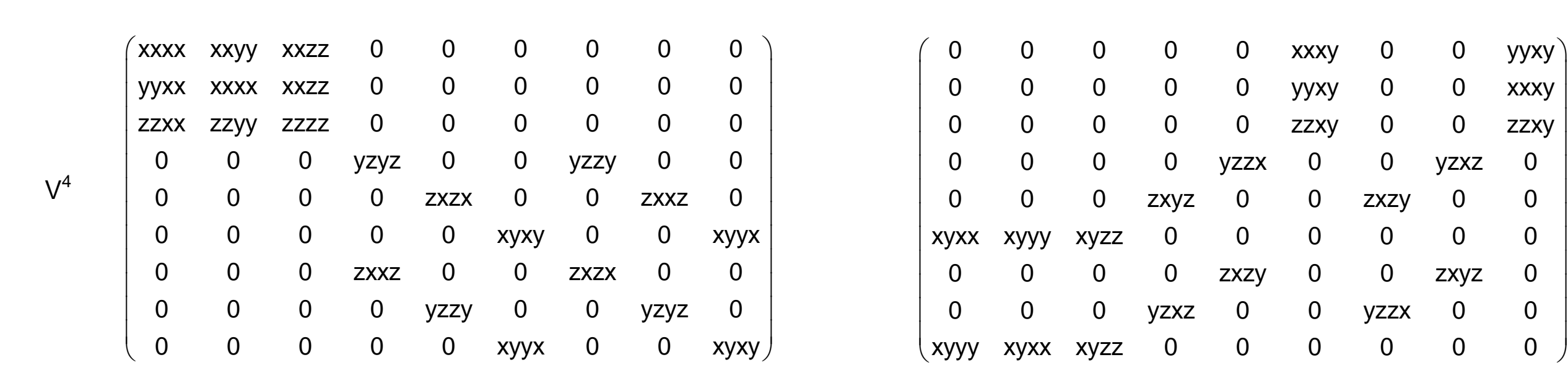

$\left[V^{4}\right] \quad\left(\begin{array}{ccccc}x x x x & 0 & 0 & y y z z & 0 \\ 0 & x x x x & 0 & y y z z & 0 \\ 0 & 0 & z z z z & x x y y & 0\end{array}\right)$

$V\left[V^{3}\right]\left(\begin{array}{cccccccccc}x x x x & 0 & 0 & x x y y & 0 & 0 & y y z z & 0 & 0 & 0 \\ 0 & x x x x & 0 & 0 & y y z z & 0 & 0 & x x y y & 0 & 0 \\ 0 & 0 & z z z z & 0 & 0 & z z x x & 0 & 0 & z z x x & 0\end{array}\right)$

$\left.V^{\prime} V^{3}\right]\left(\begin{array}{cccccccccc}0 & x x x x & 0 & 0 & y y z z & 0 & 0 & x x y y & 0 & 0 \\ 0 & 0 & z z z z & 0 & 0 & z z x x & 0 & 0 & z z x x & 0\end{array}\right)$

$\left[\left[^{2}\right]^{2}\right]\left(\begin{array}{cccccc}x x x x & x x y y & x x z z & 0 & 0 & 0 \\ & \text { yyyy } & \text { yyzz } & 0 & 0 & 0 \\ & & \text { zzzz } & 0 & 0 & 0 \\ & & & y z y z & 0 & 0 \\ & & & & \text { yzyz } & 0 \\ & & & & & \text { xyxy }\end{array}\right)$

$\left[V^{2}\right]^{2}\left(\begin{array}{cccccc}x x x x & x x y y & x x z z & 0 & 0 & 0 \\ y y x x & x x x x & x x z z & 0 & 0 & 0 \\ z z x x & z z x x & z z z z & 0 & 0 & 0 \\ 0 & 0 & 0 & y z y z & 0 & 0 \\ 0 & 0 & 0 & 0 & y z y z & 0 \\ 0 & 0 & 0 & 0 & 0 & x y x y\end{array}\right)$ $\left(\begin{array}{ccccc}0 & y \mathrm{yxx} & 0 & 0 & 0 \\ \mathrm{yxxx} & 0 & 0 & 0 & 0 \\ 0 & 0 & 0 & 0 & \mathrm{zzxy}\end{array}\right)$

$\left(\begin{array}{llllllllll}0 & \text { xyyy } & 0 & 0 & x y z z & 0 & 0 & \text { yxyy } & 0 & 0 \\ \text { xyy } & 0 & 0 & \text { yxyy } & 0 & 0 & x y z z & 0 & 0 & 0\end{array}\right)$

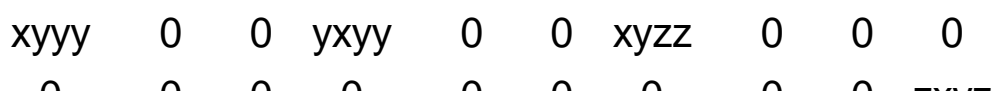

$\left(\begin{array}{cccccc}0 & 0 & 0 & 0 & 0 & x x x y \\ & 0 & 0 & 0 & 0 & x x x y \\ & & 0 & 0 & 0 & z z x y \\ & & & 0 & y z x z & 0 \\ & & & & 0 & 0 \\ & & & & & 0\end{array}\right)$

$\left(\begin{array}{cccccc}0 & 0 & 0 & 0 & 0 & x x x y \\ 0 & 0 & 0 & 0 & 0 & x x x y \\ 0 & 0 & 0 & 0 & 0 & z z x y \\ 0 & 0 & 0 & 0 & y z x z & 0 \\ 0 & 0 & 0 & y z x z & 0 & 0 \\ x y x x & x y x x & x y z z & 0 & 0 & 0\end{array}\right)$

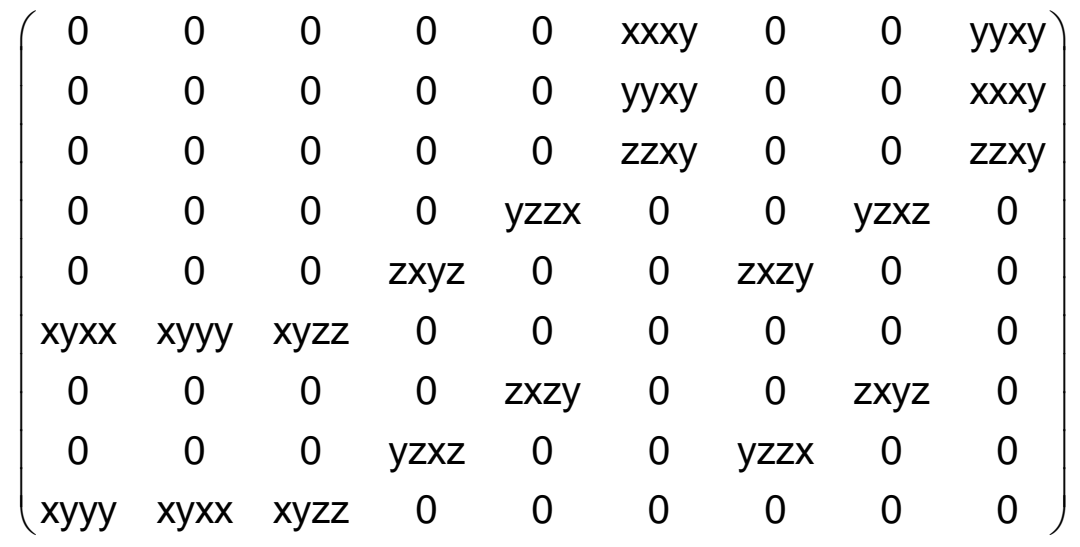

$\left(\begin{array}{ccccc}0 & y x x x & 0 & 0 & 0 \\ y x x x & 0 & 0 & 0 & 0 \\ 0 & 0 & 0 & 0 & z z x y\end{array}\right)$

$\left(\begin{array}{cccccccccc}0 & \text { xyyy } & 0 & 0 & \text { xyzz } & 0 & 0 & \text { yxyy } & 0 & 0 \\ \text { xyyy } & 0 & 0 & y x y y & 0 & 0 & x y z z & 0 & 0 & 0 \\ 0 & 0 & 0 & 0 & 0 & 0 & 0 & 0 & 0 & z x y z\end{array}\right)$

$\begin{array}{cccccccccc}x y y y & 0 & 0 & y x y y & 0 & 0 & x y z z & 0 & 0 & 0 \\ 0 & 0 & 0 & 0 & 0 & 0 & 0 & 0 & 0 & z x y z\end{array}$

$$
\left(\begin{array}{cccccc}
0 & 0 & 0 & 0 & 0 & x x x y \\
& 0 & 0 & 0 & 0 & x x x y \\
& & 0 & 0 & 0 & z z x y \\
& & & 0 & y z x z & 0 \\
& & & & 0 & 0 \\
& & & & & 0
\end{array}\right)
$$

$\left(\begin{array}{cccccc}0 & 0 & 0 & 0 & 0 & x x x y \\ 0 & 0 & 0 & 0 & 0 & x x x y \\ 0 & 0 & 0 & 0 & 0 & z z x y \\ 0 & 0 & 0 & 0 & y z x z & 0 \\ 0 & 0 & 0 & y z x z & 0 & 0 \\ \text { xyxx } & x y x x & x y z z & 0 & 0 & 0\end{array}\right)$

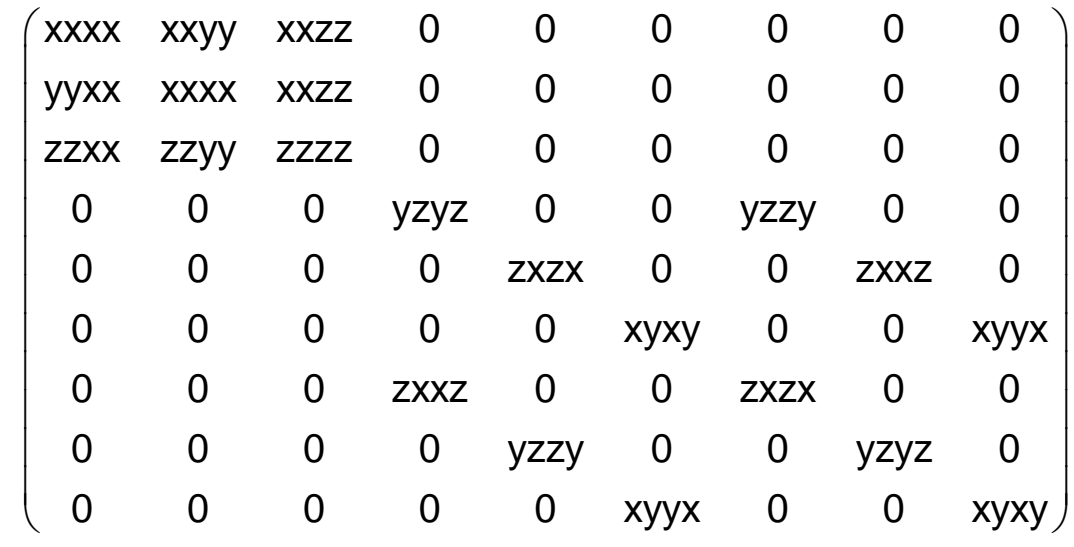

$\left(\begin{array}{ccccc}\mathrm{xxxx} & 0 & 0 & \mathrm{yyzz} & 0 \\ 0 & \mathrm{xxxx} & 0 & \mathrm{yyzz} & 0 \\ 0 & 0 & \mathrm{zzzz} & \mathrm{xxyy} & 0\end{array}\right)$

$\left(\begin{array}{cccccccccc}x x x x & 0 & 0 & x x y y & 0 & 0 & y y z z & 0 & 0 & 0 \\ 0 & x x x x & 0 & 0 & y y z z & 0 & 0 & x x y y & 0 & 0 \\ 0 & 0 & z z z z & 0 & 0 & z z x x & 0 & 0 & z z x & 0\end{array}\right)$

$\left.\begin{array}{cccccccccc}0 & x x x x & 0 & 0 & y y z z & 0 & 0 & x x y y & 0 & 0 \\ 0 & 0 & z z z z & 0 & 0 & z z x x & 0 & 0 & z z x x & 0\end{array}\right)$

$\left(\begin{array}{cccccc}x x x x & \text { xxyy } & \text { xxzz } & 0 & 0 & 0 \\ & \text { yyyy } & \text { yyzz } & 0 & 0 & 0 \\ & & \text { zzzz } & 0 & 0 & 0 \\ & & & y z y z & 0 & 0 \\ & & & & y z y z & 0 \\ & & & & & \text { xyxy }\end{array}\right)$

$\left(\begin{array}{llllll}x x x x & x x y y & x x z z & 0 & 0 & 0 \\ y y x x & x y x x & x x z z & 0 & 0 & 0\end{array}\right)$

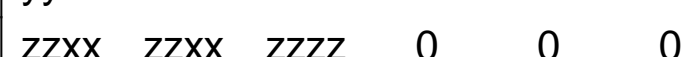

$\begin{array}{cccccc}\operatorname{zzx} x & z z x x & z z z & 0 & 0 & 0 \\ 0 & 0 & 0 & y z y z & 0 & 0\end{array}$

$\begin{array}{cccccc}0 & 0 & 0 & y z y z & 0 & 0 \\ 0 & 0 & 0 & 0 & y z y z & 0 \\ 0 & 0 & 0 & 0 & 0 & x y x y\end{array}$ 
$\left[\left(\mathrm{V}^{2}\right)\right]^{2}\left(\begin{array}{ccccccccc}\text { xxxx } & \text { xxyy } & \text { xxzz } & 0 & 0 & 0 & 0 & 0 & 0 \\ & \text { xxxx } & \text { xxzz } & 0 & 0 & 0 & 0 & 0 & 0 \\ & & \text { zzzz } & 0 & 0 & 0 & 0 & 0 & 0 \\ & & & y z y z & 0 & 0 & y z z y & 0 & 0 \\ & & & & \text { zxzx } & 0 & 0 & \text { yzzy } & 0 \\ & & & & & \text { xyxy } & 0 & 0 & \text { xyyx } \\ & & & & & & \text { zxzx } & 0 & 0 \\ & & & & & & & \text { yzyz } & 0 \\ & & & & & & & & \text { xyxy }\end{array}\right)$

$$
\begin{aligned}
& \left(\begin{array}{lllllllll}
0 & 0 & 0 & 0 & 0 & x x x y & 0 & 0 & y y x y \\
& 0 & 0 & 0 & 0 & y y x y & 0 & 0 & x x x y
\end{array}\right) \\
& \begin{array}{llllllll}
0 & 0 & 0 & 0 & y y x y & 0 & 0 & x x x y \\
& 0 & 0 & 0 & z z x y & 0 & 0 & z z x y
\end{array} \\
& \begin{array}{ccccccc}
0 & 0 & 0 & z z x y & 0 & 0 & z z x y \\
0 & y z z z & 0 & 0 & y z x z & 0
\end{array} \\
& \begin{array}{cccccc}
0 & y z z x & 0 & 0 & y z x z & 0 \\
0 & 0 & z x z y & 0 & 0
\end{array} \\
& \begin{array}{ccccc}
0 & 0 & z x z y & 0 & 0 \\
0 & 0 & 0 & 0
\end{array} \\
& \begin{array}{ccc}
0 & y z z x & 0 \\
0 & 0 \\
& & 0
\end{array}
\end{aligned}
$$

$\left(\begin{array}{lllllllll}x x x x & x x y y & x x z z & 0 & 0 & 0 & 0 & 0 & 0 \\ x y x x & x x y x & x x z z & 0 & 0 & 0 & 0 & 0 & 0 \\ z z x x & z z x x & z z z & 0 & 0 & 0 & 0 & 0 & 0\end{array}\right)$

$\left.\begin{array}{lllllllll}\mathrm{yyxx} & \mathrm{xxxx} & \mathrm{xxzz} & 0 & 0 & 0 & 0 & 0 & 0 \\ \mathrm{zzxx} & \mathrm{zzxx} & \mathrm{zzzz} & 0 & 0 & 0 & 0 & 0 & 0\end{array}\right)$

$\left[\mathrm{V}^{2}\right] \mathrm{V}^{2} \quad \begin{array}{cccccccccc}\operatorname{zzx} & \mathbf{z z} & 0 & 0 & \mathrm{yzyz} & 0 & 0 & \mathrm{zxzx} & 0 & 0 \\ 0 & 0 & 0 & 0 & z x z x & 0 & 0 & y z y & 0\end{array}$

$\left(\begin{array}{ccccccccc}0 & 0 & 0 & y z y z & 0 & 0 & z x z x & 0 & 0 \\ 0 & 0 & 0 & 0 & z x z x & 0 & 0 & y z y z & 0 \\ 0 & 0 & 0 & 0 & 0 & x y y & 0 & 0 & x y x\end{array}\right)$

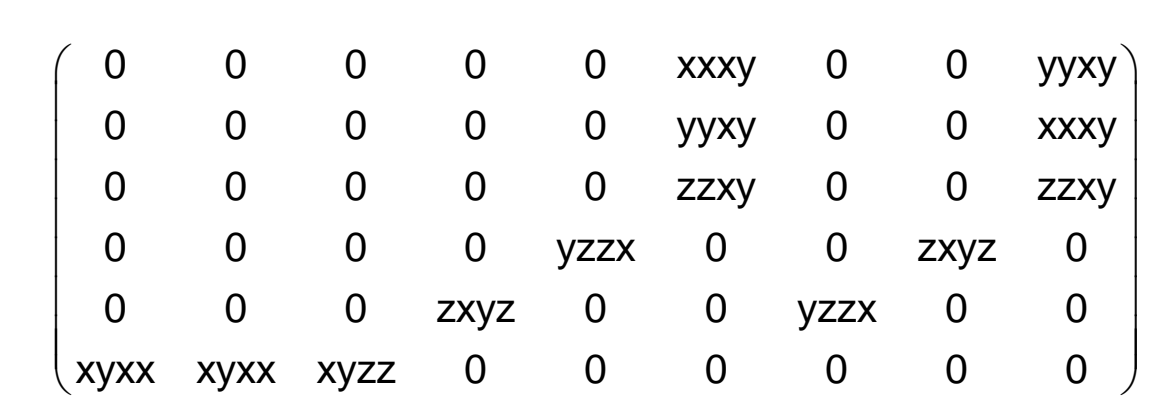

$$
\left(\begin{array}{ccccccccc}
0 & 0 & 0 & 0 & 0 & \text { xxxy } & 0 & 0 & y y x y \\
& 0 & 0 & 0 & 0 & y y x y & 0 & 0 & x x x y \\
& & 0 & 0 & 0 & z z x y & 0 & 0 & z z x y \\
& & & 0 & y z z x & 0 & 0 & y z x z & 0 \\
& & & & 0 & 0 & \text { zxzy } & 0 & 0 \\
& & & & & 0 & 0 & 0 & 0 \\
& & & & & & 0 & y z z x & 0 \\
& & & & & & & 0 & 0 \\
& & & & & & & & 0
\end{array}\right)
$$

$$
\left(\begin{array}{ccccccccc}
0 & 0 & 0 & 0 & 0 & \text { xxxy } & 0 & 0 & \text { yyxy } \\
0 & 0 & 0 & 0 & 0 & \text { yyxy } & 0 & 0 & \text { xxxy } \\
0 & 0 & 0 & 0 & 0 & \text { zzxy } & 0 & 0 & \text { zzxy } \\
0 & 0 & 0 & 0 & \text { yzzx } & 0 & 0 & \text { zxyz } & 0 \\
0 & 0 & 0 & \text { zxyz } & 0 & 0 & \text { yzzx } & 0 & 0 \\
\text { xyxx } & \text { xyxx } & \text { xyzz } & 0 & 0 & 0 & 0 & 0 & 0
\end{array}\right)
$$

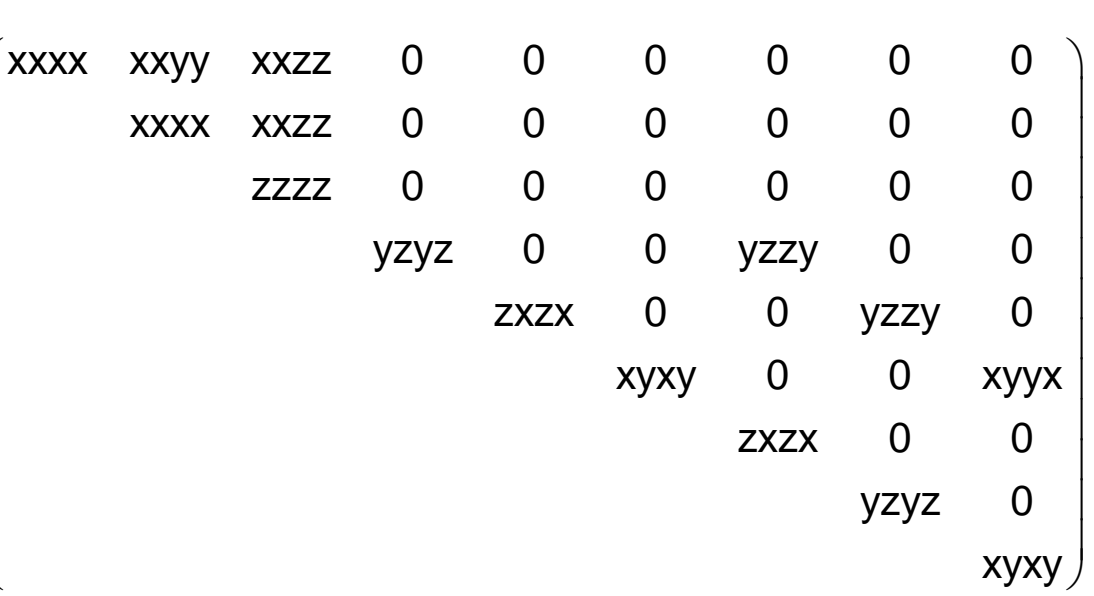

$\left(\begin{array}{lllllllll}x x x x & x x y y & x x z z & 0 & 0 & 0 & 0 & 0 & 0\end{array}\right.$ $\begin{array}{lllllllll}y y x x & x x x x & x x z z & 0 & 0 & 0 & 0 & 0 & 0 \\ z z x & z z x & z z z & 0 & 0 & 0 & 0 & 0 & 0\end{array}$ $\begin{array}{lllllllll}z z x x & z z x x & z z z z & 0 & 0 & 0 & 0 & 0 & 0\end{array}$ $\begin{array}{lllllllll}0 & 0 & 0 & y z y z & 0 & 0 & z x z x & 0 & 0\end{array}$ $\begin{array}{ccccccccc}0 & 0 & 0 & y z y z & 0 & 0 & z x z x & 0 & 0 \\ 0 & 0 & 0 & 0 & z x z x & 0 & 0 & y z y z & 0\end{array}$ 
$\left(\begin{array}{lllllllll}x x x x & x x y y & x x z z & x x y z & 0 & 0 & x x z y & 0 & 0 \\ y y x x & x x y x & y y z z & x x y z & 0 & 0 & x x z y & 0 & 0 \\ z z x x & z z x x & z z z z & 0 & 0 & 0 & 0 & 0 & 0\end{array}\right)$ $\left(\begin{array}{ccccccccc}x y y x x & x x x x & y y z z & -x x y z & 0 & 0 & -x x z y & 0 & 0 \\ z z x x & z z x x & z z z z & 0 & 0 & 0 & 0 & 0 & 0 \\ y z x x & -y z x & 0 & y z y z & 0 & 0 & y z y & 0 & 0\end{array}\right)$

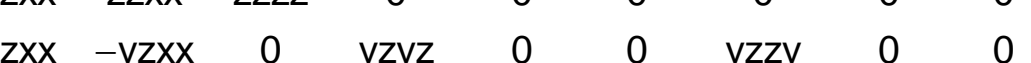
$\begin{array}{ccccccccc}\operatorname{czx} & -\mathrm{yzx} x & 0 & \mathrm{yzyz} & 0 & 0 & \mathrm{yzzy} & 0 & 0 \\ 0 & 0 & 0 & 0 & \mathrm{zxzx} & \mathrm{zyxx} & 0 & \mathrm{zxxz} & \mathrm{zyxx} \\ 0 & 0 & 0 & 0 & \mathrm{yxz} & \mathrm{xyx} & 0 & \mathrm{xxy} & \mathrm{xyy}\end{array} \mid$ $\begin{array}{lllllllll}0 & 0 & 0 & 0 & x x z y & x y x y & 0 & x x y z & x y y x\end{array}$ $\begin{array}{lllllllll}z y x x & -z y x x & 0 & z x x z & 0 & 0 & z x z x & 0 & 0\end{array}$ $\left.\begin{array}{ccccccccc}x z x x & -x z x x & 0 & 0 & y z z y & y z x x & 0 & y z y z & y z x x \\ 0 & 0 & 0 & 0 & x x z y & x y y x & 0 & x x y z & x y x y\end{array}\right)$ and: $x x x x=x x y y+x y x y+x y y x$

$\left[\mathrm{V}^{4}\right] \quad\left(\begin{array}{ccccc}3 x x y y & 0 & 0 & y y z z & x x y z \\ 0 & 3 x x y y & 0 & y y z z & 0 \\ 0 & -x x y z & z z z z & x x y y & 0\end{array}\right)$

$V\left[V^{3}\right]\left(\begin{array}{cccccccccc}3 x x y y & 0 & 0 & x x y y & 0 & 0 & y y z z & 0 & 0 & y z x x \\ 0 & 3 x x y y & 0 & 0 & y y z z & y z x x & 0 & x x y y & -y z x x & 0 \\ 0 & -z y x & z z z z & 0 & 0 & z z x & 0 & z y x & z z x x & 0\end{array}\right)$

$\left[\left[^{2}\right]^{2}\right]\left(\begin{array}{cccccc}x x x x & x x y y & x x z z & x x y z & 0 & 0 \\ & x x x x & x x z z & -x x y z & 0 & 0 \\ & & z z z z & 0 & 0 & 0 \\ & & & y z y z & 0 & 0 \\ & & & & y z y z & x x y z \\ & & & & & x y x y\end{array}\right)$

and $x y x y=1 / 2(x x x x-x x y y)$

$\left[\mathrm{V}^{2}\right]^{2}$

$\left(\begin{array}{cccccc}\text { xxxx } & x x y y & x x z z & x x y z & 0 & 0 \\ x x y y & x x x x & x x z z & -x x y z & 0 & 0 \\ z z x x & z z x x & z z z z & 0 & 0 & 0 \\ y z x x & -y z x x & 0 & y z y z & 0 & 0 \\ 0 & 0 & 0 & 0 & y z y z & y z x x \\ 0 & 0 & 0 & 0 & x x y z & x y x y\end{array}\right)$

and $x y x y=1 / 2(x x x x-x x y y)$ $\begin{array}{lllllllll}0 & 0 & 0 & 0 & 0 & 0 & 0 & 0 & 0 \\ 0 & 0 & 0 & 0 & 0 & 0 & 0 & 0 & 0\end{array}$ $0 \begin{array}{lllllllll}0 & 0 & 0 & 0 & 0 & 0 & 0 & 0 & 0 \\ 0 & 0 & 0 & 0 & 0 & 0 & 0 & 0 & 0\end{array}$

$0 \begin{array}{lllllllll}0 & 0 & 0 & 0 & 0 & 0 & 0 & 0 & 0 \\ 0 & 0 & 0 & 0 & 0 & 0 & 0 & 0 & 0\end{array}$

$0 \begin{array}{lllllllll}0 & 0 & 0 & 0 & 0 & 0 & 0 & 0 & 0 \\ 0 & 0 & 0 & 0 & 0 & 0 & 0 & 0 & 0\end{array}$

$0 \begin{array}{llllllllll}0 & 0 & 0 & 0 & 0 & 0 & 0 & 0 & 0\end{array}$

$0 \begin{array}{lllllllll}0 & 0 & 0 & 0 & 0 & 0 & 0 & 0 & 0\end{array}$

$\begin{array}{lllllllll}0 & 0 & 0 & 0 & 0 & 0 & 0 & 0 & 0 \\ 0 & 0 & 0 & 0 & 0 & 0 & 0 & 0 & 0\end{array}$

$\begin{array}{lllllllll}0 & 0 & 0 & 0 & 0 & 0 & 0 & 0 & 0 \\ 0 & 0 & 0 & 0 & 0 & 0 & 0 & 0 & 0\end{array}$

$\left(\begin{array}{lllll}0 & 0 & 0 & 0 & 0 \\ 0 & 0 & 0 & 0 & 0 \\ 0 & 0 & 0 & 0 & 0\end{array}\right)$

$\begin{array}{lllll}0 & 0 & 0 & 0 & 0\end{array}$

$\left(\begin{array}{llllllllll}0 & 0 & 0 & 0 & 0 & 0 & 0 & 0 & 0 & 0 \\ 0 & 0 & 0 & 0 & 0 & 0 & 0 & 0 & 0 & 0\end{array}\right)$

$\left(\begin{array}{lllllllllll}0 & 0 & 0 & 0 & 0 & 0 & 0 & 0 & 0 & 0 \\ 0 & 0 & 0 & 0 & 0 & 0 & 0 & 0 & 0 & 0\end{array}\right)$

$\left(\begin{array}{lllllllll}0 & 0 & 0 & 0 & 0 & 0 & 0 & 0 & 0 \\ 0 & 0 & 0 & 0 & 0 & 0 & 0 & 0 & 0 \\ 0 & 0 & 0 & 0 & 0 & 0 & 0 & 0 & 0\end{array}\right)$

$\begin{array}{lllllllll}0 & 0 & 0 & 0 & 0 & 0 & 0 & 0 & 0 \\ 0 & 0 & 0 & 0 & 0 & 0 & 0 & 0 & 0\end{array}$

$0 \begin{array}{llllllllll}0 & 0 & 0 & 0 & 0 & 0 & 0 & 0 & 0 \\ 0 & 0 & 0 & 0 & 0 & 0 & 0 & 0 & 0\end{array}$

$\begin{array}{llllllllll}0 & 0 & 0 & 0 & 0 & 0 & 0 & 0 & 0 \\ 0 & 0 & 0 & 0 & 0 & 0 & 0 & 0 & 0\end{array}$

$\begin{array}{lllllllll}0 & 0 & 0 & 0 & 0 & 0 & 0 & 0 & 0 \\ 0 & 0 & 0 & 0 & 0\end{array}$

$\begin{array}{llllllllll}0 & 0 & 0 & 0 & 0 & 0 & 0 & 0 & 0\end{array}$

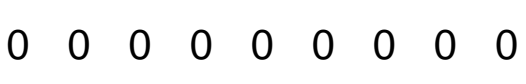

$\begin{array}{llllllllll}0 & 0 & 0 & 0 & 0 & 0 & 0 & 0 & 0\end{array}$

$\left(\begin{array}{lllll}0 & 0 & 0 & 0 & 0 \\ 0 & 0 & 0 & 0 & 0 \\ 0 & 0 & 0 & 0 & 0\end{array}\right)$

$\left(\begin{array}{lllll}0 & 0 & 0 & 0 & 0\end{array}\right)$

$\left(\begin{array}{llllllllll}0 & 0 & 0 & 0 & 0 & 0 & 0 & 0 & 0 & 0 \\ 0 & 0 & 0 & 0 & 0 & 0 & 0 & 0 & 0 & 0\end{array}\right.$ $\left.\begin{array}{llllllllll}0 & 0 & 0 & 0 & 0 & 0 & 0 & 0 & 0 & 0 \\ 0 & 0 & 0 & 0 & 0 & 0 & 0 & 0 & 0 & 0\end{array}\right)$ $\begin{array}{llllllllll}0 & 0 & 0 & 0 & 0 & 0 & 0 & 0 & 0 & 0\end{array}$

$$
\left(\begin{array}{llllll}
0 & 0 & 0 & 0 & 0 & 0 \\
& 0 & 0 & 0 & 0 & 0 \\
& & 0 & 0 & 0 & 0 \\
& & & 0 & 0 & 0 \\
& & & & 0 & 0
\end{array}\right)
$$$$
\begin{array}{lllll}
0 & 0 & 0 & 0 \\
0 & 0 & 0 & 0
\end{array}
$$$$
\begin{array}{lll}
0 & 0 & 0 \\
& 0 & 0
\end{array}
$$

0 )

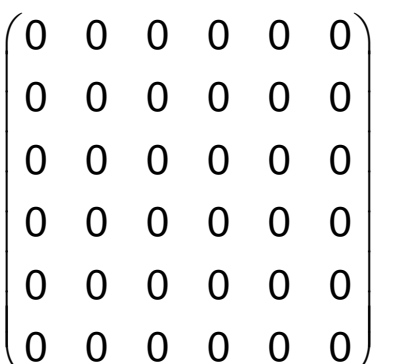

$\begin{array}{llllll}0 & 0 & 0 & 0 & 0 & 0 \\ 0 & 0 & 0 & 0 & 0 & 0\end{array}$

$\begin{array}{llllll}0 & 0 & 0 & 0 & 0 & 0 \\ 0 & 0 & 0 & 0 & 0\end{array}$

$\begin{array}{llllll}0 & 0 & 0 & 0 & 0 & 0 \\ 0 & 0 & 0 & 0 & 0 & 0\end{array}$

$\left(\begin{array}{llllll}0 & 0 & 0 & 0 & 0 & 0 \\ 0 & 0 & 0 & 0 & 0 & 0\end{array}\right)$

$\begin{array}{llllll}0 & 0 & 0 & 0 & 0 & 0 \\ 0 & 0 & 0 & 0 & 0 & 0\end{array}$

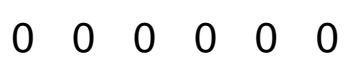

$\begin{array}{llllll}0 & 0 & 0 & 0 & 0 & 0 \\ 0 & 0 & 0 & 0 & 0 & 0\end{array}$

$\left(\begin{array}{llllll}0 & 0 & 0 & 0 & 0 & 0 \\ 0 & 0 & 0 & 0 & 0 & 0\end{array}\right.$

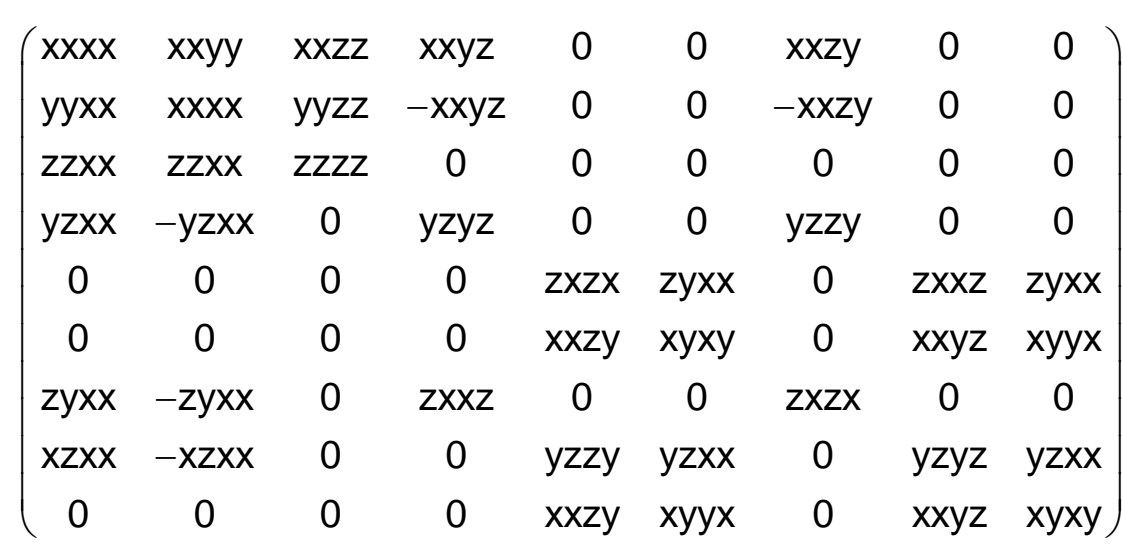

and: $x x x x=x x y y+x y x y+x y y x$

$\left(\begin{array}{ccccc}3 x x y y & 0 & 0 & y y z z & x x y z \\ 0 & 3 x x y y & 0 & y y z z & 0 \\ 0 & -x x y z & z z z z & x x y y & 0\end{array}\right)$

$\left(\begin{array}{lllll}0 & -x x y z & z z z z & x x y y & 0\end{array}\right)$

$\left(\begin{array}{cccccccccc}3 x x y y & 0 & 0 & x x y y & 0 & 0 & y y z z & 0 & 0 & y z x x \\ 0 & 3 x y y y & 0 & 0 & y y z & y z z x & 0 & x x y y & -y z x x & 0 \\ 0 & -2 y y x & z z z & 0 & 0 & z x x & 0 & z y x & z z x & 0\end{array}\right)$

$\left.\begin{array}{lllllllllll}0 & -z x y y x & 0 & 0 & y y z z & y z x x & 0 & x x y y & -y z x x & 0 \\ 0 & -z y x & z z z z & 0 & 0 & z z x x & 0 & z y y x & z z x x & 0\end{array}\right)$

$$
\left(\begin{array}{cccccc}
\text { xxxx } & \text { xxyy } & \text { xxzz } & \text { xxyz } & 0 & 0 \\
& \text { xxxx } & \text { xxzz } & -x x y z & 0 & 0 \\
& & \text { zzzz } & 0 & 0 & 0 \\
& & & y z y z & 0 & 0 \\
& & & & y z y z & \text { xxyz } \\
& & & & & \text { xyxy }
\end{array}\right)
$$

and $x y x y=1 / 2(x x x x-x x y y)$

$\left(\begin{array}{llllll}x x x x & x x y y & x x z z & x x y z & 0 & 0 \\ x x y y & x x x & x z z & -x y z & 0 & 0\end{array}\right)$

$\begin{array}{llllll}x x y y & x x x x & x x z z & -x x y z & 0 & 0 \\ z z x x & z z x x & z z z & 0 & 0 & 0\end{array}$

$\begin{array}{llllll}z z x x & z z x x & z z z z & 0 & 0 & 0 \\ y z x & -y z x & 0 & & 0 & 0\end{array}$

$\begin{array}{cccccc}y z x x & -y z x x & 0 & y z y z & 0 & 0\end{array}$

$\begin{array}{cccccc}0 & 0 & 0 & 0 & y z y z & y z x x \\ 0 & 0 & 0 & 0 & x x y z & x y x y\end{array}$

and $x y x y=1 / 2(x x x x-x x y y)$ 


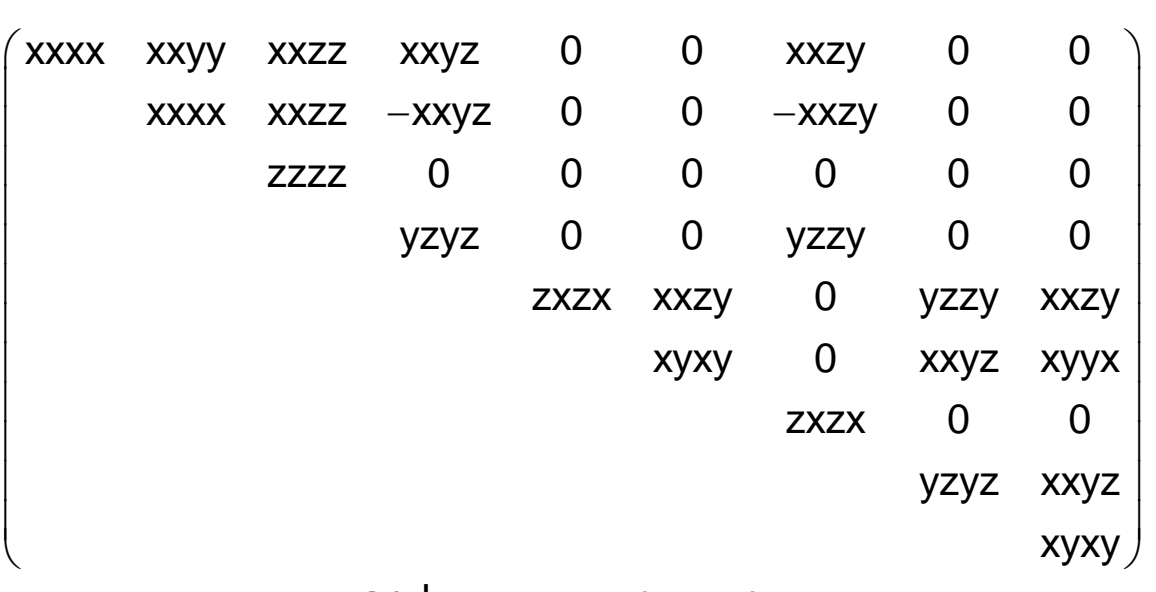

$\left(\begin{array}{lllllllll}0 & 0 & 0 & 0 & 0 & 0 & 0 & 0 & 0 \\ 0 & 0 & 0 & 0 & 0 & 0 & 0 & 0 \\ & & 0 & 0 & 0 & 0 & 0 & 0 & 0\end{array}\right)$ $\begin{array}{lllllll}0 & 0 & 0 & 0 & 0 & 0 & 0 \\ 0 & 0 & 0 & 0 & 0 & 0 & 0\end{array}$

$\begin{array}{llllll}0 & 0 & 0 & 0 & 0 & 0\end{array}$

$\begin{array}{lllll}0 & 0 & 0 & 0 & 0\end{array}$

$\begin{array}{llll}0 & 0 & 0 & 0\end{array}$

$0 \begin{array}{ll}0 & 0 \\ 0 & 0\end{array}$

and $x x x x=x x y y+y z y z+z y z y$

$\left.\begin{array}{llllllllll}x x x x & x x y y & x x z z & x x y z & 0 & 0 & x x z y & 0 & 0 \\ x x y & x x x & x x z & -x y z & 0 & 0 & -x x z y & 0 & 0\end{array}\right)\left(\begin{array}{ccccccccc}0 & 0 & 0 & 0 & 0 & 0 & 0 & 0 & 0 \\ 0 & 0 & 0 & 0 & 0 & 0 & 0 & 0 & 0\end{array}\right)$

$\left(\begin{array}{ccccccccc}x x y y & x x x x & x x z z & -x x y z & 0 & 0 & -x x z y & 0 & 0 \\ z z x x & z z x x & z z z z & 0 & 0 & 0 & 0 & 0 & 0 \\ y z x x & y z x x & 0 & y z y z & 0 & 0 & z x z x & 0 & 0\end{array}\right) \quad\left(\begin{array}{cccccccccc}0 & 0 & 0 & 0 & 0 & 0 & 0 & 0 & 0 & 0 \\ 0 & 0 & 0 & 0 & 0 & 0 & 0 & 0 & 0 \\ 0 & 0 & 0 & 0 & 0 & 0 & 0 & 0 & 0 \\ 0 & 0 & 0 & 0 & 0 & 0 & 0 & 0 & 0\end{array}\right)$ $\begin{array}{lllllllll}z z z x x & -y z x x & 0 & y z y z & 0 & 0 & z x z x & 0 & 0\end{array}$

$\begin{array}{lllllllll}0 & 0 & 0 & 0 & z x z x & y z x x & 0 & y z y z & y z x x\end{array}$

and $x x x x=x x y y+2 x y x y$ $\begin{array}{lllllllll}0 & 0 & 0 & 0 & 0 & 0 & 0 & 0 & 0 \\ 0 & 0 & 0 & 0 & 0 & 0 & 0 & 0 & 0 \\ 0 & 0 & 0 & 0 & 0 & 0 & 0 & 0 & 0\end{array}$

$\begin{array}{llllllllll}0 & 0 & 0 & 0 & 0 & 0 & 0 & 0 & 0\end{array}$ a

$\left(\begin{array}{lllllllll}0 & 0 & 0 & 0 & 0 & 0 & 0 & 0 & 0\end{array}\right)$ $\begin{array}{llllllll}0 & 0 & 0 & 0 & 0 & 0 & 0 & 0 \\ 0 & 0 & 0 & 0 & 0 & 0 & 0\end{array}$ $\begin{array}{llllll}0 & 0 & 0 & 0 & 0 & 0 \\ 0 & 0\end{array}$ $\begin{array}{lllll}0 & 0 & 0 & 0 & 0 \\ 0 & -1 & 0\end{array}$ $\begin{array}{llll}0 & 0 & 0 & 0 \\ 0 & 0 & 0 & 0\end{array}$ 0 co 0 $\begin{array}{ll}0 & 0\end{array}$$$
0)
$$

$\begin{array}{lllllllll}0 & 0 & 0 & 0 & 0 & 0 & 0 & 0 & 0 \\ 0 & 0 & 0 & 0 & 0 & 0 & 0 & 0 & 0\end{array}$ $\begin{array}{lllllllll}0 & 0 & 0 & 0 & 0 & 0 & 0 & 0 & 0 \\ 0 & 0 & 0 & 0 & 0 & 0 & 0 & 0 & 0\end{array}$ $\begin{array}{lllllllll}0 & 0 & 0 & 0 & 0 & 0 & 0 & 0 & 0 \\ 0 & 0 & 0 & 0 & 0 & 0 & 0 & 0 & 0\end{array}$ $0 \begin{array}{llllllllll}0 & 0 & 0 & 0 & 0 & 0 & 0 & 0 & 0 \\ 0 & 0 & 0 & 0 & 0 & 0 & 0 & 0 & 0\end{array}$ $\begin{array}{lllllllll}0 & 0 & 0 & 0 & 0 & 0 & 0 & 0 & 0 \\ 0 & 0 & 0 & 0 & 0 & 0 & 0 & 0 & 0\end{array}$

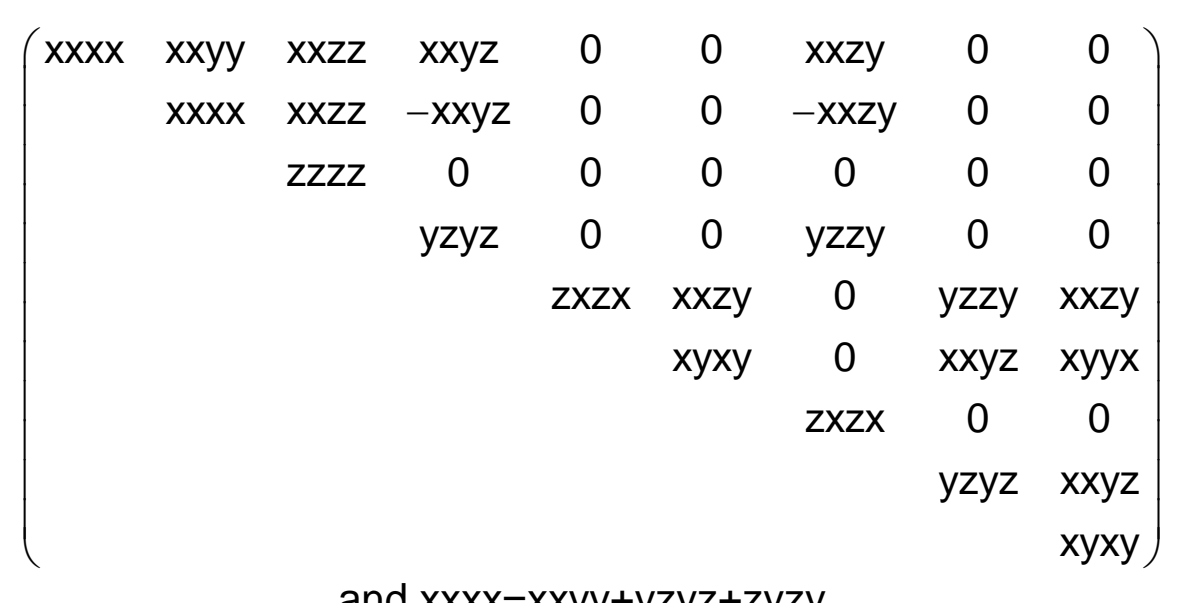

and $x x x x=x x y y+y z y z+z y z y$

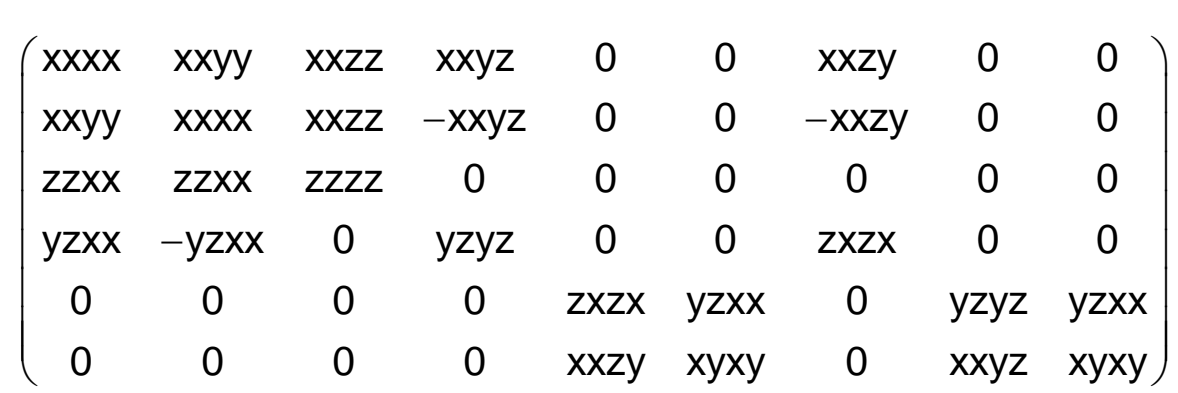

and $x x x x=x x y y+2 x y x y$ 
1

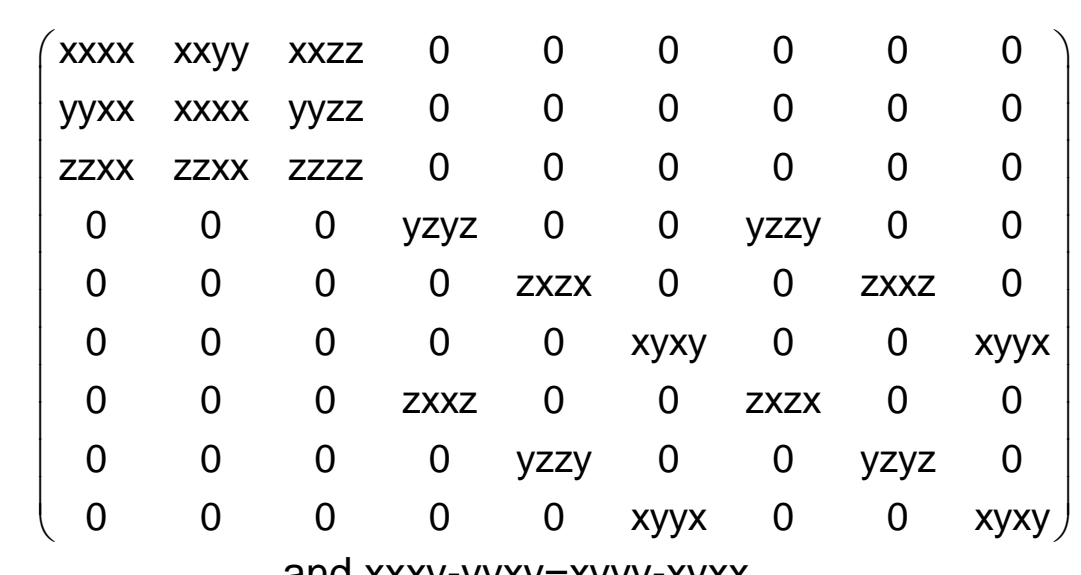

and $x x x y-y y x y=x y y y-x y x x$

$\left[V^{4}\right] \quad\left(\begin{array}{ccccc}3 x x y y & 0 & 0 & y y z z & 0 \\ 0 & 3 x x y y & 0 & y y z z & 0 \\ 0 & 0 & z z z z & x x y y & 0\end{array}\right)$

$\left.\mathrm{V}_{\mathrm{V}} \mathrm{V}^{3}\right] \quad\left(\begin{array}{cccccccccc}3 \mathrm{xxyy} & 0 & 0 & \mathrm{xxyy} & 0 & 0 & \mathrm{yyzz} & 0 & 0 & \mathrm{yyxx} \\ 0 & 3 \mathrm{xxyy} & 0 & 0 & \mathrm{yyzz} & 0 & 0 & \mathrm{xxyy} & 0 & 0 \\ 0 & 0 & \mathrm{zzzz} & 0 & 0 & \mathrm{zzxx} & 0 & 0 & \mathrm{zzxx} & 0\end{array}\right)$

$\left[\left[^{2}\right]^{2}\right]\left(\begin{array}{cccccc}x x x x & x x y y & x x z z & 0 & 0 & 0 \\ & x x x x & x x z z & 0 & 0 & 0 \\ & & z z z z & 0 & 0 & 0 \\ & & & y z y z & 0 & 0 \\ & & & & y z y z & 0 \\ & & & & & \text { xyxy }\end{array}\right)$

and $x y x y=1 / 2(x x x x-x x y y)$

$\left[\mathrm{V}^{2}\right]^{2}\left(\begin{array}{cccccc}\mathrm{xxxx} & \mathrm{xxyy} & \mathrm{xxzz} & 0 & 0 & 0 \\ \mathrm{xxyy} & \mathrm{xxxx} & \mathrm{xxzz} & 0 & 0 & 0 \\ \mathrm{zzxx} & \mathrm{zzxx} & \mathrm{zzzz} & 0 & 0 & 0 \\ 0 & 0 & 0 & \mathrm{yzyz} & 0 & 0 \\ 0 & 0 & 0 & 0 & \mathrm{yzyz} & 0 \\ 0 & 0 & 0 & 0 & 0 & \mathrm{xyxy}\end{array}\right)$

and $x y x y=1 / 2(x x x x-x x y y)$

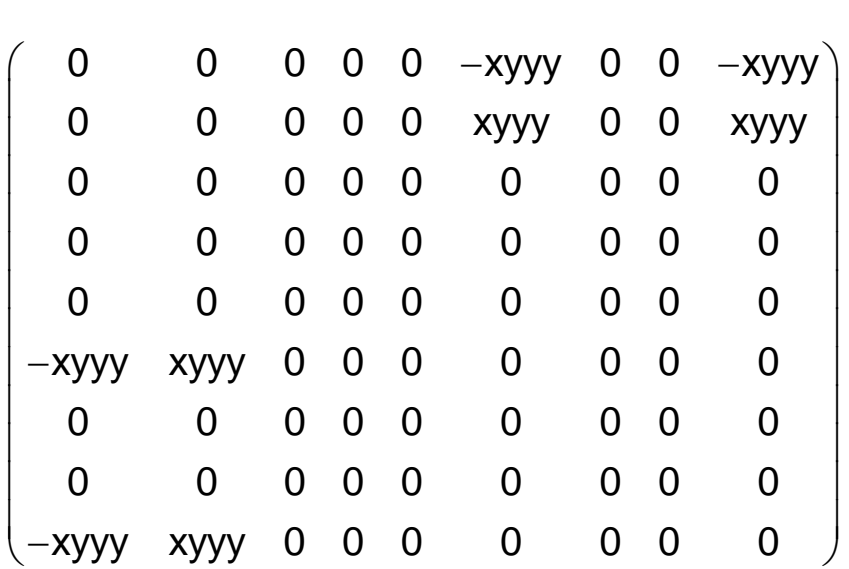

$\left(\begin{array}{ccccc}0 & x y y y & 0 & 0 & 0 \\ -x y y y & 0 & 0 & 0 & 0 \\ 0 & 0 & 0 & 0 & 0\end{array}\right)$

$\begin{array}{llllllllll}0 & \text { xyyy } & 0 & 0 & 0 & 0 & 0 & -x y y y & 0 & 0\end{array}$

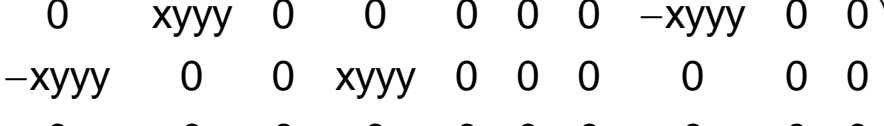
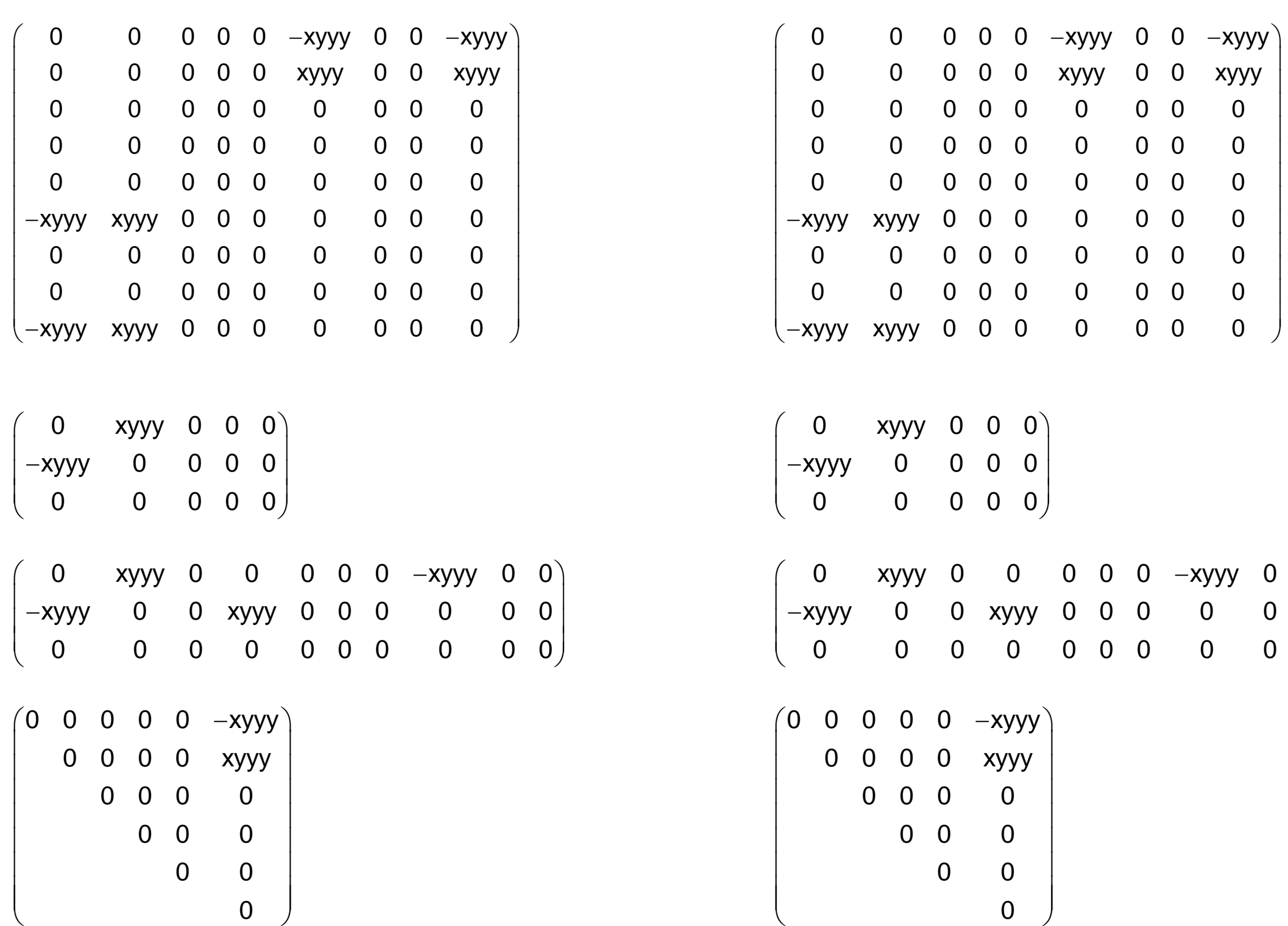

$\left(\begin{array}{ccccc}0 & x y y y & 0 & 0 & 0 \\ -x y y y & 0 & 0 & 0 & 0 \\ 0 & 0 & 0 & 0 & 0\end{array}\right)$

$\left(\begin{array}{llllllllll}0 & \text { xyyy } & 0 & 0 & 0 & 0 & 0 & -x y y y & 0 & 0\end{array}\right)$ $\left.\begin{array}{cccccccccc}-x y y y & 0 & 0 & x y y y & 0 & 0 & 0 & 0 & 0 & 0 \\ 0 & 0 & 0 & 0 & 0 & 0 & 0 & 0 & 0 & 0\end{array}\right)$

$$
\left(\begin{array}{cccccc}
0 & 0 & 0 & 0 & 0 & -x y y y \\
& 0 & 0 & 0 & 0 & x y y y \\
& & 0 & 0 & 0 & 0 \\
& & & 0 & 0 & 0 \\
& & & & 0 & 0 \\
& & & & & 0
\end{array}\right)
$$

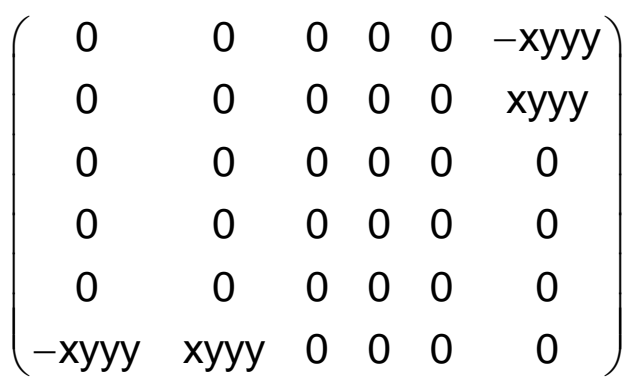

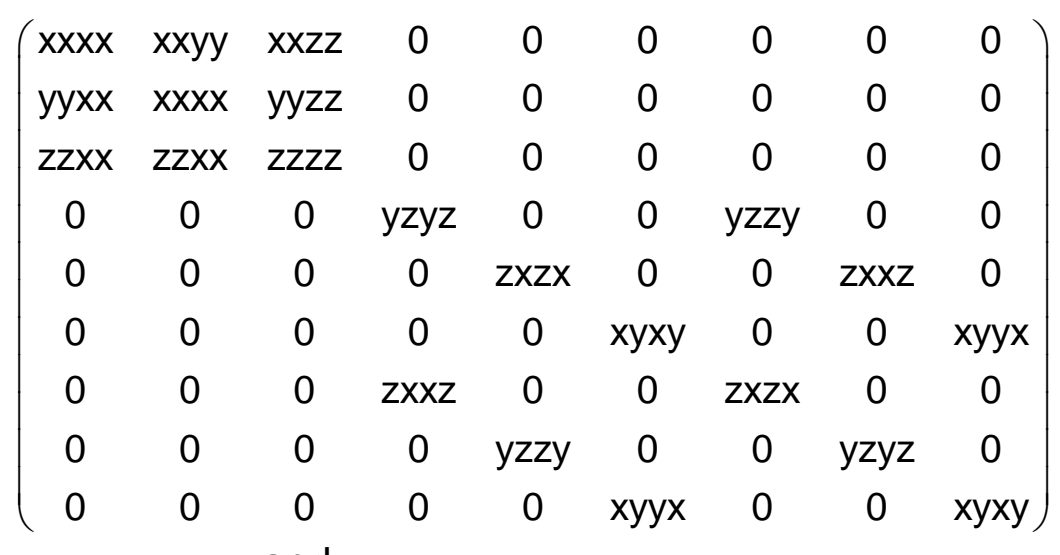
and $x x x y-y y x y=x y y y-x y x x$

$\left(\begin{array}{ccccc}3 x x y y & 0 & 0 & y y z z & 0 \\ 0 & 3 x x y y & 0 & y y z z & 0 \\ 0 & 0 & z z z z & x x y y & 0\end{array}\right)$

$\left(\begin{array}{cccccccccc}3 x x y y & 0 & 0 & x x y y & 0 & 0 & y y z z & 0 & 0 & y y x x \\ 0 & 3 x x y y & 0 & 0 & y y z z & 0 & 0 & x x y y & 0 & 0 \\ 0 & 0 & z z z z & 0 & 0 & z z x x & 0 & 0 & z z x x & 0\end{array}\right)$

$\left(\begin{array}{cccccc}x x x x & x x y y & x x z z & 0 & 0 & 0 \\ & x x x x & x x z z & 0 & 0 & 0 \\ & & z z z z & 0 & 0 & 0 \\ & & & y z y z & 0 & 0 \\ & & & & y z y z & 0 \\ & & & & & x y x y\end{array}\right)$

and $x y x y=1 / 2(x x x x-x x y y)$

$\left.\begin{array}{cccccc}x x x x & x x y y & x x z z & 0 & 0 & 0 \\ x x y y & x x x x & x x z z & 0 & 0 & 0 \\ z z x x & z z x x & z z z z & 0 & 0 & 0 \\ 0 & 0 & 0 & y z y z & 0 & 0 \\ 0 & 0 & 0 & 0 & y z y z & 0 \\ 0 & 0 & 0 & 0 & 0 & x y x y\end{array}\right)$

and $x y x y=1 / 2(x x x x-x x y y)$ 
1

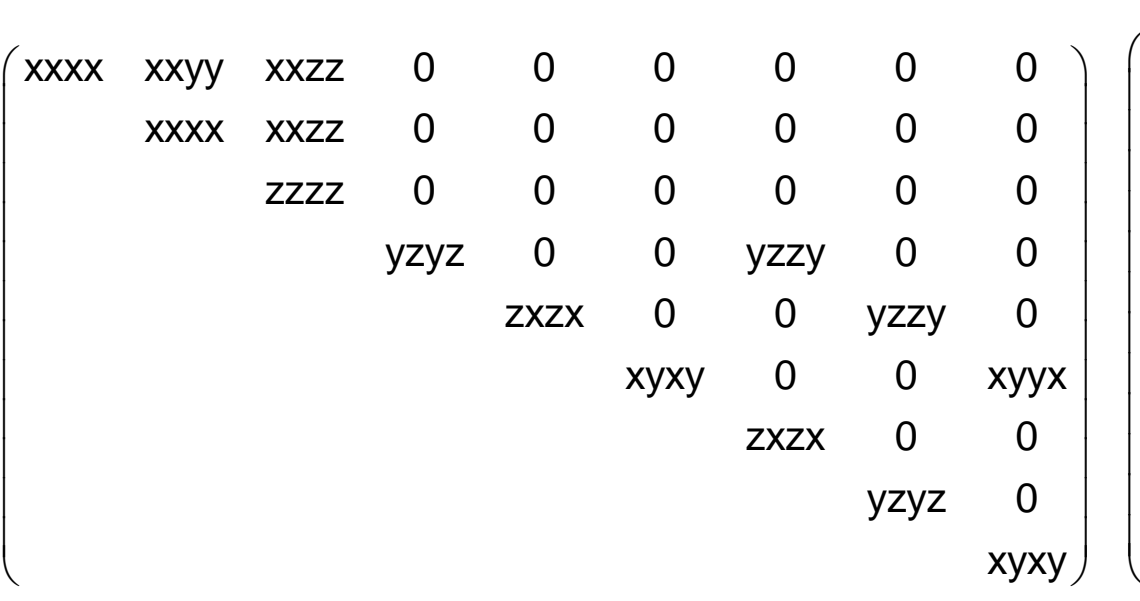

and $x x x x=x x y y+y z y z+z y z y$

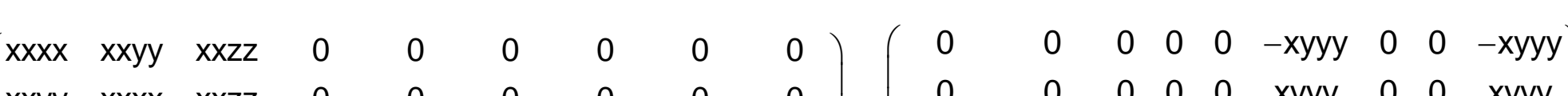

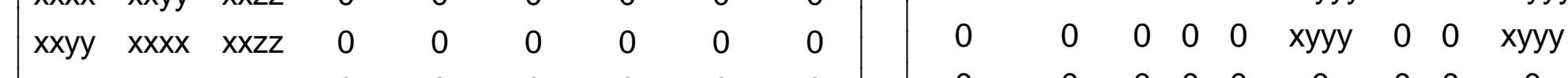

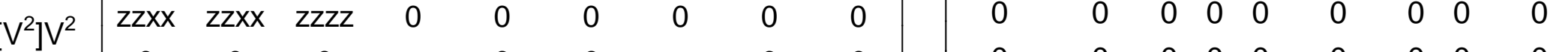
$\begin{array}{lllllllll}0 & 0 & 0 & y z y z & 0 & 0 & z x z x & 0 & 0 \\ 0 & 0 & 0 & 0 & z x z x & 0 & 0 & y z y z & 0 \\ 0 & 0 & 0 & 0 & 0 & x y y & 0 & 0 & x y y\end{array} \mid \begin{array}{lllllllll}0 & 0 & 0 & 0 & 0 & 0 & 0 & 0 & 0 \\ 0 & 0 & 0 & 0 & 0 & 0 & 0 & 0 & 0\end{array}$

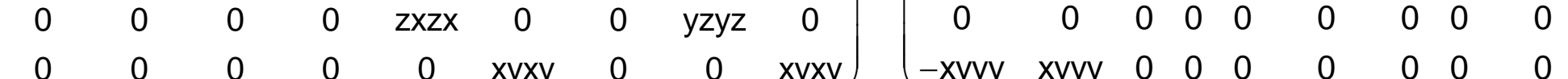
and $x x x x=x x y y+2 x y x y$ $\left(\begin{array}{lllllllll}0 & 0 & 0 & 0 & 0 & -x y y y & 0 & 0 & -x y y y \\ & 0 & 0 & 0 & 0 & -x y y & 0 & 0 & -x y y\end{array}\right)$ $\begin{array}{lllllll}0 & 0 & 0 & 0 & 0 & 0 & 0\end{array}$

$\begin{array}{llllll}0 & 0 & 0 & 0 & 0 & 0\end{array}$

$0 \begin{array}{llll}0 & 0 & 0 & 0\end{array}$

$\begin{array}{lll}0 & 0 & 0 \\ 0 & 0\end{array}$ $\begin{array}{lllllllc}0 & 0 & 0 & 0 & -x y y y & 0 & 0 & -x y y y\end{array}$ $\left(\begin{array}{lllllllll}0 & 0 & 0 & 0 & 0 & -x y y y & 0 & 0 & -x y y y\end{array}\right)$

$\begin{array}{cccccccc}0 & 0 & 0 & 0 & -x y y y & 0 & 0 & -x y y y\end{array}$

$\begin{array}{lllllll}0 & 0 & 0 & 0 & 0 & 0 & 0\end{array}$

$\begin{array}{llllll}0 & 0 & 0 & 0 & 0 & 0\end{array}$

$0 \begin{array}{llll}0 & 0 & 0 & 0\end{array}$

$\begin{array}{llll}0 & 0 & 0 & 0 \\ & 0 & 0 & 0\end{array}$

$\begin{array}{ll}0 & 0 \\ 0 & 0\end{array}$

$\left(\begin{array}{ccccccccc}0 & 0 & 0 & 0 & 0 & -x y y y & 0 & 0 & -x y y y \\ 0 & 0 & 0 & 0 & 0 & x y y y & 0 & 0 & x y y y \\ 0 & 0 & 0 & 0 & 0 & 0 & 0 & 0 & 0 \\ 0 & 0 & 0 & 0 & 0 & 0 & 0 & 0 & 0 \\ 0 & 0 & 0 & 0 & 0 & 0 & 0 & 0 & 0 \\ -x y y y & x y y y & 0 & 0 & 0 & 0 & 0 & 0 & 0\end{array}\right)$

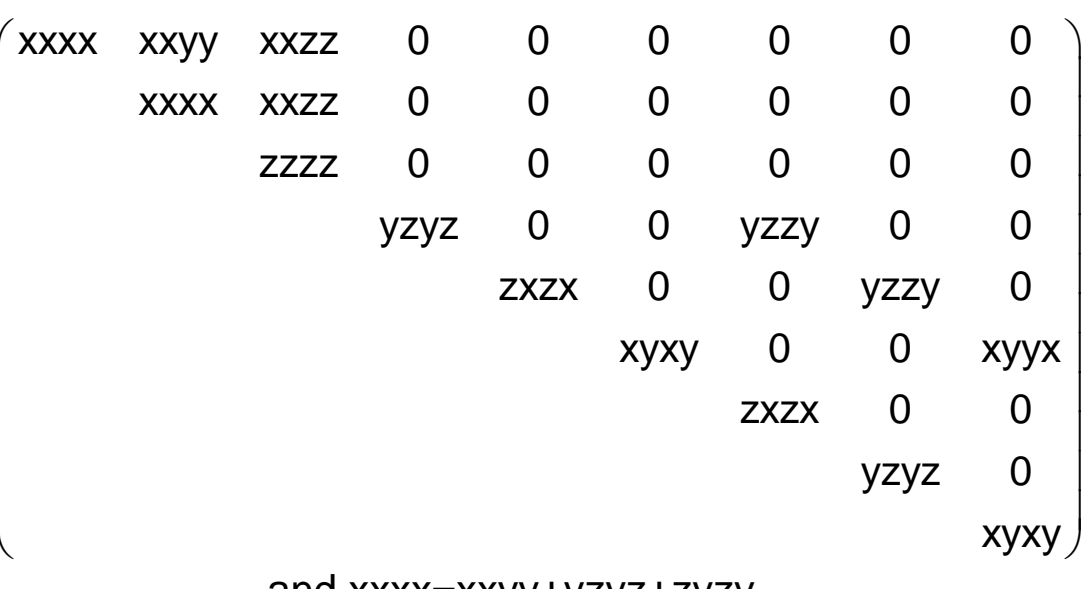

and $x x x x=x x y y+y z y z+z y z y$

$\left(\begin{array}{ccccccccc}\text { xxxx } & \text { xxyy } & \text { xxzz } & 0 & 0 & 0 & 0 & 0 & 0 \\ \text { xxyy } & \text { xxxx } & \text { xxzz } & 0 & 0 & 0 & 0 & 0 & 0 \\ \text { zzxx } & \text { zzxx } & \text { zzzz } & 0 & 0 & 0 & 0 & 0 & 0 \\ 0 & 0 & 0 & \text { yzyz } & 0 & 0 & \text { zxzx } & 0 & 0 \\ 0 & 0 & 0 & 0 & \text { zxzx } & 0 & 0 & \text { yzyz } & 0 \\ 0 & 0 & 0 & 0 & 0 & \text { xyxy } & 0 & 0 & \text { xyxy }\end{array}\right)$

and $x x x x=x x y y+2 x y x y$ 


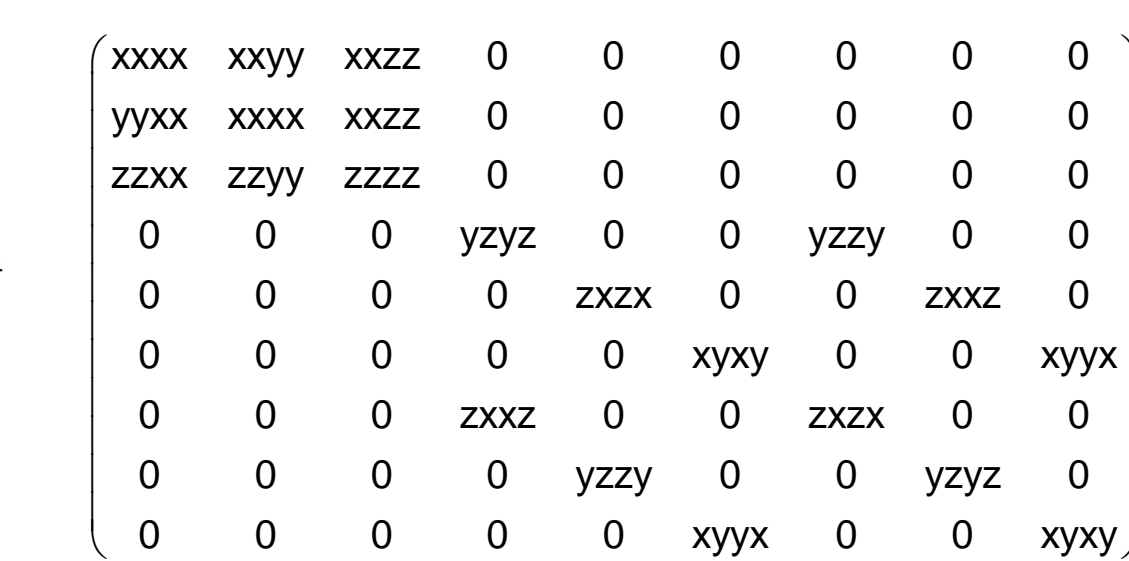

$\left(\begin{array}{lllllllll}0 & 0 & 0 & 0 & 0 & 0 & 0 & 0 & 0 \\ 0 & 0 & 0 & 0 & 0 & 0 & 0 & 0 & 0\end{array}\right)$ $\begin{array}{lllllllll}0 & 0 & 0 & 0 & 0 & 0 & 0 & 0 & 0 \\ 0 & 0 & 0 & 0 & 0 & 0 & 0 & 0 & 0\end{array}$ $0 \begin{array}{lllllllll}0 & 0 & 0 & 0 & 0 & 0 & 0 & 0 & 0 \\ 0 & 0 & 0 & 0 & 0 & 0 & 0 & 0 & 0\end{array}$ $0 \begin{array}{lllllllll}0 & 0 & 0 & 0 & 0 & 0 & 0 & 0 & 0\end{array}$ $0 \begin{array}{lllllllll}0 & 0 & 0 & 0 & 0 & 0 & 0 & 0 & 0 \\ 0 & 0 & 0 & 0 & 0 & 0 & 0 & 0 & 0\end{array}$

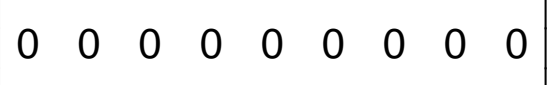
$0 \begin{array}{lllllllll}0 & 0 & 0 & 0 & 0 & 0 & 0 & 0 & 0 \\ 0 & 0 & 0 & 0 & 0 & 0 & 0 & 0 & 0\end{array}$ $\begin{array}{llllllllll}0 & 0 & 0 & 0 & 0 & 0 & 0 & 0 & 0 \\ 0 & 0 & 0 & 0 & 0 & 0 & 0 & 0 & 0\end{array}$ $\begin{array}{lllllllll}0 & 0 & 0 & 0 & 0 & 0 & 0 & 0 & 0\end{array}$ $\begin{array}{cccc}0 & 0 & 0 & x y y x \\ \text { and: } x x x x=x x y y+x y x y+x y y x\end{array}$

$\left[V^{4}\right] \quad\left(\begin{array}{ccccc}3 x x y y & 0 & 0 & y y z z & 0 \\ 0 & 3 x x y y & 0 & y y z z & 0 \\ 0 & 0 & z z z z & x x y y & 0\end{array}\right)$

$\left(\begin{array}{lllll}0 & 0 & 0 & 0 & 0 \\ 0 & 0 & 0 & 0 & 0 \\ 0 & 0 & 0 & 0 & 0\end{array}\right)$

$\begin{array}{llllll}0 & 0 & 0 & 0 & 0 \\ 0 & 0 & 0 & 0 & 0\end{array}$

$\left(\begin{array}{llllllllll}0 & 0 & 0 & 0 & 0 & 0 & 0 & 0 & 0 & 0 \\ 0 & 0 & 0 & 0 & 0 & 0 & 0 & 0 & 0 & 0\end{array}\right)$

$\left(\begin{array}{llllllllll}0 & 0 & 0 & 0 & 0 & 0 & 0 & 0 & 0 & 0 \\ 0 & 0 & 0 & 0 & 0 & 0 & 0 & 0 & 0 & 0\end{array}\right)$

V $\left[V^{3}\right] \quad\left(\begin{array}{cccccccccc}3 x x y y & 0 & 0 & x x y y & 0 & 0 & y y z z & 0 & 0 & 0 \\ 0 & 3 x x y y & 0 & 0 & y y z z & 0 & 0 & x x y y & 0 & 0 \\ 0 & 0 & z z z & 0 & 0 & z z x & 0 & 0 & z z x & 0\end{array}\right)$

$$
\left(\begin{array}{cccccc}
x x x x & x x y y & x x z z & 0 & 0 & 0 \\
& x x x x & x x z z & 0 & 0 & 0 \\
& & z z z z & 0 & 0 & 0 \\
& & & y z y z & 0 & 0 \\
& & & & y z y z & 0 \\
& & & & & x y x y
\end{array}\right)
$$

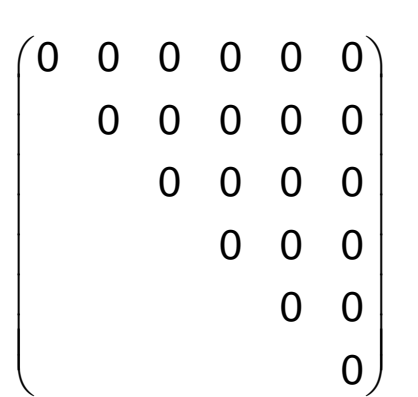

and $x y x y=1 / 2(x x x x-x x y y)$ $\left(\begin{array}{llllll}0 & 0 & 0 & 0 & 0 & 0 \\ 0 & 0 & 0 & 0 & 0 & 0 \\ 0 & 0 & 0 & 0 & 0 & 0\end{array}\right)$ $\begin{array}{lllllll}0 & 0 & 0 & 0 & 0 & 0 \\ 0 & 0 & 0 & 0 & 0 & 0 \\ 0 & 0 & 0 & 0 & 0 & 0\end{array}$ $0 \begin{array}{llllll}0 & 0 & 0 & 0 & 0 & 0\end{array}$ $\begin{array}{llllll}0 & 0 & 0 & 0 & 0 & 0 \\ 0 & 0 & 0 & 0 & 0 & 0 \\ 0 & 0 & 0 & 0 & 0 & 0\end{array}$ $\begin{array}{llllll}0 & 0 & 0 & 0 & 0 & 0 \\ 0 & 0 & 0 & 0 & 0 & 0\end{array}$ $\left(\begin{array}{lllllllll}0 & 0 & 0 & 0 & 0 & 0 & 0 & 0 & 0 \\ 0 & 0 & 0 & 0 & 0 & 0 & 0 & 0 & 0\end{array}\right.$ $\begin{array}{lllllllll}0 & 0 & 0 & 0 & 0 & 0 & 0 & 0 & 0 \\ 0 & 0 & 0 & 0 & 0 & 0 & 0 & 0 & 0\end{array}$ $0 \begin{array}{llllllllll}0 & 0 & 0 & 0 & 0 & 0 & 0 & 0 & 0 \\ 0 & 0 & 0 & 0 & 0 & 0 & 0\end{array}$ $\begin{array}{lllllllll}0 & 0 & 0 & 0 & 0 & 0 & 0 & 0 & 0\end{array}$ $0 \begin{array}{lllllllll}0 & 0 & 0 & 0 & 0 & 0 & 0 & 0 & 0 \\ 0 & 0 & 0 & 0 & 0 & 0 & 0 & 0 & 0\end{array}$ $0 \begin{array}{lllllllll}0 & 0 & 0 & 0 & 0 & 0 & 0 & 0 & 0 \\ 0 & 0 & 0 & 0 & 0 & 0 & 0 & 0 & 0\end{array}$ $\begin{array}{lllllllll}0 & 0 & 0 & 0 & 0 & 0 & 0 & 0 & 0 \\ 0 & 0 & 0 & 0 & 0 & 0 & 0 & 0\end{array}$ $0 \begin{array}{llllllllll}0 & 0 & 0 & 0 & 0 & 0 & 0 & 0 & 0 \\ 0 & 0 & 0 & 0 & 0 & 0 & 0 & 0 & 0\end{array}$

$\begin{array}{llllllllll}0 & 0 & 0 & 0 & 0 & 0 & 0 & 0 & 0\end{array}$

$\left(\begin{array}{lllll}0 & 0 & 0 & 0 & 0 \\ 0 & 0 & 0 & 0 & 0 \\ 0 & 0 & 0 & 0 & 0\end{array}\right)$

$\begin{array}{lllll}0 & 0 & 0 & 0 & 0 \\ 0 & 0 & 0\end{array}$

$\left(\begin{array}{llllllllll}0 & 0 & 0 & 0 & 0 & 0 & 0 & 0 & 0 & 0\end{array}\right.$ $\left.\begin{array}{lllllllllll}0 & 0 & 0 & 0 & 0 & 0 & 0 & 0 & 0 & 0 \\ 0 & 0 & 0 & 0 & 0 & 0 & 0 & 0 & 0 & 0\end{array}\right)$ $\begin{array}{lllllllllll}0 & 0 & 0 & 0 & 0 & 0 & 0 & 0 & 0 & 0\end{array}$

$$
\left(\begin{array}{llllll}
0 & 0 & 0 & 0 & 0 & 0 \\
& 0 & 0 & 0 & 0 & 0 \\
& & 0 & 0 & 0 & 0 \\
& & & 0 & 0 & 0 \\
& & & & 0 & 0 \\
& & & & & \\
& & & & 0
\end{array}\right)
$$

$\left(\begin{array}{llllll}0 & 0 & 0 & 0 & 0 & 0 \\ 0 & 0 & 0 & 0 & 0 & 0 \\ 0 & 0 & 0 & 0 & 0 & 0\end{array}\right)$ $\begin{array}{llllll}0 & 0 & 0 & 0 & 0 & 0 \\ 0 & 0 & 0 & 0\end{array}$ $\begin{array}{llllll}0 & 0 & 0 & 0 & 0 & 0\end{array}$ $\begin{array}{llllll}0 & 0 & 0 & 0 & 0 & 0 \\ 0 & 0 & 0 & 0 & 0 & 0 \\ 0 & 0 & 0 & 0 & 0 & 0\end{array}$ $\begin{array}{llllll}0 & 0 & 0 & 0 & 0 & 0 \\ 0 & 0 & 0 & 0 & 0 & 0\end{array}$

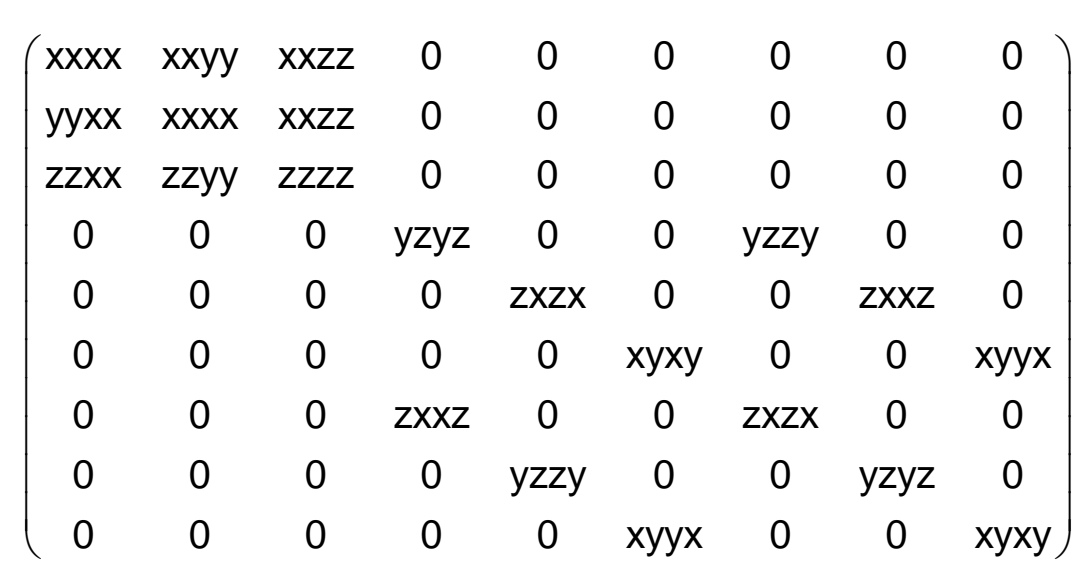

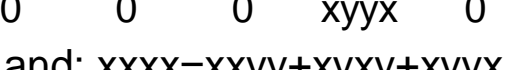

$$
\left(\begin{array}{ccccc}
3 x x y y & 0 & 0 & y y z z & 0 \\
0 & 3 x x y y & 0 & y y z z & 0 \\
0 & 0 & z z z z & x x y y & 0
\end{array}\right)
$$$$
\left.\begin{array}{cccccc}
0 & 3 x x y y & 0 & y y z z & 0 \\
0 & 0 & z z z z & x x y y & 0
\end{array}\right)
$$

$\left(\begin{array}{cccccccccc}3 x x y y & 0 & 0 & x x y y & 0 & 0 & y y z z & 0 & 0 & 0 \\ 0 & 3 x x y y & 0 & 0 & y y z z & 0 & 0 & x x y y & 0 & 0 \\ 0 & 0 & z z z & 0 & 0 & z z x & 0 & 0 & z z x & 0\end{array}\right)$ $\begin{array}{llllllllll}0 & 0 & z z z z & 0 & 0 & z z x x & 0 & 0 & z z x x & 0\end{array}$

$$
\left(\begin{array}{cccccc}
x x x x & x x y y & x x z z & 0 & 0 & 0 \\
& x x x x & x x z z & 0 & 0 & 0 \\
& & z z z z & 0 & 0 & 0 \\
& & & y z y z & 0 & 0 \\
& & & & y z y z & 0 \\
& & & & & x y x y
\end{array}\right)
$$

and $x y x y=1 / 2(x x x x-x x y y)$

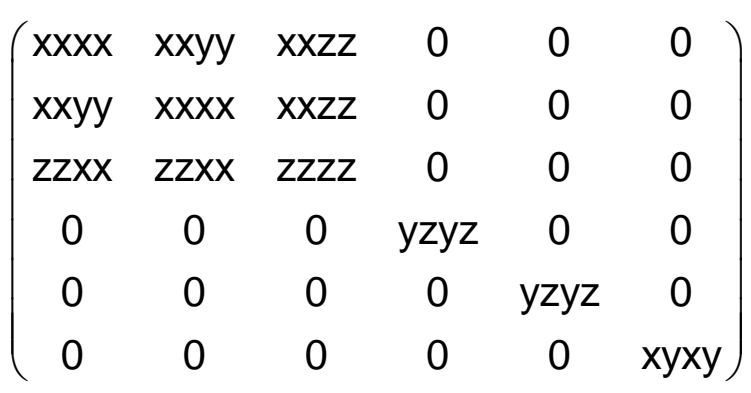

and $x y x y=1 / 2(x x x x-x x y y)$

and $x y x y=1 / 2(x x x x-x x y y)$ 
$n>4 \quad D_{\alpha h}\left(D_{\infty}\right)=\infty / m^{\prime} m^{\prime} m^{\prime}$

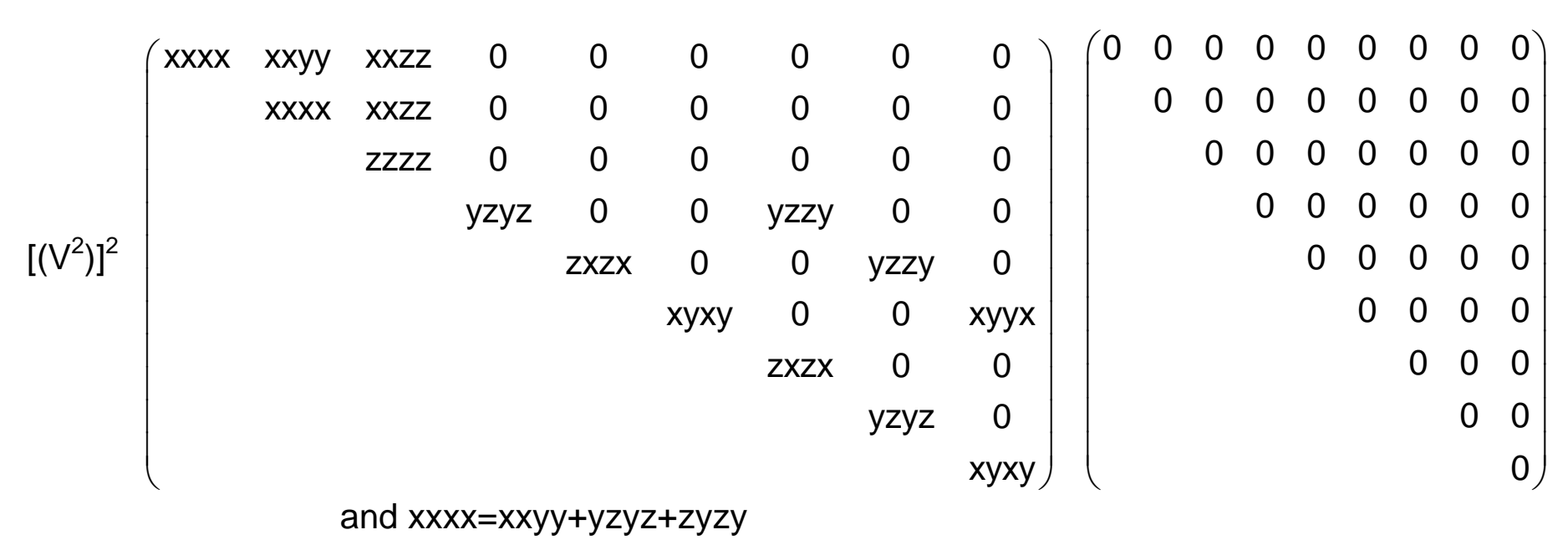

$\left[\mathrm{V}^{2}\right] \mathrm{V}^{2}\left(\begin{array}{ccccccccc}\mathrm{x} x \mathrm{x} & \mathrm{xxyy} & \mathrm{xxzz} & 0 & 0 & 0 & 0 & 0 & 0 \\ \mathrm{xxyy} & \mathrm{xxxx} & \mathrm{xxzz} & 0 & 0 & 0 & 0 & 0 & 0 \\ \mathrm{zzxx} & \mathrm{zzxx} & \mathrm{zzzz} & 0 & 0 & 0 & 0 & 0 & 0 \\ 0 & 0 & 0 & \mathrm{yzyz} & 0 & 0 & \mathrm{zxzx} & 0 & 0 \\ 0 & 0 & 0 & 0 & \mathrm{zxzx} & 0 & 0 & \mathrm{yzyz} & 0 \\ 0 & 0 & 0 & 0 & 0 & \mathrm{xyxy} & 0 & 0 & \mathrm{xyxy}\end{array}\right)\left(\begin{array}{lllllllll}0 & 0 & 0 & 0 & 0 & 0 & 0 & 0 & 0 \\ 0 & 0 & 0 & 0 & 0 & 0 & 0 & 0 & 0 \\ 0 & 0 & 0 & 0 & 0 & 0 & 0 & 0 & 0 \\ 0 & 0 & 0 & 0 & 0 & 0 & 0 & 0 & 0 \\ 0 & 0 & 0 & 0 & 0 & 0 & 0 & 0 & 0 \\ 0 & 0 & 0 & 0 & 0 & 0 & 0 & 0 & 0\end{array}\right)$

and $x x x x=x x y y+2 x y x y$

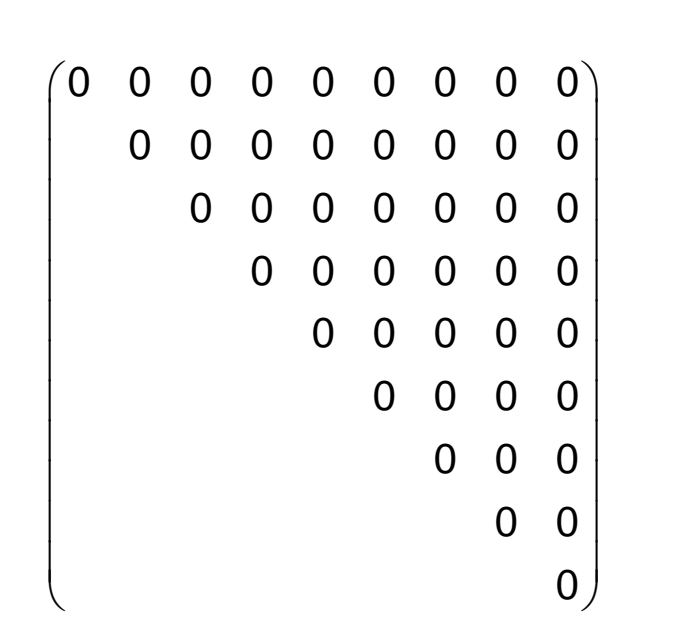

$\left(\begin{array}{lllllllll}0 & 0 & 0 & 0 & 0 & 0 & 0 & 0 & 0 \\ 0 & 0 & 0 & 0 & 0 & 0 & 0 & 0 & 0\end{array}\right)$ $\left(\begin{array}{lllllllll}0 & 0 & 0 & 0 & 0 & 0 & 0 & 0 & 0 \\ 0 & 0 & 0 & 0 & 0 & 0 & 0 & 0 & 0 \\ 0 & 0 & 0 & 0 & 0 & 0 & 0 & 0 & 0\end{array}\right)$

$0 \begin{array}{lllllllll}0 & 0 & 0 & 0 & 0 & 0 & 0 & 0 & 0\end{array}$

$\begin{array}{lllllllll}0 & 0 & 0 & 0 & 0 & 0 & 0 & 0 & 0\end{array}$

$\begin{array}{lllllllll}0 & 0 & 0 & 0 & 0 & 0 & 0 & 0 & 0\end{array}$

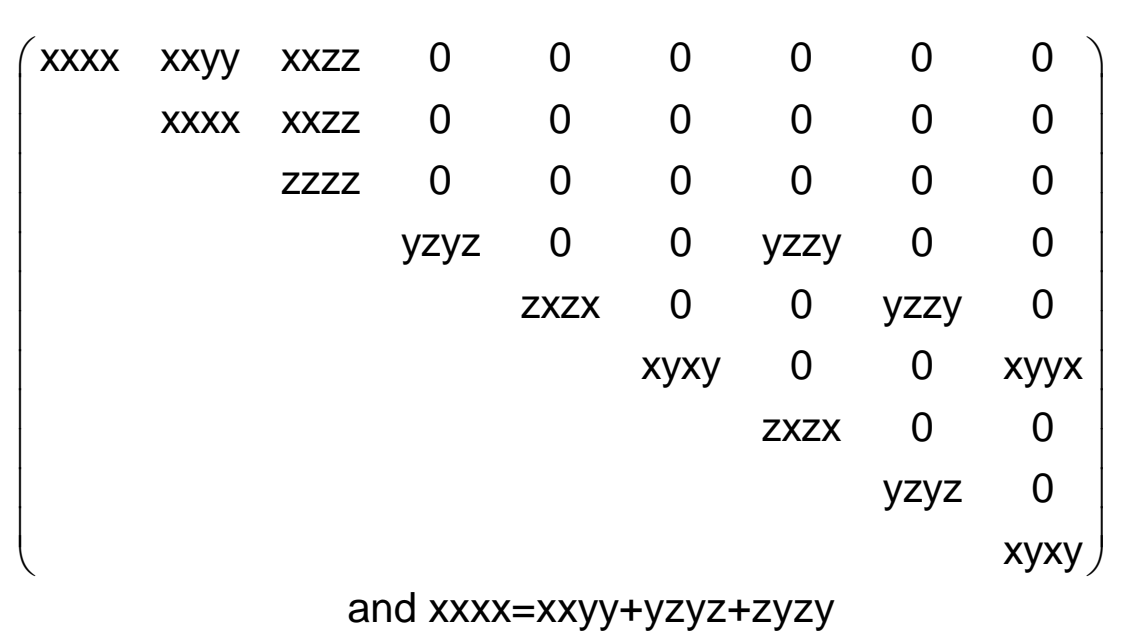

$\left(\begin{array}{ccccccccc}\text { xxxx } & \text { xxyy } & \text { xxzz } & 0 & 0 & 0 & 0 & 0 & 0 \\ \text { xxyy } & \text { xxxx } & \text { xxzz } & 0 & 0 & 0 & 0 & 0 & 0 \\ z z x x & z z x x & \text { zzzz } & 0 & 0 & 0 & 0 & 0 & 0 \\ 0 & 0 & 0 & y z y z & 0 & 0 & z x z x & 0 & 0 \\ 0 & 0 & 0 & 0 & \text { zxzx } & 0 & 0 & y z y z & 0 \\ 0 & 0 & 0 & 0 & 0 & \text { xyxy } & 0 & 0 & \text { xyxy }\end{array}\right)$

and $x x x x=x x y y+2 x y x y$ 
$\mathbb{V}^{4}\left(\begin{array}{ccccccccc}\text { xxxx } & \text { xxyy } & \text { xxzz } & 0 & 0 & 0 & 0 & 0 & 0 \\ \text { yyxx } & \text { yyyy } & \text { yyzz } & 0 & 0 & 0 & 0 & 0 & 0 \\ \text { zzxx } & \text { zzyy } & \text { zzzz } & 0 & 0 & 0 & 0 & 0 & 0 \\ 0 & 0 & 0 & \text { yzyz } & 0 & 0 & \text { yzzy } & 0 & 0 \\ 0 & 0 & 0 & 0 & \text { zxzx } & 0 & 0 & \text { zxxz } & 0 \\ 0 & 0 & 0 & 0 & 0 & \text { xyxy } & 0 & 0 & \text { xyyx } \\ 0 & 0 & 0 & \text { zyyz } & 0 & 0 & \text { zyzy } & 0 & 0 \\ 0 & 0 & 0 & 0 & \text { xzzx } & 0 & 0 & \text { xzxz } & 0 \\ 0 & 0 & 0 & 0 & 0 & \text { yxxy } & 0 & 0 & \text { yxyx }\end{array}\right)$

$\left(\begin{array}{lllllllll}0 & 0 & 0 & 0 & 0 & 0 & 0 & 0 & 0 \\ 0 & 0 & 0 & 0 & 0 & 0 & 0 & 0 & 0\end{array}\right)$ $\begin{array}{lllllllll}0 & 0 & 0 & 0 & 0 & 0 & 0 & 0 & 0 \\ 0 & 0 & 0 & 0 & 0 & 0 & 0 & 0 & 0\end{array}$

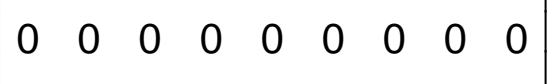
$\begin{array}{lllllllll}0 & 0 & 0 & 0 & 0 & 0 & 0 & 0 & 0 \\ 0 & 0 & 0 & 0 & 0 & 0 & 0 & 0 & 0\end{array}$ $0 \begin{array}{lllllllll}0 & 0 & 0 & 0 & 0 & 0 & 0 & 0 & 0 \\ 0 & 0 & 0 & 0 & 0 & 0 & 0 & 0 & 0\end{array}$ $\begin{array}{lllllllll}0 & 0 & 0 & 0 & 0 & 0 & 0 & 0 & 0 \\ 0 & 0 & 0 & 0 & 0 & 0 & 0 & 0 & 0\end{array}$ $\begin{array}{lllllllll}0 & 0 & 0 & 0 & 0 & 0 & 0 & 0 & 0\end{array}$ $0 \begin{array}{lllllllll}0 & 0 & 0 & 0 & 0 & 0 & 0 & 0 & 0\end{array}$

$\left.\mathrm{V}^{4}\right] \quad\left(\begin{array}{ccccc}\mathrm{xxxx} & 0 & 0 & \mathrm{yyzz} & 0 \\ 0 & \mathrm{yyyy} & 0 & \mathrm{zzxx} & 0 \\ 0 & 0 & \mathrm{zzzz} & \mathrm{xxyy} & 0\end{array}\right)$

$\left(\begin{array}{lllll}0 & 0 & 0 & 0 & 0 \\ 0 & 0 & 0 & 0 & 0 \\ 0 & 0 & 0 & 0 & 0\end{array}\right)$

$\begin{array}{lllll}0 & 0 & 0 & 0 & 0\end{array}$

$\left.V^{3}\right]\left(\begin{array}{cccccccccc}x x x x & 0 & 0 & x x y y & 0 & 0 & x x z z & 0 & 0 & 0 \\ 0 & y y y y & 0 & 0 & y y z z & 0 & 0 & y y x x & 0 & 0 \\ 0 & 0 & z z z z & 0 & 0 & z z x & 0 & 0 & z z y & 0\end{array}\right)$

$\left(\begin{array}{llllllllll}0 & 0 & 0 & 0 & 0 & 0 & 0 & 0 & 0 & 0 \\ 0 & 0 & 0 & 0 & 0 & 0 & 0 & 0 & 0 & 0\end{array}\right.$

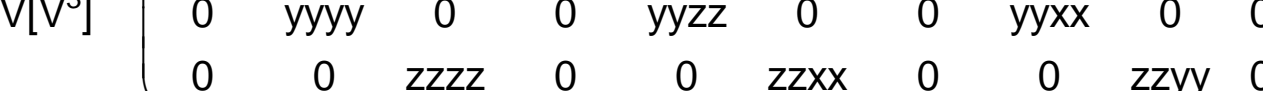

$\left.\begin{array}{llllllllll}0 & 0 & 0 & 0 & 0 & 0 & 0 & 0 & 0 & 0 \\ 0 & 0 & 0 & 0 & 0 & 0 & 0 & 0 & 0 & 0\end{array}\right)$

$\left(\begin{array}{cccccc}x x x x & x x y y & x x z z & 0 & 0 & 0 \\ & \text { yyyy } & y y z z & 0 & 0 & 0 \\ & & z z z z & 0 & 0 & 0 \\ & & & y z y z & 0 & 0 \\ & & & & x z x z & 0 \\ & & & & & \text { xyxy }\end{array}\right)$

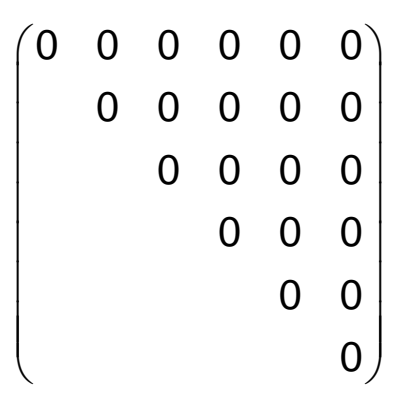

$\left(\begin{array}{llllll}0 & 0 & 0 & 0 & 0 & 0 \\ 0 & 0 & 0 & 0 & 0 & 0\end{array}\right.$

$\begin{array}{llllll}0 & 0 & 0 & 0 & 0 & 0 \\ 0 & 0 & 0 & 0 & 0 & 0\end{array}$

$\begin{array}{llllll}0 & 0 & 0 & 0 & 0 & 0 \\ 0 & 0 & 0 & 0 & 0 & 0 \\ 0 & 0 & 0 & 0 & 0 & 0\end{array}$

$\begin{array}{lllllll}0 & 0 & 0 & 0 & 0 & 0 \\ 0 & 0 & 0 & 0 & 0 & 0\end{array}$

$\begin{array}{llllll}0 & 0 & 0 & 0 & 0 & 0 \\ 0 & 0 & 0 & 0 & 0 & 0\end{array}$ $\begin{array}{lllllllll}0 & 0 & 0 & 0 & 0 & 0 & 0 & 0 & 0 \\ 0 & 0 & 0 & 0 & 0 & 0 & 0 & 0 & 0\end{array}$

$\begin{array}{lllllllll}0 & 0 & 0 & 0 & 0 & 0 & 0 & 0 & 0 \\ 0 & 0 & 0 & 0 & 0 & 0 & 0 & 0 & 0\end{array}$

$0 \begin{array}{lllllllll}0 & 0 & 0 & 0 & 0 & 0 & 0 & 0 & 0 \\ 0 & 0 & 0 & 0 & 0 & 0 & 0 & 0 & 0\end{array}$

$\begin{array}{lllllllll}0 & 0 & 0 & 0 & 0 & 0 & 0 & 0 & 0 \\ 0 & 0 & 0 & 0 & 0 & 0 & 0 & 0 & 0\end{array}$

$\begin{array}{llllllllll}0 & 0 & 0 & 0 & 0 & 0 & 0 & 0 & 0 \\ 0 & 0 & 0 & 0 & 0 & 0 & 0 & 0 & 0\end{array}$

$\begin{array}{llllllllll}0 & 0 & 0 & 0 & 0 & 0 & 0 & 0 & 0\end{array}$

$\begin{array}{llllllllll}0 & 0 & 0 & 0 & 0 & 0 & 0 & 0 & 0 \\ 0 & 0 & 0 & 0 & 0 & 0 & 0 & 0 & 0\end{array}$

$\left(\begin{array}{lllllllll}0 & 0 & 0 & 0 & 0 & 0 & 0 & 0 & 0 \\ 0 & 0 & 0 & 0 & 0 & 0 & 0 & 0 & 0\end{array}\right.$

$\left(\begin{array}{lllll}0 & 0 & 0 & 0 & 0 \\ 0 & 0 & 0 & 0 & 0 \\ 0 & 0 & 0 & 0 & 0\end{array}\right)$

$\begin{array}{lllll}0 & 0 & 0 & 0 & 0\end{array}$

$\left(\begin{array}{llllllllll}0 & 0 & 0 & 0 & 0 & 0 & 0 & 0 & 0 & 0 \\ 0 & 0 & 0 & 0 & 0 & 0 & 0 & 0 & 0 & 0 \\ 0 & 0 & 0 & 0 & 0 & 0 & 0 & 0 & 0 & 0\end{array}\right)$ $\left.\begin{array}{llllllllll}0 & 0 & 0 & 0 & 0 & 0 & 0 & 0 & 0 & 0 \\ 0 & 0 & 0 & 0 & 0 & 0 & 0 & 0 & 0 & 0\end{array}\right)$ $\begin{array}{lllllllllll}0 & 0 & 0 & 0 & 0 & 0 & 0 & 0 & 0 & 0\end{array}$

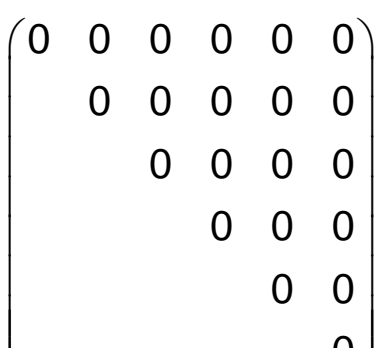

$\left(\begin{array}{llllll}0 & 0 & 0 & 0 & 0 & 0\end{array}\right.$ $\begin{array}{llllll}0 & 0 & 0 & 0 & 0 & 0 \\ 0 & 0 & 0 & 0 & 0 & 0\end{array}$ $\begin{array}{llllll}0 & 0 & 0 & 0 & 0 & 0 \\ 0 & 0 & 0 & 0 & 0 & 0\end{array}$ $0 \begin{array}{llllll}0 & 0 & 0 & 0 & 0 & 0 \\ 0 & 0 & 0 & 0 & 0 & 0\end{array}$

$\begin{array}{llllll}0 & 0 & 0 & 0 & 0 & 0 \\ 0 & 0 & 0 & 0 & 0 & 0\end{array}$

$\begin{array}{llllll}0 & 0 & 0 & 0 & 0 & 0 \\ 0 & 0 & 0 & 0 & 0 & 0\end{array}$

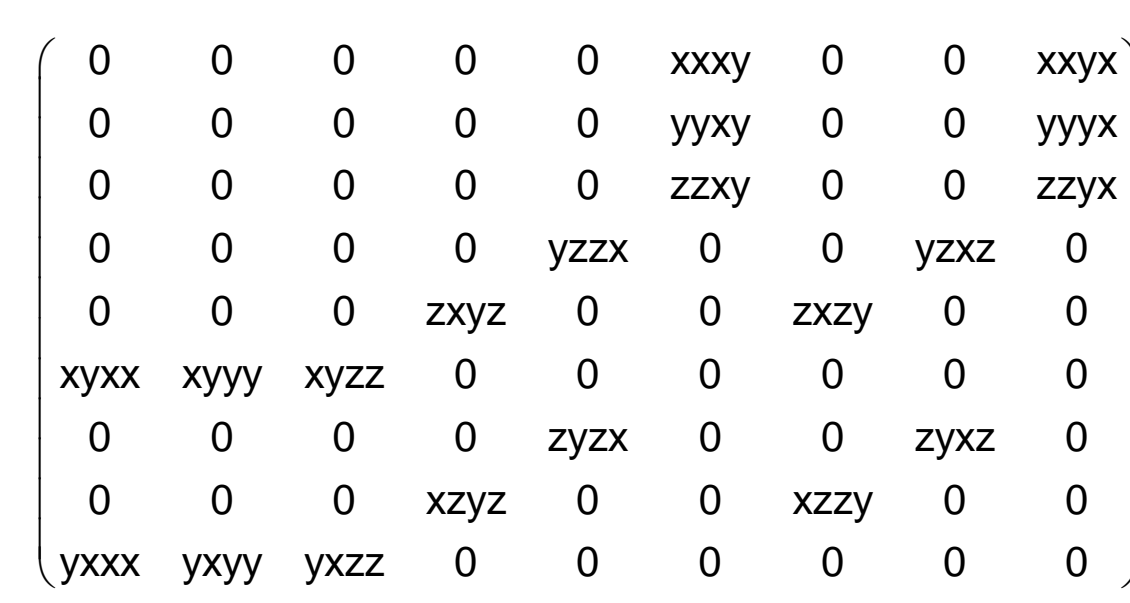

$\left(\begin{array}{ccccc}0 & x y y y & 0 & 0 & 0 \\ y x x x & 0 & 0 & 0 & 0\end{array}\right.$

$\begin{array}{ccccc}y & 0 & 0 & 0 & z z x y\end{array}$

$\left(\begin{array}{cccccccccc}0 & x y y y & 0 & 0 & x y z z & 0 & 0 & x y x x & 0 & 0 \\ y x x x & 0 & 0 & y x y y & 0 & 0 & y x z z & 0 & 0 & 0\end{array}\right)$

$\begin{array}{cccccccccc}\operatorname{yxxx} & 0 & 0 & y x y y & 0 & 0 & y x z z & 0 & 0 & 0 \\ 0 & 0 & 0 & 0 & 0 & 0 & 0 & 0 & 0 & z x y z\end{array}$

$$
\left(\begin{array}{cccccc}
0 & 0 & 0 & 0 & 0 & \mathrm{xxxy} \\
& 0 & 0 & 0 & 0 & \mathrm{yyxy} \\
& & 0 & 0 & 0 & \mathrm{zzxy} \\
& & & 0 & \mathrm{yzxz} & 0 \\
& & & & 0 & 0 \\
& & & & & 0
\end{array}\right)
$$

$\left(\begin{array}{llllll}0 & 0 & 0 & 0 & 0 & x x x y \\ 0 & 0 & 0 & 0 & 0 & x y x y\end{array}\right.$ $\begin{array}{llllll}0 & 0 & 0 & 0 & 0 & y y x y \\ 0 & 0 & 0 & 0 & 0 & z z y\end{array}$ $\begin{array}{cccccc}0 & 0 & 0 & 0 & 0 & z z x y \\ 0 & 0 & 0 & 0 & y z x z & 0\end{array}$ $\begin{array}{llllll}0 & 0 & 0 & 0 & y z x z & 0\end{array}$ $\begin{array}{llllll}0 & 0 & 0 & x z y z & 0 & 0\end{array}$ 


$$
\left[\left(\mathrm{V}^{2}\right)\right]^{2}\left(\begin{array}{ccccccccc}
\text { xxxx } & \text { xxyy } & \text { xxzz } & 0 & 0 & 0 & 0 & 0 & 0 \\
& \text { yyyy } & \text { yyzz } & 0 & 0 & 0 & 0 & 0 & 0 \\
& & \text { zzzz } & 0 & 0 & 0 & 0 & 0 & 0 \\
& & & \text { yzyz } & 0 & 0 & \text { yzzy } & 0 & 0 \\
& & & & \text { zxzx } & 0 & 0 & \text { zxxz } & 0 \\
& & & & & \text { xyxy } & 0 & 0 & \text { xyyx } \\
& & & & & & \text { zyzy } & 0 & 0 \\
& & & & & & & \text { xzxz } & 0 \\
& & & & & & & & \text { yxyx }
\end{array}\right)
$$

$\left(\begin{array}{lllllllll}0 & 0 & 0 & 0 & 0 & 0 & 0 & 0 & 0\end{array}\right.$ $\begin{array}{llllllll}0 & 0 & 0 & 0 & 0 & 0 & 0 & 0\end{array}$ $\begin{array}{lllllll}0 & 0 & 0 & 0 & 0 & 0 & 0\end{array}$ $\begin{array}{llllll}0 & 0 & 0 & 0 & 0 & 0\end{array}$ $\begin{array}{lllll}0 & 0 & 0 & 0 & 0\end{array}$ $\begin{array}{llll}0 & 0 & 0 & 0\end{array}$ $\begin{array}{lll}0 & 0 & 0 \\ & 0 & 0\end{array}$

$\left(\begin{array}{lllllllll}x x x x & x x y y & x x z z & 0 & 0 & 0 & 0 & 0 & 0 \\ y y x & y y y & y y z z & 0 & 0 & 0 & 0 & 0 & 0\end{array}\right)$ $\left(\begin{array}{llllllllll}x y y x x & y y y y & y y z z & 0 & 0 & 0 & 0 & 0 & 0 \\ z z x x & z z y y & z z z z & 0 & 0 & 0 & 0 & 0 & 0\end{array}\right)$

$\left(\begin{array}{lllllllll}0 & 0 & 0 & 0 & 0 & 0 & 0 & 0 & 0 \\ 0 & 0 & 0 & 0 & 0 & 0 & 0 & 0 & 0 \\ 0 & 0 & 0 & 0 & 0 & 0 & 0 & 0 & 0\end{array}\right)$ $\left.\begin{array}{lllllllll}0 & 0 & 0 & 0 & 0 & 0 & 0 & 0 & 0 \\ 0 & 0 & 0 & 0 & 0 & 0 & 0 & 0 & 0 \\ 0 & 0 & 0 & 0 & 0 & 0 & 0 & 0 & 0\end{array}\right)$ $\begin{array}{lllllllll}0 & 0 & 0 & 0 & 0 & 0 & 0 & 0 & 0\end{array}$

$\begin{array}{lllllllll}0 & 0 & 0 & 0 & 0 & 0 & 0 & 0 & 0 \\ 0 & 0 & 0 & 0 & 0 & 0 & 0 & 0 & 0\end{array}$

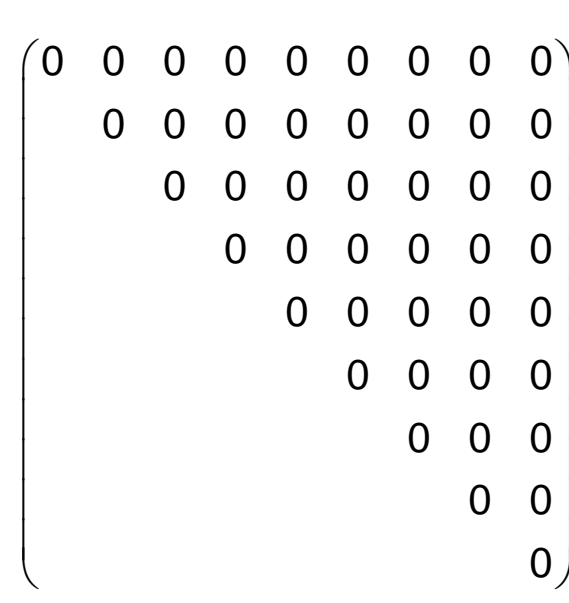

$\left(\begin{array}{lllllllll}0 & 0 & 0 & 0 & 0 & 0 & 0 & 0 & 0\end{array}\right.$ $0 \begin{array}{lllllllll}0 & 0 & 0 & 0 & 0 & 0 & 0 & 0 & 0 \\ 0 & 0 & 0 & 0 & 0 & 0 & 0 & 0 & 0\end{array}$ $\begin{array}{lllllllll}0 & 0 & 0 & 0 & 0 & 0 & 0 & 0 & 0 \\ 0 & 0 & 0 & 0 & 0 & 0 & 0 & 0 & 0\end{array}$ $\begin{array}{lllllllll}0 & 0 & 0 & 0 & 0 & 0 & 0 & 0 & 0 \\ 0 & 0 & 0 & 0 & 0 & 0 & 0 & 0 & 0\end{array}$ $\begin{array}{llllllllll}0 & 0 & 0 & 0 & 0 & 0 & 0 & 0 & 0 \\ 0 & 0 & 0 & 0 & 0 & 0 & 0 & 0 & 0\end{array}$

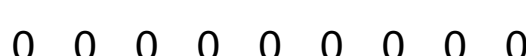

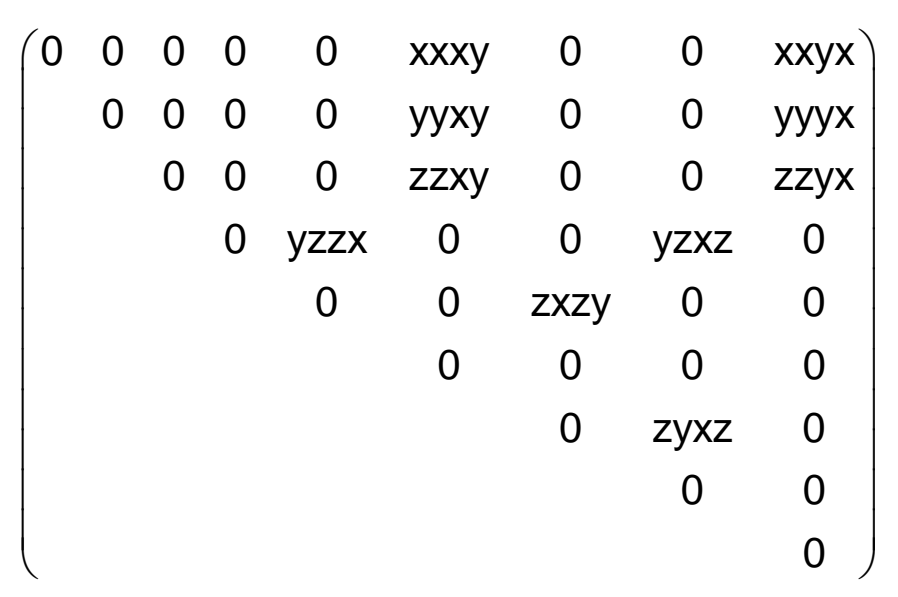

$\left(\begin{array}{ccccccccc}0 & 0 & 0 & 0 & 0 & \text { xxxy } & 0 & 0 & \text { xxyx } \\ 0 & 0 & 0 & 0 & 0 & \text { yyxy } & 0 & 0 & \text { yyyx } \\ 0 & 0 & 0 & 0 & 0 & \text { zzxy } & 0 & 0 & \text { zzyx } \\ 0 & 0 & 0 & 0 & \text { yzzx } & 0 & 0 & \text { yzxz } & 0 \\ 0 & 0 & 0 & \text { xzyz } & 0 & 0 & \text { zxzy } & 0 & 0 \\ \text { xyxx } & \text { xyyy } & \text { xyzz } & 0 & 0 & 0 & 0 & 0 & 0\end{array}\right)$ 
$\mathrm{V}^{4}\left(\begin{array}{ccccccccc}\mathrm{xxxx} & \mathrm{xxyy} & \mathrm{xxzz} & 0 & 0 & 0 & 0 & 0 & 0 \\ \mathrm{yyxx} & \mathrm{xxxx} & \mathrm{xxzz} & 0 & 0 & 0 & 0 & 0 & 0 \\ \mathrm{zzxx} & \mathrm{zzyy} & \mathrm{zzzz} & 0 & 0 & 0 & 0 & 0 & 0 \\ 0 & 0 & 0 & \mathrm{yzyz} & 0 & 0 & \mathrm{yzzy} & 0 & 0 \\ 0 & 0 & 0 & 0 & \mathrm{zxzx} & 0 & 0 & \mathrm{zxxz} & 0 \\ 0 & 0 & 0 & 0 & 0 & \mathrm{xyxy} & 0 & 0 & \mathrm{xyyx} \\ 0 & 0 & 0 & \mathrm{zxxz} & 0 & 0 & \mathrm{zxzx} & 0 & 0 \\ 0 & 0 & 0 & 0 & \mathrm{yzzy} & 0 & 0 & \mathrm{yzyz} & 0 \\ 0 & 0 & 0 & 0 & 0 & \mathrm{xyyx} & 0 & 0 & \mathrm{xyxy}\end{array}\right)$

$\left[\mathrm{V}^{4}\right] \quad\left(\begin{array}{ccccc}\mathrm{x} x \mathrm{xx} & 0 & 0 & \mathrm{yyzz} & 0 \\ 0 & \mathrm{xxxx} & 0 & \mathrm{yyzz} & 0 \\ 0 & 0 & \mathrm{zzzz} & \mathrm{xxyy} & 0\end{array}\right)$

$V\left[V^{3}\right]\left(\begin{array}{cccccccccc}x x x x & 0 & 0 & x x y y & 0 & 0 & y y z z & 0 & 0 & 0 \\ 0 & x x x x & 0 & 0 & y y z z & 0 & 0 & x x y y & 0 & 0 \\ 0 & 0 & z z z z & 0 & 0 & z z x x & 0 & 0 & z z x x & 0\end{array}\right)$

$\left[\left[^{2}\right]^{2}\right]\left(\begin{array}{cccccc}\text { xxxx } & \text { xxyy } & \text { xxzz } & 0 & 0 & 0 \\ & \text { yyyy } & \text { yyzz } & 0 & 0 & 0 \\ & & \text { zzzz } & 0 & 0 & 0 \\ & & & \text { yzyz } & 0 & 0 \\ & & & & \text { yzyz } & 0 \\ & & & & & \text { xyxy }\end{array}\right)$

$\left[\mathrm{V}^{2}\right]^{2}\left(\begin{array}{cccccc}\mathrm{xxxx} & \mathrm{xxyy} & \mathrm{xxzz} & 0 & 0 & 0 \\ \mathrm{yyxx} & \mathrm{xxxx} & \mathrm{xxzz} & 0 & 0 & 0 \\ \mathrm{zzxx} & \mathrm{zzxx} & \mathrm{zzzz} & 0 & 0 & 0 \\ 0 & 0 & 0 & \mathrm{yzyz} & 0 & 0 \\ 0 & 0 & 0 & 0 & \mathrm{yzyz} & 0 \\ 0 & 0 & 0 & 0 & 0 & \mathrm{xyxy}\end{array}\right)$

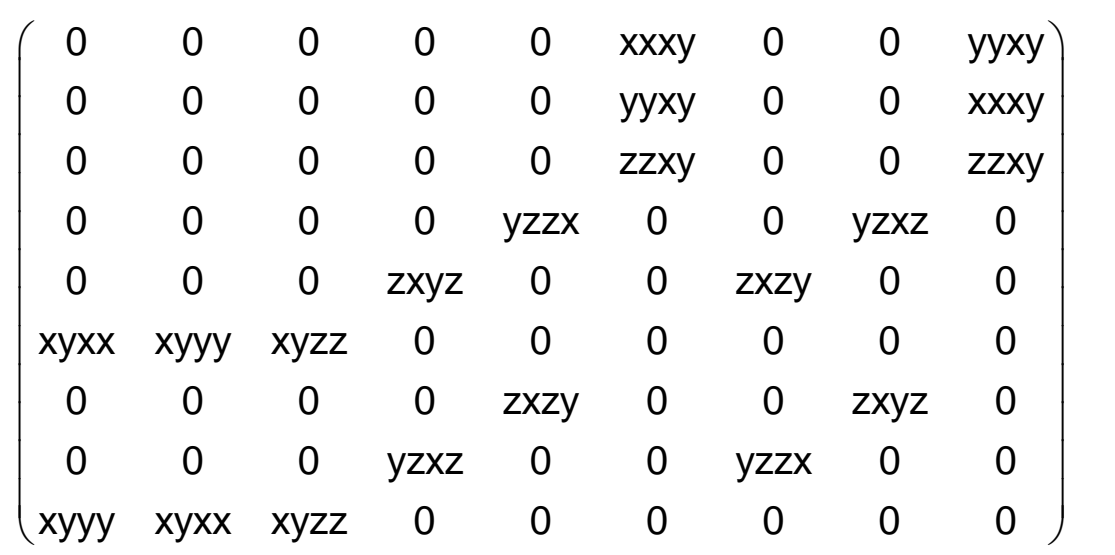

$\left(\begin{array}{ccccc}0 & y x x x & 0 & 0 & 0 \\ y x x x & 0 & 0 & 0 & 0 \\ 0 & 0 & 0 & 0 & z z x y\end{array}\right)$

$\left(\begin{array}{cccccccccc}0 & x y y y & 0 & 0 & x y z z & 0 & 0 & y x y y & 0 & 0 \\ x y y & 0 & 0 & y x y y & 0 & 0 & x y z z & 0 & 0 & 0\end{array}\right)$

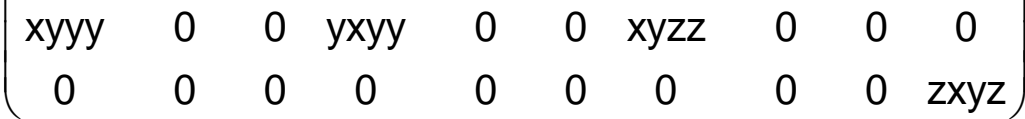

$$
\left(\begin{array}{cccccc}
0 & 0 & 0 & 0 & 0 & x x x y \\
& 0 & 0 & 0 & 0 & x x x y \\
& & 0 & 0 & 0 & z z x y \\
& & & 0 & y z x z & 0 \\
& & & & 0 & 0 \\
& & & & & 0
\end{array}\right)
$$

$\left(\begin{array}{cccccc}0 & 0 & 0 & 0 & 0 & x x x y \\ 0 & 0 & 0 & 0 & 0 & x x x y \\ 0 & 0 & 0 & 0 & 0 & z z x y \\ 0 & 0 & 0 & 0 & y z x z & 0 \\ 0 & 0 & 0 & y z x z & 0 & 0 \\ x y x x & x y x x & x y z z & 0 & 0 & 0\end{array}\right)$

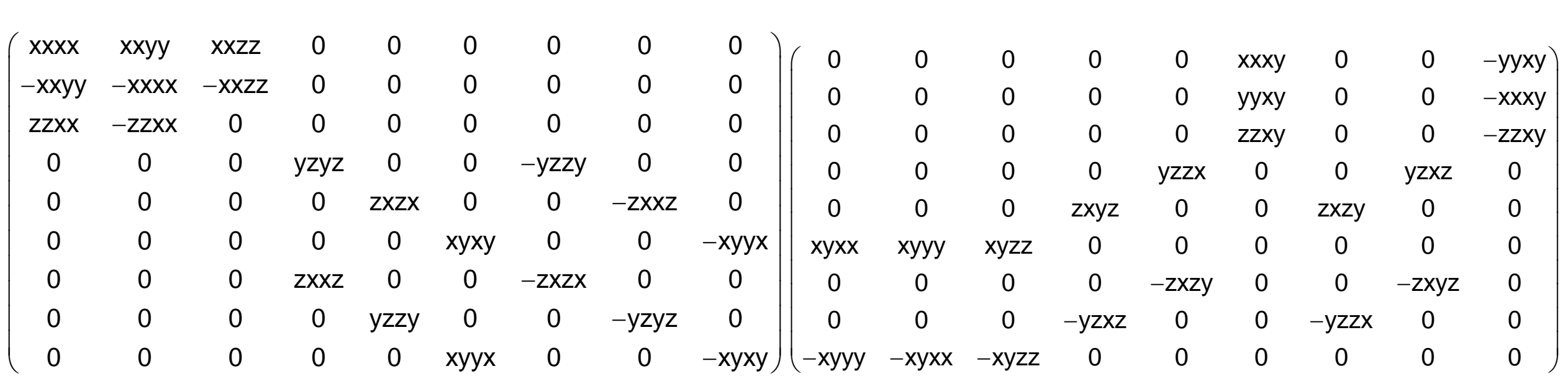

$\left(\begin{array}{ccccc}\mathrm{xxxx} & 0 & 0 & \mathrm{yyzz} & 0 \\ 0 & -\mathrm{xxxx} & 0 & -\mathrm{yyzz} & 0 \\ 0 & 0 & 0 & 0 & 0\end{array}\right)$

$\left(\begin{array}{ccccc}0 & -y x x x & 0 & 0 & 0 \\ y x x x & 0 & 0 & 0 & 0 \\ 0 & 0 & 0 & 0 & 0\end{array}\right)$

$\left(\begin{array}{cccccccccc}\mathrm{xxxx} & 0 & 0 & \mathrm{xxyy} & 0 & 0 & -\mathrm{yyzz} & 0 & 0 & 0 \\ 0 & -\mathrm{xxxx} & 0 & 0 & \mathrm{yyzz} & 0 & 0 & -\mathrm{xxyy} & 0 & 0 \\ 0 & 0 & 0 & 0 & 0 & \mathrm{zzxx} & 0 & 0 & -z z \mathrm{x} x & 0\end{array}\right)\left(\begin{array}{cccccccccc}0 & -\mathrm{y} x \mathrm{xx} & 0 & 0 & \mathrm{xyzz} & 0 & 0 & \mathrm{xyxx} & 0 & 0 \\ \mathrm{yxxx} & 0 & 0 & -\mathrm{xyxx} & 0 & 0 & -\mathrm{xyzz} & 0 & 0 & 0 \\ 0 & 0 & 0 & 0 & 0 & 0 & 0 & 0 & 0 & z x y z\end{array}\right)$

$\left(\begin{array}{cccccc}\mathrm{xxxx} & 0 & \mathrm{xxzz} & 0 & 0 & 0 \\ & -\mathrm{xxxx} & -\mathrm{xxzz} & 0 & 0 & 0 \\ & & 0 & 0 & 0 & 0 \\ & & \mathrm{yzyz} & 0 & 0 \\ & & & & -\mathrm{yzyz} & 0 \\ & & & & & 0\end{array}\right)$

$\left(\begin{array}{cccccc}0 & 0 & 0 & 0 & 0 & \mathrm{xxxy} \\ & 0 & 0 & 0 & 0 & -\mathrm{xxxy} \\ & & 0 & 0 & 0 & 0 \\ & & & 0 & 0 & 0 \\ & & & & 0 & 0 \\ & & & & & 0\end{array}\right)$

$\left(\begin{array}{cccccc}x x x x & x x y y & x x z z & 0 & 0 & 0 \\ -x x y y & -x x x x & -x x z z & 0 & 0 & 0 \\ z z x x & -z z x x & 0 & 0 & 0 & 0 \\ 0 & 0 & 0 & y z y z & 0 & 0 \\ 0 & 0 & 0 & 0 & -y z y z & 0 \\ 0 & 0 & 0 & 0 & 0 & 0\end{array}\right)$

$\left(\begin{array}{cccccc}0 & 0 & 0 & 0 & 0 & x x x y \\ 0 & 0 & 0 & 0 & 0 & -x x x y \\ 0 & 0 & 0 & 0 & 0 & 0 \\ 0 & 0 & 0 & 0 & y z x z & 0 \\ 0 & 0 & 0 & -y z x z & 0 & 0 \\ x y x x & -x y x x & 0 & 0 & 0 & 0\end{array}\right)$ 

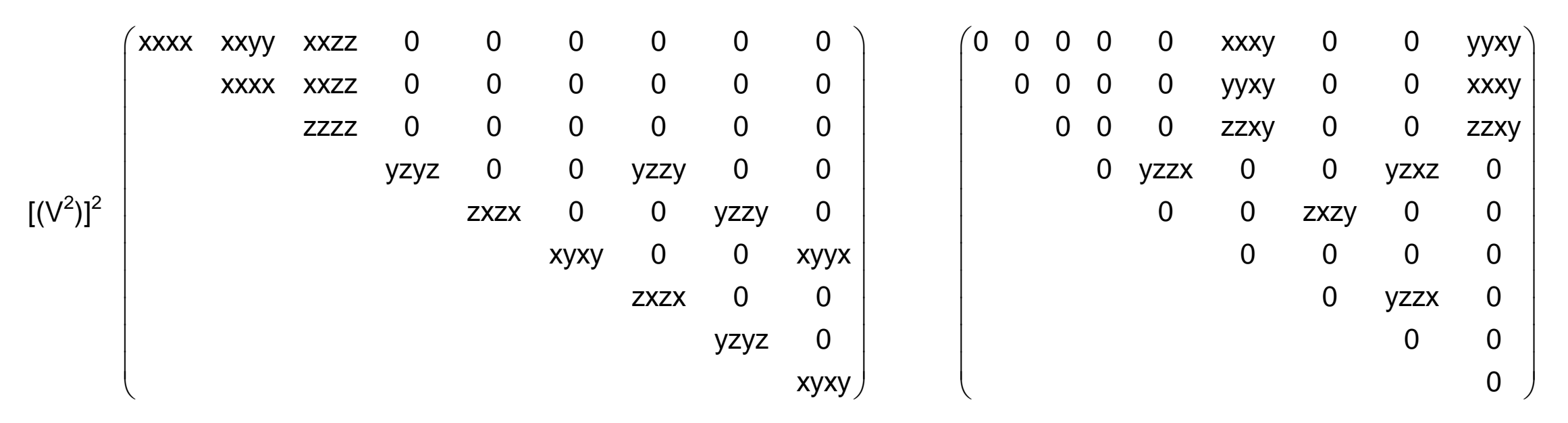

$\left[\mathrm{V}^{2}\right] \mathrm{V}^{2}\left(\begin{array}{ccccccccc}\mathrm{xxxx} & \mathrm{xxyy} & \mathrm{xxzz} & 0 & 0 & 0 & 0 & 0 & 0 \\ \mathrm{yyxx} & \mathrm{xxxx} & \mathrm{xxzz} & 0 & 0 & 0 & 0 & 0 & 0 \\ \mathrm{zzxx} & \mathrm{zzxx} & \mathrm{zzzz} & 0 & 0 & 0 & 0 & 0 & 0 \\ 0 & 0 & 0 & \mathrm{yzyz} & 0 & 0 & \mathrm{zxzx} & 0 & 0 \\ 0 & 0 & 0 & 0 & \mathrm{zxzx} & 0 & 0 & \mathrm{yzyz} & 0 \\ 0 & 0 & 0 & 0 & 0 & \mathrm{xyxy} & 0 & 0 & \mathrm{xyxy}\end{array}\right)$

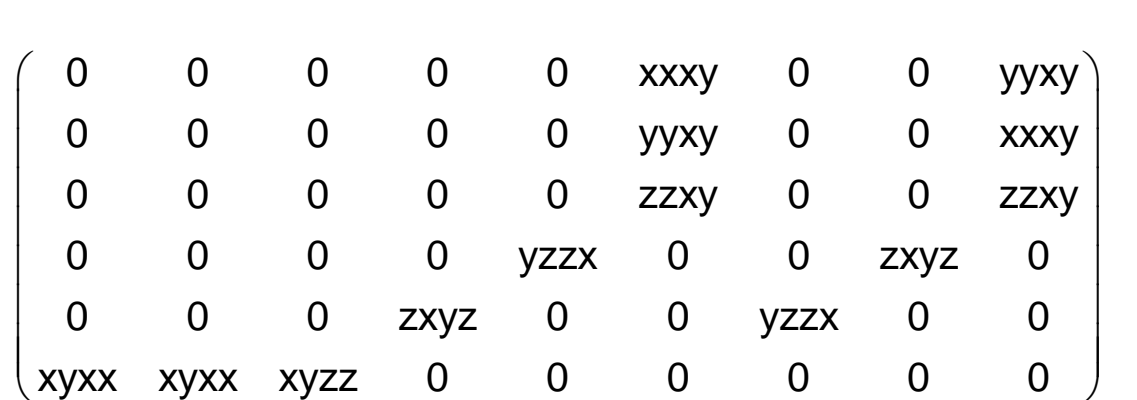

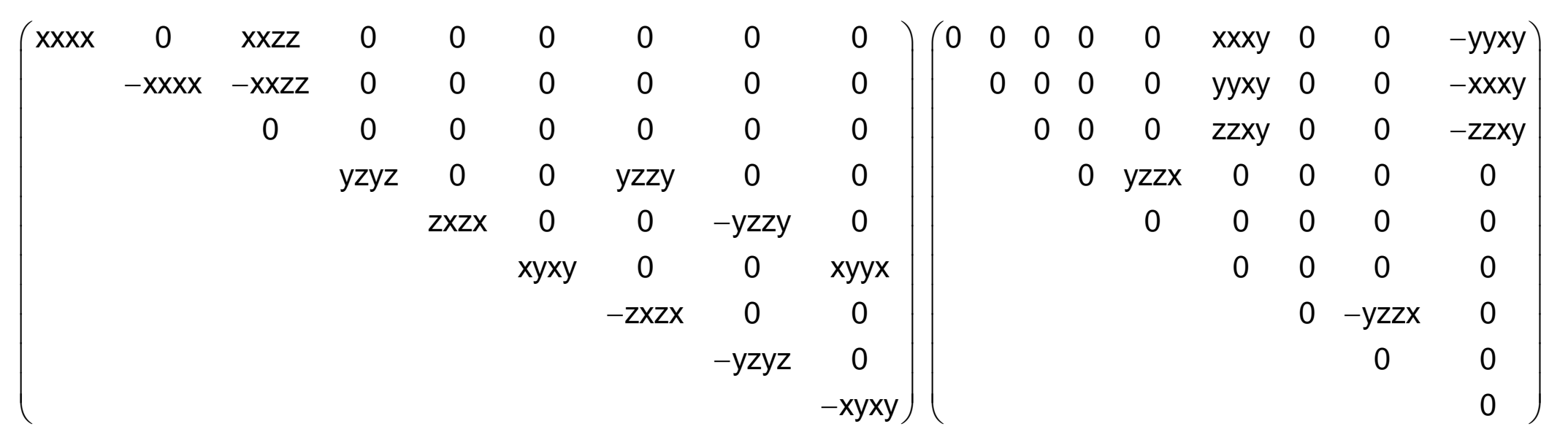

$\left(\begin{array}{ccccccccc}\text { xxxx } & \text { xxyy } & \text { xxzz } & 0 & 0 & 0 & 0 & 0 & 0 \\ -x x y y & -x x x x & -x x z z & 0 & 0 & 0 & 0 & 0 & 0 \\ z z x x & -z z x x & 0 & 0 & 0 & 0 & 0 & 0 & 0 \\ 0 & 0 & 0 & y z y z & 0 & 0 & -z x z x & 0 & 0 \\ 0 & 0 & 0 & 0 & \text { zxzx } & 0 & 0 & -y z y z & 0 \\ 0 & 0 & 0 & 0 & 0 & x y x y & 0 & 0 & -x y x y\end{array}\right)\left(\begin{array}{ccccccccc}0 & 0 & 0 & 0 & 0 & x x x y & 0 & 0 & -y y x y \\ 0 & 0 & 0 & 0 & 0 & y y x y & 0 & 0 & -x x x y \\ 0 & 0 & 0 & 0 & 0 & z z x y & 0 & 0 & -z z x y \\ 0 & 0 & 0 & 0 & y z z x & 0 & 0 & -z x y z & 0 \\ 0 & 0 & 0 & z x y z & 0 & 0 & -y z z x & 0 & 0 \\ x y x x & -x y x x & 0 & 0 & 0 & 0 & 0 & 0 & 0\end{array}\right)$ 


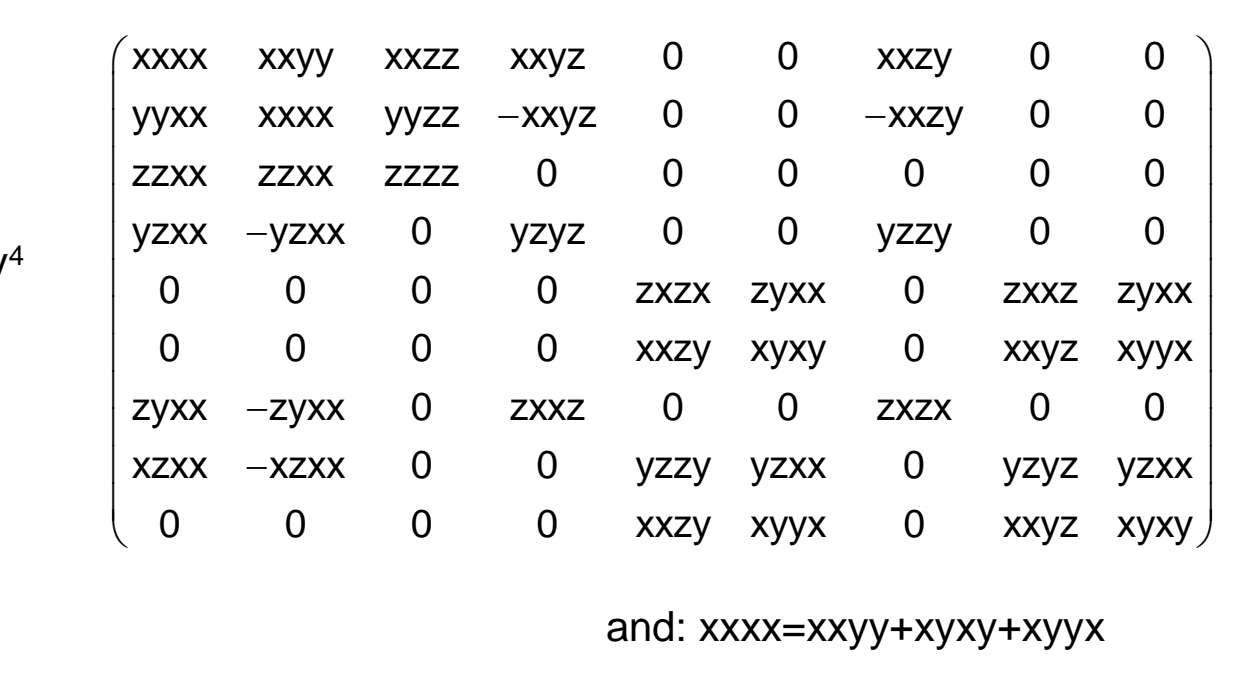

$\left[\mathrm{V}^{4}\right]$ $\left(\begin{array}{ccccc}3 x x y y & 0 & 0 & y y z z & x x y z \\ 0 & 3 x x y y & 0 & y y z z & 0 \\ 0 & -x x y z & z z z z & x x y y & 0\end{array}\right)$

$\left.V\left[V^{3}\right] \quad \begin{array}{cccccccccc}3 x x y y & 0 & 0 & x x y y & 0 & 0 & y y z z & 0 & 0 & y z x x \\ 0 & 3 x x y y & 0 & 0 & y y z z & y z x x & 0 & x x y y & -y z x x & 0 \\ 0 & -z y x x & z z z z & 0 & 0 & z z x x & 0 & z y y x & z z x x & 0\end{array}\right)$

$\left.\left[\text { [V }^{2}\right]^{2}\right]\left(\begin{array}{cccccc}x x x x & x x y y & x x z z & x x y z & 0 & 0 \\ & x x x x & x x z z & -x x y z & 0 & 0 \\ & & z z z z & 0 & 0 & 0 \\ & & & y z y z & 0 & 0 \\ & & & & y z y z & x x y z \\ & & & & & x y x y\end{array}\right)$

and $x y x y=1 / 2(x x x x-x x y y)$

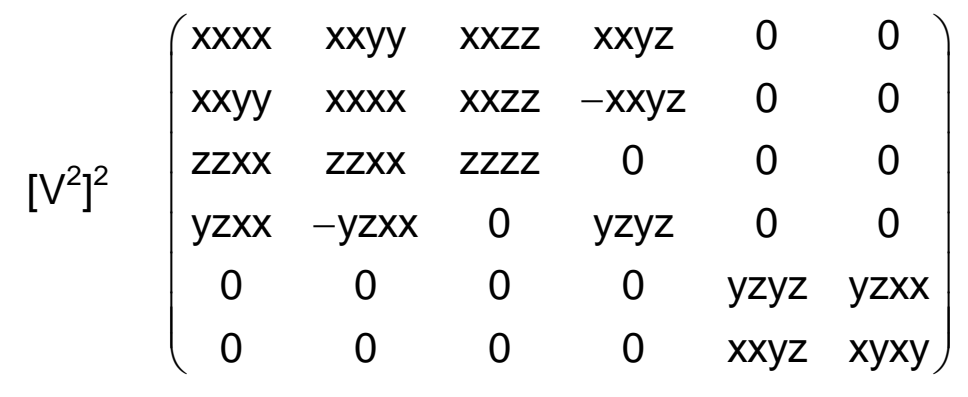

and $x y x y=1 / 2(x x x x-x x y y)$ $\left(\begin{array}{lllllllll}0 & 0 & 0 & 0 & 0 & 0 & 0 & 0 & 0 \\ 0 & 0 & 0 & 0 & 0 & 0 & 0 & 0 & 0\end{array}\right.$ $0 \begin{array}{lllllllll}0 & 0 & 0 & 0 & 0 & 0 & 0 & 0 & 0 \\ 0 & 0 & 0 & 0 & 0 & 0 & 0 & 0 & 0\end{array}$ $0 \begin{array}{llllllllll}0 & 0 & 0 & 0 & 0 & 0 & 0 & 0 & 0 \\ 0 & 0 & 0 & 0 & 0 & 0 & 0 & 0 & 0\end{array}$

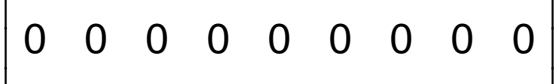
$\begin{array}{lllllllll}0 & 0 & 0 & 0 & 0 & 0 & 0 & 0 & 0 \\ 0 & 0 & 0 & 0 & 0 & 0 & 0 & 0 & 0\end{array}$ $\begin{array}{lllllllll}0 & 0 & 0 & 0 & 0 & 0 & 0 & 0 & 0 \\ 0 & 0 & 0 & 0 & 0 & 0 & 0 & 0 & 0\end{array}$ $0 \begin{array}{lllllllll}0 & 0 & 0 & 0 & 0 & 0 & 0 & 0 & 0 \\ 0 & 0 & 0 & 0 & 0 & 0 & 0 & 0 & 0\end{array}$ $0 \begin{array}{lllllllll}0 & 0 & 0 & 0 & 0 & 0 & 0 & 0 & 0 \\ 0 & 0 & 0 & 0 & 0 & 0 & 0 & 0 & 0\end{array}$ $\left(\begin{array}{lllllllll}0 & 0 & 0 & 0 & 0 & 0 & 0 & 0 & 0\end{array}\right)$

$\left(\begin{array}{lllll}0 & 0 & 0 & 0 & 0 \\ 0 & 0 & 0 & 0 & 0 \\ 0 & 0 & 0 & 0 & 0\end{array}\right)$

$\left(\begin{array}{lllll}0 & 0 & 0 & 0 & 0 \\ 0 & 0 & 0 & 0 & 0\end{array}\right)$

$\left(\begin{array}{llllllllll}0 & 0 & 0 & 0 & 0 & 0 & 0 & 0 & 0 & 0 \\ 0 & 0 & 0 & 0 & 0 & 0 & 0 & 0 & 0 & 0\end{array}\right)$ $\left(\begin{array}{lllllllllll}0 & 0 & 0 & 0 & 0 & 0 & 0 & 0 & 0 & 0 \\ 0 & 0 & 0 & 0 & 0 & 0 & 0 & 0 & 0 & 0\end{array}\right)$

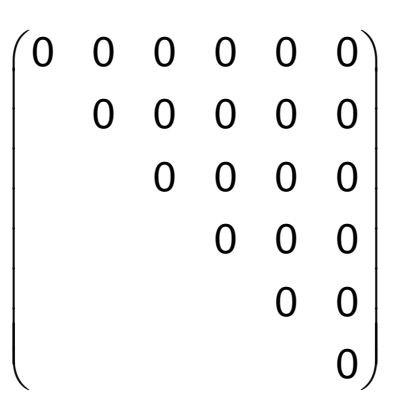

$\left(\begin{array}{lllllllll}0 & 0 & 0 & 0 & 0 & 0 & 0 & 0 & 0 \\ 0 & 0 & 0 & 0 & 0 & 0 & 0 & 0 & 0\end{array}\right)$ $\begin{array}{lllllllll}0 & 0 & 0 & 0 & 0 & 0 & 0 & 0 & 0 \\ 0 & 0 & 0 & 0 & 0 & 0 & 0 & 0 & 0\end{array}$ $\begin{array}{lllllllll}0 & 0 & 0 & 0 & 0 & 0 & 0 & 0 & 0 \\ 0 & 0 & 0 & 0 & 0 & 0 & 0 & 0 & 0\end{array}$ $\begin{array}{llllllllll}0 & 0 & 0 & 0 & 0 & 0 & 0 & 0 & 0 \\ 0 & 0 & 0 & 0 & 0 & 0 & 0 & 0 & 0\end{array}$ $\begin{array}{llllllllll}0 & 0 & 0 & 0 & 0 & 0 & 0 & 0 & 0\end{array}$ $\begin{array}{lllllllllll}0 & 0 & 0 & 0 & 0 & 0 & 0 & 0 & 0 & 0 & 0\end{array}$ $0 \begin{array}{llllllllll}0 & 0 & 0 & 0 & 0 & 0 & 0 & 0 & 0 \\ 0 & 0 & 0 & 0 & 0 & 0 & 0\end{array}$

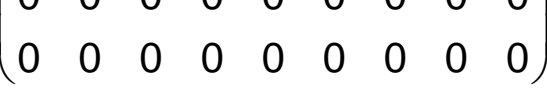

\section{$\left(\begin{array}{lllll}0 & 0 & 0 & 0 & 0 \\ 0 & 0 & 0 & 0 & 0 \\ 0 & 0 & 0 & 0 & 0\end{array}\right)$ \\ $\begin{array}{lllll}0 & 0 & 0 & 0 & 0\end{array}$}

$\begin{array}{llllllllll}0 & 0 & 0 & 0 & 0 & 0 & 0 & 0 & 0 & 0 \\ 0 & 0 & 0 & 0 & 0 & 0 & 0 & 0 & 0 & 0\end{array}$ $\left(\begin{array}{lllllllllll}0 & 0 & 0 & 0 & 0 & 0 & 0 & 0 & 0 & 0 \\ 0 & 0 & 0 & 0 & 0 & 0 & 0 & 0 & 0 & 0\end{array}\right)$

$$
\left(\begin{array}{llllll}
0 & 0 & 0 & 0 & 0 & 0 \\
& 0 & 0 & 0 & 0 & 0 \\
& & 0 & 0 & 0 & 0 \\
& & & 0 & 0 & 0 \\
& & & & 0 & 0 \\
& & & & & 0
\end{array}\right)
$$

$\left(\begin{array}{llllll}0 & 0 & 0 & 0 & 0 & 0 \\ 0 & 0 & 0 & 0 & 0 & 0 \\ 0 & 0 & 0 & 0 & 0 & 0 \\ 0 & 0 & 0 & 0 & 0 & 0 \\ 0 & 0 & 0 & 0 & 0 & 0 \\ 0 & 0 & 0 & 0 & 0 & 0\end{array}\right)$

$$
\left(\begin{array}{llllll}
0 & 0 & 0 & 0 & 0 & 0 \\
0 & 0 & 0 & 0 & 0 & 0 \\
0 & 0 & 0 & 0 & 0 & 0 \\
0 & 0 & 0 & 0 & 0 & 0 \\
0 & 0 & 0 & 0 & 0 & 0 \\
0 & 0 & 0 & 0 & 0 & 0
\end{array}\right)
$$
$0 \begin{array}{lllllllll}0 & 0 & 0 & 0 & 0 & 0 & 0 & 0 & 0 \\ 0 & 0 & 0 & 0 & 0 & 0 & 0 & 0 & 0\end{array}$

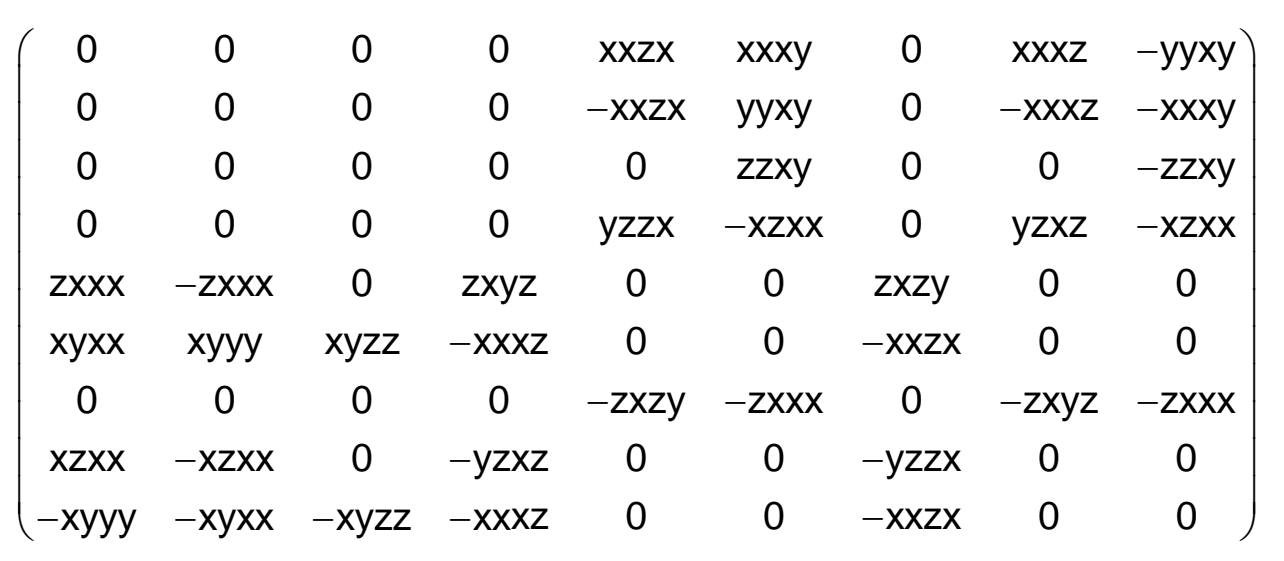

and: $x x x y-y y x y=x y y y-x y x x$

$\left.\begin{array}{ccccc}0 & 0 & 0 & 0 & 0 \\ 0 & 0 & 0 & 0 & -z x x x \\ z x x x & 0 & 0 & 0 & 0\end{array}\right)$

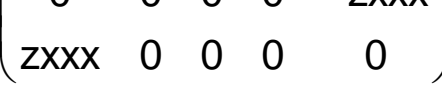

$\left(\begin{array}{cccccccccc}0 & 3 x y x x & 0 & 0 & x y z z & x z x x & 0 & x y x x & -x z x x & 0 \\ -3 x y y x & 0 & 0 & -x y x x & 0 & 0 & -x y z z & 0 & 0 & -x z x x \\ z x y x & 0 & 0 & -z x y x & 0 & 0 & 0 & 0 & 0 & 2 x y z\end{array}\right)$ $\left(\begin{array}{cccccc}0 & 0 & 0 & 0 & -y y x z & 0 \\ & 0 & 0 & 0 & y y x z & 0 \\ & & 0 & 0 & 0 & 0 \\ & & & 0 & 0 & y y x z \\ & & & & 0 & 0 \\ & & & & & 0\end{array}\right)$ 


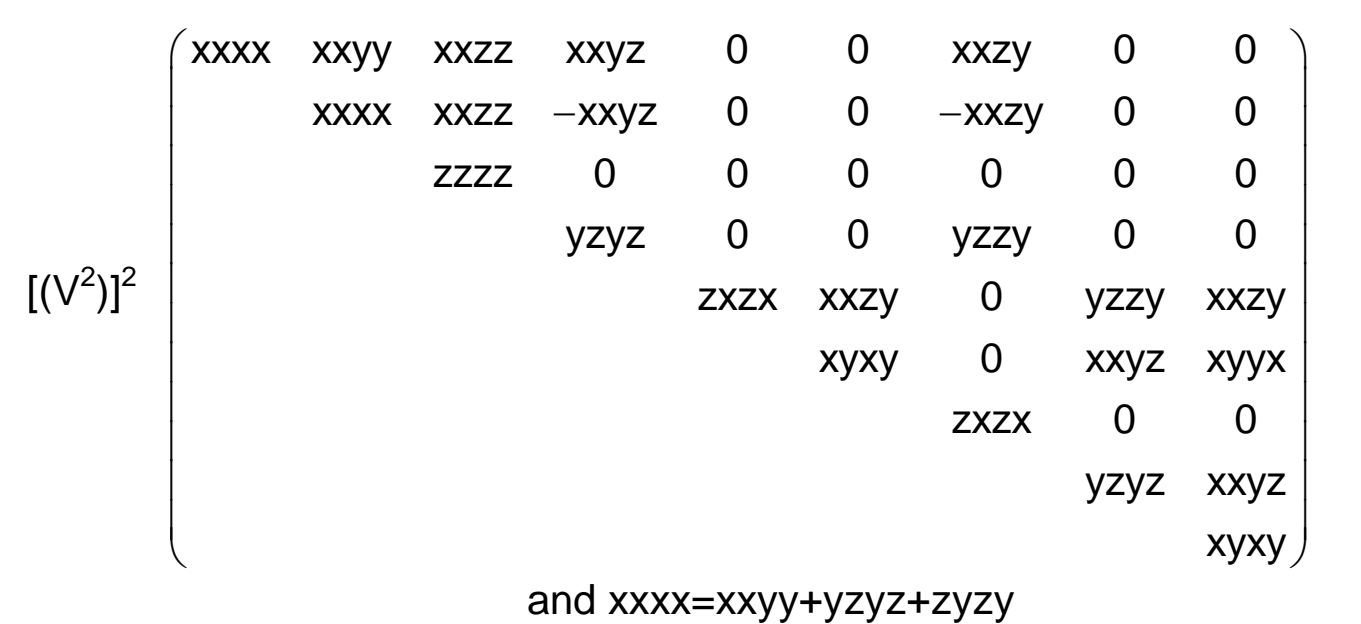

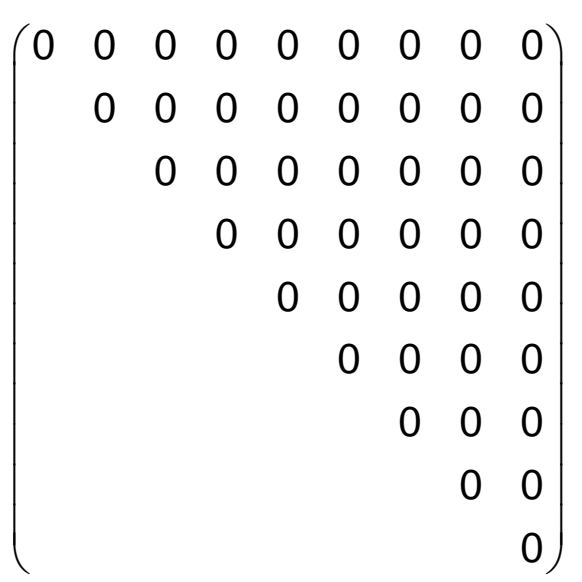

and $x x x x=x x y y+y z y z+z y z y$

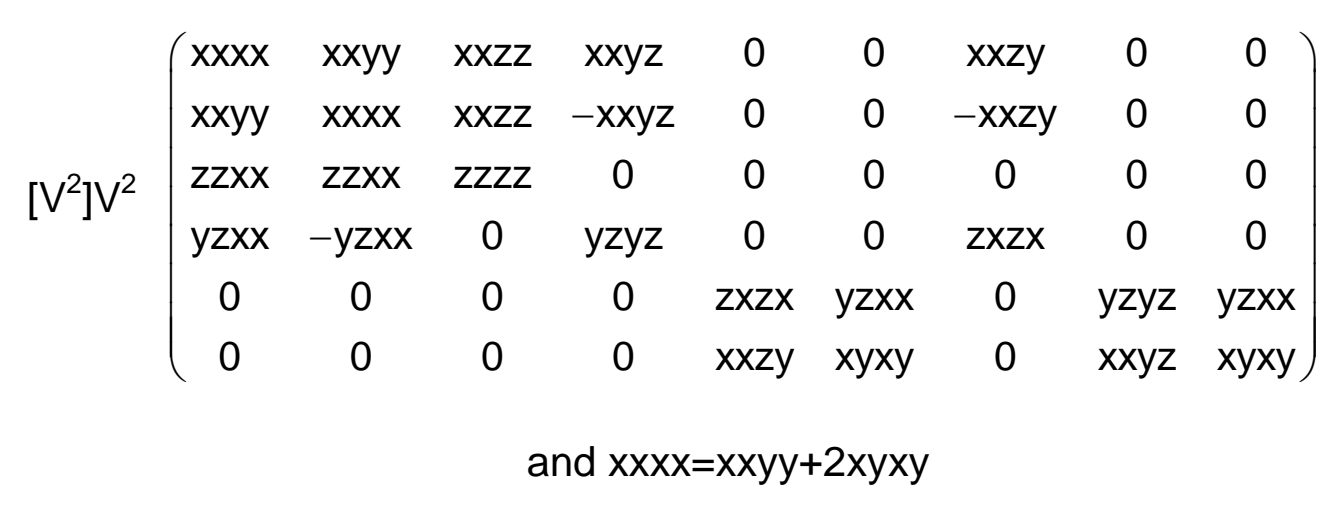

$\left(\begin{array}{lllllllll}0 & 0 & 0 & 0 & 0 & 0 & 0 & 0 & 0 \\ 0 & 0 & 0 & 0 & 0 & 0 & 0 & 0 & 0\end{array}\right.$

$\left(\begin{array}{lllllllll}0 & 0 & 0 & 0 & 0 & 0 & 0 & 0 & 0 \\ 0 & 0 & 0 & 0 & 0 & 0 & 0 & 0 & 0\end{array}\right.$

$\begin{array}{lllllllll}0 & 0 & 0 & 0 & 0 & 0 & 0 & 0 & 0\end{array}$ $0 \begin{array}{lllllllll}0 & 0 & 0 & 0 & 0 & 0 & 0 & 0 & 0 \\ 0 & 0 & 0 & 0 & 0 & 0 & 0 & 0 & 0\end{array}$ $\begin{array}{llllllllll}0 & 0 & 0 & 0 & 0 & 0 & 0 & 0 & 0 \\ 0 & 0 & 0 & 0 & 0 & 0 & 0 & 0 & 0\end{array}$ $\begin{array}{llllllllll}0 & 0 & 0 & 0 & 0 & 0 & 0 & 0 & 0\end{array}$

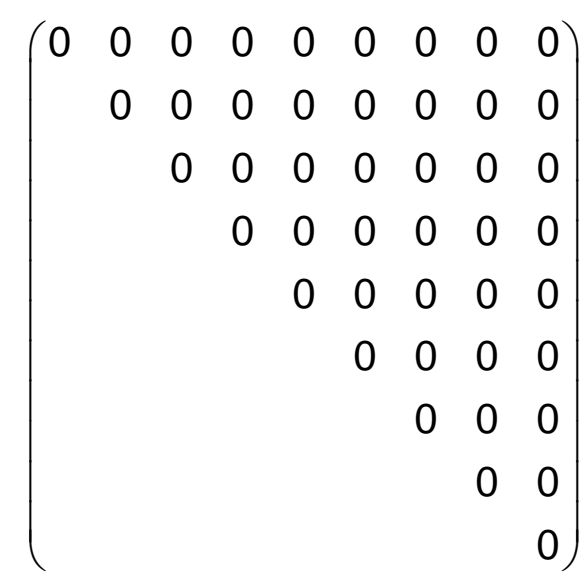

$\left(\begin{array}{lllllllll}0 & 0 & 0 & 0 & 0 & 0 & 0 & 0 & 0 \\ 0 & 0 & 0 & 0 & 0 & 0 & 0 & 0 & 0\end{array}\right.$

$\begin{array}{lllllllll}0 & 0 & 0 & 0 & 0 & 0 & 0 & 0 & 0 \\ 0 & 0 & 0 & 0 & 0 & 0 & 0 & 0 & 0\end{array}$ $\begin{array}{llllllllll}0 & 0 & 0 & 0 & 0 & 0 & 0 & 0 & 0 \\ 0 & 0 & 0 & 0 & 0 & 0 & 0 & 0\end{array}$ $0 \begin{array}{llllllllll}0 & 0 & 0 & 0 & 0 & 0 & 0 & 0 & 0\end{array}$ $0 \begin{array}{llllllllll}0 & 0 & 0 & 0 & 0 & 0 & 0 & 0 & 0 \\ 0 & 0 & 0 & 0 & 0 & 0 & 0 & 0 & 0\end{array}$ $\begin{array}{lllllllll}0 & 0 & 0 & 0 & 0 & 0 & 0 & 0 & 0\end{array}$

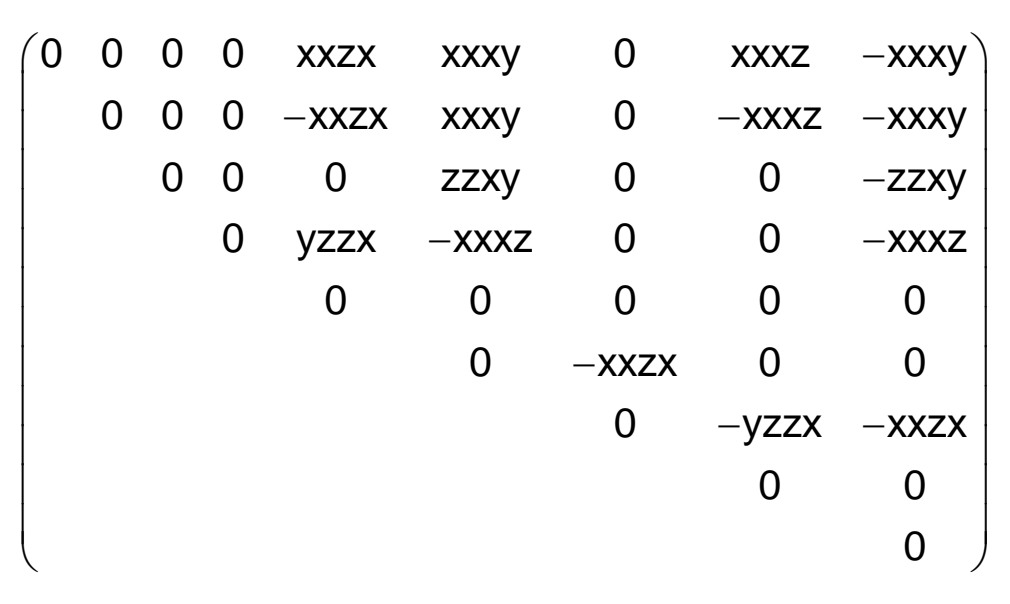

$\left(\begin{array}{ccccccccc}0 & 0 & 0 & 0 & x x z x & x x x y & 0 & x x x z & -y y x y \\ 0 & 0 & 0 & 0 & -x x z x & y y x y & 0 & -x x x z & -x x x y \\ 0 & 0 & 0 & 0 & 0 & z z x y & 0 & 0 & -z z x y \\ 0 & 0 & 0 & 0 & y z z x & -z x x x & 0 & -z x y z & -z x x x \\ z x x x & -z x x x & 0 & z x y z & 0 & 0 & -y z z x & 0 & 0 \\ x y x x & -x y x x & 0 & -x x x z & 0 & 0 & -x x z x & 0 & 0\end{array}\right)$

and: $y y x y-x x x y=2 x y x x$ 


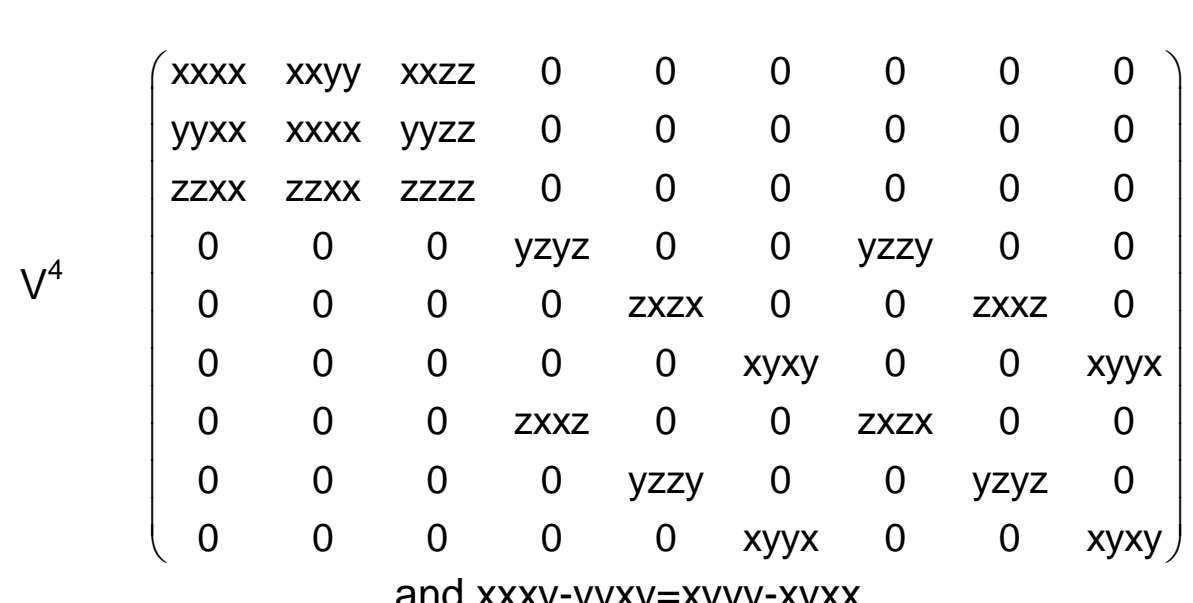

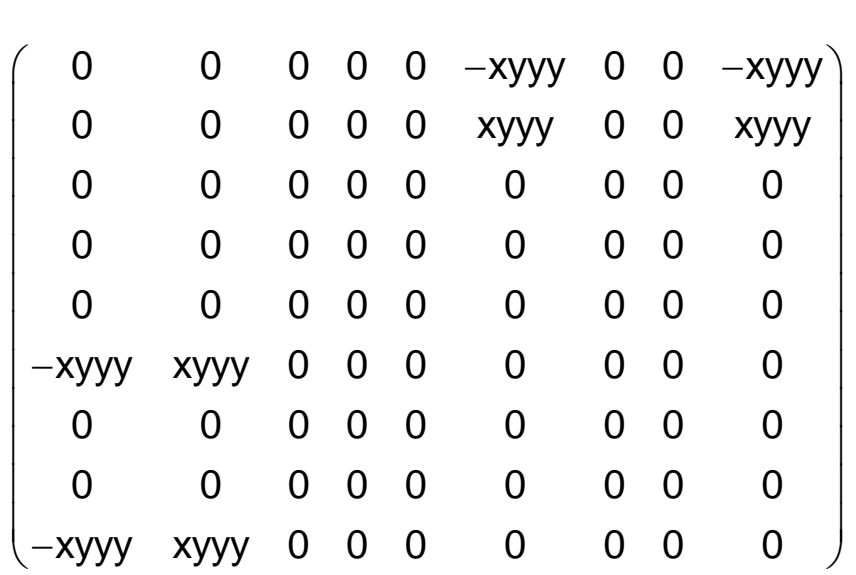

$\left.\mathrm{V}^{4}\right]\left(\begin{array}{ccccc}3 x x y y & 0 & 0 & y y z z & 0 \\ 0 & 3 x x y y & 0 & y y z z & 0 \\ 0 & 0 & z z z z & x x y y & 0\end{array}\right)$

$\left(\begin{array}{ccccc}0 & x y y y & 0 & 0 & 0 \\ -x y y y & 0 & 0 & 0 & 0 \\ 0 & 0 & 0 & 0 & 0\end{array}\right)$

$V\left[V^{3}\right] \quad\left(\begin{array}{cccccccccc}3 x x y y & 0 & 0 & x x y y & 0 & 0 & y y z z & 0 & 0 & y y x x \\ 0 & 3 x x y y & 0 & 0 & y y z z & 0 & 0 & x x y y & 0 & 0 \\ 0 & 0 & z z z & 0 & 0 & z z x & 0 & 0 & z z x & 0\end{array}\right)$

$\begin{array}{llllllllll}0 & \text { xyyy } & 0 & 0 & 0 & 0 & 0 & -x y y y & 0 & 0\end{array}$ $\left(\begin{array}{cccccccccc}0 & \text { xyyy } & 0 & 0 & 0 & 0 & 0 & -x y y y & 0 & 0 \\ -x y y y & 0 & 0 & x y y y & 0 & 0 & 0 & 0 & 0 & 0 \\ 0 & 0 & 0 & 0 & 0 & 0 & 0 & 0 & 0 & 0\end{array}\right)$

$\left.\left[\mathrm{V}^{2}\right]^{2}\right]\left(\begin{array}{cccccc}\mathrm{xxxx} & \mathrm{xxyy} & \mathrm{xxzz} & 0 & 0 & 0 \\ & \mathrm{xxxx} & \mathrm{xxzz} & 0 & 0 & 0 \\ & & \mathrm{zzzz} & 0 & 0 & 0 \\ & & & \mathrm{yzyz} & 0 & 0 \\ & & & & \mathrm{yzyz} & 0 \\ & & & & & \mathrm{xyxy}\end{array}\right)$

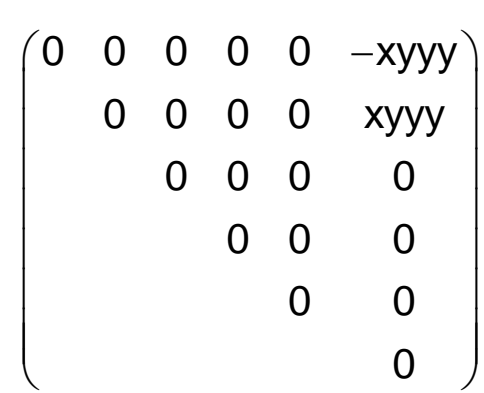

and $x y x y=1 / 2(x x x x-x x y y)$

$\left[\mathrm{V}^{2}\right]^{2}\left(\begin{array}{cccccc}\mathrm{x} x \mathrm{x} x & \mathrm{xxyy} & \mathrm{xxzz} & 0 & 0 & 0 \\ \mathrm{xxyy} & \mathrm{xxxx} & \mathrm{xxzz} & 0 & 0 & 0 \\ \mathrm{zzxx} & \mathrm{zzxx} & \mathrm{zzzz} & 0 & 0 & 0 \\ 0 & 0 & 0 & \mathrm{yzyz} & 0 & 0 \\ 0 & 0 & 0 & 0 & \mathrm{yzyz} & 0 \\ 0 & 0 & 0 & 0 & 0 & \mathrm{xyxy}\end{array}\right)$

and $x y x y=1 / 2(x x x x-x x y y)$

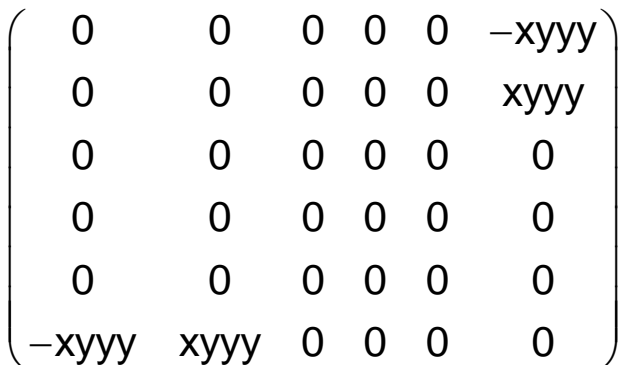

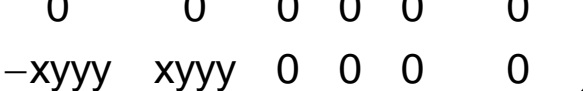

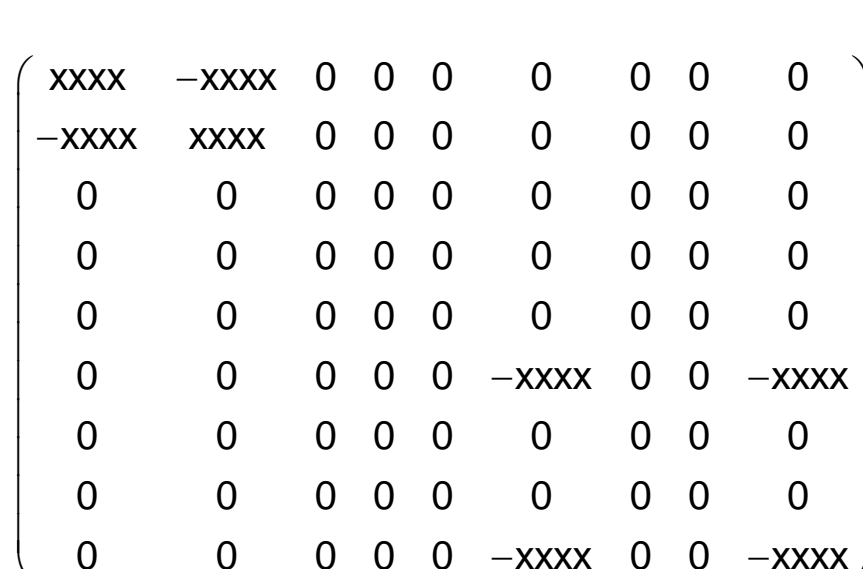

$\left(\begin{array}{ccccc}\mathrm{xxxx} & 0 & 0 & 0 & 0 \\ 0 & \mathrm{xxxx} & 0 & 0 & 0 \\ 0 & 0 & 0 & -\mathrm{xxxx} & 0\end{array}\right)$

$\left(\begin{array}{cccccccccc}\mathrm{xxxx} & 0 & 0 & -\mathrm{xxxx} & 0 & 0 & 0 & 0 & 0 & 0 \\ 0 & \mathrm{xxxx} & 0 & 0 & 0 & 0 & 0 & -\mathrm{xxxx} & 0 & 0 \\ 0 & 0 & 0 & 0 & 0 & 0 & 0 & 0 & 0 & 0\end{array}\right)$

$\left.\begin{array}{cccccccccc}0 & \mathrm{xxxx} & 0 & 0 & 0 & 0 & 0 & -\mathrm{xxxx} & 0 & 0 \\ 0 & 0 & 0 & 0 & 0 & 0 & 0 & 0 & 0 & 0\end{array}\right)$

$\left(\begin{array}{cccccc}\operatorname{xxxx} & -x x x x & 0 & 0 & 0 & 0 \\ & x x x x & 0 & 0 & 0 & 0 \\ & & 0 & 0 & 0 & 0 \\ & & & 0 & 0 & 0 \\ & & & & 0 & 0 \\ & & & & & -x x x x\end{array}\right)$

$\left(\begin{array}{cccccc}x x x x & -x x x x & 0 & 0 & 0 & 0 \\ -x x x x & x x x x & 0 & 0 & 0 & 0 \\ 0 & 0 & 0 & 0 & 0 & 0 \\ 0 & 0 & 0 & 0 & 0 & 0 \\ 0 & 0 & 0 & 0 & 0 & 0 \\ 0 & 0 & 0 & 0 & 0 & -x x x x\end{array}\right)$

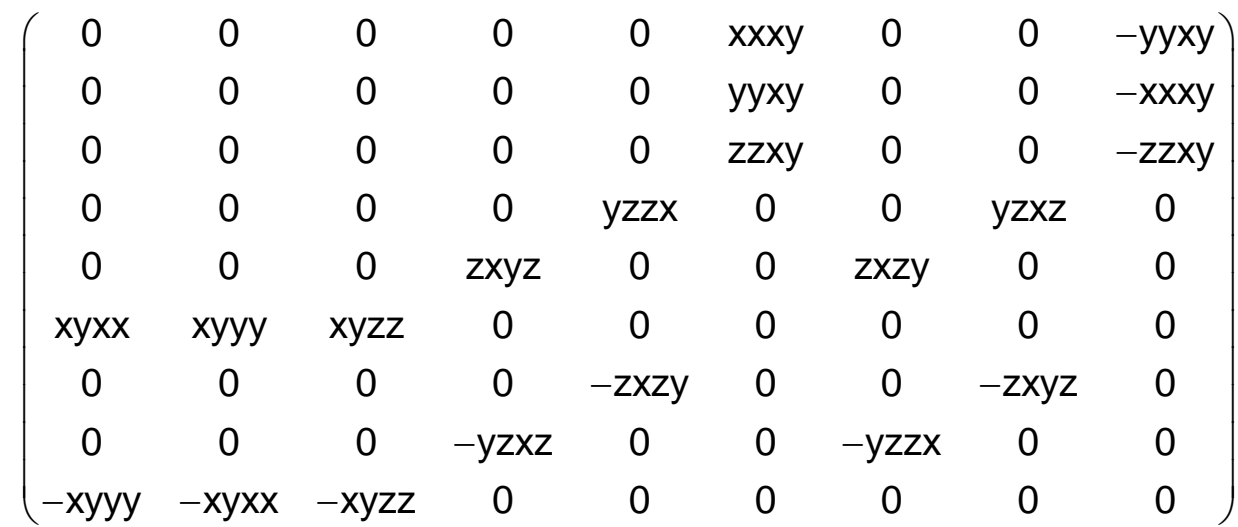

and: $x x x y-y y x y=x y y y-x y x x$

$\left(\begin{array}{lllll}0 & 0 & 0 & 0 & 0 \\ 0 & 0 & 0 & 0 & 0\end{array}\right)$

$\left.\begin{array}{lllll}0 & 0 & 0 & 0 & 0 \\ 0 & 0 & 0 & 0 & 0\end{array}\right)$

$\left(\begin{array}{cccccccccc}0 & 3 x y x x & 0 & 0 & x y z z & 0 & 0 & x y x x & 0 & 0 \\ -3 x y x & 0 & 0 & -x y x & 0 & 0 & -x y z z & 0 & 0\end{array}\right)$

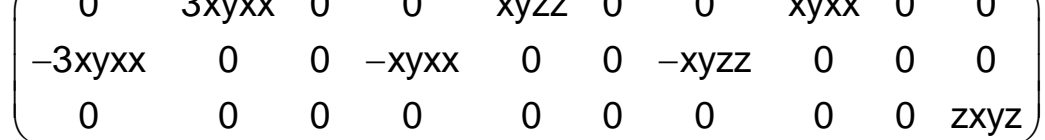

$\left(\begin{array}{llllll}0 & 0 & 0 & 0 & 0 & 0 \\ & 0 & 0 & 0 & 0 & 0 \\ & & 0 & 0 & 0 & 0 \\ & & & 0 & 0 & 0 \\ & & & & 0 & 0 \\ & & & & & 0\end{array}\right)$ $\left.\begin{array}{cccccc}0 & 0 & 0 & 0 & 0 & x x x y \\ 0 & 0 & 0 & 0 & 0 & -x x x y \\ 0 & 0 & 0 & 0 & 0 & 0 \\ 0 & 0 & 0 & 0 & y z x z & 0 \\ 0 & 0 & 0 & y z x z & 0 & 0 \\ -x x x y & x x x y & 0 & 0 & 0 & 0\end{array}\right)$ 


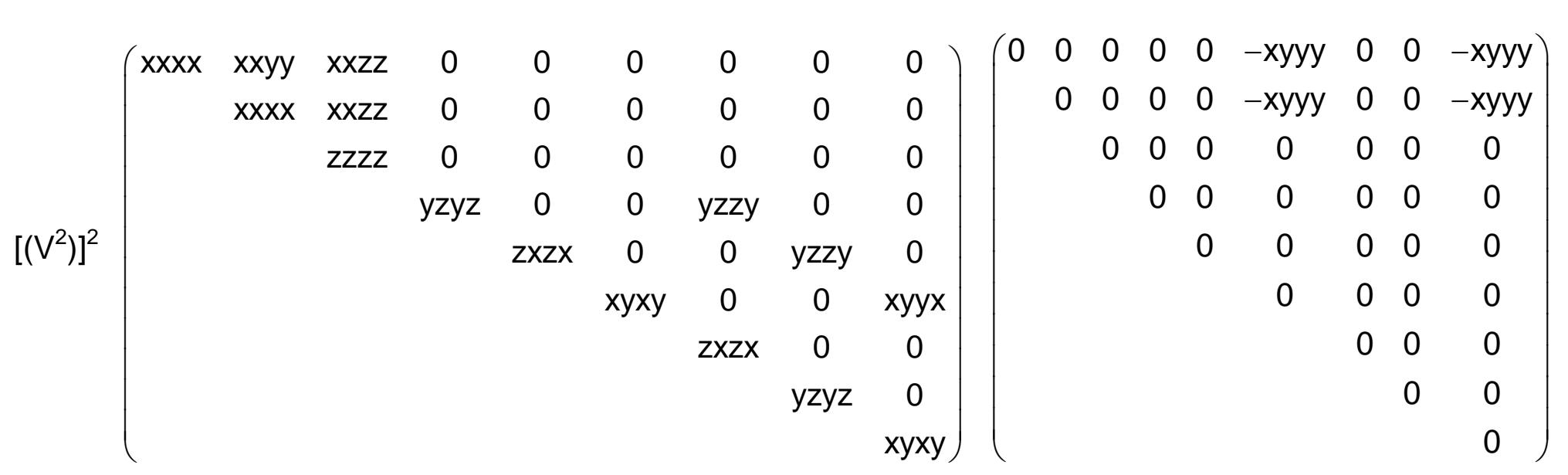

and $x x x x=x x y y+y z y z+z y z y$

\begin{tabular}{|c|c|c|c|c|c|c|c|c|c|c|c|c|c|c|c|c|}
\hline \multirow{6}{*}[\mathrm{V}^{2}]{$\mathrm{V}^{2}$} & $(x x x x$ & $x x y y$ & $x x z z$ & 0 & 0 & 0 & 0 & 0 & 0 & 0 & 0 & 0 & 0 & -xyyy & & 0 -xyyy \\
\hline & xxyy & $x x x x$ & $x x z z$ & 0 & 0 & 0 & 0 & 0 & 0 & 0 & 0 & 0 & 0 & xyyy & 0 & xyyy \\
\hline & $z z x x$ & $z z x x$ & zzzz & 0 & 0 & 0 & 0 & 0 & 0 & 0 & 0 & 0 & 0 & 0 & 0 & 0 \\
\hline & 0 & 0 & 0 & yzyz & 0 & 0 & $z x z x$ & 0 & 0 & 0 & 0 & 0 & 0 & 0 & 0 & 0 \\
\hline & 0 & 0 & 0 & 0 & $z x z x$ & 0 & 0 & yzyz & 0 & 0 & 0 & 0 & 0 & 0 & 0 & 0 \\
\hline & 0 & 0 & 0 & 0 & 0 & xyxy & 0 & 0 & хуху & $-x y y y$ & xyyy & 0 & 0 & 0 & & 0 \\
\hline
\end{tabular}

and $x x x x=x x y y+2 x y x y$
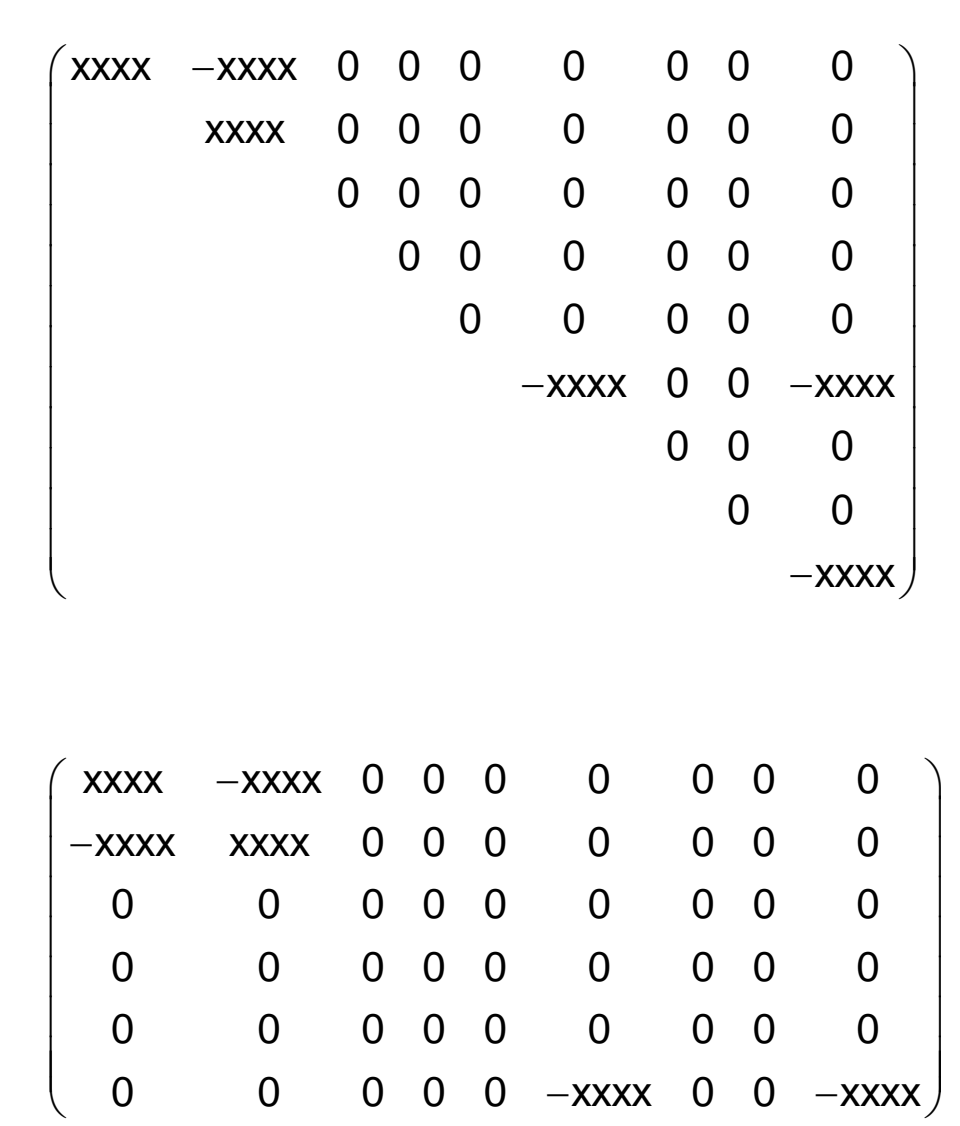

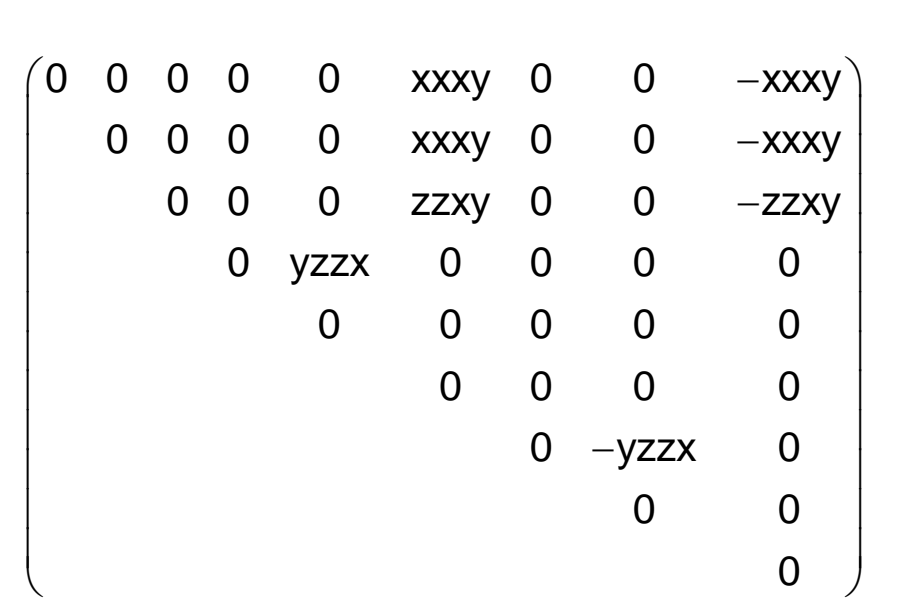

$\left(\begin{array}{ccccccccc}0 & 0 & 0 & 0 & 0 & \text { xxxy } & 0 & 0 & -\mathrm{yyxy} \\ 0 & 0 & 0 & 0 & 0 & \mathrm{yyxy} & 0 & 0 & -\mathrm{xxxy} \\ 0 & 0 & 0 & 0 & 0 & \mathrm{zzxy} & 0 & 0 & -\mathrm{zzxy} \\ 0 & 0 & 0 & 0 & \mathrm{yzzx} & 0 & 0 & -\mathrm{zxyz} & 0 \\ 0 & 0 & 0 & \text { zxyz } & 0 & 0 & -\mathrm{yzzx} & 0 & 0 \\ \mathrm{xyxx} & -\mathrm{xyxx} & 0 & 0 & 0 & 0 & 0 & 0 & 0\end{array}\right)$

and $y y x y-x x x y=2 x y x x$ 
$\left(\begin{array}{lllllllll}0 & 0 & 0 & 0 & 0 & 0 & 0 & 0 & 0 \\ 0 & 0 & 0 & 0 & 0 & 0 & 0 & 0 & 0\end{array}\right)$ $\left(\begin{array}{lllllllll}0 & 0 & 0 & 0 & 0 & 0 & 0 & 0 & 0 \\ 0 & 0 & 0 & 0 & 0 & 0 & 0 & 0 & 0\end{array}\right.$ $\begin{array}{llllllllll}0 & 0 & 0 & 0 & 0 & 0 & 0 & 0 & 0 \\ 0 & 0 & 0 & 0 & 0 & 0 & 0 & 0 & 0 & 0\end{array}$ $\begin{array}{llllllllll}0 & 0 & 0 & 0 & 0 & 0 & 0 & 0 & 0\end{array}$ $\begin{array}{llllllllll}0 & 0 & 0 & 0 & 0 & 0 & 0 & 0 & 0 \\ 0 & 0 & 0 & 0 & 0 & 0 & 0 & 0 & 0\end{array}$ $\begin{array}{lllllllllll}0 & 0 & 0 & 0 & 0 & 0 & 0 & 0 & 0 \\ 0 & 0 & 0 & 0 & 0 & 0 & 0 & 0 & 0\end{array}$

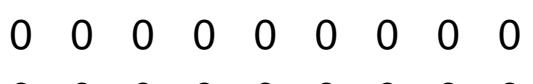

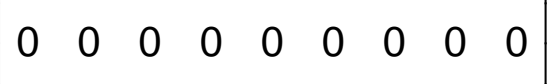

$\begin{array}{lllllllll}0 & 0 & 0 & 0 & 0 & 0 & 0 & 0 & 0 \\ 0 & 0 & 0 & 0 & 0 & 0 & 0 & 0 & 0\end{array}$

$\begin{array}{ccccccccc}z z x x & z z y y & z z z z & 0 & 0 & 0 & 0 & 0 & 0 \\ 0 & 0 & 0 & y z y z & 0 & 0 & y z z y & 0 & 0 \\ 0 & 0 & 0 & 0 & z x z x & 0 & 0 & z x y z & 0\end{array}$

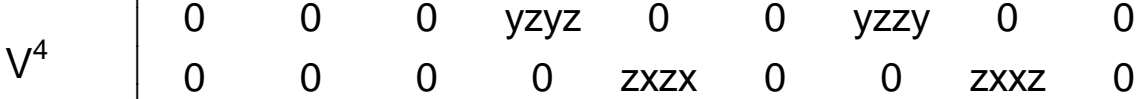

$\begin{array}{lllllllll}0 & 0 & 0 & 0 & 0 & x y x y & 0 & 0 & x y y x\end{array}$

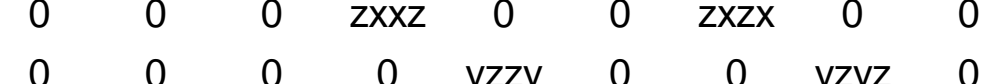

$\begin{array}{cccccccc}0 & 0 & 0 & y z z y & 0 & 0 & y z y z & 0 \\ 0 & 0 & 0 & 0 & x y y x & 0 & 0 & x y x y\end{array}$

$\left[V^{4}\right] \quad\left(\begin{array}{ccccc}3 x x y y & 0 & 0 & y y z z & 0 \\ 0 & 3 x x y y & 0 & y y z z & 0 \\ 0 & 0 & z z z z & x x y y & 0\end{array}\right)$

$\left(\begin{array}{lllll}0 & 0 & 0 & 0 & 0 \\ 0 & 0 & 0 & 0 & 0 \\ 0 & 0 & 0 & 0 & 0\end{array}\right)$

$\left(\begin{array}{llllllllll}0 & 0 & 0 & 0 & 0 & 0 & 0 & 0 & 0 & 0 \\ 0 & 0 & 0 & 0 & 0 & 0 & 0 & 0 & 0 & 0\end{array}\right)$

$\left(\begin{array}{llllllllll}0 & 0 & 0 & 0 & 0 & 0 & 0 & 0 & 0 & 0 \\ 0 & 0 & 0 & 0 & 0 & 0 & 0 & 0 & 0 & 0\end{array}\right)$

$\left.\mathrm{V}^{3} \mathrm{~V}^{3}\right]\left(\begin{array}{cccccccccc}3 \mathrm{xx} y \mathrm{y} & 0 & 0 & \mathrm{xxyy} & 0 & 0 & \mathrm{yyzz} & 0 & 0 & 0 \\ 0 & 3 x \mathrm{x} y \mathrm{y} & 0 & 0 & \mathrm{yyzz} & 0 & 0 & \mathrm{xxyy} & 0 & 0 \\ 0 & 0 & z z z & 0 & 0 & z z x & 0 & 0 & z z x & 0\end{array}\right)$

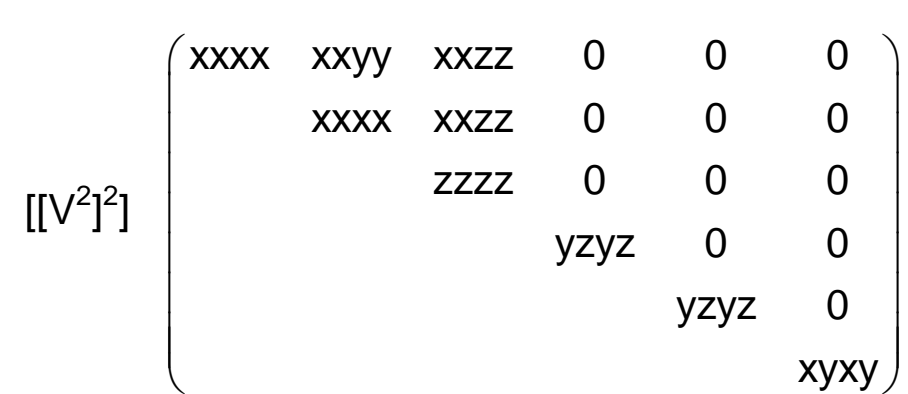

$$
\left(\begin{array}{llllll}
0 & 0 & 0 & 0 & 0 & 0 \\
& 0 & 0 & 0 & 0 & 0 \\
& & 0 & 0 & 0 & 0 \\
& & & 0 & 0 & 0 \\
& & & & 0 & 0 \\
& & & & & 0
\end{array}\right)
$$

and $x y x y=1 / 2(x x x x-x x y y)$

$\left[\mathrm{V}^{2}\right]^{2}\left(\begin{array}{cccccc}\mathrm{xxxx} & \mathrm{xxyy} & \mathrm{xxzz} & 0 & 0 & 0 \\ \mathrm{xxyy} & \mathrm{xxxx} & \mathrm{xxzz} & 0 & 0 & 0 \\ \mathrm{zzxx} & \mathrm{zzxx} & \mathrm{zzzz} & 0 & 0 & 0 \\ 0 & 0 & 0 & \mathrm{yzyz} & 0 & 0 \\ 0 & 0 & 0 & 0 & \mathrm{yzyz} & 0 \\ 0 & 0 & 0 & 0 & 0 & \mathrm{xyxy}\end{array}\right)$

$\left(\begin{array}{llllll}0 & 0 & 0 & 0 & 0 & 0 \\ 0 & 0 & 0 & 0 & 0 & 0 \\ 0 & 0 & 0 & 0 & 0 & 0 \\ 0 & 0 & 0 & 0 & 0 & 0 \\ 0 & 0 & 0 & 0 & 0 & 0 \\ 0 & 0 & 0 & 0 & 0 & 0\end{array}\right)$

and $x y x y=1 / 2(x x x x-x x y y)$ $\begin{array}{lllllllll}0 & 0 & 0 & 0 & 0 & 0 & 0 & 0 & 0 \\ 0 & 0 & 0 & 0 & 0 & 0 & 0 & 0 & 0\end{array}$

$\begin{array}{llllllllll}0 & 0 & 0 & 0 & 0 & 0 & 0 & 0 & 0 \\ 0 & 0 & 0 & 0 & 0 & 0 & 0 & 0 & 0\end{array}$

$\begin{array}{llllllllll}0 & 0 & 0 & 0 & 0 & 0 & 0 & 0 & 0 \\ 0 & 0 & 0 & 0 & 0 & 0 & 0 & 0 & 0\end{array}$

$0 \begin{array}{lllllllll}0 & 0 & 0 & 0 & 0 & 0 & 0 & 0 & 0\end{array}$

$\begin{array}{llllllllll}0 & 0 & 0 & 0 & 0 & 0 & 0 & 0 & 0 \\ 0 & 0 & 0 & 0 & 0 & 0 & 0 & 0\end{array}$

$0 \begin{array}{lllllllll}0 & 0 & 0 & 0 & 0 & 0 & 0 & 0 & 0 \\ 0 & 0 & 0 & 0 & 0 & 0 & 0 & 0 & 0\end{array}$

$0 \begin{array}{llllllllll}0 & 0 & 0 & 0 & 0 & 0 & 0 & 0 & 0 \\ 0 & 0 & 0 & 0 & 0 & 0 & 0 & 0 & 0\end{array}$

$\begin{array}{lllllllll}0 & 0 & 0 & 0 & 0 & 0 & 0 & 0 & 0 \\ 0 & 0 & 0 & 0 & 0 & 0 & 0 & 0 & 0\end{array}$

$\begin{array}{lllllllll}0 & 0 & 0 & 0 & 0 & 0 & 0 & 0 & 0\end{array}$

$\left(\begin{array}{lllll}0 & 0 & 0 & 0 & 0 \\ 0 & 0 & 0 & 0 & 0 \\ 0 & 0 & 0 & 0 & 0\end{array}\right)$

$\left(\begin{array}{llllllllll}0 & 0 & 0 & 0 & 0 & 0 & 0 & 0 & 0 & 0 \\ 0 & 0 & 0 & 0 & 0 & 0 & 0 & 0 & 0 & 0\end{array}\right.$ $\left.\begin{array}{lllllllllll}0 & 0 & 0 & 0 & 0 & 0 & 0 & 0 & 0 & 0\end{array}\right)$ $\left(\begin{array}{llllllllll}0 & 0 & 0 & 0 & 0 & 0 & 0 & 0 & 0 & 0 \\ 0 & 0 & 0 & 0 & 0 & 0 & 0 & 0 & 0 & 0 \\ 0 & 0 & 0 & 0 & 0 & 0 & 0 & 0 & 0 & 0\end{array}\right)$

$$
\begin{aligned}
& \left(\begin{array}{llllll}
0 & 0 & 0 & 0 & 0 & 0 \\
& 0 & 0 & 0 & 0 & 0
\end{array}\right) \\
& \begin{array}{llll}
0 & 0 & 0 & 0
\end{array} \\
& \begin{array}{lll}
0 & 0 & 0 \\
& 0 & 0
\end{array} \\
& 0
\end{aligned}
$$

$$
\left(\begin{array}{llllll}
0 & 0 & 0 & 0 & 0 & 0 \\
0 & 0 & 0 & 0 & 0 & 0 \\
0 & 0 & 0 & 0 & 0 & 0 \\
0 & 0 & 0 & 0 & 0 & 0 \\
0 & 0 & 0 & 0 & 0 & 0 \\
0 & 0 & 0 & 0 & 0 & 0
\end{array}\right)
$$

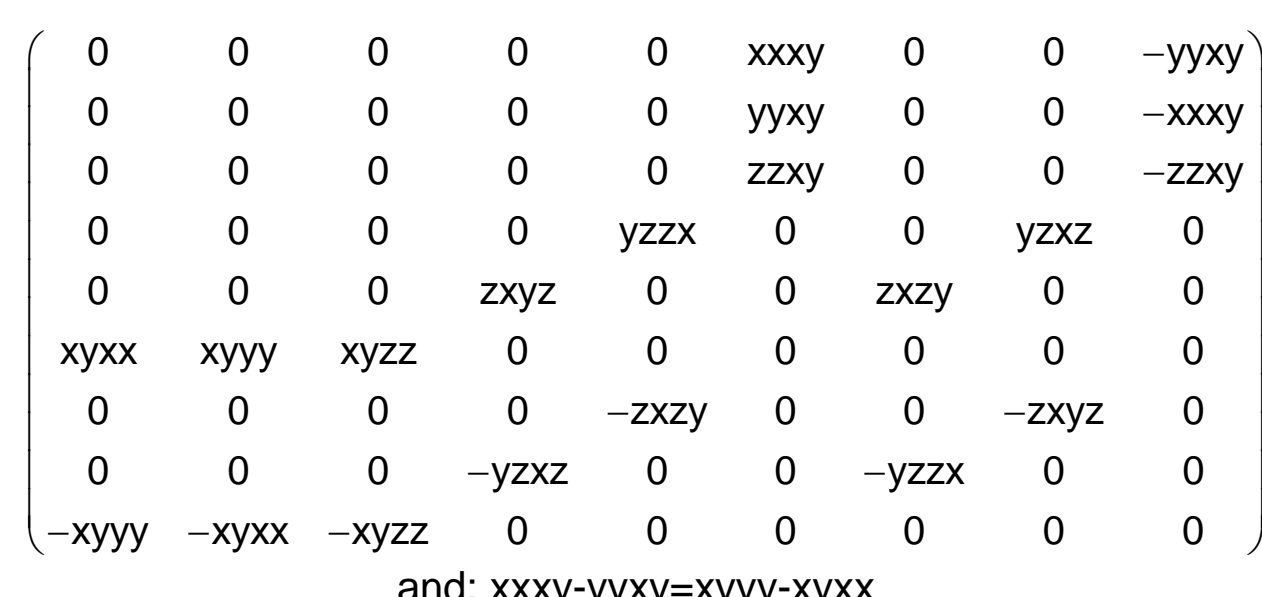

$\left(\begin{array}{lllll}0 & 0 & 0 & 0 & 0 \\ 0 & 0 & 0 & 0 & 0 \\ 0 & 0 & 0 & 0 & 0\end{array}\right)$

$\left(\begin{array}{cccccccccc}0 & 3 x y x x & 0 & 0 & x y z z & 0 & 0 & x y x x & 0 & 0 \\ -3 x y x x & 0 & 0 & -x y x x & 0 & 0 & -x y z z & 0 & 0 & 0 \\ 0 & 0 & 0 & 0 & 0 & 0 & 0 & 0 & 0 & 2 x y z\end{array}\right)$

$\begin{array}{llllllllll}0 & 0 & 0 & 0 & 0 & 0 & 0 & 0 & 0 & \text { zxyz }\end{array}$

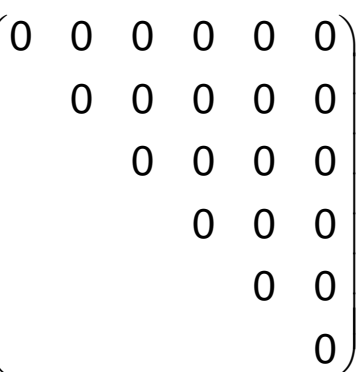

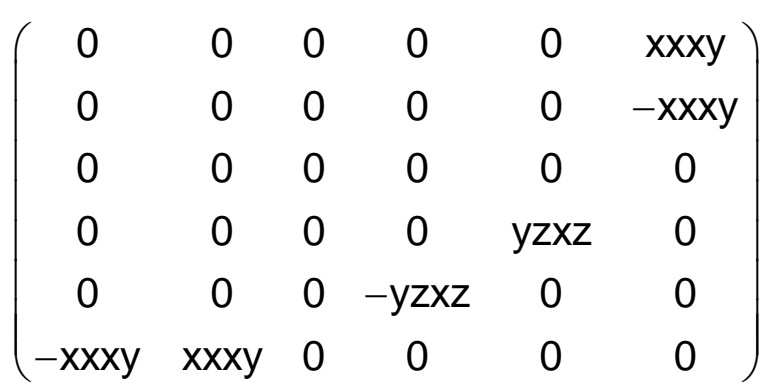




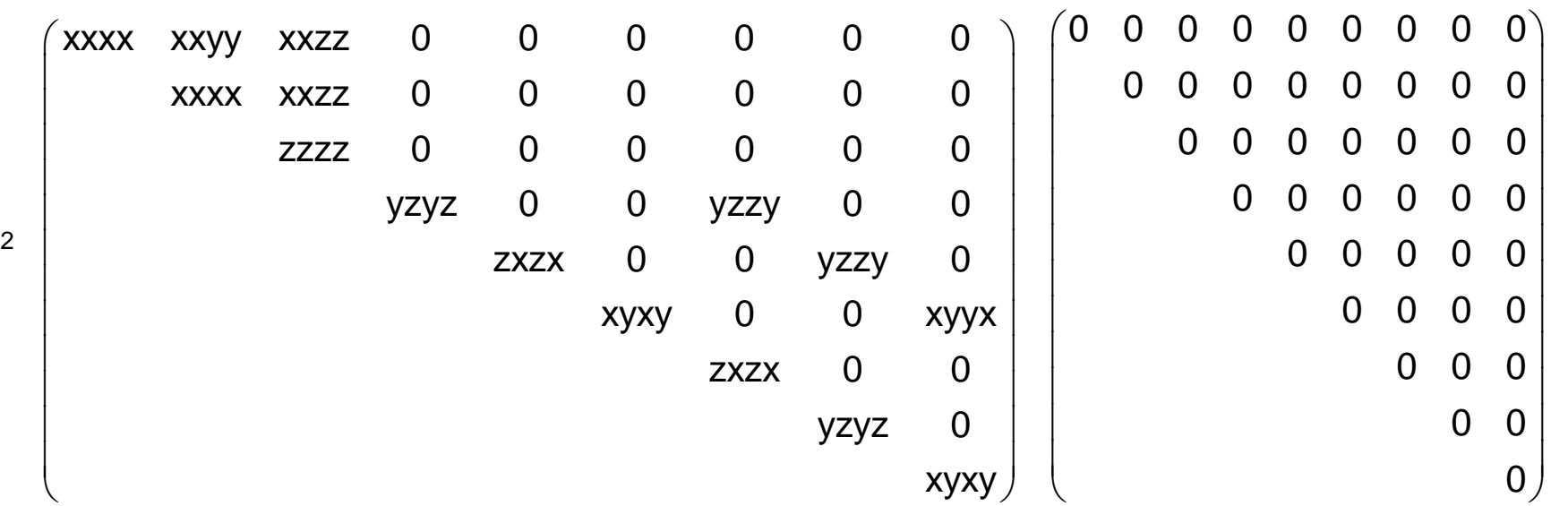

\section{and $x x x x=x x y y+y z y z+z y z y$}

$\left(\begin{array}{lllllllll}x x x x & x x y y & x x z z & 0 & 0 & 0 & 0 & 0 & 0 \\ x x y y & x x y & x y z & 0 & 0 & 0 & 0 & 0 & 0\end{array}\right)\left(\begin{array}{ccccccccc}0 & 0 & 0 & 0 & 0 & 0 & 0 & 0 & 0 \\ 0 & 0 & 0 & 0 & 0 & 0 & 0 & 0 & 0 \\ 0 & 0 & 0 & 0 & 0 & 0 & 0 & 0 & 0\end{array}\right)$

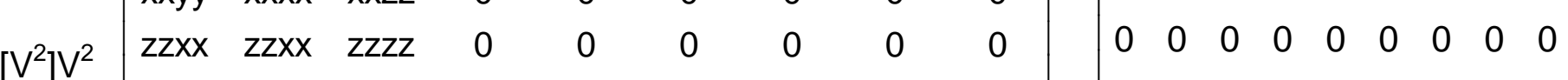

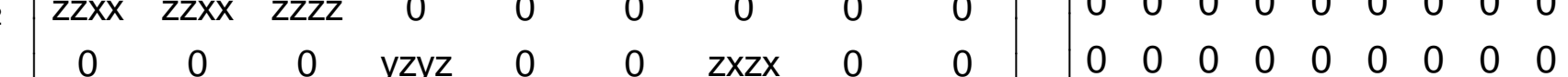

$\left(\begin{array}{ccccccccc}0 & 0 & 0 & y z y z & 0 & 0 & z x z x & 0 & 0 \\ 0 & 0 & 0 & 0 & z x z x & 0 & 0 & y z y z & 0 \\ 0 & 0 & 0 & 0 & 0 & x y x y & 0 & 0 & x y x y\end{array}\right)\left(\begin{array}{ccccccccc}0 & 0 & 0 & 0 & 0 & 0 & 0 & 0 & 0 \\ 0 & 0 & 0 & 0 & 0 & 0 & 0 & 0 & 0 \\ 0 & 0 & 0 & 0 & 0 & 0 & 0 & 0 & 0\end{array}\right)$

$\left.\begin{array}{lllcccccc}0 & 0 & 0 & 0 & 0 & x y x y & 0 & 0 & x y x y\end{array}\right)\left(\begin{array}{ccccccccc}0 & 0 & 0 & 0 & 0 & 0 & 0 & 0 & 0 \\ 0 & 0 & 0 & 0 & 0 & 0 & 0 & 0 & 0\end{array}\right)$

and $x x x x=x x y y+2 x y x y$

$$
\left(\begin{array}{lllllllll}
0 & 0 & 0 & 0 & 0 & 0 & 0 & 0 & 0 \\
& 0 & 0 & 0 & 0 & 0 & 0 & 0 & 0 \\
& & 0 & 0 & 0 & 0 & 0 & 0 & 0 \\
& & & 0 & 0 & 0 & 0 & 0 & 0 \\
& & & & 0 & 0 & 0 & 0 & 0 \\
& & & & & 0 & 0 & 0 & 0 \\
& & & & & & 0 & 0 & 0 \\
& & & & & & & 0 & 0 \\
& & & & & & & 0 & 0 \\
& & & & & & & & 0
\end{array}\right)
$$

$\left(\begin{array}{lllllllll}0 & 0 & 0 & 0 & 0 & 0 & 0 & 0 & 0 \\ 0 & 0 & 0 & 0 & 0 & 0 & 0 & 0 & 0\end{array}\right.$

$0 \begin{array}{lllllllll}0 & 0 & 0 & 0 & 0 & 0 & 0 & 0 & 0 \\ 0 & 0 & 0 & 0 & 0 & 0 & 0 & 0 & 0\end{array}$

$0 \begin{array}{llllllllll}0 & 0 & 0 & 0 & 0 & 0 & 0 & 0 & 0 \\ 0 & 0 & 0 & 0 & 0 & 0 & 0 & 0 & 0\end{array}$

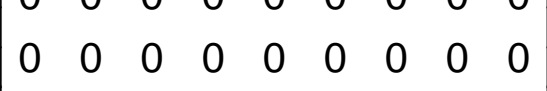

$\begin{array}{lllllllll}0 & 0 & 0 & 0 & 0 & 0 & 0 & 0 & 0 \\ 0 & 0 & 0 & 0 & 0 & 0 & 0 & 0 & 0\end{array}$

$\begin{array}{lllllllll}0 & 0 & 0 & 0 & 0 & 0 & 0 & 0 & 0\end{array}$

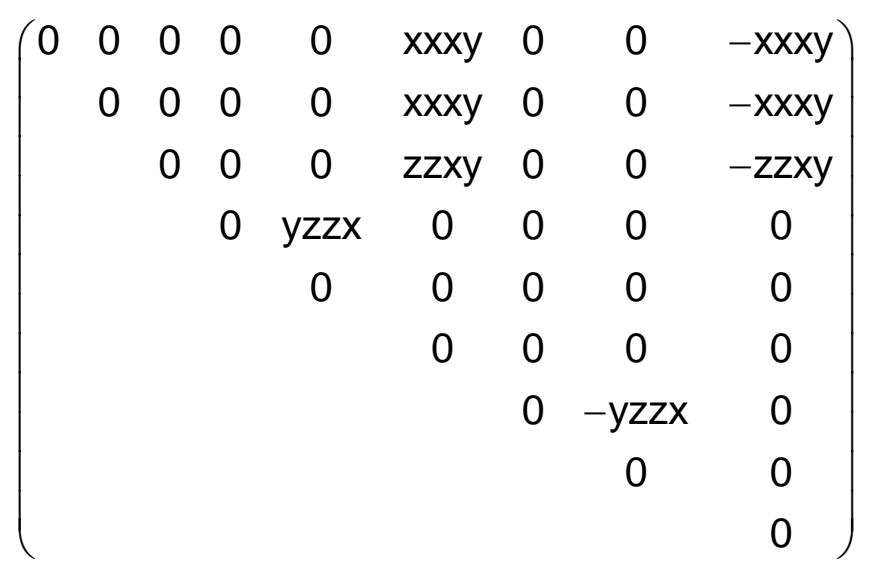

$\left(\begin{array}{ccccccccc}0 & 0 & 0 & 0 & 0 & \text { xxxy } & 0 & 0 & - \text { yyxy } \\ 0 & 0 & 0 & 0 & 0 & \text { yyxy } & 0 & 0 & - \text { xxxy } \\ 0 & 0 & 0 & 0 & 0 & \text { zzxy } & 0 & 0 & - \text { zzxy } \\ 0 & 0 & 0 & 0 & \text { yzzx } & 0 & 0 & -z x y z & 0 \\ 0 & 0 & 0 & \text { zxyz } & 0 & 0 & -y z z x & 0 & 0 \\ \text { xyxx } & -x y x x & 0 & 0 & 0 & 0 & 0 & 0 & 0\end{array}\right)$

and: $y y x y-x x x y=2 x y x x$ 
e

$\left(\begin{array}{ccccccccc}\text { xxxx } & \text { xxyy } & \text { xxzz } & 0 & 0 & 0 & 0 & 0 & 0 \\ \text { yyxx } & \text { yyyy } & \text { yyzz } & 0 & 0 & 0 & 0 & 0 & 0 \\ \text { zzxx } & \text { zzyy } & \text { zzzz } & 0 & 0 & 0 & 0 & 0 & 0 \\ 0 & 0 & 0 & \text { yzyz } & 0 & 0 & \text { yzzy } & 0 & 0 \\ 0 & 0 & 0 & 0 & \text { zxzx } & 0 & 0 & \text { zxxz } & 0 \\ 0 & 0 & 0 & 0 & 0 & \text { xyxy } & 0 & 0 & \text { xyyx } \\ 0 & 0 & 0 & \text { zyyz } & 0 & 0 & \text { zyzy } & 0 & 0 \\ 0 & 0 & 0 & 0 & \text { xzzx } & 0 & 0 & \text { xzxz } & 0 \\ 0 & 0 & 0 & 0 & 0 & \text { yxxy } & 0 & 0 & \text { yxyx }\end{array}\right)$

$\left[V^{4}\right] \quad\left(\begin{array}{ccccc}x x x x & 0 & 0 & y y z z & 0 \\ 0 & y y y y & 0 & z z x x & 0 \\ 0 & 0 & z z z z & x x y y & 0\end{array}\right)$

$V\left[V^{3}\right]\left(\begin{array}{cccccccccc}\mathrm{xxxx} & 0 & 0 & \mathrm{xxyy} & 0 & 0 & \mathrm{xxzz} & 0 & 0 & 0 \\ 0 & \mathrm{yyyy} & 0 & 0 & \mathrm{yyzz} & 0 & 0 & \mathrm{yyxx} & 0 & 0 \\ 0 & 0 & z z z z & 0 & 0 & \mathrm{zzxx} & 0 & 0 & z z y & 0\end{array}\right)$

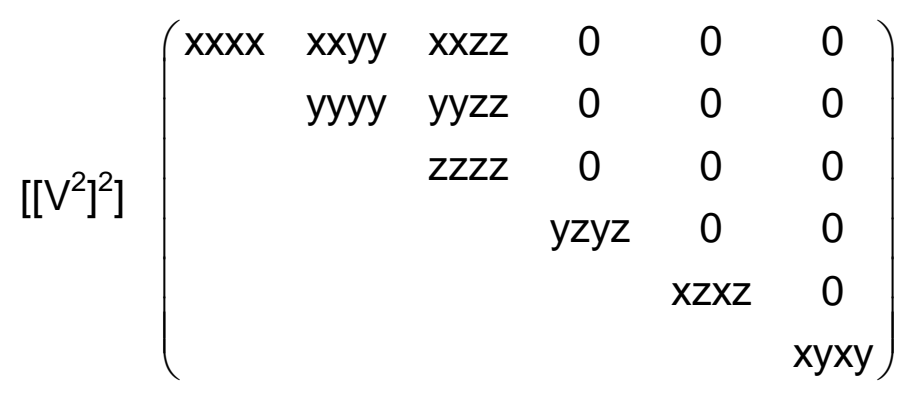

$\left[\mathrm{V}^{2}\right]^{2}$

$\left(\begin{array}{cccccc}x x x x & x x y y & x x z z & 0 & 0 & 0 \\ \text { yyxx } & \text { yyyy } & \text { yyzz } & 0 & 0 & 0 \\ \text { zzxx } & \text { zzyy } & \text { zzzz } & 0 & 0 & 0 \\ 0 & 0 & 0 & y z y z & 0 & 0 \\ 0 & 0 & 0 & 0 & x z x z & 0 \\ 0 & 0 & 0 & 0 & 0 & \text { xyxy }\end{array}\right)$

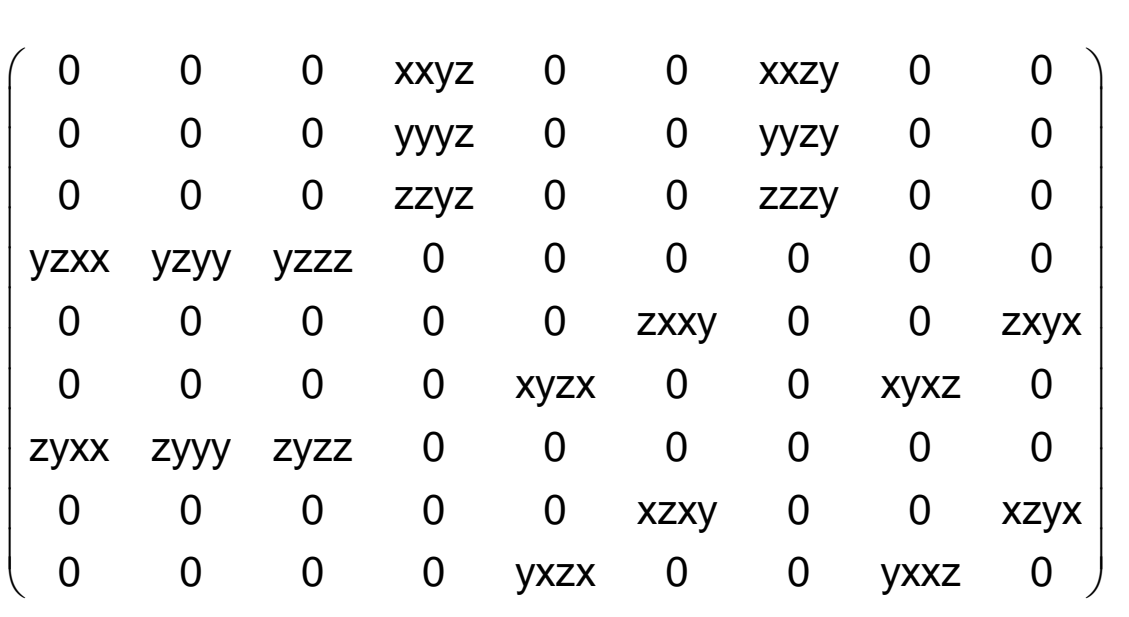

$\left(\begin{array}{ccccc}0 & 0 & 0 & 0 & x x y z \\ 0 & 0 & y z z z & 0 & 0 \\ 0 & z y y y & 0 & 0 & 0\end{array}\right)$

$\left(\begin{array}{cccccccccc}0 & 0 & 0 & 0 & 0 & 0 & 0 & 0 & 0 & x x y z \\ 0 & 0 & y z z z & 0 & 0 & y z x x & 0 & 0 & y z y & 0\end{array}\right)$ $\left(\begin{array}{cccccccccc}0 & 0 & y z z z & 0 & 0 & y z x x & 0 & 0 & \text { yzyy } & 0 \\ 0 & z y y y & 0 & 0 & z y z & 0 & 0 & z y x x & 0 & 0\end{array}\right)$

$$
\left(\begin{array}{cccccc}
0 & 0 & 0 & \text { xxyz } & 0 & 0 \\
& 0 & 0 & \text { yyyz } & 0 & 0 \\
& & 0 & \text { zzyz } & 0 & 0 \\
& & & 0 & 0 & 0 \\
& & & & 0 & \text { xzxy } \\
& & & & & 0
\end{array}\right)
$$

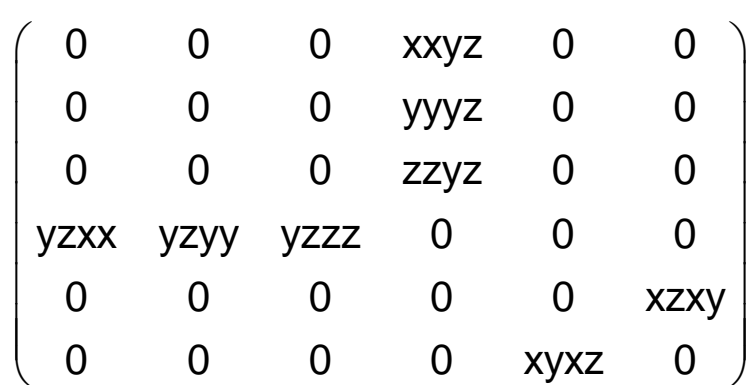

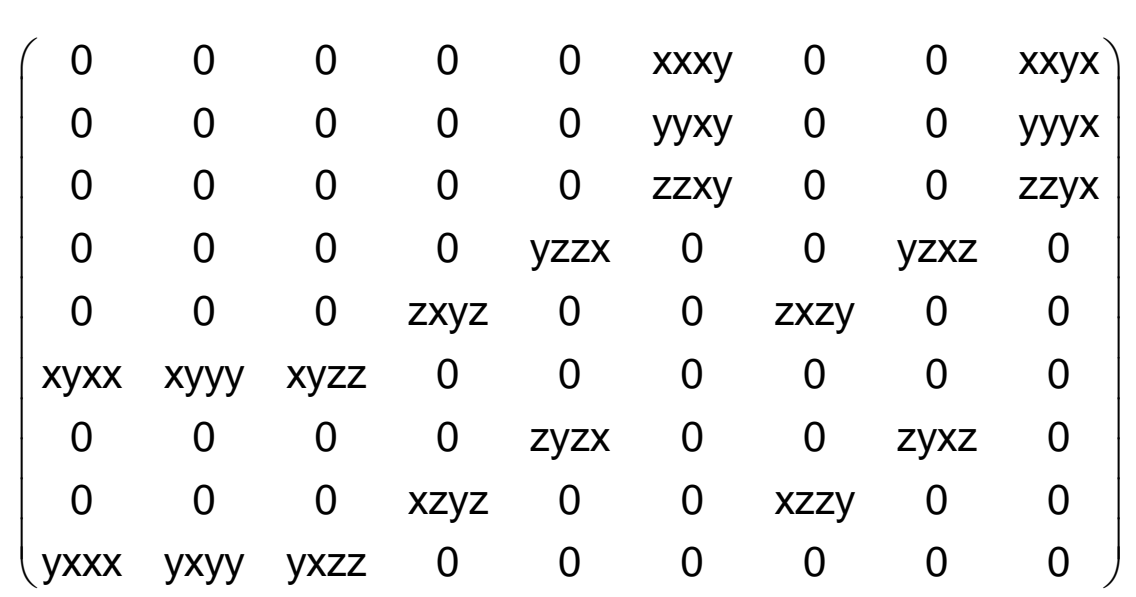

$\left(\begin{array}{ccccc}0 & x y y y & 0 & 0 & 0 \\ y x x x & 0 & 0 & 0 & 0 \\ 0 & 0 & 0 & 0 & z z x y\end{array}\right)$

$\left(\begin{array}{cccccccccc}0 & x y y y & 0 & 0 & x y z z & 0 & 0 & x y x x & 0 & 0 \\ y x x x & 0 & 0 & y x y y & 0 & 0 & y x z z & 0 & 0 & 0 \\ 0 & 0 & 0 & 0 & 0 & 0 & 0 & 0 & 0 & z x y z\end{array}\right)$

$\left(\begin{array}{cccccccccc}\mathrm{yxxx} & 0 & 0 & \mathrm{yxyy} & 0 & 0 & \mathrm{yxzz} & 0 & 0 & 0 \\ 0 & 0 & 0 & 0 & 0 & 0 & 0 & 0 & 0 & \mathrm{zxyz}\end{array}\right)$
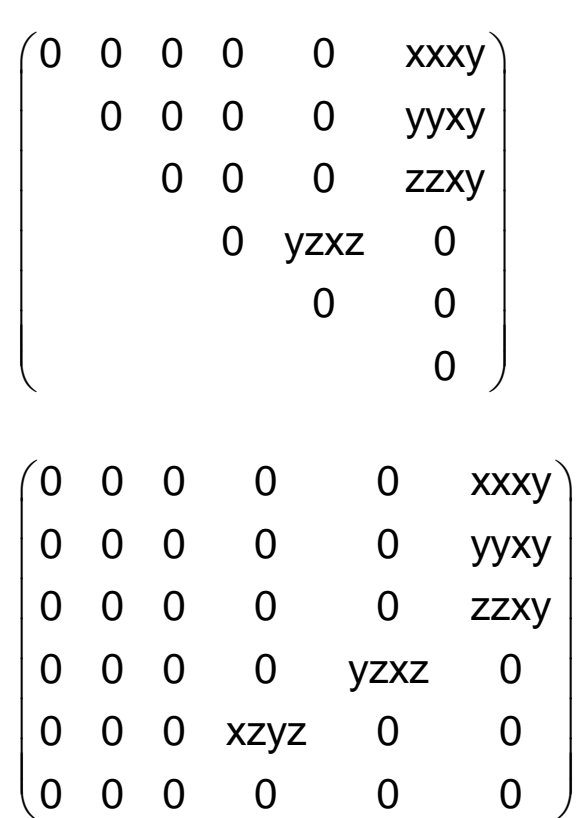

$\left(\begin{array}{ccccccccc}0 & 0 & 0 & 0 & \text { xxzx } & 0 & 0 & \text { xxxz } & 0 \\ 0 & 0 & 0 & 0 & \text { yyzx } & 0 & 0 & \text { yyxz } & 0 \\ 0 & 0 & 0 & 0 & \text { zzzx } & 0 & 0 & \text { zzxz } & 0 \\ 0 & 0 & 0 & 0 & 0 & \text { yzxy } & 0 & 0 & \text { yzyx } \\ \text { zxxx } & \text { zxyy } & \text { zxzz } & 0 & 0 & 0 & 0 & 0 & 0 \\ 0 & 0 & 0 & \text { xyyz } & 0 & 0 & \text { xyzy } & 0 & 0 \\ 0 & 0 & 0 & 0 & 0 & \text { zyxy } & 0 & 0 & \text { zyyx } \\ \text { xzxx } & \text { xzyy } & \text { xzzz } & 0 & 0 & 0 & 0 & 0 & 0 \\ 0 & 0 & 0 & \text { yxyz } & 0 & 0 & \text { yxzy } & 0 & 0\end{array}\right)$

$\left(\begin{array}{ccccc}0 & 0 & \text { xzzz } & 0 & 0 \\ 0 & 0 & 0 & 0 & y y x z \\ z x x x & 0 & 0 & 0 & 0\end{array}\right)$

$\left(\begin{array}{cccccccccc}0 & 0 & x z z z & 0 & 0 & x z x x & 0 & 0 & x z y y & 0 \\ 0 & 0 & 0 & 0 & 0 & 0 & 0 & 0 & 0 & y x y z\end{array}\right)$ $\left.\begin{array}{cccccccccc}0 & 0 & 0 & 0 & 0 & 0 & 0 & 0 & 0 & y x y z \\ z x x x & 0 & 0 & z x y y & 0 & 0 & z x z z & 0 & 0 & 0\end{array}\right)$ $\left(\begin{array}{cccccc}0 & 0 & 0 & 0 & \mathrm{xxxz} & 0 \\ & 0 & 0 & 0 & \mathrm{yyxz} & 0 \\ & & 0 & 0 & \mathrm{zzxz} & 0 \\ & & & 0 & 0 & \mathrm{yzxy} \\ & & & & 0 & 0 \\ & & & & & 0\end{array}\right)$

$\left(\begin{array}{cccccc}0 & 0 & 0 & 0 & \text { xxxz } & 0 \\ 0 & 0 & 0 & 0 & \text { yyxz } & 0 \\ 0 & 0 & 0 & 0 & z z x z & 0 \\ 0 & 0 & 0 & 0 & 0 & y z x y \\ \text { xzxx } & \text { xzyy } & \text { xzzz } & 0 & 0 & 0 \\ 0 & 0 & 0 & \text { xyyz } & 0 & 0\end{array}\right)$


$\mathrm{n}=1 \quad \mathbf{D}_{1 \mathrm{~h}}\left(\mathbf{C}_{1 \mathrm{~h}}\right)=\mathbf{2}_{\mathrm{x}} \mathbf{m}_{\mathrm{y}} \mathbf{m}_{z}\left(\mathbf{m}_{z}\right)=\mathbf{2}_{\mathrm{x}} \mathbf{m}_{\mathrm{y}} \mathbf{m}_{z}$

$\mathbf{D}_{\mathrm{nh}}\left(\mathbf{C}_{\mathrm{nh}}\right)$

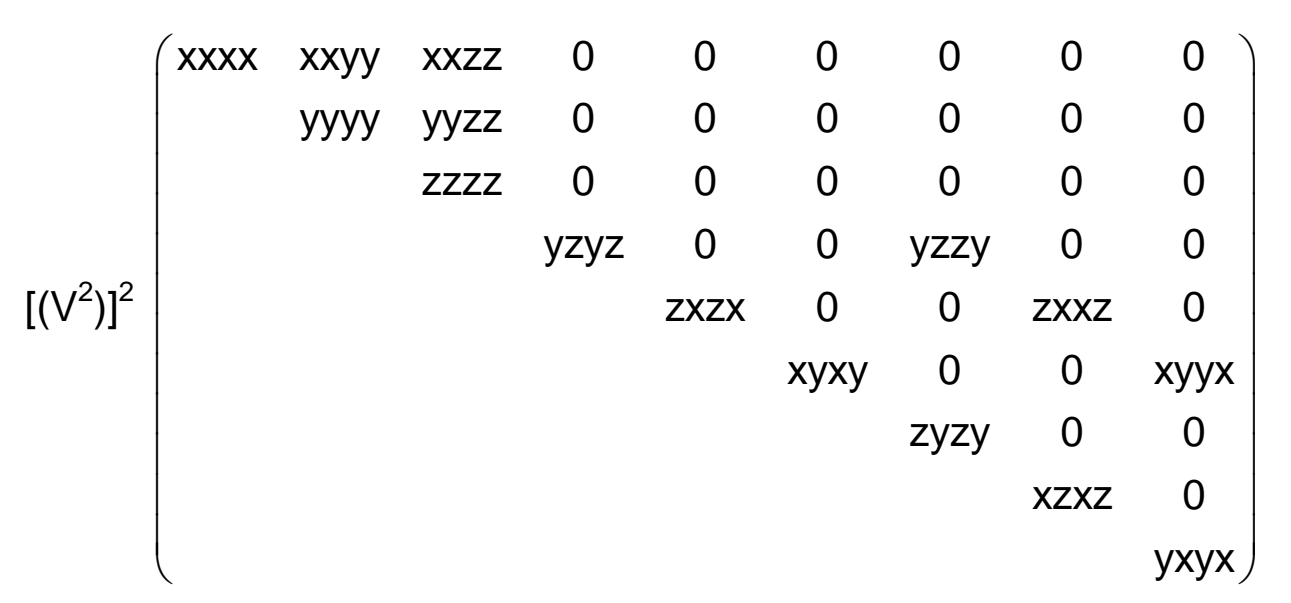

$$
\left(\begin{array}{ccccccccc}
0 & 0 & 0 & \text { xxyz } & 0 & 0 & \text { xxzy } & 0 & 0 \\
& 0 & 0 & \text { yyyz } & 0 & 0 & \text { yyzy } & 0 & 0 \\
& & 0 & z z y z & 0 & 0 & \text { zzzy } & 0 & 0 \\
& & & 0 & 0 & 0 & 0 & 0 & 0 \\
& & & & 0 & \text { zxxy } & 0 & 0 & z x y x \\
& & & & & 0 & 0 & \text { xyxz } & 0 \\
& & & & & & 0 & 0 & 0 \\
& & & & & & & 0 & \text { xzyx } \\
& & & & & & & &
\end{array}\right)
$$

$\left.\begin{array}{c}0 \\ 0 \\ 0 \\ 0 \\ \mathrm{xyx} \\ 0 \\ 0 \\ \mathrm{xzyx} \\ 0\end{array}\right)$

$$
\left(\begin{array}{ccccccccc}
0 & 0 & 0 & 0 & 0 & \text { xxxy } & 0 & 0 & x x y x \\
& 0 & 0 & 0 & 0 & y y x y & 0 & 0 & y y y x \\
& & 0 & 0 & 0 & \text { zzxy } & 0 & 0 & \text { zzyx } \\
& & & 0 & y z z x & 0 & 0 & y z x z & 0 \\
& & & & 0 & 0 & \text { zxzy } & 0 & 0 \\
& & & & & 0 & 0 & 0 & 0 \\
& & & & & & 0 & z y x z & 0 \\
& & & & & & & 0 & 0 \\
& & & & & & & & 0
\end{array}\right)
$$

$\left[V^{2}\right] \mathrm{V}^{2}\left(\begin{array}{ccccccccc}\mathrm{xxxx} & \mathrm{xxyy} & \mathrm{xxzz} & 0 & 0 & 0 & 0 & 0 & 0 \\ \mathrm{yyxx} & \mathrm{yyyy} & \mathrm{yyzz} & 0 & 0 & 0 & 0 & 0 & 0 \\ \mathrm{zzxx} & \mathrm{zzyy} & \mathrm{zzzz} & 0 & 0 & 0 & 0 & 0 & 0 \\ 0 & 0 & 0 & \mathrm{yzyz} & 0 & 0 & \mathrm{yzzy} & 0 & 0 \\ 0 & 0 & 0 & 0 & \mathrm{zxzx} & 0 & 0 & \mathrm{zxxz} & 0 \\ 0 & 0 & 0 & 0 & 0 & \mathrm{xyxy} & 0 & 0 & \text { xyyx }\end{array}\right)$

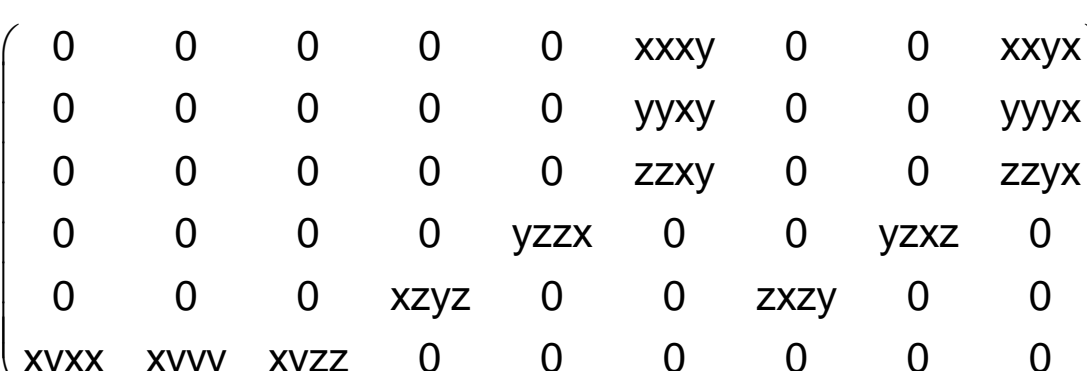

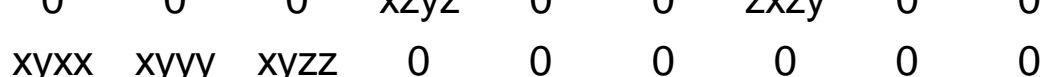

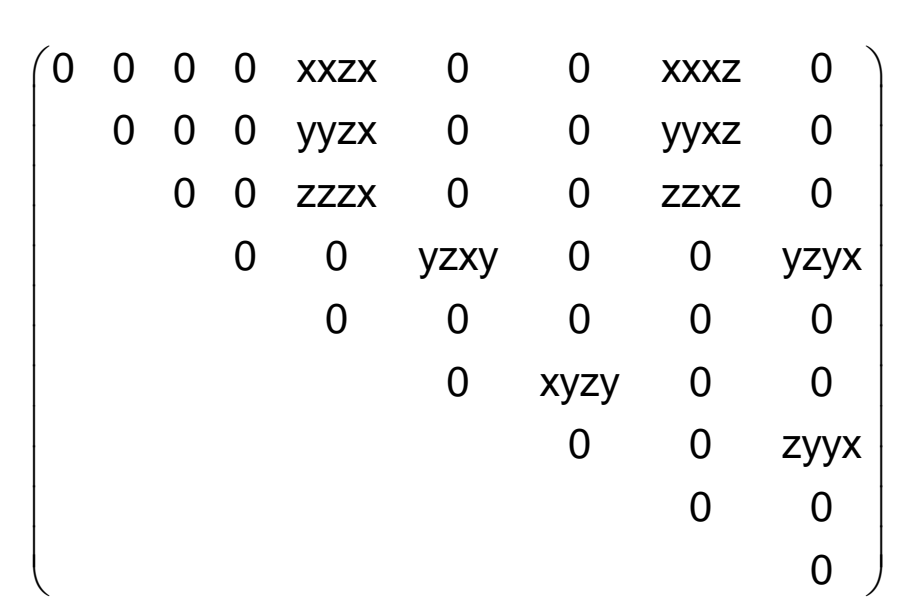

$\left(\begin{array}{ccccccccc}0 & 0 & 0 & 0 & \text { xxzx } & 0 & 0 & \text { xxxz } & 0 \\ 0 & 0 & 0 & 0 & \text { yyzx } & 0 & 0 & \text { yyxz } & 0 \\ 0 & 0 & 0 & 0 & \text { zzzx } & 0 & 0 & \text { zzxz } & 0 \\ 0 & 0 & 0 & 0 & 0 & \text { yzxy } & 0 & 0 & \text { yzyx } \\ \text { zxxx } & \text { zxyy } & \text { zxzz } & 0 & 0 & 0 & 0 & 0 & 0 \\ 0 & 0 & 0 & \text { xyyz } & 0 & 0 & \text { xyzy } & 0 & 0\end{array}\right)$ 


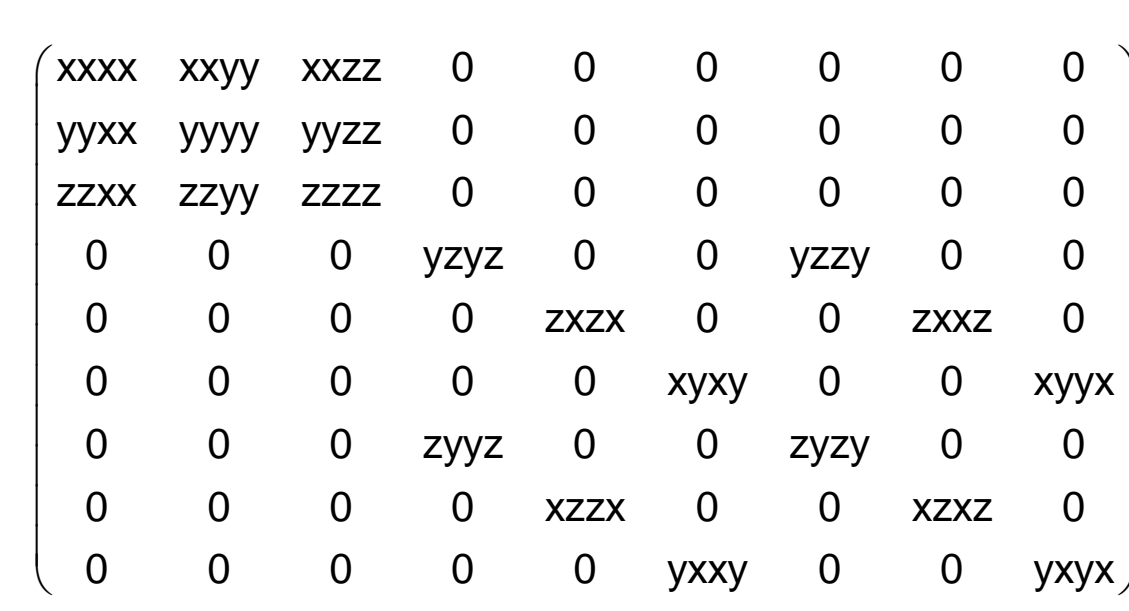

$\left(\begin{array}{lllllllll}0 & 0 & 0 & 0 & 0 & 0 & 0 & 0 & 0 \\ 0 & 0 & 0 & 0 & 0 & 0 & 0 & 0 & 0\end{array}\right.$ $\begin{array}{lllllllll}0 & 0 & 0 & 0 & 0 & 0 & 0 & 0 & 0 \\ 0 & 0 & 0 & 0 & 0 & 0 & 0 & 0 & 0\end{array}$ $\begin{array}{lllllllll}0 & 0 & 0 & 0 & 0 & 0 & 0 & 0 & 0 \\ 0 & 0 & 0 & 0 & 0 & 0 & 0 & 0 & 0\end{array}$ $0 \begin{array}{lllllllll}0 & 0 & 0 & 0 & 0 & 0 & 0 & 0 & 0 \\ 0 & 0 & 0 & 0 & 0 & 0 & 0 & 0 & 0\end{array}$ $0 \begin{array}{llllllllll}0 & 0 & 0 & 0 & 0 & 0 & 0 & 0 & 0 \\ 0 & 0 & 0 & 0 & 0 & 0 & 0 & 0 & 0\end{array}$ $0 \begin{array}{llllllllll}0 & 0 & 0 & 0 & 0 & 0 & 0 & 0 & 0 \\ 0 & 0 & 0 & 0 & 0 & 0 & 0 & 0\end{array}$ $\begin{array}{llllllllll}0 & 0 & 0 & 0 & 0 & 0 & 0 & 0 & 0\end{array}$ $\begin{array}{lllllllll}0 & 0 & 0 & 0 & 0 & 0 & 0 & 0 & 0 \\ 0 & 0 & 0 & 0 & 0 & 0 & 0 & 0 & 0\end{array}$ $\left(\begin{array}{lllllllll}0 & 0 & 0 & 0 & 0 & 0 & 0 & 0 & 0 \\ 0 & 0 & 0 & 0 & 0 & 0 & 0 & 0 & 0\end{array}\right)$

$\left[V^{4}\right] \quad\left(\begin{array}{ccccc}\mathrm{xxxx} & 0 & 0 & \mathrm{yyzz} & 0 \\ 0 & \mathrm{yyyy} & 0 & \mathrm{zzxx} & 0 \\ 0 & 0 & \mathrm{zzzz} & \mathrm{xxyy} & 0\end{array}\right)$

$$
\left(\begin{array}{lllll}
0 & 0 & 0 & 0 & 0 \\
0 & 0 & 0 & 0 & 0 \\
0 & 0 & 0 & 0 & 0
\end{array}\right)
$$

$\left.V^{3}\right]\left(\begin{array}{cccccccccc}x x x x & 0 & 0 & x x y y & 0 & 0 & x x z z & 0 & 0 & 0 \\ 0 & y y y y & 0 & 0 & y y z z & 0 & 0 & y y x x & 0 & 0 \\ 0 & 0 & z z z z & 0 & 0 & z z x & 0 & 0 & z y y & 0\end{array}\right)$

V[V] $\left(\begin{array}{ccccccccccc}0 & y y y z & 0 & 0 & y y z z & 0 & 0 & y y x x & 0 & 0 \\ 0 & 0 & z z z z & 0 & 0 & z z x x & 0 & 0 & \text { zzyy } & 0\end{array}\right)$

$\left(\begin{array}{llllllllll}0 & 0 & 0 & 0 & 0 & 0 & 0 & 0 & 0 & 0 \\ 0 & 0 & 0 & 0 & 0 & 0 & 0 & 0 & 0 & 0\end{array}\right)$ $\left.\begin{array}{llllllllll}0 & 0 & 0 & 0 & 0 & 0 & 0 & 0 & 0 & 0 \\ 0 & 0 & 0 & 0 & 0 & 0 & 0 & 0 & 0 & 0\end{array}\right)$

$\left[\left[^{2}\right]^{2}\right]\left(\begin{array}{cccccc}x x x x & x x y y & x x z z & 0 & 0 & 0 \\ & \text { yyyy } & \text { yyzz } & 0 & 0 & 0 \\ & & z z z z & 0 & 0 & 0 \\ & & & y z y z & 0 & 0 \\ & & & & x z x z & 0 \\ & & & & & x y x y\end{array}\right)$

$$
\left(\begin{array}{llllll}
0 & 0 & 0 & 0 & 0 & 0 \\
& 0 & 0 & 0 & 0 & 0 \\
& & 0 & 0 & 0 & 0 \\
& & & 0 & 0 & 0 \\
& & & & 0 & 0 \\
& & & & & 0
\end{array}\right)
$$

$\left(\begin{array}{llllll}0 & 0 & 0 & 0 & 0 & 0 \\ 0 & 0 & 0 & 0 & 0 & 0\end{array}\right.$ $\left.\begin{array}{llllll}0 & 0 & 0 & 0 & 0 & 0 \\ 0 & 0 & 0 & 0 & 0 & 0 \\ 0 & 0 & 0 & 0 & 0 & 0\end{array}\right)$ $0 \begin{array}{llllll}0 & 0 & 0 & 0 & 0 & 0\end{array}$ $\begin{array}{llllll}0 & 0 & 0 & 0 & 0 & 0 \\ 0 & 0 & 0 & 0 & 0 & 0\end{array}$ $\begin{array}{llllll}0 & 0 & 0 & 0 & 0 & 0 \\ 0 & 0 & 0 & 0 & 0 & 0\end{array}$

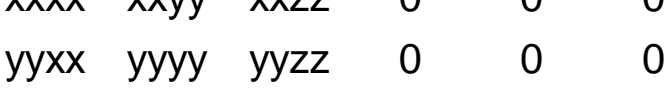
$\begin{array}{cccccc}z z x x & z z y y & z z z z & 0 & 0 & 0 \\ 0 & 0 & 0 & y z y z & 0 & 0\end{array}$

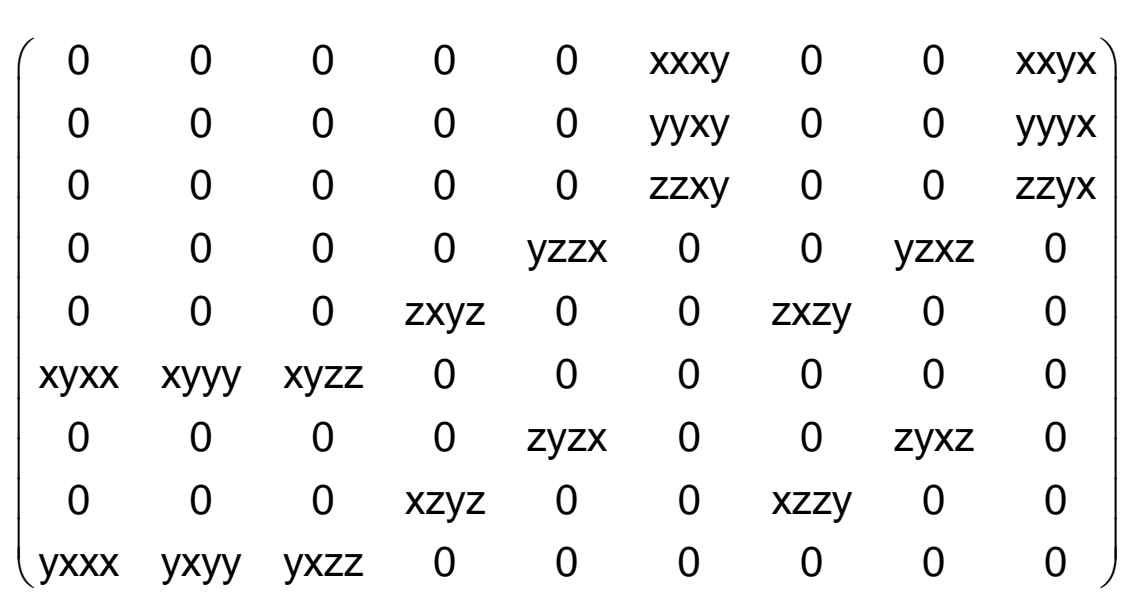

$\left(\begin{array}{ccccc}0 & x y y y & 0 & 0 & 0 \\ \operatorname{yxxx} & 0 & 0 & 0 & 0 \\ 0 & 0 & 0 & 0 & z z x y\end{array}\right)$

$\left(\begin{array}{cccccccccc}0 & x y y y & 0 & 0 & x y z z & 0 & 0 & x y x x & 0 & 0 \\ y x x x & 0 & 0 & y x y y & 0 & 0 & y x z z & 0 & 0 & 0 \\ 0 & 0 & 0 & 0 & 0 & 0 & 0 & 0 & 0 & z x y z\end{array}\right)$

$\begin{array}{cccccccccc}y x x x & 0 & 0 & y x y y & 0 & 0 & y x z z & 0 & 0 & 0 \\ 0 & 0 & 0 & 0 & 0 & 0 & 0 & 0 & 0 & z x y z\end{array}$

$$
\left(\begin{array}{cccccc}
0 & 0 & 0 & 0 & 0 & \mathrm{xxxy} \\
& 0 & 0 & 0 & 0 & \mathrm{yyxy} \\
& & 0 & 0 & 0 & \mathrm{zzxy} \\
& & & 0 & \mathrm{yzxz} & 0 \\
& & & & 0 & 0 \\
& & & & & 0
\end{array}\right)
$$

$\left(\begin{array}{cccccc}0 & 0 & 0 & 0 & 0 & \mathrm{xxxy} \\ 0 & 0 & 0 & 0 & 0 & \mathrm{yyxy} \\ 0 & 0 & 0 & 0 & 0 & \mathrm{zzxy} \\ 0 & 0 & 0 & 0 & \mathrm{yzxz} & 0 \\ 0 & 0 & 0 & \mathrm{xzyz} & 0 & 0 \\ 0 & 0 & 0 & 0 & 0 & 0\end{array}\right)$ $\left(\begin{array}{llllllll}0 & 0 & 0 & 0 & 0 & 0 & 0 & 0\end{array}\right.$ $\left.\begin{array}{lllllllllll}0 & 0 & 0 & 0 & 0 & 0 & 0 & 0 & 0 & 0 \\ 0 & 0 & 0 & 0 & 0 & 0 & 0 & 0 & 0\end{array}\right)$ $0 \begin{array}{lllllllll}0 & 0 & 0 & 0 & 0 & 0 & 0 & 0 & 0 \\ 0 & 0 & 0 & 0 & 0 & 0 & 0 & 0 & 0\end{array}$ $\begin{array}{lllllllll}0 & 0 & 0 & 0 & 0 & 0 & 0 & 0 & 0 \\ 0 & 0 & 0 & 0 & 0 & 0 & 0 & 0 & 0\end{array}$ $\begin{array}{llllllllll}0 & 0 & 0 & 0 & 0 & 0 & 0 & 0 & 0\end{array}$ $\begin{array}{lllllllll}0 & 0 & 0 & 0 & 0 & 0 & 0 & 0 & 0 \\ 0 & 0 & 0 & 0 & 0 & 0 & 0 & 0 & 0\end{array}$ $\begin{array}{lllllllll}0 & 0 & 0 & 0 & 0 & 0 & 0 & 0 & 0 \\ 0 & 0 & 0 & 0 & 0 & 0 & 0 & 0 & 0\end{array}$ $\left(\begin{array}{llllllllll}0 & 0 & 0 & 0 & 0 & 0 & 0 & 0 & 0\end{array}\right)$

$\left(\begin{array}{lllll}0 & 0 & 0 & 0 & 0 \\ 0 & 0 & 0 & 0 & 0 \\ 0 & 0 & 0 & 0 & 0\end{array}\right)$ $\left(\begin{array}{lllll}0 & 0 & 0 & 0 & 0\end{array}\right)$

$\left(\begin{array}{llllllllll}0 & 0 & 0 & 0 & 0 & 0 & 0 & 0 & 0 & 0 \\ 0 & 0 & 0 & 0 & 0 & 0 & 0 & 0 & 0 & 0\end{array}\right)$ $\left(\begin{array}{lllllllllll}0 & 0 & 0 & 0 & 0 & 0 & 0 & 0 & 0 & 0 \\ 0 & 0 & 0 & 0 & 0 & 0 & 0 & 0 & 0 & 0\end{array}\right)$ $\begin{array}{llllllllll}0 & 0 & 0 & 0 & 0 & 0 & 0 & 0 & 0 & 0\end{array}$ $\begin{array}{cccccc}0 & 0 & 0 & 0 & x z x z & 0 \\ 0 & 0 & 0 & 0 & 0 & x y x y\end{array}$ $\left.\begin{array}{llllll}0 & 0 & 0 & 0 & 0 & 0\end{array}\right)$

$\begin{array}{lllll}0 & 0 & 0 & 0 & 0\end{array}$ $\begin{array}{llll}0 & 0 & 0 & 0 \\ 0 & 0 & 0\end{array}$ $\begin{array}{lll}0 & 0 \\ 0\end{array}$

$\begin{array}{llllll}0 & 0 & 0 & 0 & 0 & 0 \\ 0 & 0 & 0 & 0 & 0 & 0\end{array}$ $\begin{array}{llllll}0 & 0 & 0 & 0 & 0 & 0 \\ 0 & 0 & 0 & 0 & 0 & 0\end{array}$ $\begin{array}{llllll}0 & 0 & 0 & 0 & 0 & 0 \\ 0 & 0 & 0 & 0 & 0 & 0\end{array}$ $\begin{array}{llllll}0 & 0 & 0 & 0 & 0 & 0\end{array}$ $\begin{array}{llllll}0 & 0 & 0 & 0 & 0 & 0\end{array}$ $\begin{array}{llllll}0 & 0 & 0 & 0 & 0 & 0 \\ 0 & 0 & 0 & 0\end{array}$ 


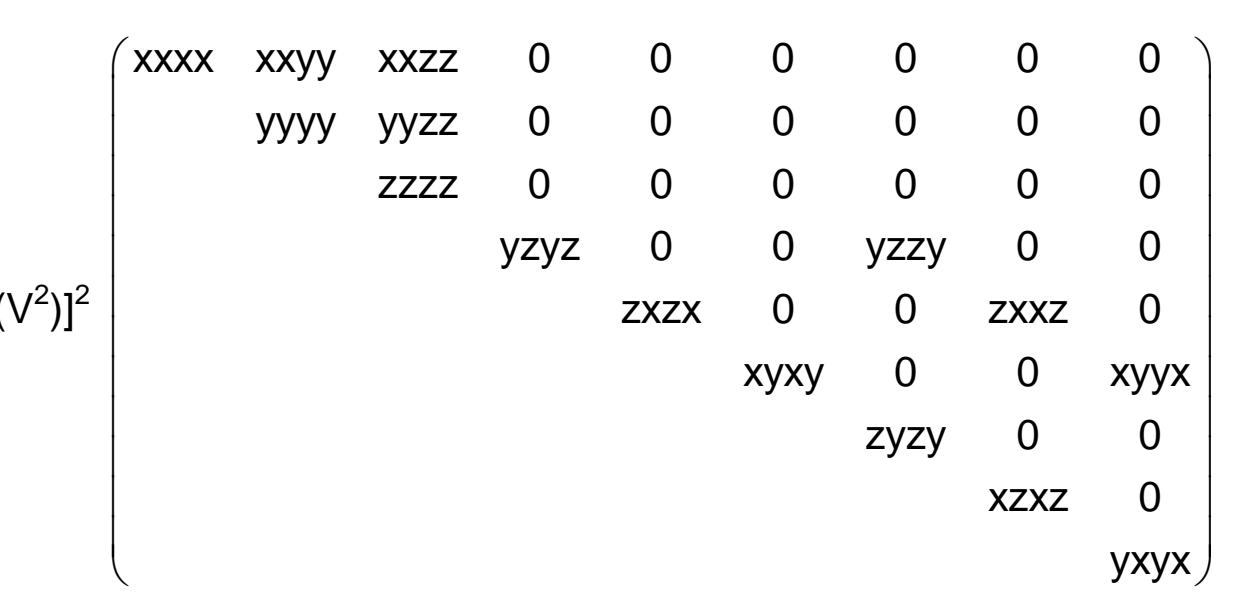

$\left(\begin{array}{lllllllll}0 & 0 & 0 & 0 & 0 & 0 & 0 & 0 & 0 \\ 0 & 0 & 0 & 0 & 0 & 0 & 0 & 0\end{array}\right)$

$\begin{array}{llllllll}0 & 0 & 0 & 0 & 0 & 0 & 0 & 0\end{array}$ $\begin{array}{lllllll}0 & 0 & 0 & 0 & 0 & 0 & 0 \\ & 0 & 0 & 0 & 0 & 0 & 0\end{array}$ $\begin{array}{lllll}0 & 0 & 0 & 0 & 0\end{array}$ $\begin{array}{llll}0 & 0 & 0 & 0\end{array}$ $\begin{array}{llll}0 & 0 & 0 & 0 \\ 0 & 0 & 0 \\ 0 & 0 & 0\end{array}$

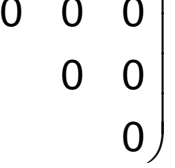

$\left[\mathrm{V}^{2}\right] \mathrm{V}^{2}\left(\begin{array}{ccccccccc}\mathrm{x} x \mathrm{x} & \mathrm{xxyy} & \mathrm{xxzz} & 0 & 0 & 0 & 0 & 0 & 0 \\ \mathrm{yyxx} & \text { yyyy } & \mathrm{yyzz} & 0 & 0 & 0 & 0 & 0 & 0 \\ \mathrm{zzxx} & \mathrm{zzyy} & \mathrm{zzzz} & 0 & 0 & 0 & 0 & 0 & 0 \\ 0 & 0 & 0 & \mathrm{yzyz} & 0 & 0 & \mathrm{yzzy} & 0 & 0 \\ 0 & 0 & 0 & 0 & \mathrm{zxzx} & 0 & 0 & \mathrm{zxxz} & 0 \\ 0 & 0 & 0 & 0 & 0 & \text { xyxy } & 0 & 0 & \text { xyyx }\end{array}\right)$ $\begin{array}{lllllllll}0 & 0 & 0 & 0 & 0 & 0 & 0 & 0 & 0 \\ 0 & 0 & 0 & 0 & 0 & 0 & 0 & 0 & 0\end{array}$

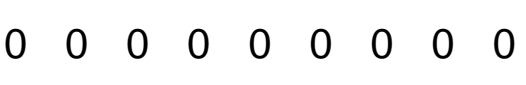
$0 \begin{array}{lllllllll}0 & 0 & 0 & 0 & 0 & 0 & 0 & 0 & 0 \\ 0 & 0 & 0 & 0 & 0 & 0 & 0 & 0 & 0\end{array}$ $\begin{array}{lllllllll}0 & 0 & 0 & 0 & 0 & 0 & 0 & 0 & 0 \\ 0 & 0 & 0 & 0 & 0 & 0 & 0 & 0 & 0\end{array}$

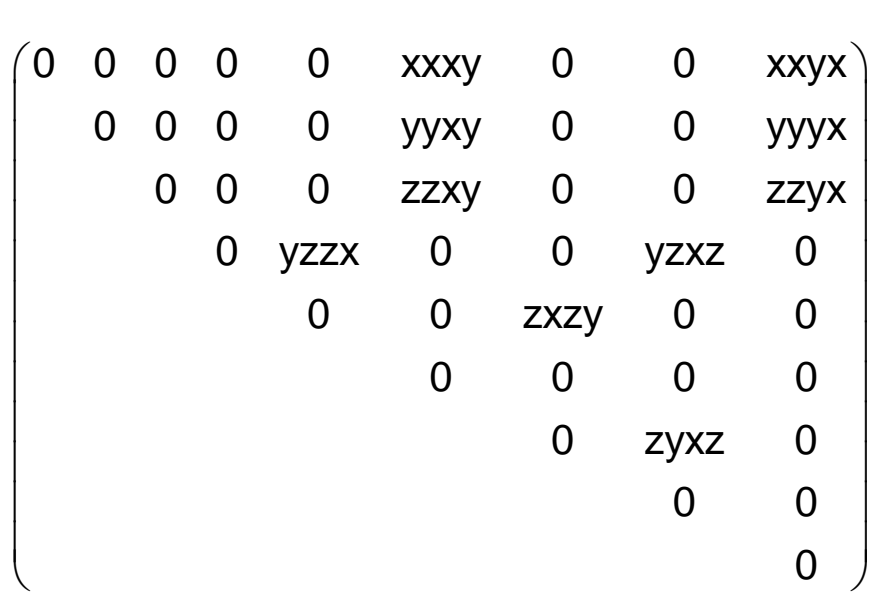

$\left(\begin{array}{lllllllll}0 & 0 & 0 & 0 & 0 & 0 & 0 & 0 & 0 \\ 0 & 0 & 0 & 0 & 0 & 0 & 0 & 0 & 0\end{array}\right)$ $\left(\begin{array}{ccccccccc}0 & 0 & 0 & 0 & 0 & \text { xxxy } & 0 & 0 & \text { xxyx } \\ 0 & 0 & 0 & 0 & 0 & \text { yyxy } & 0 & 0 & \text { yyyx } \\ 0 & 0 & 0 & 0 & 0 & \text { zzxy } & 0 & 0 & \text { zzyx } \\ 0 & 0 & 0 & 0 & \text { yzzx } & 0 & 0 & \text { yzxz } & 0 \\ 0 & 0 & 0 & \text { xzyz } & 0 & 0 & \text { zxzy } & 0 & 0 \\ \text { xyxx } & \text { xyyy } & \text { xyzz } & 0 & 0 & 0 & 0 & 0 & 0\end{array}\right)$ $\left(\begin{array}{lllllllll}0 & 0 & 0 & 0 & 0 & 0 & 0 & 0 & 0\end{array}\right)$ $\begin{array}{llllllll}0 & 0 & 0 & 0 & 0 & 0 & 0 & 0\end{array}$ $\begin{array}{llllllll}0 & 0 & 0 & 0 & 0 & 0 & 0 \\ & 0 & 0 & 0 & 0 & 0 & 0\end{array}$ $\begin{array}{llllll}0 & 0 & 0 & 0 & 0 \\ 0 & 0 & 0 & 0 & 0\end{array}$ $\begin{array}{lllll}0 & 0 & 0 & 0 \\ 0 & 0 & 0\end{array}$ $\begin{array}{lll}0 & 0 & 0 \\ 0 & 0 & 0\end{array}$ $\left.\begin{array}{ll}0 & 0 \\ & 0\end{array}\right)$

$\left(\begin{array}{lllllllll}0 & 0 & 0 & 0 & 0 & 0 & 0 & 0 & 0 \\ 0 & 0 & 0 & 0 & 0 & 0 & 0 & 0 & 0\end{array}\right)$ $\begin{array}{lllllllll}0 & 0 & 0 & 0 & 0 & 0 & 0 & 0 & 0 \\ 0 & 0 & 0 & 0 & 0 & 0 & 0 & 0 & 0\end{array}$ $\begin{array}{lllllllll}0 & 0 & 0 & 0 & 0 & 0 & 0 & 0 & 0 \\ 0 & 0 & 0 & 0 & 0 & 0 & 0 & 0 & 0\end{array}$ $\begin{array}{lllllllll}0 & 0 & 0 & 0 & 0 & 0 & 0 & 0 & 0 \\ 0 & 0 & 0 & 0 & 0 & 0 & 0 & 0 & 0\end{array}$ $\begin{array}{llllllllll}0 & 0 & 0 & 0 & 0 & 0 & 0 & 0 & 0 \\ 0 & 0 & 0 & 0 & 0 & 0 & 0 & 0 & 0\end{array}$ 


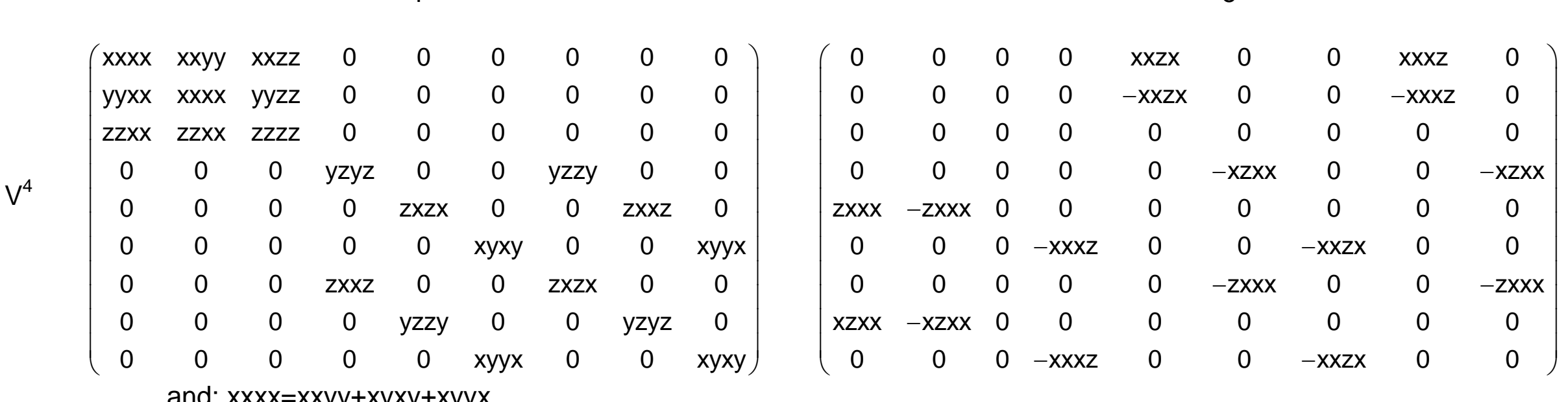
$\left(\begin{array}{ccccccccc}0 & 0 & 0 & 0 & 0 & x x x y & 0 & 0 & -y y x y \\ 0 & 0 & 0 & 0 & 0 & y y x y & 0 & 0 & -x x y y \\ 0 & 0 & 0 & 0 & 0 & z z x y & 0 & 0 & -z z x y \\ 0 & 0 & 0 & 0 & y z z x & 0 & 0 & y z x z & 0 \\ 0 & 0 & 0 & z x y z & 0 & 0 & z x z y & 0 & 0 \\ x y x x & x y y y & x y z z & 0 & 0 & 0 & 0 & 0 & 0 \\ 0 & 0 & 0 & 0 & -z x z y & 0 & 0 & -z x y z & 0 \\ 0 & 0 & 0 & -y z x z & 0 & 0 & -y z z x & 0 & 0 \\ -x y y y & -x y x x & -x y z z & 0 & 0 & 0 & 0 & 0 & 0\end{array}\right)\left(\begin{array}{ccccccccc}0 & 0 & 0 & 0 & 0 & 0 & x x z y & 0 & 0 \\ 0 & 0 & 0 & 0 & 0 & 0 & -x x z y & 0 & 0 \\ 0 & 0 & 0 & 0 & 0 & 0 & 0 & 0 & 0 \\ 0 & 0 & 0 & 0 & 0 & 0 & 0 & 0 & 0 \\ 0 & 0 & 0 & 0 & 0 & z y x x & 0 & 0 & z y x x \\ 0 & 0 & 0 & 0 & x x z y & 0 & 0 & x x y z & 0 \\ z y x x & -z y x x & 0 & 0 & 0 & 0 & 0 & 0 & 0 \\ x z x x & -x z x x & 0 & 0 & 0 & y z x x & 0 & 0 & y z x x \\ 0 & 0 & 0 & 0 & x x z y & 0 & 0 & \text { xxyz } & 0\end{array}\right)$

$\left[V^{4}\right] \quad\left(\begin{array}{ccccc}3 x x y y & 0 & 0 & y y z z & 0 \\ 0 & 3 x x y y & 0 & y y z z & 0 \\ 0 & 0 & z z z z & x x y y & 0\end{array}\right)$

$$
\left(\begin{array}{ccccc}
0 & 0 & 0 & 0 & 0 \\
0 & 0 & 0 & 0 & -z x x x \\
z x x x & 0 & 0 & 0 & 0
\end{array}\right)
$$

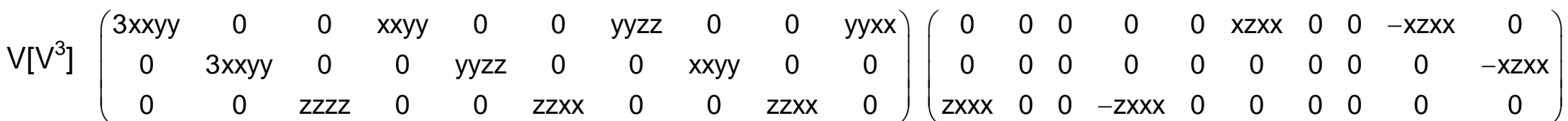

\section{$\left(\begin{array}{lllll}0 & 0 & 0 & 0 & 0 \\ 0 & 0 & 0 & 0 & 0 \\ 0 & 0 & 0 & 0 & 0\end{array}\right)$}

$\left(\begin{array}{cccccccccc}0 & 3 x y x x & 0 & 0 & x y z z & 0 & 0 & x y x x & 0 & 0 \\ -3 x y x x & 0 & 0 & -x y x x & 0 & 0 & -x y z z & 0 & 0 & 0 \\ 0 & 0 & 0 & 0 & 0 & 0 & 0 & 0 & 0 & z x y z\end{array}\right)$

$$
\left(\begin{array}{cccccc}
0 & 0 & 0 & 0 & -y y x z & 0 \\
& 0 & 0 & 0 & y y x z & 0 \\
& & 0 & 0 & 0 & 0 \\
& & & 0 & 0 & \mathrm{yyxz} \\
& & & & 0 & 0 \\
& & & & & 0
\end{array}\right)
$$

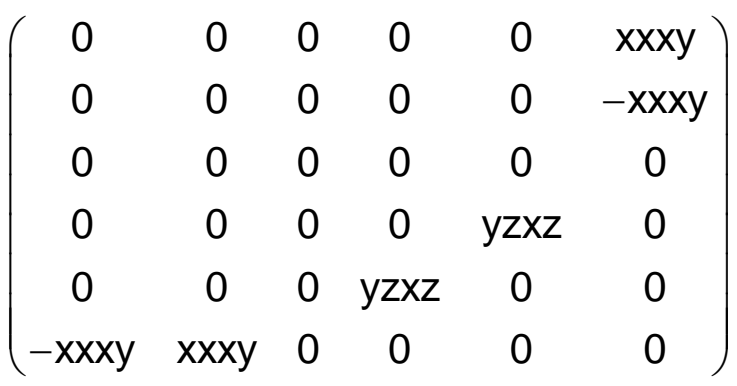

and $x y x y=1 / 2(x x x x-x x y y)$ $\left(\begin{array}{ccccc}0 & 0 & 0 & 0 & x x y z \\ 0 & 0 & 0 & 0 & 0 \\ 0 & -x x y z & 0 & 0 & 0\end{array}\right)$

$\left(\begin{array}{cccccccccc}0 & 0 & 0 & 0 & 0 & 0 & 0 & 0 & 0 & y z x x \\ 0 & 0 & 0 & 0 & 0 & y z x & 0 & 0 & -y z x & 0\end{array}\right)$ $\left(\begin{array}{ccccccccccc}0 & 0 & 0 & 0 & 0 & 0 & y z x x & 0 & 0 & -y z x x & 0 \\ 0 & -z y x x & 0 & 0 & 0 & 0 & 0 & z y x x & 0 & 0\end{array}\right)$

$\left(\begin{array}{llllll}0 & 0 & 0 & 0 & 0 & 0 \\ & 0 & 0 & 0 & 0 & 0 \\ & & 0 & 0 & 0 & 0 \\ & & & 0 & 0 & 0 \\ & & & & 0 & 0 \\ & & & & & 0\end{array}\right)$

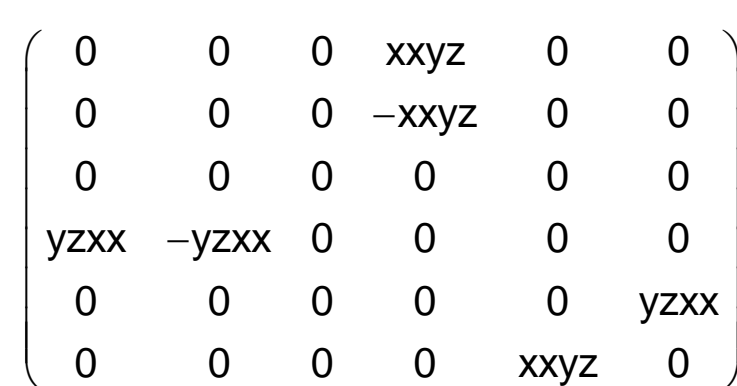

and $x y x y=1 / 2(x x x x-x x y y)$

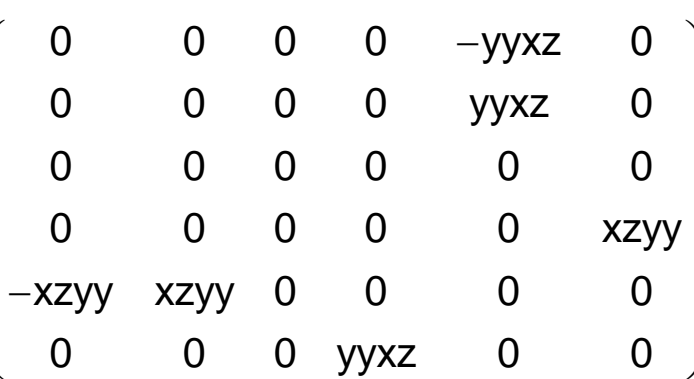

$\begin{array}{cccccc}0 & 0 & 0 & 0 & 0 & x z y y \\ 0 & 0 & 0 & y y x z & 0 & 0\end{array}$ 


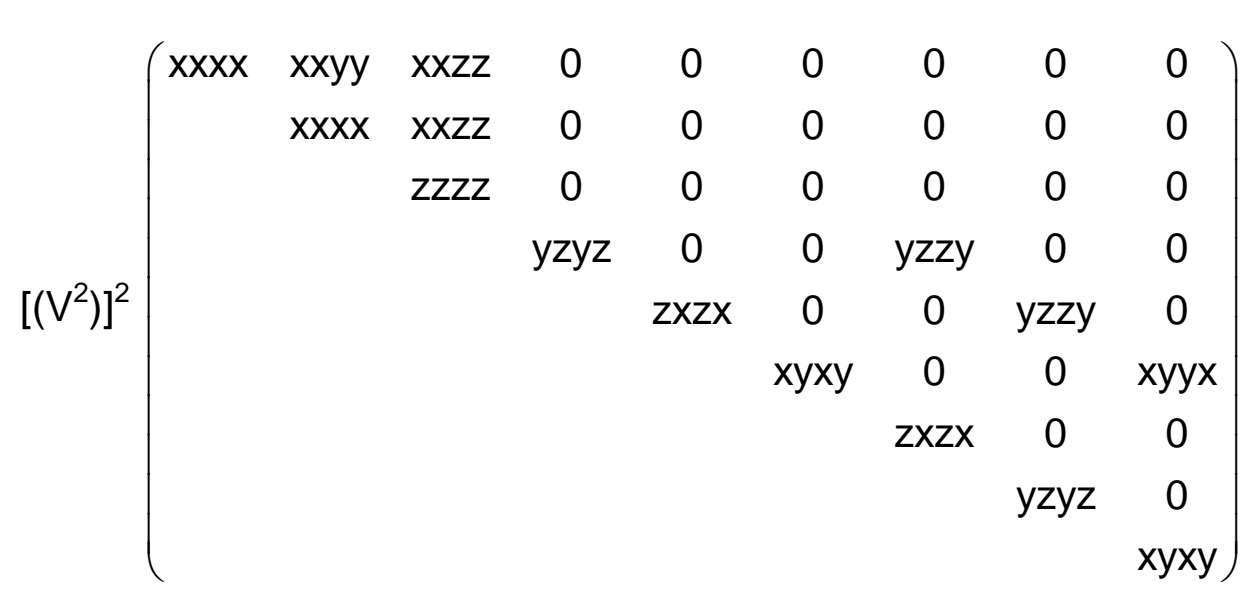
and $x x x x=x x y y+y z y z+z y z y$
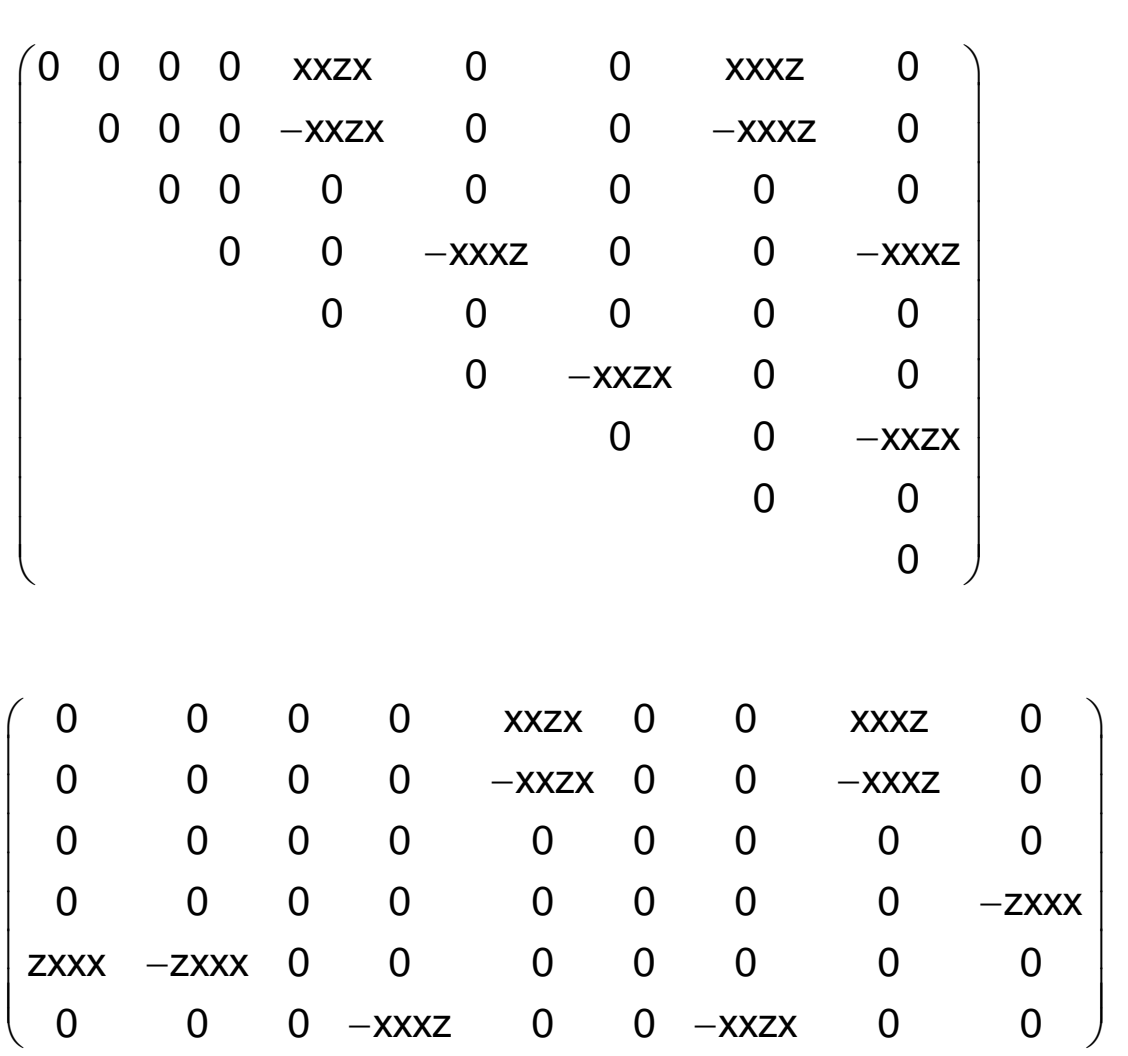
and $x x x x=x x y y+2 x y x y$
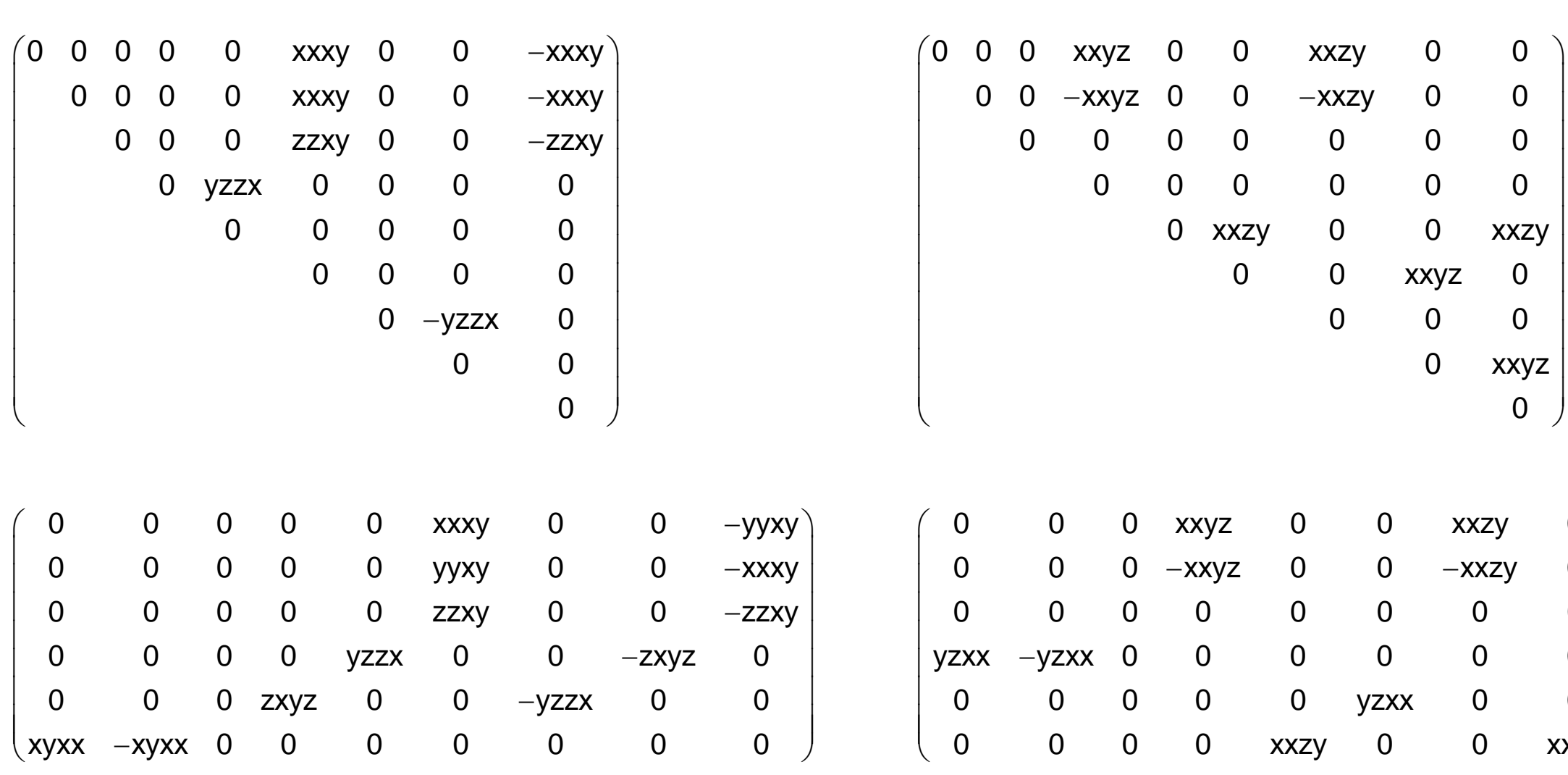

$\left(\begin{array}{ccccccccc}0 & 0 & 0 & x x y z & 0 & 0 & x x z y & 0 & 0 \\ 0 & 0 & 0 & -x x y z & 0 & 0 & -x x z y & 0 & 0 \\ 0 & 0 & 0 & 0 & 0 & 0 & 0 & 0 & 0 \\ y z x x & -y z x x & 0 & 0 & 0 & 0 & 0 & 0 & 0 \\ 0 & 0 & 0 & 0 & 0 & y z x x & 0 & 0 & y z x x \\ 0 & 0 & 0 & 0 & x x z y & 0 & 0 & \text { xxyz } & 0\end{array}\right)$

and $y y x y-x x x y=2 x y x x$ 


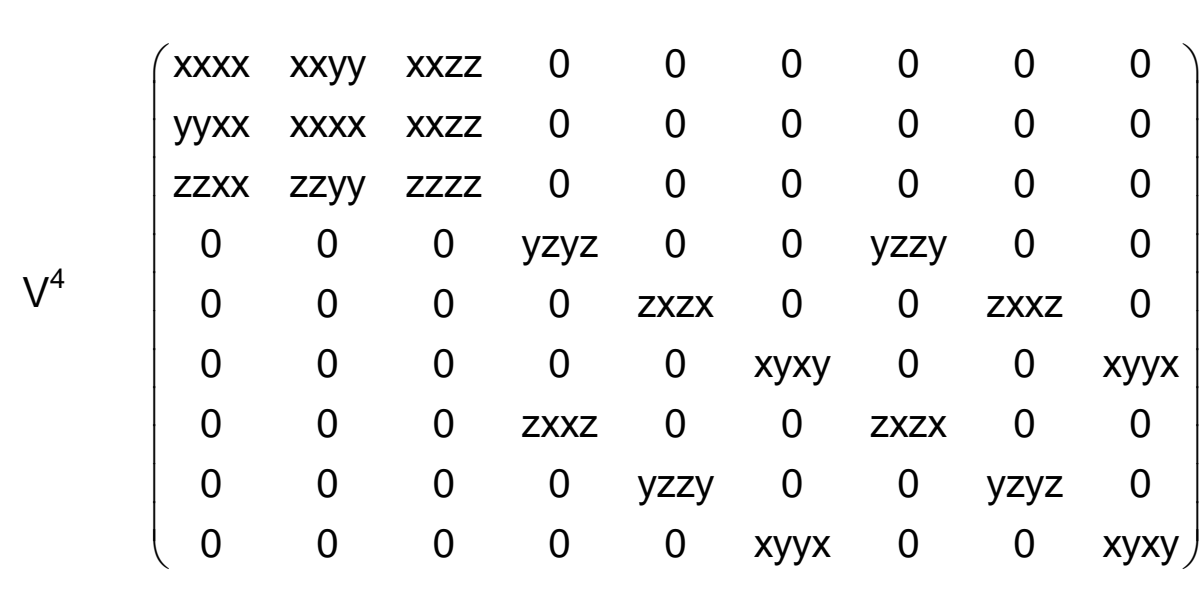

$\left(\begin{array}{lllllllll}0 & 0 & 0 & 0 & 0 & 0 & 0 & 0 & 0 \\ 0 & 0 & 0 & 0 & 0 & 0 & 0 & 0 & 0\end{array}\right)$ $\begin{array}{lllllllll}0 & 0 & 0 & 0 & 0 & 0 & 0 & 0 & 0 \\ 0 & 0 & 0 & 0 & 0 & 0 & 0 & 0 & 0\end{array}$

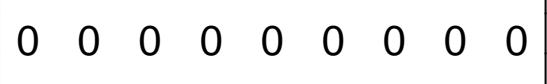
$0 \begin{array}{lllllllll}0 & 0 & 0 & 0 & 0 & 0 & 0 & 0 & 0 \\ 0 & 0 & 0 & 0 & 0 & 0 & 0 & 0 & 0\end{array}$ $\begin{array}{lllllllll}0 & 0 & 0 & 0 & 0 & 0 & 0 & 0 & 0 \\ 0 & 0 & 0 & 0 & 0 & 0 & 0 & 0 & 0\end{array}$ $\begin{array}{llllllllll}0 & 0 & 0 & 0 & 0 & 0 & 0 & 0 & 0 \\ 0 & 0 & 0 & 0 & 0 & 0 & 0 & 0 & 0\end{array}$ $0 \begin{array}{llllllllll}0 & 0 & 0 & 0 & 0 & 0 & 0 & 0 & 0 \\ 0 & 0 & 0 & 0 & 0 & 0 & 0 & 0 & 0\end{array}$ $\begin{array}{lllllllllll}0 & 0 & 0 & 0 & 0 & 0 & 0 & 0 & 0 \\ 0 & 0 & 0 & 0 & 0 & 0 & 0 & 0 & 0 \\ 0 & 0 & 0 & 0 & 0 & 0 & 0 & 0 & 0\end{array}$ $\begin{array}{llllllllll}0 & 0 & 0 & 0 & 0 & 0 & 0 & 0 & 0\end{array}$

$\left[V^{4}\right] \quad\left(\begin{array}{ccccc}\operatorname{xxxx} & 0 & 0 & y y z z & 0 \\ 0 & x x x x & 0 & y y z z & 0 \\ 0 & 0 & z z z z & x x y y & 0\end{array}\right)$

\section{$\left(\begin{array}{lllll}0 & 0 & 0 & 0 & 0 \\ 0 & 0 & 0 & 0 & 0 \\ 0 & 0 & 0 & 0 & 0\end{array}\right)$}

$\left(\begin{array}{lllll}0 & 0 & 0 & 0 & 0 \\ 0 & 0 & 0 & 0 & 0\end{array}\right)$

$\mathrm{V}\left[\mathrm{V}^{3}\right]\left(\begin{array}{cccccccccc}\mathrm{xxxx} & 0 & 0 & \mathrm{xxyy} & 0 & 0 & \mathrm{yyzz} & 0 & 0 & 0 \\ 0 & \mathrm{xxxx} & 0 & 0 & \mathrm{yyzz} & 0 & 0 & \mathrm{xxyy} & 0 & 0 \\ 0 & 0 & \mathrm{zzzz} & 0 & 0 & \mathrm{zzxx} & 0 & 0 & \mathrm{zzxx} & 0\end{array}\right)$

$\begin{array}{llllllllll}0 & 0 & 0 & 0 & 0 & 0 & 0 & 0 & 0 & 0 \\ 0 & 0 & 0 & 0 & 0 & 0 & 0 & 0 & 0 & 0\end{array}$ $\left(\begin{array}{lllllllllll}0 & 0 & 0 & 0 & 0 & 0 & 0 & 0 & 0 & 0 \\ 0 & 0 & 0 & 0 & 0 & 0 & 0 & 0 & 0 & 0\end{array}\right)$

$\left[\left[^{2}\right]^{2}\right]\left(\begin{array}{cccccc}x x x x & x x y y & x x z z & 0 & 0 & 0 \\ & \text { yyyy } & y y z z & 0 & 0 & 0 \\ & & z z z z & 0 & 0 & 0 \\ & & & y z y z & 0 & 0 \\ & & & & y z y z & 0 \\ & & & & & \text { xyxy }\end{array}\right)$

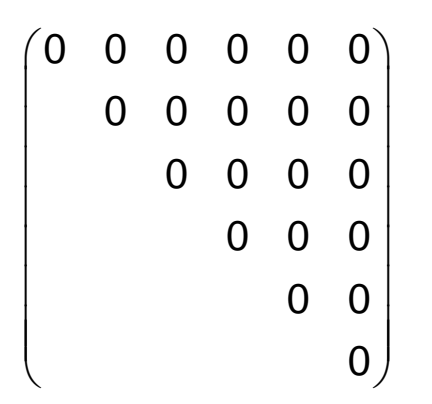

$\left(\begin{array}{llllll}0 & 0 & 0 & 0 & 0 & 0 \\ 0 & 0 & 0 & 0 & 0 & 0\end{array}\right)$ $\begin{array}{llllll}0 & 0 & 0 & 0 & 0 & 0 \\ 0 & 0 & 0 & 0 & 0 & 0 \\ 0 & 0 & 0 & 0 & 0 & 0\end{array}$ $\begin{array}{llllll}0 & 0 & 0 & 0 & 0 & 0 \\ 0 & 0 & 0 & 0\end{array}$ $\begin{array}{llllll}0 & 0 & 0 & 0 & 0 & 0 \\ 0 & 0 & 0 & 0\end{array}$ $\begin{array}{llllll}0 & 0 & 0 & 0 & 0 & 0 \\ 0 & 0 & 0 & 0 & 0 & 0\end{array}$

$\left(\begin{array}{llllll}x x x x & x x y y & x x z z & 0 & 0 & 0 \\ y y x x & x x x x & x x z z & 0 & 0 & 0 \\ z z x & z z x x & x z z z & 0 & 0 & 0\end{array}\right)$ $\begin{array}{cccccc}z z x x & z z x x & z z z z & 0 & 0 & 0 \\ 0 & 0 & 0 & y z y z & 0 & 0\end{array}$ a

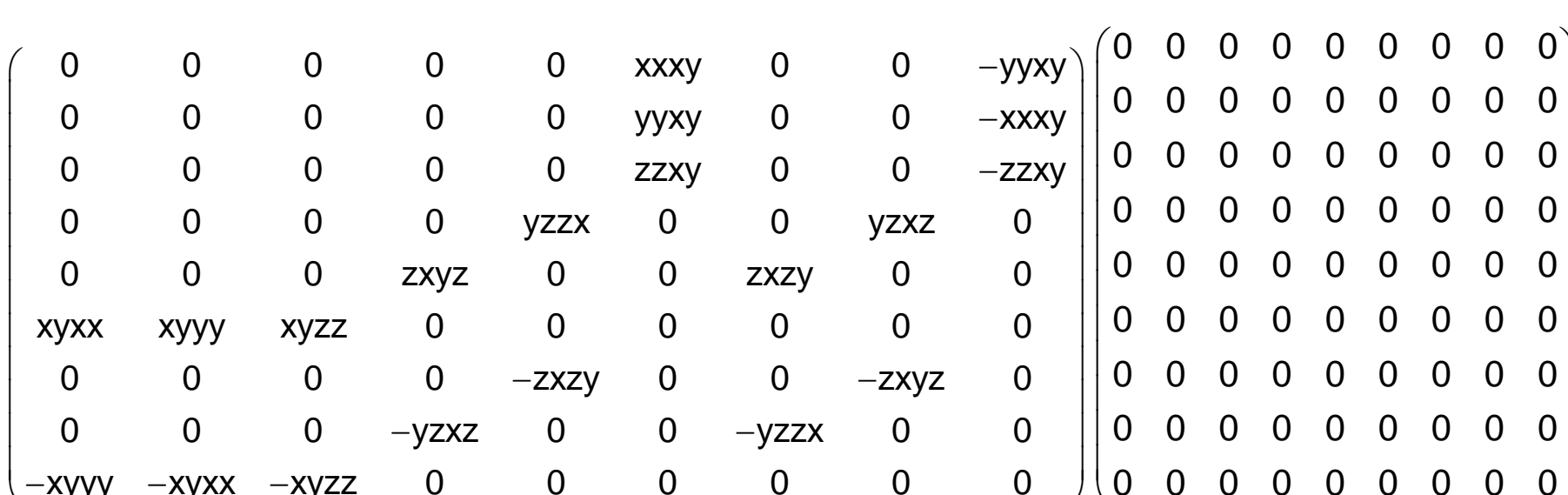
$\left(\begin{array}{ccccc}0 & -y x x x & 0 & 0 & 0 \\ y x x x & 0 & 0 & 0 & 0 \\ 0 & 0 & 0 & 0 & 0\end{array}\right)$

$\left(\begin{array}{lllll}0 & 0 & 0 & 0 & 0 \\ 0 & 0 & 0 & 0 & 0 \\ 0 & 0 & 0 & 0 & 0\end{array}\right)$ $\left(\begin{array}{lllll}0 & 0 & 0 & 0 & 0\end{array}\right)$

$\left(\begin{array}{cccccccccc}0 & -y x x x & 0 & 0 & x y z z & 0 & 0 & x y x x & 0 & 0 \\ y y x x & 0 & 0 & -x y x x & 0 & 0 & -x y z z & 0 & 0 & 0 \\ 0 & 0 & 0 & 0 & 0 & 0 & 0 & 0 & 0 & z x y z\end{array}\right)$

$\left(\begin{array}{llllllllll}0 & 0 & 0 & 0 & 0 & 0 & 0 & 0 & 0 & 0 \\ 0 & 0 & 0 & 0 & 0 & 0 & 0 & 0 & 0 & 0\end{array}\right)$ $\begin{array}{cccccccccc}y x x x & 0 & 0 & -x y x x & 0 & 0 & -x y z z & 0 & 0 & 0 \\ 0 & 0 & 0 & 0 & 0 & 0 & 0 & 0 & 0 & z x y z\end{array}$

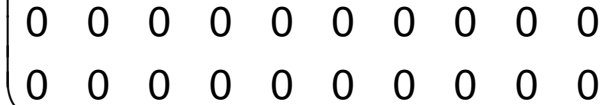
$\begin{array}{cccccc}0 & 0 & 0 & 0 & y z y z & 0 \\ 0 & 0 & 0 & 0 & 0 & x y x y\end{array}$

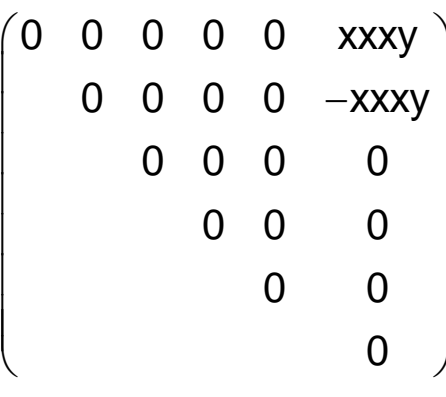

$\left(\begin{array}{cccccc}0 & 0 & 0 & 0 & 0 & x x x y \\ 0 & 0 & 0 & 0 & 0 & -x x x y \\ 0 & 0 & 0 & 0 & 0 & 0 \\ 0 & 0 & 0 & 0 & y z x z & 0 \\ 0 & 0 & 0 & -y z x z & 0 & 0 \\ x y x x & -x y x x & 0 & 0 & 0 & 0\end{array}\right)$

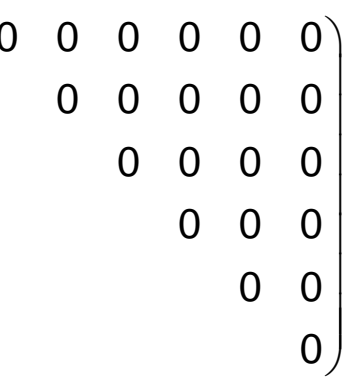

$\left(\begin{array}{llllll}0 & 0 & 0 & 0 & 0 & 0 \\ 0 & 0 & 0 & 0 & 0 & 0\end{array}\right)$ $\begin{array}{lllllll}0 & 0 & 0 & 0 & 0 & 0 \\ 0 & 0 & 0 & 0 & 0 & 0 \\ 0 & 0 & 0 & 0 & 0\end{array}$ $\begin{array}{llllll}0 & 0 & 0 & 0 & 0 & 0 \\ 0 & 0 & 0 & 0\end{array}$ $\left(\begin{array}{llllll}0 & 0 & 0 & 0 & 0 & 0\end{array}\right)$

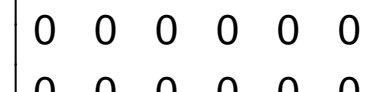




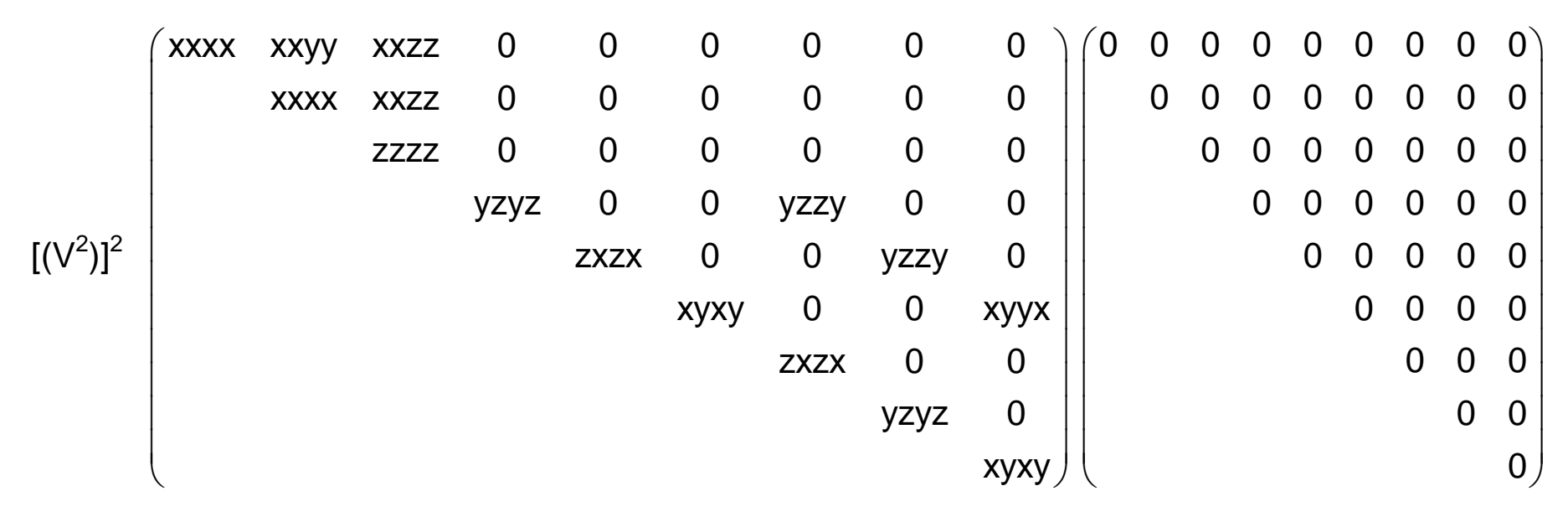

$\left(\begin{array}{lllllllll}x x x x & x x y y & x x z z & 0 & 0 & 0 & 0 & 0 & 0 \\ y y x x & x x y x & x x z z & 0 & 0 & 0 & 0 & 0 & 0 \\ z z x x & z z x x & z z z z & 0 & 0 & 0 & 0 & 0 & 0\end{array}\right)\left(\begin{array}{lllllllll}0 & 0 & 0 & 0 & 0 & 0 & 0 & 0 & 0 \\ 0 & 0 & 0 & 0 & 0 & 0 & 0 & 0 & 0 \\ 0 & 0 & 0 & 0 & 0 & 0 & 0 & 0 & 0\end{array}\right)$

$\left.\begin{array}{llllllllll}y y x x & x x x x & x x z z & 0 & 0 & 0 & 0 & 0 & 0 \\ z z x x & z z x x & z z z z & 0 & 0 & 0 & 0 & 0 & 0\end{array} \mid \begin{array}{llllllllll}0 & 0 & 0 & 0 & 0 & 0 & 0 & 0 & 0 \\ 0 & 0 & 0 & 0 & 0 & 0 & 0 & 0 & 0 \\ 0 & 0 & 0 & y z y z & 0 & 0 & z x z x & 0 & 0 & 0\end{array}\right)$

$\left[\mathrm{V}^{2}\right] \mathrm{V}^{2} \quad \begin{array}{cccccccccc}\operatorname{zzxx} & \operatorname{zzxx} & \mathrm{zzzz} & 0 & 0 & 0 & 0 & 0 & 0 \\ 0 & 0 & 0 & y z y z & 0 & 0 & z x z x & 0 & 0\end{array} \mid \begin{array}{cccccccccc}0 & 0 & 0 & 0 & 0 & 0 & 0 & 0 & 0 \\ 0 & 0 & 0 & 0 & 0 & 0 & 0 & 0 & 0 \\ 0 & 0 & 0 & 0 & 0 & z x z & 0 & 0 & y z y z & 0\end{array}$ $\left(\begin{array}{ccccccccc}0 & 0 & 0 & y z y z & 0 & 0 & z x z x & 0 & 0 \\ 0 & 0 & 0 & 0 & z x z x & 0 & 0 & y z y z & 0 \\ 0 & 0 & 0 & 0 & 0 & x y y & 0 & 0 & x y x y\end{array} \mid \begin{array}{ccccccccc}0 & 0 & 0 & 0 & 0 & 0 & 0 & 0 & 0 \\ 0 & 0 & 0 & 0 & 0 & 0 & 0 & 0 & 0 \\ 0 & 0 & 0 & 0 & 0 & 0 & 0 & 0 & 0\end{array}\right)$ $\left(\begin{array}{ccccccccc}0 & 0 & 0 & 0 & z x z x & 0 & 0 & y z y z & 0 \\ 0 & 0 & 0 & 0 & 0 & x y x y & 0 & 0 & x y x y\end{array}\right)\left(\begin{array}{lllllllll}0 & 0 & 0 & 0 & 0 & 0 & 0 & 0 & 0 \\ 0 & 0 & 0 & 0 & 0 & 0 & 0 & 0 & 0\end{array}\right)$
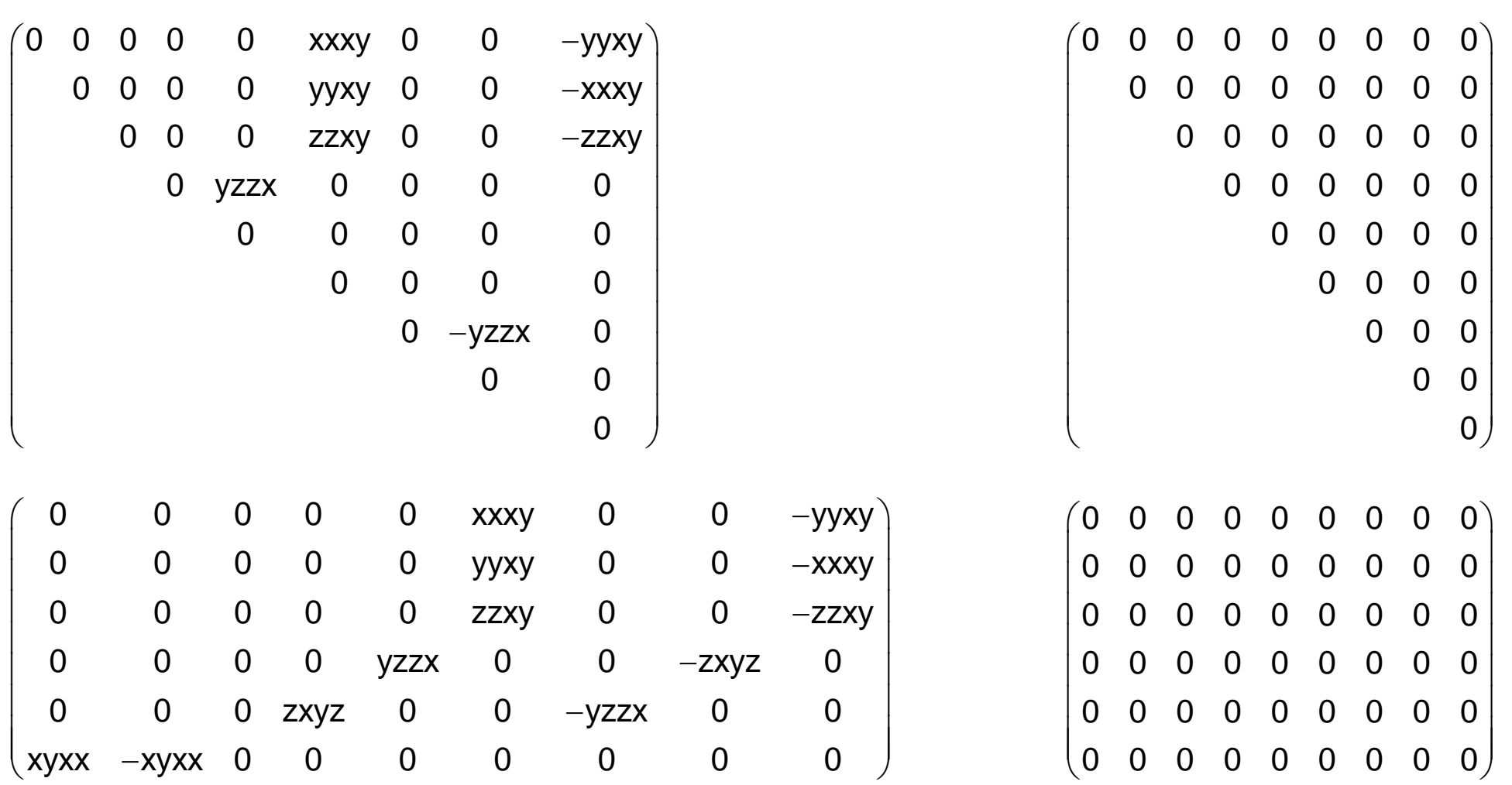

$\begin{array}{lllllllll}0 & 0 & 0 & 0 & 0 & 0 & 0 & 0 & 0 \\ 0 & 0 & 0 & 0 & 0 & 0 & 0 & 0\end{array}$ $0 \begin{array}{lllllllll}0 & 0 & 0 & 0 & 0 & 0 & 0 & 0 & 0 \\ 0 & 0 & 0 & 0 & 0 & 0 & 0 & 0 & 0\end{array}$ $0 \begin{array}{lllllllll}0 & 0 & 0 & 0 & 0 & 0 & 0 & 0 & 0 \\ 0 & 0 & 0 & 0 & 0 & 0 & 0 & 0 & 0\end{array}$ $\begin{array}{lllllllll}0 & 0 & 0 & 0 & 0 & 0 & 0 & 0 & 0 \\ 0 & 0 & 0 & 0 & 0 & 0 & 0 & 0 & 0\end{array}$ $\begin{array}{lllllllll}0 & 0 & 0 & 0 & 0 & 0 & 0 & 0 & 0 \\ 0 & 0 & 0 & 0 & 0 & 0 & 0 & 0 & 0\end{array}$ 
$\left(\begin{array}{lllllllll}0 & 0 & 0 & 0 & 0 & 0 & 0 & 0 & 0 \\ 0 & 0 & 0 & 0 & 0 & 0 & 0 & 0 & 0\end{array}\right)$ $\left(\begin{array}{lllllllll}0 & 0 & 0 & 0 & 0 & 0 & 0 & 0 & 0 \\ 0 & 0 & 0 & 0 & 0 & 0 & 0 & 0 & 0\end{array}\right.$ $\begin{array}{llllllllll}0 & 0 & 0 & 0 & 0 & 0 & 0 & 0 & 0 \\ 0 & 0 & 0 & 0 & 0 & 0 & 0 & 0 & 0 & 0\end{array}$ $\begin{array}{llllllllll}0 & 0 & 0 & 0 & 0 & 0 & 0 & 0 & 0\end{array}$ $\begin{array}{lllllllllllllll}0 & 0 & 0 & 0 & 0 & 0 & 0 & 0 & 0\end{array}$ $\begin{array}{llllllllll}0 & 0 & 0 & 0 & 0 & 0 & 0 & 0 & 0 \\ 0 & 0 & 0 & 0 & 0 & 0 & 0 & 0 & 0\end{array}$ $0 \begin{array}{lllllllll}0 & 0 & 0 & 0 & 0 & 0 & 0 & 0 & 0 \\ 0 & 0 & 0 & 0 & 0 & 0 & 0 & 0 & 0\end{array}$ $\begin{array}{lllllllll}0 & 0 & 0 & 0 & 0 & 0 & 0 & 0 & 0 \\ 0 & 0 & 0 & 0 & 0 & 0 & 0 & 0 & 0\end{array}$ $0 \begin{array}{llllllllll}0 & 0 & 0 & 0 & 0 & 0 & 0 & 0 & 0 \\ 0 & 0 & 0 & 0 & 0 & 0 & 0 & 0 & 0\end{array}$ $\begin{array}{llllllllll}0 & 0 & 0 & 0 & 0 & 0 & 0 & 0 & 0\end{array}$ $\begin{array}{cccccccc}0 & 0 & 0 & y z z y & 0 & 0 & y z y z & 0 \\ 0 & 0 & 0 & 0 & x y y x & 0 & 0 & x y x y\end{array}$

$\left(\begin{array}{lllll}0 & 0 & 0 & 0 & 0 \\ 0 & 0 & 0 & 0 & 0 \\ 0 & 0 & 0 & 0 & 0\end{array}\right)$

$\left[V^{4}\right] \quad\left(\begin{array}{ccccc}3 x x y y & 0 & 0 & y y z z & 0 \\ 0 & 3 x x y y & 0 & y y z z & 0 \\ 0 & 0 & z z z z & x x y y & 0\end{array}\right)$

$\left.\mathrm{V}^{2} \mathrm{~V}^{3}\right] \quad\left(\begin{array}{cccccccccc}3 x \mathrm{x} y \mathrm{y} & 0 & 0 & \mathrm{xxyy} & 0 & 0 & \mathrm{yyzz} & 0 & 0 & 0 \\ 0 & 3 x \mathrm{x} y \mathrm{y} & 0 & 0 & \mathrm{yyzz} & 0 & 0 & \mathrm{xxyy} & 0 & 0 \\ 0 & 0 & z z z & 0 & 0 & z z x & 0 & 0 & z z x & 0\end{array}\right)$

$\left(\begin{array}{llllllllll}0 & 0 & 0 & 0 & 0 & 0 & 0 & 0 & 0 & 0\end{array}\right)$ $\left.\begin{array}{lllllllllll}0 & 0 & 0 & 0 & 0 & 0 & 0 & 0 & 0 & 0 \\ 0 & 0 & 0 & 0 & 0 & 0 & 0 & 0 & 0 & 0\end{array}\right)$ $\left.\begin{array}{llllllllll}0 & 0 & 0 & 0 & 0 & 0 & 0 & 0 & 0 & 0 \\ 0 & 0 & 0 & 0 & 0 & 0 & 0 & 0 & 0 & 0\end{array}\right)$
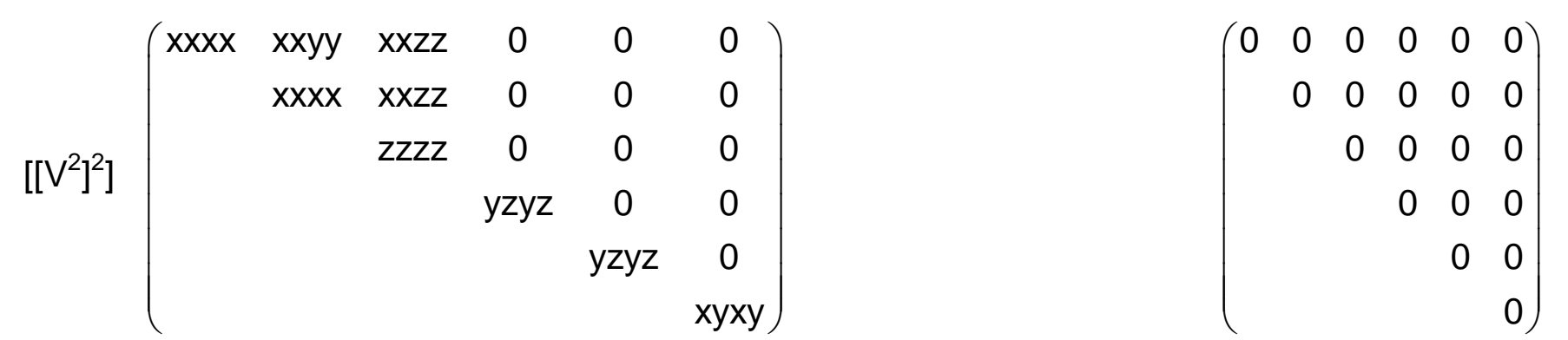

and $x y x y=1 / 2(x x x x-x x y y)$

$\left(\begin{array}{cccccc}x x x x & x x y y & x x z z & 0 & 0 & 0 \\ x x y y & x x x x & x x z z & 0 & 0 & 0 \\ z z x x & z z x x & z z z z & 0 & 0 & 0 \\ 0 & 0 & 0 & y z y z & 0 & 0 \\ 0 & 0 & 0 & 0 & y z y z & 0 \\ 0 & 0 & 0 & 0 & 0 & x y x y\end{array}\right)$

and $x y x y=1 / 2(x x x x-x x y y)$

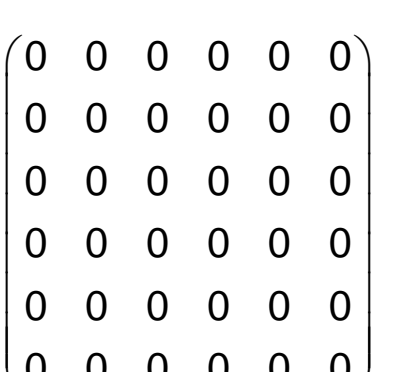

$\begin{array}{llllll}0 & 0 & 0 & 0 & 0 & 0 \\ 0 & 0 & 0 & 0 & 0 & 0\end{array}$ $\begin{array}{llllll}0 & 0 & 0 & 0 & 0 & 0 \\ 0 & 0 & 0 & 0 & 0\end{array}$ $\begin{array}{llllll}0 & 0 & 0 & 0 & 0 & 0 \\ 0 & 0 & 0 & 0 & 0 & 0\end{array}$

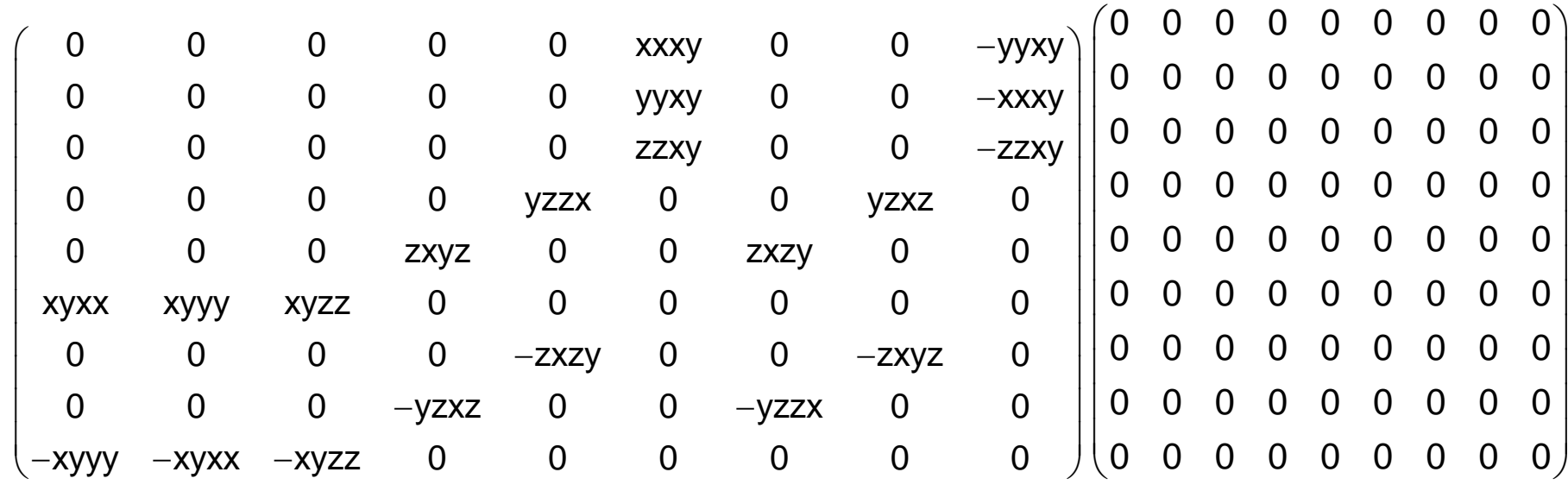

and: $x x x y-y y x y=x y y y-x y x x$

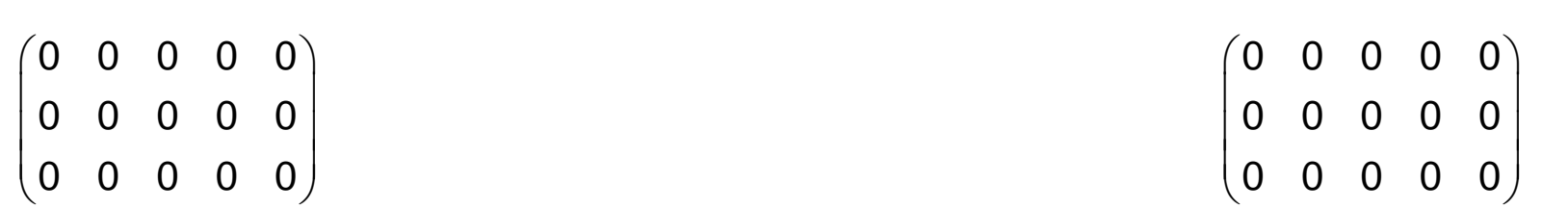

$\left(\begin{array}{cccccccccc}0 & 3 x y x x & 0 & 0 & x y z z & 0 & 0 & x y x x & 0 & 0 \\ -3 x y x x & 0 & 0 & -x y x x & 0 & 0 & -x y z z & 0 & 0 & 0 \\ 0 & 0 & 0 & 0 & 0 & 0 & 0 & 0 & 0 & 0 x y\end{array}\right)$

$\left(\begin{array}{llllllllll}0 & 0 & 0 & 0 & 0 & 0 & 0 & 0 & 0 & 0 \\ 0 & 0 & 0 & 0 & 0 & 0 & 0 & 0 & 0 & 0\end{array}\right.$ $\left(\begin{array}{llllllllll}0 & 0 & 0 & 0 & 0 & 0 & 0 & 0 & 0 & 0 \\ 0 & 0 & 0 & 0 & 0 & 0 & 0 & 0 & 0 & 0\end{array}\right)$ $\begin{array}{llllllllll}0 & 0 & 0 & 0 & 0 & 0 & 0 & 0 & 0 & z x y z\end{array}$

$\begin{array}{lllllllllll}0 & 0 & 0 & 0 & 0 & 0 & 0 & 0 & 0 & 0\end{array}$
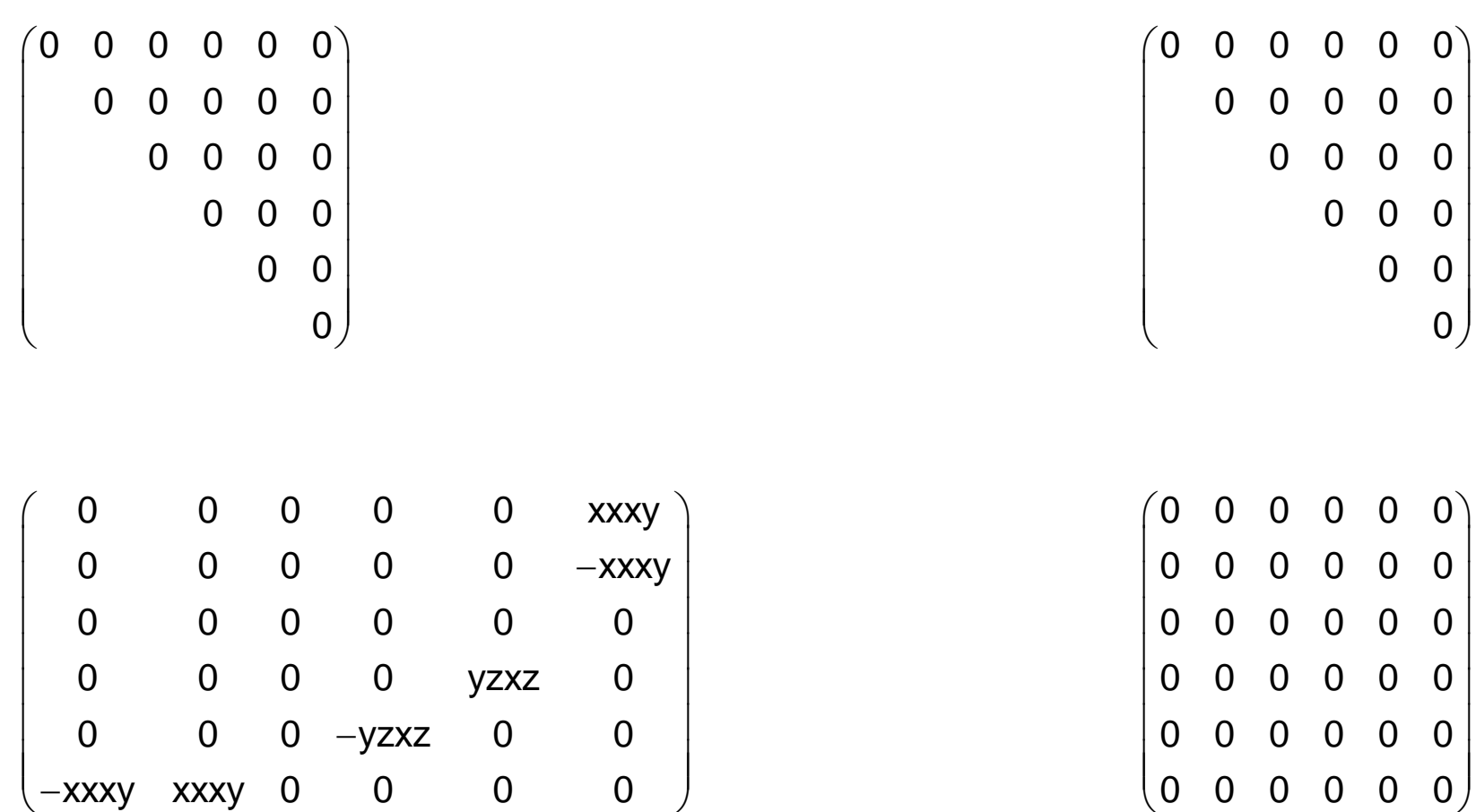

$\left(\begin{array}{llllll}0 & 0 & 0 & 0 & 0 & 0 \\ 0 & 0 & 0 & 0 & 0 & 0\end{array}\right)$ $\begin{array}{llllll}0 & 0 & 0 & 0 & 0 & 0 \\ 0 & 0 & 0 & 0 & 0 & 0\end{array}$ $\begin{array}{llllll}0 & 0 & 0 & 0 & 0 & 0 \\ 0 & 0 & 0 & 0 & 0 & 0\end{array}$ $\begin{array}{llllll}0 & 0 & 0 & 0 & 0 & 0 \\ 0 & 0 & 0 & 0 & 0 & 0\end{array}$ $\begin{array}{llllll}0 & 0 & 0 & 0 & 0 & 0 \\ 0 & 0 & 0 & 0 & 0 & 0\end{array}$ $\begin{array}{lllllll}0 & 0 & 0 & 0 & 0 & 0\end{array}$ 


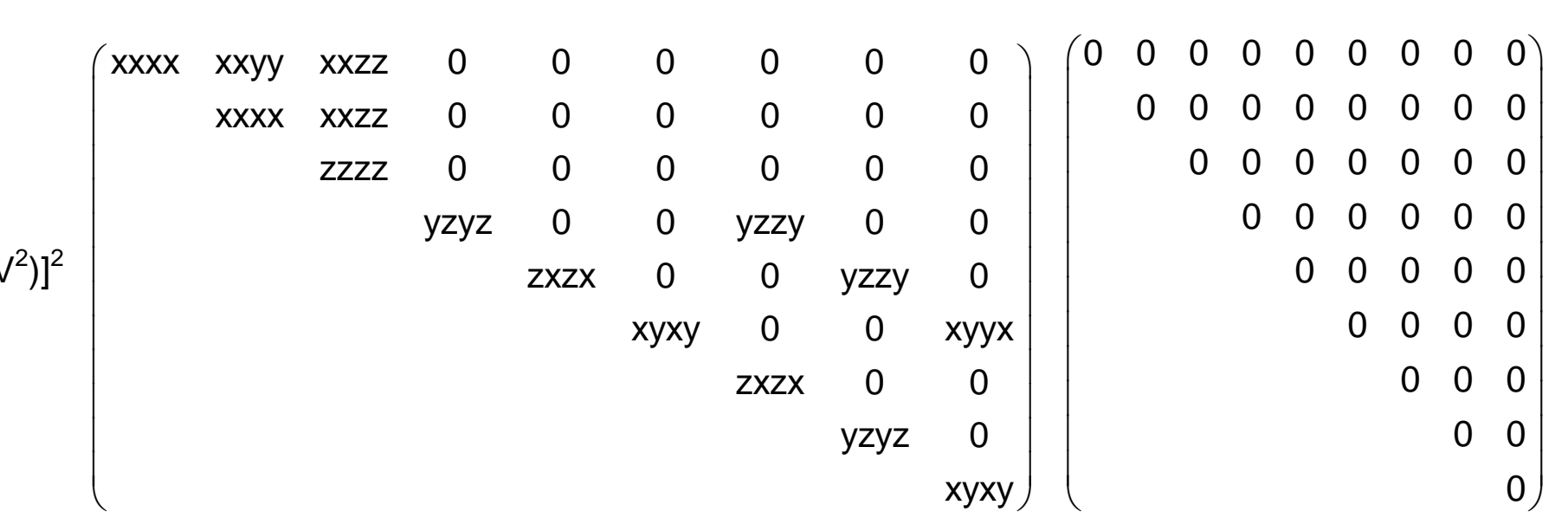

and $x x x x=x x y y+y z y z+z y z y$

$\left[\mathrm{V}^{2}\right] \mathrm{V}^{2}\left(\begin{array}{ccccccccc}\mathrm{x} x \mathrm{x} x & \mathrm{xxyy} & \mathrm{xxzz} & 0 & 0 & 0 & 0 & 0 & 0 \\ \mathrm{xxyy} & \mathrm{xyxx} & \mathrm{xzzz} & 0 & 0 & 0 & 0 & 0 & 0 \\ \mathrm{zzx} & z z x x & z z z z & 0 & 0 & 0 & 0 & 0 & 0 \\ 0 & 0 & 0 & \mathrm{yzyz} & 0 & 0 & \mathrm{zxzx} & 0 & 0 \\ 0 & 0 & 0 & 0 & \mathrm{zxzx} & 0 & 0 & \mathrm{yzyz} & 0 \\ 0 & 0 & 0 & 0 & 0 & \mathrm{xyxy} & 0 & 0 & \mathrm{xyxy}\end{array}\right)\left(\begin{array}{ccccccccc}0 & 0 & 0 & 0 & 0 & 0 & 0 & 0 & 0 \\ 0 & 0 & 0 & 0 & 0 & 0 & 0 & 0 & 0 \\ 0 & 0 & 0 & 0 & 0 & 0 & 0 & 0 & 0 \\ 0 & 0 & 0 & 0 & 0 & 0 & 0 & 0 & 0 \\ 0 & 0 & 0 & 0 & 0 & 0 & 0 & 0 & 0 \\ 0 & 0 & 0 & 0 & 0 & 0 & 0 & 0 & 0\end{array}\right)$ and $x x x x=x x y y+2 x y x y$

$$
\begin{aligned}
& \left(\begin{array}{lllllllll}
0 & 0 & 0 & 0 & 0 & x x x y & 0 & 0 & -x x x y \\
& 0 & 0 & 0 & 0 & x x x y & 0 & 0 & -x x x y
\end{array}\right) \\
& \begin{array}{llllllll}
0 & 0 & 0 & 0 & x x x y & 0 & 0 & -x x x y \\
& 0 & 0 & 0 & z z x y & 0 & 0 & -z z x y
\end{array} \\
& \begin{array}{llllll}
0 & 0 & z z x y & 0 & 0 & -z z x y
\end{array} \\
& \begin{array}{cccccc}
0 & y z z x & 0 & 0 & 0 & 0 \\
0 & 0 & 0 & 0 & 0
\end{array} \\
& \begin{array}{llll}
0 & 0 & 0 & 0 \\
0 & 0 & 0 & 0
\end{array} \\
& \begin{array}{ccc}
0-y z z x & 0 \\
0 & 0 \\
& & 0
\end{array}
\end{aligned}
$$

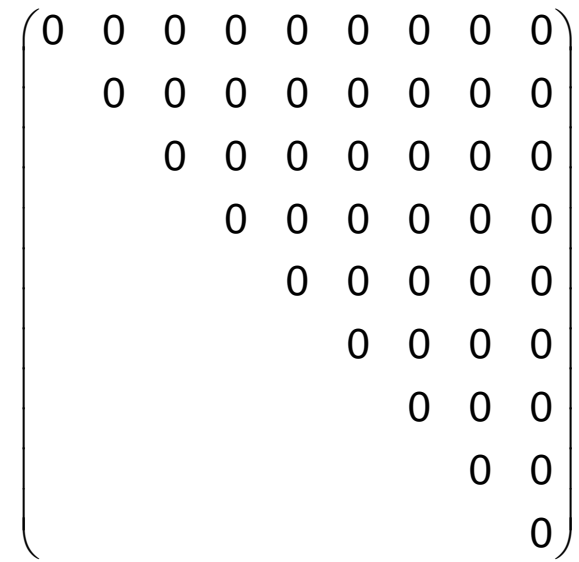

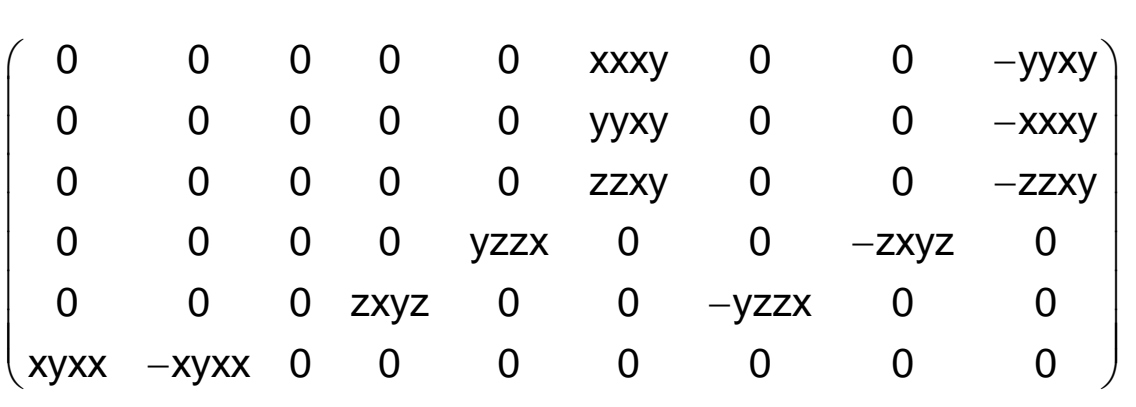

$\left(\begin{array}{lllllllll}0 & 0 & 0 & 0 & 0 & 0 & 0 & 0 & 0 \\ 0 & 0 & 0 & 0 & 0 & 0 & 0 & 0 & 0\end{array}\right)$ $\left.\begin{array}{lllllllll}0 & 0 & 0 & 0 & 0 & 0 & 0 & 0 & 0 \\ 0 & 0 & 0 & 0 & 0 & 0 & 0 & 0 & 0 \\ 0 & 0 & 0 & 0 & 0 & 0 & 0 & 0 & 0\end{array}\right)$ $\begin{array}{llllllllll}0 & 0 & 0 & 0 & 0 & 0 & 0 & 0 & 0\end{array}$ $\left(\begin{array}{lllllllll}0 & 0 & 0 & 0 & 0 & 0 & 0 & 0 & 0 \\ 0 & 0 & 0 & 0 & 0 & 0 & 0 & 0 & 0\end{array}\right)$

\section{and: $y y x y-x x x y=2 x y x x$}




\section{1}

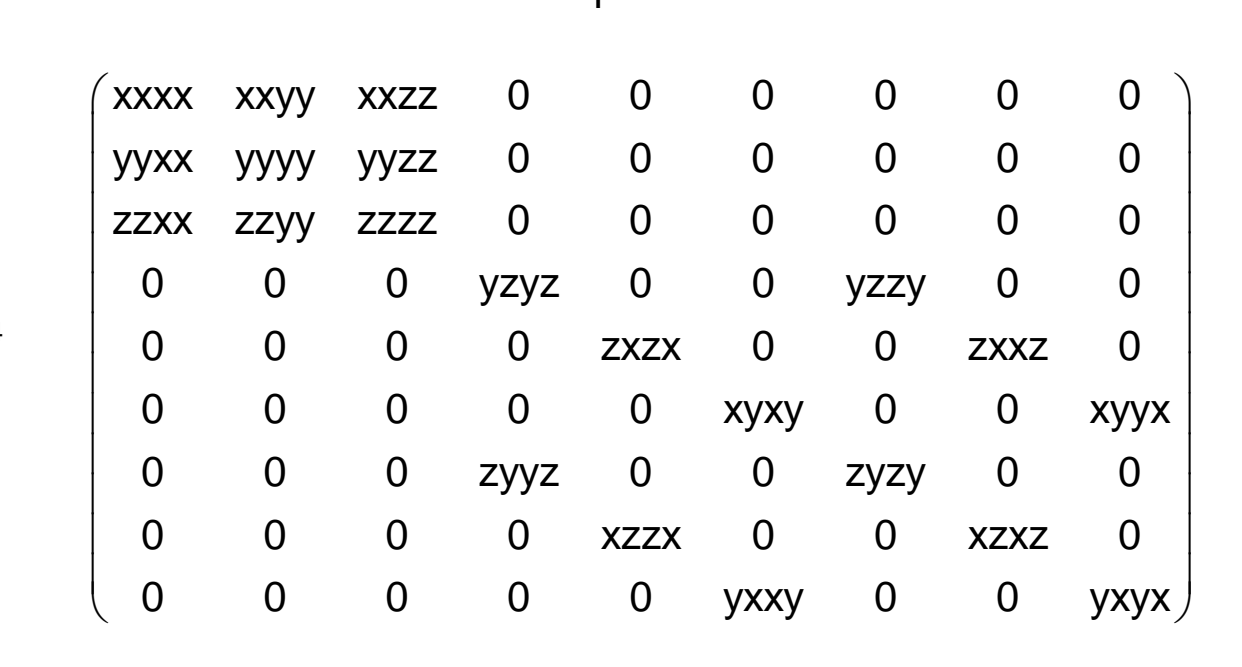

$\left[V^{4}\right] \quad\left(\begin{array}{cccccc}x x x x & 0 & 0 & y y z z & 0 \\ 0 & y y y y & 0 & z z x x & 0 \\ 0 & 0 & z z z z & x x y y & 0\end{array}\right)$

$\mathrm{V}\left[\mathrm{V}^{3}\right]\left(\begin{array}{cccccccccc}\mathrm{xxxx} & 0 & 0 & \mathrm{xxyy} & 0 & 0 & \mathrm{xxzz} & 0 & 0 & 0 \\ 0 & \mathrm{yyyy} & 0 & 0 & \mathrm{yyzz} & 0 & 0 & \mathrm{yyxx} & 0 & 0 \\ 0 & 0 & z z z z & 0 & 0 & \mathrm{zzxx} & 0 & 0 & \mathrm{zzy} & 0\end{array}\right)$

$V^{\prime}\left[V^{3}\right]\left(\begin{array}{ccccccccccc}0 & y y y y & 0 & 0 & y y z z & 0 & 0 & y y x x & 0 & 0 \\ 0 & 0 & z z z z & 0 & 0 & z z x x & 0 & 0 & z z y y & 0\end{array}\right)$

$\left[\left[^{2}\right]^{2}\right]\left(\begin{array}{cccccc}x x x x & x x y y & x x z z & 0 & 0 & 0 \\ & \text { yyyy } & \text { yyzz } & 0 & 0 & 0 \\ & & z z z z & 0 & 0 & 0 \\ & & & y z y z & 0 & 0 \\ & & & & \text { xzxz } & 0 \\ & & & & & \text { xyxy }\end{array}\right)$

$\left[V^{2}\right]^{2}\left(\begin{array}{cccccc}x x x x & x x y y & x x z z & 0 & 0 & 0 \\ y y x x & y y y y & y y z z & 0 & 0 & 0 \\ z z x x & z z y y & z z z z & 0 & 0 & 0 \\ 0 & 0 & 0 & y z y z & 0 & 0 \\ 0 & 0 & 0 & 0 & x z x z & 0 \\ 0 & 0 & 0 & 0 & 0 & x y x y\end{array}\right)$ e

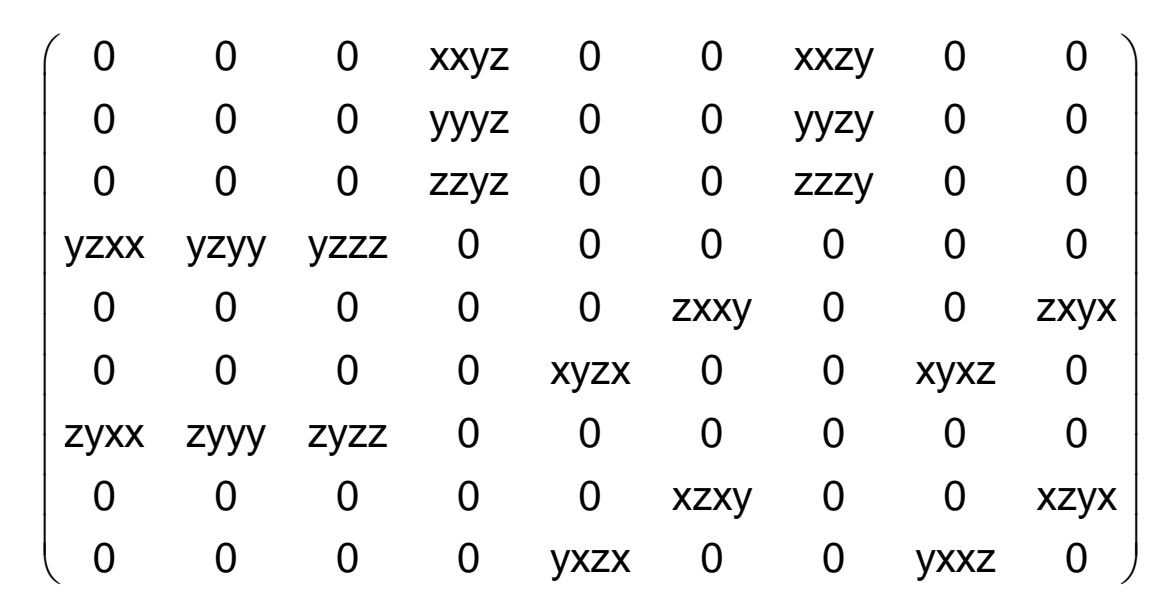

$\left(\begin{array}{ccccc}0 & 0 & 0 & 0 & x x y z \\ 0 & 0 & y z z z & 0 & 0 \\ 0 & z y y y & 0 & 0 & 0\end{array}\right)$

$\left(\begin{array}{cccccccccc}0 & 0 & 0 & 0 & 0 & 0 & 0 & 0 & 0 & x x y z \\ 0 & 0 & y z z z & 0 & 0 & y z x x & 0 & 0 & y z y & 0\end{array}\right.$ $\left(\begin{array}{cccccccccc}0 & 0 & 0 & 0 & 0 & 0 & 0 & 0 & 0 & x x y z \\ 0 & 0 & y z z z & 0 & 0 & y z x x & 0 & 0 & y z y y & 0\end{array}\right.$ $\left(\begin{array}{cccccc}0 & 0 & 0 & \text { xxyz } & 0 & 0 \\ & 0 & 0 & \text { yyyz } & 0 & 0 \\ & & 0 & \text { zzyz } & 0 & 0 \\ & & & 0 & 0 & 0 \\ & & & & 0 & \text { xzxy } \\ & & & & & 0\end{array}\right)$

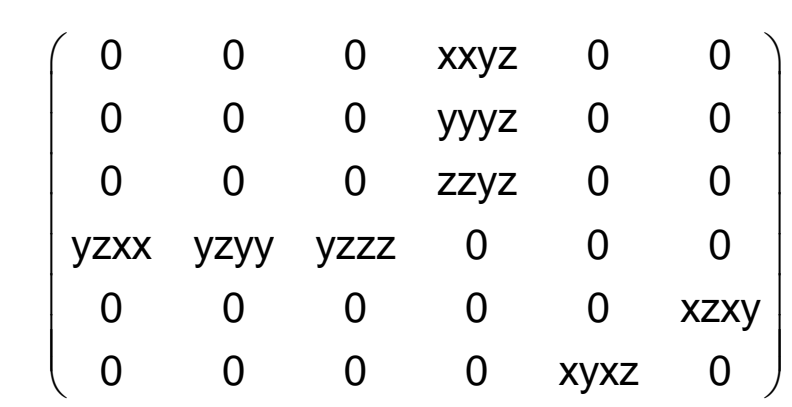

$\left(\begin{array}{ccccccccc}0 & 0 & 0 & \text { xxyz } & 0 & 0 & \text { xxzy } & 0 & 0 \\ 0 & 0 & 0 & \text { yyyz } & 0 & 0 & \text { yyzy } & 0 & 0 \\ 0 & 0 & 0 & \text { zzyz } & 0 & 0 & \text { zzzy } & 0 & 0 \\ \text { yzxx } & \text { yzyy } & \text { yzzz } & 0 & 0 & 0 & 0 & 0 & 0 \\ 0 & 0 & 0 & 0 & 0 & \text { zxxy } & 0 & 0 & \text { zxyx } \\ 0 & 0 & 0 & 0 & \text { xyzx } & 0 & 0 & \text { xyxz } & 0 \\ \text { zyxx } & \text { zyyy } & \text { zyzz } & 0 & 0 & 0 & 0 & 0 & 0 \\ 0 & 0 & 0 & 0 & 0 & \text { xzxy } & 0 & 0 & \text { xzyx } \\ 0 & 0 & 0 & 0 & \text { yxzx } & 0 & 0 & \text { yxxz } & 0\end{array}\right)$

$\left(\begin{array}{ccccc}0 & 0 & 0 & 0 & x x y z \\ 0 & 0 & y z z z & 0 & 0 \\ 0 & z y y y & 0 & 0 & 0\end{array}\right)$

$\left(\begin{array}{cccccccccc}0 & 0 & 0 & 0 & 0 & 0 & 0 & 0 & 0 & x x y z \\ 0 & 0 & y z z z & 0 & 0 & y z x x & 0 & 0 & y z y y & 0 \\ 0 & 7 y y & 0 & 0 & 7 u z & 0 & 0 & 7 w y & 0 & 0\end{array}\right.$ $\left.\begin{array}{cccccccccc}0 & 0 & y z z z & 0 & 0 & y z x x & 0 & 0 & y z y y & 0 \\ 0 & z y y & 0 & 0 & z y z z & 0 & 0 & z y x & 0 & 0\end{array}\right)$ $\left(\begin{array}{cccccc}0 & 0 & 0 & \text { xxyz } & 0 & 0 \\ & 0 & 0 & \text { yyyz } & 0 & 0 \\ & & 0 & \text { zzyz } & 0 & 0 \\ & & & 0 & 0 & 0 \\ & & & & 0 & \text { xzxy } \\ & & & & & 0\end{array}\right)$

$\left(\begin{array}{cccccc}0 & 0 & 0 & \text { xxyz } & 0 & 0 \\ 0 & 0 & 0 & \text { yyyz } & 0 & 0 \\ 0 & 0 & 0 & z z y z & 0 & 0 \\ \text { yzxx } & y z y y & y z z z & 0 & 0 & 0 \\ 0 & 0 & 0 & 0 & 0 & x z x y \\ 0 & 0 & 0 & 0 & x y x z & 0\end{array}\right)$ $\left(\begin{array}{ccccccccc}\text { xxxx } & \text { xxyy } & \text { xxzz } & 0 & 0 & 0 & 0 & 0 & 0 \\ \text { yyxx } & \text { yyyy } & \text { yyzz } & 0 & 0 & 0 & 0 & 0 & 0 \\ \text { zzxx } & \text { zzyy } & \text { zzzz } & 0 & 0 & 0 & 0 & 0 & 0 \\ 0 & 0 & 0 & \text { yzyz } & 0 & 0 & \text { yzzy } & 0 & 0 \\ 0 & 0 & 0 & 0 & \text { zxzx } & 0 & 0 & \text { zxxz } & 0 \\ 0 & 0 & 0 & 0 & 0 & \text { xyxy } & 0 & 0 & \text { xyyx } \\ 0 & 0 & 0 & \text { zyyz } & 0 & 0 & \text { zyzy } & 0 & 0 \\ 0 & 0 & 0 & 0 & \text { xzzx } & 0 & 0 & \text { xzxz } & 0 \\ 0 & 0 & 0 & 0 & 0 & \text { yxxy } & 0 & 0 & \text { yxyx }\end{array}\right)$

$\left(\begin{array}{ccccc}\mathrm{xxxx} & 0 & 0 & \mathrm{yyzz} & 0 \\ 0 & \mathrm{yyyy} & 0 & \mathrm{zzxx} & 0 \\ 0 & 0 & \mathrm{zzzz} & \mathrm{xxyy} & 0\end{array}\right)$

$\left(\begin{array}{cccccccccc}x x x x & 0 & 0 & x x y y & 0 & 0 & x x z z & 0 & 0 & 0 \\ 0 & y y y y & 0 & 0 & y y z z & 0 & 0 & y y x x & 0 & 0 \\ 0 & 0 & z z z z & 0 & 0 & z z x x & 0 & 0 & z z y & 0\end{array}\right)$

$\left(\begin{array}{cccccccccc}0 & 0 y y y & 0 & 0 & y y z z & 0 & 0 & y y x x & 0 & 0 \\ 0 & 0 & z z z z & 0 & 0 & z z x x & 0 & 0 & \text { zzyy } & 0\end{array}\right)$

$\left(\begin{array}{cccccc}\text { xxxx } & \text { xxyy } & \text { xxzz } & 0 & 0 & 0 \\ & \text { yyyy } & \text { yyzz } & 0 & 0 & 0 \\ & & \text { zzzz } & 0 & 0 & 0 \\ & & & y z y z & 0 & 0 \\ & & & & x z x z & 0 \\ & & & & & \text { xyxy }\end{array}\right)$

$\left(\begin{array}{llllll}x x x x & x x y y & x x z z & 0 & 0 & 0 \\ y y x x & y y y y & y y z z & 0 & 0 & 0 \\ z z x x & z z y y & z z z z & 0 & 0 & 0\end{array}\right)$

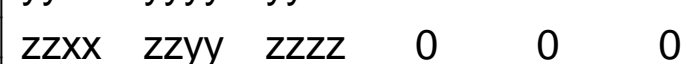

$\begin{array}{cccccc}z z x x & z z y & z z z z & 0 & 0 & 0 \\ 0 & 0 & 0 & y z y z & 0 & 0\end{array}$

$\begin{array}{cccccc}0 & 0 & 0 & y z y z & 0 & 0 \\ 0 & 0 & 0 & 0 & x z x z & 0 \\ 0 & 0 & 0 & 0 & 0 & x y y\end{array}$ 


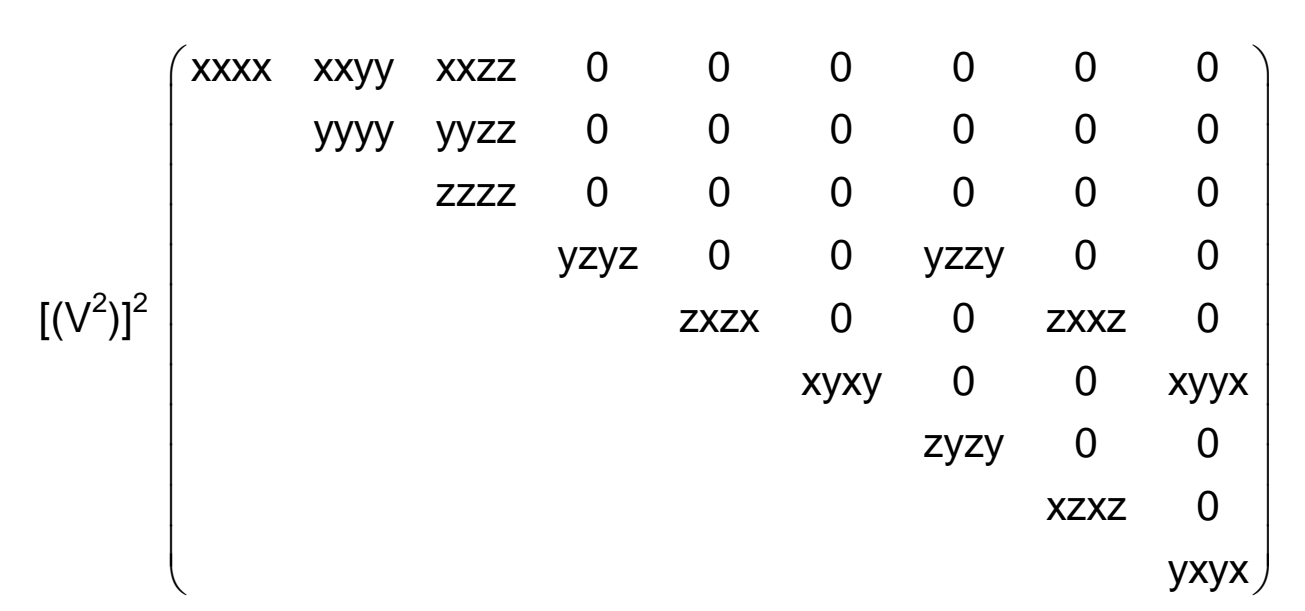

a

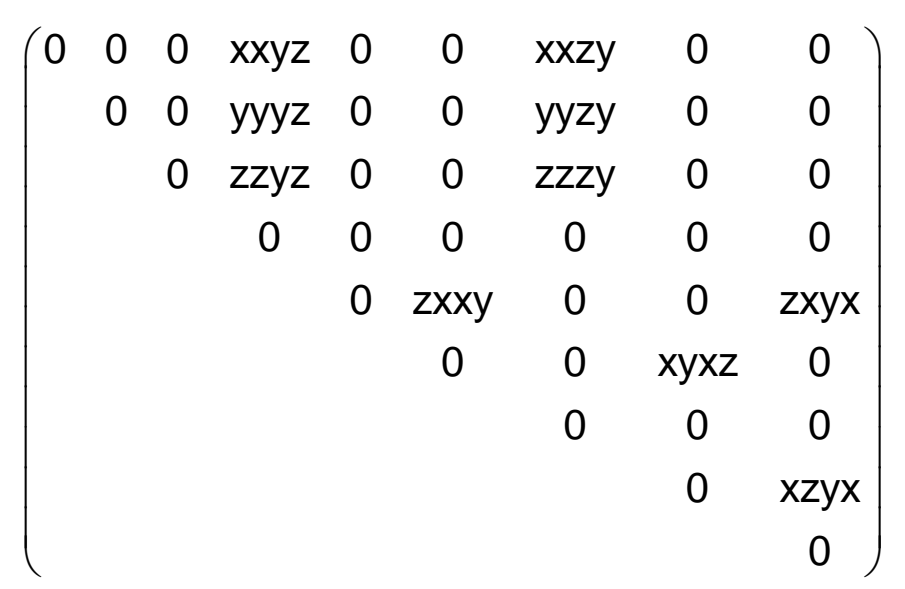

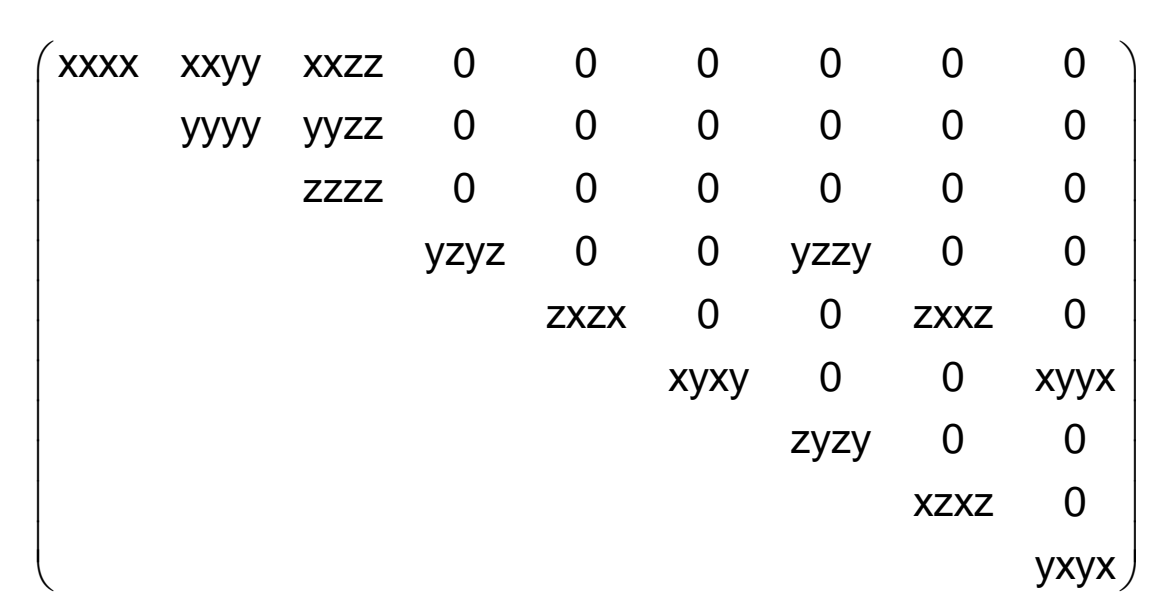

$\left[\mathrm{V}^{2}\right] \mathrm{V}^{2}\left(\begin{array}{ccccccccc}\mathrm{xxxx} & \mathrm{xxyy} & \mathrm{xxzz} & 0 & 0 & 0 & 0 & 0 & 0 \\ \mathrm{yyxx} & \mathrm{yyyy} & \mathrm{yyzz} & 0 & 0 & 0 & 0 & 0 & 0 \\ \mathrm{zzxx} & \mathrm{zzyy} & \mathrm{zzzz} & 0 & 0 & 0 & 0 & 0 & 0 \\ 0 & 0 & 0 & \mathrm{yzyz} & 0 & 0 & \mathrm{yzzy} & 0 & 0 \\ 0 & 0 & 0 & 0 & \mathrm{zxzx} & 0 & 0 & \mathrm{zxxz} & 0 \\ 0 & 0 & 0 & 0 & 0 & \mathrm{xyxy} & 0 & 0 & \mathrm{xyyx}\end{array}\right)$
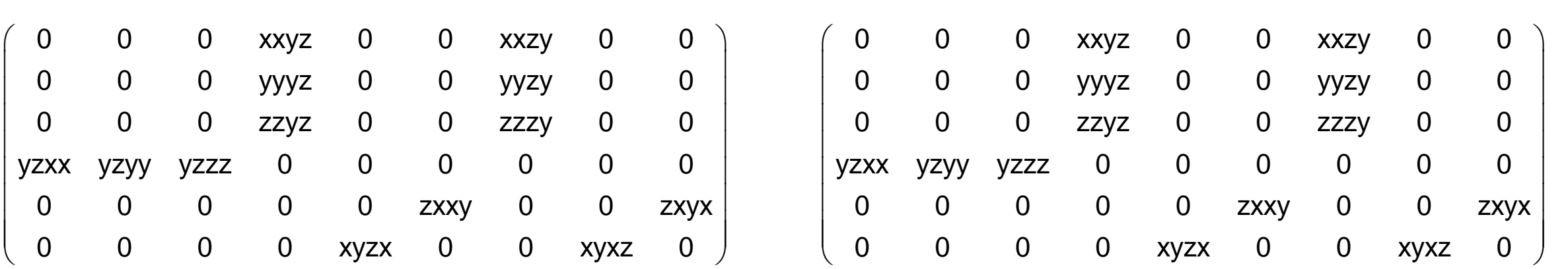

$\left(\begin{array}{ccccccccc}\text { xxxx } & \text { xxyy } & \text { xxzz } & 0 & 0 & 0 & 0 & 0 & 0 \\ \text { yyxx } & \text { yyyy } & \text { yyzz } & 0 & 0 & 0 & 0 & 0 & 0 \\ \text { zzxx } & \text { zzyy } & \text { zzzz } & 0 & 0 & 0 & 0 & 0 & 0 \\ 0 & 0 & 0 & \text { yzyz } & 0 & 0 & \text { yzzy } & 0 & 0 \\ 0 & 0 & 0 & 0 & \text { zxzx } & 0 & 0 & \text { zxxz } & 0 \\ 0 & 0 & 0 & 0 & 0 & \text { xyxy } & 0 & 0 & \text { xyyx }\end{array}\right)$ 


$\left(\begin{array}{ccccccccc}\text { xxxx } & \text { xxyy } & \text { xxzz } & 0 & 0 & 0 & 0 & 0 & 0 \\ \text { yyxx } & \text { yyyy } & \text { yyzz } & 0 & 0 & 0 & 0 & 0 & 0 \\ \text { zzxx } & \text { zzyy } & \text { zzzz } & 0 & 0 & 0 & 0 & 0 & 0 \\ 0 & 0 & 0 & \text { yzyz } & 0 & 0 & \text { yzzy } & 0 & 0 \\ 0 & 0 & 0 & 0 & \text { zxzx } & 0 & 0 & \text { zxxz } & 0 \\ 0 & 0 & 0 & 0 & 0 & \text { xyxy } & 0 & 0 & \text { xyyx } \\ 0 & 0 & 0 & \text { zyyz } & 0 & 0 & \text { zyzy } & 0 & 0 \\ 0 & 0 & 0 & 0 & \text { xzzx } & 0 & 0 & \text { xzxz } & 0 \\ 0 & 0 & 0 & 0 & 0 & \text { yxxy } & 0 & 0 & \text { yxyx }\end{array}\right)$

$\left[V^{4}\right] \quad\left(\begin{array}{ccccc}x x x x & 0 & 0 & y y z z & 0 \\ 0 & y y y y & 0 & z z x x & 0 \\ 0 & 0 & z z z z & x x y y & 0\end{array}\right)$

$\left.V^{3}\right]\left(\begin{array}{cccccccccc}x x x x & 0 & 0 & x x y y & 0 & 0 & x x z z & 0 & 0 & 0 \\ 0 & y y y y & 0 & 0 & y y z z & 0 & 0 & y y x x & 0 & 0 \\ 0 & 0 & z z z z & 0 & 0 & z z x & 0 & 0 & z z y & 0\end{array}\right)$

V[V] $\left(\begin{array}{ccccccccccc}0 & y & 0 & 0 & & 0 & 0 & x y z z & 0 & 0 & 0 \\ 0 & 0 & z z z z & 0 & 0 & z z x x & 0 & 0 & \text { zzyy } & 0\end{array}\right)$

$\left[\left[V^{2}\right]^{2}\right]\left(\begin{array}{cccccc}x x x x & x x y y & x x z z & 0 & 0 & 0 \\ & \text { yyyy } & \text { yyzz } & 0 & 0 & 0 \\ & & z z z z & 0 & 0 & 0 \\ & & & y z y z & 0 & 0 \\ & & & & x z x z & 0 \\ & & & & & \text { xyxy }\end{array}\right)$

$\left[\mathrm{V}^{2}\right]^{2}$ $\left(\begin{array}{lllllllll}0 & 0 & 0 & 0 & 0 & 0 & 0 & 0 & 0 \\ 0 & 0 & 0 & 0 & 0 & 0 & 0 & 0 & 0\end{array}\right)$

$\begin{array}{lllllllll}0 & 0 & 0 & 0 & 0 & 0 & 0 & 0 & 0 \\ 0 & 0 & 0 & 0 & 0 & 0 & 0 & 0 & 0\end{array}$ $\begin{array}{lllllllll}0 & 0 & 0 & 0 & 0 & 0 & 0 & 0 & 0 \\ 0 & 0 & 0 & 0 & 0 & 0 & 0 & 0 & 0\end{array}$

$0 \begin{array}{lllllllll}0 & 0 & 0 & 0 & 0 & 0 & 0 & 0 & 0 \\ 0 & 0 & 0 & 0 & 0 & 0 & 0 & 0 & 0\end{array}$

$0 \begin{array}{llllllllll}0 & 0 & 0 & 0 & 0 & 0 & 0 & 0 & 0 \\ 0 & 0 & 0 & 0 & 0 & 0 & 0 & 0 & 0\end{array}$

$0 \begin{array}{llllllllll}0 & 0 & 0 & 0 & 0 & 0 & 0 & 0 & 0 \\ 0 & 0 & 0 & 0 & 0 & 0 & 0 & 0 & 0\end{array}$

$\begin{array}{lllllllll}0 & 0 & 0 & 0 & 0 & 0 & 0 & 0 & 0\end{array}$

$\begin{array}{lllllllll}0 & 0 & 0 & 0 & 0 & 0 & 0 & 0 & 0\end{array}$

$\left(\begin{array}{lllll}0 & 0 & 0 & 0 & 0 \\ 0 & 0 & 0 & 0 & 0 \\ 0 & 0 & 0 & 0 & 0\end{array}\right)$

$\left(\begin{array}{llllllllll}0 & 0 & 0 & 0 & 0 & 0 & 0 & 0 & 0 & 0 \\ 0 & 0 & 0 & 0 & 0 & 0 & 0 & 0 & 0 & 0\end{array}\right.$ $\left(\begin{array}{llllllllll}0 & 0 & 0 & 0 & 0 & 0 & 0 & 0 & 0 & 0 \\ 0 & 0 & 0 & 0 & 0 & 0 & 0 & 0 & 0 & 0\end{array}\right)$

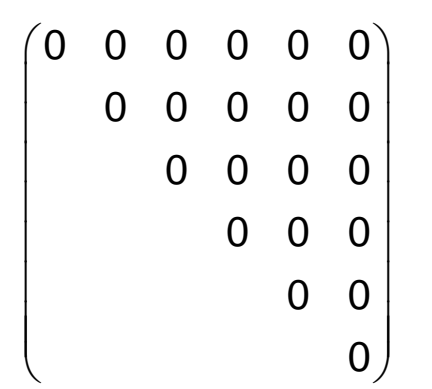

$\left(\begin{array}{llllll}0 & 0 & 0 & 0 & 0 & 0 \\ 0 & 0 & 0 & 0 & 0 & 0\end{array}\right)$ $\begin{array}{llllll}0 & 0 & 0 & 0 & 0 & 0 \\ 0 & 0 & 0 & 0 & 0 & 0 \\ 0 & 0 & 0 & 0 & 0 & 0\end{array}$ $0 \begin{array}{llllll}0 & 0 & 0 & 0 & 0 & 0\end{array}$ $\begin{array}{llllll}0 & 0 & 0 & 0 & 0 & 0 \\ 0 & 0 & 0 & 0 & 0\end{array}$ $\begin{array}{llllll}0 & 0 & 0 & 0 & 0 & 0 \\ 0 & 0 & 0 & 0 & 0 & 0\end{array}$ $\begin{array}{lllllllll}0 & 0 & 0 & 0 & 0 & 0 & 0 & 0 & 0 \\ 0 & 0 & 0 & 0 & 0 & 0 & 0 & 0 & 0\end{array}$ $\left.0 \begin{array}{lllllllll}0 & 0 & 0 & 0 & 0 & 0 & 0 & 0 & 0 \\ 0 & 0 & 0 & 0 & 0 & 0 & 0 & 0 & 0\end{array}\right)$ $0 \begin{array}{lllllllll}0 & 0 & 0 & 0 & 0 & 0 & 0 & 0 & 0 \\ 0 & 0 & 0 & 0 & 0 & 0 & 0 & 0 & 0\end{array}$ $\begin{array}{lllllllll}0 & 0 & 0 & 0 & 0 & 0 & 0 & 0 & 0 \\ 0 & 0 & 0 & 0 & 0 & 0 & 0 & 0 & 0\end{array}$ $\begin{array}{llllllllll}0 & 0 & 0 & 0 & 0 & 0 & 0 & 0 & 0 \\ 0 & 0 & 0 & 0 & 0 & 0 & 0 & 0 & 0\end{array}$ $\begin{array}{llllllllll}0 & 0 & 0 & 0 & 0 & 0 & 0 & 0 & 0 \\ 0 & 0 & 0 & 0 & 0 & 0 & 0 & 0 & 0 & 0\end{array}$ $\begin{array}{llllllllllll}0 & 0 & 0 & 0 & 0 & 0 & 0 & 0 & 0 \\ 0 & 0 & 0 & 0 & 0 & 0 & 0 & 0 & 0\end{array}$ $0 \begin{array}{llllllllll}0 & 0 & 0 & 0 & 0 & 0 & 0 & 0 & 0\end{array}$ $\begin{array}{lllllllll}0 & 0 & 0 & 0 & 0 & 0 & 0 & 0 & 0\end{array}$

$\left(\begin{array}{lllll}0 & 0 & 0 & 0 & 0 \\ 0 & 0 & 0 & 0 & 0 \\ 0 & 0 & 0 & 0 & 0\end{array}\right)$ $\begin{array}{lllll}0 & 0 & 0 & 0 & 0\end{array}$

$\left(\begin{array}{llllllllll}0 & 0 & 0 & 0 & 0 & 0 & 0 & 0 & 0 & 0 \\ 0 & 0 & 0 & 0 & 0 & 0 & 0 & 0 & 0 & 0\end{array}\right.$ $\left.\begin{array}{lllllllllll}0 & 0 & 0 & 0 & 0 & 0 & 0 & 0 & 0 & 0 \\ 0 & 0 & 0 & 0 & 0 & 0 & 0 & 0 & 0 & 0\end{array}\right)$ $\begin{array}{llllllllll}0 & 0 & 0 & 0 & 0 & 0 & 0 & 0 & 0 & 0\end{array}$

$$
\left(\begin{array}{llllll}
0 & 0 & 0 & 0 & 0 & 0 \\
& 0 & 0 & 0 & 0 & 0 \\
& & 0 & 0 & 0 & 0 \\
& & & 0 & 0 & 0 \\
& & & & 0 & 0 \\
& & & & & 0
\end{array}\right)
$$

$\left(\begin{array}{llllll}0 & 0 & 0 & 0 & 0 & 0 \\ 0 & 0 & 0 & 0 & 0 & 0\end{array}\right)$ $\left(\begin{array}{llllll}0 & 0 & 0 & 0 & 0 & 0 \\ 0 & 0 & 0 & 0 & 0 & 0 \\ 0 & 0 & 0 & 0 & 0 & 0\end{array}\right)$ $0 \begin{array}{llllll}0 & 0 & 0 & 0 & 0 & 0\end{array}$ $\begin{array}{llllll}0 & 0 & 0 & 0 & 0 & 0 \\ 0 & 0 & 0 & 0 & 0 & 0\end{array}$

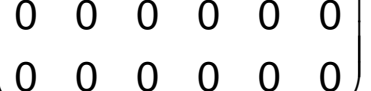

$\left(\begin{array}{ccccccccc}\text { xxxx } & \text { xxyy } & \text { xxzz } & 0 & 0 & 0 & 0 & 0 & 0 \\ \text { yyxx } & \text { yyyy } & \text { yyzz } & 0 & 0 & 0 & 0 & 0 & 0 \\ \text { zzxx } & \text { zzyy } & \text { zzzz } & 0 & 0 & 0 & 0 & 0 & 0 \\ 0 & 0 & 0 & \text { yzyz } & 0 & 0 & \text { yzzy } & 0 & 0 \\ 0 & 0 & 0 & 0 & \text { zxzx } & 0 & 0 & \text { zxxz } & 0 \\ 0 & 0 & 0 & 0 & 0 & \text { xyxy } & 0 & 0 & \text { xyyx } \\ 0 & 0 & 0 & \text { zyyz } & 0 & 0 & \text { zyzy } & 0 & 0 \\ 0 & 0 & 0 & 0 & \text { xzzx } & 0 & 0 & \text { xzxz } & 0 \\ 0 & 0 & 0 & 0 & 0 & \text { yxxy } & 0 & 0 & \text { yxyx }\end{array}\right)$

$\left(\begin{array}{ccccc}\mathrm{xxxx} & 0 & 0 & \mathrm{yyzz} & 0 \\ 0 & \mathrm{yyyy} & 0 & \mathrm{zzxx} & 0 \\ 0 & 0 & \mathrm{zzzz} & \mathrm{xxyy} & 0\end{array}\right)$

$\left(\begin{array}{cccccccccc}\mathrm{xxxx} & 0 & 0 & \mathrm{xxyy} & 0 & 0 & \mathrm{xxzz} & 0 & 0 & 0 \\ 0 & \mathrm{yyyy} & 0 & 0 & \mathrm{yyzz} & 0 & 0 & \mathrm{yyxx} & 0 & 0 \\ 0 & 0 & z z z z & 0 & 0 & \mathrm{zzxx} & 0 & 0 & \mathrm{zzyy} & 0\end{array}\right)$

$\begin{array}{llllllllll}0 & 0 & z z z z & 0 & 0 & z z x x & 0 & 0 & \text { zzyy } & 0\end{array}$

$$
\left(\begin{array}{cccccc}
\text { xxxx } & \text { xxyy } & \text { xxzz } & 0 & 0 & 0 \\
& \text { yyyy } & \text { yyzz } & 0 & 0 & 0 \\
& & \text { zzzz } & 0 & 0 & 0 \\
& & & y z y z & 0 & 0 \\
& & & & x z x z & 0 \\
& & & & & \text { xyxy }
\end{array}\right)
$$

$\left(\begin{array}{cccccc}\text { xxxx } & \text { xxyy } & \text { xxzz } & 0 & 0 & 0 \\ \text { yyxx } & \text { yyyy } & \text { yyzz } & 0 & 0 & 0 \\ \text { zzxx } & \text { zzyy } & \text { zzzz } & 0 & 0 & 0 \\ 0 & 0 & 0 & y z y z & 0 & 0 \\ 0 & 0 & 0 & 0 & \text { xzxz } & 0 \\ 0 & 0 & 0 & 0 & 0 & \text { xyxy }\end{array}\right)$ 
1

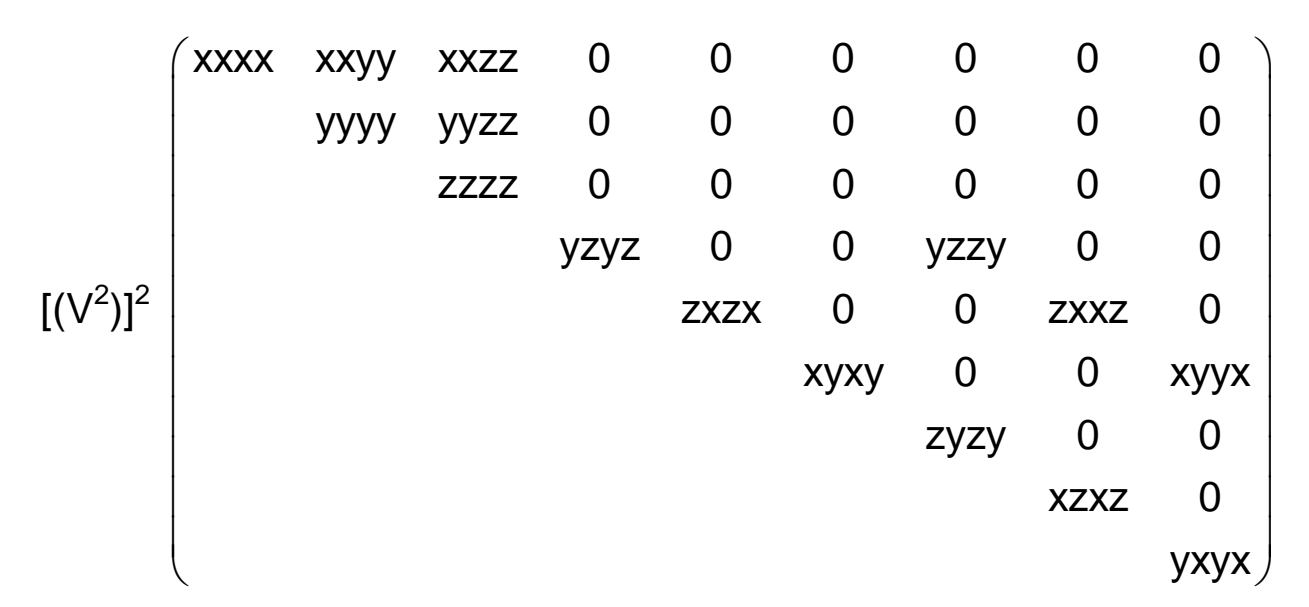

$\left[\mathrm{V}^{2}\right] \mathrm{V}^{2}\left(\begin{array}{ccccccccc}\mathrm{x} x \mathrm{x} & \mathrm{xxyy} & \mathrm{xxzz} & 0 & 0 & 0 & 0 & 0 & 0 \\ \mathrm{yyxx} & \text { yyyy } & \mathrm{yyzz} & 0 & 0 & 0 & 0 & 0 & 0 \\ \mathrm{zzxx} & \mathrm{zzyy} & \mathrm{zzzz} & 0 & 0 & 0 & 0 & 0 & 0 \\ 0 & 0 & 0 & \mathrm{yzyz} & 0 & 0 & \mathrm{yzzy} & 0 & 0 \\ 0 & 0 & 0 & 0 & \mathrm{zxzx} & 0 & 0 & \mathrm{zxxz} & 0 \\ 0 & 0 & 0 & 0 & 0 & \mathrm{xyxy} & 0 & 0 & \mathrm{xyyx}\end{array}\right)$ $\left(\begin{array}{llllllll}0 & 0 & 0 & 0 & 0 & 0 & 0 & 0 \\ 0 & 0 & 0 & 0 & 0 & 0 & 0 & 0\end{array}\right.$

$\begin{array}{llllllll}0 & 0 & 0 & 0 & 0 & 0 & 0 & 0\end{array}$ $\begin{array}{lllllll}0 & 0 & 0 & 0 & 0 & 0 & 0 \\ 0 & 0 & 0 & 0 & 0 & 0\end{array}$

$0 \begin{array}{lllll}0 & 0 & 0 & 0 & 0\end{array}$

$\begin{array}{llll}0 & 0 & 0 & 0\end{array}$

$\begin{array}{llll}0 & 0 & 0 \\ & 0 & 0\end{array}$

$\begin{array}{llllllll}0 & 0 & 0 & 0 & 0 & 0 & 0 & 0 \\ & 0 & 0 & 0 & 0 & 0 & 0 & 0\end{array}$

$\begin{array}{lllllll}0 & 0 & 0 & 0 & 0 & 0 \\ 0 & 0 & 0 & 0 & 0 & 0\end{array}$

$\begin{array}{llllll}0 & 0 & 0 & 0 & 0\end{array}$

$\begin{array}{llll}0 & 0 & 0 & 0 \\ 0 & 0 & 0\end{array}$

$\begin{array}{lll}0 & 0 & 0 \\ & 0 & 0\end{array}$

$\left(\begin{array}{lllllllll}0 & 0 & 0 & 0 & 0 & 0 & 0 & 0 & 0 \\ 0 & 0 & 0 & 0 & 0 & 0 & 0 & 0 & 0\end{array}\right)$

$\begin{array}{lllllllll}0 & 0 & 0 & 0 & 0 & 0 & 0 & 0 & 0 \\ 0 & 0 & 0 & 0 & 0 & 0 & 0 & 0 & 0\end{array}$

$\begin{array}{llllllllll}0 & 0 & 0 & 0 & 0 & 0 & 0 & 0 & 0 \\ 0 & 0 & 0 & 0 & 0 & 0 & 0 & 0 & 0\end{array}$

$\begin{array}{llllllllll}0 & 0 & 0 & 0 & 0 & 0 & 0 & 0 & 0 \\ 0 & 0 & 0 & 0 & 0 & 0 & 0 & 0 & 0\end{array}$

$\begin{array}{lllllllll}0 & 0 & 0 & 0 & 0 & 0 & 0 & 0 & 0 \\ 0 & 0 & 0 & 0 & 0 & 0 & 0 & 0 & 0\end{array}$

$\left(\begin{array}{lllllllll}0 & 0 & 0 & 0 & 0 & 0 & 0 & 0 & 0 \\ 0 & 0 & 0 & 0 & 0 & 0 & 0 & 0 & 0\end{array}\right.$ $\begin{array}{llllllllll}0 & 0 & 0 & 0 & 0 & 0 & 0 & 0 & 0 \\ 0 & 0 & 0 & 0 & 0 & 0\end{array}$ $\begin{array}{llllllllll}0 & 0 & 0 & 0 & 0 & 0 & 0 & 0 & 0 \\ 0 & 0 & 0 & 0 & 0 & 0 & 0 & 0 & 0\end{array}$ $0 \begin{array}{llllllllll}0 & 0 & 0 & 0 & 0 & 0 & 0 & 0 & 0 \\ 0 & 0 & 0 & 0 & 0 & 0 & 0 & 0 & 0\end{array}$ $\begin{array}{llllllllll}0 & 0 & 0 & 0 & 0 & 0 & 0 & 0 & 0\end{array}$ $\left(\begin{array}{lllllllll}0 & 0 & 0 & 0 & 0 & 0 & 0 & 0 & 0\end{array}\right.$

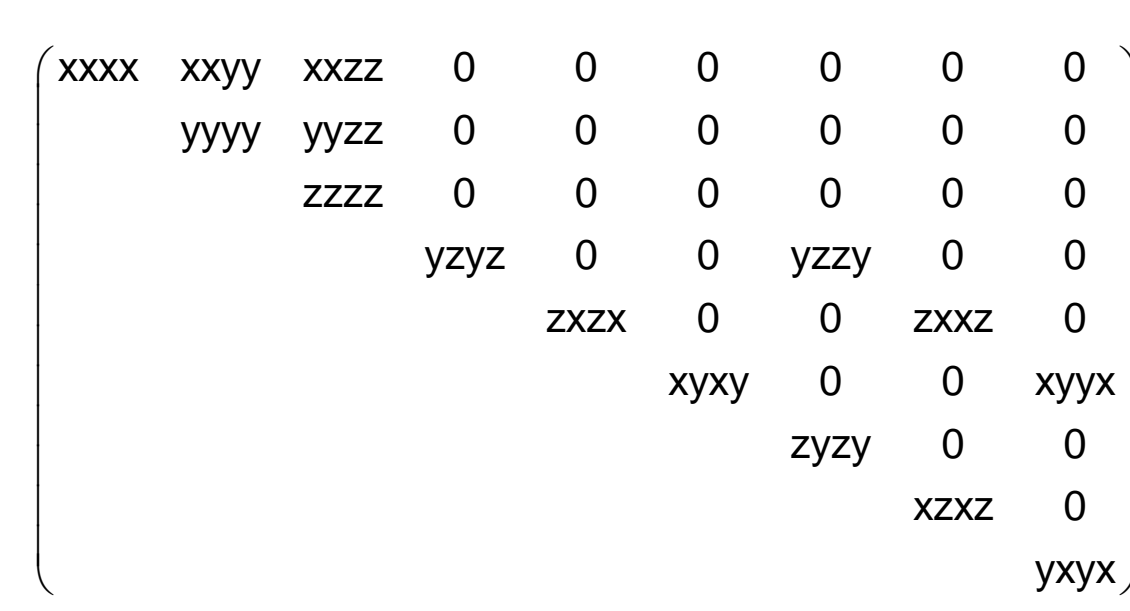

$\left(\begin{array}{lllllllll}x x x x & x x y y & x x z z & 0 & 0 & 0 & 0 & 0 & 0 \\ y & y y y & y y z & 0 & 0 & 0 & 0 & 0 & 0\end{array}\right.$ $\begin{array}{lllllllll}\text { yyxx } & y y y y & y y z z & 0 & 0 & 0 & 0 & 0 & 0\end{array}$

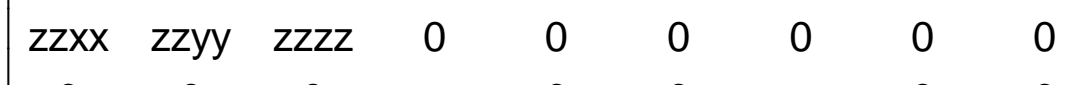
$\begin{array}{lllllllll}0 & 0 & 0 & y z y z & 0 & 0 & y z z y & 0 & 0\end{array}$ 


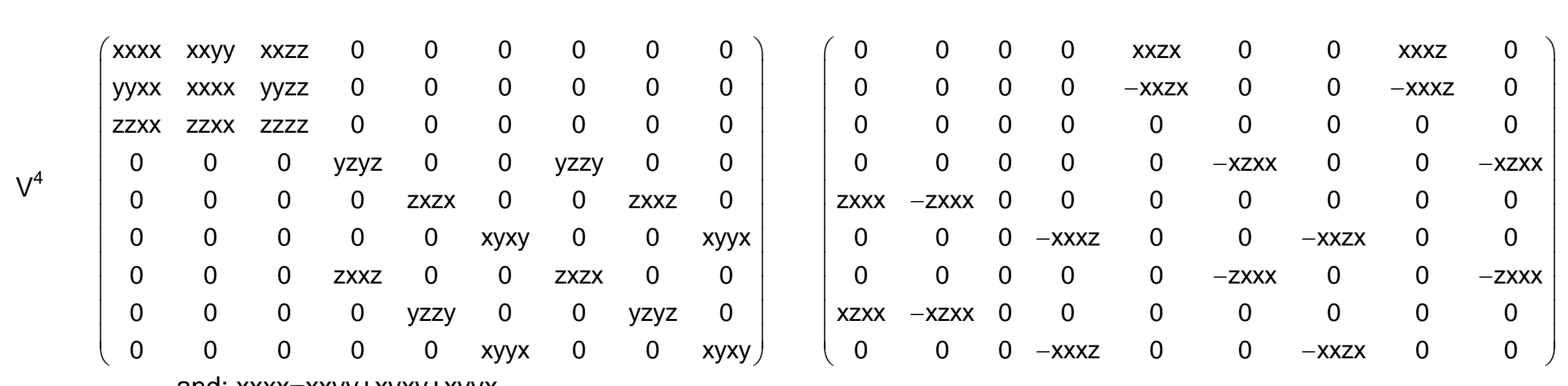
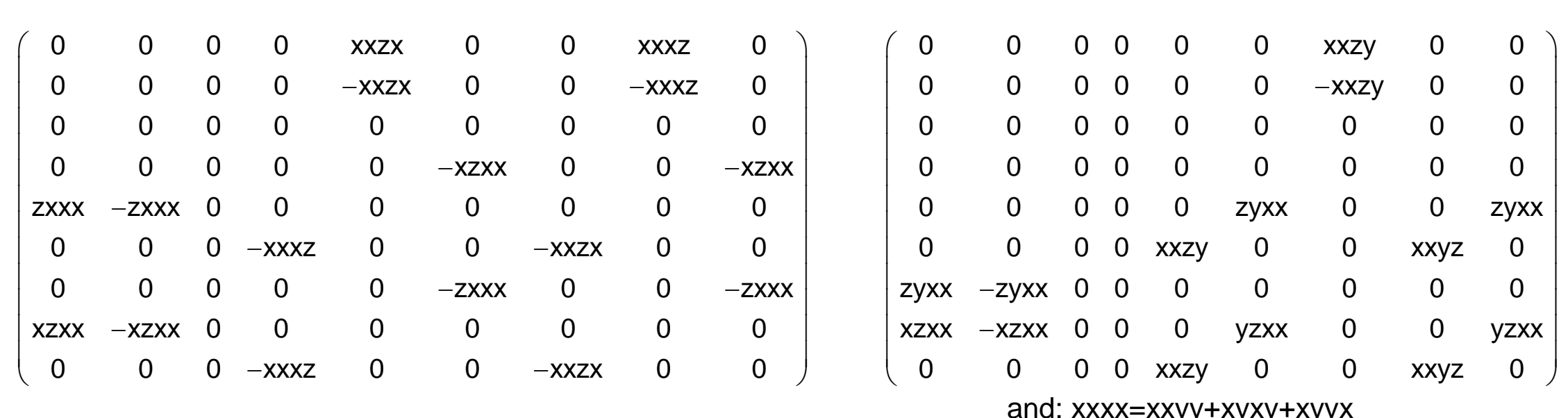

$\left[V^{4}\right]\left(\begin{array}{ccccc}3 x x y y & 0 & 0 & y y z z & 0 \\ 0 & 3 x x y y & 0 & y y z z & 0 \\ 0 & 0 & z z z z & x x y y & 0\end{array}\right)$

$$
\left(\begin{array}{ccccc}
0 & 0 & 0 & 0 & 0 \\
0 & 0 & 0 & 0 & -z x x x \\
z x x x & 0 & 0 & 0 & 0
\end{array}\right)
$$

$\left(\begin{array}{ccccc}3 x x y y & 0 & 0 & y y z z & 0 \\ 0 & 3 x x y y & 0 & y y z z & 0 \\ 0 & 0 & z z z z & x x y y & 0\end{array}\right)$

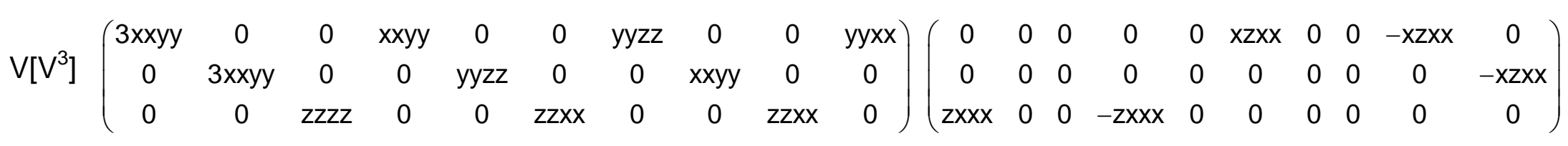

$$
\left(\begin{array}{ccccc}
0 & 0 & 0 & 0 & 0 \\
0 & 0 & 0 & 0 & -z x x x \\
z x x x & 0 & 0 & 0 & 0
\end{array}\right)
$$$$
\left(\begin{array}{cccccccccc}
0 & 0 & 0 & 0 & 0 & \mathrm{xzxx} & 0 & 0 & -\mathrm{xzxx} & 0 \\
0 & 0 & 0 & 0 & 0 & 0 & 0 & 0 & 0 & -\mathrm{xzxx} \\
\mathrm{zxxx} & 0 & 0 & -\mathrm{zxx} & 0 & 0 & 0 & 0 & 0 & 0
\end{array}\right)
$$$$
\left(\begin{array}{ccccc}
3 x x y y & 0 & 0 & y y z z & 0 \\
0 & 3 x x y y & 0 & y y z z & 0 \\
0 & 0 & z z z z & x x y y & 0
\end{array}\right)
$$

$\left[\left[V^{2}\right]^{2}\right]\left(\begin{array}{cccccc}x x x x & x x y y & x x z z & 0 & 0 & 0 \\ & x x x x & x x z z & 0 & 0 & 0 \\ & & z z z z & 0 & 0 & 0 \\ & & & y z y z & 0 & 0 \\ & & & & y z y z & 0 \\ & & & & & \text { xyxy }\end{array}\right)$

and $x y x y=1 / 2(x x x x-x x y y)$

$\left[\mathrm{V}^{2}\right]^{2}\left(\begin{array}{cccccc}x \mathrm{x} x \mathrm{x} & \mathrm{xxyy} & \mathrm{xxzz} & 0 & 0 & 0 \\ \mathrm{xxyy} & \mathrm{xxxx} & \mathrm{xxzz} & 0 & 0 & 0 \\ \mathrm{zzxx} & \mathrm{zzxx} & \mathrm{zzzz} & 0 & 0 & 0 \\ 0 & 0 & 0 & \mathrm{yzyz} & 0 & 0 \\ 0 & 0 & 0 & 0 & \mathrm{yzyz} & 0 \\ 0 & 0 & 0 & 0 & 0 & \mathrm{xyxy}\end{array}\right)$

and $x y x y=1 / 2(x x x x-x x y y)$

$$
\left(\begin{array}{cccccc}
0 & 0 & 0 & x x y z & 0 & 0 \\
& 0 & 0 & -x x y z & 0 & 0 \\
& & 0 & 0 & 0 & 0 \\
& & & 0 & 0 & 0 \\
& & & & 0 & x x y z \\
& & & & & 0
\end{array}\right)
$$

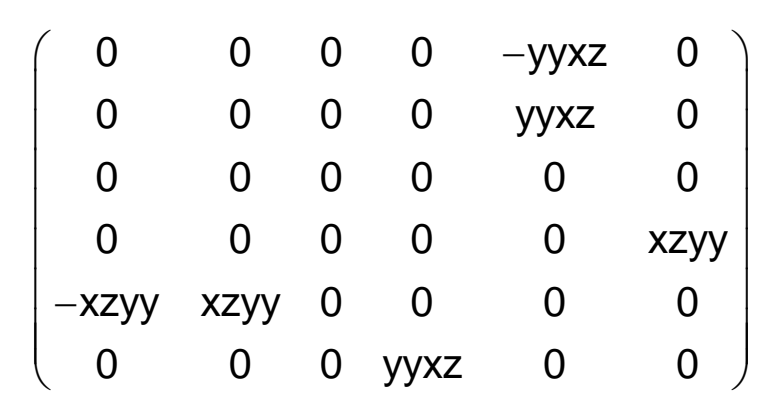

$$
\left(\begin{array}{cccccc}
0 & 0 & 0 & x x y z & 0 & 0 \\
& 0 & 0 & -x x y z & 0 & 0 \\
& & 0 & 0 & 0 & 0 \\
& & & 0 & 0 & 0 \\
& & & & 0 & x x y z \\
& & & & & 0
\end{array}\right)
$$

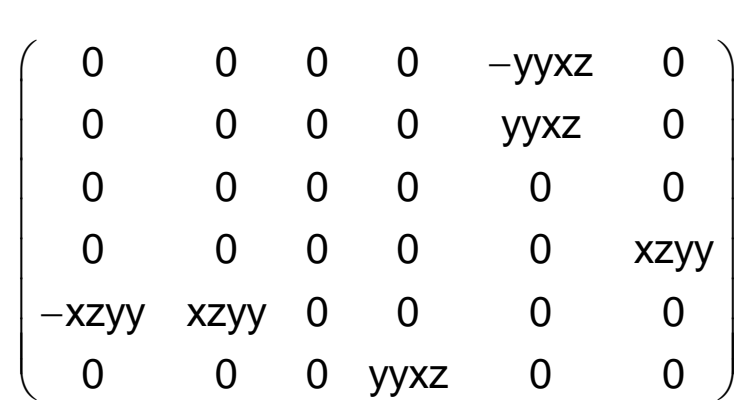

$\left(\begin{array}{cccccc}x x x x & x x y y & x x z z & 0 & 0 & 0 \\ & x x x x & x x z z & 0 & 0 & 0 \\ & & z z z z & 0 & 0 & 0 \\ & & & y z y z & 0 & 0 \\ & & & & y z y z & 0 \\ & & & & & \\ & & & & & \end{array}\right.$

and $x y x y=1 / 2(x x x x-x x y y)$

$\left(\begin{array}{llllll}x x x x & x x y y & x x z z & 0 & 0 & 0\end{array}\right.$ $\left(\begin{array}{llllll}x x x x & x x y y & x x z z & 0 & 0 & 0 \\ x x y y & x x x x & x x z z & 0 & 0 & 0 \\ z z x x & z z x x & z z z & 0 & 0 & 0\end{array}\right)$ $\begin{array}{cccccc}\operatorname{zzxx} & \operatorname{zzxx} & \mathrm{zzzz} & 0 & 0 & 0 \\ 0 & 0 & 0 & y z y z & 0 & 0 \\ 0 & 0 & 0 & 0 & y z y z & 0\end{array}$ $\begin{array}{cccccc}0 & 0 & 0 & y z y z & 0 & 0 \\ 0 & 0 & 0 & 0 & y z y z & 0 \\ 0 & 0 & 0 & 0 & 0 & x y y\end{array}$

and $x y x y=1 / 2(x x x x-x x y y)$ 
a

and $x x x x=x x y y+y z y z+z y z y$

$\left(\begin{array}{lllllll}x x x x & x x y y & x x z z & 0 & 0 & 0 & 0\end{array}\right.$

$\left.\begin{array}{lllllllll}x x y y & x x x x & x x z z & 0 & 0 & 0 & 0 & 0 & 0\end{array}\right)$

$\left[\mathrm{V}^{2}\right] \mathrm{V}^{2} \begin{array}{cccccccccc}\operatorname{zzxx} & \mathrm{zzxx} & \mathrm{zzzz} & 0 & 0 & 0 & 0 & 0 & 0 \\ 0 & 0 & 0 & \mathrm{yzyz} & 0 & 0 & \mathrm{zxzx} & 0 & 0 \\ 0 & 0 & 0 & 0 & \mathrm{z} z \mathrm{zx} & 0 & 0 & \mathrm{yzyz} & 0\end{array}$

$\begin{array}{ccccccccc}0 & 0 & 0 & y z y z & 0 & 0 & z x z x & 0 & 0 \\ 0 & 0 & 0 & 0 & z x z x & 0 & 0 & y z y z & 0 \\ 0 & 0 & 0 & 0 & 0 & x y y & 0 & 0 & x y x y\end{array}$

$\left(\begin{array}{ccccccccc}0 & 0 & 0 & 0 & 2 x z x & 0 & 0 & y z y z & 0 \\ 0 & 0 & 0 & 0 & 0 & x y x y & 0 & 0 & x y x y\end{array}\right)$

and $x x x x=x x y y+2 x y x y$
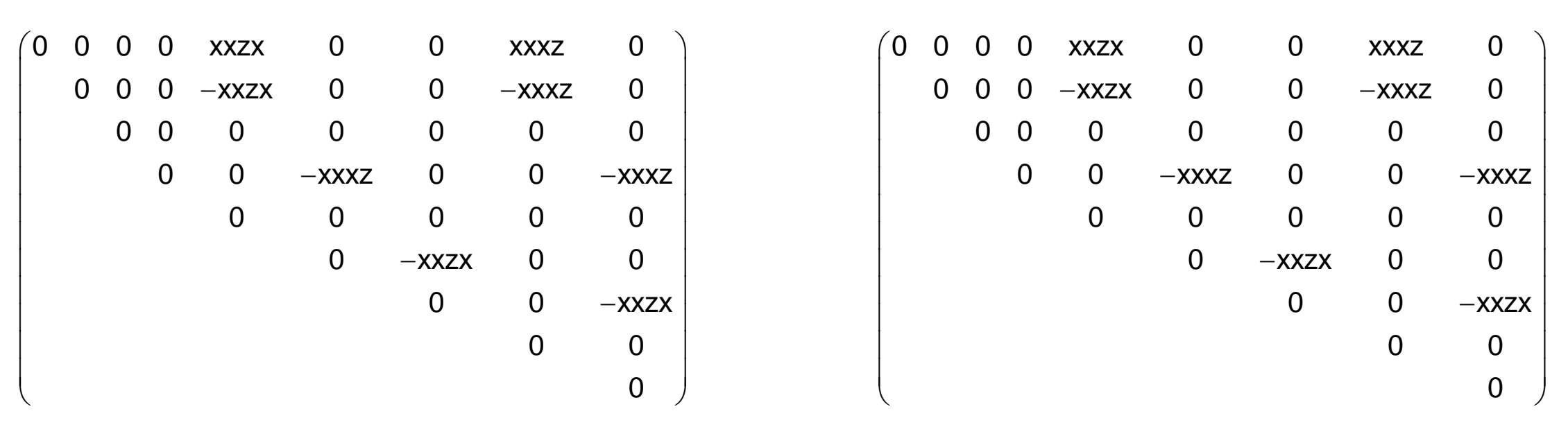

$\left(\begin{array}{ccccccccc}0 & 0 & 0 & 0 & \text { xxzx } & 0 & 0 & x x x z & 0 \\ 0 & 0 & 0 & 0 & -x x z x & 0 & 0 & -x x x z & 0 \\ 0 & 0 & 0 & 0 & 0 & 0 & 0 & 0 & 0 \\ 0 & 0 & 0 & 0 & 0 & 0 & 0 & 0 & -z x x x \\ z x x x & -z x x x & 0 & 0 & 0 & 0 & 0 & 0 & 0 \\ 0 & 0 & 0 & -x x x z & 0 & 0 & -x x z x & 0 & 0\end{array}\right)$

$\left(\begin{array}{ccccccccc}0 & 0 & 0 & 0 & \text { xxzx } & 0 & 0 & \text { xxxz } & 0 \\ 0 & 0 & 0 & 0 & -x x z x & 0 & 0 & -x x x z & 0 \\ 0 & 0 & 0 & 0 & 0 & 0 & 0 & 0 & 0 \\ 0 & 0 & 0 & 0 & 0 & 0 & 0 & 0 & -z x x x \\ z x x x & -z x x x & 0 & 0 & 0 & 0 & 0 & 0 & 0 \\ 0 & 0 & 0 & -x x x z & 0 & 0 & -x x z x & 0 & 0\end{array}\right)$

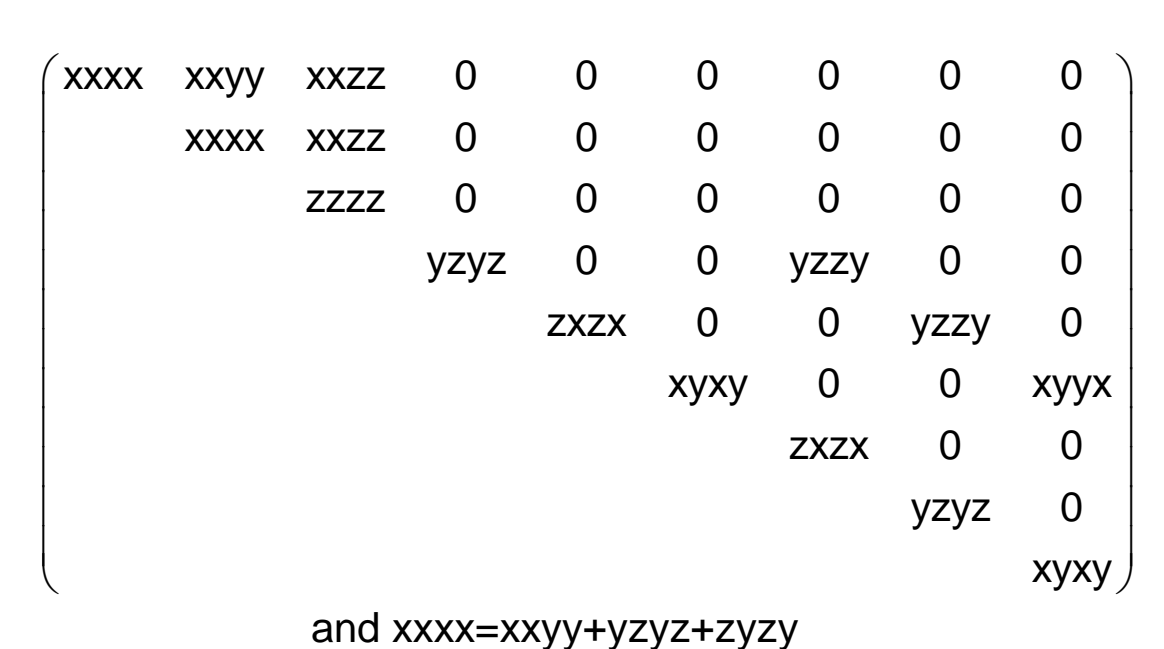

and $x x x x=x x y y+y z y z+z y z y$

$\left(\begin{array}{lllllllll}x x x x & x x y y & x x z z & 0 & 0 & 0 & 0 & 0 & 0 \\ x x y y & x x x x & x x z z & 0 & 0 & 0 & 0 & 0 & 0 \\ z z x x & x z x x & z z z & 0 & 0 & 0 & 0 & 0 & 0\end{array}\right)$ $\begin{array}{lllllllll}z z x x & z z x x & z z z z & 0 & 0 & 0 & 0 & 0 & 0\end{array}$ $\begin{array}{ccccccccc}0 & 0 & 0 & y z y z & 0 & 0 & z x z x & 0 & 0 \\ 0 & 0 & 0 & 0 & z x z x & 0 & 0 & y z y z & 0\end{array}$ $\left(\begin{array}{ccccccccc}0 & 0 & 0 & 0 & \text { zxzx } & 0 & 0 & \text { yzyz } & 0 \\ 0 & 0 & 0 & 0 & 0 & \text { xyxy } & 0 & 0 & \text { xyxy }\end{array}\right)$ and $x x x x=x x y y+2 x y x y$ 
$\left(\begin{array}{ccccccccc}\text { xxxx } & \text { xxyy } & \text { xxzz } & 0 & 0 & 0 & 0 & 0 & 0 \\ \text { yyxx } & \text { xxxx } & \text { xxzz } & 0 & 0 & 0 & 0 & 0 & 0 \\ \text { zzxx } & \text { zzyy } & \text { zzzz } & 0 & 0 & 0 & 0 & 0 & 0 \\ 0 & 0 & 0 & y z y z & 0 & 0 & y z z y & 0 & 0 \\ 0 & 0 & 0 & 0 & \text { zxzx } & 0 & 0 & \text { zxxz } & 0 \\ 0 & 0 & 0 & 0 & 0 & \text { xyxy } & 0 & 0 & \text { xyyx } \\ 0 & 0 & 0 & \text { zxxz } & 0 & 0 & \text { zxzx } & 0 & 0 \\ 0 & 0 & 0 & 0 & \text { yzzy } & 0 & 0 & \text { yzyz } & 0 \\ 0 & 0 & 0 & 0 & 0 & \text { xyyx } & 0 & 0 & \text { xyxy }\end{array}\right)$

$\left.\mathrm{V}^{4}\right] \quad\left(\begin{array}{ccccc}\mathrm{xxxx} & 0 & 0 & \mathrm{yyzz} & 0 \\ 0 & \mathrm{xxxx} & 0 & \mathrm{yyzz} & 0 \\ 0 & 0 & \mathrm{zzzz} & \mathrm{xxyy} & 0\end{array}\right)$

$\mathrm{V}\left[\mathrm{V}^{3}\right]\left(\begin{array}{cccccccccc}\mathrm{xxxx} & 0 & 0 & \mathrm{xxyy} & 0 & 0 & \mathrm{yyzz} & 0 & 0 & 0 \\ 0 & \mathrm{xxxx} & 0 & 0 & \mathrm{yyzz} & 0 & 0 & \mathrm{xxyy} & 0 & 0 \\ 0 & 0 & z z z & 0 & 0 & z z x & 0 & 0 & & \end{array}\right)$ $\left(\begin{array}{cccccccccc}0 & x x x x & 0 & 0 & y y z z & 0 & 0 & x x y y & 0 & 0 \\ 0 & 0 & z z z z & 0 & 0 & z z x x & 0 & 0 & z z x x & 0\end{array}\right)$

$\left[\left[V^{2}\right]^{2}\right]\left(\begin{array}{cccccc}x x x x & x x y y & x x z z & 0 & 0 & 0 \\ & \text { yyyy } & \text { yyzz } & 0 & 0 & 0 \\ & & z z z z & 0 & 0 & 0 \\ & & & y z y z & 0 & 0 \\ & & & & y z y z & 0 \\ & & & & & \text { xyxy }\end{array}\right)$

$\left[\mathrm{V}^{2}\right]^{2}\left(\begin{array}{cccccc}\mathrm{x} x \mathrm{x} & \mathrm{xxyy} & \mathrm{xxzz} & 0 & 0 & 0 \\ \mathrm{yyxx} & \mathrm{xxxx} & \mathrm{xxzz} & 0 & 0 & 0 \\ \mathrm{zzxx} & \mathrm{zzxx} & \mathrm{zzzz} & 0 & 0 & 0 \\ 0 & 0 & 0 & \mathrm{yzyz} & 0 & 0 \\ 0 & 0 & 0 & 0 & \mathrm{yzyz} & 0 \\ 0 & 0 & 0 & 0 & 0 & \mathrm{xyxy}\end{array}\right)$ $\left(\begin{array}{lllllllll}0 & 0 & 0 & 0 & 0 & 0 & 0 & 0 & 0 \\ 0 & 0 & 0 & 0 & 0 & 0 & 0 & 0 & 0\end{array}\right)$

$\begin{array}{lllllllll}0 & 0 & 0 & 0 & 0 & 0 & 0 & 0 & 0 \\ 0 & 0 & 0 & 0 & 0 & 0 & 0 & 0 & 0\end{array}$

$\begin{array}{llllllllll}0 & 0 & 0 & 0 & 0 & 0 & 0 & 0 & 0 \\ 0 & 0 & 0 & 0 & 0 & 0 & 0 & 0 & 0\end{array}$

$\begin{array}{lllllllll}0 & 0 & 0 & 0 & 0 & 0 & 0 & 0 & 0 \\ 0 & 0 & 0 & 0 & 0 & 0 & 0 & 0 & 0\end{array}$

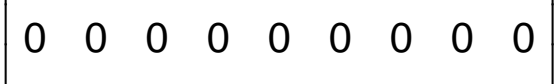

$\begin{array}{lllllllll}0 & 0 & 0 & 0 & 0 & 0 & 0 & 0 & 0 \\ 0 & 0 & 0 & 0 & 0 & 0 & 0 & 0 & 0\end{array}$

$0 \begin{array}{llllllllll}0 & 0 & 0 & 0 & 0 & 0 & 0 & 0 & 0 \\ 0 & 0 & 0 & 0 & 0 & 0 & 0 & 0 & 0\end{array}$

$0 \begin{array}{lllllllll}0 & 0 & 0 & 0 & 0 & 0 & 0 & 0 & 0 \\ 0 & 0 & 0 & 0 & 0 & 0 & 0 & 0 & 0\end{array}$

$\begin{array}{llllllllll}0 & 0 & 0 & 0 & 0 & 0 & 0 & 0 & 0\end{array}$

$\left(\begin{array}{lllll}0 & 0 & 0 & 0 & 0 \\ 0 & 0 & 0 & 0 & 0 \\ 0 & 0 & 0 & 0 & 0\end{array}\right)$

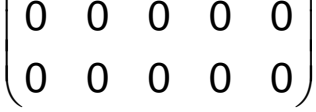

$\left(\begin{array}{llllllllll}0 & 0 & 0 & 0 & 0 & 0 & 0 & 0 & 0 & 0 \\ 0 & 0 & 0 & 0 & 0 & 0 & 0 & 0 & 0 & 0\end{array}\right)$ $\left(\begin{array}{llllllllll}0 & 0 & 0 & 0 & 0 & 0 & 0 & 0 & 0 & 0 \\ 0 & 0 & 0 & 0 & 0 & 0 & 0 & 0 & 0 & 0\end{array}\right)$

$$
\left(\begin{array}{llllll}
0 & 0 & 0 & 0 & 0 & 0 \\
& 0 & 0 & 0 & 0 & 0 \\
& & 0 & 0 & 0 & 0 \\
& & & 0 & 0 & 0 \\
& & & & 0 & 0 \\
& & & & & 0
\end{array}\right)
$$

$\left(\begin{array}{llllll}0 & 0 & 0 & 0 & 0 & 0 \\ 0 & 0 & 0 & 0 & 0 & 0\end{array}\right)$

$\begin{array}{llllll}0 & 0 & 0 & 0 & 0 & 0 \\ 0 & 0 & 0 & 0 & 0 & 0\end{array}$

$\begin{array}{llllll}0 & 0 & 0 & 0 & 0 & 0 \\ 0 & 0 & 0 & 0 & 0 & 0 \\ 0 & 0 & 0 & 0 & 0 & 0\end{array}$

$\begin{array}{llllll}0 & 0 & 0 & 0 & 0 & 0 \\ 0 & 0 & 0 & 0 & 0\end{array}$

$\begin{array}{llllll}0 & 0 & 0 & 0 & 0 & 0 \\ 0 & 0 & 0 & 0 & 0\end{array}$
$0 \begin{array}{lllllllll}0 & 0 & 0 & 0 & 0 & 0 & 0 & 0 & 0\end{array}$

$\begin{array}{llllllllll}0 & 0 & 0 & 0 & 0 & 0 & 0 & 0 & 0\end{array}$

$0 \begin{array}{lllllllll}0 & 0 & 0 & 0 & 0 & 0 & 0 & 0 & 0\end{array}$

$0 \begin{array}{lllllllll}0 & 0 & 0 & 0 & 0 & 0 & 0 & 0 & 0 \\ 0 & 0 & 0 & 0 & 0 & 0 & 0 & 0 & 0\end{array}$

$\begin{array}{llllllllll}0 & 0 & 0 & 0 & 0 & 0 & 0 & 0 & 0 \\ 0 & 0 & 0 & 0 & 0 & 0 & 0 & 0 & 0\end{array}$

$\begin{array}{lllllllll}0 & 0 & 0 & 0 & 0 & 0 & 0 & 0 & 0 \\ 0 & 0 & 0 & 0 & 0 & 0 & 0 & 0 & 0\end{array}$

$0 \begin{array}{llllllllll}0 & 0 & 0 & 0 & 0 & 0 & 0 & 0 & 0 \\ 0 & 0 & 0 & 0 & 0 & 0 & 0 & 0 & 0\end{array}$

$\left(\begin{array}{lllll}0 & 0 & 0 & 0 & 0 \\ 0 & 0 & 0 & 0 & 0 \\ 0 & 0 & 0 & 0 & 0\end{array}\right)$

$\begin{array}{lllll}0 & 0 & 0 & 0 & 0\end{array}$

$\left(\begin{array}{llllllllll}0 & 0 & 0 & 0 & 0 & 0 & 0 & 0 & 0 & 0 \\ 0 & 0 & 0 & 0 & 0 & 0 & 0 & 0 & 0 & 0\end{array}\right.$ $\left(\begin{array}{llllllllll}0 & 0 & 0 & 0 & 0 & 0 & 0 & 0 & 0 & 0 \\ 0 & 0 & 0 & 0 & 0 & 0 & 0 & 0 & 0 & 0\end{array}\right)$

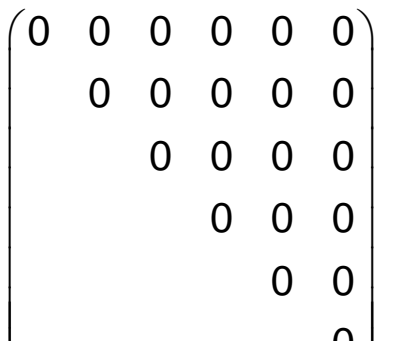

$\left(\begin{array}{llllll}0 & 0 & 0 & 0 & 0 & 0 \\ 0 & 0 & 0 & 0 & 0 & 0\end{array}\right)$

$\begin{array}{lllllll}0 & 0 & 0 & 0 & 0 & 0 \\ 0 & 0 & 0 & 0 & 0 & 0\end{array}$

$0 \begin{array}{llllll}0 & 0 & 0 & 0 & 0 & 0 \\ 0 & 0 & 0 & 0 & 0 & 0\end{array}$

$\begin{array}{llllll}0 & 0 & 0 & 0 & 0 & 0 \\ 0 & 0 & 0 & 0 & 0 & 0\end{array}$

$0 \begin{array}{llllll}0 & 0 & 0 & 0 & 0 & 0 \\ 0 & 0 & 0 & 0 & 0 & 0\end{array}$ $\left(\begin{array}{lllllllll}0 & 0 & 0 & 0 & 0 & 0 & 0 & 0 & 0 \\ 0 & 0 & 0 & 0 & 0 & 0 & 0 & 0 & 0\end{array}\right.$

$\left(\begin{array}{llllllllll}0 & 0 & 0 & 0 & 0 & 0 & 0 & 0 & 0 \\ 0 & 0 & 0 & 0 & 0 & 0 & 0 & 0 & 0 \\ 0 & 0 & 0 & 0 & 0 & 0 & 0 & 0 & 0\end{array}\right)$

$\begin{array}{lllllllll}0 & 0 & 0 & 0 & 0 & 0 & 0 & 0 & 0 \\ 0 & 0 & 0 & 0 & 0 & 0 & 0 & 0 & 0\end{array}$

$\begin{array}{lllllllll}0 & 0 & 0 & 0 & 0 & 0 & 0 & 0 & 0 \\ 0 & 0 & 0 & 0 & 0 & 0 & 0 & 0 & 0\end{array}$

$\begin{array}{lllllllll}0 & 0 & 0 & 0 & 0 & 0 & 0 & 0 & 0 \\ 0 & 0 & 0 & 0 & 0 & 0 & 0 & 0 & 0\end{array}$

$\begin{array}{lllllllllll}0 & 0 & 0 & 0 & 0 & 0 & 0 & 0 & 0 \\ 0 & 0 & 0 & 0 & 0 & 0 & 0 & 0 & 0\end{array}$

$0 \begin{array}{llllllllll}0 & 0 & 0 & 0 & 0 & 0 & 0 & 0 & 0 \\ 0 & 0 & 0 & 0 & 0 & 0 & 0 & 0 & 0\end{array}$

$\begin{array}{llllllllll}0 & 0 & 0 & 0 & 0 & 0 & 0 & 0 & 0 \\ 0 & 0 & 0 & 0 & 0 & 0 & 0 & 0 & 0\end{array}$

$\begin{array}{llllllllll}0 & 0 & 0 & 0 & 0 & 0 & 0 & 0 & 0\end{array}$

$\left(\begin{array}{ccccc}\mathrm{xxxx} & 0 & 0 & \mathrm{yyzz} & 0 \\ 0 & \mathrm{xxxx} & 0 & \mathrm{yyzz} & 0 \\ 0 & 0 & z z z z & \mathrm{x} y \mathrm{y} & 0\end{array}\right)$

$\left(\begin{array}{ccccc}0 & x x x x & 0 & y y z z & 0 \\ 0 & 0 & z z z z & x x y y & 0\end{array}\right)$

$\left(\begin{array}{cccccccccc}x x x x & 0 & 0 & x x y y & 0 & 0 & y y z z & 0 & 0 & 0 \\ 0 & x y x x & 0 & 0 & y y z z & 0 & 0 & x x y y & 0 & 0 \\ 0 & 0 & z z z z & 0 & 0 & z z x x & 0 & 0 & z z x & 0\end{array}\right)$

$\left(\begin{array}{cccccccccc}0 & \mathrm{xxxx} & 0 & 0 & \mathrm{yyzz} & 0 & 0 & \mathrm{xxyy} & 0 & 0 \\ 0 & 0 & \mathrm{zzzz} & 0 & 0 & \mathrm{zzxx} & 0 & 0 & \mathrm{zzxx} & 0\end{array}\right)$

$\left(\begin{array}{cccccc}\text { xxxx } & \text { xxyy } & \text { xxzz } & 0 & 0 & 0 \\ & \text { yyyy } & \text { yyzz } & 0 & 0 & 0 \\ & & \text { zzzz } & 0 & 0 & 0 \\ & & & y z y z & 0 & 0 \\ & & & & \text { yzyz } & 0 \\ & & & & & \text { xyxy }\end{array}\right)$

$\left(\begin{array}{llllll}x x x x & x x y y & x x z z & 0 & 0 & 0\end{array}\right.$

$\begin{array}{llllll}y y x x & x x x x & x x z z & 0 & 0 & 0 \\ z z x x & z z x x & z z z z & 0 & 0 & 0\end{array}$

$\begin{array}{cccccc}z z x x & z z x x & z z z z & 0 & 0 & 0\end{array}$

$\begin{array}{cccccc}0 & 0 & 0 & y z y z & 0 & 0 \\ 0 & 0 & 0 & 0 & y z y z & 0\end{array}$

$\begin{array}{cccccc}0 & 0 & 0 & 0 & y z y z & 0 \\ 0 & 0 & 0 & 0 & 0 & x y\end{array}$ 


$$
\begin{aligned}
& {\left[\left(V^{2}\right)\right]^{2}} \\
& \left(\begin{array}{lllllllll}
x x x & x x y y & x x z z & 0 & 0 & 0 & 0 & 0 & 0 \\
& x x x x & x x z z & 0 & 0 & 0 & 0 & 0 & 0
\end{array}\right)\left(\begin{array}{ccccccccc}
0 & 0 & 0 & 0 & 0 & 0 & 0 & 0 & 0 \\
& 0 & 0 & 0 & 0 & 0 & 0 & 0 & 0
\end{array}\right) \\
& \text { zzzz } 00 \begin{array}{lllll}
0 & 0 & 0 & 0 & 0
\end{array} \quad \begin{array}{lllllll}
0 & 0 & 0 & 0 & 0 & 0 & 0
\end{array}
\end{aligned}
$$

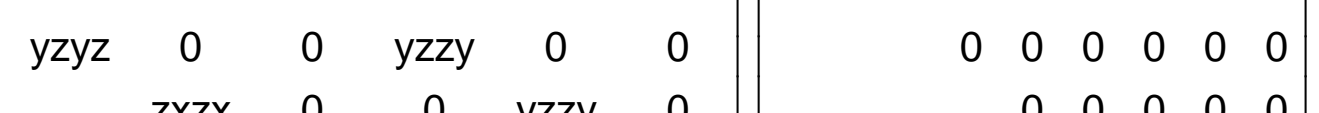

$$
\begin{aligned}
& \text { zxzx } \begin{array}{cccc}
0 & 0 & y z z y & 0 \\
x y x y & 0 & 0 & x y y x
\end{array} \mid \quad \begin{array}{lllll}
0 & 0 & 0 & 0 & 0
\end{array}
\end{aligned}
$$

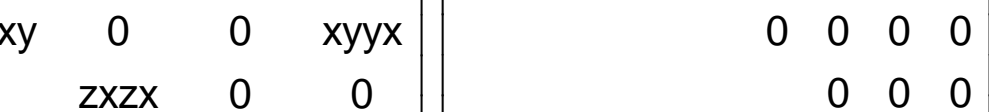

$$
\begin{aligned}
& \left.\begin{array}{cc}
y z y z & 0 \\
x y x y
\end{array}\right)\left(\begin{array}{lll}
0 & 0 \\
& & 0
\end{array}\right)
\end{aligned}
$$

$\left(\begin{array}{lllllllll}x x x x & x x y y & x x z z & 0 & 0 & 0 & 0 & 0 & 0 \\ y y x x & x x y x & x x z z & 0 & 0 & 0 & 0 & 0 & 0 \\ z z x x & z z x & z z z z & 0 & 0 & 0 & 0 & 0 & 0\end{array}\right)\left(\begin{array}{lllllllll}0 & 0 & 0 & 0 & 0 & 0 & 0 & 0 & 0 \\ 0 & 0 & 0 & 0 & 0 & 0 & 0 & 0 & 0 \\ 0 & 0 & 0 & 0 & 0 & 0 & 0 & 0 & 0\end{array}\right)$

$\left(\begin{array}{lllllllll}\mathrm{yyxx} & \mathrm{xxxx} & \mathrm{xxzz} & 0 & 0 & 0 & 0 & 0 & 0 \\ \mathrm{zzxx} & \mathrm{zzxx} & \mathrm{zzzz} & 0 & 0 & 0 & 0 & 0 & 0 \\ 0 & 0 & 0 & \mathrm{z} y \mathrm{y} & 0 & 0 & \mathrm{zxz} & 0 & 0\end{array} \mid \begin{array}{lllllllll}0 & 0 & 0 & 0 & 0 & 0 & 0 & 0 & 0 \\ 0 & 0 & 0 & 0 & 0 & 0 & 0 & 0 & 0 \\ 0 & 0 & 0 & 0 & 0 & 0 & 0 & 0 & 0\end{array}\right)$

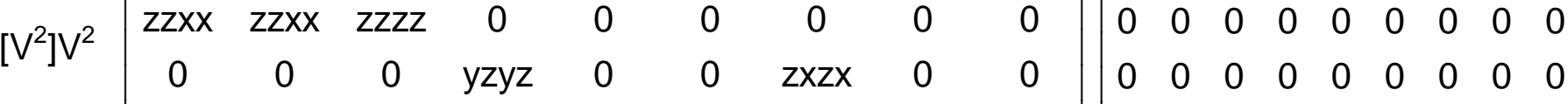

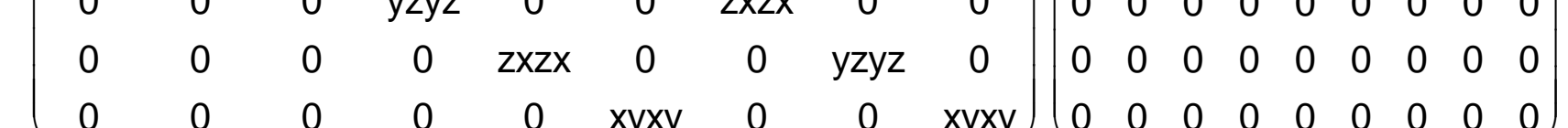

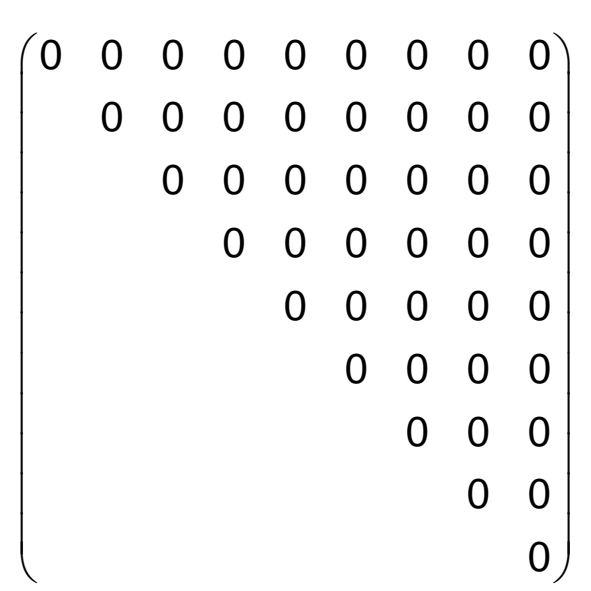

$\left(\begin{array}{lllllllll}0 & 0 & 0 & 0 & 0 & 0 & 0 & 0 & 0 \\ 0 & 0 & 0 & 0 & 0 & 0 & 0 & 0 & 0\end{array}\right)$ $0 \begin{array}{lllllllll}0 & 0 & 0 & 0 & 0 & 0 & 0 & 0 & 0 \\ 0 & 0 & 0 & 0 & 0 & 0 & 0 & 0 & 0\end{array}$

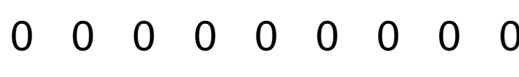

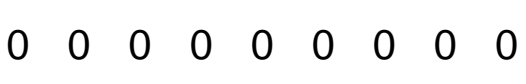
$\begin{array}{lllllllll}0 & 0 & 0 & 0 & 0 & 0 & 0 & 0 & 0 \\ 0 & 0 & 0 & 0 & 0 & 0 & 0 & 0 & 0\end{array}$

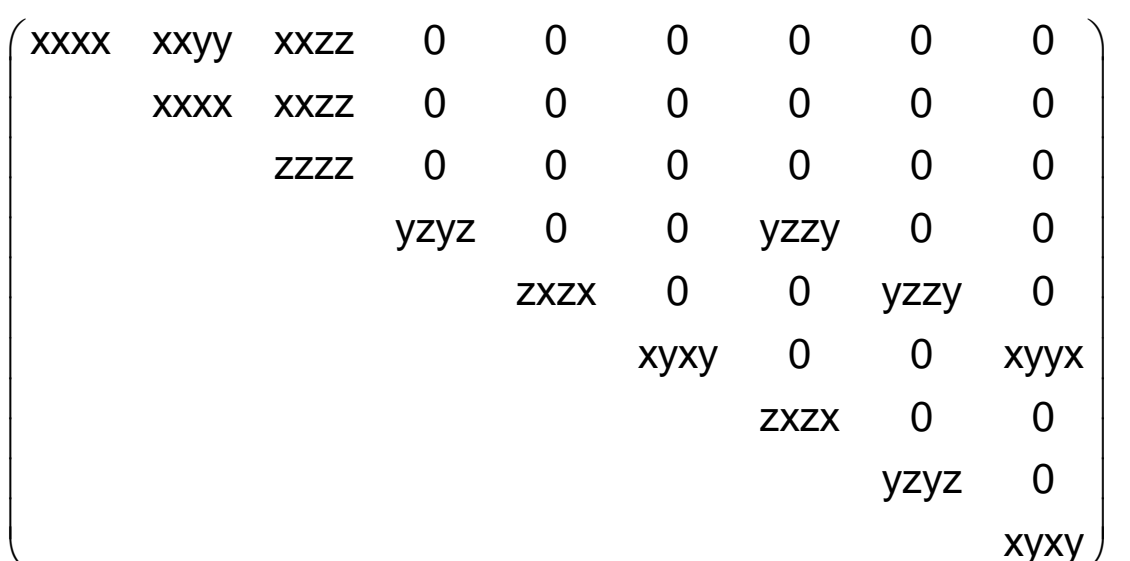

$\left(\begin{array}{ccccccccc}\text { xxxx } & \text { xxyy } & \text { xxzz } & 0 & 0 & 0 & 0 & 0 & 0 \\ \text { yyxx } & \text { xxxx } & \text { xxzz } & 0 & 0 & 0 & 0 & 0 & 0 \\ \text { zzxx } & z z x x & z z z z & 0 & 0 & 0 & 0 & 0 & 0 \\ 0 & 0 & 0 & y z y z & 0 & 0 & z x z x & 0 & 0 \\ 0 & 0 & 0 & 0 & z x z x & 0 & 0 & y z y z & 0 \\ 0 & 0 & 0 & 0 & 0 & x y x y & 0 & 0 & \text { xyxy }\end{array}\right)$ 

$\left(\begin{array}{lllllllll}0 & 0 & 0 & 0 & 0 & 0 & 0 & 0 & 0 \\ 0 & 0 & 0 & 0 & 0 & 0 & 0 & 0 & 0 \\ 0 & 0 & 0 & 0 & 0 & 0 & 0 & 0 & 0\end{array}\right)$ $\begin{array}{lllllllllll}0 & 0 & 0 & 0 & 0 & 0 & 0 & 0 & 0 \\ 0 & 0 & 0 & 0 & 0 & 0 & 0 & 0 & 0\end{array}$

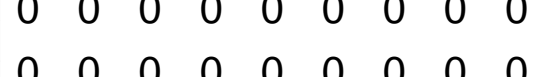
$\begin{array}{lllllllll}0 & 0 & 0 & 0 & 0 & 0 & 0 & 0 & 0 \\ 0 & 0 & 0 & 0 & 0 & 0 & 0 & 0 & 0\end{array}$ $0 \begin{array}{lllllllll}0 & 0 & 0 & 0 & 0 & 0 & 0 & 0 & 0 \\ 0 & 0 & 0 & 0 & 0 & 0 & 0 & 0 & 0\end{array}$ $\begin{array}{llllllllll}0 & 0 & 0 & 0 & 0 & 0 & 0 & 0 & 0 \\ 0 & 0 & 0 & 0 & 0 & 0 & 0 & 0 & 0\end{array}$ $\begin{array}{lllllllll}0 & 0 & 0 & 0 & 0 & 0 & 0 & 0 & 0 \\ 0 & 0 & 0 & 0 & 0 & 0 & 0 & 0 & 0\end{array}$ $\begin{array}{lllllllll}0 & 0 & 0 & 0 & 0 & 0 & 0 & 0 & 0 \\ 0 & 0 & 0 & 0 & 0 & 0 & 0 & 0\end{array}$ $\begin{array}{lllllllllll}0 & 0 & 0 & 0 & 0 & 0 & 0 & 0 & 0\end{array}$

\section{$\left(\begin{array}{lllll}0 & 0 & 0 & 0 & 0 \\ 0 & 0 & 0 & 0 & 0\end{array}\right)$ \\ $\left(\begin{array}{llllll}0 & 0 & 0 & 0 & 0 \\ 0 & 0 & 0 & 0 & 0 \\ 0 & 0 & 0 & 0 & 0\end{array}\right.$}

$\left(\begin{array}{llllllllll}0 & 0 & 0 & 0 & 0 & 0 & 0 & 0 & 0 & 0 \\ 0 & 0 & 0 & 0 & 0 & 0 & 0 & 0 & 0 & 0\end{array}\right)$ $\begin{array}{llllllllll}0 & 0 & 0 & 0 & 0 & 0 & 0 & 0 & 0 & 0 \\ 0 & 0 & 0 & 0 & 0 & 0 & 0 & 0 & 0 & 0\end{array}$ $\begin{array}{llllllllll}0 & 0 & 0 & 0 & 0 & 0 & 0 & 0 & 0 & 0\end{array}$

$\left.\mathrm{V}^{3} \mathrm{~V}^{3}\right]\left(\begin{array}{cccccccccc}3 x x y y & 0 & 0 & x x y y & 0 & 0 & y y z z & 0 & 0 & 0 \\ 0 & 3 x x y y & 0 & 0 & y y z z & 0 & 0 & x x y y & 0 & 0 \\ 0 & 0 & z z z & 0 & 0 & z z x & 0 & 0 & z z x & 0\end{array}\right)$

$$
\left(\begin{array}{llllll}
0 & 0 & 0 & 0 & 0 & 0 \\
& 0 & 0 & 0 & 0 & 0 \\
& & 0 & 0 & 0 & 0 \\
& & & 0 & 0 & 0 \\
& & & & 0 & 0 \\
& & & & & 0
\end{array}\right)
$$

and $x y x y=1 / 2(x x x x-x x y y)$ $\left(\begin{array}{llllll}0 & 0 & 0 & 0 & 0 & 0 \\ 0 & 0 & 0 & 0 & 0 & 0\end{array}\right)$ $\begin{array}{lllllll}0 & 0 & 0 & 0 & 0 & 0 \\ 0 & 0 & 0 & 0 & 0 & 0\end{array}$ $\begin{array}{lllllll}0 & 0 & 0 & 0 & 0 & 0\end{array}$ $\begin{array}{llllll}0 & 0 & 0 & 0 & 0 & 0 \\ 0 & 0 & 0 & 0 & 0 & 0 \\ 0 & 0 & 0 & 0 & 0 & 0\end{array}$ $\left.\begin{array}{llllll}0 & 0 & 0 & 0 & 0 & 0 \\ 0 & 0 & 0 & 0 & 0 & 0\end{array}\right)$ $\left(\begin{array}{lllllllll}0 & 0 & 0 & 0 & 0 & 0 & 0 & 0 & 0 \\ 0 & 0 & 0 & 0 & 0 & 0 & 0 & 0 & 0\end{array}\right.$ $\begin{array}{lllllllll}0 & 0 & 0 & 0 & 0 & 0 & 0 & 0 & 0 \\ 0 & 0 & 0 & 0 & 0 & 0 & 0 & 0 & 0\end{array}$ $0 \begin{array}{llllllllll}0 & 0 & 0 & 0 & 0 & 0 & 0 & 0 & 0 \\ 0 & 0 & 0 & 0 & 0 & 0 & 0\end{array}$ $\begin{array}{lllllllll}0 & 0 & 0 & 0 & 0 & 0 & 0 & 0 & 0\end{array}$ $0 \begin{array}{lllllllll}0 & 0 & 0 & 0 & 0 & 0 & 0 & 0 & 0 \\ 0 & 0 & 0 & 0 & 0 & 0 & 0 & 0 & 0\end{array}$ $0 \begin{array}{lllllllll}0 & 0 & 0 & 0 & 0 & 0 & 0 & 0 & 0 \\ 0 & 0 & 0 & 0 & 0 & 0 & 0 & 0 & 0\end{array}$ $\begin{array}{lllllllll}0 & 0 & 0 & 0 & 0 & 0 & 0 & 0 & 0 \\ 0 & 0 & 0 & 0 & 0 & 0 & 0 & 0 & 0\end{array}$ $0 \begin{array}{llllllllll}0 & 0 & 0 & 0 & 0 & 0 & 0 & 0 & 0 \\ 0 & 0 & 0 & 0 & 0 & 0 & 0 & 0 & 0\end{array}$ $\begin{array}{llllllllll}0 & 0 & 0 & 0 & 0 & 0 & 0 & 0 & 0\end{array}$

$\left(\begin{array}{lllll}0 & 0 & 0 & 0 & 0 \\ 0 & 0 & 0 & 0 & 0 \\ 0 & 0 & 0 & 0 & 0\end{array}\right)$

$\begin{array}{llllll}0 & 0 & 0 & 0 & 0 \\ 0 & 0 & 0 & 0 & 0\end{array}$

$\left(\begin{array}{llllllllll}0 & 0 & 0 & 0 & 0 & 0 & 0 & 0 & 0 & 0 \\ 0 & 0 & 0 & 0 & 0 & 0 & 0 & 0 & 0 & 0\end{array}\right.$ $\left.\begin{array}{lllllllllll}0 & 0 & 0 & 0 & 0 & 0 & 0 & 0 & 0 & 0 \\ 0 & 0 & 0 & 0 & 0 & 0 & 0 & 0 & 0 & 0\end{array}\right)$ $\begin{array}{lllllllllll}0 & 0 & 0 & 0 & 0 & 0 & 0 & 0 & 0 & 0\end{array}$

$$
\left(\begin{array}{llllll}
0 & 0 & 0 & 0 & 0 & 0 \\
& 0 & 0 & 0 & 0 & 0 \\
& & 0 & 0 & 0 & 0 \\
& & & 0 & 0 & 0 \\
& & & & 0 & 0 \\
& & & & & \\
& & & & 0
\end{array}\right)
$$

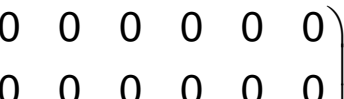
$\begin{array}{llllll}0 & 0 & 0 & 0 & 0 & 0 \\ 0 & 0 & 0 & 0\end{array}$ $\begin{array}{llllll}0 & 0 & 0 & 0 & 0 & 0\end{array}$ $\begin{array}{llllll}0 & 0 & 0 & 0 & 0 & 0 \\ 0 & 0 & 0 & 0 & 0 & 0 \\ 0 & 0 & 0 & 0 & 0 & 0\end{array}$ $\begin{array}{llllll}0 & 0 & 0 & 0 & 0 & 0 \\ 0 & 0 & 0 & 0 & 0 & 0\end{array}$

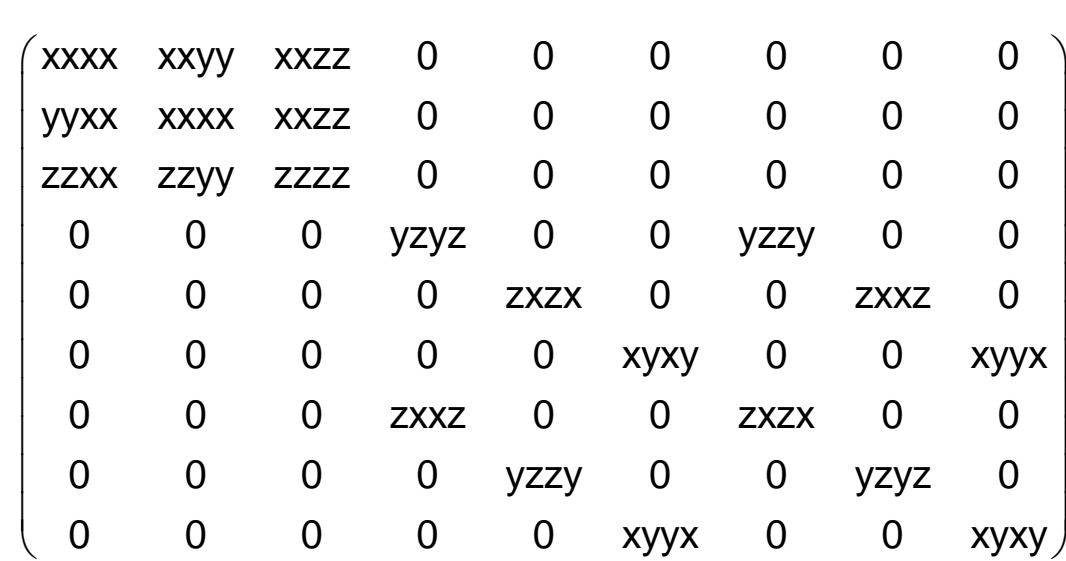

$$
\begin{aligned}
& \text { and: } x x x x=x x y y+x y x y+x y y x \\
& \left(\begin{array}{ccccc}
3 x x y y & 0 & 0 & y y z z & 0 \\
0 & 3 x x y y & 0 & y y z z & 0 \\
0 & 0 & z z z z & x x y y & 0
\end{array}\right)
\end{aligned}
$$$$
\left(\begin{array}{cccccccccc}
3 x x y y & 0 & 0 & x x y y & 0 & 0 & y y z z & 0 & 0 & 0 \\
0 & 3 x x y y & 0 & 0 & y y z z & 0 & 0 & x x y y & 0 & 0 \\
0 & 0 & 2 z z & 0 & 0 & z z x x & 0 & 0 & z z x & 0
\end{array}\right)
$$$$
\left(\begin{array}{cccccccccc}
0 & 0 & z z z z & 0 & 0 & z z x x & 0 & 0 & z z x x & 0
\end{array}\right)
$$$$
\left(\begin{array}{cccccc}
x x x x & x x y y & x x z z & 0 & 0 & 0 \\
& x x x x & x x z z & 0 & 0 & 0 \\
& & z z z z & 0 & 0 & 0 \\
& & & y z y z & 0 & 0 \\
& & & & y z y z & 0 \\
& & & & & \text { xyxy }
\end{array}\right)
$$

and $x y x y=1 / 2(x x x x-x x y y)$

$$
\left(\begin{array}{cccccc}
x x x x & x x y y & x x z z & 0 & 0 & 0 \\
x x y y & x x x x & x x z z & 0 & 0 & 0 \\
z z x x & z z x x & z z z z & 0 & 0 & 0 \\
0 & 0 & 0 & y z y z & 0 & 0 \\
0 & 0 & 0 & 0 & y z y z & 0 \\
0 & 0 & 0 & 0 & 0 & x y y
\end{array}\right)
$$

and $x y x y=1 / 2(x x x x-x x y y)$ 


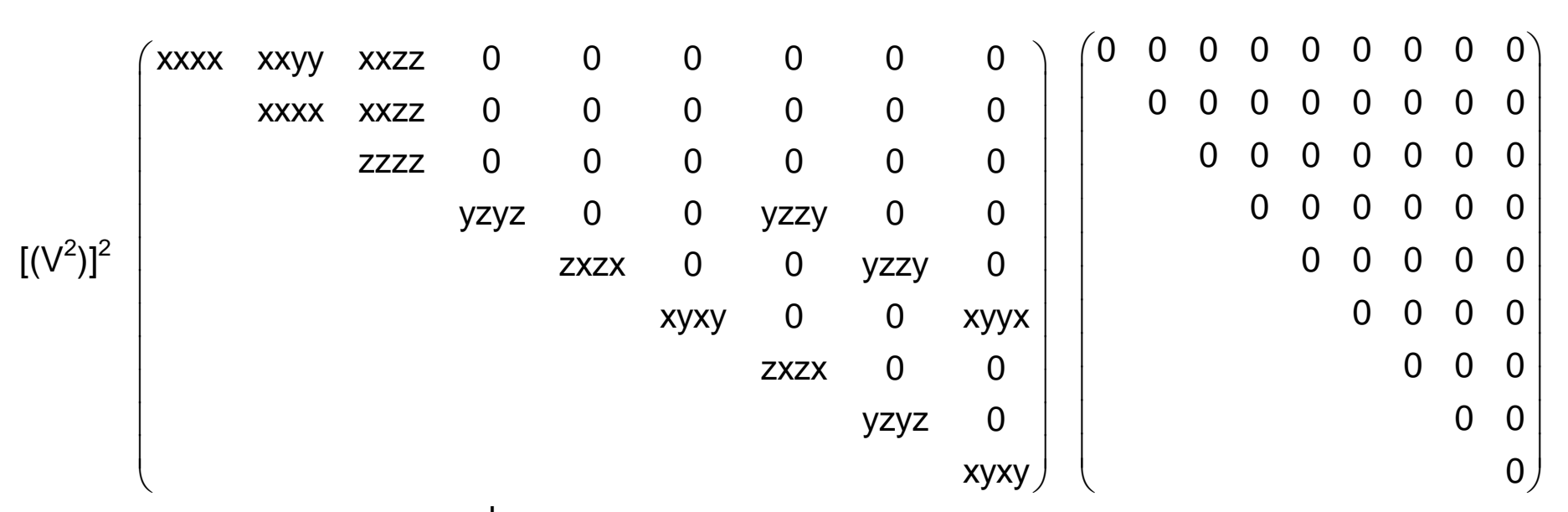
and $x x x x=x x y y+y z y z+z y z y$

$\left[\mathrm{V}^{2}\right] \mathrm{V}^{2}\left(\begin{array}{ccccccccc}\mathrm{x} x \mathrm{x} & \mathrm{xxyy} & \mathrm{xxzz} & 0 & 0 & 0 & 0 & 0 & 0 \\ \mathrm{xxyy} & \mathrm{xxxx} & \mathrm{xxzz} & 0 & 0 & 0 & 0 & 0 & 0 \\ \mathrm{zzxx} & \mathrm{zzxx} & \mathrm{zzzz} & 0 & 0 & 0 & 0 & 0 & 0 \\ 0 & 0 & 0 & \mathrm{yzyz} & 0 & 0 & \mathrm{zxzx} & 0 & 0 \\ 0 & 0 & 0 & 0 & \mathrm{zxzx} & 0 & 0 & \mathrm{yzyz} & 0 \\ 0 & 0 & 0 & 0 & 0 & \mathrm{xyxy} & 0 & 0 & \mathrm{xyxy}\end{array}\right)\left(\begin{array}{ccccccccc}0 & 0 & 0 & 0 & 0 & 0 & 0 & 0 & 0 \\ 0 & 0 & 0 & 0 & 0 & 0 & 0 & 0 & 0 \\ 0 & 0 & 0 & 0 & 0 & 0 & 0 & 0 & 0 \\ 0 & 0 & 0 & 0 & 0 & 0 & 0 & 0 & 0 \\ 0 & 0 & 0 & 0 & 0 & 0 & 0 & 0 & 0 \\ 0 & 0 & 0 & 0 & 0 & 0 & 0 & 0 & 0\end{array}\right)$ and $x x x x=x x y y+2 x y x y$

e

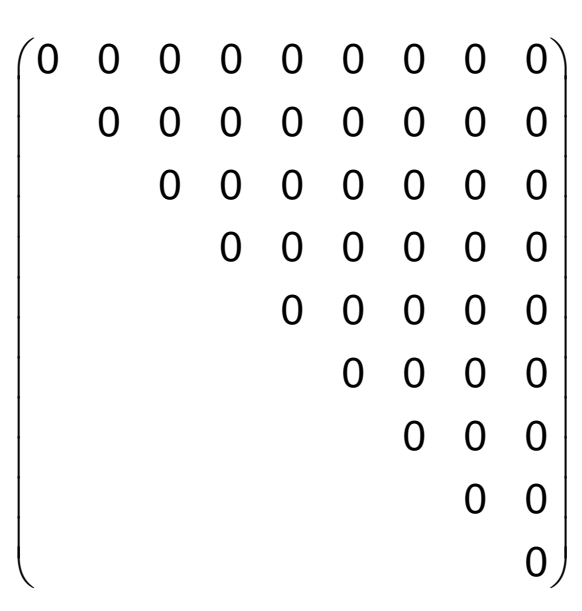

$\left(\begin{array}{lllllllll}0 & 0 & 0 & 0 & 0 & 0 & 0 & 0 & 0 \\ 0 & 0 & 0 & 0 & 0 & 0 & 0 & 0 & 0\end{array}\right)$

$\begin{array}{lllllllll}0 & 0 & 0 & 0 & 0 & 0 & 0 & 0 & 0 \\ 0 & 0 & 0 & 0 & 0 & 0 & 0 & 0 & 0\end{array}$

$\begin{array}{lllllllll}0 & 0 & 0 & 0 & 0 & 0 & 0 & 0 & 0\end{array}$

$\begin{array}{lllllllll}0 & 0 & 0 & 0 & 0 & 0 & 0 & 0 & 0 \\ 0 & 0 & 0 & 0 & 0 & 0 & 0 & 0 & 0\end{array}$

$\begin{array}{lllllllll}0 & 0 & 0 & 0 & 0 & 0 & 0 & 0 & 0 \\ 0 & 0 & 0 & 0 & 0 & 0 & 0 & 0 & 0\end{array}$ a

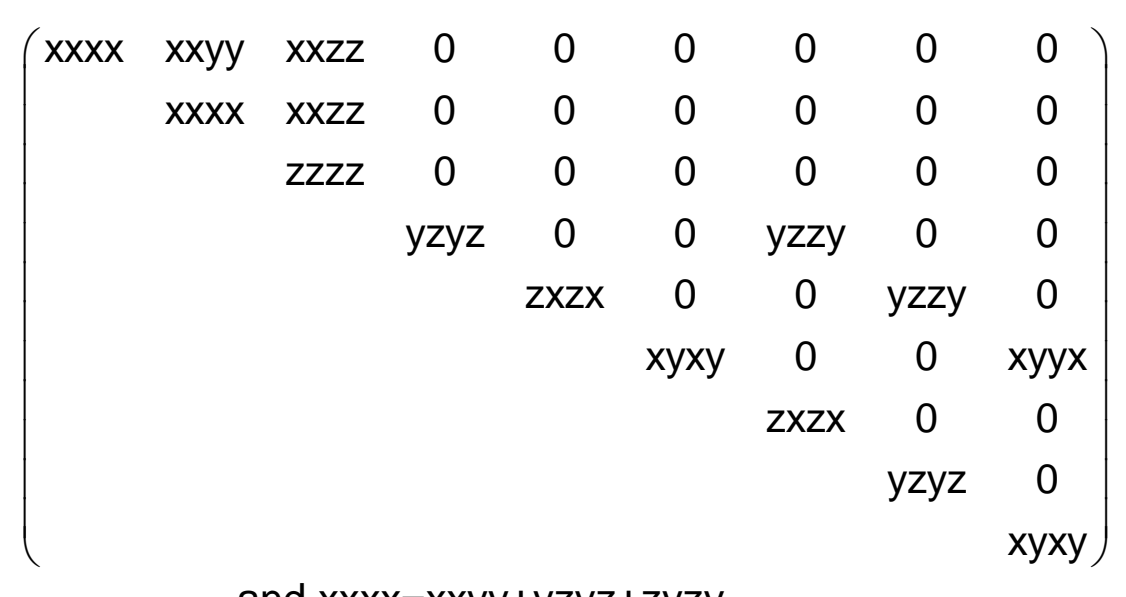

and $x x x x=x x y y+y z y z+z y z y$

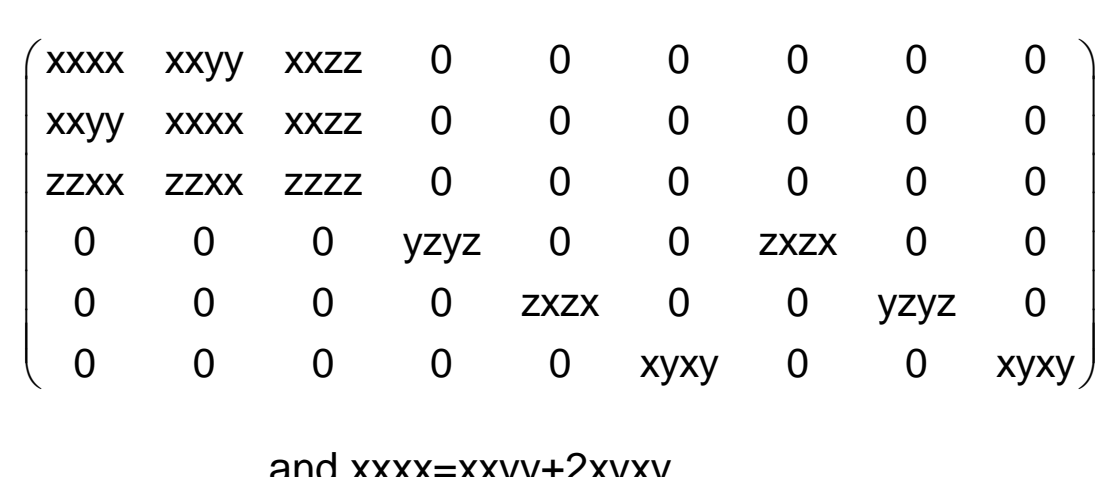


$\left(\begin{array}{lllllllll}x x x x & x x y y & x x z z & 0 & 0 & 0 & 0 & 0 & 0 \\ x & x y y & y y z z & 0 & 0 & 0 & 0 & 0 & 0\end{array}\right)$ $\left(\begin{array}{lllllllll}\text { yyxx } & \text { yyyy } & \text { yyzz } & 0 & 0 & 0 & 0 & 0 & 0 \\ z z x x & \text { zzyy } & \text { zzzz } & 0 & 0 & 0 & 0 & 0 & 0 \\ 0 & 0 & 0 & y z y z & 0 & 0 & y z z y & 0 & 0\end{array}\right)$ $\begin{array}{ccccccccc}0 & 0 & 0 & y z y z & 0 & 0 & y z z y & 0 & 0 \\ 0 & 0 & 0 & 0 & z x z x & 0 & 0 & z x x z & 0\end{array}$

$\begin{array}{llllllll}0 & 0 & 0 & z x z x & 0 & 0 & z x x z & 0\end{array}$

$\begin{array}{ccccccccc}0 & 0 & 0 & z y y z & 0 & 0 & z y z y & 0 & 0\end{array}$

$\begin{array}{lllllllll}0 & 0 & 0 & 0 & x z z x & 0 & 0 & x z x z & 0 \\ 0 & 0 & 0 & 0 & 0 & y x y & 0 & 0 & y x y x\end{array}$

$\left[V^{4}\right] \quad\left(\begin{array}{ccccc}x x x x & 0 & 0 & y y z z & 0 \\ 0 & y y y y & 0 & z z x x & 0 \\ 0 & 0 & z z z z & x x y y & 0\end{array}\right)$

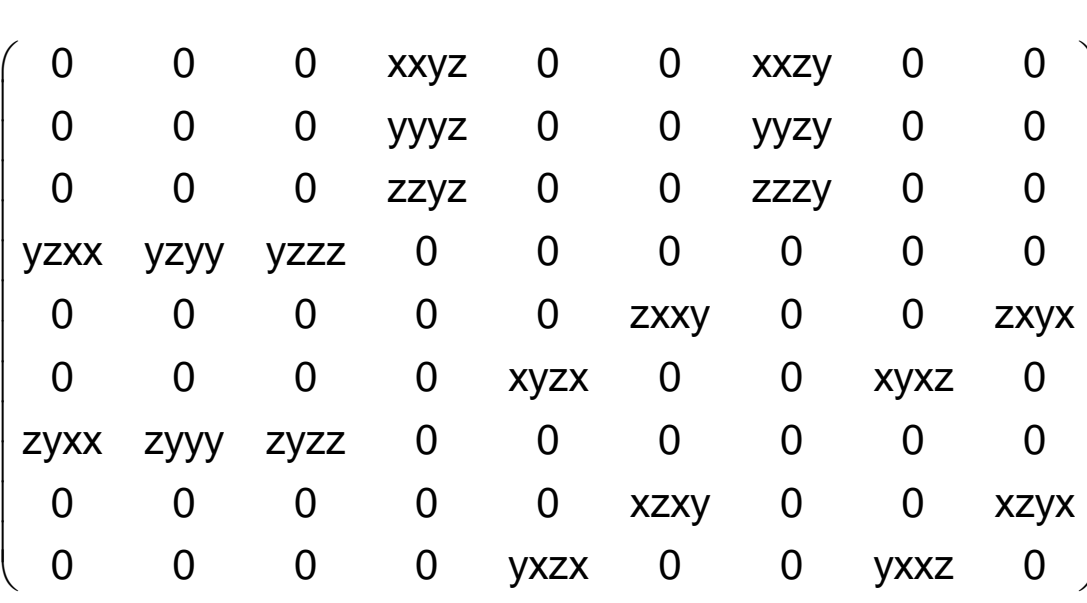

3] $\left(\begin{array}{cccccccccc}x x x x & 0 & 0 & x x y y & 0 & 0 & x x z z & 0 & 0 & 0 \\ 0 & y y y y & 0 & 0 & y y z z & 0 & 0 & y y x & 0 & 0 \\ 0 & 0 & z z z & 0 & 0 & z z x x & 0 & 0 & z z y & 0\end{array}\right)$

$\left(\begin{array}{ccccc}0 & 0 & 0 & 0 & x x y z \\ 0 & 0 & y z z z & 0 & 0 \\ 0 & z y y y & 0 & 0 & 0\end{array}\right)$

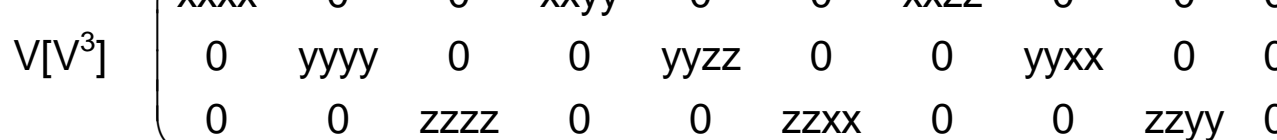

$\left(\begin{array}{cccccccccc}0 & 0 & 0 & 0 & 0 & 0 & 0 & 0 & 0 & x x y z \\ 0 & 0 & y z z z & 0 & 0 & y z x x & 0 & 0 & y z y y & 0 \\ 0 & z y y y & 0 & 0 & z y z z & 0 & 0 & z y x x & 0 & 0\end{array}\right)$ $\left(\begin{array}{cccccccccc}0 & 0 & y z z z & 0 & 0 & y z x x & 0 & 0 & y z y y & 0 \\ 0 & z y y y & 0 & 0 & z y z z & 0 & 0 & z y x x & 0 & 0\end{array}\right)$

$\left[\left[^{2}\right]^{2}\right]\left(\begin{array}{cccccc}x x x x & x x y y & x x z z & 0 & 0 & 0 \\ & \text { yyyy } & \text { yyzz } & 0 & 0 & 0 \\ & & \text { zzzz } & 0 & 0 & 0 \\ & & & y z y z & 0 & 0 \\ & & & & \text { xzxz } & 0 \\ & & & & & \text { xyxy }\end{array}\right)$

$$
\left(\begin{array}{cccccc}
0 & 0 & 0 & \text { xxyz } & 0 & 0 \\
& 0 & 0 & \text { yyyz } & 0 & 0 \\
& & 0 & \text { zzyz } & 0 & 0 \\
& & & 0 & 0 & 0 \\
& & & & 0 & \text { xzxy } \\
& & & & & 0
\end{array}\right)
$$

$\left[V^{2}\right]^{2}\left(\begin{array}{cccccc}\mathrm{xxxx} & \mathrm{xxyy} & \mathrm{xxzz} & 0 & 0 & 0 \\ \mathrm{yyxx} & \mathrm{yyyy} & \mathrm{yyzz} & 0 & 0 & 0 \\ \mathrm{zzxx} & \mathrm{zzyy} & \mathrm{zzzz} & 0 & 0 & 0 \\ 0 & 0 & 0 & \mathrm{yzyz} & 0 & 0 \\ 0 & 0 & 0 & 0 & \mathrm{xzxz} & 0 \\ 0 & 0 & 0 & 0 & 0 & \mathrm{xyxy}\end{array}\right)$

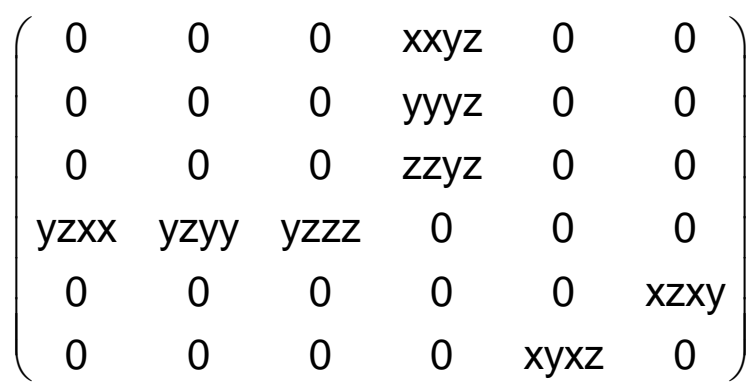

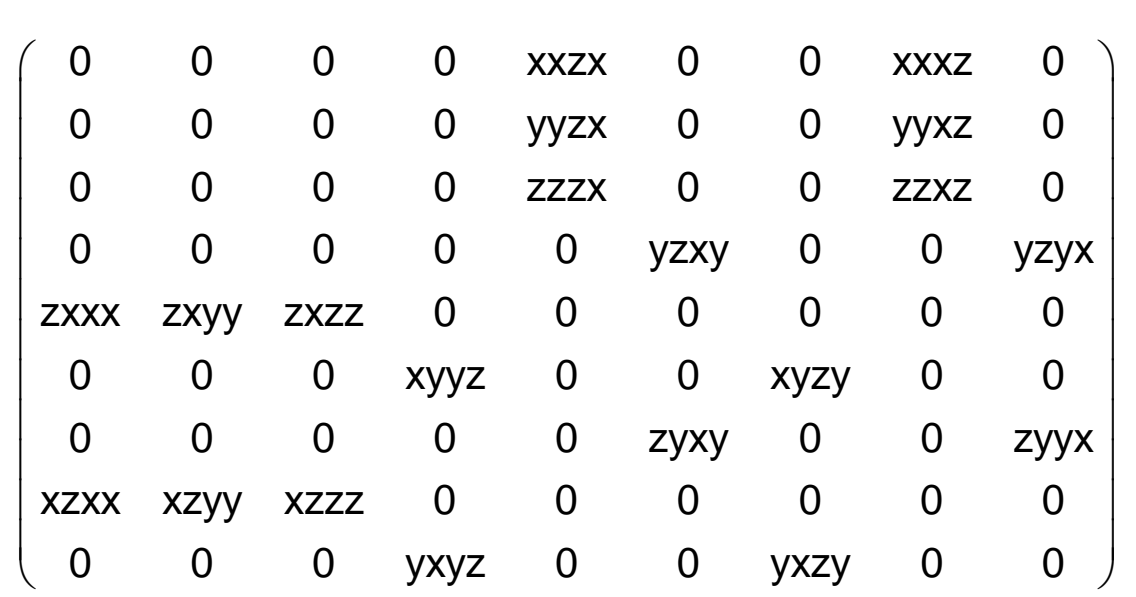

$\left(\begin{array}{ccccccccc}0 & 0 & 0 & 0 & 0 & \text { xxxy } & 0 & 0 & \text { xxyx } \\ 0 & 0 & 0 & 0 & 0 & \text { yyxy } & 0 & 0 & \text { yyyx } \\ 0 & 0 & 0 & 0 & 0 & z z x y & 0 & 0 & \text { zzyx } \\ 0 & 0 & 0 & 0 & y z z x & 0 & 0 & \text { yzxz } & 0 \\ 0 & 0 & 0 & \text { zxyz } & 0 & 0 & \text { zxzy } & 0 & 0 \\ \text { xyxx } & \text { xyyy } & \text { xyzz } & 0 & 0 & 0 & 0 & 0 & 0 \\ 0 & 0 & 0 & 0 & \text { zyzx } & 0 & 0 & \text { zyxz } & 0 \\ 0 & 0 & 0 & \text { xzyz } & 0 & 0 & \text { xzzy } & 0 & 0 \\ \text { yxxx } & \text { yxyy } & \text { yxzz } & 0 & 0 & 0 & 0 & 0 & 0\end{array}\right)$

$\left(\begin{array}{ccccc}0 & 0 & \mathrm{xzzz} & 0 & 0 \\ 0 & 0 & 0 & 0 & \mathrm{yyxz} \\ \mathrm{zxxx} & 0 & 0 & 0 & 0\end{array}\right)$

$\left(\begin{array}{ccccc}0 & x y y y & 0 & 0 & 0 \\ y x x x & 0 & 0 & 0 & 0 \\ 0 & 0 & 0 & 0 & z z x y\end{array}\right)$

$\left(\begin{array}{cccccccccc}0 & 0 & x z z z & 0 & 0 & x z x x & 0 & 0 & x z y y & 0 \\ 0 & 0 & 0 & 0 & 0 & 0 & 0 & 0 & 0 & y x y z\end{array}\right)$

$\left.\begin{array}{cccccccccc}0 & 0 & 0 & 0 & 0 & 0 & 0 & 0 & 0 & y x y z \\ z x x x & 0 & 0 & z x y y & 0 & 0 & z x z z & 0 & 0 & 0\end{array}\right)$

$\left(\begin{array}{cccccccccc}0 & x y y y & 0 & 0 & x y z z & 0 & 0 & x y x x & 0 & 0 \\ y & 0 & 0 & y x y y & 0 & 0 & y x z & 0 & 0 & 0\end{array}\right)$ $\left(\begin{array}{cccccccccc}\mathrm{yxxx} & 0 & 0 & \mathrm{yxyy} & 0 & 0 & \mathrm{yxzz} & 0 & 0 & 0 \\ 0 & 0 & 0 & 0 & 0 & 0 & 0 & 0 & 0 & \mathrm{zxyz}\end{array}\right)$

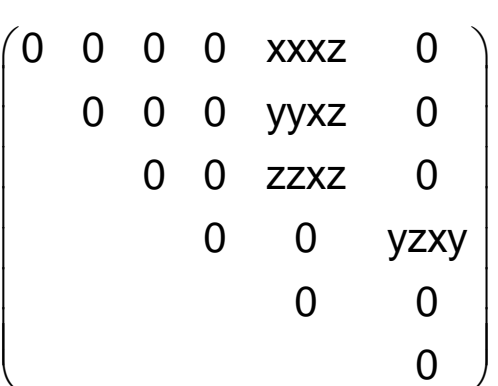

$\left(\begin{array}{cccccc}0 & 0 & 0 & 0 & 0 & \mathrm{xxxy} \\ & 0 & 0 & 0 & 0 & \mathrm{yyxy} \\ & & 0 & 0 & 0 & \mathrm{zzxy} \\ & & & 0 & \mathrm{yzxz} & 0 \\ & & & & 0 & 0 \\ & & & & & \\ & & & & & 0\end{array}\right)$

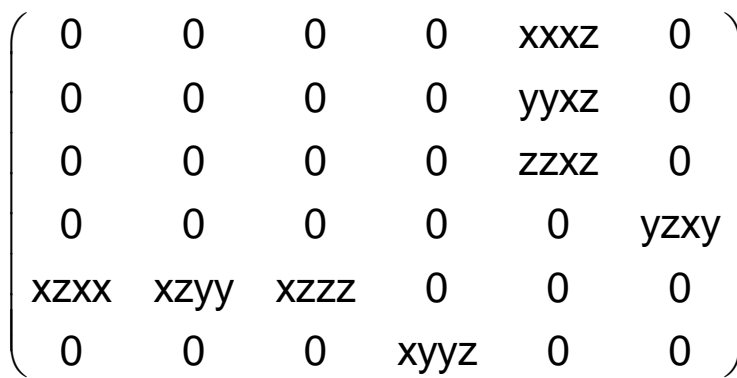

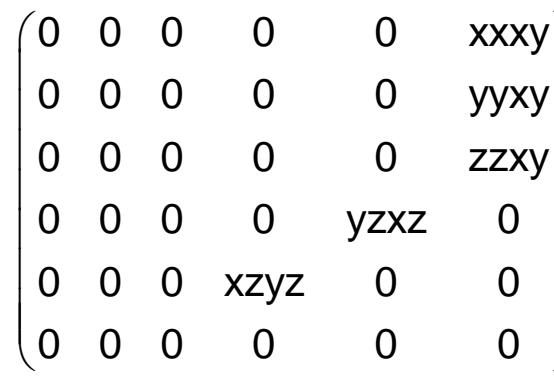




$\left(\begin{array}{ccccccccc}\text { xxxx } & \text { xxyy } & \text { xxzz } & 0 & 0 & 0 & 0 & 0 & 0 \\ \text { yyxx } & \text { yyyy } & \text { yyzz } & 0 & 0 & 0 & 0 & 0 & 0 \\ \text { zzxx } & \text { zzyy } & \text { zzzz } & 0 & 0 & 0 & 0 & 0 & 0 \\ 0 & 0 & 0 & \text { yzyz } & 0 & 0 & \text { yzzy } & 0 & 0 \\ 0 & 0 & 0 & 0 & \text { zxzx } & 0 & 0 & \text { zxxz } & 0 \\ 0 & 0 & 0 & 0 & 0 & x y x y & 0 & 0 & x y y x \\ 0 & 0 & 0 & z y y z & 0 & 0 & \text { zyzy } & 0 & 0 \\ 0 & 0 & 0 & 0 & \text { xzzx } & 0 & 0 & \text { xzxz } & 0 \\ 0 & 0 & 0 & 0 & 0 & \text { yxxy } & 0 & 0 & \text { yxyx }\end{array}\right)$

$\left(\begin{array}{lllllllll}0 & 0 & 0 & 0 & 0 & 0 & 0 & 0 & 0\end{array}\right)$ $\begin{array}{lllllllll}0 & 0 & 0 & 0 & 0 & 0 & 0 & 0 & 0 \\ 0 & 0 & 0 & 0 & 0 & 0 & 0 & 0 & 0\end{array}$ $0 \begin{array}{lllllllll}0 & 0 & 0 & 0 & 0 & 0 & 0 & 0 & 0 \\ 0 & 0 & 0 & 0 & 0 & 0 & 0 & 0 & 0\end{array}$ $0 \begin{array}{lllllllll}0 & 0 & 0 & 0 & 0 & 0 & 0 & 0 & 0 \\ 0 & 0 & 0 & 0 & 0 & 0 & 0 & 0 & 0\end{array}$ $0 \begin{array}{llllllllll}0 & 0 & 0 & 0 & 0 & 0 & 0 & 0 & 0 \\ 0 & 0 & 0 & 0 & 0 & 0 & 0 & 0 & 0\end{array}$ $\begin{array}{llllllllll}0 & 0 & 0 & 0 & 0 & 0 & 0 & 0 & 0 \\ 0 & 0 & 0 & 0 & 0 & 0 & 0 & 0 & 0 & 0\end{array}$ $\begin{array}{llllllllll}0 & 0 & 0 & 0 & 0 & 0 & 0 & 0 & 0\end{array}$ $\begin{array}{lllllllll}0 & 0 & 0 & 0 & 0 & 0 & 0 & 0 & 0 \\ 0 & 0 & 0 & 0 & 0 & 0 & 0 & 0 & 0\end{array}$

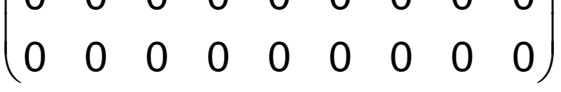

$\left[V^{4}\right] \quad\left(\begin{array}{ccccc}x x x x & 0 & 0 & y y z z & 0 \\ 0 & y y y y & 0 & z z x x & 0 \\ 0 & 0 & z z z z & x x y y & 0\end{array}\right)$

$$
\left(\begin{array}{lllll}
0 & 0 & 0 & 0 & 0 \\
0 & 0 & 0 & 0 & 0 \\
0 & 0 & 0 & 0 & 0
\end{array}\right)
$$

$V\left[V^{3}\right]\left(\begin{array}{cccccccccc}\mathrm{xxxx} & 0 & 0 & \mathrm{xxyy} & 0 & 0 & \mathrm{xxzz} & 0 & 0 & 0 \\ 0 & \mathrm{yyyy} & 0 & 0 & \mathrm{yyzz} & 0 & 0 & \mathrm{yyxx} & 0 & 0 \\ 0 & 0 & \mathrm{zzzz} & 0 & 0 & \mathrm{zzxx} & 0 & 0 & \mathrm{zzy} & 0\end{array}\right)$

$\begin{array}{llllllllll}0 & 0 & 0 & 0 & 0 & 0 & 0 & 0 & 0 & 0 \\ 0 & 0 & 0 & 0 & 0 & 0 & 0 & 0 & 0 & 0\end{array}$ $\left(\begin{array}{llllllllll}0 & 0 & 0 & 0 & 0 & 0 & 0 & 0 & 0 & 0 \\ 0 & 0 & 0 & 0 & 0 & 0 & 0 & 0 & 0 & 0\end{array}\right)$

$$
\left[\left[^{2}\right]^{2}\right]\left(\begin{array}{cccccc}
x x x x & x x y y & x x z z & 0 & 0 & 0 \\
& \text { yyyy } & y y z z & 0 & 0 & 0 \\
& & z z z z & 0 & 0 & 0 \\
& & & y z y z & 0 & 0 \\
& & & & x z x z & 0 \\
& & & & & \text { xyxy }
\end{array}\right)
$$

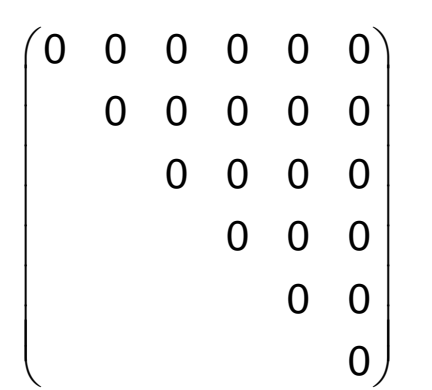

$\left(\begin{array}{llllll}0 & 0 & 0 & 0 & 0 & 0 \\ 0 & 0 & 0 & 0 & 0 & 0\end{array}\right)$ $\begin{array}{lllllll}0 & 0 & 0 & 0 & 0 & 0 \\ 0 & 0 & 0 & 0 & 0 & 0 \\ 0 & 0 & 0 & 0 & 0\end{array}$ $\begin{array}{llllll}0 & 0 & 0 & 0 & 0 & 0 \\ 0 & 0 & 0 & 0 & 0 & 0 \\ 0 & 0 & 0 & 0 & 0 & 0 \\ 0 & 0 & 0 & 0 & 0 & 0\end{array}$ $\begin{array}{llllll}0 & 0 & 0 & 0 & 0 & 0 \\ 0 & 0 & 0 & 0 & 0 & 0 \\ 0 & 0 & 0 & 0 & 0 & 0\end{array}$ $\begin{array}{llllll}0 & 0 & 0 & 0 & 0 & 0 \\ 0 & 0 & 0 & 0 & 0 & 0\end{array}$

$\left.V^{2}\right]^{2}\left(\begin{array}{cccccc}\text { yyxx } & \text { yyyy } & \text { yyzz } & 0 & 0 & 0 \\ z z x x & z z y y & z z z z & 0 & 0 & 0 \\ 0 & 0 & 0 & y z y z & 0 & 0 \\ 0 & 0 & 0 & 0 & x z x z & 0 \\ 0 & 0 & 0 & 0 & 0 & x y x y\end{array}\right)$ $\begin{array}{lllllllll}0 & 0 & 0 & 0 & 0 & 0 & 0 & 0 & 0 \\ 0 & 0 & 0 & 0 & 0 & 0 & 0 & 0 & 0\end{array}$ $0 \begin{array}{lllllllll}0 & 0 & 0 & 0 & 0 & 0 & 0 & 0 & 0 \\ 0 & 0 & 0 & 0 & 0 & 0 & 0 & 0 & 0\end{array}$ $0 \begin{array}{lllllllll}0 & 0 & 0 & 0 & 0 & 0 & 0 & 0 & 0 \\ 0 & 0 & 0 & 0 & 0 & 0 & 0 & 0 & 0\end{array}$ $0 \begin{array}{lllllllll}0 & 0 & 0 & 0 & 0 & 0 & 0 & 0 & 0 \\ 0 & 0 & 0 & 0 & 0 & 0 & 0 & 0 & 0\end{array}$ $\begin{array}{llllllllll}0 & 0 & 0 & 0 & 0 & 0 & 0 & 0 & 0 \\ 0 & 0 & 0 & 0 & 0 & 0 & 0 & 0 & 0\end{array}$ $\begin{array}{llllllllll}0 & 0 & 0 & 0 & 0 & 0 & 0 & 0 & 0 \\ 0 & 0 & 0 & 0 & 0 & 0 & 0 & 0 & 0 & 0\end{array}$ $\begin{array}{llllllllll}0 & 0 & 0 & 0 & 0 & 0 & 0 & 0 & 0\end{array}$ $\begin{array}{llllllllllllllllll}0 & 0 & 0 & 0 & 0 & 0 & 0 & 0 & 0 & 0 & 0 & 0\end{array}$ $\left(\begin{array}{lllllllll}0 & 0 & 0 & 0 & 0 & 0 & 0 & 0 & 0 \\ 0 & 0 & 0 & 0 & 0 & 0 & 0 & 0 & 0\end{array}\right)$

$$
\left(\begin{array}{lllll}
0 & 0 & 0 & 0 & 0 \\
0 & 0 & 0 & 0 & 0 \\
0 & 0 & 0 & 0 & 0
\end{array}\right)
$$

$\begin{array}{llllllllll}0 & 0 & 0 & 0 & 0 & 0 & 0 & 0 & 0 & 0 \\ 0 & 0 & 0 & 0 & 0 & 0 & 0 & 0 & 0 & 0\end{array}$ $\left(\begin{array}{llllllllll}0 & 0 & 0 & 0 & 0 & 0 & 0 & 0 & 0 & 0 \\ 0 & 0 & 0 & 0 & 0 & 0 & 0 & 0 & 0 & 0\end{array}\right)$

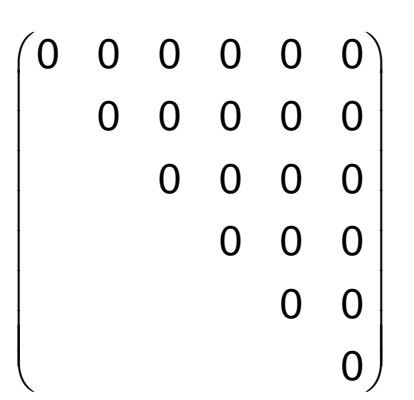

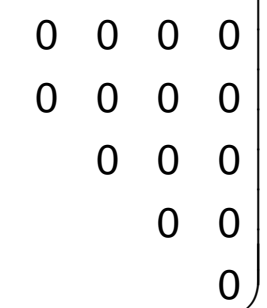

$\left(\begin{array}{llllll}0 & 0 & 0 & 0 & 0 & 0 \\ 0 & 0 & 0 & 0 & 0 & 0\end{array}\right)$

$\begin{array}{llllll}0 & 0 & 0 & 0 & 0 & 0 \\ 0 & 0 & 0 & 0 & 0 & 0\end{array}$

$\begin{array}{llllll}0 & 0 & 0 & 0 & 0 & 0 \\ 0 & 0 & 0 & 0 & 0 & 0\end{array}$ $\begin{array}{llllll}0 & 0 & 0 & 0 & 0 & 0 \\ 0 & 0 & 0 & 0 & 0 & 0\end{array}$ $\begin{array}{llllll}0 & 0 & 0 & 0 & 0 & 0 \\ 0 & 0 & 0 & 0 & 0 & 0\end{array}$ $\begin{array}{llllll}0 & 0 & 0 & 0 & 0 & 0\end{array}$ $\left(\begin{array}{ccccccccc}0 & 0 & 0 & 0 & 0 & \text { xxxy } & 0 & 0 & \text { xxyx } \\ 0 & 0 & 0 & 0 & 0 & \text { yyxy } & 0 & 0 & \text { yyyx } \\ 0 & 0 & 0 & 0 & 0 & z z x y & 0 & 0 & \text { zzyx } \\ 0 & 0 & 0 & 0 & y z z x & 0 & 0 & y z x z & 0 \\ 0 & 0 & 0 & \text { zxyz } & 0 & 0 & \text { zxzy } & 0 & 0 \\ \text { xyxx } & x y y y & x y z z & 0 & 0 & 0 & 0 & 0 & 0 \\ 0 & 0 & 0 & 0 & \text { zyzx } & 0 & 0 & z y x z & 0 \\ 0 & 0 & 0 & \text { xzyz } & 0 & 0 & \text { xzzy } & 0 & 0 \\ \text { yxxx } & \text { yxyy } & \text { yxzz } & 0 & 0 & 0 & 0 & 0 & 0\end{array}\right)$

$\left(\begin{array}{ccccc}0 & x y y y & 0 & 0 & 0 \\ y x x x & 0 & 0 & 0 & 0\end{array}\right)$

$\left(\begin{array}{ccccc}\operatorname{yxxx} & 0 & 0 & 0 & 0 \\ 0 & 0 & 0 & 0 & z z x y\end{array}\right)$

$\left(\begin{array}{cccccccccc}0 & x y y y & 0 & 0 & x y z z & 0 & 0 & x y x x & 0 & 0 \\ y x x x & 0 & 0 & y x y y & 0 & 0 & y x z z & 0 & 0 & 0 \\ 0 & 0 & 0 & 0 & 0 & 0 & 0 & 0 & 0 & z x y z\end{array}\right)$ $\left(\begin{array}{cccccccccc}\mathrm{yxxx} & 0 & 0 & \mathrm{yxyy} & 0 & 0 & \mathrm{yxzz} & 0 & 0 & 0 \\ 0 & 0 & 0 & 0 & 0 & 0 & 0 & 0 & 0 & \mathrm{zxyz}\end{array}\right)$

$$
\begin{aligned}
& \left(\begin{array}{llllll}
0 & 0 & 0 & 0 & 0 & x x x y \\
& 0 & 0 & 0 & 0 & y y x y \\
& & 0 & 0 & 0 & z z x y
\end{array}\right) \\
& \begin{array}{llll}
0 & 0 & 0 & z z x y
\end{array} \\
& \begin{array}{lll}
0 & y z x z & 0
\end{array} \\
& \begin{array}{ll}
0 \\
0
\end{array}
\end{aligned}
$$

$\left(\begin{array}{llllll}0 & 0 & 0 & 0 & 0 & x x x y \\ 0 & 0 & 0 & 0 & 0 & y y x y \\ 0 & 0 & 0 & 0 & 0 & z z x y\end{array}\right)$

$\begin{array}{lllllll}0 & 0 & 0 & 0 & 0 & x x y y \\ 0 & 0 & 0 & 0 & 0 & y y x y \\ 0 & 0 & 0 & 0 & 0 & z z x y\end{array}$ $\begin{array}{llllll}0 & 0 & 0 & x z y z & 0 & 0\end{array}$ 


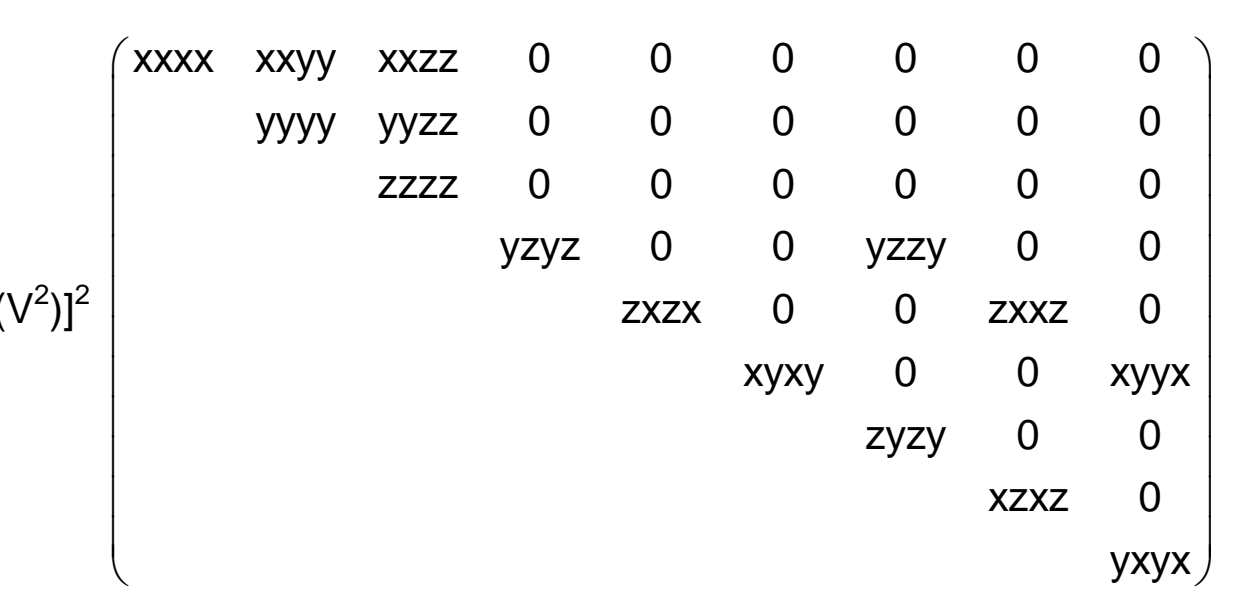

$\left(\begin{array}{lllllllll}0 & 0 & 0 & 0 & 0 & 0 & 0 & 0 & 0 \\ 0 & 0 & 0 & 0 & 0 & 0 & 0 & 0\end{array}\right)$

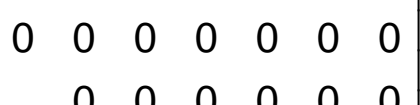
$\begin{array}{lllll}0 & 0 & 0 & 0 & 0\end{array}$ $\begin{array}{llll}0 & 0 & 0 & 0\end{array}$ $\begin{array}{llll}0 & 0 & 0 \\ 0 & 0 & 0\end{array}$ $\left.\begin{array}{lll}0 & 0 & 0 \\ & 0 & 0 \\ & & 0\end{array}\right)$

$\left[\mathrm{V}^{2}\right] \mathrm{V}^{2}\left(\begin{array}{ccccccccc}\mathrm{x} x \mathrm{x} & \mathrm{xxyy} & \mathrm{xxzz} & 0 & 0 & 0 & 0 & 0 & 0 \\ \mathrm{yyxx} & \text { yyyy } & \mathrm{yyzz} & 0 & 0 & 0 & 0 & 0 & 0 \\ \mathrm{zzxx} & \mathrm{zzyy} & \mathrm{zzzz} & 0 & 0 & 0 & 0 & 0 & 0 \\ 0 & 0 & 0 & \mathrm{yzyz} & 0 & 0 & \mathrm{yzzy} & 0 & 0 \\ 0 & 0 & 0 & 0 & \mathrm{zxzx} & 0 & 0 & \mathrm{zxxz} & 0 \\ 0 & 0 & 0 & 0 & 0 & \text { xyxy } & 0 & 0 & \text { xyyx }\end{array}\right)$

$\left(\begin{array}{lllllllll}0 & 0 & 0 & 0 & 0 & 0 & 0 & 0 & 0 \\ 0 & 0 & 0 & 0 & 0 & 0 & 0 & 0 & 0 \\ 0 & 0 & 0 & 0 & 0 & 0 & 0 & 0 & 0\end{array}\right)$ $0 \begin{array}{llllllllll}0 & 0 & 0 & 0 & 0 & 0 & 0 & 0 & 0 \\ 0 & 0 & 0 & 0 & 0 & 0 & 0 & 0 & 0\end{array}$ $\begin{array}{lllllllll}0 & 0 & 0 & 0 & 0 & 0 & 0 & 0 & 0 \\ 0 & 0 & 0 & 0 & 0 & 0 & 0 & 0 & 0\end{array}$ $\begin{array}{llllllll}0 & 0 & 0 & 0 & 0 & 0 & 0 & 0\end{array}$

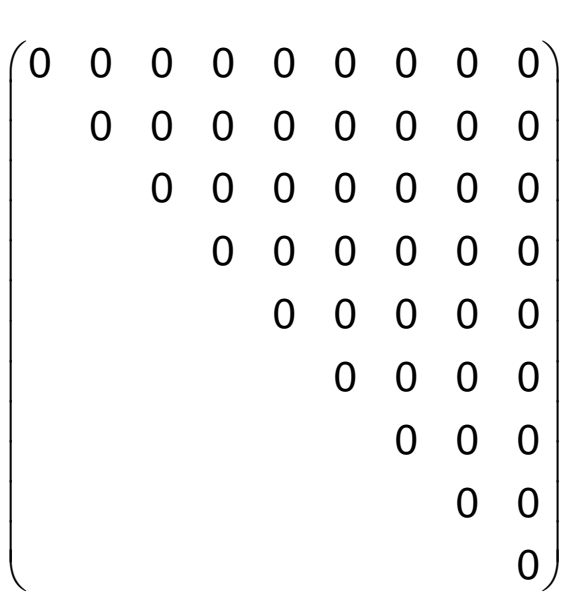

$\left(\begin{array}{lllllllll}0 & 0 & 0 & 0 & 0 & 0 & 0 & 0 & 0 \\ 0 & 0 & 0 & 0 & 0 & 0 & 0 & 0 & 0\end{array}\right)$

$0 \begin{array}{lllllllll}0 & 0 & 0 & 0 & 0 & 0 & 0 & 0 & 0 \\ 0 & 0 & 0 & 0 & 0 & 0 & 0 & 0 & 0\end{array}$ $\begin{array}{llllllllll}0 & 0 & 0 & 0 & 0 & 0 & 0 & 0 & 0 \\ 0 & 0 & 0 & 0 & 0 & 0 & 0 & 0 & 0\end{array}$ $0 \begin{array}{llllllllll}0 & 0 & 0 & 0 & 0 & 0 & 0 & 0 & 0 \\ 0 & 0 & 0 & 0 & 0 & 0 & 0 & 0 & 0\end{array}$ $\begin{array}{lllllllll}0 & 0 & 0 & 0 & 0 & 0 & 0 & 0 & 0 \\ 0 & 0 & 0 & 0 & 0 & 0 & 0 & 0 & 0\end{array}$

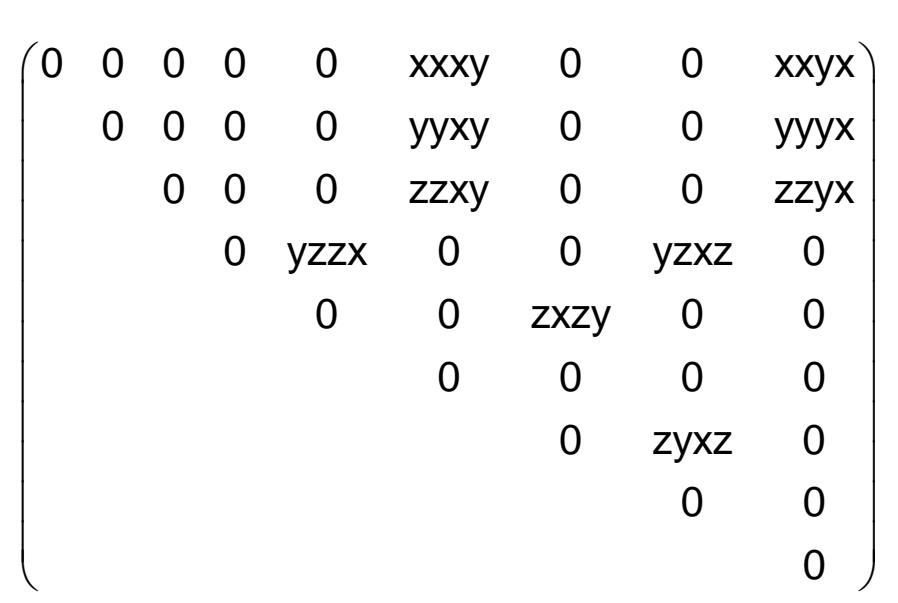
$\left.\begin{array}{lllllllll}0 & 0 & 0 & 0 & 0 & 0 & 0 & 0 & 0 \\ 0 & 0 & 0 & 0 & 0 & 0 & 0 & 0 & 0 \\ 0 & 0 & 0 & 0 & 0 & 0 & 0 & 0 & 0\end{array}\right)$ 
e
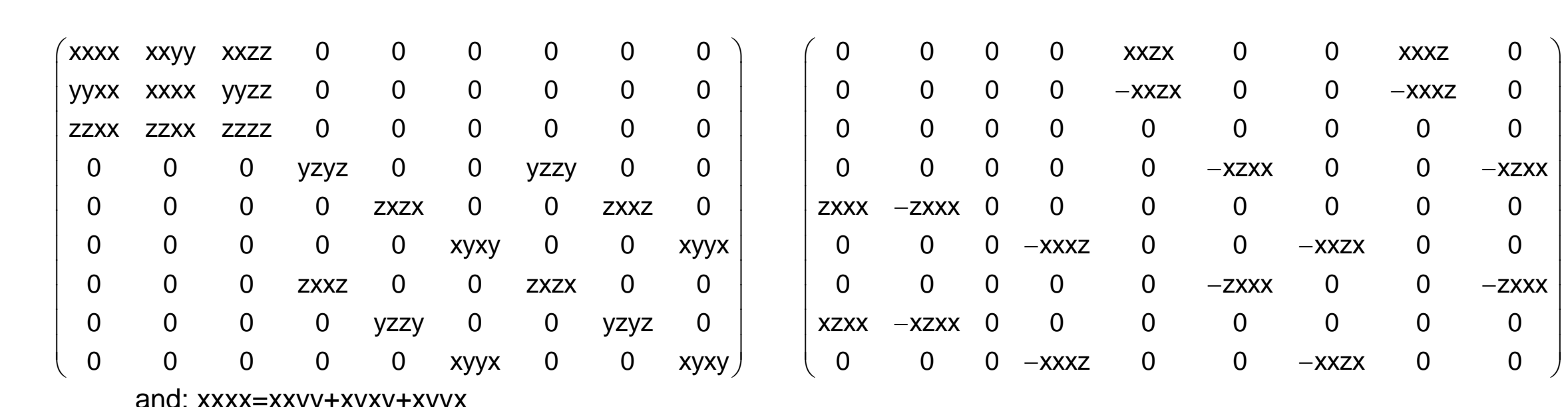

$\left[V^{4}\right]\left(\begin{array}{ccccc}3 x x y y & 0 & 0 & y y z z & 0 \\ 0 & 3 x x y y & 0 & y y z z & 0 \\ 0 & 0 & z z z z & x x y y & 0\end{array}\right)$

$$
\left(\begin{array}{ccccc}
0 & 0 & 0 & 0 & 0 \\
0 & 0 & 0 & 0 & -z x x x \\
z x x x & 0 & 0 & 0 & 0
\end{array}\right)
$$

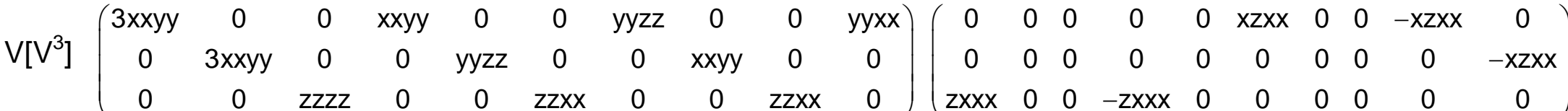

$\left[\left[^{2}\right]^{2}\right]\left(\begin{array}{cccccc}x x x x & x x y y & x x z z & 0 & 0 & 0 \\ & x x x x & x x z z & 0 & 0 & 0 \\ & & z z z z & 0 & 0 & 0 \\ & & & y z y z & 0 & 0 \\ & & & & y z y z & 0 \\ & & & & & x y x y\end{array}\right)$

$\left[\mathrm{V}^{2}\right]^{2}\left(\begin{array}{cccccc}x \mathrm{x} x \mathrm{x} & \mathrm{xxyy} & \mathrm{xxzz} & 0 & 0 & 0 \\ x \mathrm{x} y \mathrm{y} & \mathrm{xxxx} & \mathrm{xxzz} & 0 & 0 & 0 \\ \mathrm{zzxx} & \mathrm{zzxx} & \mathrm{zzzz} & 0 & 0 & 0 \\ 0 & 0 & 0 & \mathrm{yzyz} & 0 & 0 \\ 0 & 0 & 0 & 0 & \mathrm{yzyz} & 0 \\ 0 & 0 & 0 & 0 & 0 & \mathrm{xyxy}\end{array}\right)$

and $x y x y=1 / 2(x x x x-x x y y)$

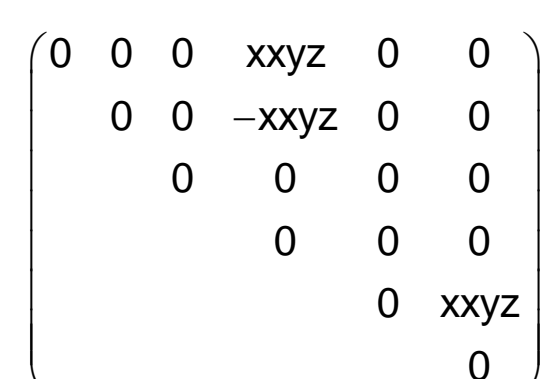

and $x y x y=1 / 2(x x x x-x x y y)$

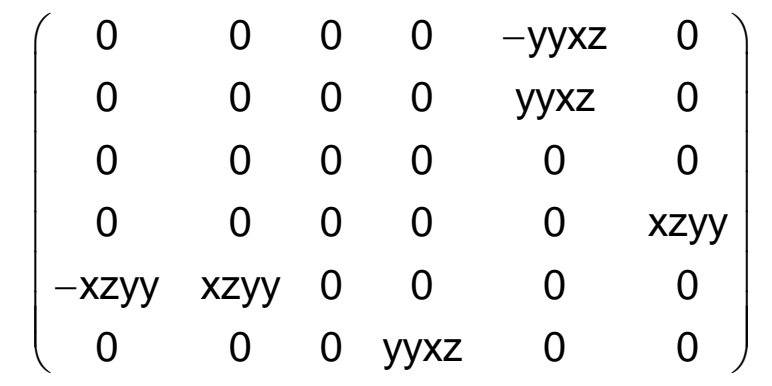

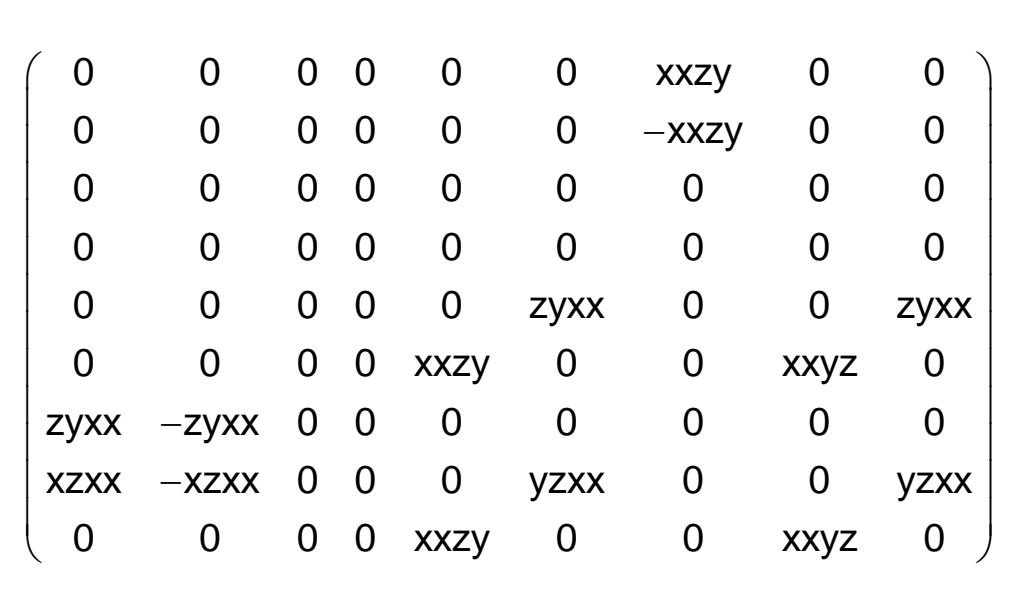

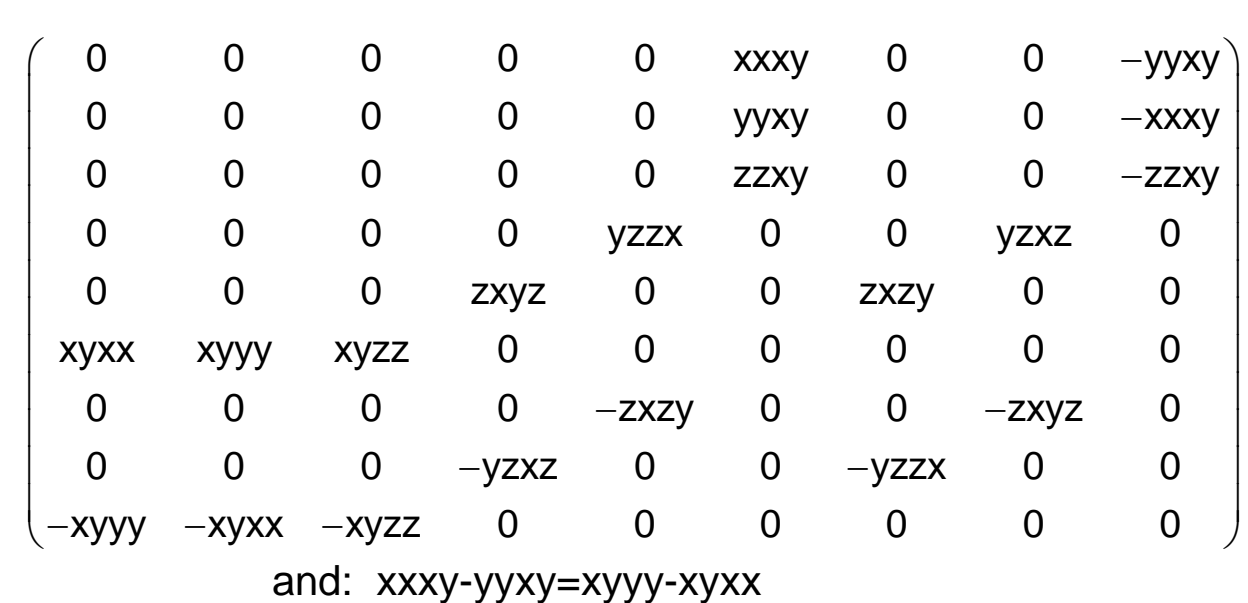

$\left(\begin{array}{lllll}0 & 0 & 0 & 0 & 0 \\ 0 & 0 & 0 & 0 & 0 \\ 0 & 0 & 0 & 0 & 0\end{array}\right)$

$\left(\begin{array}{cccccccccc}0 & 3 x y x x & 0 & 0 & x y z z & 0 & 0 & x y x x & 0 & 0 \\ -3 x y x x & 0 & 0 & -x y x x & 0 & 0 & -x y z z & 0 & 0 & 0\end{array}\right)$

$\left(\begin{array}{cccccccccc}0 & 0 & 0 & 0 & 0 & 0 & 0 & 0 & 0 & y z x x \\ 0 & 0 & 0 & 0 & 0 & y z z x & 0 & 0 & -y z x x & 0 \\ 0 & -2 y x & 0 & 0 & 0 & 0 & 0 & z y x x & 0 & 0\end{array}\right)$

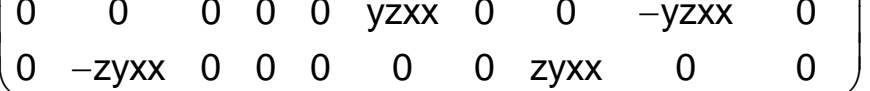

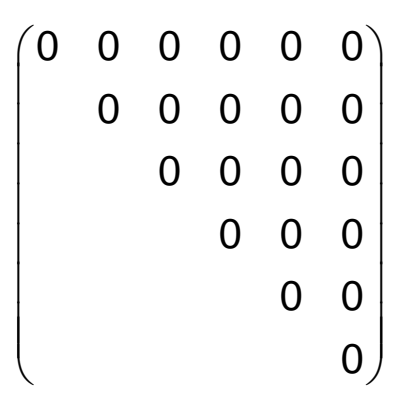

$\left(\begin{array}{cccccc}0 & 0 & 0 & 0 & -y y x z & 0 \\ & 0 & 0 & 0 & y y x z & 0 \\ & & 0 & 0 & 0 & 0 \\ & & & 0 & 0 & y y x z \\ & & & & 0 & 0 \\ & & & & & 0\end{array}\right)$

$\left(\begin{array}{cccccc}0 & 0 & 0 & \text { xxyz } & 0 & 0 \\ 0 & 0 & 0 & -x x y z & 0 & 0 \\ 0 & 0 & 0 & 0 & 0 & 0 \\ y z x x & -y z x x & 0 & 0 & 0 & 0 \\ 0 & 0 & 0 & 0 & 0 & y z x x \\ 0 & 0 & 0 & 0 & x x y z & 0\end{array}\right)$ $\left.\begin{array}{cccccc}0 & 0 & 0 & 0 & 0 & \text { xxxy } \\ 0 & 0 & 0 & 0 & 0 & -x x x y \\ 0 & 0 & 0 & 0 & 0 & 0 \\ 0 & 0 & 0 & 0 & y z x z & 0 \\ 0 & 0 & 0 & y z x z & 0 & 0 \\ -x x x y & x x x y & 0 & 0 & 0 & 0\end{array}\right)$

and $x y x y=1 / 2(x x x x-x x y y)$ 
a

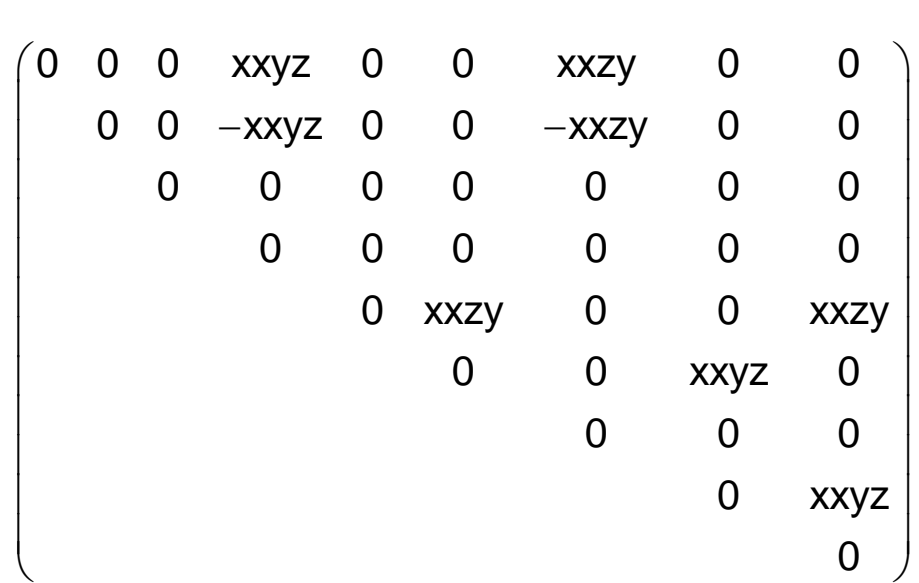

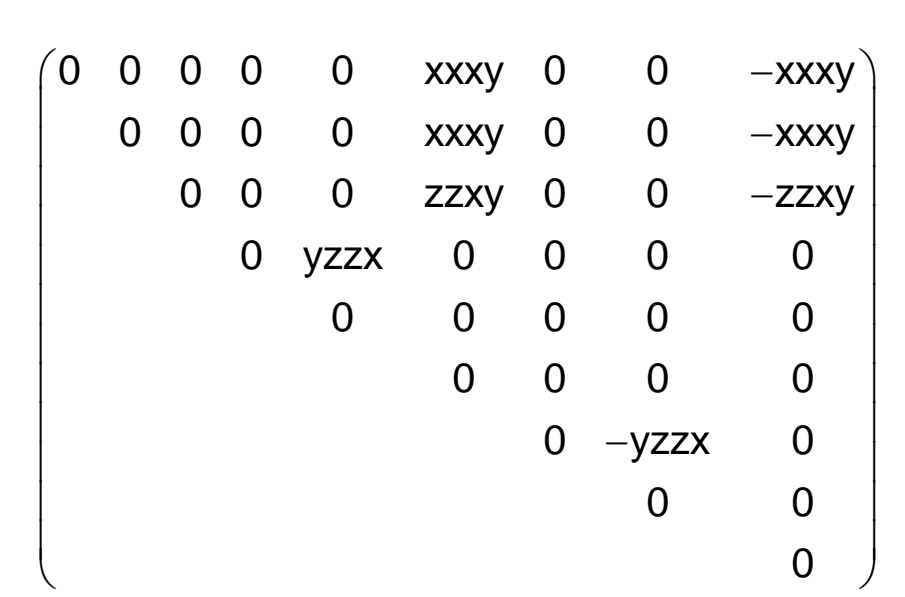

and $x x x x=x x y y+y z y z+z y z y$

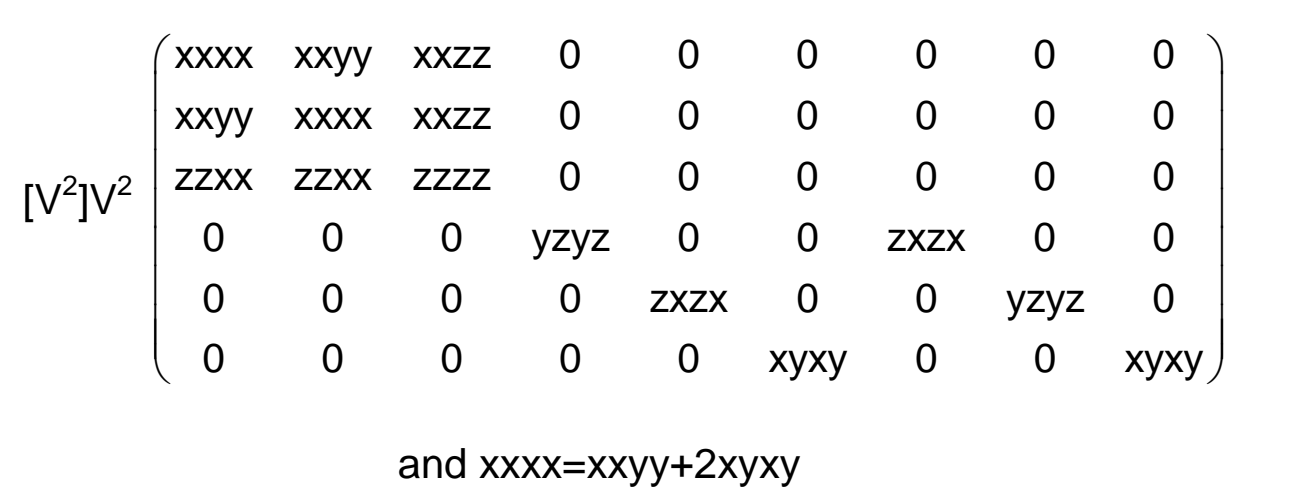




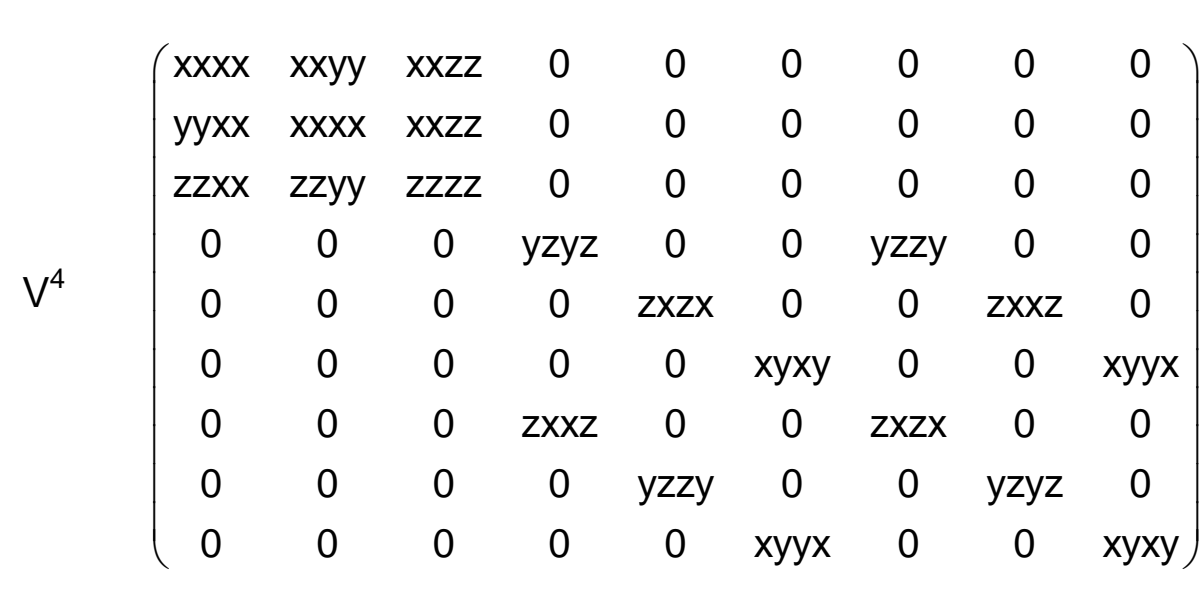

$\left(\begin{array}{lllllllllll}0 & 0 & 0 & 0 & 0 & 0 & 0 & 0 & 0 \\ 0 & 0 & 0 & 0 & 0 & 0 & 0 & 0 & 0 & 0\end{array}\right.$

$\left.\begin{array}{lllllllll}0 & 0 & 0 & 0 & 0 & 0 & 0 & 0 & 0 \\ 0 & 0 & 0 & 0 & 0 & 0 & 0 & 0 & 0\end{array}\right)$

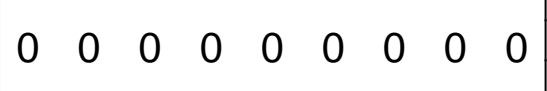

$0 \begin{array}{lllllllll}0 & 0 & 0 & 0 & 0 & 0 & 0 & 0 & 0 \\ 0 & 0 & 0 & 0 & 0 & 0 & 0 & 0 & 0\end{array}$

$0 \begin{array}{lllllllll}0 & 0 & 0 & 0 & 0 & 0 & 0 & 0 & 0 \\ 0 & 0 & 0 & 0 & 0 & 0 & 0 & 0 & 0\end{array}$

$0 \begin{array}{lllllllllll}0 & 0 & 0 & 0 & 0 & 0 & 0 & 0 & 0 \\ 0 & 0 & 0 & 0 & 0 & 0 & 0 & 0 & 0\end{array}$

$0 \begin{array}{llllllllll}0 & 0 & 0 & 0 & 0 & 0 & 0 & 0 & 0 \\ 0 & 0 & 0 & 0 & 0 & 0 & 0 & 0\end{array}$

$\left.\begin{array}{llllllllll}0 & 0 & 0 & 0 & 0 & 0 & 0 & 0 & 0 \\ 0 & 0 & 0 & 0 & 0 & 0 & 0 & 0 & 0 \\ 0 & 0 & 0 & 0 & 0 & 0 & 0 & 0 & 0\end{array}\right)$

$\left(\begin{array}{lllllllll}0 & 0 & 0 & 0 & 0 & 0 & 0 & 0 & 0\end{array}\right)$

$\left[V^{4}\right]\left(\begin{array}{ccccc}x x x x & 0 & 0 & y y z z & 0 \\ 0 & x x x x & 0 & y y z z & 0 \\ 0 & 0 & z z z z & x x y y & 0\end{array}\right)$

$\left(\begin{array}{lllll}0 & 0 & 0 & 0 & 0 \\ 0 & 0 & 0 & 0 & 0 \\ 0 & 0 & 0 & 0 & 0\end{array}\right)$

$\begin{array}{lllll}0 & 0 & 0 & 0 & 0\end{array}$

$\left.\mathrm{V}^{3}\right]\left(\begin{array}{cccccccccc}\mathrm{xxxx} & 0 & 0 & \mathrm{xxyy} & 0 & 0 & \mathrm{yyzz} & 0 & 0 & 0 \\ 0 & \mathrm{xxxx} & 0 & 0 & \mathrm{yyzz} & 0 & 0 & \mathrm{xxyy} & 0 & 0 \\ 0 & 0 & \mathrm{zzzz} & 0 & 0 & \mathrm{zzxx} & 0 & 0 & \mathrm{zzxx} & 0\end{array}\right)$

$\left(\begin{array}{llllllllll}0 & 0 & 0 & 0 & 0 & 0 & 0 & 0 & 0 & 0 \\ 0 & 0 & 0 & 0 & 0 & 0 & 0 & 0 & 0 & 0\end{array}\right.$

$\left(\begin{array}{lllllllllll}0 & 0 & 0 & 0 & 0 & 0 & 0 & 0 & 0 & 0 \\ 0 & 0 & 0 & 0 & 0 & 0 & 0 & 0 & 0 & 0\end{array}\right)$

$$
\left(\begin{array}{cccccc}
\text { xxxx } & \text { xxyy } & x x z z & 0 & 0 & 0 \\
& \text { yyyy } & \text { yyzz } & 0 & 0 & 0 \\
& & \text { zzzz } & 0 & 0 & 0 \\
& & & y z y z & 0 & 0 \\
& & & & y z y z & 0 \\
& & & & & \text { xyxy }
\end{array}\right)
$$

$\left[V^{2}\right]^{2}\left(\begin{array}{cccccc}x x x x & x x y y & x x z z & 0 & 0 & 0 \\ y y x x & x x x x & x x z z & 0 & 0 & 0 \\ z z x x & z z x x & z z z z & 0 & 0 & 0 \\ 0 & 0 & 0 & y z y z & 0 & 0 \\ 0 & 0 & 0 & 0 & y z y z & 0 \\ 0 & 0 & 0 & 0 & 0 & x y x y\end{array}\right)$

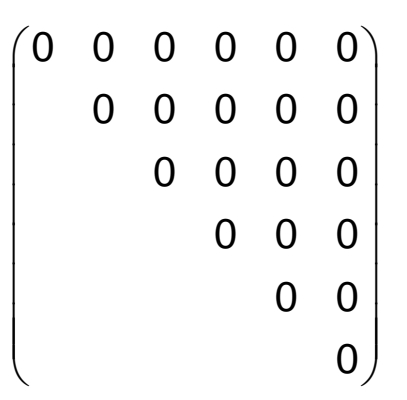

$\left(\begin{array}{llllll}0 & 0 & 0 & 0 & 0 & 0 \\ 0 & 0 & 0 & 0 & 0 & 0\end{array}\right.$ $\left.\begin{array}{llllll}0 & 0 & 0 & 0 & 0 & 0 \\ 0 & 0 & 0 & 0 & 0 & 0 \\ 0 & 0 & 0 & 0 & 0 & 0\end{array}\right)$ $\begin{array}{lllllll}0 & 0 & 0 & 0 & 0 & 0 \\ 0 & 0 & 0 & 0 & 0 & 0\end{array}$ $\begin{array}{llllll}0 & 0 & 0 & 0 & 0 & 0 \\ 0 & 0 & 0 & 0\end{array}$

$\begin{array}{llllll}0 & 0 & 0 & 0 & 0 & 0 \\ 0 & 0 & 0 & 0 & 0 & 0\end{array}$

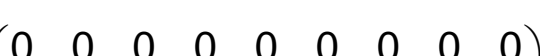

$\begin{array}{llllllllll}0 & 0 & 0 & 0 & 0 & 0 & 0 & 0 & 0\end{array}$

$0 \begin{array}{llllllllll}0 & 0 & 0 & 0 & 0 & 0 & 0 & 0 & 0 \\ 0 & 0 & 0 & 0 & 0 & 0 & 0 & 0 & 0\end{array}$ $0 \begin{array}{lllllllll}0 & 0 & 0 & 0 & 0 & 0 & 0 & 0 & 0 \\ 0 & 0 & 0 & 0 & 0 & 0 & 0 & 0 & 0\end{array}$ $0 \begin{array}{lllllllll}0 & 0 & 0 & 0 & 0 & 0 & 0 & 0 & 0 \\ 0 & 0 & 0 & 0 & 0 & 0 & 0 & 0 & 0\end{array}$ $\begin{array}{lllllllll}0 & 0 & 0 & 0 & 0 & 0 & 0 & 0 & 0 \\ 0 & 0 & 0 & 0 & 0 & 0 & 0 & 0 & 0\end{array}$ $\begin{array}{llllllllll}0 & 0 & 0 & 0 & 0 & 0 & 0 & 0 & 0 \\ 0 & 0 & 0 & 0 & 0 & 0 & 0 & 0 & 0\end{array}$ $\begin{array}{llllllllll}0 & 0 & 0 & 0 & 0 & 0 & 0 & 0 & 0 \\ 0 & 0 & 0 & 0 & 0\end{array}$ $\begin{array}{lllllllll}0 & 0 & 0 & 0 & 0 & 0 & 0 & 0 & 0\end{array}$

$\left(\begin{array}{lllll}0 & 0 & 0 & 0 & 0 \\ 0 & 0 & 0 & 0 & 0 \\ 0 & 0 & 0 & 0 & 0\end{array}\right)$

$\begin{array}{lllll}0 & 0 & 0 & 0 & 0\end{array}$

$\left(\begin{array}{llllllllll}0 & 0 & 0 & 0 & 0 & 0 & 0 & 0 & 0 & 0 \\ 0 & 0 & 0 & 0 & 0 & 0 & 0 & 0 & 0 & 0\end{array}\right.$ $\left(\begin{array}{lllllllllll}0 & 0 & 0 & 0 & 0 & 0 & 0 & 0 & 0 & 0 \\ 0 & 0 & 0 & 0 & 0 & 0 & 0 & 0 & 0 & 0\end{array}\right)$

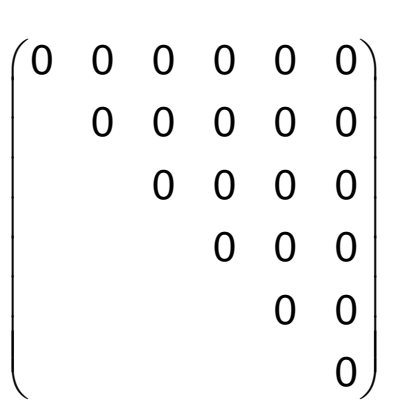

$\left(\begin{array}{llllll}0 & 0 & 0 & 0 & 0 & 0 \\ 0 & 0 & 0 & 0 & 0 & 0 \\ 0 & 0 & 0 & 0 & 0 & 0\end{array}\right)$ $\left(\begin{array}{llllll}0 & 0 & 0 & 0 & 0 & 0 \\ 0 & 0 & 0 & 0 & 0 & 0 \\ 0 & 0 & 0 & 0 & 0 & 0\end{array}\right)$ $0 \begin{array}{llllll}0 & 0 & 0 & 0 & 0 & 0\end{array}$ $\begin{array}{llllll}0 & 0 & 0 & 0 & 0 & 0\end{array}$ $\begin{array}{llllll}0 & 0 & 0 & 0 & 0 & 0 \\ 0 & 0 & 0 & 0 & 0 & 0\end{array}$

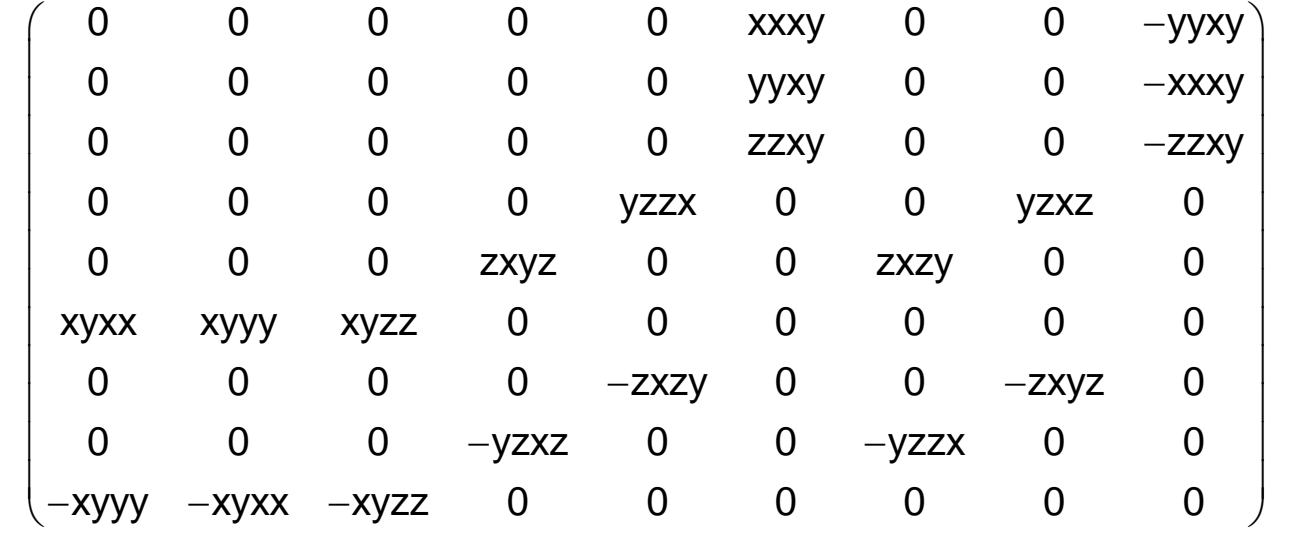

$\left(\begin{array}{ccccc}0 & -y x x x & 0 & 0 & 0 \\ y x x x & 0 & 0 & 0 & 0 \\ 0 & 0 & 0 & 0 & 0\end{array}\right)$

$\left(\begin{array}{cccccccccc}0 & -y x x x & 0 & 0 & x y z z & 0 & 0 & x y x x & 0 & 0 \\ y x x & 0 & 0 & -x y x x & 0 & 0 & -x y z z & 0 & 0 & 0\end{array}\right)$ $\begin{array}{cccccccccc}y \mathrm{yxx} & 0 & 0 & -\mathrm{xy} x \mathrm{x} & 0 & 0 & -\mathrm{xyzz} & 0 & 0 & 0 \\ 0 & 0 & 0 & 0 & 0 & 0 & 0 & 0 & 0 & \mathrm{zxyz}\end{array}$

$$
\left(\begin{array}{cccccc}
0 & 0 & 0 & 0 & 0 & \mathrm{xxxy} \\
& 0 & 0 & 0 & 0 & -\mathrm{xxxy} \\
& & 0 & 0 & 0 & 0 \\
& & & 0 & 0 & 0 \\
& & & & 0 & 0 \\
& & & & & 0
\end{array}\right)
$$

$\left(\begin{array}{cccccc}0 & 0 & 0 & 0 & 0 & x x x y \\ 0 & 0 & 0 & 0 & 0 & -x x x y \\ 0 & 0 & 0 & 0 & 0 & 0 \\ 0 & 0 & 0 & 0 & y z x z & 0 \\ 0 & 0 & 0 & -y z x z & 0 & 0 \\ x y x x & -x y x x & 0 & 0 & 0 & 0\end{array}\right)$




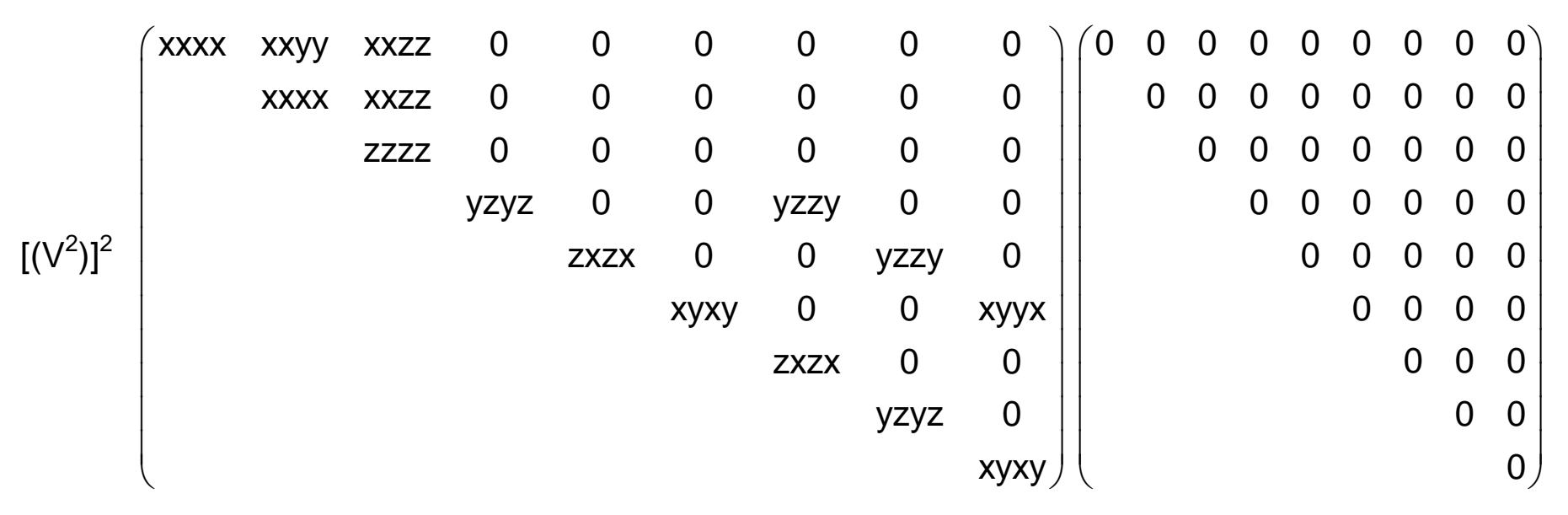

$\left(\begin{array}{lllllllll}x x x x & x x y y & x x z z & 0 & 0 & 0 & 0 & 0 & 0 \\ y y x x & x x x x & x x z z & 0 & 0 & 0 & 0 & 0 & 0\end{array}\right)\left(\begin{array}{lllllllll}0 & 0 & 0 & 0 & 0 & 0 & 0 & 0 & 0 \\ 0 & 0 & 0 & 0 & 0 & 0 & 0 & 0 & 0 \\ 0 & 0 & 0 & 0 & 0 & 0 & 0 & 0 & 0\end{array}\right)$ $\left.\begin{array}{|lllllllll}\mathrm{yyxx} & \mathrm{xxxx} & \mathrm{xxzz} & 0 & 0 & 0 & 0 & 0 & 0 \\ \mathrm{zzxx} & \mathrm{zzxx} & \mathrm{zzzz} & 0 & 0 & 0 & 0 & 0 & 0\end{array} \mid \begin{array}{lllllllll}0 & 0 & 0 & 0 & 0 & 0 & 0 & 0 & 0 \\ 0 & 0 & 0 & 0 & 0 & 0 & 0 & 0 & 0\end{array}\right)$

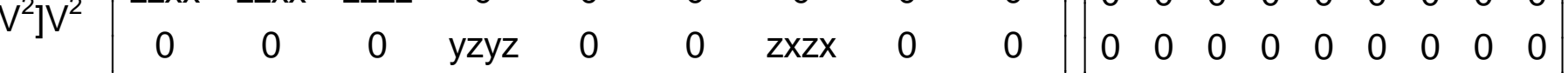
$\left(\begin{array}{ccccccccc}0 & 0 & 0 & 0 & z x z x & 0 & 0 & y z y z & 0 \\ 0 & 0 & 0 & 0 & 0 & x y x y & 0 & 0 & x y x y\end{array}\right)\left(\begin{array}{ccccccccc}0 & 0 & 0 & 0 & 0 & 0 & 0 & 0 & 0 \\ 0 & 0 & 0 & 0 & 0 & 0 & 0 & 0 & 0\end{array}\right)$

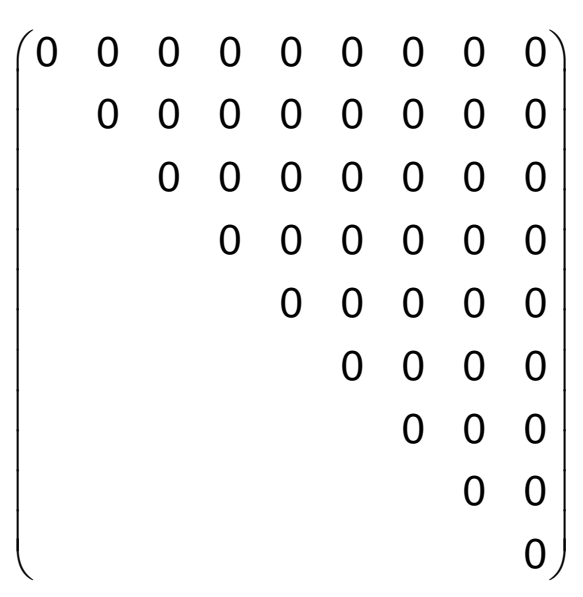

$\left(\begin{array}{lllllllll}0 & 0 & 0 & 0 & 0 & 0 & 0 & 0 & 0 \\ 0 & 0 & 0 & 0 & 0 & 0 & 0 & 0 & 0\end{array}\right.$

$0 \begin{array}{llllllllll}0 & 0 & 0 & 0 & 0 & 0 & 0 & 0 & 0 \\ 0 & 0 & 0 & 0 & 0 & 0 & 0 & 0 & 0\end{array}$

$\begin{array}{lllllllll}0 & 0 & 0 & 0 & 0 & 0 & 0 & 0 & 0\end{array}$

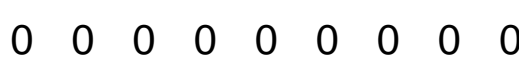

$\begin{array}{lllllllll}0 & 0 & 0 & 0 & 0 & 0 & 0 & 0 & 0 \\ 0 & 0 & 0 & 0 & 0 & 0 & 0 & 0 & 0\end{array}$

$\left(\begin{array}{llllllllll}0 & 0 & 0 & 0 & 0 & 0 & 0 & 0 & 0\end{array}\right)$

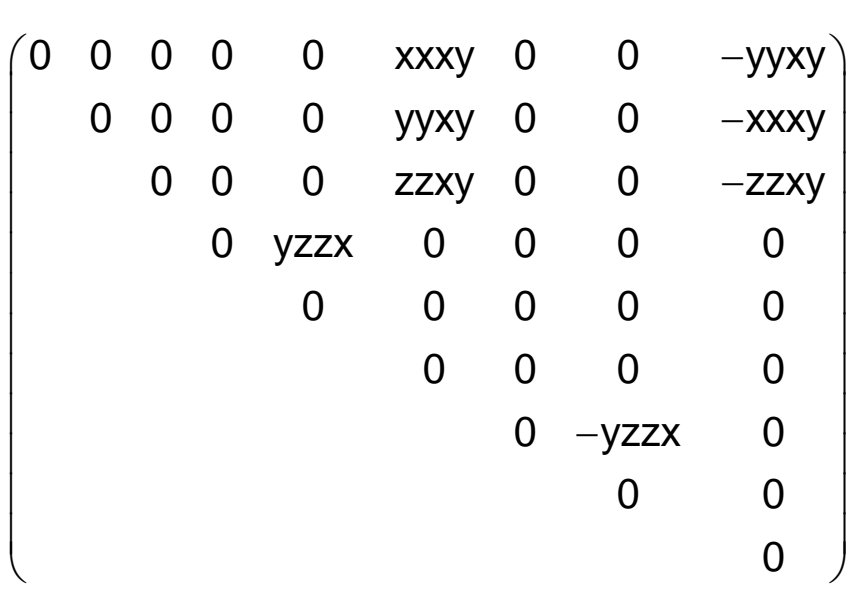

$\left(\begin{array}{ccccccccc}0 & 0 & 0 & 0 & 0 & \text { xxxy } & 0 & 0 & - \text { yyxy } \\ 0 & 0 & 0 & 0 & 0 & \text { yyxy } & 0 & 0 & - \text { xxxy } \\ 0 & 0 & 0 & 0 & 0 & \text { zzxy } & 0 & 0 & -z z x y \\ 0 & 0 & 0 & 0 & y z z x & 0 & 0 & -z x y z & 0 \\ 0 & 0 & 0 & \text { zxyz } & 0 & 0 & -y z z x & 0 & 0 \\ \text { xyxx } & - \text {-xyxx } & 0 & 0 & 0 & 0 & 0 & 0 & 0\end{array}\right)$ 

$\begin{array}{ccccccccc}z z x x & z z y y & z z z z & 0 & 0 & 0 & 0 & 0 & 0 \\ 0 & 0 & 0 & y z y z & 0 & 0 & y z z y & 0 & 0 \\ 0 & 0 & 0 & 0 & z x z x & 0 & 0 & z x y z & 0\end{array}$ $\mathrm{V}^{4} \quad \begin{array}{ccccccccc}0 & 0 & 0 & \mathrm{yzyz} & 0 & 0 & \mathrm{yzzy} & 0 & 0 \\ 0 & 0 & 0 & 0 & \mathrm{zxzx} & 0 & 0 & \mathrm{zxxz} & 0 \\ 0 & 0 & 0 & 0 & 0 & \mathrm{xyyy} & 0 & 0 & \mathrm{xyyx}\end{array}$ $\begin{array}{lllllllll}0 & 0 & 0 & 0 & 0 & x y x y & 0 & 0 & x y y x\end{array}$

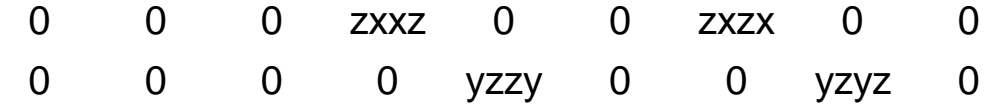
and: $x x x x=x x y y+x y x y+x y y x$

$\left[V^{4}\right] \quad\left(\begin{array}{ccccc}3 x x y y & 0 & 0 & y y z z & 0 \\ 0 & 3 x x y y & 0 & y y z z & 0 \\ 0 & 0 & z z z z & x x y y & 0\end{array}\right)$

$\left(\begin{array}{lllll}0 & 0 & 0 & 0 & 0 \\ 0 & 0 & 0 & 0 & 0 \\ 0 & 0 & 0 & 0 & 0\end{array}\right)$

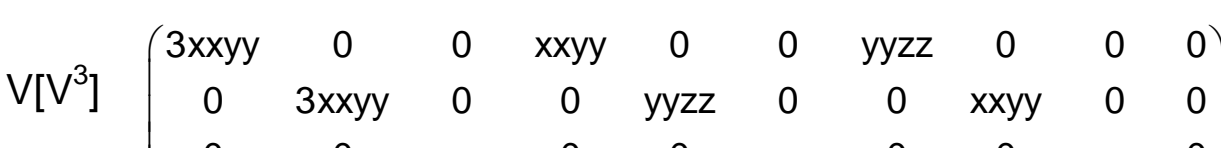

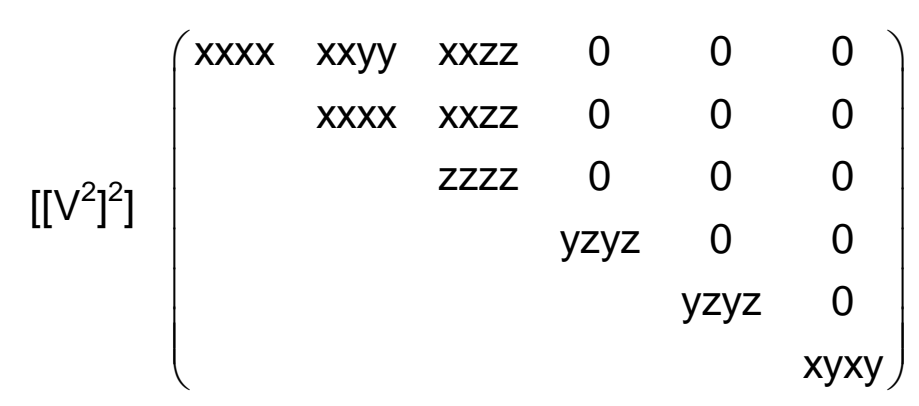

and $x y x y=1 / 2(x x x x-x x y y)$

$\left[\mathrm{V}^{2}\right]^{2}\left(\begin{array}{cccccc}x \mathrm{x} x \mathrm{x} & \mathrm{xxyy} & \mathrm{xxzz} & 0 & 0 & 0 \\ \mathrm{x} x \mathrm{y} & \mathrm{xxxx} & \mathrm{xxzz} & 0 & 0 & 0 \\ \mathrm{zzxx} & \mathrm{zzxx} & \mathrm{zzzz} & 0 & 0 & 0 \\ 0 & 0 & 0 & \mathrm{yzyz} & 0 & 0 \\ 0 & 0 & 0 & 0 & \mathrm{yzyz} & 0 \\ 0 & 0 & 0 & 0 & 0 & \mathrm{xyxy}\end{array}\right)$

and $x y x y=1 / 2(x x x x-x x y y)$ $\left(\begin{array}{lllllllll}0 & 0 & 0 & 0 & 0 & 0 & 0 & 0 & 0 \\ 0 & 0 & 0 & 0 & 0 & 0 & 0 & 0 & 0\end{array}\right)$ $\left(\begin{array}{lllllllll}0 & 0 & 0 & 0 & 0 & 0 & 0 & 0 & 0 \\ 0 & 0 & 0 & 0 & 0 & 0 & 0 & 0\end{array}\right.$ $\begin{array}{llllllllll}0 & 0 & 0 & 0 & 0 & 0 & 0 & 0 & 0 \\ 0 & 0 & 0 & 0 & 0 & 0 & 0 & 0 & 0 & 0\end{array}$ $\begin{array}{llllllllll}0 & 0 & 0 & 0 & 0 & 0 & 0 & 0 & 0\end{array}$ $\begin{array}{llllllllll}0 & 0 & 0 & 0 & 0 & 0 & 0 & 0 & 0\end{array}$ $\begin{array}{lllllllllll}0 & 0 & 0 & 0 & 0 & 0 & 0 & 0 & 0 \\ 0 & 0 & 0 & 0 & 0 & 0 & 0 & 0 & 0\end{array}$ $\begin{array}{lllllllll}0 & 0 & 0 & 0 & 0 & 0 & 0 & 0 & 0 \\ 0 & 0 & 0 & 0 & 0 & 0 & 0 & 0 & 0\end{array}$

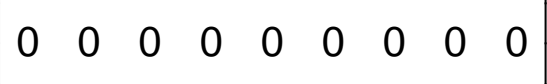
$\begin{array}{lllllllll}0 & 0 & 0 & 0 & 0 & 0 & 0 & 0 & 0 \\ 0 & 0 & 0 & 0 & 0 & 0 & 0 & 0 & 0\end{array}$

$\begin{array}{lllll}0 & 0 & 0 & 0 & 0 \\ 0 & 0 & 0 & 0\end{array}$

$\left(\begin{array}{llllllllll}0 & 0 & 0 & 0 & 0 & 0 & 0 & 0 & 0 & 0 \\ 0 & 0 & 0 & 0 & 0 & 0 & 0 & 0 & 0 & 0\end{array}\right)$ $\left.\begin{array}{llllllllll}0 & 0 & 0 & 0 & 0 & 0 & 0 & 0 & 0 & 0 \\ 0 & 0 & 0 & 0 & 0 & 0 & 0 & 0 & 0 & 0\end{array}\right)$ $\begin{array}{lllllllllll}0 & 0 & 0 & 0 & 0 & 0 & 0 & 0 & 0 & 0\end{array}$

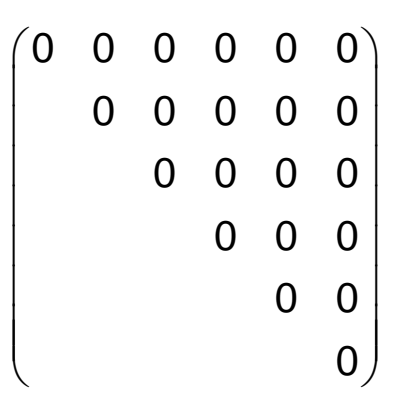

$\begin{array}{lllllllll}0 & 0 & 0 & 0 & 0 & 0 & 0 & 0 & 0 \\ 0 & 0 & 0 & 0 & 0 & 0 & 0 & 0 & 0\end{array}$

$\left.\begin{array}{lllllllll}0 & 0 & 0 & 0 & 0 & 0 & 0 & 0 & 0 \\ 0 & 0 & 0 & 0 & 0 & 0 & 0 & 0 & 0\end{array}\right)$

$0 \begin{array}{llllllllll}0 & 0 & 0 & 0 & 0 & 0 & 0 & 0 & 0 \\ 0 & 0 & 0 & 0 & 0 & 0\end{array}$ $\begin{array}{llllllllll}0 & 0 & 0 & 0 & 0 & 0 & 0 & 0 & 0\end{array}$

$\begin{array}{llllllllll}0 & 0 & 0 & 0 & 0 & 0 & 0 & 0 & 0\end{array}$

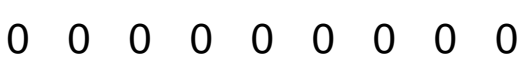

$\begin{array}{lllllllll}0 & 0 & 0 & 0 & 0 & 0 & 0 & 0 & 0\end{array}$

$\begin{array}{lllllllll}0 & 0 & 0 & 0 & 0 & 0 & 0 & 0 & 0 \\ 0 & 0 & 0 & 0 & 0 & 0 & 0 & 0 & 0\end{array}$

$\left(\begin{array}{lllll}0 & 0 & 0 & 0 & 0 \\ 0 & 0 & 0 & 0 & 0 \\ 0 & 0 & 0 & 0 & 0\end{array}\right)$

$\left(\begin{array}{llllllllll}0 & 0 & 0 & 0 & 0 & 0 & 0 & 0 & 0 & 0 \\ 0 & 0 & 0 & 0 & 0 & 0 & 0 & 0 & 0 & 0\end{array}\right)$

$\left(\begin{array}{llllllllll}0 & 0 & 0 & 0 & 0 & 0 & 0 & 0 & 0 & 0 \\ 0 & 0 & 0 & 0 & 0 & 0 & 0 & 0 & 0\end{array}\right.$

$\begin{array}{lllllllllll}0 & 0 & 0 & 0 & 0 & 0 & 0 & 0 & 0 & 0\end{array}$

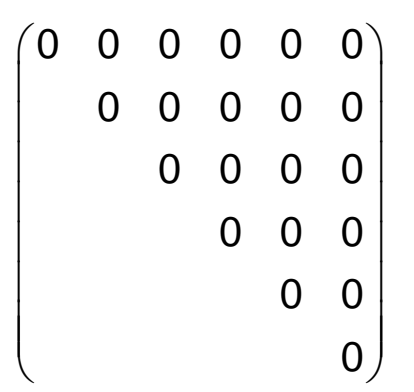

$\left(\begin{array}{llllll}0 & 0 & 0 & 0 & 0 & 0 \\ 0 & 0 & 0 & 0 & 0 & 0 \\ 0 & 0 & 0 & 0 & 0 & 0\end{array}\right)$

$\begin{array}{llllll}0 & 0 & 0 & 0 & 0 & 0 \\ 0 & 0 & 0 & 0 & 0 & 0 \\ 0 & 0 & 0 & 0 & 0 & 0\end{array}$

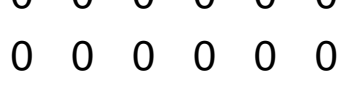

$\left.\begin{array}{llllll}0 & 0 & 0 & 0 & 0 & 0 \\ 0 & 0 & 0 & 0 & 0 & 0\end{array}\right)$

$\left(\begin{array}{llllll}0 & 0 & 0 & 0 & 0 & 0 \\ 0 & 0 & 0 & 0 & 0 & 0\end{array}\right)$

$0 \begin{array}{llllll}0 & 0 & 0 & 0 & 0 & 0 \\ 0 & 0 & 0 & 0 & 0 & 0\end{array}$

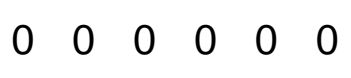

$\begin{array}{llllll}0 & 0 & 0 & 0 & 0 & 0\end{array}$

$\begin{array}{llllll}0 & 0 & 0 & 0 & 0 & 0 \\ 0 & 0 & 0 & 0 & 0 & 0\end{array}$ $\begin{array}{lllllllll}0 & 0 & 0 & 0 & 0 & 0 & 0 & 0 & 0\end{array}$

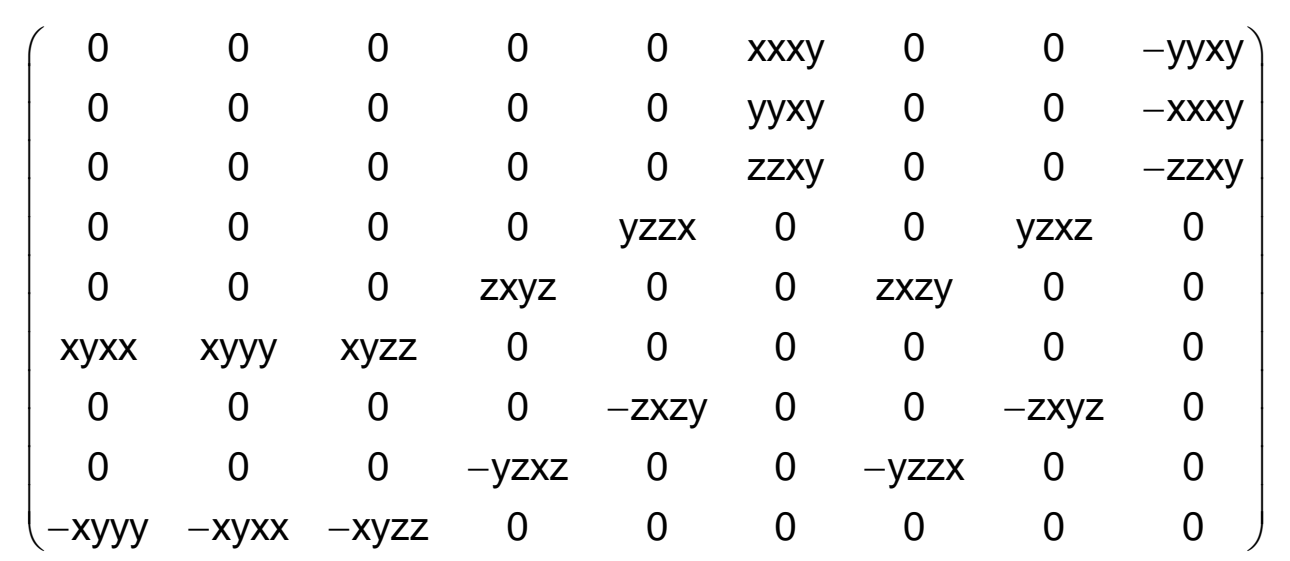

and: $x x x y-y y x y=x y y y-x y x x$

$\left(\begin{array}{lllll}0 & 0 & 0 & 0 & 0 \\ 0 & 0 & 0 & 0 & 0 \\ 0 & 0 & 0 & 0 & 0\end{array}\right)$

$\left(\begin{array}{llllll}0 & 0 & 0 & 0 & 0 \\ 0 & 0 & 0 & 0 & 0\end{array}\right)$

$\left(\begin{array}{cccccccccc}0 & 3 x y x x & 0 & 0 & x y z z & 0 & 0 & x y x x & 0 & 0 \\ -3 x y x x & 0 & 0 & -x y x & 0 & 0 & -x y z z & 0 & 0 & 0\end{array}\right)$

$\begin{array}{cccccccccccc}-3 x y x x & 0 & 0 & -x y x x & 0 & 0 & -x y z z & 0 & 0 & 0 \\ 0 & 0 & 0 & 0 & 0 & 0 & 0 & 0 & 0 & \end{array}$

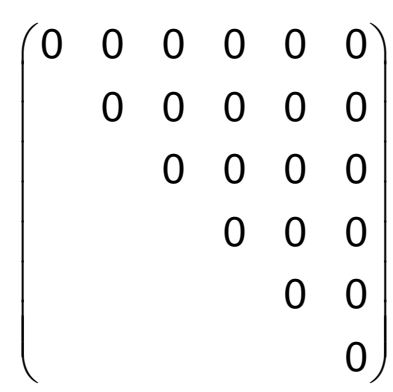

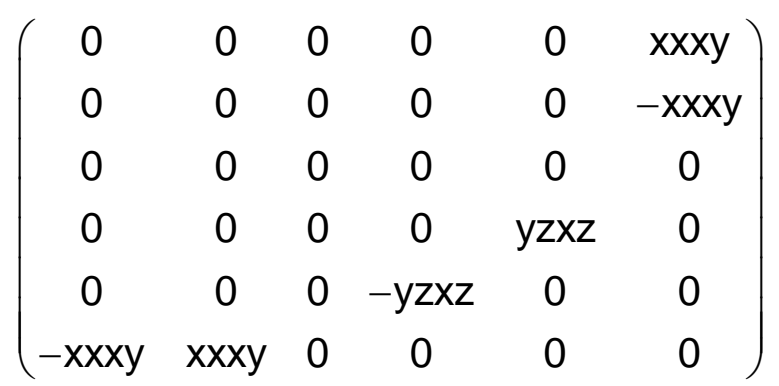


$n>4 \quad \mathbf{D}_{\alpha \mathrm{h}}\left(\mathbf{C}_{\alpha \mathrm{v}}\right)=\infty / \mathrm{m}^{\prime} \mathrm{mm}$

$$
\begin{aligned}
& \left(\begin{array}{lllllllll}
x x x x & x x y y & x x z z & 0 & 0 & 0 & 0 & 0 & 0 \\
& x x x x & x x z z & 0 & 0 & 0 & 0 & 0 & 0
\end{array}\right)\left(\begin{array}{lllllllll}
0 & 0 & 0 & 0 & 0 & 0 & 0 & 0 & 0 \\
& 0 & 0 & 0 & 0 & 0 & 0 & 0 & 0
\end{array}\right) \\
& \left.\begin{array}{llllllll}
x x x x & x x z z & 0 & 0 & 0 & 0 & 0 & 0 \\
& z z z z & 0 & 0 & 0 & 0 & 0 & 0
\end{array}\right)\left(\begin{array}{lllllllll}
0 & 0 & 0 & 0 & 0 & 0 & 0 & 0 \\
& & 0 & 0 & 0 & 0 & 0 & 0 & 0
\end{array}\right) \\
& \begin{array}{lcccccc}
x z z z & 0 & 0 & 0 & 0 & 0 & 0 \\
y z y z & 0 & 0 & y z z y & 0 & 0
\end{array} \quad \text { a } \\
& \begin{array}{ccccc}
\text { zxzx } & 0 & 0 & y z z y & 0
\end{array} \quad \begin{array}{rrrrrr}
0 & 0 & 0 & 0 & 0 & 0 \\
0 & 0 & 0 & 0 & 0
\end{array}
\end{aligned}
$$

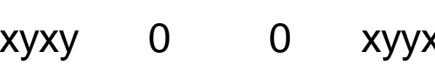

$$
\begin{aligned}
& \begin{array}{ccc}
z x z x & 0 & 0 \\
y z y z & 0
\end{array} \\
& 0 \begin{array}{lll}
0 & 0 & 0
\end{array} \\
& \begin{array}{lll}
0 & 0 & 0 \\
0 & 0 & 0 \\
& 0 & 0
\end{array} \\
& x y x y \\
& \begin{array}{ll}
0 & 0 \\
0
\end{array}
\end{aligned}
$$

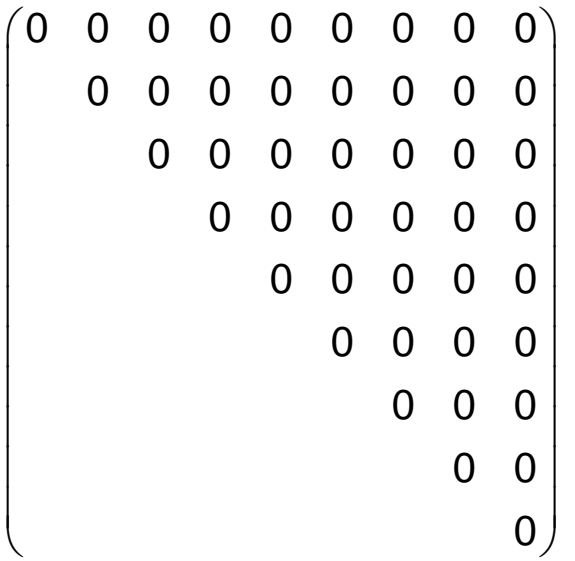

$$
\begin{aligned}
& \left(\begin{array}{lllllllll}
0 & 0 & 0 & 0 & 0 & x x x y & 0 & 0 & -x x x y \\
& 0 & 0 & 0 & 0 & x x y & 0 & 0 & -x x y y
\end{array}\right) \\
& \begin{array}{llllllll}
0 & 0 & 0 & 0 & x x x y & 0 & 0 & -x x x y
\end{array} \\
& \begin{array}{lllllll}
0 & 0 & 0 & z z x y & 0 & 0 & -z z x y
\end{array} \\
& \begin{array}{llllll}
0 & y z z x & 0 & 0 & 0 & 0
\end{array} \\
& \begin{array}{cccc}
0 & 0 & 0 & 0 \\
0 & 0 & 0 & 0
\end{array} \\
& \begin{array}{ccc}
0 & -y z z x & 0
\end{array} \\
& \begin{array}{ll}
0 & 0 \\
& 0
\end{array}
\end{aligned}
$$

and $x x x x=x x y y+y z y z+z y z y$

$\left[\mathrm{V}^{2}\right] \mathrm{V}^{2}\left(\begin{array}{ccccccccc}\mathrm{x} x \mathrm{x} x & \mathrm{xxyy} & \mathrm{xxzz} & 0 & 0 & 0 & 0 & 0 & 0 \\ \mathrm{xxyy} & \mathrm{xyxx} & \mathrm{xzzz} & 0 & 0 & 0 & 0 & 0 & 0 \\ 0 & 0 & 0 & \mathrm{zzyz} & 0 & 0 & 0 & 0 & 0 \\ 0 & 0 & 0 & 0 & \mathrm{zxzx} & 0 & 0 & \mathrm{yzzyz} & 0 \\ 0 & 0 & 0 & 0 & 0 & \mathrm{xyxy} & 0 & 0 & \mathrm{xyxy}\end{array}\right)\left(\begin{array}{ccccccccc}0 & 0 & 0 & 0 & 0 & 0 & 0 & 0 & 0 \\ 0 & 0 & 0 & 0 & 0 & 0 & 0 & 0 & 0 \\ 0 & 0 & 0 & 0 & 0 & 0 & 0 & 0 & 0 \\ 0 & 0 & 0 & 0 & 0 & 0 & 0 & 0 & 0 \\ 0 & 0 & 0 & 0 & 0 & 0 & 0 & 0 & 0 \\ 0 & 0 & 0 & 0 & 0 & 0 & 0 & 0 & 0\end{array}\right)$ and $x x x x=x x y y+2 x y x y$ $\left(\begin{array}{lllllllll}0 & 0 & 0 & 0 & 0 & 0 & 0 & 0 & 0 \\ 0 & 0 & 0 & 0 & 0 & 0 & 0 & 0 & 0 \\ 0 & 0 & 0 & 0 & 0 & 0 & 0 & 0 & 0\end{array}\right)$

$\left.\begin{array}{lllllllll}0 & 0 & 0 & 0 & 0 & 0 & 0 & 0 & 0 \\ 0 & 0 & 0 & 0 & 0 & 0 & 0 & 0 & 0 \\ 0 & 0 & 0 & 0 & 0 & 0 & 0 & 0 & 0\end{array}\right)$

$\begin{array}{lllllllll}0 & 0 & 0 & 0 & 0 & 0 & 0 & 0 & 0 \\ 0 & 0 & 0 & 0 & 0 & 0 & 0 & 0 & 0\end{array}$

$\begin{array}{llllllllll}0 & 0 & 0 & 0 & 0 & 0 & 0 & 0 & 0 \\ 0 & 0 & 0 & 0 & 0 & 0 & 0 & 0 & 0\end{array}$

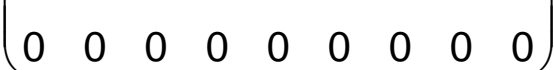

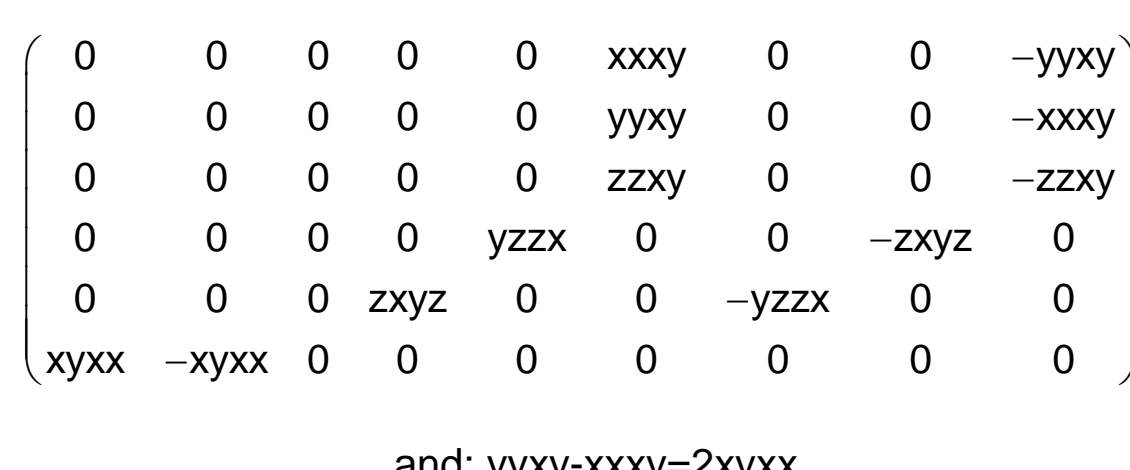

and: $y y x y-x x x y=2 x y x x$ 


$\left(\begin{array}{ccccccccc}\text { xxxx } & \text { xxyy } & \text { xxzz } & 0 & 0 & 0 & 0 & 0 & 0 \\ \text { yyxx } & \text { yyyy } & \text { yyzz } & 0 & 0 & 0 & 0 & 0 & 0 \\ \text { zzxx } & \text { zzyy } & z z z z & 0 & 0 & 0 & 0 & 0 & 0 \\ 0 & 0 & 0 & \text { yzyz } & 0 & 0 & \text { yzzy } & 0 & 0 \\ 0 & 0 & 0 & 0 & \text { zxzx } & 0 & 0 & \text { zxxz } & 0 \\ 0 & 0 & 0 & 0 & 0 & x y x y & 0 & 0 & \text { xyyx } \\ 0 & 0 & 0 & z y y z & 0 & 0 & \text { zyzy } & 0 & 0 \\ 0 & 0 & 0 & 0 & \text { xzzx } & 0 & 0 & \text { xzxz } & 0 \\ 0 & 0 & 0 & 0 & 0 & \text { yxxy } & 0 & 0 & \text { yxyx }\end{array}\right)$

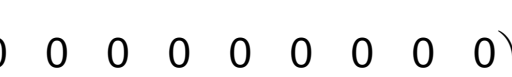
$0 \begin{array}{lllllllll}0 & 0 & 0 & 0 & 0 & 0 & 0 & 0 & 0 \\ 0 & 0 & 0 & 0 & 0 & 0 & 0 & 0 & 0\end{array}$

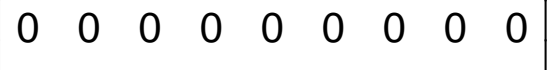
$0 \begin{array}{lllllllll}0 & 0 & 0 & 0 & 0 & 0 & 0 & 0 & 0 \\ 0 & 0 & 0 & 0 & 0 & 0 & 0 & 0 & 0\end{array}$

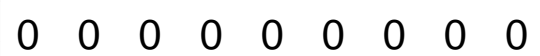
$\begin{array}{llllllllll}0 & 0 & 0 & 0 & 0 & 0 & 0 & 0 & 0 \\ 0 & 0 & 0 & 0 & 0 & 0 & 0 & 0 & 0\end{array}$ $\begin{array}{llllllllll}0 & 0 & 0 & 0 & 0 & 0 & 0 & 0 & 0\end{array}$

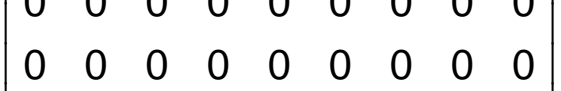
$\left(\begin{array}{lllllllll}0 & 0 & 0 & 0 & 0 & 0 & 0 & 0 & 0 \\ 0 & 0 & 0 & 0 & 0 & 0 & 0 & 0 & 0\end{array}\right)$

$\left[V^{4}\right] \quad\left(\begin{array}{ccccc}\mathrm{xxxx} & 0 & 0 & \mathrm{yyzz} & 0 \\ 0 & \mathrm{yyyy} & 0 & \mathrm{zzxx} & 0 \\ 0 & 0 & \mathrm{zzzz} & \mathrm{xxyy} & 0\end{array}\right)$

$\left(\begin{array}{lllll}0 & 0 & 0 & 0 & 0 \\ 0 & 0 & 0 & 0 & 0 \\ 0 & 0 & 0 & 0 & 0\end{array}\right)$

$\begin{array}{lllll}0 & 0 & 0 & 0 & 0\end{array}$

$\mathrm{V}\left[\mathrm{V}^{3}\right] \quad\left(\begin{array}{cccccccccc}\mathrm{x} x \mathrm{xx} & 0 & 0 & \mathrm{xxyy} & 0 & 0 & \mathrm{xxzz} & 0 & 0 & 0 \\ 0 & \mathrm{yyyy} & 0 & 0 & \mathrm{yyzz} & 0 & 0 & \mathrm{yyxx} & 0 & 0 \\ 0 & 0 & z z z & 0 & 0 & \mathrm{zzxx} & 0 & 0 & z z y & 0\end{array}\right) \quad\left(\begin{array}{cccccccccc}0 & 0 & 0 & 0 & 0 & 0 & 0 & 0 & 0 & 0 \\ 0 & 0 & 0 & 0 & 0 & 0 & 0 & 0 & 0 & 0 \\ 0 & 0 & 0 & 0 & 0 & 0 & 0 & 0 & 0 & 0\end{array}\right)$ $\left(\begin{array}{llllllllll}0 & 0 & z z z z & 0 & 0 & z z x x & 0 & 0 & \text { zzyy } & 0\end{array}\right)\left(\begin{array}{llllllllll}0 & 0 & 0 & 0 & 0 & 0 & 0 & 0 & 0 & 0\end{array}\right)$

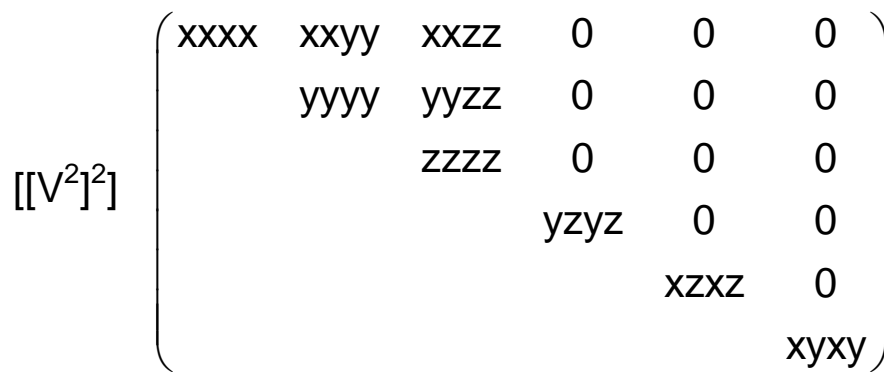

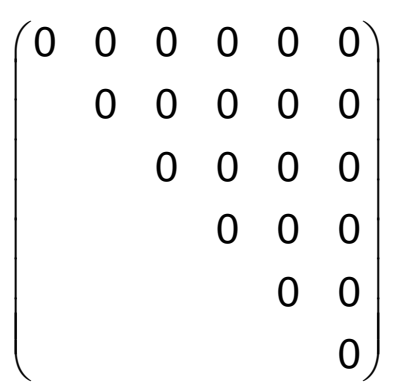

$\left.\mathrm{N}^{2}\right]^{2}\left(\begin{array}{cccccc}\mathrm{xxxx} & \mathrm{xxyy} & \mathrm{xxzz} & 0 & 0 & 0 \\ \mathrm{yyxx} & \mathrm{yyyy} & \mathrm{yyzz} & 0 & 0 & 0 \\ \mathrm{zzxx} & \mathrm{zzyy} & \mathrm{zzzz} & 0 & 0 & 0 \\ 0 & 0 & 0 & \mathrm{yzyz} & 0 & 0 \\ 0 & 0 & 0 & 0 & \mathrm{xzxz} & 0 \\ 0 & 0 & 0 & 0 & 0 & \mathrm{xyxy}\end{array}\right)$

$\left(\begin{array}{llllll}0 & 0 & 0 & 0 & 0 & 0 \\ 0 & 0 & 0 & 0 & 0 & 0 \\ 0 & 0 & 0 & 0 & 0 & 0\end{array}\right)$

$\begin{array}{llllll}0 & 0 & 0 & 0 & 0 & 0 \\ 0 & 0 & 0 & 0 & 0 & 0\end{array}$

$\begin{array}{llllll}0 & 0 & 0 & 0 & 0 & 0 \\ 0 & 0 & 0 & 0 & 0 & 0 \\ 0 & 0 & 0 & 0 & 0 & 0\end{array}$

$\begin{array}{llllll}0 & 0 & 0 & 0 & 0 & 0 \\ 0 & 0 & 0 & 0 & 0 & 0 \\ 0 & 0 & 0 & 0 & 0 & 0\end{array}$

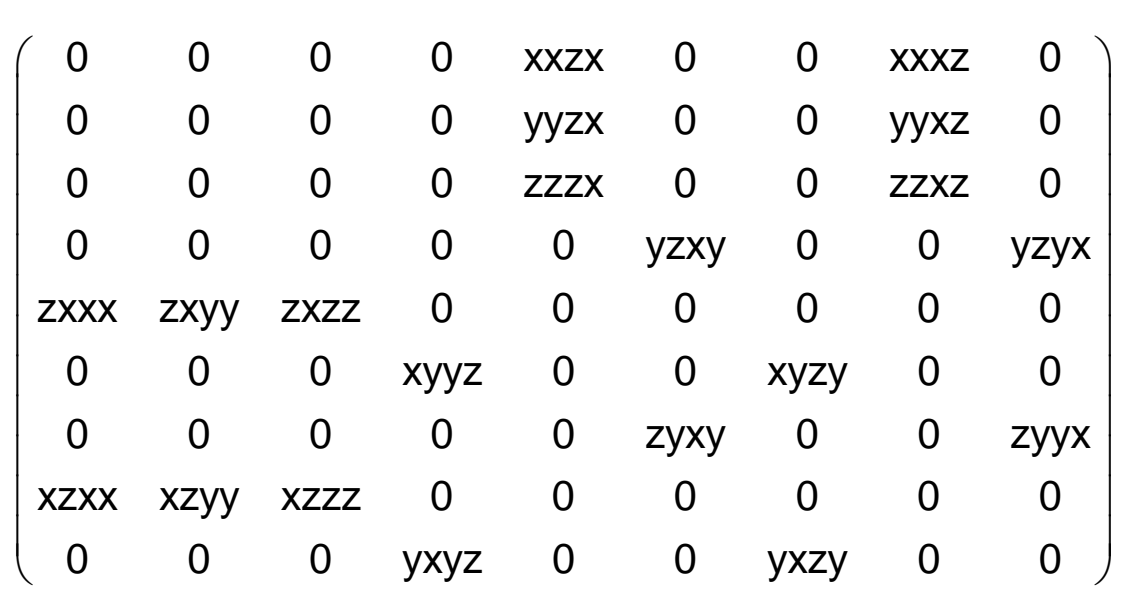

$\left(\begin{array}{ccccc}0 & 0 & x z z z & 0 & 0 \\ 0 & 0 & 0 & 0 & y y x z\end{array}\right)$

$\begin{array}{ccccc}0 x x x & 0 & 0 & 0 & 0\end{array}$

$\left(\begin{array}{cccccccccc}0 & 0 & x z z z & 0 & 0 & x z x x & 0 & 0 & x z y y & 0 \\ 0 & 0 & 0 & 0 & 0 & 0 & 0 & 0 & 0 & y x y z \\ z x x x & 0 & 0 & z x y y & 0 & 0 & z x z z & 0 & 0 & 0\end{array}\right)$

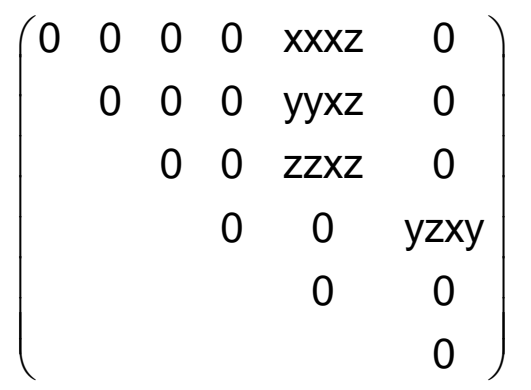

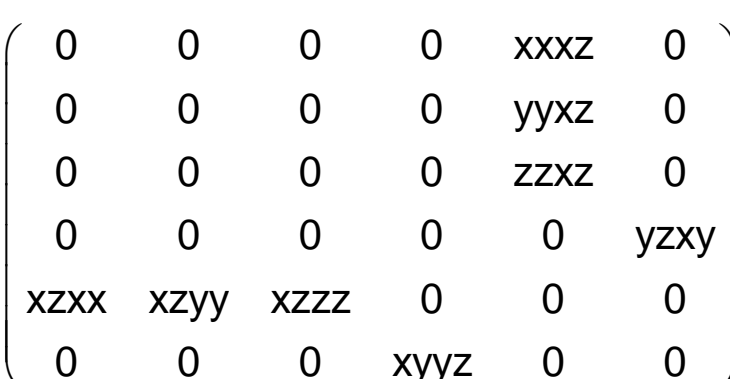

$\left(\begin{array}{llllll}0 & 0 & 0 & 0 & 0 & 0 \\ 0 & 0 & 0 & 0 & 0 & 0\end{array}\right.$

$\begin{array}{llllll}0 & 0 & 0 & 0 & 0 & 0 \\ 0 & 0 & 0 & 0 & 0 & 0\end{array}$

$\begin{array}{llllll}0 & 0 & 0 & 0 & 0 & 0 \\ 0 & 0 & 0 & 0 & 0 & 0\end{array}$

$\begin{array}{llllll}0 & 0 & 0 & 0 & 0 & 0 \\ 0 & 0 & 0 & 0 & 0 & 0\end{array}$

$\left(\begin{array}{llllll}0 & 0 & 0 & 0 & 0 & 0 \\ 0 & 0 & 0 & 0 & 0 & 0\end{array}\right.$

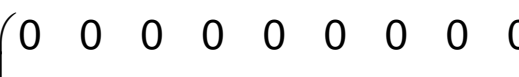
$\left.\begin{array}{llllllllll}0 & 0 & 0 & 0 & 0 & 0 & 0 & 0 & 0 \\ 0 & 0 & 0 & 0 & 0 & 0 & 0 & 0 & 0\end{array}\right)$ $\begin{array}{llllllllll}0 & 0 & 0 & 0 & 0 & 0 & 0 & 0 & 0 \\ 0 & 0 & 0 & 0 & 0 & 0 & 0 & 0 & 0\end{array}$ $\begin{array}{llllllllll}0 & 0 & 0 & 0 & 0 & 0 & 0 & 0 & 0\end{array}$ $\begin{array}{llllllllll}0 & 0 & 0 & 0 & 0 & 0 & 0 & 0 & 0 \\ 0 & 0 & 0 & 0 & 0 & 0 & 0 & 0 & 0\end{array}$ $\left(\begin{array}{lllll}0 & 0 & 0 & 0 & 0 \\ 0 & 0 & 0 & 0 & 0 \\ 0 & 0 & 0 & 0 & 0\end{array}\right)$ $\begin{array}{lllll}0 & 0 & 0 & 0 & 0\end{array}$

$\left(\begin{array}{llllllllll}0 & 0 & 0 & 0 & 0 & 0 & 0 & 0 & 0 & 0 \\ 0 & 0 & 0 & 0 & 0 & 0 & 0 & 0 & 0 & 0\end{array}\right)$

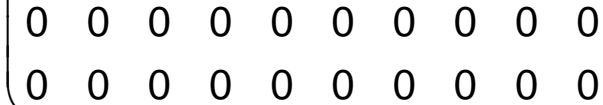

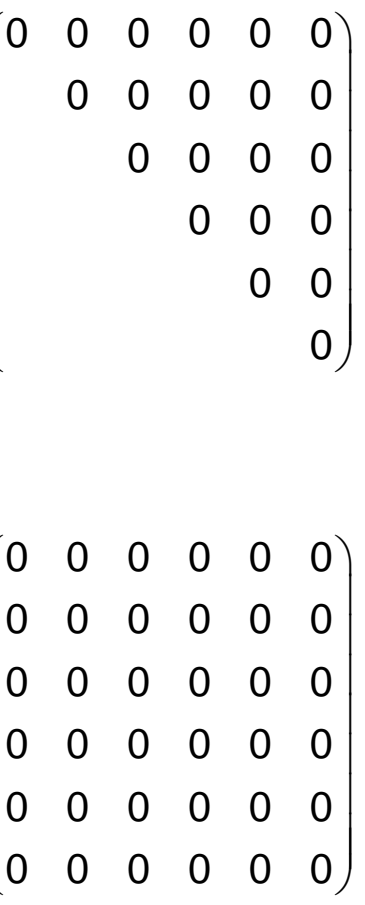




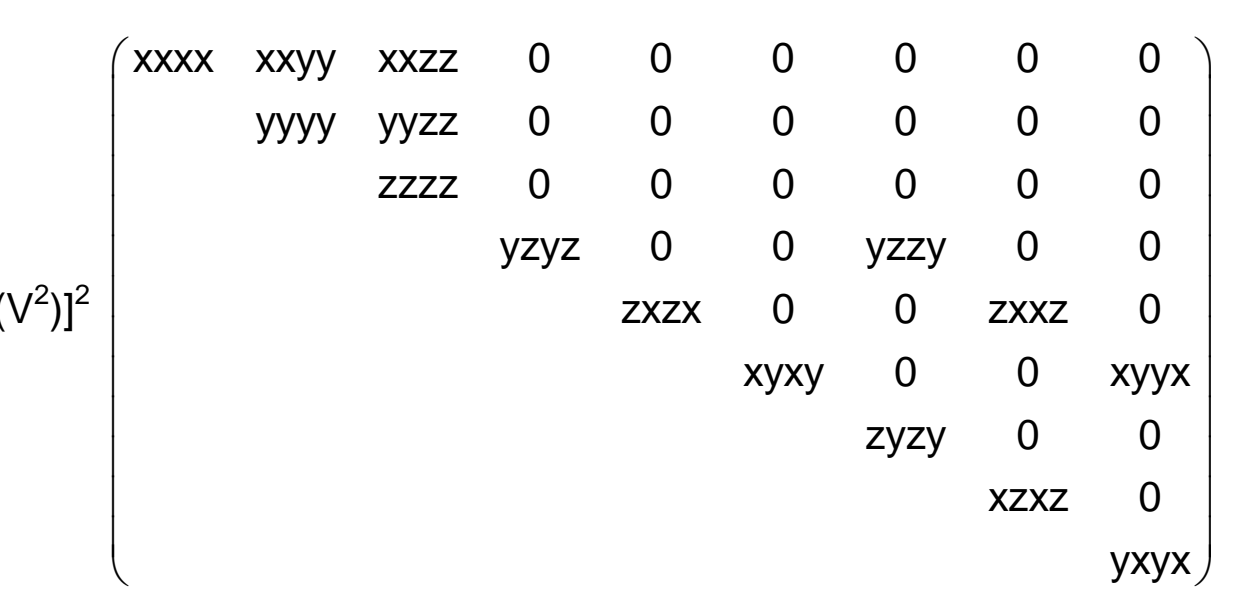

$\left(\begin{array}{lllllllll}0 & 0 & 0 & 0 & 0 & 0 & 0 & 0 & 0 \\ 0 & 0 & 0 & 0 & 0 & 0 & 0 & 0\end{array}\right)$ $0 \begin{array}{lllllll}0 & 0 & 0 & 0 & 0 & 0 & 0\end{array}$

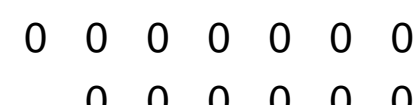
$\begin{array}{lllll}0 & 0 & 0 & 0 & 0\end{array}$ $\begin{array}{llll}0 & 0 & 0 & 0\end{array}$ $\begin{array}{lll}0 & 0 & 0 \\ & 0 & 0\end{array}$

$\left(\begin{array}{lllllllll}x x x x & x x y y & x x z z & 0 & 0 & 0 & 0 & 0 & 0 \\ y y x x & y y y y & y y z z & 0 & 0 & 0 & 0 & 0 & 0\end{array}\right) \quad\left(\begin{array}{ccccccccc}0 & 0 & 0 & 0 & 0 & 0 & 0 & 0 & 0 \\ 0 & 0 & 0 & 0 & 0 & 0 & 0 & 0 & 0 \\ 0 & 0 & 0 & 0 & 0 & 0 & 0 & 0\end{array}\right)$ $\left(\begin{array}{ccccccccc}\operatorname{yyxx} & y y y y & y y z z & 0 & 0 & 0 & 0 & 0 & 0 \\ z z x x & z z y y & z z z z & 0 & 0 & 0 & 0 & 0 & 0\end{array}\right)$ $0 \begin{array}{lllllllll}0 & 0 & 0 & 0 & 0 & 0 & 0 & 0 & 0 \\ 0 & 0 & 0 & 0 & 0 & 0 & 0 & 0 & 0\end{array}$ $0 \begin{array}{lllllllll}0 & 0 & 0 & 0 & 0 & 0 & 0 & 0 & 0 \\ 0 & 0 & 0 & 0 & 0 & 0 & 0 & 0 & 0\end{array}$

$\begin{array}{lllllllll}0 & 0 & 0 & 0 & 0 & 0 & 0 & 0 & 0 \\ 0 & 0 & 0 & 0 & 0 & 0 & 0 & 0 & 0\end{array}$

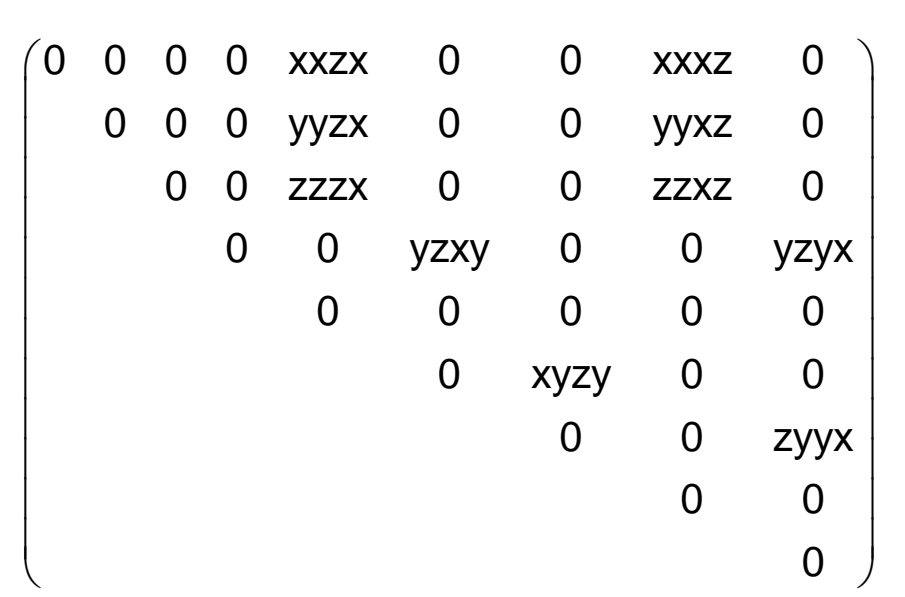

$\left(\begin{array}{ccccccccc}0 & 0 & 0 & 0 & \text { xxzx } & 0 & 0 & \text { xxxz } & 0 \\ 0 & 0 & 0 & 0 & \text { yyzx } & 0 & 0 & \text { yyxz } & 0 \\ 0 & 0 & 0 & 0 & \text { zzzx } & 0 & 0 & \text { zzxz } & 0 \\ 0 & 0 & 0 & 0 & 0 & y z x y & 0 & 0 & \text { yzyx } \\ \text { zxxx } & \text { zxyy } & \text { zxzz } & 0 & 0 & 0 & 0 & 0 & 0 \\ 0 & 0 & 0 & \text { xyyz } & 0 & 0 & \text { xyzy } & 0 & 0\end{array}\right)$ $\left(\begin{array}{lllllllll}0 & 0 & 0 & 0 & 0 & 0 & 0 & 0 & 0 \\ & 0 & 0 & 0 & 0 & 0 & 0 & 0 & 0 \\ & & 0 & 0 & 0 & 0 & 0 & 0 & 0 \\ & & & 0 & 0 & 0 & 0 & 0 & 0 \\ & & & & 0 & 0 & 0 & 0 & 0 \\ & & & & & 0 & 0 & 0 & 0 \\ & & & & & & 0 & 0 & 0 \\ & & & & & & & 0 & 0 \\ & & & & & & & & 0\end{array}\right)$

$\left(\begin{array}{lllllllll}0 & 0 & 0 & 0 & 0 & 0 & 0 & 0 & 0 \\ 0 & 0 & 0 & 0 & 0 & 0 & 0 & 0 & 0\end{array}\right)$ $\left.\begin{array}{lllllllll}0 & 0 & 0 & 0 & 0 & 0 & 0 & 0 & 0 \\ 0 & 0 & 0 & 0 & 0 & 0 & 0 & 0 & 0\end{array}\right)$ $\begin{array}{lllllllll}0 & 0 & 0 & 0 & 0 & 0 & 0 & 0 & 0 \\ 0 & 0 & 0 & 0 & 0 & 0 & 0 & 0 & 0\end{array}$ $\begin{array}{lllllllll}0 & 0 & 0 & 0 & 0 & 0 & 0 & 0 & 0 \\ 0 & 0 & 0 & 0 & 0 & 0 & 0 & 0 & 0\end{array}$ $\begin{array}{lllllllll}0 & 0 & 0 & 0 & 0 & 0 & 0 & 0 & 0 \\ 0 & 0 & 0 & 0 & 0 & 0 & 0 & 0 & 0\end{array}$ 
$\mathrm{V}^{4}\left(\begin{array}{ccccccccc}\mathrm{xxxx} & \mathrm{xxyy} & \mathrm{xxzz} & 0 & 0 & 0 & 0 & 0 & 0 \\ \mathrm{yyxx} & \mathrm{xxxx} & \mathrm{xxzz} & 0 & 0 & 0 & 0 & 0 & 0 \\ \mathrm{zzxx} & \mathrm{zzyy} & \mathrm{zzzz} & 0 & 0 & 0 & 0 & 0 & 0 \\ 0 & 0 & 0 & \mathrm{yzyz} & 0 & 0 & \mathrm{yzzy} & 0 & 0 \\ 0 & 0 & 0 & 0 & \mathrm{zxzx} & 0 & 0 & \mathrm{zxxz} & 0 \\ 0 & 0 & 0 & 0 & 0 & \mathrm{xyxy} & 0 & 0 & \mathrm{xyyx} \\ 0 & 0 & 0 & \mathrm{zxxz} & 0 & 0 & \mathrm{zxzx} & 0 & 0 \\ 0 & 0 & 0 & 0 & \mathrm{yzzy} & 0 & 0 & \mathrm{yzyz} & 0 \\ 0 & 0 & 0 & 0 & 0 & \mathrm{xyyx} & 0 & 0 & \mathrm{xyxy}\end{array}\right)$

$\left(\begin{array}{lllllllll}0 & 0 & 0 & 0 & 0 & 0 & 0 & 0 & 0 \\ 0 & 0 & 0 & 0 & 0 & 0 & 0 & 0 & 0\end{array}\right)$

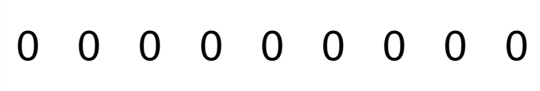
$0 \begin{array}{lllllllll}0 & 0 & 0 & 0 & 0 & 0 & 0 & 0 & 0 \\ 0 & 0 & 0 & 0 & 0 & 0 & 0 & 0 & 0\end{array}$

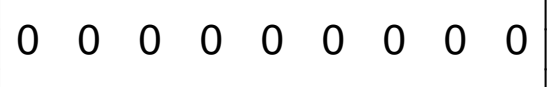
$\begin{array}{lllllllll}0 & 0 & 0 & 0 & 0 & 0 & 0 & 0 & 0 \\ 0 & 0 & 0 & 0 & 0 & 0 & 0 & 0 & 0\end{array}$ $\begin{array}{llllllllll}0 & 0 & 0 & 0 & 0 & 0 & 0 & 0 & 0\end{array}$ $\begin{array}{llllllllll}0 & 0 & 0 & 0 & 0 & 0 & 0 & 0 & 0\end{array}$ $\left(\begin{array}{lllllllll}0 & 0 & 0 & 0 & 0 & 0 & 0 & 0 & 0 \\ 0 & 0 & 0 & 0 & 0 & 0 & 0 & 0 & 0 \\ 0 & 0 & 0 & 0 & 0 & 0 & 0 & 0 & 0\end{array}\right)$ $\left(\begin{array}{lllllllll}0 & 0 & 0 & 0 & 0 & 0 & 0 & 0 & 0 \\ 0 & 0 & 0 & 0 & 0 & 0 & 0 & 0 & 0\end{array}\right)$

$\left[V^{4}\right] \quad\left(\begin{array}{ccccc}x x x x & 0 & 0 & y y z z & 0 \\ 0 & x x x x & 0 & y y z z & 0 \\ 0 & 0 & z z z z & x x y y & 0\end{array}\right)$

$\left(\begin{array}{lllll}0 & 0 & 0 & 0 & 0 \\ 0 & 0 & 0 & 0 & 0 \\ 0 & 0 & 0 & 0 & 0\end{array}\right)$

$\begin{array}{lllll}0 & 0 & 0 & 0 & 0\end{array}$

$\mathrm{V}\left[\mathrm{V}^{3}\right]\left(\begin{array}{cccccccccc}\mathrm{xxxx} & 0 & 0 & \mathrm{xxyy} & 0 & 0 & \mathrm{yyzz} & 0 & 0 & 0 \\ 0 & \mathrm{xxxx} & 0 & 0 & \mathrm{yyzz} & 0 & 0 & \mathrm{xxyy} & 0 & 0 \\ 0 & 0 & z z z z & 0 & 0 & z z x & 0 & 0 & z z x & 0\end{array}\right)$

$\begin{array}{llllllllll}0 & 0 & 0 & 0 & 0 & 0 & 0 & 0 & 0 & 0 \\ 0 & 0 & 0 & 0 & 0 & 0 & 0 & 0 & 0 & 0\end{array}$

$\begin{array}{lllllllllll}0 & 0 & 0 & 0 & 0 & 0 & 0 & 0 & 0 & 0 \\ 0 & 0 & 0 & 0 & 0 & 0 & 0 & 0 & 0 & 0\end{array}$

$\begin{array}{llllllllll}0 & 0 & 0 & 0 & 0 & 0 & 0 & 0 & 0 & 0\end{array}$

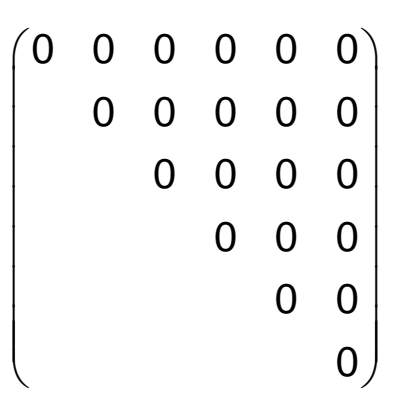

$\left(\begin{array}{llllll}0 & 0 & 0 & 0 & 0 & 0 \\ 0 & 0 & 0 & 0 & 0 & 0 \\ 0 & 0 & 0 & 0 & 0 & 0 \\ 0 & 0 & 0 & 0 & 0 & 0 \\ 0 & 0 & 0 & 0 & 0 & 0 \\ 0 & 0 & 0 & 0 & 0 & 0\end{array}\right)$ $\left(\begin{array}{lllllllll}0 & 0 & 0 & 0 & 0 & 0 & 0 & 0 & 0 \\ 0 & 0 & 0 & 0 & 0 & 0 & 0 & 0 & 0\end{array}\right.$ $0 \begin{array}{lllllllll}0 & 0 & 0 & 0 & 0 & 0 & 0 & 0 & 0 \\ 0 & 0 & 0 & 0 & 0 & 0 & 0 & 0 & 0\end{array}$ $\begin{array}{lllllllll}0 & 0 & 0 & 0 & 0 & 0 & 0 & 0 & 0 \\ 0 & 0 & 0 & 0 & 0 & 0 & 0 & 0 & 0 \\ 0 & 0 & 0 & 0 & 0 & 0 & 0\end{array}$ $\begin{array}{llllllllll}0 & 0 & 0 & 0 & 0 & 0 & 0 & 0 & 0 \\ 0 & 0 & 0 & 0 & 0 & 0 & 0 & 0 & 0\end{array}$ $0 \begin{array}{lllllllll}0 & 0 & 0 & 0 & 0 & 0 & 0 & 0 & 0 \\ 0 & 0 & 0 & 0 & 0 & 0 & 0 & 0 & 0\end{array}$ $0 \begin{array}{lllllllll}0 & 0 & 0 & 0 & 0 & 0 & 0 & 0 & 0 \\ 0 & 0 & 0 & 0 & 0 & 0 & 0 & 0 & 0\end{array}$ $\begin{array}{llllllllllllllll}0 & 0 & 0 & 0 & 0 & 0 & 0 & 0 & 0\end{array}$ $0 \begin{array}{llllllllll}0 & 0 & 0 & 0 & 0 & 0 & 0 & 0 & 0 \\ 0 & 0 & 0 & 0 & 0 & 0 & 0 & 0 & 0\end{array}$ $\begin{array}{llllllllll}0 & 0 & 0 & 0 & 0 & 0 & 0 & 0 & 0\end{array}$

\section{$\left(\begin{array}{lllll}0 & 0 & 0 & 0 & 0 \\ 0 & 0 & 0 & 0 & 0 \\ 0 & 0 & 0 & 0 & 0\end{array}\right)$ $\left(\begin{array}{lllll}0 & 0 & 0 & 0 & 0 \\ 0 & 0 & 0 & 0 & 0\end{array}\right)$}

$\left(\begin{array}{llllllllll}0 & 0 & 0 & 0 & 0 & 0 & 0 & 0 & 0 & 0 \\ 0 & 0 & 0 & 0 & 0 & 0 & 0 & 0 & 0 & 0\end{array}\right)$ $\begin{array}{lllllllllll}0 & 0 & 0 & 0 & 0 & 0 & 0 & 0 & 0 & 0 \\ 0 & 0 & 0 & 0 & 0 & 0 & 0 & 0 & 0 & 0\end{array}$ $\begin{array}{lllllllllll}0 & 0 & 0 & 0 & 0 & 0 & 0 & 0 & 0 & 0\end{array}$

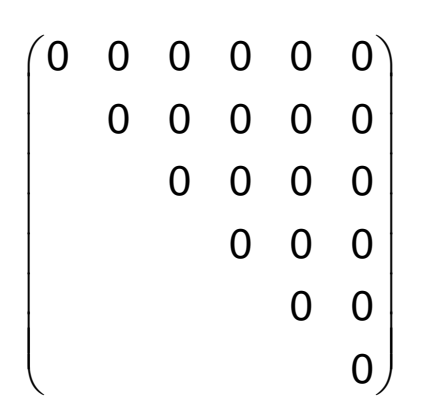

$\left(\begin{array}{llllll}0 & 0 & 0 & 0 & 0 & 0 \\ 0 & 0 & 0 & 0 & 0 & 0\end{array}\right)$ $\left(\begin{array}{llllll}0 & 0 & 0 & 0 & 0 & 0 \\ 0 & 0 & 0 & 0 & 0 & 0 \\ 0 & 0 & 0 & 0 & 0 & 0\end{array}\right)$ $\begin{array}{llllll}0 & 0 & 0 & 0 & 0 & 0\end{array}$ $\begin{array}{llllll}0 & 0 & 0 & 0 & 0 & 0\end{array}$ $\begin{array}{llllll}0 & 0 & 0 & 0 & 0 & 0 \\ 0 & 0 & 0 & 0 & 0 & 0\end{array}$$$
0
$$

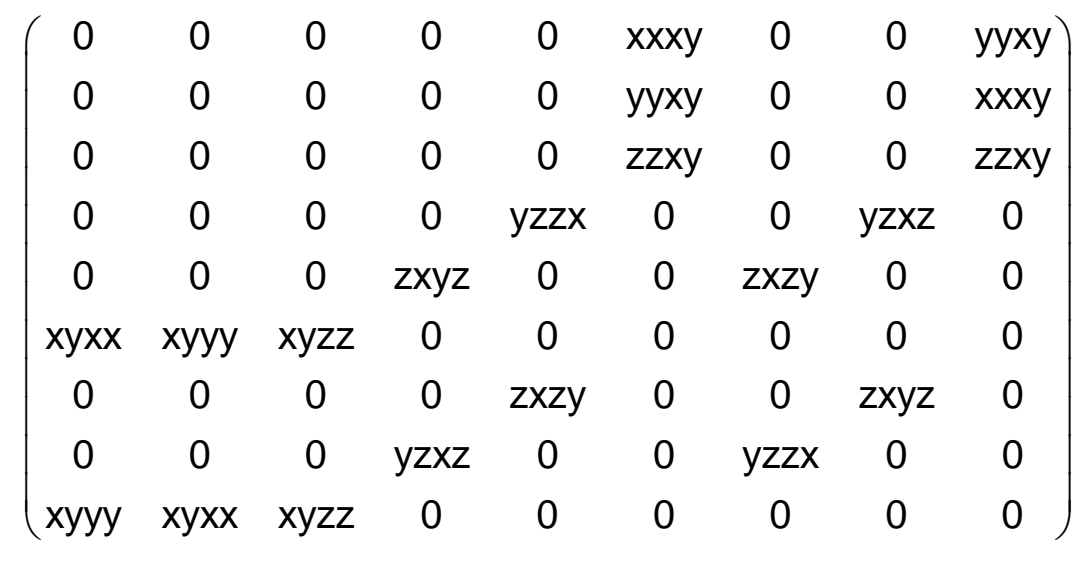

$\left(\begin{array}{ccccc}0 & y x x x & 0 & 0 & 0 \\ y x x x & 0 & 0 & 0 & 0 \\ 0 & 0 & 0 & 0 & z z x y\end{array}\right)$

$\left(\begin{array}{cccccccccc}0 & \text { xyyy } & 0 & 0 & x y z z & 0 & 0 & \text { yxyy } & 0 & 0 \\ \text { xyyy } & 0 & 0 & y x y y & 0 & 0 & x y z z & 0 & 0 & 0\end{array}\right)$ $\left.\begin{array}{cccccccccc}\text { xyyy } & 0 & 0 & \text { yxyy } & 0 & 0 & x y z z & 0 & 0 & 0 \\ 0 & 0 & 0 & 0 & 0 & 0 & 0 & 0 & 0 & z x y z\end{array}\right)$

$$
\begin{aligned}
& \left(\begin{array}{llllll}
0 & 0 & 0 & 0 & 0 & x x x y \\
& 0 & 0 & 0 & 0 & x x x y
\end{array}\right. \\
& \begin{array}{llll}
0 & 0 & 0 & x x x y \\
0 & 0 & 0 & z x y
\end{array} \\
& \begin{array}{llll}
0 & 0 & 0 & z z x y
\end{array} \\
& \begin{array}{ccc}
0 & y z x z & 0 \\
0 & 0
\end{array}
\end{aligned}
$$

$\left(\begin{array}{cccccc}0 & 0 & 0 & 0 & 0 & x x x y \\ 0 & 0 & 0 & 0 & 0 & x x x y \\ 0 & 0 & 0 & 0 & 0 & z z x y \\ 0 & 0 & 0 & 0 & y z x z & 0 \\ 0 & 0 & 0 & y z x z & 0 & 0 \\ x y x x & x y x x & x y z z & 0 & 0 & 0\end{array}\right)$



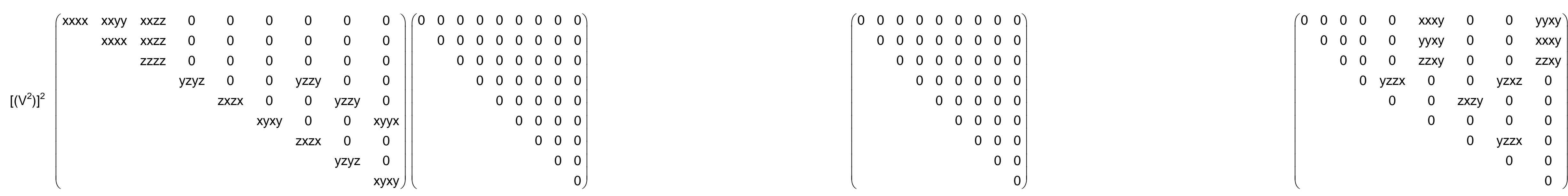

$\left[\mathrm{V}^{2}\right] \mathrm{V}^{2}\left(\begin{array}{ccccccccc}\mathrm{x} x \mathrm{x} x & \mathrm{x} x \mathrm{y} y & \mathrm{xxzz} & 0 & 0 & 0 & 0 & 0 & 0 \\ \mathrm{yyxx} & \mathrm{xxxx} & \mathrm{xxzz} & 0 & 0 & 0 & 0 & 0 & 0 \\ \mathrm{zzxx} & \mathrm{zzxx} & \mathrm{zzzz} & 0 & 0 & 0 & 0 & 0 & 0 \\ 0 & 0 & 0 & \mathrm{yzyz} & 0 & 0 & \mathrm{zxzx} & 0 & 0 \\ 0 & 0 & 0 & 0 & \mathrm{zxzx} & 0 & 0 & \mathrm{yzyz} & 0 \\ 0 & 0 & 0 & 0 & 0 & \mathrm{xyxy} & 0 & 0 & \mathrm{xyxy}\end{array}\right)\left(\begin{array}{ccccccccc}0 & 0 & 0 & 0 & 0 & 0 & 0 & 0 & 0 \\ 0 & 0 & 0 & 0 & 0 & 0 & 0 & 0 & 0 \\ 0 & 0 & 0 & 0 & 0 & 0 & 0 & 0 & 0 \\ 0 & 0 & 0 & 0 & 0 & 0 & 0 & 0 & 0 \\ 0 & 0 & 0 & 0 & 0 & 0 & 0 & 0 & 0 \\ 0 & 0 & 0 & 0 & 0 & 0 & 0 & 0 & 0\end{array}\right)$

$\left(\begin{array}{lllllllll}0 & 0 & 0 & 0 & 0 & 0 & 0 & 0 & 0 \\ 0 & 0 & 0 & 0 & 0 & 0 & 0 & 0 & 0\end{array}\right.$

$\begin{array}{lllllllll}0 & 0 & 0 & 0 & 0 & 0 & 0 & 0 & 0 \\ 0 & 0 & 0 & 0 & 0 & 0 & 0 & 0 & 0\end{array}$

$0 \begin{array}{lllllllll}0 & 0 & 0 & 0 & 0 & 0 & 0 & 0 & 0 \\ 0 & 0 & 0 & 0 & 0 & 0 & 0 & 0 & 0\end{array}$

$0 \begin{array}{lllllllll}0 & 0 & 0 & 0 & 0 & 0 & 0 & 0 & 0 \\ 0 & 0 & 0 & 0 & 0 & 0 & 0 & 0 & 0\end{array}$

$\begin{array}{lllllllll}0 & 0 & 0 & 0 & 0 & 0 & 0 & 0 & 0 \\ 0 & 0 & 0 & 0 & 0 & 0 & 0 & 0 & 0\end{array}$

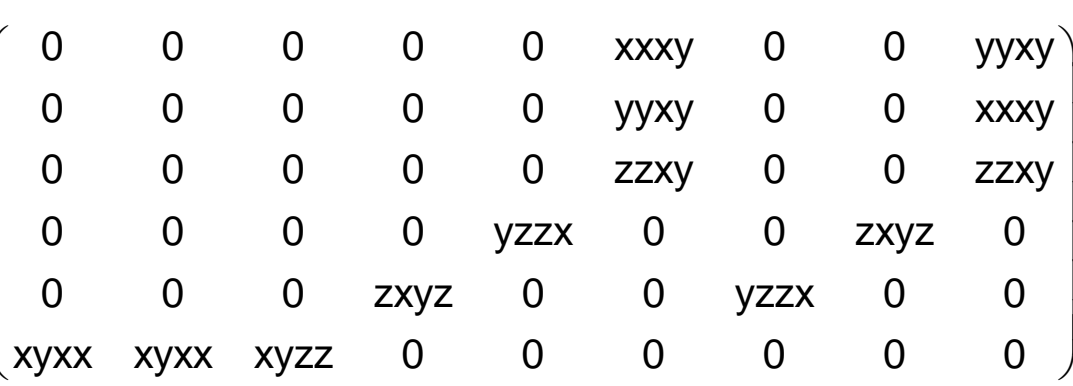




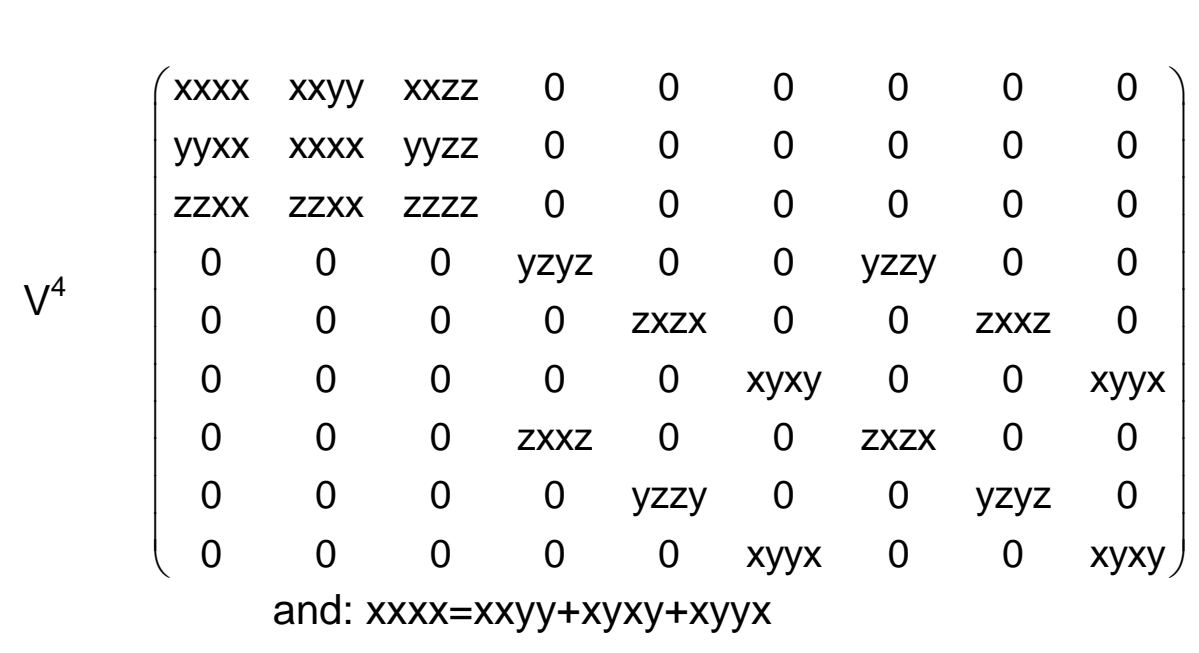

$\left(\begin{array}{lllllllll}0 & 0 & 0 & 0 & 0 & 0 & 0 & 0 & 0 \\ 0 & 0 & 0 & 0 & 0 & 0 & 0 & 0 & 0\end{array}\right)$ $\left(\begin{array}{lllllllll}0 & 0 & 0 & 0 & 0 & 0 & 0 & 0 & 0 \\ 0 & 0 & 0 & 0 & 0 & 0 & 0 & 0 & 0\end{array}\right.$ $\begin{array}{llllllllll}0 & 0 & 0 & 0 & 0 & 0 & 0 & 0 & 0 \\ 0 & 0 & 0 & 0 & 0 & 0 & 0 & 0 & 0 \\ 0 & 0 & 0 & 0 & 0 & 0 & 0\end{array}$ $0 \begin{array}{lllllllll}0 & 0 & 0 & 0 & 0 & 0 & 0 & 0 & 0\end{array}$ $\begin{array}{llllllllll}0 & 0 & 0 & 0 & 0 & 0 & 0 & 0 & 0 \\ 0 & 0 & 0 & 0 & 0 & 0 & 0 & 0 & 0\end{array}$ $\begin{array}{lllllllll}0 & 0 & 0 & 0 & 0 & 0 & 0 & 0 & 0 \\ 0 & 0 & 0 & 0 & 0 & 0 & 0 & 0 & 0\end{array}$ $\begin{array}{lllllllll}0 & 0 & 0 & 0 & 0 & 0 & 0 & 0 & 0 \\ 0 & 0 & 0 & 0 & 0 & 0 & 0 & 0 & 0\end{array}$

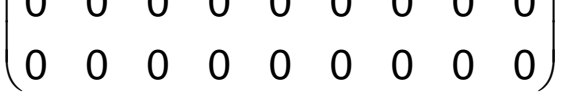
and: $x x x x=x x y y+x y x y+x y y x$
$\left[\mathrm{V}^{4}\right]$
$\left(\begin{array}{ccccc}3 x x y y & 0 & 0 & y y z z & 0 \\ 0 & 3 x x y y & 0 & y y z z & 0 \\ 0 & 0 & 2 z z & x x y y & 0\end{array}\right)$
$\left(\begin{array}{lllll}0 & 0 & 0 & 0 & 0 \\ 0 & 0 & 0 & 0 & 0 \\ 0 & 0 & 0 & 0 & 0\end{array}\right)$
$\left(\begin{array}{lllll}0 & 0 & 0 & 0 & 0 \\ 0 & 0 & 0 & 0 & 0\end{array}\right.$

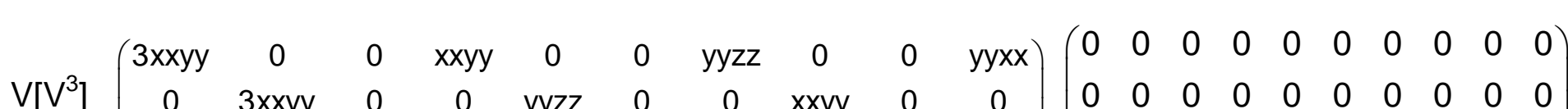
$\left.\mathrm{V}^{3} \mathrm{~V}^{3}\right]\left(\begin{array}{cccccccccc}3 x x y y & 0 & 0 & x x y y & 0 & 0 & y y z z & 0 & 0 & y y x x \\ 0 & 3 x x y y & 0 & 0 & y y z z & 0 & 0 & x x y y & 0 & 0 \\ 0 & 0 & z z z z & 0 & 0 & z z x x & 0 & 0 & z z x x & 0\end{array}\right)\left(\begin{array}{ccccccccccc}0 & 0 & 0 & 0 & 0 & 0 & 0 & 0 & 0 & 0 \\ 0 & 0 & 0 & 0 & 0 & 0 & 0 & 0 & 0 & 0 \\ 0 & 0 & 0 & 0 & 0 & 0 & 0 & 0 & 0 & 0\end{array}\right)$ $\left.\left[\mathrm{V}^{2}\right]^{2}\right]\left(\begin{array}{cccccc}\mathrm{xxxx} & \mathrm{xxyy} & \mathrm{xxzz} & 0 & 0 & 0 \\ & \mathrm{xxxx} & \mathrm{xxzz} & 0 & 0 & 0 \\ & & \mathrm{zzzz} & 0 & 0 & 0 \\ & & & \mathrm{yzyz} & 0 & 0 \\ & & & & \mathrm{yzyz} & 0 \\ & & & & & \mathrm{xyxy}\end{array}\right)$ $\left(\begin{array}{llllll}0 & 0 & 0 & 0 & 0 & 0 \\ & 0 & 0 & 0 & 0 & 0 \\ & & 0 & 0 & 0 & 0 \\ & & & 0 & 0 & 0 \\ & & & & 0 & 0 \\ & & & & & 0\end{array}\right)$ $\left[\mathrm{V}^{2}\right]^{2}\left(\begin{array}{cccccc}x \mathrm{x} x \mathrm{x} & \mathrm{xxyy} & \mathrm{xxzz} & 0 & 0 & 0 \\ \mathrm{xxyy} & \mathrm{xxxx} & \mathrm{xxzz} & 0 & 0 & 0 \\ \mathrm{zzxx} & \mathrm{zzxx} & \mathrm{zzzz} & 0 & 0 & 0 \\ 0 & 0 & 0 & \mathrm{yzyz} & 0 & 0 \\ 0 & 0 & 0 & 0 & \mathrm{yzyz} & 0 \\ 0 & 0 & 0 & 0 & 0 & \mathrm{xyxy}\end{array}\right)$

$\left(\begin{array}{llllll}0 & 0 & 0 & 0 & 0 & 0 \\ 0 & 0 & 0 & 0 & 0 & 0 \\ 0 & 0 & 0 & 0 & 0 & 0 \\ 0 & 0 & 0 & 0 & 0 & 0 \\ 0 & 0 & 0 & 0 & 0 & 0 \\ 0 & 0 & 0 & 0 & 0 & 0\end{array}\right)$

and $x y x y=1 / 2(x x x x-x x y y)$

$\left(\begin{array}{ccccccccc}0 & 0 & 0 & 0 & 0 & 0 & \text { xxzy } & 0 & 0 \\ 0 & 0 & 0 & 0 & 0 & 0 & -x x z y & 0 & 0 \\ 0 & 0 & 0 & 0 & 0 & 0 & 0 & 0 & 0 \\ 0 & 0 & 0 & 0 & 0 & 0 & 0 & 0 & 0 \\ 0 & 0 & 0 & 0 & 0 & z y x x & 0 & 0 & z y x x \\ 0 & 0 & 0 & 0 & x x z y & 0 & 0 & x x y z & 0 \\ z y x x & -z y x x & 0 & 0 & 0 & 0 & 0 & 0 & 0 \\ \text { xzxx } & -x z x x & 0 & 0 & 0 & y z x x & 0 & 0 & y z x x \\ 0 & 0 & 0 & 0 & x x z y & 0 & 0 & x x y z & 0\end{array}\right)$

$\left(\begin{array}{lllllllll}0 & 0 & 0 & 0 & 0 & 0 & 0 & 0 & 0 \\ 0 & 0 & 0 & 0 & 0 & 0 & 0 & 0 & 0\end{array}\right.$ $\begin{array}{lllllllll}0 & 0 & 0 & 0 & 0 & 0 & 0 & 0 & 0 \\ 0 & 0 & 0 & 0 & 0 & 0 & 0 & 0 & 0\end{array}$ $\begin{array}{lllllllll}0 & 0 & 0 & 0 & 0 & 0 & 0 & 0 & 0 \\ 0 & 0 & 0 & 0 & 0 & 0 & 0 & 0 & 0 \\ 0 & 0 & 0 & 0 & 0 & 0 & 0 & 0 & 0\end{array}$ $\begin{array}{lllllllll}0 & 0 & 0 & 0 & 0 & 0 & 0 & 0 & 0 \\ 0 & 0 & 0 & 0 & 0 & 0 & 0 & 0 & 0\end{array}$ $\begin{array}{lllllllllll}0 & 0 & 0 & 0 & 0 & 0 & 0 & 0 & 0 \\ 0 & 0 & 0 & 0 & 0 & 0 & 0 & 0 & 0 \\ 0 & 0 & 0 & 0 & 0 & 0\end{array}$ $\begin{array}{lllllllllll}0 & 0 & 0 & 0 & 0 & 0 & 0 & 0 & 0 \\ 0 & 0 & 0 & 0 & 0 & 0 & 0 & 0 & 0\end{array}$ $\begin{array}{lllllllll}0 & 0 & 0 & 0 & 0 & 0 & 0 & 0 & 0 \\ 0 & 0 & 0 & 0 & 0 & 0 & 0 & 0 & 0\end{array}$ $\begin{array}{llllllllll}0 & 0 & 0 & 0 & 0 & 0 & 0 & 0 & 0\end{array}$

$\left(\begin{array}{lllll}0 & 0 & 0 & 0 & 0 \\ 0 & 0 & 0 & 0 & 0 \\ 0 & 0 & 0 & 0 & 0\end{array}\right)$ $\begin{array}{lllll}0 & 0 & 0 & 0 & 0\end{array}$

$\left(\begin{array}{cccccccccc}0 & 0 & 0 & 0 & 0 & 0 & 0 & 0 & 0 & y z x x \\ 0 & 0 & 0 & 0 & 0 & y z x x & 0 & 0 & -y z x x & 0 \\ 0 & -z y x x & 0 & 0 & 0 & 0 & 0 & z y x x & 0 & 0\end{array}\right)$

$\left(\begin{array}{llllllllll}0 & 0 & 0 & 0 & 0 & 0 & 0 & 0 & 0 & 0 \\ 0 & 0 & 0 & 0 & 0 & 0 & 0 & 0 & 0 & 0\end{array}\right.$ $\left(\begin{array}{lllllllllll}0 & 0 & 0 & 0 & 0 & 0 & 0 & 0 & 0 & 0 \\ 0 & 0 & 0 & 0 & 0 & 0 & 0 & 0 & 0 & 0\end{array}\right)$

$\left(\begin{array}{cccccc}0 & 0 & 0 & 0 & 0 & 0 \\ & 0 & 0 & 0 & 0 & 0\end{array}\right)$

$0 \begin{array}{lllll}0 & 0 & 0 & 0 & 0\end{array}$

$\begin{array}{llll}0 & 0 & 0 & 0\end{array}$

$\begin{array}{lll}0 & 0 & 0 \\ & 0 & 0\end{array}$

$\left(\begin{array}{llllll}0 & 0 & 0 & 0 & 0 & 0 \\ & 0 & 0 & 0 & 0 & 0 \\ & & 0 & 0 & 0 & 0 \\ & & & 0 & 0 & 0 \\ & & & & 0 & 0 \\ & & & & & 0\end{array}\right)$

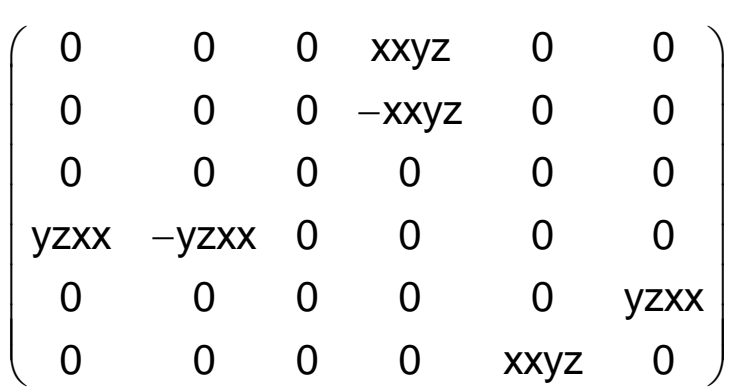

$\left(\begin{array}{llllll}0 & 0 & 0 & 0 & 0 & 0 \\ 0 & 0 & 0 & 0 & 0 & 0\end{array}\right)$ $\begin{array}{llllll}0 & 0 & 0 & 0 & 0 & 0 \\ 0 & 0 & 0 & 0 & 0 & 0\end{array}$ $\begin{array}{llllll}0 & 0 & 0 & 0 & 0 & 0 \\ 0 & 0 & 0 & 0 & 0 & 0 \\ 0 & 0 & 0 & 0 & 0 & 0\end{array}$ $\begin{array}{llllll}0 & 0 & 0 & 0 & 0 & 0 \\ 0 & 0 & 0 & 0 & 0 & 0\end{array}$ $\begin{array}{llllll}0 & 0 & 0 & 0 & 0 & 0 \\ 0 & 0 & 0 & 0 & 0 & 0\end{array}$ 


\section{a}

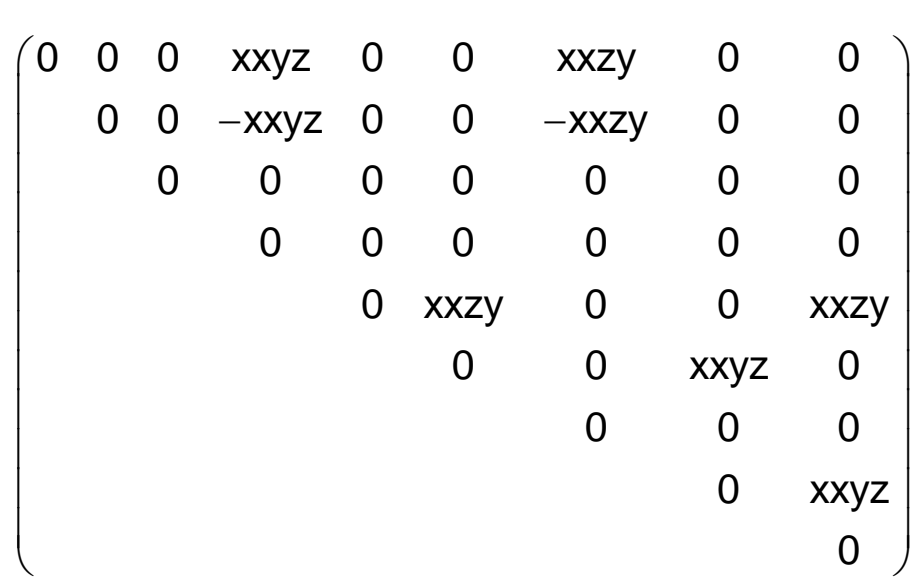

$\left(\begin{array}{lllllllll}0 & 0 & 0 & 0 & 0 & 0 & 0 & 0 & 0 \\ & 0 & 0 & 0 & 0 & 0 & 0 & 0 & 0 \\ & & 0 & 0 & 0 & 0 & 0 & 0 & 0 \\ & & & 0 & 0 & 0 & 0 & 0 & 0 \\ & & & & 0 & 0 & 0 & 0 & 0 \\ & & & & & 0 & 0 & 0 & 0 \\ & & & & & & 0 & 0 & 0 \\ & & & & & & & 0 & 0 \\ & & & & & & & & \\ & & & & & & & & 0\end{array}\right)$

and $x x x x=x x y y+y z y z+z y z y$

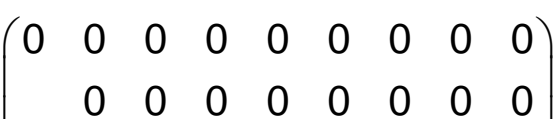

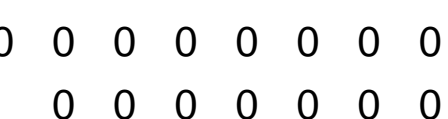

$\begin{array}{llllll}0 & 0 & 0 & 0 & 0 & 0\end{array}$

$\begin{array}{lllll}0 & 0 & 0 & 0 & 0\end{array}$

$\begin{array}{llll}0 & 0 & 0 & 0\end{array}$

$\begin{array}{lll}0 & 0 & 0 \\ 0 & 0 \\ & & 0\end{array}$

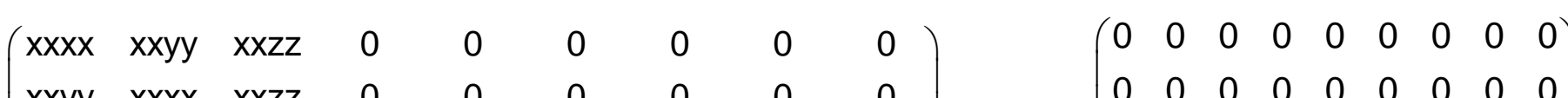

$\left(\begin{array}{lllllllll}x x y y & x x x x & x x z z & 0 & 0 & 0 & 0 & 0 & 0 \\ z z x x & z z x x & z z z z & 0 & 0 & 0 & 0 & 0 & 0\end{array}\right) \quad\left(\begin{array}{lllllllll}0 & 0 & 0 & 0 & 0 & 0 & 0 & 0 & 0 \\ 0 & 0 & 0 & 0 & 0 & 0 & 0 & 0 & 0 \\ 0 & 0 & 0 & 0 & 0 & 0 & 0 & 0 & 0\end{array}\right)$

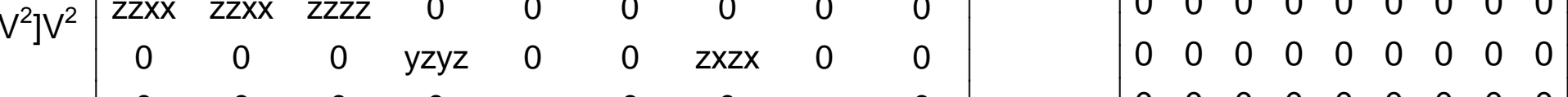

$0 \begin{array}{lllllllll}0 & 0 & 0 & 0 & 0 & 0 & 0 & 0 & 0 \\ 0 & 0 & 0 & 0 & 0 & 0 & 0\end{array}$

$\begin{array}{llllllllll}0 & 0 & 0 & 0 & 0 & 0 & 0 & 0 & 0\end{array}$

$\left(\begin{array}{ccccccccc}0 & 0 & 0 & x x y z & 0 & 0 & x x z y & 0 & 0 \\ 0 & 0 & 0 & -x x y z & 0 & 0 & -x x z y & 0 & 0 \\ 0 & 0 & 0 & 0 & 0 & 0 & 0 & 0 & 0 \\ y z x x & -y z x x & 0 & 0 & 0 & 0 & 0 & 0 & 0 \\ 0 & 0 & 0 & 0 & 0 & y z x x & 0 & 0 & y z x x \\ 0 & 0 & 0 & 0 & x x z y & 0 & 0 & x x y z & 0\end{array}\right) \quad\left(\begin{array}{ccccccccc}0 & 0 & 0 & 0 & 0 & 0 & 0 & 0 & 0 \\ 0 & 0 & 0 & 0 & 0 & 0 & 0 & 0 & 0 \\ 0 & 0 & 0 & 0 & 0 & 0 & 0 & 0 & 0 \\ 0 & 0 & 0 & 0 & 0 & 0 & 0 & 0 & 0 \\ 0 & 0 & 0 & 0 & 0 & 0 & 0 & 0 & 0 \\ 0 & 0 & 0 & 0 & 0 & 0 & 0 & 0 & 0\end{array}\right)$

and $x x x x=x x y y+2 x y x y$ 


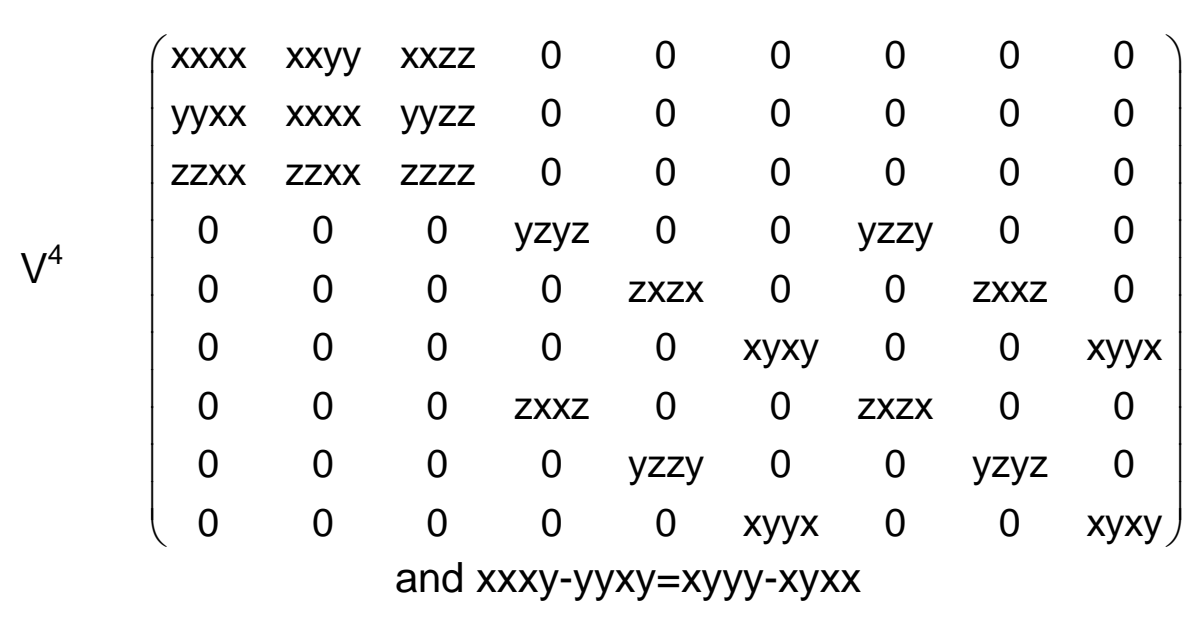

$\left(\begin{array}{lllllllll}0 & 0 & 0 & 0 & 0 & 0 & 0 & 0 & 0 \\ 0 & 0 & 0 & 0 & 0 & 0 & 0 & 0 & 0\end{array}\right.$ $0 \begin{array}{lllllllll}0 & 0 & 0 & 0 & 0 & 0 & 0 & 0 & 0 \\ 0 & 0 & 0 & 0 & 0 & 0 & 0 & 0 & 0\end{array}$ $0 \begin{array}{lllllllll}0 & 0 & 0 & 0 & 0 & 0 & 0 & 0 & 0 \\ 0 & 0 & 0 & 0 & 0 & 0 & 0 & 0 & 0\end{array}$ $0 \begin{array}{llllllllll}0 & 0 & 0 & 0 & 0 & 0 & 0 & 0 & 0 \\ 0 & 0 & 0 & 0 & 0 & 0 & 0 & 0 & 0\end{array}$ $\begin{array}{llllllllll}0 & 0 & 0 & 0 & 0 & 0 & 0 & 0 & 0 \\ 0 & 0 & 0 & 0 & 0 & 0 & 0 & 0 & 0\end{array}$ $\begin{array}{llllllllll}0 & 0 & 0 & 0 & 0 & 0 & 0 & 0 & 0 & 0\end{array}$ $\begin{array}{llllllllllllllll}0 & 0 & 0 & 0 & 0 & 0 & 0 & 0 & 0\end{array}$ $\begin{array}{llllllllll}0 & 0 & 0 & 0 & 0 & 0 & 0 & 0 & 0\end{array}$ $\left(\begin{array}{lllllllll}0 & 0 & 0 & 0 & 0 & 0 & 0 & 0 & 0 \\ 0 & 0 & 0 & 0 & 0 & 0 & 0 & 0 & 0\end{array}\right)$

$\left[V^{4}\right]\left(\begin{array}{ccccc}3 x x y y & 0 & 0 & y y z z & 0 \\ 0 & 3 x x y y & 0 & y y z z & 0 \\ 0 & 0 & z z z z & x x y y & 0\end{array}\right)$

$\left(\begin{array}{lllll}0 & 0 & 0 & 0 & 0 \\ 0 & 0 & 0 & 0 & 0 \\ 0 & 0 & 0 & 0 & 0\end{array}\right)$

$\begin{array}{lllll}0 & 0 & 0 & 0 & 0\end{array}$

V[V $\left.{ }^{3}\right] \quad\left(\begin{array}{cccccccccc}3 x x y y & 0 & 0 & x x y y & 0 & 0 & \text { yyzz } & 0 & 0 & y y x x \\ 0 & 3 x x y y & 0 & 0 & y y z z & 0 & 0 & x x y y & 0 & 0 \\ 0 & 0 & z z z z & 0 & 0 & z z x x & 0 & 0 & z z x x & 0\end{array}\right)$

$\begin{array}{llllllllll}0 & 0 & 0 & 0 & 0 & 0 & 0 & 0 & 0 & 0 \\ 0 & 0 & 0 & 0 & 0 & 0 & 0 & 0 & 0 & 0\end{array}$ $\left(\begin{array}{lllllllllll}0 & 0 & 0 & 0 & 0 & 0 & 0 & 0 & 0 & 0 \\ 0 & 0 & 0 & 0 & 0 & 0 & 0 & 0 & 0 & 0\end{array}\right)$

$\left[\left[^{2}\right]^{2}\right]\left(\begin{array}{cccccc}x x x x & x x y y & x x z z & 0 & 0 & 0 \\ & x x x x & x x z z & 0 & 0 & 0 \\ & & z z z z & 0 & 0 & 0 \\ & & & y z y z & 0 & 0 \\ & & & & y z y z & 0 \\ & & & & & \text { xyxy }\end{array}\right)$

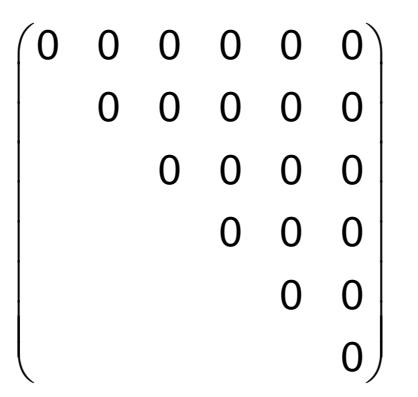

and $x y x y=1 / 2(x x x x-x x y y)$

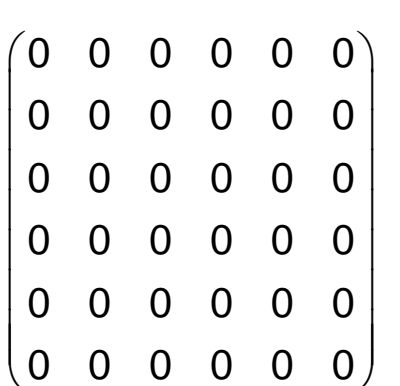

$\begin{array}{lllllllll}0 & 0 & 0 & 0 & 0 & 0 & 0 & 0 & 0 \\ 0 & 0 & 0 & 0 & 0 & 0 & 0 & 0 & 0\end{array}$ $0 \begin{array}{lllllllll}0 & 0 & 0 & 0 & 0 & 0 & 0 & 0 & 0 \\ 0 & 0 & 0 & 0 & 0 & 0 & 0 & 0 & 0\end{array}$ $\begin{array}{lllllllll}0 & 0 & 0 & 0 & 0 & 0 & 0 & 0 & 0 \\ 0 & 0 & 0 & 0 & 0 & 0 & 0 & 0 & 0\end{array} \mid$ $\begin{array}{llllllllll}0 & 0 & 0 & 0 & 0 & 0 & 0 & 0 & 0 \\ 0 & 0 & 0 & 0 & 0 & 0 & 0 & 0 & 0\end{array}$ $\begin{array}{llllllllll}0 & 0 & 0 & 0 & 0 & 0 & 0 & 0 & 0\end{array}$ $\begin{array}{llllllllllllllllll}0 & 0 & 0 & 0 & 0 & 0 & 0 & 0 & 0 \\ 0 & 0 & 0 & 0 & 0 & 0 & 0 & 0 & 0\end{array}$

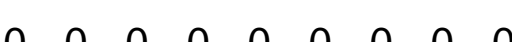
$\left(\begin{array}{lllllllll}0 & 0 & 0 & 0 & 0 & 0 & 0 & 0 & 0 \\ 0 & 0 & 0 & 0 & 0 & 0 & 0 & 0 & 0\end{array}\right)$

$$
\left(\begin{array}{lllll}
0 & 0 & 0 & 0 & 0 \\
0 & 0 & 0 & 0 & 0 \\
0 & 0 & 0 & 0 & 0
\end{array}\right)
$$

$\left(\begin{array}{llllllllll}0 & 0 & 0 & 0 & 0 & 0 & 0 & 0 & 0 & 0 \\ 0 & 0 & 0 & 0 & 0 & 0 & 0 & 0 & 0 & 0\end{array}\right)$ $\left.\begin{array}{llllllllll}0 & 0 & 0 & 0 & 0 & 0 & 0 & 0 & 0 & 0 \\ 0 & 0 & 0 & 0 & 0 & 0 & 0 & 0 & 0 & 0\end{array}\right)$ $\begin{array}{lllllllllll}0 & 0 & 0 & 0 & 0 & 0 & 0 & 0 & 0 & 0\end{array}$

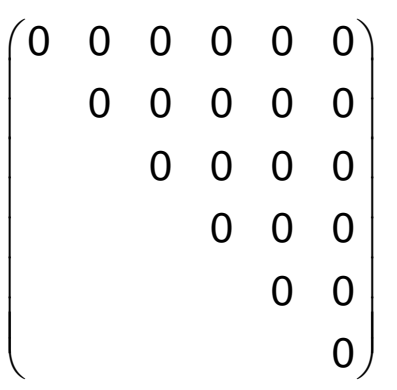

$\left(\begin{array}{llllll}0 & 0 & 0 & 0 & 0 & 0 \\ 0 & 0 & 0 & 0 & 0 & 0\end{array}\right)$ $\begin{array}{llllll}0 & 0 & 0 & 0 & 0 & 0 \\ 0 & 0 & 0 & 0 & 0 & 0\end{array}$ $\begin{array}{llllll}0 & 0 & 0 & 0 & 0 & 0 \\ 0 & 0 & 0 & 0 & 0 & 0\end{array}$

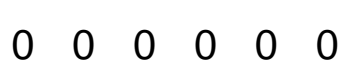
$\left(\begin{array}{llllll}0 & 0 & 0 & 0 & 0 & 0 \\ 0 & 0 & 0 & 0 & 0 & 0\end{array}\right.$

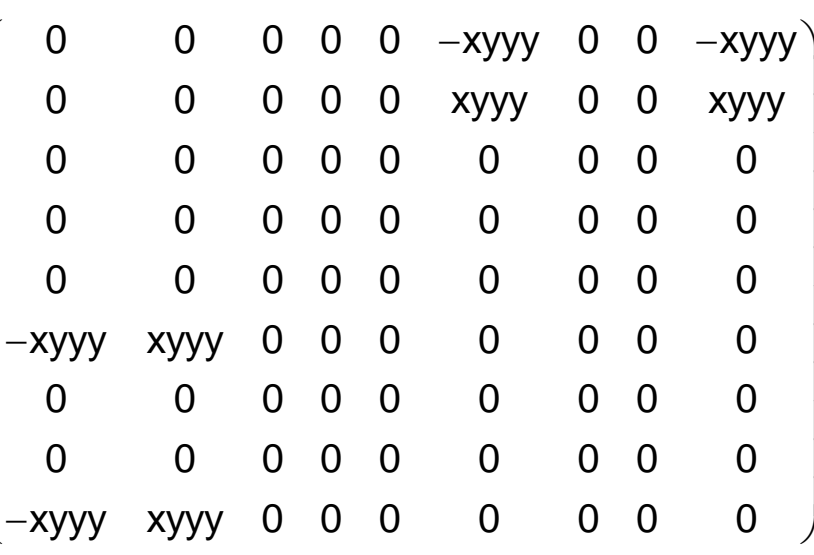

$\left(\begin{array}{ccccc}0 & \text { xyyy } & 0 & 0 & 0 \\ -x y y y & 0 & 0 & 0 & 0 \\ 0 & 0 & 0 & 0 & 0\end{array}\right)$ $\left(\begin{array}{ccccc}-x y y y & 0 & 0 & 0 & 0 \\ 0 & 0 & 0 & 0 & 0\end{array}\right)$

$\left(\begin{array}{cccccccccc}0 & \text { xyyy } & 0 & 0 & 0 & 0 & 0 & -x y y y & 0 & 0 \\ -x y y y & 0 & 0 & x y y y & 0 & 0 & 0 & 0 & 0 & 0 \\ 0 & 0 & 0 & 0 & 0 & 0 & 0 & 0 & 0 & 0\end{array}\right)$ $\left(\begin{array}{cccccccccc}0 & & 0 & 0 & 0 & 0 & 0 & 0 & -x y y y & 0 \\ -x y y y & 0 & 0 & x y y y & 0 & 0 & 0 & 0 & 0 & 0 \\ 0 & 0 & 0 & 0 & 0 & 0 & 0 & 0 & 0 & 0\end{array}\right)$

$$
\left(\begin{array}{cccccc}
0 & 0 & 0 & 0 & 0 & -x y y y \\
& 0 & 0 & 0 & 0 & x y y y \\
& & 0 & 0 & 0 & 0 \\
& & & 0 & 0 & 0 \\
& & & & 0 & 0 \\
& & & & & 0
\end{array}\right)
$$

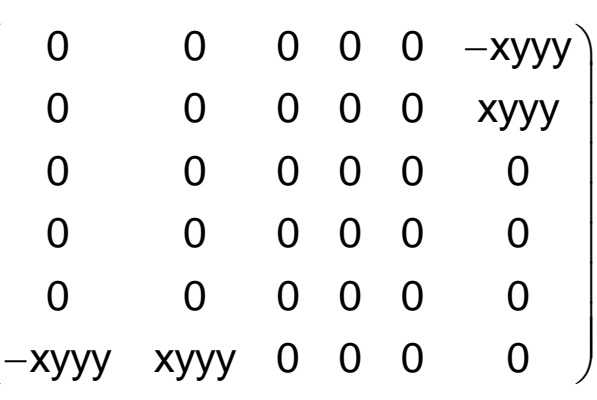

and $x y x y=1 / 2(x x x x-x x y y)$ 


$$
\left(\begin{array}{ccccccccc}
\text { xxxx } & \text { xxyy } & \text { xxzz } & 0 & 0 & 0 & 0 & 0 & 0 \\
& \text { xxxx } & \text { xxzz } & 0 & 0 & 0 & 0 & 0 & 0 \\
& & \text { zzzz } & 0 & 0 & 0 & 0 & 0 & 0 \\
& & & y z y z & 0 & 0 & y z z y & 0 & 0 \\
& & & & \text { zxzx } & 0 & 0 & \text { yzzy } & 0 \\
& & & & & \text { xyxy } & 0 & 0 & \text { xyyx } \\
& & & & & & \text { zxzx } & 0 & 0 \\
& & & & & & & \text { yzyz } & 0 \\
& & & & & & & & \text { xyxy }
\end{array}\right)
$$

and $x x x x=x x y y+y z y z+z y z y$

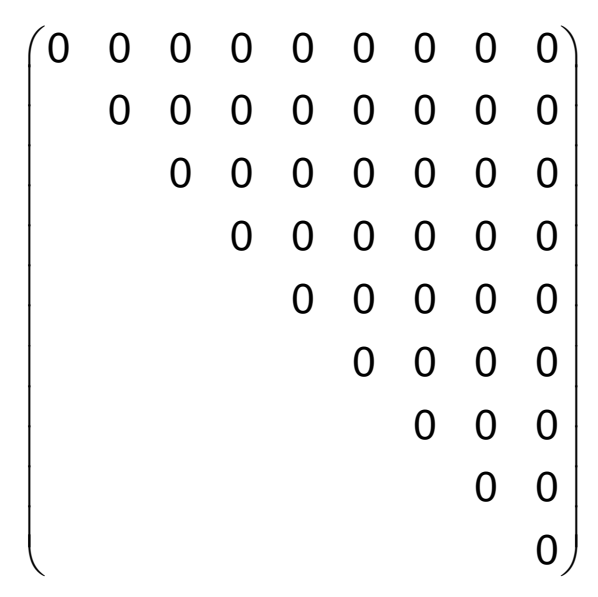

$\left.\mathrm{V}^{2}\right] \mathrm{V}^{2}\left(\begin{array}{ccccccccc}\mathrm{x} x \mathrm{x} & \mathrm{xxyy} & \mathrm{xxzz} & 0 & 0 & 0 & 0 & 0 & 0 \\ \mathrm{xxyy} & \mathrm{xxxx} & \mathrm{xxzz} & 0 & 0 & 0 & 0 & 0 & 0 \\ \mathrm{zzxx} & \mathrm{zzxx} & \mathrm{zzzz} & 0 & 0 & 0 & 0 & 0 & 0 \\ 0 & 0 & 0 & \mathrm{yzyz} & 0 & 0 & \mathrm{zxzx} & 0 & 0 \\ 0 & 0 & 0 & 0 & \mathrm{zxzx} & 0 & 0 & \mathrm{yzyz} & 0 \\ 0 & 0 & 0 & 0 & 0 & \text { xyxy } & 0 & 0 & \text { xyxy }\end{array}\right)$

$\left(\begin{array}{lllllllll}0 & 0 & 0 & 0 & 0 & 0 & 0 & 0 & 0 \\ 0 & 0 & 0 & 0 & 0 & 0 & 0 & 0 & 0\end{array}\right)$

$\begin{array}{lllllllll}0 & 0 & 0 & 0 & 0 & 0 & 0 & 0 & 0 \\ 0 & 0 & 0 & 0 & 0 & 0 & 0 & 0 & 0\end{array}$

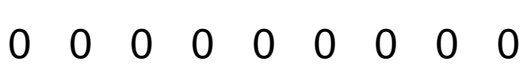

$0 \begin{array}{lllllllll}0 & 0 & 0 & 0 & 0 & 0 & 0 & 0 & 0 \\ 0 & 0 & 0 & 0 & 0 & 0 & 0 & 0 & 0\end{array}$

$\begin{array}{lllllllll}0 & 0 & 0 & 0 & 0 & 0 & 0 & 0 & 0 \\ 0 & 0 & 0 & 0 & 0 & 0 & 0 & 0 & 0\end{array}$

and $x x x x=x x y y+2 x y x y$ $\left(\begin{array}{lllllllll}0 & 0 & 0 & 0 & 0 & 0 & 0 & 0 & 0 \\ & 0 & 0 & 0 & 0 & 0 & 0 & 0 & 0\end{array}\right.$

$\begin{array}{llllllll}0 & 0 & 0 & 0 & 0 & 0 & 0\end{array}$

$\begin{array}{llllll}0 & 0 & 0 & 0 & 0 & 0\end{array}$

$\begin{array}{lllll}0 & 0 & 0 & 0 & 0\end{array}$

$\begin{array}{llll}0 & 0 & 0 & 0\end{array}$

$\begin{array}{lll}0 & 0 & 0 \\ 0 & 0\end{array}$

0

$\begin{array}{lllllllll}0 & 0 & 0 & 0 & 0 & 0 & 0 & 0 & 0\end{array}$

$0 \begin{array}{lllllllll}0 & 0 & 0 & 0 & 0 & 0 & 0 & 0 & 0 \\ 0 & 0 & 0 & 0 & 0 & 0 & 0 & 0 & 0\end{array}$

$0 \begin{array}{lllllllll}0 & 0 & 0 & 0 & 0 & 0 & 0 & 0 & 0 \\ 0 & 0 & 0 & 0 & 0 & 0 & 0 & 0 & 0\end{array}$

$0 \begin{array}{lllllllll}0 & 0 & 0 & 0 & 0 & 0 & 0 & 0 & 0 \\ 0 & 0 & 0 & 0 & 0 & 0 & 0 & 0 & 0\end{array}$

$0 \begin{array}{lllllllll}0 & 0 & 0 & 0 & 0 & 0 & 0 & 0 & 0 \\ 0 & 0 & 0 & 0 & 0 & 0 & 0 & 0 & 0\end{array}$

$\begin{array}{llllllllll}0 & 0 & 0 & 0 & 0 & 0 & 0 & 0 & 0\end{array}$
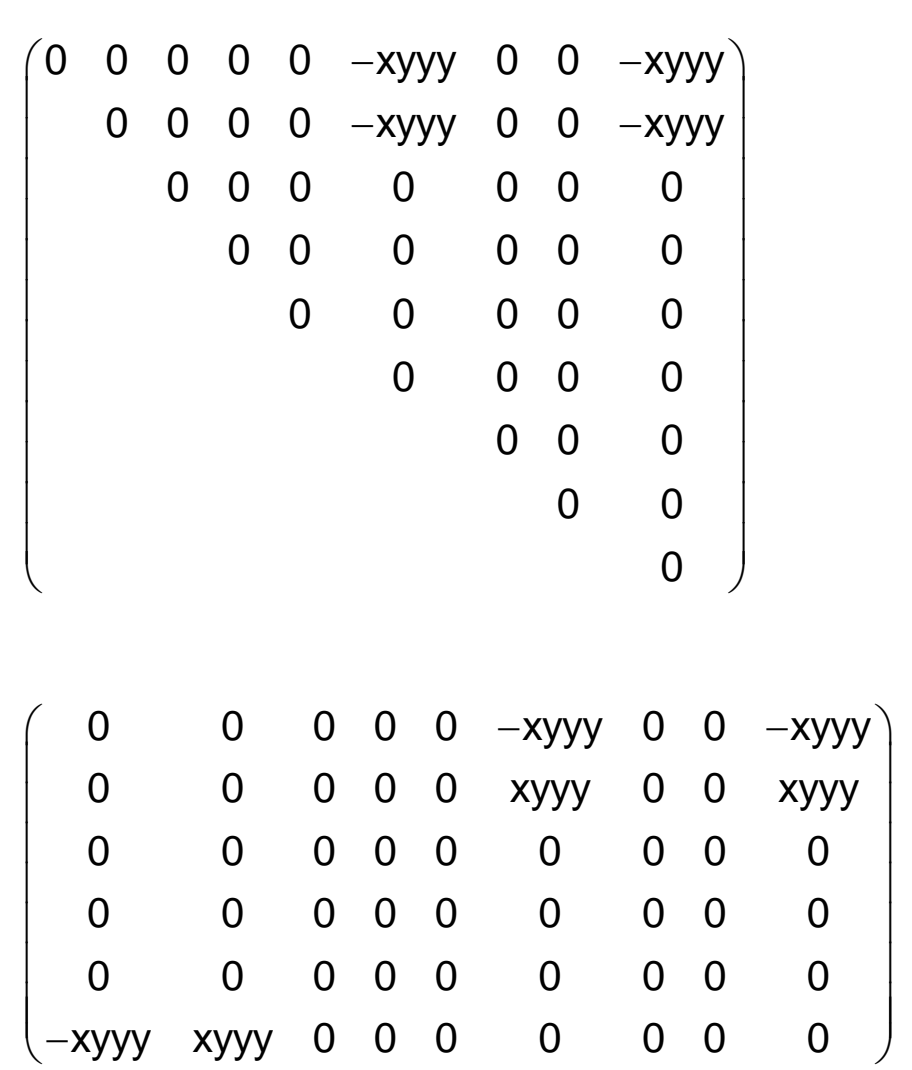
$\left(\begin{array}{lllllllll}0 & 0 & 0 & 0 & 0 & 0 & 0 & 0 & 0 \\ 0 & 0 & 0 & 0 & 0 & 0 & 0 & 0 & 0\end{array}\right)$ $\left(\begin{array}{lllllllll}0 & 0 & 0 & 0 & 0 & 0 & 0 & 0 & 0 \\ 0 & 0 & 0 & 0 & 0 & 0 & 0 & 0 & 0\end{array}\right.$ $\begin{array}{llllllllll}0 & 0 & 0 & 0 & 0 & 0 & 0 & 0 & 0 \\ 0 & 0 & 0 & 0 & 0 & 0 & 0 & 0 & 0\end{array}$ $\begin{array}{llllllllll}0 & 0 & 0 & 0 & 0 & 0 & 0 & 0 & 0\end{array}$ $\begin{array}{llllllllll}0 & 0 & 0 & 0 & 0 & 0 & 0 & 0 & 0 \\ 0 & 0 & 0 & 0 & 0 & 0 & 0 & 0 & 0\end{array}$ $\begin{array}{lllllllllll}0 & 0 & 0 & 0 & 0 & 0 & 0 & 0 & 0 \\ 0 & 0 & 0 & 0 & 0 & 0 & 0 & 0 & 0\end{array}$ $\begin{array}{lllllllll}0 & 0 & 0 & 0 & 0 & 0 & 0 & 0 & 0 \\ 0 & 0 & 0 & 0 & 0 & 0 & 0 & 0 & 0\end{array}$ $0 \begin{array}{lllllllll}0 & 0 & 0 & 0 & 0 & 0 & 0 & 0 & 0 \\ 0 & 0 & 0 & 0 & 0 & 0 & 0 & 0 & 0\end{array}$ $0 \begin{array}{lllllllll}0 & 0 & 0 & 0 & 0 & 0 & 0 & 0 & 0 \\ 0 & 0 & 0 & 0 & 0 & 0 & 0 & 0 & 0\end{array}$ $\begin{array}{lllllllllll}0 & 0 & 0 & 0 & 0 & 0 & 0 & 0 & 0\end{array}$

$\left(\begin{array}{lllll}0 & 0 & 0 & 0 & 0 \\ 0 & 0 & 0 & 0 & 0 \\ 0 & 0 & 0 & 0 & 0\end{array}\right)$

$\left[V^{4}\right] \quad\left(\begin{array}{ccccc}3 x x y y & 0 & 0 & y y z z & 0 \\ 0 & 3 x x y y & 0 & y y z z & 0 \\ 0 & 0 & z z z z & x x y y & 0\end{array}\right)$

$\left.\mathrm{V}^{3} \mathrm{~V}^{3}\right]\left(\begin{array}{cccccccccc}3 \mathrm{xx} x \mathrm{y} & 0 & 0 & \mathrm{xxyy} & 0 & 0 & \mathrm{yyzz} & 0 & 0 & 0 \\ 0 & 3 x \mathrm{x} y \mathrm{y} & 0 & 0 & \mathrm{yyzz} & 0 & 0 & \mathrm{xxyy} & 0 & 0 \\ 0 & 0 & 2 z z & 0 & 0 & z z x & 0 & 0 & z z x & 0\end{array}\right)$

$\left(\begin{array}{llllllllll}0 & 0 & 0 & 0 & 0 & 0 & 0 & 0 & 0 & 0 \\ 0 & 0 & 0 & 0 & 0 & 0 & 0 & 0 & 0 & 0\end{array}\right)$ $\left(\begin{array}{llllllllll}0 & 0 & 0 & 0 & 0 & 0 & 0 & 0 & 0 & 0 \\ 0 & 0 & 0 & 0 & 0 & 0 & 0 & 0 & 0 & 0\end{array}\right)$ $\left(\begin{array}{llllllllll}0 & 0 & 0 & 0 & 0 & 0 & 0 & 0 & 0 & 0 \\ 0 & 0 & 0 & 0 & 0 & 0 & 0 & 0 & 0 & 0\end{array}\right)$
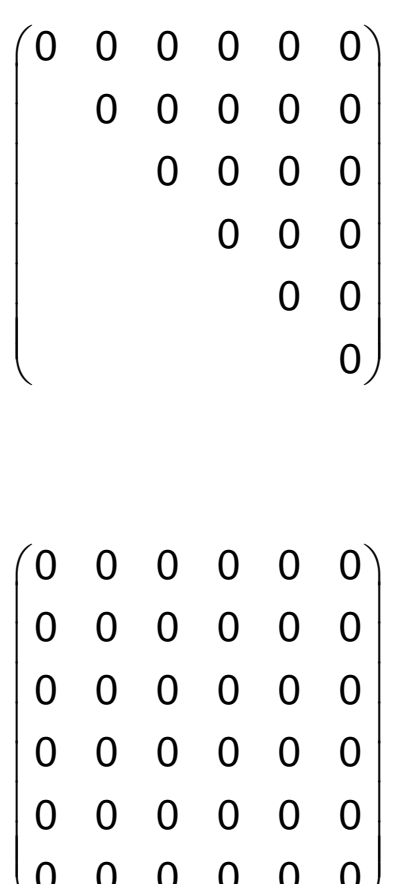

$\left(\begin{array}{lllllllll}0 & 0 & 0 & 0 & 0 & 0 & 0 & 0 & 0 \\ 0 & 0 & 0 & 0 & 0 & 0 & 0 & 0 & 0\end{array}\right)$

$0 \begin{array}{lllllllll}0 & 0 & 0 & 0 & 0 & 0 & 0 & 0 & 0 \\ 0 & 0 & 0 & 0 & 0 & 0 & 0 & 0 & 0\end{array}$

$0 \begin{array}{llllllllll}0 & 0 & 0 & 0 & 0 & 0 & 0 & 0 & 0\end{array}$ $\begin{array}{llllllllll}0 & 0 & 0 & 0 & 0 & 0 & 0 & 0 & 0 \\ 0 & 0 & 0 & 0 & 0 & 0 & 0 & 0 & 0\end{array}$

$\begin{array}{llllllllll}0 & 0 & 0 & 0 & 0 & 0 & 0 & 0 & 0\end{array}$

$0 \begin{array}{lllllllll}0 & 0 & 0 & 0 & 0 & 0 & 0 & 0 & 0 \\ 0 & 0 & 0 & 0 & 0 & 0 & 0 & 0 & 0\end{array}$

$0 \begin{array}{lllllllll}0 & 0 & 0 & 0 & 0 & 0 & 0 & 0 & 0\end{array}$

$0 \begin{array}{lllllllll}0 & 0 & 0 & 0 & 0 & 0 & 0 & 0 & 0 \\ 0 & 0 & 0 & 0 & 0 & 0 & 0 & 0 & 0\end{array}$

$\begin{array}{llllllllll}0 & 0 & 0 & 0 & 0 & 0 & 0 & 0 & 0\end{array}$

\section{$\left(\begin{array}{lllll}0 & 0 & 0 & 0 & 0 \\ 0 & 0 & 0 & 0 & 0 \\ 0 & 0 & 0 & 0 & 0\end{array}\right)$ \\ $\left.\begin{array}{lllll}0 & 0 & 0 & 0 & 0\end{array}\right)$}

$\left(\begin{array}{llllllllll}0 & 0 & 0 & 0 & 0 & 0 & 0 & 0 & 0 & 0 \\ 0 & 0 & 0 & 0 & 0 & 0 & 0 & 0 & 0 & 0\end{array}\right.$ $\begin{array}{lllllllllll}0 & 0 & 0 & 0 & 0 & 0 & 0 & 0 & 0 & 0\end{array}$ $\left.\begin{array}{lllllllllll}0 & 0 & 0 & 0 & 0 & 0 & 0 & 0 & 0 & 0 \\ 0 & 0 & 0 & 0 & 0 & 0 & 0 & 0 & 0 & 0\end{array}\right)$

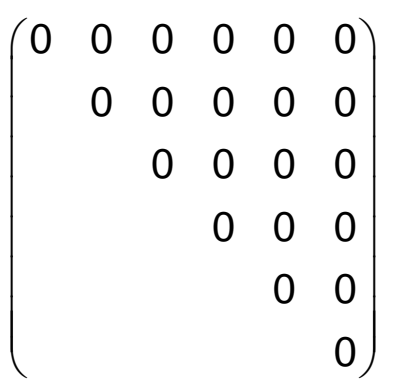

$\left(\begin{array}{llllll}0 & 0 & 0 & 0 & 0 & 0 \\ 0 & 0 & 0 & 0 & 0 & 0\end{array}\right)$

$0 \begin{array}{llllll}0 & 0 & 0 & 0 & 0 & 0 \\ 0 & 0 & 0 & 0 & 0 & 0\end{array}$

$\begin{array}{llllll}0 & 0 & 0 & 0 & 0 & 0 \\ 0 & 0 & 0 & 0 & 0 & 0\end{array}$

$0 \begin{array}{llllll}0 & 0 & 0 & 0 & 0 & 0 \\ 0 & 0 & 0 & 0 & 0 & 0\end{array}$

$0 \begin{array}{llllll}0 & 0 & 0 & 0 & 0 & 0 \\ 0 & 0 & 0 & 0 & 0 & 0\end{array}$

$\begin{array}{llllll}0 & 0 & 0 & 0 & 0 & 0 \\ 0 & 0 & 0 & 0 & 0 & 0\end{array}$ $\left(\begin{array}{lllllllll}0 & 0 & 0 & 0 & 0 & 0 & 0 & 0 & 0 \\ 0 & 0 & 0 & 0 & 0 & 0 & 0 & 0 & 0\end{array}\right.$ $\begin{array}{llllllllll}0 & 0 & 0 & 0 & 0 & 0 & 0 & 0 & 0\end{array}$ $\begin{array}{llllllllll}0 & 0 & 0 & 0 & 0 & 0 & 0 & 0 & 0 & 0\end{array}$ $\begin{array}{llllllllll}0 & 0 & 0 & 0 & 0 & 0 & 0 & 0 & 0 \\ 0 & 0 & 0 & 0 & 0 & 0 & 0 & 0 & 0\end{array}$ $\begin{array}{lllllllll}0 & 0 & 0 & 0 & 0 & 0 & 0 & 0 & 0 \\ 0 & 0 & 0 & 0 & 0 & 0 & 0 & 0 & 0\end{array}$ $\begin{array}{lllllllll}0 & 0 & 0 & 0 & 0 & 0 & 0 & 0 & 0\end{array}$ $\begin{array}{lllllllll}0 & 0 & 0 & 0 & 0 & 0 & 0 & 0 & 0 \\ 0 & 0 & 0 & 0 & 0 & 0 & 0 & 0 & 0\end{array}$

$\begin{array}{llll}0 & 0 & 0\end{array}$

$\left(\begin{array}{lllll}0 & 0 & 0 & 0 & 0 \\ 0 & 0 & 0 & 0 & 0 \\ 0 & 0 & 0 & 0 & 0\end{array}\right)$

$\begin{array}{lllll}0 & 0 & 0 & 0 & 0 \\ 0 & 0 & 0 & 0 & 0\end{array}$

$\left(\begin{array}{llllllllll}0 & 0 & 0 & 0 & 0 & 0 & 0 & 0 & 0 & 0 \\ 0 & 0 & 0 & 0 & 0 & 0 & 0 & 0 & 0 & 0\end{array}\right.$ $\left(\begin{array}{llllllllll}0 & 0 & 0 & 0 & 0 & 0 & 0 & 0 & 0 & 0 \\ 0 & 0 & 0 & 0 & 0 & 0 & 0 & 0 & 0 & 0\end{array}\right)$ $\begin{array}{lllllllllll}0 & 0 & 0 & 0 & 0 & 0 & 0 & 0 & 0 & 0\end{array}$

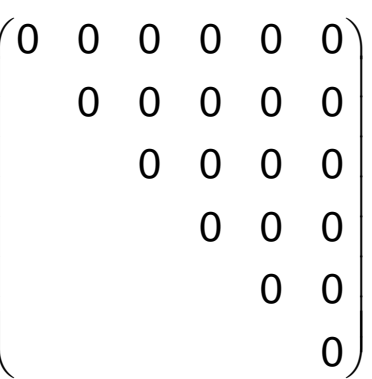

$\begin{array}{llllll}0 & 0 & 0 & 0 & 0 & 0 \\ 0 & 0 & 0 & 0 & 0 & 0\end{array}$ $\begin{array}{llllll}0 & 0 & 0 & 0 & 0 & 0 \\ 0 & 0 & 0 & 0 & 0 & 0\end{array}$ $\begin{array}{llllll}0 & 0 & 0 & 0 & 0 & 0 \\ 0 & 0 & 0 & 0 & 0 & 0\end{array}$ $\begin{array}{llllll}0 & 0 & 0 & 0 & 0 & 0 \\ 0 & 0 & 0 & 0 & 0 & 0\end{array}$ $\begin{array}{lllllll}0 & 0 & 0 & 0 & 0 & 0 \\ 0 & 0 & 0 & 0 & 0 & 0\end{array}$ $\begin{array}{lllllll}0 & 0 & 0 & 0 & 0 & 0\end{array}$

and $x y x y=1 / 2(x x x x-x x y y)$ 


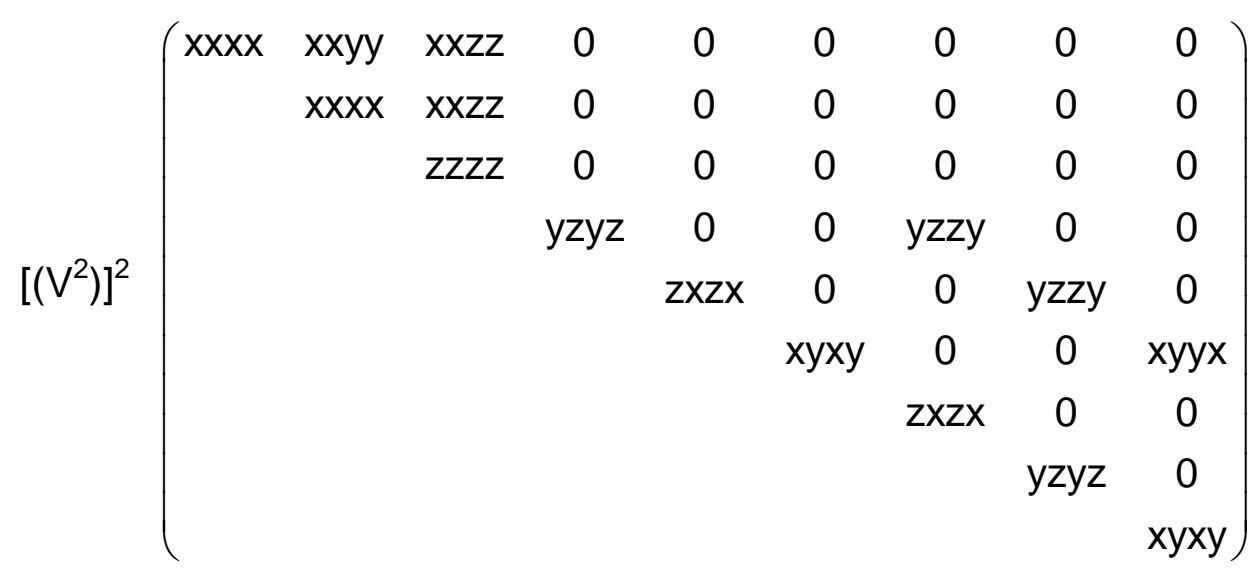

$\left(\begin{array}{lllllllll}0 & 0 & 0 & 0 & 0 & 0 & 0 & 0 & 0 \\ 0 & 0 & 0 & 0 & 0 & 0 & 0 & 0 & 0\end{array}\right.$ $\begin{array}{llllllll}0 & 0 & 0 & 0 & 0 & 0 & 0 & 0\end{array}$ $\begin{array}{lllllll}0 & 0 & 0 & 0 & 0 & 0 & 0\end{array}$ $\begin{array}{llllll}0 & 0 & 0 & 0 & 0 & 0\end{array}$ $\begin{array}{lllll}0 & 0 & 0 & 0 & 0 \\ 0 & 0 & 0 & 0 & 0\end{array}$ $\begin{array}{lllll}0 & 0 & 0 & 0 \\ 0 & 0 & 0 & 0\end{array}$ $\begin{array}{llll}0 & 0 & 0 \\ 0 & 0 & 0\end{array}$ $\begin{array}{lll}0 & 0 & 0 \\ & 0 & 0 \\ & & 0\end{array}$ and $x x x x=x x y y+y z y z+z y z y$

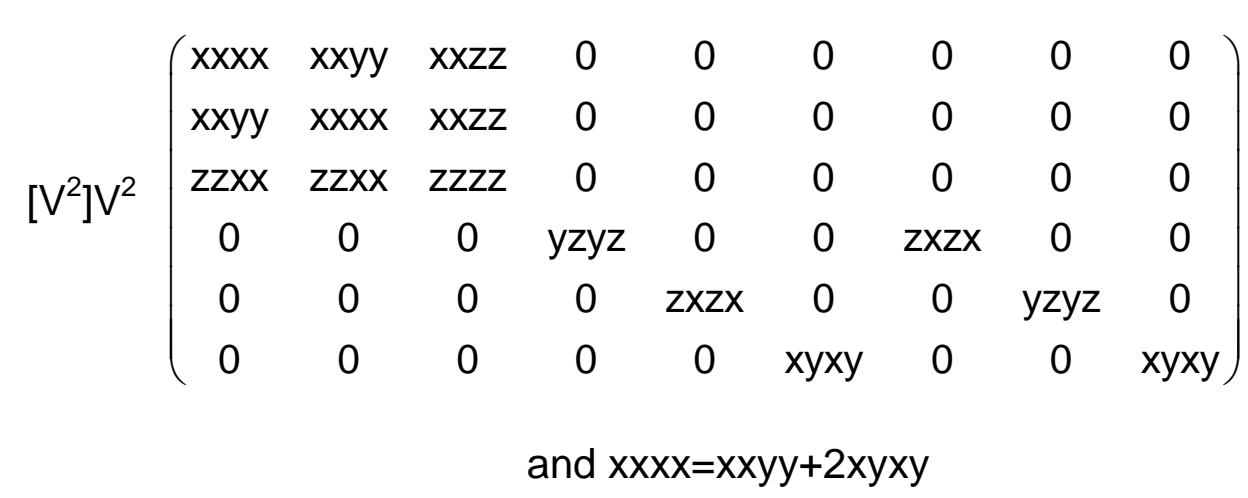

$\left(\begin{array}{lllllllll}0 & 0 & 0 & 0 & 0 & 0 & 0 & 0 & 0 \\ 0 & 0 & 0 & 0 & 0 & 0 & 0 & 0 & 0\end{array}\right)$

$\begin{array}{lllllllll}0 & 0 & 0 & 0 & 0 & 0 & 0 & 0 & 0 \\ 0 & 0 & 0 & 0 & 0 & 0 & 0 & 0 & 0\end{array}$

$0 \begin{array}{lllllllll}0 & 0 & 0 & 0 & 0 & 0 & 0 & 0 & 0 \\ 0 & 0 & 0 & 0 & 0 & 0 & 0 & 0 & 0\end{array}$

$0 \begin{array}{lllllllll}0 & 0 & 0 & 0 & 0 & 0 & 0 & 0 & 0\end{array}$

$\begin{array}{lllllllll}0 & 0 & 0 & 0 & 0 & 0 & 0 & 0 & 0 \\ 0 & 0 & 0 & 0 & 0 & 0 & 0 & 0 & 0\end{array}$

$\begin{array}{llllllllll}0 & 0 & 0 & 0 & 0 & 0 & 0 & 0 & 0 \\ 0 & 0 & 0 & 0 & 0 & 0 & 0 & 0 & 0\end{array}$

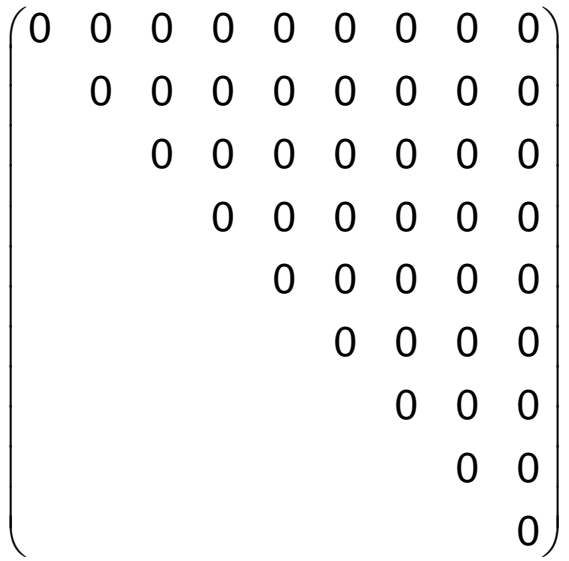

$\left(\begin{array}{lllllllll}0 & 0 & 0 & 0 & 0 & 0 & 0 & 0 & 0 \\ 0 & 0 & 0 & 0 & 0 & 0 & 0 & 0 & 0\end{array}\right)$

$\begin{array}{lllllllll}0 & 0 & 0 & 0 & 0 & 0 & 0 & 0 & 0\end{array}$

$\begin{array}{lllllllll}0 & 0 & 0 & 0 & 0 & 0 & 0 & 0 & 0\end{array}$

$\begin{array}{lllllllll}0 & 0 & 0 & 0 & 0 & 0 & 0 & 0 & 0\end{array}$

$\begin{array}{lllllllll}0 & 0 & 0 & 0 & 0 & 0 & 0 & 0 & 0 \\ 0 & 0 & 0 & 0 & 0 & 0 & 0 & 0 & 0\end{array}$

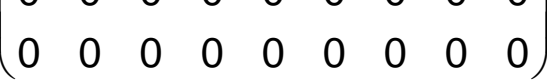

$\left(\begin{array}{lllllllll}0 & 0 & 0 & 0 & 0 & 0 & 0 & 0 & 0 \\ & 0 & 0 & 0 & 0 & 0 & 0 & 0 & 0 \\ & & 0 & 0 & 0 & 0 & 0 & 0 & 0\end{array}\right)$

$\begin{array}{lllllll}0 & 0 & 0 & 0 & 0 & 0 & 0\end{array}$

$\begin{array}{llllll}0 & 0 & 0 & 0 & 0 & 0\end{array}$

$\begin{array}{lllll}0 & 0 & 0 & 0 & 0\end{array}$

$\begin{array}{llll}0 & 0 & 0 & 0\end{array}$

$\begin{array}{lll}0 & 0 & 0 \\ & 0 & 0 \\ & & 0\end{array}$

$\left.\begin{array}{ll}0 & 0 \\ & 0\end{array}\right)$ $\begin{array}{lllllllll}0 & 0 & 0 & 0 & 0 & 0 & 0 & 0 & 0 \\ 0 & 0 & 0 & 0 & 0 & 0 & 0 & 0 & 0\end{array}$

$\left(\begin{array}{lllllllll}0 & 0 & 0 & 0 & 0 & 0 & 0 & 0 & 0 \\ 0 & 0 & 0 & 0 & 0 & 0 & 0 & 0 & 0 \\ 0 & 0 & 0 & 0 & 0 & 0 & 0 & 0 & 0\end{array}\right)$

$\begin{array}{llllllllll}0 & 0 & 0 & 0 & 0 & 0 & 0 & 0 & 0\end{array}$

$\begin{array}{lllllllll}0 & 0 & 0 & 0 & 0 & 0 & 0 & 0 & 0 \\ 0 & 0 & 0 & 0 & 0 & 0 & 0 & 0 & 0\end{array}$ 
1

$\left(\begin{array}{lllllllll}x x x x & x x y y & x x z z & 0 & 0 & 0 & 0 & 0 & 0 \\ y y x x & y y y & y y z z & 0 & 0 & 0 & 0 & 0 & 0\end{array}\right)$ $\left(\begin{array}{lllllllll}\text { yyxx } & \text { yyyy } & \text { yyzz } & 0 & 0 & 0 & 0 & 0 & 0 \\ z z x x & z z y y & z z z z & 0 & 0 & 0 & 0 & 0 & 0 \\ 0 & 0 & 0 & y z y z & 0 & 0 & y z z y & 0 & 0\end{array}\right)$ $\begin{array}{lllllllll}0 & 0 & 0 & y z y z & 0 & 0 & y z z y & 0 & 0 \\ 0 & 0 & 0 & 0 & z x z x & 0 & 0 & z x z & 0\end{array}$

$\begin{array}{lllllllll}0 & 0 & 0 & 0 & 0 & x y x y & 0 & 0 & x y y x\end{array}$

$\begin{array}{lllllllll}0 & 0 & 0 & z y y z & 0 & 0 & \text { zyzy } & 0 & 0 \\ 0 & 0 & 0 & 0 & x z z & 0 & 0 & x z x & 0\end{array}$

$\begin{array}{ccccccccc}0 & 0 & 0 & 0 & x z z x & 0 & 0 & x z x z & 0 \\ 0 & 0 & 0 & 0 & 0 & y x x y & 0 & 0 & y x y x\end{array}$

$\left[\mathrm{V}^{4}\right]\left(\begin{array}{ccccc}\mathrm{xxxx} & 0 & 0 & \mathrm{yyzz} & 0 \\ 0 & \mathrm{yyyy} & 0 & \mathrm{zzxx} & 0 \\ 0 & 0 & \mathrm{zzzz} & \mathrm{xxyy} & 0\end{array}\right)$

$\mathrm{V}\left[\mathrm{V}^{3}\right]\left(\begin{array}{cccccccccc}\mathrm{xxxx} & 0 & 0 & \mathrm{xxyy} & 0 & 0 & \mathrm{xxzz} & 0 & 0 & 0 \\ 0 & \mathrm{yyyy} & 0 & 0 & \mathrm{yyzz} & 0 & 0 & \mathrm{yyxx} & 0 & 0 \\ 0 & 0 & \mathrm{zzz} & 0 & 0 & \mathrm{zzx} & 0 & 0 & \mathrm{zzy} & 0\end{array}\right)$

$\left[\left[^{2}\right]^{2}\right]\left(\begin{array}{cccccc}\operatorname{xxxx} & \text { xxyy } & \text { xxzz } & 0 & 0 & 0 \\ & \text { yyyy } & \text { yyzz } & 0 & 0 & 0 \\ & & z z z z & 0 & 0 & 0 \\ & & & y z y z & 0 & 0 \\ & & & & \text { xzxz } & 0 \\ & & & & & \text { xyxy }\end{array}\right)$

$\left[V^{2}\right]^{2}\left(\begin{array}{cccccc}\operatorname{xxxx} & \mathrm{xxyy} & \mathrm{xxzz} & 0 & 0 & 0 \\ \mathrm{yyxx} & \mathrm{yyyy} & \mathrm{yyzz} & 0 & 0 & 0 \\ \mathrm{zzxx} & \mathrm{zzyy} & \mathrm{zzzz} & 0 & 0 & 0 \\ 0 & 0 & 0 & \mathrm{yzyz} & 0 & 0 \\ 0 & 0 & 0 & 0 & \mathrm{xzxz} & 0 \\ 0 & 0 & 0 & 0 & 0 & \mathrm{xyxy}\end{array}\right)$ $\left(\begin{array}{lllll}0 & 0 & 0 & 0 & 0 \\ 0 & 0 & 0 & 0 & 0 \\ 0 & 0 & 0 & 0 & 0\end{array}\right)$

$\begin{array}{llllllllll}0 & 0 & 0 & 0 & 0 & 0 & 0 & 0 & 0 & 0 \\ 0 & 0 & 0 & 0 & 0 & 0 & 0 & 0 & 0 & 0\end{array}$

$\left(\begin{array}{llllllllll}0 & 0 & 0 & 0 & 0 & 0 & 0 & 0 & 0 & 0 \\ 0 & 0 & 0 & 0 & 0 & 0 & 0 & 0 & 0 & 0\end{array}\right)$

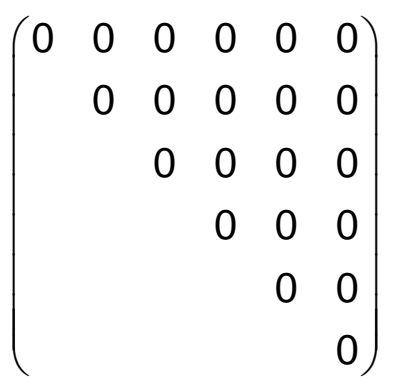

$\left(\begin{array}{lllllllll}0 & 0 & 0 & 0 & 0 & 0 & 0 & 0 & 0 \\ 0 & 0 & 0 & 0 & 0 & 0 & 0 & 0 & 0\end{array}\right)$ $0 \begin{array}{lllllllll}0 & 0 & 0 & 0 & 0 & 0 & 0 & 0 & 0 \\ 0 & 0 & 0 & 0 & 0 & 0 & 0 & 0 & 0\end{array}$ $\begin{array}{lllllllll}0 & 0 & 0 & 0 & 0 & 0 & 0 & 0 & 0 \\ 0 & 0 & 0 & 0 & 0 & 0 & 0 & 0\end{array}$ $\begin{array}{llllllllll}0 & 0 & 0 & 0 & 0 & 0 & 0 & 0 & 0 \\ 0 & 0 & 0 & 0 & 0 & 0 & 0 & 0 & 0\end{array}$ $\begin{array}{llllllllllll}0 & 0 & 0 & 0 & 0 & 0 & 0 & 0 \\ 0 & 0 & 0 & 0 & 0 & 0 & 0 & 0 & 0 \\ 0 & 0 & 0 & 0\end{array}$ $\begin{array}{llllllllll}0 & 0 & 0 & 0 & 0 & 0 & 0 & 0 & 0 \\ 0 & 0 & 0 & 0\end{array}$ $\begin{array}{llllllllll}0 & 0 & 0 & 0 & 0 & 0 & 0 & 0 & 0 \\ 0 & 0 & 0 & 0 & 0 & 0 & 0 & 0\end{array}$ $\left.\begin{array}{lllllllll}0 & 0 & 0 & 0 & 0 & 0 & 0 & 0 & 0 \\ 0 & 0 & 0 & 0 & 0 & 0 & 0 & 0 & 0\end{array}\right)$

$\left(\begin{array}{llllll}0 & 0 & 0 & 0 & 0 & 0 \\ 0 & 0 & 0 & 0 & 0 & 0 \\ 0 & 0 & 0 & 0 & 0 & 0 \\ 0 & 0 & 0 & 0 & 0 & 0 \\ 0 & 0 & 0 & 0 & 0 & 0 \\ 0 & 0 & 0 & 0 & 0 & 0\end{array}\right)$

$\begin{array}{lllllllll}0 & 0 & 0 & 0 & 0 & 0 & 0 & 0 & 0 \\ 0 & 0 & 0 & 0 & 0 & 0 & 0 & 0 & 0\end{array}$ $0 \begin{array}{lllllllll}0 & 0 & 0 & 0 & 0 & 0 & 0 & 0 & 0 \\ 0 & 0 & 0 & 0 & 0 & 0 & 0 & 0 & 0\end{array}$ $0 \begin{array}{lllllllll}0 & 0 & 0 & 0 & 0 & 0 & 0 & 0 & 0 \\ 0 & 0 & 0 & 0 & 0 & 0 & 0 & 0 & 0\end{array}$ $\begin{array}{lllllllllll}0 & 0 & 0 & 0 & 0 & 0 & 0 & 0 & 0 \\ 0 & 0 & 0 & 0 & 0 & 0 & 0 & 0 & 0\end{array}$ $\begin{array}{lllllllll}0 & 0 & 0 & 0 & 0 & 0 & 0 & 0 & 0 \\ 0 & 0 & 0 & 0 & 0 & 0 & 0 & 0 & 0\end{array}$ $\begin{array}{llllllllll}0 & 0 & 0 & 0 & 0 & 0 & 0 & 0 & 0 \\ 0 & 0 & 0 & 0 & 0 & 0 & 0 & 0\end{array}$ $\begin{array}{llllllllll}0 & 0 & 0 & 0 & 0 & 0 & 0 & 0 & 0 \\ 0 & 0 & 0 & 0 & 0 & 0 & 0 & 0\end{array}$ $0 \begin{array}{llllllllll}0 & 0 & 0 & 0 & 0 & 0 & 0 & 0 & 0 \\ 0 & 0 & 0 & 0 & 0 & 0 & 0 & 0\end{array}$ $\begin{array}{llllllllll}0 & 0 & 0 & 0 & 0 & 0 & 0 & 0 & 0\end{array}$

$\left(\begin{array}{lllll}0 & 0 & 0 & 0 & 0 \\ 0 & 0 & 0 & 0 & 0 \\ 0 & 0 & 0 & 0 & 0\end{array}\right)$

$\begin{array}{llllllllll}0 & 0 & 0 & 0 & 0 & 0 & 0 & 0 & 0 & 0 \\ 0 & 0 & 0 & 0 & 0 & 0 & 0 & 0 & 0 & 0\end{array}$ $\left(\begin{array}{llllllllll}0 & 0 & 0 & 0 & 0 & 0 & 0 & 0 & 0 & 0 \\ 0 & 0 & 0 & 0 & 0 & 0 & 0 & 0 & 0 & 0\end{array}\right)$

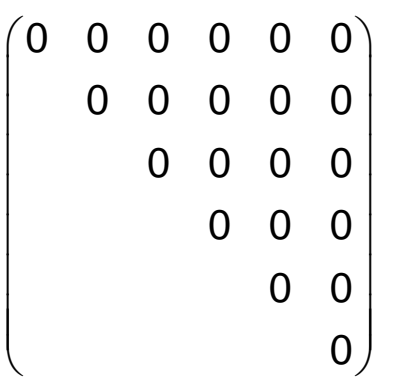

$\begin{array}{llllll}0 & 0 & 0 & 0 & 0 & 0 \\ 0 & 0 & 0 & 0 & 0 & 0\end{array}$ $\begin{array}{llllll}0 & 0 & 0 & 0 & 0 & 0 \\ 0 & 0 & 0 & 0 & 0 & 0\end{array}$ $\begin{array}{llllll}0 & 0 & 0 & 0 & 0 & 0 \\ 0 & 0 & 0 & 0 & 0 & 0\end{array}$ $\begin{array}{llllll}0 & 0 & 0 & 0 & 0 & 0 \\ 0 & 0 & 0 & 0 & 0 & 0\end{array}$ $\left.\begin{array}{llllll}0 & 0 & 0 & 0 & 0 & 0 \\ 0 & 0 & 0 & 0 & 0 & 0\end{array}\right)$

$\left(\begin{array}{ccccccccc}0 & 0 & 0 & \text { xxyz } & 0 & 0 & \text { xxzy } & 0 & 0 \\ 0 & 0 & 0 & \text { yyyz } & 0 & 0 & \text { yyzy } & 0 & 0 \\ 0 & 0 & 0 & \text { zzyz } & 0 & 0 & \text { zzzy } & 0 & 0 \\ \text { yzxx } & \text { yzyy } & \text { yzzz } & 0 & 0 & 0 & 0 & 0 & 0 \\ 0 & 0 & 0 & 0 & 0 & \text { zxxy } & 0 & 0 & \text { zxyx } \\ 0 & 0 & 0 & 0 & \text { xyzx } & 0 & 0 & \text { xyxz } & 0 \\ \text { zyxx } & \text { zyyy } & \text { zyzz } & 0 & 0 & 0 & 0 & 0 & 0 \\ 0 & 0 & 0 & 0 & 0 & \text { xzxy } & 0 & 0 & \text { xzyx } \\ 0 & 0 & 0 & 0 & \text { yxzx } & 0 & 0 & \text { yxxz } & 0\end{array}\right)$

$\left(\begin{array}{ccccc}0 & 0 & 0 & 0 & x x y z \\ 0 & 0 & y z z z & 0 & 0 \\ 0 & z y y & 0 & 0 & 0\end{array}\right)$

$\begin{array}{lllll}0 & \text { zyyy } & 0 & 0 & 0\end{array}$

$\left(\begin{array}{cccccccccc}0 & 0 & 0 & 0 & 0 & 0 & 0 & 0 & 0 & x x y z \\ 0 & 0 & y z z z & 0 & 0 & y z x x & 0 & 0 & y z y y & 0 \\ 0 & z y y y & 0 & 0 & z y z z & 0 & 0 & z y x x & 0 & 0\end{array}\right)$ $\left(\begin{array}{cccccccccc}0 & 0 & y z z z & 0 & 0 & y z x x & 0 & 0 & y z y y & 0 \\ 0 & z y y y & 0 & 0 & z y z z & 0 & 0 & z y x x & 0 & 0\end{array}\right)$
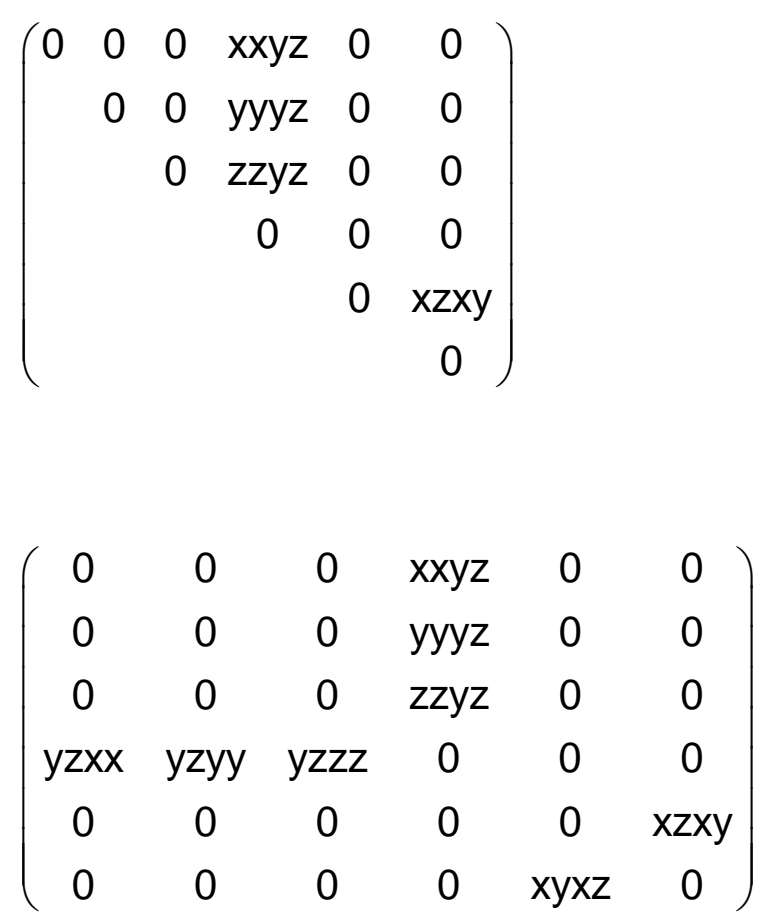


$$
\left[\left(\mathrm{V}^{2}\right)\right]^{2}\left(\begin{array}{ccccccccc}
\text { xxxx } & \text { xxyy } & \text { xxzz } & 0 & 0 & 0 & 0 & 0 & 0 \\
& \text { yyyy } & \text { yyzz } & 0 & 0 & 0 & 0 & 0 & 0 \\
& & \text { zzzz } & 0 & 0 & 0 & 0 & 0 & 0 \\
& & & \text { yzyz } & 0 & 0 & \text { yzzy } & 0 & 0 \\
& & & & \text { zxzx } & 0 & 0 & \text { zxxz } & 0 \\
& & & & & \text { xyxy } & 0 & 0 & \text { xyyx } \\
& & & & & & \text { zyzy } & 0 & 0 \\
& & & & & & & \text { xzxz } & 0 \\
& & & & & & & & \text { yxyx }
\end{array}\right)
$$

$\left(\begin{array}{lllllllll}0 & 0 & 0 & 0 & 0 & 0 & 0 & 0 & 0 \\ 0 & 0 & 0 & 0 & 0 & 0 & 0 & 0\end{array}\right)$

$\begin{array}{llllllll}0 & 0 & 0 & 0 & 0 & 0 & 0 & 0\end{array}$

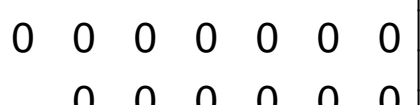
$\begin{array}{lllll}0 & 0 & 0 & 0 & 0\end{array}$ $\begin{array}{llll}0 & 0 & 0 & 0\end{array}$ $\begin{array}{llll}0 & 0 & 0 & 0 \\ 0 & 0 & 0 \\ 0 & 0 & 0\end{array}$ $\begin{array}{lll}0 & 0 & 0 \\ & 0 & 0 \\ & & 0\end{array}$ 0

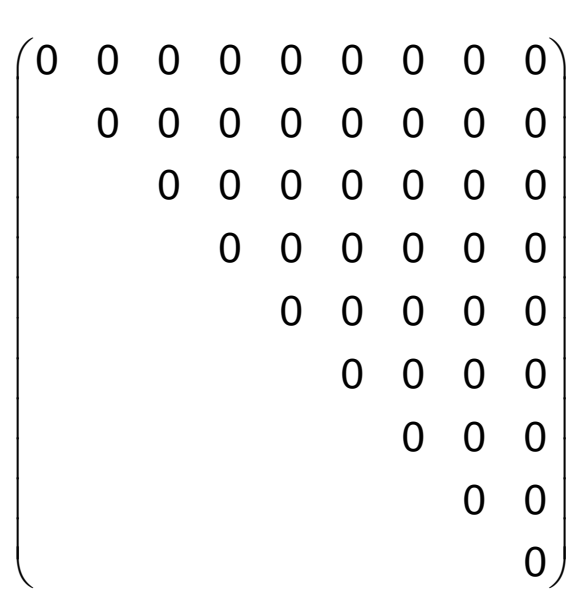

$\left(\begin{array}{lllllllll}0 & 0 & 0 & 0 & 0 & 0 & 0 & 0 & 0 \\ 0 & 0 & 0 & 0 & 0 & 0 & 0 & 0 & 0\end{array}\right.$ $\begin{array}{lllllllll}0 & 0 & 0 & 0 & 0 & 0 & 0 & 0 & 0 \\ 0 & 0 & 0 & 0 & 0 & 0 & 0 & 0 & 0\end{array}$ $0 \begin{array}{lllllllll}0 & 0 & 0 & 0 & 0 & 0 & 0 & 0 & 0 \\ 0 & 0 & 0 & 0 & 0 & 0 & 0 & 0 & 0\end{array}$ $\begin{array}{llllllllll}0 & 0 & 0 & 0 & 0 & 0 & 0 & 0 & 0 \\ 0 & 0 & 0 & 0 & 0 & 0 & 0 & 0 & 0\end{array}$ $\begin{array}{lllllllll}0 & 0 & 0 & 0 & 0 & 0 & 0 & 0 & 0 \\ 0 & 0 & 0 & 0 & 0 & 0 & 0 & 0 & 0\end{array}$

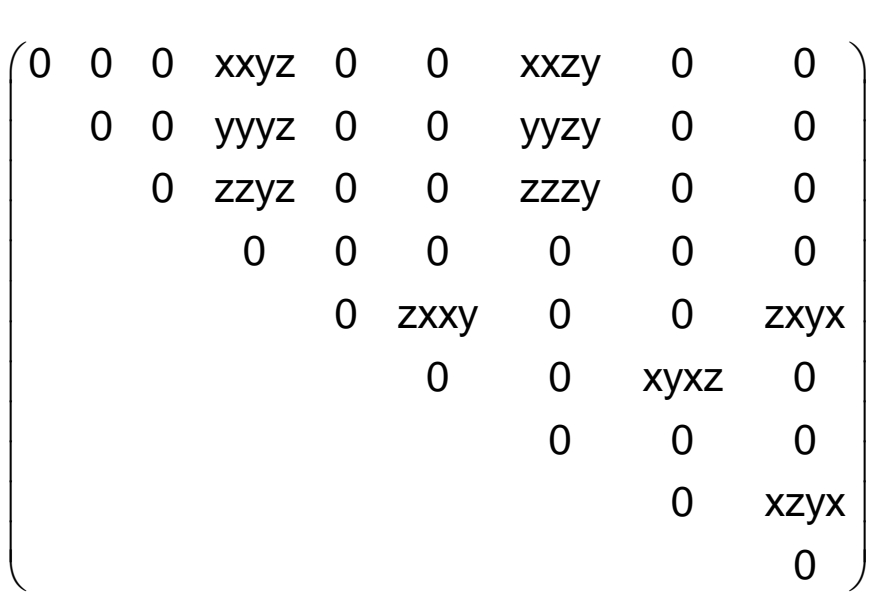

$\left(\begin{array}{lllllllll}0 & 0 & 0 & 0 & 0 & 0 & 0 & 0 & 0 \\ 0 & 0 & 0 & 0 & 0 & 0 & 0 & 0 & 0 \\ 0 & 0 & 0 & 0 & 0 & 0 & 0 & 0 & 0\end{array}\right)$ $\begin{array}{lllllllll}0 & 0 & 0 & 0 & 0 & 0 & 0 & 0 & 0 \\ 0 & 0 & 0 & 0 & 0 & 0 & 0 & 0 & 0\end{array}$ $\begin{array}{lllllllll}0 & 0 & 0 & 0 & 0 & 0 & 0 & 0 & 0 \\ 0 & 0 & 0 & 0 & 0 & 0 & 0 & 0 & 0\end{array}$ $\begin{array}{lllllllll}0 & 0 & 0 & 0 & 0 & 0 & 0 & 0 & 0 \\ 0 & 0 & 0 & 0 & 0 & 0 & 0 & 0 & 0\end{array}$

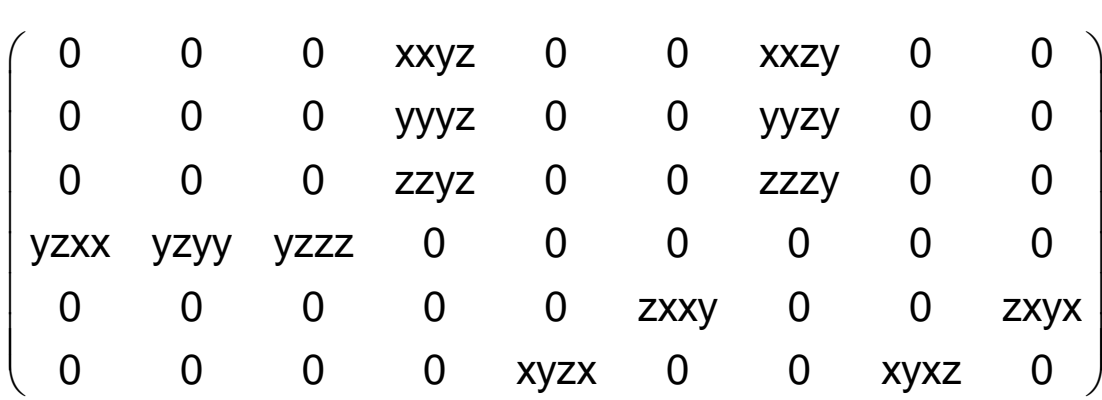


$\mathrm{V}^{4}\left(\begin{array}{ccccccccc}\mathrm{xxxx} & \mathrm{xxyy} & \mathrm{xxzz} & 0 & 0 & 0 & 0 & 0 & 0 \\ \mathrm{yyxx} & \mathrm{xxxx} & \mathrm{xxzz} & 0 & 0 & 0 & 0 & 0 & 0 \\ \mathrm{zzxx} & \mathrm{zzyy} & \mathrm{zzzz} & 0 & 0 & 0 & 0 & 0 & 0 \\ 0 & 0 & 0 & \mathrm{yzyz} & 0 & 0 & \mathrm{yzzy} & 0 & 0 \\ 0 & 0 & 0 & 0 & \mathrm{zxzx} & 0 & 0 & \mathrm{zxxz} & 0 \\ 0 & 0 & 0 & 0 & 0 & \mathrm{xyxy} & 0 & 0 & \mathrm{xyyx} \\ 0 & 0 & 0 & \mathrm{zxxz} & 0 & 0 & \mathrm{zxzx} & 0 & 0 \\ 0 & 0 & 0 & 0 & \mathrm{yzzy} & 0 & 0 & \mathrm{yzyz} & 0 \\ 0 & 0 & 0 & 0 & 0 & \mathrm{xyyx} & 0 & 0 & \mathrm{xyxy}\end{array}\right)$

$\left(\begin{array}{lllllllll}0 & 0 & 0 & 0 & 0 & 0 & 0 & 0 & 0 \\ 0 & 0 & 0 & 0 & 0 & 0 & 0 & 0 & 0\end{array}\right)$ $\left.\begin{array}{lllllllll}0 & 0 & 0 & 0 & 0 & 0 & 0 & 0 & 0 \\ 0 & 0 & 0 & 0 & 0 & 0 & 0 & 0 & 0\end{array}\right)$

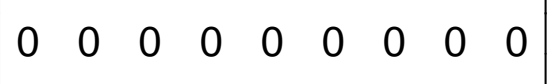
$0 \begin{array}{lllllllll}0 & 0 & 0 & 0 & 0 & 0 & 0 & 0 & 0 \\ 0 & 0 & 0 & 0 & 0 & 0 & 0 & 0 & 0\end{array}$ $0 \begin{array}{lllllllll}0 & 0 & 0 & 0 & 0 & 0 & 0 & 0 & 0 \\ 0 & 0 & 0 & 0 & 0 & 0 & 0 & 0 & 0\end{array}$ $0 \begin{array}{llllllllll}0 & 0 & 0 & 0 & 0 & 0 & 0 & 0 & 0 \\ 0 & 0 & 0 & 0 & 0 & 0 & 0 & 0 & 0\end{array}$ $\begin{array}{llllllllll}0 & 0 & 0 & 0 & 0 & 0 & 0 & 0 & 0\end{array}$ $\left(\begin{array}{lllllllll}0 & 0 & 0 & 0 & 0 & 0 & 0 & 0 & 0 \\ 0 & 0 & 0 & 0 & 0 & 0 & 0 & 0 & 0 \\ 0 & 0 & 0 & 0 & 0 & 0 & 0 & 0 & 0\end{array}\right)$ $\left(\begin{array}{lllllllll}0 & 0 & 0 & 0 & 0 & 0 & 0 & 0 & 0 \\ 0 & 0 & 0 & 0 & 0 & 0 & 0 & 0 & 0\end{array}\right)$

$\left[V^{4}\right] \quad\left(\begin{array}{ccccc}x x x x & 0 & 0 & y y z z & 0 \\ 0 & x x x x & 0 & y y z z & 0 \\ 0 & 0 & z z z z & x x y y & 0\end{array}\right)$

$\left(\begin{array}{lllll}0 & 0 & 0 & 0 & 0 \\ 0 & 0 & 0 & 0 & 0 \\ 0 & 0 & 0 & 0 & 0\end{array}\right)$

$\begin{array}{lllll}0 & 0 & 0 & 0 & 0\end{array}$

$\left.\mathrm{V} \mathrm{V}^{3}\right] \quad\left(\begin{array}{cccccccccc}\mathrm{xxxx} & 0 & 0 & \mathrm{xxyy} & 0 & 0 & \mathrm{yyzz} & 0 & 0 & 0 \\ 0 & \mathrm{xxxx} & 0 & 0 & \mathrm{yyzz} & 0 & 0 & \mathrm{xxyy} & 0 & 0 \\ 0 & 0 & \mathrm{zzzz} & 0 & 0 & \mathrm{zzxx} & 0 & 0 & \mathrm{zzxx} & 0\end{array}\right)$

$\left(\begin{array}{llllllllll}0 & 0 & 0 & 0 & 0 & 0 & 0 & 0 & 0 & 0 \\ 0 & 0 & 0 & 0 & 0 & 0 & 0 & 0 & 0 & 0\end{array}\right.$ $\left(\begin{array}{lllllllllll}0 & 0 & 0 & 0 & 0 & 0 & 0 & 0 & 0 & 0 \\ 0 & 0 & 0 & 0 & 0 & 0 & 0 & 0 & 0 & 0\end{array}\right)$

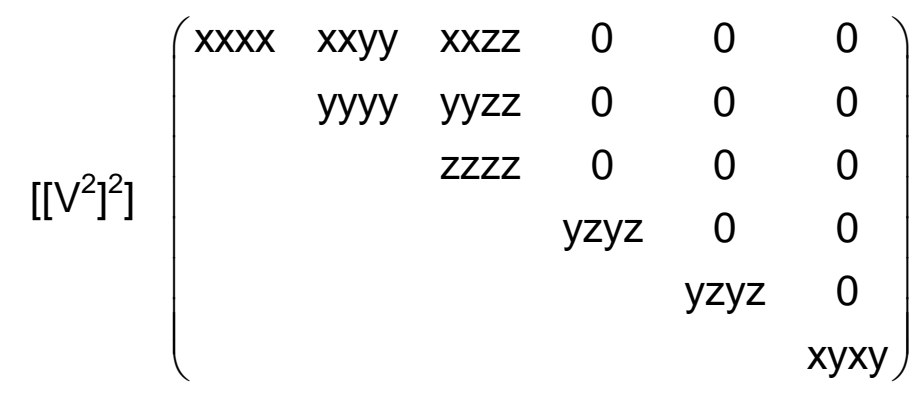

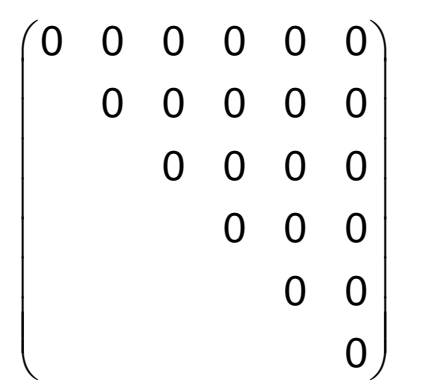

$\left(\begin{array}{llllll}0 & 0 & 0 & 0 & 0 & 0 \\ 0 & 0 & 0 & 0 & 0 & 0\end{array}\right)$ $\begin{array}{lllllll}0 & 0 & 0 & 0 & 0 & 0 \\ 0 & 0 & 0 & 0 & 0 & 0 \\ 0 & 0 & 0 & 0 & 0 & 0\end{array}$ $\begin{array}{lllllll}0 & 0 & 0 & 0 & 0 & 0 \\ 0 & 0 & 0 & 0 & 0\end{array}$ $\begin{array}{llllll}0 & 0 & 0 & 0 & 0 & 0 \\ 0 & 0 & 0 & 0 & 0\end{array}$ $\begin{array}{llllll}0 & 0 & 0 & 0 & 0 & 0 \\ 0 & 0 & 0 & 0 & 0 & 0\end{array}$

$\left(\begin{array}{cccccc}x x x x & x x y y & x x z z & 0 & 0 & 0 \\ y y x x & x x x x & x x z z & 0 & 0 & 0 \\ z z x x & z z x x & z z z z & 0 & 0 & 0 \\ 0 & 0 & 0 & y z y z & 0 & 0 \\ 0 & 0 & 0 & 0 & y z y z & 0 \\ 0 & 0 & 0 & 0 & 0 & x y x y\end{array}\right)$

$\left(\begin{array}{ccccccccc}x x x x & x x y y & x x z z & 0 & 0 & 0 & 0 & 0 & 0 \\ -x x y y & -x x x x & -x x z z & 0 & 0 & 0 & 0 & 0 & 0 \\ z z x x & -z z x x & 0 & 0 & 0 & 0 & 0 & 0 & 0 \\ 0 & 0 & 0 & y z y z & 0 & 0 & -y z z y & 0 & 0 \\ 0 & 0 & 0 & 0 & z x z x & 0 & 0 & -z x x z & 0 \\ 0 & 0 & 0 & 0 & 0 & x y x y & 0 & 0 & -x y y x \\ 0 & 0 & 0 & z x x z & 0 & 0 & -z x z x & 0 & 0 \\ 0 & 0 & 0 & 0 & y z z y & 0 & 0 & -y z y z & 0 \\ 0 & 0 & 0 & 0 & 0 & x y y x & 0 & 0 & -x y x y\end{array}\right)\left(\begin{array}{lllllllll}0 & 0 & 0 & 0 & 0 & 0 & 0 & 0 & 0 \\ 0 & 0 & 0 & 0 & 0 & 0 & 0 & 0 & 0 \\ 0 & 0 & 0 & 0 & 0 & 0 & 0 & 0 & 0 \\ 0 & 0 & 0 & 0 & 0 & 0 & 0 & 0 & 0 \\ 0 & 0 & 0 & 0 & 0 & 0 & 0 & 0 & 0 \\ 0 & 0 & 0 & 0 & 0 & 0 & 0 & 0 & 0 \\ 0 & 0 & 0 & 0 & 0 & 0 & 0 & 0 & 0 \\ 0 & 0 & 0 & 0 & 0 & 0 & 0 & 0 & 0 \\ 0 & 0 & 0 & 0 & 0 & 0 & 0 & 0 & 0\end{array}\right)$

$$
\left(\begin{array}{ccccc}
x x x x & 0 & 0 & y y z z & 0 \\
0 & -x x x x & 0 & -y y z z & 0 \\
0 & 0 & 0 & 0 & 0
\end{array}\right) \quad\left(\begin{array}{ccccc}
0 & 0 & 0 & 0 & 0 \\
0 & 0 & 0 & 0 & 0 \\
0 & 0 & 0 & 0 & 0
\end{array}\right)
$$

$\left(\begin{array}{cccccccccc}\mathrm{xxxx} & 0 & 0 & \mathrm{xxyy} & 0 & 0 & -\mathrm{yyzz} & 0 & 0 & 0 \\ 0 & -\mathrm{xxxx} & 0 & 0 & \mathrm{yyzz} & 0 & 0 & -\mathrm{xxyy} & 0 & 0 \\ 0 & 0 & 0 & 0 & 0 & \mathrm{zzxx} & 0 & 0 & -z z x x & 0\end{array}\right)\left(\begin{array}{cccccccccc}0 & 0 & 0 & 0 & 0 & 0 & 0 & 0 & 0 & 0 \\ 0 & 0 & 0 & 0 & 0 & 0 & 0 & 0 & 0 & 0 \\ 0 & 0 & 0 & 0 & 0 & 0 & 0 & 0 & 0 & 0\end{array}\right)$

$$
\left(\begin{array}{cccccc}
\mathrm{xxxx} & 0 & \mathrm{xxzz} & 0 & 0 & 0 \\
& -\mathrm{xxxx} & -\mathrm{xxzz} & 0 & 0 & 0 \\
& 0 & 0 & 0 & 0 \\
& & \mathrm{yzyz} & 0 & 0 \\
& & & & -\mathrm{yzyz} & 0 \\
& & & & & 0
\end{array}\right)
$$

$\left(\begin{array}{cccccc}x x x x & x x y y & x x z z & 0 & 0 & 0 \\ -x x y y & -x x x x & -x x z z & 0 & 0 & 0 \\ z z x x & -z z x x & 0 & 0 & 0 & 0 \\ 0 & 0 & 0 & y z y z & 0 & 0 \\ 0 & 0 & 0 & 0 & -y z y z & 0 \\ 0 & 0 & 0 & 0 & 0 & 0\end{array}\right)$

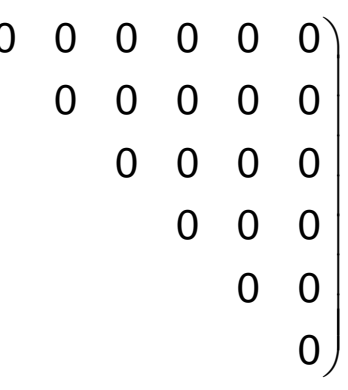

$\left(\begin{array}{llllll}0 & 0 & 0 & 0 & 0 & 0 \\ 0 & 0 & 0 & 0 & 0 & 0\end{array}\right)$

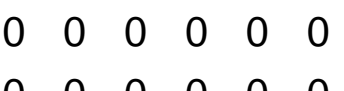
$\begin{array}{llllll}0 & 0 & 0 & 0 & 0 & 0\end{array}$ $\begin{array}{llllll}0 & 0 & 0 & 0 & 0 & 0 \\ 0 & 0 & 0 & 0 & 0\end{array}$ $\begin{array}{llllll}0 & 0 & 0 & 0 & 0 & 0\end{array}$ 


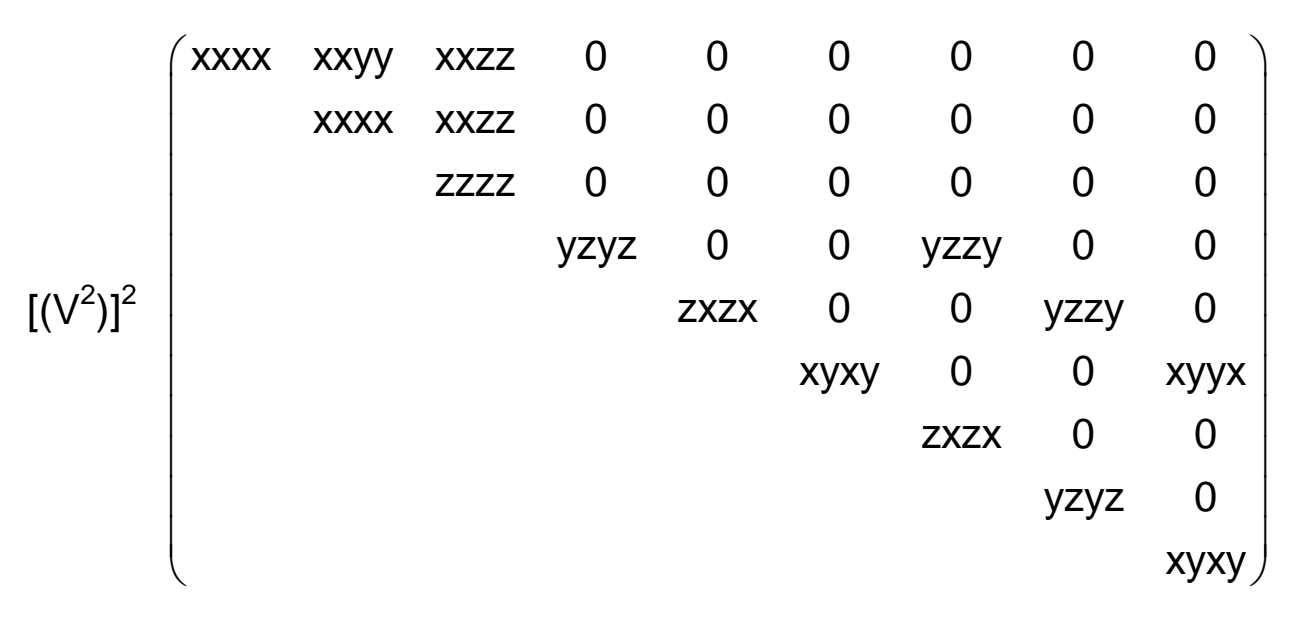

$\left(\begin{array}{lllllllll}0 & 0 & 0 & 0 & 0 & 0 & 0 & 0 & 0\end{array}\right)$

$\begin{array}{llllllll}0 & 0 & 0 & 0 & 0 & 0 & 0 & 0\end{array}$

$\begin{array}{lllllll}0 & 0 & 0 & 0 & 0 & 0 & 0\end{array}$

$0 \begin{array}{lllll}0 & 0 & 0 & 0 & 0\end{array}$

$0 \begin{array}{lllll}0 & 0 & 0 & 0 & 0\end{array}$

$\begin{array}{llll}0 & 0 & 0 & 0\end{array}$

$\left.\begin{array}{lll}0 & 0 & 0 \\ & 0 & 0 \\ & & 0\end{array}\right)$

$\left(\begin{array}{lllllllll}x x x x & x x y y & x x z z & 0 & 0 & 0 & 0 & 0 & 0 \\ \text { yyxx } & x x y x & x x z z & 0 & 0 & 0 & 0 & 0 & 0\end{array}\right)$

$\left(\begin{array}{lllllllll}y y x x & x x x x & x x z z & 0 & 0 & 0 & 0 & 0 & 0 \\ z z x x & z z x x & z z z z & 0 & 0 & 0 & 0 & 0 & 0\end{array}\right)$

$\left[\mathrm{V}^{2}\right] \mathrm{V}^{2} \mid \begin{array}{ccccccccc}\operatorname{zzxx} & \mathrm{zzXx} & \mathrm{zzzz} & 0 & 0 & 0 & 0 & 0 & 0 \\ 0 & 0 & 0 & \mathrm{yzyz} & 0 & 0 & \mathrm{zxzx} & 0 & 0\end{array}$

$\left(\begin{array}{ccccccccc}0 & 0 & 0 & y z y z & 0 & 0 & z x z x & 0 & 0 \\ 0 & 0 & 0 & 0 & z x z x & 0 & 0 & y z y z & 0 \\ 0 & 0 & 0 & 0 & 0 & x y x y & 0 & 0 & x y x y\end{array}\right)$ $\left(\begin{array}{lllllllll}0 & 0 & 0 & 0 & 0 & 0 & 0 & 0 & 0 \\ 0 & 0 & 0 & 0 & 0 & 0 & 0 & 0 & 0\end{array}\right)$

$\begin{array}{lllllllll}0 & 0 & 0 & 0 & 0 & 0 & 0 & 0 & 0 \\ 0 & 0 & 0 & 0 & 0 & 0 & 0 & 0 & 0\end{array}$

$\begin{array}{lllllllll}0 & 0 & 0 & 0 & 0 & 0 & 0 & 0 & 0\end{array}$

$\begin{array}{lllllllll}0 & 0 & 0 & 0 & 0 & 0 & 0 & 0 & 0 \\ 0 & 0 & 0 & 0 & 0 & 0 & 0 & 0 & 0\end{array}$ $\left.\begin{array}{lllllllll}0 & 0 & 0 & 0 & 0 & 0 & 0 & 0 & 0 \\ 0 & 0 & 0 & 0 & 0 & 0 & 0 & 0 & 0 \\ 0 & 0 & 0 & 0 & 0 & 0 & 0 & 0 & 0\end{array}\right)$

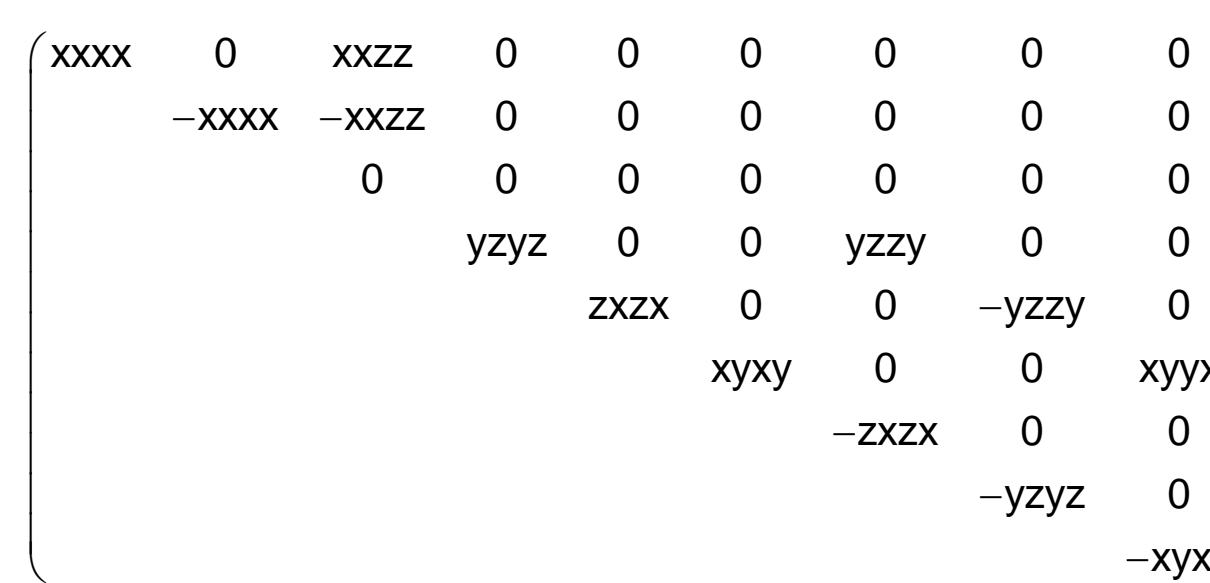

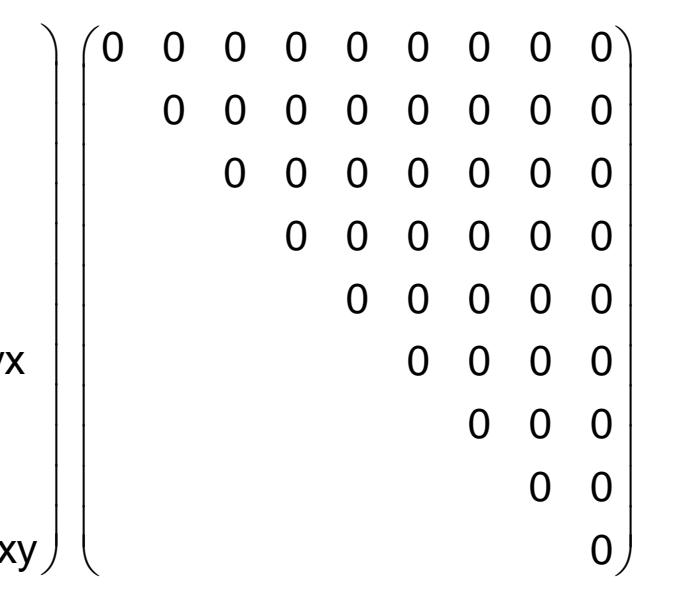

$\left(\begin{array}{ccccccccc}x x x x & x x y y & x x z z & 0 & 0 & 0 & 0 & 0 & 0 \\ -x x y y & -x x x x & -x x z z & 0 & 0 & 0 & 0 & 0 & 0 \\ z z x x & -z z x x & 0 & 0 & 0 & 0 & 0 & 0 & 0 \\ 0 & 0 & 0 & y z y z & 0 & 0 & -z x z x & 0 & 0 \\ 0 & 0 & 0 & 0 & z x z x & 0 & 0 & -y z y z & 0 \\ 0 & 0 & 0 & 0 & 0 & x y x y & 0 & 0 & -x y x y\end{array}\right)\left(\begin{array}{ccccccccc}0 & 0 & 0 & 0 & 0 & 0 & 0 & 0 & 0 \\ 0 & 0 & 0 & 0 & 0 & 0 & 0 & 0 & 0 \\ 0 & 0 & 0 & 0 & 0 & 0 & 0 & 0 & 0 \\ 0 & 0 & 0 & 0 & 0 & 0 & 0 & 0 & 0 \\ 0 & 0 & 0 & 0 & 0 & 0 & 0 & 0 & 0 \\ 0 & 0 & 0 & 0 & 0 & 0 & 0 & 0 & 0\end{array}\right)$ 


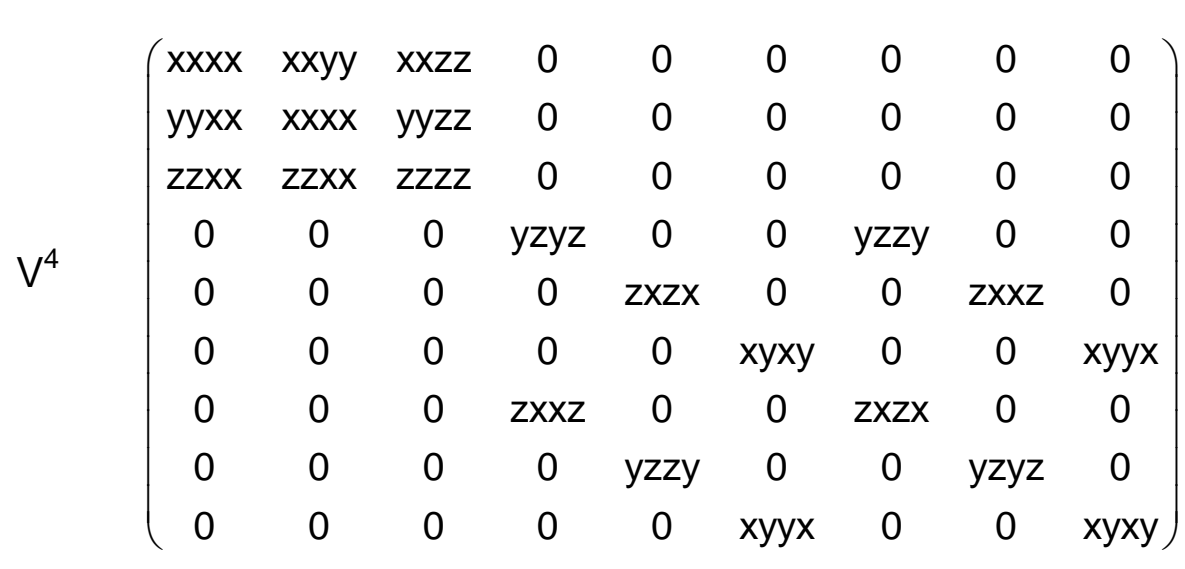

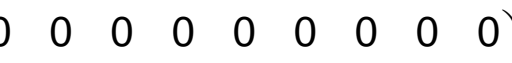
$0 \begin{array}{lllllllll}0 & 0 & 0 & 0 & 0 & 0 & 0 & 0 & 0 \\ 0 & 0 & 0 & 0 & 0 & 0 & 0 & 0 & 0\end{array}$ $0 \begin{array}{llllllllll}0 & 0 & 0 & 0 & 0 & 0 & 0 & 0 & 0 \\ 0 & 0 & 0 & 0 & 0 & 0 & 0 & 0 & 0\end{array}$ $0 \begin{array}{llllllllll}0 & 0 & 0 & 0 & 0 & 0 & 0 & 0 & 0 \\ 0 & 0 & 0 & 0 & 0 & 0 & 0 & 0 & 0\end{array}$ $0 \begin{array}{llllllllll}0 & 0 & 0 & 0 & 0 & 0 & 0 & 0 & 0 \\ 0 & 0 & 0 & 0 & 0 & 0 & 0 & 0 & 0\end{array}$ $\begin{array}{llllllllll}0 & 0 & 0 & 0 & 0 & 0 & 0 & 0 & 0 & 0\end{array}$ $\begin{array}{llllllllll}0 & 0 & 0 & 0 & 0 & 0 & 0 & 0 & 0 & 0\end{array}$ $\begin{array}{lllllllll}0 & 0 & 0 & 0 & 0 & 0 & 0 & 0 & 0 \\ 0 & 0 & 0 & 0 & 0 & 0 & 0 & 0 & 0 \\ 0 & 0 & 0 & 0 & 0 & 0 & 0 & 0 & 0\end{array}$ $\left(\begin{array}{lllllllll}0 & 0 & 0 & 0 & 0 & 0 & 0 & 0 & 0 \\ 0 & 0 & 0 & 0 & 0 & 0 & 0 & 0 & 0\end{array}\right)$ and: $x x x x=x x y y+x y x y+x y y x$

$\left[V^{4}\right]\left(\begin{array}{ccccc}3 x x y y & 0 & 0 & y y z z & 0 \\ 0 & 3 x x y y & 0 & y y z z & 0 \\ 0 & 0 & z z z z & x x y y & 0\end{array}\right)$

$\left(\begin{array}{lllll}0 & 0 & 0 & 0 & 0 \\ 0 & 0 & 0 & 0 & 0 \\ 0 & 0 & 0 & 0 & 0\end{array}\right)$

$\left(\begin{array}{lllll}0 & 0 & 0 & 0 & 0 \\ 0 & 0 & 0 & 0 & 0\end{array}\right)$

$\left.\mathrm{V} \mathrm{V}^{3}\right]\left(\begin{array}{cccccccccc}3 \mathrm{xxxyy} & 0 & 0 & \mathrm{xxyy} & 0 & 0 & \mathrm{yyzz} & 0 & 0 & \mathrm{yyxx} \\ 0 & 3 \mathrm{xxyy} & 0 & 0 & \mathrm{yyzz} & 0 & 0 & \mathrm{xxyy} & 0 & 0 \\ 0 & 0 & \mathrm{zzzz} & 0 & 0 & \mathrm{zzxx} & 0 & 0 & \mathrm{zzxx} & 0\end{array}\right)$

$\left(\begin{array}{llllllllll}0 & 0 & 0 & 0 & 0 & 0 & 0 & 0 & 0 & 0 \\ 0 & 0 & 0 & 0 & 0 & 0 & 0 & 0 & 0 & 0\end{array}\right)$ $\left.\begin{array}{llllllllll}0 & 0 & 0 & 0 & 0 & 0 & 0 & 0 & 0 & 0 \\ 0 & 0 & 0 & 0 & 0 & 0 & 0 & 0 & 0 & 0\end{array}\right)$

$\left.\left[\mathrm{V}^{2}\right]^{2}\right]\left(\begin{array}{cccccc}\mathrm{xxxx} & \mathrm{xxyy} & \mathrm{xxzz} & 0 & 0 & 0 \\ & \mathrm{xxxx} & \mathrm{xxzz} & 0 & 0 & 0 \\ & & \mathrm{zzzz} & 0 & 0 & 0 \\ & & & \mathrm{yzyz} & 0 & 0 \\ & & & & \mathrm{yzyz} & 0 \\ & & & & & \mathrm{xyxy}\end{array}\right)$

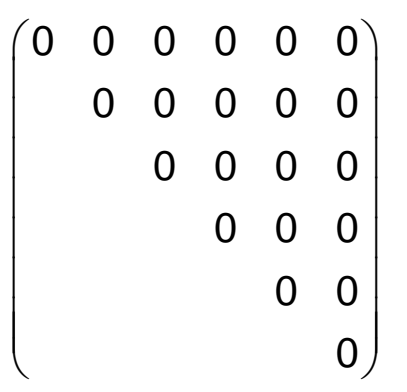

$\left[\mathrm{V}^{2}\right]^{2}\left(\begin{array}{cccccc}\mathrm{xxxx} & \mathrm{xxyy} & \mathrm{xxzz} & 0 & 0 & 0 \\ x \mathrm{xyy} & \mathrm{xxxx} & \mathrm{xxzz} & 0 & 0 & 0 \\ \mathrm{zzxx} & \mathrm{zzxx} & \mathrm{zzzz} & 0 & 0 & 0 \\ 0 & 0 & 0 & \mathrm{yzyz} & 0 & 0 \\ 0 & 0 & 0 & 0 & \mathrm{yzyz} & 0 \\ 0 & 0 & 0 & 0 & 0 & \mathrm{xyxy}\end{array}\right)$

$\left(\begin{array}{llllll}0 & 0 & 0 & 0 & 0 & 0 \\ 0 & 0 & 0 & 0 & 0 & 0 \\ 0 & 0 & 0 & 0 & 0 & 0 \\ 0 & 0 & 0 & 0 & 0 & 0 \\ 0 & 0 & 0 & 0 & 0 & 0 \\ 0 & 0 & 0 & 0 & 0 & 0\end{array}\right)$

and $x y x y=1 / 2(x x x x-x x y y)$ $\left(\begin{array}{lllllllll}0 & 0 & 0 & 0 & 0 & 0 & 0 & 0 & 0 \\ 0 & 0 & 0 & 0 & 0 & 0 & 0 & 0 & 0\end{array}\right.$ $0 \begin{array}{lllllllll}0 & 0 & 0 & 0 & 0 & 0 & 0 & 0 & 0 \\ 0 & 0 & 0 & 0 & 0 & 0 & 0 & 0 & 0\end{array}$ $\begin{array}{llllllllll}0 & 0 & 0 & 0 & 0 & 0 & 0 & 0 & 0 \\ 0 & 0 & 0 & 0 & 0 & 0 & 0 & 0 & 0\end{array}$ $0 \begin{array}{lllllllll}0 & 0 & 0 & 0 & 0 & 0 & 0 & 0 & 0 \\ 0 & 0 & 0 & 0 & 0 & 0 & 0 & 0 & 0\end{array}$ $\begin{array}{llllllllll}0 & 0 & 0 & 0 & 0 & 0 & 0 & 0 & 0 \\ 0 & 0 & 0 & 0 & 0 & 0 & 0 & 0 & 0\end{array}$ $\begin{array}{llllllllll}0 & 0 & 0 & 0 & 0 & 0 & 0 & 0 & 0\end{array}$ $\begin{array}{llllllllllllllll}0 & 0 & 0 & 0 & 0 & 0 & 0 & 0 & 0 & 0\end{array}$ $0 \begin{array}{lllllllll}0 & 0 & 0 & 0 & 0 & 0 & 0 & 0 & 0 \\ 0 & 0 & 0 & 0 & 0 & 0\end{array}$ $\begin{array}{lllllllll}0 & 0 & 0 & 0 & 0 & 0 & 0 & 0 & 0\end{array}$

$$
\left(\begin{array}{lllll}
0 & 0 & 0 & 0 & 0 \\
0 & 0 & 0 & 0 & 0 \\
0 & 0 & 0 & 0 & 0
\end{array}\right)
$$

$\left(\begin{array}{llllllllll}0 & 0 & 0 & 0 & 0 & 0 & 0 & 0 & 0 & 0 \\ 0 & 0 & 0 & 0 & 0 & 0 & 0 & 0 & 0 & 0\end{array}\right)$ $\left.\begin{array}{lllllllllll}0 & 0 & 0 & 0 & 0 & 0 & 0 & 0 & 0 & 0 \\ 0 & 0 & 0 & 0 & 0 & 0 & 0 & 0 & 0 & 0\end{array}\right)$ $\begin{array}{lllllllllll}0 & 0 & 0 & 0 & 0 & 0 & 0 & 0 & 0 & 0\end{array}$

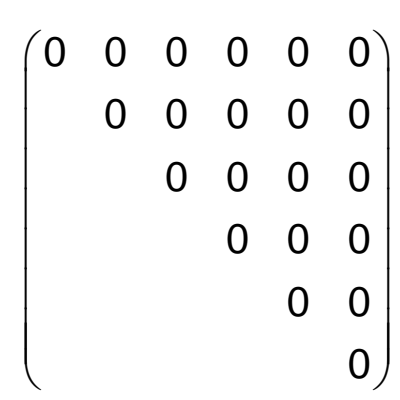

$$
\left(\begin{array}{llllll}
0 & 0 & 0 & 0 & 0 & 0 \\
0 & 0 & 0 & 0 & 0 & 0 \\
0 & 0 & 0 & 0 & 0 & 0 \\
0 & 0 & 0 & 0 & 0 & 0 \\
0 & 0 & 0 & 0 & 0 & 0 \\
0 & 0 & 0 & 0 & 0 & 0
\end{array}\right)
$$

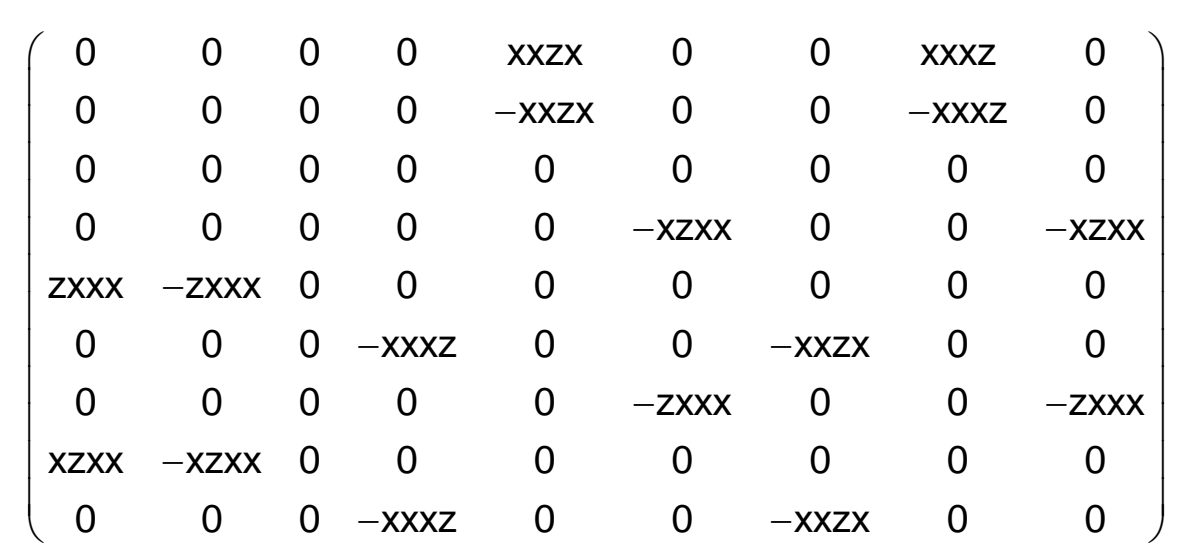

$$
\left(\begin{array}{ccccc}
0 & 0 & 0 & 0 & 0 \\
0 & 0 & 0 & 0 & -z x x x \\
z x x x & 0 & 0 & 0 & 0
\end{array}\right)
$$

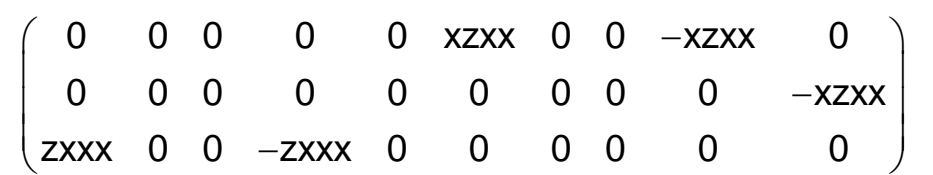

$$
\left(\begin{array}{cccccc}
0 & 0 & 0 & x x y z & 0 & 0 \\
& 0 & 0 & -x x y z & 0 & 0 \\
& & 0 & 0 & 0 & 0 \\
& & & 0 & 0 & 0 \\
& & & & 0 & x x y z \\
& & & & & 0
\end{array}\right)
$$

and $x y x y=1 / 2(x x x x-x x y y)$

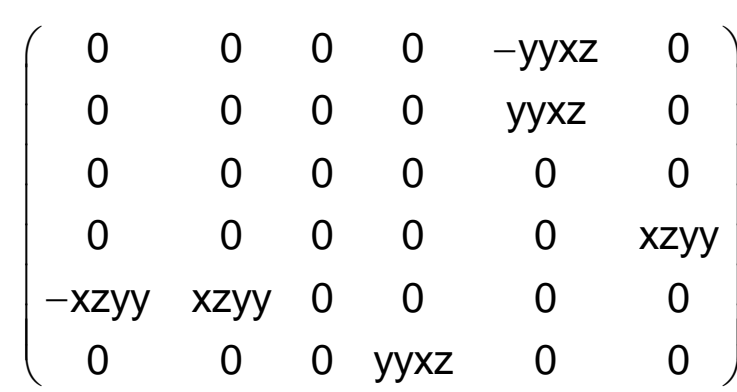




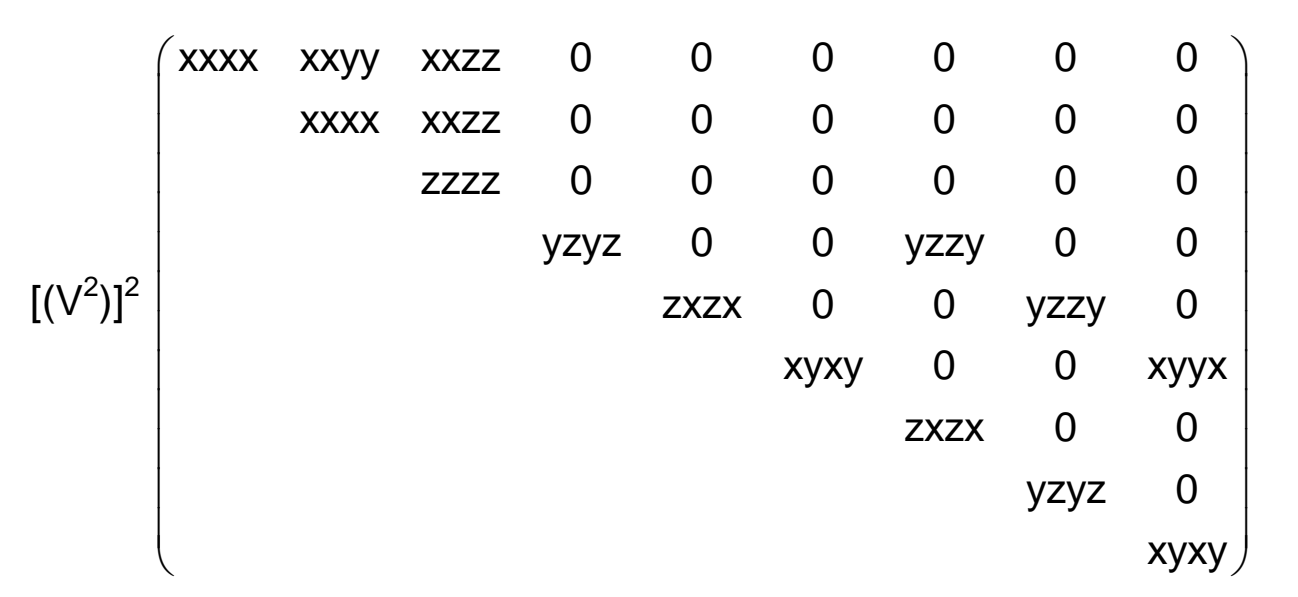

$\left(\begin{array}{lllllllll}0 & 0 & 0 & 0 & 0 & 0 & 0 & 0 & 0 \\ & 0 & 0 & 0 & 0 & 0 & 0 & 0 & 0\end{array}\right)$ $\begin{array}{lllllll}0 & 0 & 0 & 0 & 0 & 0 & 0\end{array}$ $0 \begin{array}{llllll}0 & 0 & 0 & 0 & 0 & 0\end{array}$

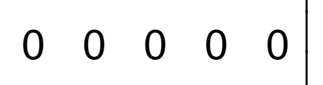
$\begin{array}{llll}0 & 0 & 0 & 0\end{array}$ $\begin{array}{lll}0 & 0 & 0 \\ & 0 & 0\end{array}$ and $x x x x=x x y y+y z y z+z y z y$

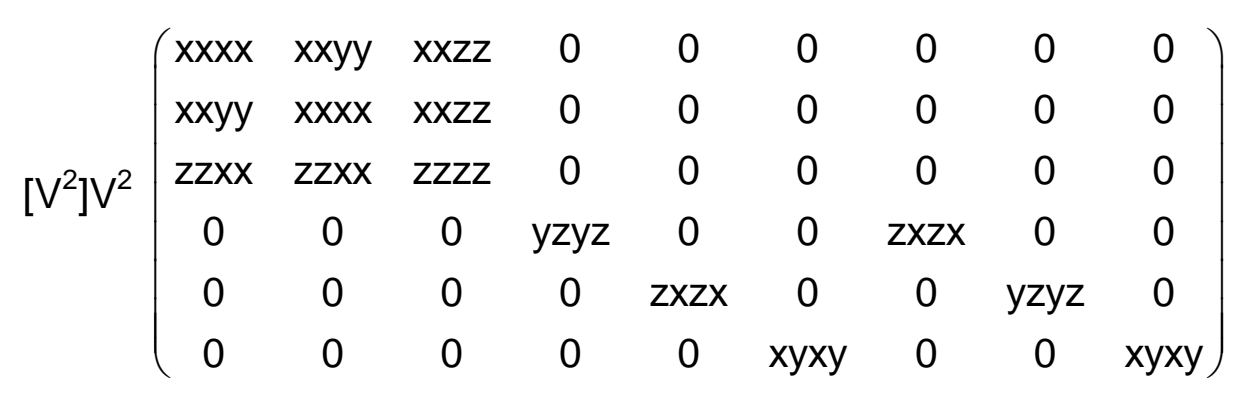

and $x x x x=x x y y+2 x y x y$

$\left(\begin{array}{lllllllll}0 & 0 & 0 & 0 & 0 & 0 & 0 & 0 & 0 \\ 0 & 0 & 0 & 0 & 0 & 0 & 0 & 0 & 0 \\ 0 & 0 & 0 & 0 & 0 & 0 & 0 & 0 & 0\end{array}\right)$ $\begin{array}{lllllllll}0 & 0 & 0 & 0 & 0 & 0 & 0 & 0 & 0\end{array}$ $0 \begin{array}{lllllllll}0 & 0 & 0 & 0 & 0 & 0 & 0 & 0 & 0 \\ 0 & 0 & 0 & 0 & 0 & 0 & 0 & 0 & 0\end{array}$ $\begin{array}{lllllllll}0 & 0 & 0 & 0 & 0 & 0 & 0 & 0 & 0 \\ 0 & 0 & 0 & 0 & 0 & 0 & 0 & 0 & 0\end{array}$
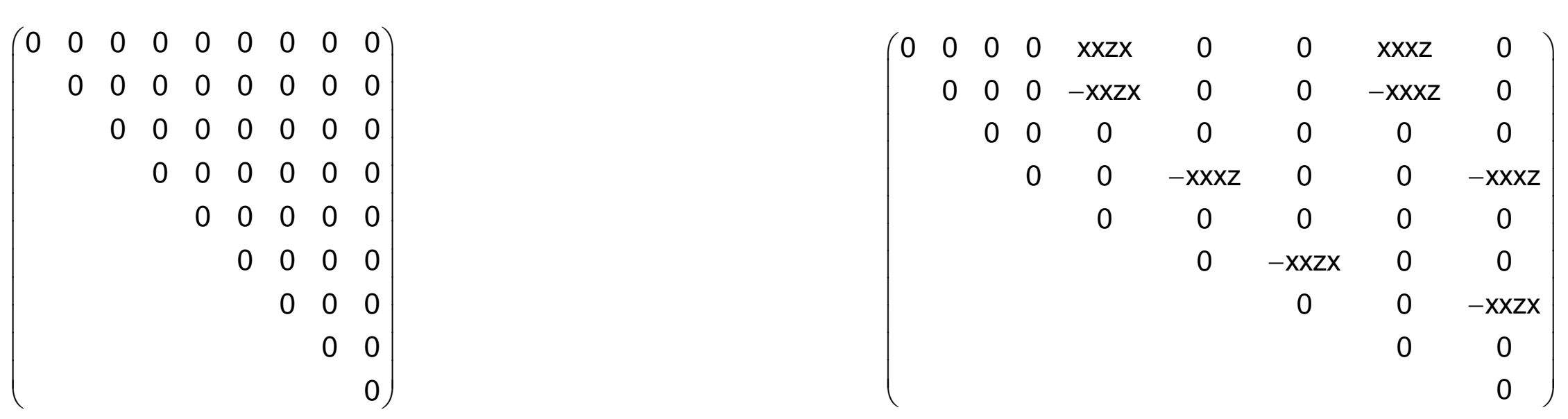

$\begin{array}{lllllllll}0 & 0 & 0 & 0 & 0 & 0 & 0 & 0 & 0 \\ 0 & 0 & 0 & 0 & 0 & 0 & 0 & 0 & 0\end{array}$ $\left.\begin{array}{llllllllll}0 & 0 & 0 & 0 & 0 & 0 & 0 & 0 & 0 \\ 0 & 0 & 0 & 0 & 0 & 0 & 0 & 0 & 0\end{array}\right)$

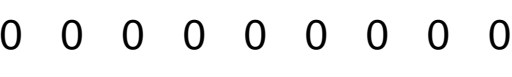
$0 \begin{array}{lllllllll}0 & 0 & 0 & 0 & 0 & 0 & 0 & 0 & 0 \\ 0 & 0 & 0 & 0 & 0 & 0 & 0 & 0 & 0\end{array}$ $\left(\begin{array}{lllllllll}0 & 0 & 0 & 0 & 0 & 0 & 0 & 0 & 0 \\ 0 & 0 & 0 & 0 & 0 & 0 & 0 & 0 & 0\end{array}\right)$ 


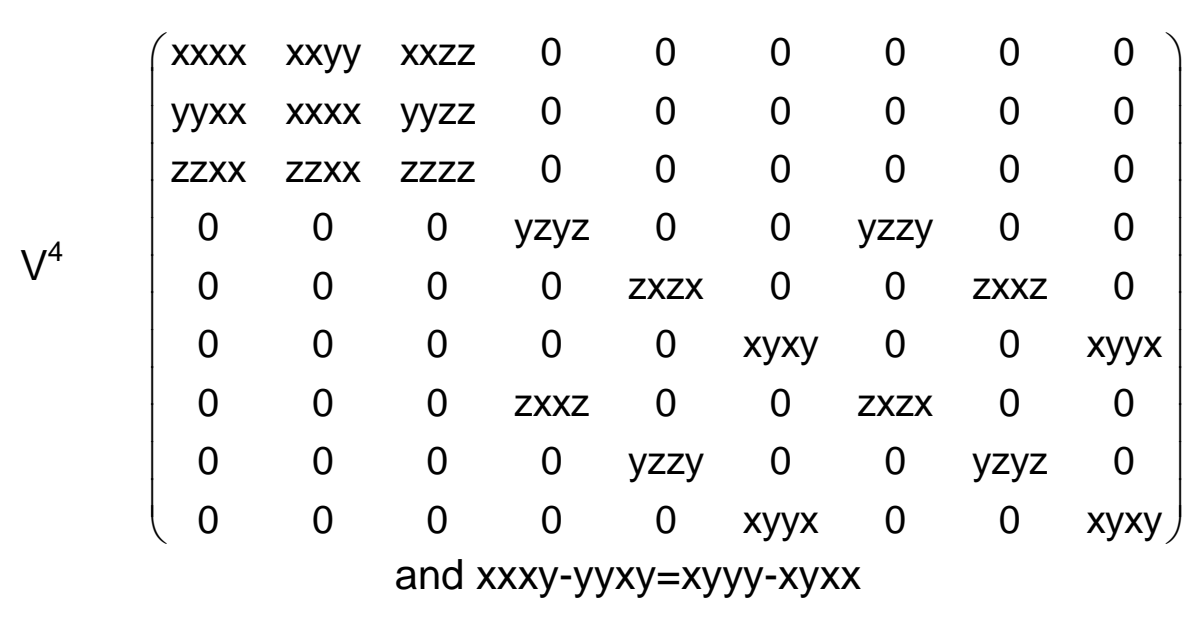

$\left(\begin{array}{lllllllll}0 & 0 & 0 & 0 & 0 & 0 & 0 & 0 & 0 \\ 0 & 0 & 0 & 0 & 0 & 0 & 0 & 0 & 0\end{array}\right)$ $\begin{array}{lllllllll}0 & 0 & 0 & 0 & 0 & 0 & 0 & 0 & 0 \\ 0 & 0 & 0 & 0 & 0 & 0 & 0 & 0 & 0\end{array}$ $0 \begin{array}{lllllllll}0 & 0 & 0 & 0 & 0 & 0 & 0 & 0 & 0 \\ 0 & 0 & 0 & 0 & 0 & 0 & 0 & 0 & 0\end{array}$ $0 \begin{array}{llllllllll}0 & 0 & 0 & 0 & 0 & 0 & 0 & 0 & 0 \\ 0 & 0 & 0 & 0 & 0 & 0 & 0 & 0 & 0\end{array}$ $0 \begin{array}{lllllllll}0 & 0 & 0 & 0 & 0 & 0 & 0 & 0 & 0 \\ 0 & 0 & 0 & 0 & 0 & 0 & 0 & 0 & 0\end{array}$ $0 \begin{array}{llllllllll}0 & 0 & 0 & 0 & 0 & 0 & 0 & 0 & 0 \\ 0 & 0 & 0 & 0 & 0 & 0 & 0 & 0 & 0\end{array}$ $\begin{array}{lllllllllll}0 & 0 & 0 & 0 & 0 & 0 & 0 & 0 & 0 \\ 0 & 0 & 0 & 0 & 0 & 0 & 0 & 0 & 0\end{array}$ $\begin{array}{llllllllll}0 & 0 & 0 & 0 & 0 & 0 & 0 & 0 & 0 \\ 0 & 0 & 0 & 0 & 0 & 0 & 0 & 0\end{array}$ $\left(\begin{array}{lllllllll}0 & 0 & 0 & 0 & 0 & 0 & 0 & 0 & 0 \\ 0 & 0 & 0 & 0 & 0 & 0 & 0 & 0 & 0\end{array}\right)$

$\left[\mathrm{V}^{4}\right]\left(\begin{array}{ccccc}3 x x y y & 0 & 0 & y y z z & 0 \\ 0 & 3 x x y y & 0 & y y z z & 0 \\ 0 & 0 & z z z z & x x y y & 0\end{array}\right)$

$\left(\begin{array}{lllll}0 & 0 & 0 & 0 & 0 \\ 0 & 0 & 0 & 0 & 0 \\ 0 & 0 & 0 & 0 & 0\end{array}\right)$

$\begin{array}{lllll}0 & 0 & 0 & 0 & 0\end{array}$

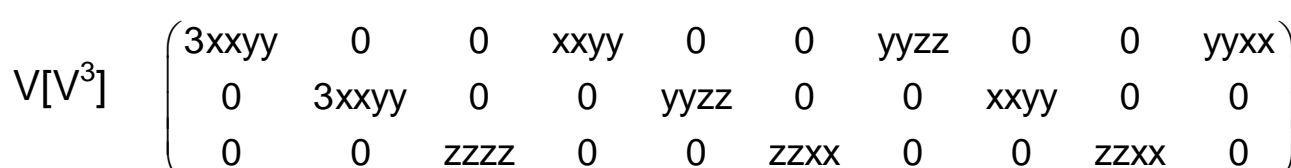

$\begin{array}{llllllllll}0 & 0 & 0 & 0 & 0 & 0 & 0 & 0 & 0 & 0 \\ 0 & 0 & 0 & 0 & 0 & 0 & 0 & 0 & 0 & 0\end{array}$ $\left(\begin{array}{llllllllll}0 & 0 & 0 & 0 & 0 & 0 & 0 & 0 & 0 & 0 \\ 0 & 0 & 0 & 0 & 0 & 0 & 0 & 0 & 0 & 0\end{array}\right)$

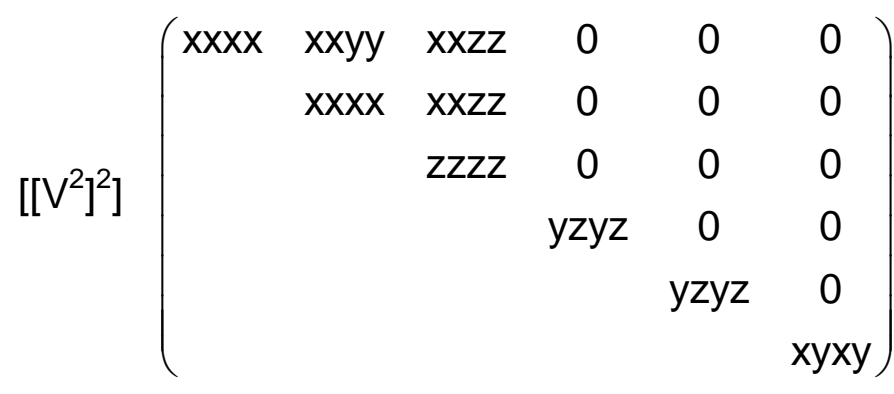

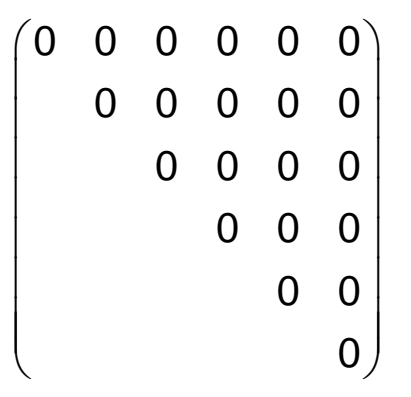

and $x y x y=1 / 2(x x x x-x x y y)$

$\left[V^{2}\right]^{2}\left(\begin{array}{cccccc}x x x x & x x y y & x x z z & 0 & 0 & 0 \\ x x y y & x x x x & x x z z & 0 & 0 & 0 \\ z z x x & z z x x & z z z z & 0 & 0 & 0 \\ 0 & 0 & 0 & y z y z & 0 & 0 \\ 0 & 0 & 0 & 0 & y z y z & 0 \\ 0 & 0 & 0 & 0 & 0 & x y x y\end{array}\right)$

$\left(\begin{array}{llllll}0 & 0 & 0 & 0 & 0 & 0 \\ 0 & 0 & 0 & 0 & 0 & 0\end{array}\right)$

$\left(\begin{array}{llllll}0 & 0 & 0 & 0 & 0 & 0 \\ 0 & 0 & 0 & 0 & 0 & 0\end{array}\right.$

$0 \begin{array}{llllll}0 & 0 & 0 & 0 & 0 & 0 \\ 0 & 0 & 0 & 0 & 0 & 0\end{array}$

$0 \begin{array}{llllll}0 & 0 & 0 & 0 & 0 & 0 \\ 0 & 0 & 0 & 0 & 0 & 0\end{array}$ $\begin{array}{llllll}0 & 0 & 0 & 0 & 0 & 0 \\ 0 & 0 & 0 & 0 & 0 & 0\end{array}$

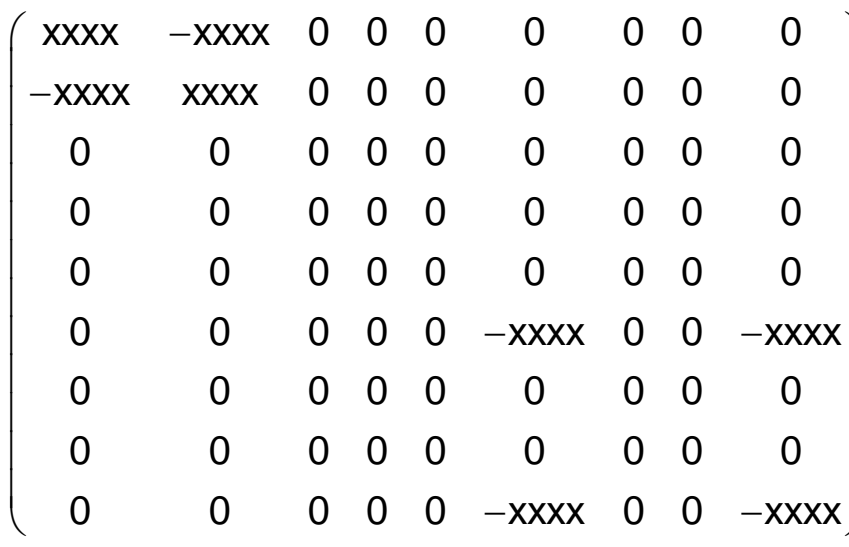

$\left(\begin{array}{ccccc}\mathrm{xxxx} & 0 & 0 & 0 & 0 \\ 0 & \mathrm{xxxx} & 0 & 0 & 0 \\ 0 & 0 & 0 & -\mathrm{xxxx} & 0\end{array}\right)$

$\left(\begin{array}{cccccccccc}x x x x & 0 & 0 & -x x x x & 0 & 0 & 0 & 0 & 0 & 0 \\ 0 & x x x x x & 0 & 0 & 0 & 0 & 0 & -x x y x & 0 & 0 \\ 0 & 0 & 0 & 0 & 0 & 0 & 0 & 0 & 0 & 0\end{array}\right)$

$\left(\begin{array}{cccccccccc}0 & \mathrm{xxxx} & 0 & 0 & 0 & 0 & 0 & -\mathrm{xxxx} & 0 & 0 \\ 0 & 0 & 0 & 0 & 0 & 0 & 0 & 0 & 0 & 0\end{array}\right)$
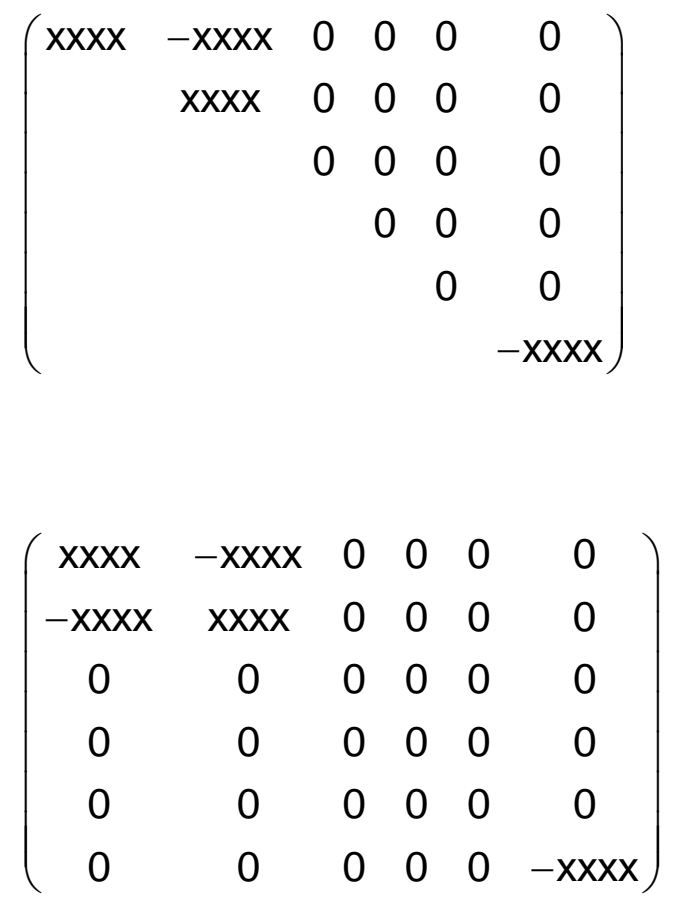

$\left(\begin{array}{lllllllll}0 & 0 & 0 & 0 & 0 & 0 & 0 & 0 & 0 \\ 0 & 0 & 0 & 0 & 0 & 0 & 0 & 0 & 0\end{array}\right)$ $\begin{array}{lllllllll}0 & 0 & 0 & 0 & 0 & 0 & 0 & 0 & 0 \\ 0 & 0 & 0 & 0 & 0 & 0 & 0 & 0 & 0\end{array}$ $\begin{array}{lllllllll}0 & 0 & 0 & 0 & 0 & 0 & 0 & 0 & 0 \\ 0 & 0 & 0 & 0 & 0 & 0 & 0 & 0 & 0 \\ 0 & 0 & 0 & 0 & 0 & 0 & 0 & 0 & 0\end{array}$ $\begin{array}{llllllllll}0 & 0 & 0 & 0 & 0 & 0 & 0 & 0 & 0 \\ 0 & 0 & 0 & 0 & 0 & 0 & 0 & 0 & 0\end{array}$ $\begin{array}{llllllllll}0 & 0 & 0 & 0 & 0 & 0 & 0 & 0 & 0 \\ 0 & 0 & 0 & 0 & 0 & 0 & 0 & 0\end{array}$ $\begin{array}{lllllllllllllllll}0 & 0 & 0 & 0 & 0 & 0 & 0 & 0 & 0 & 0\end{array}$ $\begin{array}{llllllllll}0 & 0 & 0 & 0 & 0 & 0 & 0 & 0 & 0 \\ 0 & 0 & 0 & 0 & 0 & 0 & 0 & 0 & 0\end{array}$ $\begin{array}{lllllllllll}0 & 0 & 0 & 0 & 0 & 0 & 0 & 0 & 0\end{array}$

$\left(\begin{array}{lllll}0 & 0 & 0 & 0 & 0 \\ 0 & 0 & 0 & 0 & 0 \\ 0 & 0 & 0 & 0 & 0\end{array}\right)$ $\left(\begin{array}{lllll}0 & 0 & 0 & 0 & 0 \\ 0 & 0 & 0 & 0 & 0 \\ 0 & 0 & 0 & 0\end{array}\right)$

$\left(\begin{array}{llllllllll}0 & 0 & 0 & 0 & 0 & 0 & 0 & 0 & 0 & 0 \\ 0 & 0 & 0 & 0 & 0 & 0 & 0 & 0 & 0 & 0\end{array}\right)$ $\begin{array}{llllllllll}0 & 0 & 0 & 0 & 0 & 0 & 0 & 0 & 0 & 0 \\ 0 & 0 & 0 & 0 & 0 & 0 & 0 & 0 & 0 & 0\end{array}$

$$
\left(\begin{array}{llllll}
0 & 0 & 0 & 0 & 0 & 0 \\
& 0 & 0 & 0 & 0 & 0 \\
& & 0 & 0 & 0 & 0 \\
& & & 0 & 0 & 0 \\
& & & & 0 & 0 \\
& & & & & \\
& & & & & 0
\end{array}\right)
$$

and $x y x y=1 / 2(x x x x-x x y y)$ 


$$
\text { a }
$$$$
\left(\begin{array}{ccccccccc}
x x x x & -x x x x & 0 & 0 & 0 & 0 & 0 & 0 & 0 \\
& x x x x & 0 & 0 & 0 & 0 & 0 & 0 & 0 \\
& & 0 & 0 & 0 & 0 & 0 & 0 & 0 \\
& & & 0 & 0 & 0 & 0 & 0 & 0 \\
& & & 0 & 0 & 0 & 0 & 0 \\
& & & & -x x x x & 0 & 0 & -x x x x \\
& & & & & 0 & 0 & 0 \\
& & & & & & & 0 & 0 \\
& & & & & & & & -x x x x
\end{array}\right)
$$

and $x x x x=x x y y+y z y z+z y z y$

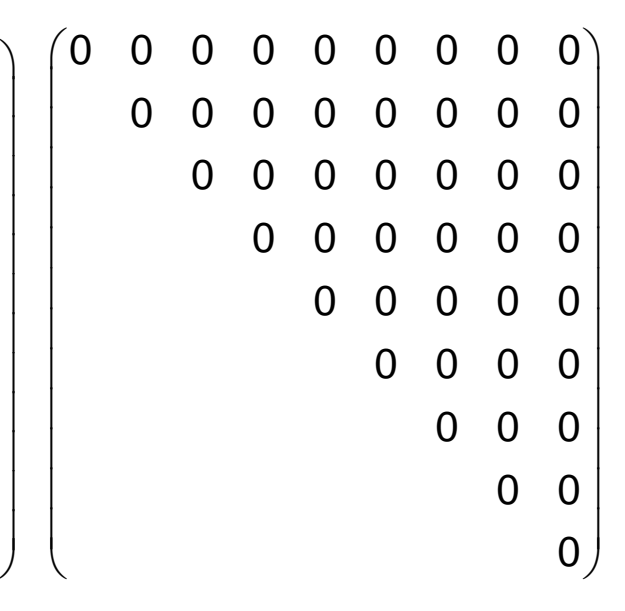

$\left(\begin{array}{lllllllll}x x x x & x x y y & x x z z & 0 & 0 & 0 & 0 & 0 & 0 \\ x y y & x x x & x z z & 0 & 0 & 0 & 0 & 0 & 0\end{array}\right)\left(\begin{array}{ccccccccc}0 & 0 & 0 & 0 & 0 & 0 & 0 & 0 & 0 \\ 0 & 0 & 0 & 0 & 0 & 0 & 0 & 0 & 0\end{array}\right)$

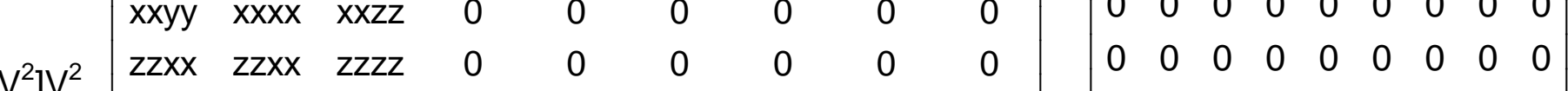
\begin{tabular}{lllllllll||lllllllll}
0 & 0 & 0 & $y z y z$ & 0 & 0 & $z x z x$ & 0 & 0 \\
0 & 0 & 0 & 0 & $z x z$ & 0 & 0 & $y$ & 0 & 0 & 0 & 0 & 0 & 0 & 0 \\
0 & 0 & 0 & 0 & 0 & 0 & 0 & 0 & 0 & 0
\end{tabular} $\left(\begin{array}{ccccccccc}0 & 0 & 0 & 0 & z x z x & 0 & 0 & y z y z & 0 \\ 0 & 0 & 0 & 0 & 0 & x y x y & 0 & 0 & x y x y\end{array}\right)\left(\begin{array}{cccccccccc}0 & 0 & 0 & 0 & 0 & 0 & 0 & 0 & 0 \\ 0 & 0 & 0 & 0 & 0 & 0 & 0 & 0 & 0\end{array}\right)$ and $x x x x=x x y y+2 x y x y$

$\left[\left(V^{2}\right)\right]^{2}$

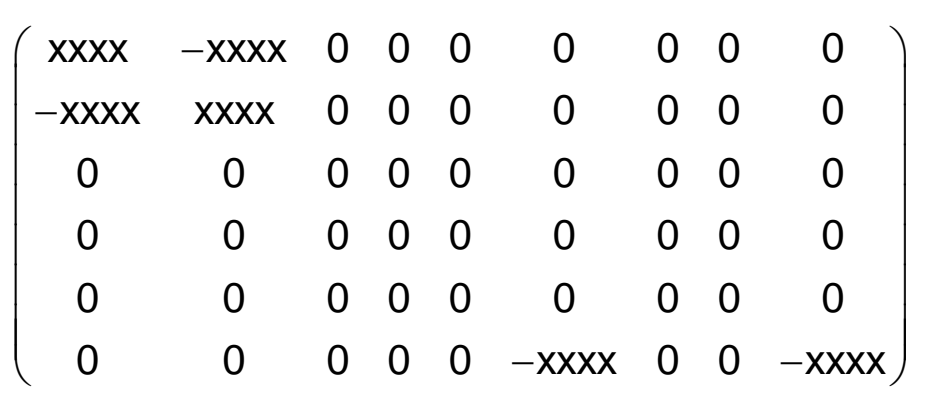

$\left(\begin{array}{lllllllll}0 & 0 & 0 & 0 & 0 & 0 & 0 & 0 & 0 \\ 0 & 0 & 0 & 0 & 0 & 0 & 0 & 0 & 0 \\ 0 & 0 & 0 & 0 & 0 & 0 & 0 & 0 & 0 \\ 0 & 0 & 0 & 0 & 0 & 0 & 0 & 0 & 0 \\ 0 & 0 & 0 & 0 & 0 & 0 & 0 & 0 & 0 \\ 0 & 0 & 0 & 0 & 0 & 0 & 0 & 0 & 0\end{array}\right)$

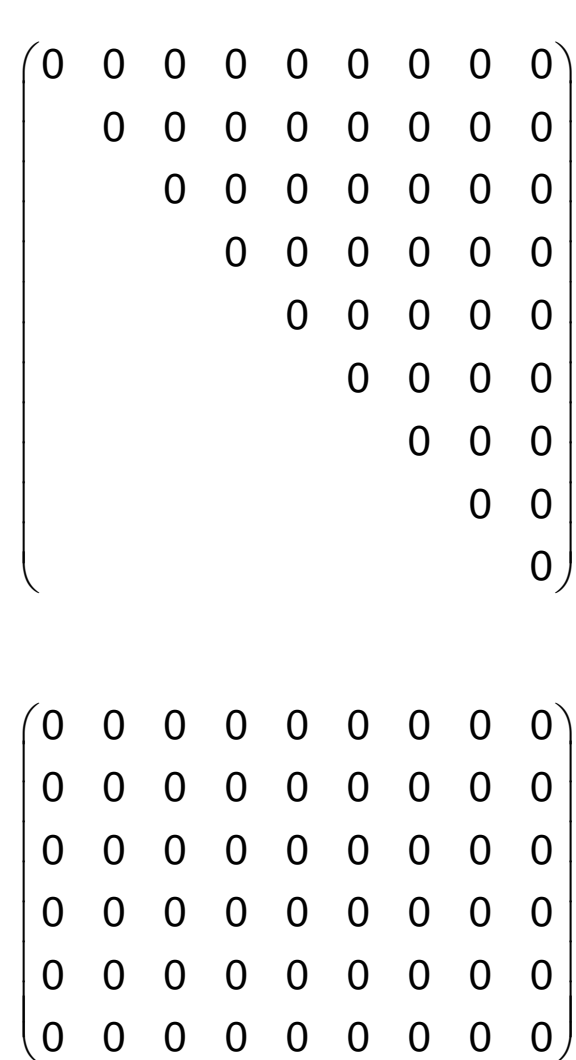




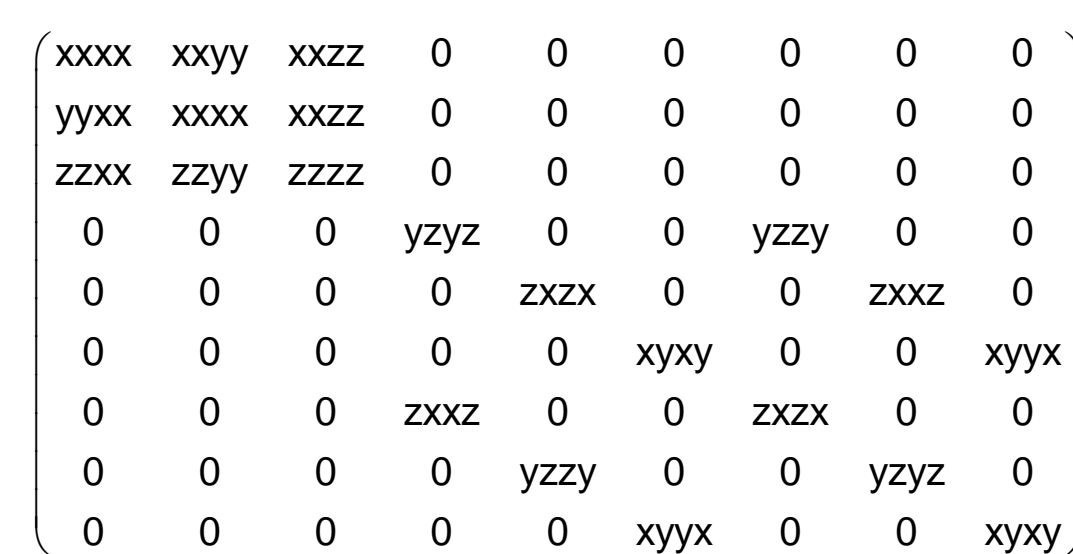

$\begin{array}{lllllllll}0 & 0 & 0 & 0 & 0 & 0 & 0 & 0 & 0 \\ 0 & 0 & 0 & 0 & 0 & 0 & 0 & 0 & 0\end{array}$ $\left(\begin{array}{llllllllllll}0 & 0 & 0 & 0 & 0 & 0 & 0 & 0 & 0\end{array}\right.$ $0 \begin{array}{lllllllll}0 & 0 & 0 & 0 & 0 & 0 & 0 & 0 & 0 \\ 0 & 0 & 0 & 0 & 0 & 0 & 0 & 0 & 0\end{array}$ $\begin{array}{llllllllll}0 & 0 & 0 & 0 & 0 & 0 & 0 & 0 & 0 \\ 0 & 0 & 0 & 0 & 0 & 0 & 0 & 0\end{array}$

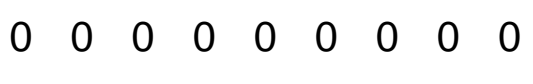

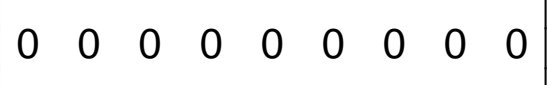
$0 \begin{array}{lllllllll}0 & 0 & 0 & 0 & 0 & 0 & 0 & 0 & 0 \\ 0 & 0 & 0 & 0 & 0 & 0 & 0 & 0 & 0\end{array}$ $0 \begin{array}{llllllllll}0 & 0 & 0 & 0 & 0 & 0 & 0 & 0 & 0 \\ 0 & 0 & 0 & 0 & 0 & 0 & 0 & 0 & 0\end{array}$ $\begin{array}{lllllllll}0 & 0 & 0 & 0 & 0 & 0 & 0 & 0 & 0\end{array}$ $\begin{array}{cccc}0 & 0 & 0 & x y y x \\ \text { and: } x x x x=x x y y+x y x y+x y y x\end{array}$

$\left[V^{4}\right] \quad\left(\begin{array}{ccccc}3 x x y y & 0 & 0 & y y z z & 0 \\ 0 & 3 x x y y & 0 & y y z z & 0 \\ 0 & 0 & z z z z & x x y y & 0\end{array}\right)$

$\left(\begin{array}{lllll}0 & 0 & 0 & 0 & 0 \\ 0 & 0 & 0 & 0 & 0 \\ 0 & 0 & 0 & 0 & 0\end{array}\right)$

$\left(\begin{array}{lllll}0 & 0 & 0 & 0 & 0 \\ 0 & 0 & 0 & 0 & 0 \\ 0 & 0 & 0 & 0 & 0\end{array}\right.$

$\left(\begin{array}{llllllllll}0 & 0 & 0 & 0 & 0 & 0 & 0 & 0 & 0 & 0 \\ 0 & 0 & 0 & 0 & 0 & 0 & 0 & 0 & 0 & 0\end{array}\right)$

$\left(\begin{array}{llllllllll}0 & 0 & 0 & 0 & 0 & 0 & 0 & 0 & 0 & 0 \\ 0 & 0 & 0 & 0 & 0 & 0 & 0 & 0 & 0 & 0\end{array}\right)$

$\left.V_{V} V^{3}\right]\left(\begin{array}{cccccccccc}3 x x y y & 0 & 0 & x x y y & 0 & 0 & y y z z & 0 & 0 & 0 \\ 0 & 3 x x y y & 0 & 0 & y y z & 0 & 0 & \text { xxyy } & 0 & 0\end{array}\right)$

$\begin{array}{lllllllllll}0 & 0 & 0 & 0 & 0 & 0 & 0 & 0 & 0 & 0\end{array}$

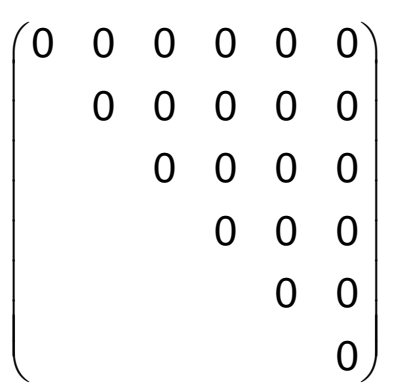

$$
\left(\begin{array}{llllll}
0 & 0 & 0 & 0 & 0 & 0 \\
0 & 0 & 0 & 0 & 0 & 0 \\
0 & 0 & 0 & 0 & 0 & 0 \\
0 & 0 & 0 & 0 & 0 & 0 \\
0 & 0 & 0 & 0 & 0 & 0 \\
0 & 0 & 0 & 0 & 0
\end{array}\right)
$$$$
\left(\begin{array}{llllll}
0 & 0 & 0 & 0 & 0 & 0
\end{array}\right)
$$

\section{and $x y x y=1 / 2(x x x x-x x y y)$}

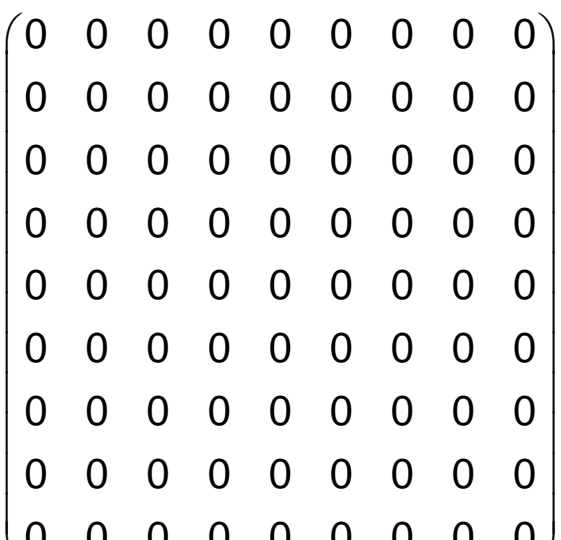

$\left(\begin{array}{lllllllll}0 & 0 & 0 & 0 & 0 & 0 & 0 & 0 & 0 \\ 0 & 0 & 0 & 0 & 0 & 0 & 0 & 0 & 0\end{array}\right)$ $\left.\begin{array}{lllllllll}0 & 0 & 0 & 0 & 0 & 0 & 0 & 0 & 0 \\ 0 & 0 & 0 & 0 & 0 & 0 & 0 & 0 & 0\end{array}\right)$ $0 \begin{array}{lllllllll}0 & 0 & 0 & 0 & 0 & 0 & 0 & 0 & 0 \\ 0 & 0 & 0 & 0 & 0 & 0 & 0 & 0 & 0\end{array}$ $\begin{array}{lllllllll}0 & 0 & 0 & 0 & 0 & 0 & 0 & 0 & 0 \\ 0 & 0 & 0 & 0 & 0 & 0 & 0 & 0 & 0\end{array}$ $\begin{array}{lllllllll}0 & 0 & 0 & 0 & 0 & 0 & 0 & 0 & 0 \\ 0 & 0 & 0 & 0 & 0 & 0 & 0 & 0 & 0\end{array}$ $\begin{array}{lllllllll}0 & 0 & 0 & 0 & 0 & 0 & 0 & 0 & 0 \\ 0 & 0 & 0 & 0 & 0 & 0 & 0 & 0 & 0\end{array}$ $\begin{array}{lllllllll}0 & 0 & 0 & 0 & 0 & 0 & 0 & 0 & 0 \\ 0 & 0 & 0 & 0 & 0 & 0 & 0 & 0 & 0\end{array}$ $\begin{array}{llllllllll}0 & 0 & 0 & 0 & 0 & 0 & 0 & 0 & 0 \\ 0 & 0 & 0 & 0 & 0 & 0 & 0 & 0 & 0\end{array}$

$\left(\begin{array}{lllll}0 & 0 & 0 & 0 & 0 \\ 0 & 0 & 0 & 0 & 0 \\ 0 & 0 & 0 & 0 & 0\end{array}\right)$

$\left.\begin{array}{llllll}0 & 0 & 0 & 0 & 0 \\ 0 & 0 & 0 & 0 & 0\end{array}\right)$

$\left(\begin{array}{lllll}0 & 0 & 0 & 0 & 0 \\ 0 & 0 & 0 & 0 & 0\end{array}\right)$

$\left.\begin{array}{lllll}0 & 0 & 0 & 0 & 0 \\ 0 & 0 & 0 & 0 & 0\end{array}\right)$

$\left(\begin{array}{llllllllll}0 & 0 & 0 & 0 & 0 & 0 & 0 & 0 & 0 & 0\end{array}\right.$ $\begin{array}{lllllllllll}0 & 0 & 0 & 0 & 0 & 0 & 0 & 0 & 0 & 0\end{array}$ $\begin{array}{lllllllllll}0 & 0 & 0 & 0 & 0 & 0 & 0 & 0 & 0 & 0\end{array}$

$\left(\begin{array}{llllllllll}0 & 0 & 0 & 0 & 0 & 0 & 0 & 0 & 0 & 0 \\ 0 & 0 & 0 & 0 & 0 & 0 & 0 & 0 & 0 & 0 \\ 0 & 0 & 0 & 0 & 0 & 0 & 0 & 0 & 0 & 0\end{array}\right)$

$\left(\begin{array}{lllllllllll}0 & 0 & 0 & 0 & 0 & 0 & 0 & 0 & 0 & 0\end{array}\right.$

$$
\left(\begin{array}{llllll}
0 & 0 & 0 & 0 & 0 & 0 \\
& 0 & 0 & 0 & 0 & 0 \\
& & 0 & 0 & 0 & 0 \\
& & & 0 & 0 & 0 \\
& & & & & 0 \\
& & & & & 0
\end{array}\right)
$$$$
\left(\begin{array}{llllll}
0 & 0 & 0 & 0 & 0 & 0 \\
& 0 & 0 & 0 & 0 & 0 \\
& & 0 & 0 & 0 & 0 \\
& & & 0 & 0 & 0 \\
& & & & 0 & 0 \\
& & & & & 0
\end{array}\right)
$$

and $x y x y=1 / 2(x x x x-x x y y)$

$$
\left(\begin{array}{llllll}
0 & 0 & 0 & 0 & 0 & 0 \\
0 & 0 & 0 & 0 & 0 & 0 \\
0 & 0 & 0 & 0 & 0 & 0 \\
0 & 0 & 0 & 0 & 0 & 0 \\
0 & 0 & 0 & 0 & 0 & 0 \\
0 & 0 & 0 & 0 & 0 & 0
\end{array}\right)
$$

$\left(\begin{array}{llllll}0 & 0 & 0 & 0 & 0 & 0 \\ 0 & 0 & 0 & 0 & 0 & 0\end{array}\right.$ $\left(\begin{array}{llllll}0 & 0 & 0 & 0 & 0 & 0 \\ 0 & 0 & 0 & 0 & 0 & 0 \\ 0 & 0 & 0 & 0 & 0 & 0 \\ 0 & 0 & 0 & 0 & 0 & 0\end{array}\right.$ $\begin{array}{llllll}0 & 0 & 0 & 0 & 0 & 0\end{array}$ $\begin{array}{llllll}0 & 0 & 0 & 0 & 0 & 0 \\ 0 & 0 & 0 & 0 & 0\end{array}$ $\begin{array}{llllll}0 & 0 & 0 & 0 & 0 & 0 \\ 0 & 0 & 0 & 0 & 0 & 0\end{array}$ 
$\left(\begin{array}{lllllllll}0 & 0 & 0 & 0 & 0 & 0 & 0 & 0 & 0 \\ & 0 & 0 & 0 & 0 & 0 & 0 & 0 & 0\end{array}\right.$ $\begin{array}{llllllll}0 & 0 & 0 & 0 & 0 & 0 & 0 & 0\end{array}$ $\begin{array}{lllllll}0 & 0 & 0 & 0 & 0 & 0 & 0\end{array}$ $\begin{array}{lllll}0 & 0 & 0 & 0 & 0 \\ 0 & 0 & 0 & 0 & 0\end{array}$ $\begin{array}{lllll}0 & 0 & 0 & 0 & 0\end{array}$ $0 \begin{array}{lll}0 & 0 & 0\end{array}$ $\begin{array}{lll}0 & 0 & 0 \\ & 0 & 0 \\ 0\end{array}$ 0 $0 \begin{array}{lllll}0 & 0 & 0 & 0 & 0 \\ 0 & 0 & 0 & 0 & 0\end{array}$ $\begin{array}{llll}0 & 0 & 0 & 0 \\ 0 & 0 & 0 & 0\end{array}$ $\begin{array}{llll}0 & 0 & 0 & 0\end{array}$ $\begin{array}{lll}0 & 0 \\ & 0\end{array}$ yzyz $\quad 0$

and $x x x x=x x y y+y z y z+z y z y$

$\left(\begin{array}{lllllllll}x x x x & x x y y & x x z z & 0 & 0 & 0 & 0 & 0 & 0 \\ x x y y & x x y x & x y z & 0 & 0 & 0 & 0 & 0 & 0\end{array}\right) \quad\left(\begin{array}{ccccccccc}0 & 0 & 0 & 0 & 0 & 0 & 0 & 0 & 0 \\ 0 & 0 & 0 & 0 & 0 & 0 & 0 & 0 & 0 \\ 0 & 0 & 0 & 0 & 0 & 0 & 0 & 0 & 0\end{array}\right)$

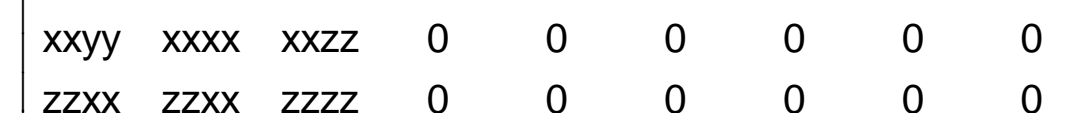
$\left[V^{2}\right] V^{2} \quad \begin{array}{llllllllll}2 z x x & z z x x & z z z z & 0 & 0 & 0 & 0 & 0 & 0\end{array}$ $\begin{array}{ccccccccc}0 & 0 & 0 & y z y z & 0 & 0 & z x z x & 0 & 0 \\ 0 & 0 & 0 & 0 & z x z x & 0 & 0 & y z y z & 0\end{array}$

$\begin{array}{llllclccc}0 & 0 & 0 & 0 & 0 & \text { xyxy } & 0 & 0 & \text { xyxy }\end{array}$

and $x x x x=x x y y+2 x y x y$ $\begin{array}{lllllllll}0 & 0 & 0 & 0 & 0 & 0 & 0 & 0 & 0 \\ 0 & 0 & 0 & 0 & 0 & 0 & 0 & 0 & 0\end{array}$

$0 \begin{array}{llllllllll}0 & 0 & 0 & 0 & 0 & 0 & 0 & 0 & 0 \\ 0 & 0 & 0 & 0 & 0 & 0 & 0 & 0 & 0\end{array}$

$\begin{array}{llllllllll}0 & 0 & 0 & 0 & 0 & 0 & 0 & 0 & 0 \\ 0 & 0 & 0 & 0 & 0 & 0 & 0 & 0 & 0\end{array}$

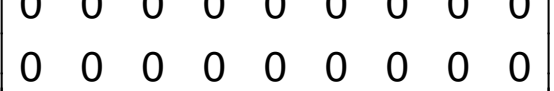

$\begin{array}{llllllllll}0 & 0 & 0 & 0 & 0 & 0 & 0 & 0 & 0 \\ 0 & 0 & 0 & 0 & 0 & 0 & 0 & 0 & 0\end{array}$ $\begin{array}{lllllllll}0 & 0 & 0 & 0 & 0 & 0 & 0 & 0 & 0 \\ 0 & 0 & 0 & 0 & 0 & 0 & 0 & 0 & 0\end{array}$ $\begin{array}{lllllllll}0 & 0 & 0 & 0 & 0 & 0 & 0 & 0 & 0 \\ 0 & 0 & 0 & 0 & 0 & 0 & 0 & 0 & 0\end{array}$ $\begin{array}{llllllllll}0 & 0 & 0 & 0 & 0 & 0 & 0 & 0 & 0 \\ 0 & 0 & 0 & 0 & 0 & 0 & 0 & 0 & 0\end{array}$ $\begin{array}{llllllllll}0 & 0 & 0 & 0 & 0 & 0 & 0 & 0 & 0 \\ 0 & 0 & 0 & 0 & 0 & 0 & 0 & 0 & 0\end{array}$ $\begin{array}{llllllllll}0 & 0 & 0 & 0 & 0 & 0 & 0 & 0 & 0 & 0\end{array}$

$\begin{array}{lllllllll}0 & 0 & 0 & 0 & 0 & 0 & 0 & 0 & 0 \\ 0 & 0 & 0 & 0 & 0 & 0 & 0 & 0 & 0\end{array}$

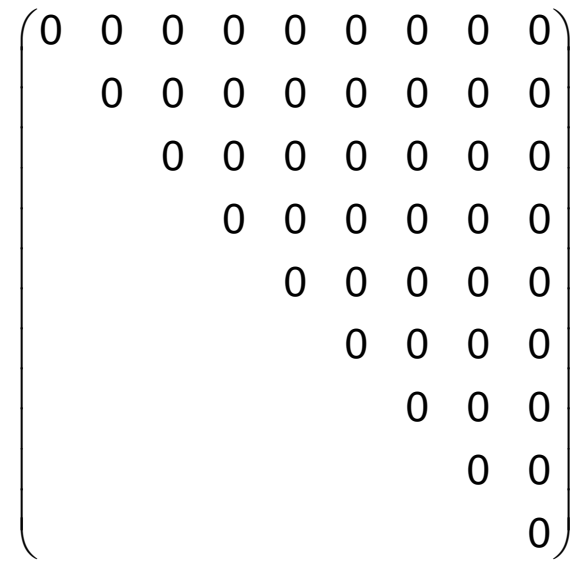

$\left(\begin{array}{lllllllll}0 & 0 & 0 & 0 & 0 & 0 & 0 & 0 & 0 \\ 0 & 0 & 0 & 0 & 0 & 0 & 0 & 0 & 0\end{array}\right.$ $\begin{array}{lllllllll}0 & 0 & 0 & 0 & 0 & 0 & 0 & 0 & 0 \\ 0 & 0 & 0 & 0 & 0 & 0 & 0 & 0 & 0\end{array}$ $\begin{array}{lllllllll}0 & 0 & 0 & 0 & 0 & 0 & 0 & 0 & 0\end{array}$ $\begin{array}{llllllllll}0 & 0 & 0 & 0 & 0 & 0 & 0 & 0 & 0 \\ 0 & 0 & 0 & 0 & 0 & 0 & 0 & 0 & 0\end{array}$ $\begin{array}{llllllllll}0 & 0 & 0 & 0 & 0 & 0 & 0 & 0 & 0 \\ 0 & 0 & 0 & 0 & 0 & 0 & 0 & 0 & 0\end{array}$ $\begin{array}{llllllllll}0 & 0 & 0 & 0 & 0 & 0 & 0 & 0 & 0\end{array}$ 